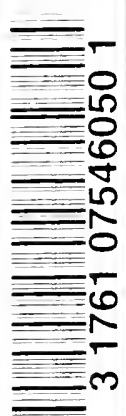



Digitized by the Internet Archive in 2008 with funding from Microsoft Corporation 



\title{
DISEASES OF THE SKIN
}

\author{
A TEXT-BOOK \\ FOR STUDENTS AND PRACTITIONERS
}

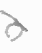

$\mathrm{BY}$

J. M? H.MACLEOD

M.A. ST. ANi,, M.D. ABERI, F.R.C.P. LONI.

Ihysician for Diseases of the Skin, Charing Cross Hospital; I'hysictan for Diseases of the Skin, London IIospital for Tropical Diseases; Physician to the Skin Department,

Victoria Hospital for Children; Lecturer on Dermatology, Charing Cross

Medical College and London School of Tropical Medicine: formerly

Editor of the Fritish Journal of Dermatulogy: Vice-President

Dermatological Section of the Royal Society of Medicine:

Nembre de la Suciété Française de Dermatologie:

Corresponding Member of the American

- Dermatological Association

23 ILLUSTR ATIONS IN COLOUR $\frac{1692}{9.2}$

\author{
I 14 PIATE.
}

435 FIGURES IN BLACK-AND-WHITE

IX PLATES AXI IX THE TEXT

\section{LONDON}

H. K. LEWIS \& CO. LTD. 

M COLLEAGLES

I I

DERIIATOLOGY 



\section{PREFACE}

THIs book, which is the outcome of many years of work in laboratory and hospital, is intended mainly for general practitioners and students, and its aim has been to furnish a practical guide to the symptomatology, pathogenesis, and treatment of skin diseases. Within recent years our knowledge of skin diseases has become so extensive that it is practically impossible to deal with the subject exhaustively within the limits of one volume. In consequence, much that is of interest in connection with the history of the subject of necessity has been omitted and the bibliographies have been restricted.

The majority of the illustrations have been selected from my own collection. For the others I have to thank a number of friends, among whom I wish to mention especially Drs. S. W. Allworthy, Wallace Beatty, Arthur Hall, R. Sabouraud, and Louis Sambon ; these I have endeavoured to acknowledge beneath the particular illustrations, but if by inadvertence I have failed to do so I would ask indulgence for any omission. I have also to thank Lt.-Colonel A. D. Jameson, R.A.M.C., for his kindness in permitting me to have a number of his cases of syphilis photographed for reproduction here.

I have to express a debt of gratitude to my friends and colleagues in dermatology for the helpful advice they have given me during the years in which this book has been in preparation; to Dr. Wilfrid S. Fox for reading the greater part of the manuscript, and for valuable criticisms, suggestions, and additional formulae; to my house physician, Miss S. G. Overton, for her assistance in preparing the index; and to my secretary, Miss E. N. Blackmore, for her indefatigable service.

In conclusion it is my pleasant duty to thank my publishers, Messrs. H. K. Lewis \& Co. Ltd., for their invariable kindness, generosity with regard to illustrations, and unfailing courtesy.

J. II. H. MACLEOD.

i i Harley Street,

LoNDON. 



\section{CONTENTS}

\section{PARTI. GENERAL \\ CHAPTER I \\ ANATOMY AND PHYSIOLOGY}

General Introdection -

EPIDERMIS

Epiderial APPENdages

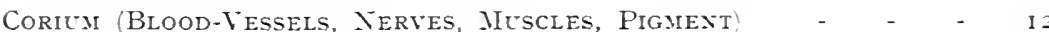

PHYSIOLOGY

\section{CHAPTER II}

GENERAL SYMPTONATOLOGY

\section{CHAPTER III}

GENERAL PATHOLOGY

Pathological Chaxges in the Epideruis -

Pathological Chasges in the Coriem

IMnUNity AND Axaphilaxis -

\section{CHAPTER IV}

GENERAL ETIOLOGY AND BACTERIOLOGY

CHAPTER V

GENERAL DIAGNOSIS

\section{CHAPTER VI}

GENERAL TREATIIENT

General Maxagenext :

Hygiene -
Employment
Rest - -
Nursing -
Clothing -
Climate -
Diet -

Diet - 
Internal. Treatment :

General Treatment

Special Treatment

Drugs

Animal Extracts

Sera and Vaccines

Saline Injections

Health Resorts

\section{CHAPTER VII}

LOCAL TREATMENT

LOCAL MEDICAMENTS

Metilods of Application

PhYsical Methods

IRADiO-TherAPEUTiCs

CHAPTER VIII

NOMENCLATURE AND CLASSIFICATION

\section{PART II. SPECIAL}

\section{CHAPTER IX}

CONGENITAL AFFECTIONS OF THE SKIN

ICHTHYOSIS

ICHTHYOSIS FOLLICULARIS

NaEVI:

Vascular Naevi :

Capillary Naevi, or Port-wine Stain - $\quad$ - $\quad$ - $\quad$ - $\quad$ I 76

Cavernous Naevi, or Raised Haemangiomata - _ _ _ . ISo

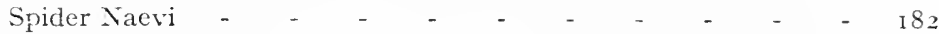

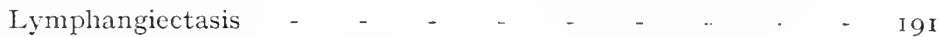

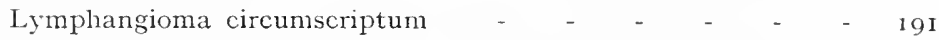
Non-Tascular Naevi:

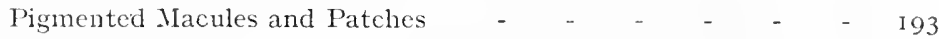

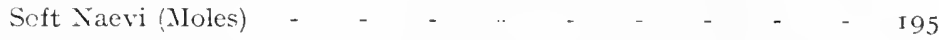

Hard Naevi :

Ichthyosis hystrix - $\quad$ - $\quad$ - $\quad$ - $\quad$ - $\quad$ - 203

Hyperkeratosis congenitalis palmaris et plantaris - - 204

Epidermolysis bullosa - - $\quad$ - $\quad$ - $\quad$ - 207

\section{CHAPTER I}

CUTANEOUS AFFFCTIONS DUE TO COLD 
LIVEdO RETICULARIS PruRitus hienalis RAYNATD'S DISFASE TRENCH FOOT -

\section{CHAPTER II}

CLTANEOLS AFEECTIONS DLE TO HEAT

INTRODUCTION

BUR:S AND SCALDS

MILIARIA -

SUDAMEN

\section{CHAPTER XII \\ CUTANEOLS AFFECTIONS DLE TO SEXLIGHT}

\section{Introduction}

StNBURN

FRECKLES

Solar Derilatitis -

HYDROA AFSTIYALE

Xeroderma pignextosty

\section{CHAPTER XIII}

DERIATITIS DLE TO ELECTRICITY, X-RAYS, AND RADILMI

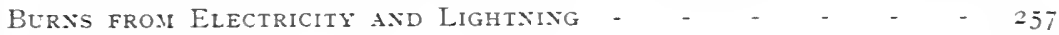
X-RAY Dermatitis - $\quad$ - $\quad$ - $\quad$ - $\quad$ - $\quad$ - $\quad$ - 260

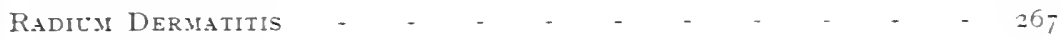

CHAPTER IIV

DERIATITIS DLE TO LOCAL IRRITANTS

Dermatitis Vexenata 269

BEDSORES

DERMATITIS ARTEFACTA -

\section{CHAPTER IV}

CLTANEOLS AFFECTIONS DUE TO STREPTOCOCCI

INTRODUCTION

IMPETIGO CONTAGIOSA

Perlèche

PeNphigts Nentatorty

Dermatitis exfoliativa infantum

EPIDEMIC ExFOLIATIVE DERMATITIS

FURFLRACEOCS IMPETIGO

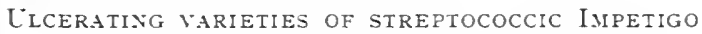

Ecthyma -

Ecthyma terebrans.

Vacciniform dermatitis

ERYSIPELAS 
Simple infantile DERMatitis

\section{CHAPTER XVI}

\section{CUTANEOUS AFFECTIONS DUE TO STAPHYLOCOCCI}

\section{INTRODUCT1ON}

IMPETIGO OF BOCKHART -

\section{Sycosis Barbae}

Sycosis nuchae (AcNe Cheloid)

Furuncles

CARBUNCLES

Vegetating Papillonata

Granuloma PYOGENicum (BOTRYOMYCOSIS) -

Multiple cutaneots Abscesses in Infants

PITYRIAS1S AND SEBORRHOIC DERMATITIS

\section{CHAPTER XVII}

CUTANEOLS AFFECTIONS DUE TO FUNGJ. (DERMATOMYCOSES)

Asconycetes:

Saccharomycetes or Yeasts. Blastomycosis

Gymnosuceae:

\begin{tabular}{|c|c|c|c|c|c|c|c|}
\hline Fungi of Ringworm & - & - & - & - & - & - & - \\
\hline Tinea circinata - & - & - & - & - & - & - & - \\
\hline Tinea cruris (Dhobi's & Itch) & & - & - & - & - & - \\
\hline Eczematoid Ringwor & & - & - & - & - & - & - \\
\hline Tinea capitis - & - & - & - & - & - & & - \\
\hline Tinea barbae - & - & - & - & - & - & & - \\
\hline Tinea unguium - & - & - & - & & & - & \\
\hline $\begin{array}{l}\text { Tinea favosa (Favus) } \\
\text { YCETES: }\end{array}$ & & - & - & - & - & - & - \\
\hline Irdia: Actinomycosis & & - & - & - & - & - & - \\
\hline otrichum: Sporotricl & nosis & - & & - & - & - & - \\
\hline 1osporum: Trichospo & rosis & - & - & - & - & - & - \\
\hline osporoides : Erythras & ina & - & - & - & - & & - \\
\hline Malassezia : Pityrias & is ver & sicolor & & - & & & - \\
\hline x $-\quad-\quad-$ & - & 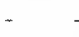 & - & . & - & . & - \\
\hline
\end{tabular}

\section{CHAPTER XVIII}

\section{CLTANEOLS AFFECTIONS DLE TO BACILLI}

STREPTOTRICHES :

Tubercle Bacillus

Tuberculosis cutis

Lupus vulgaris

Scrofulodermia

Erythema induratum -

Tuberculicles

Lepra Bacillus:

$$
\text { Leprosy }
$$




\section{CHAPTER XIX}

CLTANEOLS AFFECTIONS DLE TO PROTOZOA. SYPHILIS

\section{INTRODUCTION}

Etiologi:

Spironema pallidum -

Demonstration -

Cultivation

Inoculation Experiments

Life History

\section{Y SORE OR CHAXCRE :}

Varieties

Situation -

Diagnosis -

\section{Extra-gexital Chascres}

Histopathology

Cutaneors Syphilides -

Early Strphilides

Intermediary Syphilides

Late or Gummatous Syphilides -

Mucoes Syphilides

Concomitaxt Sraptoms

Diagrosis

Wassermann Reaction

\section{Progiosis}

Syphilis and Marriage

RE-INFECTION .

Prevention

Treatuext :

General Considerations

Salvarsan and Substitutes

Mercury

Iodides

Coxgexital Syphilis

\section{CHAPTER IX}

\section{CLTANEOUS AFFECTIONS DLE TO AXIMAL PARASITES}

ACARINA :

Sarcoptes scabiei

Ixodoidea (Ticks)

Demodex folliculorum

Leptus autumnalis (Harvest-Bug)

Dermanyssus gallinae (Fowl-Mite)

Tyroglyphus longior (Copra-itch Mite)

Pediculoides ventricosus (Grain-itch Mite) -

Chilopoda (Centipedes) 
HEXAPODA. INSECTA :

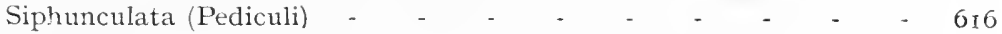

Cimex lectularius (Bed-bug) - _ _ _ _ _ - 623

Diptera :

Pulex irritans (Common Flea) - - - - - - - 625

Muscidae (Flies. Myiasis. Creeping Eruption) - . . . 625

Culicidae (Mosquitoes, Gnats) - - - - . . 630

Hymenoptera :

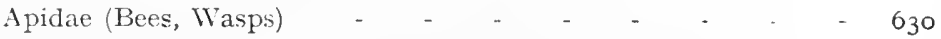

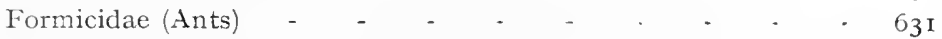

Lepidoptera (Caterpillars, Butterfies) - - . - . - 63r

Coleoptera (Beetles) - _ _ _ _ _ - . . 63I

\section{CHAPTER XXI}

PRURITUS

PruRitus iN GeNeral

PruRitus ANI

Pruritus vulvat -

\section{CHAPTER XXII}

ERITHFMATA

ERYTHEMA IN GENERAL:

Localised Erythema due to external or internal causes - . - 655

Generalised Erythema of toxic origin - - - - - $\quad 659$

Recurrent exfoliative scarlatiniform Erythema - - - • - 662

Erythema exudativum muitiforme - - _ - _ - . 662

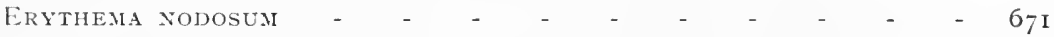

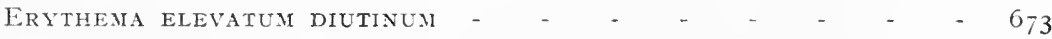

Grandloma AnNulare - $\quad$ - $\quad$ - $\quad$ - $\quad-\quad$ - $\quad-673$

Erythromelalgla - $\quad$ - $\quad$ - $\quad$ - $\quad$ - $\quad$ - $\quad$ - 677

\section{CHAPTER XXIII}

URTICARIA AND ALLIED CONDITIONS

URTICARIA

URTICARIA PIGMENTOSA -

ANGio-neurotic OEDENA

PAPULAR LRTICARIA

PRURIGO

\section{CHAPTER XXIV}

DRUG ERUPTIONS

\section{CHAPTER XXV}

\section{LICHEN}

Intronection

LICIIEN PLANUS

LiCIIENIFICATION 


\section{CHAPTER XIVI}

FCZEMIA AND ROSACEA

ECZEMA

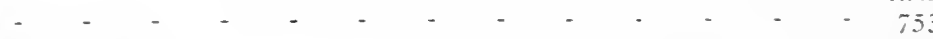

IXFAXTILE FCZEMA - $\quad$ - $\quad$ - $\quad$ - $\quad$ - $\quad$ - $\quad$ - $\quad$ - $\quad$ - 799

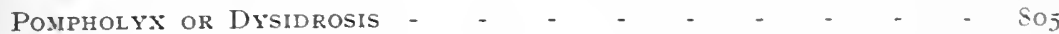

ROSACEA -

\section{CHAPTER NAVII}

SCALY AFFECTIONS

\section{PSORIASIS}

Parapsoriasis

PITYRIASIS RUBRA PILARIS

Generalised exfoliative dermatitis -

PitYriasis ROSEA

\section{CHAPTER XITIII}

VESICULAR AND BLLLOUS AFFECTIONS

\section{PeMphiges :}

Pemphigus acutus

Pemphigus chronicus

Pemphigus foliaceus -

Pemphigus vegetans -

DERMATITIS HERPETIFORMIS

HERPES :

Herpes simplex

Herpes zoster -

\section{CHAPTER NAIS}

LL“PLS ERYTHEMATOSL'S - - · 9II

\section{CHAPTER IXY}

MYCOSIS FL'NGOIDES

\section{CHAPTER XXII}

AFFECTIONS OF THE CLTANEOL'S BLOOD-VESSEIS

Purplra :

Primary -

Secondary

TELANGIECTASES

Recurring Epistaxis with multiple Telangiectases

\section{CHAPTER XXXII}

\section{A.TOMALIES OF PIGMIENTATION}

\section{Melaxoses, Coxgexital axd AcQtired}

Pigmentation fros Extraxeots Stbstaxices ix the SKIX (SILVER, 
Schamirerg's Disease

Albinisu

TATTOOING

\section{CHAPTER XXXIII}

ATROPHIES OF THE SKIN

Nervous Atrophy

Travinatic Atrophy

CONGENITAL ATROPHY

Senile Atrophy

IDIOPATHIC IIACULAR ATROPHY

IDIOPATHIC DIFFUSE ATROPHY

Krraurosis vulvaE

\section{CHAPTER XXXIV}

\section{AFFECTIONS OF THE HAIR}

Alopecia :

Premature Hereditary Alopecia

Senile Alopecia

Symptomatic Alopecia

Nervous Alopecia

Alopecia dependent on morbid conditions of the scalp

Traumatic Alopecia -

Alopecia from chemical irritants

Alopecia from Röntgen Rays -

Parasitic Alopecia

Alopecia seborrhoica

Alopecia areata -

Alopecia CICATRISATA

KNOTtED HaIRS

Fragilitas CRINIUM

MONILITHRIX -

CANITIES

HYPERTRICHOSIS

967
968
968
969
971
971
972
972
972
974
981
996
999
999
1001
1002
1005

\section{CHAPTER XXXV}

AFFECTIONS OF THE HAIR-FOLLICLFS

Folliculitis -

KERATOSIS SUPRA-FOLLICULARIS

LICHEN SPINULOSUS

\section{CHAPTER XXXTI}

AFFECTIONS OF THE SEBACEOUS GLANDS 


\section{CHAPTER XXXVII}

\section{AFFECTIONS OF THE SWEAT-APPARATLS}

Functional Affections:

Hyperidrosis - $\quad$ -

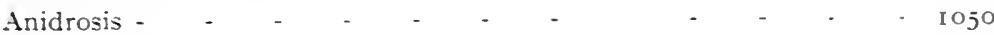

Bromidrosis - $\quad$ - $\quad$ - $\quad$ - $\quad$ - $\quad$ - $\quad$ - $\quad$ - $\quad$ - 1050

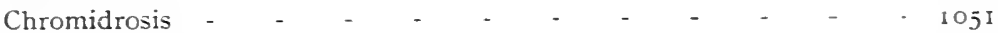

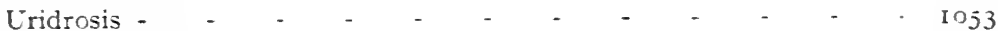

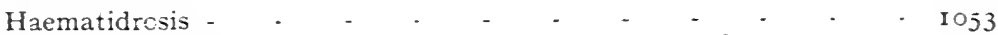

Phosphoridrosis

1053

ORGANic AfFections :

Granulosis rubra nasi

Hydrocystoma -

Hypertrophy

Atrophy

Porokeratosis -

Keratosis punctata

\section{CHAPTER XXXVIII}

\section{AFFECTIONS OF THE NAILS}

HYPERTROPHY :

Simple Hypertrophy (Onychauxis) - $\quad$ - $\quad$ - $\quad$ - $\quad$ - I06j

Hypertrophy with deformity (Onychogryphosis) - - - - - Iob6

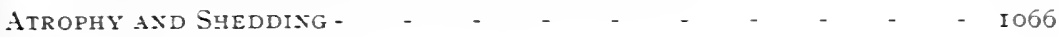

DYSTROPHY :

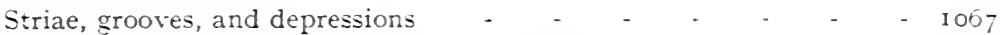

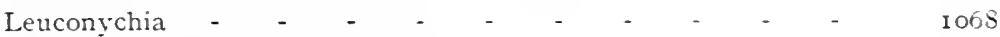

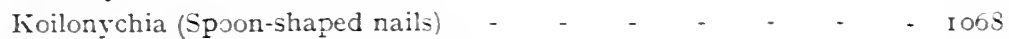

Congexital AfFections - $\quad$ - $\quad$ - $\quad$ - $\quad$ - $\quad$ - $\quad$ - $\quad$ - rojo

ACQLIRED AFFECTIONS :

Traumatic Affections

Parasitic Affections -

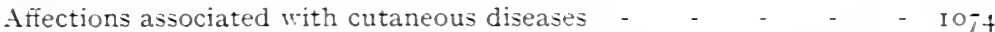

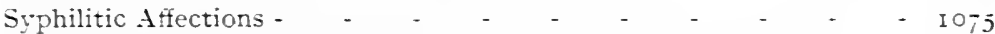

Affections due to general diseases or nerve lesions - $\quad$ - $\quad$ - I0-6

\section{CHAPTER IXXIX}

AFFECTIONS OF THE IIPS AND MOLTH

SCALY AND CRUSTED AFFECTIONS OF THE LIPS :

Perlèche

Eczema

Seborrhoic dermatitis

Cheilitis exfoliativa

Cheilitis glandularis aposteomatosa -

Fordyce's Disease

Black Harry Tongee

Trasitory circinate ert-ptios of the TONGLE 


\section{CHAPTER XL}

BENIGN NEOPLASIS OF THE SIIN

EPIDERMIS :

PAGE

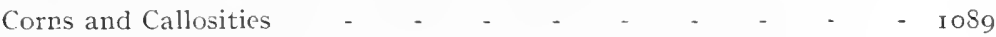

Warts :

Common Warts (Verruca vulgaris)

Plane Warts

Plantar Warts (Terruca plantaris)

Venereal Warts (Condyloma acuminatum) -

Seborrhoic Warts (Verruca senilis)

Warty Affections :

Acanthosis nigricans

Darier's Disease

Gonorrhoeal Keratosis

Cutaneous Horns

Molluscum contagiosum

Milium

Epidermal Appendages:

Benign cystic epithelioma -

Epithelioma adenoides cysticum

Hydradenoma

Sebaceous Adenoma -

CORIUM :

Fibroma and neuro-fibroma

Cheloid

Pseudo-xanthoma elasticum

Colloid Milium -

Sarcoid

Leiom yoma

Skin lesions in leucaemia and general lymphadenoma Blood-Yessels aNd Lymphatics:

Endothelioma

\section{CHAPTER X'LI}

IIALIGNANT NEOPLASIS OF THE SKIN

EPIDERMIS :

Rodent Ulcer

Squamous-celled Epithelioma

Naevo-carcinoma

Paget's Disease

CORIUN :

Sarcoma

Multiple idiopathic pigment sarcoma

\section{CHAPTER XLII}

PSEUDO-NEOPLASIS OF THE SKIN

XANTHOMA :

Santhoma palpebrarum

Xanthoma tuberosum multiplex

Xanthoma diaheticorum

$$
\begin{array}{r}
1092 \\
1093 \\
1096 \\
1097 \\
1099
\end{array}
$$

I IOO

IIO3

- $1 \mathrm{IOS}$

- $1 \mathrm{IIO}$

- III 2

III 7

I I I 8

I 120

I 122

I 123

I 126

I 129

I I 34

I 135

I 137

- $\quad$ I 38

I I 4 I

I I 42
- IIf4

- II 52

II 57

- $\quad$ I I 58

I I 64

- $\quad 1167$ 


\section{CONTENTS}

\section{SCLERODERMIA}

SCIEREMA NEONATORUM -

OEDEMA NEONATORUM

Calcareous deposits IN THE SKIN

\section{CHAPTER XLIII}

TROPICAL AFFECTIONS OF THE SKIN (Arranged alphabetically)

\section{AINHUM}

ANCylostomlasis cutanea (WATER-ITCH)

Calabar Swellings (Filaria loa)

CRAW-CRAW

Dracunculosis (Guinea-Worm)

ElEPHANTIASIS

Framboesia tropica (Yaws) -

\section{GANGOSA}

Goundou

Granuloma inguinale MYCETOMA

\section{Pellagra}

Phagedaena tropica

\section{Pinta}

Pulex penetrans (Jigger) -

\section{TINEA IMBRICATA}

TROPICAI ULCERS :

Pyogenic Ulcerations

Oriental Sore (Leishmaniasis tropica)

Espundia (Leishmaniasis Americana)

Ulcerative Dermato-mycosis (Castellani)

Veld Sore (Desert Sore)

Verruca PERUaxa -

\section{APPENDIX}

Natural Mineral Waters 


\section{LIST OF COLOURED ILLUSTRATIONS}

Piate I. Section of Skin of Palm of Hand - Facing Page II. Fig. I. PRICKLe-CElls - $\quad$ - $\quad$ - $\quad$ - $\quad$ -

2. HORN-CELLS - - - - $\quad$ - $\quad-12$

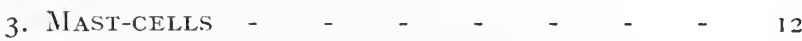

4. Plasma-Cells - $\quad-\quad-\quad-\quad-\quad-\quad-12$

5. Horn-CElls With hyalin Degeneration - 12

". III. Fig. I. IChthyosis SERPENTINA - - - - 163

2. Hyperkeratosis CONGENITA - - - -

", IV. Fig. I. Inpetigo contagiosa - $\quad$ - $\quad$ - $\quad$ - 299

2. IMPETIGO BULLOSA - $\quad$ - $\quad$ - $\quad$ - $\quad$ - $\quad 299$.

V. Elephantiasis nostras - $\quad$ - $\quad$ - $\quad$ - $\quad$ - $\quad$ - 323

VI. EczeMatold Ringworm - - - - - $\quad$ - 396

VII. Fig. I. Suppurating Chilblains - - - $\quad$ - 490

2. Papulo-Necrotic Tuberculide - - - 490

3. Lupus erythematosus - - - - - $\quad$ - 490

" Vili. Maculo-anaesthetic Leprosy - - - - $\quad 5$ II

IX. Fig. I. Erythema multiforme exudativum - - 664

2. Pemphigus chronicus - $\quad$ - $\quad$ - $\quad$ - $\quad$ - 664

X. Papulo-vesicular Utrticaria - - $\quad$ - $\quad$ - 699

" XI. Generalised Lichen planus - - - - 734

". XII. Infantile Eczema - - - - - - $\quad$ - 799

" Xili. Adenoma sebaceum - - - - - - II24

" XIV. Morphoea, or circumscribed Sclerodirmia - iis3. 


\section{PART I. GENERAL}

\section{CHAPTER I}

\section{THE ANATOMY AND PHYSIOLOGY OF THE SKIN}

\section{ANATOMY}

General Introduction.-From the embryological point of view, the human skin may be said to consist of only two layers, namely, the epidermis and the corium, dereloped respectively from the epiblastic and mesoblastic lavers of the blastodermic vesicle. These differ greatly in their minute structure for whereas the epidermis is purely cellular the corium is made up mainly of fibrous tissue. Of the two the epidermis may be regarded as the more important as by means of it and its appendages certain of the main functions of the skin, such as the protective and secretory functions, are carried on. The corium acts chiefly as a support and protection to the blood-ressels which nourish the epidermis and to the nerve endings which render it sensitive.

The Subcutaneous tissue was at one time beliered to belong to a different category from the corium, and to be developed from a different layer, but most observers are now agreed that it also is of mesoblastic origin, and should be regarded simply as a deeper layer of the corium in which some of the cells have become specialised by the deposition of fat within them.

The Superficial Architecture of the Skin.-The surface of the skin is everywhere traversed by ridges and furrows which vary in different situations in regularity, arrangement and size, and which to a considerable extent determine the shape of many of the elementary lesions of the skin. The existence of these ridges is dependent on three factors: (I) The arrangement of the fibrous bundles and elastic fibres of the underlying corium; (2) The direction of the movements to which the parts are subjected; and (3) The attachment of the skin by fibrous bundles to deeper structures.

There are two distinct rarieties of furrows on the surface of the skin: (a) Fine furrows, which tend to run parallel to one another, and are best marked on the flexor aspects of the tips of the fingers and toes. There they form more or less regular patterns which differ so 
markedly in each individual as to be of value for purposes of identification. (b) Coarse or deep furrows, which are most pronounced in the neighbourhood of joints, and are due to the fixing down of the skin by fibrous bundles to underlying structures, such as the periosteum of bone and the joint-capsules. In old age, and in wasting diseases, wrinkles or "folds of emaciation" occur, as the result of the disappearance of the subcutaneous fat.

\section{EPIDERMIS}

The epidermis is the part of the skin which is exposed to the air and which acts as a protection against injury, the harmful attacks of micro-organisms, the absorption of fluids and the baneful action of the actinic rays of the sun. In order to fulfil this purpose its outer layer has to be a highly specialised structure consisting of hard resistant horn-cells containing a fatty or waxy substance which renders the skin more or less waterproof.

For purposes of description the epidermis may be divided into five distinct layers, which are best seen in parts of the skin where the epidermis is thick, such as the sole of the foot or the palm of the hand. These layers or strata, named from within outwards, are the Stratum germinativum, the Stratum Malpighii or Rete mucosum, the Stratum granulosum, the Stratum lucidum, and the Stratum corneum. These indicate stages in the evolution of the epidermal cell from its simplest type in the Stratum germinativum till it becomes a perfect horn-cell. The epidermis as a whole is thickest on the palms and soles, and thinnest on the abdomen, elbows, forehead and cheeks. It becomes hardened and thickened from intermittent pressure, as in the soles of the feet from walking and in the palms of the hands from manual labour. (Fig. I.)

Stratum Germinatioum.-This constitutes the basal layer of the epidermis and usually consists of a single row of columnar cells with oral nuclei. These cells are arranged at right angles to the imaginary wavy line which separates the epidermis from the corium. As they are constantly dividing by mitosis and their whole function is that of reproduction, they have been named the "mother-cells of the cpidermis." Between and above them, smaller "daughter-cells" can be detected. These, except in pathological conditions, are not reproductive and do not exhibit mitotic figures; their function is that which is known as "differentiation," i.e. they are simply pushed towards the surface by the formation of new cells beneath them, and in their passage are gradually differentiated till they become horn-cells. The cells of the basal layer are not supported-as was once supposedby a basement membrane but are united by fine protoplasmic threads or fibres which, towards the corium, are collected into tufts, giving the line of demarcation between the epidermis and the corium a denticulated appearance. 



\section{PLATE I.}

\section{Section of Skin of Palm of Hand. ( $x$ about 150.)}

Stained with polychrome methylene blue and neutral orcein.
a. Stratum corneum.
b. Sweat-pore.
c. Stratum lucidum.
d. Stratum granulosum.
e. Stratum Malpighii.
f. Stratum germinativum.
g. Papilla of papillary layer.
$h$. Blood-vessel.
$i$. Corium and fibrous bundles; some cut across.
$k$. Sweat-duct.
l. Sweat-coil.
$m$. Artery and vein.
$n$. Trabecula between fat-lobules.
$o$. Septum between lobules.
p. Fat-cells.

q. Pacinian body.

(From MacLeod's Pathology of the Skin.) 


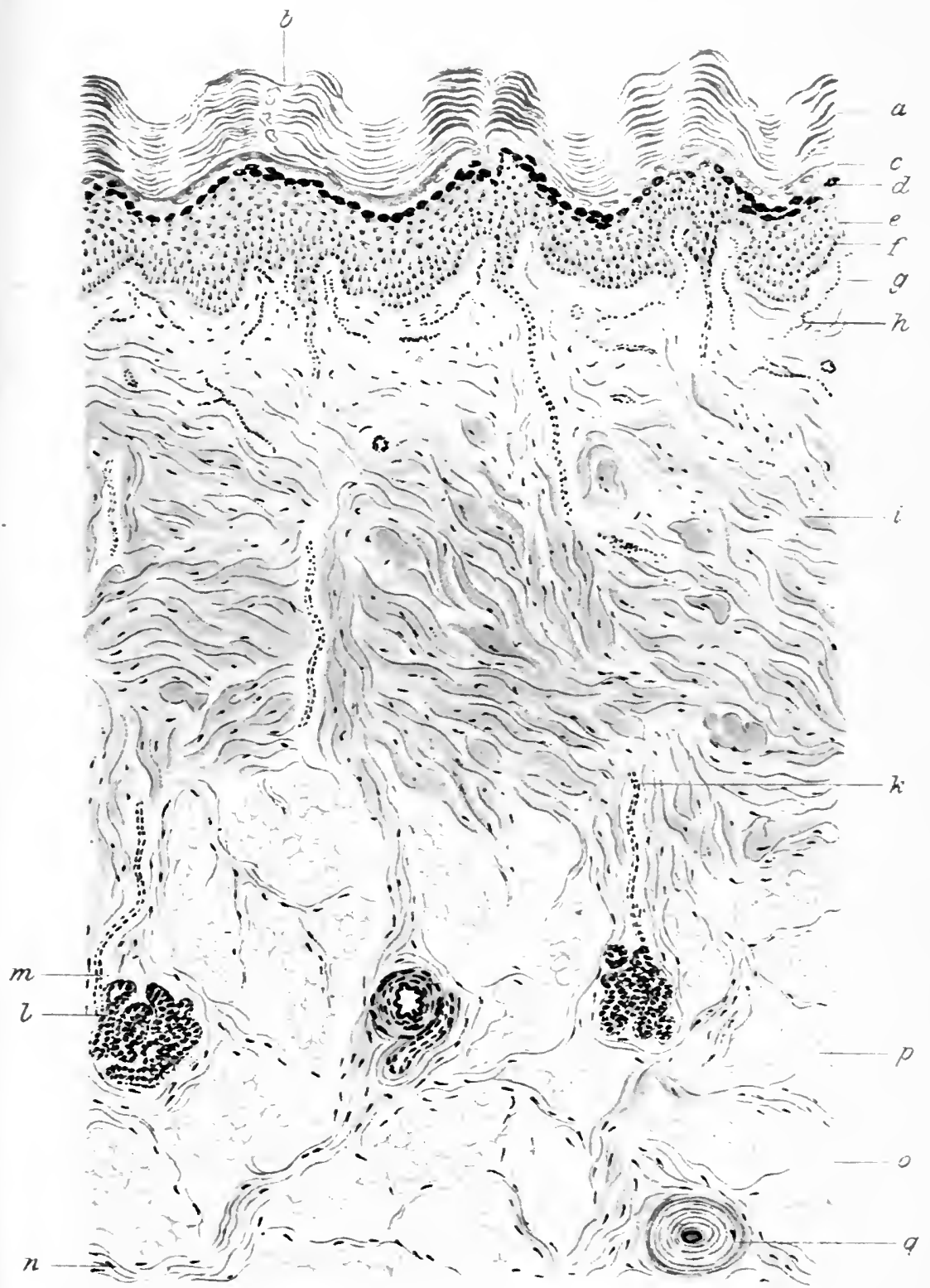



Stratum Malpighii-Rete mucosum, or Prickle-cell Layer. This layer consists of a varying number of rows of polygonal cells, which tend to become flattened towards the surface of the skin, and are built up in the form of a mosaic. They possess large, round or oval nuclei, and are united by delicate protoplasmic fibres continuous with the spongioplastic network of the cells. Owing to the presence of the intercellular protoplasmic fibres, the cells of this layer when separated present an appearance somewhat resembling a prickly pear and were

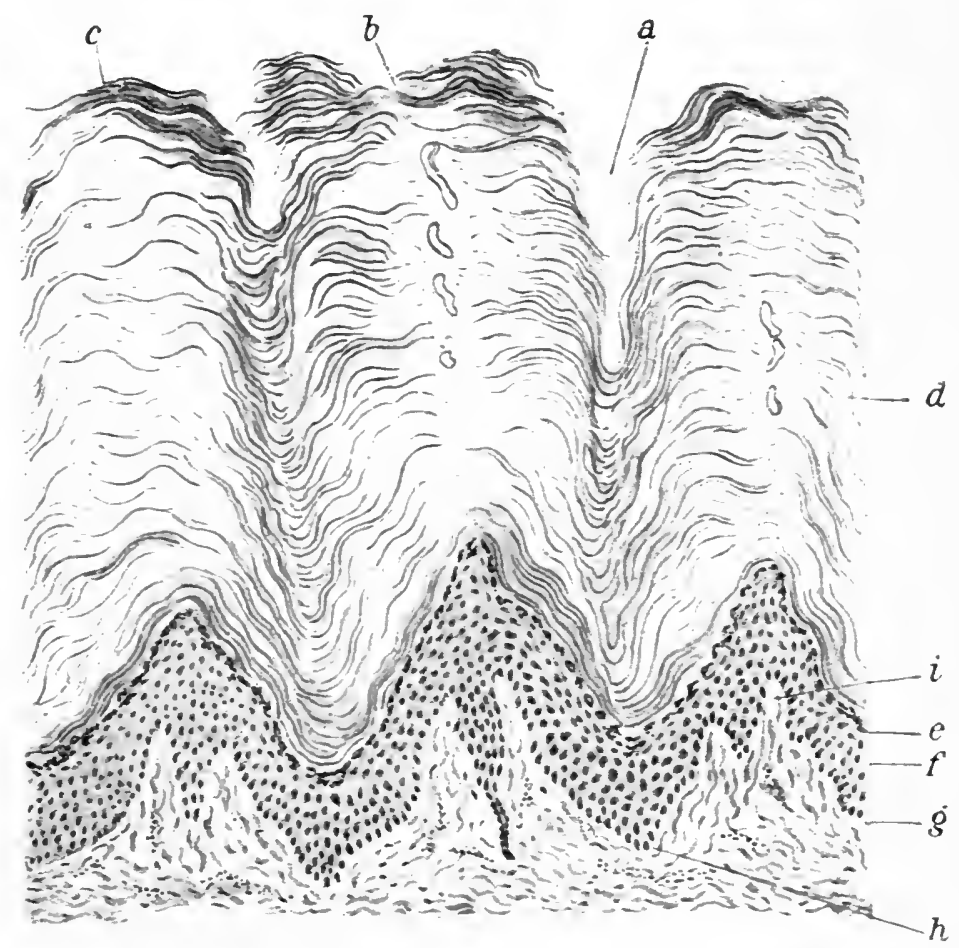

J. M. H. M. del.

FIG. I.-Vertical Section of the Skin of the Sole of the Foot. ( $x$ about I50.)

$a$. "Fine furrow," corresponding in position to an interpapillary process. $b$. Sweat pore. c. Ridge corresponding in position to two papillae. d. Stratum corneum, showing stratification, and its great thickness in this situation. e. Granular layer. $f$. Malpighian layer. g. Germinal or basal layer. h. Interpapillary process. i. Papilia.

(From MacLeod's Pathology of the Skin.)

named "prickle-cells" by Max Schultze. In spite of their delicate appearance, the protoplasmic fibres are relatively resistant structures; they are unaffected by boiling water and insoluble in weak acids. Between the cells are lymphatic spaces which are bridged over by the fibres and in which are distributed the fibres of the inter-epithelial nerve plexus. Peculiar spiral fibres situated between the pricklecells and the cells of the basal layer have been described by Herxheimer; these are generally believed to be either threads of fibrin or protoplasmic fibres passing between cells of different layers. (Plate II. I.) 
Stratum Gramulosum.-Towards the surface the prickle-cell layer merges into a layer consisting of two or three rows of flattened granular cells, with shrivelled nuclei lying in spaces, and shrunken protoplasmic fibres.

The granules are most numerous in the cell-protoplasm near the nuclear spaces. They vary in shape and size from small roundish

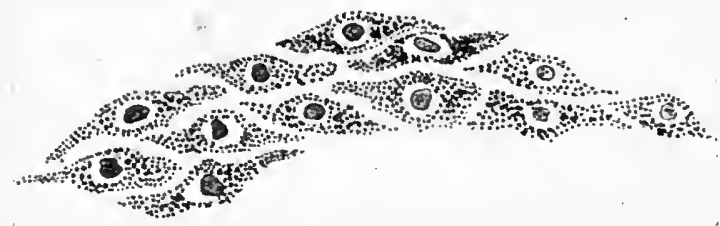

J. M. H. M. del.

Fig. 2.- Stratum Granulosum. ( $\times$ rooo.) Shows Keratohyalin Granules in the cells.

specks to coarse irregular lumps, and are strongly light-refractive. They consist of a semi-solid substance known as keratohyalin, which is insoluble in alcohol, ether, chloroform and weak acids, is digestible in pepsin and hydrochloric acid, and is not stained by osmic acid. This substance is most probably a separation-product of the protoplasm of the cell. (Fig. 2.)

Stratum Lucidum.- This layer has been so called because in unstained sections it may be seen as a semi-transparent line resembling a narrow oily streak across a sheet of paper. It consists of one or more rows of somewhat swollen irregular cells, in which the nuclei are much shrunken and frequently replaced by a mass of debris. In these cells the keratohyalin has disappeared and been replaced by an oily

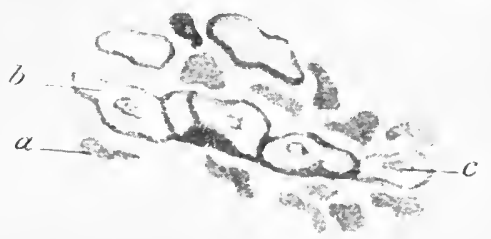

J. M. H. M. del. substance named eleidin, which is present not only within but also between the cells, and which is soluble in acids and alkalies, slightly soluble in alcohol, insoluble in ether, digestible in pepsin and hydrochloric acid, and does not stain with osmic

FIG. 3.-Stratum Lucidum and Eleidin. acid. It is probably a de$(\times 800$.

a. Eleidin around and within the cells. b. Protoplasm of the cells. c. Remains of nucieus. generative product of keratohyalin. Fat globules are also present in the cells. (Fig. 3.)

Stratum Cornemm.-The Stratum corneum is the most superficial layer of the skin, and is thickest on the palms and soles and thinnest on the face. It is composed of horn-cells which are most perfect in their structure next the Stratum lucidum, and towards the surface become more and more flattened and dried up until they form squames. 



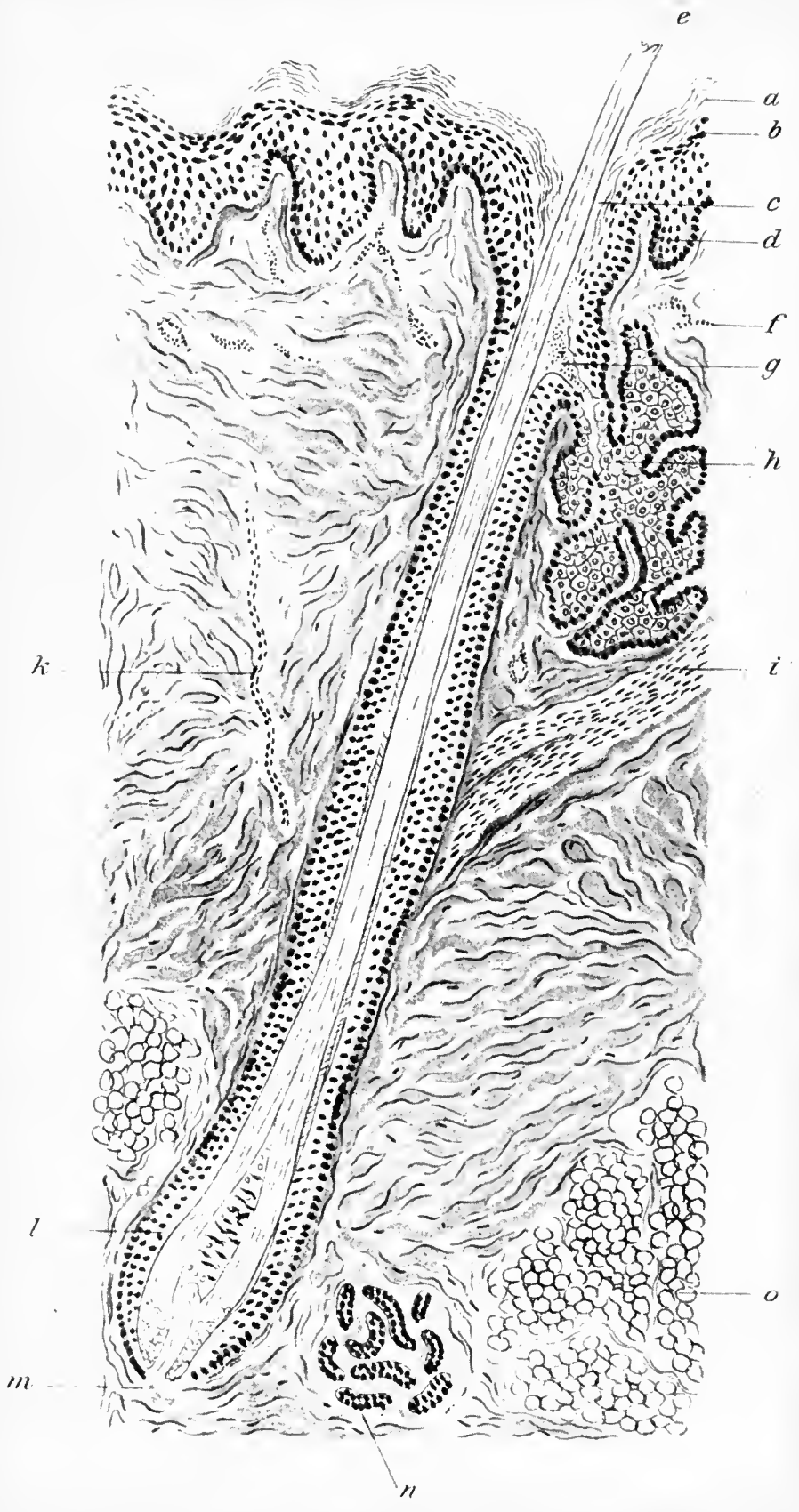

J. M. H. M. del.

FIG. 4--Section of Pilo-Sebaceous Follicle (semi-diagrammatic).

$a$. Str. Corneum, b. Str, Granulosum. c. Funnel of follicle. d. Str. Malpighij. e. Hair. f. Capillary. g. Sebaccous duct. $h$. Sebaceous gland. $i$. Arrector pili muscle. $k$. Sweat: duct, l. Hair-bulb. $m$. Papilla. n. Coil-gland. o. Fat-cells. 
A horn-cell is polygonal in shape, facetted from pressure, and presents a space in the centre from which the nucleus has disappeared. The periphery has become hardened and the prickles have persisted as dried up spicules of keratin. Eleidin can no longer be detected within the cells, but in its place there is a waxy substance.

Keratin is a highly resistant substance, the formation of which is known as the process of cornification or keratinisation. It is indigestible in pepsin-hydrochloric acid and can withstand $50 \%$ solutions of mineral acids for a prolonged period, but is readily disintegrated by alkalies. It is a somewhat complex protein material containing a considerable proportion of sulphur and on analysis is found to have in its ash a large quantity of silicates. (Plate II. 2.)

There are several theories with regard to the process of cornification. Of these the most probable is that the keratin is the result of the hardening of the protoplasmic fibres and possibly of the outer portion of the spongioplasm of the cells, and not a degenerative product resulting from the breaking down of the nuclei, nor a further stage of keratohyalin. Keratohyalin was once regarded as a precursor of keratin, but is now recognised as a degenerative product of the protoplasm of the cell and merely accessory to the process of cornification.

The presence of the waxy substance in the cells of this layer gives it the character of a waterproof coating, and the persistence of the protoplasmic fibres in the form of hard keratin spicules welding the cells together readily explains the protective power which the Stratum corneum is capable of exerting against the entrance of microorganisms and their toxins and its great ability to resist mechanical injury.

\section{EPIDERMAL APPENDAGES}

\section{HAIRS}

General Characteristics.-Hairs are present on all parts of the human skin, except the palms, soles, red portions of the lips, ungual phalanges and Glans penis. There are three types of hairs, namely :

(I) Long hairs, such as occur on the scalp, beard, moustache, axillae and pubes.

(2) Stiff hairs, or bristles, such as the eyelashes (cilia), the hairs of the nasal orifices (vibrissae) and the hairs of the auditory orifices (tragi).

(3) Lanugo, or downy hairs.

The long hairs and the lanugo hairs are implanted more or less obliquely in the skin; the bristles vertically. The shape of the hair in cross-section varies in different individuals and in different races. As a rule straight hairs are circular in section, and curly hairs oval, and the beard hairs are not infrequently angular. 
The colour of the hair is dependent on three factors :

(I) The pigment granules situated in and around the cells of the hair-cortex.

(2) The diffuse colour of the protoplasm of the hair-cells.

(3) The presence of air between the hair-cells.

The granules are believed to be responsible for black and brunette tints; the diffuse colour of the protoplasm is said to produce blonde or reddish shades; and the presence of air between the cells causes the hair to become grey or white.

In the foetus all the hairs belong to the lanugo type and are colourless except occasionally on the scalp. After birth the lanugo hairs are gradually shed, being replaced by long hairs on the scalp, and on the rest of the body by new lanugo hairs forming the downy hairs of the glabrous skin. There are cases where infants are born with long dark hair on the scalp, this is shed during the first month and replaced by lighter hair.

The shedding of the lanugo hairs towards the end of foetal life and their replacement by new hairs in the old follicles is known as the normal hair-change. This begins on the face and head of the foetus between the seventh and eighth month, and if it is not completed over the whole skin before birth, it is so during the first few months of extra-uterine life. The new hairs are not permanent, however, but only have a life of from two to four years, when they are shed and new ones form.

Besides the intermittent falling out of hairs, other hair-changes occur at periodic intervals. At birth or immediately after, the normal hair-change takes place and hair grows actively on the scalp. At puberty, hair appears in certain regions, such as the beard, axillae and about the genital organs. In middle life and later, stiff hairs or vibrissae grow about the nostrils and ears. In females, after the menopause there is not infrequently an increase of hairs on the face, especially about the lips. These hairs, which may be called the "periodic hairs," are usually coarse and pigmented, while those of the rest of the body retain their unpigmented condition. (Fig. 4.)

Structure of the Hair.-The hair consists of a shaft or stem which widens out at the lower end to form a bulbous swelling known as the root. The root and part of the shaft are situated in the hairfollicle, the remainder of the hair is free.

Shaft.-The greater portion of the shaft is formed by the haircortex, in the centre of which there is a more variable structure known as the medulla. The cortex is protected externally by a sheath or cuticle.

The cuticle consists of a single layer of flat, quadrilateral cells arranged in an imbricated manner, with their long axes directed upwards and outwards at an acute angle to the shaft.

The cortex is made up of bundles of nucleated spindle-shaped cells, which give it a fibrillated appearance. These spindles have 


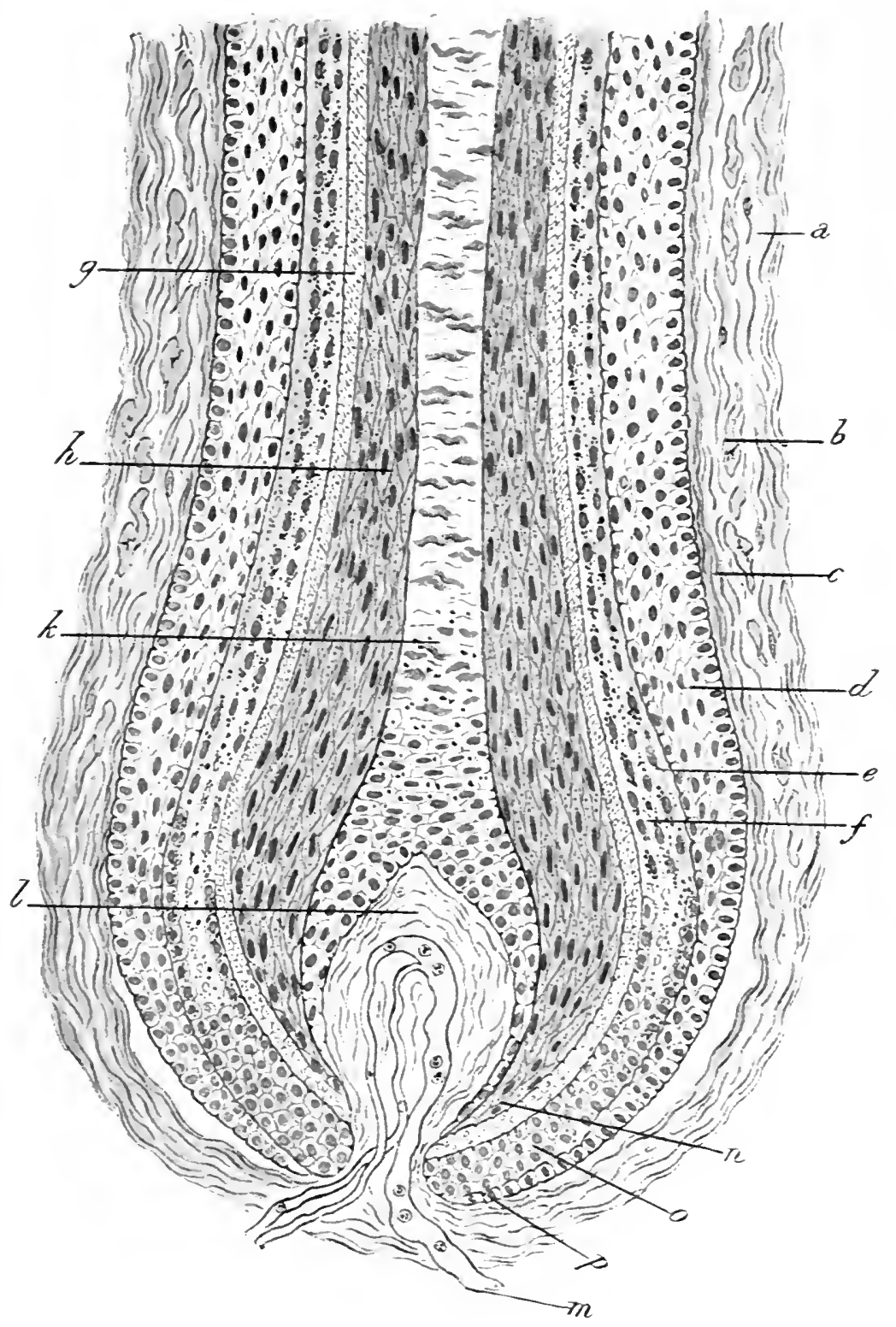

J. M. H. M. del.

FIG. 5--Longitudinal Section of a Hair-bulb and Follicle from a Beard-hair (semi-diagrammatic).

a. Outer coat of follicle, nbres longitudinally arranged, b. Middle coat of follicle, fibres transversely arranged. $c$. Inner coat of follicle, more condensed. d. Prickle-cell laver follicle. e. Outer laver of root-sheath, showing heratohvalin gramules. Inner later root-sheath; heratohvalin granules. g. Cuticles of ront-sheath and hair, is. Cortex spindle-colls with pigment granules. $k$. Iledulla; keratohtalin granules below and eleidin "lakes" above, l. Papilla. m. Capillary loop. m. Mlatrix of cortex. 0. Macrix of rotsheath. p. Matrix of prickle-cell layer of tollicle. 

ridges on the surface and interlock to form a highly resistant structure. The cells contain diffuse yellowish colouring matter, pigment granules, granules of keratohyalin, and occasionally minute air bubbles.

A medulla occurs, as a rule, only in the hairs of the scalp, beard, axillae and pubes. It is best marked in the intra-follicular portion and ceases towards the points of the hairs. It is composed of rouleaux of plates, consisting of three or four flattened cells, which, near the hairroot, contain keratohyalin granules.

Root or Bulb. - The root is softer and lighter in colour than the shaft, and is composed of the same two or three layers of cells, but these are much less differentiated and more cubical in shape than in the shaft. It is indented beneath by the upgrowing vascular papilla of the hair.

The process of cornification of the hair is analogous to that of the epidermis but the details of it are not yet fully understood. Keratobyalin granules appear in the medulla and in the cortex near the bulb and these gradually disappear as the cells become cornified. In the extra-follicular portion of the hair the component cells become cornified with the formation of hair-keratin at the periphery of the cells. Towards the free end the hair becomes hard and resistant and its component cells so closely packed together as to make the tip of the hair almost homogeneous. (Fig. 5.)

Structure of the Hair-follicle.-The hair-follicle ensheathes and protects the growing portion of the hair ; it is a simple invagination of the epidermis enveloped by a condensed layer of the connective tissue of the corium.

It may be divided for descriptive purposes into an upper third or funnel, a middle third in connection with which are the sebaceous glands and the Arrector pili muscles, and a lower third which ensheathes the hair-root and which is indented below by the papilla. In the upper third the epidermal layers lining the follicle are the same as those of the epidermis, the continuation of the Stratum corneum being next the hair. In the lower two-thirds only the prickle-cell layer and the basal layer of the epidermis persist.

The continuation of the prickle-cell layer and basal layer is generally. known as the "external root-sheath" of the follicle, and consists of several rows of polygonal prickle-cells with large round nuclei.

Between this layer and the hair there is a complicated structure named the "internal root-sheath," which is only present in the lower two-thirds of the follicle, and begins at the papilla as several layers of polygonal nucleated cells containing granules of heratohyalin. Is the cells ascend in the follicle those next the external root-sheath become cornified, while in those next the hair the cornification process is much less rapid and, owing to the difference in the degree of cornification, the layer tends to split longitudinally into two layers, the inner of which is usually known as the sheath of Huxley and the outer as the sheath of Henle. 
Within the internal root-sheath there is a cuticle formed of a single layer of elongated cells whose long axes are directed downwards and inwards. These fit in between the cells of the hair-cuticle. Hence it is almost impossible to epilate a hair without pulling out part of the root-sheath.

Enclosing the epidermal sheaths of the follicle there is a dense fibrous layer derived from the corium, which is known as the " connective tissue layer of the follicle." It is made up of collagenous and elastic fibres which externally are arranged longitudinally, while nearer the hair they tend to have a circular direction. Next the epidermal layer they become so condensed and homogeneous that they form a layer which has been described as the "hyalin or vitreous layer."

Papilla.-The papilla of the hair is a differentiated conical process of the corium analogous to a papilla of the papillary layer. It is composed of connective tissue fibres supporting a capillary loop and one or more medullated nerves. By means of it the root is nourished and any interference with its function through injury or disease causes a cessation of growth of the hair.

\section{SEBaCEOUS GLANDS}

These are small saccular glands, usually connected with the hair-follicles, but also found independently in the skin of the borders of the lips and in the areolae of the nipples. They are generally placed on the oblique side of the hair-follicle, in the angle between it and the Arrector pili muscle. They vary considerably in size, from mere appendages to the hair-follicle to structures larger than the follicle itself, and are largest about the nose, scrotum, and areolae of the nipples. They are absent in hairless parts, such as the palm of the hand and the sole of the foot. They may consist of a single saccule, or may be composed of several saccules with a common duct which opens into the neck of the hair-follicle or, in the case of the independent glands, directly on to the surface of the skin. The Meibomian glands of the eyelids, the ceruminous glands of the ears, and the smegma glands of the penis are modified sebaceous glands.

Histologically each saccule presents a connective tissue sheath inside which there is a basal layer of flat cells surrounding a mosaic of large polygonal cells with round or oval nuclei. The latter cells have undergone a change in which their protoplasm has been converted into fat droplets and their spongioplasm into a substance almost as resistant as keratin. The duct has the same minute structure as the saccule. The central cells of the saccules and duct break up and the fat droplets become discharged and collect in the lumen of the duct as a whitishfatty mass which mixed with epidermal debris forms the sebum (see page $2 \mathrm{I}$ ). 


\section{Sireat OR COIL-GLANDS}

These are single tubular glands made up of a body consisting of two or more turns forming a coil, and a duct which opens on the surface at the sweat-pore. The coil is situated in the reticular layer of the corium or in the subcutaneous tissue and opens into a duct which traverses the corium in a spiral manner and passes through the epidermis to the surface. These glands are present all over the body and are most numerous in the palm of the hand and the

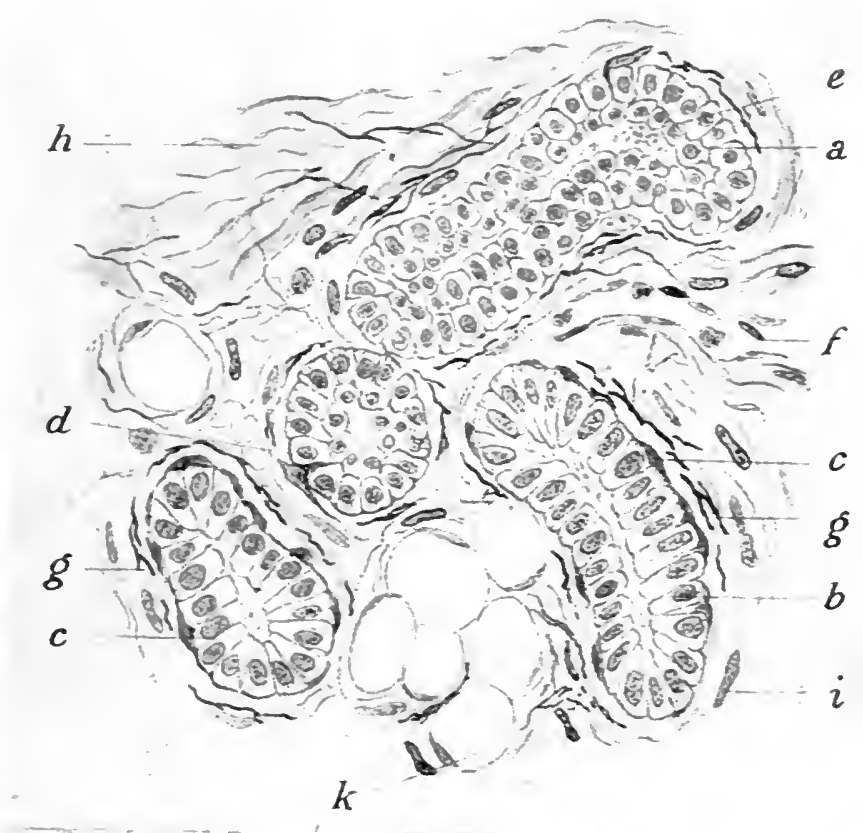

FIG. 6.-Sweat Gland. ( $x$ about joo.)

a. Coil near duct, with cubical epithelium. b. Distal part of coil with crilindrical epithelium. c. Nuclei of muscle-cells. $d$. Transverse section of coil. $c$. Debris inside the lumen. f. Capillary, g. Elastic fibres. h. Collagen bundles. i. Nucleus of collagen bundle. $k$. Fat-cell.

sole of the foot, where it has been estimated that there are as many as two thousand six hundred per square inch. They are absent on the Glans penis, margin of the lips and nail-bed. In a modified form they occur in an elliptical ring around the anus (glands of Gay) and on the eyelids (glands of Moss).

Structure.-The coil or body is composed of a single laver of cylindrical cells. These have a granular appearance when the gland is actively secreting, but this disappears when the gland is at rest. A layer of involuntary muscular cells, arranged longitudinally or spirally round the coil, encloses the cylindrical cell-layer, and outside that a fibrous capsule supporting the blood-capillaries. The lumen of the 
distal portion of the coil is wider than that of any other part of the gland, and is known as the " ampulla." Towards the duct the muscular coat disappears and the epithelium becomes more cubical, several layers being present. (Fig. 6.)

The duct in its spiral passage through the corium has a uniform calibre, and the lumen in section is stellate or forms a cleft. The tube is composed of several layers of cubical epithelium, inside which a cuticle has been described while outside there may be a connective tissue sheath, but no muscular fibres. On reaching the epidermis it passes up through an inter-papillary process, but there the duct proper may be said to end and in its further course it is represented by a spiral cleft between the prickle-cells and the horn-cells. The granular and horny layers, however, dip down into the Malpighian layer for a short distance to form a funnel for the duct. In its passage through the epidermis the sweat is mixed with the inter-epithelial lymph, which also finds exit with the sweat at the pores. A distinction is drawn by some writers between sweating and perspiring, sweat being taken to mean the secretion of the coil, and perspiration either the transudation from the blood-ressels, or the transudation and the sweat together.

\section{NAILS}

The nails are inelastic, translucent, horny plates situated on the dorsum of the distal phalanges of the fingers and toes.

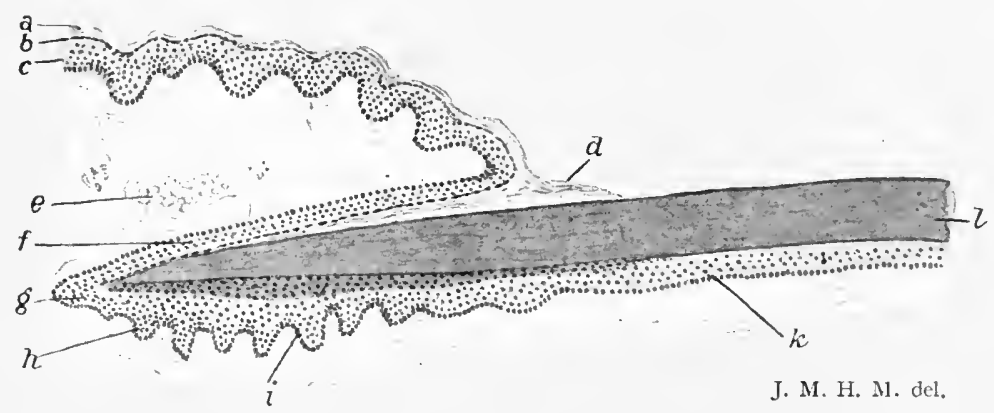

FıG. 7.-Longitudinal Section of Posterior Part of Nail and Nail-fold.

a. Stratum corneum. b. Stratum granulosum. c. Stratum Malpighii. $d$. Eponychium. e. Fat-lobes. f. Nail-fold. g. End of nail-fold. h. Nail-matrix. i. Granular cells. k. Niail-bed. i. Nail-plate.

(From MacLcod's Pathology of the Skin.)

The nail presents a posterior concave border, two almost parallel lateral borders, and a convex free edge. Its upper surface is convex transversely and to a less extent longitudinally. The whitish semilunar area occupying the posterior fifth of the nail-plate is known as the lumule. The nail is bounded by posterior and lateral nailwalls, and from the former a thin crescentic membrane passes for a short distance over the lunule. The lateral walls are slightly divergent, and have a rolled appearance. The nail-plate passes for a short 
distance beneath the lateral walls, and for a greater distance under the posterior wall, where it is known as the nail-root, and is enclosed in a structure analogous to the hair-follicle, called the nail-fold.

The nail-fold is split horizontally by the root into (I) an upper portion, forming a roof composed of the rarious lavers of the epidermis, in which the continuation of the Stratum corneum is in contact with the nail ; and (2) a lower portion named the nail-matrix. (Fig. T.)

The nail-matrix is an important structure, as it is from it that the nail grows. It extends from the posterior extremity of the nail-fold as far forward as the anterior margin of the lunule. It is composed of

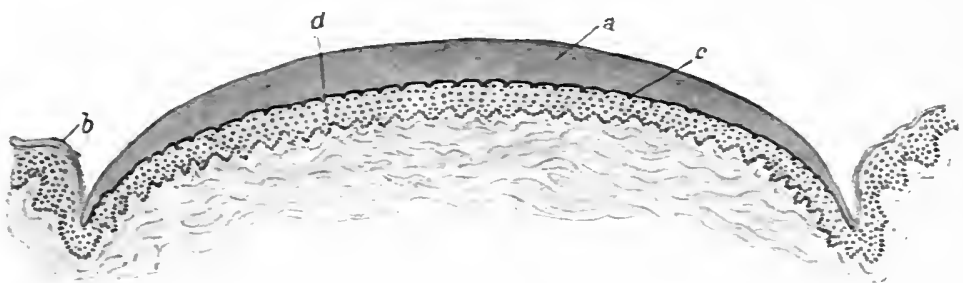

J. M. H. M. deI.

FIG. 8,-Transverse Section of Nail-plate.

a. Nail-plate. b. Lateral wall. c. Longitudinal ridges on nail-plate. d. Longitudinal papillary ridges.

(From MacLeod's Pathology of the Skin.)

a prickle-cell layer continuous behind with that of the roof of the nailfold and in front with that of the nail-bed. Above the prickle-cell layer there is a layer of several rows of flat granular cells with shrivelled nuclei, above which the true nail-cells begin to appear. It is the presence of these granular cells which gives the whitish opaque appearance to the lunule. Beneath the prickle-cell layer of the matrix there is a well-developed papillary layer in which the papillae are arranged on parallel ridges suggesting cocksccmbs. (Fig. 8.)

The nail-bed extends from the matrix almost as far as the free edge of the nail, and on it the nail-plate rests, but there is no organic connection between them. It is composed of a prickle-cell layer, beneath which is a papillary layer consisting of about sixty ridges parallel to the long axis of the nail. Under this there is a rich sub-papillary vascular plexus in which large venous sinuses are present, lined

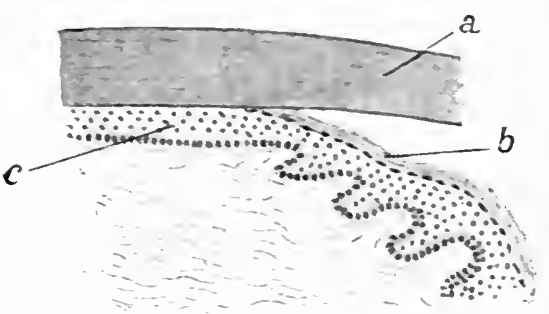

J. M. H. M. del.

FIG. 9.-Longitudinal Section of Free Border of Niail.

a. Nail-plate. b. Stratum corneum an granular layer. c. End of Nait-bed.

(From ilacLeod"s Pathoiog: of the Skirn. with endothelium, and forming an erectile tissue. (Fig. 9.)

The nail-plate, though not in organic connection with the nail-bed. is in close contact with it, since on its under surface there is a series of 
parallel ridges which fit in between those of the nail-bed and so bind the two structures together. It is made up of flat cornified cells which differ from those of the

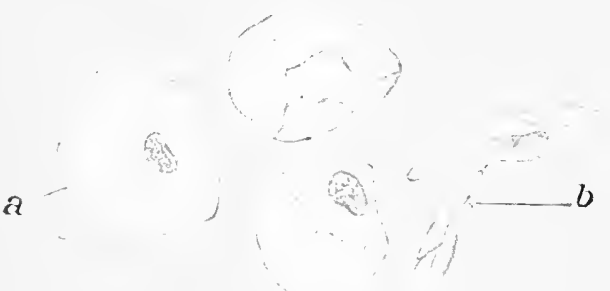

J. M. H. M. del.

FIG. 10.- Nail-cells. ( $x$ about Iooo.) From a piece of nail soaked for a week in Liquor Potassae.

a. Cells, showing facets, seen from the surface. $b$. Cells seen from the side.

(From MacLeod's Pathology of the Skin.) Stratum corneum in that the nuclei persist. These cells are arranged in superimposed lamellae, which can only be distinguished near the nail-matrix, since further forward they become knit together to form an almost homogeneous plate. (Fig. IO.)

Growth of the Nail.-If left uncut the nail does not grow indefinitely, but at a variable distance beyond the finger it becomes thin and desquamates, so that a natural free border is formed. As a rule the matrix only produces one nail, which during life is constantly growing at about the rate of $0.8 \mathrm{~mm}$. in twenty-four hours on the fingers, and $0.4 \mathrm{~mm}$. on the toes. It takes roughly about roo days for a finger nail to grow.

\section{CORIUM}

The corium, or Cutis vera as it was once called, is the dense fibrous layer of the skin which gives it strength and elasticity. It supports and protects the hair-follicles, glands, nerves and blood-vessels. Structurally it is built up chiefly of white fibrous tissue and a variable amount of yellow elastic tissue, in the interstices of which are certain well recognised cellular elements. It is customary to divide the corium into a superficial and a deep layer, known respectively as the papillary and the reticular layer. In the papillary layer the white fibrous bundles are thin, loosely packed together, and tend to have a vertical direction, while in the reticular layer they are coarse and form a dense complicated network, the meshes of which tend to be arranged horizontally.

The papillae are conical projections of the corium into the overlying epidermis, in which are found the terminal capillary loops and certain nerve-endings ; according as they support the former or the latter they are known respectively as vascular or nerve-papillae. They are situated on ridges of varying height, arranged more or less parallel to each other. The papillae may be single or more rarely compound, are longest on the flexor aspects of the finger-tips and toes, and are specially numcrous about the arcolae of the nipples.

Cellular Elements. - The cellular elements in the healthy corium consist of migratory cells and fixed cells. The migratory colls are the 



\section{PLATE II.}

FIG. 1.-Prickle-cells. ( $x$ about rooo.)

Stained with gentian-violet by the Unna-Kromayer method.

a. Large nucleus in cell with an intranuclear network and three chromatin bodies. $b$. Cell in which the surface is in focus, and the prickles seen in relief appear as fine specks. c. Exoplasm of the cell, showing protoplasmic fibres over it. $d$. Lighter endoplasm, with only a few fibres present. $e$. Small nodules on protoplasmic threads. $f$. Nuclear space.

FIG. 2.-Horn-cells from Sole of Foot. ( $x$ about 10oo.)

Stained by Gram's method.

$a$. Horn-cells with facets and covered with granules. $b$. Two cells united by keratinised epithelial fibres. $c \& d$. Cells with coarser granulations. $e$. Cell showing intracellular network.

FIG. 3.-Mast-cells. ( $\times$ about Iooo.)

Stained with polychrome methylene blue.

$a$. Spindle-shaped mast-cell with blue nucleus. $b$. Mast-cell dividing. $c$. Irregularly branched cell. $d \& e$. Mast-cells shaped like plasma-cells.

FIG. 4.-Plasma-cells. ( $x$ about rooo.)

Stained by Pappenheim's method.

$a$. Plasma-cells with reddish granulo-plasm and violet nuclei. $b$. Connective tissue cell. c. Lymphocyte. $d$. Mast-cell.

FIG. 5.-Horn-cells which have undergone Hyalin Degeneration. ( $x$ about 1000.)

Shows keratinised capsule, shrivelled nucleus, central hyalin mass. 


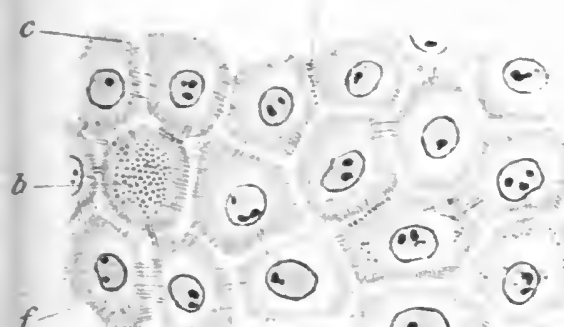

() (2) ()

Fig. .

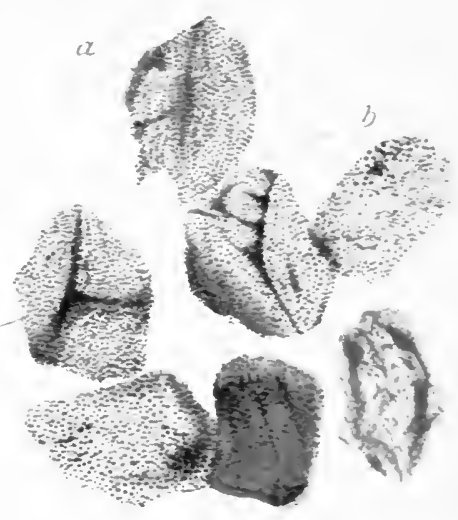

Fig. 2.

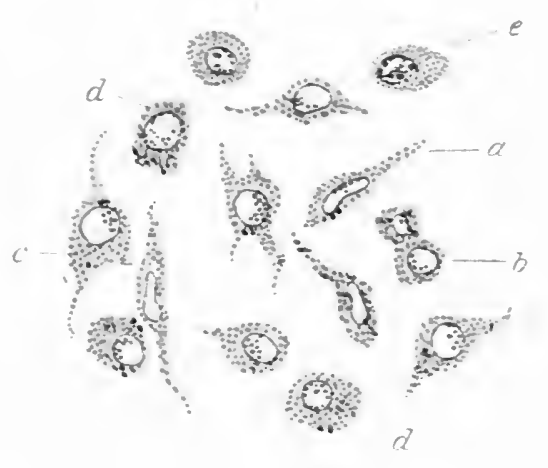

-

Fig. 3 .

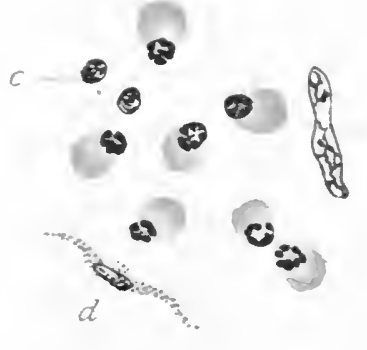

Fig.

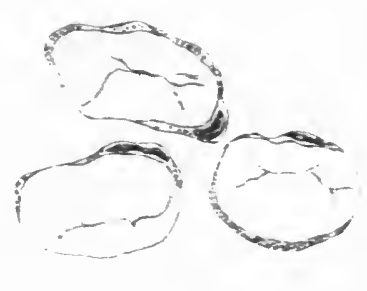

Iig. 5. 

leucocytes which are specially noticeable near the blood-ressels and in the papillary layer.

The fixed cells constitute the more important group. They are not all absolutely fixed to fibrous structures, as some are unattached and may be carried about in the lymph stream, but their movements are purely passive. There are three distinct types of fixed cells in the corium namely :

(I) Ordinary connective tissue cells which, as a rule, are spindle-shaped, but may be polygonal. These have usually long processes, tapering to fine threads, which in young connective tissue frequently unite with those of neighbouring cells to form a meshwork. The nuclei vary according to the shape of the cells, some being oral, others round, and a few polygonal. These nuclei are peculiar in having a coarse intra-nuclearnetwork with unusually open meshes, producing a "vesicular" appearance. (Fig. II.)

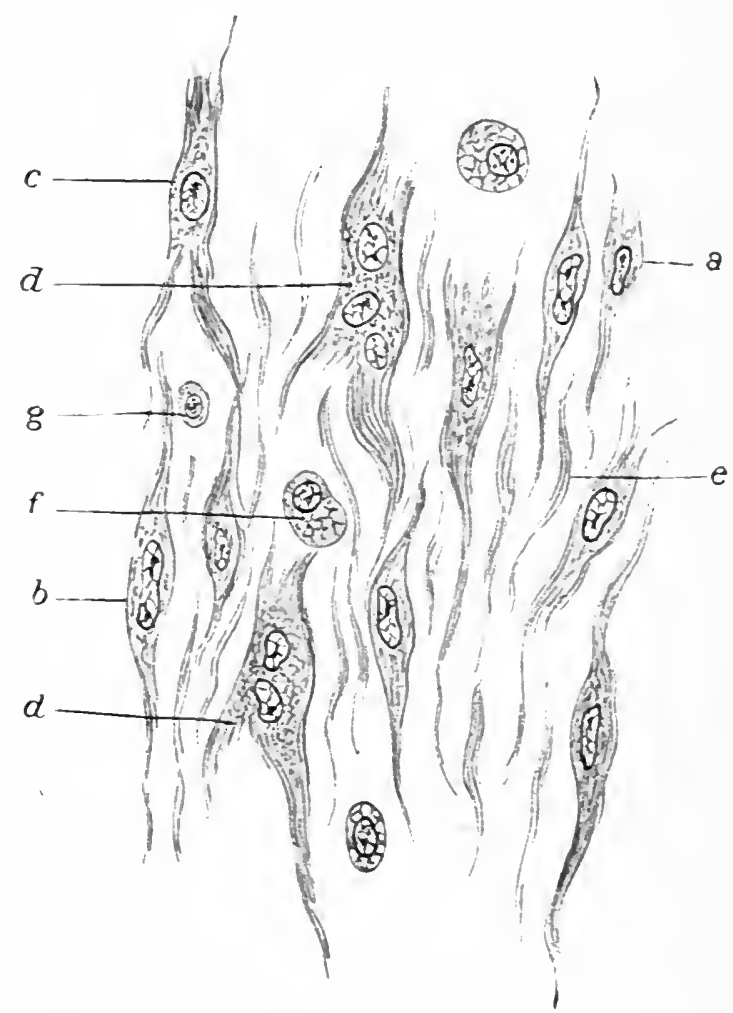

FIG. I I.-Connective Tissue Cells of Granulation Tissue.

a. Spindle-sbaped Connective Tissue cell $b$ Longer Spindle-cell rith protoplasm becoming fibrous, c. Hranching and early fibrous formation. $d$. Large Connective Tissue cells with several nuclei. $e$. Fine fibrils between the cells, probably parts of cells not shown. $f$. Connective Tissue cell with a racuolated appearance. g. Spindle-cell in transterse section.

Frequently only the nuclei of the cells can be detected, and eren with the most careful staining no protoplasm can be recognised around them. As a rule the cells or nuclei are found flattened between the fibrous bundles of the corium, and at times their processes may surround bundles and may line the lymphatic spaces between them like an endothelium.

(2) Tacuolated Cells. - These differ from the ordinary type of connective tissue cell. They have no processes, and the meshes of their spongioplasm are unusually large and rounded, giving them a racuolated appearance. The nuclei of the cells are "vesicular" in character, and mitotic figures may occasionally be detected within them. It is 
just possible that these cells are the "mother-cells" of the corium, their function being exclusively that of reproduction, and that they bear the same relation to the corium as the cells of the basal layer to the epidermis. (Fig. I2.)

(3) Mast-cells. - This type of cell is distinguished by the presence in its protoplasm of numerous coarse granules with a marked affinity for alkaline dyes. In shape and size they vary like ordinary connective tissue cells, and may be round or spindle-shaped. Their nuclei do not stain well, frequently present no nucleoli and are generally more or less obscured by the granules. (Plate II. 3.)

They occur chiefly in the neighbourhood of the blood-vessels, hair-

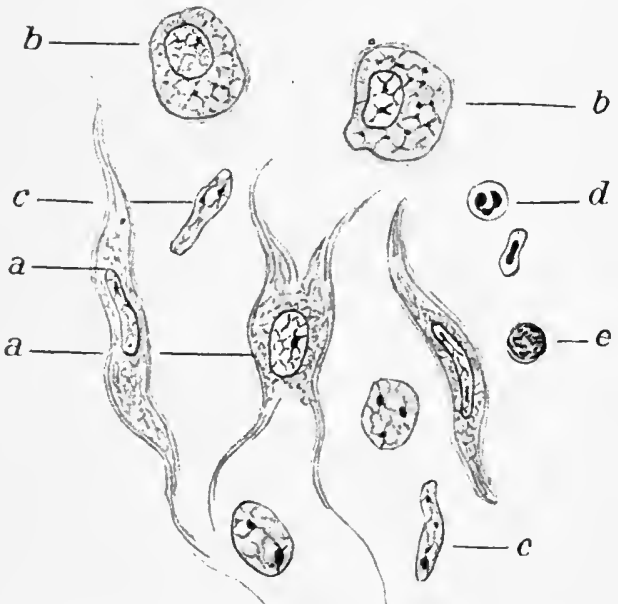

F1G. I2.-Connective Tissue Cells of the Corium.

$a$. Ordinary Connective Tissue cells, $b$. Vacuolated cells. c. Connective Tissue nuclei. $d$. Polymorphonuclear leucocyte. e. Lymphocyte. follicles, and sebaceous glands, and are found in greatest number in the skin of pigmented regions, such as the scrotum and areolae of the nipples.

The nature and origin of the mast-cell granules are still undecided. A possible explanation is that they are a separation- or degeneration-product of the protoplasm of the cells, somewhat allied to mucin.

Fibrous Elements.-(I) White fibrous bundles which are composed of numerous fibres about the breadth of a red blood corpuscle, united by a semi-fluid material.

These fibres readily split up into fine fibrils of a substance known as collagen and are incapable of branching. The bundles are flexible, slightly extensible, and when seen longitudinally present a wavy appearance which disappears when the skin is stretched.

Collagen is an albuminoid substance and is probably the anhydride of gelatine.

There are two opposing views regarding the origin of the white fibrous bundles, namely, (I) that they are the result of the direct transformation of the protoplasm of the connective tissue cells, and (2) that they are formed by the deposition of fibres in an intercellular substance secreted by the cells. Of the two the former seems to be the more probable.

(2) Elastic fibres which are arranged parallel or obliquely to the collagenous bundles and are most numerous in the reticular layer. Unlike the collagenous fibres they are capable of branching and of uniting with neighbouring fibres to form a meshwork. They envelop 


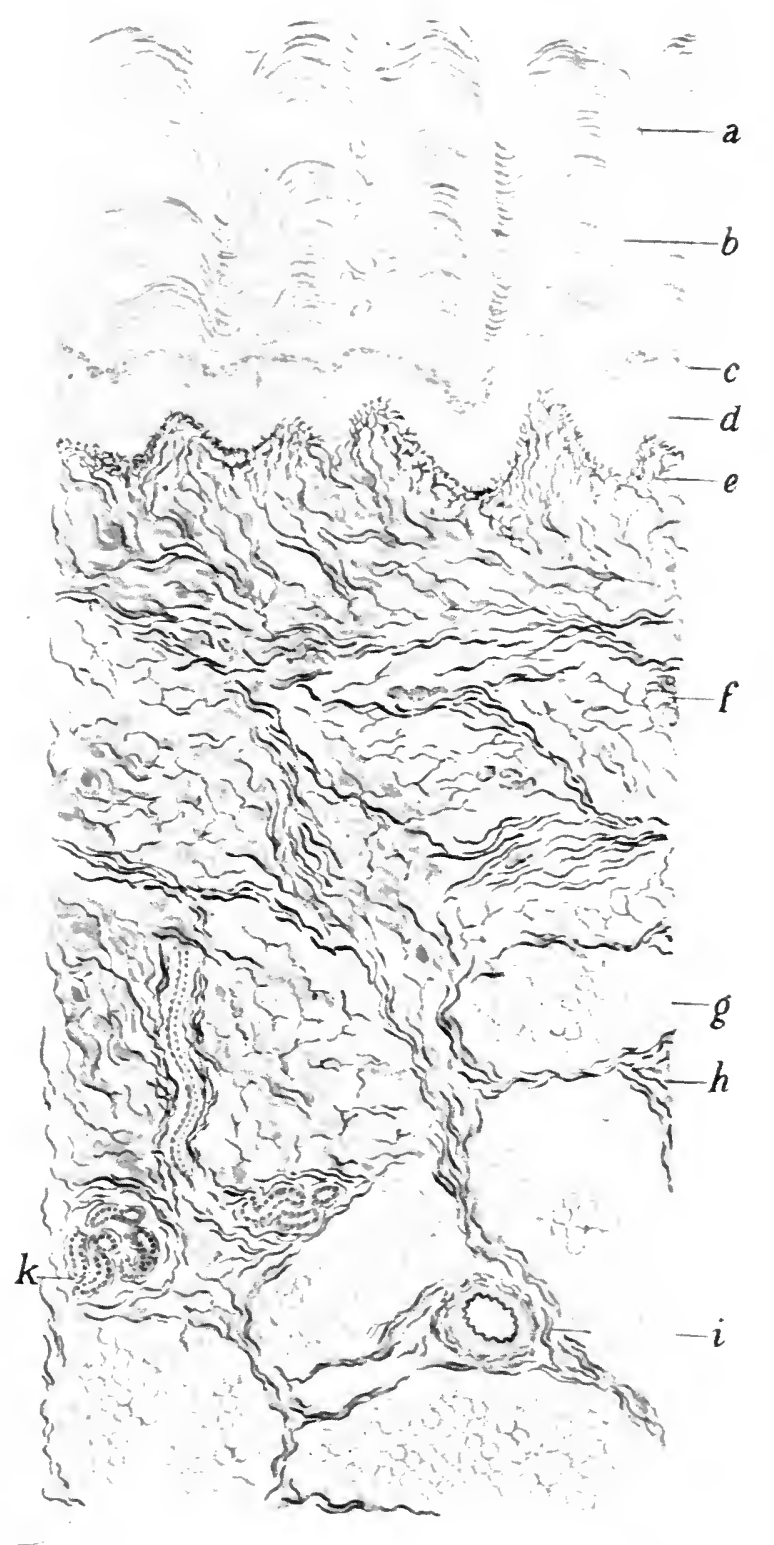

I. M. H. M. It:

FIG. I3.-Elastic Tissue. Section of Skin from Sole of Foot ( a about 60). a. Sweat-pore. b. Stratum corneum. c. Stratım granulosirn. d. Stratum Malpighii. e. Papilary layer with tine elastic tibrils passing up between basul cells of equiternis. Elastic tibrils in corium. g. Fat lobule. h. Septum composed of collazerous and ciastio abres. $i$ Small artery with elastic lamina, $k$ sweat gland with thrir tibes hetwern and around the alveoli.

(From MacLeol's Puthoingy of the Stin.) 

the coil and ducts of the sweat-glands and are present around the hairfollicles, sebaceous glands and Arrectores pilorum. The distribution of these fibres is so universal that they form a skeleton supporting the various elements of the corium. Their elasticity is not nearly so great as was at one time supposed, and is considerably less than that possessed by the collagenous fibres. In section they are angular in outline and tend to curl up on being broken across. They are composed of an albuminoid substance called elastin, which is more resistant to the action of weak acids and alkalies than collagen. The origin of the elastic fibres is undecided, and they have not been definitely proved to be capable of regeneration. (Fig. I3.)

\section{Subcutaneous Tissue}

This structure may be regarded as the deepest layer of the corium, the cells of which have become infiltrated with fat. This layer serves four important purposes:

(I) It forms a pad which protects the delicate underlying structures from being harmed by injuries to the skin.

(2) It is a bad conductor of heat.

(3) It prevents too great a loss of heat by radiation and evaporation.

(4) It forms a supporting framework for various delicate structures, such as the Pacinian bodies, nerve-trunks, coil-glands, and blood-ressels.

It is especially thick on the palms and soles, and thin on the face. It consists of a coarse fibrous meshwork enclosing large groups of fat-cells, known as fat-lobes. The fibrous walls

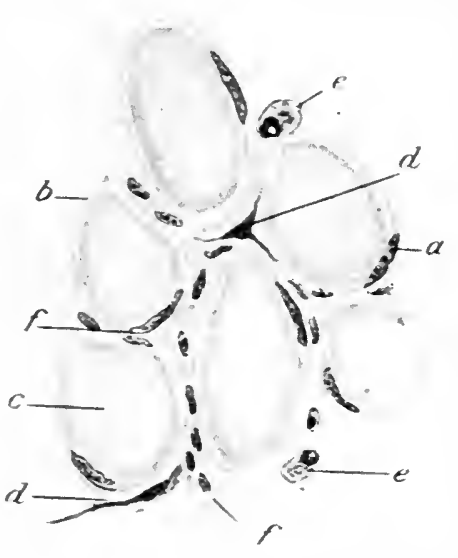

J. M. H. M. del.

Fig. I4.-Fat-Cells. $(\times 800$.

a. "Fat-cell with "mantle" and nucleus.

b. "Mantle" formed by protoplasm. corpuscle. $f$. Capillary and leucocytes. separating the lobes are named "trabeculae." The lobes are broken up by fibrous septa into lobules. The lobes, lobules and individual fat-cells are richly supplied with blood-vessels and lymphatics. which are distributed in the trabeculae and septa and form a capillary meshwork between the cells. Fat-columns (Columnae adiposae) have also been described, which pass vertically along the larger vessels from the fat-lobules to the coil-glands in the corium.

The individual fat-cells are large, oval, round, or polygonal bodies, consisting of a globule of fat encapsuled in a homogeneous mantle, the 
remains of the protoplasm of the cells; at one side of which a flattened nucleus is generally present. These cells are due to a fatty infiltration of the connective tissue cells and are not the result of a degenerative process. (Fig. I4.)

The origin of the skin-fat has been a fruitful source of controversy. The leading theories regarding it are :

I. That the skin-fat is a product of degeneration of the cell-protoplasm.

2. That it is a product of secretion of the cell-protoplasm.

3. That the subcutaneous fat, and that which occurs in the lymphatics of the skin, is derived from the coil-glands.

4. That the epithelial fat comes from the sebaceous glands.

It is generally conceded that the fat of the sebaceous glands and that which occurs in the Stratum corneum is a separation or degeneration product of the protoplasm of the cell; this explanation does not apply to the subcutaneous fat, however, for in the latter situation the cells are not degenerated and the fat is of the nature of an infiltration. The presence of fat in the coil-glands and ducts has been noted by a large number of observers, and Unna has strongly advocated the view that the subcutaneous fat is derived from that source. The lymph which bathes the coil is said by him to be loaded with fat and to be taken up, as a rule, by the small venules, and in the process filtered and the fat deposited. The fat accumulates in columns around the bloodressels, and is eventually taken up by the connective tissue cells of the subcutaneous tissue.

Other Situations in the Skin where Fat is found.-Small fat granules or globules occur in the epithelial cells of the sweat-apparatus and in the lumen of the sweat-coil and duct. They are present also in the lymphatic spaces of the corium, in the walls of the vessels and in the medullae of nerves.

In the epidermis, fat occurs in the nuclear spaces of the pricklecells, in the inter-epithelial lymphatics and in the cells of the Stratum corneum. It is also present in the epidermal cells of the hair-follicles.

\section{Blood-Vessels}

The following systems of blood-vessels in the skin begin in the subcutaneous tissue and pass towards the surface:

Blood-Vessels of the Subcutaneous Tissue.-Small arteries are present in the trabeculae between the fat-lobes; certain branches from these course in the septa between the lobules and end in capillaries between the fat-cells, while others pass up more or less vertically into the corium. Teins and venous capillaries correspond to these arteries.

Blood-vessels of the Conum.-Two somewhat horizontal vascular plexuses are generally described in the corium; a deep one situated next the subcutancous tissue and a superficial one in the sub-papillary 


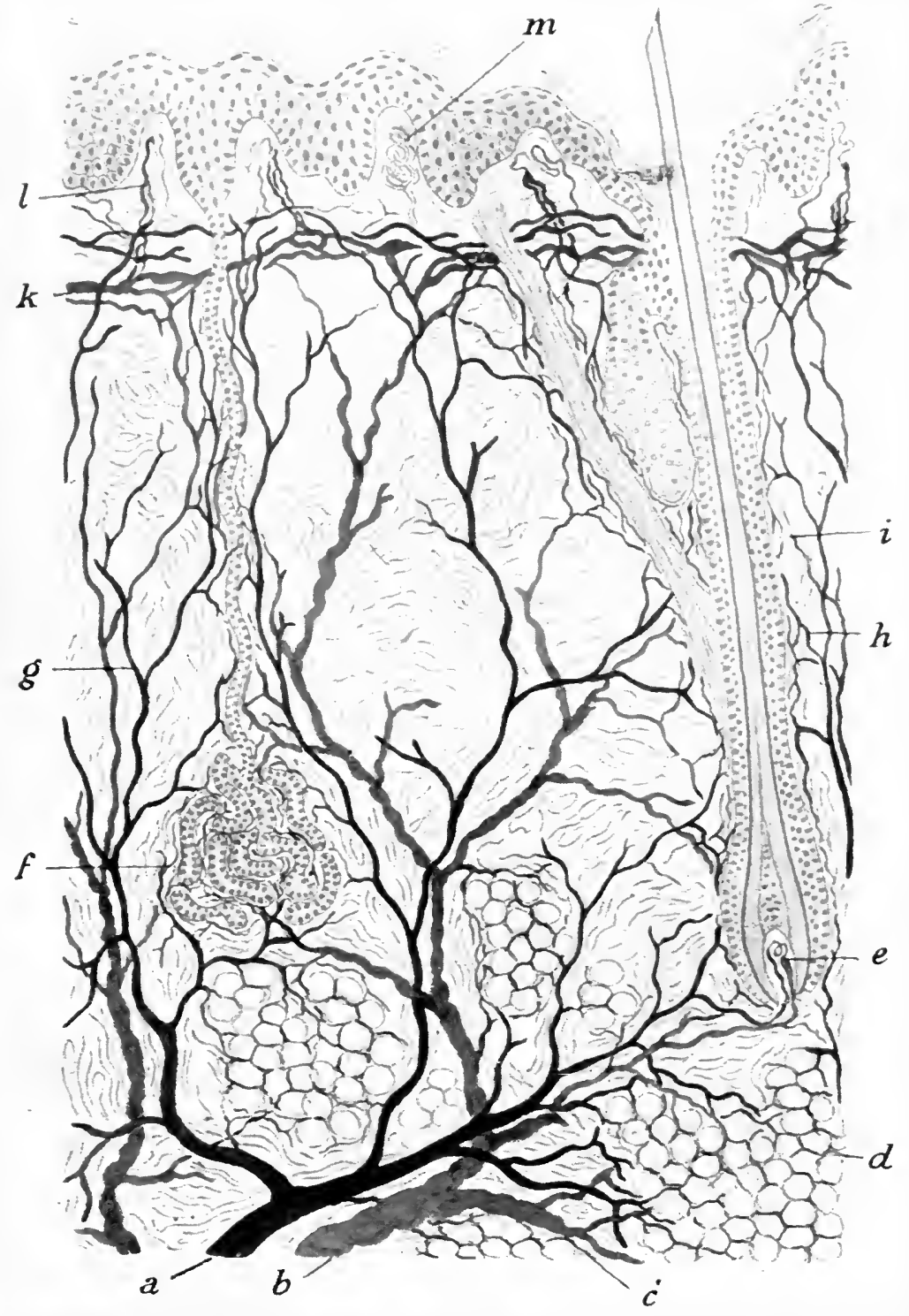

FIG. 15.-Distribution of the Skin Blood-Tessels.

$a$. Large arterial branch in subcutaneous tissue. b. Larze vein. $c$. Interlobular vein. d. Capillaries between fat-cells. e. Capillarv-loop of hair-papilla. $f$. Capillary network between sweat-coils, g. Capillaries to duct, Cipill bries to hair-follicle, Capillaries to sebaceous gland and arrector pili muscle. $k$. Sub-papillary plexus. I. Capillary-loop to 

layer. From these plexuses branches are given off to the sweatapparatus, pilo-sebaceous follicles and papillary layer. In the papillae the capillaries form loops by the junction of a single or double arterial capillary with a venous capillary several times greater in diameter. (Fig. I5.)

Throughout the corium the blood-vessels are of the nature of capillaries since they possess only an endothelial layer, and it is in the subcutaneous tissue alone that small arteries and veins occur in which a muscular wall is present. The veins of the corium are considerably larger than the arteries.

\section{LYMPHATICS}

In the skin there are comparatively few true lymphatic vessels with a distinct endothelial lining, but there are numerous spaces between the fibres of the corium and the cells of the epidermis which serve as lymphatics.

The lymphatic vessels begin as blind ends in the papillae, pass down to a lymphatic plexus in the sub-papillary layer, then course with the blood-vessels obliquely through the corium and are collected into the larger lymphatics of the subcutaneous tissue. The lymphatic vessels of the papillae are most easily seen in the large papillae of the fingers and toes. The lymph from the tissue-spaces gains entrance into the vessels either through stomata or between the endothelial cells.

The lymphatic spaces are not lined by endothelium. They are present between the fibrous bundles of the corium, around the coils and ducts of the sweat-glands, between the epidermal cells of the hairfollicles and sebaceous glands, around the Arrectores pilorum and between the prickle-cells of the epidermis. They pour their contents partly into the lymphatic vessels and partly into the veins.

\section{Nerves}

Both medullated and non-medullated nerve fibres are present in the skin :

(a) The medullated fibres form plexuses in the corium from the most superficial of which fine non-medullated fibres pass between the prickle-cells of the epidermis, forming the inter-epithelial plexus. Medullated fibres also pass to the connective tissue coats of the hairfollicles, where they lose their sheaths and split into fibres which form a plexus between the epidermal cells of the external root-sheath. A few of the medullated fibres terminate in special end-organs.

(b) The non-medullated fibres probably supply the Arrectores pilorum, the sweat-coils and the blood-vessels.

The inter-epithelial nerve plexus is composed of fibres which have lost their medullary sheaths in the sub-epithelial layer. These are 
distributed between the prickle-cells as far as the Stratum lucidum. They end either in minute swellings between the cells or pass into the cells and terminate in swellings applied to the nuclei. The fibres of the epithelial plexus are sensitive to touch-impressions and to common sensation.

\section{Special Terminal Corpuscles}

Tactile Corpuscles, or Meissner's Bodies.-These end-bulbs are situated in the papillae, are universally distributed, but are specially numerous at the tips of the fingers and toes. They are oblong or spindle-shaped, and present a transversely striated appearance owing to their being composed of superimposed layers of connective tissue between which the nerve fibrils ramify and end in small swellings. The nerve loses its medullary sheath on entering the corpuscle and splits into these fibrils.

Touch-cells of Merkel.-These are peculiar small, round or pearshaped cells situated at the termination of the medullary nerves of the papillae of the fingers and toes. Certain observers believe them to be epithelial cells, and do not regard them as sensitive.

Pacinian Bodies or Corpuscles of Vater.-In unstained sections held over a dark background these bodies appear as small milky specks situated in the subcutaneous tissue. They are most numerous on the palms and soles; they are oval or elliptical in shape and are made up of a cortex of concentrically arranged connective tissue capsules like the sheaths of an onion and a central granular core in which the fibres of the axis cylinder of the nerve terminate in a group of pear-shaped swellings, The nerves immediately below the corpuscle form a swelling known as the "stalk" and the capsules are said to be due to a splitting up of the sheath of Schwann of the nerve. The Pacinian bodies are believed to be sensitive to pressure and traction.

\section{Muscles}

These consist of the Arrectores pilorum and layers of involuntary muscle fibres such as are found in the areolae of the nipples, the Dartos of the scrotum, and the eyelids.

The Arrectores pilorum are made up of involuntary muscular fibres which arise from a neighbouring papilla and are inserted into a projection situated in the middle third of the hair-follicle. On contracting they cause an erection of the hair and at the same time a dimpling of the surface of the skin. The elastic tissue forms a framework for the muscle which is continuous with the elastic network of the corium, so that when the muscle contracts it causes an increased tension of the skin in the neighbourhood 


\section{Pigment AND Colour}

In white races very little pigment is present in the skin except in pigmented regions such as the areolae of the nipples, axillae, scrotum, around the anus, and in the hairs. The colour of the skin is, as a rule, more dependent on the subcutaneous fat and the degree of vascularity than on pigment. The pigment granules are situated chiefly in the cells of the basal layer of the epidermis. They are generally confined to the periphery of the cells while the nucleus and the zone immediately around it are free from them. They may also occur in the inter-epithelial lymphatic spaces where they form branching figures. They are sometimes present in the deeper pricklecells and more rarely in the connective tissue cells of the papillary layer. In dark races the pigment extends up as far as the granular layer of the epidermis, and is present also in pigment cells in the papillary layer of the corium which send out processes which pass up between the cells of the basal layer of the epidermis.

The true pigment of the skin, in contradistinction to the blood pigment which gives the skin its peculiar tints in bruises and purpura, consists of a substance called melanin, which is in the form of more or less fine irregular brownish-black granules. Unlike the blood pigment it never occurs as crystals and it contains no iron. It contains carbon, hydrogen, nitrogen, oxygen and sulphur, is soluble in ether, weak acids, alkalies and hydrogen peroxide, and is bleached by chlorine.

Origin of Melanin.--In spite of the profusion of literature on the subject, the origin of melanin is still undecided. There are three leading views with regard to it, (I) that it is a degeneration product of haemoglobin, (2) that it is a separation or secretion product of the protoplasm of epidermal cells, and (3) that it is derived from a decomposition product of protein.

(I) Melanin, a degenerative product of haemoglobin.-Certain observers believe that melanin is produced by the breaking up of the coarse brown granules of haemosiderin and that it is carried by certain migratory cells or leucocytes known as " chromatophores" from the blood-vessels to the epidermis. The chromatophores are supposed to obtain the melanin from the red blood-cells, to carry it to the inter-epithelial lymph-spaces and to there break down and deposit their granules, which are gradually absorbed by the epidermal cells.

Others, while agreeing that the melanin is derived from the haemoglobin, consider that it is built up from the blood by certain connective tissue cells which are capable of amoeboid movement; others again maintain that the pigment-bearing cells are neither ordinary connective tissue cells nor leucocytes, but peculiar structures derived from the mesoderm, which have been named " melanoblasts."

One of the strongest arguments in favour of this theory is that it has been proved that the transplanting of a piece of white skin on to a negro is soon followed by pigmentation of the graft, and that if a piece of negroskin be grafted on to a white man it gradually becomes depigmented.

(2) Another hypothesis is that melanin is not derived from bloodpigment, but is elaborated by the epidermal cells, and is similar to other 
products of cellular activity. According to Dyson it is a product of the nucleus and is a lipochrome in origin, being " the chromatic proteid portion after its separation from the complex lipoid granules." This view is supported by the fact that pigmentation is localised in certain regions in the white skin which are not necessarily regions with a rich bloodsupply, and that it is not most marked near the blood-vessels.

(3) A third theory is that it is a decomposition product of protein.According to this theory, the pigment is derived from decomposition products of protein, and the pigment of the skin as well as the haemoglobin of the blood may both originate from the chromogenic groups in the protein molecule. These chromogens are said to circulate in the blood and when they reach the tissue-cells to be converted into melanin by some specific oxidising ferment contained in the cell.

\section{PHYSIOLOGY}

The skin performs six important functions :

I. It is a secretory organ.

2. It is a respiratory organ.

3. It has a limited power of absorption.

4. It regulates the body temperature.

5. It is a sensory organ.

6 . It is a protective organ.

Function of Secretion.--The fluid secretions and excretions of the skin are the sweat and the sebum.

Sweat.-It is difficult to collect sweat to analyse in a pure condition as it is liable to be mixed with the products of degeneration of the epidermis, with sebum, and with the transudate from the bloodvessels which bathes the epidermal cells and passes out at the sweatpores as perspiration.

The sweat is a clear watery fluid with a saltish taste, a peculiar odour, a specific gravity of about I005, and an acid reaction. It contains water, sodium chloride, fats, volatile fatty acids, cholesterol and, in certain conditions where the kidneys are not acting properly, urea. The secretion of sweat is dependent on two sets of nerves, namely, special secretory nerves, and the nerves of the involuntary muscles of the sweat-coils. Special sweat-centres are present in the cord and medulla.

The secretion of sweat is stimulated by certain drugs, such as muscarine, nicotine, strychnine, and camphor, which act on the sweatcentres, and by pilocarpine, which acts directly on the muscles of the sweat-coils and is capable of producing its action if injected subcutaneously even when the secretory nerves are cut. It is stimulated also by certain emotions such as fright and fear, by heat, and by a venous state of the blood. It is diminished or inhibited by atropin and by the toxins of certain fever-producing micro-organisms.

The sweat has several important functions. It eliminates certain effete products from the blood and lymph such as urea and lactic acid ; it lubricates the skin as it contains fat; owing to its acid reaction it 
protects the skin from the growth of micro-organisms, but when excessive and largely diluted with the transudate from the blood-ressels it may become alkaline and its protective power cease; it helps to regulate the temperature by evaporation, as when the temperature of the body rises considerable quantities of sweat are poured out which evaporate and produce a cooling effect.

Sebum is a semi-fluid oily substance containing various fats, fatty acids, cholesterol, salts, albuminoids and water mixed up with epidermic debris. The composition of sebum, which resembles lanolin from sheep's wool, has been chiefly ascertained by analysis of the contents of sebaceous cysts, the smegma of the prepuce, or the Vernix caseosa of the foetus. On exposure to the air, it becomes cheesy in character.

The method of secretion of the sebum is not definitely decided. Unlike the sweat-glands the sebaceous glands are not under direct nervous influence and no excito-secretory nerves have been demonstrated in connection with them. The output of sebum would appear to be stimulated by an increased blood-supply to the gland and a rise of temperature. It was once believed that it was mechanically secreted by pressure caused by the contraction of the Arrector pili muscle, but this is no longer admitted and the secretion is now generally believed to be due to the vis a tergo caused by the continuous elaboration of fat by the older sebaceous cells in the centre of the lobule of the gland.

On the other hand there is a possibility that the sebum may be a true secretion and not a product of fatty degeneration, and that the cells of the gland absorb fat from the blood, change its composition, and eliminate it into the lumen of the gland. This is borne out by the observation that alterations in the quantity and quality of fat in the food have an effect on the activity of the sebaceous glands.

There would appear to be a relation between the sebaceous glands and the generative organs, for about puberty the glands become more active and their activity is increased at the menstrual period.

The sebum is most abundant where the glands are largest and numerous, as about the nose, scrotum, pre-sternal and interscapular areas. It may form an oily coating in these situations, or it may become inspissated and cheesy and form greasy scales, or it may collect in worm-like masses or "cocoons" in the funnels of the pilo-sebaceous follicles.

Its chief function is to lubricate the hairs and prevent them from becoming dry, brittle, or injuriously affected by water. It also spreads on to the skin around the mouth of the hair-follicles.

There is not infrequently present in the sebum a harmless acarus or mite known as the "Demodex folliculorum."

The Vernix caseosa is a fatty material consisting of sebum and epidermal cells. It is present over the whole of the foetus with the exception of the soles of the feet and the palms of the hands. 
Function of Respiration.-The skin is a respiratory organ and capable of eliminating water-vapour and carbonic acid gas and, possibly, of absorbing oxygen. The amount of water excreted by the skin in twenty-four hours is almost double that which is eliminated by the lungs, and is proportionate to the temperature. When the temperature reaches a sufficient height, about $33^{\circ} \mathrm{C}$., the skin becomes covered with beads of perspiration and there is an increased elimination of carbon dioxide. It has been estimated that the loss of moisture from the skin of a man clothed and at rest at a temperature of $32^{\circ} \mathrm{C}$. is about 2500 c.c. and the discharge of carbon dioxide about $7 \mathrm{grm}$. in twenty-four hours, while after active muscular exercise the loss of moisture and carbon dioxide is many times greater.

Function of Absorption.--So long as the Stratum corneum is intact the skin has little absorptive capacity, but if it be abraded absorption by the papillae readily takes place. Neither water, alcohol, nor substances dissolved in them, are absorbed by the healthy epidermis except at the orifices of the pilo-sebaceous follicles and sweat-pores. Gases, oils, fats and substances suspended in them may be absorbed by the unbroken skin, and the more nearly allied the fat is to that of the skin, the more active is its absorption. This absorption takes place especially at the sebaceous follicles and sweat-pores for there the fat mixes with the fatty secretions at these orifices. The degree of absorption of substances suspended in fats depends on their power of becoming volatilised at a low temperature. If the skin fat be removed by washing with ether, or the skin be inflamed or rubbed so that the vascularity is increased, absorption takes place more readily, and when water is in contact with the skin for some period the horn-cells gradually become sodden and swollen, especially where sebaceous glands are absent, and a certain amount of absorption may take place in consequence.

Function of Heat-Regulation.-The constant temperature of the body is maintained by the heat-regulating function of the skin. The amount of heat which is lost from the surface of the skin by radiation, conduction and evaporation, depends chiefly on the state of dilatation of the cutancous ressels and on the activity of the sweat-glands. Heat and cold cause respectively a dilatation or constriction of the cutaneous vessels, either by acting directly on the involuntary muscular fibres of the ressels of the subcutaneous tissue, or indirectly through the rasomotor centres.

Heat causes an increase in the excretion of sweat by a reflex and central stimulation of the glands, and through the evaporation of the swcat a certain amount of heat is rendered latent and the blood is cooled.

The involuntary muscles of the skin also assist in performing this function for cold stimulates them to contract with a consequent reduction of the superficies and a diminution in the loss of heat by conduction and radiation. 
The temperature of the skin varies considerably in different parts of the body, and is most constant about the trunk where it arerages $98.4^{\circ} \mathrm{F}$.

Sensory Function.-The skin is the most extensive sensory organ in the body. Oxing to the presence of peripheral nerve-endings, it is sensitive to touch, pain, heat and cold, pressure and traction. It has been demonstrated that the sensory endings in the skin are not continuous all over the surface but have an irregular grouping in sensory spots surrounded by insentient areas. These sensory spots vary in the type of sensation which results from stimulation, some being sensitive to pain and known as "pain spots," others to touch and known as " tactile spots," others to heat, cold, etc.

Protective Function.-The protective function of the skin is chiefly dependent on the Stratum corneum with its keratinised closely welded cells which enable it to resist mechanical injury and prevent the entrance of micro-organisms. The waxy fat in the horn-cells gives it the character of a waterproof coating and at the same time prevents too great an evaporation, or the absorption of toxins or poisonous gases. The fatty secretions of the sebaceous gland and the sweat gland are also protective by the greasiness which they produce in the immediate neighbourhood of the orifices of their ducts. Consequently, the epidermis, and more especially the Stratum corneum, may be regarded as the first line of defence of the skin against injury from without.

\section{REFERENCES.}

Abel and DAvis. Pigment of Negro-Skin (Journ. Exper. Med., New York, r896, i. p. 36 I).

Behrixg, Fr. Minute Anatomy of the Skin (Monats. f. prakt. Derm., Aug. 1904, p. 2ro).

BuzzI, F. Keratohyalin \& Eleidin (Monats. f. prakt. Derm., I889, viii. p. $\mathrm{I}_{49)}$.

Cedercrettz, A. Cornification of the Epidermis (Arch. f. Derm. u. Syph., I907, lxxxiv. p. I75).

Drsox, W. Cutaneous Pigmentation (Brit. Journ. Derm., I9I I, xxiii. p. 205).

MacLeod, J. M. H. Derelopment of the Human Epidermis (Brit. Journ. Derm., I 898, x. p. 233).

Structure \& Development of Horn-cells (Brit. Journ. Derm., I899, xi. p. 137).

Journ. Anat. \& Physiol., I902, xxxvi. p. 6r.

Handbook on the Pathology of the Skin, H. K. Lewis, I903.

Meirowsky-Grauderz, E. Pigment Question (Monats. f. prakt. Derm., I907, xliv. p. I66).

Merk, L. Connection of Epidermal Cells with each other and within the Corium (Monats. f. prakt. Derm., I904, xxxviii.).

Okavura, T. Die Entwicklung des Nagels (Arch. f. Derm. u. Syph., I9oo, lii.).

Pappenheim, A. Elastin, Staining of (Monats. f. prakt. Derm., I904, xxxriii.).

Pexirey, M. S. The Function of the Skin (Brit. Journ. Derm., I9Io, xxii. P. $\left.{ }^{5} j^{6}\right)$. 
REID, W. Secretion \& Absorption by the Skin (Text Book of Physiology, I 898 , p. 669).

Rosenfeld. Fat-secretion of the skin, Effect of diet on the (Zentralb. $f$. in. Med., I906, xxiii. p. 986).

Solger. Skin Pigment, Protective Action of (Derm. Zeitsch., I903. p. 329).

Thом pson, R. U. Epidermal Fibrils (Journ. Exper. Med., I906, viii. p. 4). Unna, P. G. \& Solodetz, U. Hornsubstance (Monats. f. prakt. Derm., I907, xliv. p. 399).

Die Hautfette (Bio. Zeitsch., I909, xx. p. 469).

Vörner, Hans. Tricho-Hyalin (Derm. Zeitsch., I903, x. p. 357).

White, C. J. Elastic Tissue of the Skin (Journ. Cut. Dis., I9Io, p. I63).

Winkler, F. Pigment Cells, Movements of (Arch. f. Derm. u. Syph., r9Io, ci. p. 255). 


\section{CHAPTER II}

\section{GENERAL SYMPTOMATOLOGY}

THE diseases which affect the skin differ so widely in their clinical appearances that it is almost impossible to describe their symptomatology in a general fashion, for not only is there a vast n!tmber of skin diseases, but the clinical pictures presented by them vary so greatly in different cases that practically no two are alike. In most instances skin affections are manifested by objective symptoms consisting of one or more lesions which can be seen or felt. Occasionally, however, the only evidence of a departure from the normal state may be some subjective symptom such as itching or burning. The skin disease may be a purely local affection or it may be a symptom of some general infective process such as syphilis or leprosy. It may be the only sign of a derangement of health, or it may be associated with internal disturbances, especially of the gastro-intestinal tract, liver, and nervous system.

\section{Subjective Symptoms}

These consist of itching, burning, pricking, tingling, formication, hyperaesthesia, anaesthesia and pain. One or other of these symptoms may occur more or less constantly in connection with a number of skin affections but they are of somewhat uncertain diagnostic value as differences in temperament or in the sensibility of the skin will cause them to vary greatly in individuals suffering from the same disease, and patients' statements regarding their symptoms are apt to be unreliable.

Itching is the most common of all cutaneous subjective symptoms and may even be the only indication of the skin-disorder. It is caused by the stimulation of the peripheral nerve-endings by various irritants, either acting from without, such as the sting of a nettle or the bite of an insect, or from within, such as various toxins circulating in the blood-capillaries of the corium. It varies greatly in degree and type, and may be so slight as to be negligible, or so severe as to be more intolerable than actual pain. It may be continuous, or intermittent, and may occur in more or less severe paroxysms or crises. It is present 
in association with a large number of cutaneous affections such as eczema, urticaria, Lichen planus, Dermatitis herpetiformis, Mycosis fungoides, and is one of the principal symptoms of parasitic diseases such as scabies and pediculosis.

Pain is a much rarer symptom. It differs much in character and may be lancinating or boring as in Herpes zoster, throbbing as in boils and carbuncles, or shooting as in nerve leprosy. It may be continuous, intermittent, or paroxysmal.

\section{Objective Symptoms}

Diseases of the skin are associated with more or less pronounced objective symptoms by means of which they may be distinguished. These consist of various kinds of lesions in different stages of evolution and arranged or grouped in diverse ways. There are two main types of lesions, namely, initial or primary lesions, and secondary lesions. Each skin disease has a characteristic type of initial lesion, the recognition of which is essential for diagnosis.

The initial or primary lesion is the lesion in its initial phase or at the height of its development; the secondary lesion is a late or retrogressive stage of the primary lesion, or is the result of adventitious influences acting upon it in the course of its evolution. Occasionally a type of lesion may be primary in one skin affection, and secondary in another.

\section{Primary Lesions}

The primary lesions consist of macules, papules, nodules, tumours, wheals, vesicles and bullae. The secondary lesions are scales, fissures, excoriations, crusts, ulcers and scars.

Macules.-Syn.: Spots ; Fr., Taches ; Ger., Flecke.

Macules are variously sized spots not definitely raised above the surface of the skin. In outline they may be round, oval, or irregular, and may be well-defined or may fade into the surrounding skin. In size they vary from minute specks up to patches as large as the hand or larger. In colour they differ according to their cause and may be red when due to hyperaemia and inflammation or to a dilated condition of the blood-vessels as in capillary naevi ; purple, greenish, or yellow, when due to extravasated blood as in purpura and bruises; brownish as in freckles and pigmented patches; and white as in leucodermia. They may present a variety of colours when they are caused by tattooing or by the staining of the skin by chemicals, such as silver nitrate, iodine, and aniline dyes.

Macules may be transient, as in erythematous spots; permanent, as in vitiligo; congenital, as in pigmented moles; or acquired, as in freckles. They may disappcar completely under the pressure of a 
piece of glass or " diascope," as when they are due to hyperaemia, or may not be effaced by pressure, as when they are caused by extravasated blood or pigment. As a rule they are smooth on the surface, but they may be rough and covered with fine scales. Occasionally, owing to the presence of an underlying inflammatory disturbance, they may become slightly raised, when they are known as maculo-papules.

Papules.-Sın. : Pimples; Fr., Papules; Ger., Knötchen.

Papules are small solid elevations of the skin, varying in size from a pin's head to a pea. In outline they may be round, oval, irregular or angular, and on the surface may be rounded, conical, acuminate, flat or umbilicated. They may be sharply defined and are sometimes demarcated by the minute fissures of the epidermis, or they may fade gradually into the surrounding skin. They differ greatly in colour, and may be skin-coloured as in prurigo, red as in eczema, riolaceous as in Lichen planus, yellowish-brown as in Lupus vulgaris, raw-ham coloured as in syphilis, yellowish as in xanthoma, or blackish as in sarcoma.

Papules may occur at the orifices of the sweat-ducts or the hairfollicles or independently of these structures. The surface of the papule may be smooth and shiny, occasionally with minute vessels ramifying over it as in the papule of Lichen planus, or it may be rough and surmounted by a small scale when it is known as a squamous papule, or a small vesicle when it is known as a papulo-vesicle, or a pustule when it is called a papulo-pustule. When situated at the orifice of the hairfollicle it may present a central plug or spine.

Papules may be associated with subjective symptoms such as itching and burning as in eczema, or may be unaccompanied by sensations as in Lupus vulgaris or milium. They differ greatly in their course and evolution, some persisting as papules, while others undergo changes, becoming vesicles or pustules or breaking down to form ulcers. Some disappear leaving no trace, while others are followed by pigmentation or scarring.

In consistence they may be soft and easily obliterated by pressure as in eczema, or may be replaced on pressure with a piece of glass by a brown stain indicating a cellular deposit in the corium as in tuberculosis. On the other hand they may be firm and cartilaginous as in the border of a rodent ulcer, or hard and not effaced by pressure as in small warts.

They vary in their distribution, some being discrete and irregularly disseminated as in papular urticaria, while others are arranged in groups as in Lichen scrofulosorum, or coalesce to form sheets.

Histologically, the papule may be the clinical evidence of a thickening of the epidermis as in a wart, an inflammatory cellular infiltration in the corium as in eczema, a combination of both as in Lichen planus, a granulomatous infiltration as in tuberculosis, a fibro-fatty change in the corium as in xanthoma, a plugging of the hair-follicle with a horny spine as in Keratosis pilaris, or an obstruction of the sebaceous follicle as in a sebaceous cyst. 
Nodules.-Syn. : Nodules ; Fr., Tubercules ; Ger., Knoten.

Nodules are solid well-defined elevations about the size of a pea and intermediate in size between papules and tumours. The name "tubercle" is sometimes employed as a synonym for nodule, but as it is apt to suggest a lesion of tuberculosis it is better avoided. They may be rounded, conical, or flat in shape and vary considerably in colour, being skin-coloured in fibroma, yellow in xanthoma, reddish-brown in syphilis, brown in moles, and violaceous in myomata. They differ greatly in their course, some persisting indefinitely as moles and fibromata, others disappearing spontaneously or under treatment as in syphilis. When involution takes place no trace may be left, or they may be followed by pigmentation, ulceration, or necrosis and scarring.

Histologically they may result from changes in the epidermis or its appendages, as in the lesions of Molluscum contagiosum and sebaceous cysts, or they may be due to a cellular infiltration in the corium as in the nodules of leprosy, or to fibro-cellular changes in the subcutaneous tissue as in rheumatic nodules.

Tumours.-Syn. : Fr., Tumeurs ; Ger., Geschwülste.

Tumours are circumscribed swellings larger than a pea and may be the result of an inflammatory or purulent process, as in a boil, or may be due to a neoplasm either benign or malignant. The skin over the tumour may be unaffected, as in fibroma, or red, inflamed, or purplish as in a carbuncle. They may be hard as in gummata or soft as in Molluscum fibrosum, and may be slightly elevated or much raised and even pedunculated. Certain of them persist indefinitely, while others undergo involution and disappear as the result of suppuration or necrosis.

Wheals.-Syn.: Urticae, Pomphi; Fr., Plaques Ortiées, Plaques Urticariennes; Ger., Quaddeln.

Wheals are transient raised efflorescences of varying size, firm in consistence, white or opaline in colour, and surrounded by a pink areola which fades into the neighbouring skin. The lesion produced by the sting of a nettle is a typical wheal. They may be caused by a great variety of external irritants, such as bites of insects, contact with certain plants, jellyfish, caterpillars, etc., or may be the result of toxins circulating in the cutaneous blood-vessels. In outline they may be round, oval, or irregular when due to internal causes, linear when due to scratching or the lash of a whip, or may vary indefinitely in shape when due to pressure or friction.

They vary in size from a split-pea to areas the size of the palm of the hand or even larger, as in the condition known as giant-urticaria or angio-neurotic oedema. They develop rapidly in a few seconds and may disappear almost as suddenly. They are almost invariably associated with itching or burning, which ceases when the lesion subsides.

Wheals constitute a type of reaction to toxic irritants which may be met with in any skin. Certain individuals are much more liable 
to wheal formation than others, either as an individual peculiarity or an acquired condition. As the result of an attack of urticaria a state of hypersensitiveness may be produced in which fresh wheals are readily called forth by toxic influences far weaker than those which were responsible for the original outbreak.

The pathogenesis of wheals has been a fruitful subject of controversy. At one time they were regarded as angio-neurotic in nature and due to the action of some toxic irritant on the central or peripheral vaso-motor apparatus. This was believed to lead to a dilatation of the blood-vessels supplying the affected area, a spasmodic contraction of the reins, and a consequent mechanical transudation of fluid which contained less albumin than blood-plasma. The serous fluid, collecting chiefly about the papillary layer and pressing on the underlying vessels, emptied them and gave rise to the peculiar white or opaline appearance of the lesion. When the spasm in the smaller veins passed off the circulation was restored, the fluid was absorbed, and the oedema disappeared leaving no trace except perhaps slight scaliness.

It was pointed out, however, that stimulation of the vaso-motor apparatus was incapable of producing all these changes and would only cause alterations in the calibre of the vessels, and it was then suggested that the lesions were not angio-neurotic but inflammatory and caused by the direct action of toxins in the blood-ressels and surrounding tissue; that the fluid was an inflammatory exudation; and that there was a definite cellular infiltration of an inflammatory character. In other words that the wheal was the eridence of a local reaction of the skin to some toxic substance.

Vesicles.-Syn.: Small Blisters; Fr., Vesicules; Ger., Bläschen.

Vesicles are small elevations of the epidermis varying in size from a pin's head to a split-pea and containing a clear or turbid fluid. They may arise from apparently normal skin or may be preceded by a macule or papule and be surrounded by a reddish inflammatory areola. In shape they may be rounded, conical, acuminate, or flat, and may be tense, flaccid, or occasionally umbilicated. They may be single or numerous, and may be discrete and irregularly distributed, or arranged in groups or rows. In colour they may be pale with clear contents, opaline or milky, yellow from becoming semi-purulent (vesicopustules), or red and haemorrhagic when they occur in dependent regions in states of malnutrition. Where the epidermis is thin they are usually situated superficially and tend to rupture spontaneously, or more often to be broken by the friction or rubbing to which they are subjected for the relief of itching. Where the epidermis is thick, as in the palms and soles, they present a peculiar pearly or sago-grain appearance, are not so easily broken, and tend to undernine the epidermis. They may consist of a single carity, when they are known as monolocular vesicles, or they may be multilocular. When the roof of the vesicle breaks the serum oozes out and a crust is formed which may be soft and honey-like, or dry, yellowish, green or brownish. As 
a rule when this is detached healing takes place without leaving a scar.

Vesicles are usually the result of an inflammatory disturbance, but they may occur independently in connection with derangements of the sweat-apparatus, as in hydrocystoma and sudamina. Ordinary vesicles result from the co-operation of a number of different factors, namely (I) increased pressure of lymph in the inter-epithelial lymphatic spaces of the epidermis, (2) actual oedema of the prickle-cells, (3) poisonous substances circulating in the lymph-stream causing a destruction or necrosis of the prickle-cells, (4) an initial want of vitality of the prickle-cells by which they are less able to withstand the interepithelial oedema and the poisonous substancescirculating in the lymph. All four factors are not invariably present but the first two are essential. According as the oedema of the inter-epithelial spaces or the actual oedema of the prickle-cells is in excess in the early stages of the vesicle they have been divided into two classes, namely, interstitial and parenchymatous vesicles. This division is purely arbitrary, however, as the one merges readily into the other, and it is difficult to tell whether the inter-epithelial or cellular oedema is in excess.

When the oedema in the epidermis reaches a certain degree, the intercellular fibres are no longer able to resist the constantly increasing lymph-stream and they become stretched to such an extent that a considerable space is left between the cells, and a small monolocular vesicle results around which the prickle-cells become compressed and form a sort of wall. Other monolocular vesicles are produced in the same way and may gradually coalesce and give rise to an irregular multilocular vesicle.

Where the actual cells become markedly oedematous and the meshes of their spongioplasm become reticulated or widely dilated with fluid, a number of these oedematous cells appear to coalesce and a parenchymatous vesicle results. A variety of this was described by Leloir under the heading of " alteration cavitaire," in which the nuclear spaces became dilated with fluid and so distended as to form a large cavity limited by a membrane formed by the exoplasm of the cell. The vesicles in eczema tend to pertain to the interstitial type, while those in varicella belong to the parenchymatous variety. Occasionally when the prickle-cells become separated and their inter-cellular bridges broken, they assume a roundish form and their protoplasm becomes transformed into a cloudy homogeneous mass, while the nucleus persists in the centre. Such cells are known as "balloon-cells " and have been mistaken for sporozoa; they are met with about the floor of the vesicle in Herpes zoster.

Vesicles may be situated superficially or deeply in the epidermis and more rarely may occur beneath it in the papillary layer having the whole of the epidermis as a roof. Their most common site is about the position of the granular layer. The situation in the skin is not accidental but is determined by two considerations, $(a)$ the 
resisting power of the different parts of the skin, the vesicle tending to occur in the region of least resistance which is, as a rule, between the granular layer and the Stratum corneum, $(b)$ the rapidity of the serous transudation, for if the oedema occur very rapidly the fluid tends to collect in the papillary layer and to lift up the whole of the epidermis to form a roof, while if the transudation be more gradual it will tend to pick out the situation of least resistance in the epidermis.

The cause of the umbilication or dimpling of vesicles has been much discussed and several theories have been put forward to explain it. If a vesicle forms in the epidermis around the orifice of a hair-follicle it naturally presents a central dimple, but as only a limited number of vesicles occur in this situation some other explanation is necessary.

At first sight it would seem probable that the dimpling is caused by the central portion of the roof of the vesicle being more firmly held down to the base than the peripheral part by the remains of the prickle-cells acting as guy-ropes, but this explanation cannot be accepted as these cords are so delicate that they are invariably ruptured by the rapidly collecting fluid. It would seem more probable that it is due to the oedematous process being especially active at the periphery and leading to an excessive pouring of fluid into the epidermis there, and possibly to the vesicles belonging to the parenchymatous type and the "reticulation" being more marked at the margins than at the centre.

Bullae.-Syn. : Blebs, Blisters ; Fr., Bulles ; Ger., Blasen.

Bullae are elevations of the epidermis containing serous fluid and differing from vesicles only in size, being so-called when they are larger than a pea. In outline they may be round, oval or irregular, and may be rounded or flat on the surface, and tense or flaccid. They may arise from apparently normal skin or be preceded by an erythematous patch, or, more rarely, by a wheal as in Urticaria bullosa. They are generally multilocular and contain a serous fluid with a faintly alkaline reaction in which may be bands of fibrin, polymorphonuclear leucocytes, the debris of epidermal cells and their nuclei, and sometimes microorganisms. The contents are clear at first but tend to become cloudy, yellowish, purulent and, occasionally, haemorrhagic. They may be situated superficially in the skin with only the horny layers as a covering, when they are apt to rupture spontaneously or from the slightest injury. On the other hand they may be deeply seated beneath the thick epidermis of the palms and soles and tend to spread and to undermine the epidermis before they rupture; or the contents may be absorbed, and the affected shin eventually peel off in sheets. When rupture takes place the floor of the bulla is found to be red, raw, and moist, sometimes covered with a purulent slough, and more rarely with irregular vegetations as in the disease known as Pemphigus vegetans. Occasionally the collection of fluid is slight while the areas affected are extensive and exfoliation of the epidermis takes place, as in Pemphigus foliaceus.

When the contents of the bullae are discharged, the inflammation as a rule gradually subsides and healing takes place without subsequent scarring, but when the bulla is deeply situated ulceration and scarring 
frequently result. As a rule bullae are preceded by sensations of burning or itching and when they are fully developed are generally accompanied by a feeling of tension. They may occur in association with various inflammatory diseases of the skin, such as Dermatitis herpetiformis, urticaria and pemphigus, or they may be locally produced by heat, by certain vegetable or chemical irritants like cantharides, or by the local inoculation of micro-organisms such as streptococci, and they occur readily when the prickle-cell layer is in a state of malnutrition from some pre-existing cachectic state. In bullae caused by thermal or chemical irritation, the fibrinous deposit is more marked than in those which occur in association with the skin affections mentioned above, the fibrin being caused to deposit by the action of a ferment derived from the breaking-down epidermal cells.

Pustules.-Syn. : Fr., Pustules; Ger., Pusteln.

Pustules are small elevations of the skin containing pus. They may be whitish, yellowish, brownish, or reddish in colour ; rounded, conical, flat or umbilicated on the surface ; vary in size from a pin's head to a filbert nut; and are generally situated on a raised inflammatory base. In the large majority of instances they are a further development of vesicles and in the early stage are known as vesico-pustules. Occasionally, however, the pustule is not preceded by a vesicular stage or it is passed over so quickly as not to be recognised when it is known as a "pustule d'emblée." They occur frequently about the pilo-sebaceous follicles, as in the pustules of acne and in boils. When superficial they are easily ruptured, but when the pus is situated deeply and surrounded by a profuse inflammatory cellular deposit they evacuate more slowly and may even require to be opened surgically. When rupture takes place the pus and epidermal debris dry up to form a crust or scab which may be yellowish and waxy as in Impetigo contagiosa, greenish-yellow as in boils, or brownish from the admixture of blood. Sometimes the crust, instead of being flat, is limpet-shaped and stratified from a peripheral extension of the suppurative process and the addition of new layers beneath, or it may dry at the centre and present beads of pus oozing out at the margin. Should the pustules be situated in the epidermis, healing as a rule takes place without scarring, but when they are located in the corium a scar may be inevitable and is not infrequently associated with pigmentation.

The subjective symptoms in connection with pustules vary according to the depth and degree of the inflammatory disturbance; they may be slight as in impetigo, or severe and consist of burning, throbbing, or actual pain, as in boils and carbuncles. Pustules are generally the result of the action of staphylococci but may also be caused by chemical irritants such as croton oil. 


\section{Secondary Lesions}

Scales.-Syn. : Fr., Squames; Ger., Schuppen.

Scales are exfoliations of the epidermis. Ther vary greatly in size ; some are minute and delicate and form a fine "branny" or "furfuraceous" desquamation, as in Pityriasis versicolor; others are coarser, as in eczema; others are imbricated or stratified, as in psoriasis; while others occasionally form extensive sheets of exfoliation, as in exfoliative dermatitis.

In the healthy epidermis there is a constant imperceptible shedding of the dried-up horn-cells on the cutaneous surface which is accelerated by washing and the friction of clothes, and occasionally this may become noticeable as a fine scaliness upon the extensor aspects of the limbs.

Scales may be dry, brittle, and shiny like flakes of mica, or greasy and lustreless from the admixture of sebum and sweat. In colour they may be white, greyish, Jellowish, or silvery from the presence of air between the dried cells. They are generally easily detached as flakes or lamellae, but they may be firmly adherent as in Lichen planus and Lupus erythematosus. As a rule they tend to become detached first at the free border and may be slightly everted, but exceptionally they may be adherent at the margin and break in the centre so as to form a collar around a protruding papule, as in scaly papular syphilides.

Scales occur in association with a large number of inflammatory diseases of the skin and are met with in all grades of coarseness from a branny desquamation to thick fish-like scales such as those which occur in ichthyosis. Except in physiological scaling of the effete horn-cells, they are the result of an interference with the process of cornification which may be a congenital defect, as in ichthyosis, or the result of oedema of the epidermis due to an underlying inflammatory disturbance in the corium, as in eczema and psoriasis, or of hyperaemia, as in scarlet fever. IThen the prickle-cells are oedematous they are no longer capable of going through the different stages in the evolution of a horn-cell, by which the protoplasm of the cell becomes changed into a waxy substance and the intercellular fibres into keratin, but simply dry up on nearing the surface and being exposed to the air, adhere together in fine or coarse masses, and flake off in scales; this process of imperfect cornification is known as "parakeratosis" (see p. 39).

Crusts.-Syn. : Scabs; Fr., Croûtes ; Ger., Borken ; Krusten.

Crusts are masses of dried-up serum, pus, or blood, mixed with the debris of epidermal cells. They vary in size from that of a pin's head to coarse scales several inches in diameter. They may be rellow, greenish, dirty-brown, or blackish in colour, according to the preponderance of serum, pus or blood in their formation. They may be smooth, flat or irregularly broken up, or they may be " limpet-shaped " when they cover an ulceration which is extending peripherally and to which new strata are being added from beneath, as in the "rupia" 
of syphilis. They may be soft in consistence, superficial, easily detached, and appear to be stuck on to the skin like wafers as in impetigo; or they may form dry, opaque, friable masses, as in the "scutula" of favus where they are partly composed of the mycelial threads and spores of the fungus of that disease ; or they may be thick, tough and adherent, as in the crusts covering syphilitic ulcerations, or the crusts which result from a reaction to radium.

When superficial crusts are detached the underlying surface may be dry, or red and moist, and no scarring may result, but when they are coarse and thick and cover ulcerations, more or less marked scarring generally follows.

Excoriations.-Syn.: Abrasions; Fr., Excoriations ; Ger., Hautabschürfungen, Excorationen.

An excoriation is a superficial loss of skin tissue such as is caused by a scratch by the finger nails, or by any sharp instrument, or may result from traumatism, or even from continuous friction. Excoriations vary indefinitely in size and shape and may be punctate and roundish, as where the apices of small papules have been scratched off, or somewhat larger and irregular in outline where the skin has been dug out by the nails, or linear, straight, or curved according to the agent which produced them and its line of action. They generally become covered with a crust which may be yellowish or reddish according as serum or blood preponderates in its formation, and they are usually surrounded by an inflammatory areola.

They are usually superficial, only the epidermis being injured, and heal without forming a scar. But they may extend deeper into the corium and be followed by a cicatrix which is pinkish at first but as a rule eventually becomes white. At the time of excoriation, or previous to healing, inoculation of pyogenic micro-organisms is liable to occur and to result in pustulation or ulceration not infrequently associated with enlargement of the neighbouring lymphatic glands.

Excoriations are usually met with in connection with itchy parasitic diseases, such as pediculosis, and may be accompanied by more or less deep pigmentation of the skin.

Fissures.-Syn. : Cracks, Rhagades, Rimae; Fr., Fissures ; Ger., Fissuren, Hautschrunden.

Fissures in the pathological sense, in contradistinction to the normal fissures on the cutaneous surface, are linear clefts in the skin caused by injury or disease. They vary in length and breadth, some being minute cracks, others clefts an inch or more in length. They may be superficial and the cleavage only affect the epidermis, or they may extend deeply into the corium. They may be single or multiple, and straight, wavy, irregular, or sometimes branched. They are usually associated with smarting and sometimes with actual pain when they are stretched open in movements.

They may be caused mechanically by a blow, but as a rule they are the result of movements of the skin when it is in a weakly resistant 
condition from some inflammatory disease, or when it has become thickened, inelastic, and apt to crack on being stretched or folded. Consequently, fissures usually occur on parts of the skin subjected to more or less continuous or frequent movement, such as the flexures of the large joints, the palms of the hands, the clefts between the fingers and toes, the angles of the mouth and the eyelids, and about the anus. They are commonly met with in connection with eczema of the palms of the hands, impetigo, and congenital syphilis at the angles of the mouth. Superficial fissures or " chaps" are familiar about the backs of the hands and lips from the action of water, harsh soap, imperfect drying, and exposure to cold wind.

Scars.-Syn. : Cicatrices; Fr., Cicatrices; Ger., Narben.

Scars are connective tissue new-growths which replace loss of tissue from disease or injury. They may be the result of the process of repair which occurs in the healing of an ulcer or in the making good of a breach in the skin, as in the Striae atrophicae of pregnancy, or they may be due to the replacement by fibrous tissue of the granulomatous deposit in certain affections such as syphilis or tuberculosis.

For a scar to form it is necessary that the destruction of the skin should extend down through the epidermis to the corium or subcutaneous tissue.

Scars vary indefinitely in shape and size. They may consist of punctate specks, roundish discs, linear, kidney-shaped, stellate, serpiginous, or irregular lesions. They may be smooth or uneven on the surface and soft or tough in consistence. In their early stage they tend to be pink or purplish in tinge, but may eventually become white and shiny, and in rare instances may be pigmented. The character and appearance of the scar may be of considerable diagnostic value; for example, the scars which result from small-pox are small, roundish and pitted; those which follow burns are raised, hypertrophic, corded, or fibrous; those which follow the involution of late syphilides have frequently a thin crinkled tissue-paper-like appearance ; those of Lupus erythematosus may be rough and pitted owing to the presence of gaping atrophic hair-follicles; while those of cheloid tend to spread out by irregular processes in a claw-like manner. As a rule they are not associated with subjective symptoms, but occasionally they may be intermittently itchy or painful. They are the result of a connective tissue new-growth consisting chiefly of collagen with little or no elastic tissue, and are well supplied with blood-capillaries and nerves. Glands and hairs are usually absent.

Scars are persistent structures but usually have a tendency to become less noticeable with lapse of time. Occasionally they may increase for a time and spread by sending out processes into the neighbouring tissue, as in cheloid, but after reaching a certain size they may remain stationary for an indefinite period.

Ulcers.-Sy'n. : Fr., Ulcères ; Ger., Geschwüre.

Ulcers are open sores, other than wounds, in which there is de- 
struction of the skin. In shape they may be rounded, oval, kidneyshaped, serpiginous, or irregular, and in size may vary from a split-pea to several inches in diameter. They may be superficial, only the epidermis being destroyed and the floor of the ulcer being formed by the exposed papillary layer, or they may extend deeply, reaching down into the reticular layer of the corium or even as far as the subcutaneous fasciae.

The ulcer is generally covered with a crust, but where it is indolent there may be a continuous discharge which does not tend to dry up. When the crust is removed the floor is generally found to be covered with a purulent or sanious exudation or, when healing is taking place, by more or less exuberant granulations. The edge of the ulcer is usually raised and may be markedly indurated and inflamed. It may be clean cut, giving the ulcer a punched-out appearance, or everted, or definitely undermined from an extension of the destructive process beneath it.

Ulcers may heal gradually or rapidly. They are followed inevitably by a scar and not infrequently by pigmentation. Occasionally they may refuse to heal, as in rodent ulcer. They are generally a further stage of pustulation but may be the result of the breaking down of some neoplasm or granulomatous deposit, as in rodent ulcer or in the nodules of leprosy or Lupus vulgaris. They occur readily where the resistance of the skin has been diminished, as in the indolent leg ulcer associated with varicose veins, or where the nerve supply to the skin is impaired, as in the perforating ulcers which are commonly met with in connection with spinal disease and nerve leprosy. 


\section{CHAPTER III}

\section{GENERAL PATHOLOGY OF THE SKIN}

THE pathological changes which may occur in the shin are as diverse as the clinical lesions to which they give rise. They are due to a number of different pathological processes which will be considered briefly in this chapter.

The pathological processes which involve the skin are in no way peculiar to it, but are similar to those which may occur in any other organ. They are capable, however, of being more perfectly studied in the skin than elsewhere, as by excising the lesion and examining it microscopically they can be studied in relation to the naked-eye appearances which they have produced.

The pathological processes affecting the skin may be considered under the usual headings of hypertrophy, dystrophy, atrophy and pseudo-hypertrophy.

By hypertrophy is meant an increase in the bulk of a tissue by an increase in the number or size of its tissue elements; by atrophy, a diminution in the size of a tissue from direct and continuous orerpressure; by dystrophy, an interference with the nutrition of a tissue leading to pathological changes in its elements ; by pseudo-hypertrophy an increase in the bulk of a tissue by an additional number of elements of a degenerative type.

These pathological processes may be considered briefly as they affect the different parts of the skin. Just as the skin embryologically is divisible into two distinct layers, namely, the epidermis and the corium, so from the pathological standpoint the morbid changes which occur in it may be divided into those in which the epidermis is primarily involved and those which have their seat of origin in the underlving corium.

It is a matter of great diagnostic importance to be able to distinguish whether the diseased process occurs primarily in the epidermis or the corium. Sometimes both layers may be involved but one is almost invariably attacked secondarily to the other, either as a result of the extension of the morbid process, or from some adventitious agencr rendered potent by the loss of resistance resulting from the presence 
of disease in the other layer ; for example, a tuberculous deposit in the skin which is essentially an affection of the corium may be associated with pathological changes in the epidermis in the form of warty growths or necrosis. These epidermal changes are not directly produced by the tubercle bacillus, but result from the secondary inoculation of pyogenic microbes which find a suitable soil for their growth owing to the presence and action of the tubercle bacillus in the corium.

\section{Pathological Changes in the Epidermis}

The pathological changes primarily involving the epidermis may be congenital or acquired and may affect especially one or other of the layers. They may be arranged with examples as follows :

\section{Stratum Corneum}

Hypertrophy or Hyperkeratosis.-In hyperkeratosis the horny layer becomes more or less thickened and in severe cases it may be transformed into a hard irregular horny mass. It is associated with a stronger cohesion of the individual horn-cells as well as an excessive formation. The thickening may be even, broken up by deep furrows into irregular areas, or surmounted by grotesque horny protuberances. The granular layer, though generally thickened in association with the hyperkeratosis, is not necessarily so, and may even be defective, and the underlying prickle-cell layer may exhibit no corresponding hypertrophy. Hyperkeratosis indicates that the basal or germinal layer of the epidermis has been stimulated so that an increased number of daughter-cells have been produced which undergo abnormally rapid cornification, resulting in the formation of a coherent horny mass. Hyperkeratosis may be either diffuse or circumscribed and may affect the whole cutaneous envelope or be confined to clearly demarcated areas.

\section{Congenital.}

\section{Examples of Hyperkeratosis}

I. Diffuse : Universal congenital hyperkeratosis.

II. Regional: Ichthyosis hystrix, and Keratoma palmare et plantare hereditarium.

\section{Acquired.}

I. Diffuse : Pityriasis rubra pilaris.

If. Circumscribed :

I. Simple traumatic: Corns and Callosities.

2. Associated with vascular changes: Angiokeratoma.

3. Associated with hyperidrosis: Hyperkeratosis of the palms.

4. Associated with the elimination of poisons by the sweatapparatus: Arsenical keratosis.

5. Of undetermined origin: Porokeratosis (Mibelli). 
Dystrophy.-The dystrophies may be divided into two types, namely, parakeratosis or imperfect cornification resulting from oedema in and between the prickle-cells, and dyskeratosis or anomalous cornification the exact cause of which is imperfectly understood.

Parakeratosis.-In this condition there is usually an excessive formation of horn-cells which are imperfectly cornified and form friable masses and scales such as are met with typically in psoriasis.

The essential microscopical features are:

(I) Persistence of nuclei in the horn-cells.

(2) Incomplete formation of keratin so that the cells are loosely united and allow a certain amount of air, serum and frequently leucocytes to collect between them.

(3) Moistness of the horn-cells with absence of fat so that the cells are swollen, tend to dry up on reaching the surface, adhere together in small groups or masses, and break off in the form of scales.

This change in the Stratum corneum is invariably preceded by oedema of the prickle-cell layer, which causes the cells to be swollen, the inter-epithelial lymph-spaces to be dilated and possibly to contain polymorphonuclear leucocytes, and interferes with the process of evolution of prickle-cells into horn-cells. In addition, the granular layer is defective and keratohyalin granules are absent. This condition is in the nature of a superficial catarrh and is met with in connection with inflammatory disturbances in the corium, such as psoriasis, eczema, seborrhoic dermatitis, Pityriasis alba (Hebra), scaly ringworm of the body, the desquamative stages of the acute exanthemata, etc.

The term " parakeratosis" has also been applied in a special sense to certain diseases somewhat closely allied to psoriasis.

Dyskeratosis. - Anomalies in the process of cornification are occasionally met with which give rise to the formation of peculiar structures roughly resembling psorosperms. There are several varieties of these structures; sometimes they are roundish bodies about the size of a prickle-cell with a nucleus, a granular protoplasm, and limited by a double-contoured membrane, as in the remarkable affection described by Darier under the heading of Psorospermosis follicularis vegetans. These are no longer regarded as psorosperms, but are believed to be partially cornified cells the protoplasm of which is in a state of hyalin degeneration.

In the small dimpled tumours of Yolluscum contagiosum there is an allied anomaly of cornification which results in the production of numerous oval-shaped glistening bodies which can be extruded when the tumour is squeezed. These have a facetted appearance from pressure and consist of a capsule, within which there is a more or less oval homogeneous mass and between it and the periphery the flattened remains of the nucleus. These oval bodies known as " molluscum bodies " have a superficial resemblance to oviform coccidia and were once believed to be parasites and the contagious element of the 
disease. It is now recognised, however, that they are horn-cells in which the keratinisation is imperfect so that the cells have become separated and contain a homogeneous mass of colloid or hyalin instead of a waxy substance.

Anomalies of cornification of a somewhat similar character are met with occasionally in carcinoma or malignant epithelioma of the skin and have been mistaken more than once for protozoa and regarded as being of pathogenic importance.

Atrophy and Diminished Cornification.-In all atrophic conditions of the skin the horny layer is involved and there is diminished keratinisation. In consequence of this, the protective power of the skin is impaired. It may occur as the result of continuous pressure, or in connection with any severe or prolonged illness, or where the nutrition of the skin is interfered with in nerve diseases such as Tabes dorsalis.

\section{PRICkLE-CELL LAYER}

Congenital Conditions.-There are certain congenital growths which are believed to originate in the embryonic prickle-cell layer of the epidermis, but which some observers maintain are derived from the endothelium of the capillaries in the corium (see p. I99). These belong to the class naevi. In this country we are in the habit of reserving the name naevus for congenital growths of the skin consisting of blood-vessels, but on the Continent such a restriction has been abandoned and the name is employed in a wide sense to include practically all the congenital circumscribed cutaneous anomalies such as pigmented moles, hairy moles, etc.

Acquired Conditions.-Changes in the prickle-cell layer occur in almost every form of dermatitis and many new-growths of the skin both benign and malignant have their origin in it.

Hypertrophy or Acanthosis (from $\dddot{\kappa} \alpha \omega \theta \alpha=a$ prickle).-In the true hypertrophies of the prickle-cell layer the component cells do not lose their intercellular fibres or prickles; this is an important diagnostic characteristic in distinguishing a true hypertrophy or acanthoma, which is a benign condition, from a pseudo-hypertrophy such as a carcinoma in which the cells are defective and the prickles are lost.

Hypertrophy of the prickle-cell layer is the result of an irritation of the basal or germinal layer which is not sufficient to cause either a degeneration or a malignant proliferation. In it the basal cells or " mother-cells " proliferate more actively than usual and those immediately above the basal layer, the so-called " daughter-cells," no longer become differentiated into horn-cells but assume the function of the mother-cells and proliferate also, so that mitotic figures may be detected in the cells several rows above the basal layer. The proliferation takes place not only upwards but downwards, and the interpapillary 
processes may become elongated, or thickened, or may spread out irregularly into the corium. The newly formed cells are perfect pricklecells, while the basal layer remains intact forming a limiting layer around them. Hypertrophies of this layer, as a rule, are circumscribed and result in the formation of such benign new-growths as condylomata and warts. Formerly it was customary to include such affections under the heading of "papillomata" but there is a tendency now to abandon the term "papilloma" since it is somewhat of a misnomer, for these lesions do not result from a proliferation of the papilla but from a downgrowth of the epidermis.

The following are examples of hypertrophy of the prickle-cell layer or acanthosis :

Simple Hypertrophy, where only the prickle-cell layer is involved, is met with in the cauliflower-like benign growth known as Condyloma acuminatum or venereal wart, and also in the shiny affection of the skin due to incessant rubbing known as "lichenification."

With hyperkeratosis: Sometimes the acanthosis is combined with hyperkeratosis as in common warts or in the rare condition "Acanthosis nigricans."

One of the commonest causes of acanthosis with or without hyperkeratosis of the epidermis is the presence on the cutaneous surface of micro-organisms of the staphylococcus group. It is they which are responsible for the warty growths which may be superimposed on the cutaneous lesions of syphilis, tuberculosis and elephantiasis. It is probable that both venereal and common warts are of microbic origin.

With Dyskeratosis: Hypertrophy may also be combined with dyskeratosis as in Molluscum contagiosum.

Atrophy.--In it the prickle-cell layer is diminished in thickness, or the wavy line between it and the corium is straightened out, the prickle-cells become shrivelled and the subcutaneous fat is lost so that the nerve endings are not well supported, are readily irritated, and itching is liable to occur. It may result from a number of causes of which the most important are the following :

External pressure continuously applied, for example the pressure exerted by a plaster-of-Paris dressing.

Internal pressure, as in the thinning of the skin which results from the pressure of some large internal newgrowth such as a sarcoma, or from a fibrous thickening in the corium as in sclerodermia, or from the presence of ascitic fluid.

Idiopathic atrophy. A diseased condition of the skin associated with atrophy of both the epidermis and the corium, the pathogenesis of which is not understood.

Malnutrition. Imperfect nourishment or chronic wasting disease may be associated with atrophy of the skin. 
Senile atrophy. In old age the skin is apt to become wrinkled, thin, shining and atrophic.

Neurotic Atrophy. Where the nerve supply to the skin is defective atrophy occurs in the area supplied by the affected nerve, as in the patches of maculo-anaesthetic leprosy.

Dystrophy.- Under this heading is included a number of affections in which the nutrition and development of the prickle-cells is disturbed owing to the presence of more or less oedema in and between the cells which causes them to become swollen, spongy and sometimes devitalised. This group comprises all the oedematous conditions of the skin, including vesiculation and bullous formation.

\section{Examples of Dystrophy}

Slight oedema and parakeratosis : Psoriasis, Pityriasis.

Pronounced oedema and parakeratosis: Seborrhoic Dermatitis. Vesiculation :

(a) Inter-epithelial or interstitial: Herpes zoster, Eczema, Dysidrosis, Dermatitis herpetiformis.

(b) Intra-epithelial or parenchymatous: Varicella, Variola. Bullous formation :

Dermatitis herpetiformis.

Erythema multiforme.

Urticaria bullosa.

Pemphigus.

Pemphigus vulgaris.

$\begin{array}{ll}, \quad & \text { foliaceous. } \\ , & \text { vegetans. } \\ , & \text { acutus. } \\ , \quad & \text { congenitalis (Epidermolysis bullosa). } \\ , & \text { syphiliticus (bullous syphilide) }\end{array}$

Bullae due to chemical irritants : cantharides.

Bullae due to heat and actinic rays of the sun.

Pustulation :

Primary : Impetigo of Bockhart.

Secondary to vesiculation: Impetigo contagiosa.

Ulceration :

Further stage of pustulation in weakly resistant tissues, or the result of the breaking-down of infective granulomata or of epitheliomata.

Pseudo-Hypertrophy.- Under this heading are included the malignant neoplasms of the epidermis, namely, epithelioma or Carcinoma cutis and rodent ulcer.

In this country it is customary to restrict the name epithelioma to a malignant proliferation of the epidermis, while on the Continent the 
term is used in a more rational and wider sense to include all epidermal new-growths both benign and malignant, such as carcinomata, warts, Molluscum contagiosum, etc.

$\mathrm{By}$ a malignant proliferation of the epidermis is meant one which is capable of unlimited growth, cannot be checked except by complete extirpation or destruction, and which may form growths of a similar nature in organs at a distance (Metastasis). It may take its origin from the basal or prickle-cell layer of the epidermis, the cells of the external root-sheath of the hair-follicles, the sweat-glands, and possibly from the sebaceous glands. The cells which constitute the growth differ from those from which they have sprung in that they have reverted to a simpler or undifferentiated type. If they have taken their origin from the prickle-cell layer they lose their prickles and become round or oval in shape, and unless a definite connection can be traced between them and the epidermis it may be impossible to tell whether they have developed from epidermal cells or from the cells of the corium. In this condition they cease to be able to fulfil their developmental intention and revert to the simpler function of proliferating. The degree of malignancy of the growth varies greatly in different lesions. As a rule it may be said that the greater the number of mitotic figures, and the more the basal layer has disappeared and the cellular proliferation has infiltrated the lymphatic spaces of the corium, the greater the malignancy.

In the early stages, when the cellular proliferation has just begun, it may be practically impossible to tell with certainty whether the growth is benign or malignant. Up to a certain stage all neoplasms are benign. Some may remain benign in their whole course; others, such as warts and pigmented moles which are usually benign, may become malignant; while others invariably assume malignant characteristics.

In health the function of the cells of the basal layer of the epidermis is entirely that of reproduction, while that of the prickle-cells is to become differentiated into horn-cells. If the cells of the basal layer be irritated, daughter-cells are produced with greater rapidity than normally, and these being more imperfect in type may exhibit a tendency not to differentiate but to revert to the function of the mother-cells and to divide.

Any interference with the normal relation of growth and functional activity of the epidermal cells, is liable to lead to proliferation and possibly to malignancy. At a certain age these normal relations are in a state of instability ; this usually occurs after forty years of age but may be earlier if the tissues be weak naturally or from disease, either general, or local as where malignant epithelioma develops on Lupus vulgaris. An irritation which was capable of causing a benign proliferation of the epidermis, such as a warty growth, in a young tissue might cause a malignant epithelioma in an old one, while a benign warty growth with advancing years and local irritation eventually might be 
rendered malignant. There are certain states of the skin which appear to constitute a soil on which malignant changes are liable to supervene, such, for example, as the chronic eczematoid dermatitis of the nipple known as Paget's disease, the freckled skin in Xeroderma pigmentosum, or the atrophic telangiectatic skin which results from long exposure to the X-rays. From this it will be seen that malignant growth of the epidermis is not due to one cause but may be the result of a multitude of different forms of chronic irritation, mechanical, chemical or microbic, provided the skin be in that unstable condition which renders it liable to proliferate.

Malignant growth may also take its origin in embryonic cell inclusions or "cell-rests." These are pieces of the germinal layer of the epidermis supposed to be cut off in the embryo and to lie dormant in the corium, but capable of being irritated and of giving rise to a malignant epithelioma. This is a theory of the origin of cancer which was put forward long ago by Cohnheim and it has found corroboration in the transformation of pigmented moles into cancers in the so-called naevo-carcinomata.

\section{Pathological Changes in the Epidermal APPENDAGES}

\section{HAIR}

An increase in the number of hairs on the cutaneous surface is known as hypertrichosis, defects in the structure or colour of the hair as dystrophy, shrivelling of the hair as atrophy, and absence of the hair as alopecia.

Hypertrophy or Hypertrichosis.-Hypertrichosis may be congenital or acquired. Congenital universal hypertrichosis is occasionally met with in the so-called " hairy-man," and may occur as a family trait, while congenital local hypertrichosis is found in the various forms of hairy moles.

Acquired hypertrichosis may be local or regional and is frequently met with in the face in women.

Dystrophy.-Defects in the colour of the hair, such as whitening or leucotrichia occasionally occur, and may take the form of patches or rings around the hair-shaft, believed to be the result of the presence of air between the cells of the hair-shaft and due to a temporary interference with the nutrition of the hair. Occasionally complete whitening of the hair may occur suddenly after a severe illness or following an attack of neuralgia, but this is usually temporary and the pigment eventually returns.

In the depigmented condition of the skin known as vitiligo the hairs may be involved and become white; this, like the whitening of the hair in old age, is a permanent condition.

The structure of the hair may be defective from imperfect 
nutrition and may show a tendency to become brittle and to split. There is a peculiar affection of the hair known as "Trichorrhexis nodosa " in which beadlike swellings appear at regular intervals along the hair-shaft; on microscopical examination these are found to be due to the splitting of the outer portion of the hair into its constituent fibres, which are pressed together in such a fashion as to present the appearance of two brushes stuck together end to end. This curious dystrophy is possibly the result of mechanical injury to a hair weakened by malnutrition.

Atrophy.-This may result from a break in the connection between the hair-follicle and the papilla, an interference with the nutrition at the papilla, or a constriction of the hair at the orifice of the hair-follicle. Occasionally the atrophy of the hair-shaft may be partial and the hair assume a beaded appearance as in " monilithrix."

In connection with Alopecia areata, atrophic stumps may occur in which the intra-follicular portion of the hair becomes shrivelled from an interference with the function of the papilla.

Alopecia or Baldness may be congenital or acquired.

Congenital Alopecia is a rare condition and in the majority of cases the result of an arrest in development, the hairs appearing later.

Acquired Alopecia may be due to a number of causes of which the most important are traumatism ; some form of scar-leaving dermatitis ; the presence of parasites about the hair-follicles, such as the fungi of ringworm, favus, or pyogenic cocci; general morbid conditions, such as prolonged illnesses, acute fevers, syphilis ; and morbid states of the scalp such as acute eczema, seborrhoic dermatitis, and exfoliative dermatitis.

Another form of acquired alopecia is that which is known as Alopecia areata, in which bald patches appear on the scalp independent of age or traumatism. The true cause and exact nature of this alopecia is still undecided, the two hypotheses which are at present receiving most attention being the tropho-neurotic and the toxic.

\section{HAIR-Follicles}

The hair-follicles are the seat of origin of a large number of skin diseases of an inflammatory and pustular type, chiefly because they constitute the easiest mode of entrance of micro-organisms into the deeper layers of the skin. Inflammation of the follicle is known as "folliculitis," and varies in degree from a slight congestion to a destructive suppuration. The hair-follicle is surrounded by a close network of blood-capillaries which readily become congested whenever the skin becomes inflamed, so that red follicular papules are common in many forms of dermatitis. Sometimes the inflammatory disturbance is most marked at the follicular orifices, as in the affection known as Pityriasis rubra pilaris. 
When suppurative changes supervene they may lead to the formation of a superficial pustule situated at the orifice of the hair-follicle and generally pierced by a hair, or where the suppuration penetrates more deeply into the corium a perifollicular abscess may result, as in a boil or furuncle. The suppurative folliculitis is generally caused by pyogenic micro-organisms, but it may be produced by virulent ringworm fungi or by certain chemical irritants such as croton oil. An indolent type of folliculitis is due to the presence of tubercle bacillus or its toxins, as in Acne scrofulosorum. The folliculitis may lead to complete destruction of the hair-papillae and follicles so that baldness results, as in the condition known as Folliculitis decalvans, or the destructive process may be deeper and a depressed scar be left on healing, as in Acne necrotica. Sometimes the folliculitis is associated with marked cellular hyperplasia of a granulomatous type, giving rise to indurated nodular lesions as in acne of adolescence and coccogenic sycosis of the beard; or a fibrous hypertrophy may be met with leading to a cheloidal formation, as in the rare affection on the back of the neck known as Acne cheloid.

There are certain affections of the hair-follicles which are associated with the presence of horny plugs or filiform spines. This may be an occasional occurrence and not characteristic of the disease, as in Lichen planus, Lichen scrofulosorum, seborrhoic dermatitis and miliary follicular syphilides; or it may be an essential and diagnostic feature, as in the comedones of Acne vulgaris, or in Lichen pilaris seu spinulosus, where follicular papules are surmounted by projecting spines, and Keratosis follicularis where the orifice of the follicle presents a horny plug or a scale.

Occasionally new-growths may take place from the external rootsheath or prickle-cell layer of the follicle as in the benign neoplasm known as tricho-epithelioma.

Table of Pathological Changes affecting the Hairs and HAIR-FOLLICLES

(From Handbook on the Pathology of the Skin p. 232.)

\section{Hairs}

I. Hypertrophy or Hypertrichosis :

r. Congenital :

(a) Universal : "Hairy men."

(b) Local : Hairy moles.

2 Acquired: Local or regional.

II. Dystrophy :

I. Colour-defects: Whitening of the hair (leucotrichia) may occur in patches or be universal. Hair may show patches or rings of white, or may be completely blanched. 
2. Structural defects:

(a) Dry and brittle: After wasting illnesses, etc., and in association with Pityriasis capitis, etc.

(b) Trichorrhexis nodosa.

III. Atrophy :

I. Partial : Monilithrix.

2. Total :

(a) Congenital: arrest in development.

(b) Acquired:

I. Trauniatic.

2. Parasitic.

3. Dependent on general morbid conditions.

4. Dependent on morbid states of the scalp, e.g. Seborrhoic dermatitis.

5. Alopecia areata.

\section{Hair-Follicles}

I. Hyperkeratosis of the Funnel of the Hair-Follicles occurs in a large rumber of skin-affections, such as :

I. Acne vulgaris (Comedones).

2. Hyperkeratosis follicularis et supra-follicularis (Unna) or Lichen pilaris (Bazin), Keratosis pilaris (Brocq), Lichen spinulosus.

3. Ichthyosis follicularis.

4. Psorospermosis follicularis vegetans (Darier).

II. Atrophy of the Hair Follicles :

I. Congenital : Arrest in development.

2. Acquired:

(a) Traumatic : Scars.

(b) Parasitic: Pyogenic cocci (Folliculitis decalvans).

(c) Scar-leaving diseases: Small-pox, sclerodermia, and Lupus erythematosus.

(d) Dependent on morbid states of the scalp: Seborrhoic dermatitis.

III. Folliculitis :

I. Primary folliculitis :

(a) Parasitic: (I) Staphylococcic folliculitis and perifolliculitis: Impetigo (Bockhart), furuncle, coccogenic S etc.

(2) Due to tubercle bacillus: Acne scrofulosorum.

(3) Due to fungus of ringworm (ectothrix).

(b) Chemical irritants : Croton oil. 
2. Secondary folliculitis :

(a) After keratosis and plugging of the follicle: Acne vulgaris.

(b) Associated with skin affections which do not directly involve the hair-follicle: Eczema.

\section{SEBacEOUS GLANDS}

Derangements of the sebaceous glands both functional and organic are not infrequently the cause of cutaneous diseases. The chief function of the sebum is to lubricate the hairs and provide them with a protective oily covering. If this is defective the hairs are apt to become dry and brittle from exposure to the air, or to be macerated by water when the hair is wet. The sebum also extends for a short distance around the follicular orifice so that where the hairs are close, as on the scalp, it forms a more or less continuous oily coating over the surface. In certain regions where the sebaceous glands are large as the sides of the nose, presternal and interscapular regions, the skin is naturally greasy. In these situations or when there is a pathological increase of sebum, a suitable medium is provided for the growth of certain microorganisms which normally lead a harmless existence on the cutaneous surface, and renders them virulent and liable to cause dermatitis. Decomposition changes are apt to supervene in the sebaceous material so that it becomes rancid and gives rise to an unpleasant musty odour which is noticeable in people with greasy skins who do not wash sufficiently.

Increased activity in the output of sebum is usually associated with an actual proliferation of the sebaceous glands, and it is difficult to distinguish between the two. Associated with the increased elimination there is generally an alteration in the quality of the sebum in which it either becomes more oily in consistence, as in Seborrhoea oleosa, or loses part of its fluid constituents and becomes more cheesy.

The cause of the increased output of sebum has been much discussed. It occurs naturally about puberty as a result of the development of the pilo-sebaceous system, and usually ceases in early adolescence, but in certain individuals may persist as a permanent peculiarity. It occurs also as an acquired condition in association with certain chronic inflammatory conditions of the skin associated with congestion, such as rosacea. An actual glandular hypertrophy is met with about the nose in the bulbous affection known as rhinophyma, where the proliferation of the sebaccous glands is associated with an increase in the fibrous stroma around the glands.

Cystic tumours of the sebaceous glands in the form of yellowish translucent structures may result from a plugging of the sebaceous follicles and a hypertrophy of the glands ; these are comparatively rare.

The common wen or atheroma was once believed to be a sebaceous 
cyst, but some doubt has been thrown on this and certain observers believe that it is really developed from embryonic cellular inclusions which proliferate and undergo a fatty change and that it consists of a mass of cornified cells mixed up with cholesterin and occasionally with calcareous salts.

Decrease in the output of sebum is associated with atrophy of the glands. This is found in all dry and atrophic states of the skin as in senile skin and ichthyosis. The absence of the sebaceous material renders the skin liable to injury from water and more prone to dermatitis from exposure to external irritants.

Atrophy and obliteration of the sebaceous glands are liable to occur from strangulation of the glands by fibrous tissue, as in sclerodermia and in ordinary scars.

\section{Malignant Proliferation of the Sebaceous Glands}

For a long time there has been an idea that rodent ulcers frequently take their origin from the sebaceous glands. This belief is largely attributable to the microscopical appearances in rodent ulcer where the cells may have an arrangement in acini like gland tissue. That they may be derived occasionally from sebaceous glands is generally admitted, but this is no longer believed to be invariably the case, and a definite connection may sometimes be traced with the prickle-cells of the hair-follicles or with the basal layer of the epidermis. When they originate in the sebaceous glands the growth takes place from the mother-cells and peripheral daughter-cells of the gland and not from the fatty central cells. Occasionally the cellular hyperplasia may enclose pieces of more or less normal sebaceous gland tissue, but this must not be mistaken for a growth from sebaceous gland cells.

\section{MÚscies}

The involuntary muscles of the skin may undergo pathological changes giving rise to small tumours known as leiomyomata. These develop chiefly from the dartos of the scrotum, but may do so also from the Arrectores pilorum. They may be composed partly of muscular and partly of fibrous tissue when they are known as "fibromyomata."

\section{SWEAT-GLANDS}

The affections of the sweat-apparatus may be functional where there is no definite histopathological change in the glands, or structural where there is.

Functional Derangements.- Sweat is naturally acid in reaction, but tends to become alkaline when excessive. When alkaline it forms a more suitable medium for the growth of micro-organisms, hence inflam- 
matory disturbances of microbic origin are particularly liable to occur in association with excessive sweating.

The principal functional disturbances of the sweat-apparatus are the following :

I. Hyperidrosis or excessive perspiration.

2. Anidrosis or defective perspiration.

3. Bromidrosis or foetid sweating, due to the action of the Bacterium foetidum, which causes a disintegration of the sweat after it has been excreted, and the formation of caproic and other malodorous acids.

4. Chromidrosis or pigmented sweating, in which the sweat may be purplish (cyanidrosis), reddish or greenish (chromidrosis). This may be due to the presence of amorphous granules, the growth of masses of some microbe around the hairs, or the secretion by the sweat of certain drugs such as Iodide of Potassium which gives it a pinkish tinge or copper which renders it greenish.

5. Haematidrosis in which the sweat is blood-stained. This has been met with in hysterical women and is the result of haemorrhage into the ducts of the sweat-apparatus. It has been regarded as a form of vicarious menstruation.

6. Uridrosis in which there is an increase in the output of urea in the sweat as in uraemia.

Structural Changes. - These may take the form of cysts or adenomata.

Sweat-Cysts may occur in connection with the sweat-duct proper or the channel through the epidermis, but rarely if ever in connection with the coils. A non-inflammatory cystic formation from the sweatduct known as "hydrocystoma" is occasionally met with in washerwomen who suffer from excessive sweating. In connection with rheumatism, small non-inflammatory vesicles containing sweat, known as "sudamina," may develop between the epidermal cells.

Sweat-Adenomata may arise from the epithelium of the sweat-coil or the duct and may form little benign tumours which are known as "spiradenoma," when they develop from the coil, and "syringadenoma," when they originate in the duct. Nany epithelial growths of the cutis have a glandular arrangement simulating that of the sweatcoil but these should not be regarded as " sweat-adenomata," unless a definite connection can be traced between the growth and the gland. A malignant growth arising from the sweat-apparatus has been described.

\section{NAILS}

The pathological changes which affect the nails consist of hypertrophy, deformity, atrophy and dystrophy.

Simple Hypertrophy or Onychauxis.-This is a rare condition in 
which the nail-plate is increased in size owing to an over-activity or an increase in size of the nail-matrix. It is met with in acromegaly and gigantism.

Hypertrophy with Deformity or Onychogryphosis.-Instances of this are ordinary ingrowing toe-nail, claw-nail and ram's horn nail. In it the nail-substance is peculiarly hard and opaque, of a dirtyyellowish colour, and generally uneven on the surface and traversed by longitudinal ridges. It is the result of pressure or traumatism causing a widening of the posterior nail-fold and the growth of a thicker nail. At the same time a horny thickening of the front of the nail-bed is produced which interferes with the forward growth of the nail, lifting it up and causing it to press laterally on the soft parts. The lateral pressure of the nail is apt to lacerate the soft tissues and to render them liable to become inoculated with septic organisms and to suppurate.

Atrophy.-Congenital absence or anonychia of one or more of the nails is rare. An acquired partial or total loss of the nail is, however, a frequent occurrence and depends on a more or less complete solution of the continuity between the nail-plate and the nail-matrix. The following are the chief causes:

Traumatism.-An injury to the nail causing a rupture of the blood-ressels beneath the matrix and an infusion of blood into the nail-fold frequently results in disintegration of the matrix and shedding of the nail. As a rule the matrix recovers and a new nail forms in four or five months.

Nerious Diseases. - In association with cerebral paralysis, Tabes dorsalis, Morvan's disease, or degeneration of the peripheral nerve supplying the nail-matrix, atrophy or shedding of the nail may take place. This may be due to a vaso-motor disturbance or to an interference with the trophic nerve impulses.

Blood-Stasis.-Where the circulation at the nail-matrix is impeded the nail usually becomes atrophic and assumes a grey opaque and friable appearance.

Pyogenic infection (Purulent perionychia).-Pus-inoculation about the nail-fold, as in whitlows, readily involves the nail-matrix and causes atrophy or loss of the nail.

Scar-leaving conditions.-In scar-leaving conditions, such as pustular syphilides, breaking-down gummata, and tuberculous dactylitis, a complete destruction of the nail-matrix may take place which renders the future growth of the nail impossible.

Dystrophy.- When the function of the nail-matrix is interfered with an imperfect nail-formation occurs and the nail becomes irregularly depressed, opaque, brittle and fissured, or a wedge-shaped depression may appear at the lunule. This results from imperfect cornification causing the cells to be swollen and moist and to form a soft mass which shrivels on being exposed to the drying action of the air. Occasionally the individual papillae of the matrix are affected and in place of a soft mass white opaque spots appear, which on drying give rise to minute 
punctiform depressions. When transverse lines or furrows occur, they indicate an interference with the process of cornification, and are usually due to some general illness. White discolouration of the nail, either as white specks (Flores unguium) or striae and patches (leuconychia), is due to the presence of air between the nail-cells from the shrinkage of the soft partially cornified cells.

Derangements of the nails may occur in association with a large number of chronic skin diseases, such as psoriasis, eczema and syphilis, in which the nails may show atrophic changes such as puncta, wormeaten depressions, white or brown discolourations, ridges and furrows, or deformities may supervene from an accumulation of horny matter on the nail-bed lifting up the nail. These appearances are inconstant and not sufficiently definite to warrant the diagnosis of one or other of these diseases from the nails alone without the presence of corroborative evidence elsewhere.

Parasitic Diseases or Onychomycosis. - The nail-bed and the nail are frequently attacked by the fungi of ringworm and favus, while pediculi and the acari of scabies have been known to cause disturbances by passing in beneath the nail and setting up a pustular dermatitis. In ringworm and favus the nail-plate becomes opaque, yellow, dirtybrown or black, ridged and friable, and a horny accumulation forms beneath it.

\section{Pathological Changes in the Corium}

Inflammation of the Skin or Dermatitis.-Dermatitis signifies simply inflammation of the skin and under this heading may be included the majority of skin diseases. It is the clinical evidence of a succession of changes which occur in the skin as the result of injury, but it is also a process of repair to make good the damage which has been done. The injurious agent may be situated in the epidermis primarily, -as in the case of numerous external irritants-or in the corium in the form of toxins or of micro-organisms in the blood-vessels or lymphatic spaces.

According to the intensity of the irritant, apart from its situation in the skin, a series of changes occur in the blood-vessels and fixed tissues of the corium which constitute what is known as an inflammatory reaction. This is an effort on the part of nature to withstand and destroy the injurious agent and its effects, and may be regarded as the second line of defence of the skin to the attack of micro-organisms and other harmful influences.

In the case of an irritant acting upon the epidermis it is the underlying vessels of the papillary and sub-papillary layers which are primarily involved, but when the irritant is located in the corium it is the blood-vessels in its immediate neighbourhood which react first. 
These blood-ressels become dilated, leucocytes and plasma exude from them and the blood-current is slowed. The initial stimulation of the skin causes a constriction of the vessels, but this is soon replaced by an inhibition of the raso-constrictor tone in the ressels and a dilatation supervenes. The leucocytes which extravasate from the blood-ressels infiltrate the lymphatic spaces around them and quickly make their way to the site of irritation, spreading through the tissues in virtue of their power of amoeboid movement and attracted to the irritant by the influence known as "positive chemotaxis "-a power which is responsible also for the increased exudation of plasma. Different irritants or different states of virulence of the same irritant are capable of causing different degrees of chemotactic action, so that while one irritant may produce only an exudation of plasma with a slight extravasation of leucocytes another may cause a profuse leucocytic infiltration.

In the case of virulent micro-organisms it is their toxins which constitute the chemotactic agent, and the polymorphonuclear leucocytes so attracted are capable of destroying the irritating organisms by incorporating them. This power of "phagocytosis" is increased by the presence of certain substances in the blood-serum, named "opsonins" by Wright, which render the bacteria more palatable to the phagocytes. Eosinophiles of the blood are also said to have the power of destroying irritating organisms by discharging certain secretory products in the form of granules.

When the injurious agent is chemical or thermal the chemotactic agent is either the irritant itself or poisonous products eliminated by the epidermal cells in response to it. At the same time a serous exudation takes place from the vessels and plays an important part in the inflammatory process by diluting, or in some cases by destroying, the irritant.

Vascular changes do not constitute the whole of the phenomena of the inflammatory reaction. Changes also occur in the fixed cells of the corium in the nature of a cellular hyperplasia in which are new young connective tissue cells or fibroblasts. These perform an important function in the process of repair, for they develop into new fibrous tissue to replace that which is broken down as the result of the earlier stages of the inflammatory reaction. The clinical evidences of these changes are redness and swelling and the subjective symptoms, itching, heat, and occasionally pain. Where the inflammation is slight only a dilatation of the blood-vessels with hyperaemia takes place and a red macule is produced. Where it is more marked and there is a definite cellular hyperplasia with exudation of serum, swelling occurs and a raised lesion such as a papule, nodule or plaque is formed. When retrogressive changes supervene there is generally scaliness of the epidermis, the result of more or less oedema of the epidermal cells and interference with cornification (see "Parakeratosis"), while not infrequently a true pigmentation may be produced from the 
deposition of melanin granules in and between the cells of the basal layer.

In severe degrees of inflammation the blood-stasis in the capillaries may be more persistent and red blood-cells escaping into the lymphatic spaces of the corium may there break up and deposit their bloodpigment, giving rise to the series of colours associated with a bruise. If the irritant reaches a certain degree of virulence or acts for a prolonged period the vitality of the leucocytes is destroyed and they undergo a fatty-degeneration and become pus-cells. Degenerative changes also occur in the cellular and fibrous elements of the corium and suppuration and abscess-formation supervene. The pathological changes in the corium are distinguished by the type of cellular infiltration and the alterations which occur in the blood-vessels and fibrous elements.

The types of cellular infiltration vary according to the virulence of the irritant and the time it has been acting. They are somewhat illdefined but for descriptive purposes may be considered under the following headings :

I. Cellular infiltrations of simple inflammatory conditions.

2. Cellular infiltrations of the "granulomata."

3. Cellular infiltrations of the neoplasms,-fibroma, sarcoma and endothelioma.

\section{Cellular Infiltrations in Simple Inflammatory Conditions}

When an inflammatory reaction occurs in the corium, the lymphatic spaces are more or less densely infiltrated with leucocytes, both polymorphonuclears and lymphocytes, usually collected in foci around the blood-ressels, pilo-sebaceous follicles and sweat-glands. In addition there is a proliferation of the fixed cells in the form of small round cells with deeply stained nuclei, surrounded by a faint halo of protoplasm and practically indistinguishable from lymphocytes. So close is their resemblance that considerable difference of opinion has arisen with regard to their origin, some believing that they are lymphocytes which have made their escape from the blood-vessels, while others consider that they take their origin from the fixed connective tissue cells of either the fibrous bundles or the perithelium of the blood-vesscls. As it has been suggested that lymphocytes themselves may be formed from the endothelial cells lining the blood-vessels and as they are all of mesoblastic origin, the distinction secms to be a nuatter of minor importance. It is these cells which are sometines known as "fibroblasts" and which are capable of organising into new fibrous tissue to repair any damage caused by the inflammatory process. Any of the cells of normal connective tissuc, such as large polygonal or spindle-shaped cells with vesicular nuclei, vacuolated cells, and occasionally mast-cells, may be present. (Fig. I6.)

An increase in mast-cells is found in many inflammatory con- 
ditions of the skin but its significance is not yet understood and its inconstancy renders it of little value for diagnostic purposes. It may occur in Erythema multiforme, and occasionally in Tuberculosis cutis, while in Urticaria pigmentosa it is found so constantly and to such a degree as to be of pathognomonic value.

\section{Cellular Infiltrations of the Granulomata}

Where a comparatively mild irritant has acted for a prolonged period a more persistent type of cellular infiltration may result, consisting of large cells, known as plasma-cells, and their derivatives, multinucleated cells or chorioplaques and giant-cells. This more resistant

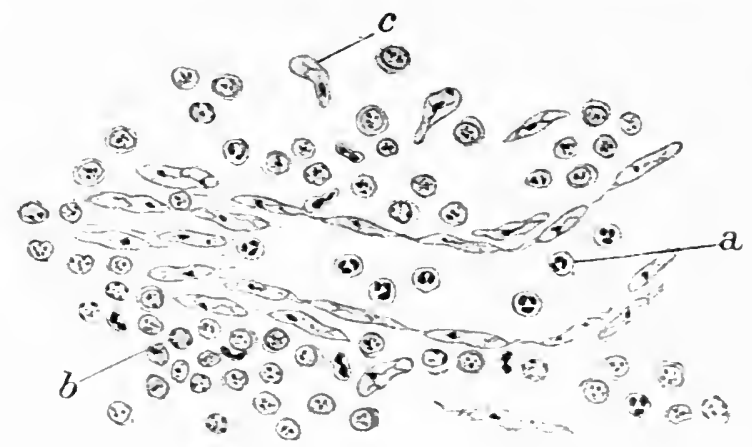

J. M. H. M. del.

Fig. I6.-Cellular Infiltration in Simple Inflammatory Conditions. Eczema. ( $x$ about 1000.)

a. Shows a longitudinal section of a capillary, with a few polvmorphonuclear leucocytes. b. Small round cells with a faintly stained halo of protoplasm around the nucleus. c. Connective tissue nucleus.

(From MacLeod's Pathology of the Skin.)

type of infiltration occurs in the lesions which have been named granulomata, from the superficial resemblance of their cellular infiltration to that in granulation tissue. This name was originally emploved by Virchow who applied it to a series of affections of an infective type, but it is a somewhat confusing term as it is not clearly defined, either clinically or histologically, and might with equal reason be made to include almost any chronic inflammatory cutaneous lesion, such as the deep-seated nodules of Acne vulgaris, the swellings which result from the presence of the large-spored fungus of ringworm, or the small tumours due to pus-cocci which the older surgeons knew as septic granulomata.

Under infective granulomata it is now customary to group a number of chronic inflammatory conditions which form a connecting link between inflammations of the skin, such as eczema and acute infective processes such as Impetigo contagiosa, and neoplasms such as fibroma and sarcoma. This group consists of Tuberculosis cutis, Syphilis cutis, 
and leprosy, and to them may be added yaws, rhinoscleroma and blastomycosis. In the majority of the members the infective virus is known, while the others present so many features in common with them as to warrant the assumption that they also have a specific virus which will be isolated in the future.

Clinically, the leading characteristic of the lesions is induration due to the presence of a dense cellular deposit in the corium.

Histologically, there is a general similarity in the architecture in all the infective granulomata, though there are certain details which generally enable the members of the group to be differentiated microscopically. The most constant feature in their histology is the presence of plasma-cells, in consequence of which the name plasmomata has been

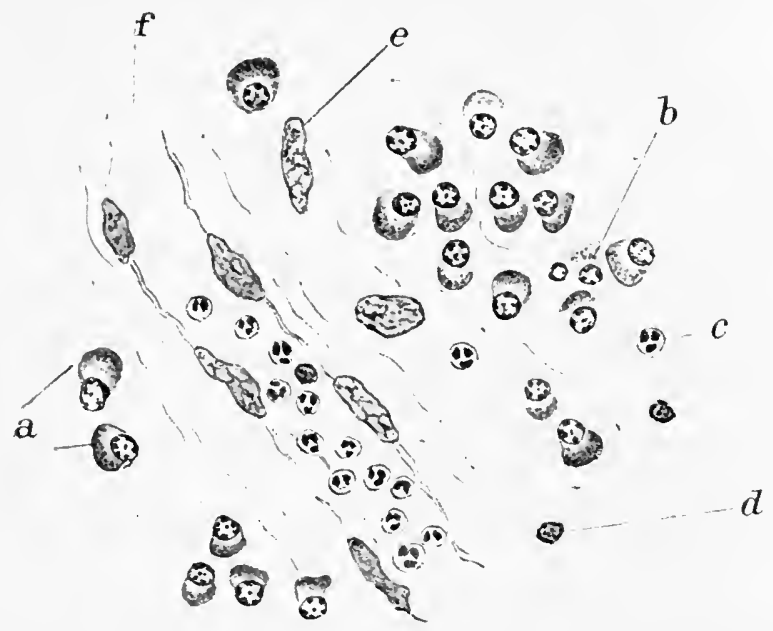

J. M. H. M. del.

Fig. I 7.-Plasma-Cells. ( $x$ about rooo.)

a. Showing several chromatin bodies around the nuclear membrane. $b$. Plasma-cell with two nuclei. c. Polymorphonuclear leucocyte. $d$. Lymphocyte. $e$. Connective tissue nucleus. $f$. Capillary in longitudinal section.

(From MlacLeod's Pathology of the Skin.)

suggested for them. In addition to plasma-cells, multinucleated cells and true giant-cells may occur and a varying number of lymphocytes and polymorphonuclear leucocytes. The fibrous elements of the corium are affected and may be in a state of rarefaction or of disintegration, as in tuberculosis, or may exhibit formative changes with the production of new fibrous tissue, as in syphilis.

The name "plasma-cell" is now generally restricted to the type of cell which Unna specially described. It is a somewhat confusing term as it suggests a close connection with blood-cells while, in reality, the name was given to it simply because it is singularly rich in protoplasm. The cell is oval, roundish or rhomboidal in shape, and 
about twice the size of a leucocyte. Its nucleus is generally placed eccentrically and varies in appearance according to the method and depth of staining. Sometimes it resembles the nucleus of a connective tissue cell with an open intra-nuclear network and a few darkly stained chromatin bodies; at other times the nuclear network is not noticeable and the nucleus appears as a clear space with six or eight chromatin bodies arranged around the periphery. The protoplasm of the cell is distinctly granular, in consequence of which the term "granuloplasm " has been applied to it. Associated with the ordinary plasma-cells are usually small round cells with nuclei like those of plasma-cells but with little or no granuloplasm around the nucleus. To these Unna applied the name of "daughter plasma-cells," which is unfortunate as it is doubtful if they are derived from plasma-cells. (Fig. I7.)

In the infective granulomata plasma-cells occur so constantly and in such numbers as to be almost pathognomonic. They are not, however, restricted to these lesions and are present normally in the lymphoid tissue of the spleen and in the bone-marrow, and pathologically in association with malignant changes of the epidermis, as in rodent ulcer, carcinoma, and Paget's disease.

The nature and origin of plasma-cells has been a fruitful subject of discussion among dermatologists during the last twenty years. According to one theory the cells are of connective tissue origin and derived from the fixed tissue of the corium, namely, the endothelium, perithelium and connective tissue near the blood-capillaries; according to another theory, they are blood-cells which have been carried to the skin by the bloodstream, have passed out of the vessels, and have become deposited in the neighbouring tissue. Numerous arguments have been advanced in favour of both these theories, and one of the most convincing in support of the latter is that the plasma-cell and the so-called daughter plasma-cell are morphologically indistinguishable from large mononuclear leucocytes and lymphocytes. It has recently been shown, however, that the large mononuclear leucocytes and the lymphocytes may be developed from the endothelium of the blood-vessels, which is itself a fixed connective tissue. In the light of this observation the problem becomies simplified and it would seem that the plasma-cells and the daughter plasma-cells are not only morphologically identical with the large mononuclear leucocytes and lymphocytes, but have a similar origin and result from the proliferation of the fixed connective ceils of the endothelium and perithelium. They appear to be called forth by the presence in the blood or the tissue spaces in the neighbourhood of the blood-capillaries of some microbe and its toxins, and their function is probably connected with the production of local immunity.

True giant-cells, as described by Langhans, which occur so frequently in tuberculosis of the skin are irregular in shape with a central degenerated and homogeneous portion enclosed by a more or less complete ring of nuclei at the periphery. This peculiar formation is the result of the presence in a cell of micro-organisms, the toxins from which cause a central degeneration of the protoplasm to form the homogeneous part and an increased vitality with proliferation of the nuclei at the 
periphery, signifying an effort on the part of nature to limit the zone of action of the toxins. True giant-cells vary in size from slightly larger than a leucocyte to many times its diameter. The outline of the cell is broken and irregular owing to small processes which project from it, and the shape is determined to some extent by the persistence of the fibrous elements, especially the surrounding elastin. The nuclei of the cells are similar to those of plasma-cells, and vary in number up to forty or fifty. Occasionally, instead of being arranged in a ring, they are grouped at the poles of the cell. The old belief that the giantcell possessed the power of amoeboid movement and was phagocytic is no longer sustained. The origin of giant-cells is still under discussion, the chief point at issue being whether they develop from a single cell, by a rapid proliferation of the nucleus without a corresponding division of the protoplasm, or are formed by the coalescence of a number of cells. The former view is the one most generally accepted.

In addition to the true giant-cells, smaller multinucleated cells occur, in which the nuclei are arranged in small groups of two to five in the centre of the cell. These are sometimes known as "chorioplaques."

\section{Cellular Infiltrations of the Neoplasms, Fibroma and Sarcoma}

In fibroma the new-growth consists of small spindle-shaped connective tissue cells and newly formed collagen bundles on which are oval or fusiform nuclei.

In sarcoma the cells vary in shape, size and structure and may be small and round like lymphocytes, polygonal and larger like endothelial cells, spindle-shaped, or multinucleated forming giant-cells. The small round-celled sarcomata are rare in the skin. The cells which compose them closely resemble small mononuclear leucocytes, but are larger and when stained present a more definite halo of protoplasm and dark chromatin bodies in their nuclei. In the large round-celled sarcomata the cells resemble endothelial cells and are probably of endothelial origin (see endotheliomata).

Endotheliomata are growths originating in the endothelium lining the lymphatics or the blood-vessels. The cells composing them are large, oval, or polygonal in shape and identical with those of a large round-celled sarcoma. When they originate from lymphatic vessels a structure may be produced which somewhat resembles a secreting gland and considerable difficulty may be experienced in distinguishing it from carcinoma. When they develop from blood-ressels they are known as haemangio-endotheliomata, and when from lymphatics as lymphangio-endotheliomata.

In the spindle-celled sarcomata the cells are identical with the spindle-cells which occur in the embryonic connective tissue. In the giant-cell type certain of the cells are large and multinucleated, which generally indicates that the sarcoma is secondary to one involving underlying bone. 
Towards the margin of the cellular mass in sarcomata plasna-cells, mast-cells, and ordinary connective tissue cells may be found and around the vessels leucocytes are usually present in varying numbers. The sarcoma cell is a mesoblastic cell derived from the connective tissue cells of the corium or the walls of the blood-ressels. The fibroblast has reverted to a simpler type, has ceased to fulfil its ordinary function of producing fibrous elements, and has assumed instead the function of reproduction. The first stage in the retrogression would be the spindle-cell and the last the small round cell, the giant-cell being simply a monstrosity caused by an imperfect power of reproduction.

\section{Degeneration of the Cells of the Corium}

Any irritant whether physical or chemical may be responsible for a degeneration of the cells if its virulence be sufficient or its action prolonged. The principal types of degeneration are fatty, hyalin, mucoid and oedematous.

Fatty Degeneration.-In all necrotic processes affecting the corium the infiltrating cells may undergo fatty degeneration, becoming swollen from the presence of an albuminous precipitate which is replaced by fat-globules. These cells gradually break down and allow the fat to infiltrate the lymphatic spaces.

Hyalin Degeneration is a homogeneous degeneration of the protoplasm which may affect the connective tissue cells as well as those of the epidermis. In it the cells become swollen from the presence of globules which give the cell the appearance of a mulberry and which may coalesce to form large homogeneous masses occupying the whole cell and pressing the remains of the nucleus to one side of it. Hyalin degeneration occurs occasionally in sarcoma, syphilis and rhinoscleroma.

Mucoid Degeneration.-In it the cells become swollen and the contents converted into a homogeneous structureless mass. A familiar example is the "lepra-cell" which results from the presence of leprabacilli in a connective tissue cell or plasma-cell causing a mucoid degeneration of the protoplasm.

Oedematous Degeneration.-In it the cells become swollen and stain badly as the meshes of their spongioplasm are dilated with oedematous fluid.

\section{Degeneration of the Fibrous Elements}

Collagen in the process of degeneration first undergoes a change in reaction, in which it ceases to attract acid aniline dyes and assumes a marked affinity for basic dyes when it is known as basophilic collagen. The degeneration generally begins in the centre of the bundle and spreads to the periphery. This is well seen in the degenerating collagen in Mycosis fungoides. 
Another degenerative product is collastin, in which the collagen is stained with acid dyes like those which stain elastin and tends to break up into irregular masses or blocks.

In addition to these are the imperfectly defined types of degeneration included under the terms " mucoid," "colloid " and "hyalin."

Elastin may also undergo a degeneration, and may become transformed into a substance named by Unna elacin in which the fibres, though retaining the outward appearance of elastin fibres, cease to attract acid dyes and take up basic dyes in alkaline solution. Elacin is found in senile skin.

\section{Pathological Changes in the Fibrous Elements White Fibrous Tissue or Collagen}

Hypertrophy.-An increase in the white fibrous stroma of the corium may be produced either by a thickening and lengthening of the pre-existing bundles of collagen or by a growth of new ones. Any attempt to draw a hard-and-fast line between these two processes is liable to be unsuccessful, however, as they generally occur together.

The hypertrophies of the collagen may be divided into (I) Diffuse, and (2) Circumscribed.

Diffuse Hypertrophy or Fibromatosis.-This condition has been studied in ordinary scar or granulation tissue. In it various types of cells are present which are stages in the development of a fibroblast into a collagenous bundle, such as spindle-cells, branching cells-larger than ordinary connective tissue spindles in which the spongioplasm forms a network the fibres of which pass out into long tapering processes-and occasionally flat wedge-shaped cells with large processes stretching out from the blunt end. The process of evolution in which the round cell becomes a spindle-cell, the spindle-cell a branched cell, and the branches of the cell become converted into collagen is generally described as the process of organisation or fibromatosis and may occur in connection with numerous inflammatory disturbances in the corium, as well as in the formation of scar tissue. It is characteristic of the cellular infiltration in many syphilitic lesions, and accounts for their marked induration.

Circumscribed Hypertrophy occurs in the benign neoplasms described as fibromata, which usually develop from the perineurium and the fibrous tissue in the interior of the peripheral nerves but which may also arise from the other white fibrous elements of the corium. Cheloids form another example of a circumscribed hypertrophy of the collagenous tissue in which there is an increase in length and breadth of the existing bundles.

Atrophy.-The pathological conditions in which atrophy of the collagen occurs are numerous ; for example, in infective granulomata, 
such as tuberculosis and leprosy, the collagen bundles are atrophied, shrivelled and eventually disintegrated, as if the cell-infiltration had developed at their expense, and in scar-leaving diseases, such as Lupus erythematosus, rarefaction and degeneration of the collagen occur. The collagen and elastin also disappear where the cellular infiltration is densest in sarcoma and carcinoma. In the various oedematous conditions of the corium - for example, in the oedematous papillary layer in eczema-the collagen becomes attenuated, and in all the necrotic processes it rapidly breaks down.

\section{Elastic Tissue}

Hypertrophy.-There is no circumscribed hypertrophy of the elastic tissue per se, though it may occasionally be increased in thickness in connection with similar changes in the collagen.

Atrophy. - Atrophy of the elastin is a common occurrence, but elastin is more resistant to toxic action than collagen and persists longer. If the elastin fibres be destroyed a scar results, for the unsupported collagen is thus enabled to expand, obliterates the superficial furrows of the skin, and gives rise to a smooth scar as in the striae of pregnancy: It is doubtful if regeneration of elastic tissue ever takes place.

\section{Table of Pathológical Chasges intolitig the Elemexts OF the Coricur, With EXaMPles}

\section{Cellular Infiltrations.}

I. Inflammatory : Eczema, Psoriasis, etc.

2. Purulent: Furuncle.

3. With necrosis: Carbuncle, Malignant pustule.

4. Plasma-cell Infiltration :

(a) Chronic inflammatory conditions, such as Acne rulgaris, Sycosis barbae, Tricophytic granuloma.

(b) In association with malignant proliferation, as in Sarcoma, Carcinoma.

(c) Infective granulomata:
I. Tuberculosis cutis.
2. Syphilis.
3. Leprosy.
4. Actinomrcosis.
5. Yaws.
6. Rhinoscleroma.
7. Mrycosis fungoides.
8. Blastomycetic dermatitis.

5. Giant-cell Infiltration.

(a) True giant-cells : Tuberculosis cutis, Tertiary syphilis.

(b) Multinucleated cells or chorioplaques: Infectire granulomata and Sarcomata.

6. Mast-cell Infiltration : Urticaria pigmentosa. 


\section{Cellular Degenerations.}

I. Fatty degeneration: Generalised Xanthoma, necrotic processes, etc.

2. Giant-cell degeneration: Tuberculosis and Syphilis.

3. Hyalin degeneration: Rhinoscleroma, Sarcoma, etc.

4. Mucoid degencration : "Lepra cells."

\section{White Fibrous Tissue or Collagen.}

I. Hypertrophy :

(a) Diffuse : Diffuse Sclerodermia, Fibromatosis.

(b) Circumscribcd : Fibroma, Neurofibroma, Cheloid, Scar.

2. Atrophy and Attenuation: Infective granulomata.

3. Degenerations of Collagen :

(a) Basophilic degeneration: Myxoedema, Mycosis fungoides, etc.

(b) Collastin : Colloid milium, Myxoedema, senile skin.

(c) Colloid : Colloid milium.

(d) Myxomatous: Sarcoma, Lipoma, etc.

(c) Oedematous: Lupus erythematosus, etc.

\section{Elastin.}

I. Atrophy : Infective granulomata, Striae of pregnancy.

2. Degeneration :

Elacin : Senile skin, Myxoedema, etc.

\section{Malignant Proliferations.}

\section{Sarcoma.}

I. Round-celled.

2. Spindle-celled.

3. Giant-celled.

\section{Endothelioma.}

I. Haemangio-endothelioma.

2. Lymphangio-endothelioma.

\section{Pathological Changes in the Blood Associated wrth Diseases of the Skin}

Changes in the blood with regard to its cellular elements, degree of alkalinity, coagulability and percentage of haemoglobin, have been recorded from tine to time in association with skin diseases. They are of comparatively little diagnostic value, however, as they vary grcatly in different cases-and cven at different times in the same patient-and their significance is not fully understood.

Red Blood Cells.--In any cachectic condition such as may be due to syphilis, leprosy, etc., the red blood cells are more or less markedly diminished. This also occurs in the eruptive period of syphilis when the disease becomes generalised. 
Haemoglobin.-The percentage of haemoglobin diminishes in cutaneous affections associated with cachexia, and in syphilis when the virus becomes generalised. When mercury is prescribed the diminution becomes more marked until the symptoms begin to disappear, when a gradual increase generally occurs and the percentage of haemoglobin may eventually reach a higher level than before the commencement of treatment.

White Blood Cells. - An increase in the number of leucocytes in the blood has been noted in diseased conditions of the skin associated with intense irritation, not so much as a general increase of all types of leucocytes but as a polymorphonuclear leucocytosis, a lymphocytosis or an eosinophilia.

A polymorphonuclear leucocytosis occurs in the acute exanthemata, erysipelas in its early stages, and sometimes in the later stages of stphilis.

A lymphocytosis occurs in leucaemia, Lymphadenoma perniciosa of Kaposi, and in the pre-mycotic stage of Mycosis fungoides, etc.

Eosinophilia has been frequently observed in association with vesicular and bullous affections. In pemphigus, for example, eosinophilia not only occurs in the blood, but also in the contents of the bullae, and a percentage of fourteen in the blood and serenty-three in the bullous fluid has been observed. It has been found that eosinophilia is never present in the contents of bullae artificially produced and is consequently a sign of some value in diagnosis. It occurs also in pruriginous diseases such as Dermatitis herpetiformis, urticaria, eczema, and scabies, when the degree of eosinophilia seems to depend more on the size of the affected area than on the intensity of the itching.

\section{Pathological Chaxges in the Blood-Vessels}

\section{Functional Changes}

The majority of skin affections are associated with a disturbance in the blood-ressels of a functional or circulatory character, such as hyperaemia, cyanosis, oedema or anaemia.

In hyperaemia there is an increased blood-flow in the skin which may be due either to a diminished resistance and an increased bloodflow (erythema) or to an increased resistance and a diminished flow (cyanosis). Active hyperaemia or erythema may result from an inhibition of the vaso-constrictor centre with a general diminution in the vascular tone and a consequent dilatation of the blood-vessels of the shin. This may result from psychical causes such as emotion, or from poisons circulating in the blood.

The erythema may be general, or localised in the form of macules or patches which may coalesce to form figures of the most diverse shapes. It may assume a retiform or arborescent appearance due to the arrangement of the blood-vessels of the papillary laver of the 
skin in cones or brushes of arterial capillaries supplied by a deep-seated central artery. The bases of the cones are next the epidermis and separated by areas in which the arterial twigs are smaller and the venous capillaries begin. The cones represent the "areas of maximum circulation" of Renaut, and between them the circulation is more sluggish. In this way a retiform appearance is produced which is noticeable in mild degrees of congestion about the legs in the form of a reddish or purplish network enclosing small areas the colour of the normal skin and corresponding to the bases of the cones. This mottled condition is frequently observed in babies' limbs from exposure to cold, in adults who suffer from a weak peripheral circulation, and in certain diseases of the skin such as the rare persistent scaly affection known as "Parakeratosis variegata."

Occasionally the venous network may become pigmented and brown from the habit of exposing the legs to the heat of a fire.

Passive Hyperaemia or Cyanosis may be local or general and is caused by some obstruction to the circulation. In it the veins are chiefly implicated and consequently the skin presents a bluish livid colour. This type of hyperaemia may go on to actual haemorrhage with the deposition of blood-pigment in the skin.

Oedema in the skin is due to a collection in it of serous fluid, the result of a blockage in the veins. It may be mechanically produced, as by a tight bandage, or may be due to a blocking of the vein, as by a thrombus, or may occur in association with inflammation owing to toxins circulating in the blood and causing spastic contraction of the veins. Sometimes the oedema is aggravated by hyperplasia of the fibrous elements in the affected area constricting the vessels.

An important factor in the production of oedema is want of nutrition of the vessel-walls, such as occurs in anaemia and in states of general debility.

The oedematous fluid varies in character according to whether the oedema is caused mechanically or is inflammatory. In the former it is known as a "transudate," has much the same composition as blood serum and contains very little albumin, while in inflammatory oedema it is known as an " exudate," is similar in constitution to blood-plasma and rich in albumin.

Haemorrhage into the Skin or Purpura.-At first the lesions are bright red in colour but, owing to the breaking up of red blood cells and the deposition of pigment, they gradually assume a yellowish and finally a greenish tinge as in a bruise. Purpura may occur in severe cases of the acute exanthemata; it may result from the ingestion of certain drugs, such as copaiba or chloral hydrate; it may be due to toxins circulating in the blood, such as that of rheumatism; it may be caused by alkaloidal poisons and be simply a further stage in the evolution of an urticarial lesion and the result of an increased virulence of the poison or a special predisposition in the individual; it may be an evidence of profound alterations in nutrition, as in scurvy; or it 
may be due to a marked increase in the intra-vascular pressure, as in the purpura which may occur in high altitudes. Purpura is not so much a disease per se as a symptom, and it is one which has not yet been satisfactorily explained. Naturally a lesion such as the rupture of a vessel-wall will allow an extravasation of blood and the production of a haematoma; but in sections of the smaller purpuric lesions no vascular defect can be detected as a rule. It has been maintained, however, that a rupture of the vessel-wall is essential and invariably occurs and that this is associated with some weakness of the resselwall, such as occurs in the malnutrition of scurvy, in fevers, in blooddiseases such as leucaemia, or as a congenital condition in haemophiliacs, or possibly from the destructive action of micro-organisms on the vessel-walls.

\section{Structural Changes}

These consist of the angiomata or benign new-growths composed of blood-vessels, and the inflammatory disturbances of the resselwalls, namely, endarteritis or endophlebitis.

The angiomata consist of (I) cavernous angiomata which are small subcutaneous tumours, composed of more or less dilated bloodvessels and connective tissue, which may be developed in the same way as erectile tissue-by a process of budding of solid rascular bodies from the walls of the vessels which become excavated and lined by endothelium-or may arise simply from a dilatation of pre-existing blood-vessels; (2) telangiectases which are acquired dilatations or new formations of capillaries resulting from a stagnation of blood in the venous capillaries extending to the arterial capillaries. They frequently occur in the face as isolated lesions or they may complicate various skin diseases, such as rosacea and Lupus erythematosus.

Vascular Naevi.- These may take the form of cavernous angiomata, port-wine stains, or the familiar spider-naevi.

Endarteritis may affect the vessels of the subcutaneous tissue and deeper parts of the corium; it occurs in syphilides, when the endothelial proliferation may block the ressel.

Endophlebitis.-The occurrence of phlebitis leads to a weakened resistance of the skin to micro-organisms and is a frequent cause of dermatitis. It may be due to the presence of tubercle bacilli in the veins, as in the condition known as "Erythema induratum of Bazin" in which the veins of the hypoderm are affected and in which necrotic nodules and ulcerations are produced about the calves of the legs. 
Table of Pathological Changes Affecting the Blood and Blood-Vessels in Association with Diseases of the Skin

\section{Changes in the Blood.}

I. Haemocytes :

Diminution: Cachectic states of leprosy, Tuberculosis, etc. Eruptive stage of syphilis.

II. Haemoglobin :

Diminution : During the course of syphilis and more marked when mercury is prescribed, till the symptoms begin to go when the haemoglobin increases.

III. Leucocytes :

Increase in the leucocytes or leucocytosis :

I. Polymorphonuclear leucocytosis: Scarlatina, Erysipelas.

2. Lymphocytosis: Leucaemia, Lymphodermia perniciosa, pre-mycotic stage of Mycosis fungoides, Measles.

3. Eosinophilia: Pemphigus, Herpes zoster, papular Eczema, Urticaria and Scabies.

\section{Circulatory Disturbances.}

I. Anaemia :

(a) Generalised : Chlorosis, Leucaemia, etc.

(b) Local : Spasm of small arteries, as in Raynaud's disease ; local pressure; organic obliteration of a certain set of vessels by a thrombus, endarteritis, or sclerodermia.

II. Hyperaemia :

I. Active hyperaemia or erythema:

(a) Generalised: Toxins circulating in the blood.

(b) Local : Reaction after cold; local asphyxia of Raynaud's disease.

2. Passive (Cyanosis)-Varicose veins.

III. Oedema :

I. Mechanical: Blocking of veins and lymphatics.

2. Inflammatory: Erysipelas.

3. Disturbances of nutrition of vessel-walls :

(a) General : Anasarca, etc.

(b) Local : Thrombosis, etc.

IV. Haemorrhage (Purpura) :

I. Acute exanthemata when severe.

2. Drugs: Copaiba, etc.

3. Alkaloidal poisons: further stage of urticaria.

4. Nutritive disturbances: Scurvy.

s. Increase in intra-vascular pressure: high altitudes, etc. 


\section{Changes Affecting the Blood-Vessels.}

I. Dilatation or New Formation, Angiomata :

I. Cavernous and cirsoid aneurism.

2. Telangiectases: Occurring alone or in association with certain skin-affections, such as Lupus erythematosus.

3. Vascular naevi : Spider-naevi and port-wine stains.

II. Endarteritis.

III. Endophlebitis: Varicose eczema, Phlebitis nodularis necrotisans (Philippson).

\section{Pathological Changes in the Lymphatics}

The lymphatic channels in the skin may be dilated or a lymphatic new-growth may occur. This leads to the formation of peculiar lesions consisting of clusters of cysts slightly resembling warts, varying in size from a pin's head to a split-pea, and containing a clear fluid. These cysts are simply dilated lymphatics lined by endothelium and occur in the condition known as "Lymphangioma circumscriptum." Occasionally there is marked cellular hypertrophy associated with the dilated lymphatics, with the result that raised patches are formed dotted over with deep-seated vesicles which have been compared to frogs' spawn. The patches are indurated, pinkish in tinge, and are met with in Elephantiasis'nostras.

Lymphoid-celled tumours are occasionally met with in which lymphocytes predominate in the cellular hyperplasia. These result from the dilatation of lymphatic spaces in the corium and their invasion by lymphocytes. To this group belong the rare skin-growths which occur in leucaemia, where the minute structure of the tumour is somewhat similar to that of a lymphatic gland.

\section{Pathological Changes in the Peripheral Nerves}

Degenerative changes in the peripheral nerves are followed by lesions in the skin in the area of distribution of these nerves, from an interference with the trophic influences which regulate the nutrition of the part and a diminution in the vaso-motor control in the bloodvessels. After an injury to a peripheral nerve or in association with peripheral neuritis, the skin in the area supplied by it may become atrophic and glossy, or bald, or vesicles, bullae, ulcers, or even gangrene, may develop on it.

Degenerative changes in the peripheral nerves have been detected in Raynaud's disease and in connection with the maculo-anaesthetic lesions in leprosy.

Inflammatory changes with haemorrhage in one or more posterior root ganglia occur in Herpes zoster in connection with the nerves 
supplying the affected area. In the majority of instances, however, where a form of dermatitis has been attributed to a disorder of the nervous system this assumption has been largely hypothetical, and the dermatitis has been the result of a functional disturbance and not associated with definite pathological lesions, either in the central nervous system or in the peripheral nerves.

Tumours of the Peripheral Nerves.-The peripheral nerves may be the seat of small tumours or neuromata. These are of two kinds, true neuromata and false neuromata. True neuromata are newgrowths caused by a proliferation of nerve tissue alone and are very rare; they generally result from an injury to a nerve and histologically are made up of ganglion cells and nerve fibrils supported by a connective tissue stroma.

False neuromata are far more common and are so called because they consist chiefly of connective tissue. They may be simply fibromata, situated on a nerve trunk, over which a few nerve fibrils are stretched; or they may result from a proliferation of the interstitial connective tissue and perineurium of the nerve together with a new formation of nerve-elements. They may occur along a peripheral nerve like beads on a rosary, or may have a plexiform arrangement. In either case they are usually painful from enclosing sensory nerve twigs. They may occur also in the form of deep-seated nodules over which the skin generally presents a violaceous tinge, and are then known as "Tubercula dolorosa" as they are frequently associated with paroxysmal attacks of excruciating pain. Von Recklinghausen has described a congenital affection characterised by multiple fibromata and pigmentary changes in the skin, associated with general nervous derangements and frequently with defective mental and physical development. In this disease nodules are present in the skin which are gelatinous to the touch and violaceous in colour and which develop in connection with peripheral nerves.

\section{Pathological Changes Affecting the Fat in the Skin}

There may be an increase of fat in the skin and subcutaneous tissue, general or local. In obesity there is an enormous increase in subcutaneous fat in which the fat lobules are not only increased in number but also in size. A local increase is seen in the fatty tumours known as "lipomata," which are chiefly regarded as the result of a fatty infiltration of connective tissue cells.

The xanthomata form another instance of fatty tumours. These are yellow or orange coloured, plane or tuberous lesions of the skin, the result of a fatty infiltration of connective tissue cells and a deposition of free fat in the lymphatic spaces. Three principal varieties of xanthoma are described, namely, Nanthoma planum affecting the eyelids, Xanthoma tuberosum multiplex situated about the elbows, 
knees, and palms, and Xanthoma diabeticorum in which the lesions are associated with diabetes.

In xanthoma there is at first a proliferation of cells about the bloodvessels in the affected area and an infiltration of the lymphatic spaces with a fatty substance which is present also in the connective tissue cells and in the endothelium of the capillaries. In some cases the cells have several nuclei and are known as xanthoma giant-cells. Surrounding the xanthoma cells are numerous fibroblasts containing fine droplets of fat. This fatty substance is a lipoid which tends to break up in the tumours into chloresterol crystals, and passes out through the capillaries of the skin to infiltrate the lymphatic spaces and gradually the cells. At the same time it causes a fibromatosis which ultimately predominates over the xanthomatous infiltration, so that the fatty tumour tends to become more and more fibrous. The same lipoid occurs in the blood in jaundice and diabetes.

A diminution of fat may occur as a general condition in emaciating diseases and in old age, or locally from wasting in the area supplied by a nerve whose functions have been impaired.

\section{Pathological Anomalies of Pigmentation}

Pigmentation due to Haematogenous Pigment.-The deposition of haematoidin and haemosiderin in the corium produces in the skin various depths of staining from purplish to green. This may follow haemorrhage or result from injury such as a bruise.

Pigmentation due to Melanin.- The following are the most important causes of an increased formation of melanin :

(I) Exposure to the actinic rays of the sun, when the pigmentation takes the form of freckles or profuse sunburn and appears in regions not generally pigmented.

(2) Flimination of certain drugs, such as arsenic, when the pigmentation chiefly involves the pigmented region but may occur elsewhere and is frequently determined by irritation and pressure of garters, corsets, etc. It consists of a brownish discolouration in which there may be points of sepia-brown colour on an earthy-pigmented base and is due to melanin in and around the epithelial cells and extending to the most superficial scales of the epidermis.

(3) Certain general diseases such as Addison's disease, when it is most evident in the naturally pigmented regions and when the melanin is found not only in the basal layer of the epidermis but throughout the prickle-cell layer, in the connective tissue cells of the corium, and in the leucocytes around the blood-vessels; syphilis, when it occurs as a café-au-lait mottling about the neck, the result of the absorption of pigment in areas in an already diffusely pigmented region, or in the neighbourhood of a healed syphilitic ulcer; and in diseases of the liver.

(4) Pregnancy and disorders of the uterus. 
(5) In association with a number of chronic dermatoses such as Lichen planus.

Pigmentation due to the deposition of metallic substances in the skin may occur, such as the general slate colour known as " Argyria," which results from the ingestion of silver and is caused by the actual deposition of fine particles of silver in the connective tissue.

Atrophy of Pigment.-A diminution of pigment may be met with as a hereditary condition in albinism, or it may be acquired as in vitiligo.

\section{IMMUNity AND ANAPHyLAXIS}

The subject of immunity, natural or acquired, in relation to diseases of the skin has attracted much attention of late years. Bound up with it is the condition known as " anaphylaxis," a statc of hypersensitiveness which occurs when the organism has reacted to some toxin or irritant and reacts more acutely to a second dose of the same toxin.

Natural Immunity is frequently met with in connection with cutaneous diseases. It is rarely absolute and is usually a relative. condition in which certain individuals are naturally less susceptible to some skin affection than others. In the case of local infections this may be due to differences in the texture of the skin, greater protective capacity of the Stratum corneum, or absence of greasiness which might form a suitable soil for the growth of micro-organisms; while if the infection be a general one, it may be the result of the presence of certain anti-bodies or protective substances in the blood.

Acquired Immunity is the result of the production of protective bodies in the blood which are called forth by some local or general infection and which render the individual, for a longer or shorter time, proof against a further attack. It has been observed that the more virulent the original infection, the more lasting the immunity. In some cases the immunity may persist throughout life as in small-pox and usually in syphilis, while in other cases it may be short-lived.

It has been stated that a local lesion such as suppurating ringworm of animal origin, which is the reaction of the skin to a virulent type of ringworm fungus, may cause a state of general immunity in which the skin becomes proof against a second infection. From cultures of the ringworm fungus, an extract has been prepared called "trichophytine" which, when injected into the skin in a patient with a deep-seated ringworm, is said to have been followed by a general reaction with rise of temperature and malaise, and a local reaction at the site of injection in the form of papules and pustules, while the actual ringworm lesion gradually subsided (Plato).

To produce immunity a somewhat severe inflammatory reaction appears to be necessary, as superficial types of ringworm, in which slightly inflamed scaly lesions are present, are multiple, while the 
deeply-seated suppurating lesions are not infrequently single as if in them a degree of immunity was set up whereby auto-inoculation did not so readily take place.

Another probable instance of acquired immunity is seen in connection with cutaneous lesions due to the inoculation of streptococci. In the infant the inoculation of these micro-organisms leads to a virulent type of bullous dermatitis known as Pemphigus neonatorum, which is sometimes fatal from a systemic invasion; in older children inoculation with the same micro-organisms is responsible, as a rule, for a common but usually harmless condition named Impetigo contagiosa; while in adults local streptococcic infections are comparatively rare. This is due to the greater delicacy and want of resistance of the infantile skin so that micro-organisms more readily penetrate and set up an inflammatory reaction, and to the gradually acquired immunity in older children and adults.

Immunity against the attacks of micro-organisms in the skin may be acquired artificially by the introduction of a ready-made antitoxin, such as one of the anti-bacterial sera, or by vaccines prepared from attenuated or killed specific micro-organisms. These vaccines do not cause immediate immunity but a lapse of several days takes place before it is produced. They act by calling forth certain anti-bodies known as agglutins, precipitins and bacteriolysins, which destroy the micro-organisms, and certain substances named opsonins by Wright which promote phagocytosis by rendering the bacteria more palatable to the phagocytes. These anti-bodies, which are responsible for the anaphylaxis, are formed as a result of the presence of the vaccine and when a second dose is given they cause its disintegration with the formation of toxic products which lead to an acute reaction. This is exemplified in the case of tuberculosis of the skin, for when an injection of old tuberculin is given in a patient with Lupus vulgaris a more or less severe general reaction and a local inflammatory reaction in the lupus patch take place. This is due to a state of anaphylaxis resulting from the presence of the tubercle bacillus in the local lesion and the formation of anti-bodies in the blood, which act on the tuberculin setting free some highly toxic bodies which cause both the general and the local - reaction.

\section{REFERENCES.}

Adansox, H. G. Modern Views on the Significance of Skin Eruptions (Goulstonian Lectures, I912).

BLoch, B. \& MAssixi, R. Studies on immunity and anaphylaxis in hyphomycetic Diseases (Zeitsch. f. Hyg. u. infekt. Krankh., I909, lxiii. p. 69).

Fordyce, J. A. Anaphylaxis in Toxic Dermatoses (Journ. Cut. Dis., I9I2, xxx. p. 128 ).

Hermax, W. J. Histology of Scars (Arch. f. Derm. u. Syph., Igro, cii. p. 65$)$. 
Hellmich, W. Epidermis pigment (Monats. f. prakt. Derm., 1907, xlv. p. 184$)$.

KRomayer, E. Epithelial cells in the Corium assuming the Characteristics of Connective Tissue-cells (Monats, f. prakt. Derm., I905, xli. p. 4I). MacLeod, J. M. H. Handbook of the Pathology of the Skin (with numerous references).

Misumi, J. Involution of Sebaceous Glands (Virchow's Archiv., I9IO, cxvii. Heft. I-3).

Rodler, C. Elacin (Arch. f. Derm. u. Syph., 1908, p. 328).

TöRök, L. Angio-neurotic \& Haematogenous Inflammatory Affections of the Skin (Derm. Zeitsch., I910, ix. p. 6I9).

Volk, R. Anaphylaxis in Dermatology (Arch. f. Derm. u. Syph., I9II, cix. p. I63). 


\section{CHAPTER IV}

\section{GENERAL ETIOLOGY}

THE causes which are responsible for diseases of the skin are numerous and varied. The skin is exposed to an endless variety of irritants and harmful agents acting on it from without, and to numerous influences of a more or less harmful character acting on it from within. Each irritant, acting either from within or from without, calls forth a type of reaction which is evident in some form of clinical lesion or eruption. The clinical appearances which result from the action of a particular agent are fundamentally similar in every individual affected but may be modified, sometimes almost out of recognition, by secondary influences such as age, the state of resistance of the skin, the region affected, scratching, the inoculation of pyogenic micro-organisms and other adventitious considerations.

There are two types of skin affections, namely, those which are symptomatic of some underlying general disturbance or systemic infection, such as urticaria or the cutaneous lesions of syphilis, and those in which the skin lesion is the only evidence of a departure from the normal state of health, as in local parasitic diseases.

The etiology of each disease will be discussed in detail in the description of it but it will be an advantage here to refer in a general manner to the more important causes. These may be grouped as follows :

\section{Predisposing Causes}

Heredity.-Heredity was once believed to play a more important part in the etiology of skin diseases than it is now known to do and many diseases of which the precise cause was uncertain were vaguely believed to be handed down from parent to child. There are comparatively few cutaneous affections, however, which are directly transmitted in this manner; in most cases it is only hereditary weakness or predisposition to subsequent infection which is transmitted. An example of a disease which may be congenital is syphilis, and of one to which only a predisposition may be inherited is leprosy. Naevi, both vascular naevi and moles, are congenital lesions which may have 
occurred in a parent or grandparent. The rare congenital condition known as Keratosis palmaris et plantaris may have been present in several generations. Sometimes the affection may be noted at birth as in Epidermolysis bullosa; at other times it may appear during the first year of life or later, as in certain naevi and ichthyosis, but in them microscopical changes are present in the skin at birth which become evident to the naked eye later. There are certain diseases also, such as psoriasis, in which heredity appears to be a factor in the etiology but it is inconstant. In one of my cases this disease was traced back for four generations and affected several members of both sexes in each family, while in another instance in which it occurred in at least three generations it was confined to the female sex.

Age.-Age is an important predisposing factor in cutaneous diseases. At birth such affections as congenital syphilis and Epidermolysis bullosa may be met with ; during the first two years of life infantile eczema, papular urticaria and ichthyosis frequently appear; puberty is the common age of incidence of Acne vulgaris and psoriasis; middle life of rosacea and Lupus erythematosus; advancing years of rodent ulcer and senile keratoses.

Sex.-Sex is an etiological factor in a certain number of skin affections. Some occur with equal frequency in both sexes, such as eczema ; others are more common in women, such as Lupus erythematosus, Erythema induratum and Paget's disease of the nipple; while others, such as sycosis of the beard and epithelioma, are more often met with in men.

Temperament.-Temperament may be a predisposing factor in certain diseases. Individuals with a neurotic excitable temperament are specially liable to suffer from diseases such as Lichen planus and attacks of it are apt to occur at times when the nervous system is in an unstable condition, as in women at the menopause. In nervous people, also, itching and other subjective symptoms are much more keenly felt than in those who are lethargic, scratching is indulged in with less restraint, and secondary inoculations with pyogenic micro-organisms are liable to take place.

General Health.- The general health of the individual has a definite bearing on certain affections. Defects in the general health or disorders of some special organ are not infrequently connected with some disturbance in the skin; derangements of the alimentary tract, for example, are apt to be associated with rosacea, diseases of the kidneys and albuminuria with Lupus erythematosus, nervous disorders with Lichen planus and pruritus, disturbances of the uterus with chloasma and Acne rulgaris, pregnancy with Dermatitis herpetiformis and sometimes with psoriasis, menstrual disorders with outbreaks of adolescent acne, and the menopause with attacks of Lichen planus.

Dentition.-The cutting of teeth in children has often been regarded as a predisposing, if not a direct, cause of certain cutaneous affections which occur about the time of the first dentition, such as infantile 
eczema and papular urticaria. Careful enquiry into this matter, how ever, has shown that the actual eruption of teeth is not necessarily associated with any cutaneous derangement. What happens is that when the infant is cutting teeth it usually becomes fretful, the digestion is impaired, and, as the result of imperfect metabolism and the circulation of toxins in the blood, various ery thematous and urticarial eruptions are apt to develop.

Reflex irritation from carious teeth has been suggested as a possible cause of Alopecia areata, but the evidence in this connection is insufficient. Bad teeth and oral sepsis, however, may act as predisposing factors to affections such as Acne vulgaris and rosacea, by causing digestive disturbances from imperfect mastication, constipation or anaemia.

Vaccination.-In the minds of the laity, vaccination is often held responsible for cutaneous eruptions such as eczema which are liable to appear about the time the infant is vaccinated. It is true that eczema and psoriasis may follow vaccination and may begin at or in the neighbourhood of the inoculation, but the relation, if any exists, is not understood, and in the vast majority of cases the association is accidental. Certain skin lesions may result from the vaccine virus, such as a generalised vaccinia, erythema, or urticaria, but they are rare; while other local conditions, such as impetigo, boils, or Lupus vulgaris, which may occur at the site of the vaccination, are due to a mixed inoculation or a subsequent infection of the unhealed lesions with staphylococci or tubercle bacilli.

Climate.-Certain skin affections such as psoriasis, tuberculosis and eczema, may occur in any climate, others are met with chiefly in temperate zones, while others are confined to tropical or sub-tropical countries and never occur in temperate climates unless as imported cases. The small-spored ringworm, for example, which is so commonly met with here, is unknown in the tropics, while vaws and mycetoma are entirely confined to tropical countries. Other diseases only occur in certain parts of the world, such for example, as Verruga peruana which is endemic on the slopes of the Andes. This is partly due to the actual variations in climate and also to some extent to differences in the customs and manner of living of the inhabitants.

Race.-The fair skin of white races is more delicate and liable to be affected by cutaneous diseases than the dark skin of the negro with its thicker epidermis and pigmentation which protect it from injury and the harmful actinic rays of light. The skin in coloured races, however, is more affected by local infective processes as it is more liable to be exposed to external injuries, such as abrasions, scratches, and bites of insects.

Habits and Environment.-Personal hygiene and general sanitary surroundings are also important factors in the causation and transmission of skin affections. This is seen specially in connection with parasitic diseases, such as ringworm, scabies and pediculosis, which are 
more prevalent in the hospital classes than in private practice as the result of want of cleanliness and overcrowding.

Season.-In a considerable number of diseases of the skin, the season of year appears to be the determining factor in their incidence, or may be a predisposing cause of recurrences. There are certain diseases which are peculiar to cold weather such as chilblains and Pruritus hiemalis, while others occur in hot weather such as Hydroa aestivale, sweat eruptions and prickly-heat. On the other hand, spring and autumn are seasons in which recurrences of certain diseases, such as psoriasis, are liable to take place, while in summer ringworm of the crutch, which may have been dormant during the cold weather, is apt to break out again.

\section{INTERNAL CAUSES}

Diathesis.-At one time, especially in France, a large number of cutaneous affections were believed to be the evidence of some special type of general derangement or diathesis such as the arthritic and lymphatic diatheses. This belief even now is firmly rooted in the minds of the laity and there is a tendency to regard cases of chronic eczema as an evidence of suppressed gout and the skin affection as a sort of safety valve. As our knowledge increases this belief is steadily losing ground. Eczema is a common disease in this country while gout is extraordinarily rare, and when they do occur together it is rather in the nature of a coincidence. In connection with rheumatism, various affections such as purpura and Erythema nodosum not infrequently occur, but these are probably toxic conditions, perhaps of microbic origin, and the result of the reaction of the skin to the same influences as are responsible for the arthritic pains. The only general diathesis which has been clearly proved to give rise to definite skin lesions is diabetes, for in connection with it xanthoma not infrequently occurs and tends to disappear without local treatment when the sugar is eliminated from the urine.

Toxins.-Lately the toxic theory of the etiology of cutaneous diseases has been revived to some extent and a number of skin affections have been shown to be due to toxic agents of different kinds, such as foodstuffs, drugs, or toxins eliminated by micro-organisms or called forth in the blood or tissues in the form of protective substances. These produce various forms of dermatitis, consisting chiefly of erythematous blotches or wheals, rashes like scarlet fever or measles, and, more rarely, of vesicles or even granulomatous lesions. As a rule it is impossible to tell from the appearance of the eruption the exact toxin which is responsible, and it has even been asserted that the eruptions do not result from the specific toxin assimilated but from certain protective bodies called forth in the blood in consequence of its presence. In this connection individual susceptibility or idiosyncrasy plays an im- 
portant part. It has been said with reason " one man's food is another man's poison," and this also applies to drugs. Certain drugs, even in minute doses, which could be taken with perfect safety by most people, are capable of causing cutaneous symptoms in a susceptible individual. The susceptibility may be a natural condition, or acquired as the result of a former dose rendering the organism hypersensitive to that particular toxin.

Certain foods, such as shellfish, are liable to cause dermatitis; others, such as pork, mushrooms, strawberries and mackerel may do so by becoming toxic from deleterious changes occurring in them; while others, such as cheese, pickles, coffee, oatmeal, eggs, etc. are poisonous only to a few. A considerable number of drugs when taken internally are capable of producing inflammation of the skin in susceptible people, either from an overdose or from imperfect elimination owing to disease of the kidneys or heart. Only a few drugs like the iodides and arsenic, produce characteristic eruptions, and in connection with the majority of them the dermatitis is in no way pathognomonic.

Not only may toxins be ingested in the form of foreign substances such as foodstuffs or drugs, but they may be produced in the organism in the form of the products which result from impaired digestion or imperfect metabolism. Eruptions caused by this type of toxin are known as the auto-intoxications. The rôle played by so-called autointoxication in connection with cutaneous diseases has probably been somewhat exaggerated, however. The products of imperfect metabolism are well known, such as indol, skatol, para-cresol, etc., but there is no evidence that any of these bodies are capable themselves of producing a dermatitis. In addition to the above there are the toxins which are eliminated by micro-organisms, either the exo-toxins which result from the activity of the microbe or the endo-toxins which are eliminated when the microbe dies and disintegrates. These may cause inflammatory reactions in the skin either directly, or indirectly by calling forth protective substances in the tissues.

Nervous Disorders.-Though many skin affections in which the etiology is doubtful have been attributed at some time or another to derangements of the central or peripheral nervous system, in only a few of them have definite changes of a degenerative type been detected in the nerve tissue. In Herpes zoster there is an inflammatory disturbance with haemorrhage in one or two posterior root ganglia, and degenerative changes have been observed in the peripheral nerves associated with more or less marked analgesic symptoms; in the maculoanaesthetic patches of leprosy the nerves supplying the affected area are in a more or less degenerated state, owing to the pressure produced by the lepromatous infiltration in the connective tissue around the nerve fibres. Lichen planus is believed to be associated in some way with derangement of the central nervous system since an attack is so often determined by psychical causes and the lesions may originate around the sweat-pores, but no definite structural changes have 
been found in the nerves supplying the skin in the seat of the eruption.

From time to time it has been observed that injury to a nerve may be followed by the formation of a bald patch in some part of the area it supplies. A localised defluvium of the hair has been experimentally produced in animals by injury to a peripheral nerve. Instances are recorded also where alopecia of the beard or scalp has occurred in association with dental caries and in which it was suggested that the loss of hair was the result of reflex irritation.

\section{External Causes}

A large number of local causes may be responsible for skin diseases. In some cases the external agent or irritant may set up a purely local reaction, as in ringworm ; in others it may be a potent factor in determining the situation of lesions which result from some general disturbance ; in others it may modify an existing lesion, as scratching and the secondary inoculation of pyogenic organisms. The external agents which may be responsible for cutaneous affections or influence their course are so numerous and varied that it would serve no useful purpose to even attempt to enumerate them. The most common may be placed in the following groups:

Traumatic Agents.-Any injury which causes a breach of the Stratum corneum renders it possible for harmful micro-organisms to penetrate the deeper parts of the skin and to set up various types of dermatitis. Even a scratch which is so superficial as to be scarcely noticeable by the naked eye may be sufficient for this.

Scratching and rubbing are familiar forms of traumatism in cutaneous diseases. Scratching lacerates the skin and is apt to lead to secondary infection by pyogenic micro-organisms; while rubbing tends to increase rather than to relieve irritation and if persisted in may produce a chronic thickening of the skin, as in the condition known as lichenification.

Heat.-Apart from actual burns, heat may be responsible for a considerable number of cutaneous lesions. It tends to produce at first only a transient flushing, but continued exposure may lead to permanent dilatation of the blood-vessels and telangiectases. It is also liable to cause excessive sweating, occasionally with the formation of sweat-cysts as in hydrocystoma. It may be responsible for the peculiar retiform mottling of the legs-usually seen in women-which results from the habit of "toasting" them before the fire.

Cold.-Cold is responsible for chilblains in people with a weak circulation, is a factor in the production of Lupus erythematosus and Raynaud's disease, and when excessive causes frost-bite. It causes a contraction of the skin muscles, an ercction of hairs, the formation of the so-called goosc-skin, and a stasis in the peripheral circulation which is well- 
marked as a bluish mottling on the limbs of babies exposed to the cold.

Sunlight.-Sunlight may have an irritating effect on the skin, causing certain forms of dermatitis and aggravating others. When concentrated, it may produce an acute inflammation of the exposed parts known as solar dermatitis; or, when acting over a long period, it may give rise to a curious atrophic pigmented condition of the shin associated with telangiectases and a tendency to warty growths which is met with not infrequently in tropical countries. These results occur quite independently of heat and are entirely due to the actinic rays at the violet end of the spectrum; pigmentation in the form of profuse sunburn or of freckles, is also due to this cause and is an effort on the part of nature to protect the organism from the harmful effect of the rays.

In addition to the dermatitis due to the actinic rays, both acute and chronic dermatitis may be caused by $\mathrm{X}$-rays and radium.

Water and Soap.-In spite of the old adage that "cleanliness is next to godliness," the former may be overdone and may lead to deleterious changes in the skin and even to the production of dermatitis. The epidermis is a protective organ and its protective function is due largely to the waxy horn-cells of the Stratum corneum; if these be removed with soap and water the skin-especially when naturally delicate-becomes more susceptible to the attacks of micro-organisms and to all forms of local' irritation. The abuse of soap and water is directly responsible for many a case of infantile eczema and is a frequent cause of dermatitis in the hands and arms of nurses and washerwomen. It is liable to aggravate all acute inflammatory diseases of the skin and the injudicious employment of it may transform a quiescent affection into an acute dermatitis. On the other hand insufficient cleanliness is equally harmful, as it allows dirt and discharges to collect, sebaceous secretion to accumulate and become rancid, and renders the surface of the skin a more suitable soil for the growth of microorganisms and more attractive to animal parasites.

Clothing.- Unsuitable or irritating clothing is sometimes the cause of cutaneous derangements. In delicate skins woollen undergarments should be avoided, as their roughness is irritating and liable to cause inflammatory papules, while being almost non-absorbent they allow perspiration to collect which keeps the skin moist and sometimes offensive. The best material to wear next the skin is lawn or fine cotton, which is both smooth and absorbent, and if necessary woollen garments may be worn over it to provide the requisite warmth.

Various articles of apparel may give rise to dermatitis or be the means of spreading it; for example, an infant's binder may chafe the skin and when damp with offensive secretions may cause irritation; starched collars when old and rough may abrade the surface of the neck and may lead to boils; in the tropics warm clothes may determine an attack of prickly-heat; the interchange of caps among schoolboys may be 
responsible for the transmission of ringworm; and the wearing of cheap dyed garments, especially stockings, may result not only in dermatitis but in dyeing the skin from the liberation of the dye by the action of the sweat.

Local Irritants.-A large number of local irritants of animal, vegetable, or mineral origin, are capable of causing a dermatitis. Some produce an inflammation on any skin, such as croton oil and cantharides; others only do so if the individual has an idiosyncrasy towards them, for example, half a dozen men may be working together in the same bakehouse and exposed to the irritating action of sugar and flour and only one of these may develop a dermatitis in consequence. This susceptibility may be a natural peculiarity or it may be an acquired condition owing to a diminished resistance of the skin from ill-health or from some pre-existing skin affection. When a dermatitis has been produced by the action of some local irritant, the individual affected becomes extraordinarily sensitive to that irritant and liable to suffer from dermatitis from a far slighter exposure to it than was necessary to produce the original attack. This hypersensitiveness or anaphylaxis is supposed to be due to the action of protective substances called forth in the blood when the first attack took place.

The vegetable irritants consist of poisonous plants, vegetable substances like croton oil, moulds, bacteria, etc., the animal irritants of bites of insects, stings of wasps, jellyfish, caterpillars, etc., and the mineral irritants of fine dust, Portland cement, certain high explosives, etc.

The important group of occupation diseases are caused by the action of local irritants, such as baker's itch from the handling of flour and sugar, tar acne in pitch workers from the exposure to tar and its fumes, acute dermatitis in carpenters from the dust of certain hard woods like teak and satin wood, and gardener's eczema from contact with poisonous plants.

Occasionally local irritants of various sorts are employed by patients themselves to produce dermatitis. This generally occurs in hysterical women and is done to elicit sympathy and avoid work. For this purpose all manner of irritants are used, but the most commonly employed are carbolic acid, vinegar, saliva, and urine.

\section{Bacteriology of the Skin}

Although the different micro-organisms which are pathogenic in the skin will be dealt with specially in connection with the diseases for which they are responsible, it will be an advantage to refer here to certain general considerations regarding them in order to avoid subsequent repetition.

After Koch discovered the tubercle bacillus and it was found that Lupus vulgaris was the result of the reaction of the skin to its presence 
locally, the importance of micro-organisms in the causation of skin diseases came to be recognised and a number of cutaneous affections, which previously had been vaguely considered to be the result of some peculiar diathesis, were definitely proved to be of microbic origin. At the same time there has been an equally well-marked tendency to assume a microbic origin for diseases which have been subsequently found to be due to other causes, and to attribute on insufficient evidence specific pathogenic properties to micro-organisms which, though occurring in the lesions, were harmless saprophytes or were present there by accident.

The skin furnishes two distinct lines of defence against the attack of micro-organisms from without, namely (I) the Stratum corneum with its closely welded horn-cells, (2) the inflammatory reaction which the presence of actively growing micro-organisms excites in the skin and which leads to their destruction. The character of the inflammatory reaction differs according to the type and virulence of the micro-organism and it is these differences which are responsible for the varied appearances of the clinical lesions of microbic origin.

The dry intact Stratum corneum is not a suitable soil for the growth of micro-organisms and even the most virulent may be present there without exerting any harmful influence; on the other hand, when the surface of the skin becomes moist and sodden, or excessively greasy, the growth of micro-orgánisms is facilitated and they may produce pathological changes. The power of resistance of the skin to the attack of micro-organisms is influenced by the following considerations : the region of the body affected, as the resistance is greatest where the skin is thick and the sebaceous glands few in number; the age of the individual, as in infancy the resistance is low, increases up to puberty when it diminishes on account of the rapid growth of the hair-follicles and sebaceous glands, increases again towards the end of middle life, and as a rule diminishes with age and the general health of the patient. The resistance may also be weakened locally by some pre-existing dermatitis or by injuries which lead to an abrasion of the surface and allow micro-organisms to penetrate into the deeper parts of the epidermis or the corium.

Micro-organisms are found in largest numbers in regions which are least exposed to friction, such as the axillae, groins, and clefts between the fingers and toes, and where the sebaceous glands are largest, as about the middle line of the back and the pre-sternal region.

The study of the bacteriology of the skin is singularly complicated and difficult. At one time or another any micro-organism may become deposited on the skin so that a complete description of the subject would necessitate a reference to every known variety of bacterium. Some of these bacteria lead as a rule a perfectly harmless existence on the skin but under altered circumstances may grow actively, form colonies, and become pathogenic; for example, the Staphylococcus 
epidermidis albus of Welch, one of the most constant of all the denizens of the skin, which, though usually harmless and saprophytic, may assume virulent properties indistinguishable from those of the Staphylococcus pyogenes albus and produce suppurative lesions. On the other hand, a micro-organism according as it acts superficially or deeply in the skin, may produce very dissimilar clinical appearances; for example, the Streptococcus pyogenes when present in the epidermis only produces superficial phlyctenular impetigo, but when present in the deeper parts of the corium it may give rise to erysipelas. The difference in type of lesion which results from the action of a microorganism in the skin depends on the state of resistance of the skin, the position of the micro-organism in it, and possibly its combined action with some other microbe (symbiosis).

The micro-organisms of the skin may be divided into two groups, namely,

(I) Those which are usually present on the healthy epidermis and only become pathogenic when they begin to proliferate actively and when their virulence increases.

(2) Those which are accidentally deposited on the cutaneous surface and occasionally assume pathogenic properties.

Many attempts have been made to classify the different species of bacteria which may occur on the healthy Stratum corneum, and complicated lists of them have been published, but differ to such an extent in the number of the micro-organisms included and in the type which are regarded as pathogenic that they are of comparatively little value and only serve to emphasise the difficulties of the subject. Thesc difficulties are in great part due to the fact that it is almost impossible to draw a hard-and-fast line between bacteria which are slightly pathogenic and those which cause no apparent disturbance.

Micro-organisms pathogenic in the skin.

(I) Staphylococcus epidermidis albus (Welch) which is probably a variant of the $\mathrm{S}$. pyogenes albus of lower virulence.

(2) Staphylococcus or Micrococcus cutis communis (Sabouraud)(Morococcus of Unna, Polymorphic coccus of Cedercreutz, Grey coccus of Sabouraud), an organism which is so closely allied to the S. epidermidis albus of Welch that some obscrvers regard them as identical, and which is believed by Sabouraud to be the cause of Pityriasis capitis or dandruff and of the scaly greasy dermatitis of the glabrous skin known as Seborrhoic dermatitis.

(3) Staphylococcus pyogenes albus which is the common cause of suppurative lesions in the skin, such as small superficial follicular pustules, coccogenic sycosis of the beard, purulent changes in vesicles and bullae, and the pustulation in Acne vulgaris. It may also be the cause of warty growths such as occur in elephantiasis, tertiary syphilis, etc.

(4) Staphylococcus pyogenes aureus which is the cause of more 
deep-seated and virulent lesions, such as boils and carbuncles, deep ulcerations, and whitlows.

(5) Microbacillus of Unna and Sabouraud (Bacillus acne of Gilchrist), a small bacillus of low virulence found in vast numbers in comedones and in the sebaceous material in seborrhoic conditions. Unna believed it to be the cause of the comedo and of the suppuration in acne, while Sabouraud regarded it as responsible for the comedo and the sebaceous flux in seborrhoea but not for the suppuration.

(6) Flask bacillus of Unna (Spore of Malassez), a small oval or gourdshaped organism, probably a yeast, with, as a rule, a large spherical portion surmounted by a small rounded process, the result of budding. It is easily found in the scales from a scurfy scalp and is believed to be pathogenic and pathognomonic of seborrhoic conditions.

(7) Streptococcus pyogenes, which is a frequent cause of pathological lesions in the skin. The type of lesion it produces depends on its situation; when present superficially it gives rise to simple phlyctenular impetigo, more deeply to erysipelas, and if in the subcutaneous tissue, to phlegmon. It is the cause of the acute inflammatory attacks and subsequent fibromatosis which occur in Elephantiasis nostras and is responsible for the superficial ulcerative condition known as ecthyma.

(\$) Bacillus of tuberculosis, Tubercle bacillus of Koch, is the cause of the different lesions of Tuberculosis cutis. This organism is no longer believed to belong to the group of the "Fission fungi" but to the class of the streptotricheae, the rods being segments of filaments.

(9) Bacillus leprae of Hansen is generally admitted to be the cause of leprosy, closely resembles the tubercle bacillus and is also believed to belong to the class of the streptotricheae.

(Io) Bacillus pyocyaneus has been found in pyodermia and in certain severe ulcerative conditions of the skin, such as "Ecthyma térébrant," bed-sores, hospital gangrene and certain cases of pemphigus. It is doubtful, however, if the bacillus is the actual cause of the lesions and is not a secondary infection.

(II) Bacillus of Matzenauer, has been detected in hospital gangrene and noma.

Micro-organisms accidentally present on the skin which are occasionally pathogenic.

(I) Bacillus diphtheriae (Klebs-Löffler bacillus) which can grow on lacerated shin causing "wound diphtheria" and has been known to produce also a bullous dermatitis, ulceration, and gangrene. The Klebs-Löffler bacillus can also cause a type of chronic sore, known as "desert sore," which was met with among European troops in the Egyptian and Palestine campaigns of the Great War and which in many cases was found to be associated with faucial diphtheria.

(2) Gas-forming organisms may be found contaminating the skin and deep wounds and proved a great source of trouble during 
the Great War. Of these the most important are the following, in the order in which they have been most commonly detected:
B. perfringens
B. sporogenes
B. oedematiens
- $66 \%$
B. oedematis maligni
- $\quad$ I $8 \%$
- $\quad 13 \%$
$-\quad 4 \%$

(3) Bacillus coli communis has been isolated from chronic ulcer of the leg, various other superficial ulcerations, and purpura. This micro-organism may be a secondary contamination.

(4) Gonococci have been found in abscesses and ulcerations in patients suffering from gonorrhoea.

(5) Pneumococci have been observed in the dilated vessels in purpura and in deep cellulitis of the neck, but that they are pathogenic on the skin is uncertain.

The majority of these micro-organisms are deposited on the surface of the skin, while a few of them, such as streptococci, may occasionally reach it through the general circulation. In the latter category may be placed also the tubercle bacillus and the lepra bacillus.

\section{REFERENCES.}

Andrewes, F. W. Evolution of the Streptococci (Lancet, I906, ii. p. I4I 7). Bolton and Brewer. Bacillus Diphtheriae (Lancet, 1905, i. p. II3I).

Cedercreutz. Investigations on a Polymorphic Coccus (Paris, J. Steinheil, I90I).

Damman. Micro-organisms of Normal Skin (Brit. Med. Journ., I892, ii. p. I22).

Fleming, A. Etiology of Acne Vulgaris (Lancet, I909, i. p. In35).

Foulerton, A. Streptotrichoses \& Tuberculosis (Lancet, I9Io, i. p. 769).

Gilchrist, T. C. Research upon Etiology of Acne Vulgaris (Johns Hopkins Hosp. Rep., ix. I 899).

Gilchrist, T. C. Bacteriological \& Microscopical Study of over 300 Vesicular \& Pustular Lesions of the Skin (Trans. Amer. Derm. Assoc., I900, p. 87).

Ivexs, M. H. F. (Lancet, Dec. 23rd, I9I6, p. I058).

Macleod, J. M. H. Micro-organisms of the Skin (Handbook of Pathology of the Skin, p. 325) ; (Encyclopaedia Medica, vol. xi.).

MacWalters. Bacillus Coli Communis (Practitioner, I909, p. 330).

Matzenauer, R. Hospital Gangrene (Tran. Congr. of Derm., Paris, p. 734).

Sabouraud, R. Le Microbacille Séborrhoique, Seborrhoée, Acnés, Calvitie, I902, p. 33 .

Sabouraud, R. Bottle Bacillus-Pityriasis et Alopécies Pelliculaires, Paris, 1904 .

Tommasoli. Flora Dermatologica (Monats. f. prakt. Derm., I889, Bd. ix., p. 49).

UnNa, P. G. Histopathology of the Diseases of the Skin (Walker's Translation, I896, p. 361).

Weinberg, M. \& Seguin, P. F. Review of Bacteriology, igi6, vol. vi. part 2, p. I9.

Whitficld, A. Bacteriology of the Skin (Allbutt \& Rolleston's System of Medicine, IgI I, vol. ix. p. 5). 


\section{CHAPTER V}

\section{GENERAL DIAGNOSIS}

THE diagnosis of cutaneous affections is usually a serious stumblingblock to the student of dermatology, and it may be so difficult as to puzzle even the expert. As a rule it can be made from the clinical appearances alone, but if these be insufficiently characteristic assistance should be obtained from a microscopical examination of discharges, scrapings, scales and hairs for bacteria or fungi, or from sections of typical lesions, or from reaction tests such as the Wassermann test for syphilis or the tuberculin test for tuberculosis.

In examining a skin disease it is essential that a good light should be available, daylight being preferable to artificial light and a side light to a top light.

It is important also that the whole of the eruption should be seen before the diagnosis is made, the patient being completely undressed if necessary. If this be omitted gross mistakes are liable to occur, as it often happens that the lesions on the exposed or easily accessible parts are not sufficiently characteristic to enable a firm diagnosis to be made and the typical lesions are only present on covered areas. It also enables the distribution and general configuration of the eruption to be studied, which are valuable aids to its recognition. With regard to distribution, where lesions are present on covered parts patients' statements are apt to be unreliable, for the patient may be in actual ignorance of them, or may be aware of them and disinclined from modesty or laziness to admit of their existence or to show them.

There is no royal road to the acquisition of skill in the diagnosis of skin diseases. It only comes from keen and patient observation and considerable experience. The student must study the diseases clinically, must see and feel the lesions which constitute them, and must learn by his own mistakes. Though text-books, illustrations and atlases are of the utmost value as an addition to clinical observation, they should be looked upon as supplementary. Some eruptions are so characteristic that little or no difficulty may be experienced in recognising them, while others are so ill-defined or anomalous that the closest observation, sometimes over an extended period, may be requisite to distinguish them. 
It is of the first importance in making a diagnosis that the student should be familiar with the groundwork of clinical dermatology, namely, the primary and secondary lesions of the skin, and should be able to distinguish between what are essential and what are merely adventitious characteristics.

The essential lesions are generally best seen in the early stages of the eruption, or at the growing edge of a lesion. They should be carefully examined : (I) by the naked eye as to their shape, size, colour, and distribution; (2) by touch as to whether they are level with the surface, raised, soft, hard, rough, or smooth ; (3) by the help of a pocket lens to ascertain finer details, such as the presence of punctate vesicles or delicate striae; (4) by a diascope to detect cellular infiltration in the corium. The diascope is a piece of glass which, when pressed firmly on the lesion, completely obliterates the redness due to dilatation of the blood-vessels but leaves a greyish or brownish semi-translucent stain should there be a cellular deposit in the corium.

A convenient form of diascope is one shaped like a pleximeter made of glass and marked in millimetres for measuring the lesions.

The following are important aids to the diagnosis of an eruption :

Manner of Evolution of the Lesions.-Certain lesions, such as warts, remain constant throughout their existence; others undergo marked changes, such as the maculo-papule of psoriasis which evolves into a bright red patch covered with silvery scales, the papulo-vesicle of eczema which breaks, weeps, and is replaced by a crust. Lesions in all stages of evolution may be present in an eruption and may combine to make up the characteristic picture.

Distribution.-Lesions may remain discrete, such as the papules in prickly heat, or may become aggregated and coalesce to form patches or peculiar figures. Sometimes they are symmetrically distributed, indicating that the virus responsible for them is circulating in the blood ; at other times they are asymmetrical, suggesting a local cause.

Manner of Involution.-Certain lesions disappear without leaving a trace, others are followed by scars or pigmentation.

Colour.--Though the colour of the lesions may be a help in diagnosis, too much reliance should not be placed upon it as it is difficult to describe colours and everyone does not see them alike. In addition the colour is apt to vary in the same disease in different patients and in different lesions in the same eruption; for example, in individuals with a feeble peripheral circulation inflammatory lesions about the extremities instead of being bright red or pink are apt to assume a deep red or purplish tinge.

Certain lesions present a characteristic tint, such as the raw-ham colour of the syphilide, the yellowish-brown translucent colour of the nodules of Lupus vulgaris, the yellowish tinge of xanthoma, the bright red tint in psoriasis, the violaceous hue of Lichen planus, the brownish stain in erythrasma, and the black colour in senile keratosis and melanotic sarcoma. 
Duration.-Certain lesions are of short duration, appearing suddenly and tending to involute spontaneously in a few days, such as those of urticaria and Herpes zoster; others run a short course if suitably treated, such as those of Impetigo contagiosa; others are singularly. chronic and persistent, such as those of Lupus erythematosus; while others persist a lifetime, such as those of ichthyosis.

Configuration.-Certain lesions tend to form characteristic figures, either by spreading peripherally and involuting at the centre or by coalescing, such as the serpiginous. lesions of tertiary syphilis, the annular lesions of ringworm and Lichen planus, and the gyrate figures of psoriasis.

Grouping.-Certain lesions tend to be grouped in clusters, such as the vesicles of Herpes zoster and Dermatitis herpetiformis ; in irregular groups, such as the papules of Lichen scrofulosorum; or in a peculiar corymbose fashion in which small papules are arranged round a large central lesion, as in certain syphilides.

Subjective Symptoms. - Interrogation of the patient with regard to the subjective symptoms or the history of the disease should not be made until the physician has examined the skin, otherwise he may be biassed as to the nature of the eruption by the opinion expressed by the patient. Too much reliance should not be placed on the statements of patients with regard to their subjective symptoms, as ther are apt to be minimised by some and exaggerated by others.

Co-existence of two Diseases.-Occasionally two distinct affections may be present at the same time, such, for example, as psoriasis and syphilis, ringworm and eczema.

General Considerations.-(a) Age.-Certain diseases almost invariably occur in infants and young children, such as papular urticaria ; others are practically confined to childhood, such as small-spored ringworm ; others rarely appear till adolescence, such as Lichen planus and Lupus erythematosus; while others are associated with old age, such as senile keratoses.

(b) Sex.-Certain skin affections, such as Erythema induratum and Lupus erythematosus, are much more common in women; others, such as epithelioma, are more frequent in men.

(c) Social Position.-Certain diseases are met with most commonly among the poorer classes, such as parasitic affections and Lupus vulgaris; others are equally common among the poor and the well-todo, such as psoriasis and rodent ulcer.

(d) Race.-It is important to ascertain the race of a patient and whether he has lived abroad, for there are certain diseases, such as leprosy, which are rarely contracted except in countries where they are endemic.

(e) Family History.-Certain diseases are hereditary, such as many cases of psoriasis; others may be congenital, such as syphilis.

(f) Occupation.-Certain occupations are apt to cause skin affections; gardeners, for example, are liable to eczematous dermatitis from 
handling various plants, tar workers to a peculiar type of acne, and washerwomen to a dermatitis resulting from the action of soap, soda, and hot water.

\section{Type of Primary Lesion as a Diagnostic Factor}

Erythematous Eruptions.-Erythematous macules or erythematous papules are the predominant lesions in a number of eruptions, such as the following: Dermatitis due to all manner of local irritants of animal, vegetable, and chemical origin or to traumatism; Eczema which, in its acute form, may appear as erythematous patches associated with oedema and be so vivid in tint as to suggest erysipelas; Erythematous eruptions due to toxins circulating in the blood, such as drugs, food toxins, bacterial toxins, autogenous toxins, and sera, which are generally most marked on the trunk and may closely simulate scarlet fever or measles.

Papular Eruptions.-The most common papular eruptions are: Acne vulgaris-follicular papules and papulo-pustules, present chiefly on the face, back or chest, and associated with comedones or blackheads; Eczema-small, itchy, rounded or acuminate papules, which usually present a minute vesicle on the apex; Erythema multiforme-papules of various sizes, which do not itch and are present generally about the backs of the hands, arms, and occasionally the face; Ichthyosis follicularis-small follicular papules, the colour of the normal skin with a horny plug or spine in the centre; Keratosis pilaris-acuminate papules with horny plugs; Lichen planus-flat lilac-tinted papules, angular in outline, smooth on the surface, occasionally umbilicated; Lichen spinulosus-papules surmounted by filiform spines; Miliaria or Prickly-heat-small red acuminate papules, associated with intense itching and profuse sweating; Milium-small white or pearly papules about the size of a millet seed, frequently situated about the eyelids; Molluscum contagiosum - pearly rounded papules, from a pin's head to a pea, with a depression in the centre and an aperture from which a mass of peculiar horn-cells can be extruded; Papular necrotic tuberculides - small red or violaceous papules which necrose, leave whitish scars and occur in tuberculous subjects; Papular syphilides-small miliary papules tending to be arranged in clusters or grouped in a corymbose fashion around a larger one, and larger papules from a split-pea to a threepenny piece, raw-ham coloured, and when pressed by a diascope leaving a reddish-brown stain; Papular urticariasmall pinhead-sized papules the colour of the skin, preceded by red papules on an erythematous base; Pityriasis rubra pilaris-small follicular papules, generally surmounted by a scale and tending to coalesce to form sheets; Prurigo-small hard papules, associated with itching, a peculiar thickening of the epidermis, or lichenification, and present chiefly on the extensor aspects of the limbs ; Warts-small flat 
or juvenile warts the colour of the shin; Yanthoma-flat papules or small plaques, yellowish or of a washleather tinge, frequently situated about the eyelids, elbows, and knees.

Nodular Eruptions. - Acne vulgaris-nodules about the face, back, or chest, associated with pustules, comedones, and often with pitted scars; Erythema induratum (Bazin)--deep-seated nodules, violaceous in colour, tending to necrose and leave depressed pigmented scars, situated chiefly on the calf of the leg in young women; Erythema multiforme-erythematous nodules, situated chiefly on the backs of the hands, forearms, and face; Erythema nodosum-oval node-like swellings, varying in size from a nut to a hen's egg, red or purplish in colour, situated chiefly on the legs; Leprosy-nodules varying in size from a split-pea to a filbert nut or larger, either persisting or tending to necrose or ulcerate, at first tender to the touch, gradually becoming anaesthetic, situated chiefly on the face and limbs but present also on the mucous membranes; Lupus vulgaris-small apple-jelly-like nodules, reddish-brown in tinge and tending to ulcerate; Rodent ulcer-small pearly nodules of a cartilaginous consistence, sometimes single, at other times forming a ringed border to a central ulceration, situated most commonly on the face; Rosacea-nodules about the face, sometimes acneiform, associated with flushing of the nose and cheeks, telangiectases and chronic indigestion; Scrofulous gummata-deep-seated nodules tending to break down and ulcerate, occurring occasionally in tuberculous children; Syphilitic cutaneous gummata-nodules or small tumours which soften, break down, and form ulcerations which tend to be serpiginous in outline and on healing to leave pigmented scars; Xanthoma tuberosum-yellowish nodules, usually situated on the elbows and knees.

Vesicular Eruptions.-Dermatitis due to local irritants-vesicular eruptions in which the resicles are situated on an inflammatory base and are associated with more or less burning and itching ; Dermatitis herpetiformis - a generalised eruption of which the most prominent feature is the occurrence of groups of inflammatory papulo-resicles, associated with marked itching and occasionally with bullae; Dysidrosis-resicles situated most commonly about the hands and feet, associated with burning and itching and not tending to rupture spontaneously; Eczema-small papulo-vesicles occurring, as a rule, in groups so closely aggregated as to form patches, tending to rupture spontaneously and weep, and associated with more or less intense itching; Herpes simplex-small groups of vesicles on a reddened base, with no marked tendency to rupture, situated most commonly about the lips ; Herpes zoster-groups of vesicles situated on inflamed skin, with a slight tendency to rupture, present in the area of distribution of some cord segment, generally unilateral, and associated with more or less severe neuralgic pain; Sudamina-small translucent vesicles, noninflammatory, due to accumulation of sweat and occurring in febrile disturbances. 
Bullous Eruptions.-Bullous syphilide-bullae in congenital syphilis, situated as a rule about the palms and soles ; Dermatitis herpetiformisoccasionally presents bullae associated with groups of papulo-vesicles and itching; Drug eruptions-bullae may be the result of poisoning from certain drugs, such as the iodides; Erythema multiforme-bullae occurring occasionally about the face, forearms, and backs of the hands ; Impetigo due to streptococci-bullae with clear contents at first, tending later to become sero-purulent, easily ruptured, the contents drying to form honey-like crusts, varying in size from a split-pea to large lesions several inches in diameter, seen most commonly in children, forming in infants the affection known as "Pemphigus neonatorum "; I.ocal irritants-may cause bullae, such as heat, cantharides, mustard plaster, poisonous plants, etc ; Pemphigusbullac apparently arising from healthy skin, irregularly distributed over the cutaneous surface, and often associated with a general disturbance Urticaria-occasionally goes on to the formation of bullae.

Pustular Eruptions.-Acne vulgaris-pustules associated with comedones ; Dermatitis - any form of dermatitis may become pustular secondarily from the jioculation of pyogenic micro-organisms; Drug eruptions - may be pustular in character, such as those due to bromides and iodides; Impetigo due to streptococci-superficial pustules occurring most commonly about the exposed parts in children, preceded by vesicles, tending to dry up and form honey-like stuck-on crusts; Pediculosis - frequently presents pustular lesions from the inoculation of pus-cocci by scratching; Pustular folliculitis, boils, and carbunclespustules occurring in connection with pilo-sebaceous follicles, due to staphylococci ; Ringworm-may occur as a pustular folliculitis about the scalp and beard, or as an aggregation of vesico-pustules on the glabrous skin; Scabies-pustules readily occur in association with scabies lesions.

Squamous Eruptions.- Scaliness almost invariably occurs in association with any acute inflammatory condition of the skin with oedema in the epidermis. Eczema-whitish scales on an inflamed base; Exfoliative dermatitis-universal desquamation of the skin in large wafer-like scales or sheets, leaving the skin vividly red, sometimes associated with loss of the hair and nails; Ichthyosis-a non-inflammatory condition appearing generally during the first year, varying greatly in severity from a fine scaliness situated chiefly on the extensor aspects of the limbs and known as "xerodernia," to coarse scales universally distributcd over the cutaneous surface like fish scales or serpent scales; Lupus erythematosus-pinkish patches, situated chiefly on the face, with adherent scales which, when raised up, show horny plugs dipping into the follicles ; Parapsoriasis-an affection superficially resembling psoriasis, characterised by more or less scaly pinkish patches, papules, or plaques; Pityriasis rosea-pinkish macules or oval patches with a fawn-coloured centre and fine branny scales; Pityriasis rubra pilaris - widely distributed scaliness on an inflamed 
and thickened skin, associated with bright red papules capped by a greyish scale; Psoriasis-profuse superficial silvery scales, easily detached, situated on a bright red inflamed base ; Ringizorm-patches on the scalp covered with fine scales and dotted over with stumps; pinkish patches or rings on the glabrous skin covered with fine scales; Seborrhoic dermatitis-yellowish greasy scales or crusts, on a slightly inflamed base, present generally on the scalp, face, sternal and interscapular regions; Squamous syphilide-scales somewhat similar to those of psoriasis but not so white and silvery, situated on a brownish or deep red slightly raised indurated base, sometimes in the form of a collar around a papule ; Tinea imbricata-a form of ringworm in which the scales are arranged in an imbricated fashion like watered-silk.

Crusts.-Crusts result from the drying up of a sero-purulent discharge mixed with epidermal scales, and are found in connection with all vesicular, bullous, and ulcerative conditions, such as eczema, impetigo, Lupus vulgaris, and syphilis.

Warts.-In addition to the ordinary warts, warty growths may occur in association with various diseases, such as carcinoma, elephantiasis, gonorrhoea, Madura foot, septic granuloma, syphilis, and tuberculosis. They are supposed to be the result of microbic infection but may be produced also by the ingestion of certain drugs, such as the iodides and arsenic.

Tumours.--The most common tumours of the skin consist of the following: Carbuncle-indolent purulent swellings; Carcinoma cutiseither in the form of rodent ulcer or malignant epithelioma ; Fibromausually soft tumours, the colour of the normal skin, sometimes pendulous, sessile or pedunculated, or multiple hard tumours ; Furuncle or boila localised swelling tending to suppurate and necrose in the centre; Cheloids-fibrous swellings; Lipoma-softish tumours of adipose tissue; Mycosis fungoides-tumour masses which tend to ulcerate, preceded by persistent pruritus, eczematisation, and infiltration of the skin; Naei -in the form of large tumour masses, either vascular naevi or soft moles; Sarcoma-melanotic sarcoma either originating in a naevus, or resulting from metastasis of a pigmentary sarcoma ; Sarcomatosis of Kaposi-purplish or blackish tumours, about the size of a pea, situated on infiltrated skin ; Xanthoma-yellowish fatty tumours of firm consistence.

Cicatricial or Atrophic Conditions.-Cicatrices follow the healing of the skin where there has been a complete destruction of the epidermis and the papillary layer of the corium by injury or ulceration, as in such conditions as Acne necrotica, leprosy, tertiary syphilides, tuberculosis, etc. In connection with Lupus erythematosus there is a peculiar superficial scarring, while after a tertiary syphilide has healed an atrophic scar is left which has been aptly compared to crinkled tissue-paper. Where the destruction is deeper, punched-out or pitted scars may remain like those of smallpox.

Pigmentation.-Acanthosis nigricans-warty pigmented patches, 
chiefly in the axillae and groins; Addison's Disease-diffuse pigmentation, ranging from bright yellow to dark brown, affecting chiefly exposed parts and parts where normal pigmentation is deepest, associated with asthenia and gastro-intestinal symptoms; Cayennepepper pigmentation - seen in connection with vascular stasis about the legs; Chloasma-pigmentation occurring in association with certain general disturbances and uterine disorders; Freckles-brown pigmented macules situated generally on the exposed parts; Lichen planus_pigmented macules or patches which persist after the lesions have involuted; Melanodermia - brownish irregular patches occurring in association with areas where the pigment is absent; Pediculosisin connection with Pediculosis corporis marked pigmentation frequently occurs on the back and shoulders; Pigmentation of haemorrhagic origin - as in bruises, purpura, haematomata, etc., in which the pigmentation of the lesions changes from purple to yellow and green; Pigmented retiform mottling of the legs - the result of continuous exposure to fire heat; Sunburn-profuse pigmentation due to the actinic rays of the sun; Syphilis-pigmentation following the healing of syphilitic ulceration, and brownish pigmentation of the neck with whitish spots; Urticaria pigmentosa-yellowish, violet, or brownish lesions suggesting xanthoma ; Varicose ulcers-ulceration of the legs and ankles associated with deep pigmentation; Xeroderma pigmentosum-freckles on the face associated with telangiectases and a tendency to warty and epitheliomatous growths.

\section{Regional Localisation as an Aid to Diagnosis}

There are certain eruptions which have a general or universal distribution, such as the secondary eruptions of syphilis and general exfoliative dermatitis; others are usually confined to definite regions, such as rosacea to the face, Erythema multiforme to the backs of the hands, arms, and face, and Pityriasis rosea to the trunk and upper parts of the arms and legs. It will be of assistance in diagnosis to refer briefly to the various lesions or eruptions which are most commonly met with in the different regions of the body.

\section{HEAD AND NECK}

Face.-The most common skin affections which occur on the face are the following: Acne vulgaris with its comedones and pustules, localised chiefly to the peripheral portion of the face, namely the forehead, cheeks, and chin, especially common between the ages of fourteen and twenty-one; Eczema, which is specially common on the face in infants and young children but may occur at all ages, tends to assume a more or less acute erythematous type associated with oedema; Erysipelas with its rose-tinted patches and well-defined borders; 
Erythema multiforme exudativum with its erythematous nodules, vesicles, or bullae, sometimes associated with similar lesions in the mucous membrane of the mouth; Impetigo contagiosa with yellow stuck-on crusts, particularly common on the face in children ; Leprosy of the nodular type, tending to give rise to a peculiar leonine appearance, the result of the presence of irregular leprous thickenings about the eyebrows; Lupus crythematosus, most commonly present somewhere in the "bat's wing area," namely the malar prominences and the bridge of the nose, presenting persistent red patches covered with adherent scales, tending to involute spontaneously and to be followed by superficial scarring; Lupus vulgaris, most commonly met with on the face, forming yellowish apple-jelly-like nodules ; Recurrent erysipeloid, like mild erysipelas but not associated with marked general disturbance; Ringworm, either in the form of distinct rings or reddish scaly patches, chiefly occurring in children; Rodent ulcer, almost exclusively present in the face, recognised by its long duration, cartilaginous border, and superficial ulceration on a hard base, commonly situated in the middle third of the face in the area extending from the nose to the ear ; Rosacea, a permanent flushing of the face most common in adult females, chiefly confined to the central area of the face-the nose and malar regions; Scaly impetigo or pityriasis, indefinite, pinkish, scaly patches due to slightly virulent progenic cocci; Seborrhoea oleosa, a greasy condition of the skin often met with about puberty, especially marked at the sides of the nose and associated with the presence of greasy plugs in the pilo-sebaceous follicles; Syphilis, reddish-brown papules or pustules belonging to the secondary period and associated with similar lesions elsewhere, or isolated ulcerative tertiary lesions, brownish-red in tint, asymmetrical, tending to be serpiginous or to simulate Lupus vulgaris but more rapid in their course and appearing later.

Lips.-The lips are not infrequently attacked by : Eczema, when they become swollen, and cracked; Extra-genital chancres, Diffuse syphilides, or Clcerative tertiary syphilides; Herpes simplex; Lupus anlgaris, when they become thickened, ulcerated, or crusted. In rare instances they may be the site of a peculiar exfoliative condition in which they become covered by a thick dirty scale or crust which, on removal, leaves the red portion of the lip thin, broken, or oozing. The upper lip is liable to be affected by chronic septic dermatitis and pustular folliculitis secondary to a septic discharge from the nose. The commissures of the mouth are occasionally the sites of fissures and rhagades in connection with congenital syphilis, and of a chronic dermatitis which may extend all around the mouth, is associated with cracks due to infection with streptococci, and is known as perlèche.

Nose and Nostrils. - The nose is especially liable to be affected by lesions of Eczema, Lupus erythematosus, Rodent ulcer, and Rosacea. The inside of the nose is prone to Impetigo, either of staphylococcic 
origin when it produces a septic rhinitis associated with folliculitis of the vibrissae, small boils, and sycosis of the upper lip, or of streptococcic origin when it may be connected with erysipeloid attacks about the cheeks. Lupus vulgaris is frequent about the edges of the nostrils when they become inflamed, swollen, and crusted, and destructive changes may result. Syphilis in its later stages is apt to attack the nose and may produce destructive changes with the formation of "saddle-nose," and ulcerative lesions may occur in connection with congenital syphilis and may appear about puberty.

In addition there are two affections which are peculiar to the nose: Lupus pernio, or chilblain lupus in which the nose becomes red, purplish, or swollen; and Rhinophyma, in which the nose becomes hypertrophic and the skin coarse and dotted over with dilated pores like the skin of an orange, due to proliferation of the sebaceous glands and an increase of fibrous tissue.

Ears.-The auricles are frequently affected by cutaneous diseases. They are apt to be attacked, especially in children, by Impetigo often secondary to a suppurative external otitis and discharge, when they become inflamed, swollen, moist, and crusted. The posterior auricular fold is liable to be affected with Impetigo or Intertrigo, when it becomes moist and inflamed, or purulent and cracked. The auricles may be the sites of Chilblains, Eczema, Cheloids, Lupus erythematosus, Lupus vulgaris, nodules of Leprosy, and Rodent ulcer.

Forehead.-The forehead may be attacked by any of the lesions which occur on the face, such as Eczema. It is a common site for Acne pustules, comedones or blackheads, flat or juvenile warts, and secondary syphilides. In Pityriasis of the scalp there is often an extension on to the forehead of pinkish scaly patches with a festooned border, known as the Corona seborrhoica.

Eyelids.--The eyelid may be the site of Xanthoma in the form of yellowish or washleather-like patches, raised or appearing as if they were let into the skin. It niay also be affected by a chronic scaly inflammatory condition secondary to a septic blepharitis. Milium in the form of small white cystic papules are common about the eyelid, especially in elderly women. Rodent ulcer may occur in the eyelid.

Eyebrows. - The eyebrows may be affected with Pityriasis in the same manner as the scalp and become inflamed and scaly. They may be attacked by Alopecia or become bald as a result of Ichthyosis follicularis. They are a common situation for Leprosy in the form of irregular nodular masses from which the hairs disappear.

Moustache and Beard Regions.-In these regions it is necessary to distinguish between diseases which are definitely follicular and those which simply involve the cutaneous surface. The most common follicular diseases of the beard are known as the Sycoses of which there are two varieties, namely, one due to pus-cocci known as Coccogenic sycosis and in which pustules are irregularly distributed about the affected area, and another caused by ringworm fungi and known as 
Hyphomy'cetic sycosis which appears as angry, lumpy lesions dotted over with superficial pustules. The skin of these regions may be attacked by any of the diseases which commonly occur about the face such as Impetigo, Lupusaulgaris, Eczema, Pityriasis, Syphilis, Lupus erythematosus and a dry scaly type of Ringworm. In women these regions may be the site of excessive hair-growth or Hypertrichosis, and in men of Alopecia areata:

Neck. - The neck may be involved by most of the common cutaneous diseases with a wide distribution such as Eczema, Psoriasis, Pityriasis rosea, Secondary syphilides and the Toxic erythemata. The back of the neck is a frequent site in children for suppurating Impetigo and Folliculitis, associated with enlarged glands, secondary to pediculi in the scalp. In men it is a common situation for Boils which may be of a singularly indolent type, for Chronic eczema and Lichenified patches in which the skin is smooth, shiny and thickened from more or less incessant rubbing. It is the site of a peculiar Pigmented syphilide which occurs chiefly in women, in which there is a diffuse pigmentation broken up by white spots and patches, and is a not uncommon situation for Scrofulodermia secondary to breaking down tuberculous glands. The nape of the neck is o:casionally affected by a peculiar condition known as Acne cheloid in which raised inflamed masses occur, dotted over with pustules, and tufts of hair.

Scalp.-The affections of the scalp are numerous, some involving the skin and others chiefly the hair-follicles. The most important of these are the following : various dry scaly conditions such as Pityriasis capitis or dandruff, the more greasy inflamed affection described as Seborrhoic dermatitis, the thick greyish scaly patches of Psoriasis. In the infant a yellowish scaly crust is often present which persists for several months, and is composed of dried up fatty material and epidermal debris. Capillary naevi are common upon the occipital region extending down to the neck and have to be distinguished from an inflammatory disturbance. In children the scalp is a common situation for Impetigo, Eczema, Boils, Pediculosis, and Ringirorm either in the form of irregularly distributed patches dotted over with stumps about quarter of an inch in length or, more rarely, of small finger-nail sized patches with short stumps that look like comedones and known as "black-dot ringworm," or of raised suppurating swellings or kerions. It is a common situation for Faun, which is easily recognised by its sulphur-like cupped crusts. It may be the site of various kinds of Syphilitic lesions such as papules, papulo-pustules of the secondary period, or tertiary superficial gummata. Bald patches are often present on the scalp and are most frequently the result of the disease known as Alopecia areata in which the patches are completely bald and shiny. Temporary bald patches may also follow some acute inflammatory process, such as an abscess. Permanent bald patches may be caused by injury or by any scarleaving condition, and occur also in the rare affection known as Psendo-pelade in the form of irregular atrophic patches on which a few 
isolated hairs may be detected, and in connection with Lupus erythematosus in which the patches are red, atrophic, and have a cribriform appearance from the presence of dilated atrophic follicles. A partial or complete defluvium of the hair may take place in association with syphilis, any severe or prolonged illness, the exanthemata, and occasionally with generalised exfoliative dermatitis.

\section{TRUNK}

The following cutaneous affections are localised chiefly on the trunk: Acnevulgaris, chiefly present about the shoulders and back ; Dermatitis herpetiformis, with its groups of papulo-vesicles and intense itching; Eczema ; Erythematous and urticarial enuptions, consisting of erythematous blotches, wheals, or lesions simulating scarlet fever or measles; Exfoliative dermatitis; Herpes zoster; Lichen planus; Lichen scrofulosontm; Melanodermia and Vitiligo; Pemphigus; Pityriasis rosea, with its pinkish slightly scaly macules or oval lesions with a fawn-coloured centre; Pityriasis rubra pilaris; Pityriasis versicolor; Psoriasis; the roseola of Syphilis; Seborrhoic dermatitis, situated in the interscapular and pre-sternal regions; and, occasionally, the peculiar hard and glossy affection of the skin called Sclerodermia.

Axillae.-Owing to the heat and moisture in this region, and the friction to which it is subjected, it is particularly prone to skin affections and forms a suitable habitat and soil for certain parasitic and microbic affections of the skin. It is a not uncommon site for Eczema, Intertrigo, Impetigo, Suppurative folliculitis, and even deep-seated Boils and Abscesses. Occasionally in nervous women it may be the seat of a localised Pruritus which induces more or less incessant rubbing and is apt to lead to the production of lichenified patches. It forms a suitable locality for the acarus of Scabies and occasionally for Pediculi. It may be attacked by body Ringworm especially of the eczematoid type and is a favourite position for Erythrasma. The sweat-glands in the axillae are usually large and active and the sweat collecting there is apt to be acted upon by certain microbes which render it offensive and foetid. Occasionally a profuse sweating is localised in one or both axillae and in rare instances the sweat may be altered in colour and the hairs gritty owing to the action of pigment-producing cocci.

Breast and Nipples. - Especially in women these are the sites of a number of cutaneous affections, such as Eczema; Impetigo; Paget's Disease of the Nipple, which begins as a chronic dermatitis and goes on to epithelioma; Scabies; Syphilitic chancres. Where the breasts are large and pendulous, Eczema, Intertrigo, and occasionally Ringworm, are liable to occur beneath them.

Umbilicus.-The umbilicus may be the site of lesions of Eczema, Erysipelas, Impetigo, Psoriasis, and occasionally of Paget's Disease.

Sacral Region.-This is a frequent position for chronic patches of 
Eczema or Psoriasis and occasionally for congenital lesions such as Pigmented or hairy moles and Spina bifida.

Lower Part of the Back and Flanks. - This area is sometimes affected by Lichen scrofulosorum. The napkin region in infants is the site of various forms of dermatitis, erythematous, papular, resicular, and superficially ulcerative. The buttocks in children and adults may be affected with Eczema, Furunculosis, Lupus iulgaris, Papulo-necrotic tuberculides, Psoriasis, and Syphilides, both papular and pustular lesions of the secondary period and late ulcerative serpiginous lesions.

Groins.-Tarious affections are commonly met with in the groin:. In infants it is a common situation for Intertrigo and in the adult for an eczematous type of Ringworm in the form of red inflamed scaly patches with a festooned border which may be raised and vesicular. Ordinary Eczema and brownish patches of Erythrasma may also occur there.

\section{Genital Organs}

Penis.-The following lesions are met with not infrequently on the penis: Eczema associated with more or less oedema, Epithelioma, Lichen planus, Psoriasis, Scabies, Sebaceous cysts, Soft sores, Syphilitic chancres.

Scrotum.-The scrotum is a farourite site of Eczema with marked oedema, Epithelioma, Sebaceous cy'sts, mucous patches of Syphilis, and, in the tropics, of Elephantiasis.

Vulva.--The vulva may be attacked by Chancres, Oedenatous eczema, Pruritus due to various causes, and mucous patches of Syphilis.

Anus.-This region is a common situation for Pruritus, which may be associated with more or less marked changes in the skin around it, generally of an eczematous type. It may be affected also with Chancres. Interirigo when it is moist, inflamed, and occasionally presents cracks extending from the anus, warty lesions of Lupus i'ulgaris, mucous patches of Syphilis, Ienereal warts, and, in tropical countries, of a condition known as Granuloma pudendi in which warty masses occur around the anus and tend to extend up in the mucous membrane.

Pubes.-The pubes is the site of a special type of Pediculosis and is a not infrequent habitat for the acarus of Scabies.

\section{LPPER Extremities}

Arms. - These may be the site of the multiform lesions of Erythema exudativum, Acute dermatitis from handling local irritants, and occasionally Tuberculous lvmphangitis in which inflamed cords appear beneath the skin with nodular tubercular swellings which tend to break down and ulcerate. The wrists are a common site for Lichen plunus. The 
extensor aspects especially about the elborrs are classical sites for Psoriasis, while Eczema and Secondary syphilides are usually more profuse on the flexor aspects.

Hands.-The hands being exposed to all manner of local irritants are extremely liable to dermatitis. Between the fingers is a specially common situation for the itchy papules and burrows of Scabies. Eczema frequently affects the hands and when it involves the palms is liable to lead to marked hyperkeratosis and the formation of fissures. There is also a common affection of the hands allied to eczema known as Dysidrosis in which small vesicles appear, chiefly between the fingers, which tend to dry up rather than to break and are associated with marked burning and itching and occasionally with large bullous lesions. Impetigo and Septic sores are frequent in the hand and may appear as pustules, superficial boils, vesicles or bullae, whitlows, warty or granulomatous lesions, and the various forms of tropical boils and sores. The hands are subject also to circulatory disturbances, such as Chilblains, Raynaud's Disease, and occasionally to a curious affection known as Sclerodactylia, in which the skin becomes hard, thickened, and waxy, leading to atrophy and destructive changes. Hyperkeratosis of the palms may occur in eczema as a congenital anomaly or from the elimination of arsenic by the sweat. In rare instances Psoriasis may be met with in the palms when it takes the form of small punctate scaly lesions or horny discs. Ringworm may attack the hands, either in the form of raised scaly patches, vesico-pustular lesions, or indolent exfoliating patches on the palms or between the fingers and due to the same fungus which causes ringworm of the crutch. The palms are a not infrequent site for late Syphilides in the form of brownish patches, sometimes presenting a slightly raised serpiginous border, usually covered with adherent scales which may exfoliate in the centre leaving a free edge towards the margin; as a rule only one hand is involved. Tuberculosis may affect the hands and take the form of scrofulodermia associated with tuberculous disease of the bones or joints, or of warty patches known as "Tuberculosis verrucosa cutis" of which the post-mortem wart is a variety. In connection with tuberculosis small papulo-necrotic lesions (Folliclis) may occur about the hands and are believed to be Tuberculides, but occur also in association with a weak peripheral circulation where no definite evidence of tuberculosis can be detected.

Nails.-The nails are subject to a number of diseases which will be described under a separate heading.

\section{LOWER ExTREMITIES}

Thighs.-There are no diseases which are peculiar to the thighs, but they may be attacked by all the ordinary dermatoses with an extensive distribution, such as Eczema, Psoriasis, Pityriasis rosea, etc. The 
extensor aspects are sometimes affected by an exaggerated and permanent condition like gooseflesh known as Keratosis pilaris.

Knees.-The knees are common situations for Ichthyosis, Nanthoma and Psoriasis.

Popliteal Spaces. - These are usual sites for Eczema.

Legs.-The legs are subject to various forms of dermatitis and ulceration especially when the resistance of the skin is lowered from oedema or the presence of varicose veins. In young women an affection known as Erythema induratum is occasionally met with, especially on the calves, in which deep-seated nodules or small tumours occur which gradually necrose or ulcerate in an indolent fashion and leave a pigmented punched-out cicatrix. Erythema nodosum is also found in the legs, chiefly on the shins, in the form of painful reddish swellings varying in size up to a pigeon's egg and generally associated with rheumatic pains. The legs may also be affected with lesions of Erythema multiforme, Purpura, Lichen planus, Syphilitic gummata, Tuberculous lesions of a warty type and in tropical countries with Elephantiasis.

Feet.-The feet may be attacked by the common diseases of the skin, such as Eczema, Psoriasis, Lichen planus, and various forms of dermatitis due to local irritation. When the sweat is excessive, it becomes decomposed and offensive, macerates the skin, and leads to a sodden cracked condition, especially between the toes.

Sole.-Owing to the thickened epidermis the sole of the foot occasionally presents large septic bullae due to streptococci. It may be the site of late Syphilitic lesions in which brownish patches occur, sometimes with a serpiginous border and associated with thick scales; or of $H_{y}$ perkeratosis which may be a congenital affection, or may be associated with various diseases such as Lichen planus and Eczema, or may be due to the elimination by the sweat of toxins such as arsenic, or may even occur in association with excessive sweating alone. An eczematous form of Ringurorm occasionally affects the feet and takes the form of exfoliating lesions, the result of the breaking of large imperfect vesicles, and is found in the soles, between the toes and occasionally spreading up as red marginated patches on the dorsum of the foot. Verruca plantaris is a painful type of wart which occurs on the sole of the foot. 


\section{CHAPTER VI}

\section{GENERAL PRINCIPLES OF TREATMENT OF CUTANEOUS DISEASES}

\section{INTRODUCTION}

THE treatment of diseases of the skin is regulated by the same general principles as are applicable to that of any organ. To be successful it must be based on a knowledge of the etiology and pathogenesis of the affection to be treated and of the therapeutic action of the different remedies both local and general which are employed. There is an old belief, which still prevails among the laity, that the curing of a skin affection drives the diseased process into the body with evil consequences. This is now recognised to be a fallacy, and should internal disturbances occur in association with the disappearance of an eruption it is known to be simply a coincidence.

For the proper treatment of a skin affection a correct diagnosis is of the first importance. It is not sufficient, however, to be able to put a name to the disease but it is necessary to appreciate its causation and the pathological changes which underlie the clinical appearances. In this way alone can treatment become rational and the long lists of empirical remedies associated with the name of the disease discarded. It is common knowledge that the more intractable a disease and the less that is known about its etiology, the longer the list of empirical remedies for it. It may happen even to the experienced, however, that a firm diagnosis is impossible, in which case the physician is thrown back on the clinical appearances and the pathological changes as guides to the proper line of treatment.

There was a time when French writers on dermatology were unanimous in believing the majority of skin affections to be symptomatic of impure states of the blood or of vague and ill-defined diatheses such as the "arthritic, strumous and lymphatic," and in consequenice their treatment was mainly constitutional. As a protest against this view Hebra and his school want to the other extreme and regarded them as due mainly to local causes and curable by external remedies alone. At the present time a middle course is generally adopted in which both local and general remedies play their part. While realising 
the importance of external treatment it is recognised that after all the skin is not an organ by itself but part of a body all the organs of which are more or less physiologically interdependent, and that even in mild forms of skin disease resulting from local causes the treatment may be impaired and the progress towards recovery retarded by the circulation of toxins in the blood or by interference with the nutrition of the skin by some general disturbance of health.

A certain number of skin diseases are purely local, independent of the general health, and curable by local treatment, such as certain parasitic affections and new-growths, and various lesions due to local irritation. Others are the cutaneous evidence of some constitutional disease, such as the pigmentation in Addison's disease or the nodules in leucaemia, when the general disease alone demands treatment. Others, such as the toxic erythemata and urticaria, are due to the absorption of toxins, chiefly from the alimentary tract, acting directly on the skin, or indirectly through the nervous system, and their treatment consists in the elimination of the toxins. Others, such as boils and carbuncles which occur in association with glycosuria, are dependent on local causes in an individual predisposed by defective nutrition or a weakened state of resistance of the cutaneous surface, and their treatment is partly local and partly constitutional.

After making the diagnosis, in many cases it is of equal importance for the physician to study the patient behind the disease, to ascertain the state of general health, and to examine thoroughly the various systems of the body, especially the gastro-intestinal and urinary systems, so as to detect any defects which might be connected with the cutaneous affection. Careful consideration should be paid to the patient's environment, as an alteration in it may sometimes be of greater value than the prescription of a medicinal regime.

The general principles and methods of treatment of cutaneous disease will be discussed under the headings of General Management, Internal Treatment, and Local Treatment.

\section{GENERAL MANAGEMENT}

The general management of cutaneous affections both acute and chronic may be of as great importance as special local and internal treatment and includes such considerations as hygienic conditions, employment, rest, nursing, clothing, climate, and diet.

\section{Hygienic Conditions}

Everything which tends to influence the general health has an indirect bearing on the skin disease.

A large number of skin affections are associated with overcrowding, and defective and imperfect sanitary conditions, and are to be found in 
their most acute forms among the poor. Local parasitic conditions such as ringworm, scabies and pediculosis, are much more common in hospital than in private practice; Lupus vulgaris and other types of tuberculosis of the skin, though not infrequent among the poor, are rare in the well-to-do. To obtain the best results from treatment it is of the first importance that the patient should be placed in as healthy surroundings as possible. In the case of children of the hospital class it may be impossible to obtain a cure so long as they are at home, neglected, dirty, and improperly or insufficiently fed, while improvement is almost immediate when they are transferred to the skilled care, proper food, light, and ventilation of a hospital ward.

\section{Employment}

Certain cutaneous diseases are the direct or indirect result of the occupation of the patient, such as the numerous forms of trade dermatitis resulting from handling irritating substances, and the skin affections which are associated with prolonged exposure to inclement weather or with sedentary occupations and confinement to badly ventilated rooms. In such cases it may be impossible to cure the patient so long as the work is continued and even after a cure has been effected re-exposure to the irritant or a return to work in the old environment may determine a recurrence. A change of employment is essential, but not necessarily a cessation from work as idleness may lead to depression and neurosis which may delay recovery.

\section{REsT}

Rest is of far greater importance in the management of skin affections than is generally recognised, and not only physical rest but mental rest. In acute widely distributed forms of dermatitis, complete rest in bed is desirable and may be imperative. The skin is a complex organ with a variety of functions, it is the principal channel of heatloss, it controls the body temperature in virtue of an elaborate nervous mechanism, it is highly sensitive, and it eliminates various waste products. In any form of dermatitis these functions are more or less interfered with, and for the patient to exert himself under such circumstances, or to be unnecessarily exposed to marked variations of temperature, may still further impair them and retard recovery.

Of course absolute rest, in the surgical sense, is impossible and even in bed the rest is only comparative, as the skin is not only always active but it is always growing and its epidermal cells are constantly going through their evolution into horn-cells. It is remarkable, however, how extensive and chronic intractable cases improve with confinement to bed apart from other treatment either local or general. The strain on the skin's activity is lessened and much of the irritation from 
friction and compression tc which it is normally subjected is aroided. Hyperaemia is reduced, as the horizontal position lessens the strain on the heart and the tension in the cutaneous blood-ressels. The temperature of the room can be regulated and the bed kept cool by light nonirritating clothes, raised off the patient by a wicker cradle and ventilated by paper shafts at each side of the bed. But one of the most important considerations of all is the facility with which local remedies can then be applied.

There are also skin affections in which, although complete rest in bed is not imperative, local rest is indicated, such as the common leg ulcer. To attempt to cure a leg ulcer, associated with varicose reins, with the leg unsupported and the patient standing or walking about, is hopeless. The leg must be rested in an elevated position and supported so that the oedema may be diminished and the congestion reliered before the ulcer itself can be successfully treated.

Almost as important as physical rest is rest from worry, anxiety, and over-work, as the effect of the mental attitude of the patient on the healing of a generalised skin affection is no fetish, but a reality. Take, for example, the case of an active professional or city man with a widely disseminated attack of acute eczema. It may be necessary to lay him up at a time when he is full of cares and responsibilities, irritable from his disease, constantly imagining that everything will go wrong in his absence, and feeling all the time that in spite of his skin complaint he is sufficiently well to be at business; to tell such a patient not to worry is useless, it is necessary to help him not to do so by suggesting some mental occupation, allaying irritation, and arranging for suitable nursing.

Another form of rest which is by no means negligible, is rest from excessive treatment. It is a common error to over-treat cutaneous diseases and one which may be followed by serious results. Not a few cases of psoriasis have been changed by excessive and injudicious treatment into exfoliative dermatitis, and cases of scabies into various degrees of sulphur dermatitis.

\section{Nursing}

In all forms of acute extensive dermatitis skilled nursing is as essential as confinement to bed, in order that the local remedies may be properly applied. Dressings may have to be changed at regular intervals, baths to be given, and itching relieved by the timely application of anti-pruritic remedies. In chronic affections, a nurse or attendant may be necessary to apply remedies to parts which cannot be reached by the patient. 


\section{Chothing}

Where the dermatitis affects the covered parts of the body the clothes should be light but sufficiently warm as, when the skin is extensively affected, the heat-regulating mechanism is impaired and the patient is liable to feel cold and to suffer from sudden variations in temperature.

Constriction or friction by tight or badly fitting corsets, rough starched collars, braces, neckbands, garters, or anything which might chafe the skin, must be avoided. The pressure of the hat on the forehead in men may be a cause of trouble by impeding the circulation in the scalp and permitting perspiration to collect and irritate the skin.

The underclothing should be soft, smooth, and absorbent, an excellent material being old linen or lawn over which woollen garments may be worn for additional warmth. Silk, or a mixture of silk and wool, may be substituted, but pure wool should not be worn next the skin as it is rough and hairy and does not readily absorb moisture and, in consequence, is apt to irritate.

\section{Climate And Change of Air}

Climate has an indirect bearing on the management of skin affections through its influence on the general condition of the patient. Certain cutaneous disorders are common in hot countries and comparatively rare in temperate zones, such as prickly heat, while others, such as chilblains, Raynand's disease, and cyanotic conditions of the extremities, are met with chiefly in cold Northern climates. Sunlight with its heat and chemical rays, sudden changes of temperature, the rare dry atmosphere of high altitudes, the dampness of swampy districts, the fragrant air of the well-wooded country, the salt-laden winds from the sea, and the protected valleys among the hills, all have their influence direct or indirect in improving or aggravating cutaneous diseases. It is difficult, however, to formulate general rules on this subject as individuals vary in the manner in which they react to climatic conditions. It may be said that all acute, and most chronic, erythematous diseases affecting the exposed parts of the body are aggravated by the wind and glare of the seasicle; while chronic skin conditions on covered parts are not infrequently benefited by a change to the coast or a sea voyage. In over-worked people whose general health is good a change to a dry, inland, moderately elevated, locality is probably most suitable; in those who are weakly and nervous, shady valleys and wooded districts are most acceptable; while in elderly people a warm, dry climate is best.

The value of a change of air is not entirely due to the altered climate, but to a large extent to the influence of different surroundings, changed life, mental rest, freedom from business and worry, pleasant occupation, and healthy out-door exercise. 


\section{DiET}

The importance of diet as an etiological and aggravating factor in skin diseases is a question upon which there is much diversity of opinion; certain observers consider its influence to be negligible while others have so ridiculously exaggerated it as to discredit its real value. Schemes of dietary containing details of the exact quantity of nitrogenous and carbohydrate food suitable in an individual case, though they may impress the patient, are of comparatively little service, but there is no doubt that certain articles of diet are capable of pruducing cutaneous eruptions of an erythematous and urticarial type, and can aggravate existing eruptions.

Unsuitable or excessive food may act deleteriously on the skin in a number of war's:

(I) It may irritate directly the nucous membrane of the gastrointestinal tract and cause flushing, especially of the face, by a reflex nerve action.

(2) It may be absorbed into the blood and produce various forms of toxic erythemata and urticaria.

(3) It may be eliminated by the sweat-glands and irritate the shin in the process.

(4) It may interfere with the nutrition of the skin, weaken the resisting power to micro-organisms, and render it prone to various forms of dermatitis.

Certain diseases are absolutely unconnected with diet, such as local parasitic conditions, others may be caused by indigestible or toxic articles, such as urticaria, rosacea, erythema, and pruritus, and others though they cannot be produced by improper food can be aggravated by it, such as eczema and possibly psoriasis.

It is impossible to make a complete list of articles of diet which should be avoided, as certain individuals have an idiosyncrasy to articles generally innocuous, while others may eat with impunity almost any form of food.

The following are the most liable to be harmful : shell-fish, such as crabs, lobsters, mussels, clams, and oysters, especially in warm weather ; oily fish, such as mackerel, herrings, eels, salmon, and sardines ; tinned meat, meat pies, and high game, from the ptomaines they contain; indigestible meats, such as salt beef, veal, and pork; cheese, pastries, spices, curries, chutney, pepper, acid fruits, strawberries, almonds, parsley, salad dressings, starchy food in infants, and oatmeal porridge in those unaccustomed to it.

As a rule it is unwise to forbid an article of diet unless there is an obvious reason for so doing, and vexatious restrictions are to be deprecated. Sydenham used to teach that "more importance was to be attached to the desires and feelings of the patient, provided they were not excessive or dangerous," and a patient when he has reached years of discretion is as a rule a good judge of what suits himself. 
On the whole it may be said that a bland nutritious diet should be adhered to and anything which is liable directly or reflexly to cause dilatation of the cutaneous blood-vessels or irritate the cutaneous nerves should be avoided.

It is probable that indigestion from excessive or hurried eating and imperfect mastication, with its consequent anaemia, constipation, and derangements of the liver, is more important in the production of cutaneous disease than the actual constituents of the diet. In acute skin affections a fluid diet of milk and beef-tea is indicated, but in chronic conditions a sensible, full, and nutritious diet has no harmful consequences.

Condiments.-Strong coffee and tea, unless in moderation and freshly infused, should be avoided but to forbid the latter may be so irksome to the patient as to vitiate its object.

Smoking.--Smoking should be indulged in sparingly as tobacco is apt to cause flushing and itching, and any soothing effect it may have is transient.

Alcohol.-As a general rule in the treatment of skin affections the less alcohol the better, as it dilates the blood-vessels, increases the force of the heart's beat, warms the skin, causes flushing, intensifies itching, and in many ways aggravates the disease. There may be special circumstances, however, in which it is permissible, as in cases where the digestion is feeble, or the patient fatigued or elderly and habituated to its use. It may then be given with meals in the form of light wines, such as dry Moselle or good claret, or in moderate quantities of sound spirits in mineral water. It is well to remember that " moderate" is a relative term, and that doses of alcohol which are moderate for one person are excessive for others. Sweet wines, port, sherry, liqueurs, and beer, should be prohibited.

\section{INTERNAL TREATMENT}

There is no branch of medicine in which internal treatment has been more abused than in dermatology. This has been due to a variety of causes, such as the intractability of some of the more common cutaneous affections, the imperfect knowledge of their causation, the old superstition that in treating a skin affection it is advisable to prescribe something which will bring out the eruption, the difficulties of diagnosis, the tendency to prescribe mercury on the off-chance that a doubtful eruption may be syphilitic or, still worse, to fall back on that last resort and much abused remedy, arsenic. The internal treatment naturally divides itself into (I) constitutional treatment of the patient, (2) special treatment of the disease by drugs believed to have a specific action on the skin. 


\section{General Constitútional Treatment}

In the majority of skin diseases constitutional treatment on general medical principles is of greater importance than the internal administration of empirical specifics. This entails not only a thorough knowledge of the skin and its diseases but also of general medicine. The systems which are most likely to be deranged in connection with cutaneous disease are the gastro-intestinal tract and the urinary system as they are closely related to the skin as emunctories, but disturbances of the blood, circulatory, nervous, and sometimes of the genital systems may also occur.

Occasionally there may be no definite derangement of any particular system but the general health may be found to be below par, the bodily functions improperly executed, and the skin, in consequence, in a state of feeble nutrition and poor resistance. In such cases a general tonic regime is indicated, with suitable food, open air cxercise, and the employment of medicinal tonics such as strychnine, quinine, iron, cod-liver oil, phosphorus, the glycero-phosphates or the hypophosphites; or a resort to some spa whose waters are ferruginous, such as Strathpeffer.

In certain cutaneous affections associated with flushing of the skin, such as rosacea, the digestion is found to be inıpaired and a carefully regulated diet, acid or alkaline digestive tonics, carminatives or small quantities of pepsin or malt with each meal, may be helpful.

Constipation is a potent factor in aggravating inflammatory disturbances of the skin and in causing pruritus, and must be treated. As a preliminary to the treatment of any acute inflammatory affection a saline aperient, such as sulphate of soda or magnesia or a teaspoonful of Carlsbad salts in a tumbler of hot water half an hour before breakfast. is of great service. It relieves the cutaneous congestion, and reduces the general irritation by causing an increased transudation of fluid from the blood-ressels and emptying the bowel. Where the constipation is more or less habitual, it should be combated by exercise or massage, and a carefully regulated diet with green vegetables, fruit, articles which leave a bulky residue such as porridge and brown bread, and sufficient liquid between meals. The continued use of saline aperients in chronic constipation is apt to be depressing and regetable laxatives should be substituted. Of these the most useful are senna, an infusion of 6 to Io pods in 3 ozs. of water taken at bedtime or stewed with a few prunes to make it more palatable, the liquid extract of cascara sagrada half to one teaspoonful with the addition of a few drops of tincture of nux romica, aloin $\frac{1}{4}$ to I grain, a teaspoonful of the compound liquorice powder, and Tamar Indien. Where the constipation is associated with marked anaemia a tablespoonful of the compound iron and sulphate of magnesia mixture is indicated, and where the liver is deranged small doses of calomel, gr. $\frac{1}{10}$ to $\frac{1}{3}$ repeated every hour and followed by a saline aperient in the morning, or podophyllin or euonymin. 
Among the newer remedies for constipation may be mentioned phenolphthalein which is a mild and harmless purgative and may be given in doses I to 3 grains in powder or capsules; sour milk; liquid paraffin ; and agar-agar, ground into small pieces and a tablespoonful taken in milk or cream in the morning and evening. The agar acts by simply adding to the bulk of the faeces, keeping them moist and aiding peristalsis. A useful preparation of agar-agar combined with liquid extract of cascara is known as Regulin and is given in doses of i) i to jss once a day with food.

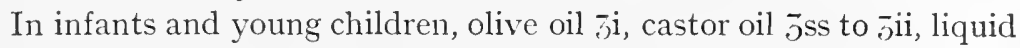
extract of cascara sagrada m. $v$ to $\bar{s}$ ss, and sulphur half a grain at nights, are useful; should these fail relief may be obtained-especially in nervous children-by ${ }_{4}^{1} \mathrm{gr}$. of belladonna given every two hours till the pupils become dilated.

Auto-Intoxication.-A number of skin affections, such as the toxic erythemata and urticaria, are due to food poisons or to auto-intoxication by the absorption of toxins resulting from fermentative processes occurring in the large intestines. In such cases repeated small doses of calomel, or a blue pill at night followed by a dose of Carlsbad salts or a Seidlitz powder in the morning, may suffice to get rid of the poison, or it may be necessary to resort to other intestinal antiseptics, such as charcoal, lactic acid, salol, or small doses of carbolic acid. In some resistant cases benefit may be derived from sour milk properly prepared from the Bulgarian bacillus, which may be made more palatable by sprinkling it with sugar or mixing it with calves' foot jelly.

Liver disorders may be responsible for certain skin affections, such as persistent pruritus, toxic erythemata, and even purpura. In such cases the direct cholagogues such as podophyllin, rhubarb, and jalap, or the indirect cholagogues such as the mercurials may be prescribed; but of equal importance is the proper regulation of the diet, out-of-door exercise, a visit to some suitable spa, and the avoidance of alcohol.

Blood derangements, such as anaemia which is often met with in connection with Acne vulgaris, etc., have to be combated by suitable dietetic, hygienic, and medicinal means ; while circulatory disturbances such as chilblains, acne, cyanosis, defective nails, and Raynaud's disease, may be improved by the judicious employment of cardiac stimulants, such as digitalis, strophanthus, ammonia, and iron.

Kidney disease.-There is a close relation between the functions of the kidneys and the skin, and when the skin is deranged an extra strain is put on the kidneys, and vice versa. In states of hyperaemia, diuretics may be of considerable benefit, either cardio-vascular such as digitalis and squills, or local diuretics such as the nitrites, spirit of nitrous ether, and the acetate or bicarbonate of potash.

Gout and Rheumatism.--The influence of gout and rheumatism as etiological factors in skin disease, though sometimes undoubted, has been greatly exaggerated. 
The so-called "gouty diathesis" may be associated with a dry scaly" condition about the extensor aspects of the limbs and the back of the neck, or with vascular derangements, such as purpura, from arteriosclerotic changes. In such cases the usual remedies should be resorted to, such as alkalies, colchicum, or a visit to a suitable mineral spa.

A certain number of cutaneous affections if not directly caused by rheumatism are generally accompanied by arthritic pains, such as Erythema nodosum and purpura. In them it is the rheumatism which demands treatment, by salicylates, salicin, alkalies, and other suitable means.

Diabetes may be responsible for a type of xanthoma and is frequently associated with pruritus, a lowered resistance of the skin to progenic micro-organisms, and a tendency to boils and carbuncles. In cases of this kind all starch and saccharine substances should be removed from the diet, and opium, codeia, or other suitable remedies, prescribed.

Worms.-In children the presence of thread-worms or roundworms is a frequent cause of pruritus. Thread-worms can best be got rid of by copious irrigation with an infusion of quassia or salt solution every day for a week; while in the case of round-worms a powder containing santonin I grain and pulv. scammonii co. 2 grains should be given at night followed by a saline aperient in the morning.

\section{SPECIAL INTtERAAL TREATMENT}

Under this heading will be considered a series of drugs which are known, or believed, to have a selective action on skin diseases. such as antimony, arsenic, ichthyol, the iodides, mercury, quinine, salicin, sulphur, thiosinamin, reast, analgesics and hypnotics, animal extracts or hormones, certain anti-bacterial sera and vaccires, and saline injections.

These have given satisfactory results in suitable cases but if used in a haphazard fashion may lead to disappointment; they are apt to be associated with the names of the diseases in which they are applicable, whereas in reality they are only useful in special phases of these diseases, and the beneficial effects are modified by individual peculiarities.

Antimony.-Antimony is employed in acute and sub-acute affections of the skin associated with hyperaemia or tension, and is administered in doses of 3 to $10 \mathrm{~m}$. of the rinum. It has a depressant action on the general circulation, lowering the tension in the cutaneous blood-ressels and allaying itching. Like arsenic it is apt to irritate the gastrointestinal mucosa, and the depression caused by it necessitates care in its employment, especially in debilitated and elderly patients with feeble digestions.

Arsenic.-Of all the internal remedies employed in connection with skin diseases, the most used and too often the most abused has been 
arsenic, and unless judiciously administered in properly selected cases it is capable of doing grievous harm. In addition to the acute exfoliative dermatitis which may result from an over-dose or from an idiosyncrasy towards the drug, its long-continued administration in small doses may cause a chronic dermatitis, with pigmentation of the skin, hyperkeratosis of the palms and soles - which has been known to become epitheliomatous-and various toxic disturbances, such as abdominal pains, vomiting, and diarrhoea.

Its manner of action is incompletely understood. It appears to be carried to the basal layer of the epidermis as a deposit of the metal and to pass gradually to the surface without becoming fixed in the tissue as an albuminate. The pigmentation associated with it, is not due to the deposit but to true pigment or melanin. Certain observers believe that its action is purely dynamic and of a respiratory character due to alternate oxidation and de-oxidation of arsenious to arsenic acid at the expense of the protoplasm of the cells, and that this has a stimulating effect on the epidermis improving cornification, and a sedative effect on the corium reducing inflammation. Others consider that its beneficial action is solely dependent on its tonic effect on the nervous system and cite as evidence in support of this view its value in small doses in urticaria and in certain vesico-bullous affections, and its tendency in large doses to cause vaso-motor paralysis with flushing and oedema.

The disease in which it is most extensively prescribed is psoriasis, for which it was at one time regarded as a specific. It has been employed also in chronic eczema, Lichen planus, urticaria, pemphigus, and Dermatitis herpetiformis, and more recently in the form of its organic preparations in syphilis. Apart from syphilis, it is the chronic scaly affections of the skin which are most benefited by its use, but its action is always uncertain and even in those cases in which definite improvement takes place it is probably as much the result of the influence of the drug on the blood and nervous system as of any specific effect on the disease.

It must never be prescribed when an eruption is coming out or in acute inflammatory affections, as it increases the pruritus and may aggravate the dermatitis. In any case it should only be given in small doses well diluted in water after meals, and should be avoided where there is the slightest gastro-intestinal irritability. To be beneficial it generally requires to be given over a period of several months.

The chief methods of prescribing it are as follows:

Fowler's Solution.-It is customary in this country to prescribe it in the form of the Liquor arsenicalis or Fowler's Solution, which is given in doses of $3 \mathrm{~m}$. t.d.s., cautiously increased to the limit of toleration. It may also be given by hypodermic injection, but in that case it is better to employ the Liquor sodii arseniatis, $3 \mathrm{~m}$. injected daily, as the latter is a more stable and less irritating composition and can be sterilised by boiling.

Asiatic Pill.-On the Continent the well-known Asiatic pill, containing $\frac{1}{2}$-grain of arsenious acid and I grain of black pepper, is still extensively used. 
Cacodylic Acid.-Is an organic combination of arsenic and methỵl radicals and is given in doses of $\mathbf{I}$ grain t.d.s. The sodium salt, Cacodylate of soda, has been extensively employed in France in doses of $\mathrm{I}$ to 3 grains by the mouth, or one-fifth of a grain by hypodermic injection; these doses are apparently large, as I grain of cacodylate of soda contains as much arsenic as I dram of Liquor arsenicalis, but it has been shown by Fraser that this salt when taken into the body is not dissociated but passes through without exerting either its toxic or its therapeutic action to the full extent. It has the great disadrantage of imparting a garlic odour to the breath

Arylarsonate Group, which consists of atoxyl or soamin, arsacetin, and orsudan, was at one time much in favour but has fallen into disuse recently as the results are no more certain than from arsenic prescribed in other forms. These drugs are given hypodermically in doses of 2 to 5 grains, dissolved in warm water and injected twice a week. They should be employed with the greatest caution, as large doses are apt to produce serious toxic symptoms, such as dizziness, headache, deafness, dry cough, retention of urine, fever, and even blindness from optic atrophy. Arsacetin and orsudan have the advantage over atoxyl of being more stable and capable of being boiled without decomposition, while in the case of atoxyl a fresh solution has to be made for each injection.

Salvarsan is the commercial name for Dioxy-Diamido-Arseno-Benzol, or the 606 of Ehrlich-Hata. This drug has a specific action on syphilis and yaws and has given brilliant results. It is a comparatively mild preparation of arsenic, but is prescribed in large doses or 0.3 to 0.6 grams according to the body-weight (I c.gr. per kilo) by intravenous injection. Its mode of administration and its substitutes will be discussed in connection with syphilis.

Ichthyol.-Ichthyol is a preparation containing sulphur which has been employed internally in urticaria, rosacea, and occasionally in extensive cases of Lupus erythematosus, as it is reputed to be capable of reducing vaso-motor excitability and hyperaemia. In certain cases of chronic urticaria it may be of benefit, but in the other conditions its value is doubtful. It is a distillation product of bitumen with sulphuric acid and is prescribed in tablets or mixed with glycerine in capsules, the dose being 2 to $5 \mathrm{~m}$., t.d.s.

Its substitutes, such as ichthalbin and ichthoform, have the advantage of being odourless and tasteless, but they are less effective and more expensive. Another substitute is thiol which can be obtained in a liquid or a solid form and may be given in pills containing $I \frac{1}{2}$ grains ; it has a somewhat bitter taste and an aromatic smell.

Iodine and the Iodides.-Iodine and the iodides have an alterative effect on the skin causing a disintegration and absorption of certain granulomatous lesions, such as those of syphilis, actinomycosis, and yaws. Occasionally individuals are met with who have an idiosyncrasy towards these drugs and in whom they produce various toxic eruptions of an erythematous, urticarial, papular, acneiform, or bullous type.

Iodide of potassium is the form in which the iodides are most commonly prescribed, but it has a markedly depressant action.

Iodide of sodium may be substituted for the potassium salt, it has a less powerful effect but is more lasting. The two may be combined with 
advantage with iodide of ammonia, which is less depressant, as in the following mixture :

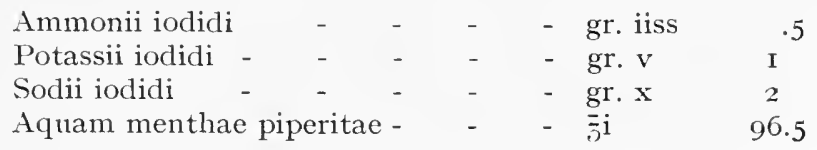

Iodide of calcium, 2 grains t.d.s., has been advocated for the treatment of ulcerating chilblains and chronic ulcers of the leg, but its value is uncertain.

In place of the iodides, certain preparations have been introduced which, while equally effective, are claimed to be less depressing, such as iodipin, iodoglidine, and collosol iodine.

Iodipin is a product of iodine and sesame oil and is made in two strengths containing Io or $25 \%$ of the element. A teaspoonful of the ro $\%$ solution corresponds to $7 \frac{1}{2}$ grains of iodide of potassium, and a teaspoonful of the $25 \%$ solution to r 8 grains. The ro \% solution is usually employed and may be given by the mouth in a little milk, or by intra-muscular injection. It can also be obtained in sugar-coated tablets containing the iodine equivalent of I grain of the potassium salt.

Iodoglidine is a preparation from vegetable albumin and is sold in tablets containing the iodine equivalent of I grain of iodide of potassium.

Collosol Iodine (Crookes). In it the iodine is in a state of fine subdivision and is readily absorbed. It is given in doses of one to four teaspoonfuls after meals.

Mercury.--The value of mercury as a specific in the treatment of syphilis has long been known and recent experiments on its effect on the Spirochaete pallida have corroborated scientifically its therapeutic value. It has not been proved to have a specific action in any other cutaneous disease.

Its methods of administration and dosage will be considered under syphilis.

Quinine-Quinine is of special value in the treatment of skin affections associated with malaria or with a depressed state of the nervous system. Good results have also been obtained from its employment in Lupus erythematosus, its effect being increased by painting the patches with tincture of iodine. It should be prescribed in doses of about 5 grains every four hours.

Salicin and the Salicylates.-Salicin and the salicylates have been strongly recommended as substitutes for arsenic in the treatment of certain chronic skin affections, such as psoriasis and Lupus erythematosus, and of various skin conditions associated with rheumatism, such as purpura and Erythema nodosum. To obtain satisfactory results salicin must be prescribed in comparatively large doses, not less than I 5 grains t.d.s., but this entails carcful supervision as in certain individuals who have an idiosyncrasy towards the drug much smaller doses are capable of giving rise to toxic symptoms and causing the appearance of a scarlatiniform rash.

Sulphur.-Sulphur has long enjoyed a reputation as a specific for certain pyogenic discases of the skin, such as furunculosis and pustular Acne vulgaris. It is believed to have a germicidal action from being eliminated by the slin in the form of a sulphide. 
In furunculosis the favourite salt is the sulphide of calcium which should be freshly prepared and prescribed in doses of one-tenth to half a grain in sugar of milk every three hours.

Thiosinamin.-Thiosinamin has been employed to reduce scar tissue, cheloids, and sclerodermia, but the results from it are uncertain. It is given hypodermically in alcoholic solution, and the injection is painful.

To obviate the pain a compound known as "fibrolysin" has been introduced; it is a combination of thiosinamin and sodium salicylate, is soluble in water, and non-irritating when injected; it is obtained in sterilised bulbs, and the dose is 5 grs. daily till improvement occurs and then at gradually increasing intervals. It is customary to inject it locally into the cheloid or the subcutaneous tissue around it, but some writers consider it unnecessary to inject at the site of the lesion and advise the gluteal muscles. It occasionally causes toxic symptoms, such as vomiting, collapse, headache, and ferer.

Yeast.-Yeast and its constituents, owing to their power of producing fermentation and leucocytosis, have gained a reputation in the treatment of certain bacterial infections of the skin, such as furunculosis and Acne vulgaris. Brewer's yeast itself is difficult to obtain fresh and pure and is unpleasant to take on account of its taste, smell, instability, and tendency to cause gastro-intestinal disturbancesespecially with carbohydrate foods. It is usually given in doses of from 2 to 3 tablespoonfuls in the day. The amount of leucocytosis it produces is slight and transitory, hence to be of real benefit it is necessary for it to be taken for several weeks continuously.

The principal constituents of yeast are nuclein and ceredin and one or other of these may be substituted with advantage for the fresh yeast as they are not so liable to cause gastric derangements and flatulence.

Nuclein is the chief nitrogenous constituent of yeast, is apparentiy identical with the proteid constituent of the nucleus, and is said to cause a mild leucocytosis, chiefly of the polymorphonuclear leucocytes, and to have a slight bactericidal action. Its chief principle is nucleinic acid, of which it contains $5 \%$. It may be obtained in solution for intra-muscular injection, the dose being Io to 60 minims with equal parts of sterile physiological salt solution, or it may be given in capsules, which contain 2 grs. of nucleinic acid, t.d.s. between meals and at bed-time.

Ceredin is a fatty constituent of yeast and has a stimulating action on the alimentary canal causing no unpleasant symptoms. It is given in pills containing $\mathrm{I}_{\frac{1}{2}}$ grs. twice daily, and acts as a mild laxative.

Other derivatives of yeast, such as zymin, furunculin, and levurin, have also been advocated, but they do not seem to have any therapeutic advantage over nuclein.

Analgesics and Hypnotics.--Analgesics and hypnotics have a comparatively limited field of utility in dermatology, though their judicious employment is occasionally of distinct value. The most important of them are the bromides, chloral hydrate, antipyrin, chloretone, opium and its alkaloidal derivatives. These are sometimes employed in 
cases of extreme cutaneous irritability and pruritus which is so severe and continuous as to interfere with sleep.

Insomnia in association with itching, not only lowers the patient's resistance, but results in scratching and an aggravation of the cutaneous condition. In such cases a dose of bromide of potassium, 30 grains in half a tumbler of water after going to bed, trional 20 grains in powder followed by a tumbler of hot milk, or chloretone 5 grains half an hour before bed-time and repeated every ten or fifteen minutes till four doses have been taken, may be sufficient to ensure six or eight hours sleep.

In children chloral hydrate is one of the most useful hypnotics and is well-borne. It may be given conveniently in the form of the official syrup of chloral, which contains Io grains to the fluid dram, the requisite dose being taken in a wineglassful of peppermint water.

These drugs should always be used with caution and under strict medical supervision as certain of them, such as the bromides, may themselves produce cutaneous lesions and it is easy for patients to acquire a drug habit from their indiscriminate use.

Antipyrin is of special value in reducing the neuralgic pains associated with Herpes zoster and sometimes gives relief from the itching of Lichen planus, Dermatitis herpetiformis, and senile pruritus.

Animal Extracts or Hormones.-Hormones, from the Greek word opuci, I arouse or excite, is a name that has been applied to the internal secretions from certain glandular structures, such as the thyroid, thymus, suprarenal, pituitary body, ovaries, testicles, etc. Recently the subject of the hormones and their relation to health and disease has aroused considerable attention and their connection with skin diseases has been carefully studied. As early as I893 Dr. Byrom Bramwell employed thyroid feeding in psoriasis and certain other scaly conditions and reported favourable results from its use. Subsequent experience of the remedy was less favourable and for a considerable period the treatment was almost entirely neglected.

It was the changes which occur in the skin in myxoedema and the dramatic manner in which they sometimes clear up under the influence of thyroid feeding which suggested the employment of the glandular extract. In myxocdema the skin is peculiarly dry, harsh, or scaly; the hair, especially that of the scalp, eyebrows, eyelashes, axillae, and pubes, tends to become scanty or prematurely grey ; and the nails may show signs of dystrophy and become opaque, ridged, and brittle. These changes are associated with a defective internal secretion from the thyroid and rapidly improve when thyroid extract is administered.

This remedy has now been employed in a considerable number of scaly skin affections, of which the most important are psoriasis, mild ichthyosis especially in children, chronic eczema, and tuberculosis of the skin. It is in psoriasis that the most favourable results have been obtained; it is most beneficial in chronic cases, but should be avoided 
in acute cases and withheld until the eruption is fully out. The general experience is that it is only useful in selected cases and that those which are most benefited are cases in which the cutaneous affection is associated with symptoms of thyroid insufficiency, such as obesity, tremors, chronic rheumatism, etc.

The dose of thyroid, like all organo-therapeutic products, should be small at first so as to accustom the system to the remedy and to detect any idiosyncrasy towards it. As a rule equally good results are obtained by comparatively small doses over a prolonged period as by pushing it to the limit of toleration. Infants and young children bear the remedy well. In infants the dose should be a half to one grain per diem ; young children of between four and fourteen may begin with one grain, increased to two and a half ; and for adults the dose ranges from two and a half to ten grains a day.

The suprarenal capsules are known to have an influence on pigmentation, for when the suprarenal hormone is defective as in Addison's disease there is a marked increase of pigment. There are two theories with regard to this phenomenon; (I) that the suprarenal secretion in some way controls the formation of pigment in the epidermal cells, inhibiting its production, and that when the secretion is diminished there is an increased output of pigment ; (2) that tyrosin is the matrix of adrenalin and that when the suprarenals are diseased tyrosin is unable to form adrenalin but accumulates in the skin and elsewhere, being converted by the action of oxydases into black pigment.

The suprarenals would also appear to exert an influence on the growth of the hair, for in one or two instances in children in whom they have been hypertrophied there has been an early sexual development with growth of the hair in the axillae and pubes, while defective suprarenals have been associated with an absence of pubic hair.

The only cutaneous disease in which suprarenal medication has been employed with reported benefit is rosacea.

The pituitary gland is closely linked up in its function with the thyroid, and hypertrophy of the pituitary follows suppression of the thyroid. In hyper-pituitaryism, such as occurs in acromegaly, there is an increase in the secretion of the cutaneous glands and the skin becomes moist and greasy and shows a tendency to hypertrichosis, but so far treatment with pituitary gland extract has not been of definite benefit in dry scaly conditions of the skin.

The exact relationship between the thymus gland and cutaneous disorders has not been established, but as it becomes functionless at puberty it is possible that the changes which occur in the skin at the same time may be causally related in some way to the loss of the influence of the thymus secretion, and may account for the scalp becoming an unsuitable soil for the growth of the fungus of ringworm, and for juvenile warts tending to disappear spontaneously.

It is not improbable that the hormones secreted by the oraries and testicles may exert an influence on the skin, especially on the hair- 
growth. With the development of the sexual organs there is a marked growth of hair, but as the sexual development increases it is not infrequently associated with premature baldness in men while, according to Sabouraud, eunuchs are rarely if ever bald. In women the cessation of the sexual function at the menopause may correspond to an excessive hair growth on the face, and about the same time certain cutaneous diseases of supposed nervous origin, such as Lichen planus, flushing, or attacks of pruritus, are liable to supervene, or, more rarely, Dermatitis herpetiformis, psoriasis, or eczema, in individuals subject to these affections. The possibility of benefiting such conditions by organotherapy is worthy of consideration and experiment.

Anti-Bacterial Sera and Vaccines.-These have been largely used in the treatment of pyogenic affections and tuberculosis. Anti-staphylococcus serum has been employed for boils and carbuncles, and antistreptococcus serum in cases of erysipelas and chronic relapsing lymphangitis ; these are polyvalent sera prepared from different strains of staphylococci and streptococci respectively.

Vaccines, ${ }^{1}$ either autogenous or from stock cultures, are used in the same type of case. In staphylococcic infections, such as boils, carbuncles, sycosis, Acne vulgaris with superficial pustules, and various forms of dermatitis secondarily inoculated with pyogenic cocci, stock vaccines may give good results; where the dermatitis is due to streptococci it is necessary to prepare the vaccine from the patient's own strain of microbe.

The conditions in which treatment by vaccines has been most successful have been suppurating staphylococcal lesions, especially acute, recent, and recurrent boils, and in them rapid involution has been caused without the assistance of any form of local treatment. In the case of chronic boils, however-especially those about the back of the neck-the results have been uncertain and often unsatisfactory. This is possibly due to acute boils being fairly well supplied with blood and the protective substances called forth by the vaccines having comparatively free access to the affected tissue; while in chronic boils where there has been healing of certain lesions and the formation of scar tissue, the circulation through the boil is impeded.

The vaccine treatment of tuberculosis of the skin has been somewhat disappointing. The old tuberculin of Koch did not fulfil its great promise, and is of little value except as an auxiliary to local treatment in ulcerated cases, or for diagnostic purposes. The new tuberculins T.R. and B.E. in small graduated doses are occasionally beneficial but cannot be relied upon. The dosage of the vaccine and the interval between the injections can generally be estimated by the improvement or otherwise of the clinical symptoms, or may be controlled to some extent by estimation of the opsonic index. After an inoculation

${ }^{1}$ For an appreciation of the value of vaccine therapy in diseases of the skin sce papers by Adamson, Whitfield, Sequeira, Topley and MacLeod in the British Journal of Dermatology and Syphilis, 1919, xxxi., Nos. 4-6. 
the opsonic index generally falls in what is known as the "negative phase," which is followed after a varying period by a rise in the index known as the "positive phase." After a time the index begins to go down again and a second injection is given, as a result of this the negative phase should become less marked, but should it be more prolonged after the second injection or no positive phase occur the dose has probably been too great.

The dosage of raccines, which is estimated largely by experience with each case, will be given in connection with the different diseases to which they are applicable; the method of injection is as follows: A sterilisable glass hypodermic syringe, either a $20 \mathrm{~m}$. or a I c.c. syringe, graduated in tens and fitted with a platino-iridium needle should be used. This is sterilised by boiling in water or oil. The oil, such as pure olive oil, is heated in a fire-proof porcelain capsule to a temperature of about I fo c., the temperature being estimated roughly by putting into the oil a small pellet of bread which turns brown at that temperature. The needle is placed in the oil and the syringe is sterilised by drawing the oil into it several times so that it comes in contact with every part of the interior. The requisite dose of the vaccine is then drawn up into the syringe and the injection made into the subcutaneous tissue of the buttock, shoulder or back, the skin being previously rubbed over with lysol. After withdrawing the needle the skin should be cleansed of every trace of $y_{\mathrm{s}} \mathrm{sol}$ and a collodion scab applied.

Human Serum.--Injections of autogenous and exogenous human serum have been employed in the treatment of psoriasis and various chronic affections such as chronic urticaria, pustular acne, etc. The treatment if given alone is of practically no value, but if combined with suitable local and general treatment is said to have a farourable influence in hastening a cure and postponing relapses. Its action is uncertain, however, and several observers who have given it a fair trial have discarded it as useless.

The serum is prepared as follows: about Ioo c.c. of blood is withdrawn from the patient's reins, this is placed in an electric centrifuge for about 30 minutes, and the serum decanted. From 20 to 30 c.c. is injected as a dose and repeated at intervals of a week or less. The injections are usually given intravenously but may also be given subcutaneously. When the intravenous method is employed caution should be exercised, as in certain individuals serious anaphylactic symptoms have been known to follow a second injection.

Horse Serum from a healthy horse has been used as a substitute for human serum and has been said to have similar effects.

Injections of Sea Water, Normal Saline, Etc.-Of recent rears the treatment of skin diseases by means of deep-sea water injections under the skin has become popular in France. The so-called "plasma" is prepared by collecting sea water at a considerable depth far out in the Bay of Biscay. Other preparations are made from a source of great purity in the Auvergne. 
This treatment has proved of temporary benefit in pruritic affections, such as Dermatitis herpetiformis and eczema, but the benefit was transient and relapses soon occurred and it is doubtful if it has any advantage over injections of normal saline solution.

Health Resorts.-Certain of the mineral spas in this country and on the Continent have long held a reputation for the treatment of skin affections. The cases which derive most benefit from them are those which, from their circumstances, cannot be thoroughly treated at home owing to the difficulty of obtaining the physical and mental rest requisite for their recovery, such as business men full of worry and anxiety which they cannot throw off so long as they are in touch with their business, people whose home existence is associated with a ceaseless round of social engagements, and plethoric patients whose sedentary occupations prevent their taking sufficient exercise to work off the accumulated waste products which result from too liberal a diet. (See Appendix.)

To be of benefit the spa must be selected carefully and the regimen and treatment carried out under expert medical supervision and not left to the patient's discretion.

The special medical regimen consists partly of imbibing quantities of minera! water and partly of some form of bath, but although these are of undoubted value the benefits derived are due, not so much to them, as to the change of climate, altered mode of life, restricted diet, and regulated exercise.

With regard to the mineral waters, in most cases it is probably the large quantity of innocuous fluid which is beneficial rather than the particular salts dissolved in it ; for in the majority of cases these waters are simply mild diuretics or laxatives and have no special effect on the skin, while even in the case of arsenical waters the benefit from them may be attributed rather to their tonic than to their specific action.

Natural mincral baths are of great value in selected cases and in conditions such as psoriasis, seborrhoic dermatitis, and parasitic affections, but grave harm may result should they be employed indiscriminately, and in no skin disease, however mild, should they be taken without being specially ordered by the physician. Acute skin diseases, especially eczema, are aggravated even by simple thermal baths, and still more so by those containing alkalies, iron, or sulphur; while a chronic eczema may be transformed into an acute weeping dermatitis by them.

For a description of the natural mineral waters and the healthresorts which are suitable in cutaneous diseases see Appendix, p. I263. 


\section{CHAPTER VII}

\section{GENERAL PRINCIPLES OF LOCAL TREATMENT}

LocAL treatment is necessary in almost every form of skin disease and in certain of them it is all that is required. To be successful it must be carefully chosen and properly applied and, owing to the varying degree of sensitiveness of the skin in different individuals, it is advisable as a preliminary to question the patient closely in order to ascertain, if possible, the manner in which his skin reacts to local remedies or the existence of some idiosyncrasy.

Under the heading of local treatment is included, not only treatment by medicaments, but also the various physical, electrical, and radiotherapeutic methods.

\section{Local Medicaments}

The local remedies employed in skin affections may be divided according to their therapeutic action into anaesthetics, anti-pruritics, antiseptics, astringents, caustics, cooling agents, detergents, keratolytics, parasiticides, protectives, reducing agents and stimulants.

\section{Therapeutic Groups}

Local Anaesthetics are sometimes employed to allay irritation, relieve pain, or produce anaesthesia for operative purposes. They consist of ethyl chloride and ether which act by refrigeration, and injections of cocain, eucain, novocain, or storain.

Anti-pruritics allay irritation and itching, and act either directly. on the sensory nerve terminations, or indirectly by evaporation or by astringing the blood-vessels. Opium, menthol, and carbolic acid, are examples of direct anti-pruritics. Menthol acts by replacing the itching with a cooling sensation.

Antiseptics are largely employed for the treatment of cutaneous diseases of bacterial origin or to prevent non-bacterial affections becoming septic. Where the skin is extensively and superficially 
involved, mild antiseptics may be used in the form of wet compresses or of antiseptic poultices such as the boro-starch poultice. Where the septic condition is localised and deeper the penetration of the antiseptic may be increased by prescribing it in an ointment, but if the epidermis be unbroken and the pathological lesion be in the corium, the local application of an antiseptic is uscless.

The most commonly employed antiseptics are boric acid, mercury, carbolic acid, resorcin, potassium permanganate, iodine, hydrogen peroxide, and sulphur.

Astringents cause the reduction of inflammatory conditions associated with oedema and exudation. They act partly on the tissue fluids, coagulating the albumin, and partly on the blood-capillaries, causing their contraction and limiting exudation. The most important are lead with or without the addition of glycerine, alum, sulphate of zinc, nitrate of silver, methylene blue, and formalin, employed in lotions; and zinc oxide, prepared calamine, and the salts of bismuth, in powders or suspended in lotions which deposit them on the skin.

Caustics may be superficial or deep in their action, and may cause every degree of effect from superficial cxfoliation of the epidermis to destruction and sloughing of the whole skin. The most powerful are nitric acid, acid nitrate of mercury, antimony, caustic potash, arsenic, ethylate of soda, nitrate of silver, and carbolic acid; and the milder are acetic, salicylic, and chromic acids, resorcin, and sulphate of copper.

The powerful caustics are used for the destruction of lupus nodules and malignant growths of the skin.

The weaker caustics remove the skin fat, coagulate the albumin, macerate the horny layer, and cause little or no discomfort when applied. They are used for the removal of horny thickenings, such as corns, callosities, and warts, and to astringe or destroy mucous patches and excessive granulations.

Of late years the employment of caustics has been largely superseded by operative or radio-therapeutic methods, but in certain malignant growths applications of arsenical paste are still advocated in spite of the pain associated with them.

Cooling Agents contain water or spirit which cools the skin by evaporation, and are useful when the skin is inflamed. 'They consist of aqueous lotions, cold creams which contain water, and spirituous lotions. The spirituous lotions evaporate more rapidly and are more effective than aqueous lotions, but should not be used on broken surfaces as they are apt to irritate.

Detergents are local remedies used for the removal from the skin of dirt, crusts, scales, or discharges. Soap and water are usually employed, but where the skin is inflamed wet dressings, boro-starch poultices, oil, or cold creams, should be substituted.

Keratolytics are applications which have the power of dissolving the keratin of the horn-cells and thus causing a reduction of horny thickenings. These are employed chiefly for the removal of scales, 
corns, and callosities, the most commonly used being salicylic acid, liquor potassae, and soft soap.

Parasiticides are employed for the destruction of the various skin parasites, both animal and regetable. The most frequently used are sulphur, hyposulphite of sodium, balsam of Peru, beta-naphthol, mercury, benzoic acid, and chrysarobin.

Protectives, as the name implies, protect the healthy or inflamed skin from the local irritation of dirt, friction, traumatism, or exposure to sunlight and wind. They consist of bland powders, thick ointments or pastes, and occlusive dressings.

Reducing Agents are local remedies which have a stimulating effect on the process of cornification and are sometimes known as "keratoplastics." According to Unna they act by abstracting oxygen from the epidermis and by causing the underlying capillaries to contract; consequently they are indicated in affections, such as psoriasis, where the cornification process is defective and the capillaries dilated. In concentrated form they act as keratolytics, macerating the epidermis and causing inflammation, oedema, and sometimes bullous formation. They consist of salicylic acid, ichthyol, resorcin, thiol, tar, pyrogallol, and chrysarobin.

Stimulants are used to improve the tone of the skin and stimulate its growth or that of its appendages, or, by producing a mild inflammatory reaction, to hasten the absorption of the pathological products of some chronic inflammatory process. They consist of such substances as tar, turpentine, acetic acid, iodine and chrysarobin, and are employed in the form of lotions or ointments.

\section{Medicaments Commonly Employed}

The number of local medicaments ${ }^{1}$ employed in the treatment of cutaneous diseases is so great that it is practically impossible to mention all, far less to discuss their value, and in this section reference wili only be made to those most commonly employed and proved to be of the greatest service. These will be described briefly in alphabetical order.

Alum is sometimes employed as an astringent either in dusting powders or lotions. It is an important constituent of an astringent lotion known as "Burow's lotion," in which it is combined with acetate of lead.

Balsam of Peru is a parasiticide which is used in the treatment of scabies, and is either painted on, or applied as a $10 \%$ ointment. Occasionally it has been known to be absorbed and to cause symptoms of intoxication.

Benzoic Acid is an antiseptic which is useful in an ointment for the treatment of ringworm of the glabrous skin.

Boric Acid is one of the most extensively used antiseptics, and is employed in the form of powders, lotions or ointments. Its action

\footnotetext{
${ }^{1}$ For prescriptions see Append'x.
} 
is mild and soothing, though cases occasionally occur in which it may prove irritating, especially to mucous membranes. It is soluble in 25 parts of cold or 3 parts of boiling water, and when finely powdered has an unctuous feeling.

B-Naphthol is an antiseptic and reducing agent sometimes employed in the treatment of scabies, ringworm, and psoriasis. It is used in ointments containing I to $5 \%$ and is almost odourless.

Carbolic Acid is antiseptic, anti-pruritic, and anaesthetic. As an antiseptic and parasiticide it is employed up to $10 \%$ in lotions and ointments or dissolved in glycerine, and is extensively used for the destruction of pediculi and for the treatment of ringworm. Lotions and ointments containing $\mathrm{I} \%$ are soothing and anti-pruritic; those containing $2 \%$ have a definitely anaesthetic and numbing effect which is succeeded by irritation ; strong solutions act as caustics, macerating the skin and even causing gangrene and sloughing.

Chrysarobin or Goa powder is a powerful anti-parasitic and stimulating remedy and is one of the most important of the so-called "reducing agents." It is used in strengths of I to Io $\%$ in ointments, pastes, and varnishes, the weaker preparations acting as irritants while the stronger preparations are liable to produce acute dermatitis. When used over extensive areas or for a prolonged period it is apt to cause exfoliative dermatitis, and has been known to be absorbed and to produce severe toxic symptoms such as vomiting, purging, fever, and insomnia, and to be excreted by the kidneys staining the urine yellow. It is particularly liable to irritate the mucous membranes and for fear of producing conjunctivitis should not be applied to the face and only with the greatest care to the scalp. It stains the skin and nals a brownish-purple tint, the hair a dirtygreenish-yellow, and the clothes a brownish colour which becomes purple from exposure to the air and washing.

It is extensively used as a parasiticide in cutaneous diseases due to fungi, such as ringworm, and as a stimulant in chronic inflammatory affections, such as psoriasis.

Anthrarobin is a substitute for chrysarobin which is not so liable to stain the skin, but is less effective.

Formalin is an astringent and antiseptic, but except in solutions of about $I \%$ it is apt to be irritating, and in stronger proportions it can produce severe dermatitis. At one time it was used frequently in the treatment of ringworm of the scalp, but it has to be employed with great care as some children have an idiosyncrasy towards it, when even in weak solutions it may give rise to severe dermatitis and even sloughing. Its astringent action is useful in the treatment of hyperidrosis of the feet.

Glycerine in weak solution is a protective and cooling agent, but when concentrated may act as an irritant from its hygroscopic action and may cause inflammation. It is added to lotions to make them less drying, or to suspend powders. In the form of the glycerine of starch 
it is a useful basis for the application of various medicaments. It is a component of certain cold creams and is sometimes used in making fluid soap.

Ichthyol is used externally as well as internally in a large number of cutaneous affections, and is antiseptic, astringent, and stimulating. It is a bituminous product containing sulphur, with an unpleasant taste and smell and a dirty-brownish appearance like tar. It is chiefly employed for the reduction of hyperaemia in inflammatory affections and acts by causing contraction of the cutaneous capillaries. It is soluble in water and spirits and may be applied in the form of ointments or lotions containing I to $5 \%$. In strong solutions it is liable to irritate the shin and is sometimes absorbed, producing severe toxic symptoms.

Various substitutes have been introduced with a less disagreeable odour, one of the most useful being thiol which also contains sulphur. It is a dark treacle-like substance which is soluble in water, and has an aromatic smell. It can be obtained also in the form of a solid brown powder.

Iodine is an antiseptic, parasiticide, and powerful irritant. It is used chiefly for the treatment of ringworm and other affections due to fungi, and is generally employed in lotions dissolved in water or spirit.

From it are prepared synthetically various other skin antiseptics, such as iodoform, iodol, and aristol. Iodoform, owing to its penetrating odour and liability to cause dermatitis, is not much used in the treatment of skin diseases. Iodol is odourless and can be prescribed in an ointment containing 5 to $10 \%$. Aristol is employed in the same manner. Both iodol and aristol contain iodine in such loose combination that it is readily given up and enabled to exert its full action on the skin.

Methylene Blue is an astringent and antiseptic, weak solutions of from I to $2 \%$ being of use in diminishing the exudation in eczema and in cleaning up ulcerated surfaces. Owing to its staining properties it is only suitable for covered parts of the body.

Mercury is a powerful parasiticide, antiseptic, and anti-syphilitic remedy. The most commonly used salts are ammoniated mercury, corrosive sublimate, calomel, the red and yellow oxides, and the nitrate. Ammoniated mercury is usually employed as an ointment in strengths of 2 or $3 \%$, which is adequate for the destruction of micro-organisms and does not injure the skin, while stronger percentages are liable to act as irritants.

Corrosive sublimate in an ointment or lotion ( $I$ to Iooo to I to 5000 ) is a valuable parasiticide.

Calomel may be used in an ointment, powder, or in the official lotion "black wash."

Permanganate of Potash is an astringent, antiseptic, anti-parasitic, deodorant, and caustic, which acts by its power of oxidising, and is employed in solutions of from I to $\mathrm{IO}^{\circ}$ according to the effect required. It has been recommended recently in the treatment of Lupus vulgaris, 
either as a powder or painted on in $50 \%$ solution. It is sometimes used as a dressing for ulcers. Its chief disadvantage is that it stains the skin.

Pyrogallol is an astringent, antiseptic, keratolytic, and reducing agent. Though consisting of colourless crystals it readily oxidises on exposure to the air and becomes black. Its antiseptic and astringent actions arc obtained in strengths of $\mathrm{I}$ to $2 \%$, while its keratolytic and caustic effects are derived from more concentrated preparations up to $10 \%$ or more. It is applied in ointments and lotions.

It is a valuable remedy for reducing horny thickenings, such as occur on the palms and soles, is occasionally employed as a caustic to destroy nodules of Lupus vulgaris, and in combination with nitrate of silver constitutes a well-known dye for blackening the hair. If applied extensively it is liable to be absorbed and to produce severe toxic symptoms, such as haemoglobinuria and collapse.

Instead of the ordinary pyrogallol, oxidised pyrogallol may be used, or various other substitutes such as eugallol and lenigallol.

Eugallol is a yellowish syrupy fluid and is either made up in an ointment or mixed with acetone and painted on the skin. It is used in the treatment of psoriasis.

Lenigallol is a white powder and is prescribed in ointments and pastes; its action is similar to that of pyrogallol but is weaker.

Resorcin is an antiseptic, astringent, keratolytic, and reducing agent, and is used in lotions and ointments. As an astringent 2 or $3 \%$ is sufficient, but if the keratolytic action is desired it has to be prescribed up to $\mathrm{I}_{5}$ or $20 \%$. It is employed to produce exfoliation of the epidermis in what is known as the "peeling cure" of acne. It is a common ingredient of antiseptic hair lotions, but should never be used where the hair is fair, grey, or white, as it stains it a greenish or yellowish rusty tint, especially after the hair has been washed with soap from the combination of the resorcin with the alkali.

Salicylic Acid is an antiseptic, keratolytic, and reducing agent. It is readily soluble in alcohol but only soluble in 600 parts of cold water, and is prescribed in lotions, ointments, and pastes, or dissolved in collodion and painted on. It nust not be used over extensive areas as it is liable to be absorbed and to cause toxic symptoms.

Sulphur is a valuable parasiticide, antiseptic, and reducing agent. It is best prescribed as the precipitate in ointments or lotions containing 2 to $10 \%$, in solutions of the hyposulphite of sodium, or as nascent sulphur made by the action of tartaric acid on the hyposulphite. Strong preparations applied externally irritate the skin and are apt to cause more or less severe dermatitis. It is largely used in the treatment of seborrhoic conditions and parasitic affections, such as scabies and ringworm.

Tannic Acid is an astringent which is of special value in the treatment of hyperidrosis of the feet. Combined with formaldehyde it forms tannoform which is a useful ingredient of astringent powders.

Tar is extensively employed in dermatological practice and is so 
valuable when properly applied that it has been said that the therapeutic skill of a dermatologist may be gauged by his success in its application. It may be used in the form of Pix liquida (wood tar), Oleum cadini (oil of juniper), Oleum rusci (oil of birch), Oleum fagi (oil of beech), the alcoholic extract known as Liquor carbonis detergens, the watery solution Liquor picis carbonis, or may be incorporated in ointments, pastes, or lotions. In weak solutions or ointments it exerts an anti-pruritic or sedative action, while in strong preparations it becomes a powerful irritant and if too extensively employed may. produce serious inflammation and șmptoms of intoxication from absorption.

Water has a stimulating and thermal action varying with the temperature ; it is also a detergent and macerating agent, destroying the superficial horn-cells and remoring the fat. It is employed as a stimulant in the form of cold baths, douches, etc. ; as a sedative in the form of warm baths or warm moist dressings ; and as a cleansing agent for the removal of dirt, discharges, and scales. It is the base of the majority of lotions.

When water is hard its macerating and deleterious effects are increased and it becomes an irritant, making the skin dry and rough. Various water-softeners may be employed to neutralise the hard lime salts, but they must be used with caution as they contain chiefly carbonate of soda, any excess of which is harmful. Where possible, rain-water or water softened by boiling should be used.

\section{Methods of Application}

Local medicaments may be applied to the skin in the form of baths, soaps, oily preparations, cold creams, liniments, ointments, pastes, plaster mulls, salve pencils, glyco-gelatines, varnishes, lotions, dusting powders and poultices.

\section{BATHS}

Baths may be classified according to the temperature of the water as follows :

Cold baths -

Cool baths -

Tepid baths

Warm baths

Hot baths -
- from 40 to $60^{\circ} \mathrm{F}$.

- from 60 to $75^{\circ} \mathrm{F}$.

- from 75 to $90^{\circ} \mathrm{F}$.

- from 90 to $100^{\circ} \mathrm{F}$.

- from 100 to $110^{\circ} \mathrm{F}$.

Cold Baths. Cold baths are stimulating and tonic in their effect. The stimulation is apt to be followed by depression and, consequently", eren in robust indiriduals they should not be prolonged for more than a few minutes, while in people with a weak circulation or a delicate constitution they are unwise. They are contra-indicated in all pruritic 
conditions, as the reaction which results from them may set up itching or may aggravate it if present.

Warm Baths.-Warm baths are most useful for cleansing purposes, owing to the macerating action of the water, but are apt to render the skin delicate and less able to resist the invasion of micro-organisms. Their cleansing effect is increased by soap, but to lather all over with soap in a daily bath, though pleasant, may have harmful results, especially if the skin be affected with any form of dermatitis.

Hot Baths.- Hot baths and the application of very hot water, or of cloths wrung out in it, have a sedative effect and relieve the irritation of local conditions such as Pruritus ani or vulvae.

After the patient comes out of a bath the skin should be thoroughly dried, not by rubbing with a rough towel, for friction is harmful, but by blotting up the water with a towel laid over the skin and rubbed on the outside.

Continuous Baths.--Continuous baths are useful in bullous conditions especially where the surface of the skin is broken, as in pemphigus and burns. The patient is immersed, all but the head, in a bath at a temperature of from $98^{\circ}$ to $100^{\circ} \mathrm{F}$; ; there he may remain for hours, days, or weeks, without interfering with his bodily health. Prolonged immersion is rarely resorted to in this country owing to its depressing effect, but it is carried out in a modified fashion at several health resorts, such as Leukerbad and Plombières.

Sea Baths.--Sea bathing is harmful in any form of acute dermatitis, especially in eczema, and may aggravate chronic cases. It is of value in local pyogenic affections such as furunculosis, and in parasitic and scrofulous conditions, owing to its tonic action on the bodily functions.

Vapour and Hot-air Baths. - Vapour baths (Russian) at a temperature of $100^{\circ}$ to $140^{\circ} \mathrm{F}$, and hot-air baths (Turkish) at a temperature of $100^{\circ}$ to $200^{\circ} \mathrm{F}$. are sometimes used in cutaneous affections but are not to be recommended. They have a stimulating effect on the skin and sweat-glands, but the subsequent depression and the rubbing by the attendant are apt to be harmful. There is a popular belief among the laity that such baths purify the blood and cause the cutaneous disease to be thrown out of the system, but the fallacy of this is too obvious to require refuting.

Wet Packs.- Wet packs may be substituted for hot baths to remove scales and crusts. They may be used for the whole body or for a limb and are of service when total immersion is inadvisable. The patient is wrapped in a sheet wrung out in warm water and is covered with a blanket; after a quarter of an hour the skin is dried and anointed with almond oil to prevent cracking.

Medicated Baths.-Medicated baths may be antiseptic, detergent, or emollient. Their action is purely local as the substances dissolved in the water are not absorbed. The bath should be kept at a temperature of about $98^{\circ} \mathrm{F}$. and the patient should remain in it from 5 to r5 minutes. 
The following are the most useful medicated baths-the quantities of the ingredients being given on a basis of 30 gallons of water.

Acid Baths. - These are made by adding an ounce of hydrochloric or nitric acid to the water and are of service in pruritic skin affections. As the acid acts on metal an earthenware bath is essential.

Alkaline Baths are made by adding sodium bicarbonate 2 to Io ounces, potassium carbonate 2 to 6 ounces, or borax 3 ounces, to the water. They are soothing in itchy affections, such as urticaria and prurigo, and are useful for softening and removing crusts and scales. Where scales are profuse and thick and the skin not inflamed the effect of the bath may be increased by scrubbing the lesions with soft soap, or by substituting a soft soap bath containing half a pound of green soap to the 30 gallons of water.

Boric Acid Baths contain 2 ounces of boric acid to the gallon of water. They are used in cases of extensive pyogenic infection, such as Pemphigus neonatorum.

Bromine and Iodine Baths are made by the addition of 20 minims of bromine, 2 ounces of potassium bromide, 30 minims of iodine, or $\frac{1}{2}$ ounce of iodide of potassium and 2 ounces of glycerine, to 30 gallons of water, and are occasionally employed in scaly and syphilitic affections.

Emollient Baths are made by the addition of 2 lbs. of bran, oatmeal, starch, linseed, gelatine, or size, to the water. The selected ingredient is put in a muslin bag and placed in the empty bath, a few gallons of hot water are poured over it and the bag squeezed so as to press out the mucilaginous substances, the remainder of the water is then added at the required temperature. In the case of the starch bath, the starch should be crushed into a powder in a basin and made into a paste with cold water, the basin is put in the bath under the hot-water tap, which is turned on and allowed to run till the water overflows and carries the starch into the bath.

Emollient baths are useful for the relief of irritation and the reduction of erythema.

Mercurial Baths are made by adding a teaspoonful each of perchloride of mercury and hydrochloric acid to the water, and are employed in cases of ulcerating syphilis. An earthenware bath must be used as the mercury acts on metal.

Permanganate of Potassium Baths are made by adding a teaspoonful of the permanganate to 30 gallons of water. They are antiseptic and soothing, but have the disadvantage of staining both the patient and the bath.

Sulphur Baths are made by the addition of $t$ ounces of sulphurate of potassium to 30 gallons of water. An earthenware bath is essential, as the sulphur acts on the metal and has also the disadvantage of staining paint. To obviate the staining a preparation known as "sulphaqua" may be employed, by which nascent sulphur and sulphuric acid are eliminated in the water, making a bath which is not so dirty and is less irritating. 
Sulphur baths are especially useful in seborrhoic dermatitis, psoriasis, and parasitic affections, such as scabies and pediculosis, but should on no account be used in eczema or in any form of acute dermatitis.

Tar Baths are made by the addition of 2 ounces of Liquor picis carbonis, 2 ounces of oil of Cade, $\frac{1}{2}$ an ounce of creolin, or $\frac{1}{2}$ an ounce of cyllin, to the water. A simple method of obtaining the effect of a tar bath is to anoint the patient with Pix liquida, or a mixture of Pix liquida 3 parts and soft soap I part, or with equal parts of Pix liquida and glycerine of starch, and then immerse him in a warm bath.

Tar baths have a stimulating effect on the skin and are especially useful in psoriasis.

Electrolytic Effects in Medicated Baths.-By passing an electric current through a medicated bath, an electrolytic action is set up which causes salts to be broken up and deposited as ions at the different poles. In this way sulphates may be made to deposit ions of nascent sulphur and chlorides ions of chlorine at the positive pole. By a suitable apparatus the patient can be made the positive or the negative pole as desired and ions of the element caused to be deposited on the skin. This procedure is said to intensify the effect of the medicaments employed.

\section{SOAPS}

A soap is formed by the combination of a fatty acid and an alkali. When the skin is washed with soap and water a certain amount of the alkali is set free, which saponifies the grease and cleanses the surface. Should an excess of alkali be present the superficial horn-cells become swollen, macerated, and destroyed, or if the fats used in making the soap be of inferior quality and in the least degree rancid irritation and inflammation may result.

Soaps are employed in everyday life simply for cleansing purposes, while in diseased conditions they are used (I) for the removal of discharges, scabs, and crusts, (2) for the stimulation of the skin, and (3) in the form of medicated soaps for curative purposes.

Though occasional cleansing of the skin with soap is of importance to enable it properly to perform its functions, to overdo it may be actually harmful. It has been asserted that the skin cannot execute its excretory function properly unless it is constantly cleansed; this is doubtless true when it becomes really dirty, as in coal-miners or sweeps, but in the ordinary individual it is a long time before sufficient dirt can accumulate to interfere with its function. The Stratum corneum is essentially a protective organ, partly from the fat in the horn-cells and partly from the horn-cells being welded together by keratinised joints. The action of soap removes the fat and softens the keratin, and so reduces the protective power of the horny layer; consequently its too free use-especially in delicate skins--may lead to eczema, chapped hands, and rough faces. 
There are three official soaps in the British Pharmacopocia, namely, Sapo durus (hard soap) made with olive oil and soda, Sapo mollis (soft soap) made with olive oil and potash, and Sapo animalis (curd soap) made with a purified animal fat (stearin). The composition of these soaps being known, it is advisable for the physician to become thoroughly familiar with their action and effects before recommending more or less attractively scented substitutes of the exact constituents of which he is ignorant.

Hard Soaps.-Hard soaps may be regarded as the type for toilet purposes, while soft soaps, being stronger, should be reserved for the removal of crusts or scales and for the maceration of horny thickenings of the skin. To be ideal, hard soaps should be neutral and should contain no excess of free unsaponified alkali, but small quantities of some perfume, such as bergamot, lavender, lemon, thyme, etc., have no deleterious effect. Neutral soaps do not form a good lather in hard water. and various plans are adopted to improve this defect. Cocoa-nut oil as a substitute for olive oil produces an abundant lather as it requires more alkali to saponify it and, consequently, a larger amount of alkali is set free when it is used, but this may be harmful to the skin. It is better to use what is known as a "superfatted soap," which contains an excess of fat.

Soft Soaps.-Soft soaps are employed chiefly for the removal of scales but are also of service in stimulating chronic affections such as psoriasis and Lupus erythematosus. For this purpose "green soap" made from linseed oil and potash is frequently substituted for the B.P. Sapo mollis.

To intensify the effect of the soap a small quantity of Liquor potassae may be added to it, or it may be mixed with alcohol, as in the classical Spiritus saponatus alkalinus of Hebra which consists of 2 parts of soft soap and I part of rectified spirit. To this may be added methyl ether to increase the penetration of the soap by enabling it to remove the grease from the skin, as in the following formula (Martindale) :

$\begin{array}{llllll}\text { Saponis mollis } & - & - & - & - & \text { jiv. } \\ \text { Alcohol }(90 \%) & - & - & - & - & \text { jiiss. }\end{array}$

Mix, and after $2+$ hours decant from the sediment, then add

$$
\text { Methylated ether - - _ - jriss. }
$$

Mollin, or Salve Soap.-This is a soft potash soap containing about I2 $\%$ of free fat. It is miscible with water and may be used as a vehicle for sulphur, ichthyol, and other drugs, but is apt to irritate inflamed surfaces.

Medicated Soaps.-A large number of medicated soaps are made, both with neutral and superfatted soap bases, and can be had in cakes or powders. Such medicaments as sulphur, mercury, carbolic acid, tar, naphthol, resorcin, salicylic acid, ichthyol, balsam of Peru, and camphor, in various proportions and combinations are incorporated in them. They are of comparatively little value so far as their specific 
action is concerned, as the drug is of neccessity much diluted and its action transient, and the chief effect obtained from them is that of the alkali of the soap and not of the medicament. It is practically useless simply to wash the skin with them, it is necessary to apply them by a brush or a piece of flannel and either to rub the lather into the skin till dry or to allow it to dry on.

\section{Oily and Greasy Preparations}

These consist of oils, cold creams, ointments, and pastes. They are indicated in skin affections: $(a)$ to replace the epidermal fat when deficient, as in dry scaly affections like ichthyosis, or in dermatitis from the too free use of soap or the action of hard water, as in washerwomen ; (b) to increase the penetration of a medicament, as an oily preparation has a greater power of penetration than an aqueous preparation ; $(c)$ to soften and aid the removal of scales and crusts. They niust beemployed with caution, for in unsuitable cases they may aggravate irritation and cause inflammation by forming a greasy coating to the skin which has the effect of damming back discharges and preventing evaporation. They are contra-indicated in acute inflammatory affections associated with nuch oedema.

Oils.-Oils are used chiefly to lubricate the skin, to protect it from friction, and to remove scales. They nay be smeared on, or applied continuously on lint or a bandage.

Almond oil is one of the most pleasant lubricants; olive oil, even in its purest form, has a disagreeable odour but is useful for the softening and removal of scales and crusts; castor oil, being soluble in alcohol, is a common constituent of non-greasy brilliantines for the hair ; turpentine oils and paraffin are frequently employed for softening the cement substance which holds nits to the hairs and for the destruction of pediculi ; codliver oil is sometimes rubbed into the skin in cases of malnutrition, or kept constantly applied on a binder, especially in tuberculosis.

Oils may be rendered more soothing by the addition of lime water to form an emulsion, as in the well-known Carron oil once extensively used in the treatment of burns, which consists of equal parts of linseed oil and lime water.

By the addition of $x_{5}$ to $20 \%$ of zinc oxide or other bland powder to an oil a soothing cream is formed, which is of great value in widely distributed inflammatory affections, as in the following formula :

$\begin{array}{llllr}\text { Zinci oxid. - } & - & - & \bar{j} \mathrm{ss} & 6 \\ \text { Calam. preparatae } & - & - & \bar{j} \mathrm{ss} & 6 \\ \text { Liquoris calcis - } & - & - & \overline{5} \mathrm{ss} & 50 \\ \text { Olei amygdalae - } & - & - & \overline{3} \mathrm{ss} & 50\end{array}$

The further addition of $\mathrm{I} \%$ of carbolic acid to the cream gives relief in pruritic conditions. 
Oily substances are employed in cutaneous diseases on account of their stimulating properties, such as the regetable tar oils which are frequently used in the treatment of psoriasis.

Oily Dressings.-For the remoral of scales in acute inflammatory conditions, pieces of lint or bandages soaked in olive oil may be substituted with advantage for watery applications. Care must be taken to use only pure oil or to add a small quantity of thymol or eucalyptus to prevent its becoming rancid.

Oleates of the Metals are useful preparations which are made by dissolving the oxides of the metals in oleic acid. Certain of them are used as powders and contain a quantity of stearin, while others are in the form of ointments. The most commonly emplored are the oleates of zinc, mercury, and lead. It is necessary that zinc oleate should be freshly prepared, as it is liable to deteriorate and blacken with age. Oleate of lead is prescribed as an ointment which is known as " diachylon ointment" and is a valuable astringent and sedative.

Cold Creams.-Cold creams are greasy applications which have a pleasant cooling effect on the skin and reduce inflammation by evaporation of the water they contain. The Lnguentum aquae rosae (B.P.) is a well-known example.

They are made chiefly from lanolin, spermaceti, wax, and almond oil, as in the following formulae:

\begin{tabular}{|c|c|c|c|c|}
\hline Cerae albae & - & - & - &.$j \mathrm{ss}$ \\
\hline Spermaceti & - & - & - & is \\
\hline Olei amỹgdalae & - & - & - & jas \\
\hline Aquam rosae ad & - & - & - & $3 \mathrm{i}$ \\
\hline Adipis lanae & - & - & - & jii \\
\hline Olei amỹgdalae & - & - & - & -jii \\
\hline Aquam aurantii & ad & - & - & \\
\hline
\end{tabular}

The addition of glycerine to the cream has a drying effect which is healing where the skin is cracked.

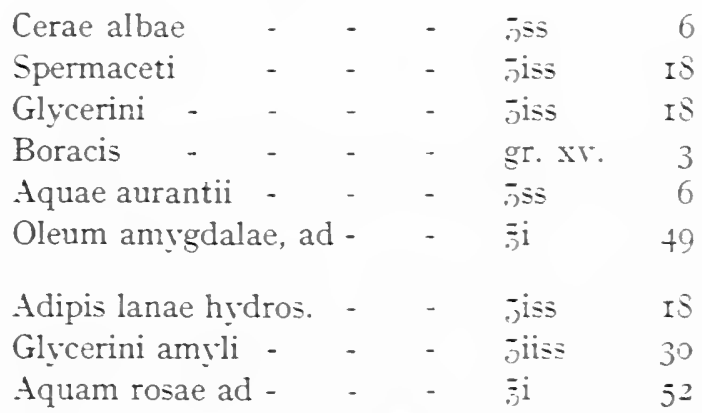

To be effective cold creams should be applied to the skin in a thick layer. When the water evaporates the oil is absorbed and the wax is left on the skin as a protective layer. 


\section{LINIMENTS}

Liniments are liquid preparations containing oil or a mixture of oil and water, the oils chiefly employed being olive, almond, and linseed. Equal parts of oil and lime water form a soothing preparation, and also an excellent basis for a cream made by the addition of $10 \%$ of zinc oxide and calamine and thickened, if desired, by $5 \%$ of lanolin.

Rubefacient liniments containing turpentine, acetic acid, camphor, or cantharides, are sometimes used for stimulation, as in Alopecia areata. (See Appendix.)

\section{Ointuents}

Ointments are the most commonly employed greasy preparations and vary indefinitely in their bases and special therapeutic ingredients.

Bases.-The choice of a proper base for the ointment is of the first importance ; to be ideal it should be non-irritating, penetrating, capable of thorough incorporation with the active ingredients and of dissociation so as to set them free in the tissues.

The common bases are Adeps benzoatus (benzoated lard), Adeps lanae (lanolin), Paraffin molle (vaselin), spermaceti, cerates, cocoabutter, cold cream, and glycerine of starch. To prevent ointments becoming rancid a few grains of thymol may be added to the base.

Adeps benzoatus, or benzoated lard, is a penetrating basis, melting at body temperature, but apt to become rancid.

Adeps lanae, or lanolin, is obtained from sheep's wool and is capable of absorbing equal weights of water, forming Adeps lanae hydros. It is unstable, but useful in combination with vaselin to give greater consistence.

Vaselin, either the ordinary yellow Paraffinum molle or the purer Paraffinum molle album, though less penetrating than lard, is stable, inert, more generally useful, and readily sets free the ingredients incorporated with it. It may prove irritating to sensitive skins, in which case a cold cream may be substituted (see page I3I).

Vasogen is a paraffin derivative somewhat similar to vaselin. It is sometimes employed as the base for iodine and mercury, but has no special advantage.

Cocoa-Butter melts at body temperature. It is a base for pomades.

Glycerine of Starch is a viscid base and has the advantage of being easily removed by washing, but it is apt to irritate delicate skins.

In order to thicken the base, bland powder such as zinc oxide may be added, which has a cooling effect by absorbing water and also prolongs the action of the special ingredient.

Special Ointments.-Ointments may be divided according to their active ingredients into bland or protective, astringent, keratolytic, antiseptic, stimulating, and sedative. (See Appendix.)

The proportion of the special ingredient varics with the required 
effect, but until the toleration of the patient is ascertained it is adrisable to begin with a low percentage. To obtain a superficial action it is only necessary to rub the ointment into the skin, but to obtain its full effect it must be spread thickly on muslin or lint and applied continuously:

Bland or protective ointments consist of the rarious simple ointments, such as the Ungt. simplex, Ungt. cetacei, and Ungt. aquae rosae. They are of value in protecting the skin from friction, moisture, or the action of the atmosphere.

Astringent ointments are prepared chiefly from zinc oxide, zinc oleate, prepared calamine, the salts of bismuth, and diachylon. Ther are soothing and reduce inflammation.

Keratolytic ointments contain salicylic acid, resorcin, ichthyol, pyrogallic acid, etc., and are of special service in reducing thickening of the horny layer of the epidermis. The extract of Cannabis indica is a useful addition to such ointments to relieve the stinging caused by them.

Antiseptic and anti-parasitic ointments contain boric acid, mercury, salicylic acid, sulphur, tar, chrysarobin, formalin, naphthol, etc.

Stimulating ointments are composed of similar ingredients to antiseptic ointments, two of the most raluable being tar and its derivatives, and chrysarobin.

Anti-pruritic and sedatice ointments contain carbolic acid, menthol, tar, opium, Cannabis indicà, etc., and are of special value in relieving local irritation, but are not applicable to extensive areas as ther are apt to inflame the skin. Their effect is transient and tends to be followed by irritation.

\section{PAstes}

Pastes are made by the addition of a quantity of some bland absorbent powder to the ointment basis and are less irritating than ointments as the powder absorbs discharges. Ther should be applied to the skin either by being rubbed in or by being spread on lint and held in position by a bandage. Should they become caked over the skin they can be remored by olive oil. Pastes are unsuitable for use on hairy parts as they are apt to mat the hairs together or to plaster them down to the skin. (See Appendix.)

The basis of the paste may consist of lanolin, raselin, benzoated lard, Carron oil, glycerine and dextrin, or glycerine and gum.

The absorbent powders used are zinc oxide, starch, talc, bismuth. kaolin, magnesium carbonate, or silicious earth.

The active ingredients are similar to those of ointments and from their ingredients pastes may be divided also into protective, astringent, antiseptic, and caustic.

Where pastes have to be applied to the face they may be skintinted by the addition of suitable quantities of prepared calamine, red bole, or turmeric. 
A simple paste is composed of equal parts of zinc oxide, starch, lanolin, and vaselin, with or without the addition of $2 \%$ of salicylic acid (Lassar's Paste). To make this more drying, lard may be substituted for the vaselin and silicious earth for the starch, as in the following preparations :

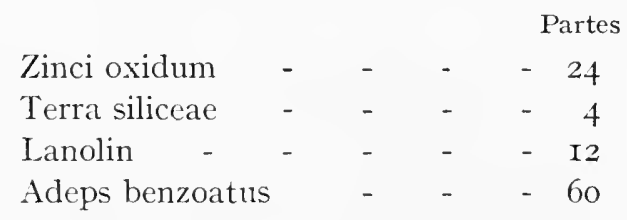

Carron oil also may be used as a base, as follows :

Partes

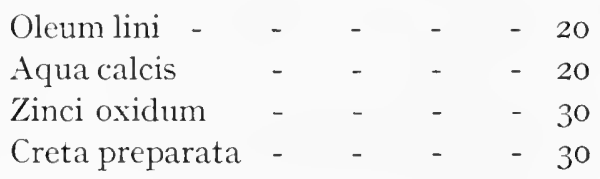

The addition of glycerine, alone or combined with dextrin or mucilage of acacia, makes a quickly-drying paste, as in the following :

Partes

\begin{tabular}{|c|c|c|c|}
\hline Zinci oxidum & - & - & - \\
\hline Glycerinum & - & - & - \\
\hline Aqua destillata & - & & - \\
\hline Zinci oxidum & - & - & - \\
\hline Dextrin - & - & - & - \\
\hline Glycerinum - & - & . & - \\
\hline Aqua destillata & - & - & - \\
\hline Zinci oxidum & - & - & - \\
\hline Acaciae mucilago & & - & - \\
\hline Glycerinum & - & - & - \\
\hline
\end{tabular}

Caustic pastes are sometimes employed for the destruction of epidermal growths, nodules of Lupus vulgaris, etc. The well-known "Vienna paste" is an example and consists of equal parts of caustic potash and quick lime; this is kept in a stoppered bottle and when required is made up into a paste with a third of its weight of methylated spirit.

\section{Plaster Mulls, Salve Pencils, Etc.}

In addition to ointments and pastes there is a series of useful preparations which are of special value where limited areas of skin are affected; namely, plaster mulls, salve mulls, and salve pencils. 


\section{Plaster Mulls}

Plaster mulls ${ }^{1}$ are non-greasy preparations in which the active ingredients are made up in the form of an adhesive plaster spread on a thin layer of gutta-percha. They can be obtained with the most frequently employed local medicaments in different proportions and combinations. The ingredients are incorporated in considerable concentration and this, combined with the presence of the impermeable gutta-percha layer behind, renders them singularly effective.

Of the many varieties of plaster mulls the most serviceable are those containing salicylic acid and creosote for the treatment of Lupus vulgaris, mercury and carbolic acid for furunculosis, and resorcin for Acne vulgaris. A piece of the plaster is cut of the required size, the layer of muslin remored, and the adhesive surface applied to the skin to which it is easily moulded owing to the thinness of the gutta-percha backing.

The chief disadvantage of the plaster mulls is that they deteriorate when they become dry, which they are very apt to do if kept for some time or exposed to the air.

\section{Salve Mulls}

Salve mulls are allied preparations which have their active ingredients incorporated in a stiff mass, chiefly consisting of benzoated mutton suet and wax, which is spread on one or both sides of a piece of muslin. When applied to the skin the basis melts and the special ingredients are set free. Like plaster mulls they quickly deteriorate if exposed to the air or allowed to become dry.

\section{Salve Pencils}

Salve pencils consist of a firm crayon-like mass composed of a mixture of lanolin 2 parts and wax I part, or of tragacanth, with which various active ingredients may be incorporated. Their field of utility is limited, the most useful being those containing chrysarobin which are of service in the treatment of small patches of psoriasis or ringworm of the scalp.

\section{Glyco-Gelatines}

Glyco-gelatines are preparations containing glrcerine, gelatine, zinc oxide, and water, which are melted and painted on to the skin and on cooling set to form a pliant protective laver. These useful preparations were introduced by Lnna, to the prototype of which he gave the

1 The ordinary official plasters in which the basis consists of resin, wax, or lead plaster, are comparatively little used in dermatology, with the exception of lead plaster itself and soap plaster which is of some value in reducing horny thickenings. 
name of "zine leim." The following are formulae for soft and hard gelatines :

$\begin{array}{lccc} & \begin{array}{c}\text { Soft } \\ \text { Glyco-gelatine } \\ \text { Partes }\end{array} & \begin{array}{c}\text { Hard } \\ \text { Glyco-gelatine } \\ \text { Partes }\end{array} \\ \text { Gelatin. - } & \text { I } 2.5 & \text { I5 } \\ \text { Glycerinum } & - & \text { I } 2.5 & \text { I0 } \\ \text { Zinci oxidum } & - & 20 & 25 \\ \text { Aqua - } & - & 55 & 50\end{array}$

The gelatine, which should be of the best quality, is melted in the water in a water-bath, and the zinc oxide and glycerine are triturated in a mortar and added with continuous stirring. When the whole is thoroughly incorporated and homogeneous it is poured into a porcelain jar and allowed to cool and set into a firm opaque jelly. (See Appendix.)

Tarious active ingredients may be incorporated with this base, such as ichthyol, tar, sulphur, and chrysarobin, in quantities from Io to $30 \%$.

The glyco-gelatine is melted by placing the jar in a saucepan of water, and painted evenly on to the skin as hot as it ean be borne. In Io to 5 minutes it dries and forms a thin pliant covering like a piece of kid-glove. To increase its consistence a thin layer of cottonwool is laid over it before it has quite set, and the superfluous wool removed when it is dry.

If properly applied to a part not subjected to friction it should last about a rreek. It may be peeled off at any time and, being soluble in water, can be easily washed away.

The great value of glyeo-gelatine is the protection it affords against the atmosphere and minor degrees of friction. As it does not prevent evaporation it may be employed in exudative conditions and is particularly useful in varicose dermatitis of the leg, and combined with a properly applied bandage forms an excellent strapping for varicose ulcers.

In the weeping stage of eczema it may be harmful by allowing some of the discharge to collect beneath and to become septic.

\section{VARNISHES}

Tarnishes are employed in cutaneous diseases both as a protective coating and as vehicles for various drugs. The simplest of them contain medicaments such as tar, ichthyol, chrysarobin, pyrogallol, etc., dissolved in spirit or acetone. When these are painted on the skin the spirit or acetone evaporates with a pleasant cooling effect and the active ingredient is left to exert its specific action.

The Linimentum exsiccans of Pick is one of the best-known varnishes. 
It is a translucent jelly, which is inconspicuous when it dries, and consists of the following :

\begin{tabular}{|c|c|c|c|c|}
\hline Glycerinum & - & & - & \\
\hline Tragacanth - & - & 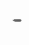 & - & \\
\hline Aqua dest. - & - & - & - & - \\
\hline
\end{tabular}

The tragacanth is pounded down in a mortar, dissolved in part of the water, allowed to stand for 6 hours, and the remainder of the water stirred in. If desired, it may be thickened by the addition of $\mathrm{IO}^{\circ}{ }_{0}$ of zinc oxide. As the preparation is apt to deteriorate if kept it is advisable to add a small quantity of thymol or carbolic acid.

Bassorin is a somewhat similar preparation and forms a good base for a varnish. It is made by mixing I part of powdered tragacanth with spirit to wet it, adding 50 parts of glycerine, and heating until clear.

Collodion, both in its contractile and flexible varieties, may be used as a vehicle for chrysarobin and salicylic acid in the treatment of psoriasis and callosities respectively. The contractile action of collodion itself is of value in reducing hyperaemia, as in hyperaemic conditions such as Lupus erythematosus.

Traumaticin, which consists of I dram of gutta-percha dissolved in I ounce of chloroform, is sometimes used as a substitute for flexible collodion as a rehicle for chrysarobin, but it has no advantages.

\section{Lotions}

Lotions act in two ways: (I) as cooling agents by evaporation, (2) as rehicles for drugs dissolved or suspended in them. To prevent them drying too quickly $I^{\circ}$ o of glycerine may be added, but is apt to irritate sensitive skins. According to their ingredients they mav be divided into astringent, sedative, antipruritic, stimulating, and antiseptic. (See Appendix.)

Astringent lotions contain tannic acid, lead, alum, etc. ; sedative and anti-pruritic lotions contain lead, sodium bicarbonate, lime water, carbolic acid, opium, and various other soothing remedies ; stimulating lotions contain tar, chrysarobin, ichthyol, etc. ; and antiseptic lotions contain mercury, boric acid, salicylic acid, and carbolic acid.

To the astringent lotions are sometimes added considerable quantities of an insoluble powder such as zinc oxide or calamine which, becoming deposited on the skin, acts as a protective and increases the astringent action. A few grains of tragacanth may be added prorided the lotion is acid in reaction.

The most common bases are distilled water, rose water, lime water, and alcohol which increases the rapidity of evaporation and the cooling effect. As a rule lotions are dabbed on the skin, but if their full effect be desired they may be employed as wet dressings. 


\section{Dusting Powders}

Dusting powders are extensively employed in cutaneous affections and may be divided into protective, absorbent, astringent, anti-pruritic, and antiseptic. To be effective they should be carefully prepared and repeatedly sifted so as to render them practically impalpable and wholly free from grit. The powder should be dusted on with a piece of cottonwool, a powder puff, or a sifter, or it should be rubbed up in the palm of the hand with a little cottonwool and then pressed into the skin.

Protective Powders.-These are of service in counteracting local irritation from the chafing of contiguous surfaces, the friction of clothes, or the action of sunlight or cold winds. The most commonly used are zinc oxide, starch, rice, licopodium, talc, kaolin, Fuller's earth, ${ }^{\mathbf{1}}$ and the salts of bismuth; others less frequently employed are zinc oleate, aristol, europhen, etc.

Toilet face powders contain combinations of the above, sometimes with the addition of orris root, and are generally so tinted as to render them inconspicuous or to increase their protective porver against the sun's rays. The tinting may be done by the addition of small quantities of prepared calamine, red bole, or turmeric.

Astringent Powders.-Astringent powders cause a contraction of the cutaneous capillaries and reduce hyperaemia. They consist of the protective powlers which are astringent in mild degrees, and others which are more active, such as tannic acid and the sub-gallate of bismuth (dermatol).

Absorbent Powders. - Absorbent powders have a cooling effect by absorbing moisture and are of value in drying the skin and reducing oedema. The most active is magnesium carbonate, which absorbs about four times its weight of water. Another powerful absorbent is Terra silicea, and this has the advantage of being less bulky and more suitable for use in pastes. Starch should not be used as an absorbent powder as it is liable to cake and ferment.

To obtain the full action of the powder it should be dusted on thickly or applied in muslin bags bandaged to the skin.

Anti-pruritic Powders.-Anti-pruritic powders are sometimes employed, such as boric acid, calomel, or sulphur; they should be used for comparatively dry surfaces and never where there is septic discharge.

\section{Poultices}

Poultices are frequently used to soften and remove crusts and as moist dressings or warm compresses for the reduction of inflammation.

${ }^{1}$ Fuller's earth is an alumirium silicate earth. As it is unsterilised it should be used with caution on abraded surfaces, a case being on record in which tetanus was transmitted by it. 
The most useful is the following well-known boro-starch poultice: Mix I teaspoonful of boric acid with + tablespoonfuls of cold water starch, make into a paste with cold water, then pour in I pint of boiling water; when cold, spread thickly on calico, cover with muslin, and apply, renewing every two hours.

The old-fashioned linseed-meal poultice should be aroided as it forms a suitable medium for the growth of micro-organisms and is apt to spread septic infection.

\section{PHYSICAL METHODS}

The physical methods in common use for the treatment of skin diseases are heat, counter-irritation, refrigeration, massage, induced hyperaemia, minor surgical procedures and cauterisation.

\section{HEAT}

Heat is employed in the treatment of skin diseases in the form of hot compresses, hot baths, and dry radiant heat baths. It is beneficial in allaying itching, reducing congestion, and improving the circulation in local inflammatory conditions such as boils.

\section{COUNTER-IRRITATION}

Counter-irritation is of service for the relief of severe localised pruritus ; for example, a mustard plaster or a blister applied over the lumbar vertebrae for the relief of pruritus about the perineum.

\section{REFRIGERATION}

Refrigeration by means of liquid air or solid carbon dioxide is useful for the destruction of naevi, warts, etc.

The value of cold as a local therapeutic agent has long been recognised, but until recently its use was limited to that of a local anaesthetic in the form of ethyl chloride or ether sprays. With the discovery of liquid air and its intense cold a valuable destructive agent was found, and its field of utility was greatly increased.

Liquid Air.-For therapeutic purposes liquid air is contained in a flask of the thermos-bottle trpe with a racuum jacket. A pad of cottonwool wrapped round the end of a stick is dipped in it and applied to the skin for a few seconds with firm pressure; this causes freezing to a depth of one-sixteenth of an inch or more, which is succeeded by an inflammatory reaction. If the application be brief, recovery will follow, if it be prolonged, destruction of the tissue with ulceration and 
sloughing will result. The curative effect is due to the inflammatory reaction, with which is associated an oedematous degeneration of weakly resistant pathological cells and a subsequent repair by a growth of epidermal cells and of new fibrous tissue in the corium.

Though the results obtained from liquid air may be satisfactory and even brilliant, it has certain disadvantages, such as its costliness and the difficulty of obtaining it pure and of applying it sufficiently accurately to restrict its action to the diseased tissue.

Carbon Dioxide.-A good substitute for liquid air

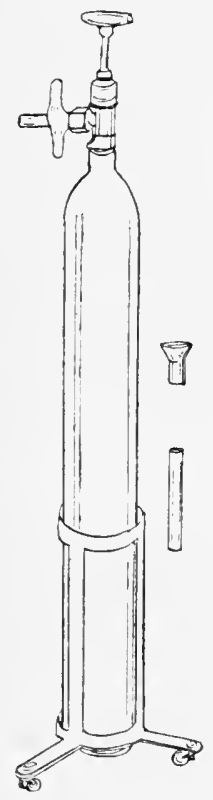

FI G. I 8 . Cylinder of Carbon Dioxide in stand, and Applicator. was found by Pusey of Chicago in solid carbon dioxide. This substance is as effective as liquid air and is cheap, easily obtainable, and capable of application in a more definite manner. It may be obtained in liquid form in a cylinder, which may be placed in a vertical position in an iron stand with castors so that it can be moved easily about the room. The nozzle of the cylinder is horizontal and has a fine bore at the free end of a calibre not much larger than a pin's head while at the proximal end it is about a quarter of an inch in diameter. (Fig. I8.)

Procedure.-The procedure is as follows: A piece of baize or chamois leather is rolled or folded into the shape of a bag and held tightly over the nozzle by the operator with his left hand, which must be protected by a glove; with his right hand he opens the valve by means of a key at the top of the cylinder, when the liquid evaporates so rapidly that its freezing point $(-80 \quad$ C. $)$ is reached and it rushes out at great pressure as a jet of vapour. In a few seconds this collects in the bag in the form of a thick deposit of carbon dioxide snow; the cylinder is turned off, the bag unfolded, and the snow scraped off with a spoon and packed into an applicator. The applicators are made of vulcanite and resemble aural specula; they are of different sizes, the bore varying from a quarter of an inch to an inch in diameter, and may be circular or square. They are fitted with rod-shaped plungers by which the snow can be pressed on to a vulcanite slab or other smooth surface so as to form a solid candle or pencil as hard as is desired. Where the lesions to be treated are very small the pencil may be scraped down to a fine point. The applicator with the solid snow is then transferred to the lesions, the speculum being held by the left hand while the right controls the plunger and regulates the amount of pressure on the skin. Where the lesions are larger than any of the applicators they may have to be treated piecemeal, care being taken to avoid overlapping.

Hore complicated forms of apparatus have been introduced with the claim that by then less snow is wasted than when it is deposited 
on baize and scraped off, but none of them is so simple as the rulcanite applicators, and carbon dioxide is cheap and the wastage in the method described above is so small as to be practically negligible.

The effect of the snow is determined by the length of exposure and the degree of pressure.

Length of Exposure.-The length of exposure ranges from 5 seconds to about 2 minutes and is partly dependent on the situation of the lesion and the delicacy of the skin. No hard-and-fast rules can be laid down with regard to it but a very short experience enables the operator to judge what is necessary in each case. After 5 to Io seconds with firm pressure the underlying skin is frozen into a solid white disc about an eighth of an inch thick; this thaws in about a minute and is followed in an hour or two by an inflammatory reaction and sometimes by the formation of a blister ; the reaction subsides and perfect healing takes place in about a week. Exposures up to 30 seconds are followed by complete healing; if prolonged to a minute or more in soft parts of the skin they are apt to be succeeded by scarring.

For the destruction of soft lesions, such as vascular naeri, the exposure should vary from Io to 30 seconds, while for hard lesions, such as warts and rodent ulcers, exposures of $\mathrm{I}$ or 2 minutes are required. In infants and young children an exposure of one-third of that for adults is sufficient. Where the skin is thick, as on the palms and soles, exposures may need to be twice as long as where it is soft and thin, as on the face and trunk ; where it is in a state of irritability from dermatitis the exposure should be short, as inflamed skin reacts strongly to the snow and heals with difficulty.

Degree of Pressure.-Where the lesions are superficial, firm contact may be all that is required; where they are hard and raised, such as warts, the pressure should be sufficient to flatten or indent the lesion and to enable the snow to exert its action on the healthy tissue immediately around.

Where the lesions are extensive the freezing may be done through a piece of thin lead cut the shape and size of the lesion and interposed between the snow and the skin; the metal conducts the cold and in this way irregularly shaped lesions can be treated exactly, but considerably longer exposure is required than when the snow is applied directly and the results are not satisfactory.

The actual application is not unbearably painful and no anaesthetic is required, the cold itself haring an anaesthetic action. The thawing may be painful and is followed by the usual discomforts associated with acute inflammation.

Should a blister occur it should be opened aseptically and the part dressed with a mild antiseptic ointment to prevent contamination with pyogenic micro-organisms.

The lesions in which this treatment has been found to give the best results are the coinmon raised angiomatous naevi of infants, pigmented and hairy moles, superficial rodent ulcers, and warts. Beneficial results 
may be obtained sometimes in Lupus vulgaris, in certain cases of Lupus erythematosus, and in port-wine stains where the lesions are superficial and the vascular network can be detected, but in them even with the greatest care it is difficult to avoid scarring.

Further details of treatment by carbon dioxide are given under diseases in which it is applicable.

\section{MASSAGE}

Massage is of occasional service in diseases of the skin, as in Alopecia areata to stimulate hair-growth, in sclerodermia to reduce fibrous thickening, and in sebaceous accumulations to express the contents. It is done with the fingers or by special instruments such as Hyde's massering ball, a cup and ball arrangement which is rolled over the skin.

In the cosmetic treatment of the face for the removal of wrinkles and the improvement of the colour and texture of the skin, massage with various creams or finely sifted dusting powders has gained an exaggerated reputation among the laity. The creams if empioyed judiciously make the skin more smooth and supple and have a cleansing effect, but are apt to aggravate or determine comedones.

\section{Induced Hyperaemia, or Bier's Suction TREATMENT}

This treatment is of some value in chronic septic conditions and circulatory disturbances of the extremities. The hyperaemia is induced either by the pressure of elastic bandages or by the suction of cupping glasses.

The pressure treatment is applied to the proximal part of the extremity by an elastic bandage 2 inches wide or an elastic band, should be sufficient to cause a feeling of fullness without discomfort, interference with the pulse, or any evidence of stasis, and should be continued for 3 to 6 hours daily. It is a useful treatment for chilblains, dystrophies of the nails, and tuberculosis of the hands and fingers.

The cupping glasses are applied alternately 5 minutes on and 5 minutes off for about an hour, and are of value in the treatment of furuncles by improving the circulation and so increasing the protective and anti-bacterial substances in the blood and by facilitating the removal of pus.

\section{Surgical Procedures}

A considerable number of minor surgical operations may be necessary, such as opening abscesses, expressing comedones and sebaceous accumulations, remoring concretions, epilation, scraping, scarification, excision, grafting, etc. The majority of them require no special de- 
scription here, while a few, such as scarification and erasion, will be referred to in the description of the diseases in which they are employed.

A large selection of special instruments can be obtained for performing these small operations, such as comedo expressors, epilation forceps, sharp spoons, curettes, fine-pointed knives, etc.

For the opening of deep-seated pustules, sharp-pointed knives similar to those employed in eye surgery, or a double-edged knife tapering to a fine point, should be used; for the remoral of sebaceous secretions a small curette with a solid cup-shaped end not much larger than a pin's head and with sharp edges, should be employed; for epilation the best forceps are those with broad smooth square ends, and for the removal of isolated stumps those with fine tapering ends carefully apposed.

\section{CALTERISATION}

Cauterisation by the Paquelin cautery or the electro-cautery is serviceable for the destruction of small cutaneous neoplasms, telangiectases, etc., and when carefully used gives excellent results. The Paquelin cautery is the more convenient of the two, the fine point being of special value in destroving small lesions with little scarring.

Hot Air.-Instead of the actual cautery, hot air is sometimes employed for the destruction of cutaneous lesions, such as the nodules in Lupus vulgaris and rodent ulcer, after the method introduced by Holländer in which hot air at a temperature of $300^{\circ} \mathrm{C}$. is played on the skin. The operation is extremely painful and requires complete anaesthesia.

The apparatus consists of a metal tube about a quarter of an inch in diameter, with a nozzle at one end and continuous at the other with an indiarubber tube fitted with an air reservoir and a rubber bulb like those of a Paquelin cauter $y^{\prime}$; beneath the metal tube a Bunsen burner is fixed and heats the air in its passage through the tube. The apparatus is so made that it can be held by a handle with the left hand, learing the right hand free to pump the air on to the skin.

Immediately on the application of the hot air the tissue appears to crackle and becomes blanched from ischaemia; when the whiteness is present over the whole of the affected area sufficient hot air has been used to destroy the diseased tissue without injuring the healthy tissue around it and recovery may take place without a marked cicatrix; if, however, the hot air be applied for a longer period absolute charring takes place and an ugly scar is left.

After the application the patch is dressed with a boro-lanolin dressing, or with a dusting powder, so as to exclude the air. Immediately after the operation the pain is severe, but this gradually passes off and the wound heals slowly by granulation. 


\section{ELECTRICAL METHODS}

The principal electrical methods of service in the treatment of cutaneous affections are electrolysis, high frequency, ionisation, galvanism, and faradism.

\section{ELECTROLYSIS}

Electrolysis is largely employed for the removal of hairs and the destrnction of telangiectases. By it hair-bulbs and hair-papillae can be destroyed, capillaries plugged, and minute cutaneous neoplasms removed with the minimum of scarring. The details of the process will be described under the heading of hypertrichosis for which it is most frequently used.

\section{High Frequency}

High frequency currents have been strongly recommended for the treatment of a large number of cutaneons diseases, but in my experience they have proved to be disappointing and of comparatively little real value. The type of electrode most commonly employed is a glass vacuum hammer-ended or pointed electrode, but metallic electrodes may be substituted. They are connected with the top of the resonator and give off high frequency sparks (fulguration), the electrode being held about an inch away from the skin for a few minutes. This causes a marked reaction with the formation of a blister.

High frequency has been used chiefly for rodent ulcer, Lupus vulgaris, Lupus erythematosus, Acne rulgaris, rosacea, port-rine stains, localised pruritus, and Alopecia areata.

\section{IONISATION, OR CATAPHORESIS}

This method of treatment has been employed extensively of late years in rodent ulcer, Lupus erythematosus, etc. By it is meant the introduction into the tissue of ions of various kinds, but chiefly of the metals zinc, copper, and mercury. They are liberated by the splitting up of a solution of the salt of the metal by the electrolytic action of a continuous current, the positively charged ions or basic radicals being attracted by the negative pole, and the negatively charged ions or acid radicals by the positive pole; in the case of zinc sulphate, for instance, the zinc being the basic radical will be attracted by the negative pole, while the sulphur being the acid radical will be repelled by the negative pole and attracted by the positive pole. The salts chiefly employed are zinc sulphate, zinc chloride, sodium clloride, copper sulphate, and perchloride of mercury. By this method it is believed that much greater penetration of the element is obtained than by any other. 
The procedure in the case of zinc sulphate, for example, is as follows: Several pieces of lint the size of the lesions to be treated are superimposed and dipped in a $2 \%$ solution of the salt in distilled water, care being taken not to handle them with the fingers to aroid contamination by chlorides. The noist lint is placed on the lesion and a flat block zinc electrode of the same size, or slightly smaller, attached to the positive pole of the battery is firmly pressed against the lint. The negative pole, consisting of a metal electrode covered in chamois leather is moistened in salt solution and held by the patient or applied to the skin at a distance from the lesion. A current of 2 to ro milliamperes is passed for ten to twenty minutes-the current averaging 2 milliamperes for each centimetre square of the positive electrode. This causes a burning sensation which may amount to actual pain but may be somewhat relieved by previous ionisation with a weak solution of cocain. When the application is made on the face, it is associated with a salt taste in the mouth. Before applying the current it is advisable to remove all grease from the shin as it is a bad conductor and apt to impede the current.

When a destructive action is desired and the lesions are raised, as in new-growths of the epidermis, a, pointed zinc needle should be substituted for the flat electrode.

If the exposure be prolonged it is necessary to move the negative pole from place to place to prevent it from exerting its destructive action and causing an intractable ulcer; rings should be removed from the fingers, as they are good conductors and may determine a sore beneath them.

The conditions most benefited by ionisation are rodent ulcer, ordinary varicose ulcer, Lupus erythematosus, and Lupus vulgaris, and great improvement may be obtained by it in suitable cases without appreciable scarring.

The employment of chlorine ions for the absorption of fibrous thickenings, such as cheloids, has been suggested, but the results from it are uncertain. In this case the lint is saturated with a weak solution of sodium chloride and the negative pole is applied to the lesion, as the chlorine passes from the negative to the positive pole.

\section{GALVANISM AND FARADISM}

Galvanism and faradism are both of service as therapeutic agents in connection with the skin and are employed chiefly to soothe pain or itching in conditions like Herpes zoster, and to relieve circulatory disturbances, such as chilblains and Raynaud's disease.

Galvanism is soothing, while faradism is stimulating, and of the two the former is the more useful.

In the case of galvanism a galvanic battery containing about Io Leclanché cells, or a continuous electrical current from the 
main with an efficient apparatus to prevent shock and give the required resistance should be used; in the case of faradism a single bichromate cell and a small induction coil are generally sufficient. The electrodes are of various sizes and shapes and are generally made of zinc covered with chamois leather. They are dipped into salt solution before being applied in order to lessen the cutaneous resistance.

For the relief of the neuralgic pain of Herpes zoster, for example, the negative pole, which should be at least a couple of inches in diameter, is applied over the spinal cord about the level of the exit of the affected nerve, the positive or soothing pole is stroked along its course, and a current of 6 to Io milliamperes, or as much as can be borne comfortably, is passed for about ten minutes. The negative pole should be moved from time to time during the passage of the current to avoid producing superficial necrosis.

For the relief of the vascular spasms in the local syncopal phase of Raynaud's disease the negative pole is placed on the arm and the positive pole is applied to the hands or fingers; while for the local asphyxial phase or for chilblains the poles are reversed and the negative pole applied to the fingers. Owing to the circulatory stasis a stimulating rather than a soothing effect is indicated in this phase and faradism may therefore be substituted for galvanism.

Electrical Baths.-Electrical baths are useful in the treatment of similar conditions and have a soothing effect. Glazed earthenware baths are employed and electrodes consisting of copper plates are hung. at the side of the bath and immersed in water about blood heat to which has been added a teaspoonful of salt to the pint of water. The moisture of the warm dilute salt solution in the bath lowers the resistance of the skin and favours the passage of the current, while the warmth increases its sedative effect.

The bath may be mono-polar or bi-polar, according as one or both poles are immersed in the water. Where one extremity has to be treated a bi-polar bath is convenient and the limb is immersed in the bath; where the trunk or face is affected a mono-polar bath is used, an electrode being applied to the affected skin and contact made by placing a hand in the bath in which the other pole is immersed. Should a soothing effect be desired the positive pole is applied to the lesion; if stimulation is required the negative pole is applied.

Where both arms or both legs require treatment, two baths may be used each containing a pole, and the patient sits with a foot or hand in each and the circuit is completed through the legs and thighs or through the arms and shoulders respectively. 


\section{RADIO-THERAPEUTICS}

Radio-therapeutics are employed in cutaneous diseases in the form of $\mathrm{X}$-rays, photo-therapy, and radium.

\section{X-RAYS}

The $\mathrm{X}$-rays are now used extensively in the treatment of cutaneous diseases and constitute one of the most powerful of all local curative agents. In suitable cases and when properly applied they are capable of giving brilliant results with perfect safety, but in unsuitable cases or if improperly administered they may produce the most disastrous effects. The question of the type of X-ray system for therapeutical purposes is a matter of opinion, and each operator has his favourite system with the working of which he is thoroughly familiar and by which he is able to obtain uniform and satisfactory results.

The following system may be described as an example of a useful type: The electrical current is obtained from the lighting main continuous current and suitable resistances interposed. A dipper mercury interrupter is used and a I2-inch coil. Connected with the interrupter is a "speed counter " which records on a dial the number of plunges of the dipper into the mercury; the dial is divided into I5 divisions, each of which is subdivided into two-each subdivision indicating 500 dips or "makes" and "breaks" of the primary circuit. The dipper is so fixed that it will plunge about $\mathrm{I} \mathrm{cm}$. into the mercury and the speed regulated to give about 1000 dips per minute. ${ }^{1}$ The tube is placed in a lead-glass shield fixed to the arm of the tube-stand, which can be moved in any direction and fixed rigidly in the required position. On one arm of the stand a milliampere meter is placed to indicate the current going through the tube, and on the other arm a spark valve or "soupape" is inserted to remove the back-lash of the make current. In the angle between the right arm and the column a lever is fixed which works on a quadrant and adjusts the spark gap between the poles. In front of the lead-glass shield there is a circular opening to which is fixed a lead-glass cylinder of a diameter corresponding to the size of the lesion to be exposed and of such a length that when the skin is placed against it it will be distant $15 \mathrm{~cm}$. from the anode of the tube. The maximum diameter of the cylinder should not exceed $7.5 \mathrm{~cm}$., i.e. half the distance between the skin and the anode, as that corresponds to the size of the section of the beam of $\mathrm{X}$-ray's and a wider cylinder would not enable a larger area to be effectively irradiated. As the tube is enclosed in a lead-glass shield and the rays are only emitted through the circular aperture the operator is in no way.

\footnotetext{
"Instead of the "Dipper break" a gas mercury rotary break of the "Zenith" type may be employed and by it the number of discharges from the tube per minute may be increased three to six times.
} 
exposed to them. The tubes employed are of the Müller pattern with a heary anode and an automatic vacuum regulator-a convenient size being $\mathrm{I}_{5} \mathrm{~cm}$. in diameter. (Fig. I9.)

Dosage.-The dosage is regulated by means of the pastille invented by Sabouraud and Noiré, which is inserted in a small metal holder at the junction of the cylinder and the shield-—that is $7.5 \mathrm{~cm}$., half the distance between the skin and the anode. The pastille is a small cardboard disc, one side of which is covered with a film of platinobarium-cyanide which, when exposed to the X-rays, becomes transformed from a greenish to a brownish tint. The maximum dose which can be given to the skin without injury and which causes a defluvium of the hair without preventing subsequent re-growth is estimated by means of a standard tint known as the tint B. Should this be exceeded and a deeper tint produced, an X-ray burn will result. If the pastilles are in proper condition they are absolutely reliable, but care must be taken in using them to judge the tint B accurately and by daylight, as when it is done by electric light the tint $B$ is reached with a threequarter dose. It is necessary also that the pastille should be backed with a piece of metal when it is in position, and unless the apparatus is in semi-obscurity it should be protected by a piece of black paper from the action of daylight which, if strong, will retard the change of colour and result in an over-dose followed by a burn. After the pastille has been used and been turned brown it will gradually recover its original tint if exposed to sunlight and may be used again two or three times, but it turns slightly more quickly each time.

The speed counter indicates the number of discharges from the tube required to turn the pastille and enables fractional doses to be estimated. With a moderately high tube with rays corresponding to about 4 degrees of penetration-as indicated by a Benoist radiometer-and a current of about half a milliampere in the secondary circuit the pastille is turned in about 12,000 discharges.

A much more accurate method of estimating fractional doses is by means of radiometers provided with a series of tints up to the full tint B, such as the Dudley Corbett radiometer.

It is usually asserted that certain individuals have an idiosyncrasy towards the X-rays and react to them more powerfully than do others. This possibility has been greatly exaggerated, but there are certain considerations which influence the dosage, such as: (I) the age of the patient, the skin of an infant for example being more delicate and reacting more readily than that of an adult ; (2) the texture of the skin whether it be coarse or fine, owing to the situation on the body or to individual peculiarities; (3) the presence of more or less pigment in the skin; (4) the condition of the skin whether it be normal or inflamed; when it is acutely inflamed or has been irritated by the local application of some drug such as iodine it reacts readily to the rays, and it is advisable to wait for the inflammation to subside before applying them. 


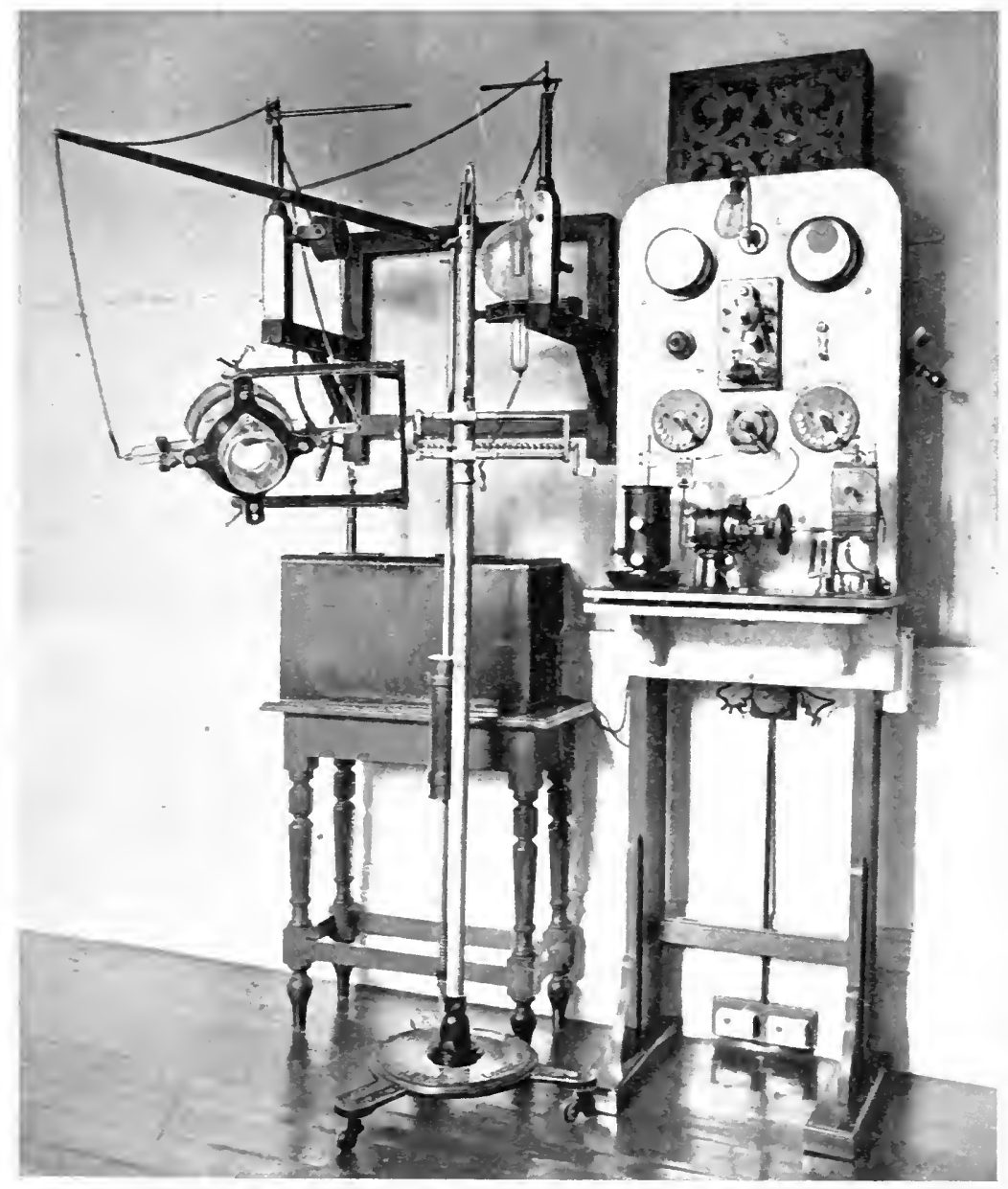

FIG. I9.-X-ray Apparatus.

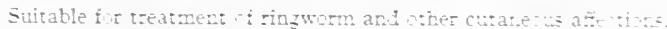



A point of extreme importance is that the space between the skin and the anode should be constant, a convenient distance being $I .5 \mathrm{~cm}$. ; as the effect of the rays varies inversely with the square of the distance it is necessary to consider eren such slight differences as the closer proximity of the summit of a convex surface in exposing the scalp.

The effects of the rays are cumulative and the influence of a dose continues for several weeks after it is given, consequently it is unsafe to repeat the maximum dose for at least six weeks.

It is customary to divide X-rays into hard or highly penetrating rays, and soft or weakly penetrating rays : these are the two extremes of the beam of X-rays, which is divisible into an infinite number of gradations from hard, through medium, to soft rars. When the vacuum in the tube is low the soft rays are in excess and the tube is known as a soft tube, when the vacuum is high the hard rays are in excess and the tube is known as a hard tube. It is generally stated that the hard penetrating rays are the chief curative rays and that the soft rays are superficial in their action and responsible for the X-ray dermatitis. This statement is misleading and doubtful; practically, it has been found that when the tube is soft a much larger number of discharges is necessary to produce the tint $\mathrm{B}$ than when the tube is hard, and that whether the tube be hard or soft the full pastille dose may be given without producing harmful results.

Where the lesion is situated beneath the shin, as in a tuberculous gland, and where it is desired to give large doses of highly penetrating rays without producing dermatitis, a screen of thick felt or of aluminium I cm. or more in thickness is used to intercept the soft rays.

Skin Affections benefited by X-rays.-The affections for which the X-rays are chiefly used are ringworm of the scalp, rodent ulcer, Lupus rulgaris, scrofulodermia, tuberculous glands, Lichen planus, lichenified patches of eczema, psoriasis, hypertrophic scars and cheloids, warty growths, comedones, Mycosis fungoides, localised pruritus, and hyperidrosis.

Histological Effects and Pathological Action.-- In small doses X-ra!s have a stimulating effect on the healthy elements of the shin and cause increased growth of the epidermis, the formation of pigment in the cells of the deeper larers, the stimulation of the sweat-glands and the hairgrowth, and an improvenent in the circulation.

In large doses, or repeated small doses, they devitalise the tissueelements and cause degeneration and interference with the process of reproduction of the cells. As a result of the direct action of an excessive dose of the rays the epidermal cells become racuolated, the riuclei shrink, and they erentually shrivel and die; the cellular elements in the corium, especially if pathological, such as giant-cells and plasmacells, undergo hyalin degeneration, the fibrous tissue especially the collagen becomes atrophic; the endothelium of the capillaries may break down and lead to the formation of telangiectatic sinuses, and the 
cells composing the hair-follicles and the sweat-coils are similarly affected.

Pathologically altered cells, whether of epiblastic or mesoblastic origin, are less resistant to the rays than healthy cells and are devitalised by smaller doses, so that while a destructive action may be taking place in the diseased elements, the neighbouring healthy elements may be actually stimulated to growth or repair.

When the cellular degeneration reaches a certain degree the toxic products of the breaking down cells are capable of setting up secondary inflammatory changes, and a reaction which is peculiar in that it occurs in a tissue the vitality of whose elements has already been impaired by the rays and which is liable to lead to destructive changes such as ulceration and necrosis.

The reaction which results from a massive dose of the $\mathrm{X}$-rays does not follow the exposure imnediately but occurs after the lapse of seven to fourteen days. Repeated small doses continued over a long period but insufficient in themselves to produce a definite reaction, lead to a devitalising of the tissue and the production of a state of instability of the skin in which it is liable to break down and ulcerate and may undergo malignant changes (see X-ray dermatitis, p. 260).

The direct bactericidal effect of the $\mathrm{X}$-rays is comparatively slight, as has been proved by exposing cultures of bacteria to the rays, when it has been found that ordinary doses may even stimulate their growth. On the other hand, the rays have the power of cleaning septic ulcerated surfaces by causing a reaction in the tissue which results in the destruction of the micro-organisms and renders the soil unsuitable for their growth.

\section{Photo-Therapy}

The application of light in the treatment of disease is an ancient practice, and as early as the fourteenth century John of Gaddesden, physician to Margaret of France the second queen of Edward I. of England, treated the young Prince Edward for smallpox by red light, by enveloping him in scarlet cloth and arranging that the bed, furniture, and hangings in his chamber should be of a bright red colour. This was said, not only to have cured the prince, but also to have prevented his being marked by the disease. In a commentary on this treatment Strickland, in her Queens of England, writes as follows: "In this childish state was the noble art of healing at the court of Margaret," and "while music and sculpture had attained some degree of perfection in England at this time, other arts and sciences were in a strange state of ignorance."

That the red rays cured the smallpox is, of course, ridiculous, but that they prevented pitting is not only possible but probable, and towards the end of last century Finsen corroborated this observation, pointed out that the action of light was injurious in smallpox, and reconımended 
that the patients should be protected against its harmful rays by placing them in a red light. This recommendation was tested in a fever hospital and was beneficial so far as the skin was concerned, but had to be discontinued on account of the irritating mental effect of the red rays on the patients and the nurses.

The harmful rays of the spectrum are the actinic or chemical raysnamely the blue, violet, and ultra-violet-which consists of vibrations of ether of small wave length and great rapidity; the rays at the opposite end of the spectrum - namely the red and orange rays-are harmless, being simply heat rays with a long wave length.

Photo-therapy is the application to the skin of the actinic and chemical rays of the spectrum. These rays have two effects: (I) they cause a powerful cutaneous reaction which destroys diseased tissue-especially in the corium-and replaces it by new fibrous tissue, (2) they are capable of killing micro-organisms on or near the cutaneous surface. They may be obtained either from sunlight or from a strong voltaicarc lamp by filtering light through a water chámber with quartz lens at each end.

The sunlight apparatus, which is only applicable in sunny climates, consists of two parts, namely a condenser and a compressor. The condenser is formed by two large flat lenses ten inches in diameter fitted into a metal frame so as to form a compartment, which is filled with water tinted blue by an ammoniacal solution of sulphate of copper and mounted on a movable stand. By this means the red, orange, and yellow heat rays are filtered from the light and the actinic rays are isolated and focussed on the diseased skin, not directly but through the compressor. The compressor is a flat disc-shaped box, with quartz sides and about the size of an ordinary watch, through which water is allowed to circulate freely by means of an entrance and an exit tube. The compressor is pressed firmly against the skin by an attendant, to render the part anaemic and allow the rays to penetrate more deeply, as it has been found that the red blood cells hinder penetration.

The artificial light apparatus originally employed by Finsen, and known as the Finsen Lamp, consisted of a large central arc lamp using a current of 50 to 60 amperes, from which radiated four telescope-like collectors with a system of quartz lenses to collect and concentrate the rays. The lower chamber of the collector was filled with distilled water and fitted with a water-jacket through which water constantly circulated. By means of a sliding adjustment the rays from the collector could be accurately focussed on the compressor. With this apparatus four patients could be treated at one time.

The Finsen-Reyn lamp was a convenient substitute for the original cumbersome apparatus, it had only a single collector and used about 20 amperes of current. Tarious other types of lamp have been introduced since then, such as the Lortet-Genoud lamp in which no separate compressor is required and the patient presses directly against the 
anterior lens; and more recently lamps with iron electrodes, and mercury vapour lamps. (Fig. 20.)

In the older lamps carbon electrodes were employed; other materials have now been substituted, such as tungsten and wolframite, which give radiations richer in actinic rays than those from the carbon arc. (Fig. 2I.)

The newer lamps, though more superficial in their action than the Finsen-Reyn lamp, are less clumsy and require shorter exposures.

With the older lamps exposures of an hour or an hour and a half were necessary, during which time the compressor was pressed firmily against the diseased area by an attendant or was fixed to the collector

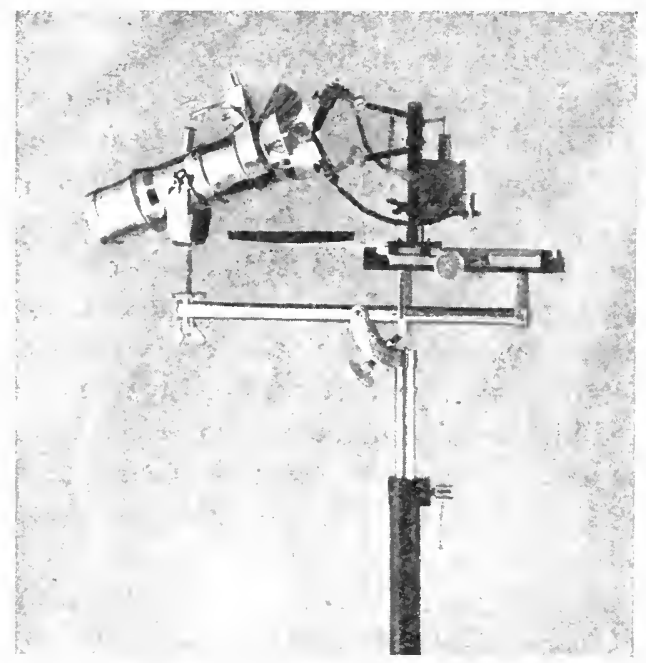

FIG. 20.-Finsen-Reyn Lamp.

at the requisite distance and the patient made to press against it ; with the more modern lamps exposures of a quarter to half an hour suffice.

This treatment was introduced for, and is chiefly employed in, the treatment of Lupus rulgaris.

Previous to the exposure any crusts are remored and the area to be exposed is cleansed with boric lotion. During the exposure the patient's eyes must be protected from the light by a pad of cottonwool covered with thick brown paper.

In from four to eight hours after the exposure a more or less acute inflammatory reaction supervenes with redness, heat, pain, and bullous formation. This lasts for about a week, during which time the part should be dressed with a simple antiseptic ointment, and when it has subsided the exposure should be repeated. The degree of reaction may be intensified in resistant patches by previously painting them with a I \% solution of erythrocene. After the first few exposures marked improvement generally takes place and the lesions become flatter and 
paler from the replacement of the superficial cellular infiltration by fibrous tissue, but beneath this generally persist some deep-seated infiltration and foci of disease which are difficult to reach.

The most suitable type of case for this treatment is an early Lupus vulgaris of the face of limited extent in which there is neither ulceration

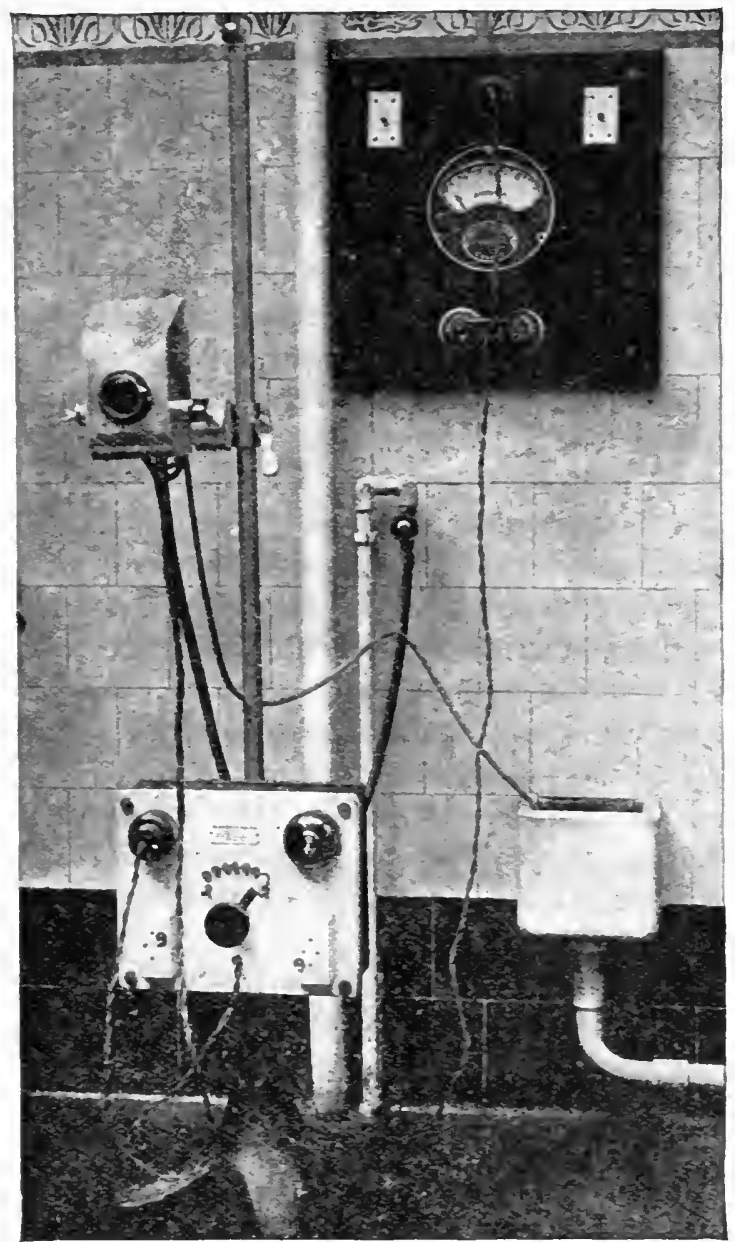

FIG. 2I.-Clltra-riolet I.amp. (Forbes type.)

nor scar tissue from previous treatment. It may also give good results in all forms of septic granulomata and in some forms of Lupus erythematosus. It is tedious and where extensive areas are involved may occupy months or even years, but the cicatrices which follow are soft, pliable, little different from the surrounding skin, and superior to those produced by any other form of treatment. A certain degree of pigmentation is caused by the light, but this disappears in course of time.

Histopathology.-The histopathological changes produced by the actinic rays consist of dilatation of the blood-vessels and an extra- 
vasation of lymphocytes and serum, followed by oedema and a spongy degeneration of the plasma-cells and giant-cells, which break down, become absorbed, and are eventually replaced by new fibrous tissue. Superficially situated bacilli are destroyed by the rays, while those more deeply-seated succumb indirectly to the inflammatory reaction.

\section{RADIUMI}

As was to be expected with such a mysterious and powerful agent as radium, its therapeutic value was greatly exaggerated at first; but though it cannot accomplish all that has been claimed for it, it is of great service in suitable cases.

It is used in the form of the bromide or the sulphate, either pure or, more usually, mixed with barium.

The bromide is a soluble salt and is applied in glass tubes which are inserted into larger metal tubes to protect them and to act as screens for certain of the rays.

The sulphate is an insoluble salt and can be spread evenly and fixed by a special varnish on metal plates, rods, or buttons, which are known as applicators. The varnish resists the action of water and of ordinary antiseptic solutions, but is partially soluble in alcohol and ether. The metal plates vary in shape, being circular, square, oblong, or triangular, and in size from a few millimetres square up to six centimetres square or more. When the proportion is I centigram of the salt to each centimetre square of surface it is known as a "full strength" plate; when it is advisable to have half- or quarter-strength plates this is done by diluting the salt to the required proportion by the addition of an inert barium salt.

Varnishing the radium on a surface has the advantage of intercepting fewer rays than when it is applied in a glass tube or an aluminium applicator, but has the disadvantage of exposing it to loss by friction and to injury by alcohol, ether, etc.

Physical Considerations. - The radiation from radium consists of three different kinds of rays, known as alpha, beta, and gamma rays.

Alpha rays are small particles resembling atoms of hydrogen, charged with positive electricity; they are slightly deflected to the left by a magnetic field, and have little power of penetration, being intercepted by a layer of cottonwool, mica, etc. They form $90 \%$ of the total radiations.

Beta rays are negatively charged electrons which are deviated to the right by a magnetic field and are alleged to be similar to cathodal rays. They are subdivided into soft, medium, and hard $\beta$ rays according to their velocity and power of penetration. They form about $9 \%$ of the rays.

Gamma ray's are vibrations of ether and resemble X-rays. They are not deflected by a magnet, and have a great velocity, probably as 
great as that of light, and an extraordinary power of penetration, being capable of passing through lead several inches thick. They constitute I $\%$ of the radiations. The greater the degree of penetration of the rays the more powerful the effect on diseased tissue, the $\gamma$ and hard $\beta$ rays being the most active while the soft $\beta$ and $\alpha$ rays have a superficial effect.

When $\gamma$ rays pass through any form of matter, secondary rays of the $\beta$ type are formed along their course, while the $\gamma$ rays themselves are supposed to result from the disintegration of the radium into $\alpha$ and $\beta$ rays.

The radio-activity of radium is estimated by means of an electroscope and is stated in terms of comparison with that of an equal quantity of uranium, that of the pure bromide or sulphate being taken at about two millions.

Choice of Apparatus. - In the choice of an apparatus it is necessary to take into consideration the weight of salt employed, its radio-activity, the total dimensions of the surface of the applicator, and the different rays emitted by it. It has been shown that radium spread out on a flat surface has much more effect on the tissue than the same quantity of the salt in a small glass tube, and it has been estimated that I cgrm. of radium salt of an actirity of Ioo,000 on a plate has approximately. the same effect as $5 \mathrm{cgrm}$. of a salt of an activity of 600,000 enclosed in a glass tube.

The applicator is not, as a rule, applied directly to the skin but is separated from it by a thin layer of gutta-percha to protect it from moisture or discharges, a piece of black paper to cut off the secondary radiations, and a screen of thin aluminium, lead, silver, etc., to intercept the less penetrating $\alpha$ and soft $\beta$ ray's.

Length of Exposure.-The length of exposure must be left largely to the experience and judgement of the operator. It varies in different cases from a few minutes to several days and is dependent on such considerations as the radio-activity of the applicator, its extent of surface, the percentage of the different rays, the screen employed, and the diseased condition for which it is applied.

Experience has taught that $5 \mathrm{mgrm}$. of pure radium sulphate varnished on a flat surface of $\mathrm{I} \mathrm{cm}$. square applied directly to the skin, after an exposure of twenty minutes can produce a severe reaction going on to ulceration, and that the same result can be obtained from three applications of ten minutes' duration on consecutive days. When screens of aluminium or lead are employed to cut off the soft rays, much longer applications may be given, even lasting several day's, before a reaction takes place. In the case of raised lesions such as small tumours, to shorten the time of exposure and to aroid an excessive superficial reaction, flat applicators may be applied on each side of the tumour so as to subject the deepest part to a crossfire of penetrating rays (Wickham and Degrais).

Action of Radium on Diseased Tissues.-The rays from radium have a more powerful effect on diseased than on healthy tissue and may be 
said to have a selective action, picking out pathological cells owing to their weaker power of resistance. The nearer the diseased cells are to the surface the more powerfully are they acted on by the rays, while the deeper cells, such as those of the corium, are less affected as the rays are to some extent screened off in passing through the epidermis.

The rays have a specific action on blood capillaries, destroying and replacing them by new fibrous tissue, and on the sensory nerve terminations, allaying itching and relieving pain. Their bactericidal effect is slight and is probably dependent on the $\alpha$ rays.

At the time of application no definite sensation or signs of inflammation are produced and the characteristic reaction does not occur, as a rule, for from ten to fourteen days. The reaction is associated with all the signs of inflammation and may be limited to the lesion itself or may spread to the neighbouring skin. In a severe reaction oozing takes place and a scab forms which is not unlike a rupial crust; this separates naturally in two to four weeks, either leaving a healed surface or being replaced by a second scab which has to separate before healing is complete. When the application has been excessive, signs of the acute reaction may appear as early as twenty-four hours and vesicles may derelop which dry up to form a scab; this falls off in a week or ten days leaving a superficial ulcer, which heals slowly and is followed by a smooth red scar covered with telangiectases like the scar of an X-ray burn.

The reaction is probably the result of an inflammatory process caused by the toxic effect of dead cells acting on a tissue partially devitalised by the rays.

Skin Affections benefited by Radium.-The cutaneous affections in which radium has given best results are rodent ulcer, cheloid, hypertrophic scars, superficial epithelioma, and vascular naevi. ' It has been used also for the destruction of seborrhoic warts, papillomata, hairy moles, nodules of Lupus vulgaris, lichenified patches of eczema, hypertrophic Lichen planus, and for the relief of pain and pruritus, but in them it has proved to be of less value than other methods of treatment.

\section{Other RADIO-ACTIVE SUBSTANCES}

The other radio-active substances which have been employed in the treatment of skin diseases are thorium and uranium, but they are of little value.

Thorium.-Thorium is mildly radio-active, its radio-activity being about a million times less than that of radium and too fecble to produce any definite effect. A quarter of a pound of hydroxide of thorium was sealed up in a flat rubber bag and placed on a patch of Tuberculosis cutis of the leg where it was kept in apposition for a fortnight; but in spite of the length of exposure it failed to set up any reaction or to be of the slightest benefit. Dusted on an ulcerated surface it secms to be as inert. 
Uranium.- Lranium is equally feeble and though in the form of calcium phosphate it has been recommended in the treatment of tuberculous affections of the skin in applications of a few hours daily, it is of no real value.

\section{REFERENCES}

\section{$X$-Ray's}

Corbetr, D. A method of standardising the tints given by the SabouraudNoiré pastille (Brit. Journ. Derm., I9I3, xxv. p. 249).

Dore, S. E. Cse, action, and dosage, of X-rays in psoriasis and other shin diseases (Brit. Med. Journ., October I9I3, p. Ior6).

MacLeod, J. M. H. Remarks on the pathological action of the Röntgen Rays (Brit. Journ. Derm., October I903, xw. p. 365 ).

Porter, C. A. Surgical treatment of X-ray carcinoma $\mathbb{E}$ other severe X-ray lesions (Journ. Med. Research, July I909, p. 35i).

Riddell, J. R. The X-rays in treatment (Brit. Med. Journ., April IgI I, P. 985$)$.

SABOURAUD, R. The Radio-therapeutics of ringworm (Brit. Journ. Derm., I906, xviii. p. I99).

Sequerra, J. H. Some experiments with X-ray filters (Arch. of the Röntgen Rays, January I9I5).

Wolbach, S. B. Pathological histology of chronic X-ray dermatitis \& early X-ray carcinoma (Journ. Med. Research, I909, p. +I5).

\section{Photo-therapy}

Hrde, J. X., Moxtgomery, F. H., \& OrMsby, O. S. A contribution to the subject of Radiotherapy \& Phototherapy in carcinoma, tuberculosis, \& other diseases of the skin (Read at the 53 rd Annual Meeting of the Amer. Med. Ass.).

Russ, S. From the visible to the Gamma ray spectrum (Brit. Med. Journ., I9I6, p. I24).

SeqLeIRA, J.H. Some experiments with the Simpson Arc Lamp (Lancet. February, 1916).

\section{Radium}

Delbet, P., \& Ledotx-Lebard, R. Radium au traitement des cancers épithéliaux (Bull. Ass. Française pour l'Etude du Cancer, I9oS).

Frxzr, X. S. Recent work in Radium, Radiology \& Electro-therapeutics (Practitioner, I9I6, p. I9I).

Kxox, R. Radium in the treatment of malignant disease (Brit. Iled. Journ., I9I3, p. II96).

LAZARCS-BARLOW, W. S. The Croonian Lectures on radio-actirity and carcinoma (Brit. Med. Journ., I909, p. I 465 ).

Pearce Gotld, A. Radium and Cancer (Idem, I9I f, p. 2).

Radium Institute Reports, London, I9I3 \& I9I4.

Wicкнам, L. Le Radium (La Presse Méd., December, igoS).

Wickhay, L., \& Degrais. Traitement des Angiomes par le Radium (Rer. de Méd., June \& July, I9o\$).

Action thérapeutique du Radium dans la Tubercle cutanée La Presse Méd., February, I90S). 


\section{CHAPTER VIII}

\section{NOMENCLATURE AND CLASSIFICATION}

\section{Nomenclature}

THE nomenclature of skin diseases has always been a stumblingblock to the student, on account of the long and complicated names employed in it and the variety of synonyms associated with each disease.

Complexity in terminology is due to several causes of which the most important are the following:

(a) Situation of the Skin.-As the skin is an external organ, morbid processes affecting it can be observed and minute differences in objective phenomena can be detected and recorded in a manner impossible in. an internal organ, and in consequence the original names employed were descriptive of objective phenomena. These names were mainly derived from Greek and Latin words, as in such names as eczema, from ' $\mathrm{E} \kappa \zeta \zeta^{\epsilon} \omega$, to boil over, and urticaria or nettle-rash, from urtica, a nettle.

(b) Differences in the Type of Lesion.-Modifications in the appearance of lesions in the same disease were distinguished by the addition of appropriate Latin adjectives, as in Eczema papulosum where the essential lesions were papules, Eczema impetiginosum where eczematous lesions had become secondarily inoculated with pyogenic cocci, Psoriasis guttata where the lesions suggested drops of mortar, and Psoriasis gyrata where they tended to evolve into gyrate figures.

(c) The Employment of Descriptive Titles. When new diseases were discovered or differentiated there was a tendency to particularise so as to make the title as descriptive as possible, as in such names as Pityriasis rubra pilaris, which signifies a scaly red affection involving the hairs.

(d) Histopathological Considerations.-As our knowledge advanced, attention came to be paid to the minute structure of the lesions and a series of new names based on their histopathology was introduced, such as Hyperkeratosis palmaris indicating a pathological process in which the horny layer of the palms is thickened, Keratosis pilaris where the hair-follicles are plugged by small horny spines, and Acanthosis nigricans 
in which there is marked proliferation of the prickle-cell layer of the epidermis.

(e) The Naming of Diseases after those who discocered them.-This method has been adopted occasionally, as in Paget's disease of the Nipple, Kaposi's disease (Xeroderma pigmentosum), Bazin's disease (Erythema induratum); but while all honour is due to the discoverer it is a method which leads eventually to confusion.

(f) Etiological Names.-The modern tendency is to name diseases so as to indicate as far as possible the underlying cause; for example, coccogenic sycosis, or Barber's itch, which is a type of sycosis due to cocci, and blastomycetic dermatitis, an inflammation of the shin due to blastomyces or yeast.

(g) Number of Synonyms. - This is due in one case to a disease having been described independently by two or more observers each of whom used his own designation, and in another to original names having proved inaccurate or misleading and having been supplanted by others which were regarded as more adequate.

At the present time an international unanimity with respect to the name which should be reserved for each disease would be an immense advantage.

\section{CLASSIFICATION}

The evolution of the subject of diseases of the skin is written largely in the systems of classification which have been employed in connection with it.

When our knowledge was mainly clinical the classification was on a purely objective basis, as in the elaborate system of Robert Willan, an English physician who lived about the beginning of the nineteenth century, which was in more or less general use till Hebra's work was published about fifty years later.

Hebra studied cutaneous affections from a pathological standpoint and divided them into twelve classes on a pathological basis, as follows : hyperaemias, anaemias, anomalies of secretion, exudations, haemorrhages, hypertrophies, atrophies, neoplasms, pseudo-plasms, ulcerations, neuroses, and parasitic diseases. This system, modified with the advance of knowledge, was adopted by the majority of writers during the latter half of last century.

It marked an epoch in dermatology and was certainly an advance upon Willan's, as it eliminated much confusion and overlapping, but it was still so far from perfect that when our French colleagues attacked the problem in connection with their large textbook, La Pratique Dermatologique (I9OI), they discarded it and resorted to the simple expedient of an alphabetical arrangement. This method also has its drawbacks, for it necessitates a knowledge on the part of the student of the name of the disease before he can employ the book for purposes of reference. 
The ideal and only satisfactory system of classification is one on an etiological basis, but in the present state of our knowledge this system is even less complete than the others. It has the advantage, however, of focussing the mind of the student on what is of the greatest importance in the understanding of the disease and its treatmentnamely the underlying cause-and it will be employed here in as far as is possible, on the same lines as were adopted by Colcott Fox in his volume on Skin Disease in Allbutt and Rolleston's System of Medicine. 


\section{PART II. SPECIAL}

\section{CHAPTER IX}

\section{CONGENITAL ANOMALIES OF THE SKIN}

THE congenital anomalies of the skin are ichthyosis, congenital hyperkeratosis of the palms and soles, and naevi. Certain of them may not be manifest at birth but may appear in early infancy or later in life and are not congenital, in the strict sense of the term, but in most if not in all of them microscopical changes are present at birth which, though insufficient to be apparent to the naked eye, increase subsequently and give rise to objective lesions.

\section{ICHTHYOSIS}

Der.: i $\chi \theta$ ús, fish.

Syn.: Ichthyosis simplex, Ichthyosis vera, Fish-skin Disease, Xerodermia, Sauriasis. Ichthyosis congenita, Harlequin foetus, Ichthyosis foetalis gravior, Hyperkeratosis universalis congenita (Unna), Keratoma malignum congenitale.

Fr., Ichthyose ; Ger., Fischschuppenauschlag.

Definition: A chronic affection of congenital origin appearing at birth or in early life, and characterised by a peculiar dryness and harshness of the skin, or the presence of scales, horny plates, or warty growths.

Introduction.-Under the heading of ichthyosis, qualified by various adjectives, a number of conditions have been grouped which differ in their nature and pathogenesis. In the first place there is Ichthyosis simplex, or ordinary ichthyosis, which does not as a rule appear till between the first and second years. Next, there is Ichthyosis foetalis gravior, or Harlequin foetus, which is a developmental anomaly of universal distribution which begins in utero probably about the fifth month, in which the infant is prematurely born, and is either stillborn or lives only a few days; this is the most severe form of the disease and is regarded by some observers as being of a different nature from ordinary ichthyosis. The two conditions seem, however, to be essentially similar in their pathogenesis and to differ in degree of severity rather than in kind. 
In addition there are certain affections to which the name " ichthyosis " has been applied which belong to a different category, such as Ichthyosis hystrix, in which grotesque warty growths appear, which should be included under the heading of naevi or circumscribed congenital anomalies of the skin; Ichthyosis cornea, a name which is sometimes used as synonymous with Ichthyosis hystrix, but originally applied by Willan to sclerodermia; Ichthyosis palmaris et plantaris, which is a congenital hyperkeratosis affecting the palms and soles; and Ichthyosis sebacea of Hebra, which is possibly the result of the persistence of an epitrichial layer which should have disappeared by the seventh month, and of an accumulation of a certain amount of fatty and epidermal debris beneath it.

Description.-True ichthyosis varies greatly in degree and distribution. In its mildest form it is known as xerodermia and appears as a peculiar dryness and harshness of the skin, the result of a thickening of the horny layer of the epidermis and a diminution or absence of the cutaneous secretions. It is present chiefly on the extensor aspects of the arms and legs, especially about the elbows and knees, and on the back, but it may be widely distributed over the cutaneous surface in a markedly symmetrical fashion. As a rule, however, the flexor surfaces of the linibs, especially the flexures of the large joints, the palms and soles, the upper parts of the thorax, and the face are spared. The affected skin is usually dirty-grey in colour and the natural lines and furrows are generally exaggerated. In the mildest cases the skin is not definitely scaly but presents a crackled appearance, from the occurrence of fine fissures dividing it up into small irregular polygonal areas, which has been aptly compared to a film of varnish or collodion which has dried and cracked. ${ }^{1}$

In a further stage of the condition there is definite evidence of scales, which are delicate and pellucid, at first adherent all over but later tending to become detached at the periphery and sometimes everted at the edges. The hair-follicles, chiefly of the extensor aspects of the limbs and buttocks, are frequently affected and become prominent, like permanent "gooseflesh," and rough to the touch like a nutmeg-grater. The prominence of the follicles in not due to the formation of inflammatory follicular papules but is the result of the presence at the orifice of a horny plug or scaly cap, beneath which is often a twisted hair. Occasionally the follicular involvement is the most marked feature of the ichthyotic process, when the condition may be described as Ichthyosis follicularis.

The affection is not as a rule associated with any marked subjective symptoms, though occasionally there may be slight itching in cold weather and the skin may be unusually sensitive to alterations in

${ }^{1}$ The peculiar greyish appearance is partly duc to the pigment in the horncells and partly to dirt which has accumulated in the fissures, while the dryness is the result of a deficiency of the secretion of both sweat and sebum and an absence of fat in the epidermal cells. 


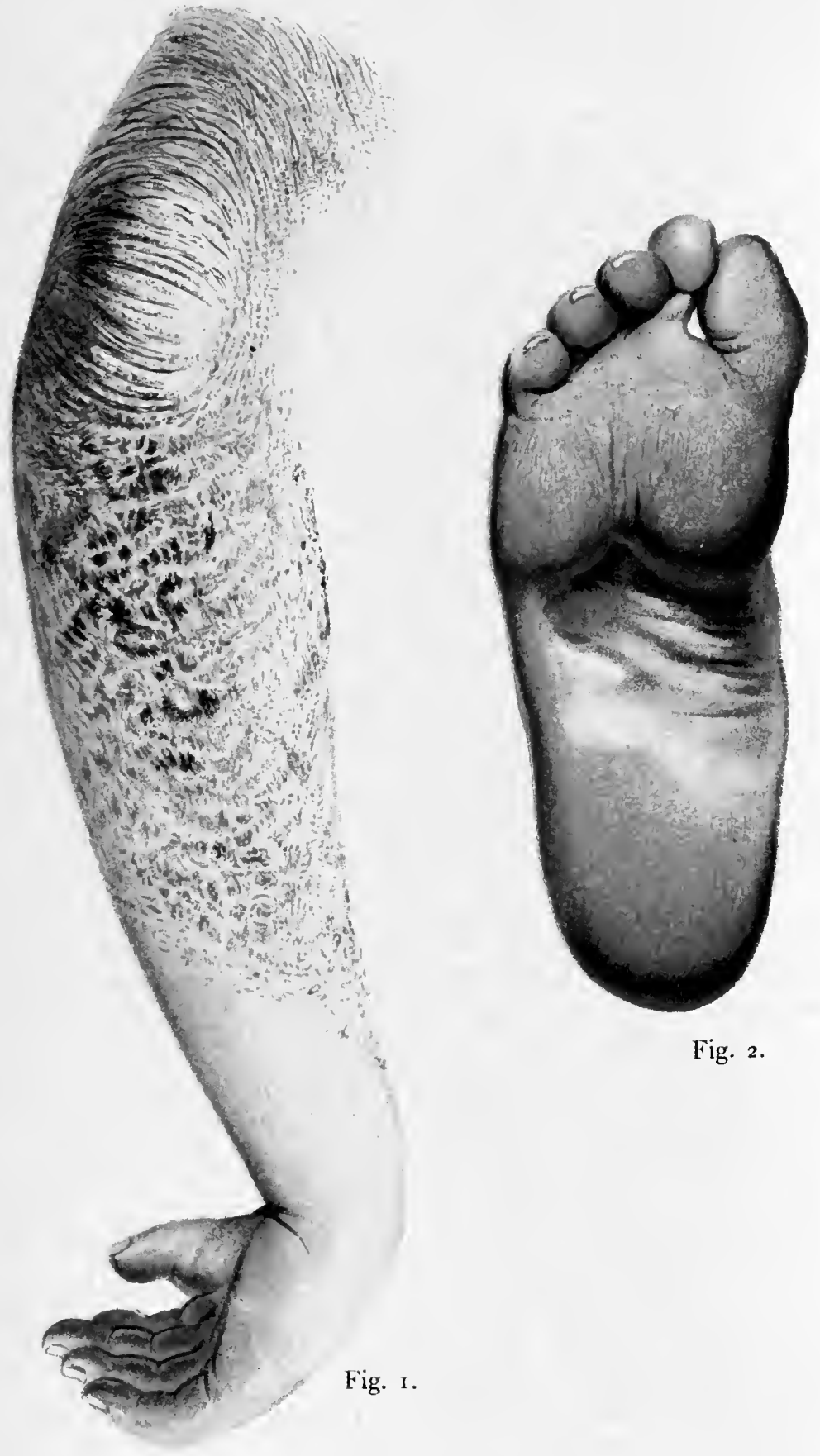

Fig. 2 . 


\section{PLATE III.}

Ichthyosis simplex vel serpentina.

FIG. I.-Serpentine scaly condition of skin of forearm.

FIG. 2.-Congenital hyperkeratosis of sole in the same case. 

temperature, especially if it has become secondarily inflamed. Where there is thickening of the horny layer, there is a decrease in the tactile sensibility.

The condition rarely appears at birth but is usually pronounced before the end of the second year, and may show itself very early as a roughening and a fine scaliness. In the most severe cases, howerer, it begins in utero and is noticeable at birth, which is generally premature. Certain observers believe that it usually begins in utero but is only apparent some time after birth, and that it is masked by the maceration of the skin by the amniotic fluid in the uterus, and the frequent use of soap and water in early infancy. Cases have been recorded where it is said not to have shown itself till puberty or later. but these are exceptional, and there is some doubt with regard to their being genuine.

The milder cases are always worse in cold weather when the skin readily becomes chapped, but improve, and may even approach the normal, in warm weather, as the result of an increased activity of the sweat and sebaceous glands which renders the skin moist, greasy and more supple. In ichthyosis, however mild, the skin becomes singularly delicate and vulnerable to local irritants, and is readily injured by exposure to cold, soap, water, attacks of micro-organisms, or any form of external irritation. Secondary changes of an inflammatory nature are liable to supervene and may lead to the production of a most intractable form of eczematous dermatitis which may persist throughout life.

Certain cases of chronic eczema which have lasted since infancy owe their chronicity to an underlying basis of ichthyosis. From the fine almost branny scales which characterise xerodermia, all degrees of coarseness may be met with in the scales till in the most serere cases they resemble a horny armour. Sometimes they are separated by fissures, at other times they overlap in an imbricated fashion. They may be delicate and pearly like the scales of a herring (Ichthyosis nacrée), or of a peculiar shiny appearance (Ichthyosis nitida), or thick like the scales of a perch or serpent (Ichthyosis serpentina). Ther vary also in colour, some being grey and dirty, and others brown or even black in tinge (Ichthyosis nigricans). They may derelop into horny plates several millimetres thick like those of a crocodile, or in advanced cases they may suggest a veritable rhinosceros hide. The scales or plates also vary in shape and may be rounded, quadrilateral, irreguilar, polygonal, or lozenge-shaped, and range in size from small scales to plates an inch or more in diameter, which are especially large in situations where there is little morement such as the front of the thighs. The plates are separated by fissures, corresponding in depth with the thickness of the plate and especially deep about the flexures of the joints. The fissures may be covered with a thin film of epidermis or they may be open, and from them may ooze a sero-sanguineous or even purulent discharge as they form an effective trap for microorganisms. (Plate III.) 
In addition to the scales or plates, ${ }^{1}$ warty masses may occur especially about the perineum and folds of the axillae. Even in advanced cases the condition does not generally attack the flexor aspects of the larger joints and the face is usually spared, though in many cases the cheeks present a peculiar shiny appearance which is almost characteristic. The perineum and genital organs are not attacked as a rule, although in severe cases the scrotum or vulva may present scales. The palms and soles when affected become thickened and horny, and the natural lines and furrows deepened. Anomalous cases are sometimes met with in which the usual distribution is not adhered to and the sites of maximum intensity may be other than those generally involved, as where the axillae are specially attacked and present warty masses.

The scalp, eyebrows, and beard region may be severely affected and become dry and scaly and the hairs lustreless and brittle.

The nails may be attacked, becoming opaque, ridged, thickened, frayed at the edges, and may be raised up from a horny accumulation in the nail-bed.

The mucous membranes are never involved.

Even in comparatively severe and extensive cases, ichthyosis does not seem seriously to interfere with the general health so long as it makes its appearance after birth, nor does it put sufficient strain on the kidneys to cause albuminuria. In one or two instances defective intelligence has been noted in connection with it. It is not associated with any developmental anomaly of the internal organs except perhaps of the thyroid gland, but that is inconstant. Occasionally, it has been found in association with asthma, but the exact relation between the two is not fully understood and may be a coincidence.

The most severe cases are those in which the skin is extensively affected in utero, and the infant at birth-which may take place at full time-is covered with a shiny horny membrane which soon becomes broken up into scales or plates, the skin changes being generally associated with atrophy of the genital organs and a peculiar drawn physiognomy. The presence of the thickened horny membrane may seriously interfere with the vital functions, so that if the infant survives for a time it generally dies young, though occasionally improvement may take place and the condition may develop into ordinary ichthyosis with no marked interference with the general health (Ichthyosis foetalis mitior).

In other cases the infant is prematurely born, either still-born or only surviving a few days (Ichthyosis foetalis gravior). In them the horny thickening is universal, involves the flexures of the joints, the

1 Bullae have been described as occurring in ichthyosis but these have either been due to secondary infection with streptococci, or if congenital were evidences of Epidermolysis bullosa and the icthyosis was not true ichthyosis but a segmental naevus of the Ichthyosis hystrix type. 
face, the palms and soles, as well as the trunk and extensor aspects of the limbs, and gives the infant the appearance of being encased in armour made up of horny plates 2 or 3 millimetres in thickness. In consequence of this wide distribution it was believed that these extensive congenital cases were of a different nature to ordinary ichthyosis and the name "Hyperkeratosis universalis congenita " was applied to them by Unna; the general trend of opinion now is that they are different degrees of a common pathological process.

At first, in utero, the horny armour is unbroken, but shortly before birth the growth and movement of the body cause the casing to crack and furrows to form. These furrows are arranged transversely on the limbs as the growth is greatest in the long axis, and in the flexures from the natural folding of the skin in movement. They are reddish or brownish in colour, vary considerably in depth, and may either be covered by a thin film of epidermis or be open and emit a discharge of sero-purulent or sanguineous fluid with an offensive odour. The horny plates are largest where there is least movement and are dirty-grey or brownish in colour, polygonal, quadrilateral or rounded in shape, of a leathery or horny consistence, sometimes smooth, at other times rough on the surface, firmly adherent except occasionally at the margin, and when forcibly detached cause bleeding. It is the presence of these plates which gives to the infant the harlequin appearance and suggested the name "harlequin foetus." The face is peculiarly repugnant owing to the thickening and contraction of the skin. The eyelids are swollen, oedematous and closed so that the eyeballs are almost invisible. The nose is flattened and only represented by the openings of the nostrils, which are generally encroached on by scales. The mouth is drawn and gaping and rhagades are generally present at the commissures, and the ears may be deformed and consist of small nodules. The limbs are semi-flexed and rigid. Should the infant be born alive, which is rare, the presence of the horny casing so interferes with the vital functions that it can neither suck nor swallow, the expansion of the lungs is interfered with, inanition rapidly supervenes, and it dies in a few days in a state of extreme marasmus or as the result of pneumonia or septicaemia.

Histopathology.- The principal microscopical changes in ichthrotic skin consist of hyperkeratosis in which the component horn-cells are perfect and present no nuclei, decrease of the granular layer, a thinning of the prickle-cell layer, and flattening of the papillary body so that the lines between the epidermis and the corium become straight or only slightly undulating. More rarely, the granular larer may be absent and the horn-cells imperfectly cornified and may adhere together in small squames. Occasionally there is acanthosis or thickening of the prickle-cell layer. (Fig. 22.)

There are no marked changes in the corium but the subcutaneous tissue is generally atrophic. Inflammatory changes have sometimes been described, such as proliferation of the endothelium and the 
perithelium of the capillaries, and a cellular infiltration containing a few plasma-cells and an increase of mast-cells, but these are probably secondarily induced. The pilo-sebaceous follicles are usually attacked and show hyperkeratosis at the mouth with the formation of horny plugs or caps beneath which a twisted hair can sometimes be detected,

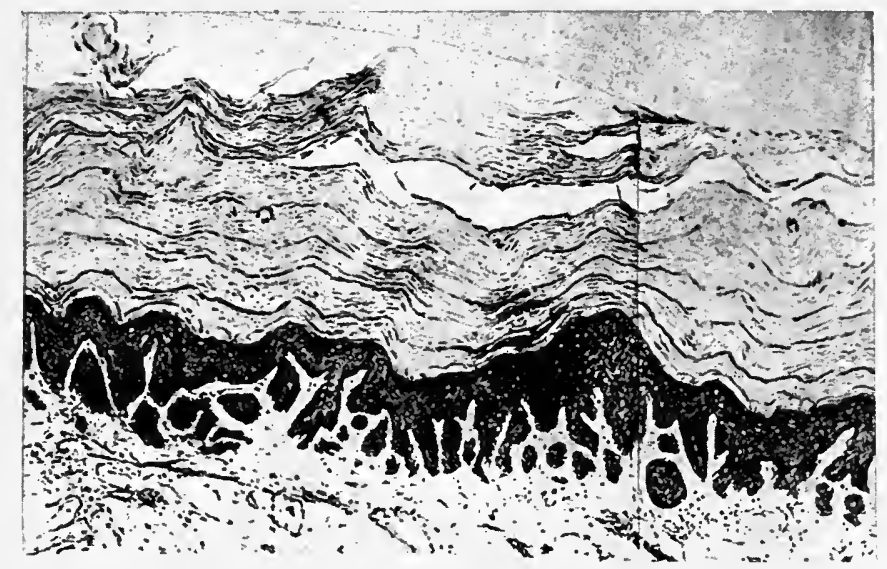

Fig. 22.-Section through Ichthyotic Area, showing Hyperkeratosis. (After Chalmers and 1nnes, Journ. Trop. Med. and Hyg., 1918.)

while the lower part of the follicle and sebaceous gland are atrophic. The sweat-glands appear to be normal though the ducts may be dilated.

Nature and Etiology.-The condition would appear to be a congenital anomaly of the epidermis associated with an activity of cornification, which though it may not be noticeable at birth probably occurs to a minor degree in utero. In view of the slight inflanımatory changes which are sometimes detected in the corium certain observers regard ichthyosis as an inflammatory disturbance of a mild type, somewhat comparable to Pityriasis rubra pilaris, and consider that the eczematous condition which so often supervenes is not secondarily induced but is a further stage of the same pathological process.

The most marked etiological factors in connection with ichthyosis are its hereditary and familial characteristics. It is the most hereditary of all skin diseases and has been known to occur in as many as six consecutive generations. Sometimes it may skip a generation or it may appear in a collateral branch. It occurs frequently as a family peculiarity, and seems capable of being transmitted by either sex, sometimes appearing in one sex only in a family, at other times affecting both sexes indiscriminately.

In a considerable number of cases it seems to appear de novo, with no history of it in an ancestor.

It has been attributed, like birth marks, to maternal impressions and the like, but there is no evidence that this is more than a fanciful hypothesis. 
Another point of interest in the etiology is the possible relation of ichthyosis to functional disturbances, organic changes, or absence, of the thyroid gland. Clinically it is difficult or impossible to detect by palpation slight changes in the thyroid in an infant, but in several cases of foetal ichthyosis in which post-mortems were obtained the thyroid was found to be absent, sclerosed, or atrophied, and in one or two instances where the ichthyosis appeared after birth the thyroid seemed to be small or not palpable. In one case a definite goitre existed, but the occurrence of ichthyosis in cretins has not yet been noted. In other cases no definite organic changes could be detected in the gland though functional disturbances may have existed.

That there is some relation between ichthyosis and thyroid deficiency is strongly suggested by the improvement which occurs in the skin condition from the careful administration of thyroid extract.

Diagnosis.-The diagnosis is rarely difficult. The peculiar dry harshness of the skin associated with keratosis of the hair-follicles, the adherent horny scales and plates with their marked predilection for the extensor aspects of the limbs, especially the elbows and knees, the absence of inflammatory changes, the appearance of the affection either at birth or soon afterwards, the frequent family tendency and the influence of heredity, are so characteristic as to make the diagnosis comparatively easy. It is only where the ichthyosis is masked by some secondary inflammatory disturbance and has become impetiginised or eczematised that any serious difficulty may be presented. But the history and the persistence of the scaliness after the inflammation has been subdued by suitable remedies, should serve to differentiate it. Chronic eczematous cases of ichthyosis are sometimes liable to be mistaken for prurigo but they are distinguished by the absence of the chronic inflammatory papules situated on the extensor aspects, the intense itching, the excoriations and scabs from scratching, the diffuse pigmentation, and the lichenification.

Prognosis. - The outlook is unfarourable unless in cases of the mildest type, for the affection tends to persist throughout life, to increase up to the age of twelve, and to show little alteration after that until senile changes supervene, when it may diminish. In mild cases remissions may take place in summer when the weather is warm and the sweat-glands become more active. The occurrence of some inflammatory process such as psoriasis or eczema in association with the ichthyosis does not appear to be followed by any definite improvement in the ichthyosis when the inflammation subsides, nor do the exanthemata as a rule cause any amelioration of the condition though cases of ordinary ichthyosis have been reported to have improved after measles and smallpox. Considerable benefit, however, may be derived from suitable treatment if carried out intelligently, the skin being rendered more comfortable, supple and elastic, and the tendency to the heaping up of scales and cracking diminished. 


\section{TREATMENT}

Internal Treatment.- Until lately internal treatment of every kind was regarded as absolutely useless, but recently the opinion has been gaining ground that the administration of thyroid gland has a distinctly beneficial effect. A number of cases have occurred which have markedly improved so long as they were under this treatment, but they have usually shown a tendency to relapse when the thyroid was discontinued. As a rule young children tolerate thyroid feeding well. In an infant doses of $\frac{1}{2}$ gr. of thyroid extract should be given daily and gradually increased to 2 grs., and in older children it may be increased up to $S$ or Io grs. In every case where it is prescribed the child should be carefully watched in case toxic symptoms such as tachycardia supervene.

Pilocarpine and jaborandi have been recommended with the object of increasing the perspiration and so rendering the skin softer, but they are of doubtful value and should any benefit accrue from their use it is of the most temporary character.

Nitro-glycerine in doses of one drop of a $\mathrm{I} \%$ solution three times a day (Buskett) and arsenic in small doses (Besnier) have also been adrocated.

Local Treatment.-Local treatment is of greater importance than general treatment, and if thoroughly carried out may cause amelioration in mild cases and may sometimes render the condition negligible, while in serere cases it may greatly add to the comfort of the patient. The object of the local treatment is to remove the scales, reduce the horny thickening, and render the skin more supple. With this end in view, daily warm baths containing borax, starch or bran should be ordered. While in the bath the scales should be removed by lathering the skin with a non-irritating super-fatted soap, or where they are more profuse and coarse with ordinary soft soap. After the bath, the skin should be mopped dry and inuncted with some emollient substance. Of these the most generally useful are glycerine either by itself or better diluted with water or rose water so as to form a Io to $20 \%$ glycerine solution, almond oil, a mixture of equal parts of almond oil and lime water, cold cream and lanolin with the addition of $2 \%$ of boric acid or resorcin to diminish the tendency to microbic infection. Where the scales are coarse a valuable application is a 5 to $10 \%$ cream of resorcin in glycerine of starch, and where they are thick and hard, as about the elbows and knees, salicylic plasters or ointments may be employed with marked benefit. The baths and local applications should be repeated as often as can be tolerated, but it should be remembercd that the treatment is only palliative and that an ichthyotic skin is particularly delicate so that the continuous application of soap and water and even mild keratolytics may set up severe inflammatory changes.

As cold has a deleterious effect on the diseased skin it is essential that the patient should wear warm clothing of a non-irritating type, and for this purpose cotton, linen-mesh, fine lawn or silk, is preferable to 
wool, and to obtain sufficient warmth a woollen garment may be worn over the undergarment.

Where the affection has become eczematous or impetiginous, the treatment is that appropriate to eczema or impetigo, and the underlying ichthyosis should be dealt with by the internal administration of thyroid extract when the inflammation has subsided.

\section{REFERENCES.}

Bland-Sutrox, J. Foetal ichthyosis (Med. Chir. Trans., I886, Lxix. p. 29I).

Bowes, J. T. Relation of ichthyosis to epitrichium (Journ. Cut. \& Gen.Urin. Dis., Dec., I 895).

ElLIOT, G. T. Intra-uterine ichthyosis (Idem, I89I, p. 20).

Meneau. Relation between foetal \& common ichthyosis (Ann. de Derm. et de Syph., I933, p. 97).

Pernet, G. Bullous ichthyosis (Brit. Journ. Derm., I9I I, xxiii. p. 344). (Idem, I9I4, P. 325.)

SHERWELL. Harlequin foetus living longer than six months (Journ. Cut. \& Gen.-Urin. Dis., Sept., I 894 ).

Starier, E. Hereditary transmission of defects in man (Thesis, Oxford, I9IO).

Winfield, J. Congenital ichthyosis, absence of thyroid (Journ. Cut. \& Gen.-Urin. Dis., I\$95, p. бт6).

\section{ICHTHYOSIS FOLLICULARIS.}

Syn.: Follicular xerodermia (Liveing), Ichthyosis cornea (Hardy-), Keratosis pilaris (Brocq), Keratosis follicularis (Kaposi), and closely allied, if not the same affection, Ulerythema ophryogenes (Taenzer).

Definition: A congenital affection of the skin characterised by the presence at the orifices of the hair-follicles of horny plugs or scales forming small acuminate papules.

Description.-The essential lesions of this affection consist of pinhead-sized, rounded or acuminate papules resulting from the presence of minute horny plugs in the orifices of the follicles. These papules are pale like the surrounding skin or of a dirty-greyish colour, and are entirely. the result of the horny plugs, which can be easily picked out, learing depressions. The papule may be pierced by a hair, but as a rule the hair is twisted up beneath the plug or scale. The skin between the papules is usually harsh and dry, and sometimes definitely ichthyotic. As a rule there are no subjective symptoms but occasionally itching may occur.

The mildest cases consist of a sort of permanent gooseflesh affecting the extensor aspects of the arms and thighs, and occasionally the trunk. In more severe cases the lesions are widely distributed orer the cutaneous surface and the face is frequently affected, especially about the eyebrows, forehead and jaws, the lesions there being more numerous, though smaller, than those on the trunk and limbs. The papules on disappearing are replaced by small cicatrices, which give the skin a 
peculiar atrophic appearance, though occasionally, instead of being white and cicatricial, the skin may be pink and inflamed, especially about the eyebrows.

In the most severe cases the scalp is affected and there is more or less complete baldness, the eyelashes and eyebrows are absent, and plugged follicles are irregularly distributed over the whole cutaneous surface with the exception of the palms and soles. The sweat-glands are not involved and the patients can perspire freely on exertion. The

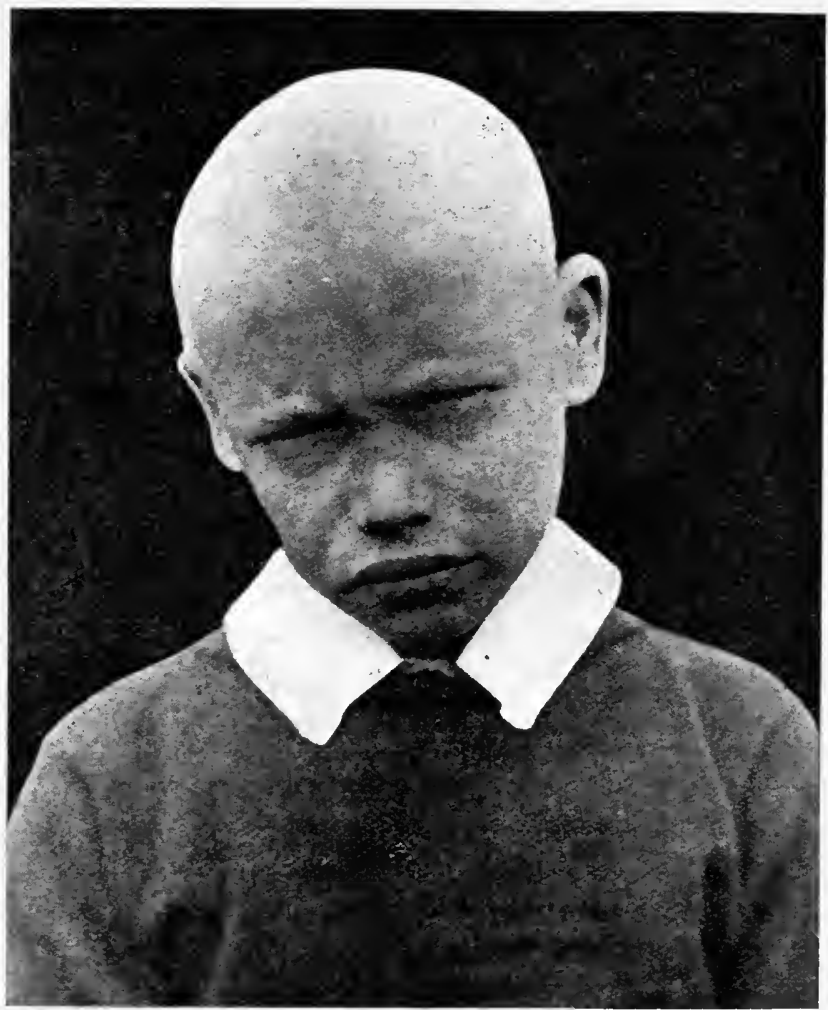

FIG. 23.- Ichthyosis Follicularis with Alopecia and Trachoma. It appeared at the age of two years.

(Author's Paper, Brit. Journ. Derm., rgog.)

skin is xerodermic and in situations such as the elbows, knees, buttocks and thighs, definite ichthyosis may be present. Cases of this type occur in families and begin in carly life. At birth the skin may appear to be healthy but within two ycars generally presents evidences of the disease, the first region to be affected being usually the eyebrows. (Fig. 23.)

What may be regarded provisionally as a still more advanced stage of this affection was described by Taenzer under the heading of A scar-leaving erythema of the eyebrow's. He observed altogether six cases of it, five of which began in carly childhood with redness of the eyebrows, followed by loss of the hair. From there it spread to the fore- 
head, cheeks, scalp and extensor aspects of the limbs. The scalp was more or less bald and the eyebrows, especially the outer halves, were hairless. The essential lesion consisted, as in the milder types described above, of acuminate plugged follicles. An additional peculiarity in Taenzer's cases was that after the disease had persisted for some years, atrophy occurred at the orifices of the follicles, resulting in the formation of depressed cicatrices surrounded by a hyperaemic halo and giving to the scalp the appearance of having recently recorered from farus.

Histopathology.--The most noticeable features in the histology of a case of Ichthyosis follicularis examined by the writer were as follows: There was marked dilatation of the funnels of the pilo-sebaceous

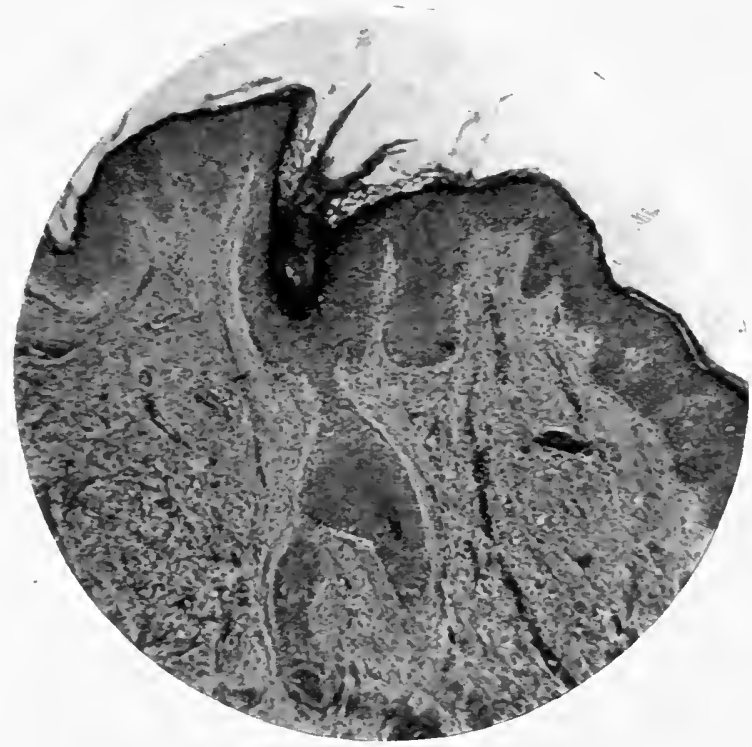

FIG. 24.-Ichthyosis Follicularis. Section under low power.

Shows dilated funnel of follicle filled with horny plug forming acuminate papule, atrophy of the lower two-thirds of the follicle, and slight hyperkeratosis between the follicles.

follicles, which were filled with horny plugs extending up beyond the level of the skin and forming acuminate papules. The lower two-thirds of the follicles were atrophied, and here and there presented pieces of atrophied hairs. The horny plug consisted of a dense central portion and a loose horny network with a tendency to a concentric arrangement at the periphery. The sebaceous glands were atrophic or absent. The epidermis between the follicles showed slight hyperkeratosis forming a loose network, the prickle-cell layer being thin and the interpapillary processes flattened out.

In the underlying corium there was slight dilatation of the bloodvessels in the neighbourhood of the follicles and an infiltration of small roundish or oval connective tissue cells around the dilated capillaries. The connective tissue fibres were normal and not rarefied as they are in inflammatory processes. (Fig. 24.) 
From the histological examination it seemed that the primary change was in the epidermis, and consisted of a process of hyperkeratosis not only affecting the funnels of the follicles but the intervening skin as well, leading to the formation of a horny network like that found in mild degrees of ichthyosis. The atrophy of the lower part of the follicles seemed to be simply the result of mechanical pressure due to the plugging. The slightly dilated blood-vessels and peri-follicular cellular infiltration, suggesting a mild inflammatory process, was probably secondary to the presence of the plug in the follicle and the friction to which it was subjected.

Etiology and Pathogenesis.-The etiology is similar to that of ichthyosis and the most important factors are its familial and hereditary traits. In a series of cases observed by Treacher Collins and the writer, it occurred in three boys out of a family of five. It has also been known to occur in children of ichthyotic parents. It would seem to be a variety of ichthyosis in which, owing to some individual peculiarity, the ichthyotic process affects the outer parts of the pilosebaceous follicles more than the intervening skin.

Sometimes follicular lesions of this type may occur as an acquired affection and manifest themselves much later. These are possibly the result of the irritation of dirt, as they are usually found in individuals who wash insufficiently; or they may be due to the elimination of toxins in a badly nourished skin, hence the name Cacotrophia folliculonum which has been applied to this type of case.

Somewhat similar follicular papules may be the result of chronic inflammation about the hair-follicles, as in the condition described by Brocq under the heading Keratose pilaire rouge.

Diagnosis.-Mild degrees of this affection resemble "goose-skin" (Cutis anserina), but goose-skin is transient and due to cold or fright, while follicular ichthyosis is permanent. In goose-skin, also, the lesions cannot be picked out like the horny plugs in Ichthyosis follicularis.

The disease with which it is most liable to be confused is Lichen spinulosus. The chief points of distinction are: (I) in L. spinulosus the lesions are invariably papules with filiform spines which project above the surface, while in I. follicularis many of them are simply acuminate follicles with a horny plug at the orifice; (2) in L. spinulosus the lesions occur in circumscribed patches or groups and are not irregularly and diffusely distributed; (3) in L. spinulosus the hairs of the scalp and eyebrows are not affected, there is no family tendency, and no xerodermia.

From Pityriasis rubra pilaris there is no difficulty in the diagnosis, as in it there is marked redness from inflammatory changes, scaly keratosis of the palms and soles, follicular plugged papules on the backs of the fingers, and a tendency for the acuminate papules to coalesce to form patches. 
Prognosis.-The condition persists indefinitely unless treated. In mild cases it can be reduced by suitable local applications; in widely distributed cases with marked ichthyosis, all that can be done by judicious treatment is to keep it in check and to render the skin as smooth as possible.

Treatment. - The treatment is similar in the main to that of ichtlyyosis. It consists of warm baths and the application of soap spirit lotion on a piece of flannel, or vapour baths. Keratolytics such as resorcin or salicylic acid, grs. 20, applied in glycerine of starch, are soothing and effective. Where the shin is very dry it should be inuncted daily with some oily application such as almond oil 2 parts and lime water I part.

\section{REFERENCES}

Lesser, F. Ichthyosis Follicularis (Ziemssen's Handbook of Skin Dis., I 885 , p. 236).

Little, Graham. Follicular Ichthyosis (Brit. Journ. Derm., I90I, xiii. p. 420 ).

MacLeod, J. M. H. Ichthyosis Follicularis with Baldness (Brit. Journ. Derm., I909, xxi. p. I65).

TAexzer, P. Ulerythema Ophryogenes (Monats. f. prakt. Derm., I Sog, viii. p. (97).

\section{ERYTHRODERMIE CONGENITALE ICHTHYOSIFORME}

\section{AVEC HYPERÉPIDERMOTROPHIE}

Under this title Brocq described, in I902, a cutaneous affection which he believed to differ from ordinary ichthyosis. This was characterised by generalised erythema, which was specially marked over the front of the neck and the flexor aspects of the large joints. It was associated with hyperkeratosis of the soles of the feet, deformity of the nails, and marked seborrhoea of the scalp and central area of the face, and in some cases with irregular flaccid bullae resembling those in femphigus, or with raised papules around the hair-follicles recalling Pityriasis rubra pilaris.

The condition was congenital in origin, tended to improve with the advance of age, and occasionally was benefited by radio-therapy:

\section{REFERENCE.}

Broce, L. Erythrodermie congénitale ichthỵosiforme arec hyperépidermotrophie (Derm. Prat., I907, ii. p. To3).

\section{NAEVI}

Der. : 'Nascor, to be born.

Definition: Circumscribed new-growths of the skin and subcutaneous tissue of congenital origin.

In this country until recently the term naevus was restricted to congenital new-growths of the blood-vessels and lymphatics of the skin. On the Continent, however, it has been used in a much wider sense to 
include all cutaneous circumscribed and regional deformities of embryonic origin, such as vascular naevi, moles, congenital warty growths and neuro-fibromata. These are not always noticeable at birth, but whenever they appear they result from the growth of an aberrant group of imperfectly developed cells which have persisted from embryonic life. There is much to be said for the Continental use of the term and it is in this wide sense that it will be employed here. From the frequency with which vascular naevi are associated with moles or other forms of congenital anomalies, and from the fact that a vascular naevus may gradually disappear and be replaced by a mole, it is evident that they are closely related and ought to be grouped under one heading, of which the most convenient is that of "naevus" or embryonic growth.

Etiology.-The cause of naevi is still unknown and little more can be said than that they are peculiar developmental anomalies. They are exceedingly common and occur in some form or other in the majority of individuals. Various theories have been advanced to explain their presence, sucl as that they are the result of some emotional disturbance in the mother during pregnancy such as shock, fright, or mental anxiety, or that they are dependent on some illness, accident or injury which she has suffered. These, however, are merely hypotheses and are not based on scientific fact.

Heredity plays an important part in their etiology and certain types of naevi may be handed down for several generations. The same type may occur in several members of a family, or in a parent and child, or the type may vary. That they are connected with imperfect development is evident from the frequency with which they are associated with physical defects such as syndactylism, webbed-fingers, hare-lip, cleft palate, hydrocephalus, meningocele, or arrest in mental development as in many cases of v. Recklinghausen's disease, and Adenoma sebaceum; and from their occurrence in positions where developmental errors are liable to be found, such as the mid-line of the body and embryonic clefts, such as the naso-facial sulcus, the oblique facial cleft, the external border of the orbit in association with dermoid cysts, and the posterior extremities of the branchial clefts. They also occur over Spina bifida occulta. Occasionally the association with developmental errors is shown by the replacement of moles by accessory mammae or their occurrence with deformities of the ear such as an accessory tragus or helical fistulae. Apart from the association of v. Recklinghausen's disease, naevi tend to occur in connection with the cutaneous nerves, especially at their point of emergence from the deep fascia-this is often seen on the face at the three points of emergence of the trigeminal nerve.

Virchow suggested that naevi had an anatomical basis and were dependent for their situation on embryonic fissures, hence the name "Fissural angiomata," but as they are by no means confined to the positions of embryonic clefts but may occur anywhere on the skin 
this explanation is only partly satisfactory. Another suggestion was advanced by Unna in connection with certain port-wine naevi, namely that they were due to inter-uterine pressure or pressure at the time of delivery. In support of this riew he cited the fact that such naevi occur not infrequently on the forehead and occiput, situations which are extremely liable to be subjected to pressure. This explanation also, though applicable to a few cases, does not explain the great majority of them.

\section{CLASSIFICATION OF NAEII}

\section{Vascular Naevi.}

(i) Derived from blood-i'essels.

(a) Capillary naevi, or port-wine stains, macules or patches.

(b) Raised haemangiomata, carernous naevi.

(c) Stellate or spider-naevi.

(ii) Derived from lymphatics.

(a) Lymphangiectases.

(b) Lymphangiomata.

\section{Non-Vascular Naevi.}

(i) Plane: pigmented macules and patches.

(ii) Raised.

A. Soft naevi Non-pigmented.

(Moles). Pigmented.

Hairy.

Giant-naevi.

B. Hard naevi

(a) Circumscribed. Verrucose.

Linear.

Ichthyosis hystrix.

(b) Regional.

Keratosis palmaris et plantaris.

C. Fibromatous naevi. (a) Fibroma molluscum

(v. Recklinghausen's Disease) (see p. II $2 S$ ).

(b) $\mathrm{N}$. lipomatodes.

D. Adenomatous naeri. Adenoma sebaceum (see p. II24). Syringo-cystadenoma (see p. I I 20). 


\section{VASCULAR NAEVI}

Vascular naevi are dilatations and new-growths of blood-vessels and lymphatics in the skin and subcutaneous tissue of congenital origin.

\section{(i) BLOO1) VASCULAR NAEVI. (N. sanguineus.)}

For purposes of description the blood vascular naevi may be divided into three main types, namely $(a)$ capillary naevi, $(b)$ raised haemangiomata and $(c)$ spider-naevi. Though in the majority of cases the naevus conforms definitely to one or other of these types, in a certain number hybrid forms occur which cannot be placed appropriately under any of these headings.

\section{(a) Capillary Naevus}

Syn.: Port-wine stain ; Naevus flammeus; Fr. Naevus vasculaire plan, Tache de feu ; Ger., Feuermal, Gefässmal.

Description.--The name " port-wine stain" though applicable to a certain number of cases of plane vascular naevi is apt to be misleading. It suggests a dark port-wine tinted discolouration of the skin, whereas in reality all grades of colour may be met with from faint pink to livid purple or plum colour. The lesions may be single or multiple and may vary from small red macules the size of a pin's head up to large patches involving extensive areas or regions of the cutaneous surface. In outline they may be well-defined or indefinite, and are usually irregular in shape. As a rule the lesion is not uniform in colour, but may be darker in the centre than at the periphery, dotted over with darker points, or present a marbled patchy appearance. When pale the network of dilated capillaries composing it can be easily distinguished, when dark it is impossible to do so and it forms a diffuse red or purplish patch. When the naevus is superficial, compression with a piece of glass brings out the capillary network and may almost completely obliterate the redness, but when it is deep-seated even firm pressure fails to remove it. The colour varies from time to time, and is rendered more vivid by exertion, crying, menstruation, pregnancy, or anything which causes congestion. The texture of the affected skin may be unaltered and soft and the naevus inappreciable to the touch, or it may be slightly raised, and in rare instances the overlying epidermis may be thickened, or even warty. To the unusual type of naevus in which warty masses occur on a red vascular basis the name Angiomes verriqueux has been applied by Rist. Sometimes a capillary naevus may undergo fibromatous changes with the development of soft fibrous tumours over its surface. 
Capillary naevi may occur anywhere on the skin but are especially common on the face and upper parts of the body. A frequent situation is the occipital region near the posterior fontanelle and about $25 \%$ of infants are found to be thus affected. They are often present about the forehead, cheeks, nose and lips, and occasionally involve the whole of one side of the face. They may form extensive patches on the trunk and limbs, and cases are met with in which they are present over almost the whole of the cutaneous surface. They may occur also in the mucous membrane of the mouth and are not infrequent at the natural orifices and about the genitalia. Occasionally the naevus may involve the whole thickness of the cheeks or lips, and be present

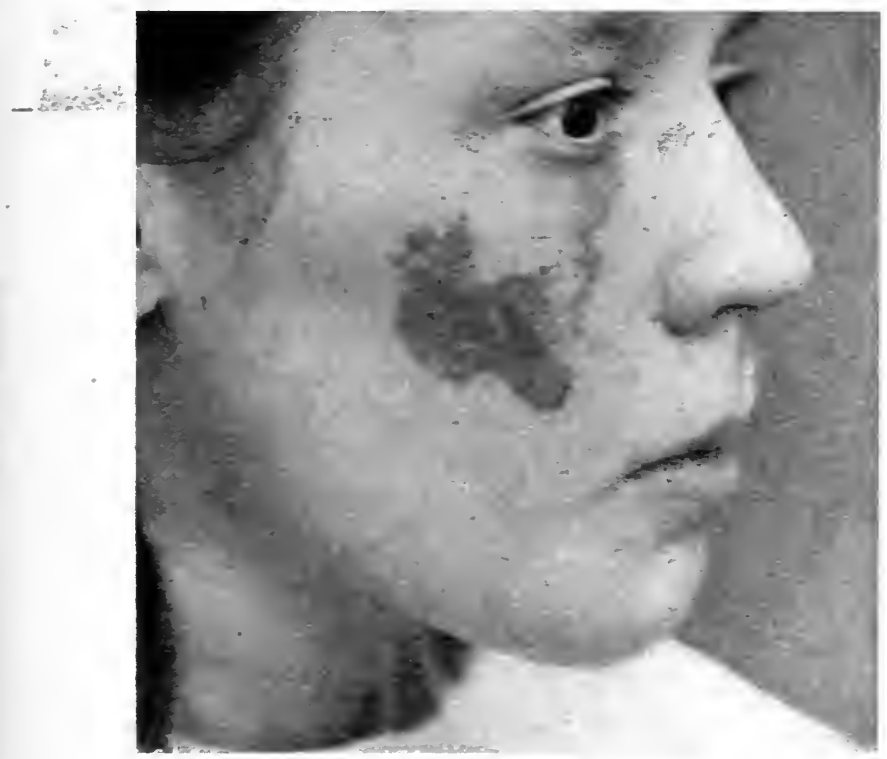

FIG. 25-Capillary Naevus.

in the mucous membrane as well as in the skin. The naevus tissue penetrating so deeply accounts for the intractability of the majority of the lesions. (Fig. 25.)

Port-wine stains are usually present at birth though they may not be noticed until a few days afterwards. They gradually increase in size with the growth of the child and not infrequently take on active growth about puberty, but after that usually remain stationary. They show little tendency to involute spontaneously but have been known in rare instances to disappear partially or completely. They are not associated with subjective symptoms, and except for the disfigurement and the mental effect rendering the patient selfconscious, or even morbid, they have no deleterious influence on health. 
Histopathology.-A port-wine naevus is the result of a capillary dilatation or new-growth chiefly of venous capillaries, and is a "congenital angiectasis." In the superficial naevi the dilatation is confined to the papillary and sub-papillary layers, while in the deeper ones the blood-vessels of the whole of the corium and subcutaneous tissue may

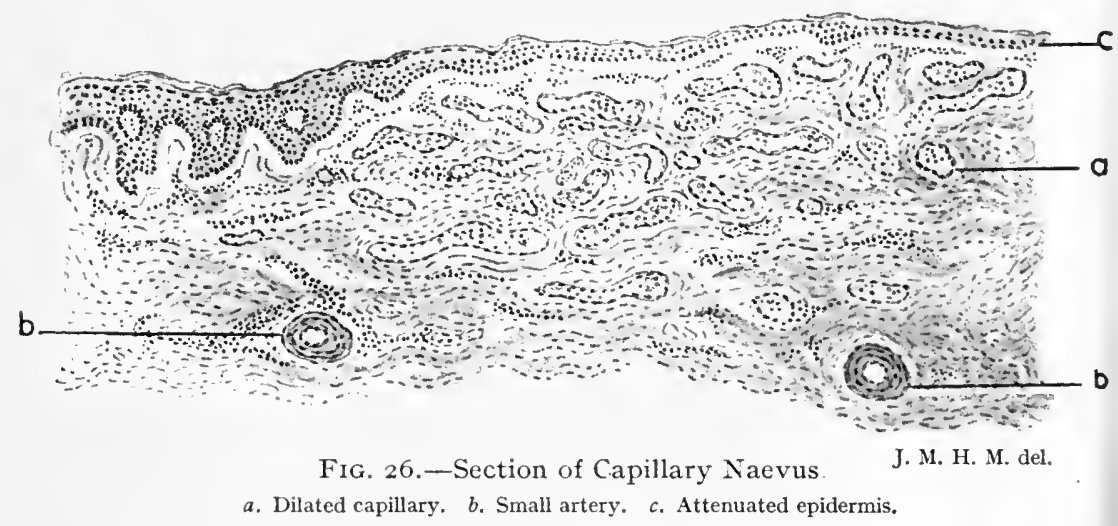

be involved. In the upper parts of the corium the capillaries are widened out into irregular cavities, while in the deeper parts they are so dilated and tortuous and their walls so thickened that they resemble the veins of the hypoderm. In some cases the vessels of the subcutaneous tissue and deeper parts of the corium may be implicated without marked changes being present in the more superficial capillaries of the papillary layer. (Fig. 26.)

\section{Angioma Serpiginosum (Crocker).}

Angioma serpiginosum is the name applied by Crocker to a peculiar type of capillary naevus which was originally described by Hutchinson under the heading of Infective angioma. Neither name is wholly adequate, but of the two that suggested by Crocker is least open to objection as the lesion is not infective. This rare condition, of which only about twenty cases have been recorded, is characterised by the presence of punctate telangiectases arranged singly, or in groups, rings, or gyrate figures and situated in one region or on different parts of the cutaneous surface, but usually with a zoniform or segmental distribution. The individual lesions vary in size from a minute speck to a small pin's head, and in colour from a faint red to a deep purple, and on fading give to the skin an appearance which has been compared to cayenne pepper sprinkled on it. The puncta first appear in small groups, which spread peripherally and clear up in the centre so as to form ringed lesions from half an inch to two inches in diameter. New foci keep on appearing beyond the ring, which go through the same stages of evolution and coalesce with neighbouring circles to form gyrate figures and map-like patterns outlined by fine red puncta and enclosing pinkish skin. The affection spreads slowly 
and intermittently with periods of activity and quiescence. The telangiectatic spots may sometimes disappear on pressure with a glass, but as a rule they cannot be obliterated. In a case described by Majocchi they were found to be situated around the orifices of the pilo-sebaceous follicles and consequently he added a third name for the affection Telangiectasis follicularis annulata.

The zoniform distribution of the lesions strongly suggests that they belong to the group of the systematised lesions of which "Naevus unius lateris" is the original type.

\section{Schamberg's Disease.}

Under the name of " a peculiar, progressive, pigmentary, disease of the skin," Schamberg describes a rare condition which presents a close resemblance to Angioma serpiginosum. The affection is characterised by the presence of numerous pin-head sized puncta, aggregated together into irregular patches, which increase by the appearance of

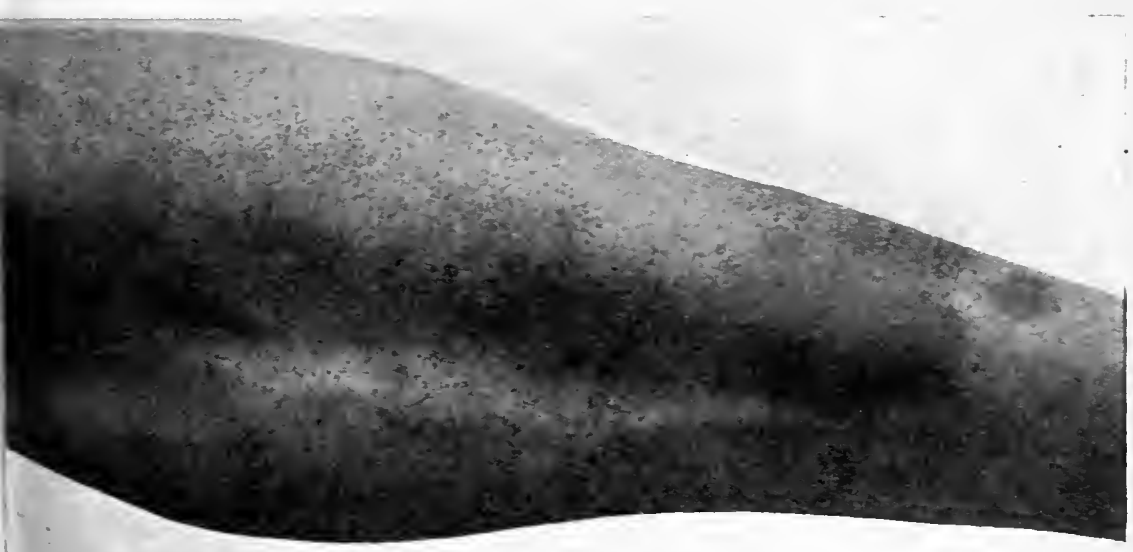

FIG. 27.-Schamberg's Disease.

fresh lesions at the periphery. At first reddish-brown in tint the puncta gradually fade till they assume a brownish-yellow colour and resemble cayenne pepper dusted over the skin. It affects chiefly the lower extremities, especially about the shins and dorsa of the feet, but has been noted also on the forearms and wrists. (Fig. 27.)

The precise nature and causation of this condition is unknown. Certain observers regard it as being of congenital origin and a variant of Angioma serpiginosum, while others consider that it is an acquired condition, of a slowly progressive inflammatory nature, and due to vascular stasis affecting the cutaneous vessels, and in support of the latter view it may be pointed out that "cavenne-pepper patches are not infrequently met with in connection with varix in the legs. 


\section{(b) Raised Haemangiomata}

Syn.: 'Strawberry birth-mark'; Cavernous angioma; Naevi vasculaires tubéreux; Spongy aneurysm; Fungus haenatoma.

These naevi are chiefly met with in infants as the familiar vascular new-growths, popularly known as strawberry or raspberry marks, but they may occur in adults in a large variety of forms in which the vascular tissue is usually mixed up with fibrous tissue and covered with a more or less warty epidermis.

The common raised angiomata of infants vary from a split-pea to a walnut, but occasionally may reach the size of the palm of the hand or larger. They may be scarcely raised above the level of the surrounding skin or may project half an inch or more. They may be flat, rounded, nodular, pedunculated, or broken up and multilobular like a mulberry

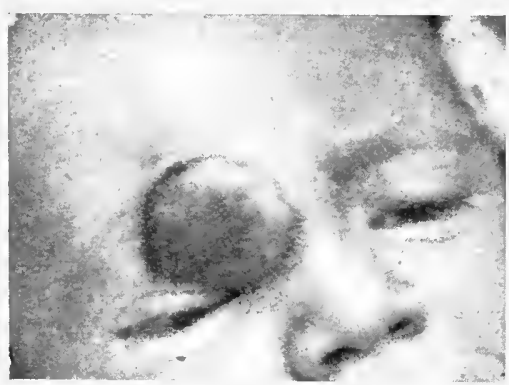

FIg. 28.-Angiomatous Naevus.

or a raspberry, and they are usually, though not invariably, hairless. In colour they vary from bright red to deep purple, and are sometimes dotted over with small telangiectases. In consistence they may be soft and compressible, spongy, or tough and almost hard; these variations are dependent on their anatomical structure and the amount of fibrous tissue present. When they are superficial the skin alone is affected and the lesion is abruptly raised above the level of the surface, where deep the subcutaneous tissue is also involved and the skin gradually raised up, the subcutaneous part of the naevus being wider than the cutaneous part. By pressure with a piece of glass the slightly raised soft lesions can be obliterated, but this is impossible when they are raised more than ${ }_{16}^{1}$ th of an inch and contain any considerable proportion of fibrous tissue. By compression also the volume of the lesion may be reduced and a certain time may be taken for it to refill with blood when the compression is relieved. The haemangiomata become more vivid in colour and prominent, even turgescent, as the result of violent exertion, or if the circulation be impeded by crying, compression of the principle vein in the neighbourhood, or other cause. Occasionally when they are situated over bony prominences pulsation has been detected in them, but there is no sound on auscultation. They may begin as soft vascular lesions and become firmer subsequently from fibromatosis. (Fig. 28.)

These naevi are most common about the face and scalp, they may occur anywhere on the skin but are rare on the lower extremities. They may be noticed at birth or not till several weeks after. They usually increase in size for months then gradually disappear spontaneously 
about the end of the second year and leave no trace or only a slightly atrophic or pigmented patch. They may, however, persist and become

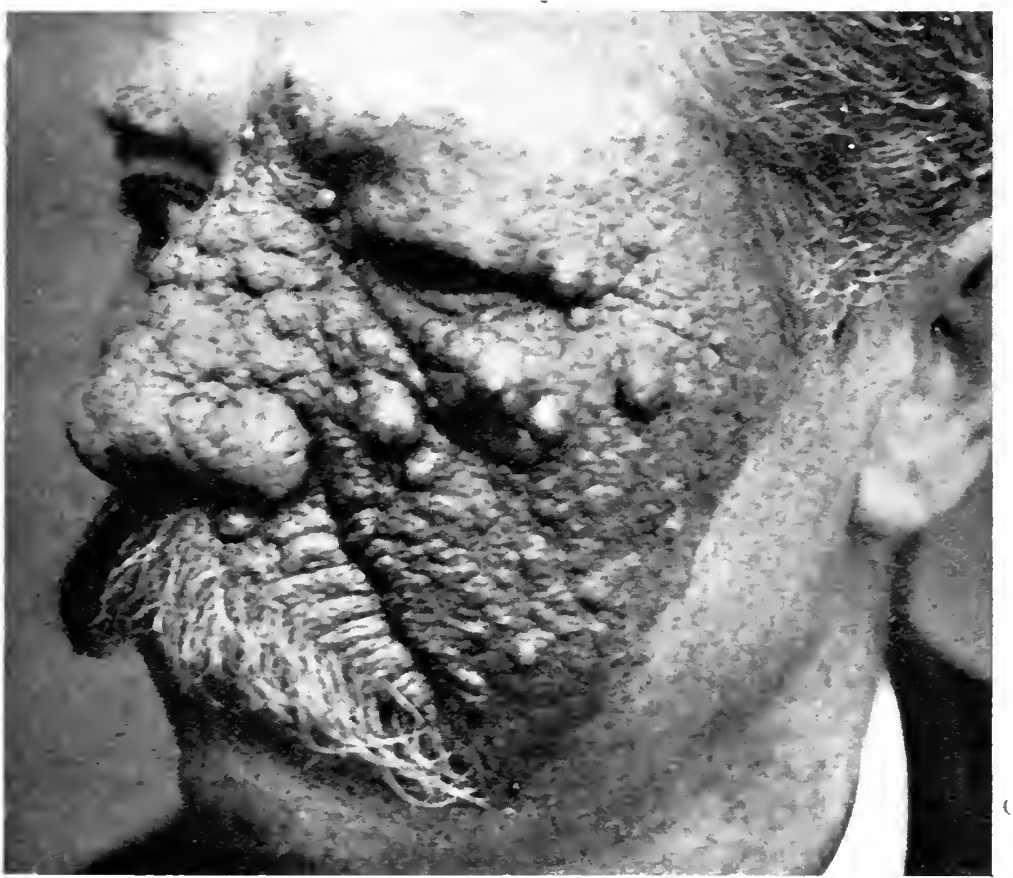

Fig. 29.--Fibro-angiomatous Naerus.

transformed into mixed fibro-vascular structures or lipomatous lesions (Naevus lipomatodes). (Fig. 29)

The "giant-vascular naevi" are a special variety of raised hae. mangiomata, consisting of large bluish tumours over which the skin is thin and covered with dilated capillaries. They may occur anywhere but are most common on the limbs and scalp. (Fig. 30.)

The haemangiomata produce no sensory symptoms and simply give trouble on account of their unsightliness, especially when situated about the face. Mothers are frequently anxious regarding them, from a

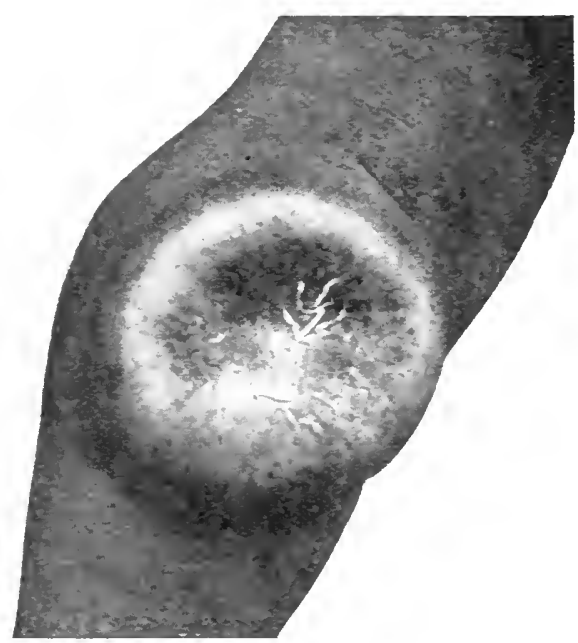

Fig. 30.-Large Subcutaneons Naerus on leg of infant 
groundless fear that they interfere in some way with the health of the child. The only danger associated with them is the possibility of a severe haemorrhage should they be injured, and this, though not fatal, is a serious occurrence in an infant. After the haemorrhage they gradually undergo retrogressive changes from clotting. Occasionally as the result of some slight abrasion and the inoculation of pus-cocci an indolent type of ulceration is produced with a viscid discharge. This is especially liable to occur in naevi about the genitalia or in intertriginous regions like the groins, but is not a serious matter, as fibrous changes consequent on the inflammation gradually lead to involution of the lesion and ultimate cure. This is taken advantage of in the old-fashioned treatment of raccinating over a naevus which is often successful in effecting a cure.

Histopathology. - The microscopical structure of haemangiomata varies with the clinical peculiarities of the lesions. When superficial the venous capillaries of the upper layers of the corium are chiefly involved, while in deeper lesions the ressels of the subcutaneous tissue or even these of the periosteum may be affected. They are dilated, tortuous, here and there widened ont to form cavernous spaces like an erectile tissue, and their walls so thickened that the capillaries of the papillary layer may resemble veins of the subcutaneous tissue. Though usually venous the sinuses occasionally may be arterial. The fibrous tissue in the neighbourhood nay be unaltered, or markedly hypertrophic surrounding the sinuses and forming a sort of capsule round the whole naevus. Evidences of the formation of new vascular tissue are present, such as budding of solid processes which become excavated and lined with endothelium to form sinuses, and the formation of a fibrous stroma whose meshes are connected with the blood-ressels. The pilo-sebaceous follicles and sweat-glands are usually atrophied. Occasionally masses of fat cells are present, either diffuse or ensheathed in a definite capsule (Naerus lipomatodes). The epidermis over the lesion may be atrophied or unaltered or it may be hypertrophied or verrucose.

\section{(c) SPIDER-NaEvus}

Syn.: Naerus araneus; Stellar naevus.

This type of naevus consists of a small central elevation from which radiate a number of dilated vessels. It varies in size from a pin's head to a split-pea. The radiating capillaries are usually connected by lateral branches and small subsidiary dilatations may be observed upon them in addition to the central elevation. Spider-naevi may be single or multiple and are most common on the face, especially on the bridge and sides of the nose, malar prominences and forehead. They may appear at birth or s'on afterwards but the majority develop later and cannot be regarded as naevi in the congenital sense of the term (vide telangiectases). 
It is doubtful whether these lesions are made up of newly formed ressels or are simply dilatations of existing capillaries. The red elevation may be produced by a dilated arterial loop and the radiating lines by venous capillaries, but more usually by a small renous loop with a congestion extending back to the arterial capillaries. The lesion is not well-defined as the radiating branches become finer at the periphery and appear to fade away into the surrounding shin.

As well as the typical stellate lesions, dilated superficial ressels of congenital origin may occur in the form of rings, streaks, plaques or occasionally small raised growths over which the epidermis may be warty.

Diagnosis.-The diagnosis of blood vascular naevi rarely presents any difficulty. Occasionally capillary naevi situated about the occiput might be mistaken for patches of eczema or ringworm, but in the naevi the texture of the scalp is unchanged, the hairs grow well over them and there is no itching nor sign of inflammation, while in ringworm the hairs are broken and form stumps, and in eczema the patch is inflamed, itchy and may be moist or crusted.

Prognosis. - The prognosis with regard to the removal of the blood vascular naevi raries with the type of naerus. In the case of the portwine stains it is uncertain and as a rule unfarourable. Where the naerus tissue is superficial it may be partially obliterated by treatment, but where it penetrates deeply, as in those cases where it involves the mucous membrane as well as the skin, the prognosis should be most guarded. On rare occasions these naevi have been knorm to undergo retrogressive changes spontaneously.

In the raised angiomata retrogression frequently takes place either spontaneously or as the result of some slight abrasion or septic inoculation. With the exception of the large subcutaneous naeri and the giant-forms known as "fungus haematomata," they can be satisfactorily removed by treatment.

In the case of spider-naeri the prognosis is excellent as they can be obliterated by suitable operative procedures.

\section{TREATMENT}

General Remarks.-Two essentials are requisite for the successful cure of a naerus, namély, that the naerus tissue be completely destroyed and that no disfigurement should result. The probability of success varies greatly according to the type of naevus. In the case of a portwine naerus the results are only occasionally satisfactory; in raised haemangionata, with the exception of the giant-forms, excellent results may be obtained by suitable treatment; in spider-naevi no great difficulty is experienced in their removal. Where practicable 
naevi should be treated as early as possible so as to remove them or abort their growth when they are comparatively small.

It will be advisable to discuss the treatment of naevi under the headings of the various types.

\section{Capillary NaEvi}

Radium.-The best form of treatment at present known for portwine stains is radium, and by it fairly satisfactory results may be obtained.

It has been found that the radiations from radium have a selective action on blood-vessels causing them to shrivel and disappear. According to the researches of Dominici and Barcat the rays cause a disintegration of the capillaries and changes in the fixed connective tissue cells in which they undergo reversion to an embryonic state. In this way the angiomatous tissue is replaced by numerous cells with a few contracted capillaries and blood-spaces among them. By the proliferation and evolution of these embryonic cells a new healthy connective tissue gradually becomes built up and replaces the naevus tissue, and a smooth, supple white scar may be obtained. The chief difficulty in the treatment with radium is to avoid producing an uneven effect and a mottled scar.

Pinch, of the Radium Institute, London, advises short unscreened exposures and if necessary gradually increasing them until a satisfactory result has been obtained. As a general rule the apparatus employed should consist of a quarter to half strength applicator of suitable size and shape. The screening should not exceed $0.02 \mathrm{~mm}$. aluminium and the exposure should range from to to 60 minutes. If the naevus be extensive and a sufficiently large applicator be not available, the surface has to be gone over seriatim and great care must be exercised to avoid overlapping. The best results are obtained when the naevus is superficial and blanches readily by gentle pressure and when there is no tendency to infiltration.

A treatment such as this to be successful requires great care and experience, and should the naevus be extensive it is exceedingly tedious unless a large quantity of radium be available.

Refrigeration. - The next best treatment is refrigeration by solid carbon dioxide. This is of value when the lesions are superficial and not too extensive. The method is described in detail in connection with raised haemangiomatous naevi for which it is specially applicable. By means of repeated freezings, fibrous changes are produced in the tissue which gradually lead to the obliteration of the vessels and the reduction of the redness. But to do this even in superficial cases a number of applications may be necessary, consequently it is only practicable in comparatively small lesions, for where large areas are involved few patients will be found who will persevere with the treatment, which, 
though not unbearable at the time, is associated with considerable pain when thawing takes place and with discomfort till the reaction has subsided and healing has occurred.

An application of the solid carbon dioxide should be given for to to 20 seconds with medium pressure. This produces a smart reaction with a tense blister, which should be allowed to dry up and separate naturally. When the reaction has subsided and healing has taken place, a further exposure may be given and so on till the redness is reduced. In extensive naevi a square applicator should be used and the affected area gone over seriatim. Great care must be taken to avoid over-exposure or too firm pressure which may lead to destruction of tissue and unsightly scars.

Electrolysis may be employed with benefit in superficial cases of port-wine stains in which the capillaries can be distinguished, but is of no value where the redness is diffuse. This treatment reduces the disfigurement by blocking the more prominent vessels and if carefully done produces no marked pitting or scarring. The method, however, is extremely tedious and requires skill. A platino-iridium needle is attached to the negative pole, the positive electrode, consisting of a metal cylinder covered with chamois leather, is held tightly by the. patient, and a current of 2 or 3 milliamperes employed. The live needle is inserted into the more prominent capillaries, kept in for a few seconds till a small bead of froth appears and the area around is blanched, and then withdrawn. The procedure is repeated till the surface has been gone over evenly. It is important to avoid electrolysing contiguous capillaries lest the zones of action of the electrolysis should overlap and result in an excessive effect with sloughing. After the electrolysis is finished the punctured area should be painted over with collodion. The treatment may be repeated after an interval of a few days.

Other methods of treatment have been suggested but the results from them are unsatisfactory. The X-rays for example have been employed, but to remove the naevus by them it is necessary to produce a definite X-ray reaction which is liable to be followed by an unsightly telangiectatic scar.

Attempts have also been made to obliterate them by ultrariolet light from the old large Finsen lamp and some of the more modern smaller patterns such as the Lortet-Genoud and the Quartz lamp. Theoretically it might be expected that this treatment would be of benefit since it produces an easily controlled inflammatory reaction which is followed by a process of repair and no appreciable scarring. Practically, howerer, it is of little value, as it is almost impossible, unless with long exposures, to obtain a sufficiently powerful inflammatory reaction to produce the necessary fibrous changes.

High frequency, by the method known as "fulguration," has been advocated but the results from it are uncertain and it is painful, requires a general anaesthetic, and is apt to produce a cheloidal scar. 
Scarification, followed by the application of a caustic such as ethylate of sodium, has been tried but this usuallyleads to the formation of a disfiguring scar.

\section{Raised Haemangiomata}

The treatment of raised haemangiomata and its results vary with the size and type of the lesion. The smaller haemangiomata up to the size of a crown-piece are readily amenable to suitable treatment, and may disappear spontaneously before the end of the second year, but in the large giant-vascular naevi or " fungus haematomata," treatment is unsatisfactory as a rule.

In the past it was customary to remove them by electrolysis or excision, which entailed a general anaesthetic, but in the case of naevi situated about the face, electrolysis was associated with the risk of shock and syncope, and excision with the production of a scar. Consequently at the present time these methods have been largely superseded by refrigeration and radium which are free from such risks.

Refrigeration by carbon dioxide snow.- In the case of the raised haemangiomata with definitely limited borders refrigeration with solid carbon dioxide, or with liquid air, gives excellent results. Carbon dioxide is the more practical, as it is less costly and its area of action can be more definitely restricted. For a description of the method of obtaining and applying the carbon dioxide see page I4o. Histologically the structure of the raised haemangiomata is a delicate one, consisting of dilatations and sinuses, connected by dilated capillaries and supported by a more or less rarefied fibrous stroma. When the naevus is frozen solid, degenerative changes occur in the endothelial lining of the dilated capillaries and, when thawing takes place and the part becomes oedematous, they tend to break down. When the reaction subsides new fibrous tissue is formed and the naevus disappears without marked scarring. The treatment is not excessively painful, as the freezing itself has an anodyne effect and neither a local nor a general anaesthetic is necessary.

Procedure.-The patient should be placed on a couch, and the skin over the naevus cleansed with soap and water, boric lotion, or ether. An applicator larger than the naevus should be chosen so as to expose about $\frac{1}{4}$ of an inch of healthy skin beyond it. When the naevus is large it is treated piecemeal by square applicators, care being taken that the individual applications do not overlap one another. The applicator is applied to the naevus with sufficient pressure to make a definite depression on the surface. The time of application varies according to the anatomical structure and situation of the naevus. In nacvi situated about the face and trunk I5 to 30 seconds should suffice, while in the tougher naevi which may occur on the scalp an exposure of I minute may be necessary. When the applicator is removed the underlying skin is frozen into a solid white disc, which thaws in about 
a minute and forms a blister in about an hour. After the application a dressing with $+\%$ boric acid ointment should be applied. When the blister becomes tense it should be opened aseptically and the dressing re-applied or be allowed to dry up and the scab to separate naturally: The reaction usually subsides in 2 to 3 weeks, after which, in successful cases, the naevus tissue may have disappeared and only a red inflamed slightly raised patch be left, or a few remnants of naerus tissue. Nothing further should be done for a few weeks, as the process of involution continues for a considerable time after the freezing.

In certain cases one application is sufficient but in many of them several are necessary.

Radium. - The treatment of raised haemangiomata by radium is much more tedious and costly, but it has the advantage of being painless, and can be carried out in an infant while asleep. By weak exposures to radium, angiomata may be dried up, decolourised, and replaced by a pale and supple scar. The same care with regard to the aroidance of reaction is not so necessary in treating these naevi as in the case of a port-wine stain, and a certain degree of reaction is an advantage. It should not be excessive, however, in case a disfiguring telangiectatic scar be left. A flat applicator of a suitable size containing sulphate of radium of half strength may be employed and exposures through a screen of $\frac{1}{10}$ millimetre of lead surrounded by black paper or French gutta-percha should be given for 2 hours on 3 successive days. This may be repeated after an interval of three weeks. In the case of markedly raised lesions resort may be made to the method described by Wichinam and Degrais as the "cross-fire" method. This consists of the application of two applicators on opposite sides of the tumour so that the interior is subjected to the bombardment of rays from both sides.

Excision is a simple method of removing raised naevi at the expense of a linear scar. It is specially applicable to superficial lesions of medium size, situated on covered parts of the body, many of which have a definite fibrous capsule which facilitates their complete remoral. The incision should be made well beyond the margin of growth, the whole of the naerus tissue remored, and the wound sutured. In deep seated naevi the operation should be done with caution as the sub cutaneous portion usually spreads beyond the cutaneous part and no capsule may be present, and a serious haemorrhage may take place. When the naerus is large it may be impossible to bring the skin together after the operation and it may be necessary to leave some of the affected shin as a flap. Where part of the naevus is in the hypoderm, haemorrhage may be avoided by compression with a clamp or by the introduction of I or 2 needles below the naevus in the healthy skin and the rinding of a piece of rubber tubing tightly beneath them. If after excision any piece of naerus skin be left it can be destrored subsequently.

Electrolysis.-By electrolysis in skilled hands angiomata of considerable size can be removed satisfactorily with comparatively little 
scarring. It has several disadvantages, however, namely (I) it is very painful and except in strong adults necessitates a general anaesthetic, (2) great skill and experience are required to obtain a good cosmetic result by it, (3) when the naevus is situated about the head or neck of an infant it is dangerous from shock. In this treatment it is best to err on the safe side by doing too little rather than too much at a time and repeating the operation if necessary.

Procedure.-The needle is usually fixed to the negative pole, an ordinary steel needle about No. 7 size or a platino-iridium needle being employed, while to the positive pole is attached a metal electrode covered with chamois leather. This is known as the unipolar method and is suitable for small naevi about the size of a split-pea.

In larger lesions the bipolar method is resorted to in which needles are attached to both poles, platinum needles being used in preference to steel ones to avoid oxidation. These needles may be manipulated separately or a special bipolar needle may be used in which they are fixed together parallel to each other, but carefully insulated, so that their points are separated about an $\frac{1}{8}$ to $\frac{1}{1}$ of an inch. For larger lesions special bipolar instruments may be used in which 4 or 5 needles are fixed, alternately positive and negative, on a horizontal bar at a distance of about a $\frac{1}{4}$ of an inch apart.

To avoid injuring the skin by the electrolytic action it has been recommended that the needles should be insulated to about a $\frac{1}{4}$ of an inch from their points. No satisfactory insulating material has yet been found, however, those in use, such as shellac and gutta-percha, being too bulky.

A general anaesthetic is invariably necessary in infants and children if the naevus be larger than a sixpence ; in adults, however, the pain of electrolysing naevi of considerable size can be borne. Before the electrolysis the surface of the naevus should be washed first with boric acid lotion, and then with spirit. If the naevus be no larger than a split-pea the unipolar method is employed, and the needle inserted at the base of the naevus and pushed horizontally across almost to the other side so as to get the full intensity of the current, while the positive electrode moistened with salt solution is placed near it. If a steel needle be used it should be sterilised in carbolic lotion before insertion, while if a platinum needle be employed it can be sterilised in the flame. A current of 6 to 8 milliamperes is slowly turned on and continued for about five minutes till the naevus becomes white and hard and the surrounding skin red. The current is then turned off and the needle withdrawn. If the whole of the naevus has not been sufficiently electrolysed, the needle may be again inserted in another position but care should be taken to avoid allowing the electrolytic zones to overlap. After the electrolysis the naevus should be bathed with boric lotion or with I in rooo biniodide of mercury solution, dried, painted with collodion, and a pad placed over it and fixed by a bandage so as to exert pressure on it for about half an hour 
afterwards. As a result of the procedure a crust usually forms which separates in about ro days.

Various modifications of the above procedure have been suggested, such as inserting the needle in the healthy skin beyond the naevus rather than into the naevus itself and making a series of insertions all round it at intervals of about a $\frac{1}{4}$ of an inch, and so cutting off the blood supply at the base; or entering the needle in the middle of the naevus pushing it towards the margin then turning on the current, partially withdrawing it and re-inserting it in a new position and so on till the naevus has been gone over in a radial fashion without withdrawing the needle altogether. Should the tissue around the negative pole become black it is an indication that necrotic changes have been produced in which case the current should be turned off the needle re-inserted in a new position and a smaller current employed.

When the naerus is larger than a sixpence the bipolar method should be adopted. This consists of inserting the two poles, each armed with a needle, parallel to each other for about a $\frac{1}{4}$ of an inch. A current of about Io milliamperes is turned on, and after 5 minutes it is turned off and the needle withdrawn and re-inserted about.a $\frac{1}{4}$ of an inch away. Instead of two separate needles it is more convenient to employ a bipolar needle insulated with sheliac to about a $\frac{1}{4}$ of an inch from the tip, as with the two needles, unless great care be taken, contact may occur and a shock be produced from short circuiting. By; means of the bipolar needle re-inserted several times the whole of the naevus may be satisfactorily electrolysed.

To be completely successful it may be necessary to have several sittings at intervals of 6 weeks or 2 months.

Cauterisation.-Cauterisation by means of a Paquelin cautery gives good results in raised naevi up to the size of a sixpence. With a sharp pointed cautery the naerus is tattooed with punctures at a distance of about a $\frac{1}{4}$ of an inch from each other until the whole surface has been gone over. Any haemorrhage which may occur is easily controlled by pressure. If the cauterisation be done carefully the scar which remains is comparatively slight and not markedly inferior to that left by electrolysis. This treatment though painful may be done without an anaesthetic even in infants.

The employment of caustics such as acid nitrate of mercury, ethylate of sodium, and fuming nitric acid, is unsatisfactory, and painful. A caustic method which has been recommended is the application of a Io \% solution of corrosive sublimate in collodion for 3 hours. This causes inflammation, suppuration, and a scab to form in about 4 days which is followed by cicatrisation. This method is liable to produce marked scarring and is inferior to the actual cautery.

Injections of coagulating fluids such as perchloride of iron or zinc chloride have been used in the past for the treatment of these lesions, but have been discontinued owing to the risk of causing fatal results by reaching the general circulation and producing pulmonary embolism. 
The old-fashioned method of vaccinating over the naevus in infants occasionally gives good results, but should never be done on the face as it leaves a vaccination scar. Care must be taken not to scarify too deeply in case the blood from the naevus should wash away the lymph.

Compression has been employed for the cure of raised haemangiomata, in the form of flexible collodion applied every other day. This is occasionally sufficient to cause the naevus to shrivel up and disappear, and may be adopted in delicate infants.

Ligature was once a favourite method of treating vascular naevi and gives good results in the case of small pyriform lesions with a definite pedicle. A strong silk thread is tied tightly round the pedicle, which causes the naevus to atrophy and fall away in the course of a few weeks.

\section{SPIDER-NAEVI}

Spider-naevi are the most easily cured of all. A useful method of dealing with them is by electrolysis. The negative pole needle is inserted into the central elevation, and a current of 2 to 3 milliamperes is allowed to pass for $\frac{1}{2}$ a minute. The current should then be turned off, the needle withdrawn, and pressure at once exerted with a pad of sterilised cottonwool to prevent the lesion from refilling with blood, after which it should be painted with contractile collodion. Should any subsidiary dilatations remain after the blanching has disappeared they may be tattooed subsequently by the negative pole. If the current be not too strong practically no scarring is left, and though a small pit may occur at first it gradually fills up. Occasionally, where these naevi are situated about the sides of the nose or forehead where the skin is thin and stretched over bones, some difficulty may be experienced in obliterating them by electrolysis owing to the delicacy of the bloodvessels and their tendency to have a cavernous arrangement, and the lesions are apt to recur after apparently successful removal. In such cases an application of solid carbon dioxide gives excellent results.

Another simple and equally successful method is cauterisation by a fine pointed thermo-cautery of the Paquelin type, or by a pointed piece of hard wood impregnated with acid nitrate of mercury or fuming nitric acid and pushed with a twisting movement into the central elevation and into any subsidiary dilatations.

\section{REFERENCES}

Crocker, Radcliffe. Angioma serpiginosum (Diseases of the Skin, I905, ii. p. 906).

Spontaneous involution of port-wine naevus (Brit. Journ. Derm., I 896 , viii. p. 44).

Pinch, H. (Radium Institute Reports, London, I9I3, I9I f).

Schamberg, J. A peculiar progressive pigmentary disease of the skin (Brit. Journ. Derm., I90I, xiii. p. I). 


\section{(ii) LYMPHATIC VASCULAR NAEVI}

Both dilatation of lymphatic vessels (lymphangiectases) and lymphatic new-growths (lymphangiomata) may occur as congenital anomalies and are met with in " Lymphangioma circumscriptum."

\section{LYMPHANGIOMA CIRCUMSCRIPTUM}

Syn.: Lupus lymphaticus (Hutchinson); Lymphangioma cavernosum (Besnier) ; Lymphangiectodés; Multiple lymphatic naevi.

Definition: A rare form of naevus characterised by clusters of deeply-seated vesicles due to dilatation and cystic formation of the lymphatic vessels in the corium.

Description: Like other forms of naevi, it usually appears in infancy, either at birth or some months later, but it may not be observed till puberty or even till adult life. It generally consists of an irregular cluster of vesicles which vary in size from a hempseed to a split-pea, are rounded or irregular in outline, translucent, and usually, of a yellowish straw colour though occasionally they may be purplish from small vascular tufts ramifying about them. In the centre of the cluster the vesicles may be closely congregated in the form of a mass which has been aptly compared to frog-spawn, while at the periphery they are scattered about in an irregular fashion, separated by areas of normal skin, and are more like boiled sago grains. Occasionally small puncta or telangiectases may be observed among the vesicles. The overlying epidermis may be smooth but is more often wrinkled or thickened and definitely warty. Sometimes the whole area of affected skin is definitely raised and irregular, owing to the presence of a more deeply-seated cavernous lymphangioma. The clusters may be single or multiple and may be in the form of irregular patches, or more rarely may have a linear arrangement. They may occur anywhere on the cutaneous surface but are most frequent on the neck and sides of the trunk, especially about the axillae. (Fig. 3I.)

The naevus tends to increase with the growth of the body and on reaching a certain size to remain stationary and to persist indefinitely. Individual vesicles usually disappear in a few months, either spontaneously or as a result of haemorrhage into them when they dry up to form a scab which on separating is apt to leave a superficial scar. As new vesicles tend to keep on coming out, however, the lesion is perpetuated.

There are no definite subjective symptoms though friction from clothing, rubbing, and other forms of traumatism, may cause more or less discomfort and occasionally bleeding. Nor are there any signs of inflammation, and those cases in which an outbreak of vesicles is heralded by an inflammatory erysipelatoid attack belong to a different category and are of streptococcic origin (see Elephantiasis nostras). 
Similar lesions have been described in the mucous membranes, and groups, sometimes of a violaceous colour, have been observed on the tongue, soft palate, insides of the cheeks, lips, and vulva. When the tongue is involved it tends to become swollen and hard and has been named "wooden tongue."

Not infrequently the lymphatic naevus is associated with other forms of vascular naevi, such as ordinary capillary naevi, cavernous haemangiomata, etc.

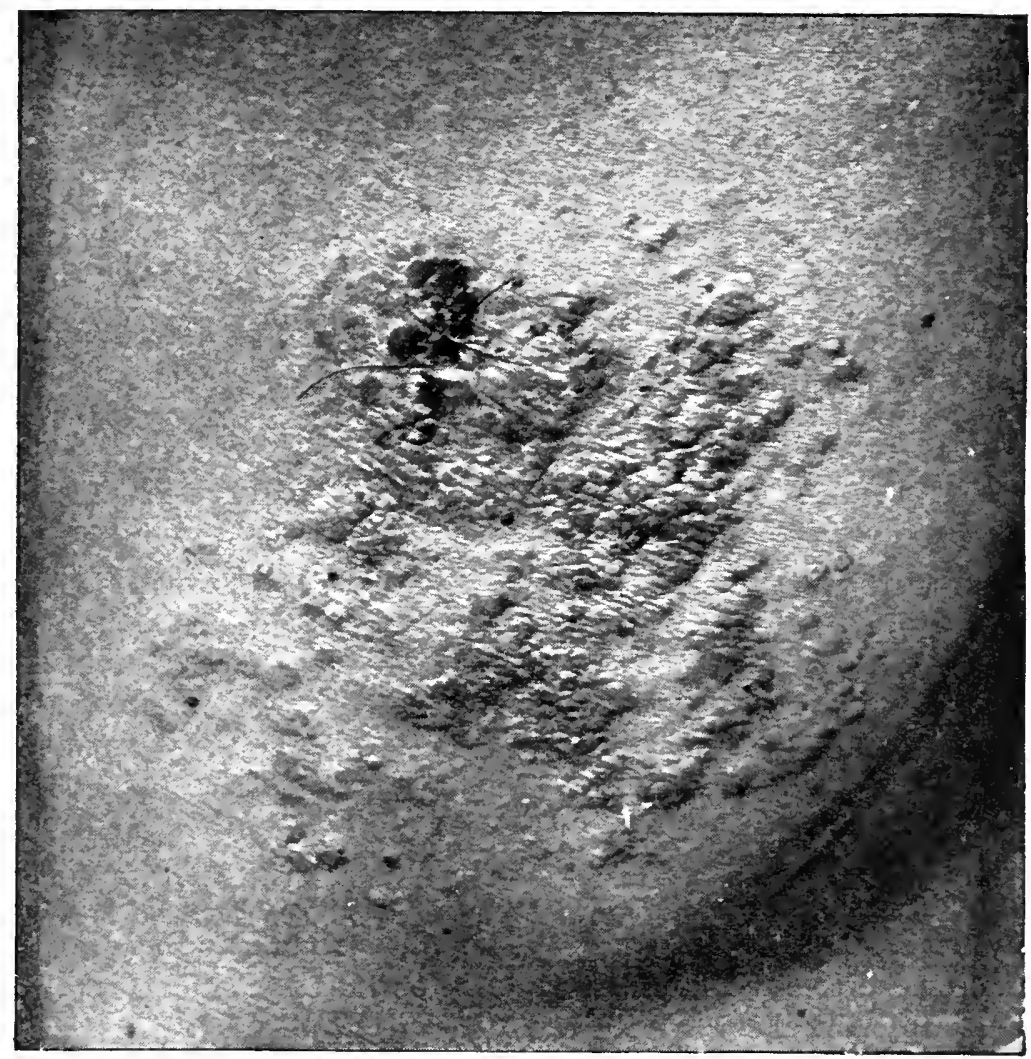

Fig. 31.-Lymphangioma Circumscriptum on the chest in a man, aged is years. Shows vesicles with clear and haemorrhagic contents.

(Dr. H. MacCormac's case, Brit. Journ. Derm., I913.)

Though it is one of the rarer forms of naevus a considerable number of cases have now been described, the majority of which have occurred in this country.

Histopathology.-Microscopical examination of sections of resicles shows that the cavities are situated in the corium, chiefly in the subpapillary layer, and that they are due to a dilatation of lymphatic ressels. Here and there contiguous dilatations have coalesced to form a multilocular cavity, the septa of which tend to break down eventually. Around the cavities the blood-capillaries are dilated. 
The overlying epidermis may be unaffected, or it may be thickened with elongation of the interpapillary processes and hyperkeratosis. The general concensus of opinion is that the dilatations liave taken place in newly formed lymphatic ressels and that the condition is a congenital lymphangioma.

Treatment.- The treatment consists of excision, which must be free to aroid recurrence, or of destruction of the vesicles by cauterisation or by freezing with solid carbon dioxide.

\section{REFERENCES}

Broce, L., \& BERNARD, L. A study of circumscribed lymphangioma of the skin and of the nucous membranes (Ann. de Derm. et de Syph., April I898, p. 305).

Fox, T. \& C. Lymphangiectodes (Path. Trans., I8 79 , xxx. p. 470).

Fraxcis, A. G. Lymphangioma circumscriptum cutis (Brit. Journ. Derm., I 893 , v. p. 33).

Hartzeli, M. B. Lymphangioma circumscriptum (Med. News, Jan. I6th, I 892).

Hutchissox. Lupus lymphaticus (Path. Trans., 1880, xxxi. p. 3+2).

Hutchissox (Junior). Histology (Idem, I885, xxxr. p. $f^{6}-$ ).

MACLEOD, J. M. H. Lymphangioma circumscriptum (Brit. Journ. Derm., I9I3, xxv. p. I90).

Török, L. On the capillary lymphangiomata of the skin and the relations of lymphangioma capillare varicosum to angiokeratoma (Monats. f. prakt. Derm., Bd. xiv. 5 March, I892).

Török, L. Lymphangioma circumscriptum (Lymphangioma capillare varicosum) (Brit. Journ. Derm., I \$9o, ii. p. 359).

\section{NON-VASCULAR NAEVI}

\section{(i) PLANE PIGMENTED MACULES AND PATCHES (NAEVUS SPILUS)}

Pigmented macules or patches of congenital origin are common. They vary in size from small specks up to a sixpence or larger, in colour from faint sepia to deep brownish-black, and in outline may be circular, oral, or irregular, and are usually well demarcated. They may be single, or multiple and occur in large numbers widely distributed over the skin. They are common on the face, neck, and back, but may be found anywhere, even on the scalp. They may be present at birth, may appear during the first few months of infancy, or may not develop till later in life; ; once present, they remain stationary and show little or no tendency to spontaneous involution. There are no subjective srmptoms associated with them. (Fig. 32.)

Histologically the shin is unaltered, except for the presence of pigment, consisting of melanin granules, situated in the cells of the basal layer, in the prickle-cells adjacent to it, and occasionally: 
in connective tissue cells (melanoblasts) in the upper layers of the corium.

Treatment.-When the lesions are small they may be destroyed by caustics such as nitric acid or ethylate of sodium applied by a glass pen or a match; this produces a scab which, on separating, leaves an area free from pigment and no appreciable scar.

They may be removed by refrigeration with carbon dioxide snow, an application of 20 to 30 seconds being given with firm pressure. This treatment is not quite satisfactory as, if the application be sufficient not only to destroy the pigment but to prevent it reappearing, scarring is liable to follow.

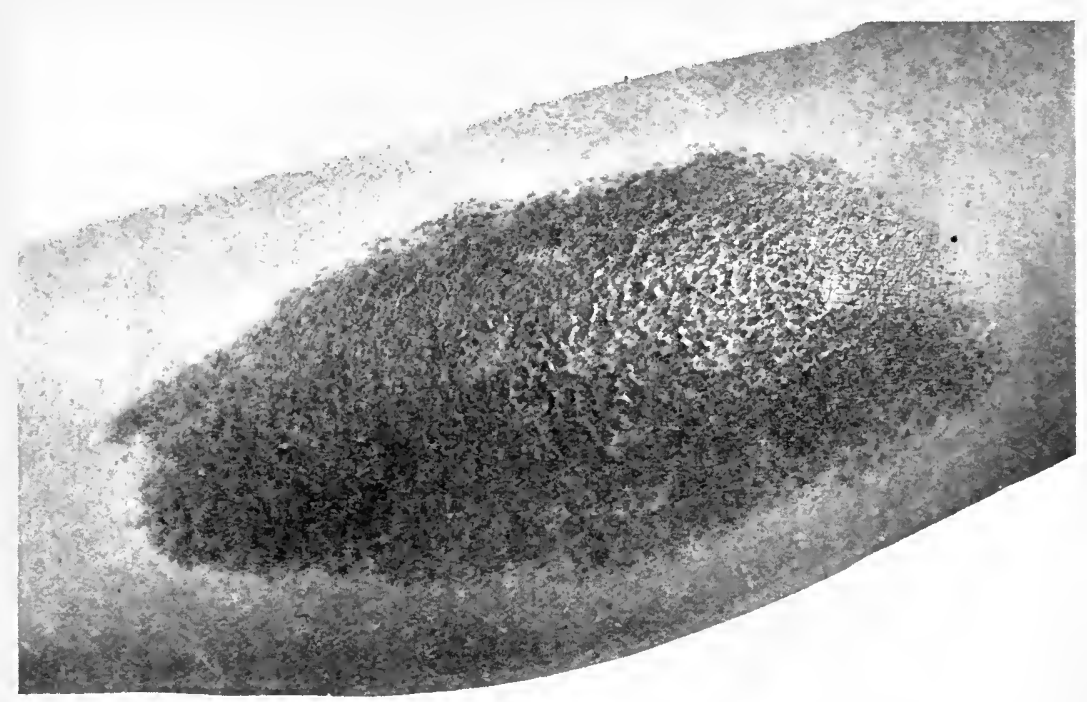

FIG. 32.--Pigmented Mole on arm.

Electrolysis may also be employed. The needle, fixed to the negative pole, is inserted diametrically across the whole lesion and a current of 2 to 3 milliamperes passed for half a minute. In larger patches the bipolar method should be employed (see page ISo). Some scarring is inevitable as a result of this treatment.

After middle life the naevi should not be treated by caustics or electrolysis as they may set up malignant changes.

In large pigmented patches refrigeration and electrolysis are unsuitable as they are liable to leave an irregular piebald appearance, and it is better to be content with less powerful measures such as bleaching with a solution of hydrogen peroxide (20 vols.) applied several times a day. 
(ii) RAISED NON-TASCULAR NAEVI

\section{A. Soft NaEvi or Moles}

Soft naevi or moles differ greatly in their clinical appearances. They are usually raised brownish growths varying in size from a lentil to an almond but are sometimes much larger and may occupy whole regions, when they are known as "Giant naevi." They may be the same colour as the surrounding skin but are more

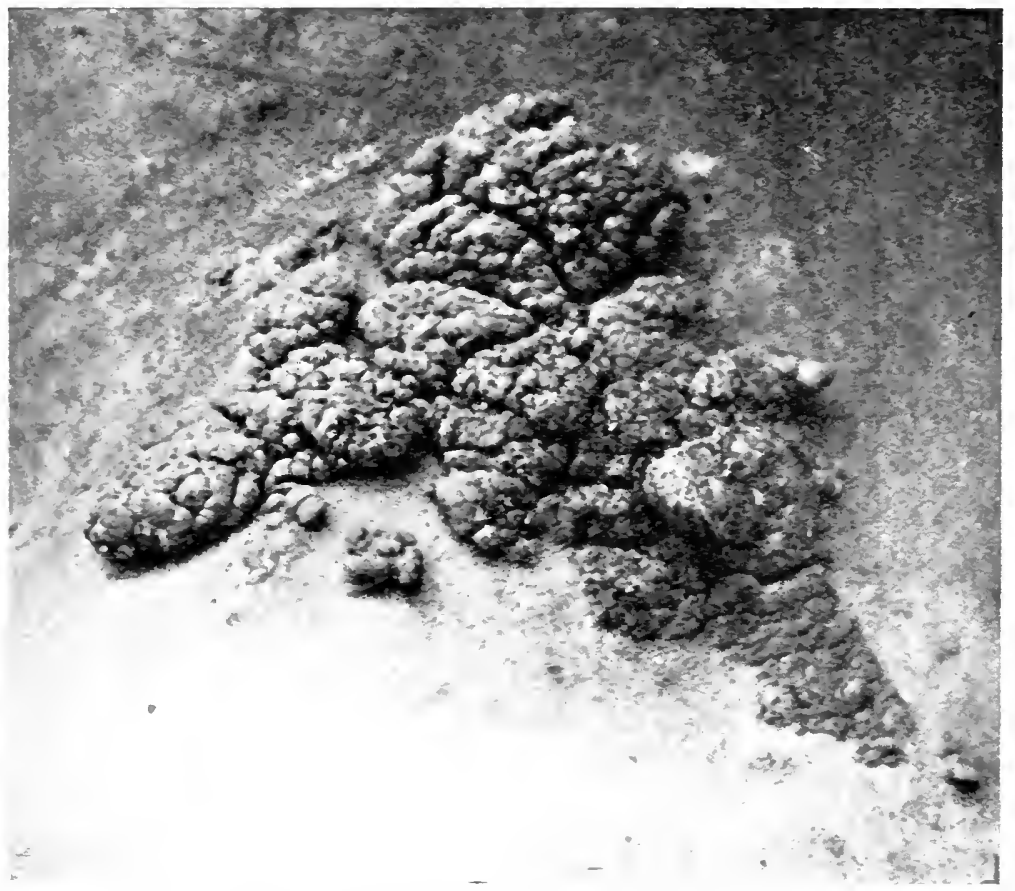

FIG. 33.-Terrucose Naevus on nape of neck.

(From D. P. G. Bayon.)

often brownish or, occasionally, may be almost black. The surface may be flat or broken up by shallow fissures into an irregular mosaic, and may be smooth or covered with fine down or dark pigmented hairs. The smaller lesions are round, oval, or irregular, in outline, and sessile, pedunculated, or raspberry-like in form. In consistence they are usually soft but in old age they may become hard and warty. (Fig. 33.)

Occasionally they are partly composed of fat-cells, forming a type of lesion known as Narus lipomatodes; at other times they are made up chiefly of sebaceous gland tissue and result from the growth of involutions of the epiblast from which pilo-sebaceous follicles should have developed, and have been named Varius sebacens. 
They may be single or multiple and may be present anywhere on the skin, but are most common on the face and neck where they are frequently found at the sites of the embryonic clefts and are beliered to be the result of inclusions when the closure took place. Sometimes they are distributed in zones or segments of the body, when they are known as Zoniform or segmental naeil. They are frequently associated with rascular naevi situated elsewhere, or occupying the same sites and forming mixed lesions. Cases are met with also where moles have occurred in the sites of vascular naevi, both developmental

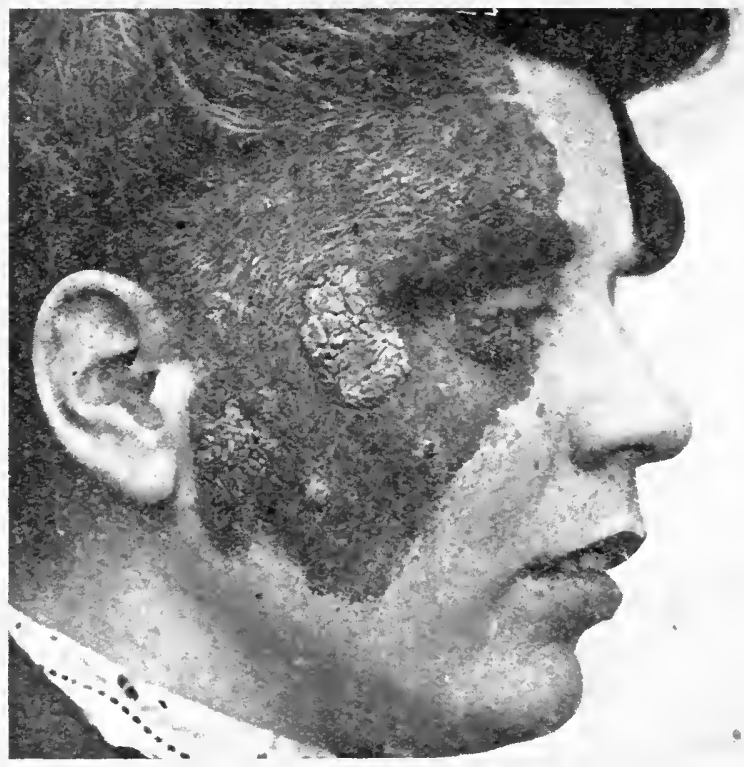

FIG. 34.-Pigmented Hairy Mole with verrucose patches.

anomalies being present at birth, the vascular naevus disappearing early and leaving the mole in its place. (Fig. 34.)

Moles may be present at birth but it is more usual for them to be first noticed in infancy, or they may not appear till adult life. They tend to increase with the growth of the body and usually become more prominent about puberty. In old age they may diminish and flatten or may undergo mollusciform or verrucose changes. Occasionally, partly as the result of local irritation, they may rapidly increase in size, become ulcerated, and assume malignant characters, invading the neighbouring lymphatics and forming what is known as a Naerocarcinoma.

The large moles or Giant naevi may be unilateral but are more often bilateral and symmetrical. They may involve considerable areas or even regions of the body - a favourite situation being the lower part of the back and buttocks, the so-called "bathing-drawers area." Occasionally they are confined to the area supplied by a cutaneous 



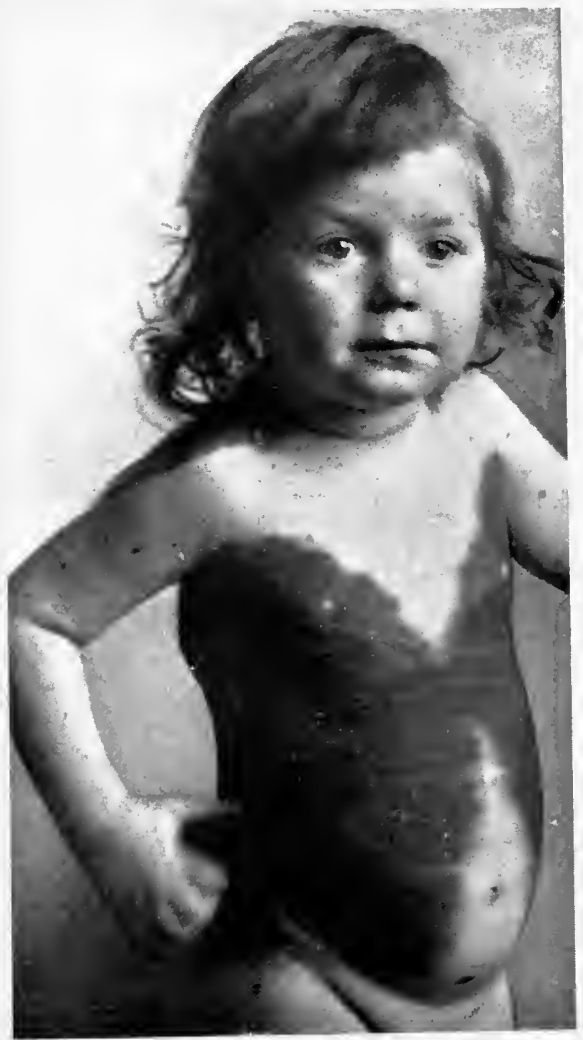

FIG. 35 .

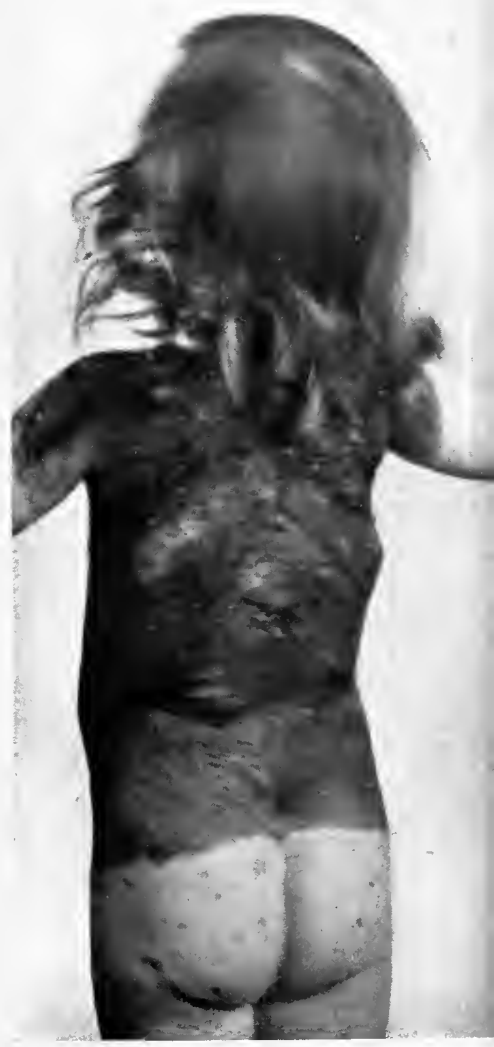

FIG. 36 .

Regional Pigmented Hairy Mole.

(Dr. Philip Burnet's case.) 
nerve or a group of nerves. They are usually of a deep sepia colour and covered with dark coarse hair. (Figs. 35 and 36.)

Histopathology.-The histological architecture of moles differs with the clinical type but however much it mar vary in detail there is always one common characteristic, namely a well-limited cellular infiltration with cells of a peculiar nature and unusual arrangement situated in the upper parts of the corium.

The epidermis may be attenuated and stretched from the pressure of the cellular deposit beneath, in which case the surface is smooth;

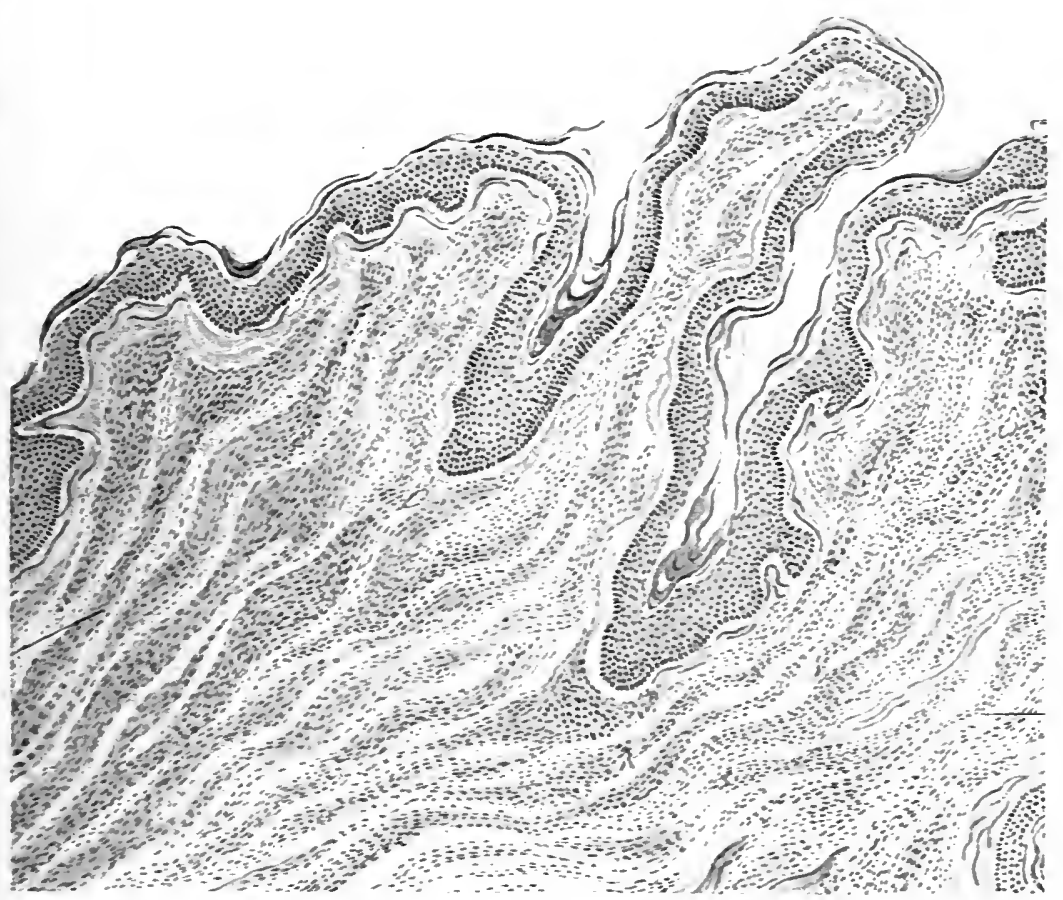

Fig. 37.--Verrucose Naevus. ( $x$ about So.) J. M. H. M. del.

Shows rows of cells spreading down obliquely into the corium.

or it may be hypertrophied and grow down in the form of long processes or show marked hyperkeratosis and the presence of horny pearls in the prickle-cell layer, in which case the surface is irregular and fissured. Melanin pigment granules are usually present in the cells of the basal layer and occasionally in the adjacent prickle-cells.

In the mollusciform or raspberry naevi the surface is markedly uneven and presents irregular furrows stretching deeply into the naevus with corresponding down-growths of the underlying epidermis.

In addition to the epidermal changes which are of secondary importance, small circumscribed groups or nests of round cells, which are of great significance, may be detected in the prickle-cell laver, as a rule near the basal layer. The groups may be contiguous with the surrounding cells or may be situated in a cyst-like space. The cells 
comprising then vary in number from a few to twenty or more and have the appearance of imperfectly developed epidermal cells; they are smaller than prickle-cells, globular in shape, with vacuolated nuclei and occasionally pigment granules but no inter-epithelial fibres.

The cellular infiltration in the corium consists of cells of a precisely similar type. It extends from near the basal layer to the level of the sub-papillary layer where it ends abruptly, while at the sides of the lesion it fades away more gradually

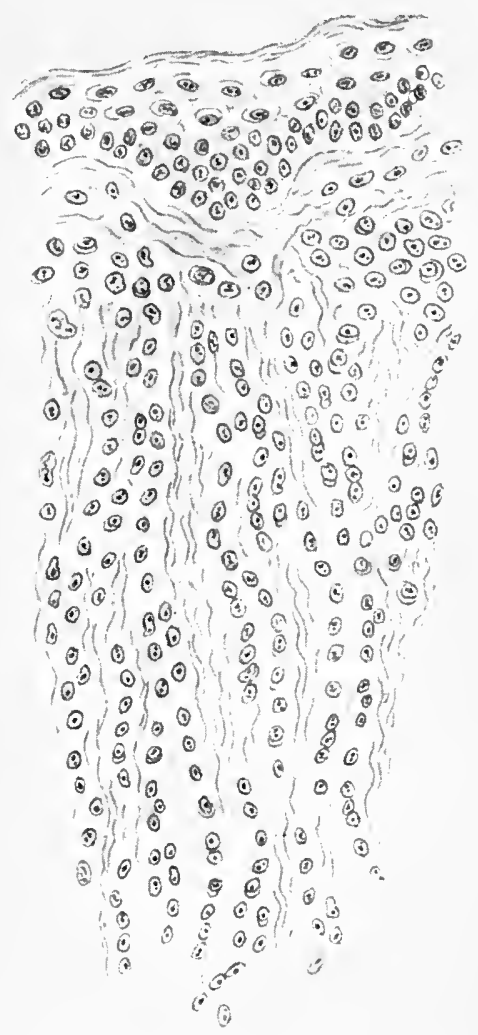

FIG. 38.-Soft Naevus.

Shows naevus-cells stretching down in rows into the corium. into the surrounding tissue. In it the cells are arranged in columns stretching down at right angles or slightly obliquely to the basal layer. In some instances these columns appear to spring from the basal layer, in others to be separated from it by a thin layer of connective tissue, and occasionally they seem to be connected with the prickle-cell layer of the hair-fcllicle. (Fig. 37.)

In a few rare cases the naevuscells are not arranged in the above manner but appear singly or in clusters throughout a certain area of the corium lying in the meshes of the collagen network. In these cases the cells occur much more deeply, spread down as far as the subcutaneous tissue, tend to become mixed up with fat-cells and have been observed to undergo fatty degeneration.

The cells composing the columns are round or cubical in shape. The cell protoplasm is homogeneous, does not stain well, and is some-

what denser at the margin than in the centre. The nuclei correspond in shape to the cells and are usually vesicular in type and here and there present deeply stained chromatin masses. The naevus-cells near the epidermis are similar to those of the " epidermal nests," while those further away lose these characteristics to a certain extent and assume ilie appearance of undifferentiated embryonic cells. Near the epidermis multinucleated cells or giant-cells may occasionally be detected in which the nuclei are not arranged around the periphery of the cell as they are in Langhans' giant-cells, but form a cluster in the centre or at one end. In the neighbourhood of the columns pigmented cells or melanoblasts may occur, these are spindle-shaped or branched and may 
be so obliterated with pigment that it is difficult to tell if the melanin is within or without the cell. Here and there pigment granules may be detected in the actual naevus-cells. (Fig. 38 .)

The collagen fibres are thin, shrunken, or broken up, and the elastin is diminished in the situations of the cellular infiltration and absent between the naevus-cells. Immediately below the infiltration the fibrous elements are normal. In time the movements and stretching of the skin elevate the naevus like a foreign body and make it more and more prominent. This process Unna graphically describes as the " birth of the naevus."

The blood-capillaries are also affected in the region of the cellular infiltration, probably as the result of actual compression. In some cases there is diminution of the hair-follicles but in others they seem to be unaffected or actually increased. Beneath the naevus-cell infiltration there may be a considerable accumulation of mast-cells but this is inconstant and does not seem to have any definite significance.

Origin of Naevus-cells.-The origin of the naevus-cells has been a subject of controversy for many years and it is not yet finally settled. In 1882 $v$. Recklinghausen declared that they were derived from the endothelium of the lymphatics, and this opinion was generally accepted till I 893 when Unna asserted that they were of epidermal origin. Since then other theories have been put forward such as:-that they originate from the endothelium or the perithelium of the blood-capillaries (Demieville, Jadassohn); or that they are embryonic connective tissue cells (Riehl, Riecke) which are capable of multiplying but not of evolving into collagen fibres. An extensive literature now exists on this subject in which numerous arguments have been advanced by the initiators and supporters of the different theories.

From the actual appearance of the cells it is impossible to come to any definite conclusion with regard to their origin, for they present characteristics which are common to imperfectly developed prickle-cells, endothelial cells, and embryonic connective tissue cells.

The only method of deciding their true nature is to demonstrate a definite connection between the naevus-cells and the epidermis or the endothelium and to trace transition stages between them and the prickle-cells or the endothelial cells; this the supporters of the respective theories claim to have done.

From the histological study of a considerable number of moles of different types it seems that the naevus-cells in the majority of specimens are epiblastic in origin. This view is strongly supported by the facts that epidermal nests of cells frequently occur in the prickle-cell layer, that columns and groups of naevus-cells in the corium may occasionally be seen to be contiguous and apparently connected with the epidermis, and that the down-growing prolongations of the epidermis may gradually merge into columns of naevus-cells. According to Unna the cells of the epidermal nests burst through the basal layer in embryonic life or infancyand are ensnared by connective tissue fibres. One of the strongest pieces of evidence in favour of this theory is seen in sections of growing moles in infants, which show the downward growth of the epidermal cells, together with the active growth of collagen and fibrous tissue encapsulating them in the corium. This same process is illustrated in later life in moles which have become malignant; if sections be cut of an early malignant growth in a mole the same down-growth of epidermal cells as is seen in the formation of the mole in infancy is repeated, and the 
carcinonia appears to be formed more from the fresh down-growing epidermal cells than from the naevus-cells already in the corinm, and the absence of the actively growing collagen and encapsulating fibrous tissue allows the malignant growth to spread. This explanation does not hold good for all cases, however, and certain specimens suggest that, though the majority of nıoles are of epidermal origin, a few of them are probably mesoblastic. In two specimens in the writer's collection there is a deposit of cells in the corium which is not arranged in columns but irregularly distributed in groups in which a number of spindle-shaped pigmented cells occur, and the general appearance presented is that of embryonic connective tissue cells; while in a case described by MacDonagh the naevus-cells were regarded as embryonic endothelial cells.

It would seem then that moles are the result of the persistence after birth in an embryonic condition of certain groups of cells, which are usually epiblastic but may occasionally be of mesoblastic origin, and which are capable of proliferating subsequently but are unable to fulfil their developmental intention and to develop into prickle-cells, sebaceous gland cells, etc. on the one hand, or into connective tissne cells on the other. Should malignant changes supervene in connection with those cells, according as the group is epiblastic or mesoblastic so the malignant tumour will be a naevo-carcinoma or a naevo-sarcoma. In the naevo-carcinoma the naevus-cells rapidly increase in number, lose their characteristics to such an extent that they are no longer recognisable, and many of them become deeply pigmented. They tend to be arranged in alveoli enclosed by spindle-cells presenting an appearance not unlike a melanotic sarcoma.

Treatment.-Though moles are not accompanied by subjective symptoms yet their unsightly appearance when present on exposed parts and their capacity for undergoing malignant changes in late life render it advisable to remove them. This should be done as early as possible as they tend to increase in size and to become more prominent. When they occur on covered parts of the body and are of moderate size the simplest method of dealing with them is excision, but when they are large, as in the case of giant naevi, an extensive plastic operation or grafting may be necessary, which requires considerable skill to ensure that the resulting scar is less disfiguring than the naevus.

When they are on the face the best treatment, both for simplicity of technique and the resultant scar, is refrigeration with carbon dioxide snow. An applicator slightly larger than the mole is chosen and an application of about 40 seconds with firm pressure is given. The blister which results from the exposure should be opened aseptically next day and a dressing of boric acid ointment applied. After the reaction has subsided, in the course of ten days or a fortnight, a further application may be given.

Electrolysis is a useful method of dealing with hairy moles about the face, the hairs being carefully epilated in the manner described for hypertrichosis; as a result the naevus tissue shrivels and the lesion becomes paler. Should any of the growth remain it can be dealt with subsequently by the unipolar or bipolar method, employing a current 
of 3 milliamperes for 30 seconds. After electrolysis the mole should be swabbed with boric lotion, dried, and painted with collodion.

Small moles may also be destroyed by a Paquelin or an electrocautery, but unless this is done most carefully the results are inferior to those obtained by the above methods.

Caustics, such as nitric acid, trichloracetic acid, or ethylate of sodium, should not be employed as they are painful, apt to give rise to disfiguring scars, and if the destruction of the mole be incomplete the irritation of the caustic may lead to malignant growth.

Attempts have been miade to obliterate moles by the $\mathrm{X}$-rays, but as this can be done only by producing a severe dermatitis and replacing the mole with an X-ray scar the treatment is to be deprecated.

\section{B. Hard or Verrucose Naevi}

Hard naevi form a complex and multiform group of congenital anomalies of the skin. They vary from small lesions the size of a hempseed covered with scales to horny masses in irregular patches, bands, or streaks, situated in different regions of the cutaneous surface (Linear naevi). A peculiar variety characterised by the presence of exuberant warty lesions and horny spines is known as Ichthyosis hystrix, and another type occurring as diffuse thichenings of the palms and soles is named Keratosis palmaris et plantaris hereditaria.

Verrucose naevi may be present at birth but more usually appear during the first year. They increase in size with the general growth and then remain stationary and show no tendency to spontaneous retrogression. They may be partially or wholly pigmented and are generally, though not invariably, hairless.

\section{Linear Naevi.}

Syn.: Systematised naevi; Ichthyosis hystrix linearis; Naerus unius lateris; Papilloma neuroticum.

The linear naevi constitute a remarkable variety of hard naevi in which the lesions are arranged in streaks several inches in length or in bands extending down the whole length of a limb. The streaks may be narrow, consisting of one or two rows of lesions, but are more usually about half an inch in breadth, widening out here and there into irregular patches, and either forming continuous bands or being broken up and separated at irregular intervals by normal skin. Their distribution varies with the region affected. On the limbs they are usually arranged longitudinally, while on the trunk they tend to be transverse or obliquely curved. They may occur anywhere on the cutaneous surface and cases have been reported where they extended from the groin to the foot, others where they formed irregular parallel bands on the abdomen, 
others where they extended from the neck across the shoulder and down the arm to the hand, etc. They most frequently affect one side of the body (Naevus unius lateris), but may be bilateral and more or less symmetrical. The bands are made up by the aggregation of small lesions of the hard naerus type and consist of raised flat papules

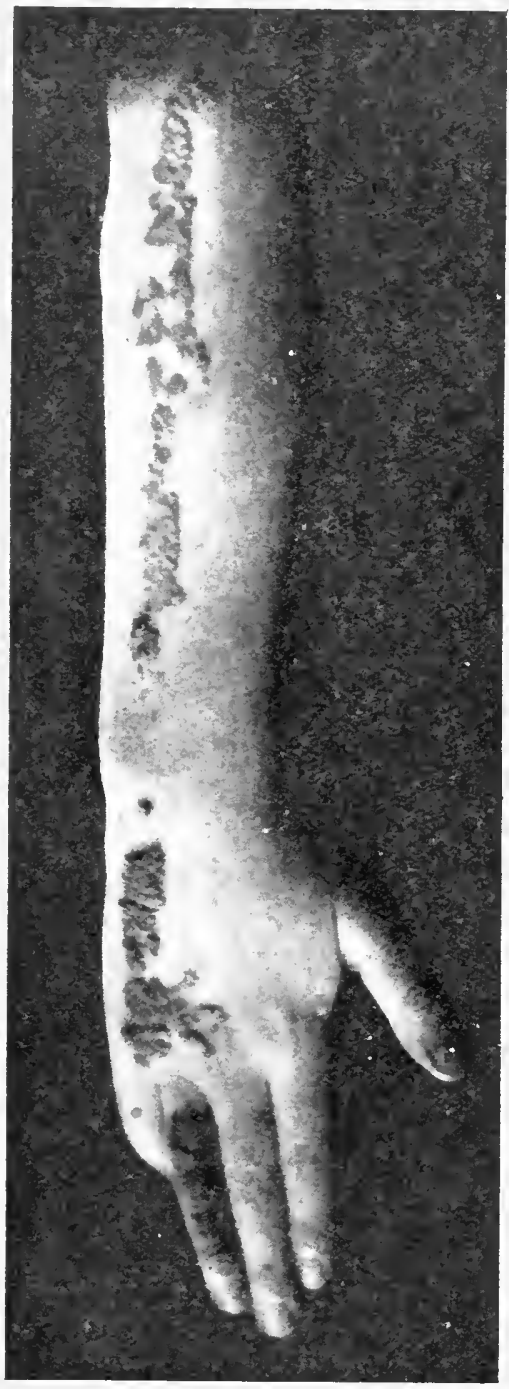
pinkish in tinge, not markedly verrucose, and simulating papules of Lichen planus, or of larger lesions of a dirty brownish colour and warty in character. (Fig. 39.)

The most striking variety of linear naevus is one which occurs especially about the extremities and consists of large horny masses forming thick spines or limpet-shaped excrescences, of a grey or blackish colour, raised $\frac{1}{2}$ an inch or more, and when torn off leaving a papillated surface. This condition has been termed Ichthyosis hystrix, or more popularly Porcupine naevus. (Fig. 40.)

In another variety described by Selhorst under the heading of Naevus acneiformis unilateris, the naevus took the form of patches or lines dotted over with large double comedones and acneiform pustules and associated with scarring.

There are no subjective symptoms connected with these lesions, and they give rise to little or no discomfort unless they occur on the face, scalp, or neck where they are disfiguring, or on the hands and feet where they are liable to become inflamed by injury, to suppurate from accidental inoculation by septic micro-organisms, or to be a source of trouble from bleeding.

Histopathology.--The histopathology of hard naevi differs from that of moles in that the most important and essential changes take place, not in the corium, but in the epidermis. According to the type of naevus either the Stratum corneum is markedly thickened (Keratoid naevus of Unna) or the prickle-cell layer is hypertrophied (Acanthoid 
naevus), or the hypertrophic changes may affect both layers. The structure varies somewhat according to the region affected, and the naevus is liable to be keratoid about the extremities and acanthoid where the skin is softer as about the face, neck and trunk.

In the corium definite naerus-cells like those of moles are rarely met with, and the only changes which can be detected are of a secondary inflammatory character from irritation and consist of the presence of

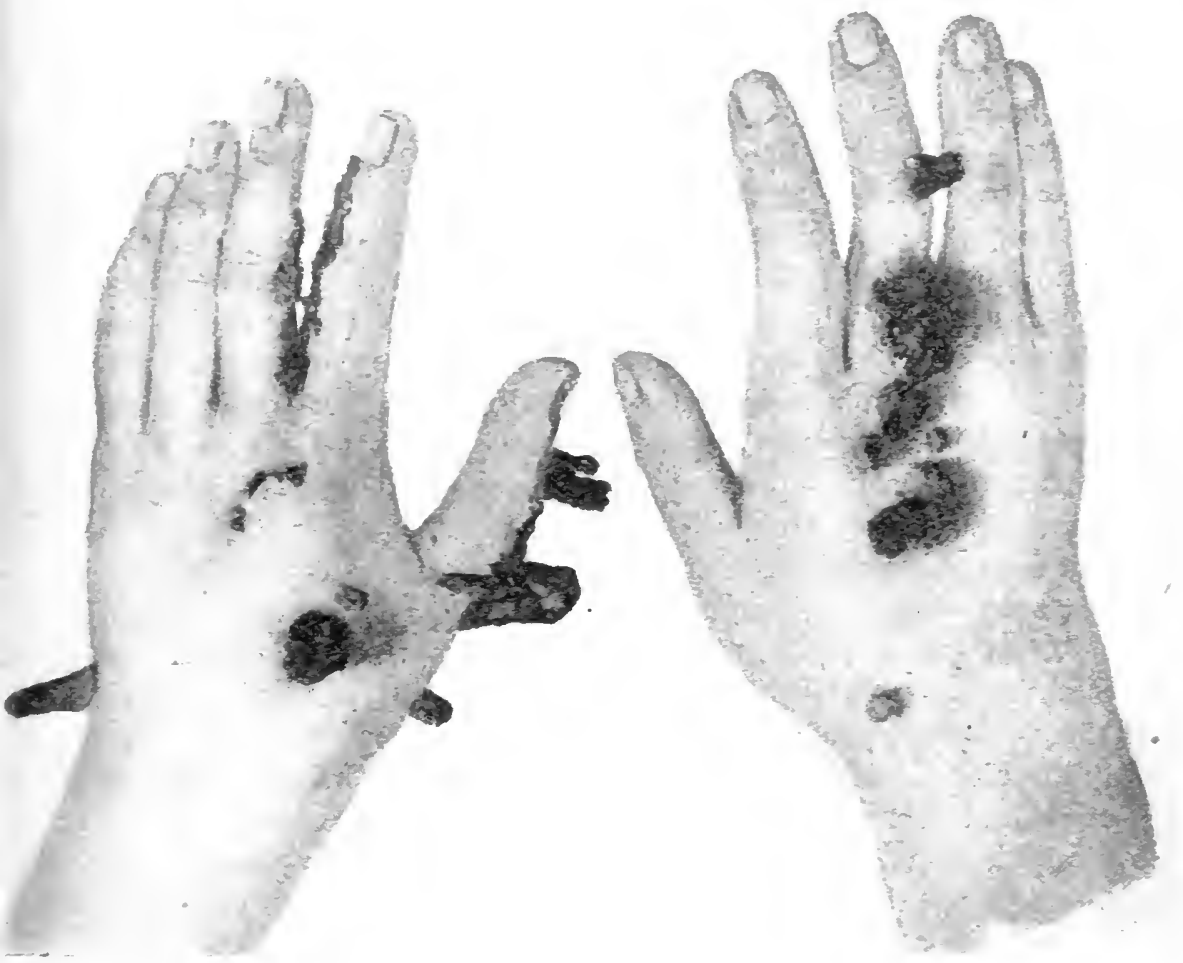

FIG. 40.-Ichthyosis Hystrix.

(Dr. J. H. Stower's Case. Brit. Journ. Derm., I9oS.)

certain groups of small cells about the vessels, and occasionally of plasma-cells and pigmented cells.

Under the heading of Naeus syringo-adenomatosi, several cases have been reported which clinically were identical with Naevus unius lateris, but histoiogically suggested a growth of the coil-glands. These presented a number of epithelial processes growing down into the corium, which either remained solid and short, or lengthened and became partially or completely canalised to form cysts sometimes containing polypi. Some of the processes bore a striking resemblance to sweat-ducts and were possibly formed from epiblastic processes similar to the embryonic rudiments of the sweat-glands.

The epidermal changes can only be explained by the presence of certain groups of cells which have persisted from embryonic life and developed in after years. 
In lesions of the Ichthyosis hystrix variety the papilla are greatly clongated and contain large dilated blood-vessels, and the interpapillary processes are correspondingly prolonged downward, with deep furrows above them into which the thickened horny layer dips down.

Etiology.--The etiology of hard naevi is uncertain. Heredity appears to play an important part, for cases have been recorded where they have occurred in several generations and in more than one member of a family.

The cause of the peculiar arrangement in linear naevi is puzzling but it seems more probable that it is due to some definite developmental error than to mere chance. It has been suggested that they follow the distribution of cutaneous nerves hence the synonym Papilloma neuroticum, or that they are due to an inter-uterine affection of a nerve ganglion (Bärensprung), or are connected in some way with Voigt's imaginary lines which separate the areas of distribution of the cutaneous nerves. Other explanations have been advanced, such as, that they are dependent on the distribution of cutaneous veins or superficial lymphatics, that they correspond with the lines of cleavage, or that they are dependent on the segmental areas or metameric segments. In short almost every conceivable explanation for them has been suggested, each capable of explaining certain of the cases but none wholly satisfactory.

Diagnosis.-The diagnosis in extreme cases presents no difficulty. Slightly marked cases have to be distinguished from linear Lichen planus, hypertrophic Lichen planus, and verrucose Lupus vulgaris. From Lichen planus they are differentiated by being congenital in origin and uninfluenced by any treatment except surgical interference ; from Lupus verrucosus by not having an underlying brownish base, by showing little or no tendency to ulceration, and by being present from birth or soon after.

Treatment.--Small lesions may be destroyed by a fine pointed cautery or by painting with salicylic collodion ( $\mathrm{x}$ in 8). Linear lesions if not too extensive may be excised, curetted, shaved off, or cut away by a pair of curved scissors, and the base touched with ethylate of sodium or with a Paquelin cautery. In extensive cases little can be done except the removal of the most prominent lesions by excision and grafting, or by curetting, and the constant use of alkaline soaps or salicylic collodion to prevent them re-forming.

\section{Hyperkeratosis congenitalis palmaris et plantaris}

Syn. : Ichthyosis congenitalis palmaris et plantaris (Thost) ; Keratoma palmare et plantare hereditarium (Unna); Tylosis palmare et plantare (Hebra).

Definition: A congenital thickening of the horny layer of the epidermis of the palms and soles. 
Description.-This rare affection occurs independently of other congenital anomalies of the skin. It consists of marked thickening of the horny layer of the epidermis of the palms and soles, symmetrical in distribution and generally affecting them to an equal degree. In a typical case these parts are covered by a thick layer, whitish-yellow or dirty brown in colour, leathery or horny in consistence, with a peculiar semi-translucent or waxy appearance, and varving in thickness up to an eighth of an inch. The surface is smooth and the finer lines obliterated, but the larger fissures are deepened and sometimes form open cracks from which oozes a serous discharge occasionally stained with blood. The thickening usually occupies the greater portion of the palms and the flexor aspects of the fingers, ends abrupty near the border, and is generally surrounded by a pinkish halo an eighth to a quarter of an inch in breadth. The affection not infrequently spreads on to the dorsa of the fingers and is especially liable to occur over the knuckles, phalangeal joints and at the borders of the nails. The nails themselves may be attacked, becoming thick, opaque, and raised up from the nail-bed by a horny accumulation beneath them. The sweatglands are not involved and in some cases show an excessive activity, rendering the thickened skin moist and sodden.

The condition is not associated with any definite subjective symptoms except slight tenderness from morements, or smarting or burning when deep fissures are present, but it may be uncomfortable as it interferes with the natural flexibility of the skin, making the hands stiff and rendering impossible finer manipulations, such as sewing or pianoplaying. Where the skin is much thickened there is a decrease in the tactile sensibility.

The soles are affected in a manner somewhat similar to the palms, the part most involred being that which is in contact with the ground in standing, while the hollow of the foot may be more or less completely spared. The thickening tends to spread up and encase the heel. The affected skin has the same semi-translucent appearance as in the hand and is waxy looking, or presents a dirty grevish or brownish tint. Cracks are generally present and secondary inoculations with pyogenic micro-organisms are liable to take place, giving rise to an inflammatory disturbance and occasionally to lymphangitis. Around the thickened horny plate there is a narrow erythematous halo. In the hollow part of the foot where the thickening is less marked, there are not infrequently small circumscribed thickenings or callosities.

The affection appears at birth and persists throughout life. In some cases during the hot weather, bullae about the size of a sixpence tend to develop beneath the thickened horny layer and may lead eventually to an exfoliation of the whole horny plate leaving a tender red surface. But the respite so obtained does not last long and the skin soon begins to thicken again.

In the island of Meleda, off the coast of Dalmatia, a somewhat analogous condition has been described which is known there as the 
Mal de Meleda and was once mistaken for leprosy. In it the thickening involves not only the hands and feet, but the knees, legs, forearms and elbows.

Histopathology.-The essential change which is found in microscopical sections is hypertrophy of the Stratum corneum or hyperkeratosis similar to that which occurs in an ordinary callosity. The granular layer appears to be normal and there is no definite change in the prickle-cell layer though occasionally there is flattening out of the papillary body. The corium is not affected except beyond the margin of the horny thickening or in connection with the fissures where it may present mild inflammatory changes.

Etiology.-It is a congenital anomaly of growth involving the process of cornification and is not influenced to any extent by local irritation, the elimination of arsenic by the skin, or other causes which tend to produce hyperkeratosis. It is hereditary and has been traced in five consecutive generations as well as in collateral branches of the family. It also occurs in families and seems to affect the sexes indiscriminately. It would appear to be more common in the poorer classes than in the well-to-do.

Diagnosis.-The principal points in connection with the diagnosis are the symmetrical involvement of the palms and soles, the absence of marked inflammatory symptoms, and the persistence of the condition from birtl. These are so characteristic as to be pathognomonic and to enable the affection to be easily differentiated from acquired hyperkeratosis the result of local irritation, the elimination of arsenic, or occurring in connection with diseases like Pityriasis rubra pilaris or eczema.

Treatment.-The affection is singularly resistant to treatment and little can be done that is of permanent value. Internal treatment is practically useless, benefit has been reported from the internal use of pilocarpine with the object of increasing the sweat and softening the skin, but this has been of the most temporary character. Some relief and comfort may accrue from suitable local measures with the object of reducing the thickening, rendering the skin more pliant, and preventing the secondary inoculation of micro-organisms. With this object the affected skin should be macerated with soft soap and hot water, or by the application of a 5 to ro $\%$ solution of caustic potash, or by a Io \% salicylic soap plaster. By these means the skin can be kept more comfortable but the thickening unfortunately recurs whenever they are discontinued. Temporary improvement can also be obtained from the careful application of X-rays.

\section{REFERENCES}

Adamson, H. G. Zoniform or Segmental Naevi (Brit. Journ. Derm., I9I 4 , xxvi. p. 379).

A case of Schamberg's Disease (Idem, r9r6, xxviii. p. 334).

Audrey, C. Naevus Zellen (Monats. f. prakt. Derm., rgoo, xxx. p. 409). 
BANDLER, V. Naevis sebaceus (Arch. f. Derm. u. Sypl., I899, xlix.)

BLAND-SutTox, J. Tumours, innocent and malignant.

Böнх, J. Keratoma hereditarium palmare et plantare (Derm. Centralblatt, March r9o4, p. I62).

Crocker, Radchiffe. Tylosis palmare et plantare (Brit. Journ. Derm., IS9r, iii. p. I69).

Decroo, T. Congenital keratosis of palms and soles (Journ. des Sci. Med. de Lille, r903, xxvii. p. Ir).

Delbaxico, E. Epithelial naerus (Monats. f. prakt. Derm., I\$96, xxii. p. 105$)$.

EIIExie. Naevi and their relation to nerve areas-an essay on pathogenesis and etiology (Nouvelle Iconographie de la Salpêtrière, I S97).

FICk, J. On soft naevi (ilonats. f. prakt. Derm., I909, pp. 397 and +43 ).

Fox, $\mathrm{W}^{\circ}$. Origin and structure of moles (Brit. Journ. Derm., I906, xviii. p. I).

HodarA, M. Weiche Naevi (Monats. f. prakt. Derm., I 897 , xxr. p. 205).

JАСов \& FClтоN. Keratosis palmaris et plantaris in five generations (Brit. Ned. Journ., July I905, p. I 25).

Johrsox. Naero-carcinoma (Journ. Cut. Dis., I905, p. ro).

ILACDoxagh, J. E. R. Naevo-xantho-endotheliomata (Brit. Journ. Derm., I9I 2, xxiv. p. $8_{5}$ ).

MACLEOD, J. M. H. Handbook of the pathology of the skin, p. I 27.

Neumaxx. Keratoma hereditarium (Nal de Meleda) (Arch. f. Derm. u. Sxph., Bd. xlii. Feb. r 896).

Prхch, H. Radium Institute Reports, London, I9I3, I9rf.

Riecke, E. On the naevus question (Arch. f. Derm. u. Syph., I903, (April), p. 65).

Selhorst, S. B. Naerus acneiformis unilateris (Brit. Journ. Derm., I896, viii. P. 4I9).

THost. Ichthyosis congenitalis palmaris et plantaris (Diss. Heidelberg, ISSO).

Uxi, P. G. Histopathology" (Walker, IS94, p. II2S).

WhiteheAd, R. H. Mlalignant tumours arising in congenital moles (Bull. of the Johns Hopkins Hosp., Sept. r 900, p. 22I).

Whitfield, A. Origin of naevus cells (Brit. Journ. Derm., I9oo, xii. p. 270$)$.

Wickha., L., \& DegraIs. Radium therapy (Dore's translation, I9ro).

IVolters. Naevi Syringo-adenomatosi (Arch. f. Derm. u. Syph. June 1904, p. 374).

\section{EPIDERMOLYSIS BULLOSA}

Syn.: Acantholysis bullosa; Epidermolysis bullosa hereditaria.

Definition: A peculiar vulnerability of the skin of congenital origin, characterised by the formation of bullae as the result of traumatism, such as blows, friction, or pressure.

Description.-This affection was observed by Tilbury Fox in I 879 , but was first described by Goldscheider in ISS2, and in the same year by Payne and Colcott Fox. Since then numerous cases have been reported. The condition, though rare, is so striking that it has attracted more than its share of attention. Its characteristic feature is vulnerability of the whole cutaneous surface, so acute that even the slightest injuries cause detachment of the epidermis and the formation of more or less tense blisters. It is a congenital anomaly and in 
one or two instances adhesions of the skin have been detected at birth indicating its pre-natal existence, but in most cases it is not noticed till a few days after birth and occasionally its appearance has been delayed to early childhood.

Cases have bcen met with where it developed in adult life as an acquired condition; these usually, though not invariably, occurred in association with chronic pemphigus and were possibly instances of secondary acantholysis (see Nikolski's sign, page 874).

In addition to the well-marked congenital cases which are extremely rare, mild degrees of it are not infrequent in which individuals are so liable to blisters from traumatism that they are debarred from handling tools or playing certain games. (Fig. 4I.)

The bullae differ from those of chronic pemphigus in being more flaccid and not arising spontaneously. They appear first on apparently normal skin but later, as their contents become purulent, an erythematous halo generally forms around the base. Occasionally the contents are more or less haemorrhagic. When the blister is broken a raw erythematous surface is left which gradually heals ; this is followed by a transient pigmentation which slowly disappears without a trace or leaves a residue of milium-like epidermic cysts. Occasionally the lesions are complicated by ulccration, crusting, and scarring, from the secondary inoculation of pyogenic cocci. The situations in which the bullae occur are naturally those which are most liable to injury, such as the hands from manual labour, the neck and wrists from the friction of the collar and the wristbands, and the waist or legs from the pressure of corsets or garters. So great may be the vulnerability of the skin that all forms of manual labour may be rendered impossible and the greatest difficulty may be experienced in finding an occupation suitable for the unfortunate sufferer. In many of the cases blebs have occurred beneath and around the nail, leading to changes in the nail-plate itself, interfering with its growth, rendering it opaque and friable, and even causing it to be shed. The mucosa of the mouth and alimentary tract may be involved, and vomiting and haematemesis have been described in connection with it, while carcinoma of the tongue has been known to occur at the early age of 25 years as a sequel to it.

After the disease has been in existence for some years, peculiar atrophic changes are liable to supervene in the skin.

The subjective symptoms are slight and consist of burning or itching, or pain in the denuded surfaces from movement. The general health is not definitely affected.

Histopathology.- The bullae may be situated either in the epidermis in the neighbourhood of the granular layer, or beneath it with the whole of the epidermis as a roof. At first they contain clear scrum and are sterile but later become contaminated with staphylococci, and fibrin and pus-cells appear. In the neighbourhood of the bullae degenerative changes have been detected in the nuclei and protoplasm of the deeper priclile-cells and cells of the basal layer of the epidermis with dilatation 


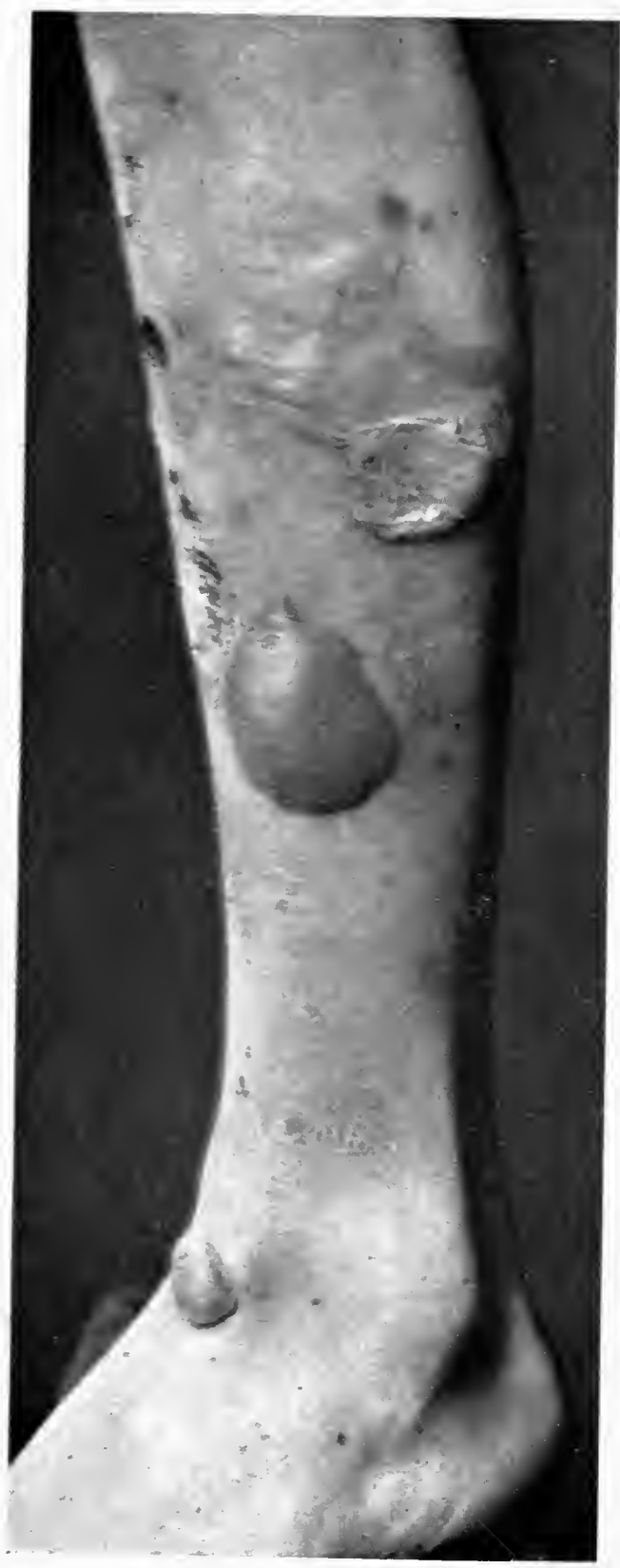

FIG. 4I.-Epidermolysis Bullosa.

(From Dr. S. W. Allworthy.) 

of the inter-epithelial lymphatic spaces. Beneath the bullae the superficial blood-capillaries are dilated and there is an infiltration of cells around them, marked oedema, and diminution of the elastic tissue.

The epidermic cysts which succeed the bullae consist of a layer of epidermal cells surrounding concentric horny lamellae, and are believed to result from the cornification of small tags of epidermis torn off from the ends of the interpapillary processes by the formation of the bleb and the lifting up of the epidermis from the corium. Certain observers have suggested that they are formed from occluded sweat-ducts.

Eosinophilia has been described both in the blood and in the contents of the bullae, but it has been so slight and variable as to be of no pathognomonic value. (Fig. 368).

Etiology and Pathogenesis. - In most cases the condition would appear to be a congenital cutaneous anomaly rather than a distinct disease and has been known to occur in association with other congenital peculiarities, such as keratosis of the palms and soles, webbed fingers, and ichthyosis. As a rule where it has developed in adult life it has been secondary to some bullous disease, like chronic pemphigus, but cases have been met with in which it appeared independently.

Heredity plays an important part in its etiology. In a group of cases recorded by Colombini, out of a family of 47 in three generations 24 were affected of which I 8 were males and 6 females; and in a family reported by Bonajuti, of 63 in five generations $3 \mathrm{I}$ were affected of which I6 were males and $I_{5}$ females. These groups both show a preponderance of cases in males. A predisposing factor in some of the cases would appear to be heat, for the affection is more marked in summer than in winter, possibly from the skin being moist from sweating.

The exact pathogenesis is not understood. It has been regarded as the result of three factors, (I) a peculiar congenital want of cohesion in the prickle-cells, in consequence of which an excess of fluid in the inter-epithelial lymphatic spaces readily leads to the separation of the cells and the formation of a bleb; (2) an excessive instability of the vaso-motor control of the cutaneous blood-vessels, in consequence of which rapid dilatation and excessive transudation of serum readily occur ; (3) a diminution or absence of the elastic tissue.

Diagnosis. - The condition is easily recognised by the vulnerability of the skin and its tendency to develop blisters on the slightest traumatism, its commencement usually in infancy, and its common occurrence in several members of a family or in several generations.

Prognosis.-In most cases, when once established, it persists throughout life, but cases have been recorded in which it gradually diminished and recovery took place.

Treatment.-There is no form of treatment, either local or general, which can effect a cure. All that can be done is to protect the skin by every possible means from traumatism, and, when blisters have occurred, to keep them clean, prevent secondary contamination, and 
stimulate healing by suitable local applications. (See treatment of pemphigus.)

\section{REFERENCES}

Adamson, H. G. Epidermic cysts (Brit. Journ. Derm., I905, xvii. p. I5). Allworthy, S. W. Epidermolysis bullosa (Idem, I9Io, xxii. p. 374).

Beatty, W. Epidermolysis bullosa (Idem, I897, ix. p. 30I).

CAne, L. B. Epidermolysis bullosa (Brit. Med. Journ., May I909, p. I I I4).

Fox, T. C. Epidermolysis bullosa (Allbutt \& Rolleston, Syst. of Med., I9I I, ix. p. 464, Bibliography).

Goldscheider. Epidermolysis bullosa (Monats. f. prakt. Derm., I882, vi. p. I63).

KAniky \& Sutton. Epidermolysis bullosa congenita (Idem, March, I9IO, p. 375).

KLAusner. Carcinoma of the tongue as a sequel to Epidermolysis bullosa. (Arch. f. Derm. u. Syph., March I9r3).

Morley, E. B. Epidermolysis bullosa hereditaria (Brit. Journ. Derm., I9I 4, xxvi. p. 35).

Petrini \& Galatz. Epidermolysis bullosa (Idem, I907, xix. p. 330).

SAvill, A. Epidermolysis bullosa (Lancet, I 906, i. p. 86).

WEISS, R. S. A case of Epidermolysis bullosa (Journ. Cut. Dis., Jan. I9I7, p. 26).

Wise \& Lautman. Epidermolysis bullosa hereditaria beginning in adult life; the acquired form of the disease (Idem, I9I5, xxxiii. p. 44I). 


\section{CHAPTER $\mathrm{X}$}

\section{CUTANEOUS AFFECTIONS DUE TO COLD}

Introduction.--The affections of the skin due to the action of cold may be divided into two classes, namely, (I) those which may occur in any one as the result of exposure to intense cold ; (2) those produced by milder degrees of cold which only occur in individuals rendered peculiarly susceptible by a feeble peripheral circulation, instability of the vaso-motor control, or a general loss of tone. In the former category may be placed frost bite and gangrene; and in the latter, chilblains, the purplish mottling of the legs so commonly seen in babies as the result of exposure to cold (Livedo annularis), an itchy condition of the skin which affects certain individuals in winter (Pruritus hiemalis), and, more rare, angio-keratoma and Raynaud's disease.

The effects on the skin vary in degree according to the intensity of the cold, the length of time of the exposure, and the susceptibility of the individual.

They consist of the following :

I. Blanching or pallor.

2. Redness-due to active hyperaemia-diffuse, mottled, or retiform.

3. Cyanosis from passive congestion.

4. Oedema, vesicular or bullous formation.

5. Superficial necrosis and ulceration.

5. Deep necrosis and gangrene.

Anatomical Considerations.-The microscopical changes produced by cold have been studied by numerous observers in the past, and our knowledge of them has recently been considerably increaser as the result of its employment as a local therapeutic agent in the form of liquid air or solid carbon dioxide.

The initial action of cold on the skin consists of the stimulation of the vaso-constrictor mechanism of the small arteries and, to a less extent, of the veins of the deeper parts of the corium and the subcutaneous tissue, and of the involuntary muscles of the hair-follicles. This results in anaemia and blanching of the exposed parts, erection of the hairs and contraction of the skin, giving rise to the familiar 
"goose-skin." At the same time the local temperature is reduced, the sensitive nerve-endings are numbed, and finer movements interfered with. If the cold be slight these effects may be counteracted by an increase of the heart's action pumping more warm blood through the skin, or by the application of warmth, or by friction and rubbing. In this way the vaso-constrictor spasm becomes inhibited, a dilatation of the vessels takes place, and the blanching is succeeded by redness associated with a feeling of warmth or tingling. The dilatation may soon pass off, and the tone of the vessels be re-established and the skin return to normal.

Should the cold be more severe, or the individual more susceptible, the dilatation may last longer and may go on to stasis, the affected part becoming dusky-red and congested. The redness may be diffuse, or broken up and mottled forming a purplish venous network enclosing paler brick-red areas corresponding to the bases of the arterial capillary cones. This degree of disturbance from cold is transitory, the part returning to normal by a process of repair in which any excess of fluid is absorbed and the damage made good by the formation of new connective tissue.

When the cold is more intense the vaso-constriction leads to complete obliteration of the lumen of the small vessels. The circulation in the cutaneous capillaries is stopped and with it the warmth of the blood, so that the skin may become deeply frozen, anaesthetic, and of a white parchment-like appearance. Should the part be slowly thawed by gentle massage or immersion in water about the temperature of the blood, complete recovery may take place, with a relaxation of the constriction, dilatation of the vessels and a flush of blood into the skin capillaries. But the circulation is apt to be impeded by the veins not having recovered sufficiently or being slightly thrombosed and a stasis may occur associated with dilatation of the cutaneous capillaries, a transudation of serum, and an extravasation of leucocytes and, occasionally, haemocytes, and as a result the part becomes swollen, reddish-purple in colour, and itches, burns, or throbs. Where the serous exudation is sudden and excessive the skin becomes raised, whitish and opaline, as in urticaria, and finally vesicles or bullae appear the contents of which are clear and serous at first but become cloudy from the presence of leucocytes and pyogenic cocci, and occasionally haemorrhagic.

In severe degrees of freezing the epidermis may be seriously injured or devitalised, the whole layer becoming homogeneous and the structure of the individual cells lost. The endothelial cells of the capillaries are also liable to be affected, and the lymph spaces between the collagen bundles to be distended with frozen serous fluid. When thawing takes place and the circulation is restored a moist slough forms, consisting of epithelial debris and coagulated sero-fibrinous exudate. This dries and separates in a few days being followed by a scar. On the other hand if the freezing has been still deeper, recovery is protracted and 
the destruction of tissue may be so great as to result in a depressed cicatrix. Where the cold has been extreme and the exposure prolonged, the vascular obliteration may be permanent and the local blood supply may be completely shut off, causing the affected skin to dry up, shrivel, and become bluish-black and gangrenous.

In short the pathological changes due to cold, if mild, are brought about by its action on the vaso-motor mechanism; if severe, by direct destruction of the tissue, associated with obliterative changes in the small blood-ressels, stoppage of the circulation, and eventual necrosis. Where the vaso-motor mechanism alone is involved complete recovery without inflammation takes place; where organic changes have occurred, either by direct action of the cold or by an excessive reaction in thawing, the recovery is incomplete and if healing takes place it is the result of a severe inflammatory reaction. In extreme cases there may be a necrosis of the whole of the frozen tissue.

\section{FROST-BITE}

Syn.: Dermatitis congelationis.

Frost-bite results either from exposure to extreme atmospheric cold or from prolonged contact with some cold object, such as ice, snow, or even a cold metal plate. It is due to cessation of the circulation in the affected part which, if not re-established in a comparatively short time, leads to necrosis and gangrene. It may occur in anyone but is naturally most common in individuals whose peripheral circulation is defective and whose general vitality is lowered by fatigue, insufficient food and clothing, or from the association of some debilitating disease such as diabetes, malaria, scurvy, or Bright's disease. It is usually met with in parts which are exposed or where the circulation is weak, such as the fingers, ears, tip of the nose, cheeks, feet, and toes, especially if the circulation be impeded by too tight boots or puttees.

At first the part affected is numb or slightly painful and assumes a bright red or livid colour, then suddenly becomes bloodless, white and translucent, and quite cold to the touch. In this stage, if dealt with at once by suitable treatment, a rapid thawing takes place, the circulation is restored, and the frozen part becomes red or purplish, hot, throbbing, and painful, and as this reaction subsides the skin gradually: returns to normal. Should the reaction be more severe it may be associated with oedema or the formation of a blister, with clear or blood-tinged contents, and healing may be delayed.

Should the freezing have been more intense the arteries rentain permanently contracted and the part becomes completely bloodless. Necrosis then sets in, with the formation of a line of demarcation, and the part either sloughs away or is surgically removed. Sometimes suppurative changes supervene about the junction of the healthy 
and the frozen skin and these may lead to severe septicaemic symptoms.

Treatment.-Treatment consists in restoring the circulation in the affected part so gradually as to avoid injuring the already weakened blood-vessels by causing too great a rush of blood into them. Any constriction, such as that caused by tight straps, garters, etc., must be relieved. On no account should the thawing be done rapidly by immersion in hot water or exposure to a hot fire as this may be followed by disastrous results, such as the formation of haemorrhagic bullae and possibly death of the part through arrest of the circulation. The reaction which follows gradual thawing and which is associated with redness, swelling, throbbing, or actual pain, should be allayed by the application of soothing and astringent remedies, such as lotions containing lead, ichthyol, tannic acid, carbolic acid, or menthol, or a paste containing $2 \%$ of ichthyol which should be spread on lint, covered with cottonwool, and kept in place by a light bandage. Relief from the pain mav be obtained also by mild application of hot air.

If vesicles or bullae be present they should not be opened but the contents allowed to become absorbed and the lesion to dry up to form a scab. If they are opened it should be done under strict antiseptic precautions and a dressing applied.

Should ulceration occur it is generally intractable. The ulcer should be dressed daily with boracic or ammoniated mercury ointment $2 \%$, or with fomentations of hydrogen peroxide (Io vols.), and if necessary should be Thiersch-grafted. Where necrosis and gangrene have taken place surgical interference is demanded. In all cases where the frost-bite has been at all severe, rest in bed is advisable and should be combined with a generous dietary and a tonic regime on general medical principles. Special vaso-dilator remedies, such as nitroglycerine, are not recommended.

\section{REFERENCES}

Fearnsides, E. G. Frost-bite (Brit. Journ. Derm., I915, xxvii. p. 33).

GHIRLANDA, E. The treatment of frost-bite with active hyperaemia (L'Ospedale maggiore, Jan. I9I6, p. I2).

Joseph \& Vieth. Treatment of chilblains (Derm. Centralb., I905, p. I62). Juliusberg. Morbid changes in the skin due to freezing (Berlin Klin. Wochenschr., I905, iv. p. 260).

MACLEOD, J. M. H. Therapeutic value of carbon dioxide snow (Brit. Med. Journ., Jan. 29th, I9Io).

Puser, W. A. Therapeutic use of solid carbon dioxide (Journ. Cut. Dis., July I9Io).

\section{CHILBLAINS}

Syn.: Pernio; Erythema pernio; Fr., Engelure; Ger., Frostbeule.

Description.-Chilblains are the red itchy swellings which are so common on the hands and feet in cold weather, especially in children. 
They are roundish or oval in shape; indefinite in outline, and vary in size from a split-pea to an inch or more in diameter. The colour may be dusky red or purplish, and may be diffuse and more intense in the centre, or pale in the middle with a darker border. They are most common on the hands, fingers, borders of the feet, toes, heels, ears, and tip of the nose. They may be single or multiple, are often bilateral, and occasionally symmetrical. They may remain discrete, or may coalesce to form irregular swellings. They are accompanied by itching,

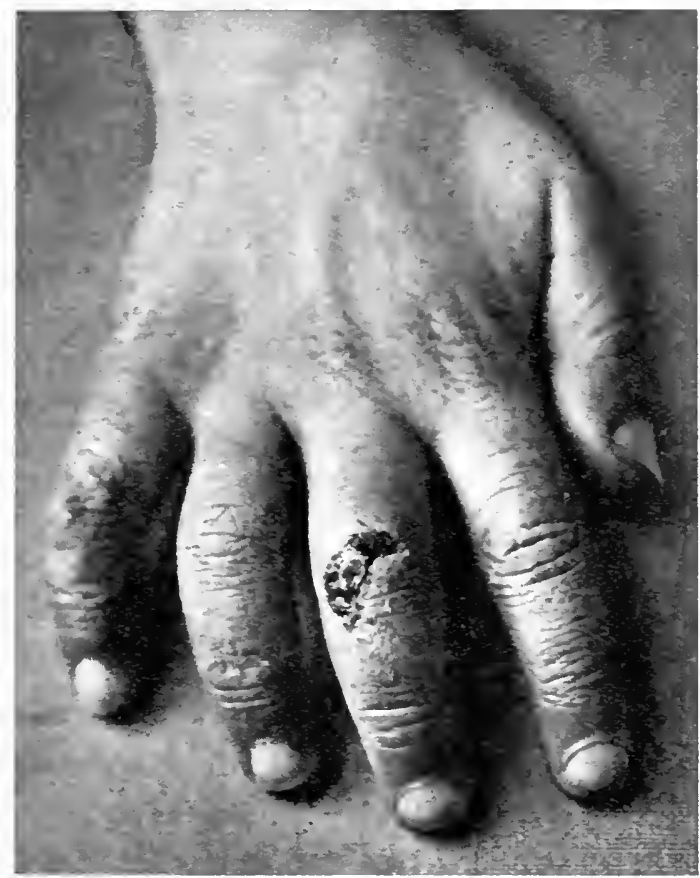

FIG. 42.-Suppurating Chilblain.

burning, or actual pain which is aggravated by warmth and, on the feet, by the confinement of the boots and their pressure in walking. (Plate VII.)

They are liable to be abraded or broken by friction and to be secondarily inoculated with pyogenic organisms causing suppuration, when they are known as suppurating or necrosing chilblains. (Fig. 42.)

The swelling is due chiefly to oedema, which in severe cases may lead to vesiculation or to the formation of bullae which may become haemorrhagic.

They may perist for a few days and disappear, or may last for weeks or months. They usually leave no trace, but when ther are broken or necrotic they heal with great difficulty and may result in scarring and disfigurement, while in the case of the ears they may be followed by atrophic changes in the skin and cartilage and permanent 
erosions. Occasionally they are succeeded by thickening of the tissues about the joints which may persist for months or indefinitely. (Fig. 43.)

Histopathology.--The microscopical changes consist of dilatation of the capillaries in the corium and of the smaller vessels of the hypo-

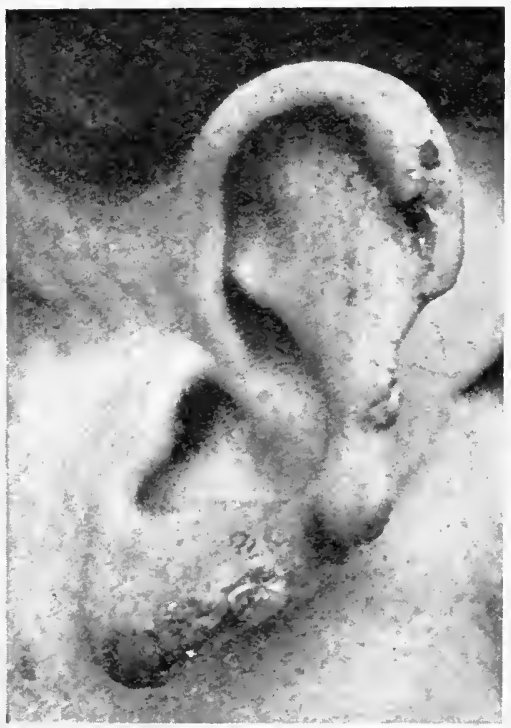

Fig. 43.--Suppurating Chilblains. derm, associated with oedema and extravasation of leucocytes, and, in severe cases, of haemocytes.

The epidermis may be unaffected, or may be oedematous, or occasionally vesicles or bullae may be present.

Suppurative changes with marked extravasation of leucocytes may be superimposed as the result of the secondary inoculation of pyogenic cocci.

Etiology.-The exciting factor in chilblains is cold, especially damp cold, which is more liable to cause them than the dry intense cold of high altitudes and they may occur as the result of even slight degrees of damp cold in susceptible individuals. They may be produced by cold weather, cold rooms, washing the hands in cold water, a breeze, or a draught.

They may occur in apparently healthy persons, but are far more common in those who are delicate.

The susceptibility may be inherited, or may be acquired in consequence of some preceding or intercurrent disease.

Of the predisposing causes the most common are the following:

(r) Age.-They may occur at any age but are especially common in children and the tendency to them, as a rule, dies out in later life. Occasionally in those who have suffered from them in childhood they recur in middle-life in association with arterio-sclerosis.

(2) Sex.--They are inore common in females than in males.

(3) Season.--They are most frequent in the cold damp weather of winter and tend to disappear in the spring, but occasionally are met with even in summer and autunin.

(4) Climate.-They prevail chiefly in the damp cold climates of 11orthern latitudes.

(5) Feeble Peripheral Circulation.-This is an important predisposing cause and is evident in diffuse redness or mottling of the skin of the extremities, cold and clammy hands and feet, swelling of the finger-tips, and dystrophy of the nails. It may result from anaemia, from weak action of the heart, or from chronic disease of the kidneys, 
and is accentuated by anything which impedes the circulation, such as garters, too tightly fitting boots, or tight gloves.

(6) Tuberculosis.--Tuberculosis or the strumous diathesis with enlarged lymphatic glands, is generally recognised as a predisposing factor and the occurrence of chilblains in such cases has been noted so frequently that certain writers have suggested that they may be due to the tuberculous toxin and are "Toxi-tuberculides."

Diagnosis.-The diagnosis is simple; except in the persistent type associated with atrophy or necrosis, when the cases may so closely resemble Lupus erythematosus that it may be almost impossible to differentiate them. As a rule chilblains are transient and disappear with warm weather, while Lupus erythematosus is persistent throughout the year and may even be aggravated in summer by the actinic rays of the sun; chilblains are raised and rounded from an exudation of serum, while the lesions of Lupus erythematosus are flat, or may be slightly infiltrated from a cellular deposit; chilblains are apt to suppurate and necrose, while the patches of Lupus erythematosus rarely do so; chilblains, unlike Lupus erythematosus, are attended by subjective symptoms.

They have to be distinguished also from papulo-necrotic tuberculides, or folliclis ; but in chilblains the lesions are larger, less numerous, occur usually in winter, die down in the spring, and tend to suppurate, while in folliclis they are small rivid red papules which may become vesicular and may dry up to form an umbilicated crust, finally ulcerate, heal slowly leaving depressed pits, and instead of occurring only in cold weather tend to come out in crops in the spring and autumn.

Prognosis.-They are readily amenable to treatment, but unless the susceptibility to them be outgrown or cured, they are liable to recur from exposure to even mild degrees of cold.

\section{TreatMent}

Both local and general treatment are necessary, but local treatment is demanded in the first instance to relieve the itching or pain and to prevent chafing or suppuration.

Local Treatment.-The special indications are to soothe and astringe, and of the numerous remedies adrocated for these purposes the most effective are: as sedatic'es, carbolic acid, menthol, and opium, and as astringents, lead, ichthyol, tannic acid, resorcin, iodine, formalin, and silver nitrate. Previous to their employment the chilblains should be bathed in hot water and nopped dry. The remedies may be applied in lotions or ointments, as in the following formulae:

Sedatives.

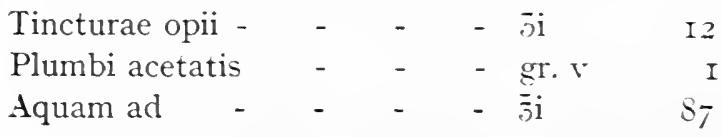




$\begin{array}{lllr}\text { Tincturae opii - }- & - & -3 \mathrm{i} & \text { I2 } \\ \text { Linimenti belladonnae } & -3 \mathrm{ss} & 6 \\ \text { Olei amygdalae - } & - & -\mathrm{m} . \mathrm{V} & \mathrm{I} \\ \text { Linimentum saponis ad } & - & \overline{3} \mathrm{i} & 8 \mathrm{I}\end{array}$

To obtain the full effect of the lotion the chilblains should be wrapped up in a piece of lint saturated in it.

A useful sedative ointment is the following :

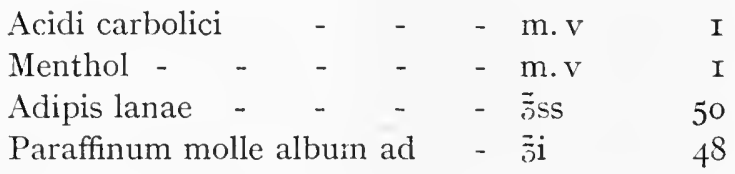

Astringents.-Lead.-In mild cases one of the most useful remedies is lead lotion. A compress soaked in a lotion containing a teaspoonful of Liq. pb. subacetatis in half-a-pint of tepid water, should be applied for an hour or more at a time, the parts then dried, and the following dusting powder applied :

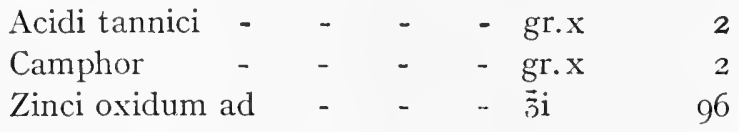

Ichthyol.-Ichthyol may be used either undiluted or as a 5 to Io \% aqueous solution, and the chilblains wrapped in lint soaked in it; or the following combination with other astringents may be substituted :

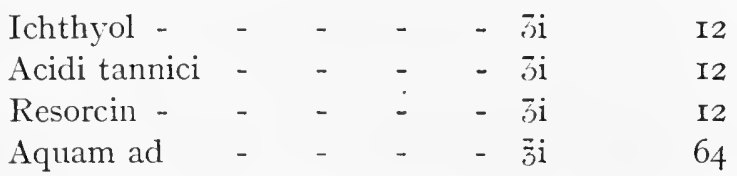

(To be painted on each night.)

A more continuous effect may be obtained by applying it in an ointment spread on lint, such as:

\begin{tabular}{|c|c|c|c|c|}
\hline Ichthyol - & - & - & - & \\
\hline Zinci oxidi & - & - & - & \\
\hline Adipis lanae & & - & - & \\
\hline Paraffinum m & blle & lbu & $\mathrm{ad}$ & \\
\hline
\end{tabular}

Should the grease prove irritating it may be counteracted by the addition of $2 \%$ of menthol.

A useful substitute for the above is a zinc-ichthyol plaster, and should objection be taken to the smell of ichthyol, thigenol may be substituted, which is odourless.

Resorcin.-Resorcin may be prescribed in a lotion containing 5 to $10 \%$ in water, or in a paste:

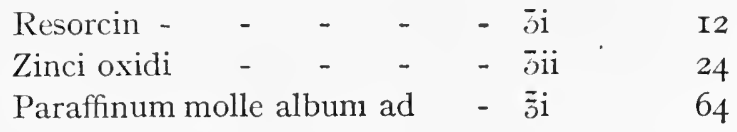

To this may be added $2 \%$ of carbolic acid as a sedative. 
Euresol (a mono-acetyl derivative of resorcin) may be substituted for it and is said to be more penetrating and effective. It is a liquid preparation and may be made up in collodion in the strength of $I$ in $S$ and painted on each night.

Formalin.-Formalin acts in much the same way as ichthyol, but it is more irritating and must on no account be used if the skin be broken. It may be employed in a lotion (ro \% in water) or as an ointment :

$\begin{array}{lllllll}\text { Formalin - } & - & - & - & - & \overline{3 i} & \text { I2 } \\ \text { Adipis lanae } & - & - & - & - & \overline{3} \text { iii } & 36 \\ \text { Paraffinum molle album ad } & - & \overline{5 i} & 52\end{array}$

Iodine.-Iodine may be used in a tincture and painted on each night, or as Io \% vasogen iodine rubbed in, or continuously applied on lint, or dissolved in collodion and painted on once or twice daily.

Balsam of Peru.-Balsam of Peru has an astringent and sedative action and is useful in combination with silver nitrate:

$\begin{array}{llllr}\text { Peruviani balsamis } & - & - & \bar{j} & \text { I2 } \\ \text { Argenti nitratis } & - & - & \text { gr. } v & \text { I } \\ \text { Paraffinum molle album ad } & - & \bar{j} & S_{7}\end{array}$

Tinct. Benzoin. Co.-Compound tincture of benzoin is soothing and astringent and may be combined with advantage with equal parts of menthol.

Antiseptics.-Where the chilblains are broken or suppurating, antiseptics such as compresses of hydrogen peroxide may be applied, or mercury or boric acid may be added to the astringents :

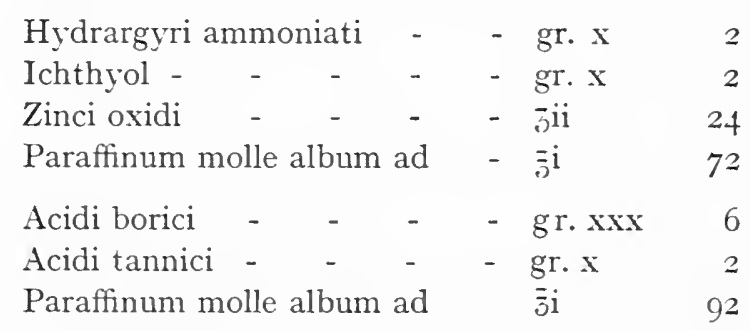

In addition to these medicaments, benefit may be derived from electric baths, both galvanic and faradic, the I-ray's and, according to certain observers, by high frequency currents. Of these, electric baths and the $\mathrm{X}$-rays are the most useful.

In the case of galvanic baths, the affected part should be placed with the negative pole in a bath containing salt and water, while the positive pole is applied higher up the limb, and a current as strong as the patient can bear comfortably should be passed for about a quarter of an hour.

The X-rays should be given in a half-pastille dose repeated if necessary at the end of the second week.

Improvement may also be obtained by gentle massage to soothe and relieve the vascular congestion. 
General Treatment and Management.-The clothes of those suffering from chilblains should be warm, over the whole length of the limbs, the boots thick, and the gloves loose in cold weather. Cold baths should be avoided, tepid water should be used in washing, and the affected parts should on no account be toasted in front of the fire. If the feet be clammy from excessive perspiration the socks should be changed frequently and the skin bathed with weak formalin or carbolic lotion and kept dry by a dusting powder containing boric acid, zinc oxide, and talc, or magnesium carbonate. The diet should be nutritious and contain a sufficiency of fatty substances and carbohydrates. Out-ofdoor exercise should be encouraged.

In almost every case of chilblains there is an underlying delicacy or predisposing cause which should be treated. There may be a feeble peripheral circulation associated with anaemia, constipation, or defective cardiac action, which require treatment on general principles. Where the amount of haemoglobin is reduced, iron is indicated; where the patient is thin, delicate, and badly nourished, cod-liver oil and malt should be prescribed; where the heart's action is feeble-especially in adults--cardiac tonics such as strychnine, digitalis, and strophanthus, are of benefit.

Certain drugs have been claimed to havé a specific action on chilblains, either by acting on the vaso-motor mechanism or by altering the quality of the blood; the most important of these are nitroglycerine, ichthyol, and chloride of calcium. The employment of calcium chloride in doses of 20 grs. t.d.s. has been strongly recommended by Sir Almroth Wright as he found that in chilblains the coagulability of the blood was defective and that they were liable to occur in association with other conditions, such as urticaria or haemophilia, in which the coagulation time was generally prolonged beyond the normal four minutes. The results from these remedies are uncertain, however, and the benefit derived from them is less than that from suitable treatment on general medical principles.

\section{ANGIO-KERATOMA}

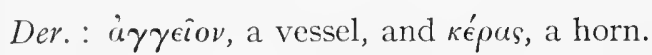

Syn.: Telangiectatic warts (Dubreuilh).

Exposure to cold is sometimes responsible in delicate-especially tuberculous-individuals with a weak circulation for a peculiar affection known as angio-keratoma, which occurs about the fingers, toes, and backs of the hands. It is characterised by punctate telangiectases, isolated or arranged in irregular groups, over many of which there is a horny or scaly thickening. Mild degrees of this condition are apt to be missed and in consequence it has been regarded as rarer than it really is. It is often associated with, or preceded by, chilblains which may have occurred for several years before it appears. 
It begins as minute telangiectases or vascular puncta dotted irregularly over the dorsal aspects of the fingers or toes, which do not disappear on pressure with a diascope, and bleed freely if pricked. The lesions tend to coalesce into irregular patches, the skin over which becomes thickened and may form small horny projections. All stages in the evolution of the lesions from minute dilated ressels to horny patches can generally be detected in the same case.

As a rule there are no definite subjective symptoms, unless the chilblain element persists and causes burning or itching. It is generally bilateral but not definitely symmetrical, usually appears in the spring, about the time chilblains subside, and is apt to persist throughout life though it has been known to disappear spontaneously.

A number of anomalous cases have been recorded in which lesions of this type have been noted elsewhere on the cutaneous surface, as on the arms, legs, and trunk, but they were not preceded by chilblains, and consisted chiefly of irregularly grouped small red spots.

Histopathology.-There is no pathognomonic feature in the histology: The most constant change consists of the dilatation of small groups of capillaries in the papillary and sub-papillary layers, which is sometimes so marked as to lead to the formation of small cavernous sinuses. Around the dilated vessels there is an infiltration of small mononuclear cells with a few polymorphonuclear leucocytes. The condition in the corium resembles a capillary lymphangioma, but in the latter instead of dilated capillaries there are small deep-seated vesicles of lymphatic origin containing clear fluid.

In addition to the primary vascular changes there is irregular hypertrophy of the prickle-cell layer and hyperkeratosis.

Etiology.-It is most common in childhood and early adolescence, and chiefly affects the female sex. It may occur in several members of a family and occasionally in a parent and child. Its exact causation is uncertain, but it would appear to be invariably preceded by chilblains and may be the result of repeated congestion of the bloodvessels leading to structural changes in the capillaries themselves. Like chilblains it is associated with a weak peripheral circulation and cold is the most important causal factor. It occurs so frequently in tuberculous subjects and in association with such tuberculous stigmata as Lupus pernio, scrofulous glands, tuberculous joints, and phthisis, that certain French writers have included it in the category of the toxituberculides, but the evidence, both clinical and histological, is insufficient to warrant this.

Diagnosis.-The diagnosis is comparatively simple. The only affection with which it might be confused is chronic solar dermatitis with its telangiectases and warty thickenings on the backs of the hands and other exposed parts, but in it there is no history of preceding chilblains and the condition is generally met with in men of middle age and upwards. 
Treatment.-The treatment is unsatisfactory. It consists of a tonic regime with a generous dietary, on the same principles as that laid down in the treatment of chilblains.

Locally, the dilated vessels may be obliterated by electrolysis or by a fine-pointed Paquelin cautery, and the horny thickenings can be reduced by radium or by the application of carbon dioxide snow. When the latter is employed care must be taken that the exposure is just enough to raise a superficial blister or scarring may result.

\section{REFERENCES}

Anderson, WV. (Brit. Journ. Derm., I898, x. p. II3).

Dubreuilh. (Ann. de Derm. et de Syph., i893, iv. p. 379).

Mrbelli. (Gior. Ital. d. Mal. Ven., I889, xxvi. p. I59).

Pringle, J. J. (Brit. Journ. Derm., I89I, iii. p. 237).

Tökök. Relation to lymphangiomata (Monats. f. prakt. Derm., r892, xiv. p. I69).

\section{LIVEDO RETICULARIS}

Syn. : Livedo annularis ; Livedo frigore.

This consists of a peculiar purplish mottling or reticulation of the skin seen chiefly in the legs of babies as the result of exposure to cold, but sometimes present in adults with a weak peripheral circulation not only on the legs but also on the extensor aspects of the arms. It is believed to occur especially in association with some defect in the general health due to tuberculosis, syphilis, malaria, etc., but it may be met with also in apparently healthy individuals. It is due to stasis in the circulation from the vaso-motor paresis which succeeds the original contraction due to cold, and the network formation is the result of the anatomical arrangement of the blood-capillaries in the skin in the form of small arterial cones, the bases of which are next the surface and are separated by areas where the circulation is naturally more sluggish. (Fig. 44.)

It generally disappears in early childhood but in people with a weak circulation it may persist throughout life. It rarely demands special treatment beyond the adoption of measures for improvement of the general circulation, such as a generous dietary, warm clothing, wearing bed-socks at night, and avoidance as far as possible of exposure to cold.

\section{REFERENCES}

ADAmson, H. G. Livedo reticularis. With a full bibliography (Brit. Journ. Derm., I9I6, xxviii. p. 28I).

\section{Pruritus Hiemalis or Winter Itch}

This form of pruritus is generally found in adults during the cold weather and as a rule affects the lower extremities, though it may involve other parts. It is usually paroxysmal and tends to come on 


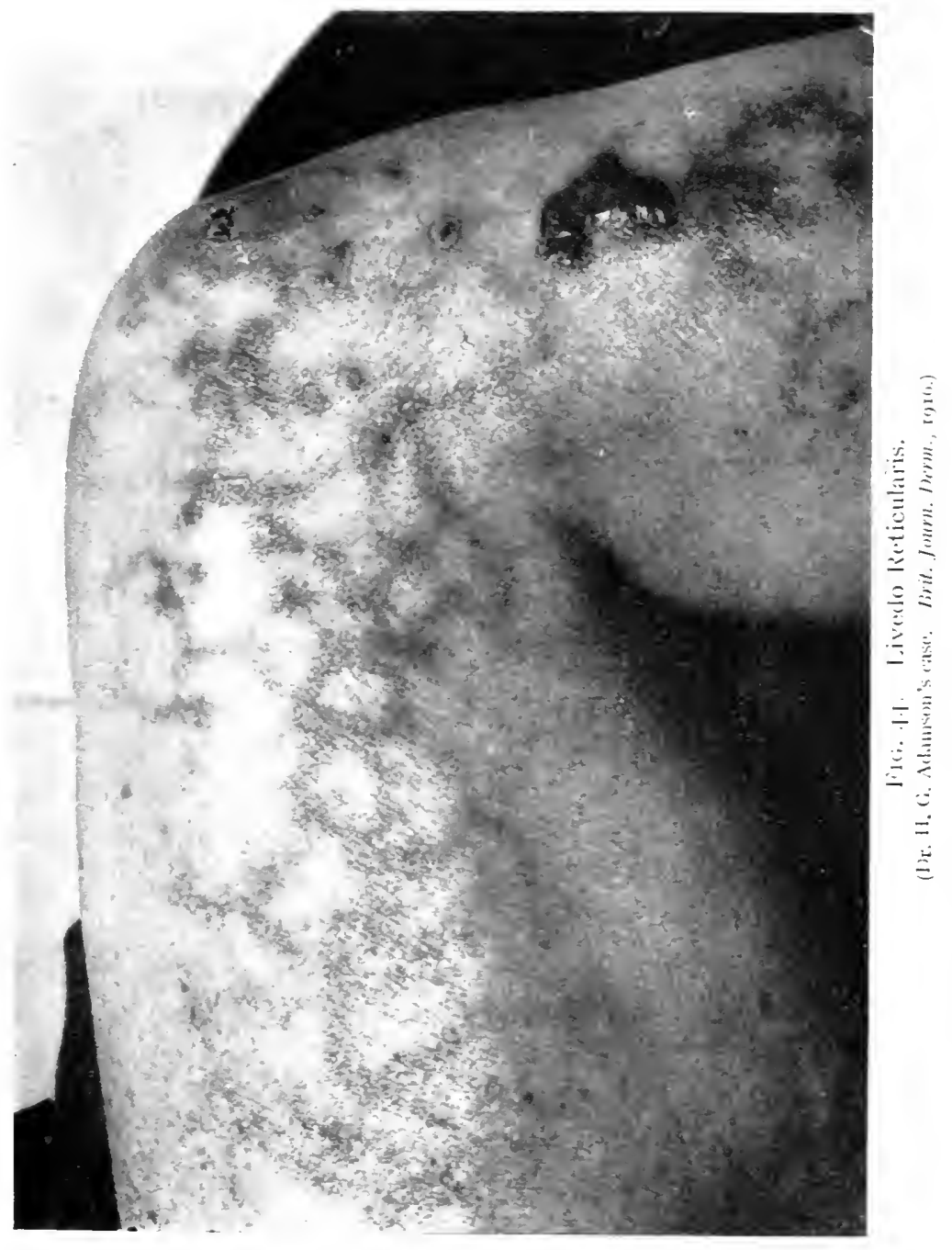

To foct p. az: 

when the patient is undressing and the shin is exposed to cold. The itching leads to rubbing and scratching and these in turn to lichenification, or eczematisation.

In some individuals cold baths give rise to paroxysms of pruritus which may last an hour or more.

\section{RAYNAUD'S DISEASE}

Syn.: Local syncope, local àsphyxia, symmetrical gangrene.

Definition: An affection occurring at the periphery of the circulation characterised by symmetrical arterial ischaemia and asphyxia, and tending to terminate in gangrene.

Description.--This triad of symptoms, grouped together by Maurice Raynaud in his thesis in I862, is usually the result of mild degrees of cold in individuals rendered susceptible from an abnormally excitable vaso-motor mechanism.

Local Syncope.-Local syncope, or arterial ischaemia, is usually the earliest phase and is met with in the form of " dead " fingers from slight exposure to cold, such as immersion of the hands in cold water, a cold atmosphere, or the handling of cold articles. It may also be produced by fine movements of the fingers, as in piano-playing, or may result from psychical causes, such as emotion or worry. Once it has been caused it readily recurs.

It is most commonly confined to one or two fingers-usually the second and third-but it may involve the whole hand and is generally bilateral and symmetrical. It may be present also in the toes, or in rare instances in the cheeks, ears, or nose.

The affected part becomes pale and waxy, cold to the touch, numb, insensitive to pain, heat, or cold, the tactile sense is lost, and finer movements of the fingers are impaired. The paroxysm may last from a few minutes to hours or even days, and may recur at rare and irregular intervals or occasionally several times in a day. When the attack disappears a reaction usually supervenes, with tingling, burning, throbbing, or shooting pains, and the pallor is replaced by cyanosis or asphyxia-the second phase of the disease.

Local syncope generally begins in early adult life and is liable to persist indefinitely. When the attacks are frequent the fingers tend to become thin and shrunken, the skin parchment-like and sclerodermatous and the nails opaque, pitted, and frayed. The association with sclerodermia has been noted most frequently in women, and may occur at the same time as the vaso-motor symptoms or may appear years after they have ceased. It may affect any part of the skin, but is most common about the hands or fingers when it is known as Sclerodactylia.

Local Asphyxia.-Local asphyxia is usually a further stage of local syncope, but it may occur independently. It is more frequent 
than syncope in situations other than the extremities, sucl as the ears, chin, nose, lips, tongue, nates, and Labia majora. It is most common in the hands and may involve both equally or one more than the other so that one hand may show only syncope while the other is cyanosed.

It is characterised by a dusky-slate or bluish-black appearance of the affected part, which is especially marked in the feet and hands and fades away on the arms and legs as a dusky red congestion or mottling.

In severe cases actual haemorrhage may occur and the asphyxiated part may become purpuric and may go through the same changes in colour as a bruise.

The cyanosis may disappear after a varying time, or it may persist as reddish-purple patches which do not fade on pressure. Unlike local syncope it rarely leads to sclerodermia, but if it attacks the fingers or toes the nails may be shed and may not grow again owing to atrophic changes having taken place in the nail-matrix.

The paroxysms have a marked tendency to recur and as time goes on become more frequent, severe, and prolonged, and associated with other signs of vascular stasis due to cold, such as Livedo reticularis.

The subjective symptoms consist of pricking and pain on pressure, which may remain long after the asphyxia has gone.

It may be associated with no definite aberration of health though occasionally the attack is ushered in by sleepiness and a rise of temperature, or it may be accompanied by internal disturbances, such as colicky pains in the abdomen, impairment of vision from changes in the fundus oculi, attacks of numbness, or even temporary paralysis.

Symmetrical Gangrene.-Symmetrical gangrene occurs in about $50 \%$ of the cases and is almost invariably preceded by local asphyxia, and often by local syncope. The necrosis may be superficial and amount to little more than desquamation or the sloughing of bullae containing serous or haemorrhagic contents; on the other hand it nıay be deep, and a mummification of the affected part take place with the formation of line of demarcation and spontaneous amputation, or the separation may result from moist suppurative changes due to pyogenic microorganisms. In this way fingers, toes, or even a whole hand or foot, may become separated, leaving an unsightly stump.

The gangrenous changes may set in a few hours after the first attack, or may not supervene till the earlier symptoms have been recurring for weeks or months.

A number of cases are on record where Raynaud's phenomena were associated with calcareous degeneration in the skin in the form of hard nodules which broke down into ulcers and discharged calcareous particles.

Etiology and Pathogenesis-It is more common in females than in males, in the proportion of about 2 to $I$; the average age of onset is about 30 , though it may begin in childhood or not till old age ; it is more prevalent in winter than in the warmer seasons. 
In males it is generally met with in those whose occupation exposes them to inclement weather and cold, such as seamen, cab-drivers, postmen, etc. ; in females a sedentary occupation seems to predispose to it.

Our knowledge of its pathogenesis is incomplete. It would seem to be a functional disturbance of the vaso-motor system of the arteries, usually of the small cutaneous arteries but occasionally of larger arteries, such as the radial, or of the veins. In all the cases there would appear to be an underlying over-excitability of the vaso-motor centres by which the slightest stimulation causes spasm of the small peripheral arteries.

The cause of the vaso-motor disturbance is uncertain. It may be due to direct stimulation of the vaso-motor centres by toxins circulating in the blood, or it may be brought about reflexly through the nervous system by agents, such as cold, acting at the periphery.

Toxic Theory.-In favour of this theory are the observations:- that the disease may be associated with toxic conditions such as jaundice, haemoglobinuria, Bright's disease, diabetes, and lead poisoning; that attacks have been observed to occur in connection with malaria and have been noted repeatedly in malarial cachexia; that in several instances it has accompanied Lupus erythematosus, certain cases of which strongly suggest a toxic erythema; and that a certain number of cases have been reported in which it has occurred in connection with syphilis, when it has been suggested that the specific toxin was responsible by causing changes in the walls of the blood-ressels.

Symmetrical gangrene has been known to appear in persons with a weak peripheral circulation as the result of an acute spasm of the peripheral arteries due to some poison circulating in the blood.

Reflex Theory.--In support of this it has been pointed out that Raynaud's phenomena are frequently connected with nervous disorders, such as peripheral neuritis, chorea, etc., or with psychic disturbances, such as mental strain, emotion, and insanity, which may cause a state of vasomotor instability.

Diagnosis.--The diagnosis presents no great difficulty; the paroxysmal character of the affection and the recurrent attacks of symmetrical syncope, asphyxia, or gangrene, forming a series of phenomena not met with in any other disease.

Local asphyxia has to be distinguished from erythromelalgia, but the latter affects males more than females, has no stage of syncope, and is not paroxysmal but remains red and painful without intermission.

Symmetrical gangrene has to be distinguished from frost-bite and from the gangrene of ergotism. Frost-bite is caused by severe cold, possibly in a healthy individual, while Raynaud's disease may be the result of mild degrees of cold and requires a special susceptibility, and in ergotism there is usually a history of having eaten ergotised rye bread, etc.

Prognosis.-Recovery from mild attacks is the rule. Some cases recover spontaneously, others under suitable treatment. There is no danger to life, but the affection is serious owing to its tendency to recur, to increase in severity, and to be associated eventually with gangrene. 


\section{TREATMENT}

The treatment is chiefly preventive and is both general and local.

General Treatment.-The principal object of the general treatment is to reduce the abnormal excitability of the vaso-motor system and the liability to paroxysms. With this aim the patient must be carefully examined and any departure from normal health treated on general medical principles. The diet should be simple and nutritious, the clothing warm, and exercise in the fresh air encouraged. Special attention should be paid to the nervous system and any defect treated. Where anaemia is present, iron, arsenic, the phosphates, and codliver oil, are indicated, and constipation should be corrected. Where the disease is associated with malaria or haemogiobinuria quinine should be given, and where syphilis is present this should be treated.

To relieve the spasm in the small arteries nitro-glycerine and amyl nitrite have been recommended, but these are useless to allay the paroxysms in ordinary doses and are only of service where angina complicates the disease. Opium and Cannabis indica have been employed to relieve the vaso-motor spasms ; opium is of special value in middle-aged and elderly people and may be prescribed in compound pills containing quarter of a grain of opium and 2 grains of the extract of hyoscyamus; Cannabis indica is given in the form of the tincture, ${ }_{5} \mathrm{~m}$. three times a day.

Everything likely to produce a paroxysm should be avoided, such as exposure to cold, cold baths, cold rooms, excitement, or cramped movements of the fingers as in playing the piano or typewriting.

Local Treatment.-The local treatment consists of various measures to improve the circulation in the affected part. In early cases friction and rubbing with turpentine or some stimulating liniment is of value. Where there is much pain sedative applications, such as lead and opium lotions, are indicated.

For the relief of the spasm the most promising treatment is galvanic baths. The negative pole is placed in a bath containing warm salt water, and in this the affected part is immersed while the positive pole is applied to the limb higher up and a current such as can be borne comfortably is passed for about a quarter of an hour.

Where dry gangrene has set in and a line of demarcation formed, spontaneous amputation may be allowed to occur, or if advisable the diseased tissue may be removed surgically.

\section{REFERENCES}

Barlow, T. Raynaud's Disease (Allbutt \& Rolleston, System of Med., I910, vii. P. I20). 
DAvis, H. Raynaud's Disease associated with calcareous degeneration (Brit. Journ. Derm., I9I2, xxiv. p. I 42 ).

Fox, H. Symmetrical gangrene (Med. Rev. of Rev., New York, 1907).

Montgomery, D. W., \& Culver, G. D. Asymmetrical Raynaud's Disease (Journ. Cut. Dis., I915, xxxiii. p. 219).

Munro, T. K. Raynaud's Disease. Glasgow, I899.

RAYXAUD, M. Sur l'asphyxie locale et la gangrène symétrique des extrémités. Paris, 1862 .

Semox, H. Raynaud's syndrome and syphilis (Brit. Med. Journ., I913, i. p. 278$)$.

WeBER, F. P. Chronic Raynaud's Disease probably on a syphilitic basis, associated with Livedo reticularis (Brit. Journ. Derm., rgr3, xxv. p. 8I).

\section{TRENCH FoOT}

This condition came into prominence in the early stages of the Great War, especially in the winter of I9I4-I5. It occurred in men who had been long in the trenches during cold weather, and was due to various causes but principally to the impeding of the circulation by the constriction of wet boots, wet puttees, etc., and the damp and cold from prolonged standing in cold weather, liquid mud, or melting snow. Arabs and negroes were found to be more susceptible to it than white men.

In its mildest form the feet became cold and macerated, or extremely painful and cyanotic; in a further stage they became swollen from oedema, and bullae and even ecchymosis occurred; while in its most advanced stage, gangrene might supervene.

A soldier who had once suffered from it was very susceptible to a recurrence and, as a rule, was useless for duty in the trenches for the remainder of that winter.

Treatment.-Prevention is very important and in those units where regular foot inspection and drill were carried out very few cases occurred. As preventives, the feet should be rubbed with lard or whale oil every day before putting on the boots, and the boots softened with dubbin or some other grease; the trenches should be kept as dry as possible by draining and planking, and every opportunity should be taken of exercising the feet to prevent then becoming numb with cold; the boots should be sufficiently large for two pairs of socks to be worn and should be taken off at least once a day and the feet and legs briskly rubbed and passive movements done; the puttees should on no account be drawn tight.

Cure.-In mild cases the patient should be confined to bed and the feet rubbed with a little oil for a few minutes every hour and wrapped in cottonwool between times.

When oedema is present the feet should be elevated and the patient told to hold up his legs vertically from time to time and to move the ankles and toes as freely as possible.

Where there is much pain or throbbing, relief may be obtained 
from hot air, steam, or galvanic baths, or from the application of a soothing cream such as :

\begin{tabular}{|c|c|c|c|c|c|c|}
\hline Tinct. opii & - & - & - & & - & $\mathrm{m}$ \\
\hline Aquae calcis & - & - & - & & & \\
\hline Oleum amygc & dala & & - & & - & \\
\hline
\end{tabular}

Ulcerations should be dressed with a I \% ammoniated mercury ointment.

\section{REFERENCES}

Blackmax, J. G. Trench Foot (Brit. Med. Journ., I9I6, i. p. 8I8).

Chalier. Treatment of Trench Foot (Le Prog. Med., February I9I 7).

Davis, G. G., \& Hilton, J. J. Trench Foot tetanus (Journ. Amer. Ass., I9I6, lxvi. p. I9I6).

Ellis, E. S. Nitro-glycerine in Trench Feet (Brit. Med. Journ., I9I7, i. p. $5^{\mathrm{I}} 3$ ).

Hughes, B. Causes and prevention of Trench Foot (Brit Med. Journ., I9I6, i. p. 7 I2).

RAymond \& PARIsot. Trench Foot. (C.R. Acad. des Sciences, Jan. 22nd, I9I7).

Smith, H. O. Treatment by subcutaneous injection of oxygen (Brit. Med. Journ., I9I 7 , i. p. 5II).

Swax, R. H. J. Trench Foot, or so-called "Frost-Bite" (Practitioner, I9I6, xc. p. 46).

Turrell, W. J. Electrical treatment of Trench Foot (Idem, p. 52). 


\section{CHAPTER XI}

\section{CUTANEOUS AFFECTIONS DUE TO HEAT}

Introduction.-The cutaneous affections due to heat may be divided into those caused by the local action of heat, namely Erythema ab igne (see p. 656), burns and scalds (Dermatitis ambustionis), and those which result from the general action of a hot atmosphere or a high body temperature, such as hyperaemia, miliaria or prickly-heat, and sudamina. In hot weather a recurrent affection is sometimes met with which is known as Hydroa aestivale or summer prurigo (see p. 250); the cause of this is doubtful but it seems probable that it is due more to the actinic rays of the sun than to the heat rays as it has been known to occur in the cold weather.

\section{BURNS AND SCALDS}

The term "burn" is generally reserved for a lesion caused by dry" heat while "scald" is employed to denote that due to moist heat. A burn is produced by dry heat of about $140^{\circ} \mathrm{F}$. and upwards and a scald by moist heat of $125^{\circ} \mathrm{F}$. and upwards.

Scalds are usually less severe than burns though the area involved is generally more extensive.

Burns vary in severity according to the temperature, the extent of the area exposed, and the duration of the exposure. In both burns and scalds the gravity is proportionate to the extent rather than to the depth of the injury and a burn or scald of considerable depth and severity may have less serious consequences than a more superficial burn where the area involved is greater.

Classification.-In France and Great Britain it is customary to classify burns after Dupuytren into six degrees, while in America a simpler classification into three degrees is generally adopted. These classifications, though purely arbitrary, are useful in drawing attention to the depth to which the burn has reached and to the tissues which have been most severely damaged.

It is the burns of the first and second degree which are of special interest to the dermatologist. 
Description.-(I) Burns of the first degree are caused by a temperature of about $140^{\circ} \mathrm{F}$. In them the skin is simply scorched but not destroyed and becomes red and sometimes slightly swollen. When the inflammation subsides it is followed by a superficial desquamation which disappears in a few days leaving either no trace or slight pigmentation.

The affected skin is tender and there is a sensation of tingling, or in more severe cases of burning which may amount to actual pain.

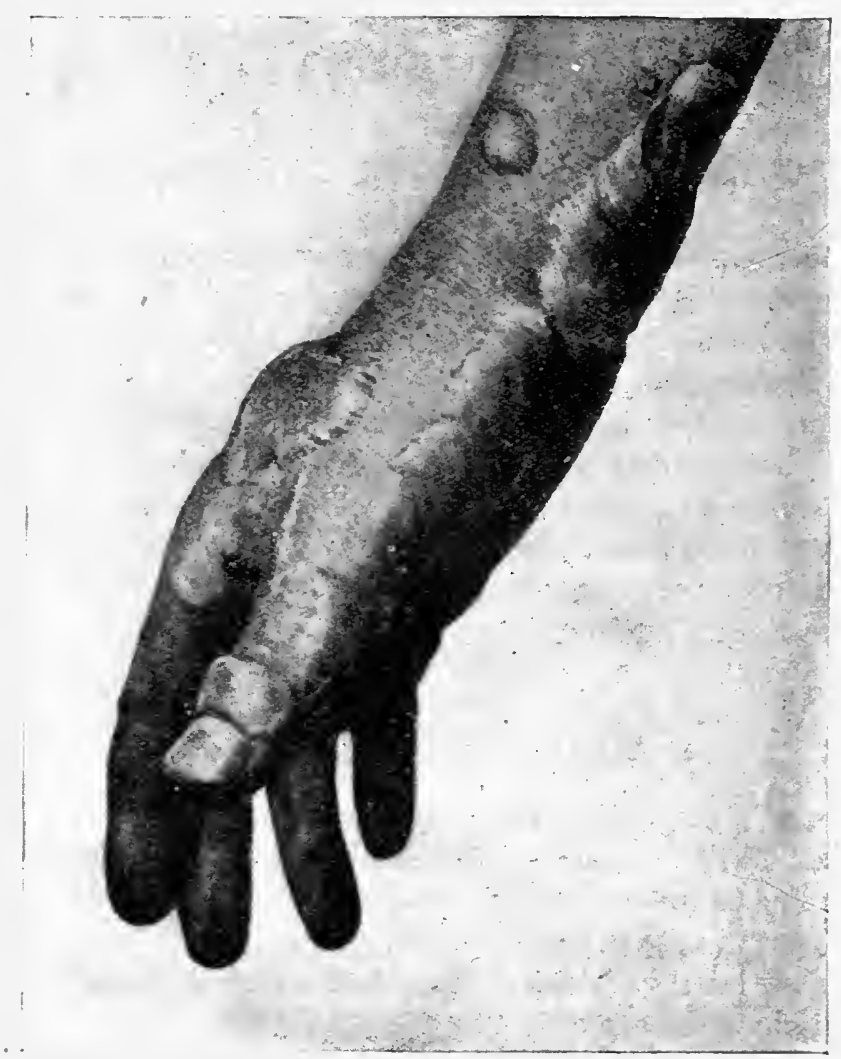

FIG. 45--Burn of Second Degree with large Bullae.

(From MacLeod's Burns and their Treatment.)

(2) In burns of the second degree, which are caused by a temperature of $160^{\circ} \mathrm{F}$. to $210^{\circ} \mathrm{F}$., the inflammation is more acute, the erythema deeper, the transudation of fluid more marked, and the burning pain more severe. Either at once or in the course of a few hours the inflamed skin becomes surmounted by groups of small vesicles, or by larger bullae which are irregular in outline and vary in size up to large blisters several inches in length. (Fig. 45.)

The bullae are readily ruptured by injury or even by increased exudation. If they are not broken the contents are absorbed or coagulate into a jelly-like mass and the bullae collapse and are gradually replaced by yellowish-grey honey-like crusts which separate in 
a week or ten days without leaving scars. On the other hand should the bleb become infected with septic micro-organisms, suppuration and ulceration may supervene followed by scarring.

In connection with burns of this degree severe constitutional symptoms may occur, the intensity of which is dependent on the extent of the burn.

(3) In burns of the third degree caused by a temperature of greater than $210^{\circ} \mathrm{F}$. the epidermis and superficial layers of the corium are more or less completely destroyed and form a dead piece of burned skin

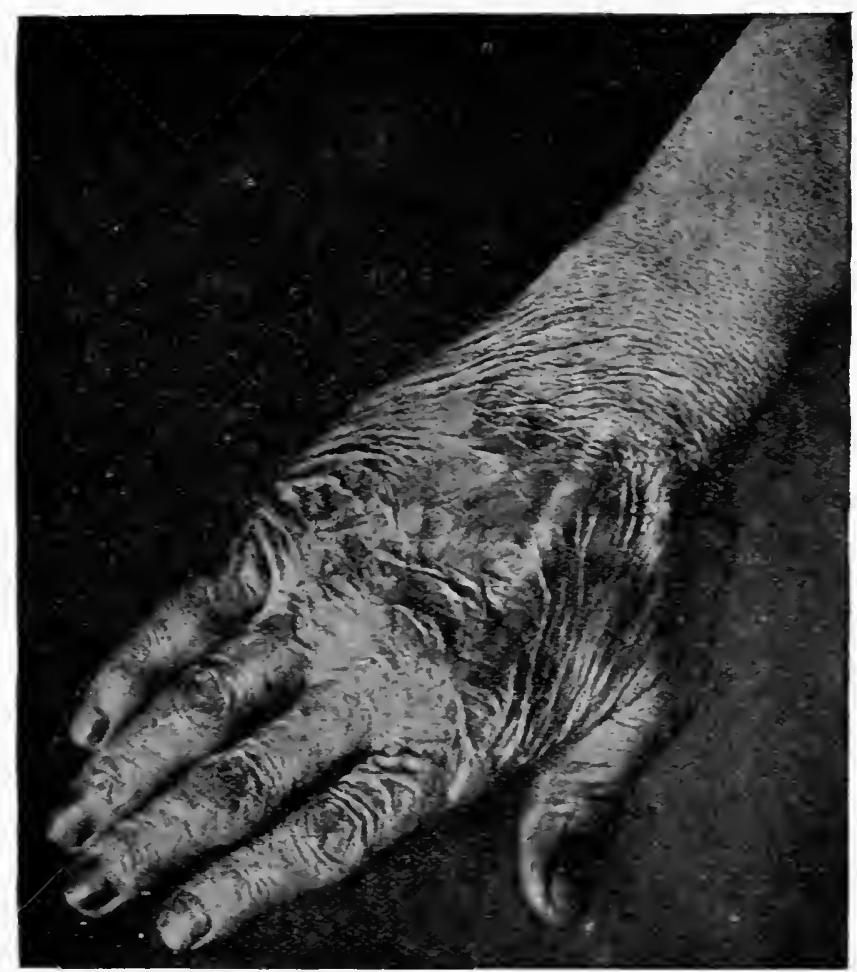

FIG. 46.-Atrophy of the Skin following a Burn of Second Degree.

(From . MacLeod's Burns and their Treatment.)

known as the "eschar." This is ashen-grey or brownish-black in colour, occasionally presents a few abortive resicles on the surface, is leathery in consistence, and as a rule is insensitive to touch. At first it is adherent to the underlying tissue but in the course of a fer days to a week a painful inflammation sets in and suppuration begins at the edges, spreads in beneath it, and causes it to slough off in about a fortnight. After the eschar comes away a raw granulating surface is left which heals slowly and is followed by scarring and not infrequently by contraction and deformity. (Fig. 46.)

At the time of the burn and for about forty-eight hours afterwards the pain may be so great as to be almost unbearable, after that it 
usually subsides for a time but returns more or less acutely with the secondary inflammation and the separation of the eschar.

(4) In burns of the fourth degree the skin is involved in its whole depth and when the eschar separates irregular cicatrices and unsightly contractures are produced.

Scars from burns occasionally become carcinomatous. A curious example of this is the "Kangri cancer" of Kashmir. The kangri is a small vessel containing burning charcoal which the natives carry about in cold weather for warmth and which they are in the habit of pressing against the thighs and lower part of the abdomen; this produces multiple burns followed by scars which, being irritated by the friction of the kangri and further exposure to heat from it, not infrequently become malignant.

(5) and (6) In burns of the fifth and sixth degrees the charring extends down through the skin and subcutaneous tissue to the muscles, even to the bone, and healing is impossible. If a considerable area be affected a fatal result is inevitable but if the burn be limited recovery may take place with loss of the burned part.

General Effects.-The constitutional effects of burns are more severe than those which follow other injuries involving an equal destruction of tissue. These consist of :

(I) Primary or immediate effects, chiefly shock.

(2) Secondary effects from the absorption of toxins, inflammatory diseases of internal organs, and sepsis.

Primary Effects.-Burns of limited extent may give rise to nervous disturbances, such as excitement and hysteria, followed by prostration ; extensive burns, on the other hand, are associated with severe shock and sometimes with fatal collapse. In the majority of fatal cases, death supervenes within forty-eight hours.

Secondary Effects.-(a) Toxaemia.-Should recovery from shock take place severe inflammatory symptoms may supervene from the absorption of chemical poisons, such as foreign proteins from the injured cells, or of actual toxic products of completely destroyed cells. This stage follows immediately on subsidence of the shock and may last until the eschar has separated.

(b) Inflammatory Diseases of Internal Organs and their Membranes. -According to the situation of the burn it may be followed by meningitis, pleurisy, bronchitis, haematemesis, tympanitis, peritonitis, nephritis, and occasionally by acute duodenal ulcer.

(c) Septicaemia.-This may occur about the time of the separation of the eschar but can be largely prevented by proper aseptic methods. Occasionally secondary infection by a virulent organism such as the tetanus bacillus or the streptococcus of erysipelas may take place.

Histopathology.-The initial action of heat is to cause dilatation of the blood-vessels throughout the corium, clinically evident in erythema, an exudation of serum, and an infiltration of leucocytes. The serous fluid passes up between the cells of the epidermis, causing them 
to be oedematous, interfering with the process of cornification, and giving rise to scaliness. When the heat has been more serere the exudation is greater, takes place more rapidly, collects in the epidermis, and forms vesicles or bullae as the result of the fluid stretching, weakening, and breaking, the inter-epithelial fibres and accumulating in the space so formed. This process is facilitated partly by the softening of the epidermal cells by the heat and the transformation of any moisture into steam which, forcing its way between the prickle-cells, separates them and prepares a passage for the fluid; and partly by the toxic action of the softened or dead epidermal cells causing positive chemotaxis and attracting more serum.

In severe degrees of burn the epidermal cells are destroyed, charred and separate as an eschar leaving a more or less deeply ulcerated surface. When the ulcer heals there is an organisation of the small round cells of the cellular infiltration into new fibrous tissue which may be so abundant as to result in a cheloidal scar.

Diagnosis.-The diagnosis of slight burns from other localised patches of erythema is rendered easy by the destruction of the hair and by the associated burning sensation. Nor need any difficulty be experienced in distinguishing the bullous stage from other bullous conditions like pemphigus or Erythema bullosum, for in the case of burns there are marked subjective symptoms, a definite history, and a localisation of the lesions to the burned area instead of an irregular distribution over the cutaneous surface.

Prognosis.-In burns, excepting in those of slight degree, a guarded prognosis should be given invariably, for the outlook is usually more serious than would appear at first sight and, though the injury may seem to be comparatively mild, if a large area be burned a fatal issue may supervene in a few days. It may be said as a generalrule that burns involving more than one-third of the cutaneous surface are apt to be followed by serious, if not fatal, consequences, while those involving one-half are almost invariably fatal. The outlook is always more grave in burns over the great serous carities, such as the chest and abdomen, than when the limbs alone are involved.

The time taken in the healing of a burn depends on the extent of the area involved, and the general condition of the patient. Burns of the first degree heal readily in a few days, those of the second degree may take several weeks and, should suppuration occur, further delay will be caused, while in burns of the third and fourth degrees the healing is generally delayed for months.

\section{Treatment}

The treatment of burns, unless in those of limited extent, is both general and local.

General Treatment.-The chief immediate danger of burns is shock and its consequences, and in severe burns before attempting to remove 
the charred clothes or to expose the burn it is imperative to deal with the shock by prompt and thorough measures on general medical principles. In extensive burns one of the best methods of combating the shock is by means of hot baths, either whole baths where the burn is extensive, or leg or arm baths where a limb only is involved. An effort should then be made to increase the blood pressure by infusions of normal saline solution, injected either into the rectum, subcutaneously, or intravenously. The value of the intravenous injections may be enhanced by the addition of adrenalin to the infusion, or by the intramuscular injection of pituitary extract-half to one c.c. of a $20 \%$ solution of the extract.

After the stage of shock has been overcome, toxic or septicaemic symptoms should be watched for and appropriately treated.

Local Treatment.-General Considerations.-An important point in the treatment of burns is to avoid, as far as possible, injuring the tissues and interfering with the new growth of epidermis over granulating surfaces by too frequent dressings. The less the wound is touched the better, provided the sero-purulent discharge is not excessive and no sign of septic absorption is present.

Until recently the favourite method of treating burns was by greasy applications, such as Carron oil. Lint saturated in it was laid on the burned surface, covered by a layer of cottonwool and a piece of oiled silk and fixed by a bandage. This type of dressing has serious disadvantages and should be discarded; the greasiness and the waterproof covering conserve the heat and increase the discomfort, while the Carron oil rather encourages than retards sepsis.

Another fallacy with regard to the treatment of burns is the old idea that it is of the first importance to exclude the air from the burned surface. This has been proved to be a complete mistake and there is no better method, where practicable, for the healing of the raw granulating surface of a severe burn than the so-called "open method" in which the burn is exposed to the air and the formation of a scab is encouraged by powdering the surface with a bland aseptic powder.

Special Treatment.-In burns of the first degree where the skin is simply scorched the principal indication in the treatment is the relief of the burning sensation. This can be done best by soaking pieces of lint in a weak solution of sodium bicarbonate, lead lotion, or a $\mathrm{I} \%$ aqueous solution of aluminium acetate, and applying them over the inflamed surface. Where the erythema is intense a $5 \%$ aqueous solution of ichthyol may be substituted.

In burns of the second degree, if clothes cover the burned surface these should be soaked off, and not torn away, so as not to break the bullae. The affected skin should then be mopped over with a solution of perchloride of mercury I in 4000, and each bulla opened aseptically in the dependent part, drained, and the cuticle left as a protective covering. The burned area should then be dressed with gauze or lint soaked in a I \% aqueous solution of aluminium acetate or in a I \% 
aqueous solution of picric acid. The dressings should be left alone as long as possible and only removed should there arise any indication of the blistered area having become septic.

In burns of the third and fourth degrees the treatment may be divided into the immediate treatment, up to the separation of the eschar, and the healing of the granulating surface.

As soon as the shock has been overcome and the charred parts removed, the burn should be cleaned by irrigation with warm boric lotion or by immersion in a warm boric acid bath. It should then be dressed with aluminium acetate or picric acid lotion, and the dressings only changed if they become markedly septic or if there be much pain. When the slough begins to separate the parts should be irrigated from time to time with boric lotion.

The principal methods of healing the granulating surface are: (a) the open method with healing under a natural scab; (b) healing

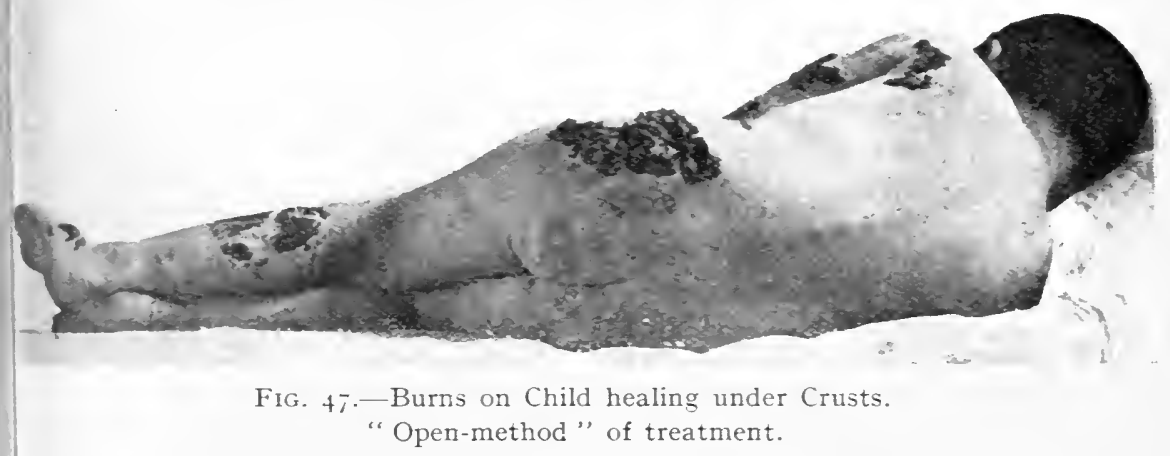

under an artificial scab, or paraffin method; $(c)$ healing under moist dressings.

(a) Open Method.-Of all the methods at present employed for the healing of the granulating surface of a burn by far the simplest, and in some way's the best, is the "open method," where healing is allowed to take place under a natural scab. This form of treatment is particularly applicable to burns of the leg or face, the leg being protected by a cage while healing.

No dressings are employed, but the raw surface is simply exposed to the air and powdered over with a bland aseptic powder which cakes up with the discharge to form a crust. The powder employed is stearate of zinc, or magnesium carbonate, or a combination of these. Should the discharge beneath the scab be excessive it may be advisable to remove a portion of the crust, bathe away the sero-purulent fluid, and re-apply the powder. Once the scab has formed it should be touched as seldom as possible but allowed to dry up, crumble, and separate naturally. (Fig. 47.)

This treatment has several great adrantages : it avoids the injurious action of antiseptics; it leares the wound, comparatively speaking, 
at rest and eliminates the ordeal of periodical re-dressings with their accompanying pain and destruction of new growing epidermis; it restrains the formation of exuberant granulations; it is followed by a pliant scar which has comparatively little tendency to contract.

(b) Paraffin Method.-The treatment of burns by paraffin was introduced in France some years ago by Dr. Barthe de Sandfort who employed a preparation known as ambrine, which is a proprietary preparation of paraffin, wax, and resin, with a melting point of $122^{\circ} \mathrm{F}$. It is melted and sprayed on the burn by a special spraying apparatus, or painted on with a broad camel's hair brush and allowed to form a waxy scab or cast under which healing takes place.

This treatment was extensively employed during the great war and. was found to be of great value in burns caused by liquid fire, petrol, etc.

Lieutenant-Colonel A. J. Hull, R.A.M.C., introduced a substitute in the form of an antiseptic paraffin preparation with a melting point of II $8^{\circ} \mathrm{F}$. to which he gave the name of "No. 7 paraffin," and which has the following formula :

\begin{tabular}{|c|c|c|c|c|}
\hline Beta naphthol & - & - & - & 0.25 \\
\hline Eucalyptus & - & - & - & 2.0 \\
\hline Olive oil - & - & - & - & 5.0 \\
\hline Paraffin molle & - & - & 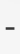 & 25.0 \\
\hline Paraffin durum & - & - & - & $67 \cdot 75$ \\
\hline
\end{tabular}

The method of use is as follows: A piece of paraffin wax is placed in the sprayer and melted over a spirit lamp, then allowed to stand till it cools down to $\mathrm{I}_{5} \mathrm{O}^{\circ} \mathrm{F}$. The burned area is cleaned with boric lotion, carefully blotted dry, and the paraffin is sprayed on; at first it is sprayed from a distance of about nine inches from the skin, when it forms a thin coating like hoar-frost, and then from about four inches from the skin till the affected area is thickly covered with the paraffin. A thin layer of cottonwool is next laid on the melted paraffin and dabbed over by a brush with more paraffin, and when this cools a waxy shell or cast is formed over the burn. This should be covered with cottonwool held in place by a loose bandage. The dressing should be left on as long as possible and only removed when the discharge becomes excessive.

When the treatment is employed for burns of the second degree in which bullae are present, the paraffin should be applied over the bullae without breaking them.

(c) Method by Moist Dressings.---This method of treatment, though a great advance on that by oily applications, is inferior to both the natural scab method and the paraffin method. In it the granulating surface is covered by a layer of gauze over which is placed a dressing of gauze or cottonwool soaked in a mildly antiseptic lotion, such as boric lotion or Dakin's hypochlorite solution, and held in place by a loose bandage ; this is kept moist by soaking the dressings from time to time in the lotion without disturbing the gauze next the raw surface. 
Where the granulations become exuberant it may be necessary to astringe them, either by touching them with nitrate of silver or by applying a compress of aluminium acetate. Where the granulating surfaces are extensive, healing may be greatly facilitated by grafting by the Thiersch method or by means of deep flaps taken from some convenient situation, the base of the flap being left uncut for about ten days to enable the circulation to be maintained in it.

Prevention and Treatment of Scarring.--It is important in the treatment of burns to do everything possible to hasten healing, as in this way hypertrophic scarring is avoided to some extent, and at the same time to see, by means of splints, etc., that healing takes place in proper position so as to avoid subsequent deformity.

Hypertrophic scarring from burns may be reduced by radium or by the $\mathrm{X}$-rays. These should be employed as early as possible as the hypertrophy is usually most marked immediately after healing has taken place, tends to diminish during the first six months, and then remains permanent. Half-strength plates of about 25 milligrammes of radium salt screened by a silver plate of $0.5 \mathrm{~mm}$. in thickness are used and exposures given of ten or twenty hours divided over several days according to the degree of hypertrophy.

The X-rays are applied in pastille doses at intervals of not less than three weeks, several exposures being necessary, as a rule, to produce any definite result. Should the slightest indication of dermatitis supervene the treatment must be stopped.

Fibrolysin has been recommended for the reduction of unsightly scars but the results from it are disappointing.

\section{REFERENCES}

Azzarelio. Pathogenesis of Death by Burns, and the Injection of Artificial Serum as a Means of Treatment (Giorn. Ital. delle Mal. Ven. e della Pelle, I898, Fasc. ii. p. II3).

Barker, C. Heavy Liquid Petrolatum in the Treatment of Burns ( $\mathrm{Y} . \mathrm{Y}$. Med. Journ., I9I6, ciii. p. II97).

Bickle, L. W. Treatment of Burns and Scalds (Med. Journ. of Australia, I9I 5, i. p. 258).

DE TARxowsky, G. Modern Treatment of Burns (Journ. Cut. Dis., I9I6, xxxiv. p. I9I).

Fauxtleroy, A. M. Report of a Case of Extensive Burn of the Third Degree (Amer. Journ. Med. Sci., June, I905, p. 985).

Gitisgs, F. C. B. Treatment of Burns and Scalds (Journ. Roy. Nav. Service, London, I9I5, i. p. 326).

Herrmax, C. The Open Method of Treating Burns (Amer. Journ. Surg., N.Y., I915, xxix. p. 63).

Holmas, C. C. Treatment of Scalds (Clin. Journ., London, I9I 5 , xliv. p. 394).

Hornus, G., and Perris, P. Traitement des Plaies de Guerre par la Méthode Carrel (Rev. de Chir., Paris, I9I6, xxxv. p. 637).

Huli, A. J. Treatment of Burns by Paraffin (Brit. Med. Journ., I9I7, i. p. 37).

MacLellax, A. "Tunnel " Skin grafting (Glasgow, Med. Journ., I9I 2, ii. p. 86). 
Macleod, J. M. H. "Hot-air" Treatment of Lupus (Brit. Journ. Derm., r899, xi. p. 346).

- Burns (Accidents in their Medico-Legal Aspect, r9ro, p. 426).

- Burns and their Treatment (Oxford War Primer, London, I9r8).

Mummery, P. Lockhart. Shock and Collapse (Latham \& English, System of Treatment, I9I2, i. p. 93).

Ormsby, Sir L. H. Burns and Scalds (Practitioner, I9II, ii. p. 433).

Parker, C. A. Treatment of Burns of Extremities with Special Reference to Prevention of Deformities, etc. (Journ. Amer. Med. Ass., I9r5, Ixv. p. I6).

- A Plea for Prevention of Deformities in Healing of Burns (Ibid., I9I6, lxvii. p. 565).

Ravogli, A. Management of Burns (Journ. Amer. Med. Ass., I9r5, lxv. p. 29I).

Will.an, R. J. Local Treatment of Burns on Naval Hospital Ship (Brit. Med. Journ., I9I6, ii. p. 318).

Woodward, C. Burns and Scalds. (Choyce, System of Surgery, I912, i. p. 29I).

\section{MILIARIA.}

Der. : Milium, millet.

Syn.: Prickly heat or Lichen tropicus; Heat rash; Red gum; Strophulus infantum. Fr., Miliare ; Ger., Schweissflechte.

Definition: An acute inflammatory affection connected with perspiration, characterised by the presence of numerous small acuminate red papules or vesicles on a red base, and associated with distressing pricking or itching.

The name miliaria has been employed in a somewhat loose manner to include two conditions which differ in their pathogenesis, namely Miliaria propria or Prickly heat, an inflammatory affection, and Miliaria crystallina or sudamina which consists of sweat-cysts situated beneath the horny layer of the epidermis and which is non-inflammatory. To avoid confusion it is advisable to restrict the use of the term to the inflammatory condition.

It has been customary to recogniseseveral types of miliaria according to the form of lesion which predominates, such as Miliaria rubra where inflammation is well-marked, Miliaria alba in which are numbers of small vesicles with white opaque contents, Miliaria papulosa in which the papular elements predominate, and Miliaria vesiculosa in which vesicles are numerous. These distinctions and the names associated with them complicate the subject and indicate simply modifications and degrees of one disease caused by peculiarities in the skin affected.

Prickly Heat.- This variety of miliaria is a distressing affection which usually appears suddenly, its onset being preceded or accompanied by more or less profuse perspiration. It is characterised by acuminate bright red papules, varying in size from a pin's point to a large pin's head, many of which when examined by a pocket lens present a minute vesicle at the apex. In addition to the papules, but in fewer numbers, 
small pin-head-sized clear vesicles situated on an inflammatory base may generally be detected. The prevalence of the papular type of lesion suggested the name " Lichen tropicus," which was once applied under the misapprehension that it belonged to the lichen group.

The lesions usually occur in enormous numbers, but however numerous or closely packed together they remain discrete and never coalesce to form red patches like those of eczema. The eruption may appear anywhere but is most common on those parts of the skin which are warmest, such as about the waist, trunk, upper part of the arms and thighs. It is absent as a rule on the palms and soles, though in severe cases it may be present there as small red macules. After a few days the papules disappear, and the contents of the vesicles become opaque, dry up and are replaced by small scales, and the attack is over.

The subjective symptoms are of the most distressing type, and consist of itching, burning, and pricking, being especially severe when the papular lesions predominate.

The affection is more troublesome than serious, unless it attacks young children or persons in ill-health. It is not associated with definite feverishness though congestion of the fauces may accompany it when extensive.

The course varies in different individuals. In some it may be transitory, the eruption lasting for a few days and then completely disappearing; but it is more usual for recurrences to take place which by overlapping may render the disease practically continuous during the hot weather. In some people the tendency to it disappears through acclimatisation; in others it recurs every hot season, and a predisposition may be established so that even if they leave warm countries to reside in temperate climates they are still liable to attacks from slight causes even in comparatively cool weather.

Owing to the intense irritation, rubbing and scratching are liable to be indulged in with the result that the skin, especially in the intertriginous regions, becomes eczematised or inoculated with pyogenic cocci and impetigo or furunculosis may supervene.

In temperate countries miliaria generally takes the form of the familiar Heat rash or " Red gum," usually met with in infants but occasionally in older children and even in adults. This is situated chiefly on the arms and chest, and appears as reddish macules, papules or small vesicles. Though itchy it is not associated with the intolerable pricking of the papular variety of the tropics. It is comparatively: rare in adults and only occurs from exposure to considerable heat or as the result of profuse perspiration from violent and unaccustomed exercise.

Histology.-A section of a typical papule or papulo-resicle of miliaria shows the chief feature in the histology to be a vesicle which is situated immediately beneath the Stratum corneum in the position of the sweat-channel. In some sections the resicle appears to be independent of the sweat-duct, but this may be due to the plane in 
which the section is cut. The vesicles may be mono-locular, or multi-locular where several have coalesced and the septa persisted; the contents may be clear, or turbid from the presence of leucocytes and broken down epithelial debris.

The epidermis in the neighbourhood of the vesicles is oedematous, the prickle-cells being swollen and spongy and the inter-epithelial spaces dilated.

The changes in the corium are slight and consist of dilatation of the blood-vessels in the papillae beneath the vesicle and an infiltration of small mono-nuclear cells in their neighbourhood. The sweat-coil appears to be normal and no definite changes can be detected in the sweat-duct in its passage through the corium. The sebaceous glands and hair-follicles are unaffected.

Etiology.-Miliaria is due to excessive heat and attacks almost every European living in tropical countries. It is not met with in natives with dark skins because they are more accustomed to heat and not subject to sudden profuse perspiration. It prevails chiefly in the hot season, and an attack may be brought about by a variety of causes, such as the wearing of unsuitable underclothing of flannel or rough woollen material, violent exercise, sudden alterations in temperature, hot rooms, excess in food or smoking, hot foods such as curries or chutney, alcoholic beverages, and, most frequent of all, hot drinks.

In this country when it occurs in infants or young children it is generally the result of their being too warmly clad. In adults it may be due to exercise in hot weather or to an occupation necessitating exposure to excessive heat such as that of cook, baker, fireman or stoker. It is more common in obese persons who perspire freely than in those of a spare habit.

Pathogenesis.-The exact manner in which the eruption is called forth is uncertain. That it is clinically associated with profuse perspiration there is no doubt, but the part played by the excessive perspiration in the production of the actual lesions is undecided. Two theories have been advanced to explain it:

(i) According to Pollitzer it is directly due to excessive sweating and the mechanism of its production is as follows: Owing to the increased fluid in the epidermis the horn-cells at the sweat-orifices are imperfectly cornified, become swollen by absorbing moisture, and block the passage of the sweat so that sweat-cysts are formed which constitute the vesicles.

(ii) The rival theory is that the lesions are inflammatory and of microbic origin and that the excessive perspiration furnishes a soil suitable for the growth of the microbe.

Neither of these theories is wholly satisfactory; the former mechanical explanation is incompatible with the amount of inflammatory disturbance and the subjective symptoms associated with it, while as regards the latter no microbe has yet been established. It may be pointed out also that the eruption is rarely present in situations like 
the palms and soles where the number of sweat-glands per square inch is most numerous, and that it is often profuse on the back where they are few.

Another explanation is possible. Sweating and perspiration are two different functions; sweating is the function of the sweat-glands, while perspiration is due to the transudation of tissue fluids. Sweat is acid in reaction, the tissue fluid which transudes is alkaline. The transudate like the sweat is eliminated at the sweat-pore, and when it is excessive the reaction of the mixture is alkaline. As a result of copious perspiration an excess of alkaline fluid is poured out at the sweat-orifice and this acting as an irritant may be responsible for the inflammatory changes and the vesicular formation.

Diagnosis.-Miliaria usually presents no great difficulty in diagnosis. The disease which it most resembles is papulo-resicular eczema. The chief points in their differentiation are the following: In miliaria the onset of the eruption is sudden and associated with perspiration, the lesions are small, have no tendency to weep or form crusts, and however numerous remain discrete; in eczema the onset is more gradual, the lesions as a rule are larger, tend to break and ooze, coalesce to form patches, and the eruption instead of being transient is persistent. It must be remembered, however, that from rubbing or scratching miliaria can be transformed easily into eczema.

In children an extensive eruption of miliaria might possibly be confused with measles or scarlet fever but the profuse sweating and the absence of constitutional symptoms should distinguish them.

Prognosis.- The prognosis in temperate climates is excellent and the eruption is easily removed by suitable treatment. In hot climates on the other hand it should be guarded as the affection may prove most intractable. In time the tendency to it may die out, but in many individuals a marked predisposition to it may be established and in spite of every care attacks may occur when the hot season comes round.

\section{TreatMent}

General Management.-The different exciting causes of prickly heat should be scrupulously avoided, such as hot drinks, long drinks, alcohol, excessive smoking, violent exercise, and unsuitable clothing. The underclothing should be made of silk, or silk and cotton, and woollen or flannel garments discarded, as they are not only unpleasantly hot but irritate the skin by their roughness. The bed-clothes should be as light as possible and may be raised off the patient by a wicker-work cradle which can be ventilated by large paper shafts on each side of the bed. In very hot weather a punka is a great addition to the comfort of the patient. Where possible a risit to a cooler climate, the hills, or a sea voyage, should be recommended. 
The diet should be light and all articles liable to produce irritation, such as black coffee, curries, chutney, etc., eschewed. Cooling drinks, provided they are not too long, are harmless and grateful to the patient, but still-lemonade or acid drinks should be taken with caution as they may determine an outbreak.

There is no internal specific, but relief may be obtained from saline aperients and diuretics by reducing the tension of the blood in the skin and making the kidneys more active.

The following formula is specially useful :

\begin{tabular}{|c|c|c|}
\hline Potassii acetatis & - & - gr x \\
\hline Spiritus aetheris nitrosi & - & $-\operatorname{mxx}$ \\
\hline Spiritus juniperis - & - & $\mathrm{mv}$ \\
\hline Decoctum scoparii ad & - & $\bar{j} \mathrm{i}$ \\
\hline
\end{tabular}

Local treatment consists chiefly of cooling lotions and dusting powders, ointments and greasy applications as a rule doing more harm than good.

Of the lotions the following are of value :

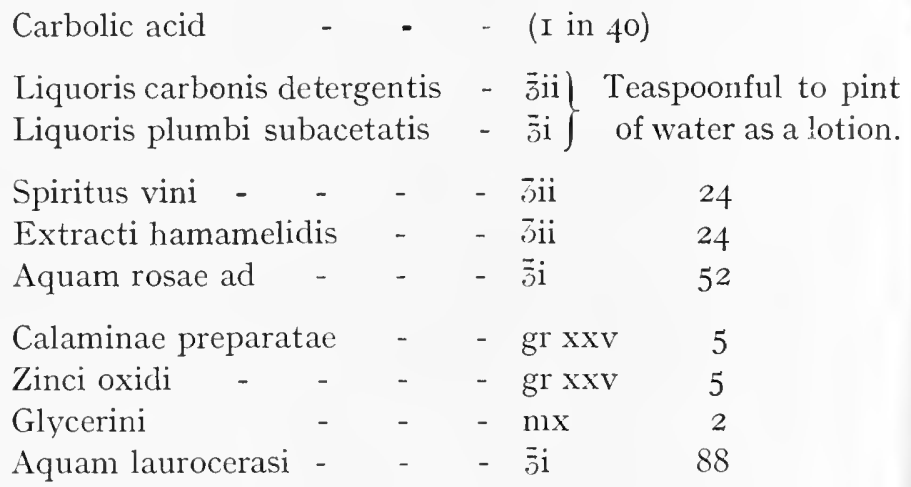

These should be applied continuously if possible, by soaking pieces of lint in the lotion and laying them on the itchy part. When the dressing is removed one or other of the following powders should be dusted on the skin :

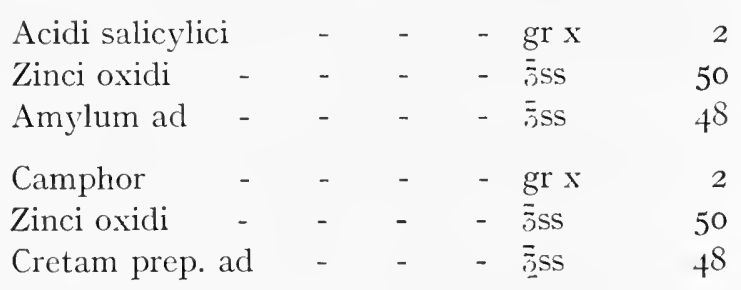

In extensive cases relief may be obtained from alkaline baths (2 ozs. each of bicarbonate of soda and borax to the 30 galls. of water at $90^{\circ} \mathrm{F}$.), bran baths, oatmeal baths, or weak permanganate baths.

In the miliaria of temperate climates and the sweat rashes of infants 
the treatment is similar to that described above, and in addition the following cream is of value:

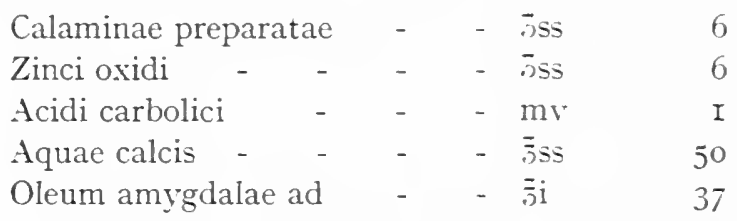

This should be rubbed into the skin and a dusting powder subsequently applied.

\section{Miliaria Fever}

Syn. : Sweating sickness; Fr., La Suette anglaise.

This is a febrile condition associated with profuse sweating and a miliaria-like eruption which has occurred in epidemic form and the exact nature of which is uncertain. It was described first in England in the fifteenth century and epidemics of it have been recorded also in the north of France and in Germany.

\section{SUDAMINA}

Syn.: Crystallina; Miliaria crystallina; Fr., Miliare crystalline; Ger., Schweissfrieselausschlag.

Definition: Ton-inflammatory small superficial resicles containing sweat.

Description.-Sudamina consist of small translucent vesicles with clear watery contents, which have been aptly compared to drops of dew. As a rule they come out suddenly, either in an irregular fashion or in crops, and are generally most profuse on the chest and back but may be present over the entire cutaneous surface. They may be few in number or exceedingly numerous, and usually remain discrete, though in rare instances they may coalesce into small builae. They are situated superficially in the epidermis but although only covered by a thin horny lamina they seldom rupture. They appear independently of inflammation, are associated with no subjective symptoms, have no erythematous base, and-unlike miliaria-have no papular stage.

In a few days the contents of the vesicles are absorbed and they dry up, scale off, and disappear without leaving a trace. The duration of an attack is usually about a week, but the affection may be prolonged by a succession of outbreaks.

The eruption is invariably associated with excessive sweating and is met with chiefly in acute febrile diseases like pneumonia, puerperal fever, or enteric fever. It has also been known to occur just before death. 
Histopathology.--The vesicles are situated between the layers of the Stratum corneum at the orifices of the sweat-ducts. They are due to blocking of the sweat-pores by swollen imperfectly cornified cells, which obstructs the outflow of sweat and causes it to collect between the horny laminae to form a vesicle.

Diagnosis.-The superficial nature of the lesions, the absence of inflammatory symptoms, and the associated pyrexia, enable it to be distinguished from other vesicular conditions, such as miliaria and eczema.

Treatment.-As it invariably disappears spontaneously and in a short time, no treatment is called for, but the application of a dusting powder of zinc oxide and talc hastens the drying up of the lesions and is cool and comforting to the patient.

\section{REFERENCES}

Conts, J. Pathology of sudamina and miliaria (Journ. Path. \& Bact., Oct. I 892).

Poli itzer, S. Prickly-heat (Journ. Cut. \& Gen.-Urin. Dis., Feb. I 893). Robinson, A. R. (Journ. Cut. Dis., I884, ii. p. 362).

Török, L. Sudamen and miliaria (Monats. f. prakt. Derm., I89 I, xiii. p. 436$)$.

Uxxa, P. G. Histopathology (Walker's Trans., Edinburgh, I 896, p. I80). 


\section{CHAPTER XII}

\section{CUTANEOUS AFFECTIONS DUE TO SUNLIGHT}

Introduction.-The specific effects of sunlight on the skin are due to the actinic or chemical rays at the violet end of the solar spectrum. They consist of pigmentation in the form of sunburn and freckles, and of dermatitis varying in degree from acute dermatitis, due to an excessive exposure to brilliant sunlight in a sensitive skin, to chronic dermatitis due to repeated exposures to rays of less intensity. The dermatitis is comparable to that caused by the X-rays, but is more superficial and has a shorter latent period between the exposure and the reaction.

The effects of light, both clinical and histological, have been carefully studied within recent years since the introduction of actinotherapy.

\section{SUNBURN.}

Sunburn is the familiar diffuse bronzing of the skin which occurs in parts exposed to sunlight. It varies in degree from slight darkening to deep bronzing and occurs more readily in fair than in dark skins. It appears rapidly after the exposure and soon fades in the absence of strong light. It is a diffuse melanosis due to an increase of melanin in the cells of the basal-layer of the epidermis and the deeper prickle-cells, and may be regarded as an effort on the part of nature to protect the sensitive and vascular corium from the harmful action of the actinic rays. It has been found that when natives with dark skins leave the tropics and come to live in Europe where the sun's rays are less intense their skins become gradually paler in tint, while if a European becomes resident in the tropics his skin becomes darker. In different races, also, the pigmentation of the skin varies directly with the intensity of the sun's rays; in Central Africa, for example, the native is almost jet-black, further away from the equator as in Abyssinia he becomes dark brown, in Morocco a lighter brown, in Southern Europe olive, in Central Europe brunette, and in Scandinaria blonde. 
Treatment.--Sunburn rarely calls for treatment but should it do so it may be reduced by bleaching with hydrogen peroxide (see treatment of freckles).

\section{FRECKLES}

Syn.: Lentigo; Ephelis; Fr., Tache de Rousseurs; Lentilles; Ger., Sommersprossen.

Definition: Small circumscribed pigmented macules situated on the parts of the skin exposed to sunlight, chiefly on the face and the backs of the hands.

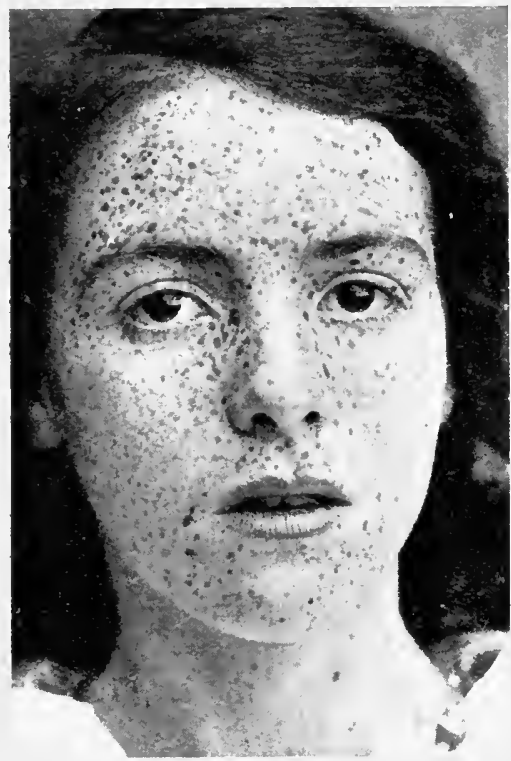

FIG. $4^{8}$ - Freckles.

Symptoms.--The macules are roundish, oval, or irregular, in outline, vary in size from a pin's head to a pea, and begin as pink or red spots which later become yellowish-brown or blackish. They may be few or extremely numerous, may be sparsely distributed or crowded together, and may sometimes coalesce to form pigmented patches the size of a bean or larger. (Fig. 48.)

They are present chiefly on the face, neck, backs of the hands, wrists, and forearms. On the face they are more or less symmetrically distributed and are especially profuse on the cheeks, forehead, and nose, but in rare instances they may be

unilateral and confined to one region.

Freckles have also been described on covered parts, such as the back, buttocks, and genital organs. These have been named "cold freckles" and are probably pigmented naevi. For such lesions it is advisable to employ the name "lentigo" and to restrict the term "freckle" to a pigmented macule caused by the actinic rays.

Freckles may appear rapidly in large numbers, or may develop slowly and insidiously. They occur at the beginning of sunmer and tend to die down in winter, except in countries like Switzerland where the winter sunlight has a high actinic value.

They cause no subjective symptoms and are an annoyance only from the disfigurement when they are profuse and dark.

They occur in vast numbers as an early symptom of Xeroderma pigmentosum - a rare affection in which there is undue susceptibility to the actinic rays. 
Histopathology.-The freckle is the evidence of an excess of melanin granules in the cells of the basal layer and in the neighbouring pricklecells of the epidermis in a circumscribed area, and occasionally in the papillary layer of the corium.

Etiology.- They are the direct result of stimulation by the actinic rays, and occur most frequently in fair delicate skins and in people with reddish hair, and are more rarely met with where the skin is coarse or naturally pigmented, as in brunettes or half-castes.

They are usually noticed first about the age of five or six years, are most profuse up to the age of twenty, tend gradually to diminish, and may disappear altogether.

They are equally common in both sexes and have a distinct tendency to occur in families, probably owing to inherited delicacy or fairness of the complexion.

It is a common observation, and one which has not yet been explained, that people who are prone to freckles do not readily become sunburnt, and vice versa.

Treatment.-As a rule freckles do not call for treatment as they tend to disappear spontaneously when the sunlight becomes less intense in winter.

Where they are dark, numerous, and disfiguring, their removal may be advisable, either by bleaching the pigment or by causing desquamation of the pigmented epidermis.

The favourite bleaching application is hydrogen peroxide. A piece of lint soaked in hydrogen peroxide (Io vols.) is applied to the freckles daily till they fade, or the freckles are scarified and the lotion dabbed on with a piece of cottonwool.

Desquamation can be brought about by the application of a I \% solution of perchloride of mercury in alcohol several times a day on a piece of cottonwool, or of a peeling paste containing resorcin or salicylic acid.

Individual freckles can be obliterated by touching them with pure carbolic acid, by refrigeration by solid carbon dioxide, or by electrolysis-but this last method is apt to leave a scar.

Nuch can be done to prevent their recurrence by avoiding the sun and by the use of brown veils, parasols, gloves, etc.

\section{REFERENCES}

Сонх, M. Anatomy of ephelides, etc. (Monats. f. prakt. Derm., is9r, xii. p. I I9).

MacLeod, J. M. H. Case of pigmented naevi-like freckles (Brit. Journ. Derm., I9I3, xxv. p. I9I).

SAALFiELd. Treatment of freckles, vitiligo, and chloasma (Brit. Journ. Derm., I 893, v. p. 63). 


\section{SOLAR DERMATITIS}

Two types of solar dermatitis may be distinguished, namely an acute and a chronic type.

\section{Acute Solar Dermatitis or Eczema Solare}

Solar dermatitis affects the exposed parts, namely, the face, neck, and hands, and the arms from having the sleeves rolled up. It is sometimes met with on the legs in children from going about bare-legged, and occasionally on the upper part of the chest and back in adults from wearing a thin white shirt or blouse which is transparent to the actinic rays. The dermatitis does not appear immediately after an exposure to excessive sunlight, but after a latent period of about 6 hours the skin begins to smart and irritate, becomes hot and tense and an erythema associated with slight oedema appears. Left to itself the inflammation usually subsides in about 48 hours, being followed by desquamation and transient pigmentation. In severe cases the oedema is excessive and may go on to vesiculation or the formation of bullae. When the face is affected the loose tissue below the eyes may become so oedematous that the eyes are almost closed up, and the pain associated with it may be as great as in erysipelas, for which the condition might be mistaken. In such cases about a week elapses before the inflammation subsides. Sometimes the healing may be prolonged and superficial ulceration occur followed by scarring. The dermatitis is quite independent of the heat rays of the solar spectrum and is more often caused by the cold reflected light from the snow-fields at a high altitude at a temperature far below zero than by the warmer sun in the tropics. The degree of dermatitis depends on the intensity of the light and the type of skin of the individual affected. People with a delicate skin and fair complexion are more prone to it and react more acutely than those whose skins are coarser.

After an attack of Solar dermatitis hypersensitiveness to sunlight is set up by which subsequent attacks are produced by far less powerful exposures than that which caused the original one.

Histopathology - The microscopical changes in acute solar dermatitis consist of dilatation of the superficial blood-vessels, exudation of serum, an infiltration of small round mono-nuclear cells most marked in the neighbourhood of the dilated vessels, and, in very acute cases, of marked oedema of the corium with dilatation of the lymphatic spaces and softening of the collagen bundles, dilatation of the inter-epithelial lymphatics of the epidermis, spongy changes in the cells, interference with the process of cornification, and sometimes the formation of vesicles or bullae. As a rule, complete recovery takes place by the repair which ends the inflammatory process. Any destructive changes which may supervene are secondary to the inflammatory reaction and 
are due to oedematous degeneration of the cellular elements or to septic inoculation and superficial ulceration.

Treatment.- Then the erythema and oedema are severe, lead lotion, consisting of half a drachm of the Liquor plumbi subacetatis to six ounces of water or milk, applied continuously in the form of a compress or lint mask, is of great service. Under this treatment the inflammation quickly subsides. If resicles or blisters be present, care must be taken to prevent them from becoming septic, and they should be bathed with boric lotion, opened and drained, and a compress of I \% aluminium acetate lotion applied. After the inflammation has subsided the skin tends to peel and to become pigmented. The pigmentation gradually disappears, and the desquamation can be counteracted by the application of cold cream. In all cases, however mild, confinement to the house is essential when the sun is out. Much can be done to prevent it in the tropics by suitable headgear, earcaps, parasols, and reils. These should be orange, red, or brown in colour and not white or green, since red materials allow only the harmless heat rays to penetrate and are opaque to the actinic rays. A useful type of parasol for this purpose is a white cotton sunshade lined with red.

In mountaineering in the snow the skin can be protected from the intense direct and reflected rays by covering it with a brown grease paint, or with lanolin dusted over with a powder containing some brownish substance such as turmeric or red bole.

\section{Chronic Solar Dermatitis}

This type of dermatitis corresponds to chronic $\mathrm{Y}$-ray dermatitis but is less severe. It is seen chiefly in middle-aged or elderly white men who have had a long open air life in tropical or sub-tropical countries, such as planters, soldiers, ranchers, etc., and been exposed day by day to the brilliant sun. It is particularly common in Australia among men on the sheep stations, and is met with occasionally in sailors, partly as the result of direct sunlight and partly from the reflected glare from the sea. It attacks the backs of the hands and the face, and is characterised by a dry atrophic and freckled state of the skin, with here and there a few telangiectases and occasionally small horny thickenings which may become malignant.

Treatment consists of heeping the skin pliant with cold cream, wearing gloves to protect it from the sun, and aroiding irritating it by the too assiduous use of soap and water. The keratoses, if small may be reduced by painting with $2-5 \%$ salicylic collodion, applying salicylic plaster or freezing with solid carbon dioxide; if large and liable to be irritated by friction or tending to develop malignant characteristics they should be excised.

\section{REFERENCE}

Dubretilh, W. Épithéliomatose d'origine solaire (Ann. de Derm. et de Syph., June I907, p. 38\%). 


\section{HYDROA AESTIVALE}

Under the heading of Hydroa aestivale (Brooke) are included two conditions, to which the names of Summer prurigo (Hutchinson) and Hydroa vacciniforme (Bazin) have been applied, and which are most probably different degrees of severity of the same affection.

Definition.-A recurrent summer eruption affecting the exposed portions of the skin and characterised by papules, papulo-vesicles and, in severe cases, by bullae, superficial ulceration, and scarring.

Symptoms. - A mild form of this affection was originally described by Sir Jonathan Hutchinson under the heading of Summer prurigo about the year 1879 , and since then a considerable number of cases have been reported. The lesions consist of small red, or dusky conical papules, varying in size from a pin's head to a lentil. When examined by a pocket lens, they sometimes present a minute vesicle at the apex, or they may appear as if about to break down and form pustules. No definite ulceration occurs in connection with them, unless they are secondarily inoculated with pus-cocci through scratching, and they simply shrivel and form a small scale which, when it separates, leaves a pigmented macule or a definite pitted scar like that from varicella.

The eruption occurs on the exposed parts of the face, neck, backs of the hands and forearms, and is most marked as a rule on the cheeks and forehead. Cases have been described in which it was present also on covered parts of the body, but it is doubtful if they belong to the same group. The lesions are irregularly distributed, usually symmetrical, and may be sparse or extremely numerous. They generally appear towards the end of spring or in the early summer and disappear completely in the winter.

In the severe cases the lesions are more acute and consist of vesicles or bullae, interspersed among which may be detected a few papules or papulo-vesicles. The bullae may be round or irregular in outline, may reach half an inch or more in diameter, and are usually surrounded by a red inflammatory areola. It is this phase of the disease to which Bazin gave the name of Hydroa vacciniforme. Sometimes in such cases the onset of the eruption is associated with malaise, loss of appetite, and pains in the joints, and may be preceded by a sensation of burning or pain in the skin. In two days the vesicles or bullae usually begin to dry up and a scab of a yellowish or brownish tinge forms. The drying process begins in the centre and extends gradually to the periphery, giving the lesion an umbilicated appearance. Where the bullae are larger they may break and their contents dry up to form an amber-coloured crust like that of impetigo, which separates, leaving a depressed scar not unlike that due to vaccination. In places where the skin is thin and stretched over bones and cartilage, as about 
the forehead, nose, auricles, and over the finger joints, pitting and disfigurement may be produced, and this is especially marked on the auricles which are apt to become shrunken and eroded at the borders. Individual lesions dry up in a few days but the attack may be prolonged for weeks by the appeal ance of successive crops.

Histopathology.--The histopathology of the lesions indicates a more or less acute inflammatory process. The blood-ressels of the corium are widely dilated, and there is an exudation of serum leading to oedema with swelling of the collagen bundles, dilatation of the interepithelial lymphatic spaces, and, in acute cases, to the formation of vesicles or bullae occupying the greater portion of the epidermis. Around the dilated ressels there is an infiltration of lymphocytes and a few polymorphonuclear leucocytes.

This is followed by drying up and necrosis of the affected part of the epidermis and the papillary layer and the formation of a depressed scar.

Etiology.-The affection generally begins in childhood and tends to recur every summer till adult life when it ceases spontaneously. Cases have been recorded, however, in which it appeared for the first time in the adult. It is equally common in both sexes, though it wis once believed to be much more frequent in bors than girls.

The exciting cause is exposure to the actinic rays of sunlight. This has been proved experimentally by exposing a susceptible individual for a short time to intense sunlight when an acute outbreak of vesicles and bullae resulted. It is essentially a summer eruption but it may appear in winter provided the sun's ray's are sufficiently intense, as in a case recorded in a patient who was wintering at St. Moritz in Switzerland. The actinic rays are not the only exciting factor, however, as an attack may be determined by strong winds or sea air even on a dull day.

For this special type of reaction to occur there must be a peculiar individual susceptibility, since it bears little resemblance to the ordinary reaction produced by the actinic rays as seen in the acute or chronic forms of solar dermatitis. The cause of this susceptibility is uncertain; it may be of congenital origin, or it may result from some obscure form of toraemia, and in support of this latter view it has been noticed that in several cases it has been associated with the presence of haematoporphyrin in the urine.

Diagnosis.-The recurrence of the affection every summer, the localisation to the exposed parts, the clinical characters of the eruption, and its tendency to leave pitted scars, render the diagnosis from other papular or bullous affections, such as Acne rulgaris and impetigo, comparatively simple.

Treatment.-The prophylaxis is similar to that of acute solar fermatitis, namely, aroidance of exposure to intense sunlight or strong xind. 
The local treatment consists of soothing the skin with an astringent lotion such as lead lotion and the application of a dusting powder. Where vesicles or bullae are present they should be punctured and a mild antiseptic ointment applied containing boric acid or ammoniated mercury. Where crusts have formed these should be softened and removed by starch poultices and the exposed surface dressed with a zinc paste, containing $2 \%$ of ichthyol or resorcin, spread on lint.

Internally quinine, arsenic, and salicin have all been recommended.

\section{REFERENCES}

Adamson, H. G. Hydroa aestivale of mild type (Brit. Journ. Derm., I906, xviii. p. I25).

BazıN. Hydroa vacciniforme (Leçons et clinique sur les affections cutanées, \&c., Paris, I 868).

Boeck, C. Hydroa vacciniforme (Bazin) (Arch. f. Derm. u. Syph., I894, xxvi. p. 23).

Buri. Summer eruption (Monats. f. prakt. Derm., I89I, xiii.).

Constantin. Bullous forms of Hydroa vacciniforme (Ann. de Derm. et de Syph., 1905, p. 92).

Fox, T. C. Slight Hydroa aestivale (Brit. Journ. Derm., i 898, x. p. 409 and I 899 , xi. p. 464 ).

Huichinson, J. Summer prurigo (Lectures on rare skin diseases, I879, p. 126).

Moreira, J. Hydroa vacciniforme seu aestivale (Brit. Journ. Derm., I 895 , p. I75).

\section{XERODERMA PIGMENTOSUM}

Syn. : Atrophoderma pigmentosum (Crocker); Angioma pigmentosum atrophicum (Taylor); Melanosis lenticularis progressiva (Pick).

Definition.-A rare affection of the skin which begins in early childhood, involves chiefly the exposed parts of the body, and is characterised at first by freckle-like pigmentation, white atrophic spots, and telangiectases; later by superficial ulcerations and warty growths; and finally by malignant epitheliomata and a fatal issue.

Description.-Xeroderma pigmentosum was first described by Kaposi in I870 and since that time about roo cases have been recorded. It is one of the most striking of all cutaneous affections both in the picture it presents and in the constancy of its course and termination. It usually begins in infancy, appearing about the sixth month or later and rarely after the second year. Cases have been recorded where it was said to have developed in old-age but these were doubtless instances of senile skin with warty and malignant epithelial growths.

There are six different types of lesions which characterise the disease in its fully developed state, namely, freckles, telangiectases, atrophic patches, warty growths, superficial ulcerations, and finally carcinomata.

The earliest lesions consist of freckles, ranging from minute specks to irregularly outlined pigmented macules the size of a threepenny 


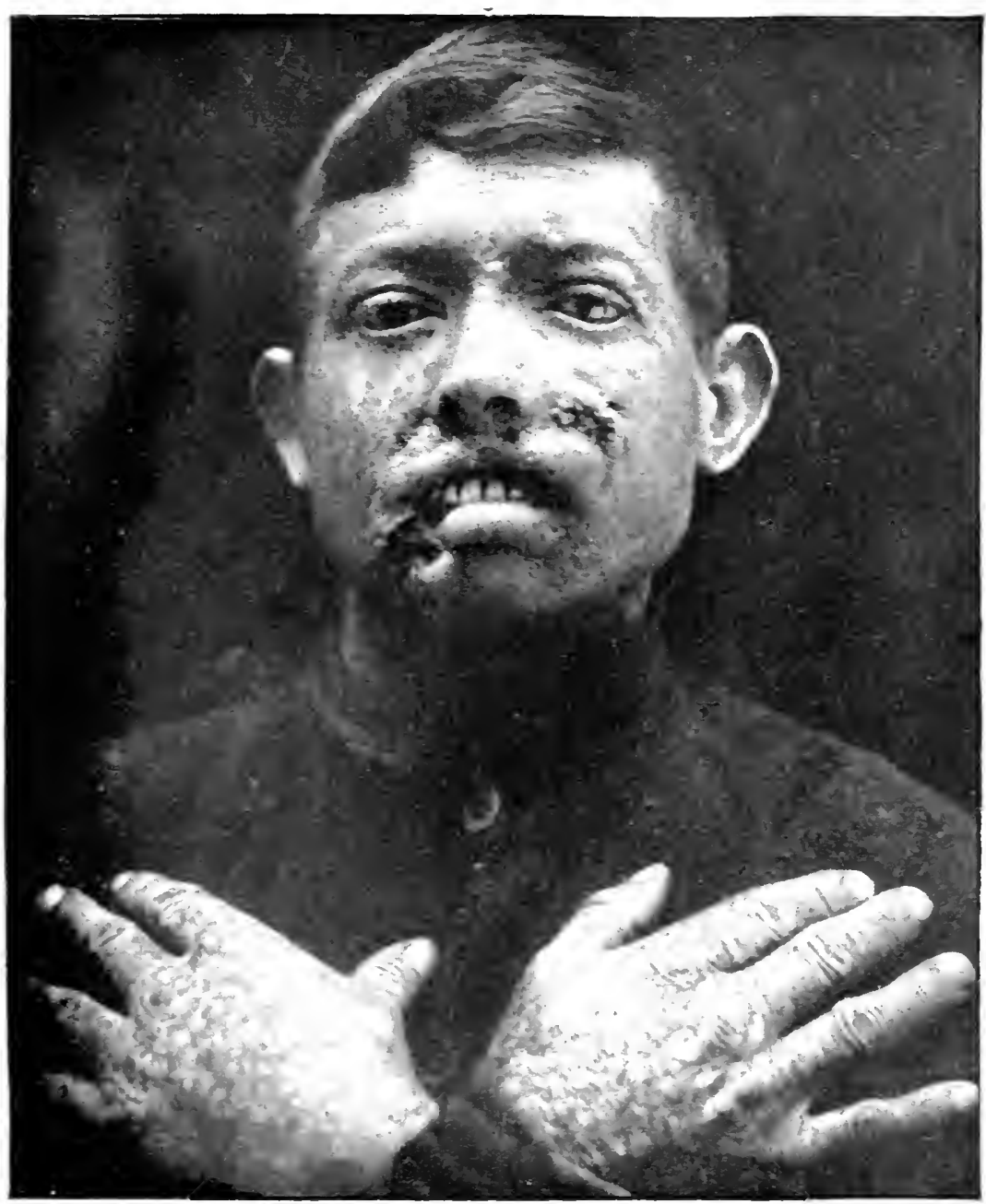

Fig. 49.-Xeroderma Pigmentosum.

Showing catcin matous mass on leet check and nose, projecting into buccal cavity, an! carcinomatols ulce involving right angle of mouth, with patchy piznentation and atoph dermia of batis i hands.

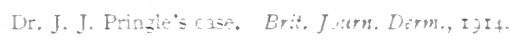



piece or larger and varying in colour from fawn to dark sepia brown, or of diffuse areas of pigmentation with freckle-like macules dotted irregularly over them. These are situated chiefly on the exposed parts, namely, the face, nech, backs of the hands, forearms, and occasionally on the scalp. They usually extend down the neck to immediately over the clavicles in front and over the shoulders behind. On the upper extremities they are most marked on the backs of the hands and decrease in number up the forearm till they finally cease about the insertion of the deltoid. The ulnar aspect of the arm is usually comparatively free. They may be present on the lower extremities on the feet and extending up as far as the knees or even higher, and in several cases a patchy pigmentation has been observed on the trunk.

The pigmented macules are at first indistinguishable from freckles due to sunlight. In several cases they have appeared in the first instance in summer and disappeared in winter, and only after several seasons have become persistent throughout the year. The affected skin is extraordinarily vulnerable to sunlight and even slight exposure may produce a more or less acute solar dermatitis, and in one or two cases the freckle-like spots are said to have followed an initial attack of erythema and oedema due to the actinic rays.

Interspersed between the freckles the second type of lesion early makes its appearance, namely, linear or stellate telangiectases and small capillary angiomata in the form of bright red, rounded or flat spots varying in size from a minute speck to a pin's head or larger.

Another feature is the presence of small atrophic spots, roundish or irregular in outline, sometimes slightly depressed, white in colour, and especially noticeable from contrast when present on the diffuse pigmented patches. In certain situations, notably about the orbits, coalescence of these atrophic macules gives rise to irregular patches resembling superficial scars with a smooth, shiny, or wrinkled surface beneath which telangiectases may be seen ramifying. It was the presence of these atrophic patches which suggested to Crocker the name of Atrophoderma pigmentosum, a title which has a definite advantage over Kaposi's criginal name in that it does not suggest a relation to xerodermia (mild ichthyosis) with which it has no connection.

After a time-it may be months or years-changes of a verrucose type occur in the skin in which it becomes rough, scaly, and shows a marked tendency to the development of horny thickenings or warty growths, of the same type as those in senile keratosis, which may take their origin in pigmented spots or independently.

In addition to the keratoses, superficial ulcerations are liable to occur, due to the secondary inoculation of septic bacteria through some abrasion in the weakly resisting skin. Should these ulcers heal they are apt to give rise to disfiguring scars and contractures and may lead to ectropion, or to a thinning and constriction at the nasal orifice, or if about the mouth to an appearance somewhat recalling an old-standing case of Lupus vulgaris. (Fig. 49.) 
Sooner or later, sometimes within a twelvemonth, at other times as long as twenty years after the onset of the disease, malignant changes supervene, and the ulcers become papillomatous, crusted and finally carcinomatous, or the warty growths develop into malignant epitheliomata. The carcinomata are usually multiple and are not infrequently present in situations where the skin and mucous membrane meet, as about the mouth, nose or orbit. Sometimes they are of the hard parement-epithelioma type, at other times of the rodent ulcer variety with a hard rolled edge. As a rule metastases are absent both in the glands and internal organs but several cases are on record in which early involvement of the lymphatic glands has occurred.

In association with the skin lesion, the eyes are frequently affected and there may be purulent conjunctivitis, corneal ulcers, and occasionally vascular pterygium.

The mucous membranes are occasionally implicated, and pigmented and telangiectatic spots have been observed on the lips and on the mucosa of the eyelids.

In the primary stages there are no subjective symptoms, but the ulcerations and carcinomatous growths of the later stages may give rise to discomfort and actual pain.

The general health is not interfered with at first but later the disfigurement, the tendency to remain indoors to avoid the irritation of sunlight, and the hopelessness with regard to a cure, cause a marked deterioration, both physical and mental, in the patient so that when the carcinomata develop there is a rapid decline and death may quickly supervene in a state of exhaustion and profound anaemia due to septic complications. The fatal result may take place comparatively soon, a few years after the onset of the disease, or it may be delayed by a long period of quiescence for fifteen years or more. By improved methods of treatment with the assistance of the $\mathrm{X}$-rays or radium a fatal issue may be still further staved off and possibly prevented.

Histopathology.--Sections of the pigmented skin show the pigment to be situated in the cells of the basal layer of the epidermis, the pricklecells immediately above it, and in the connective tissue cells of the papillary layer of the corium. In the atrophic lesions the atrophy involves, not only the epidermis, but also its appendages. (Fig. 50.)

The warty growths have nothing peculiar in their histology but beneath them the white fibrous tissue may be atrophied, while an actual increase in the elastic tissue has been described.

The carcinoma as a rule takes its origin in the prickle-cell layer and forms an epithelioma of the squamous-cell type with cell-nests. On the other hand several cases are on record where the growth originated in the basal cell layer and which conformed more to the rodent ulcer type.

Etiology.-The disease has a wide distribution in Europe but the majority of the cases have been reported from Austria and Germany. It has been known to occur in the United States of America. 
It has a marked tendency to run in families, affecting several members, usually of one sex. On the whole, however, the number of cases in both sexes are about equal. Heredity does not seem to play any part in its etiology.

The exact cause is not fully understood. The close resemblance of the lesions to those which occur in radio-dermatitis either due to the actinic rays of light or to the $\mathrm{X}$-rays, the almost complete limitation to the exposed parts, the observations that the freckling is at first worse in summer than winter and that the affected skin is hypersensitive to the actinic rays, and the improved prognosis when the skin is protected from the sun, are strongly in favour of light being an important factor in the etiology and suggest that it is the result of a congenital vulnerability to light. The occurrence of pigmented lesions, however, in corered situations, such as about the root of the neck and shoulders, is difficult to explain on this hypothesis alone, and there may be in addition some peculiar defect in the nerve supply of the skin interfering with its

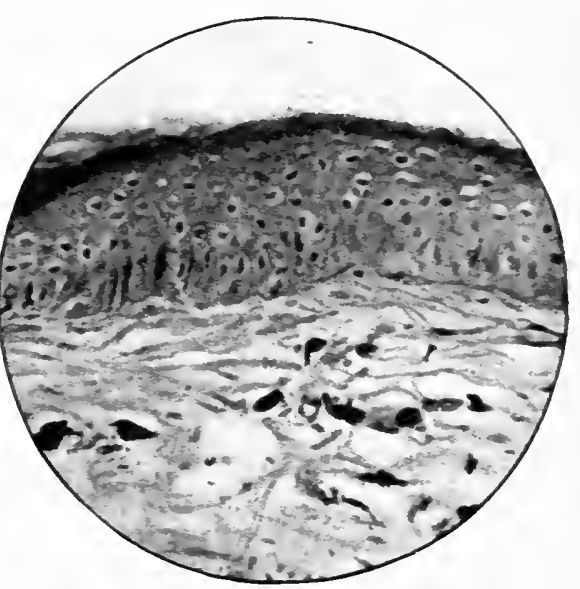

FIG. 50.- Xeroderma Pigmentosum, shows pigment in basal cells of epidermis, and in variously sized cells in corium. $\times 200$.

(After Dr. W. Dyson. Brit. Journ. Derm., Igrr.) nutrition and leading to degenerative changes and an undue susceptibility to other forms of local irritation.

Diagnosis.-In a well-marked case the diagnosis presents no diffculty and once a case, or even a satisfactory illustration of one, has been studied the disease is easily recognised again. A precisely similar state of the skin is met with in radio-dermatitis and senile skin but in them the affection does not begin in infancy and there is no family tendency.

Treatment.-It is of the first importance to protect the affected skin from sunlight. This may be done by wearing brown reils and gloves, the use of a 1 ed or brown parasol, or the application of zinc paste containing turmeric or burnt sienna, or brown grease paint.

Each type of lesion requires its own special treatment. The warty growths should be excised or curetted, the ulcers kept clean and encouraged to heal by the application of antiseptic lotions or ointments, and the carcinomata excised or treated by $\mathbf{X}$-rays, radium or refrigeration in the manner described in connection with rodent ulcer.

Where the eyes are involved the conjunctivitis may be relieved by boric lotion, the corneal ulcers healed by touching them with pure carbolic or by the use of an ointment such as yellow oxide of niercury, 
8 grs., sulphate of atropin, 2 grs., vaselin, I oz. ; and the photophobia diminished by wearing smoked glasses.

Internal treatment except on general principles has no influence on the course of the disease. Large doses of arsenic were once advised but are no longer advocated.

\section{REFERENCES}

Councilman, W. T., \& Magrath, G. B. Xeroderma pigmentosum (Journ. Med. Research, I909, xxi. p. 33I).

Crocker, R. Xeroderma pigmentosum (Trans. Med. Chir., Lond., I884, lxvii. p. I69).

- Xeroderma pigmentosum (Diseases of the Skin, 3rd. Ed., Lond., Ig05, p. 628).

Falcao. Adult cases of Xeroderma pigmentosum (Trans. 3rd Internat. Cong. Derm., Lond., r 896 , p. 280).

KAPOSI. Xeroderma pigmentosum (Lehrbuch der Hautkranklieiten, Wien, 1870 , p. I 82 ).

Kreibich. On the tumours in Xeroderma pigmentosum (Arch. f. Derm. u. Syph., I90I, p. I23).

Littıe, G. Xeroderma pigmentosum (Brit. Journ. Derm., I902, xiv. p. 94 ).

Okamura. Xeroderma pigmentosum (Arch. f. Derm. u. Syph., I900, p. 87).

Pringle, J. J. Xeroderma pigmentosum treated by radium (Brit. Journ. Derm., I9I4, xxvi. p. I44).

Rouvišre. Xeroderma pigmentosum (Ann. de Derm. et de Syph., igro, p. 34),

Taylor. Review of cases (Med. Rec., New York, I888, xxxiii. p. 26r).

West, S. Case of Xeroderma pigmentosum (Brit. Journ. Derm., I 896. viii. p. 45). 


\section{CHAPTER XIII}

\section{DERMATITIS DUE TO ELECTRICITY, X-RAYS AND RADIUII}

\section{BURNS FROM ELECTRICITY}

THE human skin, when dry, is relatively a non-conductor of electricity and though not as perfect an insulator as india-rubber still offers considerable opposition to the passage of electrical currents. Its resistance is greatly diminished should it be wet or macerated with soap or with a solution of some alkali such as common salt or bicarbonate of soda. It is the horny layer of the epidermis which is the most resistant part of the skin, the electrical current passing much more easily through the deeper layers of the epidermis and the moist corium, and it is in situations, such as the palms and soles, where the horny layer is thickest, that the resistance is at its maximum.

Burns are produced both at the points of entrance and exit of an electrical current, the injury at the entrance being, as a rule, the greater. The degree of injury to the skin increases directly with the amperage, and according to Lewis Jones if the current reach 500 milliamperes (half an ampere) not only will burns of great severity be produced but a fatal result fron shock may supervene.

The injury to the skin caused by an electrical current is probably greater when the skin is dry than when it is moist, for when moist the current more easily passes through it and becomes diffused.

The effects of electrical currents are both general and local. The initial effect of a high tension current is to produce unconsciousness which, if not fatal, may last from thirty-six to forty-eight hours; at the same time it gives rise to a powerful muscular contraction or tetanic spasm which may cause the individual to grasp the live wire or conductor and prevent his being able to let go, or may cause him to be thrown violently several feet away from the contact and involuntarily to emit a peculiar cry. (Fig. 5I.)

The injuries to the skin, which are partly due to electrolytic action and partly to intense heat caused by the current, are somewhat similar to those due to heat and vary from simple erythema and blistering to deep destruction of tissue. They differ from heat burns in presenting 
at first a peculiar dry charred appearance, in having less tendency to become septic, in being less painful, and in being far more intractable.

In mild degrees of electrical burn the skin at the contact sometimes assumes a peculiar cyanotic appearance and may be oedematous and surmounted by tense or flaccid bullae, the contents of which may be haemorrhagic. Occasionally the contact leads to a breaking up of fine particles of metal which become deposited and impregnate the skin for somedistance around, producing a brownish-grey pigmented appearance.

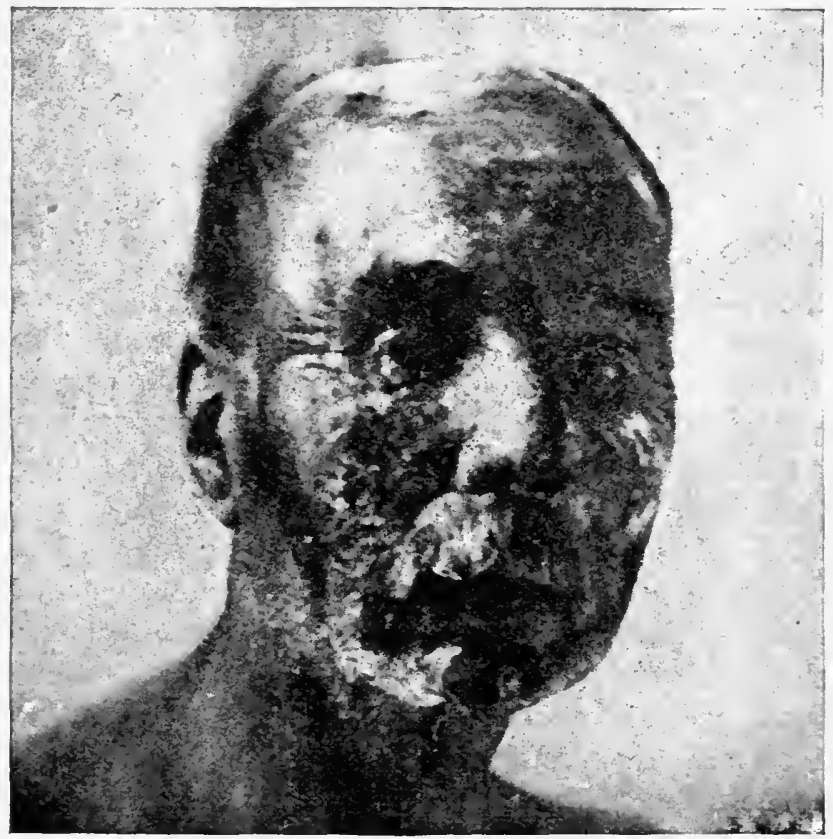

FIG. 5I.-Electrical Burn of the Second and Third Degree, showing broken bullae and charred skin.

(From MacLeod's Burns and their Treatment.)

In burns of greater scverity an eschar is formed which on separating may lead to secondary hacmorrhage and to a necrotic process which is progressive.

Sometimes the injuries, instead of suggesting burns from heat, have more the appearance of wounds produced by a blunt instrument or weapon.

The amount of injury to the skin and underlying tissues cannot be taken as a definite indication of the severity of the electrical shock, for dangerous general symptoms, and often a fatal issue, may occur with comparatively little superficial evidence, while severe local injuries may sometimes be caused without marked internal disturbances.

Treatment.-- The local treatment of electrical burns is similar to that described in connection with burns from heat. Where possible the "open method" of treatment should be adopted and the burns 
simply powdered over with stearate of zinc or magnesium carbonate. When the eschar begins to separate every precaution should be taken against secondary haemorrhage. If the burn has been severe and penetrated deeply and a whole limb has been involved, the setting in of gangrene and the formation of a line of demarcation may render amputation necessary.

The general treatment resolves itself into the treatment of shock, and any nervous symptoms which may supervene subsequently must be dealt with on general medical principles.

\section{BURNS FROM LIGHTNING}

The local effects of lightning are apt to be most severe on the exposed parts and less marked where the skin has been protected by clothes, which, if dry, offer considerable resistance to the electrical discharge. If the clothes, be damp and the person struck be sitting or lying on wet ground this may lead to a rapid diffusion of the current and to its being so quickly earthed that the actual injury to the body caused by it may be less severe than if the skin and clothes were dry.

Occasionally shock, temporary paralysis, or even death, may occur from a lightning stroke and when the body is examined practically no trace of injury may be evident on the skin.

Lightning causes destructive changes in the skin both at the entrance and exit of the current. Of these there are three different types: (I) Wounds or contusions which resemble injuries from a blunt weapon or heavy stick and are sometimes so serere as to lead not only to injury of the skin and subcutaneous tissue but even to fractures of bones.

(2) Burns of the skin resembling electrical burns caused by metallic objects worn by the individual which have acted as conductors, such as keys, coins, metal buttons, etc.

(3) Arborescent markings which are peculiar radiating red streaks spreading for some distance over the cutaneous surface from the site of entrance and exit of the current and breaking up somewhat like the branches of a tree. Occasionally they may take the form of linear wheals and in rare instances have been known to be purpuric or even necrotic. They are generally believed to be formed in the track of the discharge of electricity which has been split up in a dendriform fashion, possibly through differences in the conductivity of the tissues.

Should the burn occur in a hairy region, the hairs are generally singed.

The treatment of the local injuries due to lightning is similar to that of other burns from electricity.

\section{REFERENCES}

CoRxet, E. M., and OAKDEx, II. M. Case of Recovery after Severe Electric Burns (Proc. Roy. Soc. Med., I9I2-I 3, vi. Clin. Sec. I90).

Elder, J. M. Electrical Burns (Montreal Med. Journ., Jan. I90o, p. IS). 
Heaton, G. A Patient with Loss of Entire Upper Extremity and other Lesions after Exposure to a Strong Electric Current (Birmingham M. Rev., I9I3, lxxiii. p. 924).

Hencessy, D. Three Cases of Lightning Stroke (Brit. Med. Journ., r889, June 8th, p. 1294).

Howell, C. M. Hinds. Electrical Burns (Accidents in their MedicoLegal Aspect, I9Io, p. 258).

Jellinek, S. Atlas der Elecktropathologie (Vienna, I912).

JEx-Blake, A. J. Death by Electric Currents and Lightning. Goulstonian Lectures (Brit. Med. Journ., I9I3, i. pp. 42.5, 492, 548, 601).

JoNes, H. Lewis. Lethal Effects of Electric Currents (Brit. Med. Journ., I895, i. p. 468).

Lucas, R. C. Accidental Electrocution causing Extensive Gangrene of all the Extremities (Trans. Clin. Soc., London, 1905, xxxviii. p. 86).

MAcGregor, A. Electrical Burn successfully treated by Ionisation (Arch. Radio. \& Electrotherapy, 1915 , xx. p. 167).

Milliken, S. M. Severe Electrical Burns (Med. Rec., N.Y., I9r5, Ixxxviii. p. 589).

Oliver and Bolam. Death by Electric Shock (Brit. Med. Journ., r898, i. p. I32).

Oliver, T. Injuries by Electric Currents of High Pressure (Allbutt \& Rolleston, System of Medicine, I909, vi. p. 253).

-Pathology and Treatment of Injuries caused by Electricity (Lancet, I9tI, i. p. 363).

Tousey, S. Medical E!ectricity, 1915.

\section{X-RAY Dermatitis}

Soon after the discovery of the Röntgen rays in ISg6 it was found that they were capable of producing more or less severe dermatitis and that to aroid this it was necessary to restrict the dosage within certain limits. The dermatitis is due to an overexposure to the X-rays, either from one or more excessive doses or from the cumulative action of a series of smaller doses each in itself harmless. Over-exposure may be the result of too powerful or too prolonged irradiation or may be caused by the $\mathbf{X}$-ray tube being too near the cutaneous surface, for the effect of the X-rays on the skin varies inversely with the square of the distance of the tube from it. Individual susceptibility is also a factor in the production of X-ray dermatitis but is one of which the importance has been exaggerated.

One of the first cases which came under my observation was in rgoo, and occurred in a young man employed by a certain firm to demonstrate X-rays at an exhibition by showing the bones of his hand on a fluorescent screen. After he had done this for some weeks an acute vesicating dermatitis developed. At that time the danger of the rays was not fully appreciated, but very soon case after case occurred and the literature on the subject became loaded with reports of X-ray burns of varying degrees of severity. It was found that the X-rays themselves caused the dermatitis and not the electrical cffleuve around the tube as was once suggested. It was generally believed that the 
soft rays were mainly responsible while the hard penetrating rays with short wave lengths were considered to be harmless, but this view is open to criticism and there is another factor which is far more important, namely the quantity of the rays and the force of the irradiation.

It is customary to describe $\mathbf{X}$-ray dermatitis under two headings : (a) acute dermatitis resulting from one or more excessive doses; (b) chronic dermatitis caused by small doses repeated over a long period.

This distinction, though useful, is somewhat arbitrary as the acute forms of radio-dermatitis are liable to assume eventually the characteristics of the chronic type.

Acute X-ray Dermatitis due to one or more excessive doses. - The dermatitis caused by over-exposure to the X-rays varies in severity according to the strength and duration of the exposure. It does not appear immediately but after a latent period which varies inversely with the intensity of the dose. The dose which the healthy skin is capable of standing without harmful effects other than a temporary defluvium of the hair can be estimated conveniently by the pastille of Sabouraud and Noiré, which is now largely employed as the standard of dosage (see p. I $f$ ). Any dose greater than a pastille dose may be regarded as excessive and is almost certain to be followed by more or less severe dermatitis.

For purposes of description four degrees of dermatitis may be cited :

I. The mildest form consists of erythema associated with slight itching and burning, appears about the fourteenth day after the exposure, is transitory, and passes off in a few days either leaving no trace or being followed by slight desquamation. It is accompanied also by falling of the hairs in the exposed area, which usually grow again although sometimes imperfectly. This degree of dermatitis may result from a pastille dose in an individual with an undue susceptibility to the rays, or where a particularly sensitive area of skin had been exposed.

2. In dermatitis of the second degree the erythema is more marked, the skin is raised and oedematous, and the itching or burning more severe. It comes on about the eighth day, takes three or four weeks to disappear, is succeeded by desquamation followed by slight atrophy or sometimes by telangiectases which may not appear for months after the exposure, and by permanent loss of the hair.

3. Dermatitis of the third degree appears before the end of the first week and is of a much more acute type. It is associated with a vivid erythema, oedema, and a tendency to the formation of vesicles and bullae. This is followed by superficial ulceration which is intractable and painful, and sometimes by definite sloughing. When healing does take place-which usually is not for months-the resultant scar is shiny, hairless, atrophic, covered with telangiectases, and liable to break down and ulcerate. 
4. Dermatitis of the fourth degree begins as early as the second day and is so severe that the tissues may slough right down to the periosteum in the case of the scalp or for half an inch or more in depth in the softer tissues. An unhealthy and intractable ulcer follows, with an irregular border, which is sometimes soft, flabby, and easily digested by pyogenic cocci, at other times definitely indurated, and a base with unhealthy inert granulations covered by an adherent translucent yellowish slough. The ulcer is painful, the pain sometimes being

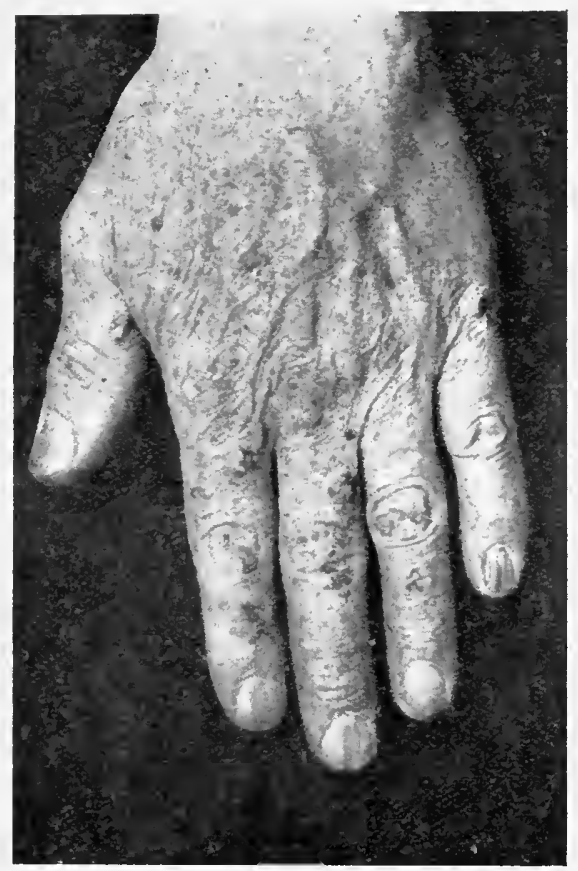

FIG. 52.-Chronic X-ray Dermatitis, shows telangiectases, small warty growths, superficial ulcerations and dystrophy of the nails. continuous and of the most agonising and stabbing type.

\section{Chronic X-ray Dermatitis from repeated small doses.-} This is usually met with in $\mathrm{X}$-ray operators or tubemakers, and chiefly in those who began to expose themselves to the rays before the danger of so doing was fully recognised; fortunately since this has been appreciated and the necessary precautions taken to avoid it, chronic X-ray dermatitis is becoming much more rare.

As a rule it affects the backs of the hands and fingers, but occasionally the face, from too great proximity in testing tubes. One of the first indications of it may be a brownish pigmentation of the skin, diffuse or patchy, from an initial stimulating action of the rays; or it may be noticed first by the skin becoming dry, smooth, and shiny, from stretching out of the natural furrows and loss of the lanugo hairs. Small telangiectases and haemorrhagic puncta subsequently appear and are characteristic of this dermatitis; they may be coarse and irregularly distributed, or fine and aggregated together in reddish patches. The haemorrhagic puncta are due to the rupture of small capillaries which may break through to the surface and give rise to haemorrhage. (Fig. 52.)

These changes in the skin are accompanied by itching or burning of an intermittent type and worse in cold weather, and by an increased sensitiveness to the rays whereby even so slight an exposure as being in the room with a live tube is definitely felt.

In advanced cases horny growths or keratoses develop in the affected area. These are greyish or blackish in colour, give the skin 
a dirty appearance, and are a great source of trouble as they may be broken off and leave superficial ulcers or may become inflamed at the base, proliferate, or become malignant. (Fig. 53.)

Owing to the diminished resistance of the skin, ulceration is apt to result from the slightest abrasion and from the secondary inoculation of pyogenic organisms. The ulcers are irregular in outline, often surrounded by a red border, and present an unhealthy-looking base covered by an adherent greyish membrane or a semi-purulent discharge. A somewhat comnion situation for them is over the joints, especially the knuckle of the middle finger, but they may occur anywhere and are usually multiple. They are peculiarly intractable and after healing tend to break down again especially in cold weather.

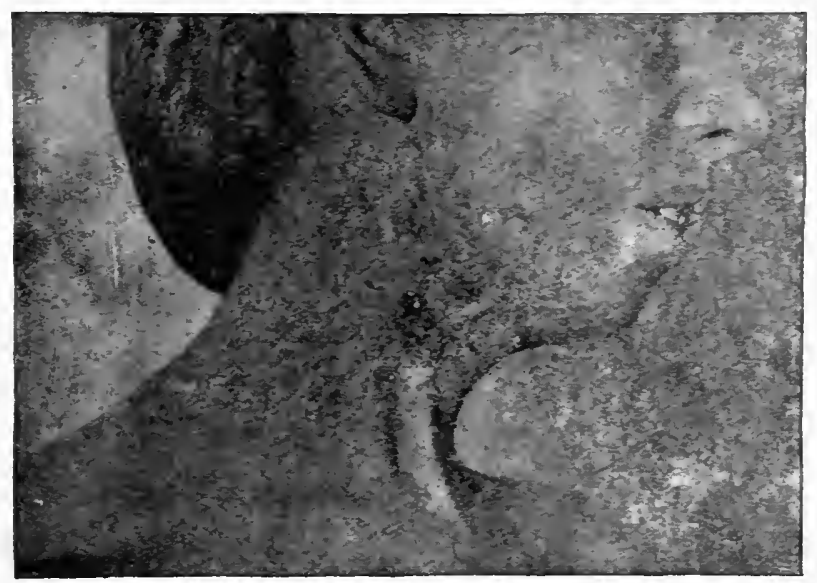

FIG. 53.-Chronic X-ray Burn, showing atrophic skin, superficial ulceration and telangiectases.

(From MacLeod's Burns and their Treatment.)

In severe cases the subcutaneous tissue may become atrophied, the fat may be replaced by fibrous tissue, the bones inay become soft and disappear and shrinkage and deformity may result, or actual gangrene may occur.

When malignant changes supervene in the keratoses or ulcers they are of a serious character and may lead to metastases and a fatal issue.

The nails are affected in nearly all the cases and become opaque, brittle, ridged, and frayed, or a suppurative perionychia may develop which may lead eventually to shedding of the nail. This is associated with pain of a characteristic type and similar to that which occurs with disease of bone or periosteum. (Fig. 54.)

Dermatitis associated with the X-rays but not directly due to their action.-There are several forms of dermatitis which are liable to occur in connection with exposures to the $\mathrm{X}$-rays which may be mistaken for true X-ray dermatitis but which belong to a different category. For 
example, a few hours after an exposure to a massive dose of X-rays for diagnostic purposes, especially where the tube has been near the skin, a patch of erythema may occur associated with slight oedema which may persist for a day. This is possibly due to electrical discharges around the tube or, more probably, to heat.

It has been observed also, especially on the scalp, that about a week after exposure to the rays small pustules may appear dotted irregularly over the exposed area. These are not due directly to the X-rays but are caused by pyogenic micro-organisms affecting skin the resist-

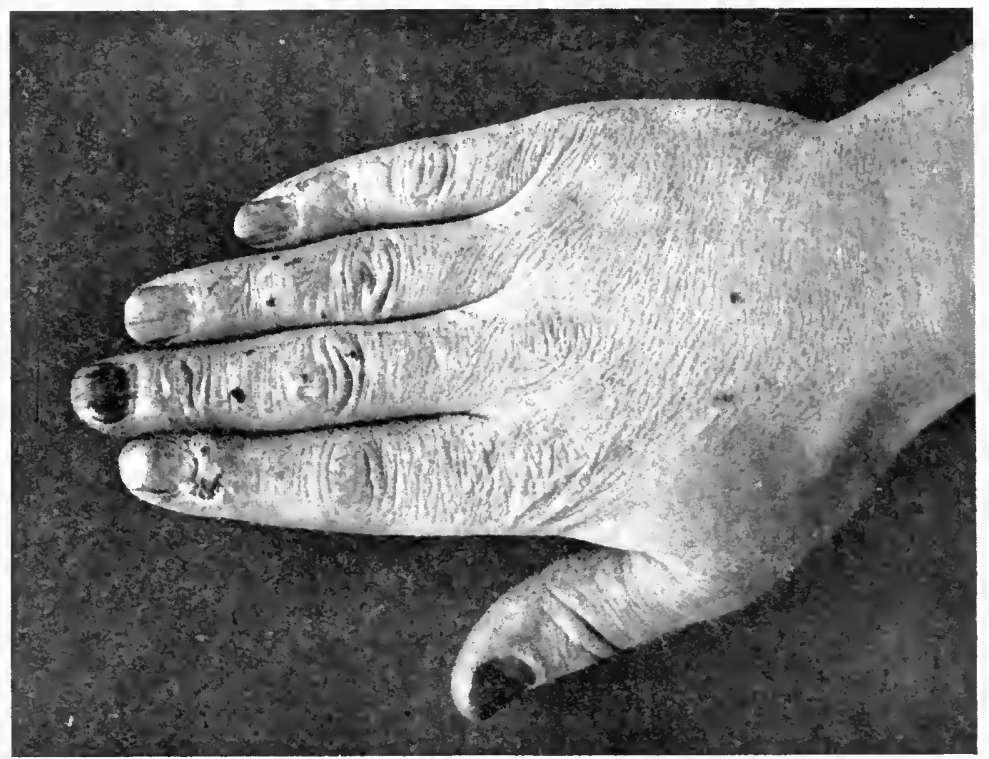

FIG. 54-Chronic X-ray Dermatitis, shows superficial ulcerations, dystrophy and discolouration of the nails.

ance of which has been lowered by the rays, and can be prevented by a course of mild antiseptic treatment before the scalp is $\mathrm{Y}$-rayed.

Pathology.-The precise action of the X-rays on the tissues is not yet fully understood. At one time it was generally believed that the rays acted mainly on the nerves and that the changes which occurred in the epidermis and blood-ressels were induced secondarily. It would seem, however, that the rays act directly on the cellular elements which come into their field of influence, causing changes which, though they develop slowly, are of a definite type, and that they act most powerfully on weakly resistant cells which have reverted to some extent to an embryonic condition, such as the cells met with in rodent ulcer. The changes which occur in the cells are essentially of a degenerative type in which they become devitalised, shrivel and eventually disintegrate. As a result of the disintegration toxic substances are produced and set up the inflammatory reaction which occurs as a rulc 
from ten days or a fortnight after the exposure. When the process of repair sets in and healing takes place permanent changes are left in the blood capillaries which are found to be dilated and sometimes carernous or at other times obliterated by new fibrous tissue. (Fig. 55.)

Prognosis.-In mild degrees of X-ray dermatitis resulting from a single orer-dose the prognosis is good and complete recorery of the skin may be expected.

If vesicles or bullae have occurred a telangiectatic scar and complete loss of the hair in the exposed area is liable to follow.

Acute burns may result in the most serious sequelae, such as sloughs, gangrene, or excruciatingly painful ulcerations which may eventually become malignant.

In the case of chronic $\mathrm{X}$-ray dermatitis, if telangiectases only are present the affection may gradually improve under suitable protective treatment; on the other hand if ulceration and keratoses have appeared the prognosis should be most guarded as the ulcers are intractable, sometimes taking months or years to heal, and liable to break down later, and both they and the keratoses may become carcinomatous.

\section{Treatment of Acute X-ray}

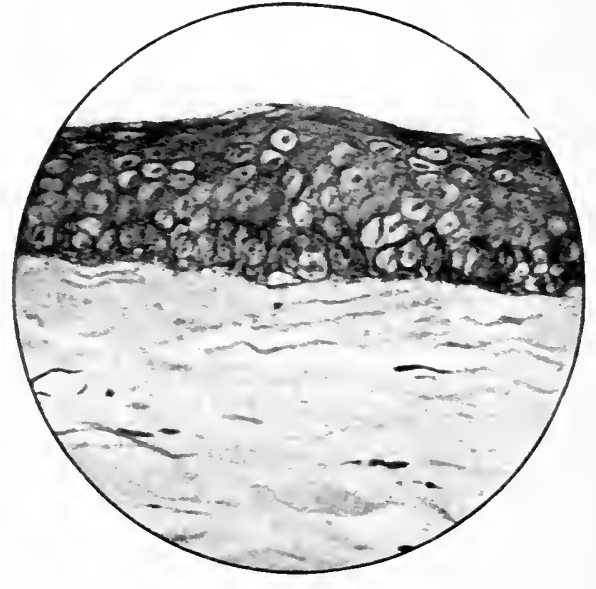

FIG. 55.-X-ray Pigmentation, shows deposit of pigment in lower epithelial cells, papillae obliterated, scar-tissue in corium. $\times 300$.

(After Dr. W. Dyson. Brit. Journ. Derm., Igrr.)

Dermatitis. - When the dermatitis consists simply of erythema, with perhaps slight oedema, soothing and astringent applications are indicated, with protective measures to prevent friction or infection from micro-organisms. For this purpose a boro-zinc powder dusted on the part and covered with lint, alternated with compresses of lead lotion, is a suitable treatment. When vesication or bullous formation has taken place the bullae should be treated in the same way as those due to heat and the surface of the skin bathed with boric acid lotion or a weak solution of biniodide of mercury, the bullae slit and drained, and a dressing of aluminium acetate applied.

Ulcerations should be kept clean by astringent antiseptic lotions, such as boric or zinc sulphate lotion, or black wash; by lotions containing oxygen-giving bodies such as hydrogen peroxide, peroxide of zinc, and potassium chlorate; or the following alum and lead lotion may be employed: Alum, gr x; Liq. pb. subacet, 万̧i ; water, ad, 亏̄i.

A soothing antiseptic ointment may be substituted, such as: Salicylic acid, gr $\mathrm{x}$; raselin, ad $\mathrm{ji}$; with the addition of menthol, gr $\mathrm{v}$; or cocain, gr $\mathrm{i}$. 
Should the pain be excessive and the ointment unsuccessful in relieving it, surgical measures may be demanded. In some cases healing may be obtained by Thiersch grafting, and even where the graft fails to take-as it often does-the edges may be stimulated to growth. Should the graft fail excision should be done where practicable, or if impossible the pain may be reduced by cutting the nerve supply to the ulcer by a deep incision above the ulcer and allowing the wound to granulate. Where there is any suspicion of malignancy, excision or amputation are indicated.

As a general principle the treatment should be conservative, as far as possible, and amputation should only be resorted to in the case of a gangrenous part after the line of demarcation has formed, for if it be done earlier it is apt to be followed by destructive changes in the scar.

Treatment of Chronic $\mathbf{X}$-ray Dermatitis. - The treatment is tedious and often disappointing. Mild cases in which telangiectases are present tend to improve under suitable treatment, but when horny growths and ulcerations have appeared the condition is extraordinarily resistant, prone to relapse, and apt to be gradually progressive. The first essential in the management of such cases is to protect the skin from external irritation. It should be wetted as little as possible and, instead of washing with soap and water, dirt should be removed from it by cold creanı or a mixture of almond oil and lime water. Gloves should be worn to protect it from cold and from the actinic rays of the sun, and for the latter purpose brown cotton gloves are particularly useful. In the case of $\mathrm{X}$-ray operators, it is wisest for them to give up working with live tubes, but should that be impossible they should be protected by gloves, opaque tube shields, lead glass behind the fluorescent screen, and by standing as far as possible behind the anticathode. The skin should be kept astringed and pliant by the daily application of an ointment such as the following: Ichthyol, gr x; zinc oxide, $\overline{5} \mathrm{i}$; and cold cream, ad, $\bar{\jmath} \mathrm{i}$. For the relief of burning or pain, fomentations of boric lotion are sometimes soothing, but in other cases they do more harm than good and compresses of lead lotion should be substituted. There is no satisfactory treatment for ramoving the telangiectases, and attempts to plug them by means of electrolysis or a needle-pointed cautery are most disappointing. Where they are fine and closely aggregated in small reddish patches, improvement may be obtained by careful exposures to radium as in the treatment of port-wine stains (see page I84).

Warty growths, if present, generally demand treatment. In situations where the skin is slack, as on the backs of the hands, the best treatment is excision. Good results may also be obtained from shaving off the top with a razor followed by the judicious application of radium. It is unwise to destroy them by caustics, the actual cautery, or by refrigeration with solid carbon dioxide, as intractable ulceration and malignant growth may result. On no account should they be picked out, as this may lead to haemorrhage and ulceration. 


\section{REFERENCES}

FRELXD, L. Radiotherapy (Trans. bỹ Lancashire, London, I9o.4).

HALl-EDwards, J. S-ray dermatitis and its prevention (Trans. Roy. Soc. Mled., Nor, 2oth, Igo8).

LAZARLS-BARLOW. Radio-activity and carcinoma (Croonan Lectures, Lancet, June 1900).

MacLeod, J. II. H. Pathological action of the N-rars (Brit. Journ. Derm., I903, xr. p. 365!.

Porter, C. A. Surgical treatment of X-ray carcinoma (Journ. Med. Research, r9Io, xxi, p. 357).

Savill, A. F. Case of X-ray ulcers cured by Hilton's Method (Lancet, I909, ii. p. I8II).

Wolbach, S. B. Histology of X-ray dermatitis (Journ. Med. Research, I910, xxi, p. 4I6).

\section{Radium Dermatitis}

The rays given off by radium are capable of producing a dermatitis somewhat similar to that due to the $\mathbf{X}$-rays, and which is believed to be caused by the Alpha and soft Beta rays. It appears about the eighth to the fourteenth day or may come out earlier after porverful exposures, the latent period varying inversely with the intensity of the irradiation.

The degree of dermatitis is dependent on the strength of the radium applicator, the area of radio-active surface, the thickness of the filter, the length of the exposure, and to a minor extent on the susceptibility of the patient.

In its mild and usual form it consists of a transient erythema with itching and burning; if more severe it may be associated with oedema, bullous formation, and superficial ulceration; and in its most severe form, deep ulceration, necrosis, and sloughing may occur. It is the evidence of an inflammatory reaction occurring in a tissue the vitality of which has been reduced by the ray's and which is set up, probably, by the toxic products of devitalised cells.

The scar which results after the dermatitis has healed is hairless, pigmented, covered with telangiectases which may not appear for six months after the exposure, sometimes depressed, and peculiarly sensitive to heat and cold.

Prognosis.-The prognosis in burns from radium as a rule is good, as the burns are usually of a mild degree and the erythema disappears in course of time without leaving a trace. In more severe burns the outlook is less favourable and when healing takes place it is liable to be followed by atrophic telangiectatic scars.

Treatment.-The treatment consists of soothing the erythema with lead lotion or dusting powder, dealing with the bullae in the same way as in $\mathrm{X}$-ray burns by drainage and aluminium acetate dressings, and treating the ulceration by antiseptic ointments.

For the removal of the telangiectases little can be done by electrolysis, but slight improvement may be obtained by the deep application 
of the penetrating Gamma rays from long exposures to radium heavily screened, which act by constricting the capillaries by stimulating the formation of new fibrous tissue.

\section{REFERENCES}

Prxсr, A. E. H. Report of the Radium Institute (Brit. Med. Journ., IGI3, i. p. I.49).

Radiu Ixstitute Report, London, I9I4.

Wicknai, L. Therapeutic actıon of radium (Brit. Journ. Derm., rgog, xxi. p. 203).

WickHam it DEgRAIs. Radium therapy (Dore's Trans., London, Igro). 


\section{CHAPTER NIY}

\section{DERMATITIS DLE TO LOCAL IRRITANTS OF ANIMAL, IEGETABLE AND MINERAL ORIGIN}

\section{DERMATITIS VENENATA}

A LARGE number of irritants of animal, regetable and mineral origin are capable of causing a more or less acute inflammatory reaction in the skin, or dermatitis. This varies in degree according to the virulence of the irritant, the length of time it has been in contact with the shin, the susceptibility of the individual, and the relative delicacy of the skin of the region affected. To this class of affections the name Dermatitis venenata is usually applied and under this heading is included the important group of the "occupation dermatoses " or "trade eczenias."

Certain irritants are capable of causing dermatitis in any skin, such as croton oil and cantharides, but the majority require an individual susceptibility or idiosyncrasy before they can exert harmful influences, and where this exists the slightest exposure may be sufficient to determine an attack. The susceptibility may be due to a congenital defect of the skin, such as ichthyosis where the skin is usually dry, scaly, and vulnerable, or Epidermolysis bullosa where superficial bullae are produced by the slightest external injury ; or it may be acquired from a lowered resistance of the skin from some general illness, or from the pre-existence of some morbid cutaneous condition, such as seborrhoea where the skin is unnaturally greasy and forms a suitable soil for the growth of progenic micro-organisms, or some inflammatory vesicular condition such as Dermatitis herpetiformis or eczema.

The influence of idiosincrasy is clearly shown in connection with dermatoses resulting from various occupations; for example, half-adozen men may be working together in the same bakehouse at the same time and only one of them may suffer from "baker's itch "-a dermatitis due to the action of flour or sugar-or a number of women mar be emplored in a laundry and have their hands and arms constantly exposed to hot water, strong soap, and soda, and in only one or two of them may a dermatitis develop. In a susceptible individual the dermatitis may come on soon after the first exposure to the irritant, or it may not appear till after repeated exposures orer a prolonged 
period; in the case of washerwomen, for example, it may be years before an attack occurs, during which time the macerating effect of the soap and soda in the water may have so reduced the protective power of the horny layer of the epidermis that a slightly excessive exposure may produce it. Once it has occurred the susceptibility may be so increased that the mildest exposure, which in the first instance would have been incapable of doing harm, will be followed by a recurrence which may be more severe than the original attack and may involve, not only parts in contact with the irritant, but also regions upon which it could not possibly have acted directly. To illustrate this increased susceptibility the following case may be cited: A laboratory attendant

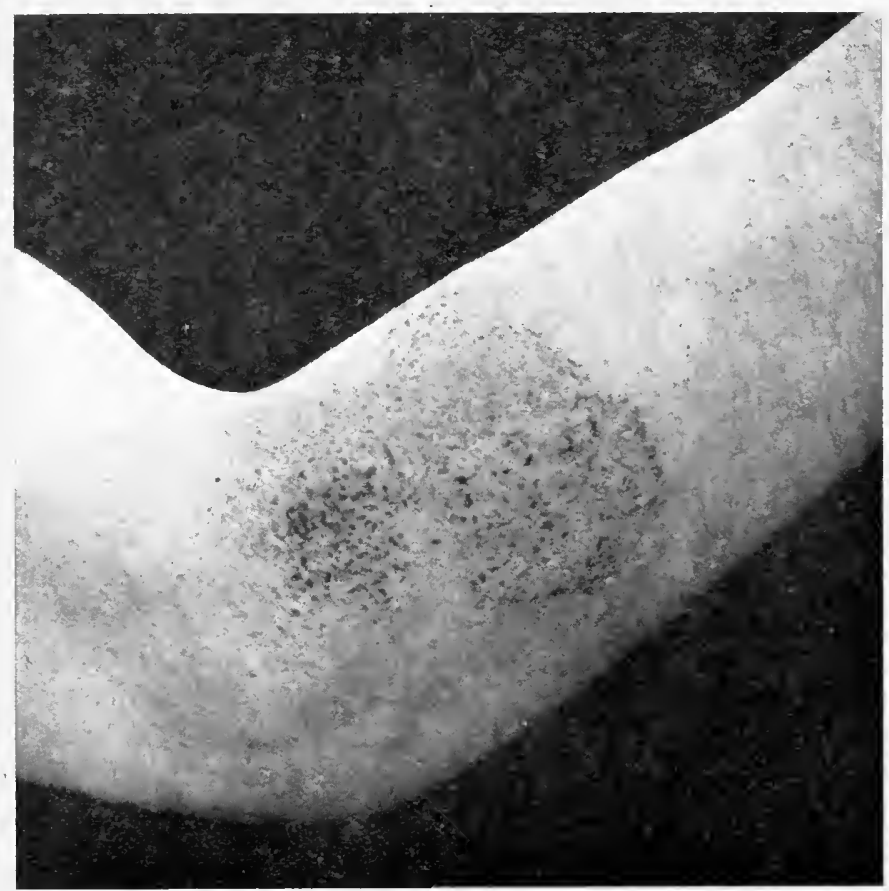

FIG. 56.-Trade Eczema. "Baker's Itch."

was in the habit of preserving specimens in a solution of formalin and had done so for over a year with impunity. One day, after being longer than usual in contact with the formalin solution, he developed a vesicular dermatitis and subsequently his skin became so sensitised to formalin that the slightest exposure to it, even to the fumes from an uncorked bottle, was liable to set up a fresh attack. On the other hand, but far more rarely, after repeated attacks of dermatitis due to some irritant a state of immunity may be established by which subsequent exposures may take place with comparative or absolute impunity. (Fig. 56.)

The severity of the dermatitis varies greatly in different cases, it may consist simply of diffuse erythema, or of papular or follicular 
lesions with burning or itching, or may be more acute and be urticarial, vesicular, or bullous in character, or in the most serere cases of all superficial ulceration or necrosis may supervene either as the result of secondary contamination with pyogenic micro-organisms or from the caustic action of the irritant itself. Provided further exposure to the irritant be avoided and suitable treatment be adopted the eruption usually runs a short course and complete healing rapidly takes place, followed, perhaps, by transient pigmentation.

The precise manner in which the dermatitis is called forth is still under discussion. Certain writers believe that it is due to the direct action of the irritant on the cells of the skin, while others maintain that it is caused indirectly by substances secreted by the epidermal cells in response to the irritant, or in other words that the cells of the epidermis eliminate an anti-body to neutralise or destroy the irritant and that it is this which acts on the endothelium of the blood-ressels and on the connective tissue cells producing an inflammatory reaction of which the dermatitis is the evidence.

In the majority of instances the dermatitis is indistinguishable clinically and histologically from eczema (see page 753). It has been customary in the past to distinguish between dermatitis and eczema and to apply the name "dermatitis" to an inflammatory affection of the skin due to some known external irritant, confined to the area of action of that irritant, and disappearing rapidly under treatment when the irritant is removed, and to reserve the name "eczema" for an affection identical clinically and microscopically with the above but in which the cause is unknown. Such a distinction seems to be untenable, as a dermatitis may persist after the irritant is remored, may occur in situations not directly acted on by it, and recurrences may take place the exciting cause of which cannot be determined-in other words a local dermatitis may become an eczema.

The most usual situations for dermatitis caused by external irritants are the face, sides of the neck, hands, and forearms, but any part of the cutaneous surface may be affected either from direct irritation or from some unknown cause after repeated exposure to the irritant has sensitised the skin. It is impossible to foretell the duration of an outbreak, but first attacks are usually more transient and amenable to treatment than those which occur subsequently.

The irritants which are responsible for dermatitis are so numerous and varied that any attempt to enumerate them here would be futile and incomplete and reference will only be made to a few of the more common.

The name Dermatoconioses has been applied to various forms of dermatitis due to the action of dusts, whether of vegetable, animal, or mineral origin, such as the dusts from hard woods, flax, silicate wool, certain explosives, etc. As a rule the dermatitis is not due to the mechanical action of the dust but to some irritant contained in it or eliminated from it by the action of the sweat, etc. 


\section{Animal Irritants}

The stings of bees, wasps, and hornets, the bites of insects such as bugs and fleas, the burrowings of acari or the larvae of certain flies, are all capable of causing dermatitis, which may be largely due to scratching for the relief of irritation and to the secondary inoculation of pus-cocci. A diffuse dermatitis, urticarial or eczematous in character results from contact with certain caterpillars of the "Woolly bear" type and with jellyfish. Cantharides causes erythema going on to vesiculation or bullous formation. Contact with wool or fur is apt to lead to dermatitis, but this is not due to the wool or fur but to some chemical, such as the bi-sulphide of arsenic, used in dressing it.

\section{VEGETABLE IRRITANTS}

A large number of irritants belong to the vegetable kingdom, such as bacteria and their toxins, fungi and numerous plants and their

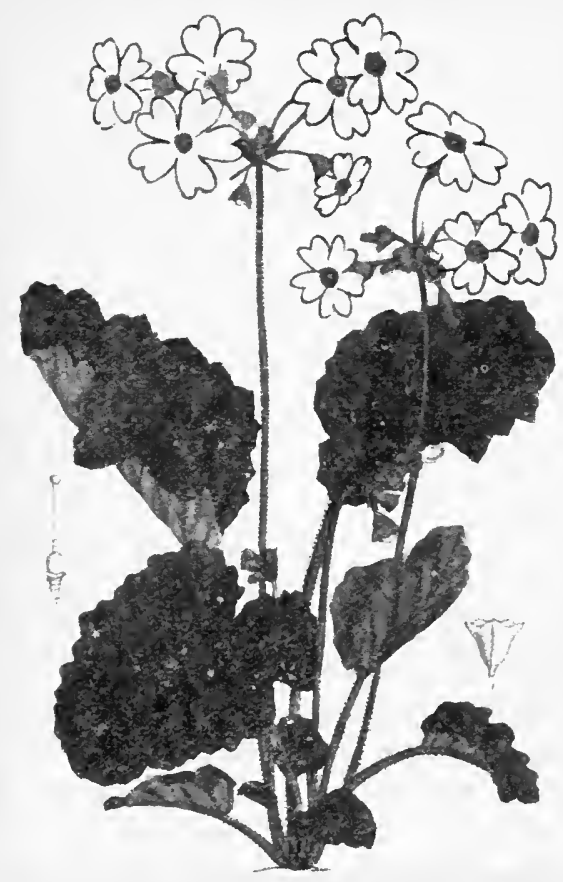

Fig. 57--Primula Obconica.

(From Liotanical Mas., IS8I. Photo by Mr. W. S. Warton.) derivatives. The dermatitis which results from bacteria is described elsewhere (see page 295).

About a hundred plants have already been found capable of setting up derma. titis and their number is gradually increasing, especially from abroad. The type of eruption varies according to the plant and the individual susceptibility, and may be erythematous, vesicular, bullous, or urticarial, and is invariably itchy. In some instances it is the acrid milky sap of the plant which is responsible for the dermatitis, in others the secretion of the glandular hairs about the stalk, leaf or flower. It may be set up not only by touching the plant but indirectly through the medium of any object which may have been in contact with it and have picked up the irritating principle.

Individuals vary in their susceptibility to irritating plants. The skin is particularly sensitive when moist from perspiration or wet from rain, and the plants themselves are more irritating when wet than dry. 
Primula obconica is one of the most common of the "poison plants" in this country, and is a primrose with whorls of small pinkish flowers. Other members of the primrose order may also cause dermatitis, such as the Primula sinensis or Chincse primrose. In susceptible individuals who have to handle these plants an attack of dermatitis is almost certain to occur every spring when the primroses are in flower. (Fig. 5\%.)

The Rhus family is another group of exotic plants which are capable of causing severe dermatitis. Of these the most irritating is the Rhus toxicodendron or "poison oak" of North America where it has long been known as a scourge to those in contact with it ; its poisonous action is the result of a milky juice or sap which becomes black on exposure to the air and contains a highly irritating volatile acid known as toxicodendric acid. Cases of Rhus dermatitis occasionally occur in this country as of late years the plant has come to be used in shrubberies. Other members of the family also irritating to the skin are the Rhus "enenata or " poison ash," the Rhus radicans or "poison ivy," and the Rhus iernix or "poison sumach," from which is obtained the black varnish known as "Japan lacquer." (Fig. $5^{8 .)}$

\section{Narcissi and daffodils}

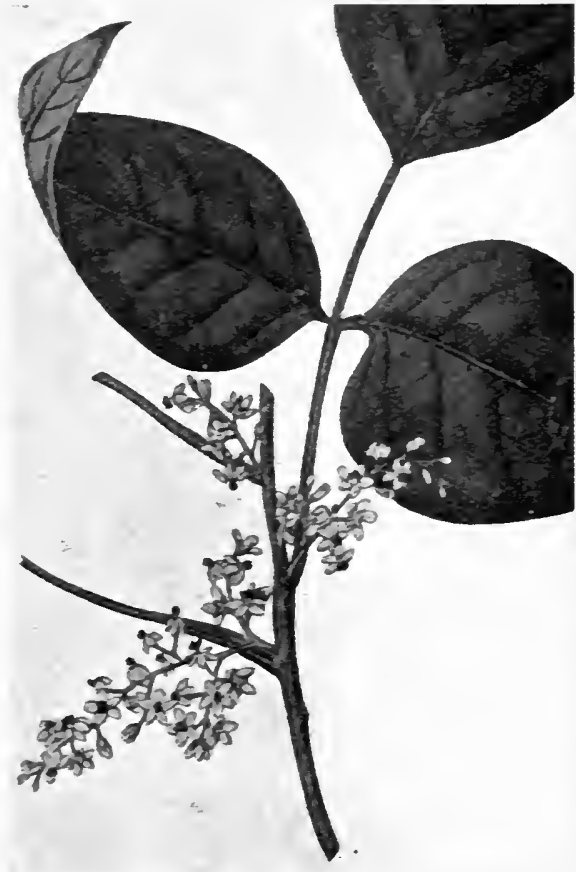

FIG. 58.-Rhus Toxicodendron (Poison Oak). (From Botanical Mag., I\$I6. Photo by Mr. W. S. Warton.) cause a dermatitis, known as "lilyrash," from the handling of the stalks or bulbs; this is believed to be due to the juice in the stalk or to crystals of oxalate of lime surrounding the bulb; it is met with chiefly in the Scilly Islands and affects the hands in the pickers and packers of the flowers. Of the other plants which may set up dermatitis in a susceptible skin only a few of the more common need be mentioned, such as hyacinths, heracleum or con-parsnip, angelica, Balm of Gilead, chrysanthemums, ampelopsis, eucalyptus, iuy, thapsia, hybiscus, fig, rue, and oleander.

The handling of canilla pods is a common cause of dermatitis, known as "vanillaism," among those engaged in packing or cleaning the pods, and may be met with also in sweet factories where the pods are used for flavouring confectionery. 
In Borneo the natives are liable to a severe eczenatous dermatitis from contact with the "Rungus tree," which is due to a resinous substance exuded by the bark.

Sitgar is apt to produce a more or less severe dermatitis which is met with in grocers, confectioners, bakers, and sugar-refiners, and in those employed in packing sweets, boiling beet-sugar, or crystallising candied fruits with syrups. The dermatitis is usually of a chronic and intractable type and may be associated with swelling of the fingertips and changes in the nails in which they become brittle, ridged, and irregular. It occurs more readily in winter than in summer as the skin is thin, apt to be dry and chapped, and in consequence is more

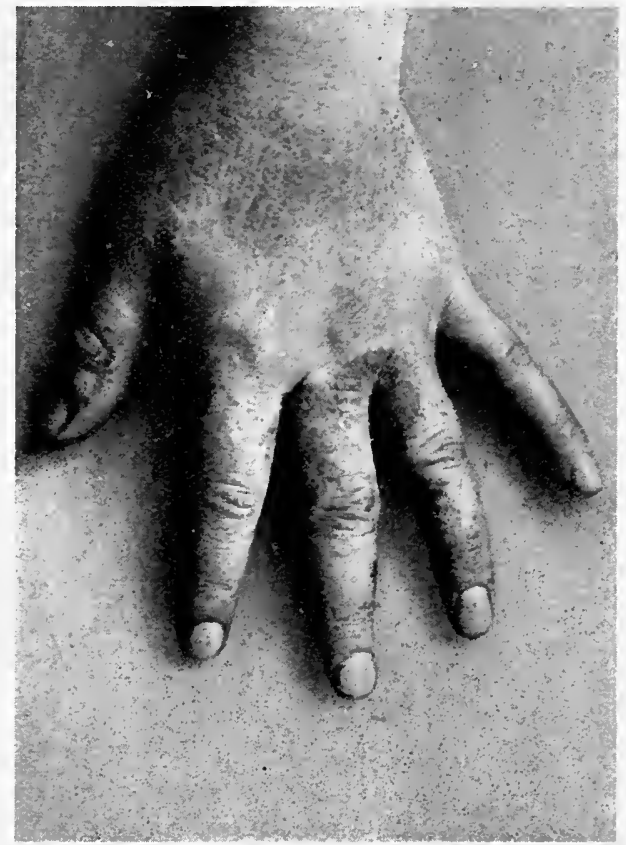

FIG. 59.-Eczematous Dermatitis caused by turpentine.

vulnerable. This dermatitis, as well as others of a cognate nature, is sometimes known as "Dry salters' itch."

Some of the well-known counter-irritants are of vegetable origin and may cause dermatitis, such as mustard, turpentine which gives rise to an erythemato-papular eruption occasionally vesicular, croton oil which causes pustulation, and arnica which may be responsible for an intensely itchy or burning papulo-vesicular rash. (Figs. 59, 60.)

The resinous dust of certain hard woods is a well-known cause of dermatitis among those working with them, such as teak, satinwood, rosewood, boxwood, and cocos-rood used in making flutes. This is due to some alkaloid or essential oil contained in the wood.

Cinchona bark possesses irritating properties and may cause a vesicular dermatitis which is met with chiefly in those employed in 
boiling the bark to make quinine. A similar dermatitis affects chemists from making other alkaloids, such as atropin.

Essential oils are sometimes responsible for eczematous eruptions ; an example of this is the dermatitis which occurs in those employed in cutting up and preserving bitter oranges.

The handling of grain and foreign barley may be associated with dermatoses known respectively as grain itch and barley itch. In the case of grain itch it is not the cereal itself which causes the

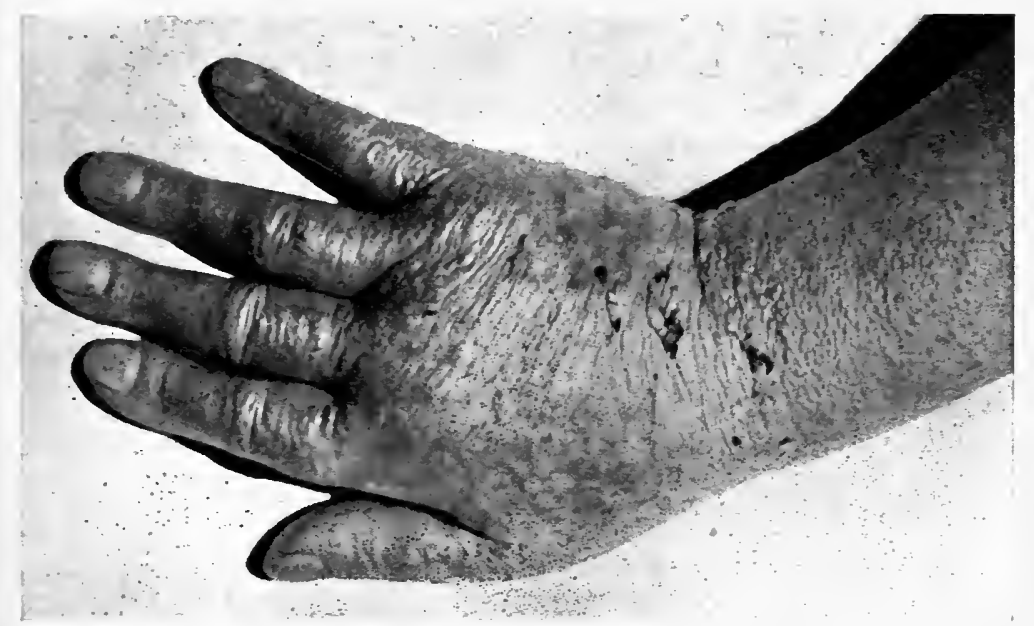

FIG. 60.-Dermatitis from arnica.

Skin inflamed, oedematous, covered with flat vesicles and intensely itchy.

dermatitis but the presence in it of a minute acarus (Pediculoides ventricosus) which on reaching the skin sets up an itchy eruption of an urticarial, vesicular, or pustular, type, and in the case of barley it results from the presence among the barley of an irritating leguminous plant known as the Mucuna pruriens.

\section{Mineral Irritants}

A large number of mineral substances are capable of irritating the skin and producing various degrees of dermatitis, of these the most common, arranged alphabetically, are the following:

Acids and Alkalies.-Acids and alkalies when concentrated have a corrosive action on the skin and set up a severe form of dermatitis which may go on to ulceration and necrosis.

Burns from corrosives may be inflicted purposely, as where vitriol or some other strong acid is thrown in the face with criminal intent to produce disfigurement or blindness; or accidentally, as where acid is spilt in the filling of carboys or where a vessel containing it is broken.

They are distinguished from burns due to heat by being more uniform in character, not giving rise to vesicles or bullae, by the resultant scarring being more marked than that from heat burns of 
the same degree, and by being accompanied by staining of the skin sometimes characteristic of the acid or alkali responsible, for example, carbolic acid causes an initial whitening and puckered appearance of the skin, glacial acetic acid stains it whitish or yellowish, hydrochloric acid greyish-yellow which fades to dirty-reddish-brown, nitric acid yellowish, and sulphuric acid reddish-brown.

Caustic alkalies, such as caustic soda and caustic potash, produce a dirty-brown stain.

The lesions due to acids are more circumscribed than those produced by alkalies, as the acid coagulates the albumin in the tissues and so limits the spread of the burn.

The treatment of burns from corrosives consists of the immediate irrigation of the burn with water so as to wash away any of the irritant which may remain, or with a neutralising solution, such as lime water where an acid has been responsible, and vinegar and water in the case of an alkali, followed by the application of a bland aseptic powder, such as sterilised stearate of zinc. Should sepsis occur any crusts should be removed, the burn irrigated with boric lotion and the powder re-applied.

Aniline dyes, especially black and red dyes, are a not infrequent cause of eczematous inflammation. Not only may the dyers be affected but the wearers of the dyed articles may suffer also and cases of dermatitis caused by dyed socks, gloves, flannel shirts, and drawers, are not uncommon.

Antimony was once used as a counter-irritant and is liable to produce an erythematous or pustular dermatitis when applied in the form of tartarate of antimony, or to have an escharotic action in the form of antimoniun chloride solution. An eczematous dermatitis is met with also among antimony smelters, affecting chiefly the head, face, lips, and beneath the nails.

Arsenic has a singularly irritating action on some skins, producing a more or less severe dermatitis in which pustules and even boils may be present. It is found in those who make arsenic, from the fine powder which is given off in the roasting of arsenical ores; in those who manufacture certain dyes such as arsenical emerald green and fuchsin which is made from arsenious acid and sulphur; among workers in tanneries from the sulphide of arsenic and lime employed to remove the hair from the hides; and among paperhangers, makers of cheap artificial flowers, and calico printers from the arsenic in the dyes. It occurs also from the wearing of cheap dyed apparel.

The sulphide of arsenic is apt to cause a persistent type of ulceration, known by the French as " pigeonneau," in the fingers of those employed in cleaning furs with it.

Aurantia.-Aurantia, or Emperor's Yellow (Ammonium hexa-nitrophenyl-amine), is a basic aniline dye which is used for colouring cheap shoes and other leather articles, yellow. It is a well-known cutaneous irritant and gives rise to a dermatitis of the type of dysidrotic eczema, 
which appears about ten days after the original contact with the dye (see hexa-nitro-diphenyl-amine).

Carbide of Calcium sometimes causes a burning eczematous dermatitis and occasionally punched-out ulcers, chiefly from handling it in the making of acetylene gas.

Chlorine causes in chlorine workers a severe type of acneiform dermatitis known as Chlor-acne, in which a profuse eruption of comedones, sometimes followed by acne pustules, occurs in workmen employed in making chlorine by electrolysis from sodium chloride. The comedones are widely distributed over the cutaneous surface being most profuse on the face, neck, back, buttocks, and genitalia. Associated with them are typical acneiform pustules or superficial cysts connected with the sebaceous glands which tend to break down and suppurate. The condition is due to the irritant local action of the chlorine gas on the skin, and usually affects the face first. As it occurs in covered parts a further explanation is necessary and this has been supplied by Sabouraud who considers that the chlorine gas is inhaled and renders the skin a favourable soil for the growth of the micro-organisms which, he believes, cause the formation of comedones and acne pustules, namely, the micro-bacillus and the Staphylococcus albus.

Chromic acid is a powerful irritant and causes a dermatitis which may go on to ulceration and necrosis. It is met with in painters from use of red or yellow chrome, in French polishers from using bi-chromate of potassium, in compositors and autotypers from the employment of chromic acid for cleaning type and plates, and in its severest form in connection with the manufacture of potassium chromate. The dermatitis may be accompanied by ulceration in the nose-the so-called " chrome ulcer"--which may be quite painless and sometimes leads to perforation of the septum.

Chrysarobin has an irritating action on skin and mucous membrane. It causes erythema of a deep red or brownish tint which extends beyond the area of application of the drug and is followed by brownish desquamation. Occasionally the symptoms may be more severe and vesication or even general exfoliative dermatitis may result. Should it reach the eye it is liable to produce an alarming conjunctivitis.

Coal tar.-Coal tar, pitch, and their derivatives anthracene, creosote, benzine, etc., frequently cause a form of dermatitis known as tar acne or pitch skin. Of this there are various grades. In mild cases erythema is produced followed by deep congestion and subsequent brownish discolouration; this is present in the exposed parts and is associated at times with a yellow discolouration of the conjunctivae. More usually, however, the lesions are acneiform in character, from the plugging of the hair-follicles by tar mixed with dust and horn-cells which leads to a retention of sebaceous material and the formation of a follicular papule or nodule. This is apt to become infected with pus-cocci and to be followed by suppuration. These 
acneiform lesions are met with chiefly on the face, neck, back, chest and arms. The constant irritation of tarry products tends to produce a condition of chronic inflammation and weakened resistance, with the result that the skin becomes prone to eczematous changes and ulceration. In addition warty growths frequently appear about the face, neck and scrotum, and the skin of the palms becomes thickened. As the result of irritation by pitch dust, tarry fumes, or some extraneous cause, such as injury or friction, the tar-warts are liable to increase in size, assume malignant characteristics, and become transformed into epitheliomatous cancer.

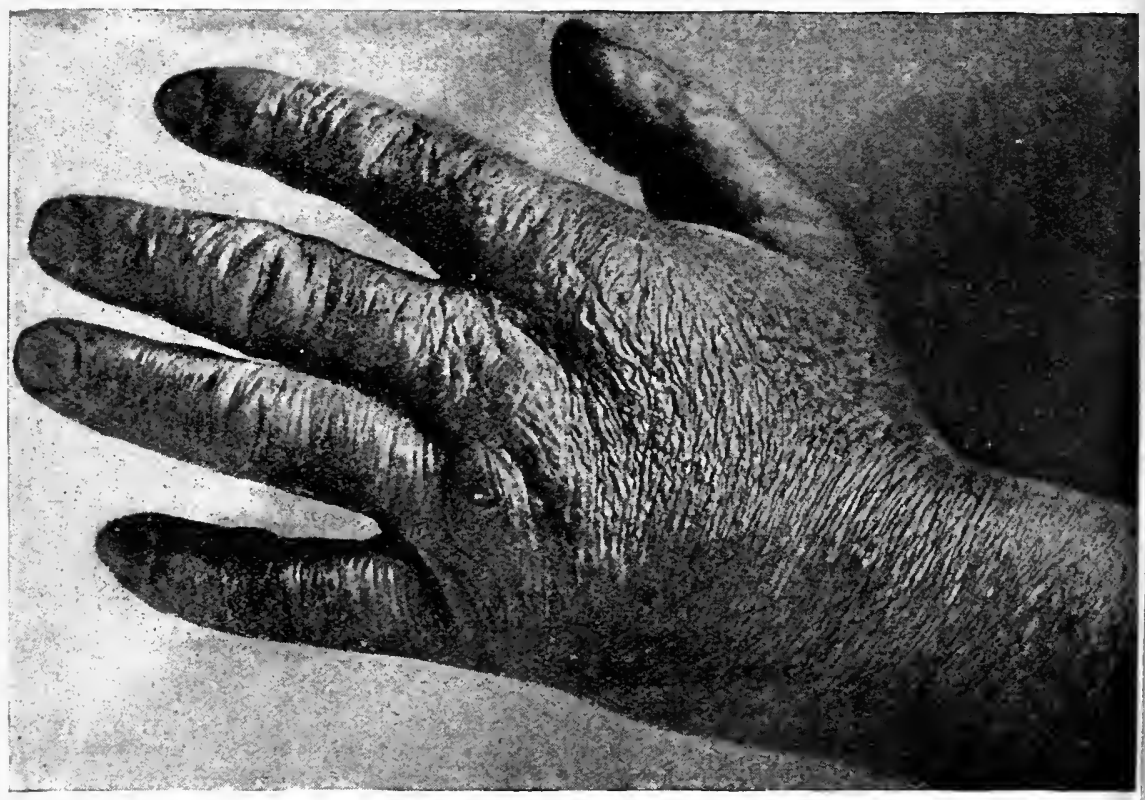

FIG. 6I.-Dermatitis from hexa-nitro-diphenyl-amine.

Ten days after contact with the powder. Sbows oedema and vesication.

(From MacLeod's Burns and their Treatment.)

Hexa-nitro-diphenyl-amine.-Hexa-nitro-diphenyl-amine is an explosive which is closely related to aurantia and is responsible for a form of dermatitis which was met with first in this country in connection with the air-raids in June I9I7, when a number of cases occurred as a result of handling a powder believed to be a mixture of this substance and trinitrotoluene which was contained in the high explosive bombs dropped in these raids. (Fig. 6r.)

In all the cases a staining of the skin took place at the time of contact with the cxplosive, while the dermatitis did not appcar until after a latent period of about ten days. On the ninth day irritation of the skin developed, sometimes so severe as to prevent sleep, and an eruption of deep-seated vesicles like those of dysidrotic eczema appeared on the palms and sides of the hands and fingers, in the inter-digital clefts, and 
sometimes on the soles and between the toes. This eruption usually reached its acme on the fourteenth day.

The vesicles were not associated with much inflammation but there was generally an oedematous swelling of the backs of the hands. They tended to coalesce to form bullae varying in size from a large pea to a hen's egg, and occasionally became septic through secondary contamination with pus-cocci. In about a week they usually dried up and the dead epidermis desquamated in flakes, leaving a shiny pink surface covered with newly-formed epidermis.

The treatment of the condition consisted of bathing the affected parts with warm water or a weak alkaline solution to remove any of the explosive powder present, followed by the application of a calamine cream or zinc paste.

Dust.-The local action of dust and dirt may cause irritation of the skin, and should an abrasion be present the dust may carry in septic micro-organisms and set up suppuration. Fine dust may act like soot and produce in time a warty dermatitis with a tendency to carcinomatous changes.

Formalin is exceedingly liable to cause an urticarial or eczematous dermatitis which may end in superficial ulceration. So sensitised to it may

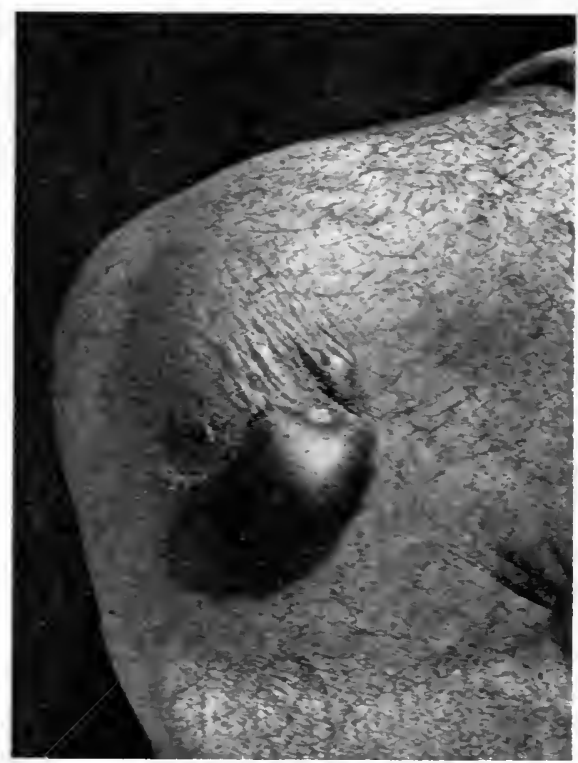

FIG. 62.-Bullous Dermatitis from mercurial in unction. the skin become that exposure to the fumes alone may be sufficient to determine a recurrence.

Iodoform, especially in the form of a powder, is apt to produce a papulo-vesicular eruption which extends beyond the area of application of the drug.

Lead, in the form of lead lotion or ointment, in rare instances causes a severe dermatitis which may be vesicular or bullous.

Lime is a not infrequent cause of eczematous dermatitis among builders, and in the form of Portland cement is particularly irritating. Should cracks or chaps be present in the skin the lime is apt to get into them and transform them into small ulcers.

Mercury, when rubbed into the skin in the form of an ointment occasionally sets up an intense inflammation which may become vesicular or even bullous. (Fig. 62.)

Fulminate of mercury is used in the making of detonators for high 
explosive shells. It is in the form of a powderwhich has an irritating effect on the skin, giving rise to an acute papulo-vesicular dermatitis about the hands and forearms and a red blotchy dermatitis on the face, chiefly affecting the forehead, cheeks, and around the eyes. The

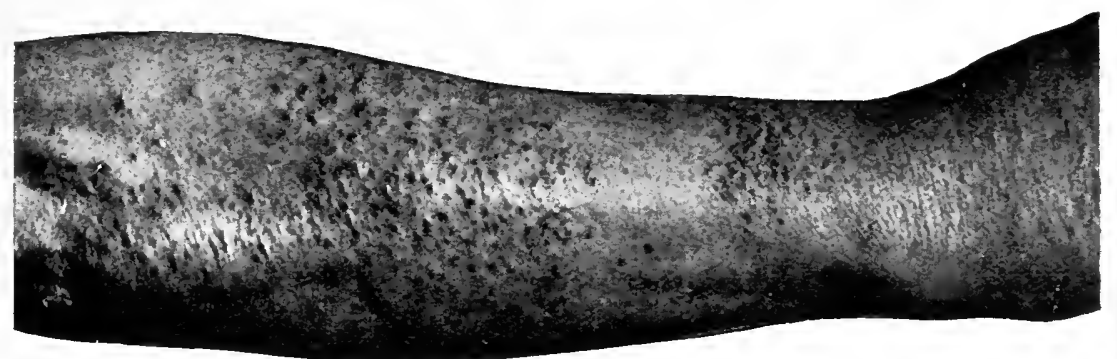

FIG, 63.-Papular Dermatitis of Forearm caused by fulminate of mercury.

(From MacLeod's Burns and their Treatment.)

powder has to be moistened and rubbed up into a paste by certain workers who are liable in consequence to a dermatitis of the hands which may be accompanied by corrosive ulcers about the tips of the fingers and destructive changes in the nails. (Fig. 63.)

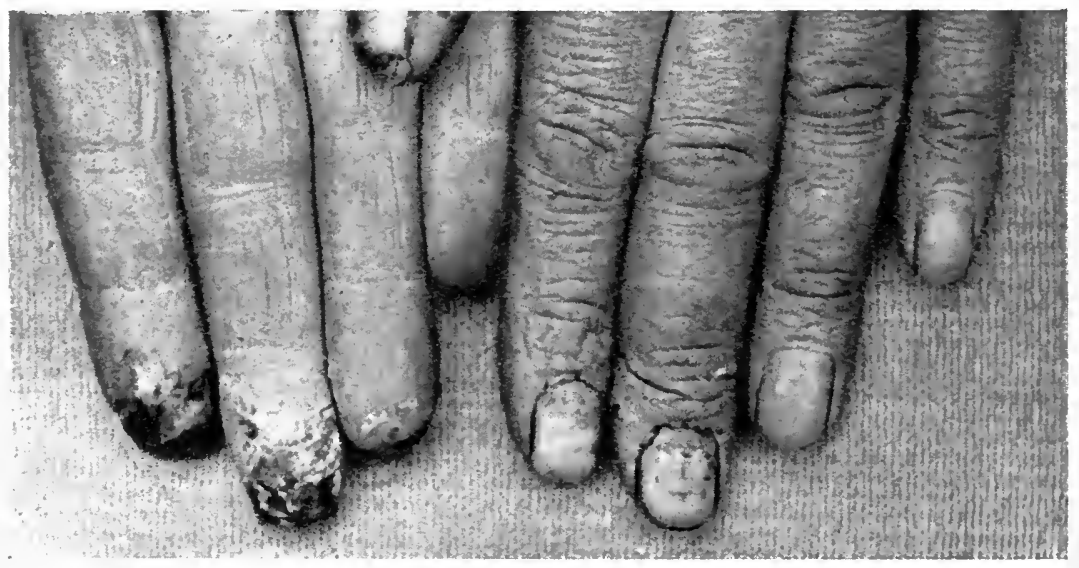

FIG. 64.-Dermatitis of Finger-Tips and Dystrophy of the Nails from oxalate of potash and metol used in developing photographs.

Metol, a chenical extensively used in developing photographs, is apt to cause an erythemato-vesicular dermatitis. (Fig. 64.)

Nitro-glycerine, which is a nixture of nitric acid, sulphuric acid, and anhydrous glycerine, is liable to produce corrosive ulcers at the tips of the fingers and beneath the nails in those making it.

Oil is liable to cause an acneiform dermatitis (Bouton d'Huile) from the plugging of the follicles with oil and dirt. This is met with in stokers, engineers, and machinery hands, from the lubricating oil and 
extremely apt to cause dermatitis, as in washerwomen. Many cases of infantile eczema are called forth or aggravated by over-zeal in its employment.

Soot from hard or stone coal may lead to a warty type of dermatitis in those constantly exposed to it, such as chimney sweeps. It occurs chiefly on the scrotum where the lesions tend to undergo malignant changes, forming the well-known " chimney-sweep's cancer."

Tetryl or "compound explosive" is a virulent skin irritant and causes a dermatitis in a considerable proportion of those working with it. This varies from a simple erythema to marked oedema or vesicular formation and is associated with itching of a continuous and distressing nature. It results from the action of a fine irritating dust which is given off in sieving the compound in the process of making it. It affects chiefly the exposed parts and is liable to be intensified about the face and eyes from rubbing them while at work. Inhalation of the powder may give rise to severe nasal irritation resulting in excessive sneezing and sometimes epistaxis. In addition to the dermatitis tetryl is apt to stain the skin a yellow or apricot tinge. Though the skin may be severely and extensively involved no internal or organic ill-effects are produced.

Thorium nitrate which is used to impregnate gas mantles may cause a painful chronic dermatitis of the hands and arms in which the skin becomes deeply coloured and oedematous.

Trinitrotoluene (T.N.T.) is another high explosive powder which has a direct irritant action on the skin causing, in mild cases, a superficial erythema and desquamation and, in severe cases, a papulovesicular eruption like dysidrotic eczema which is followed by coarse exfoliation. It affects the exposed parts, being specially marked where the skin has been chafed by tight bands as about the wrists and neck. It is most prevalent in hot weather when the skin is moist with sweat. Only some of the workers are susceptible to it but once an attack has supervened a marked idiosyncrasy is established. In addition to the skin symptoms inhalation and absorption of the T.N.T. may lead to serious toxic jaundice. These effects occur chiefly in sieving and filling canisters with the explosive, during which process a cloud of fine dust is given off which not only acts on the exposed parts but insinuates itself over the tops of stockings and socks and down the neck, and sets up a dermatitis in any covered part which it may reach. Like tetryl it tends to stain the skin a yellowish tinge which is most marked in the creases of the palms, which stand out as bright yellow lines. Owing to greater care and cleanliness in working with it the number of cases of T.N.T. dermatitis in explosive factories is now much reduced, and the serious effects which used to accrue from secondary septic complications are now almost unheard of as the dermatitis is early recognised and efficiently treated. 


\section{OCCUPATIONS IN WHICH DERMATITIS IS LIABLE}

\section{TO OCCUR}

A considerable percentage of the skin cases in adults in hospital practice are due to their occupations, and practically every one in which irritating substances have to be handled may give rise to dermatitis. This subject is of intense importance from the medico-legal aspect, for not only may the dermatitis compel the workman to give up work temporarily while a cure is being effected, but the shin may become so sensitised to the irritant that further exposure to it may be impossible without serious consequences and the patient may have to seek for some other employment at the sacrifice of the skill and experience gained in his own trade. Consequently questions of compensation under the Workmen's Compensation Act are not infrequent in this connection. The following are the more common occupations in which dermatitis is liable to occur:

Autotypers are apt to be attacked by severe dermatitis from the employment of bichromate of potash used in cleaning plates.

Bakers frequently suffer from a dermatitis caused by handling flour or sugar, known as "Baker's Itch."

Barbers are liable to an eczematous dermatitis of the hands from constant immersion in soap and water or from the irritating action of hair dyes like Paraphenylene diamine.

Bronzers suffer from dermatitis from the use of bronzing powder which is an arsenical preparation.

Builders are apt to be affected with dermatitis of the exposed parts from working with lime and cement.

Burnishers of silver may be affected by dermatitis from mercury contained in the "rouge" or strong soap used in burnishing.

Carpenters and joiners are liable to dermatitis from working with hard woods, like teak and satinwood, owing to the action of the resinous dust from the wood which contains some irritating alkaloid or essential oil.

Chemists are apt to suffer from eczematous dermatitis due to one or other of the irritants, such as the alkaloids, to which they are exposed in their work.

Compositors are subject to dermatitis from cleaning type with benzine or bichromate of potash.

Chimney-sweeps from the irritation of the soot from hard or stone coal are liable to a warty skin affection chiefly involving the scrotum. The warts have a tendency to ulcerate and eventually to become cancerous (Chimney-sweeps' cancer). The cancer may persist in the scrotum or may pass deeply and involve the testicles, and after a longer or shorter period may spread to the penis and groin glands. This condition is so prevalent that some years ago the Registrar-General estimated that chimney-sweeps were eight times more liable to die from 
cancer than were men in general; though this is possibly a larger proportion than obtains at the present time it merits grave consideration, more especially as the number of cases could probably be reduced were measures of cleanliness more carefully practised and clothes worn which afforded better protection. Fortunately the cancer is not of the most malignant type and there is a fair hope of recovery if the growth be removed early.

Coal-tar or pitch workers may suffer from a form of chronic dermatitis with warty growths-the so-called tar-warts or tar-mollusca -which are liable to develop into carcinomata of the squamous-cell type. The forearms, neck, and scrotum, are the parts generally affected. The dermatitis is due to fine particles from the tar and pitch, to fumes from the melted pitch, and to distillation products such as anthracene, creosote, and benzene. It occurs frequently in workmen in coke and anthracite works, and in manufactories of products of pressed coal such as briquettes.

Cooks may suffer from dermatitis on the face and forearms from exposure to the heat of the fire.

Cotton-winders suffer from an asphyxial condition of the fingers with anaesthetic areas and sometimes necrosis from handling the cotton thread in wet bobbin winding which has been moistened with water containing a small quantity of potash alum.

Dyers are especially liable to eczematous dermatitis, chiefly from the black and yellow aniline dyes and from the " chemic" used to wash the hands which not infrequently contains powerful irritants such as hy pochloride of lime and soda.

Electro-platers may suffer from dermatitis due to a variety of causes, such as the lime dust and olive oil used in the finishing department, the sour beer employed in the process known as "scratchbrushing," the " rouge " powder used in polishing, the " cyanide-dip " used in pickling, and the sulphate of nickel solution employed in nickelplating.

Electro-typers are liable to dermatitis from contact with copper sulphate and hydrochloric acid used in making moulds.

Fish-curers suffer from dermatitis from the action of the brine used in curing fish. It is very common among fisher-girls who sort and pack herrings, when it takes the form of an eczematous or pustular dermatitis sometimes accompanied by painful indolent ulcers about the hands.

Florists and fruiterers are liable to an eczematous dermatitis from handling irritant plants or from the acids or essential oils in fruits.

Furriers occasionally get a severe skin affection with persistent ulceration from sulphide of arsenic and lime used in curing furs.

Gardeners are exceptionally liable to dermatitis from working with irritant plants.

Grocers are subject to it from the handling of sugar and flour in their trade. 
Mill-workers, especially those in flax, jute, or cotton mills, are liable to dermatitis from the irritating action of substances used in cleansing or dying the raw material or oiling the machinery. In flaxspinners the eruption is acneiform in type and is sometimes known as " doffers' eruption," from " doff," a word used in Belfast, which means to remove the bobbins from the machine and to clean and oil them.

Munition workers frequently suffer from dermatitis from making and handling high explosives such as tetryl, T.N.T., lyddite, nitro-glycerine, fulminate of mercury, etc. (see section on Mineral Irritants).

Nurses in hospitals are prone to suffer from dermatitis from the action of antiseptic solutions and from frequently washing with soap and water.

Painters are liable to dermatitis from the aniline dyes and sulphide of arsenic in wallpapers.

Paraffin workers, especially the refiners of solid paraffin, are subject to a warty form of dermatitis from the irritating oily constituents of shale.

Photographers are frequently affected with dermatitis from the Irritating action of pyrogallic acid or its substitutes, such as metol, used in developing photographs, or from the caustic soda employed for cleaning photographic plates, which is liable to affect the nails rendering them brittle, fissured, and raised up from the nail-bed, and occasionally to produce corrosive ulcers about the tips of the fingers.

Polishers of metals are liable to dermatitis of the hands from the "rouge" which they eniploy.

French polishers are apt to suffer from it from the use of bichromate of potassium in staining, turpentine in filling-in, and oxalic acid in stripping.

Silk-winders sometimes suffer from a pustular dermatitis about the fingers from the winding of silk from cocoons softened in hot water. This is due possibly to septic infection of the macerated skin.

Soap-makers are liable to dermatitis from the strong alkalies and acids used in soap boiling.

Surgeons may suffer from dermatitis from the use of carbolic acid and biniodide of mercury specially in spirit solution.

Tanners may suffer from more or less severe dermatitis and even ulceration from bichromate of potash and hydrochloric acid used in their work.

Washerwomen suffer from an eczematous dermatitis of the hands and forearms known as Washercomen's Itch. This is papulo-resicular at first, goes on to oedema, and finally to thickening and cracking of the skin. It is due to the action of soft soap, soda, hot water, and steam. 


\section{TREATMENT}

The treatment of Dermatitis venenata depends on the degree and type of dermatitis and is similar in the main to that described for eczema (see page 776).

In mild cases where only erythema is present this can be reduced by lead lotion, calamine lotion, or calamine cream with or without the addition of I \% carbolic acid to relieve itching, followed by a bland dusting powder. ${ }^{1}$ Should the skin be broken from the rupture of vesicles or bullae a mild antiseptic ointment containing $\mathrm{I} \%$ of ammoniated mercury should be continuously applied to prevent suppuration. Where ulceration is present the crusts and discharge should be removed by warm boric lotion and the ulcers dressed with boric or ammoniated mercury ointment.

As the dermatitis is purely local, internal treatment is unnecessary as a rule.

Those exposed to irritants liable to produce dermatitis should take precautions against it by protecting the hands, where possible, by gloves, or by the application of an adhesive powder such as :

$\begin{array}{llll}\text { Zinci oxidi - } & - & - & \overline{3} \mathrm{i} \\ \text { Calam. prep. } & - & - & \bar{\Xi} \mathrm{i} \\ \text { Amyli - } & - & - & \bar{\Xi} \mathrm{i} \\ \text { Creta. prep. - } & - & - & \bar{\Xi} \mathrm{i} \\ \text { Oleum amygd. } & - & - & \bar{j} \mathrm{ss}\end{array}$

or by hardening the hands with spirit lotion. The face should bc protected by powder dusted over it or by the wearing of muslin veils. Where the irritant is in the form of finely divided dust it is advisable to wear a cap and an overall which fits closely at the wrists without a slit.

Should an attack have occurred it is imperative not to re-expose the skin to the irritant till the dermatitis is cured and the skin has returned to normal. Fven then it must be remembered that a susceptibility to the irritant has been created which may persist for a considerable time and that even slight exposure to it may determine a recurrence. In not a few cases it may be advisable to avoid further risk by seeking other employment.

\section{REFERENCES}

Adamson, H. G. Dermatitis due to explosives used in air-raids (Brit. Med. Journ., April 29th, I916, p. 618).

Bunch, J. L. Dermatitis due to Paraphenylene diamene (Brit. Journ. Derm., I9I5, xxvii. p. 348 ).

Cripps, LuCy. Properties of tetryl (Ibid., I9I7, xxix. p. 3).

Dubreullh. Des Eruptions orthoformiques (La Presse Méd., I90i, xl.).

Foerster, O. H. Primula dermatitis (Trans. Amer. Med. Assoc., June, I9IO).

${ }^{1}$ For the formulae of these applications see Appendix. 
Friedlander, A. Thorium dermatitis (Arch. f. Derm. u. Syph., rgra, cxiii, p. 359).

Halt, A. J. Muitiple ulcers of hands due to occupation (Brit. Journ. Derm., I90I, xïi. p. 209).

Hornsey, J. F. Rungus dermatitis (Brit. Med. Journ., I9r 4, p. 759).

KNowles, F. C. Occupational eczemas (Journ. Cut. Dis., I9I3, xxxi. p. $x r$ ).

MacLeod, J. M. H. Occupation dermatitis (Accidents in their MedicoLegal Aspect London, I9ro).

- Dermatitis from handling German bombs (Brit. Med. Journ., July 2rst, r9I 7, p. 8o).

Mrnistry of Musitions. Trinitrotoluene poisoning (Ibid., Dec. I6th, I9I6, p. 842).

Nixos, J. A. Cotton-seed dermatitis and its cause, etc. (Bristol Med.Chir. Journ., June 1915, p. 73).

Rolleston, H. D. The origin, symptoms, pathology, treatment, and prophylaxis, of toxic jaundice observed in munition workers (Discussion at the Roy. Soc. Med., London, Jan. I9I 7 , at which a numbe: of authorities spoke, vide Trans.).

Ruxtox, W. L. Industrial diseases due to tetryl (Brit. Journ. Derm. \& Syph., I9I7, xxix. p. I8).

Sequerra, J. H. Dermatitis due to explosives used in air-raids (Brit. Med. Journ., Aug. th, I9I 7, p, I 48 ).

SMrth, Exid. Prevention, symptoms, and treatment, of tetryl dermatitis (Ibid., April 29th, I9r6, p. 6r8).

White, J. C. Dermatitis venenata (Journ. Cut. Dis., I903, p. 44 I).

White, R. Prosser. Some new forms of occupation dermatoses (Lancet, Feb. Igth, I9r6).

- Occupational affections of the Skin, 2nd edition (Messrs. H. K. Lewis, London, 1920).

Wruls, W. K. Barley itch (Brit. Journ. Derm., I909, xxi. p. 249).

\section{BEDSORES}

Definition.-Bedsores are superficial ulcerations or necroses which result from pressure or friction on the skin when it is devitalised by some general or local disturbance interfering with the innervation and circulation of the part.

These intractable sores were once the common accompaniment of confinement to bed for any prolonged period, and were especially frequent in affections of the nervous system associated with paralysis and impairment of movement. At the present time, however, thanks to improved nursing and suitable preventive measures they are rarely met with unless in the case of old neglected bed-ridden people.

The sore begins as a dull red inflamed patcli which, through inoculation with pyogenic micro-organisms, may suppurate and form an unhealthy superficial ulcer or may undergo necrosis and separate in the form of a greyish slough. The pain associated with it is sonetimes considerable but varies greatly in different cases. The sore may be superficial and involve only the skin, or deep and lead to destruction of the underlying muscles, tendons, or even bones.

They occur chiefly in situations subject to continuous pressure from lying in the recumbent position, such as over the lower vertebrae, 
the sacrum, or the heels, but may be met with in any part of the cutaneous surface from friction or pressure, such as may be caused by an imperfectly padded or badly applied splint.

Treatment.-In this connection prevention is more important than cure and if proper preventive measures be adopted bedsores should rarely, if ever, occur. It is of the first importance that the skin should be kept scrupulously clean, as the most potent factors in the production and aggravation of these lesions are the soiling and maceration of the skin by the excreta and the inoculation of septic micro-organisms. Where the skin is not markedly inflamed the sites of pressure should be rubbed over daily with methylated spirit to harden them, and an antiseptic dusting powder, such as zinc and boric acid, or bismuth subgallate, applied subsequently. Should the skin be inflamed, the alcohol may prove too irritating and boric lotion should be substituted. Every effort should be made to relieve pressure by the use of air cushions, water beds, etc. Splints should be carefully padded and properly applied. Where possible the patient should not lie too long in one position and if incapable of voluntary movement gentle passive movements or massage may be employed to encourage the circulation.

When sores have formed they are singularly intractable and unless actively dealt with are liable to be progressive. Where ulceration has supervened compresses of boric lotion or hydrogen peroxide (Io vols.) should be applied twice daily, and the part dressed with ammoniated mercury ointment or paste. Where sloughing is considerable dead tissue should be removed, pus evacuated, and the lesions treated on ordinary surgical principles.

\section{DERMATITIS ARTEFACTA}

\section{Syn. : Dermatitis factitia; Feigned cruptions.}

It is not uncommon for lesions and injuries of the skin to be selfinflicted, either for purposes of deception, or innocently from a habit of picking the skin to remove a follicular plug or crust, or from an obsession that some foreign body or insect is present in the skin and must be picked-out to get relief.

The neurotic excoriations are generally produced by the nails, are usually present on exposed parts which are readily accessible, and are followed by scarring. One of the commonest causes is the conviction on the part of the patient that he is infested with some insect (acarophobia) which he must dig out, and it is not uncommon for such individuals to bring with them to the physician packets of dried-up scales or debris which they have picked off the skin and believe to be the remains of the insect.

The lesions caused with intent to deceive occur most frequently in hysterical girls or young women, and are usually produced with the object of exciting sympathy or avoiding work. They are by 
no means confined to women, however, but may occur for similar reasons in weaklings and malingerers of the male sex. In women they are often associated with a markedly neurotic temperament, eridenced

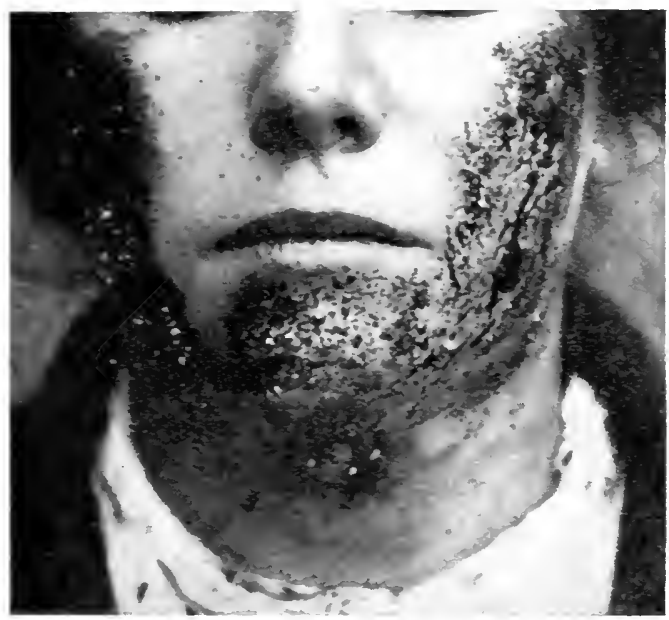

FIG. 66.-Dermatitis Artefacta.

Lesions caused br scissors.

by attacks of hysteria, anaesthetic patches in the skin or complete anaesthesia of the soft palate, and occasionally with a highly sensitive neuro-cutaneous reflex on account of which the skin reacts so readily

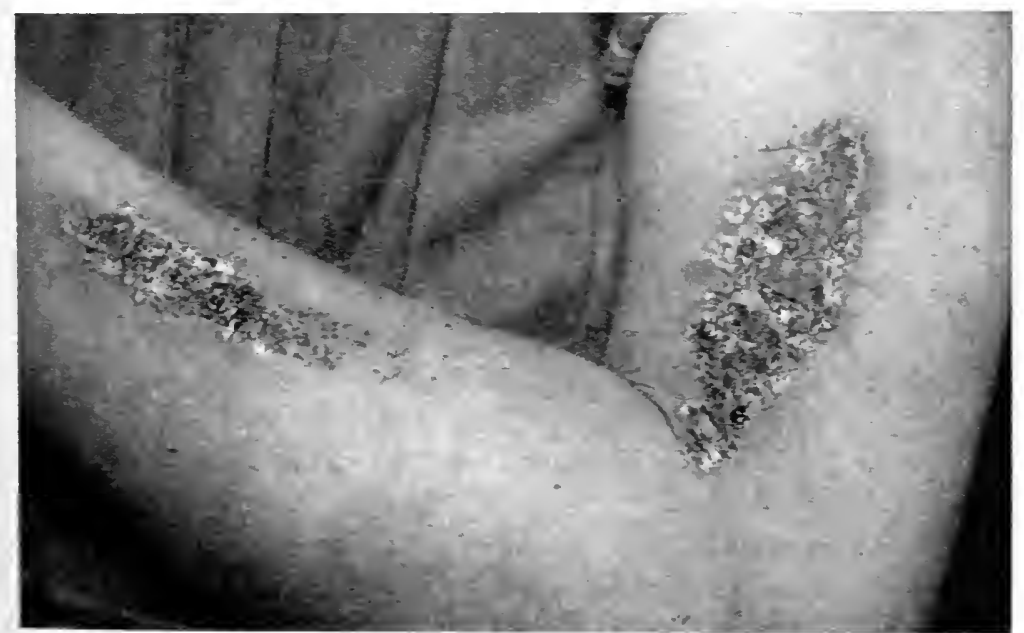

FIG. 67.-Dermatitis Artefacta.

Elongated excoriations in a neurotic girl, made by rubbing with the fingers.

(From Dr. H. G. Adamson.)

to external stimulation that artificial lesions may be easily induced without arousing suspicion. The neurosis may be natural, or acquired as the result of some debilitating illness. In other cases the patient 
may be rather phlegmatic than otherwise and may appear to be in excellent general health. (Fig. 66.)

As a rule the underlying motive is patent or easily ascertained, but there are cases in which its discovery baffles the most vivid imagination, as when it occurs in individuals who are slightly peculiar, on the border-line of insanity, or under the influence of some subtle psychical disturbance or perverted emotion. (Fig. 67.)

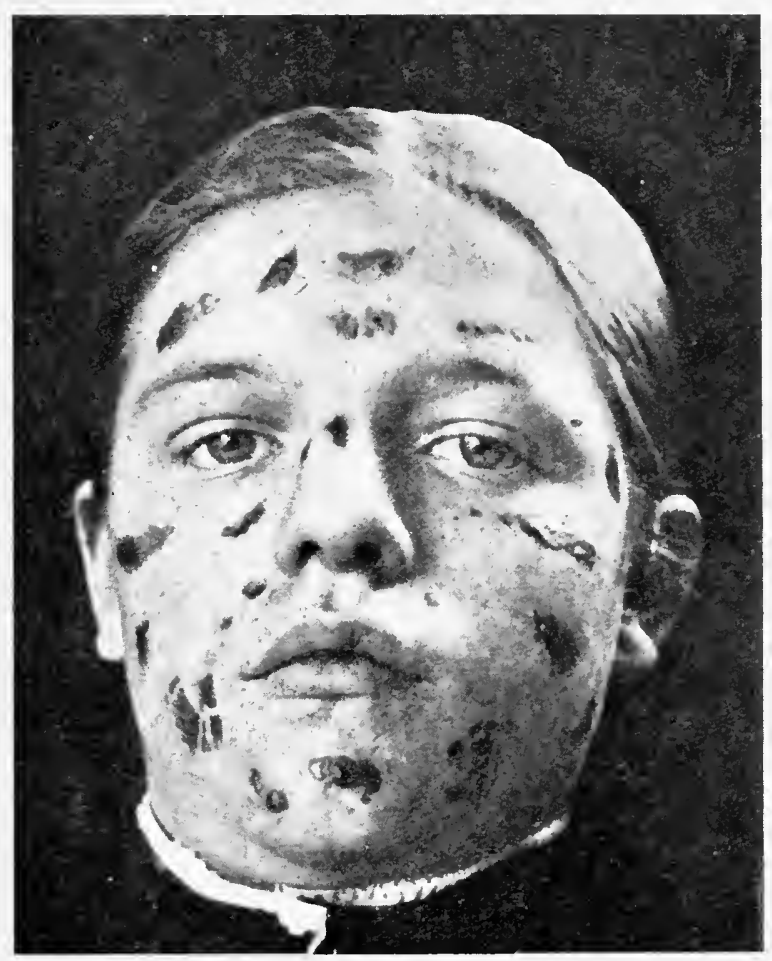

FIG. 68.-Dermatitis Artefacta.

(Dr. T. Colcott Fox's Case.)

In men the most common motives are, to shirk work, to obtain insurance benefit, or to evoke sympathy, and it is met with chiefly among artisans, labourers, sailors, soldiers, beggars, and criminals.

Artificially produced lesions are usually few in number and differ greatly in type according to the agents used to produce them. They may consist of erythematous patches suggesting Erythema multiforme, they may be vesicular and simulate herpes or eczema, or they may show a peculiar parchment-like necrosis which is very characteristic ; again they may be bullous with an inflammatory base, or they may take the form of superficial ulcers or gangrenous sores. They vary indefinitely in shape, but are not infrequently oblong with the long axis down the limb, horizontally across the chest, or slanting downwards on the 
abdomen, as in cases where they have been produced by the persistent rubbing in by the fingers of some common irritant such as spirit, salt, urine, or saliva. Occasionally they present the shape of the agent used in making them, as when they are caused by a penny soaked in vinegar or by a piece of mustard plaster. (Fig. 68.)

When they take the form of superficial ulcers they have generally a well-defined irregular border and are caused by some strong acid or alkali, while if they be gangrenous patches they are the result of some powerful escharotic. Many of the cases which have been described in the past under the headings of "neurotic gangrene" or " Dermatitis gangraenosa" belong to this category.

The most common sites to be affected are the anteroexternal aspects of the limbs, the face, more rarely the trunk, and the lesions are frequentiy limited to one side of the body and usually to parts accessible to the right hand. (Fig. 69.)

A great variety of agents may be used for this purpose and it may be difficult or impossible to identify them from the appearance of the lesions. Such crude methods may be adopted as digging out pieces of skin by the finger nails or some sharp instrument, snipping pieces with a pair of scissors, or burning with a match, hot coin, or by some other means. At other times irritants and caustics are employed, among the most com-

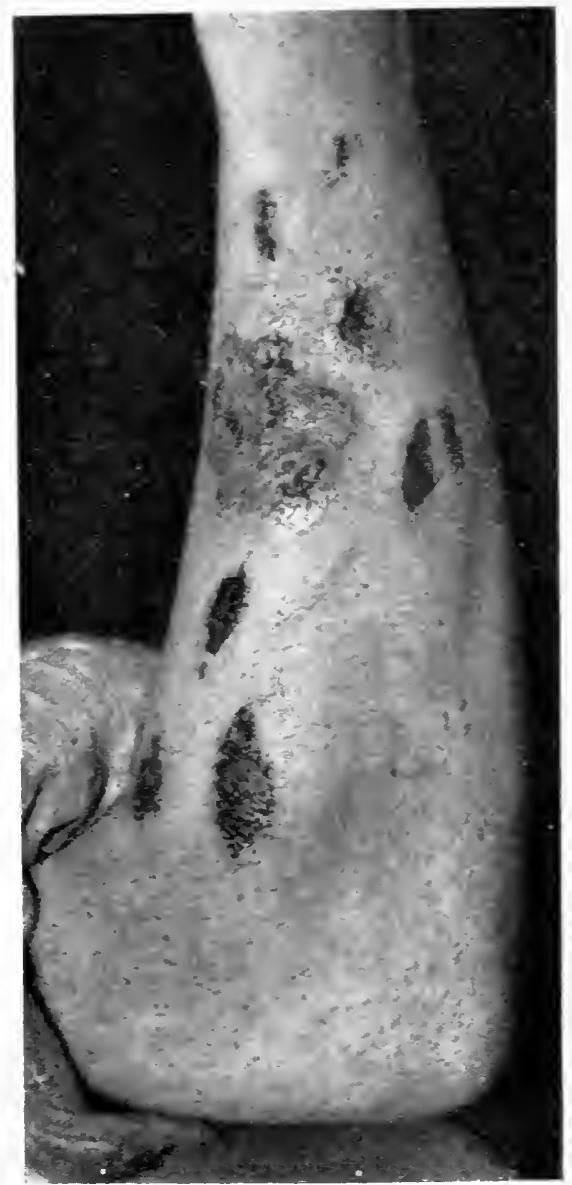

FIG. 69.-Dermatitis Artefacta.

(Dr. H. G. Adamson's Case.)

mon being carbolic acid, acetic acid, common salt, caustic soda or potash, croton oil, cantharides, turpentine, and arnica. Occasionally the idea of producing the lesions is suggested from the previous legitimate use of some irritant, which had caused a dermatitis on account of which the patient had been prevented from working and had received undue sympathy. In connection with occupations which necessitate exposure to irritating substances and where the workers are consequently liable to dernatitis, unnecessary exposure may be 
indulged in so as to perpetuate or aggravate it in order to avoid work, or to obtain compensation or sick pay. (Fig. 7o.)

Diagnosis.-As a rule there is no great difficulty in recognising a factitious dermatitis. The lesions usually have something peculiar about their characteristics, distribution, or course, which differentiates them from those of any ordinary skin disease. They tend to come out suddenly and at irregular times according as the patient has the

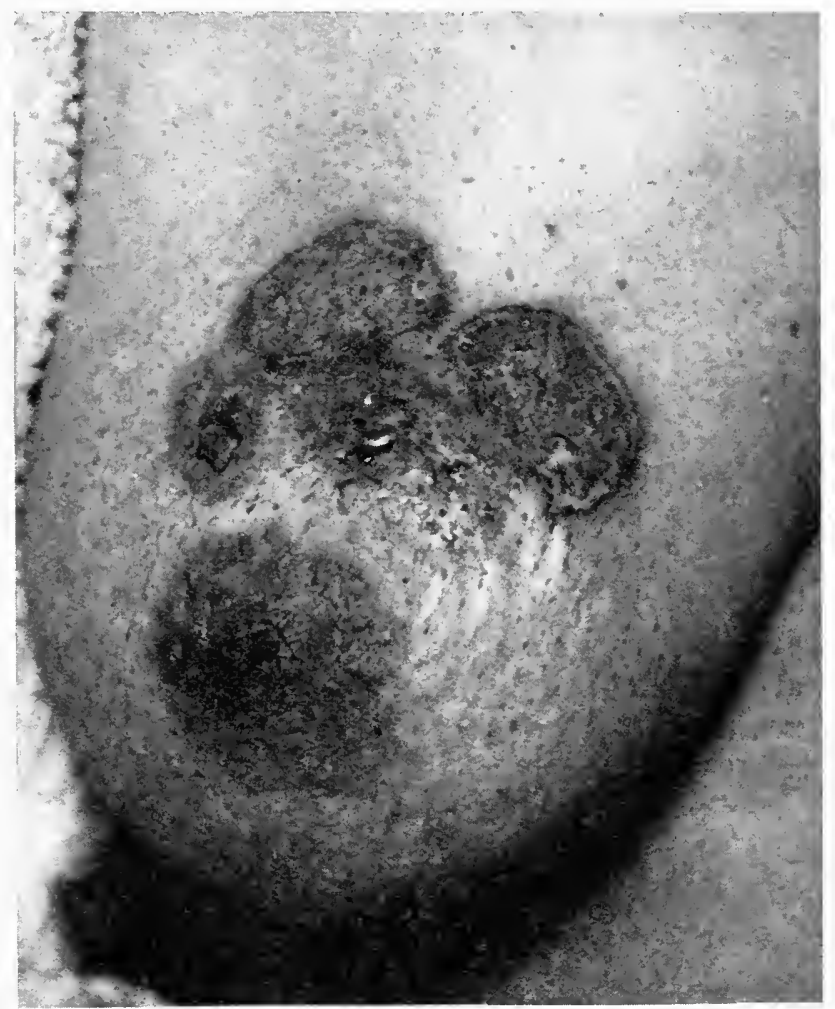

FIG. 70.-Dermatitis Artefacta.

Superficial ulcerations on breast, produced by a caustic.

(Dr. H. G. Adamson's Case.)

opportunity or the requisite privacy to produce them. As would be expected they are most liable to be caused during the night and to be found in the morning. When some caustic has been the agent its odour may cling to the lesions, or evidences of it may be seen in the neighbouring skin or clothes, or on the fingers. ${ }^{1}$ As a rule artificial lesions heal readily when covered with a fixed dressing, while fresh ones keep on appearing beyond the dressing or in some other region. In many cases the type of patient is an aid to the diagnosis for they are often

1 The reaction of the skin should be tested with a piece of moist Litmus paper in any case where the eruption resembles no known disease, as this may give a clue to the employment of some acid or alkaline caustic. 
neurotic or peculiar, have a furtive look when questioned, or may show definite signs of malingering.

Treatment and Management.-The treatment of cases of artificial dermatitis often taxes the patience and resource of the most skilful physician, for the ingenuity, perseverance, and fortitude exhibited are often remarkable and the lesions may be kept active or new ones produced indefinitely until the patient either attains some special object, or gives it up as hopeless, or is deterred by the disfigurement.

Unless the artificial nature of the lesions be obvious and the motive known it will generally be inadvisable for the physician to reveal his suspicions to the friends, as he will almost certainly have to face a denial on the part of the patient and the opprobrium of some member of the family. Even when close surveillance has enabled him to discover the agent employed, or the motive, or even to catch the patient in the act of causing them, it may be the wisest policyespecially if the patient be a neurotic girl-to promise not to disclose the nature of the affection provided no more lesions appear. Such questions must be left, however, to the discretion of the physician.

Where an artificial dermatitis is suspected but not proved, the assistance of an intelligent nurse or attendant may be of the greatest service in discovering it.

The treatment of the actual lesions is simple, varies according to the type of lesion, and consists as a rule of antiseptic applications, or occlusive dressings of which one of the most generally serviceable is zinc gelatine.

\section{REFERENCES}

Galloway, J. Feigned diseases of the skin (Allbutt and Rolleston, System of Medicine, I9II, p. 9 I).

Hy̌de, Nevixs. Bleeding stigmata (Journ. Cut. \& Gen.-Urin. Dis., I897, xv. p. 557).

Latte. Multiple neurotic gangrene (Monats. f. prakt. Derm., Igof, p. I 89).

Ormsby, O. S. Factitious dermatoses (Journ. Amer. Med. Assoc., Nor. I915, lxv. p. 1622).

Rasch. Hysterical skin affections (Derm. Centralb., I899, p. 322).

Shepherd, F. J. Cases of feigned eruptions (Journ. Cut. \& Gen.-Lrin. Dis., I 897, p. 544).

VAx HARLINGEx. Erythematous hysterical dermato-neurosis (Inter. Med. Mag., Nov. i897, p. 695).

WaLker, N. Dermatitis artefacta (Brit. Med. Journ., June, I9Io, p. I 8 I).

Wilson, Erasmus. Neurotic excoriations (Lectures on Dermatology, London, I875). 


\section{CHAPTER XV}

\section{DISEASES OF THE SKIN DUE TO VEGETABLE PARASITES}

A LARGE number of cutaneous affections are due to parasites of the vegetable or animal kingdoms. Such affections are invariably local, are curable with the destruction of the parasite and the removal of its effects, and do not interfere with the general health however extensive and widely spread they may be, except in rare instances where the local condition has passed on to a general infection and septicaemia.

In this section only the parasitic diseases which occur in temperate zones will be considered, while those which prevail in tropical and subtropical countries will be described in the section on tropical skin affections.

The vegetable parasites which give rise to cutaneous lesions belong to the group of the thallophytes which are plants with a simple cellular structure and a more or less branched thallus. They live a parasitic existence on the skin and the lesions they produce are the evidences of the reaction of the skin to their presence. Certain of them attack lower animals and may be transmitted from them to man. They can also lead a saprophytic existence on decayed or dead matter and can, as a rule, be grown on artificial media.

The principal vegetable parasites of the hurnan skin may be classified as follows :

\section{The Principal Vegetable Parasites Pathogenic on the Human Skin}

\section{Thallophyta.}

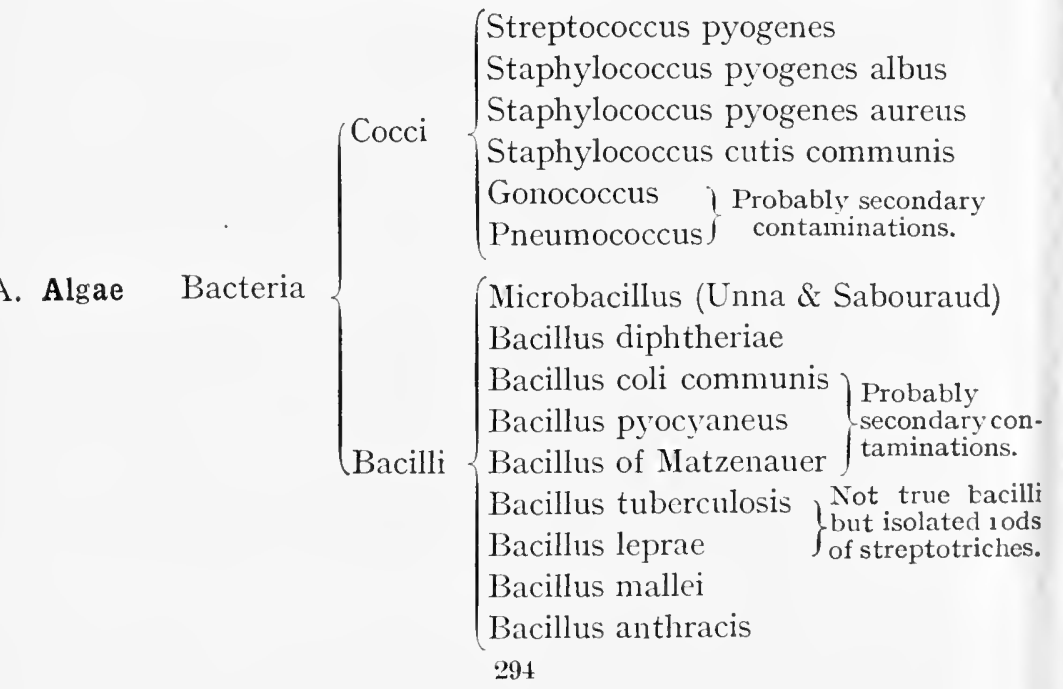




\section{CUTANEOUS AFFECTIONS OF STREPTOCOCCIC ORIGIN}

\section{INTRODUCTION}

Ever since erysipelas was found to be caused by the " Streptococcus erysipelatis" of Fehleisen the importance of streptococci in the causation of dermatitis has been appreciated, but it is only of recent date, and owing to the researches of Sabouraud and others, that the multiformity of lesions which streptococci are capable of producing has come to be recognised.

The streptococci, of which there are several main varieties, are indistinguishable from each other microscopically or in culture on

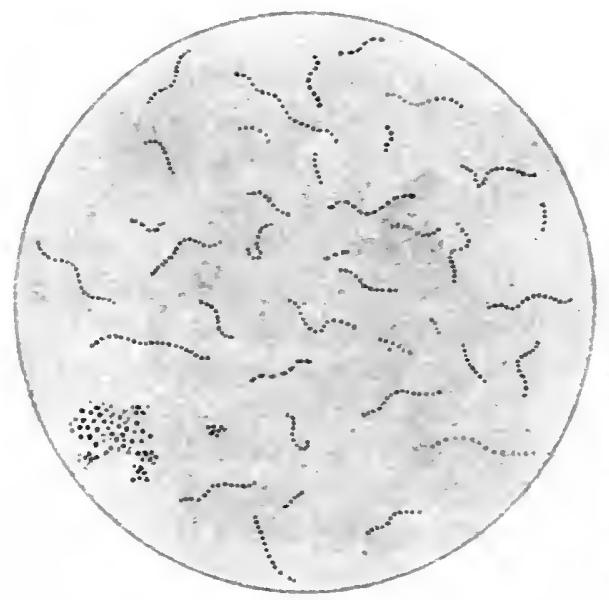

FIG. 7I.-Streptococci from Culture in Broth from vesicle of Impetigo contagiosa ( 16 hours) and colony of staphylococci.

ordinary media but they may be differentiated by the reactions which they produce in certain carbohydrate media. In this way several definite groups have been distinguished and of these the most important in connection with cutaneous disease are: the Streptococcus pyogenes with which the Streptococcus erysipelatis (Fehleisen) is known to be identical ; the S. faecalis of the intestine; the S. salivarius of the mouth and intestine, a variant of which is the S. anginosus of sore throat. Closely allied to them is the pneumococcus which may possibly be pathogenic in the skin. The streptococci may occur in the skin as long or short chains, or in a less mature form as diplococci or isolated cocci.

The Streptococcus pyogenes is responsible for a large proportion of the cutaneous affections of streptococcic origin, but the S. faecalis and the S. salivarius may, on occasion, produce similar lesions. (Fig. 7I.)

In normal circumstances, streptococci, unlike staphylococci, are not present to any extent on the cutaneous surface and when they do 
occur lead a harmless saprophytic existence, and only become pathogenic should they penetrate through a break in the horny layer either from some gross lesion or from an abrasion so slight as not to be detected. Sometimes instead of gaining entrance at the surface the microbe may reach the skin from some deep-seated focus of disease by spreading along the lymphatics, as when the skin is involved in septic periostitis. On the other hand in cases of septicaemia where a systemic invasion has taken place embolic showers of streptococci may become deposited in the skin through the general circulation.

The type of lesion produced by the streptococcus is dependent to a large extent on the precise situation of the micro-organism in the tissue. Should it be confined to the epidermis a phlyctenular impetigo results, if mainly present in the lymphatics of the corium erysipelas may be produced, while if it occurs deep down in the subcutaneous tissue it may be responsible for deep cellulitis and phlegmon. There are also other determining factors, namely the power of resistance of the skin affected, the site of infection, and the degree of virulence of the special strain of streptococcus-the more virulent the micro-organism the more actute being the type of lesion caused by it.

Pathology.-The pathological action of the streptococcus in the skin consists mainly in the production of a sero-fibrinous exudation and its consequences. When present in the epidermis the initial lesion is, as a rule, vesicular from a chemotactic action caused by the microorganism, by which a serous exudate is attracted to its site; this is an example of an effort on the part of nature to destroy the invader and counteract its effects. In the corium its presence in the lymphatic spaces causes a serous exudation with dilatation of the tissue spaces and a colliquative degeneration of the fibrous stroma. In the subcutaneous tissue a similar effect occurs in the septa between the fat lobules, with the production of cavities containing sero-purulent contents. The streptococcus does not tend to attract leucocytes towards it to the same extent as the staphylococcus, and when purulent lesions occur they are generally the result of a secondary contamination with staphylococci.

For convenience of description the dermatoses of streptococcic origin may be grouped as follows :

\section{A. Cutaneous AfFections due to the Inoculation OF STREPTOCOCCI TḦROUGH THE EPIDERMIS}

\section{Streptococci situated chiefly in the epidermis.}

r. Impetigo contagiosa or Phlyctenular impetigo.

Varieties: Penuphigus contagiosus tropicus.

Pemphigus neonatorum.

Dermatitis exfoliativa infantum.

Dermatitis exfoliativa epidemica.

Pcmphigus acutus? (See Pemphigus). 
2. Furfuraceous or Pityriasic impetigo, including certain cases of intertrigo.

3. Ulcerating impetigo of streptococcic origin, including Ecthyma, Dermatitis gangraenosa infantum, Vacciniform dermatitis.

\section{Streptococci situated in the corium and subcutaneous tissue.}

I. Erysipelas, ordinary acute type.

2. Recurrent erysipelas.

3. Persistent lymphatic oedena.

4. Whitlow.

5. Deep cellulitis and phlegmon.

Note.-The more deeply seated lesions may also result from the spread of the streptococcus to the surface via the lymphatics from some underlying septic focus.

\section{B. Cutaneous Lesions due to Streptococci Reaching the Shis via the Blood Stream in Septicaemia}

I. Haemorrhagic erythema and certain purpuras.

2. Vesicular and papulo-resicular lesions.

3. Frythematous swellings like Erythema multiforme, due to streptococcic thrombosis of subcutaneous reins.

\section{Cutaneous Affections deE to the Circliation in the Blood of Streprococcic Toxins}

Erythematous lesions of the type of Erythema multiforme or of the more generalised scarlatin:

\section{REFERENCES}

Axdrewes, F. W. Evolution of Streptococci (Lancet, I906, ii. p. If I5). Axdrewes \& HoRder. Streptococci pathogenic for man (Lancet, I906, ii. p. 708$)$.

Gordox, Mi. H. (Lancet, I905, ii. p. Ifoo).

PAxtox, P. X., \& ADAMS, S. E. Etiology of Erysipelas and allied infections (Lancet, I909, ii.).

\section{IMPETIGO CONTAGIOSA}

Syn.: Impetigo vulgaris, Streptococcic impetigo, Phlyctenular impetigo.

Definition.--An inflammatory affection of the skin characterised by the presence of superficial vesicles containing serum; these readily. rupture, and the serum coagulates to form honey-like crusts loosely. stuck on the skin like wafers.

Etiology.-Impetigo contagiosa (Tilbury Fox) is the direct resuit of the inoculation and presence in the superficial layers of the epidermis 
of the Streptococcus pyogenes. This organism may be obtained in pure culture from the serum of a superficial vesicle provided a suitable technique be employed, and the disease may be experimentally reproduced by inoculating the skin with the culture.

The technique recommended by Sabouraud for the cultivation of the micro-organism is as follows: The surface of an early unbroken vesicle is cleansed with alcohol and the serous contents removed by pricking it with a sterilised pipette. One cubic centimetre of the serum is then mixed with I c.c. of neutral peptone bouillon and incubated at $37^{\circ} \mathrm{C}$., thus reproducing anaerobic conditions similar to those which occur in the actual lesion. In twelve hours a pure culture is obtained, showing chains of streptococci a certain number of which are in diplococcic form. If the serum be taken from older lesions, in addition to the streptococci, masses of staphylococci will be found in the medium; these are secondary contaminations. If solid media be employed a culture of staphylococcus is generally the result, as that organism grows much more luxuriantly on solid media and either hides or kills the more delicate and slower-growing streptococcus. A mixed culture may be obtained, however, on solid media by the simple expedient of removing the crust, rubbing the raw surface with alcohol, inoculating a bead of the serum which exudes into the water of condensation, and then allowing it to run over the surface of the medium. After the tube has been incubated for 24 hours, small white colonies of streptococci may be seen among the larger staphylococcic colonies.

The inoculation of the streptococcus on the human skin generally takes place through a slight abrasion of the horny layer. It may possibly occur at the sweat-orifices, but this is rare as sweat under normal conditions is acid in reaction and an unsuitable soil for the growth of the microbe. It may gain entrance through cuts or other injuries, or through the pricks of pediculi, the burrowings of the acarus of scabies, or through lesions of herpes around the mouth, but is more frequently inoculated by the scratching indulged in to relieve itching. As its name suggests, the disease is highly contagious, being directly conveyed from person to person by actual contact or indirectly through the medium of infected towels, linen, shaving brushes, etc., and once it has gained a footing it is readily spread by auto-inoculation through rubbing, friction, or scratching.

Predisposing Causes.-Sex has no influence on its etiology. Age is an important factor, the disease being especially common in infancy and early childhood, owing to the delicacy of the skin. It is rare in elderly people. It is much more rife and widely distributed among the poorer classes than the well-to-do as a result of over-crowding and insanitary surroundings. It is liable to occur in epidemic form wherever numbers of people are collected together in close association, as in schools, barracks, workrooms, etc., and it is very frequently conveyed by boys to one another in the football field where it is known as scrum pox. The general state of health does not seem to have 
PIATE IV.
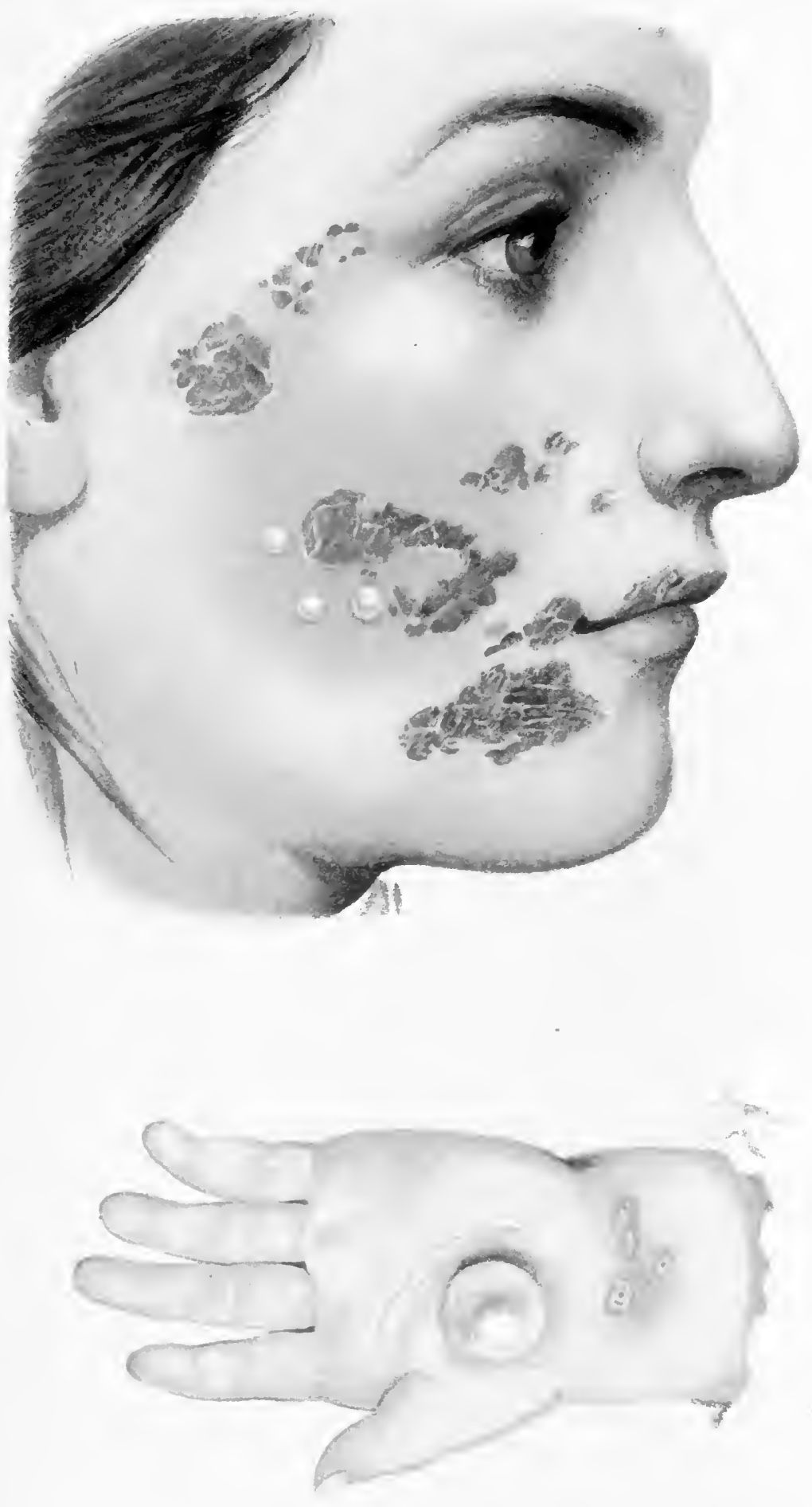
PLATE IV.

Streptococcic Infections.

FIG. I.-Impetigo contagiosa.

Shows early vesicles and "stuck-on" superficial crusts.

FIg. 2.-Bullous Impetigo.

Streptococcic bulla on palm of infant. 

any bearing on its etiology and it can attack with equal readiness the robust and the weak, but in feeble ill-nurtured children it is liable to be more chronic in its course and may lead to ulceration.

Description.-The clinical picture presented by a typical case of Impetigo contagiosa is characterised by the presence of peculiar waferlike crusts stuck over the skin in an irregular fashion, raw weeping excoriated patches where crusts have separated, and the occasional presence of small vesicles or blebs. The essential lesion is a small superficial flaccid vesicle or phlyctenule, 3 or $4 \mathrm{~mm}$. in diameter, which arises on an erythematous base and contains clear serous fluid. Sometimes larger vesicles or even bullae occur. In most situations the horny layer which forms a roof to the vesicle is so delicate that it may break spontaneously or may be ruptured by rubbing or scratching. The vesicular stage may therefore be so transient as to pass unnoticed. Should the epidermis be thicker, as on the palms and soles, the vesicles are less easily broken and their contents become cloudy and transformed into sero-pus from secondary contamination with staphylococci.

When the vesicle is ruptured, a drop of serum exudes and coagulates to form a discoid crust which is generally somewhat flat in the centre and thick at the margin and varies in colour from a yellowish to an amber tint. Beyond the edges of the crust the cuticle is not infrequently raised up and undermined with serum. At first the crust has a honey-like consistence ; on drying it becomes extremely friable and easily breaks into fissures from which serum wells up and coagulates causing irregular thickening. Should there be much secondary contamination with staphylococci the crusts become coarser and of a deep yellow or greenish colour. (Plate IV.)

The initial lesion may be single and tend to spread peripherally, or multiple lesions may occur and may coalesce and produce crusts, varying in size from a split-pea to a shilling or larger, some of which are round while others are irregular in outline. If the crust be removed soon after its formation a red, raw, oozing surface is left surrounded by a loose fringe of cuticle; if it has been present for a short time a grey fibrinous layer develops beneath it, and the crust dries up and separates naturally, being followed by a renovation of the epidermis and no scar.

The affection spreads rapidly. It may occur anywhere on the skin but is most common on the face, especially about the chin and cheeks. A not infrequent situation is behind the ears where it produces a condition sometimes known as " post-aural eczema." It may spread to the mucous membranes at the natural orifices, being associated about the lips with fissures at the corner of the mouth and the condition known as " perlèche," at the nasal orifice with crusts blocking up the nares, on the eyelids with glueing together of the eyelashes with crusts and discharge and occasionally with phlyctenular keratitis and superficial corneal ulceration. 
It may be present also at the external auditory meatus passing up for some distance into the canal where it is generally secondary to otorrhoea. It frequently occurs on the scalp and generally in association with pediculi and enlarged neck glands. In such cases the occipital portion of the scalp and the nape of the neck are, as a rule, most severely affected. Owing to the presence of the hair the typical appearances of the lesion are modified and the crusts have a thick granular appearance to which the name of Impetigo gramulata has been applied.

In association with impetigo of the scalp there is often a papular or papulo-vesicular eruption spreading down on to the neck, shoulders and back, the individual lesions being about the size of a pin's head.

Impetigo contagiosa may attack the beard region in men, usually from infection contracted at the barbers, in which case the appearances are similar to those which occur in the glabrous skin and consist of yellowish stuck-on crusts and raw oozing areas. In hairy parts such as the scalp, contamination with staphylococci is liable to result in peri-folliculitis and the formation of pustules or even boils, and in the case of the beard, streptococcic impetigo may be transformed into the more resistant staphylococcic folliculitis known as sycosis.

The next most common situation is the hands. When the lesions occur in the palms they take the form of sero-pustules over which the epidermis is thick and not easily ruptured. A common site for them is the side of the nail or the tip of the finger, where they form superficial phlyctenular whitlows. (Plate IV.)

On the trunk and covered parts of the body lesions of the classical type are comparatively rare and the disease more frequently occurs as circinate, bullous, intertriginous, papulo-vesicular, or superficially ulcerated, lesions. In rare instances, the vesicular or bullous lesions after breaking may undergo vegetative changes with the production of exuberant granulations; this is believed to be the result of a secondary infection with staphylococci.

There are no definite subjective symptoms associated with the disease except itching which may be severe in the beard region, and it does not, as a rule, interfere with the general health. A general infection from the local septic condition is extremely rare but occurs occasionally in the bullous type affecting new-born infants, known as Pemphigus neonatorum. This immunity from a systemic infection is due largely to the local protective reaction to the streptococci which occurs in the skin.

The lymphatic glands in the neighbourhood of the eruption are usually enlarged, especially the post-auricular, the posterior-cervical, and the sub-mental over which the skin may become definitely inflamed. In severe cases the glands may suppurate.

Histopathology.-A section of a fully developed vesicular lesion of Impetigo contagiosa shows the following changes: A unilocular vesicle is present in the epidermis in the situation of the granular layer, 
having for its roof the complete Stratum corneum and for its base the upper part of the prickle-cell layer. The vesicle contains serum which tends to coagulate into blocks, nuclei of polymorphonuclear leucocytes more or less broken up, chains of streptococci situated about the floor of the blister, and clusters of staphylococci immediately beneath the roof and towards the margin. The underlying epidermis is oedematous, stains badly, and shows wandering leucocytes in the dilated inter-epithelial lymphatic spaces. The vessels of the papillary and sub-papillary layer immediately beneath the vesicle are dilated and there is a slight perivascular infiltration of inflammatory cells. On examining older lesions the vesicle is found to be replaced by a crust composed of coagulated serum and leucocytic debris, and containing numerous micro-organisms, chiefly staphylococci, while beneath it a fibrinous membrane is usually present from which streptococci may be isolated. (Fig. 72.)

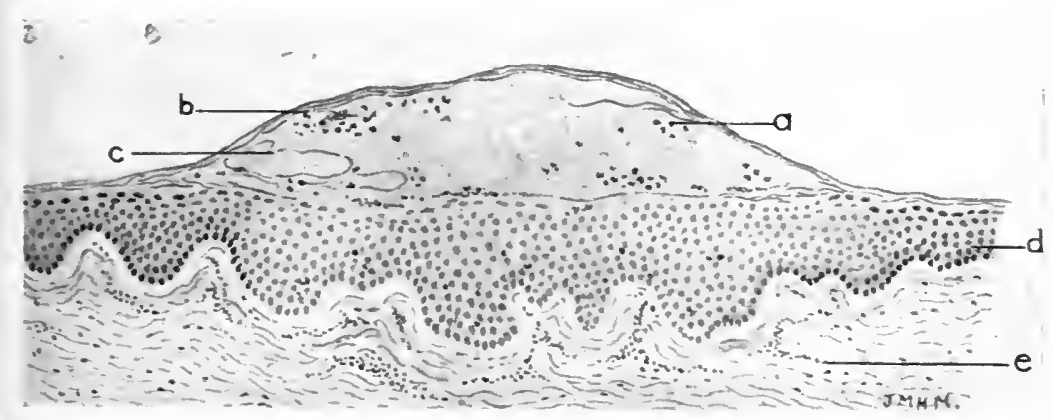

FIG. 72.-Vesicle of Streptococcic Impetigo.

a. Vesicle, b. Polymorphonuclear leucocytes and situation of secondary staphylococci. c. Dried serum. $d$. Epidermis, oedematous below vesicle with leucocytes between pricklecells. e. Dilated capillaries and cellular infiltration.

Varieties.-The clinical appearances in Impetigo contagiosa are peculiarly multiform and the disease presents a greater variety of clinical pictures than almost any other form of superficial dermatitis. The modifications in the lesions are due to differences in the age of the patient, the general state of nutrition and power of resistance of the skin affected, the region attacked, and the virulence of the special strain of streptococcus. The following are the most important varieties:

Circinate Impetigo.-In this somewhat rare type the lesions are associated with comparatively little exudation and instead of being definite vesicles have a marked tendency to spread eccentrically and at the same time to clear up in the centre, thus producing circinate lesions an inch or more in diameter, the borders of which are red, moist and edged by a fringe of cuticle sometimes undermined with serum or covered with a crust. The lesions tend to coalesce with each other to form gyrate patterns and may spread with extraordinary rapidity and may involve large areas. (Fig. 73.)

Bullous Impetigo.-Here the lesions, instead of breaking and becoming crusted, tend to develop into flaccid bullae varying in size 
from a finger-nail upwards. The covering of the bullae is thin and easily ruptured, except on the palms and soles. In this country this type of impetigo is most common in infants-the affection known as Pemphigus neonatorum being an example of it-and is rare in older children. In the tropics, however, it is prevalent both in adults and children and has been described in the East, in the Malay Peninsula and Southern China, under the heading of Pemphigus contagiosus, by Sir Patrick Manson, according to whose observations it occurs most commonly in hot damp weather, attacks Europeans as well as natives, and is not infrequently localised about the posterior wall of the axillae.

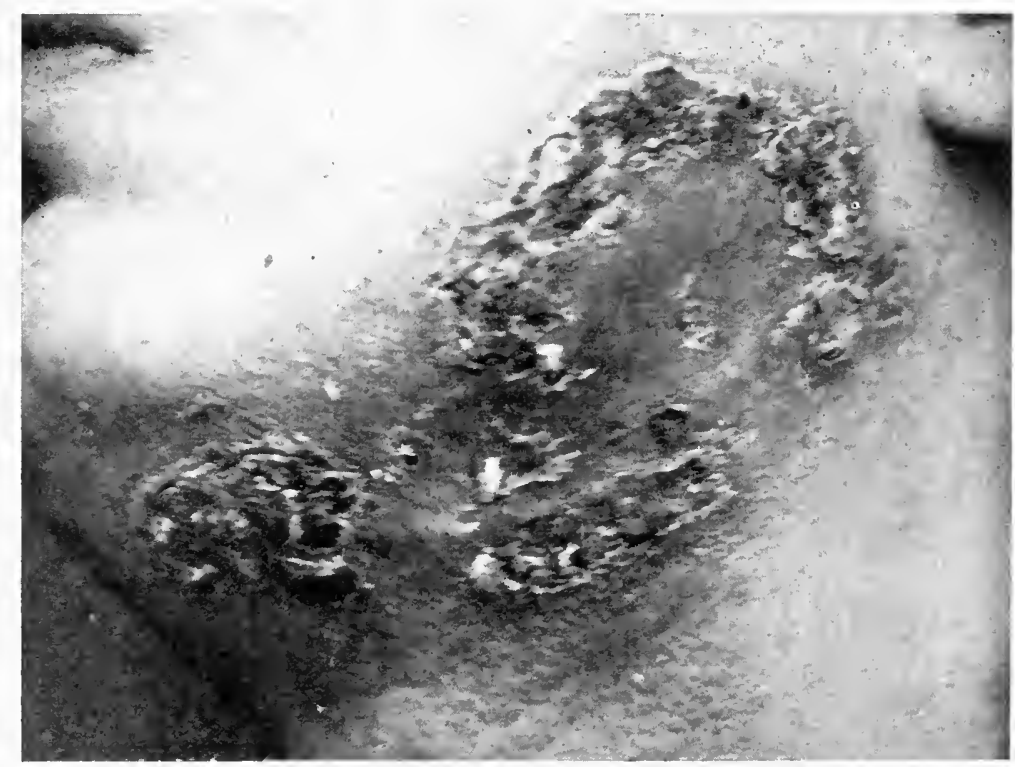

FIG. 73.-Circinate Impetigo (Streptococcic).

Ulcerating Impetigo.-In some cases the streptococcic infection, instead of being confined to the superficial layer of the epidermis, penetrates more deeply and results in ulcerative lesions followed by definite scarring. This is met with most frequently about the buttocks and legs and may be due to the S. faecalis. It is due largely to a weakened state of resistance of the skin, brought about by mal-nutrition from ill health, insufficient or improper food, or defective innervation, or by local causes, such as varicose dermatitis in adults or the sodden condition which is liable to occur about the buttocks in infants from insufficient cleanliness and the wearing of wet diapers. In such cases the initial vesicles instead of rupturing and forming superficial yellow crusts, dry up into thick dirty-looking scabs at the periphery of which a phlyctenular ring may be detected and beyond this a dusky red, angry-looking inflammatory halo. When the crust is removed a shallow ulcer is left covered with a diphtheroid membrane which extends down through the whole thickness of the epidermis and in 
which chains of streptococci can be found. To such lesions the old name ecthyma was once applied.

Intertriginous Impetigo.-This variety of impetigo presents certain clinical peculiarities owing to the fact that it occurs in situations where the skin is exposed to the friction of contiguous surfaces and liable to be moist from discharges. It is met with commonly behind the ears, between the buttocks, in the groins, between the thighs, under the breasts in fat women and at the mid-thigh folds in infants. In these situations the lesions take the form of inflamed, red, weeping surfaces at the edge of which the epidermis is generally raised up and undermined, indicating the phlyctenular origin of the lesion, and they are not infrequently associated with fissures in the foids.

Diagnosis.-In a well-marked case of impetigo the diagnosis presents no difficulty. The superficial crusts, stuck on like wafers, with their yellowish colour and honey-like consistence, the red raw surfaces where they have fallen off, the occasional early vesicle, the enlarged lymphatics. the great infectivity, and the absence of constitutional disturbance, form a group of symptoms which is not readily mistaken. The important thing with regard to the diagnosis is to realise that this condition may be secondary and mask some underlying affection such as Lupus rulgaris, eczema, pediculosis, scabies, or even syphilis.

The type of impetigo which presents the greatest difficulty in diagnosis is the bullous variety. It has to be distinguished from pemphigus and differs from it by heing more superficial, forming yellowish crusts, not having the same tendency to come out in crops, and the lesions being preceded by a red macule whereas in pemphigus they develop on apparently normal skin and there is often eosinophilia. Should any difficulty still exist it may be settled by cultivating the contents of one of the vesicles, for while in true pemphigus they are sterile, in impetigo the streptococcus can be isolated.

Prognosis. - The affection being a superficial inflammatory process resulting from the inoculation of a definite micro-organism is readily amenable to parasiticide treatment and the prognosis, as a rule, is excellent.

\section{TREATMENT}

The treatment is comparatively simple. It varies in different cases according to the extent of the disease and the resistance of the individual skin, but consists in the main of local measures for the removal of the crusts and of the application of parasiticide remedies. In children of the hospital class, whose state of nutrition is below par and who may be the subject of definite debilitating disease, the local treatment should be augmented by tonic treatment on general medica! lines.

The local treatment consists in the first instance of the removal of the scabs. This must be done thoroughly, for the application of 
parasiticide remedies with the scabs still present is useless as antiseptics are unable to penetrate the coagulated serum. On the face they may be removed by hot water and soap applied on a piece of flannel or by the repeated application of warm boric acid lotion on lint. On the scalp the hair should first be cut short over the affected parts and the crusts softened with olive oil under guttapercha tissue or by boro-starch poultices. The poultice should contain a teaspoonful of boric acid to a tablespoonful of cold water starch added to half a pint of boiling water. This is spread on cotton covered with muslin and applied for an hour or more with the muslin next to the skin. After it is taken off the scalp should be washed with soap and water and any pieces of scab still adherent removed by a blunt flat instrument such as a paper knife, the process being facilitated by clipping the hairs beneath the scabs. After they have been thoroughly removed a mild parasiticide ointment, such as Hyd. ammoniat. Io gr., Paraffin molle alb. I oz., should be applied continuously on the scalp. In the case of the glabrous skin the ointment should be thickened by the addition of $6 \%$ of oxide of zinc. Should any scabs re-form they must be removed.

In the case of the gyrate and bullous types of impetigo where a large extent of surface is involved, the application of a mercurial ointment may be contra-indicated owing to the danger of absorption. In such cases daily boric baths containing $\mathrm{x} \%$ of boric acid are of great value and should be followed by the application of zinc sulphate lotion I $\%$ or an ointment containing $2 \%$ resorcin, all the phlyctenules and bullae being opened previous to the application of the ointment.

Any complications must be dealt with appropriately. Fissures should be painted with Friar's Balsam or with I \% solution of silver nitrate in spirit, and phlyctenular keratitis should be treated by the application of dilute yellow oxide of mercury ointment, etc. The enlarged lymphatic glands require no special treatment as they rapidly resolve when the eruption is cured, but should suppuration occur it must be dealt with by ordinary surgical methods.

In all cases warning should be given of the contagiosity of the affection and every care taken to prevent the spread of the disease by contaminated towels, brushes, etc.

\section{PERLÈCHE}

The name perlèche has been extensively employed in France to designate a familiar type of impetigo which affects the lips, especially in children. It is characterised by the presence of fissures, chiefly at the commissures of the mouth, by maceration, desquamation, or crusting of the red portions of the lips, and by a red scaly form of dermatitis spreading for a short distance around the mouth. In many cases it is limited to the commissures but it may also involve the lips 
themselves and chiefly the lower one. The fissures are liable to be painful and to bleed from the movements of the mouth in speaking and eating. It is markedly contagious and may be spread by kissing or by the use of infected drinking vessels. It develops in a few days, is singularly resistant to treatment, very liable to relapse, and may continue for months. (Fig. 74.)

It is now known to be of streptococcic origin and may occur in association with other streptococcic infections, such as phlyctenular impetigo or septic stomatitis.

It has to be distinguished from Herpes labialis for which it might easily be mistaken, but its contagiosity and the absence of vesicles serre to establish the diagnosis.

The treatment consists of painting the fissures with compound tincture of benzoin, touching them with sulphate of copper or nitrate of silver, and the application of a $2 \%$ ammoniated mercury ointment. In very resistant cases

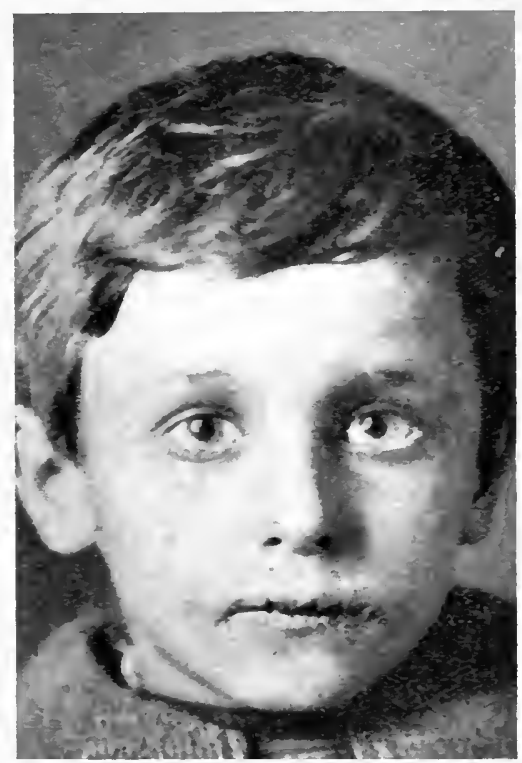

FIG. 74.-Perlèche. where the cracks are deep and the edges thickened, a cure may be effected by the $\mathrm{X}$-rays, a pastille dose being given and repeated in a month if necessary. Great relief may be obtained and healing encouraged in the case of painful deep fissures by keeping them closed by a small strip of plaster.

\section{REFERENCES}

Adaysox, H. G. Some eruptions due to streptococcal infections (St. Bart.'s Hosp. Journ., Nov. I9Io, p. I8).

Balzer \& GRIfFox. Streptococci in Impetigo contagiosa (La Presse Méd., I897).

Chalmers \& Archibald. Sudanese streptococcal dermatitis (Journ. Trop. Med. and Hyg., I9I8, xxi. p. I +5 ).

Elliot, G. Impetigo bullosa after vaccination (Journ. Cut. Dis., I 894 , xii. p. 94).

Fox, Colcotr. Dermatoses of streptococcic origin (Allbutt \& Rolleston, System of Medicine, I9I I, ix. P. I64).

Fox, Tilbury. Impetigo contagiosa. (Skin Diseases, London, 3rd Ed. I873, p. 224).

Herscheimer. Impetigo vegetans (Arch. f. Derm. u. Sỵph., I897, xxxviii).

Sabouraud, R. Impétigo (Ann. de Derm. et de Syph., Paris, I9oo, iv. p. 320. La Pratique Derm., Paris, I901, ii. p. 864$)$.

Uxxa \& Trachsler. Impetigo vulgaris (Monats. f. prakt. Derm., I899, xxvili. P. 229). 


\section{Pemphigus Neonatorum}

Definition.-A bullous impetigo of streptococcic origin attacking new-born infants.

Introduction.-The name was given before the true nature of the disease was understood, and is misleading as it has no connection with pemphigus.

The disease is now comparatively rare in this country-thanks to improved hygiene and the employment of aseptic methods in the puerperium-but not long ago it was common in epidemic form in Lying-in Institutions or in the practice of a particular mid-wife. One of the last epidemics recorded occurred at the Richmond Lying-in Charity in the autumn of 1902 ; in it there were eighteen cases, of which twelve were in the charge of one mid-wife, and eight were fatal-ming with symptoms of acute toxaemia.

It is the result of infection from the mid-wife, mother, or attendant, and in certain cases has been traced to some definite pyogenic focus, such as a whitlow or a septic finger. Its close relation to Impetigo contagiosa has been shown by instances having occurred in which an infant suffering from it has infected an older child with ordinary Impetigo contagiosa ; for example, an infant aged I2 days was brought to hospital with a widely distributed bullous eruption which had begun with a bleb on the neck, and soon after the eruption appeared a brother aged 2 years became affected with Impetigo contagiosa of the face, and the mother, who was nursing the infant, with impetigo on the breast.

The Streptococcus pyogenes is now generally admitted to be the cause, and the histological appearances of the lesions are similar to those in the early stages of Impetigo contagiosa, the position of the vesicle being in the site of the granular layer.

Description.-The disease is occasionally present at birth from infection by the mother, but more often does not make its appearance till some days or even weeks later.

There are two types or grades of it: A benign type in which the eruption, however widely distributed, is not associated with constitutional symptoms, and a malignant type in which marked general symptoms are present. In the first type the infective virus is confined to the skin, in the second it has passed into the general circulation, probably via the unhealed umbilical stump.

In a typical case the serous exudation takes place rapidly, raising up the horny layer and forming a flaccid bulla about the size of a sixpence or a shilling. This is readily broken, owing to the delicacy of the infant's skin, and leaves a red, raw, moist patch surrounded by a fringe of epidermis undermined with serum. The lesions tend to spread peripherally and to coalesce and involve considerable areas.

The original inoculation may take place anywhere on the cutaneous surface, the neck and fingers being common sites. The disease is spread subsequently by auto-inoculation from scratching or from 
the rubbing together of contiguous surfaces, such as the inner aspects of the buttocks or the flexures of the joints.

In mild cases involution may take place, with drying up of the lesions and the formation of yellowish crusts similar to those of Impetigo contagiosa. In more acute cases the lesions keep on spreading peripherally and new ones appearing until the greater part of the skin, with the exception of the palms, soles, scalp, and face, is attacked.

Occasionally secondary infection with staphylococci may occur, with the production of furuncles or abscesses, haemorrhages may take place into the bullae, or ulceration or gangrenous changes may supervene.

The mucous membranes of the mouth or eyelids may be affected, and marked conjunctivitis may be set up with beads of pus at the inner canthi.

In the malignant type the lower part of the abdomen is generally involved and the umbilical stump swollen and inflamed. Evidences of systemic infection gradually manifest themselves, such as restlessness, disinclination for food, and romiting; and later more serere symptoms, such as diarrhoea, sub-normal temperature, dyspnoea from congestion of the lungs, and cyanosis, which may lead to a fatal issue.

Diagnosis.-The affection with which it is most commonly confused is syphilis and many cases of it have been treated as such in the past. This mistake not only places an unfortunate stigma on the child and its parents, but may lead to serious delay in the employment of local antiseptic treatment. It should not occur, as the congenital bullous syphilide is practically confined to the palms and soles, parts rarely attacked in this affection, and is almost invariably associated with other signs of hereditary syphilis, such as wasting, copper-tinted macules and papules, frontal bosses, fissures at the angles of the mouth, and a peculiar earthy hue of the skin. As an aid to the diagnosis the contents of a recent bulla should be carefully examined for the Spironema pallidum or the streptococcus, and a Wassermann reaction may be done.

It has also to be distinguished from the rare affection known as Epidermolysis hereditaria bullosa, a peculiar congenital rulnerability of the skin in which flaccid bullae occur as the result of traumatism, but this is frequently associated with dystrophy or. absence of the nails, which are not involved in the streptococcic disease.

Prognosis.- Some years ago it was a particularly fatal disease and a mortality of $50 \%$ was by no means uncommon in epidemics of it. It is gradually becoming more and more rare and now that its pathogenesis is fully understood and active local treatment to prevent a systemic invasion promptly instituted the prognosis is less serious.

Treatment.-The treatment is similar in principle to that of Impetigo contagiosa. The blebs should be opened, discharges wiped away, and any loose pieces of epidermis remored. The infant should then be immersed for five to ten minutes in a bath containing $I^{\circ}$. of permanganate of potash or $5 \%$ of boric acid at a temperature of about $100^{\circ} \mathrm{F}$. After removal from the bath an ointment containing $2 \%$ of 
boric acid or I\% of ammoniated mercury should be smeared over the raw surfaces and a bland zinc and talc dusting powder applied but not allowed to cake up. This procedure should be repeated several times a day. Special attention should be paid to the umbilical stump which should be covered by occlusive dressings so as to prevent absorption taking place through it.

When systemic invasion occurs the infant should be kept warm, stimulated, and treated on general medical principles. Anti-streptococcic sera have been employed but with disappointing results; benefit may possibly be obtained from injections of an autogenous streptococcic raccine.

\section{REFERENCES}

Adanson, H. G. Pemphigus neonatorum in the light of recent research

(Brit. Journ. Derm., I903, xv. p. 447).

Broco, L. (La Prat. Derm., Paris).

Crary, G. W. Case of Acute Septic Pemphigus (Journ. Cut. \& Gen.Urin. Dis., NewY ork, I9o6, xxiv, p. I 4 ).

Freeman, W. T. Pemphigus neonatorum gangrenosus (Brit. Journ. Derm., I905, xvii. p. 376).

Kemax, R. H. Fatal case of congenital bullous eruption in an infant (Dublin Journ. Med., I90o, p. 24I).

Macleod, J. M. H. Pemphigus neonatorum (Brit. Med. Journ., I903, p. 1278$)$.

- Present state of our knowledge of pemphigus (Practitioner, 1909, p. 37).

MAGuire, G. T. Acute contagious pemphigus in the newly-born (Brit. Journ. Derm., I903, xv. p. 427).

Whitfield, A. Two cases of acute pemphigus of infants (Brit. Journ.

Derm., I903, Xv. p. 2I8).

\section{Dermatitis Exfoliativa Infantum}

Under this heading Ritter of Prague in 1878 described an infection which is most probably a variety of Pemphigus neonatorum, in which

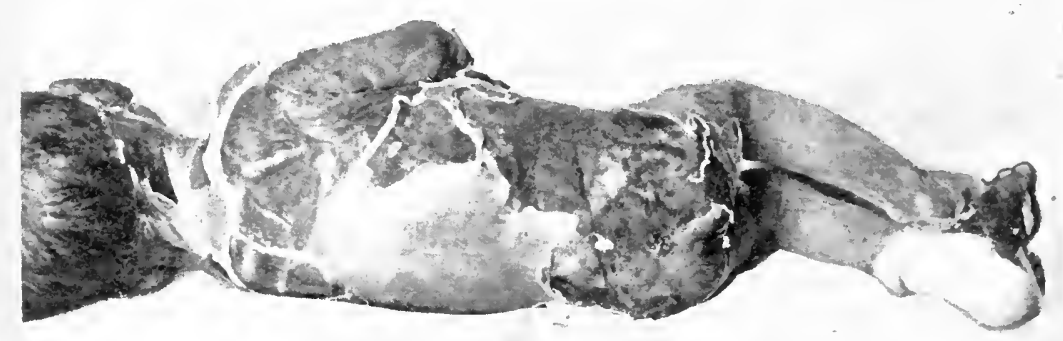

F1G. 75.-Dermatitis Exfoliativa Neonatorum.

(From Dr. E. F. Skinner.)

the vesicles and bullae are abortive, the exudation less than in the ordinary type, and the most marked feature a separation or peeling 
of the horny layer of the epidermis in scales or sheets leaving hyperaemic, moist, denuded areas. It appears in the second to the fifth week after birth and is ushered in with profuse redness which spreads rapidly and becomes more or less universal in the course of a few days, the extremities being the last parts to be involved. Almost synchronously with the hyperaemia, exfoliation of the epidermis takes place or more rarely the horny layer becomes lifted up by a slight fluid accumulation. The affection is serious and has a high rate of mortality, for out of 297 cases collected by Ritter $45 \%$ proved fatal. In a note on the histology by Skinner streptococci were detected on the surface of the denuded skin. The treatment is similar to that of Pemphigus neonatorum. (Fig. 75.)

\section{REFERENCES}

HEDINGER, E. Relation of Dermatitis exfoliativa neonatorum with Pemphigus acutus neonatorum (Arch. f. Derm. u. Siph., I9o6, lxxx. p. 349).

Ritter voi Ritiershais. (Centr. Ztg. f. Kinder, Berlin, I8 78 , p. 3).

Skinser, E. F. Histology of Dermatitis exfoliativa neonatorum (Brit. Journ. Derm., I910, xxii. p. 75).

\section{Epidemic Exfoliative Dermatitis}

Syn. : Epidemic skin disease.

In IS92 Dr. Savill, then Medical Superintendent of Paddington Infirmary, reported in detail an affection closely related to the Dermatitis exfoliativa infantum of Ritter, which occurred in London in epidemic form during the summer and autumn of IS9I-especially in July and August - chiefly among the elderly inmates of St. Marylebone Infirmary (I93 cases), Paddington Infirmary (I63 cases), and, in smaller numbers, in Lambeth Infirmary, Hanwell Lunatic Asylum, St. Mary's Hospital, and Bethnal Green Workhouse. A few sporadic cases were also reported.

Description.-There were two main types of the disease: a dry type indistinguishable from Pityriasis rubra, and a moist type resembling eczema. In a typical case the eruption might be divided into three stages, namely, a papulo-erythematous stage lasting 3 to $\delta$ days; a stage of exudation and desquamation lasting 3 to $S$ weeks; a stage of subsidence. It usually began on the exposed parts and spread from there until, in half the cases, it corered the entire skin. It appeared either as a number of papules on a congested surface with more or less oedema in the loose parts affected, or as raised blotches or rings like Erythema multiforme. The papules ran together to form sheets which flaked off in scales varying from an impalpable powder to large coarse scales, or even to casts of a whole hand. In two-thirds of the cases the scaliness was associated with a copious exudation of an eczematous type. When the inflammation subsided the skin was left thickened, had a polished brown appearance, and was sometimes cracked, 
especially about the larger joints. In severe cases the hair and nails were shed.

The subjective symptoms consisted of itching and burning and, when peeling took place, of soreness and tenderness.

There were no definite prodromal symptoms but in severe cases the eruption was accompanied by anorexia, diarrhoea, or vomiting, and occasionally by sore throat and enlarged cervical glands.

The majority of the patients were elderly inmates of the Infirmaries, of either sex but with a preponderance of males. A few of the attendants and some children also contracted the disease but in them it ran a mild course. It seemed to be a local affection due to a specific micro-organism, and Risien Russell succeeded in isolating from the scales and exudation, and also from the blood, a diplococcus which was in rod-like segments and did not liquefy gelatine.

Prognosis.-The prognosis was grave in old people and the affection was associated with a high rate of mortality. Twenty-eight of the Paddington cases died, in twenty-one of which the fatal issue was directly dependent on the disease, death supervening from profound exhaustion and being preceded by twitchings of the muscles and laboured respiration. In the cases which recovered it ran a course of about eight weeks but relapses were frequent.

Treatment.-The condition was ameliorated by local antiseptic applications, such as I \% creolin lotion which at the same time relieved the irritation.

\section{REFERENCES}

Crocker, RAdchiffe. Epidemic exfoliative dermatitis (Brit. Journ. Derm., I 895, vii. p. 395).

Echeverria, E. Histology of epidemic dermatitis (Idem, p. 9).

Russell, R. Bacteriology of epidemic skin disease (Idem, I892, iv. p. I05).

Savill, T. D. On an epidemic skin disease (Idem, p. 35).

\section{Scaly or Furfuraceous Impetigo}

Syn. : Impetigo pityrodes (Sabouraud).

In addition to the markedly exudative varieties of streptococcic impetigo with phlyctenules or bullae, an abortive type is sometimes met with in which the lesions consist of slightly inflamed patches covered with fine scales which may be so delicate as to resemble a wrinkled film on the surface of the skin. The patches vary in diameter from a threepenny piece to an inch or more, are irregular in shape, and ill-defined in outline. They are sometimes so delicate as only to be noticeable on close inspection, while at other times they are coarser, reddish-pink in tint, and easily detected. They are most prevalent in children and have been known to occur in epidemics in schools, etc. Their most common situation is the face, where they were described long ago by Alibert under the heading of Dartre iolante. They 
may be the only lesions on the face, or may be associated with ordinary phlyctenular impetigo, or with a chronic streptococcic infection and catarrh of the nasal mucosa when they may be detected on the nose, upper lip, or cheek, or with a septic discharge from the external auditory canal when they occur on the auricles, or with fissures about the lips and angles of the mouth when they affect the skin around the mouth. They may be met with also about the folds of the groin and buttocks and in the flexures of the large joints where they constitute a form of intertrigo. (Fig. 76.)

There are no constitutional symptoms in connection with the patches though occasionally there may be itching which may lead to scratching, excoriation, and even eczematisation.

Lesions of this type occurring in isolated patches on the face are often regarded by the laity as the result of cold winds, hard water, soap,

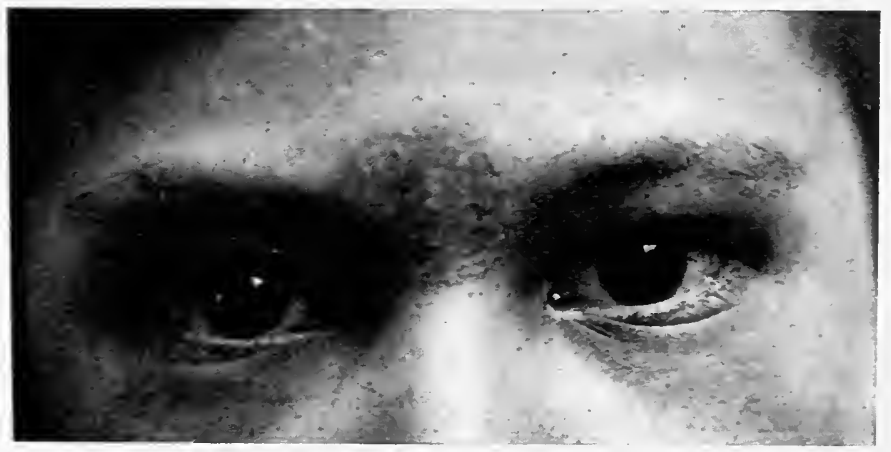

FIG. 76.-Scaly or Furfuraceous Impetigo.

or other forms of local irritation. They are now known to be a variety of Impetigo contagiosa and auto-inoculable and there is considerable evidence that they are of streptococcic origin, but patches closely resembling them may occur in association with staphylococcic lesions, which are most probably caused by the ubiquitous Staphylococcus albus.

In the production of these patches it would seem that the strain of streptococcus responsible is of low virulence, the micro-organisms possibly few in number, and the resistance of the skin high. Consequently the inflammatory reaction is slight and the serous exudation so limited that instead of collecting beneath the horny layer to form a phlyctenule it simply produces oedema of the prickle-cells, interferes with cornification, and causes the formation of scales by the process known as parakeratosis.

The treatment consists of the application of a mild parasiticide, such as a $2 \%$ ointment of ammoniated mercury and salicylic acid, or a $2 \%$ ointment of tannic acid and calomel (Sabouraud). It is more resistant to treatment than ordinary impetigo.

Under the heading of Epidermite chronique à streptocoques, Sabouraud has described a further condition characterised by the 
occurrence of erythematous patches, with papulo-vesicles on the surface, which become transformed eventually into lichenified plaques. In it the lesions have an initial acute stage, become sub-acute, and finally assume chronic characters. The earliest symptom consists of itching or smarting associated with reddening of the skin. This is followed in about 24 hours by the formation of an ill-defined patch, several inches in diameter, irregular in shape, on the surface of which papulo-vesicles may be present which become eroded by scratching and exude drops. of serum. In the final stage the serum drys up to form crusts, a definite thickening of the epidermis occurs, and the characteristic cross-hatched shiny appearance of the skin known as lichenification develops (see p. 746). Lesions of this type are frequently present on the arms, especially where the skin is thin as at the flexures of the elbow and wrist. They have been proved to be of streptococcic origin, the organisn having been demonstrated in all stages of development of the lesions.

A microscopical examination shows a definite thickening of the epidermis with elongation of the inter-papillary processes, oedema of the prickle-cells with imperfect cornification or parakeratosis, and occasionally the presence of a sub-corneal vesicle.

This condition has to be distinguished from eczema. In some cases the resemblance may be so close that only the finding of streptococci can establish the diagnosis.

\section{Ulcerating Varieties of Streptococcic Impetigo}

Ulceration of the lesions of Impetigo contagiosa has already been referred to as a rare complication. In this connection there is a series of ulcerative conditions which merits special consideration, and which may be grouped under the headings of ecthyma, Dermatitis gangraenosa infantum, and vacciniform dermatitis. In certain of them a streptococcic origin has been definitely proved, while in others the streptococcus has been found in symbiosis with other micro-organisms, such as the Bacillus pyocyaneus and the Bacillus coli.

Ecthyma.-This name was applied by Bateman in I8I7 to a pustular eruption of the extremities in which the pus tended to dry up into brownish crusts. It is now used to signify a variety of streptococcic impetigo, occurring chiefly on the lower limbs about the buttocks and thighs, in which the initial phlyctenular stages are so transient as to be rarely seen and the lesions develop into superficial ulcers covered with dark crusts. It generally occurs in infants or young children who are in a state of malnutrition from insufficient food, or enfeebled health from some wasting disease, and is the result of a weakened resistance of the skin, partly from general causes and partly from local causes such as a congested state of the circulation in the limbs. The lesions are as a rule sparsely distributed and isolated. They begin as red macules about the size of a split-pea which become raised owing 
to exudative changes in the epidermis but rarely show definite vesiculation. This is followed by a destruction of the epidermis in its whole thickness with the formation of dark dirty-looking crusts which are tough and fibrinous and when removed leave a superficial ulcer with an unhealthy diphtheroid, sometimes definitely indurated, base. Surrounding the crust there is an angry-looking inflammatory halo, the epidermis over which may be undermined with serum, indicating a peripheral extension of the lesion. Healing is slow and results in the formation of a depressed scar similar to that of vaccinia, which is surrounded at first by a pigmented halo. In some lesions the inflammatory disturbance may be so considerable as to produce a resistant type of cellular infiltration in the underlying corium, leading to induration which may persist long after healing has occurred as a reddish-brown nodule or thickening which might be mistaken for a syphilide. The lesions are inoculable and auto-inoculable and have been proved to be due to the Streptococcus pyogenes.

Dermatitis Gangraenosa Infantum or Ecthyma Terebrans.-These names have been applied to a more fulminating type of ecthyma occurring in infants and young children in a low state of health, in which an ulcerative process is superimposed upon the unhealed lesions of chicken-pox or vaccinia. Instead of healing the lesions then undergo further inflammatory changes with necrosis, crusting, and the formation of punched-out ulcers, and by spreading eccentrically and coalescing may form irregular ulcerations of considerable size. In these cases the Bacillus pyocyaneus has been found in association with the streptococcus in the local lesions and in the blood, and may be of pathogenic importance. The prognosis is grave, owing to the possibility of fatal septicaemia.

Vacciniform Dermatitis.-Under this heading Colcott Fox has described a vacciniform ecthyma in infants in which the lesions closely resemble those of vaccinia and occur usually about the anus and genitalia. They begin as erythematous macules, which may be covered with serous exudation forming a diphtheroid coating or may go on to the formation of vesicles or bullae with pustular contents. Occasionally they are in the form of papules or congested macules with a diphtheroid surface and simulate macerated congenital syphilitic papules or the lesions seen in a bromide rash. They vary in size from a splitpea to a shilling and may coalesce to form polycyclical figures. They are particularly liable to occur on adjacent surfaces, as about the genitocrural folds or between the buttocks. In certain instances involution takes place in the centre of the lesion, which becomes depressed, while the border forms a raised ring covered with a diphtheroid membrane. The Streptococcus pyogenes has been isolated in four of the cases (Colcott Fox). (Fig. 77.)

Nore.-Other names have been applied to the above condition such as Érythème Vacciniforme Syphiloỉde (Besnier), Herpès Vacciniforme (Fournier), Dermites simples infantiles (Jacquet). 
Treatment.-The treatment consists of the removal of the crusts and the bathing of the lesions with I in 2000 perchloride of mercury solution, or the painting of the base of the ulcers with tincture of iodine followed by the application of a $2 \%$ ammoniated mercury ointment. In all such cases the general health should be carefully

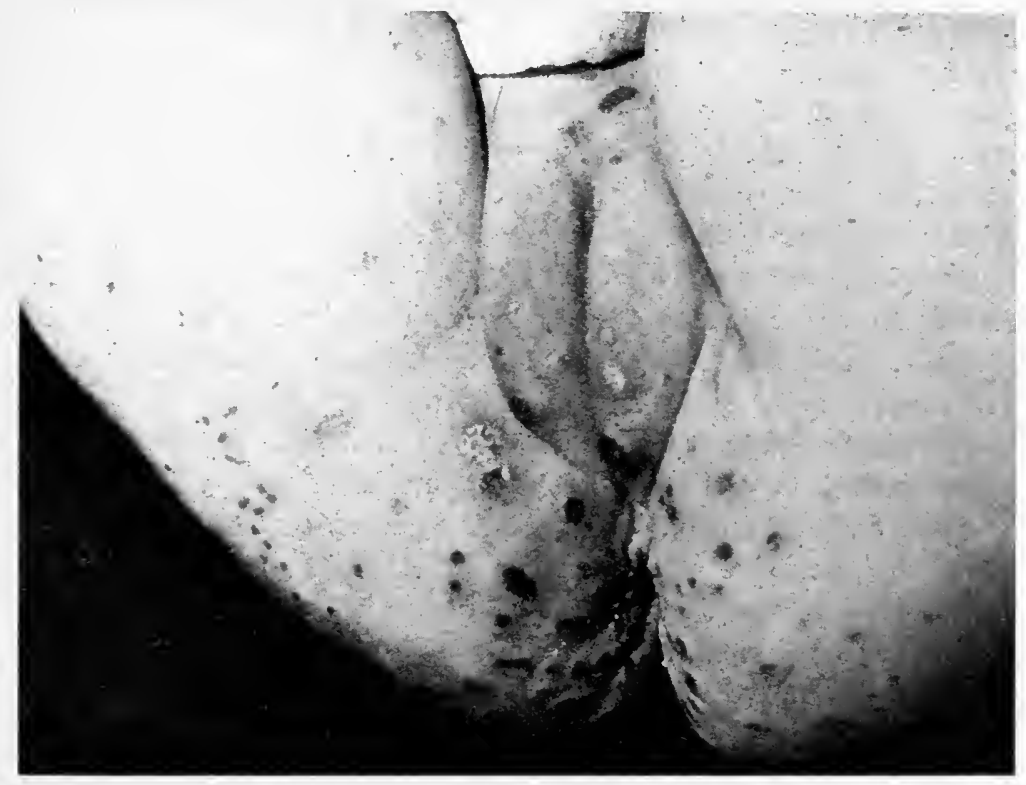

FIG. 77.-Vacciniform Eruption.

(Infantile Dermatitis of Jacquet, "Post-Erosive" Form.)

attended to on general medical lines and attention paid to such considerations as proper food, careful nursing, healthy hygienic surroundings, and a suitable tonic regime with iron, cod-liver oil, etc., prescribed.

\section{REFERENCES}

Fox, T. Colcott. Vacciniform ecthyma of infants (Brit. Journ. Derm., I907, xix. p. I9I).

Sapouraud, R. Ecthyma (La Prat. Derm., I900, i.).

\section{ERYSIPELAS}

Syn. : St. Anthony's Fire ; Fr., Êrysipèle, La Rose ; Ger., Hautrose, Rotlauf.

Definition: An acute specific inflammation of the skin characterised by redness and swelling in areas with a raised sharp border, occasionally associated with vesiculation, and usually accompanied by febrile symptoms.

Etiology and Pathogenesis.-Erysipelas is due to the presence in the skin of the Streptococcus erysipelatis of Fehleisen-now known to be 
identical with the Streptococcus pyogenes-and occasionally of other streptococci such as the S. faecalis. The streptococci are as a rule inoculated locally but they may also reach the shin from some deepseated septic focus. It is a highly contagious affection and is conveyed either directly or indirectly through the medium of infected hands, dressings, etc. It was once common, being met with in sporadic cases or in epidemics in surgical wards, lying-in institutions, etc., but now, owing to improved sanitary conditions, cleanliness, and asepsis, it is comparatively rare. The streptococci gain entrance through some breach in the continuity of the horny layer but this may be so minute as not to be detected or may have healed when sought for. It may follow some slight injury such as a prick or a scratch or occur in association with severe traumatism, surgical operation, laceration of the perineum at the puerperium, or at the unhealed umbilical stump in the new-born child. A considerable number of cases have occurred in connection with piercing the ears for earrings.

Any defect in the general health causing a diminished resistance of the skin may act as a predisposing cause, such as alcoholism, organic disease of the kidneys or other organs, or the low state of health, mental and physical, which may be associated with the puerperium in women of the infirmary class. It is most common in early adult life and statistics would seem to show that it is more prevalent in spring than in any other season of the year. It is liable to recur and any immunity which may result from an attack is readily lost, though subsequent attacks are generally mild. Recurrences may possibly be due to certain of the streptococci remaining dormant for a tinie and then reasserting themselves, an explanation which is rendered probable from cultures of these micro-organisms occasionally showing a similar tendency to die down for a period and then to undergo recrudescence.

Description.-The eruption of erysipelas is generally preceded for about 24 hours by constitutional symptoms of a somewhat vague character such as headache, sickness, romiting, chilliness, and pains in the back and limbs. These may be followed by a distinct rigor and a rapid rise in temperature to $102^{\circ} \mathrm{F}$. or more. In some cases the prodromal symptoms are mild and negligible, in others they are so severe as to be comparable to those of smallpox. The skin lesion is generally noticed first when about the size of a florin, as a single inflamed plaque with a smooth, shiny, red surface and a well-demarcated, oedematous, slightly raised border. It may be tender to the touch, feel definitely hot, and be associated with smarting or burning. On pressure with a diascope it fades to a yellowish-white. At first it is roughly circular in shape, but tends to spread peripherally in an irregular fashion by throwing out prolongations in various directions giving rise to an indented or serpiginous outline. Occasionally new foci appear beyond the original one and these spread and coalesce to form an irregular patch. 
It frequently begins on the face and spreading more or less rapidly may involve the whole region causing marked swelling and oedema where the tissue is lax as about the lips and eyelids. In some cases it avoids hairy regions, such as the scalp and beard, and tends to leave a narrow zone of unaffected skin at the hairy border. When it occurs on the scalp as a result of injury the hairs almost invariably fall out, and in a case of this type a complete defluvium of the hair took place If days after the acme of the febrile disturbance but grew again later. In other parts of the body it may occur as a localised patch or be widely distributed over a whole region or limb. It is usually unilateral, except on the face and trunk. When it affects the lower extremities the skin becomes swollen and tense, and the redness is less vivid and more dusky than elsewhere on the body. Occasionally in the early stages of the infection the lymphatics radiating from the seat of inoculation become inflamed and appear as red lines or bands on the surface. In some cases marked exudation occurs in the patch with the formation of vesicles and even bullae which may go on to ulceration. Sometimes the mucosa, especially that of the nose and mouth, may be attacked, either directly or by extension from the skin, the affected mucous membrane becoming congested, darker in colour, and glazed.

The severity of the constitutional symptoms depends largely on the extent of the eruption. The temperature varies from $100^{\circ}$ to $104^{\circ} \mathrm{F}$, rising at night and falling in the morning, or exhibiting exacerbations with each increase of activity of the local lesion, or occasionally in severe cases becoming sub-normal from sheer exhaustion. The other symptoms, such as headache and sickness, vary with the extent of the disease.

As a general rule the condition reaches its height in from 5 to 7 days, remains more or less stationary for a few days, then gradually declines. The redness fades, assumes a brownish-yellow tinge, gradually becomes yellowish-white, and in about a fortnight. disappears leaving a scaly patch. Vesicles or bullae, if present, dry up and form crusts, and, unless ulceration has taken place, fall off leaving no definite scar.

There are cases, however, which do not run this benign course, but at the end of a week the symptoms instead of declining become more severe, owing to the streptococcus having invaded not only the corium but the deeper subcutaneous tissue and there producing cellulitis or phlegmon. In the worst cases of all, abscesses containing a weak sanious pus may form and gangrenous changes supervene with deep ulceration and sloughing. These are usually associated with a profound systemic invasion, with symptoms indicating inflammatory changes in the membranes, such as pleurisy, meningitis or peritonitis, kidney disease, septic pneunınia, diarrhoea, pyaemia and mental symptoms, such as delirium, exhaustion, stupor and death.

A variant of the classical type of erysipelas is that known as Erysipelas migrans or ambulans, in which new foci or patches of disease keep on 
appearing for several weeks, or in which the disease spreads peripherally by throwing out projections in different directions and clearing up centrally, recrudescences occasionally taking place in the original situation where the disease had died down.

Histopathology.-The microscopical appearances which are present in sections of a classical case of erysipelas are the histological eridences of the reaction of the skin and subcutaneous tissue to the streptococcus in situ and consist mainly of a sero-fibrinous exudation and its consequences. More or less marked changes occur in the corium, subcutaneous tissue, and epidermis, as a result of the presence of the streptococcus in the lymphatic channels. The blood-capillaries are definitely dilated and a serous exudation takes place from them; this dilates the lymphatic spaces between the collagen bundles and is sometimes so profuse as to separate the epidermis from the underlying papillary layer and to form a flat sub-epidermal bulla. As a consequence of the presence of the serous fluid, degenerative changes occur in the collagen and elastic fibres which become split up into fibrils and rarefied or may undergo fragmentation. In this way a loose oedematous tissue results, in the spaces of which short chains of streptococci may generally be detected. There is no marked leucocritic infiltration. A certain number of polymorphonuclear leucocytes are present but are few in number in comparison with those in a staphylococcic invasion, and indicate that the streptococcus and its toxins call forth a special type of chemotactic action associated with extravasation of serum rather than of leucocytes. The sweat-glands, ducts, and pilo-sebaceous follicles in the affected region are not involved.

With the decline of the acute stage coagulative changes occur in the serum, with the formation of a fibrinous network, in the meshes of which small connective tissue cells may be detected which eventually form new collagen bundles and complete the process of repair.

As a rule the inflammation spreads deeply into the subcutaneous spaces, the lymphatic ressels in the septa between the fat cells and those which ensheathe the blood-ressels being dilated with serum in which streptococci may be detected. Changes may be noted also in the blood-ressels, especially the reins which may be thrombosed and their walls thickened.

The epidermis raries in its reaction. In mild cases the interepithelial lymphatics are dilated by the serous exudation, the prickle-cells swollen, oedematous, and difficult to stain, and the process of cornification interfered with so that scales are formed. In severe cases where the exudation is excessire it may collect beneath the horny layer to form a vesicle or bulla, similar to that of ordinary streptococcic impetigo, in which polymorphonuclear leucocytes are generally present.

Diagnosis.-The recognition of a typical case of erysipelas presents no serious difficulty; the rivid red shiny surface with its angry raised 
border, the smarting, local increase of temperature, and the associated general constitutional symptoms usually being sufficient to establish the diagnosis. It has to be distinguished from acute eczema about the face or from dermatitis due to some local irritant, such as sunlight, irritating hair-washes, poison plants, etc. In these conditions, however, the border is indefinite, papulo-vesicles may be present, there is marked itching, and, unless in infancy or early childhood, constitutional symptoms are absent.

Prognosis. - The prognosis in an ordinary case of erysipelas is good, but it depends on the extent and depth of the local inflammatory process, the severity of the constitutional symptoms, the degree of systemic invasion and the power of resistance of the patient. Debilitating conditions, such as result from alcoholism, child-birth, organic disease of the kidneys or other organs, render the outlook much more serious. When the lesions disappear, unless ulcerative or gangrenous changes have supervened, they are not followed by definite scarring and should the hairs fall out they generally grow again.

\section{TrEatMent}

In mild cases local treatment may be all that is necessary and the patient may even be allowed to go about as usual, but as a rule the affection requires to be treated much more seriously and, where constitutional symptoms are in the least degree marked, confinement to bed is necessary. Even in an attack of medium severity it is advisable to isolate the patient and this becomes absolutely essential when the case occurs in the ward of a hospital or lying-in institution.

Constitutional Treatment is based on general medical principles and is similar to that requisite in any acute febrile condition. The diet should be light and nutritious and a tonic regime prescribed. The favourite tonic remedies are iron, quinine, and strychnine, iron in the form of the Ti. ferri perchlor. half a dram t.d.s., quinine up to 30 grains per diem, and strychnine in a mixture containing the Liq. strych. hydrochlor. 5 to Io m. t.d.s. Anti-streptococcic sera have been used somewhat extensively, but with disappointing results. Occasionally benefit may be derived from an autogenous vaccine, but this is difficult to obtain. Ordinary stock streptococcic vaccines are unsatisfactory.

Local Treatment.-As a preliminary to the local treatment all crusts should be removed, abscesses opened, and pus evacuated, and if the scalp be affected the hair should be cut short.

There are two main objects in the treatment, namely, the reduction of the hyperaemia and tenseness by astringent applications, and the limitation of the disease if possible by painting some strong antiseptic beyond the border.

Ichthyol is one of the most useful astringents for this purpose. It is applied in the strength of $10-20 \%$ in an aqueous lotion, or in an 
ointment with cold cream as a base, or dissolved in collodion or traumaticin. The aqueous solution should be dabbed over the patch or lint saturated in it should be laid over it; the ointment should be spread on lint and kept continuously applied under a bandage or a mask in the case of the head or face; the collodion preparation should be painted on once or twice in the day. The dressings should be repeated every few hours.

In place of ichthyol one or other of the following may be employed: alcohol $90 \%$ solution; carbolic acid I to $5 \%$, either in aqueous solution or with the addition of an alkali as in the following formula: carbolic acid $\frac{1}{2} \mathrm{dr}$, borax 2 drs., glycerine 3 drs., water $6 \mathrm{oz}$. ; iced lead lotion containing Liq. pb. sub. I dr. to $\frac{1}{2}$ pint of iced water; picric acid, I in Iooo in water with Io parts of alcohol, painted on once every I2 hours and a dry cotton dressing applied. None of these is more effective than ichthyol, however, and some are more liable to irritate the skin.

Many more or less heroic attempts have been made to limit the spread of the disease but with doubtful success, and in an affection due to a micro-organism deeply seated in the lymphatic spaces it is somewhat futile to expect to reach it by applications painted on the surface. The spread may sometimes be prevented by the simple expedient of painting on tincture of iodine for about an inch beyond the diseased area. Stronger applications such as solutions of silver nitrate or corrosive sublimate are to be deprecated, since they are apt to lower the vitality of the skin and so interfere with its capacity for reacting naturally to the virus.

\section{Recurrent Erysipelas without Consti- TUTIONAL SyMPTOMS}

In addition to the classical erysipelas with its more or less severe onset and general symptoms, there is a mild type in which the redness is less vivid, the borders not so definitely raised and abrupt, and in which there is little or no general disturbance. It may take the form of a red blush, associated with slight oedema, or, where the tissues are lax as about the eyelids and genitals, of a definite swelling in which the redness is comparatively little marked, hence the name White erysipelas (Unna). When the swelling is the most noticeable feature it simply indicates that the serous exudation is so excessive as to obliterate the dilated blood-vessels and to mask the redness. This type usually attacks the face, where it may occur on the nose and cheek in association with chronic folliculitis of the hairs of the nostril, or with septic catarrh of the naso-lachrymal duct, or about the mouth in connection with a septic condition of the gums or carious teeth. It is met with elsewhere in association with ulcers of various types, such as varicose ulcers, ulcers due to syphilis, tuberculosis, and leprosy, discharging sinuses, and fistulae at the anus. 
The first attacks are generally transient and fade in the course of a few days. Recurrences may take place, however, at intervals of weeks or months, each successive attack becoming more and more persistent till finally the swellings acquire a doughy consistence and are not easily pitted on pressure. To such cases the names Persistent lymphatic oedema and Erysipelas perstans faciei (Kaposi) have been applied, the affection being closely allied to the elephantiasic condition resulting from recurrent attacks of erysipelas known as Elephantiasis nostras.

Histologically it is similar to erysipelas, but there is a more marked dilatation of the lymphatics or lymphangiectases, and a thickened thrombosed condition of the veins. Fibromatous changes occur around the dilated lymphaties and give the swellings their persistent doughy consistence.

The streptococcic origin of the affection has now been definitely established, and its close relation to typical Elephantiasis nostras, which is admittedly streptococcic, further corroborates it.

The treatment consists in soothing by means of a $60 \%$ solution of spirit, iced lead lotion, etc., or the time-honoured calamine lotion. Streptococcic vaccines should be tried, but there is even greater difficulty in obtaining an autogenous vaccine in these lesions than in ordinary erysipelas. It has been pointed out that cold may act as a predisposing factor to recurrence by lowering the resistance of the skin, consequently it is important to protect the patient against it.

\section{REFERENCES}

Panton, P. M., \& Adams, J. E. Etiology of Erysipelas and allied infections (Lancet, Oct. 9, I909).

Urxa, P. S. Histopath. of the Skin (Walker's Trans. Edin., I896, p. 406).

\section{Simple Infantile Dermatitis}

Under the heading of Dermites simples infantiles, Jacquet has described a not uncommon series of eruptions which occur chiefly about the prominent parts of the buttocks, thighs, and genitals, in infants, and are characterised by the presence of more or less transient red patches, on the surface of which greyish vesicles develop which become transformed through scratching into erosions or small punched-out ulcers.

The following grades or stages of the dermatitis have been described, but in the majority of cases the eruption is multiple and lesions in different stages are met with at the same time.

Simple Erythematous Stage.-In it the eruption consists of erythematous patches, ill-defined in outline, with a red shiny surface which is generally smooth but may be scaly or crusted. They are usually present on the buttocks and thighs but are occasionally met 
with on the lower part of the abdomen, scrotum, vulva, or sometimes on the heels and shoulders from pressure in lying.

Erythemato-vesicular or Erosive Stage.-In it small greyish vesicles occur, varying in size from a pin's head to a split-pea, which are transformed by friction and scratching into erosions. These may remain discrete or may coalesce to form irregular figures.

Erythemato-papular or Post-erosive Stage.-In it granulomatous changes occur in the eroded lesions with the formation of flat bright-red papules suggesting congenital syphilides.

Ulcerative Stage.--In it the erosions become transformed into small punched-out ulcers which are circular in outline and tend to coalesce into polycyclical sores.

Histopathology.--Sections of a vesicular lesion show that it occurs superficially in the epidermis in the position of the granular layer with the Stratum corneum for a roof, like the vesicle of Impetigo contagiosa. The underlying capillaries are dilated and there is an inflammatory cellular infiltration around them. In the papular stage the infiltration is more marked and there is thickening of the epidermis with elongation of the inter-papillary processes.

Etiology.--The exact causation of this affection is not established, but the superficial phlyctenule with its subsequent erosion and occasional punched-out ulceration suggests a streptococcic origin, while the granulomatous or papular phase would point to a secondary inoculation with staphylococcus. The most important pre-disposing factor is probably the local irritation of moist soiled napkins associated, in some cases, with pressure. Disturbances of the gastro-intestinal tract have been suggested as possible causes.

Diagnosis. - The eruption with which it is most liable to be confused is a papular congenital syphilide and so close may be the resemblance that Jacquet applied the name Syphiloide post-érosive to the papular stage of the dermatitis. In the syphilide, however, the palms, soles, and face, are not infrequently involved, in simple infantile dermatitis they are practically never attacked; in the syphilide the lesions have a coppery tinge, in the dermatitis they are bright red; in syphilis the skin affection is associated, as a rule, with other syphilitic stigmata, such as wasting, hoarse cry, snuffles, fissures at the angle of the mouth, etc. If any doubt still remains this can be settled by the Wassermann reaction or the therapeutic test.

Treatment.-The treatment consists chiefly of scrupulous cleanliness, the frequent changing of the diapers, and the application of mild parasiticides. In the erythematous phase, cure can be effected readily by the application of zinc-calamine cream followed by a bland zincstarch dusting powder. Where crusts are present these should be bathed away with warm water or boric lotion and the part dusted over with zinc-boro-starch powder. Should ulceration be present it is comparatively easily healed by $2 \%$ ammoniated mercury ointment. 


\section{REFERENCES}

Adamson, H. G. Eruptions of the Napkin Region in Infants (Brit. Journ. Children's Dis., 1908, v. p. I3).

Ferrand, M. Les Dermites des nouveau-nés (Histological). (Ann. de Derm. et de Syph., I908, ix. p. I93).

JacQuet, L. Dermites infantiles simples (Traité des maladies de l'enfance, I905, iv. p. 714). (La Prat. Derm., Paris, I900, i. p. 873).

\section{Elephantiasis Nostras}

This name has been applied to elephantiasis occurring in temperate climates and resulting from causes other than filaria, such as injuries, abrasions, septic inoculation, or ulceration. It presents the same essential features as tropical elephantiasis but is usually milder in character,

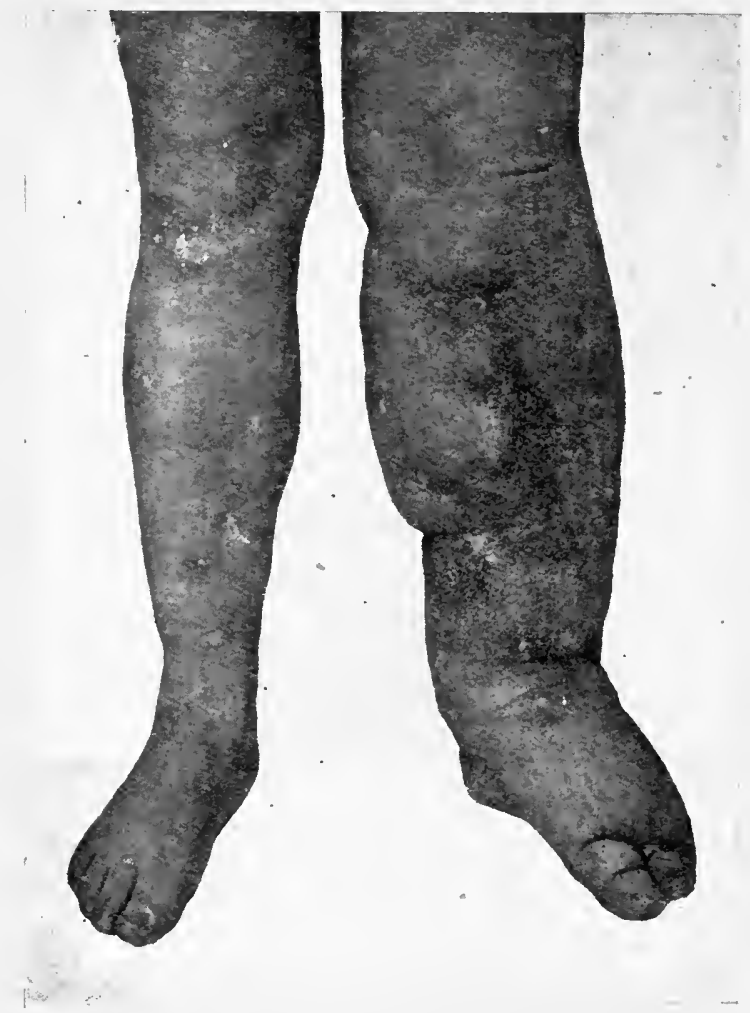

F1G. 78.-Elephantiasis Nostras.

(From Dr. E. F. Skinner.)

more limited in extent, and generally unilateral. It most commonly attacks the leg below the knee but may occur in other situations, such as the scrotum, penis, vulva, female breast, arm, hand, eyelid, or lip.

The affected region becomes swollen to several times its normal size and the skin over it thickened, brawny, warty, and generally pigmented, the thickening being preceded by recurrent attacks like 
. 


$$
8
$$




\section{PLATE V.}

\section{Elephantiasis nostras.}

Shows dusky-pink patches of lymphodermia on side of knee (Pachydermia lymphangiectatica); solid oedema and swelling of the leg; unhealthy discharging ulcer; warty masses covering foot and ankle. 

erysipelas associated with a febrile disturbance similar to " elephantoid fever." (Plate V.)

One of the first changes in the skin is the presence of raised indurated patches of a dusky pink colour, studded over with deep-seated semitranslucent vesicles, like frog's spawn, which are due to dilated lymphatics (Pachydermia lymphangiectatica). This is followed by a persistent solid oedema which finally gives place to more or less typical elephantiasis. In advanced cases the affected skin becomes warty and, from secondary inoculation, ulcerative changes with sloughing, and even gangrene, may supervene. (Fig. 78.)

Etiology and Pathogenesis.-It is most common among the poorer classes as, living in bad hygienic surroundings, they are particularly liable to secondary infection with pyogenic micro-organisms.

It may be due to any cause which leads to blocking of the lymphatics and fibrous hyperplasia. In a large number of cases it is of streptococcic origin, the streptococcus being responsible for attacks of lymphangitis followed by fibrous hypertrophy, hence the name Elephantiasis streptogenes; or it may result from blocking of the lymphatics by cicatricial changes consequent on the healing of a deep ulcer or abscess such as a suppurating bubo; it has also been known to follow the surgical removal of the inguinal glands. On the rare occasions in which it has affected both legs it is possible that the blockage may have taken place in the thoracic duct and been due to the pressure of an enlarged gland in the posterior mediastinum. It may occur also from inflammatory changes resulting froni septic inoculation of varicose ulcers and of old-standing cases of eczema of the leg.

In addition, a certain number of cases occur in connection with syphilis, tuberculosis, and leprosy. In syphilis it is comparatively common in the legs, scrotum, penis, and lips. In tuberculosis it usually affects the limbs and succeeds tubercular lymphangitis, but may occur in other parts such as the genitals and the lips. In both syphilis and tuberculosis it is preceded by acute local inflammatory attacks caused by the secondary inoculation of streptococci.

Occasionally it is congenital (E. lymphangiectatica congenita) and may be associated with other congenital vascular anomalies, such as cavernous angiomata or a diffuse red or telangiectatic condition of the skin (E. telangiectodes). In these cases the infant is generally still-born or dies soon after birth, but it may survive if the affected area be small.

Treatment.-The treatment is dependent largely on the underlying cause and is similar to that described under the heading of tropical elephantiasis.

Locally the parts should be kept clean and the ulcers healed by antiseptic dressings, the wartiness reduced by salicylic acid ointments, and the affected part massaged and bandaged. Benefit has been reported in commencing cases from galvanisin and from electrical heat baths. 


\section{CHAPTER XVI}

\section{CUTANEOUS AFFECTIONS OF STAPHYLOCOCCIC ORIGIN}

\section{INTRODUCTION}

THE part played by the staphylococcus in the causation of skin diseases is much more restricted than was supposed. At one time it

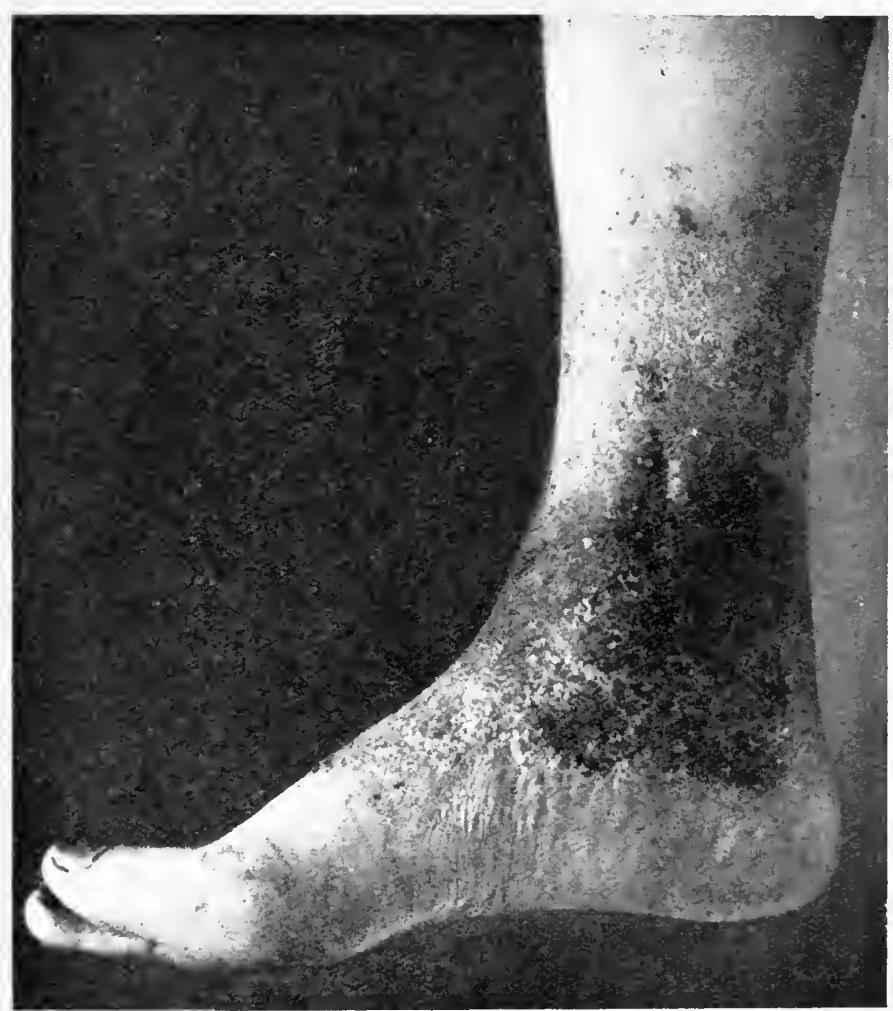

FIG. 79.--Septic Dermatitis, due to Staphylococci. Secondary to sinus from bone-disease.

was believed to be responsible for every form of impetigo, whereas the streptococcus is now known to be the cause of a large proportion of the cases. 
The skin is a more suitable soil for the growth of staphylococci than streptococci and the former are invariably present in moist warm regions, about the hair-follicles, and in the large sebaceous glands of the nose, sternal region, and mid-line of the back. As a rule they exert no harmful influence but under circumstances of lowered resistance from general or local causes, such as an excessive secretion of sweat or sebaceous material, they may multiply rapidly and assume virulent characters.

The clinical lesions produced by them vary according to the virulence of the strain of coccus and the resistance of the patient, and may take the form of a mild desquamating dermatitis on the one hand, or of a follicular pustule, boil, or carbuncle, on the other. These may be the result of staphylococci already present in the skin which have become virulent, or of an inoculation of new staphylococci. (Fig. 79.)

There is at present some diversity of opinion as to the types of staphylococci which occur in the different cutaneous lesions, and as to whether the different types are capable of causing distinctive clinical manifestations. The prevailing view is that the difference in the clinical lesions depends more on the degree of virulence of the microorganism, the power of resistance of the skin, and the region affected, than on the special strain of staphylococcus.

There are three well-known groups of pathogenic staphylococci met with on the skin, namely, the S. cutis communis (Sabouraud), the S. pyogenes albus and the S. pyogenes aureus.

\section{Staphylococcus or Micrococcus Cutis Communis}

Under this heading Sabouraud has described a staphylococcus which is a constant denizen of the healthy epidermis. It is the same micro-organism to which Unna gave the graphic title of the Morococcus, from its tendency to proliferate in mulberry-like clusters. On gelatine it grows in the form of grey streaks and shows no tendency to liquefy the medium, while on agar the streaks are buff-coloured. A smell like butyric acid is emitted by the culture, hence the name Coccus butyricus which is sometimes applied to it.

It is present in abundance in most scurfy conditions of the scalp and in the greasy yellowish scaly patches of seborrhoic dermatitis.

The S. epidermidis albus of Welch is regarded by certain observers as identical with Sabouraud's micrococcus, but as it is capable of liquefying gelatine and of curdling milk it is considered by others to be a variant of the $S$. pyogenes albus.

\section{Staphylococcus Pyogenes Albus and Aureus}

The pyogenic staphylococci are the S. pyogenes albus and aureus, which are distinct micro-organisms differing from each other in cultivation, in their capacity for liquefying gelatine and curdling milk, 
and in their reactions to certain sugar tests. The S. aureus is the more virulent and produces the more acute lesions. It is important to realise, however, that a virulent strain of $\mathrm{S}$. albus may produce a more active lesion than a mild strain of $S$. aureus and that consequently it is not always possible to tell from the appearance of the lesion the type of staphylococcus responsible for it.

The following are the more common cutaneous affections of staphylococcic origin.

I. Folliculitis and Perifolliculitis.

(a) Superficial pustular perifolliculitis (Impetigo of Bockhart).

(b) Perifolliculitis of the beard or " coccogenic sycosis."

(c) Perifolliculitis associated with granulomatous and fibrous changes, e.g. Acne cheloid.

(d) Deep-seated perifolliculitis--furuncles and carbuncles.

2. Secondary pustulation or ulceration associated with other dermatoses such as eczema, streptococcic impetigo, Acne vulgaris, scabies, pediculosis, Tuberculosis cutis, leprosy, syphilis and yaws.

3. Hypertrophic lesions.

(a) Warty growths such as occur occasionally in Tuberculosis cutis, syphilis, elephantiasis, etc.

(b) Granulomatous lesions as in the so-called septic granulomata (Botryomycosis).

4. Deep-seated abscesses. Multiple cutaneous and subcutaneous abscesses in infants.

5. Lymphangitis with subcutaneous red lines of inflamed lymphatics connecting the primary lesion or site of inoculation with suppurating or swollen glands.

6. Suppurative conditions about the nails. (Suppurative perionychia).

7. Certain whitlows.

8. Purpuric lesions resulting from the presence of staphylococci in the blood.

\section{REFERENCES}

Bockhart. Uber die Aetiologie u. Therapie des Impetigo, des Furunkels, u. Sycosis (Monats. f. prakt. Derm., r887, vi. p. 450).

Broce, L. Staphylococcie cutanée (Derm. Prat., r907, i. p. 767).

Fox, Colcotт. Dermatoses of staphylococcic origin (Allbutt \& Rolleston, System of Medicine, Igrr, ix. p. 185).

Sabouraud, R. Folliculitis (La Prat. Derm., I9or, ii. p. 65 $\mathrm{x}$ ).

Whíte, C. J. Rôle of the staphylococcus in skin disease (Boston Med. \& Surg. Journ., I899, cxli. p. 235). 


\section{SUPERFICIAL PUSTULAR PERIFOLLICULITIS OR IMPETIGO OF BOCKHART}

Definition: A superficial perifolliculitis characterised by small pustules at the orifices of the pilo-sebaceous follicles.

Description.-The lesions of this affection first appear as red specks or slightly raised roundish papules about the size of a pin's head, situated at the follicular orifices, and frequently pierced by a hair.

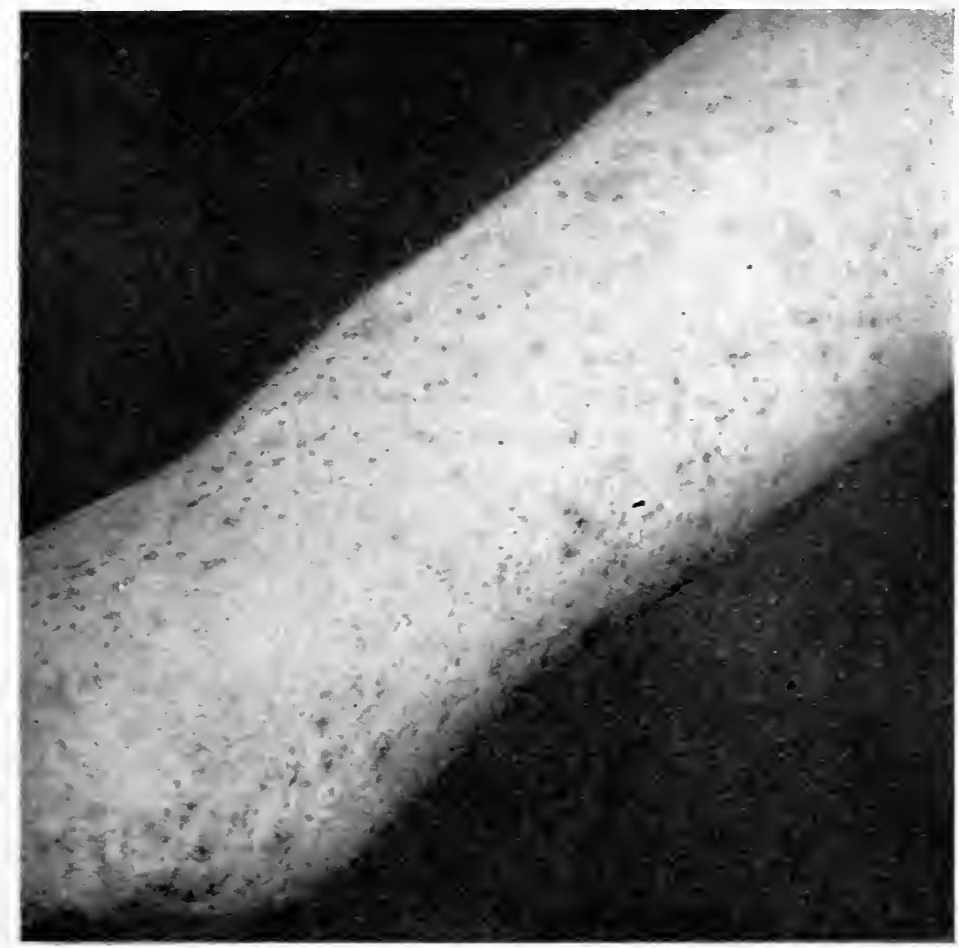

FIG. 80.-Bockhart's Impetigo.

These gradually become more prominent and in the course of a day show evidences of pus at the centre. The pustular formation is preceded by no vesicular stage such as occurs in ordinary streptococcic impetigo and the lesions are known in consequence as "Pustules ab initio" or "Pustules d'emblées." They may burst at the apex emitting a small bead of pus, but more usually dry up to form a vellowish-green scab. When the scab comes off a moist reddish macule is left which on healing does not give rise to a scar.

The pustules may occur anywhere on the cutaneous surface where hairs are present but are most common about the extensor aspects of the limbs. They may be localised to a small area, in some cases being so close together as to coalesce, or they may be widely distributed over 
the body. As a general rule they are found in greatest number in association with abscesses, suppurating wounds, boils, or other purulent lesions, but they may occur independently of any obvious focus of infection. In some cases they run an acute course and are as readily amenable to treatment as streptococcic impetigo, while in others, where the resistance of the skin is low, they may be singularly persistent and may keep coming out in crops. (Fig. 8o.)

The affection is not uncommon on the scalp especially in infants and young children. In this region it may be mild and consist of a few isolated lesions, or it may be severe and preceded by a general inflammatory disturbance of the scalp. When this occurs the scalp becomes inflamed and itchy and in a few hours an eruption of miliary follicular pustules appears more or less widely distributed over it; these dry up in a few days to form crusts, desquamation takes place and the attack is finished. As a rule, however, the original attack is succeeded by others of a similar character and the condition may persist for weeks or months. It is not infrequently associated with a scaly eczematous dermatitis beyond the hair-margin and with enlargement of the lymphatic glands in the neighbourhood.

Histopathology.- The perifollicular abscess is situated around the funnel or upper third of the pilo-sebaceous follicle, and is the result of the action of staphylococci present at first between the hair and the follicular wall and about the orifice of the sebaceous gland. The roof of the abscess is generally formed by the Stratum corneum, while the floor consists of the basal layer and lower portion of the prickle-cell layer, or, where the epidermis is more completely broken down, of the upper part of the papillary layer. As a result of the presence and action of the staphylococci, changes occur in the papillary body in the neighbourhood, consisting of dilatation of the blood-capillaries, extravasation of serum, and migration of polymorphonuclear leucocytes to the site of the invaders. In this way a dissociation of the epidermal cells is produced, necrotic changes occur, and an abscess results in which are mulberry-like groups of staphylococci situated chiefly near the roof, polymorphonuclear leucocytes, and isolated broken-up epidermal cells. (Fig. 8I.)

Etiology.-The affection is due to the Staphylococcus pyogenes, either albus or aureus, at the pilo-sebaceous follicles and can be experimentally produced by rubbing pus or even a culture of the microorganism over the surface of the skin.

In most cases it is caused by contamination of the skin in the neighbourhood of an abscess, ulcer, or sinus, by purulent discharges from the septic focus. It may, however, be the result of augmented virulence of staphylococci already present on the cutaneous surface which have been rendered active through a weakened resistance of the skin from general causes, such as impaired nutrition or debilitating disease, or from a diminished local resistance resulting from co-existent dermatitis, such as eczema, the cutaneous disturbance set up by 
pediculosis or scabies, or the lowered vitality induced by some local irritant such as tar, croton oil, chrysarobin, or the $\mathrm{X}$-rays. The occurrence of superficial pustular folliculitis in association with the defluvium of the hair caused by the X-rays in the treatment of ringworm is a complication which is not infrequent and should be guarded against by appropriate antiseptic measures.

Diagnosis. - The diagnosis is so simple that it requires no special description.

Treatment.-On the glabrous skin the treatment of this affection is comparatively easy and rapid and no scarring or disfigurement need result. It consists of the remoral of crusts and pus by boric compresses and if necessary of the evacuation of the lesions by a fine pointed tenotome or comedo expressor. Dilute ammoniated mercury ointment should then be applied continuously or, where this is impossible, it

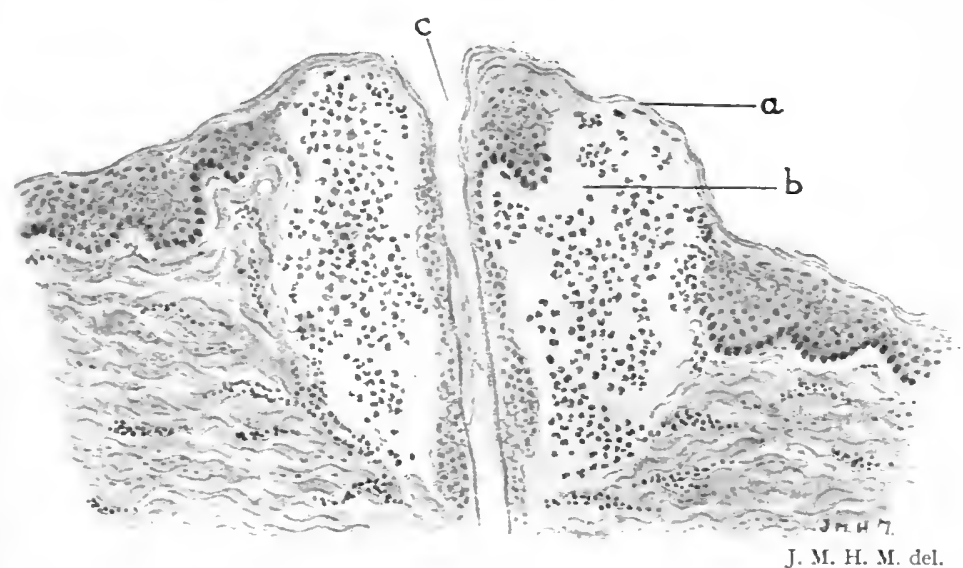

Fig. 81.-Impetigo of Bockhart. Superficial perifollicular abscess.

a. Stratum corneum forming a roof to abscess. b. Abscess. c. Hair-follicle.

should be rubbed in several times a day. A useful abortive treatment where the lesions are numerous is to paint them twice a day with tincture of iodine which causes them to dry up and desquamate in the course of a few days.

On the scalp the affection may prove most intractable, especially in girls with long hair. Where possible the hair should be cut close and the crusts and discharges removed by boro-starch poultices, or by compresses of boric acid or hydrogen peroxide (Io vols.). After the removal of the crusts, oozing points should be painted with compound tincture of bezoin or with an iodine solution containing I part of the tincture to 4 of $60 \%$ spirit. The painting should be repeated several times a day for a couple of days if necessary, the scabs being prevented by compresses. This should be followed by the continuous application of an ointment containing $2 \%$ of ammoniated mercury kept constantly applied by being spread on lint under a head bandage. 


\section{REFERENCES}

Sabouraud, R. Folliculitis (La Prat. Derm., r9or, ii. p. 65I).

UnNA, P. G. Impetigo of Bockhart (Berlin Klin., I892, xlvi. p. x).

VAN Bambere. Impetigo circumpilaire (La Semaine Méd., r9oo, p. 229).

\section{SYCOSIS BARBAE}

Syn.: Coccogenic sycosis; Mentagra; Fr., Sycose; Ger., Bartfinne.

Definition: A purulent perifolliculitis of staphylococcic origin affecting the beard and moustache, characterised by the presence of follicular papulo-pustules, inflammatory nodules, and indurated swellings, with a tendency to cicatricial changes, atrophy, and destruction of the hairs.

Description.- The earliest sign of the staphylococcic infection of the hair-follicles of the beard or moustache is the appearance of a number of discrete follicular papulo-pustules about the size of a pin's head and generally pierced by a hair. These may appear on one side of the face or on both successively or simultaneously. They are, as a rule, indolent in their course and may dry up with the formation of a yellowish crust, or may give rise to small nodules or tubercles from a cellular infiltration around a suppurating focus deeper down in the corium. When contiguous follicles are affected a confluent lesion results consisting of an infiltrated patch or swelling, the surface of which is uneven and dotted over with follicular pustules. (Fig. 82.)

The affection is kept up by new lesions appearing either isolated or in crops. The skin of the region involved gradually becomes thickened, red, and swollen, occasionally crusted or papillomatous, and is as a rule irritable, hot, and tender to the touch. In old-standing cases cicatricial changes supervene in which the skin becomes atrophic, shiny, inflamed, and the follicles destroyed. Sometimes the atrophic area is limited by an active pustular or crusted border somewhat suggesting Lupus vulgaris, hence the old name Lupoid sycosis which was once applied to this phase of it. The hairs at first remain tightly implanted in the follicle and are difficult to epilate but when suppuration sets in they come out easily with an oedematous or purulent root-sheath. In mild

Note.--The term sycosis, from the Greek word " oìкоv" a fig, was once employed in a wider sense than is customary at the present time, and was applied to any purulent folliculitis in which angry inflamed swellings occurred dotted over with yellow follicular pustules, suggesting an everted fig with its seeds. Lesions of this type are not infrequent in the beard region and are due either to staphylococci or ringworm fungi, when they are known respectively as Coccogenic and Hyphomycetic Sycosis barbae. The name was also applied to certain ill-defined scar-leaving dermatoses of the beard region, possibly of tuberculous origin. The term is now generally restricted to a staphylococcic infection of the hair-follicles of the beard, moustache, and, more rarely, of the scalp, eyelashes, inside of the nose, axillae, and pubes. 
cases only a small part of the cheek or chin may be attacked, but in severe ones the whole of the beard or moustache region may be involved. Where the disease is extensive it may be associated with small boils or even abscesses and occasionally with impetigo or eczematisation beyond the hairy border or about the auricles.

Histopathology.-Microscopically there is a perifolliculitis which extends deeply into the corium, with vascular dilatation, inflammatory cellular infiltration, and, in some cases, abscess formation about the sebaceous glands and deeper portions of the follicle. It is this cellular infiltration which is responsible for the nodules and which tends

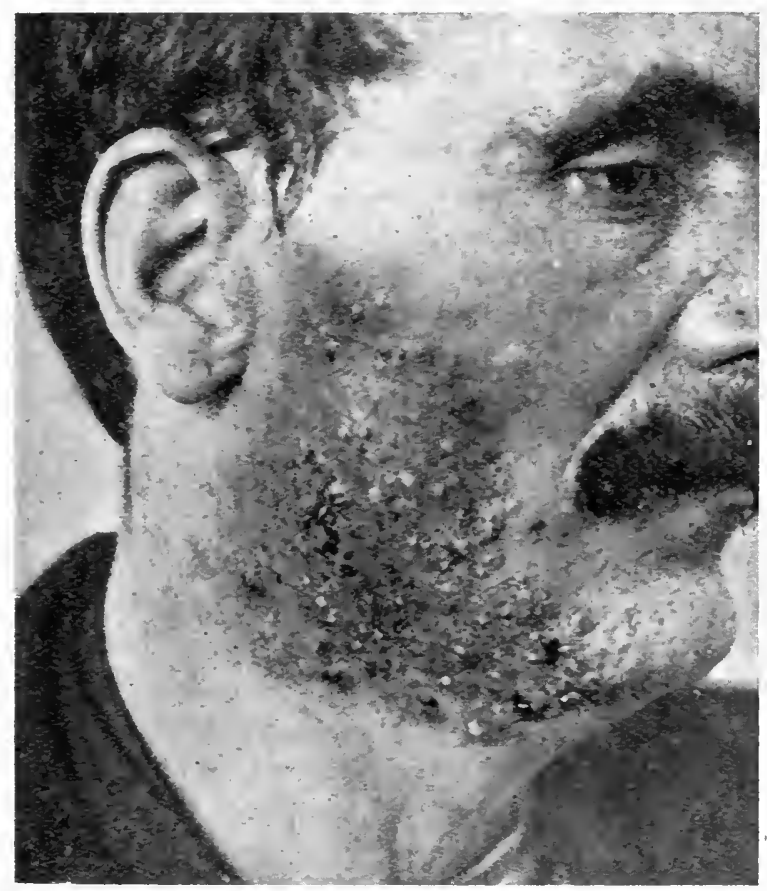

FIG. 82.-Sycosis Barbae (Coccogenic).

(From Dr. Arthur Hall.)

eventually to undergo organisation and produce cicatricial changes. The pus from the abscess spreads towards the surface around the funnel of the follicle and breaks through at the follicular orifice. As a general rule the sclerotic process does not involve the hair-papillae and re-growth of the hair takes place.

Etiology and Pathogenesis. - It is due to an inoculation of the hairfollicles with Staphylococcus pyogenes albus and aureus. This may result from a scratch, or from the employment of an infected razor, a dirty shaving-brush, etc. On the cheeks it may be due to secondary infection with staphylococci, and be preceded by some inflammatory disturbance such as eczema, streptococcic impetigo or seborrhoic dermatitis, which by lowering the vitality of the skin makes it a more 
suitable soil for the growth of the micro-organism. On the upper lip it is often caused by chronic catarrhal rhinitis and is due to infection of the sodden skin by staphylococci in the discharge from the nose.

Diagnosis.-The diseases with which coccogenic sycosis barbae is most liable to be confused are ringworm of the beard, eczema, seborrhoic dermatitis, Lupus vulgaris, and streptococcic impetigo.

From ringworm the diagnosis may be impossible without a microscopical examination of the hairs for the fungus, and in any case where there is the least doubt this should be done as a routine. As a rule the coccogenic affection is more widely and irregularly distributed over the face, while the ringworm is more localised and the lesions are more lumpy, but this is by no means invariably so and cases of ringworm of the beard occur in which there is little or no induration. The hairs in the coccogenic type are not broken and when epilated come out comparatively easily with a moist root sheath, while those of ringworm form stumps which break on epilation, are opaque, and under the microscope show the characteristic chains of fungus.

From eczema and seborrhoic dermatitis it is distinguished by being confined to the hairy region, while those conditions almost invariably spread beyond it, and present scaly, crusted or oozing patches, and the hair-follicles are not attacked.

From Lupus vulgaris it is distinguished by the absence of the reddish-brown apple-jelly nodules and the tendency to ulceration.

From streptococcic impetigo it is recognised by being essentially follicular and deep-seated while the streptococcic affection involves the skin rather than the follicles and is associated with the typical superficial phlyctenules and stuck-on crusts of Impetigo contagiosa.

Prognosis.--The affection is singularly chronic and intractable, shows no tendency to spontaneous recovery, and is apt to lead to cicatricial changes with destruction of some of the follicles and slight disfigurement from atrophy of the skin. It is amenable, however, to treatment even in the most advanced cases.

Treatment.-The successful treatment of Sycosis barbae is almost as difficult as that of ringworm of the same region, because the cocci are situated so deeply in the follicles that it is practically impossible for antiseptic remedies applied on the surface to penetrate sufficiently deeply to destroy them. As a preliminary all crusts should be removed by boro-starch poultices or other suitable means and the hairs in the affected region should be epilated. This is a somewhat painful and tedious procedure but is well worth while as it gets rid of the infected roct-sheaths, allows a freer discharge of pus, and limits the spread of the disease. Deep-seated nodules should be incised by a fine pointed scalpel and any pus evacuated, compresses of boric acid or hydrogen peroxide should then be applied for an hour several times during the day and an ointment containing $3 \%$ of ammoniated mercury, $3-6 \%$ of resorcin, or oleate of mercury ointment B.P., rubbed in at night. 
The question of shaving during the treatment is open to discussion. Its advisability depends on the extent and degree of the inflammatory thickening. Where the folliculitis is superficial it may be of benefit, but where it is deep-seated it is painful and may be impossible without cutting or lacerating the skin. Its chief disadvantage is that pustules are opened by it and the virus spread by the brush or the razor. This is avoided to some extent by the substitution for soap of an antiseptic cream, such as cold cream with the addition of $2 \%$ of ammoniated mercury, rubbed in thickly by the fingers, by dipping the razor in boiling water or I in 40 carbolic acid several times during the sharing, and by the application of a boric compress when the operation is completed.

Should the disease be persistent and the inflammatory thickening considerable, the most effective treatment is epilation by the X-rays, which should be done with the same precision as in the case of ringworm of the scalp. A full pastille dose should be given, provided the inflammation is not too acute, and repeated in six weeks if a sufficient defluvium has not taken place. Should the inflammation be intense, not more than three-quarters of a dose should be given, which may be estimated in a simple manner by comparing the pastille with the tint $B$. by artificial instead of day-light. On no account should repeated small doses be employed over a long period, as in this way a dermatitis may occur when least expected and may lead to disastrous results. After the exposure, until the hairs fall out, the skin should be anointed daily with $2 \%$ ammoniated mercury ointment and should not be shaved.

As the disease is purely local no form of general treatment is indicated. Vaccine therapy with an autogenous vaccine has been employed and the results from it are sometimes good.

\section{Sycosis ix Other Regiors}

A condition similar in its pathogenesis and analogous in its clinical features to Sycosis barbae may involve the scalp, eyelashes, eyebrows, nose, and occasionally axillae or pubes. When it attacks the scalp there is little or no tendency to the formation of indurated swellings, but destruction of the follicles is liable to occur with cicatricial atrophy, the scalp becoming red, shiny, and partially bald. The most satisfactory treatment of the scalp affection is epilation by a full pastille dose of the $\mathrm{X}$-rays to bring out the infected hairs before the destructive process has reached the papillae, the area exposed being a little bigger than the diseased area; this may cure the condition with an almost perfect re-growth of the hair.

Where the disease is present in the nose it causes considerable discomfort if not actual pain and is most resistant to treatment. In mild cases douches of hydrogen peroxide (Io vols.) or boric lotion may bring about a cure but as a rule they are ineffectual. In a few cases penefit may be derived from zinc ionisation; a solid zinc electrode 
is wrapped round with lint saturated with $2 \%$ solution of sulphate of zinc, attached to the positive pole and inserted in the nostril, the negative pole is held in the patient's hand, and a current of about 2 milliamperes is passed for about Io minutes. This procedure is repeated daily until the condition is cured. Should it fail the X-rays may be of benefit.

\section{Sycosis NuchaE}

Syn. : Sycosis framboesiformis (Hebra), Dermatitis papillaris capillitii (Kaposi), Acne cheloid; Fr., Acné chéloïdique; Ger., Nacken Keloid.

The affection to which these names have been applied is a somewhat rare inflammatory perifolliculitis which occurs most usually at the back of the neck just below the hair-margin. In a well-marked case it takes the form of a cheloidal or cicatricial thickening of the skin, irregular in outline, varying from one to several inches in its long diameter, generally situated transversely, with an uneven

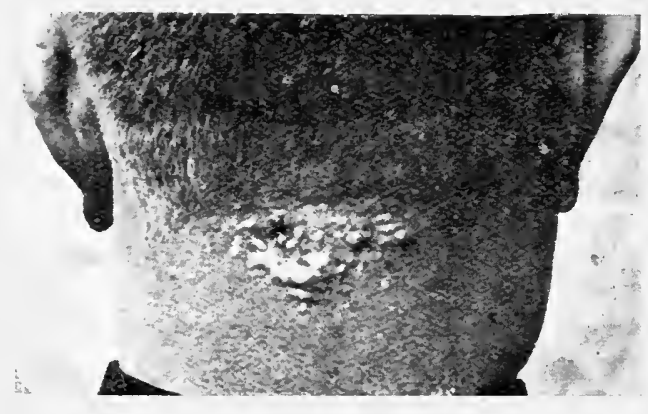

FIG. 83.-Acne Cheloid, or Sycosis Nuchae.

nodular surface which is either reddish, smooth and shiny, or presents small tufts of hairs, papulo-pustules pierced by hairs, or yellowish adherent crusts. It commences as a pustular perifolliculitis, followed by fibromatous and cicatricial changes with the formation of nodules and the destruction of the smaller pilo-sebaceous follicles. The hairs in the tufts are atrophic, bent irregularly, and come out with difficulty on epilation. Occasionally small deep-seated abscesses may be present which do not tend to necrose but from which a sanious pus may be evacuated. Here and there over the diseased area seborrhoic plugs or typical comedones may be detected blocking up the follicles, and it is their presence which gave rise to the idea that the condition was a form of acne and suggested the synonym, Acne cheloid. Comedones are of frequent occurrence in this region apart from this affection and their association with it is probably accidental rather than essential. The general consensus of opinion is in favour of regarding the condition as a sycosis analogous to sycosis of the beard, and the occasional occurrence of both simultaneously corroborates this view. (Fig. 83.) 
In certain cases the surface instead of being smooth may be covered with greasy crusts or with papillomatous vegetations which readily bleed when any attempt is made to remove them. In old-standing cases there is a tendency for the cicatricial changes to be most marked in the centre of the lesion which may become atrophic and bald, while at the periphery, where the disease is active, nodules with tufts of hair, papulo-pustules, and crusts, may be present. Though usually confined to the nape of the neck the affection may be found occasionally on the hairy scalp, the occipital region, or the vertex, either independently or by extension from the neck. As a rule there are no marked subjective symptoms though occasionally it may itch or, where small abscesses are present, it may be hot and tender.

Histopathology.-Microscopically it is found to consist of a deepseated perifolliculitis with abscess-formation around the deeper portion of the follicles and extending into the subcutaneous tissue. This is generally surrounded by a dense cellular deposit which shows signs of organising by the presence of numerous large spindle-cells and newly-formed fibrous tissue. According to Unna the new fibrous tissue is limited by the sub-papillary vascular plexus above and by the hypoderm beneath, and elastic tissue is absent from it.

Etiology and Pathogenesis.-It is probably due, like Sycosis barbae, to Staphylococcus pyogenes aureus and albus, both of which have been found in the lesions. The fibrous development is believed to result largely from the histological structure of the corium and hypoderm in the region affected, for there the tissue is naturally rich in collagen fibres and the venous state of the circulation tends to excite fibromatous changes. It occurs almost invariably in males, chiefly in early adult life, and particularly in fat-necked subjects.

Diagnosis.-The diagnosis presents no great difficulty. The cheloidal thickenings, tufts of atrophic hairs, papulo-pustules and crusts, form a complex of symptoms which is so characteristic as not to be readily mistaken.

Prognosis.-The affection is singularly chronic and intractable and shows no tendency to spontaneous cure. By mean of suitable treatment, however, especially by the X-rays, its progress may be arrested and a cure effected.

Treatment.-The condition, being purely local, does not respond to internal treatment. Vaccine therapy has been used in connection with it and both stock and autogenous staphylococcic vaccines have been employed, but the results from them thotgh occasionally beneficial have been uncertain.

By far the best treatment is the X-rays, by which not only is the fibrous thickening reduced but the hairs over the surface which may form foci for a further spread of the disease are epilated. A pastille dose is given and repeated later if necessary. If the lesion be on the hairy scalp care must be taken not to give a second dose until the effect of the first has completely worn off for fear of causing 
permanent baldness. If the lesion be in a state of acute inflammation it is advisable to reduce this by means of boric compresses before exposing it to the $\mathrm{X}$-rays.

Where the X-rays are not available the lesions should be treated on simple antiseptic and minor surgical principles; any suppurating points should be opened by a fine tenotome and the pus evacuated, crusts should be softened with oil and cleaned off, vegetations removed by the curette and bleeding points stopped by a cautery. Boric compresses should then be applied continuously. Should these prove irritating, compresses of I in 4000 corrosive sublimate may be substituted. There is no advantage in using strong antiseptic applications as they have no more penetrating power than have weaker ones and simply lower the resistance of the surface of the skin. Instead of lotions, painting with tincture of iodine diluted in 4 parts of $60 \%$ spirit may be employed, or the continuous application of $3 \%$ ainmoniated mercury ointment on lint under a bandage. After the inflammatory disturbance has completely subsided the employment of injections of fibrolysin to reduce the cheloidal thickenings and remove the cicatrix has been advocated, but the results from this procedure are unreliable. Another form of treatment which has been advocated is multiple scarification but it too is of doubtful value.

\section{BOILS}

Syn.: Furuncle ; Fr., Furoncle ; Ger., Furunkel.

Definition: An acute deep-seated circumscribed inflammatory lesion, round or acuminate in shape, painful and tender to the touch, and generally ending in central suppuration and necrosis.

Description.- The first evidence of a boil is generally a small, hard, painful thickening situated in the skin or subcutaneous tissue, the surface of which is red and inflamed. In a few days the swelling becomes more pronounced and the lesion may reach the size of a filbert nut and may be rounded or acuminate in shape. About this time the boil begins to "point," the skin near the summit becomes paler, and a central softening takes place which continues until the whole of the induration has broken down. The boil may then break and discharge itself, or the contents may dry up to form a cheesy mass which separates in a week or two as a necrotic slough. The discharge is generally greenish-yellow and may be thin, or thick and cheesy with a central pultaceous necrotic core. When the boil has been evacuated the pain and tenderness disappear and healing begins, the cavity gradually filling up with granulation tissue. The resultant scar is usually comparatively slight and consists of a red spot surrounded by a zone of pigmentation. In some cases, however, a disfiguring cicatrix may follow which is occasionally cheloidal. More rarely the lesion may commence as a superficial follicular pustule pierced by a hair, which, 
by the deep penetration of the virus, becomes transformed into a boil and goes through the same stages as those detailed above.

The course varies considerably in different cases, but the ordinary acute boil generally matures in about a week and, if properly treated, is cured in a fortnight. Boils as a rule mature more quickly in strong healthy subjects than in those with a weak constitution, simply because the boil is the clinical evidence of the local reaction of the skin to the invading micro-organisms and the reaction is much more acute in health than in disease. Occasionally the course of a boil is very slow and it may persist for weeks as a hard red nodule before softening takes place; this is known as a " blind boil."

Boils vary in size from a filbert to a walnut. They may be single or multiple and may be in close proximity or widely distributed over the body. Several boils may appear simultaneously, or they may come out successively and so perpetuate the disease for months, in which case the condition is known as furunculosis. They may occur anywhere on the skin but are especially common in situations exposed to friction such as the buttocks, inside of the thighs, back of the neck, axillae, and groins. The lymphatic glands in the neighbourhood of the lesions are frequently enlarged.

The subjective symptoms vary from itching and tenderness to excruciating pain when they occur in situations where the tissues are tense, as about the external auditory meatus. Except for the discomfort produced by them the general health is not seriously disturbed. If they are persistent, however, and widely distributed they may give rise to anaemia, malaise, and even slight pyrexia, from the absorption of toxins. In rare instances boils on the face have been known to be followed by grave consequences, as owing to the great vascularity and the free venous anastomoses, there is in this situation a possibility of thrombosis and septic phlebitis and a chance of the cocci reaching the general circulation, causing septic meningitis, pneumonia, pyaemia or even cerebral abscesses. Several cases of this type have been reported which were fatal.

Histopathology.-The microscopical appearances presented by sections of a boil vary according to the stage and extent of the suppurative process, and are similar, though much more marked, to those of the impetigo of Bockhart. The pathological changes occur in connection with pilo-sebaceous follicles, especially those of lanugo hairs where the follicular walls are weak and easily penetrated by staphylococci. They may be met with also independently of hair-follicles, in connection with the sweat-glands, or the ceruminous glands in the auditory meatus. In any case the abscess formation is situated deeply in the corium or subcutaneous tissue. The histological changes are the result of the reaction of the corium to the staphylococci and consist at first of dilatation of the neighbouring blood-ressels, more or less oedema, and a profuse migration of leucocytes; this is followed by a cellular infiltration which forms around the septic focus and 
consists of lymphocytes or small connective tissue cells and, in chronic lesions, of plasma-cells and multi-nuclear cells. This dense cellular deposit is avascular and readily breaks down to form a central necrosis and an abscess.

Etiology.-Boils are caused by the Staphylococcus pyogenes albus, aureus, or both, the aureus being the most commonly present. The micro-organism gains entrance either at the hair-follicle or through an abrasion. The lesions are spread by auto-inoculation, scratching, the chafing of the collar at the back of the neck, friction and contamination by infected clothes as in the case of oarsmen or horsemen, and, in rare instances, by cocci which have reached the skin via the general circulation as in pyaemia.

Boils may occur at any age and may affect either sex but are most common in adult males.

Predisposing Causes.--(I) The local predisposing causes consist of any condition of lowered resistance of the skin brought about by irritation, pressure, friction, the presence of pediculosis or scabies, some intercurrent dermatitis such as eczema, or excessive sweating and moisture, a factor which is noticeable in the tendency of boils to occur in the moist regions between the buttocks and in the axillae, especially in the tropics in the hot season. (2) The general predisposing causes consist of any form of lowered vitality from a defective state of the blood such as occurs in anaemia, malaria, alcoholism, prolonged illness, digestive disturbances, constipation, Bright's disease, or glycosuria. When the resistance of the individual is high, only one or two lesions may appear, while in debilitated conditions successive lesions may develop and the disease may be singularly persistent.

Diagnosis.-The diagnosis presents no serious difficulty, the only condition from which it has to be differentiated is carbuncle to which it is closely allied. The carbuncle, however, is a composite lesion with several suppurating points while the boil is single and only points in one place.

Prognosis. - The ultimate prognosis of boils is satisfactory, though the time taken for a cure to be effected varies considerably in different individuals. Where only one or two exist and the general health is good, healing may take place in the course of a week or two, but should they occur in situations where the hair is long and the tissues tough, as at the nape of the neck, they may be singularly persistent in spite of most thorough treatment. Where they are present in an indivioual whose vitality is depressed and occur in successive crops, the immediate outlook is unfavourable and a somewhat tedious and prolonged course of treatment may be necessary before the affection is finally cured. As a rule they have no deleterious effect on the general health, unless in prolonged cases or where the lesions have occurred about the face and produced serious complications from venous thrombosis. The scarring varies greatly; if the boils 
are allowed to break by themselves it may be excessive and even cheloidal, but if they are carefully incised and properly dealt with it should be negligible.

\section{TREATMENT}

The two main objects in the treatment consist of (I) destruction of the micro-organisms and the removal of their effects, (2) rendering the tissues an unfavourable soil for the further spread of the disease.

General Treatment.-Any defect in the general health, such as digestive disturbances, constipation, or derangement of the kidneys, should be sought for and dealt with on general medical principles, and in every case the urine should be examined for sugar or albumin.

Where the boils occur in anaemic over-worked people much benefit may be derived from a proper regime, with change of air, exercise, and tonics containing iron, strychnine, etc., and in those suffering from malaria quinine is indicated-in short any debilitating influence must be treated by suitable methods.

There are three internal remedies which are believed to possess a specific action on boils and furuncles, namely, sulphur, yeast, and manganese in its colloidal form.

Sulphur.-Sulphur may be prescribed in lozenges or as half to one teaspoonful of sublimed sulphur every morning. A favourite method of administration is in the form of sulphide of calcium in doses of onetenth to half a grain mixed with sugar of milk every two hours (Ringer) ; as this preparation readily becomes inert from oxidation and decomposition it should be prepared freshly every day or prescribed in coated pills. Another useful preparation of sulphur is collosol sulphur (Crookes) which is given in doses of one or two teaspoonfuls in water twice daily, and has the advantage of combining readily with protein and being easily absorbed in the stomach.

The beneficial action of sulphur is partly due to its being excreted in the sweat and acting as an antiseptic in passing through the skin, and partly to its mild laxative properties.

Yeast.-Yeast may be given at the beginning of a meal in doses of a teaspoonful or more of fresh brewer's yeast in water, milk, or beer. Larger doses should be given with caution as it sometimes tends to cause diarrhoea and colic and its smell and taste may be extremely. unpleasant.

Instead of fresh yeast its extracts, nuclein or ceredin, may be substituted, which have the advantage of being less disagreeable to the taste, more stable in composition, and capable of more accurate dosage. Nuclein should be given by subcutaneous injection or by the mouth in doses of one to two teaspoonfuls of the nuclein solution in a wineglassful of water t.d.s. between meals and at bedtime. Ceredin is a fatty constituent of yeast and is given in doses of o.I gram in a pill t.d.s. 
Collosol Manganese (Crookes).-Collosol manganese was introduced for the treatment of furunculosis by McDonagh. The results from it are sometimes encouraging both with regard to the healing of the local lesion and the general improvement of the patient. It is employed in the form of two solutions, which are mixed in the syringe and injected intramuscularly into the buttock in doses of 0.5 to $1.5 \mathrm{c.cm}$. at intervals of three days. The injections are painless and produce no local reaction.

Oxide of Tin.-In France a preparation known as stannoxyl, which is an oxide of tin, has been used extensively in the treatment of boils, acne pustules, etc. It has been claimed for it that it relieves the pain and causes the furuncle to dry up and the core to be absorbed. It is supplied in tablets of which the dose is 4 to 8 daily.

Vaccine Treatment.-Staphylococcic vaccines have been employed largely in the treatment of boils, and in acute cases this treatment probably gives better results than it does in any other skin affection. An autogenous vaccine is preferable to a stock vaccine, but the latter may be sufficiently efficacious. It is unnecessary to control the injections by the opsonic index, as should the treatment be unsuitable or the dose too large it is indicated by an eruption of fresh lesions. An initial dose of Ioo millions is given, followed in four days by 250 millions should the condition be improved or remain in statu quo, and this generally effects a cure.

In chronic cases the treatment is not so uniformly successful. An initial dose of 100 millions is given, followed in a week by 200 millions, and so on with a weekly increase of Ioo millions until a maximum of 500 millions has been reached. In these cases fresh outbreaks of lesions may occur in spite of the treatment or after temporary improvement.

The vaccine should be injected into the subcutaneous tissue of the thigh, shoulder, or back, with the usual aseptic precautions. It acts by increasing the protective substances in the blood and inducing fibrocytosis. In chronic cases failure is due to the resistance of the individual serum having been already fully stimulated from the chronicity of the disease and the continued presence of numerous staphylococci.

Local Treatment.-Local treatment may be of more importance than any form of general treatment and in all cases, even when vaccines are employed, healing may be facilitated by combining internal remedies with suitable local measures.

The local treatment varies according to the size, situation, and stage of the boil. If it be in the early indurated stage and there is no evidence of central softening or pointing it should be fomented with hot boric acid compresses changed frequently or when situated on the hands or feet by immersing the part in a hot boric lotion bath. This treatment should be continued assiduously till softening takes place and signs of pointing appear when a deep incision should be made by a fine tenotome, the pus allowed to well out, and the cavity washed 
out by injecting I in 30 carbolic acid lotion by a hypodermic syringe. The boil should on no account be opened in the hard stage nor, when opened, should it be squeezed, and the old-fashioned linseed meal poultice should be avoided as it spreads the septic condition. When it is discharging boric fomentations should still be continued, but on account of the maceration of the skin by the fomentations, every time a fresh one is applied the lesions should be bathed with I in 4000 corrosive sublimate lotion, and, to prevent the infection of the surrounding skin by the discharge, the neighbourhood for a distance of an inch or more around the boil should be painted with tincture of iodine or smeared with dilute ammoniated mercury ointment. Instead of hot boric compresses good results can be obtained by applying pads of cottonwool saturated in carbol-glycerine ( $I$ in S) and covered by guttapercha tissue.

Another treatment which is of benefit if properly carried out has been suggested by Gallois and consists of painting any unbroken boil with a solution of iodine (2 parts) in acetone ( 5 parts) and applying to the affected area a pad of lint wrung out in boiling water and saturated in glycerine. This is covered with a layer of cottonwool and bandaged, the dressing being changed twice a day.

A form of local treatment which occasionally gires good results is that by Bier's cupping glasses, which act by producing a passive hyperaemia and an increase in the amount of protective substances in the serum. When the glass is applied the degree of suction should be just sufficient to cause discomfort without actual pain. It should not be applied continuously but put on for five minutes then removed for a few minutes, re-applied, and so on for an hour once or twice a day. This treatment is of special value when the boil has matured and been incised, for by it the discharge of pus is facilitated, sloughs are separated, and healing is accelerated.

When boils occur about the face or external auditory meatus they should be promptly incised, and irrigated frequently with hot boric lotion to reduce the inflammation.

Several methods have been suggested with the object of aborting boils and causing them to resolve without suppurating but these, as a rule, give disappointing results. One of the most effective is the continuous application of mercury and carbolic plaster. Other useful methods are painting the lesions every four hours with tincture of iodine, or collodion containing $5 \%$ of iodoform, and injecting a few drops of carbolic acid into the centre.

One of the most troublesome positions for boils is the back of the neck where owing to the chafing of the collar they are liable to be spread and perpetuated. In such cases silk or flannel collars or a soft hunting stock should be worn. On the whole the most useful treatment in this situation is the persevering application of hot boric compresses. Where the skin has become markedly indurated and the affection has reached a stage of chronicity in which compresses 
seem to have lost their effect, improvement may be obtained from exposures to Finsen light given in the same manner as in Lupus vulgaris. This produces an inflammatory reaction followed by marked reduction of the induration. The X-rays are also of value in such cases, $\frac{\mathbf{3}}{\mathbf{4}}$ of a Sabouraud pastille dose being given and repeated in a month if necessary. The X-rays should not be employed in acute cases as they are liable to produce an exacerbation of the inflammation which may last several weeks before subsiding.

In every case it must be borne in mind that not only is it necessary to treat the individual lesion, but that it is equally important to render the neighbouring skin as unsuitable a soil as possible for the further spread of the disease by painting it with tincture of iodine or by rubbing in daily $3 \%$ ammoniated mercury ointment.

\section{REFERENCES}

Adamson, H. G. Boils \& carbuncles \& their treatment (Practitioner, I909, 1xxxiii. p. 5 I2).

Barozzi, J. Furunculose (La Prat. Derm., I901, ii. 710).

Desfosses. The treatment of furuncle (La Presse Méd., I902, ii. p. 653).

Lamann. Boils in auditory meatus (Monats. f. Ohrenh., I899).

Lympius. Boils on the face (Deutsch. Med. Woch., I899, Xxv. p. 474).

McDonagh, J. E. R. A new treatment for Staphylococcic infections (Med. Press \& Circ., I9I 7, p. 43I).

Morris, Sir Malcolm. Treatment of furunculosis \& other deep-seated coccogenic infections by collosol manganese (Brit. Med. Journ., rgr 8 , i. p. 445 ).

Ross, E. Treatment (Folia Therapeut., I907).

WRIGHT, A. E. Treatment by Staphylococcus vaccines (Brit Med. Journ., I904, i. p. I075).

\section{CARBUNCLE}

Syn. : Anthrax benigna; Fr., Carboncle; Anthrax bénin; Ger., Karbunkel, Brandschwär.

Definition: An acute purulent inflammation like a mass of confluent boils, forming a circumscribed flat swelling the surface of which is riddled by suppurating or necrosing points.

Description.-Clinically and histologically a carbuncle may be described as a confluent group of boils. As the suppurative process is more extensive and deep-seated than in a boil the lesions are more virulent and phlegmonous and the general symptoms associated with them correspondingly more severe.

The carbuncle is usually single, and situated most commonly near the mid-line of the upper part of the back or the lower part of the neck, but may occur anywhere on the glabrous surface. It generally begins as a painful indurated swelling, the skin over which is of a deep red colour, the inflammation spreading for some distance beyond the indurated area. In the course of a few days it may reach the size of a 
crown-piece when a number of suppurating points may generally be detected, which break down, and through which a sanious pus wells up from the multilocular abscess cavity in the lower part of the corium and subcutaneous tissue. These necrotic orifices are sometimes so numerous as to give the surface a cribriform appearance.

The central portion of the lesion generally necroses and separates as a slough, leaving an irregular punched-out ulcer. In course of time this becomes filled up with granulation tissue and healing takes place with the production of a marked cicatrix.

In an ordinary uncomplicated case occurring in a comparatively healthy patient, the whole course of the lesion may vary from a few weeks to a couple of months. In elderly debilitated people or in those run down from some cause such as diabetes, it may persist longer, be more fulminating in character, and assume larger proportions. In such cases it has been known to affect the greater part of a region and to be surrounded by a more or less extensive area of brawny oedema. In the most severe cases of all it may become gangrenous. As a rule the necrotic process is limited by the deep fasciae, but occasionally it has been known to penetrate the spinal canal when the carbuncle was on the back, the peritoneum when on the abdominal wall, and the meninges when it occurred on the face.

The pain associated with it is intense and of a gnawing or lancinating character. The general symptoms are more severe than those which accompany boils and are present from the onset. They consist of malaise, headache, pain in the back and joints, shivering, and a rise of temperature, and in the worst cases mental disturbance, delirium, and all the symptoms of fatal septicaemia.

Histopathology. - The histopathology is similar in the main to that of boils. The morbid process and abscess-formation, however, instead of being more or less circumscribed around the follicle and confined to the corium, occur more deeply in the subcutaneous tissue and spread out in the lines of least resistance between the fibrous bands and the groups of fat-cells. The staphylococci responsible gain entrance generally at the hair-follicles but occasionally at the sweat-pores. They penetrate deeply into the corium and subcutaneous tissue where they cause an abscess limited by a dense cellular infiltration which, being avascular, tends to undergo necrosis, the pus making its way upwards along the fat-columns and follicles towards the surface and eventually breaking through the epidermis.

Etiology and Pathogenesis.-The exact pathogenesis of carbuncles is not understood. Like boils they are caused by the local inoculation of the Staphylococcus pyogenes aureus, generally at the pilo-sebaceous follicles, and have been experimentally produced by. inoculating the skin with this micro-organism (Garré); but the reason why in the one case the lesions should be limited to one follicle and in the other should be more diffuse and affect several contiguous follicles, is not quite clear. It has been suggested that it may be due to a more 
virulent strain of staphylococcus inoculating several follicles at the same time, but it is probably mainly the result of an extremely low state of vitality of the skin which forms a specially suitable soil for the growth of the invading micro-organism. This lowered vitality may result from a number of causes, such as the severe constitutional depression which occurs in connection with diabetes, Bright's disease, and malaria, the convalescence from some prolonged debilitating illness, and old age. The condition is specially common in the male sex after the age of 40 .

Diagnosis.-Carbuncles are distinguished from simple boils by the multiple points of suppuration. At one time they were confused with "malignant pustule" - the skin lesion of true anthrax-hence the name which is still used for them in France, "Anthrax bénin." The malignant pustule, however, is easily distinguished from the carbuncle both in its course and clinical aspects. It begins as a vesicle which ruptures leaving a black necrotic centre surrounded by a zone of intense inflammation over which a more or less complete ring of vesicles may develop, and it occurs usually on the hand or face, situations rarely attacked by carbuncles.

Prognosis.-The prognosis should always be guarded. In most cases a cure may be effected by suitable treatment but unless radical measures be adopted the course of the affection may be indefinitely prolonged. In elderly people with diabetes or albuminuria the outlook may be extremely serious and the patient's life may be endangered from exhaustion or septicaemia. In the rare cases of malignant carbuncle about the face a fatal issue may result from septic phlebitis and its consequences.

\section{TREATMENT}

General Treatment.-The general treatment is based on ordinary medical principles. In every case the urine should be examined for sugar or albumin. Where glycosuria is detected codeia or opium is indicated in doses of I gr. t.d.s. or 2 grs. at bed-time, as it not only relieves the pain of the carbuncle and induces sleep but has a beneficial action on the diabetes. When the pain is considerable sedatives are demanded; of these bromides and chloral hydrate are of value, but opium, except in diabetics, is better avoided. Formerly when the favourite treatment was crucial incisions and a stimulating regime, it was customary to confine the patient to bed, to prescribe alcohol, in the form of burgundy and port, and to advise a specially generous diet. This treatment has now given place to a more rational one, consisting of ordinary diet, the avoidance of alcohol which only increases the tension in the lesions, fresh air, exercise, and tonics such as iron, strychnine and quinine. Sulphur and yeast have been claimed to have a specific action on carbuncles but their value is uncertain. 
Vaccine Treatment.-Vaccine treatment has given good results, the injections being similar to those employed for the treatment of boils, namely an initial injection of roo millions of a stock staphylococcic raccine followed by 250 millions in four or five days. At the same time boric compresses should be applied to the lesion and it should be protected in the intervals between the injections by pads of cottonwool. Benefit has been derived also from injections of anti-diphtheritic and anti-streptococcic sera. This does not necessarily indicate that the lesions are due to streptococci, as staphylococcic infections are also improved by anti-streptococcic serum. Recently horse serum given by the mouth has been employed and improrement recorded from its use.

Local Treatment.-By far the most satisfactory local treatment is excision or scraping, and even large lesions can be dealt with in this fashion. After the diseased tissue has been removed by the curette, scissors, or scalpel, the surface should be thoroughly swabbed over with an antiseptic lotion, such as $I$ in 20 carbolic, and allowed to granulate under suitable dressings. Various abortive measures similar to those which have been described in connection with boils have been suggested with the object of arresting the diseased process, such as painting the lesions with iodoform-collodion $\left(5^{\circ}\right)$ or the injection of carbolic acid, but these except in slight cases are of comparatively little value. When for some reason or other, such as the age or the debilitated state of the patient, a radical operation is contra-indicated, milder measures may be resorted to such as the old-fashioned treatment of applying lead plasters with a hole cut in the centre and fomenting with hot boric lotion, and, when the core has separated, of syringing the lesion with carbolic lotion and plugging it with carbolised gauze. Instead of lead plaster, mercury and carbolic plaster may be employed, or the carbuncle may be covered continuously with carbolic ointment $2 \%$ thickened with starch or zinc and spread on lint.

\section{REFERENCES}

Ashe, E. Carbuncles treated with anti-streptococcus serum (Brit. Med. Journ., ISgS, ii. p. If 2 S).

Btikler; L. D. Non-surgical treatment of boils $\&$ carbuncles, etc. (Idem, IS97, ii. p. S6S).

GARRÉ. Inoculation experiments (Fortschr. d. Med., ISS 5 , iii. p. I6j).

PAGE, H. W. Scraping of carbuncles (Brit. Med. Jo!ırn., ISSS, i. p. 635)

Parker, R. Excision of carbuncles (Idem, IS9S, ii. p. I664).

\section{VEGETATING PAPILIOMATA}

It is generally believed that staphylococci are responsible for the papillomatous regetations which sometimes occur in the granulomatous lesions of tuberculosis, syphilis, vaws and leprosy, and in connection with elephantiasis, and that the specific micro-organism of the 
infective granuloma is incapable of causing these vegetations without the staphylococcus.

Occasionally impetiginous lesions of staphylococcic origin, or the result of a mixed infection, may become warty and the purulent surface become covered with vegetations. In the illustration below, the scalp in a young woman was covered in places with papillomatous vegeta-

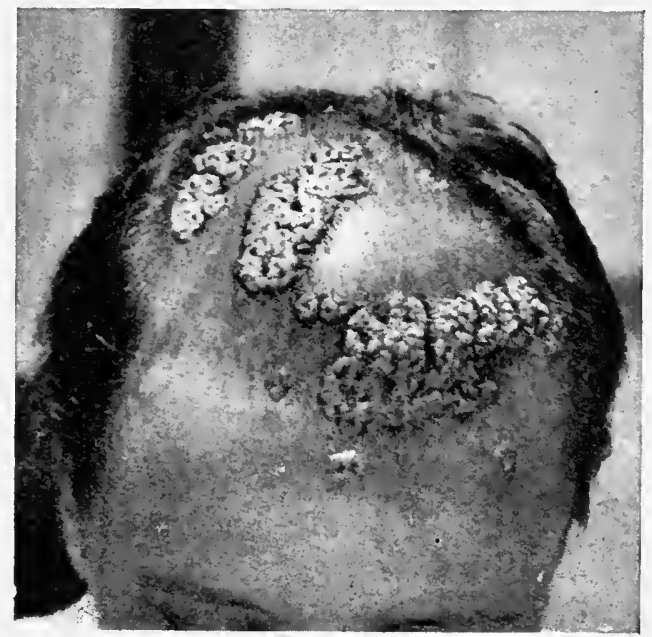

FIG. 84--Papillomatous Lesions of Septic Origin.

tions and greenish-yellow crusts. In this case the affection had originated from a septic sore on the scalp the result of an injury from a hat-pin. (Fig. 84.)

The vegetations which form the most noticeable feature in Pemphigus vegetans or Dermatitis vegetans are possibly due to staphylococci.

\section{GRANULOMA PYOGENICUM}

Syn.: Botryomycosis hominis; Septic granuloma.

The name Botryomycosis was originally applied by veterinary surgeons to granulomatous nodules which developed in connection with suppurating wounds in horses, dogs, etc., from the belief that the lesion was due to an organism known at the time as a Botryomyces. Somewhat similar lesions were observed subsequently in man and the term Botryomycosis hominis was applied to them. This view is no longer held and such lesions are now regarded as being of pyogenic origin.

The lesions are roughly circular in outline, reddish in colour, elastic in consistence, vary in size from a pea to a walnut or larger, and may be flat like a button or pad, or pedunculated. The central portion usually consists of soft exuberant granulations which are sometimes surrounded by a raised and inflamed indurated border, or the lesions may take the form of small raspberry-like excrescences. They grow slowly and on 
reaching a certain size remain stationary and unless treated may persist indefinitely. They are usually single but occasionally may be multiple. They are most frequently met with on the hands but may occur on any part of the cutaneous surface. As a rule there is no pain associated with them. The Staphylococcus pyogenes aureus has been repeatedly recovered in pure culture from them, and the organism known as Botryomyces ascoformans, which occurs in the lesions as spherical bodies of various sizes collected in clusters, is no longer regarded as pathogenic. (Fig. 85.)

Microscopical sections show a cellular deposit consisting of small connective tissue cells and plasma-cells, a formation of new connective

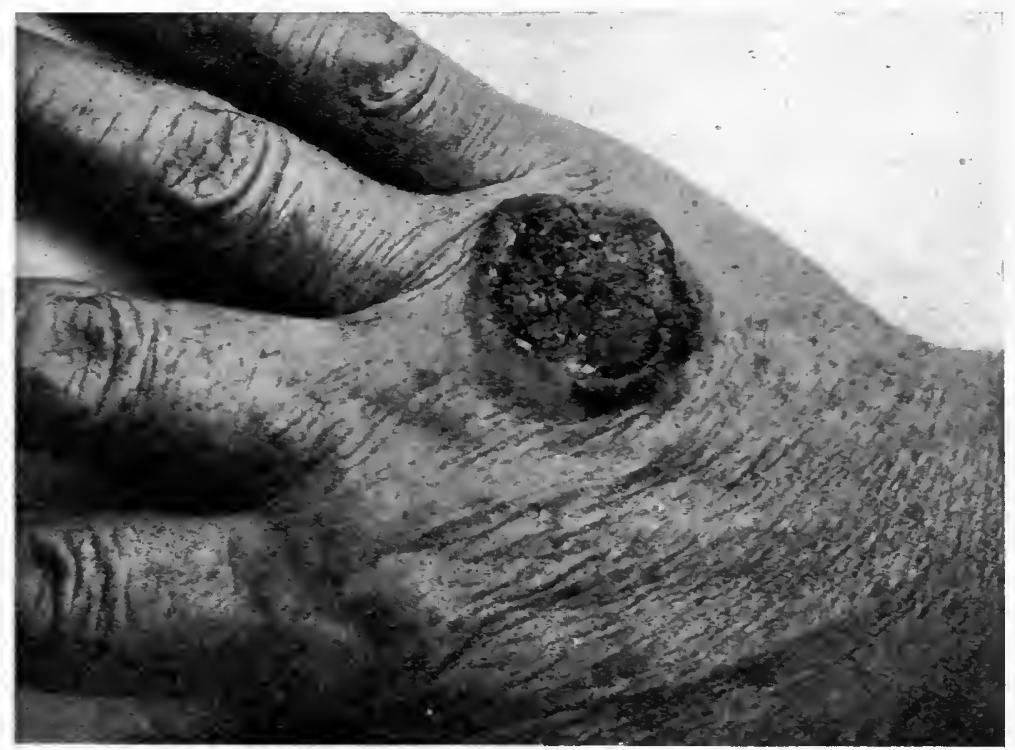

FIG, 85.-Septic Granuloma.

tissue, enlargement of the overlying papillae, and dilatation of the blood-vessels.

Treatment.-Where the lesions are small and pedunculated they may be cut off and the bleeding stopped by a cautery, where large and flat the most successful treatment is the X-rays, one or more pastille doses being given at intervals of a fortnight. Occasionally they may disappear under compresses of boric acid and dressings with ammoniated mercury ointment.

\section{REFERENCES}

Bodix, E. Botryomycose humaine (Ann. de Derm., I902, iii. p. 289). Hartzeld, .I. B. Granuloma pyogenicum (Journ. Cut. Dis., I904, xii.). Lexormant. Botryomycosis of Man (Ann. de Derm. et de Syph., Igio, p. I6I).

Poxcet \& Dor. Botryomycose humaine (Congrès de Chirurgie de Paris, I 897). 


\section{Multiple Cutaneous and Subcutaneous ABscesses IN INFANTS}

The Staphylococcus pyogenes is responsible for a rare condition which is met with in ill-fed, debilitated babies of the poorer classes and which is seen occasionally in out-patient departments of children's hospitals in large cities. It consists of cutaneous and subcutaneous abscesses more or less widely distributed over the skin, occasionally present on the scalp but most frequently about the buttocks and upper parts of the thighs. These are first noticed as small nodules not much larger than a pea, which if deep-seated may be felt before they are seen and which increase rapidly till they reach the size of a walnut, when the overlying skin becomes red, or purplish. One or two lesions generally appear first, and new ones develop by auto-inoculation; in extensive cases a considerable number may be present, even as many as a hundred having been counted in one case. The nodules tend to soften with the formation of pus which breaks through the surface and may result in an ulcer, or where the lesion is deep-seated with the formation of a discharging sinus. The glands in the immediate neighbourhood, as a rule, are enlarged and there may be marked oedema and lymphangitis where the lesions are closely aggregated. When healing takes place a scar is almost inevitable. The abscesses occur in situations subjected to friction or moist from discharges, as about the napkinregion, and are occasionally preceded by ordinary streptococcic impetigo. Where they are numerous septic absorption is liable to take place, resulting in a gastro-intestinal disturbance, a rise in temperature and, in severe cases, in a fatal septicaemia or bronchopneumonia.

The manner of inoculation of the staphylococcus is not definitely decided but it seems highly probable that the condition is a local one and that the virus gains entrance in the majority of cases through the hair-follicles; in corroboration of this view small follicular pustules may be detected not infrequently in association with the abscesses. The low power of resistance of the infant's skin seems to be the factor which is responsible for the severity of the inflammatory process. It has been suggested that the affection is caused by staphylococci conveyed to the skin via the blood stream from some deeper septic focus, but in the majority of cases there is no evidence that this is the case.

Diagnosis.-This condition has to be distinguished chiefly from tuberculous abscesses or "scrofulous gummata," but these as a rule occur in older children, are more indolent in their course, tend to necrose rather than to ulcerate, are either single or few in number, and are generally associated with other signs of tuberculosis.

Prognosis.-Where the lesions are few and the infant is in good general health the prognosis is excellent and the lesions readily respond to suitable treatment. In extensive cases in debilitated 
infants living under imperfect hygienic conditions, the outlook is grave owing to the danger of a systemic infection and the occurrence of some serious, if not fatal, intercurrent disease.

Treatment.-The treatment is based on ordinary surgical principles and consists of the incision of the abscesses, the evacuation of pus, the use of boric acid fomentations to cause the more indolent lesions to mature, and the application of a $2 \%$ ammoniated mercury ointment, or tincture of iodine I part in 4 parts of $60 \%$ spirit, to the skin around the abscesses to prevent the spread of the disease. Where the lesions are numerous boric acid baths are useful and scrupulous cleanliness with regard to diapers and dressings is essential. On no account should the old-fashioned linseed meal poultices be used as they are certain to spread the infection. The general condition of the infant should be dealt with on ordinary medical lines, special attention being paid to the feeding, the state of the digestion, and the hygienic surroundings. Small doses of staphylococcic vaccine have been known to be beneficial.

\section{REFERENCES}

Escherich. Aetiologie der multiplen Abszesse im Sänglingsalter (Münch. Med. Woch., I 886, p. 92).

Fox, Colcorr. Dermatoses of staphylococcic origin (Allbutt \& Rolleston, System of Medicine, I911, ix. p. I99).

Lewandowsky, F. Pathogenese der multiplen Abszesse im Sänglingsalter (Arch. f. Derm. u. Syph., I 906, lxxx. p. I 79).

LoNgard, S. Folliculitis abscendens infantum (Arch. f. Kinderheil., I $88_{7}$, viii. p. 369).

\section{PITYRIASIS AND SEBORRHOIC DERMATITIS}

Introduction. - The name pityriasis from the Greek mitupov (bran) was applied originally by the English dermatologist Robert Willan about the end of the eighteenth century to a group of cutaneous affections the lesions of which were characterised by delicate pellucid scales like small flakes of mica and by the absence of any obvious sign of inflammation. Under this heading he included various conditions which differed in their nature and etiology, such as the familiar scurfy state of the scalp popularly known as dandruff and the fine scaly affection of the trunk named Pityriasis versicolor. Since his time the use of the term has been extended to other scaly affections and considerable confusion has arisen owing to its association with seborrhoea. For this, Hebra and his pupils were largely responsible by teaching that in dandruff or pityriasis of the scalp the scales were not derived from the epidermis as Willan thought but originated in the sebaceous glands and were inspissated sebaceous cells. Subsequent research has shown that Willan's original view was correct and that the scales have no connection with the sebaceous glands. 
Scaly patches are common on the face and elsewhere in association with pityriasis of the scalp and are apparently of the same nature. The patches are more or less inflamed and the scales are apt to be coarse and greasy. In the medio-thoracic region they are frequently circinate or gyrate in configuration. These lesions are liable to become eczematised from scratching, and have been grouped by Unna under the heading of Seborrhoic eczema.

In the present state of our knowledge it is difficult to decide on the most suitable name. Certain writers employ the term Seborrhoic dermatitis and designate certain of the lesions Seborrhoides; this is open to the criticism that the milder forms are unconnected with seborrhoea, and that seborrhoea is not essential but only renders the skin a better soil for the growth of the micro-organisms which are believed to be responsible so that they flourish to the extent of causing inflammation. On the other hand if the non-committal name of pityriasis be used it leads to confusion as the term has been employed to designate such distinct morbid conditions as Pityriasis rosea and Pityriasis versicolor.

Bacteriology.-From the scales of pityriasis of the scalp and glabrous skin two micro-organisms may be isolated, namely the grey coccus and the Spore of Malassez or Flask bacillus of Unna.

(I) The grey coccus, or Micrococcus cutis communis of Sabouraud, has already been described (see page 82). According to Sabouraud the grey coccus is pathogenic and responsible for the inflamed patches with greasy scales where it is believed by him to be in symbiosis-with the spore of Malassez. The seborrhoea which may be associated with it he believed to be caused by the micro-bacillus (see page 325 ).

(2) The Spore of Malassez or Flask bacillus of Unna is not a true bacillus but resembles a yeast. It is about $5 \mu$ in length and varies in shape, being oval, round, or barrel-shaped, but most often shaped

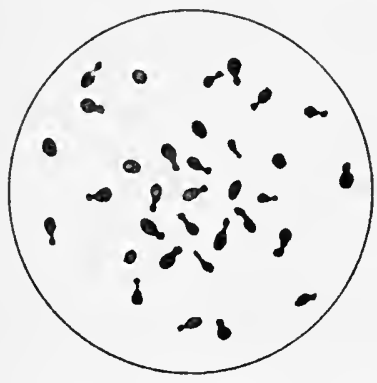

Frg. 86.-Flask-shaped Bacilli of Seborrhoea Capitis. like a flask or gourd with a large spherical portion and a small rounded process. It may be single or multiple and may be arranged in short chains or groups. It is easily demonstrated by teasing out scales and staining them with methylene blue or Gram's method. So far it has not been grown in pure culture. According to Sabouraud it is the cause of Pityriasis capitis and, in consequence, he named it the Pityro-sporon of Malassez. Other observers maintain that it has no pathogenic importance. It is so constantly present, however, in dandruff and in the patches of seborrhoic dermatitis on the glabrous skin while it is absent in other scaly conditions, such as psoriasis and Pityriasis rosea, that though it may be a harmless saprophyte it is certainly of diagnostic value. (Fig. 86.) 
The exact part played by these micro-organisms still remains undecided, the prevailing feeling at present being that the spore of Malassez and the grey coccus are causally connected with the lesions and that the micro-bacillus is of no pathogenic importance. The condition even in its mildest form is now generally believed to be a local inflammatory affection of microbic origin and a variety of impetigo.

\section{DESCRIPTION}

For purposes of description the different phases of this clinical group will be described according to the regions affected.

\section{Scalp}

Syn.: Dandruff, Pityriasis capitis, Seborrhoic dermatitis of the scalp.

In its mildest form this affection occurs as delicate pellucid powdery scales, irregularly distributed, with no obvious signs of inflammation,

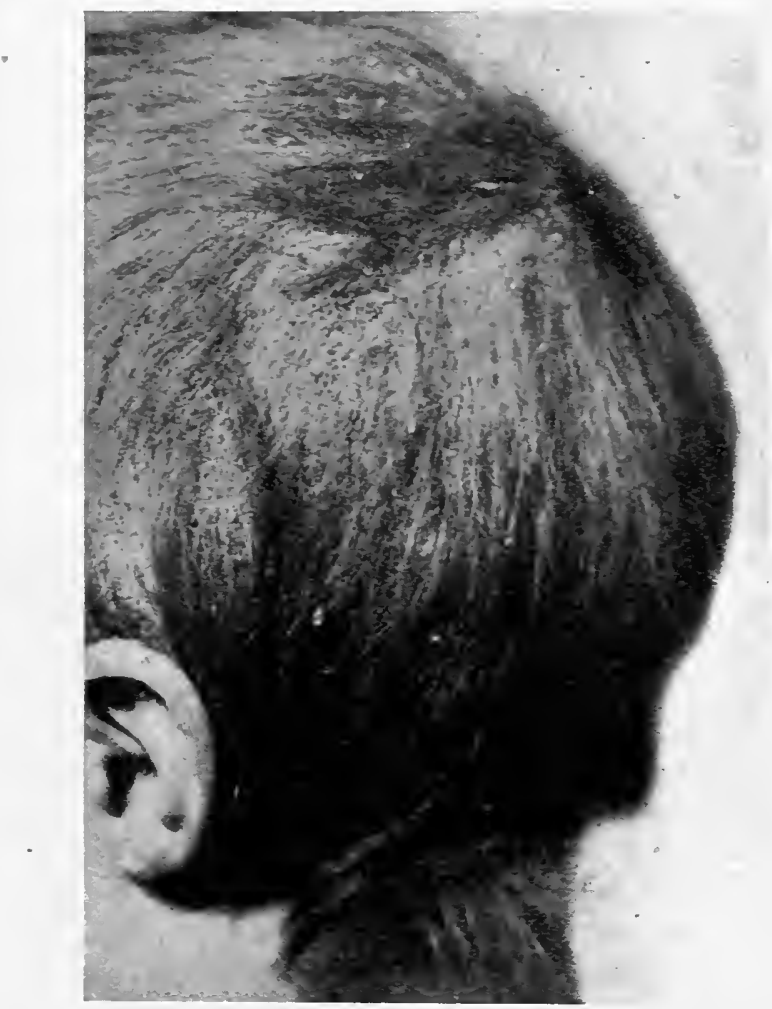

FIG. 87.-Pityriasis Capitis.

and present either between the hairs or in the form of small collarettes around them. It appears first as minute scaly patches which extend 
peripherally and tend to coalesce till the scalp is more or less completely covered. Circumscribed and diffuse varieties have both been described, but the latter is simply a further stage of the former, for the disease, if left untreated, is invariably progressive. It is extremely common and though it may not be sufficiently marked to be obvious on casually looking at the scalp, it makes itself known by the fine powdery scales which are shed when the hair is brushed, or fall down and collect on the coat collar. (Fig. 87.)

In other cases instead of being fine and powdery the scales may be coarse, greasy, and situated on a pinkish inflammatory base which

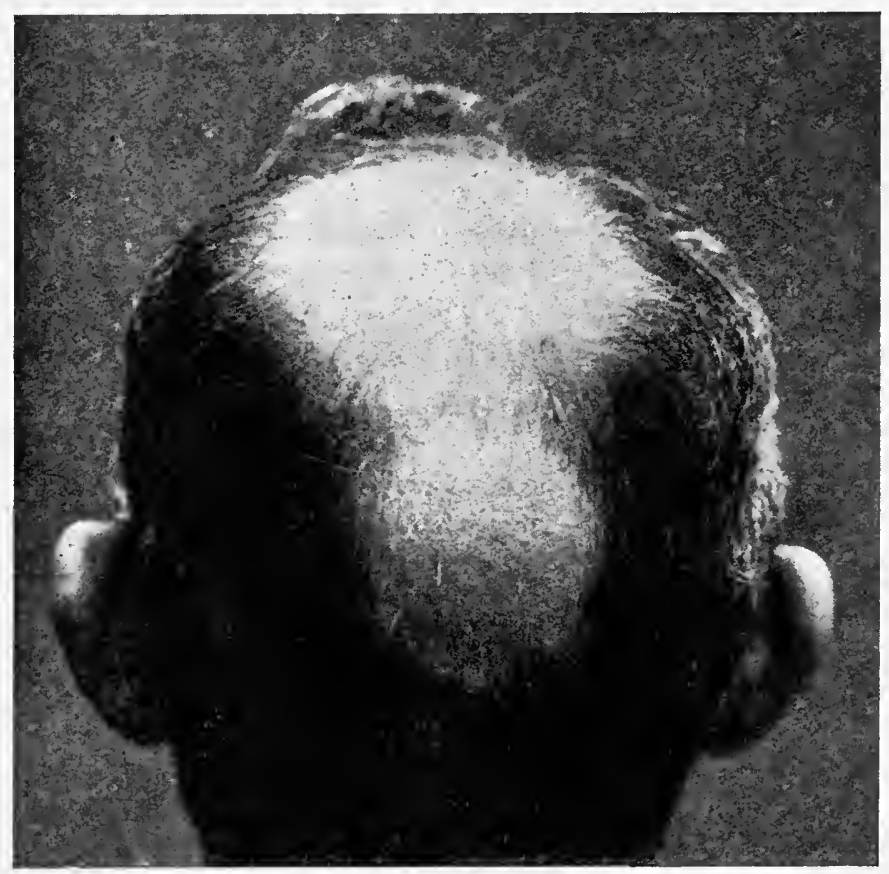

FIG. 88.-Calvities and Patch of Alopecia Areata beneath.

extends beyond the scaly areas, or may be replaced by crusts or cakes. The hair in the affected parts is lustreless, frayed, brittle, and tends to fall out, the defluvium taking place first about the vertex, and sides of the forehead, and being gradually progressive. This constitutes the type of baldness which is known as calvities, Alopecia pityrodes or seborrhoic alopecia (see p. 352), and the majority of cases of premature baldness in the male belong to this category. (Fig. 88.)

The precise relation of the scaly inflammatory condition with the loss of hair is a matter of dispute. Certain observers consider that it is the direct result of seborrhoea associated with hypertrophy of the sebaceous glands and a corresponding atrophy of the hair, but the evidence in favour of this view is unconvincing as an oily scalp has not infrequently an excellent crop of hair. Others regard it as caused 
by the micro-organisms responsible for pityriasis, but this also is debatable, for the following reasons: (I) although inflammatory disturbances of the scalp of microbic origin cause the hair to fall out, such as an attack of erysipelas or a boil, when the inflammation subsides re-growth takes place, which does not occur after pityriasis; (2) pityriasis of the scalp is exceedingly common in both sexes, while calvities is rare in women.

The subjective symptoms vary from slight itching to considerable irritation, especially when the scalp is overheated by violent exercise, a hot atmosphere, or from constantly wearing a hat or cap.

It is contagious and capable of being spread on to the glabrous skin by scales shed from the head, the majority of cases of so-called seborrhoic dermatitis commencing in or being associated with Pityriasis capitis. It may be transmitted from one person to another either directly, or indirectly by the medium of infected combs, towels, brushes, etc.

\section{Glabrous Skin}

Syn.: Pityriasis circinata; Pityriasis stéatoïde; Flannel-rash; Seborrhoic eczema (Unna); Séborrhéide eczématisante (Audrỹ); Seborrhoic dermatitis; Seborrhoea corporis (Duhring); Lichen circinatus, etc.

The above names have been applied to different phases of a scaly dermatitis affecting the glabrous skin, closely related to, if not identical with, Pityriasis capitis and frequently preceded by it. The spore of Malassez can generally be detected in the scales, and this and the frequent association with Pityriasis capitis have led to the belief that it is a microbic infection of the nature of an impetigo and that its different phases are simply due to individual peculiarities in the skin affected, differences in the situation of the lesions, the symbiosis of other micro-organisms such as the grey coccus or slightly virulent strains of the Staphylococcus pyogenes albus, and secondary changes such as eczematisation the result of rubbing and scratching. Its relation to seborrhoea has been much discussed, certain observers believing that a sebaceous flux is an essential predisposing cause, while others consider that it only intensifies the inflammatory disturbances associated with the lesions by giving the micro-organisms a niore suitable soil for their growth.

Face.-On the face, the dermatitis usually takes the form of scaly patches, not definitely raised or infiltrated, with a pinkish inflamed base which spreads beyond the scaly margin, and covered with scales which are greyish in tinge and sometimes greasy. A common site is the forehead, where they are associated with Pityriasis capitis and not infrequently stretch beyond the hair-margin in a festooned manner forming the condition sornetimes known as the Corona seborrhoica. Scaly patches of a similar nature may be met with on the cheek, chin, and about the auricles especially in the post-auricular folds. 
In other cases the lesions are not definitely scaly but occur as nummular patches of a pinkish-yellow tinge which sometimes fade in the centre forming circinate lesions. In association with the patches the skin may be yellowish and greasy, especially around the lips and at the sides of the nose. Occasionally it may begin as a pityriasis about the eyebrows, the margin of the eyelids, beard, or moustache.

Neck.-Another common situation is the nape of the neck just beyond the hair-margin. There it takes the form of a scaly reddish patch extending on to the scalp which, by rubbing and scratcning, is apt to become thickened, lichenified, and even eczematised. Such

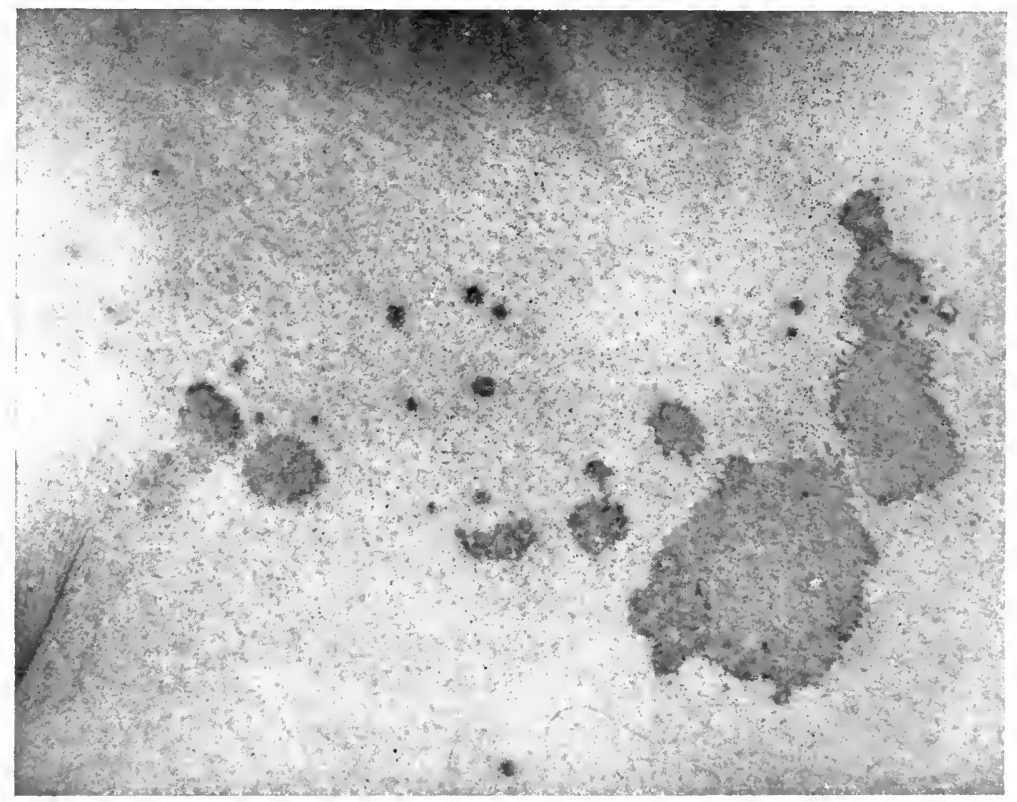

FIG. 89.-Seborrhoea Corporis.

Figured lesions with well-defined scaly borders situated on sternal and mammary regions.

patches occur chiefly in adults and elderly people and are more common in women than in men.

Trunk.--On the trunk the most frequent situations for it are the sternal and interscapular regions, the middle line of the back, the axillae, the inguinal regions, perineum, and around the umbilicus. In the medio-thoracic regions it generally takes the form of a macular, circinate, or figured, slightly scaly dermatitis which has long been known to English dermatologists as "Flannel-rash." This begins as small pinkish-yellow or brownish macules which may or may not present a thin scale on the surface and which tend to grow until they reach the size of a split-pea and to coalesce with others to form annular or gyrate figures of a brownish-yellow tinge with a smooth or slightly scaly surface. Occasionally the macules become larger, clear in the centre and form circinate lesions with a delicate reddish border which may 
be studded with minute vesicles. At other times they are follicular and coalesce to form small patches with an indefinite outline and a number of isolated lesions dotted irregularly beyond it. These patches are generally covered by greasy scales which when removed disclose a smooth shiny epidermis, pitted by open follicles. The eruption varies in different cases but the type of lesion in an individual case is generally constant. The skin of the regions affected is as a rule greasy and faintly yellow. (Fig. S9.)

Scaly patches on a more or less inflamed base may also occur in the axillae, especially when moist from excessive sweating, in the inguinal regions, perineal fold, and on the genitalia when they occasionally extend for some distance on the inner sides of the thighs. In these situations the lesions are liable to be moist and cracked at the folds, and may give place to eczema.

In some cases the eruption is widely distributed over the trunk in the form of macules, nummular lesions and variously sized patches covered with greasy scales of a dirty white tinge which, when scratched, are apt to leave a pinkish-yellow moist surface but no bleeding puncta like psoriasis. In extensive cases it may be transformed by rubbing into weeping eczema or may even develop into an exfoliative dermatitis.

In rare cases of the miliary follicular type the macules, instead of being capped by greasy scales, have definite horny plugs or spines projecting for one-sixth of an inch or more above the surface.

There is a phase of this disease which occurs about the napkin area in infants and takes the form of a red sheet of dermatitis, sometimes covered with greasy scales at other times moist and sodden, the margins of which are well-defined, and beyond which are isolated red scaly macules and patches. Similar patches may be met with about the flexures of the groins, the folds of the buttocks, and the skin around the umbilicus. It is frequently associated with Pityriasis capitis and in several instances the mother has been found to be affected with pityriasis, suggesting the possibility that the disease has been transmitted by her to the infant.

Limbs.-On the limbs the most common sites are the bends of the elbows and the popliteal spaces. There the lesions tend to coalesce to form sheets which, owing to morements of the joints, chafing of clothes, and scratching, are very apt to be excoriated and eczematised. It may occur also as follicular patches on the extensor aspects, or as macular circinate or gyrate lesions chiefly on the flexor surfaces. Occasionally the hands and feet are affected, especially between the fingers and toes. (Fig. 9o.)

Nails.-In one or two instances the nails have been observed to be pitted or fissured from the disease spreading to the nail-bed.

About puberty and adolescence it is often associated with Acne vulgaris, and in the adult with rosacea.

The subjective symptoms vary; in mild cases they may consist of slight itching when the patient becomes hot, or smarting when the 
skin is washed, while in severe cases the itching may be intense and lead to scratching, excoriation and eczematisation.

Histopathology.-Microscopical sections of a lesion show definite changes of an inflammatory type both in the epidermis and corium.

In the epidermis the interpapillary processes are elongated and widened, oedema is present in and between the prickle-cells, in some of the cells fluid has collected around the nucleus causing a nuclear space to form, and occasionally in the dilated inter-epithelial lymphatic spaces distorted leucocytes may be seen making their way towards the surface. Where the oedema is pronounced the granular layer is absent, cornification deficient, and nuclei are present in the horn-cells (para-

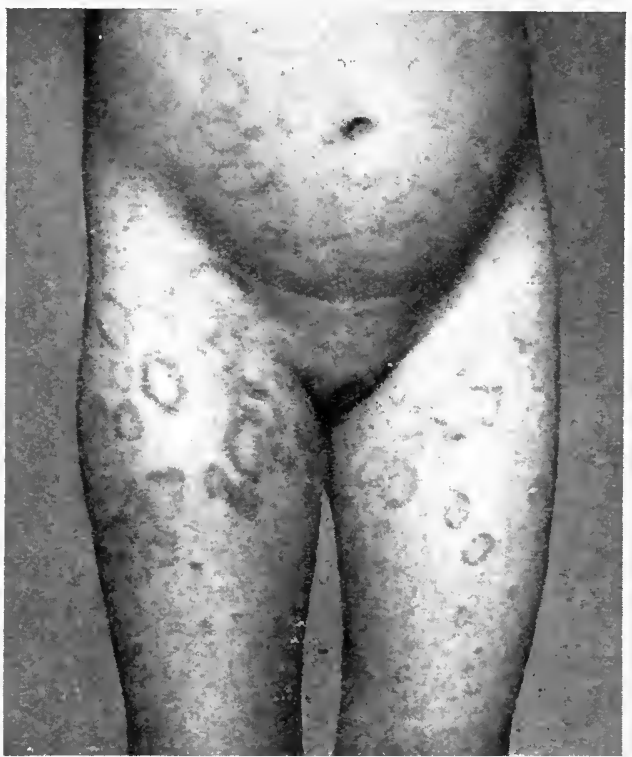

FIG. 90.-Circinate Seborrhoic Dermatitis.

keratosis) which adhere together to form scales between which is a granular debris of broken down epidermal cells, leucocytes, and coagulated serum.

In the underlying corium the papillae are elongated and the capillaries dilated, though not to the same degree as in psoriasis, and around the dilated vessels there may be a slight inflammatory cellular infiltration.

The histological picture suggests the action of micro-organisms present in the horny layer, the toxins of which have caused by chemotaxis dilatation of the capillaries, sero-fibrinous exudation, oedema in the epidermis, and parakeratosis.

Etiology and Pathogenesis.-Pityriasis capitis is found at all ages. In the infant it is often present in the form of a greasy cake or scaly patch situated over the anterior fontanelle and vertex of the scalp, which is known as the Crusta lactea. It is comparatively rare in 
childhood, more frequent at puberty, persists through adult life, and tends to disappear with advancing years. It is often believed to be more common in males than in females, but this is probably because it is more noticeable in the former than in the latter.

The corresponding affection of the glabrous skin, the so-called Seborrhoic dermatitis, is most common in infancy and, as a rule, spreads down from the scalp on to the face and trunk, a considerable proportion of the cases of infantile eczema having their origin in it. In childhood it may occur de novo or may persist from infancy, and takes the form of dry scaly patches not infrequently situated about the flexures of the large joints. It is common in the adult but diminishes in frequency with advancing years when it is liable to affect, in addition to the scalp, the sides of the nose and the genital organs.

As predisposing causes may be cited also, insufficient washing, heat from a hot atmosphere and violent exercise, or from wearing a hat or thick woolly clothes. The heat increases the sebaceous output and stimulates the sweat-glands, rendering the soil more fertile for the growth of the microbes.

There is a diversity of opinion as to whether these affections are purely local or are dependent on a peculiar constitutional state or dyscrasia, which has been described variously as the "Status exudativus" (Czerny), and the "Status seborrhoicus" (Barber \& Semon), which produces an increased susceptibility of the skin and mucous membranes by which they become unusually liable to bacterial infections.

Diagnosis. Scalp.-Pityriasis of the scalp has to be distinguished in children from ringworm. In an ordinary case of small spored-ringworm there is no difficulty owing to the presence of partially bald patches with typical stumps; in endothrix ringworm, however, where small scaly patches are dotted widely over the scalp and the stumps are less obvious, the difficulty may be considerable and only possible of solution by finding a fungus-laden stump.

In the adult it has to be differentiated chiefly from psoriasis. In psoriasis, the scales are coarser and situated on a more rivid inflammatory base, the lesions are more circumscribed, and there are generally typical patches elsewhere. Should any doubt exist scales should be examined for the spore of Malassez which is absent in psoriasis. It must be remembered, however, that psoriasis may supervene on a scalp affected with pityriasis.

Face.-The patches on the face may be difficult to distinguish from furfuraceous impetigo. In it the inflammatory disturbance is as a rule less marked and the lesions have not the same tendency to occur at the margin of the scalp.

Trunk and Limbs.-Lesions on the trunk and limbs may resemble psoriasis so closely that one phase of the dermatitis has been named Seborrhoea psoriasiformis, but the two conditions are distinct. In psoriasis the scales are dry and silvery, situated on a vividly inflamed 
base and when scraped off reveal bleeding puncta, while those of Pityriasis corporis are fawn-coloured, greasy, and when scraped off leave a shiny moist epidermis with no tendency to bleed. The distribution also is different, for in psoriasis the lesions have a predilection for the extensor surfaces of the limbs, while in this affection they are commonly met with on the flexor aspects. The spore of Malassez is absent in psoriasis but present in the scales of Pityriasis corporis.

Pityriasis rosea is another affection which it may closely simulate. In Pityriasis rosea the lesions are delicate rose pink instead of being yellowish in colour, are generally more widely distributed like an exanthem, do not occur on the scalp and rarely on the face. In Pityriasis rosea characteristic oval plaques occur, with a fawn-coloured centre and a pinkish, slightly scaly border, which are not met with in this dermatitis though the annular lesions may occasionally simulate them. The course of the affection is different, for Pityriasis rosea commences with a herald patch and is followed by an exanthem-like outbreak of macular or circinate lesions, while this dermatitis appears first as macular lesions. In Pityriasis rosea the spore of Malassez is absent.

Isolated annular lesions of seborrhoic dermatitis may sometimes be confused with Tinea circinata, but the ringworm patches are as a rule better defined, and a microscopical examination of the scales for fungus should establish the diagnosis.

There is yet another condition which may give rise to confusion, namely, the circumscribed red scaly patches which have been labelled Eczema circumscriptum, but probably belong to the Parakeratosis group. In them the colour is more vivid, the scales dry and not greasy, and the lesions are more resistant to treatment than those of Seborrhoic dermatitis.

Prognosis.-Pityriasis of the scalp, if left alone, tends to be progressive. It is singularly resistant to treatment, and liable to recur.

On the glabrous skin it is, as a rule, amenable to suitable local treatment. It is more difficult to cure when it occurs in the tropics.

\section{Treatment of Scalp}

General Treatment.- It has been stated from time to time that the peculiar constitutional state, or " Status exudativus," which is believed to underlie seborrhoic dermatitis, is associated with some general aberration of health, such as a relative acidosis, and alkalies and special diets have been recommended, but their value is uncertain and the results from internal treatment-except on general medical principles - liave not been encouraging.

Local Treatment.- The principles of the local treatment are (I) the removal of the scales, (2) the destruction of the causal micro-organisms by antiseptic applications and the stimulation of the epidermis by suitable tonic remedies. 
The details of treatment, both with regard to technique and the choice of remedies, vary according to the type of scales, the degree of inflammation, and the age and sex of the patient. As a rule the condition is resistant, and necessitates regular and thorough treatment.

1. Removal of the Scales.-Not long ago it was customary, especially in children, to remove scurf from the scalp by the emplorment of a small toothed comb; this barbarous procedure is now becoming obsolete as it does more harm than good by lacerating the scalp, permitting the entrance of micro-organisms, and spreading the disease. Where the scales are slight, one or other of the following soap preparations may be used to remove them. If the crusts are thick and adherent it may be necessary to soften them before washing, by olive oil or by rubbing in an ointment of salicylic acid $3 \%$ in cold cream.

Soap Spirit Lotion.-One of the commonest soap preparations employed for this purpose is the soap spirit lotion of Hebra.

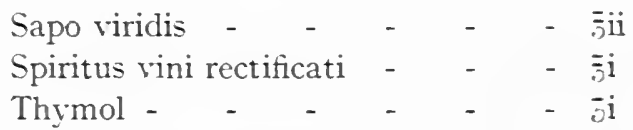

Equally effective and less sticky is the following ether soap, which is made stimulating by the addition of ammonia.

\begin{tabular}{|c|c|c|c|c|}
\hline Ether & - & - & - & - \\
\hline Liquor amm & oniae fort. & - & - & - \\
\hline Sapo viridis & $-\quad-$ & - & - & - \\
\hline
\end{tabular}

About $2 \mathrm{oz}$. of soap solution may be dissolved in $6 \mathrm{oz}$. of distilled wate1 to form a shampooing fluid, or a tablespoonful may be applied to the scalp rubbed up with hot water to form a lather, left for a few minutes and then rinsed off with more hot water. In women it is better to part the hair systematically all over the scalp and to rub in the soap with a piece of flannel or the fingers and wash it off. Should the hair be left sticky it is because insufficient water has been employed in the washing. It is important to impress on the patient that it is the scalp itself more than the hair which requires cleansing.

Where the scalp has to be washed only occasionally a pleasant substitute for soap spirit is quillaia bark or saponin. This may be employed as the liquid extract, using a tablespoonful to a quart of hot water to make a shampoo, or combined with borax as follows:

\begin{tabular}{llllll} 
Tinctura quillaiae & - & - & $-\mathrm{m} . \mathrm{xv}$ & 3 \\
Boracis & - & - & - & $-\mathrm{gr} . \mathrm{xv}$ & 3 \\
Spiritus ad & - & - & - & - & \\
\hline
\end{tabular}

The frequency of washing the head depends on the amount of scurf and the thickness of the scales; in severe cases it may be necessary to wash it daily at first, gradually reducing the frequency as improvement takes place; in ordinary cases it may be sufficient to wash it, in men once a week, and in women once a fortnight. 
Where the scalp is inflamed, oozing, and covered with greasy crusts, boro-starch poultices are preferable to spirit soap which is tooirritating.

2. Destruction of Micro-organisms and Stimulation of the Epidermis. -After the scales have been removed an antiseptic should be applied systematically once or twice a day so long as there is any tendency for the scales to re-form, the most generally employed being sulphur, mercury, salicylic acid, tar, tannic acid, resorcin, and beta naphthol. The principal stimulants employed are ammonia and capsicum.

In the choice of a remedy it must be borne in mind that certain antiseptics stain the hair, such as resorcin which turns. white or grey hair a rusty tint, and beta naphthol which darkens it. As a substitute for resorcin, euresol (monoacetate of resorcin), may be used as it has no staining properties. Tar also is a dirty preparation and may be replaced by anthrasol which is colourless.

These applications may be employed in the form of lotions, ointments, pomades, oils, or powders. The most effective method is in ointments or pomades, as they enable the remedies to penetrate more deeply than in lotions. Ointments are not popular on account of their greasiness, and in women can be used with comfort only when the hair is washed frequently.

The following are among the more useful formulae:

\begin{tabular}{|c|c|c|c|c|}
\hline Lotions. & Ammonii chloridi & - & & - gr. $\mathrm{x}$ \\
\hline & Resorcin - - & - & - & gr. $x x x$ \\
\hline & Spiritus vini rect. & - & & $\overline{3} \mathrm{ii}$ \\
\hline & Aq. dest. ad & - & - & $\overline{3} \mathrm{vi}$ \\
\hline
\end{tabular}

(In the case of men add glycerine.)

\begin{tabular}{|c|c|c|c|c|}
\hline Resorcin - & - & - & - & gr. $\mathrm{x}$ to $\mathrm{xx}$ \\
\hline Acidi tannici & - & - & & $3 i \quad$. \\
\hline Spiritus vini rect. & - & - & - & $\overline{3}$ ii \\
\hline Aq. dest. ad - & - & - & - & $\overline{3} \mathrm{vi}$ \\
\hline Acidi salicylici & - & - & - & $\bar{\zeta} \mathrm{ss}$ \\
\hline Chloral hydratis & - & & - & $3^{i}$ \\
\hline Sodii sulphatis & - & - & - & 亏ii \\
\hline Aq. dest. ad - & - & - & - & $\overline{3} \mathrm{vi}$ \\
\hline \multicolumn{2}{|c|}{ Hydrargyri perchloridi } & - & & gr. ii \\
\hline \multicolumn{2}{|c|}{ Liq. carbonis deterg. } & - & - & $3^{\mathrm{i}}$ \\
\hline Olei ricini $\quad-$ & - & - & - & $\zeta^{\mathrm{i}}$ \\
\hline Spiritus vini rect. & - & - & - & $\overline{3}$ ii \\
\hline Aq. rosae ad - & - & - & - & $\overline{3} \mathrm{vi}$ \\
\hline
\end{tabular}

(The tar is occasionally irritating and may have to be omitted.):

\begin{tabular}{|c|c|c|c|}
\hline Resorcin - & - & - & - \\
\hline vel Acidi salicylici & - & - & - \\
\hline Olei petrolei & - & - & - \\
\hline
\end{tabular}




\begin{tabular}{|c|c|c|c|}
\hline Olei citron. & - & - & - \\
\hline Spiritus vini r & ect. & - & \\
\hline Aq. dest. ad & - & - & \\
\hline Resorcin - & - & & \\
\hline Balsam of Per & & - & . \\
\hline Olei ricini & - & - & 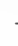 \\
\hline Spiritus vini r & ect. & & - \\
\hline
\end{tabular}

The lotions should be used once or twice daily and applied to the scalp by a brush, or on a piece of flannel, or rubbed in by the fingers.

To increase the stimulating power of the lotions Liq. ammon. fort. $2 \%$ or Ti. capsici $2 \%$, may be added.

To act as a sedative where the scalp is markedly itchy Aq. laurocerasi $2-4 \%$ may be added.

Oils.-Oils are only of service when the scalp is naturally dry and slight dandruff is present. They are not good vehicles for antiseptic remedies and necessitate almost daily washing of the scalp.

Those used chiefly are almond oil and oil of petroleum or liquid petroleum. They may be scented with a few drops of oil of lavender or bergamot.

Many of the oils on the market for application to the scalp are green in colour from the addition of green oil, which is made by adding to an oil a small quantity of chlorophyl.

Ointments. - The most useful ointment bases are: white vaselin either alone or mixed with $25 \%$ of lanolin, or cold cream (Ungt. aquae rosae).

A few useful formulae are :

\begin{tabular}{|c|c|c|c|c|c|c|}
\hline \multicolumn{3}{|c|}{ Sulphuris praecipitati } & - & & gr. $x$ & \\
\hline \multirow{2}{*}{\multicolumn{4}{|c|}{ Paraffinum molle album ad }} & & gr. $\mathrm{x}$ & \\
\hline & & & & & $\bar{j}$ & \\
\hline \multirow{2}{*}{\multicolumn{3}{|c|}{$\begin{array}{l}\text { Acidi tannici - } \\
\text { Ungt. aquae rosae ad }\end{array}$}} & - & & gr. xx & \\
\hline & & & - & & $\bar{j} \mathrm{i}$ & \\
\hline Resorcin - & - & - & - & & gr. $x$ & \\
\hline Oil of Cade & - & - & - & & m. $\mathrm{xx}$ & \\
\hline \multicolumn{3}{|c|}{ Adipem benzoat. ad } & - & & $\bar{j} \mathrm{i}$ & \\
\hline \multicolumn{3}{|c|}{ Hydrargyri ammoniati } & - & & gr. $x$ & \\
\hline Oil of Cade & - & - & - & & $5^{i}$ & \\
\hline ei olivae & - & - & - & & 5 iii & \\
\hline \multicolumn{3}{|c|}{ Lanolin anhydros. ad } & - & - & $\bar{j} \mathrm{i}$ & \\
\hline
\end{tabular}

Pomades.-The following are useful pomade bases :

$\begin{array}{llllll}\text { Cerae albae } & - & - & - & - & \overline{3} \mathrm{i} \\ \text { Olei petrolei } & - & - & - & - & \overline{5} \mathrm{iii}\end{array}$

(To be melted and allowed to cool slowly, stirring all the time.)

Olei amygdalae $\quad-\quad$ - $\quad$ zii

Olei cocao $\quad-\quad \ldots \quad-\quad-\quad \overline{\tilde{j}}$ 
Any of the antiseptics may be incorporated with these in proportions similar to those of the ointments.

The pomades are not so pleasant to use as the ointments.

Powders.-These are of service only when the scalp is dry, if used on moist or greasy scalps they readily form a disagreeable cake.

Before using them, the scalp should be well washed.

A useful formula is :

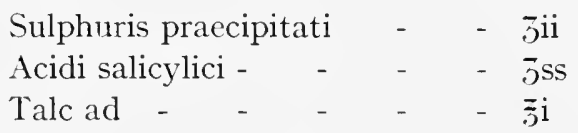

\section{Summary}

In mild cases the scalp should be washed once a week in men and once a fortnight in women by ether soap or infusion of quillaia, and resorcin, salicylic, or tar spirit lotion should be applied daily. This may be replaced if the hair is dry by a non-greasy brilliantine (formulae on page 360 ).

When the pityriasis is more extensive and the scales, greasy, an ointment should be used at night containing $2 \%$ or more of sulphur and salicylic acid and the scalp washed in the morning. The washing should be daily at first but after the scaliness has been reduced it may be less frequent and the ointment may be replaced by resorcin lotion.

Where eczematisation has occurred the affection should be treated on the principles described in connection with eczema of the scalp.

\section{Treatment of GLabrous SkIN}

As in the case of the scalp there is no specific internal treatment and any general medication which may be indicated is on ordinary lines. It is important to realise that the disease both in the scalp and glabrous skin is contagious and auto-inoculable and that measures should be adopted to prevent its being spread either directly fron one person to another or indirectly by towels, clothes, etc.

Of the various antiseptic remedies sulphur is the most generally useful and so amenable is the disease to it that in doubtful cases the rapid disappearance of the eruption under it is of diagnostic value. For the face it may be prescribed in the form of pastes, ointments, or lotions, pastes being preferable to ointments as greasy applications are not well-tolerated in this region. If an ointment be employed cold cream should be used as a base and not vaselin. The following formulae are useful :

Pastes.

$\begin{array}{lllll}\text { Sulphuris praecip. } & - & - & \text { gr. } x x x \text { to } & 6 \\ \text { Zinci oxidi }- & - & - & 5 i \mathrm{i} & 24 \\ \text { Paraffinum molle alb. ad } & - & \overline{3} \mathrm{i} & 70\end{array}$


or Unna's Zinc sulphur paste:

$\begin{array}{llllr}\text { Zinci oxidi. } & - & - & \bar{j} \mathrm{ii} & 24 \\ \text { Sulphur. praecip. } & - & - & 5 \mathrm{i} & \mathrm{i} 2 \\ \text { Terrae siliceae } & - & - & \bar{j} \mathrm{ss} & 6 \\ \text { Ad. benzoat. ad - } & - & - & \bar{j} \mathrm{i} & 5 \mathrm{~S}\end{array}$

These should be rubbed in at night so that any inflammation caused by them may subside before the morning.

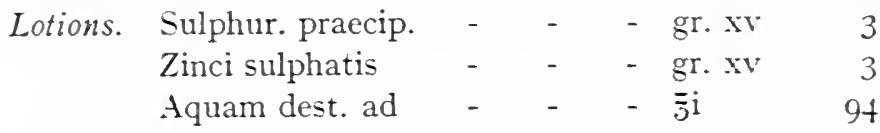

This may be dabbed on the lesions several times a day.

Instead of pure sulphur Ichthyol may be substituted and is of special value when the inflammation is marked. It can be prescribed in aqueous solution 2 to $6^{\circ}$ or as a paste or ointment as follows:

$\begin{array}{lllllll}\text { Ichthyol - } & - & - & - & - & \text { m. v to } \mathrm{xx} & 2 \\ \text { Zinci oxidi } & - & - & - & - & 5 \mathrm{ii} & 24 \\ \text { Unguentum aquae rosae ad } & - & 5 \mathrm{j} & 74\end{array}$

Previous to the application of the paste or lotion the lesions should be washed with warm water and ordinary soap. It is important to remember that sulphur is an irritant and care should be taken when employing it, especially on the face, that it does not produce a dermatitis.

When the trunk is affected and the lesions are limited to one or two regions, the scales should be washed off with soap and water and the sulphur paste or ointment rubbed in. To enable the sulphur to penetrate more deeply it may be combined with a keratolytic remedy such as 2 or $3 \%$ of salicylic acid or resorcin, as in the following formula:

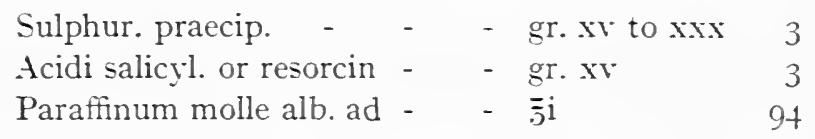

The application should be made at night and in the morning the skin should be dusted over with a powder such as the following:

\begin{tabular}{|c|c|}
\hline Sulphur. pr & ip. \\
\hline Zinci oxidi & - \\
\hline Talc ad - & - \\
\hline
\end{tabular}

In widely disseminated cases sulphur baths may be of value, either the natural sulphur baths of various health resorts such as Harrogate, Strathpeffer, Barèges, Cauterets, Luchon, or medicated sulphur baths at home, see p. 127. It is necessary, however, when the dermatitis is extensive and the inflammation acute, to be careful that they do not aggravate it or transform it into an exfoliative dermatitis. Should definite eczematisation be present it must be treated on the lines laid down for eczema. 
Lesions about the flexor aspects of the limbs call for similar treatment to those on the trunk and zinc sulphur paste generally gives good results.

Occasionally where lichenification has occurred, as about the flexures and the nape of the neck, the lesions may prove singularly resistant to treatment and in such cases a pastille dose of $\mathrm{X}$-rays is of great value, followed by the application of a bland zinc paste or of zinc gelatine as a protection against rubbing and chafing.

It is important that the underclothing should not be too heavy. Woollen materials should be avoided on account of the irritation of the wool and its non-absorbent properties and garments made of linen or cotton should be substituted.

\section{REFERENCES}

Audry. Séborrhéides (La Prat. Derm., I904).

Barber, H. W., \& SEMION, H. C. The etiology \& treatment of seborrhoic eruptions (Brit. Med. Journ., I918, ii. p. 245).

Beatty, IV. Seborrhoea (Brit. Journ. Derm., I894, vi. p. I6r).

Broce. Les eczémas séborrhéiques ou séborrhéides (La Presse Méd., I 897 , p. r62).

Brooke, H. G. Seborrhoea (Brit. Journ. Derm., I904, xvi. p. 205).

Duhring. Seborrhoea corporis (Cut. Med., Part ii.).

NacLeod, J. M. H. Seborrhoea and the seborrhoides (Practitioner, I904, p. 755$)$.

Pringle, J. J. Seborrhoea corporis (Brit. Journ. Derm., I896, viii. p. 482). (Brit. Journ. Derm., r903, xv. p. 4I).

Sabouraud. Pityriasis et Alopécies pelliculaires (Paris, 1904).

- Seborrhoea (Brit. Journ. Derm., I9or, xiii. p. 390).

-La question des Séborrhéides (La Presse Méd., June, r904).

Uxxa. Histopathology (Monats. f. prakt. Derm., I89I, xii. p. 249).

(Monats. f. prakt. Derm., I894, xviii. p. 257).

-Seborrhoic eczema (Volkmann's Klin. Vortrage, No. 79, I893). 


\section{CUTANEOUS AFFECTIONS DUE TO SACCHAROMYCETES} OR YEASTS

Certain of the yeasts are pathogenic in man and the lower animals and several of them can flourish in the skin, where they may cause either a patchy dermatitis or peculiar warty lesions and abscesses, such as are met with in the affection known as Blastomycosis.

Yeast dermatitis takes the form of irregular inflamed patches with a well-marked border, which are usually moist from exudation. They are chiefly met with in the intertriginous regions, such as the axillae, groins, and gluteal fold, and there is nothing to distinguish them from other forms of intertrigo except the presence of the yeast. It is a comparatively common affection in the tropics but only a few instances of it are on record in this country. Castellani has observed a number of cases in Ceylon, to which he gave the name of Intertrigo saccharomycetica and from which he succeeded in isolating a yeast (Saccharomycetes Samboni) which grows easily on artificial media as white colonies.

The treatment consists of bathing the affected part with warm water or boric lotion to remove the exudation, and the application of a lotion of $\mathrm{I}$ in 4000 permanganate of potash, an ointment containing $3 \%$ of salicylic acid, or a dusting powder consisting of boric acid I $\mathrm{dr}$. and zinc oxide I oz.

\section{BLASTOMYCOSIS}

Syn.: Blastomycetic dermatitis ; Oidiomycosis ; Saccharomycosis hominis ; Fr., Blastomycose cutanée.

Definition: An infection caused by certain yeast fungi, which may be cutaneous and characterised by raised verrucose lesions, or systemic and characterised by multiple abscesses and ulcerations in the skin, abscesses in the internal organs, pyaemia, and a fatal issue.

Introduction.--In 1896 Gilchrist reported a case of blastomycetic dermatitis in man, from which he isolated a yeast which he cultivated on artificial media and which was found to be capable of successful inoculation in certain susceptible animals. Since then about a hundred cases have been recorded in the United States of America, chiefly around Chicago, in Canada, in South America, Philippine Islands, Ceylon, and Japan. A few cases have been met with on the continent of Europe, but so far no authentic case has oecurred in Britain.

Closely allied, if not identical, with blastomycetic dermatitis are cases of a type originally described as a "protozoan infection of the skin" (Gilchrist), or as "Dermatitis coccidioides" (D. IV. Montgomery). These were thought at first to be due to sporozoa as the organism recovered from them appeared to increase by sporulation, but they are now known to be the result of the systenic invasion by yeast fungi which are capable of reproduction by sporulation as well as by budding. 


\section{DESCRIPTION}

Cutaneous Blastomycosis.--The lesions of cutaneous blastomycosis closely resenible the rerrucose type of Lupus vulgaris. They appear first as papules or small nodules, of a reddish-brown colour, which gradually spread and after months or years become irregular patches several inches in diameter.

The patches are raised and covered with warty projections, which may be dry and hard, or moist and soft and separated by fissures from which oozes a sero-purulent or sanguineous discharge which wells out

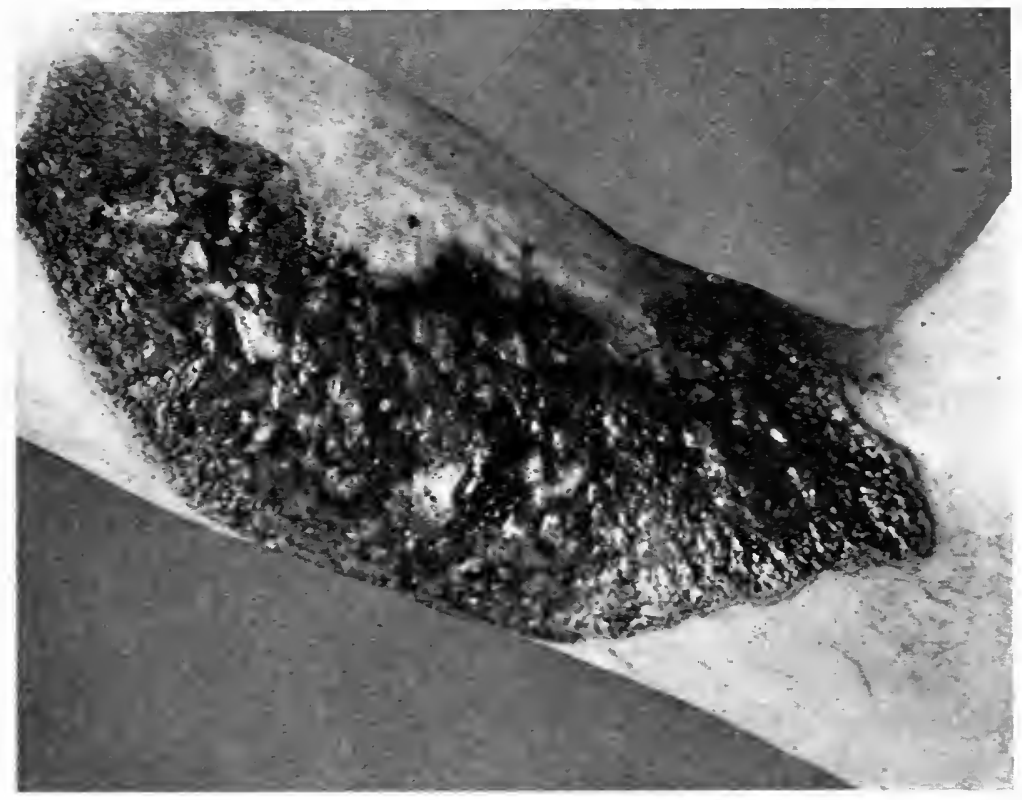

FIG. 9I.-Blastomycosis of the Arm.

(From Dr. O. S. Ormsby.)

when they are squeezed between the fingers. The discharge dries to form a dirty-yellow or brownish crust which, when removed, shows the papillomatous surface beneath. The patches vary in outline and have a well-defined dull red or purplish border which slopes down to the healthy skin and when examined by a pocket lens is found to be studded with yellow miliary abscesses. After a time retrogressive changes set in and scarring takes place with the formation of red, shiny, smooth cicatrices which may replace the whole lesion or be irregularly distributed in the form of small islands throughout the papillonatous area. From the secondary inoculation of progenic organisms the lesions may become inflamed and suppurate. The lesions are usually multiple, are spread by auto-inoculation, and are most common on the face, legs, hands, and back, but may occur anywhere on the cutaneous surface. (Fig. 9I.) 
As a rule the condition remains local and is not associated with any disturbance of the health and the neighbouring glands are not enlarged. In rare instances the fungi pass from the skin into the general circulation, a systemic infection takes place, and the internal organs become involved.

Systemic Blastomycosis.--In systemic blastomycosis the most pronounced feature is multiple abscesses situated either superficially in the subcutaneous tissue or in the internal organs.

The superficial abscesses are generally numerous and come out in crops. They appear as small subcutaneous nodules about the size of a pea, over which the skin at first is unaltered but in a few weeks it becomes red, the nodules enlarge, soften, and break down forming fistulae or superficial ulcers. The ulcers have a soft granulating or papillomatous floor, from which oozes a sanguineous or purulent discharge which dries to form crusts, and an irregular border which may be infiltrated and dotted over with miliary abscesses. (Fig. 92.)

The internal abscesses are present chiefly in the lungs, liver, kidneys, and spleen. They may occur also among the muscles and lead to necrotic changes in the bones. They vary greatly in size, and are occasionally large, especially about the abdomen, pelvis, or behind the pharynx, when they may contain a pint or even a quart of pus. The lymphatic glands in the neighbourhood are usually enlarged.

The abscess formation may be preceded for some time by evidences of a systemic invasion, such as irregular temperature and malaise.

When the lungs are involved it is indicated by wasting, cough, night-sweats, difficulty in breathing, expectoration of blood-stainer sputum in which the organisms may be detected, irregular pyrexia, and hoarseness of the voice from implication of the larynx. The lung symptoms are not generally so severe as in tuberculosis, nor are the physical signs as marked as would be expected from the post-mortem appearances.

When the kidneys are attacked there is albumin and casts in the urine, and occasionally the organism may be detected. When the intestine is affected diarrhoea is set up and the fungus may be recovered from the faeces. Sometimes the larger joints are involved and may become so painful as to suggest rheumatism. The blood shows marked leucocytosis and yeasts have been obtained in cultures from it.

The disease is progressive and characterised by exacerbations with the appearance of new crops of nodules which rapidly break down to form abscesses.

The general symptoms are similar to those of pyaemia and the patient's strength is gradually worn down till death gives a happy release, from exhaustion caused by septic absorption and the extensive involvement of the internal organs.

In the variety known as Dcrmatitis coccidioides, the symptoms are similar to those of systemic blastomycosis but the course is usually more 
rapid, as the fungi proliferate by sporulation instead of budding and the spores being smaller than the budding cells are more readily disseminated through the blood-ressels and iymphatics.

Histopathology.-Microscopically as well as clinically the lesions of cutaneous blastomycosis resemble Lupus verrucosus and are in the nature of warty granulomata.

The epidernis is markedly thickened and irregularly shaped processes somewhat similar to those which occur in renereal warts grow

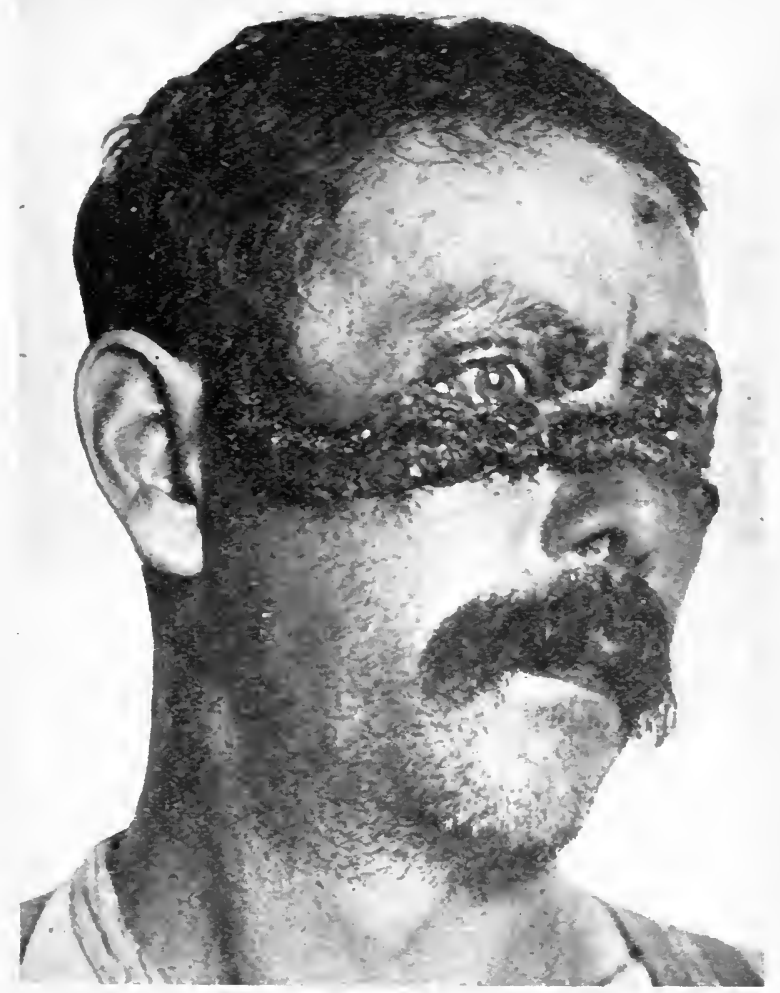

FIG. 92.-Systemic Blastomycosis.

(From Dr. O. S. Ormsby.)

down from it into the corium. The prickle-cells are oedematous, the inter-epithelial lymphatic spaces dilated, and polymorphonuclear leucocytes in various forms can generally be detected between the cells. Here and there in the epidermis miliary abscesses are present, which contain polymorphonuclear leucocytes, eosinophiles, epidermal debris, and usually one or more yeast-cells arranged singly, in pairs, or in small groups.

In the corium there is a diffuse cellular infiltration occupying the upper layers, consisting of plasma-cells, connective tissue cells, polymorphonuclear lencocytes, lymphocytes, and a few typical giant-cells. There is no definite nodular formation such as occurs in lupus but here 
and there small collections of polymorphonuclear leucocytes occur, forming miliary abscesses in which the organisms are often detected and frequently in the giant-cells. The collagen and elastin have lost their definition, stain feebly and, where the infiltration is densest, may have completely disappeared.

In systemic blastomycosis post-mortem examinations have shown abscesses in the internal organs, muscles and joints, and caries of the bones. Small miliary nodules have also been observed in the lungs, presenting a central necrotic mass with numerous polymorphonuclear leucocytes and yeast-cells surrounded by a few giant-cells containing

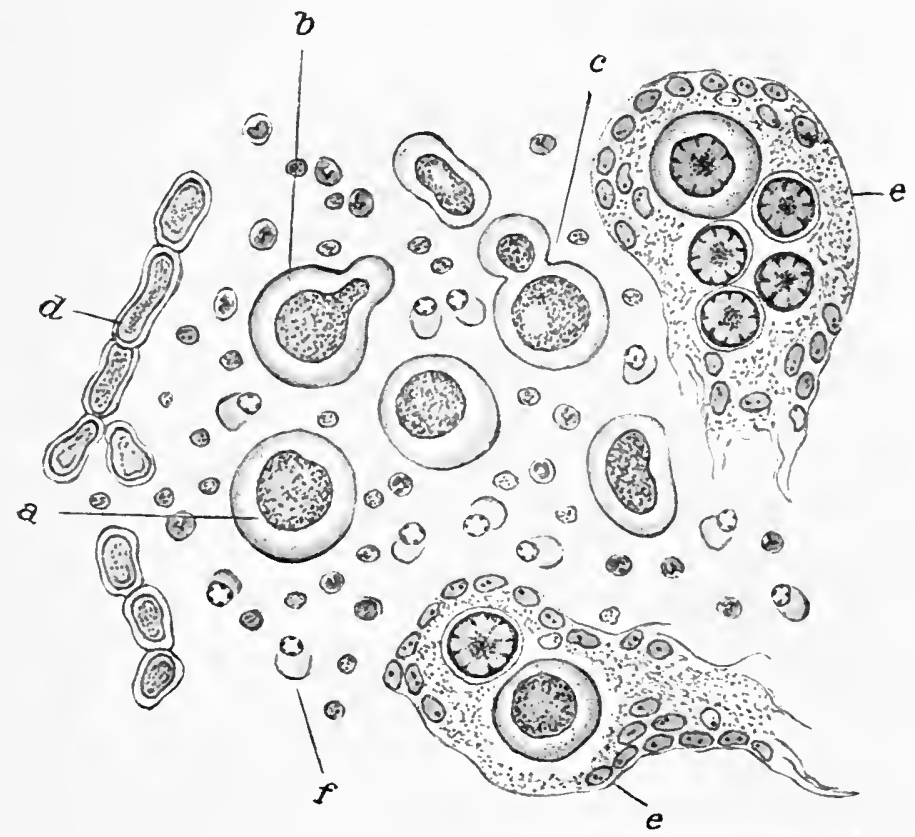

FIG. 93--Fungi of Blastomycetic Dermatitis and Dermatitis Coccidioides (semi-diagrammatic). ( $x$ about 800.$)$

a. Organism with central granular portion. $b$. Budding cell (typical of the organism of Blastomycetic dermatitis). c. Cell dividing by segmentation (typical of the organism of Blastomy"cetic dermatitis). $d$. Short jointed mycelial thread with budding. This appearance was found in a section of Dermatitis coccidioides. $e$. Giant-cell formation around organisms (Dermatitis coccidioides). $f$. Infiltration of plasma-cells and leucocytes.

(From MacLeod's Pathology of the Skin.)

the organisms beyond which is an indefinite zone of granulation tissue.

Mycology.-The affection is due to a pathogenic yeast of which there are several varieties, which may explain the varied appearances in different cases. Ricketts described four varieties, namely a blastomycetoid type in which reproduction took place by budding, a cryptococcus type in which reproduction took place in the tissues by endosporulation, an endomycetoid type in which the mycelium tended to break up into chains of endoconidia, and a hyphomycetoid type in which budding was seen occasionally. (Fig. 93.) 
The yeast is found in the sero-purulent discharge from the cutaneous lesions, in the pus from abscesses, and where the infection is generalised in the sputum, urine, faeces, or blood. It can be demonstrated easily by spreading a bead of pus on a slide and adding a drop of $5 \%$ Liquor potassae, or by staining sections of affected tissue with ordinary protoplasmic dyes.

In fresh preparations it appears as round or oval bodies from 7 to $20 \mu$ in diameter, either single or in groups, with a doubly contoured, highly refractive capsule containing granules or spores and occasionally vacuoles, and in stained preparations the capsule is generally found to be separated from the granular protoplasm by a clear space. (Fig. 94.)

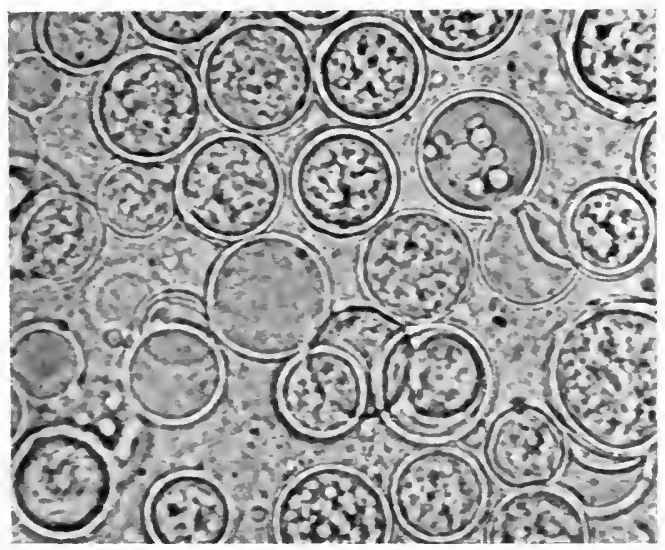

FIG. 94.-Systemic Blastomycosis. Smear from spleen.

(From Dr. O. S. Ormsby.)

It is easily grown on ordinary artificial media when it takes the form of white fluffy cultures which rary so much in different cases as to indicate the plurality of the fungus.

In the tissues it reproduces itself either by budding or sporulation; in culture, by a mycelium either with large pod-like segments containing bodies in which spores may be detected or with budding aerial hyphae.

Inoculation experiments from cultures have been successful in certain of the lower animals especially when intravenous or intraperitoneal. The organism has been found to be pathogenic in mice, rats, rabbits, dogs, and guinea-pigs, and to cause a sr'stemic infection with the formation of abdominal tumours and frequent involvement of the testicles, from which it has been successfully cultivated.

Etiology.--The predisposing factors are uncertain. Heredity does not seem to play any part in the etiology. The majority of cases have occurred in males, as they are more liable to exposure to infection than females. Age does not seem to be a predisposing factor and it has been recorded as early as $I 7$ and as late as $5 \delta$ years. Most of the cases have occurred in the poorer classes where the hygienic conditions 
were unfavourable and possibly the general resistance may have been lowered from insufficient food.

Cutaneous blastomycosis is due to local inoculation of the organism through an abrasion of the skin and in one or two cases a history of injury has preceded the appearance of the original lesion. In the systemic type, where cutaneous lesions are secondary, the infection seems to take place most frequently through the lungs and the earliest definite symptoms may simulate pulmonary tuberculosis, and yeasts may be detected in the sputurn.

Diagnosis.-Cutaneous blastomycosis has to be distinguished chiefly from Lupus verrucosus and late syphilitic lesions of the ulcerative or warty type.

In Lupus verrucosus the lesions develop more slowly and rarely reach the size which those of blastomycosis may attain, are more often single, do not show the same tendency to involve the face, being usually most marked on the hands and legs, and their surface is dry, harder, and does not present miliary abscesses. Cases of lupus frequently show other stigmata of tuberculosis and react to tuberculin but do not respond to iodide of potassium. The chief diagnostic point is the demonstration of the fungus which is usually simple.

From late syphilides the distinction is not so difficult. In the syphilitic lesions the patch is usually made up of a number of small nodules grouped in a serpiginous fashion and with a marked tendency to ulcerate. If warty lesions occur they are generally confined to the legs and feet, are of shorter duration, and react to mercury which has no influence on blastomycosis. If doubt still remains assistance may be obtained from the Wassermann reaction.

It might also be mistaken for a fungating carcinoma of the skin, but in cancer there is more induration of the base of the lesion and the neighbouring glands are generally involved.

Systemic blastomycosis where abscesses or ulcers occur is not difficult to diagnose as the organisms are easily demonstrated in the pus. In early cases where the skin is not involved to any marked extent it might be mistaken for pyaemia, tuberculosis of the lungs, nephritis, or rheumatism according as the lungs, kidneys or joints are specially affected.

Prognosis.-In cutaneous blastomycosis the prognosis is fairly satisfactory as the lesions tend to disappear under suitable treatment. If left untreated they may persist indefinitely and gradually increase, but as a rule there is a tendency to spontaneous retrogression. Occasionally systemic invasion occurs in the local cases and leads to a fatal issue.

In systemic blastomycosis the prognosis is grave and the majority of the patients die after a few weeks or months. If the diagnosis be made early and treatment instituted the outlook may be more favourable.

Treatment.-The treatment of cutaneous blastomycosis consists of the administration of iodide of potassium in heroic doses of from 200 
to 400 grns. a day. By this means the lesions can be reduced if not completely cured. The X-ray's can also cause the disappearance of the lesions, and a farourite treatment at present is a combination of both methods. Surgical procedures such as excision or scraping are not so satisfactory, and the latter is apt to be followed by recurrence.

In systemic blastomycosis the treatment consists of the administration of iodide of potassium and a general tonic regime.

Locally abscesses should be treated on ordinary surgical principles, and ulcers by antiseptic and astringent lotions among which $I \%$ sulphate of copper solution has been recommended.

NorE.-In preparing the article on blastomycosis the author is indebted to Dr. Oliver Ormsby for the illustrations and to Dr. Howard Morrow and the late Dr. Nevins $\mathrm{Hyde}$ for the pathological material from which the description of the histology was made.

\section{REFERENCES}

Buschke, A. Hautblastomylose (Handbuch der Hautkrankheiten, Mracek, Vienna, I907).

Dubretilh, IT. Blastomỹcose cutanée (Ann. de Derm. et de Syph., I904). Gilchrist, T. C. A case of Blastomycosis in man (Johns Hopkins Hosp. Rep., Baltimore, I896, i. p. 269).

- Some additional cases of Blastomycetic dermatitis (Journ. Cut. Dis., X.Y., I904, xxii. p. I0\%).

Gilchrist, T. C., \& STOKEs, W. R. A case of Pseudo-lupus vulgaris - caused bỹ blastomyces (Journ. of Exter. Med., IS98, iii.).

Hyde, HeKtoex, \& Bevax. Contribution to the study of Blastomycetic dermatitis (Brit. Journ. Derm., I 899, xi. p. 26r).

Hyde \& Richets. Blastomycosis of the skin in man (Journ. Cut. Dis., I901, xix. P. 44).

MacLeod, J. M. H. Histopathology of blastomycosis (Brit. Journ. Derm., I901, xiii. p. 301). (Brit. Journ. Derm., I902, xir. p. 302).

MÉxeat. Blastomycose cutanée (Ann. de Derm. et de Sỵph., I902, iii. p. 578$)$.

Montgomery, D. W., \& Morrow, H. Reasons for considering Dermatitis coccidioides an independent disease (Journ. Cut. Dis., August, I904).

Montgomeri, F. H., \& OrMsbi, O. S. Sỹstemic blastomỹcosis (Arch. Int. Med., August, Igo\$).

OrMsbi, O. S., \& MILIer, H. II. Systemic blastomycosis with multiple cutaneous lesions (Journ. Cut. Dis., March, I903).

RIXFORD \& GILCHRIST. Two cases of protozoan infection of the skin (Johns Hopkins Hosp. Rep., I \$96, p. 209).

\section{TINEA OR RINGWORM}

Introduction.-Ringworm is an affection due to a group of hyphomycetes or mould-fungi, which may attack the glabrous skin, hairs, nails, and in rare instances by extension the mucous membranes.

The clinical appearances vary according to the precise variety of fungus responsible, the situation of the lesions, and the individual peculiarities of the shin affected. It is customary and convenient to 
describe the clinical features of ringworm under the headings of the regions attacked, namely ringworm of the glabrous skin (T. circinata), crutch ( $\mathrm{T}$. cruris), eczematoid ringworm of the hands and feet, ringworm of the scalp ( $T$. tonsurans), beard ( $T$. barbae), and nails ( $T$. unguium), and this plan will be followed here.

As a preliminary to the clinical description the causal fungi and the pathological changes they produce in the skin and hairs will be described.

\section{The Fungi of Ringworir}

The fungi, or dermatophytes, which cause ringworm have been placed provisionally in the order of the ascomycetes, and the family gymnosaceae, but their classification is not yet absolutely settled. They are mould-fungi which can live both aerobically and anaerobically.

In the skin, where they are anaerobic, they occur in the form of a more or less dense network of mycelial threads which break up into small sections, to which the name of "spores " has been applied. They are not true spores, however, but become elongated into new mycelial threads which break up in their turn and so perpetuate the fungus. Other methods of reproduction occur if the fungus is grown anaerobically ; in hanging drop preparations, for example, reproduction takes place by endoconidia, while in cultures it takes place by chlamydospores or multilocular spindles.

The true method of fructification, however, is aerobic when they proliferate by means of aerial hyphae with terminal and lateral buds, which can be demonstrated by making an impression preparation on a cover slip from the surface of the culture.

The fungi of ringworm though closely allied to each other and sometimes so similar in appearance as to be indistinguishable microscopically, are capable of being differentiated on cultivation on artificial media. An excellent medium has been devised by Sabouraud which is known as proof agar, on which the fungi grow freely and exhibit their differential characteristics and its general employment has considerably advanced our knowledge of these fungi by enabling different observers to compare their results.

The following is the formula for proof agar :

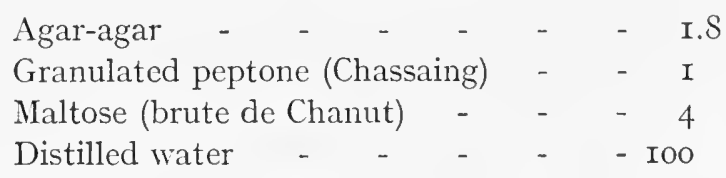

(Obtained from Cogit, 35, Boulevard Saint Michel, Paris.)

Nore.-Much work has been done on the mycology of ringworm in recent years and the literature on the subject is now considerable. Valuable researches have been carried out in connection with it in this country by Drs. Colcott Fox, Arthur Whitfield, H. G. Adamson and others, in Paris by Dr. Sabouraud, and in Ceylon by Dr. Aldo Castellani. 
Certain of the fungi of ringworm are found only on the human skin, while a considerable number occur in the lower animals and may be transmitted to man. The animals from which infection most commonly takes place in this country are the domestic animals, the cat, dog, horse and cattle, but the disease, is met with also in rats, mice, guinea-pigs, in many of the wild animals, and in rare instances in birds. The wide distribution of the disease in the animal kingdom, the characteristics of the fungi, and the ease with which they will grow on artificial media, suggest the probability that they have a saprophytic existence possibly on hay, straw, or decomposing vegetable matter, but up to the present time this has not been proved. (Fig. 95.)

There are two families of ringworm fungi which attack the human skin, namely the microspora and the trichophyta and of these there is a number of varieties. Each country has its own peculiar ringworm flora and observations on the subject, both with regard to the special fungi and their relative proportions,

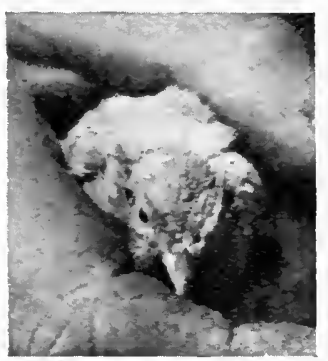

FIG. 95--Canary with bald head due to Ring. worm. only hold good for the country or district where the observations were made.

The fungi of ringworm have a double action on the skin, (I) a destructive or corrosive action on the epidermis, hairs and nails, owing to the elimination of a ferment which has the power of dissolving keratin and of rendering the surrounding tissue suitable for the nutrition of the fungus, and (2) an irritant action from their rapid growth and the toxic influence of the ferment, which results in varying degrees of inflammation, in some so mild as to be scarcely appreciable, while in others it may be so acute as to lead to vesicular, pustular and even granulomatous lesions. The degree of inflammation is dependent chiefly on the variety of fungus, and the constitutional state of the patient has comparatively little bearing upon it. There exists, however, a greater susceptibility to ringworm in some individuals than in others, consequently the same fungus may produce acute symptoms in one patient and a mild inflammatory lesion in another. Different fungi have a predilection for certain parts of the body, some attacking chiefly the hairy parts while others find a suitable soil in moist warm regions like the crutch and the axillae. Of the different fungi the most virulent are those of animal origin.

The two families of ringworm fungi, namely, microspora (small spored) and trichophyta ( $\theta \rho i \xi$, hair; $\phi v \tau o ́ v$, fungus) were originally distinguished according to the size of their spores, the term megalospora or large-spored fungi being employed as synonymous with trichophyta. It was found, however, that certain of the trichophyta had spores as small if not smaller than those of the microspora and it was 
deemed advisable to abandon the term megalospora in favour of trichophyta, the two families being distinguished rather by their morphological and cultural peculiarities than by the size of their spores.

\section{Microspora.}

Sabouraud has differentiated eleven varieties of microspora of which the following four are of clinical importance in this country, namely, the M. audouini (Grüby, Sabouraud), M. equinum (Bodin), M. caninum vel lanosum (Bodin, Sabouraud), M. felineum (Colcott Fox \& Blaxall).

Microsporon audouini.- The M. audouini is by far the most common microsporon, being responsible here for $90 \%$ of the cases of ringworm of the scalp and for a considerable number of those of the glabrous skin. In Paris it causes about $60 \%$ of the ringworms, but is unusual in Germany, Denmark and Sweden, and very rare in the Eastern countries of Europe. It is the only microsporon which is confined to the human skin ; it has been known to occur in the dog, but the infection probably took place from a child. It attacks chiefly the scalp in children and to a less extent the glabrous skin and produces comparatively little inflammation. It hardly ever attacks the adult scalp, as the scalp at puberty for some reason not fully understood becomes an unsuitable soil for its growth. A few rare cases are recorded where it has affected the adult scalp, and two such were met with in which women were infected from their children. In one of them it produced a typical dry scurfy patch near the hair-margin; in the other a suppurative lesion

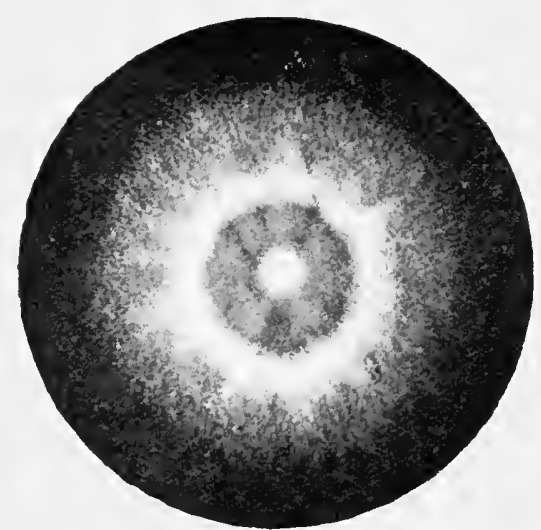

FIG. 96.-Microsporon Audouini Culture on Proof Agar.

Six weeks old. was present on the nape of the neck extending for about $\frac{1}{4}$ of an inch into the hairy scalp, while in the child the ringworm was nonsuppurative, and cultures of M. andouini were obtained from both mother and child.

On proof agar the Microsporon audouini gives rise to a delicate disc-shaped downy culture with radiating furrows. This grows slowly and in about a week after inoculation appears by transmitted light as a circular plaque with a fine margin or delicate fringe beneath the surface of the medium. In a few days the centre becomes raised in the form of a greyish white tuft or opaque knob. In about six weeks the characteristic features of the culture are fully developed, and consist of a central knob surrounded by a whitish grey cushion beyond which there is a delicate margin with a fine downy fringe. (Fig. 96.) 



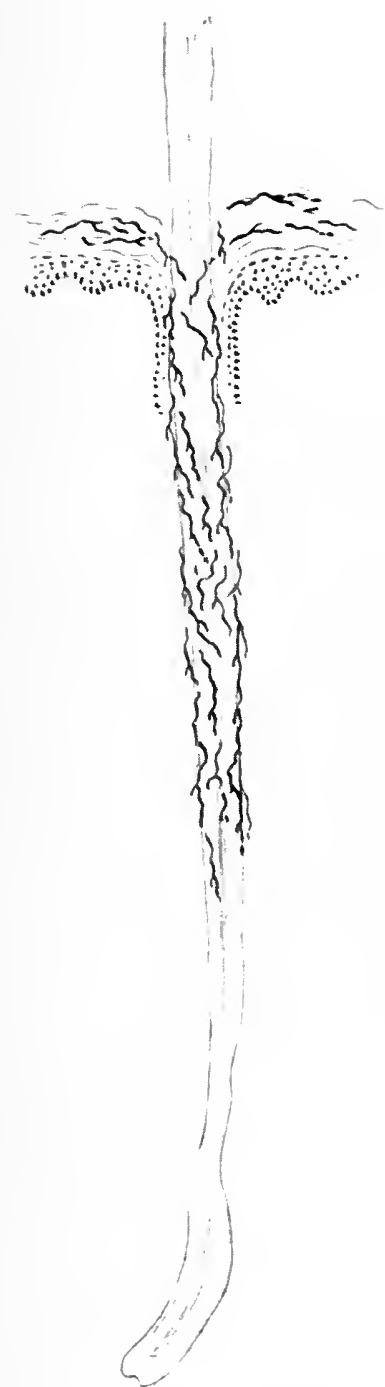

J. M. H. M. del.

Fig. 97.-First stage in the invasion of a hair by Micro. sporon Audouini, showing the fungus in the scale at the mouth of the hair-follicle, spreading down between the hair and the follicle, and forming a loose mycelial net. work ensheathing the hair.

(Semi-diagrammatic drawing.)

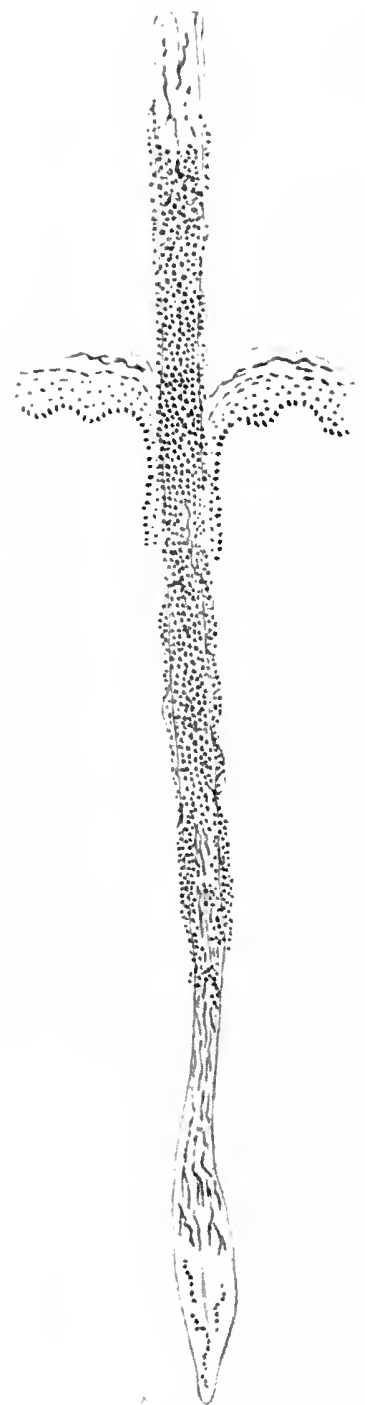

J. M. H. M. del.

F1G. 9s.-Hair fully in. fected by Microsporon Audouini. The small spores have partially stripped off the cuticle and have formed a dense mosaic ensheathing the hair. Ircelial threads are present within the hair ending in a fringe near the bulb, and a few chains of spores are seen in the bulb itself.

(stmi-diagrammatic drawing.)

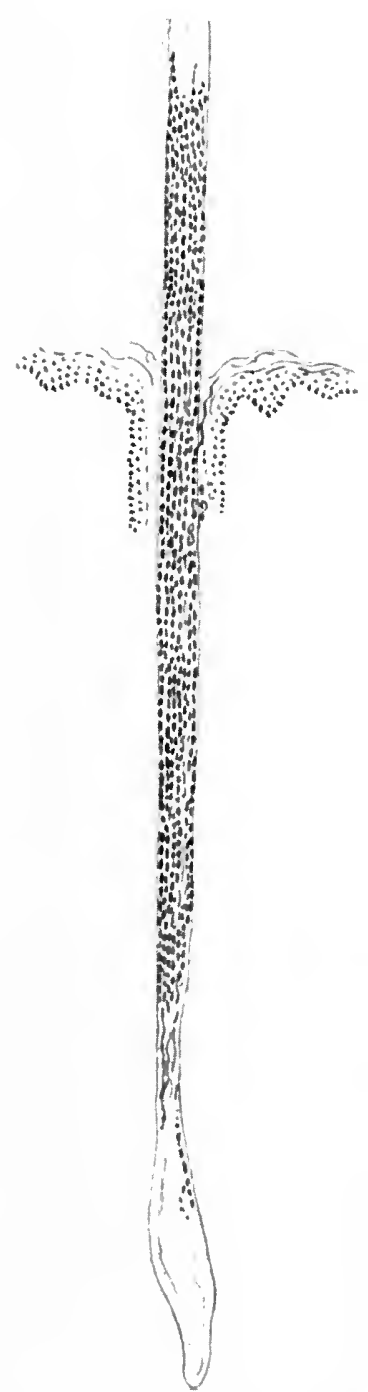

J. M. H. MI. del.

F1G. ro3.- Hair fully infected by Trichophyton Endothrix. The fungus on reaching the cuticle passes between the cells and breaks up into chains of spores which are arranged in the long axis. of the hair and densely packed together. In a fully infected hair the fungus outside the: cuticle disappears.

(Simi-diagrammatic drawing.) 
Scrapings from a patch of microsporon ringworm on the glabrous skin soaked in Liquor potassae show that the fungus consists of mycelial filaments of about $4 \mu$ in width which branch irregularly and are broken up by septa at long intervals of varying length. These are known as "plain filaments." In addition to the mycelial threads a few round or oval spores may be detected either singly or in small groups and varying in size from 2 to $t \mu$ in diameter.

The mode of invasion of the hair by the fungus is as follows: It first becomes deposited on the scalp where it causes a mild inflammation with slight redness and desquamation, from there it spreads until it meets a hair-follicle when the mycelial threads pass down between the follicle and the hair. Near the bulb they exert a corrosive action on the hair-cuticle by means of the keratolytic ferment which emanates from them. This causes the cuticle to become detached and enables the fungus to insinuate itself beneath it. The mycelium then breaks up into rows and groups of large spores, which in turn divide into small spores and these eventually become so profuse as to form a dense sheath like a mosaic enveloping the hair. Certain of the filaments grow into the hair itself and spread

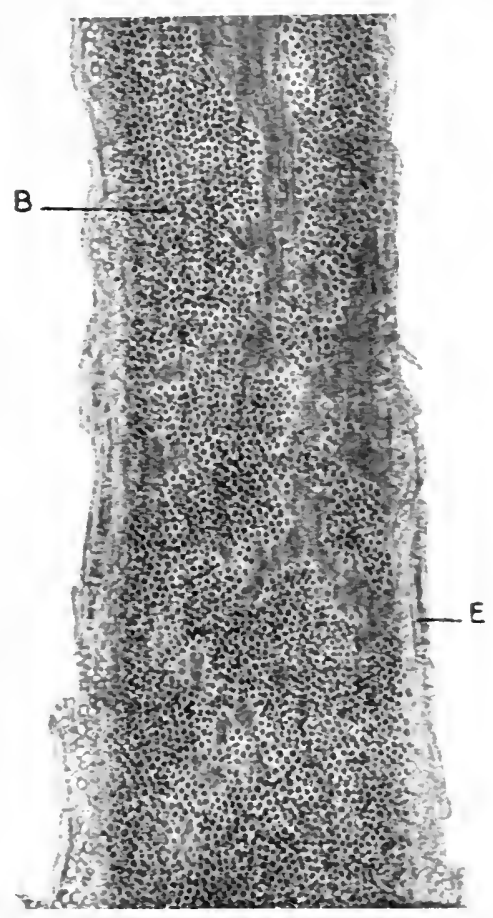

FIG. 99.-Hair infected by Microsporon Fungus forming a mosaic.

(After Dr. R. Sabouraud. Ans. Ge Derm. co de Sypis., I907.) up and down in the long axis of the shaft. (Figs. 97, 9S.)

A fully infected hair or stump presents the following appearances when soaked in Liquor potassae and examined microscopically. From near the bulb to about $\frac{1}{1}$ of an inch above the orifice of the follicle the hair is ensheathed in the mosaic of small spores; within the hair mycelial threads can be detected, ramifying in the long axis and terminating near the bulb in a fringe, the bulb itself being comparatively free as it does not provide a suitable soil for the growth of the fungus and only a few single rows of spores may be detected in it. The presence of the fungus both around and within the hair renders it so brittle that it readily breaks off at the upper level of the fungus to form a stump. (Fig. 99.) 
Other Microspora.-The other microspora, namely the M. equinum, M. felineim, and M. caninum vel lanosum, as their names indicate are of animal origin and have a wide distribution. They readily infect the human skin and produce lesions of a somewhat similar type to those caused by the M. audouini, except that the inflammatory disturbance is usually greater. On the glabrous skin they, especially the M. felineum, sometimes cause well-marked rings with a concentric arrangement, while on the scalp they occasionally give rise to suppurative patches or kerions. They may also attack the beard.

The cultures of the animal microspora are more rapid and vigorous than those of the M. audouini. They are more opaque and luxuriant, have not the transparent delicacy of the human fungus, and have a tendency to undergo pleomorphic changes and to become white and downy which masks their identity. (Figs. IOO, IOI, IO2.)

The M. caninum is comparatively common. In the cat it produces bald scaly patches and occasionally suppurative lesions. It has been known to attack the beard in man and to give rise to patches there not unlike microsporon ringworm of the scalp in children. The M. equinum produces a smooth rather than a downy culture. In the horse it causes a scaly patch over which the hair at first is disarranged and finally falls out. It has also been found in the beard in man.

\section{Trichophyta.}

The most prominent characteristic of the trichophytic family of ringworm fungi when they grow in the skin and hairs is the tendency of the mycelial filaments to break up into chains of spores like a necklace instead of forming clusters or being arranged in a mosaic. These spores are square or oblong in shape with rounded corners, are often doubly contoured, and vary in diameter from 3 to $6 \mu$. The mycelial filaments, as seen in preparations taken from the skin, exhibit a tendency to branch dichotomously.

It is customary to divide the trichopliyta into two main groups according to the precise situation of the fungus in the fully infected hair, namely $T$. endothrix where the fungus is within the hair, and T. ecto-endothrix where it is present also outside the hair. This differentiation, though of some value for descriptive purposes, is too arbitrary, for in the early stages of invasion the fungus is always outside the hair.

In a hair fully infected with $\mathrm{T}$. endothrix the fungus is found to be entirely within the hair, the original mycelial threads and chains of spores which invaded the follicle and passed down between it and the hair having disappeared. The mycelial threads enter the hair between the cells of the cuticle and break up into chains arranged in the long axis and extending from near the bulb, where the fungus ends in a mycelial fringe, to $\frac{1}{4}$ of an inch or more above the level of the scalp. In the follicular portion of the hair the spores may be so densely packed 


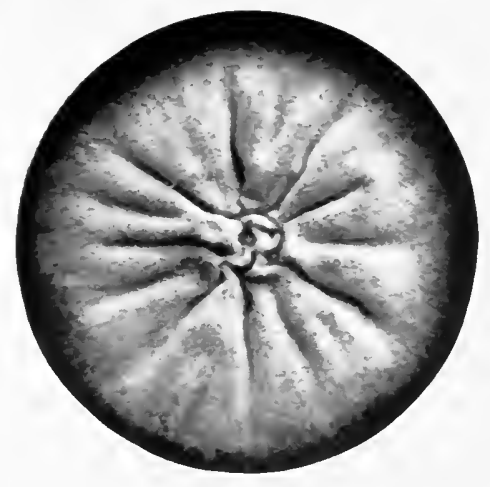

Fig. IO0.-Microsporon Equinum of Horse.

(Bodin. Ann. de Derm. et de Stpil. rgo-.

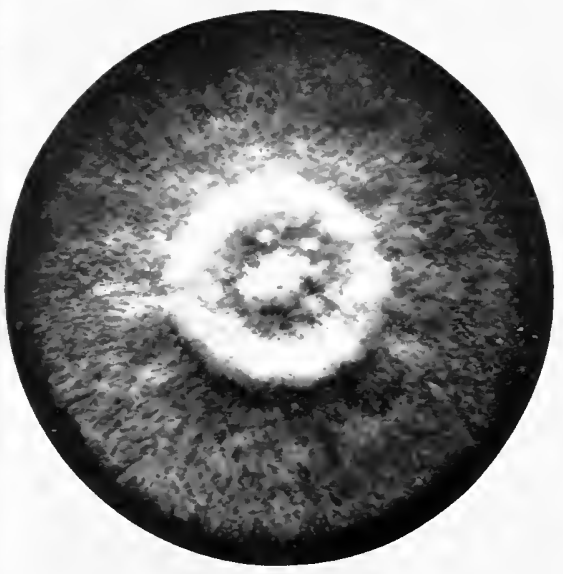

FIG. 102.-Microsporon Lanosum of Dog.

(Sabouraud. Ann. de Derm. et de Siphe, rgo-.

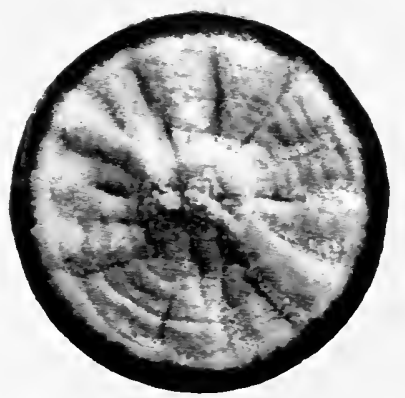

FIG. I0-.-Trichophỹton Acuminatum. Culture on prooi agar 35 days.

(From Dr. R. Sabouraud.)

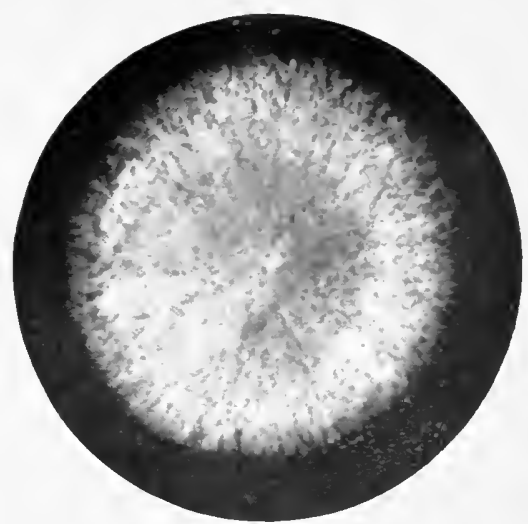

FIG. IOI.-Microsporon Fe!ineum of Cat. Colcott Fox and Blaxall. Brit. J utm. Dern... Is gó.

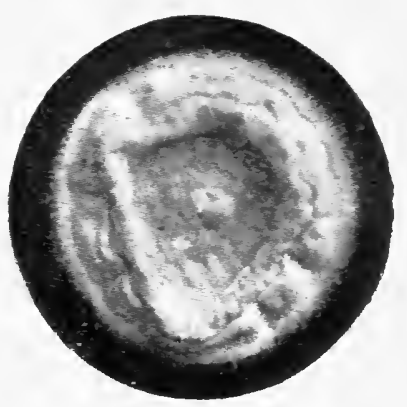

FIG. I05.-Trichophỵton Crateriforme. Culture on proof agar 35 days. (From Dr. R. Sabouraud.

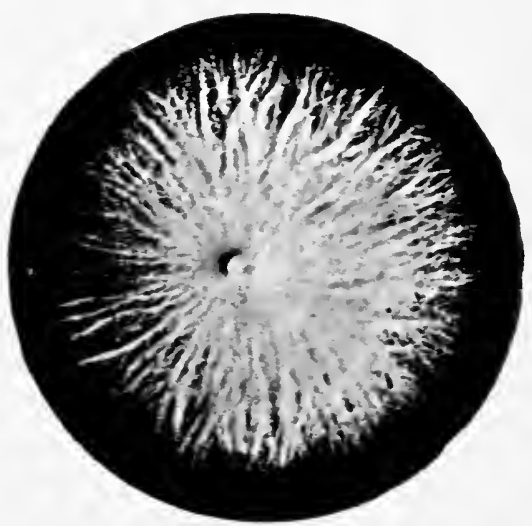

FIG. I09.-Culture of Trichophỹton Ectothrix.

Shows white central boss and coarse hyphae, forming tapering processes radiating irom the centre. This culture was made from a child, the source of intection being a borse.

(From Dr. J. Lemare Bunch.) 

as to resemble small shot in a tube, while the chain-formation is more evident in the extra-follicular portion. The presence of this dense mass of fungus renders the hairs so brittle that they are difficult to epilate and readily break off near the orifice of the follicle, leaving stumps which are frequently so short that they can only be removed by being expressed like a comedo. It is this which has given rise to the appearance aptly designated "black-dot ringworm." (Fig. I03.)

In $T$. ecto-endothrix the fully infected hair has an appearance more like that in microsporon and presents a network of fungus in the form of mycelial threads and chains of spores ensheathing the hair, and mycelial filaments and chains of spores in the long axis of the shaft ending near the bulb. This arrangement differs from that in micro-

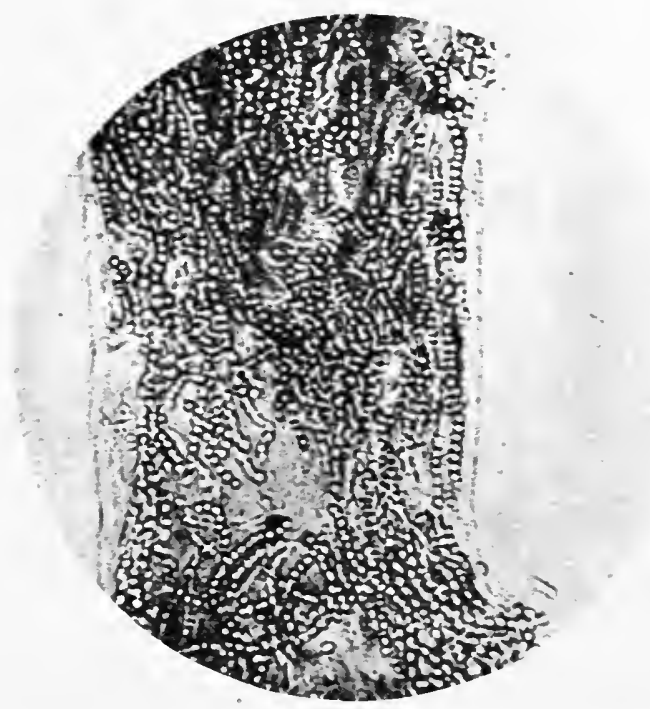

EIG. 104.- Hair fully invaded by Trichophyton Endothrix.

(After Dr. H. G. Adamson. British Journ. of Dermatology, I895.)

sporon in the absence of a mosaic of small spores around the hair and its replacement by rows of spores and mycelial threads, and from the perfect endothrix in the persistence of part of the fungus outside the hair. (Fig. I04.)

Trichophyta Endotriches.-The T. endotriches are confined to man but may be transmitted from man to the lower animals. In this country they are responsible for about $4 \%$ of the total ringworms, but are more prevalent on the Continent and in Paris give rise to between 30 and $40 \%$ of the scalp ringworms. They may attack the glabrous skin, scalp, beard, and nails. On the glabrous skin they cause plaques which are raised, scaly, and occasionally finely vesicular. On the scalp they have not the same tendency as the microsporon to die out at puberty but may persist to adult life or may attack the adult de nowo, as in a case in which endothrix fungus was present in the scalp 
of a young woman of 27 and had been contracted when she was 15 years of age.

There are three principal varieties of $T$. endothrix in this country distinguishable on proof agar as they present distinctive colours when the growth is protected from daylight, but which cannot be differentiated in microscopical specimens or by the clinical lesions which they produce. On proof agar the culture becomes noticeable about the sixth day as a small fluffy growth which gradually increases until it forms a dome with a central knob and a slight depression around it. As it grows older the centre tends to sink in like a crater, with a vermiform floor and a thick rounded wall which may become sinuous or

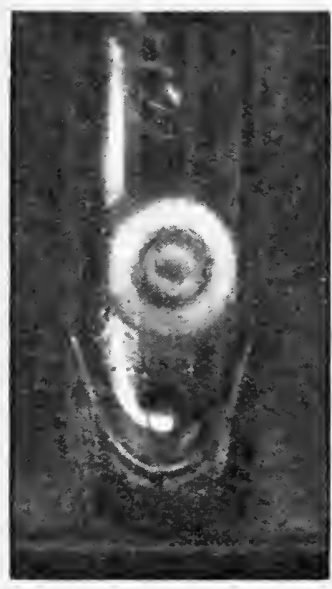

FIG. 106.--Trichophyton Sulphureum. Primrose crater.

(After Dr. T. Colcott Fox, Erit. Journ. Derm., rgog.) incised and may present buttresses and furrows extending from it irregularly.

The three varieties are:

I. A cream-coloured or white crater ( $\mathrm{T}$. crateriforme of Sabouraud) $38 \%$ of the cases. (Fig. I05.)

2. A primrose-coloured crater (T. sulphureum), 21\%. (Fig. Io6.)

3. A greyish-yellow crater (T. acuminatum of Sabouraud) $26 \%$. This begins as a knob covered with feathery spikes which were likened by Colcott Fox to "pampas grass." (Fig. I07.)

Sabouraud has described an endothrix under the heading of Trichophyton plicatile which can produce suppurating and granulomatous lesions. It forms white crateriform cultures with a powdery surface " like a silky material in folds."

In the Anglo-Egyptian Sudan, Chalmers and Marshall have described an endothrix (T. Currii) which produces a white growth with a knob-like centre on a white plateau, with or without two concentric rings and a slight fringe. It causes Tinea capitis and appears on the scalp as whitish scaly patches over which the hairs are broken off about a millimetre above the surface. It occurs almost exclusively in Sudanese boys from io to 16 years of age.

Trichophyta Ecto-endotriches.- This is the largest of the three groups of ringworm fungi. The members of it are all of animal origin and when they infect the human skin they give rise to lesions of a markedly virulent and suppurative type. They may attack the glabrous skin, scalp, beard and nails, form about $6 \%$ of the total number of ringworms in this country, and are responsible for the majority of the adult cases. According to the size of their spores they may be divided into two groups, the microides, and the megaspores. The cultures of the ecto-endotriches, of which I5 or more have been distinguished up to the present, vary in detail and have been divided by 
Sabouraud into three groups, namely, (I) powdery cultures, (2) downy cultures, and (3) corrugated cultures like favus. He gives the following list of cultures: (Fig. IoS.)

I. Large powdery or chalky cultures ( $\mathrm{T}$. gypseum) Microides. These appear on proof agar about the third day as a small white tuft which gradually assumes a powdery appearance and becomes surrounded by radiating hyphae like a star with widely divergent rays and a central opaque mass. They occur chiefly in domestic animals, such as horses and cattle, and may be transmitted readily to man, being not infrequent on the beard. Six varieties have been described.

(a) T. asteroides. Fine rays like a star.

(b) T. radiolatum. Shorter rays.

(c) T. lacticolor-cream coloured.

(d) T. granulosum-irregular chalky granulations and no peripheral rays.

(e) T. farinulentum-mealy culture and no rays.

(f) T. persicolor-star-shaped, velvety and peach-coloured. (Peach-coloured trichophyton of Adamson.)

2. Large downy cultures (T. niveum) Megaspores.

(a) T. niveum radians.

(b) T. niveum denticulatum.

These chiefly affect dogs and cats but may be transmitted to the human skin.

(c) T. equinum. (Figs. Iog, IIo.)

This fungus is rare in man but has been found in the beard.

(d) T. rosaceum. (Figs. III, II2.)

This fungus is of bird origin occurring in fowls, pigeons, and canaries. In fowls it causes a disease known as "white-crest," in which there are small crusts at the base of the feathers which remain when the feathers break away and are found to be composed of mycelial threads and spores. On the human glabrous skin it gives rise to raised plaques, scaly or covered with

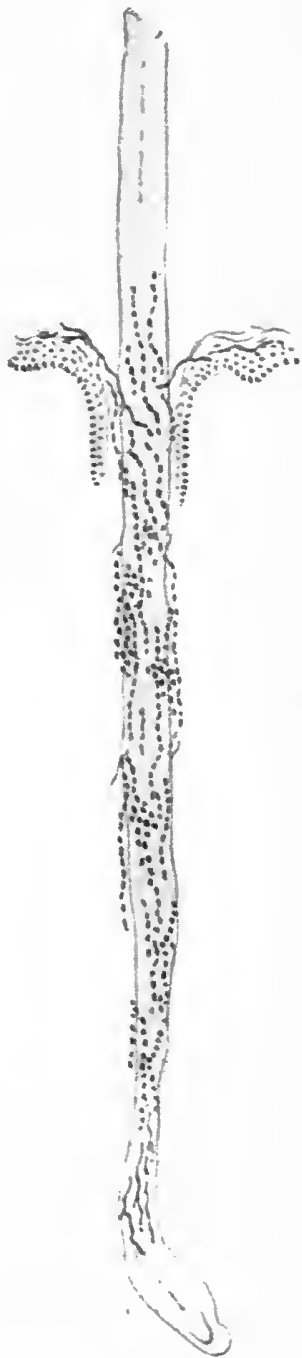

J. M. H. M. del.

FIG. Ios.-Hair in. fected with Trichophy. ton Ectothrix.

The fungus is in the form of mycelial threads and chains of spores both outside the cuticle and within the hair. resico-pustules. On the beard it may cause dry, scaly or suppurative lesions, and on the scalp kerion.

\section{Fariform cultures.}

Instead of being stellate the cultures sometime present a corrugated centre with an absence of rays and an appearance like a culture of 
favus. These cultures are peculiarly smooth and gelatinous instead of being fluffy. They are of animal origin but may be transmitted to man. The following varieties have been described by Sabouraud:

(a) T. violaceum. (Fig. II3.)

This has been recovered from suppurative ringworm of the scalp as well as from suppurative ringworm of the beard.

(b) T. ochraceum (canary yellow culture).

(c) T. verrucosum (greyish white culture).

These occur in domestic animals such as the calf and horse.

(d) T. discoides (cribriform culture with a white fringe, strongly resembling that of favus).

This fungus has been met with in France, Denmark, and recently in Egypt. It occurs in man, horse, and cattle, and has been found in the scalp, beard, and glabrous skin. It is probably a parasite of horses and is transmitted from them to man.

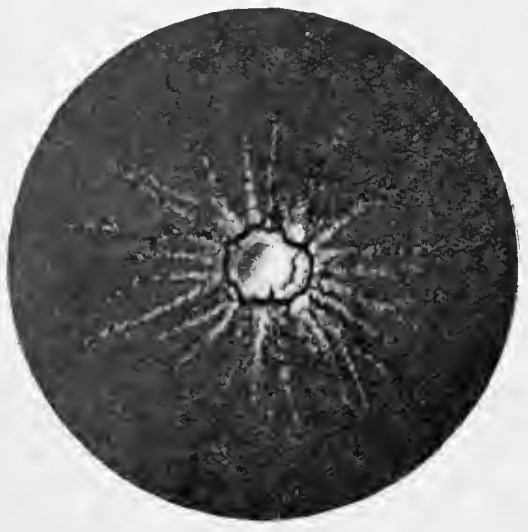

Fig. rro.-Culture of Trichophyton Ectothrix from a Horse.

Shows central mass and radiating processes, which give the culture a stellate appearance.

(From Dr. J. Lemare Bunch.

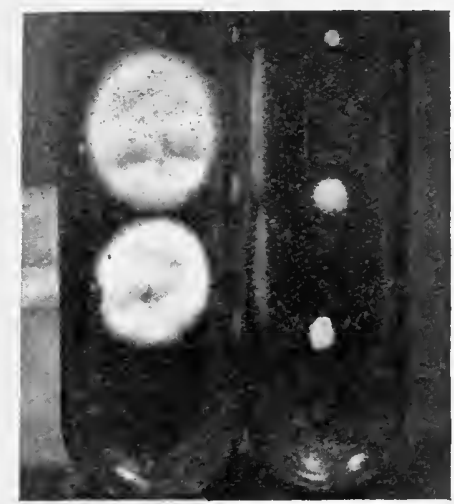

FIG. II I.-Trichophyton Rosaceum.

Culture on proof medium. (After Dr. R. A. Bolam. Brit. Journ. Derm., 1912.)

Immunity Production.-Of recent years a number of experiments have been done in connection with the production of immunity to ringworm both in man and lower animals, by Plato, Bloch, Massini, Lombardo, Adamson, and others.

Plato prepared a substance, which he called Trichophytine, by pounding up cultures from virulent ringworms and collecting the filtrate. When this was injected in cases of virulent inflammatory ringworm it caused a general reaction, a local reaction in the form of papules and pustules at the point of injection, and a gradual disappearance of the ringworm lesions.

Animal experiments were done by Bloch and Massini with a dog ringworm (Microsporon lanosum) and a horse ringworm (Trichophyton gypseum). These were inoculated on healthy dogs and horses respec- 
tively, and, where the inoculations were successful and ringworm lesions resulted, an immunity to subsequent inoculations was produced which lasted for months.

They found also that in the subjects of ringworm a cuti-reaction corresponding to that with tuberculin, occurred when a trichophytine made from broth-cultures of a virulent trichophyton was rubbed into a scratch.

Adamson, on the other hand, was unable to obtain cuti-reactions

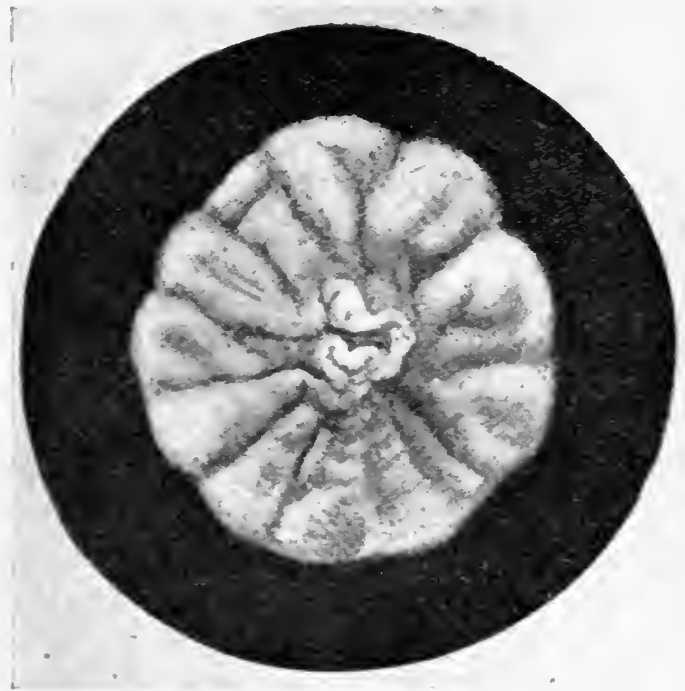

FIG. II2.-Trichophyton Rosaceum.

(After Dr. R. Bolam. British Journal of Dermatology, I912.)

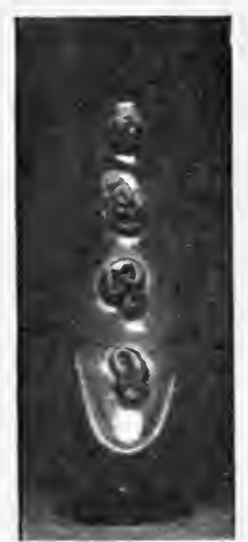

FIG. 113.-Trichophyton Violaceum. (After Dr. T. Colcott Fox, Brit. Journ. Derm., 1909.)

either in groin or scalp ringworms with trichophytine prepared after Plato's method.

There would seem, then, to be some degree of immunity produced. This occurs only in connection with acute inflammatory ringworm, however, the degree of immunity corresponding with the acuteness and extent of the inflammation, and no immunity results either in the case of superficial ringworms of the hair or of the glabrous skin. This is borne out clinically, for in a case of microsporon ringworm treated by the $\mathrm{X}$-rays a new infection occurred about three weeks after the hair had fallen out from wearing an infected cap which had been worn previous to the treatment. That it was a re-infection and not a recrudescence is vouched for as the case occurred in a ringworm clinic of the Metropolitan Asylums Board where it was successfully X-rayed and discharged as cured.

Etiology.-The ringworm fungi may be transmitted from one individual to another either directly by contact, or more often indirectly through the medium of infected combs, brushes, towels, clothes, caps or hats, the changing of caps being one of the commonest causes of 
infection in children. It is often transmitted directly from animals to man, as in the case of grooms and stablemen becoming infected from horses, cattlemen and slaughtermen from cattle, and children from dogs, cats, canaries, etc., which they have fondled as pets. It may also be communicated indirectly from animals as in a case in which a ringworm patch occurred on the hand of a man who traced it to an infection from a rug which had been previously on the back of a horse suffering from ringworm. It has been suggested that in schools where infected children may be in close proximity with one another the infection may take place by spores being blown about in the atmosphere but this mode of transmission is doubtful.

Age.-Ringworm can occur at any age, but in this country is most common in children, as $90 \%$ of the cases are due to the M. audouini which tends to die out after puberty and rarely attacks an adult.

Sex.-Either sex may be attacked indiscriminately. Animal ringworms, however, are more common in men than women as men are more liable to be exposed to infection.

Season.-The season of the year has no bearing on the incidence of ringworm. Body ringworm, however, tends to grow more actively in the warm weather and when it affects regions such as the crutch or the axillae, is liable to die down in the cold weather and to recrudesce in summer when the patient becomes hot and the skin moist with perspiration.

Station in Life.-Ringworm may attack individuals in any grade of society but is naturally much more common among the poorer classes owing to imperfect hygienic conditions and a greater liability to contagion through overcrowding.

There are no definite predisposing factors. It is questionable if the state of the general health has any bearing in enabling the individual to withstand an attack or in rendering it more severe. Doubtless certain skins form a more suitable soil for the fungus than others.

\section{RINGWORII OF THE GLABROUS SFIN}

Syn. : Tinea circinata ; Fr., Herpès circiné.

Ringworm lesions on the glabrous skin vary in appearance according to the special fungus responsible and the peculiarities of the skin affected, but when several lesions occur in the same individual they all conform to one type. They may be scaly macules, ringed or gyrate lesions, plaques, vesicular or pustular patches, and occasionally suppurative conglomerate masses or granulomatous nodules.

Macules and Patches. - These are well-defined, not raised above the level of the skin, vary in size from small macules to patches several inches in diameter, are pinkish in colour, and usually covered with scales which are easily detached. They may be irregular in outline or 
roughly oval or circular, and they may be uniform in tint or present a darker red border. They may occur anywhere on the skin. They are much more common in children than in adults and are frequently present about the face and neck in association with ringworm of the scalp. When they occur in adults they are usually caused by infection from children. The M. audouini is responsible for the majority of them but they may be caused occasionally by trichophyta. (Fig II 4.)

Ringed Lesions.-These begin as slightly raised pink scaly spots which, while extending peripherally, undergo involution in the centre so as to form rings the borders of which are usually raised and covered with fine scales. The rings gradually increase in diameter, the breadth of the border remaining constant, and after reaching a certain size may remain stationary for a time and then disappear. By the coalescence

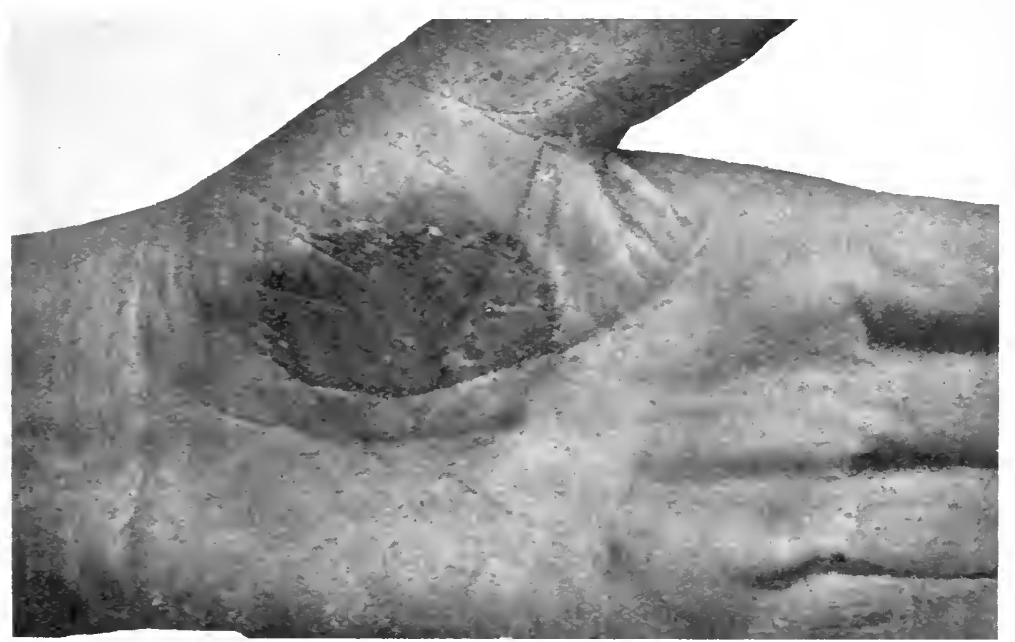

FIG. I I 4.-Tinea Circinata.

Trichophytic origin.

of neighbouring rings, festooned or gyrate figures are produced. Occasionally a recrudescence of the disease takes place in the centre of the lesions with the formation of one or more concentric rings within the original one. Circinate ringworm may be found in adults as well as in children and is most common in the exposed parts. It is caused chiefly by the microspora and where several concentric rings are present is probably due to infection from a cat or a dog. (Fig. II5.)

Plaque Lesions. -The plaques are raised, reddish in tint, circular or oval in shape, well-defined, and more or less scaly. When they occur in situations, such as the backs of the hands, where the natural folds of the skin are well-marked, these are exaggerated over the patch. The plaques may be dotted over with small resico-pustules, either irregularly distributed or forming a ring round the margin. (Fig. II6.) 
Suppurative Ringworm.-The suppurative lesions are definitely raised and have an irregular conglomerate appearance. Over the surface patulous follicles may be detected from the orifices of which oozes a yellowish serous discharge. They are not definitely painful though they may be tense and burning.

The plaques and vesico-pustular lesions are generally caused by trichophyta and where suppuration is marked the fungus is usually of animal origin. (Fig. II7.)

Granulomatous Ringworm.-Majocchi first drew attention to the condition which is known as Granuloma trichophyticum. In it the

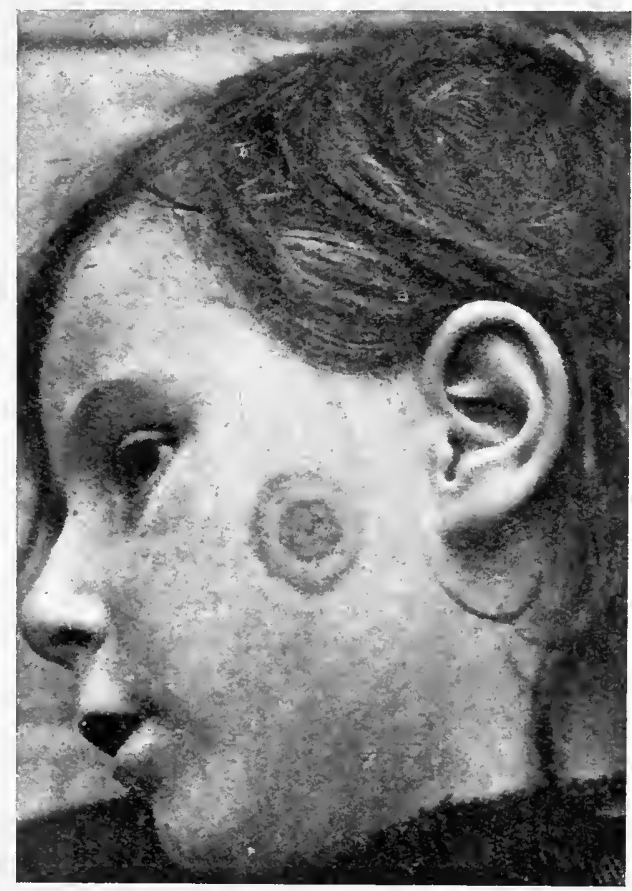

FIG. I 5 5-Tinea Circinata.

Note the concentric rings.

(From Dr. E. F. Skinner.)

ringworm lesions take the form of granulomatous nodules or plaques of a reddish-purple colour and tending to break down to form irregular ulccrations covered with a yellowish slough, somewhat resembling Tuberculosis cutis. Sections of a nodule show a thickening of the epidermis and a dense infiltration of the corium consisting of polymorphonuclear leucocytes, lymphocytes, plasma-cells, and giant-cells. Cases of this type have been observed especially in Italy and Denmark, and a case was recorded in this country by Sequeira in which was isolated the Trichophyton plicatile of Sabouraud, a fungus which is said to be the most common ringworm fungus in Denmark and is there a frequent cause of ringworm of the beard. 
Other trichophyta have been known to produce granulomatous reactions in the skin, such as the $T$. violaceum and $T$. gypseum.

Diagnosis.-Macular lesions may be confused with patches of seborrhoic dermatitis, psoriasis, or Pityriasis rosea. In seborrhoic dermatitis the scales are more profuse, yellowish, and greasy, and in psoriasis they are silvery. In Pityriasis rosea the lesions are more delicate in colour, more widely distributed, and are confined to the trunk and the upper parts of the limbs.

The final proof, however, rests with the demonstration of the fungus. For this purpose the surface of a lesion should be moistened with Liquor potassae, scraped with a scalpel, the scraping spread on a slide, a drop of Liquor potassae added, and a coverslip applied. Should ringworm

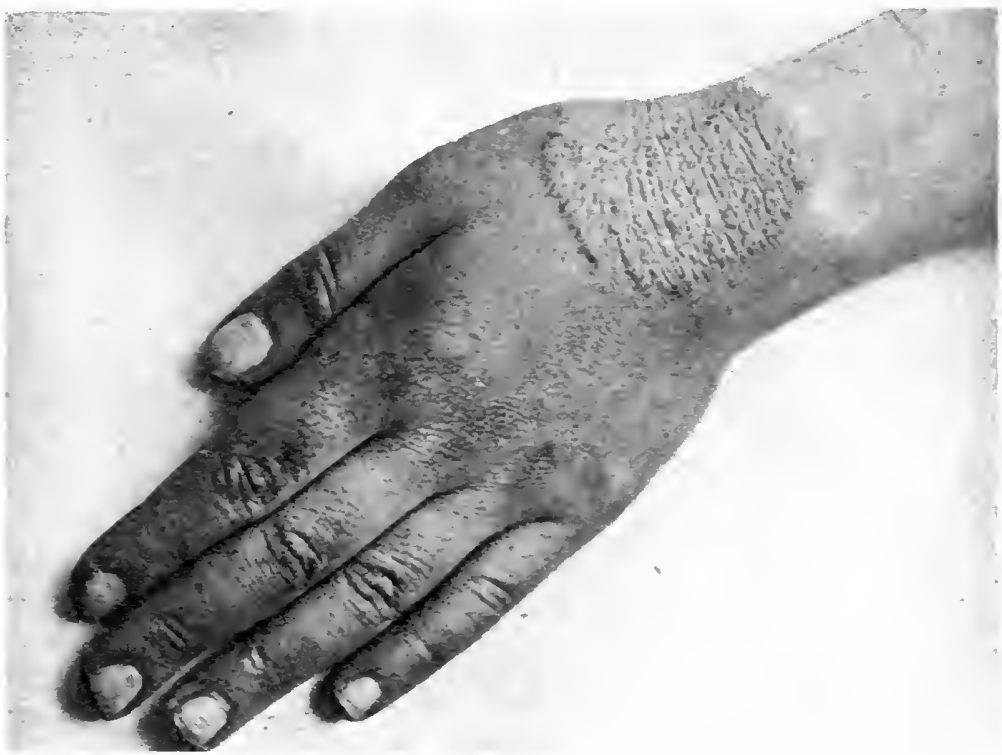

Frg. I16.- Tinea Circinata in the Hand of a Boy, infected from a canary.

be present, mycelial filaments can generally be detected in the specimen. It is important that the scraping should be taken from the growing edge of the lesion as there the fungus is most profuse.

Ringed lesions are, as a rule, easily recognised but they may be mistaken for circinate patches of Seborrhoea corporis, Pityriasis rosea, psoriasis, or even for a superficial serpiginons tertiary syphilide. The lesions of Seborrhoea corporis are yellow and greasy and are generally located on the pre-sternal and inter-scapular regions, sites not commonly attacked by ringworm. In psoriasis the circinate and gyrate figures have a broader border which is bright pink in colour and covered with silvery scales. The patches of Pityriasis rosea are delicate, oval in shape, with a pinkish border which is not raised and which encloses a characteristic fawn-tinted centre; so close may be the resemblance of Pityriasis rosea that at one time it was regarded in Vienna as a form 
of ringworm. Syphilitic lesions are differentiated by being indurated, of a deep brownish-red colour, and having no scales.

Plaques of ringworm might be mistaken for isolated patches of chronic eczema or psoriasis. Patches of eczema are less well-defined, are made up by the aggregation of papulo-vesicles, may show evidences of weeping or crusting, and are associated with more or less severe itching. It must be remembered, however, that from rubbing and scratching a patch of ringworm may become eczematised and its appearances so altered as to mask its identity.

Conglomerate pustular lesions are so characteristic as to present no difficulty in their recognition. Occasionally a septic granuloma or a

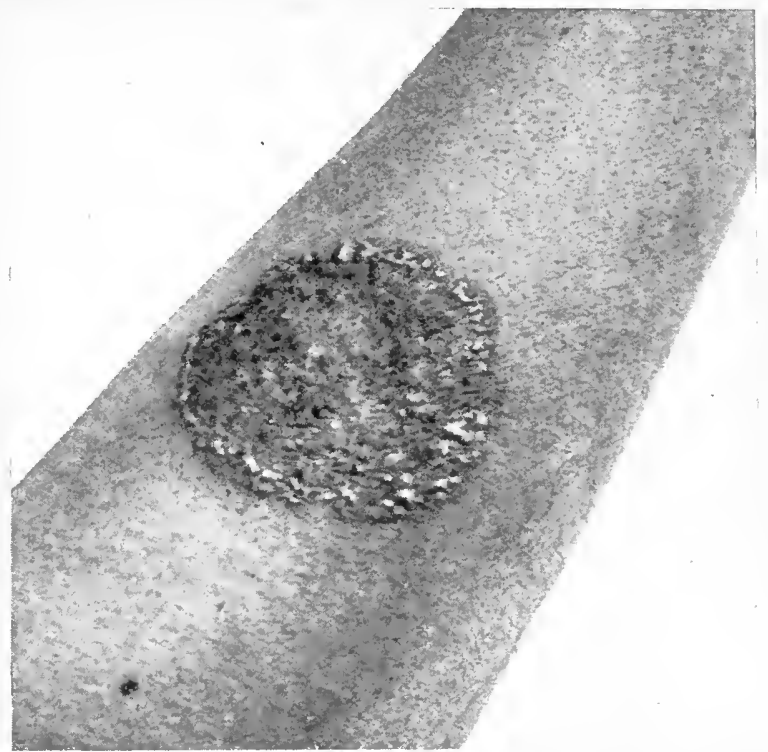

FIG. I 1 7.-Circinate Ringworm.

With vesico-pustules (Trichophytic).

hypertrophic patch of lupus about the back of the hand may show a slight resemblance, but the more rapid development of the ringworm, the acuteness of the inflammation, and the presence of vesico-pustules, crusts, or oozing patulous follicles, establish the diagnosis.

In every case it is wise to err on the safe side and to examine scrapings, scales, or infected lanugo hairs, for the ringworm fungus.

Pathology.--The fungi of ringworm on reaching the cutaneous surface gradually proliferate and the mycelial threads insinuate themselves between the cells of the horny layer of the epidermis, there they eliminate a ferment which has a solvent action on the keratin and causes a dissolution of the cells, which exerts a toxic effect resulting in a definite inflammatory reaction. This inflammatory disturbance may be regarded as the second line of defence of the skin and an effort on its part to withstand the attack of the fungus. It consists of a dilatation of 
the blood-ressels of the papillary and sub-papillary layers with extravasation of serum and possibly of polymorphonuclear leucocytes, and a cellular deposit of small round connective tissue cells.

Where the toxins produced by the fungus are rirulent, as in the trichophyta of animal origin, the serous extravasation may be so profuse as to lead to the formation of vesicles which are situated about the granular layer and which become transformed into pustules; at the same time the cellular infiltration in the corium is more marked and contains numerous plasma-cells and occasionally multi-nuclear cells or chlorioplaques. If the fungus be of low virulence the pathological process does not advance beyond the stage of slight oedema with a transient cellular infiltration and imperfect cornification, and the lesion appears as a pinkish scaly macule; should the virulence be greater the oedema and infiltration become more marked and a raised plaque is produced; if the virulence be excessive, vesication and pusformation result.

Prognosis.-The prognosis is excellent and under suitable treatment the lesions can be cured in the course of a few weeks.

Treatment.-Ringworm being a local affection without general symptoms only local treatment is required. Attenpts have been made to cure it by vaccines but so far the results from them cannot be said to be promising and in the few instances where it has been claimed that they were of benefit they were combined with local treatment. The raccines are prepared from growths on proof agar rubbed up in normal saline solution, from broth cultures, etc. As Adamson has pointed out it is unlikely that vaccines would be of use in ringwrorm, for the raison d'être of vaccine treatment is the preparation of microorganisms for destruction by the phagocrtes, and in ringworm the fungus is confined to the horny tissues and therefore cannot be reached by the phagocrites.

Dry scaly macules, patches, or rings, should be painted twice daily with collosol iodine oil, linimentum iodi of double the B.P. strength, or with tincture of iodine to which $10 \%$ of acetic acid has been added. The action of the iodine may be intensified by previously scraping the scales off the patch with a scalpel. The iodine produces irritation and leads to desquamation of the affected epidermis and destroys the fungus. After a week or ten days sufficient desquamation has generally been produced and the lesion may then be dressed with an ointment containing $2^{\circ}$ o each of precipitate of sulphur and salicyclic acid. In some individuals the iodine may prove so irritating as to cause resication and pain and may have to be discontinued.

Where the lesions are extensive, strong iodine may be too severe a remedy and it is better to employ an ointment containing salicylic acid and sulphur $3-6^{\circ}$, or benzoic acid and salicylic acid $3-6^{\circ}{ }_{0}$. The patches should be scrubbed with soft soap to remore the scales and the ointment, spread on lint, should be kept continuously applied under a bandage. 
After the ringworm has been cured a red inflamed patch is left which is easily subdued by the subsequent application of zinc paste.

Stronger measures are sometimes adopted, such as ointments containing from 3 to $6 \%$ chrysarobin or pyrogallol, but these, though effective, are unnecessary, and, as they stain the clothes and may give rise to an excessive degree of dermatitis in delicate skins, they are better avoided unless in intractable cases of ringworm of the crutch (see T. cruris).

In suppurative ringworm, painting with tincture of iodine followed by the application of an ointment containing $4 \%$ of ammoniated mercury or $2 \%$ of oleate of copper or mercury, will generally effect a cure in about ten days.

Should the ringworm have been present in covered parts of the body the underclothing which has been in contact with it should be stoved and the seams ironed with a hot flat-iron.

\section{Tinea Cruris}

Syn. : Dhobie's Itch.

Tinea cruris is a special type of ringworm of the glabrous skin which attacks moist intertriginous regions, especially the crutch, and is particularly common in warm climates such as India, where it is known as Dhobie's Itch, from the prevalent idea that it is caught from underclothes which have been infected by the native washerman. The term Dhobie's Itch is employed by laymen in the tropics to denote a number of pruriginous affections of the skin, chiefly about the groins and thighs, and under this heading have been included cases of erythrasma and impetigo as well as ringworm. It is better, however, to restrict the name to the definite type of ringworm ( $T$. cruris) which Hebra named and carefully described as Eczema marginatum.

This affection is prevalent in many warm countries and is especially common in India, Burma, and China. It was most probably introduced into this country by people coming from the East and became much more common here after the return of the soldiers from the South African War. An increase was specially noticed during the late war among soldiers who had been through the Gallipoli campaign or had been serving elsewhere in the south-eastern theatre of the war.

There are certain clinical characteristics which serve to distinguish it from ordinary ringworm of the body. It begins as slightly raised, rounded, elevated papules, situated about the fork which, owing to the moisture of the part, instead of being scaly are liable to present a raw sodden appearance. These papules spread peripherally, the border becoming raised and well-defincd, and tend to coalesce to form irregular patches with a festooned edge which is sometimes covered with thick scales and at other times dotted over with oozing papules or vesicopustules. Not infrequently the affected skin becomes broken at the folds and painful fissures result. Occasionally the patches clear up in 
the centre forming circinate lesions, or a sub-acute type may be met with in which there are red scaly areas with a well-defined slightly raised margin.

The affection may be limited to the inner surface of the thigh and the folds of the groin or it may attack the genitalia and, in extensive cases, may involve practically the whole of the bathing-drawers area. Though it may encroach on the pubic region the hairs are never affected. In the female it has been known to spread to the mucosa of the urethra and vulva. In the native it is particularly common where the loin cloth is tightest and rubs against the skin. It is not infrequently present also in the axillae, in the umbilical fold, or

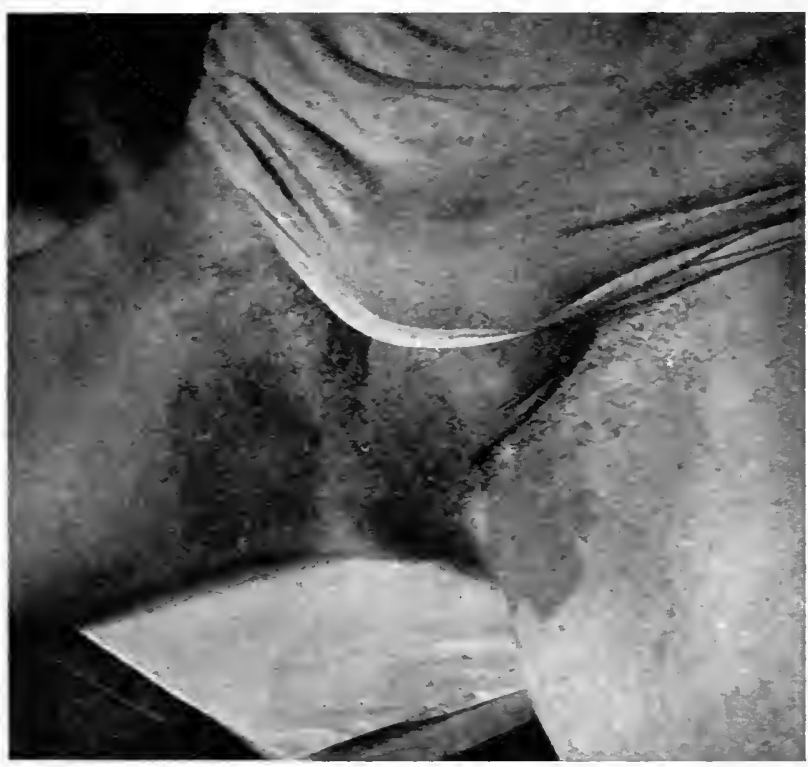

FIG. I I S. -Tinea Cruris.

(From Dr. Aldo Castellani.)

beneath the breasts in stout women, where it presents the same clinical appearances as in the groins. (Fig. IIS.)

In the warmth and moisture of these regions the fungus grows rapidly and gives rise to considerable irritation which is further aggravated by the friction of the clothes, and so sore may the affected areas become that the patient may be forced to lie up. The itching may be intense and lead to scratching and the lesions are apt, in consequence, to become eczematised or inoculated with pus-cocci and the ringworm to be complicated by septic dermatitis or boils. The irritation is naturally worse in hot weather and when the patient is warm in bed or from exercise. The affection has a tendency to improve in the cold season when it may disappear or dry up to form scaly negligible patches, but it usually reasserts itself with the return of warm weather. (Fig. II9.) 
The exact manner in which it is contracted is, as a rule, uncertain. The fungus may be transmitted directly from one individual to another by contact, as where it occurs in a husband and wife, or it may be spread indirectly from the wearing of infected clothes, the use of infected water-closet seats, etc. It has even been suggested that it may pass through the clothes to the skin. It is possible that in some cases the strong belief in India that the dhobie, or washerman, is responsible for its spread may not be ill-founded, and the native custom of

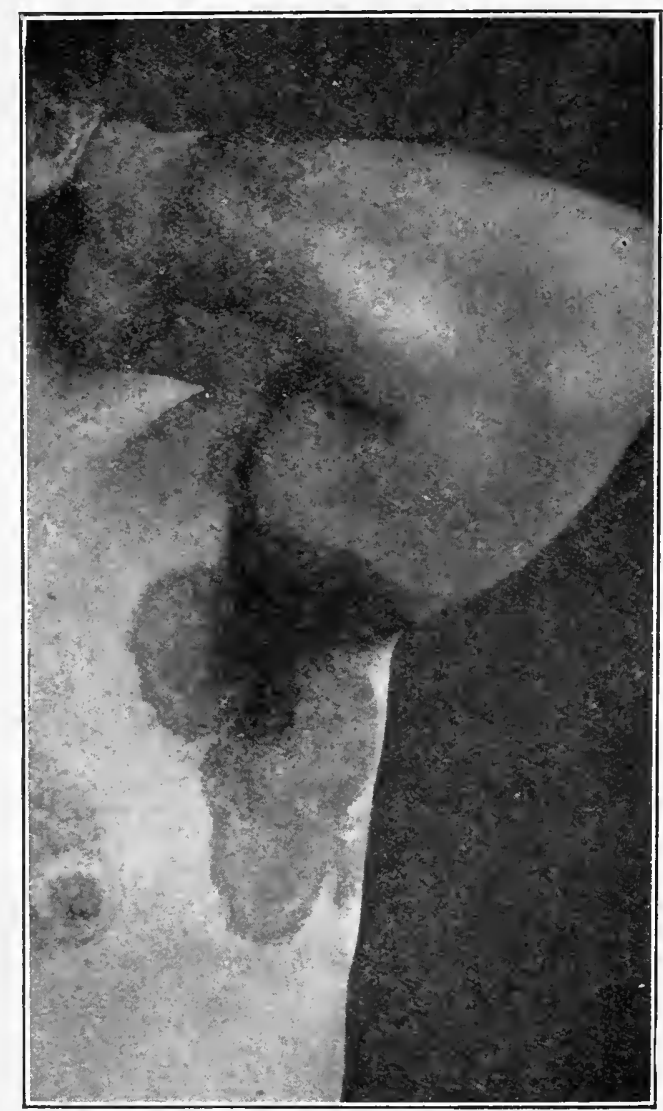

FIg. I I 9,-Tinea of Axilla.

(Dr. Aldo Castellani's Case. Trans. Soc. Trop. Med. and Hyg., I9r3.)

washing clothes in pools of stagnant water in which they may be mixed with infected clothes suggests an explanation, but the final proof of this is incomplete for up to the present the fungus has never been demonstrated in clothes returned from the wash. The objectionable habit of wearing trousers next the skin is a likely way of keeping up the infection for they are not so frequently cleaned as underclothes. In a case under the care of the writer a boy traced the infection to a pair of riding breeches which he had borrowed from his father who was suffering from the disease, and epidemics which have 
occurred in public schools have been traced to the boys promiscuously wearing one another's flannel trousers.

It is much more common in men than in women and in institutions in which both men and women are inmates it has been known to attack the men alone. This is difficult to explain, for if it were caused by contaminated water-closet seats it ought to be as common, in this country at all events, in women as in men; it is probable that a considerable number of cases do occur in women which do not come under medical observation.

Mycology.-The fungus which causes T. cruris is the Epidermophyton cruris of Castellani or Epidermophyton inguinale of Sabouraud and is peculiar in that, however widely it may spread over the skin, it never attacks the hairs. It is of human origin. It is not easily cultivated and grows slowly. On Sabouraud's proof agar medium

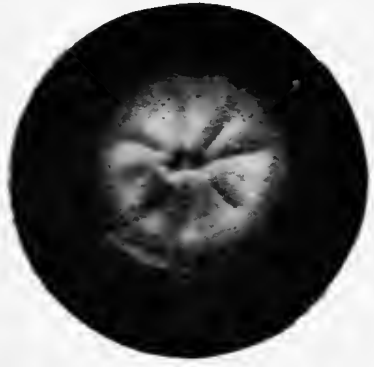

FIG. I 20.-Epidermophyton Inguinale.

I5 days' growth on proof medium.

(From Dr. R. Sabouraud. Brit. Journ. Derm., rgrr.)

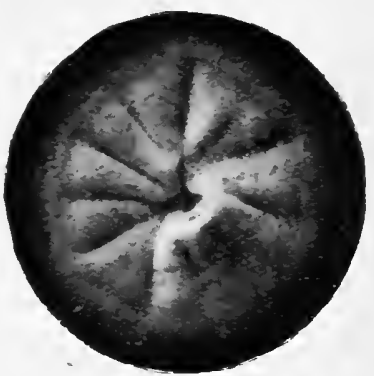

FIG. I2I.-Epidermophyton Inguinale. 25 days' growth on proof medium.

(From Dr. R. Sabouraud. Brit. Journ. Derm., IgII.)

it takes about a week to develop and appears first as a small greyishyellow powdery culture which gradually assumes an acuminate shape. A few weeks later whitish downy tufts appear which eventually overgrow the culture. (Figs. I20, I2I.)

A variety of this fungus was isolated by Castellani which gave pinkish cultures and was named Epidermophyton rubrum; this pro. duced buff-coloured scaly patches on the skin the edges of which were not so much raised as those of ordinary $T$. cruris. It was probably the same fungus as was described later under the name E. purpureum by Bang.

In rare instances other ringworm fungi have been isolated such, for example, as a fungus growing pink cultures observed by Bennet.

Diagnosis.-The diagnosis does not present any great difficulty when the lesions are well-marked and have the characteristic abrupt festooned edge, but when they are less well-defined and have become eczematised or septic, considerable difficulty may arise.

It has to be distinguished from the reddish patches of simple intertrigo-the result of chafing and the growth in the shin of a slightly virulent streptococcus-but in this condition the lesions are not definitely raised, are less well-demarcated, and are not so scaly. 
Eczema of these parts may resemble it, but the patches of eczema are more diffuse, tend to weep, and are apt to be associated with oedema and thickening of the neighbouring parts where the subcutaneous tissue is loose, such as the scrotum.

In every case the final diagnosis rests with the demonstration of the fungus. (Fig. I22.)

Treatment.-This type of ringworm as a general rule is difficult to cure and active remedies are necessary. The affected regions should in the first instance be scrubbed with hot water and soft soap to remove crusts, scales, and discharges. In mild cases this should be followed by the application of liniment of iodine or of an ointment containing $5 \%$ pyrogallic or $3-5 \%$ salicylic and benzoic acids. This treatment should be carried out at night, and in the morning the grease should be

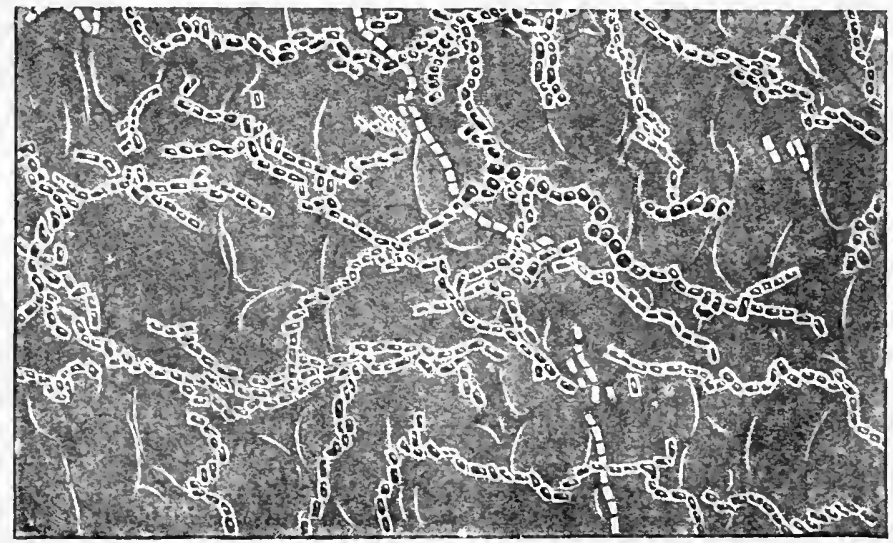

FIG. 122.-Epidermophyton Inguinale.

Unstained preparation from the groin.

(Dr. R. Sabouraud. Brit. Journ. Derm., IgII.)

wiped away and a I0\% solution of tincture of iodine in spirit applied or the skin dusted with a salicylic acid and zinc powder. Nascent sulphur treatment sometimes gives good results and consists of the application for about ten minutes of a $6 \%$ solution of hyposulphite of soda on lint covered with oiled silk, followed by painting with a $3 \%$ solution of tartaric acid.

During the treatment short cotton bathing drawers should be worn which should be changed frequently and sterilised by stoving, and a piece of lint should be inserted between the scrotum and the thigh to prevent chafing.

In resistant cases the time-honoured Vleminckx's solution may be employed and should be painted on every night for a week; this will generally be followed by recovery. To prepare this: I oz. of quicklime, 2 ozs. of precipitated sulphur, and I5 ozs. of water, are boiled together in an earthenware vessel, reduced to Io ozs., and the clear sherry-coloured fluid decanted after subsidence. 
In obstinate cases where these remedies prove ineffectual, chrysarobin should be resorted to and applied in the form of a 2 to $6 \%$ ointment rubbed in twice daily till the affected patch reacts and becomes inflamed, the applications should then be stopped as the reaction generally indicates that cure has taken place. When chrysarobin or Goa powder is used it is necessary to warn the patient of its disadvantages in staining clothes and irritating sensitive parts like the conjunctivae, and it is unsafe to place it in the hands of the ordinary native. Applications of turpentine have also been recommended, the turpentine being rubbed on in the morning and an ointment applied at night, but this remedy must also be used with caution as it is apt to produce dermatitis.

In cases where eczematisation and weeping or fissured lesions occur, chrysarobin is too irritating and is apt to aggravate the disease. In such cases, previous to the chrrsarobin treatment the condition should be soothed by the application of milder remedies such as the following:

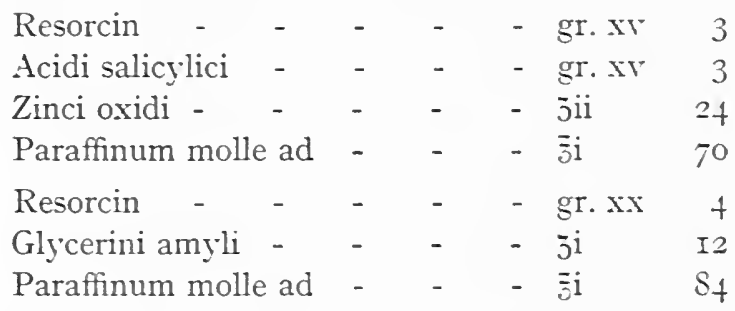

Painful fissures about the inguinal regions may be healed by painting with a $3 \%$ solution of nitrate of silver in spirit.

As in all parasitic cutaneous diseases, it is necessary that the clothing which has been worn previous to the cure of the disease should be destroyed or thoroughly disinfected.

After the affection appears to have been cured, to prevent a recrudescence the parts should be painted with a $10 \%$ solution of iodine in $60 \%$ spirit every day, and the patient recommended to wear short cotton pants or bathing drawers for some time and to powder the inside of them with equal parts of boric acid, zinc oxide, and sulphur. Without these precautions recrudescences may take place every summer for an indefinite term of years.

\section{EczeMatoid RixgWorm of the ExtRemities}

The hands and feet are occasional sites of ringworm lesions similar to those of the glabrous skin, such as dry scaly patches, rings, and raised vesicular or pustular lesions, due to fungi such as the .I. audonini, acuminatum, violaceum, etc.

In addition the extremities may be affected by an eczematoid form of ringworm which is extremely difficult to diagnose and is liable to be mistaken for dysidrotic eczema ; it is usually due to the same fungus which causes $T$. cruris, namely the Epidermophyton cruris (Castellani), and occurs in association with $\mathrm{T}$. cruris. Not infrequently the lesions 
in the groin are cured first while those in the hands and feet persist long afterwards, so that in ringworm of the extremities there is often a history of ringworm of the crutch having been present some time previously. In a case under the care of the writer a patient with ringworm of the feet gave a history of having had Dhobie's Itch in India some twenty years previously and his feet had troubled him off and on ever since; he had been told that he was suffering from gouty eczema of the feet and had undergone all manner of treatment for it, both local and medicinal, without success.

The clinical appearances presented by eczematoid ringworm of the hands and feet are fairly characteristic though they vary considerably in different cases. The essential lesion is a pinhead-sized deeply-seated vesicle which has been compared to a sago grain embedded in the epidermis. A varying number of these vesicles make their appearance, sometimes grouped, at other times irregularly and widely distributed. They are situated on heathy skin and are not surrounded by an inflammatory halo unless when they become purulent from contamination with pyogenic cocci. In a few days the vesicle dries up and the roof comes away as a scale leaving a denuded shiny, sometimes moist, surface, surrounded by a free or upturned scaly edge. Occasionally the vesicles coalesce to form blebs which may reach the size of a sixpence or larger and these in turn give rise to irregular areas of considerable extent. About the soles of the feet the fungus may produce coarse scales and horny thickenings, or between the toes may give rise to thickened white sodden skin something like parchment. (Plate II.)

Three main types of eczematoid ringworm of the extremities may be described but these merge into one another and all varieties of lesions may occur in one case :

I. An acute vesico-bullous type which tends to attack chiefly the interdigital clefts, spreading from there on to the dorsum of the foot or back of the hand in the form of erythematous patches with a more or less definite border which may be scaly or dotted over with small vesicles or vesico-pustules, or may present a sharp edge with detached undermined skin.

2. A cinronic variety which may succeed the acute type, with indefinite scaly patches, the scales of which tend to be loose at the margin and to fall off leaving a raw glazed surface.

3. A thick scaly variety affecting the soles of the feet and, more rarely, the palms of the hands, in which the horny layer is thickened in patches and sometimes fissured. The edges of the patches are irreguiar and often detached, and beyond them may sometimes be present an erythematous areola and small vesico-pustules. Between the toes the horny thickening appears as white, moist, sodden skin, and the fissures are chiefly marked in the folds of the toes. The interdigital space most frequently affected is the fourth, owing to the fourth and fifth toes being kept more closely in apposition to one another than the others and so forming a suitable place for incubating the fungus. 


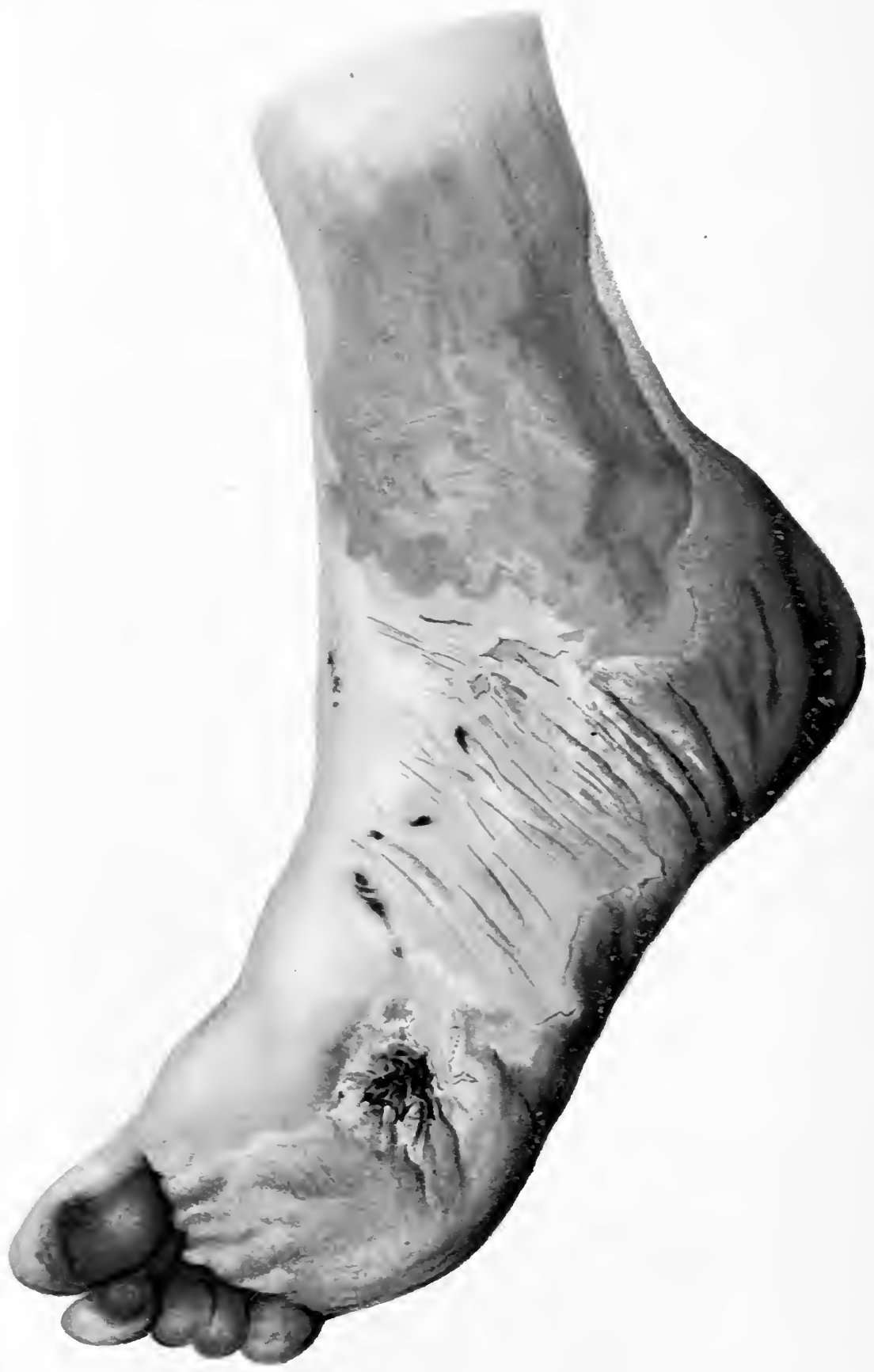

ECZEMATOID RINUWORM OF FOOT 

This thick scaly variety with fissures on the palmar and plantar surfaces is not infrequently combined with vesico-bullous lesions simulating eczema on the back of the hand or foot.

In some cases all four extremities may be involved, while in others it may be confined to the hands or the feet, and not infrequently to only one member.

In association with the skin lesions the nails are liable to be implicated and to become, in consequence, discoloured, thickened, and brittle, and sometimes raised and turned up at the free border, owing to a scaly accumulation on the underlying nail-bed.

'The affection is more common in the feet than in the hands, doubtless because the feet are less frequently and thoroughly washed, and because the interdigital clefts being closed up by the boots are more likely to harbour the fungus, and, if warm and moist, to stimulate its growth.

The subjective symptoms vary according to the acuteness of the condition. The vesicular type is usually accompanied by serere itching and when fissures are present in the folds of the toes they may be so painful as to interfere with walking. This type of ringworm is always aggravated by heat, either from hot weather or from exercise.

Diagnosis.-In the hands the acute variety has to be distinguished chiefly from dysidrosis and the squamous type from scaly eczema, while on the soles of the feet the horny desquamating type may be confused with a scaly tertiary syphilide. The final diagnosis rests with the demonstration of the fungus in the scales or the covering of the vesicles. The scales should be detached by a blunt scalpel or forceps, placed with the deep side uppermost on the microscope slide, and covered with a drop of Liquor potassae; a cover slip is applied and the specimen examined with the high power. If positive, the fungus can be seen as branching mycelial threads and chains of spores ramifying among the horn-cells. It is sometimes necessary to make a number of specimens before a positive one is obtained, when the fungus is usually found in great abundance.

Treatment.-The treatment is similar to that indicated in T. cruris and requires even more energy to effect a cure. The condition shows no tendency to heal spontaneously though it may die down and lie dormant during cold weather. When vesicles are present they should be opened, and when thickened scales occur these should be decorticated before remedies are applied. When the affection is acute or purulent, the inflammation should first be reduced by the continuous application of boric compresses, followed by the use of a dusting powder consisting of salicylic acid I part, boric acid 4 parts, and zinc oxide 4 parts. Later, a curative ointment should be applied twice daily, such as oxidised pyrogallol I5 grains, glycerine of starch 2 drachms, soft paraffin to the ounce. This ointment is effective but dirty and instead of it an ointment such as that suggested by Whitfield- 
containing benzoic acid 5\%, salicylic acid $3 \%$, in soft paraffin and cocoanut oil-gives excellent results.

The most intractable type of all is the scaly and interdigital variety in the feet with white thickened skin between the toes. In it, the affected parts should be rubbed with soft soap and warm water previous to the application of the salicylic-benzoic ointment. In some cases even this may be insufficient and it may be necessary to resort to such strong measures as the application to the whitened skin of iodine and acetic acid, Liquor potassae, a saturated solution of salicylic acid in spirit, or a salicylic plaster between the toes.

The socks should be made of white or natural coloured wool and changed twice daily, the above powder being dusted freely into them before they are put on. After being worn they should be thoroughly washed or, preferably, stoved. The boots also should be disinfected by being dusted inside with the powder, and should be swabbed out occasionally with a $I$ in 20 solution of lysol so as to destroy any fungus which may be harbouring in the moist leather, as it is possible for it to persist there and to work its way through the sock and re-infect the feet.

\section{RINGWORM OF THE SCALP}

Syn.: Tinea tonsurans; Fr., Teigne tondante.

Ringworm frequently attacks the hairy regions, especially the scalp and beard, and in rare instances the eyelashes and eyebrows.

The clinical appearances vary in different cases according to the fungus responsible, the individual peculiarities of the skin affected, and secondary considerations such as the co-existence of some other pathological condition such as psoriasis, seborrhoic dermatitis, or impetigo.

Ringworm of the scalp may be described under three main headings : (I) Microsporon ringworm of children; (2) Ringworm of the scalp caused by the Endothrix trichophyta; (3) Suppurative ringworms of the scalp, or kerions.

\section{Microsporon Ringworm, or Microsporosis}

The most common type of ringworm of the scalp in this country is due to the M. audouini. It is essentially an affection of children, most frequent between the ages of 5 and $I_{4}$ and tends to die out naturally about I5. The earliest sign of infection is a small pinkish macule or scaly patch, in which the scales are most marked at the orifices of the hair-follicles. This gradually spreads and forms a roundish, mildly inflamed lesion with a well-defined border which may be slightly raised. Except occasionally in the infant it shows no tendency to clear up in the centre and form a ring. The scales which usually cover the patch have a whitish-grey or mortar-like appearance and are easily scratched off. In addition the patch is covered with numerous short 



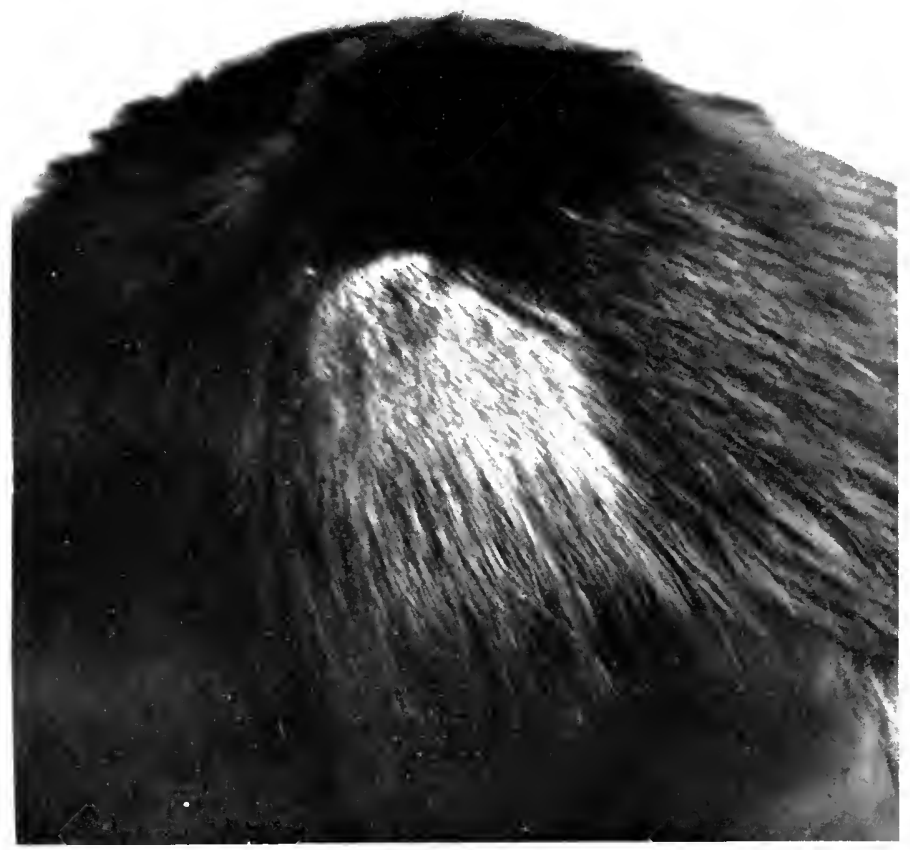

FIG. I24.--Microsporon Ringworm of Scalp. Shows scaly patch with stumps.

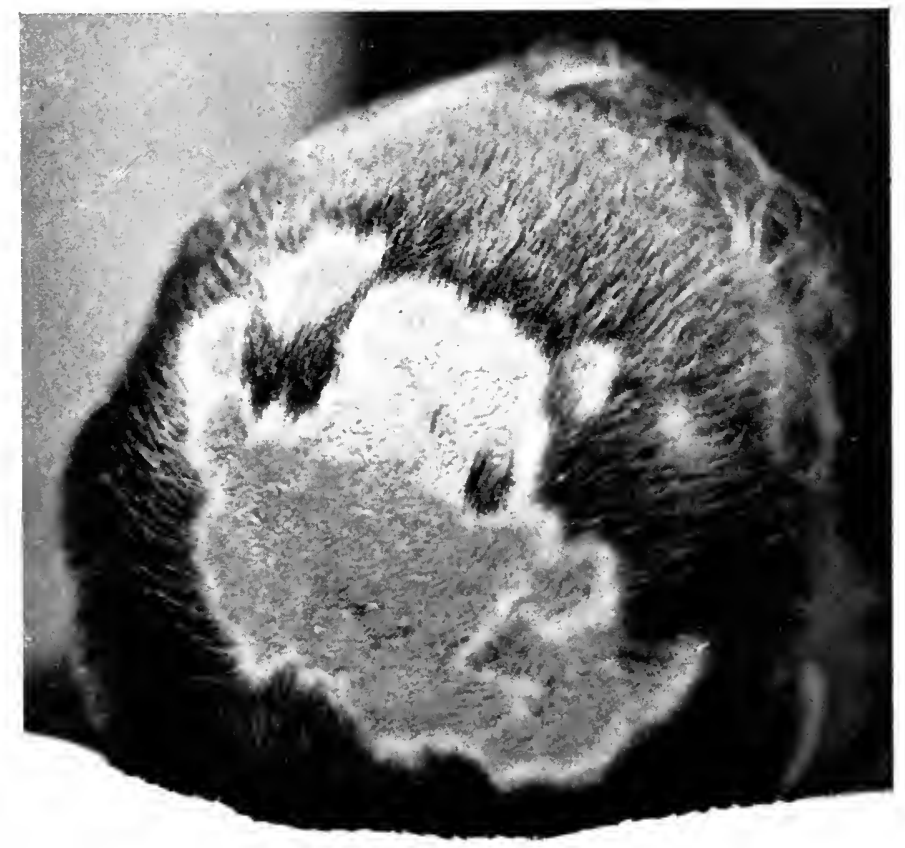

FIG. 125--Alopecia Areata following Microsporon Ringworm.

The baldness extending like a halo berond the areas affected by the ringworm. The aleperial is the result of an interference with the func tion of the papillae by the fungus in and about the hairs. 
hairs which have broken off about an eighth to a quarter of an inch above the level of the scalp, forming stumps which are thicker than the healthy hairs and present a dirty-white opaque or frosted appearance which is due to a sheath of fungus around them. These stumps are bent in all directions owing to a loss of elasticity from the presence of the fungus. At the orifice of the follicle from which the stump emerges there is a heaped-up conical scale. As a rule there are sereral such foci of ringworm and they tend to coalesce to form irregular patches. If left untreated the disease keeps on spreading till about puberty and in neglected cases may involve practically the whole scalp. (Figs. I23, I24.)

There are no constitutional symptoms associated with it and the general health does not appear to influence in any way the rapidity

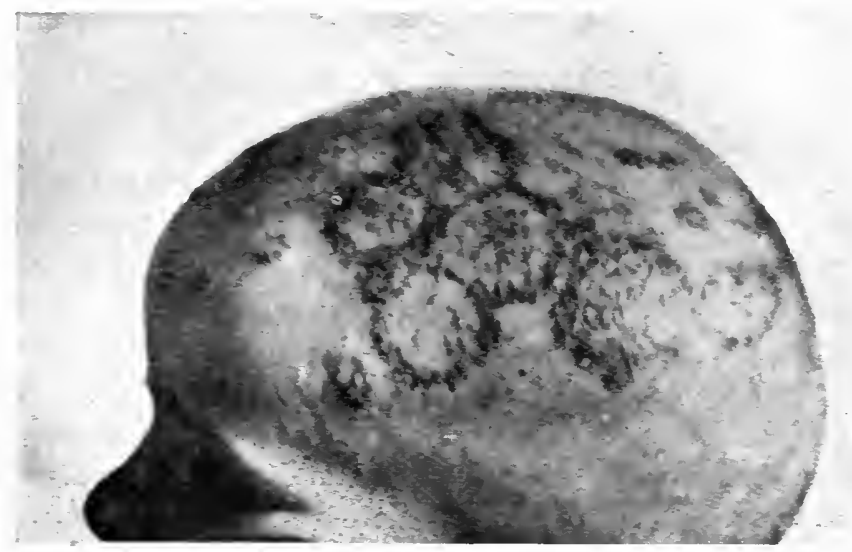

FIG. I23.-Microsporon Ringworm of the Scalp in an Infant.

Shows ringed lesions.

with which the lesions grow, or the virulence of the attack, nor does an attack confer immunity:

Modifications.-The appearance of the lesions may be greatly modified by various considerations of which the most important are the following :

(I) Treatment, by which the scales may be kept under and new healthy hairs induced to grow among the stumps, or by which the lesions may become inflamed or transformed into suppurative patches or artificial kerions.

(2) The co-existence of other skin diseases, such as seborrhoic dermatitis which renders the scales more profuse, yellowish, and greasy.

(3) Secondary' changes, such as impetigo, the result of the inoculation of pyogenic cocci, or eczematisation from rubbing and scratching.

(4) Suppurative changes either from the fungus being of animal origin or from some peculiarity of the scalp by which it reacts excessively to the fungus so that the hairs, instead of breaking off to form stumps, are shed and a bald area results (Tinea decalvans of 
Tilbury Fox) or the affected area becomes raised, inflamed, and dotted over with pustules.

(5) The association of Alopecia areata, which may develop some weeks or months after the ringworm, each patch of ringworm being gradually replaced by a bald area. This is a somewhat fortunate circumstance as when it occurs the ringworm dies out rapidly and the bald patches usually grow again in the course of six months or less. It is important to be cognisant of this possibility, especially where the $\mathrm{X}$-rays have been employed in the treatment, as baldness persisting for an unusually long period after exposure to the $\mathrm{X}$-rays may possibly be due to it and not to an excessive dose of the rays. In the case of Alopecia areata the bald areas correspond to the distribution of the ringworm, while after the $\mathrm{X}$-rays they are determined by the areas exposed. (Fig. I25.)

\section{TRICHOPHYTIC RINGWORM}

The endotriches are responsible for about $5 \%$ of the cases of scalp ringworm. They do not show the same tendency to die out at puberty as the microspora being liable to persist indefinitely and, consequently, are met with in adults as well as children. In a case under the observation of the writer an endothrix ringworm due to the $T$. sulphureum was present in a woman aged 27 , and had persisted since she was infected at the age of 15 .

The typical lesions of endothrix ringworm are small scaly patches, roundish or irregular in outline, varying in size from a split-pea to a finger-nail, sometimes few in number at other times widely disseminated over the whole scalp. Occasionally the appearances conform to those produced by the microspora but, as a rule, the lesions differ in that they are smaller and not covered with stumps of a uniform length. In certain cases stumps of about a quarter of an inch are present, but more usually the affected hairs are broken off at the orifices of the follicles forming plugs like comedones. Hence two types of endothrix ringworm have been described, namely: endothrix ringworm with stumps and endothrix ringworm with plugs, which according to Sabouraud are due respectively to the $\mathrm{T}$. acuminatum and the $\mathrm{T}$. crateriforme.

(a) Endothrix ringworm with stumps.-The stumps may be white and opaque or may resemble short atrophic hairs. They are about a quarter of an inch in length and are irregularly distributed, singly or in small groups, among healthy hairs. In the early stages of infection they are comparatively easily epilated with a succulent root-sheath, but later they become so brittle that it is only with the greatest difficulty that they can be epilated whole.

(b) Endothrix ringworm with plugs or " black-dot ringworm." - The stumps are cut off quite short and appear as small black specks like comedones. They are difficult to remove, except by a comedo expressor or with a needle or by detaching a scale covering a follicle when they 


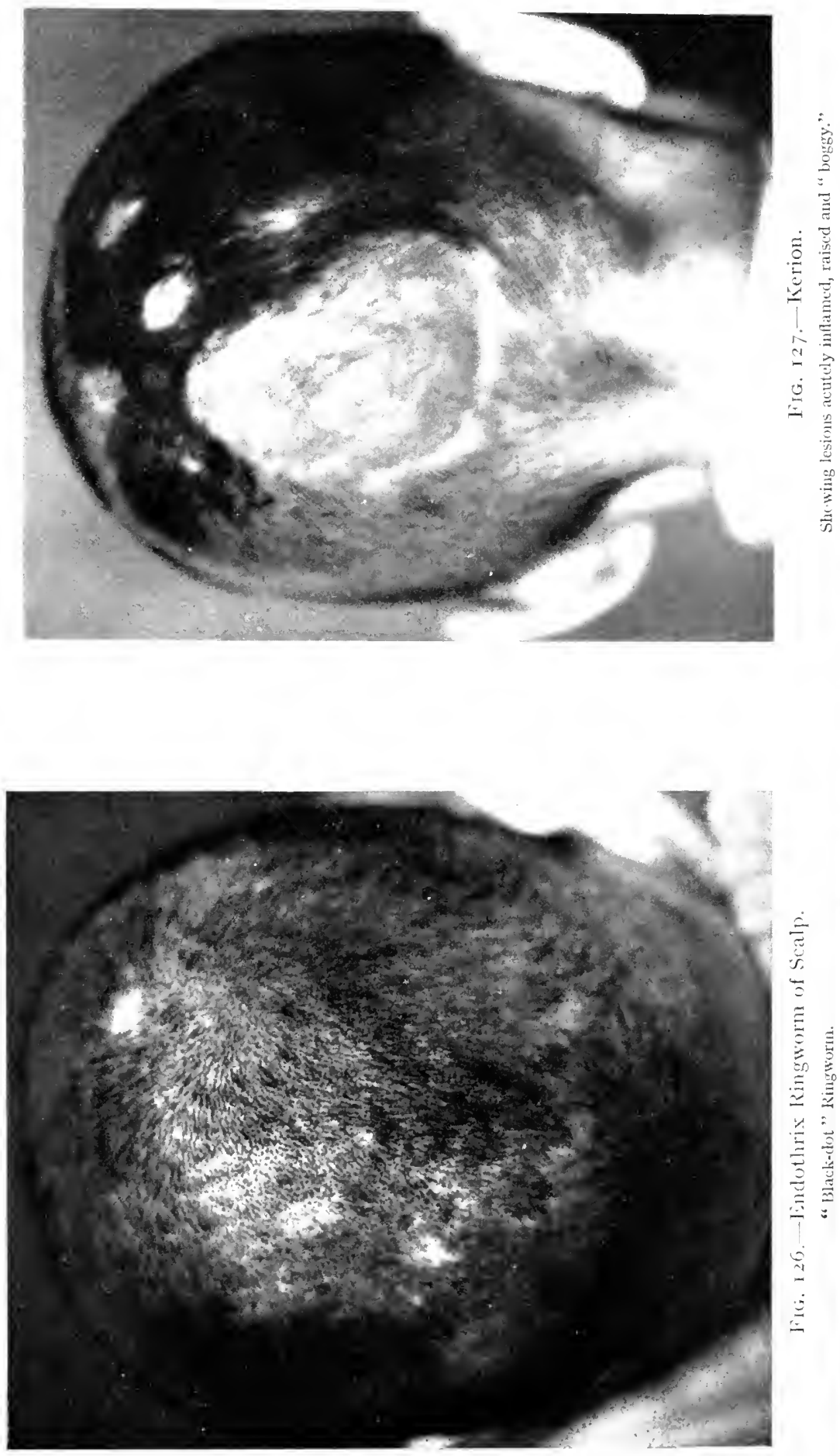
appear as a small plug on the under-surface. An examination of the plug shows it to be made up of a pigmented hair coiled like a corkscrew from the pressure of the scale capping the orifice of the follicle and the loss of elasticity due to the presence of the fungus. This type of plug is responsible for the name "black-dot ringworm." (Fig. I26.)

Endothrix ringworm is generally of human origin, and is exceedingly persistent owing to the difficulty in recognising it and its intractability to all treatment except the X-rav's.

Suppurative Ringworm of the Scalp, or Kerion.-Suppurative lesions of ringworm of the scalp are known as kerions. They are generally of animal origin and due to infection by one or other of the ecto-endothrix trichophyta though occasionally they may be caused by microspora. They may occur at any age and form the majority of ringworms of the adult scalp.

The clinical features of a suppurating ringworm of the scalp are characteristic. The lesions are well-defined, boggy, phlegmonous swellings, raised abruptly for an eighth of an inch or more above the level of the scalp, with a somewhat shiny angry-looking surface and a roundish or festooned outline. They are dotted over with small perifollicular abscesses, from which ooze beads of sero-pus, in the centre of which a loose hair can generally be detected, and between which are a few definite opaque stumps about an eighth of an inch in length. The stumps are easily epilated and on microscopical examination present mycelial threads and chains of spores around and within them. The lesions vary in size from a split-pea to a crown or larger and are, as a rule, single though they may occasionally be multiple. On palpation they have a peculiar soft fluctuating feeling which frequently leads the inexperienced to incise them in the expectation of finding pus, instead of which only a few drops of blood or serum escape. The lesions are not usually painful in spite of their angry appearance but they may be itchy or burning. At one time it was believed that the suppuration was the result of the co-existence of pyogenic cocci but it is now known to be due to the ringworm fungus itself. (Fig. I27.)

These suppurative lesions are more readily cured than the other types of ringworm of the scalp, the suppurative changes themselves leading to the destruction of the fungus. When the swelling and inflammation subside a red bald smooth patch is left which may persist for six months or incre before the hair grows again or, in rare instances, may remain permanently bald.

Diagnosis.-The early diagnosis of ringworm is of the greatest importance for not only does the disease spread rapidly but the infected person is a source of danger and may communicate it, not only directly, but indirectly through the medium of infected caps, towels, brusines, etc.

In every case a microscopical examination should be made, as the final proof rests with the demonstration of the fungus. A diseased hair or stump should be sought for and carefully epilated by epilation forceps, of which one of the best types is that designed by Professor

$2 \mathrm{c}$ 
Arthur Whitfield. It is then placed on a slide and covered with a drop of Liquor potassae, but should it be greasy it is advisable first to remove the fat by rinsing in ether. A coverslip is placed gently over the hair soaking in the potash, and the specimen is examined from time to time under a medium power of the microscope till it has cleared when it can be studied. Care should be taken to avoid pressing down the coverslip on the softened hair, as this will rupture the cuticle and alter the relation of the fungus to the different parts of the hair. In this way the fungus is demonstrated better than by staining it with aniline dyes. (Fig. I28.)

Microsporosis.-There is, as a rule, no difficulty in recognising a typical case of microsporosis of the scalp, the patches covered with grey-white scales and opaque stumps bent in different directions being quite characteristic.

It might be confused with pityriasis or even psoriasis. In pityriasis, however, there are no stumps or definite patches and the scales are more profuse and greasy ; in psoriasis the scales are dense, silvery, situated on an inflamed base which extends beyond the scaly area, and the

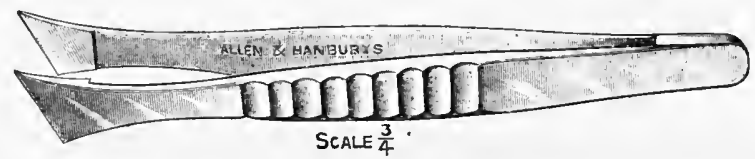

FiG. I28.- Whitfield's Epilation Forceps.

hairs are unaffected. Occasionally the diagnosis may be masked by the co-existence of ringworm with one or other of the above affections, by eczematisation from scratching, or by suppurative changes from the secondary inoculation of pyogenic cocci.

Occasionally ringworm patches may be confused with Alopecia areata but except in the case of bald patches succeeding ringworm such confusion need not occur, as the lesions of Alopecia areata are smooth, shiny, and never scaly, and any stumps which may be present at the growing edge, instead of being short opaque hairs ensheathed with fungus, are simply atrophied and when epilated present the appearance graphically described as the " point of exclamation hair."

Endothrix.-Trichophytic ringworm of the endothrix type may be difficult to recognise where only a few small scaly patches exist and the stumps are sparse and mixed up with healthy hairs. In such cases the disease may readily be missed or mistaken for pityriasis. The typical black-dot ringworm is comparatively easily diagnosed.

Suppurative Ringworms, or Kerions, have to be distinguished from impetigo or ordinary septic perifolliculitis, but here again the raised boggy lesion with its patulous oozing follicles and infected stumps presents a picture which is not easily mistaken.

Trichophytides.--In I9I I Jadassohn drew attention to the occurrence in connection with deep-seated suppurative ringworms or kerions of small follicular papules, either in groups or diffusely scattered, usually 
closely resembling Lichen scrofulosorum but occasionally presenting small horny spines like Lichen spinulosus. He considered that these eruptions, which were only met with in association with deep-seated ringworm, were the result of an intoxication taking place from the local lesion and bore the same relation to it as Lichen scrofulosorum does to Tuberculosis cutis. Since then a considerable number of cases of "lichenoid trichophytide" have been reported, especially from the clinic in Berne, the great majority of which were in children.

The eruption appears either at the acute stage of the kerion, or more often when it is about to fade, and is unaccompanied by subjective symptoms. It varies in extent and duration and is found especially on the trunk, to a lesser extent on the limbs, and rarely on the face. It may disappear in a few days, or may last several weeks. It consists of miliary follicular papules, pale pink in tint, flat or acuminate in shape, occasionally surmounted by a small crust or a horny spine. In rare instances, instead of papules, small vesicles or pustules have occurred.

There is nothing in the histopathology which is pathognomonic and sections show a perifollicular inflammation, dilatation of the follicle, and in the spinous form considerable parakeratosis in the wall of the follicle and a horny plug or spine. No fungus has been detected in the lesions.

It is distinguished from Lichen scrofulosorum by being more diffuse and evolving more rapidly, and by being associated with suppurating ringworm.

From Lichen spinulosus the diagnosis may be impossible and it has been suggested that certain cases which have been labelled Lichen spinulosus in reality belong to this category.

As no fungus has been found in the papules it seems probable that the eruption is of internal toxic origin, and may possibly be due either to toxins eliminated by the fungus and disseminated through the bloodstream or to some poisonous bodies excreted by the tissue-cells in response to the toxins.

Prognosis.-The prognosis in ringworm of the scalp varies according to the type of fungus responsible.

In microsporosis the disease tends to die out naturally at the age of about $I_{5}$ and if left alone will persist till about that time. Before the introduction of the $\mathrm{X}$-rays in the treatment of ringworm when it had to be dealt with by parasiticide applications the prognosis was uncertain and the affection so intractable that it was the opprobrium of the physician. By the X-rays any case, however widely disseminated, may be cured in from If to 2 I days after the exposure when the hair falls out, but by other means the treatment may last for months or even years before a cure is effected. From time to time cases are met with which do respond to treatment by antiseptic applications and in which a cure takes place in a few weeks. These are cases in which the scalp is peculiarly sensitive and the application of even mild antiseptics has 
the effect of producing so violent an inflammatory reaction as to kill the fungus. It is on account of cases of this type, which are unfortunately rare, that various remedies of comparatively little use have gained a false reputation.

It has been said often that delicate ill-fed children are more diffcult to cure than the strong and healthy, but there is no evidence to suggest that the constitution of the individual has any definite bearing on the spread of the disease or its response to treatment.

In endothrix ringworm where the lesions consist of small scaly patches the prognosis by $\mathrm{X}$-ray treatment is equally good, but if antiseptic ointments be employed it is more difficult to cure than microsporosis because single infected stumps or minute scaly patches are apt to be missed and the disease re-asserts itself when apparently cured.

In suppurative ringworms the prognosis with anti-parasiticide. remedies is more favourable, for the suppuration itself destroys the fungus. In such cases $\mathrm{X}$-ray treatment is unnecessary and dangerous. The red bald patch which follows the kerion may persist, however, for six months or more before the hair grows again.

With the X-rays a case may be regarded as cured whenever a complete defluvium of the affected portions of the scalp has taken place, by other methods of treatment it is more difficult to be certain that the disease is eradicated and before declaring it cured it is advisable, as a routine, to stop all treatment for a month and then carefully examine the scalp. If any marked scurfiness exists it should always be looked upon with suspicion and, if there is the slightest doubt, a further period of quarantine should be insisted upon.

\section{TREATMENT}

Preliminary Remarks. - Whenever ringworm of the scalp has been diagnosed every care should be taken at once to prevent the spread of the disease to others. The patient should be isolated in a modified fashion, a closely fitting cap of cotton or other light material continuously worn, and special brushes, towels, etc. kept for his use. In the case of children, attendance at school must be stopped until they are cured. As a preventive it would be well if all school children were periodically inspected for ringworm and if this were done, not only in board schools but in private and public schools, and infected children at once isolated and properly treated, much of the disease would be stamped out.

As a preliminary to treatment the patient should be placed in a good light and the whole scalp thoroughly examined, the hairs being turned over systematically in the opposite way to their natural direction. If after careful examination only one or two isolated patches can be detected it may be possible to save the hair, but where the diseased foci 
are numerous and widely distributed it should be entirely cut off. This should be done by a pair of patent clippers such as barbers use for the back of the neck, which is better than shaving the scalp as when it is shaved it is impossible to distinguish the healthy from the diseased hairs. The question of the cutting of the hair naturally meets with considerable opposition and the enforcing of it must be left to the discretion of the physician. In the majority of cases it is imperative and sares time in the end, as it most frequently happens that when the hair is cut short small foci of disease are revealed which were missed in the preliminary examination.

The choice of treatment depends on various considerations, such as the variety of ringworm, the age of the patient, the clinical type of the lesion, and the extent and distribution of the infection.

Microsporon ringworm of the scalp is amenable to parasiticide treatment in the infant; if between the ages of 3 and 13 years it is best treated by the X-rays; about puberty, as the disease has a natural tendency to die out at that time, the employment of the $\mathbf{X}$-rays is unnecessary and antiseptic applications suffice.

In endothrix ringworm where the lesions are scaly and consist of small foci and isolated stumps widely distributed over the scalp, the disease shows no tendency to die out at puberty and X-rays are the best form of treatment in the adult as well as the child.

In suppurative forms of ringworm, or kerions, whatever the variety of fungus responsible for them, mild antiseptic applications can generally effect a cure and X-rays are unsuitable as they are liable to cause severe dermatitis followed by baldness which may be permanent.

The difficulty of curing ringworm of the scalp is a mechanical one. Even mild antiseptics are capable of killing the fungus responsible when brought in contact with it on the glabrous"skin, but no parasiticide application short of a caustic is capable of reaching the fungus in the deeper parts of the hair-follicles or even of penetrating the hair-cuticle, hence the main object in the treatment is not so much to destroy the fungus as to cause a loosening and shedding of the hair so that it may come out and bring the fungus with it.

There are various methods of bringing the hair out, such as epilation with forceps, depilation by the X-rays, or the production of an acute inflammatory reaction by irritants. Of these, the X-ray treatment is the best method and sares much time and money in institutions. Where it is not available irritants have to be resorted to, the relative values of which are dependent on their capacity for setting up sufficient inflammatory disturbance to loosen the hairs and to cause their defluvium without injury to the skin. Epilation by forceps is unsuccessful, as even in expert hands many stumps are broken off leaving part of the infected root behind. Careful epilation in the direction of the long axis of the hair is of great assistance in accelerating a cure when the hairs have become slightly loosened by irritants. 


\section{X-Ray Treatment of Ringworm of the Scalp}

General Remarks.- The treatment of the dry scaly forms of ringworm of the scalp by the X-rays is the most rapid and effective method of dealing with the disease. In competent hands it can be done with absolute safety, not only as far as the skin is concerned but also without any deleterious effects on the general health and growth of the patient, and the fear which has been engendered in the public mind with regard to harmful effects of the treatment on the underlying brain is wholly without foundation. It is an operation which is by no means easy, and necessitates not only an X-ray system suitable for the purpose and a working knowledge of the production, mensuration, and application of the $\mathrm{X}$-rays, but also an intimate acquaintance with the disease in all its phases, as otherwise the treatment is likely to prove unsuccessful and may lead to disastrous results, such as severe dermatitis and permanent baldness. From a large experience of its difficulties the writer would advise all those attempting it to be careful and to take every opportunity of mastering the method of procedure before employing it.

The Action of the X-rays on the Scalp.-The X-rays act simply as a depilatory and do not destroy the fungus, as is seen by the ease with which a culture can be made from hairs which have fallen out as a result of exposure to the rays. There is no apparent effect on the scalp at the time of exposure nor are any sensory symptoms produced by it. Young children may be frightened by the noise and sparking of the apparatus but feel nothing and may even go to sleep during the exposure. About I4 days after the exposure the scalp becomes itchy and a transient erythema appears, followed in the next few days by defluvium of the hair in the exposed areas, which is complete by the twenty-first day and leaves the skin smooth as in Alopecia areata. Eight weeks later the hair begins to grow again as a fine down and the re-growth is complete in about six months.

The exact manner of action of the rays is not fully understood. It seems that the effect is principally on the hair-papilla, the function of which is temporarily suspended, and in consequence the nutrition and growth of the hair-bulb is interfered with and a gradual shrivelling of the intra-follicular portion of the hair takes place. It is possible also that the rays may have a direct action on the soft cells of the bulb. In If days the shrinkage has reached such an extent that the hair becomes loose in the follicle and tends to fall out. About this time a slight toxic action occurs, possibly due to toxins eliminated by the degenerated cells of the hair-bulb, and this is responsible for the erythema. In two or three weeks the papillae recover and new hairs begin to grow.

Should an excessive dose be given, various degrees of dermatitis may result. Where it is only slightly in excess the erythema may 
appear a few days earlier and the papillae may be so damaged that they are incapable of recovery and protracted or permanent baldness may follow. Should there be no re-growth in six months the chances of permanent baldness are considerable. If the dose be greater, acute inflammatory symptoms may supervene within a week, with oedema, vesication, and even ulceration, and in severe cases sloughing may take place right down to the bone. When healing occurs in such cases a disfiguring bald red scar results with a shiny atrophic surface covered with telangiectases.

The Apparatus and Dosage of the Rays. -The systems of X-ray apparatus employed for the treatment of ringworm vary according to the fancy of different operators and a comparison of their relative merits would be useless. The important point is to obtain an effective system and to work with it sufficiently to become thoroughly conversant with its peculiarities (see page I.47).

Procedure.-Before the scalp is $\mathrm{X}$-rayed any marked inflammation resulting from the prerious employment of irritating parasiticide remedies, such as iodine or chrysarobin, should be subdued by lead lotion or other sedatives. Should the scalp be exposed before the effects of these drugs have worn off a dermatitis may be caused by a dosage of the rays which otherwise would have been harmless.

As the effect of the X-rays is temporarily to reduce the vitality of the scalp, any septic condition, such as impetigo, which may be present is liable to assert itself with considerable severity and boils or even ulcerations may develop. To cbriate this a mild antiseptic ointment, containing $3 \%$ of ammoniated mercury, salicylic acid, or precipitate of sulphur, should be rubbed in for a few days before the exposure.

If the ringworm be widely disseminated and the entire scalp has to be exposed it is best done by the Kienböck-Adamson method, by which the whole scalp is given a pastille dose of the rays by exposing it in five different positions. The procedure is as follows: The hair is cut short; in the case of girls the cut-off long hair may be disinfected and made up into a wig to wear until the re-growth is complete. Five points are then marked on the scalp with an aniline pencil as follows:

Point $A, I \frac{1}{2}$ to 2 inches behind the frontal margin of the hairy scalp.

Point $\mathrm{B}, \mathrm{I}$ to $\mathrm{I} \frac{1}{2}$ inches above the centre of the flat area which forms the upper part of the occiput.

Point C, just above the lower border of the scalp at the lower part of the occiput.

Point $\mathrm{D}$, on the right side just above and in front of the ear.

Point $\mathrm{E}$, on the left side just above and in front of the ear.

The distance between any two of these points is fire inches and when the points are joined by lines these meet one another at right angles.

A special localiser is then fitted to the front of the shield, consisting of a metal rim fitted with three soft-wood tapering pegs which converge to a distance of an inch and a half from each other and are of such a 
length that when the scalp is placed in contact with the tips of the pegs it is $16 \mathrm{~cm}$. from the anti-cathode. (Fig. I29.)

The points are exposed seriatim to a pastille dose of the rays. During the exposure the glabrous skin of the forehead, ears, and neck,

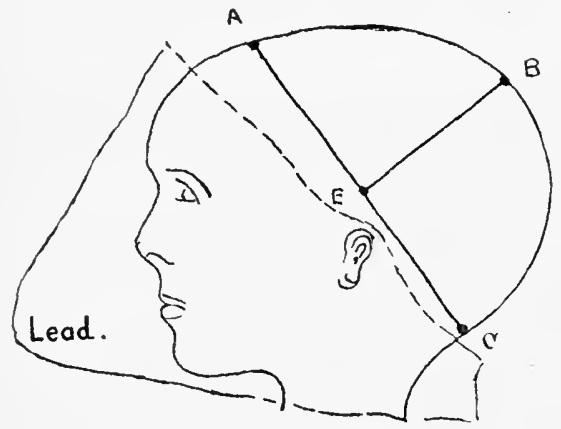

FIG. I29.-Points of Exposure. Kienbock-Adamson Iethod. is protected by lead I mm. thick, and a layer of thick felt is interposed between the pegs and the scalp. Great care should be taken when adjusting the head that the point be mid-way between the three pegs of the localiser and that the direction of each exposure be exactly at right angles to that of adjacent exposures. (Fig. I30.)

In exposing point $A$ the patient should be placed flat on his back on a couch; for $B$ and $\mathrm{C}$ he should sit on a stool with his forehead resting against the side of the couch; for $\mathrm{D}$ and $\mathrm{E}$ he should lie on his side without a pillow, his face being turned away from the tube.

When the apparatus is working properly each exposure occupies from about ten to fifteen minutes and, allowing five minutes for arranging the patient before each one, the whole time will be about an hour and a half. If a gas mercury rotary break such as the "zenith" be substituted for the " dipper" the time can be reduced safely to about one-third for each area and to three-quarters of an hour for the whole scalp. It is unwise to reduce the time further, as the margin of safety is correspondingly diminished.

By this method the whole scalp is exposed equally and each part receives only the requisite dose, the overlapping which occurs at the periphery exactly making up for the weaker irradiation.

Where the disease is not so widely distributed it may be unnecessary to X-ray the whole scalp and the exposure of one or two areas may suffice; in this case either the tripod or lead-glass localisers of suitable size may be employed. As a rule, however, it is quickest in the end to expose the whole scalp, as the ringworm may spread in the interval between the exposure and the defluvium of the hair and necessitate further exposures. (Fig. I3I.)

When the whole of the scalp has been exposed it should be left alone for a fortnight, white cotton caps being worn continuously night and day during that time. During the third week, when the hair is falling out, it should be washed every day with some non-irritating soap, such as castille soap, and hot water, the loose hairs coming out in the washing. By the end of the week the defluvium is generally complete. Occasionally it may happen that a certain number of the short diseased hairs persist after the rest of the hair has fallen out, 


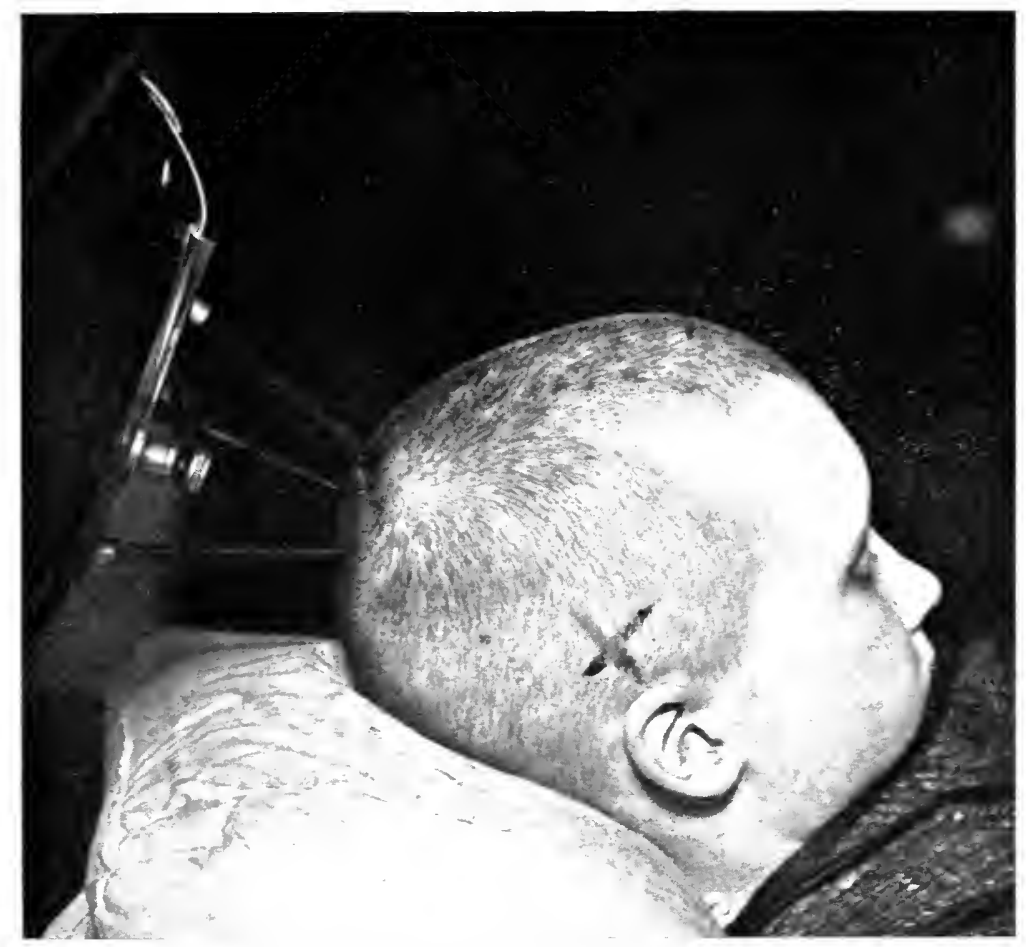

FIG. I30-- -ray Treatment of Ringworm of Scalp.

( hienbonck-tilanson Vethod.)

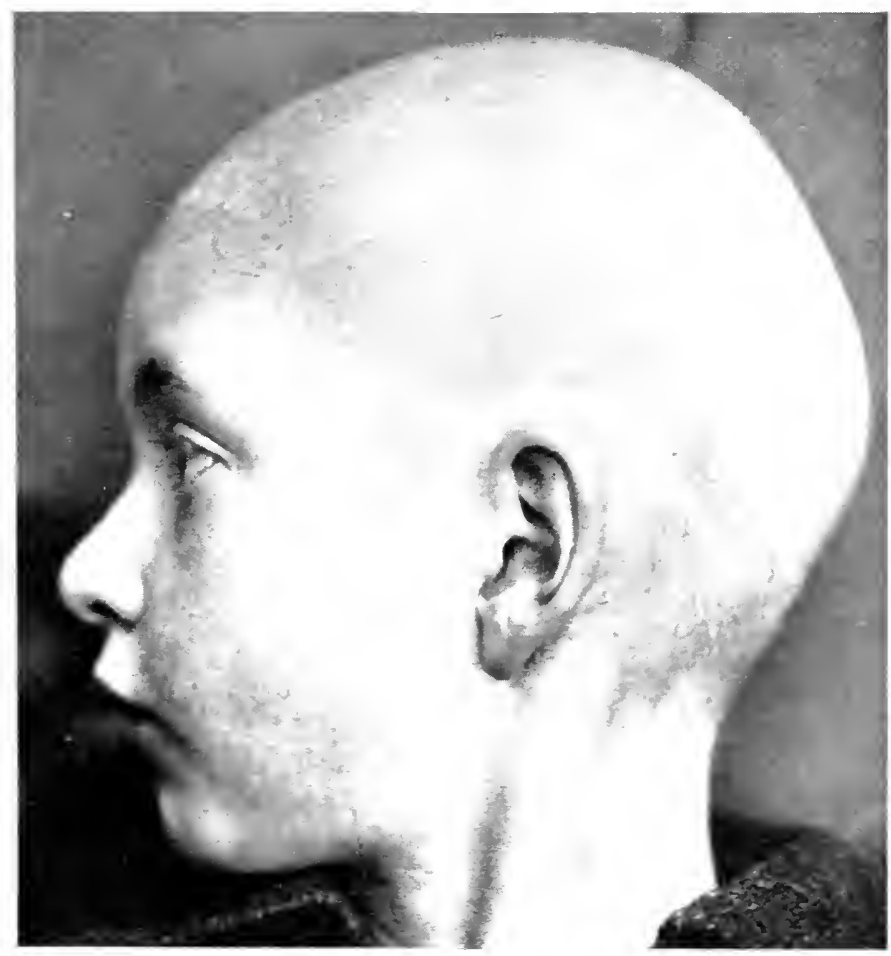

FIG. I 3 I,-Deflutium of the Hair it days after $\mathrm{X}$-ray exposure for ringworm. 


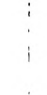


these generally come out if left alone but it is better to facilitate this by epilating them with forceps. If by the end of the fourth week no diseased hairs can be detected on the scalp the child may be pronounced cured and may mix freely with other children. After the fourth week and until the hair begins to grow, the scalp should be washed daily and gently massaged with almond oil to stimulate the circulation.

When the scalp has been only partially exposed the procedure is different because here the disease is capable of spreading beyond the exposed areas by auto-inoculation before the hair falls out. To prevent this, the scalp should be rubbed over every morning with carbolglycerine ( $I$ in S) or tincture of iodine in $60 \%$ spirit ( $I$ in S). Should these prove irritating an ointment containing $2 \%$ of salicylic acid and precipitate of sulphur may be substituted. After the fourteenth day the procedure is the same as when the whole scalp has been exposed. Should the dosage of the X-rays have been insufficient and by the end of the fourth week the hair show no signs of falling out and the scalp no evidence of erythema, the exposure may be repeated.

The hair begins to grow in as a fine down in 6 to 8 weeks and the re-growth is complete in about six months. The new hair is strong and healthy and not infrequently coarser and more curly than the old hair. At first it may be lighter or darker in tint than before but it gradually assumes its former colour.

In the above description, reference has only been made to the single dosage method. A defluvium can also be produced by repeated small doses but this is uncertain and, owing to the cumulative effects of the rays, is liable to be followed by dermatitis.

The chief causes of failure with the X-ray method of treatment are : (I) under-exposure, when an imperfect defluvium of the hair takes place and infected hairs are left; (2) omission of a focus of disease in the exposure; (3) insufficient thoroughness in the after treatment so that the disease spreads beyond the exposed areas.

It has been asserted that the treatment is attended by certain risks, such as $(a)$ severe dermatitis followed by permanent baldness; $(b)$ protracted or permanent baldness without dermatitis; (c) injury to the underlying brain and the production of epilepsy.

Should severe dermatitis follow it is the result of an orer-exposure, or of some accident, such as the use of wrong or inaccurately standardised pastilles, or the exposing of an area the resistance of which has been lowered by the application of strong irritants before the inflammation has entirely subsided. In giving the exposure it is essential that the scalp should not be allowed to come any nearer to the anti-cathode than the regulation distance of $\mathrm{I} 6 \mathrm{~cm}$. This is a matter of vital importance, for should it be any nearer there is a risk of the pastille dose producing a burn. When a cy-lindrical localiser is used, if it be of a diameter larger than $S \mathrm{~cm}$. the convex portions of the scalp are liable to get too near the tube and may be scorched.

It has been stated also that idiosyncrasy plays an important part 
in this connection and that a dose which would be safe in one child would be excessive in another, but this factor would seem to have been somewhat exaggerated. In children with fair delicate hair erythema of the scalp and defluvium of the hair occur more readily than in those with coarse dark hair ; also in certain situations, such as the lower part of the occiput, the hair comes out more readily than in the vertex. With the apparatus running so that the pastille dose corresponds to about I2,000 dips of the break, there is a certain margin of safety which makes it possible to give a full pastille dose in every case and in all situations. If larger tubes and more powerful induction coils be employed, it is possible to reduce the exposure to 7000 or 8000 ( 7 or 8 minutes) but in so doing the margin of safety is correspondingly diminished and the factor of idiosyncrasy may come into play.

Though it has been claimed that it is safe to expose the scalp of infants, it is safer to refrain from doing so until the age of $3 \frac{1}{2}$ years so as to allow the fontanelles to close, and to diminish the difficulties of keeping very young children quiet during the exposure.

With regard to protracted or permanent baldness occurring without marked dermatitis, it also would seem to be the result of overexposure, though in rare instances it might be due to idiosyncrasy in the same way as the occurrence of Alopecia areata after ringworm. Such accidents may happen to anyone, but with proper care they should be so extremely rare as not to discredit a mode of treatment possessing such obvious advantages.

With regard to injury to the brain by this treatment the writer has been unable to obtain any positive evidence of its occurrence either from his own cases, from the literature on the subject, or from anyone whom he has asked about it, and experiments which he has done in this connection strongly negative the possibility. These experiments were made to discover the actual amount of $\mathrm{X}$-rays which will penetrate the brain, when the full dose is given on the scalp. For this purpose a piece of parietal bone from the thinnest part of the calvarium of a child aged $4 \frac{1}{2}$ was procured from the post-mortem room, and it was found that only one-third of a pastille dose penetrated this extremely thin piece of bone during the exposure of a full dose.

\section{Treatment BY PARAsiticide Applications}

When the X-rays are not available one of the older methods of treatment has to be resorted to, such as applications of parasiticides or irritants to produce a sufficient inflammatory reaction to cause defluvium of the hair.

As has already been pointed out parasiticide remedies are of comparatively small value in the treatment of the dry scaly forms of ringworm of the scalp, owing to the mechanical difficulty of their reaching the fungus in the deeper parts of the follicles and of penetrating the hair-cuticle. Varions devices have been adopted to overcome this 
difficulty, such as the incorporation with the parasiticide of substances which might facilitate its penetration such as turpentine, ether, and lanolin, the constant application of the remedy under a bandage or closely fitting cap, or the attempt to drive it in by cataphoresis, but these have been of little assistance and under the most favourable conditions the average time occupied in the cure of a case by these means is more than a year. In the infantile scalp, they are of value and can effect a cure comparatively easily, while occasionally in individuals in whom the scalp is particularly sensitive or does not form a suitable soil for the growth of the fungus, the application of parasiticides sets up a marked inflammatory reaction which kills the fungus. In both these instances, howerer, the cure does not take place so much from the direct destruction of the fungus as from the inflammatory reaction.

The infinite number of parasiticides and their combinations which have been employed for the cure of scalp ringworm testifies to the intractability of the disease. To attempt to enumerate them would serve no useful purpose and it will suffice to mention only the more important, such as :

\begin{tabular}{|c|c|c|c|c|c|c|}
\hline Sulphuris praeci & bitati & - & - & & $5^{i}$ & I2 \\
\hline Acidi salicylici & - & - & - & - & gr. $x x$ & \\
\hline Hydrargyri amn & oniati & & - & - & gr. xv & \\
\hline Beta naphthol & - & - & -. & - & gr. $x$ & \\
\hline Paraffinum moll & ad & & - & & $\bar{j} i$ & 79 \\
\hline Cupri oleati & - & - & - & & $\overline{j s s}$ & \\
\hline Acidi salicylici & - & - &. & - & gr. xx & \\
\hline Paraffinum moll & $\mathrm{ad}$ & - & - & & $\bar{\jmath} \mathrm{i}$ & 30 \\
\hline Sulphuris sublim & ati & - & - & & $\bar{j} s s$ & \\
\hline Acidi carbolici & - & - & - & - & jiss & 8 \\
\hline Olei olivae & - & - & - & 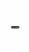 & jiss & 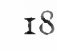 \\
\hline Cerae albae - & - & - & - & & jiss & \\
\hline Ungt. hỹdrarg. & it. ad & & - & - & $\bar{j} \mathrm{i}$ & \\
\hline
\end{tabular}

The strength of the various remedies must be altered according to the degree of toleration of the scalp; in the infant comparatively mild applications suffice. They should be rubbed into the diseased areas once or twice daily.

\section{TreatMENT BY IRRITANTS, OR THE ARTificial KERIOA METHOD}

Besides the X-rars the only treatment which promises satisfactory results in dry scaly ringworm of the scalp is the application of irritants capable of producing sufficient inflammatory reaction to cause loosening and shedding of the hair such as occurs in kerions. Considerable experience is required in carrying out this treatment to know the degree of inflammation requisite. The reaction should be of the 
type of a perifolliculitis rather than a superficial blistering. The production of pustulation is unnecessary and rather a disadvantage as it may lead to ulceration, permanent destruction of the hair-papillae, and scarring.

Different scalps vary in their readiness to react to irritants. In some even mild applications are capable of producing sufficient inflammation, while in others the strongest irritants are necessary ; consequently in all cases it is advisable to estimate the toleration of the scalp by employing mild applications at first.

The chief drawbacks are the pain or discomfort associated with it, which may be so great as to interfere with sleep, and its liability to cause swelling and tenderness of the neighbouring lymphatic glands. It requires careful supervision to avoid pushing it too far and at the same time, as it makes the affection look worse rather than better, it is necessary to encourage the patient and the parents to persevere with it sufficiently.

Of the various irritants employed the most useful are croton oil, salt, and chrysarobin, the croton oil and chrysarobin being specially applicable where the disease is limited to a few patches, and the salt where it is widely distributed. The strength and vigour with which these irritants are applied is dependent on the age of the child, the sensitiveness of the scalp, and the extent of the disease.

Croton oil.--The simplest method of employing croton oil is in a 5 to Io \% ointment with a basis of equal parts of lanolin and vaselin. The hair is first clipped short and all crusts and scales removed by washing with soft soap, the ointment is then rubbed into the patches once or twice daily, care being taken to prevent it from spreading beyond them. After a short time the patches become inflamed and certain of the stumps so loose that they can be epilated without breaking. The inflammatory reaction is kept up by further applications of the ointment and the epilation continued until the diseased areas become perfectly bald, when the condition is cured; this usually takes place in three months or less according to the vigour with which the treatment is carried out. Should the ointment produce a violent reaction with vesico-pustules, the inunction should be discontinued and boric compresses applied.

Instead of applying the croton oil in the fcrm of an ointment it may be rubbed on pure, as advocated by Aldersmith who originated the treatment. The procedure is as follows: A drop of croton oil is rubbed on to the diseased patch, the surrounding healthy skin being protected by carbolised lanolin, I in 8 . Four hours later a small soft sticky linseed poultice is applied to the patch under a piece of oiled silk. The poultice should be changed every four hours and each time it is taken off the part should be bathed with warm water and the scalp swabbed over with glycerine and carbolic. On the following day the croton oil is again applied and the poulticing continued. This procedure is repeated until sufficient inflammation has been produced to loosen the 
diseased hairs, and after these have been epilated or sponged away boric acid compresses are applied to reduce the inflammation. When this has subsided a red bald patch remains over which downy hairs grow in six or eight weeks.

Where small isolated foci or single infected stumps are present they are best treated by " needling." For this purpose a special bent needle, such as Aldersmith's, or an ordinary three-sided glover's needle is employed. This is dipped into croton oil to which $5 \%$ carbolic acid has been added to prevent suppuration, and carefully introduced into the diseased follicle by the aid of a lens held in the left hand. This causes a loosening of the hair which can then be comparatively easily: epilated by forceps. The folliculitis which follows is not so severe as to destroy the papilla and a new hair soon grows. Next to the X-rays the treatment by croton oil is the most effective method of dealing with ringworm, but it is only applicable to limited patches and isolated stumps and is so painful that it should not be employed in children under 5 years of age.

Isolated stumps may be epilated also by electrolysis, the contact being made by inserting one hand into a vessel containing saline solution in which the positive pole has been placed. This method is inferior to croton oil, more painful, and leads to destruction of the follicle and papilla.

Salt.-Ordinary sodium chloride is a powerful irritant and in some cases gives brilliant results. It is applied in an ointment containing equal parts of salt and vaselin rubbed up with a few drops of water in a mortar. This is rubbed into the patches for a few minutes twice daily. It causes considerable inflammation and, if applied over extensive areas, is painful and may prevent sleep. To relieve this the scalp should be bathed with warm water. Children vary greatly in the manner in which they react to this treatment. In some a sufficient dermatitis to cause the requisite defluvium can be produced by the salt ointment in a few weeks, while in others it may be months before this happens. It has a selective action, the inflammation being greatest in the diseased patches.

The treatment has not the risks of croton oil and may be left in the hands of the parents, the inflammation produced by it being more superficial and subsiding more rapidly.

Chrysarobin.-Chrysarobin is applied either in an ointment containing from 2 to $6 \%$ in equal parts of lanolin and vaselin, or, to restrict its action, dissolved in collodion or traumaticin ( $\mathrm{I}$ in S). Where the patches are small it may be employed conveniently as a pencil

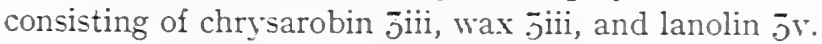

The great disadrantages of chrysarobin are: (I) its liability to be spread on to the face and to produce severe dermatitis or conjunctivitis ; (2) that it is dirty and stains the hair or anything with which it may come in contact. Consequently it must be used most carefully and is unsuitable for ordinary out-patient practice. 
Of the other irritants which have been employed, such as the oleates of mercury and copper, iodine in the form of Coster's paste (iodine 5 ii and oil of cade $\bar{\jmath}$ i), ordinary commercial izal and formalin, none is so certain in its action as those mentioned above and in the case of formalin an alarming degree of dermatitis occasionally may be produced.

Picric acid and camphor have also been advocated, the following formula being employed :

\begin{tabular}{|c|c|c|c|c|}
\hline Acidi picrici & - & - & - gr. vii & 1.5 \\
\hline Camphorae & - & - & $\overline{3}$ ss & 48 \\
\hline Spiritus recti & tus ad & - & $\overline{3} \mathrm{i}$ & 50.5 \\
\hline
\end{tabular}

this is painted on the patches twice daily but the results from it are uncertain and the preparation has the disadvantage of being inflammable (Winkelried Williams).

\section{Suppurative Ringworm of the Scalp}

The treatment of suppurative ringworm of the scalp, or kerion, is a comparatively simple matter as the suppuration itself leads to destruction of the fungus and all that is required is to leave nature alone and gradually to reduce the inflammatory process by mild antiseptic applications. The loose hairs over the patch and the healthy hairs for a short distance beyond its margin should be carefully epilated. A compress of boric lotion or hydrogen peroxide, Io vols., should te applied over the whole scalp every morning, after which the diseased areas should be dressed with $3 \%$ ammoniated mercury ointment spread on lint and kept in position by a closely fitting cap. By this means the swelling gradually subsides and a bald inflamed shiny area is left, over which the hair begins to grow in about a couple of months. It is inadvisable to employ the X-rays in these cases as they are not only unnecessary but may give rise to permanent baldness in the affected areas.

\section{RINGIVORM OF THE BEARD}

Syn.: Tinea barbae; Hyphogenic sycosis; Barbers' itch; Fr., Sycosis parasitaire; Ger., Parasitäre Bartfinne.

Ringworm of the beard is a comparatively rare affection. It may take the form of : (I) raised suppurative lesions ; (2) dry, scaly, more or less bald patches on which typical ringworm stumps may be detected among the healthy hairs.

(I) Suppurative Type.-The essential lesion of this type of ringworm is a perifollicular abscess which appears as a smooth red nodule around the dilated orifice of a hair-follicle, with a hair in the centre, from which oozes a bead of sero-pus which may dry up to form a crust. These lesions may be isolated but as a rule they occur in groups and tend to coalesce to form conglomerate masses not unlike carbuncles, which 



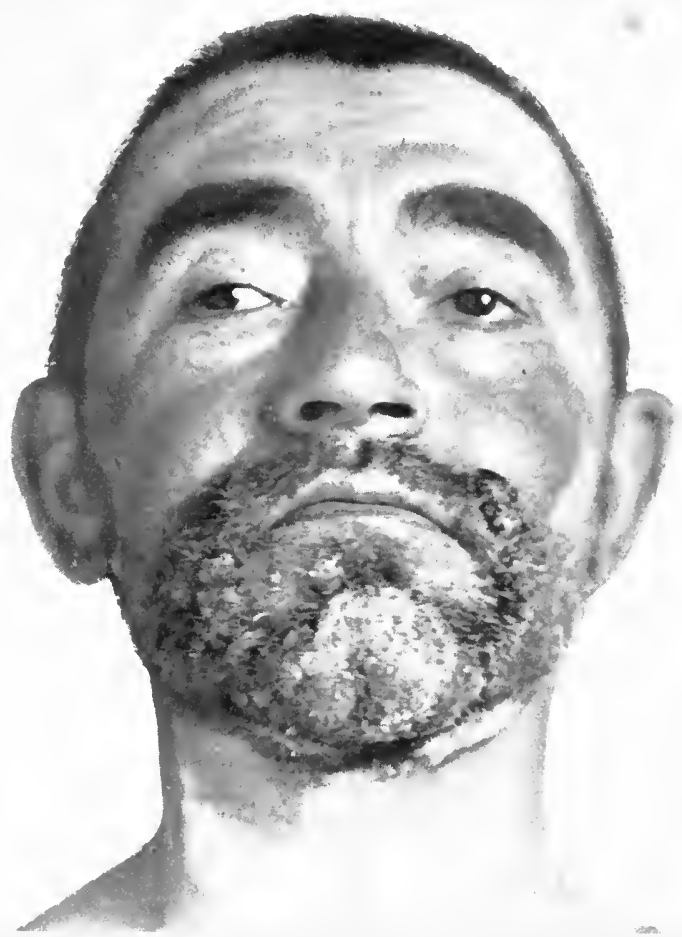

FIG. I 33.-Tinea Barbae, due to Trichophyton Rosaceum.

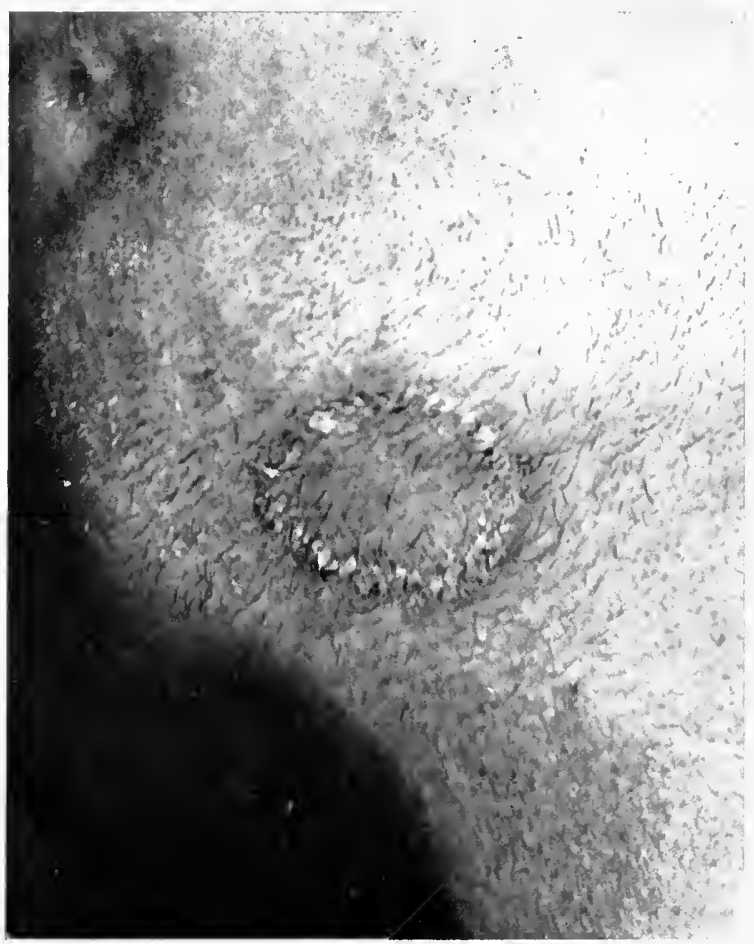

FIG. I 34-Circinate Ringworm of the Beard Region. 


\section{DERMATOMYCOSES}

are pinkish-red in colour, tense, angry-looking, sometimes painful, and dotted over with small pustules and, here and there, with opaque swollen stumps. When large and oedematous they may give a feeling of fluctuation on palpation but if incised, instead of pus, only a small bead of bloodi-stained serum oozes out. They are most common on the chin and neighbouring part of the neck but may occur anywhere about the beard. They are rare on the moustache. (Fig. I32.)

They are due to an infection by trichophyta of the ecto-endothrix type, are generally of animal, sometimes of bird, origin, and are most common in young adults, chiefly grooms, farmers, cattlemen, and

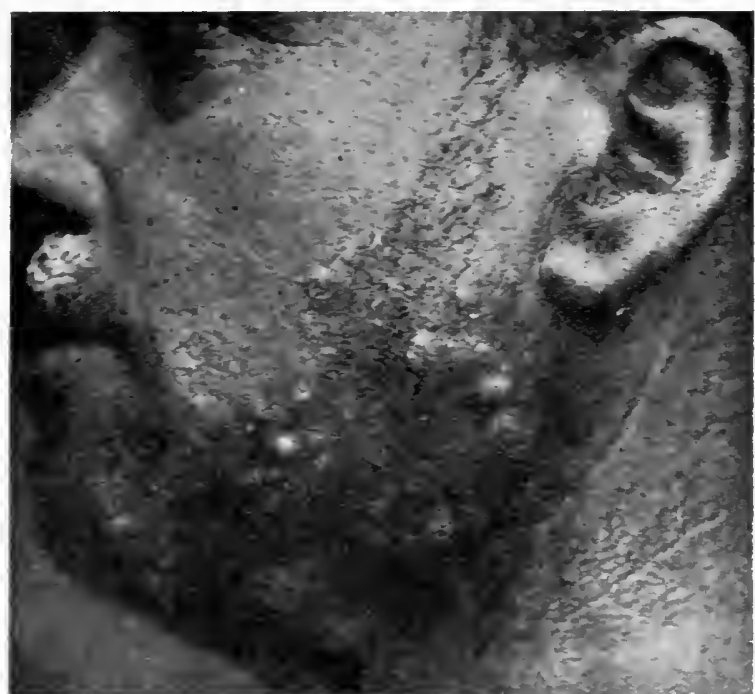

FIG. 132.-Tinea Barbae.

others whose occupations necessitate contact with infected animals. (Fig. I33.)

(2) Dry Scaly Type-This variety of ringworm of the beard begins as small pinkish scaly spots over which are a few short opaque stumps or plugged follicles such as occur in black-dot ringworm. The fungus attacks the epidermis first and spreads from there into the follicles. The patches tend to increase and coalesce to form irregular scaly areas with a well-defined border. Occasionally they may clear up in the centre and ringed lesions result. Sometimes the inflammation is more intense and the lesions become raised $u p$ into flat plaques. (Fig. I34.)

This type of ringworm is usually of human origin and not infrequently contracted in a barber's shop from an infected sharing brush. It is due to the endothrix tricophyta, the $\mathrm{T}$. violaceum, T. acuminatum, $T$. plicatile, and $T$. crateriforme, all haring been recovered from it. The $T$. violaceum is probably the most common and, in some cases, is capable of producing suppurative lesions. 
Microsporon ringworm of animal origin has also been isolated from the beard, in which case the patches resemble those of smallspored ringworm of the scalp in children.

Diagnosis.-The suppurative lesions have to be distinguished from ordinary sycosis of the beard due to pyogenic cocci but in coccogenic sycosis the lesions are perifollicular pustules, are irregularly distributed sometimes over a wide area and often present on the upper lip, while those of ringworm are inore localised, tend to form conglomerate tumours, and practically never attack the moustache.

Occasionally they may be mistaken for Impetigo contagiosa of the beard, but in it the lesions are covered by flat yellowish crusts which can be picked off leaving a moist red surface, and the affection is more superficial and of recent origin.

The dry scaly variety may be confused with pityriasis or scaly eczema, but in them the disease generally extends beyond the hairy margin and is not confined to the beard as it is in ringworm.

In every case diseased stumps should be sought for and examined for fungus.

Prognosis.-Under suitable treatment the ultimate prognosis is good, but the affection is as difficult to cure as ringworm of the scalp and if left alone is apt to persist indefinitely. When involution takes place in the raised conglomerate lesions a smooth red patch is left which may remain bald for months or over which, in rare instances, the hair may never grow again.

Treatment.-The dry type of the affection is very intractable and under ordinary parasiticide applications may persist for years in spite of the most assiduous treatment. It is best dealt with by the X-rays, the affected area being exposed to a pastille dose. Great care must be exercised with regard to localisation in the exposure for, owing to the contour of the face - especially about the chin-it is difficult, with anything but small localisers, to avoid getting some part of the convex surface dangerously near the tube. Sometimes a pastille dose is insufficient to bring out all the hairs, in which case it is best to deal with those which remain by careful epilation and parasiticide applications. It is unwise to risk a second exposure without allowing at least a month to elapse as, owing to the delicacy and vascularity of the skin of the face, it is much more prone to dermatitis than is the scalp.

The procedure is sinilar to that employed in the case of ringworm of the scalp. After the exposure, to prevent the disease spreading, the face should be rubbed over daily until the defluvium occurs with a $6 \%$ lotion of carbolic acid in glycerine. Where the $\mathrm{X}$-rays are not available, careful epilation should be resorted to, and an ointment containing oleate of copper $1 \%$, salicylic acid and precipitate of sulphur $6 \%$, or ammoniated mercury $3 \%$, should be rubbed in twice daily. If there be no marked dermatitis it is advisable to shave daily, the shaving brush being sterilised in I in 20 carbolic after use. After shaving, sulphur lotion should be rubbed into the affected arcas. 
The suppurative type is more amenable to treatment and in the $\mathrm{X}$-rays should not be used as there is a risk of causing dermatitis and permanent baldness. A cure may sometimes be effected by mild applications, such as perchloride lotion I in I000, or $3 \%$ mercurial ointment. In resistant cases the most useful treatment is to add fuel to the fire by increasing the inflammation. This is done by rubbing in an irritating ointment, such as chrysarobin $3 \%$ or oleate of copper I \%, and should be combined with careful epilation of the loose stumps and evacuation of any pus. Good results may be obtained also by exposures, repeated as often as can be tolerated, to a small Finsen lamp with a superficial action, such as the Lortet-Genoud lamp. When the pus-formation becomes excessive the ringworm fungus is usually killed and the inflammation and swelling may then be reduced by compresses of hydrogen percxide or boric acid. After the disease has been apparently cured it is necessary to watch carefully for recurrences.

\section{EYEBROWS AND EYELASHES}

The eyebrows, and even the eyelashes, may be attacked by ringworm, either primarily or by extension from the neighbouring skin, and both the dry and suppurative types may occur.

The treatment consists of careful epilation and the application of an ointment containing $3^{\circ}$ of ammoniated mercury. The employment of chrysarobin is contra-indicated, owing to the danger of its reaching the eyes and producing conjunctivitis.

In the case of the eyebrows, the X-rays may be employed.

\section{RINGWORII OF THE NAILS}

Syn.: Onycho-mycosis trichophytica; Tinea unguium.

Ringworm of the nails may be an independent affection or may occur in connection with ringworm of the glabrous skin, scalp, or beard. It is rare in this country but is comparatively common in semi-tropical countries, especially in the far east in association with body ringworm and Tinea cruris.

The nails of the hands and of the feet may both be attacked but it is more common in the hands and, in this country, is usually confined to two or three nails of one member. It is singularly persistent, cases being on record in which it has been known to have been present for at least twenty years. It has been observed in several members of a family and in more than one generation.

The infection of the nail generally takes place beneath the free border, the fungus first attacking the epidermis of the nail-bed and causing the formation of a dirty-grey scaly thickening which raises up the nail. It gradually spreads back towards the nail-matrix and finally grows up into the nail substance itself, giving rise to discoloured 
opacities and eventually leading to the formation of an irregular, thickened, spongy, dirty-looking nail, with a brittle frayed edge.

More rarely the fungus passes from the skin over the nail-fold or insinuates itself beneath the lateral borders and so reaches the nailmatrix, where it proliferates and interferes with the nutrition of the nail. When this occurs the appearances produced are somewhat different to those described above. The lunule is attacked first and becomes discoloured, depressed, uneven, and corrugated; for a time the anterior part of the nail remains unchanged, but gradually the fungus makes its way past the lunule and the whole nail is implicated and transformed into a thickened spongy mass, more or less broken up at the margin.

Ringworm of the nails is due to the trichophyta and has never been known to be caused by the M. audouini or any other form of microsporon. The T. violaceum, T. crateriforme, and a pus-forming ectoendothrix of animal origin, have been isolated from it, and in a considerable number of cases the Epidermophyton cruris of Tinea cruris has been responsible.

Diagnosis.- The appearance of the affected nails is not sufficiently characteristic to distinguish it from other forms of nail disease and the affection has generally persisted for a considerable time and is welladvanced before it is recognised. As a rule the free edge is more friable and shaggy than in psoriasis, eczema, or syphilis of the nails. The diagnosis can only be established by demonstrating the fungus; for this purpose scrapings or filings of the nails are placed in a testtube containing a small quantity of Liquor potassae, heated gently, and allowed to stand for a few hours. Specimens are then made of the softened nail debris when the fungus, if present, appears as irregularly jointed mycelial filaments.

Treatment.-The disease is singularly intractable. In the early stage of invasion where the lunule alone is attacked, it may possibly be stamped out by softening the affected portion with Liquor potassae, scraping it as thin as possible with a piece of glass, and painting it with tincture of iodine. Where the invasion has occurred beneath the anterior border and has not progressed far inward, a cure may be effected by scraping away the scaly mass from beneath the nail and painting with a $2 \%$ solution of corrosive sublimate in alcohol once or twice daily.

When the nail is completely involved cure is almost impossible except by extirpation. After the removal of the nail the thickened hyperkeratotic nail-bed should be scraped down and the diseased nailbed and matrix dressed continuously with a parasiticide ointment, such as salicylic acid gr. xxx, Hydrarg. ammoniat. gr. xv, vaselin ad $\bar{z}$ i.

Attempts have been made to cause shedding of the nails by the $\mathrm{X}$-rays, but the results have been unsatisfactory as the dosage necessary is in excess of what the skin can stand and is apt to be followed by severe dermatitis. 
Short of extirpation the most promising method of treatment is the following: The nail-plate is softened by wearing a fingerstall of rubber containing soft soap for a few days; the softened nail is then scraped down as thin as possible with a piece of glass, and this is followed by the continuous application of a piece of lint soaked in Sabouraud's iodine solution (iodine $\mathbf{5}$, Pot. iod. I, water Ioo parts), kept in position by a loose rubber fingerstall.

Another method which has given good results was introduced by Dr. A. J. Harrison. In it the nail is first scraped down with a piece of glass and then the following solution applied on lint under oiled silk for fifteen minutes, Pot. iod. jss, Liq. pot. $\bar{j} s s$, Aqua destil. $\bar{j}$ ss. This is removed and a second solution containing Hyd. perchlor. gr. v, Spirit. vini. rect. $\overline{3}$ ss, Aqua destil. $\overline{5}$ ss, applied in the same way for twenty-four hours. This procedure is repeated until a cure is effected.

Cataphoresis with icdide of potassium has been recommended and is worthy of a trial.

\section{REFERENCES}

Adauson, H. G. Immunity production in the skin eruptions due to mould fungi (Goulstonian Lectures, I 9 r 2, Bale, Sons, \& Danieisson, Ltd., London).

Bodis, E. Les Champignons parasites de l'homme (Paris, I goz).

BolaM, R. A. Trichophỵton rosaceum (Brit. Journ. Derm., I9I2, xxiv. p. I).

Buxch, J. Ringworm infection in man \& animals (Brit. Med. Journ., I 901, p. 323).

Castellaxi, A. Observations on a new species of Epidermophyton in Tinea cruris (Brit. Journ. Derm., I9IO, xxii. p. I +7 ).

Chalmers, A. J. \& Marshall, A. Systemic position of the Genus Trichophyton Malmsten, I $8+5$ (Journ, Trop. Med. \& Hy̆g., Oct. I9I p. 289.)

Chalmers, A. J. \& Marshall, A. Tinea capitis tropicalis in the AngloEgyptian Sudan (Idem, Sept. I9r f).

Chaluers, A. J. \& IArshall, A. Tinea capitis tropicalis in an Egyptian soldier, produced by T. discoides Sabouraud, I909 (Idem, March I9I5, p. 49).

Fox, T. Colcotr. The biology of ringworm (Brit. Med. Journ., ISa7, ii. p. $8 ; 6)$.

Fox, T. Colcotr. Endothrix trichophyta in London (Proc. Roy. Soc, Med., I908, ii. p. 49).

Fox, T. Colcott \& Blaxall. Plurality of the fungi of ringworm (Brit. Journ. Derm., I896, viii. p. 24 I).

Germ, A. Ueber lichenoid Trichophytie (Arch. f. Derm. u. Sỵph., IgI4, Bd. I I 8, p. 856 ).

JADASSOHx. Lichenoid trichophytides (Blatt. f. Schweizer Aertze, I9I 2 , p. 24).

Lombardo, C. Ricerche sulla Ipersensibilità in Alcune Dermatomicosi (Giornala Italiano delle Malattie Veneree e della Pelle, vol. iiı, IgI I, p. 70$)$.

MACFADYEx, A. Biology of the Ringworm Organism (Journ. Path. \& Bact., Edin., I 896, iii. p. I-6).

Majocchr. Sul granuloma tricofitico (Soc. Ital. de Derma. e Sifil., MIlan, 1906). 
PaUl, N. Occurrence of Ringworm of the Scalp in Sydney (Med. Journ. of Australia, March I916, p. 237).

Pixi. Granuloma trichophyticum Majocchi (Derm. Zeitschr., I912, p. 710$)$.

Oliver, IV. Jenkins. A case of Tinea tonsurans (Microsporon) in an adult (Brit. Journ. Derm., I9I7, xxix. p. 32).

RAsch, C. Secondary lichenoid Trichophytides in assocration with Kerion celsi. Translated from Danish by IV. Jenkins Oliver (Brit. Journ. Derm., I9I6, xxviii. p. 9).

Sabouraud, R. Les Teignes (Paris, 1910).

Sabouraud, R. Dermatomycoses common to man \& animals (Brit. Med. Journ., I908, ii. p. I089).

Sequeira, J. H. Extensive granulomatous lesions (Brit. Journ. Derm., I9I2, xxiv, p. 207).

Whitfield, A. Unusual case of Trichophytic Infection (Lancet, I908, ii. p. 237).

\section{Experimental}

Bloch \& Massini. Studien über Immunität und Überempfindlichkeit bei Hyphomyzetenerkrankungen (Zeit. f. Hyg. u. Infectionskrankheiten, Bd. lxiii. I909, p. 68).

Chalmers, A. J. \& MacDonald, N. Animal inoculations of T. discoides Sabouraud, I909 (Journ. Trop. Med. \& Hyg., June, I9I5, p. I2I).

Sabouraud, R. Nouvelles Recherches sur les Microsporons (Ann. de Derm. et de Syph., I907, pp. I6I \& 225).

\section{Treatment}

Adanson, H. G. X-ray application for the cure of ringworm (Lancet, I909, i. p. I379).

Aldersmith, H. Ringworm and Alopecia areata (1897).

MAcLeod, J. M. H. X-ray treatment of ringworm of the scalp. Special reference to suggested injuries to the brain (Lancet, 1905, i. p. I 374 ),

MacLeod, J. M. H. Treatment of ringworm of the scalp (Brit. Med. Journ., I905, ii. p. 619).

Sabouraud, R. Radio-therapeutics of ringworm (Brit. Journ. Derm., I906, xviii. p. I99).

Strickler, A. Vaccine treatment of ringworm of the scalp (Journ. Cut. Dis., 1915, xxxiii. p. 195).

Williams, Winkelried. Picric Acid and Camphor Treatment of ringworm (Brit. Journ. Derm., I9I2, xxiv. p. 2I6).

\section{Eczemiatoid Ringworm}

MAcLeOD, J. M. H. Eczematoid ringworm of the hands, feet, \& groin (Practitioner, I917, xcviii. p. I46).

Ormsis \& Mitchell. Ringworm of hands and feet (Journ. Amer. Med. Ass., I9I6. lxvii. p. 7II).

Sabouraud, R. Eczema marginatum (Arch. de Méd. Expériment., Sept. $1907)$.

Sabouraud, R. Eczematoid ringworm of the groins and extremities (Proc. Roy. Soc. Nled., I9II).

Whitfield, A. Eczematoid ringworm (Idem). 



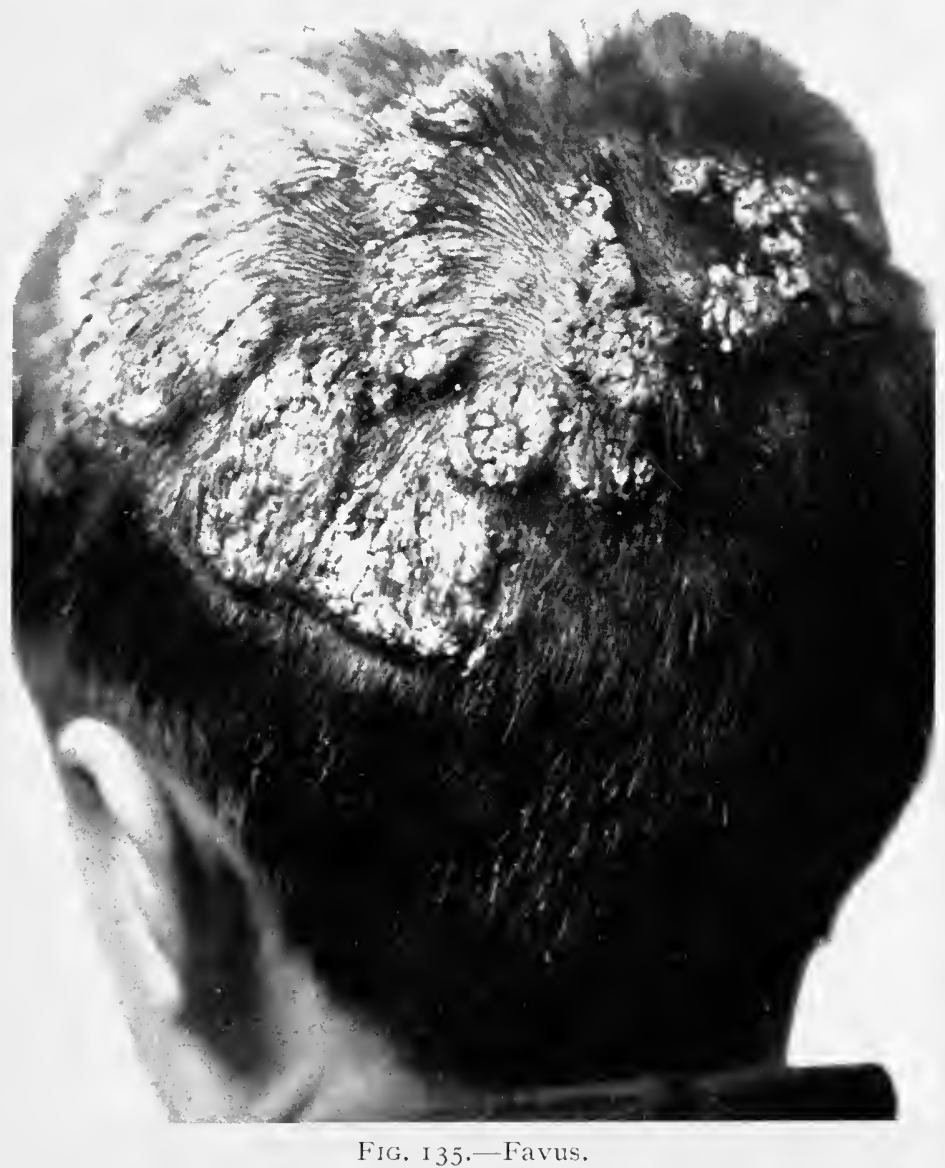

FIG. 135.-Favus.

$[$ To face $p, 42 \mathrm{I}$. 


\section{FAVUS}

Der. : Latin, Favus : honeycomb.

Syn.: Tinea favosa; Porrigo scutula; Porrigo lupinosa; Fr., Teigne faveuse.; Ger., Erbgrind.

Definition: A contagious mycotic disease due to fungi of the genus achorion, characterised by yellowish cup-shaped friable crusts which tend to coalesce to form mortar-like masses.

Description.-Favus is widely distributed over the globe, but is especially prevalent in the eastern countries of Europe, Asia Minor, Egypt, and the southern shores of the Mediterranean; it is not infrequent in Scotland, but is rare in England and the United States of America.

It most commonly affects the scalp but may attack the glabrous skin, hairs, nails, and in rare instances the mucous membranes. It has been observed on the glans penis and has been known to be present on the gastro-intestinal mucosa giving rise to diphtheritic swellings, erosions, and diarrhoea.

Scalp.-. As a general rule it appears first on the scalp and spreads from there to other parts. The characteristic lesion is known as the scutulum, and consists of a small sulphur-yellow disc with a central depression or cup usually pierced by a hair. This is first seen as a minute yellow concretion at the orifice of a hair-follicle, which gradually increases till it reaches the size of a threepenny piece or larger and is raised about a sixteenth of an inch above the level of the skin. The cupping is due in part to the disc being bound down in the centre by a hair, but in the main to the growth of the fungus being centrifugal and more luxuriant at the periphery. The upper surface is concave, sometimes whitish from being covered by a thin epidermal scale, and the under surface is moist and convex. When the scutulum is picked off the hair may come away with it, leaving a depression which is moist and sometimes purulent or bleeding. When the scutulum is of old-standing the pressure exerted by it and the inflammatory disturbance caused by the fungus lead to atrophic changes with destruction of the hair-papillae and the formation of a pitted and depressed cicatrix. Sometimes the scutula are large and coarse and present concentric furrows and ridges, at other times they form irregular yellowish-grey concretions. By the growth and coalescence of adjacent scutula honeycomb-like masses are produced which crumble like mortar and have a festooned border indicating their origin from isolated scutula. These masses are yellowish at first but later assume a dirty brown tinge. From the scutula and crusts a peculiar odour is emitted which has been aptly compared to mouse urine or musty straw. (Fig. I35.)

The hairs in the affected region become opaque, lustreless, dusty looking and tend to split longitudinally but do not show the same 
tendency to break across and form stumps as hairs infected by ringworm fungi. They are easily epilated, and when recently infected may come away with a swollen root-sheath. The scalp is attacked in an

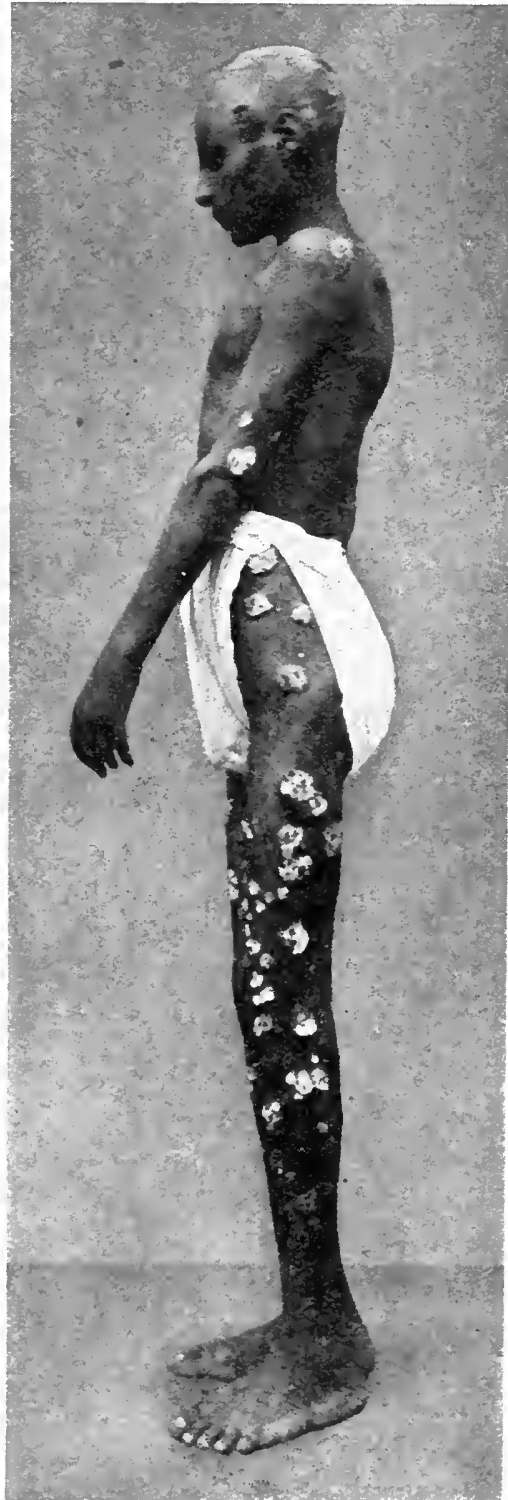

Fig. I 36.-Generalised Favus in a Native. irregular fashion and, as a rule, is not completely involved, unaffected hairs usually persisting as irregularly distributed tufts or as a fringe along the margin.

After several months the scalp becomes extensively affected and has a singularly characteristic appearance, with irregularly distributed partially bald or cicatricial areas interspersed with typical favus cups or mortar-like concretions separated by patches of healthy hair or tufts of dusty, lustreless, infected hairs. In some cases suppurative changes may supervene, in the form of folliculitis and raised boggy patches similar to the kerions of suppurating ringworm. This may be due either to a peculiar sensitiveness of the individual to the toxins eliminated by the fungus, to an exceptionally virulent strain of fungus, or to the secondary inoculation of pyogenic micro-organisms - a complication frequently due to the co-existence of pediculi in the scalp leading to scratching and the inoculation of pus-cocci.

Occasionally typical scutula are absent and the scalp presents an indefinite scurfy appearance which, at first sight, might suggest severe dandruff or psoriasis. On closer inspection, however, characteristic cicatricial patches and lustreless dusty hairs can generally be detected.

Glabrous Skin.-On the glabrous skin the lesions usually develop around the lanugo hair-follicles and consist of typical cupped discs, irregular friable honeycomb-like masses, or mortar-like crusts which, on becoming detached, leave atrophic depressed areas. (Fig. I36.) 
Occasionally it may appear as erythemato-scaly patches, roundish or irregular in outline, slightly raised, sometimes tending to clear in the centre to form a ring, and on which is a varying number of pinheadsized, or slightly larger, scutula. This type of lesion is met with in favus of mouse origin. (Figs. I37, I38.)

More rarely acute inflammatory lesions occur, in wich the erythematous base is dotted over with small vesicles or vesico-pustules (Farus herpeticus) which dry up and give place to typical scutula.

Favus does not interfere with the general health, unless it is very extensive when it may produce symptoms from the absorption of toxic products.

Nails (Onycho-mycosis farosa).When the nails are attacked they become thick, lustreless, brittle, discoloured, raised up from the nail-bed, and sometimes break away leaving an irregular crusted surface. Occasionally yellowish masses or streaks may be observed in the diseased nail-

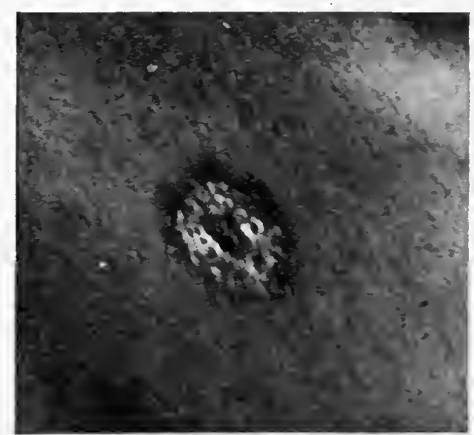

FIG. I 3\%--Mouse Favus on Child's Teck.

(Dr. H. G. Adamson's Case.) plate which are said to be characteristic, but apart from the demonstration of the fungus in scrapings and the presence of lesions elsewhere,

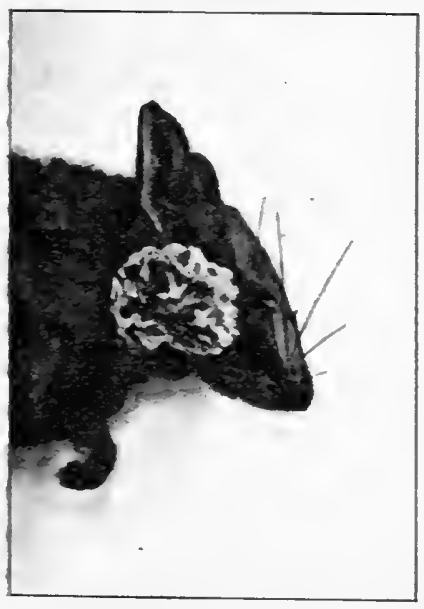

FIG. I 38 .-Favus of Mouse.

(Achorion Quinckeanum of Bodin.) (Dr. H. G. Adamson's Specimen.) it is practically impossible to diagnose the condition. The nails are generally infected from scratching an infected scalp.

Mycology.-The fungus which is responsible for at least $95 \%$ of the cases of farus in the human subject is the Achorion Schönleini. This fungus can be demonstrated easily by making extemporary preparations of a piece of the crust or an infected hair in Liquor potassae. It has a mycelium consisting of narrow, flat, branching filaments varying in length and thickness, some being thin empty tubes, others being wider and divided by transverse septa into carities containing spores. The spores are round, oval or irregular in shape and about $6 \mu$ in diameter. In preparations of the fungus from crusts the mycelium generally predominates and the spores are mixed up with amorphous granular debris. (Fig. I39.)

In the hair the shaft may be packed with mycelial threads 2 or $3 \mu$ in breadth and jointed at intervals of ro to $x_{5} \mu$. These are situated 
chiefly in the centre of the hair and lie parallel to the long axis; they divide dichotomously at very acute angles and end near the bulb in a fringe. Occasionally the mycelium splits up into several filaments ending in rows of spores known as the favic tarsus, but otherwise no spores can be detected in it. As in ringworm the infection spreads from the scalp to the hairs, the mycelial threads growing down between

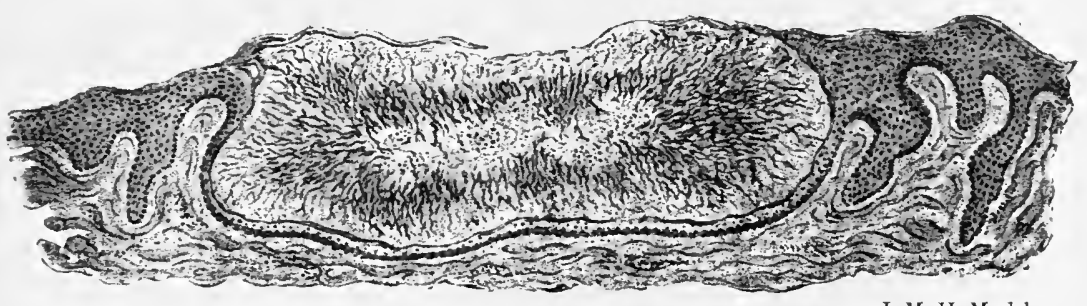

Fig. I.42.-Scutulum of Favus. ( $x$ about 8 o.)

J. M. H. M. ciel.

Shows scutulum consisting of a mycelial felt, with hyphae arranged perpendicularly, faintly stained dead hyphae at the periphery, and a central mass of spores and debris.

(From MacLeod's Pathology of the Skin.)

the inner root-sheath and the hairs, corroding the cuticle, and finally passing into the hair itself. (Figs. I40, I4I.)

In the epidermis the characteristic lesion is the scutulum. This consists of a dense mycelial felt, situated between the superficial layers

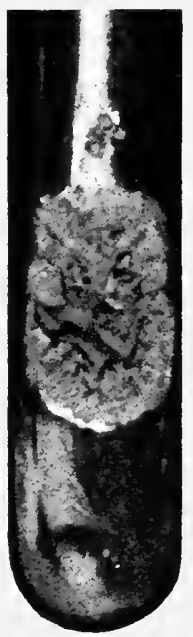

FIG. I 43.-Achorion Schönleini. of the Stratum corneum and occasionally extending down into the prickle-cell layer or even into the corium. In the centre and towards the base of the scutulum are irregular groups of spores and granular debris. From there the mycelial filaments grow towards the periphery and tend to be arranged perpendicularly to the thin horny layer which forms a covering to the mycotic mass. Near the centre the filaments are thin but towards the periphery they become thick sporulating tubes. It is this excessive peripheral growth which probably accounts for the cup-shape of the scutulum. In many instances the scutulum surrounds the orifice of a hair-follicle and is pierced by a hair. The prickle-cell layer beneath the scutulum may be unaltered or it may be thinned in the centre from pressure and may show a tendency to proliferate beyond the margin. (Fig. I 42. )

In the underlying corium there are more or (Dr. H. G. Adamson's culture.)

less marked signs of inflammation, with dilatation of the superficial capillaries, oedema, and an infiltration of lymphocytes, small round connective tissue cells and plasma-cells, which may be so marked as to merit the name of a granuloma. This is the result of the toxic action of the fungus. In some instances suppurative changes supervene and the infiltration is rich in polymorphonuclear leucocytes. 


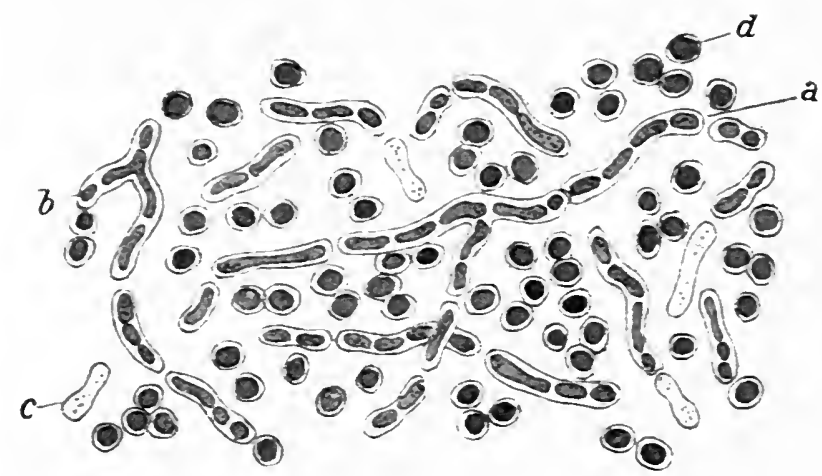

FIG. I 39.-Achorion Schönleini.

J. M. H. M. del.

Preparation in Liquor Potane.

a. Mycelium of tat. branching. truncated rlaments. b. Filames: split up by transverse septa and containing spores. c. Empty filaments. it. spores, irrezular in size and sbape.

(MacLead's Pathog: of the Skin.)

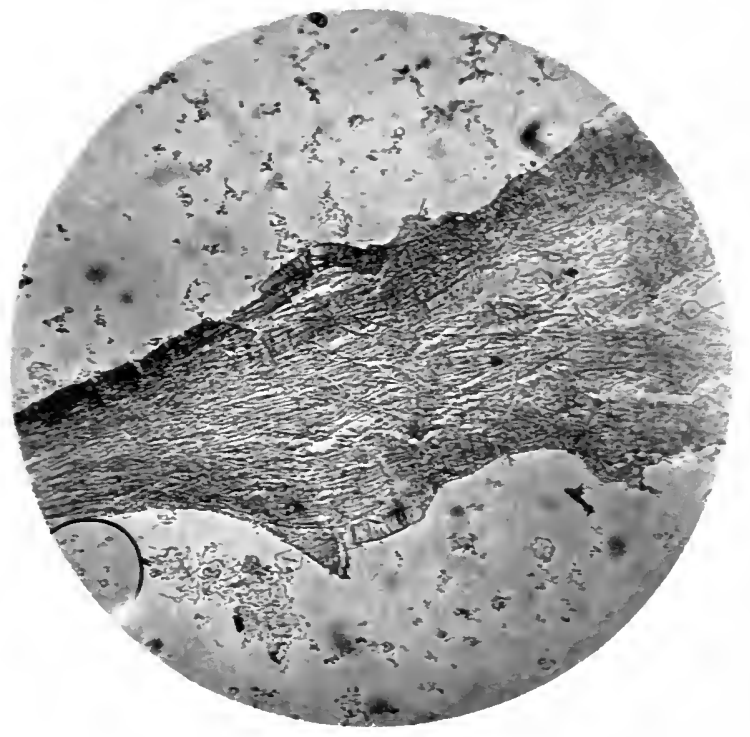

FIG. I +1 .-Hair infected with Farus. Preparation in Liquor Po:assae.

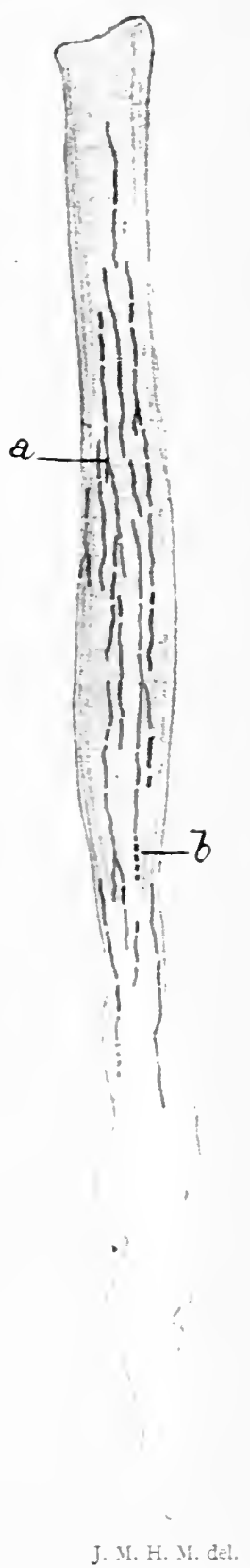

FIG. Ifo.-Hair in. fected with Fungus of Farus.

a. Chains spres trming a sheath around the hair. B. Myceliunending in a izinze at the bulb. 

When the scutulum becomes detached, healing takes place by the formation of new fibrous tissue and a cicatrix results which may be depressed.

Cultiration.--The Achorion Schönleini grows on the ordinary solid media such as maltose agar, glucose agar, and potato. On gluccse agar the growth is slow, and appears first in a week or ten days as a whitish powdery culture which soon becomes brownish in tinge and assumes a corrugated, wrinkled or vermiform appearance. It liquefies gelatine in three or four days. (Fig. I +3.)

The fructification as seen in the hanging drop shows reproduction by endoconidia, sprouting, and unsegmented spindles.

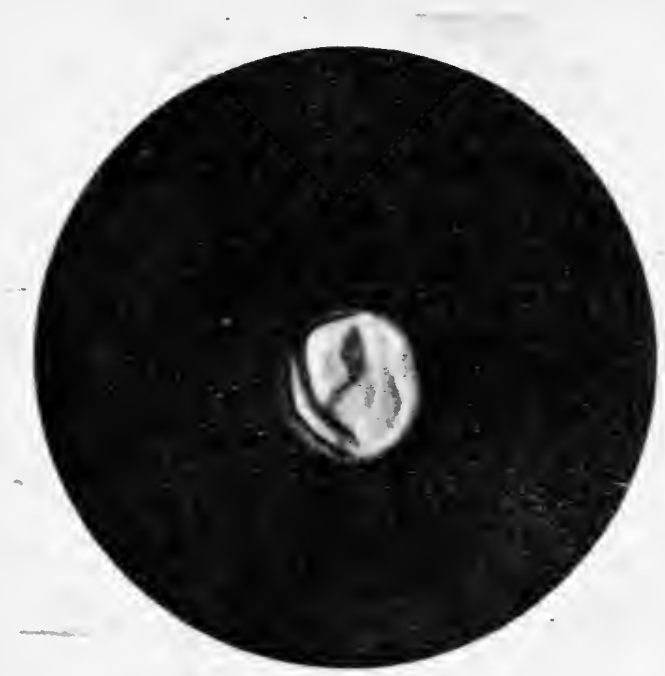

Fig. 14.-Culture of Fungus of Mouse Favus. (Anchorion Quinckeanum.)

In impression preparations from a culture on glucose agar the writer found that the fructification consisted of rods with lateral buds, occasional endoconidia, and terminal non-septate capsules.

The cultures rary considerably in detail in different cases, so much so that certain authors believe that there are several varieties of fungus which are responsible for farus in man. Sabouraud maintains, howerer, that $99 \%$ of the cases are due to the Achorion Schönleini and that the differences are due to pleomorphism rather than to variation in the fungus.

Other Fungi of Favus.-In addition tc the Achorion Schönleini, there are certain farus fungi which attack lower animals and which can be transmitted to man. These are the following:

Achorion quinckeamum (Bodin) the fungus of mouse farus. This fungus grows more luxuriantly in maltose agar than the $\mathrm{A}$. Schönleini, and forms downy white cultures which more closely resemble the cultures of microsporon ringworm than those of the ordinary achorion. 
A number of cases of infection by this fungus have been recorded in children where it occurred on the glabrous skin and was transmitted to them by mice. (Fig. Iff.)

Achorion gypseum (Bodin) occasionally affects man and can be inoculated on mice and guinea-pigs. It produces typical favus lesions in man, but on cultivation somewhat resembles the ringworm fungus known as the Trichophyton gypseum giving a luxuriant white powdery culture with an abrupt margin and a wrinkled centre. Like the A. quinckeanum it seems to form a connecting link between the fungi of ringworm and favus.

Oospora canina (Constantin and Sabrazes) the fungus which causes favus of the dog. It has been successfully inoculated on man.

Lophophyton gallinae (Mégnin) the fungus of favus in fowls; it also has been successfully inoculated in man.

Etiology.-The affection is most common in children but being difficult to eradicate is liable to persist to adult life. It may occur, however, in the adult de novo. As a rule the inoculation takes place from child to child, but it may occur indirectly through the medium of contaminated brushes, clothes, hats, etc. In rare instances it may be transmitted from the lower animals, from cats, dogs, rabbits, horses, cattle, fowls, and especially from mice. In a certain number of cases the source of origin cannot be ascertained. It is feebly infective, does not take root so rapidly as the ringworm fungi and, as a rule, gets anchored about a hair-follicle before it begins to grow. It is most prevalent where hygienic conditions are at their worst and washing is rarely indulged in, and consequently is met with almost exclusively among the poorer people who are crowded together in insanitary surroundings, hence the old French name "Teigne du Pauvre." In this country it is found chiefly in aliens, such as Russians and Poles. It is more common in rural districts than in towns, suggesting the possibility of a saprophytic existence of the fungus in vegetable matter. It is said that the fungus grows more readily in individuals who are run down in health, and especially in phthisical people whose skins are apt to be moist from perspiration.

Diagnosis.-The scutula of favus are such characteristic structures that when they are present the disease is unmistakable. Where they are not well-marked, it has to be distinguished from ringworm, seborrhoic dermatitis, and psoriasis.

From ringworm it is differentiated by the presence of powdery concretions and irregular, frequently atrophic, bald patches, and by the affected hairs being lustreless, dusty, capable of being epilated without breaking, and not forming stumps. Should any doubt exist it may be definitely settled by the demonstration of the fungus in the crusts or hairs.

From seborrhoic dermatitis and psoriasis of the scalp it is distinguished by the crusts being friable, having a yellowish appearance, and by the peculiar lustreless appearance of the hairs. 
On the glabrous skin farus can generally be recognised. Occasionally when it takes the form of erythemato-squamous lesions or is herpetic in character, the diagnosis may be difficult, or impossible unless by the demonstration of the fungus.

In favus of the nails, the nails are generally involved secondarily and lesions occur elsewhere.

As the naked eye appearances are not characteristic it is necessary to establish the diagnosis by the microscope. For this purpose scrapings should be allowed to stand for I2 hours in Liquor potassae and examined, when the fungus may be detected in the form of mycelial threads and spores insinuating themselves between the nail-cells.

Prognosis.-The affection is progressive. If left untreated it may persist indefinitely or, on the other hand, after a number of years it may undergo spontaneous cure as the result of inflammatory and cicatricial changes. On the glabrous skin it is readily amenable to suitable treatment and may be cured in a few weehs; on the scalp it is singularly intractable.

Treatment.-In farus of the scalp, by far the best treatment is the $\mathrm{X}$-rays, employed in exactly the same manner as in ringworm (see p. 406). The results, howerer, are not quite so satisfactory as in ringworm as the disease itself tends to cause atrophy of the hair-papillae and to be followed by permanently bald patches.

As a preliminary to X-ray treatment it is adrisable to destroy any nits or pediculi which may be present, and to apply a mild antiseptic ointment such as $2 \%$ ammoniated mercury or salicylic acid ointment to cure impetigo or any other septic complication. Failing the X-rays the scutula or crusts should be removed by thorough washing with soap spirit lotion, preceded if necessary by softening with olive oil, and the affected hairs should be carefully epilated. Epilation is far more satisfactory in favus than in ringworm as the hairs do not so readily break and can generally be epilated by the roots. To reliere the pain associated with the procedure a previous application of $I$ in 40 carbolic lotion crer the scalp has been recommended. A simple method of epilation is to seize a bunch of hairs between the thumb and a paper knife and to give them a tug, the affected hairs will come out while the healthy hairs, not being loose, will slip through and remain. It is more satisfactory, however, to epilate them singly with forceps.

After the epilation an antiseptic ointment should be rubbed into the scalp, the most effective being salicylic acid, resorcin, or mercury, 5 to $10 \%$ in raselin or in equal parts of lanolin and olive oil. This should be repeated daily and the ointment applied continuously at night spread on lint under a dressing.

Instead of ointment, compresses soaked in hydrogen peroxide solution (Io vols.) or in formalin are of service, but the latter should be used with caution as in certain individuals even mild solutions are liable to produce an alarming dermatitis. 
By this treatment a cure can generally be effected in a few months, but to carry it out thoroughly requires the utmost perseverance on the part of the physician and fortitude on the part of the patient. One trouble connected with it is that after the remedies have been applied for some time the scalp may appear to be cured and a careful search for diseased hairs or scales may fail to reveal the presence of fungus while all the time it is lurking about the orifice of some hair-follicle and will cause a recrudescence eventually. In consequence of this it is unwise to give a certificate of cure until the case has been without treatment for at least a month.

In the glabrous skin the treatment is much simpler and consists of the removal of the crusts by softening in oil, washing with soft soap and warm water, and the daily application of an ointment containing 5 to $10 \%$ of precipitate of sulphur and salicylic acid, or of tincture of iodine. Other remedies may be employed, such as chrysarobin, pyrogallic acid and acid nitrate of mercury.

Where the nails are affected much time is saved by removing the nail-plate so as to allow the remedies to penetrate better.

Internally sulphur has been recommended, in doses of 5 to ro grns. three times a day, as it is believed to be eliminated partly by the sweatglands and in this way to make the skin a less suitable soil for the growth of the parasite, but its value in this connection is uncertain.

In many of the cases in children the general health is below par and the general nutrition defective, consequently better hygienic conditions and suitable tonic treatment are of decided value in improving the general resistance of the skin and rendering it a less suitable soil for the growth of the fungus.

Note.-Immunity Production in Favus.-Experiments in the production of immunity in favus similar to those referred to in connection with ringworm (see page 382) have been carricd out by Bloch and Massini. They infected guinea-pigs with mouse favus (A. Quinckeanum). About 6 days after the inoculation favus cups on an inflammatory base appeared on the skin of the infected animal. It was found that from the time the lesions were established the animais were proof against further inoculation with favus and that this immunity lasted for over 18 months.

Bloch also prepared a "favin" from cultures of the A. Schönleini from which he obtained a "cuti-reaction."

Other observers who have tried to repeat these experiments have not been so succcssful.

\section{REFERENCES}

Adansox, H. G. Achorion quinckeanum (Brit. Journ. Derm., I908, xx. p. $\left.3^{65}\right)$.

Bodin, E. Les champignons parasites de l'homme (Paris, I902).

- Achorion gypseum (Ann. de Derm. et de Syph., I907, p. 586).

Darier \& Hallé. Granuloma due to favus (Ann de Derm. et de Syph., I9IO, P. I 29).

Gébrer. Favus in the new-born (Arch. f. Derm. u. Syph., October 19I2). Quincke, H. Über Favuspilze (Arch. f. exp. Path., Leipzig, I886, xxii. p. 62). 
Sabouratid, R. Dermatomycoses common to man and animals (Brit. Med. Journ., I908, ii. p. 1089).

SABRAzÉs, J. Sur le favus de l'homme, de la poule, et du chien (Paris, I893).

Srout, E. J. Favus, two indigenous cases (N.Y. Med. Journ., I900, p. IISI).

Unva, P. G. Three kinds of favus (Brit. Journ. Derm., I892, iv. p. 139).

\section{ACTINOMYCOSIS CUTIS}

Syn.: Fr., Actinomycose; Ger., Actinomykose.

Actinomycosis cutis is the name given to the lesions which occur in the skin as the result of the presence and growth in it of the actinomyces or ray fungus of Harz. It has been found, however, that in addition to the fungus described by Harz there are several other closely related organisms which can produce somewhat similar cutaneous lesions. Fungi of this type have a world-wide distribution, and cases of actinomycosis may be met with in tropical as well as temperate zones. The affection of the foot known as mycetoma, or Madura foot, which prevails in the tropics is due to allied fungi.

As a general rule the ray fungus reaches the skin by extension from an underlying diseased bone or periosteum. The most common situation is about the angle of the lower jaw or the neck, in which case the fungus has generally gained entrance through a carious tooth or an injury to the gums, set up disease in the inferior maxillary bone or periosteum, and spread from there to the skin. Another not unusual situation is over the vertebral column in connection with disease of the vertebrae. It may also invade the skin directly through any abrasion, and has been known to be implanted on the mucous membrane of the mouth and to have infected the tongue and tonsils. On the other hand the invasion of the skin may be secondary to the involvement of some internal organ, such as the pleura or the lung, when the cutaneous lesions, though extensive, are of minor importance in comparison with the visceral disease.

When the skin and subcutaneous tissue are invaded by the ray fungus, the lesion which results presents a characteristic and striking appearance. At first sight it suggests a breaking-down superficial syphilitic gumma, or the unhealthy, inflamed, indurated skin which surrounds an abscess or sinus in connection with tuberculous disease of glands, bones, or joints. It may appear as a hard swelling like a small sarcoma, of a dusky-red or purplish tint. This gradually breaks down in the centre and points in one or more places, eventually forming a number of small fistulae from which oozes a yellowish sero-purulent or sanguineous discharge containing a number of minute granules of a yellowish-grey or sulphur colour. These granules, or sclerotia as they were once called, are usually about the size of a pin's head and are composed of small masses of the fungus. (Fig. I +5.) 
More often the affected skin is uneven and lumpy from the formation of nodules on an indurated base, has the characteristic dusky-purplish colour, presents a number of oozing fistulae, the orifices of which may have an almost cartilaginous hardness and in addition, in some cases, unhealthy crateriform ulcers with overhanging borders and covered with greyish flabby granulations.

Beyond the main mass are generally small isolated foci in the form of reddish macules or small nodules varying in size from a pin's head to a lentil and presenting near the apex a yellow speck or a small fistula.

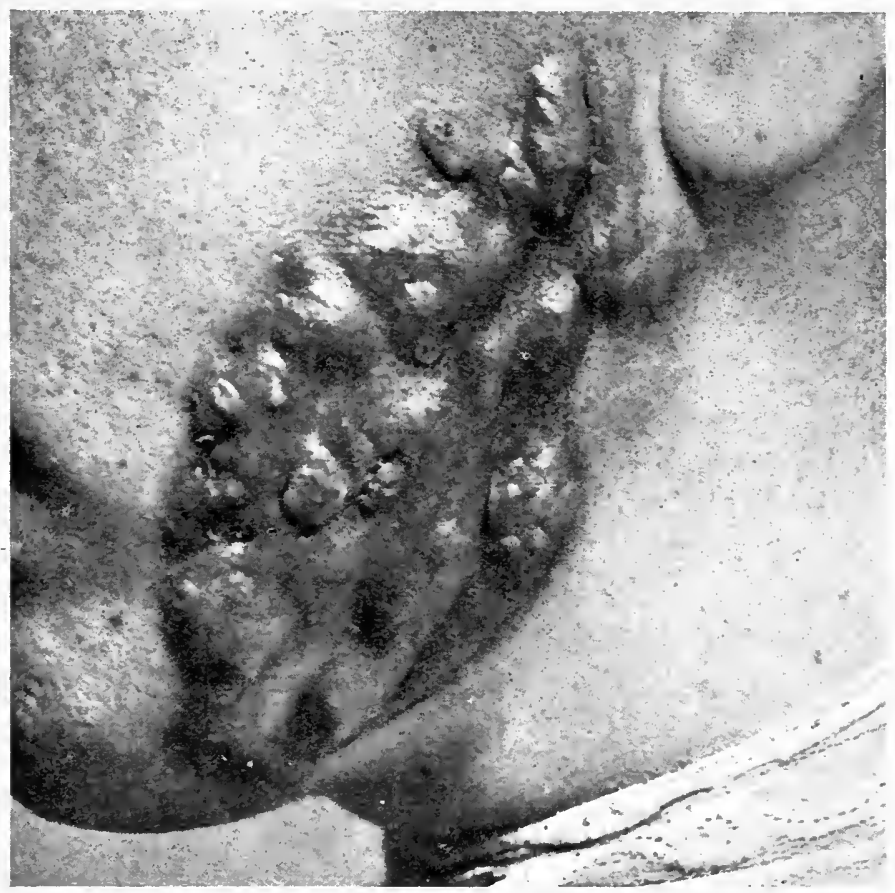

FIG. I 45.-Actinomycosis.

(From Dr. S. W. Allworthy.)

Subsidiary foci may occur at a distance from the original lesion, and even on the other side of the body.

When the affection is the result of an inoculation through the skin it is localised and limited in extent; when it is secondary to deepseated disease, either in bone or in some internal organ, much larger areas are usually involved. Even in extensive cases the lymphatic glands are rarely enlarged.

The course of the lesions varies as the result of differences in the situation affected, in the virulence of the special strain of fungus, and in the resisting power of the individual. In some cases the disease may be confined to the skin and subcutaneous tissue and of a markedly indolent type, the lesions breaking down slowly and even undergoing spontaneous healing; in others it may be acute and fulminating and 
cause destructive changes in the muscles, the viscera, and even in the bones. Occasionally the inoculation is followed by marked oedema and resication.

As a general rule the affection remains local, causes no interference with the general health, and, unless ulceration has taken place, is not associated with much pain. Should the fungus be present in some internal organ the consequences are serious and a fatal issue may result from pyaemia or from the penetration of the fungus into some vital organ, such as the brain.

Actinomycosis occurs not only in the human subject but also in cattle and horses and can be transmitted from them to man ; consequently it is particularly common among workers with animals. It may be communicated directly, or indirectly through the medium of straw, hay, etc., for the fungus is known to be capable of leading a saprophytic existence on vegetable matter, and a certain number of cases have been definitely traced to infection from a prick with a spikelet of an infected cereal, from chewing an infected straw, or from picking the teeth with an infected piece of hay.

Histopathology.-The microscopical appearances presented by sections of skin recently invaded by the fungus show an acute inflammatory reaction with the presence of a dense cellular infiltration consisting of small round cells of the lymphocytic type, plasma-cells, and, occasionally giant-cells. The blood-capillaries are dilated and there is oedema in the corium and overlying epidermis. In the tissue spaces may be detected small irregular pieces of fungus, spore-like bodies, and, occasionally, radiating forms. Sections of the skin in the neighbourhood of fistulae where disintegration has set in show small masses of ray fungus surrounded by cells, many of which are of the plasma-cell type, with here and there large connective tissue cells which are swollen and vacuolated prior to breaking up.

Mycology.-The fungus of actinomycosis belongs to the streptothrix family and there are probably sereral varieties of it, the most common being the Actinomyces bovis and the Streptothrix Israeli.

The Actinomyces boi's is the best known. During parasitic life it presents thin mycelial filaments, which are non-septate, often branch, and terminate in peculiar club-like bodies arranged in a radial manner, hence the old name of ray fungus. It is these radial clumps which form the granules in the discharge. The exact nature of the club-shaped thickenings is a matter of dispute; some observers regard them as organs of fructification, for they are only found in actively growing fungus, while others assert that they are degenerative formations.

The fungus grows readily on the ordinary culture media. On glycerinated agar it appears as granular, bright-yellow colonies which tend to coalesce. On microscopical examination of the culture, the filaments are found to have undergone fragmentation into bacillary forms. The fungus is Gram-positive, but is not acid-fast. Experimental inoculation with the cultures on animals has not been successful. 
The Streptothrix Israeli is similar to the Actinomyces bovis morphologically, but differs from it in being anaerobic. It has been successfully inoculated in man from a culture and, in consequence, it has been asserted that it is the cause of the disease and that the Actinomyces bovis is a contamination.

Diagnosis.-The clinical appearances as a rule are sufficiently characteristic to suggest actinomycosis to anyone who has seen the disease. The dusky red, or purplish, infiltrated skin, the nodules, the sinuses with their sero-purulent discharge and lemon-yellow granules, and the unhealthy ulcerations, form a clinical picture which is easily recognised. The occurrence of the lesion about the lower jaw and neck, or over the vertebrae, especially in anyone working with horses or cattle or on a farm, should arouse suspicion.

In every case the final proof rests with the demonstration of the fungus. This is usually a simple matter as the granules in the discharge are composed of clumps of mycelium. In doubtful cases, where an examination of the discharge has given negative results, a biopsy should be made at the growing edge and the sections examined for the fungus.

The affections with which it is most liable to be confounded are breaking-down syphilitic gummata, and the oedematous tuberculous skin over softening glands or diseased bone.

Prognosis. - When the disease is the result of a local inoculation with the fungus and confined to the skin and subcutaneous tissue, the prognosis is excellent and the condition is readily amenable to treatment. When it is due to extension from diseased bone the outlook is more serious. In cases where the fungus has penetrated into an internal organ, grave consequences, and even deatlı, may supervene.

Treatment.-The treatment is both general and local. Internally, iodide of potassium has been found to be of value and to have a selective action, causing an absorption of the indurated masses in the same way as it does syphilitic gummata. It is necessary to prescribe it in large doses, as much as 15 grains gradually increased to 30 grains being given three times a day according to the toleration of the patient. By this means, in certain cases, amelioration if not complete cure may be effected. Good results have been reported from injections of solutions of the iodides in cases which had resisted the employment of the drug by the mouth, several injections of a $\mathrm{I} \%$ solution of the iodide of potassium or sodium being given into the diseased area at intervals of about a week. Vaccine treatment has also been tried and improvement reported from its use. The vaccine is made from the ray fungus and given in doses of so many millicn fragments.

Locally, the treatment is on general antiseptic principles. The sinuses should be opened up, scraped with a curette, and washed out with carbolic lotion or dusted with iodoform. Where the lesion is small and definitely localised it may be excised, but as a rule this is impracticable. Exposure to the X-rays is of value in healing the 
ulcerations, relieving pain, and, to some extent, in reducing the induration, a pastille dose being given and repeated in three weeks if necessary.

A judicious combination of the above methods of treatment gives the most rapid result.

When the disease occurs about the jaw, the mouth should be examined for carious teeth and these should be remored.

Nore.-In addition to the two fungi which are responsible for Actinomycosis cutis, there are several varieties of streptotriches which may cause subcutaneous abscesses, or lesions in the skin of a somewhat similar type, such as the following:

Streptothrix hominis (Foulerton) found in multiple abscesses in the neck of a woman.

Streptothrix asteroides (Paxton) found in mycetoma and in the pus from an abscess in the neck.

Nocardia Thibiergei (Pinoy and Ravaut) found in a case of a peculiar nodular affection of the subcutaneous tissue.

Nocardia convoluta (Chalmers and Christopherson) found in Actinomycosis in man in the Sudan.

\section{REFERENCES}

Chalmers, A. \& Christopherson, J. B. A Sudanese actinomycosis (Ann. Trop. Med. \& Parisitology, Sept. I9I6).

DEAs, C. W. A case of actinomycosis successfully treated by vaccine (Brit. Med. Journ., Jan. 20th, I9I7, p. 82).

Dreyfes, W. Primary actinomycosis of skin (Munch. Med. Woch., Dec. 29th, 1903, p. 2291).

Foulertox. Case of multiple abscesses due to Streptothrix hominis (Brit. Journ. Derm., I 899 , xi. p. 4I 7 ).

Legrais, II. Actinomycosis of face (Ann. de Derm. et de Syph., Oct. I 89 I)

Merian, L. Primary actinomycosis of the skin (Derm. Wochens., Jan. I $3^{\text {th, }}$ I912).

Prixgle, J. J. Extensive case in a boy" (Trans. Roy. Med. \& Chirurg. SOC., I 894 ).

RyDigier. Injections of iodide of potassium in actinomycosis (Wien. Klin. Wochens., Sept. I 2 th, I 895$)$.

Séchard, A. Actinomycosis of finger (La Presse Méd., August Ijth, I903).

\section{SPOROTRICHOSIS CUTIS}

Introductory.-The name Sporotrichosis cutis has been applied to an affection of the shin caused by a fungus of the genus sporothrix. In ISgS Schenk first drew attention to it in America in a paper entitled "Refractory Subcutaneous Abscesses caused by a Fungus possibly related to Sporotricha." Since then so many cases have been reported, chiefly in America and France, that the affection would seem to be by no means rare though liable to escape notice on account of the similarity of its lesions to other better known conditions. Though Schenk referred to the causal fungus in his original case and recognised it as a sporothrix it was left to De Beurmann and Gougerot to describe minutely its morphological and cultural characteristics. 
Description.-There are two chief types of sporotrichosis, namely:

I. A type in which the skin affection follows an abrasion or injury, such as a cut in the finger, through which the fungus has gained entrance. This results in a chronic lymphangitis spreading along the lymphatic vessels connected with the site of the injury. In association with it small cutaneous abscesses appear, which begin as hard nodules, run an indolent course, and gradually soften and break down to form small abscesses surrounded by a red areola and sometimes suggesting actinomycosis.

2. The second type, of which a considerable number of cases have been reported in France, consists of multiple cutaneous and subcutaneous abscesses distributed anywhere on the trunk and limbs. These begin as nodules, sometimes no larger than a pea, which slowly increase and may reach the size of a tangerine orange. At first they are hard like gummata and the skin over them unaltered in colour, but gradually softening takes place and they assume a reddish or livid appearance and break down with the formation of a fistulous opening from which a yellow viscid homogeneous pus is discharged. Though these abscesses may be extremely numerous the general health of the patient is not interfered with as a rule. The lymphatic glands in the neighbourhood are not enlarged. (Fig. I46.)

In time the abscess may heal and be replaced by an irregular cheloidal scar. More frequently, the surface breaks down and a punched-out or crateriform ulcer is formed, the border of which is sometimes indurated, of a reddish-purple tinge, and studded with miliary abscesses, and the base of which is covered with pus which tends to dry up to form a crust. The lesion may simulate a superficial breaking-down gummatous syphilide or show proliferative changes with the formation of vegetations suggesting a warty tuberculous lesion.

In addition to the slin, deeper structures may be involved and abscesses may appear among the muscles, in the synovial membranes, about the periosteum, and even in the bones. It may affect the mucous membranes, especially of the larynx and pharynx, giving rise to superficial ulcerations, which are unaccompanied by the deep destructive changes which occur in tertiary syphilis, and it has been known to attack tne eye producing conjunctivitis and iritis.

In the human subject it does not appear to involve the internal organs, though it has been known to do so in the rat.

There are no subjective symptoms associated with it.

Histopathology.-The histological lesion is the result of the reaction of the skin to the sporothrix in situ and consists of a diffuse granuloma, which is in no way characteristic, situated in the corium and subcutaneous tissue, the epidermis only being involved secondarily when necrotic changes have supervened. The granuloma is composed of leucocytes, comnective tissue cells, and sometimes giant-cells. The fungus can be detected occasionally within the giant-cells or the poly- 


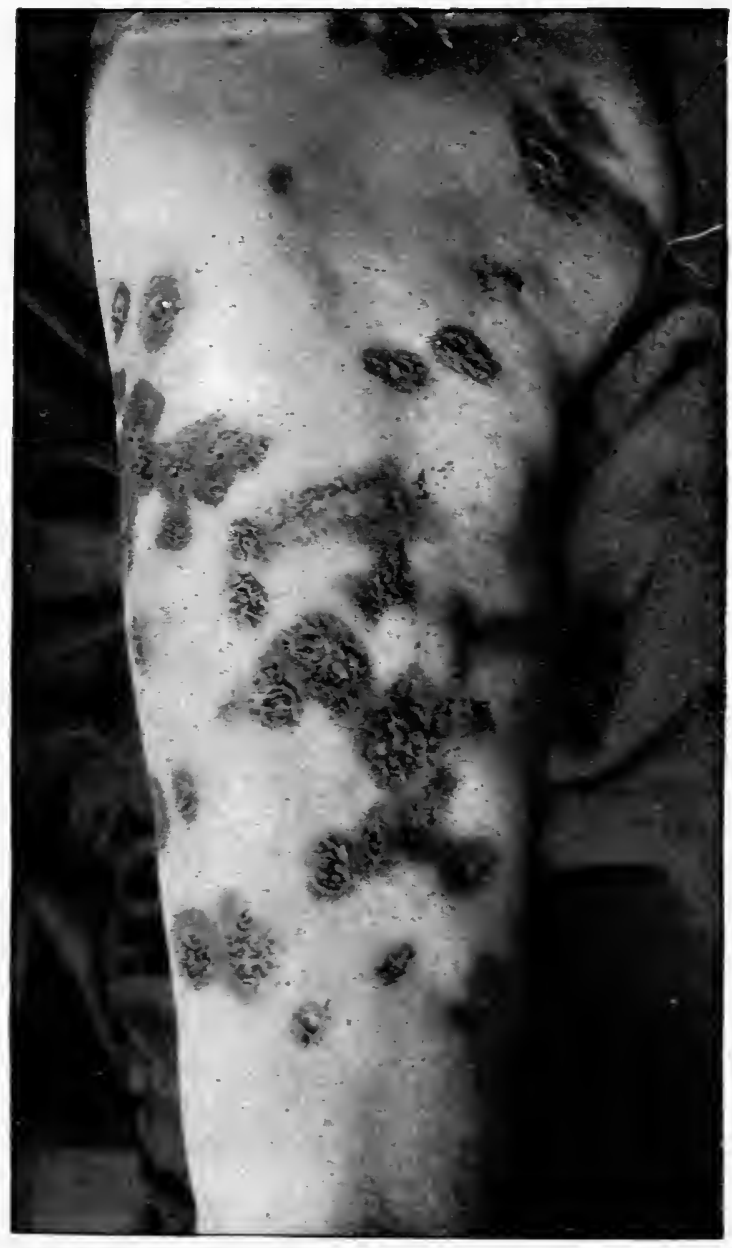

FIG. I 千6.-Sporotrichosis of Leg.

Shows multiple ulcerating gummata, deeply pigmented areas the shin over which is cribriform and covers a pocket containing pus which is discharged through the numerous small openings.

(Dr. H. G. Adamson's case. Brit. Journ. Derm., Igr3.)

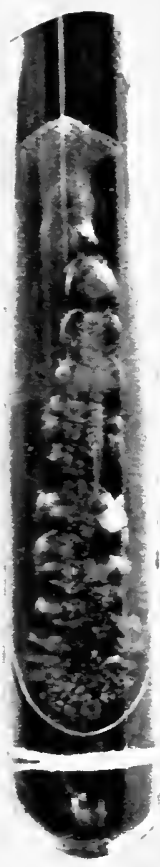

FIG. I4--Sporotrichosis. Culture of the fungus on proof medium.

(Aiter Dr. H. G. Adamson, Brit. Journ. Derm., :grr.)

[To face s. +34 . 

morphonuclear leucocytes, or free in the tissue spaces, in the form of small oblong rods from 2 to $5 \mu$ in length and $I$ to $3 \mu$ in width. According to the virulence of the fungus and the resisting power of the tissue several different types of cellular infiltration occur of which De Beurmann and Gougerot have distinguished three, namely :

(I) An epithelioid trpe in which giant-cells are present and the granuloma suggests tuberculosis; (2) a lympho-connective tissue type which is more like a syphilitic infiltration; (3) a polymorphonuclear type suggesting ecthyma.

Mycology.-The causal fungus is the Sporothrix Beurmanni, variations of which have been isolated by different observers, such as the S. Schenki found by Schenk in North America, the S. indicum found by Castellani in Ceylon, and the S. Jeanselmei. These differ slightly in the temperature in which ther grow, in their power of liquefring gelatine, and in other minor details.

The S. Beurmanni is cultivated readily on the ordinary culture media and especially well on Sabouraud's peptone-glucose-agar. It is aerobic and grows best with the cap off the tube at a temperature of about $22^{\circ} \mathrm{C}$., though some strains prefer a temperature of about $37^{\circ} \mathrm{C}$. The growth appears about the fourth day as a small white point about the size of a pin's head, surrounded by an areola formed by a delicate fringe, this increases and becomes convoluted and pigmented, and assumes a greyish-brown or blackish tinge but, after a time, tends to become white again from pleomorphism. As a rule it does not liquefy gelatine. (Fig. I47.)

In the hanging-drop, the fungus presents a delicate mycelium and clusters of spores. The mycelial filaments are about I $\mu$ thick, irregularly septate, and give off lateral branches. Connected with them are spores measuring about $2 \times 3 \mu$, either oroid in shape and arranged in small clusters of three to ten or more attached to the filaments by a lateral stalk, or pyriform and attached directly. Sometimes they are so numerous and closely aggregated as to give the appearance of a rope of spores. Permanent preparations can be made of the mycelium and spores by staining them with the ordinary aniline dyes.

The fungus is pathogenic in certain of the lower animals and recoverable from experimental lesions. It has been successfully inoculated in the rat, mouse, monkey, cat, and guinea-pig, both subcutaneously and into the peritoneum. Of these the rat has been found to be the most susceptible animal and the guinea-pig the least. Inoculated in the rat it produces widely spread subcutaneous gummatous lesions, orchitis-a symptom which has been regarded as of diagnostic value-and disease of the kidneys, liver, lungs, etc. The affection has been met with also in the dog and mule, and the fungus has been recovered in epizootic lymphangitis of the horse, a condition like glanders. The fungus is known to be parasitic on caterpillars, larvae of flies, etc., and saprophỵtic on regetable matter, such as lettuce leaves. Its life history is not fully understood. 
Serum reactions.-Widal found that the serum of an infected individual agglutinated an emulsion of sporothrix, and that a positive complement fixation was obtained in sporothrix by using a mixture of spores and mycelium as antigen.

Etiology.-The disease may occur in anyone, but is most common in adult males as they are most likely to be exposed to infection. The fungus usually gains entrance through an abrasion of the skin, such as a prick or scratch, and may be transmitted by pus from an infected animal, or in rare instances by a caterpillar or the larva of some fly, or even by contact with vegetable matter on which it was growing. It is possible that the infection can take place also through the alimentary tract, as the disease has been produced in a young guinea-pig by feeding it with milk containing the fungus.

Diagnosis.-The recognition of this affection may present the greatest difficulty and as a rule a firm diagnosis can only be made by the discovery of the fungus. As the fungus is sparsely distributed considerable perseverance may be required to find it in the pus or juice from incised nodules and it is usually easier to demonstrate it by cultivation. For this purpose the inoculation should be made from a non-ulcerated lesion which should be incised and the juice spread on a peptone-glucose-agar tube. In a well-marked case in which the nodules and abscesses are associated with chronic lymphangitis, and in which it succeeds an injury in an otherwise healthy individual, sporotrichosis is strongly suggested, but in the cases with widely spread nodules and subcutaneous abscesses, the resemblance to syphilis or tuberculosis may be so close that a diagnosis from the naked-eye appearancè is impossible.

Prognosis.-The lesions show no tendency to spontaneous cure but disappear rapidly under treatment with iodides.

Treatment.- The iodides are usually prescribed in the form of the iodide of potassium in large doses, up to one dram three time a day, and should be continued for some time after the lesions have apparently healed. To augment the action of the iodine internally the broken lesions should be swabbed with tincture of iodine, dressed with vasogen iodine ( $\mathrm{I}$ in $\mathrm{IO}$ ), or collosol iodine oil, or dusted with iodol powder. Surgical procedures, such as scraping the ulcerations, are not recommended.

\section{REFERENCES}

Adanson, H. G. Sporotrichosis: resumé of literature (Brit. Journ. Derm., rgo8, xx. p. 206).

- A case of sporotrichosis (Idem, I9 II, xxiii. p. 239).

- Sporotrichosis of the disseminated gumma type, etc. (Idem, rgr3, xxv. p. 33).

_- Sporotrichosis simulating blastomycosis (Idem, I9I3, xxv. p. 60).

BLoch, B. Sporotrichosis (Beihefte zu med. Krlin., I909, iv. p. I79).

De Beurmaxi \& Gougerot. Sporotrichosis (Ann. de Derm. et de Syph. 1907 , iv. p. 497). 



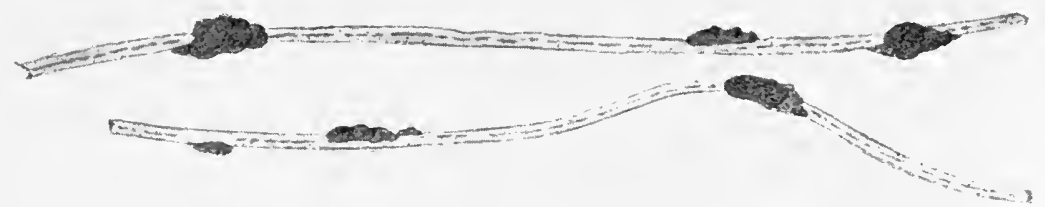

FIG. I 8. -Trichosporosis Nodosa.

J. M. H. M. del. Hairs from aboriginal Indian girl.

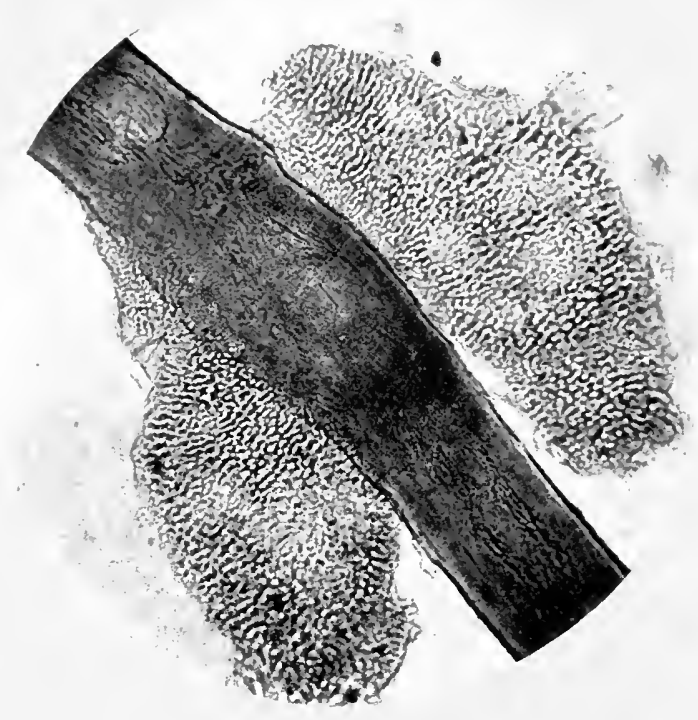

FIG. I 49.-Trichosporosis Nodosa.

Masses of spores forming concretions outside the cuticle of the hair.

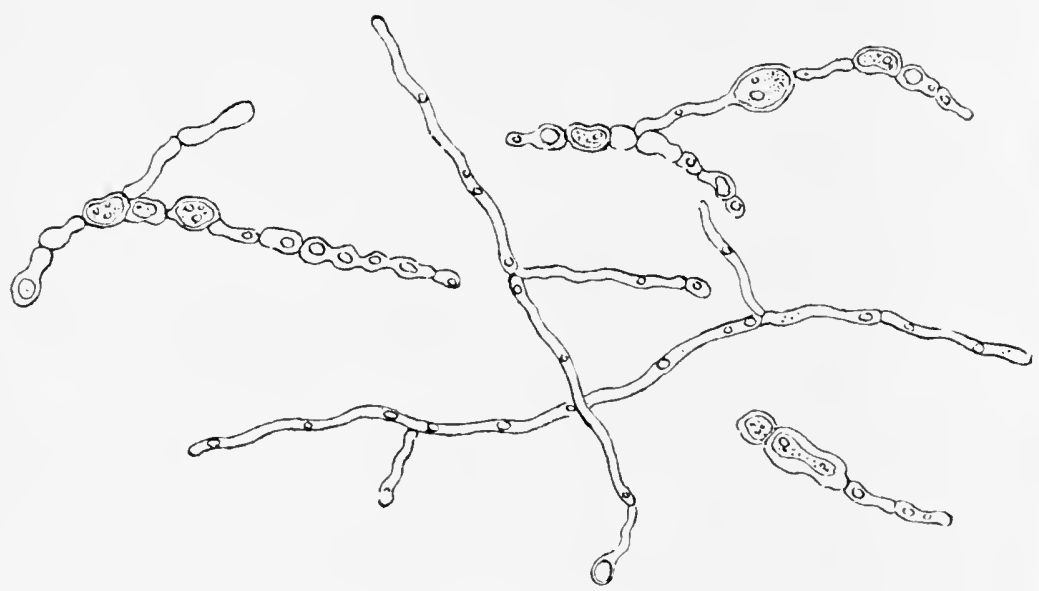

FIG. 151.-Trichosporosis Nodosa.

Shows branching mycelium, cndoconidia, from culture.

[To face $p .4 .37$. 
De Betrmanx \& Govgerot. Comparaison du Sporotrichum Jeanselmei et des Sporotrichum Voisins (Bull. Soc. Méd. des Hôpit. de Paris, Dec. 23rd, I9IO).

De Beurmani, Govgerot, \& Valcher. Sporotrichosis of the rat (Idem, I908, xxiv. p. 7 I8).

Hektoes \& Perkiss. Subcutaneous abscesses due to Sporothrix Schenki (Journ. Exp. Med., N.Y., I900, v. p. 77).

Jeanselme, E. \& Chevallier, P. Sporotrichose à foyers multiple produit par un nouveau type de sporotrichum (Presse Méd. d'Egypte, Oct. I9IO).

Scherk. Subcutaneous abscesses caused by a fungus possibly related to Sporotricha (Johns Hopkins Hosp. Bull., r 898, ix. p. 286).

Sutrox, R. L. Sporotrichosis in America (Journ. Amer. Med. Ass., I9ro, lv. p. 22I3).

WAlker, N. \& Ritchie, J. A case of sporotrichosis (Brit. Med. Journ., I9II, ii. p. I).

\section{TRICHOSPOROSIS}

The name Trichosporosis has been given to a peculiar affection of the hair characterised by the presence on the shaft of dark hard particles or nodules which are caused by a fungus known as the Trichosporon of which there are several varieties. Under this heading are included Piedra of South America or Trichosporosis tropica, Trichosporosis indica, Piedra nostras (Unna and Behrend), Tinea nodosa (Morris and Cheadle), Trichomycosis nodularis (Juhel-Renoy), and the affection described as "Chignon fungus" (Beigel and Fox).

These conditions though not identical are closely allied and probably due to different varieties of trichospora.

Trichosporosis has a wide distribution and although most common in tropical countries and chiefly in South America, has been found in temperate zones and occasionally in this country.

Trichosporosis tropica was first recognised in South America where it is known as piedra. It is most common in the valleys of Cauca in Columbia, but cases have been observed in other parts, such as British Guiana. It usually affects the hair of the head in native women, but has been observed also in the eyelashes, and on the hair of the scalp, moustache and beard in men. It is believed to be contagious. The nodules vary in size from minute specks, like particles of grit on the hair-shaft, up to small pins' heads. They tend to be oval in shape and are either situated at the side of the shaft like nits, or form concretions around it. In colour they are brownish-black and peculiarly hard, hence the name piedra (stone), and cause a crackling noise when the hair is combed. They are situated on the extra-follicular portion of the hair, vary in number from I to ro or more, and are either close to each other, or at irregular intervals. They are entirely outside the hair-shaft, but their presence eventually renders the hair so brittle that it is liable to fracture opposite them. (Fig. I48.)

On microscopical examination of affected hairs softened in Liquor potassae the nodules are found to consist of a mass of large spores of a 
fungus named by Behrend "Trichosporum giganteum." These spores have a yellowish appearance owing to diffuse colouring of the protoplasm and the presence in it of pigment granules. They have a strikingly regular arrangement which is especially marked in the smaller nodules. At the periphery of the nodule there is a palisade layer of oblong spores from Io to I2 $\mu$ in length, and situated at right angles to the hair-shaft, while next the hair the spores are polygonal in shape and are arranged in a mosaic or may present a

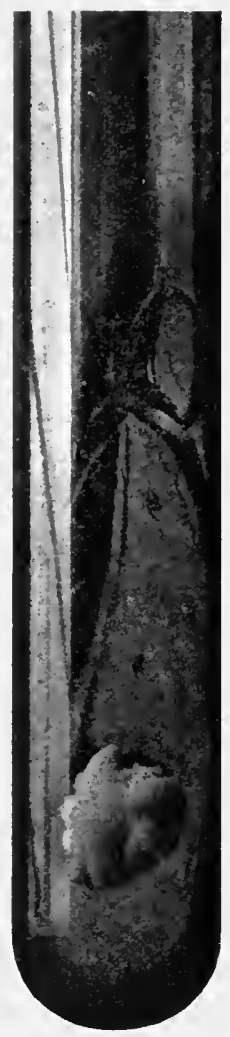

FIG. I 50.-Trichosporosis Nodosa. Culture on proof agar. peculiar fan-like grouping with rows of spores radiating from a central base. They appear to be held together in a mass by some viscid cement substance which forms a sort of capsule enclosing them. (Fig. I49.)

The fungus can be grown easily on the ordinary culture media. In broth it gives a white flocculent culture. On maltose agar the growth appears about the seventh day as a small white solid speck the size of a pin's head. This increases in the course of a week to the size of a split-pea and assumes a greyish-green tint, which spreads from the centre to the periphery, and presents a slightly powdery surface. In the course of another week the culture may reach half an inch in diameter and form a heaped-up roundish mass with a crenated nodular border and the greyish tinge becomes replaced by a brownish colour like milk chocolate. Microscopical preparations from the culture show irregularly branched septate mycelial filaments from I to $4 \mu$ in width, with spores at various intervals and endoconidia containing small spores and granules. (Figs. 150, 15I.)

The origin of this fungus is unknown. It has been suggested that it may be connected with the mucilaginous fluid resembling linseed oil with which the native women dress their hair. In British Guiana the natives attribute it to bathing in the milky or muddy water of certain rivers.

Piedra nostras was a name given by Unna and Behrend to an allied condition affecting the hairs of the moustache in Europeans. This was due in Behrend's case to a fungus with oval spores 3 to $4 \mu$ in length to which he gave the name of " Trichosporon ovoides," and which was easily grown on potato producing white colonies, the medium assuming a brownish tinge. The fungus in Unna's case was somewhat similar, grew as a yellow-white culture on agar and was named "T. ovale." In both cases the spores were considerably smaller than those of 'T. giganteum of piedra.

In the Tinea nodosa described in this country by Morris and Cheadle 
the hairs of the beard and moustache were affected, the concretions being made up of spores which were smaller than those of the trichophyta of ringworm.

Diagnosis.-Trichosporosis has to be distinguished from the more common disease of the hairs known as lepothrix, which affects the hairs of the axillae and occasionally the pubes. But in it the hairs instead of having a nodose appearance are thick, irregular, and ragged owing to the presence of concretions on the surface which are broken up into lobes or irregular masses.

Treatment.-Trichosporosis does not tend tc disappear spontaneously. The treatment is similar to that for the softening and remoral of nits but is unsatisfactory on account of the difficulty of remoring the concretions from the hairs. The hairs should first be saturated in some solvent, such as benzine, turpentine, paraffin, or petrol, and the scalp then thoroughly washed with soap and water. This procedure, however, seldom effects a cure and it is generally necessary to resort to the more drastic measures of shaving the scalp or cutting the hair short. Even then the disease is apt to recur unless some precautions are taken to destroy any spores which may be left about the scalp and, consequently, it is adrisable to apply daily some antiseptic, such as I in 8 carbol-glycerine, I in 2000 perchloride of mercury solution, or a parasiticidc ointment such as sulphur precipitate Io grns. salicylic acid Io grns. and vaselin to the oz.

\section{REFERENCES}

BEHREND. Trichomycosis nodosa (Berlin. Klin. Woch., I89o, p. f64).

Juhel-Rexoy. Trichomycosis nodosa (Ann. de Derm. et de Syph., ISgo, p. 760$)$.

MacLeod, J. II. H. Trichosporosis nodosa (Brit. Journ. Derm., IgI 2, xxiv. p. I3I).

Morris \& CheAdle. Tinea nodosa (Lancet, I879, i. p. I90. Trans. Path. Soc. Lond., I8 79 , xxx. p. $+4 \mathrm{I})$.

Pernet, G. Tinea nodosa (Brit. Journ. Derm., I900, xii. p. I32).

Trachsler, W. Piedra nostras (Monats. f. prakt. Derm., I896, xxii. p. I).

Uxis, P. G. Two cases of Piedra nostras (Lewin's Festschrift., Berlin, IS96; abs. Brit. Journ. Derm., I 896 , viii. p. I I I).

\section{ERYTHRASMA}

Erythrasma is a somewhat rare mycotic affection of the skin which is met with not only in Europe but has a wide distribution in tropical countries.

The eruption develops slowly and is characterised by well-defined reddish-yellow or brownish patches with a slightly scaly or greasy surface and sometimes so faint as to be scarcely noticeable. They are cituated chiefly about the crutch and the axillae, though they may 
occur in other moist regions such as the folds of the abdomen, the submammary folds in stout persons, or the flexures of the large joints. The condition is confined to the epidermis and shows no tendency to involve the hairs. The eruption is associated with itching which is worse when the patient becomes hot. It is slightly contagious and may be transmitted by direct infection or through the medium of watercloset seats.

It is due to a fungus of the genus Microsporoides, known as the Microsporon mimutissimum (Burchardt), which is exceedingly small and can only be seen in potash preparations with an oil immersion lens. It appears as a dense meshwork of delicate filaments about $0.7 \mu$ in thickness which tend to divide dichotomously and to break off in small

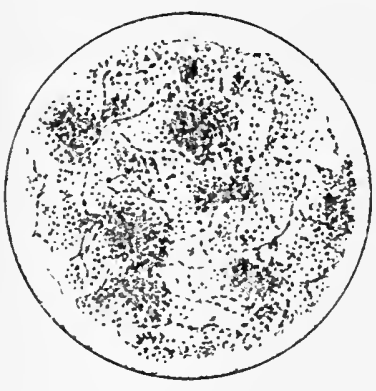

FIG. I 52.-Microsporon Minutissimum.

Nycelial threads branching dichotomously, irregularly segmented fila. ments, and spores in dense clusters. segments like spores or bacilli. So far it has not been successfully cultivated. (Fig. I52.)

Diagnosis.-The affections with which it is most liable to be confused are Pityriasis versicolor and Tinea cruris, but as a rule the diagnosis presents no real difficulty. From Pityriasis versicolor it is distinguished by the patches being brownish-yellow instead of fawn and showing no tendency to be circinate, and from Tinea cruris by its colour and by the absence of the raised festooned border. Should there be any doubt a microscopical examination will establish the diagnosis.

Treatment.-It is comparatively easily cured. The affected areas should be scrubbed with soap and water and a parasiticide remedy applied, such as an ointment containing $3 \%$ of salicylic acid and precipitate of sulphur rubbed in twice daily, or a lotion of tincture of iodine I part and $60 \%$ spirit 5 parts painted on daily until desquamation has occurred. The application of a lotion containing $\mathrm{I}$ in $\mathrm{Io00}$ permanganate of potash is also useful. In resistant cases a cure may be effected by applying a $6 \%$ solution of hyposulphite of soda on lint, covered with oiled silk, for about ten minutes, followed by painting with a $3 \%$ solution of tartaric acid; by this means nascent sulphur is formed which destroys the fungus.

\section{PITYRIASIS VERSICOLOR}

Syn.: Tinea versicolor; Dermatomycosis furfuracea; Fr., Pityriasis versicolore; Ger., Kleienflechte.

Definition: An affection due to a fungus named the Microsporon furfur, and characterised by irregular, fawn or brownish, finely desquamating macules which tend to run together to form diffuse sheets. 
Description.--The lesions appear first as small roundish or irregular macules, not infrequently situated at the orifices of the lanugo hairfollicles, which appear to be slightly raised above the surface by the presence of a fine branny or furfuraceous scaliness which is easily. scraped off and is best seen in individuals whose skins are naturally dry. They vary in colour from a pale fawn to a brownish or liver tint, hence the name "liver spots" once applied to them. The name Pityriasis versicolor is thus apt and graphic as it suggests the fine desquamation and the variations in tint of the lesions. The macules grow slowly and have usually been in existence for months before they are discovered. They coalesce to form irregular figures, which have been compared to islands and continents, or diffuse sheets involving large areas with well-defined borders which, in old lesions, are sometimes definitely raised. The patches are separated by paler areas of healthy skin which produces an appearance which at first sight might suggest leucodermia with intervening areas of melanodermia.

The affection is most common on the upper part of the trunk, especially the chest, and frequently begins about the folds of the axillae. From there it may extend upwards on the neck as far as the chin and downwards over the shoulders to the arms. It is not infrequent also about the flexures of the larger joints, in the anticubital and popliteal spaces, and especially about the genito-crural region. It occasionally occurs on the face and may extend over the beard region or beyond the hairy margin of the scalp, but it does not attack the actual hairs. In a case in a young woman under the observation of the writer, it was present in the form of brownish patches at the sides and front of the nose and around the mouth, and it has also been met with in that situation in a diffuse form suggesting chloasma. It is rare on the extremities but has been observed even on the palms and soles.

As a general rule the subjective symptoms are negligible and consist of slight itching, more marked when the patient becomes warm or perspires freely. Occasionally in especially delicate skins or where it occurs on a seborrhoic base, it produces inflammatory changes and the patches assume a pinkish tint, are slightly raised, and definitely itchy.

The disease is comparatively common in temperate zones. Similar affections occur in tropical countries and in the far east, such as the Tinea flava described by Castellani in Cerlon, which are not identical with the Pityriasis versicolor met with here but are due to allied fungi.

Mycology.-The affection is caused by a fungus of the genus Malassezia known as the Microsporon furfur. It is easily demonstrated by examining scrapings of the scales in Liquor potassae, where it is found to exist in great abundance. Microscopically it is made up of short mycelial threads, and spores clustered like bunches of grapes. The threads are I to $3 \mu$ thick, straight or sinuous, and septate at irregular intervals. The spores are roundish, vary from 2 to $6 \mu$ in diameter, and have a doubly-contoured capsule. Fructification may 
be observed occasionally in the form of sporophores containing conidia situated at the end of hyphae. It is doubtful if it has yet been cultivated on artificial media. In a few instances positive results have been claimed but these have not been verified. It has been inoculated experimentally in man and animals. (Fig. I53.)

Etiology.-It is transmitted from one person to another directly or indirectly, but it is only mildly contagious and necessitates close contact for a more or less prolonged period before infection can take place. It is unusual for it to be met with in two members of one family. It is

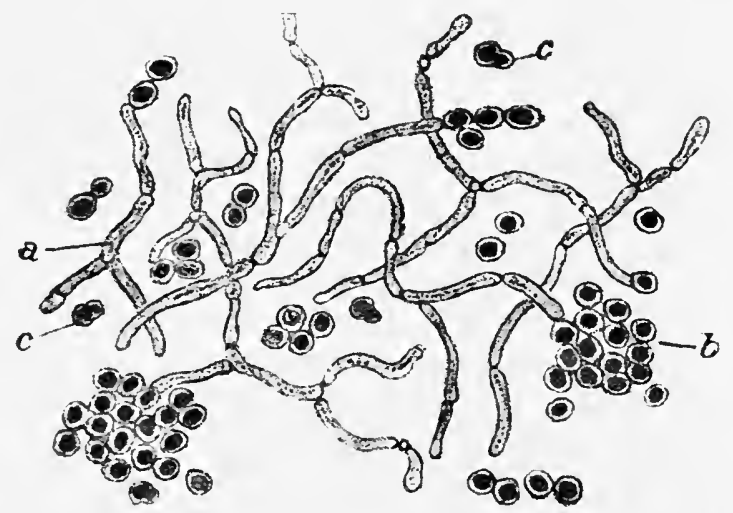

FIG. 153.-Microsporon Furfur.

J. M. H. M. del.

Preparation in Liquor Potassae.

a. Mycelium, branching and septate at irregular intervals. b. Spores grouped in clusters. c. Budding spore.

(From MacLeod's Pathology of the Skin.)

generally found in adults, is rare in childhood and old age, and is somewhat more common in males than females.

Warmth and moisture of the skin favour the growth of the fungus and it is not infrequent in phthisical subjects who sweat profusely at night, wear warm woollen or flannel underclothing during the day, and sometimes exhibit an unnecessary dread of soap and water. It is more active in summer than winter and may die down completely in cold weather and recur when it becomes warm.

Diagnosis.-The diagnosis rarely presents any marked difficulty. When it occurs about the neck it might suggest a macular syphilide but in Pityriasis versicolor the patches are larger and scaly. In rare instances on the face it has been confused with chloasma, and on the chest with seborrhoic dermatitis with which it may occur in symbiosis. A microscopical examination of scales will confirm the diagnosis.

Prognosis.-It is readily amenable to treatment but if left untreated is singularly persistent. After a time, when it has been allowed to extend over large areas, it may remain stationary but it shows no tendency to spontaneous cure.

Treatment.- The treatment is simple but to effect a cure it must be carried out thoroughly, as the fungus is liable to lurk about the natural folds and the orifices of the hair-follicles and to cause a recrudescence 
after it has been apparently cured. The affected areas should first be scrubbed with soft soap and hot water on a piece of flannel or soft nail brush and, if they are at all extensive, the scrubbing should be done in a warm bath by an attendant. After the washing the patches should be painted with tincture of iodine, which not only kills the fungus but, by staining it, brings out affected areas which were scarcely noticeable. Other effective methods are the application, for ten minutes at a time, of pieces of lint soaked in an aqueous solution of hyposulphite of soda ( $\mathrm{I}$ in 8 ) or the rubbing in of an ointment containing $3 \%$ of sulphur and salicylic acid. The treatment should be repeated daily until all trace of the affection has disappeared, which is usually in about a fortnight. Care must be taken that the remedies employed are not too active or they may set up dermatitis. If this occurs, a soothing calamine cream should be applied till the inflammation has subsided. After the disease is apparently cured it is advisable to continue treatment in a modified form for some time by the daily use of a medicated soap containing sulphur or balsam of Peru, or an occasional application of the ointment.

The underclothing worn by the patient should be baked or destroyed as it is liable to harbour the fungus and may be a source of re-infection.

\section{REFERENCES}

GotTherl. Pityriasis versicolor, on palm (Med. Rec., N.Y., I890, p. I5). Matzenauer, R. Cultivation of Microsporon furfur (Arch. f. Derm. u. Syph., I901, p. I63).

Powell, A. Tinea versicolor of face (Brit. Journ. Derm., I9oo, xii. p. I42).

SpIEtschka. Microsporon furfur (Arch. f. Derm. u. Syph., I896, xxxvii.).

\section{LEPOTHRIX}

Syn.: Trichomycosis nodosa (Patterson); Trichomycosis palmellina (Pick).

Lepothrix (from $\lambda \epsilon \pi i$ s a scale) is an affection of the hair in which the shaft is irregularly thickened by being more or less completely covered with a peculiar scaly concretion. It was originally described in England as early as I869 and has been observed in Germany and other parts of the Continent, and in Ceylon. It is comparatively common but is apt to be overlooked. It affects chiefly the hairs of the axillae where it is not infrequently associated with red sweat (see page 1052), but it may be met with also in the hairs about the genitalia. In these situations the skin is naturally moist and this seems to be an important factor in its development. To the naked eye the affected hairs are lustreless and have an irregular ragged or nodose appearance. They are singularly brittle and break on the slightest traction.

On microscopical examination with a low power the irregularity is found to be due to a concretion on the surface which may completely 
encompass the shaft. This is either continuous and broken up into lobes by fissures arranged like the barbs of a feather, or may occur at varying intervals in patches or in roundish masses sometimes as large as a pin's head. The concretions are most marked towards the free end of the hair and may be yellow, reddish, dark brown, or black. The hair-shaft itself is affected by the presence of the concretion which grows inwards, splitting up the fibres of the cortex, destroying the hair, and causing it to break with a brush-like fracture. (Fig. I54.)

The concretion appears to be composed largely of a cement substance secreted by some organism, which tends to become hard and chitinous and is somewhat of the same character as that which fixes the nit to the hair-shaft in pediculosis of the scalp.

It has to be distinguished from Trichorrhexis nodosa, an affection of the hair in which nodules due to splitting of the hair appear at

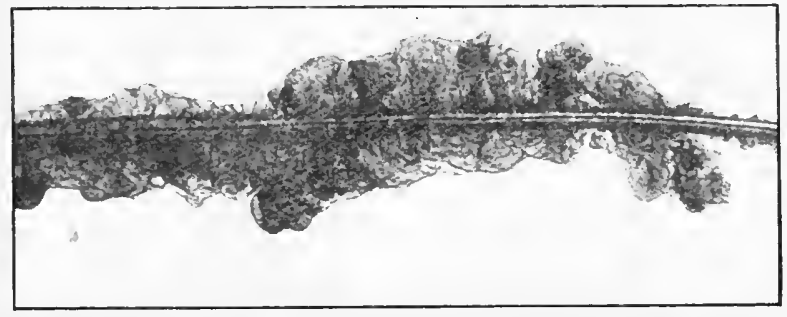

FiG. I 54.-Lepothrix.

Trichomycosis flava (Castellani).

(After Dr. Aldo Castellani. Brit. Journ. Derm., 1913.)

irregular intervals on the shaft, and from monilithrix in which the hair has a beaded appearance from partial atrophy of portions of the shaft.

Mycology.-The mycology has not been completely worked out. Various organisms have been described in connection with it, such as bacilli (Patterson and Payne), and a diplococcus (Sonnenberg and Eisner), but the most convincing were those found by Castellani in cases in Ceylon, namely, in the yellow variety a bacillus-like fungus which was probably a streptothrix or a microsporoid, and in the black and red varieties the same streptothrix growing in symbiosis with certain pigment-producing cocci-in the black variety with a coccus to which he gave the name " Micrococcus nigrescens " and in the red variety with a coccus resembling the "Micrococcus ruber" found in red sweating.

Under the heading of Trichomycosis capillitii, Winternitz has described an allied condition in the scalp of a boy, in the form of dark brown patches over which the hairs were thickened owing to the presence on them of an irregularly lobed concretion of a dark brown colour. From this he isolated a bacillus which grew as brownish colonies on agar.

Treatment.-This consists of shaving the hair and applying a lotion of resorcin I dram, spirit I oz., or perchloride of mercury solution I in Iooo, followed by an antiseptic dusting powder. 


\section{REFERENCES}

Castellani. Trichomycosis of axillae (Brit. Journ. Derm., I9I I, xxiii. p. $3+\mathrm{r}$ ).

Castellaxi. Trichomycosis flava, rubra \& nigra (Idem, 19I3, xxy. p. I.t).

Patterson, R. G. Trichomycosis nodosa (Idem, ISgo, ii. p. IOI).

Sorvexberg, E. Trichomycosis palmellina (Monats. f. prakt. Derm., I S98, xxvii, p. 538).

Wixteritiz, R. Trichomycosis capillitii (Arch. f. Derm. u. Sÿph., I 9o3, lxvi. p. 81). 


\section{CHAPTER XVIII}

\section{TUBERCULOSIS CUTIS}

\section{General Considerations}

Definition: The name Tuberculosis cutis is applied in a wide sense to all cutaneous lesions caused by the presence of the tubercle bacillus in the skin. To those which are known or thought to be due to the tuberculous toxin emanating from bacilli situated elsewhere in the tissues, the name toxi-tuberculides has been applied by Hallopeau.

Tuberculosis cutis is a protean condition, and as our knowledge of it becomes enlarged it is found that its manifestations are almost as multiform as those of syphilis. Whatever the type of clinical lesion, however, it is the result of the reaction of the skin to the bacillus in situ, and the differences in appearance are due to secondary considerations such as the degree of virulence and number of the bacilli, the situation of the lesions, and the resisting power of the individual.

For a cutaneous lesion to be established as tuberculous it is necessary that tubercle bacilli should be found in it, and that an inoculation from it should cause tuberculosis. It is not always easy, however, to obtain these proofs, for tubercle bacilli as a rule occur sparsely in the skin and may elude the most persevering search, while inoculation experiments are not absolutely certain as negative results may be obtained from tuberculous tissue where only a few bacilli are present and their virulence low. On this account the diagnosis may have to depend on certain less definite criteria, which are more in the nature of presumptive evidence than final proof yet when considered together and theirvalue properlyestimated are of undoubted assistance. Of these the most important are the clinical appearances of the lesions, their histological architecture, the fact that they react to injections of tuberculin, and the existence or evidence of past existence of tuberculosis in organs other than the skin. It will be advisable to consider these in detail in order to gauge their relative value and to avoid the error of placing too much reliance upon any one of them.

Clinical appearances.-The clinical appearances though usually typical are by no means wholly reliable for diagnostic purposes as they may be closely simulated by other granulomatous conditions, such as 
certain late syphilides, blastomycetic dermatitis and actinomycosis. Still the definite induration of the majority of the lesions, their slow evolution, reddish-brown tinge, and tendency to ulcerate on the one hand and to form warty growths on the other, are at least valuable hints towards the correct diagnosis.

Histopathology.-The histopatholcgical architecture may be an aid to diagnosis, but is not infallible, for similar microscopical appearances may be found in other cutaneous lesions, such as tertiary syphilides, and chronic nodular lesions of Acne vulgaris, while not infrequently in tuberculous lesions the characteristic histology may be absent.

Association with tuberculosis in organs other than the skin.-The association of some definite signs of tuberculosis elsewhere, such as in the glands, joints, or viscera, is of value as presumptive evidence that a cutaneous lesion is tuberculous, but it must on no account be relied upon absolutely as tuberculosis in some form or other is very common and the presence of the skin affection may be a mere coincidence and in no way etiologically connected with it.

General and local reactions to tuberculin as an aid to diagnosis.In the absence of obvious tuberculous stigmata the recognition of a patient as tuberculous may be effected experimentally by an injection of tuberculin. For diagnostic purposes the old tuberculin of Koch is employed, the newer tuberculins being less reliable. This is a concentrated solution of the extra-cellular toxins of the tubercle bacillus in glycerine. .00 I c.c. is injected in the adult, if no reaction takes place, :002 c.c. is injected two days later, and if there be still no reaction a further injection of .oof c.c. may be given. If no reaction follows the third injection the patient may be regarded as free from tuberculosis. The characteristic reaction consists of a rise of temperature of 2 or $3^{\circ} \mathrm{F}$. accompanied by a feeling of malaise, usually occurs in about 12 hours, and subsides gradually: In addition to the general reaction any tuberculous skin-lesion usually undergoes a local reaction, becoming inflamed, swollen, and tender, and sometimes bullous and ulcerated. This begins as a rule within 6 hours of the injection and subsides in about $4 S$ hours, appearing and disappearing simultaneously with the general reaction. These reactions are fairly reliable especially when a negative result is obtained, positive results being less certain as affections such as leprosy may react to the injections. A general reaction may occur without a local one and vice zersa.

Occasionally the injection in a tuberculous subject causes an eruption of Lichen scrofulosorum (see Tuberculides).

A great disadvantage of the method, and one which may amount to actual danger, is the possibility of its rendering active some latent focus of tuberculosis.

Local tuberculin reactions.-There are certain local tuberculin reactions which are of assistance in the diagnosis and are free from the danger of rendering active some internal focus of the disease. Of these 
the most frequently employed are: (a) the vaccination method (Von Pirquet), (b) the inunction method (Moró), (c) the ophthalmic instillation method (Wolff-Esner and Calmette).

The Vaccination Method of Von Pirquet is as follows:

The surface of the skin to be vaccinated is first washed with soap and water and rubbed with ether. A couple of drops of the old tuberculin are then put on the cleansed surface and vaccinated into the skin by superficial scarification. If the patient be tuberculous the scarified part becomes inflamed in 24 to 48 hours, and presents red papules or vesicles at the site of the punctures. Two days later the inflammation subsides leaving slight pigmentation. As a general rule the severity of the reaction varies inversely with that of the disease. The reaction is fairly reliable when a positive result is obtained, but a negative result is inconclusive as the latter occurs in cases of advanced internal tuberculosis. It has the great advantage over the injection and the ophthalmic methods of being harmless to the patient.

The Imunction Method of Moro consists of rubbing into the skin an ointment composed of equal parts of Koch's old tuberculin and anhydrous lanolin. A suitable site for the inunction is the side of the chest in the mid-axillary line, as there the hair-follicles which might be irritated by the procedure are small. An area of skin about 2 inches in diameter is cleansed and the grease removed by ether. About a gram of ointment is then energetically rubbed in by the patient's finger, which has been previously cleansed in the same manner. The inuncted area is covered by a piece of lint held in position by strips of adhesive plaster. The local reaction if positive develops in from 24 to 48 hours, and consists of the appearance in the inuncted area of a number of dusky red papules about the size of a pin's head on an erythematous base. According to the number of papules and the degree of the erythema the reaction is characterised as mild, medium, or strong. The papules persist for a few days then fade leaving a slight, transient pigmentation. Though the test is not absolutely reliable, a positive reaction is fairly conclusive that tuberculosis in some form or other is present, but a negative reaction is of little value in precluding it.

The Ophthalmic Method of Calmette and Wolff-Esner is carried out as follows: One drop of an aseptic glycerine-free solution of tuberculin ( $\mathrm{I}$ in IOO) is instilled into the conjunctival sac of one eye close to the internal canthus. In a tuberculous patient this produces a definite reaction which begins in 3 to 6 loours, reaches its maximum in 14 hours, and usually disappears in 36 hours. The reaction consists of reddening of the lower palpebral conjunctiva and caruncle. Should the test be negative a second instillation may be made in the other eye, but it should not be repeated in the same eye for at least a month. The test is fairly reliable but not completely so, as advanced cases of tuberculous disease may give a negative result, and a positive reaction has been obtained in other affections such as secondary syphilis. The chief disadvantage is that it may set up a 
severe reaction associated with marked photophobia and pain persisting for days or even weeks. It should on no account be employed if there be any sign of ocular disease as the inoculation would aggravate it and a reaction might occur apart from tuberculosis.

\section{Classification of the Cutaneous Manifestations of TUBERCULOSIS}

(1) Cutaneous Lesions definitely proved to be due to the presence of the Tubercle bacillus in the Skin.

Lupus rulgaris (including Tuberculosis verrucosa cutis).

Scrofulodermia (including Scrofulous gummiata and Tuberculous lymphangitis).

Miliary tuberculosis of the skin.

Erythema induratum of Bazin.

Tuberculous ulcers.

(2) Cutaneous Lesions supposed to be due to Toxins from Tubercle Bacilli situated in some Tuberculous Focus at a distance from the Skin.

Tubercuilides.

Lichen scrofulosorum.

Acne scrofulosorum or Papulo-necrotic tuberculides. (Acnitis and Folliclis.)

(3) Doubtful Toxic Tuberculides.

Lupus erythematosus.

Pityriasis rubra gravis of Hebra.

Angiokeratoma.

Psoriasiform tuberculide.

(A psoriasiform tuberculide has been described, but is probably. psoriasis occurring in a tuberculous subject.)

Distribution.-.Tuberculosis of the skin has a universal distribution prevailing in latitudes as far removed as those of Brazil and Greenland, and it affects all races though the negro seems to be particularly susceptible to it. In certain countries its occurrence is rare, for example in Egypt, the Straits Settlements, and the Malay States. In warm climates Lupus rulgaris is seldom found, though scrofulodermia is comparatively common.

CUTANEOUS AFFECTIONS DEFINITELY PROYED TO BE DLE TO THE PRESENCE OF TUBERCLE BACILLI IX THE SKIX

\section{LUPUS VULGARIS}

Syn. : Lupus; Fr., Lupus vulgaire; Lupus tuberculeux; Scrofulide tukerculeuse; Ger., Fressende Flechte.

Definition: A chronic affection of the skin due to the tubercle bacillus and characterised by reddish-brown translucent papules or 
patches with a smooth, scaly, or warty surface, and a tendency to ulceration and scarring.

It is the most common form of Tuberculosis cutis and is of frequent occurrence among the poorer classes in this country. It is essentially a disease of temperate climates and is rare in the tropics.

Description.-The initial lesion consists of a pin-head sized brownish semi-translucent macule or slightly raised papule. On casual examination it might be overlooked or regarded as a simple inflammatory lesion, but if the blood be pressed out of it by a diascope a brown stain is left which no amount of pressure will obliterate and which indicates the existence of a definite cellular deposit in the underlying corium. The papule gradually increases until it reaches the size of a split-pea, when it is found to be looser in texture, smoother and softer than the surrounding skin, and of a gelatinous consistence, assuming an appearance which has been graphically described as an " apple-jelly nodule." On the lower extremities instead of being brown it may present a livid purplish tinge. On reaching this size the nodule rernains stationary for a certain period and then tends to break down, forming a shallow ulcer surrounded by a reddish-brown border, the floor of which is covered with a semi-purulent secretion or watery exudation which dries to form a crust. As a rule several lesions occur, either in a small group, forming satellites around a larger patch, or widely separated from each other (L. nodularis). The most common site for them is the face but they may be present in other parts of the skin. Occasionally they occur at the mouth of the hair-follicles, but as a rule are independent of them. By the spreading and coalescence of the nodules irregular patches result which, though they increase very slowly, if unrestricted by treatment may in time involve large areas of the cutaneous surface.

A typical patch of Lupus vulgaris is more or less clearly demarcated and, though in its early stages it is generally roundish or oval in shape, when it reaches several inches in diameter it usually becomes irregular in outline. The centre of the patch may undergo retrogressive changes and become ulcerated, crusted, and, on healing, transformed into an uneven cicatrix, atrophic in one case, markedly fibrous and even cheloidal in another, and frequently presenting brownish-red foci of disease irregularly distributed over it. Occasionally the resultant scar is smooth, shiny, reddish-brown in colour, and has a number of dilated blood-vessels ramifying over its surface. The patches extend peripherally by the development of new nodules beyond the margin, which coalesce to form a definite raised border of a brownish-red or yellowish tint. The tendency to ulceration and necrosis is much more marked in some cases than in others; where it is a pronounced feature the old name Lupus exedens, signifying that the diseased tissue is eaten away, is appropriate. More rarely an exfoliative type of retrogression may occur in which the patch becomes scaly, degenerates, and is gradually absorbed. (Irig. I55.) 


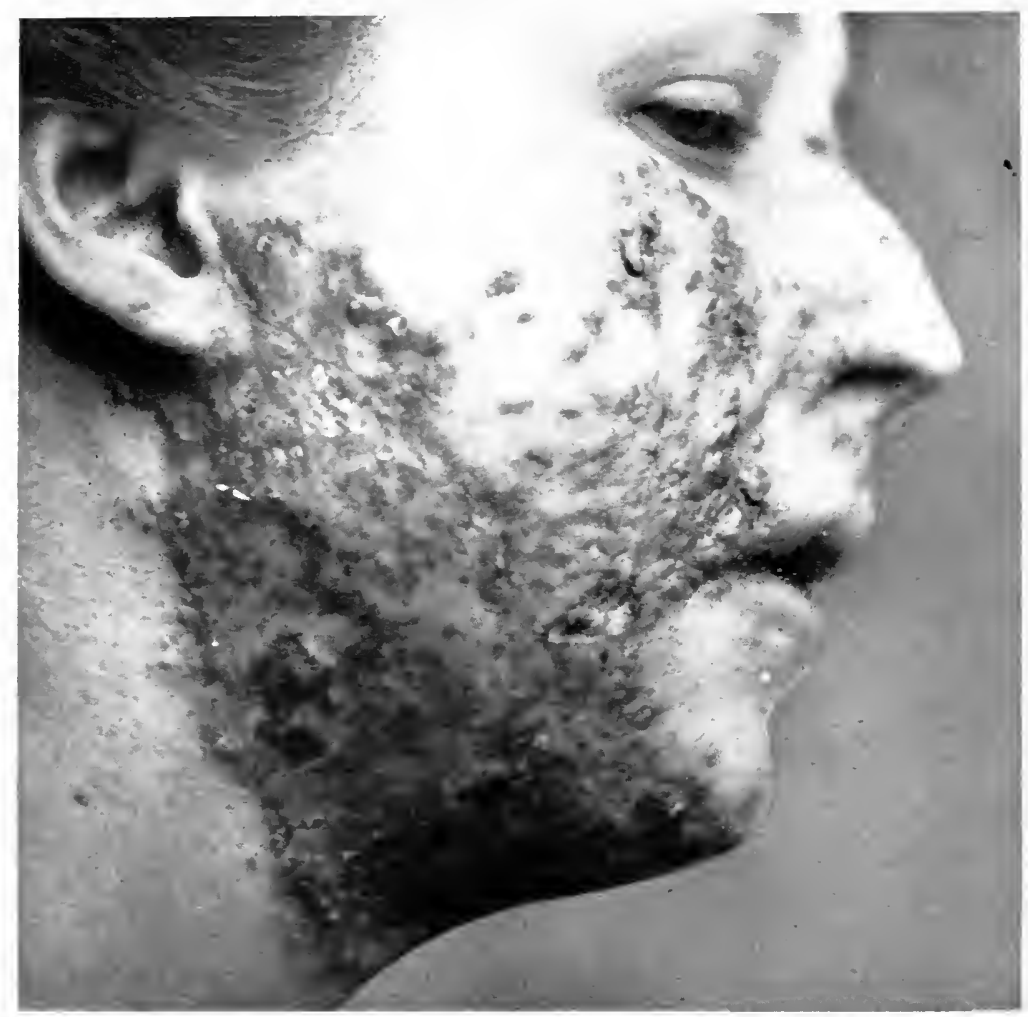

FIG. I55.-Lupus Vulgaris with superficial ulceration. 

Lupus vulgaris is most common on the exposed parts of the body; chiefly on the face. In a large number of cases it begins about the nasal orifice, especially when it commences in adult life, while another not infrequent site is about the inner canthus of the eye and over the tear duct, the disease having spread up from the inside of the nose. It rarely affects the scalp. The next most common situations are the backs of the hands, arms, legs, and buttocks. It is comparatively infrequent on the trunk and rare on the palms, soles, or genitals, unless by extension from the neighbouring skin. The mucous membranes, especially that of the nose, appear to be attacked in about $50 \%$ of the cases. (Fig. 156 .)

The amount of destruction caused by it varies greatly in different cases and depends on the degree of ulceration. It is most marked in scrofulous individuals or where the disease attacks mucous orifices. It is especially severe where the skin about the nasal orifice is involved, for there the lesions tend to become ulcerated, crusted, and covered with granulations which mask the destruction going on beneath, and end by destroying all the soft parts and even the cartilages; when healing does take place from treatment profound disfigurement is the usual result, with destruction of the greater portion of the soft

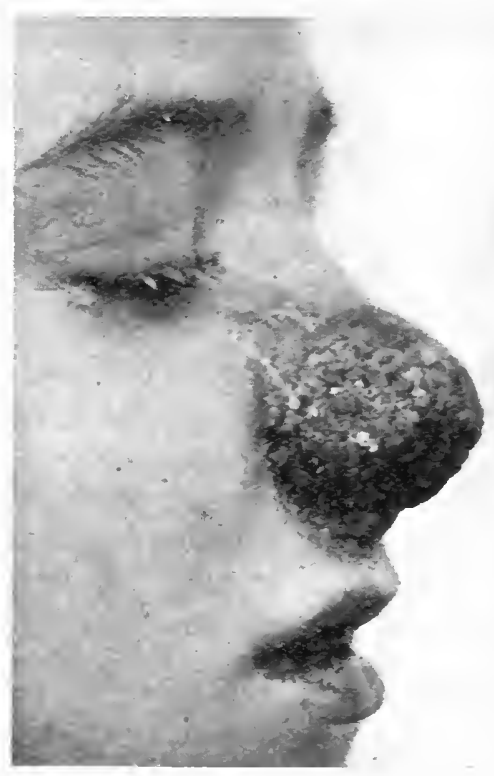

FIG. I56.-Lupus Vulgaris of Nose affecting skin and mucosa.

tissues leaving a thin eroded margin surrounding widely dilated nostrils, or with marked constriction of the orifices. When the disease attacks the lips they become swollen, oedematous, and covered with exuberant granulations and crusts, and healing is followed by cicatricial puckering, atrophic thinning, and disfiguring contraction of the opening of the mouth. When the lower eyelids are implicated the subsequent scarring is apt to result in more or less marked ectropion. When patches occur over the joints the resultant scar if fibrous and contracted may interfere with movements.

Lupus vulgaris is often associated with other manifestations of tuberculosis, such as scrofulous glands, dactylitis, tuberculous gummata, etc., or with internal tuberculosis of the lungs or other viscera.

The subjective symptoms as a rule are negligible, unless marked ulceration is present or inflammatory swelling from the secondary inoculation of pyogenic micro-organisms, when the lesions may become painful. 
Course.-The usual tendency of the disease is to be progressive and, if left alone, the patches may spread until they involve large areas or by ulceration cause marked destruction of tissue and disfigurement. Occasionally, however, the lesions may undergo spontaneous healing with the formation of atrophic or fibrous scars. As a rule the disease like other forms of tuberculosis is subject to periods of quiescence, followed by periods of activity when the resistance of the individual has become lowered or the lesions have been subjected to external irritation. In this country its progress is invariably slow and occasionally arrest may take place as a result of some local treatment insufficient to eradicate the disease.

Variations.- The clinical appearance of Lupus vulgaris varies in different cases and in different situations in the same case and a number of varicties have been described, which are determined by such considerations as the type of skin whether it is lax or stretched over bone or cartilage, the general health and resisting power of the individual affected, the existence of tuberculosis elsewhere, and the degree of secondary infection with pyogenic micro-organisms. The underlying structure is invariably the same, however, and the differences in the lesions are merely superficial and due to secondary causes.

Lupus Disseminatus. - Instead of being limited to one or two patches, multiple cases may occur in which a varying number of lesions are present widely distributed over the cutaneous surface.

For example, cases of disseminated lupus have been observed, especially on the face succeeding Herpes zoster, in which the vesicles of that disease have been replaced by a group of small lesions of L. vulgaris.

A more frequent type of multiple lupus, however, is that in children, in which a number of lesions appear almost simultaneously, irregularly distributed on the face, trunk and limbs, immediately after or during an attack of measles, or more rarely of scarlet-fever or varicella. The lesions in these cases of so-called $L$. post-exanthematicus vary in size from a split-pea to a shilling, the larger patches resulting from the coalescence of a number of small lesions. In appearance they may be smooth on the surface, slightly raised, and present the typical brownish semi-translucency of ordinary nodules of lupus; or may be scaly and bear a superficial resemblance to psoriasis ; or may be darker in colour than the apple-jelly nodules and assume a purplish-brown tinge; or, especially on the lower extremities, may have a marked tendency to become papillomatous or verrucose. They are as a rule indolent and spread slowly, some dying away spontaneously in the course of $\mathrm{I} 2$ to $\mathrm{I} 8$ months without leaving any marked scarring, while others gradually extend till they form persistent patches several inches in diameter. Cases in the adult of multiple patches situated at a distance from each other about the limbs and trunk, probably result from lesions of post-exanthematic lupus and date from childhood. 
These cases of multiple lupus occur in tuberculous subjects who, though they may have no noticeable tuberculous stigmata, such as scrofulodermia, scrofulous gummata, bone disease, etc., have some internal focus, such as tuberculous bronchial glands, and the most probable explanation of the outbreak is that this internal focus has been softened during measles and an embolic shower of tubercle bacilli has been eliminated from it, carried by the blood-stream to the skin, and there produced lesions wherever the bacilli have been arrested. A certain number of these emboli have also doubtless become deposited in the viscera, a probability which is borne out by the fact that in one or two instances an eruption of tuberculous foci in the skin has preceded death from acute tuberculosis of the lungs or from meningitis.

Note.-In addition to the typical cases of post-exanthematic multiple lupus, others have been described in which the lesions differed from those of ordinary lupus but were none the less definitely tuberculous and consisted of hempseed sized brownish papules surmounted at the apex by small vesicles, which became pustular and broke down to form smail ulcers, or of follicular lesions resembling Acne scrofulosorum in which tubercle bacilli were present in considerable numbers. Such cases suggest papulo-necrotic tuberculides rather than multiple L. vulgaris.

Lupus Verrucosus. - One of the most common varieties of L. vulgaris is that known as L. verrucosus in which the epidermis of the patches becomes hypertrophied and warty, most probably as a result of the secondary inoculation of some pyogenic micro-organisms of low virulence. When $\mathrm{L}$. vulgaris results from the local inoculation of tubercle bacilli about the hand a warty lesion is almost invariably produced, such as the familiar anatomical wart (Terruca necrogenica), or the verrucose lesions which not infrequently occur on the hands of butchers or slaughter-house men who have become infected by handling a tuberculous carcase.

Verrucose patches of lupus may occur not only as the result of accidental inoculation of the tubercle bacillus from without, but multiple verrucose lesions may be present in different situations on the skin, especially on the limbs and buttocks, in disseminate lupus of haematogenous origin. To these warty patches has been given the name Tuberculosis ierrucosa cutis. Certain writers consider it advisable to differentiate between Tuberculosis verrucosa cutis and Lupus vulgaris and describe them under different headings, but there does not seem to be any advantage in so doing as the essential structure is the same in both. (Fig. I5 7 .)

The anatomical wart (Ierruca necrogenica) occurs usually about the knuckles or on the backs of the hands and results from the inoculation of tubercle bacilli through an abrasion. It is first noticed as a reddish papule about the size of a threepenny-piece, or as a small indolent ulcer which has a tendency to ooze slightly and to become crusted over. Gradually papillomatous changes occur in the papule or the ulcer dries up and becomes verrucose. The lesion may then persist indefinitely without any marked increase in size, or more rarely 
it may undergo spontaneous involution. In rare instances where the inoculated bacilli have been unusually virulent the affection may spread up the lymphatics and a tuberculous lymphangitis result, which has been known to lead to general tuberculosis and a fatal issue.

Lesions of this type occur most frequently in pathologists, physicians and nurses from accidental inoculation from a tuberculous subject, or by auto-inoculation as in a phthisical patient infecting the back of his hand from wiping his mouth with it after coughing, but they may be met with also in butchers from bovine tubercle bacilli. They are comparatively common in coal-miners, as phthisis is prevalent among

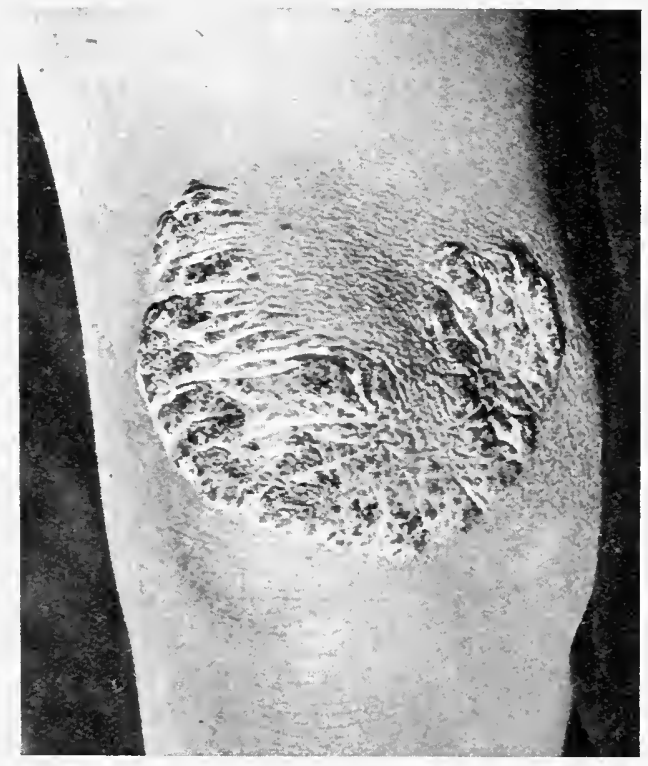

FIG. 157.-Lupus Verrucosus.

them and they are particularly liable to abrasions about the hands and forearms which become infected.

The lesions of Tuberculosis verrucosa cutis, of which the anatomical wart forms the prototype, vary in size from a sixpence to many inches in diameter. They are usually elevated and infiltrated, and covered irregularly with warty excrescences and dirty-greenish crusts, the result of the drying up of the sero-purulent discharge which oozes from sulci between the warty growths. When they are squeezed the sero-pus wells out on the surface; this reduces the tension of the lesion and gives a certain amount of relief to the patient. The base of the patch is a dull brownish-red and is surrounded as a rule by a reddish inflammatory band or halo. Spontaneous healing may take place in the centre and an atrophic scar result which may present a ringed gyrate appearance. Tubercle bacilli are found with greater ease in this than in the other varieties of Lupus vulgaris. 
L. hypertrophicus is another variety in which the patches are raised from a profuse cellular infiltration and the presence on the surface of exuberant papillary growths usually covered with dirty-greenish crusts. A common situation for this type of lesion is the upper lip and sides of the nose, but it may be met with elsewhere. (Fig. I58.)

Where the inter-papillary processes are markedly increased both downwards into the corium and on the free surface the lesions have been designated L. papillomatosus, and when the papillary excrescences are less pronounced but the lesions are swollen and smooth on the surface from a dense cellular deposit, the name of $L$. tumidus has been given to them.

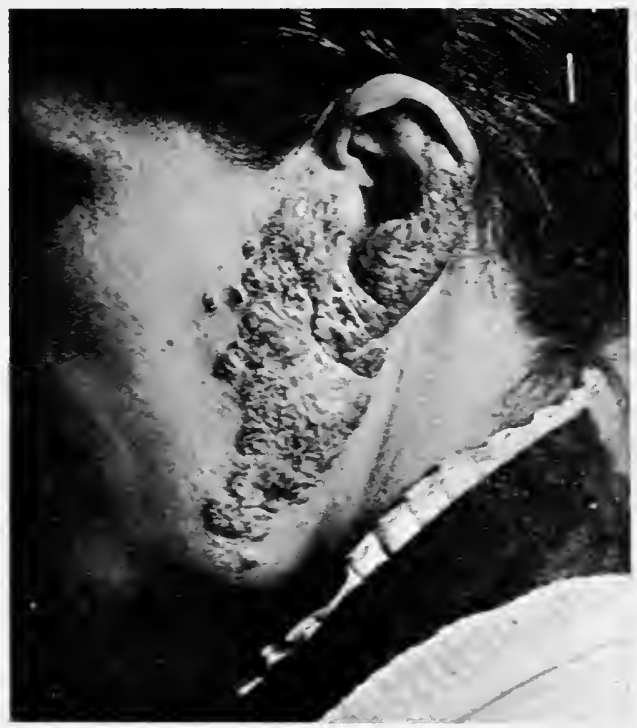

FIG. 158.-Lupus Vulgaris.

Hypertrophic type.

(From Dr. Arthur Hall.)

Various names have been employed also in connection with $\mathrm{L}$. vulgaris to signify the manner of spread of the disease. For example, where it tends to involute in the centre and to spread at the periphery it has been named L. annularis, and where the border is sinuous like that of a tertiary syphilide it is known as L. serpiginosus.

In some cases, through organisation of the small connective cells in the infiltration into new fibrous tissue, a peculiarly fibrous type of $L$. vulgaris results to which the name of $L$. scleroticus or "fibroid lupus" has been given. This variety is most common about the buttocks and is singularly resistant to treatment.

On the face in adults a peculiar superficial scaly variety is occasionally met with, which chiefly involves the skin stretched over the malar prominences and the nasal bone and closely simulates Lupus erythematosus in its clinical appearance and distribution. 
This was named L. érythématoïde by Leloir. It is distinguished from L. erythematosus by leaving a brownish stain on pressure by a diascope, possibly by the presence of a few typical apple-jelly nodules around the patches, and by the absence of the pitted adherent scales and atrophy which usually characterise L. erythematosus. (Fig. I59.)

Another variety of Lupus vulgaris is that named by Besnier Lupus pernio, which affects the nose and is characterised by diffuse purplish swelling and an occasional tendency to necrosis and ulceration. Should the congestion disappear typical nodules may become visible, while if the blood be squeezed out by a diascope a brownish stain is left, indi-

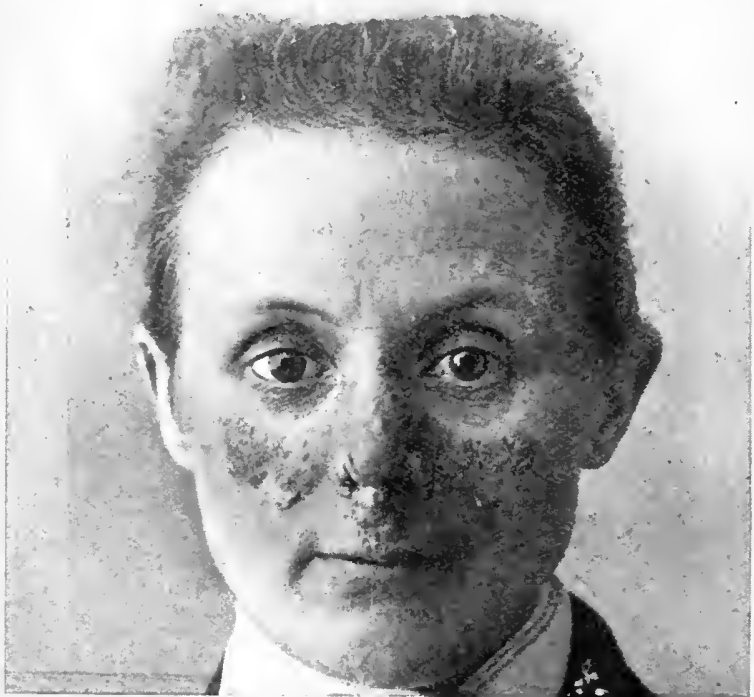

FIG. 159.-Lupus Erythematoides (Leloir).

Showing sug̣erficiai lesions of Lupus vulgaris distributed like Lupus erythematosus over the " bat's wing" area.

cating the underlying tuberculous granuloma. The cause of this variety is the occurrence of a diffuse type of $L$. vulgaris in an individual with an unusually feeble peripheral circulation, and in a situation liable to venous stasis. There is a diversity of opinion as to whether it is L. vulgaris or L. erythematosus. It is most probable that cases of both diseases have been included under the heading, and that those cases which were described by Hutchinson under the name of " chilblain lupus" in which the ears, hands and fingers were similarly involved were probably L. erythematosus, and also the cases where it was associated with typical lesions of Lupus erythematosus elsewhere on the face, scalp, or hands. But that certain of the cases are true lupus is undoubted, as all transitions can be detected from slight cases of ordinary $L$. vulgaris affecting the nose to the most 
pronounced cases where the nose is markedly swollen and of a deep purple colour.

Complications.-Occasionally in scrofulous subjects, where the resisting power is low and the infecting micro-organisms rirulent, lesions of L. vulgaris may be associated with an infection of the neighbouring lymphatics and tuberculous lymphangitis. This is most liable to occur on the limbs. Cases have also been known where a patch of lupus has been the site of inoculation of general tuberculosis. It is rare, however, for the infective virus from a lesion of lupus to penetrate deeply into the underlying tissue and to attack the glands, joints, or periosteum. When they are involved it is generally in a scrofulous individual in whom the lupus was probably the result of extension of the infection from underlying disease.

Occasionally local inflammatory attacks like erysipelas may supervene in and around patches of $\mathrm{L}$. vulgaris from secondary infection with streptococci. These may lead to the formation of new fibrous tissue in the corium blocking the lymphatics, swellings of the part affected from oedema, thickening of the integuments so that they become tough and do not pit on pressure, and, in extreme cases, to pseudo-elephantiasis. In certain instances an attack of erysipelas has been associated with healing of lupus patches, but the improvement as a rule has been temporary and the diseased process has usually reasserted itself subsequently. In scrofulous individuals where the lupus patches are extensive, the patient may be subject to occasional febrile attacks, followed by extreme prostration, and associated with inflammation of the local lesions. These are similar to the general and local reactions from the injection of the old tuberculin and are doubtless due to the absorption of toxins emanating from tubercle bacilli elsewhere in the body.

The most serious complication, and one which, though rare, is sufficiently common to be emphasised is the development on the scars of L. vulgaris of epitheliomata of a peculiarly rapid and fungating type, which, though they do not as a rule give rise to metastatic growths in the glands, are apt to lead to a fatal issue. This usually begins at the edge of the scar bordering on the healthy skin, and is probably the result of local irritation from injuries, unsuccessful efforts at local treatment, or the action of adventitious micro-organisms on an epidermis whose stability has been lowered by the underlying tuberculous deposit. It may occur at an early age but is most frequent between 40 and 50 and generally makes its appearance many years after the lupus began. It is more common in males than females, probably from their greater liability to injury, usually occurs on the face, but may be met with elsewhere, even in the mouth and larynx. Immediately upon being detected it should be excised with a wide excision and the scar carefully watched for a recurrence. (Fig. I60.)

Since the Finsen Light and X-rays have been extensively employed in the treatment of lupus there has been a tendency to blame them for 
the production of epithelioma. Though this may be just where a patch has been burned by X-rays and transformed into a telangiectatic scar which is itself liable to become epitheliomatous, in most instances the treatment was not in any way responsible for the malignant change; this serious complication was well-known before the X-rays were discovered.

Mucous Membranes.-The mucous membranes are affected in about $50 \%$ of cases of Lupus vulgaris, but if the disease is not at the actual mucous orifice it may be overlooked so that their involvement is apt to be regarded as rarer than it really is. As a rule the disease spreads

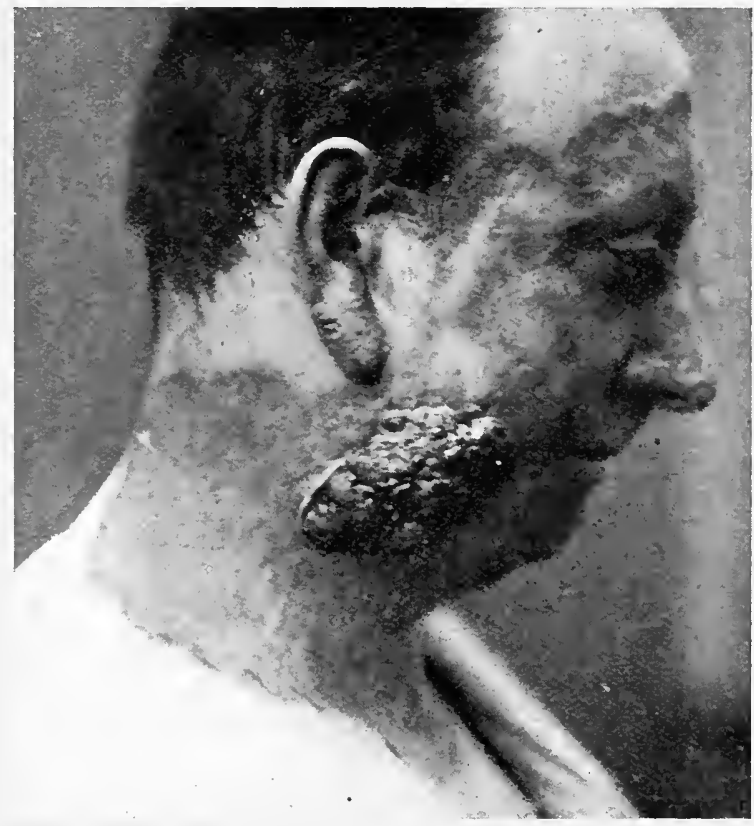

FIG. I60.--Epithelioma developing on Lupus Vulgaris.

(From Dr. Arthur Hall.)

from the mucous membrane on to the skin, but occasionally the mucosa is attacked by extension from the skin. The nasal mucosa is the part most commonly affected, especially over the lateral wall, septum, and about the anterior part of the inferior turbinated bone. In these situations it takes the form of dark red macules or nodules, not much larger than a small shot, on a lighter red base, which tend to break down, ulcerate, and to be covered with crusts. In severe cases it leads to considerable destruction of the walls and occasionally to perforation of the septum. In a large proportion of cases of lupus of the face the disease begins inside the nose, from bacilli deposited there by inhalation, which have gained entrance through abrasions caused by picking the nose, or through a mucosa inflamed by coryza. The earliest symptom of the infection suggests a nasal catarrh, which, being painless, 



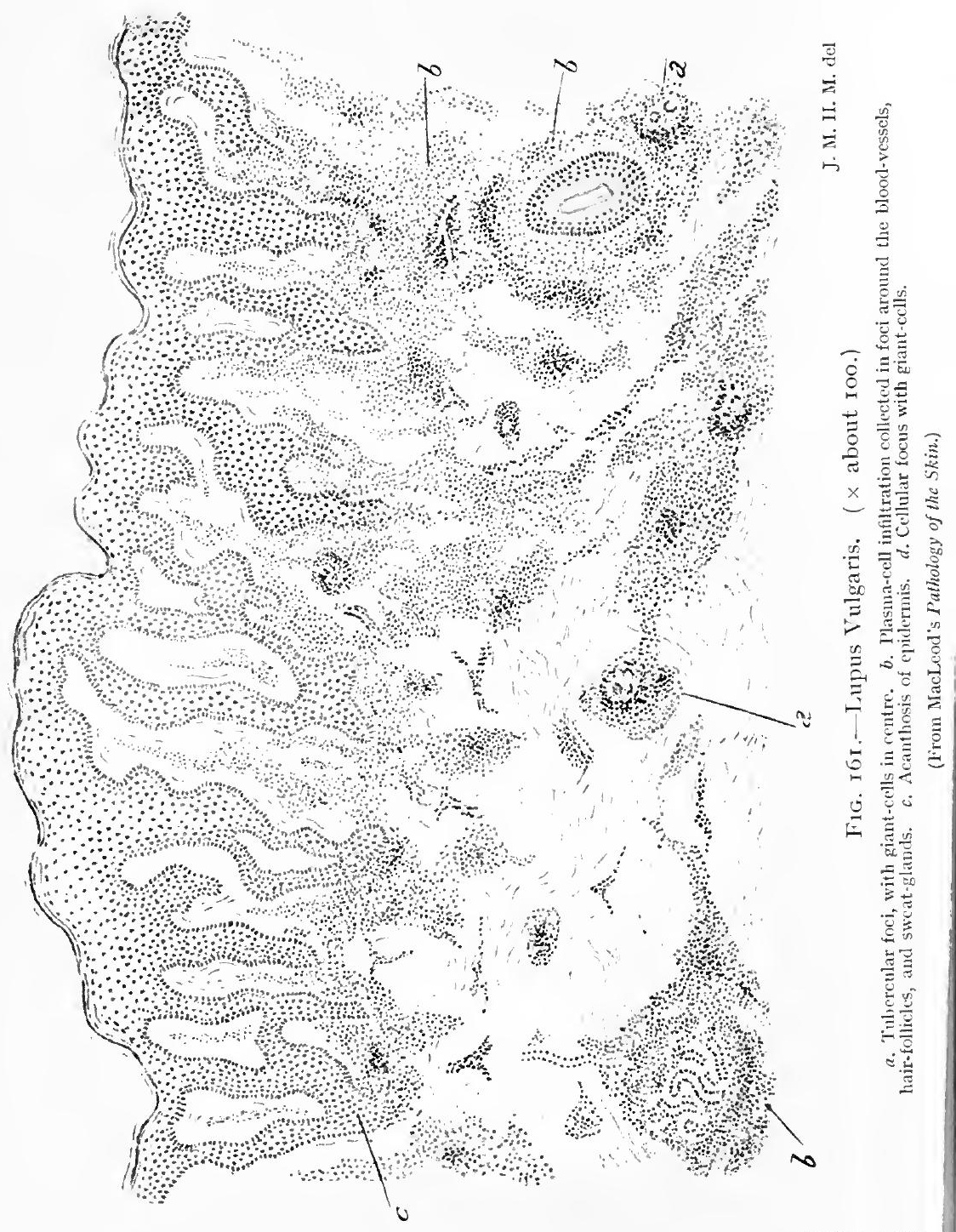

[To face $p .459$. 
may bo allowed to go on for a considerable time before its true nature is recognised, and in most cases is not discovered till the lupus has extended on to the skin.

The buccal mucosa also may be attacked, especially that of the lips, the lesions being generally of an ulcerated and hypertrophic type. It may involve the gums, soft palate, and tongue, causing brownish-red patches, ulcerated lesions, or papillary growths. On the tongue it tends to break down into an irregular or longitudinal ulcer with a flabby edge and a base studded with pale red granulations covered with a greyish-yellow exudation in which tubercle bacilli can be found readily. It may occur in the larynx, either by extension from the mouth or from the air-passages, causing ulceration about the epiglottis and the aryteno-epiglottidean folds and interfering with the voice. It sometimes attacks the mucosa of the lower eyelids and the tear-duct and from there inay spread on to the conjunctiva producing pannus-like granulations and corneal opacities. It may affect the mucous membrane of the external ear by extension from the auricle and has been known to destroy the tympanic membrane, to block the external meatus, and to impair hearing. It may occur also on the mucosa of the prepuce, vulva, vagina, uterus, and at the rectal orifice.

Histopathology.-The microscopical anatomy of a nodule of Iupus vulgaris is similar in the main to that of a miliary tubercle in the lung. It is in the corium that the essential changes take place, in the form of a more or less circumscribed cellular infiltration of a granulomatous type, the changes in the epidermis being of a secondary nature. The cellular infiltration is the histopathological evidence of the reaction of the tissues to the tubercle bacillus in their attempt to destroy the microbe and to limit its zone of influence. (Fig. r6r.)

A section of a typical lupus nodule presents the following appearances: In the corium there is a cellular infiltration, which is most marked in the deeper layers and becomes more sparse towards the papillary body, and which is not evenly distributed but tends to be aggregated in foci in the lymphatic spaces and about the capillaries, hair-follicles, sebaceous glands, sweat-coils, and ducts. The cells composing it consist mainly of small round cells of the lymphocytic type, and a varying number of plasma-cells. In the centre of the denser foci several giant-cells of the Langhans variety are usually present, with a certain number of large connective-tissue cells with vesicular nuclei (epithelioid cells) in their neighbourhood. Occasionally tubercle bacilli may be demonstrated in and between the giant-cells. Towards the centre of the foci the plasma-cells and small round cells appear to be in a condition of degeneration in which their protoplasm becomes homogeneous and their nuclei stain badly. (Fig. I62.)

The distribution of the cellular infiltration varies in different lesions according to the manner in which the bacillus reaches the skin. Should it be inoculated from without it will naturally become deposited in the lymphatics in the upper part of the corium, and the 
original foci of cellular infiltration will be found to be there. When, on the other hand, it reaches the skin from some underlying contiguous focus of disease it will naturally appear first and be most marked in the deeper layers of the corium and spread toward the surface. Should it be carried to the skin by the blood-stream the cellular foci will be found about the capillaries where the microbe has been arrested.

Where the infiltration is densest the capillaries have usually broken up and disappeared, so that the nodule is singularly avascular, a fact which is of great diagnostic value in distinguishing it from certain

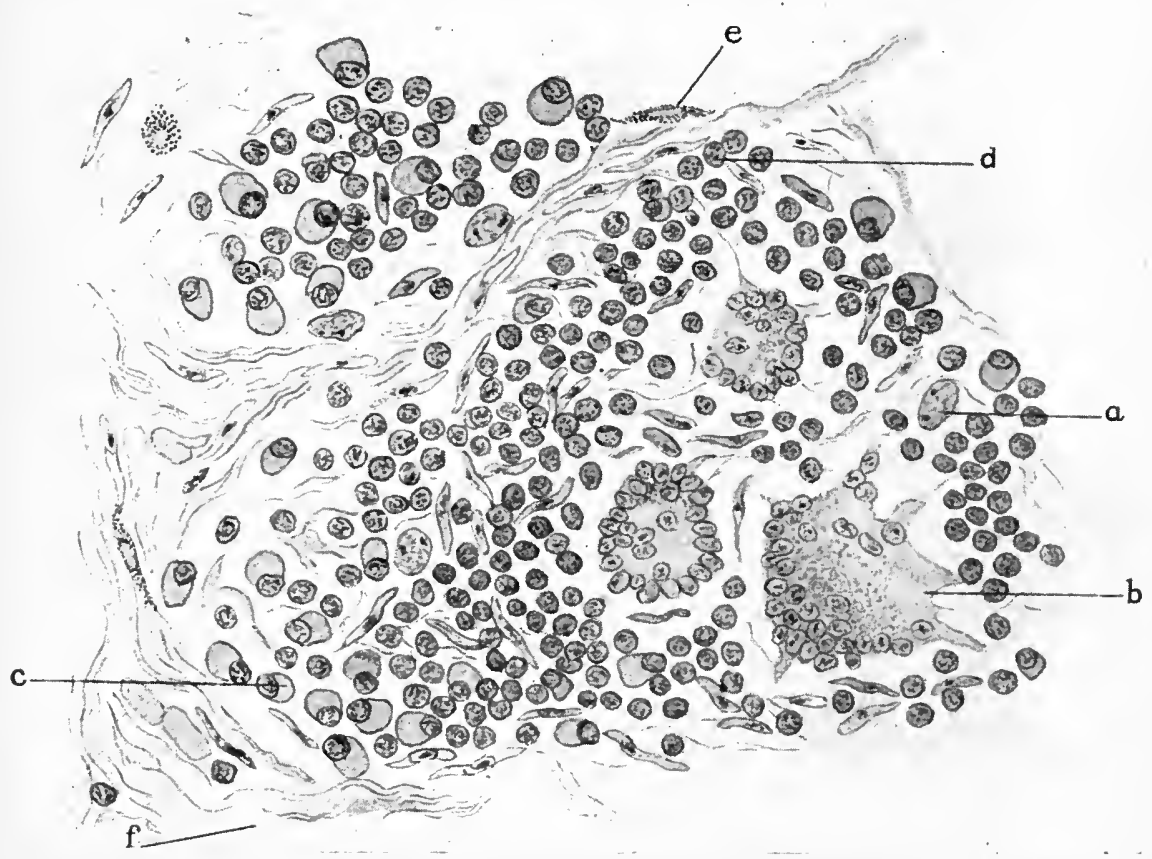

FIG. I62.-Nodule of Lupus Vulgaris. ( $x$ about Iooo.)

J. M. H. Ml. del.

$a$. Connective tissue cell. b. Giant-cell. c. Plasma-cell. $d$. Small round plasmaeell or daughter plasma-cell (Unna). e. Mast-cell. $f$. Collagen.

syphilides. In a few rare cases, however, the blood-capillaries persist and are definitely dilated so as to give the clinical appearances of telangiectases, but there is no end-arteritis as in syphilis. In the situation of the cellular infiltration the fibrous tissue has disappeared to a large extent as if the infiltration had formed at its expense. The collagen is more or less completely disintegrated and the elastin, which is more resistant, is attenuated or broken up. Beyond the zone of infiltration there may be a slight increase of fibrous tissue from concentration so that a capsule is formed. In a few rare instances there is a well-marked tendency for the cells to organise into new fibrous bundles around and separating the different foci of disease, as in the so-called sclerotic lupus, and in some cases actual hypertrophy of the 
collagen may occur as the result of attacks of acute inflammation of streptococcic origin. In none of these, however, is the elastin increased and when once destroyed it does not re-form. An unusual feature may be added in some cases by the presence of small abscesses situated about the papillae and due to the secondary inoculation of pus-cocci.

The state of the epidermis varies greatly in the different types of the disease and in different parts of the body. In an early nodular lesion it is practically unaffected. Gradually; however, as the infiltration increases it presses upwards, causing flattening of the papillary layer and stretching and atrophy of the overlying epidermal cells, and leading eventually to their destruction. In other cases hypertrophy takes place with marked increase in the inter-papillary processes which extend down into the corium and the formation of papillary excrescences on the surface; or the horny layer may be thickened and the prickle-cell layer irregularly proliferated as in the warty lesions of $\mathrm{L}$. verrucosus.

In the production of a fully developed lesion of $\mathrm{L}$. vulgaris there would seem to be two distinct processes at work: (I) An initial process caused by the presence of the tubercle bacilli, which is of a neoplastic character and results in the formation of the cellular infiltration and the destruction of the capillaries and fibrous elements; (2) A series of secondary processes due to the inoculation of more or less virulent cocci, either of the type of staphylococci causing ulceration, abscess-formation, and warty proliferation of the epidermis, or of streptococci causing attacks like erysipelas and leading to marked increase of the fibrous tissue and elephantiasic changes.

\section{Etiology ANd Pathogenesis}

Predisposing Causes. - In addition to the actual cause of the disease there are certain factors which predispose to its occurrence. Of these the most important are the following :

Sex.-It is more common in females than males in the proportion of about 3 to $I$.

Age.-It may appear at any age but usually begins in early childhood between the ages of 5 and I4, and rarely in infancy or after middle life. This is probably because the resisting power of the skin of children is low, and they are more liable than adults to be in immediate contact with tuberculous people.

Climate and Country.-It is essentially a disease of temperate climates and is rare in the tropics. Statistics seem to show that it is more common on the Continent than in Great Britain, and rarer in the United States of America and Canada.

Season.-The time of year makes no difference to its onset, but once it is established it is generally worse in cold weather and in early spring. 
Heredity.-It is seldom, if ever, a congenital affection. There is little doubt, however, that children of tuberculous parents inherit a weak power of resistance to the disease and, being more exposed to it, are more liable to infection than the children of healthy parents; the history of tuberculosis in a parent or some member of the family is surprisingly common in connection with it.

Note.-One or two instances have been recorded where infants have been born with tuberculous stigmata from infection in utero and in which tuberculosis of the uterine mucosa was found to be present in the mother.

Other Tuberculous Foci.-.The presence of other tuberculous foci predisposes to the disease, and a considerable proportion of the cases suffer from phthisis, or from local tuberculous stigmata such as scrofulous glands or tuberculous disease of bones and joints.

General Health.- In an individual whose health is enfeebled the inoculation of tubercle bacilli is liable to produce patches which spread more rapidly and are more active in type than those in a robust person, and periods of activity are liable to occur as a result of some intercurrent disease or from a general disturbance in health.

Social Station.-It is much more common among the poorer classes than among the well-to-do, as their resistance is lowered by malnutrition and bad hygienic surroundings and they are, at the same time, more exposed to infection from overcrowding and an unhealthy environment.

Occupation.-Certain occupations expose the individual to infection by tubercle bacilli, either human or bovine, such as those of pathologists, attendants in post-mortem rooms, nurses in phthisical wards, butchers, hide-sorters, and slaughter-house men.

Actual Cause.-Lupus vulgaris is due to the presence of the tubercle bacillus in the skin and is the clinical evidence of the cutaneous reaction to the bacillus and its toxins.

It may be caused not only by human tubercle bacilli, but also by bovine tubercle bacilli. The bacilli have been found in every type of L. vulgaris whether nodular, ulcerated, or verrucose, but they occur sparsely and the most persevering search may be required to find them.

The bacillus may reach the skin (I) from without, as in the numerous instances in which the infection occurs from local inoculation; (2) from within, by reaching the skin via the lymphatics from an underlying, morc or less contiguous, tuberculous focus, such as a suppurating tuberculous gland; (3) via the blood-stream, as in multiple post-exanthematic lupus.

There are several instances of local inoculation of tubercle bacilli causing L. vulgaris, the classical cxample being the verrucose lesion known as the " anatomical wart." Similar lesions may occur on the hands of butchers, or slaughter-house men from bovine bacilli. Inoculation may also take place in an unhealed vaccination sore, or 
in tattoo marks where the needled skin has been licked by a tuberculous operator, or after circumcision under the Jewish ritual, from sucking the wound to stop the haemorrhage. Cases are on record of infection on the ear from piercing for ear-rings, in nurses and hospital attendants from tuberculous sputum, and in laundresses from washing infected linen. Phthisical patients not infrequently infect themselves on the backs of the fingers and hands from wiping their moutls, and this is particularly common among coal-miners. (Fig. I63.)

Auto-inoculation may take place and new lesions be produced by scratching and rubbing.

Diagnosis.--There should be no great difficulty in recognising L. vulgaris when the characteristic, apple-jelly nodules, or the typical

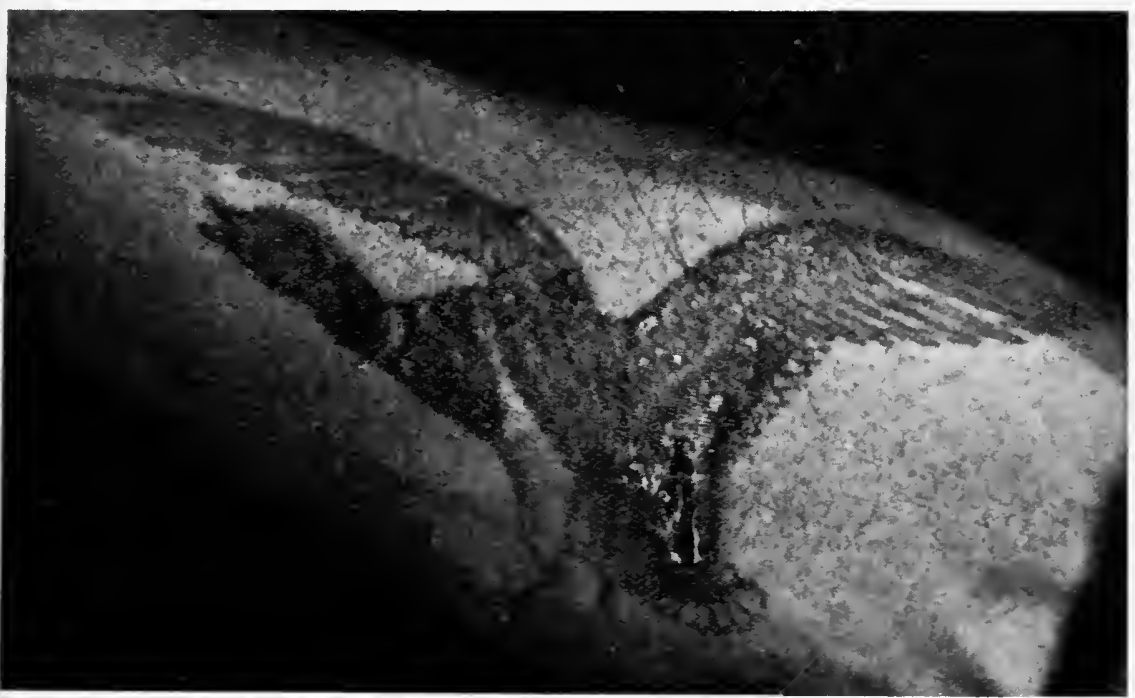

Fig. I63.-Tuberculous Ulcer and Papular Eruption following tattooing. (Dr. S. E. Dore's Case. Brit. Journ. Derm., 19rs.)

infiltrated, ulcerated or verrucose lesions are present. The indolent nature of the patches, their frequent existence since childhood, and the brown stain left on pressure with a diascope, are valuable aids to the diagnosis.

The diseases with which it is most liable to be confused are syphilis, L. erythematosus, psoriasis, and, when it is markedly verrucose, blastomycetic dermatitis.

Syphilis.-It is the superficial tertiary syphilide, either the slightly raised brownish nodule or plaque or the ulcerated superficial gummatous lesion, which is most liable to be mistaken for L. vulgaris, and cases may arise in which their differentiation is extremely difficult. The syphilide, however, is somewhat redder and more active-looking than lupus and has not infrequently a serpiginous border, while the lupus patch is brownish-yellow, indolent, and usually irregular in shape. When ulceration is present in the syphilide, it is deeper, the 
destruction of tissue greater, the discharge more profuse, offensive, and purulent, and the crusts coarser, than in lupus. The lesions of syphilis also tend to heal spontaneously, and react to mercury and the iodides, while the lupus patches rarely undergo spontaneous involution, and these drugs have no effect upon them, but, on the other hand, they react to tuberculin which the syphilides do not. The scar which results from the syphilide is soft, wrinkled like cigarette paper, and frequently surrounded by pigmentation, while that from lupus is more fibrous and irregular. Apart from the clinical appearances tertiary syphilides usually begin in middle or late life and develop quickly, while lupus lesions generally appear in early life and run an indolent course, taking several years to reach the size which a syphilide may attain in a few months.

The presence of other stigmata of either disease and the Wassermann reaction may assist in the diagnosis. Occasionally syphilis and tuberculosis may occur in the same individual-the syphilis usually being implanted on a tuberculous subject. In such cases should tuberculosis of the skin be present in any form the advent of the syphilis is liable to cause it to spread rapidly.

Leprosy.-Lesions of leprosy of the maculo-anaesthetic type, where the border is slightly raised and brownish, might give rise to confusion with lupus, but even in early leprides the sensation is more or less impaired and the nerves supplying the affected area are thickened.

Lupus Erythematosus. - In L. erythematosus the lesions are pinkish, little if at all infiltrated, pitted on the surface from the presence of dilated sebaceous orifices, and covered with adherent greyish scales, while those of $L$. vulgaris are brownish, definitely infiltrated, and either smooth on the surface or ulcerated, crusted, or covered with irregular loose scales. The superficial type known as $\mathrm{L}$. vulgaris erythematoides of Leloir most closely simulates L. erythematosus as it occurs in the same situations on the face, but in it there is usually definite infiltration and some isolated typical nodules of $\mathrm{L}$. vulgaris near the margin of the main patches.

Psoriasis.-The scaly lesions which occasionally occur in L. vulgaris of the multiple varicty may have a slight resemblance to psoriasis; but in lupus the scale is coarse and dirty while in psoriasis it is delicate, flaky, and silvery, and when removed in lupus a brownish nodule or macule is left while in psoriasis there is a thin pinkish pellicle which bleeds easily on being scratched.

Eczema.-Patches of chronic squamous eczema might be mistaken for lupus but the eczema is not so clearly defined, is more scaly, is definitely itchy, and may present points from which oozes a watery discharge.

Rosacea.-Where the nose is involved in rosacea and the skin thickened and congested it might be mistaken for lupus, but in rosacea there is no tendency to ulceration and the lesions are redder. 
Blastomycetic Dermatitis may so closely resemble L. verrucosus, that the diagnosis may be impossible without a microscopical examination and the demonstration of the blastomyces.

Rodent Ulcer might possibly be mistaken for an isolated patch of lupus, but even in its early stages the rodent papule has a peculiar pearly appearance, and the ulcer is usually surrounded by a cartilaginous rolled border.

Epitheliomatous Clcers may occasionally resemble lupus, but in them the ulceration is deeper and more active, and the base is more irregular and is enclosed by a well-defined everted edge beyond which there is a well-marked infiltration.

Prognosis.-The prognosis of $L$. vulgaris depends on the type and extent of the lesions, and on their situation. The almost invariable tendency of the disease, if left alone, is to be slowly progressive, though in a few rare instances the patches may undergo spontaneous involution; under suitable treatment, however, it may be arrested and occasionally completely cured. When mucous membranes are attacked the prognosis is more serious than when the skin alone is involved, as destruction and disfigurement are almost certain to result. As a general rule lesions which are raised and ulcerated are more difficult to eradicate than superficial patches. If the disease be recognised early it may be extirpated by excision and permanently stamped out, but if it has been allowed to extend considerably before being treated the prognosis is far less satisfactory. As it is very liable to recur after any form of treatment, except excision, the patient requires to be kept under the closest supervision, so that any new foci which may appear can be dealt with immediately; if this precaution be neglected the disease may make considerable headway before it is again seen and treated. As a rule $\mathrm{L}$. vulgaris is in no way detrimental to the general health, though in a few rare instances it may lead to a dissemination of the infection through the lymphatics and result in general tuberculosis.

\section{TREATMENT}

The successful treatment of $\mathrm{L}$. vulgaris calls for considerable resource on the part of the physician and loyal co-operation on the part of the patient. In a disease which is so multiform in its clinical types a considerable armamentarium of local measures is necessary to deal satisfactorily with its different phases, and it is on the judicious choice of the method applicable to the individual case and not on the slavish adherence to any one method upon which success depends. As it is essentially a local disease, local measures play the most important part in its treatment, though much benefit may be derived from combining them with a suitable general regime.

General Treatment.-The constitutional treatment is based largely on general medical principles and consists of improving the resisting power of the patient by suitable medical and hygienic means. It is 
of the first importance to place the patient in as healthy an environment as possible. It is no uncommon experience to find that cases which appear to be singularly resistant to local treatment while attending the out-patient department of a city hospital and living in overcrowded homes, improve markedly, apart from local treatment, when sent away to the healthier surroundings of a convalescent home in the country or at the sea-side. Sunshine, fresh air, healthy occupation, outdoor exercise, and easily digested nutritious food are of the greatest service in helping the patient to combat the disease. As an adjunct, a tonic should be prescribed for a considerable period, such as cod-liver oil, strychnine, iron, the hypophosphites, and the iodides, either in the form of the potassium salt or as the syrup of iodide of iron.

From time to time various remedies have been put forward as having a specific action on the local process, but the only one which has any definite claim to this position is thyroid extract. This remedy is incapable of curing lupus by itself but is a useful adjunct to local treatment in certain cases of the ulcerated or markedly inflamed type. In the adult it should be given at first in small doses of 2 grains t.d.s., and gradually increased till the limit of toleration is reached and the patient is taking about I5 grains per diem. To obtain benefit from the drug it is generally necessary to take it for a considerable time, but should any signs of thyroidism supervene it must at once be discontinued.

Tuberculin Injections are also of value in certain cases of lupus, especially where the lesions are extensive and ulcerated, but like thyroid extract must not be depended on exclusively. For a number of years tuberculin injections were practically abandoned in the treatment of lupus, owing to the disappointments and dangers associated with them. These were largely due to the doses being too great and the technique faulty; now that the injections are being employed in a more scientific manner the treatment is regaining some of its lost reputation. It is the new tuberculins, namely, Koch's new tuberculin T.R. and Koch's new tuberculin B.E. (bacillus emulsion), which are chiefly employed for therapeutic purposes; they are equally effective but, on the whole, T.R. is the better tolerated. T.R. is made by pulverising dried virulent tubercle bacilli, dissolving off the soluble toxins and suspending the insoluble bacterial matter in diluted glycerine. It thus differs from the old tuberculin in that its activity is dependent on the intra-cellular toxins of the protoplasm of the bacillus, and not on toxins excreted by the bacillus. It is standardised on the basis of Io $\mathrm{mg}$. of dried tubercle bacilli to I c.c. Its use is based on the rules of vaccine therapy, the object of the injection being to increase the anti-bactericidal power of the blood. The dose consists in the adult of .0002 $\mathrm{mg}$. gradually increased to $.0005 \mathrm{mg}$. The injections are made subcutaneously into the arm or buttock, with the usual aseptic precautions. It is advisable to confine the patient to bed at the time of 
the injection and to take his temperature at least erery four hours. A feeling of well-being should follow the injection but no definite rise of temperature, and the local lesion should become less inflamed and the ulceration dry up. It may be an adrantage to foment the lesions with boric compresses after the injection to cause congestion of the affected part and to bring a flood of serum and leucocrtes to it. Should definite improvement result from the first injection a slightly increased dose may be given in three weeks and this repeated again in a fortnight. It is then advisable to wait a couple of months before giving another series of injections. The clinical appearances of the lesion give definite indication as to the effect and dose of the injection, which may also be gauged by the estimation of the opsonic index. In L. vulgaris the opsonic index is usually low, about 0.6 . After the injection it generally falls a point or two during the " negative phase," and should rise again in a "positive phase" to about I.2. When the index begins to fall again a second injection should be given. As the result of subsequent injections the negative phase should become less marked; should it be more prolonged after the second injection or no positive phase occur the dose has probably been too great.

Tuberculin injections should not be given if fever or any signs of general tuberculosis are present, as even smaller doses are then capable of causing disastrous results.

Injections of thiosinamin, or its less painful substitute fibrolysin, have been advocated but they are of little use except, perhaps, for the reduction of hrpertrophic scars.

Local Treatment.-As would be expected in a disease so chronic and persistent as $\mathrm{L}$. rulgaris the number of local methods of treatment is legion, and as it would be impossible to refer to all of them only the more important need be discussed. It is well to remember that each method has its own phase of the disease to which it is applicable, and it is in the recognition of this that the skill of the physician and the probable success of his treatment lie. There are two main objects in the local treatment, (I) the complete remoral of the diseased tissue, (2) the production of as little scarring and disfigurement as possible. Where the face is involved, the cosmetic result is of the first importance, as should it be inferior it may interfere with the patient's chance of earning a livelihood and seriously-depress the mental condition.

The local measures for treating $\mathrm{L}$. rulgaris may be discussed under the headings of Surgical methods, Plissical methods, Chemical methods, and Radio-therapeutics.

\section{SÜRGICAL METHODS}

The chief surgical methods of treating I. sulgaris are excision, erasion, and scarification.

Excision.-For lesions situated on covered parts of the body, provided they are not too extensire, excision is undoubtedly the best 
treatment. On the face fairly satisfactory results from a cosmetic standpoint may be obtained by a skilful operator, though as a rule it is difficult to excise deeply enough.

The incision should be made at least a quarter of an inch beyond the apparent disease and carried down to the subcutaneous tissue, all diseased tissue dissected away, including the subcutaneous fat near the lesions as this is a frequent source of re-infection, and the edges brought together and sutured or, if the excised area be too large for this, it may be allowed to granulate or may be grafted.

Thiersch grafts are usually unsatisfactory as they are apt to produce uneven and sometimes cheloidal scars, and better results may be obtained from using strips of skin about three inches long, shaved from the front of the thigh, and applied so as to overlap each other.

Good results are also obtainable from Krause's deep grafting, which consists of transplanting a piece of skin of the exact shape of the area of tissue excised and about a quarter of an inch larger to allow for shrinkage. The graft is usually taken from the thigh, the skin of which has been thoroughly disinfected previously, and is excised as deep as the fat layer which should be scraped off. It is then placed in position and carefully sutured to the edges of the wound. Excellent cosmetic results even on the face may be obtained by this method in the hands of a skilled operator. About the mouth, eyelids and nose it may be difficult to get grafts to take, in which case resort may be had to some form of plastic operation and grafts with pedicles employed. Sometimes the grafts may shrink so much that a plastic operation is necessary to remedy the contraction; this should not be done till several months after the original operation.

Erasion.-Scraping is a favourite method of treating this disease and is specially useful for lesions on covered parts which are too extensive for excision. It is not suitable and is rarely satisfactory for lesions on the face as it may leave unsightly scars. A Volkmann's spoon or a ringed curette of suitable size may be employed for this purpose, and the scraping must be done firmly and thoroughly to be even approximately successful. The lupus tissue being soft is easily removed by the curette while the underlying healthy tissue offers considerable resistance to it. A general anaesthetic is necessary.

Unfortunately in spite of the most thorough scraping, small foci of diseased cells lurking in the interstices and pockets of the fibrous tissue are apt to be missed and to reassert themselves when the wound has healed. To lessen the chance of this, the scraped surface should be dabbed with pure carbolic acid, or ironed over with a broad-ended Paquelin cautery at a dull red heat, which has the double advantage of destroying diseased tissue and stopping haemorrhage. Nitric acid, pyrogallic acid in the form of a ro ointment, permanganate of potash as a powder, or a silver nitrate pencil, have also been employed for this purpose but they all have the disadvantage of being painful. 
The wound gradually granulates up under an antiseptic dressing such as iodoform, leaving a cicatrix which is more or less disfiguring according to the amount of tissue scraped away and the peculiarities of the individual skin. In some cases the scar is fairly satisfactory ; in others it tends to be irregular and even cheloidal, to obriate which considerable benefit may be derived from exposing the wound before it has healed to a pastille dose of the X-rays. Occasionally new capillaries may appear in the scar or raised angiomatous patches from injury to the blood-ressels of the hypoderm. It is inadvisable to graft the scraped surface, as should new foci develop beneath the graft it hides them and allows the recrudescence to assume considerable proportions before it is discovered.

After the wound has healed it should be examined periodically and carefully looked over with the assistance of a diascope for any recurrent foci of disease which should be dealt with at once. For this purpose a variety of methods may be employed, any of which will give satisfactory results if done thoroughly, such as erasion by a very small spoon, a Vidal's knife, or a dental burr, electrolysis with a coarse needle on the negative pole, cauterisation by a fine pointed Paquelin cautery at dull red heat, a silver nitrate pencil, or a piece of hard wood pointed and dipped in acid nitrate of mercury or Liquor antimonii chloridi.

The chief disadvantages of scraping, are (I) that it is an uncertain method at the best ; (2) that by it the lymphatics are apt to be opened up, leading to actual dissemination of the disease and the appearance of new foci in the neighbourhood; (3) that it usually leaves an unsightly scar.

Scarification.-For superficial non-ulcerated lesions about the face scarification is a useful method of treatment and produces the mini. mum of scarring. A fine sharp tenotome, a Vidal's knife, or a special instrument with several blades fixed together such as that derised by Pick, is employed for this purpose. When a single blade is used a number of parallel incisions are made through the diseased tissue, separated from each other by about an $\frac{1}{8}$ of an inch, followed by a second series of similar incisions crosswise so as to chop up the whole patch. The haemorrhage is then controlled by pressure and an occlusive dressing applied. When healing has taken place in about a week, the scarification is repeated, it being necessary to perform a number of scarifications before a satisfactory result is obtained. The destructive process can be intensified by applying a powder of permanganate of potassium or a $20^{\circ}$; resorcin ointment daily to the scarified surface.

This method of treatment is of considerable value in superficial patches of lupus about the nose, in which case the nares should be firmly plugged with cottonwool so as to support the sides of the nose while the incisions are being made. 


\section{Physical Methods}

The principal physical methods of treating L. vulgaris are cauterisation, refrigeration, ionisation, and congestion by cupping glasses. Of these the first two are the most important.

Cauterisation.-The actual cautery may be employed for the destruction both of isolated nodules and of whole patches of lupus, and for this purpose the Paquelin cautery is more convenient than the electro-cautery.

Where large lesions have to be dealt with the surface may be ironed over with the broad flat end of a Paquelin cautery, or cut up crosswise by a sharp-ended electro-cautery. When the cauterised area is extensive an occlusive antiseptic dressing should be applied till the eschar separates and the wound has healed. The chief disadvantages of the method are that healthy as well as diseased tissue is destroyed by it, and the scar is apt to be hypertrophic and disfiguring.

Instead of the actual cautery, hot air may be employed as the cauterising agent by means of the apparatus devised by Holländer, consisting of a metal tube, quarter of an inch in diameter, with a nozzle at one end, and an indiarubber tube at the other continuous with an air reservoir and a rubber bulb. Beneath the metal tube a Bunsen burner is fixed by which means the air pumped through the tube can be heated to about $300^{\circ} \mathrm{C}$. The hot air is allowed to play on the diseased patch for a few seconds till it crackles and becomes blanched, when the application has been sufficient to destroy the lupus tissue. If the hot air be applied for longer an absolute charring takes place from which recovery is impossible without the production of an ugly scar. After cauterisation the patch is dressed with boric ointment and bandaged so as to exclude the air. The operation is extremely painful and necessitates a general anaesthetic.

Refrigeration.-Freezing by liquid air or solid carbon dioxide is of value in the treatment of lupus, especially of the verrucose type, and the scars which result from it are eminently satisfactory. The liquid air should be applied for Io seconds, and the solid carbon dioxide for about 30 seconds with firm pressure. An acute reaction with bullous formation follows the freezing in the course of a few hours. When this has subsided under suitable antiseptic dressings, the freezing is repeated. The number of freezings which is necessary depends on the depth of the infiltration, but as a rule a considerable number is required before the diseased tissue is destroyed. The lestructive action of the freezing is much more selective than cauterisation and is produced indirectly as the result of the inflammatory reaction which succeeds the thawing.

Ionisation.--Cataphoresis or ionisation with zinc sulphate or chloride has been employed, but the results from it are uncertain. It is of some service in lesions in the nasal mucosa but to be effective it necessitates a current of $S$ to Io milliamperes and is very painful. 
Congestion.-Congestion br means of racuum cupping glasses on the principle of Bier's compression treatment has been advocated: the cupping glasses are applied intermittently orer the lesions for about an hour for periods of five minutes separated by a few minutes. The treatment, though it may produce improvement, is of little value in radically destroying the lesions.

High Frequency Currents have also been employed but are of little or no value.

\section{Chemical Methods}

A large number of chemical remedies have been used in treating lupus, the majority being caustics certain of which are believed to have a selective action. In addition there are a few sedative applicacations which are of ralue when the lesions are inflamed in reducing the hyperaemia before more radical measures are employed, such as lead lotion, lead plaster, and boro-calamine lotion. When the patches are ulcerated and crusted, as a preliminary to further treatment they should be cleaned by boro-starch poultices, boric acid compresses, or dressings of weak mercurial ointment.

The caustic remedies with the most selective action on the lesions are pyrogallic acid, salicylic acid, permanganate of potassium, arsenic, and antimony. These have been used extensively in the past as a substitute for operative procedures, but have now been largely supplanted by Finsen light or X-rar's.

Pyrogallic acid may be employed in the form of a $10^{\circ}$ o plaster or ointment spread on lint, applied continuously, and the dressing renewed several times a day. In about a week serere suppurative changes occur in the patch and the diseased tissue becomes soft and gradually sloughs while the healthy tissue in the neighbourhood is almost unaffected. The part is then dressed with boric ointment till healing takes place. By this means fairly good results have been obtained, but the treatment is tedious and painful.

Salicylic Acid is used in a similar manner either as a $10^{\circ}{ }_{0}$ ointment with the addition of creosote to deaden the pain caused by it, or in the form of salicylic-creosote plaster.

The pain produced by these caustic applications can be further deadened by previously painting the patches with $10^{\circ}$ o cocain or $5^{\circ}$ o novocain solution. The following paste spread on lint combines the action of both remedies: Pyrogallic acid ji, salicylic acid ji, resorcin jii, vaselin ad $\bar{\jmath}$.

Arsenic was once a farourite remedy in the local treatment of $\mathrm{L}$. vulgaris from its reputed selective action in picking out diseased foci, but its use has been largely abandoned owing to the pain caused by it, and the danger of its absorption. One of the best methods of applying it is in the form of Hebra's paste consisting of arsenious acid gr. $\mathbf{x x}$, cinnabar $\bar{j} \mathrm{i}$, cold cream ad $\bar{j} \mathrm{i}$. Previous to its application the diseased 
surface should be painted with a $20 \%$ solution of cocain. The paste is spread on lint and applied twice daily under an occlusive dressing for about four days. This produces an acute and painful inflammatory reaction with sloughing of the diseased tissue, after which the part is dressed with a mild antiseptic ointment till healing has taken place.

Chloride of Antimony has also been somewhat extensively used by Unna and others in the treatment of L. vulgaris. He recommends the following ointment: Salicylic acid I part, adeps benz. Io parts, to be applied with a glass rod to the patch, which is then covered with zinc oxide plaster. In the course of a few days this produces suppuration of the diseased tissue.

For isolated nodules what has been termed the " spick method " may be adopted. This consists of drilling into the nodules pointed pieces of wood impregnated with Liquor antimonii chloridi, these are cut off level with the skin and the part subsequently covered with mercury and carbolic plaster. The plaster is removed in 48 hours when the pieces of wood are found to be lying loose in pus resulting from the destruction of the nodules.

Permanganate of Potassium is also of value in this connection, either as a powder or as a 5 to Io $\%$ solution in water. In non-ulcerated cases after the surface has been cleansed the powder should be spread over the patch, covered with cottonwool and left on for I5 minutes, then removed and a moist dressing applied. The procedure is repeated daily and in a few days ulceration results, with the destruction of the diseased nodules. In ulcerated cases a Io \% solution should be painted on daily or compresses of it applied for I 5 minutes. In a few weeks softening of the nodules takes place and they can be easily wiped away. The scar after this treatment is fairly satisfactory.

A number of other remedies have been used, such as carbolic acid, zinc chloride, chrysarobin, lactic acid, oleate of mercury, and resorcin, but they have no special advantage over the caustics.

Tuberculin may be used locally, in the form of an ointment consisting of a I0\% solution of the old tuberculin in vaselin. This is rubbed into the patches for a few minutes and an occlusive dressing applied. The inunction is repeated daily for several days when an inflammatory reaction generally supervenes which, on subsiding, is followed by improvement in the lesions.

\section{RADIO-ThERAPEUTICS}

The two most valuable radio-therapeutic methods for $\mathrm{L}$. vulgaris are Finsen light and the X-rays and in lupus of the face and neck there is no treatment so satisfactory as the former, on account of the excellence of the scar which it leaves. Its chief drawback is the time it takes, which as a rule can be afforded only by the poor. 
Finsen Light Treatment or Phototherapy.-The Finsen ight method of treatment is simply the application of the actinic or chemical rays of the spectrum (blue, violet, and ultra-violet rays) to the shin. These rays have two effects ; they produce a powerful cutaneous inflammation or reaction which destroys the tuberculous infiltration and replaces it by new fibrous tissue, and ther are capable of killing micro-organisms on or near the surface of the skin. The actinic rars may be obtained either from sunlight or from a strong voltaic arc-lamp by filtering the light through a water chamber with a quartz lens at each end. Owing to the uncertainty of the sun in this country, the arc lamp is invariably employed as the source of the light. The sunlight apparatus, which is applicable in sunny climates, consists of two parts, namely, a condenser and a compressor.

The condenser is formed by two large flat lenses, Io inches in diameter, fitted into a metal frame to form a compartment, which is filled with water tinted blue by an ammoniacal solution of sulphate of copper, and mounted on a movable stand. By this means the red, orange, and yellow heat rays are filtered from the light and the remaining actinic rays are focussed on to the diseased area of the patient's skin, not directly, but through the compressor which is a flat disc-shaped box with quartz sides about the size of a man's watch. Through this the water is allowed to circulate freely by means of two small tubes. Conical shaped compressors are also obtainable to apply to situations such as the side of the nose or about the inner canthus. The compressor is pressed firmly against the skin by an attendant so as to render the part anaemic and allow the rar's to penetrate more deeply, as it has been found that the red blood-cells hinder their penetration, and at the same time the heat rays are further eliminated by the circulating water. The original Finsen lamp consists of a large central arc-lamp, using a current of 50 or 60 amperes, from which radiate four telescope-like collectors, with a srstem of quartz lenses to collect and concentrate the rays. The lower chamber of the collector is filled with distilled water and it is also fitted with a water-jacket through which water is constantly circulating. By means of a sliding adjustment the rays can be accurately focussed on to the compressor. With this cumbersome apparatus four patients can be treated at once.

A convenient substitute for the original lamp is known as the Finsen-Reyn lamp which has only a single collector and uses about 20 amperes. The chief advantages of this lamp are that, while being equally effective, it is less clumsy, much cheaper, and uses less current. Various other types of lamps have been introduced such as the LortetGenoud lamp, in which no separate compressor is required and the patient presses directly against the anterior lens, lamps with iron or tungsten electrodes, such as the Forbes lamp, and mercury vapour lamps, such as Kromayer's quartz lamp, but these are more superficial in their action than the Finsen-Rern lamp. These lamps do not 
require such long exposures as the old Finsen lamp and are useful in consequence.

Exposures of an hour or an hour and a half are necessary with the Finsen-Reyn lamp, during which time the compressor is either pressed firmly against the diseased area by an attendant, or is fixed to the collector at the requisite distance and the patient presses against it himself. Previous to the exposure any crusts are removed, and the area to be exposed is cleansed with a solution of boric acid or lysol. During the exposure the patient's eye must be protected from the light by a pad of cottonwool covered by thick brown paper. In from 4 to $\$$ hours after the exposure a more or less acute inflammatory reaction supervenes, with redness, heat, pain, and bullous formation, the value of the treatment depending largely on the depth of this reaction. It subsides in about a week, during which time the part should be dressed with a simple antiseptic ointment. The whole diseased area is gone over seriatim, the exposures being repeated whenever the reaction subsides. After the first few exposures marked improvement generally occurs in the lesions, which become flatter and paler as a result of the replacement of the superficial cellular infiltration by fibrous tissue. Beneath this, however, some deep-seated infiltration and foci generally persist which are all the more difficult to reach and act upon owing to the superficial layer of fibrous tissue. Repeated exposures will eventually penetrate to these deep-seated foci, but it saves time to destroy them by the cautery and if they are small this may be done without markedly interfering with the appearance of the scar. The degree of reaction may be intensified in resistant patches by previously painting them with carbolic acid or with $\mathrm{I} \%$ solution of erythrocene.

The treatment is tedious and, where extensive areas are involved, may occupy months or even years, still on the face and neck it is worth while as the cicatrices which result from it are soft, pliable, little different from the surrounding skin, and superior to those produced by any other method. A certain amount of pigmentation is caused by the light but this disappears in time. The type of case most suitable for Finsen light treatment is an early case of limited extent, in which neither ulceration, nor scar-tissue from previous operative treatment, is present.

The histopathological changes produced by the Finsen light consist of dilatation of the blood-vessels, extravasation of lymphocytes and serum, followed by oedema and spongy degeneration of the plasma-cells and giant-cells, which break down and become absorbed and are eventually replaced by new fibrous tissue, while the healthy cells in the neighbourhood are unaffected. At the same time the superficially situated bacilli are destroyed by the rays, while those more deeply seated succumb indirectly to the inflammatory reaction; consequently from an histopathological standpoint the Finsen light treatment is ideal. (Fig. I6.)

X-rays. - The X-rays are capable of destroying the granuloma of L. vulgaris, but, unless in most superficial lesions, only at the expense 
of a telangiectatic scar which is liable to become epitheliomatous at some subsequent period. Consequently as a routine treatment they are not to be recommended. Ther are of value, however, as a preliminary to Finsen light in ulcerated cases as they cause rapid healing of the ulceration. They are also useful in verv extensive cases to reduce the granuloma before applying the Finsen light, in cases where the patient is unable to devote the time requisite for Finsen light treatment, to check the spreading of the disease, in lesions on mucous membranes, and in situations like the evelids which cannot be dealt with by Finsen light owing to the difficulty of getting sufficient compression. They are specially useful for the reduction of verrucose lesions on the hands.

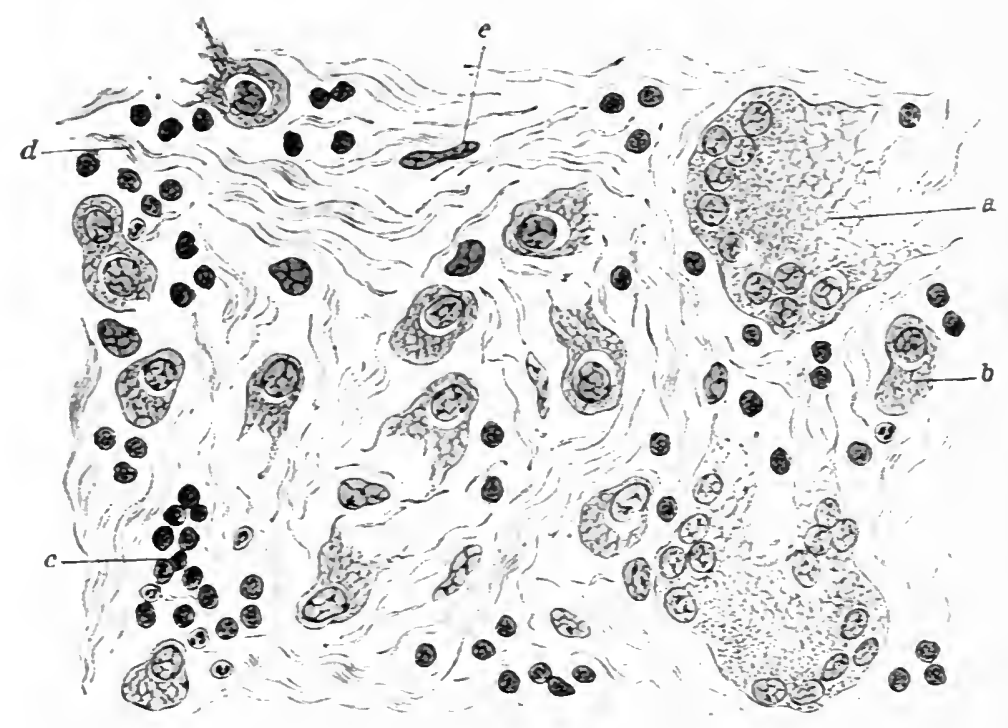

J. M. H. M. del.

Fig. I64-Granuloma of Lupus Vulgaris whth Oedematous Degeneration due to ultra-riolet light.

a. Oedemarous giant-cell. b. Oedematous plasma-cell. c. Group of leucocrtes and small round cells of doubtiul origin. d. Aitentiated aedematous ibrous stroma 2 . Connective tissue ceill.

It is usually best to begin the treatment by giving a full pastille dose screened by a layer of thick felt, and this may be repeated in three weeks.

The pathological action of the X-rays differs from that of the Finsen light in that any inflammatory reaction which may supervene is not directly due to the rays but is the result of the toxic action of cells which have been devitalised by them; consequently it occurs much later than the Finsen light reaction, appearing in about a fortnight. The $\mathrm{X}$-rays are inferior to the actinic rays in bactericidal action.

Radium.-By means of radium, lupus nodules can be made to undergo resolution and for small lesions which are not easily reached by Finsen light it is an excellent substitute. The costliness of the treatment, however, is as a rule prohibitive. Long applications of 
several hours with a powerful apparatus are usually necessary and result in an inflammatory reaction with the formation of a thick crust which heals slowly and leaves a scar which is smooth, white, and has a few telangiectases over it. Radium treatment is especially useful in the case of lesions on the nasal and buccal mucosa and on the conjunctiva.

Other radio-active substances, such as uranium and thorium, have been tried but their radio-activity is too feeble for them to be of any practical value.

Fluorescent Light has been recommended for the treatment of the disease on the face, and is of some benefit in arresting its spread. The method is simple and consists of painting the patches with a $0.5 \%$ aqueous solution of eosin and then exposing them to the sun if possible for an hour or two each day, the rest of the face being covered by a mask. To obviate staining the skin by the eosin it may be incorporated in glycerine jelly when it can be more easily removed.

\section{Selection of Method of Treatment}

In the treatment of $\mathrm{L}$. vulgaris success depends chiefly on the selection of the best method of dealing with each case, and its combination with other methods when indicated.

Where the lesions are situated on covered parts of the body and limited in extent, excision is the best form of treatment. Where they involve considerable areas, except over the knees and elbows, scraping followed by cauterisation and combined with tuberculin injections should be employed. When extensive patches are present over the elbows and knees the scarring and contraction which result from scraping may interfere with movements of the joints and one or other of the methods used on the face should be employed.

Where the lesions occur on the face and are not ulcerated Finsen light, combined if necessary with cauterisation of deep-seated foci, gives the best results. Scarification is also useful in superficial lesions. Should the lupus be present in situations which are not accessible to Finsen light, X-rays, radium, or refrigeration, should be employed.

Ulcerated lesions should be healed by X-rays as a preliminary to Finsen light treatment.

On the mucous membranes the most serviceable methods are radium, X-rays, scraping, and cauterisation with a Paquelin cautery at dull red heat.

In the case of the nasal mucosa, scraping should be followed by the early application of lactic acid or of iodine solution (iodine $\mathrm{I}$, iodide of potassium 2, and distilled water 3, parts), or the so-called two-pencil method resorted to, which consists of firmly pressing a solid zinc rod on lesions previously rubbed over with nitrate of silver. The nascent iodine method introduced by Pfannenstiel sometimes gives good results. It consists of combining sodium iodide internally with peroxide 
of hydrogen locally. Sodium iodide is given in doses of $7 \frac{1}{2}$ grains every four hours, the patient taking in this way 45 grains daily, and the nasal cavity is packed with a sterilised gauze tampon which is hept constantly moist with a $2{ }_{10}^{\circ}$ solution of hydrogen peroxide which the patient himself applies to the gauze by a pipette. Previous to the plugging of the nose all crusts and scabs should be remored. By this means free iodine is liberated and acts directly on the tubercle bacilli and diseased tissue.

On the lips and mouth the X-ray's followed by painting with the iodine solution are recommended, and for the conjunctiva the X-rays.

In every case, however limited, a suitable regime for the improvement of the general health should be adopted.

\section{SCROFULODERMIA}

Scrofulodermia is a name which has been applied in a somewhat wide sense to various forms of dermatitis occurring in tuberculous subjects, which result from the secondary involvement of the skin from underlying tuberculous disease chiefly in the lymphatic glands, bones or joints. The affected skin varies in appearance in different cases and in different situations in the same case. It is usually red or purplish in tinge, inflamed and oedematous with occasional brownish translucent patches where the oedema is slight or absent. There may be one or more discharging sinuses opening on its surface, and there is generally more or less extensive ulceration. The ulcers may be small and superficial, and either few in number or so numerous that the affected tissue seems to be riddled with them; or they may be large, with an irregular slightly infiltrated and excavated border, and an uneven base covered with viscid sero-pus which tends to dry up and form dirty-yellowish crusts. Sometimes a diseased gland undergoes dry caseation accompanied by necrosis of the overlying contiguous skin.

One of the most common situations is the side of the neck over breaking-down tuberculous glands, and from there it is liable to spread on to the face. The limbs are also frequently involved and the patches there may undergo hypertrophic changes and become irregularly covered with papillary vegetations or warty excrescences. This complication is especially common in lesions on the hands associated with tuberculous dactylitis. When the lymphatic ressels are involved cord-like thickenings of a reddish-purple tinge may be observed on the skin, and a varying number of small tense nodules in the sites of the superficial lymphatic glands, which break down and form irregular ulcers surrounded by a purplish border. The tuberculous lymphangitis may be associated with recurrent attacks of erysipelas which lead to fibrous changes in the corium, blocking of the lymphatics, and elephantiasic swelling. (Fig. I65.) 
The border-line between scrofulodermia and L. vulgaris is ill-defined and the one condition is liable to merge into the other. Yellowish-brown patches of typical lupus not infrequently occur here and there in the scrofulodermatous area, and small typical apple-jelly nodules may be detected in the neighbourhood of the patch as the result of direct inoculation with tubercle bacilli. It is no uncommon thing also for a patch of scrofulodermia gradually to dry up with healing of the ulcer and become transformed into a typical lesion of Lupus vulgaris ; on the other hand a patch of verrucose lupus may be the site of local inoculation of tubercle bacilli, which spread up the lymphatics and lead

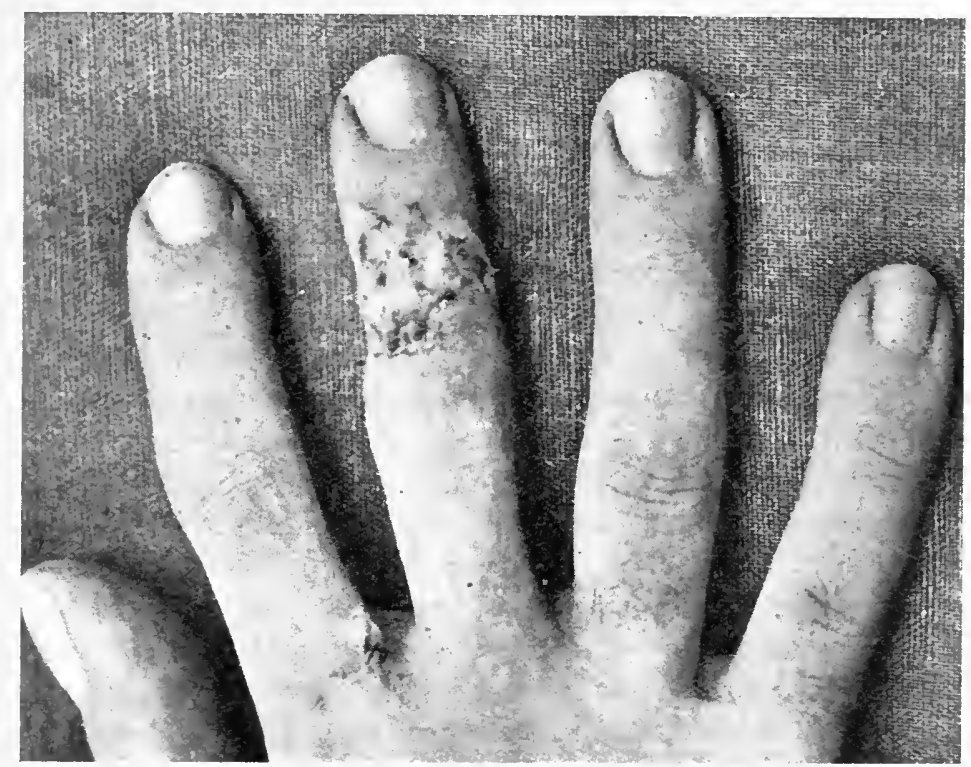

FIG. 165.-Tuberculosis Cutis with Tuberculous Dactylitis.

to the formation of tuberculous lymphangitis and scrofulodermia in the overlying skin.

The histopathology of diffuse scrofulodermia differs from that of Lupus vulgaris in that the cellular infiltration, instead of being grouped in giant-cell systems, is distributed diffusely over the whole patch. The corium as a rule is oedematous and the lymphatic spaces between the white fibrous bundles distended. The cellular infiltration is made up chiefly of small comnective cells of the lymphocytic type, plasma-cells, and occasionally multi-nucleated cells and giant-cells. It is densest near the subcutaneous tissue, and higher up in the corium it is collected in foci about the hair-follicles and sebaceous glands. The capillaries of the papillary layer are dilated and when suppurative changes have supervened small superficial abscesses may be detected. The epidermis may be atrophic or broken down, or on the other hand it may present rerrucose changes with increase of the interpapillary processes and hyperkeratosis. Tubercle bacilli may be 
found deep down in the skin or in the neighbourhood of sinuses, while pyogenic cocci are present near the surface and in the small superficial abscesses.

It is the tubercle bacilli and their toxins which are responsible for the cellular deposit, while the cocci cause the abscess-formation and the proliferation and verrucose changes in the epidermis.

Scrofulous Gummata.-There is another type of cutaneous lesion which is usually included under the heading of scrofulodermia, namely, the deep-seated nodular affection known as scrofulous gumma. This is first noticed when it is about the size of a pea as an indurated thickening with an indefinite margin situated in the hypoderm and not freely movable. At first the skin over it is unaltered so that the lesion is felt rather than seen. It gradually increases in size without any sensation until it becomes about as large as a filbert nut or even a walnut, when the skin over it becomes inflamed and assumes a purplish tinge. Meanwhile degenerative changes are going on in the lesion, either of a dry necrotic character with caseation, or more usually of a moist and suppurative nature so that the induration of the lesion is replaced by fluctuation. In any case the skin breaks down and an indolent ulcer results which gradually heals leaving a depressed cicatrix. It takes as a rule about three months for the lesion to go through these changes. Scrofulous gummata are most common in infants and young children and occur chiefly about the limbs, buttocks and trunk. As a rule they occur singly or in small numbers but in rare cases numerous small ones have been known to appear simultaneously after an attack of measles. Like other forms of scrofulodermia they may give rise to Lupus vulgaris.

Microscopical sections of an early lesion show that it originates in the subcutaneous tissue, as a focus of cellular infiltration around one of the lymphatic vessels or about a vein in the inter-lobar septa of the hypoderm, the walls of the affected vein being thickened and the lumen encroached upon. The cellular infiltration consists of small connective tissue cells, plasma-cells, and a few giant-cells. It increases gradually till it involves all the tissue in the neighbourhood and gradually spreads up into the corium, the ressels of the papillary and sub-papillary layers then becoming dilated and the epidermis stretched till it finally breaks down and ulcerates as a result of the action of progenic cocci. Tubercle bacilli have been found in and about the giant-cells and the infiltration is the result of their presence in the hypoderm. The bacilli have reached the subcutaneous tissue from some internal focus of tuberculosis, such as a tuberculous bronchial or mesenteric gland. (Fig. r66.)

The treatment of scrofulodermia is similar in the main to that which has been described in detail in connection with Lupus vulgaris. The general treatment should consist of nutritious food, hygienic surroundings, fresh air, and tonic remedies such as cod-liver oil, the syrup of iodide of iron, and the hypophosphites. Tuberculin injections should 
be employed with caution in case they should light up some internal focus of disease in the lungs or elsewhere.

Locally the first indication is to deal with the underlying tuberculous disease on ordinary surgical principles, for without so doing it is futile to attempt to cure the skin affection. The most satisfactory method is early excision, but should caseation or softening intervene they may be scraped out and thoroughly cauterised with carbolic acid. Should ulceration or wartiness persist it may be healed by X-rays and any granulomatous thickening is found to respond readily to Finsen light. It is important to treat the cutaneous affection as thoroughly as

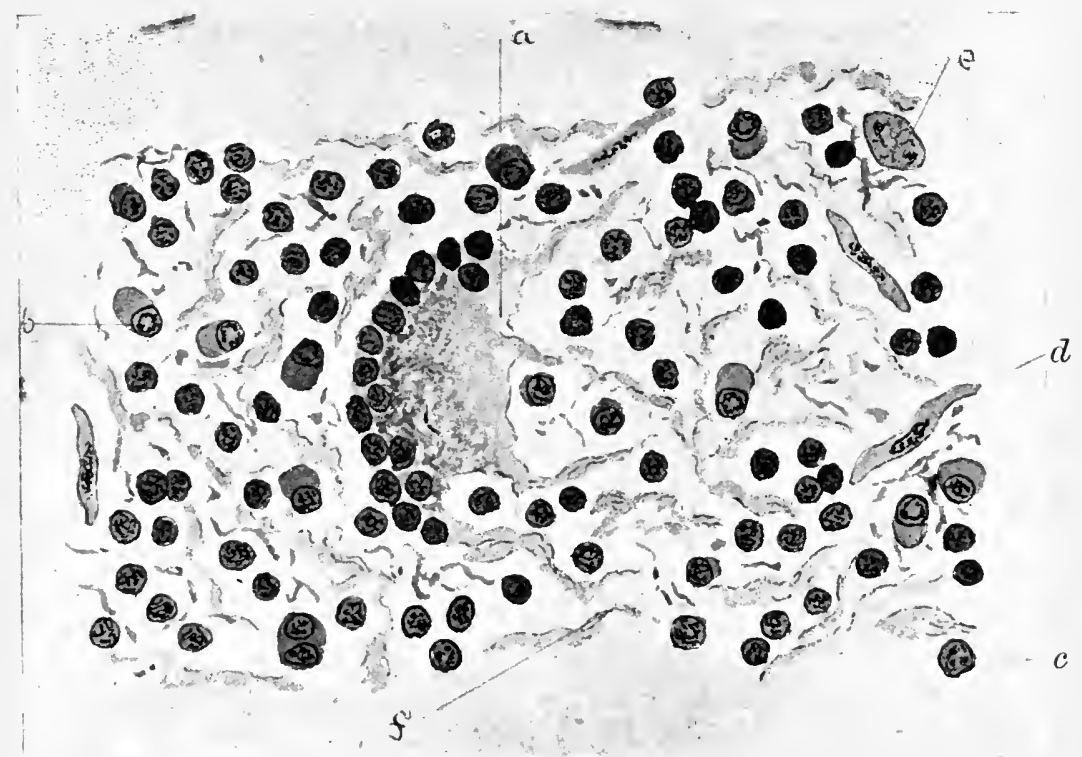

J. M. H. M. del.

F!G. I66.-Tubercle Bacilli in a Giant-cell. ( $\times$ about Iooo.)

From a case of deep-seated tubcrculous lesions on the arm.

$a$. Giant-cell with two tubercle bacilli. b. Plasma-cell. c. Daughter plasma-cell. d. Connective tissue cell. $e$. Connective tissue cell. $f$. Attenuated collagen fibres and oedema.

(From MacLeod's Pathology of the Skin.)

possible as it is liable to become transformed into ordinary $L$. vulgaris. As the result of the necrosis and ulceration the resultant scar is apt to be disfiguring and sometimes cheloidal but much can be done to obliterate it by judicious exposures to the X-rays.

\section{MILIARY TUBERCULOSIS}

This type of Tuberculosis cutis is comparatively rare, and consists of miliary tubercles, about the size of hemp-seeds, like those of visceral tuberculosis. These undergo cheesy degeneration and ulcerate, forming small oval or roundish ulcers with a flabby edge and covered 
with a scab which when remored leaves a reddish-grey floor with a sero-purulent exudation. They may remain discrete or may spread rapidly and coalesce to form large ulcers with an irregular border which is crenated from the breaking-down of contiguous miliary nodules, and a base which is covered with unhealthy granulations and dotted over here and there with yellow miliary deposits. The small ulcers are as a rule painless, while the larger ones may be definitely painful. A considerable number occur about the mucous orifices and are the result of the inoculation of the skin with infective discharges. The most common situation is about the mouth in association with ulceration of the mucosa secondary to tuberculosis of the lungs, laryns and throat, but they may be found on the genitals and about the anus. They generally, though not invariably, appear in the last stage of internal tuberculosis which is leading to a fatal issue, and cases have been recorded in which a crop of lesions of this type have appeared widely distributed over the whole cutaneous surface. Tubercle bacilli are found in considerable numbers in the lesions and inoculation experiments in susceptible animals have given positive results.

Treatment.-When the lesions appear the patient, who is invariably tuberculous, is usually so ill as to be beyond hope and the affection is a minor consideration. Should they occur in patients who are in comparatively good health they may heal spontaneously and the indications for treatment are similar to those of scrofulodermia, and consist of a general tonic regime, local treatment with antiseptic and caustic applications, such as carbolic acid or lactic acid, and erasion with a small ringed curette.

\section{TUBERCULOUS ULCERS}

Tuberculous ulcers may be primary like miliary tuberculous ulcers, or secondary as those which occur in Lupus vulgaris or scrofulodermia. They are the result of the action of progenic cocci on skin the resistance of which has been lowered by the presence of the tubercle bacillus and the granulomatous changes in the corium produced by it. They vary in size from a hemp-seed to several inches in diameter. When small they are roundish or oval in shape but when large they are generally polycrclical or crenated in outline. They may be superficial with a slightly raised edge, or deep with a flabby very undermined border. The skin immediately around them is slightly infiltrated and often presents a purplish tinge. The base of the ulcer may be smooth, greyish-red and corered with a viscid sero-purulent exudation, or uneven from the presence of soft granulations. The crusts which result from the drying up of the discharge are of a dirty-rellowish colour, and occasionally heaped up in limpet-shaped masses. The ulcers as a rule are indolent in type and take months to heal. When they heal 
a thin delicate cicatrix usually results which readily breaks down. In old people malignant changes may supervene in the ulcers or in the resultant scar. They have to be distinguished chiefly from syphilitic ulcers which are more acutely inflammatory, extend more rapidly, and have a more markedly infiltrated and serpiginous border.

\section{CUTANEOUS LESIONS WHICH ARE PROBABLY DUE TO TUBERCLE BACILLI IN THE SKIN}

\section{ERYTHEMA INDURATUM}

Syn. : "Erythème induré des scrofuleux" of Bazin.

Definition: A cutaneous disease characterised by indolent deepseated nodules, occurring chiefly on the legs in young women, which tend to undergo necrosis and leave scars.

Description.-This insidious affection is most common in girls and young women who are delicate, anaemic, or definitely scrofulous, and in a typical case appears as reddish-purple nodules, diffuse indurations, or punched-out ulcers with an infiltrated violaceous border, not unlike breaking-down syphilitic gummata, situated on the calf or the anteroextensor aspect of the leg. The lesion as a rule is detected first by palpation as a painless subcutaneous thickening about the size of a split-pea, the skin over which is unaltered or slightly reddened. This gradually increases in size till it becomes as large as a cherry or a walnut and causes the overlying skin to project and to assume a livid purplish tinge in the centre. The lesion as a rule is not well-defined but fades into the neighbouring skin. It is usually painless but may be slightly tender on pressure, or occasionally it may be associated in its early stage with definite burning or itching. After reaching a certain size it may remain stationary for a more or less prolonged period, then it either becomes gradually absorbed leaving little or no trace save a purplish stain, or more frequently it undergoes necrotic changes with the formation of an ulcer. On the surface of the lesion near the summit a small point of suppuration occurs from which oozes a sero-purulent or watery discharge and the whole nodule gradually breaks down without definite suppuration and is replaced by an ulcer, with a punched-out or undermined edge surrounded by a livid purple inflammatory zone of infiltration and oedema, and an unhealthy greyishyellow base. (Fig. I67.)

The ulcers are oval or roundish in shape, unless where two or more nodular lesions have coalesced when they become irregular with a polycyclical border. They heal slowly leaving an uneven depressed cicatrix surrounded by a pigmented areola. Their course is singularly indolent and occupies several months.

The most common situation is the leg between the knee and the ankle especially the calf and the antero-external aspect, but they have 
been observed also on the thighs, buttocks, arms, hands and ears. On the arms and hands they simulate chilblains and it is doubtful whether those which have been described on the hands and ears are papulo-necrotic tuberculides or suppurating or necrosing chilblains for the two conditions are frequently concomitant.

The lesions vary in number from one or two on each leg to a dozen or more, and are seen at all stages in their development as they tend to come out in crops. The affection may last for years and there is a marked tendency for new lesions to develop in the cold weather of winter.

It is generally associated with a feeble peripheral circulation, a marked tendency to chilblains, and a mottled congested condition of the skin of the legs. It has been known to occur also in connection with Raynaud's disease. In a considerable proportion of the cases it is associated with tuberculous stigmata, such as tuberculosis of the glands, lungs, joints, bones, scrofulous ophthalmia, a family history of tuberculosis, or with tuberculides of the papulo-necrotic type.

Histopathology.-Microscopical sections of an early lesion show that it consists of a cellular infiltration situated in the hypoderm and encroaching upon and replacing the fatcells in the neighbourhood.

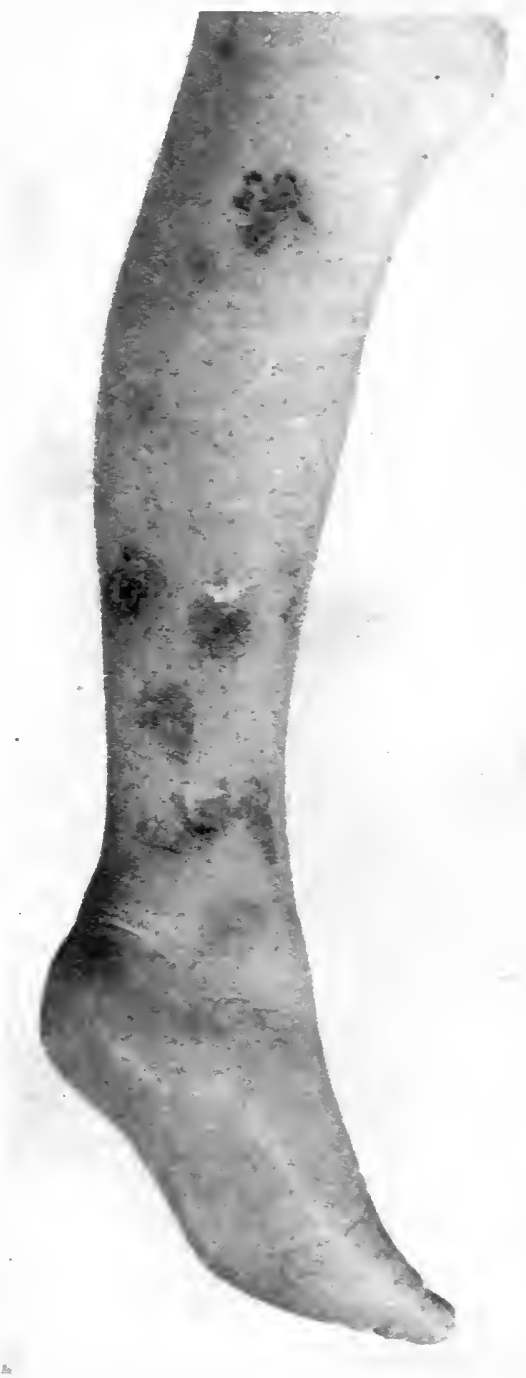

Fig. I67.-Erythema Induratum (Bazin's Disease).

It is composed of connective tissue cells both of the lymphocytic and large epithelioid type, plasma-cells and in some instances multinucleated cells and typical giant-cells.

Etiology and Pathogenesis.-Sex.-It is much more common in females than males. A few cases have been recorded in boys and one or two in men. The only one in an adult male which the writer has seen occurred in a man of 52 , haring begun at the age of 38 after an 
attack of influenza. He had no definite tuberculous stigmata but had a family history of tuberculosis and his sister also suffered from Erythema induratum.

Age.-It is most common between the ages of 12 and 20 , but has been known to appear between 6 and 7 , and to be present at 60 though it may have started much earlier. It is more common in badlynourished and insufficiently clothed young women of the working-class than amongst the well-to-do and is prevalent in those who have to stand a great deal.

Cold is an important etiological factor, as the lesions are worst in the cold weather, and once the disease is established there is a tendency for it to recur in winter.

Relation to Tuberculosis.-Though so far no one has succeeded in demonstrating tubercle bacilli in the lesions, inoculation in susceptible animals on several occasions has given positive results with the production of general tuberculosis, and the nodules have been known to react locally to injections of tuberculin. Its frequent association with symptoms of tuberculosis, its occurrence in young women with a tuberculous taint, and the histological architecture, though in themselves inconclusive, when considered collectively all point to some, if not all, of the cases being tuberculous in origin. It is more probable that it is the result of the action of the tubercle bacillus in situ, possibly in an attenuated state, than that it is due to toxins emanating from bacilli situated at a distance from the skin. In other words that the affection belongs to the scrofulodermia group rather than that of the tuberculides.

Cases have been observed where lesions identical with those of Erythema induratum of Bazin have followed inoculations with bacillary emulsion in individuals with tuberculous manifestations, such as tuberculous glands, etc. (Sequeira), and it has been suggested that they are the result of a local reaction in the skin occurring in an individual hypersensitive from the presence of a tuberculous focus and are called forth by the re-inoculation, and that tubercle bacilli in situ are not essential to cxplain their origin.

It has been maintained that there are cases, clinically and histologically indistinguishable from the tuberculous cases, which have another causation not yet fully understood, but which are in some way connected with a circulatory hypostasis.

NotE.-Philippson has described in detail changes which occur in the veins in lesions of the above type which are of a phlebitic or thrombitic nature, suggesting the possibility of the conclition having its origin in an affection of the vein. In consequence he applied the name Phlebitis nodularis necrotisans to it. (Fig. I68.)

Diagnosis. - The affections with which Erythema induratum is most liable to be confused are Erythema nodosum, syphilitic gummata, and scrofulous gummata.

From Erythema nodosum it is distinguished by being more indolent 
in its evolution, not associated with rheumatic pains in the neighbouring joints nor general symptoms such as fever, and by the lesions being usually painless, tending to ulcerate, and commonly present on the calf of the leg, while those of Erythema nodosum are usually extremely tender and painful, do not ulcerate and occur most frequently about the shins. The colour is different, for while in Erythema nodosum the lesions present the various tints associated with a bruise, those of Erythema induratum are reddish-purple. In Erythema nodosum the lesions, as a rule, come out together while in Erythema induratum they tend to appear in crops or seriatim and are present in different stages of development.

From syphilitic gummata Erythema induratum is differentiated by being usually bilateral, indolent in its course, and tending to necrose,

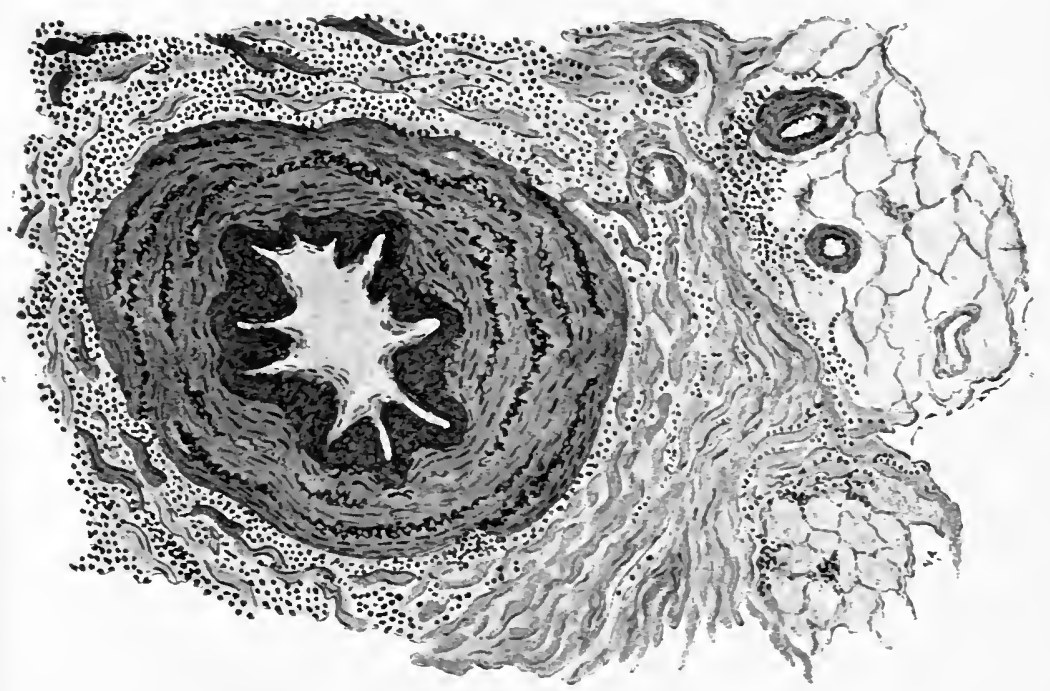

J. M. H. .I. del.

FIG. 168.-Transverse Section of Thickened Subcutaneous Vein,

From a deep-seated tuberculous nodule. Showing proliferation of the endothelium and great thickening of the coats. Around the vein is a dense cellular infiltration; the supporting stroma is attenuated and oedematous. This is the condition of the veins in Bazin's
Disease.

(From MacLeod's Pathology of the Skin.)

while a gumma if on the leg is usually confined to one limb, erolves much more rapidly and painfully, and breaks down by suppuration. It is readily amenable to iodide of potassium while Erythema induratum is uninfluenced or may be aggravated by that drug. Syphilitic gummata usually occur later in life and are equally common in men and women and are generally associated with a history of syphilis or the presence of some syphilitic stigmata.

From scrofulous gummata the distinction may be difficult. A typical scrofulous gumma is generally more rapid, attains a larger size, and is more liable to suppurate. It is not confined to the leg like Erythema induratum but may occur anywhere on the skin, and is 
essentially a disease of infants and very young children. The two affections are closely allied, signs of tuberculosis may be detected in both, and their histological architecture is practically indistinguishable. The scrofulous gumma is more rapid in its course than Erythema induratum which is essentially sluggish, but this may be because the former occurs in the delicate tissues of an infant while the latter is present later in life and in a tissue the resistance of which is diminished by circulatory disturbances. It may be that both are the result of the action of tubercle bacilli reaching the hypoderm through the bloodvessels.

The only other condition which might be mistaken for Erythema induratum is an iodide eruption of the nodular type, but the swellings which result from the drug develop more rapidly and are bright pink instead of dull and violaceous.

Treatment.-Under suitable treatment, general and local, the affection may be healed, but it is extremely resistant and in spite of the most careful supervision may tend to recur, especially in cold weather, and may persist for years. On the assumption that it is related to tuberculosis a general tonic regime is indicated with cod-liver oil, iron, quinine, or strychnine, simple easily digested food and plenty of fresh air. It is of the first importance that the legs should be rested and everything should be done to reduce the congestion by elevating the limbs and carefully bandaging them. This does more to heal the lesions than any local application. When the ulcers have broken down and ulcerated they should be irrigated with hydrogen peroxide ( $x$ in 20), carbolic acid ( $\mathrm{I}$ in 40), perchloride of mercury ( $\mathrm{x}$ in 4000 ), or methylene blue ( $x$ in IOo), and dressed with cinnabar ointment (Hydrarg. sulph. rubr. gr. $x$, Adeps benzoat. ad $\bar{j}$ i). The base of the ulcer may then be dusted with iodoform powder and dressed with a mild antiseptic ointment. The use of X-rays to heal resistant lesions sometimes gives good results.

\section{CUTANEOUS LESIONS SUPPOSED TO BE DUE TO TUBERCLE BACILLI SITUATED AT SOME DISTANCE FROM THE SKIN}

\section{TUBERCULIDES}

The name tuberculides or toxi-tuberculides has been applied by Darier and Hallopeau to a series of cutaneous affections occurring in tuberculous subjects, which are supposed to be due to toxins eliminated by tubercle bacilli situated at some distance from the skin and carried there by the blood, and in the lesions of which tubercle bacilli are absent and inoculation experiments in susceptible animals give negative results. The group in its original conception was indefinite and hypothetical and a considerable number of affections were included in it, of which the most important were the following : 
Lichen scrofulosorum.

Acne scrofulosorum or Papulo-necrotic tuberculide

Erythema induratum (Bazin).

Eczema scrofulosorum.

Pityriasis rubra (Hebra).

Lupus eræ̌thematosus.

Angio-keratoma (Mibelli).

Chilblains.

Gradually as our knowledge has increased, either by the finding of tubercle bacilli in situ, from successful inoculation experiments in lower animals, or from the realisation that the occurrence of a cutaneous lesion in a tuberculous individual may be merely an accidental association and not directly due to the tuberculous toxins, the group has dwindled to such an extent that it seems possible that in time it may completely disappear. But as eruptions of the type of Lichen scrofulosorum or simulating Acne scrofulosorum have been known to follow injections of tuberculin in a tuberculous subject, there seems some reason for still retaining the heading of " tuberculides."

In the case of Erythema induratum the evidence in favour of its being a true tuberculous manifestation is gaining ground to such an extent that it has been considered advisable to place it here tentatively under a non-committal title, while with regard to the others the evidence that they are in any way etiologically connected with tuberculosis is comparatively slight, and they will be described separately.

It has been found that cases of tuberculides almost invariably give a positive cuti-reaction, pointing to the existence of a manifest or latent focus of tuberculosis, and on this another theory of the origin of tuberculides has been based by Rist and Rolland who maintain that tuberculides in the skin are not due to the circulation of toxins or to emboli of attenuated bacilli, but are simply manifestations of allergy or hypersensibility and are reactions in the skin brought about by re-inoculation. This would explain why an injection of old tuberculin in a tuberculous subject may be followed by an eruption of Lichen scrofulosorum.

Even in the case of Lichen scrofulosorum and Acne scrofulosorum the tuberculin injection may not be directly responsible for the eruption but may be indirectly so by calling into activity attenuated tubercle bacilli lying dormant in the skin, a view which is corroborated by the lesions reacting locally to tuberculin.

\section{LICHEN SCROFULOSORUM}

Definition: A chronic inflammatory affection, occurring in tuberculous subjects, characterised by the presence of minute roundish papules of a reddish colour and arranged in groups.

Description.-It is met with chiefly in children suffering from some form of tuberculosis, such as suppurating glands, caries of bones, or 
tuberculosis of the lungs or pleurae, and may be found in association with various manifestations of tuberculosis of the skin, such as Lupus vulgaris, Erythema induratum, and scrofulous gummata.

It occurs as groups or patches of discrete, round or acuminate papules, about the size of a pin's head, which vary in colour from pinkish-yellow to reddish-brown and occasionally assume a livid or haemorrhagic appearance on the extremities. The papules may be smooth on the surface or surmounted by a small scale or even a horny spine, and sometimes a minute abscess may be detected at the apex. They are generally follicular though not invariably so, and, however close they may be to each other, have no tendency to coalesce. They are arranged in irregular groups usually about the size of finger prints, or aggregated into considerable patches not unlike slightly inflamed goose-skin. Sometimes the involution of the central papules of a group may result in a circinate or crescentic arrangement. When the papules fade a pigmented macule is left which gradually disappears, either leaving no trace or being replaced by a minute atrophic scar. The groups occur chiefly on the lowel part of the trunk about the sides and waist, are occasionally present on the shoulders, arms, buttocks, and legs, and have a marked tendency to be symmetrical. The eruption may appear quite suddenly and may persist for a considerable time and even for years, or may disappear spontaneously as the general health of the patient improves and may recur should the resistance become lowered. Occasionally as the result of friction in intertriginous situations the papules may become itchy, eczematised, and may weep, but as a rule there are no subjective symptoms.

Histopathology.- Sections of a papule show that it consists of a mass of cellular infiltration, situated in the upper part of the corium, usually around the neck of a pilo-sebaceous follicle and densest about the small capillaries of the follicles. The cells composing it are small connective tissue cells of the lymphocytic type, epithelioid cells, and mast-cells, with here and there a few plasma-cells or giant-cells. There is no sign of the grouping which occurs in a miliary tubercle, nor any definite tendency to caseation. The epidermis lining the mouth of the follicles may show proliferative changes with definite hyperkeratosis. The general appearance of the infiltrating cells would suggest that the lesion is due to some irritant of low virulence. reaching the skin by the blood-stream.

A search for tubercle bacilli in the lesions has generally been unsuccessful though on one or two occasions they have been found, and inoculation experiments in susceptible animals though usually negative have occasionally given positive results.

Etiology and Pathogenesis.-Lichen scrofulosorum being a disease which, except in well-marked cases, is apt to be overlooked, is probably more common than is generally believed. It would appear from statistics that it is more frequent in Austria and Germany than in this country and less so in France and America. It generally affects 
children and is rare under 4 and over 20, though it has been observed in infancy and adult life. It is equally common in both sexes.

Much discussion has arisen with regard to its true nature and pathogenesis. That it is a manifestation of tuberculosis is generally conceded, for not only does it invariably occur in association with definite tuberculous stigmata, but in a considerable number of instances an eruption which is clinically and histologically identical with it has resulted from the injection of tuberculin in tuberculous individuals (but not in the subjects of other diseases such as syphilis), and similar papules have been known to derelop around a patch of Lupus rulgaris which has reacted locally to tuberculin. Opinions differ, however, as to whether it is due to toxins reaching the skin from some focus of tuberculosis at a distance and is in the true sense of the word a tuberculide, or is the result of the actual presence in the skin of attenuated tubercle bacilli. In corroboration of the former view is its occurrence after tuberculin injections, and of the latter that in a $f$ instances tubercle bacilli have been found in the lesions and it has been successfully inoculated in guinea-pigs.

Were it due to toxins circulating in the blood it would be expected to occur in advanced stages of phthisis or Tabes mesenterica, when abundant toxins are probably present, but so far it does not seem to have been met with in them. That tubercle bacilli are rarely found in it is an inconclusive argument against its bacillary origin, as bacilli occur sparsely in definitely tuberculous conditions like Lupus rulgaris. The border-line between tuberculides and true tuberculous lesions is ill-defined, but it seems that the lesions are more easily explicable on the assumption of the presence of bacilli in situ, than as the result of some toxin in the blood of which there is no conclusive evidence, and the close analogy which exists between it and the miliary lichenoid syphilide, which is an active early lesion and in which spirochaetes have been found, supports this riew.

Diagnosis.-The diagnosis of Lichen scrofulosorum ma! be difficult, the affections with which it is most liable to be confused being the small lichenoid syphilide, follicular eczema, and Lichen spinulosus. From the syphilide it is distinguished by its age of incidence, history, absence of syphilitic stigmata, and distribution, the syphilide not being so confined to the trunk; from Lichen spinulosus it is differentiated by being more inflammatory, occasionally presenting miliary pustules, and being associated with signs of definite tuberculosis; and from follicular eczema it is distinguished by not being itchy, being less active and inflamed, and not tending to coalesce to form patches.

Treatment. By suitable general and local treatment the eruption may be reduced or removed, but it usually tends to recur should the resistance of the patient become reduced. In certain cases thyroid extract has a beneficial effect, but apart from this the general treatment consists of employing the well-known medicinal and hygienic measures applicable to the treatment of tuberculosis in general. 
Locally a favourite remedy has been the rubbing in of cod-liver oil or the wearing of a flannel binder saturated with it, but this treatment is dirty and unpleasant and equally good results may be obtained from rubbing the patches with the following ointment: ichthyol $\mathrm{m}$. $\mathrm{x}$, olive oil $\bar{j} \mathrm{ss}$, vaselin $\bar{j} \mathrm{ss}$, or in resistant cases from washing them with soft soap and then applying an ointment of salicylic acid gr. $\mathrm{x}$, zinc oxide $3 \mathrm{i}$, and vaselin ad $\overline{5}$.

\section{PAPULO-NECROTIC TUBERCULIDES}

Syn. : Acne scrofulosorum (Bazin), Papular and Nodular Tuberculides of intermediate size (Colcott Fox), Folliculites disséminées symétriques des parties glabres à tendance cicatricielle (Brocq), Acnitis (Barthélemy), Acne agminata (Crocker), Folliclis (Barthélemy), Hydradenitis destruens suppurativa (Pollitzer), Granulome innomine (Tenneson), etc.

Definition : A chronic inflammatory affection of the skin characterised by deep-seated small indurated nodules which tend to necrose and leave a scar.

The name Acne scrofulosorum is apt to be misleading as it suggests Acne vulgaris occurring in a scrofulous subject. The tuberculide, however, has only a superficial resemblance to Acne vulgaris and is of a totally different nature, its lesions not necessarily being follicular. The essential lesion is first noticed by palpation as a hemp-seed sized hard nodule the skin over which is unchanged, or, when more superficial, as a slightly raised rounded red papule. This gradually increases to the size of a split-pea, when it assumes a purplish colour in the centre surrounded by a reddish areola which fades into the normal skin. At the apex of some of the nodules a small vesicle may be present from which a yellow bead of serous fluid can be squeezed. (Fig. r6g.)

The nodule is unusually indolent in its evolution and it is only after several weeks that changes of a necrotic or occasionally of a suppurative character supervene. It then becomes softer, depressed in the centre, and covered by an adherent greyish-ycllow crust which is conical in shape and dips down into the central depression. When this is removed a small superficial ulcer is left, which on healing is replaced by a purplish pigmented cicatrix which eventually becomes white in the centre and in time looks not unlike a smallpox scar. The nodules are painless in their whole course, which varies from a fortnight to six or eight weeks. In a well-marked case all stages in the evolution of the lesions, from shotty nodules to pitted scars, may be observed at the same time. Certain of the lesions are definitely follicular and may present a hair in the centre, while others are independent of the hairfollicles and probably occur about the sweat-ducts. (Plate VII.)

The eruption is most marked on the extremities especially on the extensor aspects of the arms, elbows, backs of the hands, face, buttocks, 


\section{PLATE VII.}

FIG. I.-Suppurating Chilblains on back of hand in a patient with Lupus erythematosus.

FIG. 2.-Papulo-necrotic Tuberculide (Folliclis), on forearm and hand of young woman.

Fig. 3.-Lupus erythematosus of Nose. 
PIATE III

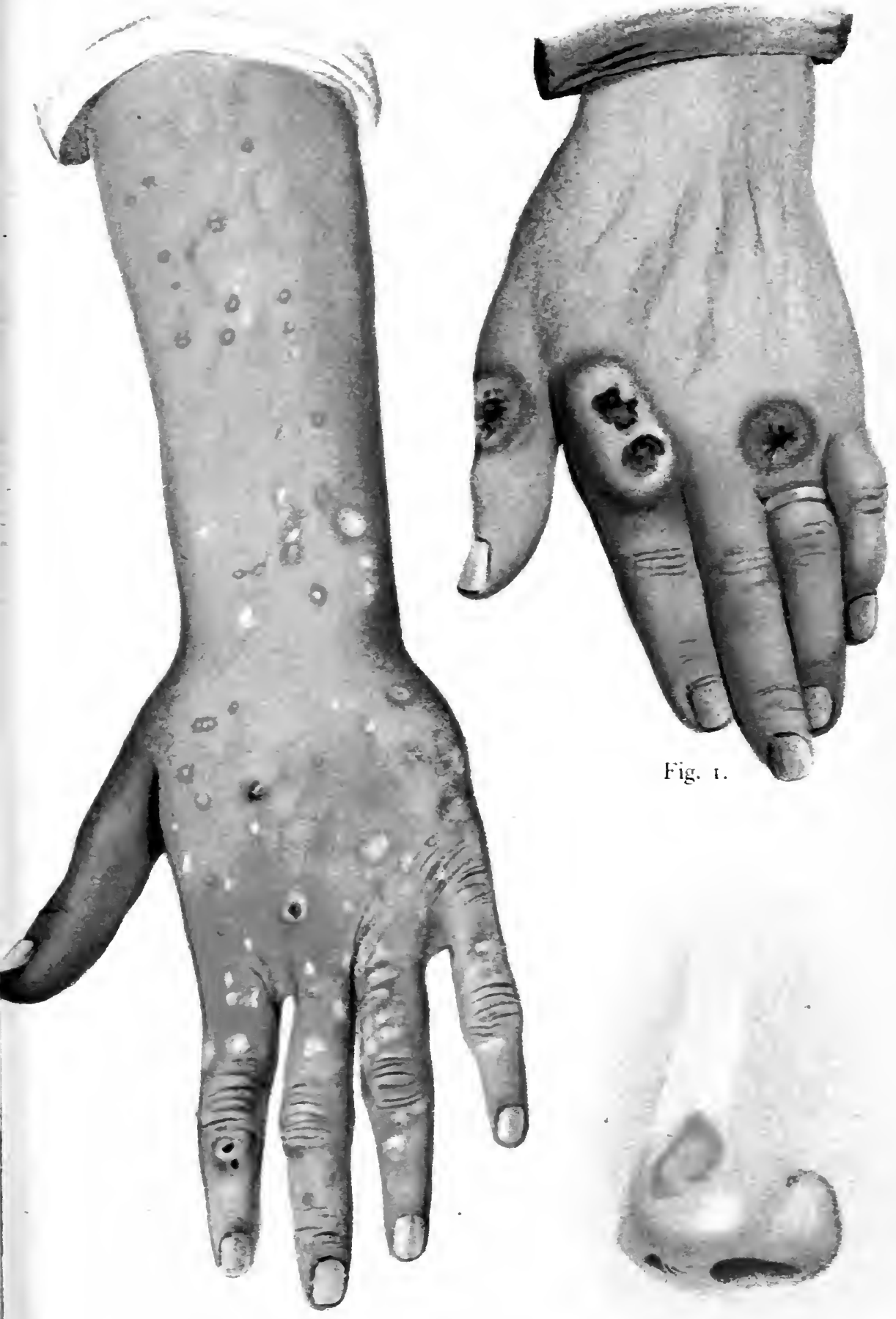

Fig. 2.

Fig. 3. 

legs, and dorsa of the feet, is less common on the trunk and ears, and extremely rare on the palms, soles and scalp. It is markedly symmetrical, and may be limited to regions such as the forearms, or may be widely distributed over the skin. The lesions are not usually grouped though they may be so on the face or the backs of the hands. They vary considerably in appearance according to their situation. On the face they are of a brownish-red tinge with a yellowish purulent apex or present a peculiar translucent waxy appearance, and they have a tendency to be grouped about the chin, malar prominences, temples and about the supra-orbital ridges, and for several lesions to coalesce to form

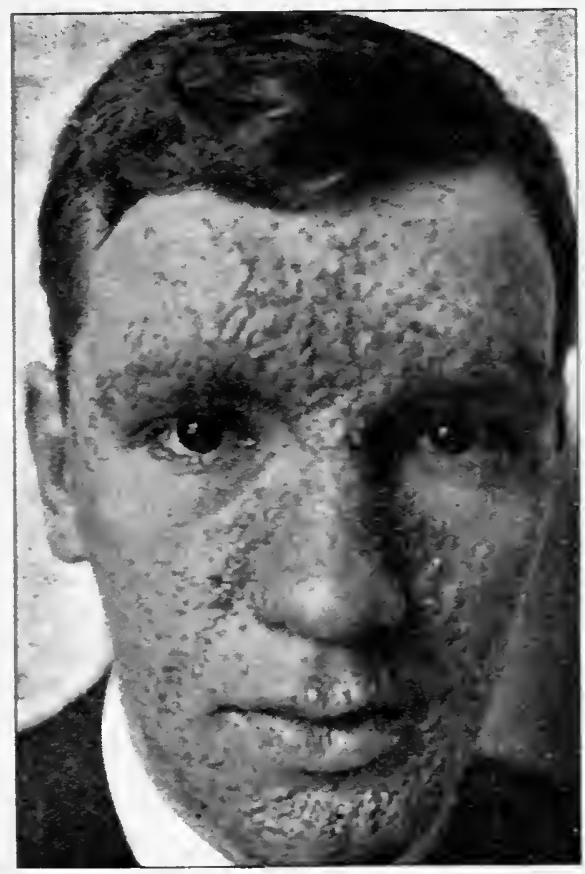

FIG. I69.-Acne Agminata.

(Dr. W. Kenneth Wills' case.)

yellowish crusted patches (acnitis, Acne agminata). On the extremities, owing to the venous stasis with which the eruption is generally associated, they tend to be livid or dark purple in colour, especially on the legs, and to aggregate to form small irregular ulcers (folliclis) situated especially between the fingers. On the trunk they may look like lesions of ordinary acne but differ in leaving a more marked scar, while on the buttocks they sometimes reach the size of a large pea and evolve like small scrofulous gummata.

The eruption generally comes out in crops and may persist for years, but is subject to intermissions and relapses, the latter frequently taking place in the cold weather. It is usually accompanied by definite signs of tuberculosis, such as phthisis, tuberculous glands, tuberculous 
disease of bones, scrofulous gummata, Lupus vulgaris, or other form of cutaneous tuberculosis, and is commonly associated also with a weak peripheral circulation, showing itself in cold hands and feet and the mottled cyanosed appearance of the limbs known as acro-asphyxia. In several instances it has been known to occur in combination with Lupus erythematosus, but this is of no great significance as one of the predisposing factors to Lupus erythematosus is a feeble peripheral circulation.

Histopathology.-The papulo-necrotic tuberculide is the clinical evidence of a cellular infiltration, situated in the hypoderm or deeper parts of the corium and most marked at first around the capillaries of the pilo-sebaceous follicles and the sweat-coils. Like Lichen scrofulosorum it consists of cells of the lymphocytic type, mast-cells, epithelioid cells, and occasionally plasma-cells and giant-cells. The character of the infiltration varies considerably in different cases, in one being diffuse and indefinite, in another collected in foci with giant-cells as in Lupus vulgaris. Tubercle bacilli have not been found so far in the lesions.

Etiology and Pathogenesis.-It is most common in adults between the ages of 20 and 30 , but is often found in young children before the ordinary acne age, and even in infants when it is liable to occur about the buttocks. It affects males and females with about equal frequency, though certain observers consider it more common in females. Cold appears to be a predisposing factor, and it is generally worse in winter and may disappear in the warm season to recur when the cold weather sets in. Cases are on record, however, in which heat appears to have been the determining factor and in which an outbreak was associated with increased activity of the sweat-glands.

Though no tubercle bacilli have been found in the lesions inoculation experiments in guinea-pigs have on several occasions given positive results. In one instance, for example, nodular lesions followed the injection of an attenuated culture of tubercle bacilli in a guinea-pig, and in another scrapings from a papulo-necrotic tuberculide mixed with glycerine and injected into a guinea-pig, both subcutaneously and into the peritoneal cavity, produced nodules containing tubercle bacilli. In the majority of cases inoculation experiments have given negative results.

The clinical appearances and indolent course of the lesions, their histology, their local reaction to tuberculin, and their occurrence in tuberculous subjects, all point to their being connected with tuberculosis and most probably due to attenuated tubcrcle bacilli in situ, which have been carried there via the blood-stream. A further argument in favour of this view is that cases have been observed in which a crop of lesions, clinically similar to papulo-necrotic tuberculides, has appeared after measles, in which tubercle bacilli have been found.

Diagnosis.-Considerable discussion has arisen as to whether the type of tuberculide of the face to which the name "acnitis " has been 
given, and that which occurs most commonly about the forearms and backs of the hands and which is known as "folliclis," are different entities or variants of the same pathological process. The general tendency at present is to regard them as having the same pathogenesis, their clinical appearances being modified by the different situations in which they occur.

The papulo-necrotic tuberculide, especially when it occurs about the trunk, might be mistaken for Acne vulgaris but it is more indolent, purplish in colour instead of reddish, does not occur as a rule around a comedo, and is usually associated with signs of tuberculosis.

From suppurating chilblains the lesions on the hands are distinguished by being smaller, more indolent, and painless, while the chilblains are actively inflamed, hot, tense and painful.

Treatment.- The general treatment is that which is applicable to all cutaneous manifestations directly or indirectly resulting from the tubercle bacilli, and consists of putting the patient in the best possible environment to improve the general health, a nutritious dietary; and the administration of tonics such as cod-liver oil, iron, and the phosphates. Tuberculin injections should be employed with great care for fear of aggravating the skin affection or rendering active some internal focus of tuberculosis.

Locally the tendency to suppuration may be diminished and the lesion aborted by scraping off the crust, touching the base of the ulcer with carbolic acid, and dusting it with iodoform or europhen. With the object of preventing secondary infection with pyogenic cocci, the affected part should be bathed daily with $I$ in 5000 perchloride of mercury solution.

\section{REFERENCES}

\section{Lupus Vulgaris}

Adamson, H. G. Multiple lupus after measles (Brit. Journ. Derm., I904, xvi. p. 366).

Ashihara. Lupus carcinoma (Arch. f. Derm. u. Syph., Igor, lvii. p. I93).

BEDford, P. V. Nascent treatment of Lupus nasi (Brit. Iled. Journ., I9I3, ii. p. 767 ).

Doutrelepoxt. Treatment of Lupus vulgaris (Brit. Journ. Derm., I910, p. 273).

KLINGMUULler, V. Contribution to tuberculosis of the skin (Arch. f. Derm. u. Syph., I904, p. I67).

Liddell, J. Lupus circumscriptus nodularis (Brit. Journ. Derm., Igoo, p. 3I9).

Little, Graham. Vaccinal lupus (Idem, Igor, xiii. p. Si).

NeIsser. Observations on therapeutic \& diagnostic value of old tuberculin (Therapie der Gegenwart, Berlin, I90o, xli. p. 22).

Reys, A, \& KJjer-Petersex. Observations on opsonins (Lancet, Igo8, i. 9 I9, p. I000).

SAALFELD, E. Lupus follicularis disseminatus (Derm. Zeitschr., Bd. viii. I90I, p. 225).

Schiele, W. Lupus from piercing auricle (Arch. f. Derm. u. Syph., Wien, 1903, lxvii. p. 337). 
Senger, E. (Derm. Centralbl., Leipzig, 1908, xi. p. 290.)

Sequeira, J. H. Seven years' experience of the Finsen treatment (Lancet, I908, i. p. 7I3).

_- Lupus carcinoma (Brit. Journ. Derm., I908, xx. p. 40).

- Treatment of intra-nasal lupus by sodium iodide and peroxide of hydrogen (Idem, I9I I, xxiii. p. 327).

- Tuberculosis of the skin (Allbutt \& Rolleston, System of Medicine, I9I I, ix. p. 466).

SPITZER. Lupus vulgaris \& Lupus erythematosus in one patient: histology (Ann. de Derm. et de Syph., Paris, 1907, viii. p. I89).

StraudberG, O. Pfannenstiel's method (Berlin Klin. Woch., I9II, p. I06).

Strebel. Treatment of lupus by high-frequency electricity (Derm. Zeitschr. Berlin. I905, xii. p. $3^{8}$ ).

Tod, H. F., \& Westers, G. T. Lupus \& tuberculous disease of ear, nose, \& throat treated by tuberculin (Practitioner, I908, lxxx. p. 703).

Walker, N. Carcinoma and X-ray treatment of lupus (Scot. Med. \& Surg. Journal, Edin., I904, xv. 5. Edin. Med. Journ., I9I I, vi. p. 295).

Westers, G. (Lancet, I907, ii. I375, I 449 ).

Wiemmans, P. Radium treatment of lupus (Monats. f. prakt. Derm., Hamburg, I906, xliii. p. 687).

WILD, R. B. Supervention of epithelioma upon Lupus vulgaris (Medical Chronicle, I907, Nov.).

Wilson, F. P. (Brit. Journ. Derm., I9Io, p. 252).

Wolters. Lupus nodularis (Arch. f. Derm. u. Syph., I904, p. 83).

\section{Miliary Tuberculosis}

Gaucher \& Druelle. (Ann. de Derm. et de Syph., Paris, I903, iv. p. 945 .)

Nobl. (Brit. Journ. Derm., I900, p. I 47. .)

Tir.eston. (Arch. Int. Med., Chicago, I909, iv. p. 2I.)

\section{Tuberculous Ulcer}

Darier, J. (Précis de Derm., Paris, I909, p. 266.)

\section{Tuberculosis Verrucosa}

ERnst, P. (Derm. Centralbl., Leipzig, i907, xi. p. 66.)

Lassar. Bovine inoculation (Derm. Zeitschr., I904, x. p. 505).

Schulze. Tuberculosis verrucosa cutis in miners (Arclı. f. Derm. u. Syph., I904, p. 329).

\section{Tuberculides}

Boeck, C. (xiii. Internat. Congrès de Méd., Paris, I90o, sect. Derm. 95.) Sarcoid (Festsclır. Kaposi, Ergänzungsband zum Arch. f. Derm. u. Syph., Wien, I900, p. I53).

Civiatte, A. Parapsoriasis type of tuberculicle (Ann. de Derm. et de Syph., I 906, vii. p. 209).

Darier \& Roussr. (Arch. de Méd. expér. et d'Anat. path., Paris, I9o6. xviii. p. I).

Fox, T. Colcott. (xiii. Internat. Con. de Méd., Paris, Igoo, sect. Derm. I22.)

Eczema seborrhoicum with spines (Brit. Journ. Derm., I906, p. 3 I 7) 
Friedlayder, D. (Brit. Journ. Derm., I9L 2, p. I3.)

Golgerot, H., \& LAROCHE, G. Experimental reproduction of cutaneous tuberculides (Arch. de méd. expér. et d'Anat. path., Igos, v. p. $58 \mathrm{I})$.

Kreibich \& Kratss. Boeck's benign miliary lupoid (Arch. f. Derm. u. Syph., I908, xcii. p. I73).

Leiners \& Spieler (Idem, i906, 1xxxi. p. 22 I).

Leredde. Les Tuberculides (Semaine méd., Paris, r9or, xxi. p. 54).

Lesseliers. (Ann. de Derm. et de Syph., Paris, I906, vii. p. S9 ${ }^{-}$.)

Macleod \& Ormsbi. (Brit. Journ. Derm., I90 I, xiii. p. 36 7 .)

Marfas, A. B. (Presse méd., Paris, I909, xvii. p. 457.)

Neunaxi. Tuberculosis of the skin (Wien. Klin. Rundschau, I903, i.).

Oliver, W. J. A case of Acne agminata (Brit. Journ. Derm., I9If, xxvi. p. 439).

Philippsox. Phlebitis nodularis necrotisans (Arch. f. Derm. u. Syph., I90I, lv. p. 215).

—Contrib. à l'étude des tuberculides (Presse méd., Paris, I902, x. p. 2 I 4 ).

PICk, W. Persistent Erythema nodosum (Arch. f. Derm. u. Syph., I904, lxxii. p. $3^{61}$ ).

RAVogLI, A. Tuberculides in relation to general tuberculosis (Journ. Cut. Dis., I909).

Riehl. (Arch. f. Derm. u. Sỵph., I90 4 , lxxii. p. $4^{6}+$.)

Rist, E., \& Rollaxd, J. Études sur la ré-infection tuberculeuse (Ann. de méd., July, I9I4).

RoxA. (xiii. Internat. Con. de Méd., Paris, r9oo, sect. Derm. 698.)

Schamberg. (Journ. Cut. Dis., I909, xxrii. p. If.)

Schaumasi, J. Études bacteriologiques sur le Lupus pernio et les Sarcoïdes cutanées (Stockholm, I9I $\gamma$ ).

Sequeira, J. H. (Brit. Journ. Derm., I90o, xii. p. Ijj. Idem, Igot, xvi. p. 38 I.)

A new conception of the tuberculides: an account of the work of Rist \& Rolland (Idem, I9I5, xxvii. p. 37I).

Thibierge \& Gastixel. (Bull. Soc. Méd. des Hôp. de Paris, igog, xxvi. p. $75 \%$.)

Whitfield, A. (Brit. Journ. Derm., I905, xvii. p. 24 I. Idem, I909, xxi. p. I).

Zieler, K. (Münch. Med. Wochenschr., I908, lv. p. I685.)

- Toxischen Tuberkulosen der Haut (Arch. f. Derm. u. Syph., I9Io).

\section{Lupus Pernio}

DARIER. Lupus pernio, a tiberculide (Proc. iv. Internat. Cong., I900).

KREIbich. (Arch. f. Derm. u. Syph., I904, xx1. p. 3.)

Kühlyañ. (Idem, Bd. c., Ht. I-3.)

Sequeira, J. H. (Brit. Journ. Derm., igo8, xx. p. I37.)

Zieler, K. Lupus pernio (Arch. f. Derm. u. Syph., Igos, xciv.).

\section{Erythema Induratum}

Kuzxitzki, E. Erỵthema induratum (Arch. f. Derm. u. Sỵpl., igio, cir.). 


\section{LEPROSY}

Syn.: Lepra Arabum; Elephantiasis Graecorum; Leontiasis; Fr., La Lèpre; Ger., Der Aussatz; Norwegian, Spedalskhed.

Definition: Leprosy is a general disease with a chronic and paroxysmal course, due to the Bacillus leprae of Hansen, and characterised by lesions in the skin, mucous membranes, nerves, and viscera. It is transmissible from man to man, but the method of infection is unknown.

History.-Leprosy, from the Greek word $\lambda \epsilon \pi \rho$ ós, scaly, has been known from very ancient times. It is referred to frequently in the Bible, in various old Japanese books and, according to certain authorities, was described in Egyptian papyri as early as 2000 B.c., but this assertion is doubted by modern Egyptologists. It is generally believed to have originated in Egypt and the East and to have spread from there in all directions, even reaching such remote parts of the world as Iceland and Australia. There are those who go so far as to believe that it was originally endemic in Egypt and that it spread from there to the Orient, but this is uncertain and all that can be definitely established is that it was known in Egypt, India, and China, long before the Christian Era. In those early times, and down to little more than a century ago, although what we now know as leprosy did undoubtedly occur, the name leprosy, owing to the imperfect knowledge of the causation of disease, was used in a wide and generic sense and included all manner of skin diseases, a fact which it is important to remember when attempting to estimate the prevalence of the disease in different countries at different times. In the "leper whiter than snow" of the Bible we now recognise the disease known as vitiligo.

References to it may be found in early Greek medical literature where it was known as elephantiasis and leontiasis. But the elephantiasis of the Greeks must not be confused with true elephantiasis, or elephantiasis of the Arabs, in which there is swelling of the legs, scrotum, etc. It was named leontiasis owing to a superficial resemblance of certain faces disfigured by leprosy to the face of a lion. It is mentioned by Hippocrates but his description suggests psoriasis rather than leprosy. Under the name "satyria" Aristotle described a disease which was probably nodular leprosy. It is to Aretaeus, however, in the first century of the Christian Era that we are indebted for the first clear description of the disease, while in the second century it was again described somewhat fully by Galen in France and Spain.

From Egypt and the East it was carried westward by the Roman soldiers, traders, and crusaders, to Europe where it gradually increased till, in the Middle Ages, it became a veritable scourge which reached its height about the thirteenth century, when it is estimated that there must have been at least 20,000 leper houses throughout Europe. At that time, and for several centuries later, there was a number of these 
leper houses in the British Islands, but it is doubtful if true leprosy ever prevailed to any extent here as the houses seem to have contained all sorts of skin and venereal diseases as well as leprosy, and were also alms houses where the indigent sick poor could end their day's. It would appear to have spread from Egypt to North and West Africa and from there to America in the train of the negro slave traffic. About the fifteenth century it began to decline rapidly in Europe owing to stringent, and too often cruel, methods of isolation which were enforced on lepers, alike by Church and State. Nor was the ban restricted to the true leper but was extended to all those who suffered from obvious skin disease and who were equally proclaimed lepers. These unfortunates were compelled to announce their uncleanliness by ringing a bell as they came along the road, were forbidden to speak to children, or to eat or drink with other than lepers and, in some instances, it is related that, by order of the Church, as soon as a leper was recognised a burial service was performed orer his person and he became officially dead. By the end of the sixteenth century comparatively ferr cases remained in France, Germany and Britain, though the disease was still common in eastern Europe, Spain, Scandinaria, the Faroe Islands and Iceland. It still prevailed until a century or two ago in the Shetland and Orkney Islands and in the Outer Hebrides but has now completely died out there. In the seventeenth century the number of lepers in the Shetland Islands was considerable. These were segregated in an island situated on the west of the Shetland group, named Papa Stour, which is separated from the mainland by a stormy sound effectively isolating it in anything but fine weather. The last case of leprosy in Papa Stour, so far as the writer has been able to ascertain from the older inhabitants, died over a century ago, and the present people, some of whom number lepers among their ancestors, are a hardy race of healthy crofters.

While leprosy has died out greatly in Europe it has broken out in other parts of the world. About the sixteenth century it spread to the West Indies and South America where it is now exceedingly prevalent. In the middle of last century a great outbreak occurred in the Hawaiian Islands where the disease was said to be of a particularly virulent type, and foci of it have been reported from other parts of the New World. During the first half of last century it was particularly common in Norway. In I 856 there were about three thousand lepers in that country but these, owing to methods of segregation and improved hygienic conditions, have gradually decreased until now there are only a few hundreds, and several of the leper asylums have been closed or converted into sanatoria for tuberculosis. The prevalence of the disease in Norway led to its being scientifically studied there and we are indebted to the Norwegians, Danielssen and Boeck, for the first comprehensive description of it, and to Armauer Hansen for the discovery of the leprosy bacillus in $18 / 3$.

Geographical Distribution.-Leprosy has a wide distribution over the globe and there is scarcely any country or latitude in which it has 
not been met with or which is beyond the range of possible infection by it.

In Europe it occurs chiefly in Russia, the Baltic Provinces, Bosnia, Herzegovina, Roumania and Turkey, the Mediterranean Islands of Corsica, Sardinia, Sicily, Crete, and Cyprus, in Portugal, the south and east coasts of Spain, Norway and Iceland. In Norway there are about 300 lepers, while in Iceland they number about 200. In France it has practically died out except for a few cases in Brittany and on the south coast. In Great Britain the disease has ceased to be endemic, though there are at present in England some 40 cases which have been imported chiefly from the West Indies and India. Only one instance is recorded in recent years where the disease was contracted in this country, namely that of a boy in Ireland who was infected by his father, a leper from Russia resident in Ireland.

In Asia it is common in Asia Minor, Persia and Arabia. In Ceylon there are about 400 cases. In India it has been estimated that at the present time there are about 200,000 lepers, but this is probably a low estimate; they are found especially along the coast, beside the great rivers, and in the valleys and districts on the lower slopes of the Himalayas. It is rife in the Malay Archipelago, throughout China, Korea, and Japan where it has been stated there are about Io,ooo lepers.

It is common in the Philippine Islands and is believed to have been introduced there from Japan about the middle of the seventeenth century. It is related that the Emperor of Japan sent a ship there containing I5o lepers to be looked after by the catholic priests and that from this it spread till thousands of the inhabitants became infected. In Australasia it is particularly common and is rife in Tahiti, Hawaii, and the Fiji Islands. In Australia itself it is somewhat rare and only small foci of it are met with, chiefly among the Chinese, in New South Wales, Queensland, and Victoria.

In South America it is prevalent in British Guiana, Brazil, Venezuela, Equador, and Mexico. It is rife in the West Indies, but there owing to segregation the numbers are being gradually reduced. In Jamaica, for example, in $\mathrm{I} 860$ there were believed to be about 800 lepers while at the present time this number has decreased to one-third although the population has nearly doubled.

In the United States it is rare and except for isolated cases only a few small foci of the disease occur in Louisiana, California, Minnesota and Florida.

In Africa it prevails along the northern littoral, in Egypt, Abyssinia, Madagascar, Central and East Africa, and to a less extent in West Africa. In South Africa it is a comparatively recent disease and was first mentioned in the middle of the eighteenth century. It was believed to have been introduced there during the early days of the Dutch colonisation by Malays imported from Dutch East India, but certain observers consider that it probably existed, before the advent of the white men, among the native inhabitants, namely the Hottentots, 
Bushmen, and Bantu races. It has apparently increased considerably in recent times in Cape Colony for in $189 \mathrm{I}$ it was estimated that there were only 600 lepers while in 1904 this number was doubled, the increase occurring both among Europeans and non-Europeans. The principal leper asylum in South Africa is the well-known penal and leper settlement at Robben Island.

\section{ETIOLOGY}

Bacillus leprae.-The Bacillus leprae of Hansen is now generally recognised to be the specific cause of the disease. Though up to the present time all the postulates of Koch have not been fulfilled by it, yet the fact that it occurs universally in leprosy in all its forms, that all the symptoms of leprosy can be explained as the direct or indirect result of a pathological process produced by it, that it is absent in all other morbid entities, and that it possesses characteristics which differentiate it from all other micro-organisms, render its position as the leprosyexciter as unassailable as that of the plasmodium in malaria.

Until recently the microorganism was believed to be one of the schizomycetes, or fission fungi, but the cladothrix arrangement and branching filamentary forms which have been met with in cultivation point to its belonging to the class of the streptotricheae.

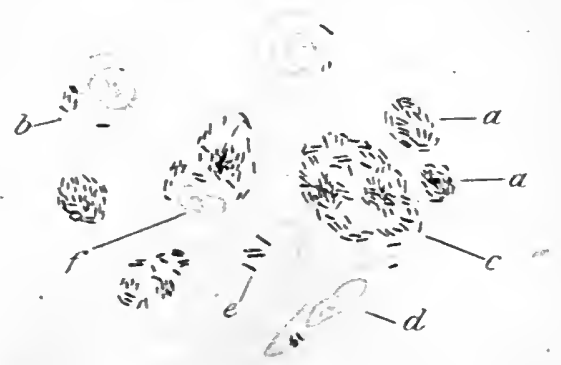

J. M. H. M. del.

FIG. I -o.-Lepra Bacilli in masses and in cells. $(x$ I000.)

a. Clump of bacilli in gloea. b. Bacilli in protoplasm of a cell. c. Clump of bacilli. d. Spindle-cell with a few bacili. $e$. Isolated bacilli. $f$. Nucleus pushed to periphery of cell.

Its exact botanical classification, howerer, is still under discussion and until this is settled it will be advisable to retain its old name of Bacillus leprae rather than the newer title of Streptothrix leproides. (Fig. I70.)

The lepra bacillus is a rod-shaped acid-fast bacterium which so closely resembles the tubercle bacillus, both in its morphology and staining reactions, that certain observers at one time regarded the two as identical. It is said to be less curved and more pointed at the ends than the tubercle bacillus and other minute differences between them have been cited but these are insufficient to render certain the recognition of isolated specimens under the microscope. It has also been found that the lepra bacillus when stained with carbol-fuchsin more readily loses the stain when treated with $20 \%$ sulphuric acid or alcohol than the tubercle bacillus, but this difference is not sufficiently marked to be of diagnostic value. The main distinction between the two organisms is that while the tubercle bacillus can be comparatively easily 
cultivated on artificial media and successfully inoculated in certain lower animals, the leprosy bacillus is exceedingly difficult to grow and attempts to cultivate it on media suitable for the growth of tubercle bacilli have given negative results, while, though several experimenters have asserted that they have succeeded in inoculating it in lower animals, their results have yet to be more widely confirmed before they can be finally accepted.

In addition to the typical short rods, other forms, such as coccoidal and club forms, have been detected in smears from nodules of leprosy, while on cultivation several observers have obtained what at first were regarded as different organisms, namely branching mycelial filaments and typical rods, but which were subsequently found to be phases of a pleomorphic organism of the streptothrix type, pointing to the acidfast rod being simply one phase in the life cycle of an organism of which the parent phase was probably the branching mycelium.

By special staining methods Unna found that he could differentiate two types of lepra bacilli. This difference in staining he regarded as due to certain of the bacilli being young and alive, while others were old and dead. He found that a large proportion of the bacilli in leprous tissue were dead and that dead bacilli occurred even in young newly formed lesions. This observation has been put forward as an explanation of the difficulty of cultivating the micro-organism.

Cultivation.-Ever since the discovery of the bacillus innumerable attempts have been made to cultivate it. In the past Hansen, Neisser, Koch, and a host of others all claimed to have succeeded, but further experiments have failed to establish the claim and have shown that either thcir technique was defective, that the cultivations had become contaminated, or that some other acid-fast micro-organism, like the tubercle bacillus, had been grown. At the present time, although a number of observers have obtained cultures which are more hopeful, still no medium has been found on which it will invariably grow. A great variety of media have becn employed, certain of which are so complicated that it is difficult to reproduce them. In the majority of instances the bacilli which have been obtained have been acidfast, though the degrec of this has varied, while in a few cases a non-acid-fast organism has been recovered which became acid-fast on injection into animals. It is important to remember in this connection that acid-fast and diphtheroid organisms are very common in man and may be contaminators.

Of the more recent attempts to cultivate the bacillus which have been claimed to be successful the following may be mentioned:

Clegg in Igog grew an acid-fast bacillus in symbiosis with amoeba and cholera vibrios, and afterwards succeeded in isolating it by heating to destroy the symbiants, and growing it on ordinary culture media. This micro-organism he believed to be the bacillus of leprosy. He found that it was pathogenic for guinea-pigs, subcutancous inoculations causing lesions rescmbling leprous lesions in the human subject. 
Duval in I9Io corroborated Clegg's work and in a similar manner obtained a somewhat different acid-fast bacillus. Later he found that he could grow this organism independently of amoeba by adding amino-acids to the culture media.

Kedrowski in Igro isolated from nodules of leprosy what he considered at first to be two distinct bacteria : (I) a non-acid-fast branching filamentary micro-organism, and (2) a slightly acid-resisting diphtheroid. These he injected into guinea-pigs and found that whaterer micro-organism was injected it resulted in the presence of acid-fast micro-organisms in the viscera of the animal, and in lesions suggesting visceral leprosy of human beings.

Bayon in IgII confirmed Kedrowski's observations and obtained a filamentary non-acid-fast micro-organism in cultures from leprous nodules and concluded that the parent form of Hansen's bacillus was a filamentary interlacing branching non-acid-fast micro-organism. With this pleomorphic micro-organism he produced in animals, such as rats, mice, and rabbits, lesions similar to those occurring in visceral leprosy of human beings. He also found on making certain serological tests that this strain was capable of reacting specifically with the serum of certain lepers, and that the inoculation of an extract of his culture caused a febrile reaction in cases of leprosy comparable to that which occurs in tuberculosis from injecting the old tuberculin.

Williams in IgII also obtained cultures of apparently different bacteria in lepers in Persia and India. These he subsequently concluded were different phases of one pleomorphic micro-organism, namely the Streptothrix leproides. According to his view the leprosy-exciter is not an acid-fast bacillus but a pleomorphic streptothrix which tends to lose its acid-fastness in artificial cultivation. $\mathrm{He}$ found the following different phases in the cycle of this micro-organism, namely,

(a) A non-acid-fast streptothrix in the mycelial stage, producing acid-fast rods.

(b) A non-acid-fast diphtheroid bacillus, producing acid-fast rods. This is really a streptothrix.

(c) An acid-fast bacillus, which is but the broken down stage of a streptothrix.

(d) An acid-fast mycelium.

By injecting the micro-organism in its acid-fast stage he succeeded in reproducing lesions resembling leprosy in guinea-pigs.

The similarity of the findings of Kedrowski, Williams, and Bayon suggest the probability that the elusive bacillus may have been cultivated at last. Against this, however, are the observations of various other workers who have attempted to repeat the above experiments and invariably obtained negative results. Fraser and Fletcher, for example, at the Institute for Medical Research, Kuala Lumpur, Federated Malay States, obtained material for cultivation from 32 nonulcerated nodular cases of leprosy and made 373 inoculations on various 
culture media, including media similar to those employed by Bayon, Duval, and Williams, and made according to their descriptions, but consistently failed to procure a culture of the Bacillus leprae though material swarming with bacilli was used on each occasion.

Inoculation Experiments on Lower Animals.-Up to the present time it may be said that no lower animal has been found in which the inoculation of leprous material will invariably produce the disease. Numerous experiments have been made on different animals, chiefly monkeys, rabbits, guinea-pigs, rats, and mice, the leprous material being inoculated under the skin, in the peritoneal cavity, in the anterior chamber of the eye, etc., but with little success.

In I909 Stanziale claimed to have inoculated it successfully into the eye of a rabbit, but no generalisation of the disease took place.

In IgII Duval reported successful inoculations into the peritoneum of two white mice, and into the subcutaneous tissue of a fullgrown monkey (Macacus rhesus).

In the same year Nicolle produced a hard swelling on the skin of a macacus monkey by injecting fragments of leprous tissue, but there was no generalisation. Other observers have also inoculated in different monkeys with doubtful success.

In I9I2 Bayon claimed to have succeeded in inoculating the disease into the testicle of a rat, and asserted that successful inoculations from human leprous nodules had been made in rabbits in at least four cases.

So far the successes have been extraordinarily few and the negative results innumerable. As an explanation of this it has been pointed out that the incubation period is probably very long and that the animal dies from other causes before the disease shows itself. But this explanation is insufficient and further experiments are necessary before it can be asserted that any lower animal is definitely susceptible.

A skin disease somewhat resembling leprosy was recently discovered in rats and has been designated "Rat-leprosy." It was first observed by Stefanski in Odessa, and later by Dean at Elstree near London, and has since been detected in various parts of the world, such as the United States, Australia, and Japan. Certain observers believe that this disease, which is due to an acid-fast bacillus resembling that of human leprosy, is true leprosy in the rat and that it probably bears the same relation to human leprosy as bovine does to human tuberculosis. It is easily transmitted from one rat to another by direct contact, through a skin abrasion, or by the subcutaneous inoculation of diseased material such as a ground-up nodule. Numerous attempts have been made to grow the micro-organism. Dean has succeeded in cultivating a diphtheroid from it, while Bayon managed to obtain a culture from the spleen of a rat which had been injected with a ground-up nodule from a spontancously diseased rat, and which produced similar glandular and visceral lesions in rats to those resulting from injection with the virus from spontaneously diseased animals. 
Mode of Invasion of the Bacillus.-Up to the present time the manner in which the lepra bacillus gains entrance has baffled research. Obviously there are various ways in which the invasion might take place, such as by the skin, alimentary tract, nose, respiratory passages, and genital organs, and it will be advisable to consider these seriatim.

Skin.-That the inoculation can take place ria the skin is borne out by a certain amount of definite evidence.-For example, there is a case on record of a medical man who became inoculated through a wound in the finger while confining a leprous woman, and Sandwith quotes the case of a Sister of Charity who became infected after pricking her finger while sewing a leper's clothes. There is also a number of authenticated instances in which the virus appears to have been introduced through vaccination and in which the bacilli have been found in the vaccine pustules.

Alimentary Tract.- It has been thought that the lepra bacilli may gain entrance through the mouth, or further down in the alimentary tract from infected water or food. This theory seems improbable owing to the extreme delicacy of the lepra bacillus and the likelihood of its being destroyed by digestive fluids, and the evidence in its favour is wholly inadequate as nothing suggestive of an initial lesion producing definite symptoms has ever been found in the alimentary canal nor has the bacillus, so far, been detected in any form of food stuff or in water.

Many articles of diet have been blamed in the past for causing it, such as rice, pork, fruits, caviare, fish, etc. Of these the one which has attracted most attention has been fish and, chiefly through the influence of the late Sir Jonathan Hutchinson, quite a number of observers believe that the disease is propagated by the eating of dried, decomposing fish. Without going into the arguments for or against this theory it is sufficient to point out that there is no evidence of a scientific nature in corroboration of it and until the lepra bacillus has been found in decayed fish the theory cannot be regarded as other than a mere hypothesis unsupported by scientific fact.

Nasal Mucosa.-Another path of infection and one which is more probable, is the nasal mucous membrane and upper respiratory tract. This is suggested by the frequency with which nasal symptoms form early manifestations of the disease, such as rhinitis, chronic coryza, ozaena, epistaxis and lepromata in the nasal mucosa, and the comparative ease with which lepra bacilli may be found in the nasal discharge, especially in the periodic exacerbations and after doses of iodide of potassium. Kolle examined the nasal mucosa of 137 lepers at Robben Island, and found bacilli in the mucus in all the +5 nodular cases, in 22 out of 30 cases of mixed leprosy, and in 2 I out of 60 maculo-anaesthetic cases. Other observers, however, have completely failed to find them in maculo-anaesthetic cases and have only succeeded where breaking-down lepromata were present in the mucosa. Should inoculation take place 
by the nose the most likely manner in which it might occur would be through an abrasion in the mucosa caused by picking it.

So far lepra bacilli have not been detected in the air and they have only been found in a few instances in dust and earth. If the bacillus were capable of being transmitted through the air the disease would be more infective than it is.

With regard to infection via the lungs, this seems improbable as it is comparatively rare to find the lungs invaded early by the lepra bacillus.

Genital Organs. - The genital organs are a possible means of entrance of the bacilli. If infection does take place in this manner it must be due to the presence of some infective sore, such as a leprous ulcer, in one or other of the genitalia, and not to the secretions, as the uterine discharges and the semen, even though the testicles may be diseased, do not contain bacilli unless secondarily contaminated.

Transmission of leprosy from man to man.-Leprosy is transmissible from man to man, either by direct contact or indirectly by some means by which the bacillus is carried from an affected to a healthy person. That this opinion is now generally accepted was evident at the Second International Conference on Leprosy held in Bergen in Igog when the first resolution passed was as follows: "Leprosy is a disease which is contagious from person to person whatever may be the method by which this is effected, and every country in whatever latitude it is situated is within the range of possible infection by leprosy and may therefore usefully undertake measures to protect itself."

Until about the middle of last century leprosy was almost invariably regarded as contagious and the most stringent measures were enforced to isolate the leper. In recent times, however, it was found that the contagiosity was in most cases extraordinarily slight, even less than in tuberculosis, and that persons might live in the closest intimacy and not infect each other. In consequence of this the harmful doctrine was spread abroad by certain writers that the disease was not contagious at all and that methods of enforced isolation were unnecessary, barbarous, and cruel.

There are several instances now on record in which deliberate attempts have been made to inoculate it on a healthy individual. There is the well-known experiment of Arning in which a criminal in the Sandwich Islands was inoculated in the arm from a leprous nodule. The man developed signs of leprosy four weeks after the inoculation and died a leper six weeks later. A doubt has been cast on the validity of this experiment, however, since the criminal came of a leprous family and was living in a country where the disease was endemic. Several earlier attempts have been reported by Danielssen, Profeta, and others, but none was definitely successful.

That the disease is communicated from person to person is proved by numerous instances where individuals previously healthy have acruired it by going to reside in a country where it was endemic and 
by living in intimate association with lepers, and these cases have, in several instances, returned home and communicated it to others who have never been abroad. There are two cases of this type on record in this country, namely, (I) the case reported by Bensen in which an Irishman acquired the disease in the West Indies, returned to Ireland, and communicated it to a brother who lived in close association with him and was said to have slept in the same bed, (2) the case already referred to in which a boy who had never been out of Ireland contracted it from his father a leper from Russia.

An instance is recorded by Jeanselme in which a native of Iyons went to live in a country where leprosy was endemic and married a healthy girl who came out to him from France. They had five children, three of whom became leprous, the source of the disease being traced to their nurse who was discovered to have leprosy and who eventually died of it.

There are several instances recorded where medical men have contracted it in the pursuance of their duties. For example there is the case referred to above in which a medical man confined a negro woman whom he recognised later to be a leper, and while doing so wounded his finger and noticed that the wound took a long time to heal. Shooting pains subsequently developed in his finger and arm and he eventually became a typical maculo-anaesthetic leper. Nurses and attendants on lepers have been known also to contract the disease from their patients.

According to the statistics of Kitasato in Japan about $7 \%$ of the children of leprous parents become infected, usually from the mother, and the proportion of cases of infection of people living in close association under one roof is 2.7 , while in the case of matrimonial infection it is about 3.8 .

In Norway, Sandes finds the proportion of children becoming infected, in whom both parents were lepers, to be $12 \%$ and Lie $39 \%$.

The manner in which the disease spreads also points to it being transmissible from man to man. In the island of Mauritius, for example, where it is now fairly common, its origin was traced to the importation of a single leper.

Mode of Transmission.-It has been thought by certain observers that the disease might be transmitted from one individual to another by some biting insect, and flies, mosquitoes, fleas, lice, mites, bugs, Demodex folliculorum, etc., have been suggested as possible carriers. With the intention of ascertaining if this be so a number of experiments have been carried out by feeding certain insects on leprous sores and examining them for lepra bacilli. Sandes obtained negative or doubtful results in the case of flies, mosquitoes and fleas. Currie on the other hand found that if mosquitoes were fed upon lepers and imbibed any body fluid containing lepra bacilli, the bacilli would be found unchanged in the alimentary tract for several hours afterwards, but that, as a rule, when the mosquito bit over a leprous nodule it did not imbibe bacilli, 
since it inserted its proboscis directly into a capillary and the blood did not contain bacilli except during a febrile exacerbation. He also ascertained that flies fed with leprous fluids contained bacilli in their intestinal tracts and faeces for several days afterwards.

In the case of the bed-bug (Cimex lectularius) several observers have obtained positive results and in consequence the insect is attracting considerable attention among leprologists as a possible carrier. Certainly the manner in which the disease is spread favours the possibility of the bug being capable of transmitting it, for the close contact which seems essential for infection, and the tendency for the disease to affect unclean people who are careless in their habits rather than the more hygienic, point to the carrier being a creeping rather than a winged insect. Goodhue of the Molokai Leper Settlement, Honolulu, found in bed-bugs acid-fast bacilli which he believed to be lepra bacilli. Long also found them in bugs that had bitten lepers, and Sandes, in a series of experiments in which he fed 75 bugs on leprous sores, found acid-fast bacilli in 20 of them in the alimentary tract, in smears made from the tissue juices, and in the macerated head and proboscis of the insect.

Attempts were made to transmit the disease from a leper to lower animals by means of leprous-fed bugs, but were unsuccessful.

Predisposing Causes.-The precise conditions necessary for infection to take place at present are imperfectly understood, but there are certain factors which seem to have a predisposing influence on it and of these the most important are the following :

Unhygienic Surroundings.-Leprosy is essentially a disease which affects the unclean and is met with chiefly in association with surroundings of squalor, poverty, overcrowding and insanitation.

Malnutrition from improper or insufficient feeding.

Sex.-Men for some reason or another seem to be more often attacked than women, in about the proportion of 3 to 2 .

Age.-The disease is not met with in infancy, but it may appear in early childhood as children are more liable to infection than adults, partly from the delicacy of their tissues, and partly from being in close contact with leprous parents or attendants. It is rare for it to begin after middle life.

Heredity.-There is no evidence of congenital leprosy and the disease has never been met with in the newly-born of leprous parents though, as is to be expected, a considerable proportion of the offspring of lepers develop the disease sooner or later. In one or two instances in which post-mortem examinations have been made on stillborn children of lepers, or those who have died soon after birth, neither the lepra bacillus nor any lesions suggestive of its presence have been detected. The placenta in leprous women has been examined for bacilli, but with negative results. 


\section{DESCRIPTION}

Incubation Period.-So great is the discrepancy in the length of the incubation period given by different observers that it proves the precise duration to be at present unknown. In some cases only a few weeks seemed to have elapsed between the presumable time of infection and the development of definite symptoms, while in others it has appeared to be years. Until the exact manner in which infection takes place has been ascertained the incubation period must remain uncertain.

Invasion. - In a considerable number of cases the disease develops insidiously, the first indication of it being some forn of cutaneous lesion: in others the invasion is marked by prodromal symptoms suggesting a general intoxication, such as progressive anaemia, vomiting, intermittent or irregular febrile attacks, mental depression or drowsiness, pains about the back and joints, a marked tendency to perspiration either local or general, epistaxis, and frontal headache. These symptoms are more or less vague and none is sufficiently constant to be of diagnostic value. They may precede the eruption by a few weeks or months, or be present for years before it appears and their nature only recognised in the light of subsequent events. They are explicable as the result of the dissemination of the bacilli and their toxins throughout the system.

Varieties.-After the prodromal septicaemic phase, local troubles develop either in the form of definite cutaneous lesions, or of peculiar sensations over certain areas, such as pricking, pain, hyperaesthesia, or itching, according as the bacilli find a suitable soil for their growth in the skin or nerres. It was on this account that Danielssen and Boeck distinguished two varieties, namely, nodular or cutaneous leprosy (Lepra tuberosa), and nerve leprosy (Lepra maculo-anaesthetica). In addition to these main groups, cases of "mixed leprosy" are met with in which nodular and maculo-anaesthetic lesions occur together. This division is purely arbitrary as cases which begin as nodular leprosy tend to develop maculo-anaesthetic lesions when they become advanced, and, to a less extent, nodular lesions may be superimposed in maculo-anaesthetic cases. Still the distinction is useful in emphasizing the prevailing features of an individual case. At the present time the precise conditions which determine that a case shall pertain to the one variety or the other are not understood. Various theories have been advanced to explain it, such as:

(I) That it is the result of a quantitative difference in the bacilli, nodular leprosy occurring when the bacilli are numerous and maculoanaesthetic leprosy when they are sparse.

(2) That it is due to a qualitative difference in the virulence of the bacilli, virulent bacilli causing nodular leprosy and bacilli of low virulence giving rise to the maculo-anaesthetic type. 
(3) That nerve leprosy is the result of an immunity in certain individuals which renders the skin an unsuitable soil for the propagation of the bacilli while the nerves remain suitable for their growth. None of these theories is altogether satisfactory.

\section{Nodular Leprosy or Lepra Tuberosa}

The first cutaneous evidence of nodular leprosy is the appearance of erythema, diffuse or more usually in patches, symmetrically distributed, chiefly on the face, extensor aspects of the arms, backs of the hands and feet, and usually associated with a rise of temperature. These lesions either disappear when the temperature declines, or become definitely raised, indurated, and transformed into nodules or plaques from a cellular infiltration of the deeper parts of the skin and subcutaneous tissue. Sometimes the plaques may be so extensive as to occupy an entire region, such as the extensor aspect of the forearm. New nodules or "lepromata" tend to come out, either singly or in crops, at varying intervals, either preceded by erythematous lesions or arising on apparently healthy skin, each outbreak being accompanied by a rise of temperature, known as "leprotic fever," which may last for a day or two. The nodules have a smooth surface, are round, oval, or irregular in shape, vary in size from a pea to a walnut or larger, have a well-defined border and, in white skins, present a dusky red or reddish-brown colour. They may occur anywhere on the cutaneous surface but are most common on the face, especially about the forehead, malar prominences, cheeks, nose, lobes of the ears, and chin, are frequent on the forearms and thighs, and rare on the scalp, palms and soles. At first they are hyperaesthetic but later tend to become anaesthetic, and may be so numerous in advanced cases as to involve practically the whole of the skin of the face. The hairs fall out wherever they occur, the eyebrows and eyelashes are generally lost but the hairs of the scalp are unaffected as lepromata are rare in that situation. On the face the nodules and infiltrated patches give rise to the characteristic disfigurement which suggested in the past such names for the disease as leontiasis and satyriasis. The forehead is irregularly thickened, studded with nodules, and broken up by deep horizontal fissures, the supra-ciliary ridges are hairless, nodular and prominent, the alae of the nose are thickened, the bridge depressed from ulceration and breaking down of the cartilage, and the whole nose coarse and flattened, the lips are thick and everted, and the ears nodular, irregular, and the lobes swollen. (Fig. I7I.)

Occasionally after an attack of leprotic fever the older nodules may improve, possibly as the result of the production of some antibody inimical to the life of the micro-organisms in the skin.

The lepromata may persist for a considerable time unaltered but tend to involute eventually. Sometimes they become hard and fibrous and may persist indefinitely, but as a rule they break down like super- 


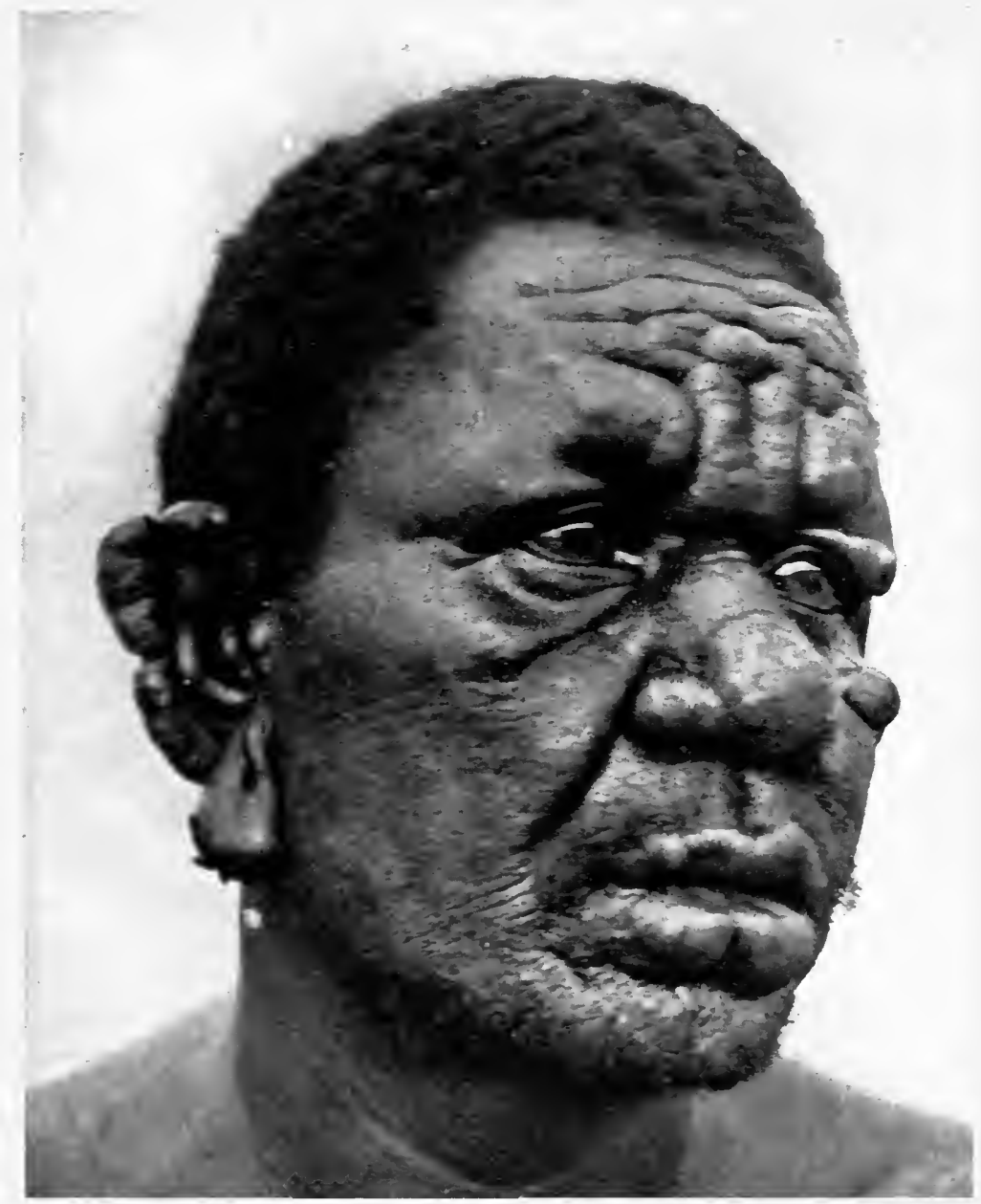

FIG. I-1.-Nodular Leprosy.

(From Photographic Collection, London School of Tropical Medicine.

[To fxie p. 50 . 

ficial gummata or undergo ulceration. The ulcers which result have an irregular border, and the floor presents a peculiar glazed appearance and is covered with a glairy sero-purulent discharge. They may heal under suitable treatment, giving rise to irregular, sometimes hypertrophic, scars, and leading to contractures, or they may burrow deeply into the underlying tissues, causing more or less severe destruction, denuding bones and tendons, and even ending in gangrene and sloughing. When the case becomes advanced the nerves are liable to be involved and lesions of the maculo-anaesthetic type to develop.

In association with the skin lesions the glands are usually affected, especially those of the neck, throat, axillae, and groin. These may become so large as to interfere with the morements of the joints, or by pressure to impede swallowing or even breathing. They have a tendency to break down and to give rise to discharging fistulae.

In the majority of cases of nodular leprosy the eyes are attacked. At first they become suffused and painful and the lachrymal secretion is increased. The conjunctiva and cornea next become the seat of small lepromata, or of a diffuse leprous infiltration. Occasionally the lepromata take the form of minute whitish specks on the cornea when the condition is known as "Keratitis punctata leprosa." The disease may then extend throughout the anterior chamber, involve the iris and uveal tract and lead to iritis and irido-cyclitis, but the retina, lens, optic nerve, and vitreous humor, are rarely implicated. As the result of the inflammatory disturbance the eye becomes swollen, and when this subsides, contraction and more or less complete blindness may supervene. At first it was thought that the ere became involved by an extension of the infection from within outwards, but the more recent idea is that the bacilli spread on to the globe from the margins of the eyelids.

The eyelids themselves are a common site for lepromata and when they ulcerate and heal cicatricial changes result, causing ectropion and the exposure of the bulb to infection by septic micro-organisms and to injury, possibly eventuating in destruction of the eye.

The mucous membranes usually become invaded by the leprous infiltration. Lepromata are frequent in the nasal mucosa, more or less blocking up the passage and involving the cartilage, and it is by the breaking down of these that the nose becomes flattened. The buccal mucosa is often attacked and lepromata may be present about the tongue, pharynx, urula and throat, interfering with mastication and deglutition, and on the epiglottis and rocal cords, causing a raucous voice and impeding respiration to such an extent that tracheotomy may be necessary.

The nails are sometimes affected but in no characteristic fashion and may present ridges, or furrows, or be thickened, brittle and opaque.

In children the development of the genital organs is retarded. In females the menstruation may be delayed or prevented and, where the 
disease has appeared later, it may become irregular and the menopause may be premature. In males an imperfect development of the testicles takes place which generally renders them impotent. It has been found that the sexual power in boys may be slightly increased at first in nodular leprosy but that this soon declines, and that consequently few children are born to nodular lepers, while those who are born do not usually live long.

Blood.-The changes in the blood are not characteristic. In oldstanding cases there is some anaemia and a diminution and destruction of red blood cells. The leucocytes are normal except during a febrile exacerbation when there may be a definite leucocytosis. Eosinophilia has been observed. Bacilli have been found in the blood both in the leucocytes and in the blood fluid during a febrile exacerbation.

Urine.-The urine shows no definite changes though an increase of ethereal sulphates has been described.

As the disease advances the face becomes more and more deformed and covered with nodules, ulcers and cicatrices. The borders of the flattened nose become purulent and crusted, and the discharge from it emits a singularly pungent foetid odour which renders nursing a painful duty. The eyes are destroyed, the sense of smell and taste are lost, and only hearing remains. Through the difficulty in swallowing and the consequent interference with nutrition the patient becomes weaker and weaker; more or less severe pain sets in owing to the involvement of nerves, the internal organs, such as the liver and spleen, become implicated, and derangements of digestion, abdominal pains, and diarrhoea supervene. But in spite of it all in many cases the brain does not seem to be impaired and even when almost every organ is affected the mind may remain clear, though it has been frequently noted that the patients become curiously sensitive, peevish, and difficult to manage; in other cases acute mental symptoms supervene. The prostration gradually increases and the human wreck dies in a state of inanition, or from some intercurrent disease, such as pleurisy, amyloid discase of the kidneys, or pneumonia.

Sometimes instead of steadily progressing the disease may become stationary for a prolonged period and then, possibly as the result of a chill or some other depressing influence, may undergo a marked exacerbation, leading rapidly to a fatal issue.

\section{Maculo-Anaesthetic or Nerve Leprosy}

Of the two forms of leprosy, the maculo-anaesthetic is the more common, especially in countries where the disease has long been endemic. In it the lepra bacilli invade the nerves and, by setting up a lepromatous hyperplasia in the connective tissuc about the nerve fibres, cause at first irritative lesions and finally anaesthetic and trophic lesions from destruction of the nerve by compression. In the irritative phase the area of distribution of the nerve affected is the site of sensory 


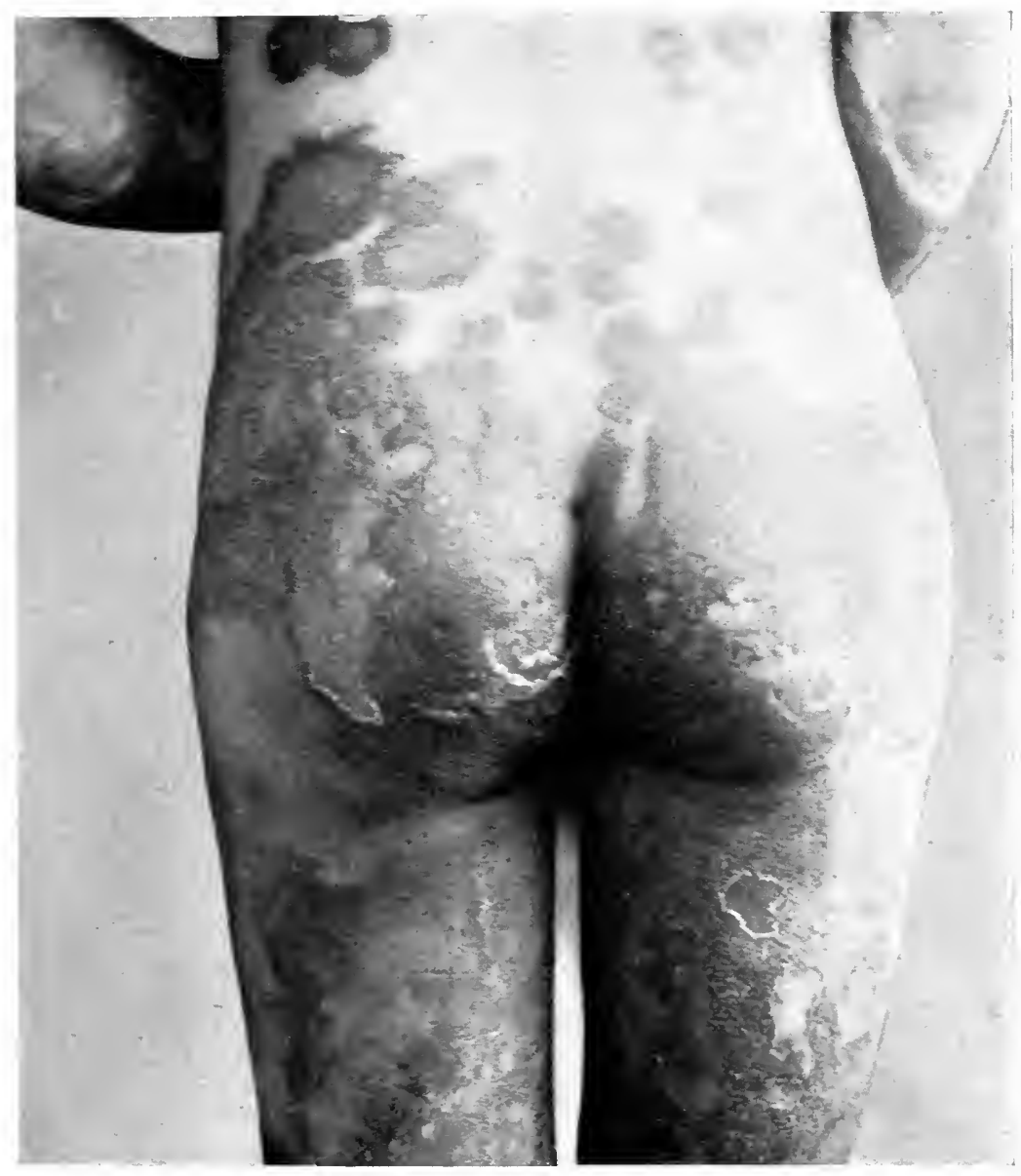

FIG. I /2.-Maculo-Anaesthetic Leprost.

(From Photographic Collection, London School of Tropical Medicine. 


PLATE VIII.

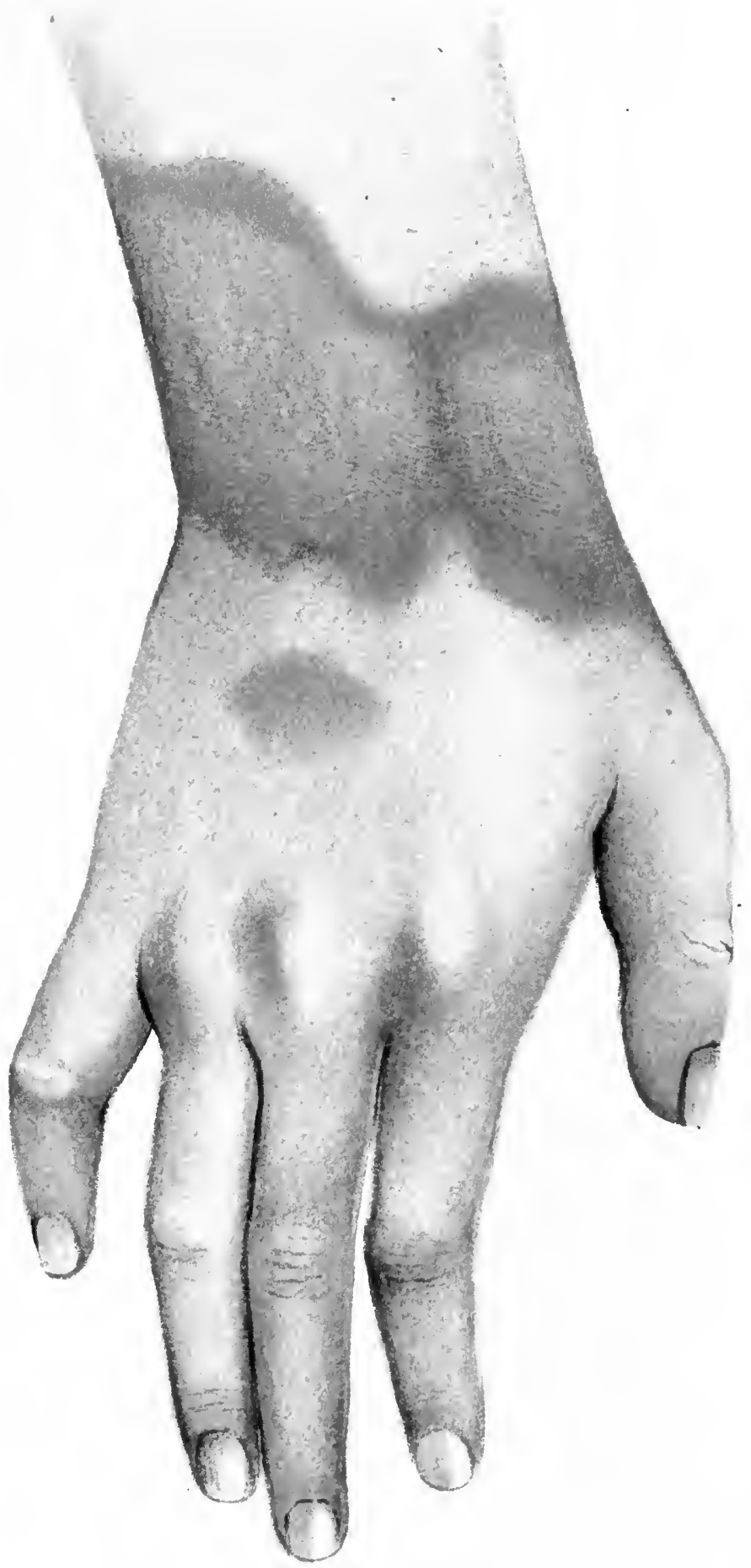

MACULO-AN HSTHETIC，LHPROSY.

Toface to.stt. 
disturbances, such as hyperaesthesia, pricking, burning, itching, or a peculiar feeling of numbness, or of raso-motor phenomena, such as flushing, or modified Raynaud's symptoms. Sometimes there is perversion of sensation, such as confusion of heat and cold, or errors of localisation, such as sensation being referred to a point outside the area excited. Not infrequently shooting or neuralgic pains may be felt along the nerve and these may be so serere and lancinating in character as to prevent sleep and to constitute a reritable crisis. Twitching of the muscles has sometimes been noted, especially when the face is involved. As the irritation continues, vesicles or small bullae may develop, the contents of which are serous at first and purulent later, and which may give rise to superficial ulcers. (Fig. I72.)

Erythematous or pigmented macules or patches next appear. These vary in size from small macules to patches several inches in diameter, are slightly raised, well-defined, smooth or scaly on the surface, and tend to involute in the centre giving rise to circinate lesions the borders of which, though slightly raised, are not definitely indurated, are duskyred or brownish-purple in tint and may be scaly or occasionally dotted over with small vesicles. In the centre of the patches the hairs are lost, the sweat-glands atrophy, and the skin becomes curiously dry and wrinkled. The coalescence of contiguous patches results in large areas enclosed by an irregular gyrate border. These macules or patches are situated about the face, back, shoulders, forearms, knees and buttocks.

Gradually the raised active border settles down and flat, faintly reddish-brown or pigmented patches persist which, in some cases, form a coarse network enclosing spaces in which the pigment is absent and suggesting ritiligo, hence the old name of "Vitiligo gravior." By this time the compression on the nerve has been sufficient to produce anaesthesia, which may not be confined to the patches but may occur along the whole distribution of the nerve, usually beginning in the case of the ulnar nerve at the tip of the little finger. The patches become insensitive to heat, cold, and pain, so that the point of a knife may be dug into them or a burn may occur on them without the patient being aware of it. For a time they remain sensitive to touch and can appreciate light stroking with a feather, while after the centre of the lesions has become anaesthetic definite hyperaesthesia may still persist at the border and for some short distance beyond it, but in time the anaesthesia becomes complete. Where the patches are large some fibres of the nerve supplying them may escape, with the result that small areas of sensibility may be present. (Plate VIII.)

The peripheral nerves most usually involved are the ulnar, peroneal, sciatic, median, radial, and the branches of the cervical plexus. The affected nerve may be uniformly thickened but more often presents fusiform swellings upon it. In the case of the ulnar nerve the enlargement can be felt behind the olecranon process, in the external popliteal nerve beneath the head of the fibula, while in the cervical plexus the 
nerves are liable to be uniformly thickened and to stand out like cords when the head is flexed.

Not only do the pathological changes in the nerves cause sensory disturbances in the areas supplied by them, but atrophic changes supervene in the muscles, accompanied by a proportionate loss of muscular power. At first the reflexes are exaggerated in the affected muscles, but later, when the reactions of degeneration set in, they become diminished and eventually disappear. The paresis of the muscles is especially noticed on the face and extremities. The muscles of the eyelids are frequently involved, especially the Orbicularis palpebrarum leading to ectropion, preventing the closing of the eye, and causing it eventually to become dry and shrivelled, or rendering it liable to injury, and septic infection. The muscles of the eye itself may become implicated and diplopia or strabismus result. The Orbicularis oris is often affected causing the mouth to droop and saliva to dribble out. Paresis of the facial muscles may occur giving rise to distortions of the face or to a staring, expressionless look. Owing to paralysis and atrophy of the extensors of the fingers a condition of permanent flexion of the middle and distal phalanges is frequently present with the production of the characteristic " main en griffe," or leper claw, while the involvement of the antero-external muscles of the limb prevents the extension of the foot, causing drop-foot and giving rise to a peculiar gait owing to the patient having to lift the foot high in walking to avoid injuring the toes. (Fig. I73.)

Trophic changes occur also in the skin, and ulcerations are common in the anaesthetic areas. On the foot, especially about the ball and heel, perforating ulcers are not infrequent as the result of pressure or injury and when they occur are liable to extend far down into the tissues, even causing destruction of bone. In the advanced stages of this variety of leprosy, necrosis and absorption of bone is of common occurrence and leads to loss of the fingers or toes, leaving small soft stumps which generally present the remains of the nail at the tip. This may spread up and involve the bones of the leg or forearm. In some instances dry gangrene supervenes and fingers and toes or portions of limbs may undergo spontaneous amputation.

In maculo-anaesthetic lepers the sexual power is retained much longer, hence more children are born to them than to nodular lepers.

This type of leprosy runs a long course and may go on for thirty years or more. In many case it tends to become arrested and, but for the anaesthesia, paralysis, and mutilations, might for practical purposes be regarded as cured, although bacilli have been found about the nerves even during thesc periods of quicscence. When death supervenes it is frequently the result of some intercurrent disease, such as tuberculosis or amyloid disease of the kidneys. 


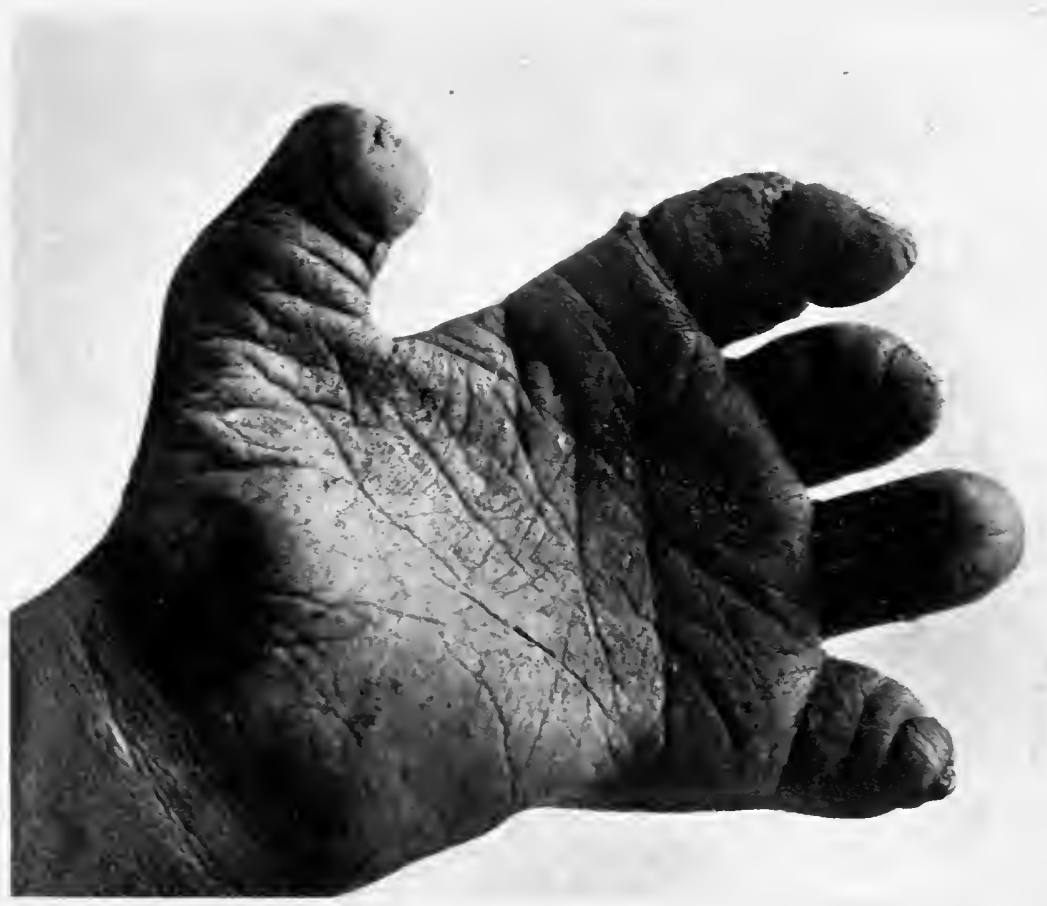

FIG. 173--Leprosy.

Shows deformity of hand and mutilated fingers.

(From Photographic Collection, London School of Tropical Medicine.) 



\section{Mixed Leprosy}

This name has been applied to those cases in which lesions of the nodular and maculo-anaesthetic type co-exist. Some cases may be mixed from the first, others commence as nodular cases and the involvement of the nerves and the appearance of maculo-anaesthetic lesions occur as late phenomena, while, more rarely, maculo-anaesthetic lesions may be present first and nodules may be added subsequently.

The term Para-Leprosis has been applied by Zambaco, Gluck, and others to various lesions occurring in descendants of lepers, such as thickenings of the nerves, paralysis of the muscles, and certain trophic changes, such as loss of the phalanges, which they believe to be hereditary and the result of the disease in their forbears. This view, however, is not generally accepted.

Pathogenesis.-After gaining entrance into the tissue the bacilli appear to lie at first in the lymph spaces and, owing to their presence there, to $\mathrm{pr}$ : duce a mild form of inflammatory reaction. In comparison with that caused by the tuhercle bacillus this reaction is slight but results in a peculiar type of infiltration which, though it has the same broad characteristics as that of the other infective granulomata, has peculiarities which render its diagnosis certain. The bacilli are disseminated through the system either by the lymph stream, or by the blood, and may be found in the nodules or lepromata in the skin, eyes, mucous membranes, lymphatic glands, viscera, and peripheral nerves, but not in the muscles, bones, and joints. According as the bacilli show a special predilection for the peripheral nerves, in contra-distinction to a more general invasion of the tissues with the formation of lepromata in the skin and viscera, so the two great types of the disease are produced -namely, the maculo-anaesthetic and the nodular type.

Histopathology. - Structure of Lepromata.-The lepromata, wherever they may be situated in the body, have the same histological architecture. At first the infiltration is non-distinctive and consists of round and spindle-shaped connective-tissue cells (derived from the fixed tissue and the adventitia and endothelium of the blood-vessels), lymphocytes, mast-cells, and occasional polymorphonuclear leucocytes should pyogenic micro-organisms be present. So far the situation of the bacilli is mainly extra-cellular. As they multiply they form colonies or globi united by homogeneous zoogloea, and the infiltration begins to show the presence of plasma-cells around the blood-capillaries. A certain number of bacilli become intra-cellular, and large characteristic cells containing vacuoles, one or more badly stained nuclei, and bacilli singly or in clumps may be noted. These are the socalled lepra-cells, as to the origin of which there has been much diversity of opinion whether (I) they are really cells derived from connective tissue cells or plasma-cells through the presence and action of the bacillus and its toxin within them, or (2) are simply clusters of bacilli round a lymphocyte or other cell. Certain of the lepra-cells 
are enlarged and contain several nuclei, forming a type of giant-cell which is different from the Langhans' giant-cell of tuberculosis in that it has neither the peripheral arrangement of nuclei nor the necrotic centre. Giant-cells simulating the Langhans' cells have been described from time to time in leprosy, but before accepting them it is necessary to be certain that a mixed infection with tuberculosis did not exist. (Fig. I74.)

The cellular infiltration leads to obliteration of the capillaries, so that the leproma is poorly supplied with blood and does not show any tendency to fibromatosis but rather to retrogressive changes and absorption.

Lepromata in the Skin.-Sections of a cutaneous nodule show that the leproma is situated usually in the corium, or more deeply in the subcutaneous tissue, and that the infiltration encroaches on the sweatglands, sebaceous glands, and hair-follicles, and finally destroys them. The changes in the epidermis are secondary and consist of an initial hypertrophy due to the irritation of the leproma and a subsequent atrophy from pressure which may be so complete as to lead to ulceration. With regard to the bacilli, these are usually confined to the corium and there is generally a thin layer near the epidermis which is free from them. One or two observers have detected bacilli in the deeper layer of the epidermis in and between the prickle-cells, and also in the corresponding layer of the hair-follicles, but it is doubtful if they exist in the sebaceous glands as they have an aversion to fat. (Fig. I75.)

More important as regards the spread of the disease is that bacilli have been found on the surface of the epidermis and in the scales. It is doubtful, however, if these bacilli have reached the surface from within, and it is much more probable that they have been deposited there from the nasal secretion or from the upper respiratory passages by sneezing or coughing. It is possible also that they may have been eliminated by the sweat, as they have been found both in the sweat and in the sweat-glands.

The pathological condition of the nerves in nodular leprosy is of the same type as that in maculo-anaesthetic cases but it appears later and spreads less rapidly, while the presence of bacilli in the small cutaneous nerves, and the changes produced by them, are responsible for the alteration in sensation on the surface of the nodule.

Lepromata in the Viscera.-In the spleen in addition to the leprous infiltrations in the trabeculae, pulp, and Malpighian corpuscles, extensive necrotic masses have been described by Arning, like those of tuberculosis with typical Langhans' giant-cells. Such cases have to be further verified from the point of view of tuberculosis before they can be finally accepted.

The involvement of the genitalia is a matter of great importance with regard to the spread of the disease, but there still remain certain gaps in our knowledge on this subject.

On the penis, nodules and flat infiltrations or ulcers with pus and bacilli have been observed from time to time. 


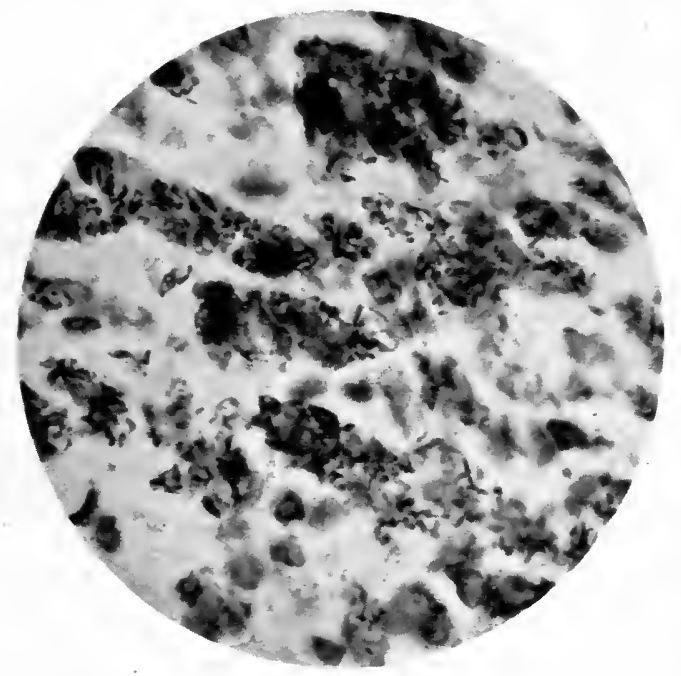

FIG. I 74.-Lepra Bacilli in clumps and "Lepra-cells." ( $x$ about 1000 .)

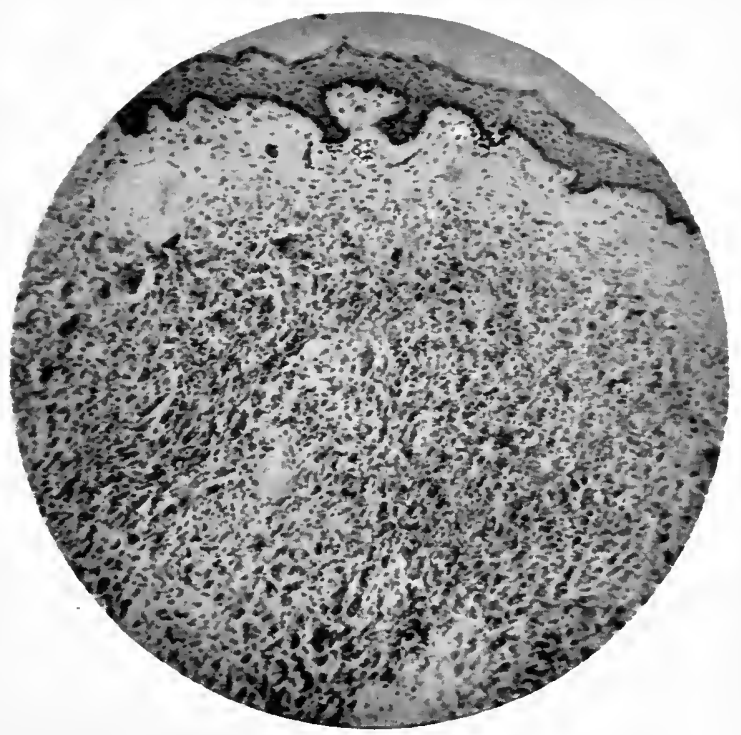

FIG. I 75.-Dense Infiltration of "Lepra-cells" and Lepra Bacilli. ( $x$ about 100.) 

In the testicles, although the connective tissue between the seminiferous tubules is markedly affected, the tubules themselves are not altered, and there is no distinct evidence that the semen contains bacilli. The atrophy which succeeds the sclerosis results in sterility.

It is much the same with the ovaries. The presence of the bacilli excites a chronic ovaritis with interstitial reaction, followed by sclerosis and atrophy. The Graafian follicles are not attacked except secondarily; and the ova are not infected with bacilli.

The mammary glands are apt to be the site of leprous infiltrations and the milk may contain bacilli.

The kidney's may be widely affected with leprous infiltrations and bacilli have been detected about the glomeruli, the epithelium of the capsule, and in the uriniferous canals, but not in the urine.

In the lungs a pure leprous infiltration may exist with small peribronchial foci, an infiltration of the peri-vascular tissue with lepracells, an occasional giant-cell of the Langhans' type, and caseation. The lesions in the lungs in leprosy are similar to those of tuberculosis, but are more solid and have less tendency to caseate.

The lymphatic glands, like the lungs, may be the seat of a mixed infection with tuberculosis and may show caseation and other signs of that disease.

In the maculo-anaesthetic cases the cutaneous lesions are not due to the presence of the bacilli in the skin, but are indirectly caused by the action of the bacilli on the peripheral nerves supplying the affected area. The lepromatous infiltration forms in the connective tissue sheaths of the nerve fibres, compressing the nerve tissue and leading to its eventual degeneration. The nerves may be similarly involved in advanced cases of nodular leprosy, but to a less extent. The bacilli may be present in large numbers and may invade the nerves in their whole course. The first indications of the involvement of the nerve is the presence of a cellular infiltration about the vessels of the perineurium and endoneurium, the bacilli lying in the connective tissue cells, plasma-cells, or free in the lymphatic spaces. They have been observed in the neurilemma and the sheath of Schwann, but have not been detected in the axis-cylinders of the nerves. Between the nerve fibres they tend to occur in rows rather than in clumps or globi. The presence of the bacilli and their toxins leads to neuritis and interstitial proliferative changes, which, by pressure on the vital nerve tissue, cause degeneration and fragmentation of the axis-cylinder.

Although the bacilli are present in large numbers in the nerves they do not occur in such masses as in lepromata.

Complications. - Leprosy may be complicated by various diseases, but the most important of them are tuberculosis, kidney disease, dysentery, and amyloid degeneration of the liver and kidneys. Occasionally it may occur in association with syphilis.

Diagnosis. - The diagnosis of a well-marked case of leprosy, whether of the nodular or the maculo-anaesthetic type, presents no serious 
difficulty to anyone who has once seen the disease. In its early stages, howere1, when the skin lesions are slight and the prodromal symptoms absent or unrecognised its diagnosis may be difficult and sometimes practically impossible. Even when severe prodromal symptoms are present, they are not sufficiently characteristic in themselves to base a diagnosis upon them. It has been asserted that the occurrence of epistaxis in association with febrile attacks is a pathognomonic symptom, but this again is not sufficiently constant to be of definite value. The occurrence of symptoms of a general intoxication in a district where leprosy is endemic and where the individual affected has been living in close association with lepers would be strongly suspicious.

It is in the maculo-anaesthetic variety that the greatest difficulty in diagnosis occurs, and in these cases the disease may have been in existence for a considerable period before its true nature is discovered.

The early erythematous patches of leprosy may closely simulate Erythema multiforme exudationm, both in appearance and distribution, but the lesions of leprosy are much more lasting and are associated with derangements of sensibility, such as hyperaesthesia or anaesthesia, which are absent in Erythema multiforme.

In nodular cases the diagnosis has to be made chiefly from syphilis and tuberculosis. From a secondary syphilide the nodules of leprosy differ in presenting a more shiny appearance as if they were oiled; in distribution, for while in syphilis the lesions are distributed indiscriminately, the lepromata are situated chiefly on the face and extremities ; in causing loss of the hair on the eyebrows and eyelids, which does not occur in syphilis. In doubtful cases the therapeutic test may establish the diagnosis, since leprosy does not react beneficially to such anti-syphilitic remedies as mercury, salvarsan, or the iodides. The Wassermann reaction has also been employed but too much reliance must not be placed upon it in this connection as a positive reaction has been obtained in certain cases of nodular leprosy. It must not be forgotten that leprosy and syphilis occasionally occur simultaneously.

From tuberculosis of the skin the diagnosis is comparatively easy. The tuberculous lesions which cutaneous lepromata most resemble are those of Lupus vulgaris, but in lupus they are confined to circumscribed areas and made up by the coalescence of small brownish-yellow. nodules, while in leprosy they are not composite, are widely distributed, usually bilateral, and more or less symmetrical.

In the maculo-anaesthetic variety the disturbances of sensibility associated with the erythematous or pigmented patches, and the enlargement of the nerves supplying the affected areas, are the most important pathognomonic symptoms. Occasionally ringed maculoanaesthetic patches may suggest ringworm, but the anaesthesia and the absence of ringworm fungus in scrapings serve to differentiate them. The pigmented patches with areas of white skin between have given 
rise to confusion with z'itiligo, but in the latter the areas are pure white and there is no loss of sensibility.

Advanced cases of nerve leprosy have to be distinguished from syringomvelia, but in the latter cutaneous lesions are absent while in the former some evidence of involvement of the skin is invariable. In syringomyelia the affection is unilateral and facial paralysis is exceedingly rare and of central origin when it does occur, "while in leprosy the affection is invariably bilateral and facial paralysis is common and of peripheral origin.

A symptom of considerable diagnostic value where the ulnar nerve is involved is hyperaesthesia, followed by loss of sensibility beginning at the tip of the little finger and spreading from there to adjacent fingers.

The final and conclusive test in every case consists in finding the lepra bacillus. In the nodular cases this is a simple matter and all that is necessary is to scarify a nodule, squeeze a drop of the tissue juices on to a slide, fix and stain by Ziehl's method, and examine under the microscope, when numerous bacilli can generally be detected. They may also be found in the nasal secretion when lepromata are present in the mucous membrane. In order to increase the chance of obtaining them in the nasal mucosa, resort has been made occasionally to the administration of full doses of iodide of potassium. In maculoanaesthetic cases bacilli cannot be found in the cutaneous lesions but are present in the nerve thickenings.

Agglutination tests have been attempted in connection with the diagnosis of leprosy but so far with imperfect success, as all acid-fast micro-organisms tend to clump when brought into contact with indifferent sera.

Attempts have been made also to obtain a cutaneous reaction by vaccinating with extract from nodules or other leprous tissue, but with negative results.

Prognosis.-The prognosis of leprosy is invariably bad and at the present time it must be confessed that no certain remedy has been found for the disease, that an absolute cure has not yet been effected, and that the old saying, "once a leper, always a leper," still holds true. On the other hand the outlook is distinctly more farourable if the patient be remored to a country where leprosy is not prevalent.

In nodular leprosy the patient may live on an arerage from six to ten vears, though occasionally death may supervene more rapidly or the disease may become quiescent for a varying period and life be prolonged for a vear or two.

The maculo-anaesthetic cases may last for twenty or thirty years and death not occur until old age, but in such cases it is questionable if life may be regarded as a blessing, for the aged leper rarely enjoys even approximately good health and becomes a miserable wreck of humanity. However quiescent the disease may become it is impossible to assert that it has been completely eradicated, and bacilli 
have been found by Lie in the nerves long after active lesions had ceased to exist and where the only evidences of the affection were atrophy, anaesthesia, and mutilations.

In about $40 \%$ of the cases death occurs as the result of the disease, the patient gradually sinking into a state of complete collapse or profound stupor. In the majority of cases, however, the end is brought about by some complication, the most common being pulmonary or renal disease. A considerable number of lepers die from tuberculosis to which they appear to be specially susceptible, partly owing to the depressing influence of the disease, and partly to the poverty and bad hygienic conditions with which they are apt to be surrounded.

\section{Treatment}

Much can be done to improve the general health and palliate the condition of the leper by suitable treatment, and though an absolute cure has not yet been found there is every hope that it may be in the future.

Internal.--Many remedies have been advocated, but their subsequent use has invariably led to disappointment. In estimating the therapeutic value of any drug or method of treatment in leprosy it is essential that it should have been employed over a long period, for the disease itself, even in countries where it is endemic, tends to undergo spontaneous remissions which may last for years and the drug or remedy which was used at the time when a remission took place is apt to get the credit for the improvement.

Of the many remedies tried the one which has given the most uniformly beneficial results has been chanlmoogra oil. This is a vegetable oil obtained from the seeds of the Taraktogenos kurzii and may be given in a variety of ways, by the mouth, per rectum, applied in the form of an ointment, or injected intramuscularly. By the mouth it is best taken in capsules, beginning with 5 to $10 \mathrm{~m}$., t.d.s., and increased to I dram or more, t.d.s., according to the toleration of the patient. Unfortunately it is particularly liable to cause digestive troubles and may have to be stopped on that account. For intramuscular injection it should be mixed with equal parts of camphorated oil and 2 c.c. of the mixture injected into the buttock once a week at first and increased to 5 c.c. every three days. The injections are very painful and may result in abscess formation at the site of injection.

Gynocardate of soda, a derivative of chaulmoogra oil, has been recommended as a substitute as it is more easily tolerated. By the mouth it is given in pills of 2 grs. beginning with one t.d.s. after food and increasing to ten or twenty a day. It may also be injected subcutaneously, in which case 2 grs. are dissolved in I c.c. of sterilised water, $\frac{1}{2} \%$ of carbolic acid added, and injected twice a week. Sir Leonard Rogers strongly recommends it to be given intravenously 
and employs a 2 or $3 \%$ solution made in distilled water or normal saline to which, after sterilisation in an autoclave, $\frac{1}{2} \%$ of carbolic acid is added, and a dose of about half a grain of the gynocardate injected into a vein in the forearm. As a result of the injections he found that a local reaction occurred in diseased tissues sometimes accompanied by fever and that there was an apparent destruction of the bacillus. The intravenous method has the advantage of being painless.

A refined preparation of chaulmoogra oil has been introduced, named " anti-leprol," which may be given by the mouth or injected intra-muscularly in doses of 3 to 5 c.c. repeated every third day. By the mouth this preparation is less liable to cause gastric disturbance than the crude oil, but when given intra-muscularly it is apt to cause pain, swelling, and even occasional abscess-formation at the site of the injection. Under this treatment many cases, especially early cases of the maculo-anaesthetic type, have improved both with regard to their general health and their leprotic symptoms, but no complete cure has been effected. To obtain benefit from the drug it is necessary to employ it over a prolonged period of at least eighteen months or two years. A collosol chaulmoogra has been prepared also and is on trial.

Other vegatable oils have been employed but with less constant results, such as gurjun oil from a plant of the Dipterocarpus family, "kauti" an oil from the Hydrocarpus inebrians, and eucalyptus.

Another substance of an oily nature which has been recommended in the treatment of leprosy is ichthyol, but the nauseating taste of this remedy and its unpleasant smell vitiate any doubtful benefit which may be derived from its use.

Iodine preparations, specially iodide of potassium, have been somewhat extensively used and have been credited with having a specific action upon the disease as they are liable to cause a general and local reaction, possibly by stimulating the activity of the lepromatous cells and leading to absorption of the toxic products of the bacteria. In spite of these reactions, however, no distinct amelioration has resulted from their use.

Another remedy which was credited at first with a specific action on the disease is anti-i'enene, but further employment of it has led to disappointment.

Mercury in the form of intramuscular injections of the soluble salts was recommended by the late Dr. Radcliffe Crocker and in a certain number of cases beneficial results have been recorded from its use.

Recently salvarsan has been tried in leprosy, both intravenously and intramuscularly, but no definite benefit has been derived from it.

In addition to the above remedies various sera, vaccines, and toxins, have been used with equally uncertain results. Soon after the old tuberculin was introduced by Koch it was given an extensive trial in leprosy. It was found that when injected a general reaction followed but that, instead of resulting in benefit, the reaction was sometimes so acute as to be actually harmful to the patient and to activate the 
disease. Since then several attempts have been made to prepare leprolins on similar lines to Koch's tuberculin from organisms which have been grown from leprous tissue. Rost, for example, in I903 prepared a leprolin from a bacillus which he cultivated from lepromata and this, when injected, gave a general and local reaction which was followed by beneficial results, such as healing of the ulcers, partial return of sensibility in the anaesthetic areas, and general improvement in the patient. Unfortunately for the specific nature of this reaction, it was found that the bacillus from which the leprolin had been made was probably a contamination. A leprolin was also made by Bayon in I9I3 from a cultivation of an organism grown from leprosy which had been described by Kedrowski.

In I9o7 Deycke introduced a substance called nastin which heclaimed to produce marked benefit, especially when injected in early cases of leprosy, by having a specific action on the lepra bacilli causing their bacteriolysis. It was prepared from a culture of a streptothrix which he obtained from a nodule of leprosy and was in the nature of an ethereal extract. This substance was rendered more active by the addition of benzoyl-chloride, which dissolved the bacillary fat, and was issued in two strengths, namely Nastin BI and B2. Though a number of cases have been said to have improved under it, the general experience of its use has been disappointing.

Stimulated by the wonderful effects of anti-diphtheritic serum, sereral attempts have been made to prepare an anti-leprotic serum on somewhat similar lines. One of the earliest of these was that of Carrasquilla in IS97. He injected the serum from certain lepers into a horse, then bled the animal, collected the serum and gave it to lepers. This was reported to have caused a definite general reaction and a softening and absorption of the local lesions. But these results caused no permanent benefit and, on consideration of the method employed to obtain the serum, there does not seem to be anything in it which could have had a specific effect. Since then sera have been made by injecting the horse with leprosy bacilli but so far the results from their use have been uncertain. In this disease the mere getting of a general and local reaction does not necessarily point to the exciter of it having any specific action on the disease, and a considerable number of indifferent substances and influences, such as preparations from micro-organisms other than the lepra exciter, inorganic substances such as iodide of potassium, and any profound disturbance such as may be caused by an injury or a severe chill, may lead to a rise of temperature, a local reaction in the old nodules of the disease, and an outbreak of new ones.

It would serve no useful purpose to dwell further on the other remedies which have been introduced as specifics and the further use of which has shown that they had no claim to that distinction. But while such remedies have, so far, been disappointing, the employment of a suitable general treatment on ordinary medical principles has been of the greatest service in staying the progress of the disease and render- 
ing the life of the patient more bearable. In the first place the nutrition of the leper must be seen to, a suitable generous dietary instituted, and such tonics as cod-liver oil, strychnine, and arsenic prescribed in order to improve the general health and increase the resistance to the bacillus. The surroundings should be made as salubrious as possible and the living apartments airy and scrupulously clean. The patients should be warmly clad, as cold is acutely felt by them and there is a marked tendencr to kidner trouble, and they should be provided with sufficient out-of-door work or exercise and mental occupation or interest to prevent them brooding on their affliction.

Any definite symptoms which may supervene should be treated. Attacks of leprotic ferer may often be reduced by quinine, and the neuralgic pains, which precede the anaesthesia, combated by salicylate of soda or salicin.

Local Treatment.- The treatment of the local lesions is of equal importance. The first indication is to keep the patient scrupulously: clean by means of daily baths, to which permanganate of potash, boric acid, or other suitable antiseptic should be added when suppurating sores are present. Ulcerating surfaces should be bathed with antiseptic lotions, of which permanganate is amongst the most useful, and suitably dressed. Antiseptic nasal douches and gargles are indicated where the nasal secretion is profuse or ulceration is present in the mouth, and when the eyes are affected they should be treated with appropriate antiseptic applications.

The X-rays have been advocated, both for healing the ulcers and causing absorption of extensive lepromata, and considerable benefit may be derived from their judicious employment.

Where gangrene appears about the extremities surgical intervention should be resorted to promptly.

Prevention.--The only possible way to prevent the spread of leprosy is by adopting some form of adequate isolation. The ideal condition is segregation in a properly controlled, medically supervised, institution, preferably a colony or settlement, perhaps on some island, where the patients could be permitted a modified type of home life and existence could be made bearable by friendly intercourse with others suffering from the same disease. It is often asserted that such measures are cruel, but this is a mistake, not only from the point of view of the public weal but also of the patient himself, for each leper who is allowed to be at large is a possible focus of infection, while to leave a leper among his friends too often ends in his becoming a nuisance and being shunned, uncared-for, and neglected. It has been found that wherever proper measures of segregation have been adopted the disease has steadily declined. As a preliminary to any form of segregation it is essential that the disease should be notifiable. In dealing with the leper the nodular cases, which present open sores or profuse nasal discharge, are naturally the most dangerous, indeed it has frequently been asserted that the risk of infection is entirely confined to such cases 
and that maculo-anaesthetic cases may be tended with inpunity, but until the exact method by which infection takes place is known such a doctrine is unsafe. Where children are born of lepers they should be separated from their parents as soon as possible and kept under observation, as the risk of infection in early life is very great. Lepers should not be allowed to follow any occupations which are dangerous with respect to contagion and should be prevented from handling or selling any food stuffs, such as milk, bread, vegetables, fish, etc. All clothing which has been worn by a leper should be disinfected and soiled handkerchiefs, dressings, etc., should be burned.

\section{REFERENCES}

BAyon, H. Culture \& identification of the germ of leprosy \& the relationship of the human disease to rat leprosy (Trans. Roy. Soc. Med., London, March, I912).

— Human and rat leprosy (Trans. Soc. of Trop. Med. \& Hyg., March I9I2).

— Clinical \& bacteriological aspect of leprosy (Brit. Med. Journ., Nov. I9I3, p. I 420).

Leprosy: a perspective of the results of experimental study of the disease (Ann. Trop. Med. \& Parasit., I9I 5, ix. p. x).

Critical review of recent experimental leprosy research (S. African Med. Rec., vol. xi. II, p. 20I).

Bensen. Leprosy (Dublin Med. Journ., I 877).

BLACK, R. S. Remarks on leprosy in Cape Colony (Lancet, April I9o6, p. $\operatorname{II67).~}$

ClegG, M. T. Cultivation of the leprosy bacillus (Philippine Journ. of Med. Sci., Oct. I9og, iv. p. 403).

CURrie, D. H. Mosquitoes \& flies in relation to the transmission of leprosy (Public Health Bull., Public Health \& Marine Hosp. Service U.S.A., No. 39).

Currie, Clegg \& Hollmann. Studies upon leprosy (Idem, Sept. I9II).

(I) The artificial cultivation of the bacillus of leprosy.

(2) Attempts at specific therapy in leprosy.

Deycke, G. A specific treatment of leprosy (Brit. Med. Journ., April $4^{\text {th, }}$ I908).

Duval, C. W. Cultivation of the leprosy bacillus (Journ. Exper. Med., March I9II).

- Experimental production of leprosy in the monkey (Univ. of Pennsylvania Bull., I9I I, p. 665).

Fraser, H. \& Fletcher, IV. The bacillus leprae (Lancet, Sept. 27th, I9I3).

Hansen, A. Heredity of leprosy (Arch. f. Derm. u. Syph., I9II, cx. p. 225).

- Decline of leprosy in Norway (Lepra, vol. iv. Fas, 4).

Heisser, V. G. Recent developments in the treatment of leprosy (New York Med. Journ., Feb. I2th, I9I6).

HoneiJ, J. A. Bone Changes in Leprosy (Amer. Journ. of Roentgenology, I9I 7 , p. 494).

Johnston, J. A. Bacteriology of leprosy (Philippine Journ. of Med. Sci., June I9I4).

Kolle. Leprosy (Deutsche med. Wchnschr., I 899, xxxix.). 


\section{RHINOSCLEROMA}

MacLeod, J. M. H. A brief survey of the present state of our knowledge of the bacteriology \& pathological anatomy of leprosy (Trans. 2nd Internat. Scientific Cong. on Leprosy, Bergen, I909).

MERCIER, C. A. Leper houses \& mediaeval hospitals (FitzPatrick Lecture, Roy. Col. Phys., London, Nov. I9I4).

Morrow, R. History of leprosy in S. Africa up to the establishment of the first leper asylum at Hemel en Aarde (S. African Med. Rec., I9I3, xi. p. I74).

MURRAY, C. F. K. The administrative side of leprosy (S. African Med. Rec., I9I3, xi. p. 183).

Neish, W. D. \& Toxkix, T. J. Leprosy in Jamaica (Bristol MedicoChirurgical Journ., March I904, xxii.).

NicolLe, C. Experimental investigations on lepra (Ann. de L'Inst. Pasteur, Maỹ I906).

Report of Government Research Pathologist on leprosy, I9r3 (S. African Med. Rec., I9I3., xi. p. I8 $)$.

ROGERS, SIR L. Intravenous injection of gynocardate of soda in leprosy. (Brit. Med. Journ., Oct. 2 Ist, I9I6).

Rost, E. R. Pathology \& treatment of leprosy (Indian Med. Gaz., May \& June I904).

SANDES, T. L. Mode of transmission of leprosy (Journ. Trop. Med. \& Hy.g. August IgI I, xiv.).

Savdwith, F. M. Two lectures on leprosy, delivered at St. Thomas' Hospital, London (Clinical Journ., Oct. 23rd, 1907, p. 26).

TEAGtE, O. Cutaneous reaction in leprosy (Philippine Journ. of Med. Sci., Oct. I909, iv.).

Verteutl. Salvarsan in leprosy (Brit. Med. Journ., Sept.23rd, I9I I).

WhitMore \& ClegG. Specific treatment of leprosy (Philippine Journ. of Sci., Dec. I910, p. 559).

Williams, T. S. B. Leprosy (Brit. Med. Journ., Dec. I9II, P. I582).

\section{RHINOSCLEROMA}

\section{Syn. : Nasal Scleroma.}

Definition: A chronic affection of the nose, upper air-passages, and adjacent tissues, characterised by indurated nodules and masses which block the airway and lead to marked deformity.

Description.-Hebra and Kaposi in I $8_{70}$ first called attention to this rare affection and observed it originally in the nose and upper lip; since then it has been found to attack not only these regions but any portion of the upper air-passages and adjacent tissues and has been seen in the hard palate, soft palate, gum, tongue, pillars of the fauces, uvula, spreading down to the larynx and trachea, up the Eustachian tube to the auditory canal, and along the lachrymal duct to the inner canthus. It begins as a rule insidiously as hard nodules situated in the deeper layer of the mucous membrane or in the corium. These nodules are at first about the size of a split-pea, flat on the surface and freely movable. As they increase in size they tend to coalesce with others to form indurated plaques, irregular in outline, clearly defined from

Note.-About 200 cases of this peculiar affection have been described and out of these 50 were observed by Kaposi. 
the surrounding healthy skin, of a peculiar stony hardness, and gradually become attached to the underlying tissue. The nodules and plaques are painless as a rule, although they may be tender on pressure, and at first present a pinkish or dusky red tinge but later tend to fade and assume a whitish appearance from cicatricial changes. The affection is most common about the nose and may begin at the junction of the skin and mucous membrane, a situation not infrequently the first to be attacked by Lupus vulgaris. Should the disease extend down to the larynx or trachea, stenosis and even suffocation may result.

When present in the nose the disease causes marked deformity and flattening, the alae become distended and the tip dragged up in a retroussé manner. The continuous growth of the masses within may lead to blocking of the nares or, by pressure, to absorption and perforation of the nasal septum. When the upper lip is involved it becones swollen, stiff, and protruding, and cracks appear at the natural fissures from which oozes a viscid discharge which dries into crusts. At the same time the upper jaw may be attacked, the gum becoming irregularly swollen and the teeth loosened from involvement of the periosteum and bone. The affected tissue does not break down, ulcerate, or necrose spontaneously like a gumma, although superficial ulceration sometimes occurs as a result of abrasion and the action of septic micro-organisms, which is followed by cicatricial or sclerotic shrinkage.

The affection is steadily and slowly progressive and has been known to last for 20 years or more. It is a purely local condition and, except for the mechanical obstruction to the air-passages and the discomfort from the deformity, it does not interfere directly with the general health. In one or two instances where a fatal issue has resulted from suffocation metastatic growths have been observed in the glands of the neck.

It has a limited geographical distribution being endemic chiefly in Austria, Southern Russia, and Poland, though occasional cases have been recorded in Switzerland, Sweden, Italy, Belgium, and Spain. It has been met with also in South America, Brazil, Salvador, and the Argentine. Cases have been observed in the United States and several in Great Britain but were all imported from some endemic area. A fow cases have been observed in India among the Hindus.

Histopathology.-It is in the corium and deeper layer of the mucosa that the essential change occurs, in the form of a dense granulomatous infiltration which, owing to certain peculiarities, has attracted considerable attention. It is made up chiefly of plasma-cells and two forms of degenerated cell known respectively as the Mikulicz cell, after its describer, and the hyalin degenerated cell. In some respects the infiltration bears a resemblance to that of leprosy, the Mikulicz cell corresponding to the so-called lepra-cell. The plasma-cells are usually present in large numbers and most dense around the blood-ressels. 
The Mikulicz cells are two or three times the size of plasma-cells, are most probably derived from them, and result from the presence in the protoplasm of a short bacillus described by ron Frisch. These bacilli are arranged in the cell in small clusters held together by a gloea, some of the larger cells containing as many as five or six such clumps. The nucleus of the cell is usually situated eccentrically and the protoplasm has undergone inucoid degeneration.

The hyalin degenerated cells are also derived from the piasma-cells but whether they are Mikulicz cells in a further stage or another type of degeneration is uncertain. They are as large as the Mikulicz cells and contain droplets of colloid material which stains readily with fuchsin.

Where the infiltration is densest the fibrous network is rarefied but as time goes on a new formation of collagen takes place, especially at the margin of the infiltration, giving hardness to the lesion and causing cicatricial shrinkage.

Any changes which may occur in the epidermis are of a secondary character. Orer the nodule it is usually stretched and the interpapillary processes flattened out, while at the edges they may grow down in an irregular manner.

Bacteriology.-A peculiar bacillus was discovered in the tissue by von Frisch and as it is invariably present in this affection and in no other it is usually regarded as causally connected with the disease. The organism has been found to be pathogenic in mice and guinea-pigs, causing abscess formation but not actually reproducing rhinoscleroma. The bacilli are short rods with rounded ends enclosed in a gelatinous capsule, arranged singly, in pairs, or in characteristic groups lying in a gloea, and are situated chiefly in the protoplasm of the Mikulicz cells or occasionally free in the lymph-spaces of the tissue. They are easilycultivated on the usual media, forming on gelatine grevish shiny pinhead sized colonies, and are gram negative. They closely resemble the pneumo-bacillus of Friedländer both morphologically and in cultivation but are recognised as being a different organism. They can be demonstrated almost as easily as lepra-bacilli by scarifying the surface of a nodule and examining the serum squeezed from it.

Etiology.-The affection is generally beliered to be caused by the bacillus of von Frisch. It attacks either sex indiscriminately, may occur at any age, and is met with chiefly among the indigent poor. The imported cases have usually been in immigrants.

Diagnosis.-It has to be distinguished chiefly from tertiary syphilis with gummatous infiltration of the naso-pharynx and upper airpassages. In rhinoscleroma the induration is greater and of a stony hardness and there is no tendency to break down and form deep ulcers as in syphilis. Should any difficulty exist in distinguishing the two conditions clinically, the diagnosis can be settled by the Wassermann reaction, a histological examination, or the demonstration of the ron Frisch bacillus. 


\section{TREATMENT}

Active treatment is essential as, if left alone, the disease is progressive and may lead to serious and even fatal results from blockage of the air-passages. The most effective treatment is the X-rays, under doses of which the diseased tissue readily becomes absorbed leaving little or no scarring. Where mechanical difficulties prevent their application, radium should be employed.

Before the introduction of X-ray treatment the prognosis was most unsatisfactory. Iodide of potassium was believed to cause some improvement but its action was uncertain. Excision or curetting were almost invariably followed by rapid recurrence, and applications of tampons and pledgets soaked in lactic acid or salicylic acid though helpful in reducing the masses only led to temporary improvement.

\section{REFERENCES}

Danziger \& Pollitzer. Rhinoscleroma cured by $\mathrm{X}$-ray treatment (Fests 2, Vier. Sift. des Deutschen Hospital, N.Y., I9I I).

KEEGAN. Rhinoscleroma in Hindoos (Ind. Med. Gaz., Jan. I889).

KraUs, A. Rhinoscleroma, metastatic growths in (Arch. f. Derm. u. Syph., I904, lxviii. p. 345).

Marshalko, T. V. Histology of rhinoscleroma (Arch. f. Derm. u. Syph., I900, p. I63).

Schridde, H. Histology of rhinoscleroma (Arch. f. Derm. u. Syph., I905, Ixxiii. p. 107). 


\section{CHAPTER XIX}

DISEASES DUE TO ANIMAL PARASITES. PROTOZOA.

\section{SYPHILIS}

Syn. : Lues venerea.

Definition: A chronic contagious disease, congenital or acquired, characterised by lesions in every tissue of the body, and due to the Spirochaeta pallida of Schaudinn and Hoffmann, or as it is now named the Spironema pallidum.

Introduction.-The cutaneous manifestations of syphilis are possibly the most striking features of the disease, yet they only constitute one phase in a general morbid process of which equally important evidences are found in the nervous and vascular systems and in the viscera. In this description only the skin lesions will be dealt with in detail, while for a more complete account of the disease, its history, manifestations in situations other than the skin, and late nervous sequelae, the student is referred to the text-books on syphilis.

It is customary to divide the course of syphilis after Ricord into three periods, namely, primary, secondary, and tertiary. This division although useful for purposes of description, is arbitrary and somewhat unscientific, as the essential underlying pathological process is the same throughout and the border-line between the periods-especially between the second and third-is ill-defined.

The primary period consists of the primary sore or initial lesion which occurs at the site of inoculation of the spirochaete, appears about twenty-six days after the inoculation, and is associated soon after its development with swelling and induration of the neighbouring lymphatic glands which drain the sore.

The secondary period is the evidence of the generalisation throughout the system of the spirochaete and manifests itself as various generalised cutaneous eruptions which appear about forty-five days after the development of the primary sore and are usually associated with a febrile disturbance.

The tertiary or late period is characterised by lesions of a more chronic type, not so widely nor so symmetrically distributed, which may appear either two or three years after the commencement of the 
secondary period-the one merging into the other-or after a latent period of indefinite duration during which no distinct evidences of the disease were present. The tertiary lesions are due to a re-awakening of the spirochaetes after a more or less prolonged period of hibernation and their presence in the skin and other tissues in a state of diminished virulence.

\section{Etiology}

Syphilis has been proved to be due to the Spirochaeta pallida (Spironema pallidum) discovered by Schaudinn and Hoffmann in I905.

Spironema pallidum.-The Spironema pallidum is a minute protozoon $4 \mu$ to $20 \mu$ in length and averaging about Io $\mu$, being slightly longer than the diameter of a red blood corpuscle. It is so small and delicate that it can be seen only by the high powers of the microscope and can be best demonstrated by $\frac{1}{1}$ th or $\frac{1}{16}$ th oil immersion lens with the aid of a darkground illuminator. It is twisted like a corkscrew, having 6 to I2 turns, and tapers at each end into minute processes which some regard as flagellae. It moves in a complicated fashion, partly by rotation on its long axis and partly by progression in an undu-

FIG. I 76. -Spironema Pallidum. ( $\times 900$. Irom scraping from chancre. Stained by Fontana's method. (Drawing by Dr. J. G. Thomson.) lating manner like a snake. At one time it was believed to be a flagellated spirillum

but is now generally regarded as a protozoon and is probably nearly allied to the trypanosomes. (Fig. I76.)

It is destroyed by a temperature of $\operatorname{II} \& \mathrm{~F} .^{\circ}$ in half an hour and it does not pass through a coarse filter.

Presence of the Spironema in the Tissues.-The Spironema pallidum is present in all the lesions of syphilis and does not occur in any other disease. It is found in the primary sore, in the indurated glands, in the roseolar spots, in all forms of secondary lesions, and in blisters artificially produced; it occurs in the blood, $15 \mathrm{mph}$, and cerebro-spinal fluid, and in the saliva where mucous patches are present; it is found also in small numbers in gummata and in diseased arteries. In congenital syphilis it is present in vast numbers in every tissue and has been found in the skin in bullous syphilides. It has been detected also in the placenta and in the umbilical cord, and has been found to be capable of penetrating the orum. (Fig. I77.) 
Its chief habitats are the walls of the blood-ressels and their immediate neighbourhood, and the lymphatics.

It can be recovered from the various lesions in apes experimentally inoculated with the disease.

Demonstration of the Spironema Pallidum.--Specimens showing the spironema may be made from any of the above lesions by the examination of a drop of serum squeezed from them.

The procedure is as follows :

(I) Primary Lesions.-The surface of the doubtful chancre should be cleansed, and superficial granulations or debris removed by a piece of cottonwool moistened in water or scraped away with a knife, and the

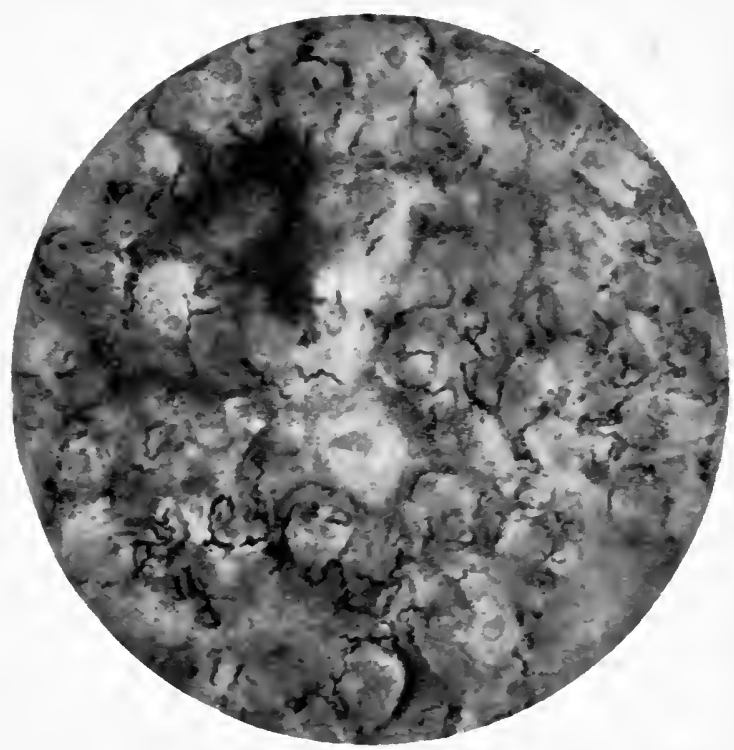

FIG. 177.-Spironema Pallidum in Suprarenal Gland, in congenital syphilis.

sore firmly squeezed so as to cause the exudation of a drop of serum. If bleeding has been caused it is necessary to wait till it has stopped before collecting a specimen. The serum is then taken up in a pipette, placed on a slide, a cover slip applied, the edges sealed with vaselin to prevent evaporation, and the preparation examined.

By this means spirochaetes may be found and a positive diagnosis made possible a fortnight or more before definite cutaneous syphilides manifest themselves. A negative result, howerer, even with the most careful technique, is inconclusive, especially in conditions in which spirochaetes are found with difficulty, such as in sores which are tending to slough or are in process of healing, or where some local antiseptic preparation has been employed.

(2) Secondary Syphilides.-In secondary cutaneous syphilides the examination for spirochaetes is made in a similar fashion. In papules 
the surface of the lesion is cleansed with alcohol and ether, the superficial layers of epidermis are removed by scraping, and a drop of serum obtained by squeezing or dry cupping; in macules a blister is artificially produced over the lesion and after the removal of the blisterfluid the serum is expressed from the raw surface and examined.

(3) Lymph Glands. - In the case of lymph glands a hypodermic needle may be used to extract the juices for examination. About 5 minims of sterile salt solution should first be injected into the gland which should then be massaged, the piston withdrawn, and the juices aspirated into the syringe and spread on a slide for examination.

The spirochaetes may be demonstrated in the preparation either by dark-ground illumination or by suitable staining.

Dark-ground Illumination. ${ }^{1}$-The dark-ground illuminator, of which there are several types, affords the simplest means of demonstrating spirochaetes. It is a sul-stage condenser which may be fitted to any microscope suitable for bacteriological work, and is so constructed as to prevent the rays of light from a specially powerful lamp coming within the objective of the field which, in consequence, appears uniformly black while the spirochaetes, owing to their high index of refraction, are rendered self-luminous and appear as bright wavy lines on the dark background.

Staining Methods.-The spironema is a difficult micro-organism to stain and the methods in general use are complicated. Among the most commonly employed are those of Giemsa, which consists of staining by a solution of eosin and azur ; of Levaditi, which-consists of impregnating the tissues in which the spirochaete is present with silver nitrate and staining it subsequently with Giemsa's method; and of Fontana modified by Tribondeau, which consists of staining with nitrate of silver solution after fixing with formol-acetic acid solution and employing tannin solution as a mordant.

These and other staining methods are so tedious that they have been superseded largely by the simpler Indian ink and Congo red methods.

Indian Ink Method.-This method is described by McDonagh as follows: A glass pipette is driven through the skin at the border of the chancre or other syphilide. A little blood runs up into the pipette which is then kept vertical for a few minutes till the serum settles. The part which contains the blood corpuscles is broken off and a drop of serum is allowed to fall on a thin clean slide. Around the drop 6 or 7 loopfuls of distilled water and the same quantity of Indian ink (Gunther \& Wagner) are placed. The three substances are then thoroughly mixed, spread out on the slide with another glass slide, allowed to dry, and examined with an oil immersion lens; the spirochaetes appear white on the dark Indian ink background.

\footnotetext{
${ }^{1}$ For a detailed description of the technique of dark-ground illumination the reader is referred to that by J. Edwin Barnard, President of the Royal Microscopical Society, in the Report of the Medical Research Committee, series 19, 19 I 8.
} 
Congo Red Method.-In this method the Indian ink is replaced by a $2 \%$ Congo red solution. When the film has dried acid alcohol is poured over it and the background assumes a semi-opaque bluish appearance while the spirochaetes remain unstained and stand out in sharp contrast.

The disadvantage of these methods is that they are not sufficiently accurate to distinguish between the Spironema pallidum and other spirochaetes which may occur in association with it, of which the most common is the Spironema refringens, a larger and coarser organism with more open spirals.

Cultivation Experiments.-Numerous attempts have been made to cultivate the Spironema pallidum, but in the majority of instances with negative results.

In I909 Schereschewsky obtained a culture on a medium prepared with horse serum, but the culture was impure and was not found to be pathogenic for animals.

In IgI2 Noguchi succeeded in cultivating it anaerobically and in reproducing lesions by it in lower animals. He did not cultivate the organism directly from human tissue but used the testicles of rabbits previously inoculated with the virus. He employed a serum water or serum agar to which had been added a piece of sterile rabbit tissue, and in this medium the organisms began to multiply after $4^{8}$ hours. $\mathrm{By}$ heating the culture up to $60^{\circ} \mathrm{C}$. he prepared a product which caused a specific cutaneous reaction in syphilitics and which he named "luetin."

The spironema has since been grown direct from human lesions in the same media.

Inoculation Experiments in Animals.-Through the researches of Metchnikoff, Roux, Neisser, and others, it has been found that monkeys, especially the anthropoid apes, are susceptible to syphilis. Out of 22 chimpanzees, for example, inoculated by Metchnikoff and Roux positive results were obtained in all. A primary sore developed at the site of inoculation after an incubation period of 30 days, and was associated with induration of the neighbouring glands; this was followed 35 days later by secondary lesions in the form of papules, ulcers, and mucous patches. Inoculations from these lesions on healthy chimpanzees produced primary sores and typical secondaries but not late tertiary symptoms.

In monkeys lower than the anthropoid apes, such as catarrhine monkey's, the inoculation resulted in a primary sore which was not definitely indurated or associated with adenitis and was not followed by secondary symptoms. In a monkey of the genus Macacus in which only a non-indurated sore followed the inoculation, a re-inoculation from the sore in a chimpanzee resulted in a typical primary sore followed by secondaries.

Inoculation experiments in animals other than monkeys have been negative or doubtful. In rabbits and dogs, keratitis has been caused by inoculation with syphilitic material from man and spirochaetes have 
been recovered from the lesions, and Noguchi claims to have inoculated the testicles of rabbits with syphilis and to have produced syphilitic orchitis.

Life History.-The life-history of the Spironema pallidum is not fully known at present but a number of suggestions have been made regarding it.

In 1906 MacLennan found in association with it a number of minute spherical bodies, some with tails, which he considered might possibly be stages in the life-history of the micro-organism.

In IgI2 E. H. Ross observed peculiar round or pear-shaped bodies with a cell-wall and a central chromatin mass, which occurred in nonulcerating primary sores and in syphilitic glands and were present both in the lymph and intracellularly in lymphocytes and epithelial cells. These he thought might be stages in the life-history of the microorganism, as he found that by placing the blood from a Hunterian chancre on specially made jelly the intracellular bodies developed into spirochaete-like structures.

McDonagh has also described a complicated life-history for the spironema. According to him the micro-organisms occur first in the form of round or kidney-shaped sporozoites about I.5 $\mu$ in diameter. These sporozoites enter connective tissue cells or lymphocytes and undergo two types of changes, one sexual and the other asexual, the sexual change resulting in the spirochaete.

These observations require further corroboration before they can be accepted.

\section{DESCRIPTION}

Primary Stage.-This is the period of the primary sore or syphilitic chancre. To this lesion various names have been applied, such as hard chancre or primary sclerosis to signify the peculiar hardness

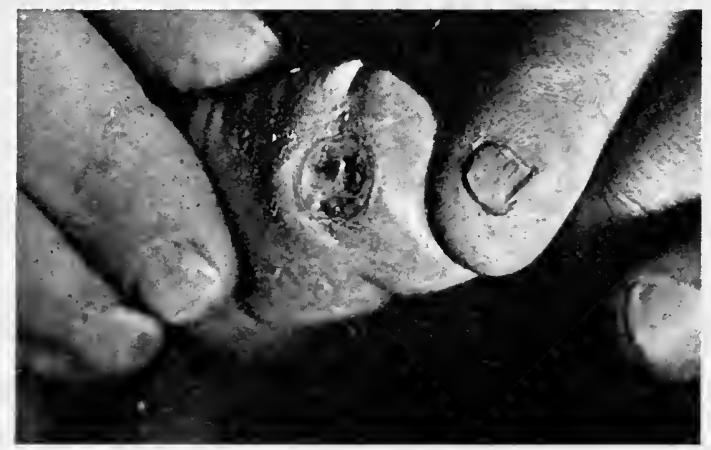

FIG. 178.-Chancre on Curona of Glans Penis.

of the lesion, Hunterian chancre, and infecting sore to distinguish it from the local venereal sore which is not associated with general symptoms and is known as a " non-infecting sore " or " soft chancre." 
The incubation period, or time between the inoculation of the spirochaete and the appearance of the chancre, is about 25 days, the maximum and minimum time according to Fournier being respectively 42 and 15 days. (Fig. I78.)

The primary sore is essentially a papule but, from friction, it tends to become eroded, or from secondary infection with pyogenic organisms to become ulcerated. It varies in appearance in different cases but is most commonly a flat erosion, circular or oval in shape, varying in size from $\frac{1}{4}$ to $\frac{1}{2}$ an inch in diameter, deep red in colour at first but later assuming a greyish tint, and either moist with a sanious discharge or covered with a thin grey membrane which when stripped off leaves a surface from which dark blood oozes. After a few days the erosion becomes raised and hard and when pinched up between the fingers feels like a disc of paper, a nodule of cartilage, or a hard ring. Though it may itch slightly at first it is associated with no definite pain. In this condition it remains for 3 or 4 weeks and then undergoes spontaneous healing and is replaced by a brownish indurated scar which may persist for months and gradually disappear, unless there has been definite ulceration when a permanent cicatrix may result. The total dura-

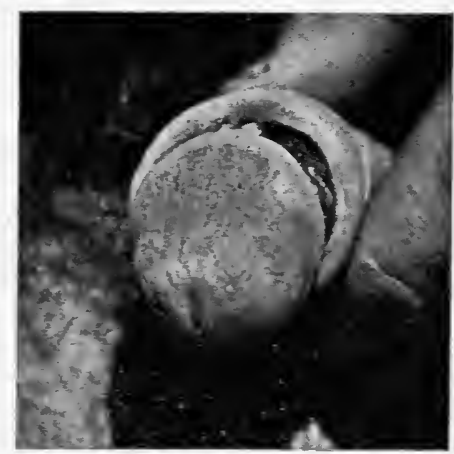

FIG. I79.-Chancre on Cervix of Glans Penis. tion of the chancre from its appearance to its complete healing is usually about 6 weeks. (Fig. I79.)

Soon after the development of the chancre the neighbouring lymphatic glands become enlarged and peculiarly hard or shotty, but they are freely movable, painless, and the skin over them is not inflamed or adherent. In this condition they may remain for a considerable time after the chancre has healed. In rare instances they may suppurate, as the result of secondary infection with pus-organisms or with the bacillus of Ducrey, forming what are known as "suppurating buboes."

\section{Varieties of Chancres}

Simple Abrasion.-Sometimes the chancre may take the form of a simple abrasion which may or may not be situated on an indurated base. Such lesions may be met with on the nipple, in the vagina, or within the anus.

Fulminating Chancre.-From secondary inoculation of pyogenic organisms the chancre may become acutely inflamed and may be associated with oedema and lymphangitis in the neighbourhood and suppuration of the enlarged glands. This acute inflammatory disturbance may be the result of excessive cauterisation, but is more 
often due to the action of irritating discharges beneath a tight prepuce.

Phagedaenic Chancre.-In debilitated subjects, especially in alcoholics or diabetics, the secondary inoculation of pus-organisms may lead to gangrene and necrosis of the chancre and the formation of a deep phagedaenic ulcer.

Mixed Chancres.-Occasionally Ducrey's bacillus of soft sore or " chancroid " may be inoculated synchronously with or about the same time as the syphilitic virus, with the result that a modified chancre known as a "mixed chancre" is produced. This begins as a sore on an inflammatory base covered with a purulent discharge, and later becomes indurated and assumes the characteristics of an ordinary chancre.

Multiple Chancres. - The chancre, as a rule, is single, but sometimes cases are met with in which two or more are present. These may be the result of several inoculations taking place at the same time from the presence of several abrasions, or possibly of a series of inoculations occurring at intervals of a few days after the original infection and before the initial sore had fully matured and immunity had been established. To such multiple chancres the name successive chancres has been applied. They must not be confused with what are known as "relapsing or recurrent chancres." In a relapsing chancre the induration after entirely disappearing under treatment reappears in the form of an ulcer or erosion which resembles the original chancre in its extent and character, or, by undergoing softening and disintegration, may suggest a breaking-down gumma It may appear a few weeks after the original chancre has healed or several years later.

\section{Situation OF CHANCRES}

Although chancres may be met with anywhere on the cutaneous surface the majority occur in the genitalia.

Genital Chancres.-Male.-About two-thirds of the chancres in the male are present in the mucous membrane of the prepuce in or behind the corona or on the surface of the glans penis, but they may be met with on other parts of the penis and on the scrotum. On the glans the chancre is usually flat, while in the balano-preputial fold it tends to be nodular. When it occurs in the urethra near the meatus it generally affects both lips and gives rise to marked induration, oedema, sometimes ulceration, and to considerable pain on micturition. When it is present on the inner surface of the prepuce in association with phimosis there may be no external evidence but it may be felt from the outside and is then known as a concealed chancre. (Fig. ISo.)

Female.-In the female the chancre may be situated on the Labia majora, the Labia minora, the clitoris, the cervix of the uterus, or at the orifice of the vagina or of the urethra. Those on the external genitalia are of the characteristic type ; those in the vagina are atypical 
and may appear as simple abrasions without definite induration, while those on the cervix may take the form of red or greyish erosions surrounded by a deep reddish areola.

In the female genitalia the chancres are apt to be more ephemeral than in the male; this is especially the case when they occur about the vagina or cervix, consequently they are liable to escape notice in those situations.

\section{Diagnosis of Genital Chancres.-} The diagnosis of genital chancres, especially in males, is easy as a rule, but should there be any difficulty it can generally be established by the demonstration of the spironema by dark-ground illumination.

Clinically the principal diagnostic points are the incubation period of 2 to $f$ weeks, induration,

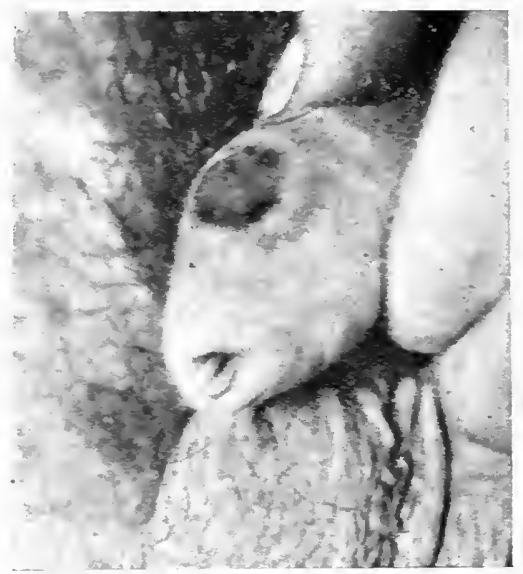

FIG. 18o.-Chancre on Prepuce. intractability to local treatment, absence of pain, and associated enlargement and hardness of the neighbouring glands.

The lesions with which a chancre on the penis is most liable to be confused are soft sore or chancroid, herpes, and scabies.

From soft sore the points of distinction may be tabulated as follows :

\section{Syphilitic Chancre.}

(I) Incubation period, 3 to 4 weeks.

(2) Usually single.

(3) Superficial erosion or ulceration, flat or elevated.

(4) Oval or circular in shape.

(5) Floor covered with a serosanious discharge or grey false membrane.

(6) Base indurated and circumscribed.

(7) Not auto-inoculable except during first Io days.

(8) Neighbouring glands enlarged, hard, and painless.

(9) Serum from it contains spironema pallidum.

\section{Soft Sore.}

A few hours to days like ordinary impetigo.

Generally multiple.

Deep punched-out ulcer.

Irregular in outline.

Covered with a purulent discharge.

Soft, or with an indefinite inflammatory thickening.

Readily auto-inoculable.

Neighbouring glands inflamed, painful, and liable to suppurate.

Discharge contains Bacillus of Ducrey. 
Herpes is not infrequent on the penis and labia; its diagnosis from chancre may be difficult, especially if vesicles are absent, but in it there is no induration and the erosion which follows the vesicles tends to be polycyclical in outline.

From scabies there should be no difficulty in diagnosis. In it the lesions may be multiple and consist of red spots which are not indurated, or of small excoriations covered with a scab on a red base. Occasionally a typical burrow may be present and there are generally evidences of the disease in the usual sites. It must not be forgotten that syphilis and scabies may co-exist and that excoriations from scratching for the relief of itching form a ready mode of entrance for the virus of syphilis.

Extra-Genital Chancres.-Extra-genital chancres are most common on the lip, mouth, fingers, and face.

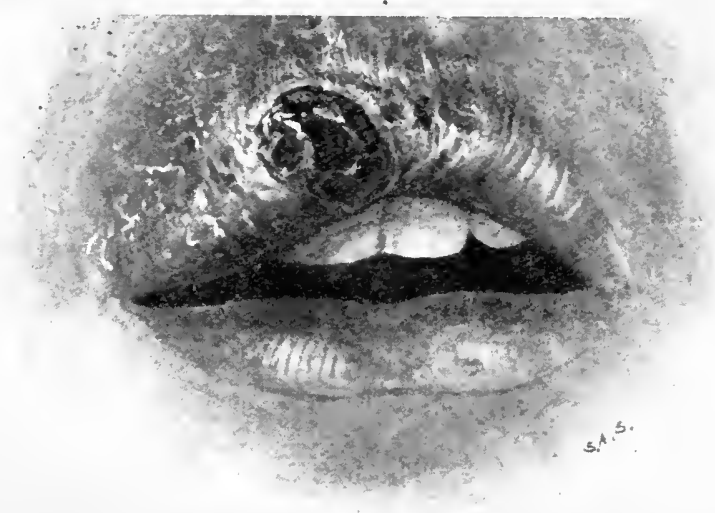

FIG. I8I.-Chancre of the Upper Lip.

With marked swelling of the lip.

(From Dr. J. E. R. IIcDonagh.)

Lip.-Syphilis may be conveyed to the lips directly by contact with a syphilitic lesion as in kissing, or indirectly by contaminated instruments and utensils such as forks, spoons, drinking-vessels, pipes, telephones, dental or surgical instruments, spatulas, etc. The resultant lesion may consist of a small fissure, erosion, or elevated ulcer with indurated edges and covered with a brown scab, and may be situated on either lip and is usually at the side. The affected lip becomes markedly swollen and oedematous and the glands beneath the chin become enlarged and hard. (Fig. I8I.)

Occasionally the chancre may resemble an epithelioma, but the latter evolves much more slowly, is more irregular in outline, is painful, usually occurs after middle-age, and the neighbouring glands, if enlarged, do not become so for 5 or 6 months.

Tongue.-Chancre of the tongue usually occurs in the anterior part and most frequently near the tip on the dorsal surface. It may take the form of a simple erosion on an indurated base, a deep ulcer, or a 
sclerotic mass, and is associated with enlargement and induration of the sub-maxillary glands. It is sometimes mistaken for an ulcer due to a carious tooth, but this is usually situated at the side of the tongue and is more painful.

Tonsil.-Chancre of the tonsil is uncommon. It may be erosive or may form a deep ulcer with abrupt edges. It is unilateral, associated with enlargement of cervical glands, especially of the gland beneath the angle of the jaw, and with swelling and induration in the fauces which cause pain and difficulty in swallowing.

Chancres may occur in other parts of the buccal mucosa, such as on the palate or gums or in the pharynx.

Nose.-Chancres may occur about the nostrils or nasal fossa and may be erosive or ulcerative. They may be caused directly or indirectly by some instrument such as a contaminated Eustachian catheter.

Eye.-Chancres about the eye are rare and when present usually occur on the eyelid or the conjunctiva. The infection may be conveyed by spitting or kissing, or indirectly by the fingers or by a contaminated towel, and they have been known to be contracted by a surgeon in examining a syphilitic throat. They may take the form of an erosion or ulcer and are generally accompanied by enlargement of the neighbouring glands. On the eyelid they may appear as a hard swelling which might be mistaken, at first, for a sty ; on the conjunctiva they are most common on the palpebral surface and at the inner angle of the eyelid and cause eversion of the lids.

Face.-Chancres on the face are often multiple and are specially common in infants. The infection may be conveyed by kissing or spitting, or indirectly by razors, shaving brushes, etc. They are associated with marked induration and enlargement of sub-maxillary and parotid glands. On the beard-region they may be mistaken for trichophytic sycosis, but the enlargement of glands and the absence of fungus would establish the diagnosis.

Breast.--Chancre of the breast is most common in women and is frequently the result of suckling a syphilitic infant with infective lesions in the mouth. It may occur on any part but is most usual on the nipple, in which situation it sometimes extends in a semi-lunar fashion round the base and might be mistaken for a simple fissure but that it is more indurated and not painful and that in association with it the glands in the axillae are enlarged, hard, and painful.

Fingers.-Chancre of the fingers is specially common among medical men, dental surgeons, nurses, and midwives, and may be contracted from examining or operating upon syphilitic patients or from handling soiled dressings, etc. The inoculation usually takes place through some abrasion, but has been known to occur on an eczematous patch or a broken chilblain. A frequent site is around the nail, where it gives rise to a painful irregular ulcer, or to a fissure with indurated edges, or may resemble a whitlow, causing the end of the finger to 
be red, hard, and swollen, and sometimes leading to separation of the nail. On the finger a chancre grows more slowly than elsewhere and in connection with it the epitrochlear and axillary glands are usually enlarged.

Anus.-Anal chancres are comparatively rare. They are most common in children as the result of criminal assaults, but may be caused indirectly by an enema syringe or some other instrument. They may be present externally or within the anal orifice; in the latter situation they are deep red in colour, usually oval in shape, not markedly indurated, and the inguinal glands are usually enlarged. They have to be distinguished from tuberculous ulcers which are more yellow in colour and the borders are better defined, and from anal fissures which are more painful and not associated with enlarged glands.

General Integument.-Chancres may occur anywhere on the cutaneous surface but have not been met with so far on the sole of the foot. The contagion may be transmitted directly or indirectly, as by tattooing with infected instruments, or by contaminated watercloset seats, towels, etc. The upper and inner aspect of the thigh is a not uncommon situation for chancres in women.

Vaccinal Chancre.-Chancres have been known to supervene on vaccination sores from the employment of lymph contaminated with the virus of syphilis. They were comparatively common when vaccination was from child to child but now, since the introduction of calf lymph, they can only occur from the employment of infected instruments in scarifying or from a secondary infection before the vaccination sore has healed.

Prognosis of Chancres. - It is impossible to foretell from the size or type of chancre the severity of the symptoms which are liable to follow it. It has been said that where the incubation period is short and the primary sore acutely inflamed or ulcerated the attack promises to be more severe than where the incubation period was longer and the chancre of a more indolent type, but this cannot be taken as a reliable guide; nor is there any difference between the severity of the symptoms which may follow a chancre on the genital organs and one situated elsewhere.

Local Treatment.-Excision of the chancre and neighbouring enlarged glands, or its destruction by the cantery, though as a rule insufficient to prevent the generalisation of the disease, yet constitutes a valuable prelude to treatment.

The lesion should be bathed with I in 2000 perchloride of mercury solution, dusted with calomel or iodoform, and kept covered with cottonwool. Where there is marked ulceration or phagedaena, warm boric compresses or a boric hip bath should be employed to reduce the inflammation, and should be followed by dusting with calomel.

Cauterisation by crude chromic acid is useful, but is so painful as to require a local anaesthetic. The application of the acid produces 
a black slough which on separating usually leaves a base of healthy granulations.

Where the prepuce is affected and phimosis is present, irrigation with biniodide solution should be resorted to, for which purpose a fine catheter may be inserted beneath the prepuce or the prepuce may be slit up to facilitate the irrigation.

Histopathology of the Chancre.--The chancre is the evidence of the local reaction of the skin to the presence of the Spironema pallidum in situ. The reactive changes occur in the corium, while the epidermis is only affected secondarily and to a minor degree. In the corium, occupying the whole of the indurated region, there is a dense infiltration of plasma-cells, frequently packed in rows between the white fibrous bundles, with a few fibro-blasts and spindle-cells indicating a new formation of fibrous tissue. It is this combination of dense cellular infiltration and new-formed fibrous tissue which gives the lesion its peculiar hardness. Within the infiltrated mass the capillaries are still present, and towards the subcutaneous tissue the small arteries show proliferative changes in the walls with an increase in the endothelium and the adventitia and occasionally an obliterative sclerosis. In the neighbourhood of the blood-vessels a few mast-cells can generally be detected, while the elastin his to a great extent disappeared throughout the infiltrated area.

In the epidermis there is proliferation of the epithelium at the margin of the lesion and flattening of the centre from pressure by the underlying granuloma. The cornification in the centre is imperfect, the granular layer being frequently absent so that there is a tendency. to desquamation and erosion.

The histology of the lesion would suggest that the Spironema pallidum was present at first in the lymphatic spaces, and there caused the profuse cellular infiltration.

\section{Cutaneous Syphilides}

The cutaneous manifestations of syphilis are so multiform that it is difficult to describe them adequately. They have certain characteristics, however, which are common to the majority and serve to distinguish them from other skin affections; of these the most important are the following:

Polymorphism.- Syphilis has been described as the " great imitator" and practically every form of primary and secondary cutaneous lesion may be simulated more or less closely by a syphilide; for example, in one case the lesions may be papular and suggest Lichen planus, in another they may be scaly like psoriasis, while in a third they may be framboesiform like yaws. Diverse types of lesions may be present from time to time in the same case, some obviously syphilitic, others so closely imitating different forms of dermatitis as to render the diagnosis difficult. 
Colour. - The majority of the syphilides have a peculiar tint which has been aptly described as " copper" or " raw ham" colour. This is explained by the histological structure, which consists chiefly of a cellular infiltration surrounded by blood-capillaries. When the syphilide is pressed by a diascope and the blood squeezed out a brownishyellow stain remains, due to the cellular deposit, but when the pressure is relieved and the blood returns the lesion assumes a redder tint somewhat resembling raw ham.

Induration.- With the exception of the early roseolar and pigmentary lesions, the syphilides as a rule are definitely indurated from the presence of the dense cellular infiltration in the corium.

Mode of Evolution.-The early syphilides come out comparatively rapidly and after persisting for a variable period disappear spontaneously. The late syphilides, on the other hand, develop more slowly and may persist indefinitely.

The course of the eruption may be interfered with by the incidence of some acute infective disease such as pneumonia or erysipelas, but when the acute infection subsides the cutaneous lesions of syphilisespecially the later lesions-generally re-assert themselves.

Configuration. - The syphilides vary in their configuration and arrangement. They usually remain discrete, but may coalesce to form circles, segments of circles, serpiginous or gyrate figures, etc. ; or a ringed lesion may develop by peripheral extension and central involution, and by a repetition of this process concentric rings may be formed (rosette syphilide). Again the lesions may be grouped in clusters, or a number may be arranged round a single larger lesion (corymbose syphilide), or they may be closely aggregated in irregular sheets (syphilide en nappe).

Distribution.-The early syphilides are usually distributed symmetrically and involve especially the face, trunk, and flexor aspects of the limbs, or they may be widely disseminated over the whole cutaneous surface; the intermediary lesions have a tendency to be grouped in a circinate or corymbose manner ; the late lesions are local, asymmetrical, and confined to one or more regions.

Subjective Symptoms.-Sensory symptoms are absent in cutaneous syphilides, unless where the lesions are irritated by friction or occur in a moist warm region, such as the perineum, when they may be associated with mild itching.

Therapentic Test.-All the cutaneous syphilides are peculiarly amenable to salvarsan and mercury internally, but local treatment has little or no effect upon them.

Spironema pallidum.-The spironema may be recovered from any of the cutaneous syphilides, but is present in greater numbers and is more easily found in the early than in the late lesions.

Wassermann Reaction.-When cutaneous lesions are present in an untreated case of syphilis the Wassermann test is almost invariably positive and can be relied upon to establish a diagnosis. A positive 
reaction is occasionally obtained in certain other diseases, such as nodular leprosy, trypanosomiasis, relapsing fever, etc., but in them it is too inconstant to be of diagnostic value.

Seierity of the Lesions. - The severity and extent of the cutaneous lesions in syphilis vary widely in different cases. Sometimes the eruption may be so mild and transient as to pass unnoticed, especially where the disease has been recognised in its early stages and treatment with salvarsan has been instituted, or where the syphilides have faded spontaneously without leaving any trace. Late syphilides, on the other hand, tend to ulcerate and to be followed by scars.

The severity of the cutaneous symptoms is dependent on the following factors :

I. The number of spirochaetes present and their degree of virulence.

2. The susceptibility of the individual. This is greatest in those who have no hereditary immunity, and it has been noted that when syphilis has been transmitted to some previously unaffected race, such as the inhabitants of some remote island of the Pacific, it has been extraordinarily virulent. Susceptibility to it may be increased by a debilitated state of health from alcoholism or from some prolonged illness, such as tuberculosis, diabetes, or malaria.

3. The severity of the cutaneous lesions may be increased locally by secondary contamination with pyogenic micro-organisms.

General Pathogenesis. - Although the cutaneous syphilides display an extraordinary multiformity in their clinical appearances, their pathogenesis is simple and the pathological process which underlies them, though differing in degree in different lesions, is uniform in type. The lesions are the result of the presence of the spironema in the corium and the clinical evidence of the reaction of the tissue to the organism. The spironema reaches the skin iia the blood-stream, and causes dilatation of the superficial cutaneous capillaries and slight proliferative changes in the endothelium, and so gives rise to the early erythematous or roseolar lesions. Later the vascular dilataticn is associated with a cellular infiltration of a granulomatous type consisting of plasma-cells and lymphocytes, and with a proliferation of fibrous tissue, which are responsible for the induration. When, after a time, the infiltration extends more deeply the ressels become blocked by proliferative changes in the endothelium and the adventitia, and the lesions tend to break down and ulcerate.

Healing takes place without subsequent scarring in the early lesions whether erythematous or papular, but where the granuloma has invaded the whole of the corium and subcutaneous tissue and has broken down scarring is inevitable.

Classification of the Cutaneous Syphilides.-Numerous attempts have been made to classify the cutaneous syphilides, but these have been so complicated and unsatisfactory as rather to increase confusion than to produce order. The usual method has been to arrange them under the headings of primary, secondary, and tertiary lesions, but this 
arrangement is defective in that lesions which generally occur early in the course of the disease and are usually placed among the secondary syphilides, in exceptional cases may not appear till much later and may occur in association with typical tertiary lesions.

For convenience of description some arrangement of the syphilides is necessary and here they will be grouped under the following headings : Early syphilides; intermediary syphilides; late syphilides. It must not be forgotten, however, that this division also is purely arbitrary.

The early syphilides consist of transient roseolar eruptions usually distributed symmetrically and present chiefly on the abdomen and chest ; pigmentary syphilides, generally affecting the neck in women; and widely distributed eruptions, usually papular but occasionally scaly, vesicular, or pustular.

The intermediary lesions have a tendency to be grouped and may break down and be followed by scars. They consist of recurrent roseolar eruptions, lesions on the palms or soles, or papules arranged in the form of rings (annular syphilides) or clustered round a central larger papule (corymbose syphilides).

The late syphilides are more localised, asymmetrical, and consist of lesions which are ulcerative or gummatous in type.

\section{Classification}

\section{Early Syphilides.}

I. Rosenlar or macular.

2. Pigmentary.

3. Papular.

4. Follicular.

5. Squamous.

6. Pustular.

The eruption, as a rule, is like an exanthem and widely distributed.

\section{Intermediary Syphilides.}

I. Grouped lesions, chiefly papular but sometimes scaly, vesicular, or pustular.

2. Palmar and plantar syphilides.

3. Recurrent roseolar eruptions.

\section{Late Syphilides.}

Ulcerative or gummatous syphilides.

\section{EARLY SyPHILIDES}

I. Roseolar or Macular.-The typical roseolar exanthem generally appears on the skin 6 or 7 weeks after the development of the chancre, which may still be present in an active state or may have healed completely and have been replaced by an indurated cicatrix. In 
severe infections or in susceptible individuals it may break out sooner ; while if the case has been recognised early and suitably treated, its appearance may be retarded or even prevented.

The eruption consists of macules, which are round, oval, or irregular, in outline, generally about the size of a split-pea or slightly larger, and of a pinkish-red tint. In some cases the lesions may be vivid and easily. recognised: in others they may be so faint as to be missed, but can usually be detected by the employment of a cobalt glass. In dependent situations, such as the legs, and in individuals in whom the cutaneous

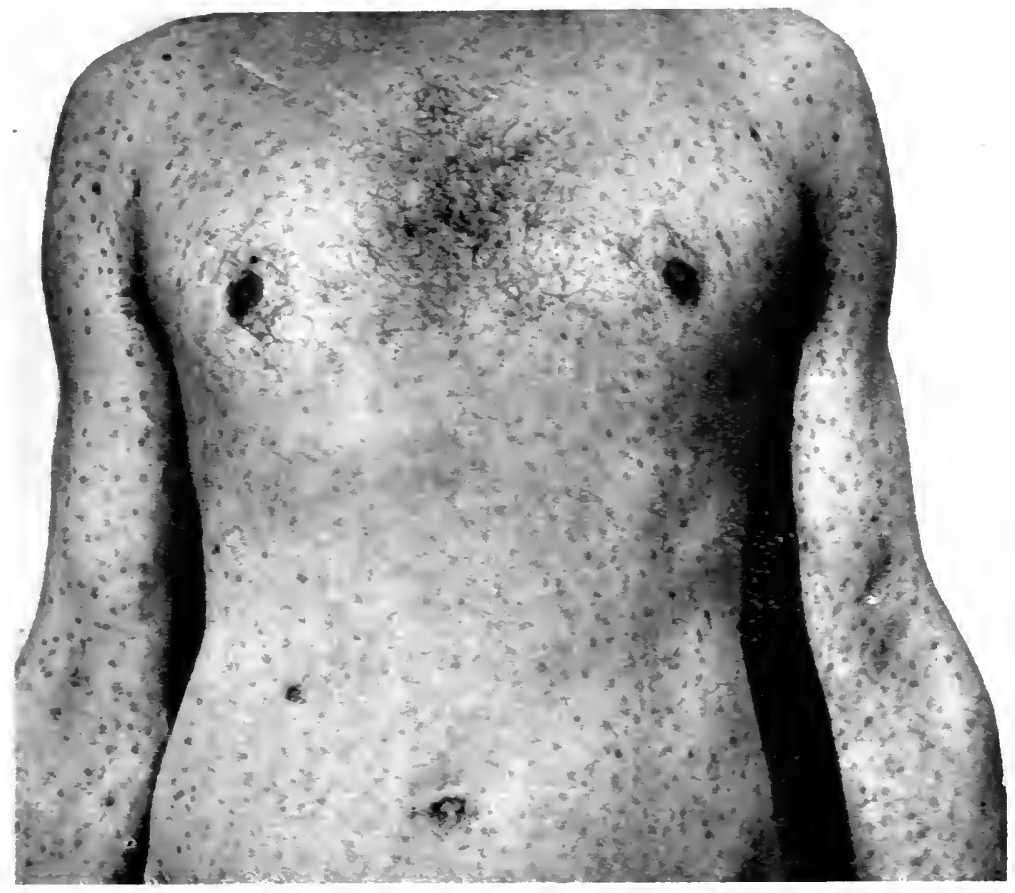

FIG. I82.-Roseolar Syphilide.

circulation is congested, they may assume a purplish tinge, but howerer dark they may be they can be made to disappear by pressure with a diascope. Ther may have well-defined borders or may be indefinite in outline merging into the surrounding skin and producing a blotchy appearance. They may remain discrete, or may coalesce into irregular patches, or mar give rise to a diffuse mottling somewhat suggesting scarlet fever. As a rule they are level with the surface of the skin, but occasionally they may be raised or even urticarial, and in rare instances have been known to be circinate. The eruption appears first on the sides of the trunk, on the central parts of the abdomen, around the umbilicus, on the flexor aspects of the arms, and on the inner surfaces of the thighs, and as a rule is most marked in these situations. In some cases it may become generalised, but the scalp and backs of the hands are rarely affected. (Fig. ISz.) 
As a rule the roseola disappears after a few days or weeks, but occasionally some of the lesions become gradually darker and may persist for a month or more. On disappearing a faint yellowish-brown stain and slight desquamation are left.

In association with the roseola there is painless enlargement and induration of the lymphatic glands, especially the posterior cervical, occipital, submaxillary, and axillary, and the glands over the mastoid.

Both the roseolar spots and the enlarged lymphatic glands are the result of the dissemination of the spironema throughout the system by the blood-serum, and the micro-organism can be found in the macules, in blisters artificially produced over them, and in the juices from the lymphatic glands.

The generalisation of the spironema is evident also in the occurrence of constitutional symptoms, such as malaise; a rise of temperatu; with evening exacerbations, which usually precedes the eruption for a day or two and disappears when it is fully out; pains in the head, most marked at night; pains in the limbs of a rheumatic type; sore throat; and albuminuria. When the throat is examined the fauces may be found to be red and congested and the tonsils to be swollen and to present either follicular ulcerations or greyish-white spots or patches, which may be detected also on the mucous membrane of the cheek and the tongue.

Histopathology.- Sections of an erythematous macule show dilatation of the superficial capillary vessels, proliferative changes in the endothelium, and a slight deposit of lymphocytes around the dilated capillaries. The Spironema pallidum has been found in the dilated vessels and among the lymphocytes which surround them.

Diagnosis.-The recognition of a well-marked roseolar syphilide presents no great difficulty, as in most cases the eruption is fairly characteristic and is associated with indications of the chancre and with enlarged glands. In women, however, the diagnosis may be more difficult as the chancre, when in the vagina, may be so insignificant and evanescent as to pass unnoticed.

It has to be distinguished from :

(I) A toxic eruption of the Erythema multiforme type, but in it the lesions are present chiefly on the face, backs of the hands, wrists, and fronts of the legs, are darker in colour, and remain discrete.

(2) Drug rashes due to copaiba, antipyrin, etc., but in them the eruption develops much more rapidly and is brighter in colour.

(3) Pityriasis rosea, but in it the lesions are scaly, in many cases the typical oval patches with a pinkish scaly border and a buff-coloured centre can be detected, and there is a history of the characteristic " herald patch." In rare instances Pityriasis rosea may develop in a patient suffering from syphilis, and should this occur in the early 



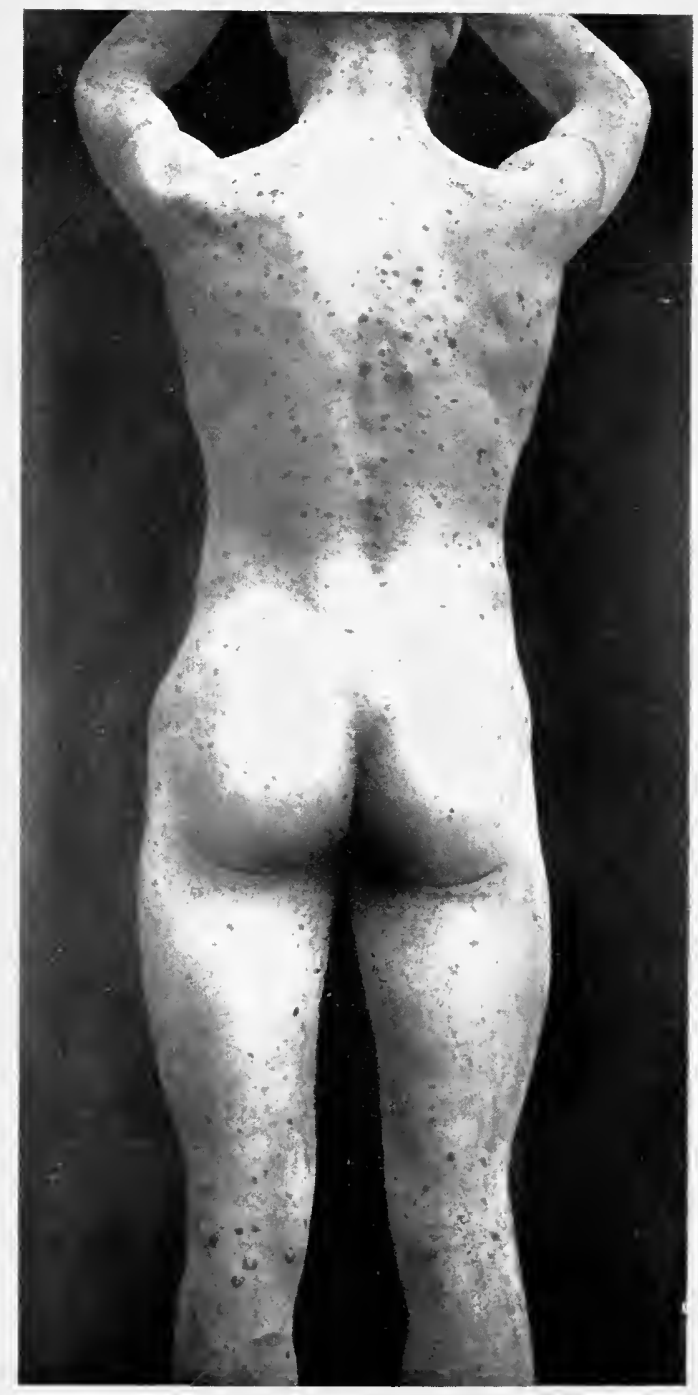

FIG. 183.-Papular Syphilide.

Showing distribution on back and limbs. 
stages of the disease when the syphilis is comparatively active the diagnosis may be exceptionally difficult. ${ }^{1}$

2. Pigmentary Syphilides.- - Ssually about the same time as the roseolar eruption, but sometimes a few months later, an irregular pigmented mottling may be observed on the back and sides of the neck, and more rarely in other situations such as the axillae, lower part of the abdomen, and thighs. It generally consists of a café-au-lait tinted irregular network enclosing areas of normal skin, but sometimes occurs as a band around the neck-the so-called "collar of Venus." It is more marked in dark than in fair skins and is found chiefly in women ; in the occasional cases in men it is less noticeable owing to the coarser texture of their skin.

The pigmentation is persistent, uninfluenced by treatment, and gradually disappears spontaneously. It is obviously a melanodermia and not due to blood pigment but the exact manner of its production is uncertain. It is generally believed that the network is the remains of the normal pigmentation or possibly the result of an increase of pigmentation, and that the white spots in the meshes are caused by the fading of the roseolar macules. Another explanation-but one which has not met with much support-is that it is a neurotic phenomenon, or "neuro-syphilide," caused by the irritation of the nerve fibrils supplying the affected area.

3. Papular Syphilides. - The most characteristic early cutaneous syphilide is papular. The papules are rounded elevations, which are reddish-pink at first but rapidly assume a raw-ham or coppery tint, while in dependent situations or in individuals with a sluggish circulation they may be purplish. They vary in size from a lentil (lenticular syphilide) to a sixpence or larger (discoid syphilide), and are usually convex or flat but occasionally are depressed. The surface may be smooth and shiny, or covered with a fine scale which is readily detached in the centre and persists in the periphery in the form of a fringe with the free border directed inwards, known as the " collar of Biett" from the - distinguished syphilographer who first described it. On being pinched up between the fingers the lesions are found to be indurated, and on pressure with a diascope they are replaced by a yellowish-brown stain. (Fig. IS3.)

The papules vary in distribution according to their size. When small they are scattered irregularly over the greater part of the cutaneous surface, being most numerous on the sides of the trunk and chest. As a rule they remain discrete, but where profuse and closely aggregated they may coalesce into patches with a brownish tint and harsh surface

${ }^{1}$ In a case in a middle-aged man Pityriasis rosea appeared i I months after the chancre. The patient had had 3 injections of salvarsan alternated with injections of mercury and followed by mercury by the mouth, and had had no marked secondary symptoms. The Pityriasis rosea was preceded by a herald patch and seemed perfectly typical, except that the lesions on disappearing left pigmented macules. At the time when the Pityriasis rosea developed the IVassermann reaction was negative. 
which are found chiefly about the gluteal region, the inner surfaces of the thighs, the perineum, and thə lower part of the face. When large they are fewer in number and more sparsely distributed, being present chiefly on the forehead (the Corona veneris), on the temples, around the mouth, at the sides of the nose, on the flexor aspects of the arms, and in the popliteal spaces. (Fig. I84.)

The papules may develop on the roseolar macules, or independently of them as reddish-pink spots which subsequently become raised and darker in tint. They come out in crops for several weeks, the out-

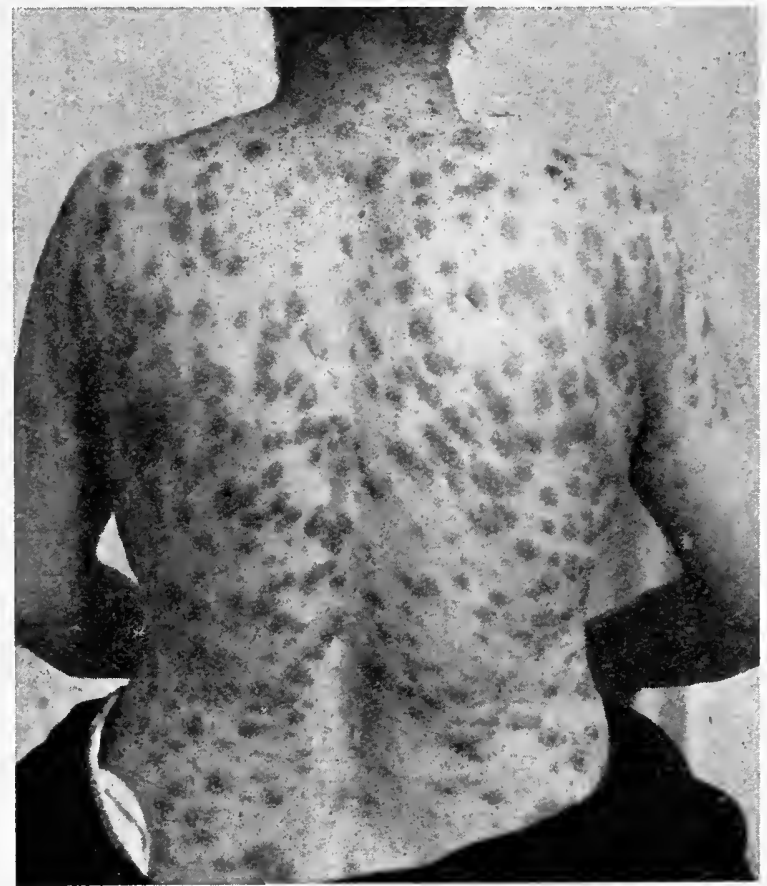

FIG. 185.--Large Papular Syphilide.

(From Dr. Arthur Hall.)

breaks sometimes being associated with a febrile disturbance, and they tend to disappear gradually after a month or two leaving a transient brownish stain. They occur independently of the hair-follicles, but may be situated around the sweat-pores. (Fig. I85.)

The appearance of the lesions may be modified by their position, by the secondary inoculation of pyogenic micro-organisms, or by the pre-existence of some other cutaneous affection such as circinate seborrhoic dermatitis. When they occur about the angles of the mouth, the edges of the nose, or between the fingers or toes, they are liable to become cracked from movements, inflamed and septic, and occasionally eczematous. On moist warm contiguous surfaces, such as about the anus, sides of the scrotum, vulva, axillac, sub-mammary region in women, etc., they become swollen and oedematous and the surface sodden and 


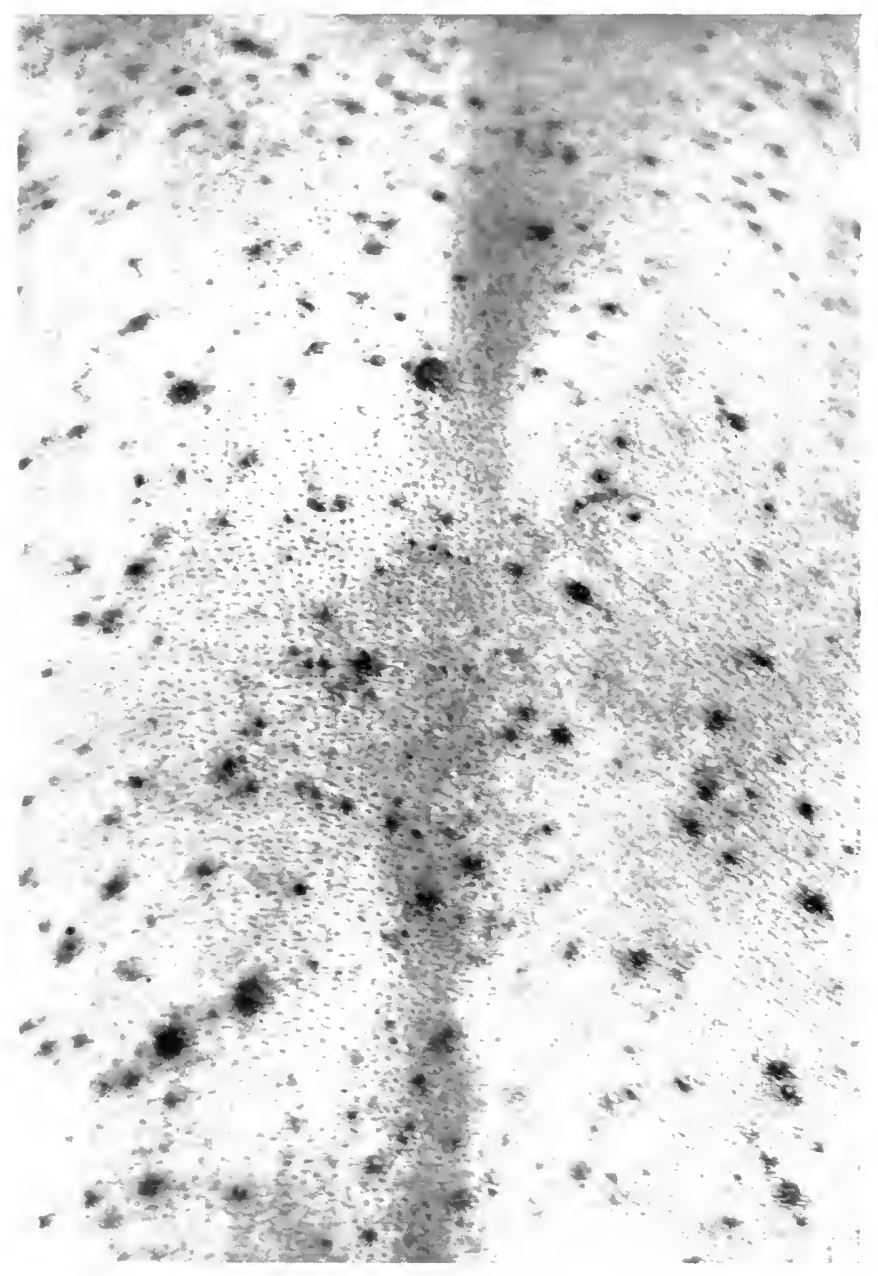

Fig. I 4 - Small Papular syphilide. 



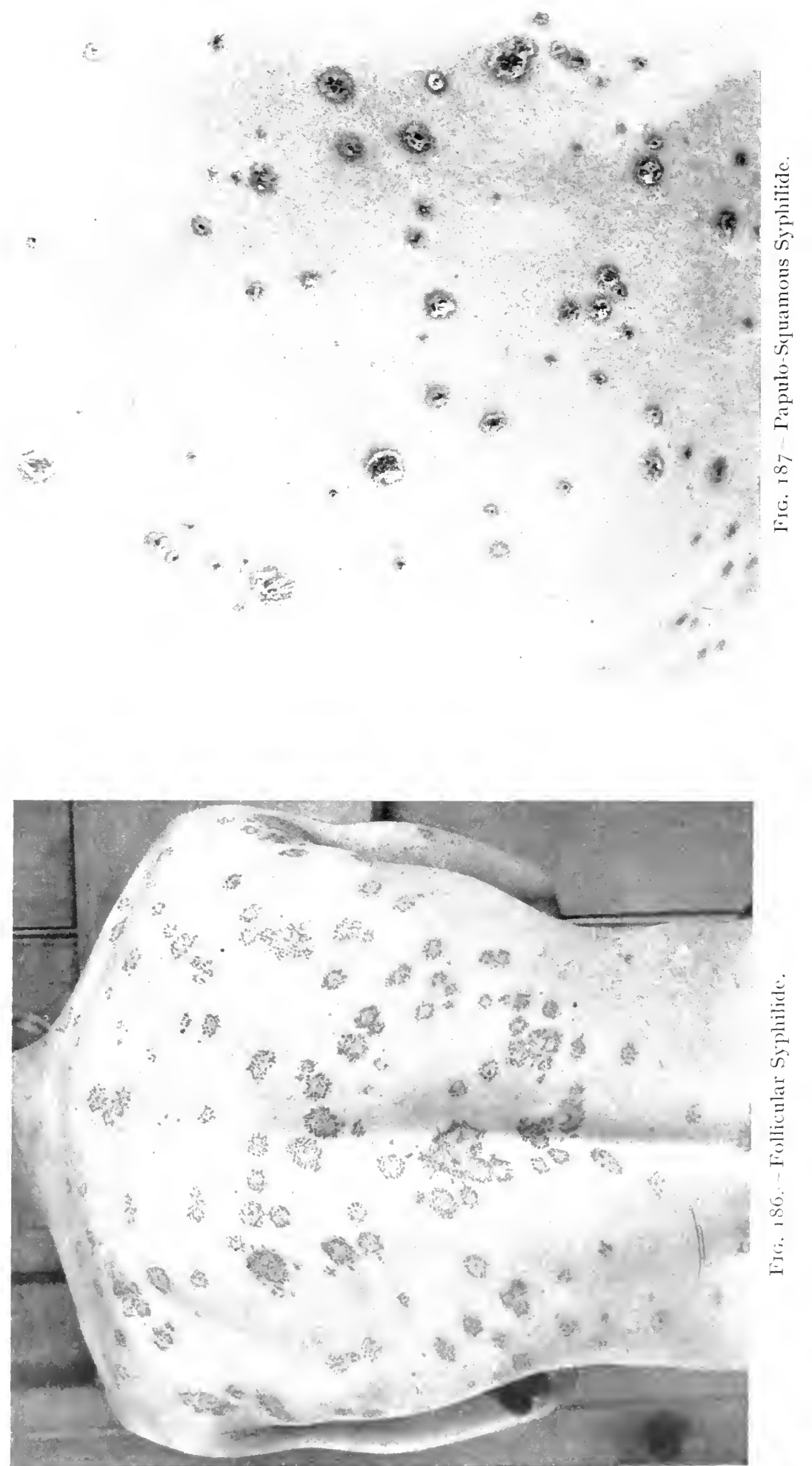

[To fuce $p .547$. 
moist from serous exudation and are known as condylomata. On the palms and soles they are not raised as a rule but tend to form rounded macules of a raw-ham tint, or small horny masses which are whitish in colour and can be picked out with the finger-nail. On the scalp they are apt to become crusted.

4. Follicular Syphilides. - A somewhat rare type of papular syphilide is follicular or acneiform and occurs at the mouth of a pilo-sebaceous follicle. The lesions are reddishbrown acuminate papules which may become capped with a small scale, horny plug, or crust, or may present an umbilicated surface. They may be widely distributed and are usuaily most marked on the face, neck, buttocks, and extensor aspects of the limbs. They may be small, about the size of a pin's head, and closely aggregated in groups like Lichen scrofulosorum (lichenoid or-miliary syphilide); or they may be larger, about the size of a split-pea, and either grouped or distributed in an irregular fashion, and may resemble Acne vulgaris but are distinguished from it by the absence of comedones and by the lesions being browner in tint, smaller, more widely disseminated, coming out more rapidly, and disappearing more quickly. (Fig. IS6.)

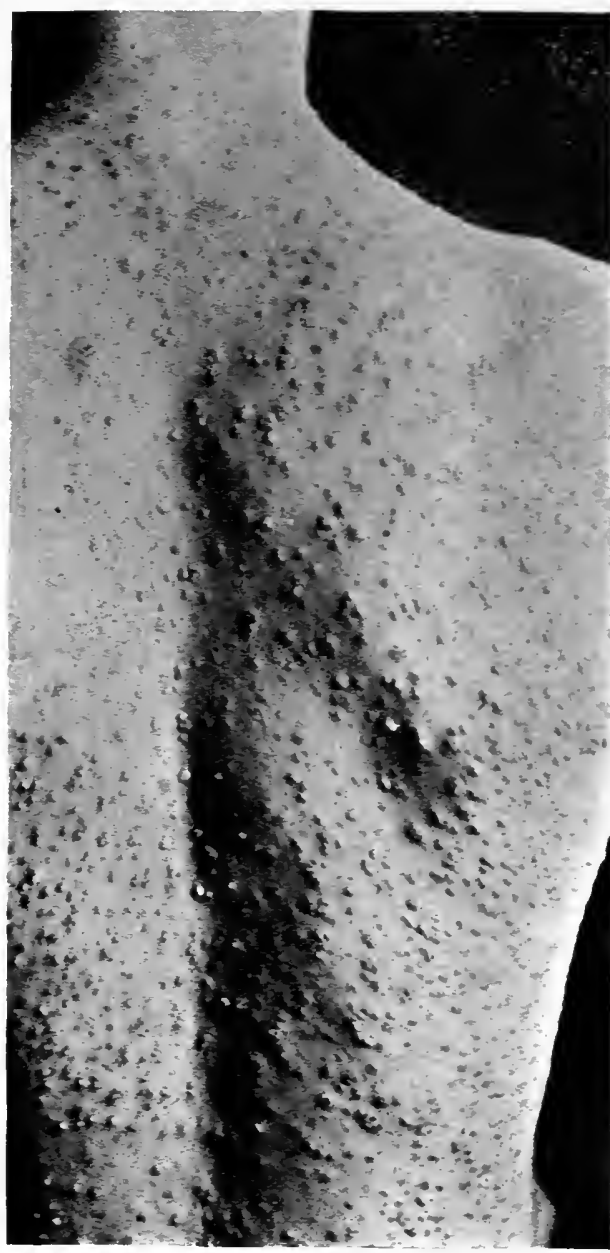

FIG. I88.-Papulo-Pustular Syphilide. Varioloid Type.

(Mr. Arthur Shillitoe's case. Brit. Journ. Derm., I904.)

5. Squamous Syphilides.-Instead of being smooth or surrounded by a scaly fringe the lesions may be covered with yellowish-white scales which are either fine like pityriasis or coarse like psoriasis. These squamous syphilides may occur anywhere on the cutaneous surface but are most common on the palms, and are met with especially in the intermediary period of the disease.

When widely distributed the squamous syphilide may be difficult 
to diagnose from psoriasis. The chief points of distinction are the following: In the squamous syphilide the scales are thin and situated on a raw-ham coloured base, while in psoriasis they are thick and silvery and the base is bright red; on removing the scale in syphilis and scratching the surface there is not the same tendency to bleed as in psoriasis; in syphilis the distribution is irregular and there is a

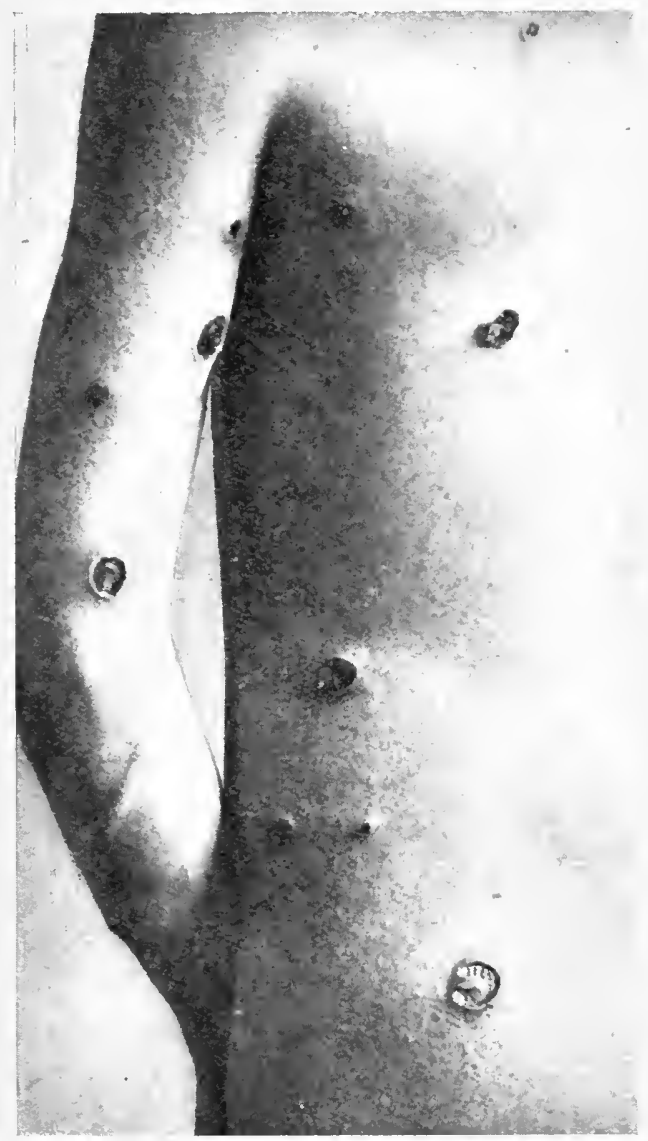

FIG. I 89.-Syphilitic Rupia. tendency for the lesions to be more profuse on the flexor aspects of the limbs, while in psoriasis the sites of predilection are the knees, elbows, extensor aspects of the limbs, and scalp; in syphilis the lesions are indurated, while in psoriasis the thickening is not due to an underlying cellular infiltration but simply to the scales. (Fig. I87.)

\section{Pustular Syphilides.-}

In the papulo-pustular syphilides the pustules are either preceded by papulovesicles or evolve so rapidly that no vesicular stage can be detected. They tend to dry up to form crusts of a dirtygreenish colour, which on separating leave either superficial ulceration (impetiginous syphilide) or a deep punched-out uleer. The ulcers heal with a pigmented cicatrix which subsequently becomes a white depressed scar surrounded for some time by a pigmented areola. (Fig. I88.)

The pustules vary in size from a millet-seed to a sixpence or larger. When small and present in large numbers they may simulate smallpox, but are distinguished from it by the absence of severe general sy'mptoms ; on the forehead they may resemble Acne vulgaris. When larger the discharge may dry up to form a dirty-green adherent scab beneath which the ulceration spreads peripherally and gives rise to new layers of scab, each larger than its predecessor, which results in the building up of a peculiar conical laminated crust like a limpet shell, which is known as a rupia. The pustular syplilides of the rupial type are 
a comparatively late manifestation, belong to the intermediary period, and appear from six to twelve months after the chancre, or even later. They occur most frequently in individuals debilitated by illness, alcoholism, or some other cause, and are especially liable to follow a virulent chancre which has become phagedaenic. (Fig. ISg.)

There is some difference of opinion with regard to the pathogenesis of the pustular syphilides; certain observers consider that the chief factor in their causation is a secondary infection with pyogenic micro-organisms, while others believe that the syphilitic virus itself is mainly responsible and in support of this view point out that they may come out in crops almost like an exanthem.

\section{INTERMEDIARY SyPHILIDES}

Under the heading of intermediary syphilides may be placed certain cutaneous lesions which, though they may occur only a few months

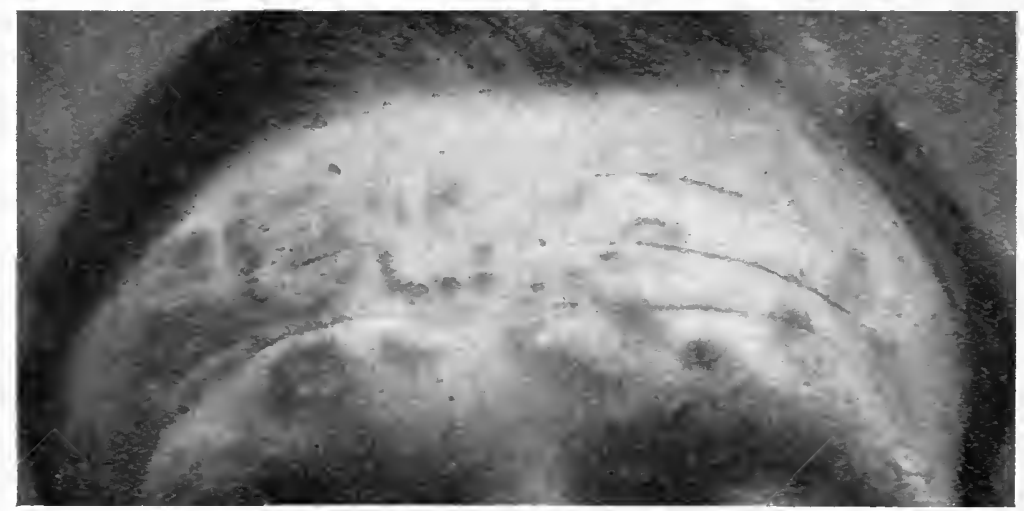

FIG. 190.-Rosette Syphilide.

after the chancre, as a rule appear considerably later and even in association with typical late syphilides. These consist of various grouped eruptions, palmar and plantar syphilides, and recurrent roseolas.

I. Grouped Syphilides.-The lesions which constitute the grouped syphilides are those which are familiar in the early stage, namely, rounded papules, acuminate or follicular papules, or more rarely papulo-resicles or pustules. They may be grouped in divers ways, of which the most conımon are corymbose grouping, grouping in patches (en placard), and circinate or annular grouping.

In corymouse gruuping the lesions are arranged in clusters around a larger central ıesıon. (Figs. I9I, I92.)

In the grouping in patches (en placard) the lesions are distributed irregularly, and usuany remain discrete though occasionally they may be 
so closely aggregated as to produce an indurated parchment-like patch the surface of which may be as harsh as a nutmeg grater.

In the annular syphilides the

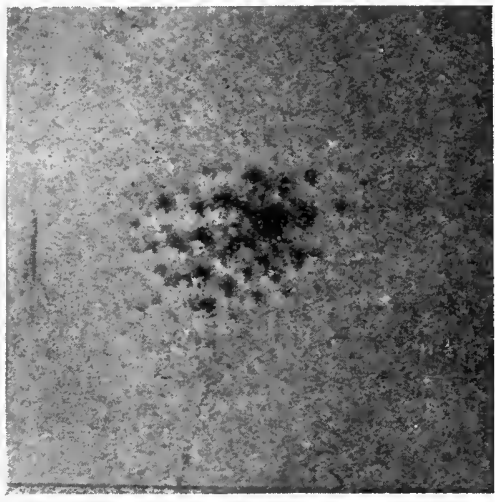

FIG. I91.-Corymbose Syphilide. ringed arrangement, though it may result occasionally from the circinate grouping of a number of small lesions, in the majority of instances originates from a single flat papule which has spread peripherally and cleared up in the centre. In this way rings are produced which vary in size up to a shilling or larger, and the borders of which are smooth or scaly and indurated. The rings may be incomplete and by coalescence may form gyrate or irregular figures, or occasionally by the development of new lesions in the centre of older ones concentric figures may result (rosette syphilide). The ringed lesions are most common

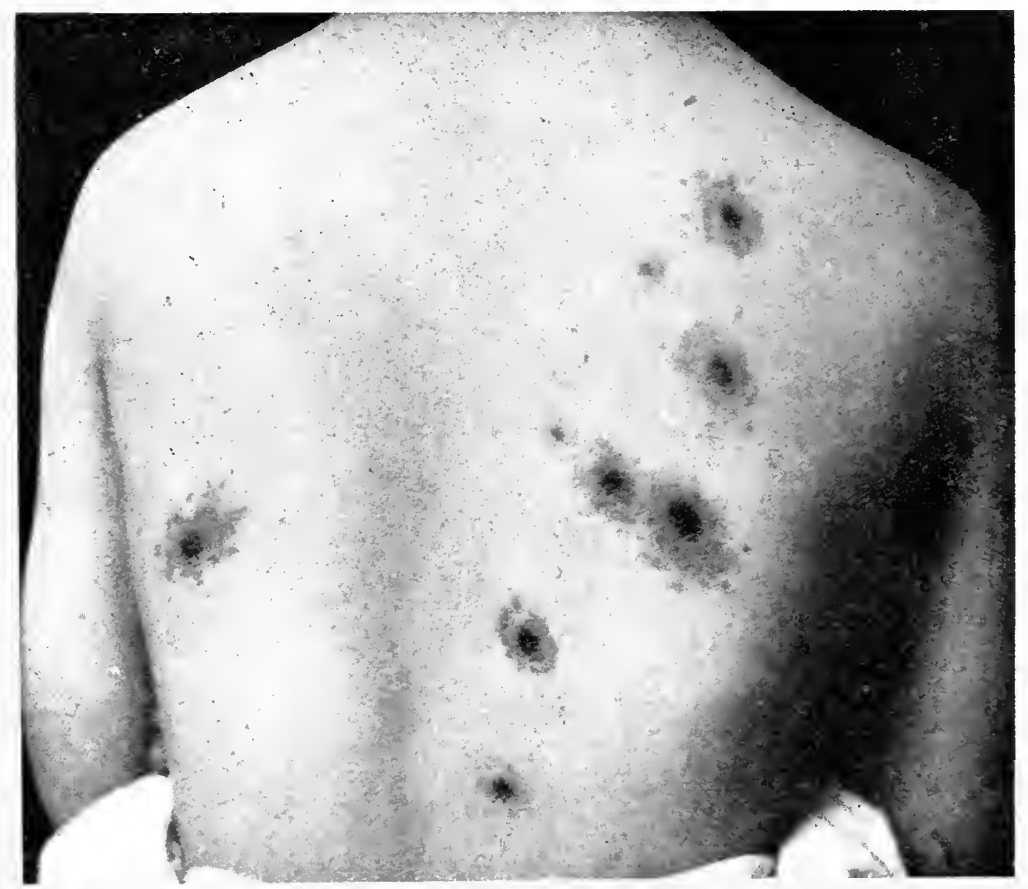

FIG. 192.-Corymbose Syphilide.

about the angles of the mouth, the chin, cheeks, and forehead, but may occur in other situations. They are most frequently met with in dark skins and especially in negroes. (Figs. I90, I93, I94.) 


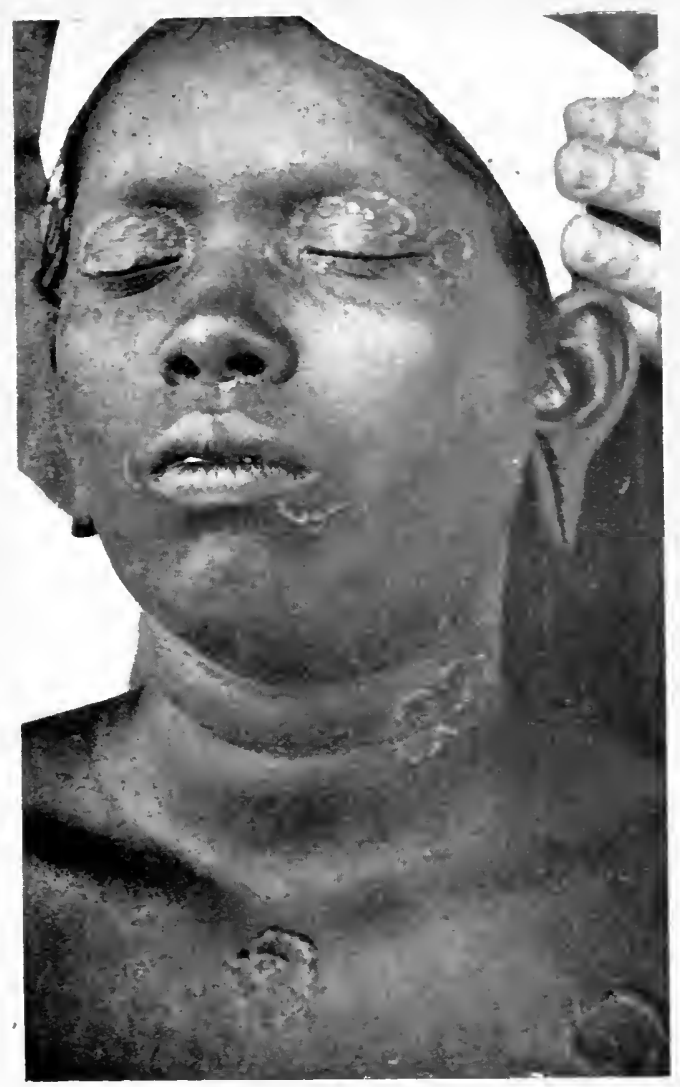

FIg. 193.-Annular Syphilide in a Tamil. (From Dr. W. T. Quaife.)

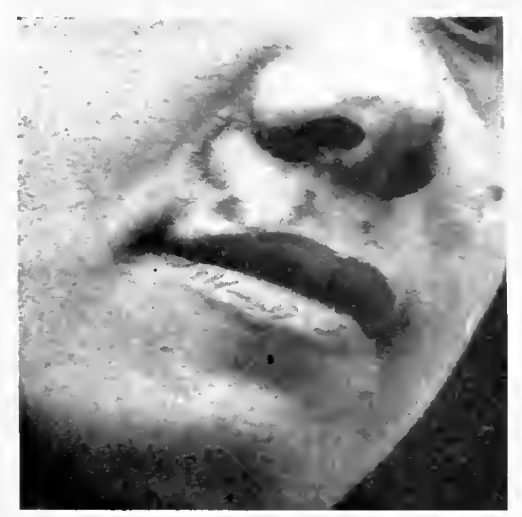

FIG. I94.-Serpiginous Syphilide. 

2. Palmar and Plantar Syphilides.-Cutaneous lesions on the palms and soles are not uncommon in the intermediary period, or may occur as a late manifestation. (Figs. I95, I96.)

On the palm they consist of yellowish-brown or raw-ham coloured macules or patches, irregular, roundish, or circinate in outline and sometimes surrounded by a slightly raised and indurated border. They may be smooth, or covered with a scale which does not usually extend to the edge of the lesion, or the surface may be thickened from hyperkeratosis and may break down to form deep painful fissures. As a rule only one hand is affected and the most common situations are the triangle of the palm and the flexures of the fingers. Palmar syphilides

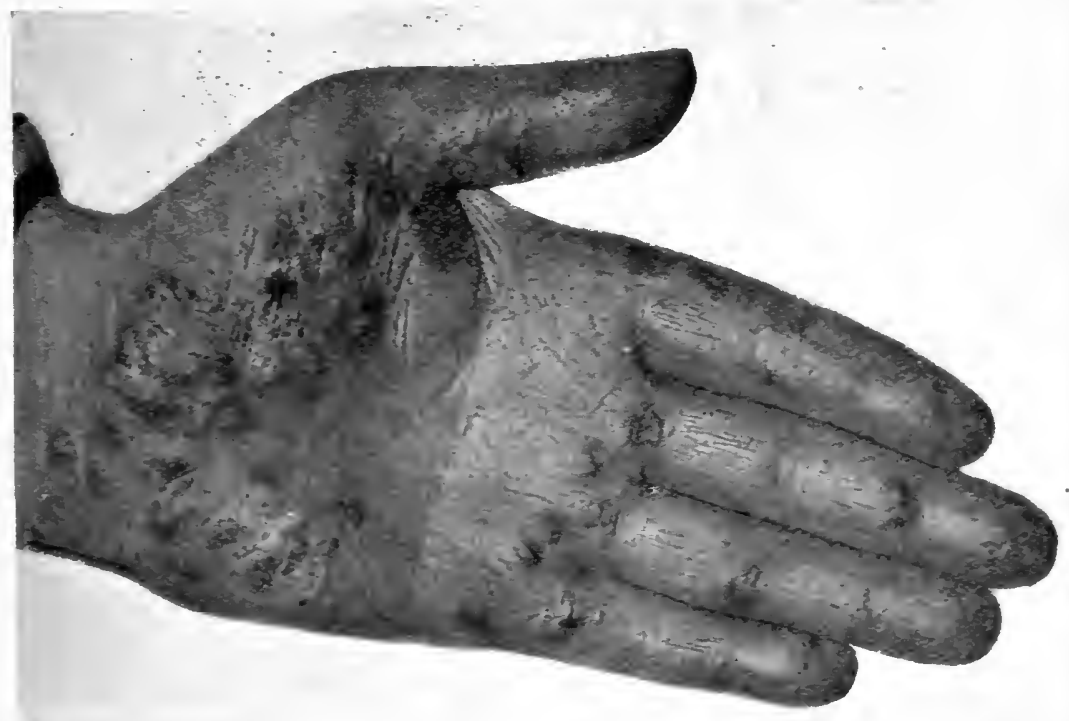

FIG. 195.-Palmar Syphilide.

are not uncommon in those who have to handle tools or instruments and have been attributed to pressure, but although pressure is unquestionably a factor in rendering them inflamed and painful it is doubtful if it does more.

Diagnosis.-Syphilitic lesions on the palm have to be distinguished from psoriasis and eczema.

Psoriasis of the palms is an exceptionally rare condition and when it does occur is associated with a profuse psoriatic eruption elsewhere and a definite history of the disease.

From palmar eczema the chief diagnostic points are the following: in syphilis the lesions are unilateral, in eczema they are generally bilateral ; in syphilis the margin is well-defined, in eczema it is irregular and broken up by islands of normal skin ; in syphilis the palm is only partially affected, in eczema it may be completely involved and the disease may extend on to the sides of the fingers or the back of the hand ; 
in syphilis the borders of the lesions may be raised and indurated and the base infiltrated, in eczema the borders are flat and the base soft; in syphilis the lesions are brownish in colour, in eczema they are reddish; in syphilis there are no associated subjective symptoms unless painful fissures are present, in eczema there is usually burning and itching.

On the soles and borders of the feet the lesions are similar to those on the palms, the most characteristic being a unilateral hyperkeratosis on a brownish base and associated with deep and painful cracks.

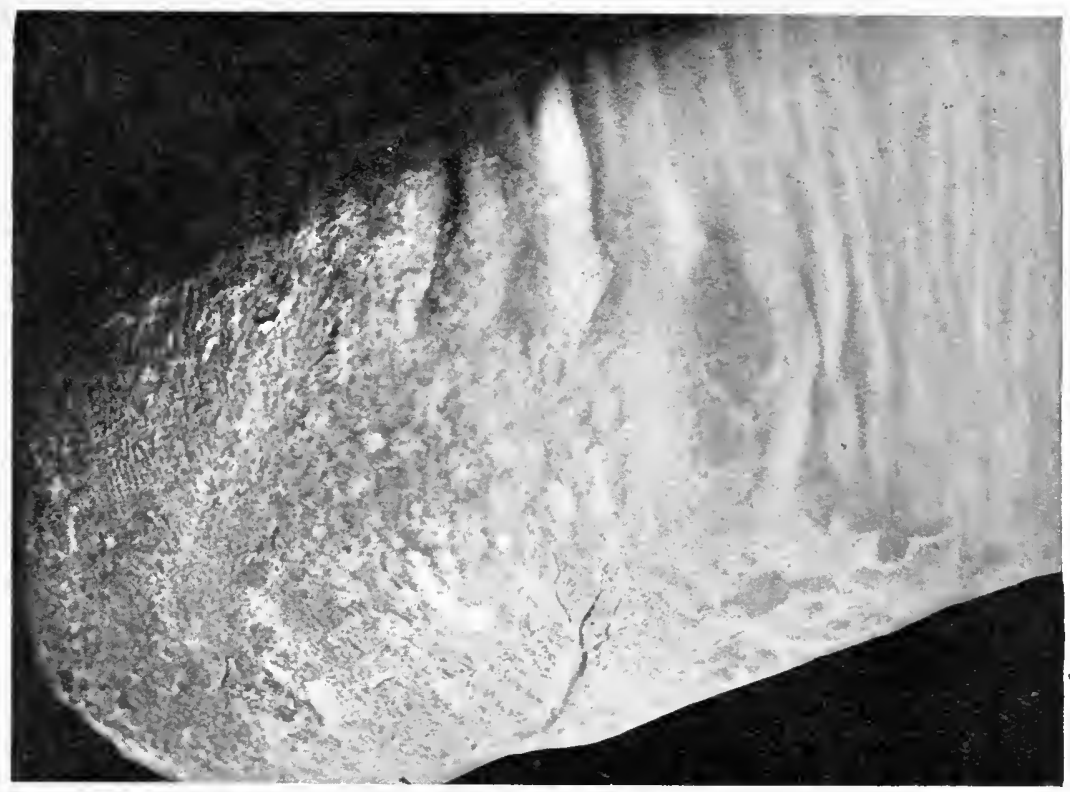

FIG. I96.-Hyperkeratosis of the Sole. Late Syphilitic Lesion.

3. Recurrent Roseolar Syphilides.-Roseolas are occasionally met with in the intermediary and even in the late stages of the disease and may recur several times in the course of an attack. The later roseolar eruptions are not so profuse as the early ones and consist of sparsely distributed macules and patches and occasionally of circinate lesions. They indicate that the spironema is still comparatively active and are believed to be due to the actual presence of the micro-organisms in the skin.

Histopathology of Early and Intermediary Syphilides.--From a histological point of view the papule may be regarded as the typical cutaneous lesion of early and intermediary syphilis, and the scaly vesicular or ulcerative lesions as variations dependent not so much on essential differences in the histological structure in the corium as on secondary changes in the epidermis. Sections of a papule show a dense cellular infiltration surrounding the capillaries in the superficial layers of the corium, which consists of plasma-cells and small connective 
tissue cells some of which are round and others spindle-shaped indicating a tendency to the formation of new fibrous tissue. Here and there multi-nuclear cells occur but in them the nuclei have not the peripheral arrangement met with in the giant-cells of tuberculosis. The walls of the capillaries are thickened from proliferation of the endothelium.

In the epidermis there is epithelial proliferation, oedema, and interference with the processes of cornification according to the extent of which the lesion is scaly or vesicular.

Local Treatment.-The early cutaneous syphilides require no special local treatment as they disappear under the internal administration of salvarsan and mercury. In the case of condylomata their infectivity may be reduced and they may be made to shrivel by dusting with calomel or by the application of a $2 \%$ chromic acid solution or of black-wash.

Palmar syphilides, when scaly or keratotic, may be benefited br the X-rays; a single pastille dose sometimes being sufficient to remore the scaliness. This should be followed by inunction with mercurial ointment. Fissures may be healed and the pain from them relieved by touching with a silver nitrate pencil. In plantar syphilides the hyperkeratosis may be reduced by the X-rays or by the application of salicylic plaster.

\section{AfFections of THE EPIDERMAL APPENDAGES IN THE EARLIER STAGES OF SyPHILIS}

Hair.-Falling of the hair is a frequent early symptom of syphilis. In rare instances the deflurium may be complete and may affect not only the scalp but also the eyebrows, beard, and moustache, but as a rule it is partial and consists of an irregular thinning in ill-defined areas which differ from those of Alopecia areata in not being completely bald but in having a few hairs irregularly scattered over them. (Fig. I97.)

Syphilitic alopecia may be due to one of two causes, either to the pre-existence of an ulcerative syphilide which has destroyed the hairfollicles and rendered re-growth impossible, or more usually to the presence of the spironema in or about the hair-papilla temporarily interfering with its action and leading to atrophy, re-growth taking place with the destruction of the micro-organism.

Nails.-The nails, especially those of the hands, and the parts immediately surrounding them, may be affected in the early stages of syphilis. Apart from a chancre about the nail the most common trpes of nail change are a dry onychia and a moist or suppurative perionychia.

Dry Onychia.-. At any period in the course of the disease the nutrition of the nail-matrix may be interfered with from the actual presence of the spironema or from the general disturbance caused by the disease. In consequence of this the nail-growth is impaired and changes occur in the nail-plate which rary in different cases but involve as a rule two 
or more of the nails on each hand. The nail-plate may become thin and flattened, or opaque, discoloured, brittle, and fissured, or rough from being dotted over with small pits or broken up by furrows. Sometimes the free end becomes thickened and a coarse opaque triangular patch with the base in front is formed. These changes, though not painful, may lead to shedding of the nail. (Fig. I98.)

Occasionally the changes in the nail-plate may be caused by the presence of one or more papules in the nail-bed, which are seen at first through the nail-plate as small red spots ; these, by upward pressure, eventually cause atrophy of the nail-substance, rendering it opaque,

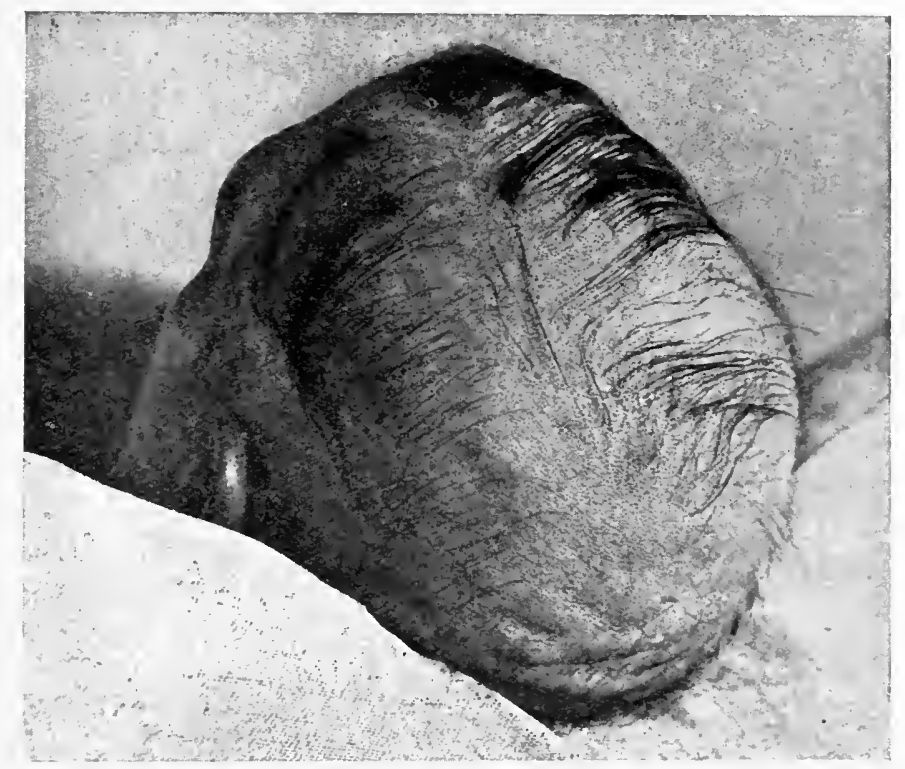

FIG. 197.- Syphilitic Alopecia of the Hair.

The defluvium took place in three weeks in the fifth month of the disease.

friable, and eroded. Sometimes the papule may occur at the free border of the nail and by encroaching on the nail-bed may give rise to a semi-circular erosion of the nail-plate. (Fig. I99.)

Moist Perionychia.-The changes met with when the parts surrounding the nail are involved are more characteristic than those in dry onychia. The nail-wall and the nail-bed itself become inflamed and indurated and the tip of the finger swollen and painful like a whitlow: Suppuration is liable to supervene, with the production of a horse-shoe shaped ulcer with red flabby granulations and covered with seropurulent discharge which oozes out at the sides of the nail. The ulceration gradually undermines the nail-plate, detaches it, and causes it to be shed. Suppurative perionychia usually affects several nails and is most frequent in the thumb and the great toc-possibly because they are specially liable to injury. (Fig. 200.) 


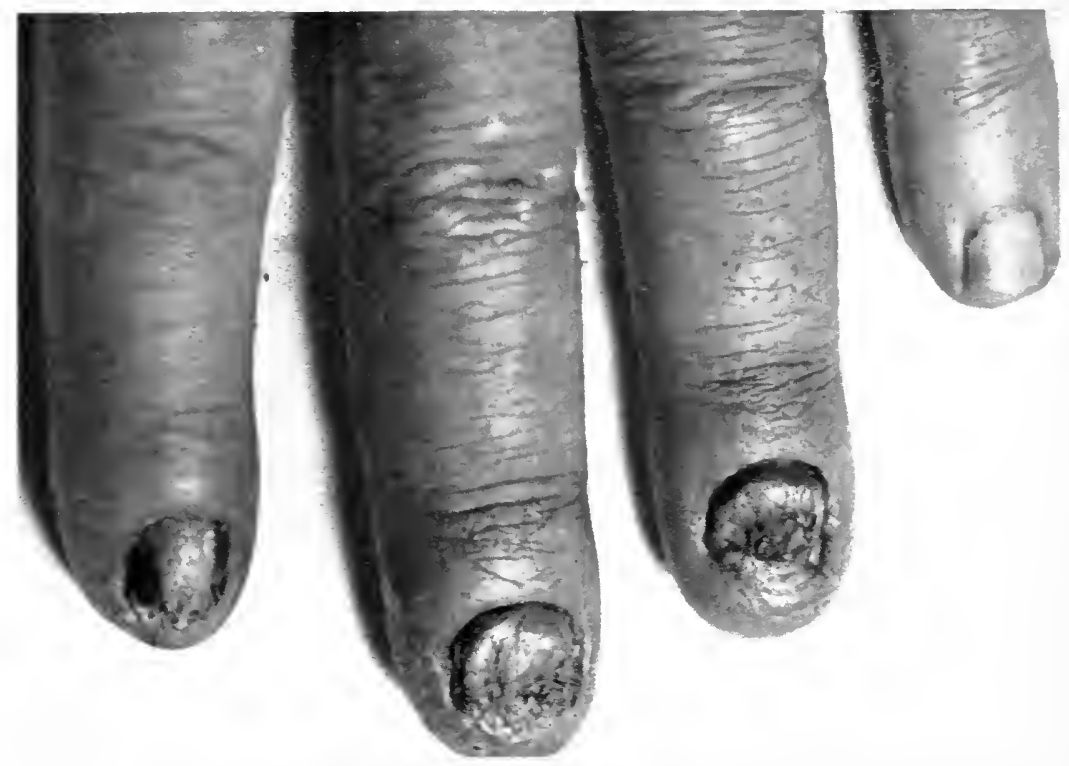

F1G. I99.-Syphilitic Onychia. Dry Type

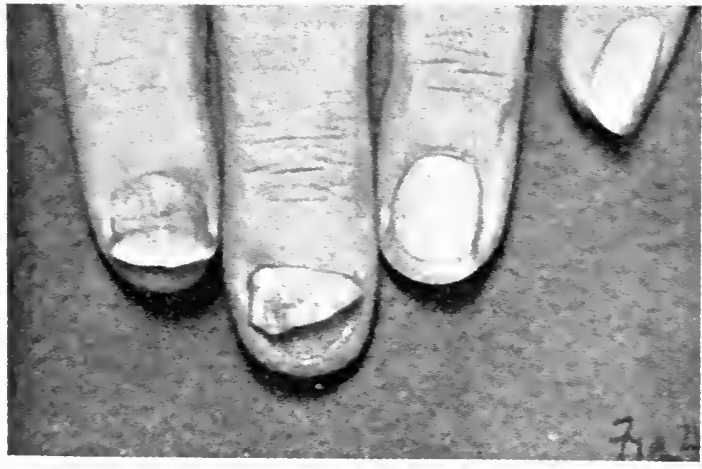

FIG. I99.-Separation of anterior part of nail from Srphilitic Papulo-pustule of Nail bed.

(From Drs. Adamson and McDonazh. Erit. Jorrm. Derm.. zyir.

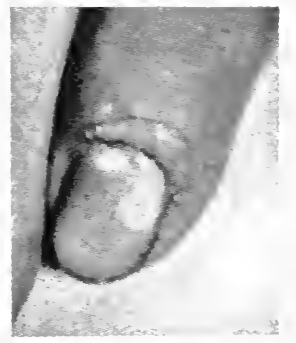

FIG. 200.-Perionychia Erphilitica.

(From D s. Alams n an? McD nagh. Brit. Jiom L) 




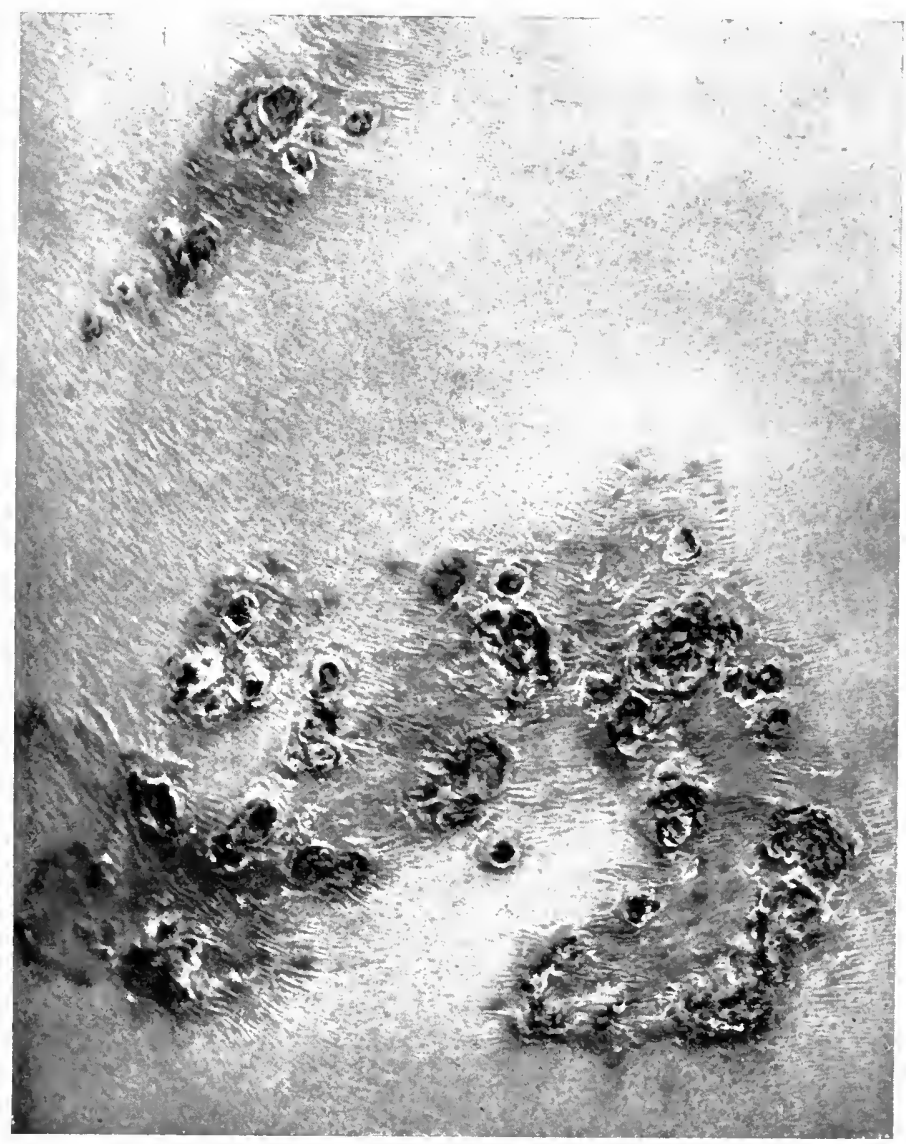

F1G. 201.-Late Superficial Ulcerating Syphilide.

$[$ To face $p .555$. 


\section{Late Cutaneous Syphilides}

The late cutaneous syphilides differ from those of the earlier stages of the disease in that they are fewer in number, asymmetrical in distribution and, as a rule, definitely localised. The essential type of lesion characterising the late period is what is known as a gumma. This consists of a fibro-cellular deposit, which tends to undergo a peculiar gummy degeneration and is either absorbed or more frequently breaks down and forms an ulcer with clean-cut edges. According as the histological lesion is situated in the corium or in the subcutaneous tissue two types of late syphilides are distinguished, namely, (I) a superficial gummatous lesion which may be nodular or may ulcerate, and (2) a deep subcutaneous gumma which usually ulcerates. (Fig. 20I.)

The gummatous lesions, as a rule, do not appear till long after the chancre, sometimes not for 20 years or more, but occasionally they may

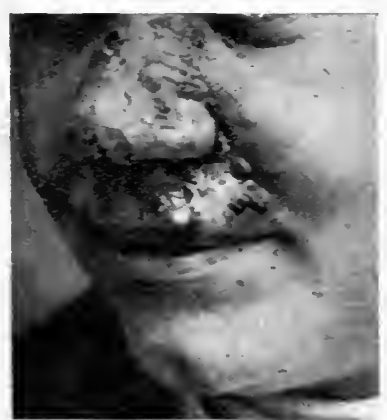

FIG. 202.-Superficial Ulcerating Syphilide of Tose and Lip.

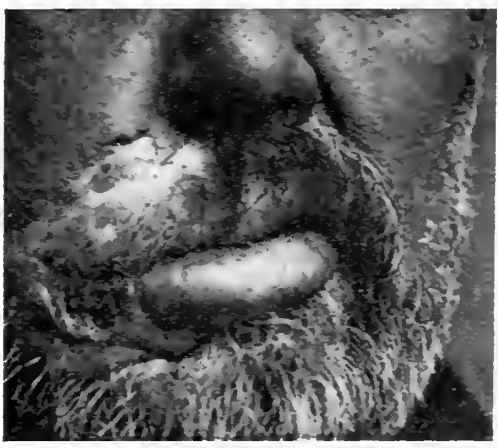

FIG. 203.-Late Clcerating Syphilide. With thickening of the lip.

occur much earlier, even a few months after the initial lesion, when they are known as precocious gummata. These are rare and are met with only in cases of syphilis which are exceptionally severe owing to improper or insufficient treatment, general debility, or other causes, or in which the chancre has undergone phagedaenic changes due to a specially virulent type of infection. They have been observed not infrequently in tropical countries in natives affected with the disease. (Fig. 202.)

Gummatous lesions are more persistent and chronic in their course than the earlier syphilides and, even when absorbed without ulceration, are followed invariably by scars which have a characteristic appearance, being peculiarly thin and wrinkled and not unlike tissuepaper which has been crumpled up and spread out again. At first pigmented, they gradually become white but are surrounded by a brown pigmented halo which may persist indefinitely. When ulceration has supervened they are depressed. (Fig. 203.) 


\section{Late Nodular Syphilides or Superficial Gummata}

The late nodular syphilides occur chiefly on the face, head, neck, shoulders, and buttocks, but may be met with anywhereon thecutaneous surface. As their position is to some extent determined by pressure they are liable to occur on the nose from the pressure of spectacles, or about the waist in women, or in the garter area. The nodules vary in size from a millet seed to a split-pea, are brownish-yellow or raw-ham coloured, and on being pressed with a diascope leave a brownish stain. They are usually definitely raised, although occasionally the infiltration is so slight as to be scarcely perceptible. They may be few in number or numerous, and tend to be aggregated in small irregular groups, or to be arranged in an annular or serpiginous fashion, or to coalesce and form small reddish-brown plaques. Not infrequently the lesions in the centre of a patch undergo spontaneous absorption or heal after ulcerating, while new lesions appear at the periphery, so that a more or less pigmented, thin, scarred area is produced which may be several inches in diameter and is bounded by a sinuous spreading margin made up of nodules or of superficial ulcerations covered with crusts. The ulcers which result from the breaking-down of the nodules as a rule are circular in outline with punched-out edges and an infiltrated border. Occasionally, from secondary infection with staphylococci, the lesions proliferate and become covered with vegetations, which gives them a framboesiform appearance. (Fig. 204.)

The nodules may heal spontaneously, or may disappear under suitable treatment, or they may form a superficial patchy infiltration which may persist for a prolonged period in spite of treatment. (Fig. 205.)

Where ulceration has occurred on the face, the contraction which results on healing may lead to deformities, such as dragging down of the lower lip, ectropion, or a peculiar pinched condition of the nose.

\section{Subcutaneous Gummata}

The subcutaneous gummata are met with most commonly on the lower limbs, especially on the upper part of the calf, but may occur elsewhere and are not infrequent on the thighs, buttocks, and scalp, their position sometimes being determined by injury. They vary in size from a pea to a walnut or larger, and at first can be felt as indurated subcutaneous nodules the skin, over which is unaltered. As they increase in size and spread nearer the surface the overlying skin assumes a dull reddish-brown or purplish tinge, and the lesion then softens in the centre or breaks down in several places and an ulcer results. The ulcers may be roundish, oval, kidney-shaped, or irregular in shape, have punched-out edges, are usually surrounded by a dull red indurated border, and present a floor which is covered with a scro-purulent or sanious discharge or, occasionally, with a dirty-greenish slough. They 

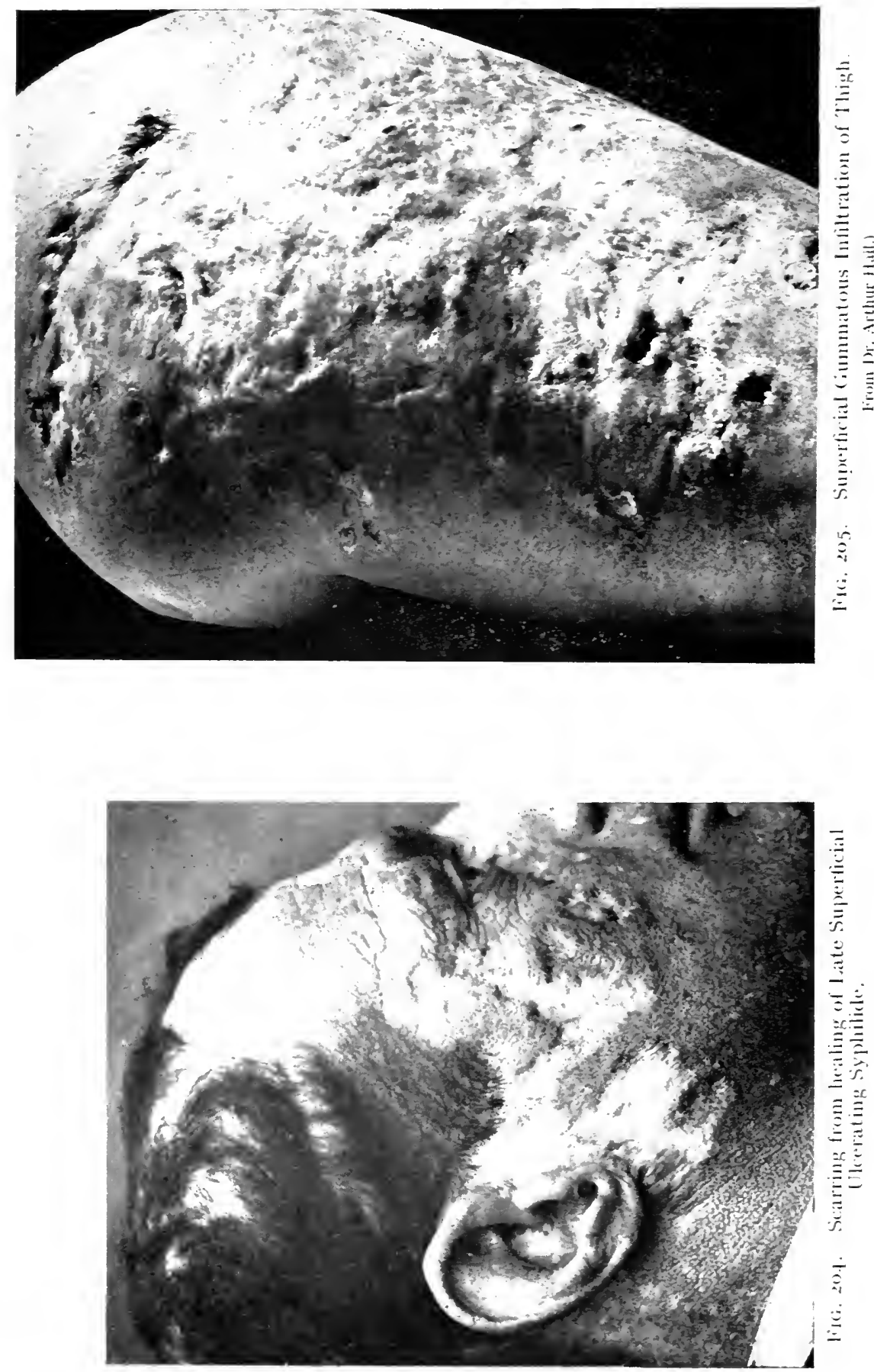

heal rapidly under suitable internal treatment with iodide of potassium, leaving a depressed cicatrix, but if untreated they are liable to cause considerable destruction of underlying structures, sometimes penetrating into the larger blood-vessels and producing serious haemorrhages, at other times causing necrosis of joints or bones, or even passing through the skull into the brain. (Fig. 206.)

Complications.-Syphilitic Elephantiasis.-Sometimes as the result of fibromatous changes, marked swelling of the parts affected by gummatous infiltrations may take place. This is seen chiefly in the legs and feet but may occur in other parts, such as the lips, penis, scrotum, vulva, nose, and ears. It is most probably the result of a secondary infection with streptococci which causes recurrent attacks of lymphangitis, succeeded by a new formation of fibrous tissue,

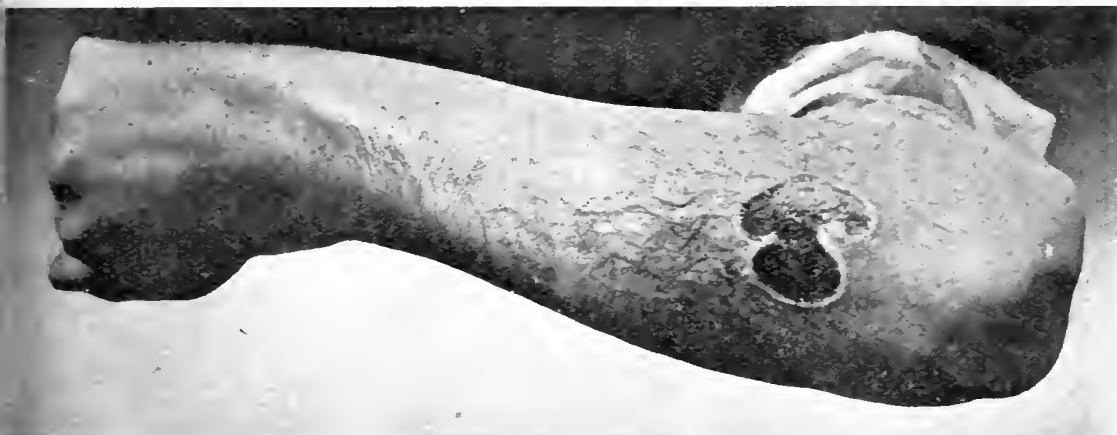

Ftg. 206.-Late Syphilitic Ulceration with well-defined Circinate Border.

blocking the lymphatics, and eventually giving rise to the elephantiasic condition. (Fig. 207.)

Phagedaenic Ulceration.-Sometimes the ulceration assumes fulminating characteristics, extending deeply and rapidly and causing serious destruction of underlying structures. This is associated with much pain locally, and not infrequently with a febrile disturbance. It occurs in individuals who are debilitated from some wasting disease or from living in unwholesome surroundings, and is probably caused by the secondary inoculation of some exceptionally virulent microorganism.

Histopathology.-The spironema has been found in late gummatous syphilides but is much more sparsely distributed in them than in the earlier lesions. It is believed that the gumma occurs on the site of a previous early syphilide which has involuted and left foci of microorganisms, possibly about the capillaries, which, after remaining in a quiescent state for years, for some reason or another renew their activity.

The histological structure is similar whether the lesion be situated superficially in the corium or deeper in the subcutaneous tissue. It 
consists of a dense cellular infiltration, most marked around the bloodcapillaries, which is made up of plasma-cells, a few imperfect giant-cells in which the nuclei tend to be in the centre rather than at the periphery, and small connective tissue cells some of which are spindle-shaped and tend to organise into new connective tissue. The fibromatosis is most marked at the periphery and forms an envelope surrounding the cellular deposit. The infiltration increases for a time, then remains stationary, and eventually undergoes degenerative changes, with the formation

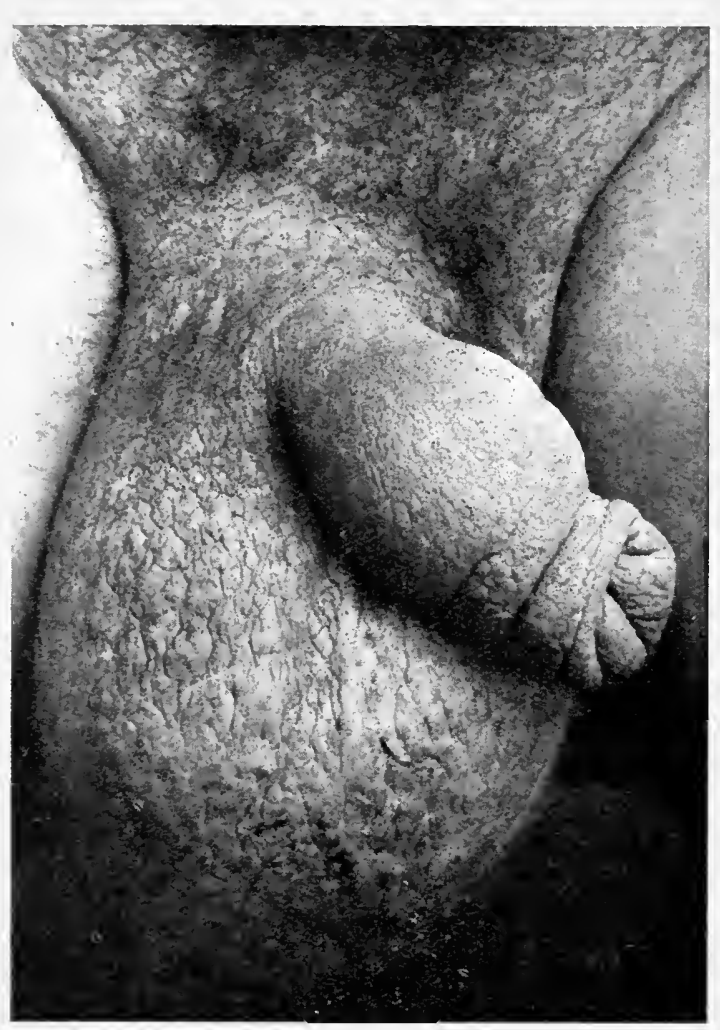

FIG. 207.-Syphilitic Elephantiasis of the Penis and Scrotum. of a gummy mass of cellular debris and a gelatinous intercellular substance, due to colloid degeneration of the fibrous tissue. This breaks down in the centre, the overlying epithelium gradually gives way, and the characteristic ulcers result.

Diagnosis. - Late nodular syphilides have to be diagnosed chiefly from Tuberculosis cutis, sycosis, Acnenecrotica,Lupus erythematosus, and rodent ulcers, while the deeper gummatous lesions have to be distinguished from varicose ulcers, and Bazin's disease. In the absence of a definite history of the disease, evidences of past sypliilitic lesions should be carefully sought, such as denuded patches on the buccal mucosa, especially on the tongue, which have succeeded mucous patches, a pigmented network on the neck in women, scars on the penis resulting from the chancre, or characteristic scars elsewhere.

Tuberculosis Cutis. - The diagnosis from Lupus vulgaris may be exceedingly difficult, and the resemblance may be so great that the clinicai differentiation may be almost impossible. The following are the principal points of distinction : 


\section{Late Nodular and Superficial Ulcerative Syphilides.}

Syphilitic lesions generally appear in adult or late life.

Syphilitic lesions evolve rapidly, and may extend in a few weeks over an area which it would take Lupus vulgaris a year or more to involve. Syphilitic nodules are of a rawham colour.

Syphilitic lesions tend to be circinate or serpiginous in their outline and manner of spreading.

Syphilitic ulcers have a welldefined infiltrated border and are deep, with punched-out edges.

Syphilitic lesions tend to heal spontaneously.

Syphilitic scars are generally thin and tissue-paper-like.

Syphilitic ulcers and nodules may clear up with anti-syphilitic treatment.

Wassermann reaction usually positive after activation with a dose of salvarsan.

\section{Lupus Vulgaris.}

Tuberculous ulcers generally begin in early life.

Tuberculous lesions evolve slowly.

Tuberculousnodules are brownishyellow and semi-translucent.

Tuberculous lesions are irregular in outline.

Tuberculous ulcers have an illdefined or ragged border and are shallow.

Tuberculous ulcers, if untreated, are progressive.

Scars from treated Lupus vulgaris are often thick and irregular and lead to contracture.

Tuberculous ulcers are unaffected by anti-syphilitic treatment.

Wassermann reaction negative.

Lupus Erythematosus.-As a rule there is no difficulty in distinguishing a late superficial syphilide from Lupus erythematosus. In old-standing cases of syphilis, however, where the lesions consist of reddish-brown patches about the face which are scarcely raised and have been replaced here and there by thin, perhaps pitted, scars, there may be considerable difficulty. As a general rule the lesions of Lupus erythematosus are pinkish and rarely infiltrated, while in syphilis they' are brownish and definitely indurated. Lupus erythematosus usually begins in early adult life, while the syphilides generally appear later. The distribution in Lupus erythematosus is characteristic as it usually affects the prominent parts of the cheek, nose, ears, scalp, and occasionally the hands, while in syphilis it is much more irregular. The scars which succeed the involuted lesions of Lupus erythematosus are, as a rule, less noticeable than those which follow the healing of an ulcerated 
syphilide. Occasionally Lupus erythematosus may occur in a patient with syphilis or vice versa, when the diagnosis may become almost impossible.

Acne Necrotica.-Acne necrotica may be confused with a superficial ulcerating syphilide but its situation is different, as it is symmetrically distributed about the forehead, sides of the scalp, sides of the nose, and, occasionally, in the sternal region. The lesions also are more inflamed and take the form, at first, of indolent acne pustules which on involuting leave pitted cicatrices like those of smallpox, while the lesions of syphilis begin as nodules, tend to coalesce into irregular or serpiginous patches, and to ulcerate, leaving thin papery scars.

Epithelioma.-Epithelioma, especially of the rodent ulcer type, might be confused with a nodular ulcerative syphilide on the face. In rodent ulcer there is a hard and cartilaginous rolled edge, either nodular or continuous, or a thickened yellowish-white opaque plaque where the ulcer has healed, and it develops much more slowly than a syphilitic ulcer, sometimes taking years to reach the size of a sixpence.

Sycosis.-Difficulty may be experienced in distinguishing between a nodular syphilide and sycosis of the beard, but the lesions of hyphomycetic sycosis are more acutely inflamed and lumpy, and the surface is studded with vesico-pustules and may be dotted over with short stumps laden with fungus.

Varicose Ulcers.-Ulcerating gummata on the legs have to be differentiated from varicose ulcers. The gummatous ulcers are usually multiple and not infrequently situated near the knee, while the varicose ulcer is usually single, more often situated near the ankle, and is associated with varicose veins. The gummatous ulcers are generally circular in outline with punched-out edges and an indurated border, while the varicose ulcer is irregular and has rounded, sometimes undermined, borders.

Bazin's Disease.-The indolent necrosing nodules of Bazin's disease or Erythema induratum,'may simulate gummatous ulcers, but the resemblance is really superficial. Bazin's disease is essentially an affection of young women and occurs at an age when gummata are extremely rare, while the lesions in it do not suppurate but necrose and, on healing, leave a depressed deeply pigmented cicatrix. Gummata, on the other hand, break down and suppurate and leave a scar of the tissue-paper type surrounded by a pigmented halo.

Local Treatment of Late Cutaneous Syphilides.-Syphilitic ulcers should be bathed with black wash ; or a piece of lint soaked in Liquor sodae chlorinatae should be applied to them; or they may be dusted with iodoform or calomel, or dressed with a dilute mercurial ointment. It is inadvisable to apply mercury locally while iodide of potassium is being administered internally, as the combination is liable to produce nascent iodide of mercury which is a powerful irritant and may increase suppuration. 


\section{Mucous Syphilides}

Lesions of a syphilitic nature may be met with in the mucous membranes during practically the whole course of the disease, and for purposes of description may be divided into early and late mucous syphilides. They occur most commonly on the buccal mucosa but may be present also on the genitalia, about the anus, and in rare instances in the nose.

Early Mucous Syphilides.-(a) Mouth.-One of the earliest symptoms of syphilis in the mouth is erythema and congestion about the tonsils and fauces. Later, more characteristic lesions usually appear somewhere on the buccal mucosa, most frequently on the tonsils, fauces, pharynx, tongue, or lips, and occasionally on the gums and cheeks.

Several types of lesions may be met with, such as superficial erosions corresponding to the roseolar macules on the skin, raised mucous patches with a denuded surface corresponding to papules, hypertrophic vegetating lesions, and superficial ulcers. They may be single or multiple and may be symmetrical.

The superficial erosion is a smooth patch resembling the base of a broken blister, of a dull red or purplish tinge, or of an opaline appearance which has been compared to milk spots or the track of a snail. They vary in size from a millet seed to a sixpence or larger, are round, oval, or irregular in outline, and may coalesce to form irregular patches. Dull red smooth patches are not infrequent on the dorsum of the tongue from a denudation of the papillae, while opaline patches are met with chiefly at the sides. On the tonsils, the milky appearance of the lesions may suggest a diphtheritic membrane.

The raised papulo-erosive lesions, or typical mucous patches, occur in the same situations as the erosions. They are slightly raised, soft lesions, covered with an offensive discharge which is virulently infective and a frequent source of contagion.

Mucous lesions are not uncommon at the angles of the mouth where they are liable to crack and form deep painful fissures which gape when the mouth is opened.

Erosions or mucous patches as a rule do not give rise to subjective symptoms, unless cracks are present or when irritated by smoking, alcohol, hot food, hot drinks, or highly seasoned dishes. They are more common in men than in women as they are to some extent determined by the use of tobacco and alcohol.

The hypertrophic vegetating lesions are comparatively rare, are willy associated with oral sepsis, and are partly due to secondary and $l_{\wedge}$ n of mucous lesions with pyogenic micro-organisms. They tubes iefly beneath the tongue in the form of whitish cauliflower-like and the 1^s.

Diagnosisore superficial and appear on the tonsils, or fauces, or on distinguished fruxue from the irritation of a jagged tooth which has 
transformed a papule into an ulcer. They are not indurated like the gummatous ulcers which occur in the mouth at a later period of the disease.

(b) Nose.-The nasal mucosa is more rarely affected than the buccal mucosa, but occasionally erosions, and even mucous patches, have been detected in the nostrils and may give rise to a nasal discharge and the formation of crusts or fissures at the edges.

(c) Genitalia.-Mucous lesions are common on the genitalia. In the male they generally occur on the glans, prepuce and scrotum, and in the female in the vulva, vagina, and about the os uteri, and in these situations are usually papulo-erosive lesions rather than simple erosions. Hypertrophic cauliflower-like excrescences are occasionally met with, chiefly in the form of dull whitish vegetations forming a more or less complete ring around the vulva.

(d) Anus.-Similar lesions may occur about the anus where they are liable to form painful cracks.

Diagnosis.-The mucous lesions in the mouth have to be distinguished from diphtheria, herpes, Dermatitis herpetiformis, mercurial stomatitis, and exfoliating marginate glossitis.

From diphtheria they are differentiated by the absence of constitutional symptoms and by the presence of the spironema instead of the Klebs-Loeffler bacillus.

From herpes of the mouth they are distinguished by herpetic lesions affecting chiefly the tongue, taking the form of small grey erosions or superficial ulcers which coalesce to form polycyclical patches, and being preceded by tingling, definite neuralgic pains, or some other sensory disturbance, while, in addition the herpetic erosion frequently presents a white collar round the margin, which is the remains of the roof of the vesicle.

Dermatitis herpetiformis may present erosive lesions in the mouth closely resembling erosive syphilides but, unless in exceptional cases, they are associated with herpetiform vesicles in the skin.

In mercurial stomatitis there is usually salivation, spongy gums, and foetor of the breath, which are not syphilitic symptoms.

The peculiar condition known variously as exfoliating marginate glossitis, geographical tongue, and wandering rash, might be confused with a syphilide; but in it the lesions have the power of altering their position, have a peculiar wavy greyish-white border from a thickening of the epithelium, and priesent no definite erosions or ulceration of the mucous surface, nor are, hey associated with cutaneous symptoms, adenitis, or other concomı its of syphilitic mucous patches.

Local Treatment of Mucous Patches.-The mucous patches shsted be cauterised daily by silver nitrate, by a 5 to Io $\%$ solution of clment. acid, or by acid nitrate of mercury applied on the end of a sium is match. Of these, chromic acid is the most useful. A suitablino produce mouth wash should be prescribed, such as borax gr. xx, gl: may increase tincture of myrrh m. $\mathrm{xx}$, and water ad. $\overline{\mathrm{j}} \mathrm{i}$, or Liquor 
m. $\mathbf{x x}$, water ad. $\overline{\tilde{z}} \mathrm{i}$, or a gargle of potassium chlorate. For resistant patches in the throat and pharynx the employment of the curette has been recommended. The hygiene of the mouth should be carefully attended to, the teeth brushed several times daily, generally put in order and any sharp points carefully ground down, and smoking should be prohibited.

Late Mucous Syphilides.--Lesions in the mucous membranes, especially in the mouth, are not infrequent in the late stages of the disease and consist mostly of gummatous infiltrations which tend to ulcerate. Sometimes the ulceration is phagedaenic, causing profound destruction not only of the soft parts but even of underlying structures such as periosteum and bone, and leading to contractures, constriction of the natural orifices, and marked disfigurement.

Mouth.-One of the most common late mucous syphilides is the so-called tertiary sclerosing glossitis. In it the tongue is at first increased in size and the mucous membrane becomes smooth and shiny from a loss of the papillae and assumes a deep red appearance. Gradually a diffuse sclerosis supervenes and the organ shrinks and becomes broken up by fissures which may become ulcerated. As a rule, erosions or superficial ulcers are present at the edges. Instead of the

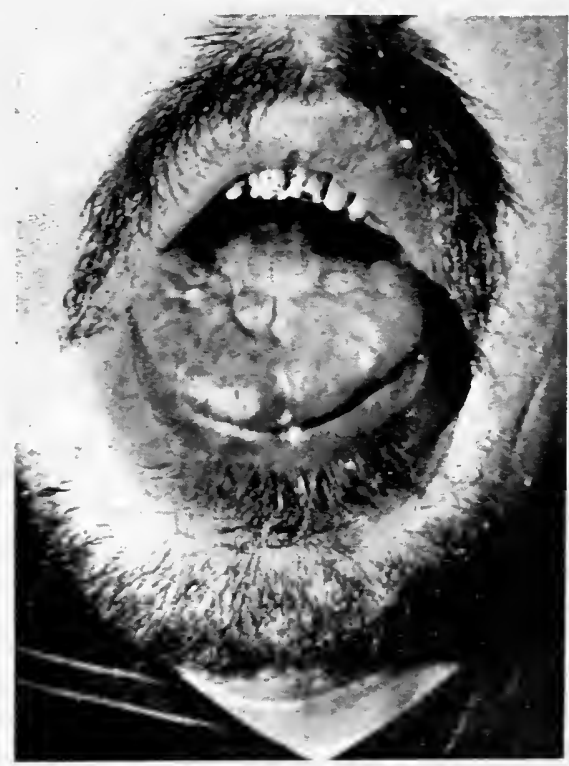

FIG. 208.-Syphilitic Glossitis.

diffuse sclerosing glossitis one or more localised gummatous nodules may occur. These vary in size from a split-pea to a filbert nut or larger, or may even involve the greater portion of the tongue. After a time they usually soften and form deep irregular ulcers, with punched-out edges, a thickened border, and a yellowish core. (Fig. 20S.)

The palate, tonsils, and naso-pharynx may all be affected by ulcerative gummatous lesions which may leat to extensive ulcerations, destruction of underlying structures, and : $: n$ to perforation. One of the most common sites for perforation: une palate, which is associated with regurgitation of food through the nose and renders the roice nasal and harsh. Sometimes the destructive process involves the Eustachian tubes and deafness results, or the nasal bones may become necrosed and the nose deformed or destroyed.

Diagnosis.-The late ulcerating mucous syphilides have to be distinguished from tuberculous ulcers. In the latter, however, the 
borders are not so indurated and the ulcers, instead of being deep and punched-out, are shallow and ragged. Tuberculous ulcers are painful, develop more slowly than the syphilitic lesions, do not react to anti-syphilitic treatment, and are generally associated with underlying tuberculosis of the larynx or lungs.

A gummatous syphilide might be mistaken for an epithelioma, but the latter is painful, much harder, generally associated with enlarged glands, and if ulcerated the edges instead of being punched-out are usually everted.

Local Treatment.-Where ulcerative lesions are present in the mouth an antiseptic gargle, such as the Liquor sodae chlorinatae $20 \mathrm{~m}$. to the ounce of water, should be employed and the lesions should be painted with tincture of iodine or with a weak solution of chromic acid. Certain writers advocate the use of the silver nitrate pencil, but this should be applied with caution as it is liable to increase the destruction. Where the soft palate is perforated, it is advisable to have some form of artificial plate made to close the opening.

\section{Concomitant Symptoms}

Although it would be out of place here to consider in detail the syphilitic changes which occur in organs other than the skin, yet it will be advisable to refer briefly to a few of the more common and obvious concomitant symptoms as they are often a valuable aid in the diagnosis. Practically every tissue of the body may be involved sooner or later, the most marked internal changes being late manifestations occurring where the infection has been severe, the resistance low, or the treatment insufficient or faulty.

Blood.--In the early stages changes of an anaemic character may be detected in the blood, with a diminution of haemocytes, a decrease in the proportion of haemoglobin, and an increase in the leucocytes. In severe cases alterations in the red blood cells may occur, of the pernicious anaemia type, and the Spironema pallidum has been found in the blood in a number of instances.

Heart.- The heart may be affected in the early stages and functional disturbances, such as palpitation, intermittent or weak action and, occasionally, angina may occur. In the later stages cardiac gummata, either circumscribed or in the form of a diffuse fibrous myocarditis and associated with pericarditis or endocarditis, may be met with, while, as a result of sclerotic changes in the coronary arteries and the aorta, Angina pectoris may supervene.

Blood-vessels. - Arteritis is one of the most common of all syphilitic phenomena. It is noticeable to a minor extent in the chancre and early cutaneous syphilides where the small blood-vessels show evidences of endo- and peri-arteritis. In the later phases of the disease, however, from the third to the tenth year, it is liable to involve the larger vessels and may be followed by the most disastrous results. Where the aorta is 
affected, for example, it may lead to Angina pectoris, aortic stenosis regurgitation, atheroma, or aneurism, or, where the cerebral arteries are attacked, it may be responsible for cerebral aneurism and haemorrhage.

The veins are similarly affected and may show signs of peri-phlebitis, endo-phlebitis, or thrombosis. Occasionally those of the legs, or more rarely of the arms, may become the seat of circumscribed gummatous infiltrations, giving rise to indolent nodules which may either break down and ulcerate or may be absorbed under treatment with mercury and iodide of potassium. This syphilitic nodular peri-phlebitis corresponds to the tuberculous condition of the veins met with in Bazin's disease (Erythema induratum).

Respiratory System.-With regard to the respiratory system, not only the nose and naso-pharynx may be the site of syphilitic lesions, but also the larynx, trachea, bronchi, and lungs. In rare instances the epiglottis may be the seat of an extra-genital chancre, mucous patches may occur on the glottis or the rocal chords, and late lesions in the form of gummata, ulcerations, and cicatricial contractions may be met with in the larynx, and lead to stenosis and sometimes to paralysis of the laryngeal muscles. Gummatous lesions may develop in the trachea followed by ulceration and cicatricial contraction, and gummatous infiltrations may be present in the bronchi. The lungs may be the site of chronic interstitial fibrosis, which is localised to certain areas and gives rise to the condition which has been described as "syphilitic phthisis."

Alimentary Tract.- In the alimentary canal, not only may the mouth be involved, but the sub-mucosa throughout its length may present gummatous thickenings usually followed by ulceration which, on healing, may lead to cicatricial contraction and more or less severe stricture. The liver may be affected in the later stages and be the seat of gummata, diffuse interstitial hepatitis, or in rare instances of yellow atrophy.

Genito-urinary System.--In addition to chancres and mucous syphilides on the external genital organs, balanitis, phimosis, and, in rare instances, sloughing of the glans penis may occur. Occasionally epididymitis may supervene in the early stages of the disease, while in the later stages three or four years after infection the testicles may be affected with interstitial fibrosis or gummatous orchitis.

In the female the rulva may be the site of elephantiasic swellings and occasionally of gummata and ulceration, and the uterus of a chancre at the cervix, or in the later stages of the disease, of parenchymatous or gummatous metritis. The ovaries are involved in the same way as the testicles, by interstitial fibrosis or by circumscribed gummata. The mammary glands may be the seat of gummata or of interstitial mastitis.

The kidneys may be affected in the earlier stages and an acute or sub-acute nephritis may occur with albuminuria, while in the later 
stages gummatous and sclerotic lesions are not infrequent and occasion ally amyloid disease may develop.

Bones and Joints. - The bones may be attacked in the early stages and ostalgia, especially of the ends of the long bones, and periostitis may occur. In the later stages there may be syphilitic osteitis and rarefaction of the bone. The gummatous osteitis is known as "syphilitic caries," and gives the bones a peculiar worm-eaten appearance, the affected part sometimes being surrounded by a thickened bony margin. (Fig. 209.)

The joints may be involved both early and late; the earlier symptoms consisting of pain or, more rarely, of effusion without marked inflammation, and the later manifestations of gummatous arthritis which most commonly affects the knee and is frequently associated with gummatous osteitis at the ends of the tibia and femur.

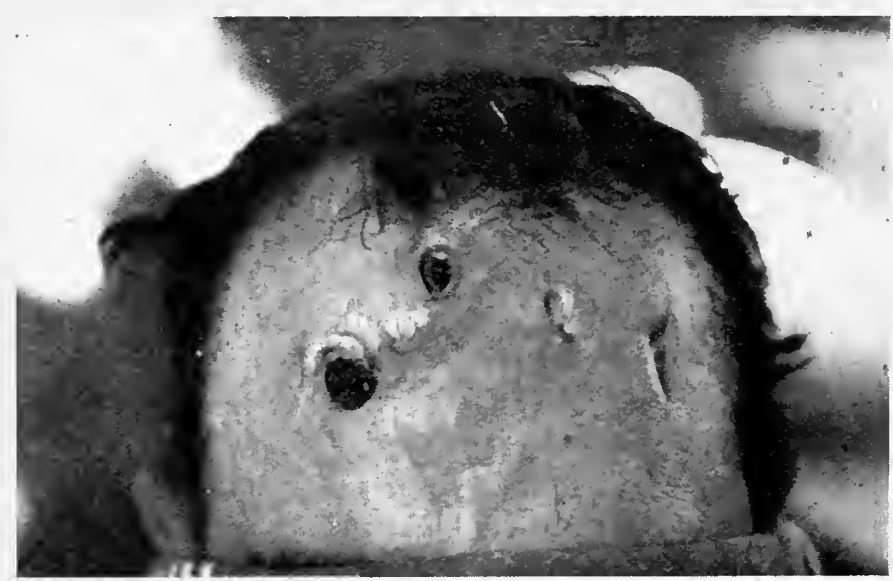

FIG. 209.-Syphilitic Necrosis of Frontal Bone.

Nervous System.-Of all the systems of the body, the one whose involvement by syphilis is liable to be followed by the most serious consequences is the nervous system, and all manner of nervous diseases are attributable to this cause. Unhappily the early phases of syphilitic disease of the nervous system may be so extremely difficult to recognise that grave morbid changes may supervene before suitable treatment is instituted. In the early periods, functional disorders of the central and peripheral nervous system are not infrequent, giving rise to frontal headache, neuralgic pains, neuritis, and occasionally to hysterical attacks. The later lesions are the result of syphilitic meningitis, of gummata in the cord and brain, or more frequently of syphilitic arteritis which, by interference with the nutrition or by haemorrhages, may lead to degeneration or destruction of the nerve tissue. The involvement of the brain may cause headaches, especially at night, epileptiform attacks, hemiplegia, paraplegia, aphasia, optic neuritis, mental disorders such as loss of memory, and actual insanity. Disease 
of the cord is evident by numbness about the feet and legs, disorders of the bladder and rectum, and spastic paraplegia with exaggerated reflexes. In addition, there are the so-called para-syphilitic manifestations, or quarternary syphilides, namely tabes and general paralysis, which are late manifestations occurring from 6 to I2 years after the infection. The Spironema pallidum has been discovered by Noguchi in the cortex of the brain in general paralysis.

Eye.-The eve may be affected at any period of the disease. Chancres may be met with on the evelids, interstitial keratitis mayoccur, especially in congenital syphilis, and iritis or gummatous infiltration of the iris is not infrequent. The cornea and sclerotic may be the seat of gummatous lesions and optic neuritis is not uncommon. The most frequent of the ocular phenomena is disseminated choroido-retinitis which is liable to lead to optic atrophy. Paralysis of the ocular muscles may be observed even in the early stages but is more frequent as a late symptom resulting from gummatous infiltrations about the brain, nerves, membranes, bones, or the globe itself.

\section{Distribution of Syphilis}

Syphilis is almost world-wide in its distribution and only a few remote regions, such as Greenland, various inaccessible districts in Central Africa, and certain rarely visited islands of the South Seas, still remain immune from it. It is common in most parts of Asia, especially in Bokhara, certain districts in India, and in China, Korea, and Japan. It is prevalent in the Straits Settlements and Borneo. In Forth America it is specially rife among the Indian population and in the South it has proved a scourge in Mexico and Brazil. In Africa it varies in virulence, in British East Africa, for example, it is comparatively rare in the interior, in the Madagascar district it is exceptionally severe and malignant, while in tropical Africa it is sometimes met with among the natives in a relatively mild form.

It has frequently been asserted that certain races are less susceptible to it than others, and this is doubtless true but is more probably the result of a higher state of morality, greater cleanliness, and better sanitation, than of racial differences.

Syphilis in the Tropics.- Syphilis in the tropics does not differ essentially from syphilis of temperate latitudes though its clinical manifestations may be considerably modified. It presents certain peculiarities, however, one of the most striking being the greater frequency of the extra-genital chancre. This is partly because the native wears little or no clothing and the skin, especially of the legs, is exposed to abrasions from the spines of prickly plants, splinters of wood, bites and stings of insects, etc., through which the spirochaete readily gains entrance. Various native customs also farour the occurrence of extra-genital chancres, such as the passing round of 
the water gourd from lip to lip and the habit of the native medicine man of sucking bleeding and ulcerating sores. Owing to secondary inoculations the chancre is apt to be infected with virulent secondary organisms and to become phagedaenic. The roseolar eruptions of the early stage cannot be detected and the papular lesions are liable to be mild in character and may even be missed. The tertiary period, on the other hand, is generally severe and associated with deep breaking-down ulcerations which, owing to the drying up of the discharge, may be covered with coarse rupial crusts; or malignant serpiginous lesions may supervene and large areas of tissue be destroyed which on healing result in contraction and disfigurement.

\section{DiAgNosis}

The differential diagnosis of the cutaneous syphilides from other affections of the skin which they may resemble has been referred to already in connection with their clinical appearances. In addition to the clinical differences there are other means by which the diagnosis can be established, such as the demonstration of the Spironema pallidum and the Wassermann complement deviation blood test or Wassermann reaction.

Wassermann Reaction.-The Wassermann reaction is a complement deviation test with a difficult and complicated technique. When properly done it is singularly reliable, a positive reaction being obtained in about $95 \%$ of cases of syphilis, but it is of no value unless carried out by an expert.

The test is not only valuable in the diagnosis of syphilis but it is also of service, and largely employed, to regulate treatment, and as it can show different degrees of positiveness it is a useful indication of the activity of the discase.

A positive reaction may be taken as a proof of the existence of syphilis; a negative reaction, on the other hand, may or may not be conclusive of its absence.

A positive reaction is usually given by the blood of a patient about 6 weeks after the coitus in which the inoculation took place-that is a fortnight after the appearance of the chancre--though it has sometimes occurred as early as the fourth week. If a negative reaction be obtained in the presence of clinical symptoms of a suspicious type the test should be repeated after an interval of about a week.

In the secondary period untreated cases invariably give a wellmarked positive reaction, while in the large majority of treated cases the reaction is also positive.

In late active syphilis where ulcerative lesions are present about the skin, throat, or tongue, a positive reaction is usually obtained, but if the case be under treatment the reaction is liable to be negative. Late syphilitic manifestations, such as aneurism and general paralysis, may give a negative result. 
It has been found that a negative reaction may become positive after an injection of salvarsan, the positive reaction being most marked about the 4 th . hour though it may be delayed till the fifth day. In cases where latent syphilis is suspected but no active symptoms are present, should the reaction be negative a provocative injection of salvarsan should be given and if the reaction become positive it is an indication that further treatment is necessary.

A negative reaction in the absence of anti-syphilitic treatment in suspected secondary syphilis may be taken as a certain proof of the absence of the disease. In the first few weets of the disease a single negative result has no value and the test should be repeated; in the late tertiary period a negative reaction should not be regarded as conclusive unless preceded by a provocative injection of salvarsan.

After appropriate treatment the reaction gradually becomes negative, to become positive again as the effects of the treatment wear off, and the longer and more powerful the courses of treatment the longer does the reaction remain negative. After courses of treatment if the reaction be permanently negative after a sufficient series of tests at regulated intervals the patient may be reasonably regarded as cured and the disease may have been so completely counteracted as to render him susceptible to re-infection.

The following series of tests has been adrocated: Once a week for four weeks, after the treatment has stopped, then once in three months till the end of the year; in the following year the test should be done twice, and in the third year once. Should all these tests be negative and all clinical signs be absent the patient may be considered to be non-infective.

The Justus Test.-This test is based on alterations in the haemoglobin in the blood and may be summarised as follows:

Syphilis, if untreated, causes a diminution of haemoglobin in the blood, varying in degree according to the severity of the attack. As the syphilis undergoes spontaneous involution the loss of haemoglobin is gradually made up. A similar decrease of haemoglobin occurs after the administration of mercury and varies according to the amount of the drug taken. The haemoglobin is restored sooner or later, but may sink again if the mercury be repeated, but if the treatment be continued the haemoglobin reaches a higher level than before mercurial treatment was begun, and the syphilitic manifestations at the same time remit. This diminution in the haemoglobin was regarded by Justus as a specific phenomenon which did not occur in the blood in healthy individuals or in other diseases. It was observed in early secondary and in all the subsequent stages of the disease, disappeared with the remission of the signs of syphilis, and reappeared with every relapse.

This test has not proved to be as raluable as was anticipated. It is uncertain in the chancre stage and negative when the disease is latent. It occurs in non-syphilitic conditions, and sometimes in healthy individuals, and occasionally there is an actual increase in haemoglobin in a syphilitic.

The Luetin Reaction.--This is a specific cutaneous reaction for syphilis which corresponds to the ron Pirquet's reaction for tubercu- 
losis. Luetin is a name given to an extract of a pure culture of the Spironema pallidum which Noguchi claims to have prepared from several different strains of the micro-organism. The cultures were made in ascitic fluid agar containing sterile placenta, the solid media culture being ground up in a mortar and the fluid media added till a liquid emulsion was obtained; this was heated to $60^{\circ} \mathrm{C}$. and 0.5 carbolic acid added to render it sterile.

The technique for the reaction is as follows:

A drop of luetin is injected intramuscularly into the arm while a drop of control emulsion, which has not been inoculated with spirochaetes, is injected into the opposite arm.

If the reaction be positive it varies in intensity, and may be papular, pustular or torpid. In the papular form it appears in about twenty-four hours as a raised, red, indurated, papule ; the induration increases for four days, then retrogression sets in, and the lesion has completely disappeared in a week. In the pustular form, which is met with chiefly in tertiary syphilis, about the third or fourth day the papule becomes transformed into a vesico-pustule which breaks, scabs, and heals in a few days. In the torpid form the reaction is delayed for some ten days and the lesion may then become papular or pustular. The constitutional symptoms associated with the reaction are negligible and consist only of a slight rise of temperature and loss of appetite.

If the reaction be negative, a small erythematous area forms around the site of the injection, which recedes in forty-eight hours without leaving induration or producing constitutional symptoms.

It has been found that this reaction is probably specific with regard to syphilis, and where it is positive syphilis may reasonably be diagnosed. It is usually absent in primary cases. It is inconstant in secondary untreated cases, but usually positive in secondary cases which have been treated, especially with salvarsan. It is positive in the majority of cases of tertiary syphilis, and a positive luetin reaction may sometimes be obtained in cases of tertiary syphilis which give a negative Wassermann. It has been found to be positive also in congenital syphilis and in late nerve syphilitic manifestations, such as tabes.

\section{PROGNOSIS}

The prognosis in syphilis invariably should be guarded as it is impossible to predict from the severity of the initial lesion or the transitoriness of the secondary symptoms whether the course will be mild, or severe and followed by grave late symptoms; but in every case if treatment be begun sufficiently early and be sufficiently thorough there is a reasonable expectation of cure.

It is the late manifestations, such as general paralysis, tabes, aneurism, and arterio-sclerosis, which are specially to be dreaded. In the past when the treatment was too often insufficient and was symptomatic and cursory, syphilis was among the most common causes of death, which resulted either directly from involvement of the central nervous system, sclerosis of the arteries, or syphilitic affections of the viscera, or indirectly by reducing the vitality of the individual so that he succumbed more readily to some co-existent disease such as pneumonia or diabetes. 
With modern methods of treatment the prognosis in each case depends more on the individual, his general state of health and habits, than on the syphilis itself, and the outlook is invariably more serious where the patient is debilitated from alcoholism, tuberculosis, or insufficient food, or is condenıned to a life in unhygienic surroundings.

Syphilis and Marriage.-With regard to the important question of syphilis and marriage it is the duty of the medical man to warn the patient of the danger of infection and to advise against marriage till the disease has been cured so far as can be ascertained by modern tests. It is a matter of opinion how soon after the Wassermann reaction becomes permanently negative marriage may be allowed, but if no active signs of the disease are present, and in primary cases should the Wassermann reaction have been negative for a minimum of one year and in secondary cases for two years after the cessation of treatmentthough in the present state of our knowledge it is unwise to give an absolute guarantee of cure-the patient may be regarded as noninfective and marriage may be permitted with safety.

Re-infection.-Although an attack of syphilis usually confers immunity, still a sufficient number of cases is now on record to show that the cure may be so perfect that immunity is lost and a second infection may occur with the development of a chancre followed in due course by secondary manifestations. A certain number of cases of so-called re-infection where no secondary lesions followed have not been real examples butinstances of recurrent chancres, pseudo-chancres, or chancriform gummata. In the true cases a considerable period has usually to elapse between the two attacks, but Mansell Moullin has recorded a case in which the interval was only $2 \frac{1}{2}$ years.

It has been found also that congenital sy.philis may not confer absolute immunity and a few instances have been reported in which a fresh infection with the disease has occurred after puberty in a congenital syphilitic who had been cured.

\section{Prevention}

In the past the measures adopted in this country for the prevention of syphilis have been wholly inadequate and the disease has been allowed to be propagated to an extent which has been a reproach on the health authority. Hypocrisy and secrecy have surrounded it like a veil, leading to its concealment and imperfect treatment, allowing it too often to assume severe and even malignant characteristics, and permitting it to be spread not only in a culpable fashion but also amongst innocent persons such as the wives and children of infected individuals, physicians, dentists, and others who have contracted it in pursuance of their duties. In addition, until recently, even in the large general hospitals the arrangements for its treatment have been insufficient and the teaching on the subject in the medical schools and 
the wards as a rule so superficial and unsystematic as to be almost valueless. To rectify this deplorable state of affairs a Royal Commission on Venereal Diseases was appointed in I9I4, and as an outcome of their findings clinics for the diagnosis and proper treatment of venereal disease have now been established in all the large centres throughout the country.

In the last few years before the war the number of cases of syphilis appeared to be somewhat on the decrease, both in hospital and private practice, and the type of case to be less virulent than formerly. At the present time, owing to the conditions associated with a great war, the incidence of syphilis in this country has increased, but with the better facilities afforded by the clinics the spread of the disease is gradually being controlled.

Syphilis is infective throughout its whole course but especially in the early stages and when active symptoms are present; the most infective lesions are chancres, mucous patches, and condylomata, but even late lesions of the gummatous type have been known to transmit the disease. Infection is most liable to take place from an open sore or an abraded mucous patch, but any breach of the surface which allows an effusion of blood may be responsible for the transmission of the Spironema pallidum.

Although syphilis is usually transmitted by sexual intercourse it must never be forgotten that it may be contracted by any means by which the spirochaete can be transferred directly or indirectly from an infected to a healthy individual. Consequently on diagnosing a case it should be the physician's first duty, not only to institute active treatment, but also to warn the patient that he is a source of infection and a danger to the public and to impress upon him to abstain from kissing or from the use of common towels, drinking vessels, knives, forks, etc., and to isolate himself as far as is compatible with the pursuance of his daily avocation.

It is sometimes spread by individuals who are unaware that they are suffering from it, either from the initial lesion being transient and not followed by well-marked secondary symptoms, or from its being situated in a concealed part such as the vagina; or by individuals in whom active symptoms are absent but who are still infective as a result of treatment having been discontinued too early-a danger which should be avoided by the judicious employment of the Wassermann reaction.

Preventive Measures.-With the object of preventing the generalisation of the disease various measures such as the destruction of the chancre, either by excision or by the actual cautery, and the removal of the enlarged glands in the groins; have been advocated in the past. It would seem, however, that the spirochaetes have already reached the general circulation before the primary sore has fully matured, consequently the results are uncertain; but still where possible the sore should be cauterised, or removed by circumcision, or attempts should 
be made to kill the spirochaetes on the surface by rubbing the sore with calomel cream or by the injection of $0.2 \mathrm{grm}$. of hectine.

Early Disinfection.-Much more hopeful results.can be obtained from disinfection as soon as possible after exposure than from destruction of the chancre after it has developed. The measures for early disinfection were based on an important experiment performed by Roux and Metchnikoff in Igo6 on a student in the Pasteur Institute in Paris. This student, who had never had syphilis, was inoculated on the balano-preputial fold with matter from a chancre; an hour after the inoculation the penis was rubbed for five minutes with a mercurial ointment containing Io grammes of calomel and 30 grammes of lanolin; as a result of the inunction he did not contract the disease. At the same time a control experiment was performed on a chimpanzee without subsequently rubbing in the calomel ointment and a typical chancre developed in due course.

Measures of early disinfection were introduced in the British Army in I9I6 and where they were carried out energetically there was distinct diminution in the incidence of syphilis; Harrison in his text-book goes so far as to regard preventive measures as a means of exterminating venereal disease. The measures he advocates consist of the employment of I in 2000 solution of Hydrarg. perchlor., and $30 \%$ calomel cream in two consistencies, one semi-fluid like ordinary cream and the other stiffer, with the following formulae:

Semi-fluid cream :

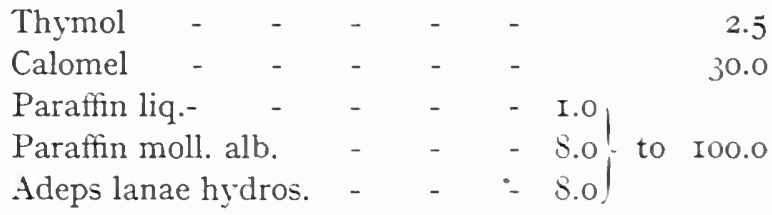

Stiffer cream :

\begin{tabular}{|c|c|c|c|c|c|c|}
\hline Thymol & - & - & - & - & \multicolumn{2}{|c|}{2.5} \\
\hline Calomel & $-\quad-$ & - & - & - & & \\
\hline \multirow{2}{*}{\multicolumn{2}{|c|}{$\begin{array}{l}\text { Paraffin moll. alb. } \\
\text { Adeps lanae hydros. }\end{array}$}} & - & - & - & I.O) & \multirow[b]{2}{*}{ to } \\
\hline & & - & - & - & $9.0\}$ & \\
\hline
\end{tabular}

He recommends the following procedure: "As soon as possible after exposure to infection:

(I) Urinate.

(2) Wash the whole genitals, pubis, and lower abdomen with soft soap and warm water. Dry.

(3) Swab the same parts well with I/2000 Hydrarg. perchlor., paying particular attention to the mouth of the prepuce, corona, and frenum, all of which are particularly apt to be abraded.

(4) Squeeze some semi-fluid calomel and thymol cream into the urethra, and finish by inuncting the parts well with a stiffer calomel and thymol cream." 
These measures are of little value later than about an hour after exposure to infection, as although the spirochaete is so delicate as to be killed easily by the antiseptics it quickly penetrates to the deeper tissues and becomes out of reach.

Preventive measures are specially applicable to men and are rarely successful in women, for anatomical reasons and also owing to the difficulty of inducing women to carry out corresponding procedures with sufficient thoroughness.

\section{TREATMENT}

The treatment of syphilis is chiefly internal. Where open sores, ulcerative lesions, or condylomata, are present local measures are indicated, but these are of minor importance and have been described in connection with the different cutaneous syphilides.

General Considerations.-As soon as syphilis has been diagnosed active measures of treatment should be instituted. The clinical diagnosis of the chancre can now be verified by the demonstration of the Spironema pallidum by the ultra-microscope and later, when the virus has become disseminated and secondary lesions have appeared, by the Wassermann reaction; once it has been definitely established, properly regulated and sufficiently prolonged treatment can usually be enforced.

In the treatment of syphilis the mental attitude of the sufferer is probably of more importance than in the treatment of any other disease except leprosy. The mere realisation of the nature of the disease, its possible consequences, the tedious treatment which it necessitates, and all the other disadvantages connected with it, are liable to cause mental depression. It should be pointed out to the patient that by modern methods of treatment properly carried out a cure may be reasonably expected and that nervous depression may interfere with the benefits from the treatment.

It is of the greatest importance that the general health should be kept up during the prolonged courses of treatment. Where possible a well-regulated life should be enjoined, with healthy out-door exercise, proper mental occupation, the avoidance of alcohol, and the restriction of tobacco. In order to keep the skin active, daily warm baths should be ordered, and during treatment the patient's weight should be taken regularly and the urine systematically examined for albumin. Where the patient is generally run down or the subject of some concomitant debilitating disease like malaria, the specific treatment should be alternated with a tonic regime and such drugs prescribed as iron, strychnine, phosphoric acid, and quinine.

Although the number of remedies which have been employed in the treatment of syphilis is legion, for practical purposes they may be reduced at the present time to three, namely, arsenic in the form of salvarsan and its substitutes, mercury, and the iodides. Of these 
salvarsan and mercury are specifics and act by destroying the spirochaetes, while the iodides simply cause absorption of the cellular hyperplasia called forth by the micro-organisms. These drugs have different indications and the best results can generally be obtained by their judicious combination. The preparations of them and their methods of employment will first be described, and this will be followed by a consideration of the manner in which they can be combined to the greatest advantage, and by a practical scheme of treatment.

Arsenical Preparations.-Arsenic has long been credited with having a beneficial effect on syphilis but it is only comparatively recently, since the introduction by Ehrlich and Hata of 606 or salvarsan, that it has been used extensively.

\section{Arylarsonates}

Certain organic arsenical compounds known as the arylarsonates, namely atoxyl, arsacetin, and orsudan, have been employed in the treatment of this disease. Atoxyl contains $27.3 \%$ of arsenic and is given on alternate days, in intramuscular injections of six grains dissolred in distilled water, until eight injections have been made. Arsacetin and orsudan are used in somewhat larger doses. The use of these preparations, though occasionally of benefit, has been discontinued owing to the grave toxic symptoms liable to be caused by them, the most serious of which are optic atrophy and blindness.

\section{Hectine}

Hectine is another preparation of arsenic which was strongly recommended by Hallopeau. It is a colourless substance containing about $2 \mathrm{I} \%$ of arsenic, is soluble in water, and is injected subcutaneously in doses of I to 2 c.c. of $0 . I^{\circ}$ o solution. It is a feeble preparation compared with salvarsan but may be employed with benefit to destroy the spirochaetes in a chancre by the injection of 0.2 gramme into the lesion.

\section{Salvarsan}

Salvarsan is an organic arsenical preparation and is the commercial name for the Ehrlich-Hata 606 (Dioxydiamino-Arseno-Benzol). It was discovered by them in Igog and since then has been extensively used with brilliant results. Its effects are many times more powerful and rapid than any of the mercurial preparations and it has a far greater influence in prerenting relapse after treatment. Under injections of this drug spirochaetes disappear from the syphilitic lesions within twenty-four hours, primary sores rapidly involute, macular syphilides fade in a few days, papular syphilides disappear, and gummatous lesions quickly heal. It acts by killing the spirochaetes, either directly, or indirectly by calling forth some toxic substances in the tissue fluids. 
At first it was thought that one or two injections sufficed to kill off the whole of the spirochaetes in the tissues and to produce the so-called "sterilisans magna," but it is now known that a considerable number of injections are-necessary before the virus is eradicated and the Wassermann reaction becomes permanently negative, and it has been found that the best results are obtained, both with regard to rapidity and permanence, by combining it with mercurial treatment.

Mode of Administration.-Salvarsan may be given by intravenous, subcutaneous, or intramuscular injection. The intravenous method

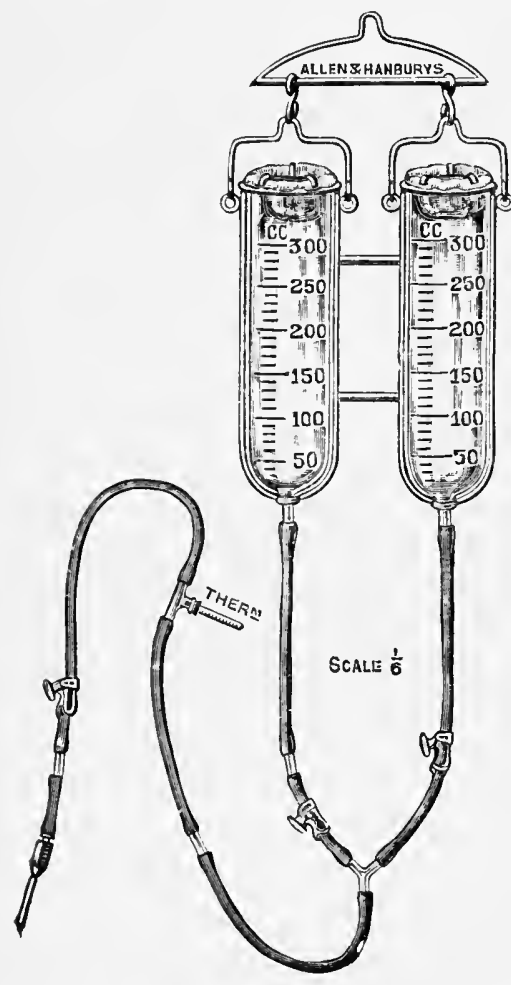

Fig. 2 Io.-Apparatus for Intravenous Injection, Army Pattern. is usually chosen as it is painless, while the other methods are exceedingly painful and liable to be followed by induration, nodosities, lameness, and sometimes by ulceration and sloughing.

Intravenous Method.-The dose of the drug when given intravenously varies from 0.2 to $0.6 \mathrm{grm}$. in the adult male and 0.2 to $0.4 \mathrm{grm}$. in the female and it is diluted for injection with about 300 c.c. of normal saline solution. A series of injections is necessary. If the dose be small it may be repeated in a week, if large a fortnight at least should be allowed to elapse before repetition. The injection is given either by a syringe or more usually by some suitable form of transfusion apparatus - either a single reservoir apparatus which acts by gravity, or a double reservoir apparatus in which one reservoir contains the drug and the other the saline solution and in which, by opening the appropriate clamp, one or other can be allowed to flow through the tube. The needle, of which there are several varieties, should be of medium bore with a short point to minimise the risk of piercing the far wall of the vein, and a base so made that it is comfortable to handle, a useful pattern being that with a square base ridged at the sides. Where a syringe is employed it should have a three-way attachment. (Figs. 2IO, 2II.)

The procedure is as follows: the drug, which is a yellow powder, is put up in a glass ampoule containing the required dose. The ampoule is cleaned and filed open and the drug shaken into a sterile glass measure containing 30 c.c. of normal saline solution at a temperature of $104^{\circ} \mathrm{F}$., the powder being added gradually and stirred with a sterile glass rod 
till a clear yellow acid solution results. This is neutralised by the addition of about 20 drops of a $15 \%$ solution of sodium hydrate, when a precipitate forms which re-dissolves. The solution is next tested with Litmus paper to see if it is slightly alkaline, and if so is filtered into a sterile glass vessel and diluted with saline solution to 300 c.c. The saline solution should be freshly prepared from chemically pure sodium chloride and must be absolutely sterile, as otherwise toxic symptoms will result from the injection.

The patient should be prepared as for a surgical operation, a purgative given the night before and no food taken for four hours previous to the injection. The veins at the elbow are usually selected, either the median basilic or the median cephalic, and the skin over them is sterilised by being rubbed with alcohol or ether or painted with iodine. The patient is placed in the recumbent position with the arm stretched out on a table, a rubber tourniquet is lightly applied on the upper arm, and the patient is instructed to open and close the fist

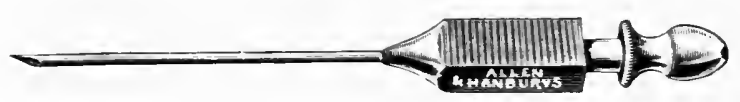

FIG. 2II.-Intravenous Needle.

several times to make the vein prominent. The needle is inserted and a few drops of saline solution introduced to see that it is lying in the vein and not in the subcutaneous tissue; if it has been properly inserted no swelling will occur from the injection, while if it is in the subcutaneous tissue the skin will become raised up. The tourniquet is then removed and a small quantity of saline solution allowed to flow into the vein, followed by the required amount of salvarsan. The injection should be made at the rate of about 30 c.c. per minute, the temperature of the solution being kept at about blood heat. After the salvarsan, a little more saline solution should be allowed to flow into the vein to wash out any traces of the drug from the needle. The needle is then withdrawn, the arm raised, and a collodion scab applied over the puncture. The patient is kept quiet, preferably resting in bed, for the next twenty-four hours and no solid food is given for four hours.

Substitutes for Saliarsan.-A number of chemically equivalent products to salvarsan have been introduced, such as Arseno-benzol or Billon which is a French substitute, and Kharsivan which is a British equivalent compound, and these are injected in the same manner.

After Effects of Saliarsan.-For two hours after an intravenous injection arsenic can be detected in the blood, but later it disappears from the blood and becomes stored in the internal organs, especially in the liver where it may remain for weeks. It is excreted by the bowel and kidneys and ten days after the injection there may be no trace of it in the urine. 
After the injection there is not infrequently a slight reaction with a rise of temperature to about $100^{\circ} \mathrm{F}$., an increase of the pulse rate, and a garlic taste in the mouth, and this may be followed by perspiration, faintness, and occasionally vomiting. As a rule it is transient and recovery soon takes place without ill-effects.

Herxheimer has described a peculiar type of reaction in which, as a result of the injection, any cutaneous syphilides present tend to become more vivid in colour and new lesions to appear. This occurs chiefly in connection with small doses of salvarsan, has been noted a few hours after the injection, reaches its height in about twelve hours, and disappears in a few days. It is believed to be a specific reaction due to endo-toxins set free from the spirochaetes by the drug.

In certain cases a severe reaction may follow the injection with disastrous and sometimes fatal consequences, but considering the number of injections which have been given the percentage of deaths has been comparatively small. This reaction is characterised by puffiness of the face, coughing, romiting, headache, rigors, and albuminuria. Cerebral symptoms may occur, such as acute headache, mental confusion, and epileptiform convulsions which may be followed by unconsciousness for a few hours and recovery or by coma and death. In certain cases jaundice of an obstructive type may be met with and death has taken place with acute yellow atrophy of the liver; it is doubtful, however, whether the salvarsan was entirely responsible for the yellow atrophy, as in certain cases it did not appear until months after the injection and may have been due to the co-existence of some liver disorder which rendered that organ more sensitive to the drug.

Cutaneous symptoms arc not infrequent and may be of an erythematous or urticarial type and either confined to the face and backs of the hands or more widely distributed. Occasionally they go on to a general exfoliative dermatitis which is associated with considerable itching, hyperkeratosis of the palms and soles, and loss of the hair and nails.

These serious toxic symptoms may be due to the direct action of the drug, either from the dose being excessive, or from the interval between successive doses having been too short and a dangerous accumulation of the drug having been allowed to occur in the system; or they may be due to defective technique and imperfect sterilisation of the water, or to faulty composition of the drug itself either in its preparation or from its partial decomposition with the formation of some highly toxic oxidation product; or they may be caused by some peculiar susceptibility on the part of the patient, either natural or acquired.

Contra-Indications to Saliarsan.-The contra-indications to the use of salvarsan consist of the co-existence of some debilitating disease, such as Addison's disease, tuberculosis, cancer, diabetes, organic disease of the kidneys, disease of the central nervous system, chronic degeneration of the cardiac muscles and endocarditis, arterial sclerosis, 
hepatic disease, and alcoholism. Where there is the slightest suspicion of organic disease of the liver, heart, kidneys, or central nervous sy'stem, salvarsan-if given at all-should be employed with caution and in small doses such as 0.2 grm. at intervals of not less than a week.

\section{Neo-Salvarsan}

Neo-salvarsan, or 9I4, is a later preparation which was introduced by Ehrlich and is a combination of formaldehyde-sulphoxylate of soda with salvarsan (606). It contains only $20 \%$ of arsenic and consequently requires $0.9 \mathrm{grm}$. to be equivalent to $0.6 \mathrm{grm}$. of salvarsan. It is readily soluble in water, forming a neutral solution, but as this solution is liable to become toxic on exposure to the air no time must be lost in injecting it.

Various chemically equivalent products to neo-salvarsan have been put on the market, such as Novarsenobillon, a favourite preparation at present, and Neo-kharsivan.

The chief advantages of the neo-salvarsan preparations are that they are less toxic than the original salvarsan, can be injected intravenously in concentrated solution, and also may be given intramuscularly and subcutaneously.

Intrazenous Injection in Concentrated Solution.-The procedure, so far as the position of the patient, the cleansing of the skin orer the vein, and the distension of the rein, are concerned is similar to that described in connection with the injection of salvarsan. The dose is dissolved in ro c.c. of distilled water in a sterile porcelain or glass measure, filtered through a piece of sterilised gauze into another ressel, and drawn into the syringe. A 20 c.c. record syringe is employedpreferably with the nozzle at the periphery-and a needlewith a medium bore and a short point. The needle may be inserted into the rein either by itself or attached to the syringe, whichever comes easier to the operator. In the latter case, in order to see that it has entered the vein, the piston should be withdrawn slightly, when a stream of blood should be seen to flow into the arsenical fluid; if it has missed the vein or has been pushed through the further wall no blood will appear in the syringe and the needle must be reinserted. When it has been ascertained that the needle is lying properly in the vein, the tourniquet is removed, the patient told to relax the fist, and the solution slowly injected, the injection taking at least one minute. After the needle is withdrawn a collodion scab or a pad and bandage should be applied over the puncture. (Fig. 2I2.)

Intramuscular Injection.-Neo-salvarsan has the adrantage over the old salvarsan that it can be injected intramuscularly without causing the same intensity of pain or being followed by disastrous consequences such as ulceration and sloughing. By this method of administration the drug is excreted more slowly, has a more protracted and powerful therapeutic effect, and, owing to its slower absorption, is 
less liable to cause serious toxic symptoms. The injection can be made safely into the buttock in the same site as that used for the intramuscular injection of mercury (see p. 595). A needle at least an inch and a half long and as sharp as possible should be employed and the neo-salvarsan may be suspended in sterile olive oil or in a creo-camph. cream base. Harrison, who strongly advocates this method, after trying various bases, including glucose and stovain, novocain solution, and creo-camph. cream, has obtained the best results by simply dissolving the dose in distilled water. By his method, which is here appended, he found that the immediate pain was largely abolished, that the local reaction which occurred 3 or + days after the injection only rendered the gluteal region stiff and tender for a few days, and that

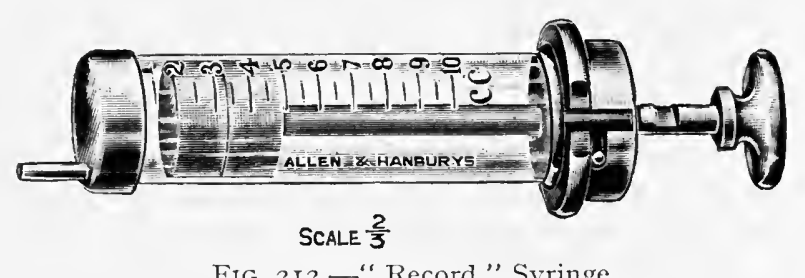

FiG. 212.- " Record" Syringe.

With peripheral nozzle for intravenous injection.

the general discomfort was so comparatively slight that in courses of seven injections at weekly intervals it was rare that a course had to be interrupted on account of the local reaction.

\section{Harrison's Technique.}

(I) Take a 2 c.c. record syringe and cap the nozzle with the base of a hollow needle, the base of which has been made up with sealing-wax.

(2) Pour ro minims of distilled water into the syringe and dissolve the " $9 \mathrm{I}_{4}$ " in it with the help of a glass rod. In doing so, hold the syringe vertically and pour the powder into the water in such a manner that as little as possible touches the sides.

(3) Refit the piston to the base of the syringe, turn the latter nozzle upwards, and push the piston into the syringe barrel.

(4) An area which is two inches square and has its postero-inferior angle two inches posterior to the tip of the great trochanter is a suitable one in which to place the injection. Find a spot in this area where no pain is caused by sharply depressing the tissues with the point of a finger pushed against the skin as if to strike the iliac bone. Here insert the needle ( $\mathrm{I} \frac{3}{4}$ inches long and 56 gauge) at right angles to the plane of the skin, pushing it in almost to its base; if the point touches bone, withdraw about two millimetres.

(5) See that no blood is oozing from the needle base.

(6) Holding the needle, in order that the position of its point may not be disturbed, fit the syringe to it and inject slowly. If the patient complains of pain running down the leg, withdraw the needle and select another site.

(7) Before removal, rotate the needle once or twice on its long axis, and as it is being withdrawn, pinch up the skin and subcutaneous tissue around the needle.

(8) Hold the skin and subcutaneous tissues thus for a few seconds after the needle has been withdrawn. 
(9) Give a hypodermic injection of morphia, gr. to $\frac{1}{4}$.

(Io) Massage the site of the "9If" injection with" a large ball of cottonwool, sewn up in lint, and repeat the massage daily for three days.

Subcutaneous Injection.-The subcutaneous injection of neosalvarsan has no definite advantage over the intramuscular. The dose is dissolved in about ro minims of distilled water and is best injected into the subcutaneous tissue immediately abore the fascia covering the gluteal muscle.

Harrison describes the technique as follows:

"The skin and subcutaneous tissue over the upper and outer quadrant of the gluteal region are pulled away from the underlying muscle with the fingers and thumb of the left hand, forming thus a kind of cone or pyramid of tissue. Into the base of this the needle, a fairly stout one, is run so as to land its point under the apex. The needle point should then be lying on the fascia, which can be verified by moring the point from side to side so as to make it scrape on the hard fascia."

\section{Other Arsenical Preparations}

Galyl.-This is an arsenical compound somewhat similar to neosalvarsan which can be injected intravenously either in a dilute or concentrated solution. It can also be administered intramuscularly, either dissolved in a solution of glucose or suspended in a fatty base. It is less powerful than salvarsan but is equally liable to be followed by toxic effects.

Disodo-Luargol.--This is a compound of salvarsan with antimony and silver prepared by Danysz. In appearance it resembles emery powder and forms a dark brown solution in distilled water. It is given intravenously in concentrated solution in the same manner as neosalvarsan and in doses of o.I5 grm. at intervals of 3 or 4 dars and gradually increased till a total dosage of 1.2 grms. has been reached. It is believed to be a more powerful agent for the destruction of spirochaetes than salvarsan itself.

Joha.-This is a preparation which consists of $40^{\circ}$ o salvarsan and iodopin and I c.c. of it contains o.t grm. of salvarsan. It has been adrocated for subcutaneous and intramuscular injection.

\section{Mercury}

Before the introduction of salvarsan mercury was described as the "sheet-anchor" in the treatment of syphilis and there are those who still believe it to be the more valuable and indispensable of the two drugs. Its exact manner of action is not fully understood. The majority of writers believe it to be directly poisonous to the spirochaete, while others assert that it acts indirectly by calling forth alexins which eventually destrox the micro-organism.

It may be administered by ingestion, inunction, intramuscular injection, and fumigation. Each of these methods has its merits and 
the choice of the one to be used is dependent on the circumstances and peculiarities of the individual case. By whatever method it is introduced into the system, mercury is eliminated in the urine, sweat, and faeces, the elimination being most rapid when it is injected intramuscularly in soluble form and slowest when it is taken by the mouth.

The maximum effects from its use are obtained from intermittent courses of small quantities given over a considerable period rather than from continuous treatment or from a few massive doses. Certain writers advocate commencing treatment with a massive dose, and Ehlers of Copenhagen, who employed an initial injection of 20 centigrammes of the benzoate of mercury for a man (or 2.5 milligrammes per kilo of body weight), followed by a course of inunction, claims to have obtained by it results equalling in efficacy and rapidity those from salvarsan.

Whichever method of administration is chosen care must be taken to avoid causing mercurialism. This may result from an excessive dosage, from a large accumulation in the tissues, or from an idiosyncrasy of the patient. The symptoms consist of salivation, stomatitis, gastro-intestinal disturbances-such as loss of appetite, diarrhoea, dyspepsia, and colicky pains-anaemia, general disorders of nutrition, and various cutaneous eruptions of an urticarial, vesicular, or exfoliative type. The gastro-intestinal disorders are most liable to occur when the drug is administered by the mouth.

In the past mercurialism was so frequent in connection with the treatment of syphilis that there were those who went so far as to prefer the disease to the cure; with proper precautions, however, a regulated dosage, and keeping the patient under observation during treatment, it need never occur.

As a preventive of stomatitis and salivation from mercury the hygiene of the mouth must be carefully attended to, defective teeth stopped, tartar removed, the teeth thoroughly brushed several times a day, and an astringent mouthwash employed such as alum grs. $x$, potassium chlorate grs. $\mathrm{x}$, and water ad. $\bar{\jmath}$ i. Should stomatitis supervene the mercury must be discontinued at once, saline aperients and hot or Turkish baths given, the gums painted with a weak solution of chromic acid, and a mouthwash such as the following frequently employed : borax grs. $\mathrm{xx}$, tincture of myrrh $\lll x x$, glycerine $m \mathrm{xx}$, and water ad. 亏i. To check salivation small doses of atropin sulphate may be prescribed, and to allay intestinal symptoms 5-Io grains of Pulv. ipecac. co.

Contra-Indications.-As mercury is eliminated by the kidneys the chief contra-indication to its employment is chronic renal disease and as a prelude to a course of treatment with it the urine should always be examined for albumin. Transitory albuminuria may occur in the early stages of syphilis from the dissemination of the syphilitic virus, but this will usually disappear when mercurial treatment is instituted; persistent albuminuria, on the other hand, is indicative of organic 
disease of the kidneys and when it is present mercury, if employed at all, must be given with the greatest caution-preferably in the form of small doses of some soluble salt such as the benzoate-and the skin kept active meanwhile by hot baths.

\section{Methods of Admisistration}

Ingestion.-In this country the most popular method of giving mercury, up till recently, has been by the mouth. This is convenient but has certain disadvantages: (I) it is less effective than either injection or inunction ; (2) it is more liable to cause stomatitis and gastrointestinal disturbances; (3) the drug is apt to be taken irregularly. Consequently ingestion has now been superseded largely by the other more efficacious methods.

The most common preparations to be given by the mouth are the " grey powder," the " blue pill," the perchloride of mercury, and the proto-iodide.

Grey Poüder.-Hydrargyrum cum creta is best given in the form of pills containing I grain of the grey powder either alone or combined with I grain of Pulv. ipecac. co. to counteract intestinal symptoms. The dose for the adult man of normal weight is 6 grains per diem, and for a woman + grains. This should be continued in courses of three months for at least three years, the courses being separated at first by a month and later by a longer interval and the daily dose gradually being reduced until the patient is only taking I grain a day.

Blue Pill.--Pilula hydrargyri contains metallic mercury and is given in doses similar to those of the grey powder. For the adult a 2 grain pill is prescribed thrice daily till active symptoms cease and then in three-monthly courses with increasing intervals, the dosage being gradually reduced till the end of the third rear. The treatment is resumed in occasional courses in the fifth and seventh years.

Perchloride of Mercury.-This is given in the form of pills containing $\frac{1}{16}$ to $\frac{1}{8}$ of a grain thrice daily.

Proto-iodide or Green Iodide of Mercury.- This is not often used as it is particularly liable to cause stomatitis and intestinal srmptoms. It is given in doses of $\frac{1}{t}$ to I gr. thrice daily and may be combined with $\frac{1}{8} \mathrm{gr}$. of opium to reduce its irritating effects.

Other preparations of mercury sometimes given by the mouth are the salicylate, tannate, and acetate, and mergal which is an organic compound of mercury and cholic acid ; but these have no adrantages over the above better-known salts.

Inunction.-When thoroughly carried out, inunction is the most effective of all the methods of administration, but it has the following disadrantages: It requires a certain amount of experience to carry it out satisfactorily ; at least an hour a day must be given up to it ; it is dirty ; it is apt to adrertise the nature of the disease from which the 
patient suffers; it is more liable than the other methods to be followed by dermatitis.

The procedure followed at Aix-la-Chapelle, where it is employed in a routine fashion and to its greatest advantage, may be taken as a model of what inunction treatment should be, but can be modified to suit the necessities of the individual case. The procedure is as follows: The patient on arrival is examined by his physician who takes notes of his case, records his weight, and instructs him to report once a week. The treatment which is carried out in the interval consists of inunction with mercurial ointments, warm baths, and mild exercise. Every morning the patient has a short walk to one of the springs and takes one or two glasses of sulphur water. This is followed by a light breakfast. Two hours later he has a warm sulphur bath at $86^{\circ} \mathrm{F}$. in which he remains for 25 minutes. After the bath his skin is well dried and 75 grains of mercurial ointment rubbed in for ${ }_{5} 5$ minutes by the bare hands of a professional rubber till the skin is quite dry. As a result of the rubbing the anointed part assumes a dirty grey colour. Different regions of the body are rubbed seriatim in the following order ; arms, forearms, chest, back, thighs, legs; the hairless parts are chosen, as dermatitis is less liable to follow than in parts where hair-follicles are present. This series of rubbings occupies six days and on the seventh it is begun again on the arms. The skin of the region to be rubbed is only washed once a week-immediately before inunction. During the course the hygiene of the mouth is carefully attended to and a mouthwash containing sulphate of alum is used frequently. The course lasts six weeks and the patient is advised to return in a year for a second course of a month's duration.

A useful ointment for inunction is equal parts of mercury and lanolin with the addition of a few drops of olive oil, $\frac{1}{2}$ to I dram of this being rubbed in at a time. It should be made freshly in small quantities as lanolin preparations readily become rancid and irritate.

Substitutes for inunction have been suggested, such as the wearing of flannel or cotton underclothing impregnated with mercury, or the employment of various forms of mercurial plasters, but these are much less effective.

Injection.-Mercury may be given by intravenous or intramuscular injection, but must on no account be injected subcutaneously as the irritation produced by it in the hypoderm may lead to acute inflammation, painful nodosities, and possibly to sloughing.

Intravenous Injection. - This method is rarely adopted as by it the drug is absorbed too rapidly and may give rise to such dangers as thrombosis or embolism, and it has no advantages over intramuscular injection. The salts which have been used chiefly are the cyanide, benzoate, and sublimate.

Intramuscular Injection.-At the present time mercury is largely administered by intramuscular injection. By this method it is less liable to cause stomatitis and gastro-intestinal symptoms than when 
taken by the mouth and it has the adrantage of absolutely accurate dosage. The injections may be of soluble salts which are given daily or on alternate dars, or of insoluble preparations, such as calomel or metallic mercury, which are given once a weet.

The injections should be made deeply into the muscles. Certain syphilologists choose as the site of injection the lumbar muscle or the depression behind the great trochanter, but the most usual situation is in the buttock around a point where a rertical line between the inner and middle thirds crosses a horizontal line between the upper and middle thirds. If the injections be made anywhere in an area about the size of a five-shilling piece with the point of intersection of these lines as the centre there is no danger of injuring large vessels or nerves and, as the injection is in the upper part of the buttock, there is no subsequent discomfort from sitting.

The injection should be done with the utmost care and cleanliness. A glass syringe should be employed and a platino-iridium needle an inch and a half long, as sharp as possible, and sufficiently strong not to break in the tissue. These should be sterilised in boiling oil. The skin in the injection area is washed and painted with iodine, and the needle, with the syringe containing the mercury attached, is plunged quickly into the muscle for about an inch and a quarter. After insertion the piston should be withdrawn and if a drop of blood comes back into the syringe it is an indication that a vein has been pierced and the needle must be reinserted. The injection is made slowly into the muscle. Care should be taken that the entire quantity of mercury is discharged through the needle, and it is a wise precaution to detach the syringe and inject a few drops of saline solution before withdrawing the needle as, should it contaminate with mercury its tract through the subcutaneous tissue, inflammation and painful nodosities are liable to follow. After the injection a collodion scab is applied.

Soluble Salts.-Injections of soluble salts are given daily in courses of about a month's duration. The most commonly employed are the benzoate, perchloride, and biniodide; these are dissolved in distilled water and about Io m injected each time.

Benzoate of Mercury.- The average dose for an adult is $\frac{1}{i}$ of a grain and the following formula is commonly employed :

\begin{tabular}{|c|c|c|}
\hline Benzoate of mercury & - & - \\
\hline Chloride of sodium & - & - \\
\hline Distilled water - & - & - \\
\hline
\end{tabular}

Perchloride of Mercury.-The average adult dose of this salt is a $\frac{1}{5}$ of a grain. Its injection is apt to be painful and consequently it has been superseded largely by the benzoate.

Biniodide of Mercury.-The average adult dose of this salt is a $\frac{1}{15}$ to a $\frac{1}{7}$ of a grain.

Insoluble Salts and Metallic Mercury.-The insoluble salts and metallic mercury are injected once a week. The most commonly 
employed salts are calomel and the salicylate and of these calomel is the more powerful, being the most effective of all the mercurial preparations used for intramuscular injection. Its chief drawback is that it is painful, but this can be corrected to a large extent by combining it with creosote and camphoric acid as in the calomel cream introduced by the late Colonel Lambkin, R.A.M.C. :

\begin{tabular}{|c|c|c|c|c|c|}
\hline Calomel & - & - & - & - & grms. \\
\hline Creosote & - & & & - & c.c. 20 \\
\hline Camphori & & & & - & c.c. \\
\hline Palmitin & & - & & - & c.c. IO \\
\hline
\end{tabular}

The cream is prepared by Oppenheimer and Io $m$ of it contain $\frac{1}{2}$ a grain of calomel; the average dose of calomel for an adult is $\frac{3}{4}$ to I grain.

Salicylate of Mercury is much less effective than calomel. It is given in doses of $\frac{1}{2}$ to $I$ grain suspended in liquid paraffin.

Metallic Mercury is a powerful remedy and second only to calomel. It is injected in the form of grey oil in which the mercury is suspended in oil of vaselin or, better, in the form of the Lambkin cream, in which it is suspended in palmitin, a netrtral fat derived from palm oil, and which is practically painless. The formula for the cream is as follows :

\begin{tabular}{|c|c|c|}
\hline Metallic mercury & - & \\
\hline Creosote - - & - & - \\
\hline Camphoric acid & - & \\
\hline almitin - & - & - \\
\hline
\end{tabular}

Io $m$ of the grey oil or cream contains I grain of metallic mercury, the average dose being I to 2 grains.

Before the introduction of salvarsan the following course of injections was largely employed in the British army (Lambkin) :

"(I) Six weeks treatment consisting of four injections of calomel and two of metallic mercury.

(2) Two months rest.

(3) Four months treatment with four injections of metallic mercury.

(4) Four months rest.

(5) Two months treatment with four injections of metallic mercury.

(6) Six months rest.

(7) Four months treatment with four injections of metallic mercury.

(8) One month rest.

(9) Two months treatment with four injections of metallic mercury."

This makes in all two years treatment consisting of four injections of calomel and eightecn of metallic mercury. By means of this series 
of injections satisfactory results were obtained. This course of treatment is only suitable for a healthy adult male and requires to be reduced in those whose general health is below par and in women.

Fumigation.-Another mode of administering mercury is by fumigation, the procedure being as follows: The patient is seated naked on a chair in a vapour bath with a sheet tied round his neck to prevent inhalation of the fumes. Under the chair is extemporised a vapourising apparatus, consisting of a metal tray on a tripod with a spirit lamp beneath it. On the tray is placed 20 grains of calomel which is rolatilised by the heat in about quarter of an lour. This is repeated daily or on alternate days.

The treatment is uncertain, is useless as a routine method, and is now practically discarded.

\section{The Iodides}

The iodides, especially iodide of potassium, are emplored in the later stages of syphilis to cause the dispersion of gummatous infiltrations and to heal ulcerations and syphilitic disease of the bones, periosteum, and nervous srstem. The drug has no specific action on the virus of syphilis but acts by causing the elimination of syphilitic products. In employing the iodides care must be taken to aroid producing toxic symptoms either from too large doses or from an idiosyncrasy towards the drug on the part of the patient. These symptoms consist of depression, coryza, buzzing in the ears, gastro-intestinal irritation, oedema of the larynx, and various types of cutaneous eruptions.

Of the iodides, the potassium salt is the most effective, but it is also the most depressing, and in consequence is better not employed alone but in combination with the less depressing ammonium or sodium salts. The dose varies according to the state of the patient and the type of syphilide, from 5 grains at first to 30 grains thrice daily or more if considered advisable.

The iodides should be given well diluted in water about an hour after meals and in intermittent courses of ten days to a fortnight. The following is a useful combined iodide mixture :

\begin{tabular}{|c|c|c|}
\hline Ammonium iodide & - & \\
\hline Potassium iodide - & - & \\
\hline Sodium iodide - & 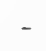 & \\
\hline rmint wat & - & \\
\hline
\end{tabular}

To be taken three times a day in a tumbler of water.

Should iodide of potassium be prescribed alone its depressant action may be reduced by the addition of 2 grains of ammonium carbonate to the dose.

Iodipin.-Certain individuals are so susceptible to iodide in any form that various substitutes have been introduced which are not so 
depressing. One of the most useful of these is iodipin, a combination of iodine and sesame oil. It is made in solutions of two strengths, namely, Io and $25 \%$, and the usual dose is half a drachm three times a day, either prescribed in capsules which are most easily taken with a glass of milk, or injected subcutaneously.

Between the courses of iodides or iodipin a tonic regime should be instituted and a mixture containing iron, quinine, or strychnine, prescribed.

A common method of prescribing the iodides is in combination with mercury and usually with the perchloride or the biniodide as in the following formulae:

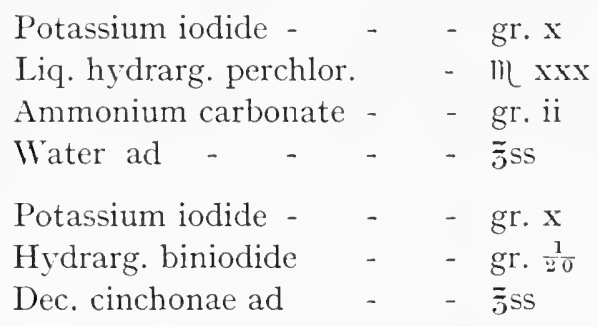

A tablespoonful in a tumbler of water between meals.

The biniodide of mercury and iodide of potassium may be given also in pills.

$$
\begin{array}{lll}
\text { Hydrarg. biniodide } & - & - \\
\text { Potassium iodide } & - & -\frac{1}{2} 0 \\
\text { gr. v }
\end{array}
$$

One before each meal and one at bed-time.

These combinations can be used with advantage about the eighth month of the disease and right on to its latest stages.

Instead of combining the iodides with mercury in the form of a mixture or pill, the iodide mixture and the mercury may be given separately, or the iodides may be given by the mouth and the mercury injected intramuscularly.

\section{Scheme of Combined Treatment with Neo- Salvarsay, Mercury, and the Iodides}

The following is an outline of a scheme of combined treatment which may be adapted to suit individual cases :

In primary cases with a negative Wassermann reaction in the adult male a minimum of six injections of novarsenobillon should be given at weekly intervals, commencing with 0.3 grm. and increasing to $0.9 \mathrm{grm}$. This should be combined with mercurial treatment in the form of inunctions or intramuscular injections of mercurial cream once a week, and followed by three-month courses of mercury by the mouth twice a year for at least two years.

In primary and secondary cases with a positive Wassermann reaction the above initial combined course should be followed in six 
weeks by a second course of four injections of novarsenobillon of 0.3 grm. increasing to $0.6 \mathrm{grm}$. and four weekly intramuscular injections of mercurial cream. Should the Wassermann reaction remain positive, further short combined courses should be given at increasing intervals. This, in turn, should be followed by three-month courses of mercury by the mouth twice a year for three years and between the courses a mixture containing potassium iodide in doses of 10 to 15 grains thrice daily for a fortnight.

In late syphilis with cutaneous symptoms a combined course of mercury and novarsenobillon should be prescribed. As a rule one or more injections of novarsenobillon will cause the involution of late lesions, but this should be followed by a short series of mercurial injections, and later by a course of iodides for a few weeks.

In pregnant women with signs of syphilis or who have been impregnated by a syphilitic, as soon as the pregnancy is detected either mercurial treatment in the form of intramuscular injections or injections of novarsenobillon should be instituted. The latter should be given in courses of 6 to 8 injections commencing with $0.3 \mathrm{grm}$. and increasing to $0.6 \mathrm{grm}$., with intervals of eight weeks between the courses and should be discontinued at the end of the seventh month of pregnancy. The two chief dangers connected with the arsenical treatment in such cases are nephritis and abortion, but these can be minimised by commencing with small doses and carefully watching the urine.

\section{Syphilis of the Cextral Nervols Systey}

Salvarsan and neo-salvarsan have been of considerable service in the treatment of syphilis of the central nervous system, especially where treatment has been instituted with as little delay after diagnosis as possible. Such conditions as Tabes dorsalis and general paralysis of the insane are singularly intractable and prolonged courses of treatment are necessary, which are controlled not only by the Wassermann reaction of the blood but also by examination of the cerebro-spinal fluid. The doses employed should invariably be small as patients suffering from nervous affections are liable to be hyper-sensitive to the drug.

The intra-spinal method has proved of greater value in this connection than the other methods of injection, as it is doubtful whether the drug ever reaches the cerebro-spinal fluid or the brain when given intravenously or intramuscularly. When injected intra-spinally the neo-salvarsan should not be dissolved in water as an aqueous solution is too toxic for this purpose, and the best method of giving the drug is to inject intra-spinally a quantity of the patient's serum from blood taken half an hour after a full intravenous dose of neo-salvarsan has been given. 
Browning and Watson describe the procedure for the intra-spinal injection of salvarsanised serum as follows :

"'The patient receives an intravenous dose of ' 606 ' or '914'; then $\frac{1}{2}$-I hour later $30-50$ c.c. blood are withdrawn from a vein with aseptic precautions and allowed to clot. The serum is then allowed to separate overnight and heated for $\frac{1}{2}$ hour at $56^{\circ} \mathrm{C}$. It may be injected intra-spinally either undiluted or after diluting with an equal volume of $0.85 \% \mathrm{NaCl}$ solution. Lumbar puncture is performed and I $_{5}$ c.c. cerebro-spinal fluid withdrawn-which should be preserved for examination-then $I_{5}-25$ c.c. of the salvarsanised serum (or 30-50 of the 50\% dilution with saline) are injected slowly. The foot of the patient's bed is kept raised for an hour afterwards. The injections may be repeated at intervals of 2-3 weeks."

\section{The Wassermane Reaction in the Control of Treatment}

In using the Wassermann test to control treatment the ideal result to be arrived at is a permanently negative reaction.

After a full course of treatment, three months should be allowed to elapse without treatment and the blood tested. If the result be negative the test should be repeated in six months and afterwards at longer intervals, and if the reaction remains persistently negative and there are no clinical signs of the disease the individual may be regarded as non-infective. If a positive result be obtained in the first or any subsequent test, treatment should be resumed at once for at least six months and the test repeated after an interval of three months without treatment.

It has been found that the majority of cases which have been subjected to prolonged and energetic treatment give a negative reaction over many years, but that in a small proportion of cases even energetic and prolonged treatment fails to produce a negative reaction--possibly as a result of insufficient treatment in the early stages of the disease.

\section{Seruir Therapy}

So far attempts to prepare an anti-syphilitic serum have been unsuccessful. It was thought that the macacus or some lower monkey might attenuate the virus of syphilis and that in this way an anti-syphilitic vaccine might be obtained, but the proof is insufficient that the virus is attenuated even although no secondary lesions succeed the primary sore. According to Neisser a serum has not yet been obtained " sufficiently strong to protect animals against syphilitic infection, nor a method which can confer active immunity, because we have not yet produced a truly attenuated virus, either by passage through an intermediate animal, or by any chemical or physical method." 


\section{CONGENITAL SYPHILIS}

Syphilis can be transmitted to the offspring at any period of the pregnancy and may result in abortion at the third month or later in a still-birth, or in a living infant which may show signs of the disease at birth and succumb almost at once or may be apparently healthy and only develop syphilitic symptoms after about six weeks or longer. Congenital syphilis is thus responsible for a high infant mortality and it has been estimated that in only about $10 \%$ of syphilitic pregnancies does the offspring survive for more than a year.

Certain observers distinguish between congenital and hereditary syphilis, meaning by congenital syphilis cases in which the disease is manifest in the child at birth or soon afterwards-the mother having been infected while pregnant and having infected the foetus through the placenta--and by hereditary syphilis cases in which the ovum was infected either by the mother being syphilitic or by the semen. Of these the hereditary cases are naturally the more serious, because the infection takes place earlier. This distinction is difficult to maintain, however, and is of comparatively little practical importance.

Mode of Transmission.--In most instances syphilis is transmitted to the ovum or the foetus by the mother being syphilitic (maternal transmission), occasionally by the semen (paternal transmission), and in rare instances by both parents being syphilitic (mixed transmission).

Certain observers deny altogether the infection of the ovum by the semen and believe that the mother is invariably infected first, and that in the cases where no signs of syphilis occur in the mother while the offspring is syphilitic the mother is herself syphilitic but the disease is latent at the time of observation. It is generally considered that the semen is infective, however, but that the spirochaetes are not carried by the spermatozoa, which are too small, but are contained in the other constituents.

It has been asserted that the foetus may be infected by the semen and in turn infect the mother who was previously healthy (conceptional syphilis), but this, if it does occur, is extremely rare.

Colle's Law.-According to Colle the mother of a syphilitic infant procreated by a syphilitic father, but who shows no signs of syphilis herself, is immune against infection and can suckle her child with safety, whereas owing to the contagiosity of the disease a healthy wetnurse suckling a syphilitic child is liable to become infected. The explanation of this well-known fact is most probably that the mother is not immune to the disease but has it in a latent form ; a view which has been corroborated recently by the discovery that mothers of syphilitic infants, even though they show no signs of the disease, usually give a positive Wassermann reaction.

Profeta's Law.-Another aphorism in connection with congenital syphilis is that known as Profeta's Law which is the converse of Colle's Law and states that an apparently healthy infant of a syphilitic mother 
can be suckled by its mother without contracting the disease. This again in the light of the Wassermann reaction would appear to be explained by the infant having the disease latent and being liable to develop it in later life.

Diday's Law of Decrease.-It has been found, as formulated by Diday, that if a syphilitic infection be virulent in character in the mother or the foetus, abortion is inevitable, but that if the mother receive appropriate anti-syphilitic treatment subsequent pregnancies may result in a still-birth, later in the birth of a child with syphilitic symptoms, later still in the birth of an apparently healthy child which

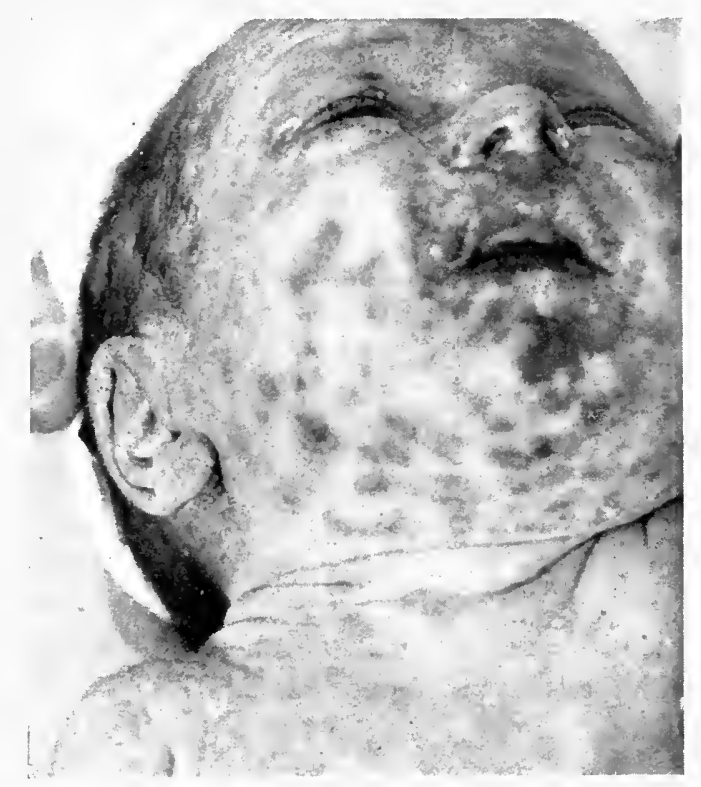

FIG. 213.-Congenital Syphilis.

shows eruption profuse about the mouth and chin, and rhagades at the corners of the mouth.

develops signs of the disease subsequently, and last of all, if the treatment has been sufficient, in healthy children.

It has been observed also that a healthy child may be born of parents one or other of whom shows definite signs of the disease if they are under suitable treatment, while should treatment be discontinued a syphilitic child is usually the result.

Description.--If the foetus be still-born from syphilis it has a peculiar shrivelled appearance with the epidermis macerated and peeling, or it may exhibit some deformity or monstrosity such as hydro-cephalus. (Fig. 213.)

If the infant be born alive but with signs of syphilis they are generally so characteristic as to be unmistakable, there is marked emaciation, the skin is wrinkled and dusky-grey, and the face is shrivelled and wizened like that of an old man or a monkey, on the 
palms and soles there are not infrequently small blebs (syphilitic pemphigus) situated on a red or brownish-red base, which are usually surrounded by a brownish halo, flaccid with sero-purulent or haemorrhagic contents, and tend to dry up and become replaced by a brownish crust. Where the bullae have broken or the crusts become detached reddish-brown raw surfaces are left. Occasionally instead of bullae abortive lesions occur such as brownish macules or small scaly patches. Where bullae form near the tip of the finger the nail-bed may be involved and the nail shed. (Fig. 2If.)

In such cases the infant usually succumbs in a few days or weeks and a postmortem examination reveals a widely-spread syphilitic involvement of the viscera with the presence of spirochaetes in vast

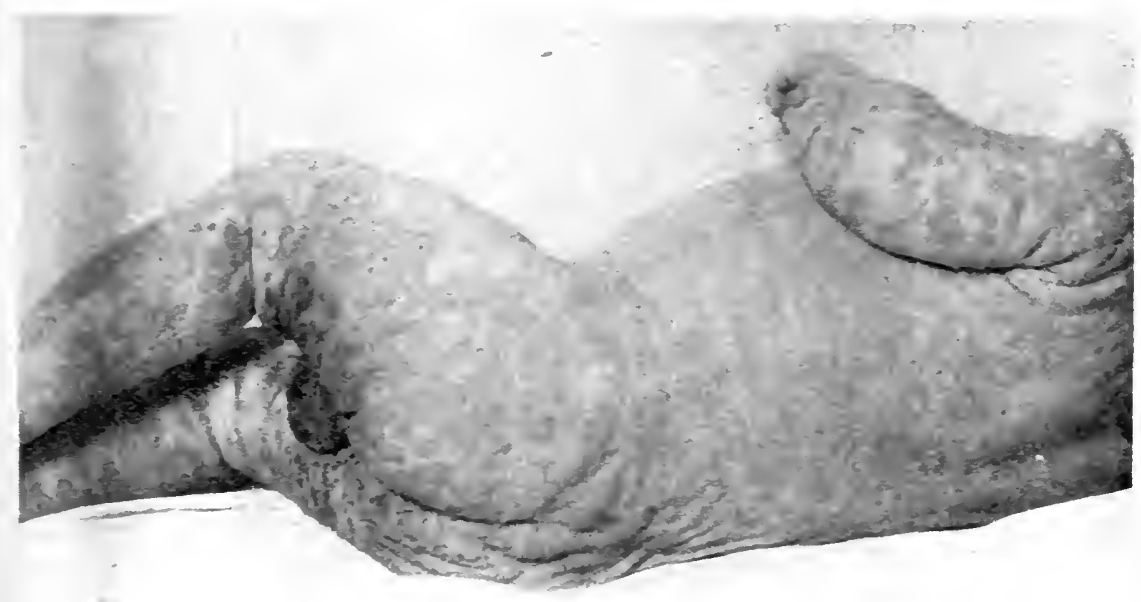

FIG. 2 I 4.-Congenital Syphilis. Macular and coarse papular eruption.

numbers in the lungs, supra-renal glands, and liver, and to a less extent in the spleen, kidners, blood, brain, and meninges.

In many cases the disease is latent at birth and the infant may appear to be perfectly healthy and symptoms only show themselves later. They usually appear about the fourth or fifth week, but may be delaved for a year or longer and occasionally even till adult life. One of the first signs in these cases is wasting and the infant begins to lose ground, takes food badly, is peerish and restless, loses weight, and becomes emaciated. The wasting is generally associated with other characteristic symptoms, such as laryngitis to which is due the harsh and strident cry of the syphilitic infant, and rhinitis which is responsible for the snuffles so commonly met with in these infants, which may interfere with breathing, cause difficulty in suckling, and may even necessitate spoon-feeding.

The most characteristic symptom is the cutaneous eruption. This consists of macules or papules varying in size from a lentil to a sixpence, 
pinkish at first but later assuming a reddish-brown or copper colour, smooth or slightly scaly, well-defined, occasionally raised, and leaving a brownish-yellow stain on pressure with a diascope. They may be round, oval, or irregular in shape, and may remain discrete or coalesce into more or less extensive sheets. They are situated chiefly around the mouth, on the forehead, about the buttocks, and on the palms and soles, but occasionally may be widely distributed over the trunk and limbs. In rare instances they may undergo involution in the centre and form rings which may coalesce and give rise to gyrate figures. Where papular lesions are present at the angles of the mouth, in the centre of the lower lip, and about the anus, owing to the movements of these parts they are liable to become cracked and septic from

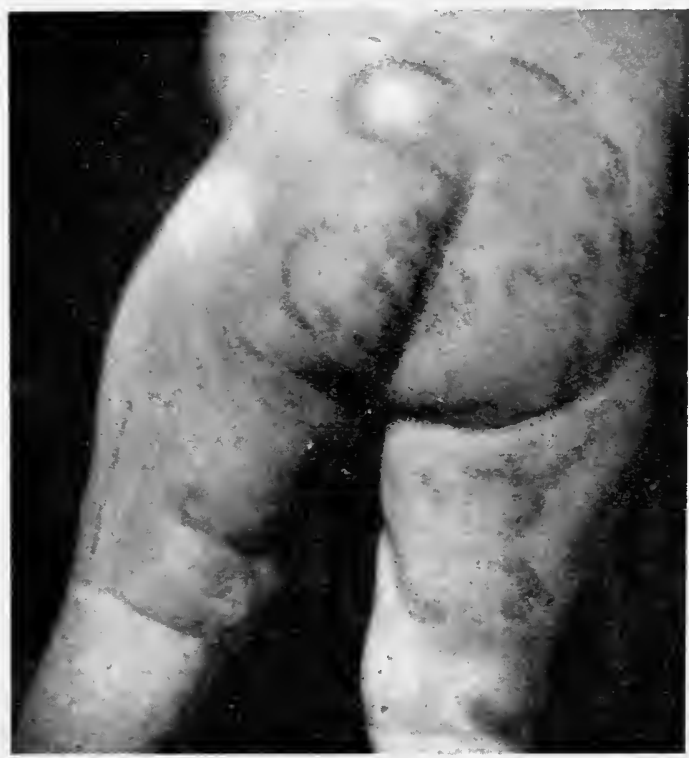

FIG. 215.-Congenital Syphilis. Eruption on buttocks and thighs.

secondary inoculation with pyogenic micro-organisms, and on healing to leave fissures or linear scars which may be observed radiating out from the angles of the mouth or spreading down from the centre of the lower lip. (Fig. 215.)

The epidermal appendages may be attacked, the nails becoming shrivelled, furrowed, and brittle, from dystrophy due to the general malnutrition, and the hair showing an irregular thinning especially marked about the vertex and occiput, and in the eyebrows and eyelashes.

In addition to the above classical symptoms there are others which are less constant, such as bone lesions, nervous disturbances, and visceral changes.

Bone Lesions.-Frontal bosses and nodes on the outer plate of the 
skull (Parrot's nodes) are not uncommon, and the ends of the long bones may be swollen from epiphysitis and may give rise to pseudoparalysis. The bones of the hands may be involved and dactylitis occur similar to that in tuberculosis. Necrosis may supervene, followed by deformities, such as depression of the bridge of the nose-or " saddleback nose" - -and the peculiar curving of the tibia which has been compared to a sabre blade.

Nerious Disturbances.-Syphilitic meningitis and brain degeneration may occur, associated with imbecility, epileptic fits, or sypphilitic plegias, and it has been shown recently that syphilis is the most frequent cause of insanity in children under fifteen years. The nervous symptoms may sometimes develop later in the form of general paralysis of the insane or juvenile tabes.

Visceral Changes.-Changes in the viscera are not infrequent, such as interstitial hepatitis, enlargement of the spleen so that it is palpable below the ribs, and enlargement of the testicles. These signs are inconstant and require to be well-marked before they give rise to objective symptoms.

Under suitable treatment the symptoms disappear rapidly, learing little or no trace except linear scars at the angles of the mouth or deformities resulting from necrosis of bone; where the treatment has been insufficient syphilitic lesions may develop vears later and may appear about puberty or early adult life; in rare instances late symptoms may be the first evidence of the disease.

Late Symptoms. - Of these the most common are condylomatous lesions about the mouth or anus which generally appear in the second year, ulceration of the rocal cords producing a husky roice, and gummata of the skin or mucous membranes which give rise to punchedout ulcers covered with a greyish slough and which, on healing, are followed by whitish scars. The so-called "triad of Hutchinson " may" also be met with, namely interstitial keratitis of the eyes, deafness, and a peculiar deformity of the second teeth in which they become pegshaped and notched. The dental condition was thought, at one time, to be due to mercury but is now known to be the result of defective development caused by syphilis. (Fig. 2I6.)

Diagnosis.-There is no difficulty in recognising a well-marked case of congenital syphilis, the marasmus, the wrinkled grey shin, the wizened features, the bullae on the palms and soles, or, where the lesions have developed later, the copper-coloured eruption about the mouth and buttocks, the fissures at the commissures of the mouth, the sunken nose, the frontal bosses, the strident cry, and the snuffles, form a picture which is unmistakable.

The bullae on the palms and soles have to be distinguished from Pemphigus neonatorum, which is a local pyogenic affection of streptococcic origin which may lead to septicaemia. In it the infant, as a rule, is well-nourished, the palms and soles are rarely affected, the eruption does not appear for some days after birth, and consists of flaccid blebs 
on a pinkish base and excoriated or crusted patches, and there is usually a history or indication of some source of infection in the nurse or attendant, such as a septic sore or a whitlow. Unless the microorganisms reach the general circulation there are no constitutional symptoms in Pemphigus neonatorum, while in congenital syphilis there are usually evidences of a general invasion and concomitant signs of the disease, such as wasting, dusky skin, shrivelled features, etc.

The copper-coloured eruption of congenital syphilis has to be distinguished from infantile eczema, vacciniform dermatitis, seborrhoic dermatitis, and simple erythema of Jacquet.

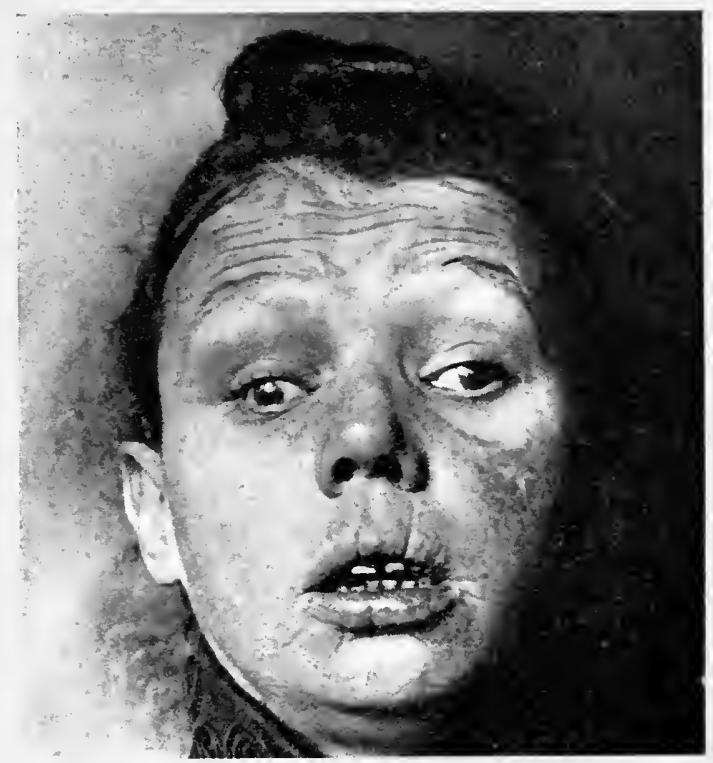

FIG. 2I6.-Congenital Syphilis.

Shows depressed bridge of nose, rhagades of the lips, "peg-teeth."

(From Dr. Arthur Hall.)

Infantile Eczema.-In infantile eczema the lesions are not raised, are ill-defined, pinkish-red in colour, made up of the coalescence of minute papulo-vesicles, and invariably itchy, while the congenital syphilide is often raised, is clearly defined, copper-coloured, and does not itch.

Vacciniform Dermatitis. - This rare condition in which vacciniform lesions are met with on the buttocks might give rise to some difficulty in diagnosis, but in it the lesions are confined to the buttocks and genital organs and do not occur around the mouth or in other situations where syphilides are apt to be present. The actual lesions commence as vesicles on a bright reddish-pink base and eventually become transformed into clearly defined superficial ulcers or excoriations covered with a yellowish or blackish scab. 
Impetigo.-Streptococcic infections of the skin about the flexures of the groins, buttocks, and lower part of the abdomen, are not uncommon and might be confused with congenital syphilis. The impetiginous lesions, however, consist of crusted patches, red moist surfaces sometimes cracked at the folds, and occasionally vesicles or flaccid bullae. The syphilide is browner in tinge and as a rule is less acutely inflamed.

Seborrhoic Dermatitis. - This condition, which occurs as pinkish patches covered with greasy yellow scales which tend to coalesce to form sheets, is not infrequent on the lower part of the abdomen and the upper part of the thighs. It is generally associated with scaly patches on the face and a greasy condition of the scalp. The lesions in it are brighter in tint than syphilides and are invariably scaly, unless in moist intertriginous regions such as the flexures of the groins and the axillae.

Simple Erythema (Jacquet).- - In infants erythematous shiny areas, flat papules, or occasionally superficial ulcers, may be met with in the napkin region, on the convex surface of the buttocks, inside of the thighs, lower part of the back, and also on the calves and heels. This condition, especially described by Jacquet, is believed by certain observers to be of toxic origin. The erythematous lesions take the form of dull red shiny areas, but have not the coppery hue peculiar to the syphilides. The superficial ulcerations are extremely difficult to recognise but are readily differentiated from congenital syphilis, in which the ulcers are punched-out.

Acquired Syphilis.-Congenital syphilis has to be distinguished also from acquired syphilis in the infant or young child where the disease has been transmitted directly or indirectly from infected parents, attendants, wet nurses, etc. In these cases the primary sore is most frequent about the lips from kissing, is not uncommon about the perineum, but may be met with anywhere on the cutaneous surface. It may be so insignificant as to pass undetected and the secondary phases of the disease may be so transient as to render the diagnosis difficult, but there are none of the classical symptoms of congenital syphilis, such as sunken nose, bullae on the palms and soles, epiphysitis, fissures at the angles of the mouth, etc.

Spironema Pallidum.-Asin acquired syphilis an early diagnosis may be made by the demonstration of the spironema, which may be found in preparations from the papules, in the contents of the bullae, or where rhinitis is present, in the nasal mucosa.

Wassermann Reaction.-In congenital syphilis the Wassermann test is not so reliable as in the acquired disease and a negative reaction may be given by a syphilitic child. In doubtful cases the mother's blood should be examined also and if a positive reaction be obtained it may be taken to apply to the offspring.

Prognosis.-In infants born with definite signs of syphilis the prognosis is invariably grave. In cases in which the disease does not 
show itself till after the fourth week, where the general health is fairly good, and proper treatment has been carried out, recovery may be reasonably expected, and this may be so complete that all immunity to the disease is lost and cases are on record in which the subjects of cured congenital syphilis have acquired syphilis in later life. It has been found that syphilitic infants invariably thrive better on the breast than when artificially reared and that the majority who survive a year are breast-fed.

Although as a whole the cases of congenital syphilis in this country would appear to be becoming milder than formerly, the number with late syphilitic manifestations of the nervous system, such as general paralysis of the insane and juvenile tabes, is still considerable.

Prevention.-As a preventive it is necessary that a mother who is syphilitic or who has been impregnated by a syphilitic father should be under careful supervision and treatment during her pregnancy.

\section{TREATMENT}

As soon as the disease is diagnosed, and even in the absence of actual signs of the disease if the child be the offspring of a syphilitic parent, anti-syphilitic treatment should be instituted without delay. Until the introduction of salvarsan this consisted almost entirely of the administration of mercury.

Mercury.-Mercury is given, as a rule, in the form of grey powder, $\frac{1}{2}$ to I grain made up with a few grains of sugar of milk t.d.s. for an infant six weeks old, and the dose gradually increased with the age of the child. Should gastro-intestinal symptoms supervene, especially diarrhoea, $\frac{1}{4}$ grain of Pulv. ipecac. co. is added to the dose. The grey powder should be given intermittently in courses of a month with a fortnight's interval, the treatment being continued for at least twelve months after all symptoms have disappeared with occasional courses during the next three years to eliminate all possibility of late nervous manifestations.

Under small doses of mercury the child thrives excellently and the drug is well borne, but it is advisable to commence with small doses as occasionally there is some idiosyncrasy towards it. During the treatment the general health of the infant should be carefully supervised, the weight taken systematically, the digestive system watched, and the urine examined from time to time for albumin.

The mercury may be administered also by inunction, about 30 grains of mercurial ointment being rubbed in on different parts of the body daily, or the ointment may be spread on a flannel binder and applied continuously to the abdomen when the movements of the child cause its absorption. The drawback to inunction is that the dose absorbed is uncertain and the ointment is apt to irritate the sensitive skin and to produce localised dermatitis. 
Where visceral lesions, ulcers in the vocal cords, or gummata, are present, small doses of iodide of potassium should be combined with the mercury.

Salvarsan.-Fxcellent results have been obtained by injections of neo-salvarsan. For infants from 2 to 6 weeks old six injections should be given at weekly intervals, commencing with a dose of $0.02 \mathrm{grm}$. and gradually increasing to $0.2 \mathrm{grm}$. The dose is dissolved in 3 to 4 c.c. of saline solution and is introduced into the reins of the scalp or in suitable cases into those at the bend of the elbow. When a vein in the scalp is chosen the child's head should be placed on a hard pillow and held firmly by an assistant who renders the vein prominent by pressing with the fingers. The needle is then inserted into the vein in the direction of the blood-stream.

The number of injections should be controlled by the Wassermann test and they should be continued for several weeks after this has become negative.

The best results are obtained by combining small doses of mercury with the salvarsan in the manner indicated for acquired syphilis (see p. 588), and treatment should be continued with occasional courses of mercury for at least two years.

Wherever possible the infant should be breast-fed by the mother. On no account should a healthy wet-nurse be employed owing to the danger of her contracting the disease. Where syphilitic rhinitis or painful lesions about the mouth are present the infant may be unable to suckle and may require to be spoon-fed.

\section{REFERENCES}

Text-Books, Reports, etc.

Brownixg \& Watson. Venereal Diseases. London, I9I9.

HaRrisox, L. WV. Diagnosis \& Treatment of Venereal Diseases in General Practice. 2nd. Ed. London, I9I9.

McDoxagh, J. E. R. The Biology \& Treatment of Venereal Diseases. London, 1915 .

McIntosh \& Fildes. Syphilis. London, I9II.

Magrax, A. C. The Practitioner's Manual of Venereal Diseases. London, I9I9.

Marshall, C. F. Syphilology. London, I906.

Power, D'Arcy, \& Murphy, Keogh. System of Syphilis. London, 1908.

Report of Royal Commission on Vexereal Diseases (First, Final and Appendix). London, I914-I916.

\section{Spirochaeta Pallida.}

Bayly, N. W. Demonstration of living Treponema pallidum (Proc. Roy. Soc. Med., Clinical Sect., I909).

McDoragh, J.E. R. Complete life history of the organism of syphilis (Brit. Journ. Derm., I9I3, xxv. p. I).

McDoragh \& Wallis. The Chemistry of the Leucocytozoon Syphilidis $\&$ of the Host's Protecting Cells (Biochemical Journ., I913, vii.). 
Medical Research Committee. Methods for the Detection of Spirochaetes. (Special Report Series, No I9, p. 35, H.MI. Stat. Off.).

Ross, E. H. An intracellular parasite developing into Spirochaetes (Brit. Med. Journ., I9I2, ii. p. I65I).

\section{J'assermann Reaction, etc.}

Fildes, P. The Wassermann Reaction (Brit. Journ. Derm., I9II, xxiii. p. I3).

McDoxagh, J. E. R. Action of salvarsan \& neosalvarsan on the Wassermann Reaction (Brit. Med. Journ., I912, i. p. 1287).

Medical Research Committees. The Wassermann Test (Special Report Series, No. I4, London, I918, H.M. Stat. Off.).

Noguchi, H. A homohemolytic system for the serum diagnosis of syphilis (Journ. Exter. Med., Balt., I9I8, xxviii. pp. 43-67).

Ploombergh, H. D. The Wassermann reaction in syphilis, leprosy, \& yaws (Philippine Journ. of Sci., I9I I, vi. p. 335).

Toplex, W. W. C. Serum tests for syphilis (Lancet, I9I8, i. p. 7I 5).

\section{Justus Test.}

Feuerstein, L. Justus haemoglobin test (Arch. f. Derm. u. Syph., I903, lxvii. p. 363 ).

Huger, W. E. Justus test for syphilis (Philadelphia Med. Journ., 1902).

Justus, J. Alterations in the blood produced by syphilis, etc. (Brit. Journ. Derm., I897, ix. p. 53).

\section{Luetin Reaction.}

Fox, Howard. Experience with Noguchi's Luetin Reaction (Journ. Cut. Dis., I9I2).

Noguchi, H. Luetin (Journ. Exper. Med., I9I2, xv. i.).

Wolfsohn. Luetin Reaction (Journ. Amer. Med. Assoc., I913).

\section{Re-Infection.}

White, C. F. Re-infection in syphilis, with observations on twenty-eight cases (Brit. Med. Journ., I9I 7, ii. p. 5I3).

\section{Treatment.}

Danysz, J. L'anti-luargol (Compt. rend. Acad. d. Sc., Paris, I917, clxiv. p. $74^{6)}$.

DORE, S.E. Administration of concentrated intravenous injections of novarsenobenzol in the treatment of syphilis (Practitioner, I9I8, ci. p. 209).

EHRLICH, P. Abhandlungen über Salvarsan (Munich, I9II).

EHRLICH \& MCDONAGH. 606 in theory \& practice (London, I9II).

Frexch, H. C. Recent developments in the recognition, treatinent, \& prophylaxis of syphilis (Hunterian Lectures, Lancet, I9I I).

Gibbard \& HARrison. Treatment of syphilis by salvarsan \& allied substances (Trans. xvii. Internat. Cong. Med., London, I9I3). Modern treatment of syphilis (Brit. Med. Journ., I9r3, ii. p. I34r).

Hallopeau, H. Hectine (Trans, xvii. Internat. Cong. Mled., London, I9I3).

LACAPÉRE. Le traitement de la syphilis par les composés arsenicaux (Paris, I9I8).

LÉvy-Bing, A. Les injections mercurielles intramusculaires dans le traitement de la syphilis (Paris, I908).

IICDonagh, J.E. R. Salvarsan \& intramine, with reflections upon chemotherapy (Lancet, I9I 7, i. p. 9I 4). 
Nersser. Treatment of syphilis with salvarsan of allied substances (Trans, xvii. Internat. Cong. Med., London, I9I3).

SEQLEIRA, J. H. Lectures on the early diagnosis and treatment of syphilis (Brit. Med. Journ., I9I7, i. pp. I \& 37 ).

TRIMBLE \& RoThWELl. A comparative study of salvarsan \& neo-salvarsan in the treatment of syphilis (Journ. Amer. Med. Assoc., I9I6, Ixvii. p. $\left.198_{4}\right)$.

\section{Preciention.}

MAY, Otro, Prevention of Venereal Diseases (Oxford Medical Publications, London, I919).

\section{Tropical Syphilis.}

Clesiox. Syphilis (The Geography of Disease, I903, p. 432).

Jeaxselue. La syphilis dans la péninsule Indo-chinoise (Ann. de Derm. et de Syph, Igor, xii. p. 8 I $\left._{\bar{\gamma}}\right)$.

Schetbe. Die venerischen Krankheiten in den warmen Ländern (Arch. f. Schiffs- u. Trop. Hỵg., I902). 


\section{CHAPTER XX}

\section{DISEASES OF THE SKIN DUE TO ANIMAL PARASITES}

\section{ACARINA AND INSECTA}

Introduction.-The human skin may be attacked by various animal parasites, such as certain acarines, various insects, and a few worms. Some of these, such as mosquitoes, fleas, and bugs, are fully mature when they settle on the skin and come there by accident or in search of nourishment; others, such as caterpillars and the larvae of certain flies, are deposited there in the larval stage, the human skin being the necessary habitat for them if they are to attain their full development.

The majority remain on the surface, but a few penetrate the skin, such as the acarus of scabies which burrows into the epidermis, and the "screw worm" or larval stage of the blue-bottle fly which makes its way into the corium and subcutaneous tissues.

The lesions produced vary in type with the parasite responsible, some being erythematous or urticarial, such as those caused by the stings or bites of insects, others being multiform, such as those due to the acarus of scabies, etc.

The following is a classification of the principal animal parasites of the skin :

Acarina.

Sarcoptes scabiei.

Ixodoidea (Ticks).

Demodex folliculorum.

Dermanyssus gallinae (Fowl-mite).

Tyroglyphus longior (Copra-itch mite).

Leptus autumnalis (Harvest-bug).

Pediculoides ventricosus (Grain-itch mite).

Chilopoda (Centipedes).

Siphunculata : Pediculi.

Hemiptera : Cimex lectularis (Bed-bug).

Hexapoda, Insecta.

Diptera: Pulex irritans (Common flea).

Pulex penetrans (Jigger or chigoe).

Muscidae (Flies).

Culicidac (Mosquitoes, gnats). 


$\begin{gathered}\text { Hexapoda, Insecta } \\ \text { (continued). }\end{gathered}$
Nematoda. $\quad \begin{gathered}\begin{array}{c}\text { Hymenoptera: Apidae (Bees, wasps). } \\ \text { Formicidae (Ants). } \\ \text { Lepidoptera: Caterpillars, butterflies. } \\ \text { Coleoptera: Beetles. }\end{array} \\ \begin{array}{r}\text { Filaria loa: Calabar swellings. } \\ \text { (See Tropical Diseases.) }\end{array} \\ \text { (See Tropical Diseases.) }\end{gathered}$

\section{REFERENCES}

General.

Castellani \& Jackson. Notes on certain insecticides (Journ. of Trop. Med. \& Hyg., I915, xviii. p. 253).

Faxthan, Stephexs \& Theobald. Animal parasites of man (Published by John Bale, Sons, \& Danielsson, Ltd., London).

KIxzoch, J. P. An investigation of the best methods of destroying lice $\&$ other body vermin (Brit. Med. Journ., June I9, I9I5, p. I038).

Maxwell-Lefroy: Measures for avoidance \& extermination of flies, mosquitoes, lice \& other vermin (Imperial College of Science \& Technology, London).

Shipley, A. E. Insects \& war (Brit. Med. Journ., IgIf \& I9I5).

\section{SCABIES}

Syn.: Itch ; Fr., Gale ; Ger., Krätze.

Definition: A contagious affection of the skin, characterised by itchy papules, vesicles or pustules, and peculiar lesions known as "burrows" due to the burrowing into the epidermis of a female mite named the Sarcoptes scabiei.

Etiology.- The female sarcoptes, or acarus of scabies, measures about $0.3 \mathrm{~mm}$. in length when impregnated, and is just visible to the naked eye as a minute greyish opaque speck. It is round or oval in shape and has four pairs of legs ; to the two anterior pairs are attached suckers, while the two posterior pairs which are concealed beneath the body; end in bristles. (Fig. 217.)

The male acarus is about

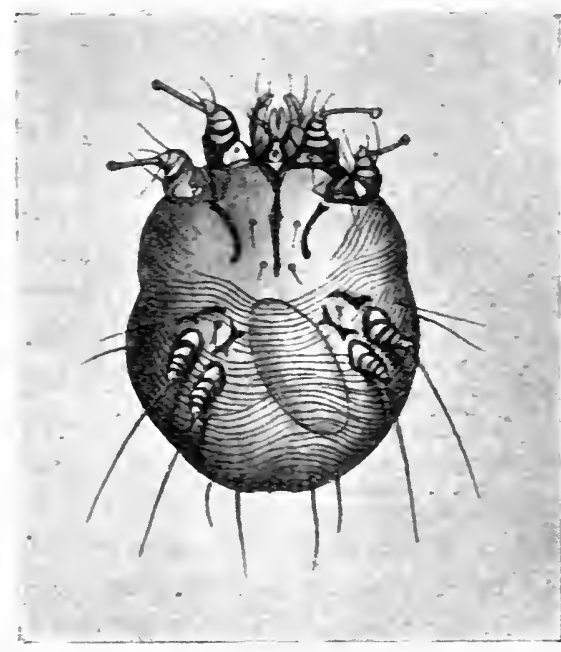

FIG. 217.-Sarcoptes Scabiei. ( $x$ I00.) (After Canestrini.)

(British Yuseum. Economic Series No. 6. Stanley Hirst. 1917.)

half the size of the female and is present on the skin only for a short time as it dies after copulation. 
After impregnation the female acarus burrows into the epidermis and works its way in a tortuous manner along the deeper part of the horny layer, raising up the overlying horn-cells to form a superficial tunnel about a quarter to half an inch in length which is known as the "burrow " or cuniculus. In it the acarus deposits its eggs and faeces, which appear as fine black granules, and at the far end it rests for a few weeks and dies, its position being indicated on the surface by a small glistening elevation or papule. (Fig. 2I8.)

In about a week the young acari hatch out, when they leave the old burrow and reach the surface, either by making punctures through

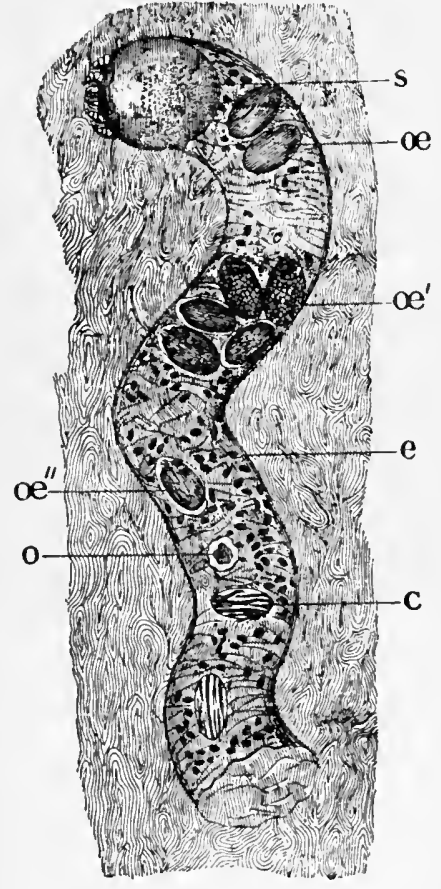

FIG. 218.-Sarcoptes Scabiei.

$s$. Adult female at end of burrow. $c$. Eggs, c. Empty egg-shell. o. Opening through which larva has escaped. e. Excrement. (From Railliet, partly after Gerlach.)

(From British Museum. Economic Series No. 6. Stanley Hirst. I9I7.)

the roof of the epidermis which appear as black specks, or through exfoliation of the horn-cells. They then move to other parts of the skin, the females become impregnated, new burrows are formed, and the process is repeated.

Scabies is usually transmitted by direct contact between an infected and a healthy subject and for it to occur the contact must be close and continuous, as in sleeping in the same bed. It may also be spread indirectly by infected clothing, sheets, blankets, etc., as acari may live on them for several days apart from contact with the body. It is rarely acquired during the day.

It has a universal distribution, occurring in tropical as well as temperate zones. It is much more common among the poor than the well-to-do as over-crowding, unhygienic surroundings, and want of cleanliness, are important factors in its dissemination. It is frequent among soldiers in barracks and camps, and wherever large numbers of people are living together in a restricted area.

Certain domestic and wild animals suffer from a similar disease due to various closely allied acari some of which are transmissible to man.

Description.-On reaching the skin, the scabies mite remains dormant for a few days or even weeks before producing symptoms. The first sign of its presence is a minute vesicle which marks the site of its penetration into the epidermis ; this is usually about the size of a pin's head, but it may be as large as a split-pea when the burrow may sometimes be seen extending on the roof of the bleb.

The burrow is the most characteristic lesion and appears on the 
surface of the skin as a ridge or line which varies in length from a quarter to half an inch, may be straight or tortuous, and presents a greyish appearance due to dark granules of faecal matter and to dirt adhering to it. In doubtful cases it can be made prominent by rubbing the surface of the skin with ink and wiping it off, as the ink adheres to the rough points of the ridge and is sucked into the burrow by capillary attraction. (Fig. 2I9.)

Burrows may occur anywhere on the cutaneous surface, except on the face and scalp, but are most common in certain situations which may be regarded as the classical sites of the disease, namely, the webs of the fingers and toes, wrists, ankles, axillae, lower part of the abdomen, umbilicus, female breast, and penis. At first the infection may be

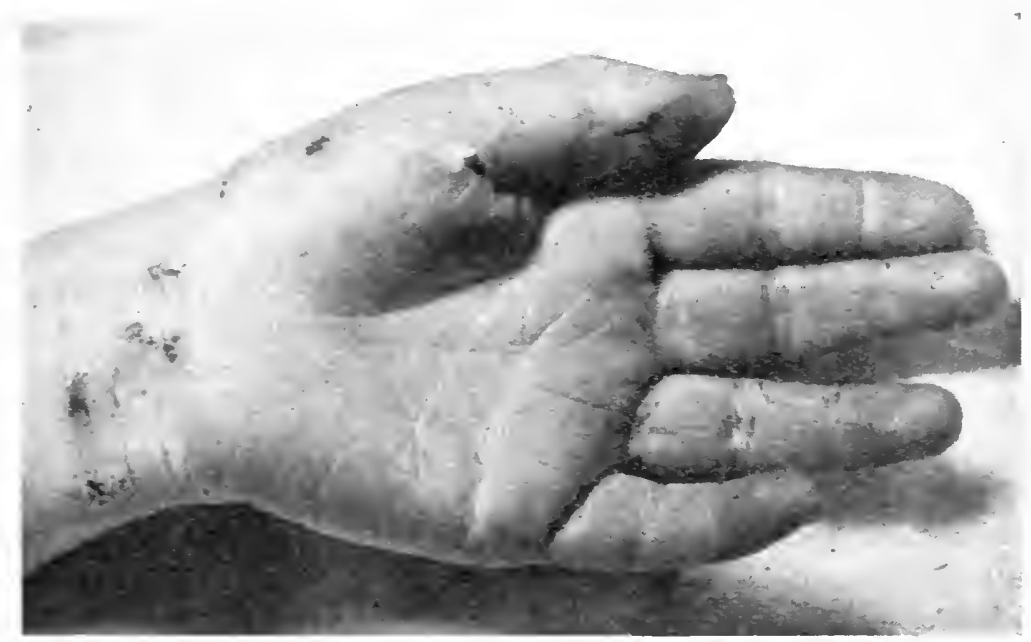

FIG. 2 I9.-Scabies.

Shows burrows on wrist and thumb.

limited to one or more of these situations, but if untreated it gradually spreads until it becomes widely distributed over the skin. In children burrows frequently occur on the buttocks and may be more marked on the feet than on the hands, while in infants they are sometimes present on the head.

In addition to the typical burrows there is a multiform eruption of itchy papules, excoriations, resicles, or even small bullae which usually contain bright greenish-yellow pus. The papules and excoriations are distributed irregularly over the skin, while the vesicles and bullae are most commonly located on the hands and feet.

From rubbing and scratching to relieve the itching, complications arise such as erythematous patches, inflamed follicles, urticarial papules, and eczematisation, and from secondary inoculation of pus-cocci, pustules, superficial ulcers, or boils-especially about the buttocks.

The itching, which usually draws the patient's attention to the disease, is constant and is always worse when he becomes warm in 
bed. It varies in degree according to the sensitiveness of the skin. At first it is localised to the sites of the acari but later, from reflex irritation, it may occur in distant parts where no acari are present.

As a rule there are no definite general symptoms, though occasionally in delicate persons interference with sleep caused by the itching may have a depressing effect and may render the patient nervous and irritable; in extensive attacks albuminuria may occur, either from the action of toxins eliminated by the acari, or from absorption of Balsam of Peru used in the treatment.

Histopathology.--Vertical sections of a burrow show that it is situated in the horny layer and that it rarely penetrates the granular layer. It usually slopes down from the surface as far as the basal layer of the Stratum corneum where it ends, but it may bend up again to the middle of the horny layer. The epidermis around the orifice is oedematous and may form a distinct vesicle. In the burrow itself minute eggs and faeces are present, while at the far end the female acarus is generally lying.

The epidermis beneath the burrow may be normal, or may show signs of oedema with dilatation of the inter-epithelial lymphatics and vesiculation. The underlying capillaries of the papillary layer may be dilated and around them there may be an inflammatory cellular infiltration.

Diagnosis.-The diagnosis of a typical case of scabies presents no difficulty; the characteristic burrows about the webs of the fingers, the itching most marked when the patient gets warm in bed, the irregularly distributed eruption of inflamed papules, and the secondary complications such as excoriations, pustules, boils about the buttocks, and occasionally superficial ulcers, forming a clinical picture which is easily recognised. On the other hand in mild cases, especially in cleanly individuals who frequently wash their hands and feet, the difficulties of diagnosis may be great and the disease may be present for a long time before it is recognised. In doubtful cases of this nature, a most careful search must be made for burrows in covered parts of the body, especially in such situations as the axillae, breasts, umbilicus, and penis.

In children it may be mistaken for papular urticaria, but in it the essential lesion is a red blotch with a central papule and not a burrow, and the eruption is confined generally to the covered parts of the body.

In every case the final diagnosis rests with the demonstration of the acarus, and with a little skill and perseverance this can usually be done. A triangular surgical needle or an ordinary darning needle should be placed on the surface of the skin and gently pushed along the length of the burrow till it reaches the small elevation at the end containing the acarus, this should be pierced by a rotary movement of the needle and opened by an upward leverage. The acarus may then be picked out on the point of the needle, when it appears as a minute 
whitish speck, and should be placed on a slide in a drop of water or glycerine and examined microscopically. (Fig. 220.)

Prognosis.-The prognosis is excellent, and the disease is readily amenable to suitable treatment.

\section{TREATMENT}

The treatment is wholly external, and consists of opening up the burrows by maceration and scrubbing with soft soap and hot water, destroying the acarus and ova by a suitable parasiticide, relieving the itching by soothing remedies, and treating secondary lesions such as pustules, ulcers, or eczematous patches, by appropriate means.

Sulphur is the most effective parasiticide for the acarus.

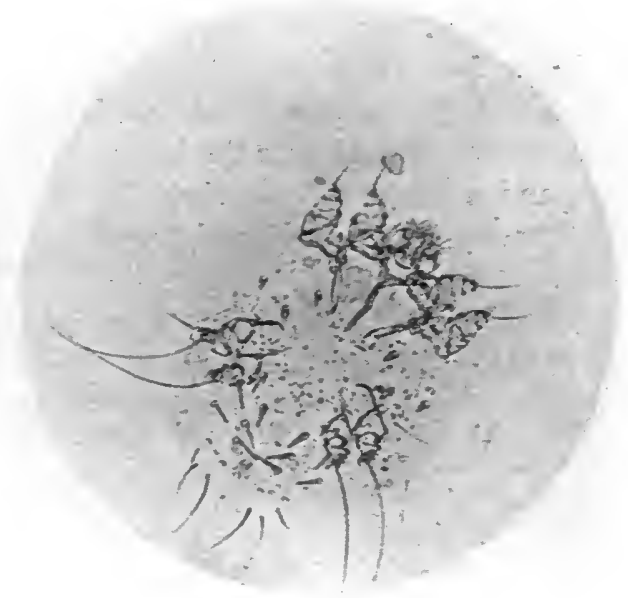

FIG. 220.-Sarcoptes Scabiei.

Specimen in glycerine.

In Paris at the St. Louis Hospital, the following drastic method of treatment was employed as a routine. The patient was first rubbed over with soft soap on a piece of flannel or soft brush for about half an hour, then put into a warm bath and the soaping continued for half an hour longer. In this way the epidermis was macerated and the burrows opened. On coming out of the bath, the following ointment was rubbed in and left on till next morning: Potassium carbonate $\frac{1}{2}$ dram, precipitate of sulphur I dram, lard to I ounce. A second bath was then given, after which the patient was believed to be cured.

This procedure, which is rather severe for ordinary sensitive skins as it is apt to result in sulphur dermatitis, may be modified with advantage as follows: A weaker sulphur ointment, such as precipitate of sulphur I dram, white vaselin to the ounce, is substituted, the patient is put into a warm bath for about half an hour, the skin rubbed with 
soft soap, and the burrows and suspicious lesions scrubbed with soap on a toothbrush. After the bath the ointment is rubbed in and left on the skin and the clothes put on. As it is impossible for the patient to reach every part of his body the inunction should be carried out by an attendant, special attention being paid to such parts as the axillae, umbilicus, inter-digital spaces, and penis. The next day the procedure is repeated, and again on the fourth day, and during this time the same underclothes and bedclothes are used.

After the first day's treatment the itching generally ceases. It is liable to recur after the fourth day from the sulphur but is then of a different character, as the sulphur causes a burning sensation rather than itching, which is amenable to calamine cream and a bland dusting powder. On the fifth day the underclothing and bedclothes should be changed.

Instead of the ordinary bath with soft soap, sulphur baths are sometimes employed (see p. I27), but they are less effective and more liable to cause sulphur dermatitis.

A valuable addition to the sulphur treatment is to dust precipitate of sulphur between the sheets and over the body, especially about the axillae and groins, and a cure may sometimes be effected by sulphur baths and sulphur powder alone, thus avoiding the unpleasantness of greasy applications.

For a week or ten days after the acari have been destroyed the skin is in such a delicate state that baths should be avoided and the skin simply dusted with a bland powder or a soothing calamine cream applied.

In resistant cases the sulphur ointment may be made more efficacious by the addition of soft soap, which causes exfoliation of the epidermis and allows the sulphur to penetrate, or the well-known Vleminckx's lotion (Liquor calcis sulphurata) (see p. 394) substituted.

Instead of sulphur inunction, scabies may be treated by means of sulphur vapour. A wooden cabinet made like a home Turkish Bath, or a simple screen arrangement, is employed and the vapour is generated from a sulphur candle. The patient is given a hot bath and scrubbed with soft soap, he then has his treatment for fifty minutes in the cabinet, after which he puts on warm clean clothes and the old clothing is disinfected. This treatment causes a slight desquamation and gives relief from the itching. The chief disadvantages are that it is liable to produce dermatitis in delicate skins and as a rule is not sufficiently thorough to eradicate the disease in one treatment. It is also apt to cause fainting.

In delicate skins and in children under three years of age, Beta naphthol is a useful substitute for sulphur. It is almost equally effective and is less irritating, but it has the disadvantage of a most unpleasant smell. It may be employed in the strength of 2 to $5 \%$ in an ointment, either alone or in combination with Balsam of Peru, soft soap, or sulphur. 
Baisam of Peru is another valuable substitute which causes less irritation, but it is too expensive for general use. It is particularly serviceable in infants and young children, but must not be employed over extensive areas as it is liable to be absorbed and to cause nephritis. It may be painted on, either pure or diluted with equal parts of spirit, olive oil, or glycerine, or it may be used as an ointment alone or in combination with sulphur. In every case where it is prescribed it is advisable previously to test the urine for albumin and to do so daily during its use.

Complications such as pustulation or eczematisation require to be treated on the lines laid down in connection with impetigo and eczema. Sometimes the irritability of the skin is so severe that it is advisable to reduce it by calamine cream before employing the parasiticide.

Disinfection.--It is of the utmost importance that all infected clothing should be disinfected, such as woollen underclothes, socks, blankets, sheets, and even gloves and wrist watches. This should be done either by steam, dry heat, or fumigation with sulphur.

Nоте.-During the late war it was found that of the skin diseases amongst soldiers on active service, excluding pediculosis, scabies was by far the most common, and from severe pyodermic complications incapacitated large numbers of men for prolonged periods.

In a paper by MacCormac and Small dealing with this subject, the following treatment was recommended:

I. On the first day the patient is given a hot bath. Before entering the bath he rubs himself thoroughly all over with soft soap, massaging it into the skin and paying particular attention to the fingers, toes, wrists, axillae, and penis. This should be continued for at least ten minutes.

He then enters the bath, which should be long enough and should contain sufficient water to permit of immersion to the neck. After steeping for fifteen minutes he scrubs himself vigorously all over with a nailbrush to open the burrows and resicles, and after the bath any vesicles which remain unruptured are opened with a surgical needle.

2. After removing the soap and drying, a liberal quantity of sulphur ointment (B.P.) is vigorously rubbed in all over the skin from the neck downwards, especially into the affected parts and the fingers, wrists, genitals, axillae, toes, and ankles.

The inunction is repeated twice daily for three days.

3. On the fourth day a second bath is given, and all the patient's clothing and bedding sterilised to prevent re-infection, even such articles as wrist-straps, strings of identity discs, gloves, etc., being included.

The majority of cases, unless there be secondary complications, are cured after three such days of treatment. Should any doubt exist, $2 \%$ B-naphthol in vaselin may be used daily for four more days.

Animal Scabies. - A number of lower animals, such as the dog, cat, horse, sheep, pig, poultry, wolf, fox, etc., are subject to a disease of the scabies type due to various acari more or less closely allied to the Sarcoptes scabiei. Certain of them, such as the scabies of the cat, rabbit, and wolf, are known to be transmissible to man. Sometimes the eruption which results is similar to that produced by the sarcoptes, but as a rule it is more diffuse and generalised, and may be scaly. 
Norwegian Scabies.-There is a type of scabies which is well-known among the lepers and vagrants in Norway which is singularly severe and associated with crusted and ulcerated lesions and with callosities about the hands and feet, and which may involve the face and scalp. This variety was believed at first to be due to neglect and secondary

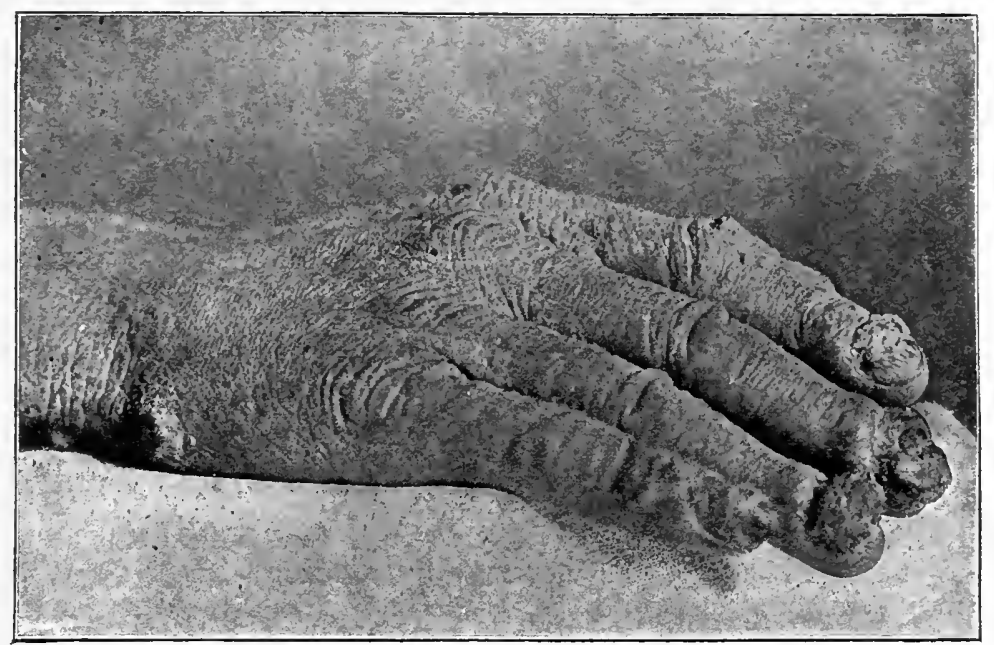

FIG. 221.-Norwegian Scabies.

(Dr. Wallace Beatty's case. Brit. Journ. Derm., I9r3.)

infection; recently it has been thought to be the result of infection with acari of animal origin, possibly from the wolf. (Fig. 22I.)

\section{REFERENCES}

Bosellini, P. N. Scabies of animal origin (Giorn. Ital. d. Mal., I905).

Bruce, J. Treatment of scabies by sulphur fumigation (Brit. Journ. Derm., I9I7, xxix. p. Ioo).

Bruce \& Hodgson. Treatment of scabies by sulphur vapour (Brit. Med. Journ., Aug. 5th, I9I6).

Crocker, Radcliffe. Canine scabies in man (Brit. Journ. Derm., i 898, x. p. 34I).

Gassmann, A. Nephritis from Balsam of Peru in cure of scabies (Münch. med. Wochen., I904, p. I345).

HARDy. Treatment of scabies (Brit. Med. \& Surg. Journ., I 889).

MacCormac, H. Skin diseases \& their treatment under war conditions (Brit. Journ. Derm., I9I7, xxix. p. I4I).

MacCormac, H., \& Small, W. The scabies problem on active service (Brit. Med. Journ., I9I7, ii. p. 384).

Schischa, A. Histology of burrows (Arch. f. Derm. u. Syph., I9oo, p. 3I3).

\section{IxODOIDEA (Ticks)}

Ticks, of which there are several species, are parasitic in animals, fowls, pigeons, and sometimes in man. They affix themselves to the 
skin by driving their probosces perpendicularly through it, proceed to suck blood for a few days, become distended to several times their ordinary size, and then drop off. This causes an urticarial lesion at the site of the puncture, which is itchy and sometimes painful, persists for several days, and may result in the transmission of diseaseas in the case of the tick (Ornithodoros moubata) which transmits the relapsing fever of tropical Africa. The female tick lays her eggs in grass or bushes and it is by coming in contact with them that the parasite is usually picked up by man. (Fig. 222.)

When the tick is discovered on the skin it should on no account be forcibly detached, as by so doing the proboscis may be broken off and left in the skin and this may lead to considerable inflammation; instead the tick should be made to let

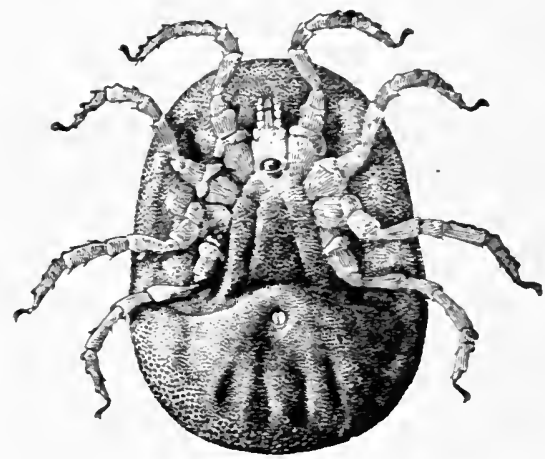

FIg. 222.-African Tick (Ornithodoros moubata, Murray).

(After Castellani and Chalmers.)

This tick transmits Relapsing Fever. The spirochaetes are found chiefly in the gut of the tick, and it is supposed that infection takes place through contamination of the wound from excreta voided by the tick whilst biting.

(British Museum. Economic Series No. 6. Stanley Hirst. I9I7.) go by dropping upon it tobacco juice or some essential oil such as clove oil. The irritation produced by the bite can be relieved by carbolic or menthol lotion.

\section{Demodex Foliculorum (Acarus folliculorum)}

Syn. : Ger., Haarbalgmilbe.

The Demodex folliculorum is a minute vermiform acarus about $0.3 \mathrm{~mm}$. in length. It has a head and eight short legs on the anterior part of the body, and a long tapering striated abdomen with a rounded tip; the male is slightly smaller than the female, and the larva has only six legs. It inhabits the sebaceous follicles of the face in man, especially where the glands are large as about the sides of the nose, the chin, and the forehead; it has been found also in those of the neck and chest. It is not uncommon, especially in coarse greasy skins, but has never been found in infants. (Fig. 223.)

It is generally believed to be a harmless denizen with no pathogenic action, but cases have been reported by De Amicis, Dubreuilh, and others, where it was associated with a fawn, or café-au-lait, pigmentation, which seemed to have its origin at the follicles which were prominent, plugged, and contained larvae, eggs, and fully developed parasites. The pigmented skin was slightly scaly and suggested Tinea versicolor, but a careful search for the fungus of that affection gave negative results. It has been suggested that the pigmentation is due 
to some toxic action exerted by the parasite, and that it is analogous to the pigmentation produced by pediculi.

The treatment consists of scrubbing the skin with soft soap and hot water, expressing the follicles with a comedo-expressor, and applying a $3 \%$ salicylic and sulphur ointment.

Other varieties of this acarus are parasitic in lower animals, such as dogs, pigs, and cattle, where they may cause considerable inflamma-

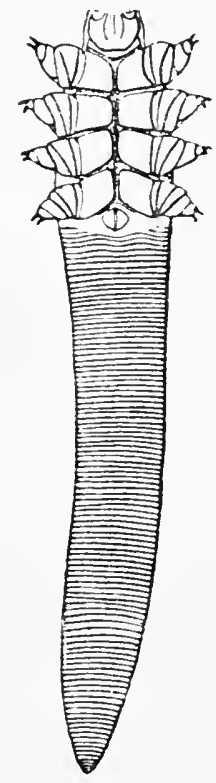

FiG. 223.-Demodex Folliculorum.

Much magnified. (After Berlese.) (British Museum. Economic Series No. 6. Stanley Hirst. I917.)

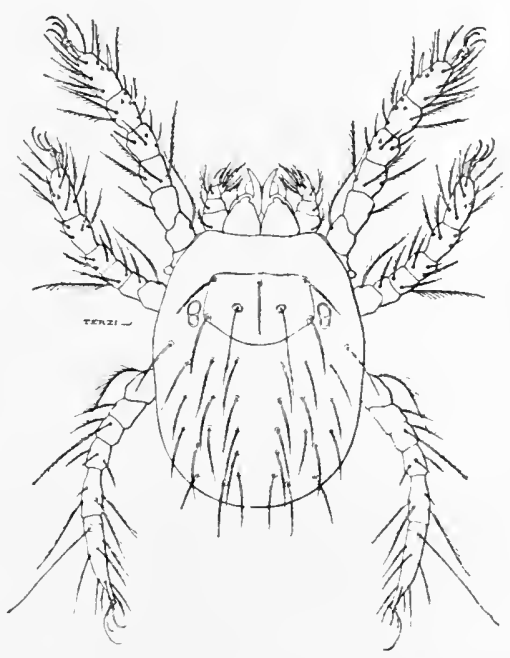

Fig. 224.- Harvest Bug (Micro. trombidium autumnale, Shaw).

The only species of "Harvest Bug " found in Great Britain.

(From British Museum. Economic Series No, 6. Stanley Hirst. $x 9^{17} 7$.)

tion. A case is on record where the demodex of a dog was transmitted to a man and caused impetiginous lesions (Lewandowski).

\section{REFERENCES}

DE Axicis. Brown patches due to Denodex folliculorum. (Giorn. Ital. delle Malat. ven. e della Pelle, I 898).

Dubreullh, W. Pigmentation due to Demodex folliculorum (Journ. de Med, de Bordeaux. Jan. I7, I90I).

Greiser, F. Demodex folliculorum of man and animals. (Archiv. f. Derm. u. Syph. Vol. xcii. p. 25, I908).

\section{Leptus Autumalis (Harvest-bug)}

This small acarus is well known in England in August and September and attacks man, and also dogs, cats, and other domestic animals, from which it may be transmitted to man. The bite produces a red swelling with itching which is apt to lead to scratching, excoriation and crusting. (Fig. 224.) 
The treatment consists of soothing the irritation by carbolic lotion ( $\mathrm{I}$ in 40), and healing the excoriations by a mild antiseptic ointment. As a preventive, dusting the skin with sulphur powder has been recommended.

\section{REFERENCE}

MacLexiar, W. Leptus autumnalis \& its skin lesions (Lancet, I905, p. $1 ; 65)$.

\section{DERMAXYSSUS AVIUM ET GalimaE (Fowl-mite)}

This insect is the well-known red mite of birds and is familiar to those who work with poultry. It is not only parasitic in fowls and

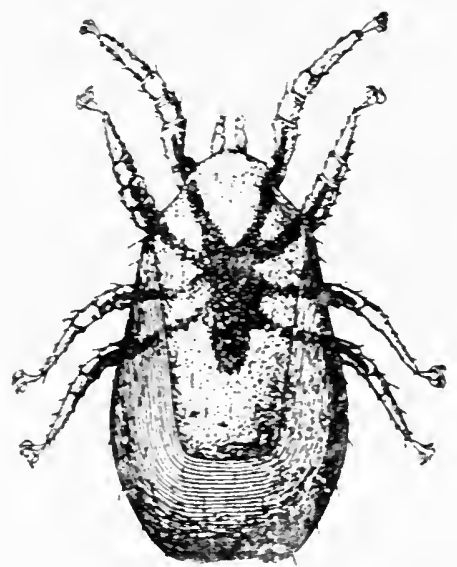

FIG. 225.-Dermanyssus Gallinae, Reid.

Parasitic on poultry, bites human beings. Female. (After Berlese.)

(British Museun. Economic Series No. 6. Stanley Hirst. 1917.)

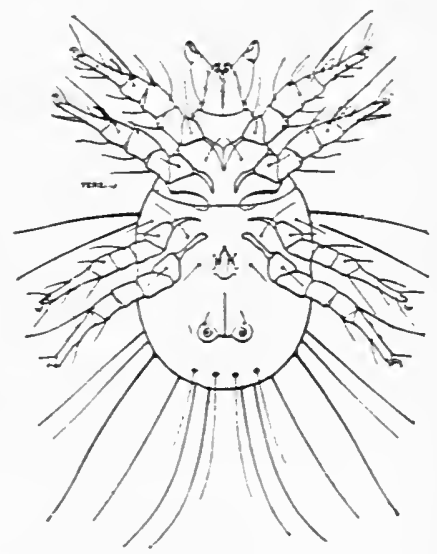

Fig. 226.-Copra Itch Acarus. Ventral aspect. (Tyroglyphus longior var. Castellanii, Hirst.)

(British Museum. Economic Series No. 6. Stanley Hirst. 19:7.

birds, but can attack the human skin and is nocturnal in its habits. It is a minute tick with an egg-shaped body, widely separated bristles, and strong mandibles. It produces a papulo-urticarial lesion which is intensely itchy and leads to scratching, excoriation, and sometimes eczematisation. The parts most liable to be attacked are the backs of the hands and forearms. (Fig. 225.)

The treatment is similar to that for the bites of harvest-bugs.

\section{TYROGLYPHCS LONGIOR (variety CASTELLANI), or COPRA-ITCH MITE}

This variety of acarus causes an exceedingly irritating form of dermatitis which has been described by Castellani in Cerlon, and cases of which have been met with in this country. It occurs in large numbers in copra, and finds a temporary habitat on the human skin, 
but does not bury itself in the epidermis like the acarus of scabies. It attacks chiefly the hands, arms, and legs, but may occur anywhere on the body, except on the face. The eruption consists of small itchy papules which become scratched and excoriated and may be secondarily inoculated with pus-cocci. It closely resembles scabies, but differs from it in the absence of burrows. It has been produced experimentally by rubbing copra dust containing the mite into the skin, and also by picking the mites out of the dust and pressing them on the skin under a covering of lint. (Fig. 226.)

The treatment consists of baths followed by the application of a $5 \%$ B-naphthol ointment which acts as an anti-pruritic and antiseptic, repelling the acarus and protecting the skin.

\section{REFERENCES}

Castellani, A. Note on copra-itch (Brit. Journ. Derm., I9r3, xxv. p. 19).

Hirst, S. Report on the mite causing the copra-itch (Brit. Journ. Derm., I9I3, xxv. p. 2I).

MacLeod, J. M. H. Acute dermatitis due to copra dust (Brit. Journ. Derm., I915, xxvii. p. I18).

\section{Pediculoides Ventricosus (Grain-itch Mite)}

Grain-itch is a well-known condition which has been recognised for the last half-century in Europe, especially in Russia and Germany,

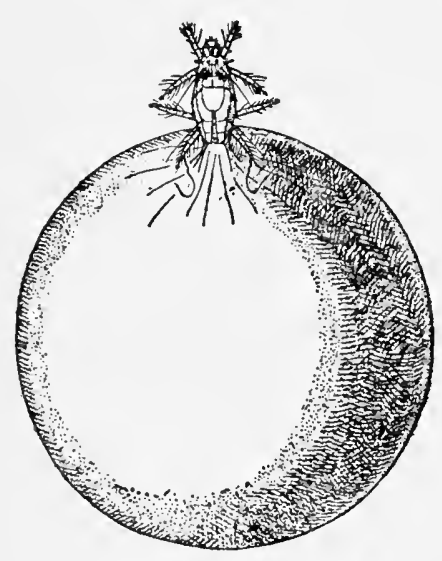

FIG. 227.-Grain-Itch Mite (Pediculoides ventricosus).

(Gravid female.) Much enlarged. (After Banks.)

(British Mruseum. Economic Series No. 6. Stanley Hirst. rgr7.)

and occasional cases of which have been met with in Great Britain. It is prevalent also in Northern Africa and various parts of America. It is due to a mite known as the Pediculoides ventricosus, the female of which has an elongated body $0.2 \mathrm{~mm}$. in length and o.or mm. in breadth. (Fig. 227.)

The eruption produced by it is papulo-vesicular in type the vesicles tending to become pustules in a few hours; it somewhat resembles papular urticaria of children. It is found chiefly in the upper parts of the body, namely the face, chest, shoulders, back, arms, and hands. It is associated with intense itching for the first day or two and occasionally with fever which may last for several days, vomiting, leucocytosis, and albuminuria.

The mite lives in the stalks of cereals and attacks men handling barley, etc., or straw in the making of mattresses or in packing. It has 
been known to be transmitted by sleeping on a new mattress in which it was present.

The treatment consists of destroying the parasite by a $5 \%$ sulphur ointment and soothing the skin with calamine lotion.

Recently a severe epidemic of it occurred in London among men who were unloading a cargo of cotton-seed (cotton-seed dermatitis). Among the seeds were numerous caterpillars of the common cotton-moth and feeding on those caterpillars were innumerable mites which were recognised by Colonel Alcock of the London School of Tropical Medicine as being the Pediculoides ventricosus. According to him the grain, straw, etc., became infected with the mite from caterpillars which, in the case of grain, are those of the grain-moth (Gelechia cerealella).

\section{REFERENCE}

Nixon, J. A. Cotton-seed dermatitis (Brit. Journ. Derm., I9I5, xxvii. p. I22).

MacLeod, J. M. H. Cotton-seed dermatitis (Brit. Journ. Derm., IgI5. xxvii. p. I26).

\section{Chilopoda (Centipedes)}

The bite of a centipede is indicated by a red spot with a haemorrhagic dark centre. Spreading from it and sometimes involving a whole limb

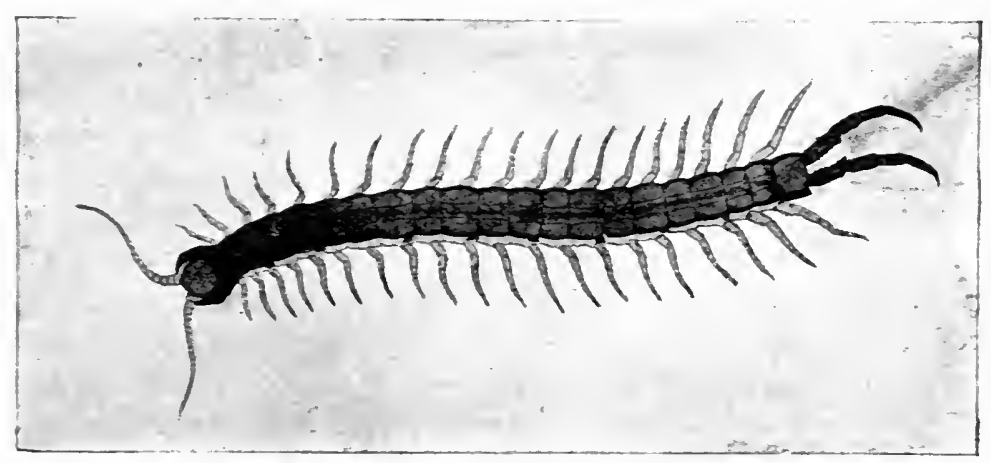

F1G. 228.-Centipede (Scolopendra morsitans).

(After Koch.)

(British Museum. Economic Series No. 6. Stanley Hirst. 1917.)

there may be oedema, lymphangitis, and swelling of the glands which drain the part, and accompanying it may be itching and sometimes pain. (Fig. 22S.)

In addition to the local symptoms there may be a general disturbance, with sickness, headache, vomiting, irregular pulse, and other signs of general toxaemia. 


\section{SIPHUNCULATA}

\section{PEDICULI (Lice)}

\section{Pediculosis.}

Syn. : Phthiriasis.

Definition: A condition of the skin which is produced by pediculi or lice.

Introduction.--Lice belong to the family of the pediculidae and are wingless insects of the order siphunculata which is closely related to hemiptera. Three varieties of lice infest the human skin which from the regions they attack are named respectively $P$. capitis, $P$. corporisor more correctly $P$. vestimentorum as the body louse has its habitat about the folds of the underclothing and only temporarily on the skinand $\mathrm{P}$. pubis or Phthirius pubis.

Pediculi have a world-wide distribution and are found not only in man but occasionally in lower animals from which they may be transmitted to man.

They attack the human skin with the object of obtaining nourishment by sucking the blood and are well-known carriers of such diseases as typhus, relapsing fever, and Trench fever; in the last instance the fever is not transmitted by the bite alone but also by the excreta - of the lice should they come in contact $¥$ ith an abraded surface. The lesions which they produce are partly the result of the injury to the skin by the penetration of the proboscis, and partly of the secretion of some toxic salivary substance the function of which is to cause a certain amount of inflammation and congestion of the part bitten so as to render it more nutritious to the louse. This toxic substance is peculiarly irritating and causes itching which leads to scratching, the inoculation of pus-cocci, and the production of a multiform secondary dermatitis characterised by inflamed patch€s, excoriations which may be roundish, irregular, or linear in shape, superficial ulceration, boils, and occasionally eczematisation. The skin, especially of the covered parts of the body, when infested with lice frequently presents a peculiar dirty appearance which has been explained as due to an impairment of nutrition caused by the toxic secretion of the lice, but is more readily accounted for by the pediculosis having occurred in individuals whose skins were ingrained with dirt owing to the unhygienic conditions in which they lived. In pediculosis of the scalp the hairs have an unhealthy appearance and lose their natural lustre.

The general health may be affected and sometimes there is a definite febrile disturbance. This has been explained as the result of toxins injected by the parasite, but such explanation is insufficient as the feverishness may occur in cases where comparatively few pediculi are present and be absent where they are numerous. It may be a coincidence. 
Parasitology.-Certain individuals are much more liable to be attacked by lice than others, their skin for some reason or another being more attractive to the insect. In appearance the scalp and body louse closely resemble one another while the $\mathrm{P}$. pubis has a distinctive shape. The P. vestimentorum is the largest and is from 2 to $5 \mathrm{~mm}$. in length and I to $2 \mathrm{~mm}$. in breadth; the P. capitis is from I to $3 \mathrm{~mm}$. in length and about I mm. in breadth. In both cases the male is considerably smaller than the female. The insects are oval in shape with a pyriform head, two prominent eyes, two antennae, mandibles with which to seize the skin, and a proboscis or sucker which is pushed into it not

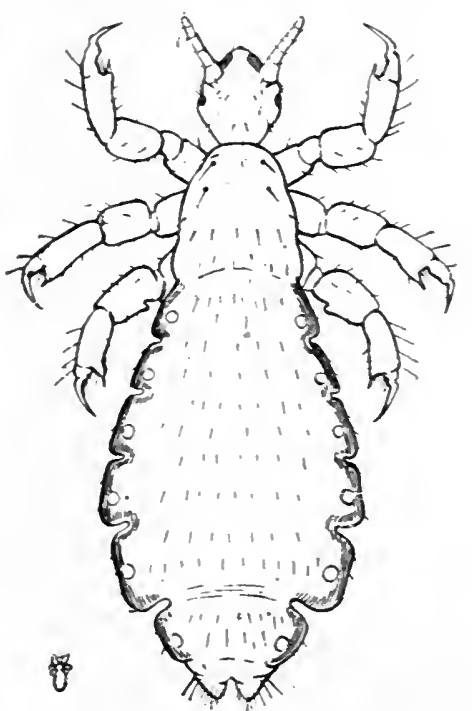

FIG. 229.-Head Louse (female). $(\times 20$.

Small figure indicates natural size.

(British Museum. Economic Series No. 2. Bruce F. Cummings. I915.)

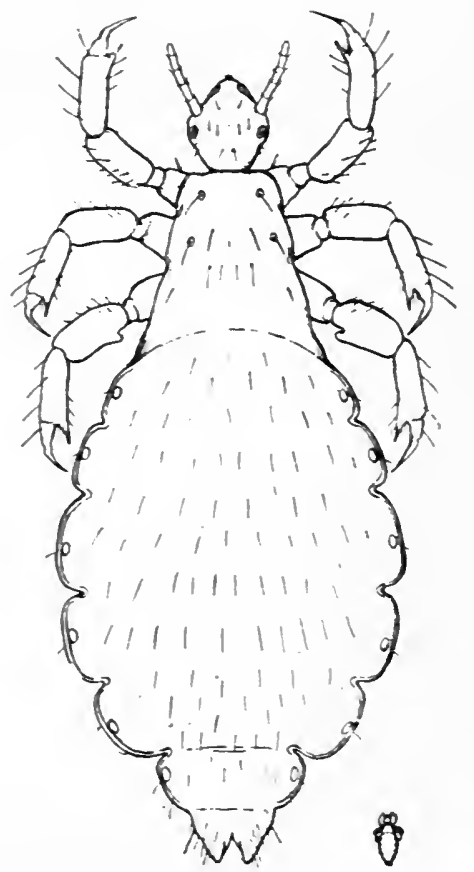

FIG. 230.-Clothes Louse (female). $(\times 20$.)

Small figure indicates natural size.

(British .luseum. Econumic Series No. 2. Bruce F. Cummings. IgI5.)

infrequently at a sweat-pore. The thorax has six legs which end in hooked extremities by which the parasite clings on to the underclothing or the hair-shaft. (Figs. 229, 230.)

Lice are dirty-greyish in colour with a darker border but become reddish when distended with blood. They vary in tint according to the colour of the skin they infest, being black in the negro skin, yellowish-brown in the Chinese, dark brown in the American Indians, and greyish in Europeans.

The P. pubis, or crab-louse, is smaller than the others and from I to $2 \mathrm{~mm}$. in length. The body is rounded in shape with no distinct 
division between the thoracic and abdominal segments and of a yellowish translucent tint. In the anterior thoracic portion are six jointed legs, while on each side of the abdomen are four or five small feet which end in bristles. (Fig. 23r.)

The reproduction of pediculi is by ova; each insect is capable of laying a large number, the $P$. capitis for instance is estimated to lay between Io and 50 eggs while the $P$. vestimentorum may lay considerably more. The ova, or nits as they are called, are oval in shape, about the size of a pin's head and easily visible to the naked eye. They are greyish in colour, have a translucent appearance, and are fixed to hairs by a chitinous gluey substance which covers about threequarters of the nit and forms a cup from which the distal end of the ovum projects. When several are present on a hair they may be

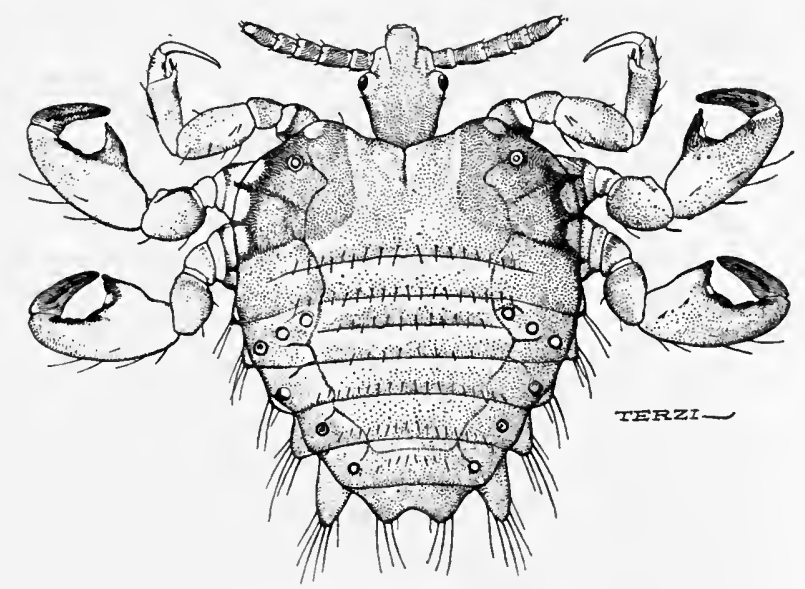

FIg. 231.-Pediculus Pubis.

situated either close together or at irregular intervals along the shaft. They are laid where the hair emerges from the follicle and get pushed further from the scalp as the hair grows. They may be attached to the hairs of the scalp, pubes, axillae, eyelashes, eyebrows, and lanugo hairs, and in the case of the $P$. vestimentorum to the hairs of the underclothing.

Under normal conditions, it has been found that eggs in the clothing take about a week to hatch, that the young pediculus reaches maturity in about a fortnight, and that the adult life is about three weeks. If the clothes are not being worn or are taken off and allowed to cool for some period every day, the eggs may not hatch for several weeks. Lice are difficult to rear apart from their natural environment but it has been found that they can exist without food for about nine days.

The clinical appearances and treatment differ according to the variety of pediculus and the region attacked. 


\section{Pediculosis Capitis}

$P$. capitis is the most common form of pediculosis in this country and is met with chiefly in children, especially in girls, and occasionally in women with long hair. The occipital region is usually attacked but the parasite may be present anywhere on the scalp and may be easily seen between the hairs. The hairs in the affected area are lustreless, powdered over with the debris of scales and crusts or matted and stuck together from dried up purulent discharge, and usually have nits attached to them. The scalp is inflamed, excoriated, and may be crusted from impetigo or superficially ulcerated, while from the constant rubbing and scratching near the hairy border the skin is liable to become

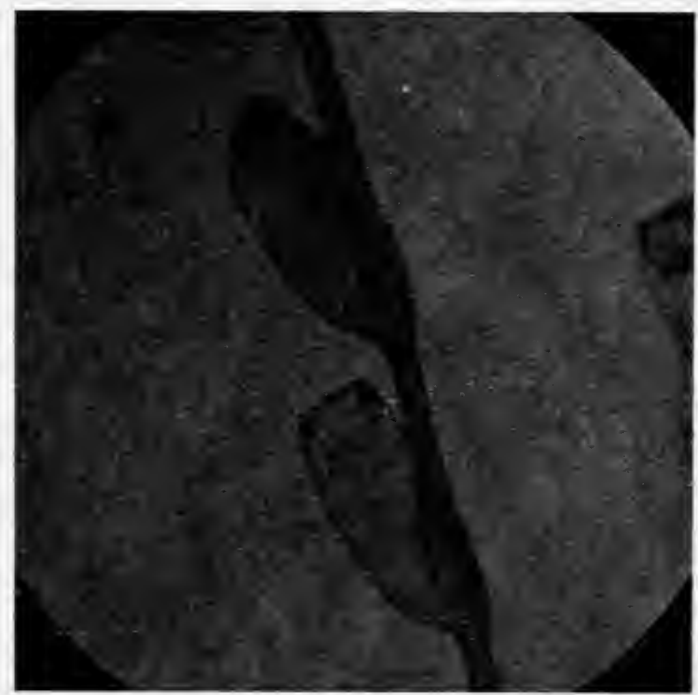

FIG. 232.-Nits on a Hair.

eczematised and crusted, especially about the nape of the neck and behind the ears. The neighbouring lymphatic glands are enlarged, particularly those of the posterior cervical region, and may be painful and in rare instances may suppurate. A peculiar musty odour emanates from the scalp which is partly due to the pediculus itself and partly to foetid crusts and discharge. (Fig. 232.)

In unkempt people with long hair the matting may be so marked as to render singularly apt the old name of " plica polonica " applied to it in Poland.

When the scalp is extensively involved and the lice have been allowed to remain unchecked for a considerable time, the general health is apt to deteriorate from septic absorption and loss of sleep due to the incessant itching.

Diagnosis.-A well-marked case of pediculosis of the scalp is easily recognised by the presence of nits on the hair-shaft, lice between the 
hairs, the impetiginous state of the scalp-especially of the occiputthe matting of the hair, and the swollen neck-glands. Small scales of dandruff may resemble nits but these are not attached to the hair and are easily brushed off.

Treatment.-The treatment is simple but must be thorough to be effective. In children where there is much matting of the hair and the scalp is extensively involved time will be saved by cutting the hair short.

The first object of the treatment is to remove the nits and to destroy the pediculi. For this purpose ordinary paraffin and turpentine are favourite applications as they dissolve the chitinous substance which attaches the nit to the hair-shaft and, at the same time, kill the pediculus. To lessen the inflammability of the paraffin it may be mixed with equal parts of olive oil. Other useful solvents and parasiticides are petrol, benzine, and xylol. Pieces of lint sufficiently large to cover the affected area are soaked in the parasiticide and applied to the scalp under a well-fitting bathing-cap, which is worn to prevent the liquid trickling down on to the face or neck and there setting up a dermatitis. This dressing is applied continuously for 6 to $\mathrm{I} 2$ hours, the scalp is then washed with soft soap and hot water, after which any nits which may remain should be removed oy a small-toothed comb. If necessary the procedure should be repeated on the following day. Where there is much crusting, the scabs should be softened and cleaned off by starch poultices.

In slight cases where the parasites are few in number, the nits may be detached by acetic acid diluted with 2 or 3 parts of water and the pediculi killed by a compress of I in IOOO perchloride of mercury solution.

Where the glabrous skin in the neighbourhood has become eczematised it must be suitably dealt with on the same lines as eczema (see page 770 ).

The enlarged cervical glands require no special treatment but readily subside when the irritation of the pediculi ceases and the secondary impetigo and pustulation are cured.

For some weeks the scalp should be carefully examined from time to time to see that no nits are left, and the hair should be washed at least once a week and combed out with a fine-toothed comb.

\section{Pediculosis Vestimentorum}

Syn. : Pediculosis corporis ; Vagabonds' Disease.

In $\mathrm{P}$. vestimentorum as the name indicates the pediculi have their habitat in the folds and seams of some garment worn next to the skin, especially where the clothing is in closest contact with the skin, as about the neck, around the waist in women, on the back, and about the genito-crural region. They are rarely found on the skin itself 


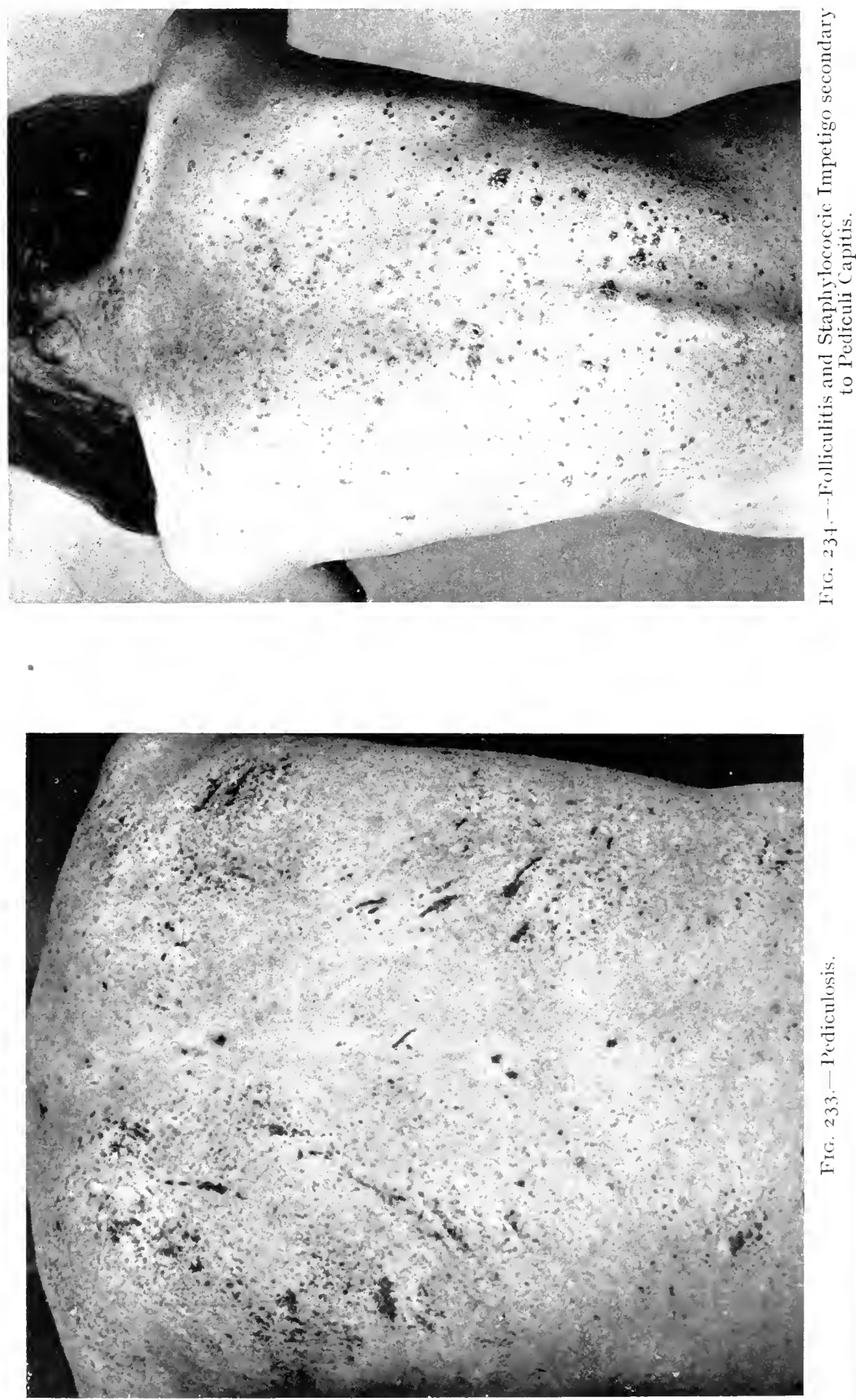
though nits may be detected on lanugo hairs and are common on the pubic hairs. (Fig. 233.)

The pediculi are anchored to the clothes by their claws and when feeding simply turn the head round to the skin and insert the proboscis. The bite thus produced takes the form of a small red macule or wheal, with a central punctum which dries into a minute black scab and persists after the inflammation has disappeared. It is accompanied by itching, which leads to scratching and the production of inflammatory patches, linear excoriations, and, from secondary infection, to superficial ulcerations, pustules, and occasionally boils. From the rubbing to which the skin is subjected it is liable to become coarse, thickened, and pigmented, especially in the upper part of the back, and to assume a dirty appearance. In sensitive skins the presence of lice may give rise to a condition of extreme irritability in which slight irritation and scratching may lead to the formation of wheals. (Fig. 234.)

It is most common in elderly people and rare in children. It is met with chiefly in the poorer classes, tramps, bedridden people, and those who live in crowded unhygienic surroundings. It is invariably present when large numbers of men are massed together, as in armies on active service.

Diagnosis.-It is easily recognised by the itching, the red puncta, the pigmentation, and the irregularly distributed linear excoriations. The absence of lesions upon the hands and feet differentiates it from scabies. Where there is any doubt the underclothes should be carefully examined for lice or nits, especially about the seams.

Treatment.-The treatment consists of warm baths, lathering the skin with soft soap, the removal of nits attached to the lanugo hairs, and the destruction of lice by the application of vaselin to which $25 \%$ of petrol has been added, by a soap solution containing $2 \%$ trichlorethylene, or by spraying the affected parts of the body with paraffin or with a 3 to $5 \%$ solution of anisol (methyl. phenate) in weak alcohol.

As a preventive, the skin and underclothing should be dusted with a parasiticide powder of which the most useful contain guaicol, iodoform, naphthalin, camphor, pyrethrum, menthol, the so-called N.C.I. insecticide which is composed of naphthalin, creosote, and iodoform, or pyrethrum powder to which has been added $10 \%$ of naphthalin and Io \% of camphor. Sulphur powder is of little use in this connection as it is a feeble insecticide.

Another useful preventive is to wear a thin buttermuslin undergarment which has been impregnated with an insecticide, such as a r $\%$ solution of naphthalin in petrol or benzine.

Where there is much itching relief may be obtained by the use of carbolic lotion, $\mathrm{I}$ in 40 , or of a powder containing menthol.

The underclothing must be disinfected, either by baking it at a temperature of about $260^{\circ} \mathrm{F}$., turning it inside out and exposing it to a jet of steam-especially at the seams-ironing it over with a hot iron, 
or immersing it in a petrol bath or in a $5 \%$ solution of cyllin. Discarded infected clothes should be burned.

NotE.-With the object of disinfecting clothes on a large scale, a simple, cheap, but absolutely efficacious, method was devised by Lt.-Col. G. F. Stammers (R.A.M.C.), and employed by Colonel William Hunter (A.M.S.) in I9I5 in Serbia, during the great epidemics of typhus and relapsing fever, and subsequently in Egypt. This consisted of what is known as the "barrel disinfector" and the "current steam railway van disinfector."

The barrel disinfector was an ordinary wine or water barrel, with one large central hole through the bottom and a circle of five or six holes around it, which stood on a circular boiler of iron. To prevent the escape of steam a narrow sausage ring filled with sand was placed between the boiler and the bottom of the barrel. The barrel was provided with a wooden lid which retarded the escape of the steam. By this means a temperature of about $212^{\circ} \mathrm{F}$. was obtained, and the clothing was disinfected in about an hour.

The principle of the current steam van disinfector was the discharge of a volume of steam from a railway engine into one or two ordinary railway vans which had been suitably adapted. This train could proceed along the line to wherever it was most required, and by means of it many thousands of articles could be disinfected daily.

\section{Pediculosis Pubis}

This variety of pediculosis is due to the P. pubis, Phthirius pubis, or crab-louse. It is characterised by itching about the pubes and genitalia, inflammation, excoriation, and sometimes pustulation. The itching is not confined to the pubic region but may occur reflexly in the neighbouring skin, and leads to scratching and sometimes eczematisation of the lower part of the abdomen or upper part of the thighs. In skins hardened from exposure or insufficient cleanliness it may be comparatively slight, but if they are sensitive or delicate it may be severe and incessant.

The louse is generally found clinging to a pubic hair with its head buried in the opening of a follicle, and the nits may be detected attached to the hairs. The lice may be present not only in the pubes but also in the axillae, about the chest and limbs in a hairy person, in the beard, and occasionally in the eyebrows and eyelashes. When present among the eyelashes they give rise to an acute inflammatory condition like eczema and along the lower lids to a peculiar reddish deposit like specks of rust which is the excrement of the pediculus.

In connection with $\mathrm{P}$. pubis peculiar small pigmented patches about the size of a pea are generally noted. These are grey or bluish in tinge, do not disappear on pressure with a diascope, and are known as Maculae caeruleae. They occur chiefly on the sides of the trunk and inner surface of the arms and thighs and are especially well-marked in individuals with fair skins. They are not due, as was once supposed, to blood-pigment but are believed to be the result of a pigment which the parasites secrete into the skin while feeding and which is believed 
to have an anaesthetic action as in cases where the maculae are numerous the itching is comparatively slight.

The affection occurs mainly in adults and is contracted in numerous ways, but chiefly by sexual intercourse. There being no body hairs in children it is only met with in them about the evebrows and eyelashes.

Diagnosis.- The diagnosis rests with the discovery of the nits or of the actual parasite. It should always be suspected when an irritating condition of the pubes or axilla is associated with excoriations and greyish-blue macules about the trunk.

Treatment.--To kill the parasite the affected region should be saturated with petrol, benzine, xylol, or paraffin, and the nits carefully removed. The skin should then be thoroughly washed with soft soap and hot water and a $2 \%$ ammoniated mercury ointment applied daily as long as it is necessary.

Where the eyelids are attacked the nits and pediculi should be removed with forceps and dilute yellow oxide of mercury ointment applied along the lids.

\section{REFERENCES}

BACOT, A. W. The louse problem (Brit. Med. Journ., I9I7, p. 296).

BRUCE, D. Trench fever-a louse-borne disease (Rep. of War Office Trench Fever Commission. Trans. Soc. Trop. Med. \& Hyg., I9I8, xi. p. I). Cummisgs, Bruce. The louse \& its relation to disease (British Museum, Economic Series, No. 2, I9I5).

Guxx, J. A. The prevention of pediculosis (Brit. Med. Journ., I9I7, p. 579).

Huxrer, W. Prevention \& arrest of lice-borne diseases by new methods of disinfection (Lancet, I9 I8, cxcv. p. 347).

JAmiesos, W. A. Treatment of Pediculosis vestimentorum (Brit. Journ. Derm., I 895, vii. p. 248).

LABBÉ, H. Treatment of phthiriasis (Journ. de Med. et de Chir. prat., June Ioth, I9I5).

Oppexherm. Pigment formed by Pediculosis pubis (Arch. f. Derm. u. Syph., I90I, p. 233).

Pelier. Pigment in Maculae caeruleae (Monats. f. prakt. Derm., I909, p. 56).

Sisor, A. Treatment of phthiriasis (Journ. de Med. et de Chir. prat., Dec. Ioth, I9I6).

\section{HEMIPTERA}

\section{Cimex Lectularies (Bed-bug)}

The bed-bug has a world-wide distribution and some variety of it is found in most countries. In Great Britain the common species is the $\mathrm{C}$. lectularius, but in warm climates it is the $\mathrm{C}$. rotundatus which is the most prevalent. The bug lives in cracks in walls, floors, ard boardings, and lurks about old wooden beds and furniture. It hides during the day and migrates on to the human skin at night in search of nourishment. It is about $5 \mathrm{~mm}$. in length and $3 \mathrm{~mm}$. in breadth, 
of a rusty-brownish colour, with a broad short head and a four-jointed proboscis containing four piercing stylets, two eyes, two antennae, and six legs ; both the male and female attack the skin, the male being the smaller of the two. Bugs have a peculiar offensive smell, which is due to a volatile liquid secreted by glands situated at the sides of the abdomen. (Fig. 235.)

On puncturing the skin with its stylets a small quantity of an irritating salivary fluid is injected, which gives rise to an urticarial lesion with a central bleeding point which develops into a haemo-

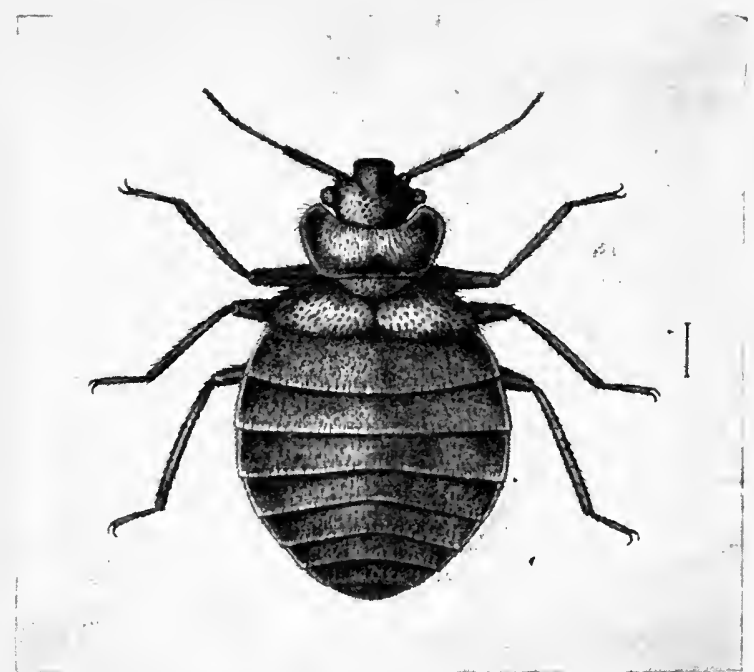

Fig. 235.-Common Bed-bug (female). Dorsal view.

The line drawn by the sicte indicates the natural size.

(British Museum Economic Series No. 5. Bruce F. Cummings. 19r7.)

rrhagic scab and lasts for days or even weeks after the surrounding inflammation has subsided. Occasionally the lesions have a purpuric appearance.

The bites, of which a number are usually caused by one insect, are distributed irregularly over the skin and are most frequent about the legs and ankles. The degree of irritation caused by them varies in different individuals; in delicate skins it may be considerable and lasting, while in the dirty and vagrant classes the skin may be so resistant and accustomed to the attacks of such pests that the bites are scarcely noticed.

Treatment.-The treatment consists of the application of a $10 \%$ menthol lotion in spirit, or carbolic lotion I in 40.

As a preventive guaicol powder should be dusted between the sheets, and cracks in the walls and other situations which may harbour the insect should be filled up or sprayed with kerosene oil. 


\section{DIPTERA}

\section{Pulex IRritass (Common flea)}

The common flea has a wide distribution in Europe where it appears to have been originally endemic and from whence it spread to the Orient and America. It is temporarily parasitic on man and may attack also domestic animals such as dogs and cats. As in the case of many other insects the male is much smaller than the female. When not present on its host it inhabits cracks and crevices in the floor and it is there that the female lays her eggs.

Fleas are important, not only from the irritation caused by their bites, but also as carriers of disease, as in the case of the rat flea (Xenopsylla cheopis) which readily attacks human beings and is responsible for the spread of plague. (Fig. 236.)

The familiar flea-bite consists of a red spot with a central haemorrhagic punctum which remains after the erythema has gone. It may be urticarial occasionally or it may be purpuric in emaciated people of the vagrant class and in dependent parts, and may even suggest purpura. The itching is due to an irritating salivary secretion ejected by the flea which retards the coagulation of the blood. It varies in different skins, and in one may be so slight

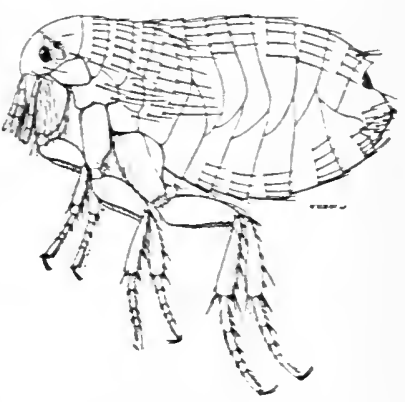

FIG. 236.--Human Flea (Female)

(Pulex irritans. $\times 25$ ).

(From British Museum. Economic Series No. 3. James Waterston. I916.) as to be almost unnoticed while in another it is so intense as to cause furious scratching and excoriation. Certain skins are more enticing to fleas than others, and while some individuals are practically immune to their attacks others invariably attract thern.

Treatment. - The treatment is similar to that for the bites of bedbugs and consists of soothing the skin by carbolic lotion, I in fo, and dusting it with powdered naphthalin or camphor as a preventive. In women liable to flea bites, a muslin bag pinned somewhere about the undergarments and containing pyrethrum powder or camphor acts as a protective.

\section{MUSCIDAE}

\section{MrIASIS}

Derication : a fly:

Definition: Myiasis is a comprehensive name applied to diseases caused by the larvae of certain flies.

The large number of cases of myiasis which liave been reported in different countries both in tropical and temperate zones show that it has a wide distribution and that, while it is most frequent in warm 
countries, it may be met with also in colder northern latitudes, such as in Norway and the Shetland Islands. Though in certain instances the causal fly is known, in many cases it has yet to be identified.

The flies deposit their eggs either on the skin or on the mucous membrane of the natural orifices, especially of the nose and ear, where they hatch in the course of a day or two and set free larvae which bore down and, according as they become situated in the skin or subcutaneous tissue, produce a dermal or a subcutaneous myiasis with more or less

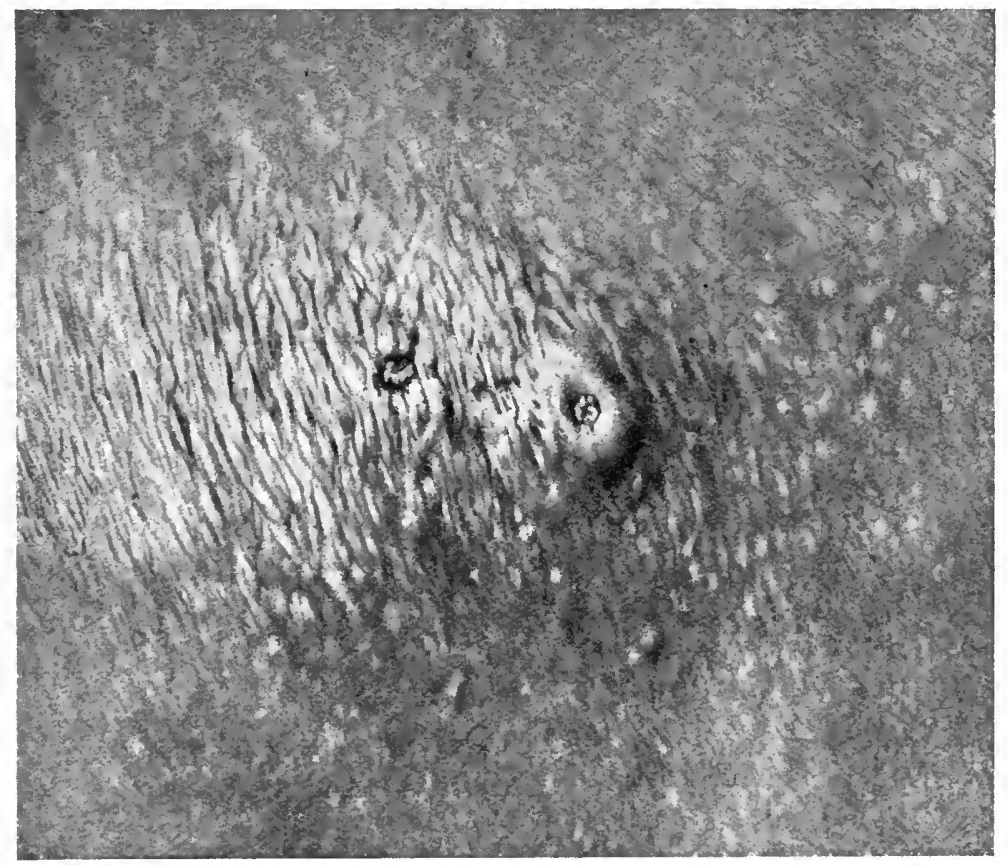

FIG. 237.-Myiasis.

Shows larvae of Dermatobia Hominis in the skin.

(From Dr. Louis Sambon.)

severe morbid changes. The best known of the pathogenic larvae are the "screw-worm," the Ver du Cayor, and the Ver Macaque. (Fig. 237.)

The so-called "screw-worm" is the larval stage of a blue-bottle fly (Musca macellaria) which occurs principally in South America and in the southern United States from July to October, but is met with also in the West Indies and in Cochin China. The fly attacks man as well as domestic animals and deposits its eggs on the skin, in wounds, or on the mucosa of the nose or auditory canal of natives sleeping in the open air. The larva is white in colour, about $\mathrm{I} 4 \mathrm{~mm}$. in length, and has a screw-like appearance owing to the presence on its surface of irregular rings of small horny spines. On the skin it produces comparatively little harm beyond giving rise to a small boil, but if it hatch in wounds or in the nose or ear it tends to burrow down through mucous membrane, 
muscle, and cartilage, till it reaches the bone. This process may produce grave inflammatory changes, with the formation either of a foul crateriform ulcer in the centre of which numbers of maggots may be seen wriggling about, or of fungating masses moist with an offensive discharge like rapidly growing epithelioma, and may be followed by a fatal issue either from septicaemia or from the penetration of larvae into the brain and the production of meningitis. (Fig. 238.)

The Ver du Cayor is the larva of the Cordylobia anthropophaga or "Tumbu fly," and is the cause of the African dermal myiasis ; it is about $\mathrm{I} 2 \mathrm{~mm}$. in length and composed of twelve segments. It has a wide distribution but prevails chiefly in the Cayor district of Senegambia

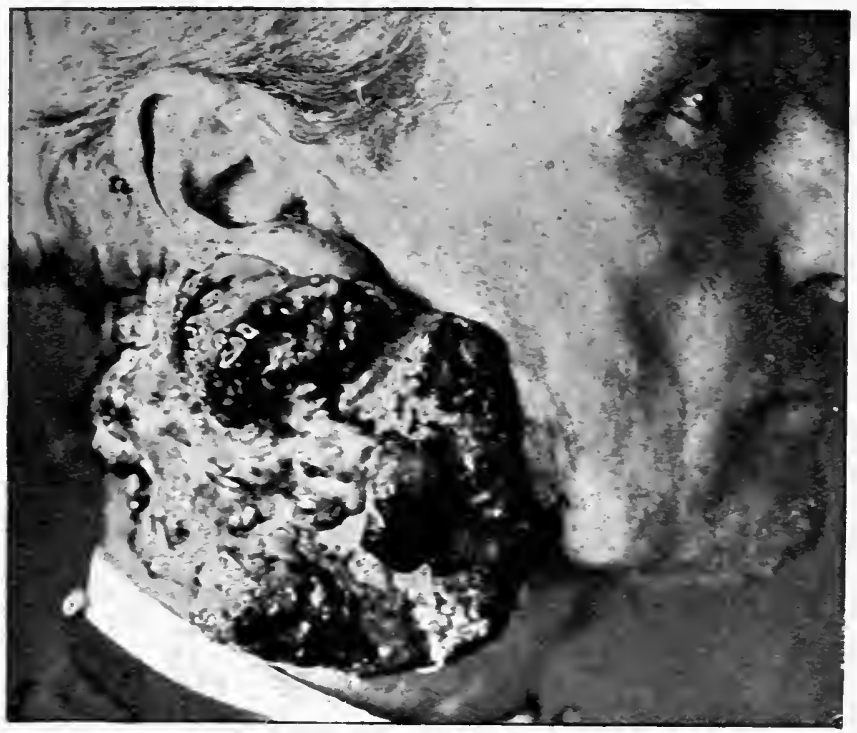

FIG. 238.-External Myiasis.

(Dr. J. M. Swan's case. Journ. Trop. Med. and Hyg., Igro.)

towards the end of the dry season. The female fly is provided with a small proboscis by which the skin of man and of various domestic animals is pierced. According to some observers the eggs are deposited in the abrasion thus produced, while others believe that they are laid on the ground or clothing and hatch there, the larvae reaching the skin subsequently. By whaterer means they gain entrance they give rise to a suppurative lesion in the nature of a painful furuncle, about threequarters of an inch in diameter, which matures in about a week and breaks, forming a central opening in which the grevish-white larvae may be detected. In Europeans the Tumbu fly abscess is frequently present on the buttocks and thighs, possibly through infection in using a latrine, but it may occur in other situations, such as the axillae and arm.

The Ver Macaque is the larva of a gad-fly (Dermatobia craniventris) which is found chiefly in Central America, Mexico, and Brazil, and is 
the cause of American dermal myiasis. This fly has two larval stages, the first of which is club-shaped, white in colour, and known as the "Ver Macaque," while the second is worm-shaped and called "Torcel." When the larvae are present in the skin they cause a painful abscess of a deep red colour which may reach the size of a pigeon's egg and is known as a "gad-fly boil." This readily breaks down and discharges a sero-purulent fluid containing the black faeces of the larvae and a

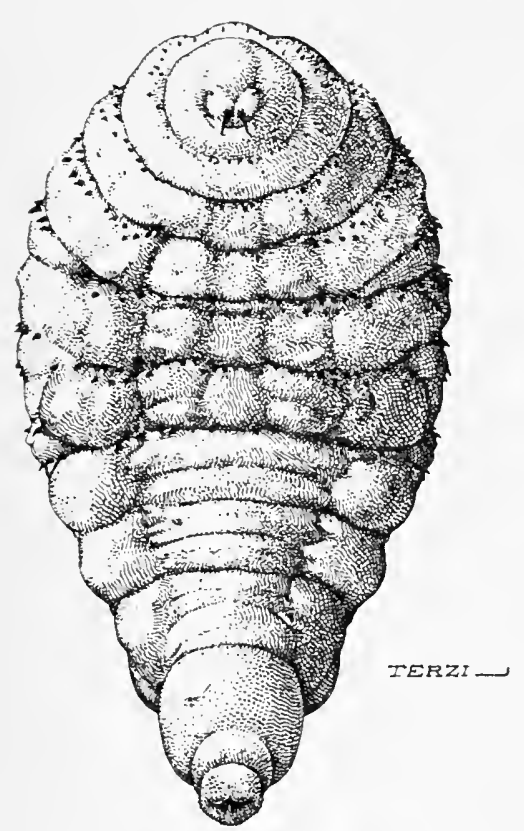

Fig. 239.-Larva of Dermatobia Hominis.

(From Dr. Louis Sambon.) few larvae which, on reaching the ground, develop into pupae and finally become imagos. (Fig. 239.)

Treatment.-The treatment, to be successful, must be prompt and thorough. Where the larvae can be seen in the centre of the boil, they should be seized with a pair of forceps and extracted. The diseased cavity should then be irrigated with I in 20 carbolic solution, or swabbed out with pure benzine. Should the larvae have reached the frontal sinus, inhalations of chloroform have been recommended to kill them, and the cavity should be opened up to allow free irrigation and the expulsion of the parasites.

Boils resulting from various larvae should be treated on ordi. nary surgical principles, freely opened, the contents expressed, and the cavity thoroughly irrigated.

In South America a favourite treatment is to allow tobacco juice to trickle into the central opening of the boil; this causes the larvae to protrude, when they can be easily expressed or extracted.

\section{REFERENCES}

Harrison, J. H. Case of Myiasis (Journ. Trop. Med. \& Hyg., I908, p. 305). Swan, T. II. Two cases of external myiasis (Journ. Trop. Med. \& Hyg., xiii. p. I).

\section{Creeping Eruption}

Syn. : Larva migrans. Myiasis linearis.

Larva migrans is a generic name which has been applied to the larvae of certain flies, such as Gastrophilus, Hypodermia bovis, etc., which burrow into the skin in a linear fashion and give rise to the so-called "creeping eruption." The condition occurs in several parts 
of Eastern Europe, especially in Southern Russia, and in Africa, Asia, and South America. It is characterised by a narrow red line, from $\frac{1}{4}$ to $\frac{1}{4}$ anch in breadth, scarcely raised above the surface, sometimes straight but as a rule curved or sinuous, which may occupy a small area or extend for a long distance on the trunk or limbs. It advances an inch or two every day, indicating the progress of the larva in the skin or subcutaneous tissue, but at the same time the opposite end gradually fades so that the line persists about the same length throughout. Its progress is associated with intense itching and scratching, and sometimes with secondary inoculation of pus-cocci. By pressure with a piece of glass the larva may sometimes be detected as a dark brownish speck about half an inch beyond the advancing end. If the larva be not destroyed it may travel actively for months or even vears. Looss has observed similar clinical appearances produced in the skin by the larvae of ankylostoma. (Fig. 240.)

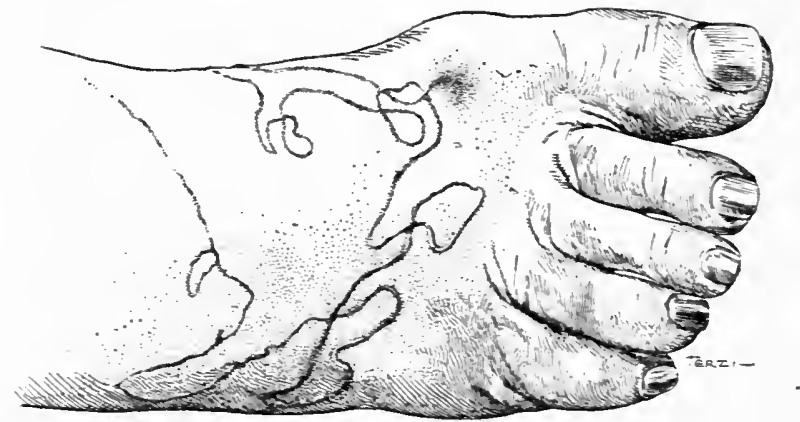

Fig. 240.-Mrviasis Linearis or Creeping Eruption.

Treatment.-The treatment is unsatisfactory unless the larva be removed or destroyed, which is by no means easy. If its exact position can be determined, an incision may be made over it and it may be extracted by forceps, and the carity irrigated with carbolic acid; or it may be got rid of by excising the small piece of skin berond the anterior end of the line which contains it ; or it may be destroyed by a thermo-cautery, after the manner of the native women of Arabia who employ a red-hot wire. Other simpler but less effective methods consist of hypodermic injections of cocain, chloroform, or carbolic acid, in the situation of the larvae. A form of treatment which has been found to be successful is freezing the progressing end of the line for about $I_{2}^{\frac{1}{2}}$ inches for one minute with solid carbon dioxide.

\section{REFERENCES}

Crocker, Radcliffe. Larva migrans (Diseases of the Skin, I905, ii. p. I309).

Castellani \& Chaluers. Larva migrans (Manual of Tropical Medicine, I9I3, p. I $\left._{5} S_{3}\right)$. 


\section{Culicidae (Mosquitoes and Gnats)}

The lesions due to the bites of gnats and mosquitoes are the result of an irritating substance which is injected under the skin by the insect before it begins to suck blood. It is probably salivary in character and is so irritating that the part bitten becomes itchy and inflamed and may rise up in the form of a wheal or even a blister, while occasionally a

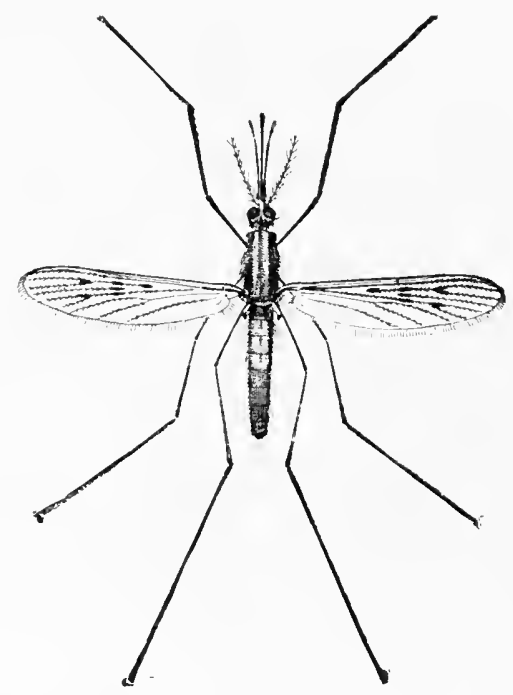

FIG. 24I.-Mosquito (Anopheles maculipennis. $\times 4$ ).

It is the carrier of malaria. Another mosquito (Culex fatigans) is the chief carrier of Elephantiasis.

(British Nluseum. Economic Series No. 4. 1. W. Edwards. 19r6.)

nodule forms which may become inoculated from scratching, may give rise to an open sore, and even lead to lymphangitis. (Fig. 24I.)

Treatment.-The treatment consists of the application of $I$ in 40 carbolic lotion or dilute solutions of ammonia such as Scrubb's ammonia; if there is much oedema and swelling it may be reduced by compresses of lead lotion or boric lotion.

\section{HYMENOPTERA}

\section{Afidae (Bees and Wasps)}

The sting of the bee or wasp is characterised by a painful red swelling, which is usually localised to the part stung and lasts about four hours. When the sting is on the face, especially where the tissues are lax as about the eyelids and the lips, it may result in oedema and swelling, sometimes alarming in degree and extending over a considerable area. 
In elderly people the sting may be associated with severe general symptoms, such as faintness, shivering, difficulty in breathing, a rise of temperature, and in rare instances a general eruption of an urticarial or morbiliform type. This is partly due to shock and partly to toxaemia from diffusion of the renom in the general circulation. Occasional instances are on record where stings have led to a fatal issue, the patient becoming pallid, collapsed, and delirious before death.

Treatment.- The treatment of stings consists of the removal of the sting when present, and the application of some soothing lotion or cream, such as calamine cream with the addition of $2^{\circ} \%$ carbolic acid, carbolic lotion ( $\mathrm{I}$ in 40 ), lead lotion, or a $2 \%$ menthol lotion in spirit. Should general symptoms supervene, stimulants, such as brandy or injections of strychnine, should be ordered.

\section{FORMICIDAE (Ants)}

Ants, especially the large tropical ants, are capable of giving a poisonous bite, which results in painful urticarial or oedematous lesions sometimes accompanied by general symptoms somewhat similar to those from the sting of a bee or a wasp.

Treatment.- The treatment is the same as that for stings of bees or wasps.

In the tropics ants are liable to infest beds and preventive measures should be taken, such as dusting the sheets with sulphur or camphor.

\section{LEPIDOPTERA}

\section{Caterpillars of Certain Butterfies}

There are several varieties of caterpillars-especially in the tropics - which when they come in contact with the skin cause a painful urticarial dermatitis; this may be so acute as to resemble erysipelas and may be accompanied by severe general symptoms.

These so-called "stinging caterpillars" are met with chiefly in Ceylon, India, and South America. They are covered with small hairs which have some irritating substance about them, and these by penetrating the skin give rise to the dermatitis.

Treatment.-The treatment is similar to that for the stings of bees.

\section{COLEOPTERA}

\section{Blister Beeties}

Various kinds of beetles produce resicles or bullae when they come in contact with the skin-a peculiarity which has been known from ancient times. 
In I9I2 Da Silva described an outbreak of dermatitis accompanied by ulceration, which occurred among the peasants in Bahia and which was traced to an insect of the order Coleoptera (beetles).

In I9I5 a somewhat similar epidemic was recorded from the Belgian Congo by Rodhain and Houssiau as a " seasonal bullous dermatitis."

In I9I7 Chalmers and King described an epidemic which occurred in Khartoum during August each year and was met with chiefly among Europeans. It was characterised by the sudden appearance of bullae or vesicles, surrounded by an inflammatory halo, single or grouped together, and with no tendency to bilateral symmetry, and affected persons in good health who, as a rule, were unable to assign any cause for the eruption. It was found to be due to beetles of the genus Epicauta and of the family Cantharidae, which reached the skin by crawling up the sleeve, up the trouser, or down the neck, and produced the lesions by excreting a blistering fluid, especially from the joints of their legs. During the month of August these blister beetles can be seen in Khartoum in the early morning on herbs, bushes, or trees, and at night, being attracted by the artificial light, they may invade the houses.

The treatment consists of pricking the blister and applying $I$ in 40 . carbolic lotion.

\section{REFERENCES}

Chalmers, A. J., \& King, H. Blister Beetles (New Orleans Med. \& Surg. Journ., 19I7, No. 5).

DA Silva (Arch. de Parasit., I9I2, xv. p. 43I).

Rodhain \& Houssiau (Bull. de la Soc. de Path. Exot., I9I5, viii. p. 587). 


\section{CHAPTER XXI}

\section{PRURITUS}

Derication: Prurire: to itcn.

Definition: Pruritus or itching is a cutaneous subjective symptom producing a desire to scratch.

Introduction.-Pruritus is a symptom and not a disease, hence it might seem to be unnecessary to describe it under a special heading. It is, however, a symptom of such importance and occurs so frequently. without any risible cutaneous lesion that it merits independent consideration. Under the heading of pruritus are grouped sereral different sensations, such as pricking, tingling, formication or the sensation of insects crawling over the skin, and itching proper.

The tendency to indiscriminate use of the terms "pruritus" and " prurigo," with their adjectives pruritic and pruriginous, as if they were synonymous has led to an unfortunate confusion; the two should be clearly differentiated-pruritus is a subjective simptom, prurigo is a papular eruption accompanied by itching.

For convenience of description the subject will be discussed under the following headings :

(I.) Symptomatic pruritus, symptomatic of different cutaneous affections.

(II.) Essential pruritus, which is not preceded by any obrious skin disease.
(I) General.
(2) Local.

Scratching and its effects.

Pruritus ani.

Pruritus vulvae.

\section{Pruritus Symptomatic of Different CrTANEOUS AFFECTIONS}

Itching occurs in a large number of skin affections. In many instances it is the symptom which first attracts the patient's attention to his disease, and but for it certain skin diseases would be negligible. It is met with in erythema, urticaria, eczema, Lichen planus, Herpes zoster, Dermatitis herpetiformis, Myoma cutis, sclerodermia, Mrcosis 
fungoides, parasitic diseases such as scabies and ringworm of the glabrous shin, and at some time or other in most forms of dermatitis.

It varies in degree according to the extent of the eruption and the nervous stability and temperament of the individual. In one case it may be mild and bearable; in another it may be severe and maddening, may lead to scratching which aggravates the underlying disease, and may result in complications from the secondary inoculation of pyogenic micro-organisms.

\section{Essential or Primary Pruritus not Preceded by Cutaneous Lesions}

Essential pruritus may be general or local.

(I) General.--Pruritus may be generalised over the whole cutancous surface, but it is rare for it to affect the entire surface equally and as a rule it is especially severe in certain areas which vary in distribution in different cases and at different times in the same case. The most careful examination may fail to reveal any pathological change in the skin which could account for it, the only objective symptoms present being the result of scratching. Rubbing and scratching may also extend pruritic areas, produce new ones, or transform a localised into a diffuse pruritus.

As in symptomatic pruritus, the itching is rarely continuous but is subject to paroxysms or crises, and sometimes to complete intermissions. The paroxysms may come on periodically from some definite cause, such as exertion, the taking of food, or the warmth of bed ; or they may be aperiodical and due to emotional disturbances, auto-intoxication, or causes of which the patient is ignorant. They may be transient lasting only a few minutes, sufficiently bearable for the patient to refrain from scratching provided he has reached years of discretion, and so slight as not to interfere with sleep. Should scratching be indulged in, however, either intentionally or reflexly during sleep, even mild degrees of pruritus may be aggravated and sleep prevented. If the paroxysms be of moderate severity but last for more than a few minutes, self-restraint generally breaks down and even the most phlegmatic and strong-willed may give way to scratching. In severe cases the paroxysms may last for an hour or more and the pain from scratching with the nails or with a rough instrument until the skin is lacerated, may be more tolerable than the incessant itching. When they are frequent and prevent sleep the patient becomes worn-out, irritable, emotional, hysterical-a mental and physical wreck-and may even seek relief in suicide.

Scratching and rubbing give temporary relief by numbing the sensory cutaneous nerres, and causing a dilatation of the capillaries and an exudation of serum. This produces a cessation of itching and a feeling of relief and comfort almost amounting to well-bcing, but 
these effects are transitory and are followed later by nervous prostration. To this sensory cycle the French have applied the graphic name of "Onanism pruritique." The relief so obtained may last for a sufficient time to enable the patient to get to sleep, but too frequently is followed by a paroxysm more serere than the preceding one.

The severity of the itching is largely dependent on the amount of scratching to which the shin is subjected. Where the pruritus is widely generalised the most itchy parts are invariably those readily accessible, and so much is this the rule that it is sometimes possible to tell if the patient be left-handed by the vigour with which the parts most easily reached by that hand have been scratched.

(2) Local.--Pruritus may be limited to certain regions or situations of the body and is then much more bearable than when it is generalised. It may be confined to one side, or to one limb, in which case it is the result of a disturbance of the sensory nerves supplying that region. The most common sites are the palms, soles, auricles, nose, mouth, tongue, anus and genitalia, and the hairy regions.

Palms and Soles.-Pruritus of the palms and soles is not infrequent in elderly people with dry skins; it is usually symmetrical, and, when it affects the soles, often involves the interdigital clefts. It is generally believed to be connected in some way with rheumatism or gout, but of this there is no definite proof and it may occur in individuals without a trace of either affection. It is sometimes present in those who suffer from a feeble peripheral circulation with cold hands and feet, or hyperidrosis.

Auricle.-It may occur in one or both auricles either in the lobe, or in the auditory canal. When it is present about the external auditory meatus it is generally due to an accumulation of wax.

Nose.-Itching and pricking sensations occasionally occur in the nasal mucosa just before the onset of a coryza, as a premonitory sign of an attack of spasmodic asthma, in connection with polypi, deflected septum, or other deformities causing obstruction to the circulation, or caused reflexly by irritation in distant parts, for example by thread-worms in the rectum in children.

Mouth and Tongue. - The buccal mucosa may be the seat of itching which may involve the lips, tongue, and the inside of the cheeks, and may occur in association with naso-pharyngeal catarrh and occasionally with toothache.

Anus and genitalia.-Pruritus in the ano-perineal region is exceedingly common. It may be present at the anus, at the orifice of the urethra, or about the scrotum, vulva and vagina. At the urethral orifice it is generally the result of urinary troubles, cystitis, diabetes, or gonorrhoea. Pruritus ani and Pruritus rulvae are so common and capable of producing such distressing symptoms that it will be advisable to consider them separately (see pages 644,649 ).

Hairy regions.-In many individuals pruritus tends to occur in the hairy regions of the body where it is known as "Trichomanie." It is 
often pronounced about puberty when the hair-growth is at its maximum. It may attack the beard, the margins of the eyelids, the axillae, the pubes, and, in persons with excessive hair-growth, the chest and arms. Apart from local causes, such as the presence of pediculi or of some form of dermatitis, it has been explained as the result of the rich innervation of the pilo-sebaceous follicles.

\section{Pathogenesis AND Etiology}

Pruritus is the result of a functional derangement of the nerves of common sensibility, in which the elcmentary sensations are exaggerated or perverted. These sensory nerves when they reach the skin form a sub-epithelial plexus, consisting chiefly of nerve fibres which have lost their medullary sheaths, and connected with which are groups of ganglia. From this plexus non-medullated nerve fibres pass up between the epithelial cells. According to Klein these fibres end in minute swellings between the prickle-cells, but Unna maintains that many of them pass into the cells and terminate in swellings applied to the nuclear membrane. It is probable that a certain degree of stimulation of these nerves is followed by itching, while more severe stimulation is painful.

The causes of pruritus vary indefinitely, and may be grouped under the headings of pre-disposing and exciting causes, and the latter divided into external and internal causes.

\section{Pre-Disposing Causes.}

Idiosyncrasy.-In certain individuals there is an idiosyncrasy or delicacy of the skin which predisposes them to pruritus. This may be inherited, may occur in families and be handed down for generations, or it may be an individual peculiarity either existent from birth or acquired as the result of ill-health, malnutrition, over-indulgence in alcohol or tobacco, or mental troubles.

Age.-General pruritus is comparatively rare in infancy and childhood, except as a symptom of some cutaneous disorder such as urticaria. It is common in adult life, and especially so in old age when the skin has undergone the degenerative changes of senile skin, in which it has become dry, atrophic, smooth and shiny, or wrinkled, partly from the disappearance of the subcutaneous fat and partly from atrophy of the corium and epidermis, with the consequence that the fine sensory nerves are badly supported and liable to injury and functional derangements. A peculiarity of this type of skin is that it does nor react so readily to rubbing and scratching as that of younger people, and cxcoriations, eczematisation, impetigo, and other effects of superficial traumatism, are rare. Certain French authors consider that senile pruritus is partly due to auto-intoxication from deficient 
kidney elimination, and that it is specially common in people suffering from renal fibrosis.

The age of the patient is an indication of the probable cause of the pruritus. In infants and young children, for example, it is commonly due to papular urticaria; in young adults to scabies; in the middleaged to diabetes; in old age to senile skin and, among the poorer classes, to Pediculi restimentorum.

\section{Exciting Causes.}

Internal Causes.-Pruritus may be produced directly by toxins circulating in the blood-ressels of the skin reaching the lymph and acting on the nerve endings, or indirectly or reflexly from disorders of the internal organs, such as diseases of the bladder and uterus, or from nerve derangements, and eren from mental impressions as when itching is called forth by the sight of someone scratching.

Blood Diseases.--It is a frequent accompaniment of blood diseases, such as general lymphadenoma, leucaemia, and chlorosis, and of certain constitutional diseases such as gout, rheumatism, diabetes, Graves' disease, Bright's disease, and obesity. In such instances it is the result of an auto-intoxication and is produced directly by the action of some toxin on the nerve-endings.

Disorders of the Alimentary. Tract such as indigestion, flatuience, and constipation are common causes, the pruritus being due to poisons resulting from imperfect metabolism which are absorbed from the alimentary tract. Numerous articles of diet are liable to aggravate pruritus and may determine an attack; of these the most important are: shell fish, tinned fish, oily fish, spices, curries, highly-seasoned dishes, mustard, cheese, sausages, strawberries, tomatoes, acid wines, malted liquors, spirits, and coffee. As a rule pruritus due to food is the result of an individual peculiarity, unless when the article of diet is taken in excess, but should an attack be produced by excess in some kind of food a susceptibility is established whereby even small quantities of it may determine a recurrence. The itching resulting from food or drink may not begin until they have been absorbed, but more frequently it occurs almost immediately and would seem to be produced reflexly. by the contact of the food with the mucous membrane of the upper part of the alimentary tract.

Diseases of the Lirer are frequently accompanied by intense pruritus. It generally occurs in jaundice and may precede it, but the severity of the pruritus is not proportionate to the degree of the jaundice.

Disorders of the Verious System. - It may be present in connection with organic diseases of the brain and cord, such as cerebral tumours, meningitis, general paralysis, and Tabes dorsalis, but is most frequently associated with functional disturbances, such as hysteria, neurasthenia, and melancholia, or with epilepsy and chorea. It may be due to an excessive excitability of the nerve-endings in the skin from functional 
or organic disturbance of the peripheral nerves, or it may be produced reflexly by some superficial or deep-seated lesion at a distance from the pruritic area.

Diseases of the Genital System.-Diseases of the uterus and ovaries are liable to produce pruritus reflexly. It may occur with menstruation, occasionally with pregnancy, and may be local or general.

Drugs.-Certain drugs which have an anti-pruritic action in one individual may cause pruritus in another, such as morphia, opium, and belladonna. This is due to idiosyncrasy.

External Causes.- - In addition to the different skin affections in which itching is a constant subjective symptom, a large number of external causes also produce it, such as the following:

Extremes of Temperature.-Cold.-In certain individuals, especially people with dry skins and mild xerodermia, itching readily occurs in cold weather and may recur each winter, when it is known as Pruritus hiemalis. A cold bath or a draught of cold air is a common cause and may aggravate it when once established; in other cases, on the contrary, the numbing effect of cold will stop the itching and relief is obtained by cold sponging or immersion in cold water.

Heat.-Pruritus is invariably aggravated by heat and is specially liable to assert itself when the patient becomes warm in bed. Persons expesed to high temperatures, such as cooks, stokers, and firemen, frequently suffer from it, and in certain individuals even hot weather may be responsible (Pruritus aestivalis).

Irritating Underclothing.- In sensitive skins itching may be produced by a hairy surface, such as by wearing woollen or flannel garments, or by sleeping between blankets.

Relief of Pressure and Constriction may be accompanied by pruritus. For example itching often occurs in women when the clothes are removed and the constriction of corsets, garters, or tight boots relieved, the itching being mainly due to dilatation of the blood-vessels.

Local Irritants.-Pruritus may be caused by an infinite variety of local irritants which may be (I) of animal origin, such as bites of ticks and mosquitoes, stings of bees, wasps, spiders, caterpillars, or jelly-fish ; (2) of vegetable origin, such as poisonous plants, certain bacteria, and the fungi of ringworm and favus ; (3) of chemical origin, such as iodoform and tar. In addition, there are the irritants responsible for the different forms of trade eczemas, such as flour in baker's itch, sugar in grocer's itch, and soda in washerwoman's eczema.

Soap may be an irritant and the abuse of it may aggravate or even produce pruritus. This is due to the free alkali eliminated when the soap is decomposed by water: It is specially liable to occur in situations like the anus and vulva where the epidermis and mucosa are apt to be abraded and the nerve-endings exposed.

Diagnosis.-The only point of importance in the diagnosis is the necessity of distinguishing between pruritus due to various internal 
causes and independent of objective skin disease, and that symptomatic of some skin affection or resulting from local irritation. In pruritus due to internal disorders or nervous derangements, the diagnosis may be masked by excoriations and impetigo, lichenification, or eczematisation, caused by scratching or rubbing. In any case of generalised itching the most careful search should be made on the skin and underclothing for parasites, or for any other source of local irritation; if the search be negative the urine should be examined for sugar and albumin, and the output of urea estimated, as diabetes, Bright's disease, and faulty metabolism are among the most common causes.

Prognosis.-The prognosis depends on the successful removal of the cause of the pruritus. Where it is a symptom of some cutaneous disorder it will persist as long as the skin disease remains uncured; where it is due to some form of local irritant, parasitic or otherwise, it will remain until the irritant be removed : and when due to internal derangements its duration will correspond with that of the underlying morbid condition. Once an attack of generalised pruritus has occurred, a vulnerability may be established by which subsequent attacks may result from irritants of less virulence than those which produced the original attack. In most cases the pruritus can be relieved, if not completely remored, by suitable treatment.

\section{TREATMENT}

The successful treatment of pruritus is dependent on the recognition and elimination of the cause ; it varies, therefore, indefinitely and each case must be treated on its own merits.

General Management.-In every case, as a prelude to treatment, it is essential to make a thorough examination of the skin for any local cause of the pruritus, such as a local irritant or a cutaneous disease; if this prove negative it should be followed by a physical examination to discover any defects in the digestive, nervous, or genito-urinary systems, special attention being directed to the liver, urine, and faeces.

Every effort should be made to reduce the sources of local irritation ; the underclothing should be of silk, silk and cotton, or linen-woollen or flannel garments with a hairy surface should not be worn next to the skin, but in cold weather they may be worn over the silk or linen.

Any defects in health should be combated with suitable remedies. Indigestion, in all its forms, should be treated on general medical principles. Constipation should be dealt with by regulated exercises, suitable diet, draughts of hot water, and salines or regetable laxatives such as cascara sagrada; aloes should not be prescribed as they are liable to irritate the rectum and may set up Pruritus ani. Any source of septic absorption, such as carious teeth and prorrhoea of the gums, should have suitable treatment.

Toxins absorbed from the lower part of the alimentary tract are 
a not-infrequent cause of pruritus ; they may be counteracted by small doses of calomel at night, followed by a saline draught in the morning, or by lactic acid 5 to Io m.t.d.s., salol 5 to Io gr. t.d.s., or ichthyol 5 gr. twice a day on an empty stomach. In certain cases of this type a course of soured milk, properly prepared from a ferment containing the true Bulgarian bacillus, may be taken with advantage. Disorders of the liver with or without jaundice should be appropriately treated.

The urine should be examined for sugar or albumin in order to detect any organic or functional defect in the kidneys, and a careful examination should be made of the blood.

The nervous system should be examined for any disorder. Apart from definite nervous lesions, the sufferer from generalised pruritus is frequently neurasthenic, irritable, depressed, and incapable of concentrated mental effort; in such cases tonics of iron, strychnine, quinine, or arsenic, are indicated, but even moreimportant is the mental and physical improvement which can be obtained by rest -in bed if necessary-or by an outdoor life, exercise without fatigue, games like golf which absorb the attention of the player, and change of air, scene, and associates.

The diet should be plain, simple, and nutritious, and anything approaching excess with regard to food must be avoided; alcohol in all its forms and coffee should be forbidden; tea, if freshly infused and weak, may usually be taken with impunity; meat should only be indulged in once a day, and some patients find it advisable to give it up altogether and to resort to a vegetarian diet with plenty of milk. Green vegetables are harmless, as a rule, but potatoes and other starchy vegetables may have to be avoided as they are difficult to digest. Considerable benefit may be derived from flushing out the system with copious draughts of slightly alkaline mineral waters and, where possible, the benefit may be increased by a sojourn at one or other of the wellknown spas, such as Harrogate, Bath, Buxton, Contrexeville, Vichy, or Aix-les-Bains. Smoking in moderation is usually permissible, provided the smoke is not inhaled; but in certain cases it is apt to produce paroxysms of itching and has to be forbidden.

Another important consideration is to keep the bedroom cool and at an even temperature. The bedclothes should be light and raised off the patient by means of a cradle, which can be ventilated by the employment of paper shafts placed on each side of the bed; the sheets should be of old well-worn linen. As a rule nightgowns are preferable to pyjamas and should be made of thin linen or lawn and not of any woollen material.

Internal Treatment.-There is no reliable specific for pruritus, though a large number of drugs have been recommended of which the most common are valerian, carbolic acid, atropin, and arsenic. Valerian may be prescribed in the form of a tincture in doses of I dram t.d.s. ; carbolic acid in pills or in a mixture containing 2 grains to the dose ; atropin in the form of Liq. atropinae sulphatis, 1 to $2 \mathrm{~m}$. ; and arsenic in doses of 2 to $8 \mathrm{~m}$. of the Liq. arsenicalis. Of these probably 
the best is valerian, but they are all uncertain and may do more harm than good.

Nerve sedatives, such as sulphonal, chloral hydrate, the bromides, and antipyrin, have been extensively used; they ease the pruritus temporarily, but lower the resistance of the patient and when their effect wears off they cause depression and decreased ability to bear the itching. Consequently they should be prescribed with the utmost caution and reserved for cases in which insomnia is a marked feature. Opium should not be given internally in any form as it may. itself produce pruritus.

On the whole, more reliance should be placed on internal treatment carried out on general principles than on any so-called internal specific.

External Treatment.-External remedies play the nost important part in the treatment and are capable of subduing, if not of completely removing, the pruritus. They may be considered under the headings of chemical and physical remedies.

Chemical Remedies. - The number of anti-pruritic remedies is legion and reference will be made only to those which have been found the most serviceable, nameiy menthol, alcohol, camphor, ichthyol, carbolic acid, opium, tar, and lead, and of these carbolic acid is the most useful.

The choice and mode of application of the remedy is determined by the degree and extent of the pruritus, the proper application being more important in many cases than the composition. Toleration to a remedy being easily established it may be necessary to change an application should it begin to lose its effect. It also happens that what will suit one case may be useless in another, or may eren be irritating ; hence it is advisable to be careful at first and, having found a suitable remedy, to continue its use so long as it is effective.

In generalised pruritus remedies may be applied in the form of lotions, creams, or dusting powders.

Lotions.-To obtain the maximum benefit from lotions they should be applied on lint which should be kept moist by spraring or pouring on the lotion every few minutes. This should not be covered with waterproof as it prevents evaporation and makes the part hot.

The following are the most useful :

Carbolic Lotions.-Carbolic lotion ( $\mathrm{I}$ in to). Carbol-glycerine ( $\mathrm{I}$ in 20).

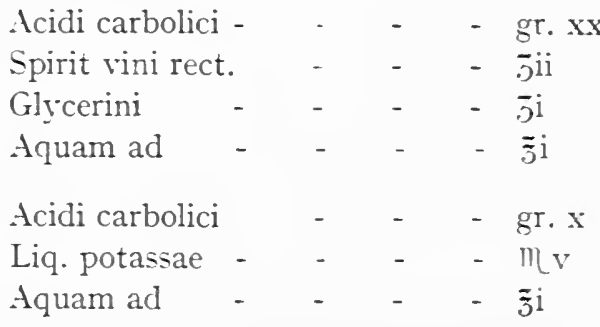


Eraporating Lotions.-These contain Eau de Cologne, lavender water, or rectified spirit, which may be undiluted or diluted with water. They act in virtue of their power of evaporating quickly and to be effective must be applied frequently.

Tar Lotions.--The simplest tar lotions are obtained from the coaltar products, namely Liq. picis carbonis and Liq. carbonis detergens, diluted with water or in Lotio calaminae in the strength of about ro minims to the ounce. The Liq. picis alkalinus (Bulkley) is also a useful remedy (see appendix).

Lead Lotions. - These are made chiefly from the Liq. plumbi subacetatis, Io to 5 minims in an ounce of water or milk. The antipruritic action of the lead may be increased by the addition of tar, a useful formula for such a lotion being :

$$
\begin{aligned}
& \text { Liq. pb. subacetatis - - - jss } \\
& \text { Liq. carb. deterg. - _ _ _ इiiss }
\end{aligned}
$$

(Sig.-A teaspoonful to half a pint of tepid water for use.)

Opium Lotions.-Opium is a valuable anti-pruritic when applied

\begin{tabular}{|c|c|c|c|c|c|}
\hline \multicolumn{2}{|c|}{ Extract of opium } & - & - & - & 川r \\
\hline Hot water & - & - & - & - & $\bar{j}$ \\
\hline Tincture of & ium & - & - & - & 川xy \\
\hline Camphor - & - & - & - & - & gr. $\mathrm{xxx}$ \\
\hline Almond oil & - & - & - & - & $\bar{j} \mathrm{i}$ \\
\hline
\end{tabular}
in lotions, such as the following:

Alkaline Lotions. - These consist of borax, bicarbonate of soda, or carbonate of potash, Io-I5 grs. to the ounce of water, and are valuable in cases where large areas are affected.

Ichthyol Lotions.-Ichthyol has a definite anti-pruritic action, drying the skin, and forming a thin protective film over it. It may be employed in lotions consisting of $\mathrm{I}-4 \%$ dissolved in water, in $50 \%$ alcohol, or in olive oil.

The anti-pruritic action of the above lotions may be intensified by the addition of hydrochlorate of cocain $2 \%$, menthol $\mathrm{I} \%$, or hydrocyanic acid I\%. Menthol lotions should be used with caution as although they have a powerful anti-pruritic action they are apt to set up inflammation.

Creams.-By applying remedies in creams or oils the effect lasts longer than if they are prescribed in lotions. In extensive cases the simplest base is olive oil or Linimentum calaninae, to either of which may be added I to $2 \%$ of carbolic acid, menthol, or ichthyol. Emollient remedies of this nature are specially useful in senile pruritus.

Powders.-Dusting powders have a cooling effect on the skin and relieve pruritus. The most useful contain as bases, oxide of zinc, magnesium carbonate, bismuth nitrate, or starch, with Io $\%$ of camphor added to render them anti-pruritic. They should be carefully powdered and put through a fine sieve to render them impalpable. 
In localised pruritus where the itching is confined to one or more patches the remedies should be employed in ointments, pastes, or varnishes, as they are more lasting in their effects than lotions. The above anti-pruritic remedies may be used in this way and to them may be added others, such as chloral hydrate, conium, and cannabis indica.

Ointments.-One of the best bases for an anti-pruritic ointment is a well-made cold cream, to an ounce of which may be added menthol 5 grs., hydrochlorate of cocain Io grs., extract of cannabis indica $\frac{1}{2} \mathrm{dr}$., extract of conium I dr., chloretone I dr., or chloral hydrate I dr. If it be desired to stiffen the ointment I or 2 drs. of zinc oxide may be added.

Pastes.-In situations exposed to friction, instead of cold cream zinc paste should be used as a base, such as that containing equal parts of zinc oxide, starch, lanolin, and raselin.

Varnishes and Gelatines are employed where the pruritic areas are small, a useful basis for a rarnish being that of Pick (see page 137), and for a gelatine that of Unna (see page I36). To these should be added $2 \%$ of ichthyol.

Physical Remedies.-Hydrotherapy.-The application of a sponge wrung out in water as hot as can be borne gives temporary relief to localised itching, while hot baths and vapour or Turkish baths may relieve a generalised pruritus. These act by causing dilatation, followed by constriction, of the cutaneous capillaries. Medicated hot baths containing bran, oatmeal, or linseed meal, 2 lbs., carbonate of soda or borax 2 ozs., gelatine $\frac{1}{2} \mathrm{lb}$, or starch I lb., to 30 gallons of water, are often of great service. Baths are not invariably successful, however, and in some cases do actual harm, being followed by an exacerbation of the itching. Tepid douches or sprays applied along the rertebral column for 5 to I 5 minutes daily are worthy of a trial.

Occasionally benefit may be derived from the employment of cold compresses, cold sprays or douches, especially when followed by the application of an evaporating alcoholic lotion or a dusting powder, but cold baths are liable to do more harm than good.

Electrical Methods.-In localised pruritus some relief may be obtained from the brush discharge from a high frequency apparatus and from faradisation from a faradic brush or bath. Of greater value than either of these is the careful application of the X-rays, about $\frac{3}{4}$ of a Sabouraud pastille dose being given and repeated in If days.

\section{SCRATCHING AND ITS EFFECTS}

The immediate effect of scratching is inflammation of the shin, with redness, and slight swelling from exudation of serum and interstitial oedema. By traumatism with the nails or some rough instrument, linear or punctate excoriations are produced, the latter being due to scratching off the tops of congested and prominent follicular papules. In some cases the irritation is so severe that relief is only 
obtained when the offending papule is dug out by the nails, leaving a raw, bleeding or oozing surface, about the size of a lentil (Prurits biopsiants of Besnier). When the exudation dries, crusts are formed, or the excoriation may become the site of inoculation of pyogenic micro-organisms and result in impetigo, furunculosis, or some other form of septic dermatitis. In old-standing cases in which the scratching has been incessant, pigmentation of the skin is liable to occur from stimulation of pigment-formation in the epidermis, and the neighbouring lymphatic glands may become enlarged.

The manner in which the skin reacts to friction and scratching varies; in young children it is more easily inflamed, lacerated, and inoculated with pus-cocci than in elderly people, while when senile it reacts with the greatest difficulty and even vigorous scratching and rubbing may leave little or no trace. Certain skins also are more delicate than others, as a peculiarity either inherited or acquired as the result of illness.

In addition to the more common and immediate changes due to scratching, certain remote effects may occur which are in the nature of reactions peculiar to the individual. In some people rubbing produces a papulo-vesicular eruption which tends to weep, to become crusted, and to assume the characteristics of eczema (eczematisation) ; in others it results in a pruriginous eruption of flat or rounded itchy papules; in others it calls forth the peculiar thickening of the skin known as lichenification.

\section{REFERENCES}

Barendt, F. H. Some Aspects of Pruritus (Med. Press \& Circ., London, I907, lxxxiii. p. 60).

Bronson, E. B. Sensation of Itching (Med. Rec., N.Y., 189o, xlii. p. 2 I).

Corletr, W. F. Pruritus Hiemalis (Journ. Cut. \& Genito-Urin. Dis., N.Y., I89I, ix. P. 4 I).

Darier, J. Précis de dermat., 1909, 465.

Hallopeau, H. Trichomanie (Réunions Clin. de l'Hôp., St. Louis, 1889).

Jacquet, L. Troubles de la sensibilité (La Prat. Derm., Paris, I904, iv. p. 341 ).

Kromayer, E. Treatment with caustic potash (Ibid., I908, xxxiv. p. 59). Veiel, T. Pruritus in kidney disease (Arch. f. Derm. u. Syph., I906, lxxx. p. 59).

\section{PRURITUS ANI}

The term Pruritus ani is usually reserved for itching at the anal orifice, extending about an inch up the rectum, and externally involving the puckered circular area corresponding to the Corrugator cutis ani muscle. As the mucous membrane and the skin at the anal orifice are richly supplied with sensory norves, this region is particularly liable to pruritus, which may be mild and transient, but is more often intense and prolonged, and in which the paroxysms may be so severe and 
frequent as to interfere with sleep, impair the general health, and cause the sufferer to shun the society of his fellows, to become neurasthenic, and even to lose all self-control.

The pruritus is rarely continuous but subject to intermissions and exacerbations brought on by a large number of external and intemal causes. The scratching indulged in, though it may give temporary relief, usually intensifies subsequent paroxysms and leads to changes in the skin about the anus, which becomes inflamed, thickened and lichenified, sodden from an offensive rectal secretion, broken up by radiating fissures, and ulcerated or warty from the secondary inoculation of pus-organisms. In old-standing cases it often becomes thin, pale, and tough like parchment or wash-leather, or the skin over the whole perineum may become eczematised, inflamed, oedematous, raw, moist and painful.

Etiology.-Pruritus ani is a symptom and not a disease. It is dependent chiefly on local causes, but it may occur in association with constitutional derangements and the most careful search may fail to reveal any local defect which might be responsible for it.

Sex.-It is more common in males than females, possibly because they are more liable to excess in eating and drinking.

Age.-It may occur at any age but is most frequent in middle life and in elderly people. The age may be some guide to the cause; in children it is frequently due to intestinal worms; in young men to sedentary occupations and congestion of the haemorrhoidal vessels; in young women to vaginal discharges; in middle-aged men to constipation, haemorrhoids and fistulae; in middle-aged women to neuroses associated with the menopause; in old age to a feeble circulation and venous congestion.

Internal Disorders.-Similar internal disorders to those which produce generalised pruritus may cause Pruritus ani ; namely, disorders of the alimentary tract especially constipation, liver derangements, impaired digestion brought about by rich indigestible foods, and general diseases such as diabetes, gout, and rheumatism. It may result also from indulgence in alcohol and coffee and from excessive smoking. It may be caused directly by congestion of the haemorrhoidal vessels due to imperfect action of the liver, or reflexly and occur almost immediately after taking coffee, alcohol, or some form of food difficult to assimilate.

Local Affections.-Disorders of the rectum are frequently responsible, such as catarrh with an offensive discharge from colitis or chronic proctitis, the presence of threadworms or masses of hard dry faeces, lesions in the mucosa such as polypi, haemorrhoids, ulcers, fistulous openings in the anal valves, and cracks between the sphincters.

Other important causes are imperfect cleanliness about the anus; incomplete emptying of the sigmoid flexure and rectum from the unnatural position in defaecation necessitated by the ordinary watercloset seats; tumours in the rectum and pelvis obstructing the 
venous circulation; and skin affections about the anus, such as boils, eczema, and psoriasis.

Diseases of the genitalia such as gonorrhoea, and leucorrhoea, and disorders of the bladder and uterus may cause it either directly or reflexly.

\section{TREATMENT}

Most cases are curable if treated early and if the cause be discovered and removed. Should treatment be delayed, however, until the skin has become thickened or eczematised, the nerves being pressed upon by the indurated skin may remain in an excitable state and the itching persist in spite of the removal of the cause. In every case as a preliminary, the anal region and the rectum should be properly examined for haemorrhoids, blind fistulae, ulcerations, polypi, or other possible local causes, as failure to do this may lead to unnecessary suffering on the part of the patient and bring discredit on the physician.

General and Preventive Treatment.-One of the most important preventive measures is scrupulous cleanliness after defaecation, with the removal of all faecal matter or mucous discharge about the anus. This cannot be accomplished satisfactorily by paper, which if coarse may itself produce irritation, but should be done by a pad of cottonwool moistened in water, and the part then dabbed dry and a simple dusting powder applied. Constipation is one of the important causes and must be treated on general medical principles. What has been said with regard to the diet in pruritus in general is equally applicable here and alcohol, coffee, and excessive smoking should be forbidden. Any obvious disorder of the liver, intestines, or genito-urinary system must be treated on general principles. When the patient is depressed, irritable and neurasthenic, a tonic regime is indicated, with change of air, healthy surroundings, and outdoor exercises, particularly riding, fishing and sea-bathing. In markedly neurotic cases in which selfcontrol is lacking, hypnotic suggestion has been recommended and is worthy of a trial.

Vaccine treatment has also been employed, with autogenous vaccines made from the Streptococcus faccalis which is usually present. The results from it have not been successful, nor can they reasonably be expected to be so, as the streptococcus is not the cause of the pruritus but a secondary complication which may give rise to ulceration.

Local Treatment.-In the first place any local lesion about the rectum must be suitably treated. Catarrh should be dealt with by washing out the rectum with weak solutions of boric acid; threadworms should be removed; haemorrhoids operated upon ; small redundant tags of mucous membrane snipped off; small fistulac opening into the rectal pockets slit up; ulcers and fissures touched with silver nitrate or lactic acid, or destroyed by the thermo-cautery ; and small warty cxcrescences cauterised or cut away. The pain associated with cauterisation may be deadened by previously injecting cocain 
behind the ulcer and inserting a morphia suppository afterwards. Unless the ulceration is slight it is advisable to confine the patient to bed for at least a week after the operation.

Other effects of scratching and rubbing, such as superficial pus-infections and eczematisation, must also be treated by appropriate remedies.

If the skin about the anus be thickened, permanent relief cannot be obtained until the induration is reduced. This may be done by painting the part with salicylic acid $6 \%$ in $50 \%$ alcohol, or with $95 \%$ carbolic acid, followed by a zinc oxide dusting powder to protect the tender surface. Ironing the part with the actual cautery at a dull red heat has been recommended, but is painful and necessitates a general anaesthetic. After the application of the cautery the part may be scothed by a lotion of bicarbonate of soda $I$ in $S$, followed by zinc oxide ointment and a cocain suppository.

In obstinate cases incision of the thickened peri-anal skin and cutting the nerves has been suggested, after which the surrounding skin is undermined, drawn over the denuded surface, and stitched to the rectal mucosa. This procedure, though sometimes successful in the hands of an experienced surgeon, is one which is rarely necessary, and is likely to be followed by serious results if attempted by a novice.

In a considerable number of cases no definite lesion can be detected either at the anus or in the rectum; in others the itching may remain after the local source of irritation has been removed, and some local application is demanded for its relief, such as preparations containing carbolic acid, cocain, menthol, tar, mercury, ichthyol, silver, and belladonna. As in ordinary pruritus what will suit one case will be ineffective in another and toleration to this or that remedy is readily established. Zinc paste with the addition of $2^{\circ}$. of salicylic acid is admirable for mild degrees of Pruritus ani, as it not only relieres the itching but protects the parts from friction or scratching by forming a coating about the anal orifice.

Carbolic acid may be prescribed in olive oil, glycerine, or vaselin, in the strength of $I$ in 20. Its anti-pruritic action may be augmented by being combined with sodium hyposulphite as in the following lotion :

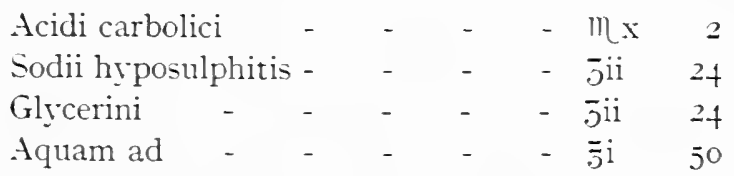

Cocain may be used as a lotion or ointment, as in the following formulae :

$\begin{array}{lllllr}\text { Cocainae hydrochloratis - } & - & - & \text { gr.x } & 2 \\ \text { Aquae laurocerasi - } & - & - & - & 5 \mathrm{iii} & 36 \\ \text { Aquan rosae ad - } & - & - & - & \overline{3} \mathrm{i} & 62 \\ \text { Cocainae hydrochloratis - } & - & - & 1 \mathrm{xv} & 3 \\ \text { Zinci oxidi - - } & - & - & - & 5 \mathrm{ii} & 24 \\ \text { Paraffinum molle album ad } & - & - & \overline{5} \mathrm{i} & 73\end{array}$


It may also be given as a suppository containing half a grain, which gives considerable relief with regard to the itching and is of special value for inducing sleep and for preventing the scratching which takes place reflexly during sleep. It is important to remember that in using cocain or morphia suppositories there is the danger of relieving the pruritus at the expense of establishing a drug habit.

Menthol may be employed occasionally as a $2 \%$ solution in olive oil or $50 \%$ alcohol, or in zinc oxide ointment, but, if persistently used, is liable to inflame the skin.

Tar is valuable in this affection and may be used as the Liquor carbonis detergens or the Liquor picis carbonis, a teaspoonful to half a pint of tepid water, and the strength increased if desired ; or in ointments such as :

\begin{tabular}{|c|c|c|c|}
\hline Oil of cade & - & - & \\
\hline Paraffinum molle ac & & - & \\
\hline Unguentum picis & - & & \\
\hline Unguentum bellado & nnae & - & 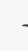 \\
\hline Unguentum aquae & rosae & - & - \\
\hline
\end{tabular}

Mercury is of value in relieving the itching where secondary inoculation of pus-cocci has taken place. It may be dusted on as calomel or applied as black-wash diluted with equal parts of lime water, or as Lotio. hydrarg. perchlor. ( $\mathrm{I}$ in IOOO), or in the form of an ointment such as ammoniated mercury $2 \%$ and vaselin.

Ichthyol is employed as a $2 \%$ lotion in water or in $50 \%$ alcohol, or in the following ointment :

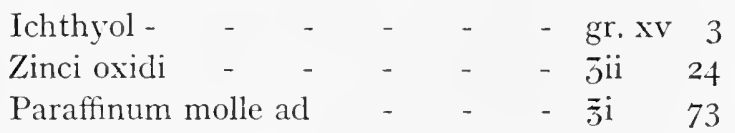

Silver--Cracks about the anal orifice may be healed by painting them with a 5 to $10 \%$ solution of nitrate of silver, and the itching relieved by the use of collosol silver suppositories.

Electrical Methods.--Various electrical measures have been employed with greater or less success, such as the X-rays, ionisation with cocain or zinc, and high frequency currents.

Of these the $\mathrm{X}$-rays have given by far the best results as they not only have a definite anti-pruritic action but also reduce the thickening of the skin. A ${ }_{4}^{3}$ Sabouraud dose should be given and this should be repeated in a month if necessary. A lead glass localiser should be used and the testicles protected.

Radium has also been found of value in the treatment of this affection. It is given in short exposures with a half-strength applicator unscreened for five to seven minutes on two consecutive days, and the series repeated later if necessary.

Zinc ionisation is of special value where superficial abrasions are present. The procedure is simple: a piece of lint the size of a penny 
is soaked in $2 \%$ sulphate of zinc solution and applied to the affected skin, the positive pole of the battery with a solid zinc electrode is pressed against the lint while the negative electrocle is held by the patient, and a current of a few milliamperes is passed for ten minutes. This has a definitely astringent action causing the abrasions to heal and relieving irritation.

Cocain is emplored in the same way, the lint being soaked in a $2^{\circ}, 0$ solution of the hydrochlorate.

\section{REFERENCES}

BrAV, H. A. Etiology \& treatment of Pruritus ani (New York Med. Journ., 1907, p. 201).

Hill, T. C. Pruritus ani (Idem, I906, p. 993).

Mason, R. D. Pruritus ani (Idem, I908, P. II5I).

Morris, Malcoly. Treatment of Pruritus ani (Brit. Med. Journ., I90, ii. p. 980$)$.

Mumery, P. L. Causes of Pruritus ani (Practitioner, I907, lxxviii. p. 686). Murray, D. H. Etiology \& treatment of Pruritus ani (Journ. Amer. Med. Assoc., I9I 8, lxxi. p. I +49).

Radicm INstitute Report (London, I9I4).

Tomkixsox, G. Etiology \& treatment of Pruritus ani (Brit. Med. Journ., I909, ii. p. 452 ).

Wallis, F. C. Causes \& treatment of Pruritus ani (Idem, I905, i. p. I029).

\section{PRURITUS VULVAE}

Pruritus vulvae merits special consideration owing to the severity of the local symptoms. The pruritus, although more or less continuous, is increased by warmth in bed and by friction from morement, and may be so intense and persistent as to prevent sleep and to cause extreme nervous depression, neurasthenia, and even hysteria. In mild cases the affected parts simply itch, in severe cases ther also feel hot, burning, or actually painful.

The first objective sign of it is inflammation of the inner surfaces of the labia and around the clitoris. This may remain localised to the vulva or may spread to the surrounding skin and may gradually involve the whole perineal region and even pass up for a short distance inside the ragina. As a result of scratching, excoriation and thickening occurs and the affected area may assume a peculiar sodden appearance due to mucous discharges from the vagina. Eczematous changes may supervene and a distressing condition be produced, with inflammation, oedema, weeping, and crusting, which is too often allowed to become far advanced before medical aid is sought. In cases of long standing, especially in elderly people, the skin undergoes kraurotic changes and becomes white and dead like parchment, and may be the seat of malignant changes of an epitheliomatous nature.

Etiology.-Pruritus vulvae may be due to a number of causes of which the most important are the following: Irritating discharges from 
the vagina and uterus such as occur in leucorrhoea, gonorrhoea, and at the end of the menstrual period, smegma collecting about the labia minora, want of cleanliness and the accumulation of dirt, superficial ulcers and tender spots about the inner surfaces of the vulva, and parasites such as Pediculi pubis or thread-worms about the anus and vagina. Skin affections involving the vulva, such as eczema and furunculosis, may also cause it but are more often secondary to the pruritus and the result of the inoculation of pus-cocci by scratching. Urine, if irritating from hyper-acidity or the presence of sugar, may produce it and it not infrequently occurs about the time of the menopause as the result of a temporary glycosuria or " climateric diabetes." Pregnancy is another potent cause owing to venous congestion.

As in Pruritus ani a certain number of cases occur in which no definite local lesion can be detected. In such there is generally marked neurosis, and in certain of them it is probable that some slight cause induced the habit of scratching and this in turn by irritating and altering the skin perpetuated and increased it.

\section{TREATMENT}

The successful treatment depends on the recognition of the cause and the removal of its effects. The urine should be tested for hyperacidity and sugar, and an examination should be made for possible local lesions such as small ulcerations, tender inflamed spots, discharges from the vagina, smegma, or parasites, which if found should be suitably treated. Small ulcerations should be touched with pure carbolic acid, silver nitrate, or the thermo-cautery, and a soothing paste subsequently applied; discharges and catarrh of the ragina should be treated by antiscptic douches, such as lotions of boric acid, potassium permanganate ( $\mathrm{I}$ in 5000), and zinc sulphate ( $\mathrm{I}$ in $\mathrm{IO00}$ ), and a vaginal tampon inserted to prevent the discharge escaping and irritating the inflamed area ; smegma should be removed and scrupulous cleanliness enjoined ; thread-worms should be got rid of, and Pediculi pubis destroyed by cutting the hairs and applying carbol-glycerine $I$ in 8 , or a dilute ammoniate of nercury ointment. Concomitant cutaneous affections such as eczema and boils must be treated by appropriate means. Where the urine is hyper-acid, alkalies should be prescribed, and where sugar is present as in the case of climateric diabctes much benefit can be derived from opium or codeia internally in doses of I gr. t.d.s. with 2 grs. at bed-time. In severe cases, especially those occurring in pregnancy or associated with ulcerations necessitating cauterisation, the paticnt should be confined to bed until the pruritus can be relieved. The diet should be of a bland character, all those foods which liave been referred to as being harmful in connection with pruritus in general should be avoided, and alcoholic stimulants and coffee forbidden. The bowels should be regulated and kept slightly loose by laxatives, and the kidneys flushed out by lithia or other alkaline mineral waters. 
Tonic treatment is indicated in those who are anaemic and run-down, and the neurasthenia which so frequently accompanies and intensifies the pruritus should be dealt with by moral persuasion, general massage, and a proper hygienic regime. When the itching is so severe as to cause insomnia, general sedatives such as chloral, paraldehyde, or bromide of sodium may be resorted to, but should be prescribed with caution in view of the risk of establishing a drug habit.

Where no definite cause can be detected or where the itching persists after the removal of some source of irritation, one or other of the various local remedies for pruritus are indicated. Hot sitz baths or sponges wrung out in as hot water as can be borne generally give relief; after drying the parts a dusting powder should be applied and the vulva covered by a pad of cottonwool fixed by a T-shaped bandage.

A large number of local anti-pruritic remedies have been employed, such as carbolic acid, menthol, chloroform, tar, chloral and cocain.

Carbolic acid may be prescribed in the form of a lotion and the following mode of application has been recommended (McCann) : A number of strips of lint three inches long are soaked in carbolic lotion ( $I$ in 20 ), and inserted for an inch into the ragina, and the remainder spread over the labia in such a way that the whole of the pruritic area is covered, a large pad of boric acid wool is then applied over the vulva and fixed with a T-shaped bandage. This may be left on for a whole night and fresh strips applied next day if necessary. In place of the carbolic lotion, menthol in a $2 \%$ solution in almond oil may be substituted and dabbed on to the affected parts. Where the skin and mucosa are raw and sore, the smarting caused by carbolic and menthol lotion may be so intense that they may have to be discontinued and milder methods resorted to. A valuable remedy where the inflamed spots arc few and the affection is confined to the mucosa is methylene blue which should be painted on in the form of a $I^{\circ}$ o solution.

In this affection ointments or pastes are more useful than lotions, as, apart from the medicament they contain, they are of benefit by protecting the parts from friction and from vaginal discharges. As a base for an ointment equal parts of soft white paraffin and lanolin may be employed, and for a paste equal parts of zinc oxide, starch, lanolin and raselin. Another useful base, especially where the skin is irritated by sugar in the urine or by discharges from the vagina, is the B.P. casein pigment which is a thick white emulsion and forms a protective coating or varnish, is porous and elastic, and dries on the skin.

As a rule the zinc paste forms a more comforting base than the vaselin and lanolin and is especially valuable when the parts have become oedematous or eczematised. To the ounce of one of these bases may be added $2{ }^{\circ}$ of carbolic acid or menthol, or $3 \%$ of ichthyol, hydrochlorate of cocain, or ammoniated mercury. Other ointments 
also give relief, such as chloroform ointment consisting of chloroform and vaselin, and tar ointments such as :

$\begin{array}{llllll}\text { Oil of cade - } & - & - & - & \overline{\mathrm{i}} & \text { I2 } \\ \text { Adipen benzoat. ad } & - & - & - & \overline{3} \mathrm{i} & 88 \\ \text { Liq. picis carbonis - } & - & - & - & \text { 川 } \mathrm{xr} & 3 \\ \text { Zinci oxidi - } & - & - & - & 5 \mathrm{ii} & 24 \\ \text { Paraffinum molle ad } & - & - & - & \overline{3} \mathrm{i} & 73\end{array}$

In Pruritus vulvae associated with diabetes the irritation and erythema are largely due to the contact of the sugar-containing urine with the vulva and the most important point in connection with the treatment is to prevent this by employing lotions or injections after micturition, such as a solution of bicarbonate of soda or Van Swieten's solution (I part of corrosive sublimate in Ioo parts of alcohol and 900 parts of water). Carnot has obtained good results from a solution of fresh brewer's yeast employed as a lotion or injection and containing a tablespoonful of the yeast to a litre of water. By it the irritating contact of the sugar is prevented and the itching is reduced as, through fermentation, a weak solution of alcohol is formed which is antipruritic.

For some time after the pruritus has subsided it is advisable to apply a bland ointment, such as zinc oxide paste, as a protective against friction.

In cases where the mucosa and slin are thickened it is necessary to reduce the induration to get rid of the pruritus; this may be done by the thermo-cautery, which necessitates a general anaesthetic, or by smearing the thickened patches with pure carbolic acid once or twice a week after drying the surface with a piece of cottonwool. In inveterate cases in elderly people where leucoplakia is present and there is a tendency to malignant changes, more radical treatment is advisable and the affected skin and mucosa should be excised and the edges brought together and sutured.

Excellent results may be obtained by exposure to the X-rays which relieves the itching and reduces the thickening of the tissues. A $\frac{3}{4}$ pastille dose may be given and repeated in six weeks if necessary:

Radium is also of value in this affection, a series of short exposures with a flat apparatus being given on consecutive days.

\section{REFERENCES}

Gibions, R. A. Pruritus vulvae, etiology and treatment (Brit. Med. Journ., I9 2 (March 2nd), p. 469).

McCanx, F. J. Pruritus vulvae (Polyclinic, London, I905, ix. p. I 30 ).

Radium Institute Report, London, I9I 4.

Siebourg. (Treatment.) (Centralbl. f. Gynak., Leipz., I909, xxv. p. 76I). Wickham \& Degrais. Treatment of Pruritus rulvae by radium (Radium-

Therapy (Dore's Trans.), r910, p. 245). 


\section{CHAPTER XYII}

\section{ERYTHEMA}

THE term Erythema, from the Greek word equítnuu a blush, signifies redness of the skin which is generally transient, disappears temporarily on pressure, and is the clinical evidence of a dilatation and congestion of the cutaneous capillaries. It has been applied in different senses by different writers to a functional disturbance of the bloodvessels, an early symptom of inflammation, or a morbid process characterised by active hyperaemia and serous exudation.

In this connection two other terms are employed, namely, Erythrodermia, a general term signifring a reddening of the skin associated with more or less infiltration, and Erythromelalgia or red neuralgia, a name given to a flushed and painful condition of the extremities, chiefly of the feet.

It is customary to include under the heading of Erythema a somewhat indefinite group of cutaneous affections which, to simplify description, may be considered under the following headings:

I. Localised erythema due to external or internal causes.

2. Generalised erythema of toxic origin and often simulating an exanthem or rash of an acute specific fever.

3. Erythema multiforme exudativum (Hebra).

The rational method of discussing this subject is on an etiological basis but there are difficulties in so doing as in certain cases the cause may be obscure and may elude the most careful search.

\section{Axatonical, Physiological axd Pathological CONSIDERATIONS}

As a preliminary to the description of this group of cutaneous affections it is advisable to refer to certain anatomical, physiological, and pathological considerations connected with the cutaneous bloodvessels.

In the corium the blood-vessels, with the exception of a few near the subcutaneous tissue, consist of capillaries whose walls are composed of an endothelial layer with neither muscular fibres nor an outer fibrous coat. Owing to their structure ther are easily dilated and readily ruptured, the dilatation being passive and the result of more blood being poured into them 
from the deeper vessels. On the other hand, the deeper vessels having a muscular coat, are regulated by the vaso-motor mechanism in which the vaso-constrictor nerves are in excess of the vaso-dilators, are constantly in action, and keep up the so-called tone. The principal vaso-constrictor centre is situated in the medulla, while subordinate centres capable of independent action are present at different levels in the spinal axis and sympathetic ganglia, and even if all three centres be cut off the muscle fibres themselves appear to have an inherent power of contraction to stimulation. When the vessels dilate it may be due to an inhibition of the vaso-constrictor tone or a stimulation of the vaso-dilator nerves, the centres for which are evenly distributed in the cord and medulla.

Erythema or active hyperaemia results from an increased flow of blood through the capillaries; cyanosis or passive hyperaemia is due to an increased amount of blood in the capillaries owing to a blockage and diminished flow in the veins.

The small arterial twigs break up into a brush of capillaries in the corium in a conical fashion, the base of the cone being in the papillary layer, and between the cones the venous capillaries pass down and mite in small veins. When the flow of blood is active through one small artery the capillaries of the cone are dilated and a roundish or oval red spot results, while if a number of small arteries are clilated a more or less diffuse redness is produced. On the other hand, if the flow be diminished, owing to slight venous congestion, a retiform appearance results due to a hyperaemic network enclosing small areas the colour of the normal skin which correspond to the bases of the arterial cones. This arrangement explains the mottling and retiform patterned hyperaemia, which is frequently observed in babies' limbs when exposed to the cold and in adults with a weak peripheral circulation.

When the increase in the blood-flow is slight, a dilatation of the capillaries alone takes place. Should the inrush of blood be greater an extravasation of serum also occurs, leading to more or less oedema in the upper part of the corium and epidermis and subsequent desquamation. Should the blood-flow be still more profuse it may result in a diapedesis of leucocytes, a rupture in the capillary wall, an extravasation of red blood cells, and the production of a haemorrhagic lesion, which, on healing, goes through the various colour changes of a bruise.

There is considerable variance of opinion as to whether an active hyperaemia or erythema should be regarded as an early stage of inflammation. Certain writers assert that in a pure ery thema there is only dilatation. of the blood-vessels, serous exudation, and an extravasation of blood-cells, while in inflammation, however slight, the exudation is sero-fibrinous and accompanied by a fixed-cell proliferation in the neighbourhood of the capillaries. Against this view it has been pointed out that cases of Erythema multiforme occur in which there are clefinite signs of inflammation and in which both a sero-fibrinous exudate and a fixed-cell proliferation may be detected.

The dilatation of the cutaneous vessels may be the direct result of the local action of some morbid agent on the blood-vessel walls, or it may be produced indirectly by the vaso-motor apparatus. As instances of morbid agents acting directly may be cited toxins from bacteria, such as streptococci, actually present in the cutaneous capillaries, and various irritants acting locally on the skin. It is much more common for the erythema to be caused indirectly, either by paralysis of the vaso-constrictors or stimulation of the vaso-dilators. It is difficult to distinguish the exact part played by the vaso-constrictors and the vaso-dilators, though it seems probable that the stimulation of the vaso-dilator nerves at some point betwcen their origin in the medulla or cord and their termination in the walls of the vessels is the more powerful factor. 
The exciting agents of the vaso-clilator mechanism are numerous and varied; they may be plysical as in heat, chemical as in poisonous drugs, toxic as in food toxins, bacterial toxins, or autogenous toxins, or psychic as in blushing from shame or anger. These will be considered in greater detail under the heading of etiology:

The type of cutaneous lesion produced by the different excitants depends on their virulence, the length of time they are in action, the sensibility of the skin, and individual idiosyncrasy. Different excitants may give rise to a more or less constant type of erythema, as is seen in recurrent attacks of a like nature called forth by different toxic causes. On the other hand, the same irritants may produce different effects in different individuals, such as a blotchy erythema in one and a raised exudative eruption in another. The element of idiosyncrasy towards any particular form of irritant has invariably to be taken into consideration. It is an evidence of vaso-motor instability and may be congenital or acquired as the result of previous attacks or of some intercurrent disease. As an example of the last is the Tache cérébral, which occurs in connection with typhoid ferer and meningitis, in which slight friction calls forth a red line or patch with a white border.

\section{LOCALISED ERYTHEMA}

Erythema often occurs in patches. These are reddish-pink in colour, pale temporarily on pressure, are roundish, oval, or irregular in outline or occasionally retiform, have a smooth surface, are sometimes slightly raised, and as a rule have an ill-defined border which fades into the surrounding skin. They vary in size according to the area of action of the irritant, may occur anywhere on the skin, and when due to internal causes tend to be symmetrical in distribution. The subjective symptoms associated with them may be negligible or may consist of heat, throbbing, or itching sufficiently marked to lead to scratching and rubbing. To the touch the reddened area feels hot so long as the hyperaemia is active.

Etiology.-Erythematous lesions vary in appearance and persistence according to their cause. Of these there is an indefinite number and any attempt to give a complete list would be futile, but the most important may be grouped under the following headings:

\section{External or Local Caluses}

(a) Physical.-I. Friction or chafing from the rubbing together of contiguous surfaces is a common cause, giving rise to the so-called Erythema intertrigo which is common in the gluteal fold, insides of the thighs, axillae, inguinal folds, and beneath the breasts in stout women. The conbination of the friction to which these parts are subjected and the moisture of the sweat causes the skin to become inflamed, tender, glazed, sometimes macerated, and occasionally: eczematised from scratching.

2. Pressure, both intermittent or prolonged, may cause erythematous patches, as seen in the red patches which occur on corered 
surfaces from lying and which are especially marked in prolonged illnesses or where the innervation is defective when, through want of cleanliness and pyogenic infection, they may be transformed into bed-sores. To pressure erythema the older dermatologists applied the name Erythema paratrimma.

3. Sunlight is a familiar cause of localised erythema, as seen in sunburn, or Erythema solare (see page 245).

4. Röntgen Rays and Radium may also produce it.

5. Heat is a common cause. A special type of heat crythema is that known as Erythema ab igne, Ephelis ab igne, or Livedo

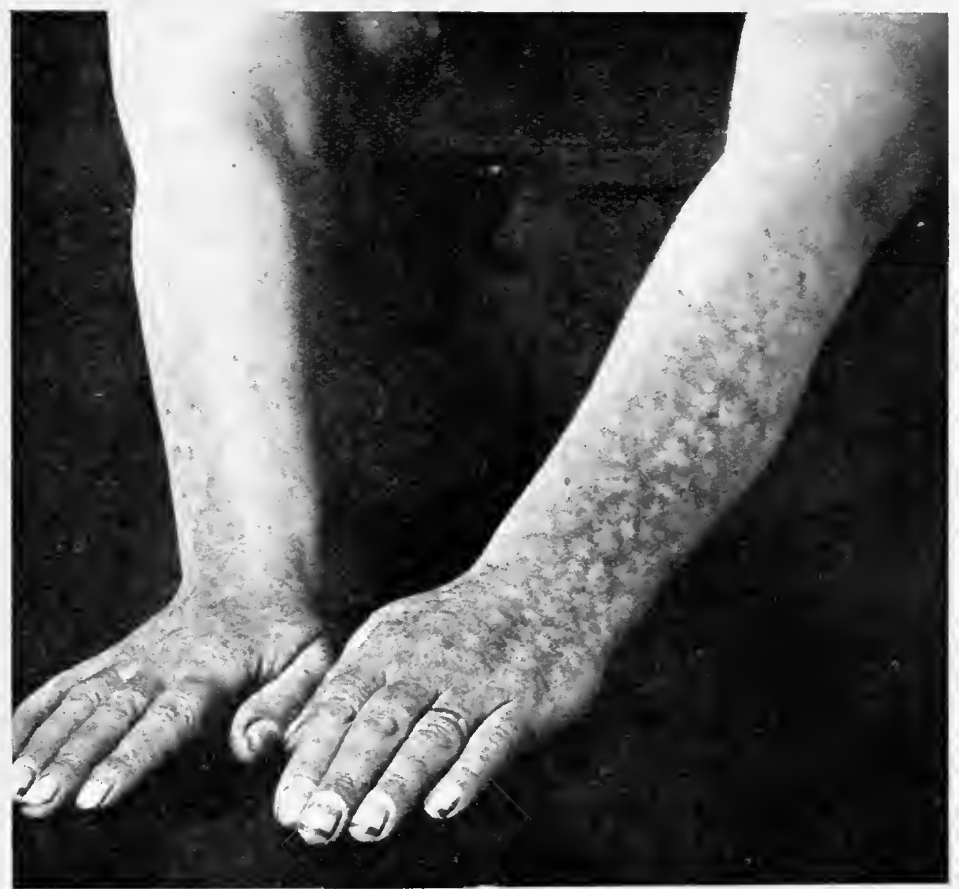

FIG. 242.-Erythema ab Igne.

(From Dr. Arthur Hall.)

reticularis which occurs in the form of a network situated on the middle or lower third of the shin, or exceptionally on other parts, and is the result of frequent or prolonged exposure to considerable heat. The network is brownish-red in colour, occasionally slightly raised, and leaves a pigmented stain on pressure with a diascope. It is the clinical evidence of congestion of the venous network enclosing pale areas corresponding to the bases of the arterial cones (see page 654 ). It is .usually caused by toasting the legs before a fire but may be produced by hot water bottles, hot compresses, etc. (Fig. 242.)

(b) Irritating and Septic Discharges may be responsible for erythema about the ears, nose, vagina, or anus, and in the napkin region in infants. 
(c) Chemical Irritants such as acids, alkalies, turpentine, mustards, etc., may cause it (see page 275).

(d) Vegetable Irritants, such as the different poison plants (see Dermatitis venenata, page $272 \%$.

(e) Bacterial Irritants, such as the toxins of septic micro-organisms.

(f) Animal Irritants, such as jellyfish, caterpillars, and the stings of insects.

\section{INTERNAL AND REFlex CAUSES}

(a) Psychical.-Mental causes, by acting reflexly on the vasodilator mechanism, may give rise to localised erythema, as in blushing from emotions such as shame, anger, etc. This is usually confined to the blush areas of the face, namely the malar prominences and the nose, but it may be more diffuse and may extend on to the neck or, occasionally in sensitive young women, may occur on the chest.

(b) Peripheral Nerve Disturbances may cause it; as in erythromelalgia where the neuralgic symptoms are associated with erythema, or in the early stages of maculo-anaesthetic leprosy where red patches appear as the result of the irritation by the leprous deposit of the nerve fibres supplying the affected skin.

(c) Reflex Irritation may be responsible for localised erythema, as in flushing after meals owing to the irritation of the sensitive mucous membrane of the stomach by food, or the flushed patches on the face and elsewhere which occur in infants in association with dentition or the presence of thread-worms, and in women with disorders of the genitalia.

(d) Toxins circulating in the Blood in specific diseases, such as pneumonia, rheumatic fever, typhoid, pellagra, etc., may produce localised erythematous blotches.

Diagnosis.-Localised patches of erythema are easily recognised. Occasionally mistakes may occur in distinguishing them from erysipelas, but the latter is usually accompanied by constitutional symptoms, the redness is more fiery and vivid, and the erythematous surface is raised and has a definite margin.

In the infant, intertrigo and simple erythema of the naphin region may be confused with congenital syphilis. The localisation is different, however, for in intertrigo the redness is limited to contiguous surfaces and in erythema the lesions are confined to the convex parts of the buttocks, thighs, genitals, etc., while in congenital syphilis they are more widely distributed, may extend down the legs to the feet, and are usually present also about the face, palms, and soles. The colour of the lesions is also different, for whereas in erythema they are reddishpink, in syphilis they have a characteristic coppery hue. In syphilis other specific symptoms are generally present, such as snuffles, hoarse cry, fissures at the lips, frontal bosses, and a peculiar grey tint of the skin. 
Prognosis.-The prognosis in localised erythema as a rule is excellent, the lesions readily disappearing when the cause is removed. In the case of psychical erythema or blushing, however, little can be done to minimise the discomfort. Where the erythema is the result of some specific toxin circulating in the blood, or of the reflex action of some irritant on the gastric mucosa, etc., the prognosis depends on the cure of the underlying disease or the removal of the irritant.

\section{TREATMENT}

The successful treatment of localised erythema is dependent on the recognition and removal of the cause.

Gastro-intestinal troubles should be corrected by careful dieting and suitable medicines. Sources of reflex irritation should be sought for and dealt with, such as thread-worms in children, decayed teeth, etc. Causes of local irritation should be eliminated, such as pressure, friction, or the action of physical, vegetable, chemical, or animal irritants.

Locally the erythematous skin should be soothed and protected by bland dusting powders or by zinc paste.

Where the erythema is the result of friction of contiguous surfaces, as in Erythema intertrigo, this must be prevented, either by rest or by the interposition of a layer of lint impregnated with powder, a muslin bag containing powder, or some other contrivance. Folds in which sweat is liable to collect must be kept scrupulously clean by frequent bathing with warm water or boric lotion, followed by the application of a drying dusting powder, such as equal parts of zinc oxide and magnesium carbonate; starch powder should not be cmployed as it is not antiseptic and is apt to become caked.

In erythema due to pressure, especially where the vitality of the skin is diminished, the most careful nursing and cleanliness are imperative to avoid bed-sores. Air-cushions and water-beds are indicated to reduce the pressure, and the skin should be bathed daily with spirit or boric lotion followed by the application of a powder containing boric acid 3 ss, zinc oxide $\overline{3} s s$, and talc $\overline{3}$ ss. Should bed-sores be present they must be treated by antiseptic lotions or dressings.

In erythematous patches and erosions in the napkin region in infants, thorough cleanliness with frequent changing of the napkins is essential and the application of mild antiseptic dusting powders, lotions, or ointments, as indicated by the individual case.

\section{Simple Erythemas of the Napkin Region IN INFANTS}

The above title was suggested by Adamson for a series of erythematous lesions not uncommon in infants-especially of the poorer classes--and which may be included under the localised erythemas. 
These occur chiefly about the buttocks, genitalia, and thighs. Jacquet (see page 320) carefully described and differentiated them under the heading of "Dermites simples infantiles" and distinguished four phases, namely:

(a) Simple erythematous.

(b) Erythemato-vesicular or erosive.

(c) Papular or post erosive.

(d) Ulcerative.

The erosive, papular, and ulcerative lesions are generally surrounded by an erythematous halo or situated on a diffuse erythematous patch.

The lesions are partly due to pressure and partly to the irritating and septic action of moist and soiled napkins. It has been suggested that gastro-intestinal disturbances may act as a predisposing factor by lowering the vitality of the skin.

\section{GENERALISED ERYTHEMA}

The types of eruption belonging to this class of erythema resemble more or less closely an exanthem of an acute specific fever and are due to toxins circulating in the blood. They vary greatly in their clinical appearances and in the extent of their distribution. In one type the eruption may be made up of red macules varying in size from a pin's point to a pin's head and so closely studded together as to form diffuse bright red areas widely distributed over the body like the bright red flush of scarlet fever; in another, the initial lesions may be macules the size of a split-pea, round, oval, or irregular, in outline, sometimes urticarial, tending to coalesce, and recalling the rash of measles; while in a third the eruption may be coarse and blotchy like a syphilitic roseola. In exceptional cases the erythema may be associated with oedema of the affected parts, or sudamina like those of rheumatic fever may be present on the reddened skin, or in rare instances the lesions may be definitely purpuric.

Generalised erythema is usually bilateral and symmetrical and is most marked on the trunk, upper arms, thighs, and face-especially about the sides of the nose. In rare instances it may be present on the hands and feet and is occasionally universal. It generally runs a fairly rapid course, reaching its fuil development in a day or two, but may not be fully out for a week. As a rule it fades in a few days, though it occasionally persists for weeks or months, and tends to recur.

Subjective symptoms may be present but are rarely pronounced. They consist of itching, pricling, tension, or burning, and are usually. most marked just before the redness appears after which they gradually diminish.

The fading of the eruption is generally accompanied by desquamation which usually commences before the redness has gone. In mild 
cases it is fine and branny, while in severe and extensive eases it is coarse, with flaky scales on the trunk and an exfoliation in thick lamellae on the palms and soles. A defluvium of the hair may oceur and also changes in the nails, such as transverse depressions at the lunules, detachment of the anterior part of the nail-plate, scaly thickening of the nail-bed, and sometimes actual shedding of the nails. In such eases it is not unusual for the sweat to be almost completely suppressed.

Occasionally the mucous membranes are attacked, the fauces and tonsils being congested, the tongue slightly furred, and the conjunctivae painful and injected.

The eruption is symptomatic of a general intoxication and is, as a rule, preceded by constitutional symptoms which may be slight but are occasionally so severe as to suggest a specific fever. Of these the most common are malaise, loss of appetite, pains in the back, joints, and limbs, headache, sickness, vomiting, griping pains, diarrhoea, insomnia, shivering, a rise of temperature to $\operatorname{roo}^{\circ}$ or $\operatorname{ror}^{\circ} \mathrm{F}$., and an increased pulse rate. They may precede the erythema by a few days and disappear when it comes out, or they may occur simultaneously with it.

Histopathology.--The microscopical appearances vary according to the severity of the erythema and consist of dilatation of the capillaries of the papillary and sub-papillary layers with serous exudation in the neighbourhood, extravasation of leucocytes, slight oedema of the overlying epidermis, and an interference with keratinisation. In some cases a decided eosinophilia and in others indicanuria have been described, but these are not sufficiently constant to be of diagnostic value.

Etiology.-Eruptions of the generalised erythema type are noncontagious. They are equally common in either sex and may oceur at any age but are most frequent between the ages of 20 and 40 . They are due to the circulation in the blood of toxins as numerous as they are various, the more common of which may be grouped under the following headings :

I. Food Toxins from foods which, when absorbed, instead of acting as nutriment, cause irritation. In this connection it is difficult to decide whether it be the food or some element of it which eonstitutes the poison, or if it be some active autogenous toxin called forth by it.

2. Autogenous Toxins produced as the result of disturbances of the liver, kidneys, gastro-intestinal tract, etc., and which, owing to defective elimination, are absorbed into the blood.

3. Drugs, such as belladomna, copaiba, opium, chloral hydrate, salicylate of soda, mercury, sandal wood, sulphonal, antipyrin, etc., may produce eruptions in certain individuals with an idiosynerasy towards them.

4. Bacterial Toxins either acting directly or indirectly by calling forth irritating anti-toxic substances in the blood, as in pyaemia, septicaemia, endocarditis, gonorrhoea, diphtheria, leprosy, ete. In 
this connection it is sometimes difficult to be sure whether it is the specific toxin or some drug taken to counteract it which is the cause of the eruption.

5. Protozoal Toxins, such as those produced by the spirochaete of syphilis, the trypanosome of sleeping sickness. or the parasites of malaria.

6. Injections of Sera and occasionally ! accination.

7. Enemas, from the irritation of soap and by causing the solution and absorption of toxic products.

8. Poisonous Effuria from Defective Drains.

Diagnosis.--The diagnosis of generalised erythema may present no serious difficulty, or on the other hand it may puzzle the most experienced clinician. The discovery of some definite toxic cause in many of the cases and the mildness or absence of constitutional symptoms generally serve to distinguish it from an exanthem of an acute specific fever; the absence of itching, oozing, and crusting, from eczema ; and the rapidity of its evolution, absence of conical papules, and want of definition of the erythematous areas, from a widely distributed case of Pityriasis rubra pilaris.

Measles can generally be excluded by the absence of coryza, cough, romiting, and other prodromal symptoms of the fever, but it is important to remember that in abortive measles such prodromal symptoms do not occur. In these cases the mouth should be examined for Koplik's spots and the distribution of the eruption should be taken into consideration, for in measles it generally begins on the forehead, face, above and behind the ears, and at the sides of the neck, and spreads downwards, while in generalised erythema it more usually attacks the trunk first. The occurrence of measles in epidemic form is a raluable aid to diagnosis.

Prognosis.-The prognosis with regard to an individual attack is excellent, as the eruption disappears without a trace and even if in severe exfoliative cases the hair and nails be shed they grow again. In a certain number of cases recurrences take place.

The affection is only serious when it is symptomatic of some severe infective process.

\section{TREATMENT}

In the first place a careful examination should be made for the cause of the eruption which, if discovered, should be suitably treated. If it be due to some drug, the drug must be prohibited; if to some food toxin this must be eliminated by suitable laxatives, diuretics, etc.; if the result of defective action of the liver, kidneys, or gastro-intestinal tract, the defective organ must be treated on general medical lines; if symptomatic of some general disease it is the latter which requires treatment.

Should the most thorough search fail to reveal any cause the health of the patient must be dealt with on general principles, hygienic, 
dietetic, and medicinal. If the skin be extensively involved the patient should be confined to bed.

The local treatment consists of the application of a dusting powder containing zinc oxide, talc, etc., or of a sedative lotion or cream to relieve itching.

The treatment of generalised exfoliative cases will be discussed under the heading of Exfoliative dermatitis.

\section{RECURRENT EXFOLIATIVE SCARLATINIFORM ERYTHEMA}

The generalised erythema of the scarlatiniform type has attracted special attention owing to the difficulties in diagnosis to which it may give rise. In it the eruption closely resembles scarlet fever and may be preceded for a few days by more or less severe throat symptoms, such as reddening of the fauces, tonsilitis, slight angina, and a furred tongue. The erythema is bright red in tint and may involve the whole cutaneous surface but is usually most marked on the trunk, arms, and thighs. About two days after its appearance, desquamation of a furfuraceous type sets in while the skin is still vividly red; the eruption may then last a few days and gradually fade or it may persist for months. Recurrences are frequent at irregular intervals, sometimes of a few months at other times of a year or longer, but as a rule are less severe than the original attack.

Diagnosis.- It is distinguished from scarlet fever by the history of previous attacks and the comparative mildness of the pre-eruptive symptoms, for eren although the fauces and tonsils may be congested the angina is seldom severe. In scarlet fever on the other hand a second attack is rare and the prodromal symptoms, especially angina and pains in the head, are acute. In scarlet fever swollen lymphatic glands and a strawberry tongue are characteristic features, while in this affection the lymphatics are rarely enlarged and the tongue, though it may at first be covered with a white fur later tends to become smooth and denuded. In scarlet fever the eruption rarely persists more than ten days while in this affection it may last for weeks or months. In scarlet fever the desquamation generally does not begin until about the sixth or serenth day when the eruption has almost, if not completely, faded, while in this affection it begins about the second or third day when the redness is still present. In doubtful cases it is advisable to isolate the patient.

\section{ERYTHEMA EXUDATIVUM MULTIFORME}

Fr., Frythème polymorphe.

Under the heading of Erythema exudativum multiforme Hebra grouped a number of erythematous eruptions in which the 
erythema was associated with more or less marked exudation of serum. These eruptions, however much they may differ in their clinical aspects, are so similar in etiology and distribution that it is reasonable to group them together and to regard them as different stages or variants of one pathological process modified according to the precise cause and the peculiarities of the individual affected. In connection with them it has been found that idiosyncrasy plays an important part and that one form of toxin may be responsible for different types of eruptions in different indiriduals and that recurrent eruptions in an individual may be constant in type though excited by different causes.

The essential lesions of the eruptions vary and may be macular, papular, circinate, urticarial, vesicular, or haemorrhagic. In the past a series of Latin names has been employed to designate the different types of eruptions according to the essential lesion which predominates, such as Erythema papulatum, E. circinatum, E.annulare, E. iris, E. tuberculatum, E. gyratum, E. urticatum, E. lividum, E. purpuricum, E. vesiculosum, E. bullosum. Such distinctive names are of little value, as the eruptions vary greatly and no two cases are alike so that any attempt to classify them according to the essential lesion is doomed to failure.

The eruptions as a rule are bilateral and symmetrical, the sites of predilection being the face, especially the forchead and around the eyes, the neck, backs of the hands, forearms, legs, knees, and dorsa of the feet. Occasionally the whole shin may be involved and not infrequently the mucous membranes.

To simplify description the eruptions may be divided into two main types, namely the maculo-papular, and the resicobullous.

Maculo-Papular Type.-In it the lesions appear first as red macules or slightly raised papules which rary in size from a pin's head to a split-pea, are roundish in outline, bright red in tint, pale temporarily on pressure, and as a rule are fairly well-defined. They tend to increase in size and to become raised and flat or rounded on the surface, and may remain discrete or may coalesce and give rise to polycrclical figures. Sometimes they take the form of deep-seated nodules or nodose swellings as in the special type known as Erythema nodosum. On palpation they may be soft but are more often of a peculiar elastic consistence. On the extremities they are apt to assume a violaceous or livid tint, especially in those with a weak circulation, and occasionally instead of being bright red they may hare an opaline or urticarial appearance, forming a connecting link between the papular and vesicular varieties. Sonetimes annular and gyrate forms are produced by the tendency of certain lesions to heal at the centre while spreading at the periphery; a remarkable example of this is Erythema iris, so-called from its superficial resemblance to the iris of the eye. In cases of unusual severity in individuals whose resistance is low the 
lesions may become purpuric and, if the haemorrhage be deep-seated may even become gangrenous. (Fig. 243.) (Plate IX.)

The eruption may develop in a few hours and be transient, lasting only a few days, or new crops may come out and prolong it for months. Sometimes individual lesions may persist for years when it is known as Erythema perstans, an annular variety of which was described by Crocker and Williams under the heading of Erythema elevatum dintinum.

When the lesions disappear they are followed by slight desquamation but neither by scarring nor pigmentation unless they have been

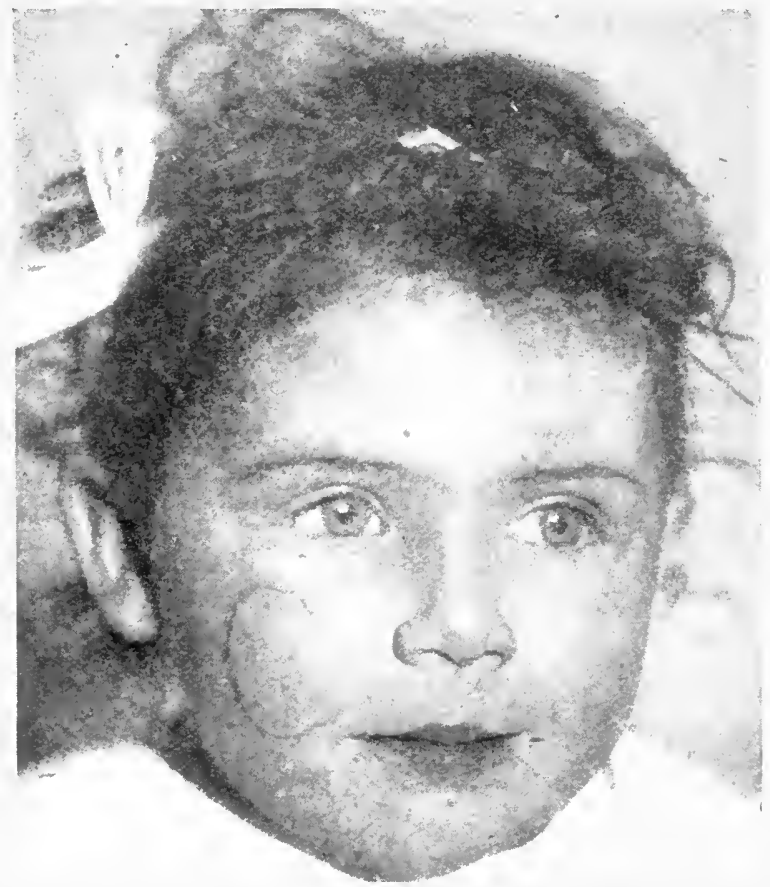

FIG. 243,-Erythema Multiforme.

Shows raised plaques on cheek and forehead.

purpuric in type when they may go through the same changes as a bruise or may be succeeded by superficial ulceration.

Vesico-Bullous Type---Here the predominating lesions are vesicles or bullae varying in size from a pin's head to an inch or more in diameter. They are generally preceded by an erythematous macule or blotch which extends beyond them as a reddish halo. They may be discrete and irregularly grouped or may be arranged in rings sometimes enclosing a red papule or central vesicle. (Fig. 244.)

The contents of the vesicles are clear at first but tend to become cloudy and purulent from infection with the ordinary skin-cocci. The vesicles are easily broken and leave a moist bleeding surface which becomes covered with a yellowish scab. They tend to come out in 



\section{PLATE IX.}

FIg. I.-Erythema multiforme exudativum.

Shows erythematous patches with well-defined borders on arm, and ringed iris lesion on hand.

FIG. 2.-Chronic Pemphigus in Infant. 

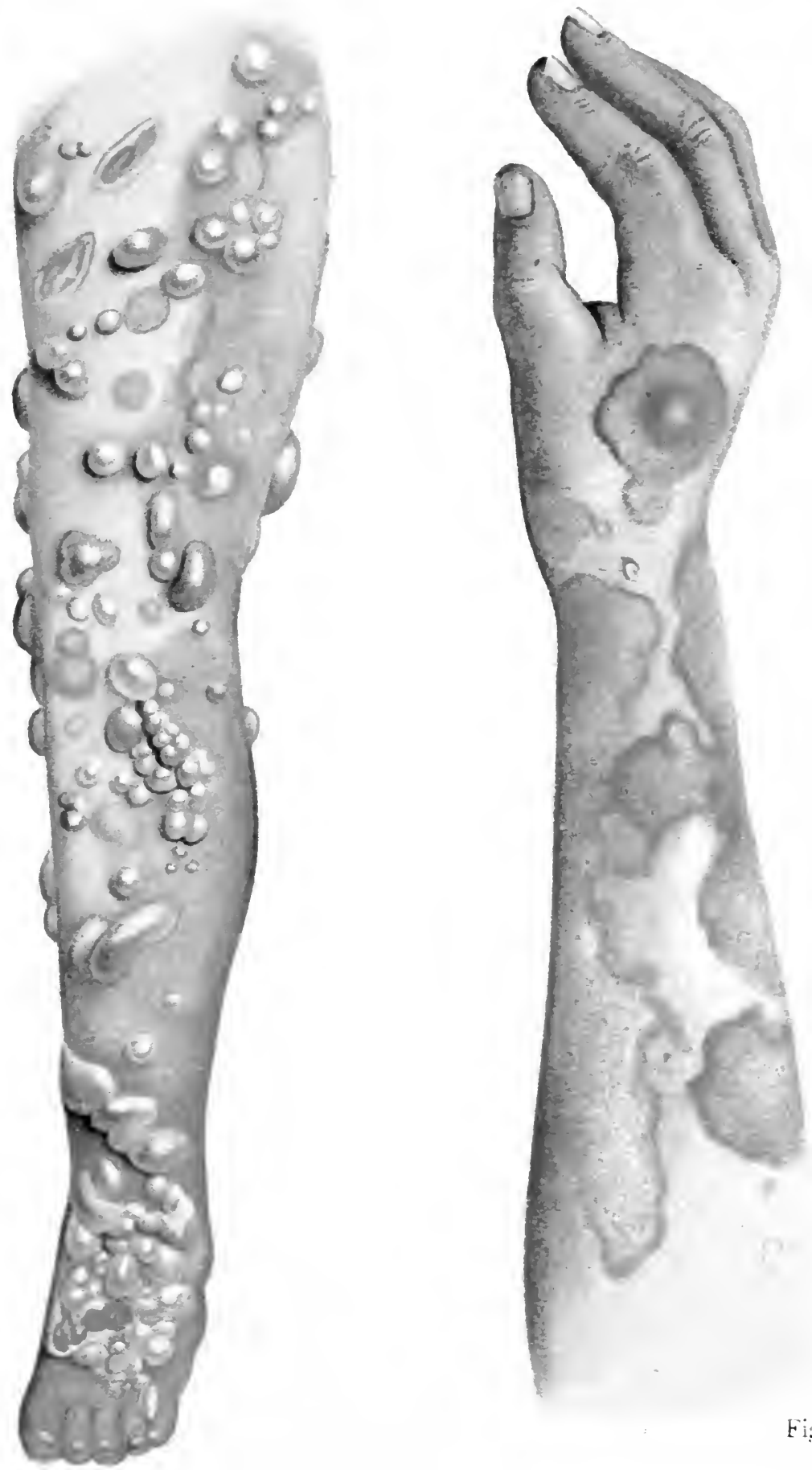

Fig. 1 .

Fig. 2 

crops and all stages in their evolution may be present at one time. Sometimes the skin on which the vesicles are situated is swollen from oedema, a complication which seems to bear no definite relation to the severity of the eruption as it may be marked when the eruption is slight and iice iersa.

Ervthema Iris is the most striking variety of Erythema exudativum. In it the lesions are annular in type. Ther begin as red macules or slightly raised papules about the size of a split-pea which tend to clear in the centre and to spread at the periphery and are limited by raised red borders. The ring keeps growing and at the same time a new central papule appears which goes through similar changes, so that a

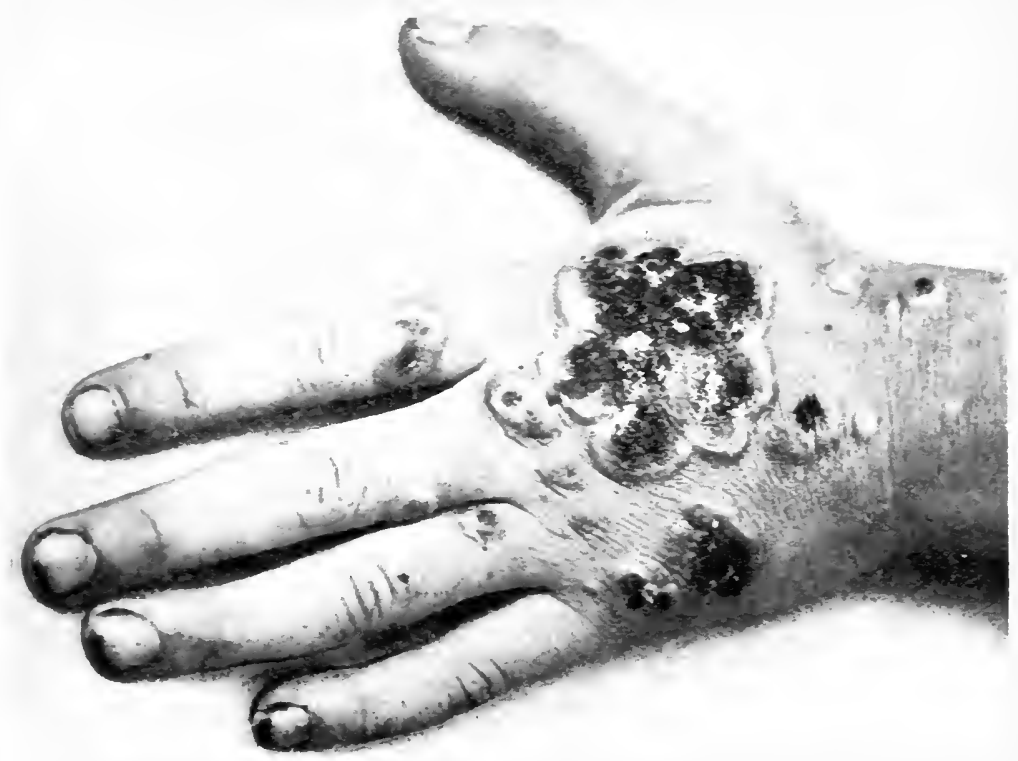

FIG. 24t.-Erythema Bullosum.

striking figure is formed consisting of two or more concentric circles surrounding a red papule. Sometimes the central portion assumes a purplish tint which is also noticeable between the rings and a target-like figure results, described by the French as "en cocarde." In some cases the rings become definitely urticalial or vesicular and the central papule is replaced by a vesicle, which dries up to form a scab which, in turn, leaves a reddish-blue surface on separating. The rings are generally broken up by a number of small resicles arranged like beads. To the vesicular phase Willan applied the name of Herpes iris and Besnier Erythema hydroa. The lesions are generally present on the backs of the hands and legs but mar occur in any of the usual situations. They last about a fortnight, fade, and leave a stain, but there is a tendency for new crops to appear and for recurrences to take place. 
Mucous Membranes.-The mucous membranes are affected in a considerable number of the cases, chiefly in those of the vesicular type, and sometimes more severely than the skin. The mouth and pharynx are most commonly involved and may present red raised patches or vesicles associated with swelling of the tongue which render the mouth so sore and tender as to cause difficulty in eating. In some cases the mucous membranes of the nose, conjunctiva, and genitalia, are attacked.

Subjective Symptoms.-These vary greatly in different cases. They may be absent, negligible, or severe, and consist of pricking, burning, tenderness, or pain on pressure, and itching which may be so great as to suggest Dermatitis herpetiformis.

General Symptoms.- There may be no premonitory symptoms or a short invasion period may occur with fever, which usually disappears

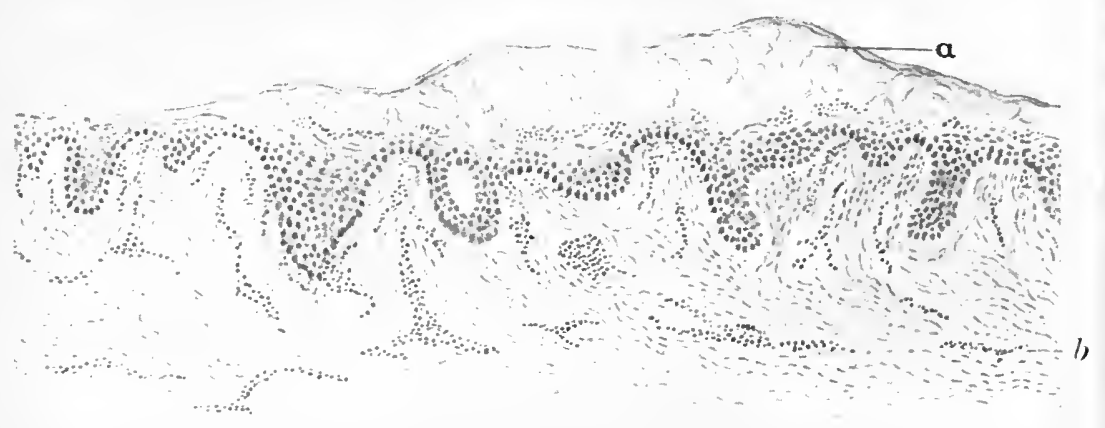

J. M. H. M. del.

FIG. 245.-Erythema Bullosum. ( $x$ about I 5 O.) a. Bulla containing coagulated serum and leucocytes. b. Capillaries of the papillary
and sub-papillary layers dilated.

when the eruption comes out, sore throat, gastro-intestinal derangements, and pains in the larger joints which may be of a severe rheumatic type and associated with swelling. Neuralgic pains have also been described in connection with it, especially in the intercostal nerves. In some cases the general health may be excellent throughout the attack, in others the patient may be seriously ill. When severe constitutional symptoms oceur they generally indicate the association of the eruption with some definite infective process, such as pneumonia, endocarditis, or nephritis with albuminuria or haematuria, and cases have been described where haematuria has appeared with each recurrence.

Histopathology.-The principal changes in the corium consist of dilatation of the blood-vessels, extravasation of serum, and diapedesis of leucocytes. These may be slight and confined to the papillary and sub-papillary layers, as in the maculo-papular lesions, or they may be more deeply-seated, occupying the whole of the corium and even extending down to the subcutaneous tissue, as in the nodular forms. When the corium is deeply involved not only lencocytes but red 
blood cells may pass out from the dilated capillaries and a proliferation of fixed-cells may be detected in their neighbourhood. (Fig. 245.)

The epidermal changes vary according to the degree and rapidity of the serous exudation. They may consist of dilatation with lymph of the inter-epithelial lymphatic spaces, oedema of the prickle-cells, and interference with cornification; or where the serous exudation is excessive the prickle-cells may become spongy and fluid may collect around the nuclei, or it may cause a rupture of the inter-epithelial fibres and the formation of a vesicle or a bulla which is generally situated about the Stratum lucidum but which, should the serous exudation have been rapid and the epidermis resistant, may form immediately beneath the epidermis in the papillary layer. The contents of the blebs are clear at first with only slight traces of fibrin, but later become cloudy or pirulent.

Etiology.-Erythema multiforme is due to toxins circulating in the blood and of these there is an endless variety.

Drug and food toxins are rare causes as they tend rather to produce a generalised erythema.

Injections of sera and toxins of bacterial origin are often responsible and in several cases micro-organisms, such as staphylococci and streptococci, have been detected in the cutaneous capillaries in the affected areas.

It may be met with also in connection with a large number of bacterial infections and septic conditions, such as diphtheria, cystitis, oral sepsis, and putrefactive changes of the intestines.

Vaccination has been followed by it in a considerable number of cases, the eruption usually being of the Erythema iris or bullous type. This has occurred even after the use of glycerinated calf $1 \mathrm{rmph}$. It has been observed that when the vaccination wounds had healed and the scabs fallen off, the raccinated area showed signs of renewed activity in sympathy with the eruption.

It may occur in connection with disturbances of the viscera, chiefly of the liver, gastro-intestinal tract, and kidners, and a form of it has been described in association with interstitial nephritis under the heading of Erythema uraemicum in which a dusky erythematous rash preceded fatal uraemic symptoms.

Its relation to rheumatism has been much discussed and certain observers consider that it may sometimes be a symptom of a rheumatic toxaemia due to a specific micro-organism, and in support of this riew point out that it frequently occurs in connection with tonsillitis, chorea, endocarditis, and other symptoms which pertain to the rheumatic group, that it may be connected with rheumatic fever, and that there is occasionally a history of rheumatism preceding or following it. Others again, take exception to this theory, believing that the rheumatic phenomena are not necessarily due to a single specific virus but may occur in a number of infective conditions. 
It is sometimes seen in epidemic form in institutions or in families, when it has been attributed to some special article of food or to sewergas from defective drains.

In not a few of the cases typical attacks may be met with in which the most careful search may fail to reveal a cause.

Predisposing Causes.-Of the predisposing factors age, sex, idiosyncrasy, and season, play a more or less important part. It chiefly attacks those under 30 years of age, is more common in females than in males, and there is a distinct tendency for initial attacks and recurrences to take place in spring and autumn.

Diagnosis.--The different phases of this protean disease may simulate a number of cutaneous affections but, as a rule, the diagnosis is not difficult and the erythematous nature and multiformity of the lesions, the sudden onset preceded by slight prodromal symptoms, and the localisation chiefly on the backs of the hands, arms, face, legs, and mouth, are generally sufficiently characteristic to establish it.

Cases of the papular type may resemble urticaria but the distribution on the extremities rather than on the trunk and the more permanent character of the lesions, enable them to be differentiated.

Annular forms may simulate ringworm but the resemblance is superficial and any doubt may be settled by a microscopical examination.

Vesicular lesions, if small and extensive, may be confused with Dermatitis herpetiformis, but in it the lesions are grouped in herpetiform clusters, the localisation is different, the symmetry is less marked, and the subjective symptoms are more severe. They might also be confused with dysidrosis but in it the vesicles tend to be confined to the hands, especially along the sides of the fingers and interdigital clefts, and are generally small, numerous, deeply-seated, and accompanied by burning and itching.

In one or two instances it has closely resembled smallpox but in the latter the eruption is more widely distributed and the lesions go through the various phases, papular, vesicular, umbilicated, and finally pustular, which are diagnostic of smallpox.

Lupus erythematosus is the affection which it most closely resembles and cases may be met with in which a firm diagnosis is almost impossible; there are observers who consider that a definite relationship exists between them and that all stages may be met with between the raised transient erythematous lesions of E. multiforme and the typical scaly atrophic patches of $\mathrm{L}$. erythematosus, hence the name Erythema atrophicans, which has been applied to the latter.

In well-marked cases they may be distinguished by the following differences:

Distribution.-In L. erythematosus the lesions are situated chiefly on the malar prominences and nose (bat's wing area) and ears, a localisation which, though occasionally met with in E. multiforme, is rare. 
Lesions.-In L. erythematosus the lesions as a rule are scaly on the surface, the scales being fine and adherent, while in E. multiforme they are smooth and on fading may be followed by a furfuraceous desquamation or a coarser cxfoliation.

Atrophy.-In L. crythematosus atrophy is a characteristic of the disease, the patches on disappearing being replaced by superficial atrophic and pitted scars, in $\mathrm{E}$. nultiforme the patches disappear without leaving a trace.

Duration.-In L. erythematosus the lesions, even in the acute forms, generally persist for months or years, while those of E. multiforme tend to disappear in a few weeks.

Prognosis.-So far as the skin lesions are concerned the prognosis is excellent and though the eruption may persist for weeks it generally disappears without leaving a scar; recurrences are not infrequent.

When it is associated with disease of the kidneys, gastro-intestinal tract, or heart, or with some definite specific infection, the prognosis is that of the underlying diseased process.

\section{TREATMENT}

As a preliminary to treatment every endeavour should be made to discover the cause. If it be a drug it must be stopped, if some article of diet or alcohol it must be prohibited, if due to a disorder of the kidneys or the liver these must be treated on general medical principles. Where it is due to some autogenous intoxication resulting from gastrointestinal disturbances, intestinal antiseptics such as small doses of calomel or salol may be tried but these are of less value than a restricted purin-free diet combined with the proper treatment of constipation where present. If it is the result of putrefactive changes or is of septic origin, vaccines of colon bacillus or staphylococcus are worthy of a trial. When it is associated with rheumatic symptoms, salicin or the salicylates are indicated, or when with malaria, quinine.

Should a thorough search fail to reveal the cause then certain drugs may be tried empirically. Where the eruption is extensive and there is much tension in the skin, relief may be obtained from small doses of the vinum antimoniale, $5 \mathrm{~m}$ every four hours. With the object of improving the tone in the vaso-motor system, quinine up to $\mathrm{I}_{5}$ grs. per diem, and ergotine have been adrocated. Where the lesions are urticarial and there is defective coagulability of the blood, calcium lactate, Io grs. t.d.s., has been recommended but the results from it are uncertain. In the prescribing of drugs in this connection, however, it is important to be careful that the remedy does not aggravate the disease and drugs, such as iodide of potassium, sulphonal, antipyrin, etc., known to be capable of producing erythematous eruptions in those with an idiosyncrasy towards them should be employed with the utmost caution or aroided altogether.

In every case a simple diet and no alcohol, with proper regulation 
of the bowels and kidneys, should be insisted upon and where the disease is extensive and there are definite constitutional symptoms it is advisable to confine the patient to bed as long as the acute symptoms last.

Local Treatment.-The local treatment is essentially protective and sedative. Protection against the ill-effects of friction or of exposure to the air and sunlight may be obtained by the use of bland zinc and talc dusting powders or zinc paste or, where possible, the lesions may be covered by a suitable dressing. Where the lesions are vesicopustular mild antiseptic applications, such as boric acid dusting powder, ammoniated mercury ointment Io grs. to the oz., etc., should be used to prevent them becoming septic. Where there is heat and itching soothing applications are indicated, such as an evaporating spirit lotion of one part of rectified spirit to four parts of distilled water, or Lotio calaminae with the addition of $\mathrm{I} \%$ carbolic acid, and where there is pain an evaporating lead lotion may be employed, such as the above spirit lotion with the addition of $\mathrm{I} \%$ of Liquor plumbi subacetatis.

\section{REFERENCES.}

Adamson, H. G. Infantile eczemas of Jacquet (Brit. Journ. Derm., I909, xxi. p. 4I). Erythema multiforme (Brit. Journ. Derm., r9I2, xxiv. p. 429$)$.

Axthosy, H. G. The toxic origin of Erythema multiforme (Journ. Cut. Dis., I9I2, xxx. p. I52).

BEARD \& BARLOw. Ery thema scarlatiniforme (Lancet, 1906, i. p. 900).

BrocQ. Erythema multiforme and polymorphic dermatitis (Ann. de Derm. et de Syph., Jan. I9I2, p. I).

DARIER, J. L'Erythème annulaire centrifuge (Erythème papulo-circine migrateur et chronique) et de quelques éruptions analogues (Ann. de Derm. et de Syph., I9I6-17, March I9I6, p. 57).

Dubreully. Recurrent Erythema scarlatiniforme (Brit. Journ. Derm., I908, xx. p. 6I).

Finger, E. Streptococci in erythema and purpura (Arch. f. Derm. u. Syph., Sonderabd., I893).

Fox, Colcott. Erythema (Allbutt \& Rolleston, System of Medicine, I9II, ix. p. 244).

Gallowar \& MAcLEOD. Relation of Erythema multiforme to general toxaemia (Brit. Journ. Derm., I903, xv. p. 8I, \& I908, xx. p. 65).

Gardiner, F. A case of Erythema scarlatiniforme (Brit. Journ. Derm., I 908 , Xx. p. 245).

Goodall, E. W. Prodromal rashes (Brit. Med. Journ., 1907, ii. p. 374).

Jacquet, L. Dermites simples infantiles (Traité des Maladies de l'Enfance, Gaucher \& Comby, I905, iv. p. 7I4).

Kirch, C. A. Erythematous and exanthematous rashes (New York Med. Journ., I908, Ixxxviii. 777).

Kreibich, K. Histology of Ery thema multiforme (Arch. f. Derm. u. Syph., I90I, P. I 25).

Osler, W. Visceral complications of Erythema multiforme (Amer. Journ. Med. Sci., I 895 ).

Polland. Exfoliative erythrodermias, clinical observations and pathogenesis (Arch. f. Derm. u. Syph., Bd. ci, hft. 2-3, I91o, p. 321).

Wende, G. W. Erythema perstans (Trans. Amer. Derm. Ass., I905, p. I 4 I). 


\section{ERYTHEMA NODOSUM}

Erythema nodosum though simply a variety of Erythema multiforme is so characteristic a type that it is customary to describe it under a separate heading. In it the eruption consists of oval ill-defined nodosities, varying in size from a hazel-nut to a pigeon's egg, generally situated on the shins with the long axis corresponding to that of the limb. The lesions come out suddenly in an hour or two, and are usually few in number, smooth on the surface, bright rose-red in. tint, and resistant to the touch. They are associated with heat, pricking, or burning, and are often tender on palpation.

The eruption is usually preceded for twenty-four hours by prodromal symptoms, such as slight fever, gastro-intestinal disturbances, malaise, and pain in the joints especially the knees and ankles. The joint pains are the most constant, are of a rheumatic type, and may be accompanied by swelling due to peri-articular inflammation and fluid in the bursae.

The eruption is usually bilateral and situated in front of the tibia on the middle and lower thirds of the legs, but it may occur on the buttocks, thighs, feet, arms, in exceptional cases on the face, but practically never on the trunk. The individual lesions may remain discrete or may coalesce to form diffuse patches and occasionally are associated with more or less marked oedema in the region affected. Each nodosity lasts about twelve hours, when retrogressive clianges set in spontaneously, the hardness decreases, the lesions become soft and almost fluctuate, and the bright rose tint becomes replaced by a violet tinge which gradually changes into blue, green, and finally yellow, like a bruise and from the similarity the affection is sometimes known as Dermatitis contusiformis. In three or four weeks the lesions have disappeared completely, being followed by a slight transient desquamation, but in some cases new crops appear and prolong the eruption for a couple of months or more. The nodules never undergo suppuration.

In association with the typical nodular lesions, papular or vesicular types are occasionally present, showing a definite connection with E. multiforme.

Histopathology.-Microscopically there is dilatation of the capillaries, exudation of serum, diapedesis of leucocytes and red blood cells, and proliferation of the connective tissue cells in the neighbourhood of the capillaries. This affects not only the cutaneous capillaries but extends down to the blood-ressels of the subcutaneous tissue. In rare instances, thrombosis has been detected in the deep-seated reins, and micro-organisms, such as streptococci and Demme's bacillus, have been found in the blood and may be pathogenically connected with the eruption. The overlying epidermis may be slightly oedematous.

Etiology.- The etiology of Erythema nodosum is the same as that of E. multiforme, the most important point being its association with pains in the joints of a rheumatic character and other morbid conditions 
related to rheumatism, such as chorea and endocarditis, and it has been suggested that it is the result of the same toxin or specific microorganism. Against this theory is the observation that Erythema nodosum does not show the same tendency to recur as other forms of Erythema multiforme, while rheumatism is usually recurrent.

It is most common in young people under 20 years of age, is about twice as frequent in girls as in boys, and is liable to appear in spring and autumn and occasionally as the result of cold.

Diagnosis.---It has to be distinguished chiefly from syphilitic gummata and from Erythema induratum of Bazin.

From syphilitic gummata it is differentiated by the gummata being less numerous, more indolent in their course, neither tender to the touch nor associated with pains in the joints or other subjective symptoms, and tending to break down and ulcerate leaving a pigmented scar.

Erythema induratum affects the calf rather than the shin, its lesions come out one at a time, are slow in their evolution, appear first as nodules that can be felt rather than seen, assume a dull reddishviolet tinge rather than bright pinkish-red, and after months undergo slow necrosis leaving a pigmented scar.

It has to be distinguished from true rheumatic nodules, which are fibrous and not erythematous, and from the painful nodules which occur in connection with varicose veins especially in youngish women and which also have a marked tendency to ulcerate and to be followed by a cicatrix and deep pigmentation of the skin.

Prognosis.-As a rule the prognosis is excellent and if any grave consequences occur they are the result, not of the Erythema nodosum, but of some underlying serious condition such as pyaemia or endocarditis. If left alone it tends to run a course of a few weeks and recurrences are rare.

\section{TREATMENT}

The general principles of treatment of Erythema nodosum are similar to those of Erythema multiforme, but there are certain special indications. In order to relieve the tenderness of the lesions and the pains in the joints, the patient should rest on a bed or couch with the legs elevated, and hot fomentations or warm lead and opium lotion should be applied, followed by a dusting powder, and the limb then covered with cottonwool under a loose bandage. This should be continued as long as the acute symptoms last.

The patient should be put on a simple diet and any underlying cause sought for and treated. Where the rheumatic pains are marked salicin in doses of 10 to $20 \mathrm{grs}$. t.d.s., is of benefit. Iodide of potassium in doses of 5 to ro grs. t.d.s., aspirin, and antipyrin, have been advocated but should be taken with caution as they may aggravate rather than improve the cutaneous lesions. 


\section{REFERENCES}

Deléard \& Halley. Erythema nodosum (L'Echo Méd. du Nord., IgI I, XV. P. 4OI).

LoIseleur \& Monziols. E. nodosum preceding a meningococcic septicaemia (Bull. et Mém. de la Soc. Méd. des Hôp., I9Io, p. 772).

O'Brian. E. nodosum, etiology of (Deutsch. Arch. f. klin. Med., IgI I, civ. p. 272).

\section{ERYTHEMA ELEVATUM DIUTINUM}

This title was given by Radcliffe Crocker and Campbell Williams to a somewhat rare type of persistent erythema possibly of toxic origin and most probably belonging to the Erythema multiforme group. In the case described by them the patient was a healthy little girl of 6 years of age. The disease began simultaneously on both knees and developed later on the buttocks, elbows and hands. The lesions were raised, well-defined, roundish or oval nodules about the size of a bean, or irregular plaques made up by the coalescence of several nodules. They were smooth on the surface, convex, purplish-red in tint, with a few dilated ressels ramifying over them, firm in consistence, tender on pressure, and not associated with any definite itching or burning. They were freely movable on the subjacent tissue and the epidermis over them appeared to be healthy. They persisted for about a year, then disappeared but whether spontaneously or as the result of the internal administration of arsenic was undecided.

Histologically they were made up of a fibro-cellular tissue in which the cellular elements predominated in one part and the fibres in another.

Somewhat similar lesions have been described in association with rheumatism, as in a case recorded by Bury in ISS9, and there is a model in the museum of the Hôpital St. Louis of an allied case with the heading of "Fibromes multiples nodulaires des extrémités, histologiquement fibromes fasciculés."

These cases are related to Granuloma annulare.

\section{REFERENCES}

Bury, J. Case of erythema with nodular thickening (Illustr. Med. News, I 889 , iii. p. I 45).

Crocker, Radcliffe \& Williams. Erythema elev. diut. (Brit. Journ. Derm., I 894 , vi. p. I).

FAvera. Erythema elev. diut. and Granuloma annulare (Derm. Zeitschr., I910, xvii. p. 5fI).

\section{GRANULOMA ANNULARE}

Syn. : Ringed Eruption (Colcott Fox), Lichen annularis (Galloway).

Definition: A chronic inflammatory affection of the skin characterised by the presence of small pearly nodules upon a slightly inflamed base, which tend to be aggregated in rings. 
Description.-This somewhat rare affection was first recognised by Colcott Fox who exhibited a typical case of it at the Dermatological Society of London in I895, in a girl aged II, under the non-committal title of " an unusual ringed eruption on the fingers." Since then a considerable number of cases have been demonstrated at the Dermatological Societies in London, France, Germany and America, and a variety of names has been applied to it.

The affection begins as a small, whitish, semi-translucent nodule about the size of a boiled sago-grain, sometimes on a faintly erythematous base, which is definitely indurated and can be made more noticeable by stretching the skin over it. A number of these lesions generally appear simultaneously or very soon after one another and tend to be arranged in a more or less perfect ring or crescent from $\frac{1}{2}$ to 2 inches in

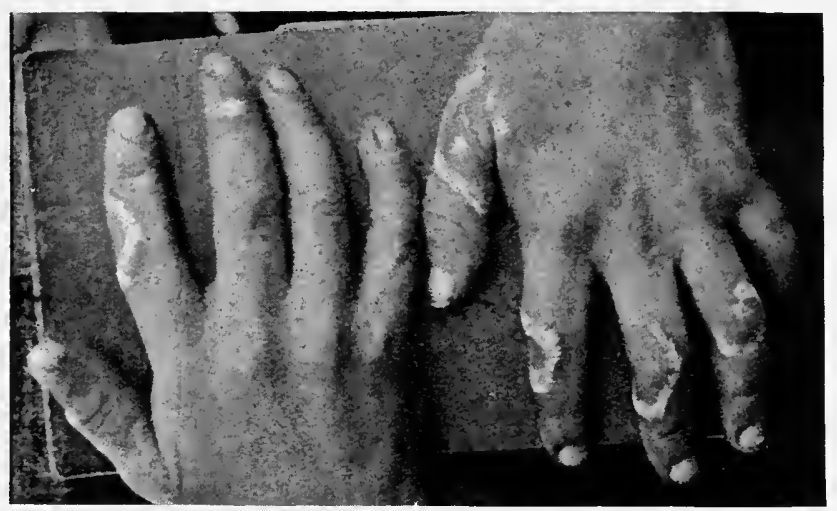

FIG. 246.-Granuloma Annulare.

(Sir James Galloway's case. Brit. Journ. Derm., xgo8.

diameter. The rings or crescents present a singularly characteristic appearance. The border is white, semi-translucent, and festooned from being made up by the coalescence of a varying number of small nodules, is from a I6th to an 8th of an inch in breadth and raised to about a I6th of an inch above the level of the skin. It is surrounded by a slightly inflamed halo and encloses an area of skin which is reddish or reddish-purple in hue and is sometimes atrophic. The individual nodules and the conglomerate border tend in time to lose their pearly appearance and to become reddish-purple. In some instances there is a small cluster of nodules instead of a ring and an appearance is produced which has been aptly likened to a pearl brooch. In rare instances the rings are formed by essential spreading of the initial nodule with involution in the centre, in which case the border is more uniform. As a rule there are no subjective symptoms associated with the lesions and the patient is generally unaware of their presence until they are noticed by accident. (Fig. 246.)

The lesions are generally few in number, varying from two or three to ten or fifteen. They are situated most frequently on the back or 
side of the fingers and next in order on the nape of the neck, wrists, feet, ankles, buttocks, thighs, calves, elbows, and ears; occasionally they occur on both sides of the body but show no definite srmmetry. They appear quite suddenly and if left alone persist for an indefinite period. They sometimes have a tendency to involute spontaneously; the border becoming broken up into separate nodules, and they finally disappear without scarring or pigmentation.

Histopathology.-A section of a typical nodule shows that the condition is the result of a more or less dense cellular infiltration chiefly localised to the deeper parts of corium adjoining the hypoderm. This infiltration is collected in foci around the sweat-coils and small vessels

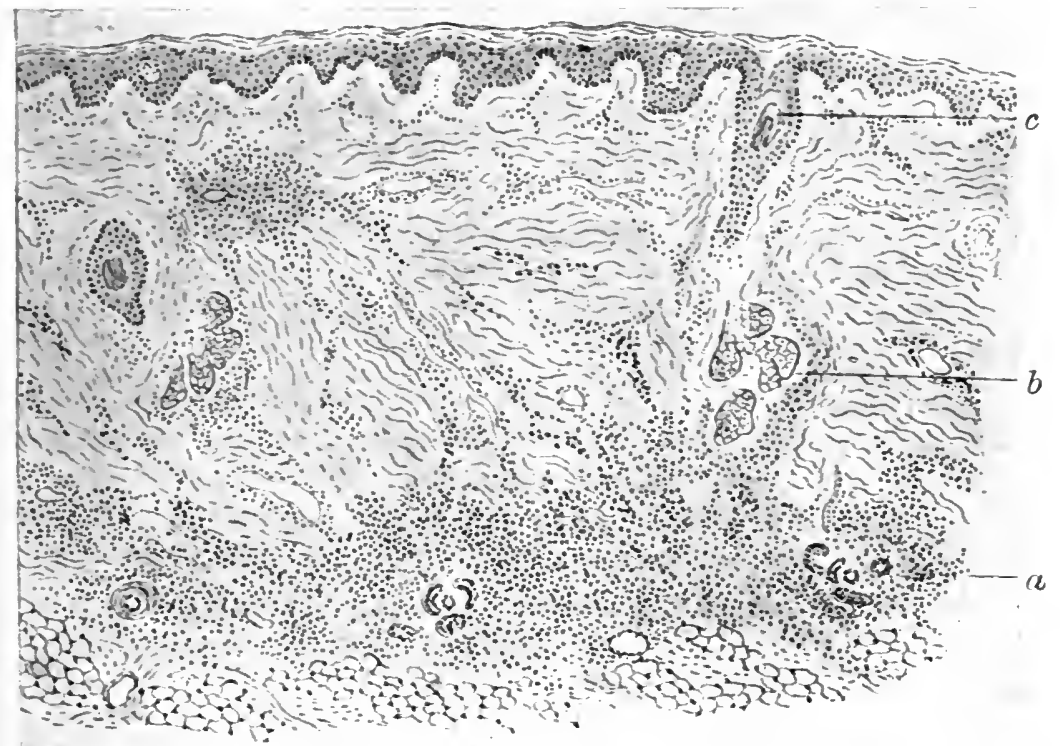

J. M. H. M. del.

FIG. 247.-Granuloma Annulare. Section of lesion. ( $x$ about I jo.)

a. Infiltration of cells collected in dense foci around the sweat-coils and small vessels. b. Sebaceous gland with cellular infiltration around it. c. Epidermis.

and tends to spread up towards the surface along the capillaries, sweatducts and pilo-sebaceous follicles. Where the infiltration is densest the fibrous elements of the corium have become attenuated and fragmented and in some cases the collagen cannot be detected. Occasionally it spreads up superficially as far as the sub-papillary or even the papillary plexus. The cells composing it consist chiefly of mononuclear cells like large lymphocytes with round nuclei and a faint halo of protoplasin, mixed up with which are small connective tissue spindlecells, a few larger cells with vesicular nuclei (epithelioid cells), and occasionally mast-cells, but there are neither plasma-cells nor giantcells. The epidermis does not show any definite changes though thickening of the horny layer has been recorded in sereral instances. (Fig. 247.) 
Nature and Etiology.-The exact nature and causation are unknown. The histological appearances suggest that it is due to a toxin reaching the skin via the blood-vessels and which is possibly eliminated by the sweat-apparatus, as the cellular infiltration is not infrequently grouped in a marked way around the sweat-coils.

There is no general toxic condition which might be regarded as in any way responsible for it though the lesions bear a slight resemblance to the nodules which occur occasionally in rheumatism. In a few cases there has been a tuberculous family history and it has been suggested that it may be a peculiar form of tuberculide, but the evidence is insufficient to warrant this assumption and the probability is that any apparent association was accidental. Galewski has described cases under the heading of " Tumores benigni sarcoidei cutis" suggesting a relationship with the sarcoids of Boeck, which are probably tuberculides.

Of the predisposing causes sex seems to have no bearing on its etiology as an equal number of cases have been reported in both sexes. Age on the other hand is a predisposing factor as it is most common in childhood and early adult life. There is some evidence that it begins most commonly in the hot weather, possibly on account of the sweatglands being more active then.

Diagnosis.- The diagnosis between badly marked cases of Granuloma annulare and the annular form of Lichen planus may present some difficulty. In Lichen planus, however, the initial lesion is not a white semi-transparent roundish nodule but a violaceous flat and angular papule, is not definitely indurated, is generally associated with itching, and frequently leaves a brownish stain. Histologically the cellular infiltration in Lichen planus is more superficial and circumscribed and there is well-marked thickening of the epidermis.

\section{TREATMENT}

The affection may disappear spontaneously but should it not do so it is generally amenable to treatment. As the precise cause is unknown there is no definite indication for internal remedies unless on general medical principles, and most cases are cured by local treatment with an ointment containing $2 \%$ or $3 \%$ of salicylic acid, kept continuously applied spread on lint under a bandage, or by painting the lesions with $2 \%$ salicylic collodion. Other remedies such as resorcin and ichthyol have given good results.

Nomenclature.-The nomenclature connected with this affection has led to considerable confusion. The name Granuloma annulare under which the majority of cases have been recorded was given to it by Radcliffe Crocker, but is an unfortunate one, as the term granuloma is a histological designation which is now generally restricted to a group of affections in which there is a cellular infiltration of a distinct type and in which plasmacells are a characteristic feature, and the cellular infiltration in this affection 
does not belong to this type and has no plasma-cells. The name Lichen annularis is equally unfortunate as it is apt to lead to confusion with Lichen planus. Cases have been recorded on the Continent under various other names, such as Erythémato-sclérose circinée du dos des mains (Audry) and Néoplasie circinée et nodulaire (Brocq). In the present state of our knowledge a " nodular ringed eruption" is perhaps the best title, as it is at the same time non-committal and sufficiently descriptive.

\section{REFERENCES}

Arxd, G. Granuloma annulare (Arch. f. Derm. u. Syph., I9I I, criii. p. 229).

AUdry, C. Erythemato-sclerosis circinée du dos des mains (Ann. de Derm. et de Syph., I904, p. 9).

BrocQ. Néoplasie circinée et nodulaire (Idem, I904, p. 1089).

Chipman, E. D. Case of Granuloma annulare (Brit. Journ. Derm., I9I I, xiii. p. 349).

Crocker, Radcliffe. Granuloma annulare (Brit. Journ. Derm., I902, xiv. p. I).

Dubreuilh. Eruption circinée chronique de la main (Ann. de Derm. et de Syph., I895, p. 355).

FAVERA. Erythema elevatum diutinum \& Granuloma annulare (Derm. Zeitschr., I9I0, xvii. p. 54I).

Fox, T. Colcotr. Ringed eruption (Brit. Journ. Derm., I895, vii. p. 9I).

Granuloma annulare (Proc. Roy. Soc. Med., I910, iii. (Derm. Sect.), p. I04).

Galloway, J. Lichen anmularis (Brit. Journ. Derm., I899, xi. p. 22I).

Krausxer. Histology of Granuloma annulare (Arch. f. Derm. u. Syph., May I9I4, cxx. p. I).

Little, E. G. Granuloma annulare: a clinical \& histological study. (Note-Comprehensive article with full references to all cases described up to I908). (Proc. Roy. Soc. Med., I908, i. (Derm. Sect.), p. 95).

\section{ERYTHROMELALGIA OR RED NEURALGIA}

This name was applied by Weir Mitchell to a rare affection chiefly of one or both lower extremities, associated with redness, local fever, and pain. It usually occurs in adults and begins with a dull burning pain, a reddish flush or purple congestion of the skin, and considerable swelling of the affected part. As a result of walking or of allowing the legs to hang down the condition is aggravated, the congestion deepens, the swelling becomes more tense, the arteries throb, and the parts may become excruciatingly painful. The affection is chronic and continuous, though subject to exacerbations in hot weather or from the dependent position, never undergoes complete remission like Raynaud's disease, and is liable to go on to gangrene. It was once believed to be a vaso-motor neurosis but since in association with it definite changes of a sclerotic and obliterative type have been observed in the larger as well as the smaller blood-vessels, it is now regarded as being 
due to them. These changes are similar to those met with in Trench foot (see page 227 for treatment).

\section{REFERENCES}

Barlow, Sir Thomas. Erythrome!algia (System of Medicine, Allbutt \& Rolleston, I9Io, iii. p. I49).

MitcheLl, Weir. Erythromelalgia (Amer. Journ. Med. Sci., I878, lxxvi. p. I7).

Weber, F. PARkes. Erythromelalgia (Brit. Journ. Derm., 1904, xvi. p. 7 o). 


\section{.}




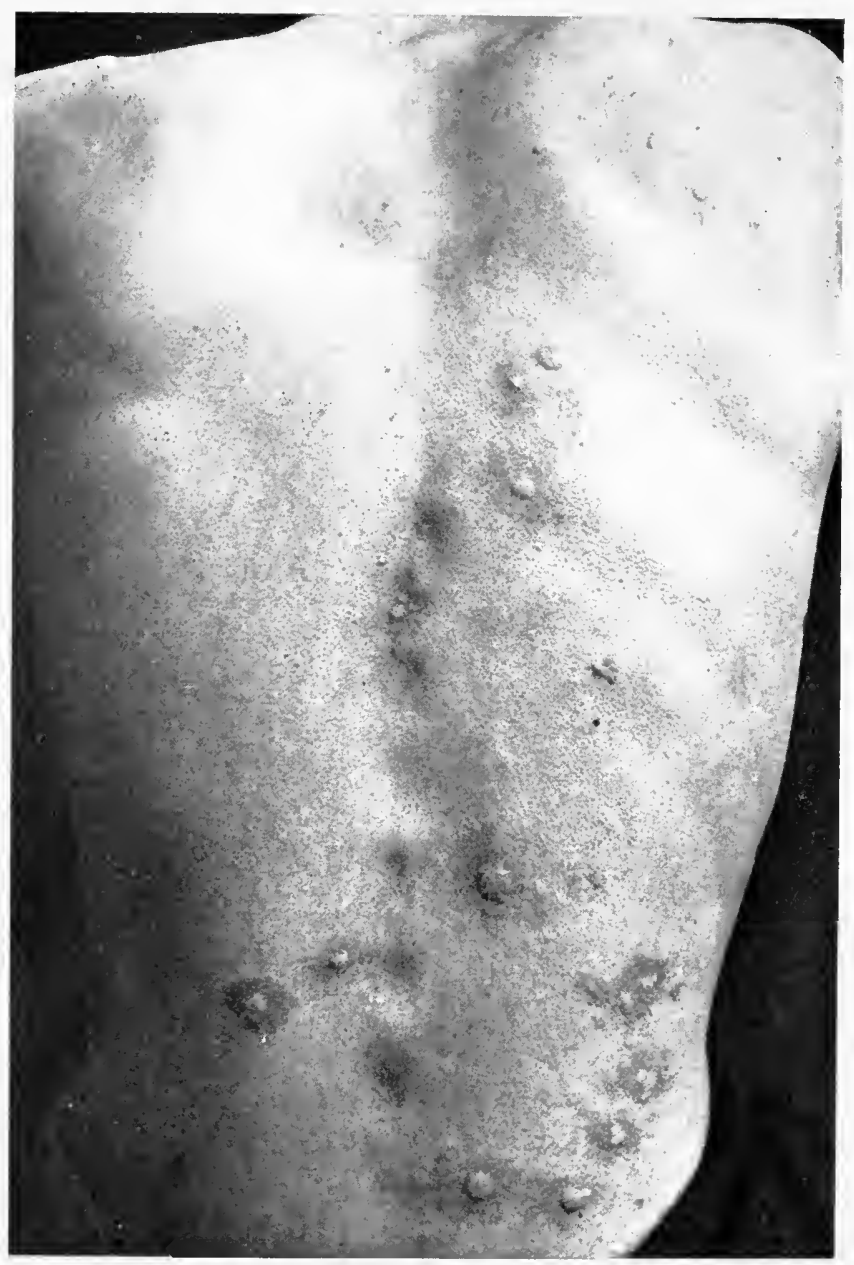

FIG. 248.-Urticaria.

With small wheals on an inflammatory base. Appeared the day after eating fried fish.

[To face p. 679. 


\section{CHAPTER XXIII}

\section{URTICARIA}

Deriv. : Urtica, a nettle.

Syn.: Nettlerash, Hives, Cnidosis; Greek Krviòn (nettle); Fr., Urticaire ; Ger., Nesselsucht.

Definition: An inflammatory affection of the skin characterised by the presence of transient whitish elevations generally surrounded by a reddish halo and similar to a wheal caused by the lash of a whip or the sting of a nettle.

Description.-Urticaria may be an essential or primary eruption due to some general or local cause, or may occur in the course of various skin affections, such as Erythema multiforme, Mycosis fungoides, and Dermatitis herpetiformis, or as a complication of itchy parasitic conditions, such as pediculosis and scabies.

The typical urticarial lesion consists of an elevation of the skin, with a flat or slightly convex surface, pinkish at first but when fully developed presenting a whitish or ivory-like tint, and generally surrounded by a reddish inflammatory halo which fades into the neighbouring skin. It is raised about a millimetre and is tense and somewhat elastic in consistence. It varies in shape according to whether it develops spontaneously when it is round, oval, or irregular, or is due to local irritation such as friction or pressure when it assumes a form corresponding to the area of action of the causal agent, and it varies in size from elevations the size of a split-pea to plaques as large as the palm of the hand or larger. The surrounding halo may be pink and faint, or reddish and vivid, and may show definite capillary pulsation. (Fig. 248.)

The lesions of urticaria are invariably associated with subjective symptoms, such as itching, burning, or tingling, which may precede their appearance and usually continue till they have faded. These may be comparatively mild, or so acute as to be almost intolerable and to interfere with sleep. They may be present continuously but are generally subject to exacerbations and tend to be worse at night.

The wheals usually come out suddenly, have a transient existence of a few minutes to several hours, and fade spontaneously. They 
may be single, or limited in number and confined to one region, or they may be numerous and more or less widely and irregularly distributed. Though they may occur anywhere in the skin they are most frequent on covered parts, especially on the trunk.

As a general rule they disappear without leaving any trace but occasionally they may be followed by slight desquamation or even by pigmentation, when they have to be distinguished from Urticaria pigmentosa which is not a true urticaria, or where they have been excoriated by scratching and septic inoculation has taken place, they may be succeeded by superficial ulceration and scarring.

The mucous membranes may be affected as well as the skin, and chiefly those of the mouth, tongue, pharynx, and glottis, and more rarely of the bronchial tubes, anus, vulva, prepuce, Eustachian tube, and middle ear. When the throat is involved swallowing and breathing may be interfered with and death may occur from suffocation; when the bronchial tubes are affected attacks of more or less severe asthma generally result and sometimes alternate with outbreaks of urticaria on the skin; when it occurs in the Eustachian tube or middle ear the hearing is impaired. Sometimes the urticaria is associated with haematemesis, diarrhoea, colitis, or melaena, pointing to an involvement of the mucosa of the gastrointestinal tract.

There are two main types of urticaria, namely an acute and a chronic type.

Acute Urticaria.-In the acute type the eruption comes out suddenly and profusely, and is preceded or accompanied by more or less severe general symptoms, such as pyrexia, sickness, vomiting, pains in the back, sore throat, and other signs of a general intoxication, or may follow a violent nerve storm such as an outburst of emotion or anger. The attack may last a few hours to a day or two and then gradually die down, or new lesions may keep on appearing and the condition become chronic.

Chronic Urticaria.-In the chronic type the eruption is perpetuated by fresh lesions coming out for months or even years. These may appear periodically, after meals from some special article of diet, from exposure to cold on removing clothes, from warmth in bed, in association with malaria, etc., or aperiodically when it may be impossible to determine the cause of the attack.

So long as the eruption continues a state of unusual sensibility of the skin is induced, by which it reacts with the greatest readiness to even slight external stimuli and wheals are easily called forth by friction or the pressure of articles of clothing, such as garters, corsets, or boots (see factitious urticaria, p. 68I).

In certain chronic cases a diminished coagulability of the blood has been noticed which is probably caused by the same factor as the urticaria; in others the coagulability may be actually increased. 
Varieties.- Instead of ordinary wheals the lesions may be papular, bullous or hacmorrhagic in type, may be abortive forming variously sized macules or blotches, or may tend to involute at the centre and spread at the periphery forming annular or gyrate figures.

In papular urticaria the lesions consist of small red papules about the size of a pin's head situated in the centre of a pinkish blotch about a quarter of an inch in diameter. This type occurs most commonly in infants, but may be met with in older children and occasionally in adults. As it differs markedly in its clinical appearances from ordinary urticaria it will be described separately (see Lichen urticatus, p. 697).

In the bullous rariety the inflammatory and oedematous process is so acute as to give rise to a resicle or bulla situated on an inflammatory basis. It is the result of an irritant of unusual virulence acting on a

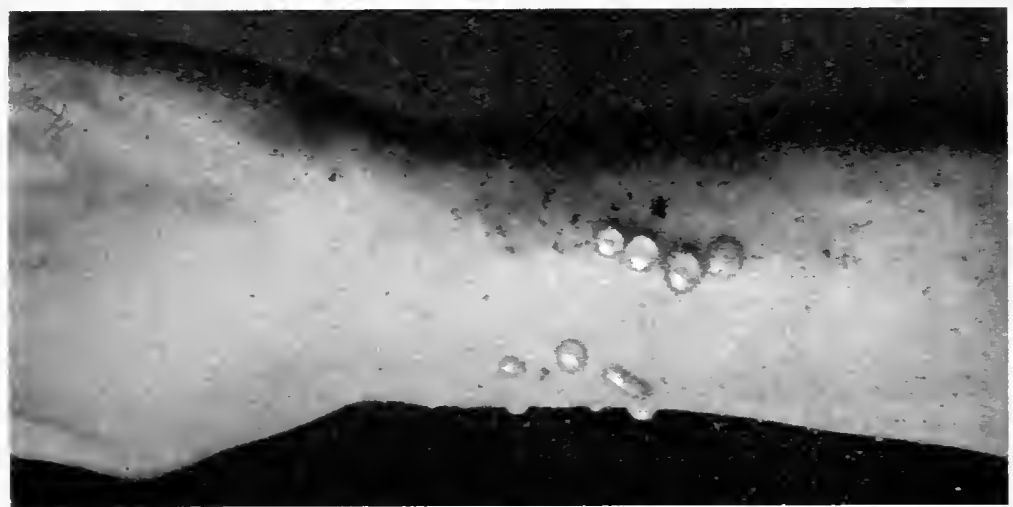

FIG. 249.--Urticaria Bullosa.

(From Dr. S. W. Allworthy.)

skin with an exceptionally sensitive vaso-motor reflex. Like papular urticaria it is more common in children than in adults. (Fig. 249.)

The haemorrhagic variety is rare and occurs in connection with severe internal disease, such as Bright's Disease, or septicaemia. Occasionally wheals may occur as a complication of purpura, in which case the bleeding occurs first and the wheal formation is superimposed (see Purpura urticans, p. 939).

There are several other rare varieties of urticaria, namely factitious urticaria, Giant urticaria, Urticaria perstans, and wandering oedema.

Factitious Urticaria.-By factitious urticaria is meant wheal formation mechanically produced by rubbing, scratching, or pressure, instead of occurring spontaneously. For its production it is necessary that the skin be hypersensitive and the vaso-motor reflex called forth with unusual ease. This hypersensitiveness is a frequent concomitant of urticaria and may persist long after the eruption has faded. It may be present from infancy or early childhood and no clear history of its inception be obtainable, but it is doubtful if it is ever congenital. It 
may occur, in association with itchy skin affections such as Dermatitis herpetiformis, parasitic conditions like scabies, or nervous disorders such as meningitis and hysteria. (Fig. 250.)

In mild cases considerable friction may be necessary to cause a wheal and a slight rub may only result in an evanescent blotch. In severe cases the slightest friction may be sufficient to produce it and if a blunt instrument, such as the end of a pen, be drawn firmly over the skin a linear wheal will result or a name may be written on the sensitive

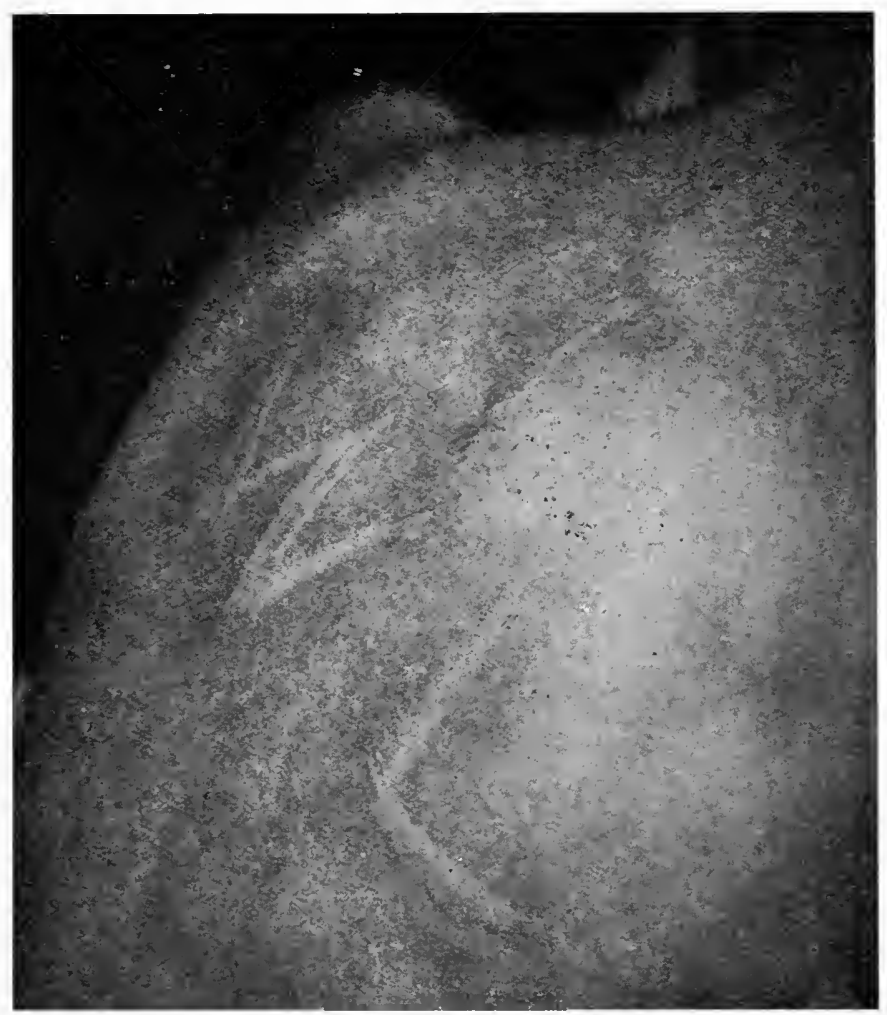

Frg. 250.-Factitious Urticaria.

surface; this is known as dermographism or autographism. It may also be produced by exposure to extreme heat or cold and can be called forth even when the patient is under the influence of a general anaesthetic. The wheal may appear a few seconds after the friction and may persist from a few minutes to an hour or longer. Where the skin is unusually' sensitive, instead of a wheal, a vesicle or bulla may be produced by the friction.

Giant Urticaria.-In giant urticaria, or Urticaria nodosa, the lesions instead of being confined to the skin involve also the subcutaneous tissue and take the form of puffy nodules or brawny swellings, the skin over which may be unaltered or reddish in tint. It occurs chiefly about the hands, forearms, feet, legs, or in situations where the tissues 
are lax as about the lower eyelids, cheeks, and lips, and may last a few hours to several days (see Angio-neurotic oedema, p. 693).

Urticaria Perstans.-In Urticaria perstans the individual lesions persist for months, and fibrous and even warty changes have been said to supervene in them. There is some doubt whether they are of the same character as ordinary wheals and it has been suggested that they are probably more of the nature of the nodules which occur in connection with rheumatism.

Wandering Oedema.-This is a name which has been apolied to peculiar cases in which the swelling, instead of being stationary, wanders up the limb. The exact nature of these cases is not fully understood but the occurrence of the puffy wandering swellings in association with typical wheals and the occasional presence of transitional forms suggest that they are closely allied to urticaria.

Histopathology.-The principle histological changes in a wheal are transient in character and occur mainly in the corium. They consist of dilatation of the blood-ressels of the papillary and sub-papillary layers, widening of the neighbouring lymphatic spaces, serous extravasation, and a slight infiltration of cells consisting of lymphocytes, polymorphonuclear leucocytes, occasional mast-cells, and oedematous connective tissue cells.

The epidermis may be unaltered or oedematous with dilatation of the inter-epithelial spaces, oedema of the prickle-cells, diminution of the keratohyalin granules, and defective cornification. In rare instances where the extravasation of fluid is excessive buliae occur either beneath the epidermis or, if the exudation has taken place more slowly, in the situation of the granular layer. The hair-follicles and sweat-glands are not affected.

Pathogenesis.-Wheal formation is a type of reaction of the skin due to a raso-motor disturbance caused by the direct action of some stimulus on the ressel walls or indirectly through the vaso-motor centres. By whatever means the stimulation takes place there is an initial contraction or spasm of the small arteries and veins at the junction of the skin and subcutaneous tissue followed by a dilatation of the arteries before the reins have become dilated, an increased flow of blood takes place into the arteries and capillaries while the outflow is impeded by the contraction of the veins, and leads to an exudation of serum. This by pressing on the dilated capillaries masks the original congestion, presses the blood out towards the periphery, and gives rise to the wheal.

Another theory is that the wheal is an inflammatory phenomenon associated with subsequent definite changes in the tissue such as occur in any process of repair after inflammation and that it is largely the result of the local action on the blood-ressels of some toxic irritant. In support of this theory it has been pointed out that numerous toxic irritants besides the juice of the nettle are capable of producing wheals when inserted into the skin, such as atropin, pepsin, cadavarin, and 
toxins from various micro-organisms such as staphylococci and Bacillus coli.

Etiology.-Urticaria may occur at any age and in either sex. The papular variety is practically confined to infancy and early childhood, while the ordinary type is rare in infancy, comparatively infrequent in old age, and somewhat more common in females than in males.

The causes are numerous and varied. Certain irritants, such as the sting of a nettle, or the ingestion of poisonous ptomaines from tinned meat or mussels, may call forth wheal formation in anyone, while others can only produce it in those who have a special idiosyncrasy or sensitiveness towards them. This predisposing sensitiveness may be an inherited peculiarity or may be acquired as the result of a previous attack of urticaria, some debilitating illness, or the abuse of alcohol or tobacco, which has lead to a weakened control of the higher nerve centres and established an unusually excitable vaso-motor mechanism.

For purposes of description the causes may be divided into external and internal.

External or Local Causes.-Of the local irritants capable of producing wheals or determining a general attack of urticaria the most important are the following :

Animal Irritants : such as bites of mosquitoes, gnats, bugs, pediculi, leeches, etc., and stings of wasps, bees, jelly-fish, and caterpillars of the woolly-bear type.

Vegetable Irritants: such as nettles, cowhage, tomato leaves, hybiscus, and Primula obconica.

Physical Irritants: Traumatism, from rubbing, friction, pressure, a sharp blow, or a lash with a whip.

Heat, from a fire, as in cooks, stokers, and firemen, from Turkish baths, or even from the warmth of bed.

Cold, from a cold bath, exposure of the skin when the clothes are removed, or chilling of the mucous membranes of the mouth and stomach by swallowing ice.

Internal Causes. - The internal causes of urticaria are usually toxic, such as food toxins, autogenous toxins, drugs, etc., but it may also be due to constitutional diseases or to disturbances of the nervous system.

Food Toxins.-Fish, such as mackerel, herrings, and fried white fish especially haddock. Shell-fish, chiefly crabs, lobsters, and mussels, in the case of mussels the toxin being an alkaloidal poison known as mytilotoxin present in the liver of the mussel. Fruits, such as strawberries and nuts. Vegetables, such as mushrooms and cucumber.

Meats, such as pork, veal, curries, high game, and tinned meats from the ptomaines present.

Foods which leave a definite amount of undigested residue, such as oatmeal, porridge, and brown bread.

Condiments, such as coffee, liqueurs, swcet and acid wines, and alcohol in any other form. 
(Note.-These irritating articles of diet may act directly on the shin by circulating in the cutaneous blood-stream or, reflexly by irritating the gastro-intestinal mucosa or possibly the nerves of taste).

Autogenous Toxins.-Foreign proteids eliminated by the cells of the mucous membrane of the alimentary tract or the liver in response to the presence of food toxins are believed by certain observers to be the invariable cause of the urticaria and not the circulation of the actual food toxins themselves. These foreign proteids are said to be more liable to be excreted when the gastro-intestinal tract is in a state of chronic irritability from indigestion, constipation, disorders of the liver, or the presence of worms in the intestines.

Constitutional Diseases: such as malaria, gout, rheumatism, septic peritonitis, or the infective fevers, the urticaria being the result of the special toxin of the disease or of some foreign proteid called forth in response to it.

Drugs: such as iodide of potassium, chloral, quinine, santonin, copaiba, sodium salicylate, and turpentine, in individuals who have an idiosyncrasy towards them.

Anti-toxic Sera: such as anti-diphtheritic and anti-streptococcic sera.

Hydatid Fluid: should the hydatid cyst burst into the peritoneal cavity or the fluid be injected hypodermically.

Disorders of the Genitai Organs,- both functional and organic, such as menstruation, parturition, and the menopause, disorders of the uterus and ovaries, and even the passing of an uterine sound.

Nervous Disturbances: Psychical, such as emotion, anger, or fright, which sometimes act by causing indigestion should the nerve storm take place soon after meals.

Disorders of nerves, such as neuralgia or Tabes dorsalis, when the urticaria may occur in the area of distribution of the affected nerve or in the site of the lightning pains.

Reflex nerve disturbances, such as may occur in connection with decared teeth, myopia, astigmatisn, or the wearing of glasses which do not properly correct errors of refraction.

Bacterial Toxins: Toxins absorbed from a focus of infection of bacteria anywhere in the body, such as $B$. coli infections of the intestine and bladder, pyorrhoea, and septic tonsilitis.

Diagnosis.-The clinical appearances of an urticarial lesion, the raised whitish elevation with its pinkish halo, its evanescent nature, and the distressing itching which accompanies it, are so characteristic as to render the diagnosis comparatively easy. Occasionally it may be difficult should no lesions be present at the time of examination while there is a history of their occurrence in the night, as in such cases even though there are usually evidences of itching, such as excoriations, these might have been caused by bug-bites or pediculi as well as by urticaria.

The puffy swelling of giant urticaria on the limbs or lower eyelids 
might be mistaken for renal disease, but the occurrence of typical urticarial lesions elsewhere and the absence of albumin in the urine serve to distinguish them.

Prognosis.-The prognosis in acute cases is excellent, as a rule, and the eruption generally fades under suitable treatment in a few days, occasionally however it may persist and become chronic. In rare instances a fatal issue has resulted from suffocation from oedema of the glottis or from septicaemia of which the urticaria was a symptom. In old people it should be regarded seriously as it may be associated with degenerative changes in the blood-vessels or with malignant disease.

In chronic urticaria the prognosis should be guarded as the disease is liable to persist indefinitely unless the exciting cause can be discovered and eliminated, and a peculiar hypersensitiveness is apt to be established whereby the slightest irritation may induce a recurrence.

\section{TREATMENT}

General Treatment.-Acute Urticaria.-In an acute attack there is generally a history of the ingestion of some substance the toxic action of which has been the direct cause of the eruption. When such is the case, if the patient be seen early it may be advisable to give an emetic, such as a dessert-spoonful of mustard in a cup of hot water, a teaspoonful of ipecacuanha wine, or a hypodermic of apomorphine; or if seen later, a purgative such as a blue pill or calomel at night and a saline aperient in the morning. These should be followed for a few days by small doses of some intestinal antiseptic, such as calomel, salicin, salol, or charcoal, and by a restricted diet and the avoidance of alcohol and smoking.

Chronic Urticaria.-In chronic cases the indications for treatment vary according to the cause, for which the most careful search must be made. Should any defect be found in the digestive organs, liver, kidneys, ovaries, or uterus, it must be dealt with by suitable means. In neurotic patients, tonics, fresh air, outdoor exercise, and change of employment, may be of the greatest value. The diet should be carefully regulated and of the simplest character and all articles prohibited which are known to be capable of determining an attack, such as shellfish, pork, curries, strawberries, coffee, alcohol, etc.

In cases where there are evidences of chronic irritability of the mucous membranes of the intestinal tract, benefit may occasionally be derived from treatment with Metchnikoff's lacto-bacilline tablets, or with sour milk freshly prepared with the Bulgarian bacillus.

When the urticaria occurs in association with malaria or rheumatism, quinine or salicin are indicated.

Should the blood-coagulability be found to be defective beneficial results have been recorded from the use of chloride or lactate of calcium to make good the deficiency of lime salts. These should be given in 
doses of Io grains three times a day for a few days, then stopped, and resumed in short intermittent courses.

In many cases in spite of the most thorough examination no definite cause for the urticaria can be found and it is necessary to fall back on certain empirical remedies to relieve the itching, induce sleep, and diminish the frequency of the attacks. Of these the most useful are chloral hydrate, phenacetin, sulphonal, potassium bromide, antipyrin, aconite, and tincture of belladonna. Phenacetin is probably the best and should be given in small doses repeated every two hours till the irritation ceases. Opium should not be employed, as it is uncertain in its action and liable to increase the itching when its effects wear off. Atropin, salol, and ichthyol, have their adrocates, and creosote has been strongly recommended in doses of 2 to $4 \mathrm{~m}$., as an initial dose, with $2 \mathrm{~m}$. every 20 minutes till the effect is produced.

In some cases benefit is derived from saline aperients, such as sodium sulphate, magnesium sulphate, or natural aperient waters; and in resistant cases a visit to some suitable spa such as Harrogate or Strathpeffer may be of the greatest advantage, not only on account of the waters but from the strict regime enforced.

In cases in which the urticaria is caused by a micro-organism like the Bacillus coli present elsewhere than in the alimentary tract autogenous vaccines may be beneficial.

Local Treatment.-The chief object of the local treatment is to relieve itching and for this purpose anti-pruritic lotions, creams and powders, are employed containing such remedies as carbolic acid, tar, lead, camphor, etc. Ointments and greasy applications are not suitable, being liable to increase the irritation.

Carbolic acid is one of the most useful of the anti-pruritics and may be prescribed as follows:

Lotions.-Solution in water ( $\mathrm{I}$ in 20 to $I$ in 40 ).

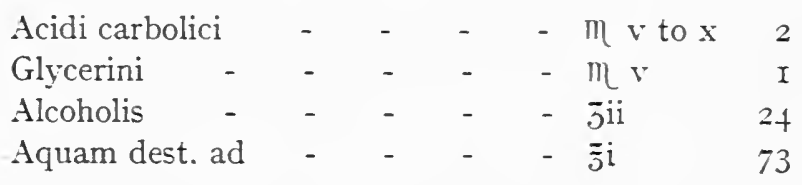

Cream.

$\begin{array}{lllll}\text { Acidi carbolici - } & - & - & - & -m v \\ \text { Zinci oxidi - } & - & - & - & -\overline{5} \mathrm{v} \\ \text { Calaminae prep. } & - & - & - & -\overline{5} \mathrm{i} \\ \text { Aquae calcis - } & - & - & - & -\overline{5} \mathrm{ss} \\ \text { Olei amygdalae } & - & - & - & -\overline{5} \mathrm{ss}\end{array}$

After the application of the lotion or cream a bland dusting powder should be used.

Tar is of service but should be employed with caution as it irritates certain skins. It may be used in the form of the Liquor picis carbonis 
a teaspoonful to half a pint of water, or as the Liquor carbonis detergens made up in the following cream :

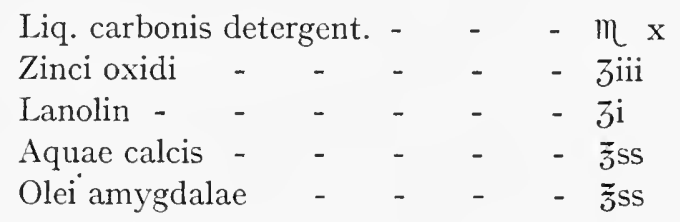

Iead is a valuable soothing remedy. It may be prescribed in the form of a lotion consisting of the Liquor plumbi subacetatis, a teaspoonful to half a pint of tepid water. It is sometimes combined with glycerine but this may prove irritating. Its action can be intensified by the addition of dilute hydrocyanic acid as in the following formula :

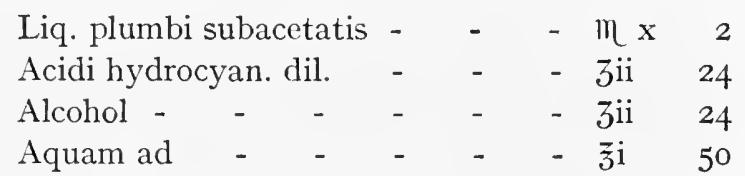

Acid lotions are soothing and of these the simplest are toilet vinegar, or one part of vinegar in 4 parts of water.

Evaporating lotions are useful, such as ordinary methylated spirit or still better the following :

\begin{tabular}{|c|c|c|c|c|}
\hline Chloral - & - & - & - & \\
\hline Chloroform & .. & - & - & \\
\hline Alcohol ad & - & - & - & \\
\hline
\end{tabular}

Ichthyol is of value and has its advocates. It is conveniently applied as an aqueous solution containing 5 to $10 \%$.

Thymol has also been recommended and the following formula is useful :

\begin{tabular}{|c|c|c|c|c|c|c|c|}
\hline Thymol & - & - & - & & & & \\
\hline Alcohol & - & & & & & & \\
\hline Liquor pe & & & & & & & \\
\hline Aquam a & - & - & - & - & & - & \\
\hline
\end{tabular}

The above are examples of the most generally useful of the antipruritic applications. Their action is temporary and it is necessary in selecting them to be careful that the remedy itself does not prove irritating. It is advisable to begin with weak solutions and gradually to increase the strength according to the toleration of the patient, as in this way any idiosyncrasy can be discovered.

Where the skin is widely affected tepid or warm alkaline baths containing borax or soda are helpful. The patient should remain in the bath about Io minutes and on coming out the skin should be dried with the least possible friction and a dusting powder applied.

Occlusive dressings have sometimes been recommended, such as painting the affected skin with Unna's zinc gelatine, but the results are unsatisfactory. 
As general measures, it is necessary to avoid over-heating by too heavy clothes in the day or too hot coverings at night, on the other hand it is important that the patient should be kept sufficiently warm as even slight cold may determine an attack.

In the case of urticaria affecting the throat and seriously impeding the breathing, sucking ice and scarification have been recommended, and where the bronchial tubes are involved the counter-irritation of local friction has been employed to bring the eruption to the surface as attacks of asthma generally alternate with outbreaks of eruption. In severe cases intubation or tracheotomy may be necessary.

\section{REFERENCES}

BARTHÉleMY. Etude sur la dermographisme (Paris, I893).

BlaIN, A. W., Jn. Urticaria following the second administration of diphtheria anti-toxin (Med. Rec., June 1908, p. 940).

Chittenden, T. H. Urticaria with recurrent haematemesis (Brit. Journ. Derm., I 898 , x. p. I 58 ).

Finch, E. B. Urticaria (Med. Rec., Feb. I9o8, p. зот).

Fox, Colcotr. Urticaria in infancy, etc. (Brit. Journ. Derm., I89o, ii. p. I33).

Gilchrist. Histology of urticaria (Journ. Amer. Med. Ass., 1896, xxvii. p. 1222).

JoAL. Urticaria produced by odours (Journ. de Méd., July Ioth, I 899.).

KreIbICh. Urticaria perstans (Arch. f. Derm. u. Syph., I899, xlviii).

MCBRIDE, W. L., \& Schorer, E. H. Erythematous and urticarial eruptions resulting from sensitisation to certain foods (Journ. Cut. Dis., I9I6, xxxiv. p. 70 ).

Mackenzie, S. Nature of urticaria (Brit. Journ. Derm., i 894, vi. p. 65). OsLer, W. Hereditary angio-neurotic oedema (Amer. Journ. Med. Soc., I $888, \mathrm{xcv}$. p. 362).

Paramore, IV. E. Experimental study of urticaria (Brit. Journ. Derm., I906, xviii. p. 239).

Philippsox. Experimental researches on urticaria (Giorn. Ital. delle Mal. Ven. e della Pelle, I899, vi. p. 675).

Török \& HARI. Pathogenesis of urticaria (Arch. f. Derm. u. Syph., I903, lxv. p. 2I).

Wills. Urticaria haemorrhagica (Lancet, I 890, i. p. 1296).

Wills \& Cooper. Acute circumscribed oedema (Brain, I 893, xvi. p. 382). Wright, A. E. Urticaria treated by calcium chloride (Brit. Journ. Derm., I 896, viii. p. 82).

\section{URTICARIA PIGMENTOSA}

Syn. : Xanthelasmoidea (Tilbury Fox).

Definition: A peculiar affection of the skin which usually begins before the sixth month and is characterised by persistent urticaria-like lesions, yellowish nodules suggesting xanthoma, and brownish-yellow stains.

Description.-This affection is one of the rarer cutaneous diseases. Originally described in England in I 869 by Nettleship it has attracted more attention in this country than elsewhere and out of ${ }_{54} 4$ cases 
collected by Graham Little (I 4 of which were personal) 62 were English, 39 German, 24 French, I6 American, and the remainder from other countries.

It usually begins in infancy as pinkish urticaria-like lesions varying in size from a pea to a nut. These may be rounded or irregular in outline but are most frequently oval with the long axis directed downwards on the limbs, downwards and forwards on the trunk, and transversely on the neck. They may be few in number and sparsely distributed or so numerous and closely aggregated as to involve nearly the whole of the cutaneous surface, and are situated chiefly on the trunk, especially on the back, and on the neck, face, and proximal parts of the limbs, occasionally on the scalp, and in extensive cases may be found anywhere on the cutaneous surface with the exception of the palms and soles. They are smooth on the surface, soft in consistence, become brighter in tint and turgescent when rubbed or when the child cries or is over-heated, and differ from the lesions of ordinary urticaria in that itching is an inconstant accompaniment and may be entirely absent. In rare instances they may be capped or replaced by bullae which are usually surrounded by a narrow inflammatory halo. They tend to come out suddenly in crops or clusters like those of ordinary urticaria, and new outbreaks occur from time to time.

Factitious urticaria or dermatographism may occur in association with it even where no itching is present and new urticarial elements may be brought out when the skin is rubbed.

The urticarial type of lesion constitutes only the initial phase of eruption and is transient, being replaced either by a brownish stain or a yellowish nodule or plaque. The stains, which are characteristic of the affection, have the same shape as the original lesion, are yellowish or brownish-yellow in tinge or may assume a violaceous hue when present in dependent positions such as the legs, especially when the circulation is feeble or the part exposed to cold. They are singularly persistent and may remain for years but finally disappear either leaving no trace or, in rare instances, slight atrophy. Recent stains may sometimes become swollen and turgescent from friction but they do not respond in this manner after they have been in existence for some time. (Fig. 25I.)

Other lesions, instead of being replaced by stains, become more solid and indurated and form fawn or reddish-yellow nodules or small plaques which bear a striking resemblance to xanthoma. The skin over them may be smooth and stretched or, where shrinkage has taken place, it may be wrinkled. In some instances they may be surrounded by a pinkish areola. After persisting for a varying time the nodules gradually involute and are succeeded by stains.

Three main types of eruption have been described: (I) A macular type which is the most common and which is characterised by pigmented macules and papules. In it the initial urticarial phase is transient and may entirely escape observation. (2) A nodular type 
which is the rarest and in which yellowish nodules predominate. (3) A mixed type which is more multiform and in which both macules and nodules are present.

In all three types the eruption presents a certain degree of multiformity owing to the presence of lesions in different stages of evolution, and with periods of quiescence and recrudescence it may persist for years but, as a rule, has disappeared by puberty.

In several instances brownish-yellow patches have been observed in the mucous membrane of the mouth but as their histology has not yet been worked out it has not been proved that they belong to the same category as the cutaneous lesions. In certain cases there is general enlargement and induration of the lymphatic glands.

The general health as a rule is not affected and there are no previous or concomitant gastro-intestinal symptoms connected with it such as

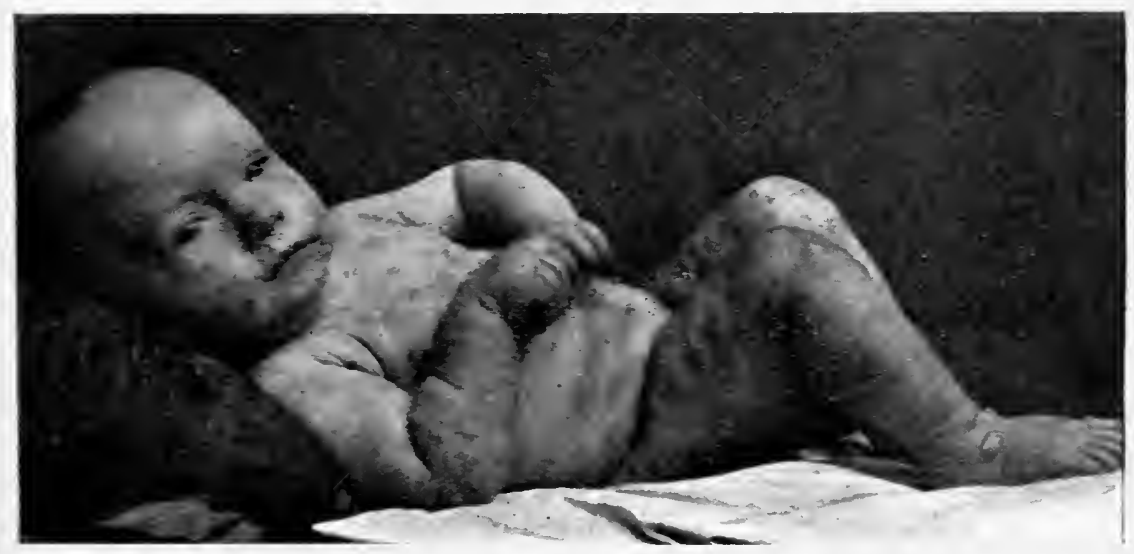

Fig. 25r.- Urticaria Pigmentosa

(After Dr. Wallace Beatty. Dublin Journ. Hed. Sci., 1906.)

are usually met with in ordinary urticaria. In rare instances feverish attacks have been known to coincide with fresh outbreaks of the eruption.

An examination of the blood has shown that, unlike ordinary urticaria, the lime content is increased and the coagulation time is sometimes shortened, also that certain alterations occur in the red bloodcells which render them more resistant to destructive agents than ordinarily.

Histopathology. - The most noticeable feature in the histology of the lesions is an infiltration of the corium with mast-cells which is so marked as to be of diagnostic value. These mast-cells are deposited chiefly around the dilated blood-vessels of the sub-papillary layer and upper portion of the corium, around the hair-follicles and sweat-glands, and in the spaces between the collagen bundles. They vary in shape and size, some being small and fusiform others large and cuboidal. They are most profuse in the nodular type of lesion which might be charac- 
terised as a " mast-cell tumour," but occur in all the types and have even been found in the apparently healthy surrounding skin. Gilchrist showed, from a series of experiments in connection with this disease, that not only were the mast-cells increased in the skin but that if a wheal were artificially produced there was a still further increase. This observation suggests the possibility of the skin being peculiar from birth in having an excess of mast-cells, and the further possibility that the increased deposits which occur in the urticarial and nodular lesions may be secondary phenomena due to local irritation, or to some internal derangement associated with marked instability of the skin so that it responds readily to slight external and internal stimuli.

As a result of the initial oedema in the urticarial stage the collagen tends to become broken upand rarefied leaving spaces in which numerous mast-cells may be detected, and the elastin has been found to be stretched or even ruptured.

There are no characteristic changes in the epidermis. In the nodular lesions it is stretched and the inter-papillary processes flattened. Pigment cells with melanin granules are present in the basal layer and the layer immediately above it, and are most abundant where the underlying mast-cells are most numerous.

Etiology.-The exact cause and nature of the disease are uncertain. It has been known to occur immediately after vaccination, to follow measles, and to develop after a fright, but these may be coincidences.

It is essentially a disease of infancy and young children and usually begins under six months, while certain cases have appeared so soon after birth as to suggest a congenital origin. A few cases have been recorded where it appeared later and it has been known to develop for the first time in adult life.

It is more common in males than in females, in the English cases in the proportion of three to two.

Diagnosis.-The diagnosis of a well-marked case presents no difficulty. It has to be distinguished from ordinary urticaria which occasionally leaves pigmented stains, from congenital syphilis, and from xanthoma.

From urticaria it is differentiated by the absence of itching, irritability, and gastro-intestinal disturbance.

In congenital syphilis the lesions are usually situated about the face, buttocks, and thighs, show no urticarial tendency, and are associated with other signs of congenital syphilis, such as rhagades at the sides of the mouth, depression of the bridge of the nose, snuffles, etc.

The nodular lesions may be confused with xanthoma, but in xanthoma the lesions are constant throughout, have a more waxy appearance, and are not associated with any urticarial tendency. 


\section{TREATMENT}

It is doubtful if treatment makes any definite impression on the course of this disease. As a rule if left to itself the eruption completely disappears by puberty and it is rare for it to occur in adult life. On general principles anything which might increase the urticarial tendency of the lesions should be aroided, errors of digestion should be corrected, the diet carefully regulated, and any departure from health suitably treated. There are no internal remedies which have any specific action on it. Salicin has been recommended but its action is uncertain. Where marked itching is present local anti-pruritic remedies, such as lotions containing spirit, lead, carbolic acid, etc., should be prescribed (see urticaria).

\section{REFERENCES}

Beatty, W. (Dub. Journ. Med. Sci., I906, cxxi. p. 422).

BонAc, C. To the knowledge of Urticaria pigmentosa (Arch. f. Derm. u. Syph., Oct. 1906, p. 49).

Brovgersma, R. G. J. (Brit. Journ. Derm., I899, xi. p. I79).

Dore, S. E. Urticaria pigmentosa in an adult (Idem, I9I5, xxvii. p. 426).

Fox, T. Colcotr. (Med.-Chir. Trans., London, I883, lxvi. p. 329).

KNowles, F. C. Urticaria pigmentosa, particularly in regard to its histology (Journ. Cut. Dis., I9I5, xxxiii. p. I $7 \mathrm{I}$ ).

Lirree, G. Contribution to the study of Urticaria pigmentosa (Brit. Journ. Derm., 1905, xvii. p. 355).

MacLeod, J. M. H. A case of Urticaria pigmentosa (Idem, I90 T, xis. p. 75).

NовL, G. Urticaria xanthelasmoidea (Arch. f. Derm. u. Syph., I905, p. $73 \&$ p. I63).

\section{ANGIO-NEUROTIC OEDEMA}

Syn.: Giant urticaria; Quincke's oedema; Acute circumscribed oedema.

Definition: A variant of urticaria characterised by sharply defined oedematous swellings situated in the skin and subcutaneous tissue and mucous membranes.

Description.--This condition, which was first carefully described by Quincke in ISS2 under the heading of acute circumscribed cutaneous oedema, is a comparatively rare affection. It is closely allied to urticaria but the lesions, instead of being superficial wheals, are deepseated oedematous swellings which are firm and elastic in consistence, do not pit on pressure, and may be tender or even painful to the touch. They may be curcumscribed and vary in size from a large nut to an apple, or diffuse when tney may involve considerable areas. The skin over them may be unaıtered, or may be pink, inflamed and hot, waxy and oedematous, occussunally moist with perspiration, and in rare instances discoloured like a viuise. They are most commonly situated on the 
face, hands, forearms, and genitalia, but may occur anywhere on the cutaneous surface. They frequently occur in mucous membranes, especially in those of the month, tongue, pharynx, larynx, stomach, and intestine. On the face, the parts usually affected are the lower lip which becomes enlarged to two or three times its normal size, or the eyelids which become so swollen as to make it difficult or impossible to open the eyes. The diffuse type is met with especially on the limbs ; in the upper extremity, for example, the whole hand and fingers may be swollen so as to resemble a boxing-glove and the arm increased to such an extent that the sleeve has to be cut to get over it.

The lesions may be single or multiple and may come out together or successively, and are sometimes symmetrical in distribution but are more often situated irregularly. They usually evolve suddenly, sometimes without warning, at other times preceded by itching or tingling or, when the buccal mucosa is attacked, by a peculiar taste in the mouth, dryness, or salivation.

The sensory symptoms as a rule are negligible and consist of a feeling of tenseness or slight itching.

The lesions may be unaccompanied by any general disturbance, or on the other hand they may be ushered in by headache, mental depression, drowsiness, and palpitation, or, where the mucous membrane of the gastro-intestinal tract is involved, by vomiting or colicky pains. Occasionally albuminuria or paroxysmal haemoglobinuria may accompany them.

The swellings most frequently appear during the night, especially during the early hours of the morning when the vaso-motor tone is at its lowest.

They are fully developed in a few hours and do not generally last more than twenty-four hours, when they subside spontaneously without leaving any trace. As a rule recurrences take place at irregular intervals, either at the site of previous lesions or elsewhere, and the tendency to them may persist for years or even indefinitely and may become a serious burden to the patient-a month rarely passing without an attack.

Typical urticarial lesions or erythematous blotches may sometimes occur in association with them and, in rare instances, factitious urticaria or even dermatographism may be present, while occasionally they may become replaced by bullae.

So far as the cutaneous symptoms are concerned the disease is not serious but when the mucous membranes are involved the outlook may be grave, for the nose may be blocked, the tongue so swollen that speaking and eating are rendered impossible, and the glottis so closed with oedema that death from suffocation may take place.

Histopathology.--The microscopical appearances are similar to those in ordinary urticaria, except that the serous exuclation is found dilating the interstitial lymphatic spaces of the subcutaneous tissue as well as of the skin and may be so profuse as to lead to bullous formation. 
Etiology and Pathogenesis.-The exact cause of angio-neurotic oedema is at present unknown. There are possibly two factors, namely, a peculiar state of vaso-motor instability, the result of some nervous derangement or shock, and some exciting cause, such as a toxin circulating in the cutaneous vessels or acting reflexly through the vasomotor centres, or some form of traumatism such as a blow or even the extraction of a tooth, or some emotional disturbance such as great excitement, anger, shock, fright, etc. It has been suggested that it is due to defective coagulability of the blood, but this is not invariably present and when it does exist may be due to the same causes as the oedema.

Of the known predisposing causes the following are the most important :

Age.-It is most prevalent in early adult life and rare in old age, but it may begin in infancy or early childhood and persist indefinitely.

Sex.-In this country it would appear to be more common in males than in females, in the proportion of about 3 to 2 .

Social Status.-The station in life does not seem to have much influence on the etiology, though it has been said to be most common among the well-to-do.

Heredity.-It may be present in severalgenerations and is frequently a family disease ; Osler, for example, refers to a family of thirty-six in five generations of whom twenty-two were affected.

Nerious Instability.-A general instability of the nervous system would seem to be a predisposing cause and it has been observed most frequently in individuals of the neurotic type who suffered from neurasthenia or hysteria. It has been known also in connection with Graves' disease.

General Diseases.-It has been met with in association with Henoch's purpura, malaria, and cirrhosis of the liver, but its relation to them is obscure.

Diagnosis.-Angio-neurotic oedema is distinguished from other forms of localised oedenia, such as occur in renal disease or venous stasis, by the rapid evolution of the swellings and their transient nature.

The condition which it is most liable to simulate is a recurrent erystpelatoid attack of streptococcic origin but there the inflammatory disturbance is much more marked.

Prognosis.-The prognosis should always be guarded as once the swellings have appeared they are liable to recur and the tendency to them may persist indefinitely. The great danger is oedema of the glottis and the patient and his friends should be warned of this possibility, which is most likely to occur when the lip is involved, and especially when the affection is a family disease; Ensor recorded 49 cases in which 12 died of nedema of the glottis and Whiting quotes from MacDowell I Io family cases in which 30 died from it. 


\section{TreatMent}

As the pathogenesis is not fully understood the treatment must be based largely on general principles. Any derangement in the gastrointestinal tract, liver, or genito-urinary organs, should be sought for and dealt with by suitable measures. Where the patient is neurotic the usual tonics, such as arsenic, quinine, iron, and strychnine, are indicated, and should be combined with a healthy outdoor regime.

In a large number of cases no defect in health has been observed and the remedies employed have been empirical in nature. With the idea that defective coagulability of the blood may be a factor in the etiology, calcium salts have been given but with no distinct benefit. Nitro-glycerine has been prescribed with the object of relieving the spasms but without success. Where the patient is neurotic, improvement sometimes follows the administration of valerian. Quinine is indicated when the swellings occur in connection with malaria.

Locally no treatment, except soothing lotions of lead or calamine, is required.

Should oedema of the glottis supervene, scarification may relieve it, but as a rule intubation or tracheotomy are necessary and it is important that someone associated with the patient should be instructed in the method of performing intubation. Ensor has reported benefit from the administration of tincture of perchloride of iron in teaspoonful doses every twenty minutes.

\section{REFERENCES}

Don, A. Henoch's Purpura \& Angio-neurotic oedema (Lancet, I909, i. p. 526).

ENSOR. (Guy's Hos. Rep., I904, lviii. p. II I).

HARBIT, F. Angioneuroses, acute paroxysmal oedema with fatal termination (Münch. Med. Wochenschr., I9I I, p. 2557).

Morichau.Beauchant. Acute circumscribed oedema of the skin and mucosa (Ann. de Derm. et de Syph., January I906, p. 22).

OsLer, W. Hereditary angio-neurotic oedema (Amer. Journ. Med. Sci., I 888, xcv. p. 362). Angio-neurotic oedema (System of Medicine, I909, vi. p. 648). (Amer. Journ. Med. Sci., I888, xcv. p. 632).

Quincke, H. Über akutes umschriebenes Handödem (Monats. f. prakt. Derm., 1882, i. p. I28).

Rosenthal, O. Angioneuroses \& the haematogenous skin-inflammations (Arch. f. Derm. u. Syph., I9Io, ci. p. 95).

Wende, G. Acute circumscribed oedema with haemoglobinuria (Journ. Cut. Dis., I 899, xvii. p. I 78 ).

Whiting, A. J. Angio-neurotic oedema as a familiar cause of sudden death (Lancet, I908, ii. p. I356).

Wills \& Cooper. Acute circumscribed oedema (Brain, I893, xvi. p. 382). 


\section{PAPULAR URTICARIA}

Syn.: Lichen urticatus; Lichen simplex aigu (Vidal); Prurigo simplex (Brocq); Strophulus pruriginosus (Hardy); Prurigo infantilis (Hutchinson).

Definition: A form of urticaria characterised by pinkish blotches about the size of the finger-nail with a central deep red papule.

Description.-This affection is one of the commonest skin discases of infancy and early childhood and is particularly prevalent in warm weather. Though it is a form of urticaria, the typical lesion is not a wheal, but a blotch or tache with a central deep red papule which persists after the blotch has faded. To such papules the name of Lichen was at one time generally applied, hence the name Lichen urticatus given by Bateman who was the first to differentiate and clearly describe the affection. The tache is pinkish-red in colour and becomes darker in tint when the skin is hot or the child cries. It is level with, and fades into, the surrounding skin but may be slightly raised or occasionally urticate; it is round or oval in shape, and varies in size from a quarter to half an inch in diameter.

The central papule is about the size of a millet seed and of a deep red tint. It is sometimes paler at the apex, indicating a tendency to the formation of a minute resicle, and in rare instances it may be capped by a small crust. (Fig. 252.)

The tache appears suddenly, and is evanescent in cliaracter lasting on an average about an hour though it may fade in a few minutes; the central papule is more persistent, shrivels and assumes a conical shape, gradually becomes the colour of the surrounding skin, and may last from twenty-four hours to a few days. The tache may precede the papule or may be synchronous with it and when it has faded the remaining papule becomes indistinguishable from a papule of prurigo, hence the name Prurigo infantilis applied to it by Hutchinson-a name which is apt to lead to confusion as prurigo is a distinct disease.

Occasionally several lesions may coalesce to form an irregular reddish blotch surmounted by a group of papules. When the lesions are rubbed they become more vivid and sometimes urticate, but factitious urticaria is rare in connection with them and if present is slight.

The lesions have a marked tendency to appear towards evening and during the night and to fade in the morning, so that if the child be examined in the daytime there may be no sign of them except a few papules or excoriations while the mother may state that at night the skin is covered with blotches or "smothered in blisters." They may be few in number, or numerous and widely distributed over the cutaneous surface but are never present on mucous membranes. They are most common on covered parts of the body, especially the lower part of the back, but may occur anywhere, even on the face. 
Intense itching occurs simultaneously with the appearance of the lesion, is most marked at the central papule and persists there after the tache has disappeared. This leads to scratching, excoriation, and, in older children, even to digging out the papules with the finger-nails, relief being obtained when the head is scratched off and it bleeds.

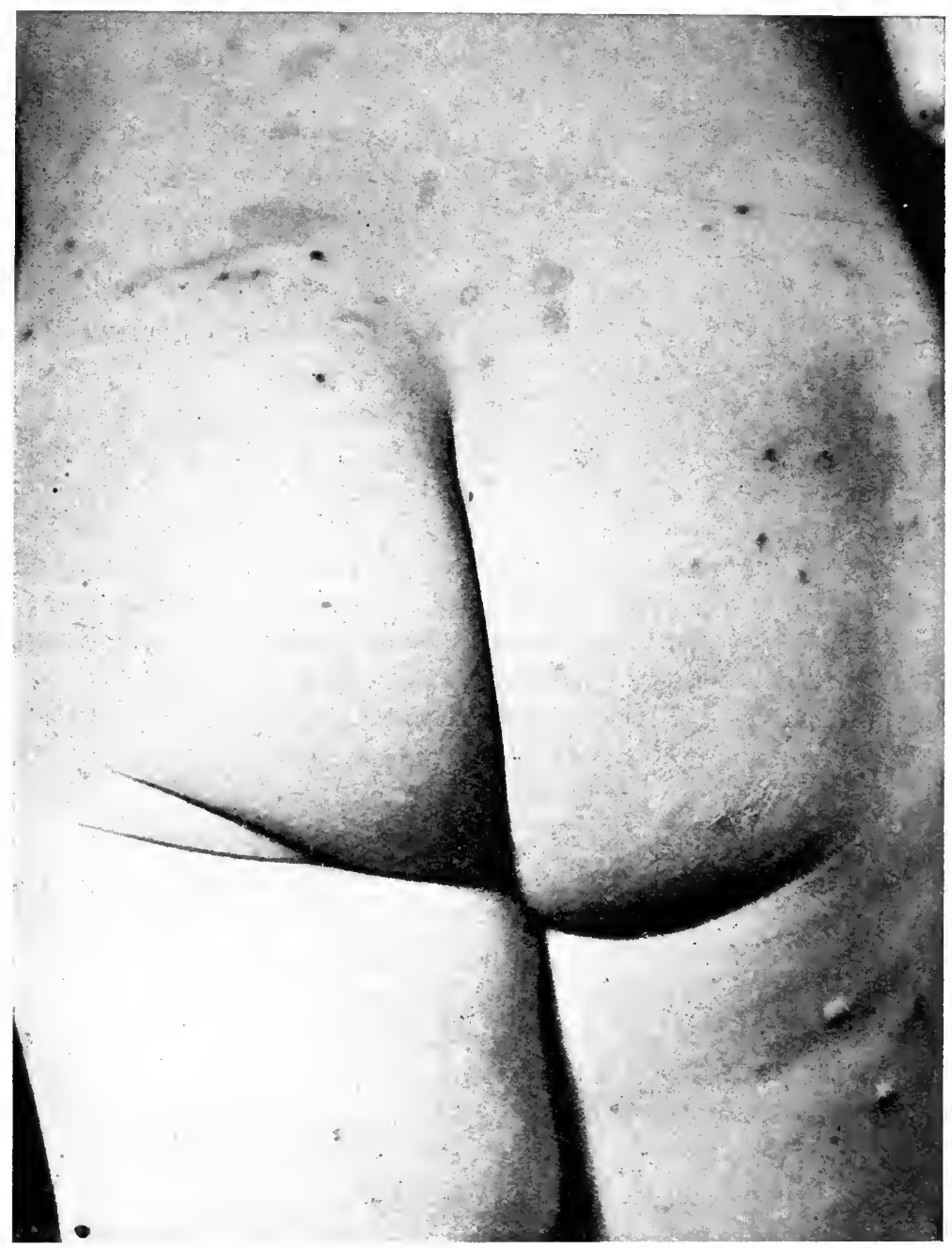

FIG. 252.-Papular Urticaria in a Child.

It has been suggested that the itching is primary and that the eruptive lesion is simply a reaction due to friction or scratching, but this view . is not generally accepted and as the lesions are liable to appear in places which are subjected to neither friction nor scratching it would seem more probable that the itching is secondary.

As the result of rubbing and scratching, inoculation of pyogenic micro-organisms may take place and give rise to pustulation, crusting, 



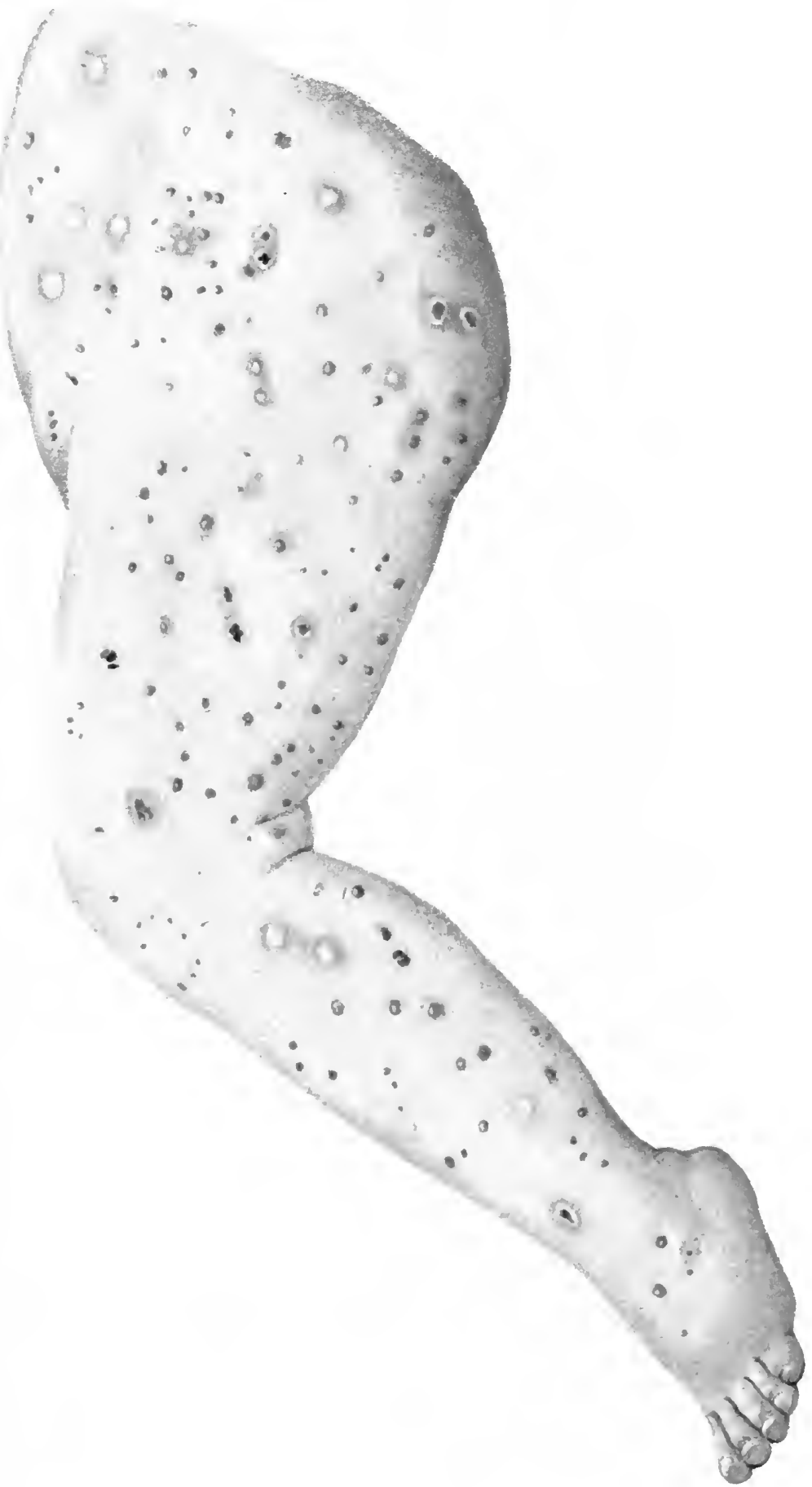


PLATE $\mathrm{X}$.

Papulo-vesicular Urticaria in child of 2 years.

Shows papules with erythematous halo, vesicles on erythematous base, older papules from which the erythema has disappeared, and excoriated lesions. 

enlargement of the neighbouring glands, and occasionally to eczematisation.

The affection is not necessarily accompanied by any general stmptoms though it is not infrequently associated with some derangement of digestion, especially constipation, and the more or less continuous and distressing itching tends to make the child restless, fretful, and sleepless, which further interferes with the digestive functions and leads to impairment of the general health.

The course varies greatly in different cases. Sometimes an acute and extensive attack may be followed, under suitable treatment, by complete recovery, but in general it tends to recur intermittently up to the age of two years or later. In severe cases it may continue in spite of the most careful treatment and be subject to exacerbations from the slightest cause.

Variations.-The central papule may be modified in the following ways :

(a) On retrogressing it may become flattened, smooth, and shiny, and resemble a papule of Lichen planus and cases of this type have been exhibited from time to time under the heading "Lichen planus infantum."

(b) Occasionally the papule may be as large as a lentil and opaline like a wheal.

(c) A further stage may be reached in which it is replaced by a vesicle or the tache becomes a bulla; this variety is known as vesicular urticaria or vesicular Lichen urticatus, and in it the lesions are generally situated on the extremities. (Plate X.)

In association with these modifications typical lesions may generally. be detected somewhere on the cutaneous surface and add to the multiformity of the clinical picture.

Histopathology.-There is nothing characteristic in the microscopical appearances of the papules. There is dilatation of the papillary blood-ressels, slight inflammatory cellular infiltration, and serous exudation in their neighbourhood, and these are associated with oedema of the epidermis which may be so severe as to lead to vesicular formation.

Etiology and Pathogenesis.-The affection is a special form of reaction of the skin almost peculiar to infants, which is called forth by some autogenous toxin or by the ingestion of some food which has acted as a poison and irritated the gastro-intestinal tract. It is most liable to occur in infancy since at that age the controlling influence of the higher centres is low and the vaso-motor reaction readily excited.

It is sometimes traceable to a definite error of diet and at other times to defective feeding continued over a long period, but it mayoccur also in infants who are being most carefully fed and eren in nurslings where the mothers are in perfect health. In some instances it affects delicate infants suffering from some debility, such as malnutrition, rickets, a dilated stomach, or a feeble digestion, or it may follow 
some debilitating condition such as varicella; on the other hand it may be met with in infants apparently in robust health.

Once an attack has occurred a tendency to the disease is established and the eruption is readily called forth by causes far less potent than that which produced the original attack. One of the most common factors in determining an attack is teething and in an infant with a tendency to it an outbreak may be expected whenever a tooth is being cut. This is due partly to nervous irritability and partly to interference with digestion caused by the teething. It often occurs also after a digestive upset which has followed a bout of crying or temper. Another not infrequent cause is the presence of intestinal worms.

Of the predisposing causes the most important are age and heat.

Age.-It is essentially a disease of infancy and usually disappears by the end of the second year, but sometimes may persist for several years and cases now and again are met with in adults. Occasionally it may appear first in older children, especially when it is of the vesicular or bullous type.

Sex.-It is equally common in males and females.

Heat.-It is especially prevalent in hot weather, and in the summer months in London among children of the hospital class it may constitute about $5 \%$ of the cases.

Social Status.-It is more common among the poor than among the well-to-do, though not so markedly so as would be expected in consideration of their greater liability to disorders of digestion and nutrition and their unhygienic surroundings.

Diagnosis.- Urticaria of the paptular type has to be distinguished chiefly from prurigo, especially where it has persisted over the second year, and from Lichen planus; the vesicular type has to be differentiated from varicella and occasionally from scabies.

From prurigo it is distinguished by the eruption not being so profuse on the extensor aspects of the limbs, by the absence of secondary changes such as lichenification and pigmentation which are so characteristic of prurigo, and by the general health not being impaired.

From Lichen planus it is differentiated by the history of the red tache in the early stage of development of the lesion and the intense itching connected with it. It is doubtful, also, if true Lichen planus ever occurs in infancy.

The diagnosis between vesicular urticaria and varicella may be extremely difficult, especially from mild varicella where the vesicles are small and have an erythematous areola. As a rule varicella is preceded by a mild febrile disturbance but this may be absent, on the other hand the urticaria may appear in association with an attack of indigestion with possibly a slight rise of temperature. The chief points of difference are: Varicella generally begins on the upper part of the trunk and spreads downward while vesicular urticaria may appear anywhere on the skin ; in varicella the lesions do not itch, while those 
of vesicular urticaria are intensely itchy; the lesions of varicella are frequently present in the mucous membranes specially of the mouth and vulva, vesicular urticaria never occurs in the mucosa; in varicella there is not infrequently a definite history of contagion.

The bullous variety of this affection, especially where the lesions are purulent and situated on the extremities, might be mistaken for scabies. In both the itching is worse at night and in connection with scabies urticate lesions are often present and occasionally slight factitious urticaria, but in scabies burrows and acari can generally be found and the affection is contagious.

Where the eruption is completely absent in the daytime or only a few papules and excoriations are present, before diagnosing papular urticaria it is necessary to eliminate the possibility of flea-bites.

Prognosis.-The prognosis is excellent and as a rule the tendency. to it does not last beyond the second year, though in the vesicular type it may persist for years. Its cure depends on the discovery of the cause and the ability to deal satisfactorily with it.

\section{Treatuent}

The treatment is both general and local.

General.- The proper management of a case of papular urticaria is of the first importance and everything should be done to regulate the diet and to counteract any sources of gastro-intestinal irritation, such as improper food, intestinal worms, etc. The general health should be carefully attended to and constitutional disorders, such as rickets, suitably treated. The clothes should not be too hot and the room should be kept at a cool even temperature at night. Where constipation is present, castor oil, liquid paraffin, olive oil, magnesia, or other suitable aperient, should be prescribed, and where the stools are loose, green, and offensive, intestinal antiseptics, such as small doses of calomel, grey powder, salol, or lactic acid, are indicated. Where the irritation is so great that the child gets no sleep by day or night, small doses of chloral hydrate may be given with advantage and will at the same time afford some respite to the tired-out mother or nurse.

Local.-Locally the indications of treatment are to soothe and protect the skin, for which purpose the following applications are of service :

Baths.-These should contain oatmeal, sodium bicarbonate, or borax. They should not be too hot and on coming out the skin of the infant should be gently blotted dry with a soft towel and a bland dusting powder applied.

Lotions.-Of the anti-pruritic lotions carbolic lotion I in 40, or the following lead and tar lotions, are the most soothing :

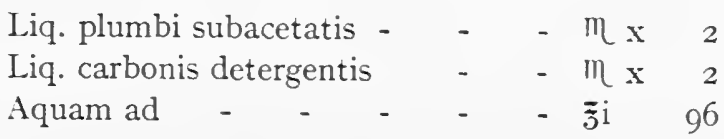


Pieces of lint saturated with the lotion should be laid over the itchy parts for about Io minutes, moistened from time to time, and then removed and a dusting powder freely applied before the lotion is completely dry.

After the application the infant should be dressed in such a way that the itchy parts are as far as possible inaccessible to scratching and rubbing.

Liniments are more lasting in their action than lotions, and of these the following calamine and carbolic liniment is especially useful :

Acidi carbolici
OR

This should be rubbed gently into the skin by the hands, a procedure which in itself is soothing, and a dusting powder subsequently applied.

Should secondary inoculation from scratching have taken place boric compresses or $2 \%$ ammoniated mercury ointment should be used till the pustulation has ceased.

\section{REFERENCES}

Funk \& Grundzach. Urticaria infantum (Monats. f. prakt. Derm., I 894, xviii. p. IO9).

Fox, Colcotr. (Brit. Journ. Derm., I89o, ii. p. I33). (Idem, I898, x. p. I 40). (Idem, I 899, xi. p. I 57).

Morris, M. (Idem, I900, xii. p. 209).

Still, G. F., \& Whitfield, A. (Idem, I902, xiv. p. 306).

\section{PRURIGO}

Deriv. : Prurire-to itch.

Syn.: Fr., Lichen agrius, Strophulus prurigineux; Ger., Juckblättern.

Definition: A cutaneous affection characterised by the presence of discrete hard papules, situated chiefly on the extensor aspects of the limbs, associated with intense itching, thickening and lichenification of the affected skin, and with enlargement of the neighbouring lymphatic glands.

Introduction.-There is considerable confusion connected with the term prurigo, as certain writers have employed it to designate a definite disease, while others have included under it several different affections characterised by the presence of more or less itchy papules. For example, under this heading certain observers have placed not only prurigo as defined above, but also papular urticaria of infants (Prurigo 
simplex of Brocq), while others have included the circumscribed lichenified patches of "Lichen simplex chronicus."

The confusion has been increased also by the use of the term in a loose fashion as if it were synonymous with pruritus. Pruritus means itching and is a purely subjective phenomenon, while prurigo is a papular disease of which pruritus is an important symptom. The adjective pruriginous has also been employed carelessly as a synonym for pruritic when, if used at all, it should be reserved to designate conditions which are prurigo-like.

Prurigo was first described in England by Willan. His description is vague and indefinite, however, and appears to include not only what we now call prurigo but a series of other itchy conditions such as pediculosis and papular urticaria. He considered that the itching was the primary phenomenon and the papular eruption was secondary. In this view he was supported later by Erasmus Wilson, who differentiated two kinds of prurigo, namely (I) a mild variety (Prurigo mitis) which could occur at any age and which he believed to be due to scratching for the relief of a peculiarly intense and neurotic kind of itching which came on in paroxysms; (2) a type which only occurred in old age (Prurigo senilis).

It is to Hebra that we are indebted for the first definite conception of the disease and, in consequence, it is generally known as Prurigo of Hebra. According to him it is a serious affection which begins in infancy as an urticaria, lasts throughout life, and usually ends fatally.

Kaposi subsequently pointed out that there was also a mild variety which was not dangerous to life and which was sometimes curable. To this he applied the name Prurigo mitis, reserving that of Prurigo of Hebra for the grave and fatal form.

Cases of the mild type continue mild throughout and never assume grave characteristics, while cases of the severe type can be recognised assuch from the first. The difference in severity is due partly to individual peculiarities whereby the skin reacts more readily to stimulation, and partly to differences in causation.

In this country prurigo is rare and when it does occur is usually comparatively mild, though grave and fatal cases have been encountered occasionally. It would seem to be much more common in the eastern countries of Europe but even there it is becoming less frequent, probably as a result of better hygienic conditions.

Description.-The essential lesion of prurigo is a small conical or rounded papule about the size of a pin's head or slightly larger. In colour it may be indistinguishable from the neighbouring skin, or may be pinkish, or even dusky red when it is present on the lower extremities or occurs in patients with a feeble peripheral circulation. The papules may be few in number and sparsely distributed, or profuse and closely congregated, but are invariably discrete. The skin between them is normal at first but is apt to become red and inflamed from rubbing and finally to undergo secondary changes such 
as thickening and pigmentation. As a rule the papules are irregularly distributed, being independent of the sweat-pores or hair-follicles, but occasionally they may be arranged in well-defined groups. An individual papule may last a few hours, or may persist for a day or longer, and then fades naturally if not extirpated by scratching, but as new ones cone out, continuously or in crops, the eruption tends to become permanent.

The papules are situated most commonly on the extensor aspects of the limbs, buttocks, lower part of the abdomen, over the sacrum, on the back and front of the chest, cheeks, sides of the neck, and occasionally on the chin and about the angles of the jaw, and are, as a rule, absent from the flexor aspects of the limbs-a point of diagnostic importance in differentiating this condition from papular eczema in which the flexures are generally involved. They are associated with itching of such a singularly intense type as to form a characteristic feature of the disease. It is constantly present, but subject to exacerbations as a result of heat, exertion, hot drinks, etc. It is focussed at the papules and relief from it is obtained only when they are excoriated so that they bleed, or are dug out by the finger-nails, the pain caused thereby being more bearable than the continuous itching. This distressing subjective symptom is largely responsible for the serious influences of the disease, as by it the patient's strength is worn down, sleep is interfered with, the neurosis, which in most cases is an important etiological factor, is intensified, and the patient may be reduced to a mental and physical wreck.

According to the earlier writers the itching is the primary phenomenon and the papules secondary and the result of rubbing. This view is still supported by the French School of Dermatologists, and in corroboration it was experimentally proved by Jacquet that if an occlusive dressing be applied and rubbing and scratching prevented, papules do not appear, although covering the skin does not prevent the itching.

In spite of this experiment, however, there are strong arguments in favour of Hebra's theory, that the papules appear first and cause the itching ; for example $(a)$ the occurrence of papules in situations which are inaccessible to scratching and in which the skin shows no signs of having been rubbed; (b) that rubbing, however much it may be indulged in, is only associated with prurigo papules in rare instances; (c) that the itching is focussed at the papule itself, instead of being diffused over a wide area.

This question of priority is one of minor importance, however, as they are both symptoms, the one objective and the other subjective, of some underlying cause, and probably manifest thenıselves simultaneously.

As a result of rubbing and scratching, which is liable to become a habit and indulged in more or less continuously by day and at odd times during the night, secondary changes inevitably occur in the skin. 
The papules may becone urticate and their base inflamed, or they may be excoriated or dug out by the nails and replaced by haemorrhagic crusts or purulent scabs; linear scratch marks may be produced, secondary inoculation of pyogenic micro-organisms may take place, pustulation or eren abscess-formation may result, and occasionally: eczematous changes may supervene. The neighbouring glands, especially those of the groin, generally become enlarged and may suppurate. The most noticeable effects, however, are a peculiar thickening of the skin in which it becomes hard and scaly with the hairs extruded, or lichenified, smooth, and shiny, with the natural furrows deepened (see p. 746). In old-standing cases pigmentation of the affected skin generally takes place. This varies from a dirty grey to a dark brown and may sometimes be so marked and diffuse as to suggest Addison's disease, but differs from it in that the pigmentation occurs in the situation of the eruption and not on the exposed parts or where the natural pigmentation is deepest.

Prurigo was said by Hebra to begin in infancy towards the end of the first year as an urticaria, and only to assume the characteristic papular form in the second year. A number of observers, however, dissent from this opinion, and consider that it never begins as an urticaria, but that the initial lesion is a papule, and that the arerage age of incidence is about the fifth year and that it may commence occasionally in adult life or even in old age.

There are no characteristic general symptoms associated with the cutaneous lesions, though in a large number of cases there may be a marked neurosis either hereditary or acquired. In severe cases the constant irritation intensifies the nervous irritability, leads to depression and eren hysteria, interferes with sleep, reduces the vitality of the patient and increases the liability to intercurrent disease.

In addition to the mild and grave varieties of prurigo, two atypical forms require to be mentioned, namely (I) a type in which the papules are small and sometimes scarcely noticeable, but in which the itching is so great that they are constantly dug out by the nails; (2) a severe type described by Vidal as Prurigo ferox.

The small papular type (Acne urticata) is rare, occurs chiefly in young women, and is associated invariably with a highly neurotic or even hysterical habit. The papules may be present on the usual sites but may occur also on the face, especially about the cheeks and forehead. In some cases they are so small as to be invisible and may pass unnoticed, but the presence of the dug-out excoriations, the lichenification of the skin, and the occasional enlargement of the groin glands, serve to establish the diagnosis. Those afflicted with this condition are usually a great trial to themselves and their friends, especially when the papules appear on the face, orring to the disfigurement and bleeding caused by the laceration of the skin by the finger-nails.

Prurigo ferox.-In this variety the lesions may reach the size of a split-pea, are reddish in colour, and may present a small vesicle at $2 \mathrm{x}$ 
the apex. They may appear anywhere on the cutaneous surface, being present not only on the covered parts but on the face and scalp, and their distribution is usually irregular. As a rule, although the itching is more intense than in ordinary prurigo, the lichenification is not so well marked. The condition occurs especially in women and has been known to begin about the menopause. This variety might be included more appropriately under the heading of papular urticaria than of prurigo.

Histopathology.- The microscopical appearances in a prurigo-papule vary according to the stage of development of the lesion and are not sufficiently characteristic to enable a firm diagnosis to be made from them. The principal changes, which are those of a spastic oedema, occur in the upper part of the corium and consist of dilatation of the capillaries of the papillary and sub-papillary layers, serous exudation, and an inflammatory cellular infiltration composed chiefly of lymphocytes and small connective tissue cells. The hair-follicles and sweat-glands are not definitely affected. The overlying epidermis may be unaltered, or the prickle-cell layer may be thickened and the prickle-cells broken down to form a small vesicle. In old-standing cases the intervening skin presents the appearances described under the heading of lichenification.

Etiology and Pathogenesis.-The precise cause of prurigo is at present unknown. It seems most reasonable to assume that it is a peculiar type of reaction of the skin occurring in a pre-disposed individual, due to some toxin of autogenous origin circulating in the blood. In the first instance a toxin of considerable virulence may be necessary to produce the reaction, but once it has occurred the organism becomes so sensitive that toxic irritants of far less potency are capable of causing recurrences, and eventually the slightest defect in metabolism or derangement in the function of some internal organ will inevitably lead to an exacerbation or a fresh outbreak, so that it becomes practically habitual. In support of this theory is the association of prurigo papules with general lymphadenoma (Hodgkin's disease) where it is probable that the same cause is responsible for the changes in the glands and for the papules and itching.

How the toxin acts is another unsolved problem, and whether it acts directly on the nerve endings and blood-vessels in the skin or indircctly must be left for future decision. It has been suggested that the toxin is of a tuberculous nature, but the evidence in favour of this view is insufficient.

Predisposing Causes.-Sex.-In children it is more common in boys than in girls, in the estimated proportion of 2 to $\mathrm{I}$, but in older cases it seems to be equally prevalent in both sexes or may even be more common in women.

Age.--The average age of incidence is about 5 years, but it has been known to commence in adult life or possibly in old age. It is doubtful if it ever begins in infancy.

Environment.-It is most commonly met with in those living in unhealthy or insanitary surroundings who are neglected, ill-nourished, 
and anaemic from bad or insufficient food and want of fresh air, and in this country the great majority of the cases occur in aliens, Polish Jews, etc. Since the hygienic conditions of the poor have been improved the frequency of the disease has appreciably diminished.

Season.-It may occur at any time of the year but is said to be worse in cold weather.

Neurotic Habit.-In almost every case an important predisposing factor would seem to be an unstable state of the nervous system, which may be evident in unusual irritability or even hysteria, and which is doubtless responsible to some extent for the intensity of the itching.

Heredity.-Heredity has no bearing on the etiology, except in so far as it may be responsible for the neurosis or for some hereditary taint such as tuberculosis or syphilis.

Diagnosis.-The recognition of prurigo rarely presents any serious difficulty, the small, discrete, white or pinkish, conical papules situated chiefly on the extensor aspects of the limbs, the intense itching, and the lichenification, excoriations, adenitis, and pigmentation, constitute a picture which is unmistakable.

The diseases with which it is most liable to be confused are papular urticaria, eczema, xerodermia, Dermatitis herpetiformis, and more rarely Keratosis pilaris and Addison's disease.

Papular urticaria of infants may be mistaken for it and certain observers still follow the teaching of Hebra that prurigo commences in infancy as an urticaria. The characteristic lesion of papular urticaria is a reddish blotch about the size of a thumb-nail with a central deeper red papule, or a small whitish wheal. This lasts for an hour or so and is replaced by a papule like that of prurigo. Towards the end of the second year, if the disease persists, the papular type of lesion predominates and it is then that the diagnosis becomes difficult and may be practically impossible. Papular urticaria, however, almost invariably disappears by the end of the third year, if not before, and is not associated with thickening of the skin or marked adenitis, while prurigo is a persistent affection and lichenification, pigmentation, and adenitis are its usual concomitants.

Eczema of the papular variety may so closely simulate prurigo as to merit the name of pruriginous eczema and the itching may be even more intense than in prurigo itself. In eczema, however, the eruption is generally more widely distributed, the inflammatory disturbance greater, and the papules are more numerous and have a tendency to coalesce to form sheets, while examination of the lesions with a pocket lens may reveal an abortive vesicle on the summit or an oozing point where a vesicle has broken.

Cases of xerodermia may resemble prurigo, but in them the lichenification is less marked and should papules occur they are generally follicular.

Cases of Dermatitis herpetiformis of the abortive papular variety may closely simulate prurigo, but in Dermatitis herpetiformis the 
itching is not so continuous, the lesions are grouped in a herpetiform manner, and the papules are generally associated with vesicles or even bullae. The affection which has been described as Prurigo gestationis is not prurigo but Dermatitis herpetiformis occurring in connection with pregnancy.

From Keratosis pilaris it is distinguished by the papules neither being follicular nor presenting the characteristic horny plugs of that disease.

Any resemblance to Addison's disease is superficial and simply due to pigmentation in an old-standing case. In Addison's disease the distribution of the pigment is different, as it is most marked on the exposed parts and about the nipples and genitalia while in prurigo it is confined chiefly to the extensor aspects of the limbs and trunk.

Prognosis.-The disease is singularly intractable, but mild cases can be improved, if not actually cured, by suitable treatment begun early and persisted in with sufficient vigour. Cases of the grave type are liable to end fatally from the gradual weakening of the patient by the incessant itching, nervous depression, sleepless nights, and interference with the functional activity of the internal organs.

\section{TREATMENT}

The treatment is both general and local and varies according to the severity of the case and the age of the patient.

General Treatment.- The aim in the general treatment is to eliminate any possible internal cause or aggravating factor and at the same time to build up the patient's strength and nervous tone to render him more resistant. Any disturbance of the digestive system, liver, kidneys, or genital organs, should be sought for and dealt with by appropriate means. The diet should be carefully regulated and all articles prohibited which are liable to have a toxic influence, such as coffee, strong drinks, rich food, curries, chutney, etc. A neurotic taint should be counteracted by an out-door life, fresh air, exercise, and a tonic regime with cod-liver oil, quinine, the phosphates, iron, or strychnine, and when the patient is weak and run down rest and confinement to bed are imperative, the benefit derived from complete rest, apart from other treatment, being sometimes remarkable.

Various remedies have been suggested for the relief of the itching at night and to induce sleep, such as salicin, Cannabis indica, antipyrin, chlorai hydrate, and opium. Of these antipyrin and chloral are the safest, but even they should be used with caution for fear of establishing a drug habit and so further lowering the resistance of the patient. According to certain observers benefit may be derived in many cases from the employment of thyroid extract, but this remedy is uncertain in its action and requires careful administration to avoid the production of toxic symptoms.

Local Treatment.-The first indication in the local treatment is to remove any complication, such as pustulation or eczematisation, by 
appropriate local remedies, and the second to relieve the itching by anti-pruritic applications and soothing baths. The most effective anti-pruritic remedies in this connection are tar, carbolic acid, and B-naphthol.

Tar may be prescribed in the form of a lotion such as a teaspoonful of the Liquor carbonis detergens to half a pint of tepid water ; or its effect may be made more permanent by combining it in the form of a cream as follows :

\begin{tabular}{|c|c|c|c|c|}
\hline Liq. carb. c & $\mathrm{ro}$ & & _ & \\
\hline Zinci oxidi & - & 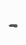 & & \\
\hline Lanolin - & - & - & - & \\
\hline Aq. calcis & & - & - & \\
\hline Ol. amygda & & 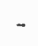 & - & \\
\hline
\end{tabular}

Carbolic acid may be used as a lotion either in water I in 40 , or in glycerine as follows:

$\begin{array}{llllr}\text { Acidi carbolici } & - & - & & \\ \text { Spiritus vini rect. } & - & - & 5 \mathrm{j} & \text { I2 } \\ \text { Glycerinum ad } & - & - & \overline{3} \mathrm{i} & \$ 6\end{array}$

or it may be added to calamine cream, and after applying the cream the skin should be mopped dry and the following dusting powder applied :

\begin{tabular}{|c|c|c|c|c|}
\hline Camphor & - & - & - & - \\
\hline Zinci oxidi & - & - & - & \\
\hline Cretae prep. & - & - & - & - \\
\hline
\end{tabular}

Beta-naphthol ointment 2 to $6 \%$ has a curative effect in some cases and is also anti-pruritic.

Occlusive dressings, such as zinc-gelatine painted on, or cod-liver oil 3 parts dissolved in white wax I part, smeared on the skin with a bandage over it (Brocq), have been recommended but their effect is uncertain and the itching often persists in spite of them.

Temporary relief may be obtained where practicable by exposure to the $\mathrm{X}$-rays and in unusually persistent cases from lumbar puncture.

\section{REFERENCES】}

Bernhardt. Pathogenesis of prurigo (Arch. f. Derm. u. Syph., Igor, p. I73).

BoAs. Relationship of Hebra's prurigo to tuberculosis (Nord. Med. Arkiv., I9I2, Abt. ii. p. 24).

Ehrers, E. Hebra's prurigo (Nord. Ned. Arkiv., r892, No. 2.4).

Fox, Colcotr. Prurigo (Allbutt \& Rolleston, System of Medicine, I9I I, ix. p. 269).

Morris, Sir M. Prurigo (Trans. Roy. Soc. Med., June I9I2. Brit. Med. Journ., 1912, p. I469).

Payne, J. F. Prurigo hiemalis (Brit. Med. Journ., I887, i. p. 985).

Zeisler, J. A case of so-called Prurigo nodularis (Journ. Cut. Dis., Nor. I912, p. 654). 


\section{CHAPTER XXIV}

\section{DRUG ERUPTIONS}

Syn.: Drug rashes; Dermatitis medicamentosa; Medicinal eruptions; Fr., Eruptions médicamenteuses; Ger., Arzneiexanthem.

Definition: Eruptions which result from the ingestion and absorption of drugs.

For convenience of description the subject will be discussed under the following headings :

I. Drug eruptions in general.

2. Drugs in common use most liable to cause cutaneous lesions.

3. Special types of drug eruptions.

\section{Drug Erruptions in General}

A large number of drugs are capable of producing eruptions. Almost every sort of cutaneous lesion may be produced by them and they may be erythematous or erythemato-papular and similar in appearance to the rashes due to food toxins, or in severe cases urticarial, vesicular, or even bullous.

Erythematous drug eruptions may be caused by a great variety of drugs, of which the most common are quinine, belladonna, copaiba, antipyrin, phenacetin, etc., but they are in no way characteristic of the drug which causes them. As a rule the eruption is most marked on the chest, back, neck, shoulders, upper arms, and thighs, and less on the face, scalp, forearms, hands, legs, and feet. It may come out after the first dose of the drug or not until it has been employed for a considerable period or the dose markedly increased. When it follows a single dose there may be a latent period between the taking of the drug and the appearance of the rash, which averages 24 to 36 hours but varies greatly in different individuals and tends to diminish as the susceptibility to the drug increases. In some cases the eruption is the only indication of ill-effects from the drug, in others it is associated with more or less severe general symptoms, such as rise in temperature, furred tongue, dry throat, congestcd fauces, gastro-intestinal irritation, headache, giddiness, etc.-symptoms which may be 
sufficiently characteristic to suggest the drug responsible. As a rule when the drug is discontinued the eruption fades and is followed by desquamation.

In addition to what may be called the non-characteristic drug rashes there are others which are peculiar to certain drugs and would appear to be caused by their specific action, such as the pustular and condylomatous lesions caused by the bromides and iodides and the horny thickening and pigmentation due to arsenic. Eruptions of this nature generally subside soon after the drug is stopped, though occasionally they may persist for weeks and in rare instances may not appear until some time after it has been discontinued.

The type of eruption caused by a drug is, as a rule, uniform but may be multiform, as in the case of quinine which may produce erythematous, purpuric, urticarial, or vesicular, lesions.

Certain drugs, if prescribed over a sufficiently long period or in large doses, are capable of producing an eruption in almost anyone. As a rule, however, an individual susceptibility to the drug is necessary and, when this is present, even minute doses, so small as to be harmless in the average individual, may cause the eruption. This susceptibility may be hereditary and affect several members of a family; or it may be acquired as the result of defective elimination by the kidneys or intestines. More frequently it is due to the individual haring taken an overdose of the drug which resulted in the eruption and was followed by an increased susceptibility and a diminution in the latent period till the rash appeared, exemplifying the well-known phenomenon of anaphylaxis. It has been observed that where there is an idiosyncrasy towards a special drug it extends also to drugs belonging to the same class, for example if an eruption is called forth by copaiba it may also be caused by cubebs and turpentine which are allied balsamic substances.

It has been said that perseverance with a drug will cause the disappearance of the eruption and establish a certain degree of tolerance towards it, but this is rarely the case and, as a rule, increased dosage is ineritably followed by exacerbation and increased susceptibility.

Etiology. - In the vast majority of cases some idiosyncrasy to the drug is present but there are other predisposing factors. It has been asserted that women and children are more liable to drug eruptions than men, and that defects in the general health, especially cardiac disease and gastro-intestinal disturbances, are recognised predisposing influences; these are of minor importance as compared with imperfect action of the kidneys, either from functional or organic disease, interfering with the rapid elimination of the drug.

Mode of Production of the Eruption.-The precise manner in which drug eruptions are called forth is not fully understood. It has been found that the large majority are the result of the ingestion of a drug through the alimentary tract and that they seldom occur if the drug be injected hypodermically. In a few rare instances a generalised eruption has resulted from the absorption of a drug applied locally ; 
for example a generalised erythema has been known to follow the application of a belladonna plaster, and that it was due to the absorption of the drug was proved by the co-existence of dryness of the throat, dilatation of the pupils, and other symptoms associated with belladonna poisoning. Iodoform and various preparations of mercury applied in dressing wounds may set up a localised dermatitis followed by a generalised eruption with the occurrence of lesions in distant parts which could not possibly have been due to the local action of the drug. These eruptions, which are generally of the erythematous or vesicular type, may be accompanied by internal symptoms, such as feverishness, vomiting, and diarrhoea, and the drug may be detected in the urine and saliva.

At one time it was thought that the eruptions were produced by the direct action of the drug on the central or peripheral vaso-motor system and that they were angio-neurotic phenomena. This view is gradually being abandoned and the tendency now is to regard the lesions as essentially inflammatory and induced by the action of some toxic substance present in the cutaneous capillaries and acting on the surrounding tissues-a view which has been strengthened by the experiments of Philippson and others who have shown that urticarial lesions may be induced experimentally by the injection into the skin of certain irritants, such as morphine, peptone, atropin, etc., even when the sympathetic nerves were cut and the sympathetic ganglia removed.

The most recent theory, and one that is gaining ground, is that the lesions are not the result of the action on the skin of the actual drug but are due to some highly toxic foreign proteid eliminated by the tissue cells, possibly by those lining the alimentary tract, which acts directly on the cells of the capillary walls and on the neighbouring cells of the corium.

Where the drug produces characteristic lesions in the skin, as in the case of arsenic, bromides, and iodides, the lesions are most probably due to the direct action of the drug.

Keratosis due to arsenic, for example, is best explained as the result of the stimulating action of the arsenic on the cells of the epidermis; the peculiar pustular condylomatous lesions caused by bromides, as directly produced by the irritant action of the salts on the epidermal cells, causing them to proliferate, and on the underlying connective tissue of the upper layers of the corium, giving rise to a granulomatous infiltration; the nodular lesions due to iodides as the result of the irritating action of the drug on the sweat-glands, sebaceous glands, and the cells in their neighbourhood.

Diagnosis.-The recognition of a drug eruption is a matter of great importance and often of extreme difficulty. In a suspected case it is sometimes possible to get a clear history of the taking of the drug but not infrequently even careful enquiry may fail to elicit the required information, especially where the drug has been taken in the form of some patent medicine. 
The majority of the eruptions belong to the erythematous type and have to be distinguished chiefly from scarlet ferer and measles. In drug rashes, as a rule, there is no temperature and though occasionally there may be slight sore throat, even furred tongue, a trace of albumin in the urine, and possibly some desquamation, still the sequence of events is not that of an acute exanthem, for there is not the same definite incubation period and the eruption comes out more suddenly and violently and generally disappears when the drug is stopped.

The diagnosis of the specific eruptions due to the iodides, bromides, and arsenic, may be difficult and will be referred to later.

Treatment.-The drug responsible must be discontinued at once, when the eruption will usually disappear in a day or two though occasionally, as in the case of bromide of potassium, it may persist for days or even weeks. Every effort should be made by diuretics and aperients to eliminate it rapidly by the kidneys or bowels, and on no account should diarrhoea caused by the drug itself be interfered with.

Local treatment is, as a rule, unnecessary.

\section{Drugs in Commox l'se yost Liable to Cause Cutaneous Lesions}

To furnish a complete list of the drugs capable of producing cutaneous eruptions would be an almost impossible task, would serve no useful purpose, and would include almost every drug as it is rare to find one towards which some individual is not unduly sensitive. Here reference will be made only to those which are in common use.

Antimony.-Antimony in rare instances causes an eruption of an erythernatous or urticarial type.

Antipyrin.-Antipyrin is liable to produce cutaneous eruptions in susceptible individuals. These usually consist of erythematous patches situated on the chest, abdomen, genital organs, and flexor aspects of the limbs, and especially well marked on the hands and feet, or they may be scarlatiniform or morbilliform in type and followed by desquamation and eren pigmentation, or occasionally may be urticarial, papular, vesicular, bullous, or pustular. In association with the cutaneous lesions there may be congestion of the conjunctivae and a definite febrile disturbance.

Arsenic.--Arsenic is one of the most common causes of drug eruptions and some years ago when it was regarded as a panacea for almost every cutaneous disease arsenical eruptions were even more frequent than they are now. It may be taken, not only medicinally, but also accidentally in some food or condiment in which it exists as an impurity. It is present, for example, in commercial sulphuric acid used in the manufacture of invert sugar for brewing, and so caused the epidemic of arsenical poisoning from beer in Lancashire in I900; in fumes from 
burning gas coke and in this way may be conveyed to chicory or malt; in certain dyes used in making confectionery, and in colouring wall-papers.

The arsenical eruptions may be divided into two classes, namely (I) those which result from acute intoxication with the drug and are due either to one or more large doses or to smaller doses in a highly susceptible individual; (2) those which occur in chronic intoxication from small doses over a prolonged period.

Eruptions in Acute Intoxication.-A number of different rashes have been observed in connection with acute arsenical poisoning. The most common are erythematous, the erythema sometimes being universal, at other times distributed as in scarlet fever or measles. The erythema is not uniform but presents here and there vivid erythematous patches and is accompanied by marked redness, pain, and swelling of the palms and soles, and oedema of the eyelids, and is followed by fine desquamation. Severe general symptoms usually accompany the cutaneous affection, such as gastro-intestinal irritation, fever, colicky pains, congestion of the conjunctivae, etc.

In severe cases the eruption may be vesicular, bullous, pustular suggesting smallpox, or even gangrenous, and in dependent situations such as the lower part of the trunk, legs, and genitalia, it may be purpuric. In rare instances it may be papular, as in a case observed by the writer in which an injection of salvarsan in a young girl of 7 years was followed a week later by a maculo-papular eruption, covered with adherent horny scales, situated on the face, scalp, chest, and arms, and accompanied by slight itching, redness and hyperkeratosis of the palms and soles, inflammation of the posterior nail-wall and hyperkeratosis of the nail-bed. The eruption gradually faded but recurred after a subsequent injection.

Occasionally an extensive generalised dermatitis may result from the local application of arsenic and may be accompanied by general symptoms indicating the absorption of the drug.

Enuptions in Chronic Intoxication.-A variety of cutaneous lesions may result from arsenic taken in small doses over a prolonged period, such as erythematous eruptions, keratosis especially affecting the palms and soles, pigmentation, Herpes zoster, hyperidrosis, and disorders of the nails. Of these the most characteristic are keratosis and pigmentation.

Erythematous Emptions.-These are of the same type as occur in acute arseincal poisoning and may be diffuse, patchy, morbilliform, or scarlatiniform, last about a week and generally end in a fine desquanuation. In rare instances the lesions may be vesicular or even bullous.

Kevatosis.-Hyperkeratosis may be said to be the most characteristic sign of chronic arsenical poisoning, it is marked where the Stratum corneum is naturally thickest as in the palms and soles, but may occur to a slighter degree anywhere on the cutaneous surface. As a rule it develops gradually and may not appear for some time after the arsenic has bcen discontinued. 



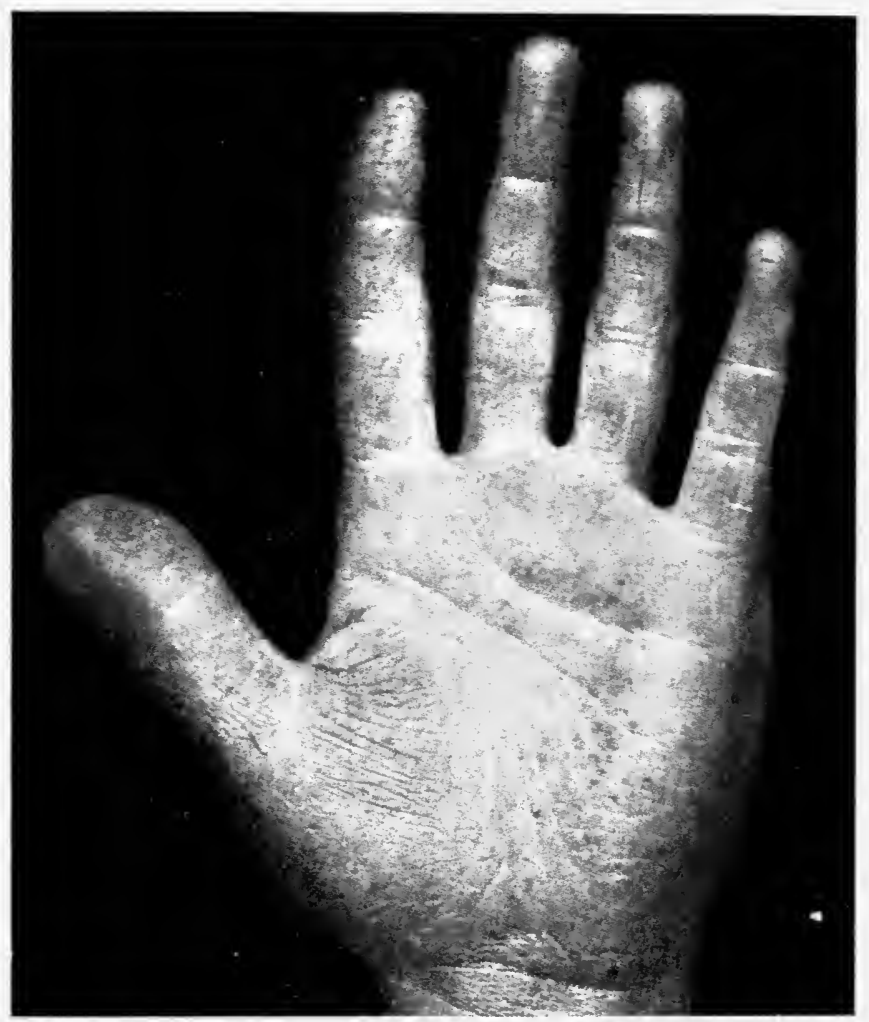

FIG. 253.-Arsenical Hyperkeratosis of Palm. (Drs. Brooke and Roberts' case. Brit. Journ. Derm., Igor.)

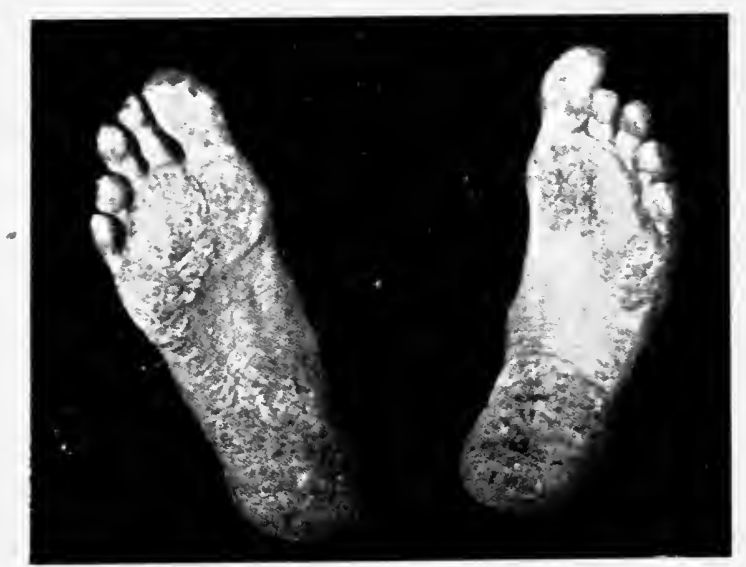

FIG. 254-Arsenical Hyperkeratosis of Feet.

(Drs. Brooke and Roberts' case. Brit. Journ. Derm., 19or.) 
On the palms the skin is thickened in an irregular manner presenting small hard callosities, chiefly situated at the periphery, tending to miss out the central triangle, and extending up the sides and between the fingers. Sometimes it is more diffuse, giving the skin a leathery appearance and interfering with the finer inovements of the hands. The horny thickenings may be situated on an erythematous base, or a diffuse horny patch may be surrounded by an erøthematous halo and erythematous lines may indicate the position of the furrows. Where the erythema is marked it is usually associated with itching. (Fig. 253.)

On the soles the keratosis frequently takes the form of corn-like lesions consisting of horny pegs which, on being picked out, leare a depression. The rest of the skin of the soles may be diffusely thichened and present a rough shagreen-like appearance. (Fig. 254.)

The keratosis is usually symmetrically distributed on the palms and soles. A definite thickening of the Stratum corneum may be present also over the joints, especially the elbows, knees, and knuckles, while over the rest of the body slight hyperkeratosis may occur and render the skin harsh, or erythematous patches or papules may be noticed with slight thickening of the horny layer giving rise to a milky appearance suggesting Lichen planus.

On the palms and soles the hyperkeratosis may be preceded or accompanied by hyperidrosis, but this as a rule disappears leaving the skin dry, thick, and parchment-like. Where the keratosis is most marked, fissures are liable to occur and these may become septic or even ulcerated from the secondary inoculation of pus-organisms, and may cause considerable pain on movement.

The keratosis would appear to result from the stimulation of the basal cells of the epidermis by arsenic, but the exact manner in which this is brought about is uncertain though it is generally believed to be due to the dynamic action of the arsenic, eliminating oxygen which acts as a stimulant. In some instances the initial stimulation is followed by atrophy of the epidermis and fatty degeneration of sweat-glands and sebaceous glands. At one time it was believed that the arsenic was secreted by the sweat-glands and produced the changes in the epidermis in passing out at the sweat-pores, but as there are no special signs of irritation or inflammation about the sweat-glands it seems more probable that the arsenic is present in the inter-epithelial lymph and even in the epidermal cells and acts directly upon them, and it has been found in the scales and in the hair.

Occasionally epithelioma has been known to follow arsenical poisoning and about $I_{5}$ authentic cases are on record. It is usually of the squamous type and has invariably occurred in connection with the taking of small doses over a prolonged period. It does not seem to be directly produced by the irritant action of the arsenic but is probably secondarily induced by extraneous influences on a soil rendered susceptible to cancer by the arsenic. In nearly all the cases 
the malignancy was either added to a hyperkeratosis or occurred on the base of some chronic cutaneous affection involving the epidermis, such as psoriasis, or pemphigus. In a number of them hyperkeratosis appeared to be an essential precursor and affected the hands and feet, situations which are liable to friction or injury. In most of them malignancy did not develop until long after the drug had been stopped, which points to its being produced by some secondary influence on a base of arsenical keratosis.

Pigmentation.-Pigmentation is another characteristic symptom and is not infrequently preceded by erythema. It is of a brownishyellow or sepia tint, sometimes becoming almost black in dark people, and is usually most marked on the neck and trunk, though it may occur

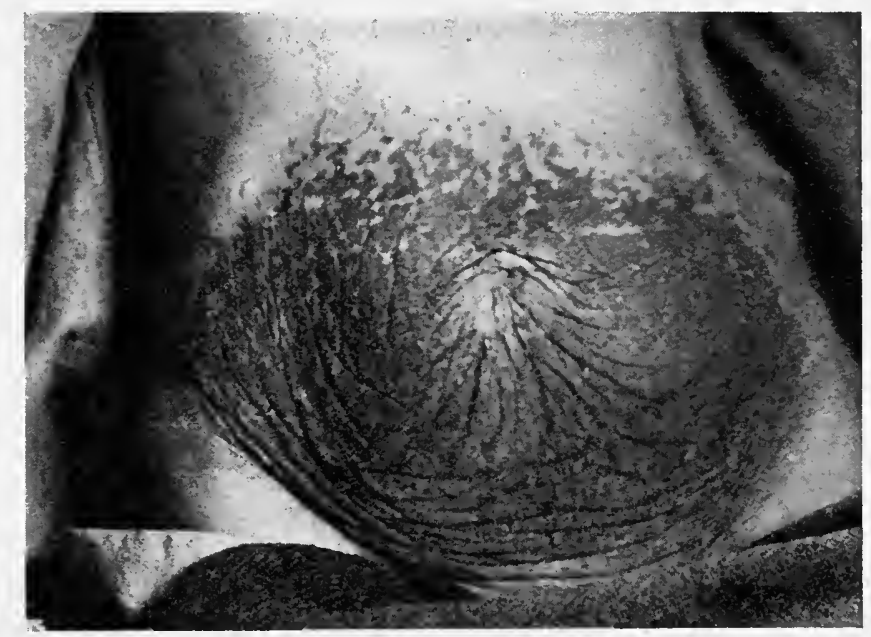

Fig. 255--Arsenical Pigmentation of Abdomen.

(After Dr. Arthur Hall. Glaisher's Forensic Medicine.)

on the limbs and face or may be widely distributed over the entire skin. It occurs in patches which may be large, but more often are small and unite to form a reticular mottling enclosing areas of normal white skin as a rule not much larger than a split-pea. It also tends to be darker in normally pigmented situations, such as the areolae of the nipples, groins, or about the axillae, and is generally well-marked in situations of pressure, as about the waist in women, or beneath the garters. Associated with it the skin is harsh and dry. (Fig. 255.)

Where the arsenic has been used in curing some chronic eruption, such as Lichen planus, psoriasis, or Dermatitis herpetiformis, the pigmentation is most marked in the sites of previous lesions, is confined to the skin, does not involve mucous membranes, and tends to fade when the drug is discontinued.

It has to be distinguished from the pigmentation of Addison's disease, but in it the pigmentation is more diffuse, the affected skin is not harsh and scaly, the mucous membranes of the mouth, con- 
junctiva, and vagina are generally involved, and the patient is usually weak and emaciated.

The pigment is non-crystalline and amorphous, and is situated in the cells of the epidermis, especially in the basal layer, but may be met with right up to the surface. It has been detected also in connective tissue cells in the papillary layer. Its exact nature is uncertain. It does not give the chemical reactions of arsenic, nor does it contain iron, suggesting that it is not derived from the blood but is probably a product of the activity of the epidermal cells.

Desquamation.-This occurs more or less all over the body. On the trunk it takes the form of thin tissue-paper-like lamellae which present a dirty appearance from pigment in the horn-cells; on the limbs, especially the extensor aspects, it is fine and furfuraceous; on the palms and soles the skin is apt to peel off in hard flakes.

Hyperidrosis.-This is a variable symptom met with chiefly on the palms and soles. In some cases it may be completely absent, in others it may consist of a slight clamminess generally accompanied by hyperaemia, while in others it may be profuse and precede or accompany the hyperkeratosis. It is due to the stimulating action of the arsenic, either on the epithelial cells of the sweat-glands or, more probably, on the secretory nerves.

Herpes zoster.-Attacks of Herpes zoster have been observed so frequently in connection with the ingestion of arsenic that they are now regarded as no mere coincidence but as the definite result of the action of the drug on the nerve supplying the affected part. This is easily understood, as arsenic has a tendency to produce neuritis and optic atrophy has resulted from time to time from the arylarsonates, such as atoxyl, soamin, etc.

Occasionally arsenic has been responsible for attacks of Herpes simplex about the lips, forehead, or prepuce.

Nails.-The nails may be affected in chronic arsenical intoxication, the nail-plate becoming striated, ridged, brittle, occasionally shed, and the nail-bed thickened from keratosis. Occasionally ulceration about the nail-walls has been observed from handling arsenical preparations. The changes in the nails are due to the direct action of the arsenic on the nail-matrix and the nail-bed, as is proved by small quantities of arsenic having been detected in the nail-parings.

Treatment. - The cutaneous symptoms of acute arsenical intoxication rapidly disappear when the drug is discontinued.

In chronic arsenical poisoning, on the other hand, although the pigmentation and erythema gradually clear up when the arsenic is stopped, the keratosis is singularly persistent and demands treatment in view of the possibility of malignant changes supervening. The horny thickenings should be remored by applications of salicylic-soapplaster, salicylic-collodion, or an ointment containing pyrogallic acid. Where ulceration is present it should be dealt with in the usual manncr. $\mathrm{X}$-rays are also useful in removing the thickened skin. 
Belladonna.-Rashes caused by belladonna are not infrequent, especially in children. They are usually scarlatiniform and associated with more or less itching, while occasionally isolated erythematous patches have occurred, or even gangrenous lesions.

Atropin has a somewhat similar effect.

Bromides.-Eruptions due to the bromides, chiefly bromide of potassium, are frequent and are among the most eharacteristic of drug rashes. They may appear after one or two large doses, or after a minute dose in a susceptible individual, or, more commonly, after the drug has been used over a prolonged period as in the treatment of some nervous disease such as ehorea or epilepsy, and are apt to persist for a considerable time after it has been discontinued. They have been known to occur in sueklings where the mother was taking bromides

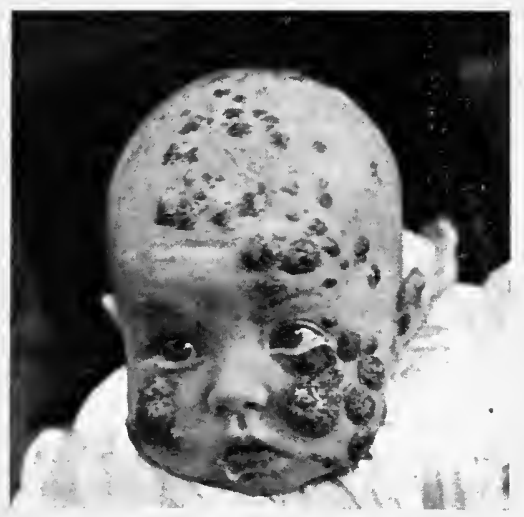

FIG. 256.--Bromide Eruption. Shows anthracoid lesions. though she herself was free from the eruption.

The most eommon type of bromide eruption is aeneiform, is sometimes known as bromacne, and is present chiefly about the face, shoulders, legs, and to a less extent on the arms.

In another not infrequent variety the lesions are peculiar, being raised, crusted, granulomatous masses, varying in size from a split-pea to a shilling or more, rounded or irregular in outline, sometimes of a dirty-brownish tint, and studded on the surface with minute pustular points from which a thick sero-purulent diseharge can be squeezed. These lesions, to which the name "anthracoid" has been applied, are especially common on the legs but may occur elsewhere. (Fig. 256.)

Various non-characteristic cruptions also may be caused by bromides, such as a vesicular rash simulating chicken-pox, erythematous blotehes, papules, urticaria, bullae, furuncles, or lesions like earbuncles.

These lesions would seem to be due to the irritating aetion of the salts causing a cellular proliferation of a granulomatous type in the corium, especially marked about the pilo-sebaceous follicles, and possibly an increase in virulenee of the ordinary skin micro-organisms leading to suppuration.

Chloral.-Eruptions from chloral are not uncommon, especially in ehildren. They may be of the Erythema multiforme type consisting of erythematous patches or papules on the face, arms, and backs of the hands, or may be more diffuse and searlatiniform. They may be aecompanied by congested conjunctivae, sore throat, and slight fever. 
Other types of eruption have been recorded in which purpuric, vesicular, bullous, urticarial, or furuncular lesions were present.

Chloralamide.-This drug has been known to give rise to erythematous eruptions associated with congestion of the mucous membranes of the mouth and conjunctivae, coryza, and fererishness.

Chloretone.-In a few rare instances chloretone has resulted in erythematous eruptions of the Erythema multiforme or scarlatiniform type.

Copaiba and Cubebs.-The eruptions due to these balsamic drugs are more characteristic than those caused by any drugs except the bromides, iodides, and arsenic. The most usual eruption is erythematous in type, consisting of variously sized elevated patches of a vivid red tinge which tend to become purplish or even black on the lower extremities. Not infrequently the hands, ankles, and backs of the feet are specially involved. The erythematous patches may be definitely raised and even urticarial and, in rare instances, may be studded with vesicles or minute pustules.

Sometimes the eruption is scarlatiniform, at other times it resembles measles, while in some instances it suggests a papular syphilide.

The copaiba rashes as a rule are associated with burning and itching and with general symptons, such as fever, sore throat, oedema about the eyelids, congestion of the conjunctivae, and some gastrointestinal disturbance. The urine has a peculiar odour and when floated on nitric acid presents a cloudy opacity, while, if allowed to stand, it may assume a violaceous tint.

Iodides.-The iodides, especially potassium iodide, are common causes of drug eruptions, partly on account of their frequent use, and partly because a large number of individuals are hypersensitive to them. A variety of different lesions are produced by them, such as acneiform lesions, erythematous patches, vesicular and bullous eruptions, purpuric lesions, pustular lesions, nodules and tuberous lesions suggesting gummata, fleshy masses like the tumours of Mycosis fungoides or sarcomata (iod-sarcoma), or condylomatous lesions studded with minute pustules like those caused by bromides, and occasionally acute oedema. (Fig. 257.)

These eruptions are most common on the parts of the body generally affected in Erythema multiforme, namely the face, forearm, and hand, but may occur on the scalp, neck, trunk, legs and feet, and are occasionally universal.

The most common iodide eruptions are acneiform (iod-acne) or furuncular and involve chiefly the face, neck, and shoulders. They result from the irritating action of the iodide on the sebaceous gland and its neighbourhood and possibly from the stimulation to growth of the cutaneous micro-organisms about its orifice. The pustules are similar to those of Acne vulgaris but there are no comedones, and the boils are like ord nary boils and may reach a considerable size, especially on the back of the neck. Not infrequently in cases of common acne, 
iodide of potassium has been prescribed, either in the form of a mixture containing the salt or in some patent medicine such as Clarke's blood mixture, with the object of " purifying the blood," and, in consequence, the acne lesions have been transformed by the iodide into large fleshy nodules or boils.

The nodular lesions occasionally suggest syphilitic gummata and as they sometimes break down and slough the resemblance is further enhanced. Owing to this mistake the dosage of the drug has sometimes been increased, and the lesions aggravated in consequence.

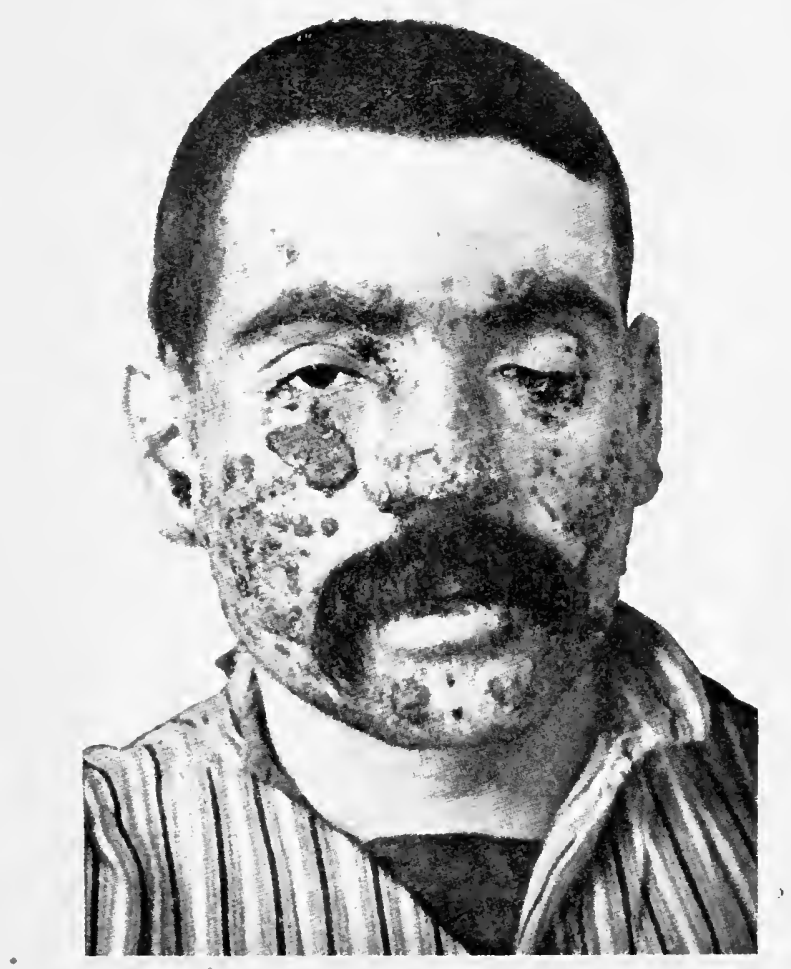

FIG. 257--Vesico-bullous Eruption due to Iodide of Potassium.

(After Dr. Arthur Hall. Edinburgh . Yedical Journal, I906.)

In addition to the above lesions which are practically indistinguishable from those due to bromides, there is a type of eruption which is peculiar to the iodides namely the vesicular or bullous type. In these cases the vesicles are situated on an erythematous base, usually contain a pale yellowish sero-pus, and present a dark centre recalling smallpox. They occur most frequently about the face, neck, hands and arms, and, where they are numerous, the skin of the affected regions may be markedly inflamed and ocdematous. When bullae occur they may have clear contents but more usually contain sero-pus, and in rare instances may be haemorrhagic and on breaking may be 
replaced by soft vegetating lesions like Pemphigus vegetans or occasionally by superficial ulcerations.

The mucous membranes may be involved, and resicular lesions, excoriations, or condylomatous lesions have been detected on the tongue, palate, fauces, and gastro-intestinal tract. These eruptions if extensive are usually associated with other symptoms of iodism, such as coryza, pharyngeal catarrh, gastro-intestinal irritation, and sometimes marked rise of temperature. Of the various iodides the most liable to produce eruptions is iodide of potassium, but they may be caused also by ammonium iodide, ferrous iodide and, more rarely, by sodium iodide. The newer substitutes for the alkaline iodides, namely iodalbacid, iodalbin, iodoglidin, and iodipin, do not seem to give rise to cutaneous eruptions.

As the iodine is eliminated to a certain extent by the kidneys the most severe examples of iodide rashes usually occur in those suffering from some disorder of the kidneys or the heart. As a preventive of iodide and bromide eruptions the addition of a few minims of Liq. arsenicalis to the mixture is sometimes of value.

Mercury.-Eruptions of the scarlatiniform, papular, or urticarial, types occasionally have been known to follow large doses of mercury, whatever the method of administration, whether by inunction, injection, inhalation of mercury vapour, or by ingestion. These may be accompanied by severe general symptoms, such as fever, diarrhoea, etc., and have been known to lead to a fatal issue.

Morphine and Opium.-Morphine or opium sometimes produce eruptions somewhat similar to those caused by antipyrin, chiefly of the erythematous type, affecting the upper part of the trunk, and accompanied by itching and desquamation. Occasionally purpuric, pustular, or urticarial lesions may be met with, and, in rare instances, vesicles and bullae may follow the use of the drug.

Quinine.-Eruptions from quinine are not infrequent and are usually of a blotchy erythematous type, though they may be urticarial, bullous, or eczematous, are generally itchy, and may be accompanied by a general febrile disturbance. The lesions are present chiefly on the face, neck, and extremities, but may occur elsewhere or may be universal. They may be followed by severe desquamation and occasionally by gangrenous lesions of the scrotum. They may be called forth by comparatively small doses of quinine or by patent medicines containing minute quantities, such as "phosferine" and "tonic water."

Rhubarb.-Rhubarb has been known to produce a scarlatiniform dermatitis and a papular rash going on to pustulation.

Salol.-Salol may cause an urticarial eruption.

Santonin.-Santonin cccasionally produces an eruption of an erythematous or urticarial type.

Silver Nitrate.--After prolonged use silver nitrate may cause a slatecoloured pigmentation of the skin known as "argyria" which was 
fairly conmon when it was customary to prescribe this drug for epilepsy. The pigmentation has a peculiar bluish-slate colour and is the result of the deposition of the metal in the skin, especially in the deeper layers of the epidermis, in the papillary layer, and about the sweat-glands. Though most marked in the exposed parts from the action of sunlight reducing the silver salt it may be universal and may affect the mucous membranes. It is permanent and treatment by eliminants, diuretics, etc., seem to have little effect upon it, though in time it may decrease slightly.

Sodium Salicylate.-Sodium salicylate, especially when chemically prepared, can give rise to eruptions which may be erythematous, urticarial, scarlatiniform, or occasionally vesicular or bullous.

Sulphonal.-Sulphonal occasionally produces scarlatiniform or erythematous eruptions followed by desquamation.

Turpentine.-Turpentine is responsible for itchy erythematous eruptions of a scarlatiniform type, somewhat similar to those produced by copaiba.

Veronal.- Teronal not infrequently causes an erythematous eruption involving the face, trunk and limbs, and consists of macules, papules, and confluent patches. The mucous membranes may be affected, the mouth swollen and painful, and the mucosa red or covered with small vesicles. Associated with it there may be a rise in temperature.

\section{Eruptions due to Animal Sera}

Injections of animal sera, especially diphtheria anti-toxic serum, are liable to be followed by general reactions accompanied by cutaneous eruptions. In certain instances they have also followed administration of serum by the mouth or per rectum. The sera are usually obtained from the horse and the eruptions may result, not only from immunised horse serum, but also from normal horse serum. The sera of different horses vary in their potency and that of the same horse may vary under different conditions; but there is another factor which determines the severity of the eruption, namely idiosyncrasy on the part of the patient. It is the serum itself which is responsible for the reaction and large doses are more liable to cause it than small ones, but the tendency to ill-effects may be reduced by the employment of highly potent sera of diminished bulk.

The serum eruption usually consists of patches of erythema which may clear in the centre and form variously sized ringed or irregular figures which may coalesce into a gyrate pattern. The patches, as a rule, begin to fade in about 24 hours and disappear in a few days with or without desquamation. The eruption is situated chiefly on the extensor aspects of the limbs, but may be more widely distributed, or in some instances may begin at the site of the injection and continue in that neighbourhood the whole time. 
In addition to the patchy type of eruption other forms may be met with, such as urticarial, scarlatiniform, or morbilliform, while in rare instances they may be purpuric, resicular, or bullous.

In connection with the shin lesions the conjunctiva is frequently. congested and the eyelids may be puffy and oedematous. More or less marked general symptoms usually occur, such as fever, pain and swellings in the joints, vomiting, nephritis, and, in exceptional cases, collapse and a fatal issue.

The serum exanthem usually appears about 6 days after the injection but it may occur immediately or be delayed for sereral weeks. Occasionally a single dose may give rise to a series of eruptions which occur at intervals of a few days and may be uniform in type or may vary, one being erythematous and a subsequent one urticarial, and may recur for years.

The phenomenon of anaphylaxis is well demonstrated in connection with serum eruptions and some of the most severe and eren fatal cases have occurred in indiriduals who have become hyper-sensitive owing to a previous injection. This hyper-sensitiveness does not occur for about If days after the first injection but may persist for years, while if a second injection be given within ro days of the first injection there may be an actual immunity against the toxic effects. When the hyper-sensitiveness is established the latent time between the injection and the appearance of the exanthem is diminished and may be reduced to a few hours or even minutes.

The treatment of serum rashes consists of relieving any irritation by weak carbolic lotion, calamine cream, or ar evaporating alcoholic lotion, and the administration of calcium salts, as it has been suggested that hyper-sensitireness to serum injections occurs largely in those with reduced coagulability of the blood.

\section{Types of ERtption AND the Drtgs which Prodece TheN}

Practically every type of cutaneous lesion may result from the toxic action of some drug. Similar types of eruption may be produced by drugs of totally different chemical constitution, while the same drug may cause different types of eruption in different individuals, or at different times in the same individual. Chloral for example may give rise to elevated erythematous patches in one person and to a papular, urticarial, petechial, vesicular, or even bullous eruption in another. Consequently to classify the different drugs in a satisfactory manner under the headings of the eruptions they produce is an impossibility, for only in exceptional cases, such as the iodides, bromides, arsenic, and copaiba, are the eruptions sufficiently characteristic. Still for diagnostic purposes it will be useful to tabulate the principal types of eruptions and to give a short list of the drugs which may cause them. 
Erythematous.-Papular.-Antipyrin ; belladonna ; chloralamide ; copaiba ; cubebs ; iodides; phenacetin ; etc.

Macular and Patchy.-Animal serum; antipyrin; arsenic ; belladonna; bromides; chloral ; chloralamide; copaiba ; cubebs ; iodides; morphine; opium; phenacetin; potassium chlorate; quinine; salicylates; turpentine.

Morbilliform.-Animal serum; antipyrin; arsenic; belladonna ; copaiba ; cubebs ; sulphonal ; turpentine.

Scarlatiniform.-Animal serum; antipyrin; arsenic; belladonna ; chloral; copaiba; cubebs ; hyoscyamus; opium ; pilocarpine ; sulphonal ; turpentine.

Urticarial.-Animal serum ; arsenic ; bromides ; copaiba ; cubebs ; iodides; mercury; opium; phenacetin; quinine; salicylate of sodium ; santonin; turpentine ; etc.

Vesicular.-Antimony ; antipyrin ; arsenic ; bromides ; chloral ; copaiba ; iodides; quinine; turpentine ; etc.

Herpetic (Herpes zoster and Herpes simplex).-Arsenic.

Bullous.-Antipyrin; bromides; chloral ; iodides; opiun ; quinine; salicylates; etc.

Polymorphous Eruptions of the Erythema Multiforme Type.-Animal serum ; antipyrin; chloral ; copaiba ; cubebs; iodides ; opium ; potassium chlorate; etc.

Pustular.-Antimony ; antipyrin ; arsenic ; bromides ; iodides ; opium; salicylates; turpentine. (Of these the most common causes are bromides and iodides).

Acneiform.-Antipyrin ; arsenic ; bromides; chloral ; iodides ; opium.

Ulceratice.-Arsenic ; bromides ; chloral hydrate ; iodides.

Condylomatous or Anthracoid.-Bromides; iodides.

Gangrenous.-Arsenic ; iodides ; quinine.

Purpuric and Petechial.-Animal serum; antipyrin; bromides; copaiba; ergot; iodides; potassium chlorate; quinine; salicylate ; sulphonal.

Keratotic.-Arsenic.

Pigmented.-Arsenic; silver nitrate.

\section{REFERENCES}

AUDRY, C. Arsenical pigmentation of the skin and mucosa (Ann, de Derm. et de Syph., Dec. I896).

BERG. Serum exanthemata (Hed. Rec., i 898 , p. 865).

Brooke \& Roberts. Epidemic of arsenical beer poisoning (Brit. Journ. Derm., I9OI, xiii. p. I2I).

Debreuilh, W. Arsenical keratosis and arsenical cancer (Ann. de Derm. et de Syph., F eb. I9 Io, p. 65).

Evans, Willmott. Bullous quinine eruptions (Brit. Journ. Derm., 1902, xiv. p. 263).

Fox, Colcotr. Arsenical keratosis (Brit. Journ. Derm., i S93, v. p. 5I). 
Gottheil, W. S. Iododerma bullosum haemorrhagicum (Journ. Amer. Med. Ass., I909, liii. p. I $\left.f^{6} 5\right)$.

HALl, A. Severe skin eruptions due to bromides and iodides (Edin. Med. Journ., March 1906).

HaMblrger, L. Arsenical pigmentation (Bull. Johns Hopkins Hosp., I900, p. S7) $_{7}$

Hutchissox, J. Iodide eruptions (New Sydenham Society Atlas).

MacLeod, J. M. H. Dermatitis following large dose of arsenic (Brit. Journ. Derm., I913, xxy. p. 86).

Ineneat, J. Arsenical dermatoses (Ann. de Derm. et de Syph., I897, vii. p. 345$)$.

PAsris, A. Sur la pathogénie des éruptions bromiques (Ann. de Derm. et de Syph., Jan. I906, vii.).

Pernet, G. Drug eruptions (Brit. Med. Journ., I903, i. p. II3I).

Reriolds, E. Arsenical poisoning (Aled. Chir. Trans., I90I, lxxxiv. p. fo9).

Stowers, J. H. Drug eruptions (Brit. Journ. Derm., I 898, x. p. 289).

WASHBours, J. W. Rashes in anti-toxin treatment of diphtheria (Guy's Hosp. Gaz., I S99).

Wechselyaxi. Antipyrin rashes (Arch. f. Derm. u. Syph., i S99). 


\section{CHAPTER XXV}

\section{LICHEN}

Definition: Lichen is an old term which was originally applied to diseases which seemed to spread on the skin as the lichen fungi on trees and was used to designate a group of skin affections in which the elements were papules and remained as such through the whole course of the disease. This restriction was not long adhered to, however, and the term came to be used by different writers for eruptions in which the papules developed later into vesicles or pustules, consequently “ Lichen" qualified by a distinctive adjective has been used to denote a number of affections which differ from each other in their nature and causation. At the present time it is reserved for a few cutaneous diseases in which the initial lesion is an inflammatory papule, and the most important of these is the Lichen planus of Erasmus Wilson. Before describing this important disease it will be of service to refer briefly to a few of the affections to which the name has been applied in the past but which have now been relegated to their proper etiological positions.

L. agrius is an obsolete term once employed to designate a papular affection, which was probably either eczema or prurigo of Hebra.

L. anmularis, circinatus, gyratus, serpiginosus are varieties of Seborrhoic dermatitis.

L. circumscriptus and L. simplex chronicus (Vidal) are now described under the heading of lichenification.

L. haemorrhagicus and L. lividus are simply papules of various forms in which small haemorrhages have occurred, or small purpuric lesions at the mouths of the hair-follicles.

L. neuroticus was a name applied by Unna to a type of case which he identified with Lichen ruber of Hebra.

L. pilaris is a dermatitis characterised by spines or plugs projecting from the hair-follicles especially of the extensor aspects of the forearms and thighs. It is a form of keratosis of the upper part of the follicle and is described under follicular diseases.

L. ruber was used by Hebra to designate a papular dermatitis unfamiliar to dermatologists of our time and the exact nature of which is uncertain. It was associated with severe general symptoms, great prostration and nerrous depression, and the large majority of the cases 
were fatal. Hebra seems to have included two distinct affections in his description, which Kaposi subsequently separated and called respectively Lichen ruber acuminatus, and Lichen ruber planus. The latter is the same as Lichen planus of Wilson, while the former is probably Pityriasis rubra pilaris of Devergie (see page 850 ). The identity of Kaposi's Lichen ruber acuminatus with Pityriasis rubra pilaris has been the subject of much discussion, but seems to have been definitely established at the Dermatological Congress in Austria in I\$92, when Kaposi on the one side, and Hallopeau, Vidal, and other French dermatologists on the other, met over a case at the Buda Pesth Hospital and claimed it respectively as Lichen ruber acuminatus and Pityriasis rubra pilaris.

$L$. scrofulosorum is a tuberculide and is described under that heading.

L. spinulosus is another peculiar keratosis of the hair-follicles in which spiny plugs occur, projecting about an $\frac{1}{8}$ of an inch above the surface of the skin; it is described under follicular diseases.

L. strofulosus is an old term and most probably referred to papular urticaria or to the papular stage of miliaria rubra.

L. syphiliticus is simply a small papular or miliary syphilide.

$L$. tropicus is a synonym for prickly heat.

L. urticatus is papular urticaria of infants and children.

$L$. variegatus is the name applied by Crocker to Parakeratosis variegata of Unna (see page 847 ).

\section{LICHEN PLANUS}

Syn. : Lichen ruber planus.

Definition: An inflammatory disease of the skin characterised by small flat glistening papules, polygona! in shape, varying in tint from bright red to lilac, and tending to aggregate to form irregular patches with a rough scaly surface.

Description.--Lichen planus, though one of the rarer skin affections, is a well-known disease in this country where it was originally described by Erasmus Wilson in I869. There are two main varieties, (I) a subacute localised type, and (2) an acute generalised type.

In most cases the initial or essential lesion consists of a flat papule, angular in outline, slightly raised, varying in size from a pin's head to a millet seed, and enclosed by the natural lines of the skin which determine its polygonal shape. It appears first as a macule but by the gradual deepening of the superficial furrows enclosing it, a cellular infiltration in the underlying corium, and a thickening of the epidermis, it becomes raised up to form a flat papule, which is pinkish-red at first but gradually assumes a peculiar lilac tint owing to the thickened epidermis casting a white shade over the blood-vessels. The surface of the papule has a glistening appearance, which has been explained as a result of the stretching of the superficial layers of the epidermis but is more probably 
clue to an alteration in the horn-cells, and in older lesions it presents whitish or milky puncta and striae which are of great diagnostic significance. On examining the papules with a pocket lens it will be seen that several of them have central horny thickenings or plugs, while others show distinct depressions or umbilication, corresponding to the sweat-pores round which many of thern have formed. (Fig. 258.)

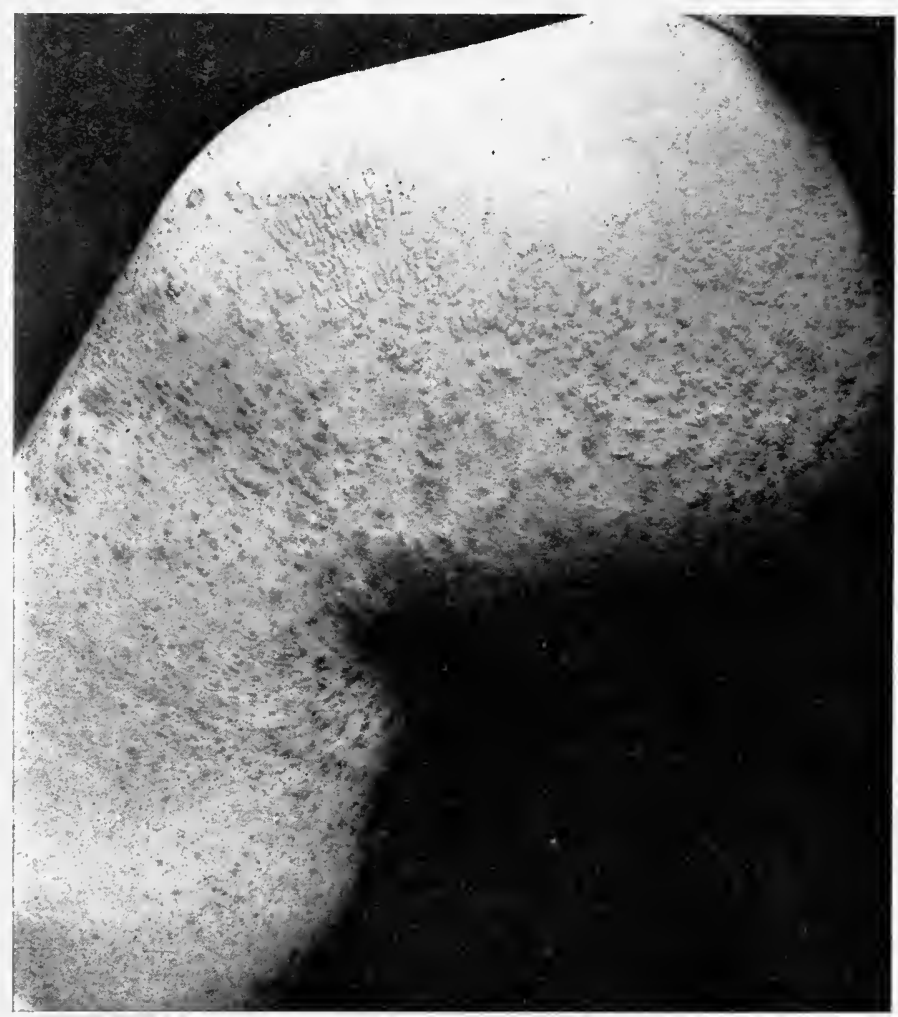

FIG. 258.-Lichen Planus.

\section{Chronic or Sub-acute Localised Type}

The sub-acute type of Lichen planus is the usual form. In it the eruption consists of a varying number of lilac-coloured papules, such as have been described, confined to certain regions and tending to be symmetrically distributed. The most common situations for them are the flexor aspects of the wrists, shins, inner sides of the knees and thighs, ankles, lower part of the abdomen, palms and soles; they are rare on the face, scalp and fingers. (Fig. 259.)

In addition to the typical flat lesions, acuminate follicular papules may occur, sometimes surmounted by spines, which protrude about an $\frac{1}{8}$ of an inch from the hair-follicles and when picked out leave a gaping orifice. These acuminate lesions are frequently situated on the limbs where they are apt to become inflamed from scratching. 
Their occurrence has probably no great significance, as similar papules may be present in other chronic skin affections such as Seborrhoic dermatitis and Lichen scrofulosorum. To such cases the name Lichen plano-pilaris (Pringle) has been given. Larger lesions, consisting of flat papules from $\frac{1}{4}$ to $\frac{1}{2}$ an inch in diameter and frequently presenting

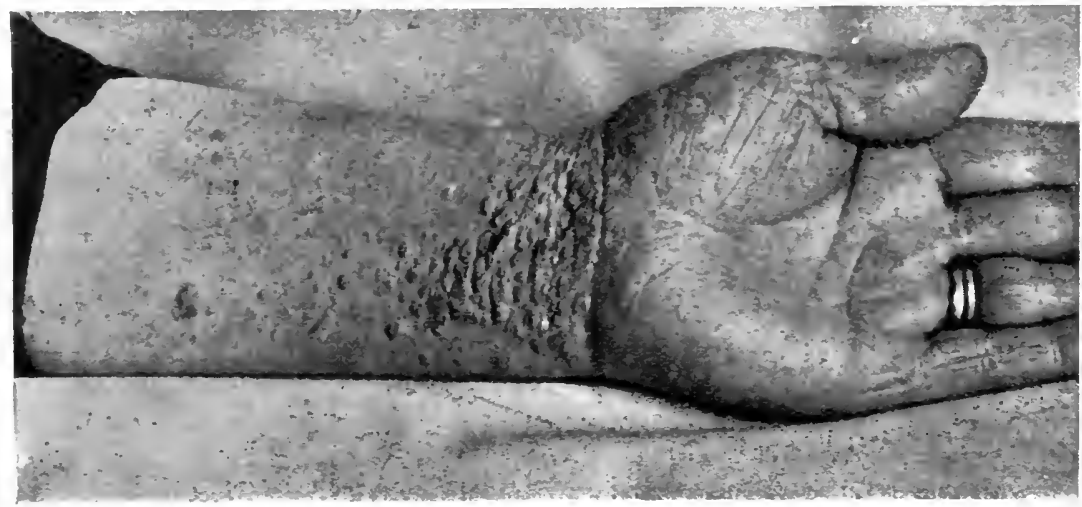

Fig. 259.-Lichen Planus.

Showing obtuse lesion on wrist.

a depression in the centre, occasionally occur and to this type the name Lichen obtusus corneus has been applied. (Fig. 26o.)

The papules either remain discrete until they fade, or coalesce to form irregular patches beyond which isolated papules usually occur.

The eruption may be confined to the region in which it originally appeared but more usually keeps on developing in new situations for

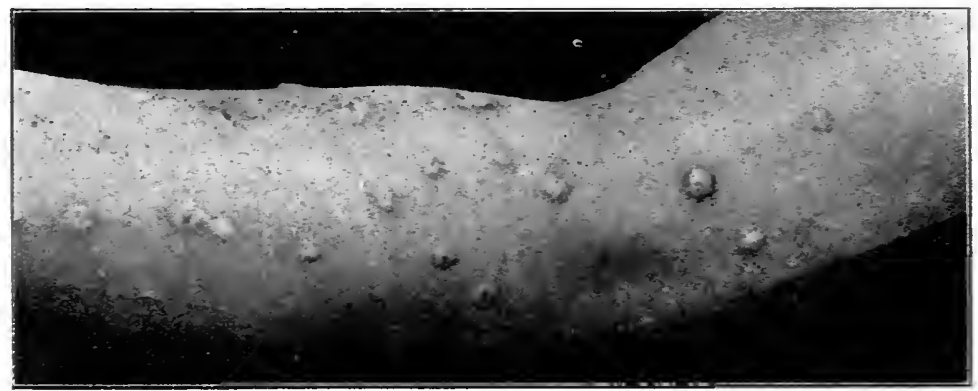

FIG. 260.-Lichen Obtusus Corneus.

(Dr. Rupert Hallam's case.)

months. When the lesions fade pigmentation is left, which raries from a sepia tint to a bluish-black and is deeper than that which occurs after any other skin affection. This may disappear in a few months and leave no trace or atrophic changes may supervene and the lesions may be replaced by slight depressions.

Subjective symptoms consisting of itching, pricking, or burning, are usually present, but vary greatly in different cases, sometimes being 
slight and negligible, at other times so intense as to be out of all proportion to the extent of the eruption.

Constitutional symptoms are usually absent in sub-acute cases, and the general health does not appear to be in any way impaired by the skin complaint, though

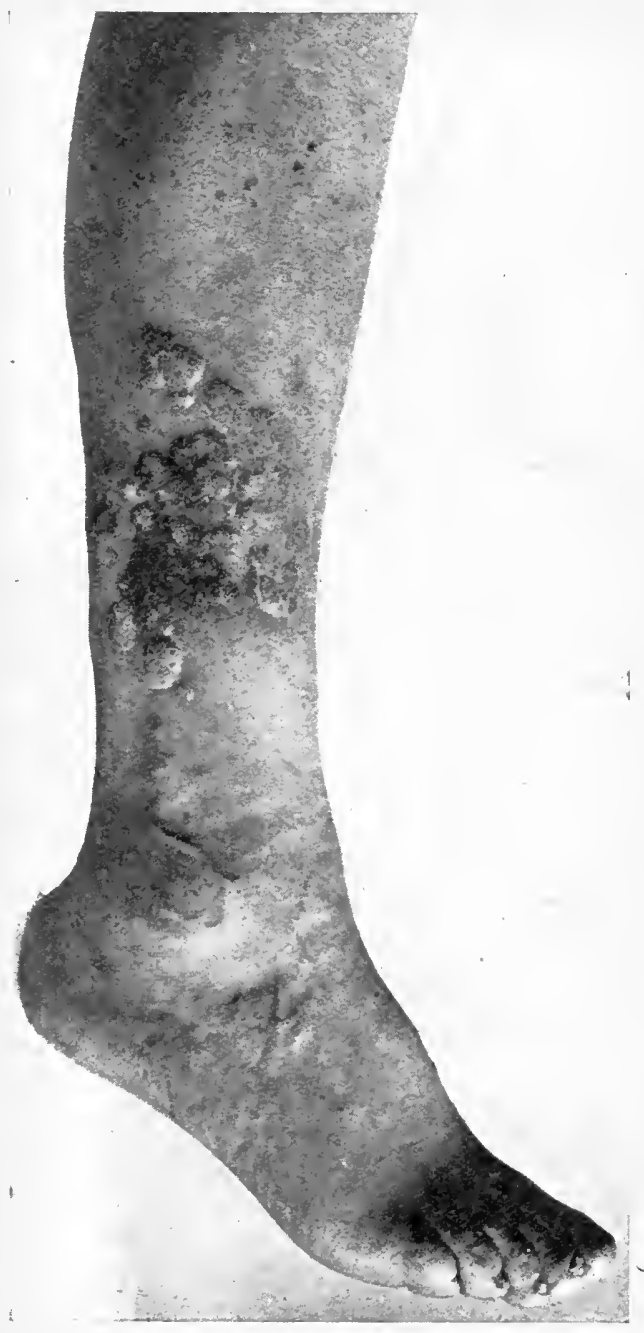

FIG. 26I.-Lichen Planus Hypertrophicus. occasionally there are evidences of nervous irritability and neurasthenia.

In a considerable number of cases the mucous membranes are affected (see page 735).

In addition to the usual sub-acute type there is a number of variations which are due to different modes of evolution and aggregation of the lesions, to which distinctive adjectives, useful for purposes of description, have been attached.

\section{VARIATIONS}

Lichen planus hypertrophicus.-In this variety the lesions coalesce to formhypertrophicpatches which differ in size from a sixpence to several inches in diameter, are well-defined, irregular in outline, and raised abruptly about an $\frac{1}{8}$ to a $\frac{1}{4}$ of an inch above the level of the surrounding skin. The smaller lesions are reddish-purple and have a cross-hatched surface, while the larger patches are a pale lilac, covered with adherent scales or horny plates or present a rough pitted appearance which has been aptly compared to shagreen and is due to the falling out of small horny plugs at the sweat-pores or mouths of the hair-follicles. On the legs the patches often become warty and assume a dirty grey appearance (Lichen planus verrucosus). Hypertrophic patches occur chiefly on the limbs but may be present also on the trunk. The itching connected with them is often considerable and 
causes them to be rubbed, scratched, and aggravated. After an indefinite time they disappear spontaneously and are followed by pigmentation. (Fig. 26r.)

Lichen obtusus corneus is the name applied by Brocy to a rare variety of hypertrophic Lichen planus in which the lesions are discrete nodules, round in shape, about the size of a split-pea or larger, and almost cartilaginous in consistence. They are pale in colour and flat or depressed in the centre where they may present a small haemorrhagic crust from scratching. They are present chiefly on the backs of the hands, forearms, legs, and ankles, and are absent on the trunk though ordinary Lichen planus papules may be present there in association

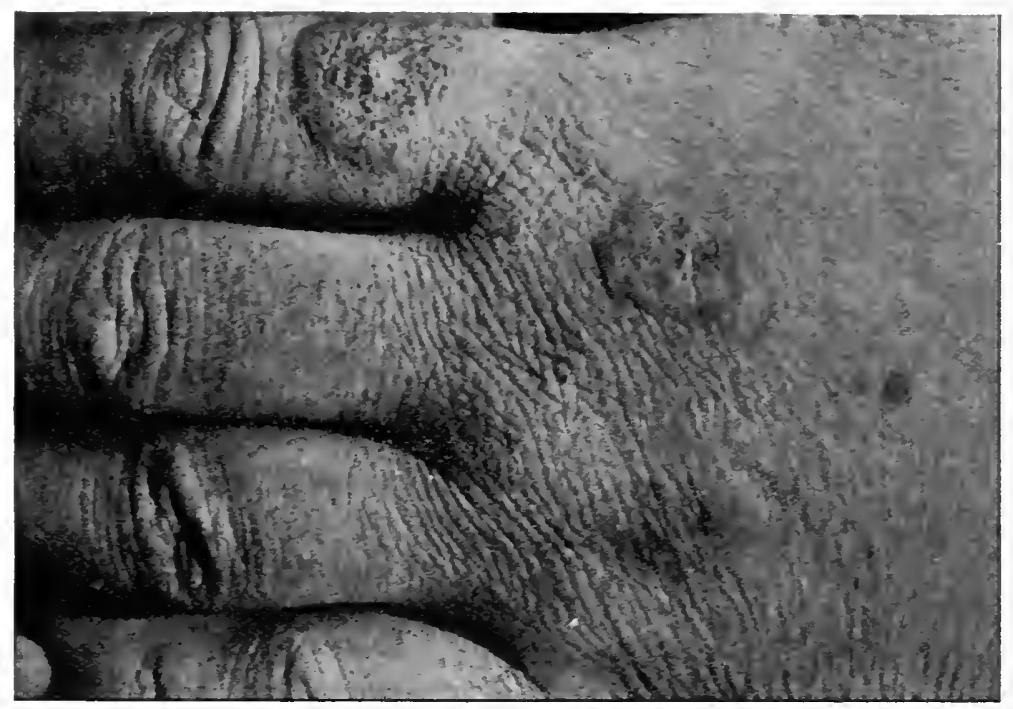

FIG. 262.-Lichen Obtusus Corneus in a Man's Hand. Shows large lesions.

with them. They may be few in number or numerous and are usually characterised by intolerable itching. (Fig. 262.)

Lichen planus annularis as the name indicates is a variety in which the lesions form rings, varying in diameter from $\frac{1}{4}$ to $\frac{3}{4}$ of an inch. The border is definitely raised, usually about an $\frac{1}{8}$ of an inch in breadth, brommish-red or purplish in colour, with a surface which is either smooth with milky puncta and striae over it or is wrinkled and covered with thin adherent scales. Occasionally the rings are incomplete or coalesce to form gyrate figures enclosing normal areas. The rings are usually: formed by the aggregation of ordinary Lichen planus papules and are generally associated with typical discrete lesions elsewhere. In a few rare instances they are developed from a single lesion by essential spreading at the periphery and clearing in the centre, in which case practically all the lesions show a tendency to become ringed.

Lichen planus atrophicus is the name applied to a variety in which marked atrophic changes occur. In such cases the papules are usually: 
flat and level with the surface, but they may present a central depression or may be raised and resemble minute cheloids. They coalesce to form small patches with a peculiar white appearance not unlike morphoea, or are lilac coloured, wrinkled on the surface, with a brownish border, and surrounded by an inflammatory halo with a few minute blood-capillaries ramifying over it. (Fig. 263.)

Lichen planus linearis is a rare variety in which the eruption occurs in the form of bands or stripes like linear naevi, varying in breadth from an $\frac{1}{8}$ to $\frac{1}{2}$ an inch or more, and composed of various types of lesions such as polygonal and acuminate papules, and small hypertrophic patches. In some cases the bands are short and broken, in others they extend in a continuous streak for a considerable distance, possibly down

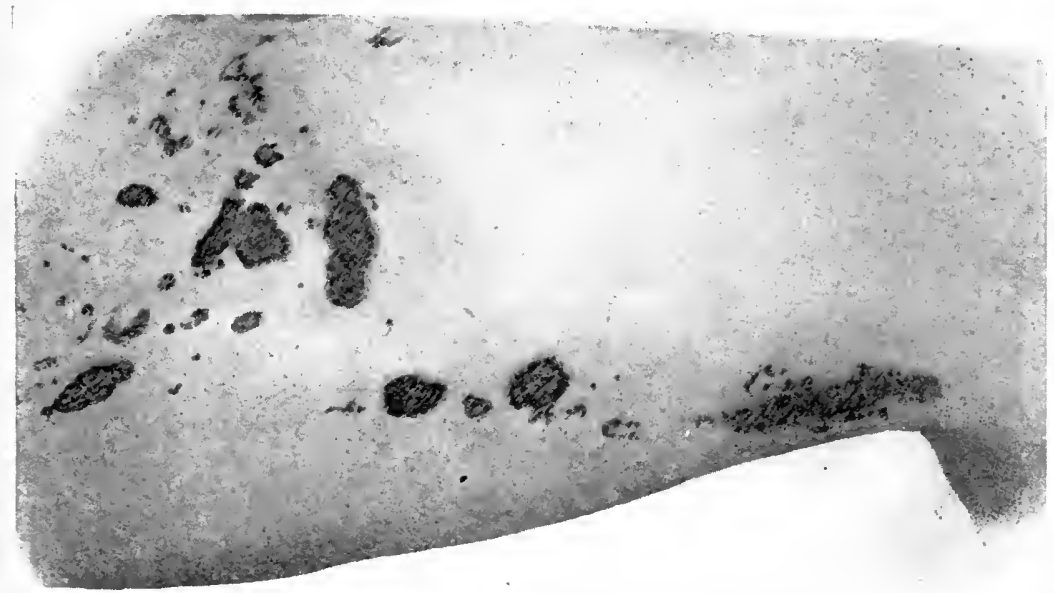

FIG. 263.-Lichen Planus Atrophicus.

the whole length of the leg or obliquely across the chest, as if they followed the course of some nerve such as the small sciatic or an intercostal. In other cases the distribution of the band does not seem to correspond with any nerve, Voigt's line, or segmental area, and the possibility of its being connected with the distribution of blood-vessels or lymphatics has been suggested. There are cases, however, which are inexplicable on any of these assumptions and it must be conceded that the exact cause of this peculiar arrangement remains unknown.

The linear lesion may come out in its whole extent simultaneously or may appear first as a small patch.

Lichen ruber moniliformis is another rare type, described by Kaposi, in which peculiar linear lesions occur like hypertrophic scars with a nodular appearance suggesting beads on a rosary. They have a yellowish or brownish waxy iook and tend to be arranged in parallel lines on the flexor aspects of the arms, the sides of the neck, and the lower part of the abdomen. In Kaposi's case they were accompanied by small typicai Lichen planus papules and pigmented macules. 
Similar cases have been recorded by other observers, such as Rolla, Dubreuilh, and Von Duhring. Though their exact nature is uncertain the general opinion is that they are the result of linear scratches in a skin affected with Lichen planus and which has a tendency to hypertrophic changes. (Fig. 264.)

Lichen planus erythematosus is an unusual variety which was described by Crocker, in which the initial papules are bright crimson in colour and exceptionally vascular and soft. In Crocker's case the patient was a healthy man of 62 and the eruption, which began in the groins, consisted of crimson red papules very like those of Lichen planus, rarely confluent in large patches, not scaly or distinctly raised, and almost completely disappearing on pressure. They were very general and diffuse in the axillae and abundant on the lower part of the abdomen where they were fainter in colour. The eruption disappeared under treatment leaving slight pitting where the papules had been.

Lichen planus bullosus.-In a certain number of cases of Lichen planus the eruption is associated with vesicles and bullae varying in size from $\frac{1}{4}$ to $I$ inch in diameter, sometimes situated on the sites of papules, and generally preceded by an inflamed patch.

They may occur about the arms and legs-situations usually attacked by Erythema bullosum. In a considerable number of cases they have appeared in patients while taking arsenic and it has been suggested that the drug was responsible for them. There is no

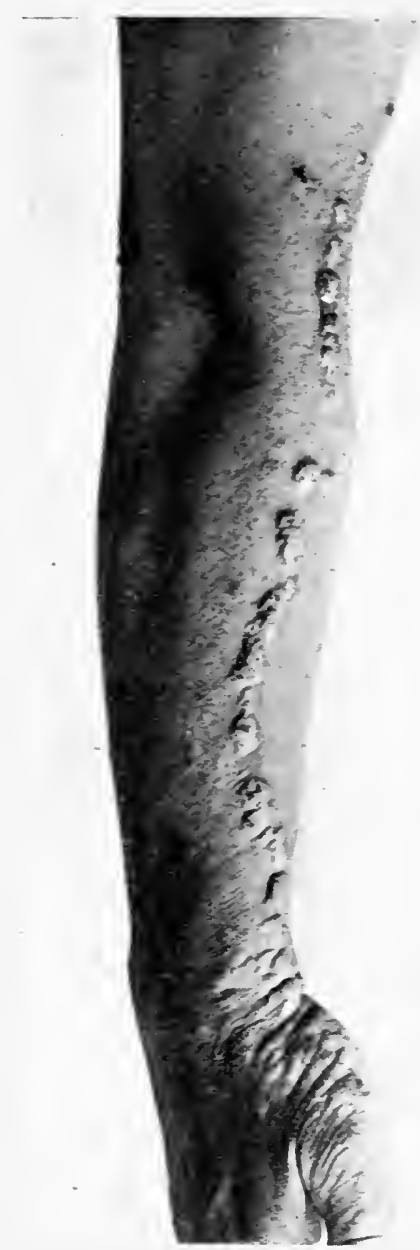

FIg. 264.-Lichen Planus. Moniliform Type.

(From Dr. Winkelried Williams.) absolute proof of this, however, as arsenic when given in other affections does not produce bullae and, though it has been prescribed in a large number of cases of chronic Lichen planus, bullae are a rare complication.

The position of the bullae in the skin varies; in some cases they are situated beneath the epidermis; in others they occur superficially at 
the level of the granular layer, while intermediate bullae may form in the prickle-cell layer. Various explanations have been put forward to account for this but it is probably caused by the rapidity of the extravasation of the serum, which when it takes place quickly collects below the epidermis and when it is extravasated more slowly passes up into the inter-epithelial lymphatics, dilates, breaks down the connecting fibres, and forms a blister.

Lichen planus haemorrhagicus.-In rare instances, in individuals with a feeble circulation and in a low state of health, the lesions of Lichen planus may become haemorrhagic. This is met with chiefly in the lower extremities and on the backs of the hands but may occur elsewhere. The lesions are bright purple and on examination with a lens show the presence of a small punctate haemorrhage in the centre of each.

\section{Acute Generalised Type}

The acute generalised type of Lichen planus is fortunately of much rarer occurrence than the chronic type as it may be accompanied by the mcst distressing itching and general symptoms and has been known to lead to a fatal issue. In it the papules are much more numerous. At first they are smooth and of a bright red colour but they gradually become scaly and assume the characteristic lilac tint. They may remain discrete, or be arranged in small groups or lines which slope downwards on the trunk, or may be aggregated to form slightly raised patches not unlike chronic eczema, irregular in shape, with a crosshatched surface like a mosaic caused by the natural lines of the skin or the conjunction of component angular papules. The skin between the lesions may be normal in colour or pinkish and inflamed. The eruption tends to be symmetrical and not infrequently appears first on the legs and spreads upwards, though it may begin on the trunk. The site of the lesions is to a considerable extent determined by scratching, rubbing, and pressure, and they are generally profuse about the waist in women from the pressure of corsets. In a severe case it may be present over the whole skin but it is unusual on the face, scalp, or fingers. (Plate XI.)

The mucosa may be affected but not so frequently or severely as in the sub-acute cases. The nails are occasionally involved and become rough, opaque, ridged, and present punctate depressions.

The generalised eruption is generally primary but occasionally it may supervene on an attack of chronic Lichen planus or it may be preceded for several weeks by a single scaly cross-hatched patch. It may take several days or weeks to reach its full development, or on the other hand it may come out so rapidly that it has spread from head to foot in 24 hours.

The subjective symptoms may be comparatively slight and the patient, in spite of the eruption, may be able to follow his occupation 


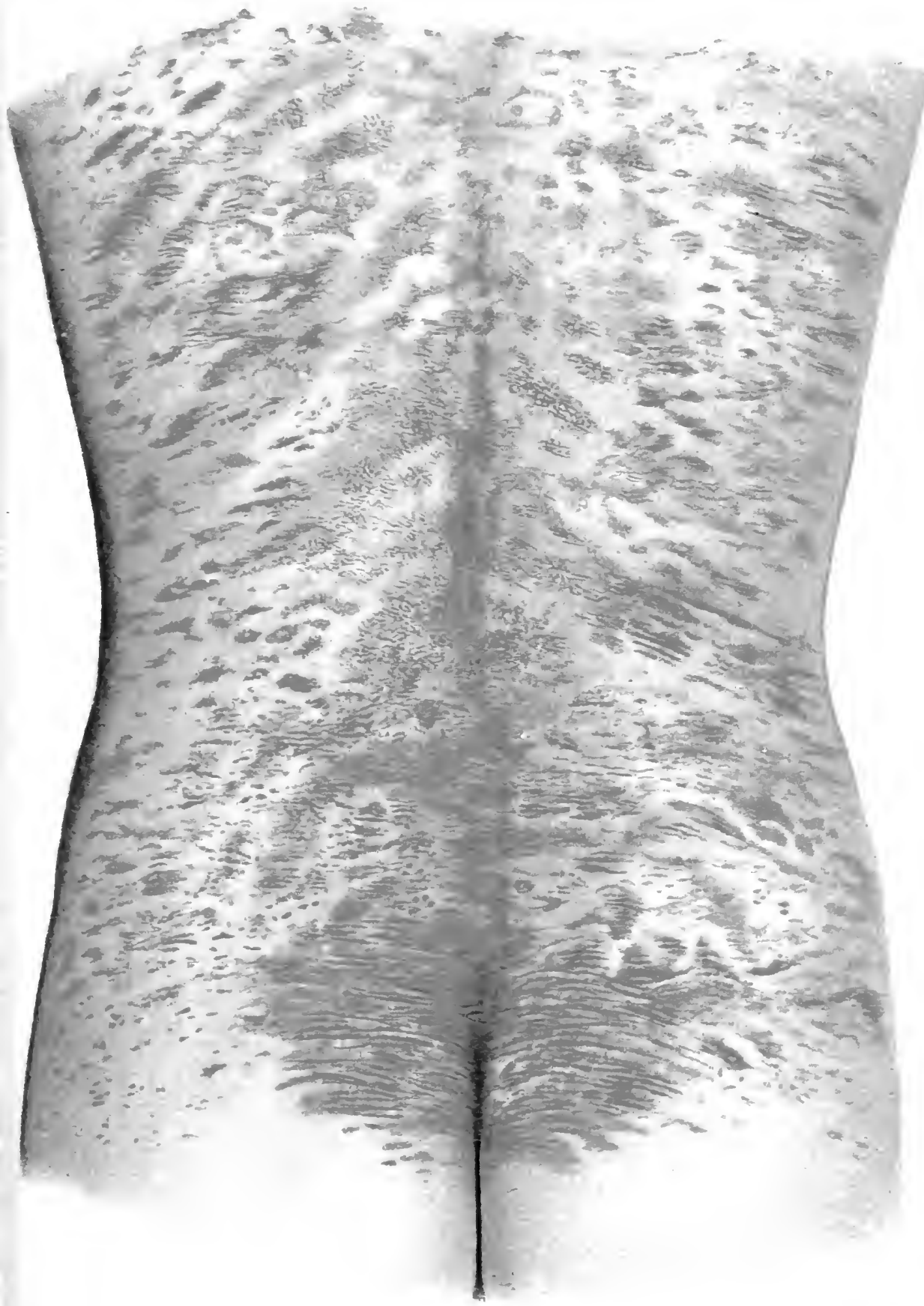

GENERALISED LICHEN PLANLS IN A WOMAN AGEL th.

Shus tiat violaceus papules agregated in irreculd patches roughly symmetrical. 

with the assistance of anti-pruritic remedies. But when the eruption is widely distributed the subjective symptoms are apt to be severe, and the itching and burning may be so continuous and distressing as to cause serious nerrous symptoms. Should the acute attack persist for several weeks the mental condition may become so disturbed that insanity, gradual marasmus, and even death may supervene. Diarrhoea may occur in connection with an acute attack and it has been suggested that this may be due to Lichen planus lesions in the mucosa of the alimentary tract (Pospelow). No definite blood-changes have been detected; eosinophilia has occasionally been described but it is inconstant. The attack may subside under suitable treatment in about 3 weeks, and as a rule with the fading of the eruption the itching diminishes. It may persist, however, for a much longer period and may leave behind it one or more chronic itchy patches about the legs or elsewhere. Relapses may take place before the attack has subsided, or recurrences after the eruption has completely disappeared. Crocker recorded the case of a lady who had suffered from the disease for I5 years and who for 5 years had had a fresh attack every July. When the eruption fades it is succeeded by a sepia or fawn coloured patchy. pigmentation which in turn gradually disappears. Arsenic has been partially blamed for this pigmentation but in some of the most marked cases arsenic had not been taken. It is not unusual to find horny plugs or small horny nodules about the palms after the acute attack has subsided.

Lichen planus of the mucous membranes.-Lesions in the mucous membranes occur in about half the cases of the sub-acute type of Iichen planus and in a smaller proportion of the acute generalised cases. The buccal mucosa is the part most commonly involved but they may occur also in the mucous membrane of the larrnx, nose, sides of the anus, urethra, glans penis, and vulva. In the mouth the lesions are usually situated on the insides of the cheeks, opposite the junction of the upper and lower jaws, and take the form of white milky rounded or oval specks, small patches with an irregular outline, or streaks with a dendriform arrangement. They may occur also on the tongue as milky macules or small irregular patches more or less symmetrically distributed and resembling leucoplakia, on the insides of the lips as small white specks, and on the palate as nilky lesions which tend to become conglomerated into small mosaic-like patches. On the glans penis if it be covered by the prepuce they take the form of whitish macules or papules, but if uncovered they are reddish or lilac-tinted like those on the skin.

Lesions on the mucous membranes give rise to no subjective symptoms except in the mouth, where they may be irritated by pungent food or smoking, nor are they any indication of the extent of the cutaneous eruption, for in some severe cases of acute generalised Lichen planus the mucosa may be scarcely, if at all, affected while in milder cases it 
may be markedly involved. The eluption on the mucosa may precede that on the skin by several weeks, or it may synchronise with it, and it may remain for a considerable time after the cutaneous lesions have faded.

\section{HistopathoLOGY}

Well-marked histological changes occur both in the corium and epidermis in Lichen planus, of a type which suggests that the corium is primarily involved. The chang:s vary according to the acuteness of the inflammatory process, the amount of irritation to which the affected skin has been subjected, and the region of the body attacked. In papules of acute Lichen planus the changes in the corium predominate while in chronic lesions of the type of Lichen planus hypertrophicus the changes in the epidermis are the most pronounced.

Corium.-The most marked feature is a cellular infiltration in the corium which is sparse immediately below the epidermis, a definite space between it and the basal layer being occasionally observed, is dense and well-demarcated, and ends about the lower level of the sub-papillary layer. At the sides of the lesion the infiltration tapers off in thin lateral prolongations. The cells composing it vary according to the type and stage of evolution of the papules, and consist mainly of mononuclear cells, some with vesicular nuclei and suggesting an origin from endothelium, others like lymphocytes and probably derived from the fixed connective tissue, and a few polymorphonuclear cells. In the older lesions plasma-cells, oval or spindle-shaped connective tissue cells, and occasional giant-cells occur, and where atrophic changes have supervened newly formed fibrous tissue may be detected.

The papillae are more or less flattened and the capillaries of the papillary and sub-papillary layers are dilated, especially at the sides of the infiltration, while towards the centre the endothelial lining has usually proliferated. Associated with the dilatation of the vessels there is oedema, rarefaction of the collagen bundles with occasionally basophilic changes, and defects in the elastic tissue which is much thinned near the epidermis where some of the fibres have broken up into granular debris and stain feebly. These changes have been observed in the corium before the epidermis has become definitely affected.

Epidermis.-In a typical polygonal papule the overlying epidermis is thickened from the proliferation of all its layers. Owing to the pressure of the oedema the interpapillary processes are flattened and the cells of the basal layer tend to become horizontally compressed and cubical. Granules of melanin occur in the cells of the basal layer, and branching pigmented cells have been detected between them and in the corium. The prickle-cell layer and granular layer are thickened and there is hyperkeratosis which is marked at the sweat-orifice, where it forms a horny plug and at the hair-follicle where it gives rise to an acuminate papule with a horny spine. (Fig. 265.) 
The horny and granular layers, by proliferating and growing down into the flattened inter-papillary processes, are the cause of the milky network of striae and puncta. In the centre of the epidermal lesion the sweat-channels may be detected beneath the horny plug, while the sweat-duct may show degeneration of the cells lining its wall and may" be dilated and contain granular debris.

The changes in the epidermis are secondary to those in the corium and result from the friction of scratching or rubbing for the relief of irritation. The inter-epithelial lymphatics may be dilated and present a few distorted leucocytes making their way towards the surface. Iri

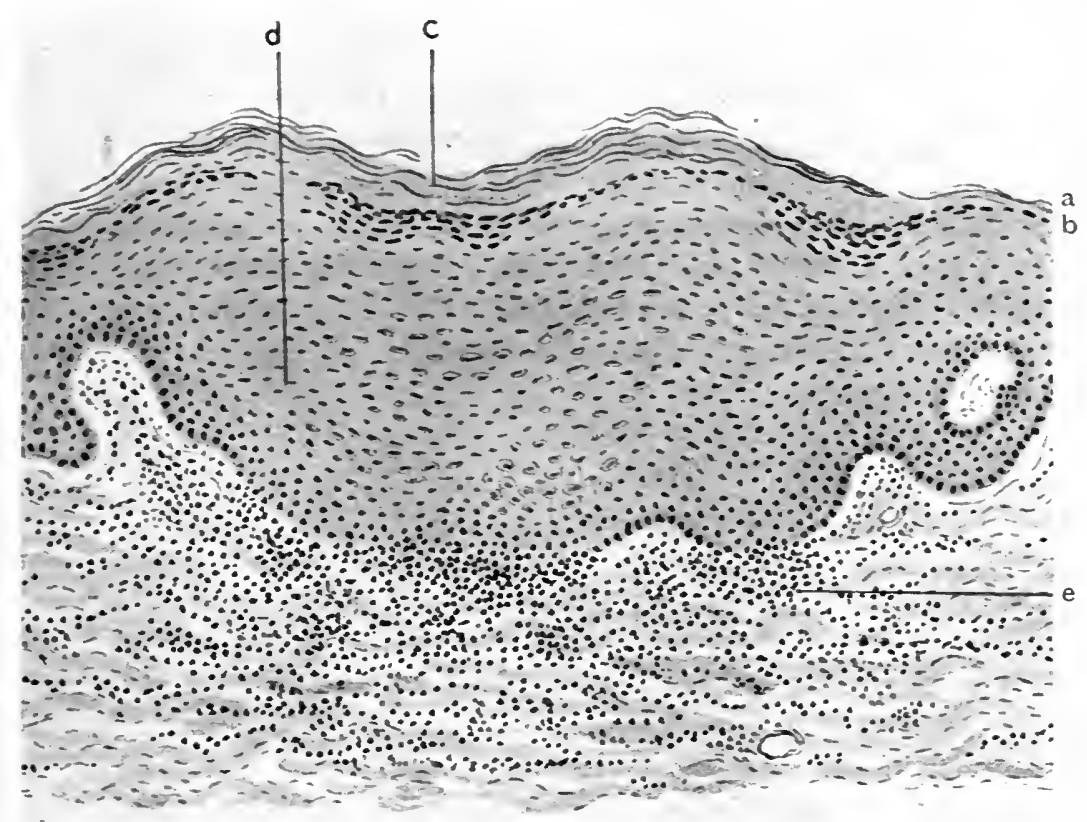

J. M. H. M. del.

FIG. 265.-Section of Papule of Lichen Planus. ( $x$ about 200.)

a. Stratum corneum. b. Stratum granulosum. c. Central depression. d. Hypertrophjed prickle-cell layer, with flattened cells. e. Cellular infiltration in papillary and sub-papillary

the hypertrophic and verrucose lesions the most marked features are proliferation of the prickle-cell layer, thickening of the granular laver, and hyperkeratosis; in the atrophic lesions there is thinning of the epidermis.

These histological changes readily explain the clinical appearances of the different lesions of the disease. The raised angular papule is due to the cellular infiltration and thickened epidermis, the glistening surface possibly to a stretching of the epidermis but more probably to a modification in the process of cornification such as is met with in Molluscum contagiosum where the horn-cells are distended with a hyalin substance, the surface striae and puncta are caused br the thickening of the horny and granular layers, the horny plug by hyper- 
keratosis at the sweat-orifices, the umbilication by the falling out of the plug or the binding down of the epidermis in the centre of it by the sweat-duct, the lilac tint and pink halo by the thickened epidermis in the centre and the dilatation of the blood-vessels and increased vascularity at the margins of the lesions. The pigmentation is due to increase of melanin in the cells at the basal layer, the occasional presence of branching pigment cells in the corium, or to small deposits of pigment from broken down cells. The degree of cellular infiltration and the extent of fibromatosis and sclerotic changes determine to what extent the lesion is raised or is depressed and atrophic.

The histological changes in lesions in the mucosa are similar to those in the skin. According to Dubreuilh, in the tongue there is a cellular infiltration which is densest opposite the ends of the interpapillary processes. These processes are elongated, which accounts for the thickening of the epidermis and for the white patches or network enclosing the filiform papillae. The Stratum corneum is not much thickened and kerato-hyalin granules are rarely present though they have been described as a characteristic feature in the histology. The blood-vessels are not so markedly dilated and the oedema may not be excessive, suggesting that the affection does not begin in or around the capillaries.

\section{Etiology}

Predisposing Causes.- Age.-Lichen planus is most common in adult life between the ages of 40 and 50 but may occur at any time from childhood to old-age. The youngest case the writer has seen was a boy aged 4 years, but several cases have been recorded in children of 3 years, and from time to time in infants. It is very doubtful if the cases in infancy were true Lichen planus ; several forms of dermatitis in infants leave lichenoid patches and the cases of so-called Lichen planus infantum are probably cases of papular urticaria, seborrhoic dermatitis, or Miliaria rubra.

Sex.-It is more common in females than males in the proportion of from 3 to 2 .

Climate.-It may occur in all climates, tropical as well as temperate, and in dark as well as in fair skins. In dark skins the early lesions appear as darker papules of the typical shape, while the older lesions become depigmented and appear as whitish macules.

Social Position.--It is said to be more common in private than in hospital practice, in the proportion of about 2 to $I$. This relative proportion is doubtful, however, as the milder forms of the disease are comparatively trivial, and hospital patients are therefore less likely to seek advice regarding them than those in more affluent circumstances.

Actual Cause.-The cause of the affection is unknown. There is much eridence in farour of its being in some way related to derangements of the nervous system but whether these nervous disturbances 
are directly responsible for it or are simply predisposing factors and the actual cause is toxic, are matters for discussion. The arguments in favour of the two theories are briefly as follows:

Theory of Nerious Origin.-(a) The disease has been observed frequently in nervous patients, in those suffering from neurasthenia, the result of some debilitating illness with nervous stigmata such as influenza, or from physical or mental overwork, worry, or anxiety, and in women at the climacteric period. Acute attacks have been known to follow closely some profound nervous shock, such as the death of a relative, or a great financial loss.

(b) The eruption is associated as a rule with subjective symptoms in the form of itching or burning, and the skin as a whole may be irritable and show a tendency to slight factitious urticaria. Occasionally in subacute cases the subjective symptoms may be out of all proportion to the extent of skin involved, while in certain acute cases they may be so severe and maddening as to lead to mental derangements and even to suicide.

(c) In Lichen planus linearis the lesions may appear to follow the line of distribution of some nerve.

(d) Herpes zoster, which is of accepted nerve origin, has been known to occur in the course of acute Lichen planus, but this might have been due to arsenic which had been prescribed for the latter disease.

No definite organic changes have been observed in the nerves supplying the affected skin, and in consequence it has been suggested that it is due to a functional disturbance. On the latter assumption the eruption would be a neuro-paralytic hyperaemia due to an interference with the vaso-motor tone. The histological appearances, however, do not bear out this theory. as a functional derangement could scarcely account for the dense infiltration and the associated epidermal changes.

Theory of Toxic Origin.-Certain observers regard Lichen planus as being due to a toxin circulating in the blood and any nervous disturbances which may precede it as predisposing factors, and they have advanced the following arguments in farour of this theory:

(a) In certain acute cases the onset of the affection is like an exanthem and suggests a general disease of which the eruption is a symptom, and in such cases general toxic symptoms such as rheumatic pain, sore throat, furred tongue, and slight pyrexia maybe present. Should nervoussymptoms occur in such a case they might be explained as the result of a state of irritability of the skin produced by the toxin, but should ther precede the eruption they cannot but be regarded as predisposing factors, lowering the vitality of the individual, and increasing the susceptibility to the disease.

(b) Cases have been described in which Lichen planus has supervened in the course of other diseases, such as diabetes, which is known to be of toxic origin. In such cases the eruption may be connected with an excessive formation of oxybutyric acid in the tissues and a large output of urea, and the nerve disturbance in the skin might be locally produced by the toxin in the same manrer as the peripheral neuritis which sometimes occurs in connection with the disease.

(c) Acute attacks of Lichen planus on several occasions have been known to succeed a chill in an individual who was over-heated and per spiring, as if the chill had lowered the vitality and so rendered the patien $t$ prone to the toxic factor.

(d) The histopathology of the lesions suggests a toxic cause and points to the poison having reached the shin wia the blood-stream and caused the proliferative changes in the corium, and the thickening of the epidermis might be explained as resulting from its presence in the lymph or the sweat acting as an irritant in much the same way as arsenic does in its passage through the skin. 
There is another factor which requires to be taken into consideration in the etiology of Lichen planus, namely the local irritating effects of scratching and rubbing. So important a part may local irritation play in determining the position of the eruption that a theory has been advanced that it is mainly the result of scratching in a predisposed individual. The facts that a row of papules may appear on the site of a linear scratch in Lichen planus, that it may occur in association with scabies from scratching, and that it is most diffuse in parts subjected to pressure from corsets, garters, collar studs, etc., have been cited in support of this theory. According to this view, Lichen planus is a peculiar type of reaction of the skin to local irritation in a predisposed individual, but exactly what this predisposition is, whether it is of toxic or nervous origin remains unsettled. An argument which is strongly against the universal application of this theory, however, is that even in extensive cases of Lichen planus there may be little or no itching.

\section{DiAgNosis}

Chronic Lichen planus papules may have to be distinguished from similar lesions in eczema, psoriasis and Seborrhoic dermatitis. In Lichen planus the papule is flat, angular in outline, shiny on the surface, pink or lilac-tinted, with a horny central plug or depression, and in certain cases presenting a milky network or puncta; in eczema the initial lesion is a papulo-vesicle with an ill-defined circular outline and more inflamed; in psoriasis it is a bright pink maculo-papule covered with a silvery scale which can be easily scratched off leaving bleeding puncta ; in Seborrhoic dermatitis it is pinkish-yellow, circular or oval in outline, softer than a papule of Lichen planus and covered by a greasy scale.

Lichen planus hypertrophicus lesions are very characteristic on account of their dull red or lilac tint, cross-hatched surface, or shagreen appearance from adherent scales and cribriform pitting. They have to be differentiated from patches of lichenification, Lupus verrucosus, and Lupus erythematosus.

Lichenification is a condition in which slightly raised cross-hatched patches occur, which are practically indistinguishable from certain conglomerate patches of Lichen planus. (See p. 746.)

Lupus vermcosus. - In it the lesions are brownish in tinge instead of lilac tinted, and the patches present a papillomatous surface with exuberant granulations or are covered with crusts, instead of being scaly and pitted like shagreen.

Lupus erythematosus.-In Lupus erythematosus the patches are scarcely if at all raised and tend to be atrophic in the centre and are surrounded by an inflammatory halo while those of Lichen planus usually rise abruptly from the surrounding skin.

Linear Lichen planus with its striate bands has to be distinguished from various forms of linear naevi. Striate Lichen planus, however, 
comes out rapidy; the whole extent of the lesion sometimes developing simultaneously, while linear naevi evolve slowly; Lichen planus lesions occur as a rule in adult life and may disappear spontaneously. or under treatment, linear naevi appear soon after birth and persist throughout life.

Acute generalised Lichen planus has to be distinguished from eczema, Pityriasis rubra pilaris and a follicular syphilide. In chronic eczema the essential lesion is different, the eruption is more inflamed, the skin between the lesions is affected, there is generally scaliness and evidence of previous weeping, and the face and hands are usually involved, while in Lichen planus the inflammation is less, the skin between the lesions may be normal, scaliness is slight, there is no weeping, and the face and hands are rarely affected.

Pityriasis rubra pilaris differs from acute Lichen planus in that the lesions instead of being angular in outline are circular and consist of round or conical papules with horny centres, the eruption is much more scaly, desquamation is an early simptom, and lesions are generally present on the dorsa of the fingers, while in Lichen planus the fingers are rarely involved.

The small follicular syphilide is an early secondary eruption and is usually associated with enlarged lymphatic glands, the remains of the chancre, and a positive Wassermann reaction, and the lesions are grouped, frequently in a corymbose manner and situated chiefly on the back.

Mucous membrane lesions have to be distinguished from leucoplakia and mucous syphilides. Leucoplakia is generally thicker, involves larger areas, and is more readily irritated by pungent substances. Syphilitic mucous patches are readily distinguished by their inflamed base, moist surface, and marked tendency to superficial ulceration.

Prognosis.-The prognosis is uncertain. In sub-acute cases it is practically impossible to predict how long it will take for the lesions to disappear, for in one case it may fade in a few weeks or months, either spontaneously or as the result of treatment, while in another new lesions may keep coming out and so prolong the eruption indefinitely.

In acute generalised cases a cure may be effected in 4 to 6 weeks, but it frequently takes much longer, and even when the greater part of the eruption has gone chronic residual patches may be left and the cure indefinitely postponed. It is not uncommon also for recurrences to take place, though not perhaps with the insistence of psoriasis, but sometimes at regular intervals or irregularly in connection with some general derangement in health.

\section{TREATMENT}

The treatment varies greatly according to whether the case is acute or chronic, and each case must be studied and treated on its own merits. 
General Treatment.-There is no drug which can be regarded as a specific. In the past arsenic has had a great reputation in this connection but its action is uncertain. In the acute generalised cases it may do more harm than good and aggravate rather than cure the affection. In sub-acute cases it is occasionally beneficial, but whether this is due to a direct specific action on the disease or simply to its action as a general nerve tonic is undecided. In every case where arsenic is prescribed, it should be employed with caution and the patient carefully watched for deleterious symptoms. It should on no account be given to patients who suffer from indigestion or other derangements of the alimentary tract, and should any symptoms such as colic or irritative diarrhoea supervene when it is being taken it must be discontinued at once. The most convenient way of administering it is in the form of the Liquor arsenicalis in doses of from 2 to $6 \mathrm{~m}$., or of the Liquor sodii arseniatis 5 to Io $\mathrm{m}$., in plenty of water, t.d.s. after meals. It may also be given by hypodermic injection of the Liquor sodii arseniatis, $3 \mathrm{~m}$. injected daily and gradually increased until the limit of toleration is reached, or of one of the organic preparations of arsenic such as atoxyl and arsacetin. Atoxyl is given in doses of $\frac{1}{2}$ to 2 grs. dissolved in warm water and injected twice a week, the dosage being regulated according to the body weight. The other members of the arylarsonate group, namely soamin, arsacetin and orsudan, may be substituted in doses of 2 to 3 grs. injected twice a week and are safer. Of these, arsacetin and orsudan have the advantage of being more stable and less liable than the others to disintegrate when kept. They all contain a large percentage of arsenium and are liable to cause grave toxic symptoms, a few cases of blindness from double optic atrophy having resulted from their employment.

Mercury on the whole seems to give better results than arsenic and that it acts beneficially is a strong argument in favour of the toxic origin of the disease. It may be given in the form of Hyd. biniodid $\frac{1}{16}$ gr., Hydrarg. cum. cret. I to 2 grs. t.d.s., Hyd. perchlor. gr. $\frac{1}{1} \overline{0}$ t.d.s., and the amount gradually increased.

Quinine and salicin also have their advocates. Salicin, in doses of Io to I5 grs.t.d.s., not only exerts its anti-toxic action but in a few cases by its depressing effect reduces the tension in the skin and calms the patient.

Antimony in the form of the vinum, 5 to ro m. t.d.s. is of value in soothing the more acute cases.

For the relief of the intolerable itching which may be associated with the eruption, antipyrin and phenacetin grs. Io t.d.s. are of service.

In the acute cases the general comfort of the patient must be carefuily considered. In certain generalised cases the irritation may be comparatively slight and the patient may be treated in an ambulant fashion and permitted to live an ordinary life. On the other hand, where the irritation is severe, to obtain good results rest in bed is necessary, the bedroom being kept at an even temperature, the 
nightdress being of some non-irritating material, such as linen, and the bed-clothes light. For cases in which the itching is severe Ravaut of Paris has employed lumbar puncture, and found that the withdrawal of a few c.c. of the cerebro-spinal fluid was sometimes followed bydiminution of the pruritus and fading of the eruption.

In chronic cases a simple tonic regime with iron, strychnine, or phosphoric acid, or the employment of fattening remedies such as milk, cream, cod-liver oil, and pea-nut butter, may do more than any specific drug. The concomitant nervous condition must be dealt with by suitable measures such as fresh air, a sea voyage, exercise, massage, cold or tepid douches, and change of air and occupation as the case indicates. Any defect in general health, such as derangements of the digestion, or disturbances of the liver and kidneys, must be treated on general medical principles.

Local Treatment.-In the acute generalised cases the first indication in the local treatment is to reduce the irritation. For this purpose ointments should be avoided and sedative anti-pruritic creams, lotions and powders employed. In the most acute cases the following calamine cream is of service:

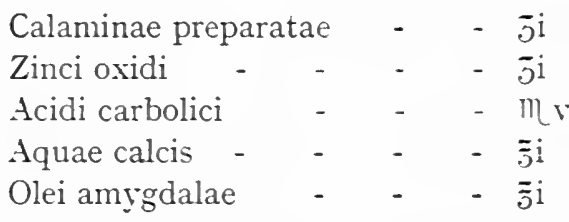

This should be smeared on the skin and followed by the application of zinc oxide and starch dusting powder to which $2 \%$ of camphor has been added. A useful substitute for the cream is olive oil with the addition of $\mathrm{I} \%$ of thymol.

Lotions of carbolic acid, lead, and tar are also of value, such as the following :

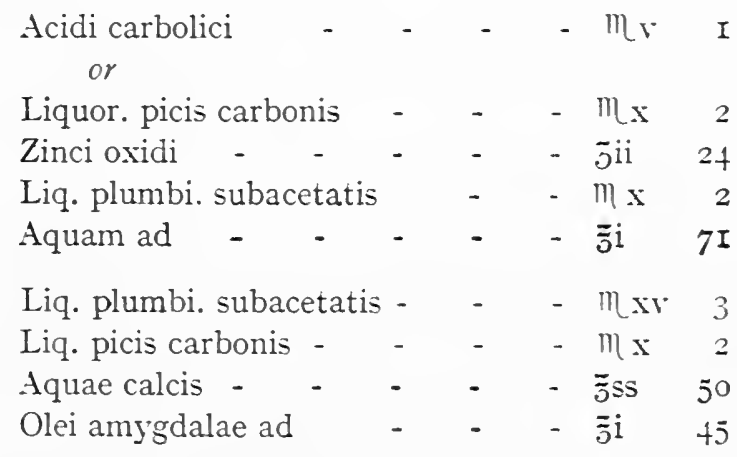

In the less acute generalised forms, relief may be obtained from medicated baths, such as creolin $\frac{1}{2}$ oz., borax and bicarbonate of soda 2 ozs., or starch and bran 2 lbs., to 30 gallons of warm water, or tepid sprays followed by cold douches. 
In the sub-acute cases ointments are permissible but should be prescribed with caution at first in case of producing irritation. A favourite ointment containing mercury and carbolic acid is the following :

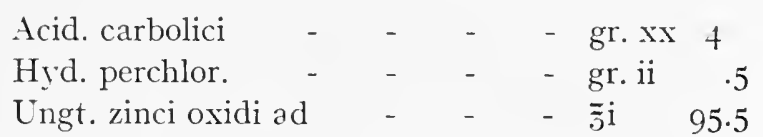

Instead of mercury, tar may be employed either in the form of oil of cade Io $\mathrm{m}$. in an ounce of lanolin, vaselin or cold cream, or in the following formula :

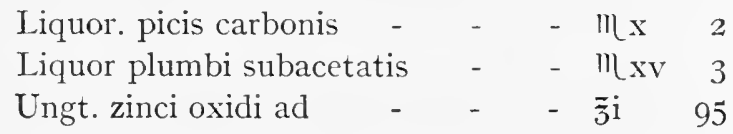

To obtain the full benefit from the ointments they should be kept continuously applied spread on lint under a bandage.

The most resistant lesions of all are the hypertrophic and verrucose patches. In treating these much stronger remedies may be employed with impunity, such as salicylic or pyrogallic acid 3 to $6 \%$ in an ounce of vaselin. A convenient substitute for the ointments are plasters containing mercury, salicylic acid, and carbolic acid.

By far the best remedy for the hypertrophic lesions is the $\mathrm{X}$-rays, which not only cause retrogression but at the same time relieve the irritation. A full Sabouraud pastille dose is given to each lesion, the healthy skin around the lesion being carefully protected during the exposures. Where the patches are only slightly raised this may suffice, but as a rule a second or even a third dose is necessary, but should not be given at a less interval than a month.

Lesions in the mucous membrane of the mouth should be treated by soothing remedies such as gargles of potassium chlorate or myrrh. On no account should an attempt be made to destroy them by caustic remedies such as chromic acid or silver nitrate for fear of irritating them and in elderly people of setting up malignant changes. Irritating foods, such as mustard, pickles, pungent substances in general, and hot drinks, should be avoided and smoking should be prohibited. The teeth should be looked to, any sharp edges dealt with, and the mouth kept in as clean and hygienic a state as possible.

\section{LiCHEN Nitidus}

Pinkus in ${ } 897$ applied this name to a peculiar eruption which somewhat resembles Lichen planus but the exact nature of which is uncertain. In 9 cases which he described it occurred in men suffering from venereal disease, and was usually confined to the penis but occasionally involved other parts such as the abdomen, chest, and arms. It consisted of small shiny papules and nodules which on the glans penis 
looked not unlike small sago grains ; they were about the size of pins' heads, or slightly larger, with a rounded surface in the centre of which was usually a depression, and had a tendency to be arranged in rows, but though very numerous and close together they never became confluent. There were no subjective symptoms associated with the eruption and as a rule the patients were ignorant of its existence until it was pointed out to them.

Histologically the lesions consisted mainly of a dense cellular infiltration of the granulomatous type, situated in the papillary and sub-papillary layers, the overlying epidermis being thin and showing eridences of parakeratosis. At the sides of the infiltration the epidermis had proliferated and grown down in long processes. The cellular infiltration was made up of round cells of the lymphocyte type, epithelioid cells, and giant-cells. Near the epidermis degenerative changes were occasionally noted with the formation of a microscopical abscess, and the collagen and elastin in the midst of the cellular infiltration were defective and showed signs of oedematous degeneration.

A case occurring in a woman suffering from Bartholinitis was described by Kyrle and McDonagh. In it a positive general reaction was obtained after an injection of I mgrm. of Koch's old tuberculin. On account of this and the histological architecture, these writers suggest that the affection is possibly of a tuberculous origin. An examination of the tissue for tubercle bacilli and inoculations in a guinea-pig gave negative results.

The condition differs from Lichen planus in that the lesions have the transparent appearance of granulomata, are all of the same type, leave no pigmentation on fading, and are not associated with subjective symptoms.

\section{REFERENCES}

Adamsox, H. G. Lichen pilaris seu spinulosus (Brit. Journ. Derm., I905, xvii. p. 39).

Broce, L. Lichen obtusus corneus (La prat. Derm., 1902, iii. p. 20I).

Lichen planus (Idem., 1907, ii. p. I94).

BRooke \& MAcLeod. Lichen planus (Allbutt \& Rolleston, System of Medicine, 1911, ix. p. 425. List of references).

Crocker, Radcliffe. Lichen planus (Int. Cong. for Derm., Paris, is89; Vienna, I892; Rome, I894).

(Int. Med. Cong., Berlin, 1900).

(Textbook, 1905, p. 379).

(Brit. Journ. Derm., I900, xii. p. 42I).

Dubreuilh, W. Histologie de Lichen plan des muqueuses (Ann. de Derm. et de Syph., 1906).

Fox, Colcotr. Lichen planus in infants (Brit. Journ. Derm., ISgI, iii. p. 20I).

KYRLE, J., \& McDonagh, J. E. R. Lichen nitidus (Brit. Journ. Derm., I909, xxi. p. 339).

LANe, E. Dangers of arylarsonates (Brit. Med. Journ., I910, i. p. 599).

Pinkes, F. Lichen nitidus (Arch. f. Derm. u. Syph., I907, Lxxr. p. II).

Reixes, S. A case of Lichen nitidus (Pinkus) (Med. Klin., I9ro).

Richard \& Sutiox. Lichen nitidus (Journ. Cut. Dis., Nov., I9Io). 
Sabouraud, R. Some points in the pathological anatomy of Lichen planus of Wilson (Ann. de Derm. et de Syph., I9Io, p. 491).

Schanberg, J. F., \& Hirschlet, R. Two cases of multiple tumours of the skin in negroes, associated with itching (Journ. Cut. Dis., I906, xxiv. p. I5I).

Sibley, W. K. A case of Lichen obtusus corneus (Brit. Journ. Derm., I9I6, xxviii, p. 329).

SNowman, J. Therapeutic applications of arsenic (Lancet, I907, ii. p. I I 49). Spiethoff. Lichen planus in pernicious anaemia (Arch. f. Derm. u. Syph. I9II, c.V., p. I69).

Thibierge, G. Lumbar puncture in pruriginous dermatoses (Comptesrendus de Xme Cong. Français de Méd. rgro).

Török, L. Anatomy of Lichen planus (Beitr. z. path. Anat. u. z. allg. Path., Jena, r889, viii.).

(Monats. f. prakt. Derm., 1889, ix. p. II5).

White, C. J. Lichen obtusus corneus (Journ. Cut. Dis., I907, xxv. p. 383).

\section{LICHENIFICATION}

Syn. : Lichénisation (Besnier).

Definition: A series of changes which occur in the skin of certain individuals when it is subjected to rubbing and scratching.

The name "Lichenification" was suggested by Brocq for this peculiar modification of the skin produced by scratching and it is to him that we are indebted for the most detailed description of it. It consists of a thickening and loss of pliability of a certain area of the skin, the surface of which when examined by oblique light shows the presence of numerous facets which are flat, glistening, and separated from each other by the natural lines of the skin which are unusually well-marked. It is invariably the result of friction, and may be due either to incessant scratching or rubbing to relieve pruritus or to intermittent pressure of a truss or of badly fitting corsets.

When the lichenification supervenes on an apparently healthy skin it is known as primary lichenification, when it is superimposed on a skin affected with eczema, psoriasis, Seborrhoic dermatitis, or other itchy condition, it is known as secondary lichenification.

According to Brocq there are two main types: (i) a circumscribed type in which the patches vary in size from a sixpence to the palm of the hand or larger; (ii) a more diffuse variety in which much of the skin may be involved. All gradations between these two main varieties may be encountered.

\section{Circumscribed Lichenification}

Syn. : Lichen circumscriptus (of the older writers); Lichen simplex chronicus (Vidal); Névrodermite ${ }^{1}$ chronique circonscrite (Jacquet); Pruritus circonscrite avec lichénification (Brocq).

'The term " névrodermite" has been applied in France to lesions produced by friction for the relief of pruritus. It corresponds to Brocq's " pruritus with lichenification." 
At first no visible changes are noticeable to indicate the underlying pruritus but, as the result of rubbing, the skin gradually becomes darker in colour, a few flat or rounded papules may be noticed dotted over it, and it becomes definitely thickened. As a rule the thickening is not clearly demarcated like a patch of hypertrophic Lichen planus but gradually fades into the surrounding skin. The most marked feature in the patch is its mosaic-like surface, which is caused by the deepening of the natural lines of the skin which cross each other and break it up in a regular manner into numerous polygonal or lozengeshaped flat and glistening plateaux each about the size of a pin's head. These flat lesions are not so definitely papular as Lichen planus and

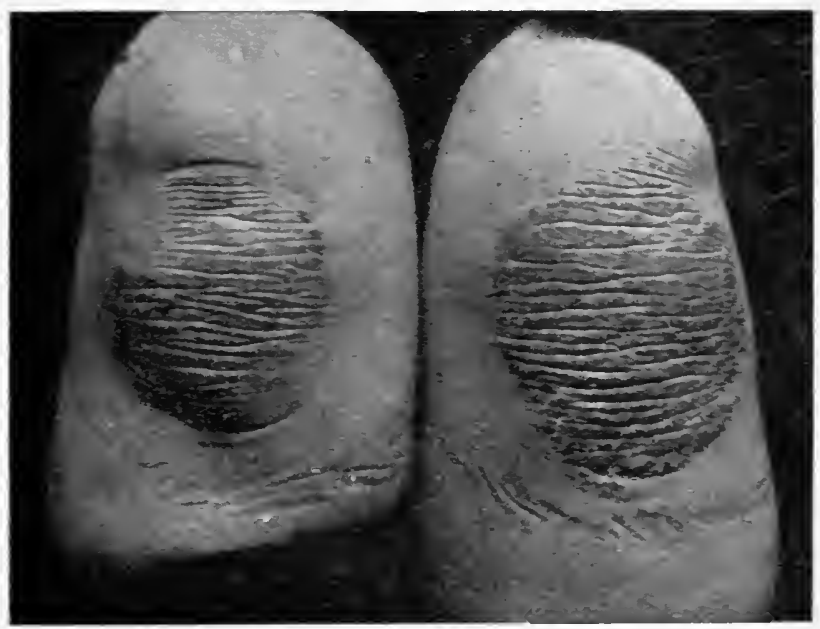

FIG. 266.-Lichenification.

Patches caused by kneeling.

have neither whitish striae nor puncta. Towards the border the plaque is slightly pigmented. (Fig. 266.)

Brocq divides the fully developed plaque into three zones:

(i) An external ill-defined zone, 2 to 3 ins. in breadth, composed of minute pigmented papules of a café-au-lait or brownish hue.

(ii) A middle zone in which the elements are polygonal or hemispherical in outline and have a shiny surface. The lesions are isolated towards the external zone and confluent towards the centre. They appear to be formed by a more marked hypertrophy than that which characterises the elements of the preceding zone.

(iii) An internal or central zone, reddish in colour or like the surrounding skin, in which the tissue is definitely infiltrated and the lichenification process is at its height. The surface is cut up by the natural lines of the skin into rectangular or lozenge-shaped facets.

The older lesions may be slightly scaly on the surface and are frequently excoriated from scratching. The patches vary considerably in shape, usually being oral but sometimes angular or irregular. The 
sensibility of the affected skin is preserved to touch, heat, and cold. The lesions occur most commonly at the nape of the neck and in the neighbourhood of the sterno-mastoid muscle, on the thighs, anteroextensor aspects of the legs, elbows, popliteal spaces, axillae, palms and soles, scrotum and vulva, and rarely on the face. They may be single or multiple and are sometimes symmetrical. The itching associated with them is usually intermittent and paroxysmal and frequently comes on about an hour after going to bed, the paroxysms sometimes being so severe as to render the patient nervous of their

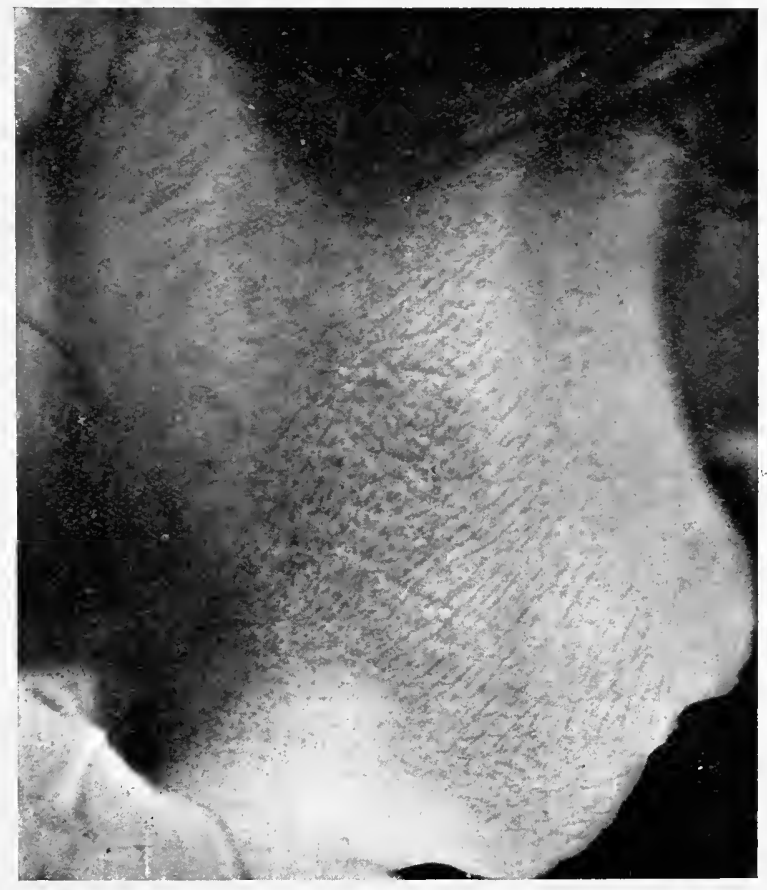

FIG. 267.-Lichenification.

Patches on nape of neck showing cross-hatching.

onset, and occasionally amounting to a veritable nerve crisis associated with violent scratching. (Fig. 267.)

Exacerbations of itching may be determined by errors of diet, cold, excitement, overheating, emotion, and various other causes dependent on individual idiosyncrasy. The lesions are seldom secondarily inoculated by pyogenic micro-organisms, but as the result of incessant scratching they may become eczematised and typical papulo-vesicles of eczema may develop on and around them followed by weeping and the formation of crusts.

It has been observed to co-exist with vitiligo. 


\section{Diffuse Lichenification}

Syn. : "Prurit diffus arec lichénification" (Brocq).

In this type various regions are affected, such as the arms, legs, abdomen, and sides of the chest, and frequently in a symmetrical fashion. As a result of the general pruritus and rubbing the skin is discoloured, slightly pigmented, irregulariy thickened, and dotted over with discrete flat shining macules, papules, or occasionally with acuminate follicular papules. Patches similar to those of the circumscribed variety may be present in situations where the pruritus is more definitely localised.

Histopathology.-Microscopically the principal changes consist of hyperkeratosis, proliferation of the prickle-cell layer with down-growth of the inter-papillary processes, and in the corium of a cellular infiltration in the papillary layer and oedema without marked rascular dilatation. These changes indicate a process produced by external irritation rather than by a toxin circulating in the blood.

Etiology.--The etiology of lichenification is bound up with that of pruritus and has been discussed in detail under that heading.

It is most common in adult life, although there is no maximum or minimum age for it, and it occurs more frequently in women than in men. Heredity may be an important factor in its etiology by determining a nervous predisposition. A general lack of tone of the nervous system is usually present and is evident in want of control, irritability and neurasthenia. There is a weakness of nerve control of the skin which may be due to the existence of some irritable skin affection such as eczema, or to auto-intoxication from digestive derangements, or from the abuse of alcohol, coffee, or tea, or from diseases like diabetes. In certain cases habit has a good deal to do with the production of the patches. In a case under the care of the writer as a result of slight irritation over the lower eyelid, a boy with chorea developed the habit of rubbing his lower lid till it became lichenified; in another a lichenified patch was present on the ulnar aspect of the left forearm in a somewhat nerrous man, who was a timekeeper in a factory and had to spend many hours every day leaning on a counter cheching the time of the workmen, and developed a habit of rubbing his arm incessantly on the counter. The habit may persist after the pruritus has gone and so perpetuate the lichenification. As in Lichen planus an attack of pruritus followed by rubbing and lichenification has been known to succeed emotional crises from great financial loss, berearement, mental anxiety, etc.

According to Brocq it is a special type of reaction which is produced easily in certain individuals, owing to lowered vitality or defective nutrition of the skin from various causes, congenital or acquired. 
Diagnosis. - The characters of lichenified skin are so obvious that it is easily recognised and the only difficulty is its distinction from Lichen planus.

At the outset it may be said that certain observers consider that the circumscribed patches of primary lichenification are badly marked patches of Lichen planus. This is undoubtedly the case sometimes, for cases occur in which a patch of lichenification is followed by an eruption of typical Lichen planus, or in which a careful search will reveal the presence of Lichen planus lesions elsewhere.

Though there is a close relation between the two conditions in their etiology, histopathology, and clinical characteristics, still it seems probable that primary lichenification in the form of patches may occur apart from Lichen planus. Brocq maintains that the initial lesion is different from Lichen planus ; the typical lesion of Lichen planus being a flat polygonal papule, frequently lilac tinted, umbilicated, and presenting milky striae and puncta on the surface, while in lichenification the lesion is a macule or a less definite flat or round papule, is yellowishpink or reddish in colour, not umbilicated, and never presents the milky puncta and striae.

Histologically the lesions differ in that the blood-capillaries are not markedly involved and no plasma-cells or other suggestion of the effects produced by toxins circulating in the blood, have been detected. It seems probable that in Lichen planus there is a predisposition to lichenification from rubbing, but that there is also another factor which determines the characteristic lesion, which is possibly a toxin circulating in the blood.

Prognosis.--With regard to the circumscribed patches, under suitable treatment the prognosis is fairly satisfactory and the patches may be removed in a month or six weeks, but if left alone they may persist for years. Sometimes the itching disappears more or less suddenly, the rubbing ceases, and the patch gradually fades leaving a pigmented area and a few isolated papules which disappear in time. On the other hand one or more patches may disappear, but new pruritic areas assert themselves, followed by rubbing and lichenification.

The prognosis, both of the circumscribed and the diffuse variety, is contingent on much the same considerations as pruritus, except that when lichenification is established a vicious circle is set up, for the thickening of the skin irritates the nerve-endings and so keeps up the pruritus, while the rubbing which is indulged in to relieve the pruritus increases the lichenification.

\section{TREATMENT}

The rational treatment of this condition has three main objectives, namely (i) removal of the cause and allaying the pruritus, (ii) increasing 
the power of resistance of the individual, (iii) removal of the thichening of the skin.

General Treatment.-The treatment for allaying the underlying pruritus is discussed in detail under pruritus. To accomplish this successfully the patient behind the disease must be most carefully studied, the functions of every organ considered, any obvious defect in general health corrected, and the resisting power increased by tonics and fattening substances. In many of the cases there is definite nervous weakness, emotionalism or hysteria, which requires to be dealt with on common-sense lines, while in others there is some general organic derangement, such as diabetes, pulmonary disease, or defects of the alimentary tract, associated with auto-intoxication from ferments.

Local Treatment.- The objects of the local treatment are to reduce the irritation and to cause retrogression of the lesion. The different anti-pruritic remedies have their special indications, and of these the most useful are carbolic lotion ( $I$ in 40), camphor $2 \%$ in spirit, tar lotions such as Liquor carbonis detergens a teaspoonful to $\frac{1}{2}$ a pint of water, and various anti-pruritic creams and ointments such as menthol or oil of cade $2 \%$ in cold cream.

In the past, various heroic operative measures have been adopted for the relief of these itchy patches, such as linear scarification, cauterisation, and incision of the nerves around the affected area. These procedures have been superseded by electro-therapeutic methods, such as static electricity, high frequency effleuves, and the X-rays.

At the present time the X-rays in suitable doses is by far the most useful means of removing the patches and allaying the itching. A single Sabouraud dose should be given and may suffice to remove the lesion, leaving only a pigmented stain. If it be insufficient a second dose should be given in a month or six weeks. After the X-ray exposure there should be no local treatment.

The most intractable of all the circumscribed patches are those occurring at the nape of the neck, especially when they spread up into the occipital scalp. From scratching or the co-existence of Seborrhoic dermatitis these patches are apt to become inflamed and even eczematised. Before applying the $\mathrm{X}$-rays in such cases it is advisable to reduce the inflammation and crusting by suitable applications, such as boric acid compresses and mild antiseptic ointments. If the scalp be involved the hair should be cut short over the affected area and a full dose of the $\mathrm{X}$-rays given, this will bring the hair out but it will grow in again in a few months.

Excellent results may be obtained by the application of radium, using a fairly large flat applicator.

After any form of treatment, however successful, it is essential to protect the skin from local irritation by painting it with zinc ichthyol gelatine, or covering it with zinc plaster. 


\section{REFERENCES}

Broco, L. Les lichénifications et les névrodermites (Ann. de Derm. et de Syph., I 896, vii. p. 779).

DARIER, J. Lichénisation (Précis de Derm., Ioo9, p. 476).

Wickнaм, L. Névrodermite circonscrite (Ann. de Derm. et de Syph., I 895 , vi. p. 537). 


\section{CHAPTER XYVI}

\section{ECZEMA}

Syn.: Fr., Eczéma; Ger., Nässende Flechte.

Definition: An inflammatory affection of the skin, characterised at first by erythema, papules, or vesicles, and later by scaly, weeping, or crusted patches associated with itching or burning.

Description.-Eczema is the most conmon and at the same time one of the most multiform of skin diseases. It may be acute, sub-acute, or chronic in its course. Its lesions and phases vary so indefinitely in different cases and at different stages in the same case that it is practically impossible to find a definition for it which is sufficiently comprehensive: nor are the different authorities at the present time at one with regard to what exactly should be included under the term. Originally the name eczema (from ' $\mathrm{E} \kappa^{\prime}\left(\epsilon^{\prime} \omega\right.$ " to boil over ") was given to various forms of dermatitis characterised by minute inflammatory. vesicles, which were easily ruptured, exuded a serous fluid which stiffened linen, and tended to form weeping patches. Subsequently it was extended to include certain dry, erythematous, papular and scaly lesions which were found both clinically and microscopically to be abortive forms or further stages of the vesicular type, and consequently. the term now includes a protean group of lesions whose varied clinical characters render its description as difficult as its definition.

The cardinal features of this group consist of erythema, superficial oedema, and an inflammatory cellular infiltration, which are responsible for papules, vesicles and exudation, yellowish crusts and scales. They are not necessarily present in the same lesion or at one time in an indiridual case, but they indicate what may be termed the different phases or stages in the pathological process. In one case the lesions may be chiefly of the erythematous type and either die down or become transformed into chronic scaly patches, in another theyare papular at first and coalesce to form infiltrated lesions, while in a third, vesicles are the predominating feature and these break, exude, and form weeping and crusted patches. It is rare, however, to find a case of eczema in which one phase of the disease is exclusively present and the so-called varieties of it simply indicate the type of lesion which 
predominates, either as the result of the special nature or virulence of the cause, the situation of the lesion, or the peculiarity of the skin of the individual.

For purposes of description the clinical features of eczema may be detailed conveniently under the headings of the erythematous, papular, and vesicular phases of the disease, and as the clinical appearances vary considerably according to the situations affected, the regional varieties of it also will be specially described with their treatment, and a section will be deroted to eczema in infants.

Erythematous Phase.-This may be regarded as the primary phase of the disease ; the typical example of it is acute erythematous eczema of the face and hands, but it may occur elsewhere on the body, and is sometimes universal, The mucous membranes are not affected to any extent though near the margin of the diseased skin they may become red and denuded of epithelium.

It begins more or less suddenly, the skin of the affected part becoming irritable, hot, and tense, and red blotches appearing upon it, which increase in size and coalesce to form ill-defined patches, sometimes markedly symmetrical, at other times irregularly distributed. The patches are smooth on the surface, raised, puffy, have a semi-translucent appearance owing to the presence of oedema in the epidermis and upper part of the corium, and in outline are ill-defined and fade gradually into the surrounding skin. The erythema is rarely universal and is generally broken up by areas of normal skin, but in severe cases of acute eczema of the face the whole region may be attacked, including the ears, and it may even spread on to the scalp. Associated with the erythema there is oedema which is most marked where the tissues are lax, as about the eyelids where it may be so severe as to close the eyes.

The colour of the patches varies in different situations. On the face they are pinkish-red and sometimes vivid like erysipelas, while on the extremities, especially in elderly people and those with a weak peripheral circulation, they may assume a dusky riolaceous tint.

The sensory symptoms vary according to the situation and extent of the lesions. When the face is involved, heat and burning are the predominating sensations, while on the limbs and covered parts of the body, itching is the most marked. These sensory disturbances may be continuous and subject to exacerbations or intermittent and spasmodic.

Under suitable treatment the disease may be arrested in the crythematous phase and subside in about a week, leaving a desquamating surface which gradually becomes normal. As a rule thickening of the skin persists, accentuating the natural furrows and occasionally giving to the face an almost lconine expression.

As the result of local irritation from scratching or improper treatment the erythematous phase is liable to pass into a chronic indolent form of dermatitis, the lesions of which are scaly and infiltrated, or lacerated, weeping, and irregularly covered with yellowish gummy scabs. 
Papular Phase.- The eczematous process maỹ go a stage further and form papules on the erythematous base; or papules may appear without any definite previous erythema, forming a more chronic type of eczema generally associated with intolerable itching. The papules are rounded or acuminate in shape and about the size of pins' heads. They may be arranged in small irregular groups, situated anywhere on the trunk, limbs, or sometimes on the face, and occasionally simmetrical, or they may be widely distributed over considerable areas, being especially common about the extensor aspects of the armis and thighs. They vary in colour according to their situation, being pinkishred on the trunk and purplish on the limbs. They are usually follicular and develop around the hair-follicles, but are occasionally independent of them. A careful examination with a lens often reveals a transparent speck at the apex indicating a minute vesicle. The lesions are the result of thickening of the epidermis, generally perifollicular, superficial oedema of the corium, and a slight inflammatory cellular infiltration.

After a short existence the papules may shrivel and be replaced by small scales, but as a rule the itching connected with them is so intolerable that they are rubbed and transformed into flat shiny lesions suggesting Lichen planus. When they are scratched and lacerated punctate oozing or bleeding takes place, which considerably relieves the irritation but may result in the inoculation of progenic cocci and the production of small purulent scabs.

On the other hand the papules may persist and either remain discrete or may coalesce to form scaly, harsh, infiltrated and exceedingly resistant patches which are readily transformed by friction into weeping crusted lesions.

Vesicular Phase.- In this phase the underlying oedema is more marked and occurs so rapidly that it floods the epidermis and causes the formation of superficial resicles. It takes place most readily where the skin is delicate, as about the flexures of the joints, forearms, scrotum, and between the fingers and toes, but may occur anywhere on the skin and even on the scalp. When the resicles are present on the palms and soles, owing to the thickness of the epidermis they are apt to run together and form small bullae. Their appearance is generally preceded by burning and itching of the affected skin, which becomes faintly red and dotted over irregularly with red macules or minute vesicles on an erythematous base. They may be irregularly scattered, or arranged in small groups which may be symmetrically distributed, and are sometimes so numerous as to form an almost continuous sheet. They gradually increase in size to that of a pin's head, the inflammatory areolae meanwhile extending peripherally and coalescing to form red patches definitely raised from oedema and covered with vesicles.

Occasionally only a single patch is present but as a rule there are several, either localised to one region or on different parts of the skin, 
sometimes appearing symmetrically on opposite sides of the body though as a rule limited to one side at first. The patches extend and join together, involving large areas and in severe cases practically the whole of the skin. (Fig. 268.)

As the vesicles are situated superficially in the epidermis they are easily ruptured, sometimes spontaneously but more often by rubbing and scratching, and a clear yellowish serous fluid exudes which tends to coagulate to form a sticky yellowish crust and has the property of stiffening linen. The exudation gradually becomes more abundant till finally an inflamed, weeping, or crusted patch results. So long as the exudation is copious the scabs are scanty, but should it dry up the crusts become marked. (Fig. 269.)

Under suitable treatment the inflammation may subside, the oedema diminish, the exudation cease, and a new horny layer be formed over the surface, which is shed several times and followed by a scaly erythematous patch which gradually undergoes involution leaving, as a rule, no trace behind it. Unfortunately this is seldom the end, for although individual lesions may disappear new crops of vesicles are liable to break out and the process to be repeated, or from scratching and rubbing a thickening of the skin is produced and the acute lesions are transformed into chronic infiltrated patches, varying in size up to large irregular areas sometimes involving a whole region, the skin over which is red or purplish, dotted with red puncta, shiny in one place, broken and exuding in another, and more or less covered with gummy yellowish crusts. Too often infection of the surface with pus-organisms takes place so that the discharge becomes seropurulent and the crusts offensive and dirty geeenish-yellow in colour. Sometimes from laceration and bleeding the scabs become brownish and haemorrhagic.

In the past various qualifying names have been applied to eczema, which are of some use in emphasising the predominating feature of the lesions in an individual case. Of these the following may be mentioned: "Weeping eczema" where exudation is a marked characteristic; "Squamous cczema," where the lesions have evolved into harsh scaly patches or extensive desquamating areas; Impetiginous or pustular eczema where secondary inoculation of pus-cocci has taken place and the exudation has become sero-purulent and the crusts greenishyellow; Tumified eczema where the skin is markedly thickened; Warty eczema or Papillomatous eczema where, as a result of the action of septic micro-organisms, the surface of the lesions and especially of those on the legs becomes covered with warty growths; Fissured eczema where superficial or deep fissures are present; Crackled eczema where, owing to the presence of irregular superficial cracks in the epidermis, the patches have an appearance suggesting crackled china - a condition which is found chiefly in old people with atrophic skins, but may be present at any age; Eczema rubrum where the lesions form large, red or violaccous, infiltrated patches, the 


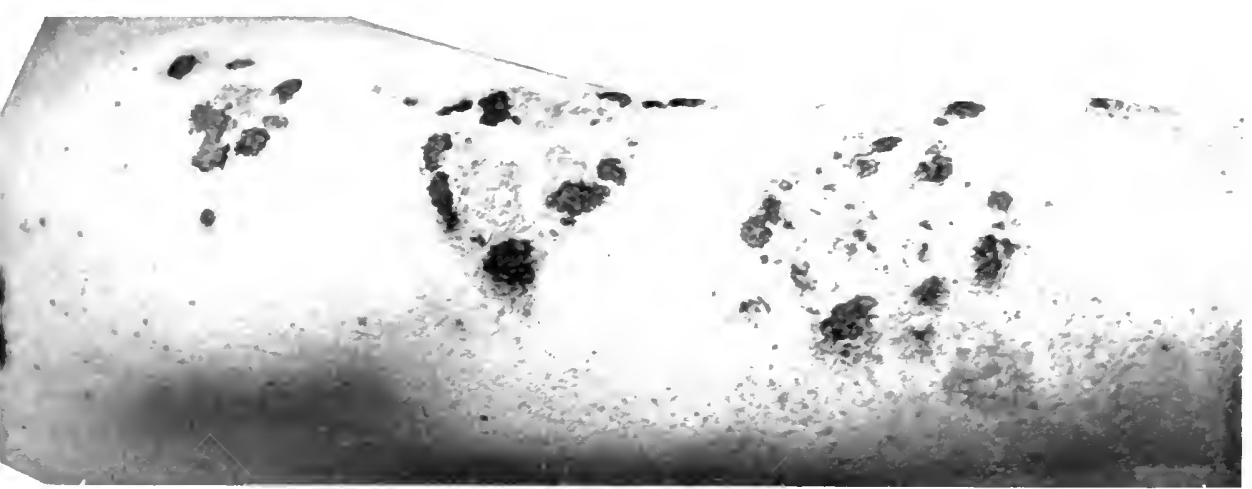

Fig. 268.-Papulo-resicular Eczema with Haemorrhagic Crusts.

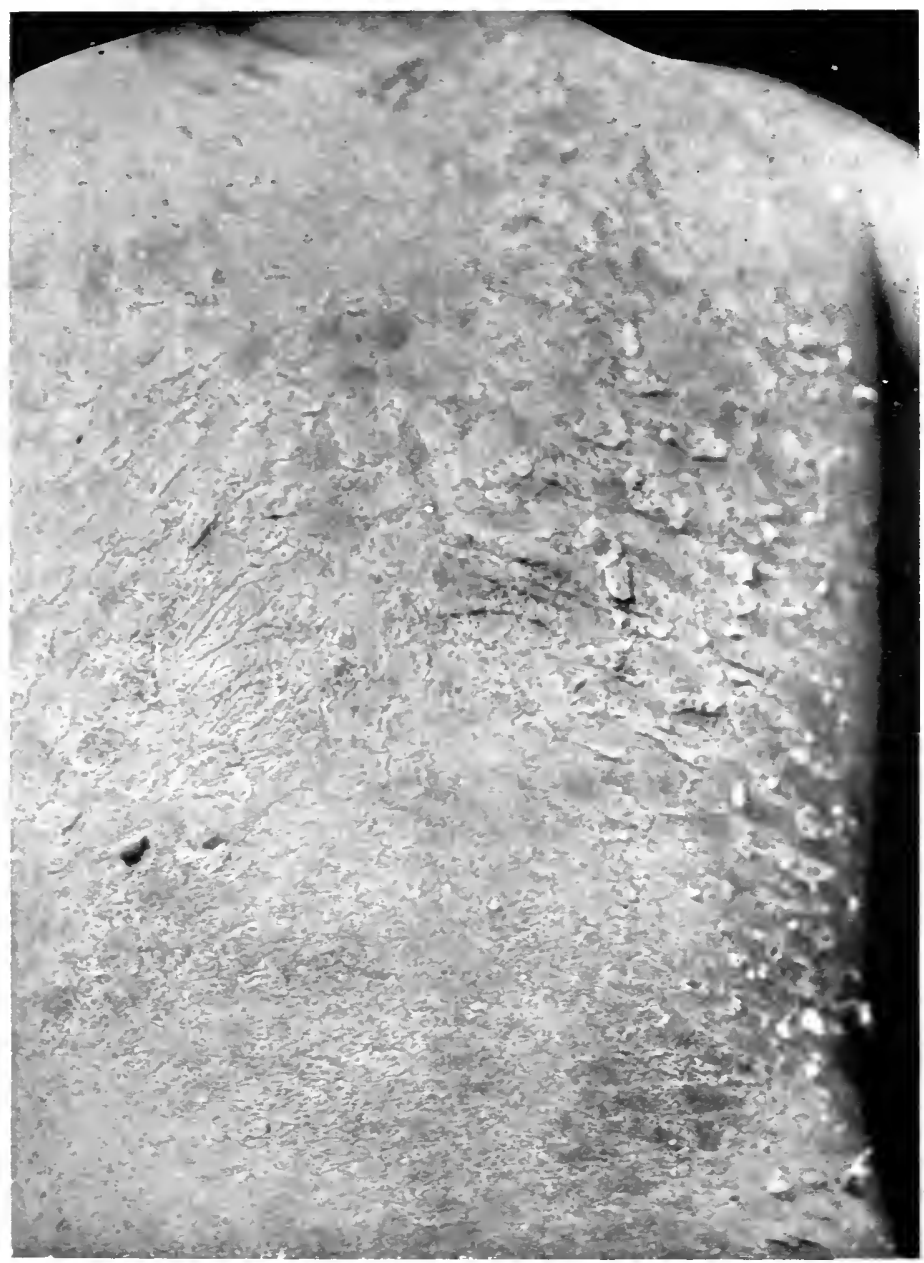

FIG. 250 -Eczema.

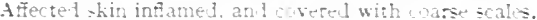



surface of which may be smooth and shiny, or raw, excoriated, weeping, and crusted.

Subjective Symptoms.-The subjective symptoms which are generally present vary in intensity and character in different cases and at different times in the same case, and consist of itching, heat, tingling, and burning, and sometimes actual pain. They are usially intermittent, and may even completely subside for a time, but tend to recur more or less acutely. In one case the itching or burning may be mild and tolerable, in another it may be so severe as to constitute a veritable crisis, driving the patient almost mad with irritation and leading to the most energetic scratching. The spasms may come on periodically, after food, when the patient becomes warm in bed, or when the clothes are removed owing to the exposure of the shin to the air and the relief of constriction; or aperiodically in association with digestive disturbances, the taking of hot drinks, alcohol, or some indigestible food, or as a result of heat, excessive exercise, or some mental disturbance such as worry or temper.

General Symptoms.-The general symptoms are comparatively slight even when the eczema is acute. Occasionally the onset of an attack may be accompanied by malaise, pains in the joints and limbs, loss of appetite, and other signs of a toxic disturbance, but as a rule these are absent and any symptoms which do occur are the result of the general irritability of the skin. In some cases the spasms of itching may be so severe as to interfere with sleep and to upset the digestion.

When an acute generalised attack subsides, it may be followed by exfoliative dermatitis, a condition which is apt to put an excessive strain on the kidneys with harmful consequences.

Complications.- The complications which occur in the lesions have already been indicated. The most important are pustulation, lichenification, warty growths, elephantiasic thickening, and in rare instances epithelioma.

The pustulation results from inoculation of pyogenic microorganisms, usually through scratching. The serous exudation is then transformed into an offensive sero-purulent discharge which dries up into dirty-greenish scabs. In rare cases superficial ulceration may supervene. Occasionally the discharge has been known to be bluish in tinge from the action of the Micrococcus pyocyaneus.

Lichenification may occur as a complication in old-standing chronic patches and is due to excessive rubbing. In it the affected shin becomes thickened, shiny, and cross-hatched, with flat rounded papules at the margin of the patch.

Papillomatous and verrucose changes may complicate an eczematous patch, especially on the legs and feet, and are probably due to the action of pyogenic staphylococci.

Elephantiasic swellings have been known to supervene in eczema of the leg associated with varicose veins, these are secondary to local 
erysipelatoid attacks of streptococcal origin resulting in blocking of the lymphatics by fibromatous changes around them.

In a few rare instances eczematous patches of long-standing in elderly people have been known to become epitheliomatous.

In addition to the local complications, general disturbances of health may occur, which, in the adult, are chiefly of a functional type and are

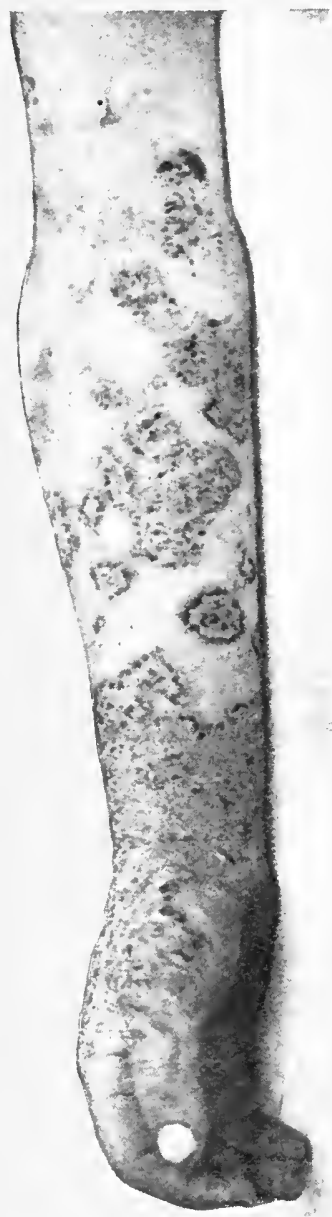

Fig. 270.-Eczematous Dermatitis secondary to Scabies.

Lesions of scabies still present. caused by nervous irritability and derangements of the kidneys.

Course.-Eczema may be acute, sub-acute or chronic in its course. Under suitable treatment an acute attack of whatever type may subside and leave no trace, but it is much more common for it to persist in certain situations and to become chronic.

As a general rule, one type of lesion prevails in an individual case and any new lesions which appear conform to it. On the other hand the course of a case may be atypical, and erythematous, papular, and vesicular phases may occur simultaneously.

The course and appearances of the disease are greatly modified by secondary considerations such as scratching, rubbing and the inoculation of pus micro-organisms.

Eczematisation.-Eczematisation is a name which has been used by certain writers as a substitute for eczema, indicating their belief that the affection is rather a type of reaction to a large variety of irritants than a specific disease, a view which will be discussed under etiology.

Provisionally, the term is useful as applied to an eczematous condition superimposed on some other cutaneous affection generally as a result of rubbing or scratching. A considerable number of different forms of dermatitis may be eczematised in this way, the most important being Seborrhoic dermatitis, various forms of impetigo of both the dry and pustular types, the irritable excoriated skin which results from scabies, dermatitis of the occipital scalp and neck associated with pediculi, dermatitis from irritant drugs, trade dermatoses, mild degrees of ichthyosis and occasionally late superficial syphilides. These by no means exhaust the list but indicate the types of dermatitis in which cczematous changes are most liable to supervene. (Fig. 270.)

Histopathology.-The microscopical appcarance of sections of eczema vary greatly according to the stage of the discase, but all possess 
certain conımon characteristics, namely, dilatation of the bloodvessels of the papillary and sub-papillary layers, slight inflammatory cellular infiltration around the dilated capillaries, oedema of the superficial parts of the corium, and elongation of the papillae. Changes also occur in the epidermis. The most important are the result of oedema, which causes dilatation of the inter-epithelial lymphatic spaces, a spongy condition of the prickle-cells, and an interference with the

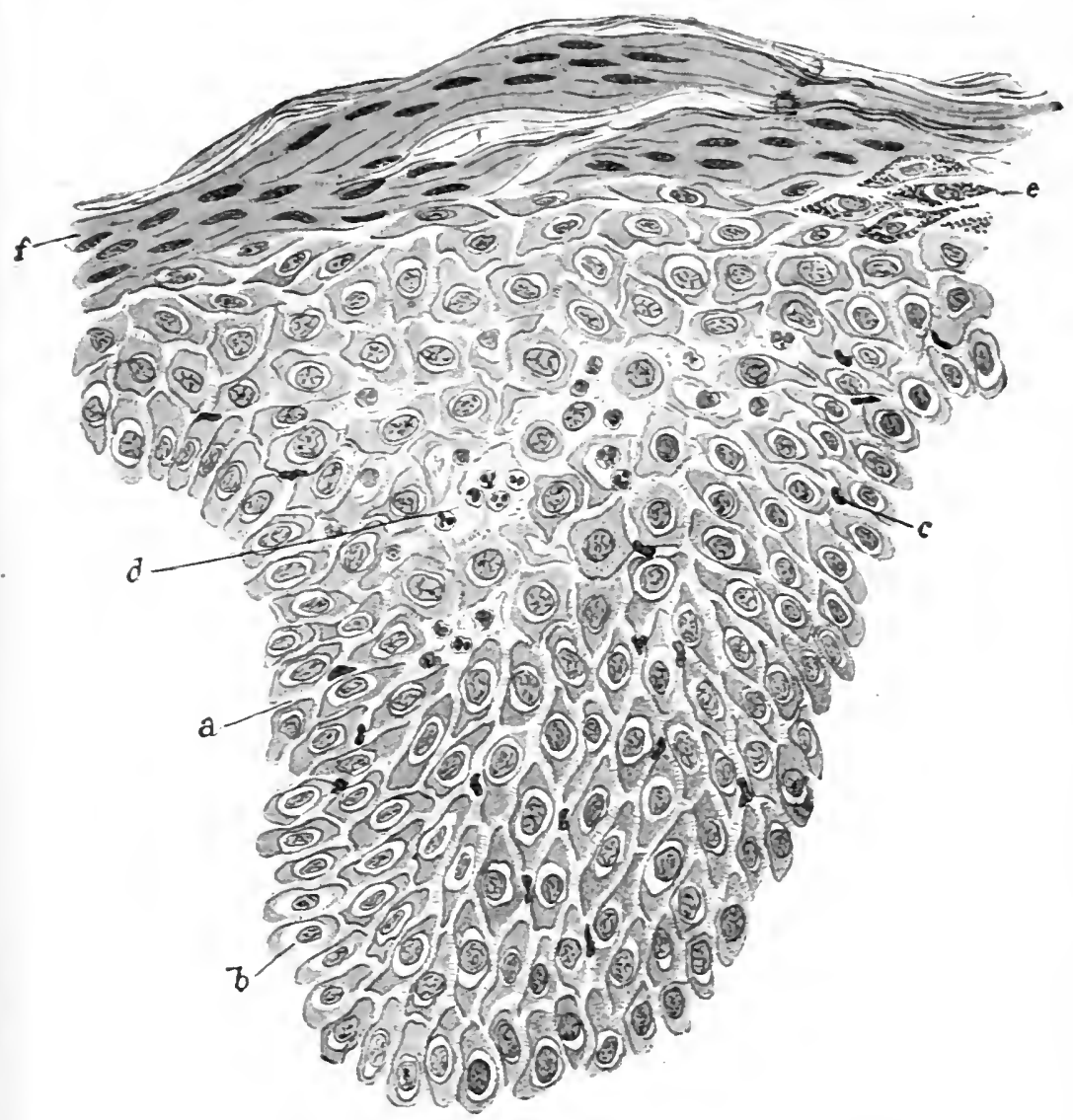

J. M. H. I. del.

FIG. 271.-Commencing Vesicle of Eczema, more deep-seated than usual.

a. Jarked inter-epithelial oedema. b. Dilated nuclear spaces. c. Leucocytes between the prickle-cells. $d$. Commencing vesicle containing leucocytes, breaking-down prickle-cells, epithelial nuclei, prickle-cells which have lost their prickles and become globular, and fibrinous debris. e. Stratum granulosum. $f$. Imperfect horn-cells with nuclei.

(From MacLeod's Pathologv of the Skin.)

process of cornification in which the granular layer is absent, and the horm-cells instead of being dry and containing a waxy substance, retain their nuclei, are moist, and only dry on reaching the surface where they form scales (parakeratosis). (Fig. 27I.)

The prickle-cell layer at the same time usually undergoes proliferative changes with elongation and broadening of the inter-papillary. processes (acanthosis). 
As the oedema increases, a time comes when the intercellular protoplasmic bridges are no longer able to hold the prickle-cells together and to resist the pressure of the fluid, so that the cells become separated and a vesicle results which appears first above the papillae where the oedema is greatest. As the fluid in it increases, the prickle-cells around become compressed and assume a spindle shape. Neighbouring vesicles gradually coalesce to form large irregular blebs which may be unilocular but are more often broken up by existing bands of pricklecells into a number of variously sized loculi. (Fig. 272.)

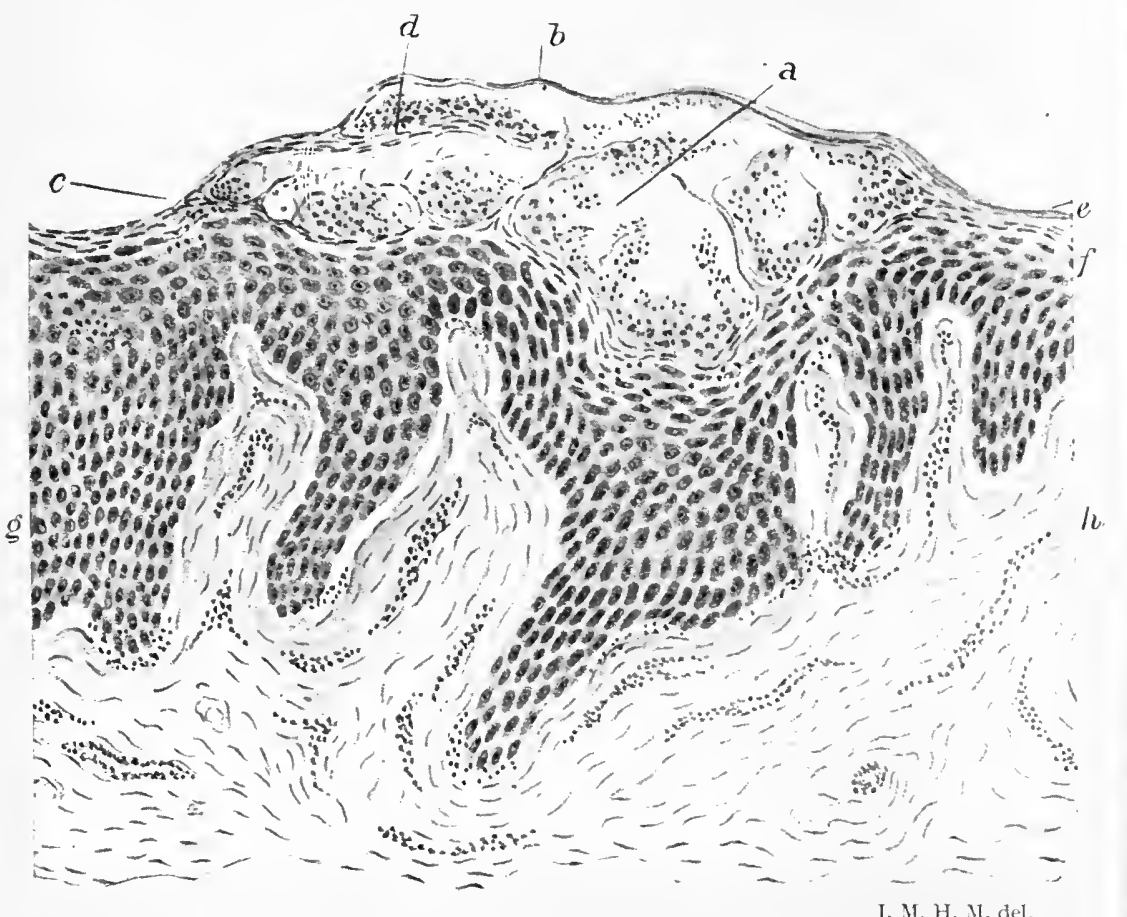

FIG. 272.- Superficial Vesicle of Eczema.

J. M. H. M. deI,

a. Vesicle broken up into imperfect loculi by septa composed of flattened epithelial cells. $b$. Roof of the vesicle formed by a tbin layer of the Stratum corneum. $c$. Floor forred by flattened epithelial cells, some of which are partially cornified. $d$. Contents of vesicle; leucocytes and epithelial debris. $e$. Imperfectly cornified layer. $f$. Stratum granulosum. g. Proliferated and oedematous Malpighian layer. $h$. Dilated blood-vessels surrounded by an inflammatory cellular infiltration.

(From MacLeod's Pathology of the Skin.)

The resicles rary in their situation in the epidermis, the majority being superficial and covered only by a thin layer of imperfectly cornified cells, while others are more deeply situated in the prickle-cell layer. They contain a coagulable fluid of the type of blood plasma, a few leucocytes, oedematous prickle-cells which have become rounded and lost their prickles, and occasional nuclei. In its early stages the resicle is found to be sterile and should micro-organisms occur they are the result of secondary infection.

The changes both in the epidermis and the corium are all explicable either (I) as the result of some irritant acting externally and causing 
the epithelial proliferation and the secretion by the epithelial cells of some poisonous substance which has produced a secondary reaction in the ressels of the papillary body and an exudation to counteract the poison, or (2) of some toxin circulating in the blood, which gives rise to primary changes in the corium, namely the vascular dilatation, cellular infiltration, and oedema, and to secondary changes in the epidermis.

\section{Etiology AND PAThogenesis}

Predisposing Factors. - Eczema forms about $25^{\circ}$, of all cases of skin disease in this country. It occurs at all ages, is particularly common in infants, diminishes in frequency up to 16 or I y years, and increases again in adult life. It affects both sexes alike. It might be expected to occur more frequently in women owing to the greater delicacy of their skins, but this is counterbalanced by the fact that men are more subjected to local irritation and exposure to various direct local causes of the disease. It prevails equally among rich and poor though in private practice, orring to the better hygienic conditions and more thorough treatment, the cases as a rule are milder in character.

Heredity does not appear to be an etiological factor of any great importance. Infants are not born with eczema nor is there any evidence that when either of the parents suffer from it the occurrence of it in the child is more than a coincidence. On the other hand an infant may inherit a delicate, defective, or unusually sensitive skin, or a state of enfeebled health which by lorrering the cutaneous resistance may render it particularly liable to the disease.

Climate has no marked bearing on the causation and eczema prevails in tropical as well as temperature zones and attacks all races. It may occur in any season of the year, but infantile eczema is more common in winter and spring than in summer.

Causation.-The exact nature and causation are not fully understood and there are three opposing theories with regard to it, namely, (I) that it is the result of a nerrous disturbance, (2) that it is caused by certain toxins circulating in the blood, (3) that it is a form of cutaneous reaction due to various local irritants acting in a predisposed indiridual.

I. The Veurotic Theory.-The theory that eczema is a nerrous manifestation was suggested. by the apparent relation of attacks of it to mental anxiety, worry, overwork, and other forms of nerve strain, and by its frequent occurrence in patients of a definitely neurotic type or at a time when the nervous system is in an unstable condition, as in women suffering from uterine derangements or at the menopause. An insuperable argument against the general acceptance of this theory is that eczema is relatively more frequent in infancy than in adult life.

It is a common experience that once the disease is established exacerbations of it may occur as the result of nervous disturbances, probably by increasing the cutaneous irritability and so inducing scratching, but there is no evidence that they are directly responsible for the disease in 
the first instance, nor have actual changes ever been detected in the peripheral nerves supplying the affected skin.

2. The Toxic Theory.-Probably the most popular theory is that eczema is due to toxic substances circulating in the blood acting on the skin either directly or reflexly through the nerves. On the one hand, various articles of diet, such as acid fruits, meat, sweet wines, etc., have been blamed, and on the other toxins resulting from imperfect metabolism and mal-assimilation in the alimentary tract.

It has not been proved, however, that improper feeding or digestive derangements are capable of producing an attack of eczema de novo, though they may aggravate it when present and may possıbly determine a recurrence. Cases of eczema occur in which there is not the slightest evidence of digestive disturbance either before or during the attack, while should such occur they may be cured without markedly improving the eczema; in the case of infants the disease is much less common in summer than in winter, and it is in summer that gastro-intestinal disturbances are most frequent.

Certain other toxins, the exact nature of which is not fully understood, have been said to cause the disease, such as those which occur in connection with gout, diabetes, rheumatism, chronic Bright's disease, and diseases of the liver.

According to certain French writers eczema is a cutaneous manifestation of a diathesis, such as the gouty, arthritic, scrofulous, or some other diathesis not yet defined. This theory was a protest against the tendency to regard skin affections too much in the light of local conditions, which view at one time predominated in the Vienna school and which, although it has been discarded frequently as being a cloak for ignorance, has been revived periodically and at the present time seems to be again reasserting itself. It is claimed by certain authorities that eczematous patients suffer from a faulty " rapport azoturique," that is to say, their waste nitrogenous extracts are not excreted in the proper form, the amount of urea compared with other substances, such as uric acid and creatinine, being below the normal percentage.

That eczema may occur in association with these different morbid conditions must be generally admitted, but that any one of them is the direct cause has yet to be established, and it is much easier to prove that gout, rheumatism, and glycosuria are predisposing than exciting causes. The view that an attack of ecżema in a gouty individual is a symptom of the disease and a sort of safety valve which prevents an attack of gout taking place, is no longer tenable and is a repetition of the old fallacy that it is dangerous to cure a skin affection in case by so doing the disease should be driver in to the system.

The connection between the toxins of these different diseased conditions and eczema is probably indirect. Their presence in the blood or elimination by the sweat-glands causes an irritability of the skin with more or less marked itching, and to relseve this rubbing and scratching are indulged in which may aggravate an already existing eczema or possibly produce the dermatitis.

3. The Local Irritation Theory.-In individuals who possess the requisite susceptibility numerous irritants, both physical and chemical, are known to give rise to a severe dermatitis which is clinically and histologically indistinguishable from eczema. Among these are the poisons secreted by certain toxic plants, various trade irritants such as sugar, flour, Portland cement, and drugs like iodoform, arnica, etc. It has been customary to differentiate these forms of dermatitis from eczema, not on account of the character of the lesions, but on the grounds that in the trade dermatitis the eruption disappears soon after the irritant ceases to operate and that the lesions do not spread beyond the area exposed to it. This distinction 
seems to be more arbitrary than real and is one which, for the simpler conception of eczema, had better be abandoned. Not only are many forms of trade dermatitis indistinguishable from acute local eczema but they may be the starting point of generalised eczema, and a patient who in the first instance had localised dermatitis due to some known irritant may develop typical eczema subsequently, new lesions appearing symmetrically and in parts remote from the direct action of the irritant. It is difficult to find a satisfactory explanation for this; it has been suggested that the lesions in remote parts result from the action of toxins produced in the initial patches, either by microbes or by the tissue cells, which are absorbed and carried to different parts of the skin by the blood-stream, and in corroboration of this view it has been pointed out that before an extensive attack of eczema develops there is occasionally some general disturbance, such as restlessness or feverishness, which might result from the presence of toxins in the blood. The weakness of the theory is the difficulty of believing that toxins could persist in the blood or the tissues long enough to be responsible for the chronic phases of the disease.

According to Brocq, eczema should be regarded not so much as a specific disease but as a special type of reaction of the skin, or neurocutaneous reflex, to a variety of causes in certain predisposed individuals, and he considers that it is more correct to speak of eczematisation the symptom than of eczema the disease.

That a predisposition or idiosyncrasy is necessary is proved by mild local irritants producing a dermatitis in certain individuals while in others even strong irritants have little or no deleterious effect on the skin.

In the same way a number of people may be working together under the same conditions of exposure to some local irritant and only one of them may suffer from dermatitis in consequence.

The skin in infancy is delicate and naturally predisposed, while in older children and adults the predisposition is hereditary, or acquired as the result of enfeebled general health or of some pre-existing disease of the skin such as ichthyosis, seborrhoic dermatitis, etc. Should an attack of eczema be produced by some local irritant a predisposition is established by which recurrences may be caused by shorter exposures or weaker doses than those which produced the original lesions.

Given the necessary predisposition numerous irritants seem to be capable of causing eczema. In the infant, in whom the etiology of the disease can be studied better than in the adult as it is under closer observation, is on a simpler diet, and possesses a more sensitive skin, external irritants seem to be entirely responsible (see infantile eczema, p. 799), and once the initial lesion is established it is kept active by rubbing and scratching.

Micro-organisms.- It has been asserted that the local irritation of micro-organisms is directly responsible and certain writers have gone so far as to regard eczema as a local parasitic disease due to a specific microbe. Various micro-organisms have been put forward, such as Unna's " morococcus," by which he claimed to have caused eczema by rubbing a culture of it into the skin. It was subsequently found, however, that it was impetigo and not eczema which had been produced and that the morococcus was not a new organism but a staphylococcus of low virulence (S. epidermidis albus) and a common denizen of the skin. Apart from the fact that no definite micro-organism has been established there are certain considerations which make it difficult to accept the theory of a local parasitic cause, such as the vesicle of eczema being sterile unless when secondarily contaminated, the occasional occurrence of outbreaks almost as sudden and generalised as an exanthem, the presence of symmetrical lesions in situations, such as the elbows, where they cannot be explained by contact, and the failure to prove the disease to be either auto-inoculable or contagious. 
Frequent comment has been made on the association and alternation which may be met with between eczema and bronchial asthma, and it has been suggested that while the one is a neuro-cutaneous reaction the other may be neuro-bronchial reflex, and that the one inhibits the other.

It has been observed also that in patients suffering from uraemia who have had chronic nephritis for years, the skin sometimes breaks out in eczema. In such cases the eczema may be a relief to the kidney action, that is to say when the eczema is active the coma and other symptoms of the renal condition are relieved, while should the eczema improve the uraemic signs tend to become aggravated.

The whole problem of the causation of eczema is still sub judice, perhaps in the light of future research it may be solved but until then it is best to study each case on its own merits and to avoid hasty generalisations.

Prognosis.-The prognosis of eczema varies greatly according to the extent and type of the lesions, their situation, the thoroughness of the treatment, and the general resisting power of the patient. Most cases are amenable to suitable treatment and a cure can generally be effected, but as an attack of eczema is liable to produce a vulnerability of the skin and a proneness to react readily in an eczematous fashion to comparatively mild irritants, fresh outbreaks are only too apt to occur and have to be carefully guarded against.

The prognosis may be said to be best in an acute attack when the skin, if sufficiently treated, may be restored to a healthy condition in a few weeks. When the eruption has been inadequately treated or aggravated by unsuitable local applications, changes of a chronic type are apt to supervene and the cure to be much delayed.

The time necessary for the healing of an individual lesion may be said to vary inversely with its acuteness; when in the erythematous phase a few days under appropriate treatment may lead to its disappearance, when it has gone on to the weeping and vesicular stage weeks of treatment may be necessary before it subsides, while should it become transformed into an infiltrated, dry, scaly or lichenified patch, were it not for the assistance of the X-rays, healing might be postponed indefinitely.

It was once believed that to cure extensive eczema was a dangerous procedure, causing the disease to be suppressed and driving it in, with evil consequences upon the general health, but this belief is now known to be a fallacy and should derangements in health occur after eczema has been cured it is simply a coincidence.

The situation of the eruption has an important bearing on the prognosis. When it occurs in the face it is apt to be aggravated by and to recur from exposure to the irritants of daily life, such as sunlight, heat, cold winds, soap and water, and in consequence may be exceptionally difficult to cure; in some cases the care which has to be exercised 
to guard against these irritants, and especially sunlight, is so great as to make life almost a burden. When the lips are affected and cracked healing may be interfered with by the movements of the mouth and the irritation of food and drink. Where the hands are involved it may be difficult to protect them from exposure to some irritant, which may have been the initial cause of the disease, and unless this can be done until the skin has recovered and for some time afterwards, treatment is of little avail.

Eczema at the flexures of the joints and in the inter-gluteal firrow is difficult to cure owing to the movements of the joints, the friction of apposing surfaces, and the irritation caused by perspiration or discharges. Eczema of the legs may be singularly intractable if it be associated with varicose veins or static congestion, and unless the patient can lie up to relieve the congestion the prognosis is most unsatisfactory.

Another important factor is the power of the patient to refrain from scratching, for should this be indulged in the most careful regime both local and general may fail to effect a cure.

When healing takes place no trace of the eczema is left, except when the leg is affected where it may be followed by pigmentation which gradually disappears.

Diagnosis.- The diagnosis of a typical case is simple. It is the atypical cases which present difficulties owing to the multiformity of the lesions and the secondary changes which may occur and mask their real nature. The most important didgnostic features considered collectively are erythema, papulo-vesiculation, exudation of serous coagulable fluid which dries to form sticky crusts, infiltration and scaliness, itching and burning, a chronic course, and a tendency to recur. For convenience of description the diseases with which it is most liable to be confused will be briefly considered in their alphabetical order.

Erythema Exudatium.-This affection may sometimes be mistaken for eczema but in it the lesions tend to appear, sometimes simultaneously, on the face, arms and backs of the hands, legs, and less frequently on the trunk, and consist of erythematous papules or plaques, smooth on the surface, sometimes urticarial at other times vesicular or bullous; the lesions of eczema are much more irregularly distributed, have not the same tendency to be symmetrical, are scaly on the surface or moist and weeping, are less markedly oedematous, and unless in exceptional cases on the palms and soles do not tend to form bullae. Itching is invariably present in eczema but absent in E. exudativum.

Exfoliatice Dermatitis. - The condition known as Exfoliative dermatitis may sometimes present difficulties in diagnosis from a widely. distributed attack of eczema. In eczema the affection is rarely. universal, areas of normal skin being generally present between the inflamed patches, while the whole skin is involved in Exfoliative dermatitis. In generalised eczema the skin may be infiltrated in one 
place, fissured, oozing, and crusted in another, while in Exfoliative dermatitis it is dry, scaly, and the infiltration slight or absent. Exfoliative dermatitis is not so much a specific disease as a universal dermatitis superimposed-generally as the result of excessive or irritating treatment-on a number of different affections, one of which is generalised eczema, so that the borderline between the two conditions is ill-defmed and the difference is more one of degree than of kind.

Lichen Planus-There is no great difficulty in distinguishing a typical case of Lichen planus of the papular type from papular eczema, for in Lichen planus the papules are flat, polygonal, dull red or violaceous in tint, and have a shiny or grey surface, while those of eczema are pinkish-red in colour, and round or acuminate in shape. In Lichen planus the papules rarely become vesicular and on disappearing leave a brown stain, while in eczema they evolve into vesicles or present a minute vesicle towards the apex.

Acute generalised Lichen planus may sometimes closely resemble eczema, especially where it is associated with itching, but in Lichen planus the eruption is invariably dry while in extensive cases of eczema a tendency to weep may generally be detected in some of the lesions. The scales of Lichen planus are delicate and adherent while those of eczema are coarser and easily picked off.

Occasionally an old-standing case of chronic eczema may assume a burnished lichenified appearance from constant rubbing, but its resemblance to a patch of Lichen planus is superficial, for the latter is more infiltrated and demarcated, has a purplish tinge, is rough and scaly on the surface, and typical Lichen papules may generally be detected in the neighbourhood.

Lupus Erythematosus.-The disseminated type of Lupus erythematosus may occasionally resemble chronic eczema. In Lupus erythematosus the patches are usually more infiltrated, better defined, either present a white punctate appearance or are covered with adherent scales with underlying prolongations which dip down into the sebaceous follicles, and frequently show central atrophy; in eczema the infiltration is less and the scales which cover the patches are white, coarse, easily picked off, and apt to leave an exuding surface. The distribution differs somewhat in the two conditions, and eczema shows little tendency to involve the "bat's wing area" of the face. Subjective symptoms as a rule are absent in Lupus erythematosus and its course is much more chronic, taking months or even years to spread over an area which eczema nay involve in a few hours.

Intertrigo.-The red hyperaemic patches which occur between the buttocks and in the axillae as the result of friction of intertriginous surfaces are liable to be mistaken for eczema, but the secretion which occurs in them is more watery and does not coagulate like that of eczema, and the localisation is different. From rubbing and scratching and the secondary inoculation of pyogenic micro-organisms the inter- 
triginous patches may alter in appearance and assume the characteristics of eczema.

Miliaria or Prickly Heat.-Both the papular and resicular types of prickly heat may be difficult to diagnose from eczema but the lesions in prickly heat are never confluent, the resicles rarely rupture, the fluid contained in them is more watery and does not tend to coagulate, and the eruption is characterised by pricking sensations rather than itching, tends to come out after hot drinks or exposure to heat, and is generally associated with marked sweating; in eczema, heat, though it may aggravate an attack, is rarely directly responsible for it. The lesions in prickly heat do not occur on an inflamed bast like those of eczema and the disease runs an acute course, while eczema tends to become chronic.

Mycosis Fungoides. - The pre-mycotic stage of this affection may suggest erythematous eczema but the lesions in it are more puffy, smoother on the surface and sometimes scarlatiniform, and the spasms of itching connected with them are infinitely more serere than those of eczema. Occasionally through scratching the pre-mycotic lesions may become eczematised and the diagnosis complicated.

Paget's Disease of the Nipple.-This rare affection is differentiated from a patch of circumscribed eczema of that region by being more definitely demarcated and infiltrated, more chronic in its course, and more resistant to treatment, and by its tendency to develop into cancer of the breast.

Pediculosis.-The scratching indulged in for relief of irritation caused by pediculi may lead to pus-inoculation and eczematisation. This occurs not infrequently about the occiput in children suffering from Pediculi capitis, about the pubes and groins where Pediculi pubis are present, and anywhere on the skin in association with Pediculi vestimentorum. A careful examination will usually reveal nits if not pediculi, and the punctate haemorrhages and linear excoriations which are generally present in connection with Pediculi restimentorum reveal the underlying cause and show that the eczematous changes are secondarr.

Pityriasis Rosea.-This affection may somewhat resemble mild scaly eczema, but the characteristic lesions of Pityriasis rosea differ from those of eczema in being more circinate, having a fawn-coloured centre surrounded bỹ a scaly superficial border, being generally limited to the trunk and arms, and having a more acute course.

Psoriasis.-Psoriasis may closely resemble chronic eczema, especially when the eczema occurs in the sites of predilection of psoriasis, such as the knees, elbows and scalp. As a general rule the lesions of psoriasis are more clearly defined, brighter red in tinge, covered with silvery scales which when remored leave a delicate pink pellicle dotted over with red puncta, which if scratched readily bleeds; in eczema the lesions are ill-defined, tend to fade into the surrounding skin, are less bright in tint, and corered with grey scales or yellowish 
scabs which when scraped off leave a harsh surface or present exuding points or cracks. Chronic scaly patches of eczema which have resulted from the aggregation of papules sometimes bear a close resemblance to psoriasis but can generally be distinguished by the above characteristics. Psoriasis has a tendency to be present on the extensor surfaces while eczema is more common on the flexor, and rarely attacks the face and palms of the hands, parts frequently affected by eczema. On the scalp, psoriasis may closely resemble eczema but eczema is generally more diffuse and extends on to the neighbouring skin, while psoriasis occurs as well-defined scaly patches and even when it encroaches on the forehead or neck is limited by a clearly demarcated border. Eczema of the scalp is frequently moist and weeps, psoriasis is always dry. Itching is rarely so severe in psoriasis as in eczema and may be wholly absent.

Ringworm.-The type of ringworm which is most difficult to distinguish from eczema is Tinea cruris or ringworm of the crutch, and so close is the resemblance that the condition was once designated Eczema marginatum. Ringworm is more acutely inflamed than eczema, better demarcated, with a raised, sometimes festooned, border which may be dotted over with vesicles or vesico-pustules, and usually presents outlying plaques or ringed patches.

This form of ringworm may also affect the axillae, hands, and feet. In the extremities it is known as eczematoid ringworm and in the past many cases of it have been mistaken for eczema. It occurs as erythematous patches dotted over with small vesicles or vesico-pustules situated chiefly in the inter-digital clefts, or as indefinite scaly patches the scales of which tend to be loose at the margin, or again as a horny thickening between the toes in which the skin is white, moist, sodden, and cracked at the folds. In those cases it is usually comparatively easy to find the fungus in the scales and so establish the diagnosis.

Ringworm of the scalp is casily differentiated from eczema by the presence on the patches of the typical fungus-laden stumps.

On the glabrous skin the ringed or raised pustular forms of ringworm are easily recognised but where the lesions consist of pinkish scaly patches the diagnosis may be more difficult. In ringworm the patches are usually sharply defined, generally raised at the border if not all over, and a careful examination of the scrapings will usually reveal the fungus.

Rosacea.-This affection might be confused with eczema of the face but the dilated capillaries, the presence of papules at the sebaceous orifices, and the limitation of the affection to the nose and the centre of the face, generally render the diagnosis simple.

Scabies.-A severe attack of scabies, with its papules, vesicles, pustules, excoriations, and purulent bullae on the palms and soles, may sometimes suggest eczema; but the existence of the pathognomonic burrow, the irregulatity of the distribution of the lesions, their presence 
in such situations as between the fingers, on the wrists, feet, genitals, and axillae, and their absence on the face and scalp, and the itching which is slight during the day but severe when the patient gets warm in bed, serve to differentiate them. The constant scratching to which the lesions are subjected may lead, however, to eczematisation of the affected skin.

Seborrhoic Dermatitis.-Patches of Seborrhoic dermatitis nay be mistaken for scaly patches of eczema, but differ in that Seborrhoic dermatitis has a predilection for the front of the chest and the interscapular region, and eczema is rarely confined to these parts, while the lesions have a pinkish-rellow tinge, are corered with greasy scales, sometimes present a circinate arrangement, and are not raised abore the surface of the skin, while those of eczema are more vivid red in tint, moist, weeping, crusted, or covered with greyish white scales, and may be infiltrated. On removing the scales in Seborrhoic dermatitis a smooth greasy surface is revealed, while if the scales of eczema are picked off exuding points may be detected or in old chronic patches a rough harsh surface may be left. Subjective symptoms are generally absent in Seborrhoic dermatitis.

In the scalp both affections are scaly, but the scales in seborrhoea are more greasy, the inflammation is slighter, and the disease tends to be limited to the hairy surface; while in eczema the patches are inflamed, the scales coarser, and the disease usually extends on to the neighbouring glabrous skin. It must be remembered, however, that it is not unusual for eczema to be engrafted on Seborrhoic dermatitis of the scalp or trunk, with the production of a compound affection to which the name Seborrhoic eczema is applicable.

Sycosis.-Eczema of the beard has to be distinguished from sycosis of both the hyphomycetic and coccogenic types, especially when they are superficial and scaly. In eczema, however, the follicles are not affected to the same degree as in sycosis, the patches may have a tendency to weep, and the disease is liable to extend beyond the hairmargin and to be present elsewhere, while in the dry scaly form of sycosis the follicles are chiefly involved and the affection is limited to the beard region.

Syphilis.-The only type of early syphilide which might be confused with eczema is the small papular variety, but in it the lesions are more widely distributed, remain discrete, if pressed by a diascope leave a brown stain, never itch, and when scratched show no tendency to weep.

Occasionally the diagnosis of squamous eczema of the palms from the palmar srphilide presents considerable difficulty ; but the syphilide as a rule is browner in tinge, more clearly-defined, more chronic in its course, and confined to one hand, while eczema is more inflamed, and diffuse, the redness fading into the surrounding shin, tends to attack the fingers as well as the palms, is generally fissured, and usually bilateral. In syphilis, while no other stigmata may be noticeable at 
the time, there is generally a positive history of the disease, while eczema of the palm is usually associated with other lesions of an eczematous type either on the arms or elsewhere on the skin.

\section{TreatMent}

Introductory.-The treatment of eczema is so comprehensive, entailing not only a thorough acquaintance with local remedies and their application but a wide knowledge of general medicine, that it is impossible within reasonable limits to do more than indicate its general principles. The multiformity of the lesions, their varying situation on the cutaneous surface, the differences in age, occupation, environment, and general health of the patient, and the peculiarities in the manner in which the skin of different individuals reacts to remedies both local and general, all combine to make the treatment so diverse that each case must be dealt with on its own merits. No hard and fast rules can be laid down which are universally applicable and slavish adherence to any one method invariably spells disaster. There is no cutaneous disease in which therapeutic skill is better brought out than in eczema, and it may be said with truth that the physician who is capable of dealing with it successfully can treat any form of dermatitis.

The treatment naturally divides itself into general, or constitutional, and local. In a few cases where the lesions are limited in extent and the general health unimpaired, local treatment is all that is required, but in the vast majority some form of general treatment or regime is of almost equal importance, and the best results can usually be obtained by the judicious combination of them both. The treatment will be described under the headings of General management, Internal treatment, Local treatment in general, and Local treatment of the different regional types.

General Management.-One of the most important considerations in the management of eczema is rest. However mild the case may be so long as the affected skin is being chafed by the friction of contiguous surfaces or improperly applied dressings, or irritated by rubbing and scratching, it is hopeless to expect any form of treatment to result in healing. In the majority of cases it may be impossible or unnecessary to obtain absolute rest, but a relative degree of rest can generally be achieved by suitable dressings and bandaging. With regard to rubbing and scratching the difficulties are far greater, for so long as the itching persists it is usually futile, even in an adult, to expect the patient to refrain from relieving it, and even although his self-control may be sufficient during the day, when night comes scratching will generally take place, either reflexly during sleep, or in the semi-conscious state before he is quite awake. To obtain rest from scratching it is necessary to allay the irritation by suitable local applications, occlusive dressings, and occasionally by sedatives or even hypnotics, and the patient should sleep in cotton gloves or should adopt some device, such as tying down 
the hands by long bandages to the foot of the bed, so that he cannot reach the itching parts.

Where the lesions are extensive and severe the patient should be kept in bed. In this way the general discomfort is reduced, the local irritation of clothes avoided, the friction and rubbing from moving about guarded against, a more or less even temperature obtained, and local remedies can be applied more thoroughly. The horizontal position also has a quieting action on the heart and reduces the tension in the skin, and at the same time the nervous system is calmed and the patient has temporary relief from the worries and anxieties of ordinary daily life. The bedroom should be kept cool and the bedclothes light, and in hot weather these should be raised off the patient by means of a cradle ventilated by paper shafts at each side of the bed. The bed should be so placed that it is not in a draught and that the light from the window does not fall directly on the patient's face. Dark curtains should be used to reduce the sunlight and where the face is attacked they should be red. The patient, not being seriously ill, should have his mind occupied by reading or other suitable amusement. In this way cases can be cured rapidly and are prevented from going from bad to worse as they not infrequently do, as the result of imperfect ambulatory treatment.

In mild cases, or in severe cases when the acute stage has passed, a change to some suitable health resort may be of great benefit, for there the patient may be prepared to undergo a more careful regime and to carry out treatment which at home would have proved irksome and possibly have been neglected. The choice of the particular health resort must depend on the type of the case. In one, benefit may be derived from a change from a bracing to a mild or even a relaxing climate, while in another a change to a more invigorating air may be advisable. As a general rule eczema, if it affects exposed parts, is aggravated at the sea-side by the salt-laden atmosphere, the fresh winds, the brilliant sunlight and the glare from the sea and sand, and at high altitudes by the glare from the sunshine and snow. In most cases a change to a well-wooded country district or protected valley is best. Various spas have a great vogue for the treatment of eczema such as Harrogate, Strathpeffer, and La Bourboule. The benefit derived from them is not the result of any special form of treatment but is largely due to the careful regime, restricted diet, flushing out of the system by copious draughts of some natural mineral water, regulated exercise, mental rest, and sufficient sleep.

Internal Treatment. - In every case, however slight, it is of the first importance to examine the patient for any underlying disturbance in general health, which, although it may not directly cause the eczema, may still be sufficient to keep it alive, to vitiate the best efforts at local therapeutics, and to interfere with progress towards recovery. The examination should be thorough, special attention being paid to the state of the gastro-intestinal tract, liver, kidneys and nerrous system. 
Although no gross derangements may be discovered yet it is frequently found that patients with eczema are below par, have lost weight, are nervous from worry and overwork, and in short are in a condition in which the general resistance and nutrition of the whole organism is defective.

It is necessary also to study the habits and type of the individual and before instituting any special line of treatment to take cognisance of such considerations as age, environment, constitution, and temperament.

In the minds of the laity and of certain of the medical profession a causal relationship between eczema and gout or rheumatism is an accepted fact, but although eczema frequently occurs in rheumatic or gouty subjects it can appear independently, and there is no proof that the toxin of either is ever directly responsible for eczema de novo. On the other hand, both gout and rheumatism are important factors in aggravating an existing eczema and in increasing the irritation, and consequently much benefit is derived from the proper treatment of such diseases by lithium, colchicum, acetate of potash, salicin, etc.

Occasionally eczema may co-exist with other diseases, such as malaria, Bright's disease, and diabetes, the effective treatment of which benefits the eczema.

Disturbances of the alimentary tract, such as constipation with its associated anaemia, flatulence, various phases of indigestion, and liver derangements, should be carefully enquired for and suitably treated. It is of the first importance for the relief of congestion of the skin and the prevention of absorption of food-toxins into the blood that the slightest tendency to constipation should be dealt with and that a free action should be obtained daily. Digestive disturbances should be treated by such well-known remedies as the acid and alkaline gentian mixtures, pepsin, pancreatin and charcoal, while liver derangements demand fractional doses of calomel, podophyllin or euonymin.

Cardiac troubles and circulatory disturbances may be important factors in aggravating itching and should be treated by suitable tonics and regular exercise.

In many cases of eczema the nervous system is at fault and there is some functional disturbance or neurosis. In some cases careful management on general medical lines, complete rest, and sedative remedies such as bromides and belladonna are indicated, while in others greater benefit may be derived from change of air, out-of-door exercise, massage, and such tonics as arsenic, iron, strychnine, phosphoric acid, and cod-liver oil.

Sometimes, in spite of the most careful search for definite underlying disease or irregularities in the function of the different systems, no derangement can be detected and the physician is tempted to fall back on one or other of the so-called internal specifics for eczema, of which a considerable number have been put forward from time to time not only by the medical profession but by the quack nostrum vendor. 
This procedure is not one to be encouraged and the indiscriminate employment of any of these remedies is calculated to do more harn than good.

Of the internal remedies the best known and most frequently employed are probably arsenic, ichthyol, salicin, and turpentine.

Arsenic was once prescribed freely in cases of chronic eczema but has now fallen largely into disrepute. It does not appear to have any specific effect on the eczema itself and probably acts chiefly as a nerve tonic. In acute cases it does actual harm by increasing the riervous irritability, upsetting the digestion, and even aggravating the local lesions, and in chronic cases it does little or no good.

Ichthyol has been adrocated as a specific in doses of 5 to $10 \mathrm{~m}$. t.d.s., and is believed to be capable of reducing hyperaemia. Its internal action, however, is uncertain and disappointing.

Salicin is chiefly of value in widely generalised cases of the chronic scaly type, or in cases associated with rheumatism. To obtain good effects it is necessary to employ doses of at least Io to I5 grains t.d.s.

Antimony has a place in the internal therapeutics of eczema for the reduction of acute tension of the shin and is best given in the form of $5 \mathrm{~m}$. of the wine at bedtime or every four hours. It should not be employed in elderly and debilitated people as its action is too depressing.

Calcium lactate has a somewhat similar effect to antimony and may. be given in doses of Io grains suspended in milk, t.d.s. before meals. Any tendency to constipation caused by the drug should be cointeracted by suitable laxatives.

For the relief of itching when it is so severe as to interfere with sleep, the judicious employment of some suitable sedative or hypnotic under the direct supervision of the physician may be not only permissible but of definite benefit. For this purpose the most useful remedies are bromide of potassium, 30 grains in a tumbler of water after going to bed, phenacetin Io grains in adults, or the syrup of chloral hydrate Io to $20 \mathrm{~m}$. in children. The stronger hypnotics, such as morphia and opium, are contra-indicated as they are apt to be followed by an exacerbation of itching when their effect has passed off.

Diet.-Wuch has been written with regard to diet in eczema. To those who regard eczema as the local evidence of some internal disturbance, errors of diet are believed to play an important part in its etiology and in consequence a most restricted diet has been insisted upon by certain physicians, in which the quantities of nitrogenous and carbohydrate foods are carefully measured according to the weight of the patient, and a long list of foods is prohibited. Such schemes of dietary, however, though they may impress the patient are unnecessary and have certain fallacies. For while two individuals may be of exactly the same weight their powers of digestion may differ considerably, and whereas in the one case, owing to a more energetic 
existence, more food is necessary to give the requisite energy, in another, owing to a sedentary life, less food is required. Again, "one man's food is another man's poison " and one individual may digest a certain article of diet which in another would cause sickness.

The rules of diet for eczema are largely those for the management of any form of illness.

In acute cases where the patient is confined to bed a light fluid diet should be prescribed consisting chiefly of milk, about 3 pints in 24 hours for an adult, and beef-tea. With convalescence the diet may be made more interesting by the gradual addition of bread and butter, milk puddings, white fish, or chicken. In chronic cases where there is no definite evidence of indigestion, an ordinary diet to which the patient has been accustomed and which he can digest comfortably may be continued and troublesome restrictions are unnecessary.

There are certain articles of diet, however, which experience has shown aggravate most cases of eczema, probably by calling forth foreign proteids from the cells lining the gastro-intestinal tract which, circulating in the blood, irritate the skin. These are shell-fish, especially crab, lobster, mussels, and oysters in the hot weather ; oily fish such as mackerel, eels, salmon, and sardines ; indigestible meats, such as veal, liver and bacon, pork, salt meat, high game, and goose; starchy foods where the digestion is feeble ; asparagus, radishes, unripe fruit, strawberries, rhubarb, mustard, curries, chutneys, rich sauces, and savouries.

The quantity of food and drink is of as great importance as the quality. Most people eat too much and drink too little. The simplest indication of the amount of food an individual can digest is that he should rise from the table feeling that he could have eaten more and is not in a state of repletion. An increased quantity of fluid should be taken to keep the kidneys active, and as a laxative a glass of plain water or of some natural uneffervescent water first thing in the morning, between meals, and at bed-time, is of undoubted benefit. Aerated waters are inadvisable as they are liable to cause flatulence.

Alcohol should be avoided in acute cases. In certain chronic cases it may be taken with impunity in small quantities, especially in patients over middle-age who are accustomed to it, and the general stimulation and improvement of the appetite produced by it may more than counterbalance any harmful effects it may have in dilating the cutaneous blood-vessels. Beer, claret, sherry, port, and the half-fermented liquors such as champagne and cider, should be prohibited. In elderly people the most satisfactory stimulant is a small quantity of brandy and water, while in the robust a glass of pure white wine, such as chablis or moselle, may be taken.

Coffee, if strong and black, is most harmful as it may cause pruritus and is only permissible as weak café-au-lait.

Weak tea, freshly infused, especially China tea, is comparatively harmless, while strong tea taken frequently is a common cause of 
indigestion, particularly in women, and aggravates the disease. Smoking in excess, especially inhaling, is harmful, rendering the patients nervous and irritable and occasionally determining spasms of itching. In moderation, however, in those accustomed to it and out-of-doors it may be of actual benefit in occupying the mind of the patient and in soothing the general irritability.

Clothes.-The question of the clothing is of considerable importance. It is essential on the one hand that it should not be too heary, as orerheating and perspiration tend to aggravate the lesions, and on the other that it should be sufficiently warm to prevent chills, as the shin in eczemais unusually sensitive to cold, and cold is liable to cause itching. It is necessary also, especially in women, to protect the shin from the actinic rays of the sun by sufficient clothing about the neck, shoulders and arms, by a reddish lining to thin blouses, shady hats, gloves, brownish veils, and red sunshades.

Woollen underclothes may irritate sensitive skins and it is better to substitute silk and wool, fine linen mesh or thin cotton, which if too cool may be worn under a woollen garment. It is important also that the skin should not be subjected to constriction or friction, such as might result from too tight garters, or badly fitting corsets.

Washing and Baths. - With regard to the advisability of washing and bathing there is the greatest diversity of opinion ; certain observers believe that water, especially if hard. owing to its detergent action is invariably harmful to eczema, while others insist on the importance of bathing the lesions to keep them as clean as possible and to prevent contamination by adventitious micro-organisms. Each case, however, must be judged separately. In acute eczema the application of soap and water is most harmful and even soft rain water without soap is better avoided, while emollient baths containing bran, oatmeal, or starch, though they may relieve the general irritability of the skin while the patient is immersed in them, are apt to be succeeded by aggravation of the condition. In chronic cases, however, it is otherwise and the judicious application of soft soap and hot water as advocated by Hebra may be of definite benefit by stimulating the skin. Where the eczematous lesions are covered with discharges and septic crusts their removal by some mild antiseptic lotion or by bathing in a weak cyllin bath (half an ounce to 30 gallons) is of the first importance, and even though the skin may be irritated in the process this is more than compensated by the reduction of the septic condition and the greater efficiency with which local remedies can be applied. In drying the skin, friction from rubbing with a rough towel should be avoided, and a soft linen towel should be laid over the part and the water blotted up.

Turkish baths and Russian baths are usually harmful and though they may promote greater activity of the skin-glands they are apt to be followed by depression and have been known to transform chronic eczema into an acute dermatitis. Medicated baths or medicated soaps 
are of little therapeutic value and may prove irritating, and seabathing is contra-indicated as it almost invariably aggravates the disease.

\section{Local Treatment}

The infinite number of remedies which have been suggested for the cure of eczema is an indication of the difficulties which beset it and of the multiformity of the lesions. To even name these remedies would be impossible nor would there be any practical gain by so doing and it is advisable to be content with referring only to those which have proved to be most generally useful, for to master the action of a few drugs is far better than to have an imperfect knowledge of a great number. In this section the general principles of the local treatment will first be described and then the detailed treatment of the regional types.

General Considerations.--(I) The local treatment of eczema should always be begun in a tentative fashion with weak applications, the strength of which can be increased gradually according to the toleration of the patient, as it is impossible to estimate the sensitiveness of an individual's skin or to foretell the existence of an idiosyncrasy towards some medicament.

(2) While under treatment, cases of eczema should be most carefully watched as the phases of the disease may alter and a local regime which may be beneficial one day may be actually harmful the next.

(3) The mode of application of a remedy is often of as great importance as the choice of the remedy itself. A medicament may be applied in the form of a powder, lotion, ointment, paste, or varnish ; as a lotion and possibly as a powder it may give good results, while as an ointment it may be harmful, or in the form of an ointment it may be of the utmost value, while if applied as a lotion it may be almost useless. Again a remedy may be dabbed on the skin, rubbed into it, applied on lint, or kept on continuously under an occlusive dressing, and the effects of these different modes of application may vary considerably. Consequently in prescribing a line of treatment it is necessary to specify the exact method of application which is to be adopted.

(4) Unless in the mildest of cases it is advisable that external remedies and dressings should be applied by an attendant and not left to the patient, as otherwisc the treatment may be carried out in a dilatory and half-hearted manner, for patients are apt to become careless and to overlook lesions in situations which are not easily accessible.

(5) As the itching is usually worse at night, local applications and dressings should be applied the last thing before going to sleep and provision should be made where possible to have the lesions redressed should the patient awake.

Local Treatment of the Different Phases of Eczema.-In order that the local treatment may be rational it is important that it should be based as far as possible on the underlying pathological condition and 
not applied in a haphazard fashion. The various phases of the disease, namely, the erythematous with its dilated blood-ressels and oedena, the papular with its cellular infiltration, the vesicular with its fluid in the epidermis and tendency to weep, the exfoliative with its imperfectly cornified cells, and the lichenified with its thickened epidermis and cellular infiltration in the corium, have all to be treated differently.

Ervthematous Phase.-In the erythematous phase, of which acute eczema of the face and hands may be taken as the type, the indications are to reduce the oedema and contract the blood-ressels: For this purpose an absorbent, astringent and sedative local treatment is demanded, and the best results are obtained by the application of suitable lotions and powders. By means of astringent lotions the evaporation of the oedematous fluid is promoted and the vessels are constringed by the coagulation of the tissue fluid, so that the hyperaemia is reduced and the part is cooled; by powders, the fluids are absorbed and the diseased skin is soothed and protected. Perhaps the most generally useful lotion for this purpose is lead lotion in the form of Liquor plumbi subacetatis, a dram to a pint of tepid water or milk. This is applied on pieces of lint or washed out buttermuslin laid over the part and kept moist, but not covered with oiled silk which prevents evaporation and keeps the skin hot. In the case of the face, lint masks should be used, which can be kept in position by being tied round the back of the head with pieces of tape. It is sometimes an advantage to apply stronger solutions of lead up to half an ounce to the pint, in which case it should be poured into a saucer and applied by a piece of cottonwool or an old shaving brush every few hours.

On covered parts of the body the addition of $2^{\circ}$ of glycerine prevents the lotion drying too quickly and may be a definite adrantage, or the Glycerinum plumbi subacetatis I in $S$ of water may be substituted, but these lotions should be used with care as, owing to the hygroscopic action of the glycerine, they are liable to prove irritating to sensitive skins.

Another useful type of lotion is the familiar calamine or the borocalamine lotion (see Appendix) which by haring a certain amount of insoluble fowder suspended in it combines the action of both a lotion and powder. It is less soothing than the lead lotion and apt to render the skin dry and harsh. In applying it a small quantity well-shaken should be poured into a saucer or dish, and a piece of lint or buttermuslin saturated in it, laid over the part, and kept moist. Should the itching be intense $\frac{1}{2}$ to $I_{0}^{0}$ of carbolic acid may be added. The employment of this lotion generally leads to a deposit on the skin which becomes caked up and requires removal from time to time. This can be done by gently rubbing the part with cold cream or bathing it with a solution of bicarbonate of soda $I_{5}$ grains to the ounce.

A weak solution of ichthyol I to $5^{\circ}$ in water is also of value in reducing erythema and should be painted on with a sharing brush every four hours. It occasionally proves irritating and if emplored 
over extensive areas may cause general symptoms; in one case in an infant it was succeeded by a temporary disturbance of the kidneys with albuminuria.

Instead of lotions, powders may be employed but they are not so effective. Of these the various bland dusting powders such as zinc oxide, starch, or magnesium carbonate, are frequently used and may be rendered antiseptic by the addition of $I \%$ of salicylic acid, or antipruritic by adding $2 \%$ of camphor or $5 \%$ of thiol powder. They should be dusted freely on the diseased skin or pressed into it by a piece of cottonwool.

In this phase of the disease ointments are liable to prove irritating and even pastes are better avoided except when the lesions occur on the limbs.

Papular Phase.-In the papular phase itching is as a rule the most prominent symptom and is more intense in this type of eczema than in any other. Consequently the first indication in treatment is to allay the irritation and so remove the tendency to scratch. This may be accomplished by anti-pruritic lotions, one of the most useful being a mixture of I part of the Liquor plumbi subacetatis and 2 parts of the Liquor carbonis detergens, I or 2 teaspoonfuls of this to be added to a pint of water. In place of this the following may be substituted :

\begin{tabular}{|c|c|c|c|c|}
\hline R Liq. carb. deterg. & 3ii & & Ti opii & $3 \mathrm{ss}$ \\
\hline Liq. pb. subacet. & $3 \mathrm{ii}$ & or & Liq. pb. subacet. & $m_{\text {viii }}$ \\
\hline Glycerini & $5 \mathrm{ii}$ & & Aquam ad & $\lesssim \mathrm{i}$ \\
\hline Zinci oxidi & $\overline{3} \mathrm{ss}$ & & & \\
\hline Aquam ad & $\overline{3} \mathrm{vi}$ & & & \\
\hline
\end{tabular}

A more useful preparation, on the whole, is a liniment of the type of the Linimentum calaminae (see Appendix) with or without the addition of $\frac{1}{2}$ to $\mathrm{I} \%$ of carbolic acid. The liniment may be applied on pieces of lint, or may be gently rubbed in, the superfluity blotted off with a towel, and a dusting powder containing $2 \%$ of camphor freely applied.

When the acute irritation has been allayed the erythema and infiltration may be reduced by the employment of zinc pastes, which are especially useful about the limbs, such as equal parts of zinc oxide, starch, lanolin and vaselin. The ordinary B.P. zinc oxide ointment is not a good preparation unless it is freshly prepared as it is made of lard and is apt to become rancid and to develop irritating properties. The zinc paste may be rendered anti-pruritic by the addition of $\frac{1}{2}$ to $\mathrm{I} \%$ of menthol or carbolic acid, but these should not be employed over a long period as they are liable to inflame the skin.

Tar in its various forms combines an anti-pruritic and reducing action and may give brilliant results, but it does not suit every case and should be employed with caution at first. It may be added to the zinc paste in the form of half a dram of the oil of cade, or Io to $30 \mathrm{~m}$. of the Liquor picis carbonis to the ounce. Anthrasol is a useful substitute for ordinary tar, being colourless and non-irritating, and may 
be added in the same small quantity. Thiol is a sulphur preparation possessing a somerhat similar action and may be added to the paste up to $10 \%$.

Ichthyol is sometimes of value in papular eczema and may be prescribed conveniently in the following cream :

$\begin{array}{lllll}\text { Ichthyol - } & - & - & - & - \\ \text { Zinci oxidi } & - & - & - & - \\ \text { Lanolini } & - & - & - & - \\ \text { Olei amygdalae } & - & - & -\bar{j} \\ \text { Aquae calcis - } & - & - & -\bar{j} s s\end{array}$

Vesicular and Weeping Phase.-In the vesicular and weeping stage the obvious indications are to dry the discharges, to prevent their becoming septic, and to reduce the underlying inflammation so that they cease. It might be thought that the simplest way to do this was by applying powders to absorb the fluid, but this is unsatisfactory as they are apt to become caked up with the gummy discharges and to form thick crusts which too often become septic. On the whole the best results may be obtained by the continuous application of mild astringent lotions such as tar, lead lotion, or the well-known Burow's lotion of lead and alum (see Appendix).

Where the weeping is excessive benefit may be derived from drying the lesions with a piece of lint and painting them over with Liquor picis carbonis, or I to $5 \%$ solution of silver nitrate, or, on covered parts, with a I\% solution of methylene blue. After the painting a bland zinc paste spread thickly on the woolly side of lint, calamine liniment, or the excellent ointment recommended by Leslie Roberts, containing glycerine of the subacetate of lead $20 \mathrm{~m}$., zinc oxide I dram, lanolin to I oz., should be applied.

Instead of silver nitrate solution, 2 to so \% solution of resorcin in water may be employed or a $5 \%$ solution of ichthyol. The disadvantage of ichthyol is that it stains underclothing.

On no account should ointments be rubbed on weeping surfaces as the grease forms a coating which prevents evaporation, dams back discharges, and generally aggravates the irritation.

If the discharge becomes septic, antiseptic lotions such as I in 2,000 perchloride of mercury, I in 300 chinosol, boric lotion, or hydrogen peroxide 5 volumes, should be employed. Pieces of buttermuslin or linen soaked in one or other of these should be laid over the surface, but only for a short time as they have an irritating action on sensitive skins. After their removal a paste containing Io grains of ammoniated mercury should be applied.

Occasionally where great difficulty is experienced in preventing sepsis, distinct benefit may be derived from vaccine treatment, with the injection of 20 to roo millions either of a stock staphylococcic, or better of an autogenous, vaccine, and repeated in a week if improvement takes place. 
Crusted Phase. - Where the lesions have become dry and crusted over with scabs it is necessary to remove the crusts before attempting any specific treatment. One of the best methods of doing this is by properly applied boro-starch poultices, the starch being spread about an inch thick on buttermuslin, laid on the part, and renewed about every four hours, the softened crusts being wiped away at cach time of changing. When particularly hard they may be softened with olive oil previous to the application of the starch poultice. They may also be got rid of by boric compresses under oiled silk. After the removal of the crusts a mild antiseptic paste with $2 \%$ of ammoniated mercury or salicylic acid should be applied.

Chronic Scaly and Thickened Phases. - Where the patches are extremely dry and chronic, a stimulating rather soothing treatment is indicated, and ointments are specially useful. An ointment with a cold cream base and containing 2 to $4 \%$ of resorcin, ichthyol, lenigallol, or salicylic acid, and $30^{\circ}$ of zinc oxide, gives good results. It may be applied continuously on lint under a light bandage or in the form of a plaster. Where the patches are exceptionally chronic and resistant it is an advantage to stimulate them daily by rubbing them with soft soap and water followed by the application of diachylon ointment.

Tar is a useful remedy for reducing chronic thickening and may be employed in the form of a paste or plaster containing 2 to $3 \%$ of Liq. picis carbonis or 6 to $12 \%$ of oil of cade. Such stimulating treatment should be carried out actively and intermittently, the part being allowed to rest under soothing applications between times.

Where the patches are markedly lichenified and itchy, improvement can be obtained from cxposure to the X-rays, a pastille dose being given and repeated in a month if necessary. Such cases have also been treated successfully by short exposures to radium employing halfstrength applicators with a large surface.

In chronic irritable patches about the leg where the surface is unbroken an extremely useful application is zinc gelatine paste painted on as hot as can be borne (see Appendix). Before applying it the skin should be bathed with boric lotion to ensure its being aseptic. By this means the diseased skin is corered by an occlusive dressing which does not quite prevent eraporation and which should last about a week. Instead of the zinc gelatine, a varnish base (see Appendix) may be substituted and rarious remedies such as resorcin $2 \%$, or ichthyol $2 \%$, incorporated with it.

\section{Regional Types of Eczema And their Treatment}

The variation in type of the lesions of eczema in different situations is brought about by such considerations as, (I) one region being exposed to the atmosphere, light, etc., while another is protected by clothes; (2) certain parts of the skin being subjected to friction from clothes or the rubbing together of contiguous surfaces; (3) in certain situations, 
such as the perineum, the skin being apt to be kept moist and irritated by discharges; $(4)$ the skin in certain parts of the body being thiclier and tougher than in others; (5) the hair-follicles being large in one region and small or absent in another; (6) the sweat-glands being more numerous, larger, and perspiration more profuse, in certain situations.

It is important to remember, however, that, although the clinical types vary in different situations, they are all phases of one disease, and that their treatment is based on the general principles already described but modified by special circumstances.

Face.-The acute ery thematous type of eczema is especially common on the face which, as a rule, is stmmetrically and extensively affected,

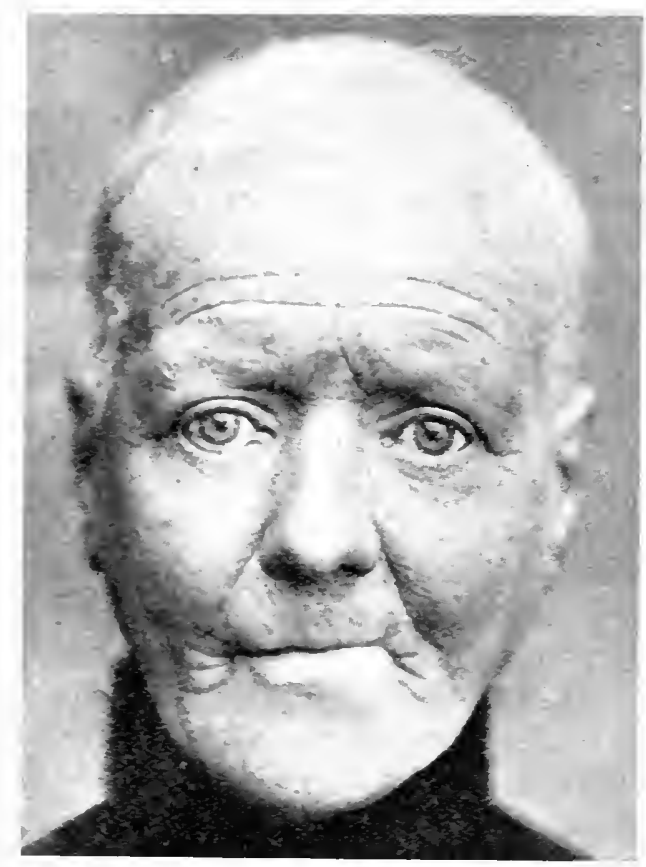

FIG. 273.-Chronic Eczema with loss of hair and thickening of the skin.

the cheeks, forehead, and nose being the parts chiefly involved. There the skin becomes red, tense, oedematous, the erelids swollen, puffy, and difficult to open, and the inflammation sometimes so severe as to resemble erysipelas. In the most acute cases the inflamed areas become covered with resicles, which break leaving a weeping or crusted surface. This acute phase may be cured under suitable treatment and the skin resume its natural appearance, or it may pass into a chronic conditicn in which the skin becomes thickened, harsh, the natural folds deepened, and a leonine appearance produced not unlike that of leprosr. (Fig. 273.)

In serere cases it may affect the whole of the face and the exposed parts of the neck, in milder cases it may be restricted to localised patches of an errthematous, papular, or vesicular character. 
Facial eczema may be not only a primary condition but may be secondary and the result of rubbing and scratching patches of seborrhoic dermatitis or impetigo or of the friction caused on the forehead by the brim of a hat.

The itching and burning in acute cases is severe and tends to come on in spasms which sometimes appear to begin at, and spread from, the bridge of the nose.

Treatment.-The treatment varies according to the type of case, acute cases require sedative treatment while chronic cases demand stimulating applications.

In acute cases where the whole face is involved it is advisable to confine the patient to bed for a day or two in a cool room at an equable temperature, and to protect the face as much as possible from the actinic rays of the sun by red blinds, red curtains, etc. A saline purge should be given, a light diet prescribed, and if the tension be great it may be reduced, in young adults, by small doses of antimony. If crusts be present they should be removed by almond oil containing $2 \%$ resorcin and applied on a lint mask, or by the judicious use of boro-starch poultices. It is inadvisable to attempt to remove them with soap as this is apt to aggravate the disease.

When the face has been cleared in this way the erythema and tension should be reduced by the continuous application of lead lotions on lint masks, which should be kept moist but should not be corered with oiled silk. The most generally useful formula for the lead lotion is Liquor piumbi subacetatis, a teaspoonful to half-a-pint of water. The glycerine preparations of lead should be used with caution on the face as they are liable to irritate. During the first twenty-four hours it is advisable to keep the mask on continuously except at meal times, when it may be taken off, the face mopped dry, and a bland dusting powder applied. It is important that the face should be dry before putting on the powder, in order to prevent caking.

Where the skin is dry and markedly inflamed, the occasional application of a $2 \%$ aqueous solution of ichthyol may be of benefit in reducing the inflammation, and where there is any tendency to weep, Burow's solution or tar lotion may be substituted for the lead.

Calamine lotion is frequently employed in acute eczema of the face, but though pleasant and cooling when first applied, it is liable to deposit and form a cake on the skin and to render the surface dry and drawn.

After a few days the acute stage generally subsides and the further treatment consists of the application of a bland zinc paste made just sufficiently thick to spread easily. If there be marked itching the following ointment is pleasant and soothing, Liquor picis carbonis Io m., zinc oxide 2 drams, and cold cream I oz. This should be spread on lint and applied continuously, being kept in place by a thin mask of buttermuslin. In this way the skin is gradually brought back to its normal condition. 
As a rule a certain amount of roughness succeeds the eczenia and the skin tends to become harsh and to peel; to obviate this the occasional application of cold cream or a lotion containing equal parts of glycerine of starch and rose-water is of service.

During the acute stage and for some time after the attack has subsided it is inadvisable to wash the face with soap and water but it may be cleansed with equal parts of milk and warm water.

An attack of eczema of the face produces a certain vulnerability and delicacy of the skin which renders it liable to flare up from slight irritation, consequently it is important to protect it from local irritation and to avoid unnecessary exposure to sunlight, cold winds, excessive heat, etc. In the case of women the best protection against the actinic rays of the sun is afforded by red sunshades, broad-brimmed hats lined with red, brown veils, and the free use of dusting powders tinted by turmeric and Armenian bole.

In the more chronic and infiltrated types of facial eczema the treatment should be stimulating, and consists of the application of zinc ointments made with a cold cream base and containing $2^{\circ}$ of salicylic acid, ichthyol, or resorcin. Where the itching is marked, relief may be obtained by the addition of $\mathrm{I} \%$ of carbolic acid or $2 \%$ Liquor picis carbonis to the ointment.

In circumscribed infiltrated patches an occasional stimulation of the skin, by rubbing it with soft soap and hot water followed by the application of zinc paste, is of value but requires to be done with caution.

Lips.-Eczema may occur on the lips in association with lesions on the face or independently. In acute cases the lips become swollen and oedematous, while, in chronic cases they become dry, inflamed, and scaly, and generally present cracks at the commissures of the mouth or vertical fissures. It may be confined to the upper lip when it is not infrequently secondary to an impetigo caused by a discharge from the nose. On the lower lip it may follow impetigo, especially that form known as perlèche, or it may be due to the local irritation of smoking cigars or cigarettes, or of some tooth-powder.

Treatment.-The treatment is difficult, as a rule, owing to the constant movements of the mouth in eating and speaking and the irritation of the saliva. When the eczema is a secondary condition it is important to discover the underlying cause and to deal with it by suitable means. If there be nasal discharge every effort should be made to stop it; where it is superimposed on impetigo, antiseptic applications are indicated; where cigarettes, cigars, or a tooth-paste are responsible they must be prohibited. So long as it continues it is advisable that liquid food should be sucked through a straw or quill.

Fissures should be painted with a solution of silver nitrate $3 \%$ or with compound tincture of benzoin, or $2 \%$ zinc sulphate dabbed on continuously, the mouth being opened wide to allow the astringent to reach the bottom of the fissure. 
When the lips are scaly and cracked the $\mathrm{X}$-rays are beneficial, a pastille dose being given and followed, if necessary, by another in the course of a month.

Where the skin is simply dry and cracked and has lost its pliability a useful application is glycerine of starch with the addition of $2 \%$ resorcin or one or other of the lip salves which generally have white wax and almond oil as a base.

Nose and Nostrils. - In extensive cases of eczema of the face the nose is generally involved and the various phases of eczema may occur on it. It is not uncommon for eczematisation to take place at the edges of the nostrils and adjacent mucosa in connection with chronic nasal catarrh or septic folliculitis about the hairs inside the nose.

Treatment.-The treatment consists of the removal of the crusts by softening them with almond oil containing $2 \%$ resorcin, healing the fissures by painting them with a solution of silver nitrate $2 \%$, followed by the application several times a day of a mild antiseptic ointment containing $2 \%$ of ammoniated mercury.

Where there is nasal catarrh it should be dealt with by astringent measures, such as $2 \%$ tannic acid introduced on a tampon or, where the condition is definitely septic, the nose should be douched with hydrogen peroxide Io volumes. Should this be ineffective, improvement may be obtained from cataphoresis with zinc ions.

Moustache and Beard.-Eczema is not uncommon on the beard and moustache in association with a widely distributed attack on the face. It may also be confined to these regions and be secondary to seborrhoic dermatitis or impetigo and, where the moustache region alone is involved, it is generally preceded by nasal catarrh. It is rarely limited to the hairy parts but generally extends on to the cheek or down the neck.

The clinical appearances vary considerably in different cases, the skin in one being dry, inflamed, and scaly, in another exuding and crusted. Though it is a superficial affection and does not involve the follicles like sycosis, it is almost as intractable.

Treatment.-The treatment consists of the removal of crusts by clipping the hair very short and applying boro-starch poultices or lead compresses.

On the whole, unless there is weeping, it is advisable to shave and this may be done satisfactorily and harmlessly by using an antiseptic cold cream or calamine liniment instead of soap. After shaving, oozing spots or cracks should be touched with a weak solution of silver nitrate and a weak zinc ichthyol ointment or paste subsequently applied, such as ichthyol to m., zinc oxide 2 drams, and cold cream to the ounce.

Occasionally where the lesions are singularly resistant the application of a mild tar ointment containing 4 to $6 \%$ of oil of cade is of service.

Where the moustache is attacked sccondarily to anterior rhinitis the treatment consists, in the first instance, of the removal of the cause 
by astringent douches, such as $\mathrm{a} \mathrm{I}_{\%}^{\circ}$ solution of permanganate of potash, alum, or tannic acid, or of cataphoresis by zinc ions.

Eyebrows.-The eyebrows are often extensively affected when eczema attacks the face, and generally symmetrically. Occasionally seborrhoic dermatitis may occur in the eyebrows and become eczematised, in which case no other eczematous lesions may be present. When attacked they become red, swollen and scaly, or weeping and crusted, and the hairs are liable to come out or to become short and irregular.

Treatment.-The treatment is similar to that described for eczema of the beard, except that it is unnecessary to clip the hairs short, and consists of the removal of the crusts, stopping the weeping, and the application of a healing salve containing resorcin or ichthyol.

Eyelids.-The eyelids may be involved and in acute cases exhibit a characteristic puffiness and swelling making it difficult for the patient to open the eyes. Occasionally the eyelids alone are affected as the result of rubbing a sensitive skin or in connection with septic blepharitis.

Treatment.-The treatment consists of the removal of the crusts and the application of a mild antiseptic ointment or cream containing $2 \%$ of ammoniated mercury or ichthyol.

Auricles.-Eczema frequently attacks the auricles and the external auditory canal. It may be part of an extensive eczema of the face, when both ears are usually affected, or it may occur in one or both ears as a secondary phenomenon superimposed on impetigo spreading on to the ears from the scalp, or be due to a septic discharge from the meatus in which case the lower part of the auricles and the retroauricular furrow are generally attacked. Eczema is not uncommon also behind the ear secondary to a septic intertrigo, when it is generally associated with fissures at the post-auricular groove and at the junction of the lobe and the face. Occasionally eczema of the lobe has been known to follow septic inoculation in piercing the ears for ear-rings. In certain individuals the ears are liable to attacks of acute eczematous dermatitis from the action of the actinic rays of the sun. (Fig. 274.)

The various phases of eczema may be found on the auricle. In one case the ears may be prominent, swollen, tense, and of a reddishpurple tinge, or cracked, oozing and crusted, in another they are thickened, red, dry, and scaly, while in a third only the lower portion and the lobe and the skin behind and around it may be affected.

Treatment.-The treatment of acute eczema of the auricles consists of the application of boro-starch poultices for the removal of crusts, astringent lotions like lead or hydrogen peroxide 5 vol. to relieve the irritation, silver nitrate solution to heal the cracks, and a soothing zinc-ichthyol paste to reduce the inflammation.

Where the eczema is connected with a discharge from the auditory meatus, or where small furuncles or crusts are present in the auditory canal, these must first be dealt with. The crusts should be softened with almond oil containing $2 \%$ of resorcin, and the canal syringed out with a $3 \%$ solution of bicarbonate of soda. Where there is much 
catarrh, benefit may be derived from the insufflation of a powder containing equal parts of zinc oxide, boric acid, and tannic acid, or from a pad dipped in $2 \%$ solution of zinc sulphate and inserted into the meatus. After the discharge has stopped zinc-ichthyol paste should be applied.

Where the eczema is secondary to septic intertrigo or to impetigo of the scalp, mild antiseptic lotions and ointments are indicated, and where small pustules are present these should be incised, and the canal syringed with boric lotion. Occasionally in chronic scaly eczema of the ear the application of a stimulating ointment containing $2 \%$ of resorcin or oil of cade is of value.

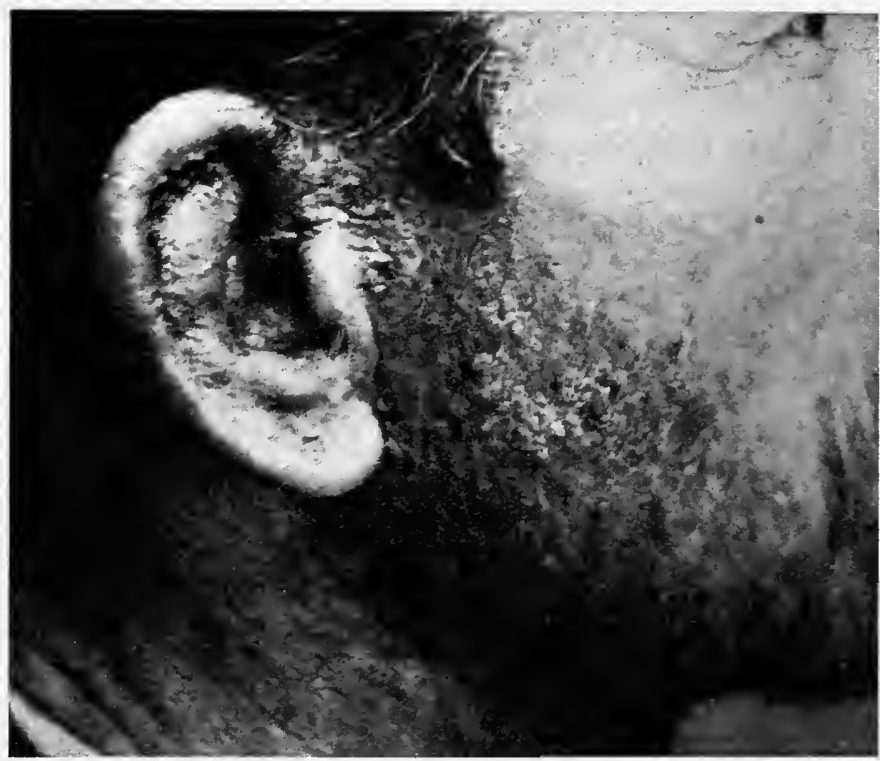

FIG. 274.-Eczema of Auricle and Beard Region.

Where the eczematous condition results from exposure to the sun, the treatment is similar to that of acute eczema and the greatest care must be taken to protect the skin from future attacks by the various means described under the heading of Solar dermatitis. Occasionally a type of eczema occurs in which the auricles are very slightly inflamed but are unusually itchy, the itchiness extending up the external auditory canal. In those cases an ichthyol cream with the addition of $\frac{1}{2} \%$ of carbolic acid gives relief.

Scalp. - The scalp is rarely affected with eczema unless the face and neck are extensively involved, and even an acute attack of facial eczema may not spread much beyond the hair-margin. On the other hand, especially in children, it may complicate a scaly impetigo or be secondary to a crusted impetigo chiefly due to pediculi, the eczematisation being largely the result of scratching and rubbing. Acute eczema of the scalp is not infrequently the result of the applica- 
tion of some injurious hair-wash or dye or the too free use of irritating soap.

In acute cases the affected skin is red and oedematous, and may present patches of papulo-resicles on an inflamed base which tend to be broken and ooze; in chronic cases the skin becomes dry, infiltrated, and scaly. If the inflammatory process be severe the hair-growth may be interfered with and permanent thinning result but, as a rule, the hair grows again when a cure is effected. The eczematisation in secondary cases is not generally limited by the hair-border but extends on to the glabrous skin of the face or neck.

Treatment. - In nearly every case the first indication in the treatment is the removal of the crusts. Where they are extensive it is simplest, except in women, to cut the hair off and this is especially the case in children when nits and pediculi are present. Where it is decided to spare the hair the nits may be removed by the help of a small-toothed comb, after softening them by alcohol, dilute acetic acid, or turpentine. After their removal and the destruction of the pediculi by turpentine, the crusts may be dealt with by the application of lint saturated in olive oil, or in I part of eucalyptus oil to 4 of olive oil. covered with a bathing cap or a piece of oiled silk, or by boric com presses under oiled silk, boro-starch poultices, or cold cream containing 2 or $3 \%$ of mercury and salicylic acid, and subsequently washed away with soap and hot water. Any weeping surface should then be astringed by painting with silver nitrate solution. According to the amount of inflammation,present and the chronicity of the eczema, the treatment must be either sedative or stimulating.

It is inadvisable to use thick ointments or pastes on the scalp as they are liable to become caked up and cause matting of the hair. The best bases are cold cream, or equal parts of lanolin and olive oil, and with these may be incorporated $2 \%$ of resorcin, salicylic acid, ichthyol, ammoniated mercury, or tar. Where ointments are being used the head should be washed about once a week with soft soap or soap spirit lotion and hot water.

Where there is much scaliness the following ointment is of value, sulphur precipitate 30 grains, ammoniated mercury 5 grains, bergamot oil 3 grains, vaselin to the ounce.

After the acute stage has passed and only slight scaliness is left, a non-greasy brilliantine may be employed with a base of castor oil I drm., to methylated spirit I ounce, to which may be added $2^{\circ}$ o resorcin, or in those with fair hair where it is inadvisable to use resorcin on account of its staining property $2 \%$ salicylic acid may be substituted. Occasionally in chronic cases a more stimulating ointment is required, and one containing $6 \%$ of oil of cade may be used.

Nape of the Neck.-The nape of the neck is a frequent site fcr eczema especially in the form of chronic lichenified patches in adults. It may be produced also by the chafing of frayed linen collars, and in children is frequently secondary to impetigo of the occipital scalp. It 
may be acutely, inflamed, oedematous and oozing, but is more commonly of the chronic type, excoriated from scratching, or infiltrated and lichenified from rubbing. The itching is sometimes intolerable, especially at night, and leads to incessant rubbing even when half asleep.

Treatment.-In acute cases the indications for treatment are similar to those described in conncction with acute eczema elsewhere on the skin.

In the case of chronic lichenified lesions the difficulties of relieving the itching are considerable. Creams, pastes and ointments containing I \% of menthol, carbolic, or cocain, give temporary relief but are usually followed by an exacerbation of the pruritus when their influence wears off. Occasionally benefit is derived from rubbing the patches with soap and hot water followed by the application of a bland zinc paste, or by employing a thick ointment containing $6 \%$ of oil of cade or $3 \%$ resorcin. Greasy preparations of any kind may prove irritating and better results may be obtained by lotions containing lead, tar, or opium, continuously applied on lint for an hour or more and followed after drying by a dusting powder.

In the lichenified type and in nearly all chronic cases the best treatment is X-rays, a pastille dose being given in the first instance and followed if necessary by another in the course of a month.

It is important that the neck should not be irritated by stiff linen collars and a silk collar or scarf should be substituted, while in women it should be protected from exposure to the actinic rays of the sun, and furs should not be worn.

Trunk.-All phases of eczema may be met with on the trunk but the papulo-vesicular type going on to the formation of weeping, crusted, or scaly patches is the most common. It may occur as isolated lesions or may involve large areas, but is rarely universal, and patches of normal skin are generally present. It frequently shows a predilection for natural folds, such as the groins, axillae, or beneath the breasts in stout women-situations exposed to the friction of clothes-and the eczema is apt to become fissured, to weep, and occasionally to bleed. It may occur on the breasts and may affect the areolae of the nipples, producing an intractable fissured type of lesion with an ill-defined border and covered with scaly or yellowish crusts. It is occasionally present about the umbilicus, and in a few cases may be confined to the lumbar region or the buttocks.

The acute stage is usually succeeded by a chronic, erythematous, scaly condition and in old-standing cases the skin tends eventually to become infiltrated, harsh, and lichenified.

Treatment.-The treatment varies according to the type and extent of the lesions.

When the eruption is acute and widely distributed it should be ticated on the principles laid down in connection with the management and treatment of an acute attack of the disease ; confinement to bed, light food, and proper nursing, are essential. 
The local treatment should consist of the removal of crusts by boro-starch poultices, the astringing of weeping surfaces by painting them with $\mathrm{I} \%$ methylene blue, or a weak solution of silver nitrate, and the application of calamine liniment or other soothing cream, which should be rubbed into the skin, the superfluity dried up, and a dusting powder applied.

One of the most intractable and itchy types of eczema on the trunk is the papular variety, which may be widely distributed or may occur in circumscribed patches. The intense irritation may be relieved by the application of lead, tar, opium, or carbolic lotions. The following lotions are useful in this connection :

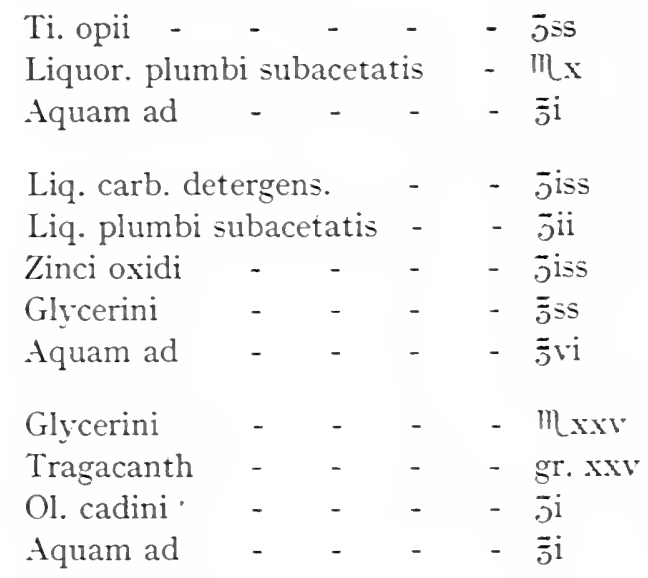

After the lotion or liniment has dried into the skin a dusting powder containing $\mathrm{I} \%$ of camphor should be applied freely. Emollient baths containing starch or bran are sometimes recommended, but on the whole are better aroided.

For isolated patches an ointment containing small quantities of tar generally gives relief.

Where an ambulatory form of treatment is permitted it is of great importance that the underclothing should be suitable, being neither hot nor irritating, and cotton, fine linen-mesh, or silk and wool, should be substituted for woollen underclothes.

Umbilicus. - When the umbilicus is the seat of eczema it is generally as part of an extensive attack, though it may be secondary to impetigo or Seborrhoic dermatitis. It generally forms a well-defined patch extending for some distance around, suggesting Paget's disease but differing from it in that there is no infiltration.

Treatment.-Any septic material or crusts should be remored by boric lotion or almond oil, and a soothing zinc paste should then be applied containing I or $2^{\circ}$ o of ammoniated mercury.

Groins.-Eczema rarely affects the groins alone, unless in the form of eczematisation secondary to impetigo, furunculosis, ringworm, and scratching; in such cases a microscopical examination should be made 
for ringworm fungi, etc., as it may be difficult to determine the cause from the clinical appearances alone. Eczema may occur in this situation as part of an extensive attack and is liable to be moist, weeping and crusted, with occasional fissures.

Treatment.-The treatment consists of the cleansing of discharges, painting of the fissures with silver nitrate solution $2 \%$, and the application of zinc paste with the addition of $2 \%$ salicylic acid.

Axillae.-The axillae are attacked in much the same way as the groins and may be affected with eczema in association with eczematous patches elsewhere, or may be the sites of eczematisation superimposed by scratching on impetigo, scabies, or ringworm. Occasionally patches of lichenification develop in the axillae, chiefly in women, which through scratching may become eczematised.

Treatment.-The treatment consists of the removal of crusts and discharges by boric lotion, boro-starch poultices, and other suitable methods. Any weeping surfaces should be astringed by the application of a $2 \%$ solution of zinc sulphate or silver nitrate, and a mild antiseptic paste applied, such as zinc paste with $2 \%$ ichthyol or salicylic acid, spread on lint and kept in position by a lady's dress-preserver with a pad of cottonwool between the flaps and fixed by a bandage.

Breast.-Eczema may affect the breast in women in association with lesions elsewhere on the trunk, or may occur independently in that situation, and be secondary to impetigo or intertrigo. In nursing mothers the nipple and areola may be involved from suckling and from excess of milk, and may become red, oozing, crusted, itchy, and associated with enlarged axillary glands. When it affects the areola of the nipple it may closely resemble Paget's disease, but this rarely occurs except between the ages of 40 and 50 and even in its early stages is generally indurated.

Treatment.-The treatment consists of the removal of crusts by suitable applications, painting the fissures with silver nitrate solution or tincture of benzoin, and the application of zinc paste or diachylon ointment.

When the folds beneath the breasts are involved the part should be cleaned with boric lotion and calamine cream applied followed by a dusting powder. Should the eczema be associated with an excessive flow of milk it is practically impossible to effect a cure without weaning the child and the various devices which have been introduced in the form of protective rubber teats are ineffective.

Genitals.-The scrotum and penis are not infrequently affected with eczema.

When the scrotum is involved it tends to become oedematous, swollen and cracked, with marked cellulitis and deepening of the normal furrows, sometimes suggesting elephantiasis. On the other hand it may be squamous and the skin thickened, excoriated and crusted.

When the penis is attacked it becomes oedenatous, swollen, and red. The itching is intense, spasmodic, interferes with sleep, and 
often produces mental depression and anxiety. It is usually part of a more generalised attack but occasionally may be the only situation affected, in which case it is generally the result of some local irritation such as friction, overheating, or the action of sugar in the urine.

Treatment.-For the intense itching, the application of a sponge wrung out in water as hot as can be borne generally gives relief. Astringent applications, such as weak silver nitrate solution, are necessary to stop weeping, followed by the application of pieces of lint saturated in calamine liniment, or zinc-ichthyol paste spread on lint, and kept in position by a suspensory bandage. Any local cause should be most carefully sought for and dealt with, such as glycosuria, or the presence of the fungus of ringworm or the acarus of scabies.

Occasionally in the dry, infiltrated type of case where the skin of the scrotum is thickened, a stimulating treatment is desirable, such as the application of ointments containing 2 or $3 \%$ of ichthyol, resorcin, or tar.

Vulva.-The vulva may be affected either by an extension of a widely distributed attack of eczema, or as the result of rubbing and scratching for the relief of Pruritus vulvae, the chafing of badly fitting clothes, or the irritation of vaginal discharges or urine containing sugar. It may be confined to the vulva or may spread on to the pubes or thighs. When it is affected the labia may become oedematous and swollen or markedly thickened and infiltrated, and may be dry on the surface, or fissured, weeping, or crusted. The itching is intense, spasmodic, and liable to interfere with sleep.

Treatment.-The treatment consists of the removal of crusts, the cleansing of septic discharges, the astringing of weeping, and the application of sedative antiseptic pastes in the acute type, and of more stimulating remedies, such as pastes containing 2 or $3 \%$ of oil of cade, in the chronic infiltrated variety.

When it is secondary to Pruritus vulvae it is essential to discover the underlying cause and to deal with it by suitable means.

Where itching is the most severe feature, relief may be obtained by sponges wrung out in hot water and followed by the application of calamine liniment containing $I \%$ carbolic acid, or of the following lotions, Liquor plumbi subacetatis I dram, glycerine I dram, hydrocyanic acid $\frac{1}{2}$ dram, lime water 2 ozs., rose water to 6 ozs., or Ti. opii m. xv, Sod. bicarb. gr. xv, Pot. bicarb. gr. x, Glycerin. in. xii, Aq. dest. ad jँi (heat before use). After the acute stage has passed zincichthyol paste may be substituted.

Anus.-Eczema may occur about the anus and inter-gluteal furrow, sometimes extending back as far as the end of the sacrum. The affected skin becomes moist, sodden, and fissured, and from incessant scratching may become thickened and rugose. In this region it is usually a secondary condition and in adults is the result of scratching for the relief of Pruritus ani and in children in connection with thread-worms. It may occur independently of pruritus, however, in association with 
eczema elsewhere or with inflamed lichenified patches on the lower part of the back.

Treatment.-As it is generally the result of scratching for the relief of Pruritus ani, no treatment is likely to cure it which does not eliminate the cause of the pruritus.

The local treatment consists of the removal of crusts and discharges by boric lotion, followed by the application of zinc paste to which $2 \%$ of Liquor picis carbonis has been added. The paste is soothing and protective and prevents the contiguous surfaces of the buttocks from chafing.

Where the affection has become chronic and the skin infiltrated, more drastic measures are necessary, such as the application of diachylon ointment and soft soap equal parts, or an ointment containing 2 to $5 \%$ of oil of cade or tincture of benzoin. In such cases the $X$-rays are often of great value both in reducing thickening of the skin and allaying itching.

For the relief of the Pruritus ani the various curative measures described under that heading are applicable, such as a sponge wrung out in very hot water, or suppositories containing opium, collosol silver, or belladonna. A mustard plaster over the lumbar enlargement is also sometimes of value.

Where the whole perineal region is crusted or scaly a sitz bath containing a small quantity of cyllin is sedative and cleansing.

Arms.- The various phases of eczema may occur on the arms, and one or both may be affected. When it is in an acute form the arms may become swollen, inflamed, and sometimes moist, weeping, or crusted, or when more chronic in type it takes the form of circumscribed, scaly, infiltrated patches frequently situated on the extensor aspects. The anti-cubital spaces are also favourite sites as the skin there is thin and subjected to considerable friction in the movements of the elbows. The forearms are apt to be involved in the eczematous forms of trade dermatitis and generally in association with the hands. In old chronic cases the skin is liable to become thickened and harsh.

Treatment.-The treatment is similar to that for the corresponding stages of the disease elsewhere and consists in acute cases of the removal of scales and crusts by boro-starch poultices, the astringing of weeping surfaces by silver nitrate solution, and the continuous application of zinc-ichthyol paste spread on lint under a bandage. Where the skin is thickened, reducing remedies such as ointments containing resorcin or tar, should be prescribed and where the patches are definitely lichenified great benefit is derived from the X-rays.

Hands.-The hands are frequently affected with eczema, sometimes symmetrically but as a rule one more than the other, and there is no part of the body where the practical difficulty of curing the disease is greater. All the different stages of eczema may be met with, from an acute weeping dermatitis with oedema, small vesicles on the backs of 
the hands and between the fingers, and large deep-seated resicles on the palms, to a more chronic papular type forming irregular excoriated patches, or an erythematous variety which is dry, wrinkled, scaly and fissured, the fissures being chiefly present at the natural folds. In some cases, owing to an increase in the horny layer, the skin of the palms becomes whitish-yellow in colour and so thichened and inelastic as to interfere with the movements of the hands and keep the fingers in a semi-flexed position, and deep gaping cracks may form on the surface. The subjective symptoms consist of itching and burning, which may be severe and troublesome. (Fig. 275.)

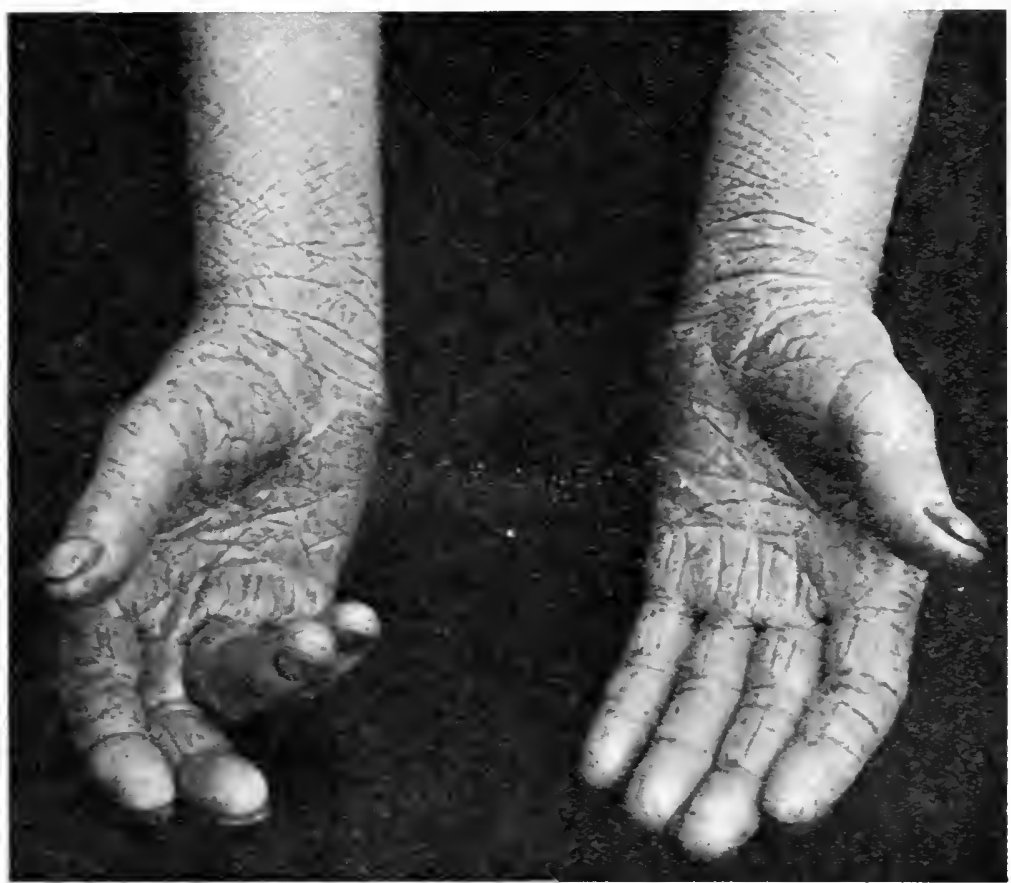

FIG. 275.-Palmar Eczema, skin thickened.

All forms of local irritation to which the hands are subjected may determine an attack of eczema, or it may occur in association with eczematous patches elsewhere, or be secondary to parasitic conditions such as impetigo, ringworm, or scabies. Occasionally it occurs on the finger-tips and on the skin around the nails as a result of certain occupations necessitating the immersion of the fingers in irritating solutions, as in developing photographs. (Fig. 2-6.)

The affection known as dysidrosis is closely allied to eczema and is possibly a variety of it, but as there is some difference of opinion on this subject it will be discussed under a special heading (see p. So j).

Treatment.- The treatment varies according to the phase of the disease. In the acute and weeping forms the hands should be bound up with boro-starch poultices or with pieces of lint soaked in Burow's 
solution to remove the crusts and astringe the surface, after which zinc-ichthyol paste or a paste containing $2 \%$ of Liquor plumbi subacetatis should be spread on lint and applied continuously day and night under a bandage, or rubbed into the skin, a piece of gutta-percha tissue pressed down upon it, and cotton gloves worn. The dressing should be changed twice daily and if the paste has become caked it may be softened and removed by almond oil.

For the treatment to be successful it is essential even in mild cases that the hands should be protected from irritants which may have caused the disease or are likely to perpetuate it, and one of the chief difficulties is that it frequently occurs in individuals who have to expose their hands to irritants in obtaining a livelihood and that

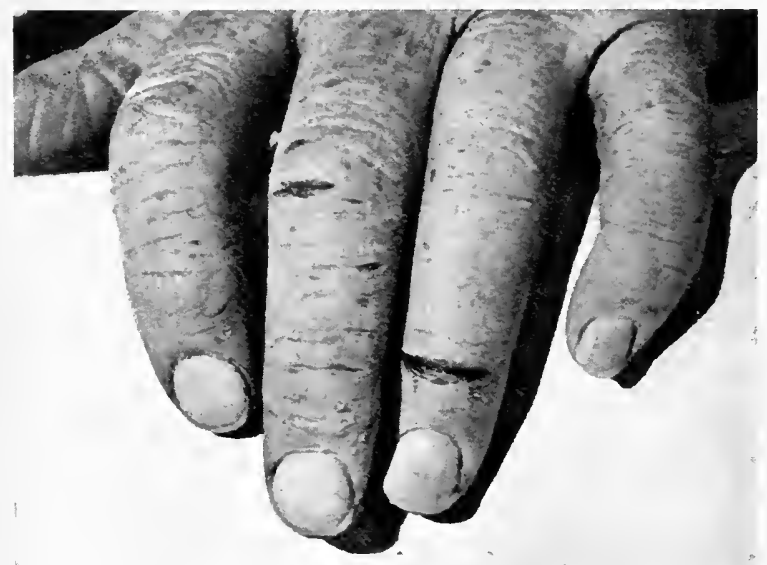

FIG. 276.--Eczema with Fissures.

even when it has been apparently cured a return to the same work is liable to cause a recurrence.

Unless in chronic cases, washing the hands with soap and water is harmful and apt to cause considerable irritation.

In chronic cases a more stimulating treatment is necessary, such as the application of ointments containing 2 or $3 \%$ of ichthyol or resorcin, the Unguentum plumbi vaselini with the addition of $2 \%$ salicylic acid, and in very resistant patches an ointment containing 2 or $3 \%$ chrysarobin followed by a bland paste. Fissures should be painted with 2 or $3 \%$ silver nitrate solution in water or in spirit of nitrous ether, or with tincture of benzoin.

Where the palms are markedly thickened and cracked it is essential to reduce the thickening in order to heal the cracks. In such cases it is useful to scrub the skin with soft soap or to dab on $20 \%$ solution of caustic potash, followed by the application of 2 or $3 \%$ salicylic acid ointment spread on lint under a bandage, ro \% salicylic acid in collodion or traumaticin, or salicylic soap plaster. Oxidised pyrogallol in the following ointment has been recommended by Jamieson, oxidised 
pyrogallol gr. $\mathbf{x}$, salicylic gr. $\mathbf{x}$, lanolin $\bar{j} \mathrm{i}$, vaselin $\bar{j}$ vii, and is a useful preparation, it has the disadvantage of blackening the skin but this gradually diminishes as the tissue heals. The most serviceable method, both for reducing the thickening of the palms and for closing the fissures, is the $\mathrm{X}$-rays, a full pastille dose being given and repeated in a month if necessary.

In cases where the skin around the nail becomes frayed, torn, and difficult to heal, the cracks should be painted with tincture of benzoin and the frayed edges plastered down with collodion.

Where the fissures are superficial, as in " chapped hands" which is simply a mild form of eczema due chiefly to the irritation of water

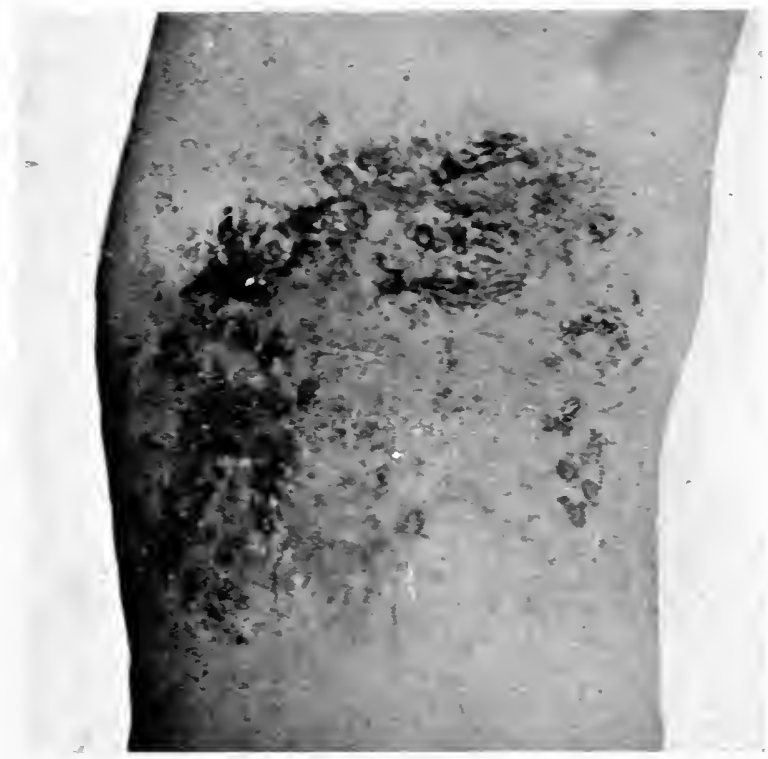

FIG. 277.-Eczema of Popliteal Space.

and soap, or to exposure of the hands to cold, the following application is useful, lanolin I dram, soft white paraffin 3 drams, glycerine 4 drams, camphor $\frac{1}{2}$ dram, or equal parts of glycerine, alcohol, and tincture of benzoin.

Legs.-Any part of the leg may be the site of eczema and in severe cases the whole limb may be affected. It may take the form of acute weeping, or chronic scaly, patches and is frequently present in the popliteal spaces, where the papulo-resicular type leading to chronic scaly lesions predominates and where, from movement and scratching, fissures and weeping excoriations are of common occurrence. It occurs also in the perineal region and about the groins, either as part of a widely distributed eczema or as a local eczematisation which has been superimposed on patches of ringworm or impetigo. (Fig. 277.)

Another farourite situation is the leg below the knee. There it may be associated with raricose veins and may give rise to the con- 
gested condition of the skin known as "varicose eczema," in which it is reddish-purple in colour, oedematous, and sometimes moist or crusted. (Fig. 278.)

The leg may be extensively affected and be red, swollen, moist, or cracked and covered with septic crusts (Eczema rubrum). This

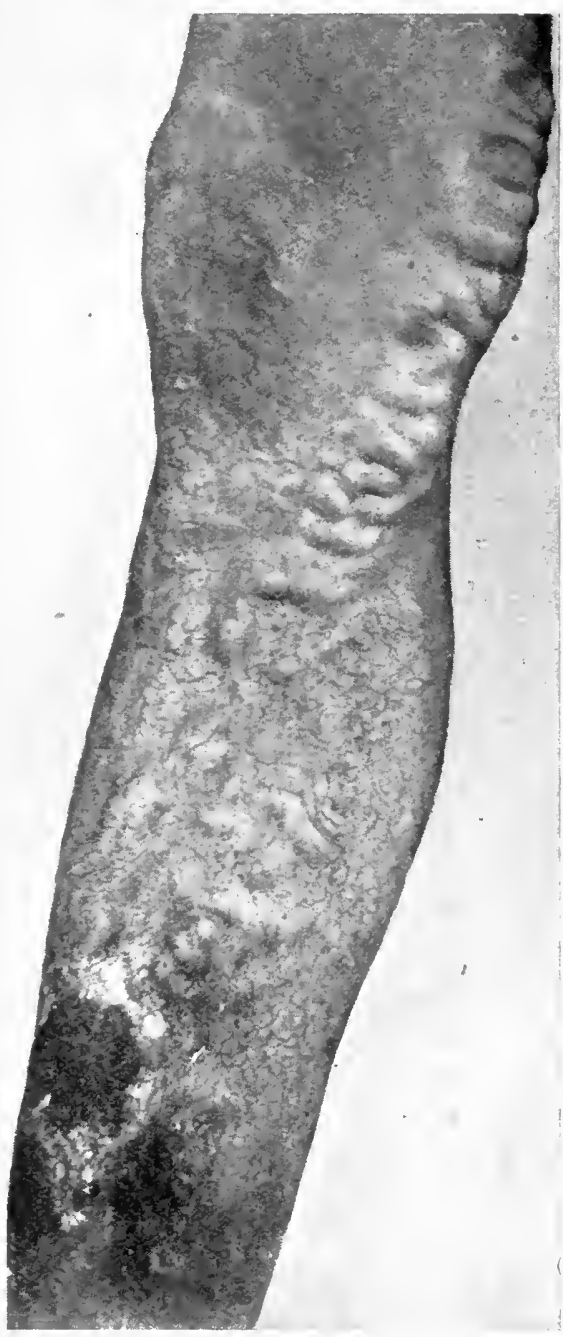

FIG. 278.-Varicose Dermatitis. condition is met with chiefly in elderly people, and as a rule affects only one leg or one more severely than the other. In old-standing cases of this type secondary septic inoculation may cause suppuration or ulceration, or may set up recurrent erysipelatoid attacks which result in fibromatous changes and finally in elephantiasic thickening. (Fig. 279.)

Occasionally chronic lichenified patches of eczema may occur about the leg from incessant rubbing for the relief of localised irritation.

Treatment.-The treatment is similar to that described for the trunk. In lichenified patches benefit is derived from exposure to the $\mathrm{X}$-rays. In the popliteal spaces the treatment is difficult, owing to the friction in walking and to their liability to be scratched. Crusts should be removed bystarch poultices, weeping astringed by painting with methylene blue solution $\mathrm{I} \%$, and fissures healed by silver nitrate solution $3 \%$, followed by continuous application of zinc-ichthyol paste, or by an ointment consisting of Oleum betulae, ichthyol, and salicylic acid, I part of each, oil of cade 5 parts, benzoate of lard 25 parts. After healing has taken place and the surface is quite dry, muslin bags filled with dusting powder may be worn beneath the underclothing to diminish the friction, or still better the patches may be painted with zinc-ichthyol gelatine. The paint should be put on as hot as can be borne and the consistency strengthened by dabbing the surface with cottonwool. It should be 
left on as long as possible and when it wears off in the course of a week it should be renewed.

In Eczema rubrum it is advisable for the patient to lie up, as otherwise it is difficult to obtain healing on account of the congestion and oedema which result from the dependent position and from walking. The treatment should be begun by boro-starch poultices to clean the

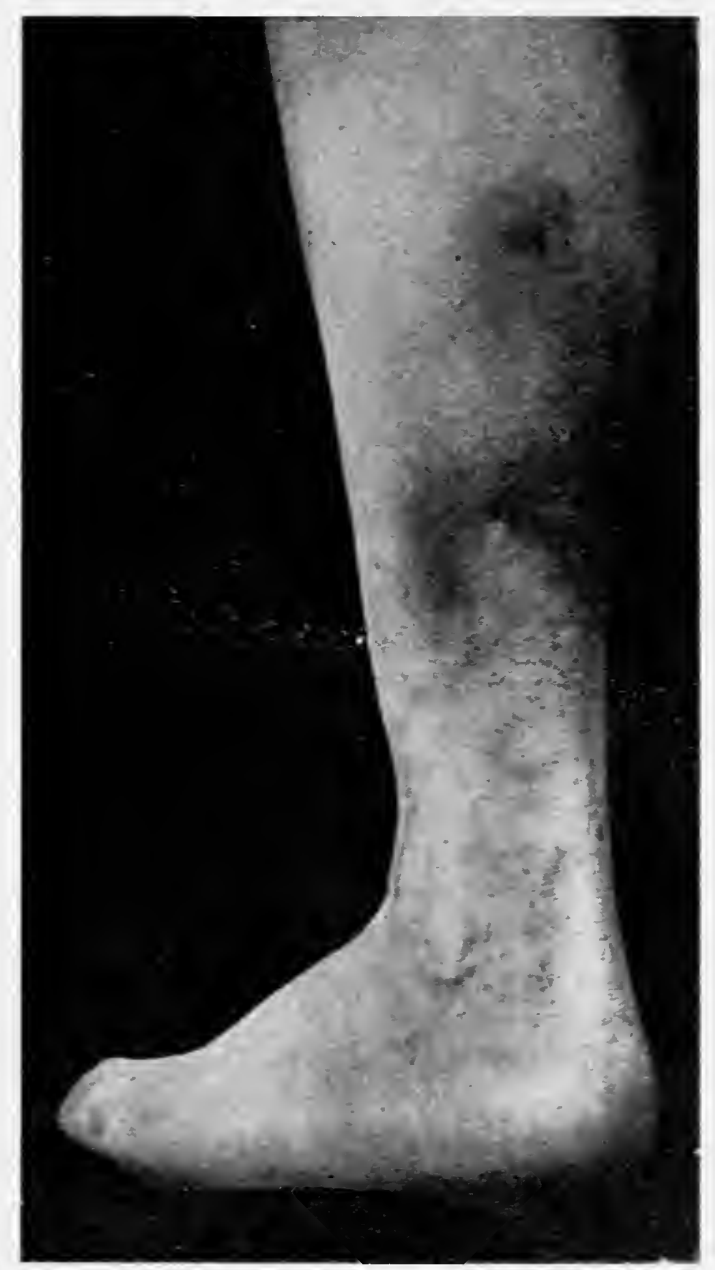

FIG. 279.-Pigmentation and Deep-seated Llcerating Jodules in Varicose Dermatitis.

surface and reduce the erythema. Diachylon ointment or ichthrol paste should then be continuously applied, thickly spread on lint under a bandage. In the course of a few days the surface instead of being crusted and weeping, becomes dry, red, and shiny. After the acute stage has passed, the skin should be cleansed with boric lotion, painted with zinc-ichthyol gelatine, a bandage carefully applied from the ankle to the knee or a well-fitting stocking worn, and the patient permitted 
to move about. To prevent recurrence the varicose veins should be dealt with on surgical principles.

Feet.-Eczema may occur on the feet, usually in association with lesions on the legs. On the dorsum it may form weeping, scaly, or erythematous patches, while on the sole there is generally marked thickening of the horny layer. It is sometimes present between the toes either as moist, fissured lesions, or as dry, inflamed, scaly patches which are markedly itchy. It has to be distinguished from eczematoid ringworm so that in every case a microscopical examination should be made for fungus.

Treatment.-For the erythematous type the application of zinc paste with the addition of salicylic acid under a bandage is a useful remedy. In the case of vesicular eczema about the toes a good application is lint soaked in Burow's solution continuously applied for a couple of hours followed by zinc paste containing salicylic acid. Where there is much thickening of the soles the various keratolytics, such as salicylic acid, resorcin, salicylic soap plaster, pyrogallic acid, or the $\mathrm{X}$-rays, may be employed. When fissures with hard horny edges are present, the edges may be rubbed down with pumice-stone and the fissures touched with $2 \%$ silver nitrate solution.

Nails.-Eczema sometimes attacks one or more of the finger-nails and in rare instances may affect them all. It may involve the nail by extension from the skin to the posterior wall and nail-matrix, when it interferes with the growth of the nail so that it becomes rough, ridged, discoloured, brittle, or even shed; or it may extend upwards and backwards from the tip of the finger, causing thickening of the nail-bed and the formation of a horny scale raising up the nail-plate. There is nothing absolutely diagnostic about the appearances in eczema of the nail. The skin around or in front of the nail is generally involved and is either red and oedematous, or dry, torn, and scaly, or cracks may occur through which septic micro-organisms gain entrance and may give rise to onychia and whitlows.

The toe-nails may be similarly affected.

Treatment.- The treatment is unsatisfactory as it has little direct influence on the nail-plate. It consists of the application of various remedies for the.eczema of the skin of the nail-wall and the tips of the fingers, and as this heals the nail-plate gradually assumes a more normal appearance. In chronic cases associated with a scaly condition of the finger-tips and horny scales beneath the free border, exposure to the $\mathrm{X}$-rays is of benefit.

Mucous Membranes.- The mucous membranes are attacked only in situations where they join the skin, as about the lips, eyclids, mouth, nose, anus, and vulva. The affected mucosa becomes oedematous and covered with a serous discharge, while the cutaneous margin is apt to become fissured and crusted.

Treatment.--This consists of the removal of the crusts after softening them with oil, and the application of $2 \%$ silver nitrate solution or 

PLATE XII.

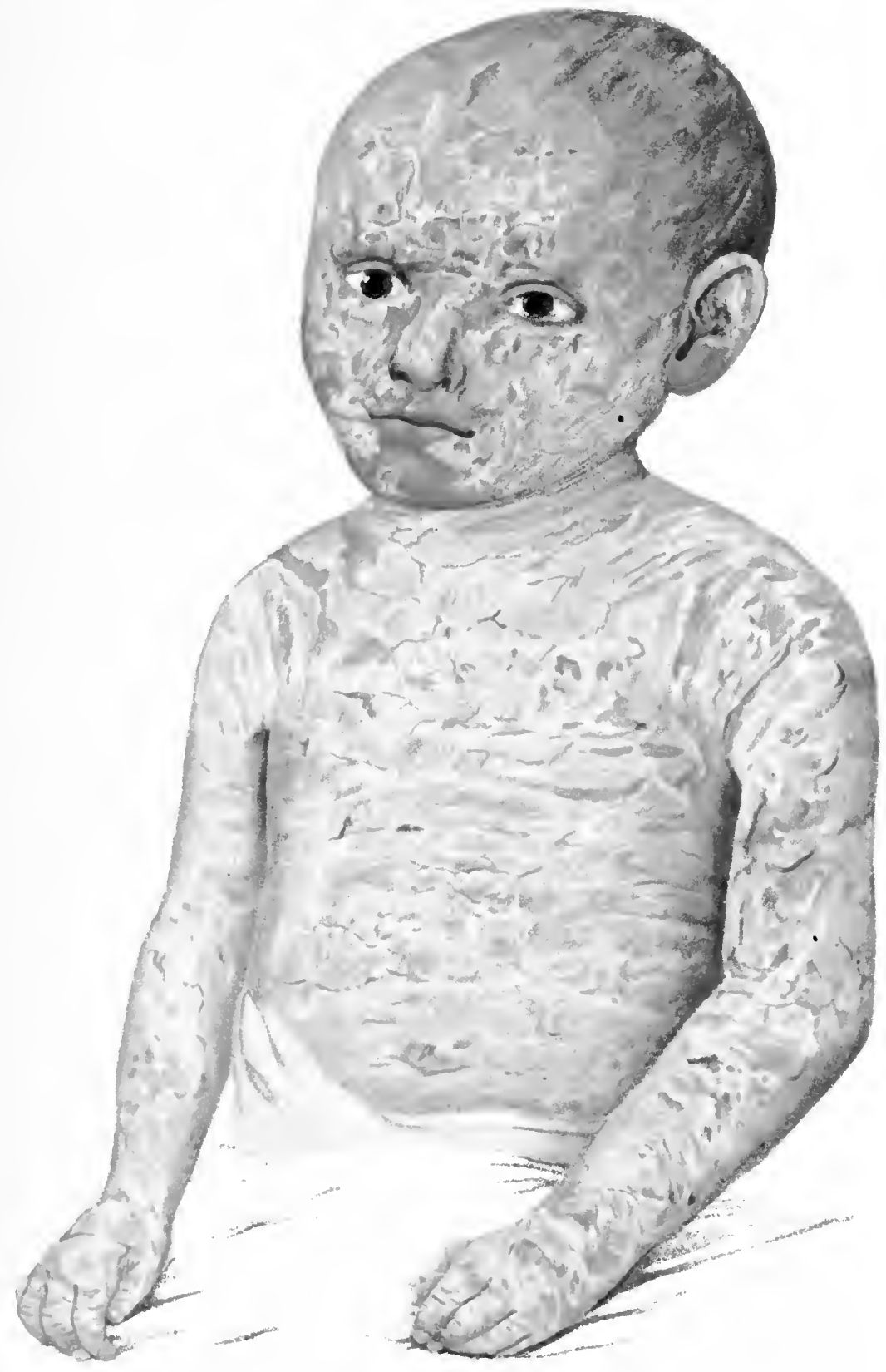

INFANTILE ECENA.

Shows acute weeping eczema and general exfoliation. 
tincture of benzoin to heal the fissures, followed by zinc-ichthyol paste. In some cases treatment by the $\mathrm{X}$-rays is of undoubted benefit.

\section{INFANTILE ECZEMA}

Introduction.-Infantile eczema is a common affection and constitutes about $25 \%$ of the cases of infantile skin disease attending a children's hospital. It is almost equally prevalent in private practice but owing to better nursing and hygiene the cases are usually milder in character. It has been considered advisable to describe infantile eczema under a separate heading for various reasons, the most important being that the special type of reaction of the skin to irritants to which the name of eczema is at present given can be seen in its purest form in the infant, as the skin is delicate and the neuro-cutaneous reflex which produces the clinical appearances which we know as eczema is unusually sensitive and readily called forth by irritants which might have little or no effect on an older skin.

The study of infantile eczema is of importance in throwing light on the etiology of the disease, for in the infant the conditions of life are such that the feeding can be more carefully regulated and watched than in the adult, the excreta noted and the possible relation of the disease to internal disturbances estimated, and such considerations as anxiety, mental worries, etc., as possible etiological factors can be eliminated. In the infant also it may be said that the element of idiosyncrasy is negligible, for while in the adult one skin may react readily in an eczematous fashion and in another may show little or no tendency to do so, in the infant it will almost invariably react in this manner to certain forms of irritation.

Description.-Infantile eczema generally commences on the face, sometimes on the scalp, not infrequently on the hands and arms, and more rarely on the covered parts of the body. It may be confined to one region, but is more usually present in several and may be universal. It begins as ill-defined erythematous patches associated with itching and burning. These fade rapidly and are replaced by an eruption of small pink papules situated on an inflamed base, which on examination by a lens are generally found to be translucent at the apex, and eventually become vesicles and weep on being scratched. In certain cases the initial erythema is transient and instead of being followed by a papular eruption is succeeded by a scaly patch. (Plate XII.)

The eruption usually comes out acutely and is not infrequently ushered in by derangement of health, a slight rise of temperature, and general fretfulness. In localised cases the irritation and itching may result in a certain amount of general disturbance which interferes with sleep and digestion. If the attack be extensive and continuous the general health may be seriously impaired, and where any of the lesions have been allowed to become septic there is danger of 
absorption and possibly of a fatal issue from septicaemia, endocarditis, broncho-pneumonia, or kidney disease. (Fig. 280.)

In some cases infantile eczema is associated with bronchial asthma or attacks of bronchitis, and it has been frequently observed that when the eczema is at its height the lung symptoms are generally in abeyance and vice versa, as if the pulmonary disturbance has some inhibitory action on the neuro-cutaneous reflex.

Etiology.-A study of the etiology of infantile eczema would lead to the conclusion that it is essentially a local condition, a form of neurocutaneous reflex due to local irritation, which, when once established, is capable of being aggravated by internal disturbances such as autointoxication from the alimentary tract, etc. The skin of the infant is extremely delicate and a considerable proportion of the cases are the result of irritation by cold, rapid changes of temperature, soap and water, dirt, perspiration on the mother's breast in nurslings, friction of towels in drying, chafing of wet soiled diapers, insufficient care in cleansing the perineum from excreta, camphor oil or turpentine rubbed on the chest as counter-irritants, etc.

Cold is one of the most important of the etiological factors, such as a cold atmosphere, cold resulting from evaporation in an imperfectly dried skin, or from taking an infant from a warm room to a cold passage or window, consequently it is more liable to occur in winter and spring than in the warmer months.

It is not infrequently secondary to impetigo or to the greasy condition of the scalp known popularly as the "milk crust."

In some cases it is superimposed on the congenital defect of the skin known as xerodermia or mild ichthyosis.

The original patches are due to local irritation and are kept alive by rubbing and scratching, but once they have developed, new lesions tend to appear symmetrically in remote parts of the skin which are not explicable as the result of the local irritant. Various suggestions have been put forward to explain these lesions, such as (I) that they are due to toxins, produced either by microbes or by the tissue-cells of the original patches, being absorbed and reaching the corresponding situations on the skin via the blood-stream, (2) that the symmetry is due to reflex nerve action.

There are certain factors which may predispose towards the affection or may aggravate it, though it is doubtful if they are capable of causing it de novo.

Heredity is occasionally cited as a causal factor, but this is doubtful, as the infant is not born with eczema though possibly with an exceptionally delicate skin which renders it susceptible.

Bad feeding and imperfect digestion are often regarded as causes, but though they may be responsible for kecping up the irritation and so aggravating the disease, it is questionable whether they can produce it.

In the popular mind there is a close relation between infantile eczema and teething and it is not uncommon for the affection to be 


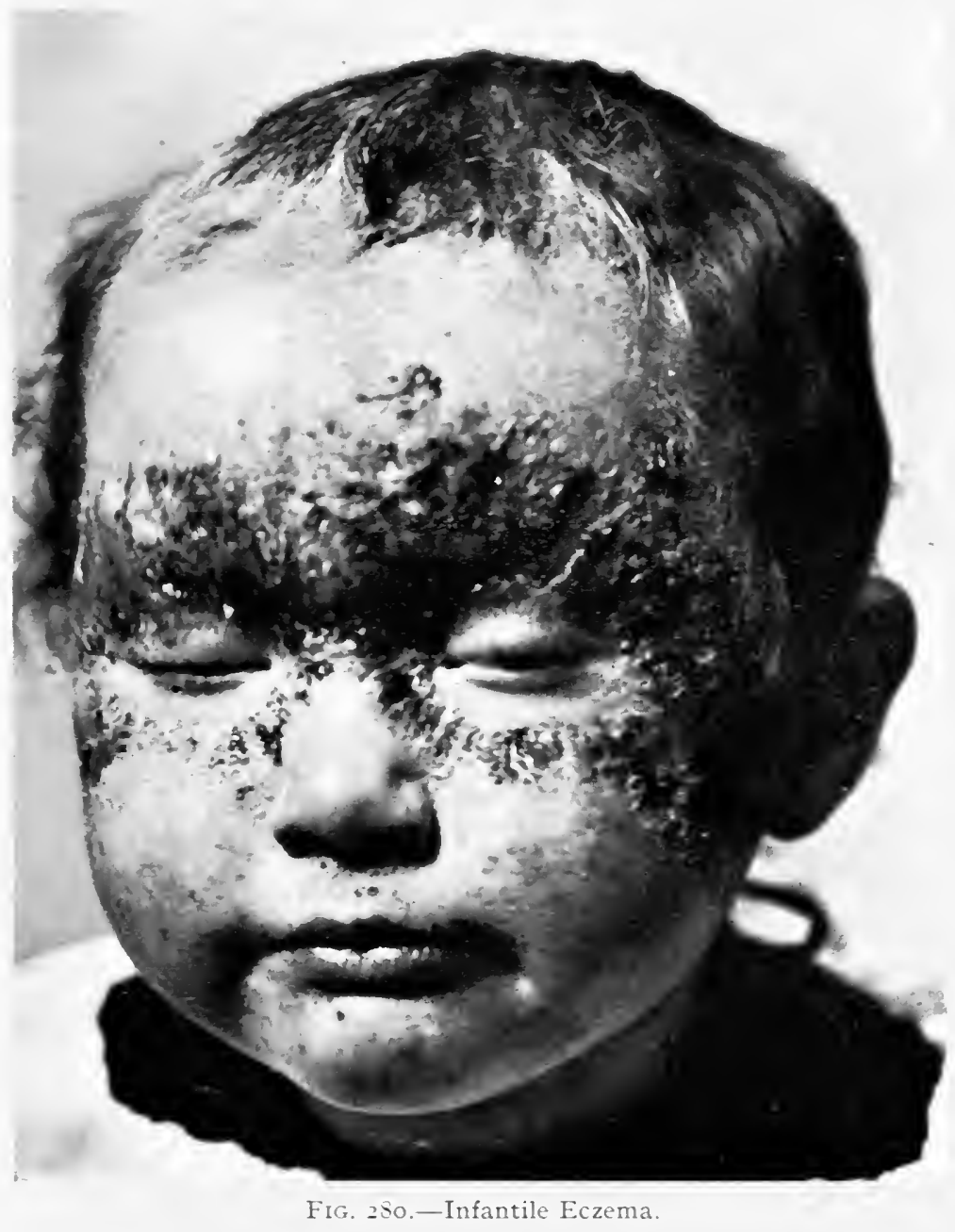

To face p. 800 . 

designated tooth rash. But tooth-cutting does not directly cause eczema, it only produces a general disturbance and a nervous irritability which leads to rubbing and scratching and so augments the disease.

Vaccination has been adranced as a cause, but careful examination of the question has shown that any connection which may exist between the two is purely accidental.

Any factors which cause irritability, such as intestinal worms, a tight prepuce, etc., may predispose towards it.

Prognosis. - The prognosis of infantile eczema and the chances of recovery are good as a rule, though it is difficult to effect a complete cure in extensive cases until the irritable period associated with toothcutting has passed. Occasionally cases occur in which the condition remains permanent, as if the eczematous neuro-cutaneous reflex were so delicate as to be called forth by the ordinary irritants of daily life.

As a rule the general symptoms are negligible, though they may interfere with the well-being of the child at the time, but in extensive cases where the eczema has become septic, a fatal issue has been known to occur from septicaemia.

\section{MANAGEMENT AND TREATMENT}

The prompt and thorough treatment of infantile eczema is of the utmost importance, for by timely interference it may usually be cured, while if left alone or treated in a half-hearted fashion it is liable to persist and become permanent.

Even in its mildest forms it may be an intractable condition, requiring the minutest attention to every detail in connection with the general health, hygienic surroundings, and daily life of the infant. and in severe cases its successful treatment may tax the resources and patience of the most shilled physician.

One of the first considerations is proper protection against cold. When the face is affected it is advisable to keep the infant indoors in frosty weather and on raw windy days, and when taken out to cover its face with a veil. A mild damp day with a soft wind is the safest for the child to go out.

Strong sunlight also acts as an irritant and has to be carefully guarded against by reils and sunshades, the latter being of a red material.

The clothing should be light and warm and the bedclothes not too heary.

With regard to diet any obvious error must be corrected. There is not sufficient evidence to warrant the assumption that defects in diet alone can cause eczema, and it may occur in babies on the breast who are being nursed regularly and taking their nourishment well, and in babies on the bottle where the feeding is all that could be desired, but any dietetic error can aggravate the condition when once established. 
The greatest care should be taken to keep the bottles clean and to feed the child at regular intervals and not simply when it cries.

Digestive disturbances must be dealt with by suitable means. Where the stools are offensive, slimy, and green, an intestinal antiseptic and an alkaline should be prescribed, such as the familiar powder containing calomel gr. $\frac{1}{2}$, and sodium bicarbonate gr. v, which should be taken in the morning so that the effects may wear off during the day and the night's rest not be disturbed. Where there is constipation a mixture containing sod. bicarb. gr. v, Tr. rhei co. m. v, Syr. zingib m. v, aq. ad. $5 \mathrm{i}$ is indicated. If there be diarrhoea it may be expedient to use sod. bicarb. gr. $v$, mist. cret. $5 \mathrm{i}$, but as a rule it should be possible to correct it by regulating the diet and giving an alkaline rhubarb mixture to get rid of the intestinal irritant.

The washing of the affected infant is a matter of great importance and one on which there are various opinions. As a general rule, it may be said that water, especially if combined with soap, owing to its macerating properties is liable to irritate the delicate skin and to aggravate the disease, so that on the whole the less water which reaches it the better, and unless specially indicated soap should be scrupulously avoided. On the other hand it is essential to remove all crusts, scales, and discharges, previous to any form of local treatment; for this purpose soap and warm water are sometimes applicable, but on the whole the best procedure is to soften crusts on the glabrous skin with almond oil and bathe them off with warm water to which has been added a teaspoonful of borax to a gallon of water. Where the scalp is thickly covered with greasy scales or crusts they should be got rid of by boro-starch poultices or by washing with soap spirit lotion.

After the thorough removal of scales and discharges the skin should be dried, by blotting up the moisture with a towel and not by rubbing, and should be powdered over with a dusting powder such as the following: Magnesium carbonate $\overline{\tilde{\jmath}} \mathrm{ss}$, zinc oxide $\overline{\tilde{\jmath}} \mathrm{ss}$, boric acid $\vec{\jmath} \mathrm{i}$. The greatest care should be taken to keep the intertriginous regions about the buttocks and genitals as clean as possible by changing the diapers whenever they are soiled.

One of the greatest difficulties is the prevention of scratching which is largely responsible for aggravating the disease and preventing healing, a few minutes' rub often being sufficient to undo all the benefit derived from a week of careful treatment. Unfortunately it is impossible as a rule, to find a local remedy which absolutely relieves the itching, and other methods have to be adopted to prevent scratching and to minimise the harm which may be done by it. In many cases there is an element of habit and the infant tends to give the affected place a rub unconsciously which immediately renders it irritable, leads to further rubbing, and establishes a vicious circle. Not infrequently if this habit can be broken and the initial rub prevented the irritation may pass off. Where the face is affected it should be protected by a mask and some method adopted to keep the hands from reaching it, 
which is by no means easy in a strong infant. Various derices have been employed with greater or less success, such as wearing cotton gloves, bandaging the hands, pinning the sleeves of the nightdress to the sheets or the mattress, tying short cardboard splints to the flexures of the arms so that the child can swing them about without being able to get at the face, or putting on a pillow-slip with a hole in the top for the head and pinning the sleeves of the nightdress to the sides of the pillow-slip while the back and front of the slip are pinned together at the fork of the legs. Another favourite method is to tie the hands to the sides of the cot by a bandage, in this case the hands should not be tied out too straight as this is apt to interfere with the breathing, but should be tied about level with the hips. Sometimes in spite of every conceivable device there is nothing for it but to walk the child up and down the room till it goes to sleep.

The local treatment varies according to the stage and character of the lesion. Where the face is chiefly involved and the eczema is dry and consists of red irritable patches, scaly, or corered with papules which even slight rubbing would break and cause to weep, the affected skin requires to be soothed and protected. For this purpose the ordinary zinc ointment is too harsh and a good substitute for it is. ichthyol gr. $r$, zinc oxide $\bar{j} \mathrm{i}$, with cold cream to the $\bar{j}$. Where the itching is severe ro $\mathrm{m}$. of Liquor picis carbonis, or tincture of camphor or 5 grains of carbolic acid may be added to the ounce of the ointment. Where the eczema is moist and oozing Io to $\mathrm{I}_{5}$ grains of acetate of lead and Io to 30 grains of magnesium carbonate may be added to the ointment, and should this fail to astringe the lesions they may be painted previously with a $2 \%$ solution of silver nitrate or with compound tincture of benzoin. The ointment should be thickly spread on lint and kept in position by a mask of thin cotton, sufficiently long to go under the chin and to extend back to the ears, with holes cut for the eyes, nose, and mouth, and kept in position by tapes tied at the back of the head. The dressings should be renewed once or twice a day, when any crusts or scales which may have formed should be removed by almond oil.

Where the face is attacked acutely and is inflamed like errsipelas, or crusted and weeping, a boro-starch poultice has a soothing effect and should be followed by the application of the abore ointment. Calamine lotion is sometimes prescribed in the acute stage, but is not satisfactory, as if kept continuously moist it is liable to macerate the skin, while if allowed to dry, it is apt to become caked up and the skin to crack.

When the acute stage has passed and all that remains is a residue of erythema, a lotion consisting of calamine $\overline{5} s \mathrm{~s}$, zinc oxide gr. $\mathrm{xr}$, glycerine m. $\mathrm{x}$, carbolic acid $\mathrm{m}$. iii, and distilled water $\bar{j} \mathrm{i}$, is a valuable application for reducing the redness and allaying the itching.

Should the facial lesions become purulent, a mild antiseptic ointment is indicated, such as Io grains of ammoniated mercurg to the ounce of soft white paraffin. 
Where the scalp is involved and covered with greasy scales these should be removed by soap spirit, followed by the application of a11 ointment containing precipitate of sulphur to grains, salicylic acid Io grains, soft white paraffin to the ounce. Where septic crusts are present an oily antiseptic preparation, such as salicylic acid Io grains, eucalyptus I dram, almond oil to the ounce, is serviceable.

In the case of eczema of the trunk, the treatment depends on the exact situation and the amount of inflammation. Where the intertriginous regions about the groins and buttocks are involved, the discharges or crusts should be softened by almond oil, and removed by bran and hot water, or by boro-starch poultices. The skin is then dried and a bland zinc ointment or paste applied or the following antiseptic.cream, prepared calamine 20 grains, zinc oxide Io grains, ammoniated mercury 5 grains, lime water $\frac{1}{2}$ ounce, almond oil I ounce.

To reduce friction it is an advantage to insert slips of cottonwool between the contiguous surfaces, and these should be kept in place by clean diapers.

When the eczema consists of itchy papular patches, the irritation may be relieved by the application of a lead and tar lotion followed by weak zinc oxide and tar ointment.

Where it is in the form of minute resicular puncta on an erythematous base and widely distributed over the back and chest-a condition which has sometimes been designated sweat eczema-one of the best applications is calamine liniment which should be gently rubbed all over the affected areas, the superfluity mopped off with a clean towel, and a dusting powder freely applied.

Where the limbs are affected the type of local remedy depends on the character of the lesions and a similar treatment is indicated to that which is applicable to the trunk.

In whatever situation the eczema may occur, it is important that the ointments or pastes which may be employed should be continuously applied thickly spread on lint under light bandages.

\section{REFERENCES}

\section{General.}

Broco, Pautrier, \& Ayrignac. Papulo-vesicular eczema (Ann. de. Derm. et de Syph., I9II, p. 513).

Cole. Studies on eczema and the pyodermites (Arch. f. Derm. u. Syph., March I9I3).

Fordyce, J. A. Anaphylaxis in eczematoid dermatitis (Journ. Cut. Dis., I9I I, P. I 29).

Hall, A. Statistical study of eczema (Brit. Journ. Derm., 1905, xvii.).

Hennans, II. J. Critical review of eczema and dermatitis, with analysis of a group of cases (Journ. Cut. Dis., I9I6, xxxiv. p. 259).

- Pathology and pathogenesis of eczema and dermatitis, the identity of the two diseases (Journ. Amer. Med. Ass., Jan. I3th., I9I7, p. 75). 
KNowles, F. C. Eczema of external origin and its relationship to dermatitis (Idem, p. 79).

Roberts, L. Eczema (Eng. Med., 2nd Ed., I9I6, vol. iv.).

White, C. J. Anaphylaxis in eczema (Prel. report) (Journ. Cut. Dis., I9I6, xxxiv. p. 5\%).

Whitfield, A. Eczema (Practitioner, 1904, lxxii. p. 202).

- Eczema (Allbutt \& Rolleston, System of Med., I9II, ix. p. 300).

Etiology.

Bulkiey. Neurotic eczema (Journ. Amer. Ass., April r6th, i898).

HodARA. (Monats. f. prakt. Derm., I $\varepsilon g \delta$, xxrii. p. $f 89$. )

Johistor, J.C. Speculations as to the causation of eczema (Journ. Cut. Dis. Jan. I9I3, p. 3).

Kxowles, F. C. The external origin of eczema (Idem, p. II).

Sabotrald. Essai critique sur l'étiologie de l'éczéma (Ann. de Derm. et de Syph., I899, x. p. 3II).

TöRök. L'éczéma est-il une maladie parasitaire ? (Idem, IS98, ix. p. Io73).

Uxxa. Morococcus (Monats. f. prakt. Derm., I899, xxix. p. 106).

\section{Treatment.}

ChAMBers, G. Observations on the etiology and treatment of eczema (Brit. Med. Journ., Oct. I 906, p. $85 \mathrm{I}$ ).

JAmbox. Treatment of eczema by coal-tar (Ann. de Derm. et de Syph., Jan. I909, p. 22).

LitTlE, E. G. The treatment of eczematous patients (Brit. Med. Journ., June I909, p. I $3+0$ ).

White, C. J. Two modern methods to be employed in the treatment of eczema (Journ. Amer. Med. Ass., Jan. I9I $/$, 1xviii. p. 8I).

\section{Seborrhoic Eczema.}

Brooke, H. G. On the seborrhoic processes (Brit. Journ. Derm., I889, i. p. $2+7$ ).

Jesionek. Seborrhoic eczema (Derm. Zeitsch., I9Io, p. 88\%).

\section{Infantile Eczema.}

Adansox, H. G. Infantile eczema ("Skin Diseases of Childhood," I90\%). HALL, A. J. Etiology of infantile eczema (Brit. Journ. Derm., I905, xvii. p. I6I).

\section{POMPHOLYX}

Syn.: Cheiro-pompholyx; Drsidrosis ; Fr., Dỹsidrose.

Definition: An inflammatory affection of the hands and feet characterised by boiled sago-grain-like resicles, or bullae, preceded by itching or burning, and sometimes associated with excessive sweating.

Introductory. - This affection was first described in England by Tilbury Fox about IS Jo, under the heading of dysidrosis (from the Greek d's and id $\rho$ ós sweat) as he believed that it was a disease of the sweat-apparatus and that the resicles were retention crsts which had formed in the sweat-ducts from blocking of the sweat-pores. This view was combated subsequently by Sir Jonathan Hutchinson who 
regarded it as an inflammatory affection quite independent of the sweat-apparatus, and named it Cheiro-pompholyx (from $\pi \circ \mu \phi o ́ \lambda \nu \xi$ a bubble and $\chi \in i \rho$ the hand) a title which is misleading as it is not invariably confined to the hands but may also attack the feet. Since then there has been much diversity of opinion with regard to its nature, for while certain observers still believe it to be essentially a disease of the sweat-apparatus, others equally emphatically assert that it is an inflammatory disturbance indistinguishable from eczema and that any relation which it may have to disorders of the sweat-apparatus is secondary. As its precise nature is not yet fully understood and since it may occur occasionally without any definite disorder of the sweatglands, or excessive perspiration, it is better to apply to it the noncommittal name of pompholyx rather than its original title of dysidrosis.

Description.--The affection is usually ushered in by more or less marked sensory symptoms which precede the eruption by an hour or two and tend to diminish when the lesions are fully evolved. These consist of itching, tingling, burning, tenseness of the skin, or even pain. The eruption generally appears on the hands, affecting both in a somewhat symmetrical fashion, though as a rule involving one more markedly than the other. It may occur on the feet and in rare instances they alone may be attacked. It has been described also on the face, arms, and trunk, but it is doubtful if the lesions in these situations belong to the same category as those on the hands, and they may possibly be hydrocystomata on the face, vesicular eczema on the limbs, and miliaria or prickly heat on the trunk.

The essential lesions consist of deep-seated vesicles about the size of a pin's head, with a whitish translucent appearance which has been aptly compared to a boiled sago-grain, and are not preceded by, or associated with, any signs of inflammation. They may occur singly, or in little groups, or in severe cases may be widely and profusely distributed over the whole part. They are most common on the palms, interdigital clefts, and sides of the fingers, and more rare on the backs of the hands and wrists. On the feet the usual situations are on the borders, between the toes, and on the soles. Where they are numerous, the affected skin though not inflamed may become tense and stiff from oedema. They have no tendency to rupture spontaneously, but dry into scales which separate in about a fortnight leaving red macules which gradually disappear without scarring. (Fig. 28I.)

Owing to the coalescence of a number of vesicles, multilocular bullae may be formed which vary up to an inch or more in diameter. These are irregular in outline, rounded and tense on the palms and soles, and flat and flaccid in other situations. Where the skin is thin, as at the sides and backs of the feet and hands, and between the fingers and toes, the bullae are readily broken, leaving raw, red, moist surfaces, while on the palms and soles they are much more resistant and the contents tend to become gradually absorbed or to dry up. 
As a rule the affection is associated with excessive sweating, clammy hands, and hyperidrosis of the feet, but this is not invariably the cast and in some instances the hands may be perfectly dry.

The lesions, especially about the feet, may become purulent from secondary contamination by pyogenic organisms, or, where they have been irritated by the injudicious employment of some strong application, a catarrhal dermatitis may be set up.

The local condition not infrequently is associated with a disturbance of the general health, and such symptoms as malaise, slight rise

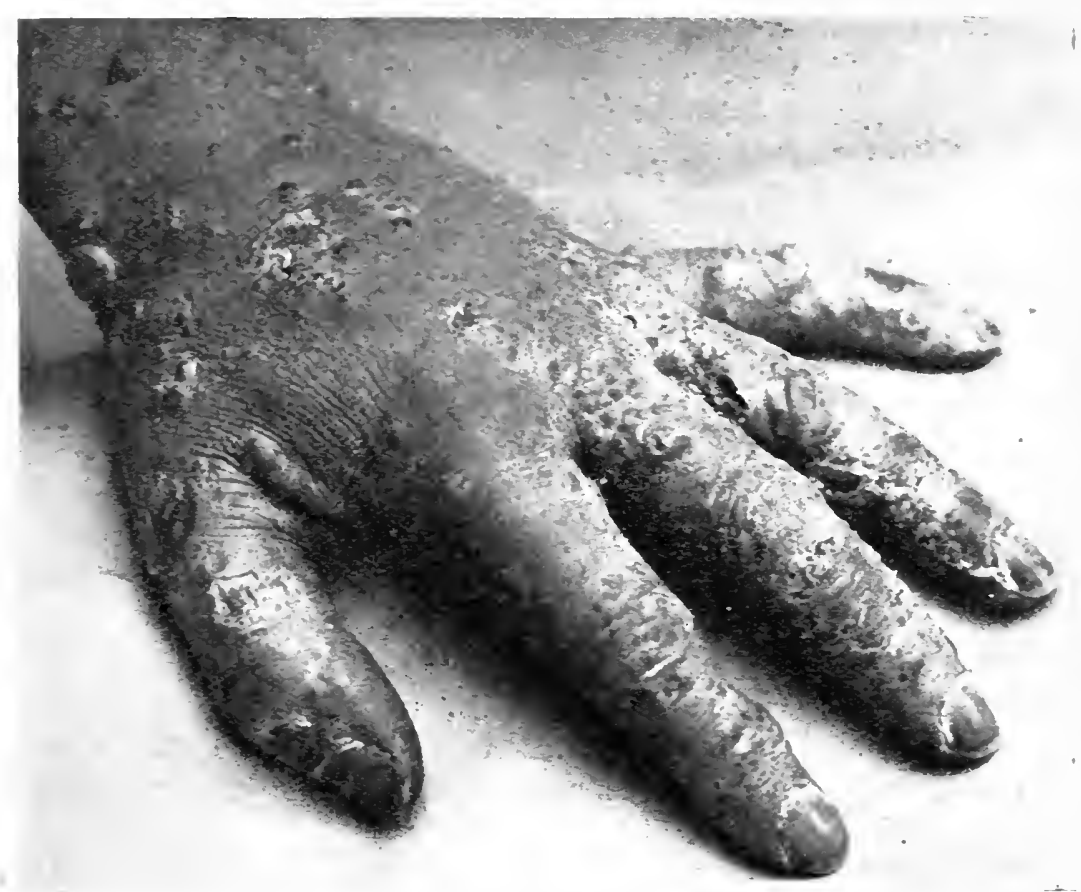

FIG. 2SI.-Dysidrosis.

of temperature, and a feeling of depression, may be observed, not only in severe cases but also where the eruption is mild and consists only of a few groups of vesicles.

The course of the disease varies, in some cases healing takes place in a few weeks, while in others fresh outbreaks may delay it for months. It has a marked tendency to recur, especially from exposure to sudden heat and sunlight, and seasonal recurrences in spring and summer are frequent.

Histopathology.--Microscopical sections show that the vesicle is situated as a rule in the prickle-cell layer in the neighbourhood of the granular layer, and is of precisely the same type as that of eczema. Around it the inter-epithelial lymphatic spaces are dilated and the prickle-cells oedematous. 
In the underlying corium the capillaries of the papillary layer are dilated and surrounded by an inflammatory infiltration of small round connective tissue cells, lymphocytes, and a few polymorphonuclear leucocytes.

Serial sections by Santi, Williams, and others, have not shown any definite connection between the vesicle and the sweat-duct, and these observers conclude that the affection is essentially inflammatory, the vesicular formation being preceded by inflammatory changes in the papillary layer.

On the other hand Nestorowsky, from an examination of a large number of serial sections, came to the opposite conclusion, namely, that the initial change was a blocking of the sweat-pores by plugs of horn-cells resulting from the irritation of excessive sweat, that the vesicle was due to the pent-up sweat dilating the inter-epithelial spaces, breaking the inter-epithelial fibrils, and finally causing necrosis of the prickle-cells, and that the inflammatory changes were secondary.

Unna found a small bacillus in the roof and contents of the vesicles, which he believed to be specific, and explained the inflammatory changes and vesicular formation as the result of the proliferation of this micro-organism in the sweat; but this view is not generally accepted.

\section{ETIOLOGY}

Predisposing Causes. - Age.-It may occur at any age but is most common in children and in early adult life and rare in infancy and old-age.

Sex.-It is more frequent in females than in males.

Circulation.-A feeble peripheral circulation, associated with excessive sweating of the extremities, is an undoubted predisposing factor.

Exciting Causes.-Nerious Derangements.-Occasionally it would appcar to be determined by mental worry, anxiety, overwork, or other psychical causes, and it seems to be particularly common in individuals with a neurotic temperament. In a case under the care of the writer in a girl of $\mathrm{I}_{5}$ with left hemiplegia who was subject to attacks in warm weather, it was always much more marked on the paralysed than on the healthy side and did not there respond so readily to treatment.

Heat.--Heat is the most frequent determining cause, either hot weather, or exposure to the heat of a fire, or the heat of violent physical excrcise. The disease is consequently much more common in spring and summer than in the colder months.

Locai Irritation.-Attacks have been reported as due to the action of local chemical irritants, such as certain dyes, explosives, etc., but such cases belong more properly to the trade dermatitis group.

Diagnosis. - The recognition of a well-marked case is comparatively simple, the itching and tingling preceding for a few hours the outbreak 
of small deep-seated resicles, like boiled sago grains, situated in groups on the palms and between the fingers, the occasional presence of bullae and the freouent association with excessive perspiration, forming a clinical picture which is easily recognised.

It has to be distinguished from scabies which it may sometimes superficially resemble, but in scabies the presence of burrows and the finding of acari will settle any doubts with regard to the diagnosis.

From eczema the diagnosis may present the greatest difficulty, and indeed certain observers regard it as a form of eczema. Those who assert that it is a disease suigeneris, point out that the essential lesion of pompholyx differs from that of eczema in being a translucent vesicle arising on apparently unaffected skin, while the typical lesion of eczema is an erythematous papule with a resic!e at the apex, and that the vesicle of pompholyx shows no tendency to rupture spontaneously but gradually dries up being replaced by a scale, while that of eczema readily breaks and weeps.

\section{TREATMENT}

The treatment is both general and local. In most cases the patient is below par, sedentary in habits, of a nerrous temperament, and has a defective peripheral circulation, so that a tonic regime on general medical principles is of undoubted benefit, and should consist of out-ofdoor exercise, an easily digested and nutritious diet, and tonics such as arsenic, quinine, and strychnine. In some cases recurrences may take place from indulgence in alcohol, coffee, or tobacco, and in consequence these should be prohibited.

Locally, the indications for treatment consist of drying up the lesions and preventing their becoming septic. For this purpose mild soothing remedies are suitable and strong irritating applications should be aroided as they are liable to transform the condition into a catarrhal dermatitis.

In mild cases the most useful application is zinc paste with the addition of $2^{\circ}$ o salicylic acid. This should be spread on strips of lint and kept applied under a bandage, the dressing being renewed once or twice a day and the dried paste cleaned off by olive oil.

In more severe cases, especially where the feet are involved, the parts should be bathed in boric lotion or weak permanganate of potash solution, followed by the application of a dusting powder of boric acid or salicylic acid $\left(5^{\circ}\right)$ in zinc oxide, or of tannoform. Where the inflammation is considerable, compresses of lead lotion are of value, or the employment of the ointment suggested by Hebra consisting of equal parts of vaselin and lead plaster. Where the moisture is excessive, a $2 \%$ solution of silver nitrate may be employed as an astringent. 


\section{REFERENCES}

Fox, Tilbury. Dysidrosis (Skin Diseases, Lond., I873, 476).

MacLeod, J. M. H. Case of dysidrosis in a girl with hemiplegia (Brit. Journ. Derm., 1917, xxix. p. 219).

Nestorowsky, W. Histopathology (Derm. Zeitschr., I906, xiii. p. I83). SAnti. Histopathology (Monats. f. prakt. Derm., I 892, xv. p. 93).

Williams, A. Winkelried. Anatomy of cheiro-pompholyx (Brit. Journ. Derm., I891, iii. p. 303).

\section{ROSACEA}

Syn. : Acne rosacea; Acne erythematosa ; Couperose.

Definition: A chronic disease of the central area of the face, characterised by temporary flushing which gradually proceeds to permanent hyperaemia, associated with telangiectases, stasis of blood, excessive activity of the sweat and sebaceous glands, and later by inflammation about the pilo-sebaceous follicles giving rise to acne-like lesions and, occasionally, to a fibro-glandular hypertrophy of the skin of the nose known as rhinophyma.

Description.-Rosacea is one of the commonest of skin diseases, is especially frequent in middle-aged women, and is the cause of much discomfort and disfigurement.

Its course may be divided into three phases or stages, namely :

(r) Phase of temporary flushings.

(2) Phase of permanent congestion usually associated with the presence of oily seborrhoea, inflammatory follicular papules, acne-like pustules, and telangiectases.

(3) Phase of hypertrophy with massive thickenings about the nose.

This division is purely arbitrary but is useful for descriptive purposes.

Occasionally by suitable preventive measures the disease may be aborted in the initial phase but, as a rule, it is allowed to reach the second more permanent stage before it attracts sufficient attention for advice to be sought regarding it. In this phase unless it is most carefully treated it is liable to persist indefinitely and may even be progressive.

It is only in rare instances that it proceeds to the third stage of hypertrophy.

I. Phase of Temporary Flushing.-The earliest sign of rosacea consists of flushing of the skin over the malar prominences and nose and in marked cases over the whole central area of the face. This flushing may last from a few minutes to an hour or longer when it usually completely disappears. It is a reflex condition due to a functional or organic disturbance in some internal organ and is most commonly the result of digestive disorders. From one cause or another the flushings become more frequent and last longer till finally the redness is constant and the condition results which is known by the French writers as Couperose. 


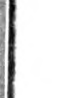

$$
\text { - }
$$




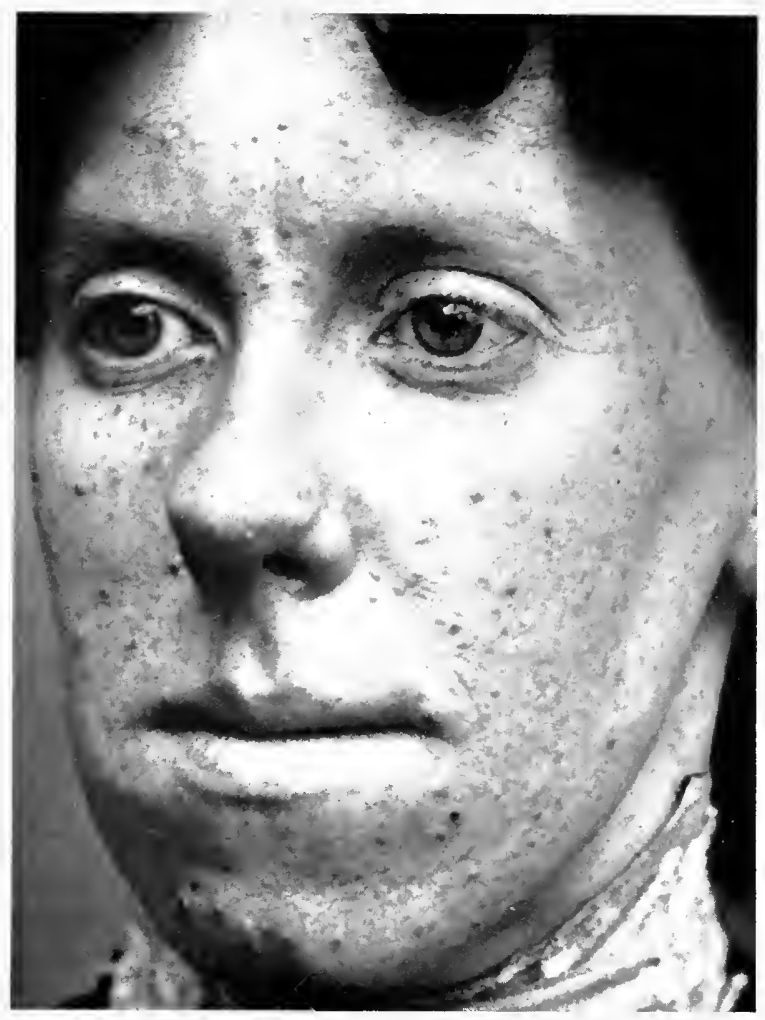

FIG. 282.-Rosacea and Papular Seborrhoide.

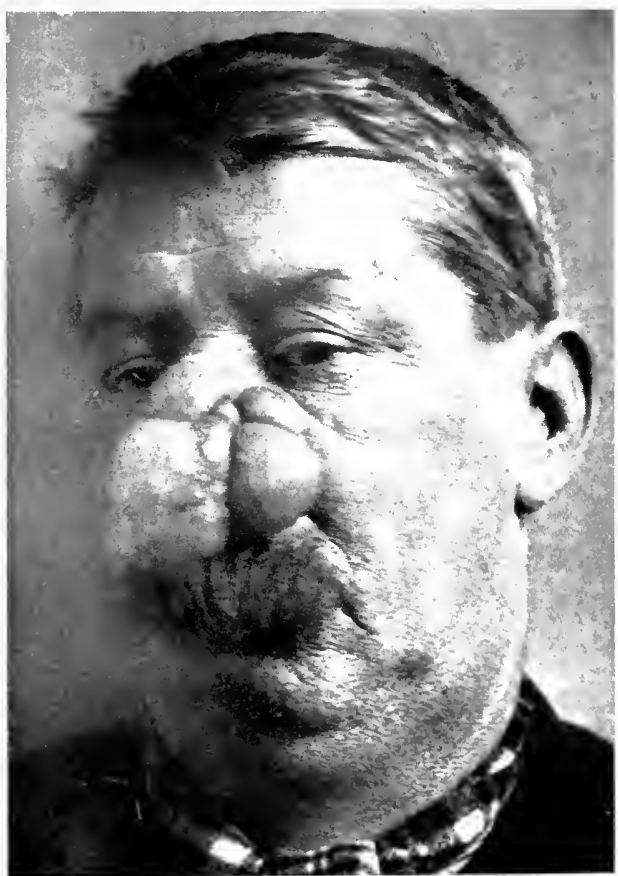

FIG, 283.-Rhinophyma.

(From Dr. Arthur Hall.) 
2. Phase of Permanent Congestion.-In this phase the redness tends to become deeper in tint and, in individuals.with a weak peripheral circulation, may even be violaceous. The superficial capillaries become permanently distended, well-marked telangiectases appear about the malar prominences, and bluish streaks are seen ramifying down the nose.

Instead of being dry, as in the earlier stage, the congested area may be moist and greasy from excessive action of the sweat-glands due to blood-stasis and oily seborrhoea. This greasy skin forms a suitable soil for the growth of the ordinary skin micro-organisms and as a result inflammatory papules appear about the pilo-sebaceous follicles, which are soft, pinkish, about the size of a hempseed, usually acuminate in shape, and sometimes present a speck of sero-pus at the apex. Occasionally they may be larger and break down in the centre to form yellowish pustules like those of Acne vulgaris-hence the old, somewhat confusing, name of Acne rosacea. When they take the form of small inflammatory papules and are associated with a considerable degree of oily seborrhoea they are often referred to as "papular seborrhoides."

In addition to the seborrhoea, rosacea may be complicated by blepharitis or phlyctenular conjunctivitis. (Fig. 282.)

3. Phase of Hypertrophy.-The final outcome of rosacea is the hypertrophic condition of the nose known as Rhinophyma and sometimes described under the misleading title of "hypertrophic acne of the nose." In it the skin of the nose is congested, coarse, thickened, greasy, becomes pale on pressure, and feels cold and clammy. At the same time lobulated or pendulous masses form, dotted over with openings of dilated follicles like the pitted skin of a tangerine orange. It is not infrequently associated with erysipelatoid attacks which spread on to the cheeks, these are due to the activity of streptococci in the lymphatics and lead to further thickening in the skin by setting up a fibrous hyperplasia, blocking of the lymphatics, and a solid oedema: (Fig. 283.)

Histopathology.- Sections of the skin in the early stages show the epidermis to be unaffected and the only noticeable change to be dilatation of the capillaries in the corium, especially marked around the hair-follicles, about the sweat-coils, and in the sub-papillary laver, with an infiltration of small cells of the lymphocytic type around the dilated ressels.

Sections of a papule show a cellular infiltration around a pilosebaceous follicle, consisting of lymphocytes and small connective tissue cells, a few polymorphonuclear leucocytes, a varying number of plasma-cells, and occasional mast-cells.

Sections of the thickened masses in rhinophyma present marked hypertrophy of the sebaceous glands, which have increased to several times their ordinary size, but no new gland formation. There is also hypertrophy and new formation of fibrous tissue. In some sections the fibrous growth predominates, in others the glandular hypertrophy. 
The blood capillaries around the enlarged sebaceous glands are dilated and there is an infiltration of cells similar to those described above. The lymphatic spaces between the connective tissue bundles are widened.

\section{ETIOLOGY}

The affection is essentially a reflex angio-neurotic condition and is due to a number of causes of which the following are the most common :

General Causes.-Digestive Disorders.--In the majority of cases it occurs in the subjects of chronic dyspepsia and although there may have been no definite indigestion when the rosacea first appeared signs of it almost inevitably develop later, such as coated tongue, unpleasant breath, discomfort or flatulence after food, etc. In some cases the flushing will occur after any article of food, in others only some definite error of diet will produce it, especially the taking of hot drinks, spiced food, alcohol, coffee, and strong tea.

Uterine Disturbances.-Another frequent cause is some uterine disturbance and it is usually aggravated during the menstrual periods and about the menopause.

Functional or Organic Disturbances in other Organs may be responsible, such as cardiac disorder or kidney trouble.

Local Causes.- Heat and Cold.--It can be determined by the action of extremes, or rapid changes, of temperature, consequently it is comparatively common among cooks, laundresses, and others who have to expose themselves constantly to heat, and among cabmen, seamen, etc., from exposure to inclement weather, especially after the skin has been flushed by hot or alcoholic drinks. Although the condition is popularly known as "grog-blossom" on no account must it be regarded as invariably a sign of alcoholism, as it is often met with in total abstainers who suffer from indigestion.

Intra-nasal Irritation.--Temporary flushing or permanent congestion of the nose may also result from intra-nasal irritation, such as is caused by the presence of polypi pressing on the septum, hypertrophic rhinitis, or cnlarged turbinate bones.

Micro-organisms.-Certain micro-organisms have been isolated in connection with it but these are of secondary importance and simply account for the inflammatory papules and pustulation. The microbacillus of Sabouraud has been found in the greasy skin and various cocci in the pus but chiefly Staphylococcus albus. Goadby isolated a peculiar bacillus which grew on agar and presented a curious beaded appearance when stained with carbol fuchsin.

Predisposing Causes.-Sex.-The disease is much more common in women than in men, in the proportion of 6 to $\mathrm{I}$, and for this there are various causes, such as,

(a) The greater instability of the nervous system in women rendering them more prone to flushing and blushing.

(b) Their greater liability to indigestion and constipation. 
(c) Their tendency to reflex disturbances from uterine disorders.

(d) The congestion produced by wearing tight clothing and corsets. Age.-It usually begins about the age of thirty, but has been known to occur in childhood and in old-age.

Weak Peripheral Circulation.-A common predisposing factor is a feeble peripheral circulation and rascular stasis about the nose and malar prominences.

Diagnosis.-Rosacea has to be distinguished chiefly from Acne vulgaris, Lupus erythematosus, and Adenoma sebaceum, but in a well-marked case the history of flushing, the concomitant signs of dyspepsia, alcoholism, or some internal disorder, the permanent redness in the central area of the face, the dilated capillaries, papules, acneiform pustules, or coarse thickening of the skin of the nose, render the diagnosis unmistakable.

Acne rulgaris.-It is distinguished from Acne vulgaris by usually commencing in adult life while acne generally appears at puberty ; by involving the central area of the face while Acne rulgaris is most marked at the periphery; by the absence of comedones which are the essential lesions of Acne rulgaris. It is necessary to remember, however, that the two affections may be concomitant and that rosacea may be superimposed on persistent Acne rulgaris.

Lupus Erythematosus. - Rosacea may closely resemble Lupus erythematosus and the same area of the face is liable to be involved in both, but in Lupus erythematosus the patches are more clearly defined, usually have a slightly raised border covered by adherent scales, and are followed by superficial scarring.

Adenoma Sebaceum-The resemblance of rosacea to Adenoma sebaceum is only superficial. In Adenoma sebaceum the redness is due to numerous pinhead-sized yellowish or reddish nodules and not to diffuse congestion, and the nodules being naevoid in origin are generally accompanied by other forms of naeroid growths such as soft moles and pigmented patches. Moreover, this disease generally begins in early life and occurs most frequently in mentally deficient or epileptic subjects.

Prognosis.-The outlook in rosacea depends largely on the stage of

the disease. If it be recognised sufficiently early and the underlying cause satisfactorily dealt with the affection may be arrested, but in the later stages it may be extremely difficult to do more than palliate it. The success of treatment is entirely dependent on the ability to cure the underlying cause and on the aroidance of all influences which produce flushing of the face.

\section{TREATMENT}

The treatment is both general and local and in the earlier stages the former is of the greater importance.

General Treatment.- In the first place it is necessary to discover the underlying disorder responsible for the flushing and to deal with it 
by appropriate means. By far the most common excitant is some disturbance of the alimentary tract. Indigestion must be treated on general medical principles, and all articles of diet which are known to be indigestible should be avoided, such as shell-fish, herrings, mackerel, and other oily fish, pork, fried eggs and bacon, buttered toast, strawberries, curries, rich spiced dishes, etc., but in each case the regulation of diet must be contingent upon the peculiarities of the individual. Alcohol, especially in the form of spirits, beer, and heavy wines, should be prohibited, while almost equally harmful are black coffee and-strong tea. Constipation must be dealt with by suitable exercises, sufficient fluid between meals or, where these are ineffective, by aperients of which the least harmful are aloes, cascara, and senna. Any departure from health must be sought for by a thorough examination of the patient, and where possible corrected. Should the rosacea be due to intra-nasal irritation, for example, no treatment will be of any permanent benefit until the cause of the irritation is removed by appropriate measures.

All influences liable to cause flushing should be avoided, such as exposure to extremes of temperature, brilliant sunlight or cold winds.

In addition to the treatment on general medical lines two drugs have been credited with exerting a specific influence on rosacea, namely arsenic and ichthyol. In the writer's experience arsenic is practically useless and liable to do harm by aggravating any concomitant digestive disturbance. Ichthyol on the other hand is sometimes of benefit, and is always worth a trial; it is supposed to soothe the nerves of the digestive tract and, in consequence, to make the reflex flushings less in degree and frequency. It should be given on an empty stomach, preferably in a capsule, in doses of from five to ten minims, twice daily, one first thing in the morning and one at bedtime.

Local Treatment.--In the stage of temporary flushing no local treatment is necessary beyond the occasional use of lead and calamine lotion.

Where the redness is permanent and associated with seborrhoea a sulphur lotion should be applied, such as sulphur precipitate 2 drms., camphor 5 grs., tragacanth Io grs., lime water I oz., water I oz., or stronger lotions such as sulphur precipitate, zinc sulphate, potassium sulphide, of each I drm., and rose water to 6 ozs., or sulphur precipitate 30 grs., resorcin I5 grs., ether 2 drms. and rectified spirit to I oz.

Where resistant scaly patches are present an ichthyol paste should be applied at night, such as ichthyol, Io m., zinc oxide 3 drms., cold cream to I oz.

As a rule greasy applications, such as ointments or creams, tend to irritate rather than soothe the congested skin and should be aroided unless the skin should become dry, tight, and scaly from the sulphur lotion.

Where acneiform pustules are present they should be opened and evacuated, and dilated capillaries and resistant papules should be destroyed by electrolysis or by a fine-pointed cautery. 
In rhinophyma of a slight degree the thickening may be reduced by the $\mathrm{X}$-rays in three-quarter pastille doses at intervals of about a fortnight, but where the condition is excessive the rays have comparatively little effect and surgical methods must be adopted. Good results may be obtained from a procedure known as decortication which consists of paring down the hypertrophic masses either by a scalpel or by a flat Paquelin cautery till a satisfactorily shaped nose results; this must be done under a general anaesthetic and care must be taken not to penetrate the nostril, a danger which may be obriated by inserting a finger during the operation. The bleeding is arrested by pressure or by the actual cautery, and the denuded surface carefully bathed with hydrogen peroxide $(20$ vols.) or biniodide of mercury lotion.

The raw surface may be grafted but, as a rule, this is unnecessary as small foci of epidermis are generally left from which a new epidermal covering gradually grows.

\section{REFERENCES}

Doнr. Rhinophyma (Arch. f. Derm. u. Syph., I \$g6, Bd. xxxri., Ht. 3).

DUBREUILH, W. Hypertrophic acne of the nose and its surgical treatment (Ann. de Derm. et de Syph., Nov. Ig03, p. 785).

Galloway, J. \& Goadbi, K. Rosacea (Brit. Journ. Derm., Igos, xx. p. 408 ).

Litrle, E. G. Rosacea (Idem, p. 265).

Solger. On rhinophyma (Arch. f. Derm. u. Syph., Sept. Igor, p. 409 '. 


\section{CHAPTER XXVII}

\section{PSORIASIS}

Der.: $\Psi$ '́pa, the itch.

Syn.: Psoriasis vulgaris; Lepra alphos (of old writers); Fr., Psoriasis; Ger., Psoriasis; Schuppenflechte.

Definition: A chronic inflammatory disease characterised by red scaly macules or slightly raised papules which spread to form circumscribed red patches covered with silvery scales.

Description.-Psoriasis is one of the most common skin affections of this country constituting between 6 and $7 \%$ of all the cases of skin disease. The characteristic essential lesion is a reddish or reddish-brown macule or slightly raised rounded papule, circular in outline, about the size of a pin's head, and gradually fading into the surrounding skin. It is covered with a fine whitish scale which if not noticeable can be made prominent by gently scratching the surface, when it assumes a distinctly silvery appearance. As soon as the red macule appears the scale may be demonstrated either by the aid of a pocket lens or by scratching, and clinically it is impossible to tell whether the erythema precedes the scaliness or vice versa, both appearing to develop synchronously. When the scale is scratched off a bright red shiny film is left, dotted over with minute red puncta which are the apices of the congested capillary loops in the elongated papillae. When the film is scratched through, bleeding from the injured capillaries occurs in the form of punctate haemorrhages, but this soon stops and the scale reforms. (Fig. 284.)

Occasionally, as a result of excessive proliferation of the epidermis and marked congestion in the papillary layer, the initial lesion takes the form of a definitely raised papule, but as a rule any thickening which may be felt is due to the scale.

The lesions extend peripherally to form more or less circular scaly plaques and may go on increasing until they reach the size of a crownpiece, but they seldom become larger, and when patches of greater size do occur, they result from the coalescence of several lesions. This tendency to increase by peripheral extension is a characteristic of psoriasis and helps to differentiate it from certain skin affections with which it may be confused, such as Lichen planus in which the patches 



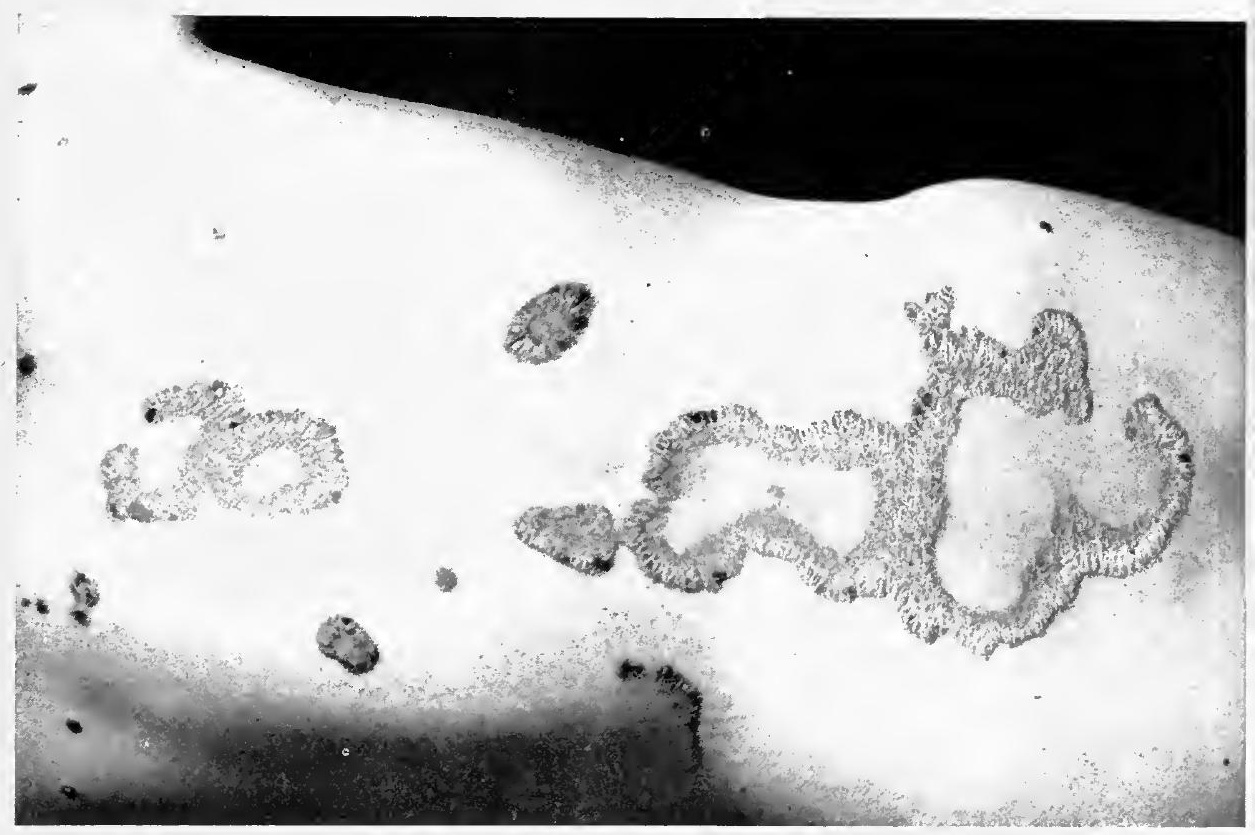

FIG. 286 -Psoriasis.

Shows gyrate lesions.

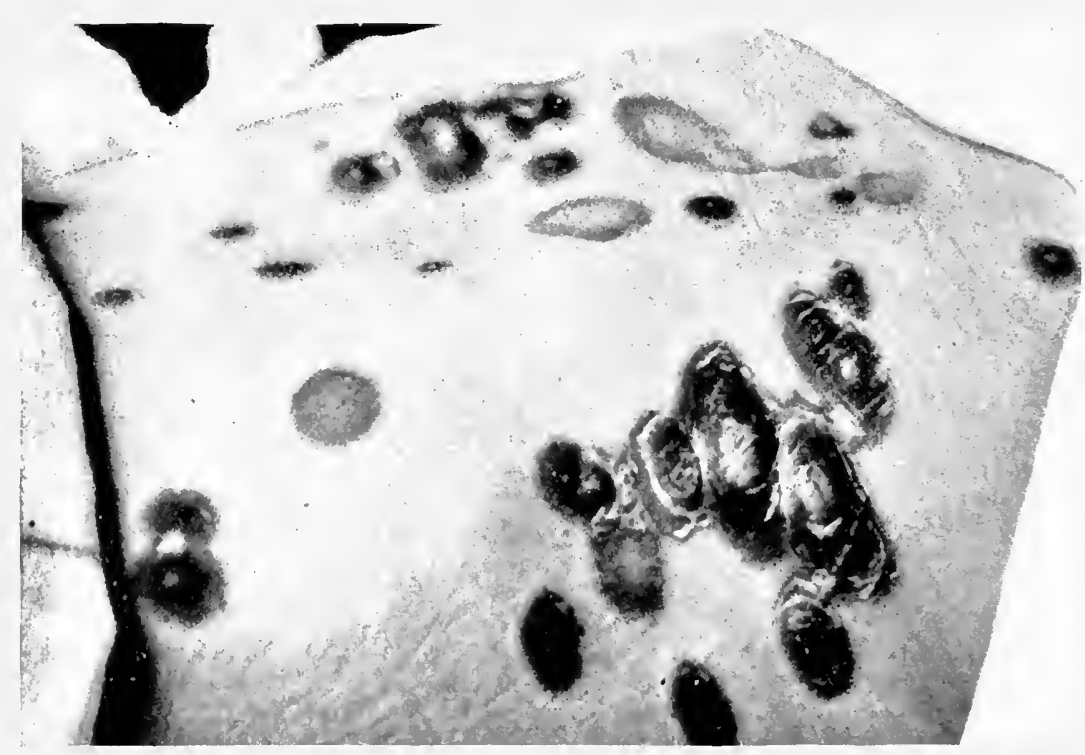

FIG. 287.-Psoriasis Rupioides.

(Sir Humphrey Rolleston's case, Brtt. Journ. Derm., Igoz.) 
are usually the result of the aggregation of a number of small initial papules. As the lesions extend new scales form beneath the original one, causing it to be thicker and more adherent in the centre than at the border, in contra-distinction to Pityriasis rosea and Tinea circinata where the scale is adherent at the edge and detached in the centre.

The large patches are circular, oval or irregular in outline, with welldefined borders, and covered by a scab or crust which is silvery from the presence of air between the squames. The older patches mày be definitely raised, indurated, and in certain situations, such as the sacral region, they may be so rough that it is possible to strike a match on them. (Fig. 285.)

The colour of the lesions varies considerably in different cases and in different situations in the same case. When the eruption is actively spreading they are a bright reddish tint, in older cases they are generally dull pink. In plethoric and alcoholic people they may assume a brownish-red or livid colour, and in dependent parts like the legs they have a cyanotic appearance.

When the patches reach the size of a crown-piece they usually cease growing and tend to fade, either all over or more commonly in the centre, forming annular or gyrate figures. When they have involuted no trace may remain, but not infrequently more or less pigmentation is left which disappears later. Arsenic has been blamed for causing the pigmentation but it may occur independently.

Variations.-No two cases of psoriasis are identical in distribution and type of eruption though they all show the same fundamental characteristics. There are several types of eruption to which it has been customary to apply distinctive Latin adjectives, but these are only of value so long as it is borne clearly in mind that they refer to differences in the manner of evolution of a single disease dependent on the peculiarities of the skin of the affected individual. The chief varieties are the following: Psoriasis punctata where the lesions are all small, not much larger than a pin's head, and occur as a rule at the sweat-orifices or the mouths of the pilo-sebaceous follicles-hence the synonym P. follicularis ; P.guttata where the majority of the lesions are like drops of mortar; $P$. nummulata where they are about the size of small silver coins; $P$. discoidea vel orbicularis where the patches do not clear up in the centre but are solid plaques; $P$. annulata vel circinata where many of the lesions are ringed; $P$. gyrata rel figurata where ringed lesions have coalesced to form gyrate figures, or more rarely have been formed by the aggregation of a number of lesions of follicular origin ; $P$. diffusa where the disease is widely distributed and large areas are involved; and $P$. rupioides, a rare variety in which the scales take the form of limpet-shaped crusts like those which occur in syphilitic rupia. (Figs. 286, 287.)

Manner of Evolution and Course.-The manner of evolution varies. The first indication may be one or more scaly patches on the extensor aspect of the leg or arm. These may remain for some time, often for 
months, and then slowly fade, or a few lesions may appear not infrequently situated on the elbows and knees, or an eruption of guttate lesions widely distributed over the trunk and limbs. Where a primitive patch occurs followed by a generalised outbreak, it suggests the possibility of a microbic cause, with the initial patch as the seat of inoculation and the gencral eruption the result of the absorption of some poison into the circulation, and the further possibility of curing the disease by thoroughly removing the primitive patch before a generalised eruption occurs.

At other times no primitive patch can be detected and the rash appears more like an exanthem, in small lesions which soon reach the size of a wafer and are widely distributed over the trunk, limbs, and scalp.

After the more generalised lesions of a primary attack have been removed by treatment, chronic more or less intractable patches may remain about the knees, elbows, and scalp, and before these have gone a fresh outbreak may take place, and in this way the disease may be kept up continuously. As a rule, however, a greater or less interval of time elapses between the original attack and a fresh outbreak. Subsequent attacks may be of the same type as the original one or of a different character. The second outbreak is as a rule more symmetrical and may be slight and confined to certain regions or widely disseminated.

Sites of Predilection.-The most common sites are the knees, elbows, scalp, extensor aspects of the limbs, and lumbar region. It may affect any portion of the skin but is least common on the face, palms and soles; it occasionally attacks the nails. It does not occur in the mucous membranes, the so-called Psoriasis linguae being leucoplakia. In forming a diagnosis, too much reliance must not be put on these so-called sites of predilection, as exceptions are frequent and it is no uncommon thing to find an attack of psoriasis in which the flexor aspects of the limbs are affected equally if not more severely than the extensor aspects, and in which lesions are present on the face. Occasionally psoriasis appears first on a scar produced by injury, operation, or vaccination.

Types of Eruption in Different Regions.-Scalp.-On the scalp it generally occurs as small patches, varying in size from a split-pea to a shilling, and tending to coalesce. These patches are clearly defined and covered with whitish or dirty-greyish scales, the scalp between them being unaffected. The growth of the hair is not interfered with even when the patches are profuse. Occasionally the earliest lesions appear on the scalp and not infrequently they persist there after the rest of the skin has been cleared, probably from the scalp having been less thoroughly treated than other regions.

Face.-In a few instances psoriasis has been known to commence on the face, but it is rare for it to be affected except when the scalp is extensively involved when indefinite red patches covered with thin scales are not uncommon on the forehead. 
The ears are occasionally attacked, especially about the concha, external meatus, and back of the ear where the skin is liable to become cracked and eczematised.

Genitalia and Perineum.--In extensive cases the scrotum and penis may be affected and the skin become cracked, inflamed, and painful. Small red spots covered with scales may appear on the glans penis, while in the female, patches may appear on the vulva. In the intertriginous regions about the anus and between the buttocks, owing to friction and moisture the lesions lose their scaliness, become macerated, and resemble patches of dermatitis due to staphylococci and streptococci.

Palms and Soles.-Psoriasis rarely attacks the palms and soles and many of the cases diagnosed as such are late scaly syphilides. When it does occur the lesions are usually symmetrical and take the form of red patches covered with scales or exfoliating in sheets, or of small thickened horny lesions, the colour of the normal skin, which can be picked out leaving a depression.

Nails.-In generalised cases the nails are usually involved, but they may be attacked when only a few lesions exist or even before the eruption develops elsewhere. All the nails may be affected or only a few. The appearances vary and the nail-plate may be opaque, thickened, of a dirty-brownish colour as if it had been stained by weak silver nitrate, and dotted over with small pits such as might have been produced by a fine knitting needle. These pits are usually filled with dirt, and may be so numerous and close together as to give the nail somewhat the appearance of a thimble. At other times, instead of pits, white spots may occur and occasionally small ecchymoses in the nail-substance owing to bleeding of congested papillae at the nailmatrix. The white spots and puncta are due to defective cornification over certain of the papillae which gives rise to the formation of small groups of moist partially cornified cells. When these dry, air replaces the moisture and white specks result, which in turn on reaching the surface become transformed into smooth puncta and finally into small pitted erosions.

When large groups of papillae are involved, white patches or striae result, which are replaced in time by depressions and furrows and the nail tends to become brittle and frayed at the free-border.

When the psoriatic process affects the nail-bed, scales form beneath the nail-plate and raise it up. These tend to crumble and break away leaving the nail detached. Occasionally the nails are so severely affected that they are partially or completely destroyed and may be shed leaving the nail-bed covered with dirty crusts.

On retracting the nail-fold a few red puncta may be seen occasionally on the posterior part of the lunule, representing congested papillae. These are analogous to the red puncta seen on removing the psoriasis scale. The nail-substance formed above them is moist and imperfectly cornified. 
Though it has been stated that psoriasis of the nails is so distinctive that the disease is capable of being diagnosed from the nail-lesions alone, this view is not generally accepted and other chronic skin affections, such as eczema and ringworm, may give rise to nail-changes practically indistinguishable from those described above.

Subjective Symptoms.--In localised and even in widely distributed attacks, the subjective symptoms may be slight or wholly absent. Occasionally when large areas are involved more or less severe itching may be present, especially in patients of a nervous disposition, and in alcoholic and gouty subjects from the circulation of toxic products.

No definite symptoms occur which would suggest that psoriasis exercises any influence on the internal organs except of a purely reflex character, as in the case of asthmatic attacks alternating with outbreaks of psoriasis.

Complications.-- Lichenification. - Where the eruption is of a chronic itchy type, as the result of continuous scratching the skin may become thickened, the natural lines deepened, the surface shiny, and the appearances produced which have been described under lichenification.

Eczematisation.-Another complication from scratching, which occurs chiefly in intertriginous regions such as the gluteal furrow or behind the ears, is the transformation of the psoriatic patches into cracked, oozing, actively inflamed and eczematised lesions. In some cases a further stage may be reached by the inoculation of pyogenic cocci and the production of pustular dermatitis.

Exfoliative Dermatitis.-The most serious complication is the transformation of the eruption into a generalised exfoliative dermatitis by the employment of too strong remedies, such as chrysarobin or tar, in a patient with a susceptible or delicate skin, or whose vitality has been diminished by some debilitating illness or from excessive indulgence in alcohol. General symptoms first occur, such as malaise, rise of temperature, a mild rigor, and generalised pruritus. An acute eruption then appears, either in patches of an erythematous or urticarial type or diffuse like scarlet fever, and may involve the whole skin, the individual psoriatic lesions being lost in the general dermatitis. The colour of the eruption gradually deepens and desquamation sets in, which varies in different cases and in different situations in the same case, sometimes being fine and branny, at other times coarse, and occasionally exfoliating in large sheets in regions like the palms and soles. When the scales come off the underlying skin is moist, shiny, and painful to the touch. As a rule the nails and hair are shed.

Under suitable treatment the desquamation generally ceases in about a month and the skin gradually becomes restored to its normal condition. 


\section{Symbiosis of Psoriasis with Other Dermatoses}

Seborrhoea.-Psoriasis may occur in a patient whose skin is oily and in whom typical lesions of Seborrhoic dermatitis are present on the scalp and trunk, when the psoriatic scales lose their silvery appearance and become yellowish and greasy. Cases of this type are intensely difficult to diagnose from Seborrhoic dermatitis and such confusing hybrid terms as "Seborrhoic psoriasis " and "Psoriasiform eczema" have been applied to them; certain writers consider that psoriasis and Seborrhoic eczema are variants of the same affection and regard psoriasis and Seborrhoic eczema as the extreme members of the group and such cases as evidences of the transition between the two. This view, however, is not generally accepted.

Syphilis.-Psoriasis occasionally co-exists with syphilis. This is generally the result of a syphilitic infection taking place in a psoriatic patient. In a case under the care of the writer in which there was a widely distributed attack of psoriasis in a man with typical syphilitic ulcerating patches on the legs, the syphilitic lesions cleared and healed under mercury and iodide of potassium leaving pigmentation, while the psoriasis pursued its course unaffected by either the syphilis or the drugs. On the other hand cases have been observed where the psoriatic lesions have become raised and slightly indurated owing to a symbiosis with syphilis.

Epithelioma has been known to develop on an old patch of psoriasis.

Histopathology.- The minute anatomy of psoriasis varies according to the type, age, situation of the lesion, and the quality of the skin affected; hence the discrepancies in the descriptions of it by different writers. There are two cardinal features in the histology about which all are agreed, namely the existence of proliferation and oedema of the epidermis, and of inflammation and elongation of the papillae in the corium. Much discussion has arisen with regard to the sequence of these changes. Clinically it seems impossible to decide whether the changes in the epidermis or the corium are primary, while histologically the difficulty is equally great, as is shown by the division of opinion between well-known observers; for example, Hebra, Kaposi, Bazin, Verotti, and others, believed that the diseased process began in the corium and was of an inflammatory nature and that the epidermal changes were secondary, while Auspitz, Unna, Neisser, Jamieson, and others, regarded the epidermal disturbance as primary. There is still a third view, which has much to commend it, namely that the changes take place synchronously.

The changes which occur are as follows:

Corium.-It is only the superficial layers of the corium which are affected in the psoriatic process, namely the papillary and sub-papillary layers. There the capillaries are dilated, the tissue around them is more or less oedematous, and there is a varying degree of peri-vascular cellular infiltration which may be present also about the sweat-glands 
and ducts and occasionally round the hair-follicles. The cells composing it are chiefly the small mono-nuclear cells, which are always present in inflammatory conditions and are indistinguishable from lymphocytes but probably take their origin from the fixed connective tissue around the capillaries and from the endothelium. In addition polymorphonuclear leucocytes may be present, varying in number according to the acuteness of the inflammatory process and the degree of secondary infection with pus-organisms, and a few oval or spindle-shaped connective tissue cells. When the oedema is considerable the collagen and elastin stain badly and are definitely rarefied. The papillae are elongated and often club-shaped and their apices extend far up into the epidermis so that only a few layers of prickle-cells exist between them and the scales, hence the readiness with which the congested capillaries are lacerated and bleed when the scale is scratched off.

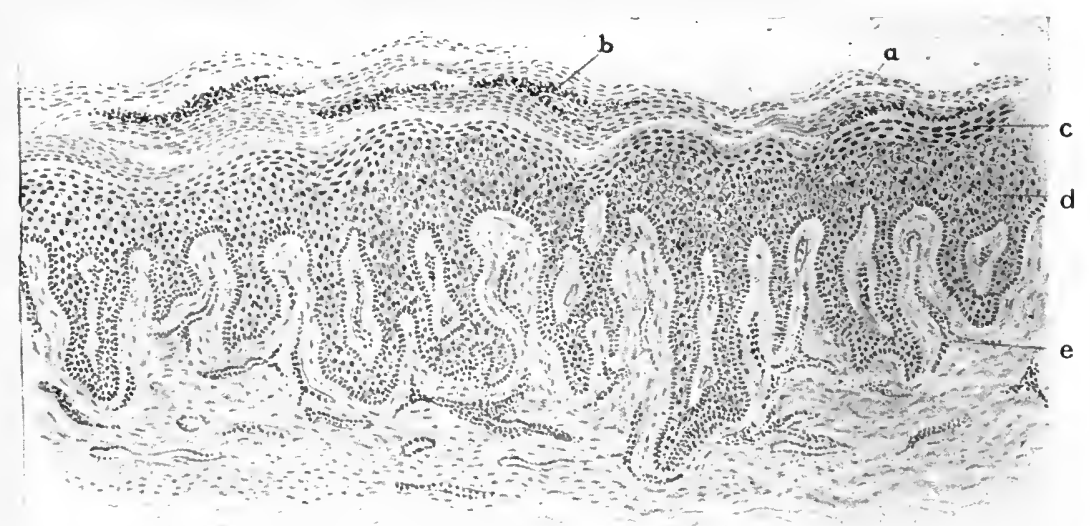

J. M. H. M. del.

FIG. 288.-Psoriasis. Section of papule. ( $x$ about I50.)

a. Imperfectly cornified scale with nuclei persisting in the horn-cells. b. Collections of broken-down leucocytes between the horny lamellae. $c$. Portion of Stratum granulosum; most of this laver has disappeared. $d$. Proliferating epidermis; prickle-cells oedematous; inter-epithelial lymphatics dilated, and leucocytes present in them. $e$. Dilated papillary and sub-papillary blood-vessels.

According to Verotti around each psoriatic lesion there is what he calls a peri-psoriatic zone which is not recognisable clinically but in which the blood-vessels of the middle and deeper layers of the corium are dilated, while the changes in the overlying epidermis are scarcely perceptible. The actual lesion he divides into a peripheral or active zone, and a central or retrogressive zone. In the peripheral zone, the papillary and sub-papillary capillaries are dilated and the infiltration is at its maximum; in the central zone, the vascular changes are less and the epidermal changes predominate. From this it would appear that the vascular changes ascend from the hypoderm to the papillary layer and diminish towards the centre of the lesion, while the epidermal changes are most marked when the vascular changes are retrogressing. These considerations suggested to Verotti that the changes in the 
corium were primary and of an inflammatory nature such as might result from a toxaemia. (Fig. 2SS.)

Epidermis.-One of the earliest changes in the epidermis is proliferation and increase of the prickle-cell layer, which tends to grow down between the papillae in long processes. The basal layer is normal but shows excessive activity in the form of an increased number of cells undergoing mitosis. According to the acuteness of the underlying inflammatory process there is more or less oedema of the prickle-cell layer, both interstitial and parenchymatous, but never sufficient to produce vesication. The inter-cellular lymphatics are dilated and may contain deformed leucocytes being carried toward the surface in the lymph. This leucocytic migration may appear before the changes in the epidermis are marked and is dependent on the degree of vascular disturbance.

The prickle-cells are swollen, moist, and stain faintly, and occasionally the oedema may cause distension of the spongioplasm so that a nuclear space is formed.

The oedema interferes with the process of cornification and, in consequence of it, the protoplasm does not form keratohyalin granules nor eleidin, and the prickle-cells instead of developing into proper horncells persist as moist swollen cells, containing the remains of a nucleus and joined to each other by partially keratinised fibres which, being soft and tough, instead of breaking away on the surface, hold the cells together so as to form squames. This impaired process of cornification, or parakeratosis, takes place unevenly and air-spaces are left between groups of cells, giving the scales their characteristic silvery appearance, while owing to the absence of keratohyalin the capillary loops can be clearly seen when the scale is removed.

Another peculiarity is the collection of masses of broken-down leucocytes and epidermal cells between the squames. These are of the nature of dry abscesses, suggest a microbic invasion, and have been regarded as the initial lesion of the disease, but this view cannot be substantiated since the abscesses are not diagnostic of psoriasis but occur also in Seborrhoic dermatitis, and no special micro-organism has been found in them but only certain well-known staphylococci.

The above are the most important histological changes in psoriasis and the chief point at issue in connection with them is whether they result from a specific virus acting first on the blood-capillaries and causing changes in the epidermis secondarily, or whether an inoculation takes place in the epidermis and the changes in the corium are produced subsequently by a process of chemotaxis. Unfortunately the histological appearances themselves do not seem capable of solving the problem, for even in the earliest lesions changes seem to have occurred both in the epidermis and the corium. 


\section{ETIOLOGY}

The true nature of psoriasis still remains unknown, notwithstanding the endless researches, clinical, microscopical, chemical, and bacteriological, which have been carried out in connection with it from the time of Hebra down to the present day. Successive generations of dermatologists in different countries have favoured this or that theory. By some it is thought to be due to a peculiar diathesis, by others to be of nervous origin and an angio-neurosis, while others again believe it to be parasitic and due to a microbe either inoculated locally or reaching the skin via the blood-stream.

Predisposing Causes.-Age.-Psoriasis may occur at any age. It rarely commences in infancy but a considerable number of cases have been reported under a year, and Rille recorded a case in an infant 5 days old. It most commonly begins between the ages of 5 and I5 and rarely after 50 , but cases are on record in old age, as in a case reported by Erasmus Wilson in which it appeared at 86 .

Sex.-In this country the bearing of sex upon the incidence of psoriasis is doubtful. On the continent, however, it is said to be more common in males in the proportion of about 3 to 2 .

Heredity.-Heredity has long been known to play a distinct part in the etiology, for the disease may attack several members of one family and frequently appears in the children of psoriatic parents. It has rarely been known to descend beyond the third generation. This hereditary influence is not the result of direct transmission but in all probability is in the nature of a family predisposition, somewhat analogous to that which occurs in tuberculosis, and points to a microbic origin and the inheritance of a suitable soil in which the parasite may readily grow.

The following table is a striking example of the bearing of heredity on the etiology and shows the disease in four generations, the sexes being affected indiscriminately. The two last cases were in the clinic at Charing X Hospital and the history was obtained from the mother. The family was Cornish and had lived at Looe for generations but not in the same house.

In the table those affected with psoriasis are marked by a cross.

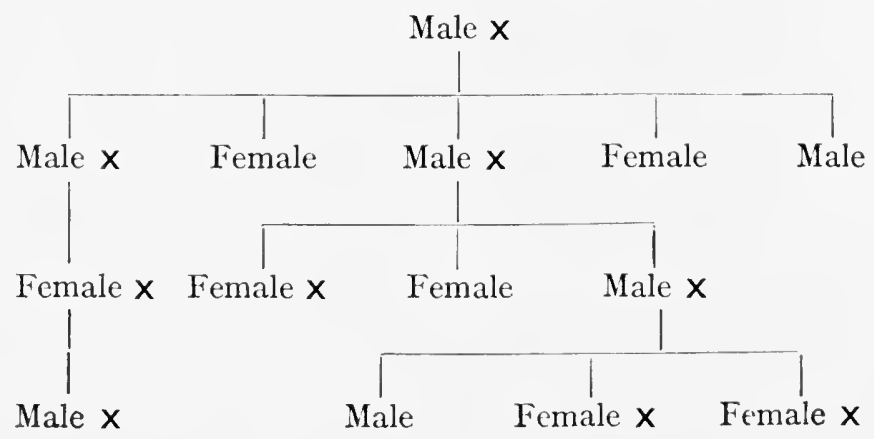


It has been suggested also that psoriasis may result from a congenital defect of the skin analogous to ichthyosis, which makes it liable to react in the form of psoriatic lessions to a variety of external and internal irritants.

General Health.-General health has a doubtful bearing on the causation. In the majority of the cases, especially in young subjects, no derangement of general health can be detected, and herice it has been described as " a disease of the healthy." On the other hand, even in young people, but more commonly in those over 30 , it is not infrequently associated with rheumatoid symptoms, anaemia, gout, indigestion, and various evidences of faulty assimilation, and occasionally with neurasthenia. Although none of these derangements can cause psoriasis they may still play an important part in reducing the general resistance and so predisposing the individual to an attack, and once the disease has been established they are liable to determine a recurrence. Many instances are on record where relapses in a psoriatic patient seemed to be the result of some serious or prolonged illness, parturition, lactation, or the menopause, or appeared to be directly determined by nervous disturbances, such as anxiety, worry, mental strain, or violent emotion.

Social Position.-Social position does not appear to influence it and it is equally common among rich and poor.

Climate.-Though it may occur in all climates, it is much more common in cold than in warm countries, and is comparatively rare in the tropics. It is more common in the Northern countries of Europe than amongst the Latin races in the South, and is more prevalent in Great Britain than in Eastern Europe. It is particularly rife in Iceland where it has been estimated as forming $8 \%$ of the skin diseases. When it occurs in dark-skinned people it assumes a milder form.

Season.-Season appears to have some influence in the inception of the disease and in causing it to recur. It not infrequently begins in spring and recurrences often take place in that season, while there is a tendency to recovery towards the end of summer. It may also begin or recur with the onset of the cold weather in autumn or winter.

Traumatism and Vaccination.-In subjects of psoriasis the situation of lesions may be determined by local injury, friction and pressure, or the stretching of the skin over bones as about the elbows and knees. The initial lesion has been known to appear on a vaccination, smallpox, or operation scar, on the site of a vesicle of Herpes zoster, and on a tattoo mark, and to spread by new lesions in the neighbourhood. In connection with the cases in which it has begun on vaccination scars, it has been suggested that a special microbe was inoculated at the time of the vaccination but, as it has occurred after vaccination both by human and bovine lymph and as animals are not known to be susceptible to it, this view is untenable. It is probable, however, that these various forms of traumatism, by locally reducing 
the vitality in a susceptible individual, render the soil suitable for the development of the disease.

Actual Cause.-The three main hypotheses with regard to the actual cause, are (I) that it is not a disease per se but is a symptom of a general diseased state or diathesis, and that it results from the action of toxins circulating in the blood; (2) that it is a tropho-neurosis; (3) that it is due to a micro-organism.

Though their advocates have cited a certain number of facts in support of each of these theories, not one of them has been definitely established, but it will be advisable to refer to a few of the arguments advanced in their favour.

I. Diathetic Theory.--It has frequently been asserted, especially by French writers, that psoriasis is an evidence of the deranged blood state to which the name Arthritic diathesis has been given and which corresponds to what is known in England as the gouty diathesis. But although the lesions of psoriasis are unusually irritable and inflamed when they are associated with gout, there is no evidence that the gout is the cause of them.

It has been said also to be a skin manifestation of rheumatism, and the not infrequent coincidence of psoriasis with various arthropathies such as arthritis and articular pains lends support to this view. But like the gouty hypothesis it is not based on any definite fact and it is generally regarded as little more than a cloak for ignorance.

The theory that it is a general disease due to the circulation of some toxin in the blood is supported by the facts that it may come out almost as suddenly as an exanthem, tends to be symmetrical, and may be followed by attacks of exfoliative dermatitis, ushered in by more or less acute general symptoms. The nature of the hypothetical toxin is unknown. It has been regarded by some writers as a product of mal-assimilation and deranged metabolism, and by others as resulting from a disturbance of the functions of the kidneys. Digestive troubles, errors of assimilation, and imperfect action of the kidneys undoubtedly aggravate an attack and may be capable of determining a recurrence, but there is no conclusive evidence that these derangements, or the toxins which circulate in the blood in association with them, can cause the disease de novo.

From time to time various blood-changes have been reported in connection with psoriasis which suggest the action of a toxin, such as a diminution in the haemocytes and haemoglobin and an increase in eosinophiles, but these are so inconstant that they are of little or no significance.

Errors of diet, and chiefly excessive meat-eating, have been advanced as a cause of psoriasis, but there seems to be no definite evidence in favour of this view. According to Pollitzer, in U.S.A. where the meat consumption is greater than that in Great Britain or any country in Europe, the percentage of cases of psoriasis is only 3, while in European countries it varies from 5 to 7 , and Audry has stated that in Toulouse, where little męat is eaten and gout is very rare, psoriasis is as frequent as in other countries.

2. Neuropathic Theory. - This theory was suggested by attacks of psoriasis having been observed to follow, and to be aggravated by, severe mental emotion, shock, grief, hysteria and anxiety; by their occurrence in association with severe neuralgias, epilepsy, chorea, etc. ; by the lesions seeming to develop in the areas of distribution of peripheral nerves or starting at points of cutaneous irritation; and by tactile and thermal sensations being diminished over the patches. It has been described as 
the result of " congenital functional weakness of the nerve centre regulating the function of the skin" (Weyl).

The association of the disease with neuropathies may however be purely accidental, and no actual pathological changes have been discovered in the nerves supplying the patches, no one has succeeded in showing why a tropho-neurosis should produce it, and there is no rational explanation why mental shock should cause such an eruption. The theory cannot therefore be accepted, except in so far that nervous disturbances may aggravate the disease once it has been established or may exert a depressing influence which may render the individual susceptible to it.

3. Parasitic Theory.-The parasitic theory demands more careful consideration than those which have been mentioned above, since the majority of cases of psoriasis are explicable by it.

There are two distinct views in this connection; (I) that the disease is a local parasitic affection, and that each lesion is due to the presence of micro-organisms locally inoculated; (2) that the lesions are due to microbes which have reached the skin via the blood-stream. There are several strong arguments against the former theory, such as $(a)$ in acute cases lesions may develop over the trunk, limbs, and scalp, almost with the suddenness and spontaneity of an exanthem, which would be difficult to explain if they were due to a purely local parasitic inoculation; and (b) the lesions tend to be distributed symmetrically and to occur on sites such as the elbows where contact is practically impossible.

The second theory that it is a general microbic disease is supported by the eruption often being symmetrical and sometimes appearing suddenly like an exanthem, and opposed by there being no general febrile disturbance such as occurs in every other constitutional infectious disease, nor any evidence of the occurrence of disease in the internal organs. It is true that it may be associated with attacks of asthma which may concur or alternate with it, but asthma is probably a neurosis and the psoriasis may act simply as an exciter or counter-irritant to it, and this argument is more in favour of the neuropathic than the parasitic hypothesis.

Clinical arguments in farour of a parasitic theory.-(a) The lesions may spread, evolve, and vary, like those of a parasitic disease such as Tinea circinata.

(b) The eruption may be removed by the local application of antiseptic remedies.

(c) Several instances are reported where apparent contagion took place. Unna describes the case of a nurse who gave the disease to three children under her charge who were free from hereditary taint. A few instances are also on record where a husband has communicated it to his wife and rice versa, or where a parent has developed psoriasis after a child has been subject to it. Destot, after a number of failures, inoculated his deltoid region by scarifying it and rubbing in psoriatic scales from an infant, and claimed to have succeeded in producing an eruption of psoriasislike lesions on the elbow I d days later. But this experiment is not quite convincing, as no lesions dereloped on the site of the inoculation and the patch of dermatitis which did appear may have been Seborrhoic dermatitis which is undoubtedly contagious. Lassar stated that he had been able to inoculate a squamous disease like psoriasis in animals, but others who have repeated his experiments have obtained negative results. On the other hand a number of failures to inoculate the disease have been recorded, so that if it be inoculable it is only under certain conditions which are at present unknown. It must be remembered, however, that some admittedly parasitic diseases such as Pityriasis versicolor are rarely if ever inoculable. Schamberg performed a series of auto-inoculation experiments on psoriatic patients in which he succeeded in obtaining a positive result only when there was a marked tendency to the development of new lesions, when the 
success could be explained as the result of traumatism. In the great majority of cases when the eruption was circumscribed or the lesions were undergoing involution the results were negative.

Though various parasites have been put forward from time to time as the cause of the disease none has yet been established. The fungus which was described by Lang and corroborated by Wolff is no longer accepted. The micrococcus of Unna has been abandoned, and the micrococci of several other writers have not been proved specific. It has been suggested that the microbe may be one of the ordinary denizens of the skin which has become virulent owing to a peculiar susceptibility of the individual, but the uniformity in character of the lesions would suggest a specific microbe.

Diagnosis.-The diagnosis in a well-marked case presents no difficulty, unless when only a few lesions are present and these are ill-defined and occur on unusual situations. In every case the initial or essential lesion should be sought for and studied, as a fully evolved patch may be so altered by adventitious circumstances, such as its existence in a moist intertriginous region, its occurrence on a greasy seborrhoic skin, or the secondary inoculation of pus-organisms from scratching, as to be almost unrecognisable. If, on the other hand, a small early lesion be found with a scale which, on being scratched off, leaves a thin membrane through which the apices of the congested papillary loops may be seen as fine red puncta, and this in turn on being lacerated leads to bleeding, the diagnosis is practically established.

The cutaneous affections with which it is most likely to be confused are the following :

Seborrhoic dermatitis.-Typical Seborrhoea corporis, or Seborrhoic dermatitis can be distinguished from psoriasis by the following characteristics :

In Seborrloic dermatitis the essential lesions are less vivid in colour, being of dirty yellowish-pink tint instead of bright red, and are irregular and ill-defined; the scales are greasy, thin and yellowish in colour instead of being dry, profuse and silvery ; the most common distribution in addition to the scalp is the sternal and interscapular regions, axillae and groins, while in psoriasis the most common sites are the elbows, knees, and extensor aspects of the limbs; the scaliness is more diffuse on the scalp (Pityriasis sicca) while in psoriasis it occurs in circumscribed patches; in the scales the flask-shaped bacilli of Malassez can usually be detected which are absent in psoriasis.

In addition to the typical cases, transitional forms may occur in which the diagnosis is difficult or practically impossible and to this class of case Crocker applied the name "Seborrhoea psoriasiforme." So similar are these cases that a doubt has been raised in the minds of certain observers whether after all Seborrhoea corporis and psoriasis are not simply extreme types of one disease ; the majority, however, still maintain that they are distinct affections.

Eczema.-As a rule there should be no difficulty in diagnosing psoriasis from eczema, but cases of chronic eczema occur in which the skin has been lichenified by scratching and weeping lesions given place 
to dry scaly patches, where the diagnosis is extremely difficult. It is further complicated by psoriatic lesions in moist regions, such as behind the ears and between the buttocks, readily becoming eczematised.

In eczema the patches are not so clearly defined, the colour is less vivid, and the scaliness is neither so profuse nor so silvery as in psoriasis. As a general rule in an extensive case of eczema the skin is more uniformly affected and it is exceptional not to find typical papulo-vesicular lesions or some evidence of punctate weeping.

Syphilis.-One of the diseases with which errors of diagnosis are most liable to occur in connection with psoriasis is syphilis. Nor is this to be wondered at when it is remembered that syphilis is the great imitator and that in the secondary period, eruptions with punctate, papular, circinate, and gyrate lesions may be present, which in their general configuration simulate the corresponding phases of psoriasis. Of these the most likely to be confused with psoriasis is the mediumsized scaly papular syphilide to which the misnomer "Syphilitic psoriasis" was once applied.

The following are the most helpful points in the differential diagnosis :

(I) In the syphilide the scales are more delicate than in psoriasis, instead of being silvery are of a dirty-whitish tint, and not infrequently break in the centre and adhere at the margin to form a collar round the lesion, while in psoriasis the scale is thicker in the centre and frequently detached at the margin. When the scale is scratched off in syphilis (by a piece of glass or a scalpel) bleeding does not take place so readily as in psoriasis.

(2) The colour of the lesions differs, for instead of being bright pink or reddish as in psoriasis, the syphilides are yellowish-brown and somewhat translucent.

(3) In psoriasis, when the scale is-removed there is little or no thickening of the tissue, while in all the scaly syphilides there is distinct induration. When the blood is pressed out of the lesion by a piece of glass or diascope, if the scale be present the psoriatic lesion is yellowish or grey, and the colour disappears under pressure if the scale be remored; in the syphilide it has a translucent yellowish-brown appearance which no amount of pressure can obliterate. In the one case the yellow tint is due to the scale, in the other the brown tinge results from the granulomatous infiltration in the corium.

(4) The syphilitic patches are more uniform than those of psoriasis and remain the same size, while those of psoriasis are more irregular and extend peripherally.

(5) When circinate figures and gyri occur in secondary syphilides they are produced by the peculiar grouping of a number of uniform small papules, while in psoriasis they result from a tendency of the lesions to fade in the centre and to spread at the periphery.

(6) The distribution differs in the two diseases for while in syphilis the face and the flexor aspects of the limbs are usual sites these are 
rarely affected in psoriasis. Too much weight, however, should not be placed on this distinction as anomalous cases of psoriasis are not infrequent in which the sites of predilection such as the elbows, knees, and scalp, are not involved.

(7) As a general rule syphilitic lesions do not itch while those of psoriasis may do so, but this is not by any means a certain criterion.

(8) The general health of syphilitics during the secondary period is frequently impaired and other stigmata of the disease may be present, such as adenitis, sore throat, mucous patches, etc., whereas in psoriasis the general health may be excellent and collateral evidences of the disease be absent.

(9) If a definite diagnosis cannot be made by the clinical appearances an examination should be made for the Spirochaeta pallida and a Wassermann reaction done.

Two other forms of syphilis require special mention in this connection, namely syphilitic rupia and palmar syphilides.

The so-called Psoriasis rupioides is distinguished from syphilitic rupia by the crusts being yellowish-white in colour while those of syphilis have a dirty-brownish tint, and by a smooth red membrane being left when the psoriatic crust is removed, which is easily done, while if the syphilitic crust be detached, which is more difficult, an ulcerated surface remains.

The late palmar syphilide not infrequently has been confused with psoriasis and the unfortunate term Syphilitic psoriasis of the palms which has been applied to it has increased this confusion. Psoriasis of the palm is a rare condition which usually occurs in the form of dense, scaly or horny thickenings of the epidermis with little or no redness, is generally bilateral, and comparatively amenable to local treatment ; the palmar syphilide on the other hand is definitely inflamed and consists of pinkish-brown patches either denuded or covered with scales which are usually delicate but may be thickened in those who do manual labour, is frequently unilateral, and is most resistant to antisyphilitic treatment and to local applications.

Pityriasis Rosea. - There should be no great difficulty in distinguishing psoriasis from this affection. Though they may both commence with a primitive patch it is rare for psoriasis to do so while it is the usual occurrence in Pityriasis rosea. The lesions of Pityriasis rosea are of a rose pink tint and covered with delicate branny scales adherent at the border, those of psoriasis are more red and vivid, and the scales coarser and adherent in the centre; the typical lesion in Pityriasis rosea is oval in shape with a scaly border and a fawn-coloured centre, while in the circinate lesions of psoriasis, the centre is of the same colour as the normal skin. The distribution is also different for in Pityriasis rosea the trunk is most affected, the disease less marked in the limbs, and the sites of predilection of psoriasis are not particularly attacked. The course of the disease is as a rule much shorter in Pityriasis rosea. 
Tinea Circinata.-Circular plaques and circinate lesions of ringworm of the glabrous skin may occasionally resemble psoriasis. The colour may be much the same in both but the scaliness is less marked in ringworm. In the absence of the small resicles at the margin of the lesions which characterise certain ringworm infections, any doubt may be put at rest by examining for the ringworm fungus.

Lichen Planus.-Chronic scaly patches of Lichen planus may sometimes simulate psoriasis. In such cases the typical shing polygonal lesions should be sought for, as they are almost invariably present in Lichen planus. The scaly patches of Lichen planus generally have a violet tinge, are harder and thicker than chronic patches of psoriasis, the scales upon them are more adherent and granular, and the surface has a shagreen-like appearance. They are often associated with intense itching and when they fade deep pigmentation is usually left.

Prognosis.-The prognosis varies so greatly in different cases that it is impossible to form any idea of what course an individual case will take. Nor is the general state of health any aid to forecasting the severity of the attack for whether the patient be debilitated or robust it may be mild and localised in one case and extensive in another. The disease as a rule does not tend to involute spontaneously; though it may do so, and if left alone it is apt to be steadily progressive.

As a general rule an attack, however extensive, can be cured in about a month by suitable treatment thoroughly carried out.

It is in the nature of the disease to recur, in some cases the attacks being practically continuous, while in others they take place at intervals of six months to several years. $\mathrm{By}$ energetic treatment and by a modified local regime in the intervals the extent and severity of the attacks may be gradually reduced and in some cases they may finally cease.

\section{TREATMENT}

The two great principles in the treatment of any disease are elimination of the cause and removal of its effects. The cause of psoriasis still remains unknown, but our clinical and histological knowledge of its effects is sufficient to enable us to treat it in a rational fashion with every hope of success. The treatment, which is chiefly local, must be thorough and should be continued till every trace of the affection has been removed, otherwise an early recurrence is almost inevitable.

General Management.-When the psoriasis is acute, actively spreading, and widely disseminated, one of the most important principles in the general management is rest, if possible complete rest in bed. By this means the skin's activity is lessened, much of the irritation from friction and compression to which it is subjected when moving about is avoided, tension in the cutaneous blood-ressels is diminished, and local treatment can be more thoroughly carried out. Equally important 
is mental rest, rest from worry, anxiety and overwork, for it is practically impossible to get rid of an extensive attack so long as the patient is harassed by mental anxiety or business worries.

Diet.--A good deal has been written with regard to food in psoriasis and various articles of diet have been prohibited. Indulgence in meat has been specially blamed for aggravating the disease and it has been suggested that a vegetarian diet may effect a cure. No one has proved, however, that it can be caused by the eating of meat and statements to that effect have been somewhat vague, such as that it occurs more frequently in those whose urine is excessively acid and its nitrogenous content increased as the result of a too generous meat diet, and that it is more common in butchers than in other trades. Excessive meat eating is bad for anyone, but there is no proof that it is specially harmful to psoriatics.

No object appears to be gained by a fancifully restricted diet, and all that is necessary is plain simple nutritious food and the avoidance of anything likely to produce toxic eruptions on the skin, such as curries, shell-fish, sardines, pickles, eels, strawberries, etc. It is well to remember also that one man's food is another man's poison, and to guard against any idiosyncrasy on the part of the patient.

With regard to condiments, strong coffee should be avoided and tea taken in moderation. Alcohol as a general rule should not be permitted as it dilates the biood-vessels, increases the heart's action, and tends to aggravate the disease. Occasionally under special circumstances, where the digestion is feeble and the patient fatigued, it may be allowed in the form of small quantities of spirits in plain or mineral water, but ale and acid or sweet wines should be prohibited invariably.

Internal Treatment.-Any definite derangement in the general health, such as disorders of the alimentary tract, liver and kidneys, asthmatic attacks, bronchitis, and cardiac defects, must be dealt with on general medical principles. The association of psoriasis with rheumatism is comparatively frequent and should be combated by salicin and the salicylates. In nervous or hysterical people with psoriasis it is often an advantage to prescribe a sedative mixture containing valerian or bromide.

Where there is much tension and irritability of the skin, antimony often gives relief and may be prescribed in the form of the vinum 5 to ro $\mathrm{m}$. twice daily, but owing to its depressing action must not be used for aged or debilitated people.

Where no definite defect in general health can be detected it is customary to resort to one or other of the so-called internal specifics, of which the most important are arsenic, thyroid extract, mercury, and salicin.

Arsenic.-Until comparatively recently, arsenic was almost universally regarded as a specific for psoriasis, and was prescribed so indiscriminately that much harm was done by it and its value largely 
discredited. Its action varies greatly in different cases, and it is almost impossible to predict when it will be beneficial. In some it appears to be so effective that no local treatment is necessary, in others even after prolonged use, it seems to have no definite effect on the disease and the eruption continues to spread in spite of the arsenic being pushed to the limit of toleration. It is specially indicated in early cases without much hyperaemia, when the eruption has reached its height and become stationary, and in first attacks in children. It should not be employed in acute cases, where there is much inflammation and the eruption is still developing. It is of little or no value for chronic patches or for psoriasis of the nails. It should on no account be given when there is any tendency to gastritis or other derangement of the alimentary tract as it will irritate the gastro-intestinal mucosa and so indirectly aggravate the skin affection. It is best given in small quantities well-diluted with water after meals, and should be stopped at once if any toxic symptoms supervene, such as dryness of the throat, conjunctivitis, pains in the abdomen, vomiting, diarrhoea, and insommia. In any case, however favourable to its use, its action is slow and as it has to be given over a prolonged period it is liable to cause pigmentation of the skin and hyperkeratosis of the palms and soles.

Its manner of action is not completely understood. Some observers believe that it has a "dynamic action" on the skin in virtue of the alternate oxidation and deoxidation of arsenious to arsenic acid at the expense of the protoplasm of the living cells, which results in stimulation of the epidermis, improvement in the process of cornification, and reduction of the inflammatory changes in the corium. Others, while granting that it is eliminated by the sweat-apparatus and so acts on the skin, believe that its beneficial effect is solely dependent on its tonic action on the nerrous system. Others again consider that it is inimical to the virus of the disease or renders the soil unsuitable for its growth.

Of the various preparations of arsenic the Liq. arsenicalis (Fowler's solution) is the one most generally employed. Small doses such as $3 \mathrm{~m}$. t.d.s. should be given at first, and increased up to $I_{5} \mathrm{~m}$. t.d.s. or whatever the limit of toleration may be. The full dose should be continued for several weeks and when improvement results should be diminished gradually. The addition of Tinct. lupuli jss to each dose has been suggested for the purpose of favouring the toleration of the drug, but this procedure is not to be commended as it only masks the toxic symptoms.

The Liq. arsenicalis may be given also by hypodermic injection of 3 to $5 \mathrm{~m}$. in a few drops of distilled water daily. This is said to be more efficacious than by the mouth and not so liable to produce gastrointestinal symptoms. Should it be decided to employ hypodermic injections, however, the Liq. sodii arseniatis should be substituted, as it is less irritating, causes little pain when injected, is more stable in its 
composition, and can be sterilised by boiling. Daily injections of $3 \mathrm{~m}$. should be given at first and gradually increased according to the toleration of the patient. In Vienna the favourite method of prescribing it was in the form of the Asiatic pill which contained $\frac{1}{1 \cong}$ of a gr. of arsenious acid with I gr. of black pepper. Kaposi used to prescribe one to four of these t.d.s. and continued until the patients had taken five or six hundred of them, only stopping when toxic symptoms supervened.

Various organic compounds of arsenic have been advocated. One of the first to be used was cacodylic acid, a combination of arsenic with methyl radicals, which was given in doses of $\mathrm{I} \frac{1}{2}$ grains t.d.s. The sodium salt known as cacodylate of sodium was also extensively employed in France, and given in doses of I to 3 grs. by the mouth or a $\frac{1}{5}$ to I gr. by hypodermic injection. These doses are apparently very large as I gr. of cacodylate of sodium contains as much arsenic as $3 \mathrm{i}$ of the Liq. arsenicalis. According to Fraser, however, the therapeutic value of this drug has been overrated and when taken into the body he considers that it is not dissociated but passes through, without exerting either its toxic or therapeutic action to the full extent.

Recently the different members of the arylarsonate group, namely atoxyl or soamin, arsacetin, and orsudan, have been employed. They are all given by hypodermic injection in doses of $\frac{1}{2}$ to 3 grs. dissolved in warm water and injected twice a week. Arsacetin and orsudan have the advantage of greater stability than the others, but their relative therapeutic merit has yet to be decided. They do not appear to give any more certain results than arsenic prescribed in any other form and as they contain a large percentage of arsenium they are dangerous to employ indiscriminately and serious results such as optic atrophy have been known to follow their use. Salvarsan and its substitutes so far have given uncertain results.

A course of the arsenical waters at La Bourboule, Roncengo, and Levico, has been recommended but though these natural springs do contain a percentage of arsenic it is a small one and any benefit which may be derived from them is probably the result of the copious draughts of the waters, the change of climate, and the general regime, rather than of the specific action of the drug.

The various methods of administering arsenic have been referred to somewhat fully, as no description of the treatment of the disease can be said to be complete without so doing, but the drug has been found to be so inconstant in its action that the old faith in it is being lost, and it has even been suggested that in the cases which have seemed to do well under its administration the improvement took place in spite of the arsenic, or as a result of its tonic action on the nervous system, rather than from any specific effect.

Thyroid Extract was introduced some years ago by Byrom Bramwell for the treatment of psoriasis and since then both it and its derivatives, iodothyrin and thyroidin, have been tried extensively. The extract 
is given in doses of $2 \frac{1}{2}$ to 5 grs. per diem and gradually increased to Io to $I_{5}$ grs. Its action is as uncertain as that of arsenic. In some cases a clearance of the eruption has been known to take place in a few weeks under its use without any other treatment, but as a rule it does little good and may do much harm. Unfortunately there are no certain indications of the exact type of case which is likely to be benefited by it, though it is said to be most useful in widespread cases in which the eruption has ceased coming out and is stationary. If large doses are employed the patient should be kept under close observation in case toxic cardiac symptoms, such as tachycardia, should superrene. On no account should it be given in patients over 50 and especially in those with weals hearts, as serious symptoms may be produced which more than counteract its doubtful utility.

Salicin and the salicylates have been strongly recommended by Crocker as substitutes for arsenic, and especially salicin as it is less liable to disagree than the sodium salt. Salicin is believed to act as a microbicide by breaking up into salicylic acid and carbolic acid. It is said to be most useful in acute widely spread cases and when given in full doses I $_{5}$ to $20 \mathrm{grs}$. t.d.s. after meals. Where depressing symptoms and headache are caused by the drug it may be combined with small doses of nux romica. While admitting that it is undoubtedly beneficial in cases associated with rheumatic symptoms experience of its use has been disappointing and it is uncertain whether it has any action on the disease except as a depressant.

Various alkalies have been employed and are useful in gouty and rheumatic subjects. Of these Liq. potassae ro m. t.d.s., acetate of potash Io to 30 grs. t.d.s., and iodide of potassium in large doses of 20 or 30 grs. t.d.s. have been adrocated chiefly. Though the large doses of potassium iodide are fairly well tolerated it is a most uncertain remedy and has no specific action.

Certain other drugs have been recommended, such as mercury, either by the mouth or by intramuscular injections of the soluble salts, in doses similar to those employed in the treatment of srphilis, and carbolic acid in I gr. pills, 3 to 6 being taken per diem (Kaposi), but these have no claim to specific action.

Serum Treatment.-Treatment of psoriasis by serum, both autogenous and foreign, and by normal horse serum, has been tried, the serum being injected either subcutaneously or intravenously. So far it has been found to be of little value when given alone but has been said to have a favourable influence when combined with suitable local and general treatment.

In conclusion it may be said that a specific for psoriasis does not exist and that the remedies which have had this reputation have gained it probably from being used at a time when the disease was fading. At the same time it must be conceded that in certain cases arsenic has a beneficial effect, probably by acting indirectly as a nerve tonic and causing a general improvement in metabolism. 
Change of Climate.-A change of scene, and change of air from a bracing to a milder climate, may be of great value in chronic resistant cases. On the other hand in young patients who are run down a change to the seaside with sea-bathing, or a sea voyage, may do more good than any form of medicinal treatment, though in certain cases the sea air seems aggravating.

It is futile to attempt to cure a widely distributed attack so long as the patient is attending to work and harassed by business, and to lay him up at home where he is in easy reach of his office and the telephone, does not help matters, for he is still in touch with his work and chafes at not being able to attend to it personally. In such cases a complete change to a continental spa with its associated regime is of the greatest benefit. The chief "cures" for psoriasis are at Aix-les-Bains where the patients are kept in warm baths for long periods, a type of treatment which is not to be recommended in elderly people as it may cause fainting; Harrogate, Strathpeffer, Aix-la-Chapelle and Barèges, which are beneficial on account of their sulphur baths and waters and especially valuable in the chronic stages of the disease; and La Bourboule, Roncengo, and Levico, which are advocated on account of their arsenical waters.

Local Treatment.- The success of the local treatment depends almost as much on the thoroughness and detail with which it is carried out as on the actual remedies. When only a few localised patches are present, they can be treated successfully by the patient himself without interference with work, but when the lesions are more extensive and in situations which are not easily got at, the remedies should be applied by an attendant, ambulatory treatment may become difficult or impossible, and it may be advisable to confine the patient to bed.

The first indication in the local treatment is the removal of the scales. Should the disease be extensive this can be done best by baths. A daily warm bath at $95^{\circ} \mathrm{F}$. to $\mathrm{IOO}^{\circ} \mathrm{F}$. with the addition of $\frac{1}{4} \mathrm{lb}$. of bicarbonate of soda to the 30 gallons of water should be given. The patient is immersed in the bath for about twenty minutes, and the patches are scrubbed with soft soap to which $5 \%$ Liq. potassae may be added to make it more alkaline. Should the lesions be unusually indolent they may be rubbed with pumice stone or sand soap.

In very acute inflammatory cases, alkaline baths may prove too strong and irritating, and emollient baths containing 2 lbs. of starch or bran to the 30 gallons of water may be substituted. Where there is marked itching the addition of an ounce of the Liq. picis carbonis to the bath is soothing, or where the skin is greasy owing to the coexistence of seborrhoea an ounce of Potassa sulphurata should be added. After the bath each patch should be rubbed with the following ointment:

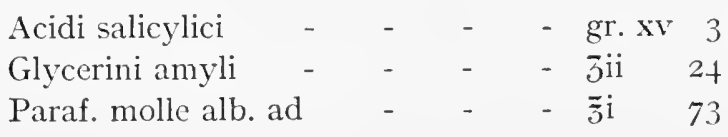


or simply with olive oil or vaselin if the lesions be painful. Other methods have been employed, such as wet packs and Turkish baths, but are less effective.

Where the lesions are few but of a chronic resistant type with thich adherent scales, more powerful measures must be adopted, such as the application of soft soap dressings covered by oiled silk. These should remain on for a few hours, after which the lesions should be scrubbed with soft soap and hot water, and then rubbed with $5^{\circ}$ o salicylic lotion in alcohol or with a creolin solution in water, and finaliy dressed with lint soaked in olive oil continuously applied under oiled silk; or salicylic soap plaster may be substituted for the lotion.

For the removal of scales on the scalp the following procedure should be adopted. The scalp should be shampooed with soap liniment containing :

\begin{tabular}{|c|c|c|c|}
\hline Saponis riridis & - & - & - \\
\hline Spiritus vini rect. & - & - & \\
\hline
\end{tabular}

A little of this liniment should be poured into a saucer and rubbed into the scalp by a piece of wet flannel, the hair being parted in different places to enable the whole of the scalp to be carefully gone over. After the washing and drying is complete the following ointment should be applied:

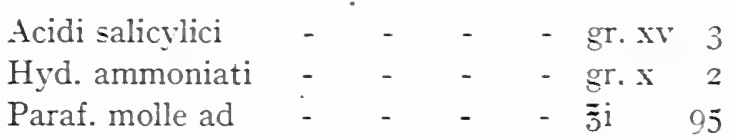

To aroid the ointment soiling the pillow at night a bathing cap may be worn. Should the scales be unusually thick and adherent they may be softened previously with olive oil. This procedure should be repeated as often as necessary. The removal of the scales in the scalp is greatly facilitated by cutting the hair short.

When the scales have been remored one or other of the so-called reducing agents should be employed with the object of attempting to improve the cornification and diminish the tendency to the formation of scales. Of these the most important are chrysarobin and tar, and of less value pyrogallol, beta-naphthol, salicylic acid, resorcin, and mercury. These reducing agents are said to act by subtracting oxygen from the tissues. Care and judgment are necessary in making a choice for several of them are powerful irritants and must be used with caution to aroid producing excessive dermatitis; they should be employed weak at first and their strength gradually increased.

In psoriasis, as in almost every form of skin disease, each case must be considered and treated on its own merits, and even different patches in the same case may require different treatment. When the patches are few and wide apart each lesion may be treated separately and strong remedies may be employed; when they are close together and numerous, it is practically impossible to treat each lesion by itself and 
the ointment or other application, having to be rubbed over large areas, requires to be much weaker.

Should the case be unusually irritable and the lesions much inflamed, before using any of these reducing agents it is necessary to allay the inflammation by dressing the parts continually with lint soaked in a solution of Glycerinum plumbi subacetatis ( $\mathrm{I}$ in 8 ) or by applying calamine cream. It must be understood, however, that the action of the reducing agents is always an irritating one and that their relative value may be said to be directly dependent on the degree of inflammatory action which they excite.

Chrysarobin is the most powerful of the reducing agents and requires to be employed with caution as, when used on an irritable skin or in individuals with an idiosyncrasy towards it, it is capable of causing serious inflammation and may transform psoriasis into exfoliative dermatitis. Great care must be exercised to prevent it reaching the eyes as it is liable to cause severe conjunctivitis, and consequently it should not be employed about the face or scalp. When used too extensively it may be absorbed and cause toxic symptoms such as pain, fever and insomnia. Another disadvantage connected with it is that it stains the hair yellow, the skin a brownish-purple, and the clothing a dirty-yellow colour which is turned by the alkali of soap in washing into an indelible purpie. In consequence of these peculiarities it is inadvisable to trust the remedy in the hands of any but the most careful patient and it is better to restrict its use to cases which are under close medical supervision.

Chrysarobin acts by exciting a powerful inflammatory reaction. This causes dilatation of the blood-vessels around the patch before those of the patch itself are affected, so that at one stage in the treatment the psoriatic lesion appears white in the centre of a purplish-red inflamed area. The treatment should not be stopped here, as the whiteness indicates that the disease is still present, but should be continued till the patch itself is inflamed. Chrysarobin is most suitable in chronic resistant patches and in extensive cases in which there is little inflammation and the disease has reached its height. It should not be used in acutely inflamed actively spreading cases, unless in a weak form such as 2 grs. in Ungt. zinci oxidi.

The procedure in an extensive case is as follows: After the scales have been removed by an alkaline bath and soft soap, a $6 \%$ ointment in vaselin is rubbed into the affected skin by a piece of fiannel or applied by a stencil brush to avoid staining the fingers. The same ointment is also spread on lint and the lesions dressed with it once a day under a bandage or a binder, a linen mask being worn except at meals to protect the face. As the ointment is apt to irritate the thin skin about the flexures of the groins, axillae, and popliteal spaces, and as lesions are seldom present there, Norman Walker recommends that they be rubbed previously with a zinc paste. In six or seven days the patches generally become blanched and surrounded by an inflamed area, and 
a few days later become inflamed. For the next day or two the chrysarobin ointment should be discontinued and calamine cream applied to soothe the skin.

As a rule the skin is now found to be cleared of the lesions with the exception of a few resistant patches usually situated about the extensor aspects of the limbs. These should be specially treated by a stronger ointment containing :

$\begin{array}{lllllll}\text { Acidi salicylici } & - & - & - & - & \text { gr. xx } & 4 \\ \text { Chrysarobin - } & - & - & - & - & \text { jss } & 6 \\ \text { Saponis viridis } & - & - & - & - & \text { jiii } & 36 \\ \text { Paraf. molle ad } & - & - & - & - & \text { ji } & 5+\end{array}$

During the treatment it is necessary to watch the skin carefully and to stop or weaken the ointment and apply a sedative cream should severe inflammation be produced.

In localised patches stronger chrysarobin ointment may be employed up to I0 $\%$, or the chrysarobin may be dissolved in traumaticin (guttapercha $\overline{3}$, chloroform $\bar{j}$ i) which is painted on with a brush daily for a week, the scabs then washed off, and if the lesion has not disappeared the painting is repeated. The chrysarobin may also be dissolved in benzine, painted on, and the lesions covered with flexible collodion. Another effective and cleanly method of employing the drug and one which is very useful for small isolated lesions is in the form of crayons, for which the following is a useful formula :

$\begin{array}{llllll}\text { Chrysarobin } & - & - & - & - & 3 \text { parts } \\ \text { White wax } & - & - & - & - & 2 \\ \text { Lanolin - } & - & - & - & - & 5\end{array}$

This is melted and poured into a mould about the size of a pencil.

Chrysarobin may be applied also in the form of plasters which can be had in various strengths and combinations from 2 to $10 \%$. These latter methods though cleaner than ointments are not so effective.

The staining of the skin from chrysarobin may be got rid of by applying the following ointment :

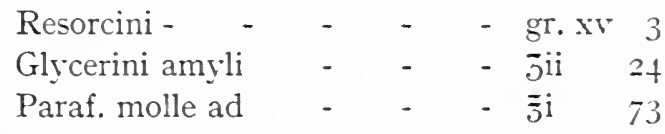

For the removal of the discolouration of the nails the best method is to soften them with soft soap and scrape them with a piece of glass.

Time alone will efface the dirty greenish staining of the hair.

In spite of its many disadvantages, in suitable cases and if properly applied, chrysarobin is by far the most satisfactory local remedy at present at our disposal.

Antharobin, a drug of much the same composition as chrysarobin, has been introduced as a substitute. It is less irritating but at the 
same time less efficacious and has the same disadvantages of staining the skin and dyeing the clothes brownish-red.

Tar is perhaps the most commonly employed reducing agent. It may be used in a number of forms all of which have their special indications. In addition to the Pix liquida and its ointment, there are the vegetable tars Ol. cadini (juniper tar), $\mathrm{Ol}$. rusci (birch tar), and $\mathrm{Ol}$. fagi (beech tar). Of these the most popular is the oil of cade because while its therapeutic action is similar to that of the others it is less irritating. The coal tar preparations are also extensively employed, namely, the alcoholic extract known as Liquor carbonis detergens, the officinal Liquor picis carbonis, and creolin.

When the disease is widely distributed and sub-acute the most convenient method of employing tar is in the form of tar baths. To make an effective tar bath add creolin ǰss, Liq. picis carbonis $\bar{z}$ ii, or oil of cade $\bar{\jmath}$ ii to 30 gallons of water at about $98^{\circ} \mathrm{F}$. Another useful plan is to paint the lesions with Pix liquida, or rub them with Pix liquida ( 3 parts) in soft soap (I part), or with a mixture of oil of cade and glycerine of starch, then immerse the patient in a warm bath and remove the tar with soft soap. Whichever form of tar bath be chosen it should be continued daily until the lesions have disappeared.

In addition to the bath it is usually necessary to apply tar to the individual lesions and the following ointments may be employed for this purpose :

$$
\text { Ungt. picis liquidae, }
$$

to which may be added $3 \%$ of salicylic acid, or $20 \%$ of soft soap to strengthen it ; or

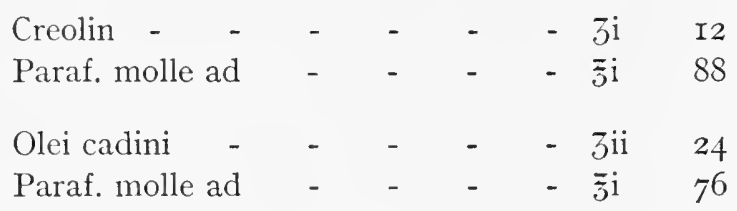

The oil of cade may be mixed with olive oil or glycerine of starch and rubbed on with lint, or in very resistant patches may be applied pure.

On the whole the coal tars are best as they are the most penetrating and anti-pruritic. In chronic patches an excellent treatment is to rub in the Liq. carbonis detergens or the Liq. picis carbonis with a nailbrush, then dress the lesions with zinc plaster or apply compresses of dilute glycerine of the subacetate of lead. The Liq. carbonis detergens can be used also as an ointment in vaselin ( $\mathrm{I}$ in 8 ).

A more active preparation of coal tar than the Liq.carbonisdetergens was introduced by Leistikow under the title of Linimentum lithanthracis, and consists of coal tar 3 parts, 95\% alcohol 2 parts, ether I part. It is very useful in old-standing resistant patches and should be rubbed on and the patches then covered with zinc plaster. 
For the face strong tar preparations should be aroided and the following ointment may be used :

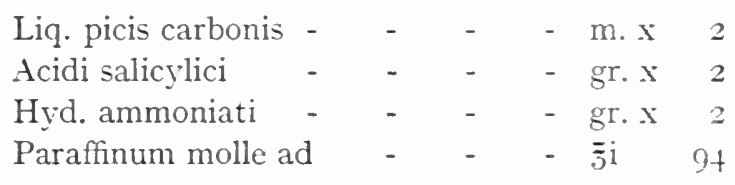

For the scalp the same ointment is useful with the addition of jii of soft soap.

Should irritation or marked acne supervene as a result of the tarapplications it is advisable to stop them, to wash the skin thoroughly with super-fatted soap, and to apply zinc ointment or calamine cream.

The disadvantages of the tar-applications are that they are dirty, have a disagreeable odour, tend to cause an acneiform eruption, and if too extensively used may produce toxic constitutional symptoms.

Pyrogallol has been recommended as a substitute for chrysarobin but is a less powerful and even dirtier remedy and stains both the skin and the linen. It cannot be used over large surfaces as it is liable to be absorbed and cause severe toxic symptoms, such as nausea, collapse, strangury, and haemoglobinuria. It is usually employed as an ointment containing 2 to $6 \%$ in vaselin, or in the following formula :

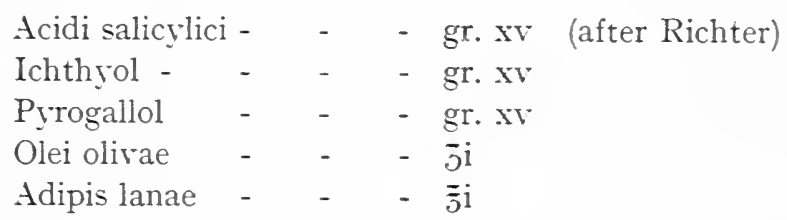

As substitutes for pyrogallol, lenigallol (triacetate of prrogallol) and eugallol (monoacetate of pyrogallol) have been recommended but they are less efficacious. They may be employed as ointments, or more effectively by painting the patches with them, either pure or diluted with acetone, and then applying zinc paste or dusting powder. This darkens the patches and a scab forms which separates in a week. One or two such applications may suffice to remove the lesions.

The other reducing agents, such as ichthyol, resorcin, and betanaphthol, are not so useful as tar or so effective in resistant cases as chrysarobin.

X-rays. - There is still another method of remoring the pzoriatic lesions, namely by the X-rays. This method is most applicable for chronic resistant patches. With the skin at a distance of $\mathrm{I}_{5} \mathrm{c} . \mathrm{m}$. from the anti-cathode, a dose of about $\frac{3}{4}$ of a Sabouraud pastille is given, and if it be insufficient, a further $\frac{1}{2}$ dose may be given in a fortnight. Though the $\mathrm{X}$-rays can remove any patch of psoriasis, like other methods of treatment they do not prevent a recurrence. 
Psoriasis of the Scalp.-After removing the scales by softening them in olive oil and shampooing with soap-spirit lotion, the following ointment should be rubbed in :

Sulphur. praecip.

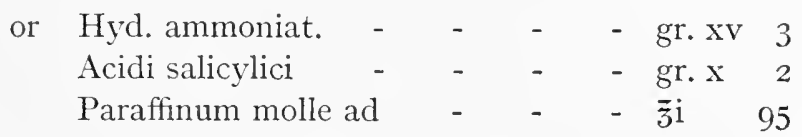

To this may be added in resistant cases Liq. picis carbonis $\mathrm{m} . \mathrm{x}$, and where the scalp is thickly covered with greasy scales instead of vaselin equal parts of soft soap and vaselin may be employed as a base.

The washing and the application of the ointment should be done daily at first, then less frequently till the scaly lesions have all disappeared. A useful procedure, especially in women, is to rub in the ointment at night, wash the scalp in the morning with the soap-spirit when necessary, and rub in daily one or other of the following lotions:

\begin{tabular}{|c|c|c|c|}
\hline Acidi salicylici & - & - & \\
\hline Ol. ricini - - & - & - & \\
\hline Spirit. vini rect. & - & - & - \\
\hline Resorcin - & - & - & \\
\hline Glycerin - - & - & - & \\
\hline Spirit. vini rect. & - & - & \\
\hline Aq. rosae ad - & - & - & \\
\hline
\end{tabular}

Psoriasis of the Nails.-When the nails are affected they should first be softened with soft soap freely applied on a piece of lint and covered by an indiarubber finger stall, or by a $5 \%$ solution of caustic potash, scraped by a piece of broken glass, and any heaped up epithelium removed from the free border. An ointment containing salicylic acid gr. $\mathrm{xv}$, and vaselin $\bar{\jmath} \mathrm{i}$ should then be rubbed over the nail, pushed up beneath it, and the fingers dressed. It is inadvisable to use chrysarobin or pyrogallol on the nails on account of their staining properties.

After Treatment.--In a favourable case thoroughly treated the eruption should be got rid of in a month or six weeks. This is far from being the case, however, in those cases in which the treatment is carried out in a half-hearted fashion, or when the patients are addicted to alcohol, such cases being so obstinate than many months may be required to clear the skin. Treatment should not cease with the removal of an attack, but should be continued in a modified form to prevent recurrence or to render future attacks milder in character. After the last spot has disappeared the patient should be advised to have a warm bath several times a week and to wash with ordinary toilet soap. Various medicated soaps, such as tar soap, sulphur and camphor soap, or beta-naphthol soap, have been strongly recommended, 
but the short time the soap is in contact with the skin and its dilution with large quantities of water render the action of the drug incorporated with it practically negligible. After the bath the skin should be rubbed with olive oil, almond oil, or cold cream, to prevent it becoming too dry. A mild tar and salicylic ointment, or a $2 \%$ beta-naphthol ointment in cold cream, should be rubbed into the sites of predilection of the disease every second night, and the patient advised to take plenty of exercise to promote perspiration. Irritating woollen undergarments shoild be replaced by silk and wool, cotton, or linen. By these means the chances of recurrence are lessened, longer intermissions may be expected, and in time there may be complete cessation of the disease.

\section{REFERENCES}

Audri, C. Psoriasis (La Prat. Derm., I904, iv. p. 88).

BRoce, L. Psoriasis (Dermat. pratiq., 1907, ii. p. 3II).

- Etiology of psoriasis (Ann. de Derm. et de Syph., I9Io, p. 156).

Brooke, H. G., \& MAcLeOD, J. M. H. Psoriasis (list of refs.) (Albutt \& Rolleston, System of Med., I9II, ix. p. 348).

BulkLey, L. D. Talue of an absolutely vegetarian diet in psoriasis (Journ. Amer. Med. Ass., Feb., I908, p. 582).

Fox, $H$. Human serum in the treatment of psoriasis and other skin diseases (Journ. Cut. Dis., I9I5, xxxiii. p. 6I5).

GottheIL, W. G. Treatment of psoriasis (Internat. Clinics, iv.).

- Value of autoserum injections in skin diseases (X.Y. Med. Journ., I9I6, ciii. p. I209; Journ. Cut. Dis., Oct. I9I6).

HaSlexd. Psoriasis: its histology and pathogenesis (Arcl. f. Derm. u. Syph., Dec, and Feb., rgr $\left.2-\mathrm{r}_{3}\right)$.

HYDE, J. N. Influence of light-hunger in the production of psoriasis (Brit. Med. Journ., Oct. 6th., I906, p. 833).

Kxowles, C. Psoriasis familialis (Journ. Amer. Med. Ass., I9I2, lix. p. 4 ( 5$)$.

Marcuse. Heredity in psoriasis (Derm. Zeitschr., I9II, xviii. p. 972).

PERRY, A. Treatment of psoriasis with horse serum (Boston Med. \& Surg. Journ., clxxiv. I916, p. 274).

PICCARDI, G. Psoriasis vulgaris of the palms of the hands (Arch. f. Derm. u. Syph., Bd. ci. hft. 203).

Pollitzer, C. Etiology of psoriasis (Journ. Cut. Dis., I909, p. 483 ).

Schamberg, J. Parasitism of psoriasis (Journ. Cut. Dis., I909, xxrii. p. 496).

Schayberg, Kolyer, \& Raiziss. A study of the germicidal activity of chrysarobin and certain other medicaments used in psoriasis (Journ. Cut. Dis., I915, xxxiii. p. I).

Sxowax, J. Therapeutic applications of arsenic (Lancet, I90 7 , ii. p. I I 49 ).

Trmble, W. B., \& Rothwolf, J. J. Treatment of psoriasis with autogenous serum (Journ. Cut. Dis., I9I5, xxxiii. p. 62I).

Walker, N. Chrysarobin in psoriasis (Scot. Med. \& Surg. Journ., Igo8, xxii. p. 3I2).

Wrifield, J. M. Psoriasis as a sequel to acute inflammation of the tonsils (Journ. Cut. Dis., I9I6, xxxiv. p. 441 ). 


\section{RESISTANT SCALY ERYTHRODERMIAS OR PARAPSORIASIS (BROCQ)}

Within recent years a number of cases of psoriasiform and lichen-like eruptions have been reported under different headings which do not conform to psoriasis, Lichen planus, or any of the ordinary recognised types of skin disease, and which though differing in minor details have so many essential features in common as to warrant their inclusion in one group. For this group Colcott Fox and the writer in Igor suggested the non-committal but descriptive heading of "resistant maculopapular scaly erythrodermias" and in the following year Brocq, who regarded them as closely related to psoriasis, gave them the simple title of "parapsoriasis." Neither of these headings is wholly satisfactory ; with regard to the former there are certain cases, though few in number, which clearly belong to this group in which maculo-papular clements are not a charactcristic feature; while with regard to the latter the relation to psoriasis does not appear to be sufficiently close to warrant the employment of a name which suggests an aberrant variety of a common disease.

According to the type and arrangement of the lesions Brocq has divided the parapsoriasis group into three varieties namely: Parapsoriasis en gouttes, P. lichénoïde, P. en plaques. This division is so convenient that it will be adhered to in the following description. Before discussing the varieties and referring to the different cases which should be included in the group, it will be advisable to describe the clinical and histological characteristics which are common to all its members.

Description.-The eruption is of a superficial inflammatory character with an almost complete absence of infiltration. The essential lesion in most cases is a macule or maculo-papule, round, oval, or angular in outline, and either smooth on the surface like a papule of Lichen planus or covered by a fine adherent scale. By the peripheral spreading and coalescence of these initial lesions in different fashions the three main varieties are produced. In the first the initial macules develop into guttate lesions, in the second they are arranged in a peculiar marbled or reticulate manner, and in the third circumscribed sharply defined patches occur, from one to three inches in diameter, the manner of evolution of which is uncertain. These lesions vary in tint from pinkish-fawn to pale red, are darker on the extremities than on the trunk, and are sometimes covered with finc scales. The eruption is distributed chiefly on the trunk, arms and legs, and more rarely on the backs of the hands and dorsa of the feet. It is absent from the scalp and face. There are no subjective symptoms associated with it, except slight itchiness from excessive heat, and it does not seem to affect the general health. The evolution of the eruption is slow, its duration long, and it is extraordinarily resistant to treatment-local applications 
which would remove the lesions of psoriasis and seborrhoic dermatitis in a comparatively short time having little or no effect upon it.

Histopathology.-Histological changes are present both in the corium and the epidermis.

Corium.-In the corium the most marked feature is dilatation of the superficial capillaries of the papillary and sub-papillary layers, associated with a cellular infiltration around the dilated resselș, and oedema and rarefaction of the connective tissue in the neighbourhood. The papillary body is flattened so as to form an undulating

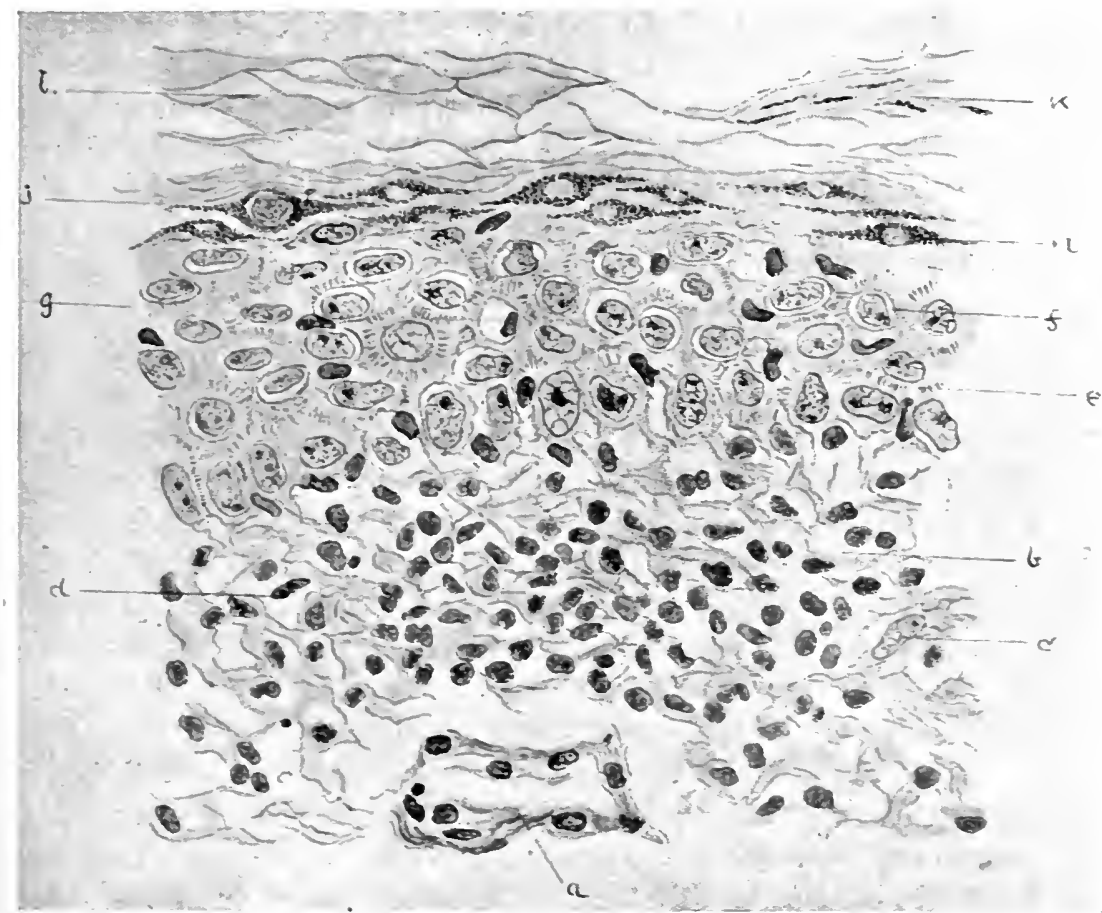

J. M. H. M. del.

FIG. 289.-Parakeratosis Variegata. ( $x$ about Ioo.) Central portion of a slightly raised papule.

a. Dilated blood-ressel. b. Dilated lymph space. $c$. Connective tissue cell. $d$. Leucocrte. Dilated inter-epithelial lymph channel. $f$. Epithelial nucleus lying in nuclear space. g. Leucocyte in nuclear space. $h$. Oedernatous prickle-cell. i. Granular layer. j. Oedematous cell of granular layer. $k$. Persisting nuclei in horn cell. $l$. Desquamating horn.cell.

(From MacLeod's Pathology of the Skin.)

line between it and the epidermis. No proliferative changes are present in the capillary walls but simply dilatation such as might be produced by congestion in the deeper reins. The collagen bundles around the dilated capillaries are oedematous, structureless, stain badly, and here and there are broken up into their component fibres. The elastin is also affected and swollen, but not to the same extent as the collagen. The cellular infiltration consists chiefly of lymplocytes. Below the sub-papillary layer the corium seems to be normal, and no changes can be detected about the pilo-sebaceous follicles or the sweat-glands. 
Epidermis.-The changes in the epidermis appear to be secondary to those in the corium and the result of oedema. The inter-papillary processes are scarcely noticeable. The basal layer is blurred from oedema and the presence of leucocytes between the cells. The pricklecells are swollen, stain indifferently, and the nuclear spaces are dilated with nuclei which are sometimes lying free in them. The interepithelial lymphatic spaces are oedematous, the inter-epithelial fibrils are stretched but not broken to form a vesicle, and leucocytes may be noted between the prickle-cells. The granular layer is defective or absent, unless where the oedema is not marked when it may appear to be normal. The Stratum lucidum is absent. The Stratum corneum varies like the granular layer, and its cells appear to be normal where the granular layer persists but show evidences of parakeratosis with persistence of the nuclei and a tendency to form scales where the granular layer is absent or defective. (Fig. 289.)

\section{VARIETIES}

(I.) Guttate Variety.-(Parapsoriasis en gouttes of Brocq); in which the lesions are allied to psoriasis.

In this variety the eruption takes the form of macules or maculopapules varying in size from a pin-head to a threepenny piece, and either smooth and shiny, or covered by a fine adherent scale which is little noticeable unless when the surface of the lesion is scratched when it becomes pearly, or by a more marked yellowish scale which is more easily detached. The lesions suggest a secondary syphilide without infiltration. They are reddish in tint and located chiefly on the trunk and proximal parts of the arms and legs, where they are irregularly distributed. They are absent from the face, scalp, and hands. There is little or no pruritus associated with them. The evolution of the eruption is slow and it is singularly resistant to treatment.

Cases conforming to this type have been reported by Jadassohn under the headings of Dermatitis psoriasiformis nodularis and " a peculiar psoriasiform and lichenoid exanthem," by Eudlitz as Psoriasis en gouttes d'aspect syphiloide, and by Juliusberg as Pityriasis lichenoid chronica.

(II.) Retiform Variety.-(Parapsoriasis lichénoïde of Brocq); in which the lesions are intermediate between psoriasis and Lichen planus.

In this variety the eruption has a peculiar mottled appearance caused by the essential lesions coalescing to form a pinkish or reddish network enclosing areas of normal skin. The meshes are comparatively regular in size, averaging 2 to $3 \mathrm{~mm}$. in diameter, and are roughly polygonal in shape. The network resembles the retiform patterning which may be seen in Erythema ab igne on the shins, in the pigmentary syphilide of the neck, and on the limbs of fat infants exposed to cold, but to it is added a superficial inflammatory condition affecting the meshwork but not the enclosed meshes, accompanied by the formation 


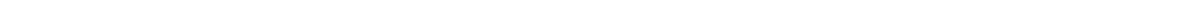




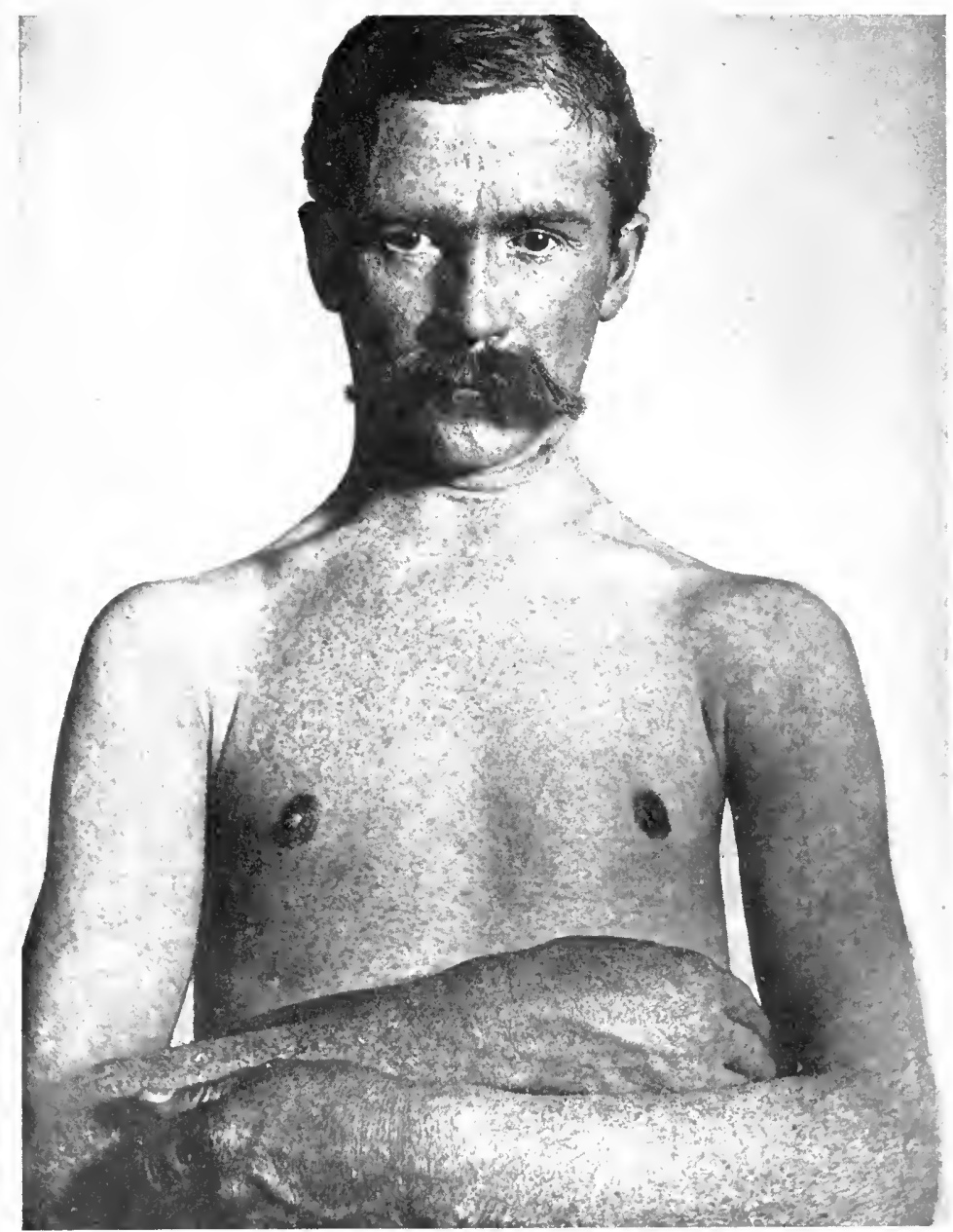

FIG, 290.--Parakeratosis Variegata.

(Colcott Fox and MacLeod's case. Photo by Whitfield.)

$\left[\right.$ To face $p .8_{47}$. 
of macules and flat papules covered with fine scales. The essential lesions consist of round or oval macules or flat papules over which the epidermis is either stretched as in Lichen planus or forms a scale. In the upper parts of the body the lesions are yellowish-pink, while on the extremities they are bluish-red from venous congestion which is most marked on the forearms, legs, and back of the hands, and produces a peculiar variegated livid appearance. On the extensor aspects of the limbs the network is coarser than on the trunk, scaliness is more marked, and the papular elements are more prominent. The shin of the palms may be thickened and fissured from hyperkeratosis as in chronic eczema. The scalp may be scurfy and the hair tend to fall out, but this is possibly the result of the association of the affection with Pityriasis alba. The nails are not affected. (Fig. 290.)

There are no definite subjective symptoms unless the patient be exposed to a high temperature when the eruption becomes slightly itchy and more inflamed.

The eruption develops slowly, is chronic in its course, is subject to spontaneous exacerbations and remissions, and is little affected by treatment.

To this variety belong the cases entitled Parakeratosis variegata by Unna, Santi, Pollitzer, Colcott Fox, MacLeod; Lichen variegatus by Crocker; and Lichen planus retiformis by Erasmus Wilson in naming a model by Baretta, which he presented to the Museum of the Royal College of Surgeons.

(III.) Patchy Variety.-(Parapsoriasis en plaques of Brocq); in which the lesions are allied to the psoriasiform variety of Seborrhoic dermatitis.

In this variety the eruption takes the form of patches varying in size from I to 4 inches in diameter, and roundish or irregular in shape. They are well-defined and vary from fawn or yellowish to reddishbrown or café-au-lait tint. They are present chiefly on the trunk and limbs and as a rule do not occur on the face, scalp or nails. On the trunk they are smooth or finely scaly and are not elevated but slight infiltration may be present. On the legs the scaliness is well-marked.

The manner of evolution of the patches is uncertain, some of them suggest that they are due to the coalescence of macules or small maculopapules, others, except for slight accentuation of the normal furrows of the skin, might be produced by a uniform inflammatory disturbance over the whole lesion.

The eruption like that of the previous types is of long duration and resistant to treatment.

To this variety belong the cases described as Erythrodermie pityriasique en plaques disseminées by Brocq, J. C. White, and others, and probably those named Xanthoerythrodermia perstans by Crocker.

Etiology and Pathology.-The cause and true nature of this group of skin affections is unknown. They seem to occur chiefly in adults 
and are probably more common in the male sex. They may affect all classes. In one or two instances they have been met with in men exposed to high temperatures such as furnace-men (Crocker) and firemen (J. C. White), but to what extent the heat was responsible is doubtful; on the other hand the writer recently had a well-marked case of the patchy type in a young man who was employed testing aeroplanes and who found that his skin was always much worse from the cold at a high altitude, and who began to suffer from the condition when he undertook this work.

Brocq considers that it is closely allied to psoriasis in its pathogenesis, but this is not of much assistance as the pathogenesis of psoriasis is not understood.

The histological features common to all the members of the group suggest that the primary changes occur in the blood-vessels and that the epidermal changes are secondary and result from oedema, but the cause of the vascular changes is unknown.

Diagnosis.-The differential diagnosis may present considerable difficulty in the first and third varieties, but in those of the second or retiform type it is comparatively easy. The diagnostic features of the eruption are the slowness of evolution of the lesions, their chronicity, their extraordinary resistance to local treatment, and the absence of subjective symptoms. The affections with which it is most likely to be confused are psoriasis, Seborrhoic dermatitis, Lichen planus, and in a few rare instances, secondary scaly syphilides.

From psoriasis cases of the guttate and patchy types differ in that the initial lesion is less bright in colour, its scales are not silvery and are more adherent and when scratched off do not leave a shiny film with haemorrhagic puncta like psoriasis; the sites of election of psoriasis are not more involved than other situations and the scalp, which is so commonly affected in psoriasis, is free. The eruption is much more persistent than psoriasis and far more resistant to treatment. Histologically there are several marked differences, of which the flattening of the papillary body is one of the most noticeable as in psoriasis the papillae are usually prominent and elongated.

From Lichen planus the initial lesions may be distinguished by being less prominent and infiltrated, not violaceous in tinge, never umbilicated, and generally scaly ; and by the absence of subjective symptoms.

From Seborrhoic dermatitis of the psoriasiform type considerable difficulty may be experienced in differentiating cases of the patchy variety. Seborrhoic dermatitis has as a rule greasy whitish-yellow scales, frequently occurs on the face, scalp, and inner surface of the joints, is usually slightly itchy, and is most amenable to local treatment; in parapsoriasis the scales are dry, whitish, and often adherent, the face and scalp are not attacked, there is practically no itchiness, and no response to anti-seborrhoic treatment.

From secondary syphilides it is easily distinguished by the absence of infiltration. 
Prognosis.-The prognosis varies considerably in different cases. It is extremely bad in the widely distributed reticulate variety and neither local nor general treatment seems to have much effect upon it. In the case of the guttate and patchy varieties improvement and even cure may be obtained by the thorough application of strong local remedies.

\section{Treatuent}

As the etiology and pathogenesis of this disease are unknown the treatment is purely empirical. As it usually occurs in those who are apparently in good health there is no definite indication for general treatment, but should any defect be detected it should be corrected on general principles. The different internal remedies which have been used in psoriasis and Lichen planus have been given in this group of diseases, but with no marked benefit. Arsenic, either taken by the mouth or injected in the form of the organic preparations, and salicin, have been tried but have produced no distinct improvement.

Locally, the comparatively mild keratolytics, such as ichthyol, resorcin, and sulphur, which are capable of curing Seborrhoic dermatitis and of greatly benefiting psoriasis, have little or no effect on these eruptions and stronger remedies are necessary. In the guttate and patchy types benefit can be derived from rubbing in an ointment containing pyrogallic acid, and a formula such as the following will be found useful :

\begin{tabular}{|c|c|c|}
\hline Pyrogallol & - & - m. $x$ to $\mathrm{xxx} 6$ \\
\hline Glycerini amyli & - & jii $\quad 24$ \\
\hline Paraffinum molle ad & d & $\bar{j} \mathrm{i}$ \\
\hline
\end{tabular}

This ointment may be strengthened by the addition of $2 \%$ of salicylic acid. After a bath and the removal of the scales by soft soap the ointment should be rubbed into the individual lesions. If the disease is very extensive, as in the reticulate variety, this procedure is contra-indicated for fear of absorption of the drug and the production of toxic symptoms. In every case where pyrogallic acid is being used extensively it is advisable to test the urine frequently and if it be discoloured to suspend the drug at once. In place of pyrogallic acid Crocker recommended vasogen-iodine $10 \%$ thoroughly rubbed in for ten minutes; this is much less toxic and equally effective.

The X-rays may be employed with benefit in isolated patches. About $\frac{3}{4}$ of a Sabouraud pastille dose should suffice to cause the patch to fade and if insufficient a second exposure may be given in a month.

In the widely distributed reticulate cases of the Parakeratosis variegata type, strong remedies are dangerous and apt to set up exfoliative dermatitis. Consequently in such cases it is adrisable to be content with milder measures and to aim rather at keeping the skin comfortable than at curing the eruption. For this purpose warm 
alkaline baths and soothing applications such as resorcin grs. $x x x$, or salicylic acid gr. $\mathrm{x}$, in an ounce of glycerine of starch will be found most serviceable.

\section{REFERENCES}

Arndt, G. Brocq's Disease with remarks on Parapsoriasis (Arch. f. Derm. u. Syph., I9Io, Bd. c., Hft. I-3).

Corlett, W. T. Parapsoriasis (Journ. Cut. Dis., I909, xxiii. p. 49).

Crocker, Radcliffe. Xanthoerythrodermia perstans (Brit. Journ. Derm., I905, xvii. p. II9).

Fox, Colcott, \& M $\triangle$ CLEod, J. M. H. Parakeratosis variegata (Ibid., I9oI, xiii. p. 319).

Heller, F. Parapsoriasis en Gouttes (Arch. f. Derm. u. Syph., IgII, p. $7 \mathrm{I})$.

MacLeOd, J. M. H. Parapsoriasis (Allbutt \& Rolleston, System of Med., I9I I, ix. p. 387).

Ravogli, A. Erythroderma squamosum (Journ. Cut. Dis., I9Io, xxviii. p. I 74).

Riecke, E. Pityriasis lichenoides chronica (Arch. f. Derm. u. Syph., I907, lxxxiii. p. 50).

Trimble, W. B. The chronic scaly erythrodermia (Journ. Amer. Ass., I909, liii. p. 264).

\section{PITYRIASIS RUBRA PILARIS}

Syn. : Lichen ruber (Hebra); Lichen ruber acuminatus (Kaposi) ; Pityriasis pilaris (Devergie).

Definition: A chronic affection of the skin characterised by small reddish-brown acuminate horny follicular papules and harsh red scaly patches widely distributed over the cutaneous surface.

Introductory.-Pityriasis rubra pilaris though one of the rarer skin diseases has excited more than its share of interest on account of the controversy which has raged with regard to its identity with Lichen ruber acuminatus.

In 1857 the disease was described in France by Devergie under the heading of Pityriasis pilaris. Three years later Hebra independently gave the name of Lichen ruber to a similar condition of which he recorded I4 cases, I2 being fatal. To avoid confusion with Lichen planus, Kaposi re-christened it Lichen ruber acuminatus and maintained with his German colleagues that it was a different affection from that described by Devergie in France. This view obtained till the Dermatological Congress met in Paris in $\mathbf{1} 889$, when the identity of the two was decided by the French dermatologists claiming a case to be Pityriasis rubra pilaris, which Kaposi asserted was Lichen ruber acuminatus.

Since that time many cases have been recorded and usually under the heading of Pityriasis rubra pilaris. They have been met with in this country, on the Continent, and in America, both in white and coloured skins. The cases have been of a far milder type than those 
.
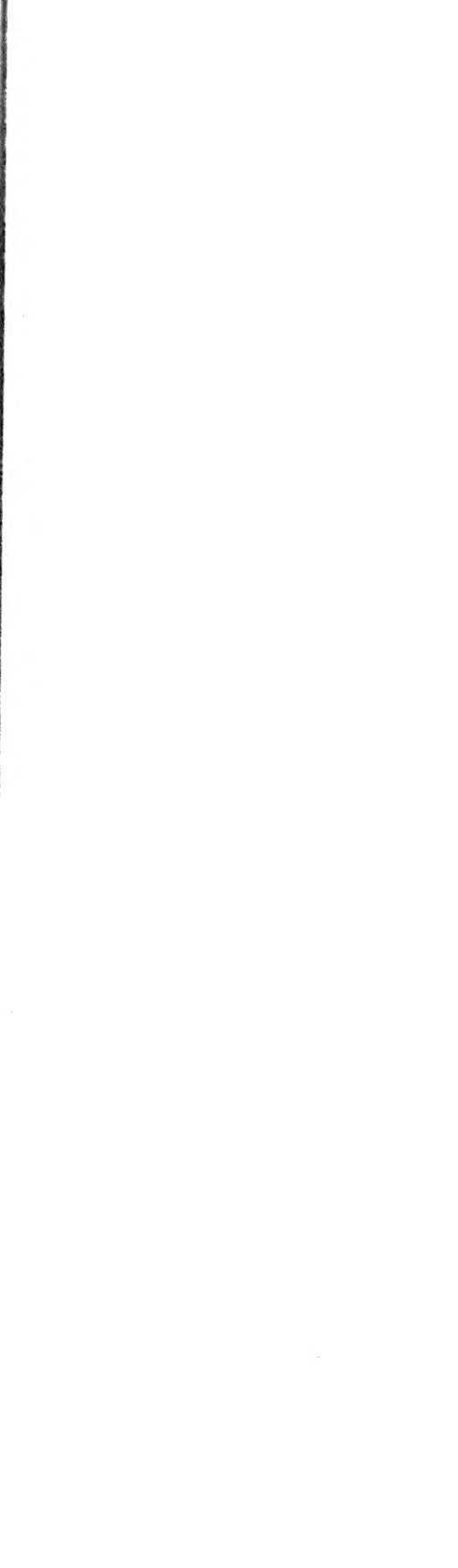


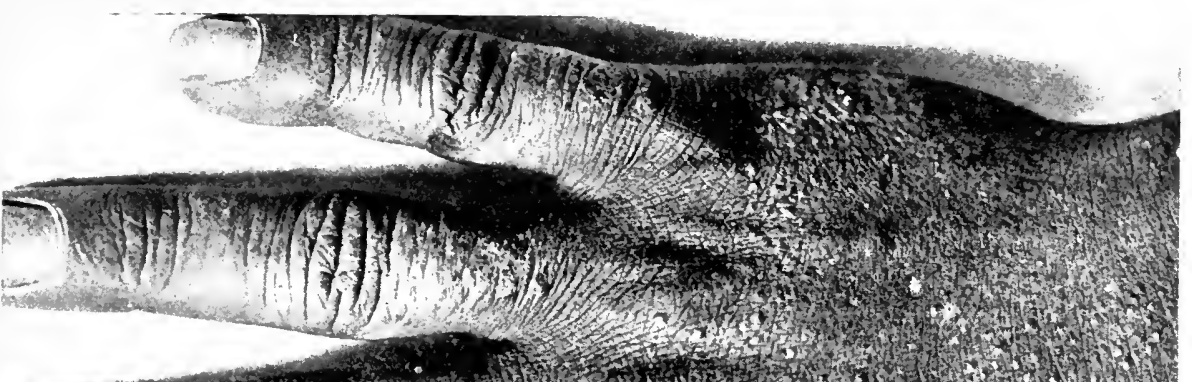

(4)

$\stackrel{5}{E}$

त

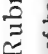

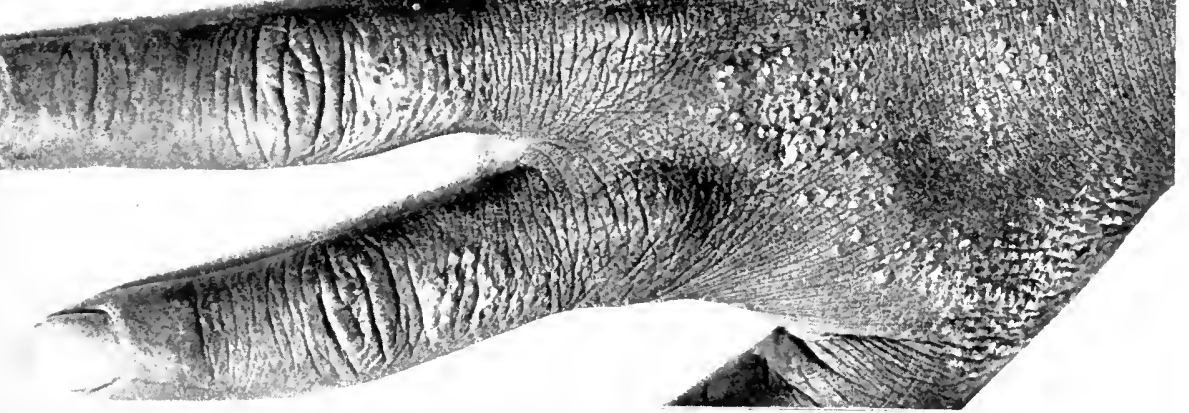

0

*

i

志
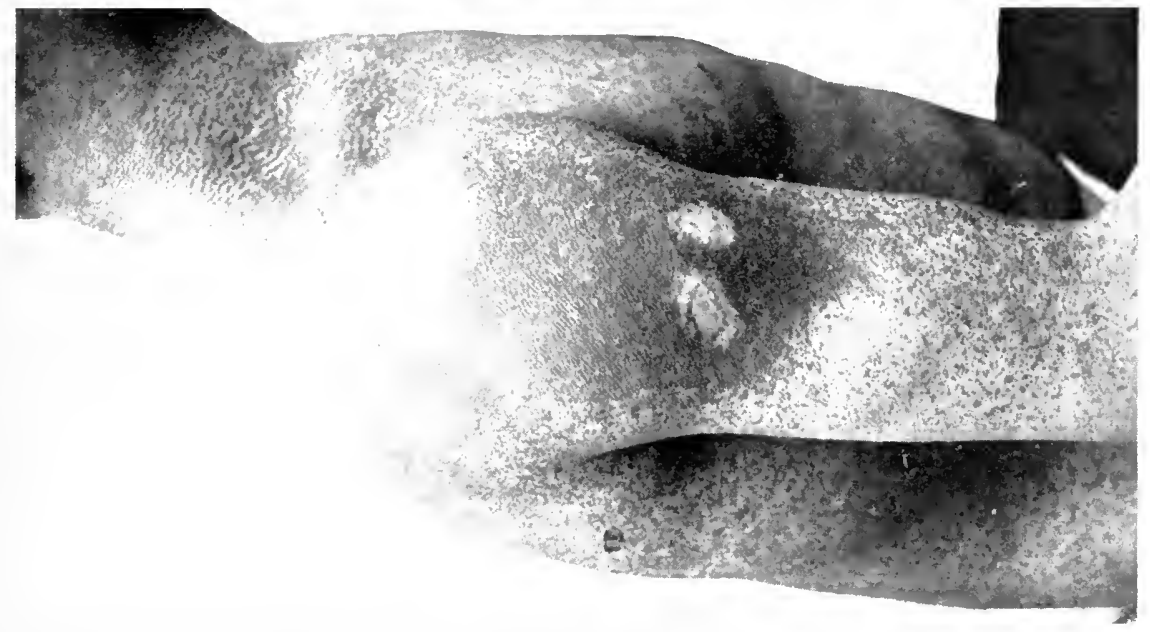

(1)

[To face $p .8$ 
described by Hebra and it has been the exception rather than the rule for the general health to be seriously impaired.

Description.-As the name indicates, the cardinal features are scaliness, redness, and papules, and the diagnostic lesions are the papules. These are invariably follicular, roundish or more usually acuminate in shape, vary in size from a pin's head to a millet seed, and in colour from skin-tint to reddish-brown. They generally have a central horny plug or may surround a hair which may be broken or doubled up. They may be sparsely and irregularly distributed, but as a rule are aggregated in sheets which present an appearance like exaggerated goose-flesh owing to the involvement of a greater or less number of contiguous follicles equidistant from one another. To the touch these sheets give a sensation which has been aptly compared to a nutmeg grater. They are most noticeable on the sides of the neck, lower part of the abdomen, and the extensor aspect of the limbs, while groups of papules, usually presenting dark horny plugs, can generally be detected on the backs of the hands and still more constantly on the dorsa of the Ist and and phalanges of the fingers. (Figs. 29I, 292.)

In addition to the papular lesions there are variously-sized red patches usually covered with fine adherent scales. These vary in size and shape from small patches to large sheets several inches in diameter, and sometimes involve nearly the whole of the cutaneous surface. They may be smooth on the surface, or broken up by the exaggeration of the natural fissures of the skin in a cross-hatched fashion like patches of lichenification. They are usually well-defined and separated by islands of normal skin, or they may gradually fade at the margin, beyond which a few discrete follicular papules can usually be detected.

The scalp is generally attacked and is covered with fine grevish scales, but it is not markedly inflamed and there is no loss of hair.

The face may be extensively involved, when it presents the appearance of exfoliating dermatitis and is uniformly red and scaly. The skin, though not definitely thickened, is harsh and sometimes so tense as to cause ectropion of the lower eyelids.

Follicular papules are absent from both the scalp and the face.

The trunk is more or less severely affected with scaly patches and with follicular sheets near the lower part of the abdomen.

On the limbs, the elbows and knees sometimes present harsh patches covered with thick plaster-like scales, while on the flexor aspects of the joints the patches are generally broken up by deep fissures due to movement.

On the palms and soles, the skin is thickened and fissured from hyperkeratosis and tends to flake off in large scales. (Fig. 293.)

The nails may be affected but in no very characteristic fashion, and may be thickened, opaque, brittle, frayed at the edge, pitted, longitudinally striated, or transversely furrowed. (Fig. 294.) 
The mucous membranes are not involved.

There are no constant subjective symptoms. Sometimes there is itching, which may be severe, and several observers have noted that when the skin is extensively involved it becomes hypersensitive to cold.

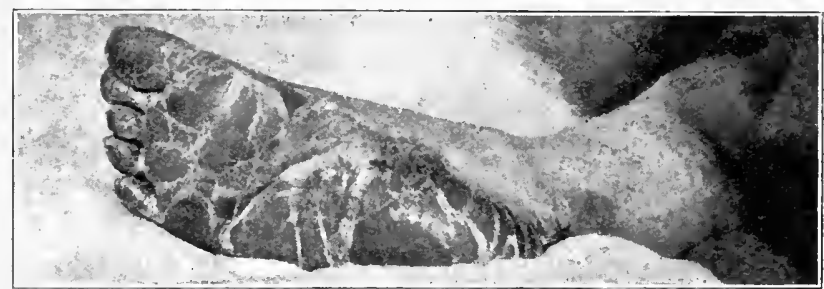

Frg. 293.-Pityriasis Rubra Pilaris.

Shows irregular exfoliation of the skin of the sole.

(Dr. Arthur Hall's case, Brit. Journ. Derm., 1903.)

General symptoms are also variable. In most cases they are completely absent, or negligible, but occasionally when the onset of the affection is unusually rapid and the course acute, there may be fever, malaise, vomiting, and other digestive disturbances. In rare instances,

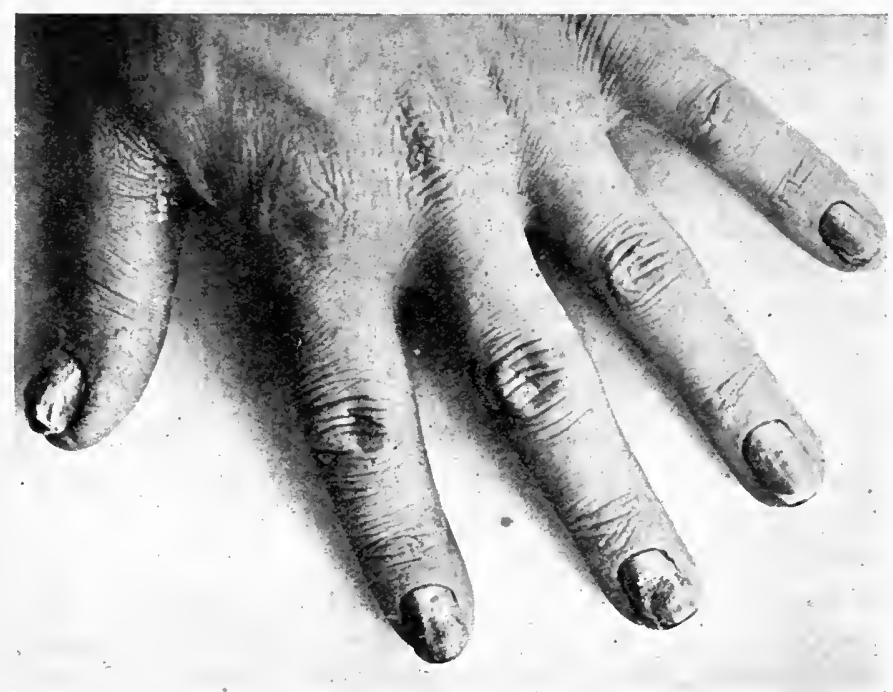

Frg. 294-- Pityriasis Rubra Pilaris.

Shows disease of the nails.

such as the cases recorded by Hebra, severe general symptoms may supervene which may lead to a fatal issue.

Onset and Course.--The affection may be slow or rapid in its course. It usually appears first with scaliness on the scalp, red scaly patches about the forehead, and thickening of the palms and soles, while later scaly patches and groups and sheets of papules appear on the trunk 
and limbs. It is singularly persistent and may remain for years subject to more or less complete remissions and exacerbations, but eventually may disappear entirely.

Histopathology.- A microscopical examination of sections of papular lesions and of the scaly patches shows the most marked feature to be hyperkeratosis chiefly involving the mouths of the pilo-sebaceous follicles but also, to a less extent, the intervening epidermis and the sweat-pores. This is associated with hypertrophy of the prickle-cell layer with increase in the width of the inter-papillary processes in the neighbourhood of the follicles. The hyperkeratosis is so marked at the mouth of the follicle as to form a plug made up of concentric horny

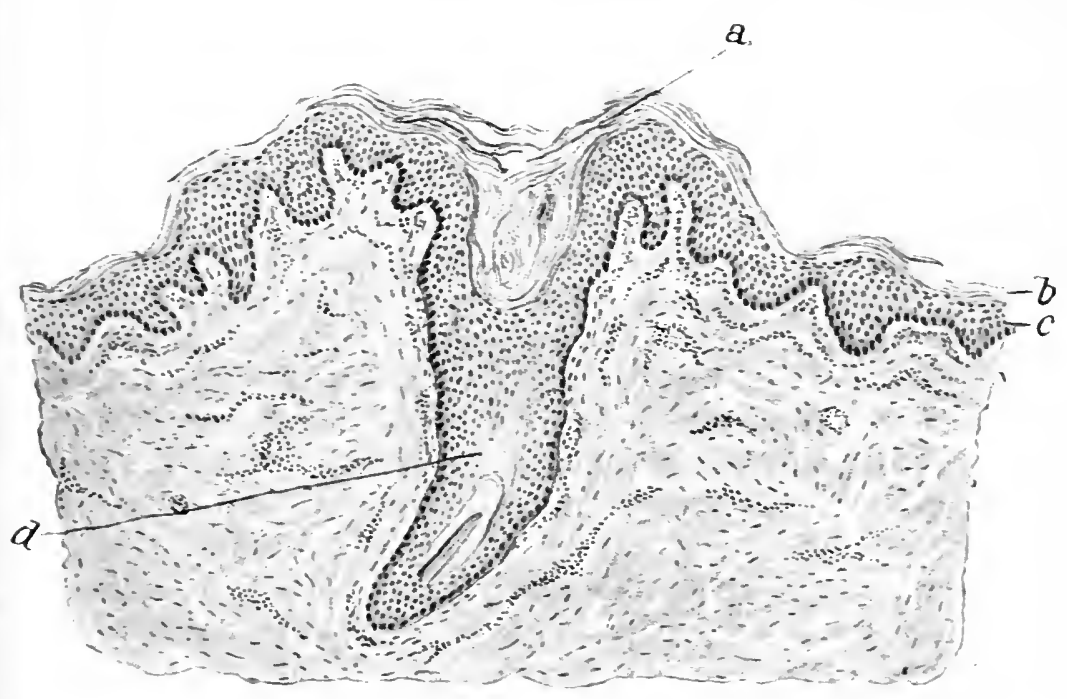

J. M. H. M. del.

FIG. 295.-Pityriasis Rubra Pilaris. ( $x$ about I00.)

$a$. Horny plug filling the funnel of the follicle. $b$. Hyperkeratosis between the papules. c. Malpighian layer. $d$. Lanugo hair-follicle.

(From MacLeod's Pathology of the Skin.)

laminae which occupy the whole of the funnel of the follicle and project for some distance above the surface. (Fig. 295.)

Beneath the epidermis the blood-vessels of the papillary and subpapillary layer are dilated and surrounded here and there by an infiltration of small round connective tissue cells with a few mast-cells, which is present also around the hair-follicles. Otherwise the corium appears to be normal, and the sweat-glands are unaffected.

The exact sequence of these changes is uncertain. Certain observers regard the hyperkeratosis as the primary change, and the alteration in the corium as secondarily induced by irritation caused by the thickened horny layer. It seems equally possible that the thickening of the epidermis is the result of some irritant which reaches the skin by the blood-ressels and sets up a mildly inflammatory change around the superficial capillaries and about the hair-follicles. 
Etiology.-The cause of the affection is unknown. As several cases have been found to react to the old tuberculin both locally and generally, it has been suggested that it is a tuberculide, but the evidence in favour of a causal connection with tuberculosis is inconclusive.

It may attack either sex, but the majority of cases seem to be in males. It can occur at any age, but not infrequently begins in childhood and has been known to appear as early as the third year (Rasch). It may be a family disease and de Beurmann records an instance in which four members of one family were affected.

Diagnosis. - The recognition of a well-marked case presents no serious difficulty. The follicular horny papules on the backs of the fingers, sides of the neck, and extensor aspect of the limbs, the sheets of papules like nutmeg graters on the lower part of the abdomen and elsewhere, the harsh, red patches covered with adherent scales and separated by islands of healthy skin, the diffuse redness and scaliness of the face, and the thickened fissured condition of the palms and soles, constitute a characteristic picture.

It has to be differentiated chiefly from psoriasis and Lichen planus.

From psoriasis it is distinguished by the scales being fine, greyishwhite and adherent, instead of coarse, silvery, laminated and flaky. In psoriasis also, the smaller patches are the result of the peripheral extension of scaly papules, while in Pityriasis rubra pilaris the follicular sheets are due to the aggregation of numerous papules. In psoriasis it is rare for the palms and soles to be affected, while it is the rule in this disease.

The type of psoriasis with which it is most liable to be confounded is the follicular or small papular type and here the whole facts of the case may have to be carefully considered before a diagnosis can be made. Several cases have been recorded where an attack of what was believed to be Pityriasis rubra pilaris was succeeded by one of psoriasis. Of course it is possible that a mistake in diagnosis had occurred but it is cqually possible that the two affections may alternate. It has also been known for Pityriasis rubra pilaris to be present in one member of a family and psoriasis in another.

From Lichen planus it is differentiated by the absence of the typical shiny, flat, violaceous papules, and of the raised, hard shagreenlike patches of the hypertrophic type of the disease. When acuminate follicular papules are present in Lichen planus the diagnosis is more difficult, but even then the acuminate lesions are generally associated with typical plane papules.

Prognosis.-The disease is singularly persistent and intractable. Periods of more or less complete retrogression may take place, but they are liable to be followed by a recrudescence. After several years it tends to disappear completely. As a rule it does not interfere with the general health, but it is well to remember that Hebra recorded 22 fatal cases, and it is unwise to treat it too lightly. 


\section{TREATMENT}

The treatment is essentially palliative. Internally there is no specific remedy and the physician is thrown back on measures for the improvement of the tone and nutrition of the patient and the correction of any obvious defect in the general health. Arsenic has been employed somewhat extensively in the past, but little or no benefit has accrued from its use, and as the drug itself is liable to cause hyperkeratosis, it appears to be definitely contra-indicated. Certain writers recommend thyroid, but though cases have been known to improve temporarily while taking it, its action is too uncertain for it to be adrocated.

Locally, considerable benefit can be obtained from suitable remedies. Where the disease is extensive the patient should have alkaline baths daily, or at frequent intervals, and the scalp should be washed with soap spirit lotion. After the bath the affected skin should be rubbed with an emollient cream containing some keratolytic remedy, such as resorcin $3 i$, to glycerine of starch $\tilde{3} i$, to which may be added 10\% of cherry laurel water, or I $\%$ of carbolic acid to relieve itching.

For the thickened patches on the elborrs, knees, palms and soles, an ointment or plaster containing salicylic or pyrogallic acid should be prescribed.

In every case care should be exercised that too strong remedies are not employed over an extensive area, partly from the danger of absorption, and partly from the possibility of transforming the condition into generalised exfoliative dermatitis.

\section{REFERENCES}

Adarsox, H. G. Pityriasis rubra pilaris (System of Med., Albutt \& Rolleston, I9I I, Vol. ix. p. 34I).

de Betriaxi, Bith \& Hecyer. Pityriasis rubra pilaris as a family disease (Ann. de Derm. et de Syph., I9I I, ii. p. II).

Hall, A. J. Case of pityriasis rubra pilaris (Brit. Journ. Derm., I903, xv. p. 403).

Milimax. Pityriasis rubra pilaris and tuberculosis (Ann. de Derm. et Syph., I906, vii. p. 1067).

\section{GENERALISED EXFOLIATIVE DERMATITIS}

Syn.: Dermatitis exfoliativa (Wilson); Pityriasis rubra (Hebra).

Definition: A persistent affection of the skin characterised by universal redness and scaliness, generally with loss of the hair and nails, occurring independently, or secondarily to some previous skin disease.

Introductory.- The subject of generalised exfoliative dermatitis is one concerning which there is considerable confusion both among English and Continental writers, and the widest divergence of opinion exists as to what should be included under the term, for while certain 
writers regard it as a morbid entity due to a single cause which is unknown, others with greater reason look upon it as a syndrome of symptoms, characterising a special type of reaction of the skin, which may be called forth in response to a number of widely differing agents.

According to the view which generally obtains in this country the affection may be spontaneous, or primary, and the result of some undetermined internal cause most probably toxic in character, or secondary and replace some previous chronic cutaneous disorder such as psoriasis, eczema, or seborrhoic dermatitis, a considerable number of cases bcing now on record in which it occurred in psoriasis as the result of the application to an unduly sensitive skin of some local irritant such as chrysarobin or tar. The propriety of including primary and secondary cases under one heading has been questioned by certain writers, especially in France, who consider that the so-called spontaneous cases belong to a totally different category from those which are superimposed on some previous skin disease.

However it maybe caused, generalised exfoliative dermatitis is a rare disease and a survey of the literature of the subject seems to show that it is becoming rarer, less severe in type, and not so liable to lead to a fatal issue. In the clinic at Charing Cross Hospital it has averaged during the last five years about one per thousand cases.

It is to Sir Erasmus Wilson in IS67 that we are indebted for the name Dermatitis exfoliativa, which he employed to designate a series of cases of universal erythema and profuse scaliness which he regarded as being similar to those described by Hebra under the heading of Pityriasis rubra. Hebra, however, would not admit of their identity and in this he was subsequently supported by Brocq and others. Although there are certain differences between the cases of the Wilson type and those of the Hebra type these appear to be of minor importance and as cases have been described exhibiting all transitions between the two types it seems questionable if there are sufficient grounds to warrant their separation as two distinct diseases.

Closely allied to Dermatitis exfoliativa, and by some regarded as an acute form of it, is " recurrent scarlatiniform exfoliative dermatitis" (see page 662).

For convenience of description Dermatitis exfoliativa of IVilson will be taken as the type and the points in which Hebra's cases of Pityriasis rubra differ from it will be referred to subsequently.

Description.- Primary Cases.-In the primary cases the affection may begin with diffuse redness, or with the appearance of several ill-defined erythematous patches or blotehes suggesting erysipelas, symmetrically distributed about the upper part of the trunk or the limbs. There may be some indefinite general symptoms, such as a feeling of tiredness, pains in the back, sickness, or fever, or such symptoms may be wholly absent and the first sign may be the eruption itself or some local burning or itching. 
The redness rapilly spreads. The patches increase in size and number; at first they are separated by areas of normal skin but these are soon obliterated by the progressing erythema and in the course of a few days the whole cutaneous surface from the scalp to the sole may be involved in a universal redness, rivid like scarlet fever in one case, of a deeper brownish hue in another, and tending in deperdent situations such as the legs to assume a purplish tinge and sometimes to be associated with oedema.

Soon after the appearance of the erythema desquamation sets in as a coarse scaliness, the scales being specially large about the trunk and limbs and finer on the face and scalp. They are irregular in outline, papery, and vary in size from small branny scales on the scalp to squames several inches in their long diameter upon the trunk. The scales are sometimes attached at the centre and loose at the margins, or they may be fixed at the base while the remainder is free, and they may overlap one another like the tiles on a roof. Ther are readily peeled off but as readily re-form, and are constantly being shed so that they collect in handfuls in the bed. When the scales are removed the underlying surface is red, glossy and dry, is rendered pale on pressure with a diascope, and presents no small red puncta such as are met with in psoriasis. Occasionally where the scales have been unusually adherent the underlying surface may be slightly moist, but on the whole one of the most striking features of the disease and one which distinguishes it from generalised eczema is its singular drỵness, and ant slight moistness which may occur in parts like the axillae and the groins is not due to discharge but to perspiration, the sweat-function not being interfered with in the earlier stages of the disease. The skin of the palms and soles is thickened and tends to become loose and to flake off in large sheets. In old-standing cases the whole skin may become definitely infiltrated and stiff and the finer movements impaired.

In most cases there is partial or complete loss of the hair and eyelashes, and the nails become opaque, brittle, striated, raised up from the nail-bed by masses of epidermis, and not infrequently detached and shed.

The mucous membranes may be involved to some extent, and occasionally transient desquamative patches have been detected in the mucosa of the inside of the cheeks, while the tongue may be bright red, swollen and fissured, and the conjunctivae sometimes markedly congested.

The subjective symptoms vary in different cases. They may be absent or negligible and consist of mild itching, burning and tenderness, or the itching may be so severe as to lead to scratching, excoriation, the secondary inoculation of pus-organisms, and even abscess-formation. As a rule the affected skin is abnormally sensitive to cold and the patient prone to chills in consequence.

Secondary Cases.--In the secondary cases the erythema appears beneath the psoriasis or other affection upon which it is superimposed, 
and gradually becomes universal, completely replacing the previous skin disease which, when the erythema begins to subside, is liable to re-assert itself.

When the erythema has involuted and the scaliness ceased there may be more or less pigmentation of the skin or discolouration due to blood pigment from venous congestion which is most marked in the legs. The possibility of the pigmentation being due to the taking of arsenic must be remembered.

The general symptoms may be indefinite and consist only of a feeling of chilliness or debility which persists for a few days and then disappears, or they may be severe and the attack may be ushered in with a definite rigor, followed by a temperature of $102^{\circ}$ to $104^{\circ}$ at night, falling somewhat in the morning and gradually returning to normal in about a week but liable to recur should relapses take place or any complication supervene. In some cases digestive disturbances are present, such as vomiting, diarrhoea, or grave disorders of the kidneys with albuminuria and decrease of urea. As a rule once the disease is established, the health rapidly deteriorates and the patient becomes nervous and irritable from the general discomfort, itching, and want of sleep, and may even develop more or less acute mental symptoms. The majority of cases recover in a few months, but relapses are apt to prolong the disease for a year or more, while sometimes recurrence follows recurrence and it may last for several years or even till life ends. About 10\% end fatally, either in a few months or after years of suffering, generally as the result of some complication such as kidney disease with uraemia, pneumonia, or tuberculosis.

In the cases described by Hebra under the heading of Pityriasis rubra the desquamation was much finer than in those of the Wilson type and was generally succeeded by atrophic changes in the skin so that it became stiff and liable to cracks and superficial ulcerations at sites of pressure. The affection frequently began in red patches covered with fine scales, situated about the flexures of the large joints, and slowly spread till after months or even years it might involve the whole skin. When this occurred the health gradually declined, feverish attacks were liable to take place and mental symptoms sometimes supervened. The general appearance of the cases when fully developed was similar to those of the Wilson type. As a rule the course was prolonged and the majority ended fatally. In a considerable number tuberculosis occurred, either in the glands or viscera, and out of eight post-mortems tuberculosis was detected in seven, but it seemed probable that it was secondary and a complication.

Histopathology.--Sections of the affected skin show that the epidermis is thin and that the cornification process is interfered with owing to oedema of the prickle-cells. The granular layer is defective and the horn-cells imperfectly cornified.

In the corium there is dilatation of the vessels of the papillary and 
sub-papillary layers and around the dilated ressels is a slight infiltration of small round connective tissue cells and leucocytes.

In sections of old-standing cases of the Hebra type fibromatosis has been described, associated with marked atrophy of the epidermis, pilo-sebaceous follicles, and sweat-glands.

These histological appearances suggest a chronic inflammatory process going on to atrophy.

Etiology.- The exact cause of the primary cases is unknown. The manner of onset and course suggest some form of auto-intoxication, and in support of this it has been pointed out that cases of a somewhat similar type have resulted from the ingestion of mercury and quinine. Alcoholism, sudden chill, or an attack of acute rheumatism have also been cited as possible causes.

In the cases of the Hebra type the affection has been regarded as a tuberculide, but the tuberculosis is more probably secondary. In a certain number of cases the onset is associated with some severe disturbance of the nervous system, such as shock, anxiety, or fright, but it is possible that these did not act directly but simply through interference with the metabolic processes and the elimination and absorption of some toxic foreign proteid.

The secondary cases are much more easily explained. Where an exfoliative dermatitis has been superimposed upon psoriasis, the cause is generally chrysarobin or tar; where it has followed other diseases, such as Dermatitis herpetiformis, seborrhoic dermatitis, or ordinary eczema, the exciting cause may be uncertain but it is usually some form of local irritation. The application of certain irritants, such as arnica or even mercury, has been known to produce it in an unduly susceptible skin, apart from previous skin disease.

Though it may occur at any age it is most frequent in middle life and old age ; it is rare in childhood but Crocker has recorded a case in a child of two months. It may affect both sexes but males seem to be specially liable to it.

Diagnosis. - The diagnosis in a well-marked case does not present any serious difficulty. It has to be distinguished from various chronic affections of the skin which may become generalised, such as psoriasis, eczema, Dermatitis herpetiformis, and Pemphigus foliaceus, but in all these conditions the eruption, however extensive, is not universal and, unless in the case of Pemphigus foliaceus, grave general symptoms rarely occur.

From psoriasis it is distinguished by the scales being more papery, larger and more easily detached, and the shin beneath them presenting no red puncta.

From eczema it is recognised by the absence of exudation, crusting and severe itching. In old-standing cases of eczema where the shin is dry, the scales are not so large and papery and there is no loss of the hair or nails.

Pemphigus foliaceus may sometimes become widely distributed and closely simulate exfoliative dermatitis; but in P. foliaceus there is 
usually moisture beneath the scales and a few flaccid bullae may generally be detected somewhere on the cutaneous surface.

Several instances are on record where the pre-mycotic stage of Mycosis fungoides has become universal and has suggested exfoliative dermatitis, and so close may be the resemblance that it may be necessary to wait until tumours develop before a firm diagnosis can be made.

Occasionally in association with lymphadenoma or lencaemia a generalised erythrodermia covered with fine scales has been described, but any difficulty in distinguishing this from exfoliative dermatitis may be overcome by an examination for enlargement of the spleen and for an increase of lymphocytes in the blood.

Exfoliative dermatitis in infants has to be distinguished from the condition described by Ritter as Dermatitis exfoliativa neonatorum, see page 308. This affection, which is probably a variety of Pemphigus neonatorum and of streptococcic origin, has occurred in lying-in institutions. It is more rapid in its course, only lasting for about a fortnight, and in many of the cases, in addition to redness and scaliness, there is exudation and even bullous formation.

Prognosis.-A guarded prognosis should be given in every case, for it is impossible to foretell at the onset the severity of the attack, its probable duration, or its outcome. About $40 \%$ of the cases have been fatal, sometimes early in the course of the disease, at other times after the lapse of years. The outlook is especially grave in children and elderly people, and in children the disease runs a more rapid and acute course than in adults.

\section{TREATMENT}

The treatinent consists in the first instance of putting the paticnt to bed so as to reduce the irritation of clothes in moving about, to prevent chills, and to enable remedies to be applied thoroughly.

The local treatment, though simply palliative, is of the greatest service in relieving the irritation, reducing the hyperaemia, and rendering the patient's existence to some extent tolerable. In this disease the skin is in an extraordinarily sensitive state and any but the most soothing remedies are calculated to do more harm than good. In the early acute stage an excellent treatment is that recommended by Sir Stephen Mackenzie, which consists of the continuous application of glycerine and lead lotion in the following manner: The patient is enveloped in pyjamas made of lint, the face covered with a lint mask and the hands and fect with lint gloves and socks respectively. This lint covering is kept constantly wet by being sponged over from time to time with the following lotion, warmed, if necessary, to avoid chilling; glycerine of the subacetate of lead $\bar{\Xi} \mathrm{i}$, glycerine $\overline{\tilde{j}} \mathrm{i}$, and water to a pint. In this way the skin is soothed and the scaling kept under. When the redness begins to diminish the lint suit may be discarded and a calamine crcam, or equal parts of liquid paraffin and glycerine of 
starch, rubbed over the skin. When the healing has further advanced zinc paste may be substituted, either rubbed gently into the skin or applied on butter-muslin. Baths have been recommended, such as simple warm baths, alkaline baths, or starch baths, but these are of doubtful benefit and must be employed with the greatest caution to avoid chilling or depressing the patient.

The internal treatment is based on ordinary generai medical principles. Any disturbance of the digestion, liver, kidneys, or other organ, which might be connected in any way with the disease, should be sought for and dealt with by appropriate means. In many of the cases there is no obrious defect and the physician is thrown back on a general tonic regime with a liberal diet.

Various drugs have been employed in the past with the idea that they might have some specific effect on the disease, such as arsenic and quinine, but they have been largely abandoned as any action they may have is tonic rather than specific, and is of less value than other tonics, such as iron, cod-liver oil, and strychnine. Where the patient is in good general health and there is much tenseness of the skin, some relief may be obtained from small doses of antimony, either a few minims of the Vinum antimoniale, or antimonium tartrate $\frac{1}{8}$ grain three times a day, but on account of its depressing effect this drug should never be resorted to when the patient is in a debilitated condition.

\section{REFERENCES}

Adamsox, H. G. Generalised exfoliative dermatitis (Allbutt \& Rolleston, System of Medicine, I9II, ix. p. 328).

Bowex, J. T. Dermatitis exfoliative (Journ. Cut. Dis., IgIo, xxviii. p. I).

BRUxSGAARD. Erythrodermia exfoliativa universalis (Arch. f. Derm. u. Syph., I903, lxvii. p. 227).

Kanitz. Pityriasis rubra-symptoms \& histopathology (Idem, I906, lxxxi. p. 259).

Kopylowski \& Wielowerski. Pityriasis rubra (Idem, I907, lxxxii. p. 255).

Mook, W. H. Dermatitis exfoliative treated with quinine (Journ. Cut. Dis., I9Io, p. 458$)$.

PARker, G. Cystic degeneration of the kidneys with generalised exfoliative dermatitis (Amer. Journ. Med. Sci., I S99).

Wolters, M. Etiology of Dermatitis exfoliativa (Arch. f. Derm. u. Syph., I9I 2, cxiii. p. 22I).

\section{PITYRIASIS ROSEA}

Syn.: Pityriasis rosé (Gibert); Pityriasis maculata et circinata (Duhring); Pityriasis rubra aigu disséminé (Bazin); Herpes tonsurans maculosus et squamosus (Kaposi).

Definition: An inflammatory disease of the skin characterised by a widely distributed eruption of pinkish scaly macules and circinate lesions. 
Description.-Pityriasis rosea was originally described by Gibert in I860 and is a comparatively rare disease, forming roughly about $0.5 \%$ of the total number of cases of skin disease in a mixed London clinic, but it is one which has attracted considerable attention owing to the suddenness with which it usually breaks out and the mystery attached to its etiology.

The eruption as a rule is widely disseminated and is usually heralded by a single scaly patch, named by Brocq the "plaque primitive," which may appear on any part of the glabrous skin but is most frequent on the neck, flanks, and thighs. This lesion is rose pink in colour, scaly on the surface, oval or circular in shape, varies in diameter from $\frac{1}{4}$ to 2 inches or larger, and has a well-defined slightly raised border. In some cases it is not unlike a resistant patch of Seborrhoic dermatitis, in others it fades in the centre to a yellowish or fawn tint and suggests a lesion of Tinea circinata. Occasionally more than one herald patch may be present, while in a certain number of cases it is absent or passes undetected by the patient.

Seven to fourteen days after the appearance of the herald patch the general eruption breaks out and as a rule it is then that the patient becomes aware of his disease and seeks advice from the physician. The outbreak is associated in many cases with various mild constitutional symptoms, such as slight fever, general enlargement of glands, pains in the limbs and joints, or sore throat from congested fauces.

The eruption comes out with the suddenness and spontaneity of an exanthem. It consists of rose-coloured macules or papules about the size of the little finger nail or smaller, with an irregular ill-defined outline, covered with wrinkled loosely attached scales, and tending to spread at the periphery and to coalesce to form irregular patches. Among the macules a certain number of circinate lesions are generally interspersed, and the two clinical types of the disease, namely Pityriasis circinata and Pityriasis maculata, are distinguished according to which type of lesion preponderates. (Fig. 296.)

The circinate lesions begin as oval or circular flat papules about the size of a threepenny piece, which increase at the periphery and fade in the centre to form ringed lesions up to the size of a shilling or larger, and may coalesce to form gyrate figures. The border is well-defined, slightly raised, pinkish, and scaly, while the central portion is fawncoloured and finely wrinkled. Sometimes the eruption is urticarial (Pityriasis rosea urticata).

It usually appears first on the neck, upper and lateral parts of the chest, and spreads down from there on to the arms, abdomen and thighs. It has been known also to commence on the arm and thighs or to come out spontaneously in all these situations. It is rarely present on the face, hands and feet, but may occur anywhere on the skin, even on the scalp. It is as a rule most profuse on the sides of the body and shows a definite tendency to be symmetrical. When oval lesions are present on the sides of the chest the long diameter usually slopes downwards 
while in the centre of the back it tends to be transverse. Sometimes from scratching and rubbing the patch may become inflamed or even eczematised. The glands in the regions where the eruption is most profuse are frequently enlarged, especially the posterior cervical glands.

The only subjective symptom is itching and this is inconstant: it may be absent, or may be slight and worse when the patient is warm in bed, or, in neurotic people and in those whose shins are irritable from toxins circulating in the blood or other cause, it may be inteinse.

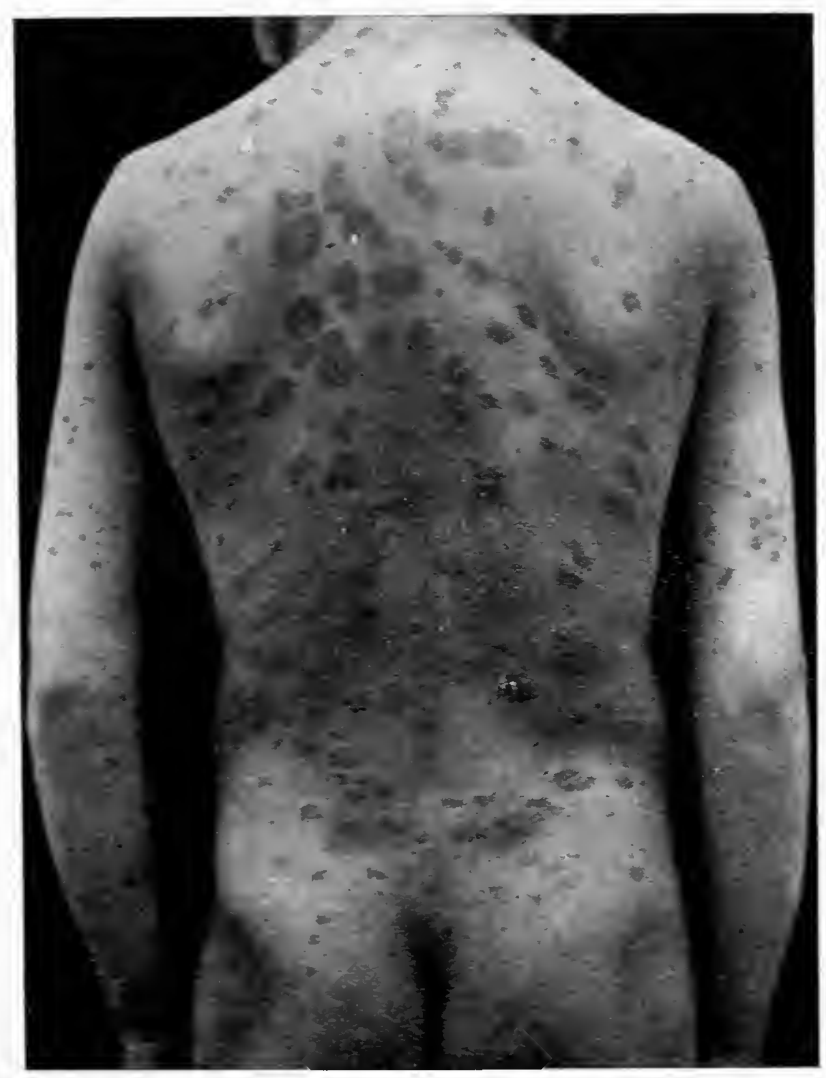

FIG. 296.-Pityriasis Rosea.

(From Dr. Wallace Beatty. Photo by Mr. J. Manby.)

In the great majority of cases the eruption gradually disappears in from 4 to 6 weeks without leaving any trace, except perhaps a temporary pigmentation, but it may persist for several months.

Occasionally the disease occurs in epidemics and cases are on record in which it has affected two or more members of a family simultaneously. It is a common observation that when one case is met with at a dermatological clinic others usually present themselves within a short time. It rarely if ever occurs twice in the same patient. These facts suggest the possibility of infectivity, but no definite instance of contagion has yet been reported. 
Histopathology.-The microscopical characteristics are somewhat similar to those of Seborrhoic dermatitis. Definite changes are present both in the epidermis and corium, which are more marked than would be expected from the clinical appearances.

In the corium they are practically confined to the papillary and sub-papillary layers, where the blood-capillaries are dilated and surrounded by a more or less diffuse cellular infiltration consisting of lymphocytes, small connective tissue cells, a few mast-cells and occasional plasma-cells (Unna). The surrounding tissue is markedly oedematous and the oedema by passing up into the epidermis is responsible for the changes there.

The amount of oedema directly deternines the extent to which the epidermis is involved; if it be slight, the changes are chiefly confined to the inter-epithelial spaces, which are dilated and show a diapedesis of leucocytes, the actual prickle-cells presenting little evidence of oedema except in so far that the process of cornification is impaired and scaliness results ${ }^{1}$; if it be excessive the prickle-cells undergo spongy changes culminating occasionally in the formation of minute vesicles near the Stratum corneum, containing distorted lymphocytes and epithelial debris.

\section{Etiology}

Predisposing Causes.-Age.-It is most common in children between the ages of 5 and ${ }_{5}$ but may occur at any age and cases have been reported in infancy and old-age. In I46 cases analysed by Iittle, $22 \%$ occurred between the ages of 5 and Io, and $44 \%$ under the age of I5.

Sex.-It has been said to be more frequent in females than in males in the proportion of about 2 to $I$, but there is considerable divergence of opinion on this point certain observers regarding it as equally common in either sex or even preponderating in males.

Season.-There is no definite agreement with regard to the seasonal incidence of the disease. It may occur at any time of the year but would seem to be more common in cold than in hot weather and to prevail in the winter and early spring.

Social Position.-Social position appears to play no part in the etiology for it attacks rich and poor indiscriminately.

Actual Cause.-The exact cause is at present unknown but the following theories have been advanced with regard to it:

Local Irritation.--Local irritants have been blamed as the cause, such as irritating under-garments, damp rooms, the wearing of damp clothes, etc., but the evidence in favour of this view is unconvincing.

Local Parasites. - So far the most careful search for pathogenic microorganisms or fungi in the scales has given negative results. At one time, when the disease was confused with ringworm, parasites of the type of

1 This is the condition which has been described more fully in connection with psoriasis in which keratohyalin granules are present in the cells of the granular layer and the nuclei persist in the horn-cells. 
the ringworm fungi were believed to be responsible, but this view has been shown to be erroneous. More recently Du Bois claimed to have found spores in the scales which he believed to be parasites of cryptogamic nature not yet identified, but other observers emploving his methods have been unsuccessful in demonstrating the organism.

Disease sui generis.-The most generally accepted view is that it is a disease sui generis but its exact nature remains uncertain.

The course of the disease, with its herald patch, general srimptoms, and cutaneous lesions, shows a certain parallelism with syphilis which would suggest a microbic origin, the herald patch being the clinical eridence of the cutaneous reaction to a micro-organism inoculated locally, and the general eruption the result of its dissemination through the circulation. On the other hand, the general invasion symptoms, period of evolution, regular course, and decline of the eruption, and the immunity. conferred by an attack, show some analogy with the exanthem of a specific fever, a view which is strengthened by the occasional occurrence of the disease in more than one member of a family, and in epidemic form.

Toxic Theory.-Another hypothesis is that it is due to a toxin circulating in the blood, and in corroboration of this theory it has been pointed out that in their histology the lesions resemble those of Erythema multiforme and that the eruption has been known to be associated with indicanuria, peptonuria, jaundice, gastric dilatation, and, in rare instances, with the puerperal state.

Diagnosis.-The differential diagnosis may present considerable difficulty, the affections with which it is most likely to be confused being scaly syphilides, Seborrhoic dermatitis, and in the case of the herald patch, Tinea circinata.

Syphilis.-The lesions of a scaly syphilide are brown in tint, usually. more raised, develop more slowly and leave a brown stain under a diascope ; those of Pityriasis rosea, on the other hand, are pinkish in tinge, slightly if at all raised, come out suddenly, and leave no stain when pressed by the diascope. In doubtful cases a microscopical examination for spirochaetes and a Wassermann test will serve to decide the question. The type of syphilide with which it is most liable to be confused is the annular secondary syphilide. It might be mistaken for a syphilitic roseola, but the roseola is not scaly and is usually concomitant with the chancre.

Seborrhoic dermatitis. - The herald patch may have to be distinguished from a single resistant patch of Seborrhoic dermatitis but as a rule this is not difficult, for in Pityriasis rosea the lesion is more circinate and has a fawn-coloured centre, while that of Seborrhoic dermatitis is more uniform and shows little or no tendencr to fade in the centre.

The eruption might also be mistaken for extensive Seborrhoea corporis with ringed lesions, but the distribution is different: in Seborrhoea corporis the lesions are most marked in the pre-sternal and interscapular regions and frequently occur on the face and scalp, while in Pityriasis rosea they are more widely distributed and rarely. affect the upper part of the face and scalp. The actual lesions differ from those of Seborrhoea corporis in which they are yellowish in 
colour, with definitely greasy scales, while in Pityriasis rosea they are pinkish and not markedly greasy. Another point of distinction is the absence from the scales of Pityriasis rosea of the spore of Malassez which is characteristic of the scales of Seborrhoic dermatitis.

Psoriasis.-No difficulty should be experienced in distinguishing it from psoriasis, for in psoriasis the lesions are more vivid in colour, the scales more silvery and less adherent and when scraped off leave bleeding puncta, and the sites of predilection are the knees, elbows, and scalp.

Tinea circinata.-So like may the herald patch be to a patch of Tinea circinata that confusion between them has existed in the Vienna school almost up to the present day, as is shown by the name Herpes tonsurans maculosus et circinatus applied to the affection by Kaposi. Clinically, however, they differ in that the herald patch always occurs on the covered parts of the body, while Tinea circinata is more frequent on exposed parts; also the ringworm lesion is more definite in outline and stronger in colour than Pityriasis rosea, and an examination of the scales usually reveals the ringworm fungus.

Prognosis.-As a rule the prognosis is excellent as the disease tends to disappear spontaneously in 6 to 8 weeks. It is uncertain whether its progress can be hastened by suitable treatment but this is of value in relieving the patient of unpleasant subjective symptoms. In a few rare cases it has been known to continue for months in spite of treatment.

\section{Treatment}

General Treatment.-On the assumption that the disease is due to the circulation of some microbe or toxin in the blood, certain anti-toxic internal remedies, such as quinine, salol, salicin, and beta-naphthol, may be prescribed, and all have their advocates. Of these salicin is possibly the most useful and should be given in doses of 10 to $15 \mathrm{grs}$. t.d.s. At the same time any obvious defects in the general health, such as rheumatism, digestive disturbances, and constipation, should be corrected by suitable means.

Local Treatment.-With regard to the local treatment, caution should be exercised in the choice of a remedy, for in a disease which clears up spontaneously in a comparatively short time it is unwise to run the risk of aggravating it, and this may be done easily by unsuitable local treatment. The injudicious employment of sulphur baths, strong sulphur ointments, and other irritating local applications, may readily do harm, increase the pruritus, and even transform the lesions into cczematous patches or produce a generalised dermatitis.

The treatment should consist of a daily warm alkaline bath containing borax 2 ozs. and sod. bicarb. 2 ozs. to the 30 gallons, in which the patient should remain for twenty minutes. Instead of the alkaline bath Jamieson recommends a permanganate of potash bath containing 
a few teaspoonfuls of Condy's fluid. After drying, one or other of the following creams should be rubbed into the skin :

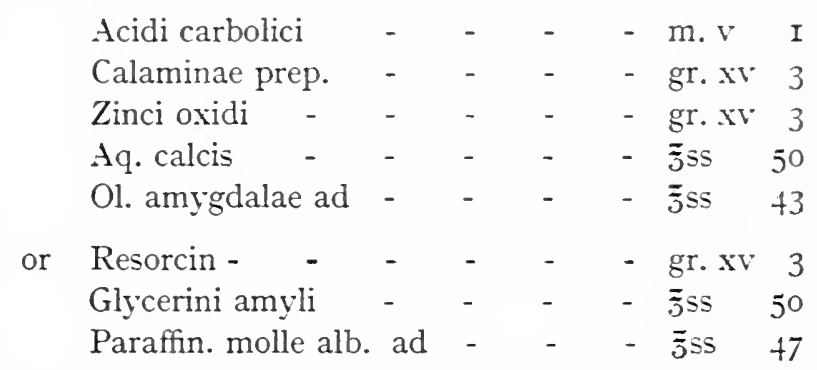

After the cream has been well rubbed in, the skin should be dusted over with the following powder :

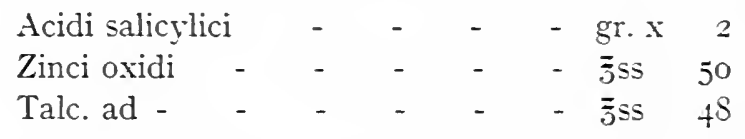

Where resistant patches are present they may be rubbed with an ointment such as the following :

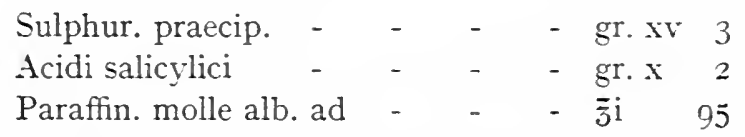

For the relief of the pruritus one or other of the following lotions may be applied :

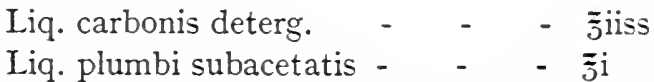

For use a teaspoonful should be added to $\frac{1}{2}$ pint of tepid water.

\begin{tabular}{|c|c|c|c|c|}
\hline Calamin. prep & - & - & - & gr. xv \\
\hline Zinci oxidi & - & - & - & gr. $x$ \\
\hline Glycerini & - & - & - & m. $\mathrm{xxx}$ \\
\hline Liq. carbonis & detergent & - & - & $\mathrm{m} . \mathrm{x}$ \\
\hline Aq. calcis & - & - & - & $\bar{j}^{\mathrm{i}}$ \\
\hline Aquam ad & - & - & - & $\tilde{\tilde{j}} \mathrm{i}$ \\
\hline
\end{tabular}

The lotion should be pressed into the skin with a piece of cottonwool and then allowed to dry in.

During the treatment the patient should wear non-irritating underclothing such as silk and cotton, rather than wool. After a week or ro days marked improvement generally results. Not infrequently the alkaline baths and the various local applications may render the skin uncomfortably dry, in which case it should be anointed, after an ordinary warm bath, with cold cream or almond oil. 


\section{REFERENCES}

Adamson, H. G. Pityriasis rosea (Albutt \& Rolleston, System of Medicine, I9I I, ix. P. 393).

Du Bors. Cryptogamic parasite in Pityriasis rosea (Ann. de Derm. et de Syph., I9I2, p. 32).

Fox, Colcotr. Pityriasis rosea (Lancet, I884, ii. p. 485).

Gibert. Traité pratique des maladies de la peau (3rd Ed., Paris, I860). Hollmanx. Pityriasis rosea, histology (Arch. f. Derm. u. Syph., I9oo, li. p. 299).

Little, G. Pityriasis rosea, discussion (Brit. Journ. Derm., I9I4, xxvi. p. II 7).

Whrte, A. J. Pityriasis rosea with pseudo-vesicles (N.Y. Med. Journ., I909, p. 962). 


\section{CHAPTER XXVIII}

\section{PEMPHIGUS}

Syn.: Fr., Pemphigus; Ger., Blasenausschlag.

Definition: An acute or chronic affection of the skin, characterised by irregularly distributed bullae arising from apparently normal or slightly inflamed skin, sometimes accompanied by serere constitutional symptoms.

Introductory.-The name "pemphigus" (from $\pi \epsilon ́ \mu \phi ı$, , a bleb, or blister) was at first applied to any eruption in which bullae occurred, but as time went on its use became more restricted, as it was found that bullae in themselves did not constitute a distinct disease but might be present in connection with various dermatoses, such as Erythema multiforme, urticaria, and erysipelas.

A large number of affections formerly placed under this heading have been deleted from the group as their true nature became more fully understood; such as Pemphigus syphiliticus which is simply a bullous syphilide, Pemphigus neonatorum which is a variety of Impetigo contagiosa and of streptococcic origin, Pemphigus contagiosus of the tropics also a variety of impetigo, Pemphigus circinatus which is a bullous form of Erythema multiforme, Pemphigus neuroticus vel hystericus a name given to bullous conditions usually occurring in hysterical young women and artificially produced either for purposes of malingering or to elicit sympathy, Pemphigus congenitalis which is a congenital vulnerability of the skin in which flaccid bullae result from traumatism.

A considerable number of cases once described as pemphigus have been relegated of recent years to the newer group of Dernatitis herpetiformis of Duhring.

At present it is customary to restrict the term pemphigus to the following conditions: Pemphigus acutus which is a severe and frequently fatal affection, Pemphigus chronicus or true pemphigus, Pemphigus foliaceus and Pemphigus vegetans which are possibly variations or further stages of Pemphigus chronicus. 


\section{PEMPHIGUS ACUTUS}

Syn.: Pemphigus malignus; Febrile pemphigus; Blister-fever.

In this rare type the bullous eruption is associated with grave constitutional symptoms, and in a large number of cases with a fatal issue. It is an acute infective disease resulting from the local inoculation of a virulent bacterium, and is met with chiefly among men who are working with animals or animal products, such as butchers, slaughterhouse men, tanncrs, etc.- the most common site of inoculation being the finger.

Description.-The onset, as a rule, is sudden. In most cases it is preceded by a wound or abrasion which may be received several months before the eruption appears. At the time of the injury or subsequently, the abrasion is inoculated by the infective virus and becomes transformed into a suppurating sore or whitlow which may have healed when the bullae develop but more frequently is still open. On the other hand, after the abrasion has been in existence for some weeks it may be replaced by a bulla, or a group of vesicles may form around it.

Several months may elapse from the time of injury to the appearance of the eruption, during which time the virus is becoming disseminated through the system. For a day or two before the outbreak of the bullae indefinite prodromal symptoms may occur, such as malaise, nausea and vomiting, rise of temperature, and sometimes a definite rigor, but in certain cases there may be no febrile disturbance until the bullae appear.

The bullae may come out anywhere on the cutaneous surface, sometimes in considerable numbers, or only a few at a time at irregular intervals, and each outburst is accompanied by a rise of temperature which may reach $103^{\circ}$ or $104^{\circ} \mathrm{F}$. They vary in size from a pea to a hen's egg and are usually oval in shape, and may be rounded and tense, or occasionally flattened and flaccid. They may develop on apparently normal skin, or may occur on an erythematous base, and are sometimes associated with erythematous blotches and vesicles which are probably abortive bullae.

The contents consist at first of clear serum which later becomes cloudy and opaque, yellow and purulent, and occasionally haemorrhagic, especially in dependent parts. When purulent, a red inflammatory halo appears around the margin of the bulla.

The bullae may break, either spontaneously or from injury, when a red, moist, denuded surface is left. By the coalescence of contiguous bullae large excoriated areas result which are tender and painful on pressure and from friction and movement. Sometimes the raw surface is covcred with purulent discharge which has an offensive foetid odour and beneath which granulations may be present. In rare instances it may becone superficially ulcerated or even gangrenous. 
The mucous membranes are generally involved, especially those of the nose, mouth, throat, larynx, and trachea ; consequently swallowing is rendered difficult and painful, breathing is impaired, the roice becomes harsh, and an irritating cough is induced. The mucosa of the stomach or intestine may be affected and romiting or diarrhoea supervene. The conjunctiva may become congested.

In the meanwhile the general health deteriorates. The patient becomes weak and exhausted from want of sleep and from insufficiency of food owing to difficulty in swallowing, mental symptoms may supervene, albumin may appear in the urine, and he may gradually sink and die in a state of collapse after a few weeks or months.

In favourable cases the lesions dry up and are replaced by crusts, fresh bullae cease to appear, the temperature goes down, the general symptoms subside, and recovery takes place. When the crusts separate, red patches are left which may be followed by transient pigmentation but no scarring. Occasional cases are on record which began acutely but gradually became less severe and finally assumed the character of chronic pemphigus.

In addition to the cases definitely traced to infection by animal products, others of a similar type have been recorded after vaccination. In a series of cases described by Howe in connection with an epidemic of smallpox in Boston in America in Igor the bullae developed about five weeks after the vaccination and the rate of mortality was so high that six of the cases ended fatally. In discussing this epidemic Bowen suggested that it might be due to infected animal lymph which had been obtained from animals just before an outbreak of "foot and mouth disease," and in support of this view may be quoted a case of acute pemphigus in a butcher who became infected in his work during an epizootic of the disease.

Small epidemics of so-called acute pemphigus have been recorded also in which the cases appeared to be contagious, but these were most probably widely distributed streptococcic impetigo and of the same class as Pemphigus neonatorum.

Histopathology.- In a fatal case of acute pemphigus in a butcher described by Pernet, Bulloch reported on the histopathology as follows: The bullae were situated between the Stratum granulosum and the prickle-cell layer of the epidermis; the underlying papillae were flattened and irregular in shape; the papillary ressels were distended and there was an infiltration of granulation cells, which in places were aggregated into masses and spread down into the deeper layers of the cutis, being specially well-marked around the sweat-glands. In the bullae was a fibrinous network which had broken down here and there and been replaced by irregularly sized granules. Entangled in the network were detached prickle-cells, irregular in outline and with feebly-stained nuclei. In addition there were small round cells with deeply stained nuclei, a few red blood corpuscles, and cocci chiefly in the form of diplococci. 
The microscopical appearances were those of a chronic inflammatory process.

Bacteriology.-From an unruptured bulla of the same case a diplococcus was isolated which resembled the gonococcus in shape but was somewhat larger and was pathogenic to a guinea-pig. At the postmortem, diplococci resembling those found in the bullae were obtained in pneumonic patches in the lungs. This diplococcus appeared to be that originally described by Demme, and also found by Bleibtreu, Wells, and others, and though it has not been definitely established as the cause of the disease, it strongly suggests that possibility. Diplococci have been isolated from the blood in similar cases.

In a certain number of cases a blood examination has revealed eosinophilia, but this has been so slight and inconstant as to be of no diagnostic value.

Etiology.-The affection is due to the inoculation of some virulent micro-organism and probably, in certain cases, to the diplococcus referred to above. The micro-organism gains entrance through some abrasion, such as a scratch from a sharp piece of bone in cutting up a carcase, or a prick or wound from a knife or other sharp instrument. The virus seems to be of animal origin and in this connection it is interesting to note that acute bullous eruptions have been observed from time to time in horses, cattle, dogs, and pigs, and have been known to be fatal. In almost all the cases the disease has occurred in young adult males who were working with animals, or with portions of dead animals. In one case the inoculation took place through an abrasion on the hand which had become infected from milking a cow with a vesicular eruption on the teats.

The exact relation of the vaccination to the animal cases is uncertain, but the clinical appearances point to an infection by some virus closely allied to that responsible for the cases in butchers, etc., and Bowen's suggestion of its possible relation to "foot and mouth" disease is of exceptional interest.

Diagnosis.-The eruption of bullae on normal skin, the grave constitutional symptoms, and the history or presence of a local sore usually in someone working with animals or cutting up carcases, constitute a clinical syndrome which is easily recognised.

Prognosis.-The outlook is invariably grave, as about $50 \%$ of the cases have died from exhaustion, septicaemia, or some complication. Occasionally the course of the disease has been rapid and death has taken place within 48 hours, but as a rule it is delayed for several weeks or even months.

\section{TREATMENT}

The internal treatment is based on general medical principles. The patient should be put to bed and symptoms, such as vomiting and diarrhoea, appropriately treated. Injections of serum have been used 
without benefit, but where a vaccine can be obtained it should be given a trial. Quinine in large doses has been recommended, and strychnine is useful where the heart's action is feeble.

The local treatment consists of disinfecting and soothing the shin. Boric acid baths are sometimes employed but are apt to be exhausting and it is generally best to keep the skin moist by some oily antiseptic application, such as equal parts of almond oil and lime water with the addition of $\mathrm{x}$ to $2 \%$ of carbolic acid.

The patient should be wrapped up in lint saturated with the oily application and, when this is removed, should be dusted with a borozinc dusting powder. A $2 \%$ ammoniated mercury ointment should be applied about the eyelids, edges of the nose and mouth, and wherever scabs and septic sores are present.

As a prophylactic it is of the utmost importance for those whose occupation renders them liable to this infection to attend promptly to any abrasion or local sore which they may have contracted in their work and to have it scrupulously disinfected, a useful application in such cases being a $6 \%$ ointment of calomel.

\section{PEMPHIGUS CHRONICUS}

Syn.: Pemphigus vulgaris; True pemphigus.

Definition: A dermatitis characterised by successive crops of bullae appearing on apparently normal skin, associated as a rule with constitutional symptoms, lasting an indefinite period, and terminating, in a large percentage of the cases, in death.

When the term pemphigus is used without a qualifying adjective it is usually taken to mean chronic pemphigus, the chief diagnostic feature of which is the appearance of blebs in successive crops without previous erythema and in an irregular fashion without any tendency to be arranged in groups. The affection is fortunately exceedingly rare and the vast majority of cases diagnosed as such are Dermatitis herpetiformis, Erythema multiforme, or, possibly, Urticaria bullosa.

Description.--The bullae are similar to those of Pemphigus acutus, vary in size from a pea to a cricket ball or larger, are round or oral in shape, and tense and distended at first and become flaccid later. On surfaces which have been denuded by a previous lesion the bullae may be imperfect. At first they contain clear serum, but this soon becomes turbid, sometimes yellow and purulent, and, in weak emaciated patients or in dependent situations, haemorrhagic. (Plate IX.)

They generally arise from apparently normal skin and it is only when the contents become purulent that an erythematous margin appears. Occasionally, however, the bleb may be preceded by a faint erythematous blotch. They may dry up in the course of a few days to form a scale or crust, or may be broken leaving a raw, moist, red surface sometimes covered with a foetid discharge or a yellowish-white 
false membrane. Occasionally, through secondary contamination, gangrenous changes and superficial ulceration may supervene.

The bullae may develop in considerable numbers at first, but more usually they come out insidiously a few at a time and by appearing in successive crops may prolong the affection indefinitely.

When the eruption has been present for some time, the skin is apt to undergo a peculiar change in which the cohesion of the epidermal cells is diminished. The position of least resistance in the normal epidermis is between the Stratum granulosum and the Stratum lucidum, and when the cohesion there becomes decreased, slight pressure may be sufficient to separate the epidermis into two layers which slide on each other, or to strip it off leaving a denuded surface. This condition, described by Auspitz as acantholysis, is known as Nikolski's sign and is especially marked in congenital pemphigus or Epidermolysis bullosa.

When the lesions dry up and the scales separate, an erythematous patch is left, which may present a transient pigmentation and is not infrequently dotted over with whitish-yellow nodules, like milium, which are small epidermic cysts formed from remnants of the interpapillary processes which have been left on the floor of the blister. Sometimes when the lesions are markedly purulent the neighbouring glands may be enlarged, and through secondary inoculation with pusorganisms, impetigo, folliculitis, abscesses, or occasionally bed-sores, may occur.

The mucous membranes are generally affected, especially those of the mouth, nose, larynx, trachea, and vulva. In some instances the lesions on the mucosa precede the bullae on the skin, or they may occur simultaneously. They appear as raw irregular areas with a deep red halo and covered by a yellowish diphtheroid membrane which, if stripped off, may lead to bleeding and to superficial ulceration.

The conjunctiva is more frequently involved than in acute pemphigus. It becomes congested and painful and a greyish membrane may appear about the inner canthus and the junction of the tarsal and bulbar conjunctivae, or may spread over the whole surface. This leads to glueing of the eyelids together, followed by cicatricial contraction, and the production of the condition known as " essential shrinkage" of the eye.

The subjective symptoms are generally slight and consist chiefly of tenderness or pain in the excoriated area from movement. Cases have been described in which there was marked itching and burning and the old term Pemphigus pruriginosus has been applied to them, but these most probably belong to the Dermatitis herpetiformis group.

The onset may be insidious and preceded by no constitutional symptoms - the first indication being onc or more bullae; on the other hand, where the bullae are numerous they may be ushered in by a definite febrile attack. 
The general health is not markedly interfered with in mild cases and recovery may take place in a few months, or even after a year or more, and the patient may suffer little beyond the inconvenience and discomfort of the local lesions. In the majority of cases general symptoms are marked from the beginning and may persist throughout the attach. They vary greatly in character and degree but consist generally of sleeplessness, nervous agitation, ferer with each fresh outbreak of blebs or from septic absorption, vomiting and, sometimes, diarrhoea. In consequence the general condition becomes lowered, there is loss of appetite and weight, and the patient may die in a state of sheer exhaustion or as the result of some intercurrent disease, such as nephritis, tuberculosis, or pneumonia.

Histopathology.- The bulla is situated in the epidermis in the region of the transitional layers, or in some cases where the serous transudation which is responsible for it has been specially rapid and excessive it may form beneath the epidermis with the flattened out papillae as the floor. It is when the blister occurs beneath the epidermis that the small epidermal tags are torn off from the apices of the inter-papillary processes and proliferate to form the milium-like epidermic cysts which occur on the skin where bullae have been.

In the corium there is marked dilatation of the blood-ressels of the papillary and sub-papillary layers, associated with oedema and an infiltration of leucocytes and small connective tissue cells. As this has been observed previous to the formation of the bullae it suggests an initial inflammatory disturbance. The contents of the bullae consist of serum in which are bands of fibrin, epithelial nuclei, the debris of epithelial cells, and polymorphonuclear leucocytes in all stages of disintegration. There is usually a marked increase of eosinophiles.

There is still considerable difference of opinion with regard to the mechanism of the formation of the bullae. According to Auspitz, they are due to diminished resistance in the prickle-cell laver in circumscribed areas so that the cells are easily separated by the rapidly collecting fluid which escapes from the dilated blood-ressels, and inflammatory phenomena if they occur are purely secondary. The riew that a pre-existing cachectic state of the epithelium is essential for the formation of the bullae is not generally accepted and the acantholysis is now believed to be a secondary phenomenon which occurs in the skin some time after the bullae have developed, probably as the result of the macerating effect of the serum which floods the epidermis or of some toxin contained in it.

The more modern view is that the bullae are due to a pre-existent inflammation with a serous transudation which, though not noticeable clinically, can be detected on microscopical examination.

Post-mortem examinations in fatal cases have not shown any-changes in the viscera which were sufficiently constant to be of etiological importance. Fatty degeneration of the liver and heart have been noted but these may be secondary. In several instances degenerative changes 
have been described in the peripheral nerves which go to the bullae, haemorrhages have been observed in both the grey and white matter of the cord and in the nerve roots, and degeneration of the multipolar cells of the anterior cornea, and sclerosis of the posterior columns have been noted. The cause of these changes is uncertain but it is possible that they are simply the result of some intercurrent disease.

Etiology.-Chronic pemphigus may occur at any age but is more common in adult life, especially after 40, than in childhood. It may attack either sex indiscriminately.

Its exact nature and cause are uncertain and none of the theories that have been advanced to explain it has yet been definitely established. Of these the principal are the toxic, the microbic and the neuropathic theories.

The Toxic Theory.-According to this theory, chronic pemphigus is a toxaemia and due to some form of auto-intoxication the nature of which is unknown. In support, it has been pointed out that the bullae are probably the result of an inflammatory disturbance suggesting the action of a toxin in the skin, which, though not apparent clinically, as it is masked by the extent and rapidity of the serous exudation, is obvious under the microscope ; that the eruption in the early stages, especially when extensive, is associated with general symptoms, such as fever, vomiting, malaise, etc.; and that the post-mortem changes which have been found, such as lardaceous disease of the liver and spleen, fatty degeneration of the heart, and parenchymatous nephritis, are such as would be produced by some toxic process acting over a prolonged period.

The Microbic Theory.-For many years the view has prevailed in certain quarters that chronic pemphigus, like the acute form, is a primary microbic affection, but the evidence in favour of it is insufficient. There is no history of a previous wound or infective sore, repeated examinations of fresh bullae for bacteria have either been negative and the contents found to be sterile, or where micro-organisms have been found it has been usually in old bullae which have probably become contaminated by the ordinary skin cocci. In some cases diagnosed as chronic pemphigus, streptococci, Bacillus pyocyaneus, and diplococci, have been found both in the bullae and the blood, but none of them has been established as the cause of the disease.

The Neuropathic Theory.-The neuropathic theory, once generally accepted, is gradually becoming obsolete. It was argued that bullae may arise in connection with disturbances of the central and peripheral nervous system, as in syringo-myelia and nerve-leprosy; that pemphigus may be associated with more or less severe mental symptoms; and that in one or two fatal cases degenerative changes have been detected in the peripheral nerves and in the spinal cord. Against these arguments it should be pointed out that where the bullae are definitely due to pathological changes in the peripheral nerves, as in leprosy, they are associated with local sensory disturbances which are absent in pemphigus; that the mental symptoms may be the result of the deterioration in health, insomnia, and general discomfort of the cutaneous lesions; and that the degenerative changes in the nerves and cord may be induced secondarily in the course of the disease.

Diagnosis.-The recognition of chronic pemphigus is exceedingly difficult and the majority of cases so diagnosed are found subsequently to belong to some other category. 
It has to be distinguished in the first instance from Dermatitis herpetiformis, which may be difficult as the first symptoms in that disease may be one or more bullae. After a time, howerer, the typical herpetiform groups of papules or vesicles, with itching or burning, usually assert themselves and the diagnosis becomes evident.

In this connection it must be noted that certain writers maintain that chronic pemphigus and Dermatitis herpetiformis are indistinguishable and are simply phases of one disease.

It has to be differentiated from the bullous type of Erythema multiforme, but in it the bullae are preceded by a definite erythematous patch, and the lesions do not occur indiscriminately over the cutaneous surface but are usually confined to sites of predilection of that disease, namely the hands, forearms, face, and sometimes the legs.

It has to be distinguished from widely distributed bullous impetigo of streptococcic origin, like Pemphigus neonatorum, but in it the bullae are more flaccid and spread by undermining the horny layer of the epidermis with serum.

In one or two cases where the initial lesions are confined to the mucous membranes of the mouth or nose they might be mistaken for syphilides, but in these cases typical bullae generally derelop on the cutaneous surface sooner or later, and the absence of concomitant evidence of syphilis usually enables a diagnosis to be made.

Prognosis.-The prognosis is invariably grave and particularly in elderly people and the gravity varies directly with the extent of the eruption. Mild cases generally recover, especially in children, and may do so in a comparatively short time. In adults the disease may persist for years and yet terminate, after numerous recurrences, in complete cure. In a large number of instances the wear and tear of the prolonged illness, the deterioration in health due to insomnia and the pain associated with the denuded surfaces, so lower the vitality that the patient becomes prone to intercurrent diseases such as pneumonia or tuberculosis, or may die from sheer exhaustion or septicaemia.

\section{TREATMENT}

As the etiology of the disease is not understood the treatment is empirical rather than specific.

General Treatment.- The object of the general treatment is to maintain the patient's strength, to lessen insomnia and pain, and to correct anaemia, digestive disturbances, diarrhoea, or any other internal disorder. The patient should be placed in the best possible surroundings with regard to hygiene and nursing, the dietary should be stimulating, and tonics should be prescribed where indicated, such as strychnine, iron, quinine in large doses, or arsenic. At one time arsenic was credited with having a specific effect and of being of special value in cases in children, but its merits seem to have been greatly. exaggerated and any benefit which may accrue from its use is probably 
the result of its tonic action on the blood and nervous system rather than of any specific action on the skin.

Intestinal antiseptics, such as mercury, salol, etc., have been advocated, especially by those who believe that it is an auto-intoxication, and, in suitable cases, undoubted benefit may be derived from their judicious employment.

Local Treatment.- The local treatment is similar to that of acute pemphigus. In the Vienna clinic in Hebra's time alkaline and mild disinfectant continuous baths were largely employed, but although they relieve the discomfort of the denuded surfaces and prevent secondary septic inoculations, they are as a rule too exhausting, and on the whole it is best to nurse the patient on a water-bed and to keep the skin as far as possible soothed and disinfected. As there is usually a considerable extent of denuded surface, care must be taken that the antiseptic employed is not used ton extensively, or in too concentrated a form, as it may be absorbed and produce toxic symptoms, as has occurred with salicylic and carbolic acids. Calamine cream with $\mathrm{r} \%$ of carbolic acid is an excellent general application. This should be rubbed all over the skin and followed by a bland dusting powder, such as zinc, talc, or magnesium carbonate, but not starch as it is apt to become septic. The skin should then be covered with butter-muslin and the limbs lightly bandaged. Ulcerated areas should be treated with a mild mercurial ointment, and where the mucosae are involved they should be dealt with by suitable disinfectant lotions, mouth washes, etc.

\section{PEMPHIGUS FOLIACEUS}

Definition: A persistent affection of the skin characterised at first by vesicles or bullae followed by universal redness and exfoliation of the epidermis in papery flakes leaving denuded areas moist with an offensive discharge.

Introductory.--The affection was described first by Cazenave in I84.4. In its fully developed state it closely resembles generalised exfoliative dermatitis, only that instead of being dry in its whole course there is usually a history of previous bullous formation and the remains of flaccid bullae or moist denuded areas are generally present. The propriety of regarding it as a distinct entity has been questioned, and the opinion is gaining ground that it is only a further stage of chronic pemphigus or of Dermatitis herpetiformis. On the other hand its clinical features are so characteristic that for descriptive purposes it is advisable to describe it as a separate disease.

Description.--It may begin either as chronic pemphigus with an eruption of bullae on an apparently healthy skin, or as a dermatitis with groups of vesicles. After a time the bullae or vesicles disappear, the skin gradually becomes reddened over the whole surface, and a general exfoliation sets in due to the separation of the epidermis by 
imperfectly formed bullae. In some cases bullae, though imperfect, still keep appearing after the stage of exfoliation has reached its height. The exfoliation, like the erythrodermia, is universal.

In a well-marked case the scalp becomes covered with greyishyellow scales or greasy crusts, and the hair is generally thinned; the face is red and puffy from oedema, and covered with brainy scales, the eyebrows are defective, and cracks usually appear at the angles of the eyes and of the mouth; the trunk presents a red patchy raw appearance, the epidermis peeling off either in large irregular flakes, in thick sodden masses, or in moist crusts compared to the bark of a tree, and leaving raw areas, covered with a foetid discharge which has a peculiar sickly penetrating odour, with here and there a few imperfectly formed vesicles or bullae between them; the skin of the limbs is similarly affected and there may be marked thickening of the palms and soles. As in chronic pemphigus the cohesion of the epidermal cells is impaired and Nikolski's sign is well-marked (see page 874). Sometimes the nails are affected and may become opaque, thickened, and brittle. The mucous membranes may be attacked as in chronic pemphigus and lesions have been described about the mouth and conjunctivae. As a rule the glands, especially those about the neck, occiput, axillae, and inguinal region, are hard and swollen but not painful or tender.

The subjective symptoms vary considerably in different cases, and may be slight and consist of itching or burning which tends to diminish when the condition becomes generalised, or intense, as in the cases which begin as Dermatitis herpetiformis.

In the majority of cases the affection is not continuous and more or less prolonged remissions occur followed by fresh outbreaks.

A peculiar complication has been described by Quinquaud under the heading of "papillomatosis" in which the skin, some time after the exfoliation, becomes thickened and wrinkled from deepening of the natural folds and presents a rough granular appearance, and there is hyperkeratosis of the palms and soles. This may be due to arsenic which was once extensively employed in the treatment of pemphigus.

The general health and nutrition at first are well-maintained in spite of the extensive involvement of the cutaneous surface, possibly owing to the subjective symptoms being slight. After the disease has existed for months or even years the patient as a rule gradually sinks into a weak state from the wear and tear of fever, diarrhoea, and albuminuria, and death generally supervenes either in a state of extreme marasmus or from some complication, such as disease of the lungs, kidneys, or heart.

Brocq in his exhaustive article on the subject in his text-book divides the cases into three groups:

I. True Pemphigus foliaceus in which there is moderate itching, the formation of blebs succeeded by erythrodermia, and desquamation which at first is in large scales and subsequently is furfuraceous. 
2. Cases in which there is only moderate itching, and in which the blebs persist after the exfoliation has developed and keep on coming out.

3. Cases of the Dermatitis herpetiformis type, preceded by crops of vesicles in herpetiform groups which keep on coming out after the exfoliation has begun and gradually lead to a fatal termination. To this class of case he gave the name "L'Herpétide maligne exfoliative."

Histopathology.-The histological changes which have been recorded in the different cases are fairly constant.

In the epidermis the interpapillary processes are thin and elongated, the prickle-cells swollen and oedematous, and the inter-epithelial lymph spaces are dilated and contain polymorphonuclear leucocytes and sometimes eosinophiles. Here and there the prickle-cells have become so separated that small vesicles have formed. The Stratum corneum is thin and the exfoliation has taken place just above the granular layer, or more rarely has involved the whole of the epidermis.

In the corium the blood-vessels of the papillary and sub-papillary layers are dilated and there is marked oedema. The white fibrous bundles are swollen and oedematous, and there is diminution of the elastic tissue in the superficial layers. Around the dilated vessels there is an infiltration of cells consisting of leucocytes, a few plasma-cells, polymorphonuclear leucocytes, and sometimes eosinophiles, while in the deeper layers of the corium an increase of mast-cells has been described. The underlying subcutaneous fat to some extent has undergone degeneration and disappeared.

The appearances suggest an inflammatory lesion of toxic origin in which the epidermal changes are secondary to those which occur in the corium.

The blood has been examined in a few cases but the findings have been inconstant and in no way characteristic. In the majority there has been a marked increase of eosinophiles but occasionally the percentage has been below normal.

Etiology.-It may occur at any age but most of the cases have been met with in young adults. Cases have been recorded in infants but these were probably instances of Ritter's disease (see page 308). It seems to be more common in females than in males and in 30 cases collected by Lansac 20 were in females.

The exciting cause is unknown but it is probably a form of autointoxication and the result of some elusive toxin or foreign proteid such as may cause chronic pemphigus or Dermatitis herpetiformis. The reason why certain cases of chronic pemphigus or Dermatitis herpetiformis progress to Pemphigus foliaceus is equally uncertain ; it may be due to a difference in the exact nature of the toxin or in the cutaneous reaction of the patient. In support of the toxic origin of the disease may be cited the general symptoms, the fever, diarrhoea, and occasional indicanuria, associated with it. 
It has been regarded by certain observers as being of microbic origin, but it does not suggest a local parasitic cause and any organisms which have been isolated, such as the Staphylococcus aureus, were probably secondary contaminations.

As in chronic pemphigus, a nerve origin has been advanced as the cause of certain cases, since in one or two instances the affection has been preceded by some severe mental shock, but the absence of marked sensory symptoms and of any disturbance of sensibility, however extensive the eruption, seems to show that any disorder of the nervous system which may be present is functional rather than organic, and if etioiogically connected, is a pre-disposing rather than an exciting cause. Various pathological changes have been recorded in the nervous system in post-mortem examinations, such as degeneration of the peripheral nerves and the ganglionic cells of the posterior horns of the cord, but these are too inconstant to be of etiological importance and are probably merely accidental or secondary.

Diagnosis. - In a well-marked case the universal redness, the extensive formation of moist greasy flakes and scales, the offensive odour, and the presence of flaccid or imperfect bullae, form a characteristic clinical picture. The condition bears a striking resemblance to generalised exfoliative dermatitis, but there the skin is dry while in Pemphigus foliaceus the scales are often moist and sodden and raw areas are generally present covered with a foetid discharge.

It has to be distinguished from generalised eczema but eczema never becomes universal and typical papulo-resicular lesions can generally be detected.

Prognosis.-The prognosis is grave and although there is a tendency in most cases to remissions these are usually temporary and succeeded sooner or later by recurrences which lead eventually to a fatal issue. Some cases, however, recover after a course of several months or even years.

The drier the case the better the chance of recovery and in the fatal cases the bullous formation has generally been well-marked and extensive moist surfaces have been present.

\section{TREATMENT}

The patient must be confined to bed and preferably to a water-bed, as the skin is exceptionally rulnerable, and should have a daily bath containing a small quantity of some deodorant, such as potassium permanganate, to clean the skin and diminish the offensive odour. After the bath, a bland protective cream or paste, such as calamine cream or zinc oxide $j \mathrm{j}$ in soft white paraffin $\bar{j} \mathrm{i}$, should be smeared orer the surface. Where denuded areas are present and have become septic it is an advantage to add I \% ammoniated mercury to the application.

Internally there are no specifics and all that can be done is to support the strength by a stimulating diet and suitable tonic remedies, 
and to treat any complication which may arise on general medical principles.

Where the lesions have become septic from the secondary inoculation of pus-cocci, a vaccine may be of benefit.

\section{PEMPHIGUS VEGETANS}

Syn. : Dermatitis vegetans.

Definition: A rare affection of the skin characterised by cauliflowerlike vegetations covered with a foetid discharge, situated chiefly about the axillae and perineum, preceded by vesicles or bullae on the floor of which they have sprouted, and associated with imperfect bullae or sodden patches on the mucosa of the mouth, rectum, or vulva.

Introductory.- - This affection was first described by Neumann, and is extremely rare, only about 75 cases having been recorded up to the present time. There has been considerable diversity of opinion as to whether it is a distinct entity, or whether the vegetations are simply an epiphenomenon which may occur in connection with any bullous disease. In support of the latter contention it has been pointed out that vegetating growths may also complicate the lesions of Erythema bullosum, Dermatitis herpetiformis, and even eczema, and that they are probably the result of a secondary microbic infection, possibly staphylococcic, the predilection which the disease exhibits for moist and warm situations such as the axillae and perineum being explained as due to the skin there affording a specially suitable soil for the growth of the micro-organism.

Description.-It usually begins insidiously without prodromal symptoms, the first indication not infrequently being some lesion on the mucous membrane of the mouth, nose, vulva, or anus, such as an imperfect blister, a denuded area covered with a white sodden diphtheroid membrane, or a superficial ulcer. In one or two instances it. has been preceded, like acute pemphigus, by a wound which had dereloped into a septic sore. Occasionally it may begin on the cutaneous surface with an outbreak of bullae, like chronic pemphigus.

When the mouth is attacked the mucosa may be extensively involved, and sodden areas may occur on the cheeks, gums, tongue, pharynx, trachea, and even the oesophagus, which render chewing and swallowing so painful that the patient dreads the taking of food and has to be fed entirely on liquid nourishment.

In typical cases it is not, as a rule, long confined to the mucosa, but after a varying time bullae or vesicles usually make their appearance somewhere on the cutaneous surface, most frequently about the large flcxures of the axillae or groins, around the anus, and about the genitalia. These bullae, at first tense and distended with clear fluid, soon become purulent and break leaving denuded areas covered with a particularly offensive discharge. Proliferative changes then super- 
vene on the raw surfaces in the form of soft condylomatous vegetations which may become exuberant cauliflower-like masses. The vegetations are kept moist with the discharge, the foetid odour of which permeates the whole room and renders nursing an unpleasant duty. In the older lesions the discharge may dry up to form greenish or haemorrhagic scabs, and beyond the margin of the regetations small blebs may sometimes be detected. Occasionally whitlow-like lesions have been recorded about the fingers, and the nails may be involved, becoming opaque, deformed, and sometimes shed.

Secondary inoculation of pyogenic organisms in situations in which bullae do not occur may lead to the formation of abscesses, impetiginous lesions about the scalp and elsewhere, and sometimes to painful phlyctenular conjunctivitis.

The subjective symptoms vary considerably in the different cases. They may be slight or absent, or there may be more or less severe itching and burning.

The extent of the eruption and the septic absorption from the purulent vegetations lead to grave constitutional symptoms; the temperature begins to rise at night, the patient becomes restless from want of sleep and the discomfort connected with the lesions, diarrhoea, albuminuria, or other grave symptoms may supervene, and death generally takes place in a few weeks or months. In some of the cases there is a marked tendency to remissions in which the vegetations shrivel and gradually disappear without leaving any trace ; but after an interval, possibly of years, the disease is apt to re-assert itself even more severely, and to lead ultimately to a fatal issue.

A few cases have been reported in which recovery took place. In them the lesions suggested a vegetating form of Dermatitis herpetiformis, but it has been doubted whether they belong to the same category as the typical cases involving the mucosa and larger flexures.

Histopathology. - The microscopical appearances and situation of the bullae are similar to those of chronic pemphigus. The diagnostic peculiarity is the presence of epidermal vegetations of the same type as those which occur in venereal warts. In them the prickle-cell layer is irregularly thickened and the interpapillary processes grow down as long pegs into the corium. The prickle-cells are swollen and oedematous, the intercellular spaces are dilated and leucocytes are present between the cells and here and there are collected to form small epidermal abscesses. The Stratum corneum is defective and the cornification process imperfect, many of the horn-cells presenting nuclei.

Histologically the condition points to the regetations being an epiphenomenon and possibly due to the action of some micro-organism, either inoculated locally or circulating in the blood.

Eosinophilia both in the bullae and in the blood has been described in a majority of the cases.

A number of micro-organisms have been isolated from the bullae and the blood, such as Staphylococcus albus and aureus, streptococci, 
diplococci, and Bacillus pyocyaneus, but none of them has been definitely proved to be the cause of the disease.

Etiology and Pathogenesis.-The affection is found chiefly in adults but has been known to occur in children, and cases have been recorded which began as early as 12 and as late as 70 years. Like Pemphigus foliaceus it would appear to be more common in females than males.

The cause is unknown. Certain observers believe that the vegetations are a complication rather than an essential feature and are probably due to the secondary inoculation of staphylococci, and that the causation is similar to that of chronic pemphigus. There is a good deal to be said in favour of this view as staphylococci may be responsible for the production of vegetations in other cutaneous lesions. Others consider that the same microbe is responsible for the bullae and the vegetations, and that it has reached the skin and mucous membranes via the blood stream, and point out that like acute pemphigus it may be preceded by some definite septic focus or may begin in mucous membranes following some septic condition such as Pyorrhoea alveolaris.

Diagnosis.-The diagnosis of a well-marked case should not be difficult, as the presence of bullae and the occurrence of vegetating moist or crusted masses about the axillae, groins, anus, and sides of the mouth, constitute an easily recognised clinical picture.

In the absence of bullae it has to be distinguished from the vegetating condition produced by iodide of potassium, but in it the lesions are not confined to the seats of predilection of Pemphigus vegetans, and there is generally a history of taking the drug.

Prognosis.-The prognosis is invariably grave and in the cases where recovery has been reported, it has usually been temporary and in the nature of a prolonged intermission and followed after a varying time by a recrudescence and a fatal issue.

Treatment.-It is similar to that of chronic pemphigus with the addition of special local applications to diminish the vegetations and, if possible, to prevent their formation. The skin should be kept scrupulously clean, the discharge thoroughly wiped away, and the crusts removed by carron oil to which $\mathrm{I} \%$ carbolic acid has been added.

The vegetations should be cleaned and astringed by lotions of hydrogen peroxide, protargol, potassium permanganate, or other suitable antiseptic, followed by the application of a powder of salicylic acid and zinc oxide $\mathrm{I}$ to 8 , or dressed with a mild mercurial or boric acid ointment spread on lint. Where the vegetating surfaces are extensive, boric baths, intermittent or continuous, may be tried. Where antiseptics are used over an extensive area it is important that they should not be in a concentrated form for fear of absorption.

The mucous membranes must be attended to by suitable lotions and mouth washes.

Internally, a tonic stimulating regime on gencral medical principles is indicated. Various drugs such as sodium iodide and arsenic have 
been recommended but any improvement from their use has been temporary as they have no specific action on the lesions.

\section{REFERENCES}

Pemphigus acutus.

Bowen, J. T. Bullous dermatitis following vaccination (Journ. Cut. Dis., 1905, xxiii. p. 79).

Custance, G. WV. Acute pemphigus (Practitioner, I9I3, xci. p. 7 Io).

Grindon, J. Acute septic pemphigus (Journ. Cut. Dis., Oct. I909).

Howe, J. S. Bullous dermatitis following vaccination (Trans. Amer. Derm., 1902, p. 23).

Landsberger. Pemphigus acutus and severe mental symptoms (Deutsch. Med. Woch., I9I2, xxxviii. p. I273).

Mook, W. H. Pemphigoid eruptions following vaccination (Journ. Cut. Dis., Oct. I9I5).

Ormsby \& Bassoe. Acute malignant pemphigus (Journ. Cut. Dis., I905, xxiii. p. 294).

Pernet \& Bulloch. Acute pemphigus (Brit. Journ. Derm., I896, viii. p. 157$)$.

\section{Pemphigus chronicus.}

Joseph, M. On pemphigus (Arch. f. Derm. u. Syph., I9II, cx. p. 399).

LESZCZYNZKI. Intravenous quinine infusions in pemphigus (Arch. f. Derm. u. Syph., Oct. I9I2).

MacLeod, J. M. H. Pemphigoid eruptions (Brit. Journ. Derm., I9I5, xxvii. p. 20I).

Michel. Pemphigus of conjunctiva (Derm. Zeitschr., I900, p. 6I7).

Petges \& Bichelonse. Septicaemia from Bacillus pyocyaneus \& chronic true bullous pemphigus (Ann. de Derm. et de Syph., x908, p. 4I7).

\section{Pemphigus foliaceus.}

Baum, H. C. Pemphigus foliaceus (Amer. Journ. Derm., I9I2, p. 356).

Broce, L. Pemphigus foliaceus (Derm. Prat., r902).

Hazex, H. Pemphigus foliaceus \& Ritter's Disease (Journ. Cut. Dis., I 9I 2, xxx. p. 325).

Leredde. Pemphigus foliaceus (Cazenave) (Ann. de Derm. et de Syph., I899, p. 60I).

Low, Cranston. Pemphigus foliaceus (Long bibliography) (Brit. Journ. Derm., I909, xxi. p. IоI).

\section{Pemphigus vegetans.}

Botrelli. A case of Pemphigus vegetans (Giorn. Ital. d. mal. Ven. e della Pelle, I9I I, lii. p. 498).

Cronguist, C. Pemphigus vegetans, clinical \& histological (Arch. f. Derm. u. Syph., I9I3, cvi. p. I43).

Fox, W. S. Vegetating pemphigus (Brit. Journ. Derm., I908, xx. p. ISI).

Hamburger \& Rubel. Pemphigus vegetans (Bacteriology) (Johns Hopkins Hosp. Bull., I903, p. 63).

Hartzell, M. B. A case of Pemphigus vegetans with special reference to the cellular elements found in the lesions (Journ. Cut. Dis., March 19I0).

Jamieson \& Welsh. A case of Pemphigus vegetans (Brit. Journ. Derm., I902, xiv. p. 287).

MacCorsiac, H. Pemphigus vegetans (Idem, IgoS, xx. p. 277).

Orisby \& Bassoe. Acute malignant pemphigus (Journ. Cut. Dis., I905). 
Pollitzer, S. Pemphigus vegetans beginning as a condylomatous patch at the anus (Festschr. z. Vierzigjahr. Stifting des Deutsch. Hosp., N.X., I9II, p. 546).

Rutherford, W. J. Pemphigus vegetans, clinical (Brit. Journ. Derm., I9Io, xxii. p. II9).

Schiedat. Pemphigus vegetans (Arch. f. Derm. u. Syph., I9Io, p. 227). Stanziale. Pemphigus vegetans: bacteriological experiments on (Ann. de Derm. et de Syph., I904, p. I5).

Winfield. Pemphigus vegetans (Record of $5^{8}$ cases published before I906) (Trans. Amer. Derm. Ass., 1906).

\section{PEMPHIGOIDS}

The name pemphigoids was introduced by Besnier to describe the group of eruptions which Tilbury Fox included under the heading of hydroa and which Duhring re-christened Dermatitis herpetiformis in I884, and Brocq named Dermatite polymorphe douloureuse in 1888 . It was made familiar in this country by Colcott Fox who employed it as a synonym for Dermatitis herpetiformis in his article on the subject in Allbutt and Rolleston's " System of Medicine."

The term is somewhat unfortunate as it simply signifies an eruption characterised at some phase or period in its course by bullae and having a resemblance to chronic pemphigus, and consequently it has been used in a loose sense by different writers to include a number of bullous eruptions, such as chronic pemphigus, acute pemphigus, Dermatitis herpetiformis, Pemphigus vegetans, Erythema bullosum, and Pemphigus neonatorum. If the names pemphigoids or pemphigoid eruptions be retained it seems best to restrict their use to the Dermatitis herpetiformis group.

\section{DERMATITIS HERPETIFORMIS}

Sy'n.: Dermatite polymorphe douloureuse (Brocq); Herpes pemphigoides ; Pemphigoid eruptions ; Pemphigoids ; P. circinatus ; P. herpetiformis ; P. pruriginosus.

Definition: A chronic recurrent polymorphous condition of the skin characterised by persistent erythematous patches, rings, or gyrate figures, prurigo-like papules and vesicles with a tendency to herpetiform ${ }^{1}$ grouping, sometimes by bullae, and associated with intense itching or burning.

Introductory.-The name Dermatitis herpetiformis was given by Duhring in 1884 to a group of eruptions which had been described previously under a great variety of names. He recognised that there was a series of affections which, though differing greatly in their clinical appearances, yet had certain common characteristics which pointed to their being closely related in etiology and variants of one morbid

1 NoTE-By herpetiform is meant grouping in clusters similar to those which occur in Herpes zoster. 
process. This had already been realised in this country by Tilbury Fox who had described them under the heading of Hydroa; his paper, however, did not attract the attention it deserved and it is by the name applied by Duhring that the group is generally known.

Previous to I880, when Fox's paper was published by his brother Colcott Fox, the cases had been described under such headings as Pemphigus circinatus, Herpes pemphigoides, Herpes circinatus, Hydroa gestationis, Impetigo herpetiformis, etc. There has been some discussion as to whether Hydroa gestationis and Impetigo herpeti-

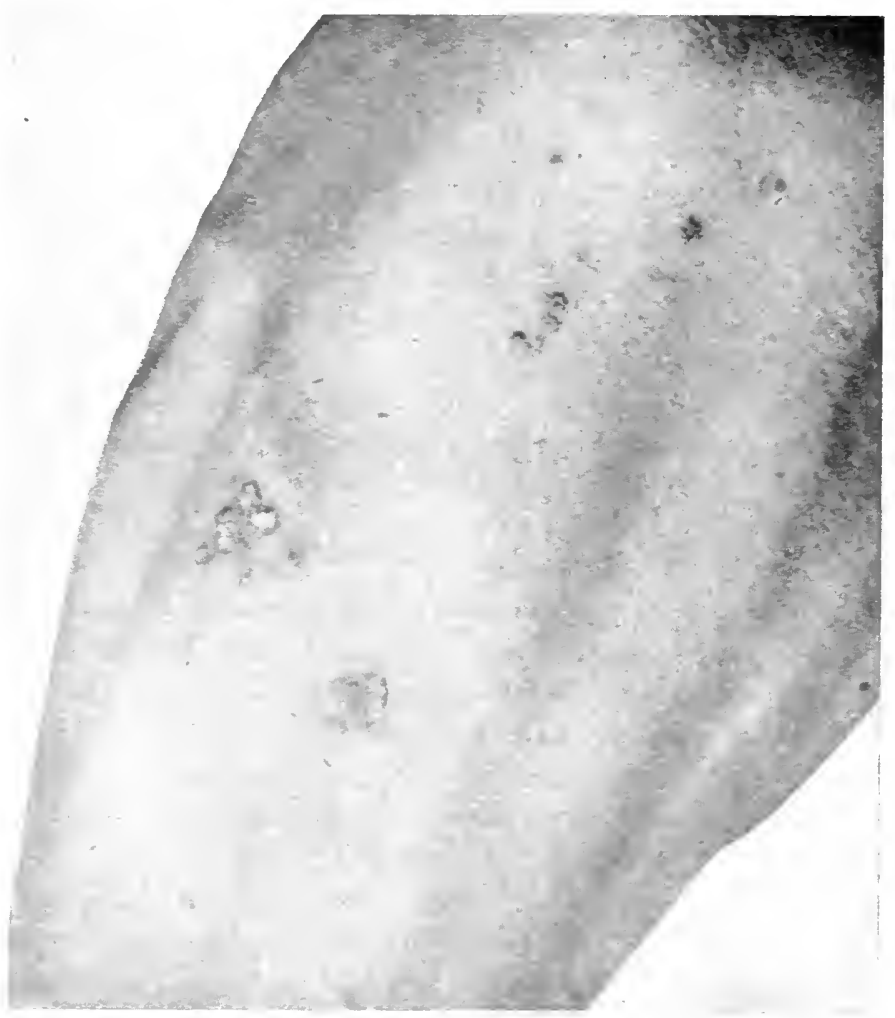

FIG. 297.-Dermatitis Herpetiformis.

Shows small groups of vesicles.

formis should be included under the heading of Dermatitis herpetiformis but the general concensus of opinion is that they belong to this group.

Dermatitis herpetiformis is a rare disease. Out of $\mathrm{I} 2,000$ consecutive cases of skin disease at Charing Cross Hospital there were only 23 cases, while out of II,000 cases at the Victoria Hospital for Children there were only 3 cases-that is $0.19 \%$ and $0.0 \mathrm{I} \%$ respectively.

Description.-To describe Dermatitis herpetiformis adequately is difficult owing to the multiformity of the lesions and eruptions. This 
is due to a number of causes of which the most important are : (a) variation in type of the initial lesion; $(b)$ occurrence of several types of lesions synchronously; $(c)$ variations in the type of lesion occurring in different attacks; (d) differences in the stage of evolution of individual lesions; $(e)$ endless differences in distribution and grouping. (Fig. 297.)

The types of initial lesions which may be met with are prurigo-like papules, papulo-vesicles, vesicles, bullae, and erythematous or urticarial patches. The patches may be level with the surface or definitely raised, are sometimes covered with papules or vesicles grouped in a herpetiform fashion, or may involute in the centre and give rise to circinate figures with rings of vesicles at the border, which may coalesce to form a gyrate patterning. (Fig. 298.)

The vesicles vary in size from a pin's head to a lentil, and may be acuminate, forming the apices of the papules, or rounded, and may appear on apparently healthy skin or develop on a red base. Sooner or later, in every case, they are surrounded by an inflammatory halo from the growth of secondary micro-organisms in the contents. The vesicles may be isolated and irregularly distributed, but as a rule they are clustered in small groups of six or eight, or, more rarely, arranged in a circinate manner at the edge of an erythematous patch like Herpes iris, or exceptionally in concentric rings like a cockade. They usually remain discrete, but occasionally may be closely aggregated to form multilocular bullae. (Fig. 299.)

The bullae vary in size from a lentil to a walnut, but are generally about the size of a small bean; they may develop on apparently healthy skin, or on an inflammatory or urticarial base, and may be regular in outline and unilocular, or irregular and multilocular. As a rule they are tense, but occasionally may be so flabby as to suggest Epidermolysis bullosa, or the fluid contents may be slight and the dissociation of the epidermis considerable, giving rise to a desquamating appearance recalling mild Pemphigus foliaceus. In rare instances, possibly through the growth of secondary micro-organisms, vegetations may grow up from the bases of the bullae, especially about the angles of the mouth, anus, vulva, or groin, and cases of this nature have been described as a mild type of Pemphigus vegetans from which recovery took place. (Fig. 300.)

The contents of the vesicles and bullae are at first clear and sterile but soon become opaque and purulent from secondary contamination with pus-organisms. The transition may be so rapid that the early clear phase may pass unobserved and it is those cases which constitute the so-called Impetigo herpetiformis.

Sometimes one type predominates, sometimes another, but as a rule several phases are present simultaneously. In some cases one type of lesion may be preserved in successive attacks, in others the type may change. In a case under the care of the writer the initial lesion was a bulla about the size of a filbert nut situated on apparently healthy skin 

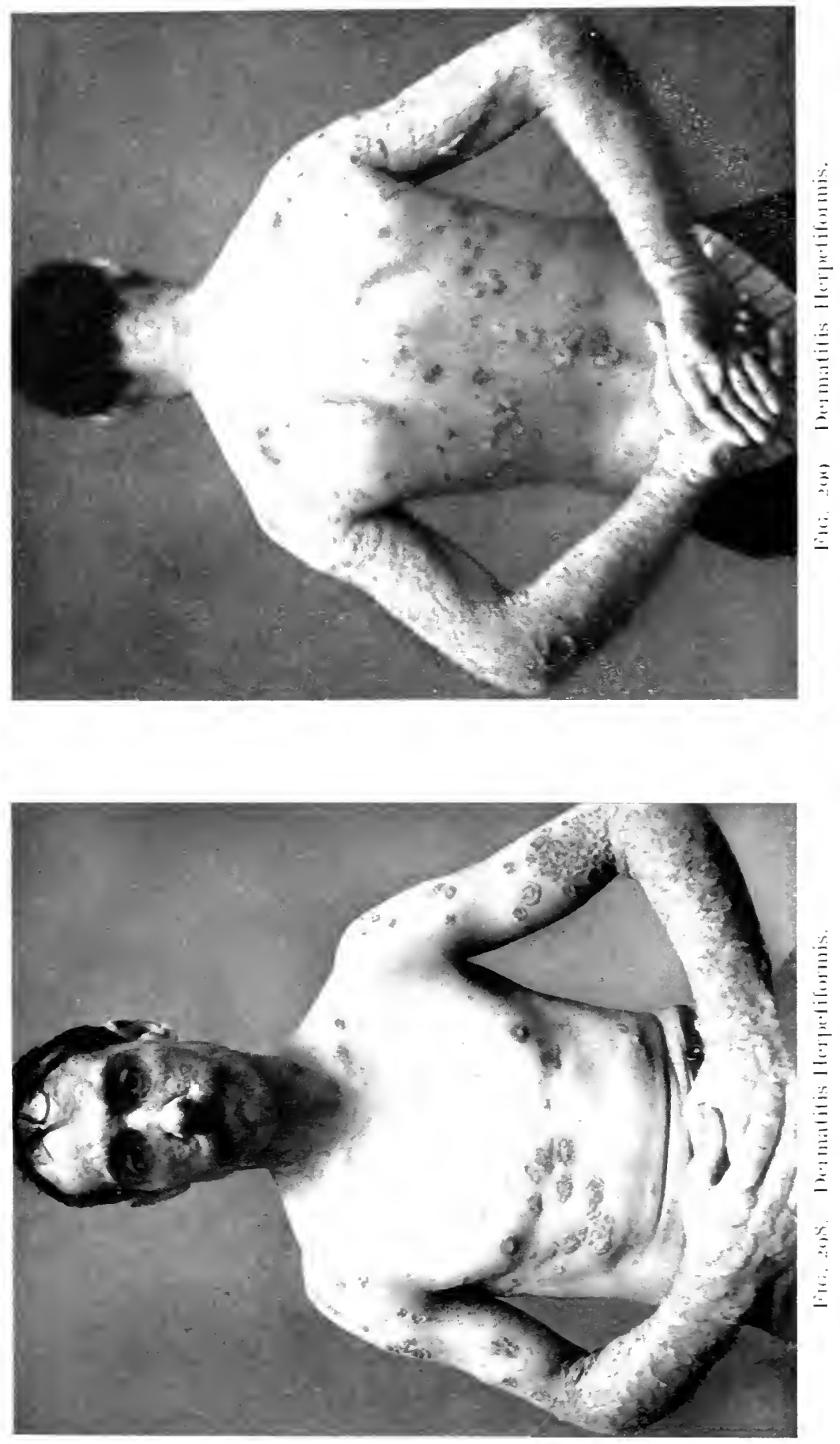

on the leg, which was followed some days later by groups of intensely itchy papulo-vesicles.

The most common lesions are vesicles and pustules. These occur at some period in the course of almost every case, while in II cases analysed, bullae were present only in $37 \%$, erythematous patches in $26 \%$, and urticarial lesions in $7 \%$.

The multiformity of the clinical picture is liable to be increased by secondary complications from rubbing and scratching and the inoculation of pus-organisms. In this way lichenification, white cicatrices, eczematisation, or pustulation, may result. Pigmentation is also liable to follow the involution of the lesions and varies in degree

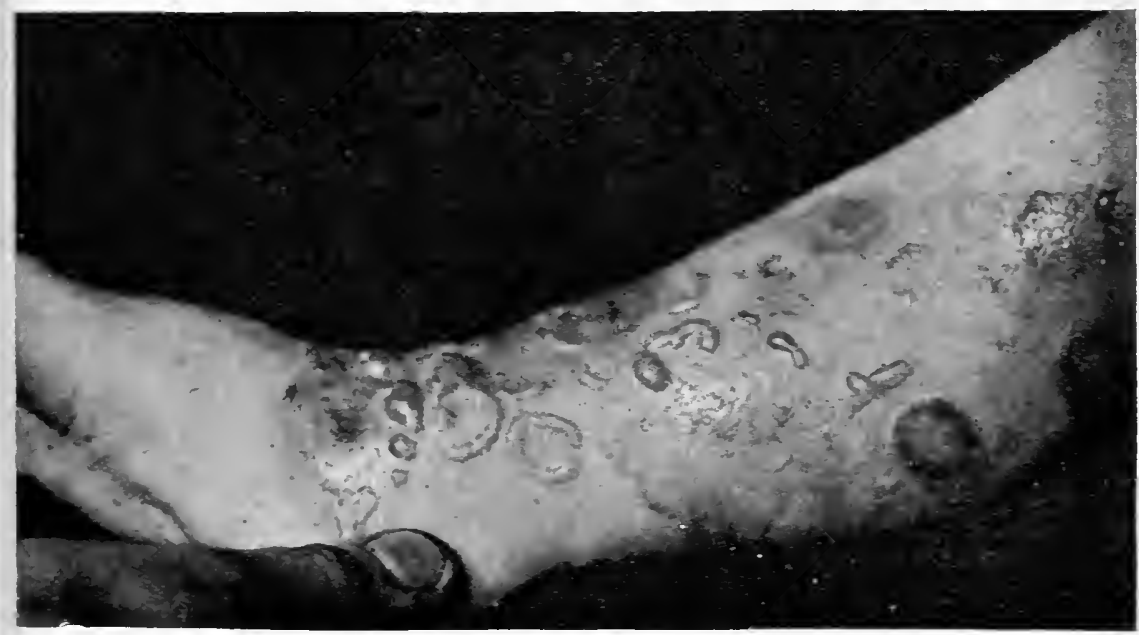

FIG. 300.-Dermatitis Herpetiformis.

Showing circinate bullae.

(Dr. Frederick Gardiner's case. Brit. Journ. Derm., rgog.)

according to the intensity of the itching ; where it is excessive it may be due to the taking of arsenic. (Fig. 301.)

The affected skin, as a rule, shows neither factitious urticaria nor the vulnerability from excessive acantholysis in which the skin slides away on pressure (Nikolski's sign).

Grouping of the papules, vesicles, and bullae, in herpetiform clusters is a constant feature and present in almost all the cases in adults. It is usually absent in the cases described as Dermatitis herpetiformis in children so that it is probable that they belong to a different category.

Mucous Membranes.-In the majority of cases the mucous membranes are not involved. Out of the II I cases analysed, the mucosa was only attacked in 22 ; in Io the mouth was affected, especially the tongue and mucous membrane of the cheeks and lips, and in 2 the condition began in the mouth; in II the mucosa of the genitals was attacked and in I it commenced in the vulva. It has been known also to occur in the conjunctiva and to lead to essential shrinkage and blind- 
ness, but this situation is not so liable to be affected as in chronic pemphigus. In several cases the mucosa of the alimentary tract has been seriously involved and both haematemesis and the passage of blood per rectum have been recorded.

In the mucous membranes it may take the form of erythematous macules, vesicles, or even bullae, leading to superficial ulcerations sometimes covered with a sodden diphtheroid membrane.

Subjective Symptoms.-The intensity of the subjective symptoms which may precede or accompany the eruption is another essential feature of the group. These consist of pricking, itching, burning, or actual neuralgic pain, and are generally paroxysmal in character. Sometimes the itching is so intense that the pain and discomfort of digging out the papules with the finger-nails are preferable to it.

General Health.-In some cases the disease is ushered in by a feverish attack with malaise and vomiting-which may recur with each successive outbreak-in others there may be no prodromal symptoms.

The general health varies greatly in different cases. At first it is almost invariably well-preserved, even when the skin is extensively involved. After the affection has been present for some time, general symptoms of a secondary character usually supervene, as the result of the gradual wear and tear from the irritating subjective symptoms, and these, when continued over a long period, are liable to have a depressing influence on the patient, both mental and physical, causing insomnia, establishing a neurotic habit, rendering him emotional and prostrate, and, in extreme cases, leading to insanity. Where the bullae are extensive and have become purulent, general symptoms may supervene as the result of septic absorption.

Blood.--Eosinophilia has been observed so often in Dermatitis herpetiformis that its presence has been regarded as of diagnostic significance and suggestive of the action of some toxin on the bone marrow. The increase of eosinophiles has been noted not only in the blood but also in the contents of the vesicles and bullae, and occasionally in the cellular infiltration in the corium. It varies from time to time in individual cases, being greatest when the eruption is at its height, tending to diminish when the itching decreases, and to disappear between the attacks. A high percentage of eosinophiles has been recorded, for example Ravogli reported $44.3 \%$ in the blood, Bushman and Williams $69 \%$ in the blood, Jamieson $24 \%$ in the contents of a bulla, and Leredde and Perrin $30-95 \%$ in vesicles.

The eosinophilia is not conștant, as out of 27 cases examined by Engman and Davis only 13 showed a definite increase of eosinophiles, while in a case under the care of the writer only $4 \%$ was noted in the blood at the height of an attack.

An increase of large mononuclear leucocytes up to $20 \%$ instead of the normal 6\% was recorded by Engman and Davis and in one case a lymphocytosis was described in the cerebro-spinal fluid. 


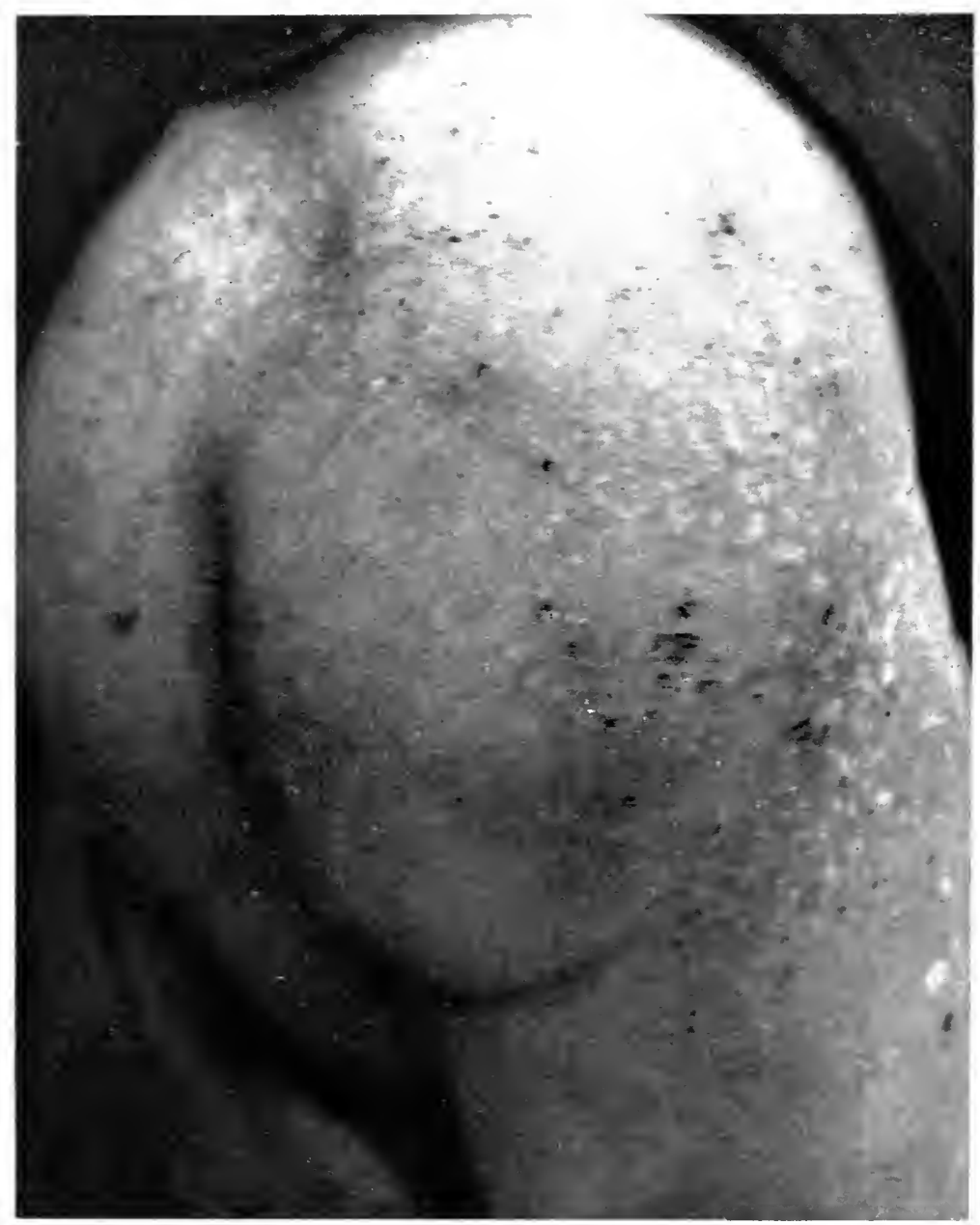

FIG. 3or.--Scarring from Scratching in Dermatitis Herpetiformis.

'To faic to soo. 

Urine.-Indicanuria is the only definite abnormality which has been recorded and it has been found to be coincident with outbreaks of the eruption, its presence suggesting auto-intoxication from putrefactive changes in the alimentary tract.

Glycosuria and a diminution of the nitrogen-content have been described in isolated cases but are probably coincidences.

Course.-The course of the affection is invariably chronic and it may last for years or indefinitely. It may be continuous but more usually is subject to periods of more or less complete remissions lasting for weeks or months.

Histopathology.-Changes occur both in the corium and in the epidermis, those in the corium being primary and of the greater importance. They consist of marked dilatation of the capillaries in the superficial layers with oedema of the surrounding fibrous tissue, rarefaction of the collagen bundles, a dense infiltration of cells, an exudation of serum, and an extravasation of lymphocytes, eosinophiles, and of polymorphonuclears if suppuration has taken place. The lymphatic spaces are dilated, especially in the papillary and sub-papillary layers. The exudation of fluid may be so rapid as to mask the initial period of congestion by the formation of vesicles or bullae. The appearances in the corium suggest an acute inflammatory disturbance the result of some toxic agent.

In the epidermis, in the papular phase the changes are comparatively slight and consist of dilatation of the inter-epithelial lymphatic channels and slight oedema of the deeper prickle-cells.

In the resicular phase there is intense intercellular oedema which leads to the formation of vesicles situated either in the epidermis itself about the granular layer, or more often immediately beneath it, the whole of the epidermis forming a roof. The vesicles are thus more deep-seated than in dysidrotic eczema. Ther contain coagulated albumin, fibrin, a debris of leucocytes, and eosinophiles. The prickle-cells in the neighbourhood of the vesicles are usually oedematous and may present a central space in which the nucleus lies, while the inter-epithelial spaces are dilated and eosinophiles occasionally may be observed in the dilated channels.

\section{ETIOLOGY}

Predisposing Causes.-Age.-It may occur at any age but is most common between 20 and 40 . It is rare in children and although typical cases have been recorded in childhood it is open to discussion whether these should not be placed under the heading of Pemphigus vulgaris, as in the majority, the vesicles showed no herpetiform grouping nor were the subjective symptoms intense. It is also probable that one or two of the cases in children were in reality Epidermolysis bullosa, while the description in others suggests vesicating urticaria or bullous erythema.

Sex.-It would appear to be equally common in males and females. 
General Health.-A low state of general health does not seem to be a predisposing cause and in most of the recorded cases it appears to have been up to the average.

Determining Causes.-Pregnancy.-Pregnancy is the most definite determining cause and the cases which have been recorded under the heading of Herpes gestationis, Herpes gravidarum, Hydroa gestationis, etc., belong to this category. It may occur as early as the third month or may not appear till after delivery. It may develop with a first pregnancy, and either recur with each subsequent one or miss one or more, or it may occur first with a later pregnancy. As a rule it does not interfere with the pregnancy nor with the health of the child, but it has been known to lead to premature birth and to death of the foetus. The severe early pustular type of Herpes gestationis probably corresponds to the Impetigo herpetiformis of Kaposi.

Disorders of Menstruation.-Several cases are on record in which it has occurred in connection with derangements of menstruation and both exacerbations and recurrences have been known to take place at the menstrual periods.

Nerve Influences.-Next to pregnancy the most important causes are nervous influences and there are certain writers who regard the disease as essentially a neurosis. Cases are on record in which an attack has been preceded or aggravated by psychical disturbances, such as emotion, anxiety, worry, fear, anger, or severe mental shock, and it has been known to be associated with hystero-epileptic fits. On the other hand, cases occur in which a neurosis, if present at all, appears to be the result and not the cause of the disease.

Drugs.-In one or two cases an eruption of this type has followed the taking of some drug, for example Mackenzie recorded a case in which it appeared in a man while taking arsenic for loss of hair.

Vaccination.-Several cases have been recorded in children as occurring after vaccination and it has been suggested that the vaccination caused it by liberating some toxin. It is possible that these cases were instances of Erythema multiforme.

Pathogenesis.-The exact nature and causation of the disease still remain unknown.

There is no evidence that it is an acute infective process due to primary microbic infection and the theory that it is a paroxysmal neurosis does not meet with general support.

The most prevalent hypothesis is that it is an intoxication from some endogenous toxin, and in favour of this theory are the occasional occurrence of general symptoms with an outbreak or recrudescence of the disease and its relationship to pregnancy and disordered menstruation, when it may be explained as a toxaemia rather than as a reflex neurosis. It has also been suggested that it may be the result of renal inadequacy and in two cases renal sclerosis was found post-mortem, but this may have been a coincidence as, except in a few instances in which indican was present, the urine has neither contained abnormal constituents nor been deficient in quantity.

The exact manner of action of the hypothetical toxin is uncertain; it may circulate in the cutaneous blood-vessels and produce the lesions 
in the skin directly, or it may act primarily on the nervous system and indirectly on the skin.

In favour of its indirect action through the nervous system it has been pointed out that vesicles may occur in connection with pathological changes in the peripheral and central nervous system, both functional and organic, and that the eruption may be explained readily as the result of the action of a toxin on the root ganglia. In support of this view attention has been drawn to the resemblance between it and Herpes zoster and the similarity in the initial lesions and in the character of the subjective symptoms accompanying them. In a case under the care of the writer in which Dermatitis herpetiformis had been present for several years an attack of Herpes zoster was superimposed, apparently as a result of a chill. In Dermatitis herpetiformis, however, no definite organic changes in the peripheral nerves, root ganglia, or spinal cord, analogous to those in Herpes zoster, have so far been detected.

In the present state of our knowledge it seems most probable that it is due to some autogenous toxin, not necessarily of a specific nature, which may be called forth by a variety of influences and which probably acts indirectly on the skin through the nervous system.

Diagnosis.-The recognition of the affection may be simple or extremely difficult. In a typical case, whether papular, vesicular, or erythematous in type, the severe sensory symptoms and the secondary effects of scratching generally form a clinical syndrome which is easily recognised. Cases which commence with one or more bullae on apparently normal skin without marked sensory disturbance may present difficulty at first, but the diagnosis is established later as groups of papules or vesicles and severe itching generally develop.

In old-standing cases of the papular type it maysimulate prurigo and the skin may become pigmented, lichenified, excoriated, and scarred, from rubbing and scratching. In some cases extreme exfoliation may supervene and the condition may resemble Pemphigus foliaceus, but the history of the disease, the previous recurrent attacks, and the sererity of the sensory symptoms, are sufficient to distinguish them.

In the past several writers seem to have confused it with the Hydroa aestivale of Crocker but the latter affection tends to stop at puberty, chiefly affects the exposed parts, is largely due to local irritation of the actinic rays of the sun, occurs almost exclusively in the male sex, and is followed by scarring.

Occasionally cases may be mistaken for eczema, and vice versa, and there is a type of eczema which occurs chiefly at middle-age, in which the itching is excessive and the lesions take the form of patches resulting from the aggregation of papulo-vesicles, which closely resembles Dermatitis herpetiformis. To such cases the name "neuritic eczema " has sometimes been applied. The chief distinction is that in them there is a greater tendency for the lesions to weep than in Dermatitis herpetiformis.

Chronic Pemphigus.-The differentiation from chronic pemphigus presents great difficulty and it has yet to be decided whether they are distinct entities or simply variants of a common morbid process. 
Though there is much room for argument on this subject it seems that in the present state of our knowledge it is advisable to regard them as distinct. The following are the principal differences :

In Dermatitis herpetiformis the eruption is essentially multiform, and the primary lesion a papulo-vesicle; in chronic pemphigus the eruption is uniform and the primary lesion a bulla.

In Dermatitis herpetiformis the bullae are rarely larger than a filbert nut ; in chronic pemphigus they may be as large as the palm of the hand.

In Dermatitis herpetiformis the lesions tend to be grouped in a herpetiform manner or arranged in rings or gyrate patterns ; in chronic pemphigus they are distributed irregularly.

In Dermatitis herpetiformis the mucous membranes are only involved in about $20 \%$ of the cases; in chronic pemphigus they are more commonly attacked.

In Dermatitis herpetiformis intense subjective symptoms are usually present ; in chronic pemphigus there are as a rule no subjective symptoms unless when the blisters have broken leaving raw painful surfaces.

In Dermatitis herpetiformis no definite changes have been found either in the internal organs or in the nervous system; in chronic pemphigus various pathological changes have been described, such as degeneration of the peripheral nerves and spinal cord, and fatty degeneration of the liver and heart.

With regard to Pemphigus vegetans it would appear that certain of the mild cases reported under that heading were vegetating types of Dermatitis herpetiformis and that the malignant cases belong to a different category - their cause still being unknown.

Prognosis. - The disease may run a mild and comparatively short course, recur once or twice and disappear without exerting any harmful influence on the general health; in the majority of cases it is more serious and persistent and by recurrences may be proionged indefinitely, gradually undermining the general health and reducing the resistance to such an extent that the patient is liable to succumb to some intercurrent disease. In some cases death has been known to supervene suddenly as the eruption disappeared.

The most fatal cases of all are of the pustular type occurring during pregnancy and these are liable to be fatal to both mother and child.

\section{TREATMENT}

The disease is singularly intractable and at the present time the treatment cannot be claimed to be more than symptomatic, aiming at the relief of the irritation, paroxysmal pain, general depression, and insomnia.

General Treatment.-The affection should be treated as an autointoxication. Everything should be done to minimise the absorption 
of foreign proteids from the alimentary tract. The diet should be simple and digestible, and restriction to a regetarian diet-which at the same time should be low, almost to the point of starvation -is sometimes of great benefit. Intestinal antiseptics may be of value, such as small doses of calomel, salol, and beta-naphthol. The kidneys should be flushed out by drinking several pints of water in the day, the nervous system should be toned by tonics and by the adoption of a regular life with sufficient exercise and fresh air and freedom from worry and anxiety.

There are no specific remedies. In the past arsenic has been largely employed but though occasionally it may have a controlling influence it cannot be said to be curative and it would appear to be less serviceable in this disease than in chronic pemphigus. In most cases it is necessary that the limit of toleration be reached before any appreciable benefit is derived from it and even then it may be ineffective, as in a case under the care of the writer where symptoms of chronic arsenical poisoning were beginning to show themselves and at the same time new groups of vesicles kept appearing on the skin. It has been found also that in cases which respond to arsenic any reduction in the dose may cause a return of the eruption. In cases where it is beneficial it would seem to act chiefly through its tonic effect on the nervous system. It would appear to be specially useful in children. It has been employed principally in the form of Fowler's solution, or as the Liq. arsenici hydrochloricus. The arylarsonates-namely, atoxyl and arsacetin-have been given a trial, but the benefit from them is doubtful and they are not to be recommended on account of the pain of the injections.

Salvarsan has been used and improvement recorded, though not cure (Russell Wilkinson).

Improvement has been reported from the employment of thyroid, but the results from it have been uncertain.

Other drugs which have been used occasionally with benefit for the relief of the irritation in extreme cases are antipyrin, phenacetin, salicin, chloral hydrate, and the bromides, but these should be employed with the utmost caution for fear of establishing a drug habit on the one hand, and of so lowering the resistance on the other, that when the effect of the remedy wears off the itching becomes more unbearable than before.

Relief has been obtained from lumbar puncture.

The local treatment should be of a mild character, soothing and antiseptic. Alkaline baths are useful in some cases, or, where there is pustulation, a weak potassium permanganate or boric acid bath should be substituted. After the bath the lesions if few in number should be covered with an occlusive paste, such as equal parts of zinc oxide, starch, lanolin, and soft white paraffin with the addition of $I \%$ salicylic acid; where the skin is extensively involved calamine cream with or without I \% of carbolic acid or tar should be rubbed in, allowed 
to dry, and the skin then dusted over with a bland dusting powder of zinc oxide or talc and boric acid. Where superficial ulcerations or septic crusts are present, an ointment containing $2 \%$ of anmoniated mercury should be employed.

Benefit has been reported in extensive cases from light baths and high frequency.

\section{REFERENCES}

Arnozan. Herpes gestationis (Arch. Clin. Bordeaux, Nov. I893).

Bowen, J. T. Bullous dermatitis following vaccination (Journ. Cut. \& Gen-Urin. Dis., I901).

- - Five cases of bullous dermatitis in children following vaccination (Trans. Amer. Derm. Ass., I90o).

Broce, L. Dermatite herpétiforme de Duhring (Ann. de Derm. et de Syph., I88I, ix. p. I).

-Des dermatites polymorphes douleureuses (Presse méd., I900).

Bushnell \& Williams. Eosinophilia in Dermatitis herpetiformis (Brit. Journ. Derm., I906, xviii. p. I77).

Duhring, L. Papers on Dermatitis herpetiformis (New Sydney Soc., I893, cxlvii. ; Cutaneous Medicine, Pt. 2).

ElliotT, G. T. Dermatitis herpetiformis developing after severe shock (Journ. Cut. \& Gen-Urin. Dis., I89I, ix. p. 42I).

ENGMAN, M. F. Indicanuria \& eosinophilia in Dermatitis herpetiformis (Journ. Cut. Dis., May I9o6).

ENGMAN \& DAvis. Some observations upon cellular elements of the blood in 300 cases of various skin diseases (Ibid., I9I 5, xxxiii. p. 73).

Fordyce, J. A. Dermatitis herpetiformis \& Impetigo herpetiformis (Journ. Cut. \& Gen-Urin. Dis., I897, xv. p. 495).

Fordyce \& GotTheil. Dermatitis vegetans in its relation to Dermatitis herpetiformis (Trans. Amer. Derm. Ass., May \& June, I906, p. I70).

Fox, T. C. Pemphigoids (Allbutt \& Rolleston, System of Medicine, I9I I, ix. p. 445).

Fox, Tilbury. Hydroa (Arch. Derm., Philad., i88o, vi. p. 6i).

Gardiner, F. Dermatitis herpetiformis in children (Brit. Journ. Derm., I909, xxi. p. 237).

Garazzini, G. A. Impetigo herpetiformis (Monats. f. prakt. Derm., I909, xlix. p. 56).

Gilchrist, T. C. Pathology of Dermatitis herpetiformis (Johns Hopkins Hośp. Rep., I896, i. p. 365).

HaAse \& Hirschler. Hydroa puerorum (Unna) (Journ. Cut. Dis., Nay 1908).

Hall, A. J. Hydroa gestationis (Quart. Med. Journ., Nov. I899, p. 29).

Hallopeau, H. Dermatite pustuleuse chronique et végétante en foyers. à progression excentrique (Internat. Atlas of Rare Skin Dis., I890).

Jamieson, W. A. Dermatitis herpetiformis (Debate on Dermatitis herpetiformis at the Derm. Soc. Lond. Brit. Journ. Derm., I 898, x. p. 73).

Leredde \& Perrin. Morbid anatomy of Herpes gestationis, etc. (Ann. de Derm. et de Syph., r895, v. p. 28I).

Eosinophiles in the vesicles of Dermatitis herpetiformis (Ibid, I896, vi. p. $45^{2}$ ).

Liddell, J. Case of Dermatitis herpetiformis (Brit. Journ. Derm., I896, vii. p. 385 ).

Mackenzie, S. Dermatitis herpetiformis (Ibid., I893, v. p. I).

MacLeod, J. M. H. Pemphigoid eruptions (Ibid., I9I5, xxvii. p. 20I).

Meynet \& Peha. Dermatitis herpetiformis in children (Ann. de Derm. et de Syph., I903, p. 893). 



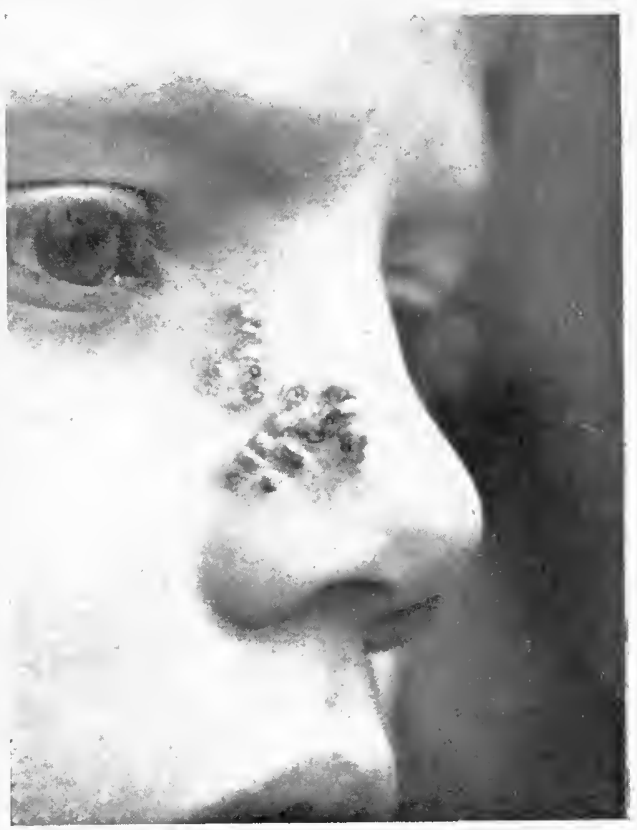

FIG. 302.-Herpes Facialis.

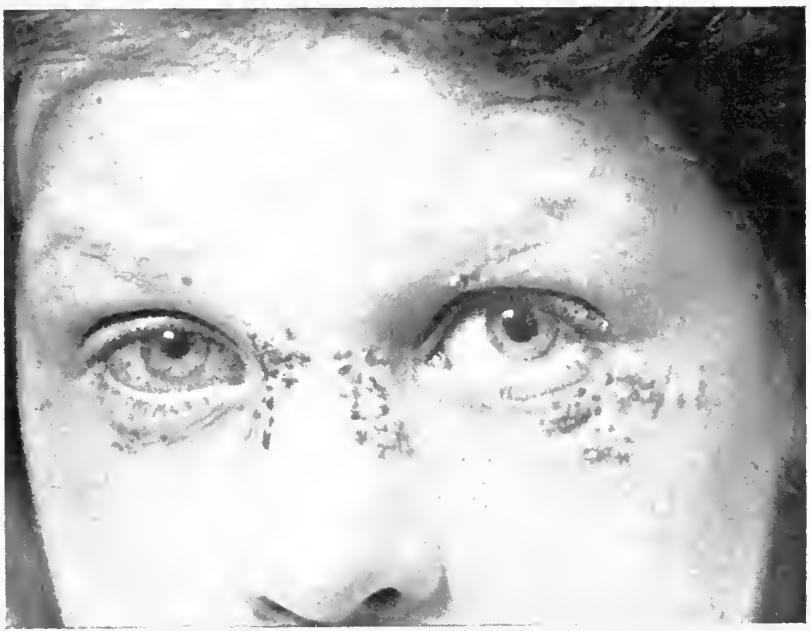

Fig. 303.-Herpes Facialis.

Shows bilateral distribution.

[To face p. 897. 
Ravogli. Eosinophilia in Dermatitis herpetiformis (Brit. Journ. Derm., I90r, xiii. p. 34).

Scheuber, G. Impetigo herpetiformis (Arch. f. Derm. u. Sỹph., I909, xciv. p. 227).

Surrox, R. L. Dermatitis herpetiformis in early childhood (Amer. Journ. Med. Sci., I 9 Io, cxl. p. 727).

WiскнAм, L. Dermatitis herpetiformis \& renal inadequacy (Brit. Journ. Derm., I 895 , vii. p. 386$)$.

Wilkissox, R. Dermatitis herpetiformis treated with salvarsan (Ibid., I9I 4, xxri. p. 96).

\section{HERPES SIMPLEX}

Syn.: Herpes febrilis; Fr., Herpes vulgaire; Ger., Bläschenflechte.

Definition: An acute inflammatory affection characterised by groups of vesicles on an erythematous base, chiefly affecting the face and genitalia, frequently bilateral, and usually recurrent.

Introductory.--Under the heading of Herpes simplex are included the familiar herpetic affection of the face known as Herpes facialis or Herpes labialis and that of the genitalia known as Herpes progenitaiis. The affection is also met with occasionally on the trunk. Though these conditions clinically resemble each other so closely as to warrant their inclusion under the common heading of Herpes simplex, still for purposes of description it will be adrisable to follow the general custom and to discuss them separately.

\section{HERPES FACIALIS}

This variety of herpes is usually ushered in by some disturbance of the general health, such as malaise, shivering, or a rise of temperature, though occasionally there may be no definite prodromal signs. It is not infrequently associated with some acute febrile disease, such as pneumonia, malaria, influenza, etc., in which case it may be preluded by a marked rise in temperature due to an exacerbation of the intercurrent disease.

Locally the first symptom is burning or itching, followed a few hours later by the appearance of an erythematous patch which may be swollen and oedematous and over which a group of papulo-resicles, or vesicles, are irregularly distributed. The resicles vary in size from a pin's head to a small pea; ther may remain discrete or, when numerous and close to each other, may coalesce to form small irregular bullae. At first they contain clear serum which rapidly becomes turbid, but only in rare instances definitely purulent. They do not usually rupture spontaneously but tend to dry up to form yellowish scabs which fall off in a few days learing a red smooth surface. Healing takes place without scarring, except occasionally where suppuration has occurred and been followed by superficial ulceration. (F:g. 302.)

3 L 
As a rule the affection does not last more than a week or ten days from its onset to the drying up and falling off of the scab, but where superficial ulceration has taken place healing may be postponed indefinitely.

The parts of the face most commonly affected are the lower lip, around the mouth, cheek, nose, auricle, buccal mucosa; and in rare instances the conjunctiva and cornea of the eye. The most common situation of all is the lower lip ; there the herpes may be extensive and involve both sides, spreading on to the cheeks and chin, or it may be limited to one side and represented by a few small vesicles or a single

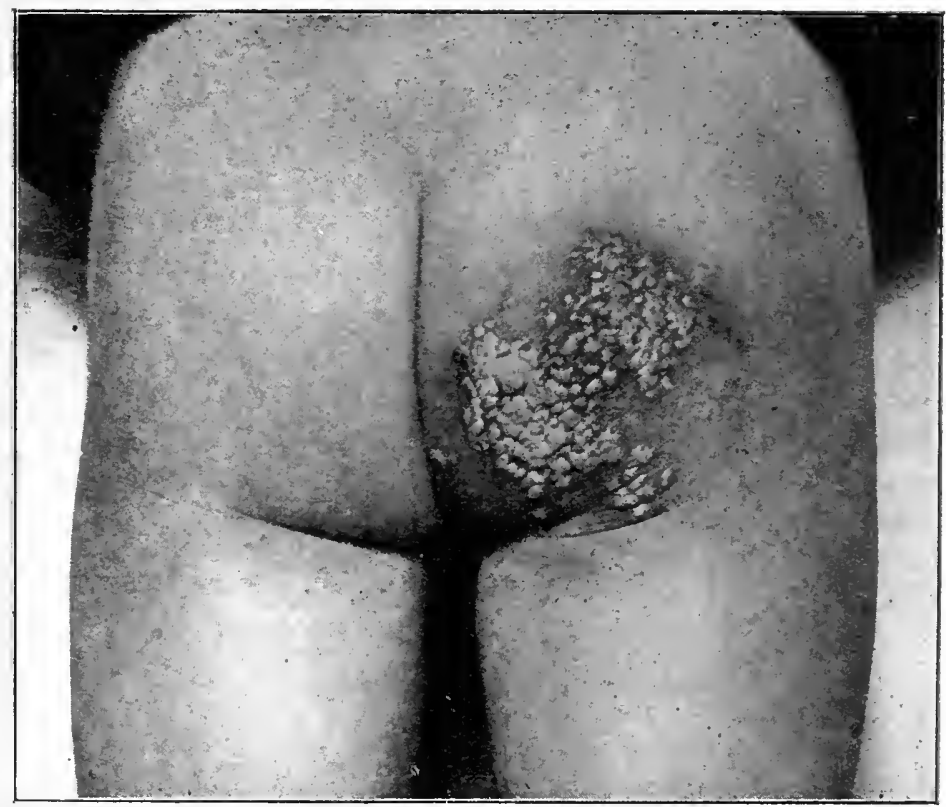

FIC. 304.--Herpes Febrilis.

(From Dr. H. G. Adamson. Brit. Journ. Derm., I9гі.)

bleb. When it occurs at the angle of the mouth it is liable to lead to cracks and to the formation of a chronic form of impetigo, sometimes known as perlèche, fron the inoculation of the cracks by cocci from the slin and saliva. (Fig. 303.)

The mucosa of the mouth is not infrequently involved. In it the vesicles are rarely detected but are replaced by superficial ulcerations, or by denuded areas covered with a whitish slough, which may be small and round or irregular in outline, or extensive and circinate and coalesce to form geographical patches. These may be present on the insides of the cheeks, palate, tongue, pharynx, larynx, or oesophagus, are generally bilateral in distribution, and usually recurrent.

In rare instances Herpes febrilis has been known to occur on the trunk or limbs, on the chest in association with an attack of pneumonia, and in other situations such as the fingers, palms, and buttocks. (Fig. 304.) 
When the affection is represented by a single bleb or a small herpetiform group it is confined to one side of the body, but when it is at all extensive it is usually bilateral. It has a marked tendency to recur, sometimes periodically as in connection with menstruation, sometimes at irregular intervals from a definite cause such as an attack of indigestion, or from some local irritation such as the passage of a catheter, or for no apparent reason. (Fig. 305.)

Epidemics have been described, under the heading of "herpetic fever " in which outbreaks of herpes occurred in association with fever. Their exact nature is not fully understood, but they have been attributed to poisoning with sewer gas.

Histopathology.- The histology of the lesion of Herpes simplex has not yet been completely worked out. In the progenital variety Unna found the vesicles situated beneath the epidermis with the papillae as a floor, while the overlying prickle-cells were in a state of coagulation

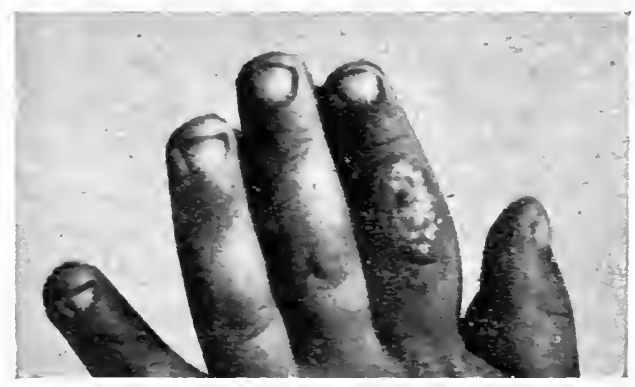

FIG. 305-- Herpes Febrilis.

(Dr. H. G. Adamson's case. Brit. Journ. Derm., rgog.)

necrosis. Balloon cells such as are characteristic of the vesicle of Herpes zoster were not detected. Beneath the vesicle the cutis was markedly oedematous and the capillaries dilated and surrounded by an infiltration of leucocytes and roundish connective cells. No organic changes have been observed in the peripheral nerves supplying the affected area nor in the root ganglia.

Etiology and Pathogenesis.-Herpes facialis may attack either sex indiscriminately and may occur at any age. It is frequently associated, and probably causally connected, with febrile disturbances such as influenza, feverish colds, tonsilitis, pneumonia, and malaria, and was once regarded as being of prognostic importance in those diseasesits presence being believed to indicate that the affection was running a favourable course, but this is no longer admitted.

Certain individuals are more predisposed to it than others, possibly as the result of a previous attack, and cases are on record in which even slight local irritation determined an attack, as where Herpes labialis has been set up by some hot substances such as mustard or pepper.

The affection strongly suggests a reflex irritation of the sympathetic ganglia and may be called forth by various forms of peripheral irri- 
tation, such as a decayed tooth, an error of refraction, or the passage of a catheter; or by some internal disturbance, such as indigestion; while in women it may precede, accompany, or follow, a menstrual period.

Diagnosis.-The diagnosis is comparatively simple. It has to be distinguished from Herpes zoster, but this latter condition is rare on the face, practically never confined to the lips, is unilateral, associated with severe sensory symptoms, and seldom recurrent.

It might be mistaken for Impetigo contagiosa, but in it there is little or no itching, and the initial vesicles become more rapidly purulent and rupture spontaneously to form the characteristic honeylike superficial crusts.

Treatment.-The treatment of the actual attack consists of drying the lesions with suitable remedies. With this object, the affected skin should be dabbed over several times daily with Eau de Cologne, spirits of camphor, or a I\% solution of chloride of zinc, or painted with a $2 \%$ solution of silver nitrate in spirit. After applying the lotion the lesions should be dusted with boro-zinc dusting powder. By this means healing is encouraged and the vesicles may sometimes be aborted. Where the lesions have become purulent or superficially ulcerated the application of a $\mathrm{I} \%$ ointment of ammoniated mercury is beneficial. Where cracks are present at the angles of the mouth, associated with persistent inflammation of the skin in the neighbourhood, the cracks should be painted with Friar's balsam, the mouth being opened widely so as to stretch them, or should this fail, a pastille dose of the X-rays will usually cause them to heal. When crusts have formed they should be softened and removed by cold cream.

The next and more important object is the prevention of recurrences. For this purpose a careful examination of the whole system should be made and any defect, such as indigestion, disease of the kidneys. uterine disorders, etc., treated by appropriate remedies. Sources of local irritation should be sought for and corrected, such as caries of the teeth, astigmatism or other error of refraction. Chills should be avoided. The internal administration of arsenic has been strongly recommended as a preventive, but though it is of undoubted benefit in some cases its action is too uncertain for it to be in any way regarded as a specific.

\section{Herpes Progenitalis}

In herpes of the genitalia, the lesions consist of a small group of inflammatory papules, papulo-resicles, or vesicles, on a slightly raised inflamed or oedematous base.

In the male they occur both on the inner and outer surface of the prepuce, on the glans, sulcus, in rare instances on the sheath of the penis, and in the urethra.

In the female they are met with on the inner surface of the labia 
majora, labia minora, clitoris, cervix of the uterus, and occasionally: on the outer surface of the labia majora and mons reneris.

The lesions are similar to those which occur on the face, but being much more liable to become irritated and sodden by friction and septic discharges they are prone to break and to form superficial ulcers with an irregular or polycyclical border. Where suppuration is marked the groin glands may be enlarged.

Wherever the lesions occur they are associated with more or less marked burning and itching, and the affection has a great tendency to recur, sometimes every few weeks, for months or years.

Pathogenesis and Etiology.-It occurs chiefly in adult life, is more common in men than in women, and is not infrequently preceded by venereal diseases such as gonorrhoea or soft sore, occurring two or three weeks after these have healed. Probably one reason why it would seem to prevail so largely in the male sex is that in them it is more liable to come under the cognisance of the phrsician. In both sexes it is liable to follow coitus. In the male a long prepuce seems to be a predisposing cause, and in the female it may occur in association with menstruation.

Indulgence in alcohol, coffee, food, etc., may cause a recurrence.

The exact cause is unknown, but it is most probably induced by a reflex irritation of the sympathetic ganglia in the neighbourhood. In a number of cases in both sexes where rerve symptoms preceded the eruption, Ravaut and Darré detected morbid changes in the cerebrospinal fluid, but their significance is uncertain.

Diagnosis.-In the early stage the affection is easily recognised, but where the resicles have broken down to form superficial ulcers or where the ulcer has been treated with caustics through mistaken zeal on the part of the phrsician, errors in diagnosis are liable to occur between it and soft sore; and in some cases a firm diagnosis between them may be impossible in the first instance. The lesions of herpes, howerer, usually heal more rapidly under suitable treatment than soft sores, the discharge from them is greater, and the groin glands are rarely. enlarged.

Treatment.-The first indication is scrupulous cleanliness. The parts should be bathed several times a day with boric lotion, then dusted with boric powder, and boric baths or douches employed after coitus. Should the lesions occur about the glans or sulcus a piece of moistened boric lint should be inserted between it and the inner surface of the prepuce. Where the prepuce is long circumcision may be advisable.

Where there is much irritation and inflammation this may be subdued by the application of a lotion containing alum gr. 20, Liq. pb. acetat $\bar{j}$ ii, water to $\tilde{\tilde{j}} \mathrm{i}$.

When ulceration has occurred the discharge should be washed off with boric lotion or black-wash and the ulcer dried and dusted with $5^{\circ}$ o calomel powder or iodoform. 
The general health of the patient should be dealt with on ordinary medical principles. Indulgence in alcohol or excessive eating should be prohibited, any defect such as indigestion, constipation, or gout suitably treated, copious draughts of water prescribed to flush out the kidneys, and a simple regime with plenty of fresh air and exercise enjoined. Where the patient is run down, arsenic may be given as a nerve tonic and is of undoubted value.

As a preventive of recurrence a plaster over the lumbar vertebrae or galvanism in the lumbo-sacral region employed in the same manner as in Herpes zoster have been recommended.

\section{REFERENCES}

Adamson, H. G. Herpes febrilis attacking the fingers (Brit. Journ. Derm., I909, xxi. p. 323).

- Recurrent herpes of the buttock (Idem, I9II, xxiii. p. 322).

Dubreullh. Recurrent herpes of buttock (Ann. de Derm. et de Syph., I905, p. 847).

Ravaut \& Darré. Nervous reaction in course of Herpes genitalis (Ann. de Derm. et de Syph., I904, p. ${ }_{4}^{81}$ ).

Rolleston, J. D. Herpes facialis in scarlet fever (Brit. Journ. Derm., I9I0, xxii. p. 309).

\section{HERPES ZOSTER}

Syn.: Zona; Shingles; Ignis sacer; Fr. Zona; Ger. Feuergürtel, Gürtelausschlag.

Definition: An acute inflammatory affection of the skin, characterised by groups of vesicles on an erythematous base, associated with sensory symptoms of a neuralgic type, and situated in the area of distribution of the nerve fibrils from a posterior root ganglion.

Introductory.-The name herpes, from the Greek word $\varepsilon_{\rho} \rho \eta \bar{s}$ meaning creeping, was once used in a much wider sense than it is now. It was applied to any condition where the eruption seemed to spread in a creeping fashion, and included a considerable number of affections which have been found to differ widely in their pathogenesis and have been relegated to the groups to which they properly belong, such as Herpes iris which is a variety of Erythema multiforme, Herpes gestationis which is a form of Dermatitis herpetiformis, and Herpes tonsurans which is ringworm of the scalp. At the present time the name is restricted to two conditions, namcly Herpes zoster and Herpes simplex with its two divisions Herpes facialis and Herpes progenitalis. Herpes zoster is a distinct affection, in which the skin eruption is associated with inflammation of the posterior root ganglion followed by secondary degencration of the posterior root and the peripheral nerves connected with the ganglion, and occurs in the area of distribution of the affected nerve fibrils. Herpes facialis and Herpes progenitalis on the other hand are allied conditions in which no definite nerve changes have yet been detected. 
Herpes zoster is comparatively common and forms about $0.3 \%$ of the cases in the dermatological clinic at Charing Cross Hospital. It most frequently occurs in the intercostal areas of the chest, forming a partial girdle, hence the name of zona, but it may be met with anywhere on the cutaneous surface. Various names have been applied to it according to the situation affected, such as Herpes zoster frontalis, H. zoster ophthalmicus, H. zoster brachialis, H. zoster sacralis, etc., but these are of no importance.

Description.-The eruption is usually preceded for several days by pain in the affected area, and by general symptoms, such as malaise, vomiting, loss of appetite, slight rigors, fever, and other signs of an acute infective process. The general symptoms are as a rule most marked in children and may be so severe as to suggest the onset of an acute specific disease, or, from their association with intercostal pain, to be mistaken for pleurisy or pneumonia.

The pain, which is located in the area of distribution of the nerve fibrils of both the anterior and posterior primary divisions from the affected root, varies in intensity in the different cases but is always of a neuralgic type. Sometimes it is comparatively mild and bearable, consisting of aching or tingling, at other times it may be severe, boring or lancinating. It is generally continuous but subject to exacerbations and partial remissions. It usually diminishes when the eruption is fully out and then gradually disappears, but in some cases it may persist long after healing has taken place. It is mildest in children,

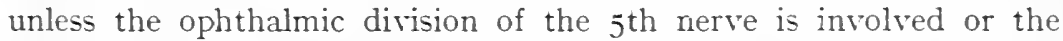
eruption has led to superficial ulceration and extensive scarring. It is most intense in elderly people in whom it may be so excruciating and intolerable as to cause deterioration of the general health, and in them may persist for months or even years. Sometimes in addition to the pain the affected skin may be hyperaesthetic and tender to the touch.

The eruption usually appears three or four days after the pain and general symptoms began, but occasionally it may develop earlier or, in rare instances, simultaneously, and with the appearance of the lesions the temperature and general symptoms usually decline. It consists, as a rule, of groups of resicles situated on an erythematous base, or of an erythematous patch on which vesicles develop subsequently. The vesicles vary in size from a pin's head to a split-pea and, when numerous, tend to become confluent and form small irregular bullae. At first they contain clear serum but this gradually becomes turbid and opaque from contamination with pus-organisms. Occasionally in severe cases the contents are definitely haemorrhagic.

The eruption as a rule develops rapidly and has reached its full extent in about ${ }^{S} \mathrm{~S}$ hours; where it has appeared early, it may develop much more slowly and new lesions may keep on coming out for about a week. In mild cases only a few groups of vesicles or abortive papulovesicles may be present, while in severe cases the vesicles may be 
numerous and irregularly distributed over the whole area supplied by the nerve root. According to Head it has its maximum intensity over and around certain spots, or " maxima," where the branches of the posterior root come to the surface. (Fig. 306.)

The vesicles show no tendency to rupture spontaneously but dry up in about a week to form small crusts which separate in course of time leaving red spots which disappear without a trace, unless the contents are purulent when the lesions may become superficially ulcerated or gangrenous and be succeeded by pitted scars. The scabs are generally yellowish in colour, but, should the contents be haemorrhagic, they may assume a dark brown tint.

Should suppuration occur, the neighbouring lymphatic glands generally become enlarged-a feature which has been noted even in

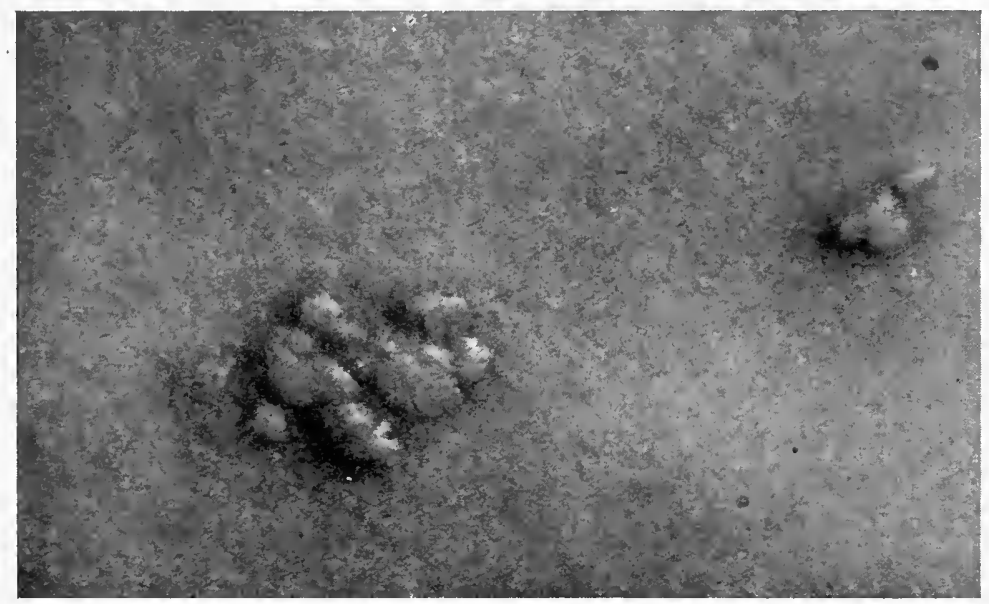

FIG. 306.--Herpes Zoster.

Group of large vesicles.

cases in which the lesions were uncontaminated by pyogenic microorganisms-and a septic temperature and fatal septicaemia have been known to ensue.

In addition to the typical groups, aberrant vesicles situated on an erythematous base can often be detected and are the result of the involvement of terminal twigs of the nerves.

A number of cases are on record in which Herpes zoster was associated with, or followed by, a general vesicular eruption (Herpes zoster generalisatus). This somewhat resembles varicella and it has been suggested in consequence that Herpes zoster is a localised form of that exanthem.

When the eruption is well out the pain generally disappears, being replaced by sensory symptoms, such as itching or burning. After it has healed the affected skin may remain tender for some time and in severe cases, where superficial ulceration and scarring have taken place, 
partial anaesthesia may supervene from degeneration of certain nerve fibrils.

The eruption is almost invariably unilateral, though it may extend for a short distance beyond the middle line both in front and behind. A few bilateral cases have been recorded in which the eruption has been at different levels of the cord on the two sides, and in one or two instances the two sides have been affected at the same level, but in these cases the Herpes zoster was probably symptomatic of an underlying myelitis.

In the vast majority of cases the affection is represented by a single attack. Occasionally cases are met with in which two or more

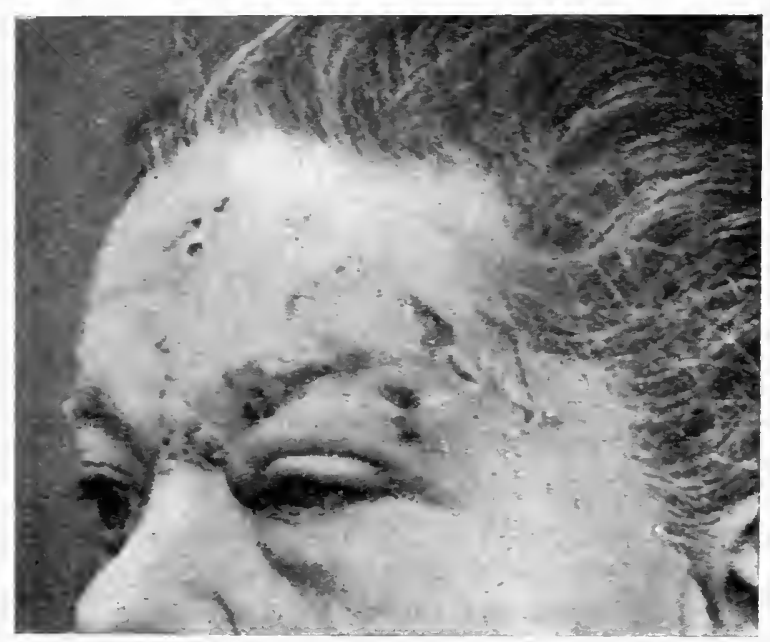

FIG. 307.-Herpes Zoster.

Supra-trochlear and Supra-orbital nerves.

attacks have occurred, but these have been invariably in different parts of the body.

The most common situation for it is the trunk from the involvement of one, or more rarely two, of the dorsal posterior root ganglia.

When it attacks the face it is generally in the area of distribution of

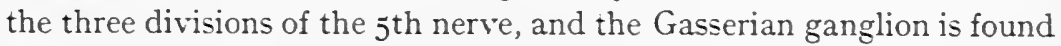
to be affected in the same way as the posterior root ganglion. The ophthalmic division is the most liable to be involved, in which case the eruption usually extends back over the forehead as far as the parietal eminence, involves the upper eyelid and not infrequently the side of the nose as far as the ala nasi, and may attack the eye and be responsible for conjunctivitis, ulceration of the cornea, iritis, and, in rare instances, pan-ophthalmitis and blindness. A case is on record where the Geniculate ganglion was implicated and in which the eruption developed over the auricle and in the auditory meatus. (Fig. 307.)

The mucous membranes of the mouth and tongue are occasionally affected, but where this has occurred the lesions have been bilateral 
and recurrent and were probably not true Herpes zoster but Herpes febrilis.

Sometimes the neighbouring motor nerve fibrils are involved and paralysis results, and cases are on record in which ptosis and ophthalmoplegia were present in connection with zoster of the ophthalmic

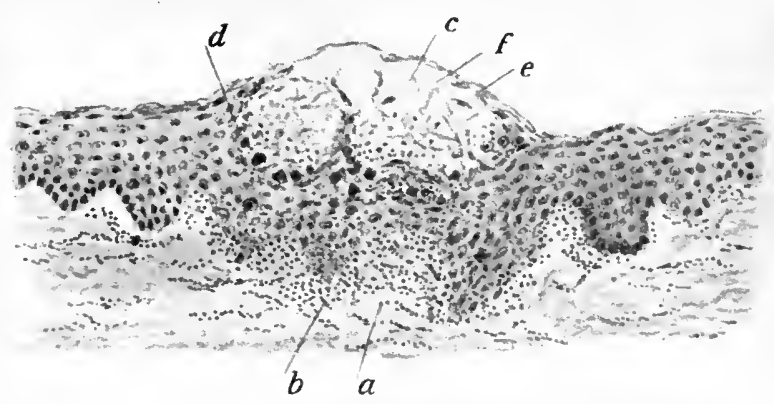

J. M. H. M. del.

Fig. 308.- Vesicle of Herpes Zoster. ( $x$ about I 50.)

a. Dilated vessel, infiltration of leucocytes, and oedema., $b$ Oedematous interpapillary process. c. Monolocular vesicle. $d$. "Balloon cells." e. Stratum corneum. f. Fibrin.

(From MacLeod's Pathology of the Skin.)

division, paralysis of the movements of the hands and fingers where the first dorsal root ganglion was implicated, and facial paralysis in association with inflammation of the 2 nd and 3 rd cervical roots.

Herpes zoster may occur in patients suffering from some other cutaneous disease, such as psoriasis, eczema, or syphilis, but the coexistence of these dermatoses in no way influences the course or severity of the zoster.

Histopathology.- The vesicle may be situated in the epidermis in the region of the granular layer, or beneath it with the papillae as a floor. At first it is unilocular but

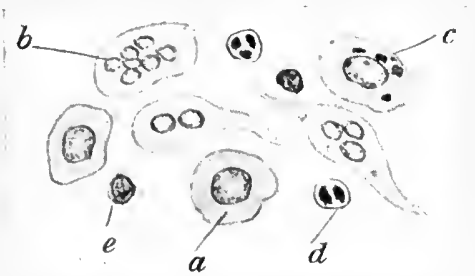

J. M. H. M. del.

FIG. 309.-Balloon Degeneration in Herpes Zoster.

$a$. Balloon-cell. b. BaHloon-cell with divided nucleus. c. Balloon-cell with remains of chromatin. d. P'olsmorphonuclear leucocyte. e. Lymphocrte.

suggesting sporozoa which Unna named "balloon cells." These are degenerated prickle-cells which have lost their prickles and the protoplasm of which has become transformed into a homogeneous cloudy mass. (Fig. 309.) by the coalescence of several vesicles large multilocular lesions may be produced; the septa between the loculi generally give way, however, and a single vesicle is again formed in which part of the septa, composed of contorted and broken-down pricklecells, may persist. (Fig. 308.)

At the base or sides of the vesicles, either lying free within them or in their neighbourhood, are peculiar swollen round cells 
The epidermis in the neighbourhood of the vesicle is not definitely thickened as in eczema, but there is marked intercellular oedema and oedema of the prickle-cells.

Associated with the epidermal changes there is acute superficial inflammation in the corium which precedes the epithelial oedema. The papillary and sub-papillary blood-vessels are dilated and there is an infiltration of lymphocytes, small connective tissue cells, and polymorphonuclear leucocytes around the dilated ressels, and oedema between the fibrous bundles.

Changes in the Nervous System.-Tarious writers, such as von Bärensprung, Charcot, Cotard, and others, have detected changes in the posterior root ganglia in association with Herpes zoster and have believed them to be causally connected with the eruption. It is to Head and Campbell however that we are indebted for the most complete description of these changes, which on post-mortem examination of the nerrous system in $2 I$ cases of Herpes zoster were as follows: In the acute cases there were haemorrhages and an inflammatory exudation into the posterior root ganglion with destruction of the ganglion cells and eventual sclerosis. Degenerative changes were detected, spreading upwards in the posterior roots and in the root fibres of the posterior columns of the cord and downwards in the peripheral afferent nerves. In the nerves in the deeper layers of the corium there was swelling of the neurilemma, degeneration of the myelin sheath, and moniliform swellings in the axis cylinders.

By noting the area of distribution of the eruption and the individual ganglia affected, Head and Campbell were able to trace out the skin areas supplied by certain of the ganglia and by filling in the others succeeded in making an approximate chart of the distribution of the nerve roots. They found that these areas did not correspond with the sensory branches of any one nerve but with several branches of nerves connected together by certain fibres passing through the same ganglion.

\section{Etiologr AND Pathogexesis}

Predisposing Causes.-Sex.-Herpes zoster is equally common in males and females.

Age.-It may occur at any age, is rare in infancy and early childhood, commonest between the ages of I2 and 25 , and gradually diminishes in frequency with advancing years.

Atmospheric Conditions.-Cases seem to occur in small epidemics under certain atmospheric conditions not fully understood.

Season.-There does not seem to be any definite seasonal periodicity though it has been said to be more prevalent in spring and autumn.

Debilitating Infuences.-Any debilitating influence may predispose to the disease, such as tuberculosis of the lung, whooping-cough, measles, diarrhoea, etc. 
Determining Causes.-The researches of Head, Campbell, and others, have shown that the cutaneous eruption is the direct result of acute inflammation in the posterior root ganglia and secondary degeneration in the peripheral nerve fibrils, but the precise cause of these changes remains unknown.

In certain cases it would appear to be symptomatic of some definite disease of the central or peripheral nervous system, such as myelitis where it usually corresponds with the upper level of the anaesthesia, Tabes dorsalis where it may occur in the sites of the lightning pains, and cerebro-spinal meningitis where herpetiform groups may be met with in different nerve root areas, and it has been known to result from injury of a peripheral nerve in connection with caries of a bone or of the teeth; but the cases connected with disease or injury of the nervous system are comparatively rare.

It may also follow some severe mental shock, or seem to be caused reflexly from peripheral irritation, such as that due to errors of refraction. It is particularly prevalent in asylums and especially in connection with General Paralysis of the Insane when it may be due to the same cause as the general paralysis or the debilitating influence of that disease may simply act as a predisposing factor.

Cold.-Cold would appear to be a determining cause in some cases and a considerable number have been known to follow a chill, as in a case under the care of the writer in which a severe attack of trigeminal Herpes zoster in an elderly gentleman commenced about 48 hours after a long railway journey in winter and was attributed by him to the skin on the affected side of his face becoming numb through travelling in a corner seat of the railway carriage with his face near the windowpane.

Arsenic.-A large number of cases are now on record where attacks have been associated with the taking of arsenic, generally over a prolonged period. For example, Herpes zoster was a marked feature in the epidemic of arsenical poisoning from drinking beer which took place in the Midlands in rgor. It is doubtful whether the arsenic is the direct cause or simply a predisposing factor, but when it is remembered that the drug has a powerful influence on the peripheral nervous system, being liable to cause a toxic neuritis, the possibility is suggested that the zoster may be directly induced by it.

Various theories have been advanced to explain the so-called spontaneous cases. It has been suggested that they are the result of an acute infective process due to some microbe, comparable to acute lobar pneumonia, and in favour of this view have been cited the prodromal symptoms, the occasional onset with a rigor, the eruption appearing about the third day, and the frequent occurrence of the cases in small epidemics.

Another theory is that it is in some way related to varicella, as cases are on record in which chicken-pox has appeared within three weeks of being in contact with Herpes zoster and also in which Herpes 
zoster has followed contact with varicella; it is most probable that these were simply coincidences.

It has been compared by Head to acute anterior poliomyelitis in which the motor cells of the anterior horns of the cord are somewhat similarly affected.

So far no microbe or toxin has been established as the cause.

Diagnosis.-The recognition of Herpes zoster in an ordinary wellmarked case presents no difficulty, the groups of vesicles on an erythematous base, situated on one side of the body and in the area of distribution of the fibrils of a posterior root ganglion or of the Gasserian ganglion, and the associated subjective symptoms, being sufficiently. characteristic to establish the diagnosis. The only condition for which it is liable to be mistaken is Herpes febrilis, but this is frequently. bilateral, or if unilateral generally consists of a single group, is usually recurrent, is not confined to any nerve area, and has no severe sensory symptoms.

Prognosis.-The prognosis is usually excellent, especially in young people, and the disease is generally cured in the course of two or three weeks.

Where the ophthalmic division of the $5^{\text {th }}$ has been attacked, serious changes in the eye, such as iritis, pan-ophthalmitis, and blindness, have been known to supervene.

Where there has been much secondary contamination, scarring and severe neuralgic pain may persist after the lesions have healed, especially in elderly people in whom it may seriously undermine the general health.

\section{TREATMENT}

At present there is no treatment which is capable of aborting an attack of Herpes zoster, though various local and general remedies have been suggested. Until the actual cause is known the treatment must be symptomatic and palliative.

The general health should be maintained by a suitable tonic regime with good food, cod-liver oil and malt, strichnine, or other tonic remedies.

Locally the affected skin should be protected by the application of zinc paste, or still better, by painting it with zinc ichthyol gelatine, or collodion, or, after wiping it orer with spirit, it should be dusted with a bland powder of zinc, talc, and boric acid, and covered with cottonwool and a light bandage.

The greatest care should be taken to aroid suppuration by bathing the skin with some weak antiseptic lotion, such as boric lotion, or hydrogen peroxide 5 volumes. When conjunctivitis is present the most careful antiseptic treatment should be instituted with boric compresses, or the application of $0.5^{\circ}$ o protargol, and if iritis occur the pupils should be kept dilated with atropin to aroid adhesions of the iris to the cornea. 
For the relief of the neuralgic pain various internal remedies have been employed, such as phenacetin, antipyrin, salicin, or other analgesic, and-most effective of all-hypodermics of morphia. Locally, compresses of opium, lotions or ointments containing $\mathrm{I} \%$ cocain or other anodyne, may be tried, but are of comparatively little service. Better results may be obtained from blisters over the nerve roots; from a galvanic current along the area of distribution of the nerve fibrils, placing the positive electrode over the spine, stroking the negative electrode along the course of the nerve, and using 2 or 3 milliamperes of current; from freezing several times daily with ethyl chloride over the ganglia and along the branches of the nerve.

\section{REFERENCES}

Campbell \& Head. Herpes zoster (pathology) (Brain, igoo, xxiii. p. 353). CARlill, H. A note on the association of Herpes zoster with arsenic (Brit. Med. Journ., 1917, p. 9).

Craxston Low, R. Herpes zoster: its cause \& association with varicella (Brit. Med. Journ., I919, i. p. 91).

FIDEHL. A case of lymphatic leukaemia with generalised Herpes zoster (Arch. f. Derm. u. Syph., I913, cxviii.).

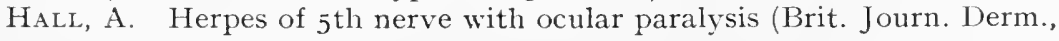
I903, XV. p. 3II).

Hartzell. Histopathology of Herpes zoster (Journ. Cut. \& Gen-Úrin. Dis., Sept. I 894).

HeAd, H. Herpes zoster (Allbutt \& Rolleston, System of Medicine, I9Io, vii. p. 470 ).

Kopytowsir. Anatomy of Herpes zoster (Arch. f. Derm. u. Syph., i 9oo, Oct., p. I7).

Minet \& Leclerco. Du zona avec éruption généralisée (La Clinique, Paris, I9I I, vi. p. IOI).

Montgomery, D. W. The course the virus of Herpes zoster takes to reach the nerve-ganglia (Journ. Cut. Dis., I913, xxxi. p. I56).

TRYB, A. Herpes zoster generalisatus (Derm. Wochenschr., I9I4, lix. p. 983).

Vogel, F. Paralysis of eye muscles in Herpes zoster ophthalmicus (Thesis, Leipzig, 19I2).

Weber, F. PARKes. Herpes zoster with paralysis of arm (Brit. Journ., I9I 5, xxvii. p. 408).

Herpes zoster with generalised eruption of varicella-like spots (Ibid., I916, xxviii. p. 13). 


\section{CHAPTER XXIX}

\section{LUPUS ERYTHEMATOSUS}

Syn.: Lupus erythematodes; Seborrhoea congestiva (Hebra); Lupus sebaceus; Ulerythema centrifugum (Unna); Erythema atrophicans (Morris). Fr. Lupus érythémateux.

Definition: A chronic inflammatory affection of the skin characterised by persistent reddish patches, with adherent grevish scales, which tend to undergo atrophic changes and to be replaced by superficial scars.

The name Lupus erythematosus was originally applied to this affection by Cazenare in $I 8_{5} I$ who recognised it as an enitity; but six years before it had been described by Hebra under the heading of Seborrhoea congestiva.

The name is somewhat unfortunate as it has led to confusion with Lupus vulgaris from which it is quite distinct, and either Frythema atrophicans or Ulerythema centrifugum (from $\dot{o} \boldsymbol{i} \lambda \dot{i}$ a scar) are preferable as they indicate two of the principal characteristics of the disease, namely the persistent erythema and the subsequent atrophy.

Description.-Lupus erythematosus is a comparatively rare affection but, owing to its intractability, cases are apt to accumulate and to make it appear to be more frequent than it really is.

There are two main types, (I) a circumscribed or discoid type, which is chronic in its evolution and course; (2) a disseminated more or less acute type. Of these the former is by far the more common, constituting about $\mathrm{So}^{\circ}{ }_{0}$ of all the cases in this country.

\section{Discoid Type (Lupus erythematosus discoides)}

This type usually attacks the face and is especially common where the skin is stretched over bones or cartilages, and where the capillaries are apt to be defective from anatomical reasons, as on the malar prominences, the bridge of the nose, and the ears-a classical situation being the "butterfly area" of the face, namely the upper part of the cheeks and the bridge of the nose. It is not infrequent on the scalp in association with lesions on the face and is sometimes present there before the face is involved. The next most common site is the back 
of the hands and the fingers. It is rare on the covered parts of the body. It may attack the mucous membranes, especially those of the mouth and nose, either by extension from the cutaneous surface or independently. In whatever region it occurs it tends to be bilateral and symmetrical. (Plate VII.)

The initial lesion consists of a rose-red macule or slightly raised papule which, when first detected, is usually about the size of a splitpea and roundish in outline. This may be diffusely red or, on closer examination, may show the presence of a dilated vascular network which disappears under the diascope but recurs when the pressure is remored. The macule extends peripherally and becomes greyishwhite and slightly depressed in the centre from the formation of a delicate adherent scale anchored to the skin by minute peg-like prolongations which dip down into the underlying pilo-sebaceous follicles. In situations, such as the tip of the nose, where the sebaceous follicles are large the plugs resemble small barley seeds and the scales are coarse, yellowish and greasy. (Fig. 3Io.)

The lesions may appear singly or in small groups and may develop simultaneously in situations at a distance from each other, such as on both hands or cheeks. Their evolution is invariably slow and it may be months or even years before the patch reaches the diameter of halfa-crown or the whole " bat's-wing" or " butterfly" area is involved. As they increase peripherally they become irregular in outline, especially where several have coalesced. (Fig. 3II.)

Retrogressive changes of an atrophic character next take place in the centre which end in the replacement of the pink scaly patch by a thin whitish scar, unless pyogenic micro-organisms have gained entrance through a crack or fissure and set up superficial ulceration which results in a more definite depressed cicatrix. The latter accident may be met with in lesions about the ears, nose, and fingers, but is rare elsewhere.

The patches may be level with the surrounding skin, or may be raised and infiltrated-the infiltration being most marked at the border.

When they are small the scaliness usually occupies the centre leaving a yellowish halo at the periphery ; when they are larger it may spread over the entire surface or take the form of whitish puncta at the mouth of the scbaceous follicles and sweat-pores, or of irregular streaks or patches. When the scale is forcibly detached the underlying thin red pellicle is usually lacerated and bleeding points result.

Where the lesions are definitely infiltrated the name Lupus érythémateux fixe has been applied to it, and where the scale is thick, yellowish and greasy and the underlying plugs are large the old names of Seborrhoca congestiva and Lupus sebaceus are applicable.

The colour varies from rose-pink to dark purple and is largely dependent on the circulation; where this is fecble, as about the nose, 


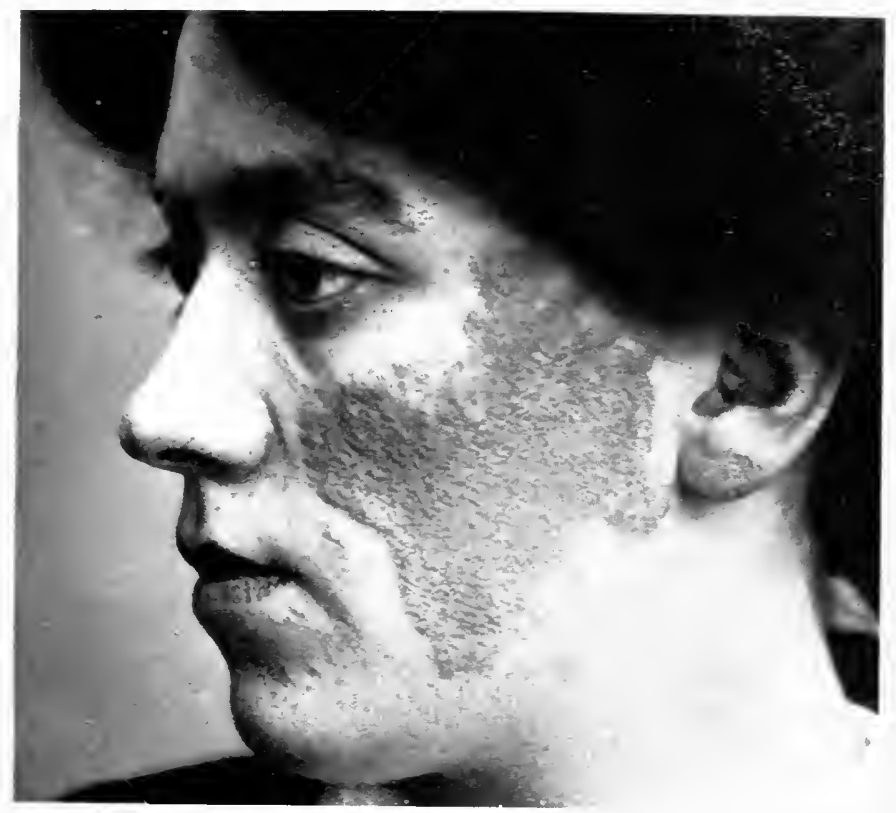

Fig. 310.--Lupus Erythematosus. Discoid type.

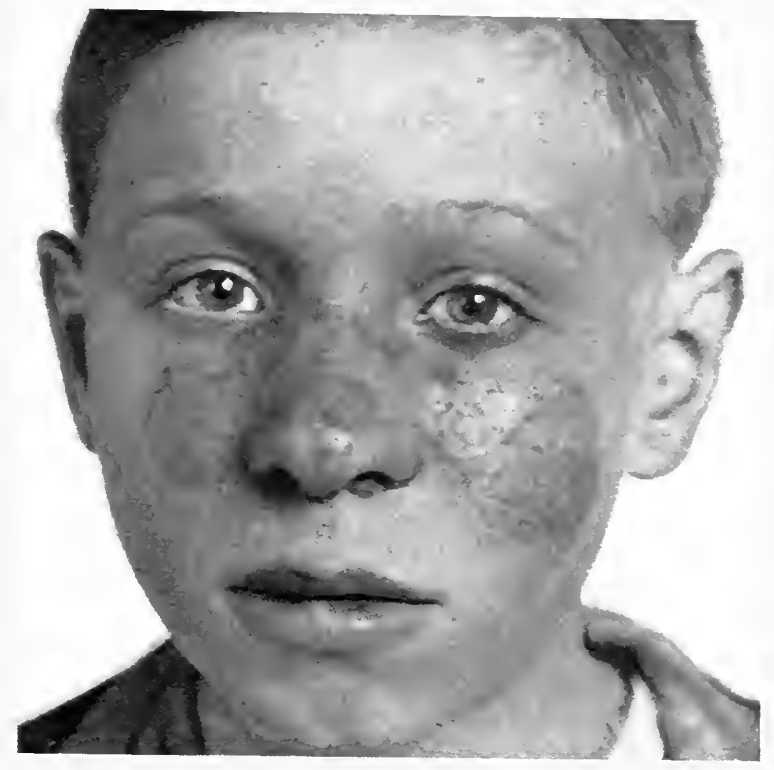

Fig. 3ri.-Lupus Erythematosus. Affecting "bat "s-wing" area. 

hands, and feet, the lesions are apt to assume a livid violaceous hue and doubtless some of the cases which have been described under the heading of " chilblain lupus" belong to this category.

The lesions vary in appearance in different parts of the skin.

On the face the classical butterfly distribution is frequent and the patches are generally rose-pink and the scales greyish-white.

On the ears it may involve the lobe, margin of the auricle, concha, and external auditory meatus, and may form scaly, atrophic, pinkish patches, groups of plugged follicles like comedones on the inside of the auricle, or an eroded border from ulceration and necrosis.

About the tip of the nose it is not infrequently of a sebaceous type.

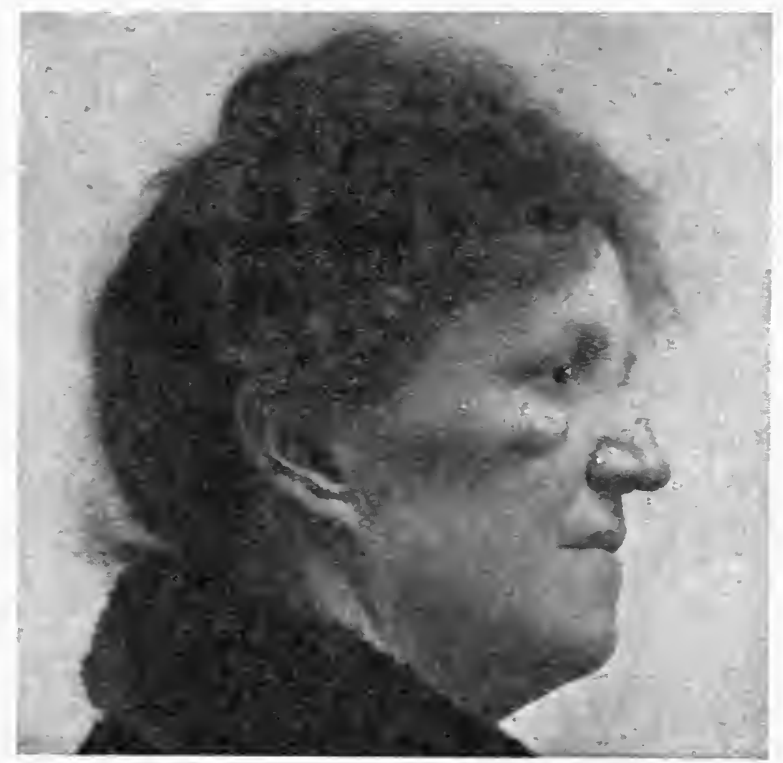

FIG. 3I2.-Lupus Erythematosus.

Indurated type followed by marked atrophy.

On the scalp the lesions are irregular in shape, are usually pink and scaly at first but gradually become pale, shiny, atrophic, and covered with telangiectases. As the atrophic process leads to absolute destruction of the hair-follicles, the orifices of which remain patent, the scar presents a peculiar pitted, or cribriform, appearance.

On the hands and fingers the lesions tend to be roundish or oval in shape, raised, oedematous, and livid purple; when ther occur orer the joints they are apt to become cracked, ulcerated, and painful, and on healing to leave depressed scars.

The nail-matrix is occasionally involved, causing a dystrophy of the nail which assumes a dusky red appearance, becomes brittle, ridged, and irregular, while the finger-tips are generally swollen and painful.

The subjective symptoms are usually slight and consist chiefly of 
itching and tingling which is most marked in the ears. Should fissures or ulcerations be present the lesions may be definitely painful.

The general health is unaffected in the discoid type though occasionally there may be evidences of weakness from anaemia, tuberculosis, or other debilitating condition.

Varieties.-In addition to the above classical discoid type the following varieties are occasionally met with: (I) A non-scaly variety in which the lesions are rose-pink in colour, raised, smooth on the surface, and resemble Erythema multiforme. In them atrophic changes may supervene in the centre which gradually lead to their disappearance, hence the name "Erythema centrifugum." (Plate VII.)

(2) A telangiectatic variety in which the most marked feature is the presence of telangiectases in the patches. This type is rare and only occurred in 2 out of 50 consecutive cases of Lupus erythematosus taken from the records of Charing Cross Hospital.

(3) An indurated variety in which tender swellings occur about the face which are slightly raised, vary in size from a sixpence to a shilling or larger, and the skin over which is normal at first but gradually assumes a pinkish-red scaly appearance. Spontaneous involution may occur in these lesions and they may disappear leaving a definite depression and comparatively little scarring. This type occurred only in I out of the 50 cases. (Fig. 3I2.)

(4) A nodular variety described by Crocker characterised by small roundish nodules varying in size from a hempseed to a bean, brownishred in tint, and not unlike Lupus vulgaris, which tend to coalesce into patches and to undergo cicatricial changes.

\section{Disseminated Type (Lupus erythematosus disseminatus)}

This type is much more acute and widely distributed than the discoid variety and the lesions more vivid in colour and less clearly defined. It usually begins in the face, and in the course of a few weeks may spread down on to the chest, arms, buttocks, even on to the legs, and in severe cases may involve the whole skin-the cutaneous lesions frequently being accompanied by severe constitutional symptoms.

There are three varieties of the disseminated type:

(I) An acute variety which supervenes in old-standing cases of the discoid type. In it a more or less sudden exacerbation takes place, generally accompanied by a febrile disturbance, malaise, and pains in the limbs; the lesions become inflamed like erysipelas (Erysipelas perstans faciei), increase rapidly, and in a few weeks appear as blotchy patches of a bright-reddish tint covered with greyish scales widely distributed over the trunk and limbs. These cases are not infrequently associated with albuminuria and death from acute nephritis. 
(2) An acute variety which appears de novo, in which the lesions burst out with the suddenness of an exanthem, at first on the face, and later widely distributed over the cutaneous surface. The lesions may appear as red macules which rapidly increase and coalesce to form patches which may be raised and oedematous or, in the most acute cases, bullous or even haemorrhagic. The eruption appears in crops, each outburst being heralded by febrile symptoms and accompanied by a profound general disturbance.

On the face the inflammatory process may be so acute as to resemble erysipelas or severe dermatitis from exposure to the sun. On the trunk and limbs it takes the form of bright-red, irregular, more or less scaly. patchesseparated byareas of normal skin. Should the hands and feet be affected they are apt to become swollen, painful, and cyanosed. Certain of the patches may disappear without a trace while others are followed by slight pigmentation or scarring. (Fig. 3I3.)

These cases are met with chiefly in young women who have not suffered previously from the disease, and they generally lead to a fatal

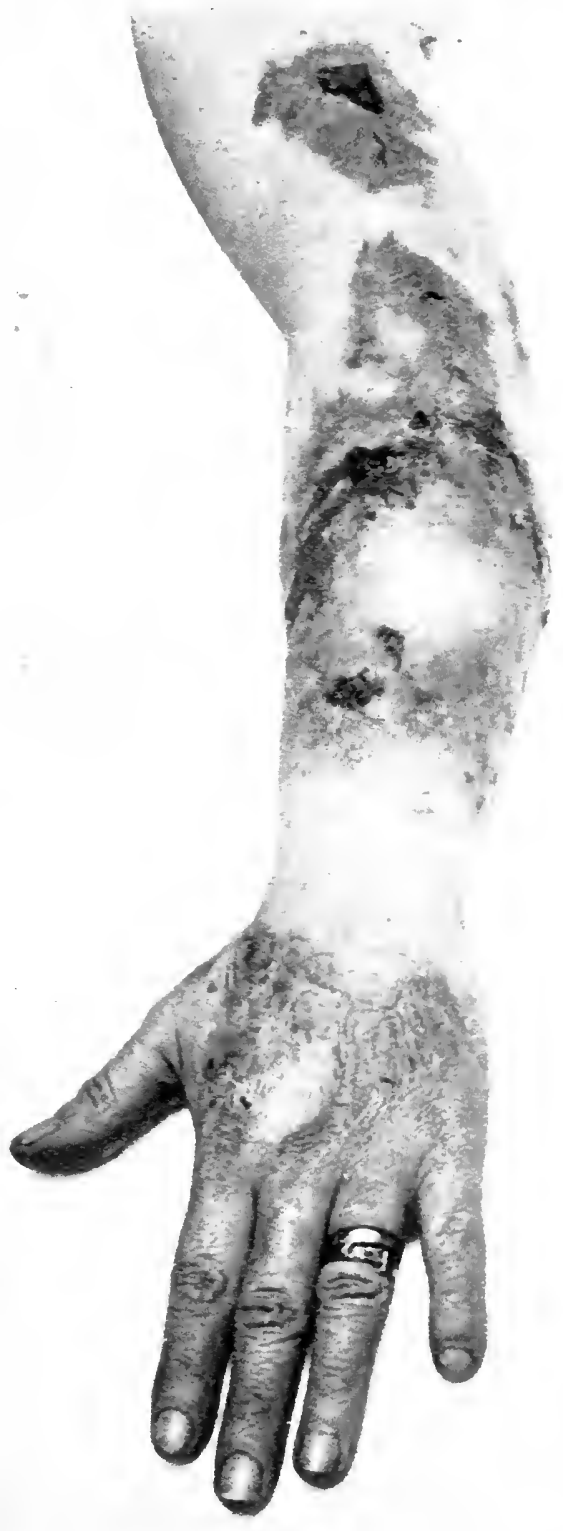
FIG. 313.-Acute Lupus Erythematosus in a

Shows superticial ulceration of the patches. issue in a few weeks from pneumonia, tuberculosis of the lungs, pleurisy, or acute kidney disease. Their onset and course are strongly suggestive of an acute toxaemia. 
(3) A sub-acute variety which occurs as a sequel of the discoid type, in which the facial patches become active, spread rapidly, and assume the appearance of an acute seborrhoic dermatitis. It occurs as pinkish patches covered with yellowish scales on the face and as irregular scaly lesions more or less widely distributed on the trunk. There is a tendency to periods of quiescence and exacerbation which may be seasonal in spring or autumn or may be determined by some general disturbance of health. When the patches fade little or no trace may be left but occasionally there is definite atrophy. In this variety the skin lesions are not accompanied by general symptoms.

Mucous Membranes. - The mucous membranes are occasionally attacked either by extension from the cutaneous surface or independently, especially those of the mouth, nose and conjunctiva.

On the lips it usually takes the form of purplish patches covered with an adherent scale or crust, the forcible detachment of which causes laceration and bleeding. The lips themselves become swollen, fissured, everted, and painful.

On the insides of the cheeks and palate it may appear as irregular dull red patches, or as grey, atrophic and parchment-like lesions, or in rare instances as superficial ulcers with a red border and covered with a greyish-yellow film.

On the tongue it may occur as irregular patches of a deep red tinge, smooth, and denuded of papillae, or may resemble a patch of leucoplakia surrounded by a reddish halo, and in rare instances may become ulcerated.

When it attacks the conjunctiva it is usually as an extension from the eyelid and appears as an ill-defined thickening covered with dilated ressels.

Histopathology.-The microscopical appearances vary according to the type and stage of evolution of the lesion. Marked changes occur both in the corium and epidermis, those in the corium being primary and essential while those in the epidermis are secondary.

Corium.- The earliest changes take place in and around the bloodressels of the sub-papillary and papillary layers and in those around the pilo-sebaceous follicles and sweat-glands. They consist of dilatation of the vessels, extravasation of serum, and a peri-vascular infiltration which consists of small round connective tissue cells, mast-cells, and occasional plasma-cells, and is most marked in the upper layers of the corium but may extend down to the hypoderm along the sweatducts and glands. Degencration of the fibrous elements takes place in the infiltrated areas, the collagen becoming rarefied and the elastin destroyed. In consequence of the ocdema and degenerative changes in the fibrous tissue a peculiar condition known as "canalisation" is produced in which the tissue spaces become widely dilated.

In older lesions the hyperaemia and oedema are less marked, the capillaries tend to become blocked with cells, the sebaceous glands to disappear, and the cellular infiltration to assume a more formative 
character with the presence of connective tissue cells and new collagen fibres indicating the occurrence of a cicatricial process. (Fig. 3I 4.)

Epidermis.-The overlying epidermis also becomes oedematous, the basal layer blurred or absent, and the inter-epithelial lymphatics dilated. The Stratum corneum is thickened, especially at the mouths of the follicles and at the sweat-pores where it forms horny plugs, and if the underlying oedema is marked there is imperfect cornification and scaliness.

The histological picture of the comparatively dry horny scaly surface and the underlying oedema has been aptly compared to a bog

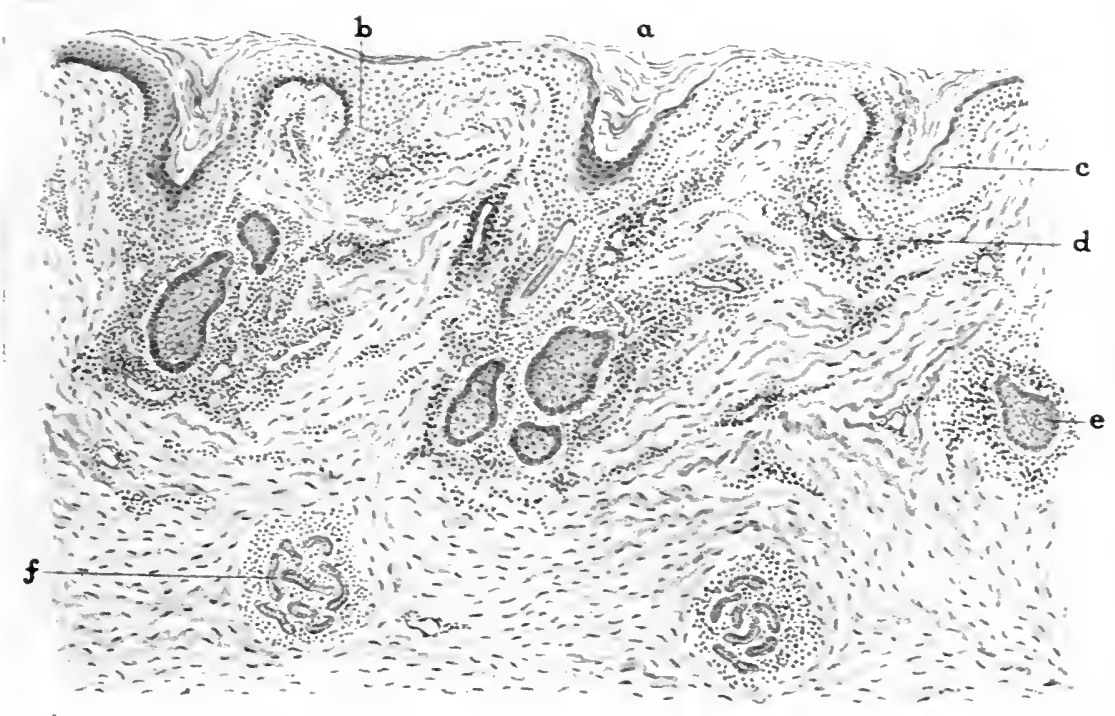

J. If. H. II. del.

FIG. 3It--Lupus Erythematosus. Section of lesion. ( $x$ about I 5o.)

$a$. Horny plug in the dilated funnel of a hair-follicle. b. Oedematous prickle-cell laver. c. Funnel of follicle. $d$. Dilated capillary surrounded by infiltration of cells. e. Sebaceous gland. $f$. Coil-gland surrounded by cellular infiltration.

covered by apparently sound mossy turf but which, if stepped upon, proves unsafe.

\section{Etiology AND PATHOGENESIS}

Predisposing Causes.-Age.-It is essentially a disease of adult life, the usual age of incidence being between 20 and 45 vears; it rarely begins in childhood or old-age.

Sex.-It is much more common in females than in males, in the proportion of rather more than 2 to $I$.

Social Status.-It is met with more frequently in private than in hospital practice. This is probably because mild cases give rise to little inconvenience and patients of the hospital class are less likely 
to seek advice about it than those with leisure and in more affluent circumstances.

Climate.-It is most prevalent in countries where the atmosphere is moist and cold, such as this country and the northern countries of Europe, and is liable to be aggravated in cold weather. It is rare in dry tropical countries.

Circulatory Disturbances.-An important predisposing factor seems to be the existence of circulatory disturbances connected with a weak peripheral circulation, such as cold hands and feet even in warm weather, a mottled cyanosed condition of the hands and forearms, a moist state of the skin of the palms and soles, and a tendency to chilblains. Various other circulatory anomalies have been observed in connection with it, such as cyanosis of the cheeks, especially marked about the malar prominences, a profusion of telangiectases in the same situation, swellings of the tip of the nose, ears, and fingers, "dead" fingers, and in a few cases the more serious phenomena of Raynaud's disease. Not only does Lupus erythematosus occur in the subjects of a feeble peripheral circulation, but the actual lesions due to a defective circulation may persist and imperceptibly become transformed into lesions which are indistinguishable from Lupus erythematosus.

Nature and Causation.-The pathogenesis of the disease still remains uncertain. It was believed originally to be an affection of the sebaceous glands and one of its first recognised characteristics was the presence of greasy plugs in the pilo-sebaceous follicles, hence the old name Seborrhoea congestiva. It was pointed out subsequently that the plugs were horny in nature and that the pathological process affected not only the mouths of the follicles but also the intervening epidermis.

It next came to be regarded as a chronic inflammatory process, the result of the action of toxins circulating in the blood. In support of the toxic theory are the following observations :

(a) Symmetry of the lesions.-This is noticeable in the circumscribed type where both cheeks, both ears, or both hands, may be involved in a symmetrical fashion, and also in the acute disseminated cases where extensive lesions may be distributed symmetrically about the trunk and limbs.

(b) The resemblance of the lesions in certain cases to those of toxic erythemata.-This resemblance, both with regard to the type of lesion and the distribution, may be so close that great difficulty may be experienced in deciding to which group the case belongs. In many of the milder cases of Erythema multiforme, discoid lesions occur about the face and hands which resemble the non-scaly variety of Lupus erythematosus. The resemblance is most marked, however, in the acute disseminated cases.

(c) Association of Lupus erythematosus with various forms of general toxaemia.-The acute disseminated type may co-exist with various forms of general toxaemia, such as Bright's disease, hepatic disease, alcoholism, etc., and in these cases it may be reasonably assumed that the Lupus erythematosus is the result of toxins circulating in the blood produced by a diseased state of the kidneys or the liver. It may also occur in association with bacterial toxins in the alimentary tract and Barber records a case in connection with an abnormal streptococcal infection of the alimentary canal. 
The toxin which has been incriminated mostly is that produced by the tubercle bacillus and certain observers go so far as to regard it as a manifestation of tuberculosis while others believe it to be a tuberculide.

Relation to Tuberculosis.-It is chiefly on clinical grounds that the tuberculous theory of the origin of Lupus erythematosus. has been based. So far tubercle bacilli have not been found in the lesions though numerous attempts have been made. Inoculation experiments of the tissue in susceptible animals have been inconclusive, and the histological architecture of the lesions does not even suggest tuberculosis.

The supporters of the theory grant this, but maintain that the lesions are due to a tuberculous toxin in the skin which has reached there via the blood-stream and has been produced by bacilli situated in some remote tuberculous focus. Of the various arguments which have been advanced in support of this theory the most important are the following:

(I) In a certain number of cases there is personal evidence or a family history of tuberculosis. Out of $9+$ cases exhibited at the Dermatological Society of London there was a record of tuberculosis in the patient in ro and a family history of tuberculosis only in 2. This corresponds with the experience at Charing Cross Hospital where signs of tuberculosis or a positive family history was only obtainable in about Io of the cases, while in the others no evidence of it could be detected. Other statistics, however, have given the proportion of tuberculosis in the cases as considerably higher. Occasionally patients with Lupus erythematosus have died of some intercurrent disease and no evidence of tuberculosis was found at the post-mortem.

(2) The clinical appearances of the lesions of Lupus erythematosus may so closely resemble those of Lupus vulgaris as to make a differential diagnosis almost impossible. This argument has been used but it is one which does not bear close scrutiny and it might be advanced with equal cogency with regard to Lupus vulgaris and certain late syphilitic lesions.

There may be considerable difficulty in diagnosing it from Lupus vulgaris of the superficial type described by Leloir as Lupus vulgaire érythématoïde, but, apart from the history and the tendency to ulceration in the latter, a microscopical examination should easily distinguish them.

(3) In a certain number of cases Lupus erythematosus occurs in association with various lesions of the skin which are generally placed in the class of the tuberculides and regarded as due to the tuberculous toxin, such as Lichen scrofulosorum.

Lupus erythematosus, however, has never been known to follow an injection of tuberculin, while a number of instances are on record in which Lichen scrofulosorum was so produced.

It has also been observed from time to time in association with Erythema induratum and Acne scrofulosorum.

It can only be conceded, therefore, that as tuberculin injections do not cause a local reaction in Lupus erythematosus nor produce the eruption de noto, there is no positive proof that the tuberculous toxin is the cause of the disease. Tuberculosis, however, may act as predisposing factor by reducing the power of resistance of the patient.

Summary.-A study of Lupus erythematosus from its many aspects strongly suggests that it is not in the true sense a pathological entity due to one specific cause, but is rather a morbid condition of the type 
of an erythema which is persistent in character and tends to be succeeded by atrophy, and which may be brought about in a predisposed individual by a variety of causes of which the following are the most important :

Predisposing Causes.-(a) Hereditary weakness.

(b) Enfeebled states of health resulting from any general morbid condition of which tuberculosis and rheumatism are probably examples.

(c) A weak peripheral circulation and various circulatory disturbances arising from anaemia, morbus cordis, etc.

(d) A delicate condition of the blood-vessels in the situations most commonly affected, owing to anatomical reasons.

Direct Causes.-(a) Local causes, of which the most common are atmospheric influences, such as cold, sudden changes from heat to cold, sunlight, traumatism, bites from mosquitoes or other insects, or local microbic action.

(b) Toxins reaching the skin via the blood-stream, such as toxins resulting from imperfect metabolism in the alimentary tract or from a defective state of the kidneys, liver, or other organs.

Diagnosis. - The diagnosis sometimes presents considerable difficulty. In a typical case of the chronic type the age of incidence, persistence, situation, and clinical characteristics, enable the disease to be recognised easily but in the acute form its differentiation may be difficult.

It has to be diagnosed chiefly from Lupus vulgaris, rosacea, seborrhoic dermatitis, and Erythema multiforme.

From Lupus aulgaris it is easily differentiated, except in the superficial variety known as Lupus vulgaire érythématoïde which resembles Lupus erythematosus in its distribution and in the superficial character of its lesions ; but, however superficial this variety of Lupus vulgaris may be, when the blood is expressed by the diascope a brown stain is left and small typical foci of the apple-jelly type may be detected.

In rosacea small papules or pustules are generally present in the inflamed area which are not met with in Lupus erythematosus, and the lesions are ill-defined, fade into the surrounding skin, are usually associated with digestive disturbances, and leave no scars.

An acute outbreak of Lupus erythematosus on the face may resemble seborrhoic dermatitis but in the latter the scales are more greasy and yellow, the base less vivid, and the border not so well-defined.

In the acute variety the patches, both on the face and covered parts, may closely simulate Erythema multiforme but may be distinguished from that condition by the persistence of the lesions and their tendency to undergo atrophic changes.

On the fingers, patches of Lupus erythematosus may resemble chilblains but differ by persisting through the summer and by usually being scaly. 
Prognosis.-The prognosis is uncertain and often unsatisfactory: If left alone the disease, as a rule, persists indefinitely and though spontaneous involution may take place in one patch, new lesions tend to develop. It is subject to periods of improvement followed by relapses either as the result of some general disturbance or from local irritation. In most cases atrophic changes and scarring follow the disappearance of the patches though lesions may occasionally clear up without leaving a trace.

In some cases treatment may effect improrement and even cure, in others it may be of little or no benefit.

In the discoid variety the lesions may undergo spontaneous healing with advancing years, but this is not invariably the case.

In the acute disseminated type the prognosis should be most guarded, for although the skin affection may disappear spontaneously. or under suitable applications, the toxaemia which is responsible for it may be the result of some profound risceral disturbance and may lead to a fatal issue.

In a few rare instances the scars of Lupus erythematosus have been known to become epitheliomatous but this is a much rarer complication than in Lupus vulgaris.

\section{TREATMENT}

The treatment is both general and local and of the two the latter up to the present has given the best results, certain cases having been undoubtedly cured by local measures alone. On the other hand cases of the acute generalised type have been known to clear up under general treatment where practically no local treatment had been employed. In most cases the best results may be obtained by the judicious combination of both measures.

General Treatment.-The general treatment of Lupus erythematosus is based largely on general medical principles. In many of the cases there is definite evidence of a lowered vitality, associated with a weak peripheral circulation, resulting from a variety of causes such as hereditary or acquired disease, disturbances of the alimentary tract, anaemia, and diseases of the heart, kidneys, or other important organs. Such defects must be carefully sought for and dealt with by appropriate means. It is important in connection with the dietary that anything which tends to cause flushing, such as alcohol, condiments, etc., should be omitted or only taken in small regulated quantities. All sources of local irritation should be scrupulously guarded against, such as cold winds, brilliant sunshine, over-heated rooms, and violent exercise.

In the acute disseminated cases it is usually necessary to confine the patient to bed and as the disease results from toxaemia, the obvious indication is to eliminate or neutralise the toxins responsible. As the exact nature of the toxin is, as a rule, uncertain, the appropriate antitoxin is not arailable but various drugs known to have an anti-toxic action have been employed. such as salicin and quinine. Salicin was 
strongly recommended by Crocker in doses of $I_{5}$ grains t.d.s. increased to 30 grains t.d.s. and sometimes gives good results, while quinine in large doses is of special value in the more acute cases. Various other drugs have been employed, such as arsenic, iodide of potassium, and ichthyol, but the results from them have been disappointing.

Local Treatment.-A large number of local remedies have been put forward for the treatment of this capricious affection, the length of the list alone, being a clear indication of the uncertainty of their action. In the choice of the treatment it is important to remember that Lupus erythematosus is much more easily aggravated than cured by local applications. Before selecting any form of treatment it is necessary carefully to study each case and lesion, and, having decided on a treatment, to begin in a cautious and tentative fashion, and gradually to increase the strength of the application according to the toleration of the patient.

There are three indications which serve as a guide to the local treatment namely, (I) the removal of the scales, (2) the drying of the lesions, (3) the reduction of the hyperaemia.

Removal of the Scales.-An effective method of removing the scales is to wash the lesions with soap spirit lotion on a piece of moist flannel, but where the scales are thick and adherent stronger measures, such as the application of a 2 or $3 \%$ salicylic ointment or plaster, may be necessary.

Drying.-For the purpose of drying the lesions, bland powders, pastes, or lotions containing an insoluble deposit, may be employed.

A useful powder consists of equal parts of zinc oxide and magnesium carbonate, the latter absorbing a larger quantity of moisture for its bulk than any other porder.

One of the best lotions is the zinc calamine lotion containing :

\begin{tabular}{|c|c|c|c|c|c|c|}
\hline Calaminae pr & par & tae & & & & gr. xv \\
\hline Zinci oxidi & - & - & - & - & & gr. $x$ \\
\hline Glycerini & - & - & 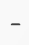 & - & - & m. $x x$ \\
\hline Aquae calcis & - & - & - & & 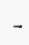 & $5^{\mathrm{i}}$ \\
\hline Aquam rosae & ad & - & - & - & - & $\bar{\jmath} \mathrm{i}$ \\
\hline
\end{tabular}

A serriceable paste is the following:

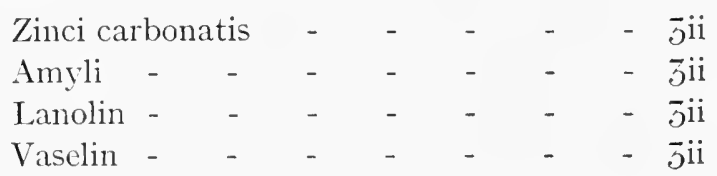

Where the lesions are active the daily removal of the scales by soap spirit followed by calamine lotion is safe and beneficial, but where they are more chronic and resistant salicylic ointment should be applied at night and followed by the lotion or paste in the morning.

Reducing Hyperaemia.-For this purpose ichthyol has a great reputation and may be employed as a 3 to $10 \%$ ointment in vaselin, 
or as a paste with the above zinc starch paste as the base, spread on lint and applied continuously. Ichthyol plasters are obtainable and are more convenient than ointments. It may be used as a 3 to $6 \%$ lotion dissolved in water or in $50 \%$ alcohol, pressed into the skin several times a day and followed by a bland dusting powder, but in this form is less effective.

Other remedies have been recommended, such as $5^{\circ} \%$ formalin ointment, and adrenalin lotion ( $\mathrm{I}$ in I000), but they are of less value and the former may irritate delicate skins.

A simple mechanical method of reducing hyperaemia which is sometimes effective is to paint the lesions two or three times a day with contractile collodion, to which $\mathrm{I} \%$ of salicylic acid may be added.

At one time a farourite method of treating the lesions was by caustic remedies, such as acid nitrate of mercury, corrosive sublimate, silyer nitrate, and carbolic acid. These are essentially destructive in their action, and though capable of remoring the lesions are apt to do so at the cost of an unsightly scar; they should never be used except for small circumscribed patches covered with a thick scale. Of the milder caustics two of the most useful are oxidised pyrogallic acid $6^{\circ}, 0$ applied daily in a paste, and resorcin $10 \%$ dissolved in spirit and painted on the lesions once a week.

In small lesions various operative measures, such as multiple scarification, electrolysis, and the actual cautery, may give good results if proper care be exercised to produce as little scarring as possible.

In addition to the chemical methods of treatment, certain physical and electrical methods have been used with greater or less success, such as ionization, refrigeration, Finsen light, $\mathrm{X}$-rays, radium, etc.

Of these, ionisation with zinc is the most reliable, probably gives the most uniformly successful results of all forms of local treatment at present at our disposal, and is suitable for all types of circumscribed Lupus erythematosus. The method is simple: a small piece of lint is saturated in a $2 \%$ solution of sulphate of zinc and applied to the lesion, and on this is pressed a flat zinc block electrode chosen according to the size of the lesion and attached to the positive pole of a galranic battery ; the negative pole, consisting of a metal cylinder covered with chamois leather and dipped in saline lotion, is grasped by the patient and a current of 2 to 5 milliamperes is passed for about $I_{5}$ minutesthe strength of the current being determined by the toleration of the patient. After the exposure a bland zinc protective paste is applied to the part. Where the lesion is large it may have to be treated piecemeal and care must then be taken to aroid overlapping. As a result of the exposure the tissue becomes blanched like parchment and continues so for a varying time according to the strength of the current employed. If the exposure be not excessive no subsequent reaction should take place, if it be too prolonged or the current too great, superficial ulceration may be produced. A practical point in connection with the treatment is that the negative electrode should be large and 
should be moved from one place to another during the exposure, on no account should it be strapped on as it may then cause an intractable necrotic ulcer. In a few cases a single application is sufficient but, as a rule, sevcral are necessary and should be given at weekly intervals.

Refrigeration with liquid air or solid carbon dioxide sometimes gives excellent results, the latter being more practical as it is cheaper and can be more accurately applied. An application of Io to $5_{5}$ seconds with medium pressure is given, care being taken when the patches are extensive and have to be treated piecemeal to avoid overlapping with the applicator. The best results from this form of treatment are obtained in lesions of the circumscribed seborrhoic type where the patches are situated on soft parts like the cheeks. Should they occur in situations where the skin is thin and stretched, as about the nose and ears, or in elderly people, the freezing is apt to produce an intractable superficial ulceration followed by scarring. It should on no account be used where the lesions are actively spreading, as then it may aggravate rather than cure.

The actinic rays sometimes give beneficial results, especially in oldstanding lesions covered with thick adherent scales. The exposures are given in the same manner as in Lupus vulgaris but require to be longer as the reaction which follows is feeble. This treatment is only applicable in chronic cases and is harmful where the patches are recent and active.

The $\mathbf{X}$-rays have been employed also, but with disappointing results. In small doses they stimulate and irritate the lesions, and in large doses they are apt to cause permanent dilatation of the vessels and may replace the lesion by an $\mathrm{X}$-ray scar.

Radium also has been used with doubtful success and, although theoretically it is known to have a sclective action on blood-capillaries causing them to disintegrate and become replaced by fibrous tissue, practical benefit in the lesions is seldom obtained.

Various electrical methods have been advocated, such as high frequency, static discharges, etc., but are of little or no value and are apt to aggravate it.

\section{REFERENCES}

BARber, H. W. A case of acute Lupus erythematosus (Brit. Journ. Derm., I9I5, xxvii. p. 365).

Bernhard, R. Co-existence of Lupus erythematosus with papulonecrotic tuberculide (Arch. f. Derm. u. Syph., I9I2, cxi. p. 53I).

BLock \& Fuchs. Lupus erythematosus \& tuberculosis (Ibid., July I9I3, cxvi.).

BoEck, C. Relation to tuberculosis (Ibid., xlii.).

Brocg. Lupus erythematosus (Traité élémentaire de Derm. Prat., I907, p. 597).

Buxch, J. L. Tuberculo-opsonic index in Lupus erythematosus (Brit. Journ. Derm., I907, xix. p. 4II).

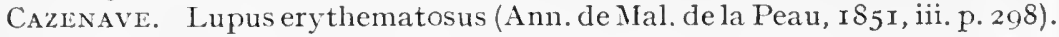

ENGMan \& Mlook. Some cases of Lupus erythematosus (Interstate Med. Journ., April 1909, p. 267). 
Freshwater, D. Etiolog: of Lupus erythematosus (Brit. Journ. Derm., 19I2, xxiv. p. 5i).

Friedlasder, D. Etiology of Lupus erythematosus, with especial reference to tuberculosis, \& a report of 13 cases tested by the Moro reaction (Journ. Cut. Dis., I9II, xxix. p. +1 I).

- Value of Much's granules \& the anti-formin method in determining the etiology of the so-called tuberculides, with especial reference to Lupus erythematosus (Brit. Journ. Derm., I 9 I 2, xxiv. p. I3).

Hartzell, M. B. Lupus erỹthematosus \& Raynaud's Disease (Amer. Journ. Med. Sci., cxliv. p. 793).

Gallowar, J., \& MACLeOd, J. II. H. Erythema multiforme d Lupus erythematosus, relationship to general toxaemia (Brit. Journ. Derm., I903, xi. p. 81).

Gotgeror. Inoculation experiments (Rer. de la Tuberculose, I898).

Kopp, K. Relationship to tuberculosis (Arch. f. klin. Med., I901, lxvi.).

McDoxagh, J. E. R. Case of acute Lupus erythematosus (Lancet, I909, P. $\left.{ }_{4} 8\right)$.

MacLeod, J. M. H. Association with Bright's Disease (Brit. Journ. Derm., I904, xti. p. 223).

- Association with fatal nephritis (Ibid., I908, xx. p. I62).

- Lupus erythematosus, its nature \& treatment (Lancet, Igos, ii. P. $127 \mathrm{I})$.

- Lupus erythematosus, nature, causation d treatment (Brit. Med. Journ., I9I3, ii.).

Mocxi, L. B. Lupus ervthematosus \& tuberculosis (Mied. Rec., I9I6, p. 983).

PARIS \& DoBRoricr. Agglutination tests (Gaucher, Diseases of the Skin, I9IO, p. 340).

PAtrRIER \& FAgE. Pathological anatomy of Lupus erythematosus of mucosae (Ann. de Derm. et de Syph., Igog, p. 6;3).

Perret, G. Le Lupus érythémateux aigu d'emblée; Etude clinique (Paris, Igos, p. I 35).

PICK, W. Relation to tuberculosis (Arch. f. Derm. u. Syph., Igor, lviii. p. 358$)$.

Poor, F. Y. Etiology of Lupus erythematosus (Derm. Zeitschr., Igor, viii.).

Pringle, J. J. Association with Raỵnaud's Disease (Brit. Journ. Derm., I 895 , vii. p. 30 ).

Multiple epitheliomata upon Lupus errthematosus Ibid., I900, xii. p. I).

Roberts, L. Acute Lupus erythematosus (Ibid., I9I I, xxiii. p. I6 - ).

Schoosheid, P. H. Histology of Lupus erythematosus (Arch. f. Derm. u. Syph., I900, P. I63).

Sequeira, J. H., \& Baleas. Clinical study of iI cases (Brit. Journ. Derm., I902, xiv. p. 36\%).

Short, T. S. Fatal case of acute Lupus erythematosus (Ibid., Igo-, xix. P. $2 \pi I$ ).

Uxxa, P. G. Histopatholog: (Walker's Translation, I896, p. Io7I).

VEREL. Relation to tuberculosis (Rep. of Internat. Derm. Congress, I 892 ).

WARDE, W. B. Association with hypertrophic rhinitis \& ozaena (Brit. Journ. Derm., 1902, xiv. p. 332).

Sclerodermic type (Ibid., I903, rv. p. 2\%).

WISE, F. The relation between Lupus erythematosus \& tuberculosis, with review of recent literature (New York Med. Journ., I 9 Is, p. I I6 4 . 


\section{CHAPTER XXX MYCOSIS FUNGOIDES}

Syn.: Granuloma fungoides (Auspitz); Fibroma fungoides (Tilbury Fox); Lymphodermia perniciosa (Kaposi); Multiple sarcoma of the skin (Hyde); Eczema tuberculatum (Erasmus Wilson); Fr. Mycosis fungoïde; Lymphadénie cutanée; Ger. Mycosis fungoides; Multiple Granulationsgeschwülste.

Definition: A chronic, generally fatal, disease of the skin, usually characterised by a persistent dermatitis which may be erythematous, urticarial, or eczematous in type and is associated with severe itching (pre-mycosic stage), and later by the development of tumours which become ulcerated or form fungoid growths (mycosic stage).

Introduction.--This disease was observed as early as I8I2 by Alibert who first described it under the name of Pian fungoïde, as he considered it to be in some way related to yaws, but later changed his opinion and renamed it Mycosis fungoides. It is exceedingly rare but, owing to its striking characteristics and high mortality, it has attracted exceptional interest and few cases have been allowed to pass without being recorded; consequently the extent of the literature on the subject is out of all proportion to the frequency of the disease.

It has a wide distribution and cases have been observed in many countries in Europe and America, in dark as well as in white skins.

Description.-The affection usually begins in an indefinite fashion. The early signs give no indication of the gravity of its nature and, as a rule, its true character is recognised only after it has been in existence for a considerable time.

Pre-mycosic Stage.--In rare instances the initial symptom consists of severe itching which may persist for months or years and may be unassociated with any obrious changes in the skin, but as a rule it is ushered in by some form of so-called pre-mycosic eruption. These pre-mycosic eruptions are characterised by their multiformity and may consist of macules, papules, plaques, wheals, bullae, or scaly patches, and may simulate so closely various forms of dermatitis, such as Erythema multiforme, psoriasis, Pityriasis rubra, Lichen planus, Seborrhoic dermatitis, or eczema, that the tumour or mycosic stage was at one time believed to be an affection sui generis, super- 
imposed on some chronic dermatitis which formed a suitable soil for its development. It is now recognised, however, that the pre-nrycosic eruption is as much a part of the disease as the chronic eczematous patch is of Paget's disease of the nipple, and that in the pre-mycosic lesions a peculiar infiltration of cells occurs in the corium the further development of which is responsible for the tumour formation.

The most characteristic lesions of the pre-mycosic stage are pad-like persistent patches which are discoid, circular, or crescentic in form, well-defined, of a reddish or dusky purplish hue, or sometimes pigmented and brownish. They may be transitory, lasting a few days or weeks then disappearing and fresh ones coming out elsewhere, or may persist for months. They vary in size up to several inches in diameter and are situated most commonly about the trunk, proximal parts of the limbs, and flexures of the larger joints, but may appear anywhere on the cutaneous surface. When present on the face they are liable to give rise to a heary leonine expression such as is met with in nodular leprosy. Occasionally they are so acutely inflamed and oedematous as to suggest erysipelas, or again they may be more indolent, and spread and coalesce with each other until the skin becomes almost universally involved and the condition known as "Homme rouge" is produced, but even in the most widely affected cases islands of apparently normal skin are usually left.

In extensive cases the lymphatic glands and spleen are liable to be enlarged, and the hair is apt to become scanty, the nails opaque, ridged, and brittle, and the lips thichened, ulcerated, and everted. Occasionally the buccal mucosa is involved. These cases possibly belong to the group described by Kaposi under the heading of Lymphodermia perniciosa.

Associated with the eruption are usually more or less severe sensory symptoms, such as paroxysmal pruritus, or burning and pricking sensations ; these are apt to lead to rubbing, scratching, secondary eczematisation, or impetigo. In rare instances subjective symptoms may be absent.

As the lesions increase in age they tend to become indurated and defined and the colour to darken to a dull brownish or violaceous tint.

Occasionally a single lesion, recalling the herald patch of Pitrriasis rosea, may precede the outbreak of a pre-mycosic eruption and may occur in various situations, such as the trunk, limbs, and face.

The pre-mycosic stage may last from a few weeks to several years and is usually followed by the mycosic or tumour stage, though in rare instances death has been known to supervene before definite tumours appear.

Mycosic or Tumour Stage.-Sometimes the tumours may be the earliest symptom of the disease ("Mycosis fungoïde à tumeurs d'emblée" ) but more commonly the tumours form on pre-existing infiltrations or on apparently healthy skin, simultaneously with or subsequently to the pre-mycosic erythematous patches. 
The tumours vary in size from a pea to a hen's egg or even larger, are roundish, oval, or irregular in shape, smooth or nodular on the surface, and of a dull reddish-brown tinge. In form they differ considerably, sometimes being hemispherical, at other times pedunculated or mushroom-shaped. They are situated most frequently on the trunk and upper parts of the limbs and more rarely on the face or scalp, and may be few or numerous.

As time goes on they gradually extend and break down in the centre forming deep sloughing ulcers with a greenish sero-purulent or haemorrhagic discharge and sometimes presenting a well-marked rolled edge. In rare instances they may disappear spontaneously without scarring or pigmentation. Associated with them the lymphatic glands may be enlarged, not as the result of metastases but most probably from septic absorption. (Fig. 3I5.)

Infiltration, superficial ulceration, or even gangrene may occur in the mucous membranes of the palate, pharynx, and larynx, and may greatly interfere with breathing and swallowing.

Changes in the viscera and metastatic deposits have been recorded post-mortem in the kidneys, lungs, suprarenals, heart, pancreas, and dura mater, but some doubt has been cast on their being true metastases, and a considerable number of careful autopsies have failed to reveal any changes except such as might occur from septicaemia.

Numerous examinations have been made of the blood, but no characteristic changes have been detected. A variable degree of eosinophilia and a slight leucocytosis have been observed in a number of cases but not more than might occur in any form of malignant disease.

In several instances the tumours appear to have been influenced by some intercurrent disease. In a case reported by Sir James Galloway and the writer, temporary improvement occurred after an attack of influenza, while cases have been recorded in which tumours disappeared after erysipelas or in the course of an attack of pneumonia (Pringle).

Death may take place from intercurrent disease, but more frequently the patient rapidly loses ground, digestive troubles supervene, diarrhoea, depression, and fever occur, and death follows from septicaemia or extreme exhaustion. In some cases, especially those of the tumeurs d'emblée type, death occurs in the course of a few months, in others it is delayed for a varying time, but generally takes place within ten years of the development of the tumours.

Histopathology.-An examination by the writer of a large number of sections from 3 cases of Mycosis fungoides in both the pre-mycosic and mycosic stages showed that the most characteristic feature in the histology was a peculiar type of cellular infiltration which persisted throughout the disease and could be detected wherever induration was present in the lesions. This infiltration resembled more closely that presented by the infective granulomata than that of a true neoplasm, such as a sarcoma or fibroma. It was seen first in the sub-papillary layer 


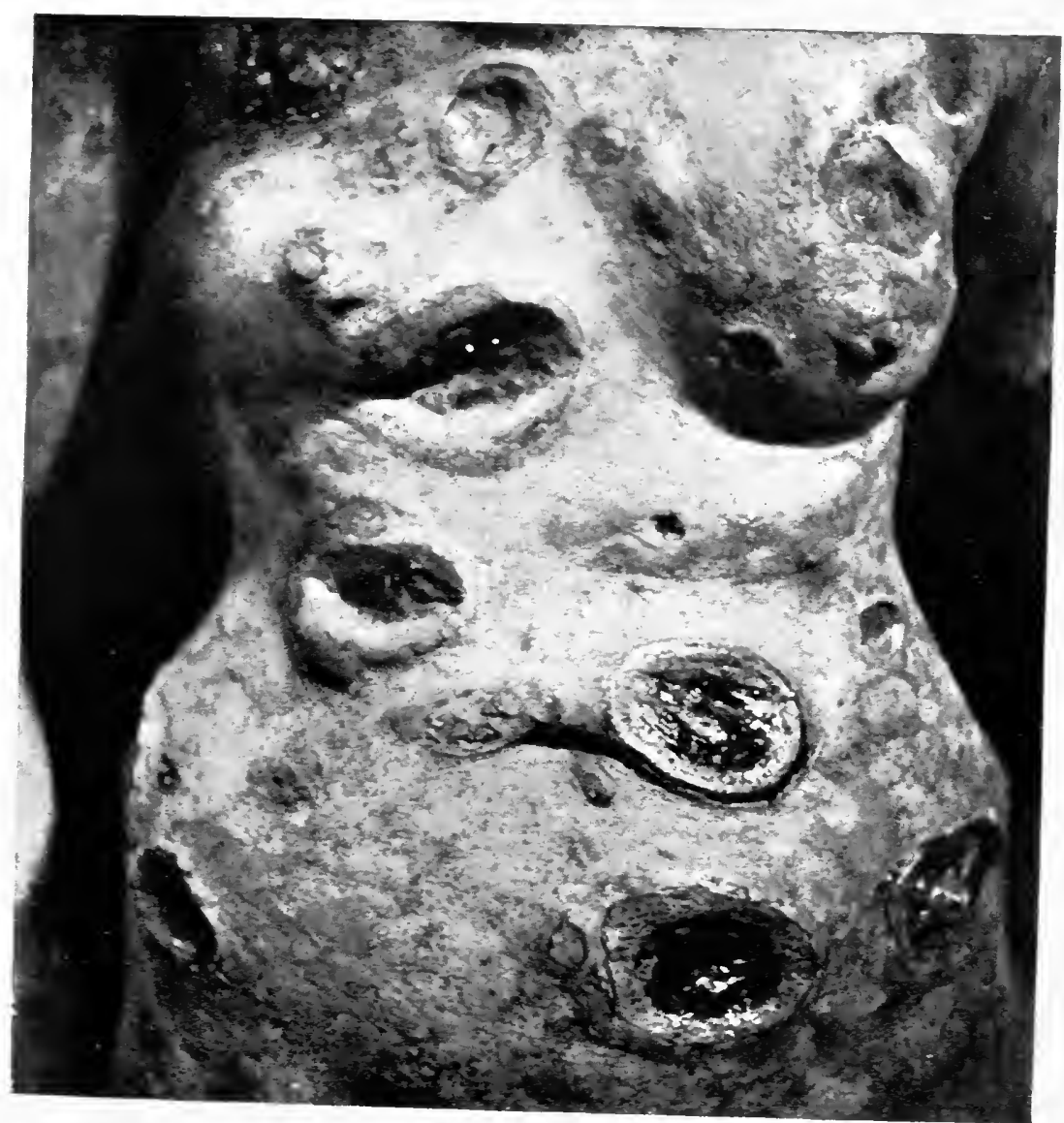

FIG. 3I 5.-Mycosis Fungoides.

Tumours and large crateriform ulcers.

(Dr. F. Parkes Weber's case. Brit. Journ. Derm., Igrs.) 

of the corium and spread from there up into the papillae and down towards the hypoderm. It was most marked around the blood-ressels of the papillary and sub-papillary layers, the hair-follicles, Arrectores pili, sebaceous glands, coil-ducts, and glands, but was observed independently of these structures spreading along the spaces between the connective tissue bundles.

The cells composing it were chiefly of connective tissue origin and showed four main types :

I. Large oval polygonal or roundish connective tissue cells containing a granular protoplasm and à large nucleus whose chromatin network and nuclear membrane were darkly stained while the nucleoplasm was no more darkly stained than the cell protoplasm. These cells appeared to be derived either from connective tissue cells or from the endothelium of bloodvessels, in earlier lesions they showed mitotic figures but later appeared to divide in an amitotic or direct manner.

2. Numerous small round cells, slightly larger than leucocytes, with nuclei similar to, though proportionately smaller than, those of the larger cells. These were probably "daughter-cells" of the first type of cell. (Fig. 3I6.)

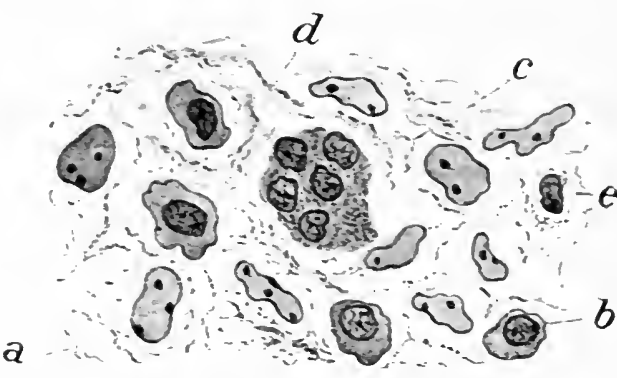

J. M. H. M. del.

FIG. 316.-Mycosis Fungoides.

( $x$ about 1000.)

Shows crenation and fragmentation of celis.

a. Connective tissue nuclei. b. Crenated ceils. c. Chorioplaque. $d$. Remains of collagen and cellular debris. e. Nucleus surrounded by mass of protoplasmic debris.

3. A few mast-cells, but considering the enormous proliferation of the other connective cells the mast-cells seemed to be relatively. diminished.

4. A few plasma-cells similar to those met with in tuberculosis, and here and there imperfect giant-cells, with eight or ten nuclei, resembling the giant-cells of a syphilitic granuloma.

The collagen and elastin were unaltered.

The epidermis presented the following changes in the pre-mycosic stage: There was an increase in the number of prickle-cells and a down-growth of the inter-epithelial processes. The inter-epithelial lymphatic spaces were dilated by oedema. In those cases where scaliness was a marked feature the transitional layers were defective, there was imperfect cornification, and the nuclei persisted in the most superficial horn-cells. The epidermal changes were not characteristic and varied greatly in different cases.

The histological changes in the tumour and breaking-down stage were as follows: The cellular proliferation had increased and spread down as far as the subcutaneous tissue. The cells composing it showed a marked tendency to break down and there was evidence of crenation, 
irregularity of the cells and fragmentation, and throughout the infiltration there was an indefinite granular debris from the disintegrating cells. The collagen and elastin were basophilic and also showed a tendency to disintegrate. The disintegrating granuloma gradually encroached on the down-growing epithelium and finally broke through the surface, forming a superficial ulcer.

From the above description it will be seen that the most characteristic features in the infiltration are the multiformity and marked fragility of the cells.

Bacteriology.-No pathogenic organism has yet been demonstrated in connection with it. Various micro-organisms, such as sarcinae, Friedländer's bacillus, etc., were found by Eyre in one case, a "short white bacillus" was isolated by M'Vail, Murray and Atkinson in another, and Gram-positive cocci were described by Stelwagon, but these were probably secondary contaminations. Inoculation experiments with the tumour tissue in animals have given negative results.

Nature and Etiology.-The true nature of Mycosis fungoides is at present unknown. The general opinion is that it is a disease sui generis, that it is an infective process due to some undiscovered virus, and that it should be placed provisionally in the group of the infective granulomata. It is believed to be quite distinct from Hodgkin's disease, true leucaemia, and Sarcomatosis cutis, with which it has sometimes been confused.

There are no definite predisposing causes. It would seem to be more common in males than females but the exact proportion is uncertain ; out of 74 cases collected by Sequeira, 46 were males and 28 females.

With regard to age, it is essentially a disease of adult life, the majority of cases occurring between the ages of 40 and 60 , the age limits recorded being 15 years in a female and 75 in a male.

It does not seem to be contagious nor is there any evidence of hereditary transmission.

In one or two instances it has followed injury, as in a case of Mycosis fungoïde d'emblée reported by Riecke. Radcliffe-Crocker also recorded a case in which it developed about a year after a severe kick on the knee which was followed by prolonged suppuration in the head of the tibia.

Diagnosis.-It is only in the early stages of the disease before the tumours have appeared that the diagnosis presents any serious difficulty. In a typical case in the advanced stage the presence of tumours varying in size from a pea to a hen's egg, hemispherical or pedunculated, ulcerated or fungating, associated with erythematous pads, eczematous patches, and paroxysms of intolerable itching, and a history of the existence of an ill-defined eruption for months or years before the tumour formation, form a clinical picture which is not readily mistaken.

In the early stages where the lesions are indefinite the diagnosis may be difficult or impossible. Gradually, however, the lesions tend to become infiltrated and this, in association with the intense itching, is 
strongly in farour of Mrcosis fungoides-a diagnosis which can usually be verified by making a biopsy and demonstrating the characteristic cellular infiltration.

A method of sero-diagnosis has been introduced by Gaucher, Joltrain, and Brin, who found that sera from mrcosic patients gave a positive complement fixation reaction with mycosic antigen, but were negative to other antigens, but further experience of this test is necessary before it can be regarded as of diagnostic value.

A certain degree of confusion in the past has occurred with regard to the diagnosis of Mrcosis fungoides from true leucaemia and lymphadenoma.

In leucaemia, apart from the characteristic blood changes, there is not infrequently a generalised prurigo which does not resemble the erythrodermia or pad-like patches of the pre-mycosic eruption, while the tumour growths which are occasionally met with are, as a rule, small nodules of a brownish or blackish tinge and not large tumours. Histologically also, the infiltration is more in the nature of a leucocrtic infiltration and not due to a fixed-cell proliferation.

In lymphadenoma there may be a generalised dermatitis with pruriginous papules, intense itching, sometimes lichenification and pigmentation, and later nodules ahout the size of a bean, which histologically have the structure of lymphatic gland tissue.

Prognosis. - The prognosis is invariably grave and a fatal issue is to be expected, which may take place in a few months, especially in cases of the d'emblée tỵpe, or may be postponed for fifteen or twenty years. A grave sign is the appearance of tumours, for once a number of ulcerating or fungating tumours have developed the end is rarely delayed for more than a couple of years.

The course varies in different cases. Sometimes the eruption undergoes spontaneous remissions followed by exacerbations, in much the same way as in leprosy, or tumours may disappear in one situation and break out in another. By means of the I-rays it is possible to cause the tumours to disappear in a most dramatic fashion, and several instances are on record in which an apparent cure has been effected. On the other hand if the tumours are extensive and the X-rays pushed there is a danger of causing septicaemia from rapid absorption of the disintegrating growths.

\section{TREATMENT}

As the cause is unknown the treatment up to the present has been empirical rather than rational. Iodide of potassium has been given with the object of causing the absorption of the tumours but without benefit, and arsenic and mercury prescribed in considerable doses have been equally disappointing. Owing to the observation that the disease has been temporarily arrested after erysipelas, injections of Colle's fluid, which contains mixed toxins of Streptococcus pyogenes and 
Bacillus prodigiosus, have been tried but though a certain amount of local reaction and a rise of temperature occurred, no permanent benefit was derived. Consequently the constitutional treatment has resolved itself into a general tonic regime on ordinary medical lines with the treatment of each special symptom as it arises.

The local treatment has been equally unsatisfactory as soothing remedies, such as calamine cream, etc., have comparatively little influence in allaying the intense irritation, while resolvent remedies, such as salicylic acid, resorcin, etc., are worse than useless as the skin is unusually sensitive and even the mildest irritant may aggravate the inflammation. Where ulcers are present they should be cleaned with mild antiseptics to prevent septic absorption.

The only treatment which at present promises any hope is the X-rays. By this means even large tumours can be made to resolve and, although a complete cure may not be effected, the condition of the patient is rendered far more bearable and the course of the disease delayed. A pastille dose should be given to each lesion and, if necessary, a second or third at monthly intervals. It has been found that the older tumours react more rapidly and favourably to the X-rays than early lesions, owing to the cellular infiltrate in the older lesions being more fragile.

\section{Lymphodermia Perniciosa}

The term Lymphodermia perniciosa was applied by Kaposi in 1885 to a type of case which has been regarded as a variety of Mycosis fungoides by certain observers, chiefly of the French school, though Kaposi himself believed it to be a manifestation of leucaemia. It began with an eczematous condition of the skin which eventually became generalised and was associated with severe pruritus; later the skin became markedly thickened, ulcerating nodules developed upon it, and the lymphatic glands and spleen became enlarged; the general health rapidly failed, and death finally supervened in a state of profound asthenia. At the post-mortem examination nodules were detected in the pleurae and the lungs, which were described as leucaemic nodes and believed to be of the same structure as the nodules in the skin.

\section{REFERENCES}

\section{Mycosis Fungoides.}

Brandweiner, A. Zur Kenntnis der Mykosis fungoides (Monatsh. f. prakt. Derm., I905, xli. 4I5).

Bushnell \& Williams. Mycosis fungoides (Brit. Med. Journ., I907, ii. I 403. References).

Fox, T. Colcott. Mycosis fungoides (System of Medicine, Allbutt \& Rolleston, I9I I, ix. p. 535).

Galloway \& MacLeod. Three cases of Mycosis fungoides (Brit. Journ. Derm., I900, xii. p. I 53).

Gaucher, Joltrain \& Brin. Sera-reactions in Mycosis fungoides (Soc. de Biol., I909, lxvii. p. 494). 
GREIG, D. M. A case of Mycosis fungoides (Brit. Journ. Derm., I904, xvi. p. $25 \mathrm{I}$ ).

Heller. Mycosis fungoides (Arch. f. Dermat. u. Syph., Wien, rgo8, xcviii. Bibliography).

Jamiesox, W. Aliax. Mycosis fungoides \& its treatment by $\mathbf{X}$-rays (Brit. Journ. Derm., I903, xv. p. I).

KNowles, F. C. Histopathology of Mycosis fungoides (Journ. Cut. Dis., I9I5, xxxiii. p. 463).

MicVail, Mltrray, \& Atkinsor. Mycosis fungoides (Glasgow Hospital Reports, IS98, i. p. 53).

Pardee, L. C. \& Zeit, F. R. Mycosis fungoides (Journ. of Cut. Dis., I9I I, xxix. p. 7).

Senear, F. E. A case of Mycosis fungoides limited to one foot (Journ. Cut. Dis., I9I5, xxxiii. p. 35I).

Sequerra, J. H. Discussion on Mycosis fungoides (Brit. Journ. Derm., I9I 4, xxvi. p. 2I3).

Stainer. X-ray treatment of Mycosis fungoides (Brit. Journ. Derm., I903, XV. 137, 2I 2).

Uxva, P. G. Histopathology (English Trans., I 896, p. 507).

\section{Lymphodermia Perniciosa.}

Hurchixsox, J., Junr. (Trans. Med. Soc. Lond., I 906, xxix. $4 \mathrm{I} 7$ ).

Kaposi. Über eine neue Form von Hautkrankheit "Lymphodermia perniciosa," zugleich ein Beitrag zur Pathologie der Leukamie (Med. Jahrb., Wien, I885, xv. r2a. Translated, Doyon. Ann. de Dermat. et Syph., Paris, I 885,2 s., vi. 400 ).

RaDAELI, F. Mycosis fungoides or pseudoleukaemia cutanea (Arch. f. Derm. u. Syph., July r906, lxxx. 323). 


\section{CHAPTER XXXI}

\section{AFFECTIONS OF THE CUTANEOUS BLOOD-VESSELS AND THE LESIONS ASSOCIATED WITH THEM}

MANY of the lesions of the skin which result from disturbances of the circulation and changes in the blood-vessels have been described under the headings of cutaneous affections due to heat and cold, such as Livedo annularis, angio-keratoma, Raynaud's disease, etc., and the important group of the vascular naevi. In this chapter will be considered :

(I) The cutaneous haemorrhages or purpuras ;

(2) The telangiectases.

\section{PURPURA}

Purpura, from the Greek $\pi \circ \rho \phi u ́ p o s$, purple, signifies an eruption of purplish spots or patches due to extravasation of blood into the skin. The term has been employed in two senses. Certain writers have restricted it to purpuric eruptions which have appeared spontaneously, with or without other symptoms, and have applied the qualifying adjective haemorrhagic to those eruptions in which the lesions are essentially erythematous, urticarial, or bullous, but occasionally become extravasated with blood, such as Erythema haemorrhagicum or Variola haemorrhagica; while others have included under it every sort of cutaneous haemorrhage, primary or secondary, except those caused by external injury, such as contusion, laceration, incision, or the bites and pricks of insects.

Both significations are open to criticism but for convenience of description the second and wider sense will be adopted here.

The lesions at first vary in colour according to the anatomical situation of the haemorrhage and are bright red or purplish if the haemorrhage be superficial and confined to the corium, and blackish if it occurs in the subcutaneous tissue. Later they gradually undergo changes in tint, becoming successively dusky blue, bluish-green, yellowish-white, and finally may disappear or may assume a brownish tinge followed by a brown stain. Unlike erythematous lesions they 
cannot be obliterated by pressure with the diascope at any period in their existence, and they persist after death.

They vary in shape and size. When small they are known as petechiae and consist of roundish, oval, irregular, sometimes stellate, well-defined macules, and range in size from a pin's head to a lentil ; when large they are called ecchymoses, are similar to traumatic bruises, and form purplish, flat or slightly raised patches; when in lines or streaks they are known as ribices; and when they occur as raised, soft and diffuse, or hard tumours, they are called haematomata.

Petechiae are the most common purpuric lesions and appear spontaneously, while ecchymoses as a rule are the result of pressure or friction and often co-exist with petechiae.

In rare instances the lesions may be flat, rounded or acuminate papules where the extravasation of blood has taken place into the epidermis or about the hair-follicles.

Purpuras may be divided into two classes; namely, (a) Primary purpuras, in which the purpuric eruption is the most marked or sole pathological feature; (b) Secondary purpuras, in which the eruption has occurred in the course of some recognised infective process or in some definite cutaneous disease, the lesions of which have become haemorrhagic. It is important to recognise, however, that such a division is purely arbitrary and that, in whatever sense the term be emplored, purpura is not a disease but a symptom and one which can be called forth by a great variety of totally different causes.

\section{A. Primari Purptras}

In the primary purpuras the eruption is generally most marked on the lower extremities and lower part of the abdomen, but it may. occur anywhere on the cutaneous surface, even beneath the nails. It may be slight and consist of isolated petechiae or ecchymoses sparsely distributed but frequently symmetrical, or extensive and the lesions so closely aggregated as to form irregular sheets, but the individual lesions are almost always discrete. Once formed they show little or no tendency to peripheral extension unless as the result of fresh extrarasation.

The eruption may come out insidiously with no subjective simptoms, and the patient be unaware of its existence until it is seen, or it may be preceded by some local inflammatory disturbance with burning or itching. As a rule fresh crops perpetuate the eruption for months. or complete remissions may take place followed by recurrences at irregular intervals.

In severe cases the mucous membranes are also involved and purpuric macules or small haemorrhagic vesicles may be detected on the tonsils, palate, and gums, and the tongue may become swollen and haemorrhagic. Bleeding is apt to occur from the mucosae, and epistaxis, bleeding from the gums, haematemesis, haemoptysis, bleeding 
from the uterus, haematuria, blood in the stools, suffusion of blood beneath the conjunctiva, and, in rare instances, retinal haemorrhages, may take place. Haemorrhages may also occur into the serous membranes, especially the pleurae and meninges, or into viscera, such as the kidneys, supra-renals, liver, spleen, and brain.

Subjective Symptoms.-There are no subjective symptoms actually connected with the cutaneous lesions, but the concomitant occurrence of pains in the joints, limbs, and back, is not infrequent and occasionally headache may occur.

Constitutional Symptoms.-In some cases the eruption may appear in an apparently healthy individual and constitutional symptoms may be absent or negligible; but as a rule definite general symptoms, varying in type and degree of severity, precede and accompany the eruption, and may be so severe as to make the purpura seem the least important feature of the case. They may consist of angina, anorexia, fever varying from IOI $^{\circ}$ to $103^{\circ} \mathrm{F}$., sickness, vomiting, diarrhoea, or of symptoms of collapse, such as a sub-normal temperature, a feeble cardiac action, and a slow intermittent pulse.

Blood.-Changes in the blood have been observed in many cases, such as a diminution in the haemoglobin, mineral salts, and other normal constituents, but these are inconstant and simply indicate defects in nutrition resulting from the presence of some toxin, autogenous, chemical, or bacterial. In certain cases-especially in the purpura of haemophiliacs - the coagulability is defective and the coagulation time prolonged from the normal four minutes to half an hour or more. Sometimes the red blood cells may be greatly reduced, even to as low as 500,000 per $\mathrm{cm}$., and the haemoglobin correspondingly diminished, while the leucocytes may be increased.

It is customary to describe four types of primary or so-called arthritic or idiopathic purpura, namely :

Purpura simplex (Willan).

Henoch's Purpura of children.

Purpura rheumatica or Peliosis rheumatica (Schönlein).

Purpura haemorrhagica or Morbus maculosus of Werlhof.

Purpura simplex includes the mild cases of purpura most commonly met with in children, in which the legs are chiefly affected. In this condition articular symptoms or slight constitutional symptoms may be present and it is frequently associated with mucous haemorrhages.

In Henoch's purpura, which also attacks children chiefly, the eruption may be purpuric, crythematous or urticarial, has a marked tendency to recur, and is associated with pains or swellings of the joints, often mild, and with gastro-intestinal crises with colic, vomiting and diarrhoea, and occasionally with nephritis which has been known to be fatal.

The name Purpura haemorrhagica is applied to cases of a severe type most common in young girls, in which an extensive purpuric 
eruption is associated with bleeding from the mucous membranes, and not infrequently with death from profound anaemia and exhaustion, or from haemorrhages into the meninges, brain or suprarenal capsules. In the most severe cases death has been known to take place in about a fortnight.

Purpura rheumatica is the term applied to cases in which the eruption, sometimes purpuric at other times ervthematous, nodular, urticarial or bullous, is accompanied by sore throat, multiple arthritis, and slight fever. So constant is the association with rarious degrees of rheumatoid arthritis, that the purpura is regarded by certain observers as an expression of rheumatism due to the circulation in the blood of a rirus which is also responsible for the joint changes. This type of case is identical with that referred to as Erythema haemorrhagicum, under the heading of Erythema multiforme exudativum.

The differentiation of primary purpuras into these groups has led to great confusion and is inadvisable. Too much stress has been laid on clinical differences and associations and too little on pathogenesis. There is no pathological lesion, either cutaneous or otherwise, which is definitely characteristic of any of them and in the present state of our knowledge it would be better to discard them and to recognise that they are not distinct diseases, but evidences of a more or less virulent infective process, usually of a septic nature, of which the purpura is only a symptom.

\section{B. SECONDARY PURPURAS}

The secondary purpuras are those in which the purpura is due to some known cause or is a symptom in the course of some recognised pathological condition. These admit of an etiological classification as follows:

I. Acute specific fevers. - In measles petechiae may occur between the lesions of the exanthem, or the lesions themselves may become haemorrhagic and bleeding may take place from the mucous membranes.

In malignant scarlet feier the exanthem may assume a dark purplish colour owing to the presence of petechiae and ecchymoses.

Vaccination in rare instances may result in haemorrhagic lesions at the site of the inoculation or in the neighbourhood, probably due to malnutrition or to debilitating disease like rickets or scurry.

In malignant small-pox, petechiae on an erythematous base may be met with in the prodromal eruption or haemorrhages may take place into the resico-pustules.

In typhus feier the mulberry eruption is essentially purpuric and consists of dusky red mottling and erythematous red papules which become petechiae.

In cerebro-spinal moningitis petechiae are common and an extensive purpuric eruption may occur, hence the name Spotted feier.

2. Infective diseases. - A purpuric eruption may occur in connection with a large number of infective diseases such as praemia, septicaemia 
- especially cardiac septicaemia, (infective endocarditis)-malaria, general tuberculosis, congenital and acquired syphilis, anthrax, and influenza.

3. Diseased conditions of the viscera, such as disorders of the liver and kidneys, may cause purpura which is believed to be due to the circulation respectively of bile and urea in the blood and the association of the eruption with jaundice and albuminuria has been frequently noted.

Purpura has been observed also in connection with disease of the supra-renal capsules and cases of fatal purpura have been reported in which haemorrhages have taken place into the medulla of the gland (Little). It has been suggested that the purpura in those cases may be the result of the diminution of adrenalin in the blood which, ceasing to exert its action in maintaining the tone in the cardiac muscle and arteries, allows dilatation and diapedesis to take place. It is more probable that the cause responsible for the visceral haemorrhage is also the cause of the purpura, and cases of fatal supra-renal haemorrhage have been recorded in which there were no changes in the skin.

4. Diseased states of the blood or the blood-vessels may be associated with purpura, such as severe anaemia, leucocythaemia, Hodgkin's disease, rickets, scurvy, or haemophilia. In haemophilia, the haemorrhages result partly from the diseased condition of the blood and partly from congenital defects in the walls of the blood-vessels.

5. Cachectic states are not infrequently associated with purpura, especially of the lower extremities, such as advanced stages of Bright's disease and the final phases of general tuberculosis or cancer. Under this heading may also be placed : (a) the purpura of senile skin which is especially marked about the arms and is probably due to degenerative changes in the vessel walls; and (2) the purpura which is liable to occur from moving parts which have been in splints or plaster for a long time, and which results from an atrophic state of the vessels.

6. Malignant disease, such as widely disseminated sarcoma, may be associated with purpuric eruptions and haemorrhages from the mucous membranes or into the viscera.

7. Certain drugs may cause purpuric eruptions in those with an idiosyncrasy towards them, such as iodide of potassium even in small doses, copaiba, chloral hydrate, potassium chlorate, sandal wood oil, quinine, antipyrin, salicylates, phosphorus, ergot, mercury, and belladonna.

S. Injections of anti-bacterial sera may cause purpura, such as antidiphtheritic and anti-streptococcic sera. Previous injections produce a state of susceptibility or anaphylaxis to subsequent injections.

9. The bites of venomous snakes also cause purpuric eruptions.

Io. Nervous influences, both central and peripheral, may be responsible for purpura, and it may be met with in mental disorders, mania, fits of passion, fright, etc., probably througl congestion, in Locomotor ataxia where the purpura takes place at the site of the lightning pains, 
and in acute myelitis and peripheral neuritis where it is possibly due to vaso-motor disturbances-an explanation which is corroborated by the occasional occurrence of haemorrhages in Rarnaud's disease.

II. Obstruction to the circulation may cause purpura mechanically from the strain on the small ressels, especially when their nutrition is defective. This may be met with in organic disease of the heart, in conrulsions of epilepsy or hysteria, and in paroxysms of whoopingcough or spasmodic asthma. Local obstruction is a not infrequent cause, as is seen in connection with raricose reins and occasionally. with venous thrombosis or pregnancy:

I2. The lesions of certain skin diseases may become purpuric, such as the vesicles of Herpes zoster, or the erythematous or bullous lesions of Erythema multiforme. It is not uncommon also for urticarial lesions to become purpuric (Purpura urticans, or Liticaria petechialis) and factitious urticaria has been known to become haemorrhagic, especially on the lower extremities, and the errthematous or urticarial streak to become transformed in the course of a couple of hours into a raised haemorrhagic wheal (purpuric dermographism).

Pathogenesis.-The extravasation of blood in purpura may be slight and confined to the corium, or extensive and involve part or whole of the subcutaneous tissue. It may take place into the epidermis, forming a haemorrhagic blister, either at the site of the granular larver or deep down with the whole epidermis as a roof. Occasionally it may occur about the hair-follicles, sebaceous glands, or sweat-glands and may escape externally by the sweat-ducts, constituting the haematidrosis occasionally met with in haemophiliacs.

The extravasation consists of serous fluid, haemocrtes, and leucocrtes, and differs from that of an inflammatory oedematous condition by the presence of red blood-cells. After it takes place, a solution of the haemoglobin occurs which is partly re-absorbed through the bloodplasma and partly crystallised in the tissues. Sometimes the haemoglobin becomes converted into pigment granules which may be free in the tissues or may be taken up by the connective tissue cells and give to the skin the grerish-brown pigmentation which sometimes succeeds purpura.

The exact manner by which the extravasation takes place has given rise to much discussion and variance of opinion and even now is not fully understood.

There are two ways in which it may occur, namely, by a leakage in the walls of the smaller blood-ressels or by a diapedesis through the intact walls. In gross extravasations, such as haematomata, rupture of the vessels can generally be detected, but in the small petechiae the most careful search may fail to reveal it. Lnna and Sack did much careful research in this connection and assert that in almost every case of cutaneous bleeding a rupture of the ressel is present and that diapedesis only occurs in a few cases. Their investigations led them to the belief that the haemorrhages usually took place from the smaller reins at the junction of the corium and subcutaneous tissue. In that situation there is a horizontal network 
of somewhat delicate veins which are in a transitional stage between vessels with an external and middle coat, and capillaries with only an endothelial layer, and which are, at the same time, imperfectly supported as the fibrous stroma of the corium is replaced there by the more open network of the subcutaneous tissues.

That diapedesis may also be a factor has been proved experimentally by Prussak, who showed that an injection of sodium chloride solution into the veins of a frog caused diapedesis of haemocytes through the unbroken vessel-wall; an observation which was subsequently corroborated by Wickham Legg. Diapedesis has also been observed in the minute punctate haemorrhages which occur in venous stasis due to cupping of the skin.

Various suggestions have been put forward to explain the haemorrhages, such as that they are the result of hyalin degeneration of the blood-vessel walls, which is a condition met with in sclerodermia, of lardaceous degeneration, or of Endarteritis obliterans. But in hyalin degeneration the vessel-walls are thickened so that the haemorrhages would be prevented rather than encouraged, and in Endarteritis obliterans, anaemia rather than purpura would be liable to be produced.

Endophlebitis and thrombosis of veins have been blamed for the rupture and haemorrhage. Where the arteries and capillaries are unaffected and there is definite venous obstruction, it is conceivable that a haemorrhage might be produced; but in actual experience thrombosis of veins rarely causes haemorrhage but rather oedema through the intact vessel-walls, unless they are in a state of mal-nutrition as in old-standing cases of varicose veins in the legs when haemorrhage may occur.

Another explanation for the haemorrhage, and one which is of greater importance than those previously referred to, is that it is the result of the presence of micro-organisms in the blood. From time to time bacillary emboli have been seen in the cutaneous capillaries in purpura; bacterial colonies have been observed in them in infantile cases, and bacilli embedded in a gelatinous plug in Purpura haemorrhagica. The organisms most frequently detected have been streptococci, but in addition to these, staphylococci, pneumococci, Bacillus coli, Bacillus pyocyaneus, diplococci, etc., have been observed. (Fig. 317.)

So often have micro-organisms been demonstrated, not only in the blood but also in the cutaneous vessels themselves, as strongly to suggest that they are etiologically connected with the purpura. These microorganisms might act mechanically by forming emboli which blocked the capillaries, or they might exert some chemical or toxic action on the blood or the blood-vessel walis.

That cocci in large numbers may be found in the skin capillaries in septic lesions which are not haemorrhagic shows that mechanical plugging alone is insufficient to explain the haemorrhage. It is much more likely that it is due to some toxic action, possibly in the nature of a negative chemotaxis, which causes a diapedesis or an actual rupture of the vesselwall according to the quantity and degree of virulence of the toxin ; it is undecided, however, whether all these micro-organisms are capable of producing toxins or only a few of them.

In the present state of our knowledge it may be said that the most potent factor in the production of cutaneous haemorrhages is the circulation in the blood of toxins, bacterial, chemical, or autogenous, and that these probably act by directly weakening the vessel-walls and causing a discharge of blood through them by a process analogous to negative chemotaxis. Malnutrition from impoverished states of the blood, with deficiency in the haemoglobin and mineral salts, may 
result in such a feeble condition of the ressel-walls that the bloodpressure itself is sufficient to cause the rupture, especially when it is increased from any obstruction to the circulation.

Diagnosis.-There is usually no great difficulty in recognising purpura as it is distinguished from any form of erythematous lesion by not paling on pressure.

Petechiae have to be differentiated from the bites of pediculi and fleas but in them there is a central bleeding point surrounded by a congested halo, whereas in the petechiae the redness is diffuse all orer.

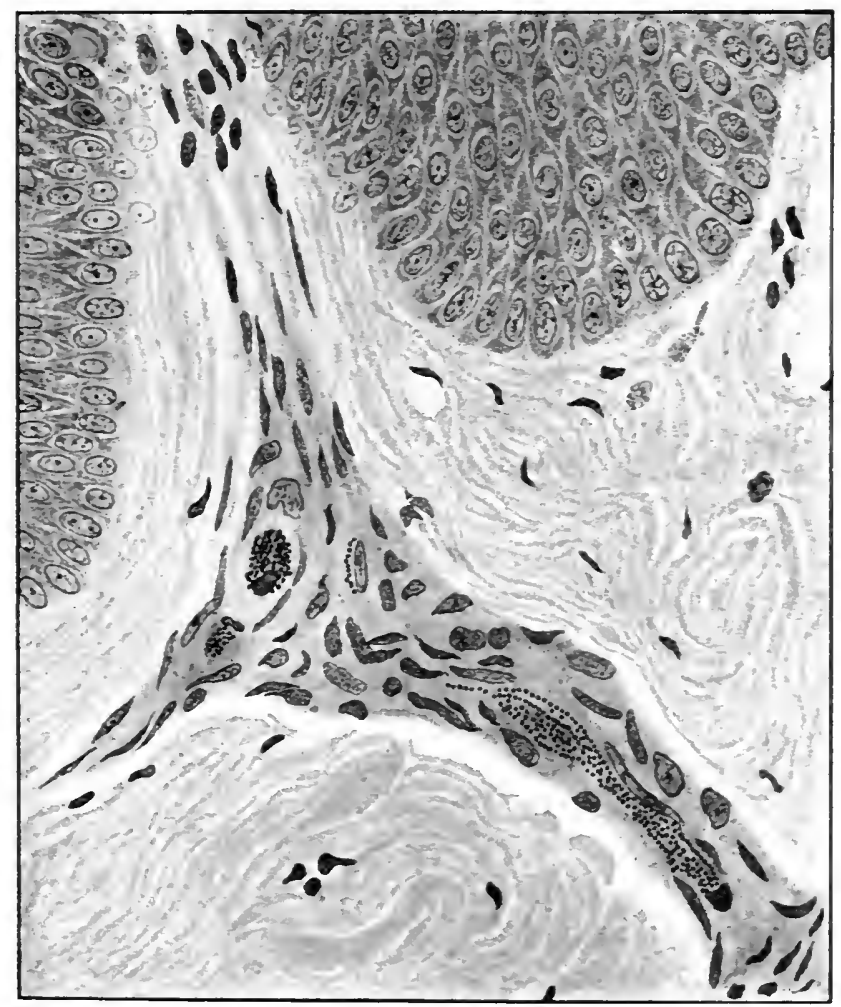

FrG. 3I7.-Purpura. Sub-papillary ressel with threads of cocci (probably Streptococci).

(After Dr. Graham Little. Brit. Journ. Derm., Igor.)

Prognosis.-The prognosis depends entirely on the cause, the actual cutaneous eruption being of no serious moment except as a symptom of the underlying pathological process. In ordinary septic purpura of a mild type, the so-called Purpura simplex, the majority of cases recover in a few weeks; on the other hand, even mild cases may assume serious characteristics with mucous or serous haemorrhages, bleeding into the viscera, and sometimes a fatal issue. In consequence it is advisable to give a guarded prognosis in every case, in view of possible developments such as internal haemorrhages or recurrences. 


\section{TREATMENT}

In order that the treatment may be rational it is necessary to dis cover the underlying cause. Every system of the body should be carefully studied, the blood and excreta examined, and any septic focus sought for, and if possible eliminated. When the source of infection has been discovered the treatment is that for its removal or counteraction on general medical or surgical principles.

As a rule it is advisable that the patient should be confined to bed and carefully nursed under good hygienic conditions, with plenty of fresh air and nourishing food. It is an advantage to elevate the legs if they are markedly affected, and to protect the skin with cottonwool under a bandage. Where there is evidence of gastro-intestinal disorder, a restricted diet with the avoidance of meat, careful regulation of the bowels, and intestinal antiseptics, such as mercury in the form of small doses of calomel, or salol, are indicated. If arthritic symptoms are pronounced, salicin or the salicylates should be prescribed. Where the coagulation time of the blood is prolonged, calcium, either in the form of the chloride or the lactate, 20 grs. t.d.s., for three or four days, may be given with benefit. With the object of improving the cardiac tone and causing vaso-constriction, adrenalin has been advocated either in doses of 5 to Io minims of $I$ in Iooo solution of adrenalin chloride by the mouth, or intravenously in doses of from 2 to 5 minims diluted with at least 20 volumes of physiological salt solution. Where an autogenous vaccine can be prepared it is worthy of a trial. Antistreptococcic sera have been recommended in septicaemic cases but should be used with great caution as they themselves are liable to produce purpura.

Where no definite cause can be ascertained, various empirical remedies have been adrocated, such as mineral acids, ergot, and turpentine. Of these, oil of turpentine is the most useful and possibly acts as an intestinal antiseptic ; it may be given in capsules containing Io or 20 minims t.d.s., and in the case of children can be combined with advantage with castor oil. In addition great benefit may be derived from tonic remedies, such as perchloride of iron, quinine, or small doses of arsenic.

\section{TELANGIECTASES}

The name telangiectasis (from the Greek $\tau \epsilon \lambda$ os end, "a $\gamma \gamma \epsilon \iota \nu$

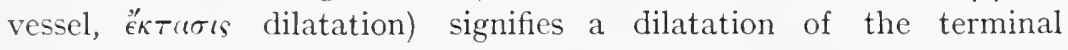
vessels either of congenital origin or acquired. It should include dilatations of lymphatics but is generally restricted to blood-vessels and will be employed in that sense in this chapter.

The congenital telangiectases have been referred to already under the heading of naevi, and here only the acquired telangiectases will be described, but it is difficult, and sometimes impossible, to distinguish 
between acquired telangiectases and those of congenital origin, since a congenital vascular anomaly may be unrecognised at birth and only. become apparent in after years.

For convenience of description the acquired telangiectases may be divided into the following groups.

(a) Primary or essential telangiectases.

(b) Secondary telangiectases, symptomatic of some cutaneous disease or internal disorder.

\section{Primari or Essential Telangiectases}

According to the type of individual lesion which predominates the primary telangiectases may be divided arbitrarily as follows :

I. Punctate lesions, single or multiple and arranged in clusters, rings, or gyrate figures.

2. Sinuous and branching lines.

3. Spider naevi with a central eleration and radiating branches.. ${ }^{1}$

4. Macules or plaques.

5. Large patches, which may be either uniform or broken up into a lacework enclosing areas of skin normal in colour, and occur frequently in the breasts, arms, and thighs, in women.

6. Raised papules or nodules, flat or rounded on the surface, varying in size from a millet-seed to a split-pea, rec to deep purple in colour, of a uniform tint, and looking like an extravasation of blood unless when examined by a lens when they are found to be made up of a dense network of fine capillaries. These lesions are most common in elderly people and have been named " senile angiomata" by Dubreuilh.

Telangiectases may be partially or completely obliterated by the pressure of a piece of glass, except in the case of the papular variety (Class 6), or where the deeper veins as well as the capillaries are dilated. The texture of the affected skin is usually unaltered but telangiectatic macules and plaques occasionally may be covered with a delicate scale, or in the hands and feet and other situations, such as the scrotum, an epithelial hypertrophy may occur with the production of a warty superstructure known as angio-keratoma. When they disappear, which they frequently do spontaneously, no trace may be left, but occasionally they are followed by atrophy and scarring.

The lesions may be single or multiple and may be present in large numbers. They may occur in any situation on the skin and have even been found on the scalp, but they are most common on the face. They may be distributed irregularly, or confined to one region, and are occasionally symmetrical. In some cases they are present not only on the skin but also in the mucosa of the lips, mouth, nose, and in rare instances in the fundus oculi.

${ }^{1}$ Note. - It is probable that the majority of the Spider naevi should be included in this group as in most cases they are acquired and may occur at any age; it is possible that some of them may be preceded by a congenital vascular defect. 
Etiology.-The causation of primary telangiectases is unknown. They seem to be more common in women than in men and to be an evidence of some anomalous condition of the terminal. blood-vessels on account of which they are liable to sudden dilatations which remain more or less permanent. In many cases heredity seems to play some part in their etiology, and they appear to be transmitted by both sexes and may occur in several members of one family.

Recurring epistaxis with multiple telangiectases of the skin and mucous membranes (Osler).- Under this heading Osler described an important group of cases occurring in families in which telangiectases appeared in middle life and were associated with more or less profuse epistaxis which had begun many years earlier. In these cases there was no evidence of haemophilia or diminution of the coagulability of the blood, but in certain of them there was a definite neurotic taint with vasomotor instability and a tendency to urticaria and dermographism, or evidences of a weak peripheral circulation. In three cases which have been described there were pre-existing vascular naevi and, in a case recorded by Hutchinson, they began soon after birth, so that they ought probably to be included under the heading of vascular naevi. In a case described by Crocker there was no suggestion of congenital origin, the patient being an adult woman in whom they appeared first at $2 \mathrm{I}$ years and were associated with mitral disease.

\section{SECONDARY TELANGIECTASES}

Symptomatic of certain Skin-affections.--Telangiectases occur as more or less important characteristics of certain skin-affections and result from local or general obstruction to the blood-flow, or from active congestion. They may form the most important feature, as in chronic X-ray dernuatitis, or may be one of several equally important characteristics, as in Lupus erythematosus, Xeroderma pigmentosum, and Adenoma sebaceum, or they may be an unimportant epi-phenomenon, as in rodent ulcer where they occur over the cartilaginous border, and in circumscribed sclerodermia or morphoea where they appear at the edges of the patches.

Symptomatic of circulatory Disturbances caused by Diseases of the Heart, Blood-vessels, and internal Viscera.-Telangiectases frequently occur where the blood tension is high and the resistance of the tissues is enfeebled through disease, as in cirrhosis of the liver. They may occur in connection with a feeble peripheral circulation and are often met with in women and children as a result of exposure to cold, when telangiectases are frequently present on the cheeks and ears or in the form of a patchy network or marbling on the legs or arms. They may accompany arterio-sclerosis and are very common in elderly people from this cause, while in younger people and children they may result from valvular disease of the heart badly compensated-especially from 
mitral stenosis-and in association with wasting and protracted diarrhoea. They have been observed as macules and patches on the face and over the thyroid gland in connection with the serious circulatory disturbance associated with exophthalmic goitre. Diseases of the lung causing an obstruction to the circulation, such as asthma, are liable to produce them. They so frequently occur with diseases of the iver, especially where gall-stones are present, that they are almost of diagnostic importance, and they are met with also in Bright's disease of the kidneys. In obese individuals a girdle of dilated venules may be observed on the chest on a level with the border of the diaphragm as a result of the feeble circulation, and the same condition has been noted in athletes from venous obstruction in the thorax. (Fig. 3I8.)

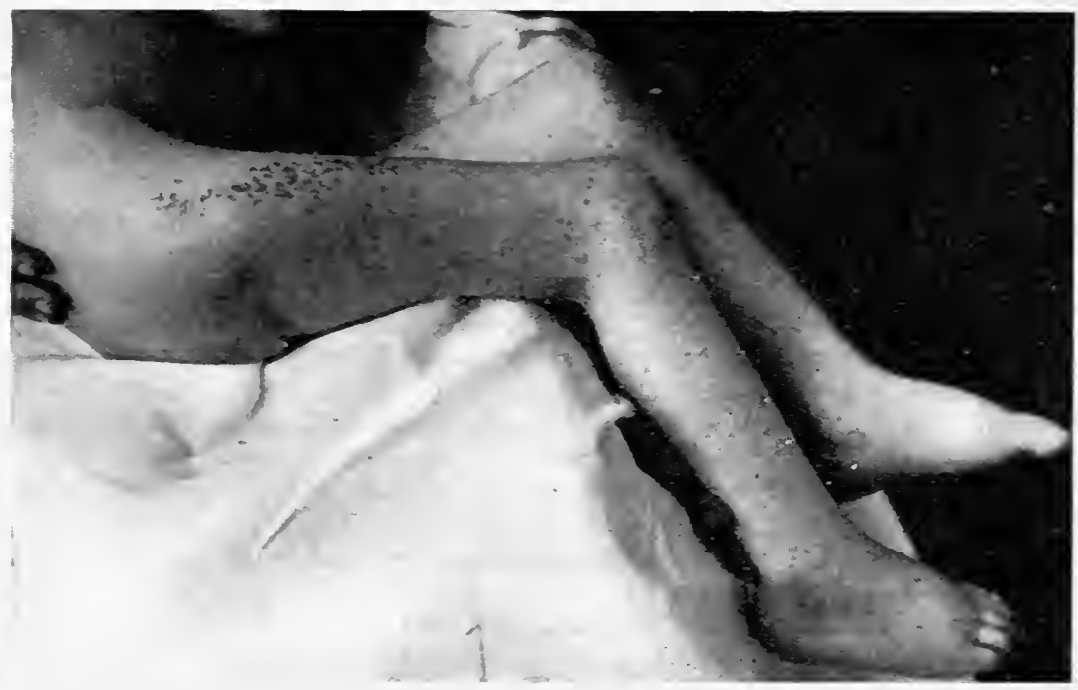

FIG. 3I8.-Telangiectases and Mottling.

Associated with wasting and protracted diarrhcea.

(Dr. E. G. Fearnsides' case. Brit. Journ. Derm., 19r2.)

Telangiectases frequently occur on the face in seamen, cab-drivers, motorists, etc., from constant exposure to inclement weather, severe cold, wind, and rain, and in stokers, bakers, cooks, etc., from excessive heat. They also result readily from flushing of the face produced by alcoholic excess or chronic indigestion.

\section{TREATMENT}

For the destruction of small telangiectases the best form of treatment is electrolysis in the manner described in connection with spider naevi, the negative pole needle being inserted into the most prominent dilatation while the positive pole is held in the hand and a current of two to three milliamperes is passed for about half a minute till frothing occurs. Larger lesions may be improved by being tattooed over with the negative pole. 
They may be dealt with also by a fine pointed cautery, by refrigeration with solid carbon dioxide, or by radium (vide treatment of portwine stains, p. I84).

When they are symptomatic of some internal disorder it is of little use treating them so long as the underlying cause remains.

For the dilated venules on the cheeks and nose in middle-aged people, careful electrolysis gives excellent results. If the case be extensive it should be done at several sittings. Any bleeding which may occur is easily controlled by pressure with a piece of cottonwool. The sites of the punctures should be painted with collodion, and the inflammation which results from the procedure may be reduced by the application of a calamine lotion. If the treatment be carefully done no scarring need be produced.

\section{REFERENCES}

\section{Purpura.}

Billings, J. S. Fatal Purpura haemorrhagica (Johns Hopkins Hosp. Bull., I894, v. p. 65).

Bruce, M., \& Galloway, J. Purpuric dermographism (Brit. Journ. Derm., I 898 , x. p. 6).

Cheyne, Watson. Micro-organisms in Idiopathic Purpura haemorrhagica (Trans. Path. Soc., London, I $88_{4}$, xxxv. p. 408).

$\mathrm{CoHs}$, E. Purpura haemorrhagica in Phthisis (Münch. Med. Wochensch., I90I, 1. p. 200I).

Copelli, M. Purpura annularis telangiectodes (Majocchi) (Giorn. Ital. d. Mal. Ven. e della Pelle, I9I5, p. 70).

CRUice, J. M. Purpura in the course of chronic pulmonary tuberculosis (Amer. Journ. Med. Sci., I912, cxliv. p. 875).

Don, A. Henoch's Purpura \& Angio-neurotic oedema (Pract., London, I $898, \operatorname{lxxx}$. p. 823).

Henoch. Lecture on Skin Diseases (New Syd. Soc., I889, ii. p. 369).

Herringham, W. P. Purpura in sarcoma, lymphadenoma, \& tubercle (St. Bart.'s Hosp. Rep., I902, p. II 7).

Hunter, William. Severest Anaemias (London, I909, Vol. I).

KLEIn. Staphylococcus haemorrhagicus (Brit. Med. Journ., I897, ii. p. $3^{85}$ ).

LEgG, Wicкнaм. Rheumatic purpura (St. Bart.'s Hosp. Rep., I883, xix. p. I77).

Little, Graham. Fatal Purpura with haemorrhage into the suprarenal capsule (Brit. Journ. Derm., I90I, xiii. p. 445).

Mackenzie, Stephen. The relationship of Purpura rheumatica to Erythema multiforme (Brit. Journ. Derm., I 896, viii. p. I I6).

Morton, A. Eruption following use of oil of sandalwood (Brit. Journ. Derm., 1 894 , vi. p. I 71).

Török, L. The Purpuras (Journ. des Mal. Cut. et Syph., April I9o3).

Unna, P. G. Haemorrlages (Histopath., Walker's Trans., i 896, p. 44).

Weber, F. PARkes. Chronic purpura of two years' duration connected with malignant endocarditis (Brit. Journ. Derm., I9Io, xxii. p. 35).

\section{Telangiectases.}

Fearnsides, E. G. Telangiectases in children, in association with wasting and protracted diarrhoea (Brit. Journ. Derm., 1912, xxiv. p. 35).

Fox, Colcott. Bilateral telangiectases of the trunk (Ibid., I908, $\mathbf{x x}$. p. 145$)$. 
Gjessisg, E. Telangiectasia hereditaria haemorrhagica (Osler) (Hospitalstidende, Nov. Ioth, I9I5).

Hutchixsox \& OlIver. Multiple telangiectases with epistaxis of the familial type (Quart. Journ. of Med., Jan. I9I6).

KELLY, A. Brow:. Multiple telangiectases of the shin \& mucous membranes of the nose and mouth (Glasgow Med. Journ., I 906, F. 4II). Majocchi, D. Telangiectasia follicularis annulata (Arch. f. Derm. u. Syph., I 898$)$.

Osler, W. Multiple telangiectases (Johns Hopkins Hosp. Bull., I90I, p. 333).

Weber, F. PARkes. Multiple hereditary developmental angiomata of the skin \& mucous membranes associated with recurring haemorrhages (Lancet, July 2oth, 1907). 


\section{CHAPTER XXXII}

\section{ANOMALIES OF PIGMENTATION}

Pigmextatios of the skin, either diffuse or in patches, may result from a large number of different causes which may be grouped under the following three headings :

(I) Pigmentation due to blood-pigments.

(2) Pigmentation due to melanin.

(3) Pigmentation due to foreign substances deposited in the skin.

\section{PIGMENTATION DUE TO BLOOD-PIGMENTS}

Pigmentation of the skin due to haematogenous pigment presents the various tints ranging from livid purple to greenish-brown which are familiar in connection with a bruise. It may take the form of patches, irregular mottling, or clusters of small puncta, and is met with in connection with any haemorrhage into the skin, such as in purpura, ecchymosis, etc., in association with severe vaso-motor disturbances such as Raynaud's disease, and in conditions of venous stasis such as occur in connection with varicose veins. It is the result of the deposition in the skin of the blood-pigments, haematoidin and haematin, which are derivatives of haemoglobin. The blood on extravasating forms a clot in the skin with a deposit of yellowish-red granules, crystals and plates of haematoidin in the centre, and amorphous ironcontaining granules of haematin at the edges.

\section{PIGMENTATION DUE TO MELANIN (MELANOSES, MELANODERMIA, MELASMA)}

The majority: of cases of pigmentation are due to an excessive formation of melanin, which may be an acquired or congenital condition.

\section{A. Congenital Melanoses}

The pigmentation which occurs in all forms of pigmented naevi (see page 193) is due to an increase of melanin, chiefly in the cells of the 
basal layer of the epidermis and in the prickle-cells immediately above it. The presence of the pigment in the epidermal cslls causes them to lose their fibrillated structure to some extent, and sets up a state of instability as a result of which they are particularly liable to proliferate rapidly and to assume malignant characteristics when irritated.

The pigmented lesions as a rule are noticeable at birth and persist throughout life, but they may appear first in early childhood or eren in adult life.

They may be raised, smooth, warty, or hairy, or may take the form of pigmented macules which may be irregularly distributed or grouped, or of patches level with the surface of the skin, which rary in tint from fawn to dark brown and may be diffuse and irregular or broken up and mottled.

Occasionally they have a zoniform or segmental arrangement. In a case in a girl under the care of the writer congenital freckle-like patches of pigmentation, about the size of lentils, were distributed on the left side of the trunk from the axilla to the buttock; in a case recorded by Adamson in a woman large patches occupied alternate segments on the two sides of the body, producing a harlequin appearance. In another case the pigmentation had a retiform arrangement in the meshes of which were small atrophic scars.

Other congenital anomalies have been described in association with the pigmented lesions, such as congenital alopecia, supernumerary nipples, vascular naeri, and multiple fibromata as in von Recklinghausen's disease.

In this group may be placed provisionally the pigmented patches known as Mongolian spots or Mongolian birthmark. This curious pigmentation has been observed chiefly in children in Japan and in various countries in the East but has been known to occur in Europeans. It consists of small patches of a darkish blue tint, which may be single or multiple, are round, oval, or irregular in shape, and vary in size from $\frac{1}{4}$ to $\mathrm{I}$ inch or more in diameter. They are generally. situated in the sacral regions but occasionally may be met with in other parts of the body ; they are noticeable at birth but are transient and usually have disappeared by about the third year.

\section{B. Acouired Melayoses}

(I) Pigmentation from Physical Agents.-(a) Actinic Ray's of Light.-Sunburn and freckles are melanoses which result from exposure to the actinic rays of sunlight.

In Neroderma pigmentosum (see page 252) there is a peculiar sensitiveness of the skin to the actinic ravs, probably of congenital origin, on account of which freckles are readily called forth on the exposed parts from the slightest exposure to sunlight. In it the freckles are associated with telangiectases, atrophic macules, and warty. growths which become malignant. 
A somewhat analogous condition occurs in the so-called "senile skin " of elderly people in which there is an increased sensitiveness to sunlight and a marked tendency to the development of freckles, telangiectases, and warty growths on the exposed parts.

(b) X-rays.-Exposure of the skin to the X-rays tends to produce melanosis. This may occur after repeated small doses or may follow X-ray dermatitis.

(c) Extremes of Temperature.-Constant exposure to extreme heat is liable to produce melanodermia in the form of macules, patches, or retiform mottling. To the pigmented macules so produced the name ephilides $a b$ igne has been applied. The reticular mottling is noticeable chiefly on the legs from the habit of toasting them in front of the fire, the reticulation being determined by the vascular network; this condition is preceded by the erythematous mottling known as erythema ab igne.

Pigmentation may result also from repeated exposure to extreme cold.

(d) Local Irritation from Pressure or Friction.-This type of pigmentation was once named Chloasma traumaticum. ${ }^{1}$ As examples of it may be cited the pigmentation met with in certain individuals from the pressure of garters, corsets, etc., or that which follows rubbing and scratching for the relief of itching in pruriginous affections such as pediculosis. In these cases the pigmentation is usually confined to the area of irritation, but occasionally may extend beyond it.

(e) Local Irritation caused by Inflammatory Agents.-This is commonly observed after the application of a mustard plaster or blister.

(2) Pigmentation due to the Ingestion of Toxins. - The most familiar example of a melanodermia resulting from the ingestion of a toxin is that caused by arsenic. As a result of the administration of arsenic the skin may become pigmented over the whole surface or in situations normally pigmented, such as the axillae, groins, neck, areolae of the nipples, etc., or in parts subjected to pressure or friction. The pigmentation may be diffuse but is more often broken up by areas of normal white skin and presents a mottled appearance. It varies in tint from fawn to deep brown and is usually associated with other symptoms of chronic arsenical poisoning, such as a harsh desquamating skin and keratosis of the palms and soles.

When pigmentation is found in connection with chronic cutaneous affections, such as Lichen planus and Dermatitis herpetiformis, arsenic should be suspected, as it is frequently prescribed in these conditions and is liable to produce deep pigmentation at the sites of the inflammatory lesions. This is not the result of a deposit of arsenic in the skin but is due to an amorphous iron-free pigment which is most probably melanin.

1 The term "chloasma" (Greek $\chi \lambda$ od j' $\omega$, to be pale green) is an old name applied to excessive pigmentation of the skin either diffuse or in patches and due to a variety of causes, hence such terms as Chloasma traumaticum, Chloasma uterinum, Chloasma caloricum. 
(3) Pigmentation associated with certain General Diseases or with Disorders of Internal Organs.-In Addison's disease melanodermia is a marked symptom. In it the pigmentation is diffuse and present chiefly in situations normally pigmented, such as the flexures of the larger joints, the areolae of the nipples, the axillae, and about the genitals, and in parts exposed to friction or pressure. It generally involves the mucous membranes of the mouth, conjunctiva, etc., and an increased pigmentation has also been described in the hair. It is due to melanin situated in the deeper parts of the epidermis, the excessive production of which is in some way connected with the defective functioning of the supra-renal glands.

Exophthalmic goitre may be characterised by more or less marked pigmentation which is sometimes so intense as to simulate that of Addison's disease. In rare instances instead of pigmentation there may be defect of pigment, or leucodermia.

In diabetes a deep bronzing of the skin is occasionally present, to which the name "diabète bronzé" has been applied, and is probably. due to the same cause as that responsible for the glycosuria. Certain observers describe it as a haemochromatosis and regard the pigment as a blood-pigment, while others believe it to be melanin. The wide distribution of the pigmentation somewhat recalls that produced by the deposition of silver in the skin in argyria, but in the latter condition it has a peculiar slaty appearance and usually involves the mucous membranes as well as the skin.

Melanodermia, either patchy or diffuse, and usually most marked in the situations normally pigmented, may be met with in a considerable number of general disturbances, such as malaria, severe forms of anaemia, cirrhosis of the liver, tuberculosis of the abdomen, the later stages of carcinoma of the abdomen, disease of the pancreas, chronic constipation, kala-azar, etc.

Various disorders of the genital organs in women are liable to be associated with pigmentation. In pregnancy, it occurs about the nipples, in a line in the centre of the abdomen, and on the face, especially about the eyes, forehead, and temples. In menstrual disorders the normal pigmentation may be increased, especially about the eyes in dark subjects, and be most marked at each menstrual period.

The exact manner in which this pigmentation is produced is unknown and certain writers regard it as toxic in origin while others attribute it to a reflex nerve disturbance.

(4) Pigmentation in Connection with Skin Affections.-In various forms of chronic dermatitis melanin may be deposited in the lesions, such as in Lichen planus, eczema, psoriasis, Dermatitis herpetiformis, Pityriasis rubra, etc., and the pigmentation may persist for a considerable time after the inflammatory lesions have disappeared. Where it is dark in tint the possibility of its being due to arsenic must be considered. 
In the rare affection known as Acanthosis nigricans patches of deep pigmentation occur about the axillae, groins, gluteal fold, etc., on which warty growths develop subsequently.

Pigmentation is frequently observed in connection with cutaneous syphilides, the most familiar example of which is the so-called " Leucoderma syphiliticum " or café-au-lait mottling which is so commonly met with during the secondary period of the disease about the neck and upper part of the thorax, especially in women. In this condition the pale areas indicate the sites of previous roseolar lesions which have been present in a situation normally pigmented.

More or less deeply pigmented patches generally associated with tissue-paper-like scarring usually follow the healing of superficial tertiary syphilitic ulcerations.

(5) Pigmentation in Cutaneous New-Growths.-A deposition of melanin occurs in pigmented naevi, naevo-carcinomata, and melanotic sarcomata, giving them a brown or black colour.

\section{PIGMENTATION DUE TO A DEPOSIT OF EXTRANEOUS SUBSTANCES}

Of these the most striking is that known as argyria in which there is a deposition in the skin of fine particles of albuminate of silver. The pigmentation is of a peculiar slaty-grey tint, is widely distributed and may involve the whole cutaneous surface with the exception of the palms and soles. It is usually present also in the mucous membranes and in the internal organs. It was at one time more frequent than it is now, from the custom of administering silver nitrate in large doses in the treatment of epilepsy. The metal may reach the skin by dissemination through the blood or it may gain entrance locally where the skin or mucous membrane has been cauterised by a nitrate of silver pencil.

Under the heading of extraneous pigmentations may be placed the so-called Maculae caeruleae or bluish-grey spots about the size of a pea, which occur about the abdomen, inside of the thighs, etc., from the bites of the Pediculus pubis. These were once thought to be due to blood-pigment but are now believed to be the result of a pigmented substance which the parasite secretes into the skin whilst fceding. The maculac are especially well-marked in individuals with a fair skin.

Diagnosis.-There is no difficulty in recognising pigmentation of the skin due to blood-pigment, owing to its characteristic range of colour.

Occasionally bluish or black staining may be met with about the feet from the action of dye from cheap stockings, especially in indiriduals with moist feet as the dye is set free by the action of the alkaline sweat. Staining of the skin is also sometimes practised by hysterical individuals for purposes of malingering. In both these conditions the 

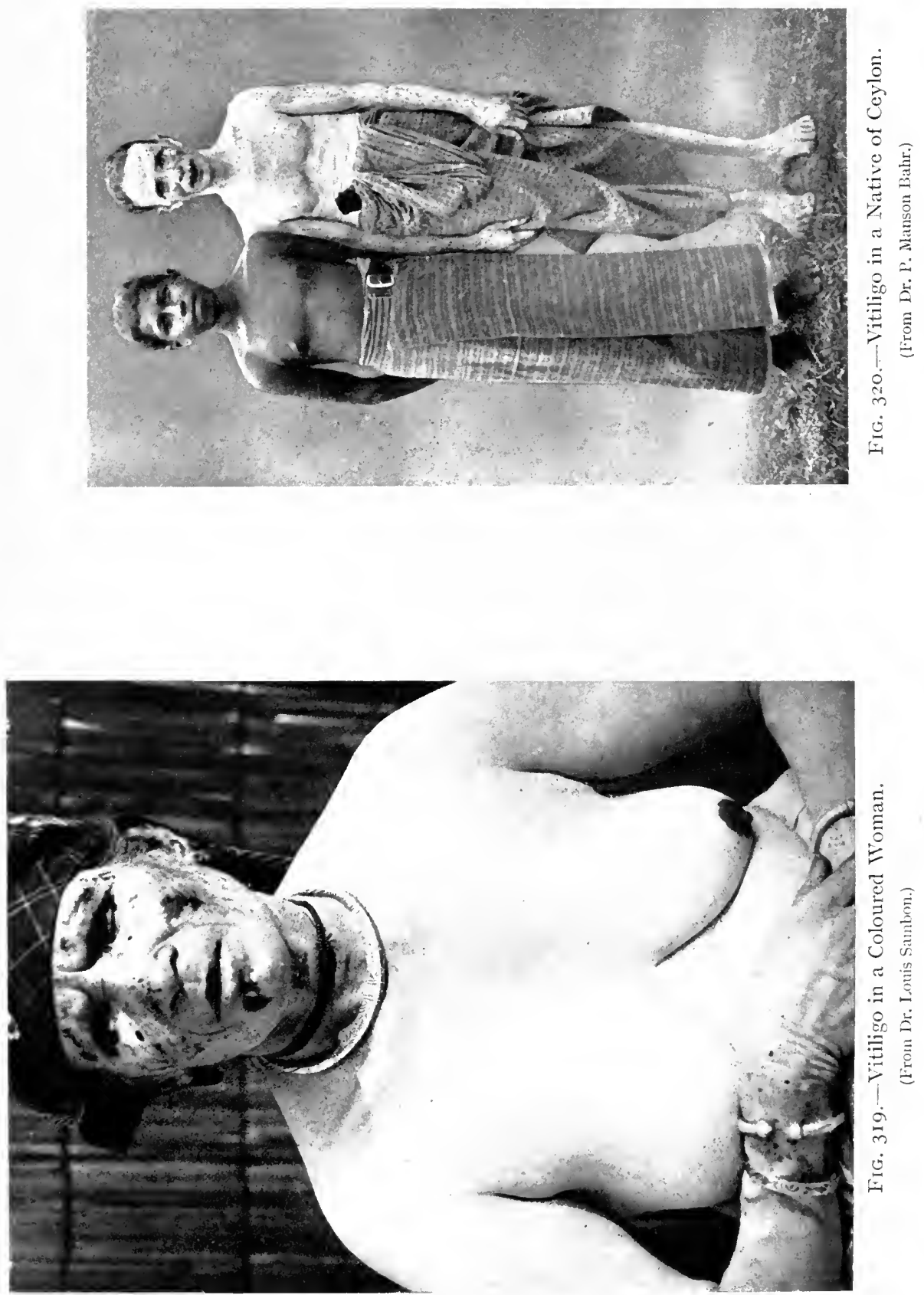

[To face $p .953$. 
staining is purely superficial, and can be remored easily by alcohol, soap and water, etc.

Pigmentation may occur from coloured sweating or chromidrosis, but this does not suggest blood-pigment.

Pigmentation due to melanin has to be distinguished from the fawncoloured staining of the skin in Tinea versicolor, and from the brownish tint in erythrasma. But in them the causal fungi can be detected easily on microscopical examination, while the skin itself is altered in texture and finely scaly.

Treatment.-Pigmentation symptomatic of some general disease as a rule disappears if the disease be cured, but unfortunately in so many of the conditions in which it occurs the disease responsible for it is progressive and incurable.

The reduction of pigmentation has been described in the treatment of freckles and sunburn.

\section{Progressive Piguentary Disease (Schamberg)}

Under this heading Schamberg described a peculiar pigmentary affection of the skin, in which the affected parts had the appearance of being dusted over with cayenne pepper (see p. I79).

\section{REFERENCES}

ADACHI. Mongolian Birthmark (Anat. Anz., Jena, I903, xxii. p. 323).

ADAmsox, H. G. Some remarks upon zoniform or segmental naeri (Brit. Journ. Derm., I9I4, xxvi. p. 379).

Crocker, Radcliffe. Pigmentation in Pitỵiasis rubra (Ibid., I896, viii. p. +4 ).

Grim, F. The pigment in the shin (Derm. Zeitsch., Bd. ii., Hft. iv., Berlin, I 895).

- MacLeod, J. M. H. Pigmented naeri-like freckles (Brit. Journ. Derm., I9I3, XXV. p. I9I).

SchamberG, J.F. A peculiar progressive pigmentary disease of the skin (Ibid., I901, xiii. p. I).

\section{LEUCODERMA}

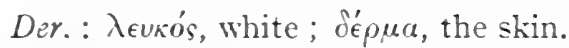

Syn.: Vitiligo; Achromia; Piebald shin.

Definition: An acquired affection of the skin characterised by white patches surrounded by pigmentation.

Description.-In the past leucoderma has been confused with leprosy, and "the leper whiter than snow" of the Old Testament was probably not leprosy but vitiligo.

In this country it is rare and only forms about $0 . \mathrm{I}_{0}^{\circ}$ of the diseases of the skin, but it is more common in natives of tropical countries and cases have occurred in which they have become almost entirely white in consequence of it. (Figs. 3I9, 320.) 
It is a disorder of pigmentation in which variously sized patches of an ivory-white tint are present on a pigmented background, giving rise to a piebald appearance. The white patches are oval or round in shape and tend to increase gradually at the periphery and coalesce, forming irregular areas with a well-defined polycyclical border.

Around the white patches the skin is pigmented a fawn or brownish tinge which is deepest in colour near the patch and fades gradually into the surrounding skin. Certain observers consider that there is definite hyper-pigmentation and that the white patches develop on a

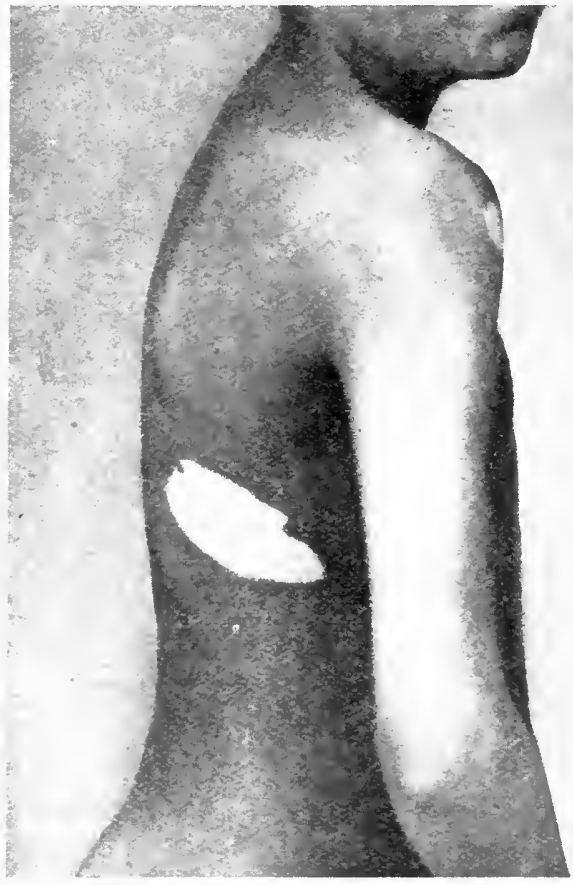

FIG. 32I.-Leucoderma and Melanoderma in a White Skin.

(From Dr. E. F. Skinner.) diffusely pigmented area; others insist that the pale areas are the essential feature of the disease and that the pigmentation is not increased but only appears so from the contrast produced by the de-pigmented patches. (Fig. 32I.)

The white skin is not altered in texture, nor is the sweat-function interfered with, but the hairs may be whitened.

It varies greatly in distribution. In some cases it is limited to small areas, in others it affects whole regions in a more or less symmetrical manner, while in others it may be so extensive as to involve practically the whole cutaneous surface including the scalp, leaving only.here and there a few irregular brownish islands. When the scalp is affected the hairs become white and in a case under the care of the writer in which the side of the face was attacked the eyebrows and eyelashes became. white.

The de-pigmented skin is hyper-sensitive to the actinic rays of sunlight and in summer it generally becomes pink and irritable and the pigmentation in the surrounding skin is increased.

In the majority of cases there are no subjective symptoms, though occasionally there may be more or less pronounced itching and peculiar sensations, such as tingling, have been known to precede the appearance of a white patch. The general health is not impaired, but where large areas are involved so much irritation may be caused by exposure to sunlight that it is said to produce giddiness. 
Under the title of Melung, Ziemann has described a form of leucoderma which is common on the palms and soles of natives of West Africa and has been observed also in India, Burma, and Ceylon. It consists of whitish areas of de-pigmentation with a marbled appearance and is usually symmetrical.

Etiology and Pathology.-The exact cause of leucoderma is unknown. The majority of observers believe it to be a neurotic condition or a tropho-neurosis and in support of this contention point out that it is most common in indiriduals with a neurotic taint, and that it is liable to occur in association with a number of conditions which are probably of nervous origin, such as Alopecia areata, migraine, and exophthalmic goitre. Others believe it to be of toxic origin and suggest that it is due to some toxin circulating in the cutaneous bloodvessels which inhibits the formation of pigment in the epidermal cells. In support of this view, a small round-celled infiltration such as might be called forth by the action of a toxin has been observed in the corium around the blood-ressels.

It is usually stated to be equally common in women and men but experience shows a distinct preponderance of cases in women. It does not usually appear till after the age of three, and rarely begins after thirty. It has been known to occur in families.

It is more frequently met with in private than in hospital practice, but this may be due to individuals of the hospital class suffering from mild degrees of it without troubling to seek advice.

On microscopic section no definite changes have been detected in the epidermis in the white areas except the complete absence of pigment, which is lacking also in the affected hairs. In the pigmented skin there is an increase of pigment in the epidermis and in certain pigmentcontaining cells in the connective tissue deposited around the bloodvessels and about the hair-follicles. Leloir has reported changes in the nerves supplying the part, such as parenchymatous neuritis in which the axis cylinder disappears and the myelin sheath breaks dorm.

Diagnosis.-As a rule the recognition of ritiligo presents no difficulty. Certain cases bear a slight resemblance to morphoea, but in it the texture of the skin is definitely altered and the lesions are either waxy and shiny or atrophic and wrinkled. In rare instances confusion might arise with patches of maculo-anaesthetic leprosy, but in leprosy there is anaesthesia and thickening of the nerve trunk supplying the affected area.

In tropical countries it has to be distinguished from certain der matomycoses, such as pinta and Pityriasis versicolor, but in them the diagnosis can usually be established by the demonstration of the fungus and the presence of slight scaliness.

Prognosis.- Titiligo is steadily progressive and incurable. Cases of apparent spontaneous recovery are either due to an extension of the de-pigmentation till whole regions are involved, or to a fading of the surrounding pigmentation and consequent reduction of the contrast. 


\section{TREATMENT}

General treatment does not appear to have any influence on the progress of the affection. On the supposition that it is a trophoneurosis a tonic regime has been prescribed but with no benefit to the local condition; while more specific measures, such as the administration of arsenic or of suprarenal gland extract to try and produce pigmentation, have been equally unsuccessful.

A diminution of the disfigurement has been attempted by various local measures, such as painting the white areas with walnut juice, weak permanganate solution, or silver nitrate lotion, or tattooing the skin with certain pigments, such as yellow or red ochre, or bleaching the pigmented patches with hydrogen peroxide.

Exposures to the X-ray have been recommended and benefit has been recorded from them, but this does not seem to be a rational treatment as the rays would be liable to increase the surrounding pigmentation and would be unlikely to stimulate the formation of pigment in the pale areas any more than actinic rays.

\section{REFERENCES}

Heidingsfeld. Vitiligo: a new instrument for its treatment, etc. (Derm. Centralblatt, Nov. I908, p. 34).

LELoIr. Pathology of vitiligo (Recherches sur les affections cutanées d'origine nerveuse, Paris, I $88 \mathrm{I}$ ).

MacLeod, J. M. H. Vitiligo (Brit. Journ. Derm., I909, xviii. p. 25I).

\section{ALBINISM}

Syn. : Congenital achromia.

Definition: By albinism is meant a congenital absence of pigment from the skin, hair, and eyes.

Description.-In albinos, owing to the absence of pigment, the affected skin becomes white, or pinkish from the blood-vessels shining through the translucent epidermis, the hair becomes white and silky, and the eyes pink from want of pigment in the iris and choroid. The albinism may be complete or partial; in the latter case it may be irregularly distributed, roughly symmetrical, or zoniform in arrangement like a segmental naevus. In the affected skin the lack of protective pigment produces an abnormal sensitiveness to light, with consequent inflammation from the slightest exposure to the sun's rays, while in the uveal tract of the eye it leads to marked photophobia associated with nystagmus and nictitation. Albinos are usually delicate, sometimes mentally deficient, and are said to be prone to tuberculosis.

The affection is congenital, occasionally occurs in families, and in rare instances has been known to be hereditary. As in vitiligo it is more common in the dark skins of natives in the tropics than in fair skins.

There is no treatment which is beneficial. 


\section{TATTOOING}

Definition: Permanent marking of the skin produced by rubbing in pigments after puncturing the skin.

Tattooing originated among savage warriors in the custom of painting the face before entering battle, but this form of adornment was temporary, as the pigment could be rubbed off, and the painting had to be repeated as occasion demanded. A more permanent method was adopted by the Polynesians and the Maoris of New Zealand who first lacerated the skin by some cutting instrument and then rubbed in either charcoal or a vegetable pigment and in this way produced hypertrophic scars in complicated curves and patterns.

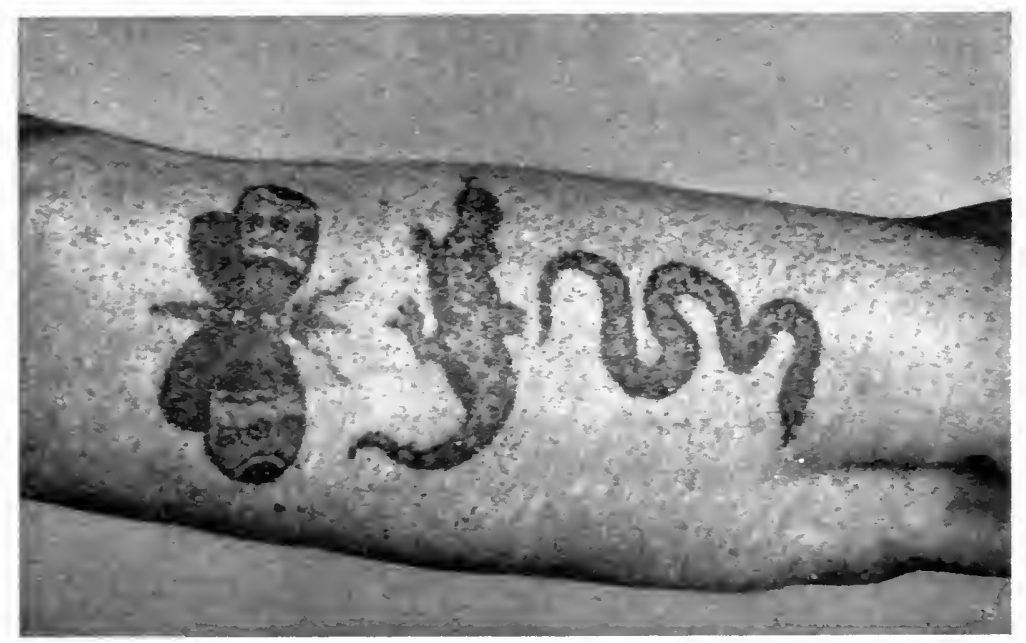

Fig. 322.-Tattooed Arm.

Tattooing has been brought to its greatest perfection among the Japanese but is practised all over the world. The chief pigments employed are indigo, vermilion, and Indian ink, which are inserted into the skin by means of more or less complicated instruments with a varying number of needles attached, by means of which a skilled operator can produce most realistic designs which are sometimes even artistic. (Fig. 322.)

Though somewhat unpleasant the actual procedure is attended with no risks, but in a certain number of cases some rirus has been inoculated accidentally during the needling process. Of these, the most important are syphilis and tuberculosis and cases are on record where the primary lesion of syphilis or a patch of Lupus vulgaris has occurred on a tattoo mark, having resulted, most probably, from moistening the part with infected saliva before the skin was punctured or from sucking the wound to stop bleeding. Occasionally a hypertrophic scar or a warty growth may be produced from the inoculation of some micro-organism. 
Tattooing of the skin may also be caused accidentally, as in cases where blue spots have appeared on the skin at the sites of hypodermic injections of morphia. These spots may be due to carbon particles introduced under the skin by the needle from the habit of disinfecting it in the flame of a wooden match, as in a case under Dr. Francis Hare ; or from iron particles derived from the needle as in a case described by Dr. F. Parkes Weber.

\section{TREATMENT}

In this country tattooing is usually indulged in in youth and regretted in maturer years when it not infrequently happens, especially in women, that there is a strong desire to get rid of the disfigurement. Unfortunately the removal of tattoo marks, unless where they are small, is unsatisfactory and usually impossible except at the expense of a disfiguring scar.

When the marks are small a neat excision is the best method of treatment; when somewhat larger shaving off the affected skin and Tiersch grafting may give a satisfactory result.

Exposures to Finsen light and freezing with carbon dioxide snow, have been suggested, but experience of both has been disappointing. In spite of repeated exposures to a Finsen or a mercury vapour lamp, each exposure being followed by a reaction, the complete obliteration of the pigmentation was not effected; while with carbon dioxide snow, unless the freezing was so deep as to be followed by a disfiguring cicatrix, no benefit resulted.

Various chemical means have been suggested for their removal, such as tattooing with a $30 \%$ solution of chloride of zinc, but the results from them have been equally unsatisfactory.

\section{REFERENCES}

Brault, M. J. Removal of tattoo marks (Ann. de Derm. et de Syph., Jan. I 895).

Dubreuilh. Removal of tattoo marks (Ibid., June r9o9, p. 367).

Heidingsfeld. A new instrument for the removal of tattoo marks (Derm. Centralblatt, Nov. 1908, p. 34).

Holland, W. Tattooing (Arch. f. Derm. u. Syph., I9I I, cx. p. 393).

VARIot. Tattoo marks (Brit. Journ. Derm., I 889, i. p. 319).

WE EBER, F. PARKes. Blue tattooing of the skin from hypodermic injections of morphine (Brit. Journ. Derm., I915, xxvii. p. 287). 


\section{CHAPTER XXXIII}

\section{ATROPHY OF THE SKIN}

ATROPHy of the skin may result from a large number of different causes of which the most important are pressure, imperfect nutrition due to some prolonged illness, interference with the nerve supply to the part, pre-existing inflammatory lesions of the skin, and senile degenerative changes. In addition, cases of primary cutaneous atrophy, both macular and diffuse, of unknown causation, have been recorded under the provisional heading of idiopathic cutaneous atrophies.

The atrophic skin may be thin, smooth and glossy, or wrinkled like crushed tissue-paper, or finely scaly, and may be white or greyish in tint, or dusky red or bluish from the shining through of the underlying capillaries. Owing to the more or less complete disappearance of the subcutaneous fat it is freely movable on the underlying tissue, while from the absence of elastic tissue it loses its resilience so that if pinched up between the fingers the folds tend to remain. This type of lax skin is sometimes known as anetodermia (Gr. "'

The hair in the affected area may become white and atrophied, or may completely disappear from the shrivelling of the pilo-sebaceous follicles; in some instances the sweat-glands may be involved and their function arrested.

For purposes of description the cutaneous atrophies may be grouped and considered under the following headings :

(I) Atrophy associated with general illness.

(2) Atrophy due to interference with the nerve supply.

(3) Atrophy associated with certain cutaneous diseases.

(4) Atrophy from pressure and traction.

(5) Congenital atrophy.

(6) Senile atrophy or Atrophodermia biotriptica.

(7) Idiopathic or primary atrophy.

(8) Kraurosis vulvae.

(r) Atrophy associated with General Illness.-As a result of interference with the nutrition of the skin, atrophic changes may be met

Note-Atrophy affecting especially the hair and nails will be described under diseases of the hair and nails. 
with in association with any prolonged illness, such as typhoid fever, pneumonia, cancer, tuberculosis of the lungs, malaria, etc.

(2) Atrophy due to interference with the Nerve Supply (Atrophodermia neuritica).--When a nerve is interfered with by injury or disease, the skin in the area supplied by it tends to become atrophic; this is seen in connection with paralysis, Tabes dorsalis, and localised nerve disturbances such as occur in leprosy.

(3) Atrophy associated with certain Cutaneous Diseases.--Atrophy may occur as a final stage in a considerable number of skin affections, such as Lupus erythematosus, morphoea, sclerodermia, Pityriasis rubra, Epidermolysis bullosa, etc., and atrophic scars usually follow superficial ulcerative lesions such as those of tuberculosis, syphilis, and leprosy, and suppurative follicular lesions such as furuncles and Acne necrotica.

(4) Atrophy from Pressure and Traction.-Continuous pressure, either external or internal, may be responsible for atrophy of the skin.

As examples of atrophy due to external pressure may be cited the atrophic patches which result from the pressure of a truss or of a plaster of Paris dressing, while the atrophic shiny skin of the abdominal wall which is met with in connection with abdominal tumours, such as sarcoma or fibroma, is an instance of atrophy due to internal pressure.

Internal pressure may lead, not only to atrophy, but also to stretching of the skin and the formation of the so-called Striae atrophicae, a familiar example of which is the Striae gravidarum or atrophic lines which occur in the abdominal wall as a result of the stretching of the shin in pregnancy. These lines have a greyish or mother-ofpearl appearance, are smooth and shiny on the surface, several inches long and about a quarter of an inch wide, and are usually situated in the long axis of the trunk parallel to each other and at right angles to the direction of the greatest tension. Similar lines may occur in the abdominal wall in ascites and in the female breast from lactation.

Striae have been described also about the buttocks and upper parts of the thighs, and in parallel lines over the elbows and knees (Striae patellares) in connection with severe illnesses such as typhoid, scarlet fever, and nephritis.

These striae are all produced mechanically by the stretching of the skin and by the rupture of the elastic tissue which acts as a skeleton holding the various parts of the corium together and which, when ruptured, allows a stretching of the white fibrous tissue and overlying epidermis to form a glossy streak. This is especially apt to occur where the skin is in a state of malnutrition through some prolonged general illness.

(5) Congenital Atrophy.-Under this heading certain cases have been reported in which the atrophy was present at birth or was noticed soon after. These are instances of defective development rather than of atrophy. 
Both diffuse and circumscribed congenital atrophy have been recorded. To the diffuse type Charcot gave the name of "Géromorphisme cutané " and a case of it has been described in an infant by Behrend in which the atrophy was associated with onycho-gryphosis. Circumscribed congenital atrophy has been recorded in the scalp in the form of bald atrophic patches noticed soon after birth, but it is possible that such cases are simply Alopecia areata.

(6) Senile Atrophy (or Atrophodermia biotriptica).-By senile atrophy from biotripsis, or "life wear," is meant the familiar wrinkled or glossy skin met with in old age. In it the skin becomes thin and lax, smooth from the absence of hair-follicles, or finely scaly, usually pigmented in patches or in freckle-like macules, and presents not infrequently small angiomata or capillary dilatations. This type of skin is liable to be the site of fibrous growths of the Fibroma molluscum type, of yellowish or brownish seborrhoic warts, and of other growths of the epidermis which, if irritated, may assume malignant characteristics and develop into carcinomata. These changes are accompanied by sensory disturbances, such as tingling and itching, which may be persistent and intolerable, but though the skin is subjected to severe scratching for their relief it does not readily react and become inflamed.

Sections of the atrophic skin show that the epidermis is thin and that the prickle-cells are wasted but that here and there are accumulations of pigment in the cells of the basal layer, which points to the conclusion that either these cells are capable of elaborating pigment even though they have lost much of their vitality or that the pigment is a product of degeneration of the cell-protoplasm.

In the corium the collagen and elastin have undergone degenerative changes, the collagen breaking up into irregular granules and fragments which eventually become a homogeneous or colloid mass which stains indifferently, while the blood-capillaries are dilated and the pilosebaceous follicles atrophied.

(7) Idiopathic or Primary Atrophy.-Two types of idiopathic atrophy have been described, namely macular and diffuse.

Idiopathic Macular Atrophy.-Syn. : Anetodermia erythematodes (Jadassohn). Under the title of macular idiopathic atrophy has been described an affection of the skin characterised by the presence of atrophic patches, round or oval in shape and varying in size from a split-pea to a finger-nail or larger, the skin of which is smooth, thin, wrinkled, and inelastic, and either the colour of the normal shin or of a bluish tinge from the shining through of the underlying venous capillaries. The lesions may be level with the surrounding skin or slightly depressed and to the touch they give the impression of a hole covered by a thin membrane. Occasionally instead of being depressed they may be raised up and dome-shaped from oedema.

The skin around the atrophic patch is unaffected and there is no halo corresponding to the lilac areola which characterises morphoea. The lesions may occur anywhere on the cutaneous surface but are 
most common about the shoulders, back, and sides of the thorax. There is no alteration of sensation in the atrophic skin but there may be tenderness on deep pressure.

Several obscrvers have described a previous inflammatory condition and asserted that the atrophy is the final stage of a chronic inflammatory lesion possibly of the nature of Urticaria perstans or Erythema multiforme. Precisely similar lesions have been observed in syphilitics occurring in the sites of previous roseolar syphilides or secondary papular lesions apart altogether from ulceration, and also in connection with tuberculosis and Acne vulgaris. In consequence it has been doubted if the affection is a separate disease and if the designation idiopathic is warranted, and it has been suggested that it is a symptom or further stage in the development of a variety of chronic inflammatory lesions.

Sections of early lesions show a peri-vascular infiltration consisting of round connective tissue cells and plasma-cells situated chiefly around the dilated capillaries. The epidermis is thin and the interpapillary processes flattened, while in the corium the elastic tissue is absent.

Idiopathic Diffuse Atrophy.-.Syn.: Atrophia cutis idiopathica progressiva; Dermatitis atrophicans (Kaposi); Acro-dermatitis chronica atrophicans (Herxheimer \& Hartmann).

Since 1876 when Taylor described the first case of diffuse idiopathic atrophy over roo cases have been recorded which may be grouped provisionally under this heading. It is doubtful, however, if the affection is a definite entity and it is more probable that the atrophy is secondary to some form of chronic dermatitis. A certain number of cases which have been included under this heading have been in reality sclerodermia.

The clinical picture presented by these cases is as follows: The affection begins usually on the lower extremities about the dorsum of the foot with the appearance of well-defined erythematous patches which may be bright red or dusky blue, smooth on the surface or covered with fine scales, and usually level with the surrounding skin or slightly raised and oedematous. Associated with them there are no definite sensations, unless there is oedema when there may be burning and itching.

By the appearance of new lesions and their gradual coalescence the whole leg may become involved as far as the hip, in the same way the upper extremity may be affected from the arm to the shoulder, while in rare instances the trunk may be attacked.

Changes of an atrophic nature gradually supervene in the chronically inflamed skin as a result of which the redness disappears, being replaced by a dirty whitish or yellowish tinge, and the skin becomes wrinkled, inelastic, folded, and freely movable on the underlying tissue owing to a diminution of the subcutaneous fat. The atrophic process also involves the hair-follicles and the sweat-glands so that 
exposure to cold does not produce goose-flesh in the affected area and injections of pilocarpine do not cause sweating.

Sections of the affected skin show that the epidermis is thin and the pilo-sebaceous follicles and sweat-glands atrophied. In the upper layers of the corium the collagen has lost its fibrillated structure and become homogeneous and the elastin has entirely disappeared. The capillaries of the papillary and subpapillary layers are dilated and surrounded by a cellular infiltration of small connective tissue cells and occasional plasma-cells. In the subcutaneous tissue the fat-cells have more or less completely disappeared.

The cause is unknown, but it is most probably an atrophy succeeding some form of chronic dermatitis and the employment of the term idiopathic in connection with it is possibly incorrect. It would appear to be somewhat more common in women than men and to bcgin as a rule in middle-age about the fortieth year.

Treatment has no effect on it, as once the atrophy has taken place it is permanent.

(S) Kraurosis Vulvae.-In this affection the tissues of the rulva, especially the Labia minora and clitoris, are shrivelled, the skin is atrophied, and patches of leucoplakia are usually present on the mucous surfaces. The condition is associated with extreme pruritus and secondary excoriation and eczematisation from scratching. It occurs after the menopause but may develop earlier and is of importance chiefly from the intolerable pruritus and the liability of the leucoplakia to develop eventually into carcinoma.

Apart from the leucoplakia the treatment is unsatisfactory. Antipruritic applications may relieve the itching but are simply palliative.

Where leucoplakia is present by far the best results are obtained from exposures to radium. The exposure should consist of halfstrength plates screened with one-tenth $\mathrm{mm}$. of lead, applied for from one to three hours according to the thickness of the lesion (Pinch).

In view of the possibility of the development of carcinoma, excision has been adrocated.

\section{REFERENCES}

Adamsor; H. G. Atrophia maculosa cutis (Brit. Journ. Derm., I9Io, xxii. p. 35r).

- Macular atrophy in a syphilitic patient (Ibid., I9I I, xxiii. p. 220).

- Macular atrophy following a secondary syphilitic eruption (Ibid., I915, xxvii. p. I32).

Bleibtret, L. Striae following scarlet fever (Münch. Med. Wochenschr., Sept. I2th, I905, p. I 768 ).

Buxch, J. L. Striae patellares (Brit. Journ. Derm., I905, xvii. p. I).

Chalmers \& DRew. Atrophodermia biotriptica in natives of the AngloEgyptian Sudan (Journ. Trop. Med. \& Hyg., May Ist, I9I5, p. 99).

Duckworth, DYce. Linear atrophy of the skin with hyper-aesthesia of adjacent parts (Brit. Journ. Derm., I893, v. p. 357).

Heuss, E. Contribution to our knowledge of Atrophia maculosa cutis (Monats. f. prakt. Derm., xxxii., Nos. I \& 2, p. 2). 
Jadassohn. Atrophia maculosa cutis (Trans. German Derm. Soc., Congress iii., Leipsic, I89I).

Little, Graham. Macular atrophy associated with Lupus erythematosus (Brit. Journ. Derm., I9II, xxiii. p. 250).

IICDONAGH, J. E. R. Idiopathic atrophy of the skin (Ibid., p. 226).

Malinowski. Idiopathic atrophy of the skin (Ann. de Derm. et de Syph., Oct. I908, p. 562).

Shepherd, F. J. Atrophia maculosa \& striata following typhoid fever (Journ. Cut. \& Gen.-Urin. Dis., Feb. I89I). 


\section{CHAPTER XXXIV}

\section{DISEASES OF THE HAIR AND HAIR-FOLLICLES}

\section{ALOPECIA}

Der. : $\dot{\alpha} \lambda \dot{\omega} \pi \eta \xi$, a fox.

Alopecia is the generic name for baldness, whether partial or complete, congenital or acquired.

\section{Congenital Alopecia}

Congenital absence of hair, or atrichia, is extremely rare, and when it occurs is frequently associated with other developmental defects, such as imperfect nails, abnormalities of the milk-teeth, opacities in the retina, defective sweat-glands, absence of mammary glands, syndactylism, and polydactylism. Cases have been recorded where infants have been born with no hair, but, as a rule, careful examination will reveal a few patches or tufts of fine downy hairs somewhere on the skin. These cases of complete congenital atrichia may be due to non-development of the hair-follicles, or to intra-uterine atrophy. In some of them the atrichia remains permanent throughout life, but in the majority hair eventually appears although sometimes not for several years.

In the foetus all the hairs belong to the lanugo or downy type, but after birth they are replaced on the scalp, eyebrows, beard, pubes, and axillae, by long hairs; in a considerable number of cases reported as congenital alopecia this hair-change has not taken place and the lanugo type has persisted. (Fig. 323.)

In addition to the generalised cases of congenital alopecia, bald patches may sometimes be observed at birth, which remain permanent. These are most common on the scalp, where they may be due to moles which usually project slightly above the level of the scalp and vary in size from a threepenny-piece to several inches in diameter. They may result also from non-development of the hair-follicles, ulceration, cicatrisation occurring in utero from amniotic adhesions, traumatism from forceps in delivery, and hydrocephalic distension of the skull in utero when the alopecia is confined to the position of the sutures. 
Congenital alopecia may affect several members of a family and, like other congenital anomalies, may occur in several generationsas in the cases reported by Nicolle where it was present in six gencrations and affected thirty-six individuals.

The prognosis should be guarded. In a certain number of cases the absence of hair is due to delayed development and growth may occur eventually, but if after the lapse of a year or two there is still no sign of hair the outlook is unfavourable. When lanugo hairs are present on the scalp at birth and there is no evidence of a "hair-change"

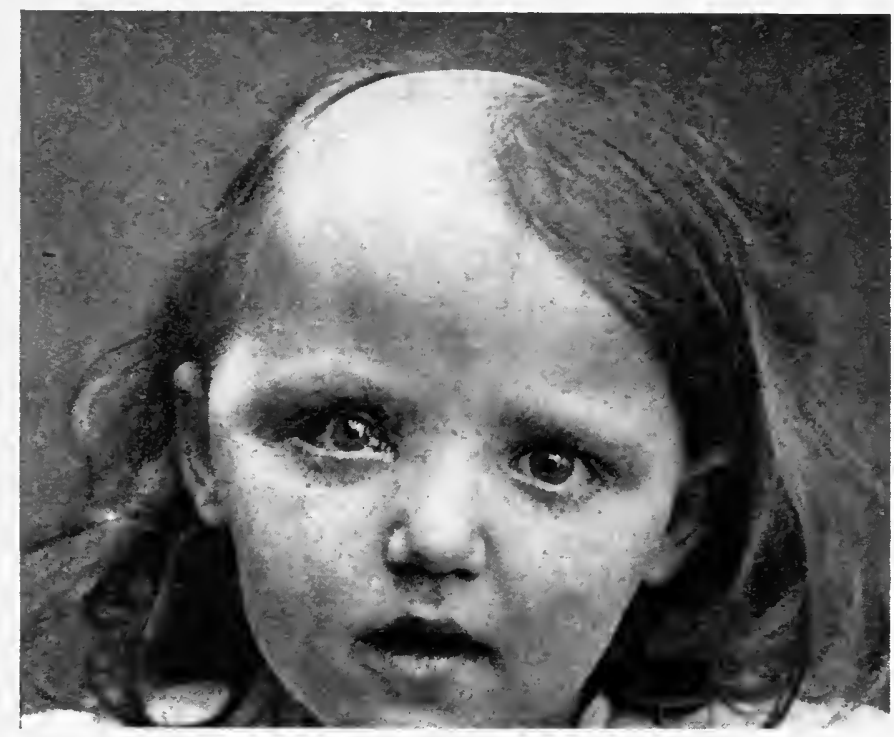

F1G. 323.-Congenital Alopecia.

Associated with mole and dermoids on right side.

taking place during the first year, it is probable that they will persist through life.

Treatment in cases of congenital alopecia is usually of no avail. When it is due to delayed development and the scalp is healthy, no treatment is required and the hair will grow naturally. When inflammation or scaliness is present this should be combated by a mild antiseptic ointment such as :

$$
\begin{aligned}
& \text { Sulphur. praecip. - - - - gr. xv } 3 \\
& \text { Acidi salicylici - - - } \quad \text { gr. x } 2 \\
& \text { Paraf. molle album ad - } \quad \text { - } 3 \text { ji } 95
\end{aligned}
$$

and the scalp washed occasionally with soap-spirit lotion.

\section{REFERENCES}

AUdry. Congenital Alopecia (Journ. des Mal. cut. et Sypl., Jan. I902). Beltmann. Congenital Alopecia (Ar h. f. Derm. u. Syph., June 1902, p. 343). 
CRocker, H. RadcllfFe. Hereditary Alopecia neurotica (Brit. Journ. Derm., I 893 , v. p. I 76 ).

Dubreuilh \& Petges. Congenital circumscribed alopecias (Ann. de Derm. et de Syph., I908 (May), p. 259).

- Congenital circumscribed alopecias (Ann. de Derm. et de Syph., I909, x. p. 455).

\section{ACQUired AlOPECIA}

The acquired alopecias form one of the most important groups of hair affections. As in congenital alopecia the baldness may be partial or complete. The two most prevalent types are the so-called Seborrhoic alopecia or calvities, and Alopecia areata; but in addition more or less complete baldness of the scalp may occur as the result of a multitude of causes which in the present state of our knowledge are difficult to classify satisfactorily.

For purposes of description the acquired alopecias, apart from Alopecia areata which is a distinct entity, may be arranged provisionally according to their etiology under the following headings :

(I) Premature hereditary alopecia.

(2) Senile alopecia.

(3) Alopecia due to or symptomatic of general diseases.

(4) Alopecia due to nervous disturbances.

(5) Alopecia due to morbid conditions of the scalp and hair-follicles.

(6) Alopecia due to traumatism.

(7) Alopecia due to chemical irritants.

(8) Alopecia due to Röntgen Rays.

(9) Alopecia due to parasites.

\section{Premature Hereditary Alopecia}

As a rule premature baldness is the result of some morbid condition of the scalp of which the most common is seborrhoea, but a certain number of cases occur in which the scalp appears to be perfectly healthy. In it the hair generally begins to fall out soon after the age of twenty, and the defluvium may either take place rapidly and the whole of the hair come out in a few months or it may be more gradual and occupy several years. The condition is more common in males than females; it is frequently hereditary; being passed down from father to son for several generations, or it may occur as a family trait.

The prognosis is unfavourable and all that can be done is to attempt to delay the process by correcting any obvious defect in general health and by prescribing a vigorous local stimulating treatment with massage and lotions containing acetic acid, cantharides, turpentine, or other well-known stimulants. Should the slightest evidence of dandruff be present it must be attacked by anti-seborrhoic applications. 


\section{Senile Alopecia}

In old age the hair-follicles and papillae, like the other tissues of the body, tend to atrophy and baldness to result. This is a gradual process and is generally accompanied by whitening of the hair. It usually begins on the vertex and temples, and spreads from there until the whole scalp is affected with the exception of a fringe round the posterior and lateral borders. The age at which it sets in varies and depends on the virility of the tissues. It is more common in men than in women, and is especially common in those whose life work has been chiefly mental and whose occupation has been sedentary.

Treatment except on general medical principles is of no avail. It is advisable, however, to prescribe some local application, which, although it has little or no effect in arresting the process, is pleasant to use and satisfies the patient, such for example as the following lotion :

\begin{tabular}{|c|c|c|c|c|c|}
\hline \multicolumn{2}{|c|}{ Ammonii chloridi } & - & - & - & gr. xv \\
\hline Resorcin & - & & & - & 3ss \\
\hline Glycerini & - & & & & $3 \mathrm{i}$ \\
\hline Eau de Co & & & & & ऊii \\
\hline Aquam de & & & - & - & इviii \\
\hline
\end{tabular}

Where the hair is white, salicylic acid should be substituted for resorcin as the latter is apt to turn white hair a rusty colour.

\section{Alopecia Symptomatic of General Diseases}

Any constitutional disease with marked lowering of the general vitality and the circulation of toxins in the blood, may be responsible for thinning of the hair and even for complete baldness. As a rule, this is temporary and re-growth takes place when health is restored. It is very common in association with the exanthemata, especially with measles and scarlet fever. In scarlet fever it usually amounts only to a slight thinning of the hair, but in severe cases there may be complete loss of hair and nails in the desquamative stage.

The defluvium is due to interference with the function of the hairpapillae, partly from malnutrition but chiefly from toxins circulating in the blood and acting directly on the hair-papillae; this has been proved experimentally in animals by the injection of toxins being followed by baldness.

In debilitating diseases, such as anaemia, pneumonia, influenza, diabetes, phthisis, and myxoedema, and in pregnancy, the hairespecially over the temporal regions-may become thin as the result of malnutrition.

Baldness has been observed in exophthalmic goitre and may be complete or localised as a frontal band extending back about threequarters of an inch from the hair margin.

In a certain number of cases of syphilis, alopecia is a well-known symptom. In the early stages it may occur as a general thinning or as 
partially bald "plucked out" patches, and in the later stages as permanently bald patches resulting from tertiary ulceration of the scalp. Total alopecia has been met with as a late manifestation of syphilis and may be associated with dystrophy of the nails.

In leprosy a general defluvium has been noted occasionally, and discrete patches of baldness in the rare instances in which lepromata have occurred in the scalp.

The prognosis in symptomatic baldness resulting from general morbid conditions is almost invariably satisfactory.

The treatment consists of attending to the general health by suitable means, medicinal, dietetic, and hygienic, so as to restore the vital tone and to eliminate the toxic factor responsible for the defluvium.

The local treatment should be stimulating and should consist of massage, friction, and the application of stimulating lotions containing spirit, turpentine, acetic acid, or cantharides, such as those described in the treatment of Alopecia areata. When much of the hair has fallen out it is often advisable to cut the remainder short to facilitate treatment. The stimulating action of the actinic rays from a Finsen lamp or a mercury vapour lamp, is also of value.

The syphilitic cases respond readily to anti-syphilitic remedies, but where ulceration and cicatrisation have taken place the hair-follicles are destroyed and the bald patches become permanent.

\section{REFERENCES}

Adansox, H. G. Onychia sicca syphilitica associated with total alopecia (Brit. Journ. Derm., I9I I, xxiii. p. 354).

BerLiNer, C. Exophthalmic goitre \& total alopecia (Monatsh. f. prakt. Derm., I I, Bd. xxiii. I896, p. 361).

Montgomery, D. W. The alopecia of hypothyreosis (Journ. Cut. Dis., 1915, xxxiii. p. 260).

\section{Alopecia due to Nervous Disturbances}

In this group may be placed two distinct types of case namely : (a) Generalised alopecia, the result of some severe nerve shock; (b) Localised alopecia, from injury to, or destruction of, a nerve, affecting the cutaneous regions supplied by that nerve.

In the generalised cases the hair may come out in masses or there may be a general thinning. It has been known to occur after some violent mental shock, anxiety, bereavement, loss of money or position, or in association with melancholia or severe persistent neuralgia. Such cases are so numerous and the deflurium may take place so soon after the nervous disturbance as to point to a causal connection between the nervous derangement and the alopecia and to negative the idea that the association is a mere coincidence.

The exact mechanism by which the fall of the hair is brought about is not fully understood. It has been explained as the result of a trophoneurotic influence, the emotion or other mental disturbance inhibiting 
the function of the papillae so that atrophy of the hair-bulb takes place with loosening and shedding of the hair. In a certain number of cases the vitality of the papillae is so impaired that the condition remains permanent but in the majority re-growth takes place after a time varying from a few months to several years. The hair frequently grows in white, but, in young people at least, this is usually temporary and the normal colour is gradually restored.

Cases of localised alopecia from injury to a nerve have been frequently recorded, the baldness as a rule being accompanied by severe neuralgia. For example, in one case the supra-orbital nerve was injured accidentally in an operation; this was followed by neuralgia of the nerve and falling of the hair of the frontal region of the same side, and when the local neuralgia disappeared the hair grew again. The same effect has been produced experimentally by Max Joseph, who resected the ganglion of the 2 nd cervical nerve in a cat and found that after several days alopecia occurred in the area supplied by that nerve, and this experiment has been corroborated by other observers. It may be explained as the result of an interference with the trophic influence of the destroyed nerve or may be due to a vaso-motor disturbance caused by it.

It is not essential that the nerve should be completely destroyed, and comparatively slight injuries to it may produce baldness not only in the area it supplies but also in other areas reflexly. For example, in a case recorded by Pontoppidan in which the carotid ganglion was removed, symmetrical bald patches occurred in the occiput in the areas supplied by the superior and inferior occipital nerves and by the posterior branch of the great auricular on the operation side.

The prognosis in localised cases is good on the whole, and even should the nerve be destroyed a collateral nerve supply is usually established and the hair eventually grows, although, as in the generalised cases, it occasionally comes in white and becomes pigmented later.

Treatment.-In generalised cases no treatment is necessary, except mild stimulation by massage and the application of a lotion containing acetic acid or turpentine in spirit. It is inadvisable to attempt to minimise the disfigurement from the white hair by means of dyes for fear of injuring the re-growth.

In localised cases a stimulating treatment should be adopted, such as menthol Io grs. in an ounce of olive oil or soft white paraffin ; this, when rubbed into the scalp, is followed by a slight inflammatory reaction.

The following lotion may also be dabbed on :

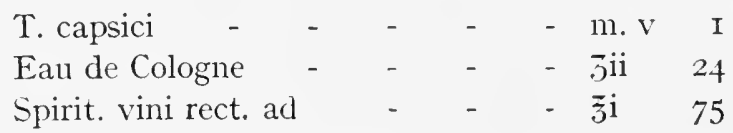

In local patches, brush discharges from a high frequency apparatus and faradisation are of value. 


\section{Alopecia dependent on Morbid States of the Scalp and Hair- follicles}

The great majority of cases of baldness due to a morbid condition of the scalp are caused by Seborrhoic dermatitis, or Pityriasis capitis; this affection is so important that it has been described under a separate heading.

Any form of acute dermatitis, such as exfoliative dermatitis, eczema, psoriasis, and erysipelas, may interfere with the hair-growth and give rise to baldness which is generally partial, irregular, patchy, and temporary - a re-growth usually taking place when the disease is cured.

On the other hand, affections such as Lupus erythematosus, tertiary syphilis, smallpox, morphoea, and localised sclerodermia, produce alopecia as a result of the atrophic cicatricial changes with which they are associated, and in these cases the destructive changes and cicatrisation extend down to, and involve, the papillae so that re-growth is impossible and the baldness is permanent.

In still another group the hairs either grow imperfectly or are absent as the result of keratotic changes at the mouth of the hair-follicles, as in monilithrix and Keratosis pilaris. In these affections the orifice of the follicle is constricted or completely blocked by horny plugs, so that constriction of the hair-shaft takes place or the eruption of the hair is prevented.

Treatment.-In Keratosis pilaris, some benefit may be derived from scrubbing the scalp with soft soap on a piece of moist flannel and the application of $3 \%$ salicylic acid ointment. On the whole, the treatment of this group is unsatisfactory.

\section{Alopecia due to Traumatism}

Localised alopecia due to injury of a nerve might be placed with propriety under this heading but it has been deemed advisable to restrict it to those cases in which the hair-papillae and follicles are directly injured.

Bald patches may follow a blow on the head, an injury from a fall, and a wound or burn of the scalp. They may result also from friction or pressure, as is seen in the occiput of babies' heads where the hair is frequently thin from rubbing, and in women from twisting or plaiting the hair too tightly, or from epilation where the papillae have been damaged by electrolysis or excessive doses of $\mathrm{X}$-ray's.

The possibility of re-growth is determined by the extent of the injury. When the alopecia is simply due to friction or traction of the hair, the re-growth should occur as soon as the cause is removed. Where the papillae have been destroyed from injury and cicatrisation has occurred, re-growth is impossible. 


\section{Alopecia due to Chemical Irritants}

Chemical irritants locally applied may cause baldness. This is familiar to all who have had experience in the treatment of ringworm of the scalp, for every now and then cases are encountered in which the application of even mild parasiticides causes inflammation and produces bald patches. Unfortunately these cases are exceptional, or the treatment of ringworm would be greatly simplified, and are the result of a peculiar idiosyncrasy of the individual on account of which the scalp reacts readily to irritants. Strong applications, such as cantharides, chrysarobin, and croton oil, if applied assiduously, usually bring out the hair.

Baldness may result also from the absorption of certain drugs ; for example, acetate of thallium when given internally has been known to cause it. This drug was at one time a favourite remedy for excessive sweating in connection with phthisis, and in several instances where intoxication took place from it a total and rapid alopecia resulted.

\section{REFERENCE}

JEANSELme. On the mechanism of alopecia produced by acetate of thallium (Ann. de Derm. et de Syph., I 898, ix. p. 999).

\section{Alopecia due to the Röntgen Rays}

If a certain dosage of the $\mathrm{X}$-rays be given to the skin the whole of the hair in the exposed region will fall out in the course of three weeks and, if the dosage be not excessive, re-growth will take place in a few months. This has been utilised for the treatment of ringworm of the scalp, where the $\mathrm{X}$-rays are employed to produce a complete defluvium of the hair and a speedy cure of the disease, but it is a delicate operation and if the proper dose be exceeded dermatitis results followed by cicatricial changes in the skin and hair-follicles and permanent baldness.

The defluvium is caused by the direct action of the rays on the soft growing hair-bulb, the cells of which die and the bulb shrivels to a tapering thread. In about a fortnight after the exposure the shrinkage of the intra-follicular portion of the hair is so great that it becomes loose in the follicle and is shed, either naturally or from friction in washing and drying.

The X-rays may also inhibit the function of the papillae, but this effect is transient provided the dosage has not been too great.

\section{Alopecia due to Parasites}

Circumscribed patches of baldness often occur as a result of the presence in and around the hair-follicles of certain hyphomycetic fungi and bacteria, such as the fungi of ringworm and favus and ordinary pyogenic cocci. Bald patches are frequent as the result of suppurating 
ringworms or kerions, but they may also succeed the common smallspored ringworm of the scalp without suppuration. The bald areas may actually replace the patches of ringworm, may be contiguous with them, or may be independent of them. In a certain number of cases they may result from the irritation of remedies for the ringworm, but they may occur before treatment is instituted, when the defluvium would seem to be due to the ringworm fungus interfering with the function of the papillae.

Occasionally a type of ringworm occurs which is due to a largespored fungus (Megalosporon endothrix), in which the stumps are so

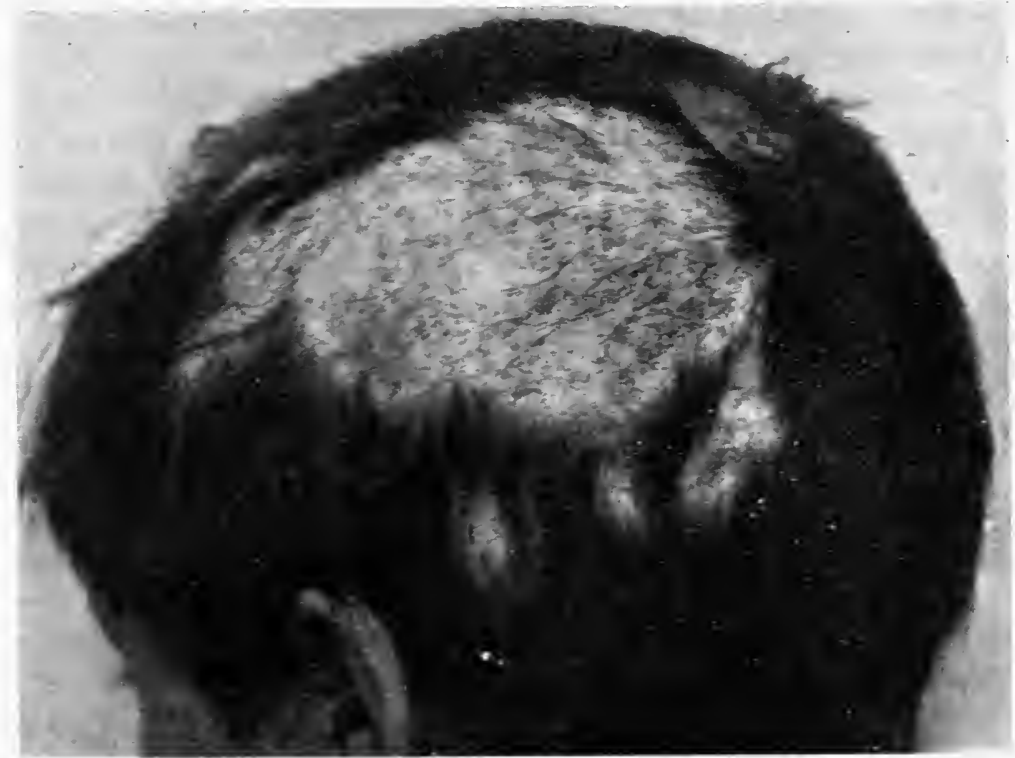

FIG. 324.-Alopecia following Severe Impetigo.

short that they appear as black specks dotted over a bald patch. This variety is often known as " bald ringworm " and has given rise to confusion in connection with Alopecia areata, and certain of the epidemics and groups of cases which were contagious and have been described as Alopecia areata were probably bald ringworm.

The presence of the fungus of farus may be responsible for bald patches, the pressure of the scutula of the disease leading to atrophy and permanent baldness.

Staphylococcic infections of the scalp, such as impetigo, folliculitis, and furunculosis, are often productive of bald patches and especially so when crusting, exudation, and ecthymatous lesions are present. In these cases the denudation occurs for some distance beyond the inflamed area and is usually transient as it is due to the inhibitive action of the micro-organisms and toxins on the hair-papillae. Under suitable treatment with boric compresses and parasiticide ointments re-growth 
takes place rapidly when the inflammation subsides, but should crusting and ulceration occur the papillae may be so injured that re-growth is retarded or permanent baldness is produced. (Fig. 324.)

A peculiar type of baldness preceded by folliculitis possibly of microbic origin has been described under the heading of "Folliculitis decalvans" (see page 46).

\section{ALOPECIA SEBORRHOICA}

Syn.: Calvities; Seborrhoic baldness; Alopecia furfuracea ; Alopecia pityrodes.

Definiticn.-Alopecia associated with seborrhoea and pityriasis of the scalp.

By far the most common type of baldness due to morbid states of the scalp is that known as Alopecia seborrhoica or calvities. At present there is some confusion as to the precise diseased conditions underlying it; there would appear to be three, namely Seborrhoea oleosa, Pityriasis capitis-distinct entities in their clinical appearances and causation - and Seborrhoic dermatitis which is a combination of the two. These are responsible for the majority of cases of progressive baldness, for practically all cases of premature idiopathic baldness, and for the larger number of those occurring in middle life. (See Fig. 88.)

This type of baldness is familiar to everyone. It begins as a rule on the vertex of the scalp and sides of the forehead and gradually spreads, the hair receding so as to leave a central tuft or toupet which in turn disappears and gives place to a large bald area occupying the crown of the head. Occasionally it may begin on the parietal regions, when triangular bald areas appear above the ears, or in rare instances it may occur as a general thinning. The hair persists longest on the sides of the head and the occiput, where it forms a fringe which lasts after the remainder of the scalp has become bald.

It may begin early in life, about the age of twenty, but more commonly does not appear till between the ages of thirty-five and forty. Once established it is slowly but inevitably progressive.

It frequently occurs in families and may be hereditary, when it has been explained as the result of a hereditary proclivity of the scalp to seborrhoca. Though it affects males more than females it is by no means confined to the male sex and a thinning of the hair on the vertex and tenıples may be observed occasionally in youngish women and more commonly in women about the time of the menopause.

In some cases the defluvium is rapid and the characteristic picture is produced in a few months, but it is more often gradual and several years may elapse before the top of the head is completely bald.

For convenience of description the types of cases which have been grouped under Alopecia seborrhoica may be discussed under the headings of the three morbid conditions responsible, but this is merely a categorical arrangement and subject to considerable overlapping. 
(I) Seborrhoea oleosa, or Steatidrosis.-In this condition, which is associated with a particularly intractable form of baldness, the scalpespecially the vertex and temporal regions-is markedly greasy, from an oily substance oozing out at the orifices of the follicles on to the hair and the epidermis in the neighbourhood. In some cases this oily substance is fluid in consistence from an excess of oleic acid, in others it is waxy from a preponderance of stearin. The actual follicular orifice is dilated and may show definite cupping. Here and there, where hairs have fallen out, the follicles present greyish greasy plugs which can be expressed easily.

It occurs usually in males but females are not exempt. It generally begins soon after puberty, but is occasionally met with in roung children and infants, and once established persists to old-age.

A rare variant of this condition has been described under the heading of Hyperidrosis oleosa, in which the thinning of the hair is more general than in ordinary steatidrosis. In it, the scalp is moist and oily, but the oiliness is diffuse and shows no definite relation to the follicular orifices and may result from abnormal activity of the sweat-glands of the scalp as the sweat is known to contain an oily substance.

(2) Pityriasis capitis, or Alopecia pityrodes.-Another and more prevalent type of case is that in which the baldness is associated with pityriasis or dandruff. In it the scalp is not obriously inflamed and the scaliness is fine, dry, almost powdery, and more or less constantly shed, especially when the hair is combed or brushed, while the hair is opaque and lustreless and near the orifice of the follicles is powdered over with fine scales. Although no inflammatory changes are noticeable yet a mild form of inflammation is responsible for the condition, which is possibly due to the action of certain micro-organisms invariably: found in association with it, namely the flask bacilli of Inna or spores of Malassez and the Staphylococcus epidermidis albus of Welch. (See Fig. ST.)

This type of baldness, though progressive, is more amenable to treatment than that associated with oily seborrhoea.

(3) Seborrhoic dermatitis.-Seborrhoic dermatitis is a combination of the first and second types and consists of Pityriasis capitis on a seborrhoic base. In it, there is a definite inflammatory disturbance, with inflamed patches, covered with greasy and waxy scales, which gradually involve the whole scalp and not infrequently spread behind the auricles and begond the hair-margin as pinkish festoons. As the result of scratching and rubbing, the inflammation may become excessive and be associated with oedema, weeping, and eczematisation. The hairs in the affected regions readily fall out, while those which remain are lustreless, unhealthy, and surrounded near the follicle by greasy scales. The condition is believed to result from the excessive growth of the microbes of Pityriasis capitis in a scalp which the presence of seborrhoea has rendered an unusually suitable soil for their proliferation. 
This type of baldness may be arrested, at least temporarily, by suitable treatment.

Etiology and Pathogenesis.-The cause of this type of alopecia is uncertain, and is bound up with that of seborrhoea, Pityriasis capitis, and seborrhoic dermatitis.

There are two main theories with regard to the nature of Seborrhoea oleosa or steatidrosis, namely, (I) that it is simply a physiological process which has become excessive owing to an individual peculiarity, and (2) that it is a pathological condition due to a specific microbe.

(I) According to the first theory steatidrosis is a physiological process which tends to become excessive as the result of an idiosyncrasy, hereditary or acquired. It is well-known that certain individuals exhibit a marked tendency to oily seborrhoea about puberty which persists in early adult life. This is evidenced by a dull greasy complexion, oily beads about the pilo-sebaceous orifices, at the sides of the nose, and in the conchae of the ears, and the presence of greasy plugs or comedones in the follicles. It is also well-marked in the scalp about the vertex and temporal regions. It would seem to be the result not only of an increased activity of the sebaceous glands with an excessive output of sebum, but also of a natural hypertrophy of the glandular tissue. In most cases it disappears soon after puberty but it may be prolonged into adult life or persist until senile atrophic changes supervene.

The sebaceous glands differ from the sweat-glands in that they possess no secretory nerve mechanism and the sebum instead of being secreted is the result of a fatty degeneration of the cells and is expelled by a simple vis-a-tergo force. The sweat-glands may become active and a marked increase of sweat occur without any definite structural changes taking place in the gland-cells, but should the sebaceous material be increased not only is the fatty degeneration more rapid but there is an augmentation in the number of cells and the glands become hypertrophied. This suggested to Leslie Roberts an ingenious theory with regard to the atrophy of the hair. He pointed out that the pilo-sebaceous follicle is a complete structure, in which a certain balance or relation is maintained between the size of the hair and that of the gland, and that normally where the hairs are long the glandular appendages are small, but that in situations where the hairs are naturally fine and downy, as on the sides of the nose and the centre of the back, the sebaceous glands are large. In steatidrosis, the glands are hypertrophied and, as would be expected, the hair-bulbs are correspondingly diminished and finally cease to grow. According to his theory seborrhoea is an individual peculiarity, in many cases of hereditary origin, and the defluvium of the hair is the result of an interference with the " natural balance" of the pilo-sebaceous follicle by hypertrophy and over-activity of the sebaceous appendage.

In the somewhat doubtful cases of the Hyperidrosis oleosa type it would seem probable that the over-activity of the sweat-glands is associated with hypertroply and over-activity of the sebaceous glands and that the latter would account for the defluvium of the hair.

This is simply a theory but it is capable of explaining a considerable number of the cases and in consequence is worthy of consideration.

(2) According to the second view steatidrosis is a pathological condition due to a specific microbic infection. Of the various micro-organisms which have been incriminated as responsible for it the one which has received the most consideration is the microbacillus of Sabouraud, which has been found invariably by him in the oily secretion and greasy plug and in consequence has been regarded as causally connected with the 
affection. The microbacillus, which is identical with the acne bacillus of Unna, enters the follicle from without and by proliferation forms a sheath around the part of the hair situated in the dilated funnel of the follicle (Utricle peladique). Keratosis of the epidermis lining the walls of the funnel of the follicle next takes place, encysting the bacilli, and forming a plug or cocoon which is attached to one side of the hairshaft. It produces at the same time hypertrophy of the sebaceous gland with an increased output of sebum and atrophy of the hair-bulb and papilla. Its presence also determines inflammatory changes, such as a lymphocytic cellular infiltration around the utricle, in the angle between the Arrector pili muscle and the follicle, and about the capillaries of the follicle and the papilla. In this way the function of the papilla is interfered with, the hair-cells cease to be pigmented and the medullary cells to form, atrophy of the hair takes place, the new hairs revert to the lanugo type, and finally even they cease to be produced and baldness results.

This ingenious theory is not yet established and there is no conclusive evidence that the microbacillus is pathogenic and more than an innocuous saprophyte existing on the sebaceous material which forms an excellent medium for its growth.

Of the two theories the first seems to be the more probable, as oily seborrhoea has never been shown to be either contagious or inoculable. With regard to Pityriasis capitis and Seborrhoic dermatitis the evidence in favour of a parasitic theory is more weighty, but the part played by the different organisms found in these conditions is uncertain and must be left for future bacteriologists to decide. Of these micro-organisms the S. epidermidis albus seems the most probable cause of Pityriasis capitis and it is quite possible that the same organism, by proliferating actively and possibly increasing in virulence by growing in the sebaceous medium, may be responsible for Seborrhoic dermatitis. The role played by the flask bacillus of Unna is unknown and it may be nothing more than a harmless saprophyte.

Prognosis. - The prognosis of seborrhoic baldness is as a rule unsatisfactory. A good deal can be done by suitable treatment to retard the process but once it has been definitely established it is usually progressive. In commencing cases if the hair has not entirely fallen out re-growth may be obtained by active treatment, but in cases where the baldness is well-advanced treatment is of comparatively little avail; still in every case thorough treatment should be instituted without delay, in the hope that if it does not arrest the deflurium it may at least retard its progress.

\section{TREATMENT}

The treatment is chiefly local, as the affection is not dependent to any marked degree on defects in the general health. Of course any obvious impairment in health will naturally increase the local process by reducing the general ritality; and should be corrected by suitable means and the patient kept as healthy as possible by a proper tonic and hygienic regime. Unfortunately there is no internal specific remedy which will make the hair grow. Occasionally pilocarpine has been 
known to do so but as its action is uncertain and it is liable to produce toxic symptoms its employment is not to be advocated.

Local Treatment.-The local treatment is of paramount importance and varies according to the type of case, but whatever the type may be the treatment to be successful must be carried out with thoroughness and perseverance. So often patients at the beginning employ local remedies thoroughly, but in the course of time through not observing any marked improvement they are apt to become half-hearted and to apply them by fits and starts, and many in their disappointment are liable to discard them altogether and to fall into the hands of the vendors of patent nostrums, not realising that they are substituting for a carefully thought-out line of treatment, some cheap and often worthless lotion fantastically labelled and sold at great profit.

The different cases of seborrhoic baldness vary so much that each must be treated on its own merits, but as a guide to the general method of treatment they may be divided for practical purposes into the above-mentioned three groups, namely, (I) those in which oily seborrhoea or steatidrosis is present; (2) those associated with dandruff or greasy scales (Pityriasis capitis); (3) those in which well-marked inflammatory lesions occur (Seborrhoic dermatitis).

(I) Seborrhoea oleosa.-The local treatment consists of the removal of the greasiness and the stimulation of the scalp, by washing with soap spirit and the application of an antiseptic alcohol lotion.

A useful formula for the soap is :

\begin{tabular}{|c|c|c|}
\hline Saponis viridis & - & - \\
\hline Spirit. vini rect. & - & - \\
\hline O1. lavandulae - & - & - \\
\hline
\end{tabular}

This is allowed to digest for twenty-four hours and then decanted. For use, the soap spirit may be mixed with warm water in the proportion of a table-spoonful to half a pint, so as to form a lotion, and the scalp washed with it. It is more effectrve, however, to rub the undiluted soap spirit into the scalp by means of a piece of moist flannel or sponge and then to rinse it out with warm water. Whicherer method be adopted the washing must be thorough ; the hair should be parted seriatim in different places and the soap rubbed into the partings till the whole of the scalp has been gone over. Women with long hair find difficulty in washing their own hair and it is an advantage for them to have it shampooed by an attendant, for unless the rinsing be done carefully the hair is apt to be left sticky and it may be necessary to cleanse it with ordinary soap. The frequency with which it should be washed depends upon the amount of greasiness, but on an average it should be done about once a week in men and once a fortnight in women.

In addition to washing, a stimulating spirit lotion should be rubbed in by a piece of sponge twice a day. This lotion should contain some drug with a keratolytic action, such as resorcin or salicylic acid, 
to remove the epithelial debris about the mouths of the follicles and to allow the spirit to penetrate more deeply, and some parasiticide to destroy or prevent the growth of micro-organisms in the oily sebum. The following will be found to be a serviceable formula:

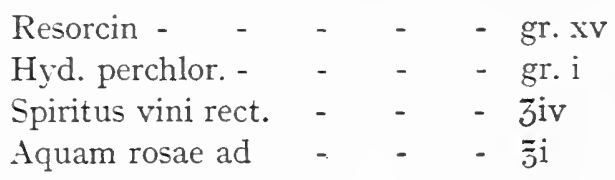

Instead of rectified spirit, Eau de Cologne may be substituted; in the case of males glycerine should be added; in fair-haired people salicylic acid should be used instead of resorcin as the latter is apt to stain the hair a rusty colour. To render the lotion more stimulating acetic acid or ammonium chloride may be added.

A lotion made on the above lines should be cooling to use and should produce a stinging feeling when applied. After the special modification of the lotion which is most pleasant to the individual has been obtained its use should be continued indefinitely.

The following nascent sulphur lotion was recommended by Crocker for these cases and gives good results:

No. I.

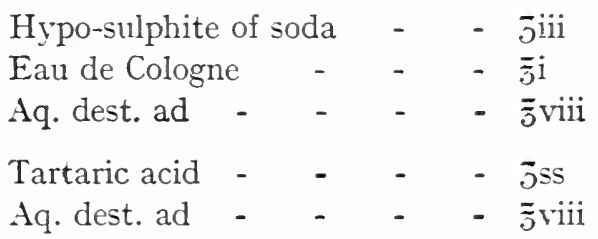

For use lotion No. I should be rubbed in with a sponge for a few minutes and No. 2 applied subsequently to the same area.

(2) Pityriasis capitis.-In the second type of case where the most marked feature is the presence of scales or dandruff, more energetic treatment is necessary.

Where the dandruff is slight it may be kept under by washing the scalp with soap spirit and applying the above-mentioned resorcin lotion. As a rule, however, lotions are insufficient and ointments have to be resorted to. The discomfort associated with the use of ointments and pomades, especially in women, is considerable so that in the first instance a non-greasy brilliantine should be tried as it is intermediate between a lotion and an ointment and less disagreeable to use.

Such a brilliantine can be made with castor oil which is soluble in spirit, and the following formula may be employed :

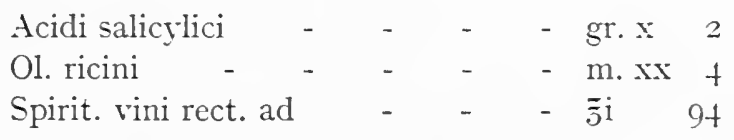

This may be made more pleasant by the addition of a few drops of Ol. ros. geran. or a teaspoonful of Eau de Cologne. The brilliantine should be rubbed into the scalp daily and the hair washed once a week. 
When the scalp is thickly covered with greasy waxy scales by far the best results are obtained by the employment of ointments containing the same active principles as the above lotions. A useful formula is :

\begin{tabular}{|c|c|c|c|}
\hline Acidi salicylici & - & - & - gr. $\mathrm{x}$ \\
\hline Sulphur. praccip. - & - & - & $-L_{\alpha r} \mathrm{x}$ \\
\hline Hydrarg. ammoniat. & - & - & $-\{\mathrm{g}$ \\
\hline Paraf. molle alb. ad & - & - & $-\quad \bar{j} \mathrm{i}$ \\
\hline
\end{tabular}

Instead of soft white paraffin a pomade base may be substituted, such as the following:

\begin{tabular}{|c|c|c|c|c|}
\hline Ol. cocoa - & - & - & - & \\
\hline Ol. amygdalae & & - & - & \\
\hline Ol. rosae gutt. & & - & - & _ \\
\hline Aq. rosae & - & - & - & \\
\hline Lanolin - & - & - & - & - \\
\hline Adipem ad & - & - & - & - \\
\hline
\end{tabular}

The pomade, being thicker in consistence than vaselin, is especially useful in hot weather.

Where the scales are thick and waxy, tar applications are of service such as:

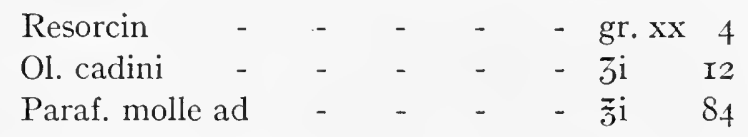

One or other of the above ointments or pomades should be thoroughly rubbed into the affected regions every night, the scalp washed- with soap spirit in the morning, dried, and the resorcin spirit lotion applied. This treatment should be continued as long as any scales are present. In moderately severe cases it is usually sufficient to apply the ointment and to wash the scalp once a week, while the lotion is rubbed in daily. In severe cases, however, it may be necessary to employ the ointment and to wash the scalp every other day. Where the scales are so thick as almost to amount to crusts it is advisable to soften them with olive oil as a preliminary to washing the scalp. When frequent washing is necessary instead of the soap spirit a weak solution of borax containing a teaspoonful to a pint of water may be employed to remove the grease.

(3) Seborrhoic dermatitis.-Where marked inflammatory changes occur and where the scalp is not only covered with greasy scales but oedematous and even eczematised, before a routine treatment with washing and the application of one or other of the above ointments is adopted it is necessary to reduce the inflammation, as should this not be done there is a danger of aggravating rather than allaying the disease. For this purpose lead lotions are specially valuable, such as glycerine of the subacetate of lead, a teaspoonful to $\frac{1}{2}$ pint of water, employed several times a day in the form of a compress. This may be 
augmented by the addition of $\frac{1}{2}$ a teaspoonful of the Liquor carbonis detergens. When the inflammation has been reduced the treatment described above should be employed.

\section{REFERENCE}

Elliot \& Merrill. Alopecia praematura \& Eczema seborrhoicum (New York Med. Journ., Oct. I 895 ).

\section{ALOPECIA AREATA}

Syn.: Alopecia circumscripta; Tinea decalvans; Porrigo decalvans; Area celsi; Fr. Pelade.

Definition: A type of baldness which occurs rapidly with complete or almost complete defluvium of the hair in limited patches, large areas, or over the whole cutaneous surface.

At the present time the exact nature of Alopecia areata is controversial and there is no unanimity either with regard to its causation or to the exact type of case to which the name should be applied. Several writers include under it alopecia due to definite causes, such as traumatism, but here it has been deemed advisable to describe these cases in a separate section (see page 97I) and to restrict the name Alopecia areata to cases conforming to the above definition which, while varying in their clinical characteristics and especially in their distribution and extent, undoubtedly have a common causationmost probably an inhibition of the function of the hair-papillae brought about by interference with their innervation and nutrition.

Alopecia areata is a comparatively common disease, forming about 2 to $5 \%$ of the cases of skin disease in England, I to $5 \%$ in Scotland, $3 \%$ in France, and 0.5 to $\mathrm{I} \%$ in America.

Description.--It usually appears as one or more bald patches about the size of a sixpence and is generally noticed first while brushing the hair. Its most common sites are, in order, the scalp especially the vertex and occipital regions, beard, moustache, eyebrows and eyelashes, but it may occur in any part where there is hair and is sometimes universal. In men it not infrequently begins at the upper part of the occiput and the sides of the scalp at the level where the hat presses.

In about half the cases careful questioning of the patient will elicit a history of abnormal sensations in the affected areas for a few days previous to the outbreak, such as itching, feelings of heat or cold, tenderness on pressure or on being touched with a brush or comb, or sometimes of more definite subjective symptoms, such as neuralgia or headache, and it not infrequently happens that the patient is able to predict the development of new lesions by the local sensations. When characteristic subjective symptoms occur in connection with one patch they are liable to be repeated with every subsequent one. Cases are also on record where each new patch has been preceded by a feeling of general malaise. 
As a rule the patch when first noticed is completely bald and smooth, the hairs either having come out suddenly over the whole patch or more gradually during several weeks. Near the margin in newly developed patches a few short hairs may be present or, more frequently, peculiar stumps which from their appearance are appropriately known as " point of exclamation hairs" (!) As a rule these are easily epilated, though they may be attached sufficiently firmly to come away with a sort of click. They vary in length from one-sixth to one-quarter of an inch, the free end being broken and frayed out like a brush while the intra-follicular portion is atrophied, depigmented, and tapered to a fine point to which is attached a small thickeningthe remains of the hair-bulb. In addition to the typical stumps, others may occasionally be noted with brush-like nodes similar to those of Trichorrhexis nodosa, or with beaded thickenings like monilithrix. (Fig. 325.)

The hairs in the immediate neighbourhood of the patch, though apparently healthy, may be affected also and come out painlessly on slight traction presenting a tapered atrophic root. Similar hairs may occur here and there over the patches when the disease is rapidly spreading, and may be an early stage of the typical stump.

The skin of the bald patch varies in different cases. At first there may be an erythematous blush and slight oedema which precedes the defluvium, persists for several days after the hair has fallen out, and then disappears, and occasionally when the patch is extending a transient inflammatory halo may be present at the periphery before the loss of the hair. In other cases there

FIG. 325.-Clavate Stump or "Point of Exclamation" Hair of Alopecia Areata.

(From MacLeod's Pathology of the Skin.) may be no signs of inflammation and the patch may appear white from the first, ivory-like, and paler than the surrounding skin. (Fig. 326.)

In some cases the follicles may close up and be scarcely noticeable even with a pocket lens, so that the surface is atrophic, smooth and glossy like a billiard ball, while in others they may remain patent and be dark and prominent from the presence of horny and greasy plugs.

Occasionally the surface may be scaly from pityriasis or greasy from seborrhoea.

In extensive cases an alteration in the consistence of the scalp may be noticed by which, through atony of the underlying tissue, it appears to be much more movable than normally and forms prominent folds on being pinched up between the fingers. To this condition Jacquet has given the name Syndrome hypotonique. 


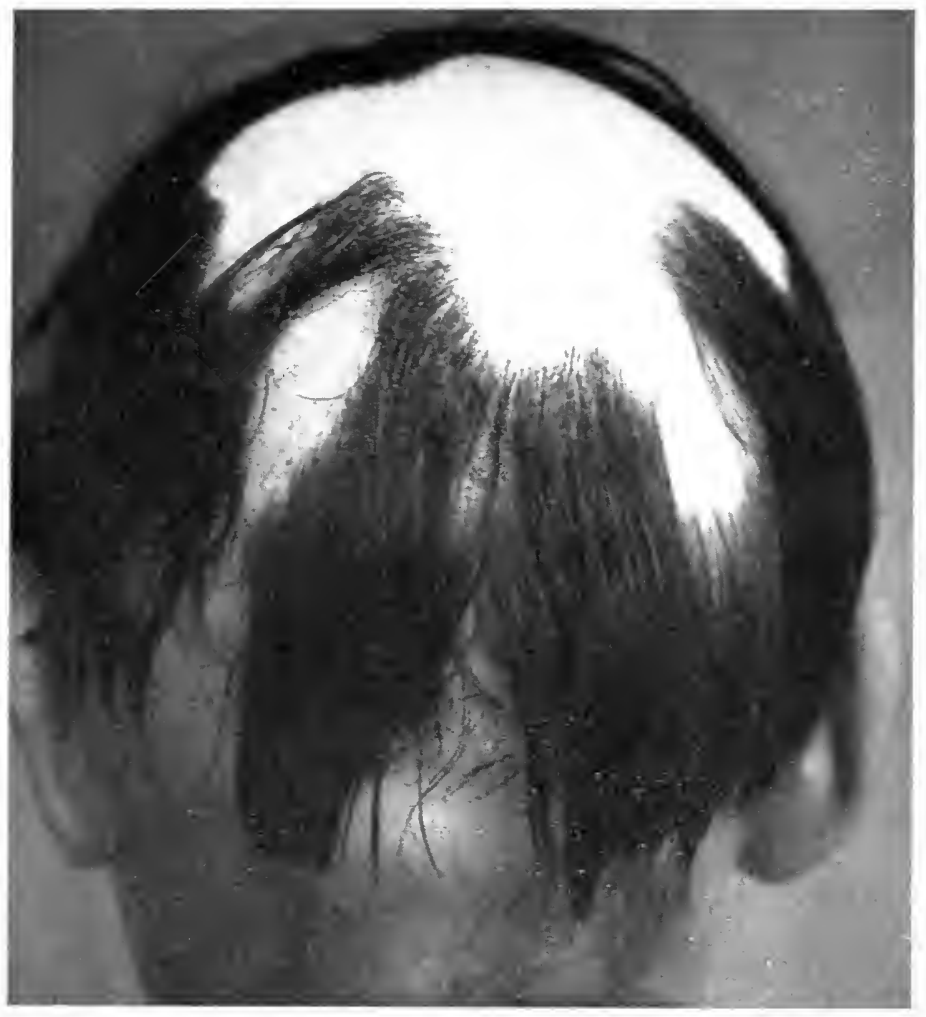

FIG. 326.-Alopecia Areata.

Symmetrical distribution of the bald areas.

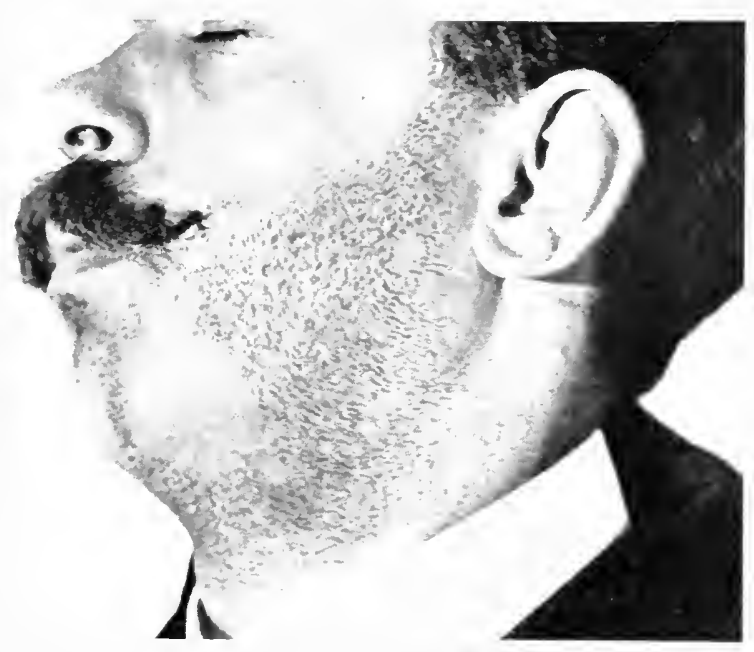

Ftg. 32,-Alopecia Areata of Beard. 

Patches of alopecia may appear simultaneously or at varying intervals and may show a rough tendency to be symmetrical. Once formed they may remain stationary or may heep on spreading, rapidly or slowly, till they reach the size of a five-shilling piece or larger, and may coalesce till the scalp becomes almost completely denuded except for a few tufts of hair irregularly distributed over it. (Fig. 327.)

The sensibility of the patches may be unaltered or even diminished, but it is more usually increased and they may be definitely tender to the touch. A vaso-motor paresis may also exist on account of which irritants do not readily produce an inflammatory reaction, and the sweat-function may be so diminished that injections of pilocarpine may not give rise to sweating.

It is rare for the patches to remain permanentlybald and spontaneous recovery is the rule, the new hairs beginning to grow after a time varying from a few weeks to several months and being seen first either in the centre of the patch, at the periphery, or all over it.

The new hair appears as fine down, which on reaching a certain size is shed and is replaced subsequently by hairs similar to those of the rest of the scalp. It is usually light in colour, and may even be white, and may remain permanently so-giving the scalp a piebald appearance -but as a rule in the course of a few months it assumes its original tint or falls out and is replaced by pigmented hairs.

Varieties.-According to the distribution of the patches in the scalp two main varieties have been differentiated by Sabouraud and others, namely, (I) a central type, the ordinary variety in which the patches are distributed irregularly in the centre of the scalp; and (2) a marginal type in which the bald areas occur around the edge of the scalp, especially at the back and sides, and gradually spread upwards leaving the central portion unaffected. The second type corresponds to what the Greeks described as "ophiasis" and was named by Sabouraud "Pelade ophiasique."

According to Sabouraud a clear distinction should be made between the two types, as he believed the central variety to be of microbic origin and to occur in adults, and ophiasis to be an infantile type of uncertain origin. This hard-and-fast rule, however, cannot be maintained, as cases of the ophiasis type are found at all ages, and mixed cases are not infrequent in which marginal and central lesions occur together.

Cniversal or generalised alopecia is a variety in which the whole of the hairy system is involved, not only the long hairs but also the fine lanugo hairs of the glabrous skin. This, with the absence of the eyebrows and eyelashes, produces a peculiar smooth appearance of the skin which is quite characteristic. In these cases the hairs usually fall out rapidly in a patchy manner and the defluvium may be complete in a few weeks. Certain of them seem to be the result of mental shock, others occur after some serious illness or pregnancy, while in some no definite cause can be assigned. In a considerable proportion there is a history of the previous occurrence of bald patches. 
Alopecia areata associated with Pityriasis of the Scalp (Alopecia seborrhoica circinata of Crocker).-In this variety, which is more common in children than in adults, the patches are scaly like the rest of the scalp. The association of these two conditions has gained special significance owing to the theory advanced by Sabouraud that Alopecia areata is an acute localised form of seborrhoea and due to the same cause, namely the microbacillus; but this view is not generally accepted and the connection between the two is uncertain and possibly accidental.

Alopecia Punctata.-Alopecia punctata is a type in which small, smooth, white patches, about a quarter of an inch in diameter are

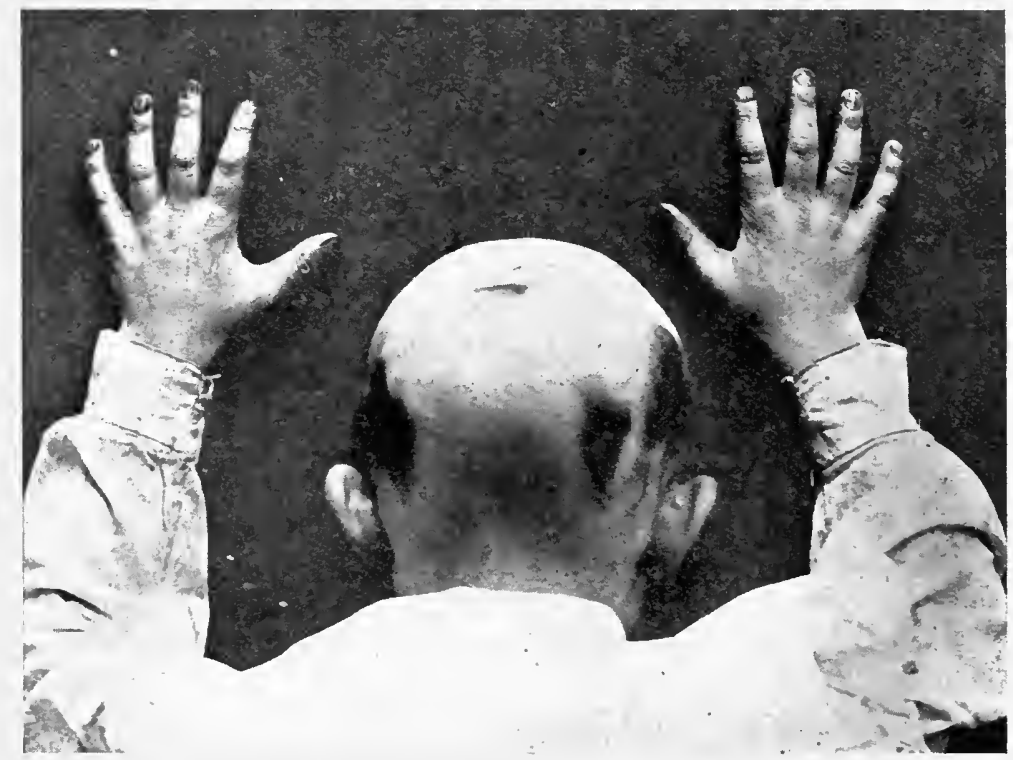

Fig. 328.-Alopecia Areata.

Associated with Dystrophy of the Nails.

distributed in considerable numbers all over the scalp. This variety is peculiarly resistant to treatment.

Nail Changes.-Alopecia areata is frequently associated with changes in the nails, especially those of the hand, several or all of which may be involved. The changes may be of an atrophic or dystrophic type, suggesting an interference with the nail production at the nail-matrix, possibly of an allied nature to that which occurs at the hair-papillae. The nails may be opaque, striatcd, or furrowed, or they may show depressions, white specks, and cven complete leuconychia. (Fig. 328.)

Histopathology.-Sections of the bald patches including the spreading edge show the following changes: The epidermis is comparatively slightly affected; it is somewhat thinned and there is a flattening of the inter-papillary processes, and an absence of pigment in the cells of the basal layer. The hair-follicles are definitely involved; in some instances they are distorted and dilated to form cysts, in 
others marked atrophic changes have supervened and they have become attenuated into thin cords of cells. The hair-papillae as a rule can be detected and lanugo-like hairs may be present in the follicles.

At the spreading edge, where the long hairs have not broken to form stumps but where intra-follicular atrophic changes have commenced, the hair-bulb is shrivelled, the medulla has disappeared, and the pigment is no longer present in the cells of the cortex except in the form of small irregular groups of granules. These changes, which begin in the papillae, spread up the shaft, and near the mouth of the follicle splitting in the cortex may be observed which precedes the breaking of the hair to form a stump. In the neighbourhood of the papillae, at the sides of the follicle, and around the intra-follicular capillaries, a slight cellular infiltration may be detected consisting of mononuclear cells of the lymphocytic type and a few mast-cells, and a dilatation and thrombosis of the capillaries of the hair-papillae have been observed. In old-standing cases evidences of fibromatosis may be noticed in the papillae and around the deeper portions of the follicle, which leads to its permanent obliteration and renders recovery impossible. The sebaceous glands undergo atrophic changes corresponding to those of the rest of the follicle, but the sweat-glands do not appear to be affected.

Etiology. - Age.-It is most common in childhood and early adult life. It is rare in infancy and under 5 years, most prevalent between the ages of Io and 20, gradually diminishes in frequency between 20 and 45 , and is rare in elderly people. It is one of the common skin-affections and forms about $2 \%$ of the cases in a skin clinique.

Sex.-It attacks both sexes equally, though it has been said to predominate in females in early life and in males after the age of 20 .

Social position.-The station in life seems to have no influence on its etiology and it is equally common in hospital and private practice.

Season.--The season of the year may have some influence in determining a recurrence or a re-growth. In some people there is a definite tendency for the hair to grow faster in summer, as is shown by the necessity of having it cut more frequently and by cases of Alopecia areata being not uncommon in which re-growth takes place in warm summer weather and new bald patches appear towards the end of autumn or in winter.

Colour of the hair.-It is said to be more frequent in dark-haired persons than in those with fair hair, but the accuracy of this observation is doubtful.

Predisposing causes. - A number of morbid conditions have been cited as possible predisposing causes, such as general debilitating diseases like exophthalmic goitre, influenza and typhoid fever ; diseases of the nervous system, such as epilepsy; chorea, Tabes dorsalis, paralysis and mental disturbances; pregnancy, ovariotomy, and disorders of the genital organs such as dysmenorrhoea; but no precise signification 
can be attached to them and their association with the disease may be accidental.

Pathogenesis.--The cause and true nature of Alopecia areata are still unknown. There are three theories with regard to its causation, namely, the microbic, tropho-neurotic, and toxic theories, each of which is capable of explaining a certain number of the cases but none is applicable to all. Certain observers consider that there is a common causation for all cases while others believe that they may be due to different causes. As the problem is still controversial it will be advisable to consider briefly the arguments in favour of each theory.

(a) Microbic Theory.-According to this view, Alopecia areata is a parasitic disease due to the presence and action of a specific microbe or fungus situated in or about the hair-follicles. If this were the case the following postulates would have to be fulfilled-(i) a pathogenic micro-organism should be found invariably present; (ii) the disease should be capable of inoculation from man to man, and possibly from man to lower animals; (iii) it should be contagious. So far none of these has been established and the arguments in favour of the theory are based on clinical observation rather than on bacteriological proof. Still it will be an advantage to review the present position with regard to the theory.

(i) Micro-organisms.- $\mathrm{Up}$ to the present time neither a microbe nor a fungus has been found in all the cases examined. From time to time different observers have discovered micro-organisms in the follicles or about the affected hairs, such as micrococci (Thin), Staphylococcus epidermidis albus (Walker and Marshall-Rockwell), cocci (Vaillard and Vincent), flask-shaped bacilli (Unna), and micro-bacilli (Sabouraud), but there is no conclusive proof, experimental or accidental, that any of them is the cause of the disease.

One of the strongest exponents of the parasitic theory was Sabouraud who regarded Alopecia areata, with the exception of those cases of the marginal type, as acute seborrhoea and due to the micro-bacillus. $\mathrm{He}$ based his opinion on finding this organism in a large number of cases and from observing that a filtered culture of it injected into a rabbit's ear was followed by total defluvium of the hair. Others have not been so successful in finding micro-bacilli, unless in cases where the Alopecia areata was associated with definite seborrhoea, and it has been noted that other bacterial toxins, when injected into lower animals, may cause loss of hair.

Out of 207 cases of Alopecia areata, WValker found seborrhoea in $\mathrm{I}_{4} \mathrm{O}$, but this is no proof that the two conditions are causally related for Seborrhoea capitis is so common that one would expect it to form a high percentage in any series of cases.

On several occasions hyphomycetic fungi have been found in supposed Alopecia areata, but these are now believed to be cases of the so-called " bald ringworm," or Tinea decalvans, due to the Megalosporon endothrix, or cases in which the Alopecia areata was preceded by, or co-existent with, the common small-spored ringworm.

(ii) Transmissibility. - Innumerable attempts have been made to inoculate Alopecia areata from man to man and to lower animals, such as dogs, rabbits, cats, etc., but except in a few doubtful instances with negative results. Jacquet made one hundred attempts to inoculate it on himself and on five of his pupils by scraping patches of Alopecia areata and rubbing the scrapings into the parts of the scalp usually attacked by the disease, or by charging a needle with them and catheterising the hair-follicles. In no instance was the result of the inoculation successful. 
(iii) Contagiosity.-In the vast majority of cases there is no suggestion of contagiosity and it is unnecessary to isolate the patient, but in a few cases the possibility of contagion has arisen where it has been met with in several members of a family or has occurred simultaneously in a parent

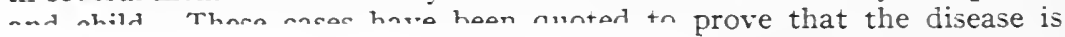

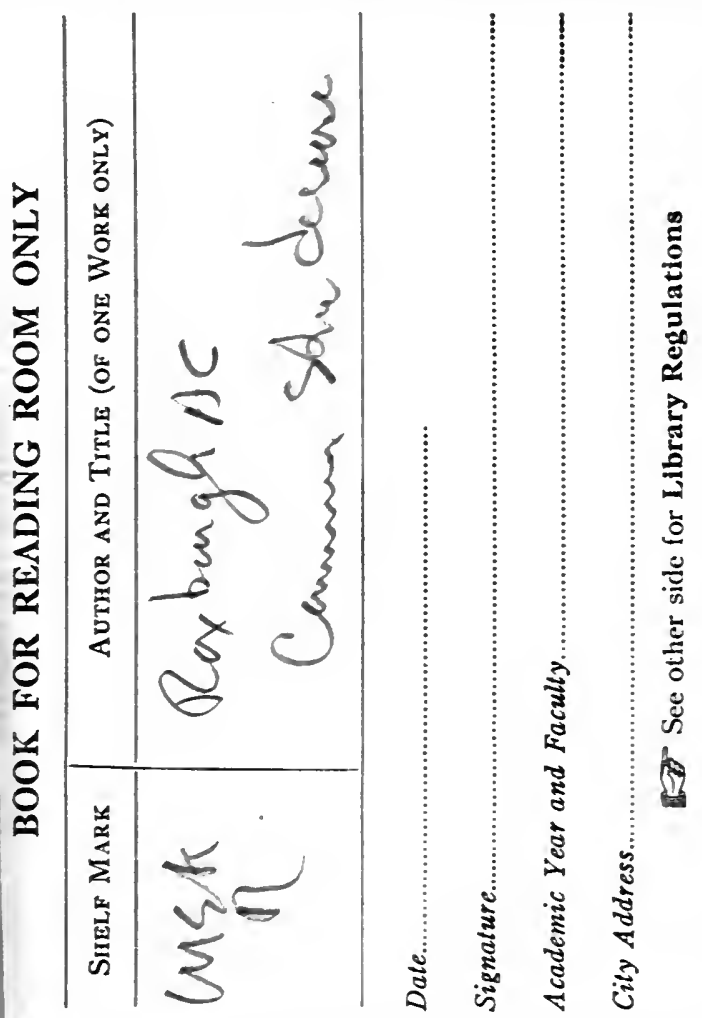
esult of hereditary prele of the evidence from currence for, as Alopecia jious such cases should be

een recorded where it has barber, and on the scalp a hired wig. Patients not I another case with which : been reported by Hillier, iers in barracks, firemen, orded by Dr. Colcott Fox $t$ Office.

$t$ be said to be absolutely on statements of patients, ? is contagious. If it were brushing and cutting the be more prevalent among

them hyphomycetic fungi obably cases of ringworm ceded that groups of cases $t$ Fox-in which the most or micro-organism, which ontagious type of Alopecia

lass of evidence is against

$t$ association of Alopecia anced as an argument in en asserted that when the disease occurs in adults it is the result of ringworm in childhood (Hutchinson). This view, however, is contrary to general experience. Alopecia areata may occur synchronously with Tinea tonsurans or it may follow it, either replacing the ringworm patches or affecting other situations on the scalp, but this does not prove that the disease is due to a fungus and it may be a mere coincidence of two common diseases of childhood. Where bald patches replace the ringworm these may be produced by irritating applications used in its treatment. On the other hand cases occur in which the alopecia was present before the treatment was begun, when the baldness is probably due to the deleterious action of toxins from the ringworm fungi acting on the hair-papillae and interfering with the growth of the hair.

It has frequently been pointed out by supporters of the parasitic theory that the falling of the hair in Alopecia areata may be preceded and accompanied by a transient erythema, which disappears soon after the hair falls out; that when a patch is spreading, an erythematous border or halo precedes the defluvium of the hair; and also that the distribution of the patches may be similar to that of small-spored ringworm. These observations are a common experience but are of little value as evidence, for while a certain number of cases have a distribution simulating that of ringworm, many have not and either show a markedly symmetrical arrangement or affect the margin of the scalp in a manner in which ringworm 
never does. So far as the transient erythema is concerned a similar erythema not infrequently occurs before the defluvium of the hair consequent upon exposure to the X-rays, in which case it can have nothing to do with micro-organisms or fungi but is probably the result of a mild inflammatory reaction due to the toxic influence of the dead cells of the hair-bulb.

On the whole the evidence in favour of a parasitic cause is inconclusive and the facts that it tends to recur and may be symmetrically distributed are in opposition to the theory.

(b) Tropho-neurotic Theory.-This hypothesis, though it seems to have more in its favour than the microbic theory, is also incapable of final proof and is only applicable to a certain number of cases. According to it, the defluvium is brought about by inhibition of the function of the papillae by disease or destruction of the nerves supplying them.

The cases which have been grouped under the headings of "Alopecia universalis " and "Alopecia localis seu neurotica" are generally admitted to be of nervous origin and to be due respectively to severe mental disturbance or to neuritis or destruction of the nerve supplying the affected area. In these cases the patches occur as a rule in the area supplied by the affected nerve but occasionally appear elsewhere, possibly as a result of reflex nerve action, and they may be symmetrically distributed.

Jacquet recently supported the nerve theory in an elaborate thesis on the subject. According to him Alopecia areata is a reflex trophoneurosis, the most common causes of which are dental defects, and auricular, bronchial, pharyngeal, gastro-intestinal, and genital, disorders. Out of 273 cases he found that in 185 it began at periods corresponding to the eruption of the permanent teeth.

According to Rousseau and Decelle certain sites of dental pain are associated with definite areas of alopecia, for example the eruption of wisdom teeth with alopecia of the neck, pain in the canines with alopecia of the chin, and dental troubles of the upper jaw with frontal and parietal alopecia-the bald areas corresponding roughly to Head's areas of referred pain from dental causes. In certain cases, when the dental defect was remedied by the extraction of carious teeth, etc., the hair grew again in a few months.

Painful trigeminal neuralgia has been followed by Alopecia areata of the same side, associated with vaso-motor phenomena such as blushing and rise of temperature.

This ingenious theory of Jacquet's has not been generally accepted. Dental troubles are common at all periods of life and their association with Alopecia areata is to be expected, but after examining a considerable number of children from this point of view the conclusion has been come to that no definite causal relation can be established between them, and it has been found that bald areas are just as frequent in association with perfect teeth as in connection with serious dental defects. Nor is there any evidence that peripheral irritation of the nerves of the larynx, gastrointestinal tract, or genitalia, can cause Alopecia areata by a reflex action. Cases are on record in which the bald patches were associated with headache from eye-strain and in which re-growth took place when the error of refraction was corrected, but such cases are rare and may be mere coincidences.

It has been observed also that in the ordinary cases of Alopecia areata, apart from the local traumatic cases, no objective signs of inflammation or degeneration have been detected in the nerves supplying the bald areas and, consequently, the tropho-neurotic theory has to be based on clinical rather than histopathological evidence.

The principal arguments in favour of the theory are:--(I) bald patches can be produced experimentally or accidentally by section of, or injury 
to, a nerve, and occur in the area supplied by that nerve or in other situations from reflex action. (2) Many cases are on record where partial or complete alopecia followed severe mental shock; in a case under the care of the writer a patient subject to slight outbreaks of Alopecia areata had serious family trouble which rendered her nervous and determined an extensive attack with complete denudation of all the hair of the body. (3) In a certain number of cases, especially in adults, the baid patches are associated with localised nerve disturbances either preceding or accompanying them, such as localised neuralgia, tenderness on pressure, itching, alterations in sensibility to heat and cold, secretory disturbances such as impairment of the sweat function so that the patch does not perspire even with injections of pilocarpine, and vaso-motor paresis on account of which the bald areas do not readily react to irritants or to the actinic rays of light. (4) Alopecia areata has been noted in connection with facial paralysis, vitiligo, epilepsy, chorea, and leucoderma. The association with leucoderma, at least, cannot be regarded as merely accidental, as leucoderma is a rare condition and yet has been observed so frequently to co-exist with Alopecia areata-sometimes preceding the baldness, at other times following it - as to point to a causal relationship. Leucoderma is generally admitted to be of neurotic origin and it is reasonable to suppose a similar cause for the alopecia.

The chief arguments against the theory are:-(I) Alopecia areata is most common in children and early adult life, at the time when worry, anxiety, and mental disorders, are rarest. (2) It frequently occurs in patients apparently enjoying perfect health and in whom there is no sign or suggestion of neurosis. (3) It is only occasionally associated with pain or alteration in sensibility of the bald patches. (4) Only in exceptional cases do the bald areas correspond to the distribution of a nerve or group of nerves.

A consideration of the above arguments for and against the nerve theory will show that it is far from being established and that at present the most that can be said for it is that a certain number of cases are undoubtedly of neurotic origin.

(c) Toxic Theory. A limited number of cases of Alopecia areata are explicable as the result of the action of a toxin either reaching the papilla by the blood-stream or locally produced in and around the hair-follicle. The baldness caused by the injection of bacterial toxins in animals, or that which results from taking acetate of thallium, which is a nerve poison, are examples of baldness produced by a toxin or poison circulating in the blood, while alopecia which occurs over and for a short distance beyond a furuncle of the scalp, and that which frequently follows a patch of suppurating ringworm, are examples of baldness caused by toxins locally produced.

That the falling of the hair may be associated with transient erythema also suggests the action of a toxin and this is borne out by the type of celullar infiltration which is present and its distribution; this infiltration consists chiefly of lymphocytes with a few mast-cells and is not confined to the papillae but occurs also about the capillaries in the neighbourhood of the follicle, whereas if it were due to bacteria in situ the infiltration would consist to a larger extent of polymorphonuclear leucocytes and the distribution would be more localised about the follicle.

In conclusion it may be said, (i) that the tropho-neurotic theory probably accounts for a larger number of cases than the other two, (ii) that a few groups of cases have occurred which appeared to be contagious and which suggested a microbic origin but in which no microorganism was found, (iii) that except for these rare cases there is no definite evidence of a parasitic origin. 
Diagnosis.-No great difficulty should be experienced in the recognition of Alopecia areata if a careful examination be made of the bald patches. The chief diagnostic points are the disappearance of hairs, the smoothness of the patches, the presence of the typical clavate stumps towards the periphery of the patch in recent lesions, and the occasional existence of a slight inflammatory halo at the spreading edge. It has to be distinguished from the following :

Ringworm of the scalp. - Small-spored ringworm is distinguished by the presence of short stumps all over the affected area, which on microscopical examination are found to be laden with fungus, and by the scaliness of the patch.

In the "black dot" variety due to the endothrix fungus, where the patches are small and the stumps broken off level with the surface, some difficulty may be experienced, but the surface is not so smooth as in Alopecia areata and the black specks dotted over it on being expressed are found, microscopically, to be the intra-follicular portion of hairs containing chains of a large-spored ringworm fungus, while any short stumps which may occur in Alopecia areata never contain fungus.

Alopecia cicatrisata (Pseudo-pelade) has to be carefully distinguished from Alopecia areata as the prognosis in it is far more unfavourable. In it the patches are much more irregular, the skin over them is atrophic, and instead of stumps single hairs or tufts of hairs persist, which are surrounded at the orifice of the follicle by an inflammatory halo and are easily epilated, the intra-follicular portion being swollen, moist, and translucent.

Lupus erythematosus may form bald patches on the scalp but these differ from Alopecia areata in being atrophic, erythematous, presenting telangiectases over the surface and cribriform pits corresponding to the mouths of the hair-follicles, and being usually associated with typical lesions on the face.

Morphoea or localised sclerodermia occasionally occurs in the scalp and may give rise to difficulties in diagnosis, but in it the lesion has a smooth waxy appearance, presents no stumps, and is surrounded by a peculiar lilac-tinted halo over which small dilated vessels can be seen ramifying.

Impetigo or abscesses of the scalp may be followed by bald patches but these are definitely inflamed and there is a previous history of the purulent affection.

Baldness produced by the Röntgen rays closely resembles Alopecia areata and were it not that the X-ray baldness is exactly localised to the area exposed it would be almost impossible to distinguish them clinically.

Prognosis.-The prognosis depends on the extent and rapidity of the defluvium, the number of previous attacks, and the age of the patient. In a first attack the prognosis as a general rule is good and the re-growth of individual patches may be expected in 
three to six months. The appearance of new patches, however, may prolong it indefinitely. Recurrences may be said to be the rule rather than the exception and there is a distinct tendency for each successive outbreak to be more extensive than its predecessors. Occasionally as the result of some severe illness or mental trouble in an individual who has been subject to Alopecia areata, a complete deflurium of the whole hairy system may take place.

The prognosis is unfavourable in elderly people, especially if they have been subject to attacks previously.

Marginal patches usually take longer to recover than central lesions and in patches about the nape of the neck re-growth may not occur for a year or more. Recovery is also prolonged in cases where the beard and moustache are affected.

In generalised alopecia the outlook is always serious and if the re-growth has not begun in a year it usually indicates that the baldness will be permanent. Even when new hair appears it is liable to be delicate and unstable and to show a marked tendency to fall out again, either completely or in patches, to the great disappointment of the patient.

\section{TREATMENT}

General Remarks.-Alopecia areata being a disease of which the exact cause is unknown the treatment is largely empirical and, as it is usually prolonged and the prognosis uncertain, considerable tact is required on the part of the physician in its management.

As there is no conclusive evidence that it is contagious there is no necessity for isolation.

Whatever the cause may be, in the majority of cases it ceases to operate spontaneously and re-growth takes place. The re-growth may be hastened by judicious treatment but may even occur in spite of unsuitable treatment. It is well to explain this to patients as it not infrequently happens that, becoming tired of the slow progress under a certain regime, they seek the assistance of patent remedies or fall into the hands of some quack or so-called " hair specialist," and should the spontaneous recovery and re-growth occur about the time that the new remedy is being tried it is unnecessary to add that it will probably get the credit.

The treatment is principally local but to obtain the best results the local applications should be combined with suitable general measures.

General Treatment.-Any defect of health which may be even remotely connected with the Alopecia areata, such as anaemia, indigestion, intestinal troubles, cardiac and circulatory affections, diseases of the kidneys, and rheumatism, should be attended to on general medical principles. It not infrequently happens that patients suffering from this disease are mentally depressed, overworked, complain of 
headaches, and are neurasthenic, and the fact of their hair falling out still further adds to their unhappiness. In such cases a tonic treatment with the usual remedies, such as nux vomica, phosphates, iron, or arsenic, and rest, general massage, change of air and surroundings; sea-bathing, and exercise, are indicated. In the case of debilitated children of the hospital class more benefit can be obtained by better food and improved hygienic conditions than from any form of local application. In view of the possibility of Alopecia areata being produced reflexly by local irritation, this should be carefully sought and treated. The hygiene of the mouth should be attended to and defective teeth removed or stopped, errors of refraction corrected, catarrh of the auditory canal or aural disease suitably treated, and adenoids removed.

There are no drugs which, taken internally, have any definite claim to be regarded as specifics for the re-growth of the hair. Arsenic has been employed in this connection but it acts as a general nerve tonic rather than as a specific remedy.

Pilocarpine has a considerable reputation and cases have been recorded in which re-growth has taken place soon after its use. It may be given in the form of one-eighth to one-quarter of a grain of the nitrate by the mouth, or one-thirtieth of a grain of the hydrochloride may be injected locally into the scalp. Although this drug occasionally gives good results, its action is uncertain and the discomfort from the excessive perspiration which it causes and the danger of its inhibiting the heart's action necessitate the utmost caution in its employment.

Local Treatment.- Of the first importance in the local treatment of Alopecia areata is the proper hygiene of the scalp. In women it should be washed about once a month, and in men once a fortnight, with any non-irritating toilet soap. If dandruff be present, soap spirit lotion, containing two parts of green soap, one part of spirit, and a few drops of thymol, should be rubbed on with a piece of sponge. Any associated diseased condition, such as pityriasis or impetigo, must be treated by appropriate remedies. Epilation of the hairs around the bald patches has been advocated with the object of arresting the progress of the disease, but is of no benefit.

If the beard or moustache be affected they should be shaved regularly as this not only reduces the disfigurement but stimulates the hair-growth.

In generalised cases it may be necessary to wear a wig, but this must be carefully fitted to avoid constricting any part of the scalp and so impeding the circulation and retarding the growth.

The local applications may be divided into antiseptics and stimulants.

Antiseptics.--Those who believe in the parasitic origin of the disease advise lotions or ointments containing mercury, carbolic acid, sulphur, resorcin, or salicylic acid, such as the following: 


\begin{tabular}{|c|c|c|c|c|c|}
\hline Hydrarg. perchlor. - & - & 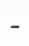 & - & gr. ii & .5 \\
\hline Spiritus vini rect. ad & & & & $\overline{3} i$ & $99 \cdot 5$ \\
\hline Resorcin $\quad-\quad-$ & & & - & $\bar{j} \mathrm{i}$ & $\mathrm{I} 2$ \\
\hline Spiritus vini rect. ad & - & - & - & $\ddot{3} \mathrm{i}$ & 88 \\
\hline Sulphur praecip. & - & - & - & gr. xx & 4 \\
\hline Ac. salicyl. - & - & - & - & gr. $\mathrm{x}$ & 2 \\
\hline Paraffinum molle ad & - & $\begin{array}{ll}- \\
-\end{array}$ & - & $\overline{3} \mathrm{i}$ & 94 \\
\hline Hydrarg. perchlor. - & - & - & 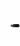 & gr. ii & .5 \\
\hline Ac. carbol. - - & - & - & - & $\mathrm{m} \cdot \mathrm{x}$ & 2 \\
\hline Paraffinum molle ad & - & - & - & $\bar{\partial} \mathrm{i}$ & $97 \cdot 5$ \\
\hline
\end{tabular}

These should be applied all over the scalp daily and rubbed in especially to the bald patches. They are chiefly valuable where dandruff is present which is due to slightly virulent micro-organisms.

A useful antiseptic lotion for general use is :

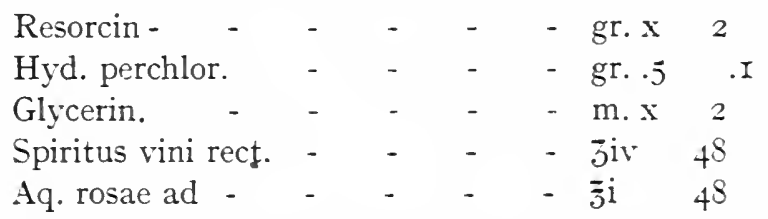

The glycerine should be omitted in the case of women as it is apt to make long hair unpleasantly sticky, and the resorcin when the hair is white or fair as it stains it a rusty tint ; in such cases the following lotion should be subştituted :

\begin{tabular}{|c|c|c|c|c|c|}
\hline Hyd. perchlor. & & & & gr. .5 & .I \\
\hline Ol. ricini - & - & - & - & m. $v$ & I \\
\hline Ac. acet. & - & - & - & m. ii & \\
\hline Spt. lavand. - & - & - & - & jii & 24 \\
\hline Alcohol. $90 \%$ ac & - & - & - & $\tilde{j} \mathrm{i}$ & $74 \cdot 4$ \\
\hline
\end{tabular}

Stimulants.-Wore valuable as local applications are the irritant and stimulant remedies, of which the most commonly emplored are acetic acid, turpentine, nitrate of silver, cantharides, carbolic acid, chrysarobin, and iodine.

At one time the customary treatment was blistering by such applications as :

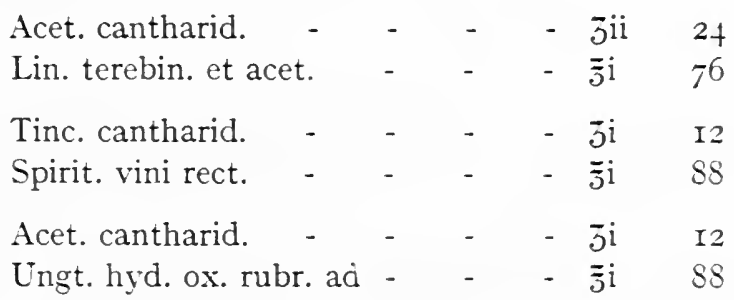

One or other of these is painted on, or rubbed into, the bald areas twice a day until blistering takes place. 
This procedure gives excellent results but it is unpleasant and milder stimulants, giving equally good results in the end, are preferable. Of these the following are recommended :

\begin{tabular}{|c|c|c|c|c|c|}
\hline Acet. cantharid. & - & - & - & - & $\operatorname{m.xxx} 6$ \\
\hline O1. limonis. - & - & - & & & 3 ii $\quad 24$ \\
\hline Spirit. vini rect. & - & & - & & $\overline{\tilde{J}} \mathrm{i}$ \\
\hline Ac. acet. - & - & - & 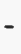 & 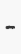 & $\operatorname{m.xxx} 6$ \\
\hline Chloroform ad & - & - & - & & $\begin{array}{ll}\overline{3} \mathrm{i} & 94\end{array}$ \\
\hline Tinc. capsici - & - & - & - & - & $\mathrm{m} \cdot \mathrm{v}$ \\
\hline Eau de Cologne & - & - & - & - & $3 \mathrm{ii}$ \\
\hline Spirit. vini rect. & - & - & - & - & $3 \mathrm{i}$ \\
\hline Lin. camphor. & - & & & & $3 \mathrm{iv}$ \\
\hline Lin. ammoniae & - & - & - & - & jiv \\
\hline Lin. chloroform. & - & - & - & - & $3 \mathrm{iv}$ \\
\hline
\end{tabular}

These should be painted on the bald patches daily or rubbed in with a piece of cottonwool.

Chrysarobin is a useful stimulant and may be employed in the form of a $5 \%$ ointment. It has to be used with great care as it is liable to set up conjunctivitis should it reach the eyes and has the additional disadvantage of staining the scalp and the hair. The best method of using it is in the form of a pencil made with wax and lanolin as follows:

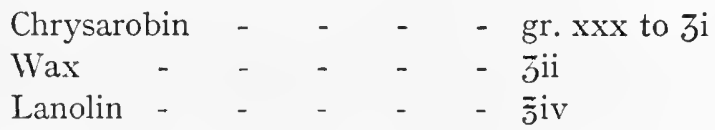

It may also be dissolved in traumaticin (gutta-percha $3 \mathrm{i}$, chloroform $\bar{j}$ ), which dries on the skin as a thin film.

Nitrate of silver is an excellent irritant and, as it stains the scalp, is also serviceable in minimising the disfigurement in patients with dark hair. It should be used in the form of a 5 to ro $\%$ aqueous solution painted on the bald patches daily so long as the scalp does not become too sore.

Carbolic acid is best employed as carbol-glycerine (2 to I) which should be painted on twice a week, then wiped off with methylated spirit.

The choice of the irritant and the strength employed depends on the degree of resistance of the individual scalp, but it is always well to begin with mild applications and to increase their strength gradually until the limit of toleration is reached.

The disfigurement from the disease may be diminished by the use of small patches of hair of a suitable tint which can be obtained from a coiffeur and stuck on, or by colouring the bald areas with charcoal or with some cosmetic.

In extensive cases where the eyebrows and eyelashes have gone, the discomfort entailed by the absence of the eyelashes, which not only 
shade the eye but protect it from the entrance of foreign bodies, may be obviated to some extent by wearing false eyelashes on the upper lids. These consist of strips or ribbons to which hairs are attached and are fixed to the lid by spirit gum. They are comfortable and realistic in appearance, their only disadrantage being their costliness.

Physical Remedies.-Various physical remedies have been used with benefit, such as massage, actinic rays of light, X-rays, and highfrequency currents.

Massage.-Massage is of undoubted value and where practicable should always be recommended, as it improves the local circulation and prevents the scalp from becoming too closely adherent to the skull ; it is specially valuable where the adipose layer is deficient. Where possible it should be carried out by an attendant.

The procedure is simple and is as follows: The fingers should first be dipped in almond oil and then, with the thumbs placed at the sides of the head, the scalp should be moved in all directions over the shull by the pressure of the fingers. The whole scalp should be gone over systematically in this way once or twice a day, about I $_{5}$ or 20 minutes being devoted to the procedure.

Instead of massage, friction with a towel or brushing with an ordinary hair-brush or with a metal brush forming an electrode in a galvanic or faradic circuit, have been recommended but none of these is as good as massage and the brushing may do harm by superficially lacerating the scalp.

Actinic Ray's.-The experience that the hair grows with greater rapidity in summer than in winter when the actinic rays of light are most intense suggested the employment of light in the treatment of this disease. The actinic rays can be obtained conveniently from a Finsen lamp or one of its substitutes. They act by producing a mild inflammatory reaction and are definitely stimulating and more penetrating than the local applications generally employed. Exposures should be given at a distance of six inches from the lamp and should be of sufficient duration to produce a mild reaction. They are repeated at intervals of a few days.

X-rays. - Mild exposures to the $\mathrm{X}$-rays have a stimulating action but their employment is too dangerous for general use.

High-frequency Currents.--Various electrical methods have been employed, such as brush discharges from a high-frequency apparatus and the sparks from a static machine; the latter to be effective are painful and, being no more certain in their action than either the actinic rays or massage, are not to be recommended.

\section{REFERENCES}

AUDRY, C. Affections of the nails in Alopecia areata (Journ. des Mal. Cut. et Syph., I900, xii. p. I6I).

Bowex, J.T. Two epidemics of Alopecia areata (Journ. Cut. \& Gen.-Urin. Dis., I 899, xvii. p. 399).

—_ Epidemic alopecia, etc. (Journ. Cut. Dis., I9I 5, xxxiii. p. $3+3$ ). 
Davis, Haldin. Epidemic Alopecia areata (Brit. Journ. Derm., I9r4, xxvi. p. 207).

Du Bors. Wassermann reaction in Alopecia areata (Ann. de Derm. e $t$ de Syph., Nov. I9Io, p. 554).

Fox, Colcott. Group of cases of Alopecia areata (Brit. Journ. Derm., I 897, ix. p. I05).

— Small epidemics of Areata alopecia (Ibid., I9r3, xxv. p. 5 I).

Hodara. Action of chrysarobin in Alopecià areata (Journ. des Mal. Cut. et Syph., Sept. r903).

JACQUet, L. A hundred attempts to inoculate Alopecia areata (La Presse Méd., Dec. 1903).

Jeanselme. Acetate of thallium in Alopecia areata (Ann. de Derm. et de Syph., r 898, ix. p. 999).

Jenkins, C. E. Case of exophthalmic goitre followed by Alopecia areata (Brit. Journ. Derm., I9r5, xxvii. p. 407).

Jones, H. EmLyn. Reflex irritation as a cause of Alopecia areata (Brit. Journ. Derm., r9r2, xxiv. p. 362).

KROMAYER. Light treatment in Alopecia areata (Monats. f. prakt. Derm., I905, p. r).

Pöhlman. Alopecia areata, etiology, investigations with thallium salts (Arch. f. Derm. u. Syph., Feb. I9r3).

Sabouraud. New researches on the causation of Alopecia areata, relation to syphilis (Ann. de Derm. et de Syph., r91o, p. 545).

— Recent researches on etiology of pelade (Ibid., I9I3, p. 88).

\section{PSEUDO-PELADE, OR ALOPECIA CICATRISATA}

Syn. : Alopécie cicatricielle (Besnier); Pseudo-pelade (Brocq); Alopecia circumscripta seu orbicularis (Neumann); Folliculitis decalvans (Crocker).

This rare form of alopecia is associated with destruction of the hairfollicles and is characterised by the presence of numerous small, atrophic, bald patches which coalesce to form irregular cicatricial areas, the borders of which are serrated by the projection into them of unaffected parts of the scalp. This disease was originally recognised as an entity by Neumann but it was first carefully described by Brocq in $I_{8} 85$.

Description.-It begins insidiously, without pain or inflammation, and is usually first noticed as several bald patches situated about the scalp or the occiput which are discovered" accidentally in brushing or combing the hair. These vary in size from a pin's head to a lentil, are round or oval in shape, white or pinkish in colour, and present a smooth, atrophic, soft, depressed surface over which the sensibility is diminished. Larger patches about the size of a fingernail may also occur which, instead of being roundish, are usually irregular in outline and tend to spread peripherally and to coalesce to form bald areas the size of the palm of the hand or larger. (Fig. 329.)

Though the disease is slowly progressive it does not result in complete baldness but a few islands or tufts of hair generally persist irregularly distributed over the bald areas, while in even the most 


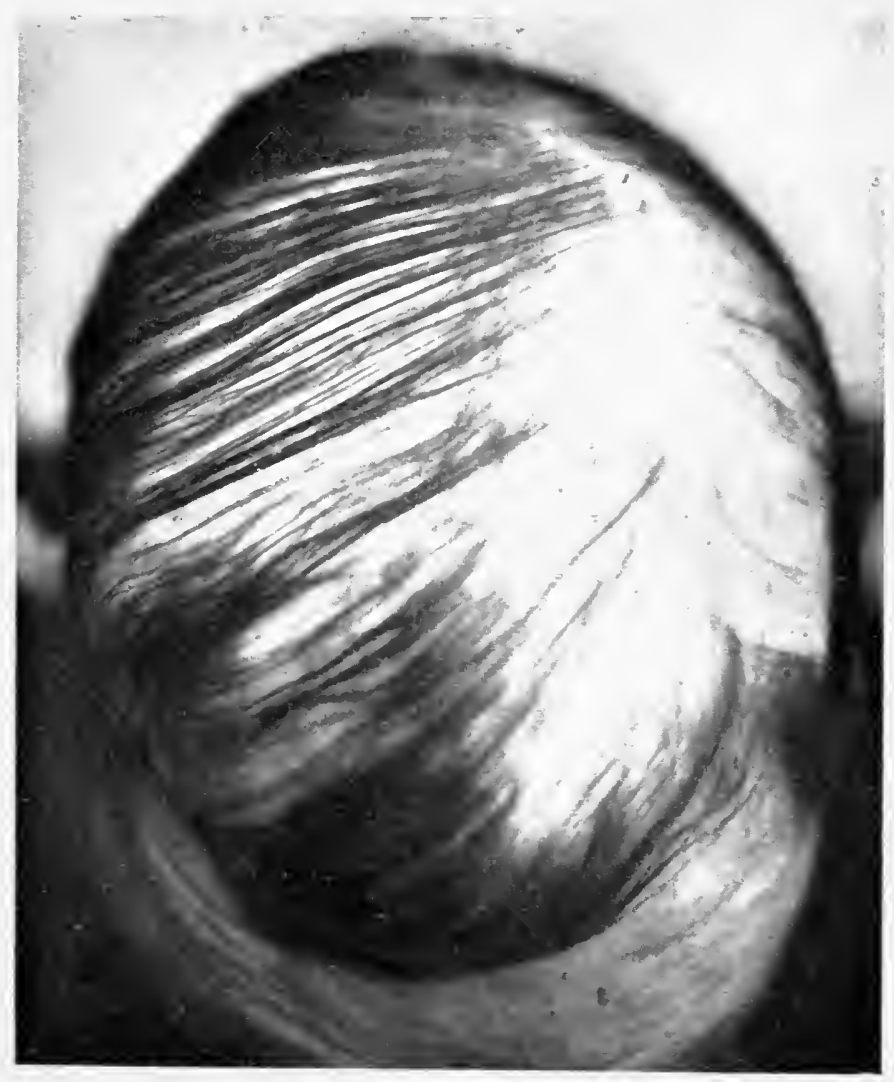

Fig. 329.-Alopecia Cicatrisata.

Showing tufts of hair irregularly distributed over the bald area.

[To face p. 936 . 

extensive cases a margin of hair remains. No stumps or broken hairs occur in the patches but they are dotted over here and there with isolated hairs or tufts which seem to have been missed over by the diseased process. These hairs come out on the slightest traction and, on epilation, the intra-follicular portion is found to be thickened and translucent, from the root-sheath remaining adherent to the root of the hair and being swollen and moist from the absorption of fluid. Where the affection has existed for some time the root-sheath dries up to form a cheesy powder, the intra-follicular portion of the hair becomes shrivelled and tapered to a fine point like the "point-ofexclamation-hairs" of Alopecia areata, and the hairs may break off leaving a short stump or plug which scarcely projects above the level of the skin. There is no definite evidence of suppuration about the follicles but there may be a faint pink inflammatory halo around them. Sometimes small scales may be detected at the follicular orifice and about the hair, which disappear when the hair is shed. Occasionally when the hairs fall out slight pits are left which disappear as the patch becomes atrophic. The hair beyond the patch is apparently quite healthy and there are no transition stages between the patch and the healthy scalp.

The sites of predilection for the disease are, in order of frequency, the vertex, upper and medium part of the occiput, and the temples, but any part of the scalp may be attacked except the margin.

The nails are not affected as a rule, though occasionally they may show signs of dystrophy, such as splitting and discolouration.

The evolution of the disease is slow. It is seldom continuous but is usually intermittent with periods of rest and activity, and sometimes after remaining stationary for several years and being limited to a few patches, it will suddenly become active and in a few months result in loss of the greater part of the hair.

Histopathology.-The histopathology of the affection has been worked out by Lenglet from a microscopical examination of three cases. According to this observer the most marked features in the histology are, (I) dilatation of the capillaries between and around the hairfollicles, which are filled with red blood corpuscles, leucocytes, and mast-cells; (2) cellular infiltration, consisting of lymphocytes and a few plasma-cells, around the dilated capillaries, massed about the upper part of the hair-follicles, and extending to the papillae in attenuated columns; (3) rarefaction of the connective tissue around the ressels and the follicles, the collagen fibres being separated by oedema and the elastin having disappeared. Another characteristic feature is the presence of numerous pigment cells in the papillary layer of the corium. The epidermis either shows no definite change or is atrophied.

The death of the hair is considered by Brocq to be brought about by the cellular infiltration enveloping the follicle and causing it to break up by the cells passing in and separating the different parts of the follicular wall. 
Repeated attempts have been made to find micro-organisms in the swollen bulb of the affected hairs but invariably with negative results; attempts to cultivate bacteria from it have been equally unsuccessful, except in one instance where a culture of a peculiar coccus was obtained (Bunch), but this observation has not been confirmed.

Etiology and Pathogenesis.-This affection occurs in adult life and usually between the ages of 20 and 45 . It is more frequent in men than women, and is most common in persons with coarse dark hair. There is no evidence that it is contagious.

Its nature and pathogenesis are unknown. It seems to be more easily explained as being of toxic origin than as due to micro-organisms in situ. It has been suggested that it is tropho-neurotic, but there is no conclusive evidence to support this hypothesis.

Diagnosis.-When the clinical characteristics are considered together there should be no difficulty in recognising pseudo-pelade.

It might be mistaken for atrophic patches resulting from fawns, but an examination of the hairs for the fungus of favus would establish the diagnosis.

It might have to be distinguished from sclerodermia in small plaques or from cicatricial alopecia due to ulceration, traumatism, or Herpes zoster, but the numerous lesions, the glossy atrophic bald skin, and the hairs with swollen root-sheaths should prevent this mistake.

From Alopecia areata it is distinguished by the absence of " pointof-exclamation "-hairs and by the existence of marked atrophy of the skin.

It might be confused with Li:pus erythematosus of the scalp but in it the bald skin is red and pitted, or covered with adherent scales, and the lesions are less numerous and are generally accompanied by characteristic patches on the skin of the face or on the ears.

The affection described by Brocq as Keratosis pilaris may resemble it, but in it small red papules occur around the hairs, which are slender and do not have a succulent root-sheath, the scalp feels rough like a nutmeg grater, and the affection is usually present also on the extensor aspects of the limbs and on the eyebrows.

The condition described by Lailler as Acné décalvante and by Quinquaud as Folliculites épilantes et décalvantes is probably simply a further development of pseudo-pelade.

Prognosis.-It is slowly progressive but in some cases spontaneous arrest takes place. It is doubtful if treatment has much effect upon it though it may delay its progress. Once any part of the scalp is affected, it remains permanently bald.

Treatment.-As the pathogenesis is unknown the treatment is more empirical than rational. Any defect in the general health should be carefully sought for and an attempt made to correct it by suitable means.

The local treatment generally adopted is based on the assumption that it is a microbic affection, and consists of epilation of the diseased 
hairs and thorough application daily of parasiticide lotions or ointments containing mercury, sulphur, resorcin, or ichthyol. Brocq advocates a lotion of perchloride of mercury $I$ in 500 , or an ointment of yellow oxide of mercury. Crocker employed an ointment of the biniodide of mercury, containing 2 grains in an ounce of vaselin.

\section{REFERENCES}

Broco, Lexiglet, Ayrigsac. Researches in alopecia with atrophy, variety pseudo-pelade (Ann. de Derm. et de Syph., I905, Jan., Feb., and March $\rangle$.

Broco, L. Pseudo-pelade (Derm. prat., I907, p. 648).

MACLeOD, J. M. H. Pseudo-pelade (Brit. Journ. Derm., I909, xxi. p. 27).

\section{KNOTTED HAIRS}

Knotting of the hair is not uncommon in connection with curly hair of the scalp or pubes. It occurs more readily in hairs which are flat on transverse section than in those which are round, as the former have a greater tendency to curl on being stretched and to form loops, which subsequent brushing or combing transforms into knots which appear as nodes on the hair and may be either true single knots or slip-knots, and may be loose or drawn tight. This condition was designated Trichonodosis by Galewsky-a name which is unsatisfactory as it is equally applicable to nodes due to other causes, such as the fungus-concretions of piedra. (Fig. 330.)

\section{REFERENCES}

Galewsky. A not-previously described disease of the hair: Trichonodosis (Arch. f. Derm. u. Syph., Sept. I906, p. I95).

MACLEOD, J. M. H. Knotting of the hair (Brit. Journ. Derm., I907. xix. p. 40).

SAalfeld, E. On Trichonodosis (Galewsky) (Arch. f. Derm. u. Syph., Oct. I906, p. 245).

\section{SPLITTING AND BREAKING OF THE HAIR}

Syn.: Fragilitas crinium. Trichorrhexis nodosa.

Splitting and breaking of the hair may occur as the result of general malnutrition from some constitutional disease or in association with some local morbid cundition of the scalp. The affected hairs become thin, lustreless, brittle, and easily broken or split in brushing or combing.

In I8 49 Erasmus Wilson applied the name Trichorrhexis nodosa to an affection characterised by splitting of the hair to form greyish- 
white nodules situated at the free end of the hair and at irregular intervals on the shaft. On microscopical examination these nodules are found to resemble in appearance the interlocking of the bristles of two brooms and to be due to the hair having split like a greenstick fracture. (Fig. 33I.)

In the eastern countries of Europe where this condition has attracted considerable attention it was met with chiefly in the hair of the beard

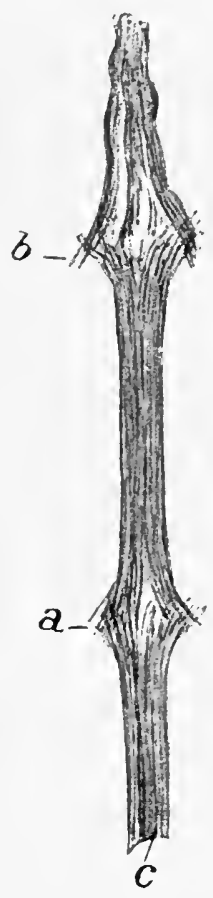

FIG. 331 -Trichorrhexis

Nodosa.

$a$. Node made up of two brushes. b. Node with the upper brush split into three. c. Hair-shaft.

(From MacLeod's Pathology of the Skin.) and moustache; in this country it has also been observed in the atrophic hairs of Alopecia areata, in morbid conditions of the scalp like pityriasis, and as a result of the presence in the hair of the ringworm fungus.

Etiology.-Certain observers have regarded it as a specific disease of microbic origin and several micro-organisms have been isolated from the nodules, such as a diplococcus (Raymond), and a bacillus (Hodara), and it has even been asserted that the micro-organism could be transmitted not only from one hair to another but also to the hairs of shaving brushes and that it could give rise to similar nodules in them.

The microbic theory is no longer accepted, however, and the general concensus of opinion is in favour of the view originally advanced by Wilson, namely, that it is simply the result of mechanical injury to hairs whose nutrition, for some reason or another, has become defective. In corroboration of this, it has been shown (I) that the condition can be produced mechanically in hairs with defective nutrition by placing them on a glass slide and tapping them with the edge of a paper-knife or other instrument (Adamson) ; (2) that it may be met with in any old shaving brush the hairs of which have been long subjected to the deleterious action of soap ; (3) that it is readily caused by brushing or combing hairs which have been rendered brittle by the action of such chemical agents as pyrogallic acid and silver nitrate used in dyeing the hair, or even by strong soaps.

Treatment.-The treatment depends on. the cause. Where the dystrophy is associated with some definite derangement in health, this should be treated on general principles. When some local affection of the scalp is present, such as pityriasis, it should be dealt with by suitable local remedies, the scalp toned by massage and the application of a stimulating lotion, and vigorous combing or brushing aroided.

Where the fraying is confined to the tips, the affected hairs should be pointed but there is no advantage in cutting them short. 


\section{REFERENCES}

ADAmsor, H. G. The etiology of Trichorrhexis nodosa (Brit. Journ. Derm., 1907, xix. p. 99).

Bruhns, C. The etiology of Trichorrhexis nodosa (Arch. f. Derm. u. Syph., xxxiii. Jan. I897).

HodARA. Bacillus of Trichorrhexis nodosa (Monats. f. prakt. Derm., I894, xix. p. 173).

LASSEUR. A contribution to the study of Trichorrhexis nodosa (Ann. de Derm. et de Syph., Nov. 1906, p. 9 II).

\section{MONILITHRIX}

Der. : Monile, a necklace.

Syn. : Beaded hair ; moniliform hair.

The name monilithrix has been applied to a rare type of atrophy of the hair which only involves portions of the shaft and gives rise to a beaded appearance. On examination with a lens fusiform thickenings or nodes are found alternating with atrophic constrictions or internodes. In the node, which is really the normal part of the hair, the medulla and pigment are present; while in the internode, which is the atrophic portion, the shaft is shrunken and the medulla and pigment are absent. Both the intra-follicular and the extra-follicular parts of the hair are affected. (Fig. 332.)

The condition, which is usually a congenital anomaly and may occur in several members of a family, is met with chiefly in the hairs of the scalp but has been observed also in the evebrows and eyelashes and even in the lanugo hairs of the limbs. In it the affected hairs tend to break off at an internode with a brush-like fracture and to remain as bristles, which may be quite short or may reach a length of 2 or 3 inches. It is usually permanent and eventually leads to complete baldness, though in occasional cases certain of the hairs escape for some time at least and may grow sufficiently long to conceal the bristles. (Fig. 333.)

The exact cause is unknown. It is not in-

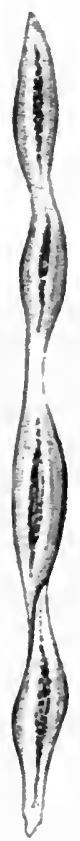

FIG. 332.-Monilithrix.

Hair twisted and beaded.

(From MacLeod's Pathology of
the Skin.) frequently associated with keratosis of the mouth of the hair-follicles which it has been suggested has something to do with the production of the beading. It is difficult to understand how pressure at the mouth of the follicle could produce it, however, and it seems more probable that it is the result of some intermittent interference with the nutrition of the air-aphpillae giving rise to defective development at the internodes. 
Treatment is of comparatively little use, as the affected follicles and papillae are so altered as to be incapable of growing normal hairs. In those cases in which keratosis is present, the employment of some keratolytic, such as salicylic acid or resorcin in an ointment, may be tried. An artificial defluvium of the hair by the $\mathrm{X}$-rays was tried by Cranston Low but without benefit.

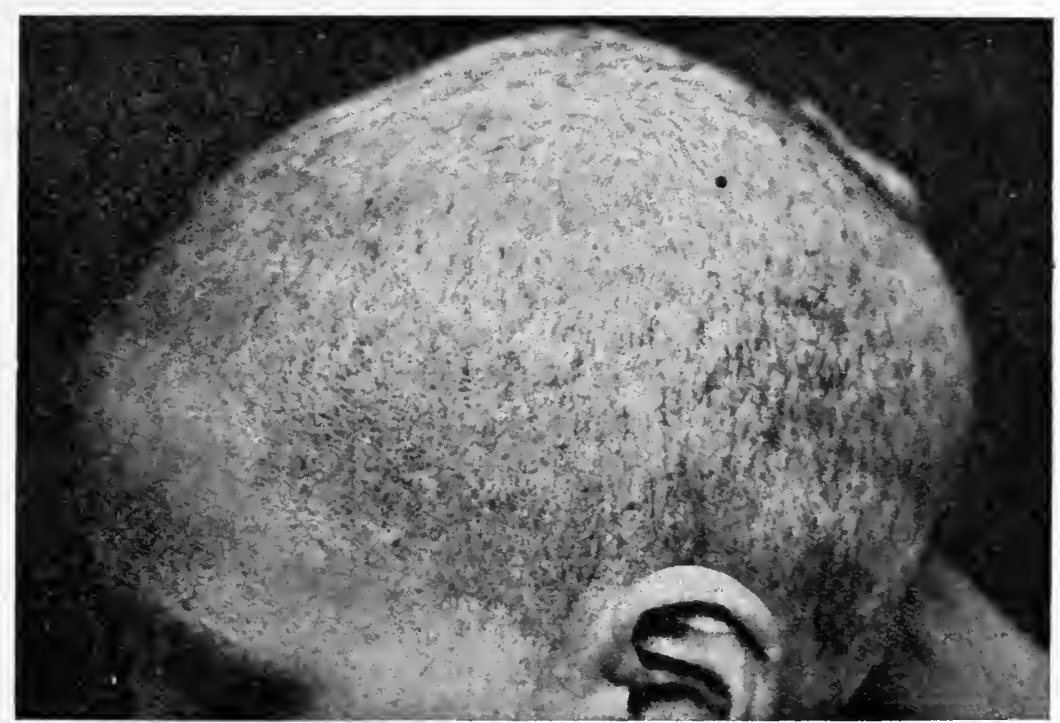

FIG. 333.-MIonilithrix.

Scalp showing baldness and prominence of follicles.

(From Dr. R. Cranston Low.)

\section{REFERENCES}

Beatry, IV., \& Scotr, J. A. Moniliform hairs (Monilithrix) (Brit. Journ. Derm., I892, iv. p. I7I).

Dore, S. E. Monilithrix (Brit. Journ. Derm., I9I I, xxiii. p. III).

Galloway, J. Alopecia areata \& Keratosis pilaris (Brit. Journ. Derm., I 896 , viii. p. 4I).

Jamieson, A. A case of monilithrix (Brit. Journ. Derm., I898, x. p. 325).

Low, R. CRanstox. Pathology and Etiology of Monilithrix (Journ. of Path. and Bact., I909, xiv. p. 230).

Schütz, J. A case of spindle-hairs (Monilithrix) (Arch. f. Derm. u. Syph., July I90o, p. 69).

\section{CANITIES}

Der. : Cani, grey hairs.

Syn.: Greyness or whiteness of the hair; Leucotrichia.

All degrees of whiteness, from the normal colour through grey to pure white, may be met with in hairs. The depigmentation is usually diffuse and may either affect the hair equally in its whole length or may be more marked in the distal than in the proximal end of the shaft ; 
on the other hand it may be patchy and give rise to a speckled appearance, or it may have an annular arrangement in which pigmented and white rings alternate (Leucotrichia annularis). (Fig. 334.)

As a rule the scalp is the part to be first affected and the whiteness appears later on the hairs of the face and body ; consequently it is not uncommon to find white hairs on the scalp with grey hairs in the moustache or beard, and normal coloured hairs on the body. The whiteness may involve the whole of the scalp, or it may occur in one or more patches and give rise to a piebald appearance.

The depigmentation generally takes place gradually and insidiously, but in not a few instances it has occurred rapidly-in about twentyfour hours-as the result of some profound mental shock.

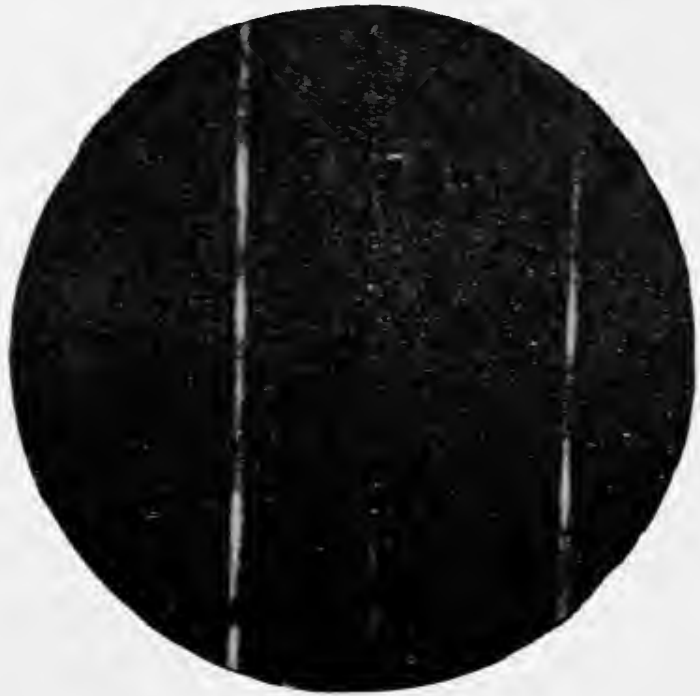

FIG. 334.-Ringed Hairs.

(Dr. Colcott Fox's case. Brit. Journ. Derm., 1906.)

The whiteness, once established, is usually permanent, but occasional cases are on record where the pigmentation has returned, as where the hair has become white owing to some severe general illness and regained its colour on recovery, or as in Alopecia areata where it has grown in white and subsequently become pigmented. There are instances also in which the hair has shown a tendency to become grey in winter and to return to its normal colour in summer, constituting a periodic canities similar to that which occurs in animals in the Arctic regions.

Pathology.-The greyness or whiteness of the hair results from the disappearance of the pigment granules situated in or around the cells of the cortex, and of the diffuse colouring matter of the protoplasm of the cells. In those cases in which it occurs suddenly it is probably due to shrinkage and atrophy of the cells of the hair-shaft and the presence of air bubbles between them. These changes would seem to be brought about by interference with the circulation and nutrition of the hair. 
papillae, in some instances from defective innervation, which tends to cause shrivelling of the soft cells of the bulb and the lower part of the shaft and allows air to collect between them.

Etiology.-In a majority of the cases canities is an evidence of senile degeneration, but in certain individuals it may develop comparatively early in life as the result of some acquired delicacy or hereditary peculiarity. It may occur prematurely with equal readiness in women or men and in dark or fair hair.

Permanent white patches or tufts may be due to a congenital defect or may develop in vitiligo.

Temporary patches or scattered grey hairs, which may recover or may be permanent, may result from some local disturbance such as Alopecia areata or Herpes zoster, or may follow a prolonged debilitating illness, some nervous disorder such as neuralgia, mental shock, worry, anxiety, or other psychosis.

Premature greyness may be due to the presence of seborrhoea of the scalp.

Whitening of the eyelashes has been known to occur in association with an error of refraction or with sympathetic ophthalmia.

The formation of the rings in Leucotrichia annularis is difficult to explain. It would appear to be the result of a rhythmical dystrophy in which either pigment does not form or air is allowed to enter between the cells, and it is possibly related in its causation to monilithrix, or may be a congenital defect. It has occurred in association with pigmentary disorders elsewhere.

Treatment.-Canities is progressive and permanent but occasionally recovery may take place, either spontaneously or from treatment.

Where the general health is defective, improvement may be obtained by a general tonic regime.

Where the whiteness is comnected with some morbid condition of the scalp, such as seborrhoea, efficient treatment of the latter may lead to recovery of the pigmentation. In this connection it is important that resorcin should not be employed, as it is liable to stain grey or white hair a dirty brownish or sandy tint. The circulation of the scalp should be stimulated by suitable tonic lotions, massage, or faradisation.

Any desire to dye the hair should be discouraged, as apart from the unsatisfactoriness of the procedure, many of the dyes employed for producing dark tints contain silver nitrate and pyrogallol which are injurious to the hair, rendering it brittle and apt to fray, while those which give the most natural results in lightish brown hair frequently contain a preparation known as hydrochlorate of paraphenylen diamen which is extremely irritating to certain skins and may produce severe dermatitis.

REFERENCES

Adamson, H. G. Ringed hairs after X-rays (Brit. Journ. Derm., Igo6 xviii. p. 404 ). 
Cheatle, G. Lexthal. Grey hair associated with nerve lesions (Brit. Med. Journ., 1912, ii. p. f;o).

Incidence of the hair's greyness (Brit. Med. Journ., July I905, ii. p. $1 ; 6)$.

Fox, T. Colcorr. Ringed hairs (Brit. Journ. Derm., I906, xviii. p. 321 ). FreEMAx. Anomalous condition of the eyelashes (Brit. Journ. Derm., I902, xir. p. 23).

Gallowar, J. Ringed hairs (Brit Journ. Derm,, I Sg6, viii. p. 437).

MEACHEx, $\Sigma$. Leucotrichia annularis with other pigmentary disorders (Brit. Journ. Derm., 1902, xiv. p. 86).

\section{HYPERTRICHOSIS, OR EXCESSIVE HAIR-GROWTH}

Syn. : Hirsuties : Hypertrichiasis ; Polytrichia.

Hypertrichosis may be congenital or acquired.

When congenital, it may be universal, the infant being born with long hair which mar either persist throughout life or may fall out soon after birth and be replaced on the glabrous skin by fine lanugo hairs; or it mar be partial, the long hairs being confined to the scalp, cheeks, or to some other region.

Partial congenital hypertrichosis occurs in various types of hairy moles which are generally deeply pigmented, sometimes wartr, vary in size from a pin's head to large lesions sometimes occupring a whole region of the body, and the hair on which is usually black.

Congenital hypertrichosis is frequently associated with other defects or deformities, such as the absence of certain teeth, club-foot, or concealed Spina bifida when the hairy patch occurs in the lumbar region.

When acquired the hypertrichosis is usually partial and is met with chiefly on the face, especially on the upper lip, cheeks, and chin, and less frequently on the chest, back, arms, and legs. It mar be the result of excessive growth of already existing long hairs, or of the transformation of lanugo into long hairs. It is doubtful if it is ever due to the formation of new hair-follicles and the increase in the number of hairs may be the result of the growth of several in the same follicle from one papilla.

Numerous cases of congenital and acquired hypertrichosis of an excessive degree are recorded in the literature, some of which have appeared as freaks on exhibition in various parts of the world, such as the well-known Russian "dog-faced man " who was corered with hairs from head to foot, women with hair reaching down to the ground, and men or women with beards several feet long.

Excessive hair-growth on the body in the male is not necessarily a sign of strength as was once believed, but may occur in weaklings and has been observed not infrequently in tuberculous subjects.

In women, it would appear to be more prevalent in brunettes than blondes, but this may be more apparent than real and due to dark hairs being more noticeable than fair ones. In some cases in which 
marked hypertrichosis occurs on the face, the growth of hair on the scalp may be scanty.

Sometimes the hairs, instead of growing long, may grow in the wrong direction, and cases have been described in which the hairs on the back and arms have been found to slant upwards instead of downwards. The most common defect of this nature is Trichiasis, in which the eyclashes instead of turning upwards bend in and irritate the conjunctiva.

Etiology.-The cause of the excessive growth is uncertain but a number of factors predispose towards it.

It may be a racial peculiarity, certain people like the Burmese and the Ainos of Japan having a profuse hair-growth while in others, like the negro, the growth is comparatively slight.

It may be a family trait and may occur in several generations.

In women it is frequently associated with functional disturbances of the genital organs and may be observed at puberty, in connection with a too early appearance of the menses, in pregnancy, at the climacteric period, and in connection with sterility. Menstrual disorders predispose towards it and it has been known to follow a temporary cessation of the menses, the long hairs falling out and being replaced by lanugo hairs when the periods returned.

In women also it frequently accompanies mental disorders, sometimes follows a severe illness, and has been met with in connection with malignant disease, especially carcinoma of the supra-renal glands, but this may be coincidence.

It is frequently asserted that it may be caused by local irritation, such as friction or blistering, and by the application of greasy substances to the skin, but the effects of these have been somewhat exaggerated and the popular fear that the application of anything greasy to the face in women is of necessity followed by an excessive growth of hair is groundless.

Prognosis.- When once it has occurred it tends to increase rather than diminish with age and only in rare cases, where it has followed a severe illness or some temporary disturbance of the female genital organs, has it been known to disappear spontaneously.

\section{TREATMENT}

Introduction.-The removal of hairs is chiefly demanded by women with hypertrichosis of the face, partly for aesthetic reasons and partly on account of the mental effect of the disfigurement which causes them to be over-sensitive and occasionally to think so much about the trouble that it becomes an obsession.

In the case of small downy hairs in women under 25 years, the patient should be advised to have nothing done and should be warned not to attempt any measures for their removal such as epilation, clipping with scissors, or the employment of depilatories, for these only make 
matters worse by stinulating the growth and transforming lanugo into long hairs or by setting up a dermatitis.

In blondes the application at night of a dusting powder containing equal parts of zinc oxide and starch has a drying effect on the skin and may retard the growth, while in brunettes the disfigurement may be reduced by bleaching the hairs with hydrogen peroxide, Io rols.

In older women, where long hairs are present, whether these be few or numerous, their removal is advisable.

In men, superfluous hairs occurring on the bridge of the nose, between the eye-brows, or on a mole, may require removal.

Treatment.- Superfluous hairs may be removed temporarily by depilatories, epilation, or shaving, but the only radical method which is approximately successful is electrolysis.

$\mathrm{X}$-rays. - Recently the X-rays have been advocated by certain writers but their use is to be deprecated because, although they are a valuable means of causing a temporary defluvium, any attempt to make this permanen $i$ is almost certain to result in atrophy of the skin and scarring. A pastille dose is the maximum which may be given to the skin without producing dermatitis, but this only causes temporary shedding of the hair, while if it be exceeded a permanent defluvium may follow but is generally accompanied by atrophic changes and the formation of telangiectases. Attempts have been made to prevent this by interposing between the shin and the tube a screen of aluminium about half a millimetre in thickness to cut off the less penetrating rays which, it is asserted, are responsible for the dermatitis, and by giving a series of pastille doses at intervals of about a fortnight. In this way it has been said that the papillae and deeper parts of the follicles may be permanently destroyed without injury to the skin, but the results from this procedure are uncertain and attended with considerable risk.

Depilatories.-Depilatories are unsatisfactory and liable to do more harm than good. The most frequently employed contain sulphide of barium, as in the following formulae:

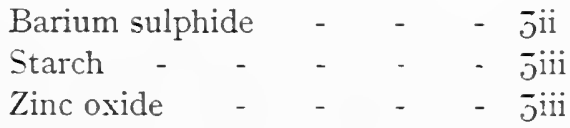

This is mixed into a paste with a little water, rubbed on the skin for five minutes, then removed, the skin washed with water, and a bland zinc paste applied.

\begin{tabular}{llll} 
Barium sulphide & - & - & - gr. $v$ \\
Starch - & - & - & - gr.xv \\
Powdered soap & - & - & - gr. xv \\
Benzaldehyde ad & - & - & - \\
\hline $\mathrm{i}$
\end{tabular}

This is applied for five minutes, then washed off.

Such depilatories simply corrode the hair as far as the follicle and have no action on the intra-follicular growing portion except to stimulate 
its growth; at the same time they are liable to inflame the skin and may cause eczematisation.

Pumice Stone.-A simple and efficacious temporary method of dealing with excessive hair-growth is by rubbing the affected skin with pumice stone. For this purpose the artificial pumice stone block is best. The hairs should be cut short and the block gently rubbed over the affected area, against the direction of the hair-growth, for a few minutes morning and evening. Should the skin become irritated by the process it should be discontinued for a few days and a soothing cold cream or calamine lotion applied.

Bleaching with Hydrogen Peroxide.-In brunettes, dark hairs on the face or limbs may be made less noticeable by bleaching with hydrogen peroxide. The affected part should first be rubbed with ether to remove any greasiness and then dabbed with peroxide (ro vols.). This also has the advantage of corroding the delicate lanugo hairs.

Various peroxide creams and soaps are on the market but are less efficacious than the pure peroxide.

Epilation should be deprecated strongly as it invariably strengthens the hair-growth and transforms the fine down into coarser hairs.

Shaving.-Of the temporary measures of dealing with hypertrichosis of the face in women where the growth is excessive and the circumstances of the patient are such as to render electrolysis out of the question, the most satisfactory method is shaving. This procedure is repugnant to most women at first but the initial prejudice is gradually overcome by custom and by the employment of a safety razor.

Electrolysis.-As already stated electrolysis is the nost reliable method of destroying hairs and if properly done should leave little or no scarring. It is, however, an operation of exceeding delicacy, somewhat painful, and is tiring to the operator. To perform it satisfactorily requires both knowledge and skill, and if done in an inferior manner it is valueless and may be responsible for disfiguring cicatrices and even for cheloidal scars.

The requisite apparatus consists of a galvanic battery of at least Io cells fitted with a reliable milliamperemeter, a cylindrical electrode covered with chamois leather, and an electrolysis needle-holder. Every operator has his own favourite needle-holder of which there are many patterns. The one used by the writer is light, and about three inches long; to one end of it is attached a thin connection cord, about two yards in length, which is soldered to the end of the holder and not fixed in the usual fashion by a screw. The needle itself is fine and tapering, bent at an angle of forty-five degrees about half an inch from the point, and made of platino-iridium which has the advantages over a fine steel sewing-needle (No. I6) that it can be bent, is slightly blunt and consequently less liablo to pierce the follicular wall in catheterising the follicle, and can be heated to red heat in the flame and so sterilised. The holder and connection cord being light neither impair the sense of touch nor interfere with the delicate manipulation on which the success 
of the operation depends. A small pair of forceps about an inch and a half long is fixed to the holder for remoring the electrolysed hairs. The holder mar be fitted with an attachment for making and breaking the current but this is unnecessary as the patient can do this by grasping or letting go the electrode. To certain of the holders a small lens is fixed, but this is of little ralue and only makes the holder unnecessarily heary. In order to see the follicular opening it is advisable, howeser, for the operator to use a lens of some kind. The ordinary watch-maker's lens has the great disadvantage of a short focal distance and necessitates an uncomfortable proximity to the patient. An ordinary pair of spectacles with a single lens of about Io dioptres or a binocular magnifier such as that derised by- Treacher Collins enables the operator to work comfortably at a distance of about 5 inches from the patient.

The patient should be seated opposite a good light in a comfortable chair, the head steadied br a head-rest or cushion, and a towel placed on the lap. The cylindrical electrode attached to the positive pole of the battery is dipped in a solution of salt and water and grasped by the patient, while the needle-holder is connected with the negative pole. The operator then selects the hair to be electrolysed, inserts the point of the needle into the orifice of the follicle on the obtuse angle side of the hair, then gently presses it down parallel with the long axis of the hair for about an eighth of an inch till the whole length of the follicle has been catheterised. The controlling handle of the battery is turned till 2 to 5 milliamperes are registered on the meter. The needle should be pushed in for another sixteenth of an inch so as to pierce the hair-papilla, and allowed to remain in the follicle for about Io seconds when a frothy bead of hydrogen may be seen at the mouth of the follicle showing that electrolysis is taking place. The current is then gradually reduced and the needle withdrawn.

The most difficult part of this procedure is the proper insertion of the needle and the passing of it through the papilla, as the follicles usually slant in an oblique direction and it is much easier to penetrate the follicular wall and to pass the needle alongside the papilla than through it.

The next important point is the method of making and breaking the current. The ideal procedure is for the battery to be worked by an attendant, who should be told to turn on the current gradually to the requisite strength after the needle has been inserted, and after a given number of seconds to reduce it to nil, when the needle is withdrawn. About a minute later the hair should be found to be loose in the follicle and easily epilated, coming away with a swollen gelatinous sheath. When an attendant is not available the patient may be asked to make and break the current by grasping and letting go the electrode, or a needle-holder fitted with an attachment for this purpose and controlled by the operator may be used. On no account should the needle charged with electricity be inserted into the follicle, as it not only $3:$ 
produces a certain amount of electric shock but owing to its corroding effect passes too easily through the wall and renders it difficult to know that it is in its proper position. In the same way the needle should not be withdrawn until the current has been turned off or an unnecessary shock will be caused. The hair should not be epilated immediately on withdrawing the needle but a minute or more should be allowed to elapse, during which time a softening and loosening takes place which enables the hair to be drawn out easily. To save time half a dozen hairs may be electrolysed one after another, and pulled out in the order in which they were treated.

The effect of the electroly'sis is to produce necrosis of the follicle, made evident by a yellowish stain surrounded by a pink zone at the orifice. Should the needle have been inserted wrongly and passed through the follicle into the corium, the pain is more acute and a blanching takes place. Soon after the electrolysis, an urticarial lesion may appear around the follicular orifice and persist for a few hours or a vesicular lesion may form. These are gradually replaced by small scabbed lesions, the scales of which separate in a few days, leaving an erythematous atrophic scar which is generally so minute as to be scarcely noticeable. If the electrolysis has been excessive a small pit may be left and sometimes a cheloidal thickening. Great care should be taken in performing the electrolysis to aroid attacking contiguous hairs, for by so doing the necrotic zones may overlap and a superficial ulcer be produced. Occasionally when the needle is withdrawn a small haemorrhage may occur but this is easily stopped by pressure with a piece of cottonwool. In dark-skinned people the procedure is frequently followed by slight pigmentation which disappears in a few months.

The requisite current varies according to the size of the hairs to be destroyed. Where the hairs are fine a current of $\mathrm{I}$ to 2 milliamperes may suffice; for hairs of a medium size about 3 milliamperes are necessary, and for strong coarse hairs for 5 milliamperes.

The length of time the current should pass ranges from five seconds in the case of fine lanugo hairs, to 20 seconds for coarse hairs, ten seconds being an average time. About forty long hairs or sixty lanugo hairs may be electrolysed at a sitting of half to three quarters of an hour ; more should not be done as it becomes irritating to the patient and tiring to the operator.

The pain or discomfort associated with the operation varies greatly in different cases and in different situations in the same case, and in sensitive neurotic women it is sometimes so marked as to make it impossible or inadrisable to electrolyse more than about 20 hairs at a sitting. It consists of a prickling pain when the current is made and a burning sensation as the electrolysis proceeds. It is, as a rule, most intense on the upper lip towards the middle line, near the lower eyelid, and on the lower lip, and is least on the sides of the chin and in the soft parts of the cheeks, but in any situation it is much more marked 
in some spots than in others. Other symptoms may be produced by the operation, such as a metallic or salt taste in the mouth or slight tingling about the teeth, but these are of little consequence.

The pain cannot be remored satisfactorily by a local anaesthetic. Attempts have been made with injections of cocain but the wide distribution of the affection and the considerable dosage of cocain which is necessary, renders it an unpractical and somewhat dangerous proceeding. Freezing with ethyl chloride has also been emplored but is unsatisfactory as it hardens the tissue too much. In cases where only a tuft of large hairs has to be remored, the injection of about I c.c. of $I^{0}{ }_{0}$ solution of norocain deadens the pain but is unnecessary.

After the operation the face should be thoroughly swabbed over with boric lotion and the patient should be told to bathe it twice daily and to apply to the inflamed area calamine lotion, or an antiseptic paste such as the following:

\begin{tabular}{|c|c|c|c|}
\hline Hyd. ammoniat. & - & - & - gr. \\
\hline Zinci oxidi - & 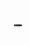 & 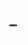 & $5 \mathrm{iii}$ \\
\hline Paraf. molle alb. ad & - & - & $\bar{j} \mathrm{i}$ \\
\hline
\end{tabular}

The probability of the success of the procedure varies with the type of case and the skill of the operator. With the utmost care the operator may consider himself fortunate if he succeeds in destroring $80^{\circ}$ of the follicles electrolysed at the sitting. Owing to the ease with which the point of the needle may slip past the papilla about $20^{\circ}$ of them are apt to be imperfectly done and the hairs will grow again in the course of a few months. It is adrisable to inform the patient of this possibility and to arrange for a second course of sittings later.

The cases which give the most satisfactory results are middle-aged women with sparsely distributed long hairs about the chin: in such cases occasional treatment by electrolysis will suflice to keep the condition well in check.

It is a different matter in cases which present large numbers of hairs amounting to a beard, whiskers, or moustache. Brocq has estimated that there are from one to nine thousand hairs on the chin, fifteen to nineteen thousand on the entire beard, and five hundred to twelve hundred on the upper lip. The difficulty of destroving such a growth is colossal but much can be done to minimise the disfigurement by courses of treatment at regular intervals of six months or a vear.

In the case of the upper lip, unless when the hairs are particularly strong and long, it is wiser to leave the condition alone: the hairs in that situation are usually fine and the follicles so small and tight that the operation is extremely difficult and painful and, should it be imperfectly done, is apt to leave disfiguring scars.

\section{REFERENCES}

Buaxd-Stitos, J. Hypertrichosis \& concealed Spina bifida (Lancet, ISS $\overline{7}$, ii. P. 5$)_{\text {. }}$ 
Broco, L. Electrolysis of hairs (Ann. de Derm. et de Syph., I897).

_- Electrolysis of hairs (Traitement des Dermatoses, I 898, p. I39).

BuLlOCK \& SEQVEIRA. On the relation of the suprarenal capsules to the sexual organs (Trans. Path. Soc. Lond., I905, 1vi. p. 189).

Freshwater, D. Hairiness or Hypertrichosis (Practitioner, I9I3, xc. p. 825$)$.

Vignolo-Lutati, C. Congenital circumscribed hypertrichosis over the vertebral column without Spina bifida (Giorn. Ital. d. Mal. Ven. e della Pelle, I9 I5, ii. p. I 72 ). 


\section{CHAPTER IXXT}

\section{DISEASES OF THE HAIR-FOLLICLES}

Introduction.-The pilo-sebaceous follicles are the seat of origin of a large number of skin affections. This is due to rarious reasons of which the most important are the following: The follicles and their protruding hairs are apt to be more prominent than the intervening skin and in consequence are more exposed to friction and more liable to become inflamed; they are richly supplied with blood, so that micro-organisms and toxins tend to be concentrated there; and, last but most important, they form the easiest mode of entrance for microorganisms to penetrate into the deeper lavers of the skin, as at the junction of the upper and middle thirds of the follicle the Stratum corneum, which extends from the surface to form a lining for the follicle, ceases, and micro-organisms, which readily collect in that situation, are no longer prevented by a horny barrier from penetrating the epidermis. In addition the contents of the sebaceous glands constitute a suitable medium for the growth of pyogenic organisms.

The diseases of the hair-follicles may be divided into two groups, namely, (I) those in which inflammation in and about the follicles is the most marked characteristic, constituting the various forms of folliculitis; (2) those in which evidences of inflammation are slight or absent and in which the leading characteristic is the presence of horny plugs or filiform spines at the follicular orifices.

\section{FOLLICLLITIS}

By folliculitis is meant inflammation in or about the hair-follicle, and this may vary in degree from slight hyperaemia to an acute suppurative process giving rise to a follicular pustule. A large number of different causes may be responsible for it of which the following are the most frequent:

I. Folliculitis due to Micro-organisms in the Follicle.--Examples of this are met with in the follicular impetigo of Bockhart, furunculosis, coccogenic sycosis from the action of pus-cocci, and in the peculiar indolent type of folliculitis known as Acne scrofulosorum, which is due to tubercle bacilli. 
Ir: Acne vulgaris the folliculitis is secondarv to the plugging of the follicle by a comedo and is due to the growth in the pent-up sebaceous material of certain micro-organisms.

In Acne cheloid the folliculitis is due to staphylococci and is associated with hypertrophy of the peri-follicular tissue.

In Acne necrotica the folliculitis is due to certain micro-organisms, the acneiform pustule being followed by necrosis and scarring.

2. Folliculitis due to Ringworm and Favus.-When these fungi are present in the hair-follicle they readily set up folliculitis which may be of an acute suppurative type, especially if the fungus be of animal origin.

3. Folliculitis due to Chemical Irritants.--Chemical irritants, such as croton oil, aconite, and tar, when rubbed on the skin are capable of causing pustulation which is most marked at the follicles. Folliculitis may also follow the ingestion of iodine and bromine, especially the potassium salts.

4. Folliculitis may occur in association with dermatoses which do not directly involve the follicles, such as rosacea, eczema, psoriasis, etc.

\section{DISEASES OF THE HAIR-FOLLICLES ASSOCIATED WITH THE PRESENCE OF HORNY PLUGS OR FILIFORM SPINES}

The subject of the follicular diseases of the skin in which horny plugs or filiform spines are present in the follicles has been rendered especially difficult by the multiformity and confusion of the nomenclature in which (i) the same name, modified perhaps by an adjective, is used to signify different conditions ; (ii) different names are used to signify the same affection; and (iii) names are employed which do not properly indicate the nature of the affection to which they are applied.

There are certain cutaneous affections in which spines or horny plugs occur occasionally in the follicles but are not a characteristic feature, such as Lichen planus, Lichen scrofulosorum, Pityriasis rubra pilaris, Seborrhoic dermatitis, and miliary follicular syphilides; in others they are an essential and diagnostic feature, such as in Acne vulgaris (comedones), Psorospermosis follicularis regetans (Darier), Keratosis supra-follicularis (Unna), and Lichen spinulosus.

\section{KERATOSIS SUPRA-FOLLICULARIS (UNNA)}

Sin. : Cacotrophia folliculorum (Tilbury Fox); Folliculitis rubra (Wilson); Keratosis follicularis (Kaposi); Kératose pilaire (Brocq).

Closely allied to it, if not the same affection: Ulerythema ophryogenes (Taenzer), Follicular xerodermia (Liveing), Ichthyosis cornea (Hardy), Ichthyosis follicularis (Lesser), Keratodermie pilaire (Thil)ierge). 
Defuntion: An affection of the shin characturised by the presence at the orifice of the hair-follicles of horny plugs or scales forming small acuminate papules.

Description.-The essential lesions of this affection consist of pinhead-sized rounded or acuminate papules resulting from the blocking of the follicles by horny plugs 0.5 to $\mathrm{I} \mathrm{mm}$. in height. The papules may be pale like the surrounding skin or of a dirty-grey colour, or they may be pinkish and covered with small telangiectases. Considerable diversity of opinion exists as to whether they are of inflammatory origin, or whether the inflammation which is occasionally present in them is secondary and due to local irritation or to adventitious micro-organisms.

In the pale lesions, at all events, the papular elevation is entirely due to the horny plug, which can be picked out easily and is simply a noninflammatory hyperkeratosis of the mouth of the follicle. Sometimes the papule is pierced by a hair but as a rule the hair is found coiled up beneath the plug. The skin between the papules is more or less harsh and dry and sometimes definitely scaly and ichthyotic ; it may be pale in colour or, in dependent parts, may be red and mottled. Subjective symptoms are generally absent though occasionally itching may occur.

The mildest type of this affection consists of a sort of permanent goose-skin affecting the extensor aspects of the arms and thighs, and occasionall $y^{-}$the trunk, and to a less extent the face about the erebrows, forehead, and jaws-the papules on the face being smaller and more numerous than those on the limbs and trunk.

In more severe cases, such as those described by Brocq as Keratosis pilaris, the lesions are more pronounced and arranged in irregular patches which sometimes enclose areas of healthy skin, or are irregularly distributed on a skin which may be normal or xerodernic. In these cases the papules on disappearing are frequently replaced by small cicatrices and the skin assumes an atrophic appearance or, in rare instances, may be pink and inflamed, especially about the evebrows.

In the most severe type the scalp is affected and there is more or less complete baldness, the erebrows and erelashes are absent, and plugged follicles are distributed irregularly over the whole skin with the exception of the palms and soles, the skin itself is rerodermic and in certain situations, such as the knees, buttocks, and thighs, may be definitely ichthrotic. The sweat-glands are not affected, so that the patients can perspire freely on exertion. These severe cases appear to be of the nature of ichthyosis of the follicles and have been described as Ichthyosis follicularis by Lesser and others. This affection usually: occurs in families and begins in early life. It is not generally noticed at birth but evidences of it may be detected within the first two rears, the eyebrows usually being first involved.

What may be regarded, provisionally, as a still more adranced stage of this affection was described by Taenzer under the heading of " scarleaving erythema of the evebrows" (Clersthemu superciliure seu 
ophryogenes). In five out of six such cases observed by him it began in early childhood with redness of the eyebrows followed by loss of the hair ; it spread from there until it affected the forehead, cheeks, scalp, and extensor aspects of the limbs, causing the scalp to be more or less bald and the eyebrows, especially the outer halves, to be hairless. As in the milder types described above the essential lesion consisted of an acuminate plugged follicle. In Taenzer's cases, after the disease had persisted for some years marked atrophy occurred at the orifice of the follicles, resulting in the formation of depressed cicatrices surrounded by a hyperaenic halo which gave to the scalp an appearance such as that met with after recovery from favus.

It has not been decided definitely whether the above types represent different stages in a common pathological process or whether they include two distinct entities, namely, (i) an ichthyotic affection in which, if inflammation occurs, it is secondary and to which the name Ichthyosis follicularis is applicable; and (ii) an acquired condition which appears later and results from chronic inflammation at the mouth of the follicles and which corresponds to the Kératose pilaire rouge of Brocq.

Cases of mild plugging of the follicles on the extensor aspects of the limbs and elsewhere may occur at any age, frequently in association with malnutrition, and are so common as to attract little attention. They may show no sign of ichthyosis and no definite evidence of inflammation, and nay be the result of simple keratosis of the follicles caused by local irritation or by the elimination of toxins by the skin.

Histopathology.- The most noticeable feature in the histology of a case of Ichthyosis follicularis examined by the writer, was marked dilatation of the funnels of the pilo-sebaceous follicles which were filled with horny plugs extending beyond the level of the skin and formed acuminate papules. The lower two-thirds of the follicles were atrophied and in some of them pieces of atrophic hairs were detected. The horny plug consisted of a dense central portion and a peripheral part with the structure of a loose horny network with a tendency to a concentric arrangement. The sebaceous glands were atrophic or absent.

The epidermis between the follicles showed slight hyperkeratosis forming a loose network, the prickle-call layer was thin, and the interpapillary processes flattened out.

In the underlying corium the blood-vessels in the neighbourhood of the follicles were dilated and there ras an infiltration of small round or oval connective tissue cells around the dilated capillarics. The connective tissue fibres were normal, and not rarefied as they are in inflammatory processes.

From the histological examination it seemed that the primary change was in the epidernis and consisted of hyperkeratosis affecting, not only the funnels of the follicles, but the intervening skin as well and leading to the formation of a horny network like that in mild degrees of ichthyosis. The atrophy of the lower part of the follicles 
seemed to bo simply the result of mechanical pressure dute to the plugging. The dilated blond-ressels and cellular infiltration suggested a mild inflammatory process resulting from the presence of the plug in the follicle which acted as a foreign body and was readily exposed to friction.

Other descriptions of the histology have been furnished by Linna, Lemoine, and Giovannini, which differ from the above in that pronounced inflammatory changes were found not only around the follicles but also between them, leading to the conclusion that the affection was of inflammatory origin.

Etiology and Pathogenesis. - The condition usually begins in child. hood, but may occur at any age, though it is rare in old people.

Mild degrees are common in those who rarely take baths and whose occupations expose them to dust and dirt. On the other hand it may be associated with malnutrition and was believed at one time to be a disease essentially of the badly nourished and scrofulous, hence the name Ichthrose anserine des scrofuleux applied to it by Lemoine in ISS2.

It has a distinct tendency to occur in families and in children of ichthyotic parents, and in a series of cases observed by Treacher Collins and the writer it affected three boys out of a family of five.

The exact nature of all the cases is uncertain. I considerable number of them are undoubtedly of congenital origin and a variety of ichthyosis in which the outer parts of the pilo-sebaceous follicles are chiefly involved; in others it may be an acquired affection and simply due to the local irritation of dirt or to the elimination of toxins in a badly nourished skin, and in them the horny plug is the primary lesion and any inflammatory changes which may supervene are secondary:

Diagnosis.- Mild degrees of this affection resemble "goose-skin" (Cutis anserina), but goose-skin is transient and due to cold or fright, while even the milder degrees of Keratosis supra-follicularis are persistent. In goose-skin, also, the lesions cannot be picked out by the nail like the horny plugs of Keratosis supra-follicularis.

The disease with which it is most liable to be confused is Lichen spinulosus and the chief points of distinction are : (I) in Lichen spinulosus the lesions are invariably papules with filiform spines projecting from them, while in Keratosis follicularis man! of them are acuminate follicles with a horny plug at the orifice which make the skin feel to the touch like a nutmeg-grater; (2) in Lichen spinulosus they are arranged in circumscribed patches or groups instead of being irregularly and diffusely distributed on the extensor aspects of the limbs: (3) in Lichen spinulosus the long hairs of the scalp and eyebrows are not affected, there is no family tendency, and the affection is not associated with xerodermia or ichthyosis.

It is distinguished from Pityriasis rubra pilaris by the presence in the latter of marked redness from inflammatory changes, scaly 
keratosis of the palms and soles, follicular plugged papules on the backs of the fingers, and a tendency for the acuminate papules to coalesce to form patches.

Prognosis.-The condition persists indefinitely unless treated. In mild cases it can be greatly reduced by suitable local treatment, in widely distributed cases with definite ichthyosis all that can be done is to keep it in check and to render the skin as smooth as possible by treatment.

Treatment.-The treatment is similar to that for ichthyosis and consists of warm baths and the application of soap spirit lotion on a piecc of flannel. Keratolytics, such as resorcin or salicylic acid, 20 grains, in glycerine of starch, I oz., are soothing and effective. When the skin is very dry it should be inuncted daily with some oily application, such as almond oil 2 parts and lime water I part.

\section{REFERENCES}

Brocg, L. Kératose pilaire (Ann. de Derm. et de Syph., 1893, p. 25).

Lewaxdowsir, F. Keratosis follicularis (Arch. f. Derm. u. Syph., r91o, ci.).

MACLEOD, J. M. H. Ichthyosis follicularis (Brit. Journ. Derm., I909, xxi. p. (65).

TAENzer. Ulerythema superciliare seu ophryogenes (MIonats. f. prakt. Derm., I 889 , viii. p. 197).

Uxis, P. G, Keratosis supra-follicularis (Histopathology, English Translation, i 896, p. 287 ).

Watsox, Chaluers. Keratosis pilaris, with special reference to the vascular changes in the skin (Brit. Journ. Derm., 1904, xvi. p. I).

\section{LICHEN SPINULOSUS (DEVERGIE)}

Sin.: Keratosis follicularis spinulosa (Unna); Lichen pilaris seu spinulosus (Crocker); Acné cornée (Hardy, Leloir); Keratose pilaire engainante (Audry).

Definition: An affection of the skin characterised by groups or patches of projecting filiform spines surmounting acuminate pinkish papules arising from hair-follicles.

Description.- Lichen spinulosus is an affection which is by no means rare in this country, especially among children. It has not attracted much attention, howerer, owing to the absence of subjective symptoms and the insignificance of the clinical appcarances. The essential lesion is a pin-head-sized, acuminate, whitish or pinkish papule, surmounted by a filiform spine which projects one-sixteenth to one-eighth of an inch from the follicle and which, if picked out, leaves a depression.

The lesions occur in large numbers but invariably remain discrete. They come out in crops and tend to form patches more or less irregular in outline and varying in size up to several inches in diameter. They may occur on different parts of the trunk and limbs but are most common on the back of the neck, buttocks, abdomen, shoulders, thighs, and extensor 



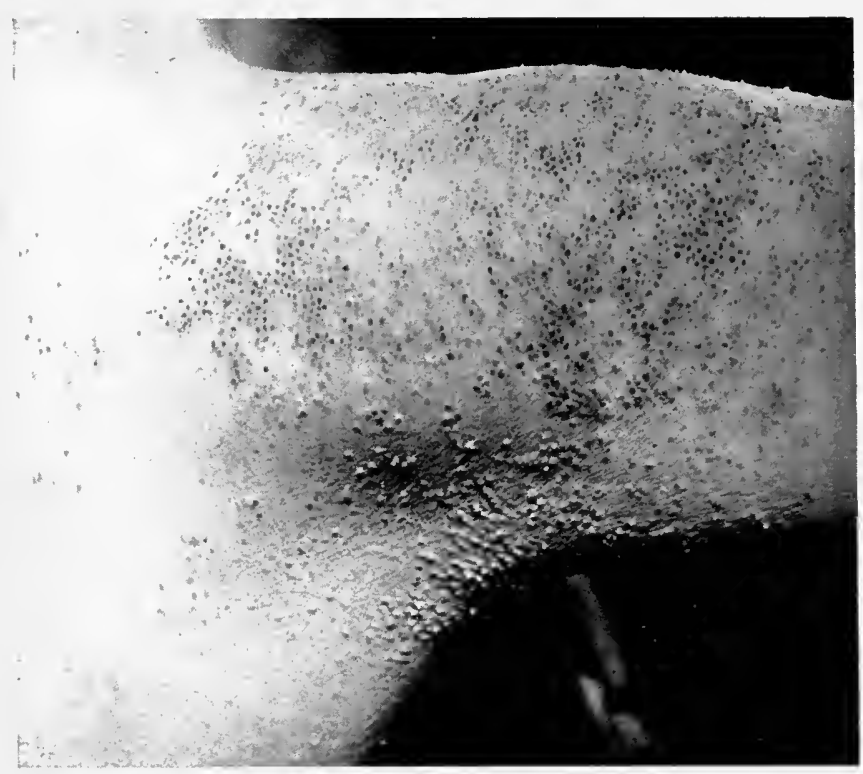

FIG. 335-Lichen Spinulosus.

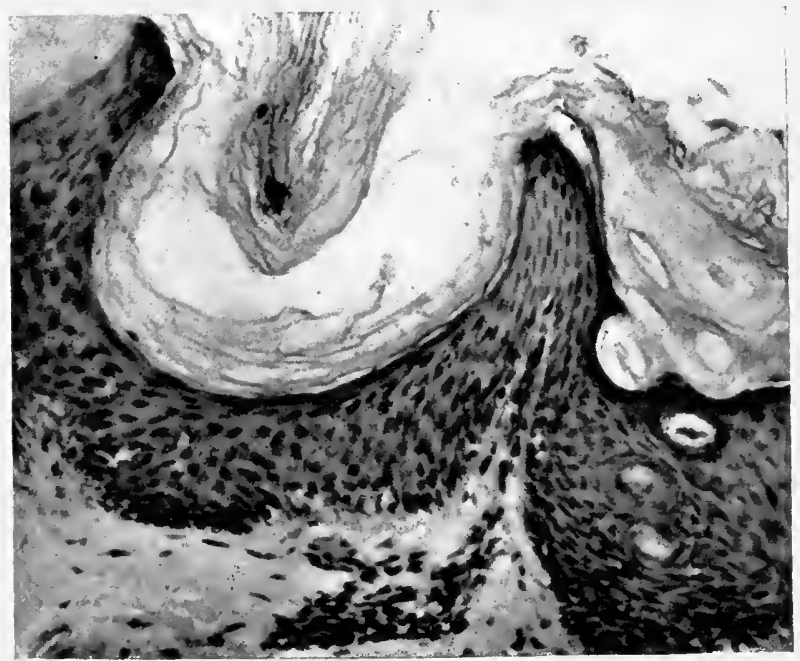

FIG. 336.-Section of I,ichen Spinulosus.

shows horny plugs in follicles with " oval bodies" beneath and small-celled infiltration around the blood-vessels and hair-follicles.

(Dr. Wallace Beatty's case. Photos by Mr. J. Manby. Brit. Journ. Derm., I915.) 
aspects of the arms and legs. They frequently show a tendency to be distributed symmetrically. (Fig. 335.)

The eruption is not associated with subjective symptoms and does not interfere with the general health.

Histopathology.- The histopathology has been minutely studied by Adamson who has given an excellent surver of the literature on the subject. He found that a horny plug made of concentric lamellae of flattened horn cells distended the follicle in its upper third and extended upwards beyond the level of the epidermis. The hair-bulb was unaltered but the sebaceous glands were atrophied. There was a slight increase in the connective tissue cells at the neck of the follicle and in the neighbourhood of the papillary vessels. (Fig. 336.)

From the histological appearances he concluded that the lesion was probably non-inflammatory in origin, and that the primary change was a hyperkeratosis at the funnel of the follicle with the formation of a horny spine.

No micro-organisms were present in the lesions.

Etiology.-The affection usually occurs in children. It is rare in adults and when it is met with in them is generally associated with acuminate or plane lesions of Lichen planus and is possibly of a different nature. It is more common in boy's than girls, and seems to be most frequent in delicate, weakly, or strumous children.

The cause is at present unknown. It is suggested on the one hand that it is primarily a hyperkeratosis of the mouth of the follicle, and that where inflammatory changes occur they are secondary to irritation (Brooke, Adamson), and on the other that it is essentially an inflammatory affection and that the epidermal changes are secondary (Crocker, Perry). If the former view be adopted it is difficult to explain the hyperheratosis; there is no evidence to suggest that it is caused by a microbe or results from irritation, and the only explanation which remains is that it is the effect of a toxin; it is well-known that toxins eliminated by the skin may produce hyperkeratosis, as in the case of arsenic, but if it were due to this cause more marked inflammatory changes around the follicle would be expected.

Diagnosis.-The disease from which it has to be distinguished chiefly is Lichen planus with acuminate spiny lesions. The actual lesions themselves may be indistinguishable, but those of Lichen planus are generally associated with plane lesions, tend to be aggregated into hypertrophic patches, are succeeded by pigmentation, and are associated with subjective srmptoms such as itching. It is possible, however, that Lichen spinulosus may be simply a rariety of Lichen planus and identical with the spiny lesions in Lichen planopilaris.

The lesions have also to be differentiated from spiny acuminate lesions of the same type which occur in Siborrhoic dermatitis and Lichen scrofulosorm These are only accidental concomitants, and typical lesions of the respective diseases are generally present. 
From Keratosis pilaris it is distinguished by the lesions in Keratosis pilaris not being grouped in patches, being usually present on the extensor surfaces of the thighs and arms, and being most common in early adult life.

From Pityriasis mbra pilaris it is diagnosed by the wider distribution of Pityriasis rubra pilaris, the inflammatory character of its lesions, the tendency to thickening and scaliness of the skin, and the presence of typical lesions on the dorsal aspect of the fingers.

Treatment.-The affection is comparatively easily cured, but if left untreated persists indefinitely. The patches should be scrubbed with soap spirit and hot water, followed by the application of $2 \%$ resorcin or salicylic acid ointment. When they are inflamed, the application of a soothing antiseptic lotion, such as boro-calamine lotion, is helpful.

As the patients frequently suffer from weak general health, suitable constitutional treatment, in the nature of fresh air, proper food, and tonics such as iron, strychnine, and cod-liver oil, should be prescribed.

\section{REFERENCES}

Adanson, H. G. Lichen pilaris seu spinulosus (Brit. Journ. Derm., 1905, xvii. p. $+\mathrm{I}$ ).

Fox, Colcotr. Lichen pilaris (Idem, IS95, vii. p. 90).

Giovacisi, S. Histology of Keratosis pilaris (Arch. f. Derm. u. Syph., I902, lxiii. p. 163).

Guibout, M. Acné sebacée cornée (Mal. de la Peau, I879, p. 662).

Hallopeau, H. Acné cornée en aires (Ann. de Derm, et de Syph., r895, vi. P. II I I).

Lewaxdowskr, F. Über Lichen spinulosus (Arch. f. Derm. u. Syph., I 905, p. 3+3). 


\section{CHAPTER XXYVI}

\section{DISEASES OF THE SEBACEOL'S GLANDS AND PILO- SEBACEOLS FOLLICLES}

\section{SEBORRHOEA}

Syn.: Seborrhagia; Steatorrhoea; Steatidrosis; Seborrhoea oleosa (Hebra) ; Hyperidrosis oleosa (Lnna); Flux sebacea (Rayer).

The term seborrhoea has been employed by different writers in such different senses that the confusion with regard to it is almost chaotic. On the, one hand it has been used in its literal meaning to signify an excessive output of sebum by the sebaceous glands-a functional disturbance rather than a disease; on the other, it has been applied to a series of morbid conditions of which a sebaceous flux was believed to be the underlying cause.

So wide has been the application of the term that a considerable number of the more common cutaneous affections related to the psoriasis group have been included under it, such as Pitrriasis capitis and all the phases of so-called seborrhoic dermatitis. Recent research, however, has shown that these affections are probably of microbic origin and that, although tbey occur as a rule in a greas. skin, the greasiness only acts "as a predisposing factor, by rendering the soil more fertile for the growth of the causal microbe.

Another cause for confusion is the teaching of Hebra and others, that under certain circumstances, not necessarily pathological, the excessive oily secretion of the sebaceous glands may become capable of setting into dry scaly masses, and that there are two forms of seborrhoea, namely Seborrhoea oleosa and Seborrhoea sicca. This riew is no longer held and only one form is now recognised, namely Seborrhoea oleosa, the Seborrhoea sicca of Hebra having been proved to be a mildly inflammatory condition of microbic origin, which has been described under the heading of Pityriasis. (See p. 35I.)

At the present time the tendency is to limit the term seborrhoea to a functional disturbance associated with increased production of sebum, either in the form of small beads of oil or of cheesy plugs which can be readily expressed from the follicles.

In white races the skin is not as a rule risibly greasy, though not 
infrequently individuals are met with in whom an obvious greasiness is present which is most marked about puberty and early adult life. In the negro, on the other hand, the stin is usually greasy, the grease probably having a protective function against the sun's rays.

The grease may be derived from three sources, namely, (I) the sebaceous glands, (2) the sweat-glands, and (3) the epidermis itself, the actual horn-cells containing a waxy substance. The exact proportion derived from these different sources is difficult to estimate and varies in different individuals and under different conditions. Where the output by the sebaceous glands is increased there is not infrequently associated with it an excessive excretion of sweat, but as the sweatglands and sebaceous glands are usually situated close to each other there is some difficulty in determining exactly the source of the grease. Unna considered that the sebaceous material formed a comparatively trivial proportion of the cutaneous fat and simply lubricated the hairs, and that the larger part emanated from the sweat-glands, and in consequence he named the condition Hyperidrosis oleosa. He based this view on the blackening with osmic acid which occurred in the cells of crusts in the ricinity of the sweat-pores, and the demonstration of fat droplets around the coil-glands and within their epithelium. His theory is not generally accepted, and it is believed that the amount of fat contained in the sweat is negligible.

Anatomical and Physiological Considerations.--In order to understand this complex subject it will be advisable to refer to the following anatomical and physiological considerations :

(a) Sebaceous glands are appendages of the hair-follicles and occur wherever hairs are present; they are found all over the cutaneous surface except on the palms, soles, and dorsal aspects of the ungual phalanges. They vary in size from minute appendages to large structures with several acini. Those connected with the long hairs are comparatively small, while those of the lanugo hair-follicles may be so large that the pilar part of the follicle may form a small appendage to the gland. This would seem to indicate:- (r) that the function of the sebum is not confined to the lubrication of the hair alone, for in that case the small hairs might be expected to have correspondingly small glands; (2) that there is a natural balance between the sizes of the pilar and sebaceous portions of the follicle.

(b) The largest sebaceous glands occur about the forehead, sides of the nose, nucha, medio-thoracic regions both sternal and interscapular, sacrum, groins, and genital organs. Where the sebaceous glands are largest there is a tendency to phrsiological greasiness, hence the above regions constitute the classical sites of seborrhoea.

(c) Sebaceous glands occur which are unconnected with hairs, such as about the prepnce and labia.

(d) The Meibomian glands of the evelids, the ceruminous glands of the auditory canal, and the areola of the nipple, are modified sebaceous glands.

(e) Sebum, which is the product of activity of sebaceous glands, is an oily substance which tends to become cheesy on exposure to the air, and which approaches in chemical composition the lanolin of sheep. It is not a true secretion but is the result of a fatty change in the cells of the gland, by a process analogous to cornification. In the epidermis the final product is a horn-cell, which is a keratinised element containing a small 
quantity of a waxy substance; in the sebaceous gland it is a cell which is not keratinised but the protoplasm of which has been almost completely transformed into a fattr mass.

When the output of sebum is excessive it means that the gland-cells are being produced at an abnormal rate by the mother-cells at the basal layer, and that they are rapidly undergoing fatty changes and being thrown off. If the cells break down an oily sebum is formed which appears as an oily bead at the follicular orifice. Should the cells be more resistant they adhere together for a longer time without disintegrating and form a fatty plug, named by Sabouraud the "seborrhoic cocoon," which blocks the mouth of the follicle; this is almost completely soluble in ether. showing that it does not contain keratin.

(f) The sebaceous gland is not supplied with a nerve apparatus for its control like the sweat-gland, and the precise causes by which the sebum is increased or diminished are not fully understood. An excessive production may be the result of an increase in size of the gland itself, while a diminished output may be due to atrophy.

Apart from structural alterations, however, there would appear to be stimulating influences which lead to more rapid formation of sebaceous cells and increased flux of sebum, such as heat, violent exercise, alcohol, or any cause which is responsible for active congestion of the capillaries about the glands. It may result also from toxins eliminated by the skin and possibly to some extent by the sebaceous glands, such as those of autogenous origin which occur in connection with disorders of the digestion, liver, and uterus.

It is also supposed that the ingestion of fatty-foods can cause an increased production of sebum.

Heredity appears to have some bearing on the actirity of the sebaceous glands and a greasy skin may occur as a peculiarity in certain families.

There appears to be some relation between seborthoea and the development and activity of the generative organs. In the infant, for example, in utero and for a short time after birth, when the developmental process is at its height, the sebaceous glands are specially active and lead to the production - with the desquamating epidermis-of the familiar greasy coating known as the Vernix caseosa, which is frequently associated with the presence of small fatty plugs in the pilo-sebaceous follicles about the face known as miliary sebaceous acne. During early childhood there is little tendency to seborrhoea, but with the development of the sexual organs at puberty the sebaceous glands become active and oily beads may appear at the sides of the nose, concha of the ear, and elsewhere, and greasy plugs in the follicles on the face. This condition usually disappears during early adult life, but may persist throughout the whole period of sexual activity as a seborrhoic disposition, or " kerosis " (Darier). In individuals who are naturally sexual the glands are specially active; and there is not infrequently an increased output of sebum in women at the menstrual periods. In old-age, when atrophic changes supervene in the glands, there is a marked diminution in the production of sebum.

Instead of a functional disorder, seborrhoea has sometimes been regarded as a morbid condition and the result of a specific microbe. In the sebaceous material are swarms of the micro-bacilli which Sabouraud believed to be the cause of the condition. This view is not generally accepted, and the absence of the bacilli from the Vernix caseosa is against it.

On the other hand, certain observers consider that sebum is a bad medium for the growth of bacteria and that it may eren be bactericidal, and this has been offered in explanation of the rarity of small-spored ringworm after puberty when the sebaceous secretion becomes active and is said to exert its antiseptic action. 
Seborrhoea in itself is of comparatively little moment, unless in exceptional cases where it causes a sallow greasy complexion with plugged prominent follicles. It is important, however, as a predisposing cause of a well-known group of cutaneous diseases, as it forms an excellent medium for the growth of micro-organisms and enables microbes, which ordinarily live a harmless saprophytic existence on the skin, to flourish even to the extent of becoming pathogenic and producing dermatitis.

The most important of these micro-organisms are: (I) the microbacillus of Sabouraud or Acne bacillus, which is believed by certain writers to be capable of transforming a sebaceous plug into a comedo, and by others of producing suppuration and the formation of an acne pustule; (2) the flask bacillus, which some consider to be the cause of Pityriasis capitis and the dry forms of the so-called seborrhoic dermatitis; (3) the grey coccus which is believed to be responsible for the inflammatory disturbance in seborrhoic dermatitis.

To the morbid conditions which occur on seborrhoic soil, French writers have given the name of Séborrhéide, which has been anglicised to Seborrhoide. Under this heading may be placed the following conditions :

I. Seborrhoea capitis (Seborrhoic baldness).

2. Seborrhoic dermatitis, Seborrhoea corporis or Flannel-rash, Seborrhoic eczema.

3. Acne vulgaris; a condition invariably preceded by seborrhoea ; the comedo, or blackhead, which is the origin of the trouble is derived from the fatty plug at the mouth of the sebaceous follicle.

4. Papular seborrhoides of the face; these consist of miliary perifollicular papules which occur in association with rosacea.

5. Seborrhoic warts; these are warty growths which are liable to become epitheliomatous and are probably of microbic origin ; they are generally believed to occur most readily on a seborrhoic basis.

\section{TREATMENT}

It is only in exceptional cases that seborrhoea requires treatment, when the scalp is oily, or the face, especially in women, is greasy, sallow, and the follicles so plugged as to be a disfigurement, or the skin of the body is so greasy and itchy that it is uncomfortable and apt to be inflamed from rubbing and from microbic infection.

As it may result in some cases from internal derangements, it is important that any defect in the general health which might be responsible for vascular congestion or for the production of autogenous toxins should be corrected, such as digestive disorders, constipation, anaemia, uterine troubles, or disturbances of menstruation. Alcohol and fatty food should be aroided. 
The local treatment is palliative rather than curative. To be effective it must be thorough and must be continued as long as the functional disturbance persists.

Scalp.-When the scalp is greasy the hair should be washed frequently with soap spirit lotion and one or other of the following lotions should be rubbed in daily :

\begin{tabular}{|c|c|c|c|c|}
\hline Resorcin & - & - & - & gr. $\mathrm{x}$ \\
\hline Ammonii chloridi & - & - & - & gr. \\
\hline Glycerini $\quad$ - & - & - & - & $5 \leq s$ \\
\hline Eau de Cologne & - & - & - & $\overline{3 i i}$ \\
\hline Aquam dest. ad & - & - & - & $\tilde{3} r i$ \\
\hline Acidi salicylici & - & - & - & gr. $\mathrm{xxy}$ \\
\hline Hydrargyiri perchlor & ridi & - & - & gr. ii \\
\hline Spiritus rectificati & - & - & - & $\bar{j} \mathrm{ii}$ \\
\hline Aquam dest. ad & - & - & - & $\overline{5} \mathrm{yi}$ \\
\hline
\end{tabular}

Face. - If there be no inflammation the face should be washed with soap spirit lotion and the sebaceous plugs should be remored by kneading or massaging the skin or by the use of a comedo expressora special roller instrument may be obtained for massaging the face but is inferior to the fingers. The skin should then be dried and calamine lotion or a bland dusting powder applied..

Chest and Back.-The grease should be washed off with spirit soap and a dusting powder containing $\mathrm{rO}_{i \mathrm{j}}^{\circ}$ of precipitate of sulphur applied. It is important that hot irritating underclothing should not be worn ; woollen garments, being non-absorbent and liable to accentuate the condition, should be replaced by linen-mesh or fine lawn next the shin, the necessary warmth being obtained by wearing thicker outer garments.

\section{REFERENCES}

AUdrr. Séborrhée- Séborrhéides (La Prat. Derm., I904, iv. p. 270).

Brooke, H. G. Clinical relationship of seborrhoea (Brit. Med. Journ., 1904, xri. p. 205).

DArier. Maladies dites Séborrhéiques (Ann. de Derm. et de Sỵph., I90\%, viii. p. 4).

Fox, T. Colcotr. Discussion on seborrhoea (Brit. Med. Journ., I90 i, ii.). Hallé \& CrVatte. The bacteriology of the sebaceous glands (Ann. de Derm. et de Syph., March 1907, p. I 4 ).

Kuzxisskr. Clinical \& experimental investigation on the question of the sebaceous secretion of the body (Arch. f. Derm. u. Syph., Feb. I9I3).

Macleod, J. M. H. Seborrhoea of the Seborrhoides (Practitioner, Igot, P. 755$)$.

Moxtgoneri, D. W. The relation of diets to seborrhoea (Journ. Cut. Dis., Dec. I916, xxxiy. p. \$29).

PRINGle, J. J. Rare seborrhoide of face (Brit. Journ. Derm., I903, xy. p. + I).

Roberts, Leslie. Seborrhoea \& its consequences (Brit. Journ. Derm., I 89 , ix. p. 219). 
Sabouraud, R. Les Maladies séborrhéiques (Paris, I902).

—- La question des séborrhéides (La Presse Méd., I904, Nos. 45 \& 50).

UNxA, P. G. Function of the sweat-glands in man (Brit. Journ. Derm., I894, vi. p. 257).

—_ Seborrhoea (Monats. f. prakt. Derm., I887, vi.).

\section{COMEDONES}

Der. : Comedo, a glutton.

Sỳn. : Blackhead; Fr. Comédon.

Definition: A small oat-shaped plug consisting of horny and sebaceous material blocking the pilo-sebaceous follicle.

The comedo when squeezed out of the follicle is found to be an oatshaped body, soft and fatty in consistence, and hard and brownishblack at the outer end where it is composed of horny material. The dark colour is only partially due to dirt and is caused chiefly by the denser cornification near the apex and the presence of diffuse pigment in the horn-cells.

The familiar blackheads are the external ends of the comedones. They are most commonly situated in the skin of the face, about the angles of the nose, cheeks, forehead, chin, inside of the auricle, neck, back, chest, and abdomen, but may be met with also on the limbs, and in rare instances on the glans penis and prepuce. They are distributed irregularly and are generally discrete, but may be arranged in groups about the cheeks and sides of the forehead, which are occasionally symmetrical. As a rule they are single, but may be double or even multiple as a result of the fusion of two or more contiguous follicles below the surface of the skin.

The blackhead may be level with the surface, depressed beneath it, or elevated to form the apex of an acuminate papule. As a result of the irritation of chemical substances or micro-organisms, the comedoplugged follicles may become inflamed and transformed into acne pustules which, on healing, may be followed by atrophic changes and scarring.

The atrophy, which is especially liable to result from large double comedones, may be so marked as to lead to permanent disfigurement. On the cheeks it may give rise to irregular pits, closely crowded together and separated by narrow ridges, sometimes associated with erythema of the affected areas. ${ }^{1}$

They generally appear about puberty-about the age of $\mathrm{I} 2$ in girls and $\mathrm{I}_{4}$ in boys - and tend to disappear spontaneously by the age of 30 . In some cases they may appear later, not infrequently about the chin in women between 30 and 40, and may even be met with in elderly people. Occasionally they are present in infants and young children, when they are usually arranged in groups.

${ }^{1}$ Advanced cases of the above type have been described under various headings, such as "Atrophodermia reticulata symmetrica faciei" (Pernet), "Folliculitis ulerythema reticulata" (MacKec), etc. 
Their presence is usually associated with oily seborrhoea of the skin which is etiologically connected with their formation.

Etiology.-The age of puberty is an important factor in their causation. At that time a growth of hair takes place and the sebaceous glands become more active, and it is suggested that the friction of the growing hair against the walls of the follicle and the irritating action of the sebum is largely responsible for the production of the horny plug.

On the other hand a microscopical examination of comedones, except those in infants, reveals the presence of a micro-bacillus which certain observers consider to be responsible. This micro-organism grows freely in sebaceous material, but it may be harmless and, if pathogenic, it cannot be the only cause of the comedones as it is absent in those which occur in infants.

Oily seborrhoea seems to be the soil on which comedones usually form and the various causes which have been cited in connection with its etiology are equally applicable to comedones (see page 976). This condition may be a congenital as well as an acquired abnormality and may be met with in several members of a family, which would explain the occurrence of comedones as a family trait.

Histopathology.-A vertical section of a comedo-plugged follicle shows that the comedo occupies the upper portion of the follicle between the opening of the sebaceous gland and the surface and dilates it into a wide funnel. The epidermis in the neighbourhood is hypertrophied and the horny layer thickened.

The comedo is the result of a process of hyperkeratosis of the lining membrane of the funnel of the follicle and is composed of concentrically arranged horny layers like the sheaths of an onion which are specially dense at the periphery and upper extremity which is dark in colour from the excessive cornification and from pigment. The central mass is cheesy and consists of sebaceous material, twisted up lanugo hairs, and the debris of horn-cells. (Fig. 337.)

In the face, where comedones generally occur, the sebaceous gland instead of being a small appendage to the follicle is larger than the follicle itself, and consequently sebaceous material predominates in the plug.

When sections of a comedo are stained with thionin or methylene blue the micro-bacilli are seen in large numbers as small rods, single or in bundles, resembling tubercle bacilli, and situated chiefly in the central part of the comedo.

In the sebaceous material about the comedo a small parasite, of the genus arachnid and known as the Demodex folliculorum, may be detected, and the flask-shaped bacillus of Unna may often be observed about the head of the comedo, but neither is of pathogenic importance.

The pressure of the comedo frequently produces atrophy of the underlying portion of the hair-follicle and of the sebaceous gland, but occasionally the lower part of the follicle may become hypertrophied. 


\section{TREATMENT}

The removal of comedones is important for two reasons: (I) the disfigurement produced by them, and (2) their tendency to become inflamed and transformed into acne pustules.

General Treatment.--When they occur in young people who are apparently in robust health no general treatment is necessary. In a large number of cases, however, they are associated with some general derangement, such as scrofula, anaemia, indigestion, constipation, and liver disturbances, which require to be treated on general medical principles.

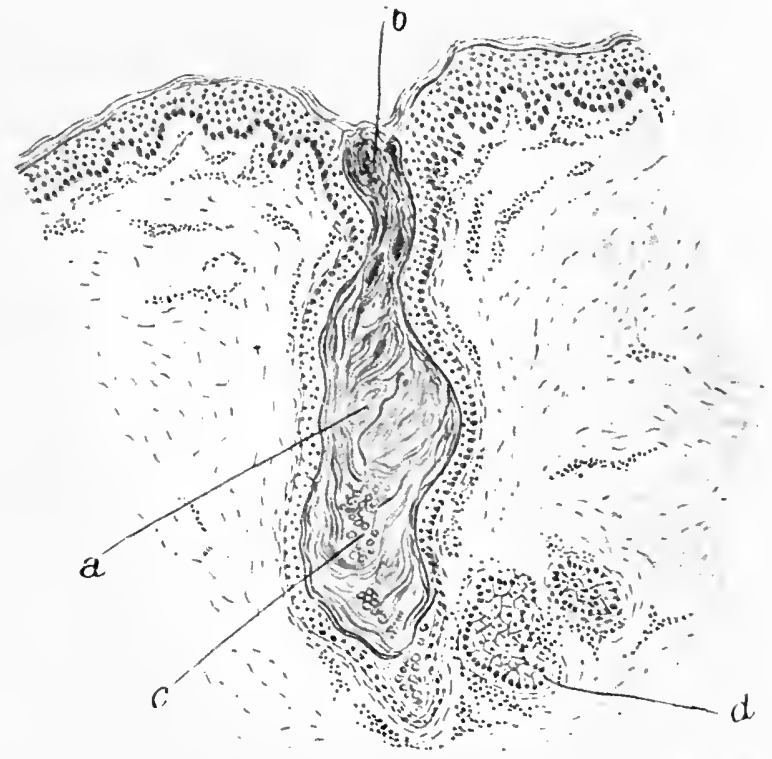

Fig. 337.-Comedo. ( $x$ about Ioo.)

J. M. H. M. del.

a. Comedo consisting of oat-shaped horny mass filling dilated funnel of pilo-sebaceous follicle. $b$. Outer third of plug darkened by diffuse horn-pigment. $c$. Sebaceous material. $d$. Sebaceous gland.

(From MacLeod's Pathology of the Skin.)

Local Treatment.-Removal of Blackheads.-Where they are few and small they may be expressed by squeezing the affected skin between two fingers or by an expressor. Where they are numerous or occur in inaccessible situations, such as the back, they should be removed by an attendant.

As a prelude to their removal the skin should be softened by hot boric compresses, by steam from a bronchitis kettle, or by lathering with soft soap and hot water. The patient should then be placed in a good light and the comedones systematically expressed. Of the various comedo expressors one of the most useful is a curved instrument with a small spoon at each end perforated by a hole, the opening at one end being slightly larger than that at the other to suit the different sizes of the comedones. The convex side of the spoon is pressed down 
firmly on the comedo which is forced out and passes up through the opening. Occasionally when the comedones are large and firmly fixed their evacuation is facilitated by freeing the apex with a fine-pointed scalpel. (Fig. 338.)

To remove small blackheads or the greasy plugs or cocoons which may be left after the more prominent blackheads have been evacuated, the skin should be rolled between the finger and thumb or massaged with the fingers dipped in soap and water. This should be followed by the application of a dusting powder containing equal parts of precipitate of sulphur and talc, or the following sulphur lotion:

$\begin{array}{llllll}\text { Sulphur. praecip. } & & - & - & - & \text { gr. } x x x \\ \text { Camphor. }\end{array}$

The $\mathrm{X}$-rays have been used for the destruction of comedones, especially about the face, and when carefully employed give good

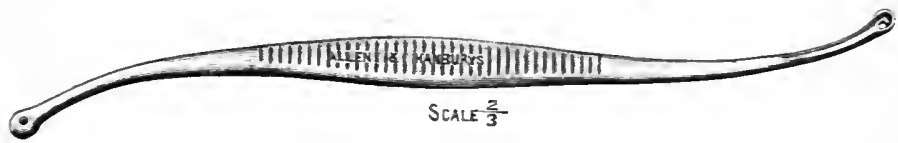

FIG. 338.-Comedo Expressor.

results. A full Sabouraud pastille dose is given, which produces temporary atrophy of the pilo-sebaceous follicles, causes shrivelling of the comedones, and reduces the thickening of the intervening epidermis.

Recently the treatment of comedones by the injection of vaccines prepared from the micro-bacillus has been employed, but with doubtful results.

\section{Grouped Comedones of Infants and Young CHILDREN}

Within late years it has been recognised that comedones may occur earlier than puberty and may even be met with in infants and very young children, especially in boys. In the infantile cases the comedones tend to be grouped in clusters and are most commonly present on the forehead, near the hair-margin and sometimes extending into the scalp, on the temples, cheeks, neck, shoulders, chest, and back. They may become inflamed or may lead to a pustular folliculitis. (Fig. 339.)

The comedones are of medium size and of the same structure as those of puberty but all attempts to find micro-bacilli in them have been unsuccessful though flask-shaped bacilli and occasional staphylococci have been detected. 
These grouped comedones are supposed to result from local irritation and it has been suggested that those on the forehead are due to the warmth and friction of a greasy dirty cap and those on the cheek to contact with the skin of the mother. In one case which came under the observation of the writer the comedones occurred on the chest of a child who, for some months previously, had been wearing over the affected part a flannel binder saturated in camphorated oil. Crocker's

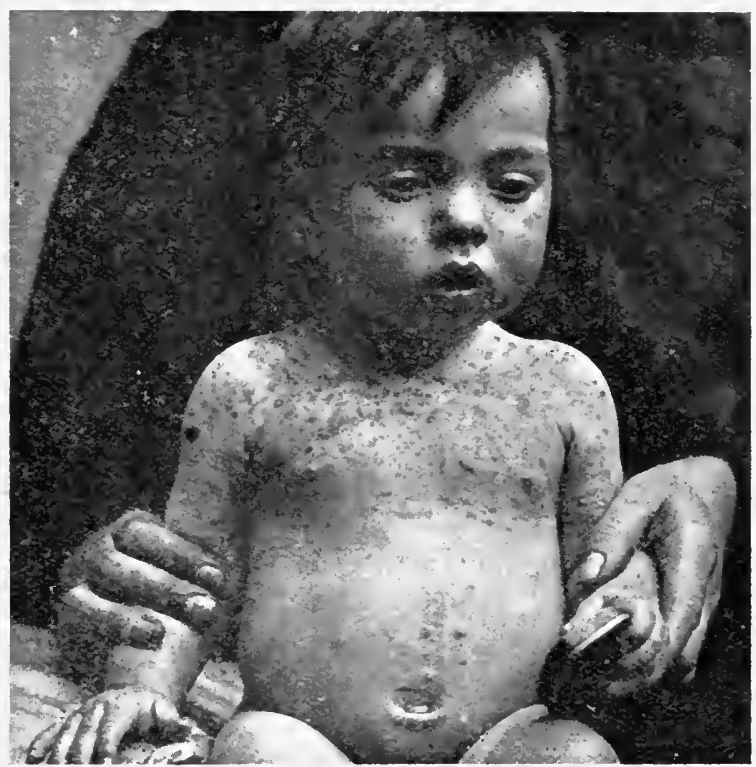

FIG. 339.-Grouped Comedones.

(From 1)r. Wallace Reatty.)

observation of the condition in several members of one family suggests a bacterial origin.

The treatment is the same as that for ordinary comedones but as a rule is more successful as they are more easily removed.

\section{REFERENCES}

Bautigny. De l'acné punctuée chez l'enfant (Thèse de Paris, 18997.

CaEsar, J. Twenty cases of comedones (Lancet, London, I 884, i. p. I I 88).

Dore, S. E. Multiple comedones (Brit. Journ. Derm., Igog, xxi. p. 220), Fox, T. Colcott. Comedones (Brit. Journ. Derm., 1895, vii. p. 213 ).

Harries, E. H. R. Some cases of grouped comedones in infancy (Brit. Journ. Derm., I9I I, xxiii. p. 5).

MACKEE, G. II. Folliculitis nlerythema reticulata (Journ. Cut. Dis,, I9I8, xxxvi. p. 339).

MACLiod, J. M. H. Grouped comedones in an infant (Brit. Jonrn. Derm., I903, Xr. p. 453 ; Idem, I907, xix. p. +24 ).

Payne, J. F. Comedones (St. Thomas' Hosp. Rep., i $88_{4}$ ). 


\section{ACNE VULGARIS}

Syn.: Acne disseminata; Acne adolescentium; Fr. Acné, Acné polymorphe, Acné juvénile; Ger. Akne.

Definition: An inflammatory disease of the pilo-sebaceous follicles, secondary to the presence of comedones, and characterised by papules, pustules, and nodules.

Description.-Acne vulgaris is one of the common skin diseases of adolescence and is so characteristic in its clinical appearances that it is easily recognised. It affects chiefly the peripheral parts of the face, namely the forehead, cheeks, and chin, the upper part of the trunk, and in extensive cases the limbs, its distribution being to some extent determined by the number and size of the sebaceous glands. (Fig. 340.)

In a well-marked case its essential features consist of comedones or blackheads and acuminate papules surmounted by blackheads, many of which are inflamed and in various stages of conversion into pustules known as acne pustules. The comedones, as a rule, are single and are eitherlevel with the surface of the skin or form the centre of an acuminate

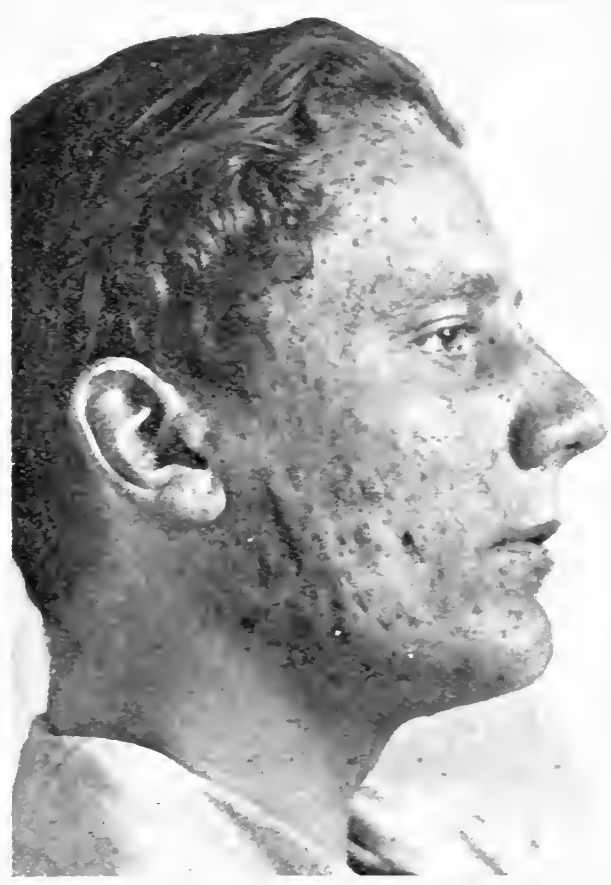

FIG. 340.-Acne Tulgaris.

Comedones and pustular lesions.

papule about the size of a pin's head; these papules may be of the same colour as the surrounding skin or may be pinkish-red from inflammatory changes, the comedo appearing as a black dot at the apex. Larger acuminate inflamed lesions about the size of a lentil are frequently present with a vellowish pustular apex in which the comedo may or may not be apparent. Hore deeply-seated nodules may also occur which vary in colour from pink to reddishpurple; these are indurated at first but tend to soften later and to form small abscesses. To complete the picture, pits, scars, and cicatricial nodules, resulting from previous lesions, are not infrequent.

The lesions usually remain discrete and are irregularly distributed, 
but occasionally they may occur in definite groups or may become aggregated to form indurated patches. (Fig. 34I.)

Pressure of the smaller lesions with a comedo-expressor causes the extrusion either of a comedo or of a bead of pus followed by the softened remains of a comedo which look like a minute cheesy wormcast, while incision of a larger nodule allows the escape of a mass or bead of yellowish-brown sero-pus with a gelatinous or mucoid consistence.

The average duration of an acne pustule is from a few days to a week, when it either breaks and discharges its contents or dries up and becomes absorbed, but the larger nodules may persist for weeks before these changes supervene.

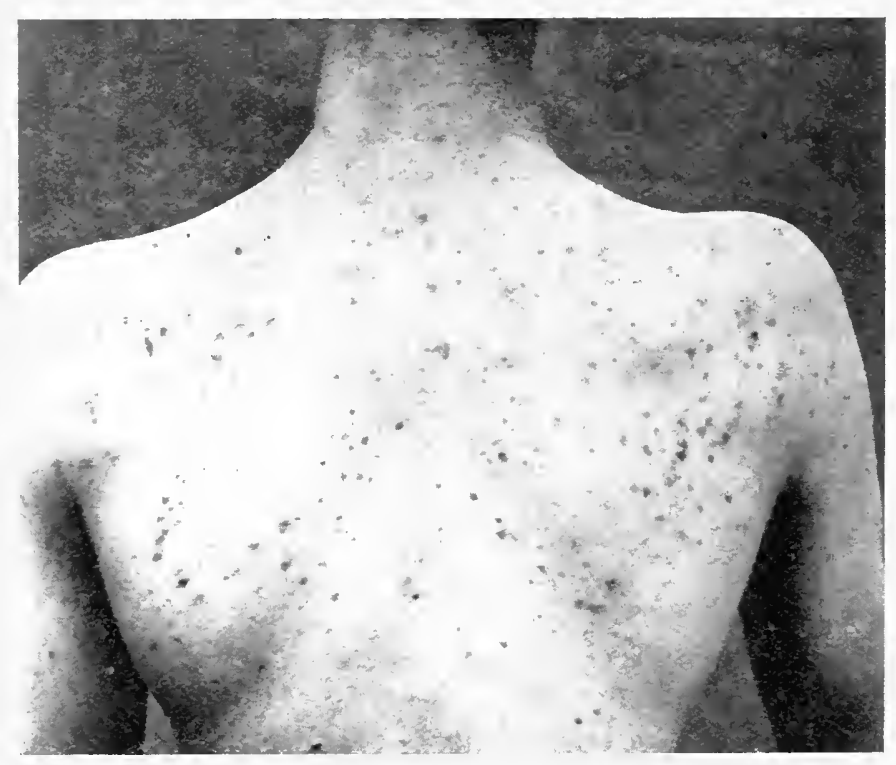

Fig. 34I.-Acne Vulgaris of Back.

As a rule the disease begins insidiously and is fairly well-marked before any notice is taken of it; at other times it comes out rapidly and is widely disseminated in a week or ten days. It is perpetuated by new lesions constantly forming and replacing those which have disappeared.

One of the most striking features of acne is its polymorphism and according to the type of lesion which predominates it is customary to distinguish the following varieties: Acne simplex where the lesions consist of papules or pustules without obrious comedones; Acne punctata vel papulosa where the chief type of lesion is an acuminate papule surmounted by a blackhead; Acne pustulosa where pustular elements predominate; Acne indurata where large indurated nodules occur; and Acne atrophica where pits are left when the lesions heal.

The texture of the skin affected is usually coarse and the surface greasy, dirty, and generally hyperaemic; this is mainly the result of 
the association of acne with oily seborrhoea or with seborrhoic dermatitis of the scalp and trunk.

The subjective symptoms vary according to the severity of the outbreak. In mild cases they may be absent, or may consist of slight itching; in severe cases considerable discomfort and even pain may be produced by the lesions, especially in situations like the shoulders where they are subjected to the pressure of clothes or braces.

Course.-The course varies in different cases. It is subject to exacerbations and remissions, but once established, unless it be cured by treatment, the patient is rarely free from it until the tendency to it dies out naturally, after months or years, when the lesions gradually dry up, diminish in number, and finally cease to appear-the recovery being synchronous with the disappearance of the comedones.

The affection usually disappears early in life, but it may be prolonged into adult life and even into old-age, especially when it occurs about the trunk.

Varieties.-In addition to the common type there are two varieties which require special notice, namely, Acne vulgaris of adult females and the so-called Acne cachecticorum of malnutrition.

Adult Form.-This is an intractable type of acne which is not uncommon in women between the ages of 25 and 40 or even later, in which comparatively few comedones are present and the most notable features are deep-seated nodular lesions situated chiefly about the chin, or indurated patches on the cheeks which are produced by the conglomeration of inflamed papules or nodules. The lesions are slow in evolving, may assume a dark purplish colour, and may be definitely painful. They are generally associated with congestive troubles arising in connection with disturbances of the alimentary tract or with utero-ovarian disorders.

Acne cachecticorum.-This is a name which has been given to a severe type of acne which occurs in individuals whose resistance is exceptionally low from malnutrition or other cause. In it comedones are only slightly noticeable, while nodules, cyst-abscesses, and in the worst cases haemorrhagic lesions like those of scurvy, are marked features. It is not confined to the face but may appear in any situation where pilo-sebaceous follicles are present.

Histopathology.-The different lesions of Acne rulgaris result from changes which take place in the comedo, in the comedo-plugged follicle, and in the neighbouring corium, from the presence of the acne bacillus and certain progenic staphylococci, and consequently their pathogenesis is intimately bound up with that of the comedo. When the micro-organisms proliferate and exert their toxic influence on the comedo it tends to break down and soften in the centre, and if a section of it be examined at this stage large numbers of acne bacilli can generally be found in the central softened parts while groups of staphylococci may be detected between the outer layers. In the simple papulo-pustular lesions the sebaceous glands are frequently 
atrophied, possibly owing to the pressure of the comedo, while in the lower third of the follicle below the comedo the external root-sheath or prickle-cell layer may be hypertrophied.

Surrounding the follicle are evidences of inflammation, the blood capillaries are dilated and there is an inflammatory cellular infiltration consisting of lymphocytes, small connective tissue cells, polymorphonuclear leucocytes varying in number according to the virulence of the inflammatory process, and a certain number of plasma-cells.

The histology of the nodular lesions is different; in them there is a dense cellular infiltration in the corium around the broken comedo, which is of a more chronic type and in which a large number of plasmacells may be present with a certain number of multi-nucleated cells or giant-cells, forming a histological picture somewhat resembling that of miliary tubercle. In the older cellular infiltrations there may be newly-formed spindle-shaped conncctive tissue cells which may result in the formation of cicatrices and even of small cheloidal thickenings.

Where the nodule has softened a small abscess cavity may be formed, either in the situation of the comedo or replacing the sebaceous gland which, if it occur deeply, is usually encysted in a dense mass of cellular infiltration.

It is probable that the more chronic granulomatous infiltration which is met with in connection with the nodular lesions is due to the action of a less virulent microbe, or to the soil being unsuitable for the proliferation of the micro-organism.

\section{ETIOLOGY}

Predisposing Causes.-Sex.-Acne vulgaris attacks young people of both sexes with equal frequency.

Age.-In the vast majority of cases it commences about puberty, in girls about I 2 and in boys about I4, and tends to clear up in early adult life. It may appear first in adult life, especially in women between the ages of 25 and 40 . In rare instances it occurs in infants and young children as the result of inflammatory changes supervening in the grouped comedones of infancy.

Climate.-Climate does not seem to have any bearing on its causation as it is common in the tropics as well as in temperate regions.

Season.-It is doubtful if the season of the year has any influence on its etiology and course, though occasionally exacerbations appear to occur in spring.

Social Position.-It prevails in rich and poor alike. Some of the worst cases met with by the writer have been in private patients; but in the poor, debilitating circumstances, such as unhealthy surroundings and malnutrition, by lowering the resistance prolong the disease, render it especially severe, and facilitate the formation of chronic nodular lesions and abscesses such as those which characterise Acne cachecticorum. 
Morbid States of the Skin.--Of these the most important is oily seborrhoea. This condition, which is functional rather than pathological, forms the most suitable soil for the development of comedones which constitute the base of ordinary acne.

Local Irritation.-Another factor of considerable importance in the causation of acne is local irritation from dust and dirt, or from the action of irritants such as oily substances, turpentine, tar, chlorine, etc.

General .Morbid Conditions. - The changes which take place in the comedo-plugged follicle and result in the formation of the acne pustule are of an inflammatory nature and due either to the acne bacillus or to certain staphylococci. Consequently any influence which promotes the growth of these micro-organisms may act as a predisposing cause, such as digestive troubles, disorders of menstruation, and defective states of the blood.

Digestive troubles have a decided influence in lowering the resistance of the skin to the growth of micro-organisms, and even slight disturbances of the digestion may determine an outbreak of acne in an individual subject to it. This is one reason why acne is so apt to appear at puberty when digestive defects are particularly common from eating too fast, from errors in diet, indulgence in sweets, constipation, etc.

Menstrual, ovarian, and uterine disturbances, by producing hỵperaemia, flushing, and a toxic state of the blood, are liable to aggravate acne by enabling the micro-organisms to proliferate more freely. A fresh outbreak of lesions is common just before the menstrual period in women and there is a marked tendency for it to improve between the periods.

Defective states of the blood, such as are present in anaemia, chlorosis, diabetes, tuberculosis, scurvy, after any prolonged illness, from a sedentary occupation, or in connection with a feeble peripheral circulation, are liable to prevent the skin from reacting to microorganisms and thus aggravate or prolong the course of the disease.

Certain drugs taken internally and eliminated by the skin, such as iodides and bromides, tend to produce acneiform lesions and to transform acne pustules into granulomatous nodules. This is not infrequently met with as the result of taking iodide of potassium in some patent medicine advertised to purify the blood.

Actual Cause.-All observers are agreed that the inflammation and pustulation which characterise the lesions of acne are due to the action of micro-organisms in the follicle around the comedo, but there is considerable diversity of opinion as to the precise microorganisms.

The bacillus, known as the Acne bacillus or micro-bacillus, is invariably present in the comedo, even in the early stages before inflammatory changes have supervened, and in the pustular lesions staphylococci are present in addition. It has been found that the bacilli tend to be situated near the centre of the comedo while the staphylococci predominate at the periphery. 
At present there is a difference of opinion as to whether the pustular changes are due (I) to the bacillus, (2) to the bacillus in combination with staphylococci, or (3) entirely to staphylococci of which the Staphylococcus pyogenes albus is the most constant. The third view seems the most probable, as acne bacilli occur with equal frequency in comedones which show no signs of inflammation and in those which are broken down in the centre of a pustular lesion.

The Acne bacillus was described and named by Unna in I893, who found it to be invariably present in sections of comedones and in pus from acne lesions. It was described again in I 897 by Sabouraud under the title of the micro-bacillus and he succeeded in cultivating it. He believed it to be the cause of oily seborrhoea and comedones but considered that the acne pustules were due to secondary infection with staphylococci. It varies from I to $4 \mu$ in length and is about $0.5 \mu$ in breadth. It exists in two forms, namely, young and old bacilli. The young bacilli are punctiform, oval, or barrel-shaped, have an envelope which stains with gentian violet, and have a superficial resemblance to cocci. The older bacilli are longer, sigmoidshaped, occur singly or arranged in chains or bundles, and somewhat resemble the diphtheroid bacillus of superficial ulcers. They stain readily with basic aniline dyes. By Gram's method the young bacilli stain evenly and darkly, while in the older bacilli deeply stained dots occur at the poles or in a chain throughout their length.

It is anaerobic and flourishes on glucose agar under anaerobic conditions. Sabouraud grew it with difficulty on a peptone-agar medium rendered slightly acid with acetic acid and at a temperature of $37^{\circ} \mathrm{C}$. Fleming cultivated it on the following medium: ordinary agar neutralised by $2 \%$ hydrochloric acid with the addition of $\frac{1}{4}$ of its volume of an emulsion made by shaking ro parts of ascitic fluid with I part of oleic acid; to this was added a sufficient quantity of a solution of neutral red to give it a distinct red colour. The microorganism grew in 24 to 48 hours in the form of white colonies which showed up well on the reddish medium.

The organism does not appear to be pathogenic to guinea-pigs. When rubbed on the skin it has been said to be capable of producing a pustular dermatitis (Fleming), and subcutaneous inoculations of it have been claimed to produce baldness.

Diagnosis.-The diagnosis presents no difficulty. The clinical features in a well-marked case, namely the comedones, the inflamed acuminate papules, with a comedo at the apex or with a yellowish white speck of pus, the larger pustules, the reddish or purplish nodules in various stages of softening, the associated greasy or congested skin and occasional evidences of Seborrhoic dermatitis, and the resultant scars and pits, serve to make up a somewhat complex picture which cannot be mistaken.

Rosacea.-It has to be distinguished in the first place from rosacea and confusion has arisen in this connection owing to the employment 
of the unfortunate term Acne rosacea. Rosacea is an erythematous condition with obvious dilatation of the vessels of the central portion of the face, especially the nose, central part of the forehead and malar prominences, while Acne vulgaris affects the periphery. When nodules and pustules occur in rosacea they are secondary to the congestive condition and are not dependent on comedones which, as a rule, are absent. The nodules tend to break down to form abscesses from which ordinary sero-pus is emitted and not the peculiar mucoid brownish substance which can be expressed from deep-seated abscesses in acne. Rosacea generally occurs after the age of 30 , while acne is most common between If and 20 .

Syphilis.-An acneiform pustular syphilide of the face may occasionally resemble Acne vulgaris. The syphilide, however, is not limited to the face or trunk, but is more or less generalised, is associated with other signs of syphilis, does not occur on the base of a comedo, has a brownish instead of a pinkish tinge, and tends to be grouped rather than irregularly distributed.

Acne iarioliformis has to be distinguished from Acne vulgaris, but in it the lesions are localised to the forehead, upper part of the face, and scalp, and invariably leave pitted scars like smallpox.

Acne agminata is a tuberculide which somewhat resembles Acne vulgaris, but is distinguished from it by characteristic groups of yellowish translucent papules.

Variola.-An acute outbreak of acne pustules on the trunk might possibly be confused with smallpox. The history and the absence of constitutional symptoms serve to differentiate them, and while acne is usually confined to the face, back, and chest, variola, however mild, is met with on the legs, forearms, and hands.

Prognosis.-In Acne vulgaris the ultimate prognosis, as a rule, is excellent, and the disease may clear up spontaneously in early adult life or may be cured after a few months treatment. On the other hand it may prove singularly intractable and considerable patience and perseverance may be required to effect a cure, and in some cases in spite of the most thorough treatment it may linger on with occasional outbreaks for years. This is especially the case where the back is affected with indurated nodules and abscesses, or where the disease occurs about the chin in women.

If it has been properly dealt with it usually leaves no trace, but disfiguring pits and scars are almost ineritable where the patient has dealt with the lesions, where deep-seated nodules or abscess-cysts have been allowed to break without surgical assistance, or where itching has led to vigorous scratching or to the lesions being dug out by the finger-nails.

\section{TREATMENT}

General Remarks.-It has been said of Acne vulgaris that it is one of the most intractable of skin affections. This is doubtless true in a 
few cases but it is the exception rather than the rule and usually means that the case has been improperly treated in the first instance and the disease allowed to become deeply-seated and widely distributed before it was thoroughly taken in hand. If rational treatment be adopted and thoroughly carried out there is no skin affection in which the response is better.

The treatment should be based on the etiology and pathogenesis and should have the following objects: (I) the removal of the comedones, the evacuation of the pus, and the cleansing of the affected surface; (2) the improvement of the tone of the skin by general measures, so as to render it more resistant to micro-organisms and to reduce the tendency to seborrhoea; (3) the removal of after-effects, such as thickening of the skin and hypertrophic scars.

It may be considered under the headings of local, internal, and vaccine treatment.

Local Treatment.-The local treatment consists of: (I) operative treatment to remove the comedones and to get rid of pus ; (2) treatment by parasiticide applications; (3) treatment to reduce scarring; (4) after-treatment to prevent recurrence.

\section{(I) Operative treatment}

(a) Remozal of Comedones.- The first aim in the treatment of acne is the removal of the comedones (see page I028).

(b) Evacuation of Pus.--The next procedure is to let out the pus, not only from the superficial pustules but also from the indurated nodules and abscess-cysts. Each pustule should be opened and evacuated. In the case of deep-seated indurated lesions the position of the pus may generally be revealed by pinching the nodule between the finger and thumb when the part of the surface over the pus becomes paler in tint.

To prevent scarring it is important to open the lesions with a suitable knife and not to allow them to be pricked with a needle or left to break of themselves. Various forms of small lancets may be employed for this purpose, such as a linear cataract knife, a small tenotome, or a double-edged knife. with straight blades tapering to a fine point; a special instrument, known as an acne lancet, which has shoulders to prevent its penetrating too deeply, may also be used, but the shoulders may be a disadvantage where the pus is deeply situated. The instrument is pushed vertically into the pustule or nodule till the pus is reached, and the contents are then squeezed out. This operation, if skilfully performed and with a sharp knife, is practically painless and leaves no mark.

After the pus has been expelled the small abscess cavities may be syringed with $\mathrm{I}$ in fo carbolic lotion by a hypodermic syringe, or the carbolic may be inserted by a pointed match or a glass pen with spiral ridges, and in this way the remaining microbes destroyed and the 
cavity prevented from re-filling. The use of carbolic should be reserved for lesions on the covered parts as it is apt to cause scarring.

Dry-cupping has been suggested as a means of evacuating the pus and reducing the inflammation, the cup being removed every few minutes to allow a fresh influx of blood; but this method is of comparatively little value.

When the pustular lesions have been opened a boric acid fomentation or a compress of hydrogen peroxide (Io vols.) should be applied for a quarter of an hour and followed by a powder containing equal parts of boric acid, precipitated sulphur, and starch.

The above operative procedures, which may be termed the "toilet of acne," should be repeated frequently, daily if necessary, till the pustulation is checked and comedones cease to appear.

\section{(2) Treatment of the skin by parasiticide applications}

In addition to the operative treatment, parasiticides should be applied to the shin in an attempt to destroy the micro-organisms responsible for the pustulation and possibly for the comedones. So long as these exist on the surface of the shin mild antiseptics are quite effective, but when they are present in the depths of the follicles even strong antiseptics may be of no avail.

Of the various parasiticides which may be used, such as carbolic acid, mercury, resorcin, sulphur, and ichthyol, sulphur is the most generally useful and may be employed in a lotion, powder, medicated soap, or ointment.

Lotions.-The following formulae for sulphur lotions are serviceable:

\begin{tabular}{|c|c|c|c|}
\hline Sulphur praecip. & - & - & - \\
\hline Zinci sulphatis & - & - & - \\
\hline Potassii sulph. & - & - & - \\
\hline Glycerini - & - & - & - \\
\hline Aquam rosae ad & - & - & - \\
\hline Sulphur. praecip. & - & - & - \\
\hline Camphor. - & - & - & - \\
\hline Pulv. tragacanth. & - & - & - \\
\hline Aquae calcis - & - & - & - \\
\hline Aquam dest. ad & - & - & - \\
\hline Sulphur. praecip. & - & - & - \\
\hline Glycerini - & - & - & - \\
\hline Alcoholis - & - & - & - \\
\hline Aquam dest. ad & - & - & - \\
\hline
\end{tabular}

For use, pour a little of the lotion into a saucer, press it into the skin with a piece of cottonwool, or apply it on a piece of lint, and let it dry on. This should be done each night, and any deposit washed off in the morning. 
Sulphur lotions are not always well-borne and may set up dermatitis followed by desquamation. This, provided it is not excessive, is no disadvantage as it helps to reduce the keratosis at the mouth of the follicle which is responsible for the blackheads; but in delicate skins it may be so severe that it may be necessary to stop the sulphur and to reduce the inflammation by soothing remedies such as calamine cream or lotion.

As a substitute for sulphur, various antiseptics may be used, such as ichthyol $20 \%$ in water or rectified spirit; resorcin $5 \%$ in rectified spirit; salicylic acid $2 \%$ in rectified spirit; perchloride of mercury $5 \%$ in rectified spirit ; boric acid $5 \%$ in rectified spirit; and camphor $5 \%$ in rectified spirit.

Considerable experience is required in the choice of the remedy suitable for the individual case and no hard and fast rules can be formulated, as individuals vary in their toleration, and skins affected with acne are peculiarly sensitive. One general principle may be laid down, namely that in every case mild applications should be employed in the first instance, and the degree of toleration of the skin ascertained before the strength is increased.

In acne of the trunk stronger remedies may be tolerated than on the face, such as 10\% Liquor calcis sulphurata or $40 \%$ formaldehyde. These, after being applied for a few days, produce a dermatitis which should be subdued by dusting powders or by a bland zinc paste. In this connection it is important that where there is marked pustulation the underclothes should be disinfected.

Powders.-Dusting powders are of service in reducing the greasiness of the skin and in soothing irritation, are of special value in the treatment of acne of the back, and should be applied after the lotion has dried on the skin. Of these precipitated sulphur is an admirable powder, and may be combined with zinc oxide and $2 \%$ of camphor added to allay itching. Dusting powders containing boric acid, salicylic acid, and magnesium carbonate, are also useful. (See Appendix.)

Soaps.-Sapo mollis, Sapo viridis, and the different medicated soaps are helpful in the treatment of acne, especially where the back or chest are involved, as they cleanse the skin of grease and, by macerating the superficial layers of the epidermis, enable the parasiticides to reach the micro-organisms. Instead of the green soap, soap liniment may be employed and to this may be added $2 \%$ of resorcin or salicylic acid. The most serviceable medicated soaps are those containing sulphur combined with camphor and Balsam of Peru.

The soap should be rubbed into the skin twice a day by a piece of flannel moistened in hot water, or it should be lathered on thickly with a shaving brush and allowed to dry on, but the lather is apt to be irritating and should only be left for a few minutes at first and the time gradually increased as the toleration to it is established.

Ointments.-Greasy applications should be avoided in the early stages of acne, unless the patient complains of the skin being uncom- 
fortably dry when the occasional use of cold cream may be permitted. Various ointments have been recommended, containing sulphur, salicylic acid, resorcin, or mercury, in a base of lanolin or vaselin ; but these, though they penetrate more deeply than lotions, do not get deep enough to reach the micro-organisms situated in the lower part of the follicles, and their greasiness may increase the inflammation by stopping evaporation and may even cause oedema and eczematisation.

Plasters.-Plasters containing sulphur, mercury, carbolic acid, etc., are useful substitutes for ointments and are especially indicated where much pustulation is present. They are more effective than ointments as they keep the medicament continuously applied.

\section{(3) Methods of removing thickening and disfigurement due to scarring}

In the final stages of the disease, when little is left except thickening and irregularities of the shin and the majority of the comedones are removed, considerable improvement can be produced by peeling the skin. This treatment consists in the frequent application of an irritating ointment of the keratolytic class, which produces an inflammatory reaction and reduces the hyperkeratosis not only of the mouths of the hair-follicles but of the intervening skin. One or other of the following ointments may be employed for the purpose:

\begin{tabular}{|c|c|c|c|}
\hline Beta-naphthol & - & - & - \\
\hline Sulphur. praecip. & - & - & \\
\hline Saponis viridis & - & - & - \\
\hline Ungt. aq. rosae & - & - & - \\
\hline
\end{tabular}

This is rubbed into the skin twice a day for three days, and the inflammation which it causes is then reduced by the following bland paste :

\begin{tabular}{|c|c|c|c|c|}
\hline Zinci oxic & & - & & \\
\hline Amỹli & - & - & & \\
\hline Lanolin. & - & - & & - \\
\hline Vaselin. & - & - & & \\
\hline Ac. salicy & Vlici & - & & - \\
\hline
\end{tabular}

When the inflammation has subsided the irritant ointment is applied again if necessary.

Instead of the beta-naphthol, resorcin may be substituted:

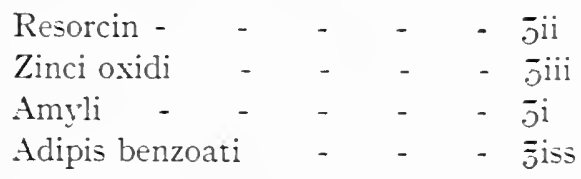

Should these applications prove too irritating the percentage of beta-naphthol or resorcin may be reduced, or a lotion consisting of 
resorcin in spirit may be substituted, till the skin becomes inflamed and glossy. This form of treatment gives good results if properly carried out but is extremely unpleasant and should not be undertaken without explaining to the patient the discomfort it entails and the necessity of confinement to the house for about a fortnight.

Röntgen Rays.-For the reduction of hypertrophic scarring in oldstanding cases applications of the $\mathrm{X}$-rays give good results. They are also of value where the comedones are unusually hard, prominent, and numerous, and the intervening skin is thickened, when a full pastille dose will reduce the thickening and cause the comedones to shrivel and disappear. It is preferable to give a single full dose, as repeated partial doses are liable to inflame the affected skin. The X-rays should on no account be employed while the skin is inflamed by local applications, nor should any irritating lotion or ointment be applied for at least a month after exposure to them.

In the local treatment of a case of acne it is necessary to deal at the same time with the scurfy condition of the scalp with which it is so frequently associated, as the scurf, which harbours both micro-bacilli and staphylococci, by being shed on to the face may perpetuate the disease. It should be treated on the lines laid down for the treatment of Seborrhoic dermatitis of the scalp, namely by the application of sulphur-salicylic ointment, washing with soap spirit lotion, or the use of the following non-greasy brilliantine :

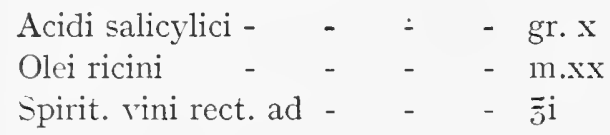

\section{(4) After-treatment}

After the disease has been actively attacked and its marked features destroyed, it is important to continue a mild form of local treatment to prevent recurrence. The comedones should be expressed in a routine fashion, and the skin bathed daily with a little hot water to which has been added a few drops of tincture of benzoin or a teaspoonful of Eau de Cologne, or washed with some mild medicated soap containing sulphur, camphor, or Balsam of Peru. A lotion of boric acid $5 \%$ or resorcin $I \%$ in rectified spirit, may be dabbed on the skin once a day with advantage, followed by the application of a powder containing equal parts of zinc oxide, starch, and precipitated sulphur or of a bland zinc paste.

Internal Treatment.--Acne is essentially a local disease but as its course, if not its existence, is largely influenced by the general health of the patient, general as well as local treatment is necessary.

There are no absolute specifics, though certain remedies if judiciously employed are of such value as to rank as partial specifics, such as sulphur and yeast. 
Sulphur is credited with haring a deterrent action on pustulation and has obtained a somewhat exaggerated reputation in the past in the treatment of this disease. It is best prescribed in the form of the sulphide of calcium, a quarter of a grain t.d.s., or of the more recent colloidal preparation known as collosol sulphur (Crooke's).

Yeast also acts by checking pustulation and is usually employed in the form of fresh brewers' yeast, a teaspoonful three times a day in water before meals. In this form the smell and taste of the yeast are obnoxious, and it has a tendency to cause gastro-intestinal disturbances such as diarrhoea and flatulence. These disadvantages can be orercome by the substitution of one or other of the newer preparations of yeast such as nuclein and ceredin (see treatment of boils).

Arsenic has its adrocates in this connection but its tendency to upset the digestion makes it apt to do more harm than good.

Apart from these partially specific remedies, internal treatment should be carried out on general principles and any defect in health sought for and corrected where possible, such as indigestion, constipation, derangements of the liver, etc.

Weak digestion should be treated by acid tonics, bismuth, pepsin, and other recognised remedies; fermentation in the lorrer part of the alimentary tract may be corrected by salol, salicylate of bismuth, naphthol, or by a course of sour milk ; and constipation should be dealt with on general principles.

With regard to diet no detailed rules are necessary. It should be light and easily digested and articles which tend to produce urticaria, such as cheese, shell-fish, pickles, sweet wines, beer, and coffee, should be aroided. When the digestive organs are acting properly almost any food can be assimilated, but if they be weak articles of dietary which can usually be taken with impunity; such as oatmeal, sweets, pastry, etc., should be eschewed.

The commencement of the menstrual period in girls is coincident with a toxic state of the blood and is frequently associated with inflammation of existing comedones and new crops of pustular lesions. This tendency can be reduced br prescribing a saline aperient with the addition of a small quantity of iron, sufficient to cause two eracuations per diem, to be taken a day or two before the period is expected.

Fresh lesions are apt to appear also in connection with dysmenorrhoea, and the correction of the latter disturbance is of value in combating the acne.

Scrofulous individuals are apt to suffer from acne as their shins are thick and oily and they have large lymphatic glands, a profuse growth of hair, and are more or less anaemic. In such cases improvement of the acne can be obtained by treatment on general medical principles.

In certain cases where no definite derangement of the general health can be detected but the patients themselves appear to be below par, a tonic regime is indicated with iron, hypophosphites, and nux 
vomica, massage, sea bathing, cold douching, and other strengthening measures.

Vaccine Treatment.- The vaccines are prepared from the microbacillus and the Staphylococcus pyogenes aureus and albus, and are obtained either from autogenous or stock cultures. The precise vaccine is chosen according to the type of case; where comedones predominate a vaccine of micro-bacilli is employed, where indurated and pustular lesions are present a mixed vaccine, and where pustulation is the most marked feature a staphylococcic vaccine. It is unnecessary to employ an autogenous vaccine except in resistant cases. The dose suggested is about 5 millions of the micro-bacillus and about I25 millions of the staphylococcus, the injections being given about once a week, and the dosage so regulated as to show no negative phase.

In a certain number of cases this treatment produces an amelioration of the pustules so long as the resistance of the individual is high, but should a negative phase assert itself or the resistance be lowered by the treatment being discontinued, the pustulation may recur more intensely than before. This treatment by itself does not appear to stop the formation of comedones and it has yet to be proved that the comedo is due to the micro-bacillus. On the whole the pustulation can be dealt with more effectively by surgical procedures.

\section{REFERENCES}

Bexiaxs, T. H. C. The relations of the Staphylococcus albus \& the Acne bacillus to the epidermis, \& the excretions of the skin-with special reference to the lesions of Acne vulgaris (Brit. Journ. Derm., I9I5, xxvii. p. 393).

Fleming, A. Etiology of Acne vulgaris \& its treatment by vaccines (Lancet, I909, i. p. I035).

Gilchrist, T. Etiology of Acne vulgaris (Journ. Cut. Dis., March r903). Lasseur. Treatment by vaccines (Ann. de Derm. et de Syph., July igro, p. 377).

Lovejoy, E. R. Treatment by vaccines (Amer. Journ. Med. Sci., cxliii. May I912, p. 693).

Morris \& DORE. Acne vulgaris with keloid scars (Brit. Journ. Derm., I909, xxi. p. 329).

Sabouraud. Acne vulgaris (Ann. de l'Inst. Pasteur, 1897, xi. p. 134).

- Seborrhée acné, calvities (Paris, I902).

Westers. Vaccine treatment of Acne vulgaris (Brit. Journ. Derm., I9I0, xxii. p. 6).

\section{ACNE NECROTICA}

Syn.: Acne varioliformis; Acné nécrotique varioliforme (Sabouraud) ; Acné à cicatrices déprimées (Besnier and Doyon); Acne frontalis seu varioliformis (Hebra).

Definition: A superficial acneiform eruption situated most frequently about the forehead and scalp and characterised by indolent 
papulo-pustules which on involuting leave depressed scars resembling the pits of smallpox.

Description.-The lesions consist of rounded or acuminate papules, pinkish or dusky red in colour, and about the size of a split-pea. After a few hours the central part of the papule assumes a yellowish tinge owing to a rapid process of necrosis, and an adherent scab gradually forms of a brownish colour which tends to sink in the centre, giving rise to an umbilicated appearance. Healing takes place beneath the scab and when it is remored or comes away naturally a superficial pit is left. (Fig. $3+2$.)

The lesions are situated most commonly on the forehead, along the borders of the scalp, on the scalp itself, and about the temple; ther may be met with occasionally elsewhere on the face, neck, back, and, exceptionally, on the limbs. They have a tendency to form groups, to be distributed in a somewhat symmetrical fashion, and to come out in crops.

The course of the affection is singularly chronic and it may persist indefinitely. There is usually.

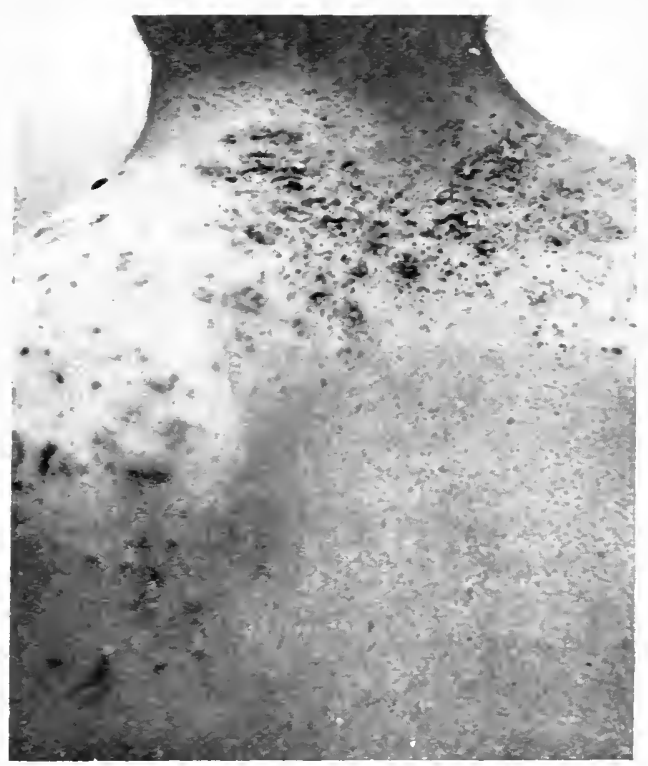

FIG. 342.-Acne Necrotica Scrofulosorum.

(From Dr. Kenneth Wills.) a marked tendency to periods of more or less complete remission, followed by relapses which occasionally would appear to be determined by seasonal influences as they have been known to recur regularly in winter.

There are no definite subjective symptoms, though occasionally slight itching may be present.

Histopathology.-In its early stages the lesion consists of an inflamed pilo-sebaceous follicle with a seborrhoic plug blocking its dilated orifice, surrounded by oedema, dilated capillaries, and an inflammatory cellular infiltration. Tecrotic changes appear later which lead to destruction of the follicle, the papilla, and the epidermis in its immediate neighbourhood. On healing, the growing epidermis insinuates itself under the necrotic area so that the latter becomes encased in a capsule of new epidermis, forms a crust, gradually gets thrown off, and leaves a pit.

According to Sabouraud, who has worked out the pathogenesis 
of this affection, it invariably arises on a base of seborrhoea, the seborrhoic plug being infected primarily with the micro-bacillus of acne. On this soil staphylococci are implanted subsequently and, by making their way into the plug and the surrounding follicular walls, give rise to the necrotic abscess.

Etiology.-The precise cause is not known. It has been said to occur with special frequency in individuals suffering from some form of gastric derangement, a predisposing factor which it has in common with Acne vulgaris.

The similarity of its pitted scars to those of Acne scrofulosorum has suggested the possibility of a tuberculous origin, but up to the present this has not been established.

It is more common in men than women and rarely appears under the age of 25 .

Diagnosis.-The conditions with which it is most liable to be confused are the small acneiform or pustular syphilide, and the rare affection known as Acne urticata.

The syphilide can usually be distinguished by the wider distribution of the lesions, the presence of concomitant signs of syphilis, and the manner in which the eruption disappears under anti-syphilitic treatment.

Occasional cases of Acne urticata affecting the face, where intensely itchy papules have been dug out by the nails and left a pit, might be confused with it; but in Acne urticata the pits are coarser, irregular, and have a tendency to be linear, and the condition usually occurs in neurotic women, whereas in Acne necrotica the pits are roundish in outline and the disease is generally met with in men.

Treatment.-The treatment is both general and local. The general treatment is on ordinary medical principles and consists of improving any debilitated condition which may be present by a tonic regime and dealing appropriately with any definite disorder of health such as a gastric derangement. Benefit has been reported from the employment of an autogenous vaccine.

Locally, the treatment consists of the application of ointments containing parasiticides and keratolytics, such as ammoniated mercury, sulphur, salicylic acid, and resorcin.

\section{REFERENCES}

Allen, C. W. Cases of Acne necrotica (Trans. Amer. Derm. Assoc., r898). Boeck, C. Acne necrotica (Norsk. Mag. f. Loegevidensk., Christiania, I888, 4 R., iii. p. 793).

Dubreuilh, IT. Acne necrotica (Arch. clin. de Bordeaux, I894).

Sabouraud. Acné necrotique varioliforme (Maladies du Cuir Chevelu, Paris, 1902). 


\title{
CHAPTER XXNVII
}

\author{
DISEASES OF THE SITEAT-GLANDS
}

\section{Functional Disorders}

\section{HYPERIDROSIS}

Syn.: Excessive sweating; Sudorrhoea.

Sweating in such excess as to be a morbid condition may be local or general.

Local hyperidrosis is met with chiefly in situations where the sweatglands are naturally large, such as the axillae, the palms and soles, between the toes, and about the genitalia, and is usually srmmetrical. It may occur elsewhere, either as a congenital peculiarity or as an acquired affection, in some area or region corresponding to the distribution of a peripheral nerve which has been stimulated directly or reflexly by injury or some other excitant.

General hyperidrosis may be met with, from dampness of the skin to pouring out of sweat so profuse as to soak the clothes. As the sweating is solely due to stimulation of the secretory nerves of the sweat-glands and independent of increased rascularity; it may not be accompanied by redness, and the skin, though moist, may be pallid, as in the cold sweats associated with pain and fear; on the other hand it may be associated with hyperaemia and a rise of temperature in the skin, as in the sweating in rheumatic fever. The moistness which accompanies hyperaemia may not be due to sweating but to a transudation of water from the blood and an exaggeration of the perpetual insensible perspiration of the skin which does not come from the sweatcoils.

Hyperidrosis is liable to be complicated by various secondary changes. On the palms and soles the sweat has a stimulating action on cornification so that hyperkeratosis may occur in which the skin becomes thickened, opaque, sodden, and apt to peel off in sheets. The damp skin is readily inflamed and even eczematised by friction, especially in intertriginous regions such as the upper part of the thighs or beneath pendulous breasts in women, or from the growth of microorganisms it may become the site of impetigo or pustular folliculitis. 
Excessive sweating is responsible also for a number of cutaneous affections, such as sudamina, hydrocystoma, Granulosis rubra nasi, etc.

Etiology.-Hyperidrosis may be due to a great variety of causes.

Local Sicating.-Localised sweating may be caused by lesions in the central nervous system, such as a tumour in the brain or an injury to the cord, while injury to a peripheral nerve may directly or reflexly lead to hyperidrosis of the area which it supplies. Even functional sensory disturbances, such as neuralgia, may cause sweating in the area of distribution of the affected nerve and in certain individuals some peculiar smell or taste has been known to produce it reflexly. Paralysis of the sympathetic nerve may be followed in the first instance by sweating on the paralysed side but this is gradually replaced by cessation of sweating, or anidrosis.

General Sweating.-General sweating may be a congenital affection and may occur in several members of a family. It is not uncommon in obese individuals with a sluggish general circulation. It may be due to psychical causes, as in the cold sweats due to fright or the sweating which is occasionally associated with emotional flushing. It usually accompanies a high temperature, owing to the stimulation of the sweatapparatus by heat. It may result from the stimulation of the toxins of certain diseases, such as rheumatism and tuberculosis, or of certain chemical toxins, such as lead, alcohol, or arsenic. Arsenic is a frequent cause and one of the most constant symptoms of chronic arsenical poisoning is hyperidrosis of the palms and soles.

Prognosis.-The prognosis varies greatly with the cause. In congenital cases the outlook is hopeless, but where the hyperidrosis is dependent on some definite deviation from health, improvement in the general condition may lead to its decrease or complete cessation. It is liable to be increased by all influences which tend to cause sweating naturally, such as violent exercise, high temperatures, etc. Occasionally it has been known to disappear spontaneously after many forms of treatment had been tried in vain.

Treatment.-In most cases it is associated with some defect in the general health and this should be carefully sought for and combated by every means, dietetic, hygienic, and medicinal. Anything which is liable to cause excessive sweating, such as alcohol, over-indulgence in tobacco, tea, or coffee, should be strictly limited or forbidden. Tarious specific remedies, such as atropin, belladonna, salicylate of soda, ergot, and sulphur, have been employed from time to time but they are all uncertain and only of temporary benefit.

In cases of hyperidrosis associated with a sluggish circulation, a general want of tone, or a tendency to obesity, improvement may be obtained from sea-bathing, salt baths, cold or tepid douching, and regulated exercises.

The local treatment consists of drying the skin and applying a dusting powder containing magnesium carbonate, bismuth, or zinc 
oxide, with the addition of 2 or $3^{\circ} .0$ of salicylic acid. Sponging with astringent lotions, such as a watery lotion containing $2^{\circ}$. of alum or tannic acid, is also useful, or the employment of baths containing an ounce of alum to the 30 gallons of water.

In the case of localised hyperidrosis about the trunk, and especially. the axillae, the most effective treatment is the I-rays. By them the most intractable cases may be benefited if not permanently cured. A pastille dose should be given, followed by a second after an interval of a month; care should be taken to aroid an over-exposure, as the skin in the axillae is delicate and sensitive and pigmentation is liable to follow even a single pastille dose, and should the dose be excessive telangiectases invariably result. These dangers can be minimised by the employment of screens of aluminium.

Failing the X-rays, a useful treatment is the application of a sponge wrung out in as hot water as can be borne, the skin afterwards being dried and a salicylic dusting powder applied or a lotion containing I or $2 \%$ of quinine or tannic acid in alcohol or Eau de Cologne. The parts should be kept scrupulously clean and great attention paid to the clothes which come in contact with them as they absorb the sweat and retain the offensive odour.

In the case of the feet and hands the treatment depends on the degree of inflammation associated with the hyperidrosis and whether the extremities are cold or hot.

In mild cases it is, as a rule, sufficient to bathe the feet once or twice daily with boric lotion, then rub them over with a quinine and alcohol lotion, and finally to apply a dusting powder, especially between the toes, such as a salicylic powder or tannoform I part and talc or zinc oxide 2 parts. The socks should be changed frequently and an astringent powder dusted into them before they are put on. White sochs are best as the dye from coloured socks is apt to be freed by the action of the sweat and to stain the skin.

In severe cases where the sweat is offensive, the feet should be bathed daily in $\mathrm{I}^{\circ}{ }_{0}$ solution of potassium permanganate or $5^{\circ}{ }_{0}$ solution of formalin. Where the feet are hot and the skin inflamed and tender, a useful application is zinc paste containing $2^{\circ}$ of ichthyol, or diachylon plaster spread on lint and applied continuously. Under these conditions the feet should not be washed but should be kept dry by the use of some dusting powder. Where the feet are cold, stimulants, such as acetic acid or camphor, should be added to the lotions or ointments. Occasionally the hyperidrosis appears to be connected with flat foot and in such cases definite improvement may be obtained from wearing suitable boots and supports for the soles.

\section{REFERENCES}

Kaposi. Hyperidrosis spinalis superior (Arch. f. Derm. u. Sỵph., i 899 , xlix. p. 32 I).

Penbrey, M. Functions of the Skin (Brit. Journ. Derm., I9I0, xxii. p. 225). 
Pringle, J. J., \& Adamson, H. G. Diseases of the Sweat Apparatus (Allbutt \& Rolleston, System of Medicine, I911, ix. p. 633).

Vörner. Hyperidrosis unius lateris congenita (Deutsch. Med. Wochenschr., I907, xxxiii. p. 2090).

\section{ANIDROSIS}

Syn.: Defective sweating; Fr. Anidrose.

In certain morbid conditions the quantity of sweat secreted may be diminished or wholly suppressed. This may be a local, regional, or general condition and may be congenital or acquired.

Congenital anidrosis is due to lack of development of sweat-glands and may either be unaccompanied by any other abnormality of the skin or may be associated with ichthyosis.

Acquired anidrosis may be due to a variety of causes. It may be the result of atrophy or destruction of the sweat-coils or ducts from some fibrous growth, such as sclerodermia, cheloid, or a scar. It may occur in association with certain cutaneous affections, such as psoriasis, the sweat-function returning when the dermatosis is cured. It is liable to supervene in old age from atrophy of the glands. It may be due to both functional and organic disturbances of the nerves from implication of the secretory fibrils, as in the maculo-anaesthetic patches of leprosy, in paralysis, and even in severe neuralgia.

General anidrosis may also be symptomatic and may accompany various morbid conditions, such as pyrexia, diabetes, tuberculosis, and chronic nephritis.

Treatment.- Where it is associated with some curable skin affection, it is the latter which requires treatment. Where it is the result of some nervous disorder of a functional type, the secretion may be restored by counter-irritation, massage, the faradic current, or the external application of warmth from Turkish baths or radiant heat, or by sweat-excitants, such as hot drinks, jaborandi by the mouth, or pilocarpine by hypodermic injection; where the coils and glands are atrophied or destroyed treatment obviously is useless.

\section{BROMIDROSIS}

Syn.: Osmidrosis; Fr. Bromidrose.

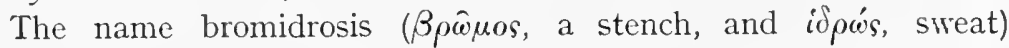
signifies sweat with a foetid odour. This is met with chiefly in the feet and armpits and about the perineum and groins, and is due either to some alteration in the sweat previous to its secretion, or to decomposition after secretion from the action of various bacteria-notably the Bacterium foetidum-which break up the sweat and eliminate certain evil-smelling fatty acids. 
It occurs in the feet most commonlyamong those whose occupations necessitate much standing or walking, and is generally associated with hyperidrosis. When first secreted the sweat is not offensive, but from decomposition it soon emits a penetrating rancid odour.

In the axillae, on the other hand, the sweat is odoriferous on secretion and becomes more so from subsequent decomposition-the odour being more stuffy than rancid, but none the less unpleasant.

Individuals with a greasy skin are especially liable to suffer from it and it is more common in brunettes and those with reddish hair than in blondes. In women it is often associated with disorders of menstruation, especially with suppression of the menses, and is apt to be accentuated at the menstrual periods.

Etiology.-Important factors in the causation of bromidrosis are want of cleanliness both with regard to the skin and the clothes, wearing of thick non-absorbent underclothes or stockings, and prevention of evaporation by the use of waterproof dress-protectors or thick boots.

In connection with ordinary bromidrosis reference may be made to the peculiar odours given off by the sweat in certain diseases, such as diabetes and rheumatic fever; to those which occasionally result from the eating of certain pungent vegetables, such as onions or garlic, or from the ingestion of certain drugs, such as asafoetida or valerian; and to the distinctive odour of the sweat from the negro shin.

Treatment.-The treatment is that described in connection with hyperidrosis.

Where the feet are affected the socks and stockings should be changed at short intervals and the inside of the boots should be dusted with a powder either of boric acid or of salicylic acid and tannoform in the ratio of I to $S$. The feet should be bathed once or twice a day, either in warm boric lotion, formalin 2 to $5 \%$ in an aqueous or alcoholic solution, a weak lotion of potassium permanganate ( $I$ in $I 000$ ), or in methylated spirit, and this followed by application of the astringent dusting powder.

In bromidrosis of the axillae the underclothes should be frequently. changed and attention should be paid to the outer garments as weil as to the underclothes. The treatment consists of cleansing the skin with soap and water, bathing it with alcohol or Eau de Cologne, and finally applying a dusting powder of salicylic acid and tannoform.

\section{CHROMIDROSIS}

Syn. : Seborrhoea nigricans; Fr. Chromidrose.

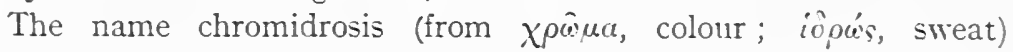
signifies secretion of coloured sweat. This has been described in various parts of the body but most commonly on the face, especially about the orbits, and in the axillae, crural, and perineal regions, and occasionally 
on the hands and feet. In the majority of cases the sweat is black or dark brown (melanidrosis), or bluish (cyanidrosis), from the presence in it of dark amorphous granules, and produces a diffuse or punctate discolouration which can be partially removed by rubbing with a cloth.

In addition to the cases in which the sweat is darkly pigmented others are on record in which it was green, red, or yellow.

About $80 \%$ of the cases have occurred in women and in a considerable number of them it has been associated with disorders of menstruation or with severe constipation. It has been observed most frequently in women of a highly neurotic temperament who suffered from hysterical attacks, and a certain number of the cases have been produced artificially with the object of eliciting sympathy - a proceeding which may sometimes be accomplished with such cunning as to render detection difficult.

Pseudo-chromidrosis may be due to aniline dyes which were not sufficiently fast and were eliminated from the underclothing or stockings by the chemical action of acid sweat. This has been observed in the feet by Arthur Hall who recorded a series of cases of blue toes in which the discolouration resulted from the wearing of cheap black stockings the aniline dye of which became dissolved and stained the epidermis to some depth.

True chromidrosis would seem to be an exceedingly rare condition but there are several authentic cases on record, such as that described by Colcott Fox in a female in whom amorphous granules were present in the sweat and gave rise to dark patches around the orbits, which case was thoroughly investigated by a special committee and accepted.

The composition of the amorphous granules in the true cases is uncertain, and various theories have been put forward with regard to it, such as :

(I) That the granules are chemically related to indigo.

(2) That they are melanin granules which have been washed out by the sweat in its passage through the epidermis.

(3) That the colour of the sweat is due to indican.

It was pointed out by Radcliffe-Crocker and corroborated by others that in certain of the cases the pigment granules were present not only in the sweat but in oily or fatty material which appeared to be derived from the sebaceous glands, hence the synonym Seborrhoea nigricans.

In association with coloured sweat, pigment has been detected in other secretions and excretions, such as the saliva, urine, and faeces (Teevan, Neligan, Quinan).

Red or yellow chromidrosis is usually met with in the axillae and is due to the Bacillus prodigiosus, which forms reddish or yellowish zooglea masses around the hairs, which are washed off in the sweat and so produce the colouring.

Cases of coloured sweating have been reported occasionally from ingestion of certain drugs. For instance a case is on record in which 
a man with syphilis had his white beard stained pink by the coloured sweat which resulted from taking iodide of potassium; blue sweating has been known to follow the taking of iron; green sweating has been observed from time to time in copper workers as the result of the ingestion of copper, from the exposure of the skin to copper fumes, or from the contact of a copper electrode with an abraded cutaneous surface.

Treatment.-The treatment consists mainly in dealing with the underlying general condition, such as the neurosis or constipation. Where the staining is a local condition and the result of aniline dres or bacteria, the cleansing of the skin with soap and water and the application of a mild antiseptic lotion should suffice to remove it.

\section{URIDROSIS}

Definition: The secretion by the sweat of an excess of urea.

In normal health a small amount of urea is eliminated by the sweat and in rare instances this is increased to the extent of making the sweat smell of urine and causing a deposit of urinary salts on the surface of the skin in a fine crystalline powder. This condition is met with almost exclusively in the later stages of severe kidney trouble, but has been described also in comnection with cholera and as the result of injections of pilocarpine.

\section{HAEMATIDROSIS}

$\mathrm{By}$ haematidrosis is meant haemorrhage into the ducts or coils of the sweat-apparatus so that the sweat becomes blood-stained. This has occurred in several instances in hysterical young women and been regarded as a form of vicarious menstruation. It is not definitely related to haemophilia, nor must it be confused with haemorrhage from the skin.

The sebaceous flux may be similarly affected.

\section{PHOSPHORIDROSIS}

Phosphoridrosis is the name applied to sweat which is phosphorescent. It has been said to result from the ingestion of phosphorus, either as a medicine or in fish, or from the presence of phosphorescent bacteria. It has been described in connection with tuberculosis of the lungs, scurry, and cancer.

\section{REFERENCES}

Bromidrosis.

Moxis. Sur les odeurs du corps humain (Ann. de la Soc. de Méd. d'Anvers, Paris, $\mathrm{I} \mathrm{S}_{5}$ ).

Thrx. Bromidrosis foetidum (Practitioner, ISSI, xxrii. p. 210; Brit. Med. Journ., ISSo, xriii. p. 463 ). 


\section{Chromidrosis.}

Dubreutly, Red chromidrosis (Arch. Clin. de Bordeaux, January, I 894). Fox, Colcotr. (Trans. Clin. Soc., Lond., I88I, xiv. p. 2 I I).

Hall, A. J. Note on causation of blue toes (Brit. Journ. Derm., I902, xiv. p. 4 I 8 ).

Neligan \& Quinax. Pigment in the secretions (Dublin Quart. Journ., I 853 , p. 295).

Putxam, J. W. Black chromidrosis with hysterical paralysis (N.I. Med. Journ., July $4^{\text {th }, ~ 1903, ~ p . ~ 26) . ~}$

TeEvax. Pigment in the secretions (Med. Chir. Soc. Trans., Lond., I845, xxviii. p. 6II).

\section{ORGANIC DISORDERS}

\section{GRANULOSIS RUBRA NASI}

Syn.: Peri-syringitis chronica nasi (Jadassohn) ; Dermatitis micropapulosa erythematosa hyperidrotica nasi (Jadassohn); A peculiar inflammatory dermatitis of the nose in young individuals with sweating (Herrmann) ; False acne rosacea of children (Audry) ; A peculiar form of acne with changes in the sweat glands (Luithlen).

Definition: A peculiar affection of the skin over the cartilaginous portion of the nose in children, characterised by persistent hyperidrosis, diffuse redness, and the presence of inflammatory micro-papules chiefly situated at the sweat-orifices.

Description.-Luithlen first drew attention to this peculiar condition in I90o and in the following year Jadassohn described a number of cases in detail and gave it the name Granulosis rubra nasi, by which it is now generally known. About forty cases have been recorded since then, the reports of which are all more or less a repetition and amplification of Jadassohn's description, while others have no doubt been recognised without having been published.

The disease chiefly affects the skin of the cartilaginous portion of the nose, and extends in front from the tip to the bridge, and spreads out laterally over the alae nasi, as a rule exempting the column and the edges of the nostrils. It may spread on to the skin of the cheeks, and over the canine fossae on to the upper lip, but it never involves the nasal mucosa. The affected skin may be pale pink, red, or bluish and livid, the colouring being diffuse, clearly limited at the sides, and fading gradually into the normal skin above. A few small telangiectases may be detected orer it with a lens, and sometimes even by the naked eve. (Fig. 343.)

In spite of being red, the nose is gencrally cold to the touch, is moist and glistening from hyperidrosis, and at the sweat-pores small beads of clear sweat can usually be detected which, in a case observed by the writer, had an alkaline reaction. In addition, the skin is irregularly dotted over with macules and micro-papules, which are reddishbrown in colour and somewhat resemble small foci of Lupus vulgaris 
but differ from that affection in that they disappear under pressure with a diascope; they are rounded or acuminate in shape, frequently situated at the sweat orifices, and occasionally present a small sweat bead on their summit. It is the presence of the micro-papules which gives to the tip of the nose the characteristic granular appearance which suggested the name granulosis.

In some cases small vesicles have been detected which are indistinguishable from those of hydrocystoma, are about the size of a pin's head, are clear or bluish in tinge, and on being punctured emit a drop of clear fluid. Deep-seated pseudo-resicles have also been observed

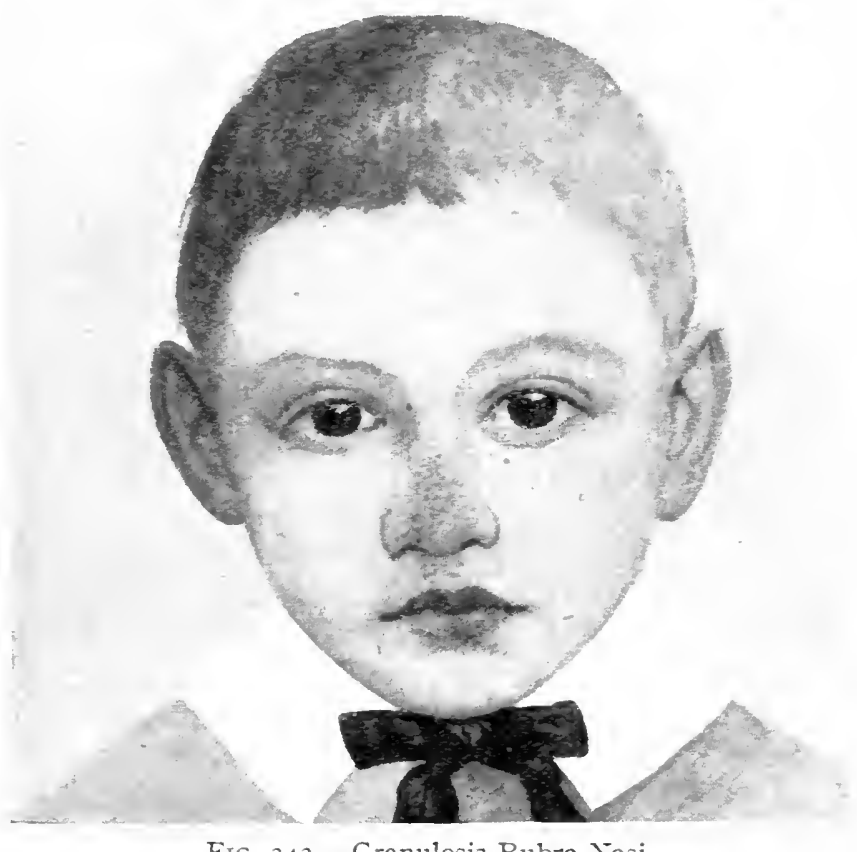

FIG. 343.-Granulosis Rubra Nasi.

which were dried up and usually presented a small scale on the surface.

Subjective symptoms are absent as a rule, but there may be slight itching and a feeling of coldness. It may be associated with evidences of a weak peripheral circulation, such as chilblains, congested and clammy hands and feet, and occasionally marked hyperidrosis of the palms and soles.

The disease begins insidiously in early life and when once established persists, more or less uninfluenced by treatment or by heat, cold, etc., till puberty; it then gradually disappears, though recently cases have been reported where traces of it were detected in adult life (Pinkus).

Histopathology.- The epidermis is scarcely altered, though it may be thickened or parakeratosis may be present about the sweat-orifices. 
The superficial blood capillaries are dilated and around them, and also around the sweat-ducts, there is a cellular infiltration consisting of polymorphonuclear leucocytes and fibro-blasts with a few plasma-cells and mast-cells, and it is these deposits of cells which are responsible for the micro-papules. Beneath the infiltration the sweat-duct may be dilated and, if the infiltration be marked and fibromatosis has occurred, it may be pressed on and even obliterated and a retention sweat-cyst formed; hence the lesions like hydrocystoma.

Etiology.-Age.-It is essentially a disease of childhood and may be observed first in infancy. It usually disappears about puberty though two cases are on record in which it persisted in adult life.

Sex.-Sex does not seem to play any part in its etiology, for out of thirty cases collected by the writer seventeen occurred in boys and thirteen in girls.

Heredity.-Hereditary weakness may be a predisposing factor and various general defects have been noted in the parents, such as the presence of tuberculosis or syphilis. A case has been recorded in which the mother of the patient was also affected (Lebet), and cases have been reported in a brother and sister (Pringle).

Personal History.-The disease usually attacks weakly children who are delicate-looking, badly nourished, occasionally of the scrofulous type, and who generally exhibit signs of a weak peripheral circulation.

Season.-The temperature has considerable influence on some of the cases, the condition being most marked either in very cold or very hot weather; in other cases, however, changes of temperature do not affect it.

Pathogenesis. - The pathogenesis of the condition is still sub judice. It is obviously related to a weak peripheral circulation, but it is a matter for dispute whether the congested state of the blood-vessels precedes the hyperidrosis, or whether the sweating is the initial disturbance and determines the vascular dilatation and inflammatory infiltration.

Diagnosis.-There is little or no difficulty in recognising the affection. It has to be differentiated from Lupus vulgaris, Lupus erythematosus, Acne vulgaris, hydrocystoma, rosacea, and eczema. It is distinguished from Lupus wulgaris by the lesions disappearing on pressure and showing no tendency to ulcerate; from Lupus erythematosus by the absence of atrophy, scarring, and adherent scales; from Acne iulgaris by the pilosebaceous follicles not being involved; from hydrocystoma by the localisation and the occurrence of the discase in childhood; from rosacea by the age of incidence and the absence of changes in the sebaceous glands; from eczema by the absence of weeping, crusting, and subjective symptoms.

Treatment.-It is singularly chronic and unyielding to treatment so long as the hyperidrosis is present, but tends eventually to disappear spontaneously. 
The internal treatment is on purely general principles with the object of improving the tone of the patient or of counteracting some obrious defect. Cod-liver oil, iron, fresh air, good food, etc., should be freely prescribed.

The local treatment is thoroughly unsatisfactory and although astringent lotions, dusting powders containing alum, tannoform, etc. and pastes containing tannic acid, ichthyol, and the like, may produce temporary benefit they do not effect a cure and a relapse usually follows their intermission. Beneficial results have been recorded from repeated superficial scarification of the affected area.

\section{REFERENCES}

Adamsox, H. G. Granulosis rubra nasi (Brit. Journ. Derm., I907, xix. p. $T$ I).

Brooke \& Savatard. Granulosis rubra nasi (Brit. Journ. Derm., 19II, xxiii. p. 369).

-Fox, Colcorr. Granulosis rubra nasi (Brit. Journ. Derm., I906, xriii. p. 320$)$.

Herrmaxx. Granulosis rubra nasi (Arch. f. Derm. u. Syph., I902, lx. p. - 7). JADAssohx. Granulosis rubra nasi (Arch. f. Derm. u. Syph., I9or, lviii. P. I +5$)$.

Lebet, A. Contribution to the study of hydrocystoma (Ann. de Derm. et de Syph., I903, iv. p. 273).

Little, G. Granulosis rubra nasi (Brit. Journ. Derm., I906, xviii. p. 2IS). Luithlex. Granulosis rubra nasi (Kaposi's Festschrift, I900, p. 709).

MacLeod, J. M. H. Granulosis rubra nasi (Brit. Journ. Derm., I903, xv. p. I97).

Malherbe, H. Granulosis rubra nasi (Journ. des Mal. Cut. et Syph., 1905, xvii. p. 97).

PICk, W. Granulosis rubra nasi (Arch. f. Derm. u. Syph., I902, lxii. p. I05).

PIxkes. Case in an adult male (Derm. Zeitschr., I90 4 , ix. p. 6ł2).

Pringle, J. J. Cases in brother \& sister (Trans. Derm. Soc. Lond., I 894). RICARD, L. Thesis on Granulosis rubra nasi (Toulouse, I904).

\section{HYDROCYSTOMA}

Definition: An affection of the skin of the face, characterised by deep-seated pseudo-vesicles, believed to be due to cystic dilatation of the sweat-duct or sweat-gland.

Description.-Hydrocystoma, which was first described by A. R. Robinson of New York in $I S S_{4}$, is a rare condition superficially resembling dysidrosis but differing from it in being non-inflammatory and confined to the face.

The eruption consists of deep-seated pseudo-resicles, which appear on apparently normal skin and are situated chiefly about the forehead, nose, orbit, cheeks, and chin, and occasionally on the neck. They are round or ovoid in shape, vary in size from a pin's head to a pea, and contain a clear or slightly opaque fiuid. The larger lesions have a bluish tinge, most marked at the periphery, and present an appearance which has been aptly compared to boiled sago grains. If punctured 
they exude a ciear or slightly granular fluid which is acid in reaction, but they have no tendency to rupture spontaneously and if left alone gradually dry up to form white specks, like milium, which scale off in a few weeks leaving either no trace, or occasionally slight pigmentation.

They vary in number in different cases from two or three up to sereral hundred, but even when closely crowded together remain discrete.

The eruption is not accompanied, as a rule, by inflammation nor by any subjective symptom beyond a mild degree of smarting, but it is invariably associated with profuse perspiration, and the lesions become more abundant as perspiration increases. It is usually symmetrical in distribution but Jamieson has recorded an instance in which it was entirely confined to one side of the face, the perspiration also being more marked on the affected side.

Histopathology and Pathogenesis.-The microscopical anatomy has been described by various writers (Robinson, Adam, Lebet). The lesion consists essentially of a cyst situated in the corium, lined with several layers of epithelium, and containing a clear or slightly granular fluid. Its appearance and position suggest that it has taken its origin from the sweat-apparatus, either from the sweat-duct immediately above the coil or from one of the glomeruli of the coil itself. The overlying epidermis is not altered, except secondarily by pressure, and the pilo-sebaceous follicles are unaffected though they may be pushed aside. There are no other changes except occasionally slight cellular infiltration around the neighbouring blood-capillaries.

There is no concensus of opinion with regard to the precise origin of the cyst. According to Robinson it is due to a dilatation of the sweat-duct near the coil-gland, but if this be the case it is curious that even in careful serial sections the remains of the duct above the cyst have never been detected nor any connection between the cyst and the sweat-pore on the surface of the skin.

Another hypothesis is that it arises from a dilated glomerulus, and is associated with a general hypertrophy of the gland in which there is not a corresponding enlargement of the duct to carry off the excessive secretion.

It has been suggested also that it is a retention cyst in the sweatduct, the result of constriction of the duct produced by new fibrous tissue and secondary to a slight inflammatory infiltration in the neighbourhood of the duct caused by excessive sweating.

Still another theory is that it is a cystic adenoma of congenital origin, but the age at which it develops is strongly against this view.

Etiology.-Hydrocystoma has been known to appear in old-age but is more usual in middle-life. It is far more common in women than men, and the majority of cases have been in middle-aged women constantly exposed to a hot moist atmosphere, such as washerwomen. The condition is most marked in hot weather and tends to disappear partially or completely in winter and to recur in summer. 
The principal determining cause seems to be excessive activity of the sweat-glands, and consequently the affection occurs mostly in people who sweat readily from exposure to heat or from some other cause possibly nervous. In a case recorded by Hutchinson in which the sweating was limited to one side of the face, it was associated witl neuralgia on the same side.

Diagnosis.-Hydrocystoma is distinguished from other yesicular conditions, such as eczema, sudamina, and miliaria, by the exclusive localisation to the face, the annual recurrence, the greater depth of the lesions, and the absence of all tendency for them to rupture spontaneously. It may have a superficial resemblance to Epithelioma adenoides cysticum but in that condition the small nodules are more solid and are not associated with excessive perspiration.

Treatment.-The treatment consists of counteracting the tendency to perspiration by change of occupation and attention to the general health. Locally, the vesicles should be punctured and the skin bathed with some antiseptic lotion followed by the use of an antiseptic dusting powder, such as salicylic acid and zinc oxide ( $I$ in S).

\section{REFERENCES}

Adan, J. Hỵdrocysstoma (Brit. Med. Journ., Dec. IS93; Brit. Journ. Derm., I 895 , vii. p. I69).

Hutchissox, J. Case illustrating the neurotic origin of hydrocystoma (Brit. Journ. Derm., I895, vii. p. I37).

Lebet, A. Histopathology of hỹdrocystoma (Ann. de Derm. et de Syph., I903, iv. p. 273).

Morton, A. Hydrocystoma (Brit. Journ. Derm., I895, vii. p. 245).

PINkUs. Relation of hydrocystoma to Granulosis rubra nasi (Derm. Zeitschr., r904, xi. p. $6+2)$.

Robisson, A. R. Hỹdrocystoma (Trans. Amer. Derm. Assoc., I884; Journ. Cut. \& Gen.-L̈rin. Dis., August I 893 ).

Thibierge, G. Hydrocystoma (Ann. de Derm. et de Syph., Nor. I895).

\section{HYPERTROPHY}

Hypertrophy of the sweat-glands may be either congenital or acquired. In it the glands may be increased in number or in size by the growth of new convoluted tubes forming adenomata.

Congenital hypertrophy may be a general or a localised condition. When general, it is due to an increased development of sweat-glands, most marked in situations in which they are normally most profuse, such as about the palms, soles, axillae, etc. ; when local or regional, it is a form of naevus-in the broad sense of the term.

Acquired hypertrophy may occur in connection with excessive activity of the glands in much the same way as muscular development results from exercise, and is met with in morbid conditions associated with excessive sweating, such as rheumatic ferer and tuberculosis of the lungs, and in hyperidrosis of the palms, soles, or other regions. 


\section{ATROPHY}

Absence or atrophy of the sweat-glands may be congenital or acquired. When acquired it may be associated with various forms of atrophy of the skin, such as senile atrophy, or local atrophy due to continuous pressure such as occurs after the removal of a plaster of Paris dressing, or it may follow interference by disease or injury with the nerve supply to the sweat-coils, as in the maculo-anaesthetic patches of leprosy or in the various forms of paralysis from injury to the peripheral nerves or spinal cord.

\section{POROKERATOSIS (MIBELLI)}

Syn.: Hyperkeratosis eccentrica (Respighi); Keratodermia eccentrica.

Definition: A rare affection of the skin, of hereditary origin, and characterised by horny excrescences which spread eccentrically to form irregular figures with an atrophic centre surrounded by a well-defined horny ridge.

The affection was first described in Italy in IS93 by Vittorio Mibelli and Respighi independently. The name porokeratosis was given to it by Mibelli as he considered that it was essentially a hyperkeratosis occurring about the sweat-pores. About 60 cases are on record and it has been observed in this country, in France, Germany, and America.

Description.- - It can occur anywhere on the cutaneous surface, and has been known to spread on to the red portions of the lips. It appears first as a small horny elevation which increases, assumes an acuminate shape, and becomes surrounded by a horny collar or ring. This gradually extends centrifugally, becomes flat in the centre, advances in a festoon manner, and gives rise to irregular lesions consisting of a well-defined ridge enclosing an area of slightly atrophic skin which presents the normal lines of the skin-somewhat widened-is dry from atrophy of the sweat-glands and smooth from disappearance of the lanugo hairs. The most characteristic feature of the lesion is the horny ridge, which is yellowish or brownish in tint, triangular in transverse section, and usually presents a furrow or cleft along the border. In rare instances the base may be slightly inflamed from friction. The ridge is especially marked in positions which are subject to rubbing, as about the ankles and dorsa of the feet, and more delicate where the skin is soft and smooth, as about the face and the flexures of the forearms. On the buccal mucosa it takes the form of irregular patches limited by a whitish opaque linear elevation. (Fig. 344.)

The affection does not interfere with the general health, is not associated with subjective symptoms, and has no disadvantage except unsightliness. As a rule it begins in childhood and is slowly progressive 
throughout life, new lesions developing from time to time, but cases are on record in which it did not appear until adult life or old-age.

Histopathology.--Sections of the horny ridge show hyperkeratosis around the sweat-orifices and blocking of the epidermal portion of the duct with horny material. It is not a perfect hyperkeratosis, as the horn-cells are swollen and slightly moist, and it has been described as a "para-hyperkeratosis." The underlying prickle-cell layer may. be increased and the inter-papillary processes elongated.

Any changes which occur in the corium would appear to be of a secondary nature and consist of a slight dilatation of the capillaries, an

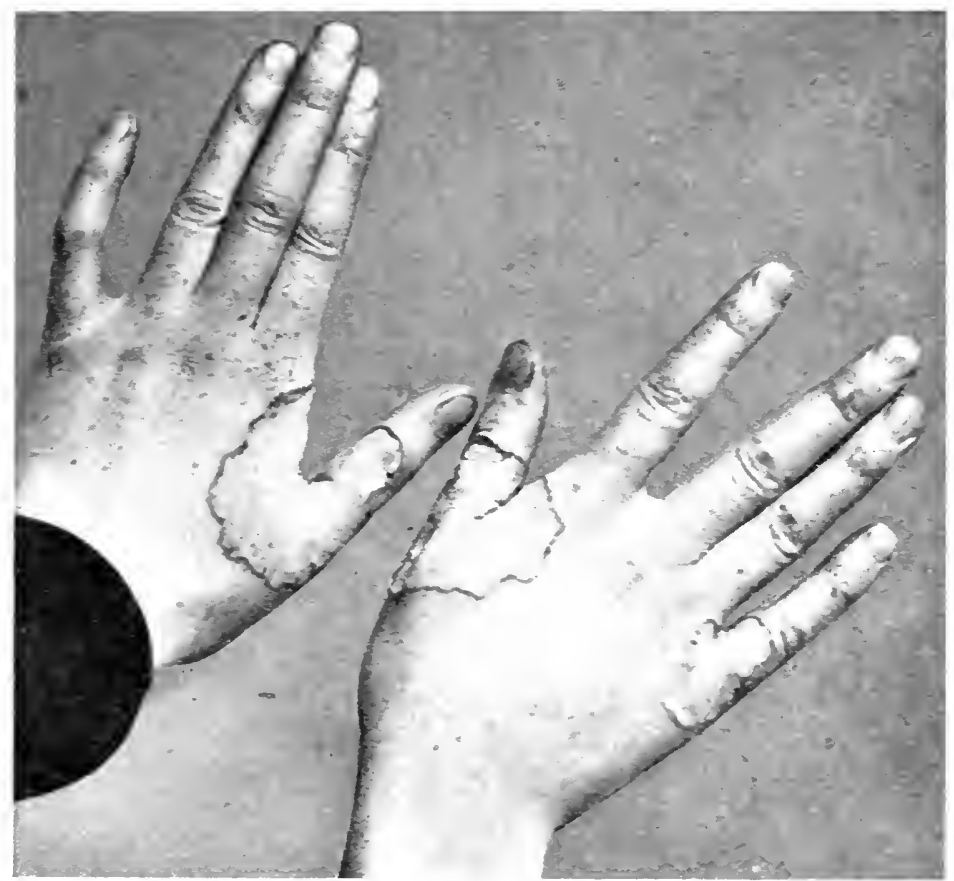

F!G. 344.- " Porokeratosis " or "Hyperkeratosis Eccentrica."

(From Sir James Galloway.)

infiltration of mono-nuclear cells, a diminution in the collagen, and a disappearance of the elastic network. The presence of the hyperkeratosis leads to atrophy of the underlying sweat-coils and, where the process has involved the orifice of the pilo-sebaceous follicle, it leads to atrophy of the hairs and sebaceous glands.

Etiology.-The nature and causation of the affection are not fully understood. The most important factor in its etiology would appear to be heredity, and it may show a family prevalence. It has been hnown to occur in several generations and Mibelli recalls an instance in which three brothers, a paternal uncle, and a paternal grandfather, all had it. It is generally explained as being essentially a hyperkeratosis about the orifices of the sweat-ducts, but whatever the cause of this there is no 
evidence that it is an infective condition and no micro-organism has been isolated in connection with it. Its general characteristics, appearance in early life, indefinite persistence, and occurrence as a hereditary peculiarity, seem to suggest that it is an anomalous type of verrucose naevus in which the seat of origin is located in the epidermis chiefly about the sweat-orifices.

Diagnosis.- The affection has to be distinguished from Lichen annularis, but in the latter there is no horny ridge with a characteristic groove but the border is smooth, flat, or rounded, definitely infiltrated, and microscopic sections show a cellular infiltration in the underlying corium.

Treatment.-The treatment is wholly unsatisfactory. The border may be smoothed down by keratolytic applications, such as pyrogallic or salicylic acid plasters or pastes, but it rapidly reasserts itself when the applications are stopped. Curetting is of temporary benefit but the only really efficient methods of dealing with it are excision, or refrigeration with solid carbon dioxide.

\section{REFERENCES}

DuCrey, A., \& Respighi, E. The presence on the buccal mucous membrane of the affection improperly called Porokeratosis (Ann. de Derm. et de Syph., I 898, ix. p. I).

Galloway, J., \& Macleod, J. MI. H. Porokeratosis (Brit. Journ. Derm., I90I, xiii. p. $262 \& 300)$.

Josteph, M. Porokeratosis (Arch. f. Derm. u. Syph., I897, xxxix., Hft. 3).

Matsumato, S. Porokeratosis Mibelli, histology of (Japan, Zeitschr. f. Derm. u. Urol., I9I6, xvi. p. 293 ; Journ. Cut. Dis., I9I6, xxxiv. p. 489; Ibid., I918, xxxvi. p. 379).

Mibelli, V. Porokeratosis (Internat. Atlas of Rare Skin Dis., I892-I895). Two new cases of Porokeratosis (Ann. de Derm. et de Syph., June I 905 , p. 503).

Respighi, E. Hyperkeratosis eccentrica (Il Giorn. Ital. delle Mal. Ven. e della Pelle, I $\$ 95$, i.).

\section{KERATOSIS PUNCTATA}

Syn. : Keratodermia punctata (Chalmers \& Kamar) ; Porokeratose papillomateuse palmaire et plantaire (Mantoux).

Definition: A peculiar keratosis of the palms and soles, characterised by a pitted appearance of the affected skin and a patchy thickening of the horny layer.

Description.-In r $89 \mathrm{r}$ Hallopeau and Claisse described a case of this rare affection; in 1903 Mantoux reported a second case, and referred to a morlel in the St. Louis Hospital Museum of a case from Besnier's clinic labelled "Symmetrical erythematous keratodermia of the extremities, punctate form of keratosis localised to the sweat orifices, palm of hand." Cases have been described also by de Bcurmann and Gougerot, and by Chalmers and Kamar who have given a detailed account of its histopathology. 
The most noticeable feature of this condition is the presence on the palms and palmar aspects of the fingers, and on the soles, of numerous small rounded pits, or crateriform depressions, with here and there a definite thickening of the epidermis in the form of keratotic patches and ridges which tend to become cracked at the folds of the joints. Associated with it there is neither hyperidrosis nor definite sensory symptoms, though the skin around the pits may be erythematous. (Fig. 345.)

According to Chalmers and Kamar the pits and keratotic patches are brought about as follows: Small rings of heaped up horn-cells

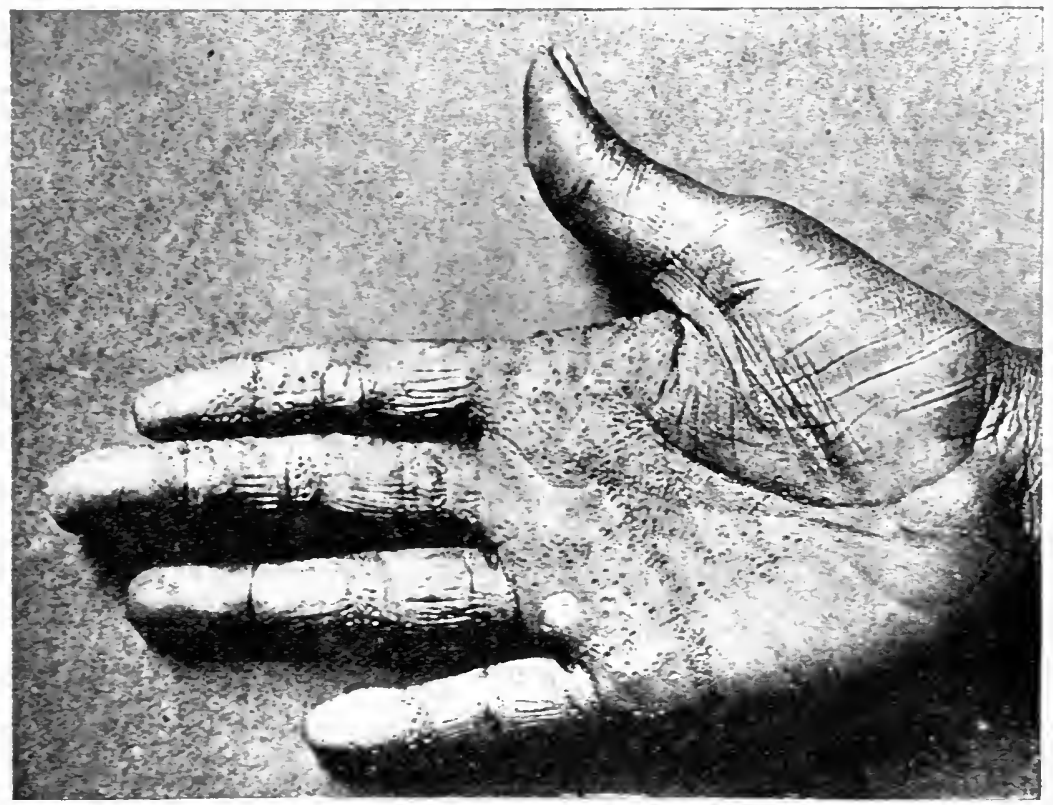

FIG. 345.-Keratodermia Punctata.

(Drs. Chalmers and hamar's case. Journ. of Trop. Mel., I9I\%.)

develop around the orifices of the sweat-ducts, these join together to form an irregular-shaped swelling or translucent nodule in the centre of which a cone-shaped horny plug appears, which comes away and leares a deepish cavity with a reddish base, steep walls, and undermined horny edges. The carity or depression gradually becomes filled up from the bottom with horn-cells and when completely filled forms a thickened area which, when joined to similar areas, gives rise to a horny patch or ridge on which the sweat-pores are found still to be patent.

Histopathology.-There is a general thickening of the Stratum corneum, with here and there corn-like bodies of closely welded nucleated horn-cells. These bodies are cone-shaped with the apex beneath, and press down like an ordinary corn on the subjacent structures; on their deep aspect they are firmly fixed but are loosely attached towards 
the surface. The prickle-cell layer is normal, except under the apex of the corn where it is compressed and thin. (Fig. 346.)

In the corium the papillae beneath the corn are compressed and the ressels dilated, while in the sub-papillary layer there is a cellular infiltration consisting of small connective tissue cells, cells which appear to originate in the endothelium of the vessels, and a few plasma-cells. Both the blood capillaries and the lymphatics are partly obstructed by

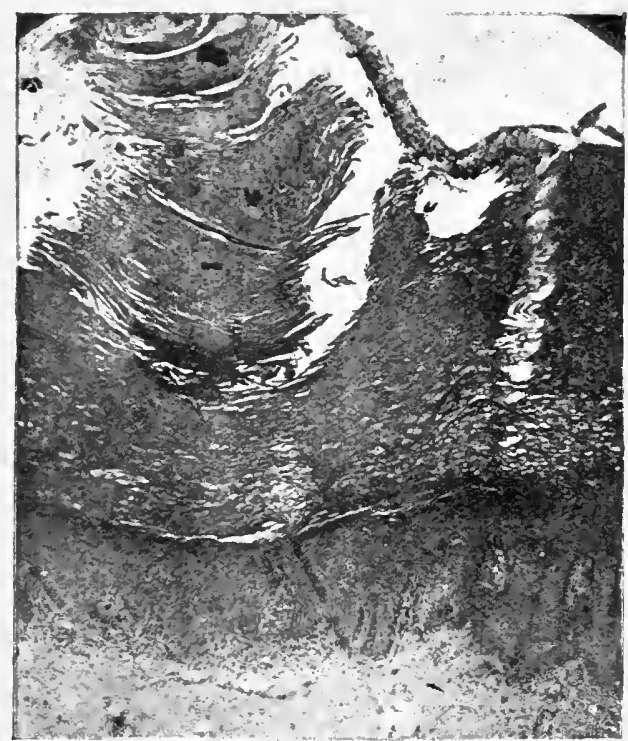

FiG. 346.-Section of Keratodermia Punctata.

Shows blocking of sweat-duct by horny plug and passage of a neighbouring duct through horny layer.

(Drs. Chalmers and Kamar's case. Journ. Trop. Med., I917.) fibrinous clots or by an inward proliferation of the endothelial cells (Chalmers \& Kamar).

Etiology.-It may occur at any age and in either sex. As it is somewhat similar to the keratosis met with in the palms in chronic arsenical poisoning and which appears to begin around the sweatorifices, it is possible that it may be an acquired condition and due to the elimination in the sweat of some toxic substance, either derived from the alimentary tract or the result of imperfect metabolism. On the other hand it has been suggested that it may be of congenital origin and Thibierge has described cases of " disseminated congenital porokeratosis" which resemble it, while Hallopeau and Claisse believed it to be a form of naevus appearing in adolescence.

Treatment.-It is singularly resistant to treatment, and though the thickening may be reduced and the pitting partially obliterated by salicylic plaster or ointment, they are liable to recur on the cessation of treatment.

\section{REFERENCES}

Chalmers, A. J., \& Kiamar, A. Keratodermia punctata (Journ. Trop. Med. \& Hyg., I9I7, June, p. I2I).

De Bevruanix \& Gougerot. (Ann. de Derm. et de Syph., r 905, vi. p. 629) Galloway, J., \& AdAusox, H. G. Punctate Keratosis, with histological notes \& bibliography (Brit. Journ. Derm., I9I 8 , xxx. p. I23).

Hallopeau \& Clalsse (Bull. de la Soc. Française de Derm. et de Syppl.,. I 89 I , P. I I 6 ).

Maxtoux (Ann. de Derm. et de Syph., I903, iv. p. I5).

Tumbierge (La Prat. Derm., i904, ii. p. 8+3). 


\section{CHAPTER XXYTII}

\section{DISEASES OF THE NAILS}

Syn.: Fr., Maladies des Ongles; Ger., Krankenheiten der Nägel.

Introduction.-An abnormal condition of one or more of the nails is a common occurrence and may be met with in association with a number of cutaneous affections and with various general diseases, or may result from traumatism, physical or chemical irritants, or from parasites such as progenic cocci or the fungi of ringworm or farus.

Sometimes the nail-plate is involved primarily, but more often it is implicated secondarily as the result of morbid changes in the nailmatrix, nail-bed, or surrounding soft tissues.

From the appearance of the diseased nail it is difficult or impossible to come to a definite conclusion with regard to the underlying cause, since sinilar appearances may be produced by totaliy different causes. In most cases a firm diagnosis can only be arrived at from the presence of concomitant symptoms of some cutaneous disease or of some general morbid condition, such as a cardiac affection or a weak peripheral circulation, by the microscopical demonstration of some parasite, or from a history of injury or local irritation.

When only one nail is involved, or several in an astmmetrical manner, the presumption is that some local cause is responsible; when nails on both sides are attacked more or less s.mmetrically it points to some general disturbance.

Pathological Considerations.-To understand the pathological changes which involve the nail it is essential that the student should be thoroughly conversant with its normal structure and derelopment. They may be grouped under the headings of hypertrophy, atrophy and dystrophy.

\section{Hypertrophy}

Simple Hypertrophy (Onychanxis).-This is a rare condition in which the nail is enlarged, thickened, and hardened from overactivity or an increase in size of the nail-matrix. As a rule it affects all the nails of the hands and feet and is met with in acromegaly and gigantism, and in the clubbed cyanotic fingers of congenital and acquired 
Morbus cordis and certain diseases of the lungs when it probably results from venous congestion.

Hypertrophy *ith Deformity (Onychogryphosis). - Irregular thickening and deformity of the nail is much more common than simple hypertrophy and is due either to pressure on or injury to the nail causing a widening of the nail-fold and the formation of a thicker nail, or to a horny or scaly thickening of the nail-bed acting as a barrier to its forward growth, raising it up, and leading to deformity. In this way such conditions as ingrowing toe-nail, claw-nail, and ram's horn nail, are produced-names which indicate the shapes which are assumed.

\section{Atrophy and Shedding of the Nail}

Congenital Anonychia.-Congenital absence of one or more nails is rare but may occur occasionally in association with other maldevelopments. It may be the result of delayed development and the nails, although absent at birth, may appear later.

Acquired Anonychia.-This condition is much more common than congenital anonychia. For complete loss of the nail to take place a breach of continuity between the nail and the nail-matrix is essential. Pathological conditions affecting the nail alone do not as a rule produce it, as the nail is only mechanically united to the nail-bed by the fitting together of the longitudinal ridges on the under-surface of the nail and the corresponding grooves on the nail-bed.

The breach of continuity at the nail-matrix may be due to a number of causes, of which the following are the most important:

(i) Pyogenic Infection. The infection of the soft parts about the nail with pyogenic cocci, giving rise to what is known as purulent perionychia, may lead to shedding of the nail. As a rule when the purulent condition is cured the nail grows again, but in rare instances it is no sooner re-formed than it is undermined and shed (Onychia maligna).

Sometimes the separation is partial and occurs at the nail-matrix while the connection between the nail and the nail-bed is still retained. A hiatus forms between the nail and the nail-matrix which may be covered with crusts or may leave the posterior part of the nail-bed exposed. This space gradually increases and within it, in due course, an imperfect new nail-growth may be detected, which may be followed in turn by a perfect nail and by the shedding of the old one.

The nail may also be raised up and separated from the nail-bed while still connected with the nail-matrix, from the presence of various lesions on the nail-bed, such as warty thickenings or scaly excrescences caused by eczema, psoriasis, ringworm, or farus.

(ii) Febrile Conditions. During any acute fever a temporary cessation of growth at the nail-matrix is liable to occur, but this does not lead to shedding of the nail. In convalescence the nails grow rapidly, but the newly-formed nail is usually thin, owing to imperfect 
action of the nail-matrix and narrowing of the posterior nail-fold by the pressure of the turgescent vessels in the extremities before the circulation regains its proper tone.

(iii) Scarring Conditions. Smallpox, pustular syphilides, gummata, etc., affecting the nail-matrix or the nail-bed may lead to destruction of the matrix and loss of the nail, and the subsequent scarring may be so severe that re-growth is prevented.

(iv) Traumatism. Severe injuries to the nail, such as bruises or blows, may lead to disintegration of the matrix and shedding of the nail by causing a sudden rupture of the blood-ressels underlying the matrix and an effusion of blood in the posterior nail-fold. In those circumstances recovery generally takes place and a new nail grows.

(v) Nervous Diseases. In cerebral paralysis, Tabes dorsalis, syringomyelia, and nerve leprosy, the diseased state of the nerves which supply the nail-matrices may so interfere with their function as to lead to shedding of the nails. In connection with cerebral lesions it is the nails of the great toe which are most often involved. Sometimes the nails grow again, but where the nerves have been severely affected or where the division of a large nerve, such as the median, has taken place the prognosis is unfavourable.

(vi) Cutaneous Affections. In diseases of the skin in which there is marked desquamation, such as Pityriasis rubra, general exfoliative dermatitis, and Pemphigus foliaceus, shedding of the nails may occur. It has been met with also in connection with complete alopecia.

\section{Dystrophy}

By dystrophy of the nail is meant the changes which occur in its texture and colour from an interference with the function of the nailmatrix from imperfect nutrition. It may occur in acute and chronic diseases, wasting conditions, intoxications, certain cutaneous diseases, from injury or disease of the nerves supplying the nail-matrix, and in parasitic affections.

The following are the principal alterations which may be met with :

Striation.-One of the first evidences of imperfect nail-growth may be an accentuation of the longitudinal striae normally present. Sometimes the longitudinal ridges are coarse and separated br furrows, or there may be a single median ridge which may divide like a $Y$ twothirds up the nail. Such reeded nails are exceedingly liable to split and become fraved at the free margin, the splitting sometimes extending for a considerable distance towards the lunule (onychorrhexis). The cause of the striation is believed to be an accentuation of the parallel papillary ridges of the nail-matrix, owing to congestion and imperfect circulation.

Transierse Grooies. - Single or multiple transverse furrows constitute a common dystrophy of the nail. These are due to an interference with the proper growth of nail-plate so that it becomes soft, the cells 
composing it being only partially cornified. These cells are at first swollen and moist and give rise to a transverse opacity in the nail, but later they tend to shrivel and a depression or groove is produced. The groove is first noticed in the position of the lunule but gradually advances with the growth of the nail, losing its opacity and finally assuming the colour of the anterior portion of the nail-plate. These grooves are usually determined by some general illness, and repeated attacks of illness may be indicated by a series of parallel transrerse grooves. From the position of the grooves it is sometimes possible to make an approximate guess at the date of an illness. The nail-growth from the lunule to the free edge takes about five monthsthe nail growing somewhat faster in summer than in winter-and should the groove be present about the middle of the nail it would

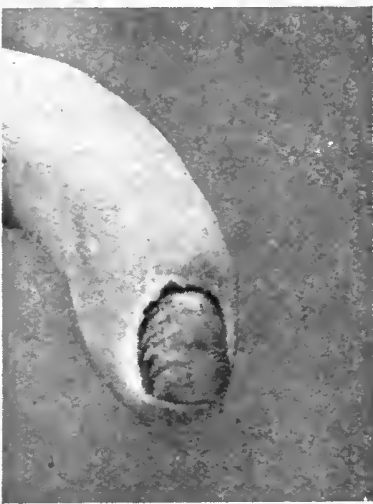

FIG. 347.-Dystrophy of Nail due to Inflammation of Nail-wall.

(Trom Dr. Wallace Beatty.) suggest an illness the acme of which had occurred two or three months previously. (Fig. 3+7.)

Punctiform Depressions.-The presence of minute puncta, resembling pin-pricks on the nail, is not uncommon and is met with in connection with a number of cutaneous diseases. It is the result of imperfect nail-formation in individual papillae of the matrix. In severe cases it may give rise to a peculiar cribriform or worm-eaten appearance.

Lenconychia.-The name leuconychia has been applied to white specks (Flores unguium or "gifts"), irregular white patches or bands, and complete whiteness of the nail. The patches vary from a translucent bluish-grey to an opaque white like chalk. They are generally explained as the result of imperfect nail-growth with the formation of moist cells which, on drying, shrivel, the spaces left being filled with air which produces the white opacity. This explains those cases in which the opacity is followed by shrinkage and the formation of depressions or grooves, but not the cases in which the nail, though whitened, does not appear to be altered in texture and shows no tendency to shrivel, and these suggest some peculiarity of cornification by which an opaque form of nail-cell is formed $a b$ initio. (Fig. 348 .)

Spoon-shaped Nails (Koilonychia).-Sometimes the nails, instead of being convex on their long axis, may become detached, turned up at the free edge, and assume a concave shape to which the name "spoon-shaped nails" has been applied; or instead of being completely concave only one of the anterior corners may be detached. This may result from thickening of the horny layer of the nail-bed near the free border impeding the forward growth of the nail and raising it up at the free margin. Minor degrees of it may be met with 
in association with various skin affections; or it may occur independently of cutaneous disease from no obvious cause, and be due to some error in the growth of the nail-matrix or peculiarity of the nail-bed. It has been known to occur as a congenital anomaly and to affect several members of a family or several generations.

The opposite of this condition, in which the nail-plate instead of being concave presents an increase in its convex curvature, is sometimes met with in clubbed fingers.

Ingrowing Toe-nail or Claunail (Onvichogryphosis). - This form of dystrophyand deformity usually attacks the big toe-nail. In it the nail substance is peculiarly hard, thickened, opaque, of a dirtybrownish colour, and roughened by the presence of wary longitudinal transverse ridges. Owing to its hardness and size the nail is apt to presson the soft parts at the side of or beneath the free border, causing

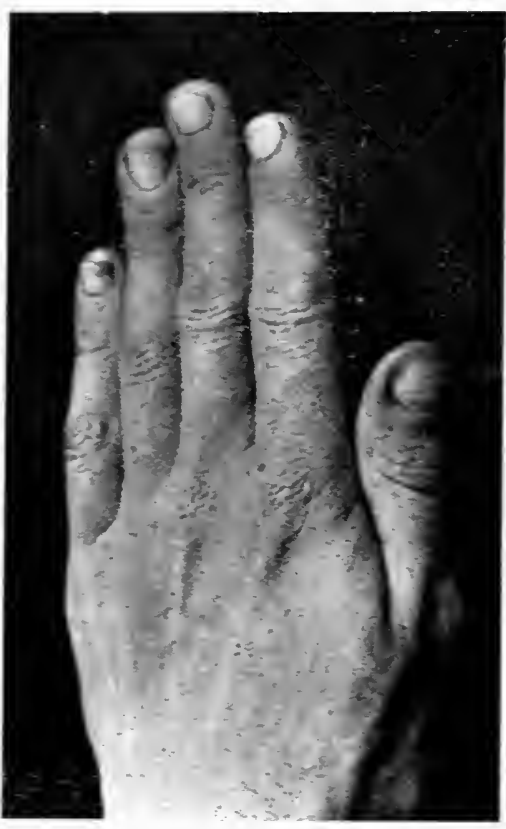

FIG. 348.-Leuconychia. Affected all the nails of the hands. pain which is usually intermittent, but sometimes constant, and may be so severe as to render walking impossible. Occasionally the pain

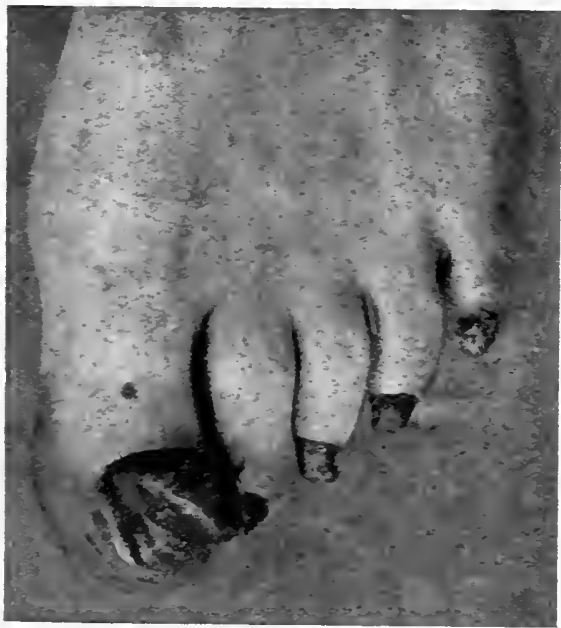

FIG. 349.-Onychogryphosis. is the result of the exposure of the sensitive nail-bed from the nail being raised up at the free border. The pressure of the nail reduces the resistance of the soft parts pressed upon, rendering them prone to infection with progenic organisms which may give rise to suppuration, oedema, and sometimes to gangrene and sloughing of the nail. (Fig. 349.)

Instead of the ordinary ingrowing toe-nail, the nail may become curved and twisted and assume the various shapes graphically described as clawnail, ram's horn nail, etc.

Treatment.-In the milder cases the ingrowing nail should be cut as short as possible and pledgets of gauze impregnated with boric lotion 
inserted between the edge of the nail and the inflamed skin. If the lateral pressure be considerable it is necessary to press down the skin of the nail-fold before the pledget can be introduced. Any bleeding points or granulations should be touched with silver nitrate or the actual cautery. Strips of adhesive diachylon plaster should then be wound tightly round the ungual phalanx and covered by a rubber cot. The plaster dressing should be removed in twenty-four hours, the feet bathed in boric lotion, and a new dressing applied. By this treatment the patient is gradually enabled to wear boots and to walk about, and the possibilities of complete recovery are considerable.

Another useful method is to moisten the nail with warm Liquor potassae applied with the frayed-out end of a piece of cane. This softens the nail, which can then be scraped down with a piece of glass till it is as thin as paper and the ingrowing edges lifted up and cut with scissors.

In the severer forms, namely claw-nail and ram's horn nail, the above treatment is useless and complete extirpation is necessary. After the removal of the nail the skin should be stitched over the nail-bed, since in these cases the matrix is damaged irretrievably and incapable of forming a healthy nail.

\section{DISEASES OF THE NAIIS ARRANGED ON AN ETIOLOGICAL BASIS}

The affections of the nails may be grouped under the following headings based on our present knowledge of their etiology :

I. Congenital affections.

2. Diseases of the nail due to physical or chemical irritants :

(a) Traumatism from mechanical injury.

(b) X-rays, actinic rays, etc.

(c) Chemical irritants.

3. Parasitic diseases of the nails :

(a) Progenic infections.

(b) Mrcotic infections due to the fungi of ringworm and farus (Onychomycosis).

(c) Acari of scabies.

4. Affections of the nails in cutaneous diseases.

5. Affections of the nails in syphilis.

6. Dystrophies due to

(a) General diseases, circulatory disturbances, etc.

(b) Nerve lesions.

\section{Congenttal AfFections}

Congenital anonychia may be a hereditary or family condition and may occur in association with congenital alopecia. Cases are on record in which at birth at full-time the nails, though seemingly normal in other 
respects, presented no free border, but by the end of the first month the thin plate in front of the anterior groove had desquamated and disappeared and the nails had assumed a natural appearance. This condition may be due to persistence of the epitrichium.

Various dystrophies and deformities may also occur as congenital or family peculiarities, such as spoon-shaped nails, twisted nails, ingrowing nails, and raising up of the nail from the presence of horny thickening on the nail-bed (sub-ungual hyperkeratosis). It is possible, however, that certain cases of so-called dystrophy of the nails which have been reported in families and have developed after birth may be of parasitic origin and due to the ringworm fungus. (Fig. 350.)

Congenital deformities of the nail may be associated with other congenital anomalies of the skin, such as ichthrosis and congenital hypertrichosis.

In certain individuals rudimentary nails have occurred, which were thin and soft like those of an infant, and these have persisted to adult life. The

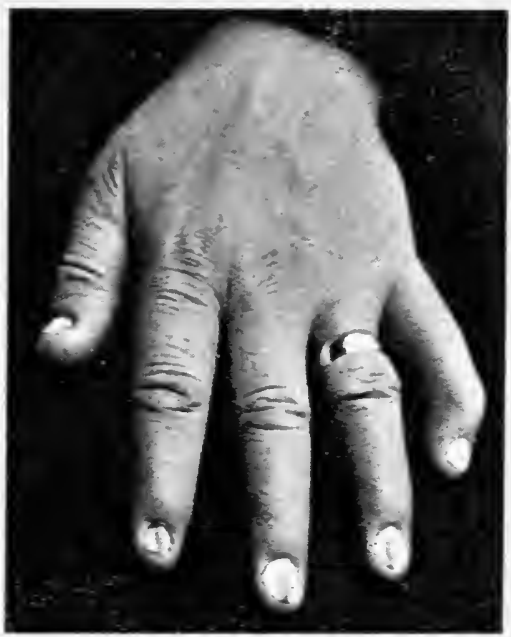

FIG 350.-Congenital Deformit! of Nails.

It was associated with absence of patellae. (From Dr. Dougla, Firth.) nails may be defective or absent in connection with the hereditary affection Epidermolysis bullosa.

\section{Diseases of the Nail dé to Physical oR CheMICAL IRRITANTS}

(a) Traumatism.-The nails may be severely injured as the result of traumatism, blows, crushing, etc. The free edges also mar be worn down and may even become concave rather than convex from manual labour, or from incessant scratching, or they may become irregularly shortened from being constantly bitten. I not infrequent cause of injury is too assiduous manicuring and such forcible pressing back of the thin film of skin which stretches down over the lunule that the lunule and underlying nail-matrix are injured and the nail grows irregularly or presents opacities or depressions.

(b) X-rays, Actinic Rays, etc.-In connection with chronic X-ray dermatitis of the fungers the nails are liable to be severely involved and may present rarious degrees of dystrophy, from reeded, fraved nails, to splitting and even shedding. Small warty growths are also liable to develop in the nail-bed beneath the free border; these are 
painful and tend to raise up the nail. At the same time the resistance of the soft parts around the nail is diminished by the rays so that they are liable to become infected by pyogenic micro-organisms, and suppuration and painful ulceration may supervene.

These changes are due to the action of the X-rays on the nailmatrix and the soft growing part of the nail adjoining it, which interferes with the nutrition and leads to imperfect nail-growth. The action of the rays on the nail-matrix is comparable to that on the hair-papillae and, if a sufficient dose were given, would lead to shedding of the nail. Unfortunately the dose of X-rays necessary to cause a defluvium of the nail is in excess of the Sabouraud pastille dose which brings out the hair safely, and as it cannot be given without injuring the skin and neighbouring soft tissues its employment is inadmissible.

The treatment is unsatisfactory but benefit may be derived from soothing antiseptic applications to relieve pain and reduce the inflammation, and from short exposures to radium to heal superficial ulcerations around the nail and remove painful warty excrescences.

A somewhat similar condition, though not so severe, may occur as the result of long exposures to the actinic rays of sunlight and may be seen on the hands of those affected with chronic solar dermatitis.

(c) Chemical Irritants.-The nails are liable to be more or less severely involved in association with the various forms of occupation dermatitis resulting from the handling of irritating substances. They may be partially destroyed, or rendered friable and discoloured, by actual contact with such irritants as formalin, sugar, tar, metol, etc. ; or various dystrophies, such as opacities, reeded nails, fraying at the margins, or the presence of transverse or longitudinal furrows, may be secondarily induced by inflanmatory processes occurring in the nailmatrix, nail-bed, or nail-wall.

The treatment of these conditions consists in aroiding further exposure to the irritant, either by a change of occupation or by protecting the fingers with gloves. Inflammatory affections of the shin surrounding the nails should be dealt with on the principles laid down for the treatment of eczema of the nails.

\section{Parasitic Diseases of the NaIls}

(a) Pyogenic Cocci.-One of the most frequent causes of diseased conditions about the nail and the surrounding tissue is septic infection due to the ordinary pyogenic cocci. When the nail and the nailbed alone are attacked the inflammatory condition which results is known as onychia, when the surrounding tissues are involved as perionychia, and when the inflammation extends more decply as panaritium or whitlow'.

The most common type of onychia is an acute painful condition caused by the Streptococcus pyogenes. This is met with not infrequently in children in association with Impetigo contagiosa, for which 
the same organism is responsible, and in doctors and nurses from direct infection of the fingers. It appears first as a small blister somewhere around the nail but most frequently on the lateral nail-wall, this becomes purulent and causes inflammation and oedema of the nailwall beneath which pus may be seen oozing out. The pus may insinuate itself beneath the nail and so reach the nail-matrix, loosen the continuity. between the nail and the matrix, raise up the nail from its bed, and eventually cause it to be shed. Is a rule the nail grows again when the septic condition is cured.

In the most severe cases of all, which are known as Onychia maligna, the purulent infection tends to lead to the formation of ulcers, at the margin of or under the nail, covered with granulations or a necrotic slough. The ulceration, spreading beneath the nail, gradually reaches the nail-matrix and causes such destructive changes in it that re-growth of the nail is improbable, and even where it does take place the new nail is rapidly undermined and shed. In these cases the inflammatory disturbance is liable to spread up the sides of the finger and involve the neighbouring lymphatics, and may lead to serious lymphangitis and possibly to septic absorption.

Sometimes the shedding of the nail is more gradual, owing to the matrix being inrolved chiefly while the nail is comparatively unaffected. This leads to dystrophy and irregular growth of the nail, or to a gradual shedding in which a space is sometimes left between the nail-plate and the posterior nail-wall, which is usually covered with a scab or a purulent discharge and in which occasionally a portion of the nail-bed may be exposed. Should the nail-matrix recover, a new growth in the form of a thin plate gradually pushes itself forward and replaces the discoloured and irregular original nail.

An infection about the nail may be caused also by the Staphylococcus pyogenes aureus (Staphylonychia). In it, one or more small abscesses form beneath the free border of the nail which dry up into small scaly crusts, keep recurring, and cause discomfort or actual pain. This condition is apt to be produced by biting the nails.

In many cases of infective onychia, whether due to streptococcus or staphylococcus, there is some predisposing cause, such as lowered vitality of the soft tissues about the nails from exposure to local irritation from chemical or physical irritants, or from nerve injury or disease interfering with the vitality of the nail-matrix, or from pressure on the soft tissues from an ingrowing toe-nail.

Treatment.-The treatment of infective onychias consists of the continuous application of antiseptic lotions in the form of compresses of boric acid or hydrogen peroxide to clean up discharges, and the astringing of exuberant granulations with nitrate of silver, or sulphate of copper. Where the foot is affected it should be immersed in boric acid and any diseased tissue or slough removed by a curette. If these means be persevered with it is rarely necessary to extirpate the nail. 
(b) Fungi of Ringworm and Favus (Onychomycosis).--The affections caused by the growth in or about the nail of the fungi of ringworm or favus have been described on $\mathrm{pp} .4^{\mathrm{I}} 7,423$. When the fungus of ringworm attacks the nail, alterations are produced in its colour, form, and hardness. Owing to the spreading of the fungus into the nailmatrix the nail-growth is interfered with and deformities result, while keratosis in the nail-bed tends to raise up the nail.

In the case of favus, on the other hand, the fungus does not as a rule invade the nail-plate nor spread into the nail-matrix, but is present chiefly in the nail-bed, where it causes the formation of friable crusts beneath the nail and may lead to a crumbling of the nail itself and the production of a honeycomb appearance.

The ultimate diagnosis of these affections is only possible by a microscopical examination.

(c) Scabies.-The nails are involved in the severe cases of scabies of the type met with from time to time in Norway. In these cases the acarus may burrow into the nail-bed, causing irregular cavities in the overlying nail in which eggs are deposited, and leading to opacities and irregularities of the nail and sometimes to suppurative perionychia. (See p. 6ro.)

\section{Affections of the Nails in Cutaneous Diseases}

The nails are liable to become involved in connection with most forms of chronic dermatitis. They may be shed in general exfoliative dermatitis and Alopecia areata, and more or less seriously implicated in eczema, psoriasis, Pityriasis rubra, pemphigus, etc. From the appearance of the affected nails it is impossible-apart from corroborative symptoms elsewhere-to come to a definite conclusion with regard to the diagnosis.

(a) Eczema.-The nails are frequently affected in eczema but the appearances produced vary so greatly that there is no condition of the nails which can be regarded as in any way characteristic of the disease.

In acute eczema involving the ungual phalanges, an exudation about the nail-matrix may occur and may set up such a disturbance that the nail-plate is actually shed, or a sero-purulent perionychia may develop in which the nail-wall becomes swollen, tender, and painful, and thə nail itself soft, yellow, and opaque.

In the more chronic forms of dry eczema the nail is liable to become rough and indented with transverse grooves, or may present white specks, punctate pittings, or a peculiar worm-eaten appearance; or there may be thickening and scaly parakeratosis of the nail-bed separating the nail and causing the tip or sides to be everted.

These conditions are exceedingly chronic and as they are apt to persist after the eczema elsewhere has been cured it may be months or years before a normal growth of nail takes place. Occasionally where 
the eczema has seriously involved the nail-matrix a perfect nail may never grow but only an irregular thickening of horny tissue.

Treatment.- The treatment in the acute cases consists of the application of compresses of boric acid or hydrogen peroxide to reduce sepsis, followed by zinc paste.

For the treatment of chronic dry eczema of the nails see p. 798 .

(b) Psoriasis. - The nails are implicated in about I0\% of cases of psoriasis, but the changes which occur vary so much in different cases and are so similar to those in other chronic dermatoses that the diagnosis of psoriasis from the appearance of the nails alone is apt to be fallacious. It occasionally happens that only the nails are attacked and the diagnosis is made good by the history of previous attacks of the disease or its subsequent development elsewhere. It is unusual for all the nails to be affected, and there is no tendency to symmetry.

The most common changes consist of a greyish or dirty-yellowish discolouration and the presence of opaque specks or punctate pits like the depressions on a.thimble, which result from minute psoriatic papules on the nail-bed. Where a small patch of psoriasis is present on the nail-bed a corresponding opacity appears on the nail. In addition to pitting, transierse grooves and irregular furrows are not uncommon, and the presence of scales on the nail-bed may cause the nail to become detached, sometimes to such an extent that a quill may be passed up under it as far back as the matrix.

Treatment.-For the treatment of psoriasis of the nail see p. $8_{f 2}$. Where the nail is severely affected it should be softened by soft soap applied under a finger-stall and the usual remedies for psoriasis should be inserted beneath the nail.

(c) Alopecia areata.-In association with Alopecia areata various forms of dystrophy of the nails are not uncommon, such as leuconychia, striation, splitting, and occasionally shedding. These changes are comparatively rare, and in the majority of cases in which they have been recorded it is doubtful if they were definitely connected with the alopecia or were a coincidence. It is possible, however, that the same causes, probably acting through the nerrous system, which interfered with the nutrition at the hair-papillae and caused the defluvium of the hair, might in like manner have affected the nail-matrices and have led to imperfect nail-growth.

(d) Other Chronic Dermatoses.-Changes in the nails may be met with in connection with pemphigus, Epidermolysis bullosa, extensive Lichen planus, Pityriasis rubra pilaris, etc., but they are not characteristic of the disease and are simply the result of its extension to the nail-fold, nail-bed, or nail-matrix.

\section{Affections of the Nails ix Syphilis}

The nails may be attacked in both congenital and acquired syphilis. 
In congenital syphilis, bullae occurring about the nail-bed and matrix may cause partial destruction or total loss of the nail or may lead to suppurative perionychia and various types of dystrophy.

In acquired syphilis the nail-bed may be the seat of a primary sore, or of papules, pustules, or even gummata, but the appearances which result in the nail are not characteristic.

Chancres about the nail or nail-fold are met with from time to time, especially among doctors and nurses. They may be indistinguishable from ordinary suppurative onychia, or may take the form of a dry swelling about the nail-fold, a slight scaly lesion beneath the nail itself, or a persistent indurated fissure. The appearance of the nail is not sufficient to warrant a diagnosis of syphilis unless there are other concomitant signs, such as marked enlargement of the neighbouring glands, a syphilitic eruption, and a positive Wassermann reaction.

Papular lesions about the nail-bed and matrix may give rise to opacities and erosions of the overlying nail-substance and may render the nail brittle and frayed.

Another rare manifestation of syphilis is a moist ulcerating perionychia, usually present in the big toe and associated with so much pain that walking may be impossible. In it the soft tissues around the nail may break down giving rise to an ulcer, which may extend around the nail, may spread down even to the bone, and may lead eventually to detachment of the nail-plate. In some instances the purulent perionychia may have started from some local injury, such as a prick or an abrasion, and may be the result of a septic infection on syphilitic tissue.

Various forms of dystrophy may be met with in connection with syphilis, in which the nails both of the hands and feet may be involved in a symmetrical fashion. Though the name "Onychia sicca syphilitica" has been applied to this condition it is by no means characteristic of syphilis and is simply a dystrophy due to interference with the nutrition caused by the general infection.

Treatment.-The treatment of syphilitic infections of the nails is that of syphilis in general. Where marked suppuration is present a useful local treatment consists of the application of compresses of perchloride of mercury solution followed by the winding round the ungual phalanx of a strip of mercurial plaster.

\section{Dystrophies}

(a) General Diseases, Circulatory Disturbances, etc.-Dystrophies of the nails may be present in connection with any general disease, from interference with the nutrition at the nail-matrix. Circulatory disturbances due to heart disease or a feeble peripheral circulation also give rise to imperfect nail-growth. (Fig. 35I.)

In association with hyperidrosis and a weak peripheral circulation a condition of the nails has been described by Nevins Hyde under the 
name of "egg-shell nail," in which they became translucent, thin detached from the nail-bed, turned up, and concave.

(b) Nerve Lesions.-Dystrophies may result from interference with the nerve supply to the nail-matrix by disease or injury. At the same time the resistance of the skin around the nail is liable to be impaired by the defective innervation and to become prone to infection with progenic cocci so that ulceration and suppuration may supervene in the surrounding tissues.

Treatment.-The treatment of nail-dystrophies is on general principles and varies with the peculiarities of each case. The state of the peripheral circulation should be attended to and venous stasis about

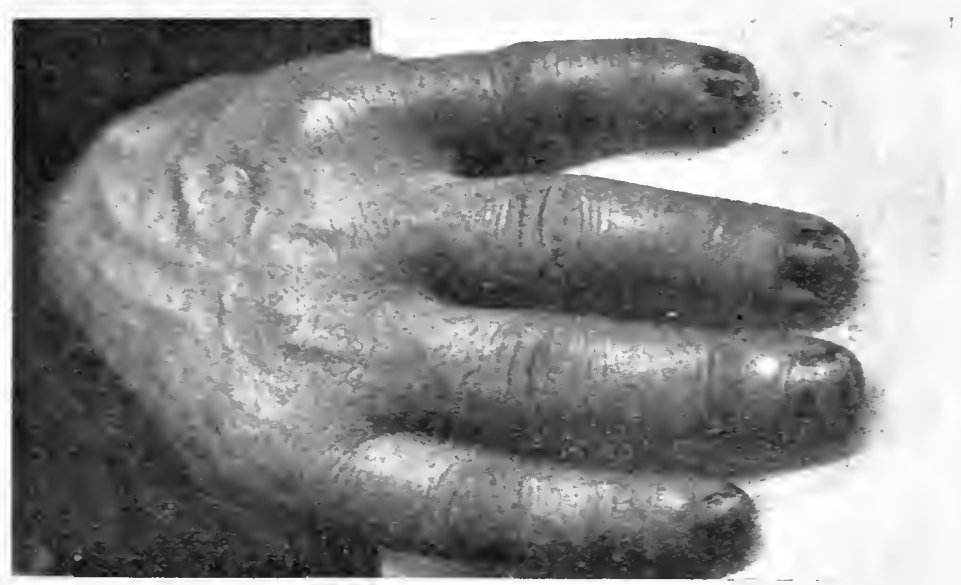

F1G. 351.-Haemorrhage into Nail-bed and Nail-matrix.

the extremities relieved by appropriate means. Splitting of the nails may be dealt with by painting the edges with collodion or by wearing a finger-stall to prevent the ragged nails catching and tearing.

\section{REFERENCES}

\section{Parasitic.}

Adansox, H. G. Diseases of the Nails (Allbutt \& Rolleston, System of Medicine, I9I I, ix. p. 733).

ADArsox \& McDoxagh. Two unusual forms of syphilitic nails with general remarks on syphilis of the nails (Brit. Journ. Derm., I9I I, xxiii. p. 6S).

Bosellixi. Dýstrophy of the nails in malaria (Giorn. Ital. d. Mal. Ven. e della Pelle, I9I $5, v$ p. + I3).

Dubretilh \& Frèche. Eczéma des ongles (Journ. de Med. de Bordeaux, I90I, Xxxi. p. 249).

Deckworth, D. Observations on some conditions of the nails in constitutional diseases (St. Bart. Hosp. Rep., Lond., I909, xir. p. 5).

Flower, $X$. Evidence of recent severe illness afforded by the nails (Brit. Med. Journ., I9II, i. p. 869).

Grixidox, J. (Trans. Amer. Derm. Assoc., Igor, p. II I). 
Hutchinsox, J. Diseases of the nails with special reference to significance as symptoms (Brit. Journ. Derm., I 899, xi. p. 30I).

Hyde, J. Nevins. Egg-shell nail (Amer. Derm. Assoc., Dec. I905, p. 98).

MAcLEOD, J. M. H. Diseases of the nails (Quain's Dictionary of Medicine, I902).

Sabouraud, R. Septic onychias (Bull. Soc. Franc. de Derm. et Syph., Paris, 1896, vii. p. 2).

\section{Congenital.}

MacLeod, J. M. H. On the development of the human epidermis \& its appendages (Brit. Journ. Derm., I898, x. p. I83).

Müller, C. Congenital onychogryphosis (Münch. Med. Wochenschr., Dec. 1904, p. 2180 ).

Simth, IV. R. A case of abnormality of the finger-nails (Journ. Anat. \& Phys., I892, xxvi.).

WAELSCH, L. On "Koilonychia" \& "Platyonychia" hereditaria (Arch. f. Derm. u. Syph., I903, lxvii. p. 250). 


\section{CHAPTER XXXIX}

\section{DISEASES OF THE LIPS AND MOLTH}

\section{Scaly AND Crusted Affections of the Lips}

THE red portion of the lips may be the seat of various scaly and crusted affections, generally associated with more or less marked

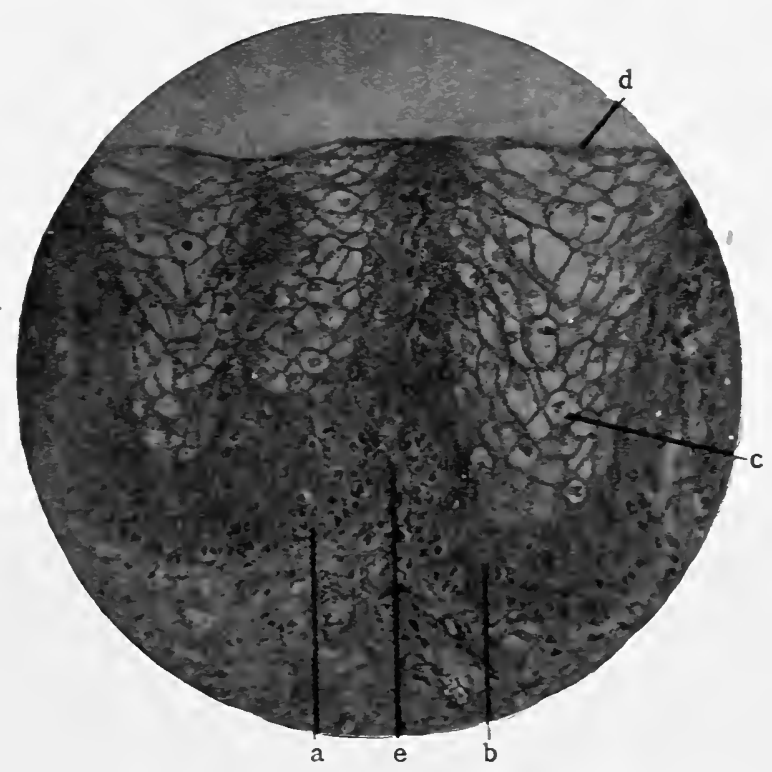

FIG. 352.-Section of Aormal Mucosa from Inner Surface of Lower Lip.

a. Basal proliferating layer. b. Daughter-cells hardly yet differentiated. c. Daughtercells, showing change into cells with small nucleus and large perinuclear space. d. Superficial layer of cells. e. Papilla,

(After Dr. W. T. Quaite. Brit. Journ. Derm., IgoS.)

inflammation and oedema. Of these the most important are the following :

Perlèche.-This scaly condition, resulting from a streptococcal infection, has already been described on p. 304 .

Eczema.-An eczematous affection of the lips may occur in association with eczema elsewhere, or as a purely local condition due to some irritant, such as the mouthpiece of a pipe or of a wind instrument, like a flute or a cornet, or to the action of certain dentifrices. (Fig. 352.) 
Seborrhoic Dermatitis.-The lips are occasionally affected in association with seborrhoic dermatitis of the scalp or glabrous skin and become covered with the yellowish greasy scales or crusts which are characteristic of seborrhoea. As a rule the scaliness is limited to the red portion of the lips but slight inflammation may spread a short distance beyond it. Various degrees of it have been described, from a slightly scaly affection to coarse yellowish crusts which are fairly adherent and which, when forcibly detached, are liable to leave an excoriated oozing surface. Special attention has been paid to the condition in France where it has been given the names "Seborrhoee des lèrres" (Brocq), and "Eczéma exfoliant des lèrres" (Besnier and Doyon). It has been known to form the basis of epithelioma of the lip.

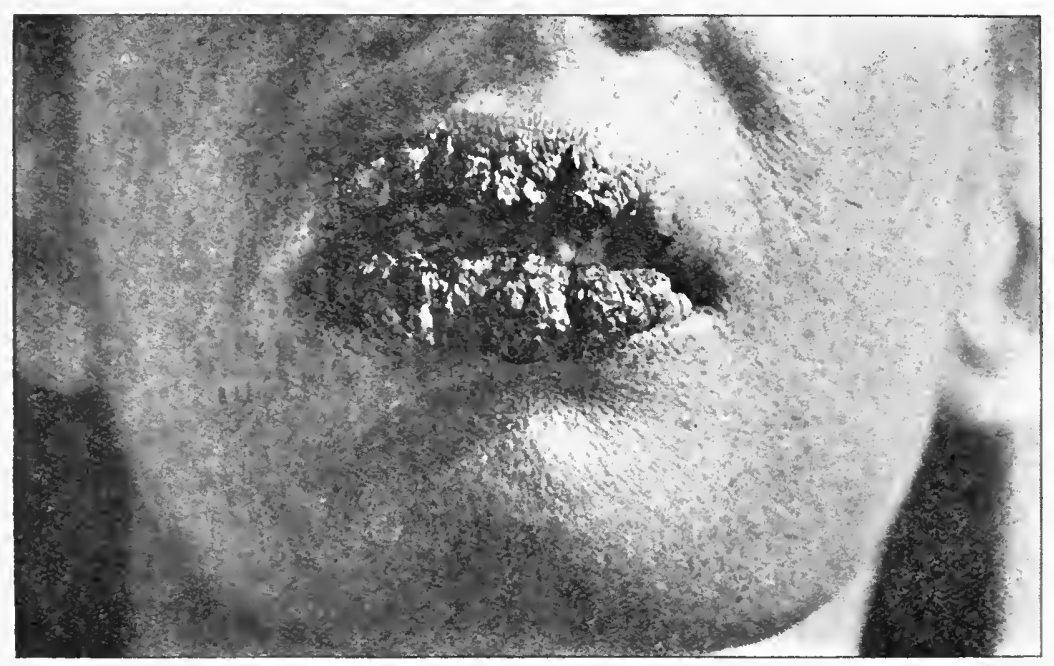

FIG. 353.--Chronic Exfoliation of the Lips.

Associated with a septic state of the mouth.

It is singularly resistant to treatment but benefit has been recorded from anti-seborrhoic applications, such as sulphur and resorcin ointments and lotions, and trichloracetic acid, as well as from exposures to X-rays and radium.

Cheilitis exfoliativa.-Under this non-committal heading may be described a much more marked and severe type of exfoliation, usually confined to the lower lip. In it the lip is swollen, tender, protruding, and more or less completely covered with a large coarse crust which is firm in consistence, yellowish-grecn or blornish in colour, and generally extends from the cutaneous margin over the lip and gradually fades away in the mouth. It is loosely adherent and can be picked off easily leaving the lip dry and glazed, except here and there where it may be attached so firmly that its removal may lcad to laceration with oozing or bleeding. When the crust is removed a new one gradually forms. (Fig. 353.) 
The precise cause of this peculiar affection is unknown. It is possible that certain of the cases are simply an adranced stage of seborrhoic dermatitis of the lips but others appear to belong to a different category.

Several of the cases have occurred in women of a markedly neurotic habit, and in a case recorded by the writer in a young and nervous woman the lip condition was associated with Pyorrhoea alveolaris, the teeth being covered with tartar, the gums sodden, the salivary and mucous secretions riscid, and the mouth and tongue dry. In this case it appeared to begin as a desquamation of the lip, the result of some local septic infection possibly spreading from the mouth. In course of time this was transformed into a coarse crust, which was probably due to the patient's habit of sucking and working the lip under the teeth and so inducing a riscid secretion of salira and mucus which, mixing with the desquamated epithelium, formed a scab.

The treatment is unsatisfactory, but some improrement mar be obtained by cleaning the teeth and the mouth, remoring the scab, painting the surface with a $3 \%$ solution of silver nitrate, and applying a I \% mercurial ointment daily. The best treatment is exposures to a radium plate. Where this is unobtainable the X-rays should be tried but are less effective.

Cheilitis glandularis aposteomatosa.-- - nder this heading Tolkmann in IS7o described a peculiar condition, chiefly involving the lower lip, in which the lip was swollen, tense, and studded with hempseedsized nodules which had formed about the orifices of the mucous glands. From these orifices, which were dilated, a muco-purulent discharge was emitted which tended to dry up and form crusts and to cause the lips to be glued together in the nornings. Cases of this type have been described by other observers, such as Purdon, Sutton, and Schamberg.

The exact nature of Volkmann's disease is unknown though it is generally believed to be a catarrhal affection of the glands of the lip and has been found to be associated with catarrh of the nose and throat. According to Sutton, who studied its histopathology, there is hypertrophy of the mucous glands which he considered might possibly. be of congenital origin.

The treatment is unsatisfactory but some benefit has been recorded from the X-rays.

\section{REFERENCES}

DAvis, H. Cheilitis exfoliativa (Brit. Journ. Derm., I9I I, xxiii. p. I 49).

GALLOWAY, J. Chronic exfoliating inflammation affecting the lower lip (Brit. Journ. Derm., IS95, vii. p. II3).

Kaposi, Bescier, \& Dorox. Maladies de la peau (Paris, isgi, Vol. i. p. 665 ).

Macleod, J. M. H. Cheilitis glandularis (Brit. Journ. Derm., I9o\%, xix. p. 3ss).

MoNtgomeri, D. Seborrhoea of the lower lip \& its relationship to epithelioma (Journ. Cut. Dis., I913, xxxi. p. S2).

Cheilitis exfoliativa, treated by radium (Ibid., I9IS, xxxvi. p. 363). 
Purdon, H. S. Four cases of Cheilitis glandularis (Brit. Journ. Derm., I 893, v. p. 23).

Surton, R. L. Cheilitis glandularis aposteomatosa of R. Volkmann (Journ. Cut. Dis., April, I909).

\section{FORDYCE'S DISEASE}

In I8g6 Fordyce drew attention to a peculiar affection of the mucous membrane which takes the form of small whitish or yellowish opacities situated on the red parts of the lips and in the mouth along the line of the teeth. The lesions are irregular in outline, vary in size from minute specks to a pin's head, are flat and level with the surface, not perceptible to the touch, and give rise to no subjective symptoms. When present in slight degrees, which is not uncommon, little or no attention is paid to it, but where well marked it is noticed readily as the lesions may be so numerous and closely aggregated as to produce the appearance of a yellowish band or streak on each lip. On the upper lip, where the band is usually most marked, it is narrow in the middle, spreads out symmetrically on either side till it reaches about a quarter of an inch in width, then tapers off towards the angles of the mouth.

According to Fordyce the lesions are due to degenerative changes in the epidermis, but more recently it has been suggested that they are the result of imperfectly developed sebaceous glands.

The condition must not be confused with Lichen planus of the buccal mucosa, in which the lesions are whiter, generally situated on the insides of the cheeks, and usually arranged in streaks in a dendriform fashion.

The treatment is unsatisfactory.

\section{REFERENCES}

Fordyce. A peculiar affection of the mucous membrane of the lips $\&$ oral cavity (Journ. Cut. \& Gen.-Urin. Dis., I896, p. 4I3).

MacLeod, J. M. H. Fordyce's Disease (Brit. Journ. Derm., I904, xvi. p. I 45).

\section{BLACK HAIRY TONGUE}

Syn.: Hyperkeratosis linguae; Lingua nigra ; Melanoglossia.

This rare affection is characterised by the presence on the dorsum of the tongue of a blackish or brownish fur, the result of the elongation and darkening of the filiform papillae, which may be lengthened to a quarter or even half an inch and give rise to a hairy appearance, hence the name " hairy tongue." As a rule the condition is most marked in the central portion of the tongue in front of the circumrallate papillae but occasionally it may be most pronounced towards the tip. The discolouration is blackest at the centre and fades to a brownish tinge towards the periphery.

It appears gradually, persists for weeks or months, then disappears spontaneously. There are no subjective symptoms associated with it. 
Vollmer, who made a microscopical examination of it, described a hyperkeratosis of the epithelium of the papillae and attributed the dark colour to pigmentation in the horn-cells which was most marked in the older cells.

The treatment consists of the employment of alkaline mouthwashes containing potassium chlorate or borax, or of painting the patches with $I_{0}^{0}$ salicylic lotion.

\section{REFERENCES}

Butitx \& Spexcer. Hairy black tongue (Diseases of the Tongue, rgoo. p. $I+5)$.

Coffix, A. Case of melanoglossia (Journ. des Mal. Cut. et Syph., Feb. I900, P. 65).

Vollyer, E. The nature of black hair-tongue (Arch. f. Derm. u. Sỵph., Oct. I $89 S)$.

\section{TRANSITORY CIRCINATE ERUPTION OF THE TONGUE}

Syn.: Wandering rash; Glossitis exfoliativa marginata; Gyrate glossitis; Geographical tongue.

This condition, which is comparatively rare, is met with chiefly in children, but has been known to occur also in adult life, either de noio or persisting from childhood. It is characterised by the presence on the tongue of yellowish-white rings or gyrate figures enclosing roundish or irregular areas of smooth red mucous membrane which are sometimes slightly depressed. It occurs on the dorsal aspect in front of the circumvallate papillae, is most marked towards the tip, and mayspread over the border to the under surface.

It begins as a small reddish eleration which soon assumes a yellowishwhite appearance from desquamation of the epithelium; this increases peripherally and clears up in the centre to form a ring. By the coalescence of neighbouring rings, gyrate figures are produced and appearances suggesting the outlines on a map-hence the name geograplical tongue. The individual rings are transitory, reaching their full size in about a week and then gradually disappearing spontaneously; but the affection is kept up by the constant development of new lesions, sometimes in the centre of old ones, at other times independently of them. There are no definite subjective symptoms.

A histological examination by Parot showed a thickening of the epithelium and an inflammatory cellular infiltration in the papillary and sub-papillary layers of the corium.

The nature and causation of the affection are uncertain. Various organisms and even spirochaetes have been found in the desquamating epithelium, but none has been shown to be pathogenic and it seems more probable that it is a sub-acute inflammatory process symptomatic of some digestive disturbance. 
From time to time it has been confused with superficial syphilitic glossitis, but in the syphilide there is definite ulceration.

It is singularly resistant to treatment and may persist for months or even years in spite of all manner of local applications. As the lesions tend to disappear spontaneously while new ones keep developing, there is nothing to be gained by attempting to destroy them by severe remedies such as caustics, which may lead to harmful results by irritating the epidermis, and only soothing mouth-washes containing borax, potassium chlorate, and the like, should be employed. A careful dietary should be prescribed and any digestive disability sought for and treated.

\section{REFERENCES}

BEsiter. Wandering rash on the tongue (Arch. de Derm. et de Syph., April IS89).

Butlix \& Spexcer (Diseases of the Tongue, I9oo, p. 94).

Fox, Colcott. Geographical tongue (Lancet, $188_{4}$, p. $8+2$ ).

MacLeod, J. M. H., \& Leathexi, A. N. Gyrate glossitis (Brit. Journ. Derm., I908, xx. p. 86).

\section{LEUCOPLAKIA}

Syn.: Lencoplasia; Leukokeratosis; Ichthyosis glossae; Psoriasis buccalis.

Definition: A thickening of the epithelium of mucous membranes giving rise to variously sized greyish-white patches.

Description.-Lencoplakia, as the name indicates, consists of whitish plaques situated on mucous membranes and due to thickening and keratisation of the epithelium. The plaques vary in shape, being round, oval, or irregular in outline, and either remain discrete or coalesce to form larger patches, diffuse sheets, streaks or an irregular network. Sometimes they appear as a thin whitish opaline pellicle, or they may be coarse and silvery or parchment tinted, or occasionally may be more or less deeply pigmented. The surface is generally smooth, but may be rough and warty, and occasionally may come off in flakes. The parts most usually affected are the dorsum and sides of the tongue, the palate, inner surface of the cheeks, commissures of the mouth, and lower lip, but it may occur also on the glans and prepuce of the penis and on the rulva.

When the patches are large they are usually broken up into irregular facets, and in situations subjected to movement, such as the angles of the mouth, painful fissures are liable to form which may go on to ulceration. Associated with the leucoplakia, sclerotic changes may supervene in the underlying fibrous tissue and lead to cicatricial contraction and shrinkage of the part; this may occur on the tongue, but is much more common on the vulva where a condition is produced which is known as Kraurosis vulvae in which the labia are markedly 
shrunken, the mucous surfaces more or less covered with whitish patches, and the opening of the ragina contracted.

Leucoplakia is a chronic affection and liable to persist indefinitely, but it may sometimes disappear spontaneously or from treatment. It is liable to be a precursor of cancer and, from the continued irritation of smoking, a jagged tooth, or other local cause, is apt to become epitheliomatous.

Histopathology.-The affected epithelium is found to be thickened and the surface layers of cells to have become keratinised like the hornylayer of the skin. Beneath the epithelium there is an infiltration of small connective tissue cells which is most marked around the capillaries. In old-standing cases where sclerosis has supervened, spindle cells and newly formed connective fibres may be detected.

Etiology.-This affection has been long believed to be closely related to syphilis, indeed certain writers have gone so far as to include it among the late mucous syphilides while others have regarded syphilis as an essential predisposing factor. This riew is gradually losing ground, however, and there are at present a number of observers who do not consider it to be syphilitic, or even a definite morbid entity, but believe it to be due to a variety of causes, such as the local irritation of heary smoking, or the cherring of tobacco, or of spices, highly seasoned dishes, irritating dentifrices, etc.

In the case of the female genitalia it is the result of the action of irritating discharges, while on the penis it may succeed balanitis. It is much more common in men than women.

Diagnosis.-Leucoplahia has to be distinguished from exfoliative marginate glossitis, but in the latter the lesions have a definite war. border, are transient instead of persistent, and clear up in one place to develop in another.

It has to be differentiated also from Lichen planus of the mucous membranes, but Lichen planus is most common on the inner surface of the cheeks opposite the last two molars, has a tendency to appear in whitish streaks with a characteristic dendriform arrangement, and is usually associated with trpical plane lesions on the skin.

Treatment.-The most effective methods of treatment are br X-rays or radium. In the case of the X-rays a pastille dose is giren and repeated if necessary in the course of a month, while if radium be employed, exposures to an unscreened radium plate of half strength should be given for half an hour at intervals of a fortnight.

Caustic remedies, such as nitrate of silver, acid nitrate of mercury, or the actual cautery, were once employed somewhat extensively in it: treatment but they should be used with the utmost caution for fear of producing malignant changes and, on the whole, are better aroided. Where fissures are present these may be healed by a silver nitrate pencil, or by being touched with $10^{\circ}$ chromic acid applied on a match.

Every care should be taken to aroid irritating the affection, by strict attention to the teeth and the hrgiene of the mouth in general, 
and the avoidance of tobacco, alcohol, or other irritants; in the case of the genitalia scrupulous cleanliness should be insisted upon.

Internal remedies have little or no effect upon it unless it occurs in association with syphilis. The internal administration of arsenic has been recommended, but its action is uncertain.

\section{REFERENCES}

Bонас, C. Leukoplakia \& kraurosis (Arch. f. Derm. u. Syph., I9r I, cv. p. I79).

Filaretopoulo, G. Buccal leukoplakia (Journ. des Mal. Cut. et Syph., I905, ii. P. 8I).

Gaucher, Perrin, Barthélemy, \& Fournier. Leucoplasia (Discussion, Internat. Cong. Med., Paris, I900).

Perrin, L. Leucoplasia of vulva \& anus (Ann. de Derm. et de Syph., Igor, P. 2I).

Rosenheim, S. Leukokeratosis buccalis (Bull. Johns Hopkins Hosp., 190, p. 47). 


\section{CHAPTER XL}

\section{NEOPLASMS OF THE SKIN}

Introduction.-The tumours of the skin may be divided into two groups, namely, primary cutaneous growths, and secondary or metastatic growths. The first group is far the larger and includes all the benign growths, while the second comprises only a few, such as certain sarcomata and carcinomata.

The word "tumour" is ill-defined, as it simply means a swelling and might be extended to include all new-formations in the skin, chronic inflammatory thickenings, the more resistant cellular deposits met with in the infective granulomata, such as the nodules of lupus, the papules of syphilis, etc., and eren abscesses or cysts. The term "neoplasm" is preferable, as it is more concise and indicates an excessive and permanent increase of some constituent element of the skin existing either in foetal life or after birth.

The cutaneous neoplasms may be divided into benign and malignant, though it is practically impossible to draw a hard-and-fast line between them. Up to a certain stage all neoplasms may be regarded as benign, while those which are usually benign may assume malignant characteristics-such as the naero-carcinomata, in which a mole which is generally benign has undergone proliferative changes of a malignant type and become a carcinoma.

A malignant neoplasm differs from one that is benign in being, as a rule, capable of unlimited growth and in having the power of invading surrounding tissue, but certain of the malignant growths are only locally malignant and incapable of giving rise to metastases.

Histologically one of the most important distinctions between a benign and a malignant growth is that in the former there is a simple hypertrophy due to an increase of the normal elements of the tissue from which the growth is derived, whereas in the latter the elements consist of cells many of which have reverted to a simpler type. As an example of the former the common wart may be cited, which is a benign neoplasm of the epidermis in which the growth is due to a proliferation of normal epidermal cells, and as an example of the 
latter a carcinoma of the skin, the cells composing which may be so altered as to render it impossible to tell from their appearance alone from which layer of the skin the growth originated.

It is customary to classify the cutaneous neoplasms on a histological basis.

\section{CLASSIFICATION}

\section{A. Benign Neoplasms}

Epidermis.

I. Corns and callosities.

2. Warts-Common warts, Verruca vulgaris. Plane warts.

Plantar warts, Yerruca plantaris.

Venereal warts, Condyloma acuminatum.

Seborrhoic warts, Verruca senilis.

3. Warty affections.

Acanthosis nigricans.

Darier's Disease.

4. Cutaneous horns.

5. Epidermal naevi (see p. 193).

6. Molluscum contagiosum.

7. Milium.

Epidermal Appendages.

Benign cystic epithelioma.

Epithelioma adenoides cysticum.

Tomato tumours of the scalp.

Sebaceous adenoma.

Hydradenoma.

Corizm.

Fibroma and neuro-fibroma.

Cheloid.

Pseudo-xanthoma elasticum.

Colloid milium.

Sarcoid.

Leiomyoma.

Skin lesions in leucaemia and pseudo-leucaemia.

Blood-ressels and Lymphatics.

Angioma (see p. 65).

Lympliangioma circumscriptum (see p. I9r).

Endotheliomata.

\section{B. Malignant Neoplasms}

Epidermis.

Rodent ulcer.

Squamous celled epithelioma.

Naero-carcinoma.

Paget's Disease.

Corium.

Sarcoma.

Mlultiple idiopathic pigment sarcoma. 


\section{LACLASSIFIED AFFECTIONS}

Xanthoma.

X. palpebrarum.

X. tuberosum multiplex.

X. diabeticorum.

Sclerodermia.

Sclerema neonatorum.

Oedema neonatorum.

Calcareous deposits in the skin.

\section{CORNS}

Syn.: Clarus (a nail) ; Fr., Cor ; Ger., Leichdorn.

Definition: A corn is a circumscribed horny thickening, roughly conical in shape, with the base on the surface and the small end pressing on the capillaries and nerves of the corium.

Corns are usually situated on the toes at the sites of friction and intermittent pressure. Ther occur as a rule where the skin covers a bony protuberance, such as on the external aspect of the little toe and the dorsum of the second toe, but ther are not infrequently present between the toes and, in rare instances, on the hands.

It is customary to classify them into two varieties, namely (I) hard corns, such as occur on the external surfaces of the toes, (2) soft corns which are present between the toes and are softened by the macerating action of the sweat.

In the hard corn the surface is shing and polished and when the superficial laver is shaved off presents a whitish or grevish opaque appearance. In the centre is a horny peg or core which is the densest part of the structure and this, by pressing on the underlying sensitive nerves in the papillary laver, is responsible for the pain which is the troublesome feature of the growth. Hard corns vary in size from a split-pea to a threepenny piece.

Soft corns have a greyish or whitish appearance, and are often depressed in the centre at the site of the core. A not uncommon situation for them is between the fourth and fifth toes, where the friction of the nail of the little toe is largely responsible.

As a rule the surrounding skin is normal, but sometimes it shows signs of inflammation, and occasionally of suppuration and ulceration resulting from infection by a dirty knife in cutting the corn.

The pain associated with them is of a dull boring character, but may be sharp and lancinating. As a rule it is only present when the corn is subjected to pressure in standing or walking, but it may occur independently of pressure, especially in wet weather, and in many indiriduals pain in corns is as certain an indication of coming rain as a falling barometer. This is due to the corn being composed of horny material which is hrgroscopic and tends to become swollen when the air is moist. 
Sometimes the pain is increased by the formation of a bursa beneath the corn. A bursa may also form over a joint subjected to friction apart from any corn formation, such lesions usually occurring on the ball of the big toe and being known popularly as bunions.

Corns and bunions are both due to friction or distortion of the feet caused by badly fitting or wrongly shaped boots. The most common faults in a boot are that it is too short, or that the line on the inside is not sufficiently straight, so that the big toe is bent.outward, a large and prominent angle is produced at the ball, and the second and third toes are raised up.

Histopathology.- Tertical sections of a corn show that the Stratum cornetim is hypertrophied to form a flat cone the apex of which points downward. The underlying prickle-cell layer may be normal or definitely atrophied from pressure, while the inter-papillary processes are more or less completely flattened out. Towards the periphery the papillae are sometimes hypertrophied. Unless inflammatory changes are present there is no marked alteration in the corium other than flattening of the papillae.

\section{TREATMENT}

The first indication in the treatment is to eliminate friction and intermittent pressure, and when this is done the corns tend to disappear, and they have often been observed to do so spontaneously in the course of a prolonged illness. Where rest is impossible every effort must be made to ensure that the boots fit properly, and in this connection it is well to remember that boots which are too large are just as liable to produce corns as those which pinch. Of equal importance is the fitting of the socks, for if they be too big the consequent folds may cause pressure or friction.

One of the most common methods of treatment is to pare the corn with a sharp knife and to pick out the centre. This is an unsatisfactory procedure, however, as although it reduces the thickening it also exposes the underlying delicate part to friction, which causes the corn to grow again. Another disadrantage is the danger of infecting the corn with the knife should the epidermis be penetrated and the corn be made to bleed.

Complete excision with a sharp scalpel under strict aseptic precautions may give good results and can be done easily under a local anaesthetic.

Various macerating and keratolytic agents are also commonly employed, such as cold water compresses, soaking the feet in water containing sodium bicarbonate, or the application of salicylic acid-which forms the base of the majority of the so-called " corn cures" in a formula such as the following:

Acidi salicylici, $5 i$.

Collodii, $\overline{3}$ i.

To this may be added extract of Cannabis indica $2 \%$ to relieve pain. 
The foot should be soaked in soda and water, and any loose epidermis exfoliated from the surface or picked away. It should then be dried, the corn painted with salicylic collodion, and a circular adhesive corn plaster applied to relieve pressure. The painting should be repeated day and night for a week, the foot again soaked in soda and water, and the softened corn picked away. When most of the corn has been removed in this manner, the cure may be completed by touching the centre with a silver nitrate pencil or by painting it with trichloracetic or lactic acid.

Another method is to cauterise the central core by a fine pointed thermal or electric cautery. This may be done without causing any great discomfort under a local anaesthetic, such as norocain.

When the corn is inflamed this should be reduced by lead lotion or a diachylon plaster.

Should suppuration supervene it must be dealt with actively, as fatal septicaemia has been known to originate from neglecting a suppurating corn. In such cases the treatment consists of ordinary antiseptic applications, such as compresses of hydrogen peroxide and boric acid, followed by the application of suitable dressings and the protection of the parts from friction or pressure.

Soft corns should be treated by applying $2 \%$ salicylic ointment and separating the toes by inserting a pad of cottonwool soaked in castor oil. An important preventive is to keep the feet dry and hard. With this object they should be bathed daily, especially before hard exercise, with some astringent lotion, such as equal parts of spirits of wine, extract of hamamelis and water, followed by the application of an antiseptic dusting powder, such as salicylic acid ro grains, boric acid 2 drachms, and talc I ounce.

The treatment of bunions consists of the removal of the friction and pressure responsible for them, the reduction of inflammation by lead compresses, etc., and the destruction of any overlying corn or horny thickening in the manner indicated above.

\section{CALLOSITIES}

Syn.: Callus (hardened skin); Tylosis (from the Greek, hard skin); Tyloma; Fr., Durillon; Ger., Verhärtung.

Callosities are the familiar horny thickenings produced on the hands, feet, and other situations, by constant friction, and differ from corns in having no central cores to render them painful. They are the result of general hypertrophy of the horny layer and represent an effort on the part of nature to protect underlying tissues from local irritation. They are greyish-yellow or brownish in colour, usually elevated in the centre, and fade into the surrounding skin.

They are common on the feet in those who do much walking or go bare-footed, on the hands from using tools and from manual exercise, 
on the finger-tips in harpists and violinists, and on the ischial tuberosities from sitting on hard chairs, but they may occur in any situation where the skin is subjected to pressure or friction against some underlying bony protuberance.

Treatment.-They tend to disappear spontaneously when the cause is removed. They can be reduced by the X-rays, or by 1o \% salicylic plaster, followed by soaking the part in soda and water, and scraping away the softened horny tissue or rubbing it down with pumice stone.

\section{REFERENCE}

Joxes, H. Lewis. Corns treated by ionization (Brit. Med. Journ., I9r3, ii. p. 938).

\section{WARTS}

Syn.: Verruca vulgaris.

Two varieties of common warts are usually distinguished, (I) the familiar raised warts so commonly met with about the hands ; (2) the

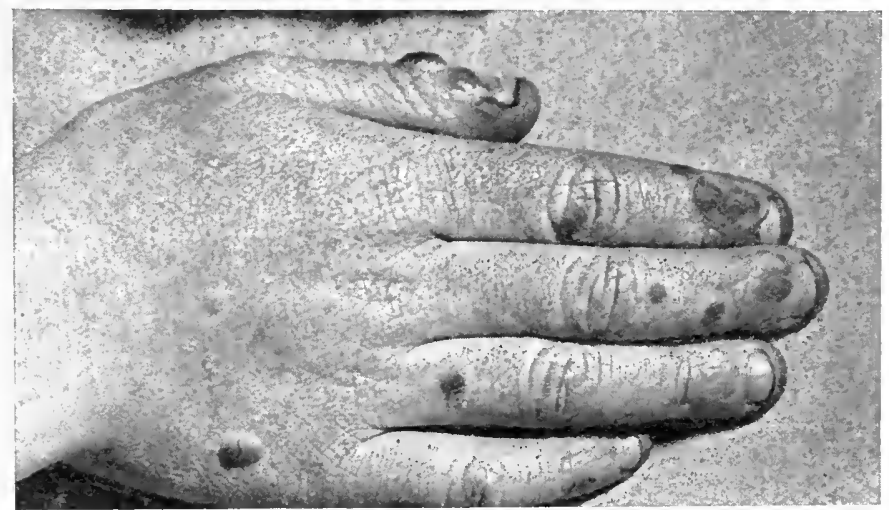

F1G. 354.- Verıuca Vulgaris.

flat, or plane, warts which occur chiefly in children (Verruca plana seu jurenilis).

There is no essential difference between these varieties and both may be met with in the same individual, the plane jurenile wart being sometimes an early stage of the raised flat-topped wart. (Fig. 354.)

Common raised warts may be single or multiple and may be irregularly distributed or grouped, sometimes in a cluster around a larger central one. They are situated most frequently on the backs of the hands and fingers, and more rarely on the face, scalp, arms, and genitalia; occasionally they occur about the nail-wall or at the tips of the fingers, and may extend beneath the nail. In size they vary from a pin's-head to a pea or larger, and may coalesce to form irregular masses. They appear first as small greyish-yellow or pinkish trans- 
lucent papules practically the colour of the normal skin, the translucency being due to thickening of the epidermis. After a time they become raised and flat-topped, the surface being grerish or blackish from dirt and rough from the presence of small horny points which can be seen easily with a pocket lens. The warts may become red and inflamed from friction. (Fig. 355.)

Plane warts are usually multiple and may occur in groups closely aggregated together. They are situated chiefly on the face, hands, and forearms, and are most common in children. They are small, roundish or irregular in outline, smooth on the surface, the

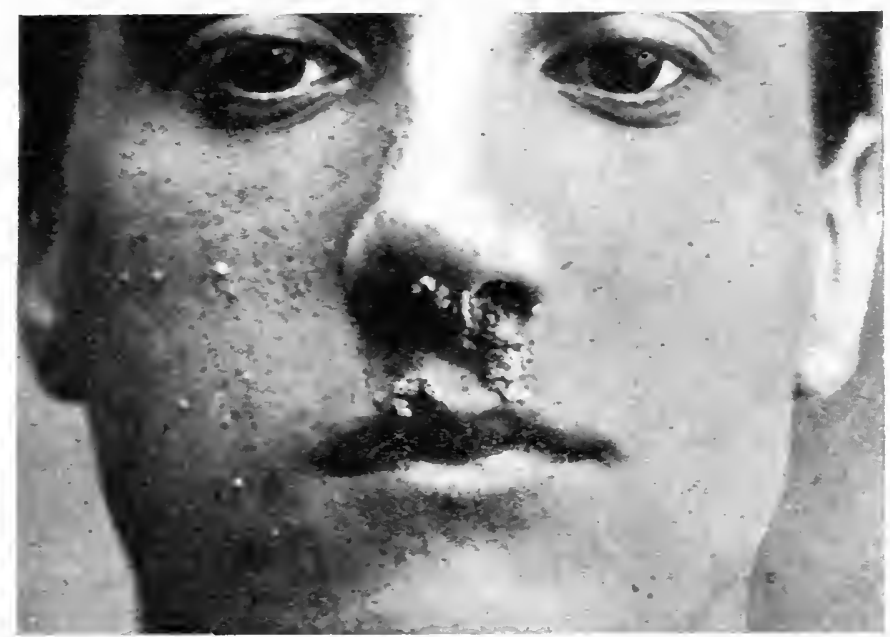

FIG. 355.--Verruca Vulgaris.

Warts on nose. upper lip and cheek.

colour of the surrounding skin, and often bear a resemblance to the papules of Lichen planus-which disease, however, is rare on the face and is unusual in children. (Fig. 356.)

Warts give rise to no subjective symptoms but are inconvenient for aesthetic reasons, especially when present on the face. When they occur about the fingers they may interfere with fine movements, such as piano playing, sewing, knitting, etc.

Histopathology.-Sections of warts show that the new-growth consists of a circumscribed thickening of the prickle-cell layer of the epidermis (acanthoma) with irregular growth of the inter-papillary processes and corresponding elongation or irregularity of the papillae. Throughout the prickle-cell layer mitotic figures can be detected here and there in the cells. The overlying Stratum corneum is thickened, especially over the papillae, giving rise to the horny points. The underlying corium is unaffected, unless from secondary inflammation.

Etiology - The cause of warts is at present unknown, but they are generally believed to be due to some micro-organism, as they are auto. inoculable, often occur on contiguous surfaces, are transmissible from 
one individual to another, and have been inoculated experimentally. The virus would appear to enter readily through an abrasion, as not infrequently rows of warts have been observed along the line of a scratch.

They are more common in children than in adults, and appear to grow readily on moist clammy hands.

\section{TREATMENT}

The treatment is chiefly local, and the value of general measures is largely a matter of opinion. Warts may persist for a prolonged period and gradually disappear; on the other hand they may be short-lived and vanish spontaneously in a few weeks or months, and it is on account of this latter tendency that treatment by charms,

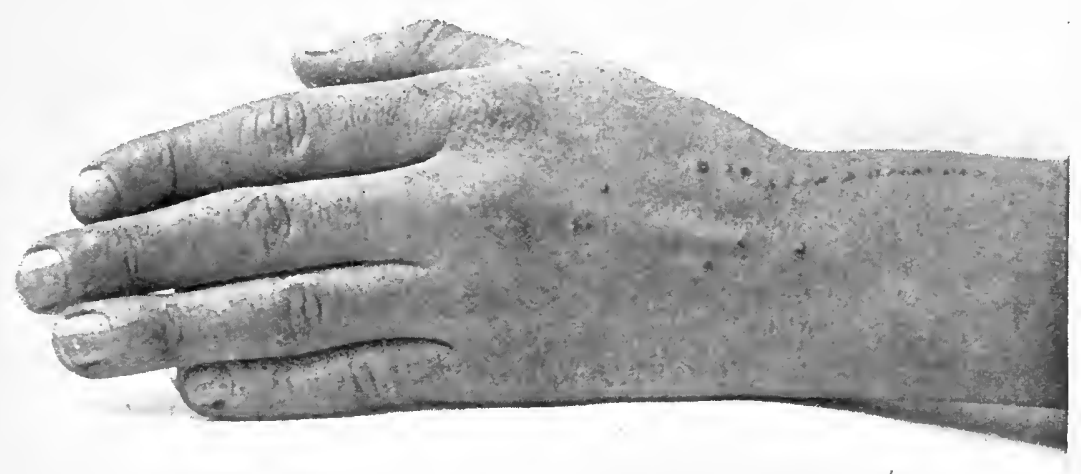

FIG. 356.-Verruca Plana.

Row of flat warts following a scratch.

suggestion, incantations, and the like, has so long held vogue even among educated persons.

General Treatment.-There are certain internal remedies which have been followed so often by a rapid disappearance of warts as to suggest cause and effect. Of these the most important are the salts of magnesium, lime salts, and mercury.

Magnesium sulphate is prescribed in doses of from to to 30 grs. t.d.s., according to the age of the patient, a sufficient quantity being given to ensure three loose motions in the day.

Chloride of calcium is given in doses of from 5 to Io grs. t.d.s.

Mercury has been specially recommended in the form of the green iodide, one-sixth to one-quarter of a grain t.d.s.

The manner of action of the salts is uncertain, but it has been suggested that warts may be associated with a deficiency of lime in the tissues and that when this is made good the skin becomes an unsuitable soil for the growth of the specific microbe.

Other internal remedies have been employed, such as thyroid gland and arsenic, but their value is doubtful. 
Local Treatment. - Warts may be removed satisfactorily by various forms of local treatment, such as refrigeration, caustics, curettage, etc.

Refrigeration.-Freezing by solid carbon dioxide is a radical and comparatively painless method of treating warts. Exposures of a minute or longer-according to the size of the wart-should be given with firm pressure, the skin being frozen for about one millimetre beyond the base of the wart. After the exposure, a reaction takes place and a blister develops which is sometimes haemorrhagic and the roof of which is formed by the wart. This should be punctured aseptically, the fluid evacuated, and a dry boracic dressing applied. After a week or ten days the wart separates, leaving a healed smooth surface. Should the exposure be too long, superficial ulceration may result followed by scarring. Occasionally after complete removal of a wart by this treatment a ring of new ones may form at the margin of the exposed area.

Carstics.-One of the most common methods of treatment is the application of some caustic on a glass rod or a match, such as acid nitrate of mercury, nitrate of silver, glacial acetic acid, trichloracetic acid, $30 \%$ chromic acid, fuming nitric acid, and ethylate of soda. By repeated applications of one or other of these, most warts can be made to disappear, but the treatment is tedious and often painful.

In using trichloracetic acid the acid should be made fluid by adding a drop of water to a few crystals in a watch glass. After applying it, the surface of the wart should be washed with boric lotion to prevent the acid acting too deeply.

Curetting.-Warts can be removed by curetting after freezing them with ethyl-chloride, but this treatment is painful and may be followed by scarring. It is sometimes useful in the case of warts on the palmar and plantar surfaces of the fingers and toes which have been softened previously by wearing a piece of salicylic plaster (Io \%) over them for a week.

Cauterisation.-Warts can be rapidly and effectively removed by an electro-cautery or a Paquelin cautery. Should the wart be large, or several be present, it is advisable to inject a local anaesthetic, such as novocain and adrenalin, or eucain, beneath the wart. Previous to the cauterisation the affected skin should be painted with tincture of iodine and after the removal of the growth the charred area should be dusted with boracic powder.

X-rays. - Treatment of warts by the X-rays as a rule is effective and painless. Several pastille doses are generally necessary and should be given at intervals of three weeks, care being taken to protect the surrounding skin with lead so that only the wart is exposed. Occasionally, after the lapse of a varying period, a re-growth of the wart may take place on the original site.

Similar results may be obtained by radium.

Ionisation.-Ionisation with zinc sulphate gives good results, but is rather painful. A solid zinc electrode is attached to the positive 
pole of a battery, a piece of lint is saturated in a $3 \%$ solution of sulphate of zinc and pressed against the wart by the electrode, and the negative electrode is held by the patient. A current of from 3 to 7 milliamperes -according to the toleration of the patient-is made for twenty minutes, as a result of which the wart may be destroyed with practically no scarring.

Electrolysis.-This method is useful in the case of small warts, the negative pole being pushed into the base of the wart, or a bipolar needle being employed.

Sea-water.-The macerating action of sea-water is sometimes useful in the reduction of warty thickenings and corns. It is most effective in the form of compresses of warm sea-water applied two or three times a day for ten minutes.

Plane juvenile warts can generally be removed by painting them daily with salicylic collodion ( $\mathrm{I}$ in 8 ), or with $4 \%$ perchloride of mercury in collodion, or rubbing them with the following ointment: Resorcin 30 grs., salicylic acid 30 grs., soft white paraffin I oz. After a few days the parts should be washed with soda and water and the surface of the wart scraped off. This procedure should be repeated as often as necessary.

\section{VERRUCAE PLANTARES OR PLANTAR PAPILLOMATA}

The sole of the foot, and occasionally the palm of the hand, is subject to a peculiar type of wart, which is a hybrid between a corn and a wart and may be so painful in standing or walking as to force the sufferer to lie up. The lesions are usually present in the sites of pressure near the roots of the toes or beneath the heel, and consist of horny thickenings varying in size from a split-pea to an inch or more in diameter, rounded or irregular in outline, and single or multiple. The typical lesion is even on the surface and of a greyish-yellow tinge. When the superficial layer is peeled or shaved off it is found that there is a central depression surrounded by a hard horny ring which spreads to the periphery of the wart. The opening widens out beneath to a base covered with a whitish opaque papillomatous growth which is tough but bleeds readily on being picked with a scalpel. Sometimes the lesions are conglomerate and present a number of these central depressions.

Their cause is uncertain. Certain observers have suggested that they may be due to microbic infection combined with the friction of walking and that they are in reality sensitive warts ringed round by a callosity ; others believe them to be due to local injury.

Microscopical sections show hyperkeratosis at the periphery, and marked acanthosis and elongation of the interpapillary processes in the centre. Towards the centre of the processes the prickle-cells 
tend to be racuolated, and present peculiar refractile intercellular bodies like protozoa which are believed to be a form of nuclear degeneration.

\section{TREATIENT}

In some instances plantar warts disappear readily under the continuous application of a ro salicylic plaster, but in others this treatment is unsatisfactory. In resistant cases it is useful to soften the lesions with salicylic plaster, pick off the superficial layers which form a lid to the central depression, and expose the lesion to a pastille dose of $\mathrm{X}$-rays, which may be repeated in three weeks if necessary. By this means the pain in the lesion is relieved and the epidermal hypertrophy reduced.

Another method which is usually successful is freezing with solid carbon dioxide, an exposure of a minute or longer with firm pressure being necessary. The disadrantage of this treatment is the severe pain associated with it.

Cataphoresis with zinc sulphate has been recommended. A piece of lint is saturated with a $2 \%$ solution of zinc sulphate and applied over the lesion; on this is placed a solid zinc electrode attached to the positive pole while the negative pole is held by the patient, and a current of about 2 milliamperes, or more if the patient can bear it, is then passed for about quarter of an hour.

In addition to these drastic measures temporary benefit may be derived from paring down the horny ring and touching the centre with silver nitrate or glacial acetic acid.

Should these forms of treatment prove unsuccessful, it may be advisable to resort to excision.

\section{ACUMINATE WARTS}

Sy'n. : Condyloma acuminatum; Tenereal warts; Filiform warts.

Though most common about the genitalia and in the gluteal fold, acuminate warts may be found also on the scalp, especially in association with seborrhoea and pityriasis. They may be single or multiple. and are small filiform growths, generally constricted at the base, and vary in size from a pin's head to irregular exuberant cauliflowerlike masses which may reach an inch or more in diameter. In moist situations, such as about the vulva or anus, the warts are soft, macerated, pinkish in colour, covered with a glairy discharge, and readily bleed; on the scalp they are harder, and the filiform projections are sometimes corered with a horny cap. They occur frequently in association with renereal disease, especially gonorrhoea, hence the synonym "renereal wart," but are probably due to some organism present in the discharge and not directly to the gonococcus. On the 
scalp they may be caused by some organism which flourishes on a greasy seborrhoic soil.

Histopathology.-Sections of acuminate warts show circumscribed hypertrophy of the prickle-cell layer (acanthosis) with or without thickening of the Stratum corneum, and down-growth of the interpapillary processes into the corium in irregular projections. The prickle-cells are enlarged to several times their ordinary size, retain their fibrillated structure, and mitotic figures can be detected in them as far up as the granular layer. The inter-epithelial lymphatics

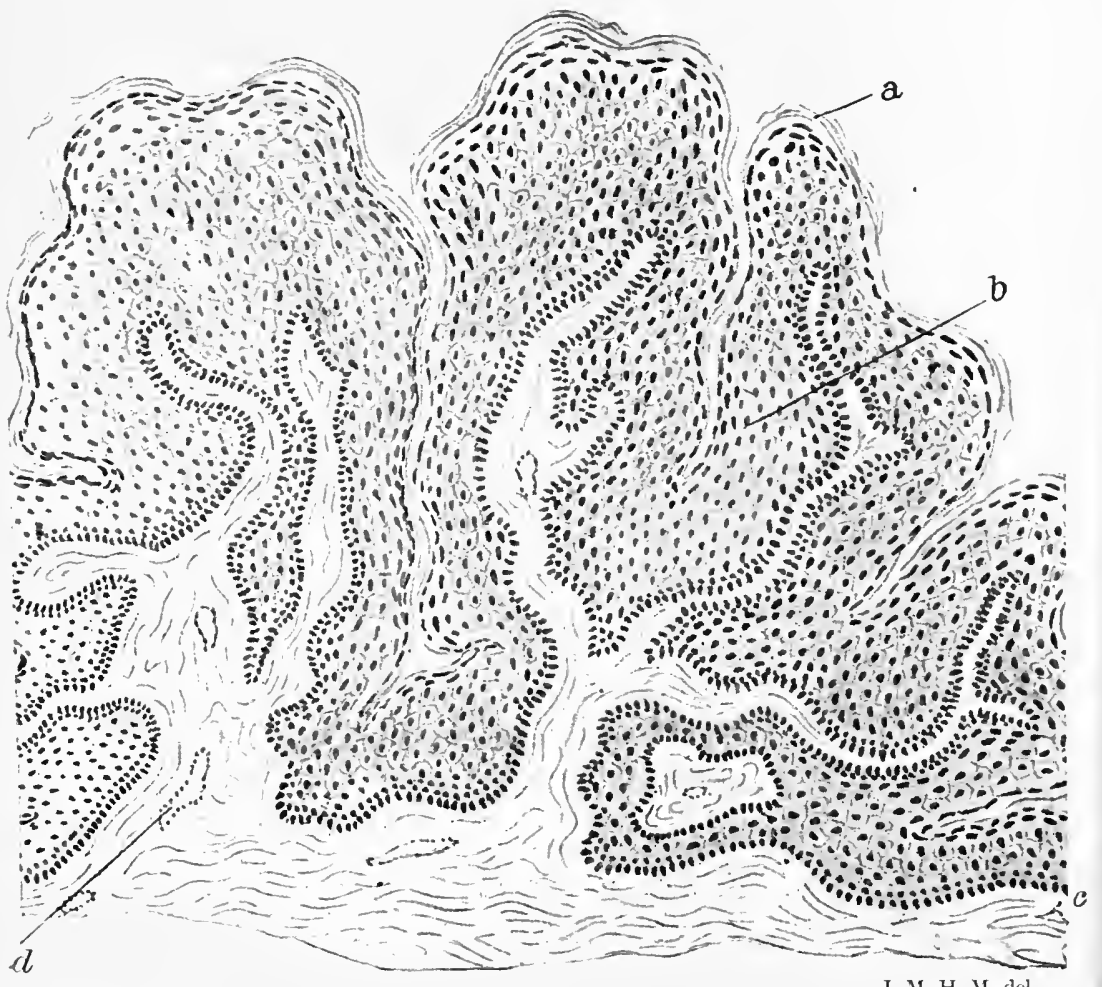

J. M. H, M. del.

FIG. 357.-Section of Condyloma Acuminatum (Acanthosis).

$a$. Stratum corneum not thickened. $b$. Prickle-cells actively proliferating and unusually large. c. Basal layer intact. $d$. Dilated blood-vessel.

(From MacLeod's Pathology of the Skin.)

are dilated and polymorphonuclear leucocytes may be detected between the prickle-cells. Secondary dilatation of the superficial capillaries and an inflammatory cellular infiltration are usually present in those about the genitalia as the result of the irritating action of discharges. (Fig. 357.)

Treatment.-When present on the genitalia acuminate warts can be removed by the thermo-cautery, by curetting, by the X-rays, or by touching them daily with a pledget of cottonwool soaked in Io per cent. solution of formaldehyde; when on the scalp cauterisation is usually the best form of treatment, and should be followed by 
the application of resorcin-salicylic ointment to remove the predisposing pityriasis and seborrhoea.

Note. Warty growh of the acuminate type are commonly known as papillomata, but this term is somewhat loose and misleading as these lesions are essentially benign epitheliomata and any changes which occur in the papillae are secondary.

\section{SEBORRHOIC OR SENILE WARTS}

As the name indicates this type of wart occurs chietly in elderly people, but it may be met with at an earlier age in association with a

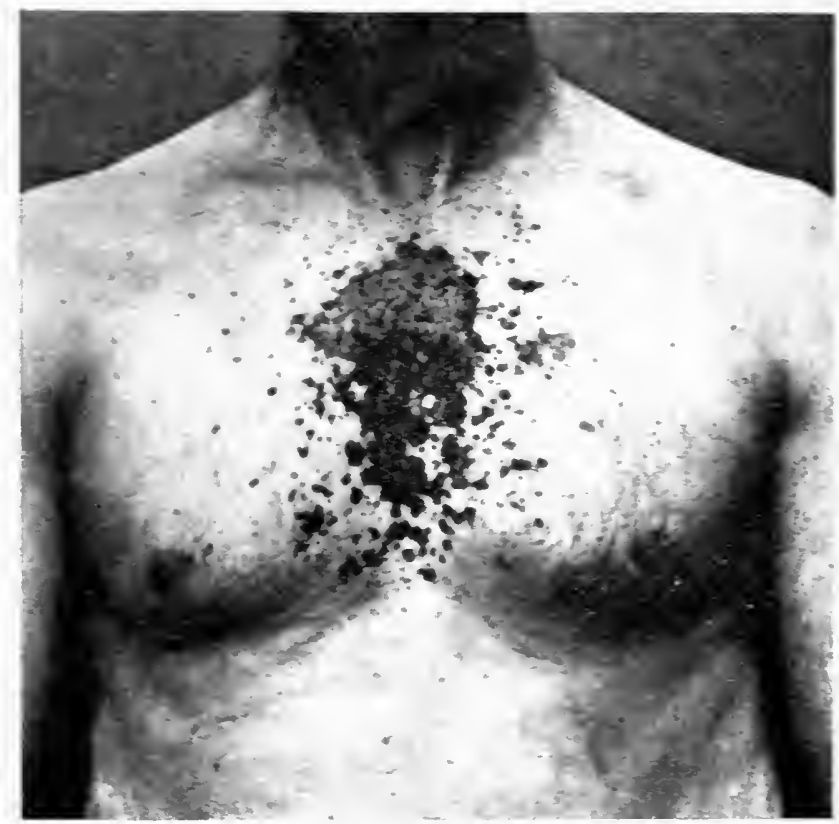

FiG. 35S.-Seborthoea Corporis with flat black warts. (From Dr. E. F. Skinder.)

greasy seborrhoic shin. The lesions are circular or irregular elevations of a grevish-yellow or blackish tint, which is due either to dirt or to a deposit of pigment in the deeper lavers of the epidermis. Ther vary in size from a pea to a shilling and occur chiefly on the trunk and occasionally on the face, backs of the hands, and forearms. In the older lesions the surface is usually hard and horny, and when scraped small tough particles may be separated, leaving bleeding points. In elderly people seborrhoic warts mav undergo malignant changes as the result of friction or imperfect treatment, forming the so-called sebaceous epitheliomata (Epithelioma sebacée). (Fig. 35s.)

Microscopical sections show much the same structure as that which occurs in acuminate warts. 
Treatment.-Seborrhoic 'warts can be removed by any of the methods described in connection with common warts. As a rule they respond readily to the $\mathrm{X}$-rays. They are amenable also to formaldehyde solution, which should be applied daily by a piece of cottonwool, care being taken not to let the solution come in contact with the surrounding healthy skin. Caustics and the actual cautery should be employed with caution for fear of causing malignancy.

\section{REFERENCES}

Common Warts.

HALL, A. Treatment of simple warts by internal remedies (Brit. Journ. Derm., I906, xviii. p. I06).

Lixtle, G. Warts and chilblains (Practitioner, r910, 84, p. 554).

Montgomery, D. W., \& Culver, G. D. Treatment of warts (California State Journ. Med., I918, xvi. p. 250).

Pagliaro. Gonococcic Warts (Clin. Dermosif., Nov. 1905).

WARD, E. Multiple pigmented warts in pregnancy (Brit. Journ. Derm., I9I3, xxv. p. I53).

White, C. J. Use of mercury in Verrucae planae juveniles (Journ. Cut. Dis., r915, xxxiii. p. 738 ; r916, xxxiv. p. 36r).

\section{Plantar Warts.}

Bowes, J. T. Verrucae plantares (Boston Med. \& Surg. Journ., I907, clvii. p. $78 \mathrm{I})$.

Dubreuilh, WV. Verrucae plantares (Ann. de Derm. et de Syph., r895, vi.). Surton, R. L. Verrucae plantares (Amer. Journ. Med. Sci., I912, cxliv. p. $7 \mathbf{I})$.

\section{WARTY AFFECTIONS}

\section{ACANTHOSIS NIGRICANS}

Syn.: Dystrophie papillaire et pigmentaire (Darier); Keratosis follicularis; Keratosis nigricans.

The name Acanthosis nigricanș was given by Unna in ISS9 to a peculiar skin affection, characterised by pigmentation and warty thickenings which were present chiefly about the neck and the flexures of the larger joints, and generally associated with malignant disease in the abdomen. Though an exceedingly rare disease its striking appearance has attracted considerable attention and detailed accounts of about thirty cases may be found in the literature.

Description.-The affection, as a rule, develops insidiously, and is first noticed as a roughness or discolouration of the skin which is sometimes preceded by pricking or itching. It appears first about the nape of the neck, anterior borders of the axillae, breasts, and umbilicus, and varies in colour from pale brown to a dirty-grey or black. At the same time the skin tends to become thickened and dotted over with small warty growths, some sessile others pedunculated, which vary in size from a pin's head to a pea or larger. In moist regions, such as 
around the anus, in the axillae, about the genitalia, and at the commissures of the mouth, the warty growths become exuberant and form irregular cauliflower-like vegetations, sometimes accompanied by painful fissures which emit a sero-purulent or sanguineous viscid discharge.

The nape and sides of the neck are usually markedly involved; the skin, from the hairy border to the root of the neck behind and as far down as the clavicles in front, is deeply pigmented, leathery, and thickened, and is broken up by deep transverse furrows extending downwards and forwards and by more superficial vertical furrows dividing it into irregular polygonal areas and producing a corrugated appearance something like that of a contracted scrotum. Similar pigmented and verrucose patches are usually met with in the flexures of the elbows, the popliteal spaces, perineum, over the backs of the hands and fingers, and on the dorsa of the feet. The skin of the palms and soles is harsh, thickened, and inelastic, and finer morements are interfered with. The hair of the scalp and of the affected areas of the body tends to fall out, and dystrophic changes may occur in the nails rendering them ridged, fissured, and thickened.

The mucous membranes, especially those of the mouth and rulva, are generally involved, and become covered with epithelial growths, but the pigmentation is not increased.

The insides of the lips, the gums, and the inner surface of the cheeks, may present a granular appearance, the tongue may be swollen and relvety from hypertrophied filiform papillae, and the palate may present occasional excrescences suggesting - venereal warts. The palpebral conjunctiva is liable to be affected and granular, in which case the eyelashes generally fall out and a sero-purulent discharge is present which collects at the margins of the lids.

The pigmentation and warty thickenings do not appear to be dependent on one another, as warty growths may occur which are not pigmented and pigmented macules or patches which show no definite verrucosity.

The subjective symptoms are variable, sometimes being completely absent, at other times consisting of itching or pain, due to irritation from friction, the secondary inoculation of micro-organisms, or the presence of fissures.

The actual skin affection gives rise to no general symptoms, but, as it is usually associated with serious internal disease, the health gradually deteriorates and, after a few months or several years, the patient dies in a state of profound cachexia.

Histopathology.-Sections of the pigmented skin and warty excrescences show no changes of a pathognomonic character. The pigmentation is the result of a deposit of melanin in the cells of the basal larer and, to a less extent, of pigmented cells in the connective tissue of the upper layers of the corium. The warty thickenings are similar in structure to ordinary common warts and present hypertrophy of 
the prickle-cell layer, a well-marked granular layer, and a varying degree of hyperkeratosis.

In the corium the papillae may be elongated, and the superficial blood-capillaries dilated and surrounded by an infiltration of lymphocytes, small round connective tissue cells, and a few mast-cells.

Etiology.-Its cause and true nature are at present unknown. In at least $30 \%$ of the cases it has been associated with malignant disease in the abdomen or pelvis, and in one instance it followed cancer of the female breast; post-mortem examinations of several of these cases have revealed nothing definite to account for the cutaneous lesions.

The superficial resemblance of the pigmentation to that in Addison's disease has suggested the possibility of its being in some way connected with defective suprarenal secretion, but no changes have been found in the suprarenals, except in one case (Kuznitzky) in which they were swollen and inflamed. On the other hand it has been suggested that it may be caused by pressure of the cancerous growth on the suprarenal nerves or on some other part of the abdominal sympathetic system.

It is more common in women than men, in the proportion of about 2 to I, usually occurs about the age of 40 , but has been known to begin in childhood and to be unconnected with intra-abdominal cancer.

Diagnosis. - The picture presented by a well-marked case is unmistakable. The only conditions with which it might be confused are Darier's disease (Psorospermosis follicularis vegetans) and Addison's disease.

In Darier's disease the lesions consist of crusted papules closely aggregated together, rather than warty growths; on microscopical examination these are found to be the result of a dilatation and blocking of the funnel of the pilo-sebaceous follicles with a horny mass, while the prickle-cell layer in the neighbourhood presents the characteristic round bodies or pseudo-coccidia. In Darier's disease there is no associated malignancy.

In Addison's disease the distribution of the pigmentation is different, as it occurs chiefly in situations normally pigmented, such as the face, neck, anus, and genitalia, spreads later on to the abdomen and legs, and finally affects the mucous membranes; and the skin, however deeply pigmented, is never harsh, thickened, or warty.

Prognosis.-The outlook in Acanthosis nigricans is invariably grave owing to cancer. In the rare cases in which there is no malignancy the course of the disease is generally slow and inconstant; occasionally the growths disappear spontaneously, and in several instances the pigmentation has diminished temporarily and recurred later.

Treatment.-Where there is no sign of intra-abdominal cancer, improvement may be obtained from the application of $5 \%$ resorcin or salicylic acid in glycerine of starch, from salicylic soap plaster, or from washing with soda.

Exuberant warty growths, if inconvenient, may be removed by the curette, by carbon dioxide snow, or by exposure to the X-rays. 



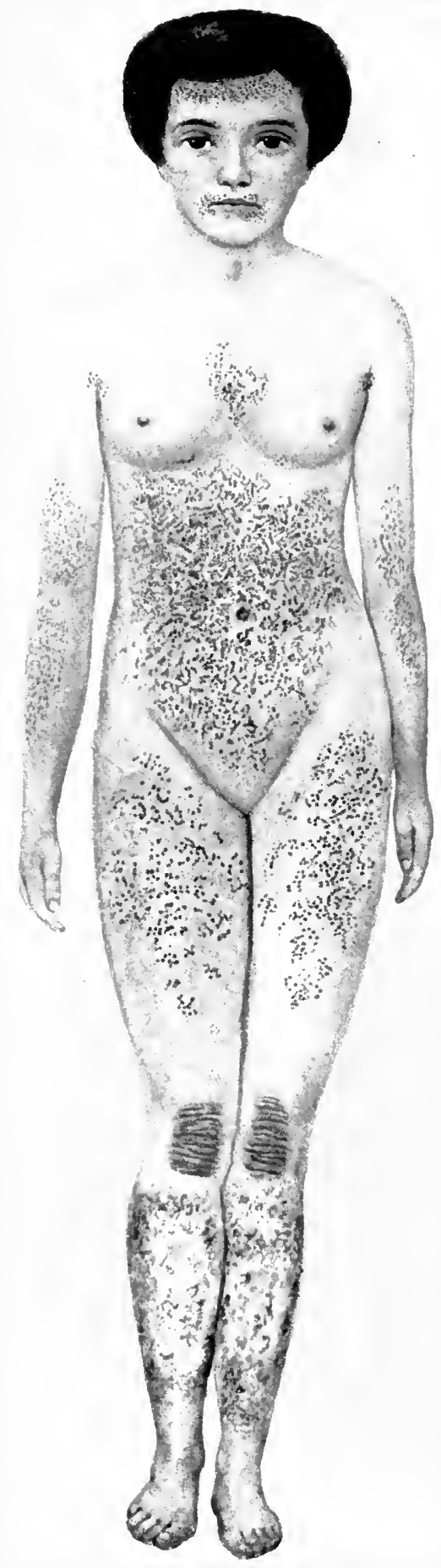

FIG. 359--Psorospermosis Follicularis Vegetans (Darier's disease.)

Shows the characteristic distribution, also secondary superficial ulceration and crusts on the legs,

[To face p. IIO3. 
In cases associated with cancer, treatment is of little value exeepi for the relief of local symptoms.

\section{REFERENCES}

BÉrox, B. Acanthosis nigricans (Monats. f. prakt. Derm., Ioon, xlix p. 2).

DARIER, J. Dỹstrophie papillaire et pigmentaire (Ann. de Derm. et de Syph., I 893, ir. p. S6j).

Lirrle, E. G. Acanthosis nigricans (Brit. Journ. Derm., I 9oI, xiii. p. f2I).

Ibid. (Brit. Journ. Derm., I902, xiv. p. 26).

Nixox. Acanthosis nigricans (Brit. Journ. Derm., I9I f, xxri. p. 9 i).

White, J. C. Acanthosis nigricans (Journ. Cut. Dis., I9I 2, xxx. p. I 791.

WickhaM, L. Letter from Paris (Brit. Journ. Derm., IS9\%, ix. p. 27

\section{DARIER'S DISEASE}

Syn.: Psorospermose folliculaire végétante; Keratosis follicularis (White \& Bowen); Keratosis regetans (Crocker).

Defunition: A rare affection of the skin characterised by dirtybrownish papules, corered with a horny crust, which tend to coalesce to form crusted patches, distributed in various regions of the body and usually in a symmetrical fashion.

Description.-The affection was first described by Darier in IS\$9, and since then between fo and jo authentic cases have been reported. It usually begins in the face, but has been known to appear first on the arms, umbilicus, or back. When fully- developed it is generally present on the face, scalp, back, abdomen where it frequently forms a girdle at the level of the umbilicus, flexures of the limbs, axillae, inguinal regions, and about the anus and genitalia, and is symmetrical in arrangement. (Fig. 359.)

The initial lesion is a papule, rarying in size from a pin's head to a millet seed, irregular in shape, of a dirt $y$-brown colour, and covered by a greasy crust which, when removed, presents on its under-surface one or more horny plugs and leaves a red weeping surface which may bleed. In the centre of some of the lesions there is a black dot which mag correspond with the opening of a sweat-duct, while in or beside others are lanugo hairs or irregular openings from which pus or sebaceous material can be expressed, producing an umbilicated appearance. Certain of the initial lesions suggest papules of Lichen planus, others lesions of Molluscum contagiosum covered by dirty scabs. Sometimes the papules may remain discrete and form clusters, stripes, or rows, especially noticeable about the axillae, naso-labial folds and inguinal regions, or they may coalesce into irregular greasy crusted patches. The seat of the papule may be about the orifice of a pilosebaceous follicle, at a sweat-pore, or in the intervening epidermis.

Besides the patches due to the coalescence of papules, in serere cases and in a late stage of the disease papillated excrescences are liable to occur in moist regions, such as the groins, axillae, or about 
the anus and genitalia, and occasionally pedunculated growths have been observed. (Fig. 360.)

Apart from the actual papules and patches the skin of the affected region may be diffusely thickened and dirty-grey in colour.

In most cases there is a scaly or crusted condition of the scalp, in which the scales are rather thicker than those of seborrhoic dermatitis, and the hairs are dry and lustreless.

The nails also are usually affected, becoming dry, thickened, brittle, longitudinally striated, and occasionally raised up from the nail-bed by a horny thickening.

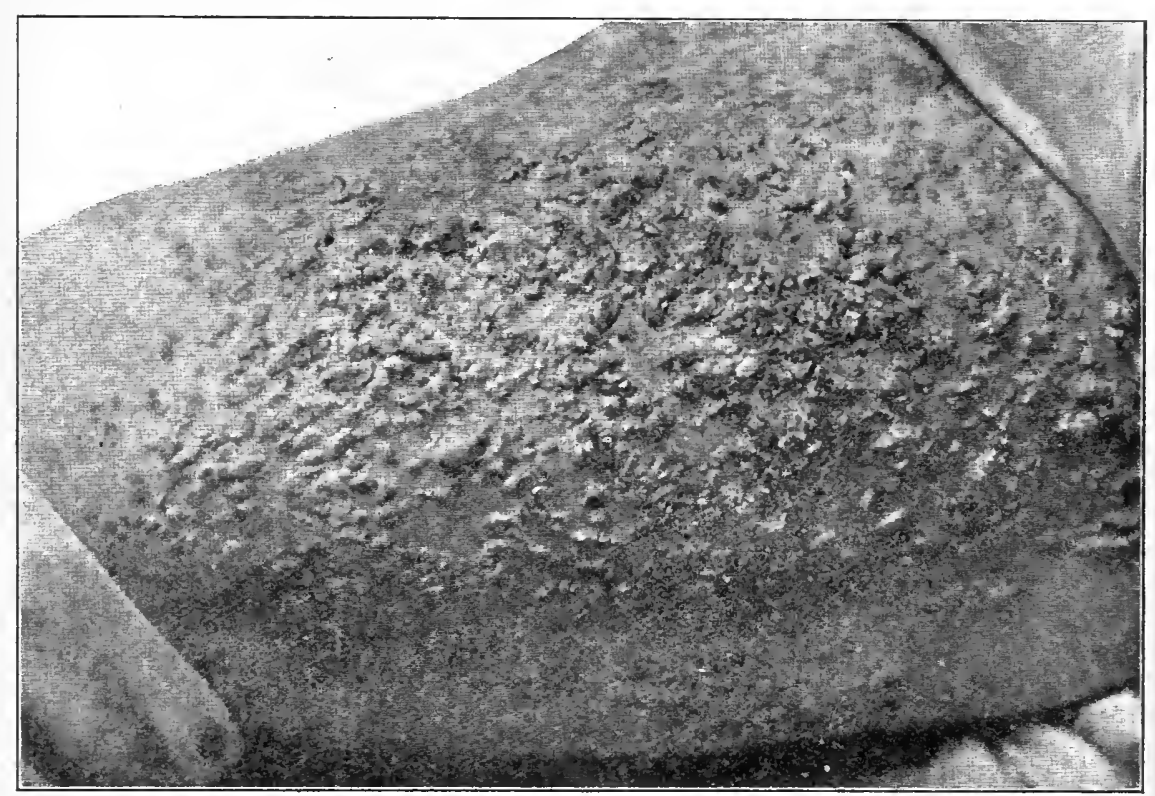

FIG. 360.-Psorospermosis Follicularis Vegetans (Darier).

(Dr. W. Allan Jamieson's case. Edin. Med. Journ., I908.)

In a few cases small papular lesions have been present in the buccal mucosa, especially on the tongue and lips, but as a rule the mucous membranes are not involved.

Subjective symptoms may be absent or negligible: on the other hand there may be considerable irritation.

The disease is chronic, progressive, and liable to acute exacerbations with the appearance of numerous fresh lesions. However extensive the cruption may be it does not seem to interfere in any way with the general health.

Histopathology.-In lesions from a typical case examined and reported upon by the writer, the following changes were noted, which corresponded in the main to those originally described by Darier :

The epidermis was thickened in all its layers and the inter-papillary processes were widlened and had sent down blunt prolongations into 
the corium into which the granular layer dipped down, producing an appearance not unlike a commencing growth of Molluscum contagiosum. Above the granular layer were thick horny plugs consisting of an irregular mass and network of imperfectly cornified cells. The funnels of the pilo-sebaceous follicles were dilated and plugged with imperfectly cornified and partially degenerated horny material, and horny plugs were present also in the situations of the sweat-pores. The basal layer was irregular and blurred and pigment granules were present in the cells. (Fig. 36I.) Near the granular layer certain of the prickle-cells had undergone a peculiar degenerative change, had become separated from each other, assumed a round shape in consequence of the breaking

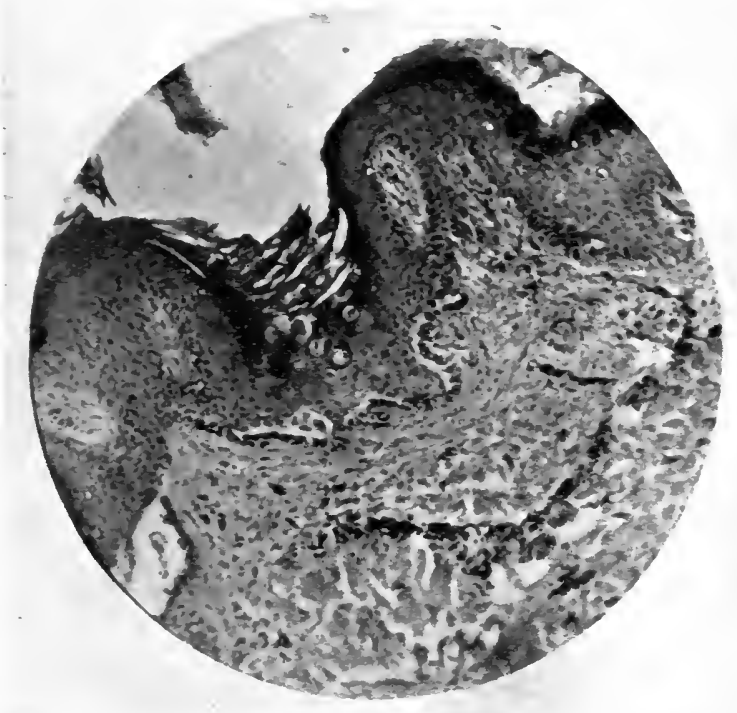

FIG. 361.-Section of Psorospermosis Follicularis Vegetans. (Low power.)

Shows "Round Bodies" situated about the Stratum granulosum in a lesion sornewhat resembling Ifolluscum contagiosum.

of the inter-epithelial fibrils, and the protoplasm had passed to the periphery and condensed to form a cell-mantle, while the nucleus lay in a space in the centre or was compressed towards the periphery in the form of a crescent. These round degenerated cells were named "corps ronds" by Darier, who at one time believed them to be psorosperms. Among the horn-cells was a large number of peculiar round or oval structures which were homogeneous or granular and occasionally presented a nucleus in the centre, these also were first described by Darier who named them "grains." Both the "corps ronds" and the " grains " may be demonstrated easily in the fresh state by macerating crusts or scrapings of the epidermis in Liquor potassae. Occasionally cell-nests were detected, one or more of the grains sometimes forming a nucleus to the nest. In short the condition was that of a 
dyskeratosis associated with a peculiar cellular degeneration which might be located at the follicles or be independent of them. (Fig. 362.)

In the corium the blood-vessels of the papillary and sub-papillary layers were dilated and surrounded by a cellular infiltration consisting of lymphocytes, mast-cells, a few connective tissue cells, and an occasional plasma-cell-appearances suggesting an inflammatory disturbance resulting from the pressure or irritation of the overlying thickened epidermis or from the presence of micro-organisms. The papillae between the thickened epidermal processes were elongated and in places the apices had come near the surface of the skin. The fibrous elements seemed to be healthy.

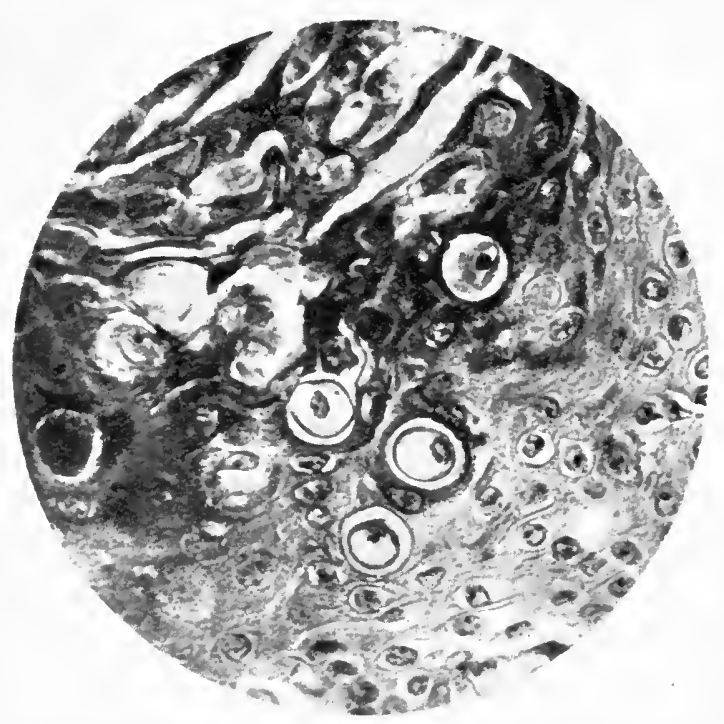

FIG. 362.-Psorospermosis Follicularis Vegetans. (High power.) Shows "Round Bodies " in the epidermis and "Grains" in the Stratum corneum.

Etiology.-The cause of the disease is unknown. Darier's original view that the "corps ronds" and "grains" were intra- and extracellular psorosperms was abandoned subsequently by himself and is no longer accepted and these peculiar bodies are now recognised as due to imperfect cornification and hyalin degeneration.

There are several points of homology between Darier's disease and Molluscum contagiosum, and the "grains," though smaller, bear a striking resemblance to " molluscum bodies." This homology and the greasy seborrhoic condition on which the papules develop suggest the possibility of the disease being an infective process superimposed on a seborrhoic soil, but so far no specific micro-organism has been discovered in connection with it.

It begins as a rule in early life, sometimes in the first few years, more usually in the second decade, and rarely after zo. The majority 
of the cases occur in males, in the ratio of about 2 to I. Heredity seems to play some part in the etiology, and it has been observed on several occasions to affect a parent and one or more of the offspring, and in one instance it appeared in three generations.

It has been suggested that it is a congenital affection and a form of naevus which develops sometime after birth, like many cases of ichthyosis.

Diagnosis. - The two diseases with which it is most liable to be confused are Acanthosis nigricans and Keratosis follicularis contagiosa of Brooke.

In Acanthosis nigricans the distribution is somewhat similar but the mucous membranes are more severely affected, the cutaneous lesions more verrucose, and the skin more deeply pigmented. Histologically, the " corps ronds " and "grains " are absent. Acanthosis nigricans does not affect families, begins usually after the age of to, attacks females more than males, and is usually associated with malignant visceral disease; Darier's disease frequently occurs in families, begins in early life, is more prevalent in males, and is not associated with visceral changes.

Keratosis follicularis contagiosa is so strikingly similar with regard to the age at which it occurs and the onset, its presence in more than one member of a family, its distribution and general characteristics, that it suggests a mild variant of the same affection, but in Brooke's disease the " corps ronds " were not observed and the initial lesion was invariably follicular.

Treatment.-The affection is chronic and intractable, and any improvement which may take place from the judicious application of local remedies is temporary. Considerable benefit may be derived from the application of salicylic and sulphur ointment to reduce the horny thickening and the greasy seborrhoea, from 5 to $\mathrm{IO}^{\circ}{ }_{0}$ resorcin in glycerine of starch and other keratolytics, and from the thorough cleansing of offensive discharges by antiseptic lotions. Exuberant vegetations may be removed by the curette, and haemorrhage controlled by the actual cautery.

General treatment has no influence on it.

\section{REFERENCES}

Atdry, C., \& Dalous, E. Notes \& reflections with respect to a new case of Darier's Disease (Journ. des Mal. Cut. et Syph., I 904, xvi. p. Sor).

Boeck, C. Four cases of Darier's Disease (Arch. f. Derm. u. Syph., ISgI, xxiii. p. 857 ).

Bukorskr, J. Psorospermosis follicularis vegetans (Darier) (Arch. f. Derm. u. Syph., I905, p. 279).

DARIER. Psorospermose folliculaire végétante (Ann. de Derm. et de Syph., I889, x. p. 597).

Psorospermosis follicularis vegetans (Internat. Atlas of Rare Skin Diseases, I893, Pls. xxiii. \& xxir.).

Note on the pathological anatomy of a new case of Psorospermosis follicularis vegetans (Ann. de Derm. et de Syph., ISg6, vii. p. 7t2). 
Fox, IV. Darier's Disease (Brit. Journ. Derm., I9I I, xxiii. p. 359).

GILCHRIST. The so-called protozoa occurring in Psorospermosis follicularis vegetans (Darier) (The Johns Hopkins Hosp. Rep., I 896, i. p. 29I). Huber. Darier's Disease with identical lesions on the mucous membrane (Arch. f. Derm. u. Syph., I903, lxv. p. 127).

Jamieson, W. A. Darier's Disease (Edin. Med. Journ., I907, p. 32).

Lustgarten. On Psorospermosis follicularis (Journ. Cut. \& Gen.-Urin. Dis., I 89I, p. 7).

MacLeod, J. M. H. Darier's Disease (Brit. Journ. Derm., I909, xxi. p. 256). Pohlmann. Darier's Disease in three generations (Arch. f. Derm. u. Syph., xcvii. p. 195).

Scheer, M. Darier's Disease in its early stages (Journ. Cut. Dis., Dec. I9I6, xxxiv. p. 837).

White, J. C. A case of Keratosis (Ichthyosis) follicularis (Journ. Cut. \& Gen.-Urin. Dis., I 889, p. 201).

\section{GONORRHOEAL KERATOSIS}

Syn.: Keratodermia blenorrhagica ; Fr.: Kératodermie blenorrhagique.

This affection is a rare manifestation of gonorrhoea. It may appear from two to eight weeks after the disease was contracted, in cases where

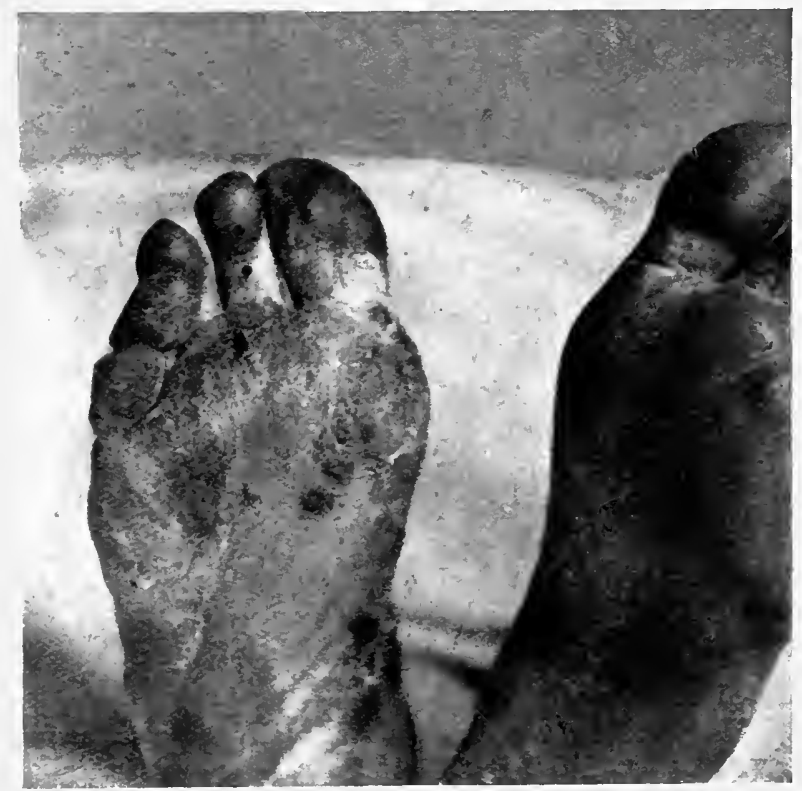

FIG. 363.-Gonorrhoeal Keratosis.

Shows thickening of epidermis on pressure areas and horny masses.

(Dr. Winkelried Williams' case. Brit. Journ. Derm., I910.)

a general dissemination of gonococci has taken place and in association with pyrexia, gonorrhoeal arthritis, tenosynovitis, debility, or other signs of gonorrhoeal septicaemia. 
Vidal first drew attention to it as early as IS93, and in I9Io cases were demonstrated in this country by Sequeira and Winkelried Williams. Since then cases have been recorded on the Continent, in America, and in this country. (Fig. 363.)

The keratosis may be widely distributed orer the cutaneous surface, but is more frequently confined to the soles of the feet, palms of the hands, and about the elbows and knees. It may occur as a diffuse thickening of the horny layer of the palms and soles which is dotted over with irregular horny thickenings; more commonly it takes the form of transparent yellowish or brownish-yellow cone-shaped

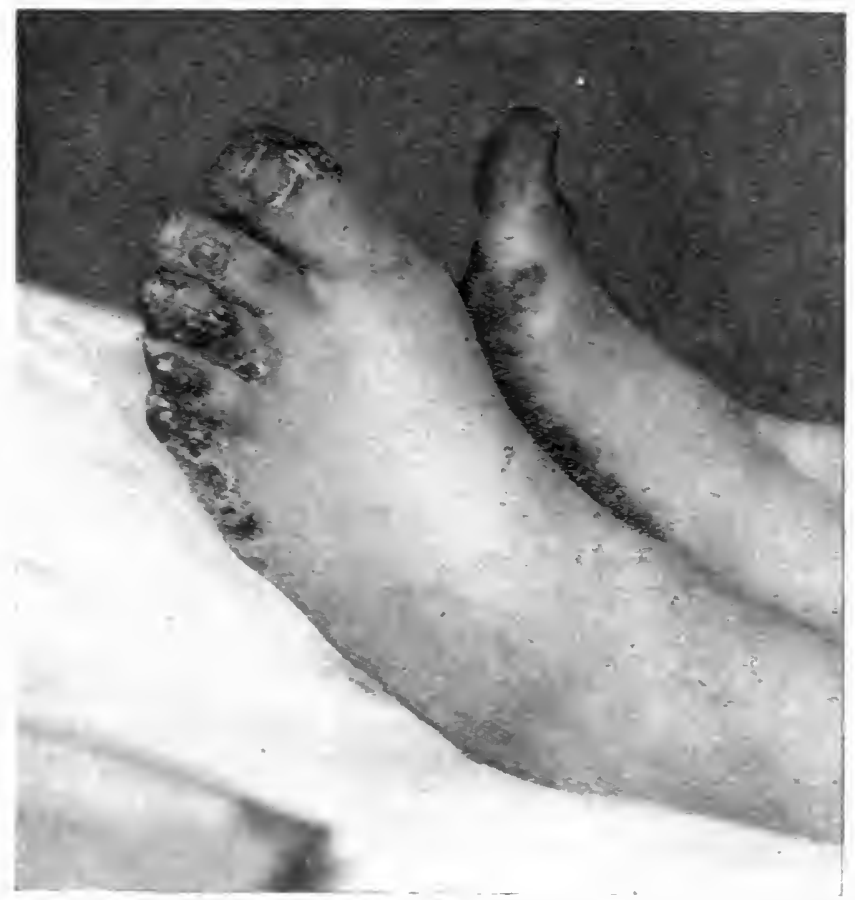

FIG. 364 -Gonornhoeal Heratosis.

Shows horny masses on toes and side of the foot.

(Dr. Winkelried Williams' case. Brit. Journ. Derm., I9 ro.)

horny crusts, which begin as small red patches, or occasionally as resicles, and rapidly grow into horny cones situated on a horny base, varying in size from one-quarter to three-quarters of an inch in diameter, and sometimes surrounded by a reddish or purplish areola. These conical excrescences are specially marked on the soles and sides of the feet, and, in the latter situation, are liable to coalesce to form horny ridges surmounted by dome-shaped crusts giving rise to an appearance which has been aptly compared to the mountain peaks on a relief map.

The nails are liable to be involved as the result of horny crusts about the nail-beds, and are apt to become opaque and sometimes detached. (Fig. 364.) 
In course of time the horny material tends to dry up, either spontaneously or under the influence of suitable treatment, to separate at the base, and to become detached, leaving a moist red area which, on healing, is replaced by pigmentation. If the crust be forcibly separated before it has completely dried up, the under surface is found to be covered by a greyish-white material, like putty, which can be scraped off readily.

Histopathology.--Sections of an early horny lesion show thickening of the whole of the epidermis, with well-marked hyperkeratosis and an increase of the prickle-cell-layer with elongation of the inter-papillary processes. The underlying papillae are oedematous and there is a cellular infiltration consisting of lymphocytes, plasma-cells, polymorphonuclear leucocytes, and occasional eosinophiles. In the deeper parts of the corium the cellular infiltration is situated chiefly around the blood-vessels.

Repeated examination of the horny crusts and of the slimy material beneath them for gonococci has given negative results.

Treatment.-The treatment of the condition is that of gonorrhoea, and the lesions readily dry up and separate when the general infection subsides. Marked improvement has been recorded under gonococcal vaccines.

\section{REFERENCES}

Brown \& Davidson. A case of gonorrhoeal keratosis (Brit. Med. Journ., I9I 7 , ii. p. 453).

Chauffard, A. Keratodermie blenorrhagique (Ikongraphia Dermatologica, Fasc. 5, I910).

I. Demany. Skin changes in gonorrhoea (La Presse Méd., x897, No. 50, p. 282).

Little, E. G. Keratodermia blenorrhagica (Practitioner, 1916, p. 531).

Rотн, V. "Hyperkeratotic" gonorrhoeal eruptions (Münch. Med. Wochenschr., May 3oth, I905).

Sequeira, J. H. Keratodermie blenorrhagique (Brit. Journ. Derm., I9IO, xxii. p. I39).

Vidal. Keratodermie blenorrhagique (Soc. Derm., Jan. I th i893).

\section{CUTANEOUS HORNS}

Syn. : Cornu cutaneum; Fr. : Corne cutanée ; Ger. : Hauthorn.

Cutaneous horns are peculiar horny growths, sometimes closely resembling the horns of anirials, which are occasionally met with in the skin in human beings. The situations in which they have been found most frequently are the scalp and the face, especially about the forehead, but in rare instances they may occur elsewhere. They are usually single, but may be multiple and occasionally may be present in considerable numbers. They vary in size from small horny nodules to large excrescences a foot or more in length and several inches in circumference. 
The horn is situated on a base which is usually convex, but in some instances the surrounding skin may have proliferated and grown up around the proximal end so that it becomes enclosed in a cup or sheath of warty skin. The whole structure, both horn and base, is entirely cutaneous and morable, thus corresponding to the horn on a rhinoceros and differing from an ordinary horn, which has a bony attachment.

In shape cutaneous horns vary indefinitely; they may be small, rounded or irregular, tuberous excrescences; they may be long, straight, curved, or spirally twisted like a ram's horn and pointed at the distal end ; in section they may be round, oval, or angular. They are usually rough on the surface, sometimes present longitudinal or transverse ridges, and in colour are generally dirty-brcwn or green. As a rule they are peculiarly tough, though not quite so hard as bone, but occasionally they may be brittle and friable.

At first their growth is rapid but later it becomes slow and intermittent, and after reaching a certain size ceases, when the horn may be permanent or may become loosened at the roots, detached, and a new one begin to grow. Sometimes the skin around the base becomes inflamed and infected, in which case suppuration may supervene and lead to separation of the horn. Owing to the irritation caused by the presence and morement of the horn the warty base readily assumes malignant changes and tends to become epitheliomatous. If the horn be forcibly removed from the base, bleeding from the torn capillaries of the underlying elongated papillae is liable to occur.

Histopathology.- The horn is similar in structure to hard verrucose naevi of the Ichthyosis hystrix type. At first there is a proliferation of the prickle-cell layer (acanthosis), associated with hyperkeratosis with which it is commensurate, and later the acanthosis ceases while the hyperkeratosis progresses to form the horn. The horny mass is made up of cells which show a rough tendency to a concentric arrangement. There is more or less irregular downgrowth of the interpapillary processes and corresponding elongation of the papillae. The changes in the superficial capillaries of the corium are induced secondarily.

Etiology.-The cause of these growths is unknown. They may occur at any age but are most frequent in elderly people. They are met with in either sex, but are believed to be more common in women than in men. In certain instances they have followed an injury, in others they appeared to grow on some previous epidermal lesion such as a wart or a sebaceous cyst. In several cases they have been known to take their origin in an acuminate wart on the penis.

Treatment.-The simplest form of treatment is excision. Where this is not practicable an equally efficacious method is to dissect away. the horn from, the base and to destroy the base by exposure to the I-rays or by freezing with solid carbon dioxide; it is unwise to attack it with caustics as unless the base is completely destroyed by this 
means re-growth of the horn is almost certain and malignant changes may supervene.

\section{REFERENCES}

Dore, S. E. Case of Cutaneous Horn (Brit. Journ. Derm., I902, xiv. p. 423). Gould, A. P. Horn growing from the glans penis associated with Epithelioma (Trans. Path. Soc., Lond., I887, xxxviii. 355).

\section{MOLLUSCUM CONTAGIOSUM}

Syn. : Epithelioma molluscum ; Epithelioma contagiosum.

Definition: Small epidermal growths, varying in size from a pin's head to a split-pea or larger, of a pearly-grey or pinkish tint, rounded or flat on the surface, with a central depression from which can be expressed a whitish caseous substance.

Description.-This affection is one of the rarer cutaneous diseases. It has a wide distribution in Europe and America, and may be met with in any race. In London it occurs in the proportion of about I per mil cases, and out of 6,700 consecutive cases in the skin department at Charing Cross Hospital there were only six cases of it. Crocker in the edition of his text-book published in I905 gives the proportion as 2 per mil, but included in this were the returns from a children's hospital, which would raise the percentage as the disease is more common in children than in adults.

It appears first as one or more minute rounded elevations of the epidermis, the colour of the ordinary skin, or more often of a greyishpearly or pinkish hue. These, on reaching the size of a large pin's head, become dimpled at the summit and the surface more or less flattened, and even at this stage with the aid of a pocket lens a minute opening may be detected in the centre of the depression. The lesions grow comparatively slowly and may take months to attain the size of a split-pea. To the touch they are semi-solid and elastic, and present a translucent cartilaginous appearance but are not so hard as cartilage. When squeezed between the fingers or with a pair of forceps a peculiar white caseous substance can be expressed, which is an important characteristic and consists of imperfectly cornified cells in a state of hyalin degeneration. In older lesions the mass instead of being cheesy may become soft and creamy in consistence like white lead, or it may become hard and chalky. (Fig. 365.)

If left alone the growths may undergo spontaneous involution, dry up, and disappear. On the other hand, from traumatism and the inoculation of septic organisms, suppuration or superficial ulceration may supervene and may be followed by scarring.

Occasionally they may reach the size of a small filbert nut, when they tend to become pedunculated and to assume a yellowish waxy appearance with, not infrequently, small dilated vessels coursing. over the surface. 


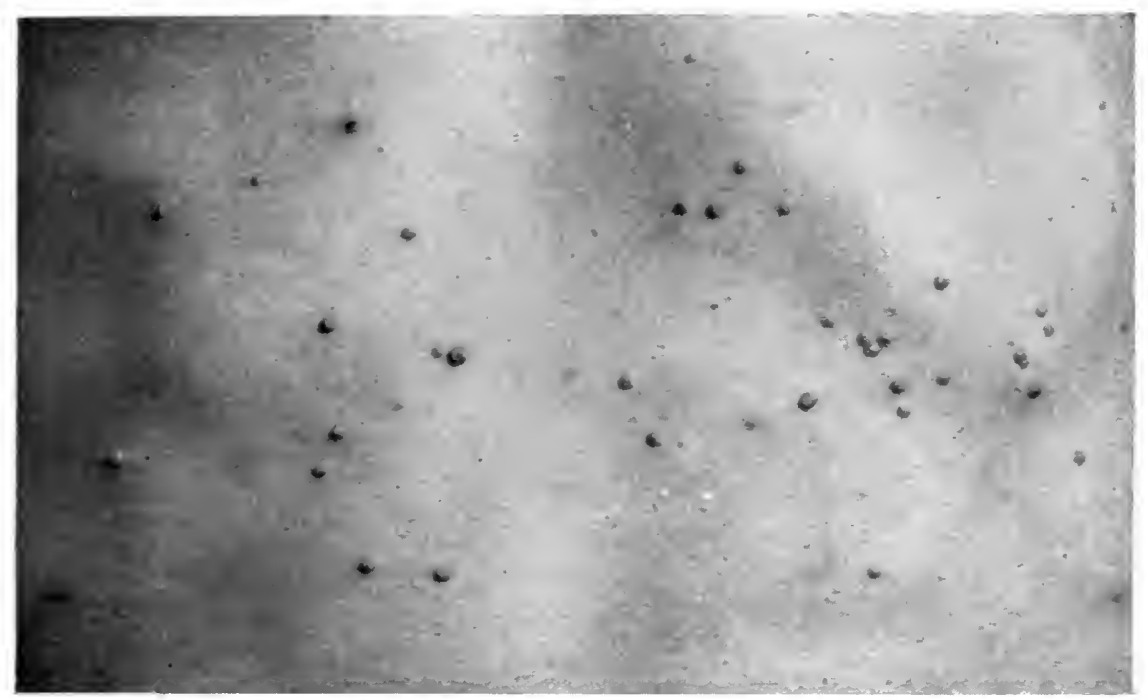

FIG. 355.-Molluscum Contagiosum on Chest.

Shows small umbilicated pearly lesions.

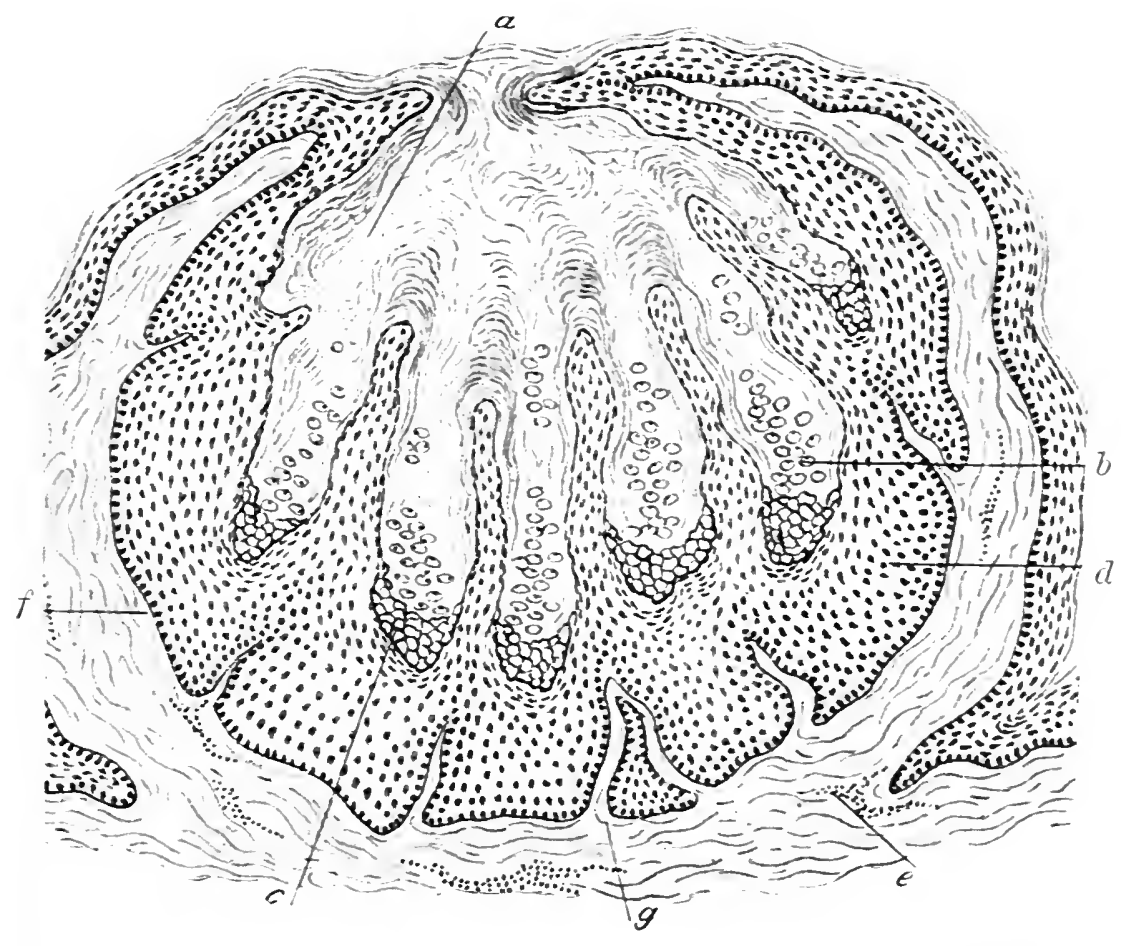

J. W. $\mathrm{H}$, II del.

FIG. 366.- Section of Molluscum Contagiosum. (y about I 20 .

a. Dimple and cavity liner and almost completely nilled with horn-cells. b. .. I slluscum bodies." c. Transitional layers. “i. Proliferated Malpizhian laver. ¿. Dilated blool-vessel. f. Condensed ribrous capsule. g. Papillae tlattened or persisting as a cleft, givitig to the tumour a lobulated appearance. 

In rare instances ther increase to form tumours the size of a walnut or even of a small tomato (Molluscum giganteum), reddish in colour, rounded or flattened on the surface, and haring a central crater with an irregular base which may be covered with an offensive yellowish slough. The larger lesions tend to persist indefinitely:

The growths may occur anywhere on the cutaneous surface, except on the palms and soles, and are particularly common on the face, especially about the margins of the eyelids, and on the nech and scalp. They are met with not infrequently on the hands, trunk, genitalia, female breast, and upper part of the thighs, and one or two instances are on record in which they have been observed on the lips and mucous membrane of the mouth, where they presented a slight resemblance to leucoplakia.

As a rule they are limited in number, varying from two to about ten, but sometimes mar be numerous and widely scattered, as in a case under the care of the writer in which over one hundred were counted. They are usually discrete and irregularly distributed, but occasionally may occur in close clusters suggesting the spread of the disease by contiguity. About the groins and thighs, they may coalesce into irregular patches or sheets, or occasionally, on the scalp, they may form exuberant conglomerate masses which have been mistaken for malignant epitheliomata.

When they occur about the groins, perineum, and genitalia they tend to lose their translucent pearly aspect and to become flat and covered with a layer of sodden epithelium due to maceration by discharges in that region.

As a rule there are no subjective symptoms, unless they are irritated by rubbing or inflamed by secondary septic infection. When they occur about the genitalia, they may be actually painful from the chafing of the clothes in moving; if they are present about the edges of the erelids they are apt to be accompanied by irritating conjunctivitis.

Histopathology.-Sections of commencing Molluscum contagiosum show that the lesion consists of an epithelial growth which originates in the prickle-cell layer of the epidermis and spreads down into the corium, causing a condensation of the underlying white fibrous bundles to form a capsule. The epithelial mass is rounded in shape and broken up into lobules by fibrous septa which pass in from the surrounding corium. In its growth the small tumour becomes raised up, dragging with it the epidermis at the sides and flattening out the interpapillary processes. In the centre there is an opening which extends down in finger-like projections into the lobules, forming an irregular carity lined with peculiar imperfectly cornified cells which may completely block it and which form the cheesy contents of the lesion. The whole appearance of the growth with its central carity and orifice resembles a gland and its duct so closely as to have given rise to the idea that it took its origin in a sebaceous gland. It is now known, 
however, that it is not a glandular structure but derived from the prickle-cell layer of the epidermis, or possibly, in rare instances, from the prickle-cell layer of the hair-follicle. (Fig. 366.)

Towards the centre of the cavity the peculiar cclls, which are known as "molluscum bodies," may be found lying free, while at the margins they are closely packed together. When free they are oval or rounded in shape, have a glistening appearance, and present a capsule containing a homogeneous mass, and a flattened nucleus, or the granular remains of one, between the mass and the capsule. These oval bodies have a superficial resemblance to protozoa of the " coccidium oviforme" type, and were once believed to be parasites and the cause of the disease, but are now known to be imperfectly cornified cells consisting mainly of a homogeneous mass of hyalin or colloid-a degenerative product of the protoplasm of the cell-enclosed in an envelope of keratin. The hyalin degeneration commences in the prickle-cells, where globules of it may be found pushing aside the nucleus of the cell,

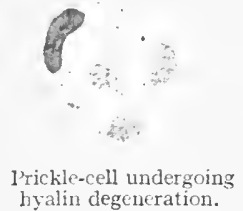

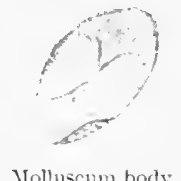

Fig. 367.-MIolluscum Contagiosum.

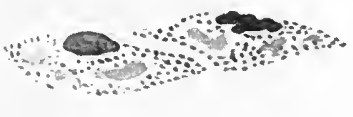

Granular cells undergoing hyalin degeneration.

and is further developed between the granules of keratohyalin and in the cells of the Stratum lucidum. (Fig. 367.)

Etiology.-The disease is more common in children than in adults. It is said to occur more frequently in the poorer classes than in the well-to-do, but in the writer's experience the number of cases in hospital and private practice are about equal. A point of interest in this connection is that the great majority of the hospital cases were in children while the private cases were mostly in adults, three in one year being attributable to Turkish baths.

As its name denotes the affection is contagious, auto-inoculable, and capable of transmission accidentally and experimentally from one individual to another. It has been inoculated successfully in a considerable number of cases, and where negative results have been obtained these may have been due to a want of susceptibility of the individual inoculated or to the incubation period being so long as to admit the possibility of the virus of the disease being eliminated before the growths developed. Instances of accidental transmission are numerous; Brocq quotes a case in which a physician, after squeezing out the contents of a lesion in a patient, inadvertently scratched his own face with his nail contaminated with the cheesy material, and thus inoculated the virus which was followed by typical molluscous growths in about six weeks. A case is also on record where a surgeon suffering from it on the fingers infected an opcration wound. 
It is not uncommon to meet with it in husband and wife or in several members of a family, and cases are on record where an infant infected the breast of the mother. A number of epidemics have been described in various institutions, such as schools, reformatories, etc., and it is not infrequent in connection with public baths, where it may be due to towels, wooden seats, or to the rings, parallel bars, or other gymnastic apparatus which may form part of the establishment. A considerable number of cases have occurred after Turkish baths, possibly through the agency of an infected dressing-gown, towel, couch, or even the hands of the attendant or rubber ; probably the softening of the skin by the bath renders it particularly vulnerable to the contagion. It has been reported to be particularly cominon in Aberdeen among the fishing girls who gut herrings, etc., but the source of the virus in those cases is uncertain.

A similar affection occurs in certain domestic birds, such as pigeons, fowls, pheasants, etc., in which lesions are present about the head, beak, and claws. These growths are not identical with the human Molluscum contagiosum but can be inoculated successfully on the human skin.

The virus of the disease is unknown but is believed to be exceedingly small and may be a minute protozoon. It can be filtered through a Berkfeld filter, and the filtrate, though bacteriologically sterile, can communicate the disease.

The incubation period is long. Where the inoculation was done by rubbing cheesy material from a lesion into the skin, with or without previous scarification, it varied from six weeks to three months or longer; in cases where the infiltrate was employed it was three months.

Diagnosis.-In children, small lesions of Molluscum contagiosum have to be distinguished chiefly from milia; but milia are white and opaque, have not the pearly translucent appearance, are not umbilicated, and the contents cannot be expressed by squeezing.

In the adult the early lesions may suggest commencing rodent ulcer or benign crstic epithelioma, but these growths are both harder than Molluscum contagiosum, are never umbilicated, and do not have expressible contents.

Any further difficulty in diagnosis may be set at rest by an examination of the cheesy contents for the "molluscum bodies," or by a microscopic examination.

\section{TREATMENTT}

The treatment is simple and effective, and has as its object the destruction of the lesions without scarring.

The smaller lesions may be removed by squeezing out the contents, when the growth collapses and the walls shrivel up.

Where the lesions vary in size from a buckshot to a split-pea the best treatment, and one that is comparatively painless, is curetting, 
the affected skin being painted over previously with a weak solution of iodine. After curetting a little pressure will stop the bleeding and the surface of the lesion may be dusted over with boracic powder or painted with collodion. By this means healing takes place, as a rule, in a few days, leaving no scar.

Where they are situated about the margins of the eyelid it is better to snip them off with a pair of curved scissors and to touch the base with a sharpened match dipped in tincture of iodine.

Where they are the size of a small filbert nut they are usually pedunculated and may be cut across at the pedicle and the base scraped out, or if sessile they may be incised and the contents expressed.

The large tomato-like growths should be freely excised.

Another useful method of treatment, but one that is more painful, is the employment of a Paquelin cautery with a point about the size of a small steel knitting needle. Excellent results can be obtained from it but care must be taken not to cauterise beyond the limit of the growth or scarring will follow.

Other methods have been advocated, such as electrolysis, or the insertion into the orifice of a sharpened match impregnated with acid nitrate of mercury, pure carbolic acid, or tincture of iodine ; but none of them has any advantage over curetting or the actual cautery.

When numerous growths have been removed, it is advisable for some time afterwards to apply a I in 2,000 solution of perchloride of mercury daily to the skin, or in regions like the axillae or the groins to use a $2 \%$ salicylic acid and sulphur ointment. By these measures incubating lesions may be rendered abortive. It is important also to sterilise the underclothing, as the virus conceivably may be harboured there and give rise to re-infection.

\section{REFERENCES}

Casagrandi. Molluscum contagiosum (Ann. de Derm. et de Syph., I9o8, p. 455).

Fox, Colcott. Molluscum contagiosum (Brit. Journ. Derm., I898, x. p. 2 I6).

Knowles, F. C. Epidemic of Molluscum contagiosum (Journ. Amer. Med. Assoc., I909, p. 671).

Kreibich. Etiology of Molluscum contagiosum (Arch. f. Derm. u. Syph., Jan. I9I3).

KUZnitzky, MI. Cell changes in Molluscum contagiosum (Arch. f. Derm. u. Syph., Bd. xxxii. Hft. I, p. 2).

Little, E. G. Molluscum contagiosum (Brit. Journ. Derm., I9Io, xxii. p. I8I).

MAALEOD, J. M. H. Observations on Molluscum contagiosum (Brit. Journ. Derm., I9I5, xxvii. p. I2).

Pringle, J. J. Molluscum contagiosum of the scrotum, perineum, and contiguous parts (Brit. Journ. Derm., I898, x. p. I98).

Molluscum contagiosum (Idem, p. 4I 8).

Westminster Hospital Reports. Seven cases in which operation wounds were infected with Molluscum contagiosum (I909, vol. xvi.).

White, C. J., \& Rober, W. N. Molluscum contagiosum (Journ. Med. Research, April I902, p. 225). 


\section{MILIUM}

Syn.: Grutum.

Definition: Small, opaque, whitish or yellowish, non-inflammatory lesions, situated on the upper part of the face, due to the presence of horny cysts in the corium.

The term milium has been applied in the past in a somewhat loose fashion to three distinct types of lesions which, though resembling one another in appearance, differ in their origin and pathology; these are :

(I) Small rounded yellowish projections about the size of a pin's head, which form in connection with the sebaceous glands and contain

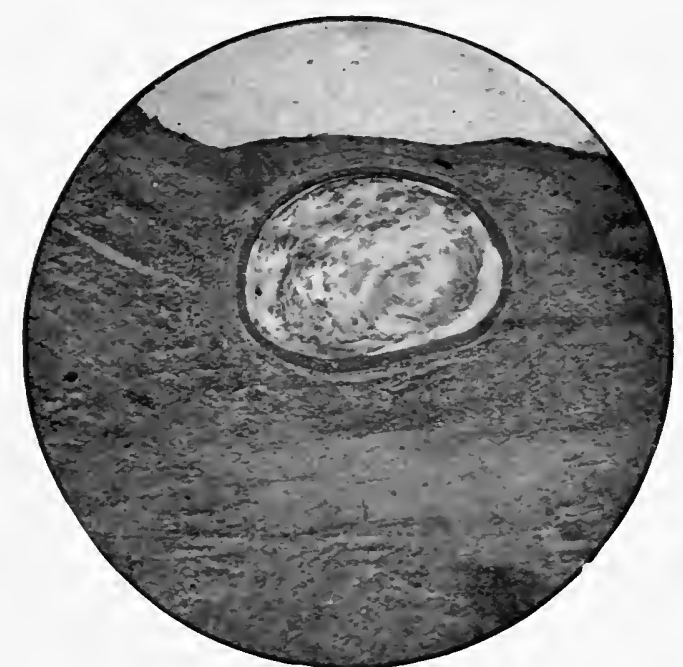

FIG. 368.--Epidermal Inclusion Cyst in Herpetic Scar.

(Dr. Harvard F. Warner's case. Brit. Journ. Derm., 1907.)

sebaceous material and epidermal debris. They are met with occasionally in infants about the face and are not uncommon about the genitalia (see sebaceous cysts).

(2) Small opaque white lesions about the size of a pin's head, which occur in association with rodent ulcer, benign cystic epithelioma, and in the scars of herpes, pemphigus, and Epidermolysis bullosa. These are not true milia but are due to epidermal cysts which have originated from some remnant of epidermis which has been included in the scar. To them the name pseudo-milia may be suitably applied. (Fig. 368.)

(3) True milium, in which the lesions are small, opaque, whitish or pearly bodies, smooth, rounded, and projecting, and vary in size from a mere speck to a pin's head or larger. They are usually discrete, but occasionally several may be aggregated together to form a small irregular whitish patch. They vary in consistence, sometimes being soft and difficult to feel, at other times being hard and almost stony. They are usually situated in the upper part of the face, especially about 
the forehead, eyelids, and cheeks, and may be few in number and attract little or no attention, or so numerous as to be a disfigurement. They grow slowly, persist indefinitely, and give rise to no subjective symptoms. They may be of congenital origin.

Sections of milia show that they are peculiar cyst-like structures, with walls formed by several layers of flattened epidermal cells, and contain a horny mass in loose concentric layers. The cyst is situated in the corium and, as a rule, is completely separated from the overlying epidermis by a thin layer of fibrous tissue.

There are several riews with regard to their origin, but the prevailing opinion is that they arise from the prickle-cell layer of the lanugo hair-follicle and that in certain cases they may be naevoid and may take their origin in embryonic cell-rests either of the epidermis or of hair-follicles.

Treatment.-When the lesions are sufficiently numerous to require treatment the epidermis over them should be incised and the horny cyst shelled out; this is a simple and effective procedure, the cyst coming away easily on the point of the scalpel as a small round white body.

\section{REEERENCES}

Robinsox. Observations on some cutaneous cyst formations (New York Med. Journ., I900, I 79, p. I I25).

\section{BENIGN CYSTIC EPITHELIOMA ${ }^{1}$}

The name "benign cystic epithelioma" has been employed to designate a group of small benign epithelial growths, most frequently met with about the face but occurring also on the neck and trunk. They consist of nodules, which are roundish or oval in shape, vary in size from a pin's head to a split-pea, are of a semi-translucent neutral or bluish tinge, and have a smooth shiny surface over which a small network of capillaries may sometimes be detected. These nodules are usually benign, grow slowly and, after reaching a certain size, persist indefinitely; occasionally they have been known to break down, become locally malignant, and to assume the characteristics of ordinary rodent ulcer to which they are closely allied in their histopathology.

A number of cases are on record in which round reddish tumours witl a shiny surface and varying in size from a pea to a round lobulated lesion like a medium-sized tomato have been observed on the scalp. These growths have a tendency to disappear spontaneously, hence the name "withering sarcoma" which has been applied to them. At one time the "tomato tumours" were believed to be endotheliomata

"The name "epithelioma" is used here in its wider and more correct sense to mean a tumour of the epithelium, and not in its more usual restricted sense of a malignant epithelial growth. 


\section{.}




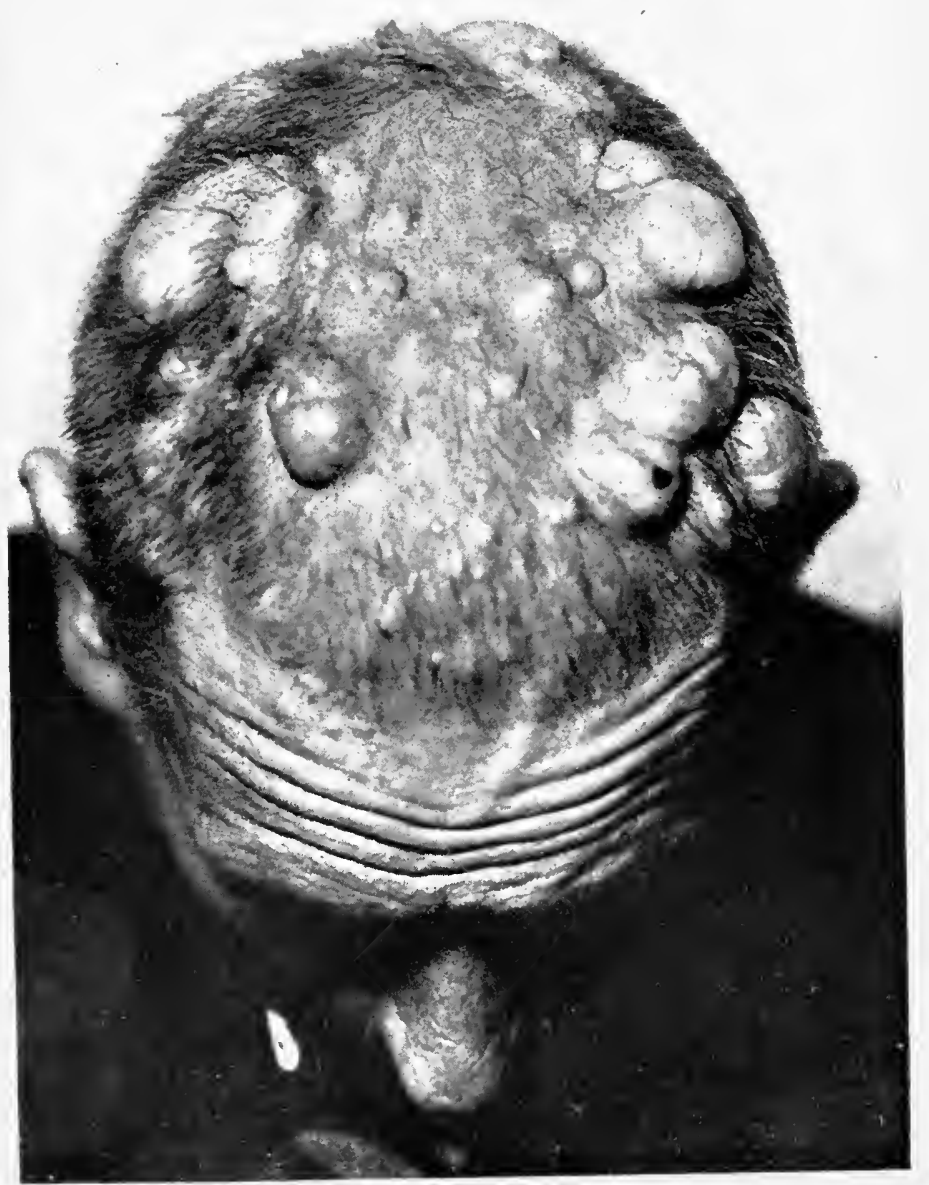

FIG. 369.-Multiple Benign Basal-cell Epithelioma of the Scalp. (Dr. H. G. Adamson's case. Brit. Journ. Derm., 1918.) 
but recent observations show that ther probably belong to the class benign cystic epitheliomata and consist of masses of cells of epithelial origin enclosed in capsules of fibrous tissue. They may occur in either sex. (Fig. 369.)

A microscopical examination shows that the tumour consists of a circumscribed epithelial downgrowth, limited bỹ a capsule of fibrous tissue, roughly suggesting an adenoma. The downgrowth is made up of branching finger-like projections, here and there dilated into crstlike structures which either contain a homogeneous colloid material or

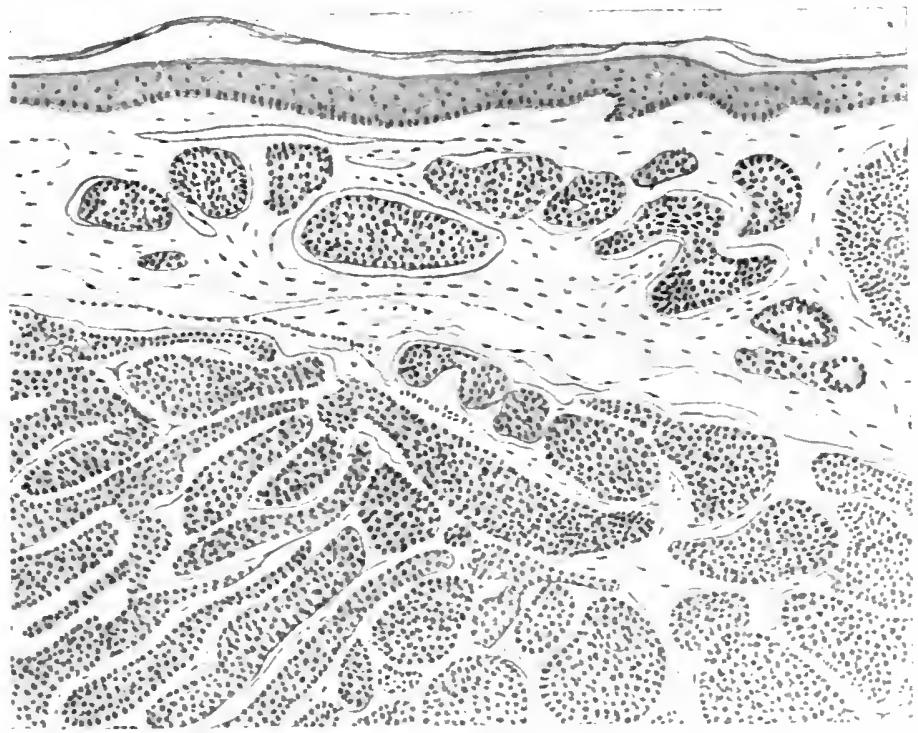

FIG. 37o.-Section of Muitiple Benign Basal-cell Epithelioma of the Scalp.

Shows group of rounded and oval cells chararteristic of basal-cell epitheliuma.

(After Dr. H. G. Adamson, Brit. Journ. Derm., I915.)

present concentric horny layers, like the horny cell-nests of malignant epithelioma growing from the prickle-cell layer of the skin.

They may take their origin from the epidermis itself, from the prickle-cell layer of the hair-follicle (external root-sheath), or possibly. from rudimentary sweat-follicles, and they differ in their histological detail according to whether they originate in the simplest form of epidermal cells, such as are met with in the basal layer of the epidermis or the external root-sheath, or from the more highly specialised cells of the prickle-cell layer. It seems probable that they are derived from groups of embryonic cells (cell-rests of Cohnheim) which, if they had fulfilled their developmental intention, would have developed into either epidermis, a pilo-sebaceous follicle, or a sweat-gland, and that they belong to the class naevi, and may be designated " naeri crstepitheliomatosi." Certain observers in the past have believed them to be mesoblastic and to originate in endothelium, but this view is no longer accepted. (Fig. 3\%o.) 
Considerable confusion exists with regard to these tumours, owing to the diversity of opinion as to their precise nature and the part of the epidermis from which they originate, and to the variety of names which have been applied to them, such as :

Adenoma of the sweat-glands (Perry).

Syringo-cystadenoma (Török, Unna).

Epithéliome kystique bénin (Jacquet).

Cylindroma of the skin (Nicolau).

Tricho-epithelioma papulosum multiplex (Jarisch).

Naevi epitheliaux kystiques (Besnier).

Haemangendothelioma cutis papulosum (Jarisch).

Epithelioma adenoides cysticum (Brooke).

\section{Epithelioma Adenoides Cysticum (Brooke)}

Under the heading of Epithelioma adenoides cysticum Brooke described in IS92 a series of cases which belong to the category of the

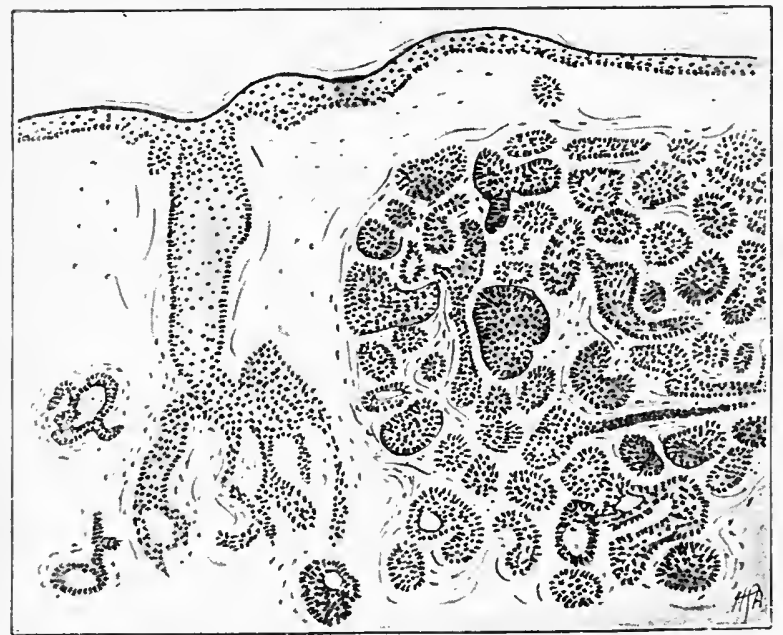

FIG. 37I.-Section of Epithelioma Adenoides Cysticum (Brooke).

Shows the cellular growth from the hair-follicle (Tricho-epithelioma).

(After Dr. H. G. Adamson. Brit. Journ. Derm., 1916.)

benign cystic epitheliomata. The lesions consisted of small shiny nodules, present chiefly on the face and distributed more or less symmetrically on the forehead, between the eyes, at the sides of the nose and on the naso-labial folds, auricles, temples, and chin, and occasionally on the scalp and neck. They were movable in the skin, firm to the touch, associated with no definite subjective symptoms, usually appeared first about puberty, increased slowly with occasional accelerations, and persisted indefinitely. Here and there minute milium-like bodies were detected in them, or a few milium-like lesions were dispersed among them 



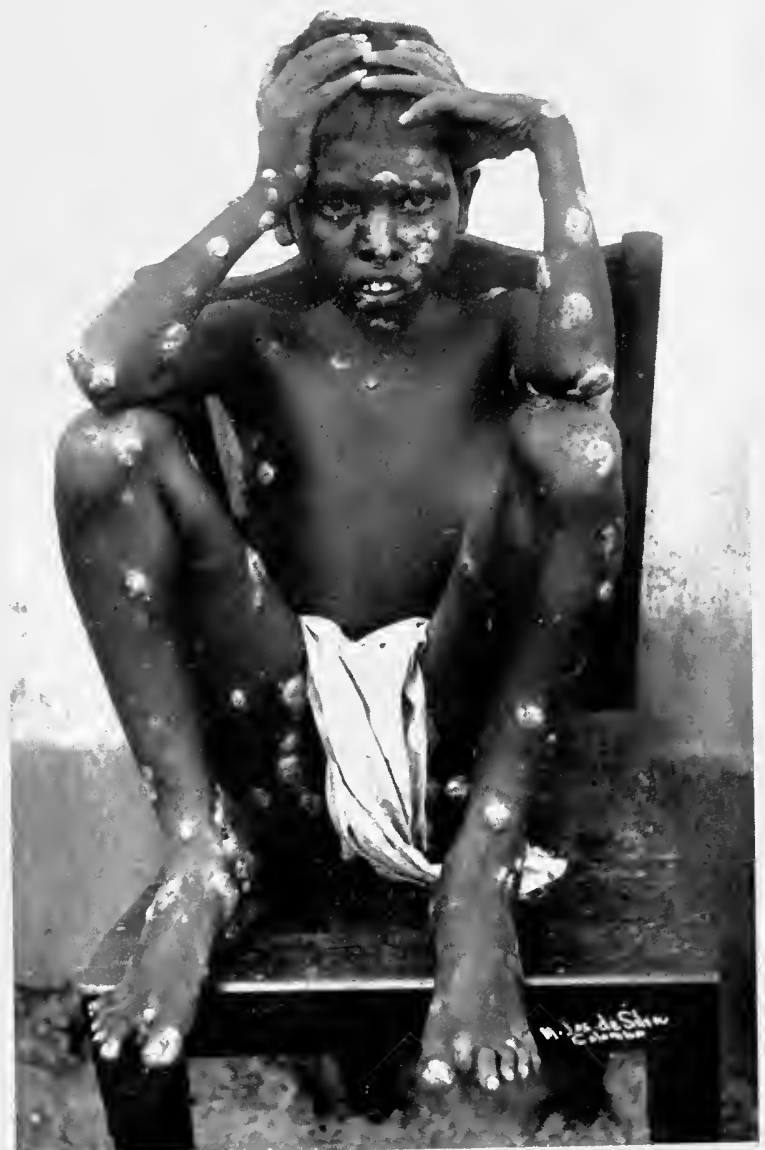

Fig. 409.-Yaws.

(From Dr. Aldo Castellani.)

[To face $p$. 1121 . 
The cases were almost exclusively in the female sex. In certain instances several members of a family were affected, and in one it occurred in a mother and daughter and was probably of congenital origin.

Histopathology.-The histopathology is similar to that described above, the finger-like epithelial processes of the growth being surrounded by a well-marked basal layer of regular columnar celis enclosing a mass of cells which have lost their fibrillated structure. Typical colloid cysts and horny pearls are present, and, in addition, small cysts containing sebaceous material which constitute the milium-like bodies.

The growths take their origin partly from the epidermis, and partly from the prickle-cell layer of the hair-follicle, as is evident by the occasional presence of lanugo hairs in the projections or coiled up in the cysts. (Fig. 37I.)

Cases of a somewhat allied type have been described, in which the lesions were confined to the trunk and showed a more marked tendency to come out in eruptive outbreaks. It is possible that in them the growth originated in embryonic sweat-glands (see hy-dradenoma).

Treatment.-The treatment is simple and consists of curetting, cauterisation, or the application of $\mathrm{X}$-rays. In the larger nodules exposure to the $\mathrm{X}$-rays should be preceded by curetting.

\section{REFERENCES}

Adansox, H. G. Epithelioma adenoides cysticum (Lancet, Oct. I 7 th I908, p. II33; Trans. Roy. Soc. Med., March I9I 4).

Brooke, H. G. Epithelioma adenoides cysticum (Brit. Journ. Derm., I 892 , iv. p. 269).

CsillaG, J. Contribution to the knowledge of Epithelioma adenoides cysticum (Arch. f. Derm. u. Syph., June I906, p. I63).

DoHI, S. Syringoma (Lymphangioma tuberosum multiplex Kaposi) (Arch. f. Derm. u. Syph., I907, lxxxviii.).

Dore, S. E. Multiple benign cystic epitheliomata (Brit. Journ. Derm., I9I 2 , xxiv. p. I90).

Fordyce, J. A. Multiple benign cystic epithelioma of the skin (Journ. Cut. \& Gen.-Urin. Dis., Dec. I 892).

Hartzell, M. B. Benign cystic epithelioma (Amer. Journ. Med. Sci., Sept. I902).

Benign cystic epithelioma (Brit. Journ. Derm., I904, xvi. p. 36I).

Litrle, E. G. Two cases of Epithelioma adenoides cristicum (Brit. Journ. Derm., I9I4, xxvi. p. I73).

Miller, J. W. Multiple benign cystic epithelioma (Journ. Cut. Dis., I9I5, xxxiii. p. $4^{62}$ ).

Nicolau, S. Cylindroma of the skin (Arch. de Med. exper. et Anat. path., Nov. I903, p. 796).

Perry, E. C. Adenoma of the sweat glands (Internat. Atlas of Rare Skir. Diseases, No. ix.).

PICK, W. On Epithelioma adenoides cysticum (Brooke) \& its relation to adenoma of the sebaceous glands (Arch. f. Derm. u. Syph., Oct.-Nov'. I90I, p. 20I). 
Robinson, A. R. Tricho-fibro-epithelioma (New Tork Med. Journ., June I909).

Sutton, R. L. Multiple benign cystic epithelioma \& Adenoma sebaceum in the negro (Journ. Cut. Dis., Sept. I9II).

\section{HYDRADENOMA}

Syn.: Adenoma of the sweat-glands or ducts; Syringadenoma; Syringoma ; Fr., Hydradénome éruptif.

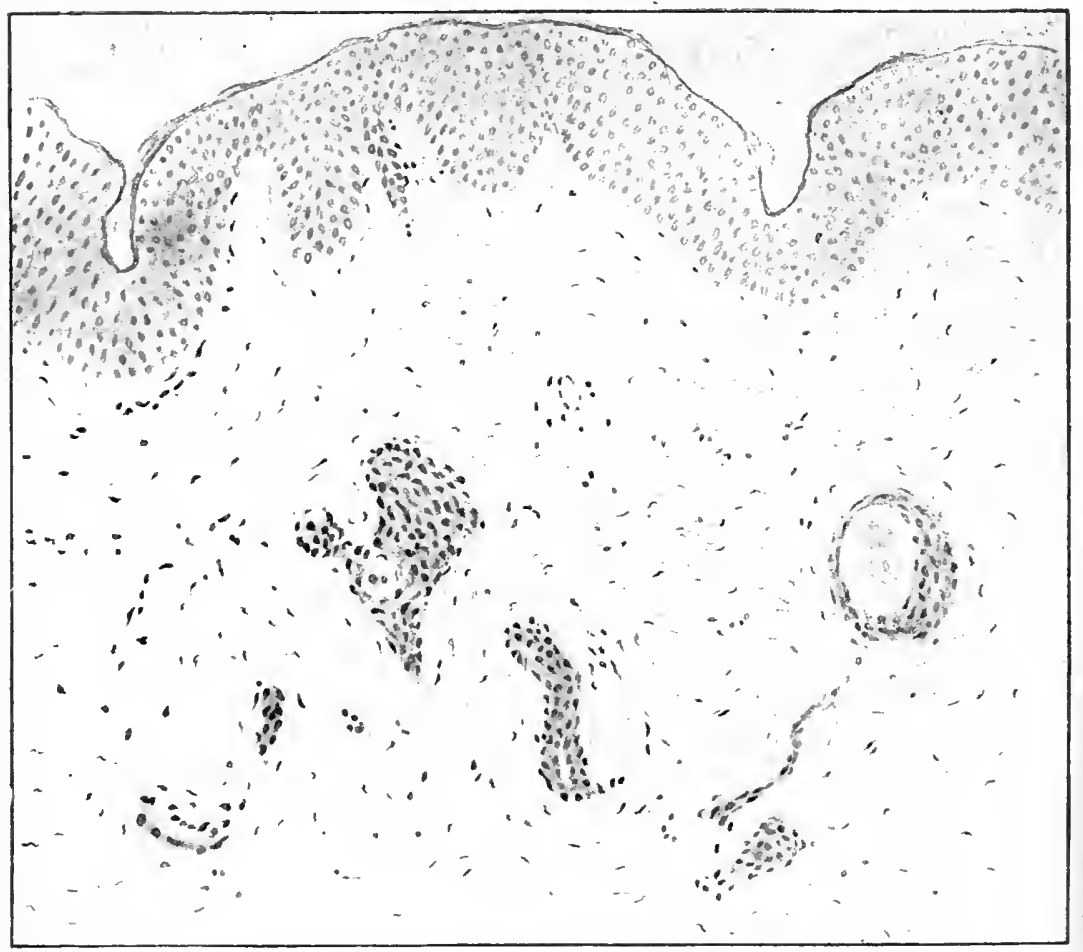

FIG. 372.-Section of Syringoma.

(Mr. McDonagh's case. Brit. Journ. Derm., IgI2.)

Hydradenomata consist of small nodules somewhat similar to those of Epithelioma adenoides cysticum but pinker in colour and sometimes surrounded by an inflammatory halo. They have been observed about the trunk, shoulders, and upper part of the arms, and tend to come out in crops.

Microscopically the tumour is made up of epithelial processes or tubes, which become dilated to form ampullae like those of the sweatcoils. Cysts lined with a layer of cubical epithelium and containing a colloid substance have also been described in connection with them.

The growths are probably of congenital origin and arise from embryonic cell-rests. Certain of the cases possibly belong to the 
group of the benign cystic epitheliomata, while in others the origin from the epithelium of the duct or coil has been more definite and the new-growth has simulated a sweat-coil. (Fig. 372.)

The treatment is the same as for benign cystic epithelioma.

Under the heading of "Adeno-cystoma intracanaliculare," Elliot described a peculiar case of Naerus unius lateris, which on microscopical section presented cystic cavities connected with the sweat-glands and ducts and lined with cubical epithelium.

A curious tumour of the face has also been described by Rolleston, which on microscopical examination showed a sweat-duct dilated in the form of a cyst within which was a definite papillomatous growth. The growth was about the size of a threepenny piece, was raised and slightly warty, and occurred on the left side of the face in a woman.

\section{REFERENCES}

BRaUss, T. Widely distributed sweat-gland adenoma with cystic formation (Arch. f. Derm. u. Syph., March I903, p. 347).

Eliot, G. T. Adeno-cystoma intracanaliculare occurring in a Naevus unius lateris (Journ. Cut. \& Gen.-Urin. Dis., May r893).

Rollestos, H. D. A dilated sweat-duct with intracystic papillomata (Brit. Journ. Derm., 1902, xiv. p. 83).

\section{ADENOMA OF THE SEBACEOUS GLANDS}

Sebaceous cysts, or atheromata, are frequently met with, especially in the scalp and scrotum. They are tense, painless nodules, from which inspissated sebaceous material can be squeezed, are easily enucleated, and are found to be composed of collections of sebaceous material and partially cornified epithelial cells. They were at one time regarded as retention cysts of the sebaceous glands but a more recent theory is that they are of embryonic origin and due to the growth of epidermal cellular inclusions which form sebaceous material.

Small sebaceous cysts about the size of pins'-heads are not infrequently present on the rulva and penis and occasionally, in infants, on the face (Strophulus albidus of Willan). These have been confused with milium and included in that group (see p. III $/$ ), but they are really retention cysts forming in connection with sebaceous glands and containing sebaceous material and the debris of epidermal cells. In them the sebaceous duct is dilated, and although there is usually an opening at the surface this may be plugged with debris.

Small isolated tumours rather smaller than a pea have been described which result from hypertrophy of the sebaceous glands, but these are exceedingly rare. 


\section{ADENOMA SEBACEUM}

Syn. : Naevi vasculaires et papillaires (Vidal); Naevus sebaceus.

Definition: A rare naevoid affection of the skin, characterised by pin's-head-sized yellowish or reddish nodules situated usually in the central two-thirds of the face, and generally associated with other congenital anomalies.

Description.--This affection is of congenital origin, may appear at birth, during the first few years of life, about puberty, or, in rare instances, not until early adult life. In a typical case such as that described by Pringle in I 890 the lesions consist of small rounded or oval nodules, the size of a pin's-head or slightly larger, varying in tint from yellowish-white to vivid red or reddish-brown, sometimes waxy at other times translucent, and with a smooth convex surface. Over the paler lesions is a fine network of capillaries which, when in excess, gives rise to the vivid red tint and masks the yellow which is the natural hue of the lesions. The nodules are confined to the face and are markedly symmetrical and constant in their distribution; they occur chiefly on the cheek, sides of the nose, and naso-labial folds, irregularly dotted over the forehead and chin, and occasionally on the scalp. In the naso-labial folds they are usually so profuse and contiguous as to coalesce to form irregular patches. (Plate XIII.)

In addition, independent telangiectases may be present about the nose and cheeks, and various forms of naevoid growths, such as soft moles, pigmented patches, and Fibroma molluscum, may be met with on the cutaneous surface, while in rare instances congenital anomalies, such as a supernumerary nipple, have been observed. In a certain number of the cases the skin has presented a peculiarly coarse texture, with dilated pilo-sebaceous follicles which were sometimes blocked with large comedones; these may occur singly or in clusters, as in a case reported by the writer where there was a small patch of inflamed follicles plugged with comedones situated over the lumbar spine.

The majority of the cases have occurred in mentally deficient or epileptic children and have been met with chiefly in imbecile asylums; occasionally there has been no mental disturbance.

The affection may be hereditary and instances are on record where it occurred in several members of a family.

The lesions usually increase in number during childhood, and a definite exacerbation sometimes takes place about puberty. After a varying time the condition remains stationary and tends to persist indefinitely, though occasionally individual lesions may involute spontaneously with or without atrophic scarring.

In addition to the typical cases in which the lesions are confined to the face, others have been recorded in which the nodules were distributed irregularly and asymmetrically over the surface of the skin, varied in size from a hempseed to a split-pea or larger, and presented a whitish-yellow translucent appearance. 


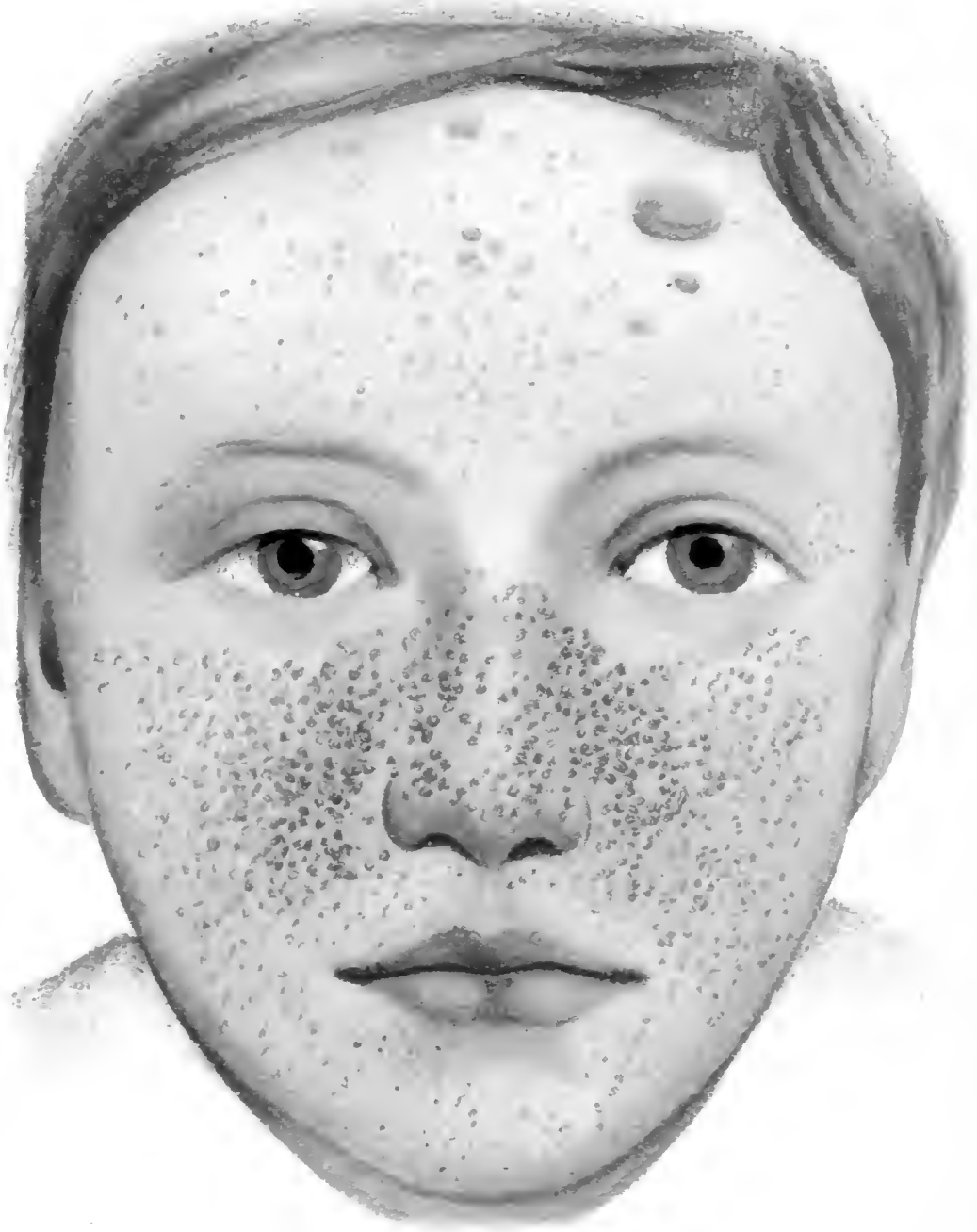

AOENOMA SEBAOELY.

Shows soft naevi or mules on torehead and sma!l angiomatit in centlal ar*a of face. 

Histopathology.- Sections of a yellowish waxy lesion show that the growth is a hyperplasia of sebaceous glands, while in the red lesions this is associated with a more or less marked formation of capillaries with, in some cases, an increase in fibrous tissue, proving that the lesions are mixed sebaceous and rascular naevi. The sections also show that the growth is not clearly defined, as in Molluscum contagiosum, but that the abnormal tissue spreads out laterally in an indefinite manner.

In addition to the sebaceous gland tissue Crocker noted hyperplasia of the sweat-glands and designated the lesions in consequence "pilo-sebaceous hydradenomata."

Diagnosis.-Adenoma sebaceum has to be distinguished chiefly from colloid milium, the small nodules of which may present a close resemblance to the yellowish translucent lesions. In colloid milium, however, the lesions are much less numerous, are not associated with telangiectases, other forms of naeroid growth, or mental deficiency, and are distributed mainly about the forehead and orbit and not so much in the central area of the face.

It may also present a superficial resemblance to rosacea, as small red papules and telangiectases occur in both affections and the distribution is similar; in rosacea the red papules tend to suppurate and are associated with flushing of the face and digestive derangements.

Treatment.-Like any other form of naevus, it is purely local, and the only treatment either external or internal which has any effect is the actual destruction of the lesions. This can be done most satisfactorily in small nodules by electrolysis or a fine-pointed Paquelin cautery, and in the larger naevoid growths by refrigeration with solid carbon dioxide. The degree of scarring from the treatment is dependent on the skill and judgment of the operator.

\section{REFERENCES}

AdAsox, H. G. Adenoma sebaceum in mother and son (Brit. Journ. Derm., I9I I, xxiii. p. I09).

Buschke. On Adenoma sebaceum (Derm. Zeitschr., Bd. xi. Hft. p. +67$)$.

Jamesor, W. A., and Huie, L. H. Molluscum fibrosum pendulum of Adenoma sebaceum (Brit. Journ. Derm., I906, xriii. p. 379).

Korнe, R. To our knowledge of the tumours of the sebaceous glands (Arch. f. Derm. u. Sỵph., I904, pp. 33 \& 359).

Little, E. G. Adenoma sebaceum (Brit. Journ. Derm., Igog, xxi. p. $327)$.

MAcLeod, J. M. H. Adenoma sebaceum (Brit. Journ. Derm., Igo6, xviii. p. 2I 8).

Poòr, F. Clinical characters \& anatomy of so-called Adenoma sebaceum (Monats. f. prakt. Derm., April i905, p. 379).

Pringle, J. J. Adenoma sebaceum (Brit. Journ. Derm., i 890 , ii. p. I).

TAYLOR, G. G. S., \& BARENDT, F. H. Three cases of Adenoma sebaceum in one family (Brit. Journ. Derm., I893, r. p. 360 ). 


\section{CORIUM}

\section{FIBROMATA}

Fibromata are comparatively rare in the skin; they are benign neoplasms consisting of white fibrous tissue or collagen, usually take their origin from the perineurium or interstitial tissue of the peripheral nerves, and are now generally designated " neuro-fibromata."

Apart from the neuro-fibromata, fibrous growths in the skin occasionally develop from the connective tissue fibres of the corium itself, such as the fibrous thickenings sometimes met with in Adenoma sebaceum of congenital origin. Under this heading in its wider sense may also be included the fibrous hypertrophy in cheloids and hypertrophic scars.

The fibromata appear either as pinkish or whitish nodules or as flat thickenings, and are usually smooth and shiny on the surface.

Microscopical sections show that they are made up of thickened collagen bundles which, by interlacing irregularly with each other and forming a dense fibrous mass, push aside the capillaries and pilosebaceous follicles and cause them to atrophy. In the early stages of the growth delicate new collagen fibres and numbers of young round, oval, or polygonal connective tissue cells (see p. I3) may be detected and the lesion is softer to the touch than in the later stages when the fibres have fully developed.

Neuro-fibromata.-The neuro-fibromata are of two kinds, (I) superficial cutaneous lesions, and (2) more deeply-seated growths connected with a peripheral nerve either in the corium or subcutaneous tissue.

Superficial Cutaneous Neuro-Fibromata.-These growths vary in size from a hempseed to an orange or larger and are generally known as Fibroma molluscum or Fibroma simplex. At first they are sessile, but as they increase in size they usually become pedunculated and flabby and tend to fall down in the form of pendulous baggy lesions, sometimes weighing several pounds (Fibroma pendulum), which may be superimposed one upon another like the flounces on a lady's dress. As a rule they have a gelatinous feeling, the sessile lesions becoming easily invaginated on pressure as if the contents had been pushed down into the subcutaneous tissue, while the larger pendulous growths can be rolled between the fingers. The skin over the growths may be normal in tint, but more often assumes a violaceous or brownish hue from capillary congestion; at the same time it is usually altered in texture, tending to become coarse and irregularly covered with dilated pilo-sebaceous follicles plugged with comedones. Beneath the pendulous masses there may be inflammation from friction and the action of micro-organisms, and the skin may become red, moist, fissured, and sometimes painful. (Fig. 373.) 


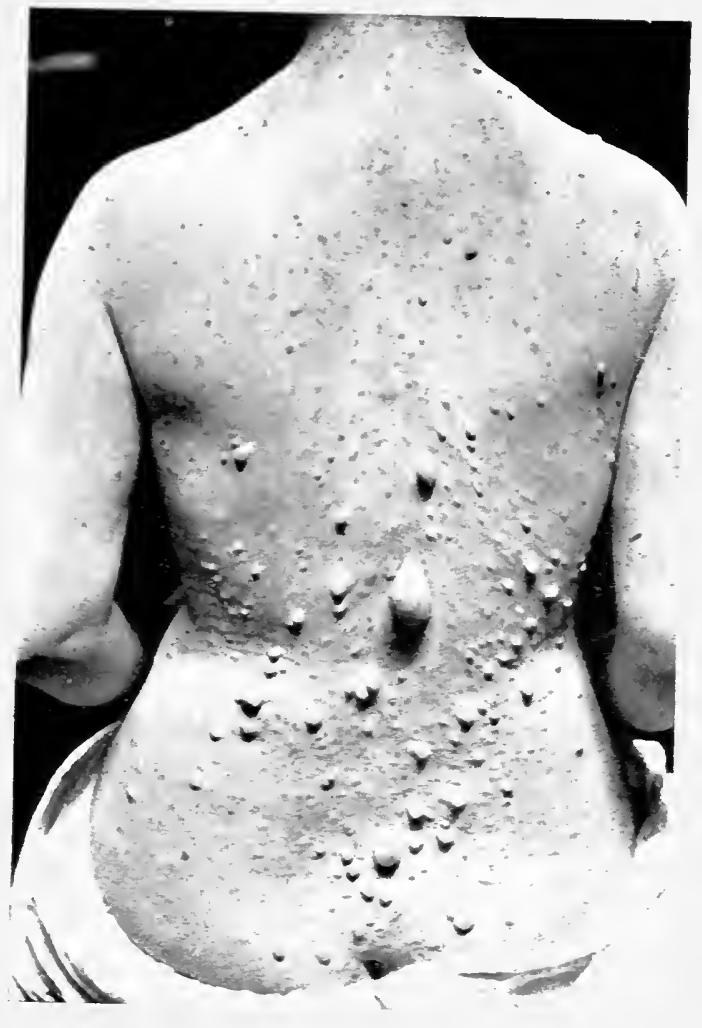

FIG. 373.-Fibroma Molluscum.

(Dr. Wallace Beatty's case. Photo bry Mr. J. Manby.)

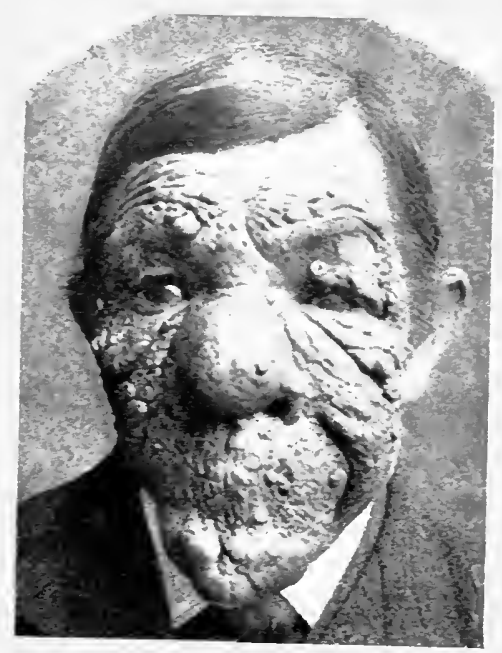

FIG. 374.-Fibroma Molluscum. (From Dr. H. C. G. Baron. 

The lesions are multiple, as a rule, and may be few and sparsely distributed, or numerous, and sometimes universal. In distribution they are usually irregular but in rare instances may have a linear arrangement from having formed along the course of a peripheral nerve. They are most common about the face, neck, and shoulders, but may be present in varying numbers on the trunk, scalp, and limbs, while in one or two instances peculiar thickenings have been described in the mucous membrane of the mouth which were believed to be of the same nature. (Fig. 374.)

There is no pain or other subjective symptom associated with them unless fron dermatitis beneath the pendulous growths.

The lesions are of congenital origin and belong to the group of naevi; they may be met with in several members of a family and are sometimes hereditary. They are seldom present at birth but usually appear in childhood, especially about puberty, and tend to increase in size and number as age adrances. In some instances, however, they do not make their appearance till comparatively: late in life. As a rule they are permanent but occasionally have been known to disappear, either spontaneously or as the result of ulceration or sloughing from the action of septic micro-organisms which have gained entrance through some

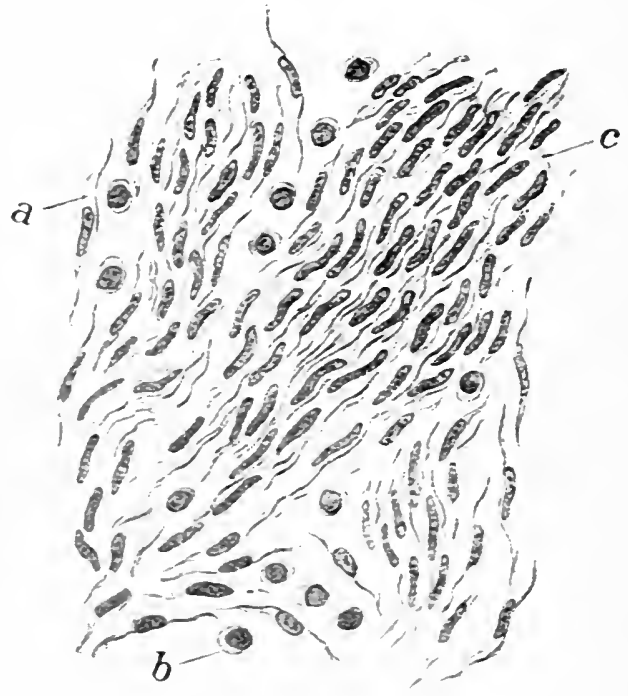

FIG. 375.-Neuro-fibroma Cells. ( $x$ about I ooo.)

a. Spindle-shaped connective tissue cell. b. Round and polygonal cells. c. Mlass of spindle cells.

abrasion. Sometimes the molluscous growths are associated with other congenital anomalies, such as vascular naevi, warty naevi, hairy moles, and bony deformities. Ther mav occur in all races, but are said to be especially common among the Chinese.

Neuro-Fibromata connected with Peripheral Veries.-In them the fibrous growths may occur as nodules along a peripheral nerve like the beads of a rosary, or, especially about the face and head, they may have a plexiform arrangement owing to a general thickening of the fibrous tissue of the nerves of some small plexus (Elephantiasis nervorum). Occasionally the enclosure within the growth of sensory nerve twigs or the stretching over it of nerve fibrils, may give rise to pain of so severe and paroxysmal a character as to justify the synonym "Tubercula dolorosa." 
Histopathology.-Microscopical sections of the molluscous growths in their various stages show a fairly constant histology. The soft gelatinous material so characteristic of them consists of a fine connective tissue stroma of new fibres of collagen, between which are a mucoid substance and a cellular deposit of oval or spindle connective tissue cells and mast-cells. Here and there fine nerve fibrils may be detected surrounded by oval or spindle-shaped connective tissue cells, pointing to the origin of the growth in the connective tissue of the nerve. Towards the periphery of the lesions the stroma becomes much more profuse and the cellular deposit decreases. (Fig. 375.)

Treatment.-The treatment is surgical and consists of excision, or of ligature in the case of growths with a narrow pedicle. In the large pendulous growths, vessels of a considerable size enter at the pedicle and these should be tied before they are cut in order to avoid unnecessary haemorrhage.

Internal treatment makes no impression on the lesions but benefit has been claimed from injections of fibrolysin.

\section{Von Rechlinghausen's Disease, or Neuro- Fibromatosis}

In I882 von Recklinghausen wrote an important paper on multiple fibromata of the skin in which he co-ordinated a triad of symptoms which occur so constantly together as to suggest that they form a distinct type of disease. This triad consists of :

(I) Neuro-fibromata of the skin, in the form of molluscous growths of the sessile or pendulous type.

(2) Neuro-fibromata, either nodular or plexiform, connected with the peripheral nerves.

(3) Pigmentation, in the form of freckle-like lesions, varying in tint from fawn to sepia brown, and irregular patches of a café-au-lait colour. (Fig. 376.)

In addition to the typical cases in which all three symptoms are present a certain number of incomplete cases has been recorded in which one or two of them were absent. For example, there may be molluscous growths of the skin with no definite neuro-fibromata of the nerves or pigmentation ; or the freckles, which are really pigmented naevi, may be the only sign of the disease.

Sometimes the pigmented lesions are noticed at birth or soon afterwards, while the neuro-fibromata may not develop for a number of years.

As a rule, in cases of neuro-fibromatosis the intelligence of the patient is below the average and there may be defective physical development.

\section{REFERENCES}

Bagshawe, F. Case of multiple skin tumours (false neuromata) (Brit. Journ. Derm., I893, v. p. 307). 


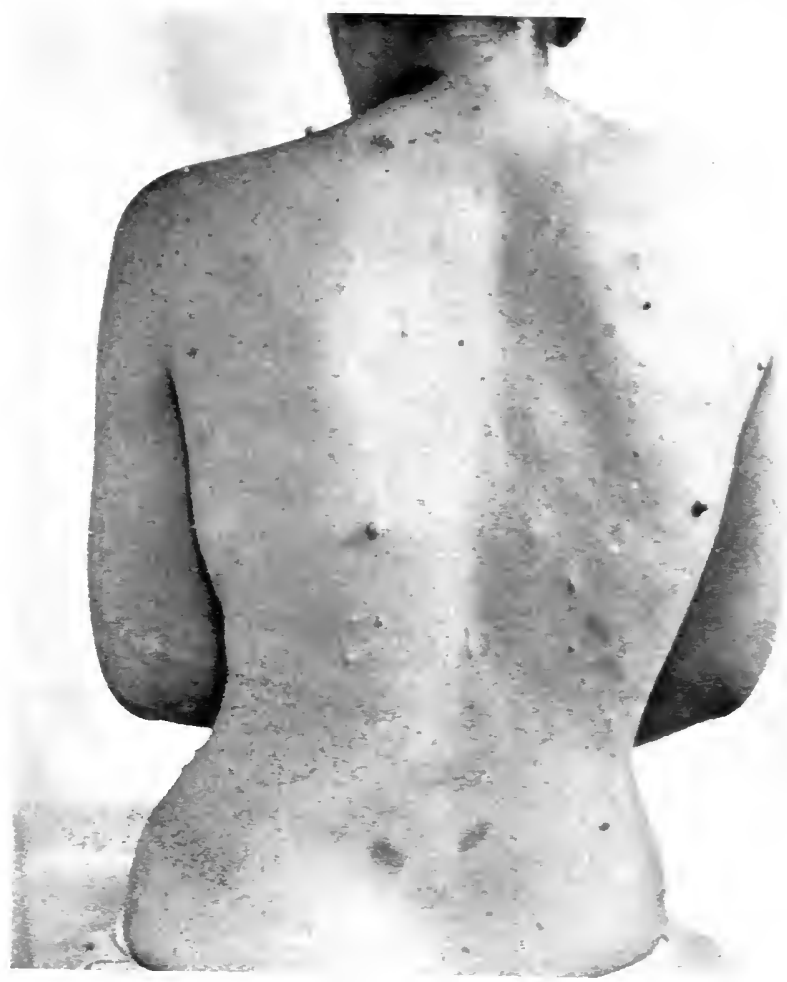

FIG. 3,-6.-Ton Recklinghausen's Disease.

Shows neuro-fibromata and pigmented patches.

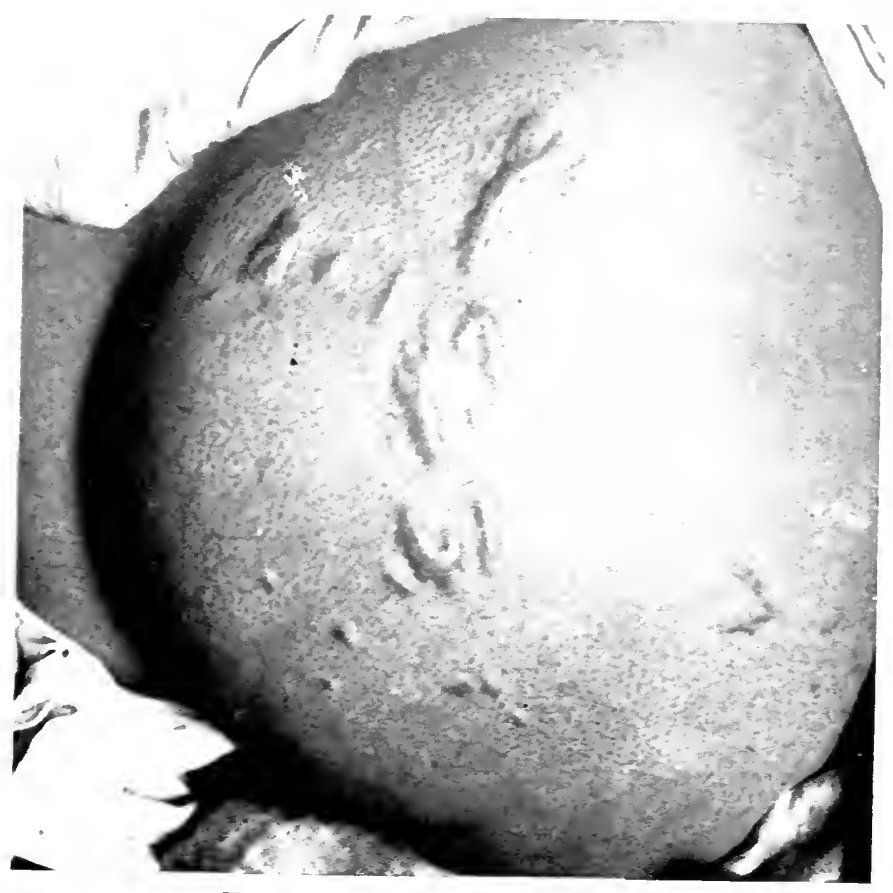

FIG. 37\%-Cheloids on Buttock.

(From Dr. Arthur Hall.) 

Gallowar, J. Development of Molluscum fibrosum (Brit. Journ. Derm., I 896 , viii. p. 27\%).

Thomsor, A. Neuroma in neuro-fibromatosis (Edinburgh, Ig,oo).

Vox Recklisghacsex. Leber die multiplen Fibrome der Haut (Berlin. I 882 ).

WEBER, F. PARkes. Cutaneous pigmentation as an incomplete form of Rechlinghausen's Disease, etc. (Brit. Journ. Derm., Ig09, xxi. p. 49).

\section{DERMATOLYSIS}

Sy'n.: Elastic skin.

Dermatolysis is a name which has been applied to skin which is loosely attached to the subcutaneous tissue and can be pulled for a considerable distance in various directions. The condition may be congenital or acquired, - -as where it occurs in the skin of the abdomen or about the female breasts after parturition or lactation,-and is largely due to the absence of a perfect connective tissue stroma and its replacement by delicate fibres of collagen to form a network in the meshes of which there mar be fluid.

\section{CHELOID}

Syn. : Cheloid of Alibert; Fr. : Cheloide; Cancroïde.

Definition: Cheloids are variously sized elevations of the shin which are irregular in shape and not infrequently elongated with claw-like prolongations, firm in consistence, smooth on the surface, and due to a new growth of fibro-cellular tissue in the corium.

Introduction.-The name Cheloid from $x \eta \lambda \eta$ a claw was applied to these growths by Alibert about ISI5. He described two trpes, namely "true or idiopathic cheloid" which he believed to be spontaneous, and "false or scar cheloid" which he regarded as being the result of some definite canse such as an abrasion or other injury. This distinction was adhered to until comparatively recently; but at the present time it is generally considered that there is no real difference between them and that in every instance the cheloid is due to some local injury, or possibly to the action of some micro-organism in a susceptible individual.

The cheloid of Alibert has to be differentiated from the heloid of Addison (from knis a blemish), which name was applied by him to what is now known as sclerodermia.

Cheloids have to be distinguished from ordinary hypertrophic scars, the principal point of distinction being that in the hypertrophic scar the hypertrophy is limited to the original scarred area, while in the cheloid the fibrous growth spreads beyond it and invades the surrounding tissue.

Description.-The cheloid appears first as a pinkish or reddish elevation, roundish or oral in shape, and smooth and glistening on the 
surface. This increases slowly and may assume a variety of shapes, forming a band, a cord, or an irregular flat elevation the surface of which may be even and convex, flat, or concave, or uneven and nodular. Cheloids tend to spread by sending out projections into the neighbouring tissue and where several small ones have coalesced they may form irregular figures enclosing areas of unaffected skin. (Fig. 377.)

The most characteristic type is a raised band at the end of which are claw-like projections, simple or branched, which spread out and anchor the cheloid to the adjoining skin. The surface is even, or occasionally intersected by cords which stretch longitudinally from the projections on one side to those on the other.

When fully developed the growth is invariably well-defined with sloping, vertical, or occasionally overhanging, edges. In colour it is generally pinkish or pearly white, but may be purple or brownish, and when actively growing is not infrequently surrounded by a reddish inflammatory halo. Over the surface, and especially near the margin, branching blood-vessels may be detected and in rare instances the vascularity may be so marked as to suggest erectile tissue. In consistence it may be tough like india-rubber or hard and cartilaginous, and is firmly situated in the skin though not attached to the underlying structures.

There may be no subjective symptoms, but not infrequently there is aching and occasionally actual pain.

As a rule only one or two are present, though sometimes they may be in considerable numbers, especially where they have developed on the scars of acne or smallpox.

They may occur anywhere on the cutaneous surface, are most common over the sternum, and comparatively rare on the face and extremities. They grow slowly and after months or years reach a certain size when they tend to remain stationary for an indefinite period. Occasionally they may involute spontaneously, becoming soft, flabby, and wrinkled, and finally disappearing. In rare instances they may ulcerate and have been known to become malignant and to develop into sarcomata.

Histopathology.-The cheloid is the clinical expression of a newgrowth of fibrous tissue in the corium. Sections of a lesion show a fibrous mass occupying the sub-papillary and reticular layers. In the centre of the mass are long coarse bundles of collagen lying parallel with the surface or here and there arranged vertically, while between the bundles are numerous elongated connective tissue cells. Towards the edge of the mass the collagen may show a tendency to form a capsule round the central portion. The hair-follicles, sebaceous glands, sweat-ducts, and coil-glands, are pressed aside by it, and in the mass itself the blood-vessels may be completely absent while beyond it there may be a loose new connective tissue formation and the blood-vessels may be unusually large. 
Around the capillaries a deposit of round or oval connective tissue cells has often been observed, with occasionally a few plasma-cells and mast-cells, which points to a chronic inflammatory process. On this account it has been suggested that the claw-like prolongations have their shape determined by fibrous tissue forming round small bloodvessels. These vascular changes may be observed for some distance beyond the main mass, which explains the tendency to recurrence after removal should the excision not have been sufficiently free.

The elastic tissue is usually absent and it is believed that the lack of the scaffolding afforded by it may be partly responsible for the hypertrophic fibrous growth.

The epidermis may be normal or may be involved secondarily. Where the pressure of the underlying mass is considerable it may cause flattening of the inter-papillary processes and stretching of the prickle-cell and horny layers.

Etiology.-The precise cause is uncertain. It is now generally believed that there is no such thing as a spontaneous cheloid and that each is the result of some injury to the skin, though it is admitted that there is some peculiar idiosyncrasy which renders the skin prone to react in this fashion.

In one or two instances they have occurred as a hereditary peculiarity and have been observed in more than one member of a family. They would appear to attack certain races more than others, especially those with dark skins. Some tribes are proud of this susceptibility and are in the habit of adorning their bodies with cheloidal growths and hypertrophic scars - which are produced artificially by lacerating the skin with a sharp stick impregnated with mud or with the juice of some irritating plant-in much the same way as other tribes decorate themselves with paint.

They may follow injuries to the skin caused by an endless variety of agents. Sometimes the injury may be so slight as to pass almost unnoticed, such as a pin-prick or the bite of an insect; at other times it may be severe, such as wounds, stitches, burns, ulcerations, tattoomarks, or excoriations of the skin from scratching or from digging out urticarial papules with the finger-nail. In one or two instances symmetrical cheloids have been known to follow piercing the lobes of the ears for ear-rings, pnssibly from microbic infection.

They may occur in the healing of ulcerating infective processes, such as Lupus rulgaris and late cutaneous syphilides, and may succeed furuncles, lesions of Acne rulgaris, and smallpox, but in these cases they are more often hypertrophic scars than true cheloids.

In several instances Seborrhoic dermatitis has been known to be a precursor of cheloid, probably from the scratching of the skin for the relief of the itching and from the inoculation of some organism which flourished in the greasy surface. It has been suggested that Seborrhoic dermatitis is responsible for cheloids occurring over the sternum, as the sternal region is the most common site for Seborrhoea corporis. 
Diagnosis.-Cheloids, as a rule, are easily recognised; they have to be differentiated from hypertrophic scars but, as has already been pointed out, the hypertrophic scar is not prolonged into the surrounding tissue but is confined to the original healed area.

Prognosis.-They tend to persist indefinitely, rarely disappear spontaneously, and are singularly resistant to treatment.

\section{TREATMENT}

The most efficacious method of dealing with them is by radium, and by it not only can the irritation of the cheloid be removed but the growth may be caused to involute to an appreciable degree, if not completely, without subsequent scarring. A full-strength radium plate is used, screened with I mm. silver, and an exposure of I8 to 30 hours employed, spread over 3 to 5 days, according to the thickness and size of the lesion.

Somewhat similar, though less brilliant results, may be obtained by judicious employment of the X-rays. Doses of three-quarters of a pastille dose are given at intervals of not less than a fortnight, the three-quarter tint in the pastille being estimated by using a radiometer.

Finsen Light has also been recommended and, as a result of the inflammatory disturbance caused by it, a gradual absorption of the cheloid sometimes takes place. This method, however, is much less effective than either radium or X-rays.

Excision has been tried in a number of cases but the results have been disappointing, as the cheloid is liable to recur and may even assume greater proportions than before.

A surgical procedure which has given favourable results in careful hands is that of multiple scarification, followed by the rubbing in of some strong irritating ointment such as a $5 \%$ resorcin ointment. This excites a strong inflammatory action and inay cause the resolution of the cheloid.

Various plasters containing salicylic acid, ichthyol, soap plaster, etc., have been employed and occasionally have caused improvement, but any benefit which may be derived from their use is dependent on the degree of inflammatory reaction they set up and this can be produced more effectively by Finsen Light.

Applications of carbon dioxide snow are of value in destroying small cheloids but, in order to prevent the disfiguring depressed scar which is apt to result, great care must be taken that the exposure is not too long or the pressure too great.

Another method of treatment for small cheloids is by electrolysis. By a current of about 5 milliamperes and the needle pushed in at the margins of the lesion the dilated blood-vessels may be obliterated, the spread of the cheloid prevented, and the lesion reduced, but there is a risk of new cheloids forming at the punctures. 
Some years ago injections of thiosinamin in the neighbourhood of the lesion were strongly advocated and were said to cause diminution of the cheloid. The following solution was employed: thiosinamin $\$$ grains, glycerin $20 \mathrm{~m}$., distilled water 2 drachms. This was gently heated until the solution cleared and ro $\mathrm{m}$. injected once or twice a week. The results from it are uncertain and disappointing. The chief objection to its use is the extreme pain associated with it; to obviate this a modification of thiosinamin, known as fibrolysin, has been introduced which is less painful but is also less active.

Internal treatment has no influence on cheloids; certain observers claim that thyroid has a specific action on them, but this is not the usual experience.

\section{HYPERTROPHIC SCARS}

Hypertrophic scars differ from cheloids in that the hypertrophy does not spread into the surrounding tissues but is limited to the scarred area. They may occur after any abrasion or injury of the cutaneous surface, or may be superimposed on the scars of ulcerative conditions, such as syphilis, tuberculosis, burns, boils, etc. Hypertrophic scars, especially those after burns, are much more transitory than cheloids and may undergo spontaneous healing.

They develop on granulation tissue but their exact cause is uncertain. It seems most likely that they result from some secondary contamination, possibly from the inoculation of some coccus.

Histologically their structure is similar to that of the cheloid, but the collagen bundles are less densely packed and there is not the same tendency to the formation of a fibrous capsule.

Treatment, when called for, is chiefly for cosmetic reasons and to reduce contractures, and is similar to that for cheloids.

\section{Pitted Scars}

Pitted scars such as occur after small-pox not infrequently demand treatment. They may be reduced by scarification and rubbing in a $2^{\circ}$ ointment of resorcin, by peeling the skin with strong resorcin paste, or by careful refrigeration with solid carbon dioxide.

\section{REFERENCES}

Alibert (Monographie des Dermatoses, Ed. 2, I\$35; Précis théorique et pratique sur les maladies de la peau, I860).

ARLexov, M. A. On spontaneous \& scar keloid (Russ. Journ. of Skin d Ven. Dis., 1902).

Balzer \& Mousseatx (Annales, r898, p. 454).

Block (Journ. Cut. Dis., I895, p. Io7).

Crocker, RadCliffe (Brit. Journ. Derm., I899, p. 297).

Crocher, Radcliffe, \& Pernet, G. (Brit. Journ. Derm., IS99, p. 43I).

HaRris, G. H. Removal of hypertrophied scar tissue by means of the Röntgen Raỹs (Australian Med. Gaz., April I9or, p. 133). 
Hutchinson (Edin. Med. Journ., r897, xliii. p. 5).

Joseph, M. (Arch. f. Derm. u. Syph., r899, p. 277).

Kirsch, H. Two cases of spontaneous keloid (Arch. f. Derm. u. Syph., 1906, p. 255).

Krzysztalowicz, F. A contribution to the knowledge of the pathogenesis of keloid (Monats. f. prakt. Derm., I909, p. 38r).

Laurence (Brit. Med. Journ., I 898, ii. p. I5r).

MACLEOD, J. M. H. Cheloids from piercing ears (Brit. Journ. Derm., I9I 2 , xxiv. p. 316).

Rerss. On spontaneous multiple keloids (Arch. f. Derm. u. Syph., I9or, p. 323).

SснӥTz, J. A case of so-called true cheloid combined with scar-cheloid (Arch. f. Derm. u. Syph., xxix. Hft. I).

UNNA (The histopathology of diseases of the skin, I896).

\section{ELASTIC TISSUE TUMOUR}

Syn. : Xanthoma elasticum (Balzer); Pseudo-xanthoma elasticum (Darier).

In I884, under the heading of Xanthoma elasticum, Balzer described a condition which he regarded as a peculiar form of xanthoma, in which the elastic tissue was swollen, degenerated, and broken up into irregular fragments. Subsequent observations of similar cases by Darier and others have proved that the condition is distinct from xanthoma and due entirely to elastic tissue changes.

The affection is exceedingly rare, and only about half-a-dozen cases have been fully described in the literature, but as a firm diagnosis is impossible without a microscopical examination and the condition gives rise to no subjective symptoms a certain number of cases have doubtless been missed.

The lesions consist of flat papules or nodules, slightly raised above the level of the skin, varying in size from a hempseed to a pea, round or oval in outline, fawn or yellowish in colour, soft in consistence, and resembling small masses of butter embedded in the corium with the overlying epidermis unaffected. They tend to be arranged in clusters or in a linear fashion, and are either discrete or coalesce to form irregular nodules. They have been found chiefly on the front of the abdomen, sternal region, sides of the neck, flexor aspects of the limbs, and about the flexures of the larger joints.

The time of onset has varied in the different cases and it has been known to begin in infancy, about puberty, and in early and middle adult life. It has been recorded in several members of a family suggesting that it may be a congenital anomaly.

The cause is unknown; in four of the cases the paticnts suffered from pulmonary tuberculosis but this may have been a coincidence.

Microscopical sections show that the nodulc is due to the presence of a mass of swollen and fragmented elastic tissue fibres situated in the reticular part of the corium, which gives the staining reaction either of healthy clastin or of its degenerative product elacin. In 
several cases giant-cells have been observed in which the nuclei were not arranged in a ring or horse-shoe fashion, as in the giant-cells of tuberculosis, but formed a cluster within a mass of faintly stained protoplasm. It has been suggested that these cells are definitely related to the elastic tissue changes and may be responsible for the destruction of the elastic fibres.

\section{REFERENCES}

Balzer. Xanthoma elasticum (Arch. de Physiol., I884, p. 65).

Bodis, E. Pseudo-xanthoma elasticum (Ann. de Derm. et de Syph., Oct. I900, P. I073).

D̈̈BENDORFER, E. Pseudo-xanthoma elasticum \& colloid degeneration in scars (Arch. f. Derm. u. Syph., Feb. I903, lxiv. p. I75) .

Litile, E. G. Pseudo-xanthoma elasticum (Brit. Journ. Derm., I908, $\mathrm{xx}$. p. 194).

Werther. Pseudo-xanthoma elasticum (Arch. f. Derm. u. Syph., March I90.4, P. 23).

\section{COLLOID MILIUM}

Syn. : Colloid degeneration of the skin.

Definition: A peculiar affection of the skin, due to colloid degeneration in the corium, and characterised by the presence of yellowish translucent nodules which are usually situated in the upper part of the face.

This condition was described first in I866 by Wagner under the title of colloid milium. It is rare, only about 25 cases having been reported, but probably occurs more frequently than the records would suggest as the condition is not well known and mild degrees of it may be missed. On the other hand a certain number of cases reported as colloid milium were possibly pseudo-milium, such as occurs in the scars of pemphigus, Acne agminata, or herpes.

At first sight the characteristic lesions suggest a vesicle as they are flat lemon-yellow or brownish nodules, varying in size from a pin's head to a hempseed, with a peculiar translucent appearance, but when punctured they are found to contain no fluid but a transparent jelly-like substance. They are usually discrete, and are situated chiefly. on the upper parts of the face, especially on the forehead, about the eyes, malar prominences, bridge of the nose, ears, and occasionally on the backs of the hands. Lesions, in the form of yellow streaks or patches, have been observed also on the cornea, ocular conjunctiva, and mucosa of the lips. When the nodules are few and sparsely distributed they may be little noticeable, but when they are numerous and closely aggregated they give rise to an appearance which has been compared to smearing the face with yellow clay, and produce considerable disfigurement. After reaching a certain size the nodules become stationary, but occasionally they may break through the epidermis, shell out, and leave a superficial scar, or they may shrivel spontaneously. 
In addition to the yellowish lesions there is usually diffuse pigmentation in the affected areas which is believed to be due to exposure to sunlight.

There are no subjective symptoms.

It has been met with chiefly in men over 40 , but has been known to occur in children and an instance is on record where it affected two brothers aged respectively 9 and $\mathrm{I} 2$.

The cause is unknown; exposure to the actinic rays of the sun has been suggested, also senile degeneration, but the latter is discounted by the cases which have occurred in childhood.

Histopathology.- Sections of the nodules show a fairly constant histology. The epidermis is only affected secondarily, being more or less thinned from pressure of the underlying colloid mass, but in one or two instances degenerative changes have been described in the cells of the basal layer and the lower prickle-cells.

The principal changes are met with in the corium and consist of an amorphous or granular mass of colloid material occupying the papillary and sub-papillary layers, and either separated from the epidermis by a thin band of fibrous tissue or reaching right up to the basal cells. The colloid material is a degenerative product of both the collagen and elastin, and the various stages in the degeneration can be demonstrated by suitable methods of staining. The cellular elements remain immune from the degeneration and apparently normal connective tissue cells and nuclei are usually present in the midst of the amorphous debris. The piio-sebaceous follicles and sweat-glands are not affected; but colloid changes have supervened in the connective tissue of the walls of the blood-vessels, not only in the diseased area but for some distance beyond it, suggesting that the degeneration may be due to some toxic substance circulating in the blood which attacks the blood-vessels first and the neighbouring connective tissue subsequently.

Treatment.-The treatment consists of curetting or incision of the epidermis and shelling out the nodule. Satisfactory results may be obtained also by electrolysis or by the use of a fine-pointed cautery.

\section{REFERENCES}

Balzer. Pseudo-milium colloïde (La prat. Derm., I90o, i. p. 629).

Besnier. Dégénérescence colloïde du derme (Ann de Derm. et de Syph., I $879, \mathrm{x}, \mathrm{p} .46 \mathrm{I})$.

Hartzell, MI. B. Colloid degeneration of the skin (Journ. Cut. Dis., I9I4, xxxii. p. 683).

Philıppson, I. Relationships of colloid milium (Brit. Journ. Derm., I89 I, iii. p. 35).

Wagner. Colloid milium (Arch. d. Heilk., I866, vii. p. 463).

White. Colloid degeneration of the skin (Journ. Cut. \& Gen.-Urin. Dis., I902, $\mathrm{xx}, \mathrm{p} .49)$. 


\section{SARCOID}

The name sarcoid has been employed by various writers, such as Darier, Roussy, and Boeck, to designate certain nodular lesions of the skin which some observers now describe as hypodermic tuberculides.

The Sarcoid of Darier-Roussy does not differ materially from the so-called "multiple benign sarcoid" of Boeck and is somewhat akin to the "Erythème induré des jeunes filles" of Bazin. The name is an unfortunate one as it suggests a relationship with sarcoma, from which it is differentiated readily by its histology. It is characterised by groups of subcutaneous nodules about the size of a pea, some scarcely perceptible to the touch while others are distinctly raised. They are reddish-brown in colour and usually situated on a circumscribed, infiltrated, purplish patch, irregular in outline, and varying in size from one to several inches in diameter. The lesions show no tendency to ulcerate but may necrose and leave depressed pigmented scars. They are usually present on the extremities and trunk and, more rarely, on the face. However extensive the eruption the general health does not seem to be affected by it and some of the patients have appeared to be in robust health, but in a number of instances it has been associated with severe pains in the joints and a feeble peripheral circulation with cyanosis about the hands, feet, and legs. The majority of cases have been in women about forty years of age.

The lesions differ from those of Erythema induratum in that the nodules are grouped, distributed on the extremities, trunk, and face, instead of being confined to the legs, and rarely break down and ulcerate.

In a case microscopically examined by Adamson the nodules showed a round-celled and epithelioid-celled infiltration around the bloodvessels of the deeper parts of the corium and the subcutaneous tissue; other observers have noted the presence of giant-cells in the infiltration. No tubercle bacilli were found in the lesions.

Although there is no absolute proof, certain writers believe the lesions to be tuberculides and injections of old tuberculin have been known to give a marked local reaction in them. Most of the patients, however, have seemed to be in excellent health and have presented none of the stigmata of tuberculosis.

The Multiple Benign Sareoid of Boeck is closely allied to that of Darier-Roussy, and may be the same disease. Boeck's affection is characterised by firm benign nodules, varying in size from a hempseed to a bean, bright red in colour in their early stage, assuming a dusky bluish tinge and finally becoming yellowish-brown, widely distributed in a more or less symmetrical fashion on the head, trunk, and extensor aspects of the extremities, tending to disappear spontaneously after a varying time by a process of necrosis and absorption-rarely by ulceration-and leaving a brownish, pigmented, depressed scar which 
eventually becomes white. Where the lesions are numerous and small the name " miliary sarcoid" has been applied to them.

Microscopic sections showed large connective tissue cells and a few giant-cells.

The condition has been said to be amenable to arsenic internally and to improve under small doses of tuberculin.

\section{REFERENCES}

Adamson, H. G. Subcutaneous "sarcoid" (Brit. Journ. Derm., I9I2, xxiv. p. 393).

BoEck, C. Further observations on Multiple benign sarcoid (Arch. f. Derm. u. Syph., r905, lxxiii. pp. 7 I \& 301).

Johnston, J. C. Sarcoma \& the sarcoid growths of the skin (Brit. Journ. Derm., I90r, xiii. p. 258).

PAwlofF, P. A. On the so-called benign sarcoidal growths of the skin (Boeck) (Monats. f. prakt. Derm., I904, p. 469).

Scharmann, J. Lupus pernio \& its relationship with sarcoids (Ann. de Derm. et de Syph., r91 7, vi. p. 357).

Sequeira, J. H. Non-ulcerating tuberculide, Boeck's miliary sarcoid (?) following Lupus vulgaris (Brit. Journ. Derm., I9I I, xxiii. p. 85).

Sarcoid (Ibid., I9I4, xxvi. p. 420).

Thibierge \& Bord. Note on two cases of subcutaneous sarcoids (Ann. de Derm. et de Syph., I907, p. I13).

Urban, O. Boeck's sarcoid (Arch. f. Derm. u. Syph., ci. Hft. I).

\section{MYOMA}

Syn.: Leiomyoma ; Dermato-myoma.

The myomata constitute a rare group of neoplasms in which the new-growth is made up chiefly of smooth muscle fibres, hence the

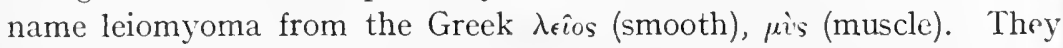
are of two types, (I) multiple cutaneous myomata, (2) subcutaneous leiomyomata, which are usually single.

The multiple cutaneous leiomyomata, though rarer than the more deeply-seated solitary growths, are of more interest to the dermatologist than the subcutaneous lesions which concern the surgeon.

\section{Multiple Cutaneous Leiomyomata}

The multiple cutaneous leiomyomata are among the rarest of cutaneous new-growths, only between 30 and 40 cases having been recorded in the literature. They appear as firm nodules, usually about the size of a pea, fixed to the skin but freely movable on the subjacent tissues, rounded or ovoid in shape, smooth on the surface, pinkish or more often dusky reddish-brown in colour, or sometimes violaceous when present in dependent positions. They may have a distinctly yellowish hue suggesting xanthoma but are not so waxy, or they may have a 
semi-translucent appearance not unlike the "frogspawn nodules " which occur in connection with Elephantiasis nostras. They are usually few in number, about a dozen arranged in a group, but they may be more numerous and irregularly distributed. As they take their origin from the smooth muscular fibres of the Arrector pili muscles they may be met with wherever hairs occur, but they have been observed most frequently on the face, neck, trunk, and limbs, especially on the extensor aspects. They may be discrete and separated by apparently healthy skin or may coalesce to form a conglomerate raised patch. They may appear at any age, either one at a time or several simultaneously, slowly increase in number and size for a few years, and then remain stationary. (Fig. 378 .)

As a rule they are associated with more or less marked subjective symptoms, but in some cases these have been wholly absent. Occasionally they are heralded by a sensation of itching or pain which may

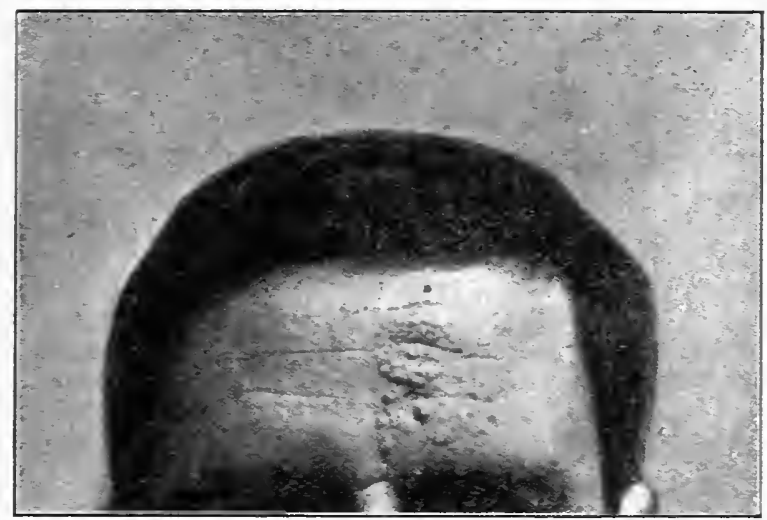

FIG. 378.-Leiomyomata.

(Dr. Wallace Beatty's case. Brit. Journ. Derm. Igo-.)

first attract the patient's attention to them, or they may exist for some time and attain the size of a pea before they become sensitive. In the majority of cases they become definitely painful, the pain being of a severe and paroxysmal type, lasting from a few minutes to an hour or more, and probably being the result of pressure on sensitive nerve endings or of the stretching of nerve fibrils by the growth. They are tender or even painful on pressure and spasms of pain are liable to be set up by friction or exposure to extremes of temperature, in some cases the sensitiveness to cold being so marked that bathing with cold water may be followed by a paroxysm.

Histopathology.-Sections of a nodule show a mass of smooth muscle fibre situated in the corium. As a rule it is separated from the overlying epidermis by a thin laver of connective tissue but has no definite connective tissue capsule. Elastic fibres occasionally may be observed spreading for a short distance into it. The component muscle-cells have typical strap-shaped nuclei and lie in all directions, 
surrounding hair-follicles, and passing in between the saccules of the sebaceous glands or among the sweat-coils. The muscular growth takes place from the Arrectores pilorum. (Fig. 379.)

Diagnosis.-It is impossible to make a firm diagnosis of the growths without a microscopical examination, but the grouping, size, slow

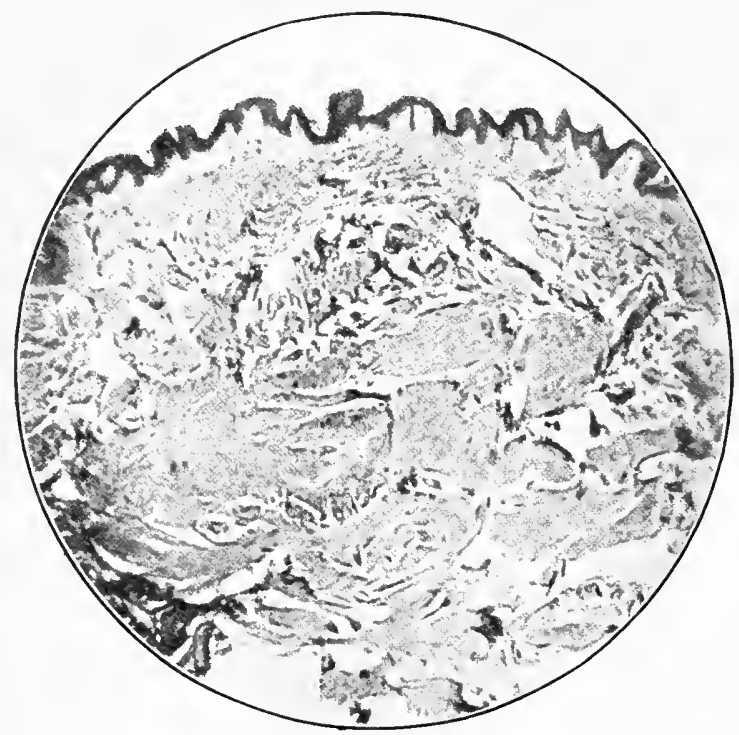

FIG. 379. - S ction of Leiomyoma.

Shows bundles of muscle-cells forming a branched mass in the corium. (Dr. Wallace Beatty's case. Photomicrograph by Prof. J. Alfred Scott.)

development, dusky red appearance, and associated paroxysmal pain, are strongly suggestive of multiple myomata.

Treatment.-The treatment consists of destruction by electrolysis or the cautery, or of excision in the case of the larger lesions.

\section{Subcutaneous Leiomyomata}

The subcutaneous myomata are usually solitary, vary in size from a pea to a filbert nut or larger, and are met with chiefly on the scrotum and labia majora where they are developed from the muscle cells of the dartos or from those immediately under the skin of the labia. They may also take their origin in the muscular layer of some small bloodvessel, or they may possibly arisc from embryonic muscle cell-rests and may belong to the class naevus. Histologically they may not be pure leiomyomata, but may be composed partly of fibrous tissue forming a fibromyoma, or of blood-vessels constituting an angiomyoma (Myoma telangiectodes of Virchow), or of lymphatics forming a lymphangiomyoma.

\section{REFERENCES}

Beatty, W. Multiple leiomyomata of the skin (Brit. Journ. Derm., I907, xix. p. I). 
CRocker, RADCLifFe. Myoma multiplex of the skin (Idem, i 897 , ix. pp. I \& 47).

Macleod, J. M. H. Multiple leiomyoma of the skin (Idem, Igo8, xx. p. 84 ).

Roberts, Leslie. Superficial cutaneous myomata (Idem, I900, xii. p. I I5).

SoвotKa. A contribution to the study of Myoma cutis \& sub-cutis (Arch.

f. Derm. u. Syph., March I9I3).

\section{SKIN LESIONS IN LEUCAEMIA AND GENERAL LYMPHA- DENOMA (HODGKIN'S DISEASE).}

Lesions may occur in the skin in leucaemia (Leucaemia cutis) and in Hodgkin's Disease (Lympho-granulomatosis pruriginosa).

\section{LEUCAEMIA}

The most characteristic cutaneous lesions in leucaemia are nodules varying in size from a pea to a raisin, usually discrete, rarely confluent, and either the colour of the normal skin, or dusky-red, slaty-grey, or plum-coloured. They may be few in number and irregularly grouped, or numerous and more or less symmetrically distributed. A favourite site is the front and sides of the trunk, but they may be met with anywhere on the cutaneous surface. In appearance they are practically indistinguishable from secondary sarcomatous growths, are firm to the touch, and although sometimes tender on pressure give rise to no definite subjective symptoms.

Occasionally there may be a diffuse infiltration of the skin similar to that which is responsible for the nodules.

Sections of a nodule show a cellular deposit in the corium, with secondary changes in the epidermis from pressure, such as flattening of the inter-papillary processes. The cellular infiltration occupies the central part of the corium, is not well-defined, and may spread up as far as the epidermis. It has roughly the appearance of a lymphfollicle of a lymphatic gland, being made up chiefly of lymphocytes, a few mast-cells, and occasional plasma-cells, contained within the meshes of a delicate fibrous reticulum. At the present time there is some difference of opinion as to whether the lymphocytes are merely deposited in the skin or are formed there from the endothelial cells.

\section{GENERAL LYMPHADENOMA}

In Hodgkin's disease the cutaneous lesions consist of prurigo-like papules which are associated with intense pruritus. They are bright red or dusky in hue, distributed irregularly about the trunk and limbs, and come out in crops, each crop being accompanied by an exacerbation of the pruritus. Occasionally the lesions may be urticarial in type or 
even vesicular. In addition, the affected skin generally presents a pigmented mottling with brownish macules where papules have involuted and, as a result of scratching, is liable to secondary changes, such as excoriations, lichenification, and even eczematisation.

The eruption may be accompanied by enlargement of the lymphatic glands, on the other hand it may precede the lymphadenoma by months or even years and in consequence may constitute an important early symptom of the disease.

The lesions are distinguished from the nodules of Leucaemia cutis by the intense itching which accompanies them.

The eruption closely simulates scabies and cases are apt to be subjected to a course of parasiticide treatment before their true nature is discovered.

A case is on record in which the disease was complicated by generalised exfoliative erythrodermia.

\section{REFERENCES}

McDonagh, J.E. R. The role played by a lymphocyte in inflammation $\&$ its probable relationship to sarcoma (Brit. Journ. Derm., I9I4, xxvi. p. 283).

Neuberger. Lymphatic leucaemia with leucaemic tumours of the skin (Trans. Germ. Derm. Soc. Cong. iii., held at Leipsic, I890).

Pinkus, F. Lymphatic leucaemic \& pseudo-leucaemic skin affections (Arch. f. Derm. u. Syph., Oct. I899).

RiehL, G. Leukaemia cutis (2nd Internat. Derm. Cong. ; Brit. Journ. Derm., I 892 , iv. p. 3i6).

Rolleston, H. D. Pruritus in Lymphadenoma (Brit. Med. Journ., I909, ii. p. 852$)$.

Rolleston, H. D., \& Fox, W. Myeloid leukaemia with nodular infiltration of the skin (Brit. Journ. Derm., 1909, xxi. p. 376).

Shaw, H. Batty, \& Loughlin, S. Case of Leucocythaemia cutis (Brit. Journ. Derm., 19I7, xxix. p. 36).

Steven, J. L. Acute lymphatic leukaemia with numerous subcutaneous lymphatic nodules (Glasgow Med. Journ., July I903, p. I).

Weber, F. P., \& Dore, S. E. Case of Pruritus in Hodgkin's Disease (Brit. Journ. Derm., I9I8, xxx. p. I5).

\section{ENDOTHELIOMATA}

Endotheliomata occasionally occur in the skin, but they are extremely rare. At one time a number of cutaneous new-growths were said to be of endothelial origin, but the work of Dubreuilh and others has shown that the majority really belong to the class " benign cystic epitheliomata." In true endotheliomata the lesions are reddish-blue papules or nodules, sessile or pedunculated, varying in size from a pin's head to a pea or larger, sometimes single but more often multiple, and irregularly distributed anywhere on the cutaneous surface.

Microscopical sections show a cellular growth, originating in the endothelium lining a blood-ressel or lymphatic, the cells of which are 
roundish or polygonal in outline with large oval nuclei which stain deeply and present one or more nucleoli and a deeply stained nuclear network. The endothelium being of mesoblastic origin the growth is mesoblastic, closely related to a sarcoma, and quite distinct from any form of epithelioma, the actual cells composing it resembling those of a large round-celled sarcoma. Sometimes the cellular proliferation takes place around the lumen of the vessel, at other times it forms a mass of cells blocking the lumen and spreads from there into the surrounding inter-fascicular spaces.

Wolters has described a case in which the endothelial proliferation blocked the blood-vessels and formed a dense infiltration in the corium in which, here and there, were clumps of cells like endothelial cells, which had undergone hyalin degeneration in the centre and so formed cyst-like structures. This condition he named "haemangio-endothelioma," and the analogous growth from the lymphatic endothelium, "lymphangio-endothelioma."

Though closely allied to the angio-sarcomata there is a difference, in that the cellular proliferation in the endothelioma takes place at the expense of the ressels from which it originated, so that no vessels can be seen ramifying between the cells, while in angio-sarcoma the growth is vascular and a new formation of capillaries occurs.

\section{REFERENCES}

FIck, J. On endothelioma (Monats. f. prakt. Derm., Narch I909, pp. I99 \& 25I).

Haslt:id, P. Multiple endotheliomata of the scalp (Arch. f. Derm. u. Syph., 1906, pp. $247 \& 323$ ).

Kerrle, E. H. Tumours arising from endothelium (Proc. Roy. Soc. Med., May I9I 8 , xi. No. 7 ).

LAZARLS-BARLOW, W. S. Histological diagnosis of the endotheliomata (Glasgow Med. Journ., 1907, iv. p. 265).

McDoragh, J. E. R. Spontaneous disappearance of an endothelioma (Naevo-xanthoma) (Brit. Journ. Derm., 1909, xxi. p. 254).

Congenital xanthoma (endothelioma) (Ibid., I9II, xxiii. p. II5).

Wolters. Haemangio-endothelioma tuberosum multiplex and haemangiosarcoma cutis (Arch. f. Derm. u. Syph., I90o, liii. p. 269).

Woolley \& Whire. Malignant vascular tumour of the shin (Perithelioma) (Journ. Cut. \& Gen.-Lrin. Dis., October I902, p. 453). 


\section{CHAPTER XLI}

\section{MALIGNANT NEW-GROWTHS}

\section{EPIDERMIS AND ITS APPENDAGES}

THE malignant epitheliomata may be divided into two classes, namely: (I) those which are locally malignant and which, though they may spread laterally and deeply into the surrounding tissues, do not give rise to metastases; (2) those which are generally malignant, and invade organs and tissues at a distance.

The locally malignant epitheliomata consist of the so-called rodent ulcers, of which there are several clinical varieties; the generally malignant epitheliomata include the squamous epitheliomata of the skin and secondary carcinomata due to invasion of the skin from deep-seated cancer.

\section{RODENT ULCER}

Syn.: Jacob's ulcer; Ulcus exedens; Noli me tangere: $F r$. Ulcère rongeant; Ger., der flache Hautkrebs.

Definition: A chronic ulcer of the skin, usually present on the face, which if not arrested by treatment is slowly progressive, destroying underlying tissues and sometimes leading to a fatal issue.

Description.-This type of ulcer, originally described by Jacob of Dublin in I827 and known at one time as "Jacob's ulcer," is comparatively common. It is more prevalent in this country than on the Continent or in America, and consequently the bulk of the literature is British. Until lately it was believed here to be a different disease from cancer, but this view is no longer held, as recent observations of its histology have amply established the continental opinion that it is simply a variety of Carcinoma cutis, - - several cases being on record in which it developed into squamous epithelioma,-and it is now included under the heading of the malignant epitheliomata.

It begins either as a small reddish-brown or bluish-grey pearly nodule with a smooth shiny surface over which small capillaries may be seen ramifying, or as a minute epidermal elevation covered by a soft adherent crust. It is entirely confined to the skin, movable on the 


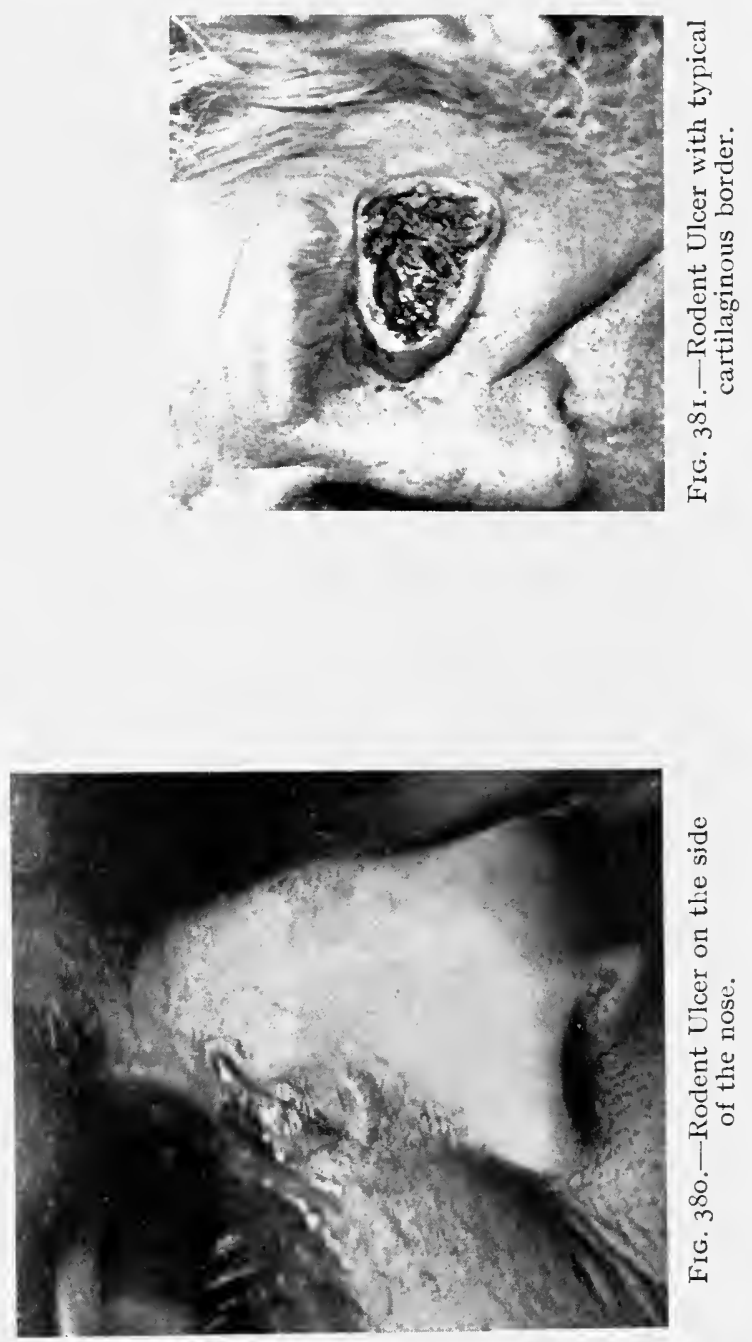

[To face $p$. II 45 . 
subjacent tissues, and feels hard and almost cartilaginous to the touch. As there are no subjective symptoms, it has usually existed for some time and reached about the size of a split-pea before notice is taken of it. It grows exceedingly slowly and may take sereral years to attain the size of a threepenny piece; it then not infrequently assumes the form of a button-shaped pearly growth with a depressed centre and a rounded border. Occasionally subsidiary nodules may appear and these, on growing, coalesce, so that irregular figures are produced with the same characteristic pearly aspect. (Fig. 38o.)

As a result of rubbing, scratching, or some slight traumatism, the surface is liable to become abraded and to break down in the form of a superficial ulcer, with a yellowish or brownish scab which, when picked off, reveals a bright red base, covered with a glairy secretion and irregular from the presence of small nodules or granulations. Surrounding the ulcer is a well-defined hard border or rolled edge which is similar in tint to the original nodule, slightly raised above the level of the ulcer, and usually uneven and beaded from thickenings at irregular intervals. (Fig. 3 SI.)

After persisting for some time in this comparatively benign state the diseased process usually becomes more active, and a rapid and serious development is liable to occur. It may spread gradually and form a large irregular ulcer with an uneven surface and covered by an offensive glairy or haemorrhagic discharge; or it may become still more active and penetrate into the underlying tissues, destroring muscle, cartilage, periosteum, and even bone, perforating soft parts such as the eyelid or the alae of the nose, invading the neighbouring mucous membrane, and giving rise to deep irregular carities replacing the orbit or nose or eating away the side of the face. When the orbit is involved, blindness is inevitable, but the sclerotic is peculiarly resistant to the growth. However extensive the destructive process may be, the neighbouring lymphatic glands are not affected and metastases do not occur. Occasionally the growth may reach the size of a filbert nut or larger before ulceration takes place.

At first, eren though the ravages of the disease are considerable, the general health is in no way affected; later a state of exhaustion generally supervenes from the pain caused by the involvement of sensitive structures and the interference with sleep, and the patient, after years of tedious discomfort and suffering, passes away in a state of profound cachexia. Occasionally the end may be hastened by the diseased process assuming the characteristics of a squamous epithelioma and giving rise to metastatic growths.

Rodent ulcers are most common on the face, especially in the region limited by lines from the erebrow to the top of the auricle and from the upper lip to the lobe of the ear, the most frequent situations being the neighbourhood of the inner canthus and the side of the nose. They may be met with also on the forehead, about the auricle, 
and occasionally on the scalp, and in rare instances have occurred on the neck, back of the hand, sternal region, back, groin, umbilicus, and leg.

In the vast majority of cases the lesion is single, but sometimes may be multiple. In a case under the care of the writer six small rodent ulcers were present on the face of a sailor aged 55 years, and a case is on record in which as many as 20 were counted. So far the multiple cases which have been reported have been in men.

In addition to typical rodent ulcers there are several varieties which, although they have a similar histology, are sufficiently different clinically to warrant a separate description. These result from variations in the texture of the skin, differences in the part of the epidermis from which they grow, and degenerative changes in the lesions. The most important are the following :

(I) A superficial cicatrising variety.

(2) A card-like variety.

(3) A cystic variety.

(I) Superficial cicatrising variety. This is met with most commonly where the skin is stretched tightly over bone, as on the forehead, scalp, and over the malar and mastoid processes. In it the nodule breaks down early and becomes a superficial ulcer, which spreads rapidly, cicatricial changes occurring in the centre while the ulceration progresses in an irregular fashion at the edges. The cicatricial area is surrounded by a sinuous border made up of nodules or of ulcerated grooves usually covered with scabs, and presents in the centre translucent pearly nodules or irregular pigmented patches. From its appearance it might be mistaken for an old-standing patch of Lupus vulgaris or for a superficial serpiginous syphilide. Sometimes these ulcers occupy a considerable area, as in a case under the care of the writer in a man aged 50 in whom there was a cicatrising lesion on the forehead and scalp, which was roughly circular in outline and measured a little over 3 inches in diameter.

(2) Card-like variety. In this variety the new-growth takes the form of a flat thickening of the skin which has been likened to a piece of playing-card let into it. The thickening has a waxy or ivory-like appearance, the surface is smooth and usually has a few dilated capillaries ramifying over it, and there is a marked tendency for it to break down and ulcerate. Histologically it has the same essential structure as a nodule. In some cases the ivory-like plaque is practically indistinguishable from localised sclerodermia or morphoea.

(3) Cystic variety. In it cystic degeneration occurs, with the formation of soft bluish semi-translucent cysts, varying in size from a small shot to a pea or larger, the skin over which is smooth and glossy. When punctured, a colloid or gelatinous fluid can usually be expressed. The cystic formation may be primary, but is more often met with in recurrences after unsuccessful treatment. (Figs. 3S2, 383.) 
Etiology.-According to statistics the affection would appear to be more common in men than women; according to Bowlby's statistics, based on 66 cases, it was in the proportion of 5 to 3 , while out of 20 consecutive cases in the skin department at Charing Cross Hospital it was 12 to 8 . The earliest case in my experience was at It years, while Sequeira has recorded one which began at I2; it is rare under 30, usually begins after 40, and may be regarded as essentially a disease of the middle-aged and elderly.

In most instances no cause can be suggested for it and the lesion is usually discovered by accident. On the other hand cases are on record where it followed some slight injury, such as a prick or sting, or originated in a mole which had been irritated or imperfectly treated. In a number of cases of Carcinoma cutis, possibly of the

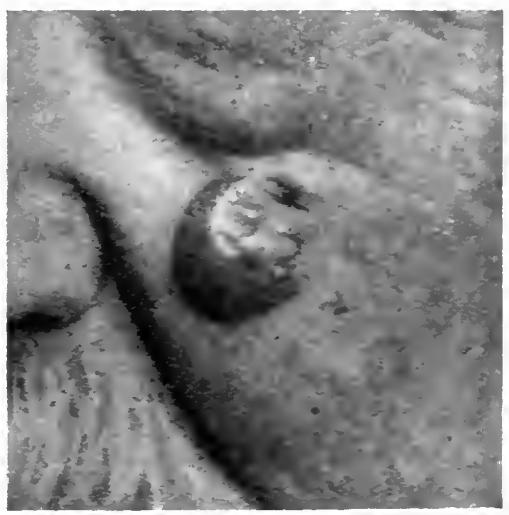

FIG. 332.-Cystic Rodent Llcer on Cheek. rodent ulcer type, Borrel discovered numerous Acari folliculorum in openings of the pilo-sebaceous follicles, but whether these had any causal connection with the epithelioma is doubtful.

Histopathology.-The microscopical appearances of an early nodule are characteristic and consist of an epithelial downgrowth in the corium, which reaches down to

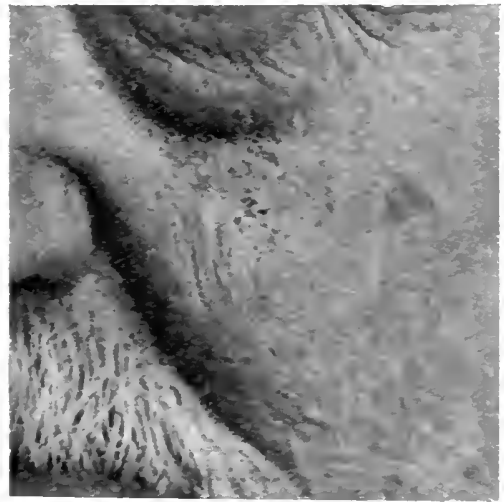

Fig. 383.-Cystic Rodent Ulcer after treatment with radium. the hypoderm and is made up of irregular branching projections, sometimes presenting an alveolar appearance from small variouslyshaped islands of epithelium being cut off from the processes in the section. The epithelial processes are limited by a regular row of columnarcells forming a basal laver which is usually unbroken, and within which the cells are small, densely crowded together, oval or roundish in shape, with well-marked nuclei occupying nearly the whole cell, and, as a rule, have lost their

fibrillated structure. Here and there in the projections are usually small cysts containing a degenerated colloid material or a hyalin substance, and in rare instances cell-nests with concentric horny laminae. At the margin of the epithelial growth there is a well-marked cellular infiltration in which plasma-cells predominate. (Fig. 34.4 .) 
The whole epithelial mass is surrounded by a capsule of fibrous tissue, due to the condensation of the fibrous stroma caused by the downgrowth, and is divided up by fibrous trabeculae. This capsule is not so dense as that which occurs in benign cystic epithelioma.

Serial sections have shown that the epithelial growth takes its origin most frequently from the cells of the external root-sheath, usually about the level of the sebaceous gland, and is in most cases a trichoepithelioma.

Sections of an ulcerated rodent show destruction of the superficial epidermis and inflammatory changes in the corium, consisting of a

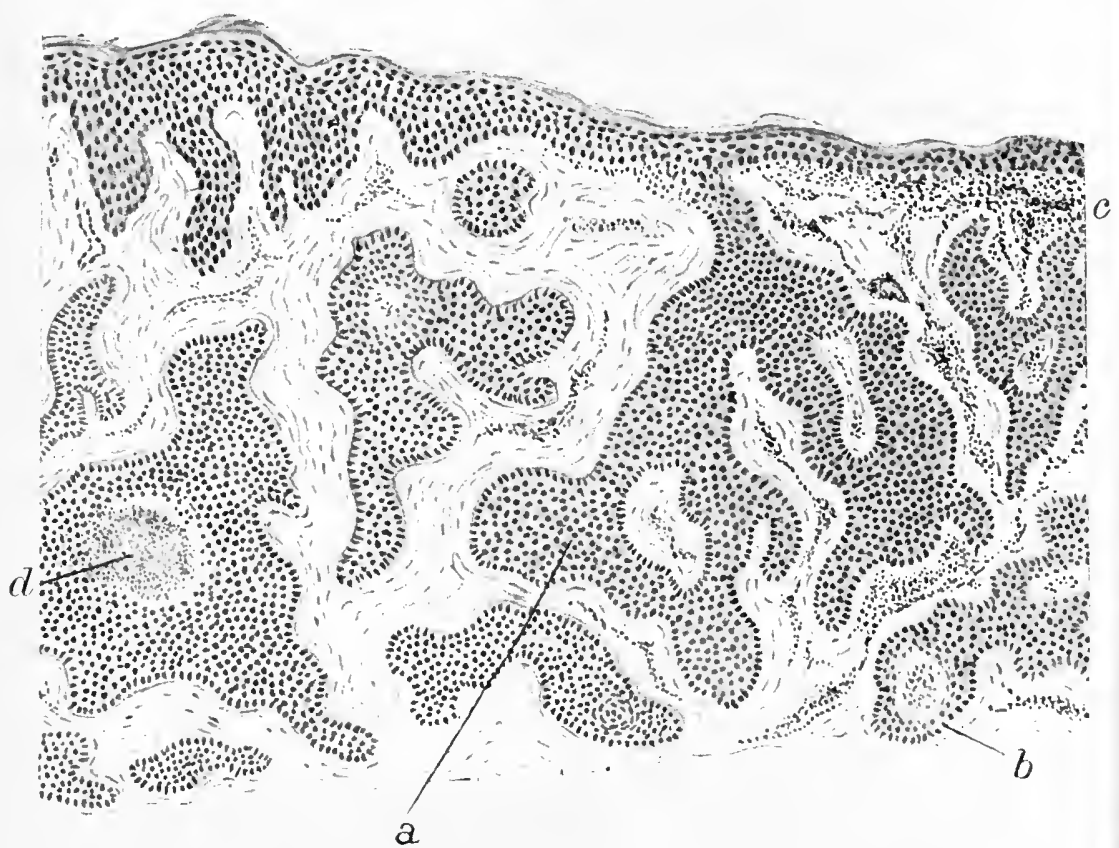

J. MI. H. MI. del.

FIG. 384.- Section of Rodent Ulcer. ( $x$ about Ioo.)

Section of rodent ulcer in the nodular stage before the centre has broken down completely to form an ulcer. Left side shows a section of raised " cartilaginous" border, while right side is part of a depressed central portion which is beginning to break down.

a. Maiignant proliferation of epidermis of tubular type; cylindrical processes and acini present. $b$. Basal limiting layer persisting almost universally. c. Cyst containing brokendown cellular debris. $d$. Cellular infiltration in corium, consisting of leucocytes, plasma-cells, and mast-cells; the infiltration is specially abundant towards right side.

(From MacLeod's Pathology of the Skin.)

dilatation of the superficial blood-vessels and a cellular infiltration made up of leucocytes and plasma-cells with a few mast-cells deposited around them.

Diagnosis.-The diagnosis as a rule presents no serious difficulty, the pearly nodule, or superficial ulcer, with a rolled cartilagincus border, usually situated about the inner canthus or side of the nose, being characteristic.

The cicatrising variety may be mistaken for a patch of Lupus vingaris or a superficial tertiary syphilide; but in lupus the affection 
has usually begun in early life and typical apple-jelly-like nodules may be detected at the spreading edges, while in syphilis the rapidity of development of the lesion, the peculiar tissue-paper-like pigmented scar, and the brownish-red soft nodules or ulcerations at the margin, are sufficient to establish the diagnosis.

The card-like variety may be difficult to diagnose from morphoea, as in both there is an ivory-like or waxy plaque with vessels ramifying over it; but in morphoea there is less tendency to break down and ulcerate, and the lesions are usually multiple.

Prognosis.-The prognosis is always grave, and recurrences are liable to take place after any form of treatment. There is every hope of a complete cure if the disease be confined to the skin and suitably and thoroughly dealt with while in its early stage; but when it is in its later stages and has invaded mucous membranes or involved muscle, periosteum, or bone, and has formed a large ulcerating carity, the outlook is most serious and no treatment is of permanent benefit. With increased familiarity with the early stages of the disease it should be recognised in time and such a catastrophe averted.

Every case which has been treated should be kept under observation and inspected periodically at intervals of not longer than a year, as should the growth recur it is apt to progress rapidly.

\section{TREATMENT}

The treatment is invariably a serious matter, and every endeavour should be made completely to eradicate the growth in its early stages. Insufficient or unsuitable treatment is liable to irritate rather than to cure, and any improvement which may follow it is apt to be temporary and to be succeeded by a rapid recurrence. The consequences of imperfect treatment have been so disastrous in the past that the older writers dreaded interfering with it, hence the name "Noli me tangere."

Of the various forms of treatment the following are the most important :

Caustics. One of the oldest methods was by caustics, such as arsenic, zinc chloride, caustic potash, pyrogallol, resorcin, etc., and of these arsenic and caustic potash have given the best results.

Arsenic.-Arsenic is used in the form of a powder, such as arsenious acid and powdered gum arabic equal parts, or arsenious acid $\bar{j}$ i. bi-sulphide of mercury grs. $\mathrm{xl}$, and calomel $\mathrm{j}$ i. The powder is mixed with sufficient water to form a stiff paste, which is spread on lint and applied over the ulcer for about twenty-four hours. This causes an acute inflammatory reaction which results eventually in sloughing of the diseased tissue. After the paste is removed, boric compresses or boro-starch poultices should be applied to reduce the inflammation and to encourage healthy granulation. Sometimes several applications of the paste may be necessary. Should the 
ulcerated area be large and refuse to heal, it may be advisable to resort to Thiersch grafts.

It is claimed that the arsenic has a selective action, eating away the diseased tissue and leaving the healthy, and consequently it was regarded at one time as an ideal treatment. A great drawback is the intense pain associated with it, and to reduce this the arsenious acid may be mixed with an equal quantity of orthoform, or the arsenical powder may be made up into a paste with a saturated solution of cocain instead of water.

The scar which results from this method is comparatively slight.

Caustic potash. Caustic potash is used in the form of a solid stick which is rubbed firmly over the growth for several minutes, the surrounding skin being protected by a thick layer of vaselin. After the application the action of the caustic should be stopped by a drop of vinegar. This treatment is painful at the time but the pain is transient.

The employment of caustics for the destruction of rodent ulcers has fallen largely into disuse and has been superseded by less painful methods. It is still serviceable, however, in the late stages and occasional cases are on record where, combined with curetting, it has been of marked benefit when other methods had failed.

Cautery. By means of a Paquelin or an electric cautery small nodules, or the nodular border, can be destroyed without much scarring ; but this procedure seldom eradicates the growth and a recurrence is almost inevitable.

Excision. Where possible the best treatment is free and wide excision, but this is often impracticable owing to the situation of the lesion and the serious disfigurement and retraction which may result. When a large ulcer has been excised it is impossible to draw the edges together, and it may be necessary to resort to grafting to facilitate healing and to reduce the scar.

X-rays. The X-rays are useful in the early stages and cause the nodules to disappear and the ulceration to heal with a negligible degree of scarring. Three or four pastille doses at intervals of about three weeks are usually necessary. In the later stages the X-rays are of little or no value, except to lessen pain.

This treatment is specially applicable in elderly people as it is painless, but the results from it are rarely permanent and recurrences are liable to take place in a year or eighteen months. Where there is much nodular thickening, treatment by $\mathrm{X}$-rays, as well as by radium, may be facilitated by previously curetting the lesion.

Radium. At the present time, with the exception of excision, radium is the most successful method of treating rodent ulcer and in the early stages of the disease the results from it are brilliant, the growth being destroyed comparatively painlessly, the resultant scar being supple and little noticeable, and the tendency to recurrence being less than by any other method. Radium is also useful in arresting progress in the later stages of the disease. It loses its value when the 
mucous membranes are involved, especially those of the nose and mouth, but may sometimes cause healing where the orbital mucosa is affected.

The procedure is as follows: A full-strength radium plate (i.e. Io mig. of pure radium sulphate or bromide to the square centimetre) covered with two layers of gutta-percha tissue to cut out the alpha rays and so prevent too active a reaction, is applied unscreened for one, two, or three hours, according to the thickness of the growth. Where such a powerful applicator is not available longer exposures are necessary.

The reaction from it takes place in about a fortnight and is associated with redness, oedema, and a diffuse discharge which dries up to form an adherent crust like a rupial scab. Frequently a secondary reaction follows, probably owing to the tissues themselves becoming radio-active, and a second scab forms which separates in about a month. The cure is then complete.

Telangiectases are rarely present in the scar unless the exposure has been too prolonged or a long series of short exposures has been given instead of a single massive one.

Ionisation. Cataphoresis with zinc sulphate has been adrocated, a $3^{\circ}$ solution being employed and a current of 5 to ro milliamperes, according to the toleration of the patient, passed for fifteen minutes at a time. The application results in blanching of the affected part, followed by a reaction and the formation of a crust which, on separating, leaves a fairly satisfactory scar. This treatment is painful and unreliable, recurrences after it are the rule rather than the exception, and the results from it are far less satisfactory than those from either radium or X-ray's.

Refrigeration. Solid carbon dioxide can be employed to destroysmall rodent ulcers, but unless the freezing be sufficiently drastic to cause total destruction of the diseased tissue a recurrence is inevitable, while if the destruction be complete the resultant scar is liable to be unsightly.

To sum up it may be said that, where possible, excision is the most satisfactory treatment, and failing that radium or the X-ray's, combined if advisable with curetting. The selection of the method of treatment, however, must depend on the peculiarities of the individual case.

\section{REFERENCES}

Adamsox, H. G. Two cases of multiple rodent ulcer (Lancet, Igos, p. I I33). Iultiple ulcerating basal-cell epithelioma with zoniform distribution and possibly of sweat-gland origin (Brit. Journ. Derm. \& Syph., I9I 7 , xxix. p. I).

BORREL. Acarus folliculorum in early carcinoma of the skin (Ann. de l'Inst. Pasteur, Paris, rgog, xxiii. p. 97).

CARLES. Three cases of rodent ulcer, clinical, histological, \& therapeutic indications (Ann. de Derm. et de Syphl., July I905, p. 593).

Darier, J. Des naevocarcinomes (Rep. Xil Internat. Cong. Med., London, I9I3!. 
Davis, H. Epithelioma \& rodent ulcer occurring in the same patient (Brit. Journ. Derm., I9I3, xxv. p. I49).

Fordice, J. A. Epithelioma of the skin, benign \& malignant (Rep. XVII Internat. Cong. Med., London, I9I3).

GRAY, A. MI. H. Rodent ulcer treated with arsenic paste (Brit. Journ. Derm., I9I3, xxv. p. I63).

van Harlingen, A. Treatment of epithelioma by means of caustic potash (Journ. Cut. Dis., Aug. I906).

Jadassohn, J. Die benignen Epitheliomata (Rep. XVII Internat. Cong. Med., London, I9I3).

Little, E. G. Rodent ulcer, its nature \& some methods of treatment (Practitioner, I9I5, p. 58).

McDonagh, J.E. R. A classification \& description of cutaneous epitheliomata (Rep. XVII Internat. Cong. Med., London, I9I3).

MacLeod, J. M. H. Cystic rodent ulcer (Brit. Journ. Derm., I9o9, xxi. p. 349).

Morris, iI. Some physico-therapeutic methods in dermatology (Brit. Journ. Derm., I9I2, xxiv. p. I69).

\section{SQUAMOUS-CELLED CARCINOMA CUTIS}

Syn.: Pavement epithelioma; Squamous-celled epithelioma ; Prickle-cell carcinoma.

The squamous-celled epitheliomata differ from rodent ulcers by growing more rapidly and by giving rise to metastases in neighbouring glands and tissues. They may be : (I) Primary, developing either on apparently normal skin or on some benign growth usually of a warty character; (2) secondary to some underlying cancer, from an extension of the disease to the skin via the lymphatics.

In primary cases it may be impossible to decide from clinical appearances whether the cancer developed on healthy skin or on some previous warty growth ; in secondary carcinomatosis the appearances are usually so characteristic as to be easily recognised.

Primary squamous epithelioma.- It appears first as a small hard brownish-red elevation, which becomes surmounted by an adherent, rough, horny scab, and bleeds readily if this be picked off. It grows more rapidly than a rodent ulcer and in a few months may reach the size of a shilling or larger. It may then appear either as an irregular protruding mass or as a raised, flat, hard plaque, one or two millimetres in height, with abrupt edges beneath which the induration spreads out into apparently healthy skin. At this stage the surface usually breaks down to form an ulcer, the margins of which are irregular and sometimes undermined, and the base of a bright reddish hue, uneven from epithelial projections, and covered with a glairy or sanious discharge which dries to form a haemorrhagic crust. (Fig. 385.)

In situations where the mucous membrane and skin meet, as about the mouth, anus, and genitalia, the cancer tends to assume vegetating or fungating characteristics and sometimes to form irregular cauliflower-like excrescences.

As the growth increases, the neighbouring lymphatic glands become 

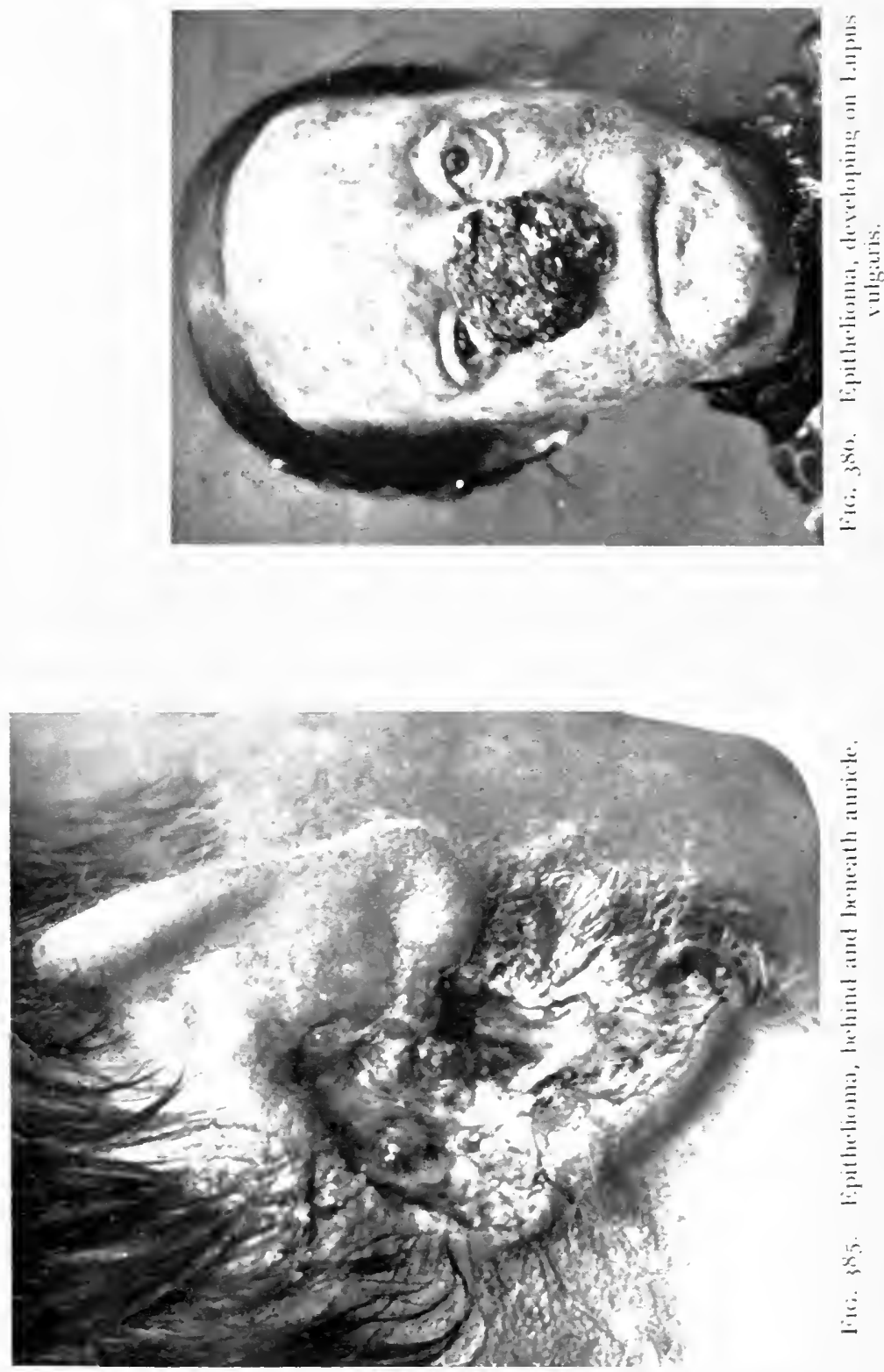

\begin{tabular}{l}
0 \\
\hline
\end{tabular} 

swollen and indurated from metastases and new cancerous foci may appear in the adjacent shin or at a distance. The further spread of the disease requires no special description. If left unchecked it will inevitably progress, sometimes comparatively slowly at other times with alarming speed, destroying the tissue locally and giving rise to metastatic growths; these gradually interfere with the vitality of the patient and lead to a fatal issue in a state of profound cachexia from pressure on vital structures, interference with nutrition, exhaustion from pain, and secondary septic troubles.

In the large majority of cases squamous-celled carcinomata develop on some previous epidermal growth, and in the comparatively few instances in which no previous abnormality was detected it may have been in existence and have passed unnoticed, or there may have been some microscopical defect, such as an embryonic cell inclusion or a cell-rest which had not fulfilled its developmental intention.

The cutaneous lesions most liable to develop into squamous-celled carcinomata are the following:

(a) The hard warty growths which result from long exposure to the actinic rays of the sun and are met with in chronic solar dermatitis.

(b) The warty thickenings which occur in Ierodermia pigmentosaa condition in which there is a congenital vulnerability to actinic rays.

(c) The keratoses and superficial ulcerations of chronic X-ray dermatitis.

(d) The warty growths associated with telangiectases and atrophy which characterise the type of skin known as "sailor's skin," which is similar to that in chronic solar dermatitis, and results from prolonged exposure to light, wind, salt spray, etc.

(e) The seborrhoic warts which occur in senile skin and are liable to develop into a type of cancer known as "Epithelioma sebaceum."

(f) Cutaneous horns.

$(g)$ Scar tissue, especially scars from burns, as in " Kangri cancer " of the abdomen (see page 232).

(h) The warty thickenings from prolonged contact with tar and its products (tar cancer), soot (chimney-sweep's cancer of the scrotum), and paraffin. The cancers which result are known as "trade cancers."

(i) Certain long-standing cutaneous diseases, such as Lupus vulgaris, Lupus erythematosus, and Darier's disease. In a certain number of the cases in which carcinoma has developed on Lupus vulgaris, excessive treatment by the $\mathbb{X}$-rays has been responsible. (Fig. 386.)

(j) The keratotic condition of the palms and soles which occurs in connection with chronic arsenical poisoning.

(k) Patches of leucoplakia on the mucosa of the mouth and lips, and Kraurosis vulvae.

Clinically it may be impossible to tell if a warty growth is benign or has become malignant; but the onset of malignancy is usually associated with evidences of inflammation, such as redness about the 
base of the growth and, possibly, itching, or the appearance of fissures which become infected with bacteria and ulcerated.

Secondary squamous epithelioma.-Secondary carcinomatosis, as this is sometimes called, is usually multiple and most frequently occurs in cancer of the female breast or of the prostate gland, and comparatively rarely in cancer of the abdominal or pelvic organs.

In it the skin lesions consist of nodules or paques which are dullreddish, livid, or yellowish in tinge, smooth on the surface, domeshaped or flat, and peculiarly hard to the touch. These tend to coalesce to form sheets occupying large areas and sometimes extending from the breast to the shoulder, down the side, and even round to the back (cancer en cuirasse). The surface of the plaques may be smooth, but is usually broken up here and there by fissures which are particularly liable to become infected by septic bacteria and to break down into extensive ulcers.

In connection with cancer of the breast the involvement of the skin may be out of all proportion to the area of the breast affected, for while a large surface of the skin may be implicated there may be only a small hard cancerous nodule about the nipple the skin over which is puckered and immovable.

Histopathology.-In this type of-carcinoma the horny layer is increased and passes down into the openings of the pilo-sebaceous follicles in the form of horny plugs. The tumour mass consists of a proliferated prickle-cell layer which grows down into the corium in irregular branching projections with an acinous arrangement, or spreads along the lymphatic spaces in the form of a coarse network; from the projections being cut in making the section, islands of epithelial cells of various shapes and sizes are also present. The downgrowth is limited near the epidermis by a well-marked basal layer of columnar or cubical cells, but this is usually lost towards the lower end of the processes. (Fig. 387.)

The cells composing the epithelial downgrowth are large and polygonal with vesicular nuclei which stain faintly and are sometimes situated in a space or vacuole. The prickles are preserved in the cells for a considerable distance from the epidermis and can be seen bridging the inter-epithelial lymphatic spaces, but deeper down they generally disappear and the cells become round or ovoid and irregularly crowded together. Mitotic figures can usually be detected in a considerable number of the cells throughout the growth.

Here and there are usually present the so-called horny pearls, so characteristic of this type of epithelioma. These consist of concentric horriy laminae which result from the cornification of groups of pricklecells around a single horn-cell, from a deposit of cellular debris, or from one or more cells in a state of hyalin degeneration.

In addition, cyst-like cavities from mucoid degeneration may occur, in the centre of which may be detected a few cells undergoing hyalin degeneration. 
Towards the upper part of the growth peculiar cellular formations and inclusions may be noted which have been mistaken for protozoa or yeast cells. These are prominent round structures, sometimes with a double contour, which result from imperfect cornification, hyalin degeneration, endogenous new cell formation, or the invagination of one cell within another.

In the corium there is no thickening of fibrous tissue to form a capsule to the growth, such as occurs in the benign epitheliomata and to some extent in rodent ulcer, the collagen bundles are rarefied from oedema, and the elastin has disappeared. At the edges of the growth

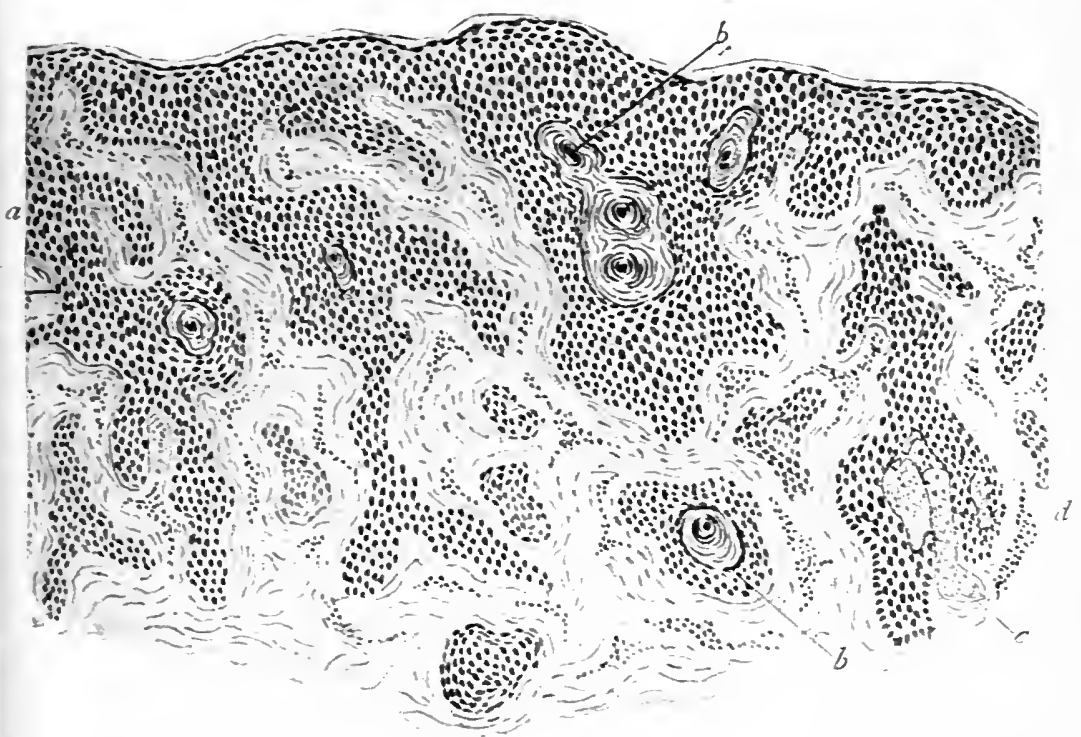

J. MI. H. M. del.

FIG. 387.-Section of Pavement Epithelioma. ( $x$ about Ioo.)

a. Basal layer has disappeared and the cancerous cells are invading the lymphatic spaces of corium. $b$. Cell-rests; concentric lavers of horn-cells surrounding a central core. $c$. Disappearing sebaceous gland. d. Cellular infiltration in corium.

(From MacLeod's Pathology of the Skin.)

the lymphatic spaces are blocked with oroid epithelial cells,-indicating an active spread of the disease,- - the blood-vessels are dilated, and there is a cellular infiltration consisting of plasma-cells, lymphocytes, and mast-cells, with occasional young connective tissue cells which show a tendency to organise into new fibrous tissue such as is met with in scirrhous cancer of the breast.

The malignant character of the growth is shown by the large number of irregular mitotic figures distributed throughout the epidermal cells, the absence of a basal layer towards the free end of the processes, the spreading of the epithelial cells along the lymphatic spaces to invade the surrounding tissue, and the absence of a fibrous capsule.

Etiology.-Squamous-celled carcinoma is most common after the age of 40 , but it may occur earlier, especially about the hands. It 
is more prevalent in men than in women, as they are more exposed by their occupations to conditions liable to give rise to keratotic growths which subsequently become malignant.

The primary carcinomata of the skin are due to a variety of irritants, chemical, physical, toxic, or bacterial, acting on a skin the vitality and resistance of which have been lowered as the result of senile changes, or by the presence of some abnormality or pre-cancerous lesion, such as a seborrhoic wart or an X-ray scar.

It is still a point for discussion whether the malignant change results from the continued action of the cause responsible for the original keratosis, or is due to some fresh excitant; in most cases it would seem that some new irritant is superimposed. In carcinoma of the skin due to chronic arsenical poisoning, for example, the action of the arsenic on the skin causes hyperkeratosis of the palms and soles; this may persist for a prolonged period and finally disappear spontaneously, or, as the result of traumatism or movement of the part, cracks may appear which become secondarily infected with septic bacteria and give rise to ulcers which eventually become malignant. In this case the arsenic causes the keratosis-an unstable condition in which malignant changes are liable to supervene-and the traumatism and septic inoculation do the rest.

In the same way, in a cancer developing on an old patch of Lupus vulgaris it is not the tubercle bacilli which are responsible for the epithelioma but some extraneous irritant, such as X-rays, secondary infection with septic micro-organisms, traumatism, etc.

On the other hand the same cause may give rise to the early benign stage and to the malignancy, as in lip cancer resulting from continued irritation from smoking a clay pipe.

It is possible that in some instances the cancer may be of bacterial origin and due to an infective virus allied to that which causes certain warts. Up to the present time, however, this has not been established and although various parasites, both animal and regetable, have been described in connection with it they have proved to be either secondary contaminations or structures due to degeneration or abnormal cornification of epithelial cells.

In certain cases the cancer may take its origin from embryonic cell-rests which have proliferated in later life as the result of irritation.

The possibility of heredity being a predisposing cause is worthy of consideration, as certain individuals seem to be born with a type of skin which is prone to epitheliomatous growths.

Prognosis and Treatment.-The prognosis is invariably grave and is especially so when the neighbouring glands and tissues have been invaded by the disease. If the lesion be in a state of local malignancy and of a slow-growing type, a wide excision may effect a cure; but if the neighbouring glands are involved, even if they be thoroughly cleared the outlook is much less hopeful. 
Failing excision, radium may be of some value in destroying the local growth, and X-rays in drying up ulcerations and relieving pain. On no account should caustics or the actual cautery be resorted to as they do much more harm than good and tend to irritate the growth to further activity.

\section{REFERENCES}

Allworthy \& Pernet. Multiple carcinomata of the skin (Brit. Journ. Derm., I900, xii. p. 350).

BECK \& KrompeCKER. The finer architecture of primary carcinoma of the skin (Leopold Voss, I903).

Boxsey, V. The Hunterian Lectures on the connective tissue in carcinoma $\&$ in certain inflammatory states that precede its onset. Lecture 2 (Lancet, May I9o8, p. I 465 ).

Carless, A. Carcinoma of the breast (Brit. Journ. Derm., I 899, xi. p. 29). ETcheverry. Syphilis \& cancer. (Ann. de Derm. et de Syph., I90 f, p. 797).

FORDYCE, J. A. Pathology of malignant epithelial growths of the skin (Journ. Amer. Med. Ass., I9Io, lv. pp. I624-I630).

MacLEOD, J. M. H. Epithelioma developing on an X-ray scar in Lupus vulgaris (Brit. Journ. Derm., I906, xviii. p. I04).

Morris, M. Cancer en cuirasse (Brit. Journ. Derm., I895, vii. p. 393).

Cancer \& its origin (Bradshaw Lecture, Dec. I903).

Nutt, Beattie, \& Pye-Smith. Arsenic cancer (Lancet, Aug. I9I3).

Plumuer, H. G. The parasitic theory of cancer (Brit. Med. Journ. I903, Xv. P. I 5 II $)$.

Pollitzer. Cancer en cuirasse (Journ. Cut. Dis., April, I909).

RavogLI, A. Cancer of the skin (Urologic \& Cut. Rev., I9I7, xxi.).

SAVATARD, L. Malignant growths on lupus scars (Med. Chronicle, July $\left.I_{9} I_{4}\right)$.

SequeirA, J. H. Lupus carcinoma (Brit. Journ. Derm., I908, xx. p. 40).

WiLd, R. B. Supervention of epithelioma upon Lupus vulgaris (Al. Chronicle, Nov. I907).

Wile, U. J. Arsenical Cancer (Journ. Cut. Dis., I9I 2, xxx. p. I92).

\section{Naevo-Carcisiona}

The name naevo-carcinoma has been applied to cancer which takes its origin in a mole, usually of the pigmented type, and is the most rapidly malignant of all forms of cancer. The malignant changes usually do not take place until after middle-age and may seem to develop spontaneously but more often are the result of friction, traumatism, or some other form of local irritation.

When a mole is assuming malignant characteristics it increases rapidly, becomes more deeply pigmented, and tends to break down and ulcerate in the centre and to show signs of inflammation around it, the neighbouring glands early become involved and metastatic growths occur in deeper structures or in the skin itself.

An interesting case was recorded by Wilfrid Fox in which two independent naevo-carcinomata were present in the same individual, the one pigmented and the other non-pigmented; the former originated in a freckle-like patch, and the other in a non-pigmented soft mole. 
Microscopically the growth consists of masses of cells which have an alveolar arrangement or form a coarse network from invading the lymphatic spaces. The cells are rounded or oval in shape, with large nuclei which stain faintly, and contain melanin granules. The surrounding fibrous tissue is rarefied and has no tendency to form a limiting capsule.

At one time pigmented growths of the skin of this nature were believed to be melanotic sarcomata but, as it is generally recognised that the naevus cells which compose them are of epidermal origin, they are now included in the group of the melanotic carcinomata.

The treatment consists of early and wide excision.

\section{REFERENCES}

Darier, J. Des Naevocarcinomes (Rep. XTIIth Internat. Cong. Med., London, r9r3).

Fox, IV. Two independent primary new-growths on the face (Brit. Journ. Derm., I9I I, xxiii. p. 358).

Lillex, C. H. Naevo-carcinoma (melanotic) of the scalp (Idem, I9I2, xxiv. p. 4II).

Pollitzer. Naevus-cancer. (Trans. Amer. Derm. Ass., May I900).

UNA, P. G. Naevi \& naevo-carcinoma (Berlin Klin. Wochenschr., I893), Whitehead, R. H. Malignant tumours arising in congenital mole. (Bull. Johns Hopkins Hosp., Sept. I900, p. 22I).

\section{PAGET'S DISEASE}

Syn.: Malignant dermatitis (Thin); Eczematcid epitheliomatosis (Besnier).

Definition: A peculiar form of carcinoma of the skin, usually affecting the nipple and areola of the breast in women, but occasionally occurring elsewhere, which appears as a chronic indurated eczematous patch and leads eventually to duct cancer of the mammary gland.

Description.-This affection, originally described by Paget in I874, though one of the rare diseases of the skin possesses an unusually large literature and has been the subject of much controversy. In his initial communication based on I $_{5}$ cases Paget described it as " a disease of the mammary areola preceding cancer of the mammary gland." He did not consider that the cutaneous condition was carcinoma but believed it to be independent and to be separated from the carcinoma of the gland by a layer of apparently healthy tissue. This view is no longer held and the opinion is gaining ground that the lesion in the skin is malignant from the outset and that the cancer in the gland is a further development of it or an associated growth from the epithelial cells of the lactiferous ducts.

In a typical case it appears first as an inflamed patch covered by adherent scales, situated on the areola of the nipple and stretching for a short distance beyond it. As time goes on the patch assumes a vivid red colour, and becomes raw, flat from the more or less complete 
disappearance of the nipple, dotted over with whitish islands of sodden epithelium, and moist from an alkaline sticky secretion which tends to dry up to form yellowish scabs beneath which are small areas of superficial ulceration. It is definitely indurated, and on being pinched up between the fingers feels not unlike a penny beneath a piece of cloth. In shape it may be round, oval, or polycyclical, and it has a well-defined sometimes raised, border. It does not as a rule extend far beyond the areola of the nipple, but cases are on record in which nearly the whole of the skin of the breast was affected. (Fig. 358 .)

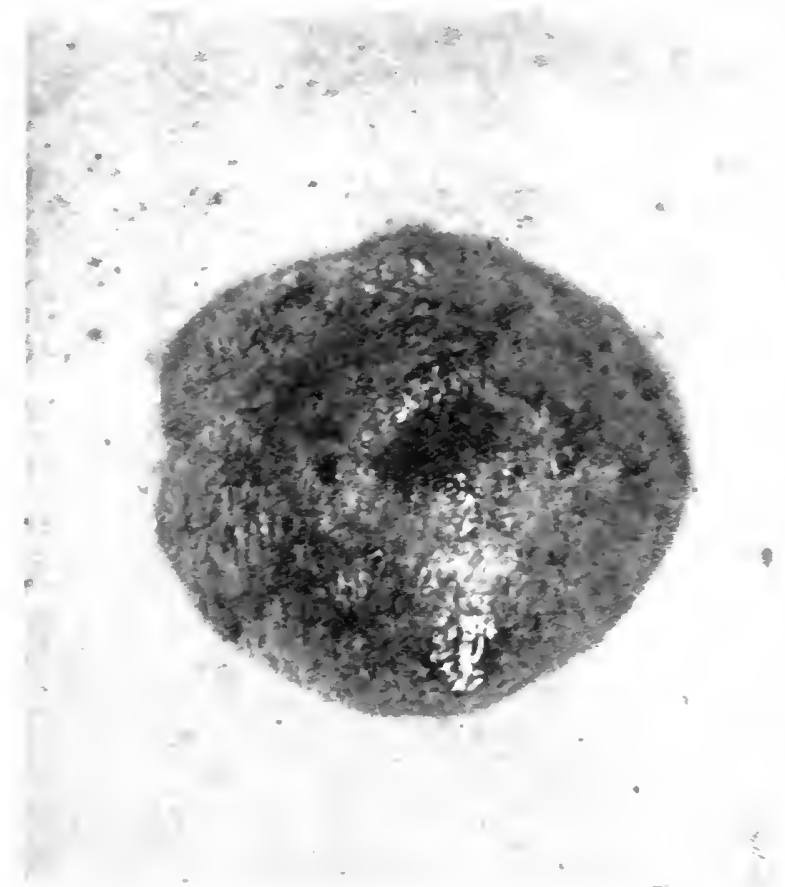

Fig. 388.-Paget's Disease of Nipple.

The lesion persists in this comparatively benign condition for a considerable time, as a rule for a few years but sometimes for as long as $I_{5}$ or 20 years. Changes of a malignant character ultimately supervene in the breast, the first signs of which are the occurrence of an indurated lump beneath the diseased skin and retraction of the nipple. The induration may spread gradually until the whole gland becomes involved and metastatic growths usually develop in the neighbouring axillary glands.

The subjective symptoms are slight, and consist of burning, pricking or itching, and occasionally pain or tenderness on pressure. It is a purely local condition and in its early stages does not interfere with the general health.

As a rule it appears in women between the ages of 40 and 60 and affects one breast, but cases are on record in which it developed on the 
vulva, pubes, and umbilicus. It may be met with also in males, in whom it has been known to occur in the umbilicus, scrotum, penis, and anus, but so far it has not been observed in the male breast. (Fig. 389.)

In the non-breast cases the diseased skin, both in its naked eye and histological appearances, is similar to that in the typical breast cases, and the skin lesion is associated with cancer of the underlying tissues.

Histopathology.-The microscopical appearances have been minutely described by a number of observers and these descriptions, which closely resemble one another, show that the histology is both constant and characteristic. (Fig. 390.)

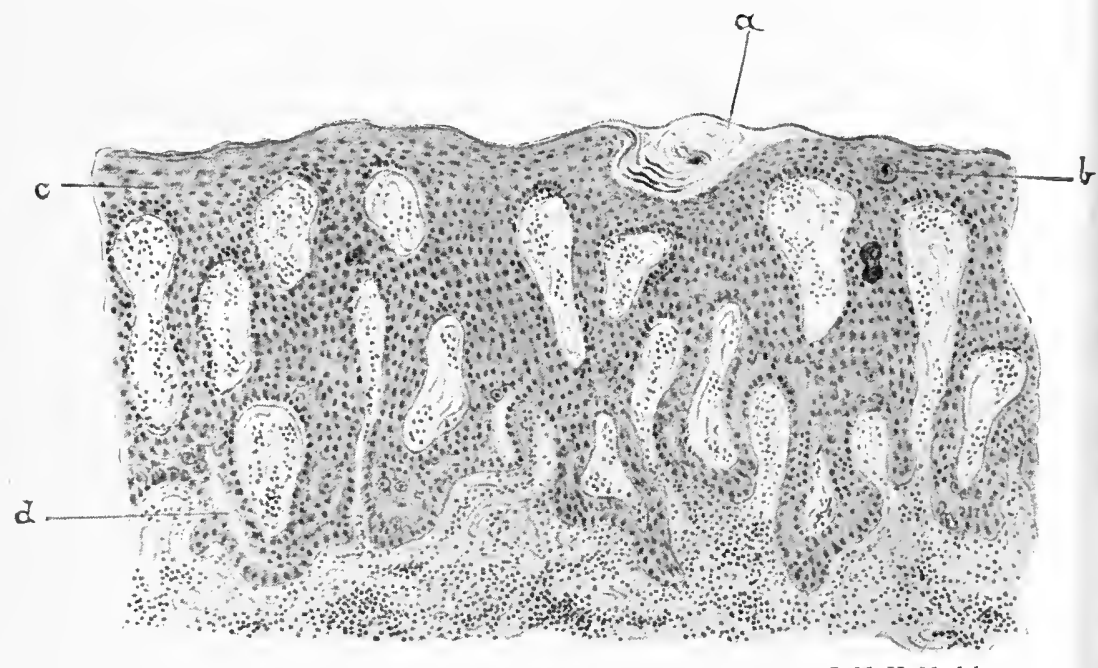

J. M. H. M. del.

F16. 390.- Section of raised border in Paget's Disease of the Umbilicus.

$a$. Cornified plug with layer of granular cells heneath. b. Horny cell-nest. c. Malpighian

layer. $d$. Oedematous degeneration of prickle-cells.

In the epidermis there is proliferation and downgrowth of the interpapillary processes which are widened and lengthened. The Stratum corneum is irregular, defective in one place and increased in another, and here and there extends down in plugs into the openings of the pilo-sebaceous follicles. The granular layer is defective, and the basal layer broken through in places by the proliferating prickle-cells. The prickle-cells are oedematous and swollen, with pale nuclei situated in distended nuclcar spaces, and some of them have broken down and given rise to irregular cavities. In spite of the oedema, mitotic figures may be detected in some nuclei, showing that the cells are still capable of proliferation. (Fig. 39r.)

The most characteristic feature is the presence of rounded structures about the size of a prickle-cell or larger, which are distributed irregularly throughout the epidermis, some being situated near the horny layer, others deep down towards the basal layer, while the majority occur 


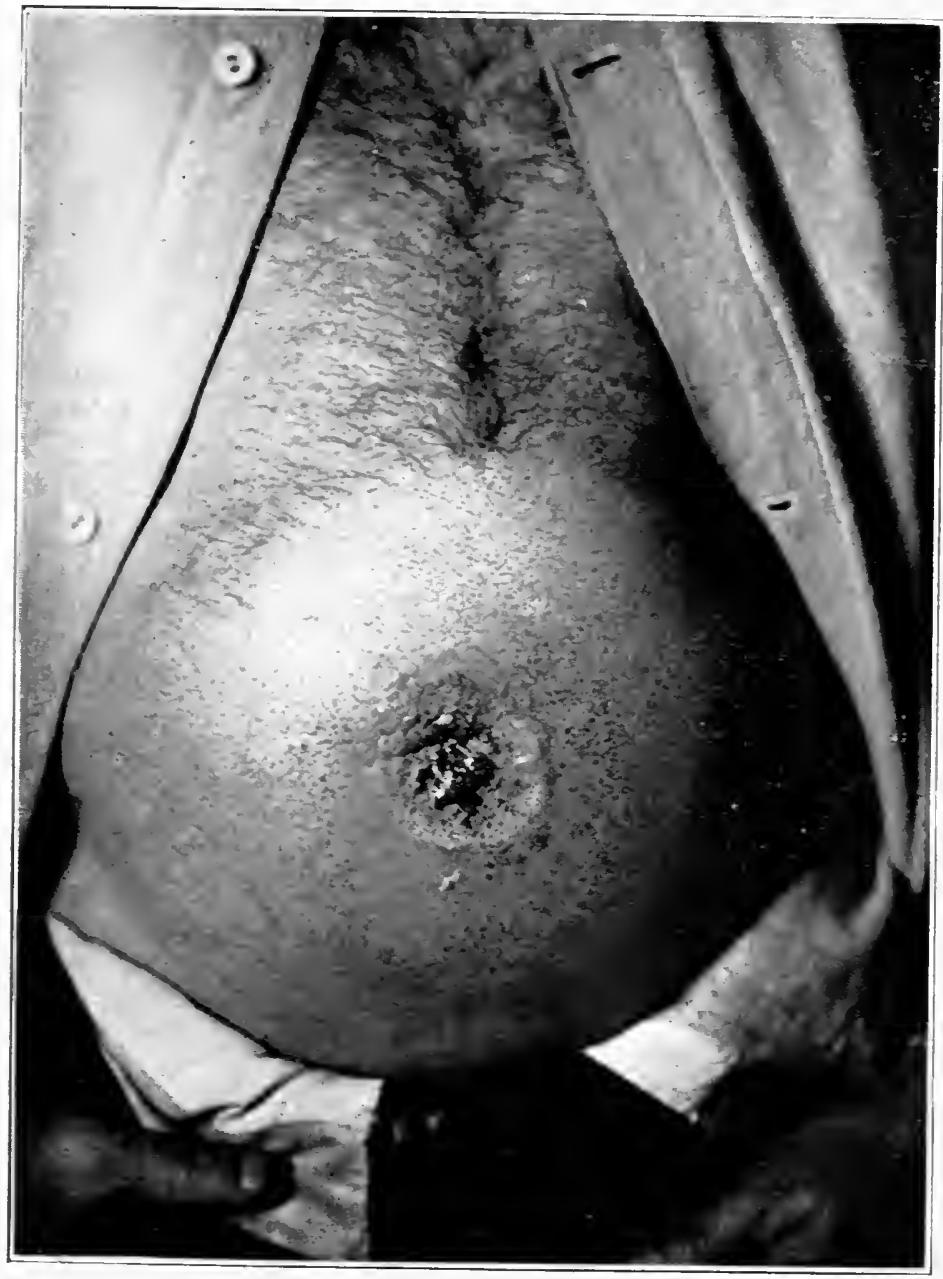

FIG. 389.-Paget's Disease.

Affecting the umbilicus.

(Case of Drs. Colcott Fox and MacLeod. Brit. Journ. Derm., I304-i 

about the middle. Those which lie immediately below the Stratum corneum resemble coccidia and are surrounded by a hard keratinised coating, or ectoplasm, which stains darkly ; others are doubly-contoured

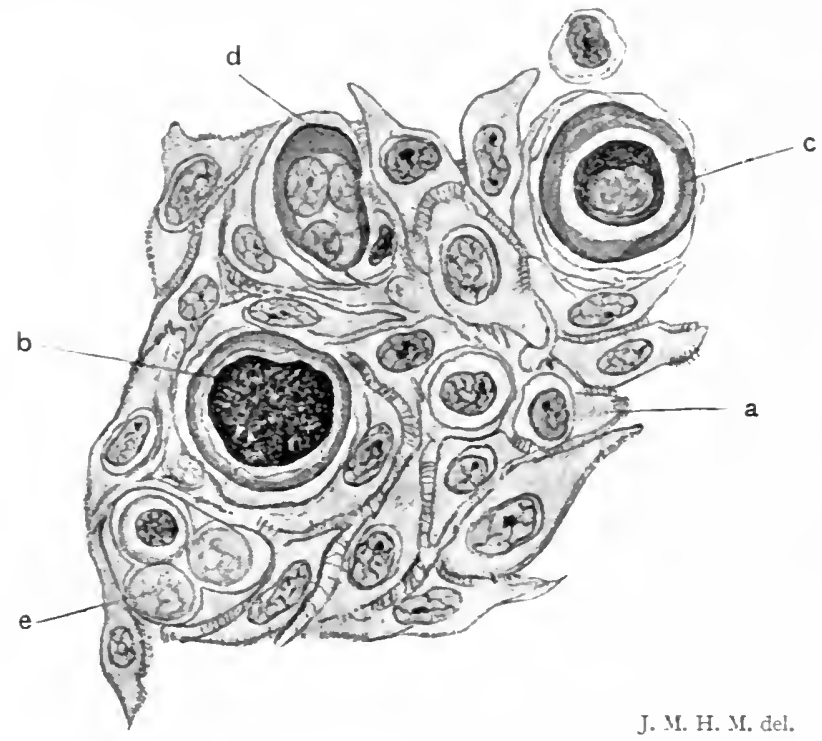

FIG. 391.-Paget's Disease. Section of border.

a. Prickle-cells, b. Partially cornified swollen cell with central degeneration. c. Similar cell with bardened ectoplasm. A. Multinuclear oedematous cell. c. Multinuclear cel enclosing newly formed "round cell.",

and more like yeast cells, with occasionally a flattened crescentic nucleus between the concentric borders, and may be due to the ency'sting of one cell within another or may be an endogenons cellular formation, as is suggested by certain of them being multi-nucleated. On account of these superficial resemblances the round bodies were once regarded as parasites and the cause of the disease, but are now known to take their origin in prickle-cells which have lost their fibrils, undergone hyalin degeneration, and assumed a homogeneous globular appearance. They are somewhat similar to the round bodies met with in the epidermis in Darier's disease (Psorospermosis follicularis), or the molluscous cells of Molluscum con-

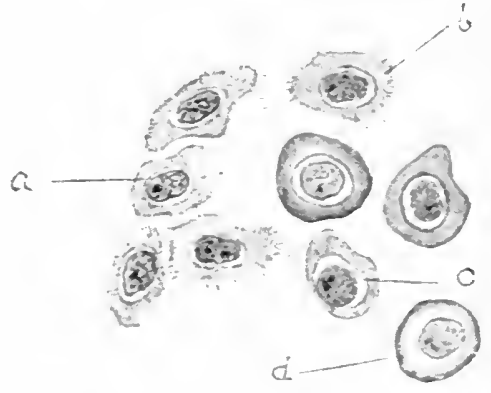

Fig. 392.-Changes in Prickle-cells in Paget's Disease of the L'mbilicus.

a. Prickle-cell with nucleus in space. b. Pricklecell with broken-up spongioplasm. c. Prickle-cell from which the prickles have disappeared. $d$. Prickle-cell in which the protoplasm has become homogeneous, the ectoplasm condensed, and the cell rounded like a coccidium. tagiosum. They are a peculiarity of Paget's disease and can be demonstrated comparatively easily by macerating a piece of the adherent scale or sodden epithelium in Liquor potassae and examining it with a high power. (Fig. 392.) 
In the corium the most marked change is a dense sheet of cellular infiltration, occupying the superficial layers and chiefly collected around the papillary and sub-papillary blood-vessels which are usually dilated. It consists largely of plasma-cells and lymphocytes and has been described as a bulwark limiting the downgrowth of the epithelium. In the area of cellular infiltration both the collagen and elastin are defective.

Even in the early stages of the disease there is proliferation of the epithelial cells of the lactiferous ducts. These cells eventually block the ducts, break through the walls, invade the lymphatic spaces in the gland, and give rise to an alveolar carcinoma which tends to become scirrhous. This invasion of the breast may take place early and may be well advanced before the clinical evidences of cancer become obvious.

There is some difference of opinion with regard to the origin of the cancer. At one time it was believed that the changes in the epidermis were cancerous from the outset and that, though they were kept in check for a considerable time by the plasma-cell infiltration in the corium, they gradually spread into the mammary gland and there set up a carcinoma. Another view, and one which has been. recently supported by Sampson Handley, is that the carcinoma of the gland is primary and that there are no definite malignant changes in the surface epithelium. According to him, the cutaneous lesion is due to blocking of the lymphatic vessels by extension of the carcinomatous growth along the ducts, which causes lymphatic oedema of the skin and the eczematous and other changes.

In the non-breast cases also alveolar cancer has been recorded, and this may have originated in some duct, such as a mucous gland duct. In a case described by Colcott Fox and MacLeod, in which the umbilicus was affected and the skin lesion was successfully excised and an invasion of the deeper tissues prevented, sections of the skin showed numerous gland follicles similar to Lieberkuhn's follicles of the small intestine, indicating that a portion of Meckel's diverticulum had been included, and it seemed probable that had general malignant changes with deep invasion of the underlying tissue occurred they might have taken their origin in the epithelium lining these follicles.

Etiology.-There is little known with regard to the causation of Paget's disease. It usually appears mysteriously without any history of traumatism or local irritation and the large majority of cases occur in women between the ages of 40 and 60 .

Diagnosis.-It has to be distinguished chiefly from eczema, which is not uncommon about the nipple in connection with lactation or as a consequence of scabies or impetigo. In eczema the affection is usually bilateral whereas in Paget's disease it is unilateral ; eczema may occur at any age while Paget's disease usually appears after 40 ; eczema is less constant in its course, nore subject to remissions and exacerbations and more amenable to treatment; in eczema the nipple is not altered in shape but simply inflamed, while in Paget's disease it is 
flattened or retracted; in eczema the patch is irregular and ill-defined, and there is no marked induration, whereas in Paget's disease the border is well demarcated and sometimes raised, and there is pronounced induration ; in eczema the surface of the lesion is not so brilliantly red as in Paget's disease.

Should any doubt still exist with regard to the diagnosis it may be settled by an examination of a scale for pseudo-coccidia or by a biopsy.

Treatment.-In breast cases, as the disease tends early to invade the deeper tissues, a radical operation with removal of the breast is the wisest course. Excellent results have been recorded both from radium and X-rays in the destruction of the cutaneous lesion but there is little gained by this so long as the disease is left in the underlying breast.

In non-breast cases a free and wide excision of the affected skin, or treatment by radium, may suffice.

\section{REFERENCES}

Butis, J. The finer anatomy of two breasts, the areolae of which had been the seats of long-standing eczema (Med. Chir. Trans., I8 76 , ix. p. I0 7 ).

Darier \& Couriliatd. Paget's Disease (Ann. de Derm. et de Syph., IS93, iv. p. 33).

Dubrelily. Paget's Disease (Brit. Journ. Derm., I9or, xiii. p. fo-).

Eraxs, Willyott. Paget's Disease (Brit. Journ. Derm., I909, xxi. p. 306).

EAbri, J., \& Traltmaxx, H. Paget's Disease (Arch. f. Derm. u. Syph., I 904, p. 37).

Fisse. Contribution à l'étude de l'epithéliomatose de Paget (Thèse de Toulouse, IS97).

Fordice. Paget's Disease of the genital region (Journ. Cut. Dis., Dec. I903).

Fox, Colcott, \& MacLeod, J. II. H. Paget's Disease of the umbilicus (Brit. Journ. Derm., I904, xvi. p. 4I).

Haxdiey, W. S. Paget's Disease of the nipple (Hunterian Lecture, Feb. I9I7; Lancet, April I9I7, p. jI9).

JAcksox, G. T. Paget's Disease (Journ. Cut. Dis., May I903, p. 20I).

Milligax, W. A. Paget's Disease of the umbilicus (Brit. Journ. Derm., I9II, xxiii. P. fII).

PAGET, J. Disease of the mammary areola preceding cancer of the mammary gland (St. Bart.'s Hosp. Rep., I 7 $_{7}$ ).

RAvogli. 'Paget's Disease (Internat. Med. Cong., Rome, IS94).

Roliestox \& Huxt. Paget's Disease (Trans. Path. Soc., London, I897).

Rosenberg, E. J. Paget's Disease (Monats. f. prakt. Derm., I909, p. 235).

TARxowsky. Paget's Disease of the penis (Ann. de Derm. et de Syph., I89I, P. +II).

Thix. Paget's Disease (Brit. Med. Journ., ISSI, pp. 433, 760, 79S).

Wichism, L. Contribution to the study of the cutaneous psorosperms if certain forms of cancer (Internat. Cong. Derm., Paris, IS89; Ann. de Derm. et de Sỵph., Jan., ISgo).

IIrffeld. Paget's Disease of the lip (Monatschr. f. prakt. Derm., I 896 , xxii. p. $3 I_{4}$ ). 


\section{CORIUM}

\section{SARCOMA}

Sarcoma of the skin is comparatively rare ; it is usually secondary to sarcoma elsewhere, but occasionally may be primary, arising on apparently healthy skin or developing on some previous pathological condition such as a scar or a boil. When it appears spontaneously it may be a naevo-sarcoma and originate in certain cells deposited in the tissue in embryonic life, which had not fulfilled their developmental intention.

Primary sarcoma of the skin is usually of the large round-celled type, though several instances are on record in which it was spindlecelled or belonged to the comparatively benign group of the fibrosarcomata.

Giant-celled sarcomata in the skin are invariably secondary to an osteo-sarcoma.

Pigmented or melanotic sarcomata in the skin are also secondary and arise from sarcoma of some pigmented structure such as the choroid. The majority of cases of so-called melanotic sarcomata, in which the growth has developed from a mole, are really melanotic carcinomata (see naevo-carcinoma).

Cutaneous sarcomata may be single or multiple. They appear as elevations of the skin, which vary in size from a pea to a walnut or larger, are round or oval in shape, usually sessile, skin-tinted, duskyred, or violaceous in colour, and firm to the touch unless in the rare instances in which they have undergone myxomatous degeneration. As a rule they grow rapidly, but occasionally may undergo spontaneous involution and wither or, as the result of traumatism and the inoculation of septic cocci, they may ulcerate and break down. Their degree of malignancy is usually great and they are liable to cause death in six months or a year, from septic complications or from sheer exhaustion following the involvement of vital organs.

Sarcomatosis cutis is a somewhat vague term which has been applied by Perrin and others to cases in which numerous sarcomatous growths, believed to be primary, are widely distributed over the cutaneous surface and appear to take their origin in a single tumour in the skin. This condition must not be confused with the disease described by Kaposi as Multiple idiopathic haemorrhagic sarcoma which is a much more benign affection and differs in its histopathology from a true sarcoma.

Histopathology.-The majority of cutaneous sarcomata are of the large round-celled type, though small round-celled, spindle-celled, giant-celled, and melanotic sarcomata, have all been described. The growth consists of a dense cellular hyperplasia, situated in the corium and separated from the epidermis by a thin layer of healthy fibrous tissue. The infiltration seems to have taken place at the expense 
of the fibrous elements as both the collagen and the elastin are diminished, the collagen persisting as delicate broken fibrils between the cells and the elastin having disappeared almost completely. In addition to the remains of the old collagen, a fine fibrous network, known as the "sarcoma reticulum," has been described by. White and Johnstone which is believed by them to be a product of the activity of the cells.

The blood-ressels in the affected area as a rule are dilated and where they are especially numerous and tortuous the name " angiosarcoma " has been applied to the growth.

The cells which constitute the infiltration rary like the cells in the classical types of sarcoma.

In the small round-celled type they resemble lymphocytes but when stained by suitable methods show a more definite halo of protoplasm around a darkly stained nucleus.

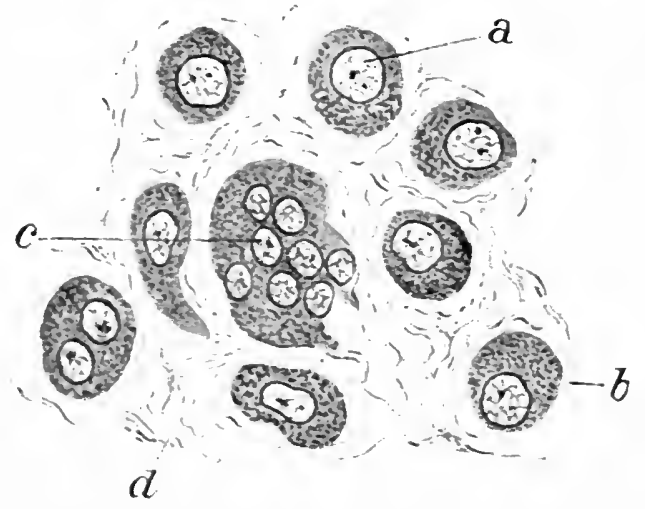

FIG. 393.--Large Round-celled Sarcoma. (x about rooo.)

a. Large round cell with coarsely granular protopiasm. b. Polygonal cell. c. Chorioplaque. 4. Attenuated fibrous stroma.

In the large round-celled variety they are round or polygonal from pressure, the protoplasm is usually granular, while the nucleus, which is situated either in the centre of the cell or eccentrically like that in a plasma-cell, stains somewhat faintly. (Fig. 393.)

In the giant-celled variety the characteristic cells are not like the giant-cells of tuberculosis but are simply multi-nucleated cells containing as many as 50 or more nuclei.

In the spindle-celled sarcomata the spindle-cells are identical with those found in embryonic tissue, some being small with fine tapering poles while others are oat-shaped or fusiform. The nuclei are oval or spindle-shaped, rich in chromatin, and stain vividly. (Figs. 394, 395.)

In the pigmented sarcomata granules of melanin are present within and between the cells. The melanin has to be distinguished from blood-pigment, which may occur in any of the varieties of sarcomata and is responsible for their brownish or violaceous colour.

The degree of malignancy of the growth may be estimated to a large extent by the histology, and it may be said in a general way 
that the more the cellular is in excess of the fibrous element, the greater the proliferative activity of the cells, the smaller the size of the cells, and the more abundant the blood supply-the greater the malignancy.

Treatment.-Primary single growths should be freely excised. Should operation be impracticable, benefit may be derived from treatment by radium, especially by burying tubes within the growth.

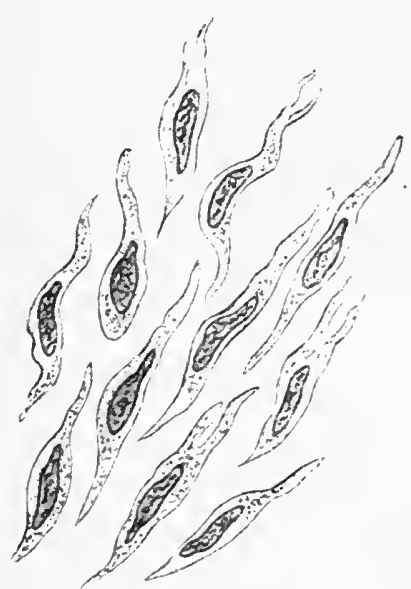

FIG. 394.-Large Spindle-celled Sarcoma.

Protoplasm of cells. Coarsely granular.

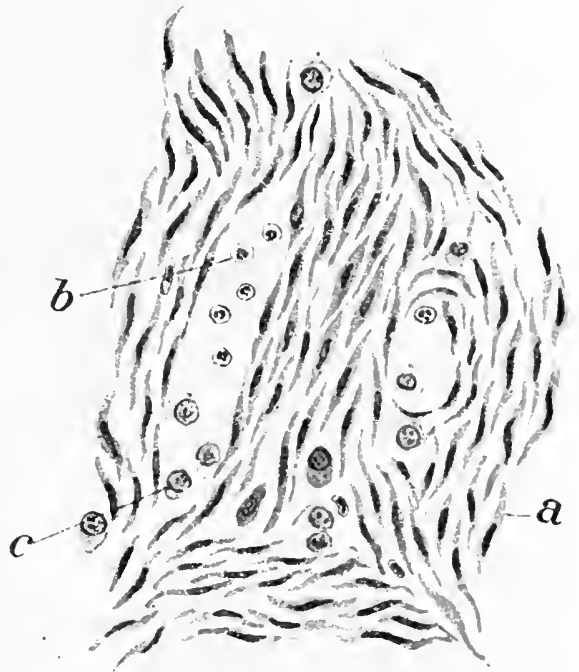

Fig. 395.-Small Spindle-celled Sarcoma. a. Spindle-cell. b. Leucocyte. c. Polygonal cell.

In Sarcomatosis cutis benefit has been claimed from the X-rays and the internal administration of arsenic, either in the form of large doses of Fowler's solution or of injections of atoxyl.

\section{REFERENCES}

De Anicis. Sarcomatosis cutis (xii. Internat. Med. Cong., Moscow. Monatschr. f. prakt. Derm., I897, xxv. p. 309).

Fordyce, J. A. Melanomas \& some types of sarcoma of the skin (Journ. Amer. Med. Assoc., I9Io, liv. p. 9I).

Harrison, A. J. Primary sarcoma of the skin (Brit. Journ. Derm., I902, xiv. p. 222).

Johnston, J. C. Sarcoma \& sarcoid growths of the skin (Brit. Journ. Derm., I90I, xiii. p. 24I).

MicDonagh, J. E. R. Relationship of lymphocyte to sarcoma, etc. (Brit. Journ. Derm., I9I4, xxvi. p. 283).

Perrin. Sarcoma (Prat. de Derm., Paris, igoo, iv.).

Prxi, G. Primary sarcoma of the skin (Arch. f. Derm. u. Sypl., I902, p. IO3).

SEQUEIRA \& TURNBUll. Sarcoma of the skin, secondary to tumour of the foot (Brit. Journ. Derm., I9I 4 , xxvi. p. 4 II). 


\section{MULTIPLE IDIOPATHIC PIGMENT SARCOMA}

Syn.: Multiple idiopathic haemorrhagic sarcoma (Kaposi) ; Acrosarcoma multiplex cutaneum telangiectodes (Unna).

Under the heading of Multiple idiopathic pigment sarcoma Ḱaposi, in IS 72 , described a rare cutaneous neoplasm which he believed to be a variety of sarcoma. In his description he seems to have included at least two conditions; namely (I) a malignant affection, in which multiple growths more or less widely distributed over the cutaneous surface were followed by metastases in the internal organs and a fatal issue after a course of from two to six years ; (2) a much more benign condition affecting the extremities, especially the legs in men, in which the growths were capable of disappearing spontaneously, did not give rise to metastases in neighbouring glands or internal organs, and did not materially interfere with the general health.

The former is probably primary sarcomatosis cutis, while the latter, which will be described here, is not a sarcoma but a peculiar connective tissue neoplasm possibly of angiomatous origin.

Vultiple idiopathic sarcoma has been met with chiefly in Italy and eastern Europe, several cases having been reported from Poland and Galicia. There have been cases also in this country, which have been demonstrated at the various dermatological societies. Practically all have occurred in men, and the few which have been reported in women are doubtful and possibly belong to the first category.

It generally appears between the ages of to and 5o, but may develop later. It is usually confined to the legs and feet, but lesions may be met with on the arms and elsewhere, and in one case the glans penis was involved. It may be bilateral and more or less symmetrical, or may be limited to one side and occasionally has been found on the arm as well as the leg.

It begins, as a rule, as discoloured patches or flat infiltrations of a brownish or purplish hue. After a time, possibly several years, painless nodules appear on the patches, which vary in size from a hempseed to a pea, are rounded or flat, soft and red like angiomata or more dense and tough and either skin-tint, purplish, or almost black from venous stasis or blood pigmentation. They may be smooth on the surface or rough and scaly, and are liable to bleed on slight injury. They grow slowly, some remaining sessile while others become pedunculated or sometimes pendulous; after reaching a certain size they may persist indefinitely, or may disappear spontaneously, or if pedunculated they may be broken off. Sometimes they become infected with pyogenic organisms and may form ulcers which, on healing, leave pigmented depressed scars, or they may develop into fungating growths. In several instances small vesicles or bullae containing blood have been observed interspersed among the nodules. (Fig. 396.)

In the majority of cases another feature is added sooner or later, 
namely diffuse oedema and thickening of the skin of the affected region which may suggest elephantiasis and greatly interfere with finer movements, especially of the hands:

The condition may persist indefinitely, for while certain of the growths may disappear, either by withering, by being broken off, or by ulceration, others remain and new ones form. In a considerable number of cases it has cleared up spontaneously after a variable period, which has been as long as twenty years.

The general health does not seem to be in any way impaired by it, and should death supervene it is the result of some intercurrent disease.

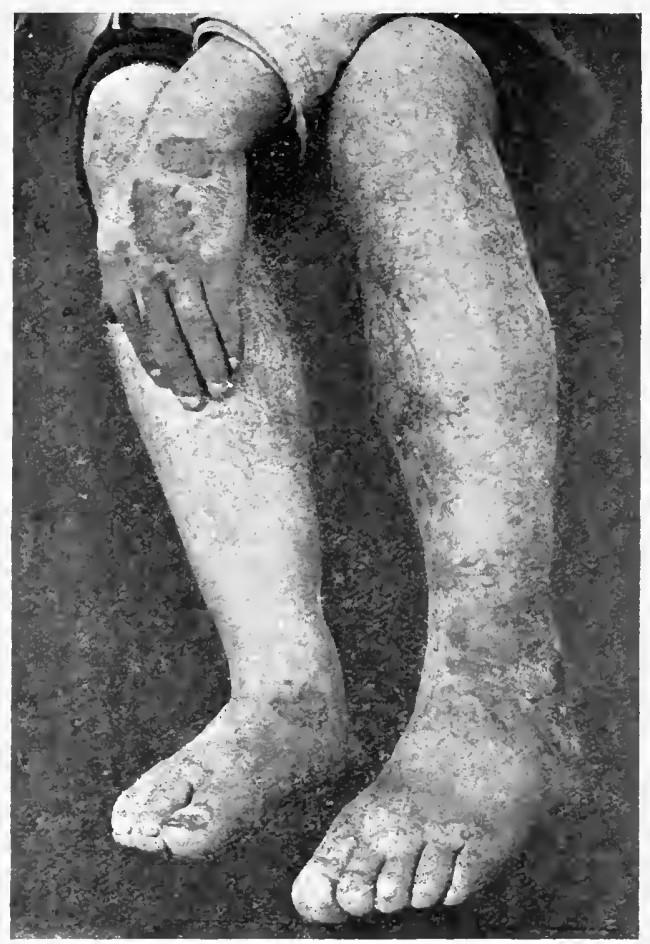

FIG. 3@6.-Multip`e Idiopathic Haemorrhagic Sarcoma.

(Dr. F. Parkes Weber's case. Brit. Journ. Derm., I916.)

Histopathology.-In I905 the writer had an opportunity of reporting on the histopathology of a typical case and as the changes found there were in the main similar to those described by previous and subsequent observers this report will be freely quoted.

Sections were made of a small early lesion and of an older reddishpurple growth about the size of the kernel of a filbert nut, which were excised from the leg. These showed that the changes in the epidermis were secondary and consisted simply of attenuation and flattening of the inter-papillary processes, due to pressure of the underlying tumour mass in the corium, some interference with cornification, and 
lengthening and proliferation of the inter-papillary processes at the sides of the growth from irritation. (Fig. 397.)

The tumour mass was situated in the middle of the reticular layer of the corium and was fairly well circumscribed by a dense layer of fibrous tissue. It was a loose fibro-cellular structure, markedly vascular, with dilated capillaries which were occasionally widened out into small sinuses containing broken down blood-cells and lined by endothelium which here and there had proliferated. The collagen fibres were thin and separated by oedema, and in the spaces between them were deposits of haemocytes and blood-pigment of a yellowishbrown colour and entirely extra-cellular. The fibrous stroma had the appearance of young connective tissue and consisted of slender bundles and fibres of collagen, and the elastic tissue had disappeared in the centre of the mass.

The cellular elements consisted of connective tissue cells with large vesicularnuclei, some spindle-shaped others with branching processes, and mixed up with them were numerous smaller polygonal cells, with deeply stained nuclei, which appeared to be fibroblasts. Around the dilated capillaries a few mast-cells were present, but there were no plasmacells such as have been described in other cases. The pilo-sebaceous follicles and sweat-glands were absent from the tumour mass, but this may have been accidental as other writers have noted the formation of the growth around the hair-follicles.

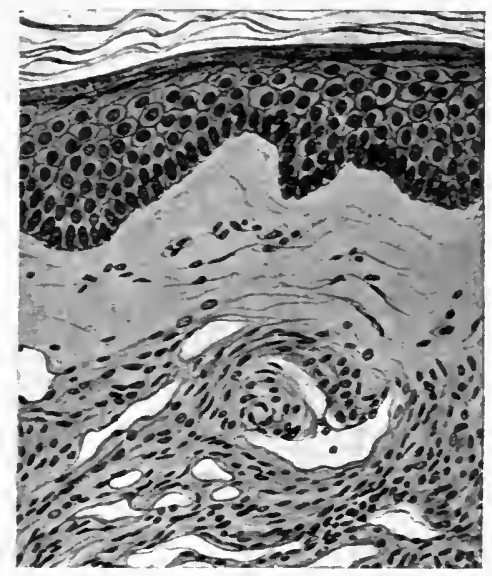

FIG. 397-- Section of Multiple Idiopathic Haemorrhagic Sarcoma.

Shows dilatation of skin-capillaries, proliferation of interstitial connective tissue, spindle-cells, and new fibrous tissue.

(Dr. F. Parkes Weber's case. Brit. Journ. Derm., Ig16.)

The appearances were of an organising connective tissue associated with marked vascular dilatation, oedema, and deposit of blood-pigment, were similar to what occurs in the healing stage of inflammation prevented from completely organising by vascular stasis, and did not correspond to any form of sarcoma. Certain observers consider that there is a new formation of blood-capillaries and that the growth is really a form of angioma, the spindle cells taking their origin from the endothelium ; but whether this is so or whether the growth is of chronic inflammatory origin has yet to be decided.

Etiology.-The cause of the condition is unknown. Repeated attempts to discover a micro-organism in connection with it have given negative results. Several of the cases have been associated with attacks of gout which have been regarded as being of etiological importance, but as evidences of gout have been absent in the large majority of cases the association was probably a coincidence. It has been 
known to commence from an injury; as in a case in which the first lesion appeared in the site of a wound as a small growth, like a septic granuloma, which was followed by bluish nodules.

Treatment.-Internal treatment does not seem to have any definite effect on it. Arsenic has been tried, both in massive doses and in smaller doses over a prolonged period, but without benefit. Locally improvement has been recorded from exposures to the X-rays.

\section{REFERENCES}

Fordyce, J. A. Multiple pigmented sarcoma of the skin (Journ. Cut. \& Gen.-Urin. Dis., Jan. I 89I).

Hutchinson. Clinical Illustrations of Surgery, vol. i., and Arch. of Surg., vol. v., p. 236.

Kaposi. Idiopathic multiple pigment sarcoma (Arch. f. Derm. u. Syph., I 872 ).

Idiopathic multiple pigment sarcoma (Path. u. Therap. der Hautkrankheiten, 5th ed., p. 922).

MacLeod, J. M. H. Notes on the histo-pathology of multiple idiopathic haemorrhagic sarcoma (Brit. Journ. Derm., I905, xvii. p. I73).

SEMENow. Sarcoma idiopathicum pigmentosum multiplex cutis (XII. Internat. Med. Cong., Moscow; Monatschr. f. prakt. Derm., I897, xxv. p. 539).

Sequeira, J. H., \& Bulloch, W. Idiopathic multiple pigment sarcoma (Brit. Journ. Derm., I90I, xiii. p. 20I).

Sequeira, J. H. Multiple idiopathic pigment sarcoma (Brit. Journ. Derm., I908, xx. p. 376).

Multiple idiopathic pigment sarcoma (Idem, I9Io, xxii. p. 356).

Iultiple idiopathic pigment sarcoma (Idem, I9I3, xxv. p. 35I).

Weber, F. P., \& DASER, P. Idiopathic multiple pigment sarcoma of Kaposi (Brit. Journ. Derm., I905, xvii. p. I35).

Weber, F. P. Three cases of so-called Multiple idiopathic haemorrhagic sarcoma (Brit. Journ. Derm., I9I6, xxviii. p. 309). 


\section{CHAPTER XLII}

\section{PSELDO-NEOPLASMS}

\section{XANTHOMA}

Syn.: Xanthelasma (Wilson); Vitiligoidea (Addison \& Gull); Plaques jaunâtres des paupières (Rayer); Fibroma lipomatodes (Virchow).

Definition: A fatty fibrous change in the skin giving rise to peculiar yellowish plaques, nodules or tumours.

Introduction.-It is to Addison and Gull in $I 8_{5}$ I that we are indebted for the earliest clear description of this affection to which they gave the name of "vitiligoidea." Previous to this, however, Raver had referred to a case in which the eye-lids were affected under the designation of Plaques jaunâtres des paupières.

The name xanthoma, from the Greek $\xi^{a v} \theta$ ós yellow, is the one which is now generally employed.

It is customary to describe three varieties of Xanthoma, namely (r) X. planum, in which the lesions consist of macules or flat, slightlyraised papules or plaques, which when situated on the eyelids are known as $\mathrm{X}$. palpebrarum; (2) $\mathrm{X}$. tuberosum multiplex in which the lesions form flat or rounded nodules or tumours, and are generally multiple and widely distributed; (3) X. diabeticorum which occurs in association with glycosuria.

Certain observers maintain that the lesions of $\mathrm{X}$. palpebrarum differ from those of $\mathrm{X}$. tuberosum multiplex; others oppose this view and point out that plane lesions indistinguishable from those of $\mathrm{X}$. palpebrarum have been observed in the eyelids in association with nodules and tumours of $\mathbb{X}$. tuberosum multiplex elsewhere, and with X. diabeticorum.

It has been asserted also that $\mathbb{X}$. diabeticorum is distinct from $\mathrm{X}$. tuberosum multiplex, as in the former the lesions are more rapid in their evolution, more transient and tend to die away spontaneously; while those of $\mathrm{X}$. tuberosum multiplex are liable to persist indefinitely.

While admitting such minor differences, these seem to be of degree rather than of kind and the pathogenesis of the affections seems so similar as to suggest that they are variants of a common 
pathological process. To simplify the description, it will be advisable to adhere to the conventional clinical sub-division.

The affection described by Balzer as Pseudo-xanthoma elasticum (see p. II34), belongs to a different category and is histologically distinct.

\section{Xanthoma Palpebrarum}

This somewhat rare condition is met with chiefly in private practice, not because it attacks one class more than another but because unless it is extensive it causes comparatively little inconvenience and the average hospital patient takes no notice of it.

In it the lesions consist of yellowish specks or plaques situated in the skin of the eyelids. These may be level with the surface or slightly raised above it, are soft to the touch, and the skin over them is smooth or slightly wrinkled. They have been compared to thin slices of cheese embedded in the corium beneath the epidermis.

In size they vary from small specks no larger than a pin's head to irregular patches the size of a finger-nail. The larger patches result from the coalescence of numerous small lesions and their conglomerate nature can be detected readily with the aid of a pocket lens.

In shape the smaller lesions are round, oval, or angular while the larger plaques are irregular, festooned, or elongated, and invariably are well-defined.

In colour they vary from fawn or chamois leather tint to deep orange, or occasionally to dirty-brown or purple, and not infrequently present a minute red speck in the centre.

They usually begin near the inner canthus of the eye, on either lid, and as a rule affect the lids of both eyes symmetrically. In extensive cases the eyelids may be almost completely involved and become thickened, yellow, and heavy, and by overhanging the eyes may interfere with sight.

The lesions on the eyelids may be associated occasionally with plane lesions elsewhere on the face, or even in the mucous membrane of the mouth, and sometimes with tumour lesions on the body or limbs. As a rule there are no definite subjective symptoms, except perhaps slight itching.

It occurs chiefly in adults, but may begin in childhood in which case it would suggest a naevoid condition, and has becn met with in several generations. It is more common in women than in men and is not infrequently associated with a sallow skin, dark eyes, attacks of migraine, a tendency to jaundice from a disordered state of the liver, or with uterine or ovarian disturbances.

\section{Xanthoma Tuberosum Multiplex}

The lesions in this type of xanthoma differ greatly in shape and size. They may consist of small specks or flat papules scarcely raised 


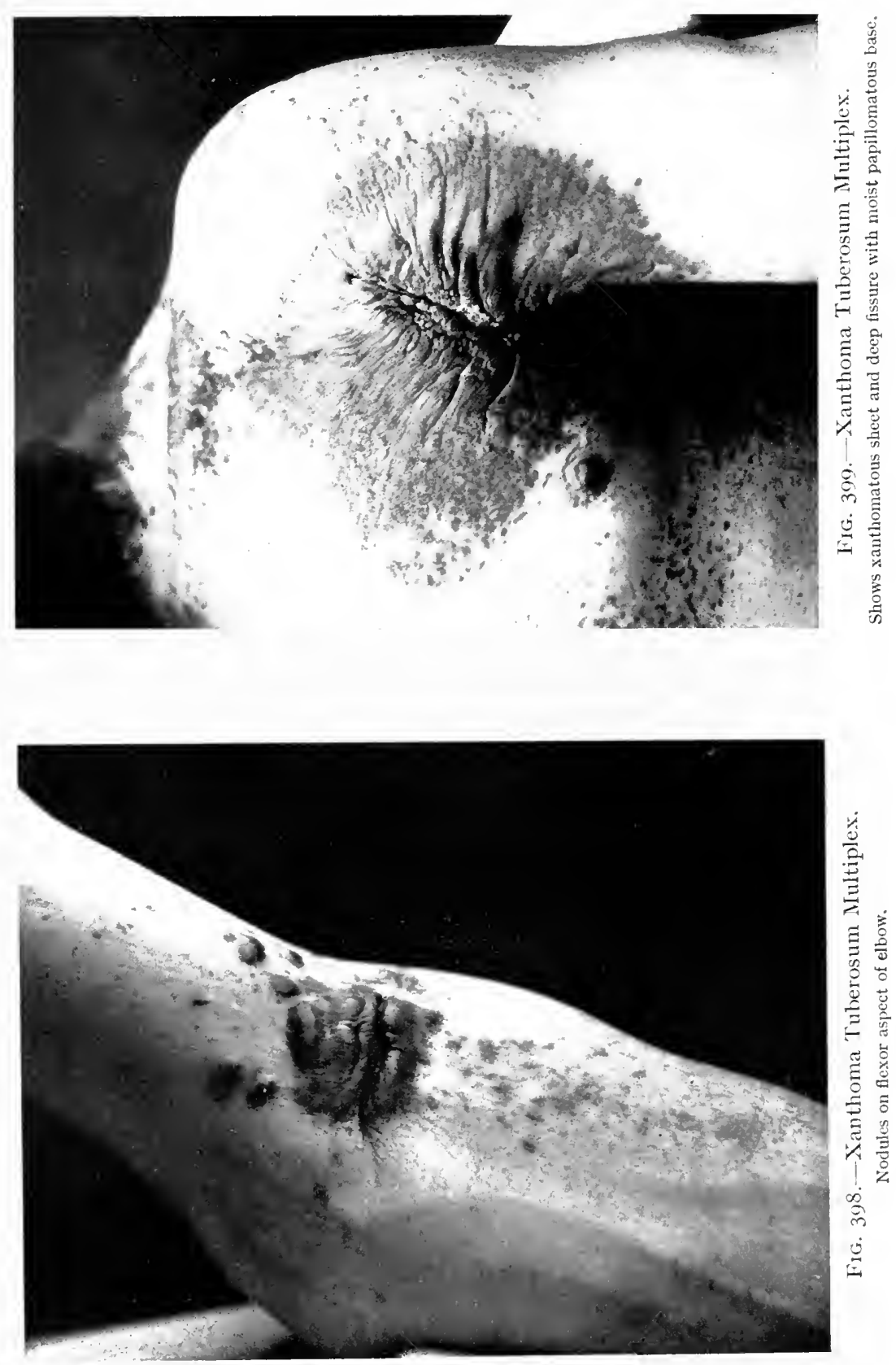

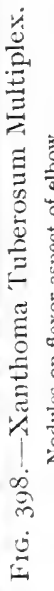

To face $p$. II 73 . 
above the level of the skin, or of rounded nodules, tumours as large as a tangerine orange, or conglomerate patches involving considerable areas. In outline they may be rounded, festooned, or irregular, the larger lesions as a rule being made up by the coalescence of smaller ones and generally presenting a lobulated appearance. In colour they vary from whitish-yellow to orange or brown, and in consistence may be soft, hard, or even cartilaginous. The surface may be smooth or wrinkled, and the sweat-pores over it so distinct as to suggest orange peel. (Fig. 398.)

The lesions may be few in number and situated chiefly about the larger joints, such as the elbows and knees, or they may have a wide distribution and attack almost any part of the skin, but they invariably show a predilection for situations subjected to movement. They may occur on the face, about the forehead, eyelids, cheeks, on the ears, scalp, flexures of the arms, borders of the axillae, and even on the penis and vulva. They are occasionally present in the palms as stripes or streaks over the larger folds, and similar linear lesions are sometimes met with in the webs of the fingers, sides of the neck, soles of the feet, and around the anus.

They usually commence as a reddish-brown speck or a slightly raised flat papule which is soft, almost imperceptible to the touch, and does not totally fade on pressure with the diascope but leaves a reddish stain like purpura or presents dilated capillaries ramifying over it. These reddish specks gradually become more prominent and when about the size of a small lentil assume the yellowish tinge and firm consistence of typical xanthoma lesions. They may grow into nodules as large as a bean, or may coalesce and give rise to lobulated tumours or conglomerate plaques. The larger lesions about the joints are liable to be broken up by folds from movements, or cracked from stretching so that deep fissures form which allow the secondary inoculation of pyogenic micro-organisms and lead to suppurative changes and the formation of purulent scabs. (Fig. 399.)

Their evolution is usually slow, though cases are on record where they have developed extensively in a few weeks. As a rule the lesions keep on appearing and gradually increasing for months or years, then become stationary and may persist indefinitely. Should involution occur no trace may be left, but in most cases the skin is altered in texture and slightly wrinkled and there may be superficial scarring.

Xanthoma lesions may occur in connection with tendon sheaths and periosteum, where they give rise to deformities and swellings of a cartilaginous consistence, which may reach the size of a hen's egg and are occasionally associated with loss of adjoining bony tissue.

They may occur on the mucous membranes of the mouth, oesophagus, bronchi, and larynx, and on the cornea of the eye.

They have been found post-mortem in the liver under the capsule of Glisson and about the bile ducts, in the intima of the aorta and 
great arteries, in the endocardium, in the peritoneum and around the spleen.

There are practically no subjective symptoms, except slight tingling and itching, but where the affection occurs about exposed parts it is most disfiguring, and when present to any extent about the joints may impede movement.

This type of xanthoma is met with generally in adult life and frequently in connection with jaundice due to some disorder of the liver, such as cirrhosis, or xanthomatous formation about the liver, bile ducts, or gall bladder. In addition to ordinary jaundice the skin may present a curious yellow pigmentation from xanthoma due to a slight fatty deposit which is not sufficient to cause a tumour formation or to render the skin definitely opaque (Xanthoma chromia).

When it occurs in infancy and early childhood it may be of congenital or naevoid origin.

\section{Xanthoma Diabeticorum}

Xanthoma lesions are not infrequent in association with glycosuria, both in Diabetes insipidus and Diabetes mellitus. They generally take the form of small nodules, varying in size from a millet-seed to a lentil or bean, and are usually located about the extensor aspects of the forearms, elbows, legs, and buttocks, but may occur elsewhere in the skin and in rare instances have been found in the mucosa. They are pale in colour, usually fawn tinted, smooth on the surface, soft in consistence or hard and tough, and are occasionally surrounded by a pink congested halo. Sometimes they are reddish with a yellow apex and suggest pustules but when punctured prove to be solid. When the lesions are small and papular the condition is sometimes described as Lichen diabeticorum. (Fig. 40o.)

The nodules may be few in number or numerous and usually remain discrete though occasionally they may coalesce, especially about the elbows and knees, into conglomerate patches or lobulated masses. They develop rapidly, become fully evolved in a few days, and after persisting for a varying time-which may be weeks or monthstend to disappear either spontaneously or in consequence of successful treatment of the glycosuria. The affection is thus far more transitory than Xanthoma tuberosum multiplex, but as new lesions keep on coming out it may persist for years and even after it has completely gone it may recur.

The lesions are not actually painful, but touching them may produce a tingling sensation which may last from several minutes to an hour.

When they disappear no trace may be left or only slight scarring.

It is met with in young people suffering from Diabetes mellitus, but is much more common in connection with the glycosuria of hepatic origin occurring in middle-aged well-nourished adults in whom the existence of sugar in the urine may be first suspected from the presence 
of the eruption. The xanthoma does not appear to bear any relation to the amount of sugar, for it may occur where only a grain or two of glucose to the ounce is present or where it amounts to 7 or $8^{\circ}$.

Occasionally albumin as well as sugar has been found in the urine in association with it.

Histopathology.-The descriptions of the microscopical appearances in xanthoma differ in detail ; there is a general unanimity with regard

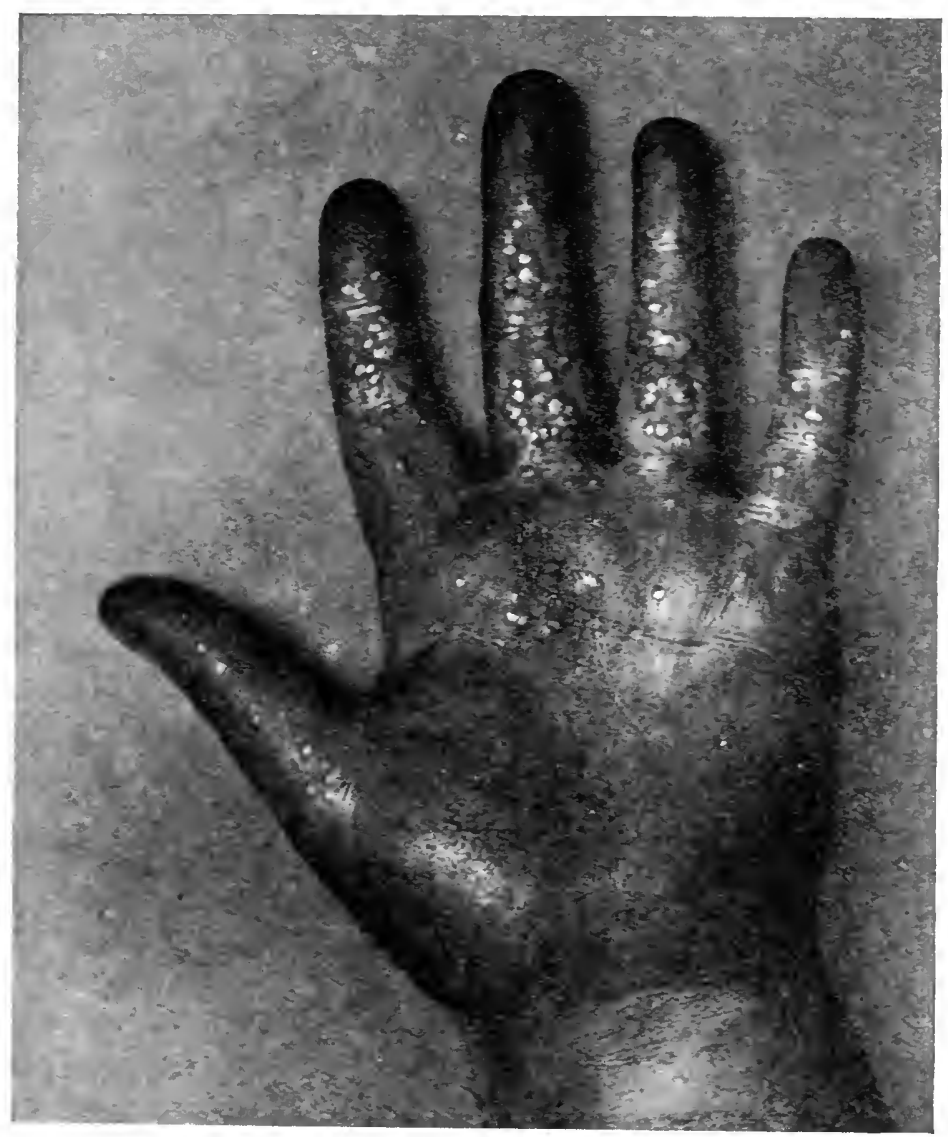

FIG. 400.-Xanthoma Diabeticorum.

(Dr. G. H. Lancashire's case. Brut. Journ. Derm., I907.)

to the principal changes which occur but there is considerable diversity of opinion with regard to their interpretation.

The pathological process inay be confined to the corium, or may spread down to the subcutaneous tissue. It is of a fatty-fibrous nature and consists of a cellular and fatty infiltration and a fibrous-hyperplasia-the lesions becoming hard in relation to the degree of the fibro-cellular change.

In the initial reddish spots there is dilatation of the capillaries of the sub-papillary layer in the affected area, associated with proliferation of the endothelium, and a cellular infiltration around the 
dilated capillaries consisting of round cells, oval or small fusiform cells of connective tissue origin, and occasional mast-cells. (Fig. 40r.)

In older lesions, large cells known as " xanthoma cells" or " xanthoma giant-cells" with two or more nuclei are generally present.

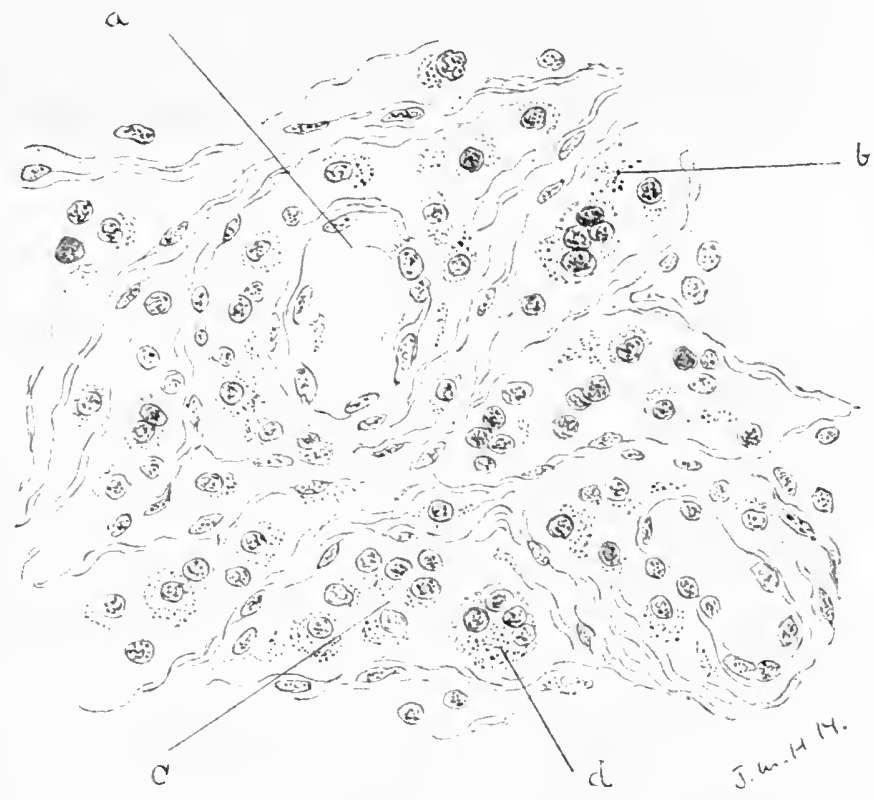

FIg. 40I.-Xanthoma Tuberosum Multiplex.

$a$. Dilated capilaries. $b$. Fatty infiltration of connective tissue cells. c. Xanthoma cells. $d$. Xanthoma giant-cells.

These are derived from the round or oval connective tissue cells or from the endothelium, and are probably the result of the irritative action of fat which has infiltrated the protoplasm of the cells. They may be widely distributed throughout the

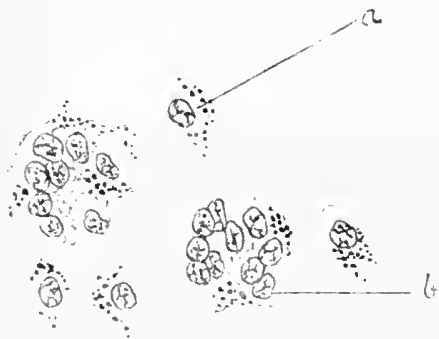

FIG. 402.-Xanthoma Tuberosum Multiplex.

$a$. Xanthoma cells, $b$. Xanthoma giant-cells. whole fibro-cellular mass, but are more often arranged in small groups about the capillaries-the groups not infrequently being surrounded by fusiform cells and newly-formed connective tissue fibres and suggesting nodules of tuberculosis. As time goes on the tissue tends to become more and more fibrous and permanent. (Fig. 402.)

Sections specially stained for fat with osmic acid or Sudan 3 show the presence of fatty granules in the dilated capillaries, in the endothelial cells, in the xanthoma cells, and lying free in the interfascicular lymphatic spaces. The fatty deposit is most marked immedi- 
ately around the capillaries and may extend down to the subcutaneous tissue. When it is removed from the xanthoma cells by ether or alcohol a wide spongioplastic network is left.

The precise nature of the fat is uncertain but it is believed to be a lipoid which exists in the blood in the form of a cholesterol-fattyacid-ester and which has been found in association with jaundice and glycosuria.

In addition. to the fatty granules, crystals of cholestrine can generally be found, either free in the lymph spaces or in the xanthoma cells.

The fat, which occurs in the form of granules or droplets, infiltrates the cells in much the same way as it does in the formation of adipose tissue, except that the granules in the xanthoma cells show no tendency to join together to form globules.

The sweat-glands are not involved, unless the fibro-fatty deposit is excessive when, by encroaching upon them, it causes them to atrophy.

The overlying epidermis may be little, if at all, affected except for a deposit of yellowish fat granules in the cells of the basal layer, but where the fibro-fatty changes are considerable it may be stretched and the inter-papillary processes flattened.

In Xanthoma tuberosum multiplex of the plane variety the fibrofatty changes are confined to the corium beneath the papillary layer and do not extend so far as the subcutaneous tissue; in the nodular variety the papillary layer is affected and the pathological changes spread into the subcutaneous layer.

The appearance of the lesion suggests that the fatty deposit is the fons et origo of all the pathological changes and by its irritative action produces the dilatation of the capillaries, the cellular infiltration, and the fibrous hyperplasia.

In Xanthoma diabeticorm the histology of the lesions is similar, except that the fibrous formation is less marked in the tissue and the cellular infiltration relatively greater than in Xanthoma multiplexpointing to its being more definitely inflammatory. In it there is a tendency for the affected tissue to break down in the centre to form an amorphous mass from granular degeneration of the cells and fragmentation of the collagen. The elastic tissue, on the other hand, is not affected and consequently appears to be proportionately increased.

The histology of the plane lesions of Xanthoma palpebrarum is said to differ from that of Xanthoma tuberosum multiplex. In it cord-like masses of fat have been described lying parallel to the fibres of the orbicularis muscle; these, it has been suggested, are the result of a fatty degeneration of the muscle fibres and not of a fatty infiltration of the cells.

In short it would seem from a study of the histology of the different forms of xanthoma that they are simply variations of one morbid process and that any alterations in the appearance of the 
lesions depend on the precise lipoid responsible, the degree of irritation caused by it, and the situation in the skin. The reason why the larger joints and the eyelids are specially liable to the fatty deposit is that the movements are excessive there and consequently the fat is more apt to get pumped out of the capillaries and deposited in the skin.

Various theories have been advanced to explain the pathogenesis of xanthoma, such as, that it is an inflammatory disturbance of toxic origin, resulting in a fatty degeneration wherever the toxin is excessive ; that it is a form of naevus, the xanthoma cells being of embryonic origin; that it is due to some toxin in the blood which causes a deposition of the lipoid which infiltrates the skin. The last view is the one which seems most probable and according to which the different facts of the disease are most readily explained.

Diagnosis.-No great difficulty should be experienced in the recognition of this affection, the yellowish, opaque, well-defined macules, nodules, or tumours, with the smooth skin stretched over the surface being so characteristic as to render the differential diagnosis obvious.

In infants multiple xanthoma has been mistaken for Urticaria pigmentosa. In that affection, however, the lesions are more multiform, some being pink, others yellowish or brownish, while some are definitely urticarial and may be associated with factitious urticaria.

Xanthoma palpebrarum might be mistaken for milium but in it the lesions are whiter, roundish in shape, and when opened the white contents may be shelled out.

Prognosis.-The prognosis in Xanthoma multiplex and palpebrarum is usually unsatisfactory, and when once the lesions have appeared they tend to persist indefinitely. In Xanthoma diabeticorum, on the other hand, the lesions may involute should the sugar disappear from the urine, but are apt to recur if the sugar returns.

\section{TREATMENT}

Treatment is chiefly demanded when the lesions interfere with the movements of the joints or the eyelids, or for aesthetic reasons when they occur in exposed parts. Unfortunately it is almost invariably unsatisfactory.

Small lesions in the eyelids may be destroyed by electrolysis; the needle forming the negative pole is inserted horizontally along the length of the xanthomatous macule or plaque and a current of 3 milliamperes is passed for half a minute. This is repeated until the whole lesion has been gone over. It is a painful procedure and the results are not sufficiently certain to make it worth while except where the lesions are small and insignificant.

Another method is to touch them several times with acetic acid. This produces an inflammatory reaction which masks the xanthoma for a time but which, on subsiding, as a rule leaves the condition much as before. 
When the patches round the eves are large the most satisfactory method is excision, which must be done carefully to aroid giving rise to epiphora or ectropion.

For xanthomatous lesions elsewhere the application of keratolytics, such as salicylic plaster or salicylic collodion, may be of benefit. The salicylic collodion, of a strength of $I$ in 8 , should be painted on several times a day.

Exposures to $\mathbf{X}$-rays give better results than the above procedure and are capable of reducing, if not of remoring, the patches. Pastille doses of the X-rays should be given at intervals of not less than six weeks.

Benefit may be derived also from freezing with solid carbon dioxide.

Except in Xanthoma diabeticorum internal treatment is of no benefit and there is no drug which can be said to have a specific action on the lesions. It is well, however, that any obrious defect in the general health, such as jaundice or uterine disturbances, should be treated. In Xanthoma diabeticorum the eruption may be made to disappear should the glycosuria be successfully dealt with by diet or by the administration of arsenic, codein, or other appropriate remedy.

\section{REFERENCES}

Xanthoma palpebraram.

Uxxa, P. G. Histopathology (Walker's Trans., I896, p. 945).

Yanthoma tuberosum multiplex.

MacLeod, J. .I. H. (Brit. Journ. Derm., I9I3, xxv. p. 344).

Politizer, S. (Brit. Journ. Derm., I $893, v$ p. 367).

Pollitzer \& Wile (Journ. Cut. Dis., May I9I2, p. 235).

TöröK, L. (Ann. de Derm. et de Syph., I893).

Weber, F. Parkes (Brit. Journ. Derm. \& Syph., I9I7, xxix. p. 202).

\section{Yanthoma diabeticorum.}

Bessier (Ann. de Derm et de Syph., I889, v.).

Crocker, RadciffFe (Brit. Journ. Derm., i 892 , iv. p. 253).

Morris, M., \& Clarke, Jacksox (Brit. Journ. Derm., I892, iv. p. 237).

Peser, W. A., \& Johnstox, O. P. (Journ. Cut. Dis., Dec. I9oS).

Robixsox, A. R. (Brit. Journ. Derm., IS9I, iii. p. I06).

\section{SCLERODERMIA}

Der. : $\sigma \kappa \lambda \eta \rho o ́$, hard; and $\delta \varepsilon^{\prime} \rho \mu \alpha$, the skin.

Syn.: Scleriasis; Sclerema adultorum; Sclerodactylia; Norphoea; Fr., Sclérodermie.

Definition: A chronic cutaneous disease which is diffuse or circumscribed, and characterised by peculiar changes in which the affected shin becomes smooth and hard and tends to undergo atrophic changes and to become bound down to underlying structures.

This somewhat rare affection has been known under such a variety of names that considerable confusion has resulted in the past with regard 
to it. One of the earliest was " Ichthyosis cornea " given to it by Willan in 1808 ; this name was subsequently applied by Alibert to verrucose naevi of the type of Ichthyosis hystrix, and he substituted for it "sclerema," under which he included not only sclerema of adults but also sclerema of infants-an affection which it is advisable to describe separately. In I854 Addison re-christened the circumscribed variety the unfortunate and confusing name of " true keloid," and later Erasmus Wilson gave it the name of " morphoea." It has been described also under the heading of "vitiligo" and, from the incorrect supposition that it was connected with leprosy, as " Lepra vitiligo."

For convenience of description sclerodermia is divided into generalised or diffuse sclerodermia and circumscribed sclerodermia. Although these differ in their evolution, their clinical and histological appearances closely resemble each other and, as all gradations between them may be met with, it seems probable that they are variants of one pathological process.

The generalised type is the more rare and comparatively few authentic cases of it have been recorded in this country; out of I0,000 cases of skin disease Radcliffe Crocker met with only 2, while out of 27,000 general medical cases seen by Byrom Bramwell, there were only 9.

\section{Generalised or Diffuse Sclerodermia}

This type of sclerodermia may appear without prodromal symptoms and for no apparent reason; on the other hand it may be preceded for a few weeks by some general disturbance, such as fever, chilliness, pains in the joints or limbs. It may develop acutely and be ushered in by erythema, urticaria, or even by bullous formation; as a rule it is much more insidious in its onset and is felt before it is seen, being brought to the notice of the patient by a feeling of tightness or immobility of the skin.

It generally appears first as a pseudo-oedematous condition of the skin of the upper part of the body, especially of the neck or shoulders, but it may commence on the face, trunk, or extremities. From there it gradually spreads in a more or less symmetrical fashion, until it sometimes involves the greater part of the cutaneous surface. Associated with it, according to the part affected, there is stiffness of the neck, immobility of the chest, difficulty in opening the mouth or eyes, rigidity of the limbs, etc. When the extremities are involved it may be accompanied by rheumatic pains or rheumatoid arthritis.

The affected skin presents marked changes. It becomes smooth and glossy from the disappearance of the natural ridges and furrows, more or less hairless, white or yellowish in tinge, and presents dilated capillaries ramifying over it. It is thickened as if it were oedematous, but it is not a true oedema, and pits with the greatest difficulty on pressure. In appearance it has been aptly compared to a bladder 
distended with lard. In some cases it is pigmented a yellowish-brown tint which is either diffuse or patchy and, when present in symmetrical areas about the face, axillae, or around the waist, suggests the pigmentation of Addison's disease. A peculiar complication observed by the writer was the presence of warty pigmented patches of a blackish tint situated about the elbows, axillae, groins, and neck.

At first the skin can be pinched up in folds, but as time goes on it becomes adherent to the underlying muscles or bones, causing immobility and disfigurement. The face becomes smooth and expressionless, the wrinkles of the forehead and cheeks disappear, and the lips and eyelids become so thickened that there may be difficulty in moving the jaws or opening the eyes; from involvement of the neck the head becomes fixed, though not twisted as in torticollis, and the movements of the larynx impeded so that swallowing is difficult; in the thorax, the furrows between the ribs become obliterated and the whole region encased in a cuirass of indurated skin which impairs respiration; the female breasts become hard, shrunken, and conical; if the arms be affected on the extensor aspect flexion is interfered with, while if on the flexor aspect the limb becomes fixed in a semi-flexed position; if the legs be attacked there is difficulty in walking.

The condition is slowly progressive though subject to periods of intermission, and may take months or years before it reaches its full development. It may remain stationary for a time, undergo retrogressive changes, and the skin gradually return to normal ; but as a rule atrophic changes supervene, in which the yellowish tinge is replaced by a dirty-grey colour, the surface becomes flaky, owing to the disappearance of the subcutaneous fat the skin becomes bound down to the underlying structures, a state of complete immobility results, and the affected areas assume an appearance which has been compared to solid wax or to wood covered with parchment. The continuous pressure of the hard skin leads to atrophy of muscles and bone, and to shrinkage and deformity. The face becomes withered and hard and the skin bound down to the bone; the lips become thin and tight so as to interfere with eating and speaking; the nose becomes shrivelled and elongated as in the cicatricial phase of old-standing lupus; the eyelids become retracted and the eyeballs shrunken, and a blank staring appearance results; the ears becone withered, dragged back, and fixed over the mastoid processes, and sclerotic changes may occur in the drum itself causing deafness; the limbs become so shrunken and fixed that flexion is impossible without breaking the skin; and the thorax and abdomen become so rigid as to cause disastrous effects on the general health, impeding the respiration and circulation, interfering with the functions of the viscera, and leading to a progressive malnutrition followed by extreme marasmus and a fatal issue from inanition or from some intercurrent disease.

The mucous membrane may be involved and atrophic patches may appear on the inside of the cheeks, palate, and tongue, while the 
teeth may become loose and fall out; the mucous membrane of the vagina may also be affected.

The sweat-glands in the diseased areas are destroyed and in consequence, a severe strain may be put on the kidneys resulting in uraemic symptoms.

The vitality of the affected skin is so lowered that even slight injuries, especially about prominent parts like the elbows, knees, or over the malleoli, are liable to be followed by breaking of the skin, intractable ulceration, and even local gangrene.

There are no marked changes in sensibility, though occasionally there may be slight burning, itching, or numbness, and in rare instances rheumatic or neuralgic pains.

In rare instances sclerodermia may be complicated by the presence of subcutaneous calcareous deposits, which would appear to be a terminal phase of the fibrosis as they occur in the midst of the dense connective tissue. These deposits gradually come to the surface, there set up ulceration, and are finally expelled. They are most marked about joints, and though first present on the hands may occur later on the feet and may eventually develop in other parts of the skin. Chemically, the concretions are found to consist of carbonate and phosphate of calcium.

\section{Sclerodactylia}

Sclerodactylia is a variety of diffuse sclerodermia which begins in the hands and tends to spread from there on to the arms, neck, face, and chest. It commences with symptoms similar to those of Raynaud's disease and attacks several fingers or the whole hand and may affect both hands more or less symmetrically. In it the vaso-motor paroxysms increase in frequency until at last a permanent asphyxial phase occurs ; this is followed by progressive sclerotic changes in which the skin becomes thickened, occasionally ulcerated and cicatrised-especially about the finger-tips and knuckles-and the nails become dystrophied, opaque, discoloured, sometimes narrowed and distorted like the claw of a bird, or completely obliterated. The fingers become hard, stiff, and shiny, and either permanently distended or semi-flexed with the joints ankylosed, the terminal phalanges may undergo spontaneous necrosis and absorption, the fingers may be transformed into hard shrivelled stumps, and all grades of disfigurement may be met with, from a claw-like deformity similar to the "main en griffe" of leprosy to a mutilated hand with atrophic stumps.

There is considerable pain, at first in connection with the asphyxial crises and later from attempts to move the rigid joints or from ulceration.

Occasionally it may commence in the feet, in which case the Raynaud's phenomena are not so definite or may be absent. 

PLATE SII.

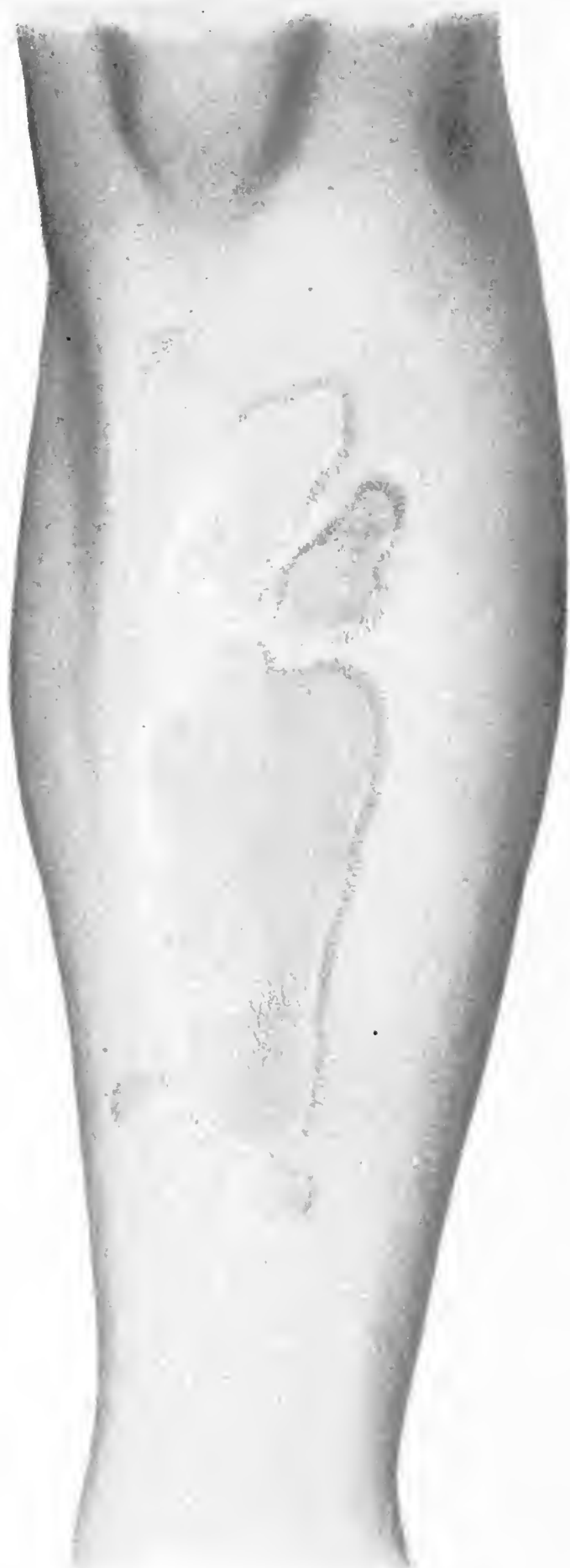

MOKPHOEA, UK CIRT 'USCKIBED SCLERODKRMIA.

Shows ivory-like sclerodermic patches, with telangiectases, and surrounded by at pinkish intammatory halo. 


\section{Circumscribed Sclerodermia or Morphoea}

In it the sclerodermia is limited to one or several patches, which tend to increase peripherally and may involve considerable areas but are never as widely distributed as in the diffuse type. It may occur in the form of plaques, bands, or guttate lesions-all of which may be present in the same case.

(i) Plaque Variety.-The plaques of sclerodermia are the most characteristic and common lesions of the disease. They may be preceded for weeks or months by an itchy patch of a red or violet tinge. This gradually fades and is replaced by a sclerotic lesion, surrounded by a lilac border which on close examination is found to be made up of small dilated blood-ressels. As a rule the sclerotic changes first draw attention to the lesion and it is noted accidentally as a glossy, indurated patch which is difficult to pinch up between the fingers. When fully developed, owing to obliteration of the natural furrows of the skin and disappearance of the hairs and sweat-glands, the plaque is smooth and shiny like polished bone, or from the presence of minute patulous follicles it may resemble parchment. In consistence it feels like a bladder filled with lard ; in colour it may be bluish-white like the ground of Chinese porcelain or yellowish like old ivory, and it may be uniform or mottled, while small dilated capillaries may often be observed ramifying over the surface. The margin is well-defined and occasionally surrounded by a paler halo or, more often, by a pinkish or lilac border of dilated capillaries about a quarter of an inch in breadth with, in many cases, a yellowish-brown pigmented halo beyond. The plaques are round, oval, or irregular, and may be definitely raised and suggest cheloids, may be level with the skin and resemble a piece of visiting card or parchment let into it, or may be depressed. In rare instances the surface may be uneven and nodulated. (Plate XIT.)

They may occur in any situation but are particularly frequent on the neck, the breasts in women, the intercostal regions, and the shins and thighs, and are often symmetrical. On the chest and limbs they tend to be oval, with the long axis in the direction of the ribs or of the limbs. On the scalp they are singularly hard and adherent, and are generally depressed, bald, and atrophic. They may occur also on the mucous membranes of the lips, tongue, inside of the cheeks, pharynx, or vagina.

The plaques extend slowly until they reach a certain size, remain stationary for months or years, and then undergo retrogressive changes, either spontaneously or under treatment. The hardness may diminish, the lilac border disappear, the pigment fade, and the plaques re-assume the appearance of the normal skin ; or they may be replaced by brownish patches without much alteration in the texture of the skin, forming the type of the disease known as Morphoea nigra, or by atrophic changes in which the lesions become greg, depressed, flaky, and not infrequently ulcerated. 
There may be no subjective symptoms, but occasionally itching and sometimes actual pain may occur. As a rule constitutional symptoms are wholly absent, unless extensive plaques about the chest interfere with respiration.

In some cases the sclerodermia may be localised to one side of the face and involve the chin, cheek, side of the nose, forehead, and may even extend on to the scalp chiefly in the area supplied by the supraorbital nerve. On the affected side the skin becomes smooth, glossy, shrunken, and bound down to the bone, the subcutaneous tissue atrophied, and the scalp completely bald, producing an appearance which once seen cannot be readily mistaken. This is known as hemiatrophy of the face and may occur by itself or in association with circumscribed sclerodermia elsewhere.

(ii) Band Variety.-In this variety, which is far more rare than the plaque type, the lesions are in the form of stripes or bands which may occur alone or in association with plaques. The bands, which may be single or multiple, are found chiefly on the limbs and vary in length from about an inch to the whole extent of a limb, and in width from quarter to half an inch or more. They may appear first as red streaks or may not be noticed till sclerotic changes develop. In consistence and colour they are similar to the plaques and, like them, are surrounded by a lilac border or pigmented zone, and may be raised, level with the skin, or depressed and atrophic. They are sometimes associated with itching or with paroxysms of cramp-like pains, and may interfere with movement.

(iii) Guttate Variety.-In it the lesions are smooth, shiny, whitish macules which vary in size from a pin's head to a lentil, are roundish or irregular in outline, are not definitely raised, and are occasionally surrounded by a lilac border. The surface may present a central punctum due to a blocked follicle, may have a few telangiectases ramifying over it, or may be dotted over with black specks.

The macules may be discrete and arranged irregularly, or in groups, or in rows in the lines of cleavage of the skin, or they may coalesce to form irregular patches or a network. They are most common on the upper part of the thorax and neck and behind the ears, and tend to be symmetrical. Occasionally they involute and become covered with a white scale which when removed reveals definite atrophy. Where no lilac border is present they appear as white spots on an apparently healthy skin and the condition has been described as white-spot disease.

Histopathology.-There is considerable diversity of opinion with regard to the histopathology, which has been worked out chiefly in the circumscribed variety. In it changes occur in the corium and subcutaneous tissue, and secondarily in the epidermis.

In the corium there is a cellular infiltration, consisting of connective tissue cells, situated chiefly around the sub-papillary vessels and about the hair-follicles. The collagen is found to be markedly 
increased, but the elastin appears to be normal. Beyond the sclerotic area the blood-ressels are dilated and surrounded by a cellular infiltration.

In the subcutaneous tissue the blood-ressels show signs of endoand peri-arteritis or peri-phlebitis, with proliferation of the endothelium and of the middle and external coats.

In advanced lesions the hypertrophy of the collagen is more marked and leads to obliteration of the capillaries and lymph-spaces, hairfollicles, and sweat-glands. In the final stages atrophic changes are present with granular degeneration of the collagen, the more resistant elastic tissue being unaffected and consequently appearing to be excessive, and the subcutaneous fatty tissue disappearing and being replaced by a fibrous stroma.

The cutaneous nerves have been examined for degenerative changes, but with negative results; sclerosis in the grey matter at the anterior horn of the cord and degeneration of the simpathetic nerres have been noted, but have not been corroborated.

Etiology and Pathogenesis.-The affection is more common in women than in men in the proportion of about 2 to $I$. Its chief age of incidence is about 30 years, though it has been known to occur as early as I3 months and in old-age.

Its precise cause is unknown though several theories have been advanced to explain it :

It has been known to follow a chill from exposure to damp and cold and a causal connection has been suggested between them.

According to Bramwell it is relatively frequent in stone-masons; out of his nine cases five were in stone-masons and were attributed by him to the holding of a chisel in the hand during cold weather, as in these cases the condition commenced in the hand.

Its occurrence in connection with rheumatism, disorders of menstruation, pregnancy, and alcoholism, has suggested possible causes.

It has been found not infrequently in association with thyroid insufficiency, such as hypertrophy, atrophy, or sclerosis, of the gland, or with myxoedema or Graves' disease, and certain observers regard the thyroid disease as responsible for it.

According to Unna the essential lesion is hypertrophy of the collagen and the atrophic changes are the result of an obliterative endarteritis; others look upon the vascular changes as primary and regard the condition as an infective process of toxic origin and in support of this view cite the condition of the blood-vessels, the symmetry of distribution, and the occasional occurrence of constitutional prodromal symptoms, and assert that the majority of the changes can be explained as the result of arterial, venous, or lymphatic stasis.

Another theory is that it is of nervous origin and a form of trophoneurosis, and in support of this riew it has been pointed out :

(a) That bands or patches of circumscribed sclerodermia sometimes correspond in distribution to the area supplied by a cutaneous nerve, such as the supra-orbital, some other branch of the fifth, an intercostal, or one of the nerves of the extremities; and that in other cases the lesions seem to correspond roughly in shape and extent to the projection on the shin of a nerve root or a metameric segment of the cord;

(b) That the patches may be associated with sensory symptoms suggesting a neuritis ; 
(c) That in sclerodactylia the sclerosis is preceded by Raynaud's phenomena, which points to a functional disturbance of the vaso-motor nerves, and that its resemblance to syringo-myelia suggests an organic lesion of the cord.

Against the nervous theory it has been pointed out that post-mortem examinations in cases of generalised sclerodermia have failed to disclose definite pathological changes in the peripheral or central nervous system, and that degenerations which have been reported in the cord and sympathetic nerves have not been corroborated.

Diagnosis.-Generalised sclerodermia, in the early oedematous or indurated stage, has to be distinguished from the oedema of Raynaud's disease or cardiac disease, but in them the oedema is more localised and varies from time to time, while the affected tissue is less firm, pits easily on pressure, and does not tend to atrophy.

It has to be differentiated from myxoedema, but in it the tense and waxy condition of the skin is associated with peculiar flushing of the cheeks and with mental deficiency.

Occasionally where the pigmentation is marked it may suggest Addison's disease, but in the supra-renal affection the pigmentation is deepest in the exposed parts and about the areolae of the nipples and genitals and there is marked cachexia ; or it may be confused with melanodermia, but there the skin is not altered in texture and the areas between the pigmented patches are pure white.

Sclerodactylia may resemble leprosy, but in the latter there is marked anaesthesia, the nerves in the neighbourhood-especially the ulnar and median-are generally found to be thickened, and there are usually other obvious signs of leprosy. It may also resemble syringomyelia, especially of the Morvan type, but in syringo-myelia the atrophic changes are generally limited to one side and there is paresis, analgesia, and a tendency to the formation of perforating ulcers, and necrosis of bone. The differentiation of sclerodactylia from Raynaud's disease may arise, but it is of minor importance as typical Raynaud phenomena may go on to sclerodactylia.

Circumscribed sclerodermia or morphoea is easily diagnosed by the glossy surface of the lesions, their hardness and resistance, lilac border, pigmented halo, and telangiectases. It may resemble cheloid, but in it the border is more irregular, the surface raised, and the lesion movable instead of being bound down to underlying structures.

Guttate lesions may be confused with papules of Lichen planus, but in them the outline is angular and the tint violaceous instead of white.

Prognosis.-The prognosis in generalised sclerodermia should be guarded. A certain number of cases in the acute pseudo-oedematous stage may undergo spontaneous recovery but where atrophic changes have supervened a fatal issue is almost inevitable, either from malnutrition and marasmus due to interference with the functions of the vital organs, or from some intercurrent disease. Even progressive cases of sclerodactylia may prove fatal. 
In the circumscribed type the prognosis is more favourable and unless in extensive cases the skin affection has no harmful influence on the general health. The actual lesions may undergo spontaneous cure or sometimes may be removed by treatment, but occasionally they may persist indefinitely.

\section{TREATMENT}

The treatment of generalised sclerodermia is unsatisfactory and there is no internal specific. Thyroid extract has been given in a considerable number of cases, both by the mouth and by implantation of the gland-tissue, but the results from it-though sometimes encouraging-are uncertain. Pilocarpine has been recommended with the object of retarding the spread of the disease by promoting sweating, but its action is disappointing.

The general treatment should consist of a tonic regime with good food, fresh air, and ordinary remedies such as strychnine or iron, or salicin or the salicylates, where it is associated with rheumatism.

Locally the patient should be protected from cold by warm clothes, and the skin should be kept active by Turkish baths, etc., and pliant by massaging with almond oil.

In circumscribed lesions local treatment is of definite value, especially massage; this should be done regularly every day, each patch being rubbed for about ten minutes with warm oil either by the patient or by an attendant. Other methods have been tried, such as galvanism and injections of fibrolysin, but in my experience the results from them have been disappointing.

The $\mathrm{X}$-rays seem to retard the spread of the disease and soften the plaques and are worthy of a trial.

Guttate lesions may be destroyed by electrolysis or by freezing with carbon dioxide, but scarring is apt to follow. Painting with a saturated solution of resorcin in alcohol, and the application of mercurial plasters, have been recommended.

\section{REFERENCES}

Branwell, B. Diffuse sclerodermia, its frequency, occurrence in stonemasons, etc. (Edin. Med. Journ., I9I 4 , xii. p. $3^{8}{ }_{7}$ ).

Brissat'd. Pathogenesis of sclerodermia (La Presse Méd., I897, p. 285).

Fox, Colcort. History of sclerodermia in England (Brit. Journ. Derm., I 892 , iv. p. IOI).

HoffA. Pathological anatomy of sclerodermia (Münch. med. Wochenschr., I 892).

Huxter, W. K. Sclerodermia with subcutaneous calcareous deposits (Glasgow Med. Journ., I9I3, p. 2+I).

Johrstor, J. C., \& Sherwell, S. White-spot disease (Journ. Cut Dis., July I903).

Joxes, F. S., \& Ttrábll, H. M. Sclerodermia guttata (Brit. Journ. Derm., I915, xxvii. p. 450 ). 
JuliusberG, F. On the White-spot disease (Derm. Zeitschr., I909, XV. p. I2).

KRETZMIER. " Small-spot sclerodermia circumscripta," etc. (Arch. f. Derm. u. Syph., I9I3, cxviii. p. I48).

Lewin \& Heller. Die Sclerodermie (Berlin, I895).

Meneau, J. Sclerodermia, a clinical study (Journ. des Mal. Cut. et Syph., I 898$)$.

Musro, T. K. Diffuse symmetrical sclerodermia (Glasgow Med. Journ., I903, p. 4I7).

Osler, W. Diffuse sclerodermia (Journ. Cut. Dis., I898, p. 49).

Ravogl, A. On a rare form of sclerodermia (Journ. Cut. Dis., Jan. I9I 7).

Rogtes. Opotherapeutic treatment of sclerodermia (Ann. de Derm. et de Syph., July r9 Io, p. 383).

Scholefield, R. E., \& Weber, F. P. Sclerodactylia with subcutaneous calcareous concretions (Brit. Journ. Derm., I9II, xxiii. p. 276).

Thibierge, G. Sclérodermie (La Prat. Derm., I904, iv. p. 24I).

Westberg, F. White-spot dermatitis (Monats. f. prakt. Derm., I90I, xxxiii. p. 355).

Whitehouse, H. H. Wassermann test in sclerodermia (Journ. Cut. Dis., Dec. 1909).

Zarubin, V. Sclerodermia (Arch. f. Derm. u. Syph., I900, p. 49).

\section{SCLEREMA NEONATORUM}

Syn. : Scleroderma neonatorum; Fr., Sclérème des nouveau-nés; L'endurcissement athrepsique (Parrot); Algidité progressive (Hervieux); Ger., Fettsklerem.

Sclerema of the new-born is a very rare affection; it is related to ordinary sclerodermia of the adult but is generally believed to be etiologically distinct. Like sclerodermia it occurs in two forms: (I) a diffuse and sometimes universal type which is almost invariably fatal ; (2) a circumscribed type which may not interfere with life and may undergo spontaneous recovery.

The diffuse variety is extremely rare, but as its striking peculiarities lead to most cases being reported it would appear to be more common than it really is. A considerable number of the cases have been described in France, where it is apparently more frequent than in this country.

It may be present at birth but more often appears on the third or fourth day. It is generally noticed first on the lower extremities and from there spreads rapidly on to the trunk, arms, and face, reaching its full development in a few days.

The affected skin is tense, glossy, stretched over prominences, hard like wood, and so firmly adherent to the underlying muscles or bones that it does not pit on pressure and cannot be pinched up between the fingers. In colour it is dirty-yellow and sometimes mottled, while on the lower extremities it is purplish or livid and becomes pale on pressure. The sensibility is unaltered but the skin feels cold to the touch.

The hardness and rigidity, which may involve not only the skin but also the subcutaneous tissue and muscle, give the infant the 
appearance of a statue or of a corpse in rigor mortis. The immobility induced by it interferes with vital functions, renders suckling difficult or impossible, impairs digestion, impedes respiration, enfeebles the heart's action, lowers the pulse rate, and causes the temperature to fall to $S 0^{\circ}$ or less. In consequence the infant becomes drowsy and more and more shrunken; diarrhoea or jaundice may occur, and death generally takes place in the course of a week in a state of extreme marasmus or from some intercurrent disease of the lungs or kidners.

In the circumscribed variety the lesions consist of hard, sometimes irregularly raised, plaques which are most commonly situated about the calves, buttocks, and lower parts of the back. The affected skin is tense and waxy, reddish or purple on the lower extremities and paler in shade or yellowish elsewhere, the colour fading on pressure but slowly returning when the pressure is remored. The plaques are generally well-defined, are difficult to pinch up, and feel like a piece of wood or hard indiarubber let into the skin.

It may appear soon after birth but more often develops about the end of the first month, and may spread and coalesce to involve considerable areas or may undergo retrogressive changes in about six months. Unless it is extensive it does not interfere materially with the health of the child.

It is questionable whether this circumscribed variety is distinct from circumscribed sclerodermia in an infant.

Histopathology.- The histology has not been satisfactorily worked out and there is some discrepancy of opinion regarding it. The most definite changes are of an atrophic type and involve the skin and subcutaneous tissue, which may become transformed into a compact and homogeneous layer covered by a thin stretched epidermis. An increase of connective tissue has been described in the corium, and a relative diminution in the subcutaneous tissue owing to the disappearance of the fat.

Etiology.-The causation is unknown. It seems to occur chiefly in association with malnutrition, due to bad hygienic surroundings, a syphilitic taint, exposure to cold, or a debilitated condition of the mother, and it has been met with in infants prematurely born.

Diagnosis.-It has to be distinguished from Oedema neonatorum, but in it the affected skin is not hide-bound but raised, pits on pressure, and the immobility is much less marked.

It has to be differentiated from generalised congenital hrperkeratosis or " harlequin foetus," in which there is universal thickening of the horny layer giving the infant the appearance of being encased in an armour of horny plates separated by deep furrows. A harlequin foetus, if born alive, rarely survives more than a day.

Treatment.- In the diffuse cases the outlook is bad and all that can be done is to keep the infant warm by wrapping it in cottonwool, applying hot bottles, or placing it in an incubator, and by administering stimulants and nourishment by a tube by the mouth or per rectum. 
In the circumscribed cases good results have been reported from thyroid and thymus gland; locally the skin should be kept as soft as possible by massage with warm almond oil.

\section{REFERENCES}

Ballantyne. Sclerema and Oedema neonatorum (Brit. Med. Journ., I 890 , i. p. 403).

Carpenter, G., \& Neave, S. Sclerema neonatorum (Lancet, I9o6, ii. P. 158$)$.

Fox, Colcotr. Case of Sclerema neonatorum (Brit. Journ. Derm., I904, xvi. p. I9).

Little, E. G. A case of Sclerema neonatorum (Brit. Journ. Derm., I9I4, xxvi. p. 360).

LotTA, M. Sclerema neonatorum (Journ. Cut. Dis., I909, xxvii. p. 87).

Mackenzie, S. Case of Sclerema neonatorum (Brit. Journ. Derm., I907, xix. p. 293).

PARRot. L'Athrepsie (Paris, I877).

Pringle, J. J. Case of Sclerema neonatorum (Brit. Journ. Derm., I899, xi. p. 290).

\section{OEDEMA NEONATORUM}

In this affection the oedema generally commences on the lower extremities on one or both feet, and spreads from there on to the trunk, arms, and face; occasionally it begins on the penis, sc:otum, or vulva. It is progressive but rarely universal and generally takes the form of irregular circumscribed swellings. It may appear at birth but as a rule is not noticed till a few days after. Instances are on record where it has not only been congenital in origin but has attacked more than one member of a family.

The affected skin is raised, tense, doughy in consistence, and pits on pressure; it is dusky-yellow or reddish in colour, cyanosed and mottled on the lower extremities, and is sometimes associated with purpura and epistaxis.

When extensive it is apt to be fatal, the patient becoming drowsy, the cardiac and pulmonary action weak, the temperature sub-normal, and some intercurrent disease supervening in the lungs or the kidneys. Before death the oedema may disappear leaving the skin wrinkled and shrunken.

When it is confined to the limbs and shows no great tendency to peripheral extension, retrogressive changes may set in with spontaneous recovery.

Histopathology.--The principal changes consist of a serous oedema throughout the corium and subcutaneous tissue, with an increase of the subcutaneous fat which becomes solidified and of a brownish tinge.

Etiology.-In a few of the cases cardiac disease is believed to have been responsible, and in others thrombosis in a large rein such as the inferior vena cava; cases have also occurred in which there was no 
evidence of cardiac, pulmonary, or renal, trouble and the cause of which is uncertain. Like Sclerema neonatorum it usually occurs in debilitated infants who are badly nourished or prematurely born, though circumscribed lesions have been met with in infants apparently healthy.

Diagnosis.--It has to be distinguished from Sclerema neonatorum, but in it the affected skin is not raised and oedematous but hard and hide-bound.

Treatment.-The treatment, which is generally of little arail in extensive cases, is similar to that for sclerema and consists of keeping the child warm and nourished. Small doses of calcium salts may be given with the object of improving the coagulability of the blood and reducing the tendency to serous extrarasation.

\section{REFERENCES}

Blacker, G. C. Oedema neonatorum (Brit. Journ. Derm., I898, x. p. 87). Little, E. G. A case of Oedema neonatorum (Ibid., I9I2, xxii. p. 400 ).

\section{CALCAREOUS DEPOSITS IN THE SKIN}

Calcareous deposits may occur both in the skin and subcutaneous tissue. They are met with in connection with various cutaneous neoplasms, such as fibromata, sarcomata, lipomata, and carcinomata, and from the calcification of some cutaneous vein forming a phlebolith.

In elderly persons, chiefly women, numerous hempseed-sized concretions of calcium salts may sometimes be detected on the legs, especially over the anterior surface of the tibia, these are believed to be due to calcification of fat lobules in the subcutaneous tissue.

Calcification may occur in connection with old-standing cases of Lupus vulgaris, as it is known to do in association with caseation in tuberculosis elserwhere. In a case under the observation of the writer, small calcareous nodules appeared in a patch of lupus on the face which was undergoing superficial ulceration.

In benign cystic epitheliomata calcareous deposits have been detected which appeared to be the result of calcification of the contents of the cysts. Cases are also on record where calcification appeared to affect sebaceous glands; Ualherbe described a series of twelve such cases under the heading of "calcified epithelial tumours of the sebaceous glands," and similar cases have been recorded in this country by Eve and Jonathan Hutchinson (Jnr.), but whether theywere true sebaceous adenomata or benign cystic epitheliomata is uncertain.

Another peculiar group of cases in which calcification occurs has been described under such names as "chalk gout," "subcutaneous calcareous granuloma," etc. In them chalky deposits are found in the centre of a granulomatous infiltration in which giant-cells may be 
detected-the most common site for the concretions being the extensor aspect of the extremities. In a case in a middle-aged woman at Charing Cross Hospital the parts affected were the feet, ankles, fronts of the legs, elbows, and extensor aspects of the forearms. Red patches first appeared which were tender to the touch and felt hot; after several months these became excessively painful, chalk stones became visible in the centre, suppuration supervened, the chalk stone separated, and the patches healed with a scar. There was a history of several attacks of pain and swelling of the right big toe, suggesting gout, and there was sclerodactylia of the left hand. A chemical examination of the concretions showed that they were not tophi and that they did not contain urate of sodium but were composed of calcium carbonate and calcium phosphate.

The association of sclerodactylia, or more widespread sclerodermia, and multiple calcareous concretions has been observed in a number of cases by Scholefield, Parkes Weber, and others.

The cause of the deposition of lime salts in the tissue is not clear. In neoplasms it would appear to be in the nature of a secondary degenerative process in the cells of the new-growth; in fibromata it is just possible that it may be primary and that the fibromatosis may result from the irritation caused by the concretions.

Various theories have been advanced to account for the concretions: it has been suggested that they are primary and the result of some defective metabolism; on the other hand they have been regarded as secondary to the sclerosis and obliterative changes in the small blood-vessels and a final stage of the sclerotic process; or a third view has been advanced that the chalky deposits are in some way connected with tuberculosis and the presence of giant-cells in the lesions has been cited in support of the theory, but although a case has been described in which they occurred in a tuberculous patient the evidence of a causal connection is insufficient and it seems more probable that the granulomatous formation was secondary to the chalky deposit, than due to tubercle bacilli.

The treatment is unsatisfactory. In view of the close relationship between the metabolism of lime and phosphoric acid a case was treated by Briscoe with phosphoric acid but the results were inconclusive.

\section{REFERENCES}

Beñett, E. H. On calcification of adipose tissue (Dublin Journ. Med. Sci., I 878 , lxv. p. I9).

Chitari, O. MI. Uber die herdweise Verkalkung und Verknocherung des subcutanen Fettgewebes (Zeit. für Heilkunde, I907, xxviii. Supplementheft, P. I).

EVE, F. Adenoma of the sebaceous glands partly calcified (Trans. Path. Soc., Lond., I882, xxxiii. p. 335).

Huxter, WV. K. Sclerodermia with subcutaneous calcareous deposits (Glasgow Med. Journ., April I9I3, p. 24I).

Hutchinson, J. (Jnr.). Calcifying adenoma of the skin (Trans. Path. Soc., Lond., I 890 , xli. p. 275). 
Lowexbach. Calcification in skin in tuberculous patient (Arch. f. Derm. u. Syph., 1904, lxx. p. 450).

MacLeod, J. M. H. [lcerations due to chalk stones (Brit. Journ. Derm., I9I 4 , xxri. p. 364 ).

Malmerbe. Researches on calcified epithelioma of the sebaceous glands (Trans. Internat. Med. Cong., Lond., I88I, i. p. fo\$).

Thibierge, G., \& Weissexbach, R. J. Concrétions calcaires souscutanées et sclérodermie (Ann. de Derm. et de Syph., IgII, ii. pp. I $29-155$ ).

Weber, F. Parkes, \& Scholefield, R. E. Case of sclerodactylia with subcutaneous calcareous concretions (Brit. Journ. Derm., I9II, xxiii. p. 276 ).

Wildbolz. Phosphatic \& calcareous concretions (Arch. f. Derm. u. Syph., I904, lxx. p. 435). 


\title{
CHAPTER XLIII
}

\section{TROPICAL DISEASES OF THE SKIN}

\begin{abstract}
AINHUM
Syn.: Guduram (Iljes, a tribe of African negroes); Faddidite (Madagascar); Banko-kerendé (Sudan); Quigila (Brazil) ; Sukha pakla (India); and other native names.

Definition: An affection of the toes, especially the little toe, characterised by the formation of a furrow at the digito-plantar fold which gradually deepens, as if from the action of a ligature, and ends in loss of the toe.
\end{abstract}

History and Distribution. - The condition was described in I860 by Clarke who had seen it among the natives of the Gold Coast and regarded it as a manifestation of yaws. Subsequently, it was observed in Brazil by Da Silva Lima who named it ainhum from a Nagos word meaning to saw or cut.

It has a wide distribution in Africa, and cases have been recorded from the West coast, Madagascar, Zambesi, Transvaal, Algiers, Egypt, and the Sudan. In South America it has been met with chiefly in Brazil and occasionally in the Argentine and British Guiana. Cases have been observed in North America and the West Indies. It is not uncommon in India, especially among the Hindus. Instances have been recorded also from the Antilles, New Caledonia, and the Gilbert Islands.

It occurs only in dark-skinned races, chiefly in negroes, and has not been observed so far in a European.

Description.--It is met with usually in the fifth toe but may occur in the fourth-according to Da Silva Lima the little toes are affected in 45 out of 50 cases and the fourth toe in 5 out of 50 ; more rarely it may occur in the great toe, and even in the fingers. It may attack both little toes simultaneously or after a varying interval.

It first appears as a shallow transverse crack or groove at the digitoplantar fold, this deepens from a progressive constriction in the corium like an ever-tightening ligature, and slowly extends upwards and outwards till the two ends meet, either on the dorsum or on the outer surface of the toe. The skin of the strangled portion is not greatly altered in appearance but the tissues feel soft like a lipoma. After a time, as a result of the pressure, the distal portion of the toe becomes 
swollen, rounded, and bulbous and assumes the appearance of a small cherry or potato attached to the foot by a narrow pedicle. The bone and ligaments included in this pedicle become gradually absorbed, and finally the digital portion of the toe is cast off. The resulting wound heals readily but occasionally there is left for some time a small indolent ulcer in which bone may be detected. Not infrequently the spontaneous amputation is anticipated by the patient in order to rid himself of the pain and inconvenience caused by the tendency of the swollen toe on its limp pedicle to double under the foot in walking or to be injured by catching on various objects.

The constriction generally corresponds with the first inter-pharyngeal joint, though it may pass through the shaft of the phalanx itself. Ulceration may be present in the constricting groove as a result of injury and secondary septic infection and may lead to inflammation and even gangrene of the distal part. The progress of the disease is slow and it may be ten rears before amputation takes place. In rare instances the process has been known to repeat itself in the stump.

Histopathology.-Longitudinal sections show hypertrophy of the epidermis at the constriction, a dense fibrous ring in the underlying corium, and a rarefying osteitis of the bone beneath. At the proximal side of the constriction there is an inflammatory round-celled infiltration about the small blood-ressels which are partially obliterated from endo-arteritis. In the distal portion the tissue is oedematous and in a state of fatty degeneration as a result of cutting off the blood supply. The histological appearances have been compared to what occurs in a tumour when it is ligatured.

Etiology.-It is more frequent in males than in females, and is essentially a disease of adult life but has been observed occasionally in children. It has been said to be hereditary and familial, but this is doubtful.

The exact cause is unknown. It has been suggested that it is due to self-mutilation or is the result of continuous pressure caused by the wearing of tight metal rings on the toes, but these explanations are inadequate. Other hypotheses are that it is tropho-neurosis, a form of sclerodermia, or is in some way connected with leprosy.

The most likely explanation is that it is an infective condition of parasitic origin and due to an abrasion and the inoculation of some micro-organism in the digito-plantar fold which leads to a contracting fibromatosis. This view is strengthened by its occurrence in natives who, going about barefooted, are especially liable to abrasions of the skin and to local infections.

Treatment. - In the early stages benefit may be derived and the strangulation arrested by a longitudinal incision through the constricting ring. When it is far advanced and the toe has become useless and a source of annoyance and pain it is best to amputate at the pedicle. 


\section{REFERENCES}

Clarke (Trans. of the Epidemiological Soc., I 860, i. p. I05).

Da Silva Lima (Gaz. Medica de Bahai, I852).

Moreira, J. A new pathologico-anatomical \& clinical contribution to our knowledge of ainhum (Monatsch. f. prakt: Derm., xxx., 8, p. 36I).

\section{CUTANEOUS ANCYLOSTOMIASIS}

Syn.: Ground-itch; Water-itch; Coolie-itch ; Pani-ghao.

Ground-itch is a form of vesicular dermatitis which attacks coolies working in the tea gardens of Assam in the wet season. It occurs also in the West Indies, British Honduras, and in the southern United States. It is an important disease so far as the tea planter is concerned since after heavy rain it may incapacitate as many as $5 \%$ of the coolies. It almost invariably attacks the feet but is occasionally present on the ankles and legs.

It usually begins with itching, followed by swelling of the feet, which gradually increases up to the second day when walking becomes painful and the eruption appears. This consists of red macules or papules which rapidly become vesicles about the size of lentils, situated either singly or in herpetiform groups about the sole, dorsum of the foot, or interdigital clefts. The vesicles become purulent, break, and form small ulcers, which in unfavourable conditions may become gangrenous. The affection is frequently associated with anaemia.

Etiology.--It occurs almost exclusively in the wet season and is at its worst after a heavy rainfall when the plantations are covered with liquid mud. The coolies working in the parts of the plantations near the huts suffer more from it than those on the outsides, owing to the greater contamination of the mud with human excreta.

It is believed to be due to the penetration of the skin by ancylostoma embryos and it has been proved experimentally that rubbing the skin with earth in which the embryos are present is followed by an eruption like water-itch. In the tea gardens in Assam a large percentage of the coolies suffer from ancylostomiasis and their excrement is impregnated with ova, these are hatched out in the wet weather and the embryos easily penetrate the skin of the coolies' feet which is macerated and sodden from tramping about in the mud. The ulceration and phagedaena associated with it are due to secondary pyogenic inoculation.

Treatment.-As a preventive Barbadoes tar has proved of great value in the West Indies. Every morning the feet and legs of the coolies are painted over with the tar and they are then made to walk through fine sand or sawdust which adheres to the tar and forms an impermeable covering to the skin. In certain tea gardens the coolies are made to wear kurrams or sabots with high heels at each end which raise the feet from the ground and prevent contamination with the infected mud. 
The treatment consists of opening the vesicles and dusting on boroiodoform powder. Should open sores be present, boric compresses, followed by dressings with a mild mercurial ointment, should be employed. In addition the feet should be bathed daily in I in to carbolic and the coolies confined to hospital until the lesions have healed.

\section{REFERENCES}

Browe. Pani-ghao or Sore Foot of Assam (Journ. Trop. Med., Igor, iv. p. 56).

Castellail \& Chalyers. Ground-itch (Man. of Trop. Med., and Ed., London, 1913, p. 556).

DALGetTy. Water-itch, or Sore Foot of Coolies (Journ. Trop. Med., Igor, iv. p. 73).

Schatdix:. Entrance of Ankylostoma larvae by the Skin (Dentsch. med. Wch., I904, p. 1338).

Suith. Uncinariasis in the South (Journ. Amer. Med. Assoc., I904, ii. p. 592).

\section{NEMATODA}

\section{FILARIA LOA}

\section{CALABAR SWELLINGS}

Syn. : Tropical swellings; Ger., Kamerungeschwulste.

Definition: Diffuse swellings of the shin probably due to the presence of the Filaria loa in the subcutaneous tissue.

Description.-This condition, first described under the heading of "Calabar swellings" in I895 by Argyll Robertson, occurs chiefly in the west coast of Africa, and especially in southern Nigeria, the Cameroons, and Old Calabar.

The swellings are diffuse, raised, red and inflamed, roundish or oval in shape, and about the size of half an egg. The skin over them feels hot and they may be itchy or even actually painful. They may be single or multiple ; they are most common on the legs, ankles, or arms, but may be met with on the feet, hands, and even on the face. Ther appear suddenly, last a few days, and gradually fade. They are accompanied occasionally by a disturbance of the general health with headache, nausea, and romiting, and there is usually marked eosinophilia in the blood which may reach as high as fo or $50^{\circ} .0$. The affection may be temporary and cease after one or more attacks; on the other hand it may be extraordinarily persistent and recur for years, even after the patient has left the district in which it was endemic.

Etiology and Pathogenesis.-The swellings may appear in either sex and at any age. They are generally believed to be due to the presence in the subcutaneous tissues of the adult Filaria loa. Though, up to the present, the adult worn has not been found in the swellings there is the strongest circumstantial eridence that it is there, for its 
embryos (Microfilaria diurna) have been found in the lymph in the swellings and occasionally in the peripheral blood during the day, and the worm has appeared in patients with swellings in the skin of the scalp, penis, or finger, or wriggling in the conjunctiva of the eye or in the anterior chamber, when it causes pain, blurred vision, and photophobia. (Fig. 403.)

It has been suggested by Manson that the swellings are the evidence of an inflammatory disturbance caused by the expulsion of the microfilaria from the adult worm, and by Ward that they are the result of the irritative action of the excreta of the worm.

The life-history of this worm is not yet fully understood so that the manner of transmission of the disease is still uncertain, but various

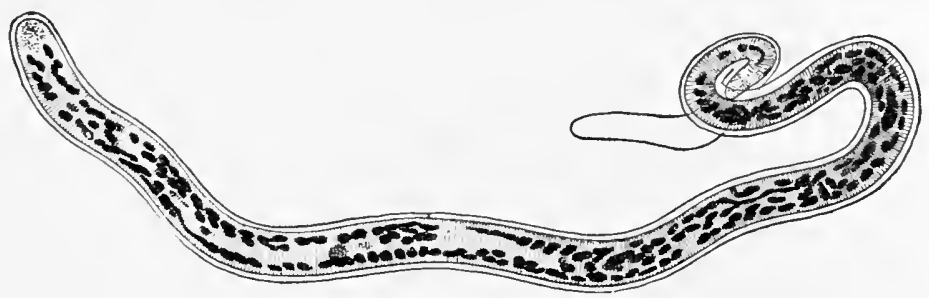

Fig. 403.-Microfilaria Loa. ( $x$ about I 500.)

(Castellani \& Chalmers' Manual of Tropical Medicine.)

flies have been recognised as possible intermediary hosts, such as the mango-fly and the glossinae.

Treatment.-The local discomfort from itching or pain should be soothed by means of lead or calamine lotions, etc. A careful look-out should be kept for the worm in case it should appear in the skin or conjunctiva, when a small incision should be made and it should be carefully extracted. If its position be uncertain it may be destroyed by injections of perchloride of mercury (I-Ioth gr.) into the swellings.

Until the life-history of the worm is understood no special indication can be given as to prophylaxis.

\section{REFERENCES}

Burrows, D. Relationship of Microfilaria diurna to Filaria loa (Journ. Trop. Med. \& Hyg., I9Io, xiii. p. 49).

Castellani \& Chalmers. Calabar swellings (Man. Trop. Med., London, I913).

Low, G. C. Filaria loa infection (Journ. Trop. Med. \& Hyg., I912, p. 38). WARD. Calabar swellings (Journ. Infective Dis., 1906, p. 37).

\section{CRAW-CRAW}

Craw-craw is a generic name which has been applied indiscriminately in West Africa both by white men and natives to various skin affections generally characterised by itching and pustulation. In I 875 
O'Neil described under this heading a resico-pustular affection which suggested scabies in its clinical characters and distribution. According to Bennett the uneducated natives of Calabar employed the name for practically all skin diseases, while the more intelligent natives limited its application to three conditions, namely leprosy or bad craw-craw, Tinea circinata or Krooboy's craw-craw, and craw-craw proper, a papulovesicular disease which he beliered to be pustular eczema. Inder the same heading Plehn has described among the natives on the Cameroon coast a papular dermatitis which chiefly attacks the inside of the thighs. Emily has used the name in the French Congo for a chronic pustular disease which began as a reddish-brown spot, was excoriated by scratching, and transformed into a superficial ulcer the floor of which gradually became covered with pale granulations secreting thich tenacious pus.

The above are a few examples of the wide use of the term, but there are many others and except as an interesting native name it might well be abandoned.

\section{REFERENCES}

Benvetr. Craw-craw (Journ. Trop. Med., I90i, iv. p. 55).

Evily. Treatment of Craw-craw (Arch. de Méd., Nor. IS99, lxxi. p. 56). O NeIl. The presence of filaria in Craw-craw (Lancet, I87j, i. p. 26j).

\section{DRACUNCULOSIS}

Syn.: Dracontiasis.

Definition: The infection of man with the Dracunculus medinensis, or guinea-worm.

Description.-Dracunculosis is a tropical disease which is met with chiefly in the west coast of Africa, but is known to occur also in India, Persia, Asia Minor, Fiji Islands, and in South America in Brazil and British Guiana.

In some cases the outline of the worm can be seen clearly under the shin, in others the infection is characterised in the skin by a peculiar type of ulcer. Sometimes there is only one ulcer and one worm, but as a rule there are several. It appears first as a thickening of the shin about the size of a halfpenny, this becomes a vesicle which, on bursting, forms an ulcer from which exudes a clear fluid containing the larvae of the worm, and in the opening at the bottom of which the vaginal orifice of the worm may be detected. Later the anterior end extrudes through the opening and the worm can then be extracted. The most common sites for the ulcers are the leg, ankle, or foot, but they occur occasionally on the upper extremity, trunk, scrotum, perineum, and in rare instances in the eyelid and tongue. (Fig. 404.)

In addition to the characteristic skin lesions, the presence of the worm may give rise to an urticarial eruption, symptoms of general 
intoxication-such as pain and ferer-and marked eosinophilia in the blood which may reach $50 \%$.

Etiology.-The female guinea-worm is from 50 to So $\mathrm{mm}$. in length and from 1.5 to $1.7 \mathrm{~mm}$. in diameter, while the male, which is rarely seen, is about $22 \mathrm{~mm}$. in length. The anterior end of the worm is rounded and has a triangular mouth-opening, while the tail carries a bent chitinous hook.

The larvae of the worm infect cyclops - a small crustacean which is present in drinking water-and reach the human body by this means. On being swallowed and entering the stomach the cyclops is killed and the larva escapes, pierces the wall of the stomach, and, on reaching the connective tissue about the mesentery, develops into an adult. When

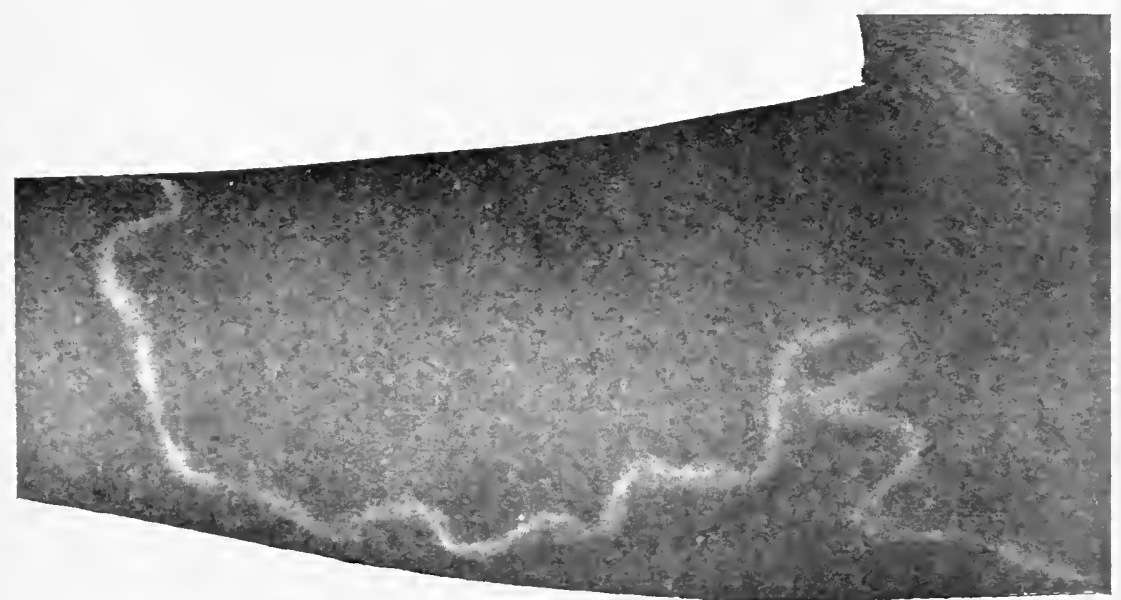

FIG. 404.-Guinea-worm.

(From Dr. P. Manson-Bahr.)

about a year old the female makes its way to the surface in order to reach water in which to deposit its larvae, and so causes the cutaneous lesion.

Treatment.-The treatment consists of extracting the worm whole, after which the ulcer heals rapidly and antiseptic dressings are rarely necessary. This may be done in about a fortnight after the manner of the native, by twisting the protruding anterior portion round a stick and giving it a turn or two every day. Great care must be taken not to extract too vigorously for fear of breaking the worm and leaving a dead piece behind which may cause serious inflammation. Another method is to douche the ulcer frequently with water, which causes the worm to expel its embryos, a process which is complete in about three weeks, after which it can be extracted easily or becomes absorbed. The worm may be hilled also by chloroform, or by corrosive sublimate I in Iooo, injected into the swelling or into the worm itself fixed by a ligature (Emily). 
The prophylaxis consists of drinking only boiled or filtered water, or of rendering the water sterile by passing steam into the wells.

\section{REFERENCES}

Castellaxi \& Chalmers. Dracontiasis (Man. Trop. Med., London, I9I3, p. I 4 I).

Faxthay, Stephexs, \& Theobald. Dracunculus medinensis (Animal Parasites of Man, London, I916, p. $3^{S_{5}}$.

\section{ELEPHANTIASIS}

Syn.: Elephantiasis Arabum, E. Indica, E. Nostras, Barbadoes Leg, Cochin Leg, Pachydermia, Spargosis, Hypersarcosis, Shlipada; and numerous other native names.

Definition: A chronic inflammatory condition of the skin and subcutaneous tissue, associated with lrmph stasis and connective tissue hyperplasia, leading to swelling, induration, and deformity of the affected parts.

Introduction.-In early times the term elephantiasis was employed with a wider signification than it is now. Celsus used it to designate leprosy-possibly because in certain cases of leprosy there may be elephantine thickening of a limb-and it was not until the seventeenth century that the two diseases were clearly differentiated and named E. graecorum which was leprosy, and E. arabum which is what we now mean by elephantiasis.

In the past it has been confused also with mrcetoma, or Madura foot, and certain native names are still used indiscriminately for both.

Distribution.-It is essentially a tropical disease and is extremely common anywhere between the latitudes $35^{\circ}$ north and $35^{\circ}$ south. It occurs in abundance in Oceania, S. America, India, China, Japan, and Africa, and is so common in certain Islands of the Pacific as to affect about half the population.

It is more common near the coast and about the banks and mouths of large rivers than in the interior, probably because the population there is greater, and it is more prevalent in some districts than in others, seeming to be endemic in certain regions-possibly from the presence of large numbers of mosquitoes which act as intermediary hosts for the filaria now known to be causally connected with the disease.

It is met with to a less extent in temperate zones, in this country and in other parts of Europe, where it is knomn as E. nostras. This type as a rule is milder than tropical elephantiasis and, while similar in its clinical features and histology, has a different pathogenesis. It has been described already among the affections of streptococcic origin (see p. 320).

Description.-In the large majority of cases the parts affected are the leg and scrotum, but more rarely it attacks the rulva, female breast, $4 G$ 
arm, and scalp. In some cases it is confined to one region, while in others it may involve several.

The local condition is preceded for a varying period by attacks of acute inflammation accompanied by a general febrile disturbance known as "elephantoid fever" which might be mistaken for an attack of influenza or malaria. This may be ushered in with a definite rigor and a rise of temperature to $103^{\circ}$ or more, or it may be more gradual in its onset and accompanied by headache, loss of appetite, pains in the back and limbs, nausea, and vomiting. It may abate in the course of a few days with profuse sweating or it may take weeks to subside.

The local inflammation appears spontaneously and is of the nature of an acute lymphangitis, being associated with swelling from oedema, throbbing, sometimes actual pain and tenderness, and occasionally with a milky discharge or oozing from the affected skin and with enlargement of the glands and reins in the neighbourhood.

It passes off gradually and the swelling may disappear completely, but, as a rule, some residual thickening and alteration of the tissue is left.

Recurrences usually take place at intervals of weeks or months, especially in summer, and with each fresh attack the thickening becomes more marked until finally, after several years, the stage of true elephantiasis is reached and the febrile attacks cease.

At first the swelling of the affected region is smooth and soft ( $E$. glabra) but in time it becomes hard and warty (E. verrucosa), and the sensibility is diminished.

The appearances of the local condition vary according to the region attacked.

Leg.-Both legs are usually involved and most commonly below the knees. The thickening may be so great that the calf may reach a girth of 20 inches or more and may hang down in a massive swelling, with large deep folds at the ankles from which oozes a moist and offensive discharge. The dorsum of the foot becomes rounded and swollen, the sole covered with horny masses, the toes square and flattened against each other, and the nails thickened and distorted. The swelling may end abruptly at the knee or it may decrease gradually; on the other hand it may spread up and involve the thigh or even the buttocks from which occasionally heavy pendulous masses hang down. The surface, though smooth at first, gradually becomes rough and horny like the hide of a rhinoceros, and may be covered with dirty crusts or uicers. As a result of the swelling over the joints the mobility is impaired, though a certain amount of movement is permitted by the deep folds.

The general health is not greatly interfered with, except when the growth is so great that the patient is unable to get about, or when secondary septic inoculation takes place and febrile disturbances result. (IFig. 405.) 


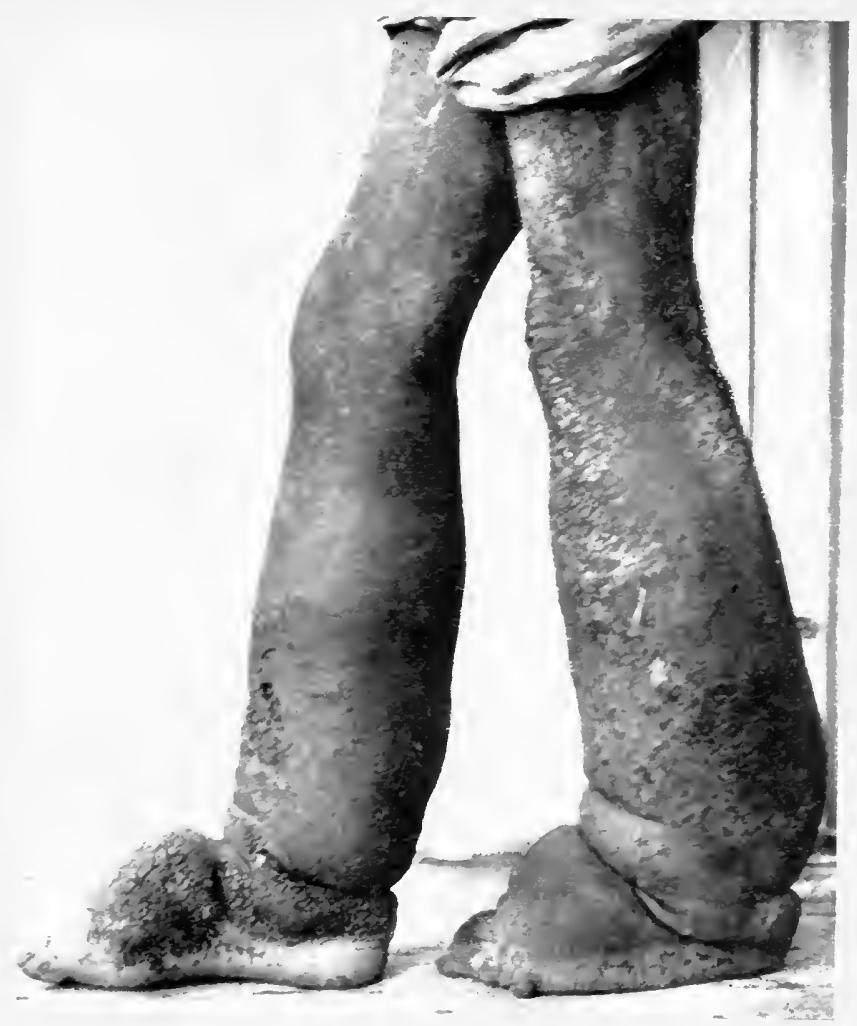

FIr. 405-Filaria! Elephantiasis of Legs

(From Dr. Louis Sambon.)

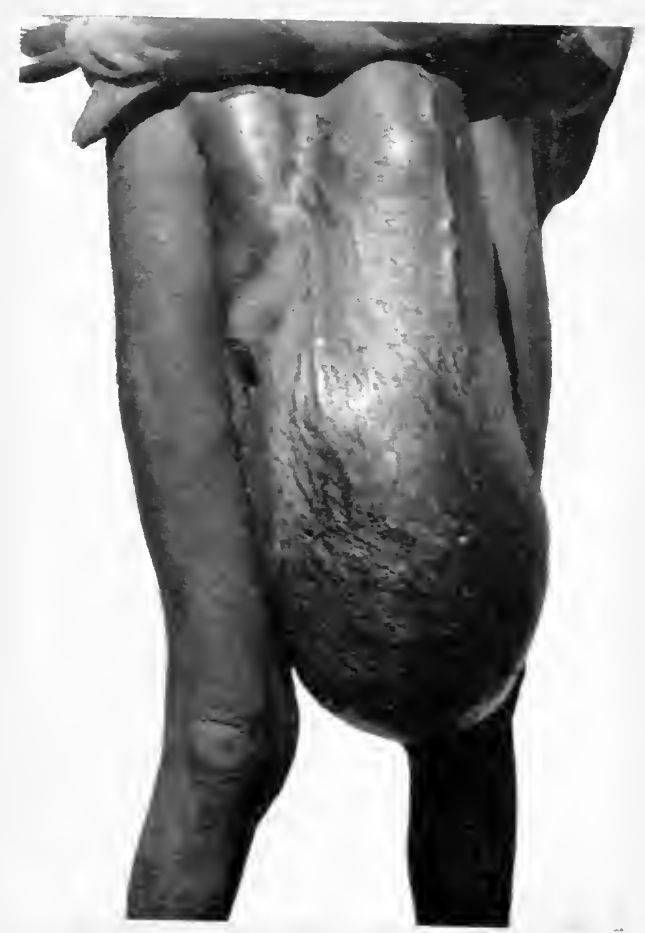

FIG. 406.-Elephantiasis of Scrotum. (From Dr. Louis Sambon.) 

Scrotum.-When the scrotum is involved it tends to grow down as a pear-shaped tumour with the base downwards, which may reach as far as the ankles and may weigh roo lbs. or more. The skin over it is rough and hard, the natural furrows are exaggerated, and in old cases it is liable to septic inoculation with the formation of ulcers and gangrenous sloughs. The penis is retracted to the base of a tunnel which opens below the middle of the growth, the testicles are pulled down, often surrounded by hydroceles, and occasionally atrophied, and the spermatic cords are stretched giving rise to a dragging pain. (Fig. fo6.)

The affection of the scrotum may begin with attacks of lymphangitis leading to lymph scrotum, in which the skin presents semitranslucent deep-seated vesicles containing a milky lymph and occasionally filaria.

Vulva.-In the case of the vulva one or other of the Labia majora is generally attacked and forms a tumour which hangs down and may weigh 6 or 8 lbs.

Breast.-If the female breast be attacked a pendulous mass results which may hang down as far as the knee.

Armis.-One or both arms may be affected, either in the deltoid region or extending on to the forearm and hand, and occasionally pendulous growths may form weighing $30 \mathrm{lbs}$. or more.

Scalp.- In the case of the scalp the thickening may take the form of an irregularly folded mass like an unusual style of dressing the hair, the mass being hard, and several inches thick, and the hair orer it scanty. (Fig. 407.)

Blood.-The blood is not characteristically altered; as in other parasitic diseases, during the febrile attacks there may be leucocrtosis and eosinophilia, but these are not of diagnostic value. Microfilariae may be detected in the blood in fresh cases; they should always be sought for by night as during the day they retire into the ressels of the lungs.

Pathology.-When the affected part is cut into it is found to be peculiarly hard, from a dense superficial fibrous stroma which, deeper down, becomes more open and coarse. From the cut surfaces a serosanguineous fluid or clear lymph exudes, while beneath it is an oily gelatinous substance like blubber. The lymph glands are enlarged and hard, the walls of the subcutaneous ressels proliferated, the fasciae between the muscles hypertrophied, the muscles themselves degenerated, and the bone thickened from exostoses.

Microscopically the principal changes are found in the corium and subcutaneous tissue. In the early stages there is a cellular infiltration, consisting of small round cells and spindle-cells about the capillaries, sebaceous glands, and coil-glands, and spreading into the lymphatic spaces; this infiltration is of a formative type and the cells composing it show eridences of organising into new connective tissue. The white fibrous tissue is hypertrophied, while the elastic tissue is unaffected. 
The lymphatics in the corium and superficial parts of the subcutaneous tissue are varicosed and form cavernous dilatations lined with endothelium, while in the deeper lymphatic vessels there is a fibrous thickening of the walls and dead or partially calcified filariae have been detected within them blocking the lumen. The lymphatic glands themselves may show fibrous changes, involving especially the central portion and obliterating the afferent vessels. Proliferative changes may be observed also in the walls of the subcutaneous veins, and occasionally thrombosis.

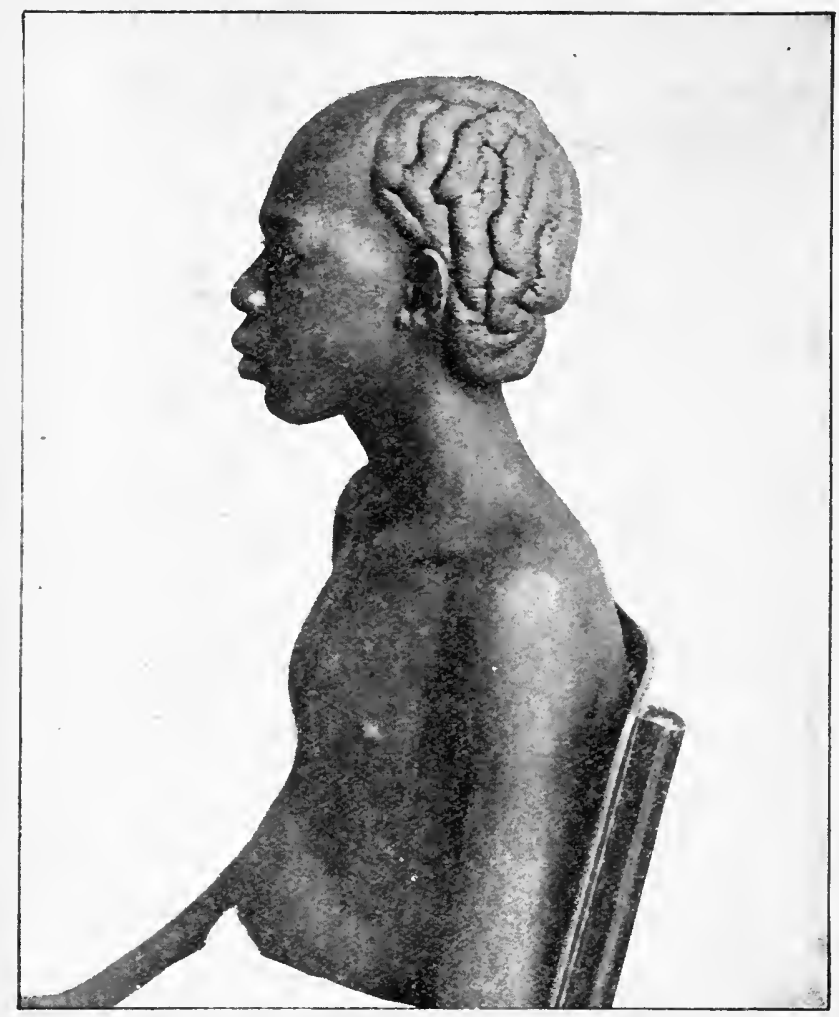

FrG, 407.-Elephantiasis of Scalp.

(Dr. G. A. MacDonald's case. Journ. of Trop. Med., I905.)

The papillae may be unchanged but are usually elongated and broadened and clumps of pigment may be detected in the papillary layer. No definite alterations are noticeable in the nerves supplying the part. The sweat-glands and hair-follicles are unaffected at first but in time become obliterated by the fibrous hyperplasia.

The epidermis shows more or less marked hypertrophy, irregular thickening of the prickle-cell layer, and hyperkeratosis.

Pathogenesis.-The most important fcatures in the histological picture consist of lymph stasis, varicosity of the lymphatics, and hypertrophy of the fibrous tissue, but the precise manner in which 
these changes are brought about is not yet fully understood. It is generally believed that the presence of filariae in the lymphatics is directly or indirectly responsible for them. Manson, who enunciated this theory, pointed out that the geographical distribution of elephantiasis and of Microfilaria nocturna (the larval stage of the F. Bancrofti) closely correspond, and that elephantiasis so frequently accompanies or succeeds lymph-scrotum, chyluria, and other accepted filarial affections, and the local inflammatory attacks are so indistinguishable from filarial lymphangitis, that little doubt is left that it is a stage of filariasis.

The exact manner in which the filariae bring about the lymph stasis and the fibromatosis is uncertain. They might do so in one of two ways, either (I) directly; by mechanically blocking the smaller lymphatic ressels and glands, or (2) indirectly, by setting up an inflammatory disturbance leading to fibromatosis and obliterative changes in the lymphatics and reins.

(I) Theory of Direct Blockage.--In support of the view that the stasis is the result of direct blockage of the lymphatics Manson pointed out that under normal conditions the microfilariae can pass along the smaller lymphatics and through the glands without difficulty, but should an injury to the adult female take place in the lymphatic ressel, which is liable to happen in exposed situations like the leg, the female may abort and instead of giving birth to sinuous active embryos may produce spherical ova which are launched into the lymph-stream and are passively carried to the nearest lymphatic glands and plug the afferent vessels. Even the adult filariae themselves, by forming a coil or cluster, might block the larger lymphatics, and in corroboration of this, dead filariae have been found in the lymphatic ressels and glands in elephantiasis (Manson-Bahr).

(2) Theory of Inflammatory Reaction.-It has been urged on the other hand that blockage of the lymphatics alone is incapable of producing complete stagnation of lymph, that it requires also blockage of the reins, and that there is probably another factor in the production of lymph stasis, namely inflammatory reaction. The latter causes a fibrous hyperplasia throughout the corium and subcutaneous tissue, proliferative changes in the walls of the lymphatic vessels, and occasionally- thrombosis and obliteration of the veins. These are the outcome of local inflammatory attacks which may be due either to chronic irritation set up by the filaria or its embryos in the lymphatics and to periodical discharges of microfilariae from the parental uterus, or to some local injury to a tissue whose vitality has been lowered from the presence of a lymph stasis, or to the secondary inoculation of septic micro-organisms such as streptococci, as in E. nostras. Against the last suggestion an examination of the lymph during an attack has proved it to be sterile (Manson-Bahr).

While admitting that streptococci may aggravate the condition in tropical elephantiasis it seems much more probable that the filariae themselves are entirely responsible for it, both by blocking the lymphatics and by causing the inflammatory changes which lead to fibrous hyperplasia and obliteration of the lymphatics.

Against the filarial theory it has been urged that microfilariae are found only rarely in the blood of persons suffering from elephantiasis. It is not to be expected, however, that they would be found readily when the lymphatic glands are blocked, as they would thus be prevented from reaching the blood, and filariae have been detected in the lymphatic glands by 
gland puncture without corresponding microfilariae being found in the blood.

It is rare to find filariae in the lymphatics in elephantiasis of the legs, but in the scrotum they have been found repeatedly both in the adult and larval forms.

The filariae are transmitted to man by the bites of mosquitoes, of which a considerable number are known carriers or intermediary hosts, such as Culex fatigans, Pyretophorus costalis, Stegomyia fasciata, and S. pseudo-scutellaris, and penetrate the skin after the withdrawal of the stylets of the mosquito.

The warty changes which occur in old-standing cases are probably the result of secondary inoculation of pyogenic micro-organisms, chiefly staphylococci.

Etiology.-It is more common in males than in females in the proportion of 3 to $\mathrm{I}$, and may occur at any age but is most prevalent in early and middle life.

The high temperature of the tropics would appear to be a predisposing factor by favouring the occurrence of the mosquito and the passage of the filariae through the proboscis.

Prognosis.-As a rule elephantiasis however severe is not dangerous to life, but it tends to render the patient's existence a burden, from the discomfort or actual pain of dragging about an unwieldy and cumbersome mass and from interference with mobility. If treated early it may possibly be aborted or kept in check.

\section{Treatment}

As there is no means of destroying the filariae in the tissues the treatment must be on general principles. Prophylactic measures should be adopted, such as removal to districts where the disease is not endemic and the employment of mosquito nets or other methods of preventing mosquito bites.

Attacks of acute lymphangitis should be soothed by means of fomentations or the application of lead and opium lotions, and the febrile disturbances should be dealt with by diaphoretics, quinine, and other well-known measures.

The local treatment varies according to the region involved.

In the case of the leg treatment is unsatisfactory, on the whole. In the early stages Castellani reports beneficial results from rest in bed with the leg elevated, massage once or twice a day, daily injections of fibrolysin, and the application of continuous pressure by means of rubber or flannel bandages. Fibrolysin, which is a painless substitute for thiosinamin, is given in doses of 2 to 4 c.c. injected locally or into the buttocks every day for about six months, with occasional intermissions of a weck or more. It has a reputation for causing the disintegration and absorption of fibrous tissue but the results from it are disappointing. 


\section{ELEPHANTIASIS}

Greater benefit can be derived from massage and the application of a Martin's rubber bandage, which reduces the thickening and the roughness of the skin and renders it soft and pliable. After the bandage is remored puttees or elastic stockings should be worn to prevent a recurrence of the swelling.

Where warty growths are present they may be reduced by salicylic ointment or salicylic soap plaster, and where ulceration or septic discharges occur they should be dealt with by antiseptic applications, such as boric lotion, and dressings of mercurial ointment, and by scrupulous cleanliness.

In severe cases various surgical procedures have been adrocated. Ligature of the main artery has been tried repeatedly with the object of cutting oft the nutrition and so bringing about degeneration and absorption of the diseased tissue, but, though theoretically sound, the results from it are usually transitory even if at first beneficial.

Excision of redge-shaped or elliptical pieces of tissue, followed by careful apposition and suturing, has resulted in distinct benefit in oldstanding cases where the disease has reached its height, but this method is useless so long as the condition is progressive and associated with attacks of lymphangitis.

The procedure known as "lymphangio-plasty" - which consists of aiding the circulation of the lymph by inserting pieces of silk along the subcutaneous tissues by means of probes through small incisionsor the tapping of the part by Southey's tubes to drain it, have been employed but usually with disappointing results.

In extreme cases amputation may be advisable.

In the case of the scrotum, relief may be obtained in early cases by the support of a suspensory bandage or by bandaging up to the abdomen. In severe cases excellent results have been obtained from amputation leaving the penis and testicles; for a description of the operation see Castellani and Chalmers' Manual of Tropical Medicine, I9I3, p. I It4.

\section{REFERENCES}

Bahr, P. IIAxsox. Filariasis in Fiji (Trans. Soc. Trop. Med., I9I 2, י.). CAHILL, J. Tuberculous lymphangitis (Brit. Journ. Derm., I S95, vii. p. I). Castellaxi, A. Treatment of Elephantiasis (Journ. Trop. Med. \& Hyg., I90-, X. P. 297).

Castellaxi \& Chalmers. Elephantiasis (Man. Trop. Med., 2nd Ed., London, I9I3, p. II3j).

Fraxcrs, A. G. Elephantiasis and tertiary syphilis (Brit. Journ. Derm., IS94, vi. p. 225).

Jesisemie, E. Elephantiasis des Arabes (Derm. Exot., Paris, I904, p. 329).

Macdoxald, A. Elephantiasis of the scalp (Journ. Trop. Med., I 905 , p. 9ך).

Maxsox, P. (Textbook of Tropical Diseases; and Allbutt \& Rolleston's System of Medicine, 190-, Vol. ii., Pt. 2, p. 945. References).

SABOtRACD, R. Sur la parasitologie de l'éléphantiasis nostras (Ann. de Derm. et de Sỵph., IS92, p. 592).

[xis, P. G. Histopathology (Walker's Trans., IS96, p. $78_{3}$ ). 
Volmmer, E. Elephantiasis lymphangiectatica congenita (Arch. f. Derm. u. Syph., 1903, lxv. p. 345).

Whitehead, W. Lymphangiectasis (Brit. Med. Journ., I902, 1. p. 75).

\section{FRAMBOESIA TROPICA (YAWS)}

Syn. : Pian (French Colonies), Dubi (Gold Coast), Tetia (Congo), Parangi (Ceylon), Buena (Burmah), Coco (Fiji), Tonga (New Caledonia), Puru (Malay States), Boubas (South America); and a number of other native names.

Definition: A general contagious disease of the tropics, characterised by a framboesiform (raspberry-like) eruption, generally believed to be due to the Treponema pertenue (Castellani).

History.-It was about the time of the discovery of America that this disease first became known among physicians in Europe. In the micldle of the fifteenth century it was described by Oviedo y Valdez. In the seventeenth century Breton and Labat referred to it in the West Indies, where it was known among the natives as "pyans" and where it was so common among the slaves working in the plantations that in almost all the larger centres special hospitals were built for the treatment and segregation of its victims. It was next described in the Dutch East Indies under the name of " anboyna pox." It is said to have been carried to Scotland in I769 by sailors from the West Indies who were wrecked on the coast near Wigtown, and was named there "sibbens" or " sivvens" from the Celtic word " sivvi" a raspberry. It is also believed to have occurred last century in Ireland, where it was known as " button scurvy."

Geographical Distribution.-Iaws has a wide distribution in the Tropics and is rarely if ever met with in temperate zones.

In America it occurs chiefly in the West Indies, Mexico, Brazil, Venezuela, and British Guiana.

In Africa it is most common on the West Coast for about Io degrees on each side of the Equator, and is specially prevalent in Angola, Congo Free State, Mozambique, and Madagascar. It has been observed also in the Sudan, Tripoli, and occasionally in Rhodesia and South Africa among Kaffirs.

In Asia it is particularly rife in Ceylon which is the nearest endemic focus to this country, and is common also in the Nalay Peninsula, Java, Assam, and Siam. It is present in China and the Philippine Islands, and occasional cases have been recorded in India, but it has not been observed in Japan.

In Australasia it is most common in the New Hebrides, Fiji, Samoa, New Caledonia, and the Gilbert and Ellice Islands, and is sometimes met with in North Australia.

In preparing the description of yaws the author is greatly indebted for many details to the important article on the subject by Castellani and Chalmers in their "Manual of Tropical Medicine" (1913), and to various articles by the former in the journals. 
Etiology.- Yaws is due to a specific micro-organism discovered by Castellani in I905 and named by him the Treponema pertenue. It is highly contagious, the infection taking place either directly through an abrasion in the skin, or indirectly by contact with contaminated articles or by transmission by some biting insect, such as a fly, tick, bug, etc.

The possibility of the fly being a carrier has attracted considerable attention lately, as yaws prevails mostly where sanitation is absent and in conditions of squalor and overcrowding where flies abound and are liable to swarm on the sores of the disease. Apart from imbibing the virus the flies readily pick it up on their legs and could easily transmit it to a healthy person through a scratch or a wound. In corroboration of this possibility Robertson in I90 7 found the Treponema pertenue and various septic microorganisms in centrifugalised sterile water in which flies infesting the yaws houses and in contact with yaws sores had been washed for twenty-four hours.

Treponema pertenue. - This micro-organism is a protozoon and was found in scrapings from papules of yaws. Previous to this, various micro-organisms had been detected in the lesions; Powell, for example, found a veast fungus in ISg6, Breda a bacillus, and Nicholls and Watts a micrococcus in IS99, but none of them was proved to be specific.

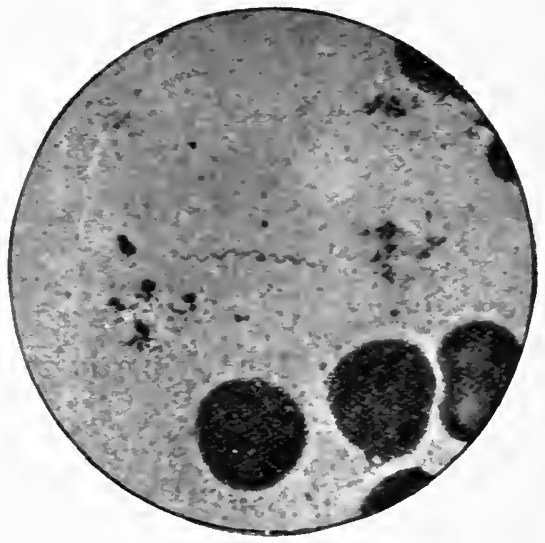

FIg. 408.-Treponema Pertenue of Yaws (Castellani).

(From Dr. Aldo Castellani. Journ. Trop. Med. and $H_{\text {j.g., Ig0o.) }}$

The Treponema pertenue is so similar, both morphologically and in its staining reactions, to the Spirochaeta pallida (Treponema pallidum) of syphilis that Schaudinn himself could not differentiate them. Certain observers have now detected minute differences between them and have pointed out that the treponema of yaws is even more slender and delicate than that of syphilis. That they are two perfectly distinct organisms has been proved by Charlouis' successful experimental inoculation of syphilis in a native suffering from yaws (ISSI), and by the observation that monkeys inoculated with the one do not become immune to the other. (Fig. fo8.)

The treponema is a delicate spiral with some $S$ to Io coils and moves with a slow corhscrew motion. Its life history is not yet ascertained, but longitudinal divisions have been noted in it and Castellani has found some peculiar bodies within leucocytes and lying free between them, oval or roundish in shape and containing chromatin dots, which he thought might possibly be stages in its development.

In unbroken lesions it is the only organism present, but when the lesions break and develop into open sores it becomes associated with 
various cocci, bacilli, and spirochaetes, which are secondary contaminations. It is found also in the lymphatic glands, spleen, and bonemarrow, but has not yet been demonstrated in the blood, although its presence there is indicated by inoculations of infected blood in monkeys giving rise to yars lesions in which it can be found. It is not present in the cerebro-spinal fluid, and inoculations with the fluid into four monkeys were negative.

Inoculation on Man.-It is readily inoculable from man to man. In I88 I Charlouis inoculated 32 Chinese prisoners with material from yaws and in 28 of them produced the disease, which began at the seat of the inoculation and developed into a typical general eruption. He also succeeded in inoculating syphilis in a native with yaws, and there are cases on record where syphilis has occurred naturally in patients suffering from yaws.

Inoculation on Monkeys.-Certain monkeys, such as those of the Genus macacus, are susceptible to yaws and when inoculated either subcutaneously or through a surface abrasion develop typical yaws lesions at the site of the inoculation from the serum of which the organism can be recovered. No general eruption has yet followed the inoculation but evidences of generalisation have been recorded, such as the presence of the organisms in the spleen and lymphatic glands. The incubation period varies from a minimum of $\mathrm{I} 9$ days to a maximum of 92 days.

Predisposing Causes.-Yaws is extremely rare in Europeans and almost entirely confined to natives, amongst whom it is most frequent in the lower classes as it is fostered by overcrowding and insanitary habits.

It may occur at any age, but is most common in children. As it is highly contagious, the majority of children in an endemic area become infected and later become partially immune, but the immunity conferred by an attack of yaws is not nearly so absolute as that by syphilis and second attacks are not infrequent. It is not congenital and is rarely seen in infants under six months of age.

Sex has no bearing on its etiology.

Description.--For purposes of description the symptoms of yaws may be arbitrarily divided into three stages corresponding to the classical stages in syphilis, namely (I) a primary stage characterised by a lesion at the site of inoculation; (2) a secondary stage or eruptive period in which typical yaws lesions are more or less widely distributed over the skin; (3) a tertiary stage associated with ulcerations and the presence of gummatous nodules.

Primary Stage.-The incubation period is from two to four weeks and is characterised by symptoms of general intoxication, such as headache, pains in the joints, fever, and general malaise. The initial lesion forms at the site of inoculation and consists of a papule, or a group of papules which tend to coalesce. In about a week the papules become moist and a vellowish secretion oozes from them; this dries 
up to form a crust which, when removed, leares an ulcer with a grant1lating base. Sometimes the ulcer heals, at other times it may develop into a granulomatous nodule like a lesion of the eruptive stage, when it is known as the "mother-yaw:" The glands in the neighbourhood of the sore may become enlarged.

Certain observers have denied the existence of the primary lesion, but it is now generally believed to occur, though when small it may pass unnoticed. It may be situated anywhere on the cutaneous surface but is almost invariably extra-genital. It appears at the site of some abrasion, such as a scratch, wound, unhealed raccination sore, ulcer, pustule, or insect bite, the most common sites being the arms, legs, and dorsa of the feet, where natives are particularly liable to injury from their limbs being uncovered. It is not uncommon for a mother to be infected with yaws by her child, the inoculation taking place on the nipple, or above the hip from carrving the child astride, or on the upper arm from resting the child's head there.

The sore may disappear in a few weeks and before the general eruption develops, but more often it is present then and may persist for months. When it heals, a scar remains which is usually flat and smooth, or occasionally raised and hypertrophic, and which is white at first and subsequently becomes pigmented.

Secondary or Eruptive Stage.-The eruption does not appear as a rule until a month or six weeks after the primary sore, and it is usually ushered in with an exacerbation of the general simptoms. It begins as rounded papules about the size of pins' heads which appear in different parts of the body and keep coming out in crops. Some of these persist for weeks, then disappear slowly, and are replaced by a transient scaliness; others increase in size, become raised up, and develop into soft warty masses, moist on the surface with a seropurulent discharge which dries up to form a vellow or brownish friable crust, on removing which a raw papillomatous surface is exposed. (Fig. fog.)

The papillomatous lesions are the typical yaws. They vary in size from a quarter of an inch to an inch or more in diameter, and are usually distributed on the face and extremities, rarely on the scalp though they may be met with on the border of the hair. As a rule they are discrete, but sometimes may be aggregated in a circinate fashion (ringworm yaws), or may coalesce to form irregular condylomatous masses which are present chiefly about the flexures of the large joints, such as the axillae and groins, or about the nostrils, lips, anus, and genitalia. A frequent situation is the soles of the feet, where they may ulcerate or where, by undermining the thich epidermis and pressing on the nerves, they may give rise to so much pain that they cause lameness and a peculiar crab-like gait, hence the name "crab raws." (Fig. 110.)

They may occur also about the borders of the nail, or on the nailbed, and may lead to changes in the nail itself, rendering it opaque, 
ridged, brittle, and sometimes causing it to be shed. All stages in development from the slightly raised papule to the fully developed crusted lesion, may be met with simultaneously.

The mucous membranes are not markedly involved, though small granulomata have been observed on the nasal mucosa and about the tongue. Occasionally the lymphatic glands are enlarged, especially those about the neck and groin, but they do not suppurate. The eyelids may be affected, but it is rare for the eye itself to be attacked though cases are on record in which iritis occurred.

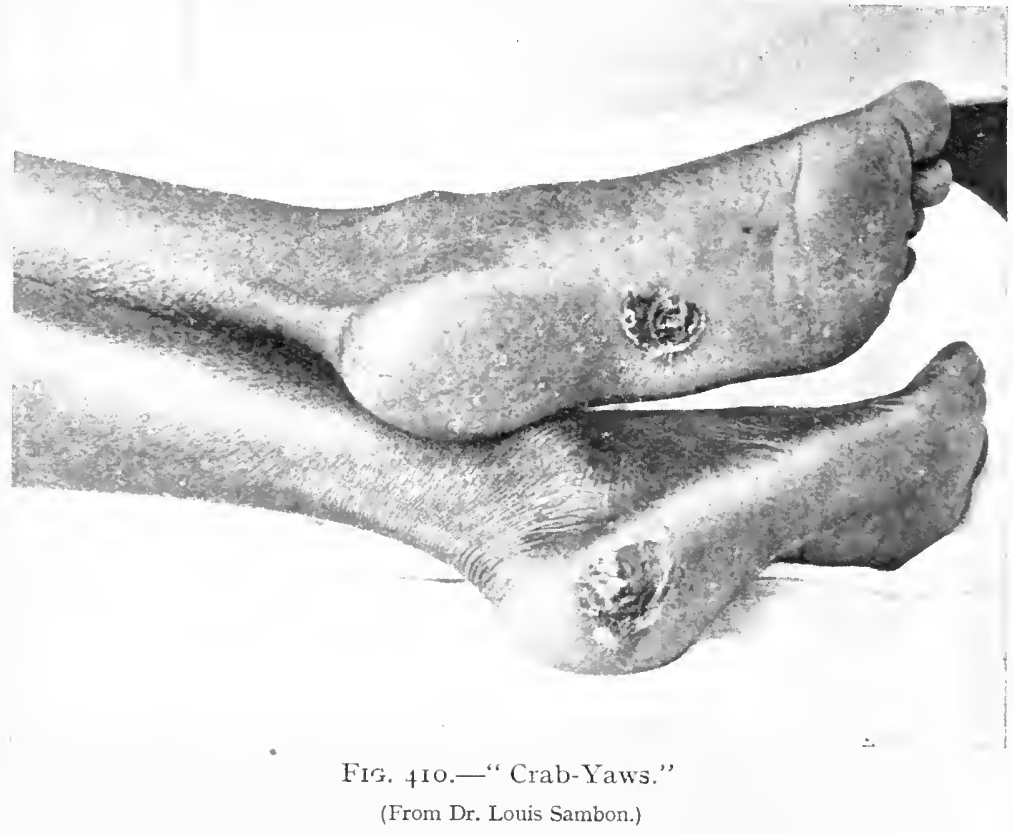

There are $n$ o characteristic changes in the blood except, in some cases, sl:ght anaemia.

With regard to subjective symptoms, the lesions are usually painless, except where ulceration has occurred or where they are present beneath the thick epidermis of the palms or soles or about the nails, but they may be accompanied by considerable itching. In oldstanding cases a peculiar fusty odour is exhaled by the affected skin, which is believed to be due to the action of some contaminating bacilli as it can be removed by applying antiseptic lotions to the lesions.

After six or eight weeks the individual lesions shrink, disappear and leave whitish discolourations which gradually become pigmented, but the eruption as a whole, from occurring in successive outbreaks, may last for a year or longer. In children it is usually over in about six months.

A peculiar pitted appearance about the palms and soles has been observed as a late manifestation and is the result of hyperkeratosis and 
the formation of hard nodules with central horny plugs which, when removed leave depressions. Another not infrequent sequel is periostitis which is liable to occur about the digital phalanges and may gire rise to marked dactrlitis. Neuritis is another troublesome simptom which may follow in the train of vaws, and may be associated with marked pain along the course of the nerve which may be so severe in the lower extremities as to cause lameness and trophic disturbances in the muscles.

Tertiary Stage.-The occurrence of a tertiary stage corresponding to that in syphilis has been doubted by many writers and where marked ulceration and necrosis have occurred in patients who have had yaws these have been attributed by them to syphilis. Castellani and others, however, are convinced of the occurrence of tertiary lesions years after the disappearance of the vaws eruption. These consist chiefly of gummatous nodules which break down and discharge; ulcers with clear-cut margins or thick undermined edges, which, on healing, leave hypertrophic scars and which may be present anywhere on the skin and occasionally on the mucous membranes of the nose, palate, and pharynx; lesions of bones, such as painful nodes, chronic periostitis, and occasional necrosis of the palate and nasal bones, and sometimes perforation of the septum; contractures or indurations of various muscles.

In Fiji, where yaws is very common, these late lesions have been observed frequently and they cannot all be attributed to stphilis as the latter is an exceedingly rare disease in those islands.

Histopathology.-In Igor, through the kindness of the late Sir William Kynsey, the writer had an opportunity of making a histological examination of the different lesions of yaws, the tissue being sent from Ceylon. The following is a summary of the microscopical appearances: The initial change in the earliest lesion appeared to be in the bloodressels of the papillary and sub-papillary layer, which were dilated and tortuous but had no thickening of the ressel-walls or endothelial proliferation. Around the ressels there was an extravasation of leucocytes which passed from them into the tissue-spaces and eventually reached the epidermis. Here and there in the neighbourhood of the dilated vessels were groups of plasma-cells.

In the epidermis the inter-papillary processes were elongated and thickened and the prickle-cells were enlarged and oedematous. The Stratum granulosum was defective and there was imperfect cornification.

In the older lesions the plasma-cells were much more numerous and were arranged in diffuse sheets occupving the upper layers of the corium and spreading down towards the hypoderm. They were densely clustered around the blood-ressels, follicles, and glands. A few of the larger plasma-celis presented two nuclei. Numerous polymorphonuclear leucocytes were present throughout the infiltration and a number of large connective tissue cells. There was no newly-formed 
fibrous tissue such as is found in a syphilide, and the blood-vessels in the midst of the infiltration were unaffected. (Fig. 4II.)

In the epidermis there was marked proliferation and the interpapillary processes had grown down for a considerable distance into the corium. The epidermis over the elongated papillae was not thickened, so that the tortuous vessels came near the surface and rendered the yaw liable to bleed. The cornification was imperfect and there was heaping-up of incompletely cornified cells into a horny mass, broken up into lamellae between which were layers of

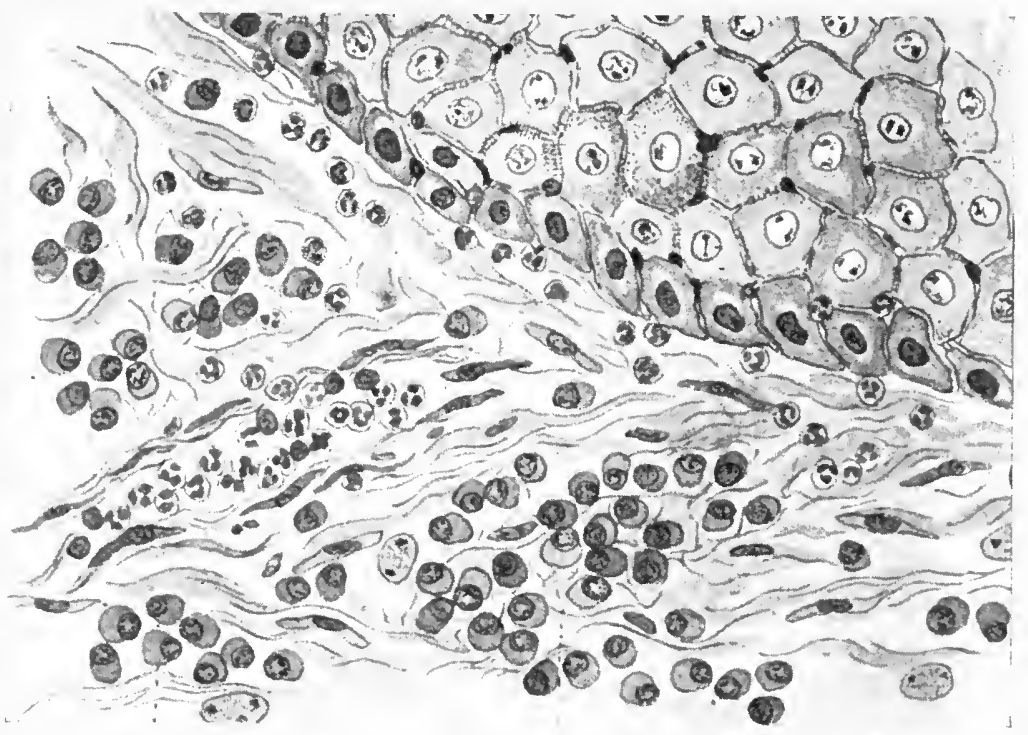

FIG. 4I I.-Commencing Granuloma of Saws.

J. MI. H. MI. del. ( $x$ about 8oo.)

Showing prickle-cells, swollen and oedematous ; a blood-capillary dilated and filled with leucocytes; plasma-cells.

leucocytic debris and fibrin which gave the horny crust its peculiar crumbly and cheesy consistence. (Fig. +12.)

Diagnosis. - The disease with which yaws is most likely to be confused is syphilis and so close may be the resemblance that certain well-known observers in the past have gone so far as to regard yaws as being syphilis in a dark skin. That they are two distinct diseases is now generally acknowledged.

Clinically they differ in that the primary lesion in yaws is extragenital and the eruption essentially uniform though lesions in different stages of development may be present at the same time, whilst in syphilis the chancre is usually genital and the eruption multiple, different types of lesions being met with in different cases: yaws is never congenital, while syphilis may be ; a second attack of yaws is not uncommon, while a second attack of syphilis is exceedingly rare; yaws is confined to the tropics, while syphilis is pandemic. 
Histologically they differ in that in raws the plasma-cells of the cellular infiltration are not arranged so definitely in rows or clustered round the blood-ressels as in syphilis, and there are no large multinuclear cells (chorio-plaques) or true giant-cells, the rarefaction of the collagen is more marked than in syphilis, and there is no organisation or colloidal degeneration such as occurs in syphilitic summata. In the blood-ressels there are no distinct proliferative changes in the vessel-walls or endothelium such as occur in syphilis. In the epidermis there is marked proliferation and down-growth with great thickening of the horny layer (due to hyperkeratosis or parakeratosis), which is unusual in șphilis.

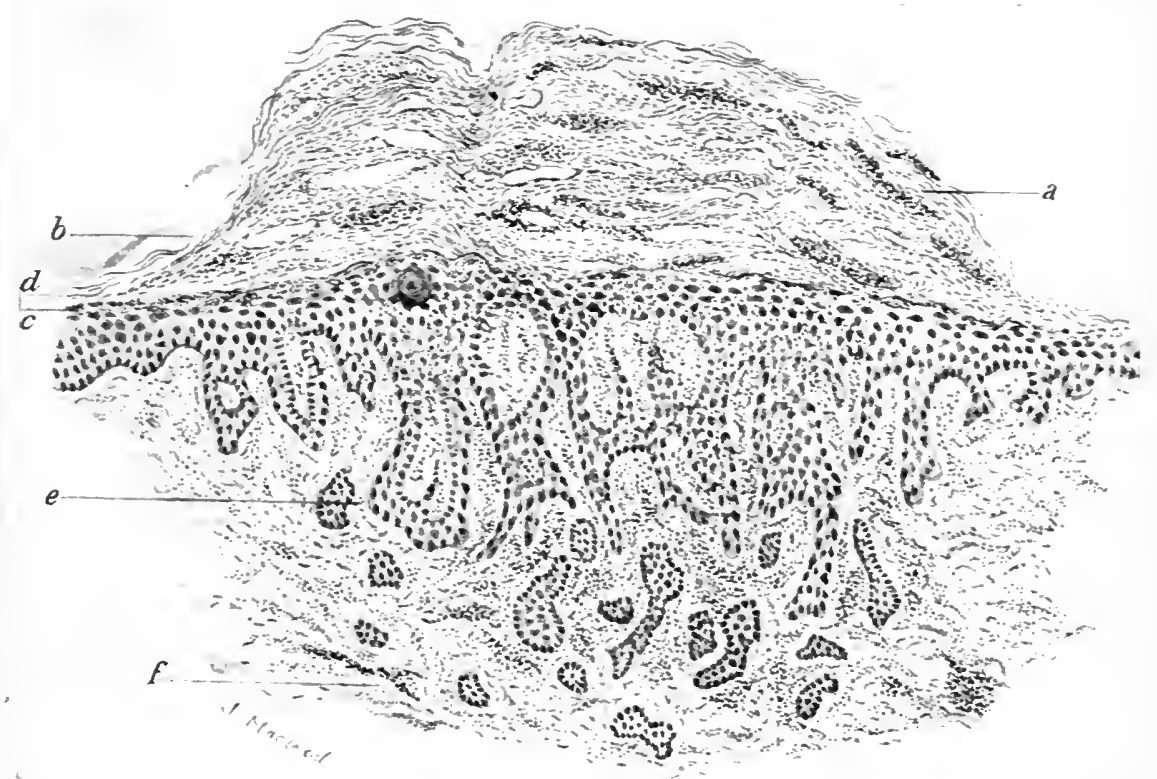

Fig. fI2.-Crusted Papule of laws, ( $x$ about 250.)

a. Crust. b. Space between partially cornified lamellae. c. Granular Jayer. d. Horny" pearl. e. Down-growing epithelial processes. $f$. Dilated blood-vessels.

More convincing than either the clinical or histological differences, an individual suffering from yaws may contract syphilis, and yaws can be inoculated experimentally both in man and in monkeys suffering from syphilis ; in other words the one disease does not confer immunity to the other.

Yaws has to be distinguished also from Verruga Peruviana, but in the latter there is severe fever, the lesions bleed more readily, and the affection is confined to certain valleys and slopes of the Andes. It has been suggested that Verruga Peruviana is a combination of two diseases, namely yaws and enteric fever, but this view is not generally. accepted.

Prognosis.-The prognosis is usually good. The disease itself does not lead to a fatal issue, unless in rare instances where the sores have 
been extensive, the purulent secretion profuse, and absorption and septicaemia have supervened. When death does take place it is almost invariably the result of some intercurrent disease, such as pulmonary tuberculosis. The long course of the affection and the anaemia and debility associated with it render it of serious economic importance.

\section{TREATMENT}

General Treatment.- The most effective remedy at present known is salvarsan or its substitutes, administered in the same way as in syphilis. The drug acts almost like a charm and a single dose may be sufficient to clear up the eruption though, occasionally, relapses may occur. It has been observed also that the serum of patients treated with salvarsan has a curative effect when injected in patients suffering from yaws. (Figs. 4I3, 4I.).)

Another drug which has been used with favourable results, is iodide of potassium. To obtain benefit it must be given in large doses, such as $\mathbf{5}_{5}$ grains or more three times a day in an adult. Castellani recommends its combination with tartar emetic and sodium salicylate in the following mixture: Pot. iod. I dr., tartar emetic I gr., sod. salicyl. Io grs., sod. bicarb. I5 grs., to I oz. of water ; three doses are given daily, diluted in four times the amount of water, to adults.

Nercury in any form has no specific action on the disease and no improvement is obtained even after its prolonged use.

Any general defect, such as anaemia or debility, should be treated on ordinary medical principles with nourishing food, and tonics, such as iron and cod-liver oil.

Local Treatment consists of scrupulous cleanliness, and regular baths or sea bathing. Open sores should be cleansed by antiseptic lotions, such as weak permanganate of potash, carbolic acid I in 40, or perchloride of mercury I in Iooo, or they should be dusted over with some antiseptic powder such as boric acid or iodoform, or dressed with a mild mercurial ointment. Painful yaws on the soles should be scraped with a Volkmann's spoon and packed with iodoform gauze till healing occurs. To prevent the spread of the disease patients should be isolated until the eruption has disappeared. Their huts should be thoroughly disinfected and the clothes they have worn should be burned. In a district where the disease is endemic every care should be taken to heal abrasions in order to prevent them becoming a channel of infection.

\section{REFERENCES}

Ashburn, P. MI., \& Craig, C. F. Observations upon Treponema pertenue Castellani of yaws \& the experimental production of the disease in monkeys (Phil. Journ. Sci., Vol. ii., No. 5).

Castellani, A. Is yaws syphilis? (Journ. Trop. Med. \& Hyg., Jan. I9o6, p.r.) Framboesia tropica (Journ. Cut. Dis., April \& May I908).

The nternal treatment of yaws (Journ. Trop. Med. \& Hyg., March I9ri5, p. 6r). 

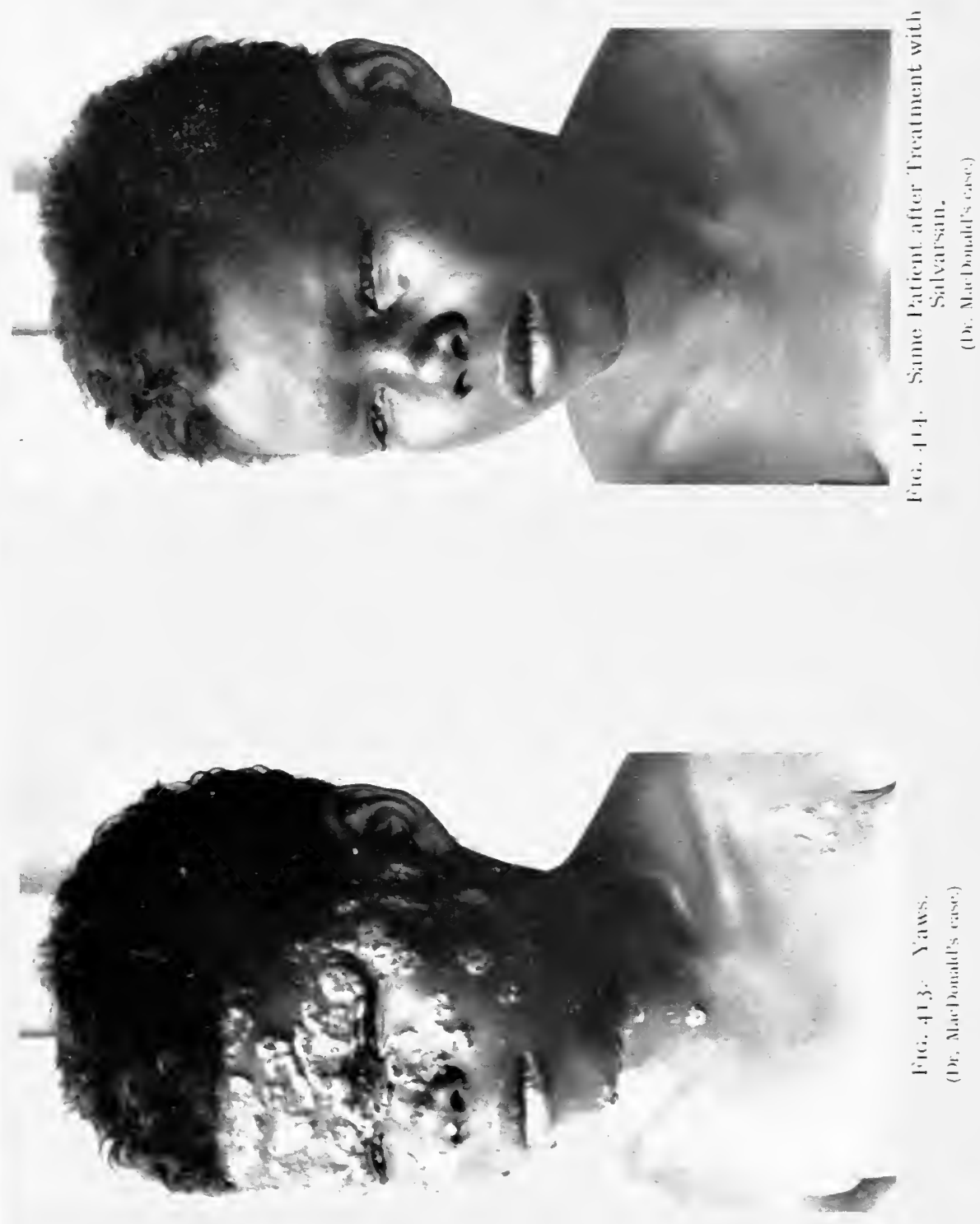

Castellani \& Chaluers. Yaws (Man. of Trop. Med., London, 1913).

Charlocis, II. Polypapilloma tropicum (Viertelj. f. Derm. u. Syph., ISS 1, viii.).

Cockis, R. P. The treatment of twenty-two cases of yaws by salvarsan (Journ. Trop. Med. \& Hyg., Sept. I9I 2, p. 27\%).

Gimlette, T. D. The Puru of the Malay Peninsula (Journ. Trop. Med., May 1906, p. I 49).

HARPER, P. $5 t^{2}$ cases of yaws treated bỵ kharsivan \& arsenobillon (Trans. Soc. Trop. Med. \& Hyg., Feb. I9i 7 , p. 82).

MACLEOD, J. M. H. The histopathology of yaws (Brit. Med. Joum., Sept. Igor).

Montagee, A. A. Tertiary yaws (Journ. Trop. Med. \& Hyg., Vol. xiii,, No. II).

Nicholls, H. Yaws (2oth Cent. Pract. Med., IS99, New York, xvi.).

Powell, A. Yaws in India (Brit. Journ. Derm., I 896, p. 457).

Rivas, F. A. The spirochaete of yaws (Tesis de docterado, Lniversitad de Venezuela, 1906).

Robertsos, A. Bacteriology \& treatment of yaws (Brit. Med. Journ., Oct. I90\%, p. \$68).

SPAAR, E. C. Three cases of Parangi treated with Dr. Castellani's mixture (Journ. Trop. Med. \& Hyg., Aug. 1915, p. r 70 ).

Strosg, R. P. The specific cure of yaws by dioxydiamido-arsenobinzol (Trans. Soc. Trop. Mec.. \& Hyg., April I9I I, p. 153).

White, C. J., \& Trzzer, E. E. A case of framboesia (Journ. Cut. Dis., March I9II).

\section{GANGOSA}

Syn. : Rhinopharyngitis mutilans; Kaninloma (Fiji).

Definition: A progressive ulcerative affection of the throat. soft palate, and nose, which destroys cartilage and bone, gives rise to deformity, and tends to spread on to the skin.

Description.--The name gangosa is from a Spanish word meaning nasal roice and denotes one of the characteristic features of the disease. The condition was first described in 1882 by the Spanish Commission to the Ladrone Islands and is endemic chiefly in the island of Guam, where it is said to affect about two per thousand of the population; cases have also been reported from Fiji, the Caroline Islands, British Guiana, Dominica, Panama, and Italy, and it may have a still wider geographical distribution as a somerwhat similar condition has been observed by Castellani and Chalmers in Cerlon and by Seligman in New Guinea.

It occurs almost exclusively in natives, only one case having been reported so far in a white man, a sailor who had recently come from Guam (Stitt).

It usually commences in the throat or soft palate as a nodule, which soon becomes a superficial ulcer covered with a grerish slough and emitting an offensive odour. This ulcer, by spreading forward and destroying in turn the soft and hard palate and the nasal septum and cartilages, causes the nose to fall in or to become eroded, and the mouth and nose to become transformed into a single cavity. Later it 
may extend on to the face and attack the upper lip, may spread to the eyes, destroying the eyelids and cornea and interfering with vision, or may extend down to the larynx. The ulceration may occur also on the uncovered parts of the body, especially on the hands and feet. It is progressive and may last for months or years, usually being subject to periods of activity and quiescence. When the ulcers heal they are followed by disfiguring scars somewhat like those caused by burns and lead to more or less marked contracture and deformity. The disease is not fatal in itself and does not greatly interfere with the general health. (Fig. 4I5.)

Microscopical examination of sections of the spreading margin of the ulcer show a cellular infiltration, consisting mainly of lymphocytes with an occasional giant-cell, and a marked dilatation of the bloodvessels. Various micro-organisms have been detected on the surface,

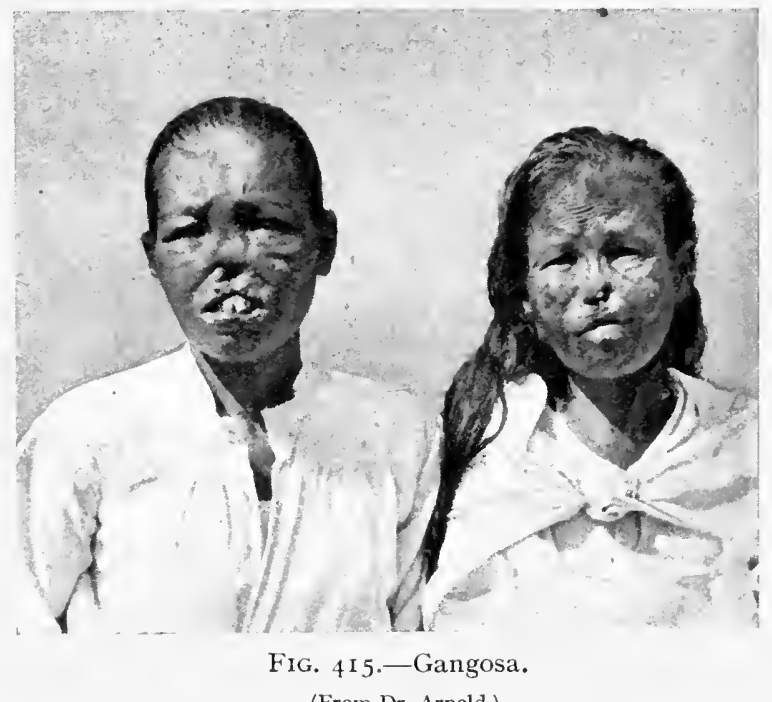

(From Dr. Arnold.)

such as micrococci and bacilli, but none of them has been proved to be pathogenic.

Etiology.-The true nature and causation of gangosa are uncertain but the opinion is gradually gaining ground that it is a distinct entity. It has been suggested by Daniels that it is a tertiary form of yaws, but other observers dissent from this view and point to the fact that it may be absent in countries where yaws is common.

Diagnosis.-A diagnosis has to be made from ulcerating tertiary syphilis, which it may closely resemble clinically, but a negative Wassermann reaction and the absence of the Spirochaeta pallida and of any reaction to mercury and iodide of potassium distinguish them.

Treatment.-So far no method of treatment has been discovered by which the progress of the ulceration can be arrested, but antiseptic 
applications, such as tincture of iodine or ammoniated mercury ointment, are of value in preventing the secondary growth of pyogenic organisms, while the employment of a solution of permanganate of potash is useful as a deodorant.

\section{REFERENCES}

Axgeny (New Orleans Med. \& Surg. Journ., I9I2).

BRANCH (Journ. Trop. Med., I906, ix. I56).

FORDYCE \& ARNold (Journ. Cut. Dis., I906, xxiv. p. I).

LEYS (Journ. Trop. Med., I906, ix. p. 47).

Mink \& MCLeaN (Journ. Amer. Med. Assoc., I906, xlvii. p. I I66).

Musgrave \& Marshall (Philippine Journ. of Science, I907, ii. p. 387).

Sritr (U.S. Naval Med. Bulletin, 1907, i. p. 96).

\section{GOUNDOU}

Syn.: Gros-nez; Henpuye or " dog-nose" (Gold Coast) ; Anákhré or "big-nose" (French Ivory Coast).

Definition: A peculiar affection of the nose, characterised by symmetrical swelling of the sides resulting from exostosis of the nasal processes of the sub-maxillary bone.

This remarkable condition first described by Macalister in I882 under the title of " horned men " is met with among the natives of the West Coast of Africa, having been reported chiefly from the Gold Coast and the French Ivory Coast. It is so prevalent in certain districts of the Ivory Coast that it has been estimated that on the lower banks of the river Comoë between I and $2 \%$ of the natives are affected. Cases have been recorded also in Sierra Leone, Angola, Uganda, and Zanzibar, and outside the African continent in the West Indies, Malay Peninsula, Sumatra, and China. It usually affects natives but has been observed in a European. Recently it has been found in certain of the higher apes.

Description.-The disease usually commences in childhood but may not appear till later and may not develop until adult life. It is generally ushered in by frontal headache associated with a sero-sanguineous discharge from the nose, which persists for about six months and then gradually disappears. During this time hard pea-sized swellings develop on each side of the nose just below the level of the inner canthus, which slowly increase in a downward and outward direction and tend to assume an oval shape. About the age of puberty they may have reached the size of an almond, while at the age of twenty they may be as large as half a pigeon's egg or larger. As a rule the condition is symmetrical but it has been known to be unilateral, or one tumour may develop before the other. In any case the growth is invariably slow and may be continuous through life or may cease about puberty or later. The skin over the tumours is normal in texture and freely movable in the subjacent tissue. There is no 
inflammation associated with it and the lesions show no tendency to break down like gummata. To the touch they are of a bony hardness and fixed, and except in the early stages, are not tender. Occasionally they have been known to become painful in wet weather. (Fig. 4I6.) They cause a bulging in the walls of the nose and constrict the nasal cavity but do not encroach on the septum or the nasal cartilage nor do they prevent breathing. The sense of smell is not impaired but the nasal mucosa is swollen and sensitive and a nasal voice may be produced. When they reach a certain size they interfere with rision and may necessitate bending the head to see over them, while if they become sufficiently large they may displace the eye and may even destroy it by pressure. (Fig. 417.)

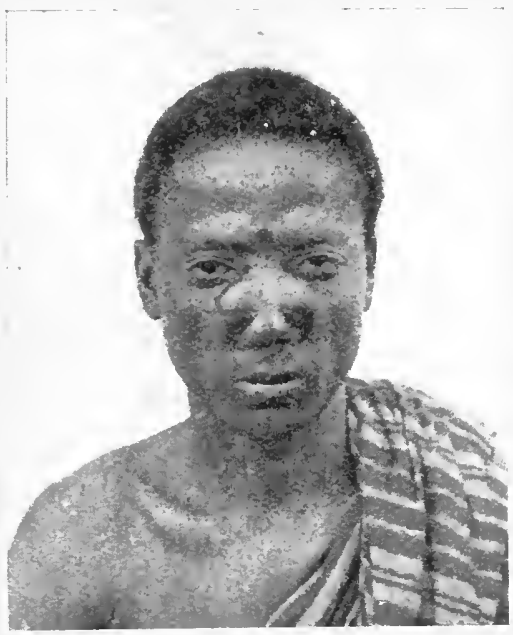

Fig. 4I6.--Goundou.

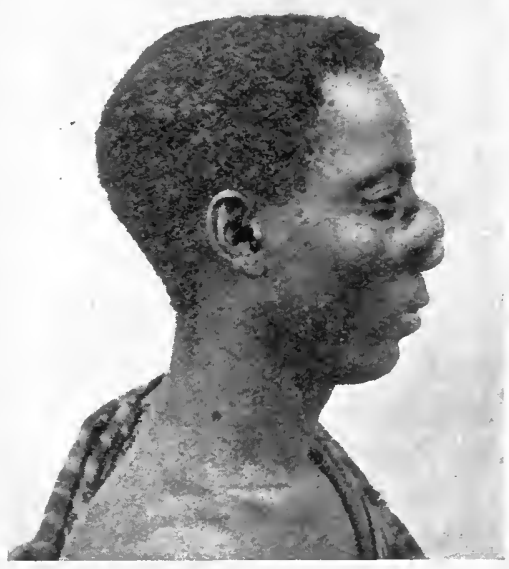

Fig. 4I7.--Goundou.

When cut into, they are found to consist mainly of soft cancellous bone with a thin covering of compact bone, which has formed beneath the periosteum of the nasal process of the superior maxilla. The infraorbital plate sometimes may be invaded.

Etiology.--The condition is now generally beliered to be the result of a chronic sub-periostitis of the nasal process and infra-orbital plate of the superior maxilla, the cause of which is at present unknown.

A hypothesis which has attracted considerable attention is that it is caused by the presence under the periosteum of the larvae of certain flics which have penetrated the nasal process from the nose, but this riew is not gencrally accepted.

It is said to be more common in males than in females. High temperature may have something to do with its etiology as almost all the recorded cases have occurred in the tropics.

Treatment.- The only satisfactory method of treatment is excision, the incision being made in the long axis, the skin retracted, and the bony growth remove 1 by bone forceps. 


\section{REFERENCES}

Braddox, W. Notes on the occurrence of Goundou or Gros-nez in the Malay Peninsula (Journ. Trop. Med., Igor, is. p. I jo).

Brimpt. Goundou (Bull. Soc. de Path. Exot., 1909).

Chamers, A. J. Report on Henpuye in the Gold Coast Colony (Lancet, I900, i. p. 20).

Friedrichsex. Goundou (Journ. Trop. Med., I903, vi. p. 62).

GrahaM, J. C. Goundou or Anákhré (Journ. Trop. Med., Igoo, iii. p. I I).

Leger. Goundou (Bull. Soc. de Path. Exot., 19I2).

Macalister, A. Further evidence as to the existence of horned men in Africa (Proc. Roy. Irish Acad., and series, vol. iii. (Science), I $88_{3}$. p. $77 \mathrm{I})$.

Marchoux \& Mesill. Goundon (Bull. Soc. de Path. Exot., I912).

Maxwell. Goundou (Journ. Trop. Med., I90o, iii. p. Iro).

Nell, A. Goundou: its relation to yaws (Journ. Trop. Med., I903, vi. p. $34^{8)}$.

Rexier, WV. A case of Goundou or Anákhré (Journ. Trop. Med., I9oo, p. $\mathrm{I}_{45}, \& \mathrm{I}_{90}$, p. $2 \mathrm{I}_{4}$ ).

Rogues \& Bouffard. Goundou (Bull. Soc. de Path. Exot., I9o8).

Scheube. Goundou (Diseases of warm climates, I902, p. 550).

Strachax, H. Bony overgrowths or exostoses in West Indian negro (Brit. Med. Journ., I 894 , i. p. I 89 ).

\section{GRANULOMA INGUINALE}

Syn. : Ulcerating granuloma of the pudenda.

Definition: A chronic granulomatous affection of the genitalia, perineum, and inguinai regions.

Attention was first drawn to this condition in India by Kenneth MacLeod and Maitland under the heading of "serpiginous ulceration of the genitals" and in r 896 by Conyers and Daniels in British Guiana where it was known as "the lupoid form of groin ulceration." The modern names of Granuloma inguinale or Granuloma pudendi are more accurate, as ulceration is not an essential characteristic and may not be even a marked feature.

It is confined to the tropics and only imported cases have been observed in temperate zones. It occurs chiefly in British Guiana, the West Indies, West Africa, India, South China, and western Australia, and mostly among natives and negroes, the cases in white men being comparatively rare.

Description.--It appears first as a small papule or nodule usually situated on the penis or pubis, and, in the female, on the labia minora. This gradually increases until it reaches the size of a sixpence or larger, is definitely indurated, pinkish in colour, and smooth and shiny on the surface. From the formation of new nodules and their coalescence irregular raised patches or masses result. (Fig. +18.)

The epidermis of the lesions, being stretched, is liable to be abraded and readily bleeds, and from the raw surface and in the grooves and folds between individual nodules a serous discharge of a singularly 
offensive odour is emitted. Occasionally there are proliferative changes of a secondary character with the formation of warty exuberant vegetations. (Fig. 4I9.)

It is usually most marked about the pubes-where it is particularly active about the pilo-sebaceous follicles and liable to cause shedding of the hairs-and in moist regions such as the folds of the groins and perineum. It is apt to extend up the mucous orifices and block the urethra, or to pass up into the rectum and vagina and cause a rectovaginal fistula.

The lesions spread by peripheral extension and by the production of new lesions by auto-inoculation through contact. They have a

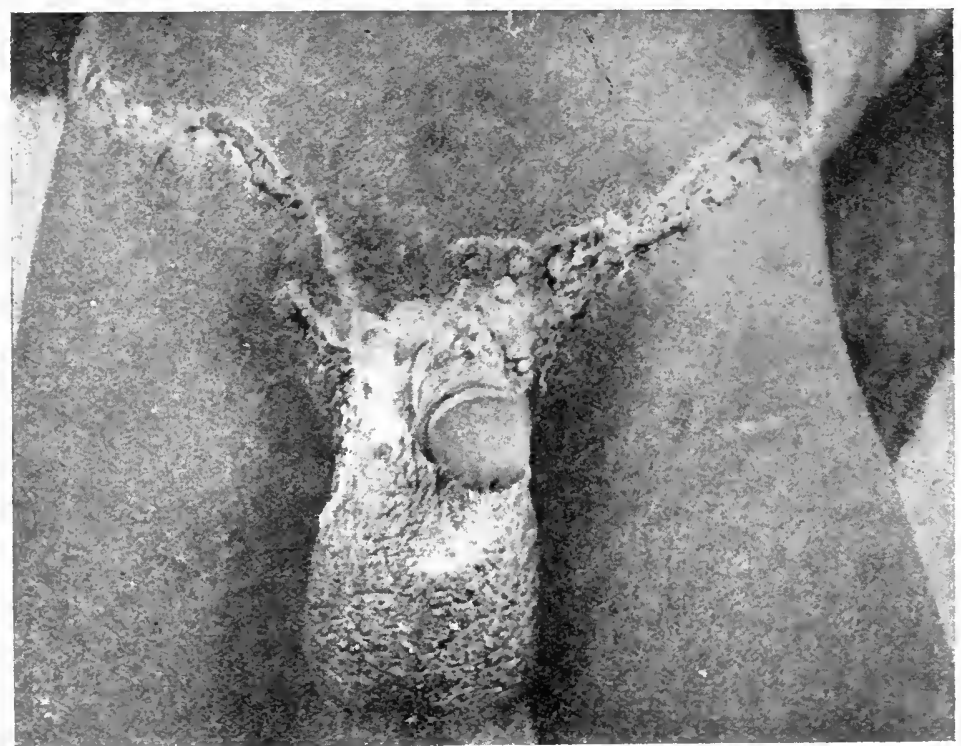

FIG. 418.-Granuloma Inguinale.

(After Conyers and Daniels.)

decided tendency to heal spontaneously, leaving hard, irregular, deeply pigmented scars. On the other hand, superficial ulcerations may result from abrasions and the secondary inoculation of pyogenic micro-organisms. The fibromatosis evident in the scar-formation is liable to obstruct the lymphatics and may lead to considerable oedema and to an clephantiasic condition of the genitalia.

It is not associated with either pain or definite itching, and does not appear to affect the general health directly.

Histopathology.-Sections of a growth show that the essential feature is a cellular infiltration in the upper layers of the corium, consisting of lymphocytes, polymorphonuclear leucocytes, plasma-cells, mast-cells, and connective tissue cells. The white fibrous tissuc and elastin have disappeared and been replaced, to a great extent, by the 
infiltration. In the older lesions there is a considerable number of fibroblasts among the cells and new connective tissue in various stages of development. The overlying epidermis is thickened and the interpapillary processes may be irregularly elongated.

Etiology.-It may occur in either sex indiscriminately. It has never been observed under puberty and may not appear till old age but usually begins in adult life. It is auto-inoculable, contagious, and believed to be transmitted generally by sexual intercourse, but the contagiosity does not seem to be high.

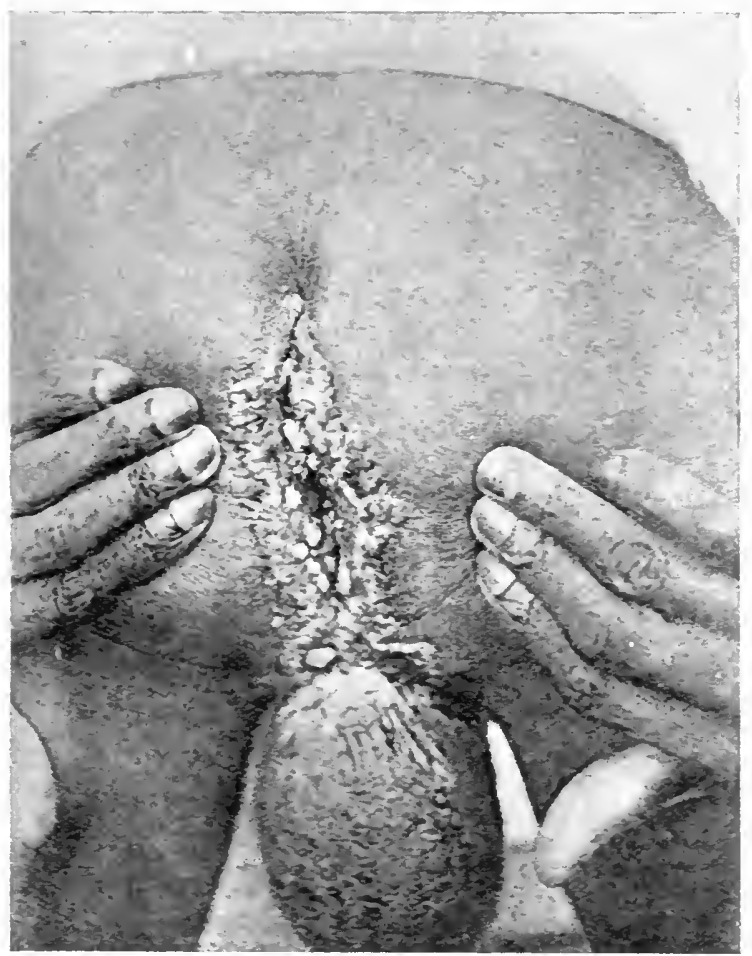

FIG. 419.- Granuloma Inguinale.

(After Conyers and Daniels.)

The exact cause is not yet certain. Spirochaetes have been found in the scrapings from the surface but were probably accidental contaminations. Donovan has observed a coccus-like body, singly or in groups, in the mononuclear cells scraped from the surface and this may be etiologically connected with it.

Experimental inoculation in lower animals so far has been unsuccessful.

Diagnosis.-There is no great difficulty in diagnosing a welldeveloped case. Where it has not extended beyond the penis it might be mistaken for a squamous-celled carcinoma but in Granuloma inguinale the neighbouring lymphatics are not enlarged. 
It might be mistaken for tertiary syphilitic ulceration but for the absence of a positive Wassermann reaction, the failure to react to the therapeutic tests for syphilis, and the absence of any other signs of syphilis.

\section{TREATMENT}

The only satisfactory treatment is complete eradication of the discased tissue. When the lesion is small and circumscribed the best method is excision of the affected skin and underlying hard subcutaneous tissue; should this be impracticable, the curette may be resorted to, but it is difficult to get rid of the tissue by this means owing to its tough fibrous characteristics. After the curetting the surface should be cauterised by the actual cautery or by nitrate of silver. A useful aid to treatment especially in cases in which there is marked proliferation of the epidermis, is the X-rays. Onc or more pastille doses of the rays should be given at intervals of three weeks. In a case so treated by the writer the lesion became withered and friable after two exposures, was easily scraped away by the curette, and was followed by a fairly satisfactory scar.

One of the chief difficulties in the treatment is the tendency of the affection to extend up the mucous orifices, and in the case of the urethra in order to keep an open meatus it may be necessary to amputate the glans penis up to the distance implicated by the growth.

Milder local measures are of little value except for the reduction of the offensive discharge and the prevention of ulceration. With this object the surface may be cleansed with solutions of permanganate of potash I in 50 , or perchloride of mercury $I$ in 2000.

Internal treatment is of service on general medical principles where the patient is run down from some cause such as malaria or any other lowering disease.

Mercury would appear to have no influence on its course, but improvement has been claimed from large doses of iodide of potassium.

Encouraging results from intravenous injections of Antimonium tartaratum have been recorded by different observers. Low and Newham have reported a case which was successfully treated by this means and another which was refractory to the treatment.

\section{REFERENCES}

Bonve, C. Three cases of venereal granuloma treated witl tartar emetic (Journ. Trop. Ned. \& Hyg., I9I 7, xx. p. Iog).

Castellait \& Chalmers. Granuloma inguinale (Man. of Trop. Med., 2nd Ed., London, I9I3, p. I57I).

Cllland, J. 13., \& Hickinbothan, J. R. Etiology of ulcerative granuloma of the pudenda (Journ. Trop. Med. \& Hyg., Nay I909, p. I+3).

Conyers, J. H., \& Daxiels, C. W. Granuloma inguinate (Brit. Guiana Med. Ann., I 896, p. I3).

Galloway, J. Granuloma inguinale (Brit. Journ. Derm., 1897 , ix. p. 134). 
Low \& NEwнA. A case of ulcerating granuloma successfully treated by intravenous injections of antimony (Brit. Merl. Journ., IgI0, 2, p. $\left.3 S_{7}\right)$.

A case of ulcerating granuloma refractory to intravenous injections of antimony (Trans. Soc. Trop. Med., Igi 7 , April, p. Iog).

Wise, H. S. Granuloma inguinale (Brit. Med. Journ., I906, I, p. I27f).

\section{MYCETOMA}

Syn.: Madura foot; Fungus foot of India; and rarious native names such as Slipada in Bengal, Hatty-ka-pung (large foot or elephant foot) in Deccan, Keerengrah (dwelling of worms) in Raj Putana. and Ghootloo Hahdee (insect-egg) in Bellary.

Definition: Mycetoma is a disease which usually affects the foot, and is characterised by swelling and deformity, with degeneration of the affected tissues, and the formation of cysts with fistulous openings on the surface which contain an oily putrid fluid in which mycotic aggregations are suspended. ${ }^{1}$

Geographical Distribution.-Wretoma was believed originally to be confined wholly to India but is now known to have a far wider distribution. It is endemic chiefly in Madras, Madura, Trichinopoli, and Bellary, and occurs also in Bombay, Bengal, the Punjaub, and the North-West Provinces, but cases have been recorded in Cerlon, Egypt, the Sudan, Yemen, Madagascar, Senegambia, Morocco, Algeria, and Cyprus, and in Canada, the Lnited States, South America, the West Indies and Cuba, and in Europe in Italy. It is peculiarly limited in distribution, and occurs in districts the country around which may be practically immune.

Description.-It is due to certain mould-fungi which gain entrance through some abrasion in the skin. As is indicated by the sinonym Madura foot it usually affects the foot, where the primary inoculation may take place on the sole or, more rarely, on the inner side of the foot or between the toes, but cases have been reported in which it attacked the knee, leg, trunk, neck, and jaw.

After the primary inoculation there is an incubation period which varies but is probably long-in Musgrave and Clegg's successful experimental inoculation of a monkey's foot with the disease Discomyces asteroides) it was found to be between Io and I6 dars.

The initial lesion develops in the form of a circumscribed indurated swelling about the diameter of a threepenny piece, but medical men rarely see it in this stage as the native usually does not present himself for treatment until it is well adranced. This nodule runs an indolent

${ }^{1}$ Chalmers and Archibald include under the mycetomas net only the Maduromycoses, but also the Actinomycoses. They classify the Maduromycrses into three groups, namely, (I) the Black Maduromycoses with black grains: 2) the White or Iellow Maduromycoses with white or yellowish grain:; 3) the Red Maduromycoses with red grans. These they further sub-divide according to the geographical distribution and the causal organism. Vide Journ. Trop. Med. and Hyg., Iors, xxi. p. I zo.) 
course and may or may not be associated with pain. After a time a vesicle may form on the surface, and in a couple of months it generally begins to soften and eventually breaks down in the centre to form a fistulous opening from which oozes an offensive purulent oily discharge, in which are suspended granules of different colours according to the variety of the disease. The granules, or " sclerotia," may be whitish or yellow, vary in size from mere specks to aggregations as large as a pea, and somewhat resemble fish-roe; may be brown or black, and hard or brittle, and suggest coarse gunpowder; or, in exceptional cases, may be red, soft, and mulberry-like.

It used to be customary to divide the disease into three varieties, according to the colour of the granules, namely, (i) the white, yellow, or ochroid variety, (ii) the black or melanoid variety, (iii) the red variety; but the modern classification is based on the different species of causal fungi.

Gradually new nodules appear which are similar to the primary lesions and which go through the same stages of degeneration, giving the foot a peculiar irregular tuberculated appearance. They vary in size from a small shot to a filbert nut and may be seen in all stages of evolution, some being solid while others are soft and converted into fistulous openings blocked by vegetations.

The foot becomes swollen and assumes a deformed appearance which is characteristic of the disease. It increases in breadth, the sides become rounded giving it an oval shape, the arch swells up and disappears, the sole becomes convex, and the toes raised so that the patient is unable to place the heel and toes on the ground at the same time. The toes themselves are not generally affected and the patient is able to separate them. The ends of the tibia and fibula are usually thickened and the ankles swollen. The affected skin is dark, dirty, and more or less covered with a viscid discharge which oozes out with the movements of the foot. To the touch the diseased tissue has a peculiar elastic feeling somewhat like indiarubber. If a probe be inserted into one of the fistulous openings it penetrates deeply, even down to the bone, and may be passed in various directions almost without pain. The whole foot eventually assumes the appearance of a homogeneous mass, the result of the process of softening having attacked all the tissues, with the exception of the tendons and fasciae, and even the bones may be honeycombed with cavities. This peculiar degenerative softening is a characteristic feature and shows that the tissues, when once affected, do not react in the normal way to the invading fungus but simply degenerate beyond repair. Above the diseased area the leg becomes thin and atrophic and the glands of the groin and popliteal region may become enlarged from septic absorption. The foot gradually becomes useless and the native is compelled to walk on his heels or adopt some mode of locomotion which does not necessitate putting the foot to the ground. There is comparatively little pain, except occasionally in cold weather. (Fig. 420.) 


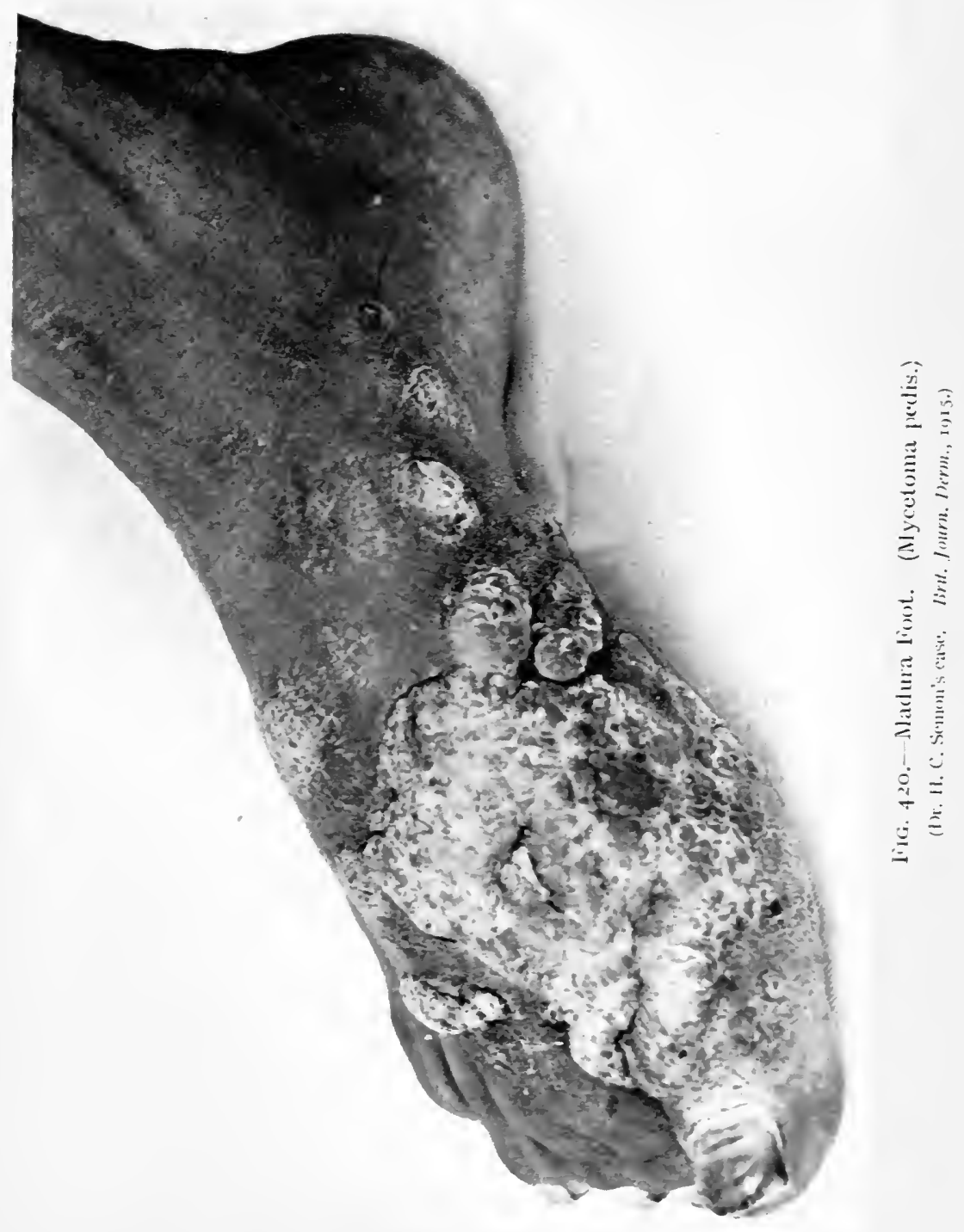

iT fave p. Iวะ6. 


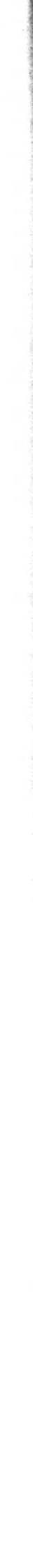


The course of the disease is slow and it does not interfere to any extent with the general health till it is well-advanced. It is a purely local condition and unlike actinomycosis rarely shows any tendency to invade the internal organs, but after a lapse of ten to fifteen years usually results in death from septic absorption, from some intercurrent disease, or from exhaustion and inanition due to anaemia, porerty, and starvation from loss of work.

Pathology.-In section in an adranced case the whole foot is found to have become softened and the tissues to be fused into an almost homogeneous mass which cuts with the consistence of cheese. The cut surfaces present a network of canals and cysts varying in size from a pin's head to a pigeon's egg, opening on the surface in the centre of a nodule or in funnel-shaped fistulous orifices, lined with fibrous tissue and containing, in the white rariety small cheesy masses

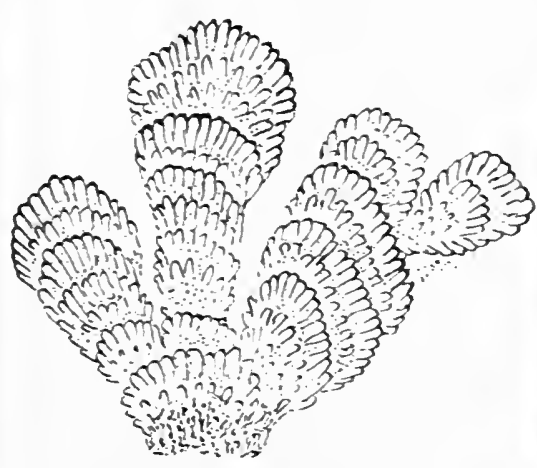

FIG. 42I.-Diagrammatic Drawing of the Fungus of Mycetoma.

Shows the imbricated processes arising fan-shaped fonm a central base or stalk.

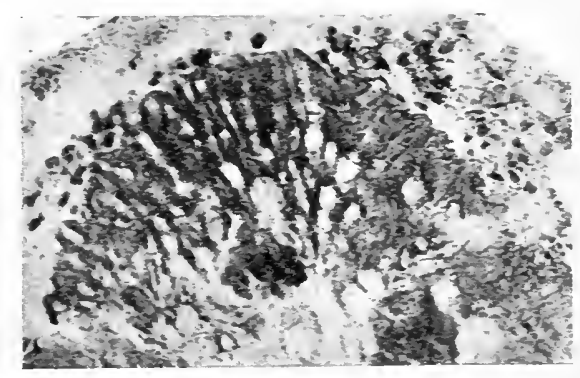

Fig. 422.-Maduromycosis.

Shows fungal hyphae in cortical portion.

(Drs. Chalmers and Archibald. Ann. Trop. Meit. ant Parasit., I9I6.)

varying from a hemp-seed to a pea and not unlike fish-roe, in the black variety dark brownish masses like coal particles, and in the red variety either pinkish aggregations or an oily fluid without granules. The cysts are situated chiefly about the subcutaneous tissue but may be found in the muscles and even in the bone.

Microscopical sections of the nodules before they have softened present an architecture somewhat like tuberculosis. In the centre there is a mass of fungus which is oval or roundish in shape or in the form of a crescentic rosette. Surrounding it is a dense infiltration of small cells, consisting chiefly of leucocytes and small connective tissue cells, and a few giant-cells, with here and there a deposition of pigment, and enclosing the whole a capsule of fibrous tissue. This formation suggests an attempt on the part of the tissues to limit the spread of the parasite. Around the nodules the tissues are oedematous and the blood-ressels show evidences of endarteritis, thrombosis, and periarteritis. Sections of softened nodules show degeneration of the fibrous bundles and the cellular infiltration, and replacement of the 
granulomatous structure by a dense deposit of polymorphonuclear leucocytes and debris, forming an oily putrid fluid in the midst of which are the granular masses.

On staining with safranin or haematoxylin the granules or sclerotia are found to consist of the aggregation of a number of systems of fungi. Each system has a central or basal mass of mycelial threads or debris, above which is a regular series of radiating club-shaped hyphae with a fan-shaped arrangenient. Several of these systems are grouped together, sometimes on a common base, at other times in an imbricated or concentric manner, and occasionally with a mulberrylike arrangement. (Figs. 42I, 422.)

The colour of the granules is due to pigment secreted by the fungus, the exact nature of which is uncertain.

Fungi.--The Mycetomas are due to fungi which belong to the orders of: (I) the Ascomycetes, in which reproduction takes place by asexual spores, (2) the Hyphomycetes, or Fungi

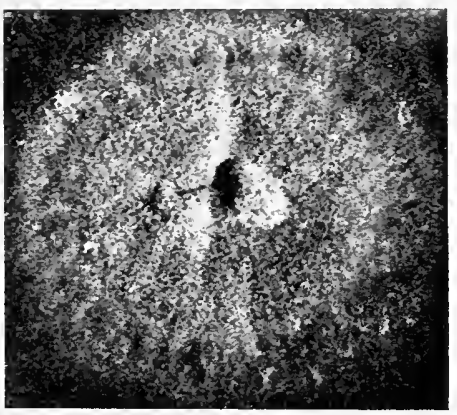

FIG. 423.-Sudanese Maduromycosis.

Culture on proof agar.

(Drs. Chalmers and Archibald. Ann. Trop. .Med. and Parasit., Igr6.) imperfecti, the botanical position of which is uncertain and in which reproduction takes place by conidia. At least nine species of causal fungi have been recognised and there are probably others. (Fig. 42.3.)

The granules are due to aggregations of the fungi and the differences in colour indicate differences in the pigment secreted by the respective fungi which compose them.

The precise habitat of these fungi is uncertain, though it is most probable that they have a saprophytic existence in the soil or in decaying vegetable matter-one variety (the Discomyces bovis) having been detected in the spikelets of various cereals.

Etiology.--It is essentially a disease of agricultural districts and attacks chiefly bare-footed natives in the fields and plantations. It rarely affects Europeans, owing to the better hygienic conditions in which they live and to the protection of their feet by boots.

It is more prevalent in men than in women and children, as would be expected in a disease which occurs chiefly in agricultural labourers.

The fungus gains entrance through abrasions caused by bites or stings of insects, pricks from spines of plants or splinters of wood, or cuts from knives, sharp stones, etc.

Diagnosis.-It is rare for the diagnosis to present difficulty. In early cases it might be confused with clephantiasis, syphilis, tuberculosis, or leprosy, but the derelopment of the characteristic deformity and the formation of the fistulous openings with discharge laden with mycotic aggregations will establish the diagnosis. 
From actinomycosis the diagnosis nay be more difficult, but actinomy.cosis occurs in temperate latitudes, runs a rapid course, affects internal organs and mucous membranes, and has yellow granules in its discharges, while mycetoma is met with chiefly in tropical or subtropical countries, runs a slow course, does not become generalised, and has gramules of various colours.

Should the clinical appearances fail to render the diagnosis certain an examination of the mycotic aggregations microscopically and culturally will decide it.

Prognosis.-The prognosis is unfarourable and only surgical removal seems to stop the progress of the disease when once it is established. Cases have been recorded (Brumpt) in which healing has taken place early but these are exceptions.

Treatment.-There is no specific medicinal treatment, and iodide of potassium, which is of undoubted value in actinomycosis, has no beneficial effect on it. Early cases may be arrested by cauterisation, curetting, or excision of the initial lesion, but when it is well-adranced partial or complete amputation is necessary, the incision being made in the healthy tissue beyond the lesion.

As preventive measures the feet should be protected where possible by boots or sandals, and every precaution should be taken when a wound occurs to keep it aseptic and to have it properly dressed till it has healed.

\section{PARAMYCETOMA}

The nane " paramycetoma" has been employed by Chalmers and Archibald to include " all growths and granulations producing enlargement, deformity and destruction in any part of the tissues of man or animals, which are caused by the presence of fungi of any nature whatsoever, but in which 'grains' are either entirely absent or are so few in number and small in size as to escape observation without prolonged search."

\section{PSELDOMYCETOMA}

The name "pseudonycetoma" is used by the above authors for a growth which, though clinically resembling typical mycetoma in the presence of swelling, ulceration, and discharge, differs therefrom in the absence of "grains" in the tissues and the discharge.

\section{REFERENCES}

ADAMI d KirkPATRICK. Notes on a case of Madura foot occurring in

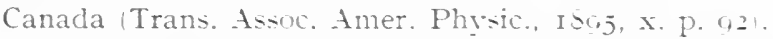

Boccaro. An analyis of one hundred cases of mycetoma Lancet, iso3. ii. P.,$(-$,$) .$ 
Brumpt, Bouffard, \& Chabaneix. Note sur un cas de mycétome observé à Djibouti (Arch. de Parasit., I9or, iv. p. 563) .

Castellani \& Chalmers (Manual of Trop. Med., 2nd Edit., London, I913. p. 1527 ).

Chalmers, A. J., \& Archibald, R. G. A Sudanese Maduromycosis (Ann. Trop. Med. \& Parasit., I9I6, x.).

Mycetoma \& Pseudomycetomatous formations (New Orleans Med. \& Surg. Journ., I9I 7 , lxx.).

The Classification of the Mycetomas (Journ. Trop. Med. \& Hyg., I9I8, xxi. p. I2I).

Paramycetoma (Journ. Trop. Med. \& Hyg., I9I8, xxi. p. I77).

MAdDen. Two cases of the pink variety of Mycetoma (Journ. Trop. Med., I902, v. p. 243).

Sutron, R. L. Mycetoma in America (Journ. Amer. Med. Assoc., I9I3, lx. p. I339).

\section{PELLAGRA}

Syn. : Erythema endemicum, Lombardian Leprosy; Fr., Mal de Misère, Mal de Saint Amans, Mauvais Dartre; Ital., Malattia della Miseria, Risipola Lombarda, Lepra Italica Maïdica, Elephantiasis Italica, Mal del Sole; Spanish, Mal de la Rosa ; Egyptian, Inshup, Gofar, etc.

Definition: An endemic disease, characterised by cutaneous lesions situated chiefly on the exposed parts of the skin, and by gastrointestinal and nervous symptoms which generally appear first in spring and recur in spring or autumn.

Introduction.--Pellagra, derived from the two Italian words pelle (skin) and agra (rough), though probably known even in the time of Hippocrates was not definitely recognised as a disease sui generis until 1735. It is to Gaspar Casal, Court Physician to Philip V. of Spain, that we are indebted for the first clear description of it and for the recognition that the cutaneous, gastro-intestinal, and nervous symptoms were all part of one disease. In 1755 Thiéry, who had met Casal in Madrid and had there seen cases of pellagra, wrote an account of it which was published in Paris. It was known in Italy about $x 770$ and carefully described by Francesco Frapolli in his work named "Animadversiones in Morbum Vulgo Pelagram." It was subsequently studied and written up by Lombroso in I880, and since 1905 has been the subject of much investigation.

Distribution.-As the disease becomes more familiar it is found that its distribution is almost world-wide and that it may be met with in all climates, tropical, sub-tropical, and temperate alike.

In Europe, though the early cases were chiefly observed in Spain and Italy, it has been seen in Portugal, France, Austria, Hungary, Russia, Serbia, Roumania, Turkey, Bulgaria, and Greece. In Great Britain it was known as early as 1860 when a case was reported by Brown in Scotland, and in recent times a number of cases have been observed both in England and Scotland where it has been found as far 



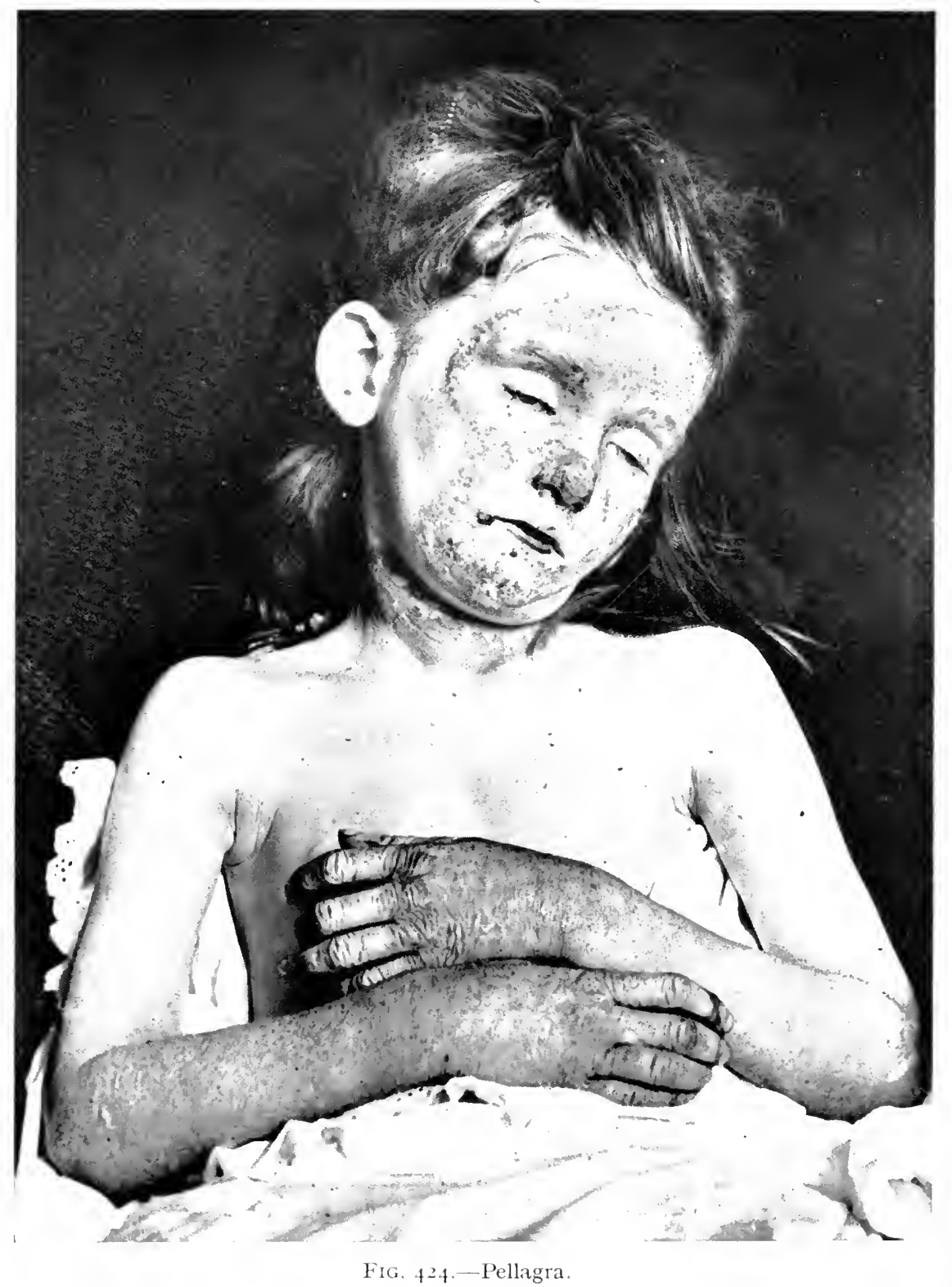

Typical eruption on face, bands and forearms.

(From Dr. Louis Sambon.) 
north as the Shetland Islands. In France it was recognised by Hameau as early as ISIS and had been known previously in Gascony; especially about the banks of the Ciron and Leyre rivers, and in the valleys of the Pyrenees.

In Africa it has been observed in Egypt, Algeria, Rhodesia, Central and South Africa.

In Asia it has been met with in Persia, Asia Minor, India, and the Straits Settlements.

In America it has been known since IS34 but it was not until I907 that it was discovered to be a comparatively common disease in the United States, it being estimated in IgIo that there were about I0,000 cases widely distributed over 30 of the States. Cases have been observed in South America, the West Indies, and in Oceania in the Philippine Islands and the Sandwich Islands.

Sambon, who has made a special study of the epidemiology of pellagra, has found that the majority of cases are met with in country districts, in the hills as well as in the plains, chiefly in habitations in close proximity to running streams, and that cases in the towns are comparatively rare and occur, as a rule, in individuals who have come to the town from the country. In certain districts it has been found that pellagra is gradually diminishing and simultaneously with it malaria, probably as the result of improved drainage.

Description.-Pellagra is a general infection in which the chief symptoms occur in the skin, gastro-intestinal tract, and nerrous system.

The incubation period is uncertain, but that it may be comparatively short is shown in a case reported by Sambon in which a child, born in an Italian gaol and nursed by its own mother in gaol until five months old, developed pellagra in two weeks on being sent out to live with some peasants in a pellagrous district.

The lesions in the skin are the most constant and characteristic, and in mild cases may be the only symptoms. They are limited at first to the exposed parts, namely the backs of the hands, face, neck, upper part of the chest, forearms, and dorsa of the feet in those who go about barefoot, and they are usually symmetrical. In rare instances they may be present later on covered parts, such as the elbows and knees, or about the anus and genitalia. (Fig. 424.)

When they appear first they resemble patches of acute dermatitis, like those produced by the sun, the affected skin becoming bright red in colour, raised and tense from oedema, covered with vesicles or bullae, and hot, painful, and tender to the touch. The acute dermatitis usually subsides in about a week, being followed by desquamation. The affected areas then assume the characteristic appearances of pellagrous skin, they are rough, thickened in places, or wrinkled, atrophic, and pigmented, are well-defined, and usually limited by a reddish border which is especially well-marked and raised on the wrists, 
and lower part of the neck where it is known as " the necklace of Casal." (Fig. +25 .)

The skin may recover from these changes and become normal again when the original attack has been mild, as it is sometimes in children; as a general rule, however, the texture of the skin is altered and after repeated attacks it becomes pigmented, atrophic, wrinkled, or parchment-like, and in places thickened and scaly.

In association with the cutaneous lesions the mucosa of the mouth may be involved and stomatitis, swelling and denudation of the tongue, and vesicles and superficial ulcerations on the palate and lips, may occur. Occasionally salivation with enlargement of the parotid glands may be met with and has been observed especially in the Egyptian cases.

The gastro-intestinal symptoms are less constant, vary in different cases, and consist chiefly of dyspepsia, vomiting, constipation, or diarrhoea.

Of the nervous symptoms one of the earliest and most frequent is rertigo, which is specially noticeable when the patient rises from the recumbent position or gets up quickly from a chair. In mild cases the giddiness may occur only in the mornings, but in severe cases it may come on at any time and may render walking difficult and any form of work impossible. Tremblings in the limbs may supervene with exaggeration, and later diminution, of the knee-jerk, which may be followed by spasmodic contraction of the muscles and paralysis of the limbs or eyelids. Tenderness over the spine and at the sides of the dorsal and lumbar cord is sometimes met with, and occasionally lightning pains or peripheral neuritis.

Mental symptoms usually develop; at first they may be slight, consisting of irascibility, anxiety, hysterical attacks, emotionalism, and insomnia; but later they are apt to be more severe, and melancholia, dementia, maniacal symptoms, or a suicidal tendency, may supervene - the favourite method of suicide being drowning. It is on account of these mental symptoms that cases of pellagra are liable to be met with in asylums and several of the earliest cases observed in this country were asylum cases.

No definite changes have been detected in the blood, with the exception of a mononuclear leucocytosis which is inconstant.

The subjective symptoms consist of burning and tingling; they are intensified by the sun's rays but may persist independently and may continue after the patient is in bed at night.

Recrudescences of the eruption are liable to take place in the spring or, less frequently, in the autumn.

Course.-The course varies greatly in different patients. If the attacks be mild complete recovery may take place under suitable treatment but.sooner or later recurrences are apt to supervene which lead to gradual weakening, both physical and mental, and to a fatal isulle after a course which may be as long as ten or fifteen years. $\mathrm{O}_{n}$ 


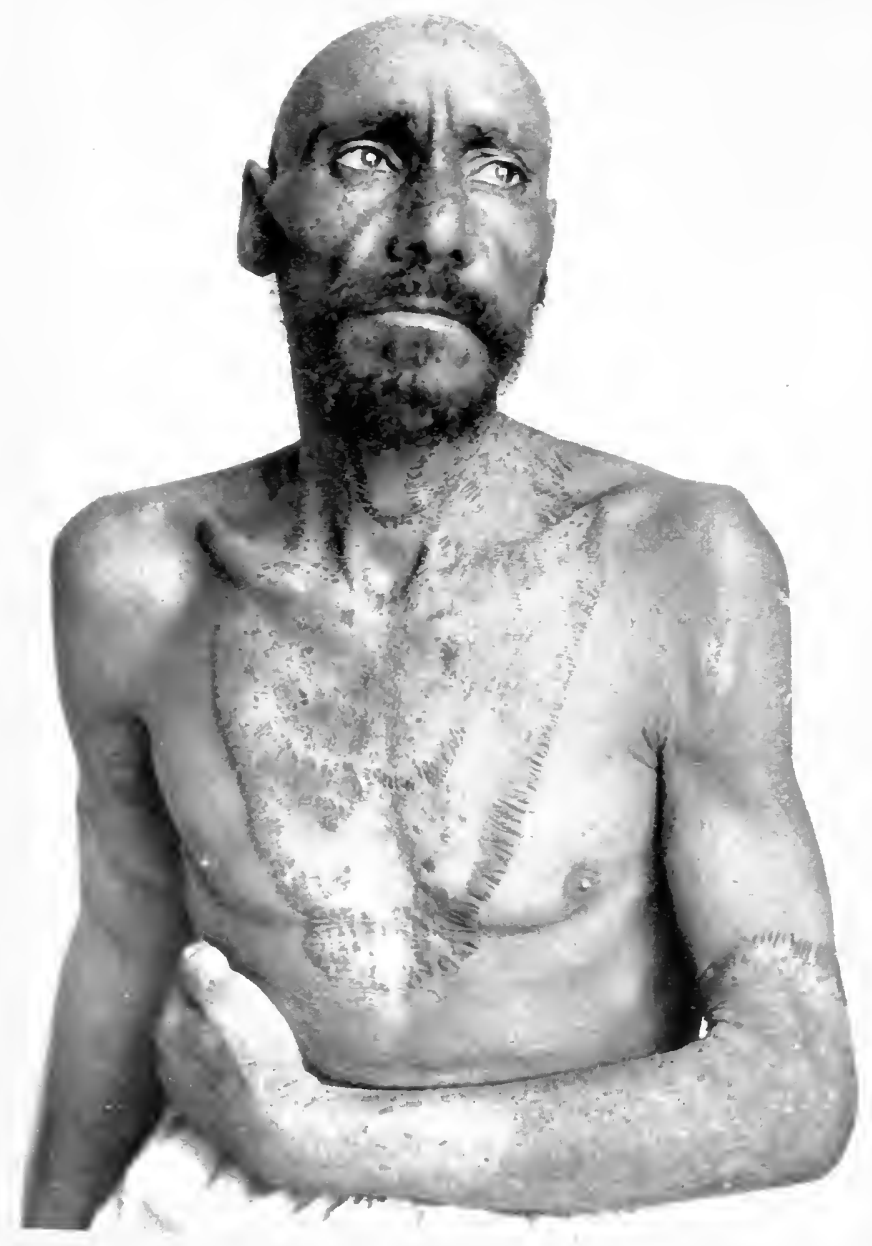

FIG. 425--Pellagra.

Shows "Necklace of Casal."

(From London School of Tropical Medicine, Collection. 

the other hand the disease may be much more acute, and death take place in a few months from involvement of the kidneys or from some intercurrent disease.

Histopathology.--Sections of the affected shin present a variety of changes according to the stage of the disease. In the early lesions the epidermis is thickened and shows evidences of parakeratosis, while in the later stages it is thinned. In the corium there is an inflammatory cellular infiltration around the superficial capillaries.

The histopathology of the nervous lesions has been reported on by Mott who examined the brain and cord in a fatal case and found changes indicating the condition to be in the nature of a chronic toxaemia. The changes were observed in sections of the cerebrum, cerebellum, pons, medulla, spinal cord, and spinal ganglia. He was unable to detect the presence of chronic meningo-encephalitis and meningo-myelitis such as are commonly met with in protozoal diseases. "All the posterior spinal ganglion cells showed in varying degrees a marked chromatolysis, swelling of the cell, disappearance of the Nissl granules except at the periphery, and frequently an eccentric position of the nucleus. All the anterior horn-cells and their homologues in the medulla and pons showed varying degrees of perinuclear chromatolysis ; in some instances the cells were so markedly swollen, and the nucleus was so eccentric, that it appeared as if the cell were dead. There was a marked chromatolysis of the celis of Clarke's column. The Betz cells of the cortex showed similar changes but not so marked; likewise the cells of Purkinje; the pyramidal cells of the cerebral cortex did not appear to be markedly affected."

Etiology.-The true nature and causation of pellagra as vet remain unknown but the recent observations of Sambon and others suggest that it may be an infective disease due to some specific virus not yet discovered. There are three well-known theories with regard to its causation, namely the maize theory, the deficiency theory, and the parasitic theory.

Maize Theory.-Pellagra has for so long been attributed to the eating of maize that this theory of its origin has become an almost fixed idea and difficult to supplant. But, just as in the case of the fish theory of the causation of leprosy, the evidence of a scientific nature in its favour is inadequate. Among the arguments against it are:-(I) That the disease has been found in districts in which the people have no opportunity of eating maize. (2) That where the disease is endemic and the authorities have prohibited maize, the number of cases does not seem to have been reduced appreciably.

Various hypotheses have been advanced to explain the action of maize in producing the disease :-(I) That certain individuals exhibit a peculiar susceptibility to maize and develop pellagra from its ingestion, while insusceptible individuals may eat it with impunity.

(2) That the cereal must be in a damaged or mouldy condition before it can exert a pathogenic action and that it is not the maize itself which causes pellagra but some contamination growing on it, such as a mould.

(3) That the toxic action of the cereal is due to a poison eliminated by it from the action of the Bacillus coli communis. 
(4) That the ingestion of maize causes the elimination of certain substances known as photo-dynamic substances, which become transformed into toxins under the influence of sunlight.

Deficiency Theory. - The deficiency theory explains pellagra as a result of the absence of some nitrogenous constituent in the food. This theory seems improbable, as pellagra may be met with in individuals who are well-nourished and in whose dietary there is no nitrogenous defect.

Parasitic Theory.- One of the earliest exponents of the parasitic theory was Tizzoni, who isolated from maize a streptobacillus which he found also in the blood and cerebro-spinal fluid in pellagrins after death, but his observations have not been verified.

Sambon has suggested that the specific virus may be a protozoon transmitted by certain biting flies known as the simuliidae. He found that pellagra occurred chiefly in the neighbourhood of running water in which the larvae of these flies could be discovered invariably, and observed that the seasonal incidence of the disease in spring and autumn coincided with the appearance in swarms of these flies which readily bite men and animals.

Predisposing Causes.-The seasonal incidence of pellagra is an important characteristic of the disease and is of diagnostic value. The original attack is generally in spring or early summer and recurrences usually take place in spring or autumn. Exceptional cases have been recorded in which the incidence was in winter.

Sex.-The disease seems to affect both sexes indiscriminately.

Age.--It may occur at any age, having been noted as early as three months. It is not infrequent in children but is liable to be missed in them, as the synuptoms are often singularly mild, and may even be mistaken for ordinary sunburn.

Social Position.-Social position does not seem to have any effect upon the etiology and pellagra is met with among the rich and wellnourished and the poor and ill-fed alike. Once the disease is established starvation or any lowering influence is liable to cause exacerbation of the symptoms.

Sunlight.-The actinic rays of the sun would seem to be an important factor in the production of the cutaneous lesions, which have all the appearance of a dermatitis resulting from exposure to the sun's rays, and exposure to the sun calls them forth in the first instance and aggravates them when present; but that there is some other factor is evident from the acute manner in which the skin reacts even to the slightest exposure to the actinic rays and the occasional occurrence of lesions in covered parts, especially about the genitalia.

Diagnosis. - The recognition of pellagra, especially in isolated cases existing in districts where the disease is not endemic, may present considerable difficulty unless the typical syndrome of cutaneous, gastro-intestinal, and nervous symptoms is pronounced.

The cutaneous eruption has to be distinguished from Erythema multiforme, ordinary solar dermatitis, and chronic eczema.

From Erythema multiforme, to which it bears a certain resemblance in distribution and in the involvement of the mucous membrane 
of the mouth, it is distinguished by the seasonal incidence, the changes in the skin which persist after the acute attachs have subsided, and the concomitant nervous and gastro-intestinal symptoms.

From solar dermatitis it is distinguished by the presence of lesions in the mouth.

From chronic eczema the distinctive features are the well-marked line of demarcation and the absence of itching.

Prognosis.-The prognosis should be guarded, as recurrences may take place even after an interval of years and may lead to a fatal issue. Mild cases may be given a farourable prognosis provided they can be removed to a non-pellagrous district and there put under a proper general regime and suitable treatment instituted.

The outlook is specially grave where the pellagra is complicated by some intercurrent disease, such as tuberculosis or typhoid fever.

Treatment.-The patient should be removed from the pellagrous area as soon as possible and placed under the best hygienic conditions. Not infrequently pellagrins are ill-nourished and much can be done to relieve their symptoms by a suitable and generous dietary.

There is no drug at present known which can be claimed to have a specific action on the disease. Arsenic has been adrocated but the results from it are inconstant. It has been given chiefly in the form of Liquor arsenicalis by the mouth and of atoxyl by intramuscular injertion, and it is recommended that it should be begun as early as possible and continued for at least a month after the acute symptoms have disappeared.

Any symptoms which may be prominent, such as indigestion, constipation, or diarrhoea, should be dealt with on general medical principles. Where the nervous symptoms are marked and associated with insomnia it may be advisable to resort to sedatives or hypnotics, such as the bromides or chloral hydrate, but these should be prescribed with the utmost caution for fear of establishing a drug habit-which is specially liable to occur on account of the depression, melancholia, and other mental derangements, associated with the disease.

Locally, the dermatitis may be reduced by lead or calamine lotion and the skin should be protected by the application of ichthyol paste or by the use of a dark brown dusting powder. Every care must be taken to aroid exposing the skin to the actinic rays of the sun and to protect it by the use of shady hats, veils, gloves, etc.

Note-In preparing this section on pellagra the author has been greatly indebted to the writings of Dr. Sambon, to the Report on the Illinois Commission, and to the exhaustive chapter by Castellani and Chalmers in their "Manual of Tropical Medicine," I9I3.

\section{REFERENCES}

Boxd, H. E. Causation \& treatment of pellagra (Journ. Trop. Med. \& Hy.g., I9I5, xviii. p. 229).

Box, C. R. Fatal Pellagra in two English boys (Brit. Med. Journ., I9I3, 2, p. 2).

Pellagra in England (Trans. Soc. Trop. Med., London, I9I3). 
Castellaxi \& Chalmers (Man. of Tropical Medicine, I9r3, 2nd Edit., p. I230).

Creightox Welliax. On "Winter cases" of pellagra (Journ. Trop. Med. \& Hyg., May, I9 12, p. г3I).

Hamoxd, J. A. B. Case of pellagra in England (Brit. Med. Journ., I9I3, 2, p. I2).

Low, Cranston. Case of pellagra from the Shetland Islands (Edin. Med. Journ., I909).

MAcneal, WV. J. Pellagra (First Progress Rep. of the Thompson-McFadden Pellagra Commission, I913).

Mort, F. W. Nervous system in pellagra (Trans. Soc. Trop. Med. \& Hyg., London, I9I3).

Histological changes in the nervous system of Dr. Box's case of pellagra (Brit. Med. Journ., I9ז3, 2, p. 4).

Sambon, L. W. Exposition of the parasite fly theory of the etiology of pellagra (Progress Rep. on the Investigation of Pellagra, London, I9I0).

The natural history of pellagra (Brit. Med. Journ., I9I3, 2, p. 5).

Pellagra in Great Britain (Idem, p. I19).

Sambon \& Chalmers. Pellagra in the British Islands (Brit. Med. Journ., 1912. For work done in Roumania vide leading article; Journ. Trop. Med. \& Hyg., Dec. I5th, I9II; and for the work done at Burano, the same journal \& article for Oct., I9I2).

SANDwith, F. II. Is pellagra a disease due to deficiency of nutrition? (Trans. Soc. Trop. Med. \& Hyg., April I9I3: \& discussion).

Viswalingam, A. Pellagra (Journ. Trop. Med. \& Hyg., I9x8, xxi. p. I 53).

\section{TROPICAL PHAGEDAENA}

Syn.: Tropical ulcer; Aden ulcer; Yemen ulcer; Guiana ulcer ; Malabar ulcer ; Annamite sore ; Mozambique sore ; Fr., Ulcère phagédénique des pays chauds; Ger., Tropische Phagedenismus.

Definition: Tropical phagedaena is a form of ulceration which is endemic in tropical and sub-tropical countries and is characterised by the presence of a chronic sloughing sore with a grey false membrane, a foetid exudation, and a tendency to infiltrate and destroy underlying tissues.

We are chiefly indebted to French writers for our knowledge of tropical phagedaena. In I862 Le Roy de Méricourt and Rochard suggested the French name of "Ulcère phagédénique des pays chauds" as a generic name for a number of sloughing ulcers previously described by observers in different tropical countries and variously named by them according to the district in which they occurred but believed by Rochard to be identical, such as the sloughing ulcer so familiar among the coolies in Mozambique and among the galley-slaves in French Guiana, and the ulcer known as Annamite sore from which many of the soldiers suffered in the French expeditionary force sent out to Cochin China.

Geographical Distribution.-The affection has a wide distribution in all tropical and sub-tropical regions, but is met with chiefly in lowlying swampy districts about the mouths of rivers and on the sea- 
coast. In tropical Africa it prevails on the east coast in Mozambique, Madagascar, and Zanzibar, and on the west in Gambia and the Gold Coast. In sub-tropical Africa it is met with in Egypt, Abrssinia, and the Sudan. In Asia it is encountered in the province of lemen and on the shores of the Red Sea. In Indo-China it is so prevalent that Jeanselme has described this region as its place of election. It is very common in the Straits Settlements, and the southern littoral of China, and cases have been reported in the Malay States, Borneo, Sumatra, Fiji, and the Solomon Islands. It is met with also in tropical America and the West Indies.

Description.--The typical ulcer has a characteristic appearance which is easily recognised: it is roundish or oral in outline; raries in size from one to several inches in diameter; the floor is bathed in a foetid exudation of a brownish colour which has been likened to winedregs, and is usually covered by a pulpr greyish false membrane which, when removed, exposes a more or less deep concave depression or excavation; the margins are not definitely raised or thickened, nor is there any punched-out appearance or undermining except in oldstanding cases; it is surrounded by a dusky-red areola, the shin of which is oedematous and painful on pressure. As a rule the pain connected with it is slight, or may be absent.

It appears soon after the inoculation as an inflamed papule or papulo-resicle, of a reddish colour, which may be itchy. In the course of a few days this develops into a definite vesicle or bulla, filled with sero-sanguineous fluid and situated on an inflamed base, and it is the breaking of this resicle which results in the unhealthy ulcer.

It is situated most frequently on the dorsum of the foot or the front of the leg, but is not confined to the lower extremities and may occur on the hands and arms of coolies, especially when working at the rice harrest, and has been known occasionally to attack the face and even the covered parts. It is single in the majority of cases, and may be chronic and mild in type or may assume acute characteristics and result in the formation of terrible gangrenous sloughs.

In mild cases healing may take place under suitable treatment in the course of a few weeks or months, but the resulting scar is unsatisfactory, almost invariably disfiguring, and liable to break down from the slightest injury. On the other hand when the virus is unusually powerful or the individual affected is in a low state of health, the ulcer may take on fulminating phagedaenic characters and spread rapidly, the gangrenous process attacking layer after layer of the soft parts. When the slough separates the muscles are frequently laid bare and decomposed, blood-ressels and nerves are dissected out, joints denuded, disarticulation occurs, and eren the periosteum may be affected and the bone exposed.

Associated with the local changes are usually general symptoms, such as febrile attacks, insomnia, and pain, and a fatal issue may result from exhaustion or from various complications, such as septi- 
caemia, haemorrhage from an e:oded vessel, or suppuration of an affected joint. Should recovery take place permanent disfigurement is apt to follow from contracture and ankylosis.

Histopathology.--Sections of an ulcer show that the epidermis is destroyed and replaced by a granular debris in which are masses of leucocytes in various stages of disintegration and in which bacilli and spirochaetes may be detected. Immediately beneath the ulcer the fibrous tissue is oedematous, while deeper down it may be dense and show evidences of fibromatosis. At the edge of the lesion the epidermis is thickened.

Etiology.--Tropical ulcer usually follows an abrasion of the skin from traumatism, bites of insects, or other causes, or it may be a complication of some pre-existing sore of yaws, syphilis, or tuberculosis. It bears a striking resemblance to hospital gangrene, with which certain observers believe it to be identical. It has been found to be auto-inoculable and cases are not infrequent in which several small satellite ulcers have occurred around a central sore. It has been stated to be directly contagious, but there is not sufficient evidence to prove this and so far inoculation experiments in man, guinea-pigs, and monkeys, have been unsuccessful. It is transmitted possibly by some intermediate bearer, such as a blood-sucking insect, and has been known to occur at the site of a leech-bite.

One of the most important factors in its causation is a hot moist climate. It occurs chiefly in the rainy season and prevails in lowlying swampy districts, imperfectly drained fields, alluvial beds of rivers, and on the marshy lands about the sea coast; it is rare in the highlands of the interior and in towns. It has been suggested by various writers that the pathogenic virus has its habitat in the mud and mouldy vegetation of the marshes, but it seems more probable that the marshy districts form the breeding ground for some insect which is the carrier of infection.

Of the pre-disposing causes, the most important is a weak state of general health, such as may be brought about by exhaustion and want of food, as in slave-convoys on the march, badly-fed coolies, pioneers opening up marshy land, or soldiers during an arduous campaign, or by wasting diseases, such as scurvy, dysentery, and malaria. So close is the association between it and malaria that the name " malaria ulcer" has been applied frequently to it.

There is no racial immunity, but it is more common in natives than in Europeans, partly because the native is more insanitary in lis habits, and partly because his bare legs and feet are more exposed to abrasions and to the bites of infection-carriers. Malaria is usually responsible when it attacks Europeans and it has been noticed that the gangrenous process is liable to spread with each attack of fever.

It is more common in male adults than in women and children.

Bacteriology.-The clinical characters and course point to the conclusion that it is the result of the inoculation of a specific microbe 
in a susceptible individual, but the exact micro-organism is uncertain. A number of micro-organisms have been found, both in the false membrane and in the exudation, but none of them has been proved to be specific. In ISS Le Dantec, for example, described a bacillus which was 7 to ro $\mu$ in length, gram-negative, did not grow on ordinary culture media, and with which inoculation experiments gave negative results. In rgoo a closely allied bacillus was described by Matzenauer in connection with hospital gangrene. spirochaetes were found in the lesions by Vincent, and in I9o 7 Prowazek described a spirochaete, which he named Spirochaeta Schaudinni and which he beliered to be the cause. This spirochaete varied in length from ro to $20 \mu$, had only: a few coils, a well-marked undulating membrane, a delicate flagellum at one extremity, and was somewhat similar to a spirochaete found by him in balano-posthitis.

Diagnosis.-Tropical phagedaena has to be distinguished from the sores of yaws, srphilis, leprosy and tuberculosis, and from the various ulcers now grouped under the heading of Oriental sore. The pathognomonic sign which distinguishes it from those lesions is the adherent grey false membrane.

In yaws the lesions are multiple and a microscopical examination may reveal the Treponema pertenue of Castellani.

In syphilis the ulcer has more definitely punched-out edges and reacts to anti-specific treatment.

In oriental sore the lesion is as a rule much smaller and the Leishman body (Leishmania tropica) can be isolated from it.

It must be remembered that any of these sores may be contaminated by the specific virus and transformed into tropical phagedaena, and on the other hand that the tropical ulcer may form the seat of inoculation of yaws or syphilis.

There is considerable diversity of opinion with regard to its relation to hospital gangrene. Certain writers assert that the two conditions are identical, basing their belief on the clinical similarity of serere cases and the fact that closely allied bacilli have been isolated in both ; others regard them as separate diseases, and point out that in many of the cases of tropical ulcer there is a tendency to self-limitation which in hospital gangrene is unusual, and that there is less definite evidence of contagiosity than in hospital gangrene.

Prognosis. - The prognosis under suitable treatment is farourable and the affection may be eradicated in the course of a few weeks or months. Spontaneous healing is rare and if left alone it is liable to assume severe fulminating and destructive characteristics and may not only destroy the underlying structures but, through pyaemia or haemorrhage, may have a fatal result.

\section{TREATMENT}

Prophylaxis. - As tropical phagedaena is capable of incapacitating large numbers of native labourers and soldiers campaigning in a country 
where it is endemic, every effort should be made to prevent its occurrence. It is of the first importance to protect the legs and feet; it is difficult to induce natives to wear boots, even if they can be provided, but adequate protection may be obtained from sandals or puttees of some light material. When cases occur they should be isolated as far as possible and to avoid overcrowding in the hospitals the patients may be treated in temporary huts or under canvas.

General Treatment.-The general treatment consists of placing the patient under as satisfactory hygienic conditions as possible, with good food and plenty of fresh vegetables, and dealing with any obvious defects in health on general medical principles. In cases of malaria quinine should be prescribed, and in syphilis mercury and iodide of potassium. Opium in full doses has been strongly advocated by Manson for the double purpose of reducing the phagedaena and relieving the pain. In the case of Europeans a change of climate to a district where it is not endemic or a sea-voyage may be followed by rapid healing. Where the sores are extensive it is important that the patient should lie up and that the affected limb should be properly bandaged and kept elevated.

Local Treatment. - The local treatment to be successful must be energetic and thorough. It is necessary in the first instance to remove the false membrane and the sloughing tissue. This may be done by a thermo-cautery, by which means an occlusive scab is produced which separates in about a week and leaves a clean granulated surface. It is better, however, to scrape it away with a sharp spoon, if necessary under a general anaesthetic. After thorough curetting, the floor of the ulcer should be swabbed with pure carbolic acid or irrigated with sublimate solution I in I000, or potassium permanganate $I$ in 2000 , and finally dusted with an astringent antiseptic powder, such as equal parts of iodoform and boric acid. An antiseptic dressing, such as I5 grs. of ammoniated mercury in an ounce of vaselin, or protargol 5 to $20 \%$ in vaselin (Castellani) spread on lint, should be placed over the sore under a carefully applied bandage. Instead of the dressing, it may be irrigated continuously with a warm antiseptic lotion.

In mild cases Tschudnowsky has recommended the application of the following solution which forms an occlusive dressing by solidifying on exposure to the air :

\begin{tabular}{|c|c|c|c|c|c|}
\hline Iodol & - & - & - & - & gr. iv \\
\hline Glycerine - & - & - & - & - & Il v \\
\hline Gum arabic & - & - & - & - & gr. $x$ \\
\hline Alcohol - & - & - & - & - & $\overline{5} \mathrm{i}$ \\
\hline
\end{tabular}

When the healing is slow Le Dantec has suggested cutting down the raised edges and applying strips of diachylon plaster to draw the edges together and to exert continuous pressure on the granulations.

In severe and extensive cases Thiersch grafting may have to be resorted to or the edges dissected up and brought together by sutures. 
orer the wound. Where excessive gangrenous changes have supervened free amputation of the diseased tissue may be necessary.

\section{REFERENCES}

BLAISE. L'ulcère phagédénique des payss chauds en Algérie Gaz. hebd. de Néd. et de Chir., IS97, p. 951 ).

Bonet. De l'ulcère phagédénique observé au Tonkin (An:. de Derm. et de Syph., I S90, pp. 210, 307).

Castellaxi \& Chalmers. L̈lcus tropicum (Man. of Trop. Med., IgI3, 2nd Ed., p. 1560).

CRoss. Malarial ulcers in British Central Africa (Journ. Trop. Med., I900, iii. p. 85).

Jeanselie (Cours. de Derm. Exot., I904, p. 216).

LE DAxTEC. Phagedaenism of warm countries, its identity with hospital gangrene, pathology, stmptoms, and treatment (Arch. de IIed. Nar., IS99, Ixxi. p. I33; Trans. by Falcke, Journ. Trop. Med., I 899 , i. p. 308).

Maxsos (Tropical Diseases, I903, p. 6/3).

Matzexaler, The etiology of hospital gangrene (Arch. f. Derm. u. Syph., IgOI, 1vii. p. 229).

Rochard. De l'ulcère de Cochin China (Arch. gén. de méd., I862, xix. p. $66 \%$ ).

Tschudxowshr. Contribution à la géographie méd. de l'Archipel. Malais (Thèse de Doctorat, Paris, IS99).

\section{PINTA}

Syn. : Spotted sickness, Peint, Caraté, Mal del Pinto, Cute, Catiri, Pannus Carateus (Alibert).

Definition: Under the heading of Pinta, Caraté, and various other native names, a peculiar group of closely allied epiphrtic shin diseases of the tropics has been described, which is characterised by the appearance of spots and patches of different colours due to several species of chromogenic fungi.

Geographical Distribution.-It is endemic chiefly in the tropical countries of the western hemisphere, such as Mexico, Central America, Venezuela, Chili, and Peru, and is particularly rife in Columbia where in certain districts it affects I in Io of the population. It occurs also in Guatemala and Honduras. It has been described in the Philippine Islands, and a somewhat similar condition has been observed in Algiers, Egypt, and the Gold Coast. The exact relation or identity of the cases described in these different countries is uncertain. but they appear to be so similar as to suggest that they are caused by closely allied fungi.

Description.-The eruption generally appears on the uncorered parts of the body and may attack the face, neck, forearms, wrists, legs, and elbows. It comes out slowly, after a more or less prolonged incubation period in which the skin may be definitely itchy, is not preceded by constitutional symptoms, and is a purely local condition. 
It begins as one or more roundish, coloured spots, which enlarge and coalesce to form patches, irregular in outline, ill-defined, covered with fine scales, and not definitely raised above the surface. New lesions form by auto-contagion and join older ones, until the whole of the glabrous skin except that of the palms and soles may be involved in a patchy fashion, giving rise to a piebald appearance.

Pinta has been divided into varieties according to the colour of the patches, namely blue, violet, black, red, grey, or white pinta. These variations in tint are due to different species of fungi and, to a slight extent, to the situation of the fungi in the epidermis, for when the lesions are black or blue the fungus is generally situated more superficially than when grey. The colour varies also in different patients and in different situations in the same patient; the black variety is found chiefly in dark skins, the red in white skins, and the white is a retrogressive phase.

There are two stages in the erolution of the disease, namely, an active stage of pigment formation (stage of hyperchromia) and a late atrophic stage characterised by the disappearance of pigment (stage of achromia). The latter stage is due to the action of the fungus upon the melanin and is noticed first over bony prominences subjected to friction, such as the elbows. Beginning from the centre of the lesions and spreading towards the periphery the process eventually results in complete disappearance of the cutaneous pigment and replaces the coloured patches with white areas.

The affected skin is dry and coarse; in some cases it emits an offensive odour which has been compared to that of a mangy dog or of dirty linen, but this odour is not specific and is simply the result of perspiration and lack of cleanliness.

Though the palms and soles are not pigmented they are occasionally subject to much thickening of the horny layer and the consequent formation of deep cracks which readily gape. Neither the nails nor the hair are attacked as a rule, but if the scalp be affected the hairs tend to become atrophic and lustreless and may fall out.

In long-standing cases the mucous membranes of the mouth, prepuce, and vagina, may show pigmentation.

The eruption is not associated with constitutional symptoms but may be a source of annoyance on account of the itching, which is most marked when the scaliness is excessive and at night and is apt to lead to scratching, excoriation, eczematisation, and the secondary production of pyogenic lesions.

It runs a chronic course, is slowly progressive, does not tend to heal spontaneously, and unless thoroughly treated may persist throughout life.

Etiology.-It is due to the presence in the epidermis of some chromogenic fungus which can be transmitted from man to man, and a considerable number of species of fungi have been found in it; Montoya in his well-known thesis on the subject in 1898 , described as many as 
twenty varieties. It is probable that the flora of pinta vary like those of ringworm not only in different countries but in different districts in the same country, and that certain of the species which have been described may have been secondary contaminations. So far the principal fungi observed have belonged to the genera Aspergillus, Penicillium, Monilia and Montoyella; they can be found easily by scraping the surface and soaking the scales in Liquor potassae, when they appear as mycelial threads with dichotomous branching. They are distinguished by differences in their organs of fructification and in their cultures on ordinary media, such as Maltose agar. They have been inoculated successfully on the human subject.

Predisposing Causes.-One of the most important predispnsing causes is a hot moist climate, and in countries where it is endemic it is found mainly in marshy lands about the banks of rivers and prevails chiefly in the rainy season.

Another predisposing cause is the want of resistance and eczematisation of the skin of natives working in mines where they are exposed to water containing a large quantity of sulphates or other mineral salts.

It attacks the poor rather than the rich and well-fed, as want of cleanliness, lack of clothing, bad hygienic conditions, and insufficient food are all farourable to its development.

It rarely affects the white population, but is rife among Indians, negroes, and half-castes. In British Honduras, for example, the Europeans escape almost entirely, while about $60 \%$ of the Caribs are attacked (Cran).

Age and sex have no bearing on its etiology:

Diagnosis.--The skin affections with which it is most liable to be confused are vitiligo and chloasma, and from time to time cases of vitiligo have been described under the heading of pinta or caraté. Vitiligo differs from pinta in being more symmetrically distributed, neither scaly nor itchy, and not due to a fungus. Chloasma is distributed symmetrically, and is not scaly.

Occasionally cases of pinta have been mistaken for leprosy, but apart from other differences the patches in pinta are not anaesthetic like those of the more serious disease.

It has to be distinguished from the different varieties of Pityriasis versicolor, namely, Tinea nigra and Tinea flava (Castellani), but in these conditions the fungus is similar to the Microsporon furfur, is situated more superficially in the skin, does not involve such large areas, and is not associated with itching.

\section{TREATMENT}

The treatment is similar to that of ringworm of the glabrous shin or Pityriasis versicolor. When it occurs extensively the patient should 
be immersed in a warm bath and scrubbed with soft soap, and an antiparasitic ointment applied such as the following:

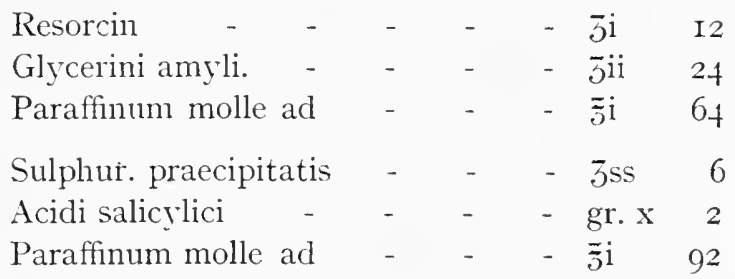

Instead of ointments, nascent sulphur lotion may be used. This is formed by applying a solution of hyposulphite of soda $6 \%$ on lint covered with oiled silk for Io minutes, and subsequently painting the areas with a $3 \%$ solution of tartaric acid. When large areas are involved, they should be treated seriatim, as it is inadvisable to apply these renedies too extensively at one time.

When the eruption is confined to a few localised patches, stronger applications such as Ti. iodi. pyrogallol and chrysarobin may be used. Chrysarobin should be rubbed on in the form of an ointment containing 20 to 30 grs. to the oz. of Ungt zinci oxidi, or dissolved in traumaticin (guttapercha $\bar{\jmath}$ i, chloroform $\overline{3}$ ).

When patches occur on the face they should be treated by the application of dilute ammoniated mercury ointment or of the following:

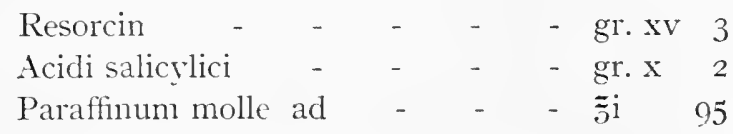

For some time after they have been apparently cured the affected areas should be rubbed daily with a $2 \%$ sulphur and salicylic acid ointment, and a powder containing salicylic acid $\overline{5} \mathrm{i}$, zinc oxide $\overline{\bar{j}} \mathrm{ss}$, and starch $\overline{\tilde{J}}$ ss should be dusted on the skin and on the inside of the underclothing.

\section{REFERENCES}

Castellani \& Chalmers. Pinta (Man. of Tropical Medicine, znd Ed., I9I3, p. I5I3).

Cran. Pinta in British Honduras (Journ. Trop. Med., Igoo, 2, p. Io). Jeanselae. Caratés (Cours de Derm. exot., I904, p. 267).

Montoya. Note sur les Caratés (Ann. de Derm. et de Syph., Paris, 1897, viii. p. 464 ; I898, ix. p. 673).

Sandwith, F. M. Pinta (Brit. Med. Journ., I905, 2, p. I270).

\section{PULEX PENETRANS}

Syn.: Dermatophilus penetrans; Sand-flea; Jigger ; Chigoe.

The sand-flea is one of the parasitic diptera and gives rise to a distressing affection known as dermato-philiasis. This flea was confined originally to tropical America and the West Indies but was 
carried in ships from Brazil to West Africa, where it spread rapidly along the trade routes from west to east and now prevails in many parts of that continent; from there it was convered to India, and is steadily making its way round the world. (Figs. $426,+27$.)

The jigger is smaller than the ordinary flea and inhabits sandy soil and the dust of native huts and kraals. It readily attacks any warm-

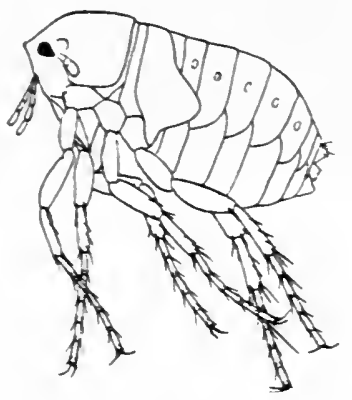

FIG. 426.-Dermatophilus Penetrans. Ioung Female.

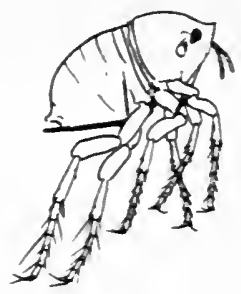

FIG. 427.-Dermatophilus Penetrans. Male.

blooded animal and has been found also in birds. The female, which is about I mm. in length and of a reddish-brown tinge, when impregnated burrows obliquely into the skin of its host until only the two posterior segments are visible and there remains nourishing itself until the ova mature. Meanwhile the abdomen swells and the insect becomes round like a pea with two small projections, the head and thorax in front and the last two abdominal segments behind. The eggs are expelled a few at a time through the opening in the skin by muscular action or by pressure, fall to the ground, and hatch out larvae which gradually go through their developmental cycle. (Fig. +28 .)

The presence of the insect in the skin gives rise to a nodule about the size of a pea, in the centre of which the posterior segments of the parasite are visible as a black dot. Sometimes the inflammation caused

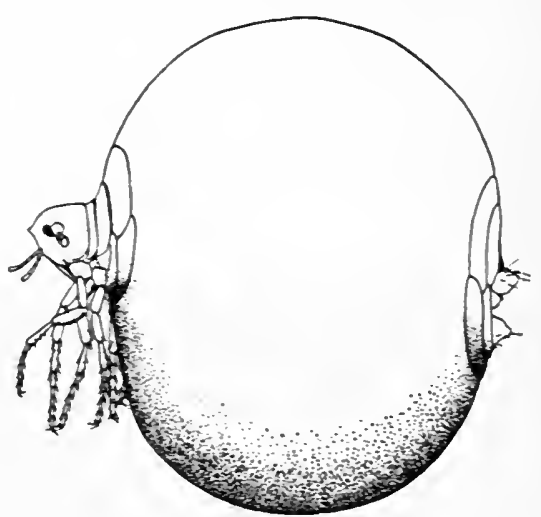

FIG. 428.-Dermatophilus Penetrans. Gravid Female.

by it is so acute as to lead to the formation of a blister which on breaking reveals the insect in the centre.

It chiefly attacks the soles of the feet, the interdigital clefts, and the skin beneath the nails and about the nail-walls, but may be found in other parts, such as the face, genitals, and limbs. When present about the feet, the itching and pain may interfere with walking.

After expelling all its eggs the jigger dies, shrivels up, and is sloughed off leaving a small ulcer, but as a rule no serious consequences follow, 
unless secondary inoculation has taken place when phagedaenic sores, erysipelas, tetanus, or even loss of a limb may result.

\section{TREATMENT}

The treatment consists of removing the insect, intact if possible--an operation which the native becomes very skilful in performing. It can be picked out by a sterile needle or other sharp instrument, after which the sore should be washed with I in 40 carbolic lotion and an antiseptic dressing applied, or, if any portion of the flea remain, it should be touched with pure carbolic. When jiggers are present about the fingers it may be necessary to remove the nail in order to reach them.

As prophylactic measures in districts where the flea is prevalent, the rooms and huts should be carefully swept to remove the dust which harbours the insect, and poultry and all domestic animals should be kept outside. The feet should be examined frequently for jiggers, especially under the nails, and should be protected in the house by stockings and sandals or, still better, by high boots. Native children with bare feet which are liable to be infected should not be permitted to enter European habitations.

A useful preventive is to anoint the skin with oil of cloves and to sprinkle the floor with Jeyes' fluid, naphthalin, lysol, infusion of native tobacco, or other insecticide.

\section{REFERENCES}

Wellman, F. C. The Chigger in South-west Africa (Published by the Amer. Soc. Trop. Med.).

\section{TINEA IMBRICATA}

\section{Syn.: Tokelau Ringworm; Pita ; Cascado ; Gogo.}

Tinea imbricata is the name given by Manson to a peculiar type of tropical ringworm or dermato-mycosis, which is endemic chiefly in the moist, tropical climates of the Malay Peninsula and the South Sea Islands where it attacks large numbers of the population. Of the various native synonyms the best known is Tokelau ringworm from the Tokelau Islands where it is specially rife. From the South Sea Islands it spread to the East Indies, China, the Straits Settlements and the Philippine Islands and recently has been observed in Brazil and Ceylon. It is confined mainly to the Papuans and Malays, but it may attack other natives, and where natives are crowded together in insanitary surroundings it may be so common as to be almost universal.

The clinical peculiarity of this type of ringworm is the imbricated arrangement of the scales which are greyish-white in colour and have an appearance which has been likened to tissue-paper. They vary in size and thickness, some being delicate while others are coarse, and are 
arranged in concentric circles with the base of the scales towards the periphery and the free margin in the centre, or in way parallel lines like watered silk. When they are removed they leave dark concentric or wavy marks. (Fig. 429.)

About a week after the inoculation a slightly raised brownish patch becomes visible which tends to clear up in the centre by the loosening and shedding of the scale, but keeps on spreading peripherally. New lesions then appear in the centre and erolve in the sanie manner till finally a concentric sistem is produced composed of rings of scales which

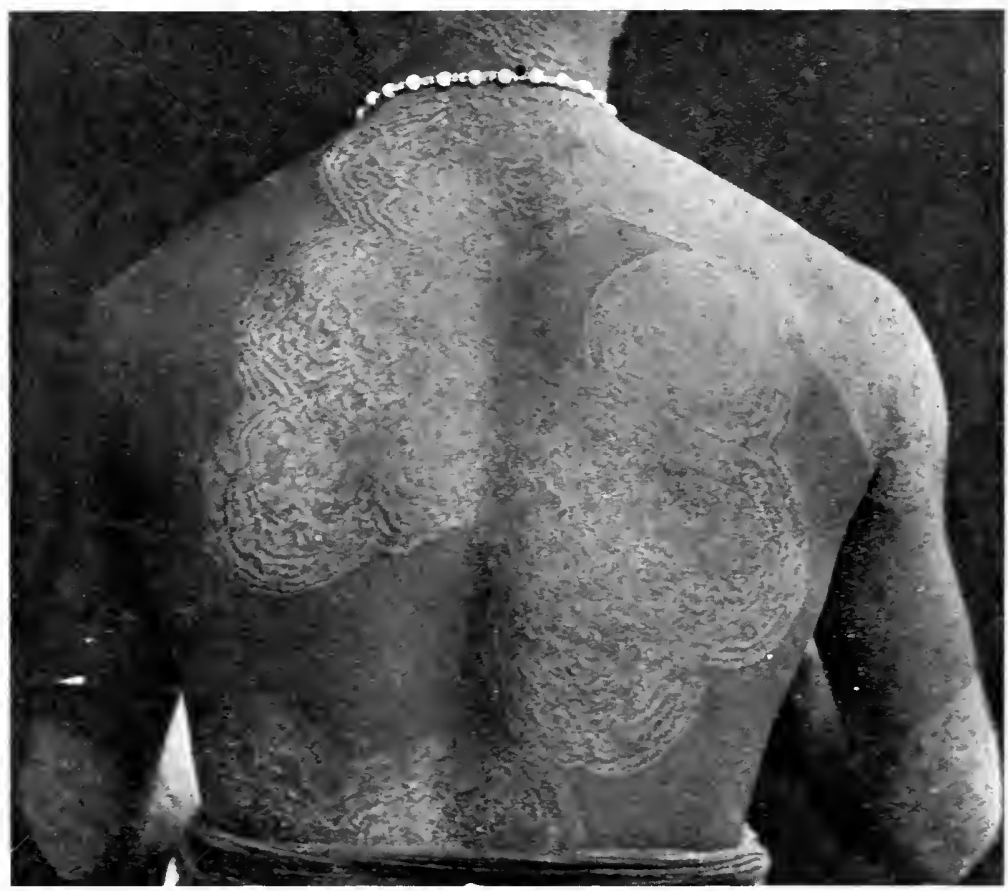

FrG. 429.-Tinea Imbricata.

(From London Tropical School Collection.)

overlap in an imbricated fashion. By the coalescence of adjoining systems elaborate figures are produced and large areas of the cutaneous surface become implicated; in severe cases practically the whole of the skin is attacked, even the palms and soles. The nails are not infrequently involved and become brittle, discoloured, opaque and striated. The scalp is sometimes, though rarely, affected but the actual hairs are not attacked. The disease may produce a loss of pigment in places, giving rise to leucodermic areas and to a piebald appearance.

There are no constitutional symptoms but there may be more or less severe pruritus which leads to scratching, followed by eczematisation or by impetigo or furunculosis from abrasion of the surface and the inoculation of psogenic cocci. 
Like other forms of tropical ringworm it is at its worst in the hot season and tends to become more bearable, and even quiescent, in cold weather. (Fig. 430.)

It is highly contagious and has been inoculated experimentally from man to man. It is due to a special hyphomycetic fungus known as the Endodermophyton concentricum (Blanchard) which is present in great abundance in the epidermis and the scales. When a scale or scraping of the surface is examined in Liquor potassae or stained with methylene blue the fungus appears as a network of mycelial filaments about $3 \mu$ in breadth which branch dichotomously, have septa at irregular intervals, and tend to break up into round, oval or quadrilateral spores which are either arranged in definite. rows or in small

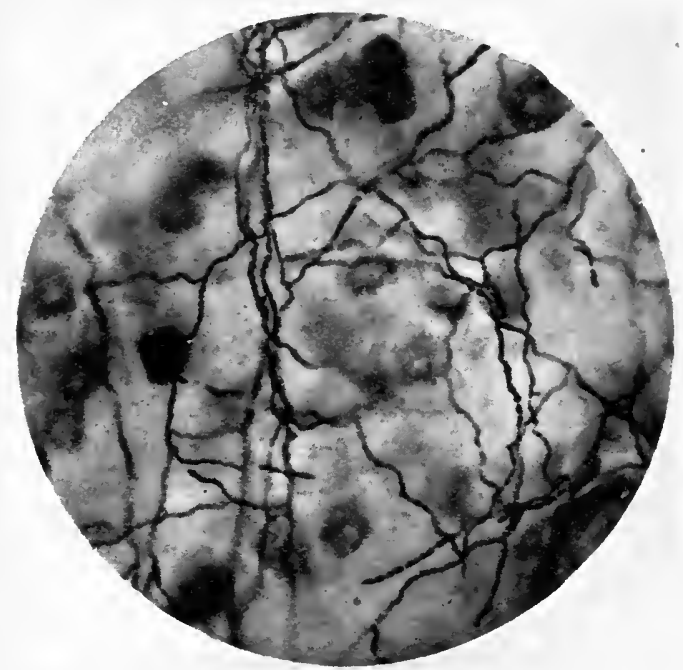

FIG. 430.-Fungus of Tinea Imbricata in Scale.

clusters. Castellani succeeded in growing this fungus on various artificial media. On glucose agar it forms an abundant yellowish or ambercoloured growth with a cerebriform surface. On maltose agar (proof) the growth is mostly submerged and has a central knob which is not covered by a duvet. (Fig. 43I.)

Castellani isolated another variety of fungus which he has named Endodermophyton indicum ; its microscopical appearances are identical with those of the other but the culture has an orange tint and is covered by a delicate duvet; thus establishing the plurality of the causal fungi. (Fig. +32.)

\section{TREATMENT}

The disease if left alone shows no tendency to spontaneous recovery and persists indefinitely. It is exceedingly intractable and after being apparently cured, tends to reassert itself. As a preliminary to treat- 
ment the skin should be scrubbed with soft soap or sand soap and hot water to remove the scales, and this should be followed by the application of a parasiticide ointment or lotion. Of these a large number have

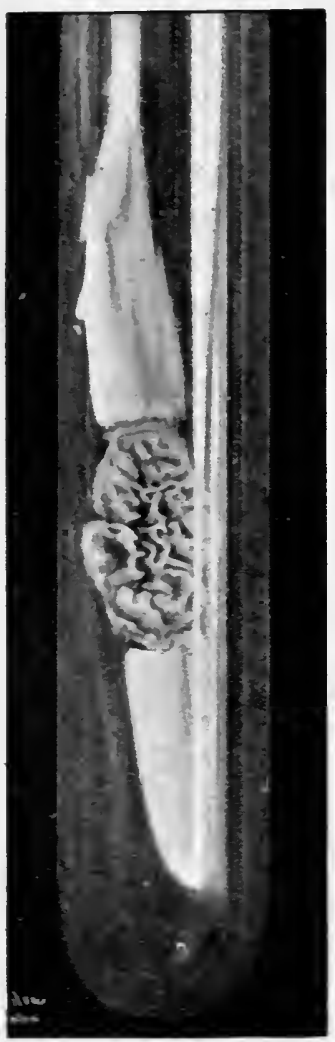

FIG. 43I.-Tinea Imbricata.

Culture of Endodermophyton Con. centricum (Blanchard).

(After Dr. Aldo Castellani. Brit. Journ. Derm., I913.) been tried, with more or less indifferent success. Castellani recommends resorcin, 60 to I20 grains, dissolved in compound tincture of benzoin, freely applied once or twice a day to the affected regions. Should the whole body be involved this procedure is too drastic, owing to the danger of absorption of the resorcin, consequently only one half of the body should be treated at a time, the two halves being dealt with on alternate days. Manson has suggested an iodine liniment of double the strength of the B.P. liniment, which gives excellent results where the disease is limited to a few patches but is too severe to be employed over whole regions.

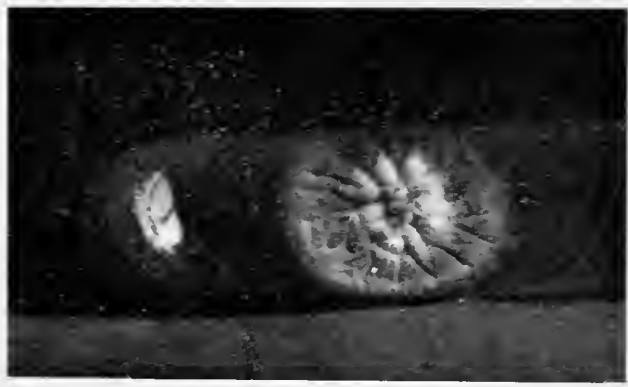

FIG. 432.-Tinea Imbricata.

Culture of Endodermophyton Indicum (Castellani).

Formalin $40 \%$ solution and chrysarobin $6 \%$ in vaselin are sometimes used, but these also are applicable only to localised patches and over extensive areas are apt to be painful and may produce severe dermatitis and symptoms of absorption. On account of the irritating properties of these remedies, it is unsafe to place them in the hands of the ordinary native.

\section{REFERENCES}

Castellavi, A. Fungus of Tinea imbricata (Journ. Trop. Med. \& Hỹg., I9Io, p. 370; Idem, I9II, p. 8I).

Tinea imbricata (Brit. Journ. Derm., I913, xiv. p. 377).

Jeanselye. Tokelau (La Prat. Derm., 1903, p. 455).

MAssox, P. Tinea imbricata (Tropical Diseases, I903, p. 69I).

Paranhos. Tinea imbricata in Brazil (Journ. Trop. Med. \& Hyg., Igo4. vii. p. I53). 
Paranhos \& Leme. Note on Tinea imbricata in Brazil (Journ. Trop. Med. \& Hyg., 1906, p. 129).

Pijper, A. Tinea imbricata in South Africa (Journ. Trop. Med. \& Hyg., I9I 8 , xxi. p. 45).

\section{TROPICAL ULCERS}

In tropical and sub-tropical countries ulcerations of the skin are particularly common. They occur more often among natives than white men for the obvious reasons that natives, not being so well protected by clothing, are more exposed to abrasions, bites of insects, and other means of inoculation by micro-organisms, are usually insanitary in their habits, and are crowded together in huts and compounds.

Certain types of ulceration are met with both in tropical and temperate zones, such as those which result from syphilis, tuberculosis, leprosy, and the common pyogenic infections; others are peculiar to tropical and sub-tropical zones, and of these the most important are the so-called Tropical ulcer, Oriental sore, and Veld sore.

\section{Pyogenic Ulcerations}

Boils of staphylococcic origin are common in tropical and subtropical countries and may be single or multiple. Occasionally they have been given special names according to the country or district in which they occurred, such as the so-called Nile boils of the AngloEgyptian Sudan which have been shown to be due to the Staphylococcus pyogenes aureus.

Ecthymatous sores due to an infection with streptococci subsequently contaminated by staphylococci are not uncommon among natives, and are specially liable to occur when the resistance is lowered as the result of some prolonged illness or in association with malnutrition from improper or insufficient food.

Under the heading of Tropical ecthyma, or Dermatitis cupoliformis, Castellani has described nodular cutaneous lesions, of a dusky red colour, varying in size from a pea to a cherry, which break down in the centre to form an ulcer with undermined edges. These are met with in Ceylon, chiefly among Europeans, and he found them to be due to a special strain of staphylococcus, which is Gram positive and does not liquefy gelatine, and which he named the S. tropicalis.

\section{ORIENTAL SORE}

Syn.: Delhi boil; Aleppo boil; Oriental boil; Biskra bouton; Clou de Gafsa; Cutaneous Leishmaniasis; Kandahar sore; Lahore sore ; Pian bois or forest yaws (British Guiana) ; Puru (Malay States) ; and a variety of other native names. 
Definition: An indolent ulcer with an indurated border and unhealthy granulations, due to Leishmania tropica (Wright).

In the past the name Oriental boil was used in a somewhat loose sense to include a number of ulcerative lesions met with in the tropics and due to various causes, but at the present time it is restricted to a nodulo-ulcerative condition due to a definite protozoon-the Leishmania tropica.

History and Distribution.-It was not until the Indian Mutiny in I857 that the Oriental boil attracted much attention in Europe, although it had been carefully described in 1756 under the heading of Aleppo boil. In I875 a commission was appointed and it was thoroughly investigated by Lewis and Cunningham, and recently it has again attracted attention owing to the discovery of its etiological connection with Leishmania tropica.

The Oriental boil has a fairly wide distribution in tropical and sub-tropical countries and has been met with occasionally even in temperate zones. It is usually limited to certain well-defined districts in a country where it is endemic, but it is not confined to crowded areas in towns or about the mouths of rivers but may be met with in sparsely populated districts and on high ground.

It is prevalent in India, especially about Delhi, Lahore, Kandahar, Multan, Raj Putana States, and the valley of the Indus. In Africa it has been observed most frequently in the northern countries along the Mediterranean littoral, Egypt, and the Sudan, but cases have been recorded in East, West, and South Africa. In Asia it occurs chiefly in Asia Minor, Persia, Mesopotamia, Syria, and the Caucasus; in South America in Brazil, Peru, Bolivia, and British Guiana; in Europe cases have been recorded in the near East, especially in Cyprus, Crete, and Greece.

Description.-The incubation period between the inoculation of the protozoon and the appearance of the lesion varies from a few weeks to several months but averages about two months. It has been said to be associated with irregular attacks of fever, and when the organism has been inoculated experimentally it has given rise, before the derelopment of the indurated sore, to general symptoms consisting of fererishness, headache, etc.

The ulcers may be single or multiple and may occur anywhere on the skin, but are most common on exposed parts of the body. They begin as small red spots like mosquito bites, which become definitely raised and shotty in the centre-the central portion being surrounded by an inflamed areola which becomes indurated later. As time goes on the central nodule assumes a deep purple colour, and when it has reached the size of a pea it begins to desquamate on the surface. Subsequently it becomes moist and crusted and a few yellow points appear, indicating the breaking down of the underlying tissue. When the scab is remored an irregular ulcer is revealed with eroded edges, an indurated border, and a base covered with unhealthy granulations 
moist with a sero-purulent secretion. The tissue around may be definitely oedematous or may present yellow papulo-pustules from an extension of the disease. As a rule the ulcers are from half to one inch in diameter but may be larger, and they are irregular in outline where several have coalesced. There is usually no pain, and the neighbouring lymphatics are not involved unless from secondary pusinfection. (Fig. 433.)

The ulcers are peculiarly indolent and intractable and in spite of the most active treatment may persist for weeks or months. Occasionally they may spread more rapidly, either from their occurrence in persons who are run down in health from starvation or some depressing influence such as malaria or syphilis, from being caused by a more

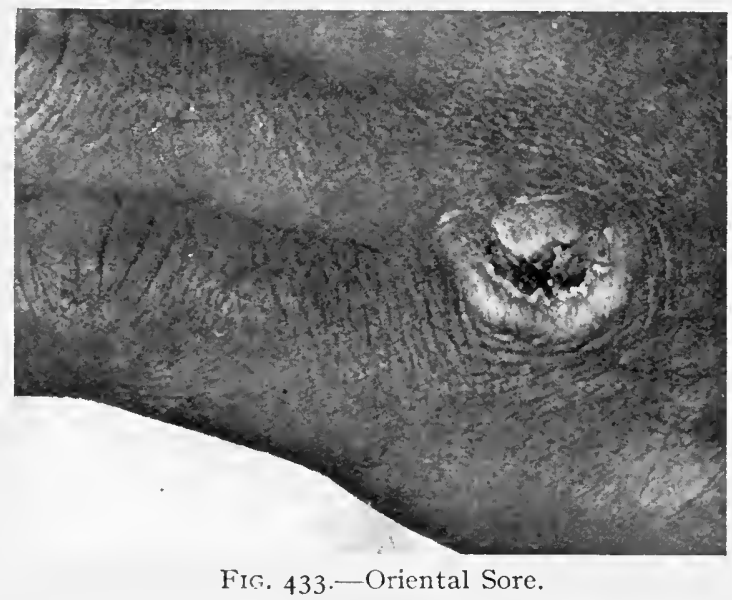

virulent strain of Leishmania, or from the symbiosis of pyogenic cocci with the protozoa. On healing they are followed by a whitish or purplish depressed scar.

In addition to the ulcerative type a nodular variety has been described, in which the lesions do not show the same tendency to break down-possibly from a higher power of resistance in the affected skin.

Histopathology.- Sections of an early lesion show it to be a granulomatous condition in which there is a dense cellular infiltration in the corium, consisting of lymphoid cells, polymorphonuclear leucocytes, plasma-cells, and numerous cells of an endothelial type. At the margins of the ulcer there is definite proliferation of the epidermis with the formation of cell-nests, while in the centre the epidermis and superficial part of the corium have disappeared owing to the upward pressure of the infiltration. The lymphoid and endothelial cells of the infiltration have a peculiar coarse granular appearance from the presence in the cytoplasm of numbers of the Leishmania tropica; these may occur singly, in pairs, or in groups, and are sometimes present between the cells. 
Parasite.-The infective parasite is known as the Leishmania tropica. It appears in the tissues as round, oval, or pyriform bodies, varying in length from 2 to $3.5 \mu$, and in breadth from $I .5$ to $2 \mu$, and can be obtained from the ulcer by scraping the surface, spreading on a slide, and staining with Giemsa's or Leishman's stain. It is never found in the peripheral blood. It is composed of granular protoplasm and contains two masses, one of which is rounded and stains faintly, known as the nucleus, and the other rod-shaped and stains deeply, known as the blepharoblast. This parasite has been successfully cultivated in human blood serum where it developed into flagellate forms, showing that it is simply a stage in the evolution of a flagellated organism. Inoculation experiments on aninals have given

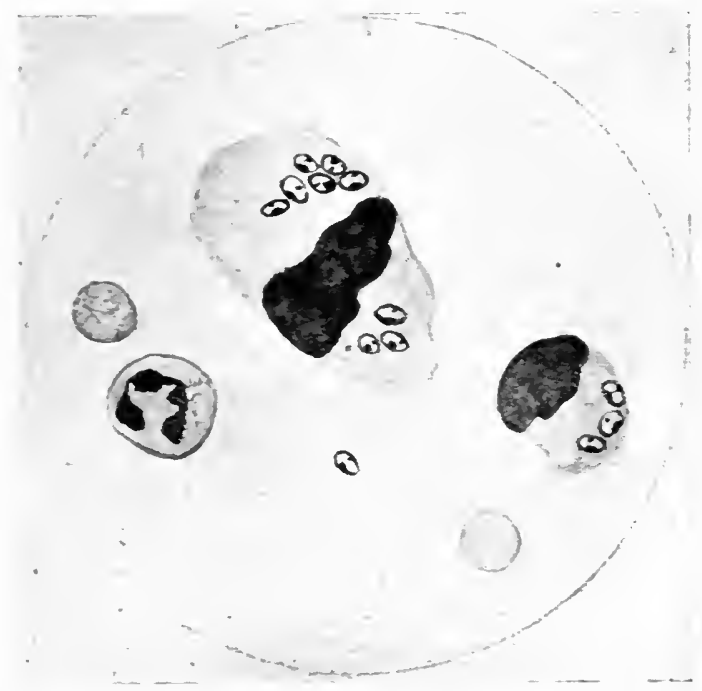

FIG. 434.-Leishmania Tropica ( $\times$ 900) from Case of Oriental Sore. Parasites are found in the cytoplasm of the endothelial cells and mononuclear leucocytes. (Drawn by Dr. J. G. Thomson.)

negative results, except possibly in monkeys. Row has recorded a successful inoculation in Macacus sinicus, and Nicolle has claimed to have inoculated monkeys with Clou de Gafsa but appeared to recover a different parasite from that described by Row. (Fig. 434.)

The condition is auto-inoculable and inoculable from one individual to another, but it is uncertain whether it is transmitted directly through an abrasion or ulceration, or indirectly by flies or other insects.

The parasites are indistinguishable from those obtained in Indian Kala-azar and it has been suggested that it may be the primary sore of Kala-azar; this view is not generally accepted, however, and the Leishmania Donovani of Kala-azar is believed to be different from the Leishmania tropica of Oriental sore.

Etiology.-The disease may occur in anyone exposed to the contagion, irrespective of age, sex, or nationality, but it is especially likely to 
attack individuals who have recently come to reside in a district where it is endemic. It has been said to be more prevalent in the tropics in the beginning of the cool weather and in sub-tropical countries in the autumn.

Certain observers maintain that once the ulcer has healed absolute immunity is conferred against the development of future lesions, but others assert that it is in no way protective. This difference of opinion may be explained by the possible existence of different varieties of the parasite and the probability that the sore of one district does not give immunity to that of another district.

Diagnosis.-There is no difficulty in recognising the condition in countries where it is endemic. It has to be distinguished from a breaking-down syphilitic gumma, but the history, failure to react to specific remedies, and negative Wassermann reaction, serve to distinguish them. Occasionally it may be difficult to differentiate from the ulceration of yaws, but any doubts can be overcome by examining a scraping for the parasite.

\section{TREATMENT}

The treatment is unsatisfactory and the sores are singularly intractable. They should be kept as clean as possible, and after the necrotic tissue has been curetted they should be irrigated with hydrogen peroxide, Io vols., or I in 2000 perchloride of mercury.

Other treatments which have been recommended are powdering the surface of the ulcer with permanganate of potash which, when moist, eats away the diseased tissue; washing the sores with $5 \%$ solution of protargol (Castellani); applying tincture of iodine $10 \%$; dressing with a $2 \%$ antimonium tartaratum ointment (Low) ; chlorine ionisation and hypochlorite dressings (Lyster and McKinstry).

Benefit may be derived from the $\mathrm{X}$-rays, a pastille dose being given, and repeated, if necessary, in six to eight weeks.

The general health should be attended to and healing may be aided by a more generous diet, a tonic regime, the suitable treatment of malaria, syphilis, or other concomitant disease, and, in India, by a change to the hills.

\section{REFERENCES}

Cardamatis, J. P. Leishmanioses en Grèce (Bouton d'Orient) (Bull. Soc. Path. Exot., Paris, May I 2th, I909).

CAR'IER, R. M. Oriental Sore of Northern India, a protozoal infection (Brit. Med. Journ., Sept., 1909).

Castellani, A. Dermatitis cupoliformis (Journ. Trop. Med. \& Hyg., I9I 6 , xix. p.4I).

FirTh, R. H. (Allbutt \& Rolleston's System of Medicine, I907, ii.).

James, S. P. Oriental or Delhi Sore (Scientific Memoirs by Officers of the Mledical \& Sanitary Departments of the Government of India, I905, new series No. I3). 
Low, G. C. Oriental sore treated by antimonium tartaratum (Tartar emetic) locally (Journ. Trop. Med. \& Hyg., r9r 5, p. 258).

Lyster, C. R. C., \& McKinstry, W. H. Oriental Sore (Lancet, Feb. 23rd., r9r 8).

NAtTAN-Larrier, L., \& Bussière, A. Répartition des Leishmania dans le Bouton d'Orient. (Bull. Soc. Path. Exot., Paris, Jan. I909).

Nicolle \& Maxceaux. Oriental Boil (Ann. de l'Inst. Pasteur, Sept. r910).

Row, R. Further observations on Leishmania tropica of Criental Sore of Cambay, India (Brit. Med. Journ., I910, p. 867).

Stephexs, J. W. W., \& Christophers, S. R. Practical study of Malaria, etc. (3rd Edition, r 908 ).

Thomson, D. B., \& Balfour, A. Non-ulcerating "Oriental Sore" (Wellcome Research Labs., Khartoum).

Wexyon, C. M. Oriental Sore in Bagdad (Parasit., Igrr, iv.).

Wright, J. H. Protozoa in a case of Tropical Ulcer (Journ. Med. Research, Dec. 1903).

\section{ESPUNDIA}

Syn.: Naso-oral Leishmaniasis; Breda's Disease; American Leishmaniasis.

Definition: An ulcero-granulomatous condition of the skin and mucous membrane of the mouth and nose due to Leishmania tropica (variety Americana of Laveran and Nattan-Larrier).

This affection is endemic in South America, chiefly in Peru and Brazil, and has been observed also in the Sudan and in Italy in emigrants returning from Brazil where it is known as Boubas Braziliana. The parasite responsible is a variant of the Leishmania tropica of Oriental sore and was first found in the lesions by Bueno de Miranda and Splendore. It is believed to be transmitted by some bloodsucking insect such as a bug or a louse. It is capable of being cultivated and of successful inoculation in monkeys.

After an incubation period of uncertain length it usually begins on some exposed part of the body as a nodule, which breaks down to form an ulcer like Oriental sore. Before, or shortly after, this has healed ulcero-granulomatous lesions make their appearance in the buccal or nasal mucosa, and cause destruction of nasal cartilages and deformity of the nose. The pharynx and larynx may be involved and speaking and swallowing interfered with. The affection is progressive and leads eventually to profound cachexia-death usually taking place from some intercurrent disease.

The condition is believed to be different from ordinary Oriental sore, in which the mucous membranes have been known to be affected in only a few instances.

Treatment.-The initial lesion should be destroyed, either by excision or the cautery, and the ulceration in the mouth treated by antiseptic mouthwashes, such as dilute permanganate of potash solution, or painted with $10 \%$ chromic acid. Christopherson has 
obtained good results from intravenous injections of antimony tartarate in doses of from $\frac{1}{2}$ to 3 grains in distilled water.

\section{REFERENCES}

Castellani \& Chalmers. (Manual of Tropical Medicine, 2nd Ed., London, I9I3, p. I 556).

Christopherson, J. B. Notes on a case of Espundia (Naso-oral Leishmaniasis) treated by the intravenous injection of Antimonium Tartaratum (Journ. Trop. Med., I917, xx. p. 229).

De Miranda, Bueno. Espundia (Arch. de Soc. de Med. de S. Paulo, I9io). Laveran \& Nattan-Larrier. Espundia (Bull. Path. Exot., I9I2).

Susu, B. J. Espundia in the Anglo-Egyptian Sudan (Journ. Trop. Med., I $917, x x$. p. I46).

\section{Ulcerative Dermato-Mycosis}

Under this heading Castellani has described in Ceylon a peculiar dermatomycosis of hyphomycetic origin, and cases of it have been met with also in the Malay States. It is characterised by ulcers over the body, except on the face, scalp, and palms; these are well-defined, roundish or oval in shape, with a red granulating base and an abundant purulent secretion which sometimes dries up to form thick yellow crusts. Gummatous nodules and furuncular lesions may also be present.

The fungus is not seen microscopically in scrapings but can be grown on glucose agar, where it gives rise to amber-coloured colonies which coalesce into a knotty mass, or occasionally to cultures which suggest endothrix ringworm.

The treatment consists of keeping the ulcers clean by dressing them with weak perchloride of mercury lotion, and internally of iodide of potassium in full doses.

\section{REFERENCE}

Castellani, A. A new ulcerative dermato-mycosis (Brit. Med. Journ. I9I6, ii. p. 486).

\section{VELD SORE}

Syn.: Barcoo Rot; Natal Sore; Gift Zeer.

The typical Veld sore is a superficial ulcer, edged by a fringe of exfoliating epidermis, surrounded by an inflammatory areola, and peculiarly resistant to treatment.

It came into prominence during the South African war (1899-1902), when it was the source of much discomfort and invaliding among British troops. It was well known to the Boers under the names of Gift Zeer (poison sore) in the Transvaal, Brand Zeer (burn sore) in the Orange River Colony, and Natal sore, and is identical with a local 
sore which is prevalent in the Barcoo River district of North Queensland and named Barcoo Rot. It was prevalent amongst the Australians stationed in Egypt during the late war.

It begins as a small itchy papulo-vesicle, or group of vesicles which coalesce into a blister, and this breaks down to form a superficial ulcer the red floor of which somewhat resembles that of a cantharides blister. The edges are not definitely raised and consist of an irregular festooned fringe of broken epidermis. The lesions vary in size from a threepenny piece to a florin and are frequently multiple, when they are usually present in different stages of evolution. As a rule, suppuration is not a marked feature but it may occur from secondary pyogenic infection, and occasionally from the same cause the lesions may assume a raised granulomatous appearance and the neighbouring lymphatic glands may become inflamed.

Veld sores are usually present on the backs of the hands and extensor aspects of the forearms, but are sometimes found on the feet and legs. They do not tend to heal spontaneously and may persist for several months in spite of treatment. They not infrequently have the appearance of healing and a scab may form over the surface, but if this be removed sero-pus will generally be found beneath it. When they do heal little or no cicatrix may be left and the hairs may grow on the affected area, but if there has been much secondary infection and ulceration a depressed scar may result.

Etiology.-The ulcer is of microbic origin and doubtless due to a micro-organism, which has gained entrance at the hair-follicles or through some abrasion caused by injury or insect bites. The clinical appearances suggest a local coccic infection and Bishop Harrnan isolated from cases among the troops in the South African War a diplococcus which he was able to grow on artificial media, and produced a typical Veld sore on his arm by inoculating himself with it. To this organism he gave the name Micrococcus vesicans. Martin, who studied the affection in Egypt in IgI6 among the Australian troops, found that it was a staphylococcic infection and that the predominating microorganism was the S. albus. He observed that the hair-follicles were infected with this organism, especially the connective tissue sheaths, and he considered that the infection took place through the hairfollicles and that it was spread by them.

Most observers agree in regarding a low state of health as a predisposing factor. In the Boer War it was noted that it was more common in the cavalry than in the infantry, because the cavalrymen were not in such good condition as they were further away from their commissariat, and were more liable to injuries of the hands and arms from handling their trappings and waggons. It was also observed that it was more prevalent in battalions on the march and men out on the veld than in men in towns or camps, and that it rarely attacked men until they had been out for a sufficient time to become run-down from hardship, fatigue, and shortness of rations. 


\section{Treatment}

The treatment is both general and local, the general treatment consisting of improving the condition of the patient by suitable measures, dietetic, hygienic, and medicinal.

Locally, it is necessary in the first place to clip or scrape away all the exfoliating epidermis at the margins, for it has been observed that the infective microbe penetrates the epidermis in advance of the blister. At the same time the hairs on the floor and at the margins of the ulcer should be carefully epilated. The surface of the lesion should be thoroughly irrigated with I in IOoo corrosive sublimate solution, or with hydrogen peroxide ( 5 vols.). Calomel, or boro-iodoform powder, should then be dusted on and an antiseptic dressing of dilute ammoniated mercury ointment applied under pieces of adhesive strapping. This treatment should be repeated daily till the sore has healed.

\section{REFERENCES}

Bishop Harman. Clinical \& pathological characters of Veld Sore prevalent amongst the troops in S. Africa (Journ. Path. \& Bact., I903, ix. p. I). Martin. Observations on the pathology of Barcoo Rot (Brit. Med. Journ., 1917, p. 761).

Ogston, A. Veld Sore (Brit. Med. Journ., I9or, I, p. 95I).

Pridmore. Veld Sore (Brit. Med. Journ., I 90I, I, p. 2 II ).

\section{DESERT SORE (Diphtheritic sore)}

Under the heading of "desert sore" Craig has described a superficial ulcer, believed to be due to the Klebs-Löffler bacillus, which was met with during the Egyptian and Palestine campaigns in the late war, being especially prevalent among mounted troops.

The sore, which was of a similar type to the Veld sore, had a sudden onset and appeared first as a painful vesicle on an acutely inflamed base, which varied in size from a pea to half an inch or more. It soon burst, forming a shallow ulcer the floor of which was dry, red, and glazed, and frequently covered by a pearly grey membrane. The edges of the surrounding skin became undermined and the ulcer spread peripherally, then became stationary and assumed a characteristic appearance--punched-out and circular in outline, with undermined edges and thickened margins, and the base covered with grey-coloured debris beneath which a tough and adherent membrane was frequently present. (Fig. 435.)

The ulcers occurred invariably on exposed parts and mainly on those covered by hairs around which they appeared to develop, and were present chiefly on the dorsa of the hands, forearms, elbows, knees, and exposed parts of the legs, and in rare instances on the face.

Besides the ordinary organisms of suppuration, the Klebs-Löffler bacillus was found to be constantly present and was believed to be the cause. 
The ulcers were commonly associated with faucial diphtheria and it was found that there was a close correlation between the incidence of diphtheria of the nose and throat and the occurrence of the sores.

With the usual local treatment they healed with difficulty, but the diphtheria anti-toxin proved an absolute specific and sores which had resisted treatment for months healed in a few days with an average dose of 4000 units.

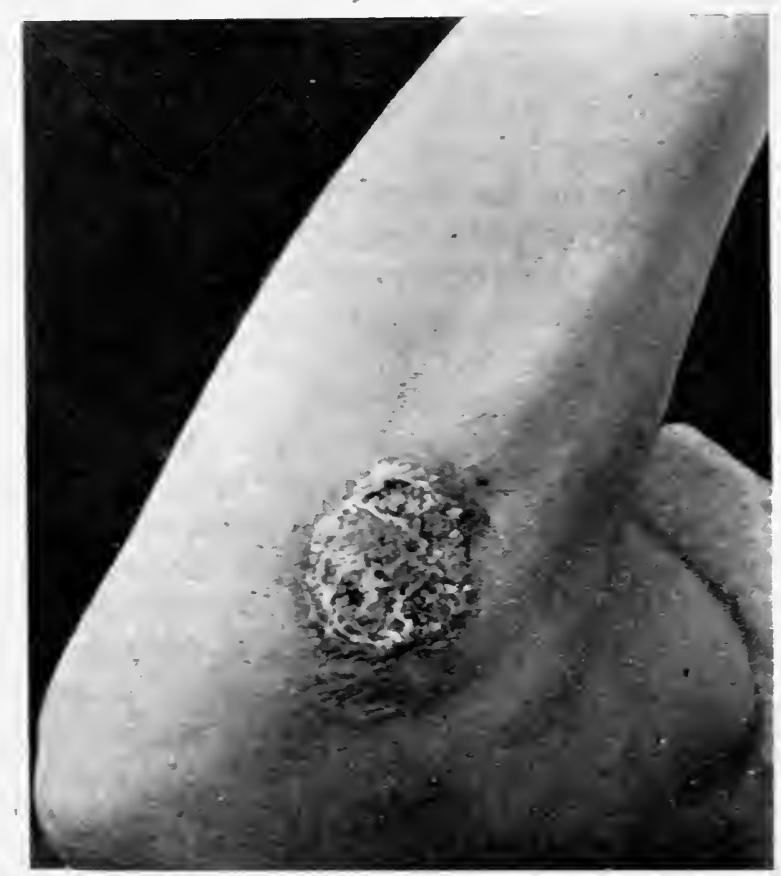

FIG. 435.-Diphtheritic Sore.

Which healed with two injections of anti-diphtheritic serum.

(From Dr. P. Manson-Babr.)

\section{REFERENCE}

Craig, Colix M. "Desert," Septic, or Veldt Sore amongst European troops (Lancet, Sept. I3th, I9I9).

\section{VERRUCA PERUANA}

Syn.: Verruga Peruviana, Bouton des Andes, la Maladie de Carrion, Fièbre de la Oroya.

Definition: An endemic infectious disease, characterised by irregular ferer associated with anaemia and rheumatoid pains, and followed by an eruption of granulomatous swellings on the shin, mucous menbranes, and internal organs.

Introduction.-As early as the sixteenth century references to this disease appeared in Spanish works on Peru, but it was not until the 
nineteenth century that it was described clearly, when it attracted special attention owing to a serious outbreak of the fever which occurred in I870 among the workmen making the railway between Lima and Oroya. At this time two forms of the disease were recognised, a malignant and a benign.

The malignant form was an irregular remittent or intermittent fever known as "Oroya fever." It had an incubation period of 2 I days and was accompanied by a progressive anaemia, so severe that the red cells might fall in a few days to less than one million per c.mm., by vertigo, restlessness, air-hunger, and a high mortality, and in a small percentage of the cases in which recovery from the fever took place by an eruption of nodules.

The benign form was characterised by a peculiar eruption of granulomatous nodules and warty excrescences (verrucas). In it the fever and anaemia might be slight or even absent.

There has been much discussion as to the relation of the fever and the eruption. As early as I885 Carrion, a student of medicine at Lima, tried to settle the question by vaccinating his arm with blood from a verruca tumour. Six months later he became a martyr to science and succumbed to an attack of Oroya fever but without the development of verrucas. Since his time much work has been done on the subject and the prevalent opinion at present is that Oroya fever is distinct from the verruca eruption and that it is related to the para-typhoid group of fevers. Those cases in which both the fever and the eruptive stage occur are regarded as examples of patients suffering from verucca having become infected with a variant of para-typhoid.

Geographical Distribution.-In its distribution it is limited to valleys at altitudes of 3000 to 6000 feet on the western slopes of the Peruvian Andes, and is never met with on the plains or on the coast. Its chief endemic areas are in the Department of Lima and in the Province of Huaraz.

Descrintion.-The eruption appears first like an exanthem on the face, neck, extensor aspects of the arms and legs, and occasionally on the conjunctivae, lips, and buccal mucosa. It consists of small pinkishred macules, which become papules, and later develop into nodules about the size of a split-pea but sometimes considerably larger. These have a tendency to undergo warty changes. In addition to the superficial warty lesions, subcutaneous nodules are also met with which may reach a considerable size. At first these are freely movable beneath the skin but in time they become adherent to it and finally break down to form fungating ulcers.

The eruption comes out in crops, in some cases each crop being preceded by an attack of fever. After persisting about six months the nodules disappear, either leaving no trace or, where they have become ulcerated, being followed by a scar.

The mortality from the malignant form is heavy and may reach $90 \%$. It was so great during the construction of the Oroya railway 
that one of the bridges crossing the river is known to this day as the "Pont de Verrugas." In the benign type it is fatal in about 10\% of the cases among natives and 40\% among whites, death being caused by the presence of the nodules in internal organs, anaemia from haemorrhages, and complications such as septicaemia, malaria, syphilis, tuberculosis, or infection by bacilli of the B. coli communis type (Barton's bacillus).

Etiology.-The disease may attack all races and neither age nor sex confers any immunity towards it. Up to the present time its cause is unknown; Barton and Biffi have isolated several strains of the typhoid colon group of bacilli in patients suffering from verrucas, but these micro-organisms-though most probably responsible for the fever-do not appear to cause the eruption. . Barton has also described certain bacillus-like bodies in the erythrocytes in a number of cases and has observed that their disappearance from the peripheral blood coincides with recovery, but their exact nature and relation to the verrucas is uncertain.

The disease is usually contracted at night and is generally believed to be transmitted by the bite of some suctorial insect, such as a mosquito or tick, which bites at night. This was borne out in Igog during the construction of several bridges on the Oroya railway when the workmen were made to sleep outside the endemic areas, with remarkable success in the prevention of infection.

Histopathology.-Sections of nodules showed swelling of the connective tissue fibres and an infiltration, consisting of embryonic connective tissue cells, mononuclear cells, and polymorphonuclear cells, situated around the capillaries which were dilated and, in places, cavernous. The epidermis was affected secondarily.

Diagnosis.-The eruption has to be differentiated chiefly from yaws, with which at one time it was confused. It is distinguished by not being contagious, by its absence from the trunk, by the tendency to haemorrhage of the lesions, and by the absence of the Treponema pertenue of Castellani.

\section{TREATMENT}

No specific remedy for it is known and all that can be done is to increase the resistance of the patient by proper nourishment and the usual tonic remedies and, whenever possible, by removal to a district where the disease is not endemic-preferably to the plains or the coast. When febrile symptoms are pronounced the cases should be treated in much the same way as typhoid ferer.

Iodide of potassium and injections of arsenic have been employed but with doubtful success. From time to time the natives have used decoctions from various plants, such as maize, Buttneria cordata, etc., but any benefit derived from them was probably simply from their diaphoretic action. The natives also believe that chills and cold baths 
should be avoided as they consider that they do serious harm by preventing the eruption from coming out and so endangering the life of the patient-a repetition of the old fallacy of eruptions being better out than in.

For the actual destruction of the local lesions, which are liable to give trouble from haemorrhage, the old-fashioned plan of ligaturing the base should be adopted where possible. When ulceration superrenes it is advisable to bathe the lesions with some antiseptic lotion, such as I in 4000 corrosive sublimate, and to dress them with ointments containing boric acid or ammoniated mercury.

\section{REFERENCES}

Barton, A. L. Descripción de elementos endo-globulares hallados en los enfermos de fièbre verrucosa (Crón. Méd. Lima, I909, xxvi, p. 7). Bassett-Smith. The pathology of the blood in verruga (Brit. Med. Journ., I909, 2, p. 783 ; Trans. Soc. Trop. Med., I9I3).

Brffi (Arch. f. Schiffs. u. Tropen. Hyg., I908, xii. p. I).

CARrion, D. Verruga Peruana (Monitor Ned. Lima, I885, p. I62).

Castellani \& Chalmers. Verruga Peruviana (Man. Trop. Med., 2nd Ed., I9I3, p. I I96).

Darling, S. T. Verruca Peruana (Journ. Amer. Med. Assoc., I9I I, lvii. p. 207I).

Firth, R. H. Verruga (Allbutt's "System of Medicine," I907, 2, p. 704). Odriozola, E. La Maladie de Carrion (Paris, I 898, Carré et Naud).

Tomayo, M. Verruga Peruana (Crón. Méd., Lima, I905). 


\section{APPENDIX}

\section{NATURAL MINERAL WATERS}

THE following are the most important of the natural mineral waters, but, with the exception of the arsenical and bromo-iodine waters, none can be claimed to have any specific action on the skin. Some of them can be obtained bottled; others deteriorate by keeping and should be drunk at the spring at their natural temperature.

Alkaline Waters.-The principal alkaline waters are those of Contrexéville, Carlsbad, Marienbad, Vichy, and Vals. These owe their alkalinity to the presence in them of bicarbonate of soda and are speciallyuseful in gouty and rheumatic conditions and in chronic skin affections associated with them, such as psoriasis.

The Carlsbad Sprudel contains sulphate of soda combined with bicarbonate of soda, and has a laxative action; a teaspoonful dissolved in a large tumbler of hot water is taken in the morning before breakfast.

Arsenical Waters.-These are chiefly tonic and are employed in the same type of cases as ferruginous waters, being specially indicated in cutaneous affections associated with anaemia. The best known are those of Levico, La Bourbole, Royat, and Roncegno.

Bromo-iodine Waters. - These are sometimes prescribed in late syphilides, but otherwise have no special indication in connection with cutaneous disease. The strongest of them is Woodhall Spa, which contains about 5 grains of bromide of sodium and 2 grains of iodide of potassium per gallon. Iodine is contained also in the waters of Kreuznach and Box.

Fernuginous Waters. - The chalybeate waters contain carbonate of iron, have a definite tonic action, and are of special value in anaemic patients. They are not unpalatable, owing to the presence in them of carbonic acid gas, and certain of them are aperient which is useful in counteracting the constipating tendency of the iron. Among the best known are those of Homburg, Schwalbach, Spa, Kissingen, and St. Moritz.

Saline Waters. - These owe their effect to the presence in them of the sulphates of magnesia and soda. Some are simply laxative, while othersknown as "bitter waters"- - contain a larger proportion of sulphates and are definitely purgative. As examples of the milder salines may be mentioned Püllna and Friedrichshall, and of the purgative bitter waters Hunyadi Janos and Aesculap. They are useful for the relief of the congestion in inflammatory conditions of the skin occurring in plethoric and constipated patients and should be given in tepid water before breakfast.

Sulphur Waters. - These have a mild laxative action, and are useful in cutaneous diseases associated with constipation, such as acne and rosacea. They contain varying quantities of sulphate of sodium, sulphuretted hydrogen gas, and common salt. Sulphur springs are numerous, some of the best known being Strathpeffer, Harrogate, Llandrindod, Schinznach, Aix-la-Chapelle, and certain of the Pyrenean spas such as Barèges and 
Bagnères-des-Luchon, Challes in Savoy, and Uriage in Isère. As a rule sulphur waters keep badly in spite of the most careful bottling and should be drunk at the spring itself.

\section{SPAS}

The number of spas which attract patients suffering from skin disease is great and increases year by year. The following are among the best known in this country and the Continent, arranged for convenience in alphabetical order :

Aix-les-Bains (France), is situated in the Alpine Savoy district at an altitude of about 800 feet. It has a mild and pleasant climate, being surrounded by hills and mountains. Its season is from May to September. The springs are saline and sulphurous, contain also traces of iodides and bromides, and issue at a temperature of $I I 4^{\circ}$ to $I I 7^{\circ} \mathrm{F}$. These waters are indicated in acne, psoriasis, and syphilis, especially when associated with chronic rheumatism or gout.

Aix-la-Chapelle, or Aachen (Rhenish Prussia), is one of the largest watering-places in Europe. It is situated at an altitude of 730 feet, and is open all the year round, the season being from May to September. Its waters are strongly sulphurous and hot, varying from $116^{\circ}$ to $160^{\circ} \mathrm{F}$. The treatment there is indicated especially in syphilis, psoriasis, and acnethe favourite anti-syphilitic treatment being mercurial inunctions.

Bath (Somerset), has mildly saline springs at a temperature of about $120^{\circ} \mathrm{F}$. They are employed chiefly as baths and are of value in psoriasis.

Bourbole (France) is situated in the Puy de Dôme on the Dordogne and stands at an altitude of about 2500 feet in an open valley protected to the north by mountains. It possesses a sunny and invigorating climate and is chiefly frequented in July and August. It is noted for its hot alkaline arsenical springs at $\mathrm{I}_{4} 0^{\circ} \mathrm{F}$. The spring known as the "Source des Fièvres " is the richest of all the natural arsenical waters and contains 28 milligrammes of arseniate of soda to the quart of water, being equivalent to about $2 \mathrm{I}$ drops of Liquor arsenicalis; it is a limpid fluid with a salt taste and is employed as baths in late syphilis, psoriasis, acne, and tuberculosis of the skin.

Buxton (Derby), is situated amid the beautiful scenery of the Derbyshire Wye at about rooo feet above sea-level and has a bracing climate. Its waters are slightly alkaline and contain carbonate of lime, and have a temperature of $80^{\circ} \mathrm{F}$. In baths they are emollient and pleasant from the presence of nitrogen. They have no special indication in connection with skin diseases.

Carlsbad (Austria), is situated about $\mathrm{I} I 2$ feet above sea-level in a narrow valley and is open all the year, the season being from April to October. Its hot saline springs are advocated for the treatment of plethoric conditions, liver disturbances, gout, and chronic constipation, and are useful in psoriasis and other chronic skin affections associated with the plethoric habit, disease of the kidneys, and sluggish action of the liver.

Contrexéville (France), is situated in the Vosges Mountains and its season is from June to September. Its springs are mildly saline and cold and are useful in liver derangements and, owing to their diuretic action, in flushing out the kidneys. They have no special indication in cutaneous diseases, but have been recommended in cases of pruritus associated with disturbances of the liver in which no local lesion can be found.

Droitwich (Worcester), has saline springs which are ten times salter than the sea and so heavy that it is impossible to sink in the swimming 
baths. The waters are harmful to any but the healthiest and strongest skins and are employed chiefy in rheumatic and gouty conditions.

Ems (Germany), is picturesquely situated in the valley of the Lahn amid well-wooded hills. The season is from May to September. It has hot alkaline springs which have no special indication in skin diseases though the baths may be of benefit in psoriasis.

Harrogate (Yorks), is one of the most popular of English health resorts and is situated in a moorland at an altitude of about 400 feet. It has a dry bracing climate, and its chief springs contain sulphur, magnesia, and iron. In chronic skin affections, such as psoriasis and acne, the sulphurous and saline baths are of value, but as in the case of all spas, it is important that they should be taken under medical supervision.

Kreuznach (Prussia), is near Bingen and situated on the Nahe. Its season is from May to October and its springs are saline and contain a small proportion of bromide and iodide of magnesium. The treatment there is of value in syphilis and in scrofulous affections.

Leukerbad, or Louche (Switzerland), in the Valais Canton, is situated at an altitude of about 4000 feet at the foot of the famous Gemmi. Its season is from May to October. The waters are mildly mineralised and are used chiefly as continuous baths at a temperature of about $95^{\circ} \mathrm{F}$., which are of service in bullous affections of the skin and also in psoriasis to macerate the scales.

Levico (Austria), is a Tyrolese village situated at the entrance of the Sugana Valley at an altitude of about 4500 feet. The season is from April to September. The waters, which are brought down from the mountains in pipes, contain arsenic and iron in considerable quantities and are dark in colour. They are given in doses of a dram in milk or plain water. The baths contain the same spring water diluted and are recommended in psoriasis and chronic eczema, especially when associated with rheumatism.

Llandrindod (Radnorshire), is situated in the centre of a plateau sheltered to the east by the Radnor Forest and has a bracing climate. It possesses sulphur and iron springs which are useful in scrofulous conditions and in anaemia.

Marienbad (Austria), is situated in a broad valley surrounded by pineclad hills at an altitude of about 2000 feet and is about twenty-five miles from Carlsbad. The climate is bracing during the summer. It has cold alkaline sulphurous springs which contain a small quantity of iron. They are more purgative than Carlsbad water and are useful in chronic scaly affections associated with constipation and liver disturbances.

Plombières (France), is situated in a narrow valley in the Vosges Mountains at an altitude of about $I_{400}$ feet and is much frequented by the French, especially women. Its waters are mildly mineralised and vary in temperature from $60^{\circ}$ to $160^{\circ} \mathrm{F}$. A special feature is prolonged baths, but they have no special indication in cutaneous diseases.

Roncegno (Austria), is situated in the province of Trient in the south Tyrol. Its waters are arsenical and ferruginous and contain small quantities of nickel. They are given in doses of $\mathrm{I}$ to $\&$ teaspoonfuls, and are especially useful in anaemia and cutaneous affections associated with it.

Royat (France), in the Puy de Dôme district, lies in the valley of St. Mart on the lower slopes of the Auvergne Mountains. The season is from June to September. Its waters are saline but contain small quantities of iron and arsenic. They are specially useful in anaemia and rheumatism, but have no special indications in skin affections unless associated with general disorders.

Schinznach (Switzerland), in the Argovia Canton is situated at the base of the Wülpelsberg at an altitude of Iroo feet. The season is from May to October. It is famous for its hot sulphate of lime springs at 
$95^{\circ} \mathrm{F}$. and its baths are useful in acne, psoriasis, seborrhoic dermatitis, and various parasitic affections of the skin.

Spa (Belgium), is situated in a deep sheltered valley protected by pine-clad hills. It has cold ferruginous springs which are useful in chlorosis and anaemia, but are of no special value in cutaneous diseases.

Strathpeffer (Cromarty), is beautifully situated at the base of Ben Wyvis. It has a pleasant climate and is sheltered from almost every wind, except that from the sea, and is open all the year, the season being from May to September. Its chief springs are sulphurous, but there are also iron springs. It is useful in gouty and rheumatic conditions and in psoriasis and chronic eczema associated with them.

Vichy (France), is situated in a valley on the river Allier near the Auvergne Mountains at an altitude of 780 feet. It is one of the most popular French health resorts and its season is from May to October. Its waters are alkaline and useful in gouty conditions. 


\section{FORMULAE}

\section{CATAPLASM}

Boro-starch Poultice.

Add a teaspoonful of boric acid powder to a tablespoonful of cold water starch, mix with a little cold water, then pour in a pint of boiling water, and stir till melted; let stand till cold and spread thickly on pieces of cotton, cover with muslin, and apply to the part. Change the poultice every few hours.

\section{COLLODIONS}

Collodium Acidi Salicylici.

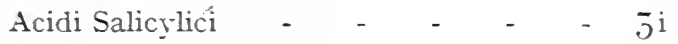

Ext. Cannabis Indicae - $\quad-\quad$ - $\quad$ - gr. xx

Collodium Flexile ad - $\quad$ - $\quad \frac{5}{3} \quad$

For corns, callosities, etc.

Collodium Chry'sarobini.

Chrysarobini - -

Acidi Salicylici - $-{ }_{-}$

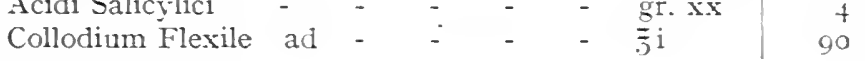

For psoriasis.

\section{CREAM}

\section{Cremor Lanolini.}

\section{Lanolini -}

Cerae Albae -

Olei Amygdalae

Aquae Rosae -

Soothing cream and hase.

\section{GELATINES}

Glycogelatin-zinci.

\begin{tabular}{|c|c|c|c|c|c|}
\hline Gelatini - & - & - & - & $-\mid 2.5$ & $15)$ \\
\hline Glycerini & - & - & - & -12.51 Soft & ro (Hard . \\
\hline Zinci Oxidi & - & - & - & -20 jelly & 25 contractile \\
\hline Aquae - & - & - & - & -55 & jol jelly \\
\hline
\end{tabular}

Melt the glycerine and water, then stir in the zinc oxide and dispense in a deep tin. 
Place the tin in a basin of boiling water, melt to the consistence of cream, then paint on and cover with a thin film of absorbent wool.

To this may be added $2 \%$ ichthyol, $5 \%$ tar, etc.

Especially useful as a protective on the legs and may be left on for a few days.

\section{LINIMENTS}

Linimentum Acidi Salicylici.

\begin{tabular}{lllllll|r} 
Acidi Salicylici & - & - & - & - & - & gr. x & 2 \\
Resorcin - & - & - & - & - & - & gr. xx & 4 \\
Olei Eucalypti & - & - & - & - & - & m xx & 4 \\
Olei Olivae ad & - & - & - & - & - & $\vdots \mathrm{j}$ & 90
\end{tabular}

Keratolytic: useful in seborrhoic dermatitis.

\section{Linimentum Cadini.}

\begin{tabular}{llllllll|r} 
Olei Cadini & - & - & - & - & - & - & $3 \mathrm{i}$ & 6 \\
Olei Olivae & ad & - & - & - & - & - & 3 &
\end{tabular}

For psoriasis.

\section{Linimentum Calaminae.}

Calaminae Preparatae - $\quad$ - $\quad$ gr. xv $\mid 3$

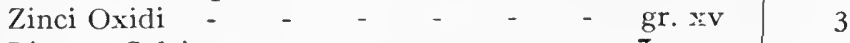

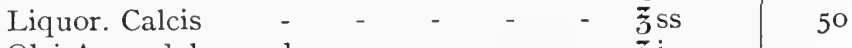

Olei Amygdalae ad - $\quad$ - $\quad$ - $\quad$ -

Sedative: useful in Lichen planus, erythema, urticaria, etc.

\section{LOTIONS}

Lotio Acetici.

\begin{tabular}{llllll|c} 
Hydrargyri Perchloridi & - & - & - & - & gr. $\frac{1}{2}$ & $\cdot$ I \\
Olei Ricini - - & - & - & - & - & mii & $\cdot 5$ \\
Acidi Acetici - - & - & - & - & - & mv & I \\
Spiritus Lavandulae & - & - & - & - & $3 \mathrm{ii}$ & 24 \\
Alcohol 90\% ad - & - & - & - & - & 3 &
\end{tabular}

Stimulant for Alopecia areata.

\section{Lotio Acidi Borici.}

A saturated solution of boric acid in cold water and contains about I 5 grains of boric acid in the fluid ounce $(3 \%)$.

Mild antiseptic.

\section{Lotio Acidi Carbolici.}

Acidi Carbolici Liquefacti I

Aquam (at $50^{\circ} \mathrm{C}$.) - $\quad$ - $20,40,80$ or 100 parts.

Antiseptic and antipruritic.

\section{Lotio Acidi Carbolici Co.}

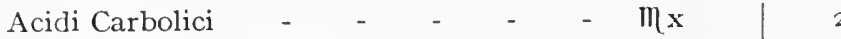

Glycerini - - $\quad-\quad-\quad-m_{\mathrm{v}}$

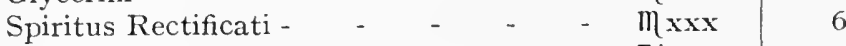

$\begin{array}{llllllll}\text { Aquam ad } & - & - & - & - & - & \overline{3}^{\mathrm{i}} & 9 \mathrm{I}\end{array}$

Antipruritic: useful in pruritus, urticaria, etc. 
Lotio Acidi Lactici.

Acidi Lactici -

Spiritus Vini Rectificati ad - $\quad-\quad-\bar{j}^{i}$

For Alopecia areata.

Lotio Acidi Salicylici.

Acidi Salicylici

Olei Ricini -

Olei Rosmarini

Spiritus ad -

$\begin{array}{llllll}- & - & - & - & - & \mathrm{gr} \times \mathrm{xv} \\ - & - & - & - & - & \mathrm{m} \times \mathrm{xx} \\ - & - & - & - & - & \mathrm{q} . \mathrm{s} \\ - & - & - & - & - & \bar{j} \mathrm{i}\end{array}$

Useful for scurfy conditions of the scalp.

\section{Lotio Acidi Tannici.}

Glycerini Acidi Tannici -

Aquam ad .

Astringent.

Lotio Aluminis Co.

\begin{tabular}{llllll|r} 
Aluminis - & - & - & - & - & gr. $x$ & 2 \\
Liq. Plumbi Acetatis & - & - & - & - & $\overline{5} \mathrm{i}$ & 12 \\
Aquam ad - & - & - & - & - & $\overline{3} \mathrm{i}$ & 86
\end{tabular}

Astringent: useful in removing crusts.

Lotio Ammoniae Fortior.

Liquoris Ammoniae Fort

Spiritus Rosmarini -

Aquae Mellis - $\quad$ - $\quad$ - $\quad$ - $\quad$ - jiss

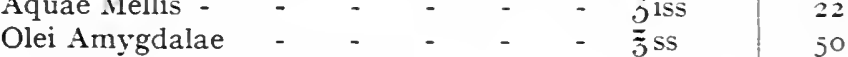

Stimulant for hair-growth.

\section{Lotio Bicarbonatis Co.}

\begin{tabular}{lllllll|r} 
Tinct. Opii - & - & - & - & - & - & $\prod \times v$ & 3 \\
Sodii Bicarbonatis & - & - & - & - & - & gr. $x v$ & 3 \\
Glycerini - & - & - & - & - & - & gr. $x$ & 2 \\
Aquam ad & - & - & - & - & - & $\prod_{x}$ & 2 \\
& & & & & & $\overline{3} \mathrm{i}$ & 90
\end{tabular}

Sedative and antipruritic.

\section{Lotio Bismuthi.}

\begin{tabular}{|c|c|c|c|c|c|}
\hline Bismuthi Subnitratis & - & - & - & $\therefore$ & gr. xv \\
\hline Zinci Oxidi - - & - & - & - & - & gr. $x$ \\
\hline Spiritus Camphorae & - & - & - & - & $m x$ \\
\hline Glycerini - - & - & - & - & - & $m x x$ \\
\hline Aquam ad - & - & - & - & - & $\overline{\tilde{z}}^{\mathrm{i}}$ \\
\hline
\end{tabular}

Sedative.

\section{Lotio Boracis.}

Glycerini Boracis -

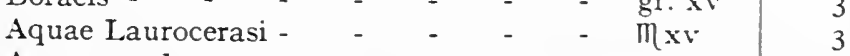

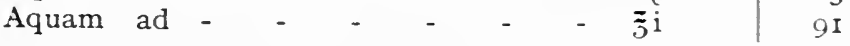

Sedative for irritable scalp. 


\section{Lotio Calaminae.}

\begin{tabular}{lllllll|r} 
Calaminae Preparatae & - & - & - & - & gr. xv & 3 \\
Zinci Oxidi & - & - & - & - & - & gr. x & 2 \\
Glycerini & - & - & - & - & - & Mxxx & 6 \\
Aquae Calcis - & - & - & - & - & - & Mlxxx & r 6 \\
Aquam Rosae ad - & - & - & - & - & $\overline{5} \mathrm{i}$ & 73
\end{tabular}

Sedative for inflammatory affections.

Lotio Calaminae cum Plumbi.

\begin{tabular}{|c|c|c|c|c|c|c|c|}
\hline Calaminae $\mathrm{I}$ & rep & atae & - & - & - & gr. xv & 3 \\
\hline Zinci Oxidi & - & - & - & - & - & gr. $\mathrm{x}$ & 2 \\
\hline Glycerini & - & - & - & - & - & $m x$ & 2 \\
\hline Liq. Plumbi & Sul & cetatis - & - & - & - & Mii & \\
\hline Aquam ad & - & - $\quad-$ & - & - & - & $\overline{3} \bar{i}$ & \\
\hline
\end{tabular}

Sedative and antipruritic.

Lotio Calcii Sulphuratis (Vleminckx' Solution).

I ounce of quicklime, 2 ounces of precipitated sulphur, and $\Upsilon_{5}$ ounces of water, are boiled together in an earthenware vessel, then reduced to Io ounces, and the clear sherry-coloured fluid is decanted after subsidence.

Antiparasitic : useful in Tinea corporis, etc.

\section{Lotio Cantharidis.}

\begin{tabular}{llllll|r} 
Aceti Cantharidis - & - & - & - & - & mxxx & 6 \\
Glycerini - & - & - & - & - & Mxxx & 6 \\
Spiritus Rosmarini - & - & - & - & - & $3 \mathrm{ii}$ & 24 \\
Aquam Rosae ad & - & - & - & - & $\overline{3} \mathrm{i}$ & 64
\end{tabular}

Stimulant for alopecia.

Lotio Cantharidis cum Acidi Acetici.

\begin{tabular}{llllll|r} 
Hydrargyri Perchloridi & - & - & - & - & gr. 5 & ·I \\
Acidi Acetici Glacialis & - & - & - & - & gr. v & I \\
Resorcin - - & - & - & - & - & $\operatorname{gr} . x$ & 2 \\
Tinct. Cantharidis - & - & - & - & - & Mxxx & 6 \\
Spiritus Vini Rectificati & ad & - & - & - & $\overline{3} \mathrm{i}$ & 90
\end{tabular}

For Alopecia areata.

Lotio Cantharidis cum Ammoniae.

Tinct. Cantharidis

Liquoris Ammoniae Fortis

Glycerini

Aquam ad -

Stimulant for alopecia.

\section{Lotio Evaporans.}

\begin{tabular}{lllllll|c} 
Ammonii Chloridi & - & - & - & - & - & gr. xii & $2 \cdot 5$ \\
Alcohol $90 \%-$ & - & - & - & - & - & Mxxxv & 7 \\
Aquam ad & - & - & - & - & - & $\bar{\jmath} \mathrm{i}$ & $90 \cdot 5$
\end{tabular}

Cooling lotion.

\section{Lotio Formalini.}

Formalini

Benzoli -

Olei Geranii - $\quad$ - $\quad$ - _ _ .

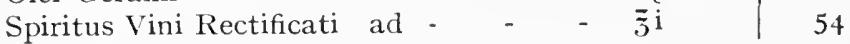

Parasiticide for the scalp. 
Lotio Ichthyol Co.

Ichthyol - $\quad-\quad-\quad-\quad-5^{\mathrm{i}}$

Acidi Tannici - $\quad-\quad-\quad-\quad-\quad-5 i$

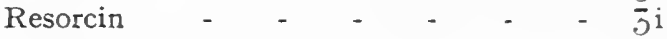

Aquam ad - - - - - $\quad-\quad \bar{j}$

Lotion for chilblains.

Lotio Jaborandi.

Tinct. Jaborand

Tinct. Cantharidis -

Acidi Salicylici

Chloroformi

Spiritus Vini Rectificati ad .

For stimulating hair-growth.

\section{Lotio Menthol.}

Menthol -

Spiritus Vini Rectificati - _ _ _ _ .

Acidi Acetici dil. - $\quad$ - $\quad$ - $\quad$ - $\quad$ - jiv

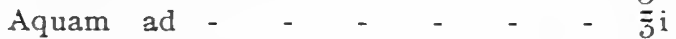

Sedative and antipruritic.

\section{Lotio Picis Alkalinus.}

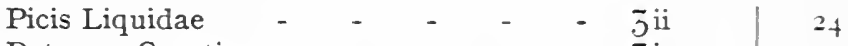

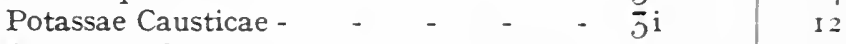

Aquam ad - $\quad$ - $\quad$ - $\quad$ - $\bar{j}^{\mathrm{i}} \quad 64$

Half a teaspoonful to an ounce of water for use as a lotion. Antipruritic and detergent.

Lotio Picis Carbonis.

\begin{tabular}{llllll|r} 
Liquor Picis Carbonis & - & - & - & - & Mxv & 3 \\
Aquam ad - & - & - & - & - & $\overline{3}$ & 97
\end{tabular}

Detergent and reducing : useful in weeping eczema.

\section{Lotio Picis Co.}

Liquor. Picis Carbonis - $\quad$ - $\quad$ - $m_{x} \mid 2$

Spiritus Rectific ti - $\quad$ - $\quad$ - $\quad$ - $m x x$

Mucilaginis Tragacanthae - $\quad$ - 3 ss 6

Aquam ad - - - - - - $\bar{j}$ i 90

Detergent and antipruritic : useful in eczema and pruritus.

\section{Lotio Plumbi Detergens.}

Liq. Plumbi Subacetatis Fortis - - $m_{x} \mid \begin{aligned} & 2 \\ & \text { L }\end{aligned}$

Liq. Carbonis Detergentis - $\quad-\quad$ -

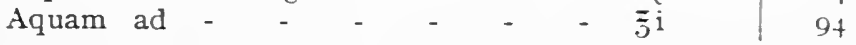

Antipruritic.

\section{Lotio Plumbi Evaporans.}

Liq. Plumbi Subacetatis Fortis

Alcohol 90\% vel Spiritus Methylati - $-5 i$

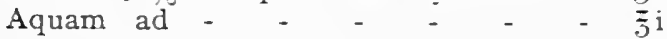

Sedative and astringent. 
Lotio Plumbi cum Opii.

Liq. Plumbi Subacetatis - $\quad$ - $\quad$ - $m x \quad 2$

Glycerini - $\quad-\quad-\quad-\quad-m_{x}$

\begin{tabular}{llllllll|r} 
Tinct. Opii & - & - & - & - & - & - & mxv & 3 \\
Aquam ad & - & - & - & - & - & - & $\bar{\jmath} \mathrm{i}$ & 93
\end{tabular}

Sedative and antipruritic.

Lotio Quillaiae.

$\begin{array}{lllllll}\text { Tinct. Quillaiae } & - & - & - & - & 3 \mathrm{i} & \text { I2 }\end{array}$

Liquor. Ammoniae Fortis - - - $\quad 3$ ss $\quad 6$

Spiritus Vini Rectificati ad - - - $3 \mathrm{j} \quad 82$

Shampoo lotion.

Lotio Resorcin.

\begin{tabular}{llllllll|r} 
Resorcin & - & - & - & - & - & - & gr. xx & 4 \\
Glycerini & - & - & - & - & - & - & $\prod_{x}$ & 2 \\
Eau de Cologne & - & - & - & - & - & $3 \mathrm{ii}$ & 24 \\
Aquam ad - & - & - & - & - & - & $3 \mathrm{i}$ & 70
\end{tabular}

Useful in mild seborrhoea.

Must not be used in white or grey hair.

Lotio Resorcin Co.

Resorcin

Olei Petrolei

Olei Citronis - $\quad$ - $\quad-\quad-\quad-\quad$ -

Spiritus Rectificati - $\quad-\quad-\quad-$

Aquam Dest. ad - $\quad$ - $\quad$ -

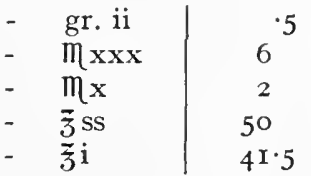

Useful in mild Seborrhoea capitis.

Lotio Saponis cum Ammoniae.

Liquor. Ammoniae - - - - - - $m l x v$

Saponis Viridis $\quad-\quad-\quad-\quad-\quad-\quad m_{x v}$

\begin{tabular}{lll|l} 
Spiritus Vini Rectificati ad - & - & 亏i & 88
\end{tabular}

Dry shampoo.

Lotio Sodii Bicarbonatis.

Sodii Bicarbonatis -

Boracis - -

Acidi Hydrocyanici Dil. - _ _ $\quad-\quad-g r$.

Aquam ad - - $\quad$ - $\quad$ - $\bar{\jmath} \mathrm{i}$

Sedative and antipruritic.

Lotio Sodii Hyposulphitis.

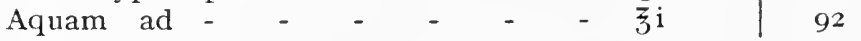

Useful in parasitic skin affections.

Lotio Sulphuris.

Sulphuris Praecipitati - $\quad$ - $\quad-\quad$ - gr. $\mathbf{x x} \mid 4$

Alcohol 90\% - - - - $\quad$ - $\quad$ - $m_{x v}$

Glycerini - $\quad-\quad-\quad-\quad-\quad-m_{x}$

$\begin{array}{llllllll}\text { Liquor. Calcis } & - & - & - & - & - & 5 \mathrm{ii} & 24\end{array}$

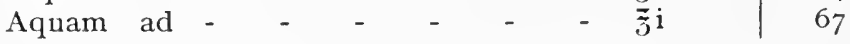

Antiseptic: useful in Acne vulgaris and rosacea. 
Lotio Sulphuris Co.

\begin{tabular}{llllll|r} 
Sulphuris Praecipitati & & & - & - & gr. $x$ & 2 \\
Zinci Sulphatis - & - & - & - & - & gr. $x$ & 2 \\
Potassae Sulphuratae & - & - & - & - & gr. $x$ & 2 \\
Aquam ad - - & - & - & - & - & Ji & 94
\end{tabular}

Antiseptic: useful in seborrhoic affections, Acne vulgaris, and rosacea.

Lotio Tannici Co.

Acidi Tannici -

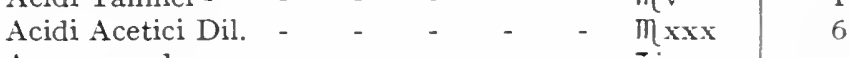

$\begin{array}{llllllll}\text { Aquam ad } & - & - & - & - & - & 5 & \end{array}$

Astringent: useful in hyperidrosis.

Lotio Zinci Detergens.

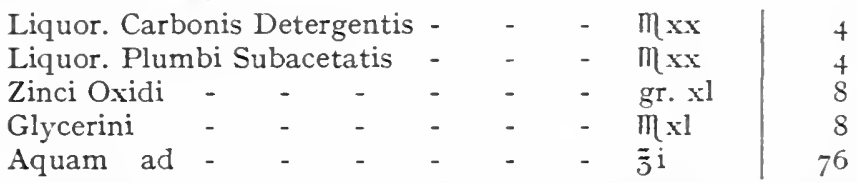

Sedative: useful in papular eczema, Lichen planus, etc.

Lotio Zinci Sulphatis ("Red lotion").

Zinci Sulphatis - $\quad$ - $\quad$ -

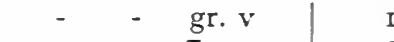

Aquam Camphorae ad $\quad-\quad-\frac{3}{3} i$

Astringent.

\section{PASTES}

Pasta Zinci.

\begin{tabular}{|c|c|c|c|c|c|c|c|}
\hline Zinci Oxidi & - & - & - & - & - & - & \\
\hline Talc - & - & - & - & - & - & - & partes \\
\hline Adipis Lanae & $-\quad-$ & - & - & - & - & - & aequales. \\
\hline Paraffini Moll & is Albi & - & - & - & - & - & \\
\hline
\end{tabular}

Sedative and astringent: useful base.

Generally add salicylic acid $2 \%$.

\section{PLASTERS}

Plaster mulls are used for prolonged application of drugs and may be had containing carbolic acid, salicylic acid, creosote, chrysarobin, mercury, ichthyol, etc., in different strengths.

\section{POWDERS}

Pulvis Acidi Borici Co.

\begin{tabular}{|c|c|c|c|c|c|c|c|c|}
\hline Acidi Borici & - & - & - & - & - & - & - & - \\
\hline Zinci Oxidi & - & - & - & - & - & - & - & - \\
\hline Pulv. Amyli & - & - & - & - & - & - & - & - \\
\hline
\end{tabular}

Pulvis Acidi Salicylici cum Zinci Oxidi.

Acidi Salicylici

Zinci Oxidi

Antiseptic. 
Pulvis Acidi Tannici cum Iodoformi.

Acidi Tannici

Iodoformi aa. partes aequales.

Astringent and antiseptic.

Pulvis Calomel Co.

Calomel -

Zinci Oxidi

Pulv. Amyli -

Antiseptic.

Pulvis Camphorae Co.

Camphorae - $\quad-\quad-\quad-\quad-\quad-\quad-\quad-\quad$ I

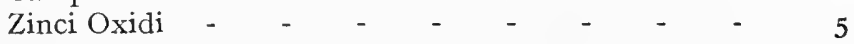

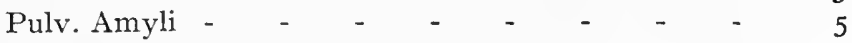

Sedative.

Pulvis Sulphuris cum Zinci Oxidi.

Sulphuris Sublimati

Zinci Oxidi aa. partes aequales.

Useful for scabies.

\section{SOAPS}

Sapo Liquidus.

Saponis Mollis

Spiritus Vini Rectificati -

Olei Lavandulae

Thymol vel

Wet shampoo.

Sapo ${ }_{\text {* Picis. }}$

Liquor. Picis Carbonis

Saponis Viridis aa. partes aequales.

For psoriasis.

\section{OINTMENTS}

Ungt. Acidi Carbolici Co.

\begin{tabular}{lllllll|r} 
Acidi Carbolici & - & - & - & - & - & gr. xl & 8 \\
Olei Olivae - & - & - & - & - & - & m $1 \times x x$ & I 6 \\
Cerae Albae & - & - & - & - & - & gr. xc & I 8 \\
Sulphuris Sublimati & - & - & - & - & Jii & 24 \\
Ungt. Hydrarg. Nitratis ad & - & - & - & Ji & 34 \\
For ringworm of the scalp.
\end{tabular}

Ungt. Acidi Salicylici cum Acidi Benzoici.

Acidi Salicylici - $\quad$ - $\quad-\quad$ -

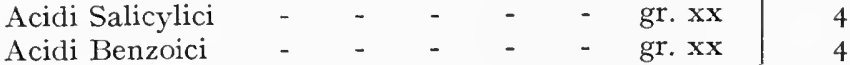

\begin{tabular}{lllll|l} 
Paraffinum Nolle ad & - & - & - & - & 5
\end{tabular}

For ringworm of the glabrous skin.

Ungt. Balsami Peruviani.

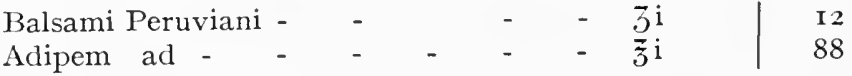

For scabies, etc. 
Ungt. Beta-naphthol.

\begin{tabular}{lllllll|r} 
Beta-naphthol & - & - & - & - & - & gr. $x l$ & 8 \\
Saponis Viridis & - & - & - & - & - & $3 \mathrm{ii}$ & 24 \\
Cretae Praecipitatae & - & - & - & - & gr. $\mathrm{xxx}$ & 6 \\
Adipem ad - & - & - & - & - & $3 \mathrm{i}$ & 62 \\
$\quad \begin{array}{l}\text { Antiparasitic. } \\
\end{array}$
\end{tabular}

Ungt. Betulae Co.

\begin{tabular}{llllllll|r} 
Olei Cadini & - & - & - & - & - & - & $3 \mathrm{iss}$ & $20^{\circ}$ \\
Resorcin & - & - & - & - & - & - & gr. $\mathrm{x}$ & 2 \\
Ichthyol & - & - & - & - & - & - & $\mathrm{gr} x$ & 2 \\
Olei Betulae & - & - & - & - & - & - & $\prod_{\mathrm{x}}$ & 2 \\
Adipern Benzoatum & ad & - & - & - & $\overline{3} \mathrm{i}$ & 74
\end{tabular}

For psoriasis.

\section{Ungt. Carbolici.cum Salicylici.}

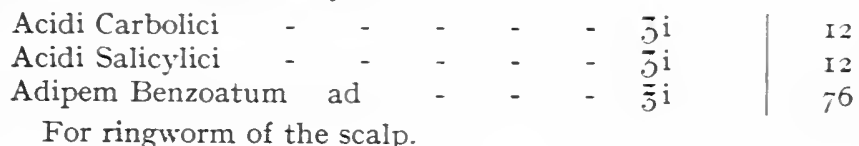

\section{Ungt. Chrysarobini.}

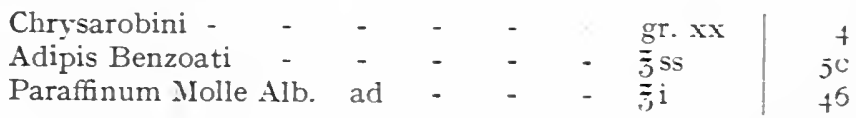

For psoriasis.

Ungt. Diachyli.

$\begin{array}{llllllll}\text { Emplastrum Plumbi } & - & - & - & - & - & - & \text { I } \\ \text { Paraffini Mlollis - } & - & - & - & - & - & - & 2\end{array}$

Sedative: to be spread thickly on lint and applied continuously.

Ungt. Hydrargyri Ammoniati Dilutum.

\begin{tabular}{llllll|r} 
Hydrargyri Ammoniati - & - & - & - & gr. $\mathrm{xv}$ & 3 \\
Paraffinum Molle ad & - & - & - & $\frac{3}{\mathrm{i}}$ & 97
\end{tabular}

Useful antiseptic.

Ungt. Hydrargyri cum Picis.

Hydrargyri Ammoniati -

Liquor. Picis Carbonis

Adipem Benzoatum ad

For psoriasis. 2 6 
Ungt. Oleatorum.

Ungt. Zinci Oleati

Ungt. Hydrargyri Oleati

Ungt. Diachyli aa. partes aequales.

For eczema.

Ungt. Picis Carbonis.

\begin{tabular}{llllll|l} 
Liquor. Picis Carbonis & - & - & - & - & $3 \mathrm{ii}$ & 24
\end{tabular}

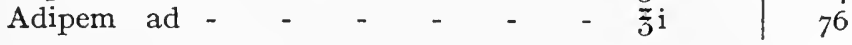

For psoriasis.

Ungt. Picis cum Acidi Salicylici.

Liquor. Picis Carbonis - $\quad$ - $\quad$ - $m_{x x x}$

Acidi Salicylici - $\quad-\quad$ - $\quad$ - $\quad$ gr. xv 3

Hydrargyri Ammoniati - $\quad$ - $\quad$ - $\quad$ gr. v

Paraffinum Molle Alb. ad - - - $\mathrm{j}^{\mathrm{i}} 90$

For psoriasis.

Ungt. Plumbi cum Calaminae.

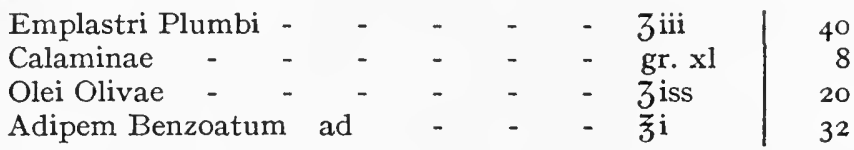

For acute inflammatory conditions.

Ungt. Resorcin cum Zinci.

Resorcin

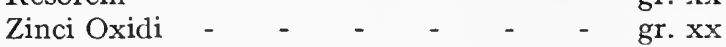

Amyli - - - - $\quad-\quad$ - $\quad$ - gr. $x x$

\begin{tabular}{lllll|l} 
Paraffinum Molle Alb. ad & - & - & & &
\end{tabular}

For seborrhoic dermatitis, ichthyosis, etc.

Ungt. Salicylici.

Acidi Salicylici

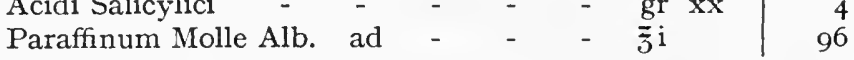

Keratolytic.

Ungt. Salis.

Sodii Chloridi.

Paraffini Mollis Alb. aa. partes aequales.

For ringworm of the scalp.

Ungt. Sulphuris.

Adipem ad -

For scabies.

Ungt. Sulphuris cum Acidi Salicylici.

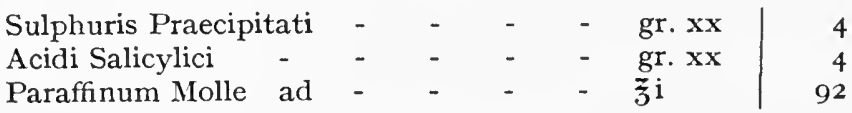

For seborrhoic conditions and pityriasis. 
Ungt. Zinci cum Bismuth.

\begin{tabular}{lllllll|r} 
Zinci Oxidi - & - & - & - & - & - & gr. xx & 4 \\
Bismuthi Oxidi & - & - & - & - & - & gr. xl & 8 \\
Paraffinum Molle & ad & - & - & - & - & 亏i & 88 \\
Protective. & & & & & & &
\end{tabular}

Ungt. Zinci cum Ichthyol.

\begin{tabular}{lllllll|l} 
Ichthyol & - & - & - & - & - & $\overline{3} \mathrm{iii}$ & 36 \\
Zinci Oxidi & - & - & - & - & - & $\overline{3}$ & 12 \\
Paraffinum Molle & ad & - & - & - & - & $\overline{3} \mathrm{i}$ & 52
\end{tabular}

For eczema.

Ungt. Zinci cum Picis.

\begin{tabular}{llllll|r} 
Zinci Oxidi - & - & - & - & - & $\bar{j}$ & I \\
Liquor. Picis Carbonis & - & - & - & - & $\prod_{x}$ & 2 \\
Ungt. Aquae Rosae ad & - & - & - & $\overline{3} \mathrm{i}$ & 86 \\
Soothing ointment in eczema.
\end{tabular}

\section{VARNISH BASES}

Tragacanth Base

\begin{tabular}{llllllll|r} 
Tragacanthi & - & - & - & - & - & - & gr. $\mathrm{xxx}$ & 6 \\
Glycerini & - & - & - & - & - & - & $\prod_{x}$ & 2 \\
Aquam ad & - & - & - & - & - & - & $\tilde{J}^{\mathrm{i}}$ & 92
\end{tabular}

Traumaticin Base.

\begin{tabular}{lllllll|l} 
Guttaperchae - & - & - & - & - & - & $\bar{j}$ & I2 \\
Chloroformum ad & - & - & - & - & $\overline{5} \mathrm{i}$ & 88
\end{tabular}

Useful bases, to which may be added salicylic acid, ichthyol, sulphur, zinc oxide, chrysarobin, etc.

To be painted on and allowed to dry. 


\section{INDEX}

Abrasion (see Excoriation), 34.

Abscesses, multiple cutaneous, in infants, 348 .

- diagnosis of, 348 .

- prognosis of, 348 .

- treatment of, 349 .

Absorption by the skin, 22.

Acantholysis bullosa (see Epidermolysis bullosa), 207.

Acanthosis, 40.

- daughter-cells in, 40 .

- etiology of, 4 I.

- mother-cells in, 40.

- nigricans, 1 Ioo.

- and cancer, I IOI.

- diagnosis of, $\mathrm{IIO}_{2}$.

- etiology of, I IOz.

- histopathology of, I IOI.

- treatment of, I IO2.

Acarus folliculorum, 6 I I .

- scabiei, 603.

Achorion gypseum (Bodin), 426 .

- quinckeanum (Bodin), 426.

- Schönleini, 423.

- cultivation of, 425 .

Achromia (see Leucoderma), 953.

- congenital (see Albinism), 956.

Acid, benzoic, I 2 I .

- boric, I 21 .

- carbolic, I22.

- dermatitis from, 275 .

- salicylic, 124.

- tannic, 124 .

Acne adolescentium, Io33.

- agminata (Crocker), 490.

- atrophica, 1032.

- bacillus, I 036 .

- cachecticorum, I033.

- cheloid, 46, 334 .

- disseminata, ro3I.

- erythematosa (see Rosacea), 8 ro.

- frontalis seu varioliformis, I044.

- hypertrophic, of the nose, 8 I I.

- indurata, 1032 .

- necrotica, 46I, IO44.

- diagnosis of, $\mathrm{IO}_{4} 6$.

- etiology of, $\mathrm{IO}_{4} 6$.

- histopathology of, I045.

- -treatment of, 1046.

- punctata, ro32.
Acne pustulosa, 1032.

- scrofulosorum, 46, 490 .

- tar, 277.

- urticata (see Prurigo), 705.

- varioliformis (see Acne necrotica), I044.

- vulgaris, Io3 I.

- - after-treatment of, 1042 .

- bacteriology of, 1036 .

- course of, I033.

- diagnosis of, 1036 .

_- etiology of, I033.

- histopathology of, 1033.

-. prognosis of, 1037 .

- - treatment of, I037.

- - general, 1037 .

- - internal, $\mathrm{IO}_{42}$.

- - local, I $_{3} 8$.

- - vaccine, I044.

- - X-ray, $\mathrm{IO}_{42} 2$.

Acnitis (Barthélemy) (see Papulonecrotic tuberculides), 490.

Acro-dermatitis chronica atrophicans, 962.

Actinic rays, effect of, on skin, 245 .

Actinomyces bovis, 43r.

Actinomycosis cutis, 429 .

- diagnosis of, 432 .

- fungi of, 43 I.

- - Actinomyces bovis (Ray fungus), 43 I.

- Nocardia convoluta, 433.

- - - thibiergei, 433.

__ - Streptothrix asteroides, 433.

- - - hominis, 433 .

- - - israeli, 432 .

prognosis of, 432 .

sclerotia of, 429 .

- treatment of, 432 .

- - general, 432 .

- local, 432 .

Adamson, H. G. Dermatitis artefacta, 289.

- Favus, mouse, 423.

- Immunity production in ringworm, $3^{8} 3$.

- Simple erythemas of napkin-region, $65^{8}$.

- Sporotrichosis cutis, 433 .

- X-ray exposure of scalp for ringworm, 407 . 
Addison's disease, bronzing of skin in, 69.

Adeno-cystoma intracanaliculare (Elliot), I 123.

Adenoma of sebaceous glands, I I 23.

- of sweat-glands, I I 2O, I I 22.

- sebaceum, I I 24 .

- - diagnosis of, I 125 .

- histopathology of, I I 25.

- treatment of, I I 25.

Ainhum, I 194.

- distribution of, I 194 .

- etiology of, I 195.

- histopathology of, I I95.

- treatment of, II95.

Aix-la-Chapelle, inunction treatment of syphilis at, 584 .

Albinism, 956 .

Alcohol and skin affections, Io6.

Aldersmith, $H$. Croton oil treatment of ringworm, 4 I 2 .

Alkalies, dermatitis from, 275.

Alkaloids, dermatitis from, 275 .

Alopecia, 45, 965.

- acquired, 967 .

- - from chemical irritants, 972.

- from nervous disturbances, 78 , 969 .

- - from parasites, 972 .

- from traumatism, $97 \mathrm{I}$.

- from X-ráys, 972.

- - in general diseases, 968 .

- - in skin affections, $97 \mathrm{I}$.

- - premature hereditary, 96\%.

- - senile, 968 .

- - treatment of, 968, 970, 97I.

- areata, 45, 981.

- contagiosity of, $98 \%$.

-..- description of, $98 \mathrm{I}$.

- - diagnosis of, 990 .

- - etiology of, 985 .

- - actual cause, 986.

- - microbic theory, 986.

- - - toxic theory, 989 .

- _ - tropho-neurotic theory, 988.

- - predisposing causes, $9 \$_{j}$.

- histopathology of, $98_{4}$.

- nail-changes in, $9 S_{4}$.

- - prognosis of, 990.

- - relation to ringworm, 400, 987.

- - transmissibility of, 986.

- treatment of, $99 \mathrm{I}$.

- - internal, 991 .

-.. - local, 992.

- - - by actinic rays, 995 ; antiseptics, 992; high frequency, 995 ; massage, 995 ; stimulation, 993.

- - varieties of, 983 .

- - central, $98_{3}$.

- - - generalised, 983 .

- - marginal, $98_{3}$.

- - punctate, $9 \$_{4}$.

_- with Pityriasis capitis, 934 .

- cicatrisata, 996.

- - diagnosis of, 998 .
Alopecia, cicatr'sata, etiology of, 998.

- - histopathology of, 997.

- prognosis of, 998 .

- treatment of, 998 .

- circumscripta, $9 S \mathbf{I}$.

- congenital, 965 .

- - prognosis of, 966 .

- treatment of, 966.

- furfuracea, 974 .

- pityrodes, 352, 974.

- seborrhoic, 352,974.

- - etiology of, 976 .

- - in Pityriasis capitis, 975.

- - in Seborrhoea oleosa, 975.

- - in Seborrhoic dermatitis, 975.

- prognosis of, 977 .

- - treatment of, $97 \%$.

- circinata (Crocker), $9 s_{4}$.

Alum, use of, I2I.

Analgesics, treatment bỹ, I I 3 .

Anaphylaxis, 70 .

Anatomy, general, of the skin, I.

Ancylostomiasis, cutaneous, I 196.

Anetodermia, 959.

- erythematodes (Jadassohn), $96 \mathrm{I}$.

Angio-keratoma, 220.

- diagnosis of, $22 \mathrm{I}$.

- etiology of, 221 .

- histopathology of, $22 \mathrm{I}$.

- treatment of, 222 .

Angioma, 65 .

- cavernous, 65 , ISo.

- fissural, I74.

- infective, 1,8 .

- pigmentosum atrophicum, 252 .

- senile, $9+3$.

- serpiginosum, $1-3$.

Angiomes verruqueux, $I>6$.

Angio-neurotic oedema, 693.

Anidrosis, 1050 .

Aniline dyes, dermatitis from, $2-6$.

Anonychia, 5I, 1066.

Anopheles maculipennis, 630 .

Antharobin (see Chrysarobin), I 22.

Anthrax, benigna (see Carbuncle), 342 .

Antimony, dermatitis from, $2,6,7-13$.

- treatment by, Iog.

Anti-pruritics, is 9.

Antipyrin, eruptions from, - I 3 .

Antiseptics, I 9 .

Ants, bites from, 631 .

Appendix, 1263.

Area celsi (see Alopecia areata), 98I.

Argyria, 70, -21.

Arnica, dermatitis from, 274 .

Arrector pili, I 8 .

Arsacetin (see Arylarsonates), I I I.

Arsenic, action of, Iog.

- contra-indications to, 109.

- dermatitis from, $276,7-13$.

- - acute, 7 I 3 .

- chronic, $7 \mathrm{I}_{4}$.

- - treatment of, 7 I 7 .

- desquamation from, $7 \mathrm{r} 7$.

- epithelioma from, - I4.

- erythema from, 7 If. 
Arsenic, Herpes zoster from, 7 I 7.

- hyperidrosis from, 717 .

- indications for, rog.

- keratosis from, 714.

- nail-disease from, 717 .

- pigmentation from, III, 716 .

- preparations of, I 09, 575 .

Arseno-benzol in syphilis, 577.

Arylarsonates, II I.

- in syphilis, 575 .

Asiatic pill, I IO.

Astringents, I 20.

Atheroma (see Wen), $4^{8}$, I 123.

Atoxyl (see Arylarsonates), III.

Atrophodermia biotriptica, 96I.

- neuritica, 960 .

- pigmentosa, 252 .

- reticulata symmetrica faciei (Pernet), I026.

Atrophy of skin, 959.

- congenital, 966.

- from general illness, 959.

- from interference with nerve supply, 960 .

- from pressure, 960 .

- in skin diseases, 960 .

- primary, 96r.

- - diffuse, 962 .

- macular, 96I.

- senile, 96I.

Aurantia, dermatitis from, 276.

Autographism, 682.

Auto-intoxication in skin diseases, I08.

Bacillus, acne, 83 .

- coli communis, 84 .

- diphtheriae, 83 .

- flask (Unna), 350.

- leprae (Hansen), 83

- oedematis maligni, 84 .

- of Matzenauer, 83 .

- perfringens, 84 .

- pyocyaneus, 83 .

- sporogenes, 84 .

Bacteriology of skin, 80 .

Baldness (see Alopecia), 965.

Balloon-cells, 30 .

- in Herpes zoster, 906.

Balsam of Peru, use of, I2 I.

Barbadoes leg (see Elephantiasis), I 20I.

Barbers' dermatitis, 283.

- itch, 4I 4.

Barcoo rot (see Veld Sore), I 256.

Barley itch, 275.

Basal layer (see Stratum germinativum), 2.

Basophilic collagen, 59 .

Bassorin varnish, 137 .

Bateman, Ecthyma, 312.

Baths, I25.

- electrical, 146.

- electrolytic effects in, 128.

- emollient, 127.

- medicated, I 26.

Bayon, H. Lepra bacillus, cultivation of, 525 .
Bazin's disease (see Erythema induratum), 482 .

Beatty, Wallace. Leiomyoma, II 39.

Bed-bug, 623.

- sores, 287 .

- - treatment of, 288.

Belladonna eruptions, 718.

Beta-naphthol, use of, I22.

Birth-marks (see Naevi), 40, 65, I 76 .

Biskra button, 1250.

Blackheads (see Comedones), roz6.

Blastomycetic dermatitis (see Blastomycosis), 366 .

Blastomycosis, 366 .

- cutaneous, 367 .

- diagnosis of, 372 .

- etiology of, 37I.

- mycology of, 370 .

- prognosis of, 372 .

- systemic, 368 .

- treatment of, 372 .

Blister (see Bulla), 3 I.

— beetle, 63I.

- fever (see Pemphigus acutus), 870.

Bloch and Massini. Immunity production in favus, 428 .

- Inoculation experiments in ringworm, 382 .

Blood, pathological changes in, 62 .

- vessels of corium, 16.

- - of subcutaneous tissue, $x 6$.

- - pathological changes in, 63 .

Bockhart's impetigo, 327.

Bodin, E. Favus fungi, 425.

Boeck, C. Leprosy, 507.

Boil, 336.

- Aleppo (see Oriental sore), I 250.

- Delhi (see Oriental sore), I250.

- description of, 336 .

— diagnosis of, 338 .

— etiology of, 338 .

- histopathology of, 337 .

- oriental, 1250.

- prognosis of, 338 .

- treatment of, 339.

- - general, 339 .

- - by ceredin, 339; manganese, collosol, 340 ; nuclein, 339 ; stannoxyl, 340 ; sulphur, 339 ; tin oxide, 340 ; yeast. 339 .

- local, 340 .

- - - by carbolic injections, 34I ; cupping glasses (Bier's), 34I ; fomentations, 34I ; iodine, 34I ; iodoform, 34I ; plasters, $34 \mathrm{I} ; \mathrm{X}$-rays, $34^{2}$.

- - vaccine, 340 .

Botryomycosis hominis (see Granuloma pyogenicum), 346 .

Boubas (see Framboesia tropica), I208.

Bristle (see Hair), 5.

Brom-acne, 7 I 8.

Bromide eruptions, 718 .

Bromidrosis, 1050.

— etiology of, I05I. 
Bromidrosis, treatment of, $105 \mathrm{I}$.

Brooke, H. G. Epithelioma adenoides cysticum, I I 20 .

Browning and Watson. Intraspinal injections of salvarsanised serum, 590.

Buboes, suppurating, 533.

Bug, bed-, 623 .

- harrest, 612 .

- treatment of bites from, 6i3, 624.

Bullae, definition and characters of, 3 I.

- in Pemphigus foliaceus, 31 .

- in Pemphigus vegetans, 3 I.

Bunion, I ogo.

- treatment of, IO9I.

Burns, from acids, 2,6 .

- from corrosives, 275 .

- from electricity., 257 .

- - treatment, 258 .

- from heat, 229.

- classification of, 229 .

- degrees of, 230.

- description of, 230 .

- diagnosis of, 233 .

- - general effects of, 232 .

- histopathology of, 232.

- prognosis of, 233 .

- - scarring from, 237 .

- - prevention of, 237 .

- treatment of, $23 \%$.

treatment of, 233 .

- - general, 233 .

- - local, 234 .

- - special, 234 .

- - - moist dressings, 236 .

- - open method, 235 .

- - - paraffin method, 236 .

- from lightning, 259 .

- from radium (see Radium dermatitis), $26 \%$.

- from X-rays (see X-ray dermatitis), 260 .

Burow's lotion, I 21 .

Button scurvy (see Framboesia tropica), I 208 .

Cacodylic acid, I I I .

Cacotrophia folliculorum, 1,2, ror 4 .

Calabar swellings, I I9\%.

- etiology of, I 197.

- Filaria loa and, 1197.

- treatment of, I I 98 .

Calcareous deposits in the skin, I I9I.

- etiology of, I 192.

- treatment of, I I92.

Calcium carbide, dermatitis from, 27\%

Callosity, Iogi.

- treatment of, 1092.

Callus (see Callosity), I ogI.

Calmette's reaction in tuberculosis, $44^{8}$.

Calvities, 352, 974 .

Cancer (see Carcinoma cutis), I 152 .

- en cuirasse, II 54 .

- Kangri, 232.

Canities, 1002.

- etiology of, 1004 .
Canities, pathology of, I003.

- treatment of, $100_{4}$.

Cantharides, dermatitis from, 272 .

Caraté (see Pinta), I $2+1$.

Carbon dioxide (solid), length of exposure, I 4 I.

- mode of application, I fo.

- treatment by, I 40 .

Carbuncle, 342 .

- diagnosis of, $3+4$.

- etiology of, 343 .

- histopatholog! of, 343 .

- prognosis of, 344 .

- treatment of, 344

- general, $3+4$.

- local, 345 .

- - vaccine, 345 .

Carcinoma cutis, I I 52 .

- etiology of, I 155 .

- histopatholog: of, 1 I 54 .

- naevo-, 1 i $5 \%$.

- squamous, primary, i I 52.

- secondary, I I 54 .

- skin-affections predisposing to, I 153.

- treatment of, I I 56 .

Carcinomatosis, I I 54 .

Carpenters' dermatitis, 283 .

Castellani, A. Dermatomýcoses, 365.

- Epidermophyton cruris, 393, 395 .

- - rubrum, 393.

- Treponema pertenue. I 209.

Cataphoresis (see Ionisation), I 44 .

Cataplasms, I $26 \%$.

Caterpillars, dermatitis from, $272,63 I$.

Caustics, 120 .

- burns from, 2-6.

Cauterisation, $\mathrm{I}+3$.

- by hot air, I+3.

Cedercreutz, polymorphic coccus of, $\$ 2$.

Cell, connective tissue, I 3 .

- fixed, It.

- mast, It.

- migratory, I 2.

- multi-nuclear, 55 .

- rests, 44 .

- vacuolated, I 3, 54.

Centipedes, bites from, 6 I 5 .

Ceredin (see least), I I 2.

Ceruminous glands, 8 .

Chalmers, A. J. Classification of Dermatomycoses, 365 .

- Maduromycosis, 122\%.

- Trichophyton currii, 3 so.

Chancre, hard (see Syphilis), 532.

- soft (see Sore, soft), 535 .

Chancroid, 534 .

Chapped hands, 795 .

Cheilitis exfoliativa, Ioso.

- glandularis aposteomatosa, IoSI.

Cheiro-pompholyx, soj.

Cheloid, 35, 60, I I 29.

- description of, 1129.

- diagnosis of, I I 32 .

- etiology of, I I 3 I.

- false, I 129.

- histopathology of, I I 3 I. 
Cheloid, idiopathic, I 129.

- scar, I 29.

- treatment of, I 132

- true, I I 29.

Chemists' dermatitis, 283 .

Chemotaxis, positive, 53 .

Chigoe, I 244.

Chignon fungus (see Trichosporosis), 437.

Chilblain, 2 I 4 .

- diagnosis of, 217.

- etiology of, 216.

- histopathology of, 2 I 6.

-lupus, 456, 9 I3.

- necrosing, 2 I 5 .

- prognosis of, 217.

- suppurating, 215 .

- treatment of, 217 .

- - general, 220 .

- local, 2 I 7.

Chimney sweeps' cancer, 282.

Chloasma traumaticum, 950.

Chlor-acne, 277 .

Chloral eruptions, 718 .

Chloretone eruptions, 7 I 9.

Chlorine, dermatitis from, 277 .

Chloresterol, 2 I.

Chorioplaques, $55,5^{8}$.

Chromatophores, I9.

Chrome ulcer, 277.

Chromic acid, dermatitis from, 277.

Chromidrosis, $105 \mathrm{I}$.

- etiology of, 1052.

- pseudo-, I052.

- treatment of, 1053 .

Chrysarobin, 122.

- dermatitis from, 277.

- disadvantages of, 122.

Cicatrix (see Scar), 35.

Cilia (see Hair), 5.

Cimex lenticularius, $6 \geq 3$.

Cinchona bark, dermatitis from, 275 .

Classification of skin diseases, 159.

Clavus (see Corn), ro89.

Climate and skin affections, $\mathrm{IO}_{4}$.

Clothing and skin affections, 104.

Cnidosis (see Urticaria), 679.

Coal tar, dermatitis from, 277 .

- derivatives of, 277 .

Coccus, butyricus, 325 .

- grey, $35^{\circ}$.

Coco (see Framboesia tropica), 1208.

Cocoons (see Sebum), 2 I .

Coil-glands (see Sweat-glands), 9.

Cold, creams, 131 .

- effect of, on skin, 2 I I .

- pathological changes due to, 2 I 2 .

Collagen, 14.

- pathological changes in, 59 .

Collar of Biett (see Syphilide, papular), 545 .

_- of Venus (see Syphilide, pigmentary), 545.

Collastin, 6o.

Colle's law in congenital syphilis, $59 \mathrm{r}$.

Collodions, 1267 .
Colloid milium (Wagner), I I 3.5.

- histopathology of, I 136.

- treatment of, i I 36 .

Colour of the skin, 19.

Columnae adiposae, 15.

Comedones, 1026.

- etiology of, 1027.

- grouped, of infants, I029.

- histopathology of, 1027 .

- pitting from, 1026 .

- treatment of, 1028 .

Condiments and skin affections, to6

Condyloma acuminatum, 4r, 1097.

-C description of, I097.

Cones, arterial, 64.

Connective-tissue cells, I 3 .

Constipation and skin diseases, I07.

Copaiba eruption, 7 I 9 .

Copra-itch mite, 6i 3 .

Corbett's radiometer, $4_{4} 8$.

Cordylobia anthropophaga, 627 .

Corium, cellular elements of, I 2

- fibrous elements of, I 4 .

- pathological changes in, 52 .

- structure of, I 2.

Corn, ro89.

- hard, 1089 .

- histopathology of, Iogo.

- soft, Io89.

- treatment of, rogo.

Cornification, anomalous, 39.

- diminished, 40.

- imperfect, 39 .

- of hair, 7 .

- process of, 5 .

- theories of, 5 .

Corona seborrhoica, 353 .

- Veneris, $54^{6}$.

Cortex of hair (see Hair), 5 .

Coster's paste, in ringworm, 4 I 4 .

Counter-irritation, treatment by, I 39

Couperose (see Rosacea), 8 io.

Cracks (see Fissure), 34.

Craw-craw, I 198.

Creams, 131, 1267.

Creeping eruption, 628 .

Crocker, Radcliffe. Angioma serpiginosum, i 78 .

Croton oil, dermatitis from, 274 .

Crust, definition and characters of, 33 .

Crusta lactea, 356 .

Crystallina (see Sudamina), 243.

Cuticle of hair (see Hair), 5 .

Cuti-reaction, in favus, 428 .

- in leprosy, 517 .

- in ringworm, 383 .

- in syphilis, 53I.

Cutis anserina (see Goose-skin), I62.

- vera (see Corium), I 2.

Cyanidrosis, 1052.

Cyanosis, 64 .

Cylindroma, I 20.

Daffodils, dermatitis from, 273 .

Dandruff (see Pityriasis capitis), 351.

Danielssen. Leprosy, 507 . 
Darier's disease (see Psorospermosis follicularis vegetans), I I 3 .

- toxi-tuberculides, 486 .

Dartos muscle, is.

Dartre volante, 3 Io.

Daughter-cells of epidermis, 2 .

De Beurmann and Gougerot. Sporotrichosis, 433 .

Delhi boil, I 250 .

Demodex folliculorum, 21, 6 I I.

- lesions from, 6i I.

- treatment of, 612.

Dermanyssus avium, 613 .

Dermatite polymorphe douloureuse (Brocq), 886.

Dermatitis, artefacta, 288 .

- causal agents of, 291 .

- characteristics of, 290.

- diagnosis of, 292.

- - treatment of, 293.

- atrophicans (Kaposi), 962 .

- autotypers', 283 .

- bakers', 283 .

- barbers', 283.

- beetle-, 631.

- blastomycetic (see Blastomycosis), 366.

- bronzers', 283 .

- builders', 283.

— burnishers', 283.

- carpenters', 283.

- caterpillar-, 631 .

- caused by, external irritants, 272 .

- - animal, 272 .

- - mineral, 275 .

- - vegetable, 272 .

- chemists", 283 .

- chimney-sweeps', 283 .

- coal-tar workers', 284.

- coccidioides (see Blastomycosis), 366.

- compositors', 283 .

- congelationis (see Frost-bite), 2 I 3.

- cooks', $28+$.

- cotton-seed, 6ij.

- cotton-winders', $2 S_{4}$.

- cupoliformis (Castellani), 1250.

- dyers', $2 S_{4}$.

- electroplaters", 284 .

- electrotypers', 284 .

- epidemic exfoliative, 309.

- - description of, 309.

- prognosis of, 310 .

- - treatment of, 310 .

- exfoliativa, 855 .

- - diagnosis of, 859 .

- etiology of, 859 .

- histopathology of, 859 .

- - infantum, 303 .

- - prognosis of, 860 .

- primary, 856 .

- - recurrent scarlatiniform, $s_{5} 6$.

- - secondary, $85 \%$.

- treatment of, 860 .

- factitia, 288 .

- fish-curers', $28+$.

- florists', $28_{4}$.
Dermatitis, fruiterers', $2 S_{4}$.

- furriers', 384 .

- gangraenosa, 291 .

- - infantum, 313

- gardeners', $28+$.

- generalised exfoliative, $S_{55}$.

- herpetiformis, $\$ 86$.

- - description of, 88\%.

- - diagnosis of, 893 .

-__ - from Pemphigus chronicus, 893.

eosinophilia in, 8yo.

etiology of, S9I.

- histopathology of, 80 I.

- - mucous membrane lesions in, \$89.

- pregnancy and, 892.

- - prognosis of, S94.

- - symptoms, subjective of, 890 .

- - treatment of, 894 .

_- - internal, 894 .

- - - by arsenic, 895 .

- - local, 895.

- infantile, 320 .

- malignant (see Paget's disease), I I 58 .

- medicamentosa (see Drug eruptions). Tro.

- micropapulosa erythematosa hyperidrotica nasi, ro5t.

- mill-workers', 285 .

- munition workers', 285.

- nurses', $2 S_{5}$.

- occupational, 268.

- painters', 285 .

- papillaris capillitii, 334 .

- paraffin workers', 285.

- pathology of, 52 .

- photographers', $2 S_{5}$.

- polishers', 285 .

- psoriasiformis nodularis (Jadassohn), $8_{4} 6$.

- radium, 26;.

— seborrhoic, 349 .

- bacteriology of, 350 .

- - diagnosis of, $35 \%$.

— - etiology of, 356 .

- histopathology of, 356 .

- - of face, 353 .

- - of limbs, 355 .

- - of nails, 355 .

- of nech, 35t

_ - of scalp (see Pityriasis capitis), $35 \mathrm{I}$.

- of trunk, 354 .

- - prognosis of, $35 \mathrm{~s}$.

- treatment of, $35 \mathrm{~s}$.

- - general, 358 .

—- local, 362 .

- silk-winders', $2 S_{5}$.

- soap makers', 235.

- solar, 79,248 .

- - acute, $2 f^{8}$.

- - histopathology of, $2+8$.

- - treatment of, $2+9$.

-. chronic, $2+9$.

- - treatment of, 249 . 
Dermatitis, surgeons', 285 .

- tanners', 285.

- vacciniform, 313 .

- varicose, 797.

- vegetans (see Pemphigus vegetans), 882 .

- venenata, 269 .

- - treatment of, 286 .

- washerwoman's, 285 .

- X-ray, 260 .

Dermatoconioses, $27 \mathrm{I}$.

Dermatobia cyaniventris, 627 .

- hominis, 626 .

Dermatolysis, I 129.

Dermatomycosis, $36_{5}$.

- classification of fungi, 365 .

- furfuracea (see Pityriasis versicolor), 440 .

- ulcerative (Castellani), r 256 .

Dermatophiliasis, 1244 .

- geographical distribution of, 1244 .

- treatment of, 12.46 .

Dermatophilus penetrans, 1244 .

Dermites simples infantiles (Jacquet), 320.

Dermographism, 682 .

- purpuric, 939.

Desert sore, 83,1258 .

- Klebs-Loeffler bacillus in, 1258 .

Detergents, 120.

Dhobie's itch (see Tinea cruris), 390.

Diabetes and skin disease, rog.

Diagnosis, general, 85,99 .

Diascope, 86.

Diathesis and skin disease, 76 .

Diday's law in congenital syphilis, 592.

Diet in skin diseases, 105.

Doffers' eruption, $28 \mathrm{x}, 285$.

Dominici and Barcat. Action of radium, 184 .

Dracontiasis, I 199.

Dracunculosis, r r 99.

- etiology of, r 200 .

- treatment of, 1200.

Drug eruptions, 7 ro.

- diagnosis of, 712 .

- etiology of, 7 I I.

- mode of production of, 7II.

- treatment of, $7 \times 3$.

- types of, 723 .

Dyers' dermatitis, 284 .

Dysidrosis (see Pompholyx), 805.

Dyskeratosis, 39.

- in Darier's disease, 39.

— in Molluscum contagiosum, 39.

Ecchymoses, 935.

Ecthyma, 303, 312.

- terebrans, 313 .

- tropical (Castellani), I 250.

Eczema, 753.

- clothing in, 775 .

- complications of, 757 .

- course of, 758

- crackled, 756 .

- description of, 753 .
Eczema, diagnosis of, $76_{5}$.

- diet in, 773 .

- erythematous, 754 .

- etiology of, $76 \mathrm{r}$.

- - actual cause, 76r.

- - - irritation theory, 762 .

- - neurotic theory, 76r.

$\longrightarrow$ parasitic theory, 763 .

- - toxic theory, 762 .

- - predisposing causes, $76 \mathbf{r}$.

— fissured, 756 .

- histopathology of, 758 .

— impetiginous, 756 .

- infantile, 799 .

- - description of, 799.

- - etiology of, 800 .

- - prognosis of, 8or.

- - treatment of, 8or.

- marginatum (seo Tinea cruris), 390.

- of anus, 791 .

- of arms, 792.

- of auricles, 785 .

- of axillae, 790 .

-_ of beard region, 784 .

— of breasts, 790 .

- of eyebrows, 785 .

- of eyelids, 785 .

- of face, $78 \mathrm{r}$.

- of feet, 798 .

- of genitalia, 790 .

- of hands, 793 .

— of legs, 795 .

— of lips, 783 .

- of mucous membranes, 798 .

- of nails, 798 .

- of neck, 797 .

- of nose, 784 .

- of scalp, 786 .

- of trunk, 788 .

-- papular, 754 .

- prognosis of, 764 .

- pustular, 756 .

- rubrum, 756 .

- seborrhoic, $350,353$.

- solare (see Solar dermatitis), 79.

- squamous, 756 .

- symptoms of, 757 .

—- general, 757 .

- - subjective, 757 .

- trade, 269.

- treatment of, 770 .

- - internal, $77 \mathrm{r}$.

- local, 776 .

- - of regional types, 780 .

- tuberculatum (see Mycosis fungoides), 926 .

- varicose, 796 .

- vesicular, 755.

- washing and baths in, 775 .

Eczematisation, 738 .

Elacin, 6o.

Elastic tissue, I 5 .

- tumour, II 34 .

Elastin, 15.

- pathological changes in, 60 .

Electrical baths, I 46 . 
Electrical treatment, I 4 .

— - by faradism, I 45 .

- by galvanism, I 45 .

Electrolysis, I 4 .

- in hypertrichosis, roos.

Eleidin, 4.

Elephantiasis Arabum, I 20 .

- - etiology of, I 206.

- - geographical distribution of, I 201.

- - of genitalia, I 203 .

- of leg, 1203 .

- - of scalp, 1203 .

- - pathogenesis of, I 204.

- - blockage by flaria theory, I 205 .

- - inflammatory theory, I 205.

- pathology of, I 203

- - treatment of, I 206.

- - by fibrolysin, I $20 \%$.

- - - by surgical methods, $120 \%$.

- glabra, I 202.

- Graecorum (see Leprosý), 496.

- Indica, I 201 .

- lymphangiectatica congenita, 323.

- nervorum (see Neuro-fibroma), i 126.

- nostras, $320,322$.

- etiology of, 323 .

- treatment of, 323 .

- pseudo-, and tuberculosis, $45 \%, 477$

- streptogenes, 323 .

- syphilitic, $55 \%$.

- telangiectodes, 323 .

- verrucosa, I 202.

Elephantoid fever, 323, I 202.

Endodermophyton concentricum (Blanchard), $124^{8}$.

- Indicum (Castellani), I $24^{8}$.

Endothelioma, I I 42 .

- haemangio-, $5 s$, I I 43 .

- lymphangio-, 58 , I I 43 .

Eosinophilia, 63.

- in Dermatitis herpetiformis, 390.

- in Pemphigus chronicus, $8 ; 5$.

Ephelis (see Freckle), 246 .

- ab igne, 656 .

Epidermis, appendages of, 5 .

- layers of, 2.

- thickness of, 2.

- pathological changes in, 38 .

Epidermite chronique à streptocoques (Sabouraud), 3 I I.

Epidermolysis bullosa, 20\%.

- diagnosis of, 209.

- etiology of, 209.

- histopathology of, 203.

- prognosis of, 209.

- treatment of, 209 .

Epidermophyton cruris (Castellani), 393.

- inguinale (Sabouraud), 393.

- purpureum (Bang), 393.

Epithelioma adenoides (Brooke), I I 20.

- benign cystic, I I 8 .

- contagiosum (see Molluscum contagiosum), I I I 2.
Epithelioma, from arsenic, - I 5 .

- maligna (see Carcinoma cutis), I I 52.

- molluscum (see Molluscum contagiosum), I I I 2 .

- patholog! of, +2 .

- pavement, I I 52 .

— sebaceum, rog9.

- squamous-celled. I 52 .

- tricho-, I I 20.

- histopathology of. I I 2 I.

- - treatment, I I 21 .

Epitheliomatosis, eczematoid (Besnier), I 15 S.

Epitrichial layer, persistence of, I62.

Eruptions, feigned (see Dermatitis artefacta), 288.

Erysipelas, 324 .

- ambulans, 316.

- and tuberculosis, $45 \%$.

- description of, 315 .

- diagnosis of, 317 .

- etiology of, 3 I 4 .

- histopatholog! of, 3 I $\%$.

- migrans, 3 I 6.

- perstans faciei, 320 .

- prognosis of, 3 I 3 .

- recurrent, 3 I 9.

- treatment of, 3 I 8 .

Erythema, 653 .

- ab igne, 229,650.

- anatomy and physiology of, 653 .

- annulare, 653 .

- atrophicans (Morris), 9 I I.

- bullosum, 663 .

- centrifugum (see Lupus erythematosus), 9 I 4 .

- circinatum, 663 .

- elevatum diutinum, 673.

- endemicum (see Pellagra), I230.

- generalised, 659.

- - diagnosis of, $66 \mathrm{I}$.

- - etiology of, 650 .

- histopathology of, 660 .

- prognosis of, $606 \mathrm{I}$.

- treatment of, 66r.

- giratum, 663.

- haemorrhagicum (see Purpura), 937.

- hydroa, 665 .

- induratum (Bazin), 4\$2.

- - diagnosis of, 454 .

- etiology of, $4^{5} 3$.

- histopathology of, $4 s_{3}$.

- relation to tuberculosis, 434 .

- - treatment of, 456 .

- iris, 665 .

- local, 659.

- - diagnosis of, $65 \mathrm{I}$.

- etiology of, 655 .

- - external causes of, $65 j$.

_- - internal causes of, $65 \mathrm{I}$.

- prognosis of, $65 \mathrm{~s}$.

- - treatment of, 653 .

Erythema multiforme 662 .

exudativum,

- - diagnosis of, 665

- etiology of, 66;. 
Erythema multiforme, histopathology of, 666 .

- mucous membrane lesions in, 666

- - prognosis of, 669 .

- - treatment of, 669.

- nodosum, 67I.

_- diagnosis of, $67 \mathrm{I}$.

-. etiology of, 671 .

- - histopathology of, 671 .

- prognosis of, $67 \mathbf{I}$.

- - treatment of, $67 \mathrm{I}$.

- of napkin-region in infants, 658 .

- papulatum, 663.

- paratrimma, 656 .

- pathology of, 63 .

- pernio (see Chilblain), 2 I 4 .

- perstans, 664 .

- faciei (see Lupus erythematosus), 9 I 4 .

- purpuricum, $66_{3}$.

- recurrent exfoliative scarlatiniform, 662.

- solare, 245 .

- uraemicum, 667 .

- urticatum, 663.

- vesiculosum, 663 .

Erythrasma, 439.

- diagnosis of, 439 .

- fungus of, $44^{\circ}$.

- Microsporon minutissimum, $44^{\circ}$.

- treatment of, 440 .

Erythrodermia, 653 .

- resistant scaly (Colcott Fox and Macleod), 844 .

Erythrodermie congenitale ichthyosiforme (Brocq), I 73 .

- pityriasiques en plaques disseminées (Brocq), 847.

Erythromelalgia, 653, 677 .

Eschar (see Burn), 229.

Espundia, I 255.

- geographical distribution of, I255.

- treatment of, 1255 .

Eugallol (see Pyrogallol), I 24.

Excoriation, 34.

Faddidite (see Ainhum), I I94.

Fat, cells, I 5 .

- columns, i 5 .

- origin of, 16.

- situations of, I 6.

Favic tarsus, 42.4.

Favin (see Immunity production in favus), 428

Favus, 42I.

- diagnosis of, 426 .

- distribution of, $42 \mathrm{I}$.

- etiology of, 426 .

- fungi of, 423 .

- Achorion gypseum (Bodin), 426.

_... quinckeanum (Bodin), 426.

_- Schönleini, 423.

- - cultivation of, 425 .

- Lophophyton gallinae (Mégnin), 426.
Favus, fungi of, Oospora canina (Constantin and Sabrazés), 426.

- herpeticus, 423 .

- histopathology of, 424 .

- immunity production in, 428 .

- in lower animals, 426 .

- mouse, 425 .

- of glabrous skin, 422 .

-.. of nails, 423 .

- of scalp, $42 \mathbf{I}$.

- prognosis of, 427 .

- scutulum, $42 \mathbf{I}$.

- structure of, 424 .

- treatment of, 427 .

- - general, 428 .

- local, 427 .

- - by parasiticides, 427 .

- - by X-rays, 427 .

Fibres, elastic, 14.

- - situations of, I 5 .

- - structure of, 15.

Fibroblasts, 53 .

_ in sarcoma, 59.

Fibrolysin, treatment by, I I 3 .

Fibroma, 58, 60, I 126.

- lipomatodes (see Xanthoma), i i 7 I.

- molluscum, I 26 .

- neuro-, I I 26.

- - histopathology of, I I 28 .

- - subcutaneous, II27.

- - superficial cutaneous, I 26.

- - treatment of, I I28.

- pendulum, i 126.

- simplex, I 126.

Fibromatosis, 60 .

- neuro- (see von Recklinghausen's disease), I 128 .

Fibromyoma, 49.

Fibrous bundles, origin of, I 4 .

- structure of, I4.

- white, I 4 .

Finsen light (see Photo-therapy), I 50.

- - Reyn lamp, I 52.

Fish-skin disease (see Ichthyosis), I6r.

Fission fungi, 83, 499.

Fissure, definition and characters of, 34 .

Fixed cells of corium, I4.

Flannel rash (see Dermatitis, seborrhoic), 349.

Flask-shaped bacillus (Unna), 83 .

Flea-bites, 625 .

Flies, diseases from larvae of, 625 .

- Tumbu, 627 .

Flores unguium, 52, 1068.

Folds of emaciation, 2.

Folliclis (see Papulo-necrotic tuberculides), 490.

Folliculitis, 45, I O 3 .

- decalvans, 46, 974, 996 .

- from chemical irritants, IOI 4.

- hyphomycetic, IOI 4 .

- in skin affections, IOI 4.

- microbic, IOI 3 .

- rubra, IOI4.

- superficial pustular (see Bockhart's impetigo), 327 . 
Folliculitis, suppurative, 46 .

- ulerythema reticulata (Machee), I0 26.

Forbes' lamp, I 53 .

Fordyce's disease of lips, 1082.

Formalin, 122.

- dermatitis from, 279 .

Formulae, I 267.

Fowler's solution (see Arsenic), rog.

Fox, Colcott. Classification of skin disease, I60.

- Paget's disease, I I 60.

- Ringed eruption (see Granuloma annulare), 673 .

- with Blaxall, ringworm fungi, 376 .

Fox, Tilbury. Dysidrosis, 805.

- Epidermolysis bullosa, 207.

- Tinea decalvans, 400.

- Xanthelasmoidea, 689.

Fox, Wilfrid. Naevo-carcinoma, I 157.

Fragilitas crinium, 999.

Framboesia tropica, I 208.

- cutaneous lesions of, I 2 IO.

- - in primary stage, I 210.

- - in secondary stage, I 2 I 3.

- - in ulcerative stage, I 2 I 3.

- diagnosis of, $\mathrm{I}_{2} \mathrm{I}_{4}$.

- etiology of, I 209.

- predisposing causes, I 2 ro.

- Treponema pallidum (Castellani), I 209.

- geographical distribution of, 1208.

- histopathology of, I 2 I 3.

- treatment of, I 2 I 6 .

- local, I 2 I 6.

- salvarsan, I 2 I 6.

Freckles, $2+6$.

- etiology of, 247.

- histopathology, 247 .

- treatment of, $2+7$.

French polishers' dermatitis, 285 .

Frost-bite, 21 3 .

- etiology of, 2 I 3 .

- treatment of, $2 \mathrm{I}_{4}$

Functions of the skin, 20-23.

Fungus foot (see Mycetoma), I 225.

- haematoma, I So.

Fur, dermatitis from, 272, 284 .

Furrows, of the skin, I.

- causation of, I.

- varieties of, I.

- on nails, 52 .

Furuncle (see Boil), 336.

Furunculin (see least), I I 2.

Furunculosis, 33i.

Gad-fly boil, 628 .

Galloway, J. Lichen annularis, 675.

- Mycosis fungoides, 928 .

Galyl in syphilis, $58 \mathrm{I}$.

Gangosa, I 2 I \%

- diagnosis of, I 2 IS.

- etiology of, I 2 is.

- geographical distribution of, I 2 I 7 .

- treatment of, 1218.
Gangrene, symmetrical (see Raynaud's disease), 223 .

Gardeners' dermatitis, 254 .

Gay's glands, 9.

Gelatines, I $26 \%$.

Giant-cells, 5\%.

- origin of, $5^{8}$.

Gilchrist, T.C. Blastomycetic dermatitis, 366 .

- . Iast-cells in urticaria pigmentosa. 692.

Glossitis exfoliativa marginata, $\mathrm{IOS}_{3}$.

- syphilitic, 563 .

Glycerine, I 22 .

Glyco-gelatines, I 35 .

Gnat-bites, 630 .

Goa powder (see Chrysarobin), I 22.

Gonococcus, $S_{4}$.

Gonorrhoeal keratosis, I IoS.

Goose-fiesh, permanent, I62, I69.

- transient, $I_{7} 2$.

Goundou, I 219.

- etio'ogy of, 1220 .

- treatment of, I 220 .

Gout, in skin diseases, ros.

Grain-itch, 275.

Granuloma, 55 .

- annulare, 6,3.

- description of, 674 .

- - etiology of, 676 .

- histopathology of, 6,5.

- nomenclature of. $67 \%$.

- - treatment of, 6,5.

- calcareous, subcutaneous, I I9I.

- fungoides (Auspitz), 926.

- infective, 55 .

- inguinale, 1221 .

- - diagnosis of, 1223 .

- etiolog: of, I 223 .

- histopathology of, I 222 .

- - treatment of, 1222 .

- pudendi, 122 I .

- pyogenicum, 346 .

- treatment of, $3+7$.

- septic, $3+6$.

- trichophyticum, 386.

Granuloplasm (see Plasma-cell), 56.

Granulosis rubra nasi, I054.

- diagnosis of, 1056 .

- etiology of, 1056 .

- histopathology of, 1055.

- treatment of, I056.

Greek fire, 28I.

Grey coccus of Sabouraud, 82.

Grocers' dermatitis, $2 \mathrm{~S}_{4}$.

Gros-nez (see Goundou), I 2 I 9.

Grutum (see Vilium), I I 7 .

Guduram (see Ainhum), I I94.

Guinea-worm infection, I 200 .

Gumma, scrofulous, 4;9.

- syphilitic, 550 .

Haematidiosis, 1053.

Haematoma, 935.

Hair, 5.

- atrophy of, 45 . 
Hair, beaded, roor.

- change of, 6 .

- colour of, 6 .

- cornification of, 7 .

- dystrophy of, 4.4 .

- favus of, $42 \mathrm{I}$.

- grey, 1002 .

- hypertrophy of, 44 .

- infected by Achorion Schönleini, 424 .

- Microsporon, 376 .

- - Trichophytous, 378 .

- knotted, 999.

- moniliform, IOOI.

- pathological changes in, 44 .

- periodic, 6 .

- point of exclamation (see Alopecia areata), 982.

- ringed, 1003 .

- shape of, 5 .

- situations of, 5 .

- splitting of, 999 .

- structure of, 6 .

- varieties of, 5 .

Hair-follicle, 45 .

- diseases of, 1013 .

- pathological changes in, 45 .

- structure of, 7 .

Hansen's bacillus leprae, 500.

Harlequin foetus, $\mathbf{I}_{5}$.

Harman, Bishop. Veld sore, 1257.

Harrison, A. J. Tinea unguium, treatment, 4 I 9 .

Harrison, L. W. Intramuscular injection of neo-salvarsan, 579 .

Harvest-bug, 6I 2 .

- treatment of bites from, 613 .

Harz, Ray fungus of, 429 .

Health-resorts, treatment at, i $18, \mathrm{I}_{2} 64$.

Heat, effect of, on skin, 229 .

— rash (see Miliaria), 238.

- regulation by the skin, 22 .

- treatment by, 139 .

Hebra, F. Arsenical paste of, 471.

- Classification of slin diseases by, 159.

Hectine in syphilis, 575 .

Hemi-atrophy of the face (see Sclerodermia), i 84.

Henle's sheath, 7 .

Herpes, 89\%.

- circinatus (see Dermatitis herpetiformis), 886 .

-_ facialis, 897 .

- febrilis, 897 .

- gravidarum (see Dermatitis herpetiformis), 886 .

— iris (see Erythema iris), $66_{5}$.

- maculosus et squamosus (see Pityriasis rosea), $86 \mathrm{I}$.

- pemphigoides (see Dermatitis herpetiformis), 886 .

- progenitalis, 900 .

- - diagnosis of, 901 .

- - etiology of, $90 \mathrm{I}$.

- treatment of, 901 .
Herpes, simplex, 897 .

- diagnosis of, 900 .

- - etiology of, 899 .

- - histopathology of, 899 .

treatment of, 900 .

- tonsurans (see Ringworm), 398.

- zoster, 902 .

- - arsenic and, 908.

- - diagnosis of, 909 .

- - etiology of, 907 .

- - generalisatus, 904 .

- - histopathology of, 906 .

- - nervous system, changes in, 907 .

- - prognosis of, 909 .

- - treatment of, 909 .

— - varicella and, 904, 908 .

Herpetic fever, 899 .

Heredity in skin diseases, 73 .

Herxheimer's spiral fibres, 3 .

Hexa-nitro-diphenyl-amine dermatitis, 278 .

High-frequency, treatment by, I44.

Hirsuties, 1005.

Hives (see Urticaria), 679.

Holländer's hot air apparatus, I 43.

- in Lupus vulgaris, $47^{\circ}$.

Hormones, I I 4 .

Horn-cells, 5 .

- waxy substance in, 5 .

Horns, cutaneous, I I IO.

— etiology of, I I I I.

- histopathology of, I I I I.

- treatment of, IIII.

Hull, A. J. Paraffin treatment of burns, 236 .

Hutchinson, Jonathan. Infective angioma, 178 .

- Leprosy, Fish-eating theory of, 503 .

- Summer prurigo, $25^{\circ}$.

- Syphilis congenital, triad of symptoms in, 595 .

Huxley's sheath, 7 .

Hyalin layer of hair-follicle, 80.

Hyde's massering ball, I 42 .

Hydradenitis destruens suppurativa (Pollitzer), 490.

Hydradenoma, I 22.

Hydroa aestivale, $25^{\circ}$.

- - diagnosis of, $25 \mathrm{I}$.

- - etiology of, $25 \mathrm{I}$.

- histopathology of, $25 \mathrm{r}$.

- treatment of, 250 .

- gestationis (see Dermatitis herpetiformis), 886 .

- vacciniforme, 250 .

Hydrocystoma, 30, 50, 1057.

- description of, 1057 .

- diagnosis of, 1059.

- etiology of, 1058 .

- histopathology of, 1058.

- pathogenesis of, 1058 .

- treatment of, I059.

Hyperaemia, active, 63 .

- passive, 64 .

Hyperidrosis, 1047.

- general, $\mathrm{IO}_{47}$. 
Hyperidrosis, local, $\mathrm{IO}_{4} 7$.

- - etiology of, $\mathrm{IO}_{4} \mathrm{~S}$.

- prognosis of, $\mathrm{IO}_{4} 8$.

- - treatment of, Io 48 .

- - by X-rays, Io+9.

- oleosa (see Seborrhoea oleosa), 975. 1021.

Hyperkeratosis, 38

- acquired, 38 .

- congenital, $3^{8}$

- palmar and plantar, 204

- - diagnosis of, 206.

- - etiology of, 206.

- - - histopathology of, 206.

_- - treatment of, 206.

- eccentrica (see Porokeratosis), I060.

- linguae, $\mathrm{IOS}_{2}$.

Hypertrichosis, 44, 1005.

- acquired, I005.

- - etiology of, I 006.

- prognosis of, I 006.

- - treatment of, roo6.

_ — - by bleaching, I008; depila. tories, I007; electrolysis, Ioo8; epilation, Ioos; pumice stone, I008; shaving, Ioos; X-rays, 1007.

- congenital, roo5.

Hypnotics, in skin diseases, I I 3 .

Ichthalbin (see Ichthyol), I I I.

Ichthoform (see Ichthyol), II .

Ichthyol, treatment by", I I I, I 23.

Ichthyosis, I6 I.

- congenitalis palmaris et plantaris, I $62,204$.

- cornea, I62, I69, IOIt, I I So.

- description of, I 62 .

- foetalis mitior et gravior, 163,164 .

- follicularis, I69, IOI 4 .

- description of, I 69.

- - diagnosis of, $I 72$.

- etiology of, I 72 .

- - histopathology of, I 7 I.

- prognosis of, I 73 .

- treatment of, I73.

- glossae (see Leucoplakia), Io\$ $_{4}$.

- hystrix, 201 .

- nacrée, I63.

- nigricans, 163 .

- nitida, I63.

- sebacea, I62.

- serpentina, 163.

- simplex, I6I.

- - diagnosis of, I67.

- etiology of, 166.

- histopathology of, 165.

- - prognosis of, 167 .

- - treatment of, I 63.

- - varieties of, 163 .

Immunity, acquired, 70 .

- natural, 7o.

Impetigo, Bockhart's, 327.

- diagnosis of, 329 .

- ctiology of, $32 \mathrm{~S}$.

- histopathology of, 328 .
Impetigo, Rockhart's, treatment of, 329.

- bullous, 301 .

- circinate, $30 \mathrm{I}$.

- contagiosa, $29 \%$.

- description of, 298.

- - diagnosis of, 302 .

- etiology of, 297 .

- prognosis of, 303 .

- treatment of, 304 .

- varieties of, 301 .

- furfuraceous, 3 Io.

- herpetiformis, 89 ;.

- intertriginous, 303

- phlyctenular, $29 \%$.

- pityrodes, 3 ro.

- scaly, 310.

- streptococcic, 297 .

- ulcerating, 302 .

- vulgaris, 297 .

Infiltration, cellular, 54 .

Inter-epithelial nerve plexus, 17 .

Intertrigo, 790 .

- saccharomycetica (Castellani), 366.

Iod-acne, , I 9 .

- sarcoma, 7 I9.

Iodide eruptions, 7 I9.

Iodides, treatment by, I I I.

- in syphilis, $5^{8} 7$.

Iodine treatment by, I I I, I 23.

- collosol (Crookes), I I2.

Iodipin (see Iodine). I I I.

- in syphilis, 587 .

Iodoform, I 23 .

- dermatitis from, 279 .

Iodoglidine (see Iodine), I I I.

Iodol (see Iodine), I I .

Ionisation, treatment by, I 44 .

Irritants, dermatitis caused by, so.

Itch (see Scabies), 603.

- bakers', 269.

- barbers', 414.

- barley, 275 .

- coolie-, I 196.

- copra-, 613.

- dhobie's, 390.

- dry salters', 274 .

- grain, 275, 614.

- ground, I 196.

- washerwomen's, 285 .

- water-, I 196.

Itching, 25 .

Iry, dermatitis from, 273 .

Jacquet, L. Dermites simples infantiles, 320 .

- Syphiloỉde post-érosive, 32 I.

Jadassohn. Trichophytides, 402.

Jelly-fish, dermatitis from, $2 \% 2$.

Jigger, I $2+t$.

Jones, Lewis. Electrical burns, 257.

Justus test in syphilis, 569 .

Kaposi. Erysipelas perstans faciei, 320.

- Multiple idiopathic sarcoma, I 6 ;.

- Rhinoscleroma, 523 . 
Ǩaposi. Xeroderma pigmentosum, 320. Keloid (see Cheloid), 35, I I 29.

- of Addison, I I 29.

Keratin, 5 .

- in hair, 7 .

Keratinisation (see Cornification), 5 .

Keratitis punctata leprosa, 509.

Keratohyalin granules, 4 .

- in hair, 7 .

- relation to eleidin, 4 .

Keratodermia blenorrhagica (see Gonorrhoeal keratosis), i io8.

- eccentrica (see Porokeratosis), Io6o.

- punctata (see Keratosis punctata), 1062.

Keratolytics, I 20 .

Keratoma malignum congenitale (see Ichthyosis), I $6 \mathbf{r}$.

Keratoplastics, I 2 I.

Keratose pilaire rouge, 172.

Keratosis follicularis, I69, I I OO, I Io3.

- spinulosa, 1 о 8 .

- gonorrhoeal, 1 108.

- - pathology of, I I IO.

- - treatment of, i I Io.

- nigricans (see Acanthosis nigricans), I IOO.

- palmaris et plantaris hereditaria, 201, 204.

- pilaris, I 69.

- punctata, 1062.

- etiology of, 1064 .

- - histopathology of, 1063 .

- - treatment of, I 064 .

- supra-follicularis, IOI 4.

- - diagnosis of, IoI 7 .

- etiology of, ror 7 .

- histopathology of, IOI 6.

- - prognosis of, IoI8.

- - treatment of, Iог 8 .

- vegetans (Crocker), I Io3.

Kerion, 398, 401.

- diagnosis of, 402 .

- fungi of, for.

- prognosis of, $4 \mathrm{O}_{4}$.

- treatment of, $4 \mathrm{I} 4$.

Kharsivan in syphilis, 577 .

Kidney disease and skin affections, so8.

Kienböck, X-ray treatment of ringworm, 407 .

Klebs-Loeffler bacillus, 83 .

- in desert sore, I 258 .

Koilonychia, ı 068.

Koplik's spots, 66I .

Kraurosis vulvae, 963.

Lambkin's cream, 586 .

Langhan's giant-cells, 57 .

Lanugo hair (see Hair), 5 .

- shedding of, 6 .

Larva migrans, 628 .

Lead, dermatitis from, 279.

Lentigo (see Freckle), 246.

Leiomyoma, 49 (see Myoma), 1138.

Leishmaniasis Americana (see Espun(ia), 1255 .
Leishmaniasis, cutaneous (see Oriental sore), I $25^{8}$.

- naso-oral (see Espundia), 1255.

Lenigallol (see Pyrogallol), I 24.

Leontiasis (see Leprosy), 496.

Lepothrix, 443.

- fungi of, 444 .

- Micrococcus nigrescens (Castellani), 444 .

- - ruber (Castellani), 444 .

Lepra arabum, 496.

- bacillus, 499.

- maculo-anaesthetica, 5 I o.

- tuberosa, 508.

- vitiligo (see Sclerodermia), I I 79.

Lepromata, 508 .

Leprosy, 496.

- anaesthetic, 510 .

- bacillus (Hansen), 500.

- cultivation of, 517 .

- - demonstration of, $5 \mathrm{I} 7$.

- distinction from tubercle bacillus, 499.

- morphology of, 500.

- staining of, $5 \mathrm{I} 7$.

- children of leprous parents, 522 .

- complications of, 5 I 5 .

- contagiousness of, 504 .

- death in, cause of, 5IO, 5I2, 5 I 8 .

- diagnosis of, 5 I 5 .

- - agglutination tests, 5I7.

- cuti-reaction, 5 I 7 .

- Wassermann reaction, 516 .

- etiology of, 500.

- bacillus, 500 .

- fish-eating theory, 503 .

-.- predisposing causes, 506 .

- geographical distribution of, 497.

- histopathology of, 5 I 3 .

- history of, 496 .

- inoculation experiments, 502 .

- - in man, 504 .

- - in lower animals, 502 .

- International Conference (I 909), resolution, $5 \mathrm{O}_{4}$.

- invasion, 507 .

- - possible site of, 503 .

- - alimentary tract, 503 .

_- genitalia, 504 .

_- nasal mucosa, 503.

- - skin, 503 .

- symptoms of, 507 .

- isolation in, 521 .

- lepra-cells, 513 .

- origin of, $5 \mathrm{I} 3$.

- lepromata, 508 .

- histopathology of, 513 .

- leprotic fever, 508.

- Lombardian (see Pellagra), 1230.

- maculo-anaesthetic, 5 IO.

- course of, 512 .

- nerve lesions in, 511, 515 .

- skin lesions in, $5 \mathrm{II}$.

- - atrophy of hairs and sweatglands, $51 \mathrm{I}$.

- - bullae, $5 \mathrm{II}$. 
Leprosy, maculo-anaesthetic, skin lesions in, pigmentary changes, 5 II.

- ulcers, $5 \mathrm{II}$.

- neuralgic pains in, 5 I I .

- - paresis in, 512 .

- prognosis of, 517 .

- - sensory symptoms of, 511 .

- trophic changes in, 512 .

- - claw-hand, $5 \mathrm{I} 2$.

- - muscular atrophy, 512 .

- - mutilations, 512 .

- mixed, 507, 513 .

- nerve (see Maculo-anaesthetic), 5 ro.

- nodular, 508 .

- course of, 510.

- - description of, 509.

- - histopathology of, 513

- leontiasis, 508 .

- lesions in eyes, 509.

$\ldots \ldots$ in genitalia, 509 .

- - in glands, 509 .

_ - in nails, 509 .

- - in skin, 509 .

- prognosis of, 517 .

- notification of, 521 .

- para-leprosis, 513 .

- pathogenesis of, 513 .

- prevention of, 521 .

- prognosis of, 517 .

-- - climatic effect on, $51 \%$.

- remissions in, 518 .

- satyriasis, 508 .

- segregation in, 521 .

- transmission of, 504 .

- treatment of, $51 \mathrm{~S}$.

- - internal, 5 I8.

- - by anti-venene, 519; chaulmoogra oil, 518; gynocardate of soda, 518; ichthyol, 519 ; iodides, 519; leprolin, 520; mercury, 519; nastin, 520; sera, 520; tuberculin, 519; vaccines, 519 .

- - symptomatic, 521 .

- local, 52I.

— - by antiseptics, 521 .

- - by X-rays, 521 .

- tubercular (see Nodular), 508.

- varieties of, $50 \%$.

Leprotic fever, 508 .

Leptus autumnalis, 6 I 2.

Lesions, primary, 26.

- secondary, 33.

Leucaemia cutis, I I 4 .

Leucoderma, 953.

- diagnosis of, 955 .

- etiology of, 955 .

- prognosis of, 955 .

- syphilitic, 545,952 .

- treatment of, 956 .

Leuconychia, 52 .

- description of, I 068.

Leucoplakia, I084.

- diagnosis of, I085.
Leucoplakia, etiology of, 1085.

- histopathology of, 1085 .

- treatment of, $\operatorname{IoS}_{5}$.

Leucotrichia, 44, 1002.

- annularis, 1003 .

Levurin (see least), I I 2.

Lice (see Pediculi), 616.

Lichen, agrius (see Prurigo), 702.

- annularis (see Granuloma annulare), 673.

- circinatus (see Seborrhoic dermatit:s), 353

- circumscriptus, 726.

- diabeticorum (see Xanthoma), I I 74 .

- lividus, 726 .

- nitidus, 744

- pilaris, 726 .

- - seu spinulosus (Crocker), ror 8.

- planus, $72 \%$.

- - acute generalised, 734 .

- annularis, $73 \mathrm{I}$.

- atrophicus, 73I.

- bullosus, 733 .

- chronic, 728 .

- diagnosis of, ifo.

- - erythematosus (Crocker), 733.

- etiology of, 738 .

- - actual cause, theories, 738 .

- - predisposing causes, 738 .

- haemorrhagicus, 734 .

- - histopathology of, 736 .

- - inpertrophicus, 730 .

- infantum (see Papular urticaria), $69 \%$.

- linearis, 732 .

- moniliformis (Kaposi), 732 .

_- mucous membrane lesions in,, 36 .

- obtusus corneus (Brocq), 731 .

- - retiformis (see Parapsoriasis), 847 .

- - treatment of, $74 \mathrm{I}$.

- ruber (Hebra), 850 .

- ruber acuminatus, 727,850

- scrofulosorum, 457 .

- - diagnosis of, 489 .

- etiology of, 488 .

- histopathology of, 488 .

- treatment of, $4^{\text {Sg }}$.

- - tubercle bacilli in, 489 .

- simplex aigu (Vidal), 697.

- simplex chronicus (Brocq), 703, 726.

- spinulosus (Devergie), 727, IOIS.

- diagnosis of, IOI9.

-. etiology of, rorg.

- histopathology of, rorg.

- - treatment of, 1020.

- tropicus (see Miliaria), 238.

- urticatus (see Papular urticaria), 697.

- variegatus (see Parapsoriasis), 847 .

Lichenification, $7+6$.

- circumscribed, i 46 .

- diagnosis of, 750 .

- diffuse, $7+9$.

- primary, $74^{6}$.

- prognosis of, 750 .

- secondary, 746 .

- treatment of, $75 \%$. 
Lime, dermatitis from, 279 .

Liniment, 132, I 268.

- exsiccans (Pick), 136.

Lipoma, 68.

Lips, diseases of, 1079.

- eczema of, 1079 .

- Fordyce's disease, ro82.

- seborrhoic dermatitis of, 1082 .

Liquid air, treatment by, I39.

Little, E. Graham. Pityriasis rosea, 864 .

- Urticaria pigmentosa, 690.

Livedo annularis, 222.

- frigore (see Livedo reticularis).

- reticularis, 222, 656 .

Liver, disorders of and skin diseases, 108.

Lophophyton gallinae (Mégnin), 426.

Lortet-Genoud lamp, I 5 I.

Lotions, 1 37, 1268

- action of, 137 .

- varieties of, 137 .

Low, R. Cranston. Monilithrix, 1002.

Luargol, in syphilis, $5^{8} \mathrm{I}$.

Lues venerea (see Syphilis), 527.

Luetin (see Syphilis), $53 \mathrm{I}$.

— reaction, 569 .

Lunule (see Nail), I I.

Lupus annularis, 455 .

- disseminatus, $45^{2}$.

- erythematoides (Leloir), 456, $9 \mathrm{II}$.

- erythematosus, $9 \mathrm{I} \mathrm{I}$.

- - discoides, $9 \mathrm{Ir}$.

- - - situations of, $9 \mathrm{I} 3$.

- - - symptoms of, 913.

- - disseminatus, $9 \mathrm{I}_{4}$.

—— acute, 914

_ _ _ primary (Aigu d'emblée), 9 I 5 .

_ _ - secondary to chronic lesions, $9 \mathrm{r} 4$.

- - sub-acute, 9 I 6 .

— diagnosis of, 920 .

- - etiology of, 917 .

- - - actual cause of, 9 r 8 .

- _ - sebaceous gland theory, 9 I 8 .

- - - toxic theory, 9 I 8 .

— — - tuberculosis theory, 919.

- - predisposing causes of, 917 .

—— - summary of, 919.

— histopathology of, 9 I 6 .

- mucous membranes, lesions in, 916.

- prognosis of, $92 \mathrm{I}$.

- - treatment of, $92 \mathrm{I}$.

- - - general, 921

- - local, 922.

__ _ - by actinic rays, 924 ; caustics, 923 ; freezing with carbon dioxide snow, 924; ionisation, 923 ; radium, 924 ; X-rays, 924 .

_ _ _ drying of lesions, 922 .

_ _ - reduction of hyperaemia, 922 .

_ _ - - removal of scales, 922 .
Lupus erythematosus, varieties of, 9 I 4 .

- - indurated, $9 \mathrm{r} 4$.

- - nodular, 914 .

- - non-scaly, 914 .

- - telangiectatic, 914 .

— fibroid, 455 .

- hypertrophicus, 455.

- lymphaticus (see Lymphangioma circumscriptum), 191.

- nodularis, 450 .

- papillomatosus, 455 .

- pernio, $45^{6}$.

- post-exanthematicus, 452 .

- scleroticus, 455.

- sebaceus, 911 .

- serpiginosus, 455 .

- tumidus, 455 .

- verrucosus, 453.

- vulgaris, 449 .

$\longrightarrow \longrightarrow$ and erysipelas, 457 .

- - association with other tuberculous lesions, 451 .

— - bovine tubercle bacilli and, 462 .

- complications of, 457 .

- - - adenitis, 457 .

- - epithelioma, 457 .

_- - - relation to actinic and $\mathrm{X}$-ray treatment, 457 .

- - lymphangitis, 457 .

$\longrightarrow-$ pseudo-elephantiasis, 457 .

_- - secondary infection, 457 .

— contagiousness of, 463 .

- course of, $45^{2}$.

- - description of, 450 .

- - destruction of tissue by, $45 \mathrm{I}$.

- diagnosis of, 463 .

- etiology of, 461 .

- - - actual cause, 462 .

- _ - - modes of invasion of skin by tubercle bacilli, 462 .

_- - predisposing causes, $46 \mathrm{r}$.

- - general health and, 465 .

- - histopathology of, 459 .

- measles and, 452 .

- - of mucous membranes, $457,45^{8}$.

- - buccal, 459.

- - nasal, $45^{8}$.

- - prognosis of, 465 .

- - scarring from, 450 .

- - situations of, $45 \mathrm{I}$.

- transmissibility of, $\$^{62}$.

- - by tattooing, $4^{6} 3$.

- - - by vaccination, 462 .

- treatment of, 465 .

- - choice of method, 476 .

- - general, 466 .

_ _ internal, 466 .

— - - by thiosinamin, 467 .

_ _ - by thyroid extract, 466 .

- - - by tuberculin, 466 .

- - local, 467 .

- _ - by Bier's suction glasses, 47I; caustics, $47 \mathrm{I}$; cauterisation, 470 ; curetting, 468; excision and grafting, 468 ; 
fluorescent light, 476 ; high-frequency currents, 47 ; ionisation, 470 ; keratolytics, 47 I.

Lupus vulgaris, treatment of, local, by phototheraps, 473 .

\section{- _ - histological} caused by, 474 .

changes

- - - by radium, 475; refrigeration, $47^{\circ}$; scarification, 469 ; scraping, 468 ; "spick" method, 472 ; tuberculin, 472 ; X-rays, 474 .

- - of mucous membranes, 476 .

- varieties of, 452 .

Lymphadenoma cutis, I I 4 I.

Lymphangiectodes (see Lymphangioma circumscriptum), I9I.

Lymphangioma cavernosum, I91.

- circumscriptum, 67, 191.

- description of, I9I.

- - histopathology of, 192.

- treatment of, 193.

Lymphatic spaces, $I 7$.

- vessels, I $\%$.

- pathological changes in, 67 .

Lymphodermia perniciosa (Kaposi), 932.

Lympho-granulomatosis pruriginosa, I I $f$.

MacCormac, H. Lymphangioma circumscriptum, 192.

- Scabies, treatment of, 609.

Mackenzie, Stephen. Exfoliative dermatitis, treatment of, 860 .

Maculae caeruleae, 622.

Macule, definition and characters of, 26.

Maculo-papule (see Macule), 26.

Madura foot (see Mycetoma), I 225.

Maduromycosis (see Mycetoma), I225.

- sclerotia in, 1225 .

Majocchi. Granuloma trichophyticum. 386.

- Telangiectasis follicularis annulata, I 79 .

Mal de Meleda, 206.

Malassez, spore of, $350,353$.

Manson, Sir P. Elephantiasis, 1205.

Massage in skin diseases, $1_{42} 2$

Mast-cells, increase of, 54 .

- in Urticaria pigmentosa, 55, 69 r.

- origin of, I4.

- situations of, I4.

- structure of, I4.

- tumour, 69i.

I'Donagh, J. E. R. Lichen nitidus, $7+5$.

- Spirochaeta pallida, life-history of, 532.

Medicinal eruptions, 7 Io.

Medulla of hair (see Hair), 6.

IIegaspores (see Trichophyton ectoendothrix), 3 So.
Meibomian glands, 8 .

Meissner's bodies (see Tactile corpuscles), 18.

Melanidrosis, 1052.

Melanin, 19, 54 .

- origin of, 19.

Melanoblasts, I9.

— in pigmented naevi, 194.

Melanodermia (see Melanoses), 948.

Melanoglossia, Iosz.

Melanosis, 94 S.

- acquired, 949.

- - association with cutaneous neoplasms, 952.

—- general diseases, $95 \mathrm{I}$.

- - skin diseases, 951 .

- from actinic rays, 949 .

- from heat and cold, 950.

- from local irritation, $95^{\circ}$.

- from toxins, 950 .

- from X-rays, 950.

- congenital, $94 \mathrm{~S}$.

- lenticularis progressiva, 252.

Melung, 955.

Mentagra (see Sycosis barbae), 330.

Mercurial eruptions, 721 .

Mercury, dermatitis from, 279 .

- fulminate of, 280 .

- treatment by, $112,123$.

Ietchnikoff and Roux. Inoculation of syphilis in monkeys, 531 .

Methylene blue, 123.

Metol, dermatitis from, 280.

Vicrobacillus (Sabouraud and Lnna), $8_{3}$.

Micrococcus cutis communis (Sabouraud), $S_{2}, 325,350$.

Microides (see Trichophyton ecto-endothrix), 3 So.

Microsporon, Audouini, 3,6 .

- cultivation of, 376 .

- - invasion of hair by, 377 .

- caninum, 376,378 .

- equinum, 376,378 .

- felineum, 376 .

- furfur, $4+1$.

- lanosum, 3,6.

- minutissimum, 440 .

Microsporosis, 398 .

- diagnosis of, 402 .

- prognosis of, 403 .

Migratory cells, 12.

Mikulicz cells in rhinoscleroma, 525 .

Miliaria, $23 \mathrm{~S}$.

- alba, $23 \mathrm{~S}$.

- crystallina (see Sudamina), 243.

- diagnosis of, $24 \mathrm{I}$.

- etiology of, 240.

- fever, 243 .

- histopathology of, 239.

- papulosa, $23 \mathrm{~s}$.

- prognosis of, 241 .

- propria, 238.

- rubra, 238 .

- treatment of, 241 .

- - general, 24 I. 
Miliaria, treatment of, local, 242.

- vesiculosa, 238 .

Milium, I I 7 .

- colloid (see Colloid milium), I 135.

- treatment of, I I 18 .

- varieties of, I I I 7 .

Mineral waters, natural, ${ }_{2} 26_{3}$.

Mites, copra-itch, 6I3.

- fowl, 6 I 3 .

- grain-itch, 6I3.

- itch, 603 .

Mitosis, of mother-cells, 2.

- of vacuolated cells, I 3 .

Moles (see Naevi), 195.

Mollin, 129.

Molluscum bodies, 39, I I I 4 .

- contagiosum, I I 2 .

- - diagnosis of, I I I 5 .

- - etiology of, I I i 4 .

- - histopathology of, I I I 3 .

- - treatment of, I I 15.

- giganteum, I I 13 .

Mongolian spots, $9+9$.

Monilithrix, 45, I OOI.

- description of, IOOI.

- etiology of, r IOOI.

- treatment of, 1002 .

Montgomery, D. W. Dermatitis coccidioides, 366 .

Moro's reaction in tuberculosis, $44^{8}$.

Morococcus (Unna), 82, 325.

Morphoea (see Sclerodermia), I 183.

- nigra, I 183 .

Mosquito-bites, 630 .

Moss' glands, 9 .

Mother-cells of corium, 14.

—of epidermis, 2.

Mulls, plaster, I 35 .

- salve, I 35.

Munition-workers' dermatitis, 285 .

Iusca macellaria, 626 .

Muscles of the skin, 18.

- pathological changes in, 49 .

Mycetoma, I 225 .

- diagnosis of, 1228.

- distribution of, I 225 .

— etiology of, 1228.

- fungi of, 1228 .

- ascomycetes, 1228 .

- hyphomycetes, I 228 .

- para-, I 229.

- pathology of, 1227 .

- pseudo-, I 229.

- sclerotia in, 1226 .

- varieties of, 1226 .

Mycosis fungoides, 926 .

_- bacteriology of, 930 .

- - diagnosis of, 930 .

— - etiology of, 930 .

- - histopathology of, 928 .

- prognosis of, $93 \mathrm{I}$.

— stages of, 926 .

— - mycosic or tumour, 927 .

- - pre-mycosic, 926.

- - treatment of, 931 .

_- by X-rays, 932.
Myiasis, 625 .

- description of, 625 .

- linearis, 628 .

- - description of, 629.

— - treatment of, 629 .

- parasitology of, 626 .

- treatment of, 628 .

Nyoma, angio-, I I 40.

- cutaneous, I I 38 .

- - diagnosis of, I 140 .

- - histopathology of, I I 39.

- - treatment of, II 4 O.

- lymphangio-, I I 4 O.

- subcutaneous, I I 4 O.

- telangiectodes, I I 40 .

Naevo-carcinoma, I96, I I 57.

Naevus, 40.

- acneiformis unilateralis, 202.

- application of term, I74.

- araneus, I 82 .

- capillary, i 76 .

- - description of, I 76 .

- histopathology of, 178 .

- situations of, 177 .

- - treatment of, 184 .

- - by carbon dioxide snow, I 84 ; electrolysis, I 85 ; high-frequency, 185 ; radium, I 84 ; scarification, I86 ; ultraviolet rays, $18_{5} ; \mathrm{X}$-rays, $18_{5}$.

- varieties of, $r 78$.

- cells, I98.

- origin of, 199.

- classification of, I 75 .

- cyst-epitheliomatosi, I I 19.

- fibro-angiomatous, I 81 .

- flammeus, 176 .

- giant, 195, 196.

- giant-vascular, I8I.

- hard, 201.

- linear, zor

—-diagnosis of, 204 .

— - etiology of, 204.

- histopathology of, 202 .

- - treatment of, 204.

- lipomatodes, I81, 195.

- lymphatic, I9I.

- non-vascular, I93

- pigmented, I93.

- - histopathology of, I 93.

- treatment of, 194.

— - by bleaching, 194.

__ - by carbon dioxide snow, I94.

- - by caustics, 194 .

- - by electrolysis, I94.

- porcupine, zoI

- raised haemangiomatous, 180 .

- - histopathology of, I 82 .

- situations of, I 80 .

- treatment of, 186.

$\ldots \ldots$ by carbon dioxide snow, I 86 ; cauterisation, I 88 ; compression, I90; electrolysis, I 89 ; excision, I 87 ; liga. ture, I90 ; radium, I87. 
Naevus, sebaceus, I95, I I 24.

- segmental, I96.

— soft non-vascular, I95.

- histopathology of, 197.

- - treatment of, 200.

_- by carbon dioxide snow, 200 ; caustics, 2OI ; cauterisation, 201 ; electroly*sis, 201 ; excision, 200.

- spider, 182.

- - treatment of, I90.

- - - by cauterisation, 190 ; electrolysis, I90.

- spilus, I93.

- stellar, i 82 .

- subcutaneous, 181 .

- syringo-adenomatosi, 203.

- systematised, 201 .

- unilateral, $20 \mathrm{I}$.

- vascular, $65,176,1124$.

- verrucose, 201.

- zoniform, 196.

Nail, atrophy of, 5I, I066.

- - acquired, ro66.

- - from cutaneous affections, $106 \%$.

— - from febrile conditions, I066.

— - from pyogenic infection, 1066.

- - from scarring conditions, 106\%.

—- from traumatism, I067.

- congenital, Io66.

- bed of, 1069.

- claw deformity of, I 069.

- disease of, 1065, I07I.

- acquired, IO $\mathrm{T}$.

- - - from chemical irritants, 1072.

- - from favus, Ioj4.

- - from physical causes, ro

- - from pyogenic cocci, 10 2 .

- - from ringworm (see Tinea unguium), $41 \%$, $10 \pi 4$.

- - from scabies, I0 7 .

- - from X-rays, Io 7 .

- - in Alopecia areata, 1075.

- - in eczema, Io 74 .

- - - in circulatory diseases, 10 6 .

- - in nervous affections, 1077 .

- - in psoriasis, 1075 .

- - in syphilis, I075.

- congenital, Io 7 o.

- dystrophy of, 51, 1067

- egg-shell deformity of, 1077.

- fold of, I I.

- grooving of, 1067.

- growth of, I 2.

- hypertrophy of, 50, 1065.

- ingrowing toe-, ro69.

- matrix of, II.

- pathological changes in, 50 .

- plate of, II.

- punctiform depressions of, I068.

- ram's horn deformity of, 1068.

- striation of, I06-

- structure of, II.

- whiteness of (see Leuconychia), ro6.
Tarcissi, dermatitis from, 273.

Teisser. Syphilis in monkeys $53 \mathrm{r}$

Neo-kharsivan in syphilis, $57 \%$.

Teoplasms, cutaneous, ro5;

- - benign, Ioss.

- classification of, ross.

- - malignant, I I +4 .

- pseudo-, I I 7 I.

Neo-salvarsan, 5-9.

Nettle-rash (see Lrticaria), 6-9

Nerves, cutaneous, I-.

- medullated, 17 .

- non-medullated, I 7 .

- - pathological changes in, 67 .

- tumours of, 68 .

Neuroma, false, 68.

- true, 63.

Seurotic excoriations, 285 .

- gangrene, 291.

Térrodermites, 746 .

Nikolski's sign, 8\%4.

- in Dermatitis herpetiformis, Ssg.

- in Epidermolysis bullosa, 208.

- in Pemphigus foliaceus, 879.

Vitroglycerine, dermatitis from, 2 So.

Nits (see Pediculosis capitis), 619.

Nocardia convoluta. 433 .

- thibiergei, 433 .

Nodules, definition and characters of. 28 .

- histology of, 25.

Jogucni, cultivation of Spironema pailidum, $53 \mathrm{I}$.

Nomenclature, i 58 .

Novarsenobillon in stphilis, 579.

Nuclein (see Yeast), II3.

Nurses' dermatitis, 285 .

Oedema, acute circumscribed, 693.

- angio-neurotic (Quincke), 693

- - description of, 693.

- - diagnosis of, 695 .

- - etiology of, 695 .

- histopathology of, 694 .

- - prognosis of, 695 .

- treatment of, 696.

- neonatorum, I I 90.

- - diagnosis of, I I 1 I

- - etiology of, I 190 .

- histopathology of, I I 90.

- - treatment of, II9I.

- pathology of, 64 .

- persistent lymphatic, 320 .

- wandering, 683 .

Oils, acneiform dermatitis from, 250 .

- essential, dermatitis from, 275 .

- indications for use of, I 30 .

-varieties of, I 30 .

Ointments, I 32 .

- bases of, 132 .

- formulae of, 1274 .

- special, I32.

Oleates, use of, I 3 I

Oleum, cadini, $1 \geq 5$.

— fagi, I 25.

- rusci, 125. 
Onychauxis, 50, 1065.

Onychia, I072.

- maligna, 1073.

- syphilitica, 1076.

- treatment of, 1073.

Onychogryphosis, 51, I069.

Onychomycosis (see Tinea unguium), 417,1074 .

- favosa (see Favus of nails), 423.

Onychorrhexis, 1067.

Oospora canina (Constantin and Sabrazés), 426.

Ophiasis (see Alopecia areata), 983 .

Opium eruptions, 721 .

Opsonins, 53, 71 .

Oriental boil, I 250 .

- sore, 1250.

Ormsby, O. S. Blastomycosis, 367 .

Ornithodoros moubata, $61 \mathrm{I}$.

Oroya fever (see Verruca Peruana), I 259.

Orsudan (see Arylarsonates), I I I.

Orthoform, dermatitis from, 281 .

Osmidrosis (see Bromidrosis), I050.

Pacinian bodies, 18 .

Pachydermia lymphangiectatica, 323 .

Paget's disease, i I 58 .

- diagnosis of, I 162.

- etiology of, 1162 .

- histopathology of, i 160.

- treatment of, 1163 .

l'ain spots, 23 .

Panaritium, 1072.

Pani-ghao (see Ancylostomiasis cutaneous), I 196 .

Papillae, hair-, 8.

- nerve, 12.

- vascular, 12.

Papillary layer (see Corium), I 2.

Papilloma neuroticum (see Naevus, linear), 201.

- vegetating, $3+5$.

Papule, definition of, 27.

- histopathology of, 27.

- squamous, 27 .

Papulo-pustule, 27.

- vesicle, 27.

Paraffin, dermatitis from, $28 \mathrm{I}$.

- - workers' dermatitis, 285 .

Parakeratosis, 33, 39 .

- variegata, 847 .

Parangi (see Framboesia tropica), I 208.

Paraphenylen diamen, dermatitis from, 281,1004 .

Parapsoriasis (Brocq), 844 .

- diagnosis of, $84^{8}$.

- etiology of, 847 .

- histopathology of, 8.4 .

- prognosis of, 849 .

- treatment of, 8.49 .

- varieties of, $8{ }_{4} 6$.

- en gouttes 846 .

- en plaques, 847 .

- lichenoïdes, 846 .

Parasites, animal, 602 .
Parasites, animal, cutaneous diseases from, 603 to 632 .

— vegetable, 294.

Parasiticides, I 2 I.

Pastes, I 33, I 273.

Pediculoides ventricosus, 275, 6I4.

Pediculosis, 6I 6.

- capitis, 619.

- description of, 619.

- - diagnosis of, 619 .

- - treatment of, 620 .

- corporis (see Pediculosis vestimentorum), 622 .

- disinfection of clothes, 622 .

- parasitology of, 617.

- pubis, 622.

- description of, 622 .

- - diagnosis of, 623 .

- - treatment of, 623 .

- vestimentorum, 620 .

- - description of, 620 .

- diagnosis of, 621 .

- - treatment of, $62 \mathrm{I}$.

Pediculus, capitis, 617.

- pubis, 6r 8 .

- vestimentorum, 617 .

Pelade (see Alopecia areata), 98I.

Peliosis rheumaticus (Schönlein), 936.

Pellagra, I 230.

- course of, 1232.

- diagnosis of, 1234 .

- etiology of, 1233 .

- - deficiency theory of, I234.

- - maize theory of, 1233 .

- - parasitic theory of, I 234 .

- - predisposing causes of, 1234. .

- - sunlight, 1234 .

- geographical distribution of, I 230.

- necklace of Casal, I232.

- pathology of, 1233.

- treatment of, I 235.

Pemphigoids, 886.

Pempligus, 869.

- acutus, 87o.

- - bacteriology of, 872 .

- description of, 870 .

- - diagnosis of, 872 .

- etiology of, 872 .

- - histopathology of, $87 \mathbf{1}$.

- - prognosis of, 872 .

- - treatment of, 872 .

- chronicus, 873 .

- - diagnosis of, 876 . etiology of, 8,6 .

- - microbic theory, 876 .

- - neuropathic theory, 876 .

- - toxic theory, 876 .

- histopathology of, 875 .

- treatment of, 877 .

- circinatus, 886 .

- congenitalis (see Epidermolysis bullosa), 207.

- contagiosus, 302 .

- epidemic, 871.

- febrilis, 8\%o.

- foliaceus, 878 . 
Pemphigus, foliaceus, diagnosis of, $88 \mathrm{I}$.

- etiology of, 880 .

- histopathology of, 880.

- papillomatosis in, 879.

- - prognosis of, $S 8 \mathrm{I}$.

- - treatment of, S8I.

- varieties of (Brocq), 879.

- herpetiformis (see Dermatitis herpetiformis), 886 .

- hystericus (see Dermatitis artefacta), 288.

- malignus, 87 o.

- neonatorum, 302, 305.

- - diagnosis of, $30 \%$.

- prognosis of, 307 .

- - treatment of, 307 .

- neuroticus (see Dermatitis artefacta), 288.

- pruriginosus (see Dermatitis herpetiformis), 886 .

- syphiliticus (see Syphilide, bullous), 592.

- vegetans, 882 .

- - diagnosis of, $88_{4}$.

- - etiology of, $88_{4}$.

- - histopathology of, $88_{3}$.

- - prognosis of, $88_{4}$.

- treatment of, $88_{4}$.

- vulgaris, 8\%3.

Pencils, salve, 135 .

Perionychia, 51, 1072 .

Perlèche, $30_{4}$.

- treatment of, 305 .

Pernet, G. Pemphigus acutus, 37 I

Pernio (see Chilblain), $2 I_{4}$.

Petechiae, 935.

Pfannenstiel's nascent iodine in Lupus vulgaris, 4,6 .

Phagedaena tropica, I 237.

- - bacteriology of, I 238 .

- - diagnosis of, I 239.

- - etiology of, 1238 .

- - geographical distribution of, r 236.

- - histopathology of, 1238.

- - prophylaxis of, I 239.

- - treatment of, I 239.

Phagocytosis, 53 .

Phenyl hydrazine hydrochloride, dermatitis from, $28 \mathrm{I}$.

Phlebitis nodularis necrotisans (Philipp. son), $67,4^{8}+$.

Phlyctenular impetigo (see Impetigo contagiosa), $29 \%$.

Phosphoridrosis, I053.

Phosphorus, dermatitis from, $23 \mathrm{I}$.

Photographers' dermatitis, 285.

Photo-therapy, 50.

- diseases benefited bỹ, I 52 .

- histological effects of, 153.

- history of, I 50.

- indications for, 152 .

- length of exposure, I 52.

- sunlight apparatus, I 5 I.

- varieties of lamps, I 5 I.

Phthiriasis (see Pediculosis), 6 I6.

Phthirius pubis, 616.
Physiology of the skin, 20.

Pian (see Framboesia tropica), I 208.

Pick's linimentum exsiccans, I 36.

Piebald skin (see Leucoderma), 953.

Piedra, 437 .

- nostras (Lnna and Behrend), $+3^{8}$.

Pigeonneau, 2,6 .

Pigment, composition of, I9.

- in blood, I9.

- of hair, 6 .

- situations of, I9.

Pigmentary disease, prosressive (Schamberg), 953.

Pigmentation (see Melanoses), 948 .

- anomalies of, $69,94 \mathrm{~s}$.

- from arsenic, I I I, $;$ I 6 .

— from silver salts, $; 2$ I, 952 .

- situations of normal, i9.

Pinch, A. E. H. Radium treatment, I 8 .

Pinta, I $24 \mathrm{I}$.

- diagnosis of, $12+3$.

- etiology of, $12+2$.

- geographical distribution of, I 24 I.

- treatment of, 1243 .

Pituitary gland, treatment by, II5.

Pityriasis, 349 .

- capitis, $35 \mathrm{I}, 975$.

- - diagnosis of, $35 \%$.

- eriology of, 356 .

- - prognosis of, 358 .

- - treatment of, 358,979 .

- circinata, 353 .

- lichenoides chronica (Juiiusberg), $8+6$.

- maculata et circinata (Duhring), 86I.

- pilaris (Devergie), 850 .

- rosea, 861.

- - diagnosis of, $86 \mathbf{5}$.

- etiology of, 864 .

- herald patch of, 862 .

- - histopathology of, 864 .

- prognosis of, 866 .

- - treatment of, $\$ 66$.

- urticata, 862.

- rubra (Hebra), 855 .

- rubra pilaris, 850 .

- - course of, 852 .

- - diagnosis of, $8_{54}$.

- - etiology of, 854 .

- - - histopathology of, 853 .

- - prognosis of, 854 .

- - treatment of, 855 .

- stéatoïde, 353 .

- versicolor, 440 .

- description of, $t+\mathrm{I}$.

- - diagnosis of, $4+2$.

- etiology of, $+t^{2}$.

- fungus of, +4 .

- - Microsporon furfur, $f+\mathrm{I}$.

- prognosis of, $t+2$.

- treatment of, $4+2$.

Pityro-sporon of Malassez, 350.

Pix liquida (see Tar), I 24

Plasma-cells, 56 . 
Plasma-cells, daughter, 57.

- in infective granulomata, 57 .

- in sarcomata, 59.

- origin of, 57 .

Plasmoma, 56 .

Plaster-mulls, I 35, I 273.

- of Paris, dermatitis from, 28I.

Plato's trichophytine, 382.

Plica polonica, 6 I 9.

Poison plants, dermatitis from, 272.

Pollitzer. Miliaria, cause of, 240.

Polymorphic coccus of Cedercreutz, 82 .

Pneumococcus in skin, 84 .

Pomphi (see Wheals), 28.

Pompholyx, 805.

- diagnosis of, 808 .

- etiology of, 808 .

- histopathology of, 807 .

- treatment of, 809 .

Porokeratose papillomateuse palmaire (Mantoux), 1062.

Porokeratosis (Mibelli), r o6o.

- diagnosis of, 1062 .

— etiology of, 1061 .

- histopathology of, ro6r.

- treatment of, ro62.

Porrigo decalvans, $98 \mathrm{I}$.

- lupinosa (see Favus), $42 x$.

- scutula (see Favus), 421.

Port-wine stain (see Naevus, capillary), I 76 .

Poultices, I 38.

Powders, I 38, I 273.

Prickle-cell, 3 .

- layer, 3 .

- atrophy of, 4 I.

- - dystrophy of, 42 .

- - hypertrophy of, 40.

- - protoplasmic fibres of, 3 .

- pseudo-hypertrophy of, 42 .

Prickly-heat (see Miliaria), 238.

Primulas, dermatitis from, 273.

Pringle, J. J. Xeroderma pigmentosum, 253.

Profeta's law in congenital syphilis, 59 I.

Protective function of the skin, 23.

Prurigo, 633.

- diagnosis of, 707 .

- etiology of, 706 .

— ferox (Vidal), 705 .

- histopathology of, 706 .

- infantile (Hutchinson), 697.

- mitis, 703.

- prognosis of, 708 .

- senile, 703 .

- simplex (see Urticaria, papular), 697.

- summer (see Hydroa aestivale), 250.

- treatment of, 708.

Pruritus, 633.

- aestivalis, 638 .

- ani, 644.

- - etiology of, 645 .

- - treatment of, 646 .

- diagnosis of, 638 .

- etiology of (general), 636 .
Pruritus, etiology of (general), exciting causes, 637 .

- - predisposing causes, 636 .

- hiemalis, 222,638 .

- primary (essential), 634 .

- - general, 634 .

- local, 635 .

- - of auricles, 635.

- - - of hairy regions, 635 .

- - - of mouth, 635 .

- - of nose, 635 .

- - of palms, 635 .

- prognosis in, 639 .

- secondary (symptomatic), 633.

- senile, 636 .

- treatment, $6_{40}$.

- - general, 640 .

- - local, 64I.

- - by electricity, 643 .

- - by hydrotherapy, 643 .

- vulvae, 649 .

- - etiology of, 649 .

- treatment of, $65^{\circ}$.

Pseudo-pelade (see Alopecia cicatrisata) 996.

Psoriasis, $8 \mathrm{I} 6$.

- after-treatment of, 843 .

- buccalis (see Leucoplakia), 1084 .

- chrysarobin in, 838 .

- circinata, 8I 7 .

- climate in, 836 .

- complications of, 820 .

- course of, 8I 7 .

- description of, 816 .

- diagnosis of, 828 .

- diet in, 832 .

- diffusa, 8I 7 .

- essential lesion of, 8I6.

- etiology of, 824.

- - actual cause, 826 .

- - - diathetic theory, 826 .

- - neuropathic theory, 826 .

- - parasitic theory, 827 .

- - predisposing causes, 824 .

- figurata, 8I 7 .

- follicularis, 8I 7 .

- guttata, 8I 7 .

- heredity in, 825 .

- histopathology of, $82 \mathrm{I}$.

- nummulata, 8I 7 .

- of face, 8I 8 .

- of genitalia, 819 .

- of nails, 8I9.

- - treatment of, 842 .

- of palms, 8I9.

一 of scalp, 8 I 8 .

- - treatment of, 842 .

- of soles, 8r9.

- orbicularis, 817 .

- prognosis of, $83 \mathrm{I}$.

- punctata, 8I 7 .

- rupioides, 817 .

- season in, 825 .

- sites of predilection of, 818 .

- symbiosis with other diseases, 82I.

- symptoms, subjective, of, 820 . 
Psoriasis, tar in, $8 \Varangle 0$.

- treatment of, $83 \mathrm{I}$.

- - internal, 832 .

- by alkalies, 835 ; arsenic, 833 ; salicin and salicylates, 835 ; serum. 835 ; thyroid extract, 834 .

- local, 836 .

- - - reduction of lesions, 837

- - - by chrysarobin, 838 .

- - by pyrogallol, 8 4 I.

- - - by tar, 840 .

- - by X-rays, 8 I.

- variations of, $8 \mathrm{r} 7$.

Psorospermosis follicularis vegetans, I IO3.

- diagnosis of, I ro7.

- etiology of, I 106.

- histopathology of, I IO4.

- treatment of, I Io-

Pulex irritans, 625 .

- penetrans, 1244 .

Purpura, 934.

- diagnosis of, 94 .

- haemorrhagica, 936.

- Henoch's 936. .

- pathogenesis of, 64, 939.

- primary, 935 .

- prognosis of, $94 \mathrm{I}$.

- rheumatica, 936 .

- simplex, 936.

- secondary, 937.

- treatment of, 942 .

- urticans, 939.

Puru (see Framboesia tropica), I 208.

Pusey, W. A. Carbon dioxide snow, I 40 .

Pustule, definition of, 32 .

- d'emblée, 32.

- symptoms associated with, 32.

Pyrogallol, 124.

- substitutes for, 124 .

Quincke's oedema (see Angio-neurotic oedema), 693.

Quinine, treatment by, I 12 .

- eruptions, $72 \mathrm{r}$.

Radio-therapeutics, in shin disease, 147 .

Radium, I 54.

- alpha rays, I54.

- apparatus, $\mathbf{I} 55$.

- beta rays, I 54 .

- dermatitis from, 267 .

- - prognosis of, $26 \%$.

- treatment of, $26 \%$.

- gamma rays, 154.

- histological effects of, 155 .

- length of exposure to, 155 .

- skin affections benefited by, I 56 .

Ray fungus (see Actinomycosis), 431 .

Raynaud's disease, 223 .

- diagnosis of, 225 .

- etiology of, 224 .

- local asphyxia, 223 .

- local svncope, 223.
Raynaud's disease, prognosis of, 225.

- symmetrical gangrene, 224 .

- treatment of, 220 .

Refrigeration, treatment by, I 39.

Renaut's areas of maximum circulation, 64 .

Resorcin, I 24.

Respiratory function of skin, $2 ?$.

Rete mucosum (see Prickle-cell layer), 3.

Reticular layer (see Corium), 12.

Ricketts, Pathogenic yeasts, 37o.

Rhagades (see Fissures), 34.

Rheumatism and shin disease, 109.

Rhinophyma, $4^{8}$, SII.

Rhinoscleroma, 523 .

- bacillus (ron Frisch), 525 .

- diagnosis of, 525 .

- etiology of, 525 .

- geographical distribution of, 524 .

- histopathology of, 524 .

- Mikulicz cells in, 525 .

- treatment of, $5 \geq 6$.

- - by X-rays, 526.

Rhus, dermatitis from, 273 .

Ridges of the shin. I.

Rima (see Fissure), 34.

Ringworm, 373.

- animal origin of some cases, 375. $379,38 \mathrm{I}$.

- association with Alopecia areata. too.

- bird origin of some cases, 375 .

- black-dot, foo.

- cuti-reaction in, $3 \$_{3}$.

- demonstration of fungi, for.

- diagnosis of, foo (see also Tinea), 373.

- eczematoid of extrenities, 395.

-- diagnosis of, $39 \%$.

- treatment of, 397 .

- - varieties of, 396 .

- etiology of, 383 .

- fungi of, $3,7.4$.

- cultivation of, $3 \pi 4$.

- microsporon, $3-6$.

- trichophyton, $37^{8}$.

- - ecto-endothrix, 3 so.

- - endothrix, 379

- granulomatous, 386 .

- immunity production in, 382.

- mycology of, 3it, 393.

- of axillae, 390 .

- of beard (see Tinea barbae), fI 4 .

- of crutch (see Tinea cruris), 390.

-- of eyebrows and eyelashes, $4 \mathrm{I} 7$.

- of feet. 395 .

- of genital region (see Tinea cruris), 390.

- of glabrous skin (see Tinea circinata), 384 .

- of hands, 395 .

- of mucous membranes, 373, 391 .

- of nails (see Tinea unguium), fI 7 .

- of scalp (see Tinea tonsurans), 398 .

- prognosis of, 403 (see also Tinea), 373. 
Ringworm, suppurative, 386, 39I, 40 (see also Kerion), 398, 40I.

- Tokelau (see Tinea imbricata), 1 246. - treatment of (see Tinea), 373.

Rist, Angiomes verruqueux, 176 .

Ritter's disease, 308 .

Rodent ulcer, I I 44 .

- - diagnosis of, I I 48 .

- etiology of, 1147 .

- bistopathology of, 1977 .

- - prognosis of, I I 49.

- - treatment of, I I 49.

- - by arsenical paste, II49; cautery, Ir 5o; excision, I 5 o ; freezing with carbon dioxide, I I 5 I ; ionisation, II 5 ; radium, II5O ; X-rays, I I 50 .

- varieties of, I 146.

- - card-like, I I 46 .

-.. cystic, I $1_{4} 6$.

- - superficial cicatrising, I 146 .

Rogers, Jeonard, Treatment of leprosy, 5 r8.

Röntgen rays (see $\mathrm{X}$-rays), $\mathrm{I} 47$.

Root-sheath of hair, external, 7 .

- internal, 7 .

Rosacea, 8 Io.

- diagnosis of, 813.

- etiology of, 812.

- histopathology of, 8I I.

- prognosis of, 81 3 .

- stages of, 8ro.

- treatment of, 813.

Rungus tree, dermatitis from, 274.

Rupia, 33 .

Sabouraud, R. Pastille, $x_{4} 8$.

- Cultivation of streptococcus, 298.

- Epidermophyton inguinale, 393.

- Micrococcus cutis communis, 350 .

- Microspora, 376 .

- Proof-agar, 374.

Saccharomycetes, cutaneous affections due to, 366 .

- - treatment of, 366 .

- samboni, 366 .

Saccharomycosis hominis (see Blastomycosis), 366 .

Salicin, treatment by, I 2.

Salicylates, eruptions from, 722 .

- treatment by, I 2 .

Salvarsan, r r r.

— in Framboesia tropica, 1208.

- in syphilis, 575 .

Sambon, L. Pellagra, I233.

Santonin eruption, 721 .

Sarcoma, 58 .

- angio-, I 165.

- giant-celled, I I 64 .

- histopathology of, I I64.

- melanotic, I I64.

- multiple idiopathic pigment, I 167.

- - etiology of, I I 69.

- - histopathology of, I 168 .

- treatment of, ri 70 .
Sarcoma, osteo-, I 164 .

- pigmented, I 164 .

- primary, I 64.

- reticulum, I 165 .

- round-celled, i 164 .

- secondary, I 64 .

- treatment of, 1166.

- withering (see Epitheliomia, benign cystic), I I 8 .

Sarcoid, I 137.

- miliary, I 138 .

- multiple benign (Boeck), I 137 .

- of Darier-Roussy, I 137.

Sarcomatosis cutis, I 64 .

Sarcoptes scabiei, 604 .

Sauriasis (see Ichthyosis), I6r.

Savill, T. D. Epidermic exfoliative dermatitis, 309 .

Scabies, 6o3.

— complications of, 609 .

- diagnosis of, 606 .

- disinfection of clothes, 609.

- etiology of, 603 .

-- histopathology of, 606.

- Norwegian, 6 ro.

- of animal origin, 609.

- sarcoptes of, 604 .

- treatment of, 607 .

Scab (see Crust), 33 .

Scald (see Burn), 229.

Scale, definition and characters of, 33 .

Scar, definition and characters of, 35 .

- hypertrophic, Ir 33 .

- pitted, 1133.

Schamberg's disease, I 79, 953.

Schenk, Sporothrichosis, 433.

Schereschewsky, Cultivation of Spironema pallidum, 531 .

Schultz, Max. Prickle-cells, 3.

Sclerema adultorum (see Sclerodermia), I 79 .

- neonatorum, i 88 .

- - diagnosis of, x 189 .

- - etiology of, i i 89 .

—- histopathology of, I 189.

- - treatment of, 1 I $8 \mathrm{a}$.

Scleriasis (see Sclerodermia), I I 79.

Sclerodactylia, 223, I I 82 .

- Raynaud's phenomena in, I 182 .

Sclerodermia, I 179.

- circumscribed, 1 I 83 .

- band variety, I I 84 .

- - guttate variety, I 8.4 .

- plaque variety, $\mathrm{r}$ I 84 .

- diagnosis of, I 186.

- etiology of, 1 I 85 .

- nervous theory, I I 85 .

- - toxic theory, I 85 .

- generalised, r 180 .

- - calcareous deposits in, I I 82 .

- histopathology of, I 184 .

- neonatorum, i 88.

- pathogenesis of, r 185 .

- prognosis of, i 186.

Scleroma, nasal (see Rhinoscleroma), 523. 
Sclerosis, primary (see Syphilis), 532. Scratching and its effects, 643 .

Screw-worm, 626.

Scrofulodermia, 477 (see Tuberculosis cutis), $+4^{6}$.

- description of, 477 .

- histopathology of, 478 .

- treatment of, 479 .

- tubercle bacilli in, 479 .

Scutula of favus, 34 .

Sebaceous cyst, 1123 .

- glands, ${ }_{4} 8$.

- - a trophy of, 49 .

- calcified epithelial tumours of, I I I.

- cystic tumours of, +8 .

- - malignant proliferation of, 49 .

- - situations of, 8 .

- - structure of, 8 .

Seborrhoea, 1021 .

- anatomy and physiology of, 1022.

- bacteriology of, 976, 1024 .

- congestiva (see Lupus erythematosus), 911 .

- corporis (Duhring), 349 .

- nigricans (see Chromidrosis), 1051.

- oleosa, $102 \mathrm{I}$.

- - etiology of, 976 .

- - of scalp, 975 .

- - treatment of, 978

- - treatment of, I024.

- psoriasiformis, $35 \mathrm{I}$.

- sicca, 1021 .

Seborrhoic baldness, 975 .

- dermatitis, 3.9 .

- eczema, 353.

- warts, 1024 .

Seborrhoides, 350,1024 .

- papular (in Rosacea), 81 I, ro24.

Sebum, 20.

- composition of, 21 .

- function of, 21 .

- output of, $4^{8}$.

- secretion of, 21 .

Selhorst. Naevus acneiformis unilateris, 202.

Sequeira, J. H. Gronorrhoeal keratosis, 1 log.

Sera, eruptions from, 722 .

Sheath of Schwann, 18.

Shlipada (see Elephantiasis), I 20 I.

Shingles (see Herpes zoster), toz.

Sibbens (see Framboesia tropica), I 208.

Silver nitrate pigmentation, 721 .

Smegma, 21 .

- glands, 8 .

Smoking and skin affections, ro6.

Soamin (see Arylarsonates), 1 I I.

Soap, dermatitis from, 281 .

- effect of, on skin, 79 .

- makers' dermatitis, 285 .

- medicated, I 29.

- use of, 118 .

- varieties of, 129,1274 .

Soot, dermatitis from, $2 S_{2}$.
Sore, diphtheritic (see Desert sore), I 258 .

- hard (see Syphilis), 532.

- infecting, 532 .

- Natal (see Veld sore), 1256.

- non-infecting, 532, 535 .

- oriental, 1250.

- - diaguosis of. 1254 .

- - etiology of, 1253 .

- - geographical distribution of, $125 \mathrm{I}$.

- - histopathology of, 1252 .

- Leishmania tropica and, 1253

— - parasitology of, 1253.

- - treatment of, 1254 .

- soft, 535 .

- tropical (see Phagedaena tropica), I 237 .

Spas, list of, I 264 .

- skin affections benefited at, 1264 .

Spargosis (see Elephantiasis), I 201.

Spider-naevus, 182.

Spiradenoma (see Sweat-adenoma), 50.

Spirochaeta pallida (see Spironema pallidum), 528 .

Spiritus saponatus alkalinus (Hebra), 129.

Spore of Malassez, 83, 350, 353 .

Sporotrichosis cutis, 433 .

- description of, $+3+$.

- diagnosis of, $\$ 36$.

- fungus of, 435 .

- - sporothrix of Beurmann, 435 .

- - Castellani, +35 .

- - - Jeanselme, 435 .

- - Schenk, 435 .

- histopathology of, +34

- proginosis of, +36 .

- serum reactions in, +36 .

- treatment of, +36 .

- types of lesions, 434 .

Stannoxyl, in boils, $34^{\circ}$.

Staphylococcus, cutaneous affections due to, 324,326 .

- cutis communis, 325,82 .

- epidermidis albus, $325,8 z$.

- pyogenes albus, 325, $8 z$.

- pyogenes aureus, 325, 82.

Status exudativus (Czerny), 35\%.

- seborrhoicus (Barber and Semon), 357.

Steatidrosis (sce Seborrhoea oleosa), 975,1021 .

Stimulants, local, I $\geq 1$.

Stings, from bees and wasps. 630.

Stowers, J. H. Ichthyosis hystrix, 203

Stratum corneum, 4 .

- - atrophy of, to.

- - dystrophy of, 39 .

- bypertrophy of, 38 .

- - pathological changes in, 35 .

- germinativum, 2.

- granulosum, $t$.

- lucidum, 4 .

- Malpighii (see Prickle-cell layer), 3.

Streptococcus, anginosus, 295.

- erysipelatis, 295 . 
Streptococcus, faecalis, 295.

- lesions due to, 295 .

- pathological action of, 296.

- pyogenes, 82, 83, 295.

- cultivation of, 298 .

- salivarius, 295.

Streptothrix, $8_{3}$.

- asteroides, 433 .

- hominis, 433 .

- isracli, 432 .

- leproides (see Bacillus leprae), 499.

Striae atrophicae, 35, 6I, 960.

- patcllares, 960 .

Strophulus albidus (see Sebaceous cyst), 1123.

- infantum, $238^{\circ}$.

- pruriginosus (Hardy), 697.

Subcutaneous tissue, I 5 .

- - development of, I.

- - function of, 15.

- - structure of, I5.

- - thickness of, I 5 .

Suction treatment (Bier's), $I_{42}$.

Sindamina, 30, 50, 243.

-.. deseription of, $2+3$.

- diagnosis of, 2.4.

- histopathology of, 244 .

- treatment of, 2.44.

Sugar, dermatitis from, 274.

Sukha-pakla (see Ainhum), 1 I 94.

Sulphaqua, 127.

Sulphonal eruptions, 722 .

Sulphur, baths, I 27.

- use of, I 2 , I 24 .

Sunburn, 245.

- treatment of, $24^{6}$.

Sunlight, effect of, on skin, 245 .

Supra-renal gland, treatment by, I 5 .

Surgical treatment of skin affections, I 42 .

Sweat, 20 .

- adenoma I I 22.

- centres, 20.

- - action of drugs on, 20 .

- coil, so.

- - ampulla of, Io.

- composition of, 20.

- cyst, 50 .

- duct, Io.

- functions of, 20 .

- glands, 9.

- - atrophy of, I 060.

- - diseases of, 1059.

- functional disorders of, 50, 10.46.

- - hypertrophy of, 1059.

- - acquired, 1059.

—_- congenital, I059.

- - situations of, 9 .

- structural changes in, 50. - structure of, 9.

pore, 9 .

Sweating sickness (see Miliaria fever), $2+3$.

Sycosis, barbae, coccogenic, 330 .

- -.. - diagnosis of, 332. etiology of, $33 \mathrm{I}$.
Sycosis, barbae, coccogenic, prognosis of, 332 .

- - - treatment of, 332 .

- - hyphogenic (see Tinea barbae), 414.

- framboesiformis (see Sycosis nuchae) 334.

- lupoid, 330 .

- nuchae, 334 .

- - diagnosis of, 335 .

- etiology of, 335 .

- - histopathology of, 335 .

- - prognosis of, 335 .

- treatment of, 335 .

-- of axillae, 333.

- of eyebrows, 333 .

— of eyelashes, 333 .

- of moustache, $33^{\circ}$.

- of pubes, 333 .

- of scalp, 333 .

Syndrome hypotonique, 982 .

Syphilis, 527 .

- alimentary tract, disease in, $56_{5}$.

- alopecia in, 553 .

- blood changes in, 564 .

- blood-vessel changes in, 564 .

- bone and joint disease in, 566 .

- chancre, hard, 532.

- - duration of, 533 .

- Listopathology of, 539.

- induration of, 532 .

- - prognosis of, 538 .

- - situation of, 534 .

- - genital, 534 .

_- _ diagnosis from soft sore, 535 .

_..... extra-genital, 536 .

- - - usual sites of, 536 .

- - treatment, local, 538.

— - varieties of, 533 .

- - abrasion, 533 .

- - concealed, 534 .

_- fulminating, 533 .

- - mixed, 534 .

- - multiple, 534 .

- - phagedaenic, 534 .

- - - recurrent, 534 .

- - relapsing, 534.

- - - successive, 534 .

- complications of, 557 .

- condylomata, 547 .

- congenital, $59 \mathrm{I}$.

- - bone lesions in, 594 .

_ Colle's law in, 59 I.

_ cutaneous lesions in, 592.

- - description of, 592 .

- - cliagnosis of, 595 .

- Diday's law in, 592.

- late lesions of, 595 .

- nervous disturbances in, 595 .

- prevention of, 598 .

_- Profeta's law in, 59I.

_- prognosis of, 597 .

- treatment of, 598 .

— - by mercury, 598 .

_ _ - by salvarsail, 599 .

— - transmission of, $59 \mathrm{I}$. 
Syphilis, congenital, triad of Hutchin. son, 595 .

- - visceral changes in, 595 .

- Wassermann reaction in, 597 .

- cutaneous lesions in, 539.

- classification of, 539 .

- - common characteristics of, 539.

- early, 542 .

- - discoid, 545 .

- - follicular, 547 .

- - histopathology of, 552 .

- - impetiginons, $54 \mathrm{~S}$.

- - lenticular, $5+5$.

- - lichenoid, 547 .

- - macular, 542 .

- - - miliary, 547.

- - papular, 545 .

- - pigmentary, 545 .

- - - pustular, 547 .

- - roseolar, $54^{2}$.

- _ rupial, $54^{8}$.

- - squamous, 547 .

- - varioloid, 547 .

- - intermediary, 549 .

_- - annular, 550 .

- - circinate, $55^{\circ}$.

- - corymbose, 549 .

—- histopathology of, 552 .

- - palmar, $55 \mathrm{r}$.

- - plantar, 551 .

- - - recurrent roseolar, 552 .

- - - rosette, 550 .

- - serpiginous, 550 .

- - treatment, local, $55^{2}$.

- late, 555 .

- - diagnosis of, 558 .

- - elephantiasis in, 557 .

- - gummata, 556 .

- - - subcutaneous, $5 \mathbf{5} 6$.

- - - superficial, 556 .

- - histopathology of, 557 .

- - phagedaenic ulceration in, 557

- - treatment, local, 560 .

- etiology of, 528 .

- eye disease in, 567 .

- genito-urinary disease in, 565 .

- geographical distribution of, 567 .

- glands, enlargement of, 533 .

- heart disease in, 564 .

- hereditary (see congenital), $59 \mathrm{r}$.

- incubation period of, 533 .

- Justus test in, 569 .

- Iuetin reaction in, 531, 569 .

- marriage and, $57 \mathbf{r}$.

- mucous membrane lesions in, 561 .

- - early, 561 .

- - diagnosis of, 562 .

- - situation of, 561 .

- - treatment of, 563 .

- - late, 563 .

- - - diagnosis of, $5^{6} 3$.

- - situation of, 563 .

- - treatment of, $56_{4}$.

- - patches, 561 .

- nail disease in, 553 .

- nervous disease in, 566 .
Syphilis, onychia in, 553 .

- drỹ, 553 .

- - suppurative, 554 .

- pathogenesis of, $54 \mathrm{I}$.

- prevention of, 572 .

- prognosis of, $5 ; 0$.

- prophylaxis of, $57 \mathrm{r}$.

- re-infection with, $57 \mathrm{x}$.

- respiratory disease in, $5 \overline{5}$.

- rupia, $54^{8}$.

- severity of, 541 .

- Spirocliaeta pallida (see Spironema pallidumi).

- Spironema pallidum, 528 .

- - cultivation of, $53 \mathrm{r}$.

- demonstration of, 529 .

- - - by dark ground illumination, 530 .

- - by staining, 530.

- - - Congo red method, 531 .

- _ - Indian ink method, 530 .

- - - in cutaneous syphilides, 529.

- - in lymph glands, 530 .

- - - in primary lesions, 529 .

- - inoculation experiments with, 531 .

- - - in anthropoid apes, $53 \mathrm{I}$.

- - life-history of, 532 .

- - morphology of, 528 .

- presence in tissues, 528 .

- treatment of, 574 .

-.. by arsenical preparations, 575 .

- - arylarsonates, $\mathbf{5 7 5}$.

- - galyl, $58 \mathrm{r}$.

- - hectine, 575 .

- - - joha, 581 .

_- - luargol, $5 \mathrm{Sr}$.

- neo-salvarsan, 579.

- _ - - intra-muscular injection of, 579 .

- - - intra-venous injection of, $5 \% 9$.

- - - - subcutaneous injection of, $58 \mathrm{r}$.

— - - substitutes for, 579 .

- - - neo-kharsivan, 579.

— _ _ - novarsenobillon, 579.

- - salvarsan, 575 .

- - - after-effects of, 577 .

- - - combined with mercury, 588 .

- - contra-indications to, 578 .

- - - Herxheimer's reaction from. 578 .

- - - intra-venous injection of, 576.

- - - substitutes for, $577,579$.

- _ - - arseno-benzol (Billon), $57 \%$.

- kharsivan, $57 \%$.

- - by iodides, $58 \%$.

- - by iodipin, $58 \%$.

— by mercury, $58 \mathrm{r}$.

- - - administration of, $5^{83}$.

- - - by fumigation, $58 \%$.

- - - by ingestion, 583 . 
Syphilis, treatment of

- by mercury, administration, by injection, 584 .

— - - by inunction, 583 .

- - contra-indications to, 582 .

- - by serum, 590 .

_- of central nervous system, 589 .

— - by salvarsanised serum, 590 .

- of preguant women, 589 .

- tropical, 567 .

- Wassermann reaction in, 540, 568 .

- as guide to treatment, 590 .

- - value of, 569 .

Syringadenoma, 1122 .

Syringo-cyst adenoma, 1120.

Syringoma, I1 22.

Tactile corpuscles, 18.

- spots, 23.

Tacnzer, P. Scar-leaving erythema of eyebrows, 70 .

Tanners' dermatitis, 285 .

Tannoform, 124.

Tar acne, 277.

- baths, 128 .

- mollusca, 284.

- preparations of, 125 .

— warts, 278.

Tattooing, 957.

- diseases conveyed by, 957 .

- removal of, 958 .

- tuberculosis cutis caused by, 463 .

Teak, dermatitis from, 274 .

Teigne du pauvre (see Favus), 421 .

Telangiectasis, 65,942 .

- follicularis annulata, 178 .

- nultiple with epistaxis (Osler), 944.

- primary, 943 .

- secondary, 944.

- treatment of, 945.

Tetryl, dermatitis from, 282 .

Thiol (see Iclithyol), in.

Thiosinamin, 113 .

Thoriun nitrate, dermatitis from, 282.

- treatment by, 156 .

Thyroid treatment, I I4.

- - in ichthyosis, 167 .

- - in psoriasis, 834 .

Ticks, bites from, 610 .

Tinea, 373 .

- barbae, 414.

_- diagnosis of, 416 .

- - fungi of, 415.

- - prognosis of, +16 .

- - scaly type, 415 .

- - suppurative type, $4 \mathbf{I} 4$.

- - treatment of, 416 .

- - by actinic rays of light, 417 .

_ — by parasiticides, 417 .

— - by X-rays, $4 \mathrm{I} 6$.

- circinata, 384 .

- - diagnosis of, 387 .

- pathology of, 388 .

- - prognosis of, 389 .

- - treatment of, 389 .

- - types of lesions, 384 .
Tinea, cruris, 390 .

— - diagnosis of, 393.

- - fungi of, 393.

- - treatment of, 394 .

- decalvans, 400, 981 .

- favosa (see Favus), 421.

- flava, 4.1 .

- imbricata, 1246.

- - description of, 1246.

- - fungi of, 1248 .

- - geographical distribution of, 1246 .

- - treatment of, 1248 .

- nodosa (Morris and Cheadle), 437 , 439 .

- tonsurans, 398.

- - and Alopecia areata, 400.

- - demonstration of fungi, 402.

- - diagnosis of, 401 .

— - nicrosporon, 398.

- - - diagnosis of, 402 .

_ - modifications of, 399.

- - - by Alopecia areata, 400.

- - - by other skin affections, 399 .

— - — by secondary changes, 399.

- - - - by treatment, 399.

_ - - prognosis of, 403 .

- - - treatment of, $4 \mathrm{O}_{4}$.

_ _ _ by irritants (artificial kerion method), 4II.

- - - - chrysarobin, 413 .

- - - - Coster's paste, 414.

- - - - croton oil, 4I2.

- - - - salt, 4I3.

_ _ - by parasiticides, 410.

_ _ - by X-rays, 406 .

_ - _ - - action on scalp, 406.

- - - - apparatus, I47.

_ _ _ _ causes of failure of, 409.

- _ - - dangers of, 409.

_ - _ - dosage of, 148 .

- - - - method, 407.

- - - - discussion of, 404 .

- - suppurative, 401, 404, 4I4 (see also Kerion), 401.

- - trichophyton, too.

- - ecto-endothrix, for.

- - endothrix, 400 .

- - - - diagnosis of, 402.

- - - - prognosis of, 404 .

- - - - treatment of, 404 .

- inguium, 417 .

- - diagnosis of, 418 .

- - fungi of, 418 .

- - treatment of, 418.

- versicolor (see Pityriasis versicolor), $44^{\circ}$.

Tokclau ringworm, 12.46 .

Tongue, black hairy, 1082.

- cliseases of, ro82.

- geograplical, 1083.

- transitory circinate eruption of, 1083 .

Touch-cells of Merkel, 18.

Toxins, in skin cliseases, 76 .

Toxi-tuberculides, $217,221,486$.

Trabeculae (see Subcutaneous tissue), 15. 
Tragi, 5 .

Traumaticin, 137 .

Treatment, general principles of, Ioo.

Trench foot, 227 .

Treponema pertenue (Castellani), I 209 - - inoculation of, in man, I210.

- - - in monkeys, I 210.

- pallidum, 528 .

Tricho-epithelioma, $4^{6}$, I 120.

Tricho-keratin (see Hair-keratin), 7 .

Trichomanie, 635 .

Trichomycosis (see Lepothrix), 443 .

- nodularis (see Trichosporosis), 43\%.

Trichophytides (Jadassohn), 402.

- lichenoid, to2.

Trichophytine, $70,3_{2}$.

Trichophyton, acuminatum (Sabour and), 380 .

- asteroides, ${ }_{3} \mathrm{SI}$.

- crateriforme (Sabouraud), 3So.

- currii, 380 .

- denticulatum, 38 I.

- discoides, 382.

- ecto-endothrix,. 378 .

- endothrix, 378 .

- equinum, $38 \mathrm{r}$.

- farinulentum, 381 .

- granulosum, $38 \mathrm{I}$.

- gypseum, $3^{8} \mathbf{I}$.

- lacticolor, $38 \mathrm{I}$.

- niveum, $3 \mathrm{~S}$ I.

-ochraceum, 382.

- persicolor, $3 \mathrm{SI}$.

- plicatile (Sabouraud), 3 So.

- radians, $38 \mathrm{I}$.

- radiolatum, $3 S_{I}$.

- rosaceum, $38 \mathrm{I}$.

- sulphureum, 3 So.

- verrucosum, 382.

- violaceum, 382 .

Trichorrhexis nodosa, 45, 999.

- - etiology of, Iooo.

- - treatment of, rooo.

Trichosporosis, 437 .

- diagnosis of, +39 .

- indica, 437 .

- nodosa, 437 .

- treatment of, 439 .

- tropica, 437 .

- - fungus of, $+3 \mathrm{~s}$.

Trichosporum giganteum (Behrend), $43^{8}$.

- ovale (Unna), $+3 s$.

- ovoides (Behrend), 438 .

Trinitrotoluene (T.X.T.), dermatitis from, 282.

Tubercula dolorosa (see Neurofibroma). $68,1127$.

Tuberculides, $449,4^{86}$.

- nature of, 486 .

- origin of, $48 \%$.

- papulo-necrotic, 490 .

- - diagnosis of, 492 .

- - etiology of, 492.

- - histopathology of, 492.

- treatment of, 493 .
Tuberculides, varieties of, 486 .

Tuberculosis cutis, 446 .

- definition of, +46 .

- diagnosis of (general), $44^{6}$.

- - by clinical appearances, 46 .

- - by histopathology, +47 .

- - by tuberculin reactions, $44 \pi$.

- - general, $4+7$.

- - local, tti.

- - - inunction (Moro), ${ }_{44} \mathrm{~s}$.

_ - - ophthalmic (Calmette), ${ }_{4} 8$.

- - - vaccination (von Pirquet), $44^{8}$.

- geographical distribution of, 449 .

- lesions of, $4+9$.

- - classification of, 44 .

- - due to toxins from other tuberculous foci, 449 .

- - due to tubercle bacilli in the skin, 449 .

- miliary, + so.

- scrofulodermia, 477 .

- treatment of, $+8 \mathrm{r}$.

- verrucosa, 453 .

Tumour, characters of, $2 S$.

- lymphoid-celled, 67.

- tomato (see Epithelioma, benign cystic), I I IS.

Turpentine, dermatitis from, 274,722 .

Tylosis (see Callosity), IO9I.

- palmare et plantare, 2o,

Tyroglyphus longior (Castellani), 6I 3 .

Ulcer, 35 .

- Aden, 1236.

- exedens, I I 44.

- Jacob's (see Rodent ulcer), I I 4 .

- Malabar, I 236.

- rodent, II 4 .

- tropical, I 236.

- tuberculous, $48 \mathrm{I}$.

- Yemen, I 236.

Ulerythema centrifugum (L'nna), gi 1 .

- ophryogenes (see Ichthyosis follicularis), I69, IOIt.

- superciliare, Ior 5.

Enna, P. G. Flask-bacillus, 350.

- Morococcus, 325.

- Naevus-cells, origin of, I98.

- Plasma-cells, 56 .

- Seborrhoic eczema, 350 .

Uranium, treatment by, $15 \%$.

U'ridrosis, 1053.

Urticae (see Wheals), $2 S$.

Urticaria, 6-9.

- acute, 680.

- bullous, 68r.

- chronic, 68o.

- description of, 6 -9.

- diagnosis of, $68_{5}$.

- etiology of, $68_{4}$.

- factitious, $6 \mathrm{SI}_{\mathrm{I}}$.

- giant, 682,003.

- haemorrhagic, $68_{\mathrm{I}}$.

- histopathology of 683 .

- mucous membrane lesions in, 680 . 
Urticaria, nodosa, 682, 693.

- papular, 697.

- - diagnosis of, 700 .

— - etiology of, 699 .

- - histopathology of, 699.

- prognosis in, $70 \mathrm{I}$.

- - treatment of, 7or.

- varieties of, 699 .

- - bullous, 699 .

- - - vesicular, 699.

- pathogenesis of, 683 .

- petechialis, 939 .

- perstans, 683 .

- pigmentosa, 689 .

- - diagnosis of, 692 .

- etiology of, 692.

-. histopathology of, 69I.

—- mast-cells in, $69 \mathrm{I}$.

_ - treatment of, 693 .

- prognosis in, 686 .

- treatment of, 686.

Utricle peladique (Sabouraud), 977.

Vaccination and skin disease, 75 .

- and lupus vulgaris, 462 .

Vaccines, treatment by, I 6.

Vacuolated cells, I3.

Vagabond's disease (see Pediculi vestimentorum), 620 .

Vanillaism, 273.

Varnislies, $136,1277$.

Vater's corpuscles (see Pacinian bodies), I 8.

Veld sores, 1256.

- etiology of, 1257 .

- treatment of, I 258 .

Ver du Cayor, 627.

- Macaque, 627.

Vernix caseosia, 2 I.

Veronal eruption, 722 .

Verruca (see W'art), rog2.

- acuminata, $100-$.

- juvenilis, ro92.

- necrogenicá, 453 .

-. l'eruana, I 259 .

-...- diagnosis of, r $26 \mathrm{r}$.

-_- etiology of, I 261 .

- - geographical distribution of, I 260.

- treatment of, I $26 \mathrm{I}$

- plana, I092.

- plantaris, Iog6.

- seborrlioica, 1099.

- senilis, rog9.

Verruga P'eruviana, I 259.

Vesicle, 20.

- formation of, 30

- interstitial, 30 .

- monolocular, 29.

- multilocular, 20.

- parencliymatous, 30.

- site of, 30 .

- umbilication of, $3 \mathbf{I}$.

Vesico-pustule, 29, 32 .

Vibices, "35.

Vibrissac, 5 .

Virchow, R. Granulomata, 55.
Vitiligo (see Leucoderma), 953.

- gravior (see Leprosy), 5 Ir.

Vitiligoidea (see Xanthoma), I I 7r.

Vitreous layer (see Hair-follicle), 45.

Vleminckx's solution, 394 .

von Frisch's bacillus of rhinoscleroma, 525.

von Pirquet's reaction in tuberculosis, $44^{8}$.

von Recklinghausen's disease, I 28.

Wart, acuminate, I097.

- - histopathology of, 1098.

- - treatment of, Io99.

- common, I092.

_ - etiology of, 1093.

- - histopathology of, I093.

- - treatment of, I094.

—- - internal, ro94.

—— local, rog5.

_ - - by caustics, 1095 ; cauterisation, ro95; curetting, r095; electrolysis, 1096; freezing with carbon dioxide, ro95; ionisation, I095; sea-water, I096 ; $\mathrm{X}$-rays, 1095 .

- filiform, 1097.

— juvenile, I093.

- plane, rog3.

- plantar, I096.

— - treatment of, 1097.

- seborrhoic, I099.

- - treatment of, I Ioo.

- senile, rog9.

- telangiectatic, 220 .

- venereal, I097.

Washerwomen's itch, 285 .

Wassermann reaction in leprosy, $5 \mathrm{I} 6$.

- - in syphilis, $540,568,590$.

Water, effect on skin of, 125 .

Weber, F. Parkes. Multiple idiopathic sarcoma, 1 I 69 .

- Mycosis fungoides, 928 .

Welch, Staphylococcus epidermidis albus, 325.

Wen, 48 .

Wheal, 28.

- pathogenesis of, 29.

White-spot-disease (see Sclerodermia), I 8 .

Whitfield, A. Epilation forceps, 402.

- Ringworm ointment, 397.

Whitlow, 1072.

Wickham and Degrais, Cross-fire radium exposure, $155,187$.

Willan, R. Classification by, I59.

- Pityriasis, 349.

Willianı, Winkelried. Gonorrhoeal keratosis, I Io9.

- Ringworm treatment, 4I 4.

Wilson, Lirasmus. Lichen planus, 727 .

Winter itch (see Pruritus hienalis), 222.

Woods, hard, dermatitis from, 274.

Worms, and skin disease, I09. 
X-ray apparatus, 147 .

- dermatitis, 260.

- acute, $26 \mathrm{I}$.

- chrónic, 262.

- pathology of, 264 .

- - prognosis of, 265 .

- treatment of, 265 .

- dosage of, ${ }_{4} 8$.

- histopathological effects of, I 49.

- skin affections benefited by, I 49.

- treatment by, I 77 .

Xanthelasma, I I 7 .

Xanthelasmoidea (see Urticaria pigmentosa), 689 .

Nantho - erythrodermia perstans (Crocker), 847 .

Xanthoma, I I 7 I.

- cells, I I 75 .

- chromia, I I74.

- diabeticorum, I I 74 .

- diagnosis of, I 78 .

- elasticum (Balzer), I I 34 .

- giant-cells, 69 , i 176 .
Xanthoma, histopathology of, II 75 .

- palpebrarum, 'II 72.

- pathology of, 68.

- prognosis of, $\mathbf{I} \mathbf{1} 78$.

- treatment of, II 78 .

- tuberosum multiplex, I 72 .

Xeroderma, I62.

- follicular, 69.

- pigmentosum, 252.

- - diagnosis of, 255 .

- - etiology of, 254 .

- - histopathology of, 254.

- - treatment of, 255 .

Yaws (see Framboesia tropica), I208.

- crab-, I 2 II.

- mother-, I2II.

- ringworm-, I2II.

least, treatment by, I I3.

Zymin (see Yeast), II 3.

Zona (see Herpes zoster), 902. 


$$
\text { - }
$$

.

का से 2 



\section{PLEASE do NOT REMOVE \\ CARDS OR SLIPS FROM THIS POCKET \\ UNIVERSITY OF TORONTO LIBRARY}

$$
\text { Eioned }
$$


